\title{
UNIVERSITY OF EDINBURGH
}

Initiation, Propagation and Resolution of Inflammation

\author{
Professor Adriano Giorgio Rossi \\ BSc (Hons), PhD
}

Doctorate of Science

(Volume II)

MRC Centre for Inflammation Research

Queen's Medical Research Institute

University of Edinburgh Medical School

47 Little France Crescent

Edinburgh,

EH16 4TJ,

Scotland, UK

January 2010 
62 Rossi AG, Cousin JM, Dransfield I, Lawson MF, Chilvers ER, Haslett C. Agents that elevate cAMP inhibit human neutrophil apoptosis. Biochem Biophys Res Commun. 1995 Dec 26;217(3):892-9. (Major)

Hannah S, Nadra I, Dransfield I, Pryde JG, Rossi AG, Haslett C. Constitutive neutrophil apoptosis in culture is modulated by cell density independently of $\beta_{2}$ integrin-mediated adhesion. FEBS Lett. 1998 Jan 9;421(2):141-6. (Moderate)

Rossi AG, McCutcheon JC, Roy N, Chilvers ER, Haslett C, Dransfield I. Regulation of macrophage phagocytosis of apoptotic cells by cAMP. J Immunol. 1998 Apr 1;160(7):3562-8. (Major)

65 Ward C, Chilvers ER, Lawson MF, Pryde JG, Fujihara S, Farrow SN, Haslett C, Rossi AG. NF- $\mathrm{\kappa B}$ activation is a critical regulator of human granulocyte apoptosis in vitro. $\mathrm{J}$ Biol Chem. 1999 Feb 12;274(7):4309-18. (Major)

66 Liu Y, Cousin JM, Hughes J, Van Damme J, Seckl JR, Haslett C, Dransfield I, Savill J, Rossi AG. Glucocorticoids promote nonphlogistic phagocytosis of apoptotic leukocytes. J Immunol. 1999 Mar 15;162(6):3639-46. (Major)

67 Ward C, Wong TH, Murray J, Rahman I, Haslett C, Chilvers ER, Rossi AG. Induction of human neutrophil apoptosis by nitric oxide donors: evidence for a caspase-dependent, cyclic-GMP-independent, mechanism. Biochem Pharmacol. 2000 Feb 1;59(3):305-14. (Major)

68 Pryde JG, Walker A, Rossi AG, Hannah S, Haslett C. Temperature-dependent arrest of neutrophil apoptosis. Failure of Bax insertion into mitochondria at $15^{\circ} \mathrm{C}$ prevents the release of cytochrome c. J Biol Chem. 2000 Oct 27;275(43):33574-84. (Moderate)

69 Rossi AG, Ward C, Murray J, Martin MC, Fujihara S, Dransfield I, Haslett C. Transcriptional regulation of eosinophil apoptosis. Respiratory Medicine. 2000 94: 12591262. (Major)

70 Giles KM, Ross K, Rossi AG, Hotchin NA, Haslett C, Dransfield I. Glucocorticoid augmentation of macrophage capacity for phagocytosis of apoptotic cells is associated with reduced p130Cas expression, loss of paxillin/pyk2 phosphorylation, and high levels of active Rac. J Immunol. 2001 Jul 15;167(2):976-86. (Moderate)

71 Martin MC, Dransfield I, Haslett C, Rossi AG. Cyclic AMP regulation of neutrophil apoptosis occurs via a novel protein kinase A-independent signalling pathway. J Biol Chem. 2001 Nov 30;276(48):45041-50. (Major)

72 Taylor EL, Megson IL, Haslett C, Rossi AG. Dissociation of DNA fragmentation from other hallmarks of apoptosis in nitric oxide-treated neutrophils: differences between individual nitric oxide donor drugs. Biochem Biophys Res Commun. 2001 Dec 21;289(5):1229-36. (Major) 
Fujihara S, Ward C, Dransfield I, Hay RT, Uings IJ, Hayes B, Farrow SN, Haslett C, Rossi AG. Inhibition of nuclear factor- $\mathrm{\kappa B}$ activation un-masks the ability of TNF- $\alpha$ to induce human eosinophil apoptosis. Eur J Immunol. 2002 Feb;32(2):457-66. (Major)

74 Ward C, Dransfield I, Murray J, Farrow SN, Haslett C, Rossi AG. Prostaglandin $\mathrm{D}_{2}$ and its metabolites induce caspase-dependent granulocyte apoptosis that is mediated via inhibition of $\mathrm{I} \kappa \mathrm{B} \alpha$ degradation using a peroxisome proliferator-activated receptor- $\gamma$ independent mechanism. J Immunol. 2002 Jun 15;168(12):6232-43. (Major)

75 Wickenden JA, Clarke MC, Rossi AG, Rahman I, Faux SP, Donaldson K, MacNee W. Cigarette smoke prevents apoptosis through inhibition of caspase activation and induces necrosis. Am J Respir Cell Mol Biol. 2003 Nov;29(5):562-70. (Minor)

76 Murray J, Ward C, O'Flaherty JT, Dransfield I, Haslett C, Chilvers ER, Rossi AG. Role of leukotrienes in the regulation of human granulocyte behaviour: dissociation between agonist-induced activation and retardation of apoptosis. $\mathrm{Br} \mathrm{J}$ Pharmacol. 2003 May;139(2):388-98. (Major)

77 Murray J, Walmsley SR, Mecklenburgh KI, Cowburn AS, White JF, Rossi AG, Chilvers ER. Hypoxic regulation of neutrophil apoptosis role: of reactive oxygen intermediates in constitutive and tumor necrosis factor $\alpha$-induced cell death. Ann N Y Acad Sci. 2003 Dec;1010:417-25. (Moderate)

78 Walker A, Ward C, Sheldrake TA, Dransfield I, Rossi AG, Pryde JG, Haslett C. Golgi fragmentation during Fas-mediated apoptosis is associated with the rapid loss of GM130. Biochem Biophys Res Commun. 2004 Mar 26;316(1):6-11. (Moderate)

79 Kirkham PA, Spooner G, Rahman I, Rossi AG. Macrophage phagocytosis of apoptotic neutrophils is compromised by matrix proteins modified by cigarette smoke and lipid peroxidation products. Biochem Biophys Res Commun. 2004 May 21;318(1):32-7. (Major)

80 Heasman SJ, Giles KM, Rossi AG, Allen JE, Haslett C, Dransfield I. Interferon $\gamma$ suppresses glucocorticoid augmentation of macrophage clearance of apoptotic cells. Eur J Immunol. 2004 Jun;34(6):1752-61. (Minor)

81 Arruda MA, Rossi AG, de Freitas MS, Barja-Fidalgo C, Graça-Souza AV. Heme inhibits human neutrophil apoptosis: involvement of phosphoinositide 3-kinase, MAPK, and NFкB. J Immunol. 2004 Aug 1;173(3):2023-30. (Moderate)

82 Taylor EL, Rossi AG, Shaw CA, Dal Rio FP, Haslett C, Megson IL. GEA 3162 decomposes to co-generate nitric oxide and superoxide and induces apoptosis in human neutrophils via a peroxynitrite-dependent mechanism. $\mathrm{Br} \mathrm{J}$ Pharmacol. 2004 Sep;143(1):179-85. (Moderate)

83 Fujihara S, Jaffray E, Farrow SN, Rossi AG, Haslett C, Hay RT. Inhibition of NF-кB by a cell permeable form of $\mathrm{I} \kappa \mathrm{B} \alpha$ induces apoptosis in eosinophils. Biochem Biophys Res Commun. 2005 Jan 21;326(3):632-7. (Moderate) 

Phosphoinositide- 3 kinases critically regulate the recruitment and survival of eosinophils in vivo: importance for the resolution of allergic inflammation. J Leukoc Biol. 2005 May;77(5):800-10. (Moderate)

85 Ward C, Murray J, Clugston A, Dransfield I, Haslett C, Rossi AG. Interleukin-10 inhibits lipopolysaccharide-induced survival and extracellular signal-regulated kinase activation in human neutrophils. Eur J Immunol. 2005 Sep;35(9):2728-37. (Major) Sawatzky DA, Willoughby DA, Colville-Nash PR, Rossi AG. The involvement of the apoptosis-modulating proteins ERK $1 / 2, \mathrm{Bcl}-\mathrm{x}_{\mathrm{L}}$ and $\mathrm{Bax}$ in the resolution of acute inflammation in vivo. Am J Pathol. 2006 Jan;168(1):33-41. (Major)

Rossi AG, Sawatzky DA, Walker A, Ward C, Sheldrake TA, Riley NA, Caldicott A, Martinez-Losa M, Walker TR, Duffin R, Gray M, Crescenzi E, Martin MC, Brady HJ, Savill JS, Dransfield I, Haslett C. Cyclin-dependent kinase inhibitors enhance the resolution of inflammation by promoting inflammatory cell apoptosis. Nat Med. 2006 Sep;12(9):1056-64. (Major)

Arruda MA, Barcellos-de-Souza P, Sampaio AL, Rossi AG, Graça-Souza AV, BarjaFidalgo C. NADPH oxidase-derived ROS: key modulators of heme-induced mitochondrial stability in human neutrophils. Exp Cell Res. 2006 Nov 15;312(19):393948. (Minor)

89 Taylor EL, Li JT, Tupper JC, Rossi AG, Winn RK, Harlan JM. GEA 3162, a peroxynitrite donor, induces Bcl-2-sensitive, p53-independent apoptosis in murine bone marrow cells. Biochem Pharmacol. 2007 Oct 1;74(7):1039-49. (Minor)

90 Pinho V, de Castro Russo R, Amaral FA, de Sousa LP, Barsante MM, de Souza DG, Alves-Filho JC, Cara DC, Hayflick JS, Rommel C, Ruckle T, Rossi AG, Teixeira MM. Tissue- and stimulus-dependent role of phosphatidylinositol 3-kinase isoforms for neutrophil recruitment induced by chemoattractants in vivo. J Immunol. 2007 Dec 1;179(11):7891-8. (Minor)

91 Farnworth SL, Henderson NC, Mackinnon AC, Atkinson KM, Wilkinson T, Dhaliwal K, Hayashi K, Simpson AJ, Rossi AG, Haslett C, Sethi T. Galectin-3 reduces the severity of pneumococcal pneumonia by augmenting neutrophil function. Am J Pathol. 2008 Feb;172(2):395-405. (Moderate)

92 Bournazou I, Pound JD, Duffin R, Bournazos S, Melville LA, Brown SB, Rossi AG, Gregory CD. Apoptotic human cells inhibit migration of granulocytes via release of lactoferrin. J Clin Invest. 2009 Jan;119(1):20-32. (Major)

93 Michlewska S, Dransfield I, Megson IL, Rossi AG. Macrophage phagocytosis of apoptotic neutrophils is critically regulated by the opposing actions of pro-inflammatory and anti-inflammatory agents: key role for TNF- $\alpha$. FASEB J. 2009 Mar;23(3):844-54. (Major) 
Shaw CA, Webb DJ, Rossi AG, Megson IL. Cyclic GMP protects human macrophages against peroxynitrite-induced apoptosis. J Inflamm (Lond). 2009 May 7;6:14. (Moderate)

95 Sousa LP, Carmo AF, Rezende BM, Lopes F, Silva DM, Alessandri AL, Bonjardim CA, Rossi AG, Teixeira MM, Pinho V. Cyclic AMP enhances resolution of allergic pleurisy by promoting inflammatory cell apoptosis via inhibition of PI3K/Akt and NF-kB. Biochem Pharmacol. 2009 Aug 15;78(4):396-405. (Moderate)

96 Li HN, Barlow PG, Bylund J, Mackellar A, Björstad A, Conlon J, Hiemstra PS, Haslett C, Gray M, Simpson AJ, Rossi AG, Davidson DJ. Secondary necrosis of apoptotic neutrophils induced by the human cathelicidin LL-37 is not proinflammatory to phagocytosing macrophages. J Leukoc Biol. 2009 Oct;86(4):891-902. (Moderate)

97 Duffin R, Leitch AE, Sheldrake TA, Hallett JM, Meyer C, Fox S, Alessandri AL, Martin MC, Brady HJ, Teixeira MM, Dransfield I, Haslett C, Rossi AG. The CDK inhibitor, Rroscovitine, promotes eosinophil apoptosis by down-regulation of Mcl-1. FEBS Lett. 2009 Aug 6;583(15):2540-6. (Major)

\section{3b Relevant Reviews, Chapters and additional Publications}

98 Rossi AG, Haslett C. 'Inflammation, cell injury, and apoptosis' in Pro-inflammatory and anti-inflammatory peptides Ed. Said S.I) in the Lung Biology in Health and Disease series (Ed. Lenfant C). 1998 Vol. 117, Chapt 2, pp 9-24. IBSN 0-8247-0120-8. (Major) Chilvers ER, Rossi AG, Murray J, Haslett C. Regulation of granulocyte apoptosis and implications for anti-inflammatory therapy. Thorax. 1998 Jul;53(7):533-4. (Moderate)

100 Hart SP, Rossi AG, Dransfield I. You can lead a macrophage to an inflammatory site but can you make it eat? Biochem Soc Trans. 1998 Nov;26(4):650-2. Review. (Moderate)

101 Mecklenburgh K, Murray J, Brazil T, Ward C, Rossi AG, Chilvers ER. Role of neutrophil apoptosis in the resolution of pulmonary inflammation. Monaldi Arch Chest Dis. 1999 Aug;54(4):345-9. Review. (Moderate)

102 Ward C, Dransfield I, Chilvers ER, Haslett C, Rossi AG. Pharmacological manipulation of granulocyte apoptosis: potential therapeutic targets. Trends Pharmacol Sci. 1999 Dec;20(12):503-9. Review. (Major)

103 Giles KM, Hart SP, Haslett C, Rossi AG, Dransfield I. An appetite for apoptotic cells? Controversies and challenges. Br J Haematol. 2000 Apr;109(1):1-12. Review. (Moderate)

104 Taylor EL, Megson IL, Haslett C, Rossi AG. Nitric oxide: a key regulator of myeloid inflammatory cell apoptosis. Cell Death Differ. 2003 Apr;10(4):418-30. Review. (Major)

105 Walker A, Ward C, Dransfield I, Haslett C, Rossi AG. Regulation of granulocyte apoptosis by hemopoietic growth factors, cytokines and drugs: potential relevance to allergic inflammation. Curr Drug Targets Inflamm Allergy. 2003 Dec;2(4):339-47. Review. (Major)

106 Ward C, Rossi AG, Haslett C, Dransfield I. Apoptosis: future perspectives. Essays Biochem. 2003;39:155-62. Review. (Moderate) 
107 Rossi AG, Ward C, Dransfield I, Haslett C. 'Apoptosis of inflammatory cells' in Middleton's allergy: principals and practice ( $6^{\text {th }}$ Edition) Eds. Adkinson NF et al., Mosby-Harcourt, 2003 Chapt 26 (in press-August).pp 412-424. ISBN 0323014259. (Major)

108 Heasman SJ, Giles KM, Ward C, Rossi AG, Haslett C, Dransfield I. Glucocorticoidmediated regulation of granulocyte apoptosis and macrophage phagocytosis of apoptotic cells: implications for the resolution of inflammation. J Endocrinol. $2003 \mathrm{Jul} ; 178(1): 29$ 36. (Moderate)

109 Gilroy DW, Lawrence T, Perretti M, Rossi AG. Inflammatory resolution: new opportunities for drug discovery. Nat Rev Drug Discov. 2004 May;3(5):401-16. Review. (Major)

110 Dransfield I, Rossi AG. Granulocyte apoptosis: who would work with a 'real' inflammatory cell? Biochem Soc Trans. 2004 Jun;32(Pt3):447-51. Review. (Major)

111 Rossi AG, Ward C, Dransfield I. Getting to grips with the granulocyte: manipulation of granulocyte behaviour and apoptosis by protein transduction methods. Biochem Soc Trans. 2004 Jun;32(Pt3):452-5. Review. (Major)

112 Ward C, Walker A, Dransfield I, Haslett C, Rossi AG. Regulation of granulocyte apoptosis by NF-кB. Biochem Soc Trans. 2004 Jun;32(Pt3):465-7. Review. (Major)

113 Farahi N, Cowburn AS, Rossi AG, Chilvers ER. Eating their way out of trouble: selective uptake of apoptotic eosinophils by bronchial epithelial cells. Clin Exp Allergy. 2004 Oct;34(10):1503-6. (Moderate)

114 Walker A, Ward C, Martinez-Losa M, Rossi AG. 'Neutrophils and Apoptosis' in "The neutrophils: New outlook for old cells" 2nd Edition, Ed. Gabrilovich, D.I., Imperial College Press, London. 2004 pp153-168. (ISBN: 1-86094-472-8). (Major)

115 Shaw CA, Taylor EL, Megson IL, Rossi AG. Nitric oxide and the resolution of inflammation: implications for atherosclerosis. Mem Inst Oswaldo Cruz. 2005 Mar;100 Suppl 1:67-71. (Major)

116 Walker A, Ward C, Taylor EL, Dransfield I, Hart SP, Haslett C, Rossi AG. Regulation of neutrophil apoptosis and removal of apoptotic cells. Curr Drug Targets Inflamm Allergy. 2005 Aug;4(4):447-54. Review. (Major)

117 Dransfield I, Rossi AG, Brown SB, Hart SP. Neutrophils: dead or effete? Cell surface phenotype and implications for phagocytic clearance. Cell Death Differ. 2005 Nov;12(11):1363-7. (Moderate)

118 Riley NA, Ward C, Sawatzky DA, Sheldrake TA, Dransfield I, Haslett C, Rossi AG. Granulocyte apoptosis and macrophage clearance of apoptotic cells as targets for pharmacological intervention in inflammatory diseases. Anti-inflammatory and Antiallergy Agents in Medicinal Chemistry. 2006 5(1) 3-12. (Major) 
119 Shaw CA, Megson IL, Rossi AG. (2006) Apoptosis and atherosclerosis: The Role of Nitric Oxide. Anti-inflammatory and Anti-allergy Agents in Medicinal Chemistry. 2006 5(1) 27-33. (Major)

120 Serhan CN, Brain SD, Buckley CD, Gilroy DW, Haslett C, O'Neill LA, Perretti M, Rossi AG, Wallace JL. Resolution of inflammation: state of the art, definitions and terms. FASEB J. 2007 Feb;21(2):325-32. Review. (Moderate)

121 Lister MF, Sharkey J, Sawatzky DA, Hodgkiss JP, Davidson DJ, Rossi AG, Finlayson K. The role of the purinergic P2X7 receptor in inflammation. J Inflamm (Lond). 2007 Mar $16 ; 4: 5$. corresponding author. (Major)

122 Rossi AG, Hallett JM, Sawatzky DA, Teixeira MM, Haslett C. Modulation of granulocyte apoptosis can influence the resolution of inflammation. Biochem Soc Trans. 2007 Apr;35(Pt 2):288-91. (Major)

123 Michlewska S, McColl A, Rossi AG, Megson IL, Dransfield I. Clearance of dying cells and autoimmunity. Autoimmunity. 2007 Jun;40(4):267-73. Review. (Moderate)

124 McColl A, Michlewska S, Dransfield I, Rossi AG. Effects of glucocorticoids on apoptosis and clearance of apoptotic cells. ScientificWorldJournal. 2007 Aug 17;7:116581. Review. (Major)

125 Taylor EL, Rossi AG, Dransfield I, Hart SP. 'Analysis of neutrophil apoptosis' in Methods in Molecular Biology; Neutrophil Methods and Protocols, Eds, Quinn MT, Deleo FR and Bokoch GM, Humana Press (New Jersey), 2007, ISBN: 978-1-58829-7884. (Moderate) also cited as Taylor EL, Rossi AG, Dransfield I, Hart SP. Analysis of neutrophil apoptosis. Methods Mol Biol. 2007;412:177-200. (Moderate)

126 Hart SP, Dransfield I, Rossi AG. Phagocytosis of apoptotic cells. Methods. 2008 Mar;44(3):280-5. Review. (Major)

127 Hallett JM, Leitch AE, Riley NA, Duffin R, Haslett C, Rossi AG. Novel pharmacological strategies for driving inflammatory cell apoptosis and enhancing the resolution of inflammation. Trends Pharmacol Sci. 2008 May;29(5):250-7. Review. (Major)

128 Leitch AE, Duffin R, Haslett C, Rossi AG. Relevance of granulocyte apoptosis to resolution of inflammation at the respiratory mucosa. Mucosal Immunol. 2008 Sep;1(5):350-63. Review. (Major)

129 Leitch AE, Haslett C, Rossi AG. Cyclin-dependent kinase inhibitor drugs as potential novel anti-inflammatory and pro-resolution agents. $\mathrm{Br} \quad \mathrm{J}$ Pharmacol. 2009 Oct;158(4):1004-16. 


\section{List of additional publications not presented in this thesis (published abstracts not listed)}

130 Rossi AG, MacIntyre DE, McMillan RM. Human neutrophil activation: cytosolic free calcium-thresholds for aggregation, degranulation, leukotriene $\mathrm{B}_{4}$ synthesis and phosphatidate formation. Biochem. Soc. Trans. 1988;16 (1): 37-38. (Major)

131 Cousin JM, Haslett C, Rossi AG. Regulation of granulocyte apoptosis by PKC inhibition and elevation of $\left[\mathrm{Ca}^{2+}\right]_{\mathrm{i}}$. Biochem Soc Trans. 1997 May;25(2):243S. (Major)

132 Ward C, Hannah S, Chilvers ER, Farrow S, Haslett C, Rossi AG. Transforming growth factor- $\beta$ increases the inhibitory effects of GM-CSF and dexamethasone on neutrophil apoptosis. Biochem Soc Trans. 1997 May;25(2):244S. (Major)

133 Ward C, Murray J, Bruce L, Farrow S, Chilvers ER, Hannah S, Haslett C, Rossi AG. Interleukin-10 does not directly affect the constitutive rate of human neutrophil or eosinophil apoptosis. Biochem Soc Trans. 1997 May;25(2):245S. (Major)

134 Cousin JM, Haslett C, Rossi AG. Effect of the protein phosphatase inhibitors, okadaic acid and calyculin A, on dexamethasone-mediated inhibition of neutrophil apoptosis. Biochem Soc Trans. 1997 May;25(2):246S. (Major)

135 Rossi AG. Focus on chronic obstructive pulmonary disease- Inflammation 2003- $6^{\text {th }}$ World Congress. IDrugs: The Investigational Drugs Journal. 2003 6(9):838-840. (Major)

136 Ward C, Walker A, Rossi AG. 6th World Congress on Inflammation. Expert Opin Biol Ther. 2003 Dec;3(8):1291-4. (Major)

137 Sawatzky DA, Megson IL, Rossi AG. Sildenafil offers protection against NSAID-induced gastric injury. Br J Pharmacol. 2005 Oct;146(4):477-8. (Major)

138 Soares R, Azevedo I, Sawatzky DA, Rossi AG. Apigenin: Is It a Pro- or AntiInflammatory Agent? Am. J. Pathol. 2006 168(5): 1762 - 1763. (Major)

138 Rossi AG, Sawatzky DA - Preface. Progress in Inflammation Research (series Ed, Parnham M.J.); The resolution of Inflammation, Eds Rossi, A.G., and Sawatzky D.A., Birkhauser (Berlin) 2008, ISBN 978-3-7643-7505-8 (Major)

139 Duffin R, Shaw CA, Rossi AG. Sildenafil reduces alcohol-induced gastric damage: just say 'NO'. Br J Pharmacol. 2008 Feb;153(4):623-4. (Major) 


\title{
AGENTS THAT ELEVATE cAMP INHIBIT HUMAN NEUTROPHIL APOPTOSIS
}

\author{
A.G. Rossi ${ }^{1}$, J.M. Cousin, I. Dransfield, M.F. Lawson, E.R. Chilvers, and \\ C. Haslett
}

\author{
Respiratory Medicine Unit, Department of Medicine, Rayne Laboratory, \\ University of Edinburgh Medical School, Teviot Place, Edinburgh, EH8 9AG, UK
}

Received October 30, 1995

SUMMARY: Neutrophil apoptosis, determined after $20 \mathrm{~h}$ in culture using standard criteria and shedding of cell surface CD16 (FcYRIII), is dramatically inhibited, in a concentration-dependent manner, by the cAMP analogs, dibutyryl-cAMP and 8-Br-cAMP, and the adenylyl cyclase activator, forskolin. Furthermore, the stable receptor-directed $\mathrm{PGD}_{2}$ mimetic, $\mathrm{ZK} 118.182$, and the $\mathrm{PGE}_{2}$ mimetic, 11-deoxy $\mathrm{PGE}_{1}$, similarly inhibited apoptosis. The DP-receptor antagonist BW A 868 C blocked the effect of ZK 118.182 and the protein kinase A inhibitor $\mathrm{H}-89$ reversed the inhibition of apoptosis induced by dibutyryl-cAMP. These results clearly show that neutrophil apoptosis is markedly attenuated by cAMP elevating agents. This nucleotide second messenger may play a fundamental role in controlling neutrophil longevity and pharmacological regulation of cAMP levels or actions may influence neutrophil apoptosis in vivo. Q !995 Academic phess, :te.

Neutrophils have been specifically implicated in the pathogenesis of a variety of inflammatory diseases. Their granules contain a large number of agents with the capacity not only to injure tissues but also to initiate and amplify the inflammatory response. It had been assumed that extravasated neutrophils inevitably disintegrate (or underwent necrosis) before being removed from tissues, although this process as a rule would be potentially injurious to healthy local tissue (1). However, we have identified an alternative fate whereby the neutrophil undergoes apoptosis (2,3). During this process the cell remains intact and retains its granules but loses its ability to mount secretory responses in response to external stimuli (4). The apoptotic neutrophil is phagocytosed by macrophages using highly effective and novel phagocyte recognition mechanisms (3) which fail to trigger macrophage pro-inflammatory responses (5). Moreover, apoptosis may be the key mechanisms controlling the functional longevity of granulocytes, the constitutive rate being profoundly influenced by inflammatory mediators (6-9), a number of which are known to modulate intracellular levels of cAMP $(10,11)$.

Thus apoptosis may be a centrally important process for the control of neutrophil biology and in the resolution of the inflammatory response thereby limiting tissue injury and promoting wound repair. In this study we investigate the effects of agents that modulate cAMP levels on

\footnotetext{
${ }^{1}$ Corresponding author: Fax: 01316506528.
}

$0006-291 \times 195 \quad \$ 12.00$

Copyright (c) 1995 hy Academic Press, Inc. All rights of reproduction in any form reserved. 
neutrophil apoptosis. Numerous studies have shown that agonist-induced human neutrophil activation is inhibited by cAMP analogs and cAMP-elevating agents such as the adenylyl cyclase activator forskolin, phosphodiesterase inhibitors, and receptor-directed prostaglandins (e.g., PGD2 and $\mathrm{PGE}_{2}$ ). In particular, the stable $\mathrm{PGD}_{2}$ mimetic ZK 118.182 (12,13), acting via DP receptors, and 11-deoxy $\mathrm{PGE}_{1}(14)$, acting primarily via $\mathrm{EP}_{2}$ receptors, suppress neutrophil activation. Although cAMP promotes apoptosis in a variety of other cell types (15-25) the mechanism by which cAMP influences apoptosis and the effect of cAMP-elevating agents on neutrophil apoptosis is currently unknown. In this study we show that cell permeant cAMP analogs, forskolin, and stable PG mimetics all act to inhibit neutrophil apoptosis in vitro as determined by standard criteria $(4,8,26)$ and by changes in cell surface CDI6 (FcyRIII) expression (26). Hence, cAMP, as well as rendering neutrophils less responsive to secretagogue agonists, also inhibits apoptosis thereby promoting neutrophil survival. We propose that cAMP plays an important role in the intracellular control of neutrophil apoptosis and that PGs may serve as physiological regulators of neutrophil survival as well as function. Modulation of cAMP levels may therefore have important consequences in the treatment of acute and chronic inflammatory conditions.

\section{MATERIALS AND METHODS}

Materials. The hybridoma clone $3 \mathrm{G} 8$ (CDI6) was from Dr. J. Unkeless, Mount Sinai Medical School, NY and mAb 3G8 (anti-CD16) was purified as described (26). FITC-conjugated F(ab') goat $\mathrm{Ab}$ to mouse Ig was purchased from the Dako Corp., High Wycómbe, UK. ZK 118.182 was from Dr. F. McDonald, Schering AG, Berlin, Germany and BW A868C was from Dr. B. Whittle, Wellcome Plc, UK. 11-deoxy $\mathrm{PGE}_{1}$ was purchased from Cascade Biochem, Reading, UK. Forskolin, dibutyryl-cAMP, and 8-Br-cAMP, were from Sigma Chemical Co, Poole, UK and H89 was from Calbiochem-Novabiochem, Nottingham, UK.

Neutrophil isolation and culture. Human neutrophils were purified as previously described (27). The neutrophils ( $>95 \%$ pure and $>99 \%$ viable) were resuspended $(4 \times 106 / \mathrm{ml}$ ) in Iscove's modified Dulbecco's medium (Life Technologies, Paisley, UK) containing 10\% autologous serum, $100 \mathrm{U} / \mathrm{ml}$ penicillin, and $100 \mu \mathrm{g} / \mathrm{ml}$ streptomycin. All reagents tested for their effects on neutrophil apoptosis were sequentially diluted, from stock solutions, in the above medium, placed in duplicate wells of a flat bottomed 96 well polypropylene plate (Becton Dickinson, Oxford, UK) and the volume adjusted to $75 \mu \mathrm{l}$. Aliquots of cells $(75 \mu \mathrm{l})$ were added to the wells to give a final volume of $150 \mu \mathrm{l}$ and cell concentration of $2 \times 10^{6} / \mathrm{ml}$. The plates were incubated at $37^{\circ} \mathrm{C}$ in a $5 \% \mathrm{CO}_{2}$ atmosphere for $20 \mathrm{~h}$.

Assessment of cell viability and recovery. At time 0 and $20 \mathrm{~h}$, aliquots $(40 \mu \mathrm{l})$ of cells were counted on a haemocytometer and cell viability assessed by exclusion of the vital dye trypan blue.

Morphological assessment of apoptosis. At time $20 \mathrm{~h}$, aliquots $(150 \mu \mathrm{l})$ of cell suspension were used for cytocentrifuge preparations, fixed in methanol and stained with Wright-Giemsa and examined for morphological features of apoptosis (nuclear condensation and vacuolation) using oil emersion light microscopy. A minimum of 500 cells/slide were examined.

Assessment of apoptosis by measurement of CD16 shedding. Aliquots $(50 \mu \mathrm{l})$ from one of the duplicate treatments were placed in wells of a round bottomed 96 -well polypropylene plate and washed in ice cold PBS containing $0.2 \%$ BSA and $0.1 \%$ sodium azide. Cells were incubated in $30 \mu \mathrm{l}$ of saturating concentrations of primary Ab (mouse mAb anti CD16;3G8) for 30 min on ice. The cells were then washed twice with the above buffer and incubated in $30 \mu$ of a 1/40 dilution of FITC-conjugated $\mathrm{F}\left(\mathrm{ab}^{\prime}\right) 2$ goat $\mathrm{Ab}$ to mouse $\mathrm{Ig}$ for $30 \mathrm{~min}$ on ice and washed twice before flow cytometric assessment using an EPICS Profile II (Coulter Electronics, Luton, UK).

Statistical methods. Data were expressed as mean \pm SEM and were analysed by one way analysis of variance followed by the Newman-Keuls procedure. P values $<0.05$, indicated by an $*$, were considered to be significant. 


\section{RESULTS AND DISCUSSION}

Neutrophils cultured in vitro for $20 \mathrm{~h}$ undergo spontaneous apoptosis as assessed by standard criteria $(4,8,26)$ and by loss of surface $\mathrm{CD} 16$ ( FcrRIII)(26). Our data confirm the close correlation that exists between these assessment methods; for example, $59 \pm 5 \%$ of neutrophils cultured for $20 \mathrm{~h}$ exhibited typical morphology of apoptotic cells and $62 \pm 7 \%$ of the neutrophils shed their surface $\mathrm{CD} 16(\mathrm{n}=6)$. Treatment of neutrophils with ZK $118.182\left(3 \times 10^{-5} \mathrm{M}\right), 11$-deoxy PGE $1\left(3 \times 10^{-5}\right.$ M), dibutyryl-cAMP $\left(2 \times 10^{-3} \mathrm{M}\right)$ and 8-Br-cAMP $\left(2 \times 10^{-3} \mathrm{M}\right)$ dramatically inhibited apoptosis (79. 54,92 , and $70 \%$ inhibition respectively)(Fig.1). The marked inhibition of apoptosis induced by dibutyryl-cAMP (as determined by shedding of $\mathrm{CD} 16$ ) is exemplified by the representative flow cytometric analysis depicted on Fig.2. The CD16 "low" peak in the control $20 \mathrm{~h}$ cells represents apoptotic cells that have shed their surface CD16; the percentage of cells with "low" CD16 was markedly reduced when compared to cells that had been treated with dibutyryl-cAMP whereas the corresponding CD16 "high" peak was increased. Together, these results demonstrate that morphological and surface alterations that accompany apoptosis are inhibited by cAMP elevating agents. When neutrophils were incubated in the presence of the cAMP analogs, dibutyryl-cAMP and 8-Br-cAMP (Fig.3A), and the adenylyl cyclase activator, forskolin (Fig.3B) there was a concentration-dependent inhibition of apoptosis. Similarly, incubation of neutrophils with the PG mimetics, ZK 118.182 and 11-deoxy PGE $_{1}$ (Fig.4A) also caused a concentration-dependent inhibition of apoptosis. Furthermore, the DP receptor antagonist, BW A868C inhibited the effect of $\mathrm{ZK} 118.182$ suggesting that the $\mathrm{PGD}_{2}$ mimetic is indeed interacting with specific DP receptors (Fig.4B). ZK 118.182 and dibutyryl-cAMP were the most effective inhibitors of neutrophil apoptosis. None of the reagents used were toxic to the cells as determined by the exclusion of trypan blue. Apoptosis and its inhibition by cAMP elevating agents was verified using a DNA

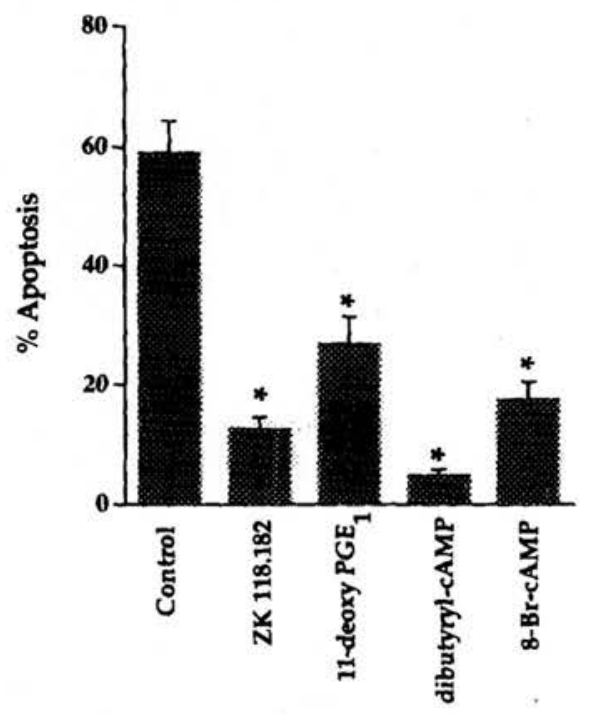

Figure 1. Effect of PG mimetics and cAMP analogs on neutrophil apoptosis as assessed morphologically after $20 \mathrm{~h}$ culture. All treatments were significantly different from control level of apoptosis $(n=3-6)$. 


\section{A Control $(20 \mathrm{~h})$}

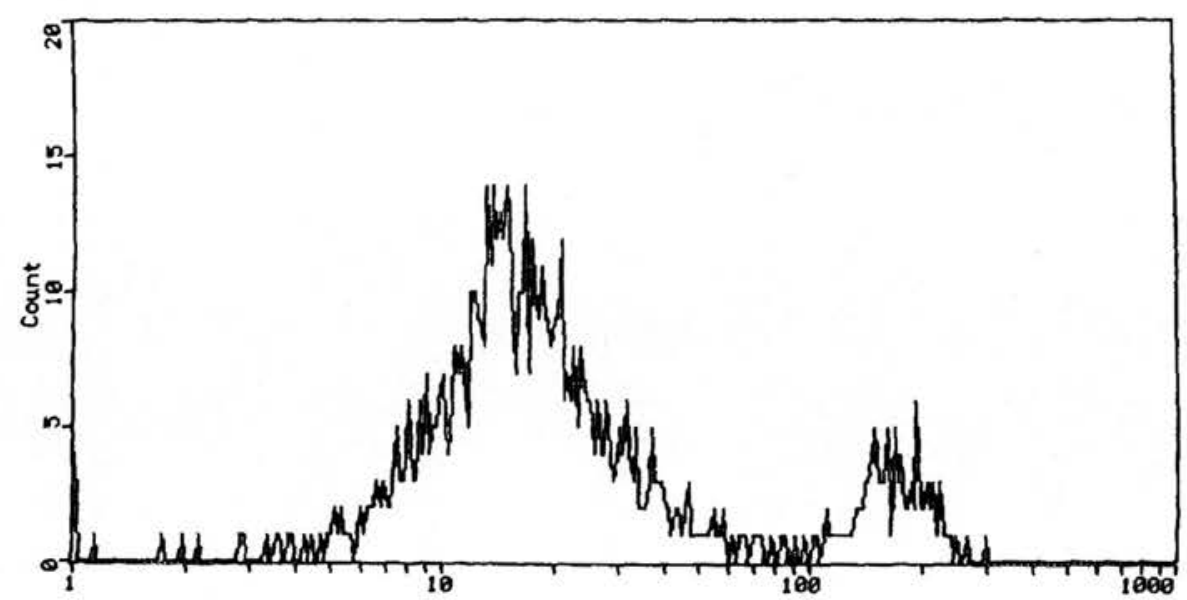

B dibutyryl-cAMP treated $(20 \mathrm{~h})$

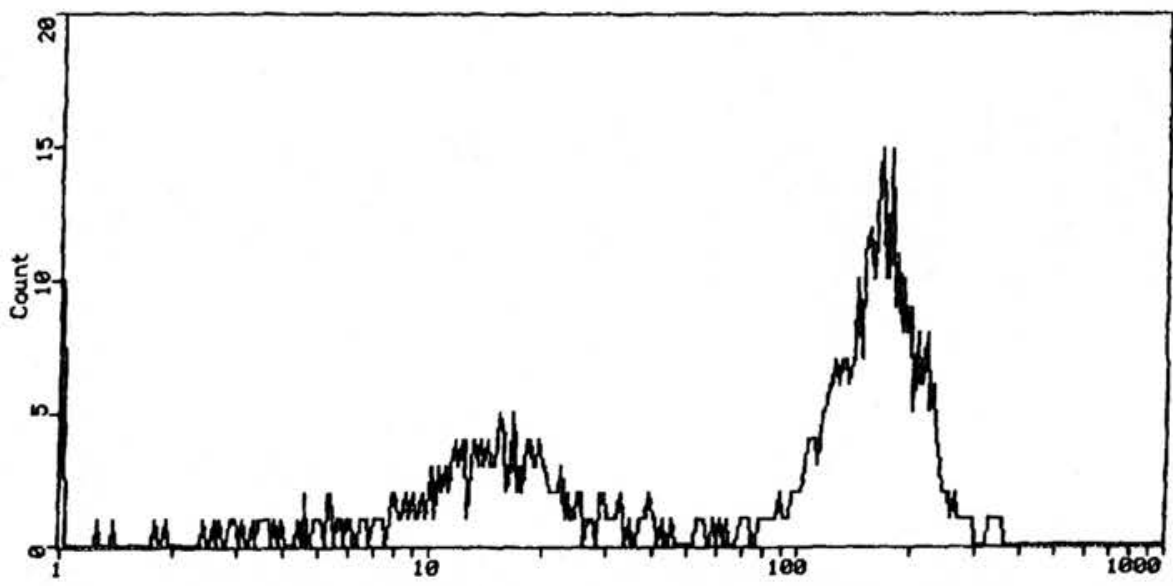

Figure 2. Effect of dibutyryl-cAMP upon levels of surface CD16 (FcyRIII) expression during $20 \mathrm{~h}$ in vitro culture. The results are representative flow cytometric traces of control cells (A) and cells treated with $2 \times 10^{-3} \mathrm{M}$ dibutyryl-cAMP (B).

fragmentation technique (8) and by propidium iodide staining of DNA (26) (data not shown). These results demonstrate that cell permeable cAMP analogs (dibutyryl-cAMP and 8-Br-cAMP), elevation of cAMP by the receptor agonists (ZK 118.182 and 11 -deoxy PGE $_{1}$ ) and direct activation of adenylyl cyclase (forskolin) all result in suppression of neutrophil apoptosis. Inhibition of apoptosis mediated by dibutyryl-cAMP treatment was completely prevented when the cells were cocultured with the PKA inhibitor H-89 (Fig.5) suggesting that cAMP-mediated inhibition of apoptosis is mediated by PKA activation. $\mathrm{H}-89$ per se did not promote apoptosis at $20 \mathrm{~h}$ suggesting that basal turnover of cAMP may not be an important factor in regulating neutrophil apoptosis; a 

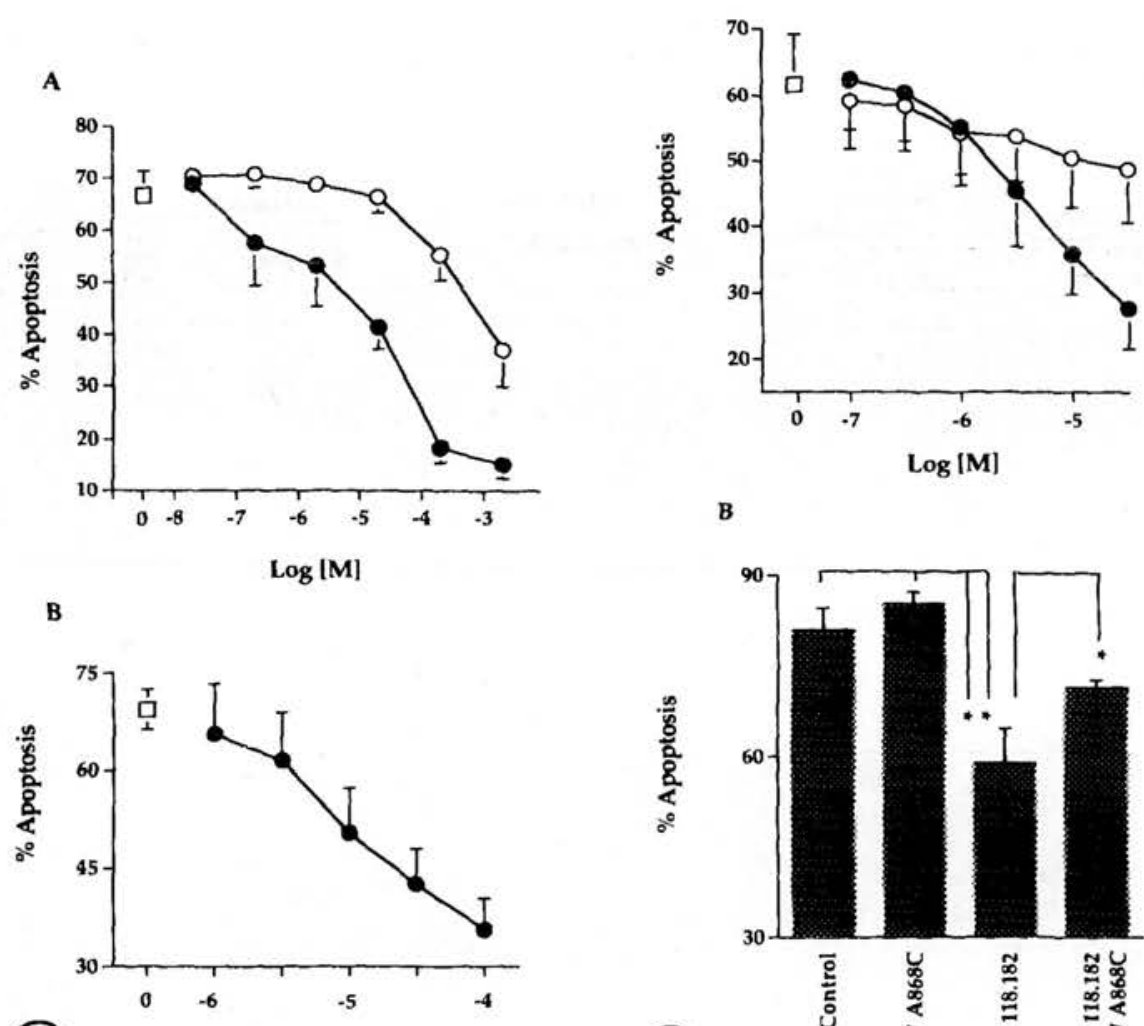

(3)

$\log [\mathbf{M}]$

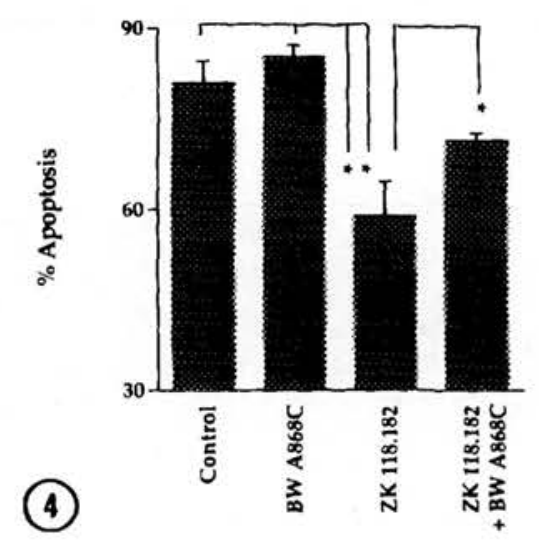

Figure 3. Effect of cAMP analogs (A) and forskolin (B) on neutrophil apoptosis as assessed by CD16 expression after $20 \mathrm{~h}$ culture $(n=4-6)$. Control level of apoptosis is represented by an unfilled square in both panels, dibutyryl-cAMP by filled circles (A), 8-Br-cAMP by open circle (A), and forskolin by filled circles (B).

Figure 4, Effect of PG mimetics (ZK 118.182 and 11-deoxy PGE 1) on neutrophil apoptosis (A) and the effect of BW A868C on ZK 118.182-induced inhibition of apoptosis (B). Apoptosis was assessed by the levels of expression of surface CD16 after $20 \mathrm{~h}$ culture $(n=5-6)$. In panel $A$, control level of apoptosis is represented by an unfilled square, ZK 118.182 by filled circles, and 11-deoxy PGE 1 by open circles.

possibility requiring further investigation. Although we have not directly monitored cAMP levels in this study it is highly likely in view of the documented effects of these agents on intracellular cAMP that prostaglandins are exerting their inhibition of neutrophil apoptosis by interacting with distinct plasmalemmal receptors to cause an elevation of cAMP (28). In addition, direct ligand binding (29) and pharmacological (30) studies have correlated PG-induced inhibition of function with the existence of DP and $\mathrm{EP}_{2}$ receptors on human neutrophils.

Our observations show that cAMP-elevating agents inhibit neutrophil apoptosis whereas in a number of other cell types cAMP accelerates apoptosis (15-25). These results indicate that 


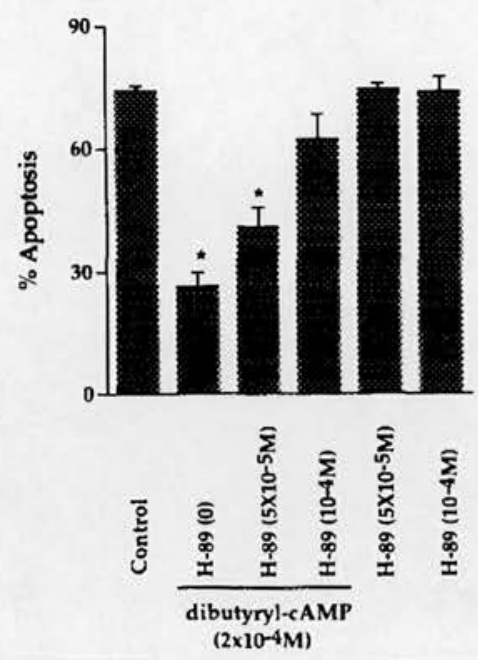

Figure 5. Effect of H-89 on dibutyryl-cAMP-induced inhibition of neutrophil apoptosis as assessed by the levels of expression of surface CD16 after 20 h culture ( $n=5$ ).

neutrophil apoptosis is regulated differently from other cells. Indeed, other studies have shown that agents that inhibit protein synthesis retard thymocyte apoptosis (31) whereas they promote neutrophil apoptosis (32) and elevation of cytosolic free calcium concentration retards neutrophil apoptosis (33) but promotes thymocyte apoptosis (31). We have also shown recently that hypoxia inhibits apoptosis in the neutrophil (34) whereas an identical stimulus is pro-apoptotic in a number of cell lines (35-39). Interestingly, eosinophil survival has been reported to be enhanced in the presence of airway epithelial cells, an effect possibly mediated by PGE 2 as well as GM-CSF (40). It has recently been reported that dibutyryl-cAMP, forskolin and the type IV phosphodiestease inhibitor, rolipram, slightly shorten human neutrophil survival (41); however, the cells were cultured in the absence of serum and apoptosis per se was not determined for neutrophils treated with these agents. These differences most likely explain the apparent discrepancies between that study and the clear inhibition of neutrophil apoptosis by cAMP-elevating agents reported in this study.

Although the mechanisms by which cAMP influences apoptosis are unknown it is possible that cAMP mediates changes in expression of key regulatory genes, transcription factors and proteins reportedly associated with regulation of apoptosis. Elevation of cAMP has been reported to modulate neutrophil production and effects of cytokines known to influence the rate of neutrophil apoptosis (e.g., TNF $\alpha$ and GM-CSF) $(11,42,43)$. cAMP has also been shown to alter the reorganisation of microfilament components of the actin cytoskeleton (44), a fundamental process in cell responses such as chemotaxis and phagocytosis but also likely to be important in the formation of apoptotic bodies (45). Our study shows that agents that elevate cAMP retard neutrophil apoptosis thereby promoting their survival and increasing their functional longevity. Furthermore, receptor-directed agonists such as PGs, which have been shown in vivo to be either pro- or anti-inflammatory depending on the site of action (13), can also suppress programmed cell 
death. Thus pharmacological manipulation of cAMP levels or actions may be expected to exert modulating influences : $\mathrm{u}$ iuflammatory conditions where the neutrophil plays a key role.

Acknowledgments: We thank the Medical Research Council, UK, the Wellcome Trust, UK and the University of Edinburgh for their generous support. ERC is a Wellcome Senior Research Fellow in Clinical Science. We thank Dr. R.A. Armstrong for helpful discussions.

\section{REFERENCES}

1 Hurley, J.V. (1983) In Acute Inflammation (J.V. Hurley, Ed.), Chapter 11, pp. 109-117, Churchill Livingstone, London, UK.

2 Savill, J.S., Wyllie, A.H., Henson, J.E., Walport, M.J., Henson, P.M., and Haslett, C. (1989) J. Clin. Invest. 83, 865-875.

3 Savill, J., and Haslett, C. (1994) In Immunopharmacology of Neutrophils (P.G. Hellewell and T.J.Williams, Eds.), Chapter 14, pp. 295-314. Academic Press, London, UK.

4 Whyte, M.K.B., Meagher, L.C., MacDermot, J., and Haslett, C. (1993) J. Immunol. 150. 5124-5134.

5 Meagher, L.C., Savill, J.S., Baker, A., Fuller, R.W., and Haslett, C. (1992) J. Leukoc. Biol. 52, 269-273.

6 Colotta, F., Re, F., Polentarutti, N., Sozzani, S., and Mantovani, A. (1992) Blood 80, $2012-$ 2020.

7 Brach, M.A., de Vos, S., Gruss, H-J., and Herrmann, F. (1992) Blood 80, 2920-2924.

8 Lee, A., Whyte, M.K.B., and Haslett, C. (1993) J. Leukoc. Biol. 54, 283-288.

9 Murray, J., Condliffe, A.M., Haslett, C., and Chilvers, E.R. (1996) Biochem Soc. Trans. 24, $80 \mathrm{~S}$.

10 Verghese, M.W., Fox, K., McPhail, L.C., and Snyderman, R. (1985) J. Biol. Chem. 260 , 2679-6775.

11 Nathan, C., and Sanchez, E. (1990) J. Cell Biol. 111, 2171-2181.

12 Darius, H., Michael-Hepp, J., Thierauch, K-H., and Fisch, A. (1994) Eur. J. Pharmacol. 258, 207-213.

13 Pons, F., Williams, T.J., Kirk, S.A., McDonald, F., and Rossi, A.G. (1994) Eur. J. Pharmacol. 261, 237-247.

14 Armstrong, R.A., and Talpain, E. (1994) Prostaglandins 48, 221-234.

15 McConkey, D.J., Orrenius, S., and Jondal, M. (1990) J. Immunol. 145, 1227-1230.

16 Lanotte, M., Riviere, J.B., Hermouet, S., Houge, G., Vintermyr, O.K., Gjertsen, B.T., and Doskeland, S.O. (1991) J. Cell. Physiol. 146, 73-80.

17 Suzuki, K., Tadakuma, T., and Kizaki, H. (1991) Cell. Immunol. 134, 235-240.

18 Brown, D.M., Warner, G.L., Ales-Martinez, J.E., Scott, D.W., and Phipps, R.P. (1992) Clin. Immunol. Immunopath. 63, 221-229.

19 Dowd, D.R., and Miesfeld, R.L. (1992) Mol. Cell. Biol. 12, 3600-3608.

20 Mastino, A., Piacentini, M., Grelli, S., Favalli, C., Autuori, F., Tentori, L., Oliverio, S., and Garaci, E. (1992) Dev. Immunol. 2, 263-271.

21 Ackerman, R.C., and Murdoch, W.J. (1993) Prostaglandins 45, 475-485.

22 Vintermyr, O.K., Gjertsen, B.T., Lanotte, M., and Doskeland, S.O. (1993) Exp. Cell Res. 206, $157-161$.

23 Aharoni, D., Dantes, A., Oren, M., and Amsterdam, A., (1995) Exp. Cell Res. 218, $271-282$.

24 Lomo J., Blomhoff, H.K., Beiske, K., Stokke,T., and Smeland, E.B. (1995) J. Immunol. 154, 1634-1643.

25 Mentz, F., Mossalayi, M.D., Ouaaz, F., and Debre, P. (1995) Eur. J. Immunol. 25, 17981801 .

26 Dransfield, I., Buckle, A-M., Savill, J.S., McDowall, A., Haslett, C., and Hogg, N. (1994) J. Immunol. 153, 1254-1263.

27 Haslett, C., Guthrie, L.A., Kopaniak, M.M., Johnston, R.B., and Henson, P.M. (1985) Am. J. Pathol. 119, 101-110.

28 Coleman, R.A., Smith, W.L., and Narumiya, S. (1994) Pharmacol. Rev. 46, 205-229.

29 Rossi, A.G., and O'Flaherty, J.T. (1989) Prostaglandins 37, 641-653.

30 Wheeldon, A., and Vardey, C.J. (1993) Br. J. Pharmacol. 108, 1051-1054.

31 Wyllie, A.H., Morris, R.G., Smith, A.L., and Dunlop, D. (1984) J. Pathol. 142, 67-77.

32 Haslett, C., Savill, J., and Meagher, L. (1990) Biochem. Soc. Trans. 18, 225-227. 
33 Whyte, M.K.B., Hardwick, S.J., Meagher, L.C., Savill, J.S., and Haslett, C. (1993) J. Clin. Invest. $92,446-455$.

34 Hannah, S., Mecklenburgh, K., Rahman, I., Bellingan, G.J., Greening, A., Haslett, C., and Chilvers, E.R., (1995) FEBS Lett. 372, 233-237.

35 Rosenbaum, D.M., Michaelson, M., Batter, D.K., Doshi, P., and Kessler, J.A. (1994) Ann. Neurol. 36, 864-870.

36 Muschel, R.J., Bernhard, E.J., Garza, L., McKenna, W.G., and Koch, C.J. (1995) Cancer Res. 55, 995-998.

37 Shimizu, S., Eguchi, Y., Kosaka, H., Kamiiki, W., Matsuda, H., and Tsujimoto, Y. (1995) Nature 374, $811-813$.

38 Jacobson, M.D., and Raff, M.C. (1995) Nature 374, 814-816.

39 Yao, K-S., Clayton, M., and O'Dwyer, P.J. (1995) J. Natl. Cancer Inst. 87, 117-122.

40 Masuda, T., Suda, Y., Shimura, S., Maruyama, N., Aizawa, T., Tamura, G., Sasaki, H., and Takishima, T. (1992) Respiration 59, 238-242.

41 Aoshibi, K., Nagai, A., and Konno, K. (1995) Antimicrob. Agents and Chemother. 39, 872877.

42 Brandt, E., Petersen, F., and Flad, H-D. (1992) J. Immunol. 149, 1356-1364.

43 Ottonello, L., Morone, M.P., Dapino, P., and Dallegri, F. (1995) Clin. Exp. Immunol. 101, 502-506.

44 Downey, G.P., Elson, E.L., Schwab, B., Erzurum, S.C., Young, S.K., and Worthen, G.S. (1991) J. Cell Biol. 114, 1179-1190.

45 Martin, S.J., and Cotter, T.G. (1990) Cell Tissue Kinet. 23, 545-559. 


\title{
Constitutive neutrophil apoptosis in culture is modulated by cell density independently of $\beta_{2}$ integrin-mediated adhesion
}

\author{
$\rightarrow$ \\ Sharon Hannah*, Imad Nadra, Ian Dransfield, James G. Pryde, Adriano G. Rossi, \\ Christopher Haslett
}

Rayne Laboratory, Respiratory Medicine Unit, Department of Medicine (R.I.E.), University of Edinburgh Medical School, Teviot Place, Edinburgh, EH8 $9 A G$, Scotland, UK

Received 5 December 1997

\begin{abstract}
Although inflammatory mediators modulate the rate of constitutive neutrophil apoptosis in vitro the effects of microenvironmental conditions have not been fully investigated. In this study, we demonstrate that the rate of constitutive neutrophil apoptosis is affected by the number of cells per unit surface area, with enhanced survival at high cell density. Furthermore, the presence of protein or serum in the culture medium also enhances neutrophil survival. These effects were independent of $\beta_{2}$ integrin-mediated adhesion and were not influenced by specific adhesion to extracellular matrix components. Thus, the rate of neutrophil apoptosis is fundamentally influenced by microenvironmental conditions and indicates that factors such as cell density and extracellular protein concentration must be considered when investigating mechanisms regulating inflammatory cell apoptosis in vitro.

(c) 1998 Federation of European Biochemical Societies.
\end{abstract}

Key words: Neutrophil; Apoptosis; Adhesion; Micro-environment

\section{Introduction}

Tissue infection by pathogens initiates an acute inflammatory response characterised by rapid neutrophil recruitment $[1,2]$ and release of neutrophil granule contents with anti-microbial activity to facilitate pathogen destruction [3]. Following elimination of the inflammatory insult, resolution of acute inflammation requires that recruited neutrophils are removed before they cause 'inappropriate' damage to tissue due to cytotoxic effects of granule contents [4-6]. Neutrophil apoptosis (programmed cell death) characterised by morphological and biochemical alterations, e.g. condensation and cleavage of nuclear chromatin [9] together with functional alterations that limit stimulus-driven release of cytotoxic granule contents $[7,8]$ provides one mechanism for limiting the tissue destructive potential of the neutrophil. Furthermore, specific neutrophil membrane alterations signal macrophage recognition and phagocytosis of apoptotic neutrophils [10-12] via mechanisms which do not provoke pro-inflammatory mediator release, consistent with a role for apoptotic neutrophil death in resolution of inflammatory processes [13].

\footnotetext{
*Corresponding author. Fax: +44 (131) 650-4384.

E-mail: s.hannah@ed.ac.uk
}

Abbreviations: mAb, monoclonal antibody; MDM, modified Dulbecco's medium; db-cAMP, dibutyryl adenosine- $3^{\prime}, 5^{\prime}$-cyclic monophosphate; fMLP, $N$-formyl-methionyl-leucyl-phenylalanine
The rate at which neutrophils undergo apoptosis is likely to represent a critical factor determining the absolute tissue load of neutrophil products at inflammatory sites. The presence of inflammatory mediators such as granulocyte-macrophage colony-stimulating factor (GM-CSF) and bacterial lipopolysaccharide (LPS) can prolong neutrophil longevity in vitro $[14,15]$, and also potentiate neutrophil effector function. In addition, local environmental conditions such as hypoxia suppress the apoptotic programme in neutrophils [16] and it is likely that micro-environmental factors profoundly influence progression of the inflammatory response.

Recent studies indicate that in addition to the critical role of neutrophil adhesion in efficient recruitment of cells during development of the inflammatory response [17], adhesion plays an important role in regulation of cellular survival. Neutrophil apoptosis is potentiated following adhesion to fibronectin in a $\beta_{2}$ integrin-dependent manner when neutrophils are cultured on interleukin-1-stimulated endothelial cells [18]. A role for the $\beta_{2}$ integrin CD11b/CD18 (Mac-1) in the regulation of neutrophil apoptosis was further supported by studies in CD11b/CD18 deficient mice [19] where neutrophil apoptosis was found to be accelerated following engagement of $\mathrm{CD} 11 \mathrm{~b} / \mathrm{CD} 18$ and assembly of the NADPH oxidase during phagocytosis. This pathway was suggested to provide a regulatory feedback mechanism for elimination of phagocytically active neutrophils, although these studies suggested that the constitutive rate of neutrophil apoptosis in suspension culture was largely CD18-independent. Furthermore, a recent study showing that cross-linking of $\mathrm{CD} 11 \mathrm{~b} / \mathrm{CD} 18$ potentiated TNF $\alpha$ induced human neutrophil apoptosis again suggesting a role for $\beta_{2}$ integrins in the rate of activated neutrophil apoptosis [20].

In the present study, we have defined the environmental parameters which affect the rate of constitutive apoptosis of monolayers of neutrophils during in vitro culture. The rate of apoptosis was found to be decreased in a manner that is inversely related to the cell density per $\mathrm{cm}^{2}$ of the culture vessel, apparently independent of adhesion to the culture vessel. In addition, neutrophil survival was enhanced when the cells were cultured in medium containing protein. The results of experiments presented in this paper together with our previous work raises the possibility that localised hypoxic conditions provide one mechanism for increased neutrophil survival at high cell density. Furthermore, our data demonstrate that careful consideration must be given to the design of culture conditions when studying the effects of agents that modulate apoptosis. 


\section{Materials and methods}

\subsection{Materials}

All chemicals were purchased from Sigma, Dorset, UK except dexamethasone which was from David Bull Laboratories, Warwick, U.K. Cell culture media were from Life Technologies, Paisley, UK, dextran T-500 and Percoll from Pharmacia, Milton Keynes, UK, Diff Quick from Baxter Healthcare, Glasgow, UK and cell culture plastic was from Becton Dickinson, Leics., UK. Monoclonal antibodies (mAb): KIM 127, CD18 function promoting antibody [21] (gift from Martyn Robinson, Celltech); TS1-18, anti-CD18 (American Type Tissue Culture Collection); ICRF44, anti-CD11b [22] (gift from Nancy Hogg, ICRF); BBA-2, anti-CD62-E (R and D Systems, Oxon, UK); and VLA-3, anti-CD49c (clone 11G5; Serotec, Oxford, UK). Extracellular matrix components: fibronectin (Sigma); vitronectin (CalbiochemNovobiochem, Nottingham, UK), and collagen types I and VI (Life Technologies).

\subsection{Neutrophil isolation}

Neutrophils were isolated from citrate anti-coagulated blood as previously reported [23]. Briefly, cells were separated from whole blood by centrifugation at $220 \times \mathrm{g}$ for $20 \mathrm{~min}$ and the upper platelet-rich plasma layer removed. Leukocytes were separated from the erythrocytes in the cell pellet by differential sedimentation using $0.6 \%$ (w/v) dextran T-500. Granulocytes were separated from the mononuclear cells through a discontinuous isotonic Percoll gradient. Granulocytes were harvested from the $63 / 73 \%$ interface of the gradient and were $>95 \%$ neutrophils as determined by morphological analysis and $>99 \%$ viable as determined by trypan blue dye exclusion.

\subsection{In vitro culture of neutrophils}

Neutrophils were suspended in Iscove's MDM containing penicillin $(100 \mathrm{U} / \mathrm{ml})$ and streptomycin $(100 \mu \mathrm{g} / \mathrm{ml})$ and supplemented with either autologous serum (recalcified autologous plasma; $0.00008-2.4 \%$ (w/v albumin) corresponding to $0.001-30 \%$ (v/v serum)) or $0.001-0.1 \%$ (w/v) BSA. In a separate series of experiments, the neutrophils were suspended in Iscove's MDM containing penicillin $(100 \mathrm{U} / \mathrm{ml})$ and streptomycin $(100 \mu \mathrm{g} / \mathrm{ml})$ and supplemented with either $10 \%(\mathrm{v} / \mathrm{v})$ autologous serum or $0.1 \%(\mathrm{w} / \mathrm{v}) \mathrm{BSA}$, in the absence or presence of $2 \mathrm{mM}$ db-cAMP or $1 \mu \mathrm{M}$ dexamethasone. The neutrophils were dispensed into either flat-bottomed 96-well polypropylene plates or Teflon pots (Pierce, Chester, UK) at various densities and volumes and incubated at $37^{\circ} \mathrm{C} / 5 \% \mathrm{CO}_{2}$.

\subsection{Assessment of apoptosis}

Triplicate cytocentrifuge preparations were made for each treatment and time point, stained with Diff-Quick, and apoptotic neutrophils identified by their darkly stained, condensed nucleus and cytoplasmic vacuolation [7]. In addition, neutrophils were labelled with FITC-conjugated $\mathrm{CD} 16 \mathrm{mAb}$ and samples analysed by flow cytometry with CD16 'low' expressing neutrophils representing the apoptotic population [23].

\subsection{Antibody blocking experiments}

Neutrophils were suspended at a density of $0.5 \times 10^{6} / \mathrm{ml}$ in Iscove's MDM supplemented with autologous serum, penicillin $(100 \mathrm{U} / \mathrm{ml})$ and streptomycin $(100 \mu \mathrm{g} / \mathrm{ml})$ and dispensed into flat-bottomed 96-well polypropylene plates $(0.1 \mathrm{ml} /$ well $)$ containing either PBS or test antibody (final concentration $20 \mu \mathrm{g}$ of $\mathrm{IgG} / \mathrm{ml}$ in PBS). After culture for $20 \mathrm{~h}$ at $37^{\circ} \mathrm{C} / 5 \% \mathrm{CO}_{2}$, apoptosis was assessed morphologically.

\subsection{Extracellular matrix}

Extracellular matrix proteins $(10 \mu \mathrm{g} / \mathrm{ml})$ were adsorbed to flat-bottomed polypropylene 96 -well plates $(0.1 \mathrm{ml} /$ well $)$ overnight at $4^{\circ} \mathrm{C}$ and the wells washed with PBS. Neutrophils $\left(1 \times 10^{6}\right.$ cells $/ \mathrm{ml}$ or $8 \times 10^{6}$ cells $/ \mathrm{ml}$ ) in Iscove's MDM supplemented with $10 \%(\mathrm{v} / \mathrm{v})$ autologous serum, penicillin $(100 \mathrm{U} / \mathrm{ml})$ and streptomycin $(100 \mu \mathrm{g} / \mathrm{ml})$ were dispensed into the coated wells or uncoated, control wells $(0.15 \mathrm{ml} / \mathrm{well})$. The samples were then cultured for $20 \mathrm{~h}$ at $37^{\circ} \mathrm{C} / 5 \% \mathrm{CO}_{2}$, after which the percentage of apoptosis assessed as described above.

\subsection{Supernatant transfer experiments}

Neutrophils were suspended at densities of either $0.5 \times 10^{6} / \mathrm{ml}$ (low density) or $4 \times 10^{6} / \mathrm{ml}$ (high density) in $4 \mathrm{ml}$ Iscove's MDM supple- mented with $10 \%(\mathrm{v} / \mathrm{v})$ autologous serum, penicillin $(100 \mathrm{U} / \mathrm{ml})$ and streptomycin $(100 \mu \mathrm{g} / \mathrm{ml})$ then cultured overnight in Teflon pots. A positive control culture of high density neutrophils aged in the absence of serum and other exogenous protein supplements was also prepared. After culture for $20 \mathrm{~h}$ at $37^{\circ} \mathrm{C} / 5 \% \mathrm{CO}_{2}$, the supernatants were collected and added to fresh neutrophils from the same donor at low density $\left(0.5 \times 10^{6} / \mathrm{ml}\right)$. The serum-free supernatant was supplemented with serum to $10 \%(\mathrm{v} / \mathrm{v})$, and a further control culture consisting of fresh neutrophils cultured in fresh medium supplemented with $10 \%(\mathrm{v} / \mathrm{v})$ serum was prepared. The neutrophils were cultured for $20 \mathrm{~h}$ at $37^{\circ} \mathrm{C} / 5 \% \mathrm{CO}_{2}$, after which triplicate cytocentrifuge preparations were made.

\subsection{Statistics}

All data are presented as mean \pm S.E.M. for $(n)$ separate experiments. Values were compared using the Student's $t$-test or where appropriate by one-way analysis of variance followed by the Newman-Keuls procedure. Differences were considered significant when $P<0.05$.

a.

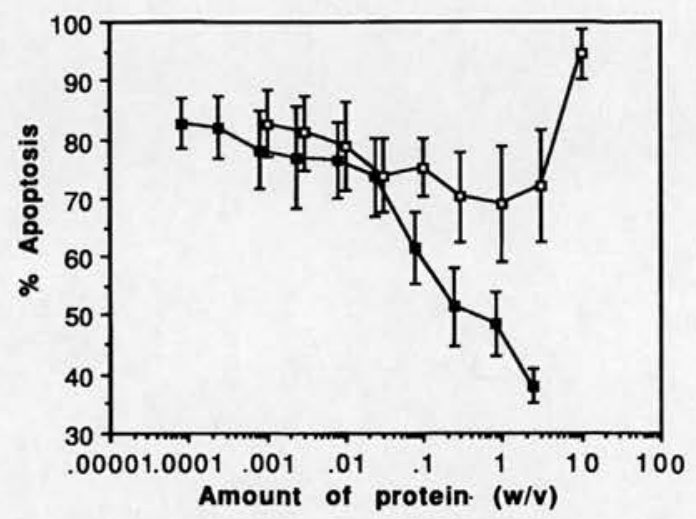

b

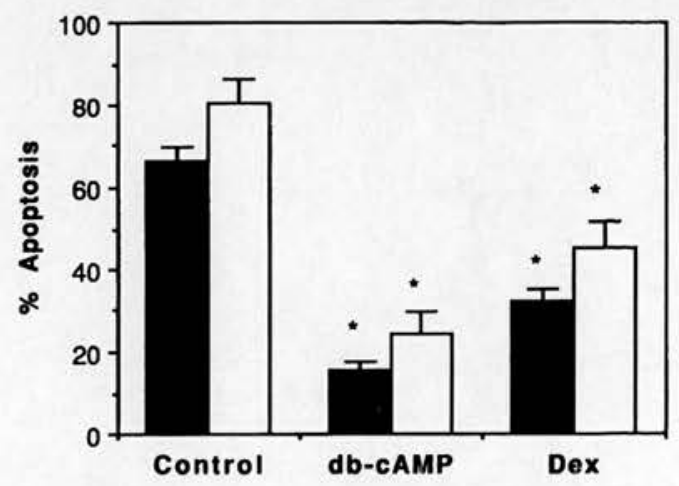

Fig. 1. The effect of serum and BSA on neutrophil apoptosis. Neutrophils $\left(5 \times 10^{6} / \mathrm{ml}\right)$ were cultured for $20 \mathrm{~h}$ in flat-bottomed 96-well polypropylene plates after which the percentage apoptosis was assessed morphologically as detailed in Section 2. a: Neutrophils $(0.2 \mathrm{ml})$ were suspended in the presence of either Iscove's MDM supplemented with $0.001-30 \%$ (v/v) serum (equivalent a serum albumin protein concentration of $0.00008-2.4 \%(\mathrm{w} / \mathrm{v})$; closed symbols) or $0.003-10 \%(\mathrm{w} / \mathrm{v})$ BSA (open symbols) (data represent mean \pm S.E.M.; $n=5$ ). b: Neutrophils were suspended in medium supplemented with either $10 \%(\mathrm{v} / \mathrm{v})$ serum (closed bars) or $0.1 \%(\mathrm{w} / \mathrm{v})$ BSA (open bars) and cultured in the presence or absence of $2 \mathrm{mM}$ $\mathrm{db}-\mathrm{cAMP}$ or $1 \mu \mathrm{M}$ dexamethasone (data represent mean \pm S.E.M.; $n=5 ; P<0.05)$. 
a.

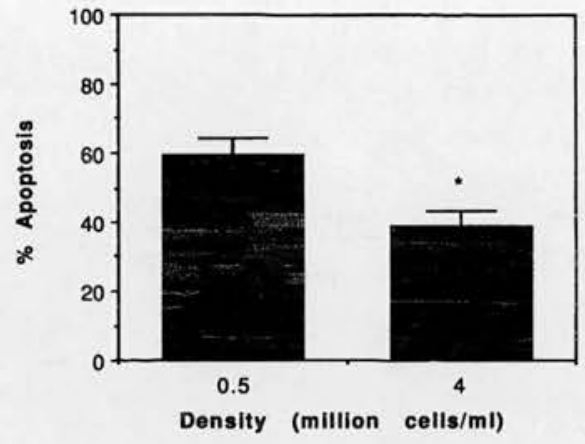

b.

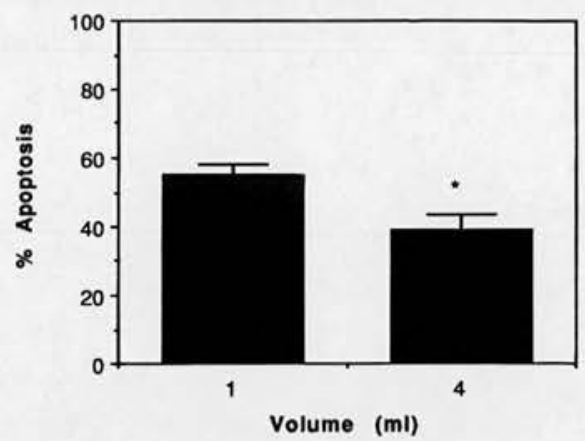

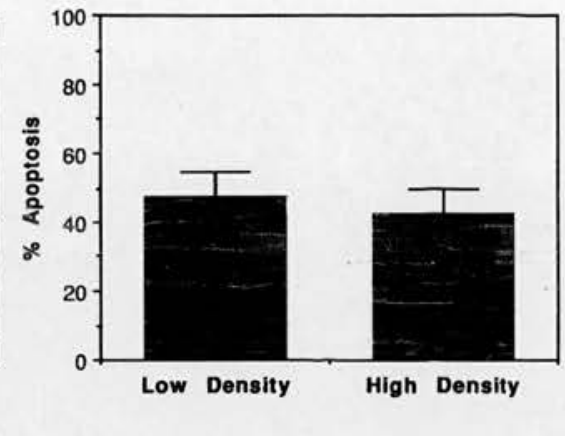

d.

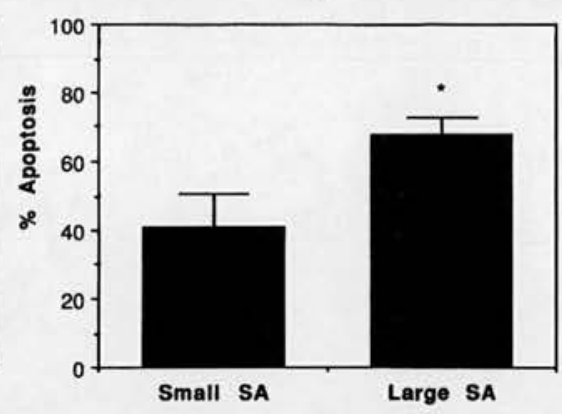

Fig. 2. The effect of volume, cell number and surface area on the rate of neutrophil apoptosis. Neutrophils were cultured for $20 \mathrm{~h}$ in Teflon vessels after which the percentage of apoptosis was assessed morphologically as detailed in Section 2. a: Neutrophils were suspended at either $0.5 \times 10^{6} / \mathrm{ml}$ or $4 \times 10^{6} / \mathrm{ml}$ in a constant volume of $4 \mathrm{ml}$ (data represent mean \pm S.E.M; $n=6 ; P<0.05$ ). b: Neutrophils were suspended at a constant density of $4 \times 10^{6} / \mathrm{ml}$ and cultured in volumes of either $1 \mathrm{ml}$ or $4 \mathrm{ml}$ (data represent mean \pm S.E.M.; $n=4 ; P<0.05$ ). c: The total number of neutrophils cultured in each vessel was constant $\left(16 \times 10^{6}\right)$ but they were suspended at either a low density (32 ml) or a high density $(4 \mathrm{ml})$ in large Teflon containers (data represent mean \pm S.E.M.; $n=3)$. d: Neutrophils $\left(4 \times 10^{6}\right)$ were cultured in Teflon containers with different surface areas. The surface area of the small and large containers were $201 \mathrm{~mm}^{2}$ and $1385 \mathrm{~mm}^{2}$ respectively (data represent mean \pm S.E.M.; $n=5 ; P<0.05)$.

\section{Results and discussion}

Unlike a number of other cell types, neutrophils undergo constitutive apoptosis when cultured in vitro [3]. The rate at which a population of neutrophils become apoptotic can be influenced by the addition of cytokines [14-16] or altered levels of second messengers within the cell [24] and by manipulating the extracellular environment [16]. However, in view of conflicting reports relating to the effects of certain mediators such as fMLP $[15,25]$ and cAMP $[26,27]$ on neutrophil apoptosis we have determined how the rate of neutrophil apoptosis is influenced by culture conditions.

We first assessed the effects of different serum concentrations upon constitutive neutrophil apoptosis during in vitro culture. In these experiments, neutrophils were cultured in the absence or presence of various concentrations of autologous serum. These experiments revealed that in the absence of exogenously added protein, neutrophils cultured for $20 \mathrm{~h}$ underwent secondary necrosis with large numbers of trypan blue positive cells present (data not shown). In contrast, the addition of $0.008-0.1 \%(w / v)$ concentrations of serum was sufficient to maintain neutrophil plasma membrane integrity, although a large proportion of cells underwent apoptosis (Fig. la). We next tested whether inclusion of bovine serum albumin (BSA) also provided survival in the absence of human serum components and whether survival was still augmented by db-cAMP or dexamethasone in the presence of BSA. As shown in Fig. 1a, addition of $0.1-1 \%(w / v)$ BSA was sufficient to rescue significant numbers of neutrophils from necrosis in the absence of other serum factors. However, in the presence of high concentrations of BSA $(10 \% \mathrm{w} / \mathrm{v})$ increased neutrophil apoptosis was observed. Neutrophil apoptosis in the presence of BSA could be inhibited by the addition of exogenous mediators suggesting that db-cAMP and dexamethasone survival effects are independent of other serum components (Fig. 1b). In contrast to the effects of high concentrations of BSA, serum $(0.1-3 \% \mathrm{w} / \mathrm{v})$ had a profound inhibitory effect upon neutrophil apoptosis suggesting the presence of additional survival factors. One possible explanation for the pre-survival effects of inclusion of protein in the culture medium is that respiratory burst activation and release of reactive oxygen species occurs in the absence of protein and engages 'activation-dependent' apoptosis pathways in neutrophils, accelerating the rate of apoptosis [28].

Having established that neutrophil survival factors in vitro required the presence of protein, but not necessarily serum, we next investigated the influence of the type of culture vessel. In this series of experiments, we cultured the neutrophils in the presence of $10 \%(\mathrm{v} / \mathrm{v})$ autologous serum, allowing for direct comparison with other studies. Neutrophils were cultured for $18 \mathrm{~h}$ at various densities $\left(0.5 \times 10^{6} / \mathrm{ml}\right.$ or $\left.4 \times 10^{6} / \mathrm{ml}\right)$ in Teflon containers. Results shown in Fig. $2 \mathrm{a}$ indicate that the rate of neutrophil apoptosis was inversely proportional to the cell density (density being defined as the number of cells per unit volume) suggesting that neutrophil survival was increased at high cell density. The observed density-dependent effect was 
investigated further by varying the volume of cell suspension at a constant cell density in Iscove's MDM containing 10\% autologous serum. Results presented in Fig. 2b suggested that the proportion of neutrophils undergoing apoptosis during in vitro culture was affected by volume. However, under these culture conditions neutrophils in suspension settle onto the surface of the culture vessel. When we attempted to keep cells in suspension they went necrotic suggesting a sensitivity to mechanical shear in vitro (data not shown). We then investigated the possibility that the absolute number of cells placed in a culture vessel of defined size may be of greater significance than either the cell density or the total volume of cell suspension. In order to test this suggestion we placed a constant number of cells in different volumes of culture medium in identical sized culture vessels. We found that under these conditions, the rate of apoptosis was unaffected (Fig. 2c) suggesting that cell concentration per se was not a critical factor. Since under these experimental conditions the surface area of the vessel was kept constant, we examined whether culturing a

a.

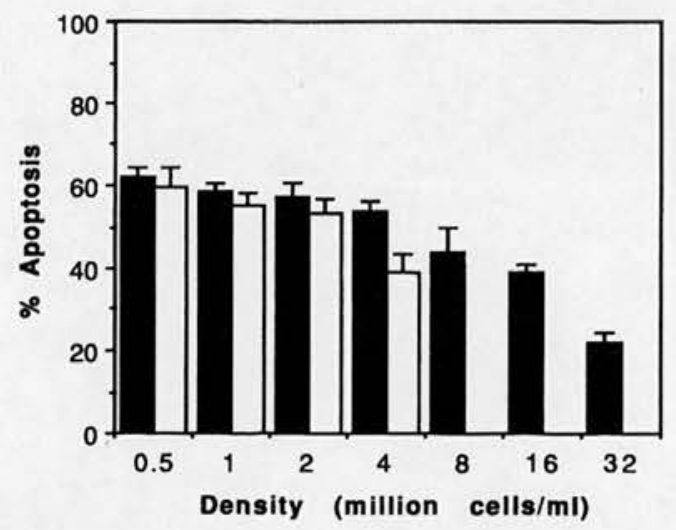

b.

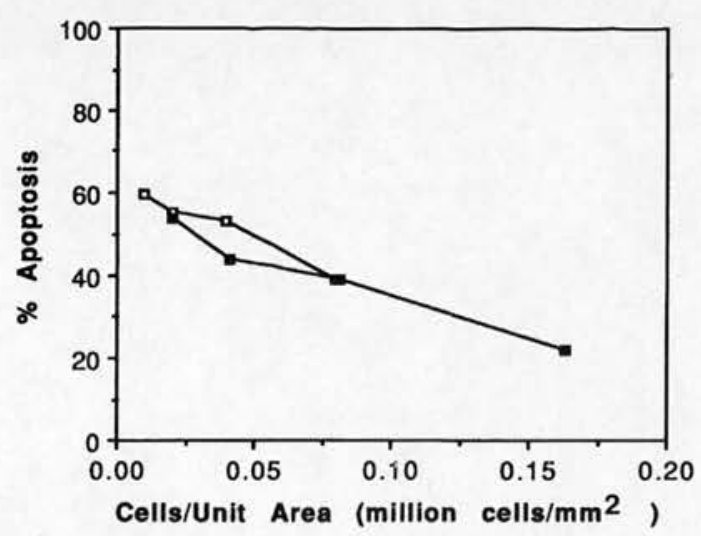

Fig. 3. Comparison of neutrophil apoptosis after culture in Teflon or polypropylene vessels. a: Neutrophils were cultured in Teflon (open bars) or polypropylene (closed bars) containers at a variety of different cell densities and a constant volume of $4 \mathrm{ml}$ in Teflon containers and $0.1 \mathrm{ml}$ in polypropylene containers. The percentage of apoptosis after $20 \mathrm{~h}$ was assessed morphologically as detailed in Section 2. Data represent mean \pm S.E.M.; $n=3$ separate experiments. b: The same data were expressed as the percentage of apoptosis per unit surface area of the culture vessel. The surface area of the Teflon and polypropylene containers were $201 \mathrm{~mm}^{2}$ and $19.6 \mathrm{~mm}^{2}$ respectively. a.

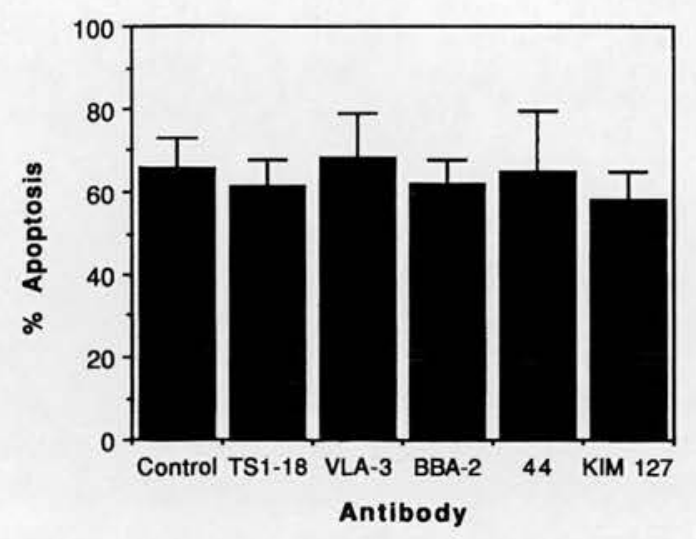

b.

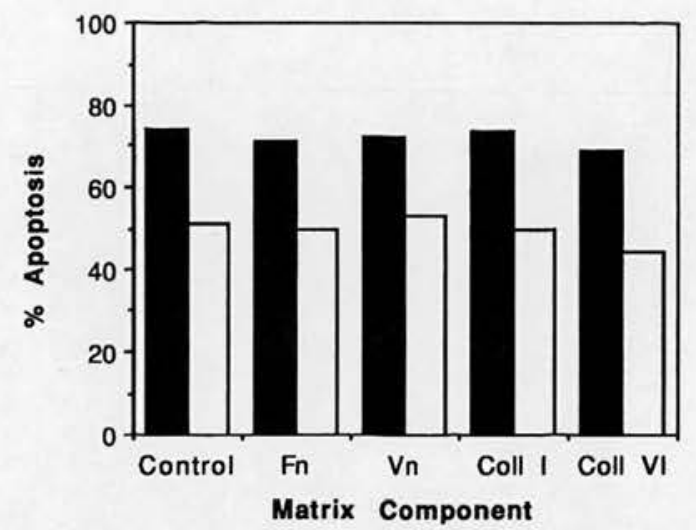

Fig. 4. The effect of culturing neutrophils in pre-coated polypropylene vessels. a: Neutrophils were cultured in polypropylene vessels for $20 \mathrm{~h}$ in the presence of a panel of antibodies as detailed in Section 2. The percentage of apoptosis after culture was assessed morphologically as previously detailed. Data represent mean \pm S.E.M.; $n=3$ separate experiments. b: Neutrophils were cultured in polypropylene vessels which were pre-coated with matrix proteins at low (closed bars) and high densities (open bars). The percentage apoptosis was assessed after $20 \mathrm{~h}$ of culture. Data show a representative experiment, which was confirmed by labelling with FITC-conjugated CD16 mAb as detailed in Section 2.

constant number of cells in vessels with different surface areas affected the rate of apoptosis. From results of experiments shown in Fig. 2d it was evident that the rate of apoptosis was inversely proportional to the number of cells per unit surface area. We next investigated whether cell density per unit area of culture vessel influenced the rate of apoptosis when cells were cultured on polypropylene, a surface to which neutrophils will adhere (data not shown). Although the density per unit volume of neutrophils which exhibit higher rates of apoptosis in polypropylene was higher than that in Teflon (Fig. 3a), when the data were expressed in terms of the number of cells per unit area (Fig. 3b) there appeared to be little difference between the two culture conditions, suggesting that adhesion to a culture vessel was not influencing the apoptosis rate under these culture conditions. Whilst the use of culture conditions that place neutrophils in contact with 2-dimensional surfaces to investigate regulation of apoptosis may fail to mimic the environment of neutrophils at inflammatory sites, 


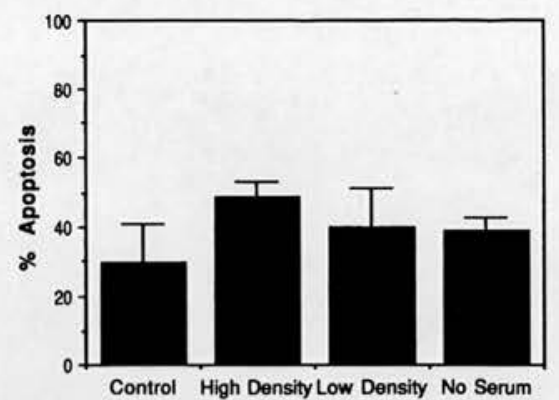

Fig. 5. The effect of culturing low density neutrophils in previously prepared supernatants from high and low density neutrophils. Neutrophils were cultured at $0.5 \times 10^{6} / \mathrm{ml}$ (low density) or $4 \times 10^{6} / \mathrm{ml}$ (high density) in the presence of $10 \%(\mathrm{v} / \mathrm{v})$ serum or at high density in the absence of added serum in Teflon pots for $20 \mathrm{~h}$ as detailed in Section 2. The supernatants were collected and used as the culture media for fresh neutrophils from the same donor which were cultured at low density for $20 \mathrm{~h}$. Control cultures containing fresh neutrophils cultured in fresh medium were also prepared. The percentage of apoptosis after culture was assessed morphologically as previously detailed (data represent mean \pm S.E.M.; $n=3$ ).

these experiments establish that cell density per unit surface area represents a critical factor determining the rate of neutrophil apoptosis during in vitro culture.

In view of evidence that adhesion provides regulatory signals determining cell survival we next investigated whether specific adhesion via $\beta_{2}$ integrins affected the rate of apoptosis of neutrophils cultured in polypropylene. Neutrophils were cultured in the presence of CD18 blocking monoclonal antibodies $(\mathrm{mAb})$. Although CD18 mAb efficiently blocked neutrophil adhesion to plastic in static adhesion assays (data not shown), we did not observe alterations in the rate of neutrophil apoptosis in the presence of CD18 mAb (Fig. 4a). Moreover, addition of the $\beta_{2}$ integrin function promoting $\mathrm{mAb}$ KIM 127 did not influence the rate of apoptosis, indicating that $\beta_{2}$ integrin-mediated adhesion events may not provide regulatory signals determining commitment to apoptosis in unstimulated cells. In addition, neutrophil apoptosis at either low $\left(1 \times 10^{6} / \mathrm{ml}\right)$ or high $\left(8 \times 10^{6} / \mathrm{ml}\right)$ cell densities was not altered by pre-coating the culture vessels with extracellular matrix proteins fibronectin, vitronectin or collagens (Fig. $4 \mathrm{~b})$. Together, these data suggest that engagement of specific adhesion receptors on neutrophils does not influence the constitutive rate at which neutrophils undergo apoptosis in vitro.

The possibility that neutrophils secrete factor(s) which at high cell density provide autocrine survival signals was addressed in a series of supernatant transfer experiments. Neutrophils were suspended at high and low densities in the presence of serum in Teflon pots and cultured for $20 \mathrm{~h}$. The supernatants were collected and used to culture, at low density, freshly isolated neutrophils from the same donor (Fig. 5). Neutrophils cultured in the presence of supernatants from 'high density' neutrophils were not protected against apoptosis. Moreover, the supernatant from neutrophils cultured in the absence of serum did not cause any significant effect on the extent of apoptosis of fresh neutrophils cultured in vitro. Although cellular depletion of media constituents may complicate interpretation of these results, it seems likely that neutrophils are not capable of secreting survival factors at levels sufficient to rescue neutrophils cultured in vitro at low density from apoptosis.
In summary, examination of physical parameters which influence neutrophil apoptosis in culture revealed that survival is dependent on the number of cells per unit area of the culture vessel, consistent with the suggestions of Raff [29], that cellular communication is a key determinant of commitment to apoptosis. In addition, the presence of protein in the culture medium also augmented neutrophil survival. However, our data did not support a role for the principal neutrophil adhesion receptors, $\beta_{2}$ integrins, in providing regulatory signals that determine rates of apoptosis. Moreover, at low cell density, plating neutrophils onto a variety of extracellular matrix components failed to provide 'rescue' from accelerated apoptosis. Although supernatant transfer experiments do not exclude the possibility that high local concentrations of autocrine factors are present prior to harvesting of the supernatant, it is equally likely that high neutrophil density per unit area of culture vessel gives rise to local hypoxic conditions and $\mathrm{pH}$ changes which exert protective effects [16]. Furthermore, our findings that micro-environmental conditions influence neutrophil survival have important implications for study design when assessing the role of exogenous factors in regulation of the apoptotic programme in neutrophils.

Acknowledgements: This work was supported by a Medical Research Council (UK) Programme Grant and a Wellcome Trust studentship awarded to I.N.

\section{References}

[1] Malech, H.L. and Gallin, J.I. (1987) New Engl. J. Med. 317, 687694.

[2] Weiss, S.J. (1988) New Engl. J. Med. 320, 365-376

[3] Haslett, C., Savill, J.S., Whyte, M.K.B., Stern, M., Dransfield, I. and Meagher, L.C. (1994) Philos. Trans. R. Soc. Lond. B 345, 327-333.

[4] Savill, J. and Haslett, C. (1994) in: Immunopharmacology of Neutrophils (Helliwell, P.G. and Williams, T.J., Eds.) Ch. 14, pp. 295-314, Academic Press, London, UK

[5] Grigg, J.M., Savill, J.S., Sarraf, C., Haslett, C. and Silverman, M. (1991) Lancet 338, 720-722.

[6] Cox, G., Crossley, J. and Xing, Z. (1995) Am. J. Respir. Cell Mol. Biol. 12, 232-237.

[7] Savill, J.S., Wyllie, A.H., Henson, J.E., Walport, M.J., Henson, P.M. and Haslett, C. (1989) J. Clin. Invest. 83, 865-875.

[8] Whyte, M.K.B., Meagher, L.C., MacDermott, J. and Haslett, C. (1993) J. Immunol. 150, $5124-5134$

[9] Wyllie, A.H., Kerr, J.F.R. and Currie, A.R. (1980) Int. Rev. Cytol. 68, 251-305.

[10] Savill, J., Henson, P.M. and Haslett, C. (1989) J. Clin. Invest. 84, 1518-1527.

[11] Savill, J.S., Dransfield, I., Hogg, N. and Haslett, C. (1990) Nature $343,170-172$.

[12] Savill, J., Fadok, V., Henson, P. and Haslett, C. (1993) Immunol. Today 14, 131-136.

[13] Meagher, L.C., Savill, J.S., Baker, A., Fuller, R.W. and Haslett, C. (1992) J. Leuk. Biol. 52, 269-273.

[14] Colotta, F., Re, F., Polentarutti, N., Sozzani, S. and Mantovani, A. (1992) Blood 80, 2012-2020.

[15] Lee, A., Whyte, M.K.B. and Haslett, C. (1993) J. Leuk. Biol. 54, 283-288.

[16] Hannah, S., Mecklenburgh, K., Rahman, I., Bellingan, G.J., Greening, A., Haslett, C. and Chilvers, E.R. (1995) FEBS Lett. $372,233-237$.

[17] Zimmerman, G.A., Prescott, S.M. and McIntyre, T.M. (1992) Immunol. Today 13, 93-100.

[18] Ginis, I. and Faller, D.V. (1997) Am. J. Physiol. 272, C295-C309.

[19] Coxon, A., Rieu, P., Barkalow, F.J., Askari, S., Sharpe, A.H., von Andrian, U.H., Arnaout, M.A. and Mayadas, T.N. (1996) Immunity 5, 653-666. 
[20] Walzog, B., Jeblonsk, F., Zakrzewicz, A. and Gahtgens, P. (1997) FASEB J. 11, 1177-1186.

[21] Robinson, M.K., Andrew, D., Rosen, H., Brown, D., Ortlepp, S., Stephens, P. and Butcher, E.C. (1992) J. Immunol. 148, 10801085.

[22] Malhotra, V., Hogg, N. and Sim, R.B. (1986) Eur. J. Immunol. $16,1117-1123$.

[23] Dransfield, I., Buckle, A-M., Savill, J.S., McDowell, A., Haslett, C. and Hogg, N. (1994) J. Immunol. 153, 1254-1263.

[24] Whyte, M.K.B., Hardwick, S.J., Meagher, L.C., Savill, J.S. and Haslett, C. (1993) J. Clin. Invest. 92, 446-455.
[25] Herbert, M.J., Takano, T., Holthofer, H. and Brady, H.R. (1996) J. Immunol. 157, 3105-3115.

[26] Rossi, A.G., Cousin, J.M., Dransfield, I., Lawson, M.F., Chilvers, E.R. and Haslett, C. (1995) Biochem. Biophys. Res. Commun. 217, 892-899.

[27] Aoshiba, K., Nagai, A. and Konno, K. (1995) Antimicrob. Agents Chemother. 39, 872-877.

[28] Nathan, C., Xe, Q.W., Halbwachs-Mecarelli, L. and Jin, W.W. (1993) J. Cell Biol. 122, 243-256.

[29] Raff, M. (1992) Nature 356, 397-400. 


\title{
Regulation of Macrophage Phagocytosis of Apoptotic Cells by CAMP ${ }^{1}$
}

\author{
Adriano G. Rossi, ${ }^{2}$ Judith C. McCutcheon, Noémi Roy, Edwin R. Chilvers, \\ Christopher Haslett, and Ian Dransfield
}

Regulation of macrophage capacity to remove apoptotic cells may control the balance of apoptotic and necrotic leukocytes at inflamed foci and the extent of leukocyte-mediated tissue damage. Although the molecules involved in the phagocytic process are beginning to be defined, little is known about the underlying regulatory and signaling mechanisms controlling this process. In this paper, we have investigated the effects of treatment of human monocyte-derived macrophages with PGs and other agents that elevate intracellular cAMP on phagocytosis. $\mathrm{PGE}_{2}$ and $\mathrm{PGD}_{2}$ specifically reduced the proportion of macrophages that phagocytosed apoptotic cells. Similar results were obtained with the membrane-permeable cAMP analogues dibutyryl-cAMP and 8-bromo-cAMP but not with the cGMP analogue dibutyryl-GMP. Consistent with the observation that phagocytosis was inhibited by cAMP elevation, treatment of monocyte-derived macrophages with $\mathrm{PGE}_{2}$ resulted in rapid, transient increase in levels of intracellular cAMP. These effects were not due to nonspecific inhibition of monocyte-derived macrophage phagocytosis given that ingestion of Ig-opsonized erythrocytes was unaffected. Elevation of cAMP induced morphologic alterations indicative of changes in the adhesive status of the macrophage, including cell rounding and disassembly of structures that represent points of contact with substrate containing actin and talin. These results strongly suggest that rapid activation of cAMP signaling pathways by inflammatory mediators regulates processes that limit tissue injury and that modulation of cAMP levels represents an additional therapeutic target in the control of resolution of inflammation. The Journal of Immunology, 1998, 160: 3562-3568.

$\mathrm{T}$ he ultimate fate of the large numbers of recruited neutrophil granulocytes during inflammatory responses has been widely assumed to involve necrosis and disintegration in situ before cellular fragments are cleared by local phagocytes (1). However, the capacity of many granule contents to cause cellular injury (2) and cleave tissue matrix proteins into chemotactic fragments (3) implies that necrotic cell death would damage healthy tissues and exacerbate inflammatory processes. There is now compelling evidence that neutrophil apoptosis, which is associated with preservation of granule contents and down-regulation of effector function, including granule secretion $(4,5)$, limits the potential for neutrophil-mediated tissue damage during progression of inflammatory responses (reviewed in Ref. 6). An important consequence of the apoptotic process is cell surface alterations that lead to rapid recognition and phagocytosis of apoptotic neutrophils $(4,7)$. In contrast to macrophage phagocytosis of particles opsonized with $\mathrm{C}$ or Ig, this does not provoke the release of proinflammatory macrophage mediators (thromboxane $\mathrm{B}_{2}$ or $N$-acetyl- $\beta$-D-glucosaminidase) (8), contributing to limitation of tissue injury and promotion of resolution of inflammation. There is clear histologic evidence for the involvement of this process in acute inflammation (9), and necrotic

Respiratory Medicine Unit, Department of Medicine (RIE), Rayne Laboratory, The University of Edinburgh Medical School, Edinburgh, U.K.

Received for publication August 15, 1997. Accepted for publication December 9, 1997.

The costs of publication of this article were defrayed in part by the payment of page charges. This article must therefore be hereby marked advertisement in accordance with 18 U.S.C. Section 1734 solely to indicate this fact.

${ }^{1}$ This work was supported by the Medical Research Council, U.K., and the Faculty of Medicine at the University of Edinburgh.

${ }^{2}$ Address correspondence and reprint requests to Dr. Adriano G. Rossi, Respiratory Medicine Unit, Department of Medicine (RIE), Rayne Laboratory, The University of Edinburgh Medical School, Teviot Place, Edinburgh, EH8 9AG Scotland, U.K. E-mail address: a.g.rossi@ed.ac.uk neutrophils are observed at chronically inflamed sites; thus, the tissue load of apoptotic cells is likely to be a critical factor in determining the outcome of inflammation.

Potential control mechanisms for regulation of the recognition and phagocytic pathways by which apoptotic cells are removed include prolongation of myeloid cell functional longevity, such as phagocytic and secretory responses by inflammatory mediators and growth factors $(10-12)$. Inhibition of the rate of apoptosis in these cell populations at inflamed sites would serve a dual purpose of regulating the potential for neutrophil-mediated damage at these sites and the numbers of neutrophils that are available for recognition by macrophages. Inflammatory mediators also act to regulate phagocytosis of apoptotic cells by macrophages. Multiple receptor pathways are implicated in the phagocytosis of apoptotic cells, although specific pathways may be dominant in certain environmental conditions $(13,14)$. For human monocyte-derived macrophages, we have proposed a model in which macrophage $\alpha_{v} \beta_{3}$ and CD36 act in concert to allow apoptotic cell recognition $(7,13)$. Modulation of receptor expression during monocyte differentiation may determine the phagocytic ability of macrophage populations at different anatomic sites. Induction of $\alpha_{v} \beta_{3}$ occurs concomitantly with the acquisition of the capacity to phagocytose apoptotic cells during in vitro culture of monocytes (15). For murine macrophages, different phenotypes may be induced by local microenvironmental stimuli. Bone marrow-derived macrophages, in contrast to those isolated from the peritoneal cavity which utilize a phosphatidylserine based pathway, use an $\alpha_{\mathrm{v}} \beta_{3}$ integrinbased recognition mechanism (16). In addition, phagocytosis of $\beta$-1,3-glucan particles can induce a switch between these two pathways (17). There is also clear evidence that macrophage phagocytic responses are also influenced by integrin-mediated signal transduction pathways (18). Thus, control of apoptotic cell removal may be achieved by regulation of macrophage phagocytic potential. 
In this study, we have investigated whether modulation of protein kinase activity had a regulatory role in the process of macrophage recognition of apoptotic cells. In particular, we have examined the effects of short term treatment of human monocytederived macrophages with PGs, which elevate intracellular cAMP. Our results suggest that specific inhibition of phagocytosis of apoptotic neutrophils following cAMP elevation may involve disassembly of cytoskeletal elements involved in cell-substratum interaction without inducing cell detachment. These data support a model in which the matrix microenvironment exerts control over the macrophage capacity for clearance of apoptotic cells. Thus, pharmacologic targeting of elements of the PKA pathway may represent a novel strategy for the modulation of macrophage capacity for the clearance of apoptotic cells.

\section{Materials and Methods}

\section{Abs and other reagents}

Iscove's DMEM, HBSS, and culture supplements were from Life Technologies, Paisley, U.K., and Falcon tissue culture plastic was from A. \& J. Beveridge, Edinburgh, U.K. Rhodamine-phalloidin was obtained from Molecular Probes (Eugene, OR). Dextran T500 was from Pharmacia, Milton Keynes, U.K. Normal rabbit serum was from the Scottish Ab Production Unit, Lanarkshire, U.K. Rabbit anti-human erythrocyte membrane Ags and FITC-conjugated $\mathrm{F}\left(\mathrm{ab}^{\prime}\right)_{2}$ fragments of goat anti-mouse Ig were from Dako (High Wycombe, U.K.), MAb all of IgG1 isotype, 23 C6 (CD51/CD61 complex) and 13C2 (CD51), were a gift from Dr. Mike Horton, Imperial Cancer Research Fund, London, U.K. PM6/13 (CD61) was a gift from Dr. Mike Wilkinson, Royal College of Surgeons, London, U.K. FA6-152 (CD36) was selected from $\mathrm{Ab}$ panels obtained from the $\mathrm{V}$ th International Workshop on Leukocyte Differentiation Ags. PGs were purchased from Cascade Biochem., Reading, U.K. All other chemicals were obtained from Sigma Chemical, Poole, U.K.

\section{Cell isolation}

Mononuclear cells and neutrophils were isolated from peripheral blood as described $(4,19)$. Freshly citrated blood was centrifuged at $400 \times g$ for 20 $\mathrm{min}$, and the platelet-rich plasma supernatant was used to prepare autologous serum by addition of calcium chloride ( $10 \mathrm{mM}$ final concentration) Leukocytes were isolated after removal of erythrocytes by sedimentation using $6 \%(\mathrm{w} / \mathrm{v})$ dextran T500 in saline by fractionation on a discontinuous gradient of isotonic Percoll solutions made in $\mathrm{Ca}^{2+} / \mathrm{Mg}^{2+}$-free PBS (CMFPBS) Percoll concentrations of $49.5,63$, and $72.9 \%$ at $700 \times g$ for $20 \mathrm{~min}$ Mononuclear cells were aspirated from the $49.5 \% / 63 \%$ interface and neutrophils from the $63 \% / 72.9 \%$ interface and washed three times in HBSS before culture. Neutrophils were cultured at $4 \times 10^{6} / \mathrm{ml}$ in Iscove's DMEM containing $10 \%$ autologous serum at $37^{\circ} \mathrm{C}$ in a $5 \% \mathrm{CO}$, atmosphere for 18 to $24 \mathrm{~h}$. Neutrophils used in the phagocytosis assay were $>50 \%$ apoptotic as determined by morphologic appearance, CD16 "low" expression, annexin $\mathrm{V}$ positivity and $>98 \%$ of cells retain membrane integrity as assessed by the vital dye trypan blue. Monocytes were enriched from the mononuclear cells by selectively attaching them to 24-well plates for $1 \mathrm{~h}$ at $37^{\circ} \mathrm{C}$. The nonadherent lymphocytes were removed and the adherent monocytes were washed twice in PBS. Monocytes were then cultured for 5 to 7 days in Iscove's DMEM containing $10 \%$ autologous serum at $37^{\circ} \mathrm{C}$ in a $5 \% \mathrm{CO}_{2}$ atmosphere. Their maturation into macrophages was assessed by flow cytometry using a panel of myeloid-specific and activation markers including CD16 and CD51/CD61 (data not shown).

\section{Assay for macrophage phagocytosis of apoptotic neutrophils}

Phagocytosis of apoptotic neutrophils was assayed by minor modifications of previously described methods $(4,7)$. Neutrophils that had been cultured in vitro were washed once with Iscove's DMEM and resuspended at $4 \times$ $10^{6} \mathrm{cells} / \mathrm{ml}$ in Iscove's DMEM alone. Adherent macrophages were washed in Iscove's DMEM before addition of $1 \mathrm{ml}$ of the above neutrophil suspension to each well. After $30 \mathrm{~min}$ of incubation at $37^{\circ} \mathrm{C}$, the wells were washed four times with ice-cold CMF-PBS, fixed in $2.5 \%$ glutaraldehyde/ PBS for $10 \mathrm{~min}$, and then stained for myeloperoxidase at $37^{\circ} \mathrm{C}$ using 0.1 $\mathrm{mg} / \mathrm{ml}$ dimethoxybenzidine and $0.03 \%(\mathrm{v} / \mathrm{v})$ hydrogen peroxide in PBS The percentage of macrophages that had phagocytosed myeloperoxidasepositive apoptotic neutrophils was quantified microscopically by examination of randomly selected fields and counting at least $500 \mathrm{cells} / \mathrm{well}$. Results from each experiment were expressed either as the mean percentage of phagocytic macrophages of triplicate wells or as the percentage phagocytosis relative to untreated controls.

\section{Macrophage phagocytosis of IgG-opsonized erythrocytes}

Erythrocytes were washed and resuspended at $2.5 \times 10^{7} / \mathrm{ml}$ in Iscove's DMEM and incubated at $4^{\circ} \mathrm{C}$ with rabbit polyclonal anti-human erythrocyte $\mathrm{Ab}(1: 100)$ for $30 \mathrm{~min}$. Opsonized erythrocytes were then washed and resuspended at $1 \times 10^{6} / \mathrm{ml}$ in Iscove's DMEM and incubated with macrophages at a macrophage-erythrocyte ratio of $1: 2$.

\section{Flow cytometry and immunocytochemistry}

Flow cytometry was performed as described previously $(19,20)$, with all incubations conducted on ice. Six-day-old monocyte-derived macrophages were detached from cell culture plates by vigorous pipetting after incubation on ice with PBS for $30 \mathrm{~min}$. Macrophages $\left(1 \times 10^{5}\right)$ were washed with ice-cold PBS containing $0.2 \%(\mathrm{w} / \mathrm{v}) \mathrm{BSA}$ and $0.1 \%(\mathrm{w} / \mathrm{v})$ sodium azide and preincubated with $20 \%(\mathrm{v} / \mathrm{v})$ normal rabbit serum to block "nonspecific" binding to Ig Fc receptors. Cells were then incubated with saturating concentrations of $\mathrm{mAb}$ for $30 \mathrm{~min}$. After washing, cells were incubated with FITC-conjugated $\mathrm{F}\left(\mathrm{ab}^{\prime}\right)_{2}$ goat anti-mouse $\mathrm{Ig}(1: 25)$ for $30 \mathrm{~min}$ and washed twice before flow cytometric analysis using an EPICS Profile II flow cytometer (Coulter Electronics, Luton, U.K.) (20). For indirect immunofluorescence analysis, macrophages were resuspended at $1 \times 10^{6} / \mathrm{ml}$ of Iscove's DMEM and $10^{5} /$ well added to multispot microscope slides and allowed to adhere by incubation for $1 \mathrm{~h}$ at $37^{\circ} \mathrm{C}$. After rinsing with Iscove's DMEM, cells were fixed in $1 \%$ formaldehyde in PBS for 10 min. Cells were permeabilized using $1 \%$ Triton X-100 in PBS for $5 \mathrm{~min}$ and then washed thoroughly in PBS. Slides were blocked in PBS containing $20 \%$ normal rabbit serum for $10 \mathrm{~min}$ before incubation with primary $\mathrm{Ab}$ optimally diluted in PBS containing normal rabbit serum. Bound primary Ab was detected using $\mathrm{F}\left(\mathrm{ab}^{\prime}\right)_{2}$ FITC-conjugated rabbit anti-mouse Ig Ab. For visualization of actin, rhodamine-phalloidin $(0.15 \mu \mathrm{M})$ was included. Slides were mounted in $50 \%$ glycerol in PBS and examined using an Olympus BH-2 microscope with a fluorescent lamp attachment.

Treatment of monocyte-derived macrophages with agents that elevate intracellular CAMP

Macrophages were washed once with Iscove's DMEM and incubated for various times as stated in text at $37^{\circ} \mathrm{C}$ with dbcAMP, ${ }^{3} 8$-Br-cAMP, or PGs diluted in Iscove's DMEM. For cAMP determinations, $\mathrm{PGE}_{2}$ was added in the presence of a $2.5 \mathrm{mM}$ concentration of the phosphodiesterase inhibitor 3-isobutyl-1-methylxanthine (IBMX). The treated cells were then washed once with Iscove's DMEM before assessment of macrophage phagocytosis or immunocytochemistry.

\section{Determination of CAMP concentration}

Macrophages were cultured adherent to six-well tissue culture plates for 6 days as described above. Macrophages were washed once with Iscove's DMEM and incubated with Iscove's DMEM alone or Iscove's DMEM containing $0.5 \mathrm{mM}$ IBMX and $3 \mu \mathrm{M} \mathrm{PGE}_{2}$ for $0.5,2,5,10,15$, or $45 \mathrm{~min}$. The medium was then aspirate and $600 \mu \mathrm{l}$ of ice-cold $0.5 \mathrm{M}$ trichloroacetic acid were added. Macrophages were extracted on ice for $20 \mathrm{~min}$ and then scraped off, and material was transferred to Eppendorf tubes. The samples were then vortexed and centrifuged $(10,000 \times \mathrm{g}, 5 \mathrm{~min})$, and $500 \mu \mathrm{l}$ of the supernatant were added to $125 \mu \mathrm{l}$ of $10 \mathrm{mM}$ EDTA $(\mathrm{pH} \mathrm{7.0)}$ and $500 \mu \mathrm{l}$ of freshly prepared 1,1,2-trichlorotrifluoroethane-tri- $n$-octylamine $(50: 50$, $\mathrm{v} / \mathrm{v})$ (21). After further vigorous mixing, the samples were centrifuged $(10,000 \times g, 2 \mathrm{~min})$, and $500 \mu \mathrm{l}$ of the aqueous layer were removed and neutralized with $100 \mu \mathrm{l}$ of $6 \mathrm{M}$ sodium hydrogen carbonate. Cyclic AMP measurements were made on triplicate $50-\mu \mathrm{l}$ aliquots from each sample. Each tube contained $50 \mu \mathrm{l}$ of buffer ( $50 \mathrm{mM}$ Tris- $\mathrm{HCl}, 4 \mathrm{M}$ EDTA, pH 7.5), $50 \mu \mathrm{l}$ of sample, or standard containing known amounts of cAMP (doubling dilutions from 0.125 to $16 \mathrm{pmol}$ with $250 \mathrm{pmol}$ of cAMP used to define nonspecific binding) and $100 \mu \mathrm{l}\left[{ }^{3} \mathrm{H}\right] \mathrm{cAMP}$ (88,000 dpm; DuPont (U.K.), Stevenage, U.K.). The reaction was initiated by addition of 150 $\mu \mathrm{l}$-cAMP-binding protein (final concentration, $0.85 \mu \mathrm{g}$ protein $/ \mathrm{ml}$ ) and incubated overnight at $4^{\circ} \mathrm{C}$. The reaction was terminated by the addition of $250 \mu \mathrm{l}$ of $0.5 \%$ activated charcoal. After $3 \mathrm{~min}$, the samples were centrifuged $(10,000 \times \mathrm{g}, 4 \mathrm{~min})$ and $200 \mu \mathrm{l}$ of the supernatant were transferred to $5-\mathrm{ml}$ scintillation vials. Five milliliters of Flo-Scint IV scintillation fluid (Canberra Packard, Pangbourne, U.K.) were added to each vial, and the samples were analyzed on a liquid scintillation counter.

${ }^{3}$ Abbreviations used in this paper: dbcAMP, dibutyryl-cAMP; dbcGMP, dibutyrylcAMP; IBMX, 3-isobutyl-1-methylxanthine; 8-Br-cAMP, 8-bromo-cAMP; PKA, protein kinase $A$. 


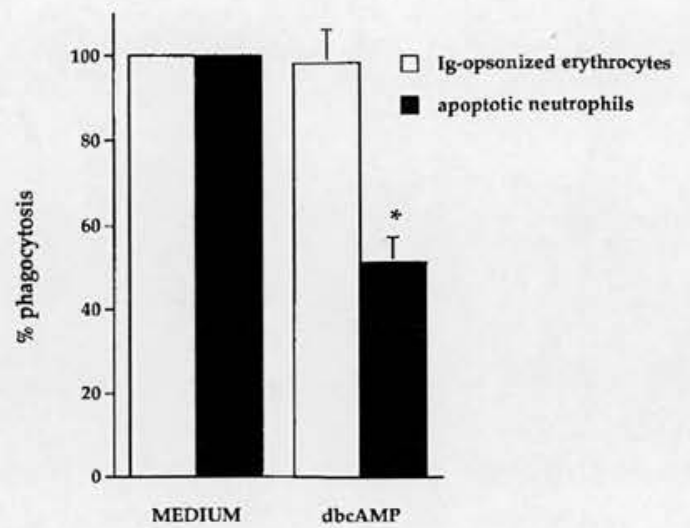

FIGURE 1. Effect of dbcAMP on macrophage recognition of apoptotic neutrophils and opsonized erythrocytes. Human monocyte-derived macrophages pretreated with dbcAMP $(2 \mathrm{mM})$ for 15 min were assessed for their ability to phagocytose in vitro cultured neutrophils ( erythrocytes $(\square)$. Relative percentage phagocytosis is expressed compared with control medium pretreatment cells representing mean \pm SEM of 14 separate experiments. In these experiments, the percentage of cells ingesting apoptotic neutrophils under control conditions was $20 \pm 4 \%$ and following dbcAMP treatment was $10 \pm 3 \%$. The percentage of control macrophages ingesting opsonized erythrocytes was $30 \pm 3 \%$, whereas in dbcAMP-treated macrophages the value was unchanged $(31 \pm 4 \%) .{ }^{*} p<$ 0.05 compared with control.

\section{Analysis of results}

Results are expressed as the mean \pm SEM and $n=$ number of independent experiments using macrophages from different donors. Results were analyzed by one-way analysis of variance followed by the Newman-Keuls procedure to determine significant differences between groups. $p$ values $<$ 0.05 were considered to be significant where * represents $p<0.05$.

\section{Results}

Elevation of cAMP specifically inhibits macrophage phagocytosis of apoptotic neutrophils

Human monocyte-derived macrophages were pretreated with the membrane-permeable analogue of cAMP, dbcAMP, to mimic elevation of intracellular cAMP levels, and phagocytosis of apoptotic neutrophils was assessed. When compared with preincubation in medium alone, $2 \mathrm{mM}$ dbcAMP decreased the percentage of monocyte-derived macrophages that phagocytosed apoptotic neutrophils (Fig. 1). The low proportion of phagocytic macrophages following dbcAMP treatment made assessment of the effects of dbcAMP on the number of apoptotic neutrophils phagocytosed per macrophage (phagocytic index) difficult, particularly since under control conditions the phagocytic index was $\sim 1.4$. In a series of experiments, maximal effects of $2 \mathrm{mM}$ dbcAMP treatment were apparent following $15 \mathrm{~min}$ of pretreatment with no further inhibition observed with longer incubation times (data not shown). All subsequent experiments were therefore performed using 15-min pretreatment times. Since elevation of cAMP might result in functional alterations downstream of the receptors mediating macrophage recognition of apoptotic neutrophils that would affect all phagocytic processes, we assessed whether dbcAMP had similar effects on Fc $\gamma$ R-mediated phagocytosis. In contrast to the observed inhibition of monocyte-derived macrophage phagocytosis of apoptotic cells, pretreatment of macrophages with dbcAMP did not inhibit recognition of Ig-opsonized erythrocytes (Fig. 1). Thus, treatment of macrophages with an activator of PKA does not nonspecifically inhibit all phagocytic pathways. Furthermore, modulation of phagocytosis of apoptotic neutrophils following cAMP

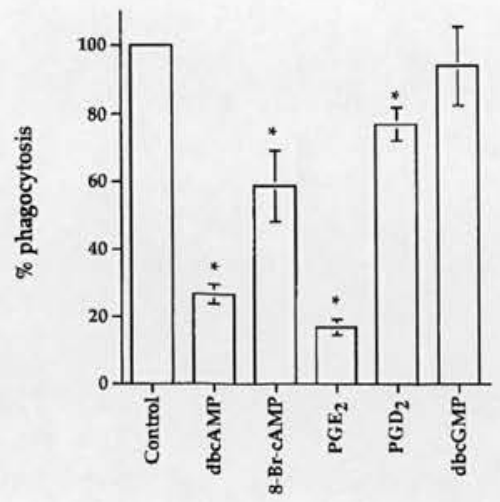

FIGURE 2. Fffect of prostanoids, dbcAMP, 8-Br-cAMP, and dbcGMP on macrophage recognition of apoptotic cells. Human monocyte-derived macrophages pretreated with $\mathrm{PGE}_{2}(10 \mu \mathrm{M}), \mathrm{PGD}_{2}(10 \mu \mathrm{M})$, dbcAMP $(2$ $\mathrm{mM}), 8$-Br-cAMP ( $2 \mathrm{mM})$, and dbcGMP $(2 \mathrm{mM})$ for $15 \mathrm{~min}$ were assessed for their ability to phagocytose in vitro cultured neutrophils. The percentage of macrophages ingesting apoptotic neutrophils is expressed as the mean \pm SEM of 3 to 12 separate experiments. In this series of experiments, $24 \pm 2$ of control macrophages phagocytosed apoptotic neutrophils. ${ }^{*} p<$ 0.05 compared with control.

elevation suggests that the potential of monocyte-derived macrophages to phagocytose apoptotic cells can be rapidly and dynamically regulated.

\section{Effect of inflammatory mediators that elevate cAMP}

Arachidonic acid metabolites of the PG series are key inflammatory mediators that interact with specific cell surface receptors to activate adenylate cyclase resulting in an elevation of intracellular cAMP. We therefore assessed the effects of a number of different PGs for their ability to inhibit monocyte-derived macrophage phagocytosis of apoptotic neutrophils. Macrophages were pretreated with either $\mathrm{PGE}_{2}(10 \mu \mathrm{M})$ or $\mathrm{PGD}_{2}(10 \mu \mathrm{M})$ for 15 min before testing their phagocytic ability. We also compared the effects of elevation of cGMP using dbcGMP $(2 \mathrm{mM})$ in comparison with the positive controls, dbcAMP $(2 \mathrm{mM})$ and $8 \mathrm{Br}$-cAMP (2 $\mathrm{mM}$ ), as described above. We found that all the PGs tested were capable of inhibition of macrophage phagocytosis of apoptotic neutrophils (Fig. 2). In contrast to the effects of elevation of intracellular cAMP, raising cGMP levels did not inhibit monocytederived macrophage phagocytosis. These results suggest that macrophage phagocytosis can be regulated by intracellular signals and that the levels of intracellular cAMP may have a role in determining macrophage capacity for phagocytosis of apoptotic neutrophils.

Concentration-response effects of $P G E_{2}, P G D_{2}$, and dbcAMP on macrophage phagocytosis of apoptotic neutrophils

Since the above data suggest that macrophages express multiple receptors for different PG species, we next determined the relative potency and efficacy of $\mathrm{PGE}_{2}$ and $\mathrm{PGD}_{2}$ in comparison with $\mathrm{db}$ cAMP for inhibition of phagocytosis of apoptotic neutrophils. Results shown in Figure 3 indicate that half-maximal inhibition $\left(\mathrm{IC}_{50}\right)$ is observed at $\approx 0.5 \mathrm{mM}$ for dbcAMP treatment. Maximal inhibition of macrophage phagocytosis was observed following pretreatment with $10 \mu \mathrm{M}$ concentrations of both $\mathrm{PGE}_{2}$ and $\mathrm{PGD}_{2}$. However, $\mathrm{PGE}_{2}$ (to $\mathrm{IC}_{50} \approx 1 \mu \mathrm{M}$ ) was much more potent that $\mathrm{PGD}_{2}(10 \mu \mathrm{M}$ inhibited phagocytosis only by $\approx 25 \%)$. 


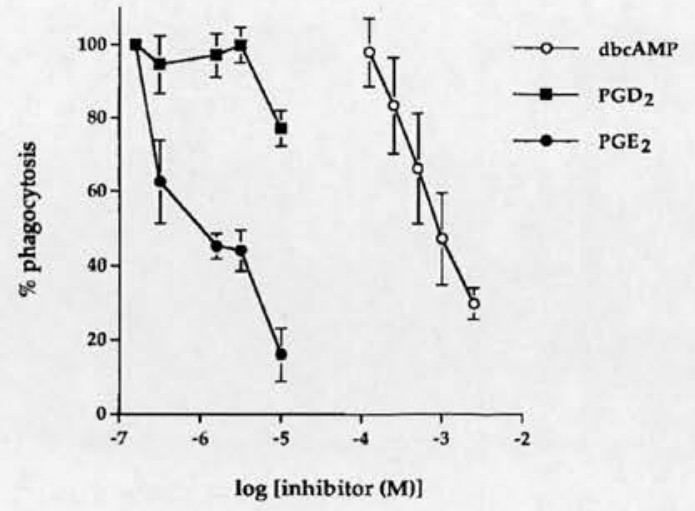

FIGURE 3. Concentration-response effects of dbcAMP, $\mathrm{PGE}_{2}$, and $\mathrm{PGD}_{2}$ on macrophage recognition of apoptotic cells. Human monocytederived macrophages pretreated with varying concentrations of dbcAMP (O) $\mathrm{PGE}_{2}(\boldsymbol{\theta})$, and $\mathrm{PGD}_{2}(\mathbf{\square})$ for $15 \mathrm{~min}$ were assessed for their ability to phagocytose in vitro cultured neutrophils. The percentage of macrophages ingesting apoptotic neutrophils is expressed as the mean \pm SEM of 5 to 12 separate experiments.

\section{Effect of $P G E_{2}$ on intracellular cAMP concentrations in macrophages}

To determine whether pretreatment of monocyte-derived macrophages with PGs exerted inhibitory effects via elevation of cAMP, we next measured intracellular concentrations of CAMP in macrophages following treatment with $\mathrm{PGE}_{2}$ using a radioreceptor assay. As shown in Figure 4, $\mathrm{PGE}_{2}$ acted rapidly to elevate cAMP, increasing cAMP levels 10 -fold after $5 \mathrm{~min}$. Interestingly, observed elevation was transient, returning to basal levels within 30 min. In contrast, inhibition of phagocytosis of apoptotic neutrophils following $\mathrm{PGE}_{2}$ treatment was prolonged with inhibitory effects observed after $1 \mathrm{~h}$ (data not shown), implying that elevation of cAMP initiates signaling pathways that ultimately determine phagocytic responses.

\section{Effects of cAMP elevation on macrophage adhesion}

One possible mechanism for the rapid regulatory effects of cAMP elevation would be altered expression of surface receptors involved in macrophage recognition of apoptotic cells. However,

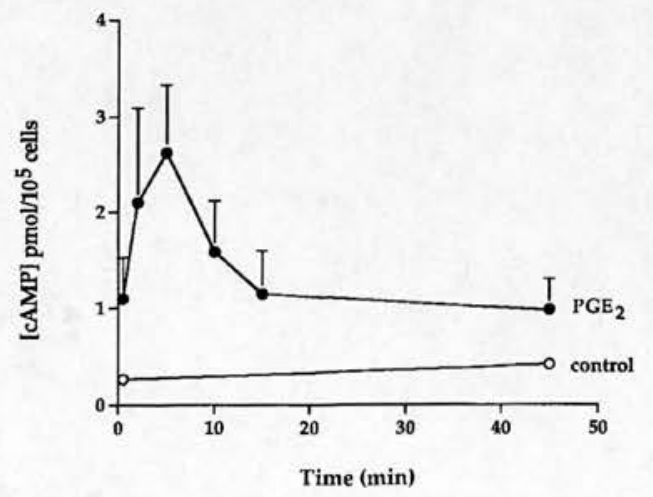

FIGURE 4. Effect of $\mathrm{PGE}_{2}$ on intracellular levels of macrophage cAMP. The intracellular concentrations of cAMP in human monocytederived macrophages pretreated with $\mathrm{PGE}_{2}(3 \mu \mathrm{M})$ in the presence of IBMX $(2.5 \mathrm{mM})$ for the indicated times were determined. The results are expressed as picomols of cAMP $/ 10^{5}$ cells representing mean \pm SEM of four separate experiments. flow cytometric analysis indicated that there was no significant alteration in surface expression of CD51, CD61, and the $61 \mathrm{D} 3 \mathrm{Ag}$, as assessed by changes in mean fluorescence intensity, following dbcAMP pretreatment (data not shown), suggesting that the observed effects did not involve modulation of surface receptor expression. We therefore examined the effects of cAMP elevation on the distribution of receptors involved in the recognition process. Indirect immunofluorescence analysis of $\beta_{3}$ integrins and $\mathrm{CD} 36$ on macrophages pretreated with dbcAMP for 15 min revealed that the cellular distribution of CD36 was similar on untreated macrophages and those pretreated with dbcAMP. Although heterogeneity in terms of the pattern of distribution of the integrin $\beta_{3}$ subunit was observed in both untreated and treated macrophage populations and treatment of macrophages with dbcAMP resulted in an increase in the proportion of macrophages with a uniform $\beta_{3}$ distribution (data not shown), no clear association between $\beta_{3}$ distribution patterns and phagocytic capacity was apparent.

It has previously been demonstrated that elevation of cAMP induces phenotypic alterations in macrophages and other cell types. Microscopic examination of human monocyte-derived macrophages treated with dbcAMP (Fig. 5, $A$ and $B$ ) and $\mathrm{PGE}_{2}$ (data not shown) revealed clear morphologic alterations that were suggestive of altered adhesion. However, quantitative analysis revealed that elevation of cAMP did not alter the numbers of adherent macrophages (104 $\pm 4 \%$ of adhesion of untreated macrophages, $n=3$ separate experiments). These data also demonstrate that observed inhibition of phagocytosis is not due to selective detachment of phagocytic macrophages. Moreover, additional experiments indicated that pretreatment of macrophages with dbcAMP did not alter the ability of macrophages to adhere to substrate (data not shown). Although dbcAMP did not affect the percentage of adherent cells, when we examined the intracellular distribution of cytoskeletal elements, we found that colocalization of actin and talin into discrete structures (representing contact points with substrate) was altered by dbcAMP treatment (Fig. 5, $C-F)$. Similar results were found when we examined vinculin, paxillin, and tyrosine phosphorylation patterns (data not shown), suggesting that dbcAMP induces morphologic alterations by uncoupling adhesion receptors from cytoplasmic cytoskeletal elements, including potential signal transduction pathways (22).

\section{Discussion}

The regulatory mechanisms that underlie macrophage recognition, phagocytosis, and removal of apoptotic cells from inflamed sites are central to our understanding of the control and resolution of inflammation. Perturbation of the balance between neutrophil recruitment from the circulation, phagocytic removal of apoptotic neutrophils by macrophages (or other phagocytes), and neutrophil disintegration or necrosis may be critical determinants of whether inflammation ultimately resolves (6). A proportion of human monocyte-derived macrophages constitutively recognize and phagocytose apoptotic, but not freshly isolated, neutrophils (13), indicating that the apoptotic cell provides a phagocytic signal to the macrophage. However, since peripheral blood monocytes and some macrophage populations (e.g., alveolar macrophages; our unpublished observations) are relatively inefficient at phagocytosis of apoptotic cells, differentiation- or microenvironment-dependent phenotypic alterations may also regulate this process. Evidence for further regulatory mechanisms include the finding that treatment of macrophages with proinflammatory cytokines, notably GM-CSF, augments macrophage phagocytosis independently of altered receptor expression (23). More recently, we have demonstrated that 

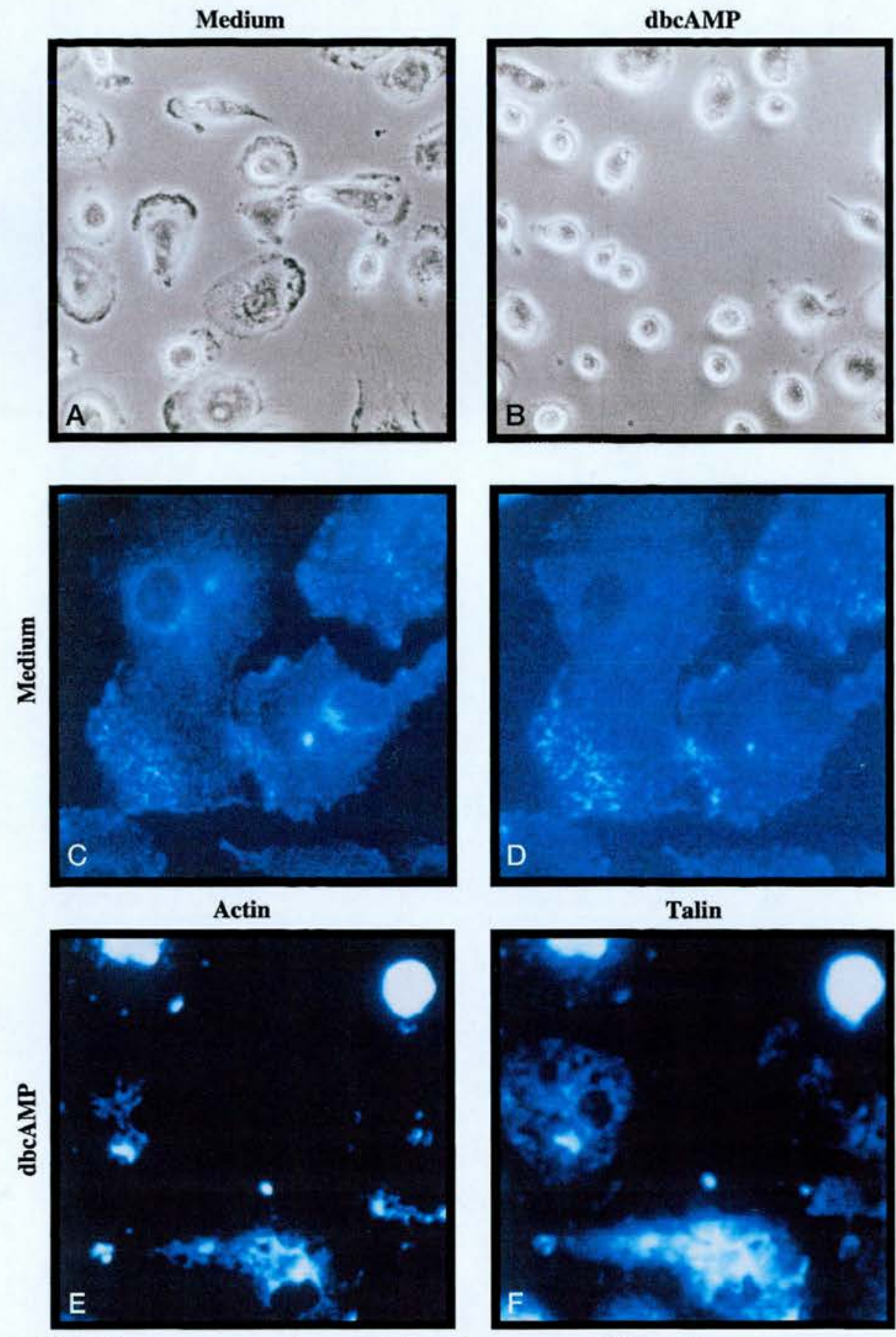

FIGURE 5. Effect of dbcAMP treatment on macrophage cytoskeleton. Untreated or dbcAMP-treated human monocyte-derived macrophages were washed twice before fixation and $(A$ and $B)$ examined using an inverted microscope $(\times 20$ objective) or stained for actin $(C$ and $E)$ using rhodamine phalloidin and talin $(D$ and $F)$ using dual labeling techniques. Scanning confocal laser microscopy ( $\times 60$ objective) was then used to examine intracellular distributions of these proteins. Untreated macrophages (Medium) show morphologic features typical of motile cells $(A)$. In contrast, following dbcAMP treatment, many more macrophages appear rounded $(B)$. There is clear colocalization of actin and talin in untreated macrophages $(C$ and $D)$ possibly representing areas of contact with substrate. In dbcAMP-treated macrophages, actin staining is more disordered $(E$ and $F)$.

cross-linking of CD44 rapidly and specifically augments phagocytosis of apoptotic neutrophils, suggesting that macrophage phagocytic capacity can be dynamically regulated (20).

In this study, we demonstrate that elevation of cAMP within monocyte-derived macrophages specifically reduces macrophage potential to recognize and phagocytose apoptotic neutrophils but not Ig-opsonized erythrocytes, indicating that cAMP does not block all phagocytic pathways in macrophages. Elevation of cAMP and activation of PKA may signal rapid, subtle responses to microenvironmental stimuli, resulting in reduced capacity for phagocytosis of apoptotic cells by macrophages. The prostanoids $\mathrm{PGE}_{2}$ or $\mathrm{PGD}_{2}$ have antiinflammatory effects on neutrophil 
function in terms of granule secretion (24-26). However, our observation that macrophage removal of apoptotic cells is inhibited by these agents raises the possibility that they may increase the tissue load of apoptotic cells at inflamed sites. Inflammatory mediators may therefore have complex effects on processes involved in resolution of inflammation. One possibility is that PG-induced cAMP elevation may provide counterregulatory signals to proinflammatory mediators or CD44 cross-linking. Preliminary experiments indicate that dbcAMP treatment inhibits CD44 augmented monocyte-derived macrophage phagocytosis of apoptotic neutrophils (CD44 treated (20), $50.1+9 \%$ phagocytosis; CD $44+2 \mathrm{mM}$ dbcAMP treated, $18.6+8 \% ; n=5$ ). However, since dbcAMP also inhibits phagocytosis under control conditions (see Figs. 1-3), it is difficult to determine the precise relationship between cAMP elevation and CD44-mediated signals in control of phagocytic capacity. We are currently further investigating the effects of PGs and other antiinflammatory agents on macrophage phagocytic responses in combination with GM-CSF or other proinflammatory cytokines.

The observations reported here may be of great significance for removal of apoptotic neutrophils in situ. A variety of inflammatory mediators (including PGs) that stimulate adenylate cyclase activity and activate PKA (27) are likely to decrease apoptotic cell removal. All monocyte-derived macrophages may be capable of phagocytosis of apoptotic cells, providing that a certain activation "threshold" is reached, with PKA playing a regulatory role in limiting phagocytic responses. For instance, some monocyte-derived macrophage subpopulations, e.g., multinucleated giant cells, show low levels of phagocytosis of apoptotic cells but are readily able to phagocytose Ig-opsonized erythrocytes, suggesting that phagocytic competence is not the basis for the lack of apoptotic cell uptake. One possibility is that differential PKA activity in giant cells accounts for observed low level responsiveness to apoptotic cell stimuli.

Data presented in this article indicate that there are multiple levels of control for macrophage removal of apoptotic cells from inflamed sites. Although the mechanism by which cAMP elevation down-regulates macrophage phagocytosis of apoptotic cells remains to be determined, we have observed that there are rapid alterations in the distribution of cytoskeletal components (actin, talin, vinculin, and paxillin) in macrophages following dbcAMP treatment. These changes are accompanied by cell "rounding" when macrophages are examined by light microscopy and show parallels with the reported effects of PGs on murine macrophage adhesion, spreading, and motility $(28,29)$. Membrane ruffling and filopodia extension were inhibited by prolonged PG treatment in a reversible manner, resulting in altered macrophage adhesion. Together with recent evidence that in cytotoxic T cells PKA is able to phosphorylate Rho (30), a key regulator of cytoskeletal organization, these data indicate that intracellular cAMP levels may also have a pivotal role.

We have investigated the effects of agents that disrupt microfilament and microtubule organization (cytochalasin B and nocodazole). Although we did find inhibition of recognition of apoptotic cells when macrophage were treated with nocodazole $(2.5$ $\mu \mathrm{g} / \mathrm{ml}$ ), it was difficult to assess the effects of cytochalasin B (5 $\mu \mathrm{g} / \mathrm{ml}$ ) because the washing procedure used for assessment of macrophage phagocytosis resulted in significant detachment of cells in three of five experiments. These findings suggest that treatment of macrophage with dbcAMP differs from disruption of microfilaments in two ways. First, unlike cytochalasin B treatment, dbcAMP does not inhibit phagocytosis of Ig-opsonized erythrocytes (Fig. 1) $(31,32)$. Indeed, Newman et al. (32) have previously demonstrated that cAMP does not inhibit FcR-mediated phagocy- tosis. Second, although we have noted disassembly of structures that may represent areas of contact with substrate and cell "rounding," treatment of macrophages with dbcAMP does not result in detachment, and cells remain firmly adherent through multiple washes that are necessary for assessment of phagocytic capacity. Adhesion of macrophages to extracellular matrix components, e.g., fibronectin, has previously been shown to modulate C or Ig receptor-mediated phagocytosis $(33,34)$, and it is tempting to speculate that the extracellular matrix composition of inflamed sites exerts indirect regulatory effects on the process of removal of apoptotic cells. One possibility is that intracellular levels of cAMP are altered following integrin-mediated adhesion to extracellular matrix components as found for human neutrophils (35).

In summary, elevation of cAMP, using cell permeable analogues of cAMP (dbcAMP, 8-Br-cAMP) or by receptor-directed stimuli $\left(\mathrm{PGE}_{2}, \mathrm{PGD}_{2}\right)$, in macrophages specifically inhibits phagocytosis of apoptotic cells but not Ig-opsonized erythrocytes. Modulation is independent of changes in the percentage of adherent macrophages, although a specific, rapid redistribution of cytoskeletal elements was observed, suggestive of changes in the adhesive status of macrophages. These data indicate that intracellular cAMP may have a key role in controlling the capacity for removal of apoptotic cells by macrophages at inflamed sites during the resolution of inflammatory processes.

\section{Acknowledgments}

We thank Drs. Mike Horton, Nancy Hogg, and Mike Wilkinson for generously providing mAbs. Thanks to Dr. Stewart Fleming and Scott Cunningham for help with the scanning laser confocal microscopy and to Fiona Rossi for technical support.

\section{References}

1. Hurley, J. V. 1983. Termination of acute inflammation. I. Resolution. In Acute Inflammation. J. V. Hurley, ed. 2nd ed. Churchill Livingstone, London, p. 109.

2. Henson, P. M., and R. B. Johnston. 1987. Tissue injury in inflammation: oxidants, proteinases and cationic proteins. J. Clin. Invest. 79:669.

3. Vitario, T., H. Seppa, and A. Vaher. 1981. Susceptibility of soluble and matrix fibronectins to degradation by tissue proteinases, mast cell kinase and cathepsin G. J. Biol. Chem. 256:471.

4. Savill, J. S., A. H. Wyllie, J. E. Henson, M. J. Walport, P. M. Henson, and C. Haslett. 1989. Macrophage phagocytosis of aging neutrophils in inflammation: programmed cell death in the neutrophil leads to its recognition by macrophages. J. Clin. Invest. 83:865.

5. Whyte, M. K. B., L. C. Meagher, J. MacDermot, and C. Haslett. 1993. Impairment of function in aging neutrophils is associated with apoptosis. J. Immunol. 150:5124.

6. Haslett, C., J. S. Savill, M. K. B. Whyte, M. Stern, I. Dransfield, and L. C. Meagher. 1994. Granulocyte apoptosis and the control of inflammation. Phil. Trans. R. Soc. Lond. B 345:327.

7. Savill, J., I. Dransfield, N. Hogg, and C. Haslett. 1990. Vitronectin receptormediated phagocytosis of cells undergoing apoptosis. Nature 343:170.

8. Meagher, L. C., J. S. Savill, A. Baker, R. W. Fuller, and C. Haslett. 1992. Phagocytosis of apoptotic neutrophils does not induce macrophage release of thromboxane $\mathrm{B}_{2}$. J. Leukocyte Biol. 52:269

9. Jones, S. T., M. J Denton, P. J. L. Holt, and A. J. Freemont, 1993. Possible clearance of effete polymorphonuclear leucocytes from synovial fluid by cytophagic mononuclear cells: implications for pathogenesis and chronicity in inflammatory arthritis. Ann. Rheum. Dis. 52:121.

10. Collota, F., F. Re, N. Polentarutti, S. Sozzani, and A. Mantovani. 1992. Modulation of granulocyte survival and programmed cell death by cytokines and bacterial products. Blood 80:2012.

11. Stern, M., L. Meagher, J. S. Savill, and C. Haslett. 1992. Apoptosis in human eosinophils: programmed cell death in the eosinophil leads to phagocytosis by macrophages and is modulated by IL-5. J. Immunol. 148:3543.

12. Lee, A., M. K. Whyte, and C. Haslett. 1993. Inhibition of apoptosis and prolongation of neutrophil function longevity by inflammatory mediators. J. Leukocyte Biol. 54:283.

13. Savill, J., V. Fadok, P. Henson, and C. Haslett. 1993. Phagocyte recognition of cells undergoing apoptosis. Immunol. Today 14:131.

14. Hart, S. P., C. Haslett, and I. Dransfield, 1996. Recognition of apoptotic cells by phagocytes. Experientia 52:950.

15. De Nichilo, M. O., and G. F. Burns. 1993. Granulocyte-macrophage and macrophage colony-stimulating factors regulate $\alpha_{\mathrm{v}}$ integrin expression on cultured human macrophages Proc. Natl. Acad. Sci. USA 90:2517. 
16. Fadok, V. A., J. S. Savill, C. Haslett, D. L. Bratton, D. E. Doherty, P. A. Campbell, and P. M. Henson. 1992. Different populations of macrophages use either the vitronectin receptor or the phosphatidylserine receptor to recognize and remove apoptotic cells. J. Immunol. 149:4029.

17. Fadok, V. A., D. J. Laszlo, P. W. Noble, L. Weinstein, D. W. Riches, and P. M. Henson. 1993. Particle digestibility is required for induction of the phosphatidylserine recognition mechanism used by murine macrophages to phagocytose apoptotic cells. J. Immunol, 151:4274.

18. Brown, E. J. 1992. Complement receptors, adhesion, and phagocytosis. Infect. Agents Dis. 1:63.

19. Dransfield, I., A.-M. Buckle, J. S. Savill, A. McDowall, C. Haslett, and N. Hogg. 1994. Neutrophil apoptosis is associated with a reduction in CD16 (FcyRIII) expression. J. Immunol. 153:1254.

20. Hart, S. P., G. J. Dougherty, C. Haslett, and I. Dransfield. 1997. CD44 regulates phagocytosis of apoptotic neutrophil granulocytes, but not apoptotic lymphocytes, by human macrophages. J. Immunol. 159:919.

21. Downes, C. P., P. T. Hawkins, and R. F. Irvine. 1986. Inositol 1,3,4,5-tetrakisphosphate and not phosphatidylinositol 3,4-bisphosphate is the probable precursor of inositol 1,3,4-trisphosphate in agonist stimulated parotid gland. Biochem. J. $238: 501$.

22. Sastry, S. K., and A. F. Horwitz. 1993. Integrin cytoplasmic domains: mediators of cytoskeletal linkages and extra- and intra-cellular initiated trausmembrane signalling. Curr Opin. Cell Biol, 5:819.

23. Ren, Y., and J. Savill. 1995. Proinflammatory cytokines potentiate thrombospondin-mediated phagocytosis of neutrophils undergoing apoptosis. J. Immunol. 154:2366.

24. Cox, J. P., and M. L. Karnovsky. 1973. The depression of phagocytosis by exogenous cyclic nucleotides, prostaglandins, and theophylline. J. Cell Biol. 59. 480.

25. Marone, G., L. L. Thomas, and L. M. Lichtenstein. 1980. The role of agonsts that activate adenyl cyclase in the control of cAMP metabolism and enzyme release by human polymorphonuclear leukocytes. J. Immunol. 125:2277.
26. Rossi, A. G., and J. T. O'Flaherty. 1989. Prostaglandin binding sites in human polymorphonuclear neutrophils. Prostaglandins 37:64l

27. Taylor, S. S., J. A. Buechler, and W. Yonemoto. 1990. cAMP-dependent protein kinase: framework for a diverse family of regulatory enzymes. Annu. Rev. Biochem. 59:971.

28. Canterow, W. D., H. T. Cheung, and G. Sundharadas. 1978. Effects of prostaglandins on the spreading, adhesion and migration of mouse peritoneal macrophages. Prostaglandins 16:39.

29. Oropeza-Rendon, R. L., V. Speth, G. Hiller, K., Weber, and H. Fischer. 1979 Prostaglandin E1 reversibly induces morphological changes in macrophages and inhibits phagocytosis. Exp. Cell Res. 119:365.

30. Lang, P., F. Gesbert, M. Delespine-Carmagnat, R. Stancou, M. Pouchelet, and J. Bertoglio. 1996. Protein kinase A phosphorylation of RhoA mediates the morphological and functional effects of cyclic AMP in cytotoxic lymphocytes. EMBO J. 15:510.

31. Axline, S. G., and E. P. Reaven. 1974. Inhibition of phagocytosis and plasma membrane mobility of the cultivated macrophage by cytochalasin B. J. Cell Biol. 62:647.

32. Newman, S. L., L. K. Mikus, and M. A. Tucci. 1991. Differential requirements for cellular cytoskeleton in human macrophage complement receptor- and $\mathrm{Fs}$ receptor-mediated phagocytosis. J. Immunol. 146:967.

33. Wright, S. D., L. S. Craigmyle, and S. C. Silverstein. 1983. Fibronectin and serum amyloid $\mathrm{P}$ component stimulate $\mathrm{C} 3 \mathrm{~b}$ - and $\mathrm{C} 3 \mathrm{bi}$-mediated phagocytosis on cultured human monocytes. J. Exp. Med. 158:1338.

34. Pommier, C. G., S. Inada, L. F. Fries, T. Takahashi, M. M. Frank, and E. J. Brown. 1983. Plasma fibronectin enhances phagocytosis of opsonised particles by human peripheral blood monocytes. J. Exp. Med. 157:1844.

35. Nathan, C., and E. Sanchez. 1990. Tumor necrosis factor and CD11/CD18 ( $\beta 2)$ integrins act synergistically to lower cAMP in human neutrophils. J. Cell Biol. III:2171 


\title{
NF- $\kappa$ B Activation Is a Critical Regulator of Human Granulocyte Apoptosis in Vitro*
}

(Received for publication, June 19, 1998, and in revised form, October 12, 1998)

\author{
Carol Ward $\ddagger$, Edwin R. Chilvers§, Mark F. Lawson, James G. Pryde, Satoko Fujihara, \\ Stuart N. Farrown, Christopher Haslett, and Adriano G. Rossi \\ From the Respiratory Medicine Unit, Department of Medicine (RIE), Rayne Laboratory, University of Edinburgh Medical \\ School, Teviot Place, Edinburgh, EH8 9AG, United Kingdom and १The Cell Biology Unit, Glaxo-Wellcome, Gunnelswood \\ Road, Stevenage, Herts, SG1 2NY, United Kingdom
}

\begin{abstract}
During beneficial inflammation, potentially tissuedamaging granulocytes undergo apoptosis before being cleared by phagocytes in a non-phlogistic manner. Here we show that the rate of constitutive apoptosis in human neutrophils and eosinophils is greatly accelerated in both a rapid and concentration-dependent manner by the fungal metabolite gliotoxin, but not by its inactive analog methylthiogliotoxin. This induction of apoptosis was abolished by the caspase inhibitor zVAD-fmk, correlated with the inhibition of nuclear factor-kappa $B$ $(\mathrm{NF}-\kappa \mathrm{B})$, and was mimicked by a cell permeable inhibitory peptide of $\mathrm{NF}-\kappa \mathrm{B}, \mathrm{SN}-50$; other $\mathrm{NF}-\kappa \mathrm{B}$ inhibitors, curcumin and pyrrolidine dithiocarbamate; and the proteasome inhibitor, MG-132. Gliotoxin also augmented dramatically the early $(2-6 \mathrm{~h})$ pro-apoptotic effects of tumor necrosis factor- $\alpha$ (TNF- $\alpha$ ) in neutrophils and unmasked the ability of TNF- $\alpha$ to induce eosinophil apoptosis. In neutrophils, TNF- $\alpha$ caused a gliotoxin-inhibitable activation of an inducible form of NF- $\kappa \mathrm{B}$, a response that may underlie the ability of TNF- $\alpha$ to delay apoptosis at later times (12-24 h) and limit its early killing effect. Furthermore, cycloheximide displayed a similar capacity to enhance TNF- $\alpha$ induced neutrophil apoptosis even at time points when cycloheximide alone had no proapoptotic effect, suggesting that NF- $\mathrm{B}$ may regulate the production of protein $(\mathrm{s})$ which protect neutrophils from the cytotoxic effects of TNF- $\alpha$. These data shed light on the biochemical and molecular mechanisms regulating human granulocyte apoptosis and, in particular, indicate that the transcription factor NF- $\mathrm{B}$ plays a crucial role in regulating the physiological cell death pathway in granulocytes.
\end{abstract}

Neutrophilic and eosinophilic granulocytes originate from a common myeloid precursor; neutrophils are particularly active in the defense against invading micro-organisms whereas eosinophils serve in anti-parasitic defenses and play a role in allergic inflammation. The normally beneficial acute inflammatory response can become dysregulated and result in chronic inflammatory conditions where tissue damage arises in part

* This work was supported in part by Medical Research Council Program Grant G9016491 and the Wellcome Trust. The costs of publication of this article were defrayed in part by the payment of page charges. This article must therefore be hereby marked "advertisement" in accordance with 18 U.S.C. Section 1734 solely to indicate this fact.

$\doteqdot$ Supported by a Glaxo-Wellcome studentship.

$\S$ Wellcome Senior Research Fellow in Clinical Science.

$\|$ To whom correspondence should be addressed: Respiratory Medicine Unit, Dept. of Medicine, Rayne Laboratory, University of Edinburgh Medical School, Teviot Place, Edinburgh, EH8 9AG, Scotland, United Kingdom. Tel.: 44-131-651-1323; Fax: 44-131-650-4384; E-mail: a.g.rossi@ed.ac.uk. due to the inappropriate liberation of inflammatory cell-derived histotoxic products. We have previously described a granulocyte clearance mechanism likely to be important in the normal control and resolution processes of inflammation whereby granulocytes must first undergo apoptosis (programmed cell death) before being phagocytosed and cleared by macrophages in situ $(1,2)$. Apoptosis also causes functional down-regulation of granulocytes and the retention of proteolytic granule contents to further limit the potential for granulocyte-mediated tissue damage $(3,4)$. While little is known about the physiological mechanisms involved in controlling granulocyte apoptosis, many in vitro studies have now shown that an array of pro-inflammatory cytokines and inflammatory mediators known to be present at inflamed sites inhibit the process of apoptosis in granulocytes (2-9). This has led to the suggestion that such agents act both to attract and activate inflammatory cells and also delay their removal. A notable exception to this rule, however, is TNF- $\alpha,{ }^{1}$ which, at early time points in neutrophil culture, causes acceleration of the constitutive rate of apoptosis (10).

Many inflammatory mediators regulate gene expression in target cells by influencing the activities of transcription factors such as nuclear factor- $\kappa \mathrm{B}$ (NF- $\kappa \mathrm{B})$. NF- $\kappa \mathrm{B}$ is composed of homoor heterodimers of the Rel family proteins (p50/NF $\kappa \mathrm{B} 1, \mathrm{p} 52 /$ $\mathrm{NF}_{\kappa} \mathrm{B} 2$, p65/RelA, and $\mathrm{cRel}$ ) which are sequestered in the cytoplasm by physical association with inhibitor proteins referred to as $I_{\kappa} \mathrm{B}$ (11). Upon activation, the $\mathrm{I}_{\kappa} \mathrm{B}$ subunit is rapidly phosphorylated leading to its proteolytic breakdown permitting $\mathrm{NF}-\kappa \mathrm{B}$ to translocate to the nucleus $(12,13)$ where it regulates the activity of many genes involved in the inflammatory response, including those for pro-inflammatory cytokines. In a number of cell systems TNF- $\alpha$ has been shown to induce rapid activation of $\mathrm{NF}-\kappa \mathrm{B}$, a response known to mediate a number of TNF- $\alpha$ induced cellular responses (reviewed in Refs. 14-16). However, whether the activation of NF- $\kappa \mathrm{B}$ is involved in either the pro- or anti-apoptotic effects of TNF- $\alpha$ in granulocytes is currently unknown.

Whereas the inhibition of NF- $\kappa \mathrm{B}$ has been shown to induce apoptosis in murine B cells (17), a completely opposite effect has been observed in other cell types where apoptosis is associated with activation of $N F-\kappa B(18)$. In addition, several groups have reported that inactivation of NF- $\kappa \mathrm{B}$ increases the cytotoxic effects of TNF- $\alpha$ (19-21). In granulocytes, it remains uncertain whether NF- $\kappa \mathrm{B}$ can be activated by inflammatory mediators, or is indeed present, in human neutrophils. For

${ }^{1}$ The abbreviations used are: TNF- $\alpha$, tumor necrosis factor- $\alpha$; EMSA, electrophoretic mobility shift assay; LPS, lipopolysaccharide; NF- $\kappa \mathrm{B}$, nuclear factor- $\kappa$ B; PDTC, pyrrolidine dithiocarbamate; zVAD-fmk, benzyloxycarbonyl-Val-Ala-Asp-fluoromethylketone: DMEM, Dulbecco's modified Eagle's medium. 
example, while MacDonald et al., (22) reported that lipopolysaccharide (LPS), TNF- $\alpha$, and the chemotactic peptide $N$ formyl-methionyl-leucyl-phenylalanine all cause a marked activation of NF- $\kappa \mathrm{B}$, Browning et al., (23) found no such activity in these cells despite obvious $\mathrm{NF}-\kappa \mathrm{B}$ activation in peripheral blood mononuclear cells.

Gliotoxin, a member of the epipolythiodioxoperazine family of compounds (24), exhibits immunosuppressive activity both in vivo and in vitro. For example, gliotoxin has been shown to inhibit mitogen-induced proliferation of both $\mathrm{T}$ and B cells, induce macrophage and osteoclast apoptosis in vitro $(25,26)$, and cause thymocyte and spleen cell apoptosis in vivo (27). However, the biochemical and molecular mechanisms underlying these effects remain uncertain. Gliotoxin has, however, recently been shown to be a potent and specific inhibitor of $\mathrm{NF}-\kappa \mathrm{B}(28)$. We therefore used gliotoxin as a pharmacological tool to investigate the involvement of $\mathrm{NF}-\kappa \mathrm{B}$ in the regulation of granulocyte apoptosis. We demonstrate that gliotoxin causes a rapid and major induction of apoptosis in human peripheral blood granulocytes in vitro and up-regulates TNF- $\alpha$-induced apoptosis in both neutrophils and eosinophils. In addition, we present evidence that these effects occur via a specific, nontoxic and caspase-controlled mechanism that is mediated by the ability of gliotoxin to inhibit an inducible form of NF- $\kappa$ B. The ability of other NF- $\kappa \mathrm{B}$ inhibitors to cause a similar induction of apoptosis provides further evidence supporting the involvement of $\mathrm{NF}-\kappa \mathrm{B}$ in granulocyte apoptosis. Interestingly, the pro-apoptotic effect of TNF- $\alpha$ is enhanced by protein synthesis blockade suggesting that NF- $\kappa \mathrm{B}$ activation results in the generation of an unidentified survival protein. These data therefore strongly suggest that NF- $\kappa \mathrm{B}$ plays a key role in regulating both constitutive and TNF- $\alpha$ stimulated human granulocyte apoptosis.

\section{EXPERIMENTAL PROCEDURES \\ Neutrophil and Eosinophil Isolation and Culture}

Neutrophils and eosinophils were isolated from the peripheral blood of normal donors by dextran sedimentation followed by centrifugation through discontinuous plasma-Percoll gradients $(2,29)$. Only neutrophil preparations with a neutrophil purity of $>98 \%$ were used. Eosinophils were separated from contaminating neutrophils using an immunomagnetic separation step with sheep anti-mouse IgG-Dynabeads (Dynabeads M-450, Dynal, Merseyside, United Kingdom) coated with the murine anti-neutrophil antibody 3G8 (anti-CD16; a gift from Dr. J. Unkeless, Mount Sinai Medical School, New York). Cells were mixed with washed 3G8-coated Dynabeads at a bead:neutrophil ratio of 3:1 on a rotary mixer at $4{ }^{\circ} \mathrm{C}$ for $20 \mathrm{~min}$, and the beads removed magnetically by two 3-min stationary magnetic contacts (Dynal Magnetic Particle Concentrator, MPC-1) to yield an a eosinophil population of $>98 \%$ purity. After purification, cells were washed twice in phosphate-buffered saline without calcium and magnesium and once in phosphatebuffered saline before resuspending in Iscove's DMEM (Life Technologies, Paisley, UK) with $10 \%$ autologous serum. Both cell types were cultured in flat-bottomed Falcon flexible wells (Becton Dickinson, Oxford, UK) at $37^{\circ} \mathrm{C}$ in a $5 \% \mathrm{CO}_{2}$ atmosphere; neutrophils at a concentration of $5 \times 10^{6} / \mathrm{ml}$ and eosinophils at $2 \times 10^{6} / \mathrm{ml}$. Cells were cultured in the absence or presence of test agents as described in the figure legends. All experiments were performed at least 3 times and each treatment done in triplicate.

\section{Assessment of Granulocyte Apoptosis}

Morphology-Cells were cyto-centrifuged, fixed in methanol, stained with Diff-Quik ${ }^{\mathrm{TM}}$, and counted using oil immersion microscopy $(\times 100$ objective) to determine the proportion of cells with highly distinctive apoptotic morphology $(5,7,10)$. At least 500 cells were counted per slide with the observer blinded to the experiment conditions. The results were expressed as the mean $\%$ apoptosis \pm S.E.

Annexin V Binding-A separate and independent assessment of apoptosis was performed by flow cytometry using fluorescein isothiocyanate-labeled recombinant human annexin $\mathrm{V}$ that binds to phosphatidylserine exposed on the surface of apoptotic cells. Stock annexin V (Bender MedSystems, Vienna, Austria) was diluted 1:200 with binding buffer and then added $(25 \mu \mathrm{l})$ to $75 \mu \mathrm{l}$ of the recovered cell samples. Following a 10 -min incubation at $4{ }^{\circ} \mathrm{C}$, these samples were fixed by the addition of $100 \mu \mathrm{l}$ of $3 \%$ paraformaldehyde in phosphate-buffered saline before analysis using an EPICS Profile II (Coulter Electronics, Luton, UK).

DNA Fragmentation Assay-DNA was extracted as described previously (10). Briefly, $2 \times 10^{6}$ neutrophils were taken after the indicated treatment and lysed in $500 \mu \mathrm{l}$ of lysis buffer $(6 \mathrm{M}$ guanidine hydrochloride, $50 \mathrm{~mm}$ Tris- $\mathrm{HCl}, \mathrm{pH} 8.0$, and $0.1 \% \mathrm{~N}$-lauroyl sarcosine) at $4{ }^{\circ} \mathrm{C}$ and the nucleic acids extracted by the addition of an equal volume of $10 \mathrm{~mm}$ Tris- $\mathrm{HCl}, \mathrm{pH} 8.0$-saturated phenol:chloroform mixture $(50: 50, \mathrm{v} / \mathrm{v})$. The resulting emulsion was centrifuged at $12,000 \times \mathrm{g}_{\mathrm{av}}$ for $10 \mathrm{~min}$ at room temperature and the aqueous phase removed and precipitated with 0.6 volumes of isopropyl alcohol at room temperature. The precipitated nucleic acids were then pelleted by centrifugation at $10,000 \times g_{a v}$ for 5 min and re-dissolved in $50 \mu \mathrm{l}$ of TE buffer (10 mM Tris-Cl, 1 mM EDTA, $\mathrm{pH} 8.0$ ) containing $50 \mu \mathrm{g} / \mathrm{ml}$ RNase A. The fragmented DNA was separated by agarose gel electrophoresis on a $1.4 \%(\mathrm{w} / \mathrm{v})$ agarose (Flowgen, UK) $0.5 \times$ TBE (10 mM Trizma (Tris base), $10 \mathrm{~mm}$ boric acid, and $1 \mathrm{~mm}$ EDTA, pH 8.3) gel. The gel was run for $2 \mathrm{~h}$ at $75 \mathrm{~V}$ and stained using ethidium bromide $(0.5 \mu \mathrm{g} / \mathrm{ml})$. The UV transilluminated image was printed by digital thermal printing using a GS7600 gel documentation system (UVP Products, UK).

\section{Assessment of Cell Membrane Integrity}

Since apoptotic neutrophils and eosinophils maintain the integrity of their plasma membrane, assessment of granulocyte necrosis can be determined by the ability of cells to exclude the vital dye trypan blue and also by flow cytometry using propidium iodide staining. Samples $\left(150 \mu \mathrm{l}\right.$ of cells at $2 \times 10^{6} / \mathrm{ml}$ ) were centrifuged and resuspended in 150 $\mu \mathrm{l}$ of propidium iodide solution $(33 \mu \mathrm{g} / \mathrm{ml}$ propidium iodide in phosphate-buffered saline containing $1.67 \mathrm{mg} / \mathrm{ml}$ RNase). The profiles of heat-treated (necrotic cells) from the same samples were used as controls.

\section{Electrophoretic Mobility Shift Assay (EMSA)}

EMSAs were carried out as described by the manufacturer (Promega Corp, Southampton, UK). Nuclear extracts were prepared from $5 \times 10^{6}$ cells using a modification of the method of Dignam et al. (30). Briefly, pelleted cells were resuspended in $200 \mu \mathrm{l}$ of hypotonic buffer (buffer A: $10 \mathrm{~mm}$ Tris- $\mathrm{HCl}$, pH 7.8, $1.5 \mathrm{~mm}$ EDTA, $10 \mathrm{mM} \mathrm{KCl,} 0.5 \mathrm{~mm}$ dithiothreitol, $1 \mu \mathrm{g} / \mathrm{ml}$ aprotinin, leupeptin, and pepstatin A, $1 \mu \mathrm{M}$ 4-(2-aminoethyl)benzenesulfonyl fluoride, $1 \mathrm{~mm}$ sodium orthovanadate, $0.5 \mathrm{~mm}$ benzamidine, and $2 \mathrm{~mm}$ levamisole) and placed on ice for $10 \mathrm{~min}$. Following the addition of 0.1 volumes of $10 \%$ Nonidet P-40 (W/v) the cells were vortexed briefly and centrifuged at $12,000 \times g_{\text {av }}$ for 2 min at $4{ }^{\circ} \mathrm{C}$. The supernatant was discarded and the pellet washed in $100 \mu \mathrm{l}$ of buffer A minus Nonidet $\mathrm{P}-40$ and re-centrifuged. The pelleted nuclei were then resuspended in $50 \mu \mathrm{l}$ of hypertonic buffer (buffer B: $20 \mathrm{~mm}$ Tris- $\mathrm{HCl}, \mathrm{pH}$ $7.8,150 \mathrm{~mm} \mathrm{NaCl}, 50 \mathrm{~mm} \mathrm{KCl}, 1.5 \mathrm{~mm}$ EDTA, $5 \mathrm{~mm}$ dithiothreitol, 1 $\mu \mathrm{g} / \mathrm{ml}$ aprotinin, leupeptin, and pepstatin A, $1 \mu \mathrm{M}$ 4-(2-aminoethyl)benzenesulfonyl fluoride, $1 \mathrm{~mm}$ sodium orthovanadate, $0.5 \mathrm{~mm}$ benzamidine, and $2 \mathrm{~mm}$ levamisole) and stored at $-80^{\circ} \mathrm{C}$ until use.

Nuclear extracts (approximately $2 \mu \mathrm{g}$ of protein, $7 \times 10^{5}$ cell equivalent, in $7 \mu \mathrm{l})$ were incubated in binding buffer $(4 \%$ glycerol, $1 \mathrm{~mm}$ $\mathrm{MgCl}_{2}, 0.5 \mathrm{~mm}$ EDTA, $0.5 \mathrm{~mm}$ dithiothreitol, $50 \mathrm{~mm} \mathrm{NaCl}, 10 \mathrm{~mm}$ Tris-HCl, pH 7.5, with $50 \mu \mathrm{g} / \mathrm{ml}$ poly(dI-dC) poly(dI-dC) (Pharmacia Biotech, UK) with $17 \mathrm{fmol}$ of $\gamma_{-}{ }^{32} \mathrm{P}$-labeled double stranded oligonucleotide containing the decameric $\kappa \mathrm{B}$-binding site $(3000 \mathrm{Ci} / \mathrm{mmol}$; Promega Corp., Southampton, UK) at $22^{\circ} \mathrm{C}$ for 20 min prior to addition of $5 \mu \mathrm{l}$ of loading buffer $\left(0.01 \%(\mathrm{w} / \mathrm{v})\right.$ bromphenol blue, $20 \%(\mathrm{w} / \mathrm{v})$ Ficoll $400^{\mathrm{TM}}$, and $1 \mathrm{~mm}$ EDTA). Samples were loaded onto an $8 \%(\mathrm{w} / \mathrm{v})$ native acrylamide gel (Protean IIxi, Bio-Rad, Hemel Hempstead, UK) in $0.5 \times$ TBE buffer and run at $250 \mathrm{~V}$ for $2 \mathrm{~h}$. The gel was then dried onto $3 \mathrm{MM}$ paper (Whatman UK, Maidstone, UK) and BioMax MS-1 x-ray film (Kodak, Anachem, Luton, UK) was exposed to the gel. Processed films were analyzed using the GrabIt and GelPlate software (UVP, Orme Technologies, UK) and the data expressed as a percent of the control value for each experiment.

\section{Materials}

Further specific materials were obtained as follows: curcumin, gliotoxin, LPS (Escherichia coli 0127:B8), methylthiogliotoxin, and PDTC (Sigma Co., Poole, UK); recombinant human TNF- $\alpha$ (R \& D Systems, Abingdon, Oxon, UK); zVAD-fmk (Bachem (UK) Ltd., Saffron Walden, UK). The proteasome inhibitor MG-132 (N-cbz-Leu-Leu-leucinal), and the NF- $\kappa$ B inhibitory peptides SN50 and SN50M (Biomol, Affinity Re- 
A

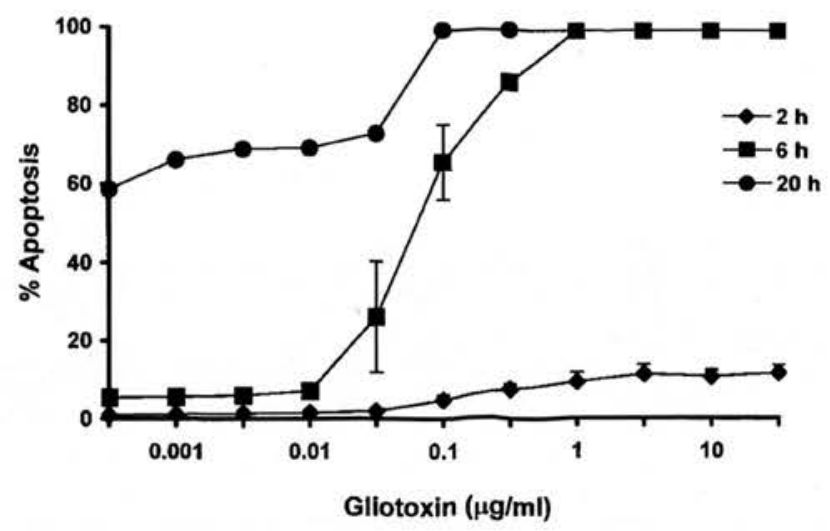

B

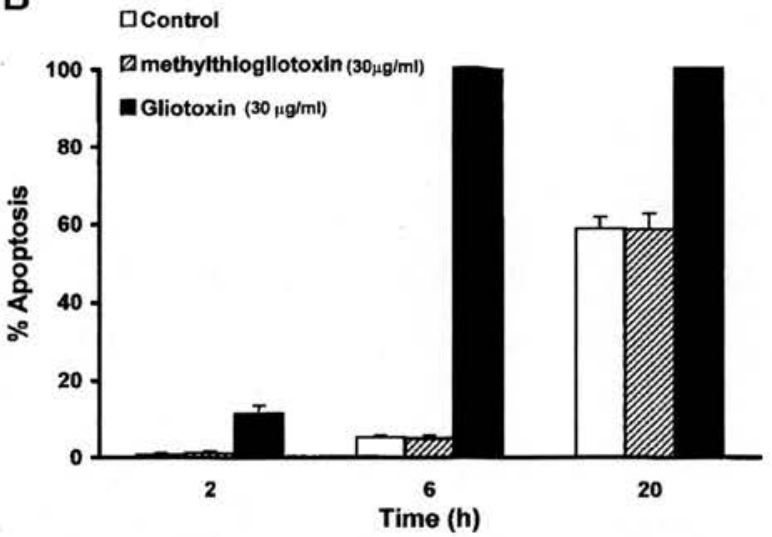

FIG. 1. Time course and concentration-response curve for the effect of gliotoxin on human neutrophil apoptosis. Human neutrophils $\left(5 \times 10^{6} / \mathrm{ml}\right)$ were cultured at $37^{\circ} \mathrm{C}$ in Iscove's DMEM containing $10 \%$ autologous serum and treated with the indicated concentrations of gliotoxin. At the time periods indicated, the cells were resuspended and cytocentrifuge preparations made. These were fixed and stained, and apoptosis was assessed morphologically. $A$, represents the effect of gliotoxin $(0.001-30 \mu \mathrm{g} / \mathrm{ml})$ on neutrophil apoptosis after 2 , 6 , or $20 \mathrm{~h}$ of culture. $B$, represents the effect of methylthiogliotoxin (30 $\mu \mathrm{g} / \mathrm{ml})$ or gliotoxin $(30 \mu \mathrm{g} / \mathrm{ml})$ on neutrophil apoptosis after 2,6 , or $20 \mathrm{~h}$ culture. All values represent mean \pm S.E. of $n=3$ experiments, each performed in triplicate. Where not shown, S.E. values are less than $2 \%$ of the mean.

search Products, Mamhead, UK). All other reagents were obtained from Sigma, UK, and were of the highest purity.

\section{Statistical Analysis}

The results are expressed as mean \pm S.E. of the number $(n)$ of independent experiments each using cells from separate donors with each treatment performed in triplicate. Statistical analysis was performed by ANOVA with comparisons between groups made using the Newman-Kuels procedure. Differences were considered significant when $p<0.05$.

\section{RESULTS}

Effect of Gliotoxin on Neutrophil Apoptosis-As shown in Fig. 1 gliotoxin caused a rapid and profound induction of neutrophil apoptosis in vitro which was both concentration (e.g. at $6 \mathrm{~h}$ $\mathrm{EC}_{50}=76.1 \pm 22.1 \mathrm{ng} / \mathrm{ml}$, Fig. $1 A$ ), and time-dependent (Fig. 1, $A$ and $B$ ). Hence using a maximally effective gliotoxin concentration of $1 \mu \mathrm{g} / \mathrm{ml}$, apoptosis was readily apparent within $2 \mathrm{~h}$ and reached $100 \%$ by $6 \mathrm{~h}$. At $20 \mathrm{~h}$, when the rate of constitutive neutrophil apoptosis was $58.7 \pm 2.9 \%$, gliotoxin caused $100 \%$ apoptosis at all concentrations greater than $0.1 \mu \mathrm{g} / \mathrm{ml}$. The inactive analogue of gliotoxin, methylthiogliotoxin, did not af- fect the constitutive rate of neutrophil apoptosis at any of the time points studied (Fig. 1B). Neither gliotoxin nor its inactive analogue, methylthiogliotoxin, caused cell necrosis since less than $1 \%$ of the cells were permeable to the vital dye trypan blue.

Gliotoxin Acts Synergistically with TNF- $\alpha$ to Stimulate Neutrophil Apoptosis-In contrast to many other hematopoetic cells, human neutrophils appear highly resistant to the induction of apoptosis induced by certain agents, for example, incubation with $\mathrm{Ca}^{2+}$ ionophores $(31,32)$, cAMP elevating agents (33), corticosteroids (9), and LPS (7) causes inhibition of apoptosis as does hypoxia (34). Furthermore, while TNF- $\alpha$ and Fas-L can induce neutrophil apoptosis, this effect is modest and transient, and in the case of TNF- $\alpha$ abolished if the cells are initially primed with platelet-activating factor or LPS $(10,35)$. We therefore sought to determine the effect of gliotoxin on TNF- $\alpha$-induced apoptosis in neutrophils. These experiments were performed deliberately at a very early time point $(2 \mathrm{~h})$ when the independent pro-apoptotic effects of even a maximally effective concentration of TNF- $\alpha(10 \mathrm{ng} / \mathrm{ml})(10)$ and gliotoxin (1 $\mu \mathrm{g} / \mathrm{ml}$; Fig. $1 A)$ are only just apparent. As shown in Fig. 2A, a major synergy was observed between these agents for the induction of apoptosis which was apparent even at gliotoxin concentrations as low as $3 \mathrm{ng} / \mathrm{ml}$. Hence, a concentration of 0.1 $\mu \mathrm{g} / \mathrm{ml}$ gliotoxin in combination with TNF- $\alpha(10 \mathrm{ng} / \mathrm{ml})$ caused almost $100 \%$ apoptosis at $2 \mathrm{~h}$. With gliotoxin alone, only $6 \%$ apoptosis was noted at $2 \mathrm{~h}$, with just over $65 \%$ at $6 \mathrm{~h}$ (Fig. $1 \mathrm{~A}$ ). Again, methylthiogliotoxin had no effect on constitutive apoptosis or TNF- $\alpha$-induced apoptosis at $2 \mathrm{~h}$ (Fig. $2 B$ ). The level of necrosis in cells from each treatment was assessed by trypan blue exclusion; all values were $<1 \%$ (data not shown).

The genuine nature of both the intrinsic pro-apoptotic effect of gliotoxin and the dramatic synergy with TNF- $\alpha$ was assessed by comparing the quantitative morphological effects of these agents with their effects on annexin V binding and DNA fragmentation. The changes from normal cell morphology to apoptotic morphology are clearly seen in Fig. $3 A$; where non-apoptotic neutrophils contain a multilobed nucleus and the apoptotic cells have a shrunken appearance with pyknotic nuclei. These data can be compared with Fig. $3 B$, where the annexin V "low peak" represents non-apoptotic cells and the annexin V "high peak" represents apoptotic cells since the fluorescein isothiocyanate-labeled annexin $\mathrm{V}$ binds in the presence of $\mathrm{Ca}^{2+}$ to phosphatidylserine exposed on the outer membrane of apoptotic cells. Although control cells at $2 \mathrm{~h}$ exhibit low rates of apoptosis, the small increase in annexin $\mathrm{V}$ positive cells observed with TNF- $\alpha$ and gliotoxin alone is again dramatically augmented when the cells are cultured in the presence of both reagents together. Analysis by DNA fragmentation also demonstrates that cells cultured alone or in combination with the above reagents exhibit the classical "ladder" of DNA fragmentation associated with apoptosis (Fig. $3 C$ ).

Combined Gliotoxin and TNF- $\alpha$ Treatment Does Not Cause Necrosis-Although our initial studies using trypan blue as a marker of plasma membrane integrity indicated that gliotoxin, both in the presence and absence of TNF- $\alpha$, induced a purely apoptotic form of cell death, we felt it was important to validate this further by assessing necrosis in an independent manner using propidium iodide staining detected by flow cytometry. Fig. $3 D$ shows the profile of neutrophils $4 \mathrm{~h}$ following treatment with gliotoxin $(0.1 \mu \mathrm{g} / \mathrm{ml})$ and TNF- $\alpha(10 \mathrm{ng} / \mathrm{ml})$ where, despite apoptotic rates of $100 \%$, almost all cells showed low fluorescence indicating that the cell membrane had remained intact. As a positive control, cells cultured initially with TNF- $\alpha$ and gliotoxin were then heat-treated $\left(60{ }^{\circ} \mathrm{C}, 5 \mathrm{~min}\right)$ to ensure $100 \%$ necrosis. This resulted in a uniform and major increase in 


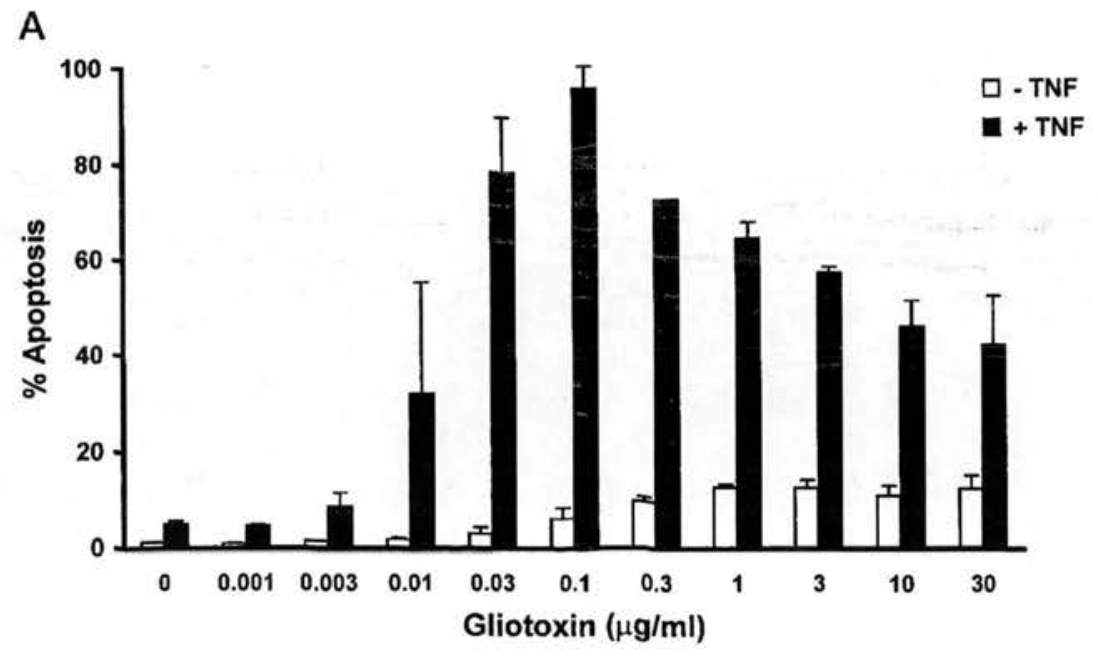

Fig. 2. The effect of gliotoxin, methylthiogliotoxin, and TNF- $\alpha$ on human neutrophil apoptosis. Human neutrophils $\left(5 \times 10^{6} / \mathrm{ml}\right)$ were cultured at $37^{\circ} \mathrm{C}$ in Iscove's DMEM containing $10 \%$ autologous serum and treated with the indicated concentrations of gliotoxin, plus or minus TNF- $\alpha$. After $2 \mathrm{~h}$ of culture, the cells were resuspended and cytocentrifuge preparations made. These were fixed and stained, and apoptosis was assessed morphologically. A, represents the effect of gliotoxin $(0.001-30 \mu \mathrm{g} / \mathrm{ml})$ on TNF- $\alpha$ $(10 \mathrm{ng} / \mathrm{ml}$ )-induced neutrophil apoptosis. $B$, represents the effect of methylthiogliotoxin $(30 \mu \mathrm{g} / \mathrm{ml})$ or gliotoxin $(30 \mu \mathrm{g} / \mathrm{ml})$, plus or minus TNF- $\alpha(10 \mathrm{ng} / \mathrm{ml})$. All values represent mean \pm S.E. of $n=3$ experiments, each performed in triplicate. Where not shown, S.E. values are less than $2 \%$ of the mean.

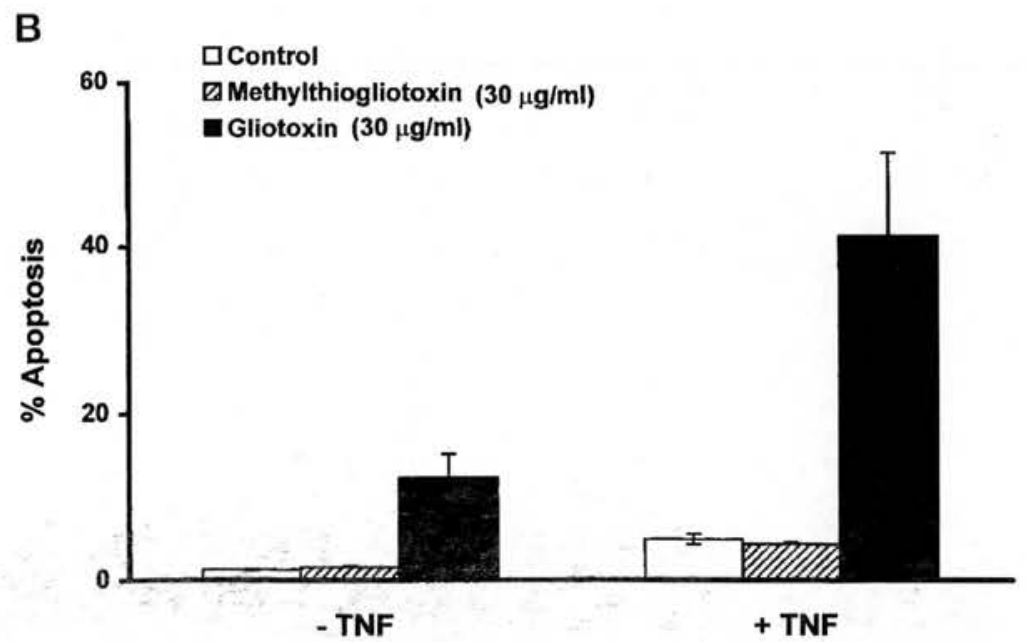

propidium iodide staining (Fig. $3 D$ ). These data coincide completely with the results obtained with trypan blue staining and confirm that these cells had undergone apoptotic cell death and were not necrotic.

Gliotoxin Inhibits the Survival Effect of LPS-To investigate whether gliotoxin could modulate the effects of LPS on the rate of neutrophil apoptosis we performed a series of experiments where neutrophils were cultured for $2,3,4$, and $20 \mathrm{~h}$ in the presence of LPS, gliotoxin, and a combination of LPS plus gliotoxin and apoptosis assessed morphologically (Fig. 4). As reported previously (7) LPS caused an inhibition of neutrophil apoptosis at $20 \mathrm{~h}$ when compared with control cells. Interestingly, the suppressive effect of LPS was prevented by the strong pro-apoptotic effect of gliotoxin. In addition, unlike coculture of gliotoxin plus TNF- $\alpha$, no synergistic induction of apoptosis was observed when LPS was cultured in the presence of gliotoxin (Fig. 4).

Gliotoxin Unmasks the Ability of TNF- $\alpha$ to Induce Eosinophil Apoptosis-To explore whether the pro-apoptotic effect of gliotoxin was restricted to neutrophils, a similar set of experiments were performed using human peripheral blood eosinophils isolated from mildly atopic individuals. While these cells display a similar capacity to undergo constitutive apoptosis when aged in vitro, this process is much slower than that observed for the neutrophil and is differentially regulated being, for example, stimulated rather than inhibited by corticosteroids (9). We therefore investigated the effect of gliotoxin on eosinophil apo- ptosis at $4 \mathrm{~h}$ in the presence and absence of TNF- $\alpha$. The results, shown in Fig. 5, $A$ and $B$, demonstrated that gliotoxin caused a similar induction of apoptosis in eosinophils $\left(\mathrm{EC}_{50}=0.37 \pm\right.$ $0.22 \mu \mathrm{g} / \mathrm{ml}$ ) and caused a synergistic increase in the rate of apoptosis when the cells were co-cultured with TNF- $\alpha$. This latter observation was all the more striking since in eosinophils, TNF- $\alpha$ treatment alone had no effect on the rate of apoptosis (Fig. $5 B$ and data not shown). Hence, almost $100 \%$ apoptosis was observed using a gliotoxin concentration of 0.1 $\mu \mathrm{g} / \mathrm{ml}$ plus TNF- $\alpha$ at a time point of $4 \mathrm{~h}$. In comparison, eosinophils cultured in the absence of gliotoxin would normally show only $40 \%$ apoptosis at a 40 -h time point $(2,36)$. Necrosis in these cells was $<2 \%$ and the inactive analogue of gliotoxin had no effect on either the constitutive rate of apoptosis alone or in conjunction with TNF- $\alpha$ (data not shown). As with the neutrophils, eosinophils demonstrated classic apoptotic morphology when treated with these agents.

Gliotoxin Causes Inhibition of an Inducible Isoform of NF. $\kappa B$-Recent studies have indicated that NF- $\kappa \mathrm{B}$ may play an important role in regulating the rate of apoptosis in certain transformed cells $(17,18)$. Hence, because gliotoxin has been reported to act as a specific inhibitor of NF- $\kappa \mathrm{B}(28)$ experiments were designed to identify and characterize the expression of this transcription factor in human neutrophils and determine if gliotoxin could inhibit such activity. Preliminary time course data established $90 \mathrm{~min}$ as the optimal time to examine basal, gliotoxin, and TNF- $\alpha$ regulated NF- $\kappa \mathrm{B}$ activity in these cells 
A

FIG. 3. The effect of gliotoxin and TNF- $\alpha$ on neutrophil morphology, phosphatidylserine expression, and cell membrane integrity. $A$, cytocentrifuge preparations of human neutrophils incubated for $2 \mathrm{~h}$ in Iscove's DMEM at $37{ }^{\circ} \mathrm{C}$ containing $10 \%$ autologous serum alone (control), plus TNF- $\alpha$, plus gliotoxin, or with both TNF- $\alpha$ and gliotoxin at the concentrations shown. Neutrophils treated with both reagents all clearly show apoptotic morphology. $B$, after $2 \mathrm{~h}$ in culture at $37^{\circ} \mathrm{C}$, cells treated with Iscove's DMEM, TNF- $\alpha(10 \mathrm{ng} / \mathrm{ml})$, gliotoxin $(0.1 \mu \mathrm{g} / \mathrm{ml})$, or with both TNF- $\alpha$ plus gliotoxin were resuspended and incubated with fluorescein isothiocyanate-labeled recombinant human annexin $\mathrm{V}$ to determine phosphatidylserine expression. The cells were then fixed and analyzed using an EPICS Profile II. Mean fluorescence values are shown for a minimum of 5,000 cells for each condition. $C$, DNA fragmentation in human neutrophils treated with TNF- $\alpha(10 \mathrm{ng} / \mathrm{ml})$, gliotoxin $(0.1 \mu \mathrm{g} / \mathrm{ml})$, or both reagents together for $2 \mathrm{~h}$. DNA was prepared as detailed under "Experimental Procedures"; lane 1, DNA marker (1-kilobase ladder); lane 2, freshly isolated neutrophils; lane 3 , control; lane 4 , TNF- $\alpha$; lane 5 , gliotoxin, $2 \mathrm{~h}$; lane 6 , co-culture with gliotoxin and TNF- $\alpha$; lane 7 , DNA marker (1-kilobase ladder). $D$, to assess cell membrane integrity, cells treated with both TNF- $\alpha(10 \mathrm{ng} / \mathrm{ml})$ and gliotoxin $(0.1 \mu \mathrm{g} / \mathrm{ml})$ for $4 \mathrm{~h}$, which induced $100 \%$ apoptosis even by $2 \mathrm{~h}$, were resuspended and incubated with propidium iodide, fixed, and analyzed using an EPICS Profile II (solid line). An aliquot of rells from this preparation was heated as indicated under "Experimental Procedures" to produce $100 \%$ necrosis (dotted line). Mean fluorescence values are shown for a minimum of 5,000 cells for each condition.
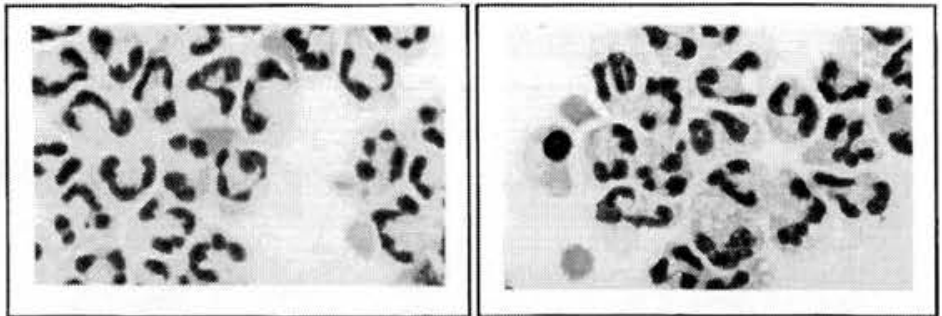

Gliotoxin (0.1 He/ml) 2 h Apoptesis (11.3\%)

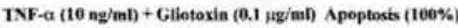
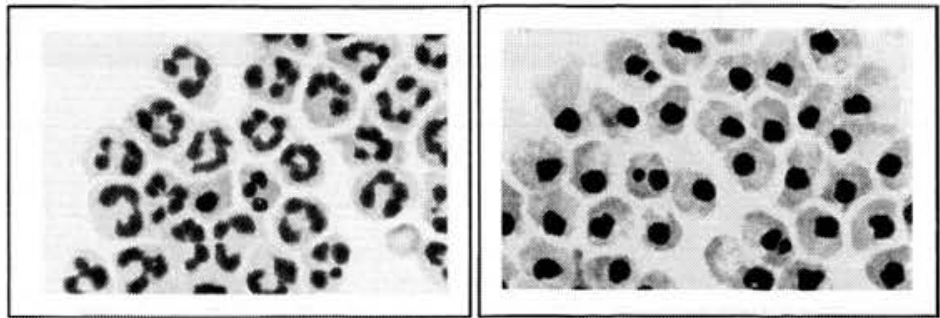

B
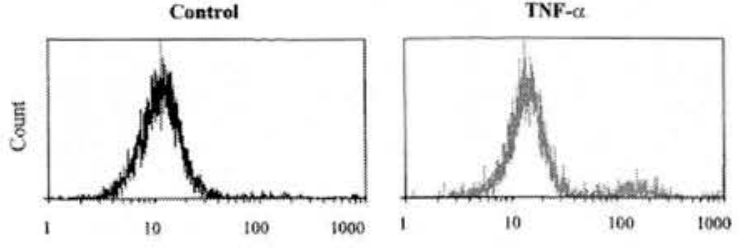

Gliotoxin
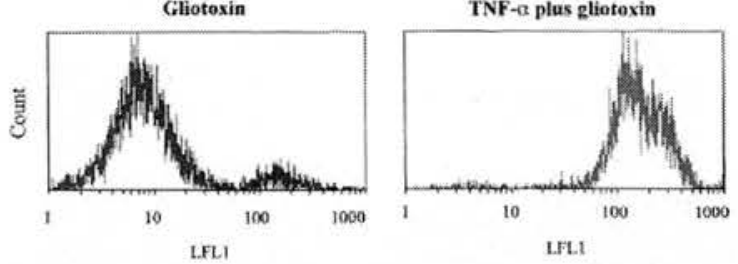

C

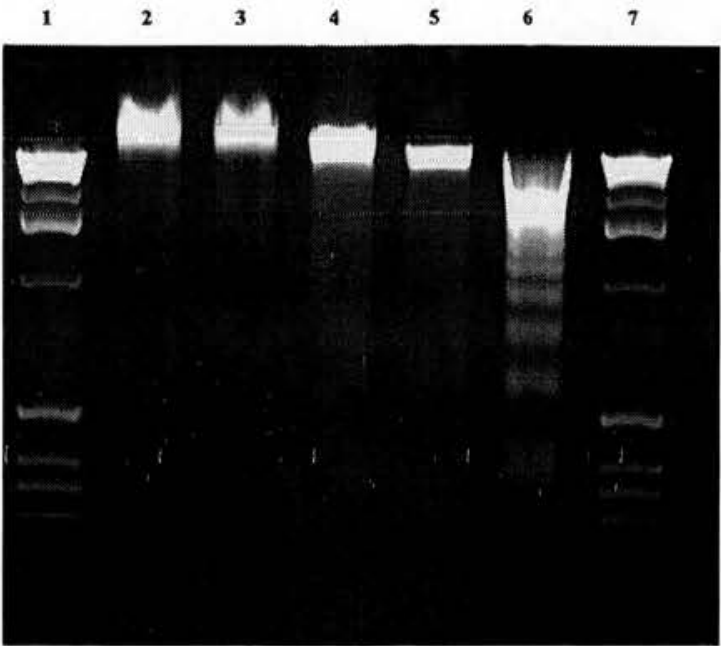

(data not shown). Of note, this time point also coincided with the onset of the biologically observable effect of gliotoxin. As shown in Fig. 6, A-C, NF- $\kappa$ B EMSAs performed on neutrophil nuclear extracts indicated the presence of 3 discrete bands in these gels. To ascertain which of these bands were specifically $\mathrm{NF}-\kappa \mathrm{B}$, an excess of unlabeled probe was included in the label- ing reaction to displace specific binding; as shown in Fig. $6 C$, two NF- $\kappa \mathrm{B}$ bands were identified and designated $\mathrm{A}$ and $\mathrm{B}$.

In both TNF- $\alpha(10 \mathrm{ng} / \mathrm{ml}, 0-90 \mathrm{~min})$ and LPS $(1 \mu \mathrm{g} / \mathrm{ml}, 0-120$ min) treated cells, no change in the intensity of band B was observed (Fig. 5, $A$ and $B$, and data not shown). This, together with its strong expression in freshly prepared untreated neu- 



FTG, 4. The effect of gliotoxin alone and in the presence of LPS on human neutrophil apoptosis. Human neutrophils $\left(5 \times 10^{6} / \mathrm{ml}\right)$ were cultured in Iscove's DMEM at $37^{\circ} \mathrm{C}$ containing $10 \%$ autologous serum alone and treated with gliotoxin $(0.1 \mu \mathrm{g} / \mathrm{ml})$, with or without LPS $(1 \mu \mathrm{g} / \mathrm{ml})$. At the time periods indicated, the cells were resuspended and cytocentrifuge preparation made. These were fixed and stained, and apoptosis was assessed morphologically. All values represent mean \pm S.E. of $n=3-5$ experiments, each performed in triplicate. Where not shown, S.E. values are less than $2 \%$ of the mean.

trophils suggests that this band represents a form of constitutively active NF- $\kappa \mathrm{B}$. However, band A was markedly up-regulated by TNF- $\alpha$ (Fig. 6, $A$ and $C$ ), and as shown in Fig. $6 A$, gliotoxin caused both a concentration-dependent inhibition of this NF- $\kappa$ B activity and abolished the TNF- $\alpha$ stimulated increase in this band. As shown in Fig. $6 D$, densiometric analysis of these data confirmed that co-treatment of neutrophils with TNF- $\alpha$ and gliotoxin at a maximal effective functional concentration of $0.1 \mu \mathrm{g} / \mathrm{ml}$ (Fig. 2A) inhibited this band more than treatment with gliotoxin alone. The results in Fig. 6, $A$ and $D$, confirm that the basal level of NF- $\mathrm{B}$ activity in control samples at $90 \mathrm{~min}$ was inhibited by gliotoxin treatment; a finding that was observed at all other time points tested.

Further evidence that strongly supports the suggestion that NF- $\kappa \mathrm{B}$ inhibition is linked to the induction of neutrophil apoptosis is provided by the fact that the cell permeable NF- $\mathrm{B}$ inhibitory peptide, SN50 (37), also increased the rate of constitutive neutrophil apoptosis despite the fact that less than $5 \%$ of the peptide reportedly enters the cell (37). Hence at $6 \mathrm{~h}$, the SN50 peptide increased neutrophil apoptosis from $4.7 \pm 1.2$ to $15.0 \pm 3.2 \%(n=3 ; p<0.05)$, whereas the less active peptide SN50M only increased apoptosis from $4.7 \pm 1.2$ to $5.6 \pm 0.8 \%$ $(n=3)$. Similar effects on neutrophil apoptosis were seen at $20 \mathrm{~h}$ and eosinophil apoptosis at 20 and $40 \mathrm{~h}$ (Table I and data not shown). Other agents known to inhibit NF- $\mathrm{B}$ similarly

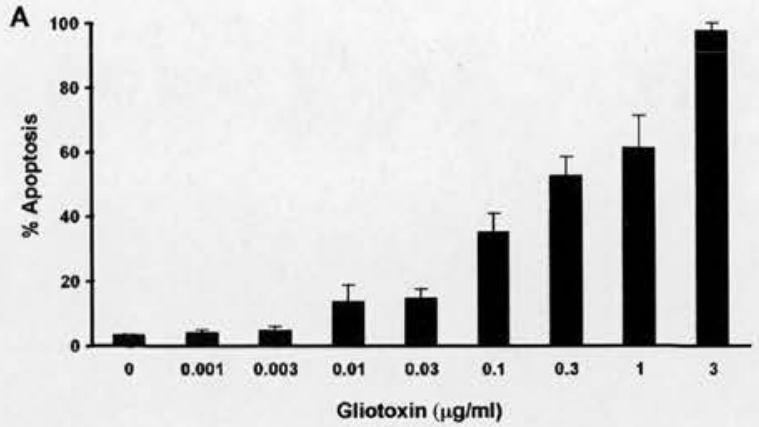

B

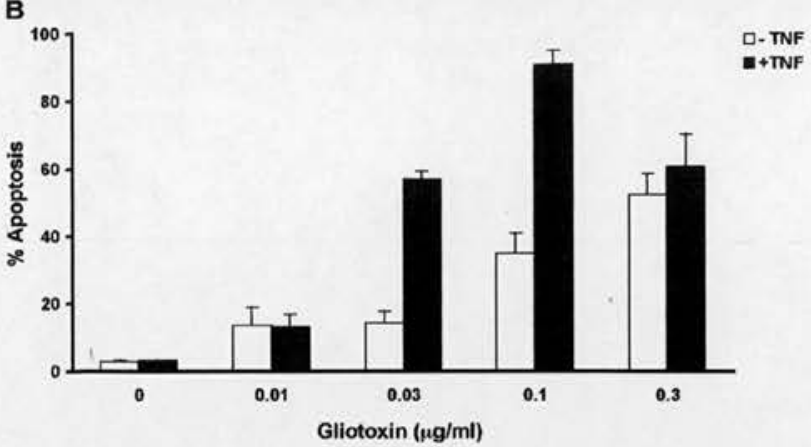

FIG. 5. The effect of gliotoxin alone and in the presence of TNF- $\alpha$ on human eosinophil apoptosis. Human eosinophils $(2 \times$ $10^{6} / \mathrm{ml}$ ) were cultured in Iscove's DMEM at $37^{\circ} \mathrm{C}$ containing $10 \%$ autologous serum alone and treated with the indicated concentrations of gliotoxin with or without TNF- $\alpha$. At the time periods indicated, the cells were resuspended and cytocentrifuge preparation made. These were fixed and stained, and apoptosis was assessed morphologically. $A$, represents the effects of gliotoxin $(0-3 \mu \mathrm{g} / \mathrm{ml})$ on eosinophil apoptosis after $4 \mathrm{~h}$ of culture. $B$, represents the effects gliotoxin $(0-0.3 \mu \mathrm{g} / \mathrm{ml})$ with or without TNF- $\alpha(10 \mathrm{ng} / \mathrm{ml})$ on eosinophil apoptosis after a 4 -h culture. All values represent mean \pm S.E. of $n=3$ experiments, each performed in triplicate. Where not shown, S.E. values are less than $2 \%$ of the mean.

induced neutrophil apoptosis. The proteasome inhibitor, MG132 (38) and the NF- $\mathrm{kB}$ inhibitor curcumin (39) caused a timedependent induction of neutrophil apoptosis (Fig. 7A). PDTC, that acts as both a radical scavenger and inhibitor of NF- $\kappa$ B activation (40), also caused a significant induction of apoptosis when cultured with neutrophils for $20 \mathrm{~h}$ (Fig. $7 B$ ). Furthermore, treating neutrophils with LPS $(100 \mathrm{ng} / \mathrm{ml}, 20 \mathrm{~min})$ which we have previously reported to induce a profound inhibition of neutrophil apoptosis (7) was found to cause the appearance of this inducible isoform of NF- $\kappa \mathrm{B}$ (Fig. $6 B$ ); and this induction could be inhibited by gliotoxin $(0.1 \mu \mathrm{g} / \mathrm{ml})$.

Induction of Apoptosis by Gliotoxin Is Dependent on Activation of the Caspase-cascade Pathway-We have recently demonstrated that the early pro-apoptotic effects of TNF- $\alpha$ in human neutrophils requires activation of both TNF-55 and TNF-75 receptor subtypes and thereby differs significantly from the priming effect of TNF- $\alpha$ which is signaled via the TNF-p55 receptor alone (10). To determine whether the proapoptotic effects of gliotoxin and the marked synergism displayed by TNF- $\alpha$ and gliotoxin were mediated via activation of the caspase pathway, we co-incubated neutrophils with TNF- $\alpha$, gliotoxin, and zVAD-fmk. At $2 \mathrm{~h}$, zVAD-fmk completely inhibited the increase in apoptosis induced by gliotoxin, TNF- $\alpha$ and by both factors together (Fig. $8 A$ ). This demonstrates that apoptosis induced by both factors alone, or together, is dependent on caspase activation.

Gliotoxin May Enhance TNF- $\alpha$-induced Apoptosis by Inhibiting Production of a Survival Factor-Taken together, the 
A
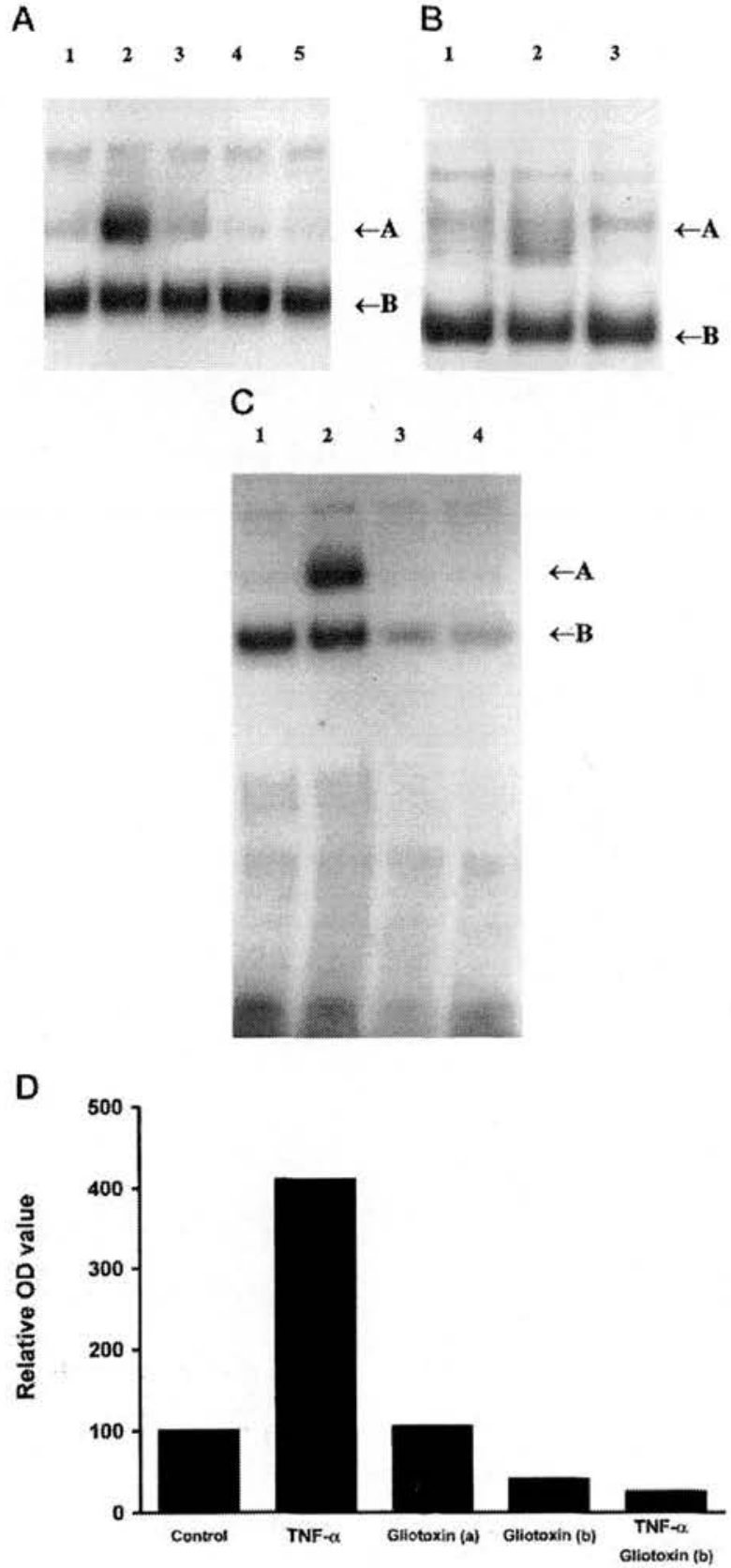

FIG. 6. Effect of gliotoxin and TNF- $\alpha$ on NF- $\mathrm{BB}$ mobilization. $A$ EMSA of nuclear extracts from neutrophils treated with control buffer: lane 1 , TNF- $\alpha(10 \mathrm{ng} / \mathrm{ml})$; lane 2 , gliotoxin $(1 \mu \mathrm{g} / \mathrm{ml})$; lane 3 , gliotoxin $(0.1 \mu \mathrm{g} / \mathrm{ml}) ;$ lane 4 , and TNF- $\alpha(10 \mathrm{ng} / \mathrm{ml})$ plus gliotoxin $(0.1 \mu \mathrm{g} / \mathrm{ml}) ;$ lane 5 , for $90 \mathrm{~min}$ at $37^{\circ} \mathrm{C}$. B, EMSA showing the up-regulation of the inducible isoform (band A) by LPS (a known inhibitor of neutrophil apoptosis) after $20 \mathrm{~min}$ culture: lane 1, control; lane 2 , LPS $(100 \mathrm{ng} / \mathrm{ml})$; and lane 3 , LPS $(100 \mathrm{ng} / \mathrm{ml})$ plus gliotoxin $(0.1 \mu \mathrm{g} / \mathrm{ml})$. C, EMSA showing displacement of specific NF- $\mathrm{\kappa B}$ bands by excess cold oligonucleotide probe; lane 1 , control; lane 2 , TNF- $\alpha(10 \mathrm{ng} / \mathrm{ml})$; lane 3 , TNF- $\alpha$ (10 $\mathrm{ng} / \mathrm{ml})$ plus 50 -fold excess cold oligonucleotide; lane 4 , TNF $-\alpha(10 \mathrm{ng} / \mathrm{ml})$ plus 100-fold excess cold oligonucleotide. Only the bands marked A and $\mathrm{B}$ are specific. $D$, densiometry scanning of band A from the EMSA shown in $A$. This shows the reduction of an inducible isoform of NF- $\kappa \mathrm{B}$ by gliotoxin $(a=1 \mu \mathrm{g} / \mathrm{ml} ; b=0.1 \mu \mathrm{g} / \mathrm{ml})$, and further inhibition by co-treatment with TNF- $\alpha$ plus gliotoxin (b)

above results suggest that activation of an inducible form of $\mathrm{NF}-\kappa \mathrm{B}$ may inhibit or restrain the pro-apoptotic effects of TNF- $\alpha$ which are mediated by the parallel activation of the
TABLE I

The effect of $N F-\kappa B$ inhibitory peptides on human neutrophil apoptosis

Human neutrophils $\left(5 \times 10^{6} / \mathrm{ml}\right)$ were resuspended in Iscove's DMEM without autologous serum and treated with control buffer, SN50 (100 $\mu \mathrm{g} / \mathrm{ml})$, and SN50M $(100 \mu \mathrm{g} / \mathrm{ml})$. After $15 \mathrm{~min}$ at $37^{\circ} \mathrm{C}, 10 \%$ autologous serum (final concentration) was added, and after 6 and $20 \mathrm{~h}$ of culture, the cells were resuspended and cytocentrifuge preparations made. These were fixed and stained, and apoptosis was assessed morphologically. All values are from $n=$ three separate experiments, each performed in triplicate.

\begin{tabular}{lccc}
\hline \multicolumn{1}{c}{ Treatment } & Time & Mean & S.E. \\
\hline & & \% apoptosis \\
Control (buffer) & $6 \mathrm{~h}$ & 4.7 & 1.2 \\
SN50 (active) & $6 \mathrm{~h}$ & $15.0^{a}$ & 3.3 \\
SN50M (less-active) & $6 \mathrm{~h}$ & 6.8 & 5.6 \\
Control (buffer) & $20 \mathrm{~h}$ & 58.9 & 7.7 \\
SN50 (active) & $20 \mathrm{~h}$ & $81.4^{a}$ & 2.7 \\
SN50M (less-active) & $20 \mathrm{~h}$ & 70.3 & 8.2 \\
\hline
\end{tabular}

${ }^{a}$ Represents significance differences $(p<0.05)$ from the appropriate control.

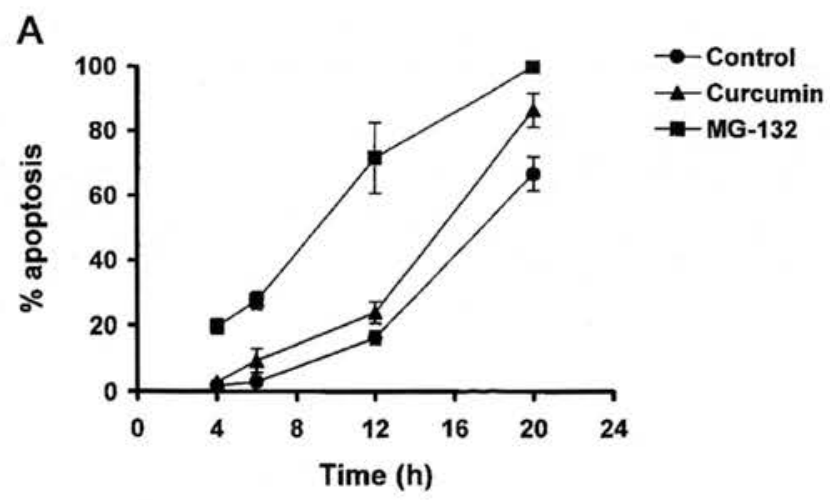

B

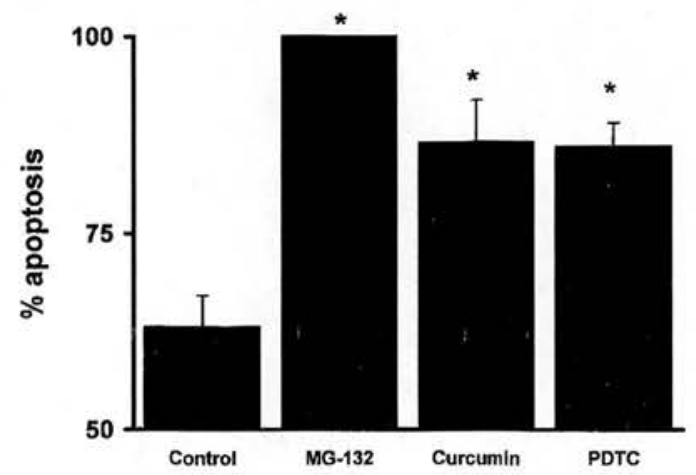

FIG. 7. Effect of other NF-kB inhibitors on neutrophil apoptosis. Human neutrophils $\left(5 \times 10^{6} / \mathrm{ml}\right)$ were cultured at $37^{\circ} \mathrm{C}$ in Iscove' DMEM containing $10 \%$ autologous serum and treated with the indicated reagent. $A$, neutrophils were treated with MG $132(20 \mu \mathrm{M})$ and curcumin $(20 \mu \mathrm{M})$ at the time periods indicated; $B$, neutrophils were treated with MG-132 $(100 \mu \mathrm{M})$, curcumin $(20 \mu \mathrm{M})$, and PDTC $(300 \mu \mathrm{M})$ for $20 \mathrm{~h}$. After incubation, the cells were resuspended and cytocentrifuge preparations made. These were fixed and stained, and apoptosis was assessed morphologically. All values represent mean \pm S.E. of $n=$ three to six experiments, each performed in triplicate. Where not shown, S.E. values are less than $2 \%$ of the mean.

caspase pathway. The possibility that this most likely reflects the production of a protein or proteins which act to suppress the activation of the caspase pathway and thus protect granulocytes from the cytotoxic effects of this cytokine was investigated by incubating neutrophils with TNF- $\alpha$ and cyclohexi- 

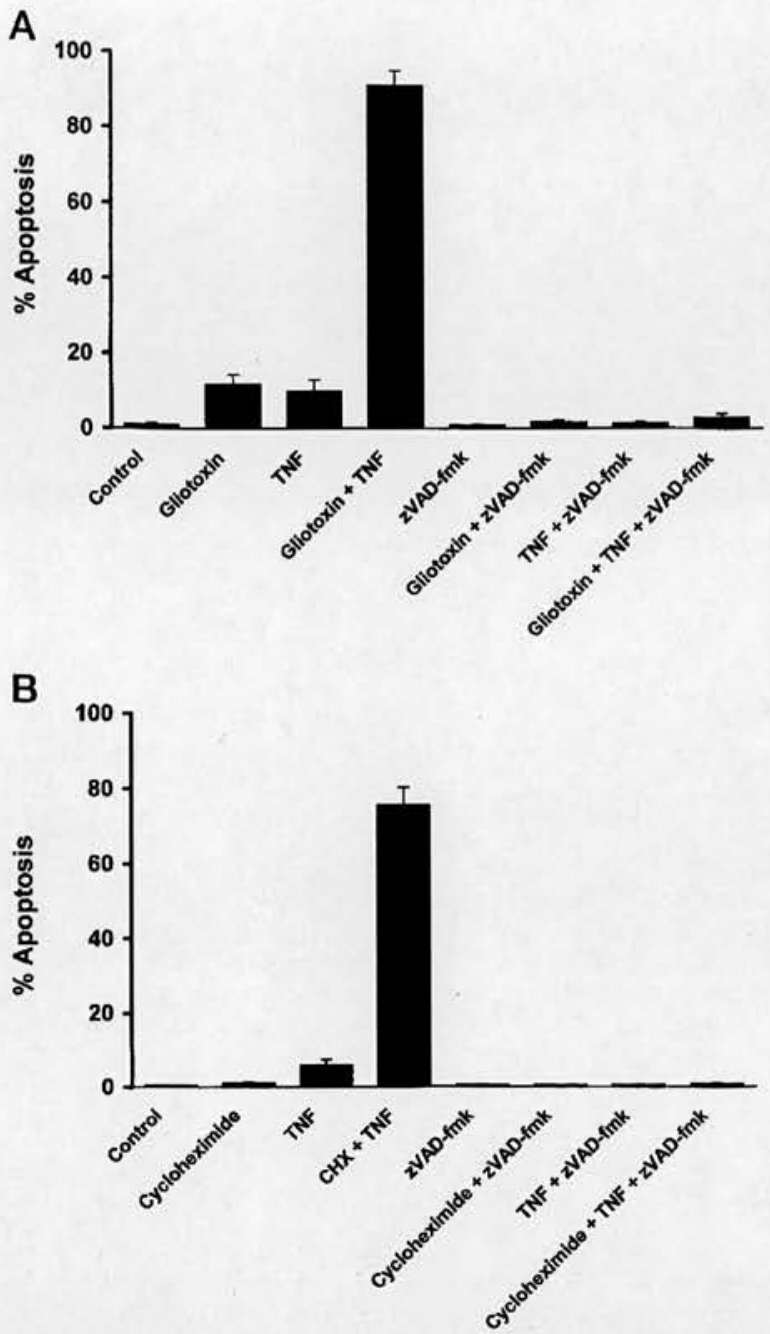

Fig. 8. Effect of the caspase inhibitor, zVAD-fmk, and the protein synthesis inhibitor, cycloheximide, on gliotoxin, TNF- $\alpha$, or gliotoxin plus TNF- $\alpha$-induced human neutrophil apoptosis. $\mathrm{Hu}$ man neutrophils $\left(5 \times 10^{6} / \mathrm{ml}\right)$ were cultured in DMEM containing $10 \%$ autologous serum and treated with the indicated reagents. After $2 \mathrm{~h}$ of culture, the cells were resuspended and cytocentrifuge preparation made. These were fixed and stained, and apoptosis was assessed morphologically. A, represents the effects of zVAD-fmk $(100 \mu \mathrm{M})$ on glio toxin $(0.1 \mu \mathrm{g} / \mathrm{ml})-$, TNF- $\alpha(10 \mathrm{ng} / \mathrm{ml})-$, or gliotoxin plus TNF- $\alpha$-induced neutrophil apoptosis. $B$, represents the effects of cycloheximide $(5 \mu \mathrm{M})$ on gliotoxin $(0.1 \mu \mathrm{g} / \mathrm{ml})$-, TNF- $\alpha(10 \mathrm{ng} / \mathrm{ml})$-, or gliotoxin plus TNF- $\alpha$ induced neutrophil apoptosis. All values represent mean \pm S.E. of $n=$ three experiments, each performed in triplicate. Where not shown, S.E. values are less than $2 \%$ of the mean.

mide, a protein synthesis inhibitor. As illustrated in Fig. $8 B$, cycloheximide used specifically at a concentration $(5 \mu \mathrm{M})$ that alone had almost no effect on neutrophil apoptosis at $2 \mathrm{~h}$, caused a synergistic increase in the level of apoptosis when neutrophils were co-cultured with TNF- $\alpha$. This supports the view that TNF- $\alpha$ treatment indeed results in the generation of a survival protein that protects these cells from the TNF receptor-caspase-dependent induction of apoptosis by TNF- $\alpha$.

\section{DISCUSSION}

We have demonstrated that gliotoxin, but not its inactive derivative methylthiogliotoxin, $(a)$ induces a direct time- and concentration-dependent increase in the rate of constitutive apoptosis in both neutrophils and eosinophils, (b) enhances the pro-apoptotic effect of TNF- $\alpha$ in neutrophils, and $(c)$ reveals the cytotoxic effects of TNF- $\alpha$ in eosinophils. In these studies extreme care was taken to ensure that gliotoxin, at all time points and concentrations studied, was non-toxic and caused genuine apoptosis that was indistinguishable from later constitutive apoptosis. The similar effects of gliotoxin in both neutrophils and eosinophils and the same concentration of gliotoxin $(0.1$ $\mu \mathrm{g} / \mathrm{ml}$ ) required for maximal enhancement of the pro-apoptotic effects of TNF- $\alpha$, suggests that an identical underlying mechanism is regulating the induction of cell death in both these cell types. However, although inhibition of basal NF- $\kappa$ B activity may be involved in neutrophil apoptosis when induced by gliotoxin alone (Fig. 1) since gliotoxin appears to block basal levels of NF- $\kappa \mathrm{B}$ activity (Fig. 6, $A$ and $D$ ), only the expression of the inducible NF- $\kappa \mathrm{B}$ isoform is down-regulated before the onset of cell death driven by the combined effects of TNF- $\alpha$ and gliotoxin (Fig. 6). Even in control neutrophils incubated for $20 \mathrm{~h}$, where the constitutive rate of apoptosis is approximately $70 \%$, the density of the constitutive NF- $\mathrm{kB}$ band was unaffected (data not shown). These differences in the inducible and constitutive forms of NF- $\kappa \mathrm{B}$ most likely reflects differential regulation of activation, for example, by the involvement of different isoforms of the inhibitory $1 \kappa \mathrm{B}$ subunit, or that the constitutively active NF- $\kappa \mathrm{B}$ is formed from a different set of dimers from the classical RelA/p50 heterodimer. It has recently been demonstrated that neutrophils contain c-Rel, p50, and p105 (the p50 precursor protein) as well as Rel-A $(22,41)$. The inducible band we observed has also been reported to be upregulated in neutrophils by phagocytosis of IgG opsonized yeast particles (42), and has been shown to consist mainly of Rel-A/ p50 heterodimers and possibly a small amount of c-Rel (42). In that study, phagocytosis of these particles did not affect the activity of the constitutive complex. In addition, it has recently been reported that $\mathrm{NF}-\kappa \mathrm{B}$ becomes activated, via a mechanisms not involving oxidant generation, when neutrophils phagocytose bacteria (43).

Because NF- $\kappa \mathrm{B}$ is also activated by certain pro-apoptotic stimuli such as TNF- $\alpha$, this transcription factor has been considered as a possible regulator of cell death. Hence, in some T cell clones, activation of NF- $\kappa \mathrm{B}$ appears to correlate with the onset of apoptosis (18). However, NF- $\kappa \mathrm{B}$ activation has clearly been shown to be anti-apoptotic in HT 1080 fibrosarcoma cells (21) and TNF- $\alpha$ induced NF- $\kappa$ B activation prevents cell death in HeLa and MCF7 cells (44). Here we show for the first time that in a non-transformed cell namely the neutrophil, inhibition of an inducible form of NF- $\kappa \mathrm{B}$ is related to the induction of apoptosis.

Several mechanisms, aside from NF- $\mathrm{B}$ inhibition, have been proposed for the pro-apoptotic actions of gliotoxin in other cells. Sutton et al. (45) have shown that although this fungal metabolite did not affect intracellular calcium levels, there was a correlation between increases in cAMP levels and apoptosis in gliotoxin-treated splenocytes. However, we and others have previously demonstrated that agents that elevate intracellular cAMP inhibit apoptosis in both neutrophils and eosinophils (33, 46). It has also been suggested that protein kinase A-dependent phosphorylation of histone H3 correlates with gliotoxin-induced apoptosis in thymocytes (47), but again in neutrophils, activation of protein kinase A inhibits apoptosis (33). Although gliotoxin has been reported to inhibit protein synthesis (48) it is highly unlikely that this mechanism is directly responsible for its pro-apoptotic effects: first, gliotoxin induces apoptosis in thymocytes whereas inhibition of protein synthesis by cycloheximide inhibits thymocyte apoptosis. Second, since NF- $\kappa$ B activation is involved in the control of multiple genes, many of which encode for inflammatory mediator synthesis, inactivation of NF- $\kappa \mathrm{B}$ would therefore be expected to inhibit protein 
synthesis. Third, while protein synthesis inhibitors do up-regulate the rate of constitutive cell death in granulocytes, the kinetics of this response are very different to those observed with gliotoxin. For example, Whyte et al. (49) have reported that cycloheximide $(50 \mu \mathrm{M})$ and actinomycin D $(1 \mu \mathrm{M})$ induce apoptosis in approximately $30 \%$ of neutrophils by $6 \mathrm{~h}$. In our experiments, gliotoxin $(0.1 \mu \mathrm{g} / \mathrm{ml})$, induces a rate of almost twice this, whereas $1.0 \mu \mathrm{g} / \mathrm{ml}$ gliotoxin induced $100 \%$ neutrophil apoptosis by this time point (see Fig. 1A). Likewise, our own results with cycloheximide indicate that protein synthesis inhibition alone does not affect the rate of neutrophil apoptosis at $2 \mathrm{~h}$ whereas gliotoxin alone produced almost $15 \%$ apoptosis over this period (Figs. $1 A$ and $8 B$ ). While gliotoxin inhibits $\mathrm{NF}-\kappa \mathrm{B}$, cycloheximide and actinomycin $\mathrm{D}$ have been shown in several systems to activate this transcription factor $(50,51)$. Although both cycloheximide and gliotoxin give a similar synergistic pro-apoptotic response with TNF- $\alpha$, this suggests that different mechanisms must be involved. However, while gliotoxin may prevent synthesis of a protective protein inducible by NF- $\kappa \mathrm{B}$ activation, cycloheximide would also preclude synthesis of such a protein so that in both cases the cells would be sensitive to the pro-apoptotic effects of TNF- $\alpha$. It is of interest to note that granulocytes do have the capacity to synthesize proteins, albeit in a limited capacity (49). We believe that this synthetic capacity will be directed toward resolution of the inflammatory response with the generation of protein(s) that affect the apoptotic program of inflammatory cells.

Our results indicate that the inducible isoform of NF- $\mathrm{BB}$ disappears from the gliotoxin-treated granulocyte nucleus just before the onset of stimulated apoptosis. The possibility that these events are causally related is supported by the following observations: (i) the synthetic cell-permeable peptide SN50 (37), a known inhibitor of NF- $\mathrm{BB}$, also induces apoptosis in neutrophils and eosinophils; (ii) other agents that inhibit NF-kB activation, namely PDTC and curcumin as well as the proteasome inhibitor MG-132 also cause an induction of granulocyte apoptosis; (iii) the kinetics for gliotoxin-mediated inhibition of NF- $\kappa \mathrm{B}$ match those for the onset of induction of apoptosis; (iv) LPS which stimulates NF- $\mathrm{kB}$ activity prolongs neutrophil and eosinophil survival; and (v) that gliotoxin sensitizes both neutrophils and eosinophils to the pro-apoptotic effects of TNF- $\alpha$. Indeed, our studies provide the first plausible explanation for the modest and temporally constrained apoptotic response of neutrophils to TNF- $\alpha$ and the observation that pretreatment with LPS, PAF or granulocyte/macrophagecolony stimulating factor, abolishes the cytotoxic effect of this cytokine (10). Indeed, this latter point is of particular relevance when investigating the pro-apoptotic effect of TNF- $\alpha$ in neutrophils since pre-treatment of these cells causes a rapid decrease of both TNF- $\alpha$ receptors subtypes from the surface membrane (10). This phenomenon, together with the fact that the effects of SN50 are, at best, modest due to limited access of the peptide to its intracellular target (37) and the requirement for pretreatment with the peptide, precluded accurate assessment of SN50 on TNF- $\alpha$ induced apoptosis in neutrophils.

When neutrophils were co-cultured with LPS and gliotoxin, gliotoxin failed to render LPS pro-apoptotic despite the fact that LPS induced survival was inhibited by gliotoxin (Fig. 4). These results suggest that LPS does not trigger a death pathway in neutrophils but stimulates a NF- $\kappa \mathrm{B}$-mediated survival pathway i.e. when NF- $\kappa \mathrm{B}$ activation is blocked, LPS is no longer capable of delaying apoptosis. The precise intracellular mechanisms by which the NF- $\kappa$ B inhibitors used in this study induce apoptosis are unknown and is the subject of further investigation. For example, it would be of interest to perform an in-depth analyses of the effect of gliotoxin and the other agents on the degradation of the inhibitory subunit $\mathrm{I}_{\kappa} \mathrm{B}$, especially since $\mathrm{Pahl}$ et al. (28) reported that gliotoxin appeared to prevent $I_{\kappa} B$ degradation rather than mediate its effect at the level of DNA binding.

In a number of immune cells NF- $\kappa \mathrm{B}$ activation by agents such as TNF- $\alpha$ has been shown to play a central role in regulating the genes for inflammatory cytokines such as granulocyte/macrophage-colony stimulating factor and TNF- $\alpha$ itself (14). The importance of this response in vivo is that many of these factors inhibit granulocyte apoptosis and may therefore delay inflammatory resolution by enhancing the longevity of these cells. Indeed, a positive-feedback loop may exist since many of these inflammatory mediators which protect against apoptosis in neutrophils and eosinophils also activate NF- $\kappa \mathrm{B}$ (7). Conversely, we have recently shown that NO, a known inhibitor of NF- $\kappa \mathrm{B}(52,53)$, is a potent inducer of apoptosis in neutrophils (54). Our current results suggest that the activation of an inducible form of NF- $\kappa$ B represents a powerful survival mechanism in granulocytes, and that when this pathway is inhibited, in both neutrophils and eosinophils, these cells undergo a greatly augmented rate of apoptotic cell death. It is possible that $\mathrm{NF}-\kappa \mathrm{B}$ performs a similar function in other cell types that undergo apoptosis in response to gliotoxin.

Enhanced cytotoxic responses to TNF- $\alpha$ have also been demonstrated in cells where NF- $\kappa$ B is genetically deficient or inactivated (19-21) and hepatocytes from Rel-A null mice are known to undergo apoptosis causing death in utero (55). Embryonic fibroblasts and macrophages from Rel-A-deficient mice also showed dramatic loss of viability when treated with TNF- $\alpha$ leading to the suggestion that Rel-A regulates a protective mechanism against the cytotoxic effects of TNF- $\alpha$. It would be of interest to investigate the effects of TNF- $\alpha$ and gliotoxin on granulocytes isolated from mice deficient in $\operatorname{Rel~A;~it~would~be~}$ reasonable to predict that TNF- $\alpha$ will induce a rapid cell death and gliotoxin and other NF- $\mathrm{NB}$ inhibitors would not have a dramatic effect on the rate of granulocyte apoptosis. Although our experiments indicate that gliotoxin does not inhibit the constitutive form of NF- $\kappa \mathrm{B}$, at least at early time points, it does inhibit the activation of an inducible isoform of NF- $\kappa \mathrm{B}$, which most likely consists of heterodimers containing the Rel-A/p65 protein and therefore could perform a similar anti-apoptotic function in neutrophils and eosinophils. While in our hands TNF- $\alpha$ does not produce significant cytotoxic effects in eosinophils, co-treatment with gliotoxin caused these cells to become highly responsive to this cytokine producing greatly increased levels of apoptosis. This suggests that both of these inflammatory cell types could be stimulated to undergo apoptosis and hence be cleared rapidly by phagocytes at sites of inflammation if activation of the inducible NF- $\kappa \mathrm{B}$ isoform were inhibited.

The mechanism whereby inactivation of $\mathrm{NF}-\mathrm{\kappa B}$ induces granulocyte apoptosis and increases the cytotoxic response to TNF- $\alpha$ is currently unclear. Since gliotoxin and TNF- $\alpha$ driven apoptosis are both inhibited by the caspase-inhibitor zVADfmk, this, together with the synergy for apoptosis observed with these agents, implies that NF- $\kappa$ B or an NF- $\kappa$ B regulated step influences granulocyte apoptosis at an intermediate step between the TNF- $\alpha$ receptor and caspase activation. The possibility that NF- $\kappa \mathrm{B}$ controls the transcriptional activity of a gene(s) which induces the synthesis of survival proteins is supported by the observation that cycloheximide also increases apoptosis in granulocytes (49). This suggests a strong link between inducible NF- $\kappa \mathrm{B}$ activation and the control of TNF- $\alpha$ induced apoptosis, possibly via the production of a protein inhibitor of this pathway. Indeed, as we have shown, protein synthesis inhibition enhances the pro-apoptotic effect of TNF- $\alpha$ as early as $2 \mathrm{~h}$ of culture. Indeed, one possible candidate for this 
protein has already been suggested: A20, a protein induced by TNF- $\alpha$ activation of NF- $\kappa \mathrm{B}(56,57)$, has been shown to protect against TNF- $\alpha$ induced cell death by acting at the level of the TNF- $\alpha$ receptor-associated proteins TRAF-1 and TRAF2 (58). Although A20 has yet to be demonstrated in neutrophils or eosinophils, this would represent an attractive candidate protein to fulfill such a role.

The ability of gliotoxin to enhance the cytotoxic effects of TNF- $\alpha$ and itself produce a rapid onset of apoptosis in inflammatory cells such as neutrophils and eosinophils may suggest $\mathrm{NF}-\kappa \mathrm{B}$ inhibition as a logical therapeutic target in the treatment of inflammatory conditions. In a rat model of lung inflammation, suppression of NF- $\kappa$ B activity has already been shown to block the development of neutrophil lung inflammation by inhibiting the synthesis of chemotaxins (59). Our results suggest that NF- $\kappa$ B inhibition may also be of benefit in enhancing the resolution of inflammation by allowing a more rapid clearance of granulocytes. We therefore propose that granulocyte apoptosis is regulated by an inducible form of the transcription factor NF- $\mathrm{KB}$ and suggest that inhibition of this transcription factor may be exploited for therapeutic benefit in inflammatory conditions where granulocytes play a prominent role.

\section{REFERENCES}

1. Savill, J. S., Wyllie, A. H., Henson, J. E., Walport, M. J., Henson, P. M., and Haslett, C. (1989) J. Clin. Invest. 83, 865-875

2. Stern, M., Meagher, L., Savill, J., and Haslett, C. (1992) J. Immunol. 148, 3543-3549

3. Whyte, M. K. B., Meagher, L. C., MacDermot, J., and Haslett, C. (1993) J. Immunol. 150, 5124-5134

4. Stern, M., Savill, J. S., and Haslett, C. (1996) Am. J. Pathol. 149, 911-921

5. Her, E., Frazer, J., Austen, F., and Owen, W. F., Jr. (1991) J. Clin. Invest. 88, 1982-1987

6. Colotta, F., Re, F., Polentarutti, N., Sozzani, S., and Mantovani, A. (1992) Blood 80, 2012-2020

7. Lee, A., Whyte, M. K. B., and Haslett, C. (1993) J. Leukocyte Biol. 54, 283-288 8. Takanaski, S., Nonaka, R, Xing, X., O'Byrne, P., Dolovich, J., and Jordanna, M. (1994) J. Exp. Med. 180, 711-715

9. Meagher, L. C., Cousin, J. M., Seckl, J. R., and Haslett, C. (1996) J. Immunol. 156, 4422-4428

10. Murray, J., Barbara, J. A. J., Dunkley, S. A., Lopez, A. F., Van Ostade, X. Condliffe, A. M., Dransfield, I., Haslett, C., and Chilvers, E. R. (1997) Blood 90, 2772-2783

11. Henkel, T., Machleidt, T., Alkalay, I., Kronke, M., Ben-Neriah, Y., and Baeuerle, P. A. (1993) Nature 365, 182-185

12. Traenckner, E. B., Wilk, S., and Baeuerle, P. A. (1994) EMBO J. 13, 5433-5441

13. Finco, T. S., Beg, A. A., and Baldwin, A. S., Jr. (1994) Proc. Natl. Acad. Sci. U. S. A. 91, $11884-11888$

14. Baeuerle, P. A., and Baichwal, V. R. (1994) Adv. Immunol. 62, 1192-1198

15. May, M. J., and Ghosh, S. (1998) Immunol. Today 19, 80-88

16. Baldwin, A., Jr. (1996) Annu. Rev. Immunol, 14, 649-681

17. Wu, M., Lee, H., Bellas, R. E., Schauer, S. L., Arsura, M., Katz, D., FitzGerald, M. J., Rothstein, T. L., Sherr, D. H., and Sonenshein, G. E. (1996) EMBO J. $15,4682-4690$

18. Grimm, S. Bauer, M. K. A., Baeuerle, P. A., and Schulze-Osthoff, K. (1996) J. Cell Biol. 134, 13-23

19. Beg, A. A., and Baltimore, D. (1996) Science 274, 782-784

20. Van Antwerp, D. J., Martin, S. J., Kafri, T., Green, D. R., and Verma, I. M. (1996) Science 274, 787-789
21. Wang, C., Mayo, M. W., and Baldwin, A. S. (1996) Science 274, 784-787

22. McDonald, P. P., Bald, A., and Cassatella, A. A. (1997) Blood 89, 3421-3433

23. Browning, D. D. Pan, Z K. Prossnitz, E. R. and Ye, R. D. (1997) J. Biol. Chem. 272, 7995-8001

24. Braithwaite, A. W., Eichner, R. D., Waring, P., and Müllbacher, A. (1987) Mol. Immunol. 24, 47-55

25. Ozaki, K., Takeda, H., Iwahashi, H., Kitano, S., and Hanazawa, S. (1997 FEBS Lett. 410, 297-300

26. Waring, P., Eichner, R. D., Müllbacher, A., and Sjaarda, A. (1988) J. Biol. Chem. 263, 18493-18499

27. Sutton, P., Newcombe, N. R., Waring, P., and Müllbacher, A. (1994) Infect. Immunol, 62, 1192-1198

28. Pahl, H. L., Krauß, B., Schulze-Osthoff, K., Decker, T., Traenckner, E. B., Vogt, M., Myers, C., Parks, T., Warring, P., Mühlbacher, A., Czernilofsky, A-P., and Baeuerle, P. A. (1996) J. Exp. Med. 183, 1829-1840

29. Haslett, C., Guthrie, L. A., Kopaniak, M. M., Johnston, R. B., Jr., and Henson, P. M. (1985) Am. J. Pathol. 119, 101-110

30. Dignam, J. D., Lebovitz, R. M., and Roeder, R. G. (1983) Nucleic Acids Res. 11, $1475-1489$

31. Whyte, M. K., Hardwick, S. J., Meagher, L. C., Savill, J. S., and Haslett, C. (1993) J. Clin. Invest, 92, 446-455

32. Cousin, J. M., Haslett C., and Rossi A. G. (1997) Biochem. Soc. Trans. 25, 243 33. Rossi, A. G., Cousin J. M., Dransfield I., Lawson M. F., Chilvers E. R., and Haslett C. (1995) Biochem. Biophys. Res. Commun. 271, 892-899

34. Hannah, S., Mecklenburgh, K., Rahman, I., Bellingan, G. J., Greening, A. Haslett, C., and Chilvers, E. R. (1995) FEBS Lett. 372, 233-237

35. Hachiya, O., Takeda, Y., Miyata, H., Watanabe, H., Yamashita, T., and Sendo, F. (1995) Microbiol. Immunol. 39, 715-723

36. Ward, C., Murray, J., Bruce, L., Farrow, S., Chilvers, E. R., Hannah, S. Haslett, C., and Rossi, A. G. (1997) Biochem. Soc. Trans. 25, 245S

37. Lin, Y., Yao, S., Veach, R. A., Torgerson, T. R., and Hawiger, J. (1995) J. Biol. Chem. 270, 14255-14258

38. Adams, J., and Stein, R. (1996) Annu. Rep. Med. Chem. 31, 279-287

39. Singh, S., and Aggarwal, B. B. (1995) J. Biol Chem. 270, 24995-25000

40. Schreck, R., Meier, B., Mannel, D. N., Droge, W., and Baeuerle, P. A. (1992) J. Exp. Med. 175, 1181-1194

41. Druker, B. J., Neumann, M., Okuda, K., Franza, B. R, and Griffin, J. D. (1994) J. Biol. Chem. 269, 5387-5390

42. MacDonald, P. P., and Cassatella, M. A. (1997) FEBS Lett. 412, 583-586

43. Vollebregt, M., Hampton, M. B., and Winterbourn, C. C. (1998) FEBS Lett. 432, $40-44$

44. Lui, Z., Hsu, H., Goeddel, D. V., and Karin, M. (1996) Cell 87, 565-576

45. Sutton, P., Beaver J., and Waring, P. (1995) Biochem. Pharmacol. 50, 2009-2014

46. Hallsworth, M. P., Giembycz, M. A., Barnes, P. J., and Lee, T. H. (1996) Br. J. Pharmacol. 117, 79-86

47. Waring, P., Khan, T., and Sjaarda, A. (1997) J. Biol. Chem. 272, 17929-17936 48. Waring, P. (1990) J. Biol. Chem. 265, 14476-14480

49. Whyte, M. K. B., Savill, J., Meagher, L. C., Lee, A., and Haslett, C. (1997) J. Leukocyte Biol. 62, 195-202

50. Newton, R., Adcock, I. M., and Barnes, P. J. (1996) Biochem. Biophy. Res. Commun. 218, 518-523

51. Faggioli, L., Costanzo, C., Merola, M., Furia, A., and Palmieri, M. (1997) Biochem. Biophy. Res. Commun. 233, 507-513

52. Matthews, J. R., Botting, C. H., Panico, M., Morris, H. R., and Hay, R. T. (1997) Nucleic Acids Res. 24, 2236-2242

53. Peng, H., Libby, P., and Liao, J. K. (1995) J. Biol. Chem. 270, 14214-14219 54. Wong, T., Rossi, A. G., and Chilvers, E. R. (1997) J. Clin. Sci. 92, 7

55. Beg, A. A., Sha, W. C., Bronson, R. T., Ghosh, S., and Baltimore, D. (1995) Nature 376, 167-170

56. Krikos, A., Laherty, C. D., and Dixit, V. M. (1992) J. Biol. Chem. 267, 17971-17976

57. Opipari, A. W., Hu., H. M., Yabkowitz, R., and Dixit, V. M. (1992) J. Biol. Chem. 267, 12424-12427

58. Song, H. Y., Rothe, M., and Goeddel, D. V. (1996) Biochemistry 93, 6721-6725 59. Blackwell, T. S., Blackwell, T., Holden, E. P., Christman, B. W., and Christman, J. W. (1996) J. Immunol. 157, 1630-1673 


\title{
Glucocorticoids Promote Nonphlogistic Phagocytosis of Apoptotic Leukocytes ${ }^{1}$
}

\author{
Yuqing Liu, ${ }^{2 *}$ Joanne M. Cousin, ${ }^{2 \dagger}$ Jeremy Hughes, ${ }^{*}$ Jo Van Damme, ${ }^{\ddagger}$ Jonathan R. Seckl, ${ }^{\S}$ \\ Christopher Haslett, ${ }^{\dagger}$ Ian Dransfield, ${ }^{\dagger}$ John Savill, $^{3 *}$ and Adriano G. Rossi ${ }^{\dagger}$
}

Phagocyte recognition, uptake, and nonphlogistic degradation of neutrophils and other leukocytes undergoing apoptosis promote the resolution of inflammation. This study assessed the effects of anti-inflammatory glucocorticoids on this leukocyte clearance mechanism. Pretreatment of "semimature" 5-day human monocyte-derived macrophages (M $\phi$ ) for 24 h with methylprednisolone, dexamethasone, and hydrocortisone, but not the nonglucocorticoid steroids aldosterone, estradiol, and progesterone, potentiated phagocytosis of apoptotic neutrophils. These effects were specific in that the potentiated phagocytosis of apoptotic neutrophils was completely blocked by the glucocorticoid receptor antagonist RU38486, and glucocorticoids did not promote 5-day M $\phi$ ingestion of opsonized erythrocytes. Similar glucocorticoid-mediated potentiation was observed with 5-day $M \phi$ uptake of alternative apoptotic "targets" (eosinophils and Jurkat $\mathbf{T}$ cells) and in uptake of apoptotic neutrophils by alternative phagocytes (human glomerular mesangial cells and murine $\mathrm{M} \phi$ elicited into the peritoneum or derived from bone marrow). Importantly, methylprednisolone-mediated enhancement of the uptake of apoptotic neutrophils did not trigger the release of the chemokines IL-8 and monocyte chemoattractant protein-1. Furthermore, longer-term potentiation by methylprednisolone was observed in maturing human monocyte-derived $\mathbf{M} \phi$, with greater increases in 5-day $\mathbf{M} \phi$ uptake of apoptotic cells being observed the earlier glucocorticoids were added during monocyte maturation into $\mathrm{M} \phi$. We conclude that potentiation of nonphlogistic clearance of apoptotic leukocytes by phagocytes is a hitherto unrecognized property of glucocorticoids that has potential implications for therapies aimed at promoting the resolution of inflammatory diseases. The Journal of Immunology, 1999, 162: 3639-3646.

I nflammatory responses, which evolved to eliminate invading microorganisms and repair damaged tissues, become undesirably persistent in a number of disease states. Although there is strong evidence that leukocytes can mediate tissue injury in inflammatory disorders (1), little is known of the mechanisms that promote the resolution of inflammation by eliminating leukocytes from tissues. An important factor in the successful resolution of inflammation is the recognition, uptake, and degradation by phagocytes of intact leukocytes undergoing deletion by apoptosis (2-9). Not only are tissues protected from the noxious contents of leukocytes, but apoptotic cells are also rapidly phagocytosed and degraded by professional phagocytes (macrophages $(M \phi)^{4}$ ) and by semiprofessional phagocytes (e.g., glomerular mesangial cells, fi-

*Division of Renal and Inflammatory Disease, School of Medical and Surgical Sciences, University Hospital, Nottingham, United Kingdom; 'Rayne Laboratory, Respiratory Medicine Unit, University of Edinburgh Medical School, Edinburgh, United Kingdom; ' ${ }^{\dagger}$ Katholieke Universiteit Leuven, Rega Institute, Leuven, Belgium; and

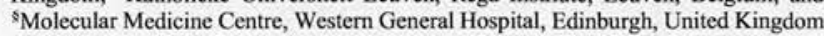
Received for publication August 5, 1998. Accepted for publication December $15,1998$.

The costs of publication of this article were defrayed in part by the payment of page charges. This article must therefore be hereby marked advertisement in accordance with 18 U.S.C. Section 1734 solely to indicate this fact.

${ }^{3}$ This work was supported by the Wellcome Trust ( 047273 and 039108$)$, the Medical Research Council (G9016491), and the University of Nottingham. Y.L. was supported by the National Kidney Research Fund, J.M.C. was supported by the University of Edinburgh, and A.G.R. was supported by the Medical Research Council.

${ }^{2}$ Y.L. and J.M.C. contributed equally to this work and are joint first authors.

${ }^{3}$ Address correspondence and reprint requests to Prof. John Savill, Department of Medicine, Royal Infirmary of Edinburgh, Lauriston Place, Edinburgh HE3 9YW, U.K. E-mail address: J.Savill@ed.ac.uk

${ }^{4}$ Abbreviations used in this paper: $\mathrm{M} \phi$, macrophage(s); EIgG, IgG-opsonized E; MPO, myeloperoxidase; PMNs, polymorphonuclear leukocytes; MCP, monocyte chemoattractant protein. broblasts) without inciting proinflammatory secretory responses $(6,10-12)$.

Although several molecular pathways by which phagocytes recognize apoptotic cells have been identified (reviewed in Refs. 13 and 14), the mechanisms that control phagocytic capacity for the clearance of apoptotic cells are less well understood. The modulation of phagocyte capacity for apoptotic cell clearance represents a potential therapeutic target in the control of inflammatory disease. Although CD44-mediated increases in the M $\phi$ uptake of apoptotic neutrophils may hold some therapeutic promise (15), it is unlikely that there is clinical utility in the observation that proinflammatory cytokines promote $\mathrm{M} \phi$ ingestion of apoptotic cells (16). Therefore, we sought to investigate further the regulatory mechanisms that might control phagocyte clearance of leukocytes undergoing apoptosis.

It is well established that glucocorticoids are powerful antiinflammatory agents that suppress many phlogistic responses including inflammatory cell recruitment and activation (17-20). However, there have been relatively few studies of the effects of these steroids on the resolution phase of inflammation. We and others have shown that glucocorticoids delay constitutive apoptosis in neutrophils, whereas eosinophil apoptosis is accelerated (21-23), providing an attractive explanation for the therapeutic efficacy of glucocorticoids in eosinophilic inflammation. However, implicit in this observation is the need for nonphlogistic clearance of an increased tissue load of apoptotic eosinophils, implying that glucocorticoids might also upregulate the phagocytic capacity for apoptotic granulocytes so that greater numbers are safely removed. Moreover, although glucocorticoids delay neutrophil apoptosis, these agents are effective in suppressing inflammatory responses characterized by intense infiltration of tissues with neutrophils (24) that will eventually undergo apoptosis, implying that glucocorticoids may also up-regulate the phagocyte capacity to clear apoptotic neutrophils. 
To test the hypothesis that glucocorticoids potentiate phagocyte capacity for the nonphlogistic clearance of apoptotic leukocytes, we have undertaken the first study of the effects of glucocorticoids upon this process, which is important in the resolution of inflammation. We report that glucocorticoids specifically promote safe clearance by various phagocyte types of apoptotic leukocytes from different lineages, establishing that glucocorticoids have hitherto unrecognized but beneficial regulatory effects upon phagocytes, which may promote the safe termination of inflammatory responses.

\section{Materials and Methods Materials}

All reagents were obtained from Sigma (St. Louis, MO) unless otherwise stated. Culture media (Iscove's modified DMEM, RPMI 1640, and HBSS) and supplements $(100 \mathrm{U} / \mathrm{ml}$ penicillin, $10058 / \mathrm{ml}$ streptomycin, $2 \mathrm{mM}$ glutamine, and FCS) were obtained from Life Technologies Laboratories (Paisley, U.K.). Brewer's thioglycollate medium was obtained from Difco (Detroit, MI). Methylprednisolone was obtained from Upjohn (Birmingham, U.K.); dexamethasone, hydrocortisone, aldosterone, progesterone, and estradiol were obtained from Sigma (Poole, U.K.). RU38486 was kindly provided by Dr. Ian Hall (University of Nottingham). Percoll was obtained from Pharmacia Fine Clienicals (Piscataway, NJ). Sterile tissue culture plasticware was obtained from Falcon Plastics (Cockeysville, MD).

\section{Granulocyte isolation and induction of apoptosis}

Human neutrophils ( $>98 \%$ pure on May-Giemsa-stained cytopreparations) were isolated from fresh, citrated blood of healthy volunteers by dextran sedimentation and discontinuous plasma-Percoll (Pharmacia Fine Chemicals) density gradient centrifugation $(2,25)$. The neutrophils were aged in tissue culture at $37^{\circ} \mathrm{C}$ and $5 \% \mathrm{CO}_{2}$ for $24 \mathrm{~h}$ in Iscove's DMEM with $10 \%$ autologous platelet-rich, plasma-derived serum to undergo apoptosis verified by typical morphology (2). Only aged neutrophils with a viability (assessed by trypan blue dye exclusion) $>98 \%$ were used.

Human eosinophils (95-98\% pure on May-Giemsa-stained cytopreparations) were isolated from the blood of mildly eosinophilic but healthy human volunteers by using a combination of discontinuous plasma-Percoll density gradient centrifugation to obtain eosinophil-enriched granulocyte populations followed by immunomagnetic depletion of neutrophils using $3 \mathrm{G} 8 \mathrm{CD} 16 \mathrm{mAb}$, essentially as described $(6,26)$. Purified eosinophils were then cultured for up to $72 \mathrm{~h}$ in conditions identical to those for neutrophils to undergo apoptosis verified by typical morphology (26). Only aged eosinophils with viability $>98 \%$ by trypan blue dye exclusion were used.

\section{Preparation of human monocyte-derived $M \phi$}

Human monocytes were prepared by well-established methods from the mixed mononuclear cell band of the discontinuous plasma-Percoll gradients used to prepare neutrophils (2). Mononuclear cells were suspended in Iscove's DMEM at $4 \times 10^{6} / \mathrm{ml}$, and $100 \mu \mathrm{l}$ was added to each well of a 96-well plate, which was then incubated at $37^{\circ}$ for $1 \mathrm{~h}$. Nonadherent cells, including contaminating lymphocytes, were then washed off, and adherent monocytes were cultured in Iscove's DMEM with $10 \%$ autologous platelet-rich, plasma-derived serum for 5 days to mature into monocyte-derived $\mathrm{M} \phi$.

\section{Preparation of murine $M \phi$ populations}

Ten-week-old BALB/c mice were purchased from the University of Nottingham Biomedical Facility and were housed and treated under U.K. Home Office-approved conditions. Thioglycollate-elicited inflammatory peritoneal $\mathrm{M} \phi$ and bone marrow-derived $\mathrm{M} \phi$ were prepared as described (27). Briefly, inflammatory peritoneal exudate $\mathrm{M} \phi$ were elicited into the peritoneal cavity of 10 -wk-old female BALB/c mice by injection of $3 \%$ Brewer's thioglycollate and harvested 5 days later after humane killing by peritoneal lavage with DMEM medium alone. Exudate cells were adhered to 96 -well plates for $1 \mathrm{~h}$ at $0.1 \times 10^{6} \mathrm{cell} / \mathrm{well}$, and nonadherent cells were washed off and replaced with DMEM containing 10\% FCS plus supplements as above. Peritoneal $\mathrm{M} \phi$ were used within $48 \mathrm{~h}$ after isolation. Bone marrow-derived $\mathrm{M} \phi$ were isolated from femurs of humanely killed mice, cut at both ends, and the bone marrow expelled by flushing with a 25 -gauge needle with DMEM with $10 \%$ FCS supplements as above and $10 \%$ conditioned medium from L929 cells as a source of monocyte-CSF. M $\phi$ were employed after 7-10 days culture.

\section{Culture of human mesangial cells}

Mesangial cells were prepared using standard methods of serial culture/ trypsinization in tissue culture flasks of adherent outgrowth cells from glomeruli obtained by sieving diced human normal renal cortex as described previously $(3,11)$. Cells were cultured in RPMI 1640 medium with $10 \%$ FCS and $5 \mathrm{ml} / 500 \mathrm{ml}$ of insulin/selenium/transferrin growth supplement from Life Technologies Laboratories (Grand Island, NY) and were used between passages four and six after subculture into 96-well plates. Great care was taken to verify the purity and phenotype of mesangial cells, as previously described $(3,11)$. Cells were uniformly smooth muscle actinpositive and CD45-negative (by immunofluorescence). Cells did not take up acetylated low-density lipoprotein or opsonized zymosan particles (excluding $\mathrm{M} \phi$ contamination) and were cytokeratin and factor VIII-related Ag-negative (by immunofluorescence), excluding contamination with glomerular epithelial or endothelial cells.

\section{Culture of Jurkat $T$ cells and induction of apoptosis}

Cells of the Jurkat T cell line (a gift from C. Gregory, University of Nottingham) were grown in suspension culture in RPMI 1640 with 5\% FCS and supplements. To induce morphologically verified apoptosis, cells were deprived of serum with cycloheximide at $50 \mu \mathrm{g} / \mathrm{ml}$ for $4 \mathrm{~h}$ before being washed and employed in interaction assays with $M \phi$.

\section{Interaction assays}

A microscopically quantified phagocytic assay of $\mathrm{M} \phi$ phagocytosis of aged polymorphonuclear leukocytes (PMNs) was used, which has been described and illustrated in detail before $(2,28)$. Minor adaptations for $\mathrm{M} \phi$ cultured in 96-well plates have been described (16). Briefly, PMNs aged for $24 \mathrm{~h}$ in culture, to undergo apoptosis, were washed once in HBSS, suspended in Iscove's DMEM, and $0.5 \times 10^{6}$ aged PMNs in $50 \mu \mathrm{l}$ of medium were added to each washed well of $\mathrm{M} \phi$. After interaction for 30 min at $37^{\circ} \mathrm{C}$ in $5 \% \mathrm{CO}_{2}$ atmosphere, the wells were washed in saline at $4^{\circ} \mathrm{C}$ to remove noningested apoptotic PMNs, fixed with $2 \%$ glutaraldehyde in $0.9 \%$ saline, stained for myeloperoxidase (MPO; present in PMNs but not $\mathrm{M} \phi)$ using hydrogen peroxide and dimethoxybenzidine as substrate as previously described $(2,29)$, and then the proportion of $M \phi$ ingesting neutrophils were counted by inverted light microscopy, exactly as described $(2,28)$. Because it was also important to determine whether the phagocytic capacity of individual $\mathrm{M} \phi$ had been increased, the number of PMNs within 100 randomly selected $\mathrm{M} \phi$ in each well was counted in some experiments. These counts were possible because the duration of the phagocytic assay was sufficiently short for ingested PMNs to remain intact $(2,28,29)$. The $\mathrm{M} \phi$ uptake of aged apoptotic eosinophils, $0.1 \times 10^{6}$ in $50 \mu \mathrm{l}$ medium for each well of $\mathrm{M} \phi$ or of IgG-opsonized human erythrocytes (EIgG), $0.1 \times$ $10^{6}$ in $50 \mu \mathrm{l}$ medium for each well of $\mathrm{M} \phi$, was determined by similar means, as described $(26,28)$, because these cell types also stain for peroxidase. The uptake of apoptotic Jurkat T cells was assessed by staining with Haemalum (BDH, Poole, U.K.), as described (4).

To quantify mesangial cell phagocytosis of apoptotic PMNs, we used a previously described, reproducible, microscopically quantified assay in which the uptake of apoptotic PMN was shown by electron microscopy and susceptibility to inhibition by colchicine to be a result of active phagocytosis (3). Culture medium was aspirated from the mesangial cells, and 50 $\mu \mathrm{l}$ of a $20 \times 10^{6} / \mathrm{ml}$ suspension of neutrophils in RPMI 1640 with $10 \%$ FCS was added to each well together with $50 \mu$ l of Iscove's DMEM. The two cell types were cocultured in $5 \% \mathrm{CO}_{2}$ at $37^{\circ} \mathrm{C}$ for $3 \mathrm{~h}$, and the interaction was then stopped by the addition of cold normal saline and the removal of nonadherent cells by manual washing with a Pasteur pipette. The mesangial cell monolayer was then trypsinized, and a separate cytocentrifuge preparation was prepared for each well. These were fixed with $2 \%$ glutaraldehyde and stained for MPO and finally counterstained with Haemalum. The proportion of mesangial cells containing brown MPOpositive globules wholly within the outline of the cell was then counted by light microscopy with a minimum of 500 cells per slide being counted.

\section{Effect of steroid hormones}

Before interaction with apoptotic leukocytes, $\mathrm{M} \phi$ or mesangial cells were preincubated for varying durations of time in medium plus supplements/ serum, as described above, containing the desired final concentration of steroid hormone. Control wells were left undisturbed in medium plus supplements/serum. However, preliminary experiments (not shown) demonstrated that the replacement of medium after aspiration at the preincubation times used did not, in itself, alter the phagocytic signal. 


\section{Effect of glucocorticoid receptor antagonist RU38486}

The effect of glucocorticoid receptor antagonist RU38486 was assessed using final concentrations of RU38486 or ethanol-based vehicle control $>10$-fold those employed for glucocorticoids. Medium was aspirated from phagocytes $24 \mathrm{~h}$ before assay of phagocytosis and was replaced by $180 \mu \mathrm{l}$ of RU38486 at 11.1-fold final steroid concentration in medium plus supplements/serum as above. Twenty minutes later, $20 \mu \mathrm{l}$ of medium plus supplements/serum containing no steroid (as a control) or steroid at 10-fold final concentration was added, and the phagocytes were incubated for the desired period until interaction with apoptotic leukocytes.

\section{Effect of cycloheximide}

The protein synthesis inhibitor cycloheximide was included at $2.5 \mu \mathrm{M}$ for $12 \mathrm{~h}$ in medium plus supplements/serum \pm steroid before assay of phagocytosis of apoptotic leukocytes.

\section{Assessment of $M \phi$ and mesangial cell response following the} ingestion of apoptotic PMNs

Monocyte-derived M $\phi$ in 24-well plates were either incubated in Iscove's DMEM alone or interacted with apoptotic PMNs or opsonized zymosan. After $30 \mathrm{~min}, \mathrm{M} \phi$ were carefully washed, and $250 \mu$ l Iscove's DMEM was added to each well. After $24 \mathrm{~h}$ coculture with $\mathrm{M} \phi$, the medium was aspirated, centrifuged, and stored at $-80^{\circ} \mathrm{C}$. M $\phi$ phagocytosis of neutrophils or zymosan was quantified by fixing three of the wells on each plate with $2 \%$ glutaraldehyde and, in the case of neutrophils, by staining for MPO as outlined previously. The proportion of $\mathrm{M} \phi$ ingesting apoptotic neutrophils or opsonized zymosan was counted by inversion light microscopy (28).

Mesangial cells in 96-well plates were either cultured in the presence of RPMI 1640 with $10 \%$ FCS with or without $10 \mathrm{ng} / \mathrm{ml} \mathrm{IL-1} \mathrm{for} 6 \mathrm{~h}$ or interacted for $3 \mathrm{~h}$ with apoptotic PMNs as previously outlined. Mesangial cells were then carefully washed with normal saline, and the medium was replaced with $50 \mu \mathrm{l}$ of RPMI 1640 without $\mathrm{FCS}$ and incubated at $37^{\circ} \mathrm{C}$. The medium was aspirated after $24 \mathrm{~h}$, centrifuged, and stored at $-80^{\circ} \mathrm{C}$. Cytocentrifuge preparations from 4 wells on each plate were used to quantify mesangial cell phagocytosis of apoptotic PMNs.

Measurement of IL-8 and monocyte chemoattractant protein-1 $(M C P-1)$ levels in tissue culture supernatants

IL- 8 and MCP-1 levels were measured by ELISA using a peroxidase conjugate for detection. Briefly, assay plates were coated with protein A-purified goat polyclonal $\mathrm{Ab}$ against pure natural IL-8 (30) or rabbit polyclonal $\mathrm{Ab}$ against recombinant MCP-1 (31). Samples were tested at $1 / 10$ dilution. Immunoreactivity was specifically measured using mAbs against IL-8 and MCP-1 (R\&D Systems, Abingdon, U.K.), respectively, as secondary Abs. Detection was obtained by peroxidase-conjugated, affinity-purified goat antimouse IgG. The ELISA for IL- 8 and MCP-1 were specific because natural forms of other chemokines including MCP-2, MCP-3 (32), granulocyte chemotactic protein-2, ENA-78, GROa, inflammatory protein-10, NAP-2 (33), or cytokines (IL-1, IFN- $\gamma$, IFN- $\beta$ ) were not detectable. The sensitivity of the MCP-1 and IL-8 ELISA were $<1 \mathrm{ng} / \mathrm{ml}$ and $<100 \mathrm{pg} / \mathrm{ml}$, respectively.

\section{Statistical methods}

All values are expressed as mean \pm SEM of the indicated number of experiments. Statistical significance (defined as $p<0.05$ ) was evaluated using Student's $t$ test and where appropriate by one-way ANOVA with comparison between groups using the Newman-Keuls procedure.

\section{Results}

Glucocorticoids specifically promote phagocytosis of apoptotic neutrophils by semimature monocyte-derived $M \phi$

To facilitate the detection of possible potentiating effects upon $\mathrm{M} \phi$ phagocytosis of apoptotic neutrophils (16), we studied "semimature" cultures of human monocyte-derived $\mathrm{M} \phi$ matured for 5 days in adherent culture on plastic because maximal capacity for the uptake of apoptotic cells generally requires maturation for around 7 days (29). Pretreatment of 5-day $\mathrm{M} \phi$ with the glucocorticoid methylprednisolone (Fig. 1) or dexamethasone (data not shown) for different periods of time up to $24 \mathrm{~h}$ caused a progressive increase in the proportion of $\mathrm{M} \phi$ that were capable of phagocytosing apoptotic neutrophils and in the number of apoptotic neutrophils ingested per $\mathrm{M} \phi$. For example, methylprednisolone at $200 \mathrm{nM}$ for

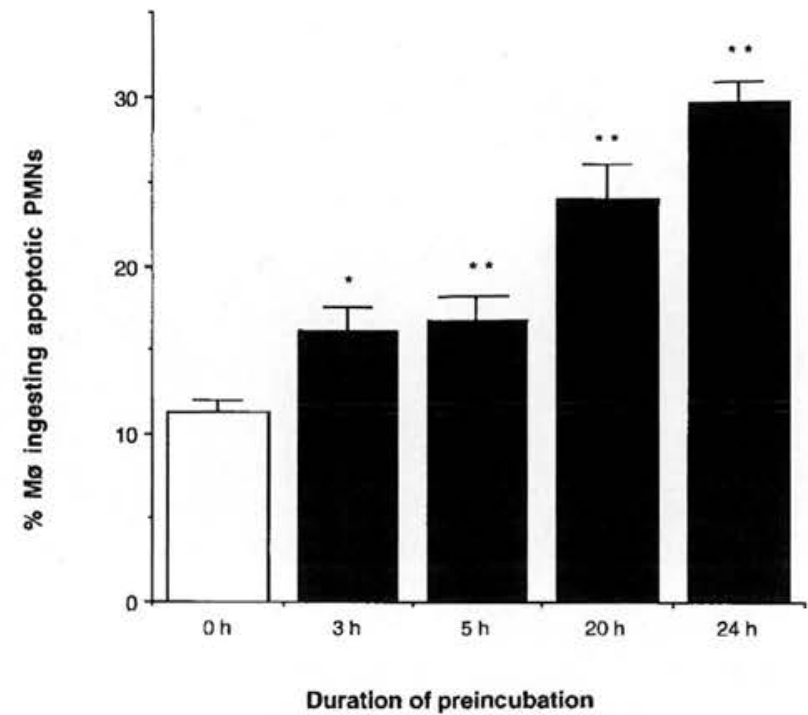

FIGURE 1. Time course of potentiation by $200 \mathrm{nM}$ methylprednisolone of human monocyte-derived $\mathrm{M} \phi$ phagocytosis of apoptotic PMNs. Note increase in methylprednisolone-treated $\mathrm{M} \phi$ (filled bars) vs control $\mathrm{M} \phi$ (open bar) as early as $3 \mathrm{~h}$. In all conditions, phagocytosis was assayed at the same time, with differing preincubation times. Data are mean $\pm \mathrm{SEM}, n=$ 17. $*, p<0.05$ and $* *, p<0.001$ vs control values.

$24 \mathrm{~h}$ augmented the number of apoptotic neutrophils ingested per 100 phagocytically active $\mathrm{M} \phi$ from $138.9 \pm 4.1$ to $209.6 \pm 5.1$ $(p<0.01 ; n=9)$. Short preincubation times $(<3 \mathrm{~h})$ were without effect (Fig. 1), excluding the remote possibility that there was a "carry over" effect of glucocorticoid treatment of $\mathrm{M} \phi$ upon added apoptotic neutrophils. The specificity of glucocorticoid effects upon $\mathrm{M} \phi$ phagocytosis was further investigated by comparison of the effects of pretreatment of $\mathrm{M} \phi$ for $24 \mathrm{~h}$ with a number of different steroids. These studies demonstrated that methylprednisolone, dexamethasone, and hydrocortisone acted in a concentration-dependent manner to augment phagocytosis (Fig. 2), whereas the mineralocorticoid aldosterone at $5 \mathrm{nM}$, a concentration 10-fold above that inducing maximal effects on sodium transport in vitro (34), and the sex steroids progesterone $(1 \mu \mathrm{M})$ and estradiol $(1 \mu \mathrm{M})$ were without effect (Table I). The specificity of the observed augmentation of $\mathrm{M} \phi$ phagocytosis of apoptotic neutrophils by glucocorticoids was further demonstrated by the failure of glucocorticoid pretreatment to induce either binding or phagocytosis of nonapoptotic neutrophils (data not shown) and the lack of effect of methylprednisolone on the phagocytosis of EIgG; in a series of nine experiments, pretreatment for $24 \mathrm{~h}$ with methylprednisolone at $200 \mathrm{nM}$ increased the proportion of 5-day M $\phi$ ingesting apoptotic neutrophils from $14.1 \pm 1.5 \%$ to $29.6 \pm 3.2 \%$ (mean $\pm \mathrm{SE}, p<0.001$ ) but had no significant effect on the proportion of 5-day $\mathrm{M} \phi$ ingesting EIgG (untreated 5-day $\mathrm{M} \phi 52.7 \pm$ $6.4 \%$ vs methylprednisolone-treated 5-day $\mathrm{M} \phi 54.3 \pm 7.1 \%$ ).

Glucocorticoid promotion of $M \phi$ uptake of apoptotic neutrophils is specific for the glucocorticoid receptor and inhibitable by cycloheximide

To confirm that glucocorticoids were acting via $\mathrm{M} \phi$ steroid receptors, we assessed the effect of the specific steroid receptor antagonist RU38486 upon glucocorticoid-promoted 5-day $\mathrm{M} \phi$ phagocytosis of apoptotic neutrophils (Fig. 3). RU38486 used at a 10fold greater concentration than the glucocorticoids completely blocked increased phagocytosis stimulated by 24 -h treatment with 


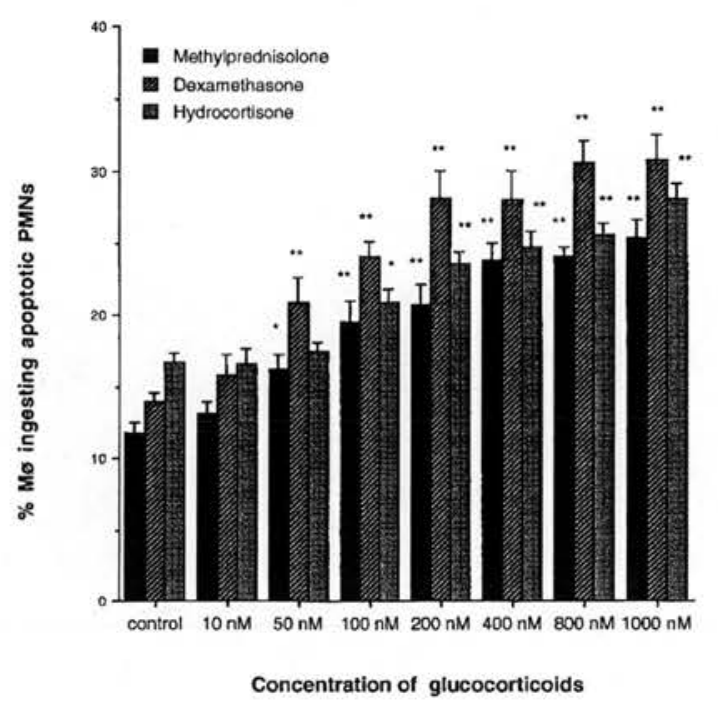

FIGURE 2. Concentration-related potentiation of human monocyte-derived M $\phi$ phagocytosis of apoptotic PMNs by $24-\mathrm{h}$ treatment with glucocorticoids. Methylprednisolone, dexamethasone, and hydrocortisone exhibited similar effects. Logistic considerations required a separate series of experiments for each glucocorticoid explaining variation in controls for each compound. Data are mean $\pm \mathrm{SEM}, n=12$ for each compound. $*, p<$ 0.05 and $* *, p<0.001$ vs control values.

methylprednisolone, dexamethasone, and hydrocortisone without exhibiting any independent inhibitory effect upon $\mathrm{M} \phi$ phagocytosis of apoptotic cells (Fig. 3). Together with the lack of effect upon $\mathrm{M} \phi$ phagocytosis of EIgG cited above, these data argue strongly against a nonspecific "membrane" effect on $\mathrm{M} \phi$. Furthermore, methylprednisolone failed to promote phagocytosis of apoptotic neutrophils by $\mathrm{M} \phi$ pretreated for $12 \mathrm{~h}$ with the protein synthesis inhibitor cycloheximide (a reversible blocker of mRNA translation) at $2.5 \mu \mathrm{M}$, a concentration previously shown to inhibit $>95 \%$ of protein synthesis in myeloid cells and to inhibit $\mathrm{M} \phi$ phagocytosis of apoptotic cells without toxic effects on the $M \phi$, as evidenced by retained ability to ingest opsonized erythrocytes (35, 36 ). Thus, in a series of seven experiments in which $15.7 \pm 1.7 \%$ (mean $\pm \mathrm{SE}$ ) of 5-day $\mathrm{M} \phi$ ingested apoptotic neutrophils under control conditions and 12 -h pretreatment with methylprednisolone at $200 \mathrm{nM}$ increased recognition to $38.3 \pm 4.2 \%(p<0.001$ vs control), such glucocorticoid pretreatment was unable to reverse the inhibitory effect of concomitant 12 -h pretreatment with cycloheximide at $2.5 \mu \mathrm{M}$, which significantly reduced the proportion of $\mathrm{M} \phi$ ingesting apoptotic neutrophils whether $\mathrm{M} \phi$ had been treated with methylprednisolone $(4.9 \pm 0.9 \%, p<0.001)$ or not $(5.7 \pm$ $1.4 \%, p<0.001)$.

Table I. Effect of 24-h treatment with nonglucocorticoid steroid hormones on human monocyte-derived M\$ uptake of apoptotic PMNs ${ }^{a}$

\begin{tabular}{lc}
\hline \hline \multicolumn{1}{c}{ Hormone } & $\begin{array}{c}\text { M } \phi \text { Uptake of Apoptotic PMNs } \\
(\% \text { of control; mean } \pm \text { SE; } n=10)\end{array}$ \\
\hline None & $100.0 \pm 4.0$ \\
Aldosterone $(5 \mathrm{nM})$ & $108.5 \pm 6.1$ \\
Estradiol $(1 \mu \mathrm{M})$ & $112.5 \pm 9.3$ \\
Progesterone $(1 \mu \mathrm{M})$ & $97.4 \pm 14.3$ \\
\hline
\end{tabular}

${ }^{a}$ No statistically significant differences were observed. In these experiments, $22.1 \pm 1.8 \%$ of $\mathrm{M} \phi$-ingested apoptotic PMNs were used.

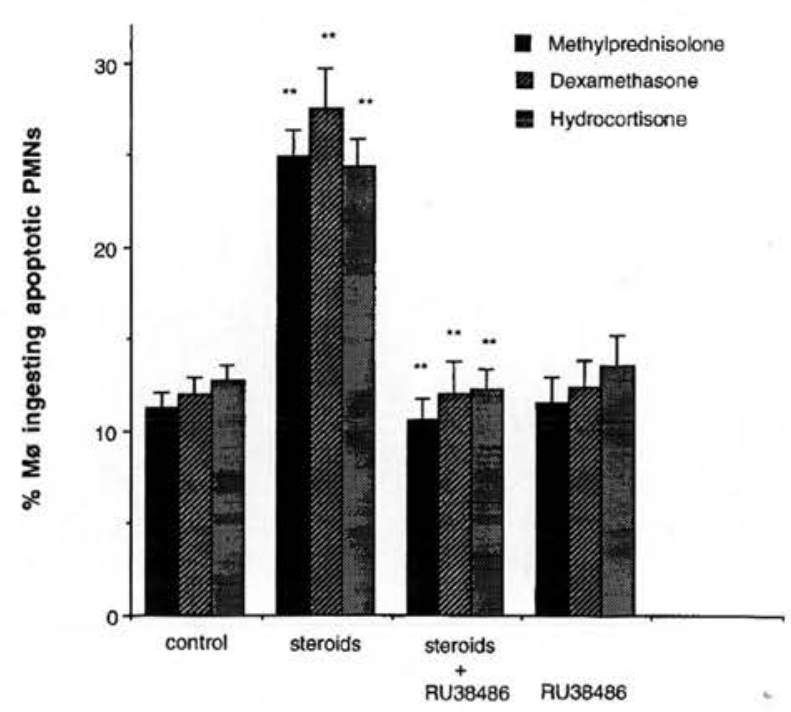

FIGURE 3. Specificity of glucocorticoid enhancement of human monocyte-derived M $\phi$ phagocytosis of apoptotic neutrophils; abrogation by competitive antagonism of steroid receptor. Note RU38486 at 10 times the glucocorticoid concentration completely inhibited potentiation by methylprednisolone at $200 \mathrm{nM}$, dexamethasone at $1 \mu \mathrm{M}$, and hydrocortisone at 1 $\mu \mathrm{M}$ without exerting an independent inhibitory effect on phagocytosis; controls included RU38486 vehicle. Data are mean \pm SEM, $n=10$. **, $p<0.001$ vs control values.

Glucocorticoids also promote phagocytosis in other models of phagocyte clearance of apoptotic cells

In view of the observation that ligation of $\mathrm{M} \phi \mathrm{CD} 44$ specifically promotes phagocytosis of apoptotic neutrophils, having little effect upon uptake of apoptotic cells of other lineages (15), it was of interest to determine whether the prophagocytic effect of glucocorticoids upon $\mathrm{M} \phi$ was restricted to uptake of apoptotic neutrophils. This proved not to be the case in that methylprednisolone also promoted phagocytosis of apoptotic Jurkat cells from $13.4 \pm 1.4 \%$ of $\mathrm{M} \phi$ under control conditions to $24.2 \pm 1.5 \%$ after 24 -h culture with $200 \mathrm{nM}$ methylprednisolone (mean $\pm \mathrm{SE}, n=6, p<0.001$ ). Furthermore, 24-h incubation of $\mathrm{M} \phi$ with $1 \mu \mathrm{M}$ dexamethasone increased $\mathrm{M} \phi$ phagocytosis of apoptotic eosinophils from $49.0 \pm$ $3.9 \%$ to $67.8 \pm 5.1 \%$ (mean $\pm \mathrm{SE}, n=6, p<0.001$ ).

We were also concerned to establish whether the prophagocytic effect of glucocorticoids was limited to the human monocyte-derived $\mathrm{M} \phi$, because previous work has established that other phagocyte populations recognize apoptotic cells by mechanisms distinct from the $\alpha_{\mathrm{v}} \beta_{3}$ vitronectin receptor/thrombospondin/CD36 system characterized in the human monocyte-derived $\mathrm{M} \phi$ (reviewed in Ref. 13). Human mesangial cells, "semiprofessional" glomerular phagocytes, which usually recognize apoptotic neutrophils by a CD36-independent, $\alpha_{\mathrm{v}} \beta_{3}$-mediated mechanism (11) also exhibited increased phagocytosis of apoptotic neutrophils when treated with glucocorticoids (Fig. 4). Furthermore, this effect was not limited by phagocyte species in that glucocorticoids exhibited a similar promotion of phagocytosis of apoptotic neutrophils by murine bone marrow-derived $\mathrm{M} \phi$ populations and murine thioglycollateelicited peritoneal inflammatory $\mathrm{M} \phi$ (Fig. 5), which normally employ $\alpha_{\mathrm{V}} \beta_{3}$-independent phosphatidylserine receptors in the uptake of apoptotic cells (27).

Glucocorticoid-promoted uptake of apoptotic cells does not promote proinflammatory secretory responses by phagocytes

Growing evidence supports the concept that phagocyte clearance of leukocytes undergoing apoptosis is an injury-limiting leukocyte 
disposal mechanism likely to promote the resolution of inflammation (reviewed in Ref. 37). Strong support is provided by data that demonstrate that $\mathrm{M} \phi$ and mesangial cells phagocytosing apoptotic cells are not stimulated to secrete proinflammatory eicosanoids, granule enzymes, or cytokines $(6,10,11)$. The wide-ranging antiinflammatory effects of glucocorticoids suggested that the promotion of phagocyte clearance of apoptotic cells would be unlikely to provoke proinflammatory responses from ingesting phagocytes, but it was clearly necessary to test this assumption. We found that increases in $\mathrm{M} \phi$ uptake of apoptotic neutrophils induced by methylprednisolone pretreatment did not invoke the release of the proinflammatory chemokines IL- 8 and MCP- 1 by M $\phi$ (Table II) or mesangial cells (Table III). However, methylprednisolone at 200 $\mathrm{nM}$ failed to abrogate IL- 8 and MCP- 1 secretion triggered by $\mathrm{M} \phi$ phagocytosis of opsonized zymosan particles (Table III), indicating that glucocorticoids were not acting to suppress production of these chemokines by $\mathrm{M} \phi$.

During monocyte maturation, earlier and more prolonged exposure to glucocorticoid results in greater potentiation of $M \phi$ phagocytic capacity at 5 days

Although human monocyte-derived $\mathrm{M} \phi$ are able to ingest senescent neutrophils undergoing apoptosis, freshly isolated human peripheral blood monocytes lack this capacity, which is progressively acquired over several days as adherent monocytes cultured with autologous serum differentiate into $\mathrm{M} \phi$ (29). Because the mechanisms responsible are very poorly understood, we considered it possible that early exposure of maturing monocytes to glucocorticoids might undesirably disrupt acquisition of the capacity to ingest apoptotic neutrophils. In fact, the earlier maturing monocytes were exposed to $200 \mathrm{nM}$ prednisolone throughout subsequent culture, the greater (compared with control) the potentiation of the uptake of apoptotic neutrophils at 5 days (Fig. 6).

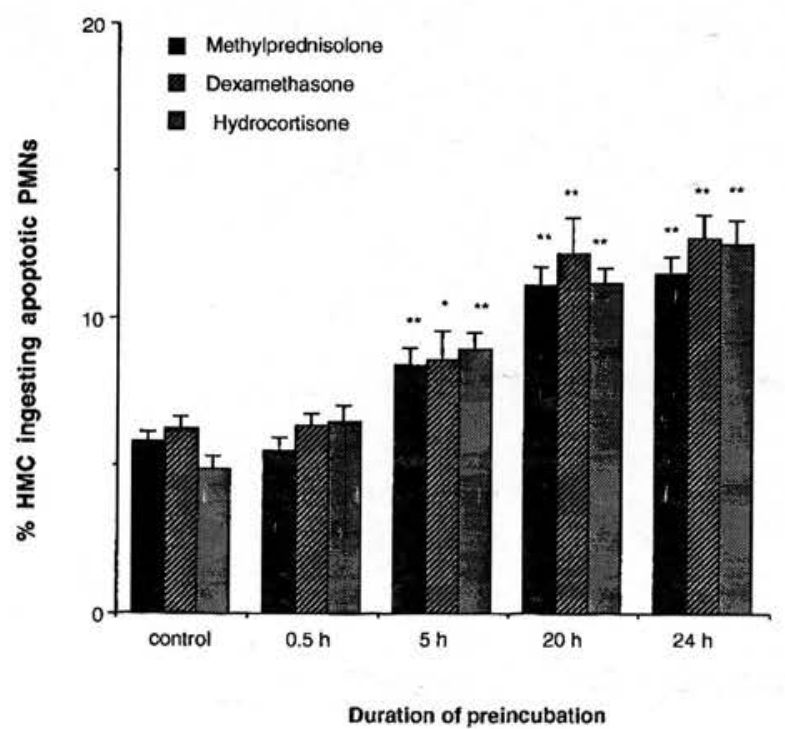

FIGURE 4. Time course of glucocorticoid potentiation of human glomerular mesangial cell (HMC) phagocytosis of apoptotic neutrophils. Methylprednisolone, dexamethasone, and hydrocortisone, all at $200 \mathrm{nM}$, exerted similar effects. Under all conditions, HMC phagocytosis of apoptotic PMNs was assayed at the same time; preincubation times varies as shown. Data are mean \pm SEM, $n=6 . *, p<0.05 ; * *, p<0.001$ vs control values.
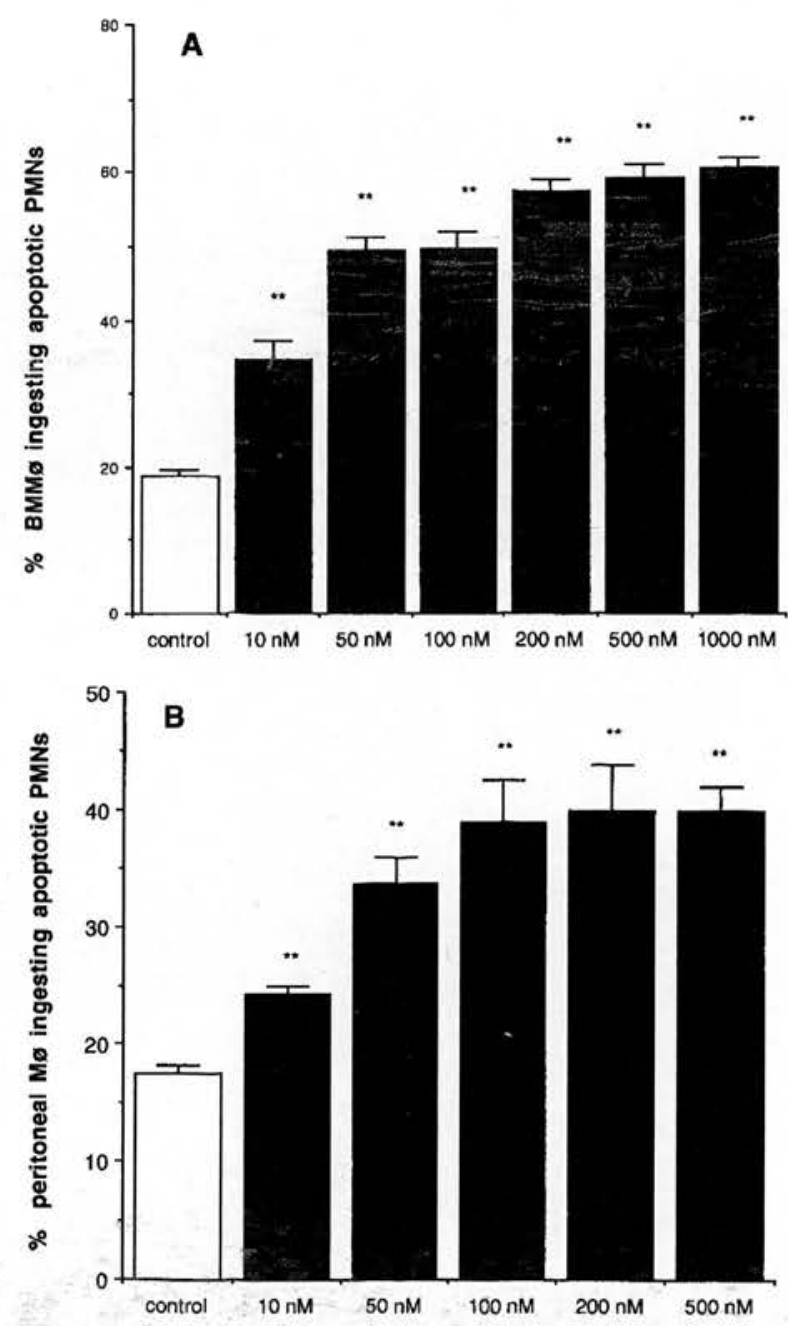

FIGURE 5. Concentration-related potentiation of murine $M \phi$ phagocytosis of human apoptotic neutrophils by $24-\mathrm{h}$ treatment with methylprednisolone. $A$, Murine bone marrow-derived $\mathrm{M} \phi(\mathrm{BMM} \phi) . B$, Thioglycollate-elicited peritoneal $\mathrm{M} \phi$. Data are mean $\pm \mathrm{SEM}, n=6 . * *, p<0.001$ vs control values.

\section{Discussion}

This report establishes the capacity of glucocorticoids to promote nonphlogistic phagocytosis of cells undergoing apoptosis. This effect was specific in that in human monocyte-derived $M \phi$ treated for $24 \mathrm{~h}$ from 4 days' maturation it was mediated via the RU48686-inhibitable steroid receptor, not induced by the mineralocorticoid aldosterone or by sex steroids, and not observed for FcR-mediated phagocytosis. Furthermore, there was evidence of a generally relevant promotion of phagocyte clearance of apoptotic cells in that glucocorticoids promoted $\mathrm{M} \phi$ phagocytosis of apoptotic Jurkat T lymphocytes and apoptotic eosinophils as well as promoting the uptake of apoptotic neutrophils by both human glomerular mesangial cells and two different populations of murine $\mathrm{M} \phi$. Finally, the promoting effect of glucocorticoids was not limited to "semimature" 4-day $\mathrm{M} \phi$ because earlier exposure to glucocorticoid during $\mathrm{M} \phi$ differentiation resulted in a still greater capacity for phagocytosis of apoptotic cells at 5 days.

The data provide new insights into the anti-inflammatory effects of glucocorticoids, demonstrating a hitherto unrecognized mechanism by which these agents may ameliorate inflammatory injury (the promotion of safe phagocytic clearance of leukocytes being 
Table II. Effect of 24-h treatment with $200 \mathrm{nM}$ methylprednisolone upon $I L-8$ and $M C P-1$ release from human monocyte-derived $M \phi$ ingesting particulate stimuli ${ }^{\circ}$

\begin{tabular}{llcc}
\hline \hline \multicolumn{1}{c}{ Condition } & \multicolumn{1}{c}{ Stimulus } & $\begin{array}{c}\text { IL-8 Concentration } \\
(\mathrm{ng} / \mathrm{ml} \text {; mean } \pm \text { SE; } n=4)\end{array}$ & $\begin{array}{c}\text { MCP-1 Concentration } \\
(\mathrm{ng} / \mathrm{ml} \text { mean } \pm \text { SE; } n=4)\end{array}$ \\
\hline Control & None & $0.7 \pm 0.1$ & $1.8 \pm 0.5$ \\
Control & Apoptotic PMNs & $0.5 \pm 0.0$ & $1.7 \pm 0.4$ \\
Control & Opsonized zymosan & $53.0 \pm 2.1^{\dagger}$ & $7.4 \pm 2.6^{*}$ \\
Methylprednisolone & None & $0.7 \pm 0.2$ & $2.0 \pm 0.6$ \\
Methylprednisolone & Apoptotic PMNs & $0.8 \pm 0.2$ & $2.4 \pm 0.8$ \\
Methylprednisolone & Opsonized zymosan & $53.5 \pm 7.2^{\dagger}$ & $6.1 \pm 2.6^{*}$ \\
\hline
\end{tabular}

${ }^{a}$ In these experiments, $10.8 \pm 0.5 \%$ of $\mathrm{M} \phi$-ingested apoptotic PMNs under control conditions were used, a figure that increased to $17.8 \pm 1.6 \%$ after methylprednisolone treatment. $*, p<0.05$ and ${ }^{\dagger}, \mathrm{p}<0.01$ vs $\mathrm{M} \phi$ not receiving phagocytosed particles.

deleted by apoptosis). Data from in vitro studies emphasize that glucocorticoids may coordinately promote the deletion of certain leukocyte types by engaging apoptosis $(22,23,38)$, while at the same time up-regulating the capacity for phagocytic clearance (this study). Recently published observations that steroid treatment of asthma is associated with the resolution of eosinophilic inflammation and increased evidence of eosinophil phagocytosis by broncho-alveolar $\mathrm{M} \phi$ provide evidence that similar mechanisms may operate in vivo in man (8). Furthermore, although glucocorticoids slow constitutive apoptosis in neutrophils $(21,23)$, glucocorticoidtreated neutrophils eventually undergo apoptosis and therefore require phagocytic clearance in vivo to prevent secondary necrosis with the resultant release of toxic granule contents. Further studies will be required to establish whether these findings can be extrapolated to inflammatory conditions in different tissues in vivo. However, it appears likely that the observed increases in both the proportion of $\mathrm{M} \phi$ ingesting apoptotic neutrophils and in the number of ingested cells per phagocytically active $\mathrm{M} \phi$ will equate to a significant increase in clearance capacity in vivo.

Nevertheless, our observation that glucocorticoid-mediated enhancement of $\mathrm{M} \phi$ phagocytosis of apoptotic cells was not achieved by a costly loss of the teleogically appropriate lack of proinflammatory response by the phagocyte is of fundamental importance. Methylprednisolone-enhanced phagocytosis of apoptotic cells failed to stimulate IL- 8 and MCP- 1 release from either M $\phi$ or mesangial cells. By contrast, under the conditions employed, methylprednisolone did not suppress M $\phi$ release of IL- 8 and MCP-1 after phagocytosis of opsonized zymosan. These findings were underscored by comparable observations when mesangial cells were employed as the phagocyte, where IL-1 was employed as a positive control stimulus $(11,30)$ instead of opsonized zymosan. Therefore, failure to elicit a proinflammatory response did not merely reflect a general suppression of chemokine synthesis by glucocorticoids, which has been reported in other in vitro systems (reviewed in Ref. 24).

The modulation of phagocyte capacity for uptake of apoptotic cells by glucocorticoids exhibits interesting differences from previous reports on the regulation of phagocytosis of these and other particles. In contrast to CD44-mediated enhancement of $\mathrm{M} \phi$ uptake of apoptotic neutrophils (15), the prophagocytic effect of glucocorticoids required several hours of treatment and was unable to reverse inhibition by cycloheximide, consistent with a requirement for new protein synthesis. Moreover, unlike CD44 ligation, prophagocytic effects were not restricted to apoptotic target cells of the neutrophil lineage. Furthermore, in other phagocytic systems glucocorticoids have been reported to inhibit rat alveolar $\mathrm{M} \phi$ ingestion of carbon particles (39) and murine $\mathrm{M} \phi$ phagocytosis of heatkilled Saccharomyces cerevisiae (40-43). Nevertheless, freshly isolated human monocyte phagocytosis of $\beta$-glucan particles prepared from the same species of yeast was enhanced by glucocorticoids such as dexamethasone at $200 \mathrm{nM}$ (44), and there are reports that glucocorticoids up-regulate $\mathrm{M} \phi$ receptors for cytokines, including those for granulocyte-M $\phi \operatorname{CSF}(45)$, which can promote $\mathrm{M} \phi$ uptake of apoptotic cells (16). Consequently, the current data are not necessarily inconsistent with previous studies of the glucocorticoid regulation of phagocytosis.

Further studies will be needed to define mechanisms by which glucocorticoids potentiate phagocytosis of apoptotic cells. Our preliminary immunofluorescence flow cytometry studies (data not shown) on 5-day human monocyte-derived $\mathrm{M} \phi$ treated with 200 $\mathrm{nM}$ methylprednisolone for $24 \mathrm{~h}$ have shown no detectable difference in $\mathrm{M} \phi$ surface expression of components of the $\alpha_{\mathrm{y}} \beta_{3}$ vitronectin receptor/thrombospondin/CD36 recognition mechanism (35) and a modest down-regulation of CD14 consistent with previous reports (46), suggesting that up-regulated expression of these surface receptors is unlikely to account for the potentiating effects

Table III. Effect of 24-h ireatment with $200 \mathrm{nM}$ methylprednisolone upon $I L-8$ and MCP-I release from stimulated human mesangial cells ${ }^{a}$

\begin{tabular}{llcc}
\hline \multicolumn{1}{c}{ Condition } & \multicolumn{1}{c}{ Stimulus } & $\begin{array}{c}\text { IL-8 Concentration } \\
\text { (ng/ml; mean } \pm \text { SE; } n=4)\end{array}$ & $\begin{array}{c}\text { MCP-1 Concentration } \\
(\mathrm{ng} / \mathrm{ml} \text { mean } \pm \text { SE; } n=4)\end{array}$ \\
\hline Control & None & $15.2 \pm 1.65$ & $22.5 \pm 3.0$ \\
Control & Apoptotic PMNs & $14.5 \pm 1.7$ & $31.3 \pm 1.25$ \\
Control & IL-1 $(10 \mathrm{ng} / \mathrm{ml})$ & $350.0 \pm 60.0^{*}$ & $133.5 \pm 8.5^{*}$ \\
Methylprednisolone & None & $17.4 \pm 0.4$ & $21.8 \pm 1.75$ \\
Methylprednisolone & Apoptotic PMNs & $29.0 \pm 5.5$ & $42.5 \pm 7.0$ \\
Methylprednisolone & IL-1 $(10 \mathrm{ng} / \mathrm{ml})$ & $425.0 \pm 85.0^{*}$ & $188.5 \pm 16.5^{*}$ \\
\hline
\end{tabular}

${ }^{a}$ In these experiments, $7.4 \%$ of mesangial cells ingested apoptotic PMNs under control conditions, a figure that increased to $14.8 \%$ after methylprednisolone treatment.

${ }^{*}, p<0.01$ vs unstimulated mesangial cells. IL-8 and MCP-1 release from methylpredisolone-treated mesangial cells was not significantly different from controls. 


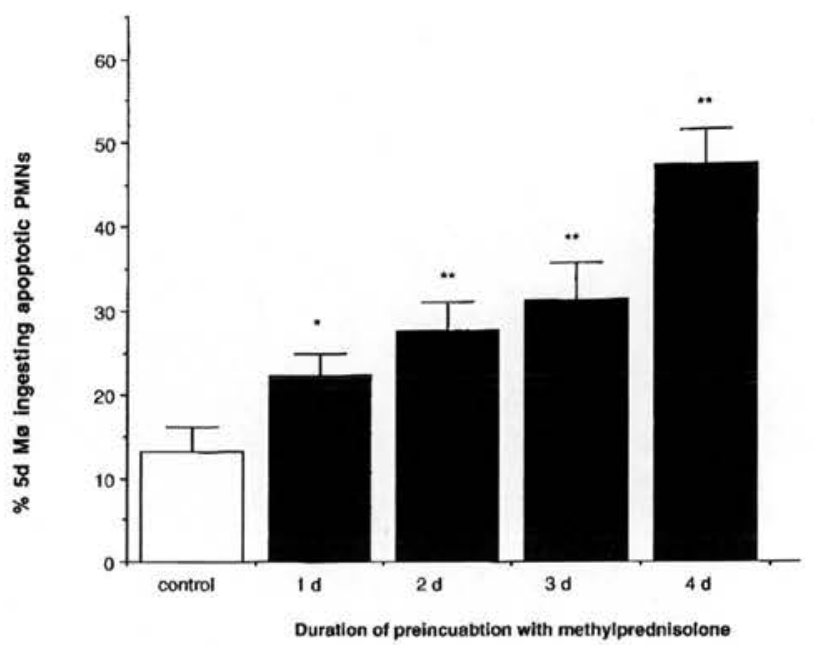

FIGURE 6. Progressively earlier inclusion of methylprednisolone at $200 \mathrm{nM}$ in cultures of maturing monocytes leads to greater potentiation of 5 -day $\mathrm{M} \phi$ phagocytosis of apoptotic neutrophils. Compare potentiation by 1-day treatment with that for 4-day treatment (i.e., methylprednisolone included from 1 day of monocyte (M $\phi$ culture). Data are mean \pm SEM, $n=6 . *, p<0.05 ; * *, p<0.001$ vs control values.

of glucocorticoids. This work on surface receptor expression will need to be expanded, and possible effects of glucocorticoids on cytoskeletal elements (47) should also be considered.

Lastly, although hydrocortisone and dexamethasone might appear to be equipotent in our studies, comparisons of concentrations at which different glucocorticoids exert a similar effect in vitro should be made with considerable caution because $\mathrm{ABC}$ transporters such as MDR1A can expel synthetic glucocorticoid substrates such as dexamethasone from within leucocytes (48), with the result that the effective concentration of glucocorticoid at the intracellular receptor is lower than the extracellular concentration applied to the cell.

In conclusion, we report that glucocorticoids specifically enhance the nonphlogistic phagocytic uptake of apoptotic leukocytes of differing lineages by human and murine $\mathrm{M} \phi$ populations and also "semiprofessional" phagocytes, glomerular mesangial cells. The data add new emphasis to the anti-inflammatory properties of glucocorticoids and suggest that further work could identify novel therapeutic approaches toward promoting the safe resolution of inflammatory conditions.

\section{Acknowledgments}

We thank Prof. M. Horton (St Bartholomew's Hospital, London, U.K.), Dr. Ian Hall, and Prof. Chris Gregory (University of Nottingham, U.K.) for the gifts of reagents. We also thank Judith Hayes and Dorothy May for expert secretarial assistance.

\section{References}

1. Malech, H .D., and J. I. Gallin. 1988. Neutrophils in human diseases. N. Engl. J. Med. 317:687.

2. Savill, J. S., A. H. Wyllie, J. E. Henson, M. J. Walport, P. M. Henson, and C. Haslett. (1989). Macrophage phagocytosis of aging neutrophils in inflammation: programmed cell death in the neutrophil leads to its recognition by macrophages. J. Clin. Invest. 83:865.

3. Savill, J. S., J. Smith, Y. Ren, C. Sarraf, F. Abbott, and A. J. Rees. 1992. Glomerular mesangial cells and inflammatory macrophages ingest neutrophils undergoing apoptosis. Kidney Int. 42:924.

4. Akbar, A. N., J. Savill, W. Gombert, M. Bofill, N. J. Borthwick, F. Whitelaw, J. Grundy, G. Janossy, and M. Salmon. 1994. The specific recognition by macrophages of $\mathrm{CD}^{+}, \mathrm{CD}^{+} 5 \mathrm{RO}^{+} \mathrm{T}$ cells undergoing apoptosis: a mechanism for $\mathrm{T}$ cell clearance during resolution of viral infections. J. Exp. Med. 180:1943.
5. Cox, G. J. J. Crossley, and Z. Xing. 1995. Macrophage engulfment of apoptotic neutrophils contributes to the resolution of acute pulmonary inflammation in vivo. Am. J. Respir. Cell. Mol. Biol. 12:232.

6. Stern, M., J. Savill, and C. Haslett. 1996. Human monocyte-derived macrophage phagocytosis of senescent eosinophils undergoing apoptosis: mediation by $\alpha_{v} \beta_{3}$ / CD36/thrombospondin recognition mechanism and lack of phlogistic response. Am. J. Pathol. 149:911.

7. Tsuyuki, S., C. Bertrand, F. Erard, A. Trifilieff, J. Tsuyuki, M. Wesp, G. P. Anderson, and A. J. Coyle. 1995. Activation of the Fas receptor on lung eosinophils leads to apoptosis and the resolution of eosinophilic inflammation of the airways. J. Clin. Invest. 96:2924.

8. Woolley, K. L., P. G. Gibson, K. Carty, A. J. Wilson, S. H. Twaddell, and M. J. Woolley. 1996. Eosinophil apoptosis and the resolution of airway inflammation in asthma. Am. J. Respir. Crit. Care. Med. 154:237.

9. Milik, A. M., V. A. Buechner-Maxwell, J. Sonstein, S. Kim, G. D. Seitzman, T. F. Beals, and J. L. Curtis. 1997. Lung lymphocyte elimination by apoptosis in the murine response to intratracheal particulate antigen. J. Clin. Invest. 99:1082.

10. Meagher, L. C., J. S. Savill, A. Baker, and C. Haslett. 1992. Phagocytosis of apoptotic neutrophils does not induce macrophage release of Thromboxane B2. J. Leukocyte Biol. 52:269.

11. Hughes, J., Y. Liu, J. Van Damme, and J. Savill. 1997. Human glomerular mesangial cell phagocytosis of apoptotic neutrophils: mediation by a novel CD36independent vitronectin receptor/thrombospondin recognition mechanism that is uncoupled from chemokine secretion. J. Immunol. 158:4389.

12. Fadok, V. A., D. L. Bratton, A. Konowal, P. W. Freed, J. Y. Westcott, and P. M. Henson. 1998. Macrophages that have ingested apoptotic cells in vitro inhibit proinflammatory cytokine production through autocrine/paracrine mechanisms involving TGF- $\beta$, PGE2 and PAF. J. Clin. Invest. 101:890.

13. Savill, J. Recognition and phagocytosis of cells undergoing apoptosis. 1997. Br. Med. Bull. 53:1.

14. Savill, J. 1998. Phagocytic docking without shocking. Nature 392:442.

15. Hart, S. P., G. J. Dougherty, C. Haslett, and I. Dransfield. 1997. CD44 regulates phagocytosis of apoptotic neutrophil granulocytes, but not apoptotic lymphocytes, by human macrophages. J. Immunol. 159:919.

16. Ren, Y., and J. Savill. 1995. Pro-inflammatory cytokines potentiate thrombospondin-mediated phagocytosis of neutrophils undergoing apoptosis. J. Immunol. 154:2366.

17. Goulding, N. J., and P. M. Guyre. 1993. Glucocorticoids, lipocortins and the immune response. Curr. Opin. Immunol. 5:108.

18. Schleimer, R. P. 1993. An overview of glucocorticoid anti-inflammatory actions. Eur. J. Clin. Pharmacol, 45:S3.

19. Farsky, S. P., P. Sannomiya, and J. Garcia-Leme. 1995. Secreted glucocorticoids regulate leukocyte-endothelial interactions in inflammation: a direct vital microscopic study. J. Leukocyte Biol. 57:379.

20. Tailor, A., A. M. Das, S. J. Getting, R. J. Flower, and M. Perretti. 1997. Subacute treatment of rats with dexamethasone reduces ICAM-1 levels on circulating monocytes. Biochem. Biophys. Res. Comm. 231:675.

21. Cox, G. 1995. Glucocorticoid treatment inhibits apoptosis in human neutrophils J. Immunol. 154:4719.

22. Her, E., J. Frazer, K. F. Austen, and W. F. Owen, Jr. 1991. Eosinophil haematopoetins antagonise the programmed cell death of eosinophils: cytokine and glucocorticoid effects on eosinophils maintained by endothelial cell-conditioned medium. J. Clin. Invest. 88:1982,

23. Meagher, L. C., J. M. Cousin, J. R. Seckl, and C. Haslett. 1996. Opposing effects of glucocorticoids on the rate of apoptosis in neutrophilic and eosinophilic granulocytes. J. Immunol. 156:4422.

24. Barnes, P. J. 1998. Anti-inflammatory actions of glucocorticoids: molecular mechanisms. Clin. Sci. 94:557.

25. Haslett, C., L. A. Guthrie, M. M. Kopaniak, R. B. Johnston, and P. M. Henson. 1985. Modulation of multiple neutrophil functions by preparative methods or trace concentrations of bacterial lipopolysaccharide. Am. J. Pathol. 119: 101.

26. Stern, M., L. C. Meagher, J. S. Savill, and C. Haslett. 1992. Apoptosis in human eosinophils: programmed cell death in the eosinophil leads to phagocytosis by macrophages and is modulated by IL-5. J. Immunol. 148:3543.

27. Fadok, V., J. S. Savill, C. Haslett, D. L. Bratton, D. E. Doherty, P. A. Campbell, and P. M. Henson. 1992. Different populations of macrophages use either the vitronectin receptor or the phosphatidylserine receptor to recognise and remove apoptotic cells. J. Immunol. 149:4029.

28. Savill, J. S., P. M. Henson, and C. Haslett. 1989, Phagocytosis of aged human neutrophils by macrophages is mediated by a novel "charge sensitive" recognition mechanism. J. Clin. Invest. 84:1518.

29. Newman, S. L., J. E. Henson, and P. M. Henson. 1982. Phagocytosis of senescent neutrophils by human monocyte-derived macrophages and rabbit inflammatory macrophages. J. Exp. Med. 156:430.

30. Van Damme, J., B. Decock, R. Conings, J. P. Lenaerts, G. Opdenakker, and A. Billiau. 1989. The chemotactic activity for granulocytes produced by virally infected fibroblasts is identical to monocyte derived interleukin 8. Eur. J. Immunol. 19:1189.

31. Van Damme, J., P. Proost, W. Put, S. Arens, J.-P. Lenaerts, R. Conings, G. Opdenakker, H. Heremans, and A. Billiau. 1994. Induction of monocyte chemotactic proteins MCP-1 and MCP-2 in human fibroblasts and leukocytes by cytokines and cytokine inducers: chemical synthesis of MCP-2 and development of a specific RIA. J. Immunol. 152:5495.

32. Van Damme, J., P. Proost, J. P. Lenaerts, and G. Opdenakker. 1992. Structural and functional identification of two tumor-derived monocyte chemotactic proteins (MCP-2 and MCP-3) belonging to the chemokine family. J. Exp. Med. 176:59. 
33. Proost, P., A. Wuyts, R. Conings, J. P. Lenaerts, A. Billiau, G. Opdenakker, and J. Van Damme. 1992. Human and bovine granulocyte chemotactic protein-2complete amino acid sequence and functional characterization as chemokines. Biochemistry 32:10170.

34. Christ, M., K. Douwes, C. Eisen, G. Bechtner, K. Theisen, and M. Wehling. 1995. Rapid effects of aldosterone on sodium-transport in vascular smooth-muscle cells. Hypertension 25:117.

35. Savill, J., N. Hogg, Y. Ren, and C. Haslett. 1992. Thrombospondin cooperates with $\mathrm{CD} 36$ and the vitronectin receptor in macrophage recognition of neutrophils undergoing apoptosis. J. Clin. Invest. 90:1513.

36. Whyte, M. K. B., S. J. Hardwick, L. C. Meagher, J. S. Savill, and C. Haslett. 1993. Transient elevations of cytosolic calcium retard neutrophil apoptosis in vitro. J. Clin. Invest. 92:446.

37. Savill, J., and C. Haslett. 1995. Granulocyte clearance by apoptosis in the resolution of inflammation. Semin. Cell. Biol. 6:385.

38. Kawabori, S., K. Soda, M. H. Perdue, and J. Bienenstock. 1991. The dynamics of intestinal eosinophil depletion in rats treated with dexamethasone. Lab. Invest. $64: 224$.

39. Lortie, C., G. M. King, and I. Y. R. Adamson. 1990. Effects of dexamethasone on macrophages in fetal and neonatal rat lung. Pediatr. Pulmonol. 8:138.

40. Becker, J., and R. J. Grasso. 1985. Suppression of phagocytosis by dexamethasone in macrophage cultures: inability of arachidonic acid, indomethacin and nordihydroguaiaretic acid to reverse the inhibitory response mediated by a steroid-inducible factor. Int. J. Immunopharmac. 7:839.

41. Grasso, R. J., T. W. Klein, and W. R. Benjamin. 1981. Inhibition of yeast phagocytosis and cell spreading by glucocorticoids in cultures of resident murine peritoneal macrophages. J. Immunopharmac. 3:171.
42. Grasso, R. J., L. A. West, R. C. Guay, Jr, and T. W. Klein. 1982. Inhibitions of yeast phagocytosis by dexamethasone in macrophage cultures: Reversibility of the effect and enhanced suppression in cultures of stimulated macrophages. J. Immunopharmac. 4:265.

43. Grasso, R. J., L. A. West, R. C. Guay, Jr, and T. W. Klein. 1983. Modulatory effects of heat-labile serum components on the inhibition of phagocytosis by dexamethasone in peritoneal macrophage cultures. Int. J. Immunopharmac. 5:267.

44. Kay, J., and J. K. Czop. 1994. Enhancement of human monocyte $\beta$-glucan receptors by glucocorticoids. Immunology 81:96.

45. Hawrylowicz, C., L. Guida, and E. Paleolog. 1994. Dexamethasone upregulates granulocyte-macrophage colony-stimulating factor receptor expression on human monocytes. Immunology 83:274.

46. Nockher, W. A., and J. E. Scherberich. 1997. Expression and release of the monocyte lipopolysaccharide receptor antigen CD14 are suppressed by glucocorticoids in vivo and in vitro. J. Immunol. 158:1345.

47. Wu, Y.-C., and H. R. Horvitz. 1998. C. elegans cell-corpse engulfment and cellmigration protein CED-5 is similar to human DOCK180 and Drosophila MBC. Nature 392:501.

48. Schinkel, A. H, J. J. M. Smit, O. Vantellingen, J. H. Beijnen, E. Wagenaar, L. Vandeemter, C. A. A. M. Mol, M. A. Vandervalk, E. C. Robanusmaandag, H. P. J. Teriele, A. J. M. Berns, and P. Borst. 1994. Disruption of the mouse MDR1A P-glycoprotein gene leads to a deficiency in the blood-brain-barrier and to increased sensitivity to drugs. Cell $77: 491$. 


\title{
Induction of Human Neutrophil Apoptosis by Nitric Oxide Donors: Evidence for a Caspase-dependent, Cyclic-GMP-independent, Mechanism
}

\author{
Carol Ward, Terence H. Wong, Joanna Murray, Irfan Rahman, Christopher Haslett, \\ Edwin R. Chilvers* and Adriano G. Rossi† \\ Respiratory Medicine Unit, Department of Medicine (RIE), Rayne Laboratory, University of Edinburgh \\ Medical SchOOL, Edinburgh, EH8 9AG, U.K.
}

\begin{abstract}
This study investigated the regulatory effects of the major inflammatory mediator, nitric oxide (NO), on human neutrophil apoptosis in vitro. Co-culture of human neutrophils with the NO donors GEA 3162 (1,2,3,4-oxatriazolium,5-amino-3-(3,4-dichlorophenyl)-chloride) (10-100 $\mu \mathrm{M})$ and 3-morpholino-sydnonimine (SIN-1) $(0.3-3 \mathrm{mM})$ caused a dramatic and concentration-dependent induction of apoptosis. However, $\mathrm{N}$-formyl-methionyl-leucyl-phenylalanine (FMLP)-induced neutrophil activation (actin reorganization and chemotaxis) was inhibited by GEA 3162 treatment. The pro-apoptotic effects of the NO donors were (i) unaffected by the soluble guanylate cyclase inhibitor LY-83583 (6-anilino-5,8-quinolinedione; $100 \mu \mathrm{M}$ ), (ii) antagonized by superoxide dismutase $(6 \mu \mathrm{g} / \mathrm{mL}$ ), (iii) mimicked by exogenous peroxynitrite (at concentrations $>100 \mu \mathrm{M})$, and (iv) inhibited by the caspase inhibitor Z-Val-Ala-DL-Asp-fluoromethylketone $(100 \mu \mathrm{M})$. The pro-apoptotic effect of the NO donors was not mimicked by the cell-permeable cyclic nucleotide analogue, $N^{6}, 2-\mathrm{O}$-dibutyrylguanosine- $3^{\prime}, 5^{\prime}$-cyclic monophosphate (dibutyryl-cGMP) at concentrations $\leq 0.2 \mathrm{mM}$. Indeed, at high concentrations ( $\geq 2 \mathrm{mM}$ ), dibutyryl-cGMP caused an inhibition of apoptosis. These results suggest that NO-mediated apoptosis, although caspase-dependent, is mediated by a cGMP-independent mechanism and involves the concurrent generation of oxygen free radicals and, potentially, peroxynitrite. Our data reveal a unique role for $\mathrm{NO}$ in inflammatory responses with differential effects upon neutrophil activation and survival, with important implications for the successful resolution of inflammation. BIOCHEM PHARMACOL 59;3:305-314, 2000. (C) 1999 Elsevier Science Inc.
\end{abstract}

KEY WORDS. neutrophil; apoptosis; nitric oxide; inflammation; caspase

Apoptosis represents a physiological form of programmed cell death that permits efficient phagocytic removal of intact senescent cells. In contrast, cell death by necrosis is typified by the loss of membrane integrity and hence represents a potentially pro-inflammatory and injurious mode of cell deletion $[1,2]$. In the neutrophil, apoptosis is characterized by a set of distinct morphological and biochemical changes [3-6] which results in functional downregulation [5] and the rapid ingestion and removal of these cells by a process that uniquely fails to incite an inflammatory phagocytic response [7]. In view of the enormous capacity of the neutrophil to release an array of histotoxic products during necrotic cell death, apoptosis therefore offers a physiological, efficient, and non-inflammatory mechanism for neutrophil removal. Granulocyte apoptosis

* Present address: Respiratory Medicine Unit, Department of Medicine, University of Cambridge School of Clinical Medicine, Addenbrooke's Hospital, Cambridge CB2 2QQ, U.K.

$\dagger$ Corresponding author: Dr. Adriano Rossi, Respiratory Medicine Unit, Department of Medicine (RIE), Rayne Laboratory, University of Edinburgh Medical School, Teviot Place, Edinburgh, EH8 9AG, U.K. Tel. (44)

131651 1323; FAX (44) 131650 4384; E-mail a.g.rossi@ed.ac.uk Received 20 April 1999; accepted 3 August 1999. is thus regarded as a crucial process underlying the control and successful resolution of inflammation.

Despite clear evidence that NO $¥$ plays an important role in a number of physiological and pathological processes, displaying pro-inflammatory or anti-inflammatory effects depending on its effective concentration at the inflammatory site [8-10], the regulatory effects of $\mathrm{NO}$ on human neutrophil apoptosis are unknown. Furthermore, the signaling events regulating neutrophil apoptosis remain ill defined. Many of the known modulators of apoptosis in other cell types have very different effects on neutrophil apoptosis; for example, the glucocorticoid dexamethasone and agents that elevate cytosolic free $\mathrm{Ca}^{2+}$ or intracellular cAMP inhibit neutrophil apoptosis [11-15], yet induce apoptosis in other myeloid cells $[13,14,16,17]$. Inflamma-

\# Abbreviations: db-cAMP, $N^{6}, 2-0$-dibutyryladenosine-3',5'-cyclic monophosphate; db-cGMP, $N^{2}, 2$-O-dibutyrylguanosine-3',5'-cyclic monophosphate; MDM, modified Dulbecco's medium; FMLP, $N$-formyl-methionylleucyl-phenylalanine; GEA 3162, 1,2,3,4-oxatriazolium,5-amino-3-(3,4dichlorophenyl)-chloride; HBSS, Hank's balanced salt solution; LY. 83583, 6-anilino-5,8-quinolinedione; $\mathrm{NO}$, nitric oxide; $\mathrm{ONOO}^{-}$, peroxynitrite; $\mathrm{O}_{2}^{*}$, superoxide anion; sGC, soluble guanylate cyclase; SIN-1, 3-morpholino-sydnonimine; SOD, superoxide dismutase; and ZVAD-fmk, Z-Val-Ala-DL-Asp-fluoromethylketone. 
tory mediators also differentially regulate neutrophil apoptosis; for example, tumor necrosis factor induces apoptosis at early time points after culture [18], whereas granulocytemacrophage colony-stimulating factor, $\mathrm{C} 5 \mathrm{a}$, and lipopolysaccharide all inhibit the rate of apoptosis $[4,19]$. Therefore, these data relating to the effects of $\mathrm{NO}$ on apoptosis in other cell types cannot be extrapolated to the neutrophil.

We therefore sought to define the role of $\mathrm{NO}$ in regulating human neutrophil apoptosis in vitro. We examined the direct effects of the well-characterized NO donors GEA 3162 [20] and SIN-1 [21], together with the cellpermeable cyclic nucleotide analogue db-cGMP, LY-83583, an inhibitor of the cGMP-protein kinase G pathway [22], exogenous $\mathrm{SOD}$, and $\mathrm{ONOO}^{-}$(a product of $\mathrm{O}_{2}$ and $\mathrm{NO}$ interaction). We present evidence demonstrating that in contrast to its effect on neutrophil activation NO mediates a profound pro-apoptotic effect on neutrophils. These data represent a further investigation of our initial preliminary observations of NO-mediated apoptosis in neutrophils [23], which have been confirmed in recent reports by Blaylock et al. [24] and Fotenberry et al. [25], who also demonstrated that NO induces apoptosis in human neutrophils. Our results describe additional mechanistic data on the NOmediated pro-apoptotic effect. We report for the first time that $\mathrm{NO}$ induces apoptosis in human neutrophils by a mechanism involving a caspase-dependent pathway that is not mediated by the guanylate cyclase/cGMP pathway. Our studies suggest that NO-mediated effects may have significance in inflammatory conditions where the mechanisms controlling neutrophil activation and survival are normally tightly regulated.

\section{MATERIALS AND METHODS \\ Reagents}

Sodium citrate was purchased from Phoenix Pharmaceuticals Ltd. Percoll and dextran were obtained from Pharmacia Biotechnologies. Iscove's MDM, PBS, HBSS, and supplements (penicillin and streptomycin) were from Life Technologies. Sterile tissue culture plasticware and flexiwell plates were purchased from Corning-Costar and Falcon. Methanol was purchased from Fisons Scientific. Diff-Quik stain was obtained from Baxter. db-cAMP, db-cGMP, SOD, SIN-1, $N^{\mathrm{G}}$-nitro-L-arginine methyl ester (L-NAME), FMLP, and trypan blue were purchased from Sigma Chemical Co. Ltd. GEA 3162, $N^{\mathrm{G}}$-monomethyl-L-arginine monoacetate (L-NMMA), LY-83583 were from Alexis Corporation. ZVAD-fmk was from Bachem.

\section{Neutrophil Preparation}

Human neutrophils were isolated and purified from peripheral venous blood taken from healthy adult volunteers as previously described [26]. In brief, aliquots $(40 \mathrm{~mL})$ of blood were anticoagulated with $4 \mathrm{~mL} 3.8 \%$ sodium citrate and centrifuged $\left(300 \mathrm{~g} ; 25^{\circ} ; 20 \mathrm{~min}\right)$. The leukocyte-rich fraction was separated from erythrocytes using dextran sedimentation and the neutrophils separated from mononuclear cells using discontinuous PBS-Percoll gradients. The purified neutrophils were washed in platelet-poor plasma, followed by HBSS without $\mathrm{Ca}^{2+}$ and $\mathrm{Mg}^{2+}$, and finally resuspended in HBSS with $\mathrm{Ca}^{2+}$ and $\mathrm{Mg}^{2+}$. Cell purity was determined by examining cytocentrifuged preparations (fixed in methanol and stained with Diff-Quik) and viability assessed by trypan blue exclusion. The neutrophils were routinely $>95 \%$ pure $(<0.5 \%$ monocyte contamination) and $>99.5 \%$ viable.

\section{Assessment of Neutrophil Apoptosis by Cytology}

Neutrophils $\left(5 \times 10^{6} / \mathrm{mL}\right)$ were suspended in Iscove's MDM supplemented with $10 \%$ autologous serum, $10 \mathrm{U} / \mathrm{mL}$ penicillin, and $50 \mu \mathrm{g} / \mathrm{ml}$ streptomycin (unless otherwise stated). Iscove's MDM was replaced with PBS containing $\mathrm{Ca}^{2+}$ and $\mathrm{Mg}^{2+}$ for cultures with $\mathrm{ONOO}^{-}$(see below). Neutrophils $\left(3.75 \times 10^{5}\right)$ were cultured in the presence or absence of the reagents indicated in a final volume of 150 $\mu \mathrm{L}$ at $37^{\circ}$ in a humidified $5 \% \mathrm{CO}_{2}$ atmosphere in flatbottomed 96-well flexiwell plates. At 4, 6, and $20 \mathrm{hr}$, neutrophils were harvested from culture and assessed for recovery, viability (trypan blue exclusion), and apoptosis. Apoptosis was assessed morphologically by oil immersion light microscopy $(\times 100$ objective $)$ of fixed and stained cytocentrifuge preparations of the recovered neutrophils. Apoptotic neutrophils were defined as cells containing one or more hyperchromatic pyknotic nuclei [3]. All treatments were performed in triplicate with at least 500 cells counted per slide and the observer blinded to the experimental condition. Results are expressed as the percentage of cells displaying characteristic apoptotic morphology.

\section{Assessment of Neutrophil Apoptosis by Annexin V Binding}

A separate and independent assessment of apoptosis was performed by flow cytometry using fluorescein isothiocyanate-labeled recombinant human annexin $V$ that binds to phosphatidylserine exposed on the surface of apoptotic cells [27]. Neutrophils $\left(5 \times 10^{6} / \mathrm{mL}\right)$ were cultured as described above and at the time indicated an aliquot $(25 \mu \mathrm{L})$ of recovered cells was added to $75 \mu \mathrm{L}$ of a 1:200 dilution of annexin V (Bender Med Systems). Following a 10-min incubation at $4^{\circ}$, samples were analyzed using an EPICS Profile II (Coulter).

\section{Neutrophil Shape Change}

The effects of NO donors on FMLP-induced shape change were examined by preincubating neutrophils $(450 \mu \mathrm{L}$ at $2 \times 10^{6} / \mathrm{mL}$ in PBS containing $\mathrm{Ca}^{2+}$ and $\mathrm{Mg}^{2+}$ in the absence of serum) with the corresponding NO donor for 45 min at $37^{\circ}$ in a gently shaking water bath before the addition of $10 \mathrm{nM}$ FMLP for a further $10 \mathrm{~min}$. Incubations were terminated by the addition of $500 \mu \mathrm{L} 2.5 \%$ gluteral- 
dehyde. Samples were analyzed for shape change by flow cytometry (Coulter EPICS Profile II; Coulter) as previously described [28].

\section{Neutrophil Chemotaxis}

FMLP (100 nM in $35 \mu \mathrm{L}$ Iscove's MDM containing 10\% autologous serum) was placed in the bottom wells of a Neuroprobe 96-well chemotaxis chamber (Porvair Filtronics), with neutrophils $\left(225 \mu \mathrm{L}\right.$ at $3 \times 10^{6} / \mathrm{mL}$ in Iscove's MDM containing $10 \%$ autologous serum) that had been incubated in the presence or absence of the indicated concentration of the NO donor for $45 \mathrm{~min}$ at $37^{\circ}$ added to the top wells. The chamber was then incubated for $90 \mathrm{~min}$ at $37^{\circ}$ in a humidified $5 \% \mathrm{CO}_{2}$ atmosphere. The polycarbonate filter (with $3-\mu \mathrm{m}$ pores) was then removed, the top surface scraped to remove adherent cells, and washed $(0.9 \%$ saline), dried, fixed and stained (Diff-Quik) prior to reading in a Dynatech MR5000 ELISA plate reader for O.D. at 550 $\mathrm{nm}$. Results are expressed as a percentage of the control well O.D. values. Neutrophils that had migrated through the filter into the bottom wells were also counted under light microscopy using a hemocytometer for verification.

\section{Peroxynitrite $\left(\mathrm{ONOO}^{-}\right)$Synthesis}

$\mathrm{ONOO}^{-}$was synthesized by the method as detailed by [29]. It is important to note that, due to the chemical instability of $\mathrm{ONOO}^{-}$when incubated under neutral or acidic conditions, all experiments examining the potential role of $\mathrm{ONOO}^{-}$in mediating NO-induced apoptosis, including control incubations, were performed in PBS containing $\mathrm{Ca}^{2+}$ and $\mathrm{Mg}^{2+}$ in the absence of serum at a $\mathrm{pH}$ of 8.0.

\section{Analysis of Results}

The results are expressed as means \pm SEM of the number $\mathrm{N})$ of independent experiments each using cells from different donors. Statistical analysis was performed by ANOVA with comparisons between groups made using the Newman-Keuls procedure. Differences were considered significant when $P<0.05$.

\section{RESULTS}

\section{Effects of NO Donors on Neutrophil Apoptosis}

Neutrophil apoptosis is accompanied by dramatic morphological changes; non-apoptotic neutrophils contain multilobular nuclei, whereas apoptotic neutrophils exhibit hyperchromatic pyknotic nuclei ([3] and Fig. 1). It is important to note that neutrophils cultured in vitro undergo constitutive apoptosis; for example, in the current experiments, $2.8 \pm 0.6 \%$ and $61.5 \pm 4.5 \%$ of neutrophils cultured for 6 and $20 \mathrm{hr}$, respectively exhibited apoptosis even in the absence of NO donors $(\mathrm{N}=11)$. Cellular necrosis at $20 \mathrm{hr}$ was negligible with $>98 \%$ cell viability, assessed by trypan blue exclusion. Non-apoptotic neutrophils cultured for $6 \mathrm{hr}$ are shown in Fig. 1A (control). In comparison, the apoptotic morphology of neutrophils is demonstrated in Fig. 1B, where the cells had been cultured for $6 \mathrm{hr}$ with the NO donor GEA $3162(100 \mu \mathrm{M})$. The induction of apoptosis by GEA 3162 was confirmed by analyses of annexin $\mathrm{V}$ binding to the cell surface of apoptotic neutrophils, where the NO donor caused a concentration-dependent increase in the number of cells binding annexin $\mathrm{V}$ after $6 \mathrm{hr}$ culture (Fig. 1, C, D, and E). These findings indicate that GEA 3162 causes cell surface changes (exposure of phosphatidylserine molecules) as well as the nuclear changes (Fig. 1B) associated with apoptosis. The effects of two structurally distinct NO donors, GEA 3162, and SIN-1, on neutrophil apoptosis at 6 and $20 \mathrm{hr}$ is shown in Fig. 2. GEA $3162(10-100 \mu \mathrm{M})$ (Fig. 2A) and SIN-1 (0.3-3 mM) (Fig. 2B) caused a concentration-dependent induction of apoptosis at both time points. There was little evidence of necrosis under any condition except after treatment with GEA 3162 $(100 \mu \mathrm{M}$ ) for $20 \mathrm{hr}$ (control, $99 \pm 1 \%$ viable, $100 \mu \mathrm{M}$ GEA $3162,54 \pm 15 \%$ viable, as assessed by trypan blue exclusion). The markedly different potency and effectiveness of the two NO donors probably reflects the lipophilic nature of GEA 3162 and the fact that it is a highly effective NO donor when used in intact cells in comparison to SIN-1 $[30,31]$.

\section{Cyclic GMP-independent Effect of NO Donors on Neutrophil Apoptosis}

To investigate whether the pro-apoptotic effects of NO donors are mediated via a cGMP-dependent pathway, we (i) examined the effects of the cell-permeable cGMP analogue db-cGMP on the constitutive rate of neutrophil apoptosis and (ii) co-cultured cells with $\mathrm{NO}$ donors in the presence of the sGS inhibitor LY-83583. As shown in Fig. $3 \mathrm{~A}$, addition of db-cGMP at 0.2 or $2 \mathrm{mM}$ failed to induce apoptosis, but a concentration of $2 \mathrm{mM}$ inhibited apoptosis at 6 and $20 \mathrm{hr}$ to a similar extent as that observed with $\mathrm{db}-\mathrm{cAMP}$ [12]. Concentrations of db-cGMP $<0.2 \mathrm{mM}$ also had no effect on the rate of apoptosis (data not shown). Figure $3 \mathrm{~B}$ shows that a concentration of $100 \mu \mathrm{M} \mathrm{LY}-83583$, which inhibits agonist-induced cGMP-dependent protein kinase activation and degranulation in human neutrophils [22], did not significantly affect SIN-1 (3 mM)- or GEA $3162(30 \mu \mathrm{M})$-induced apoptosis assessed at $6 \mathrm{hr}$. Taken together, these results suggest that $\mathrm{NO}$ donors trigger apoptosis independently of cGMP signaling pathways. Interestingly, when neutrophils were co-cultured with GEA 3162 in the presence of the caspase inhibitor ZVAD-fmk $(100 \mu \mathrm{M})$, NO-induced apoptosis was inhibited by $>70 \%$ (Fig. 3C). This experiment was performed at the earlier time point of $4 \mathrm{hr}$, when the constitutive rate of apoptosis was extremely low. This ensured that the NO-induced apoptosis was blocked rather than constitutive apoptosis. 

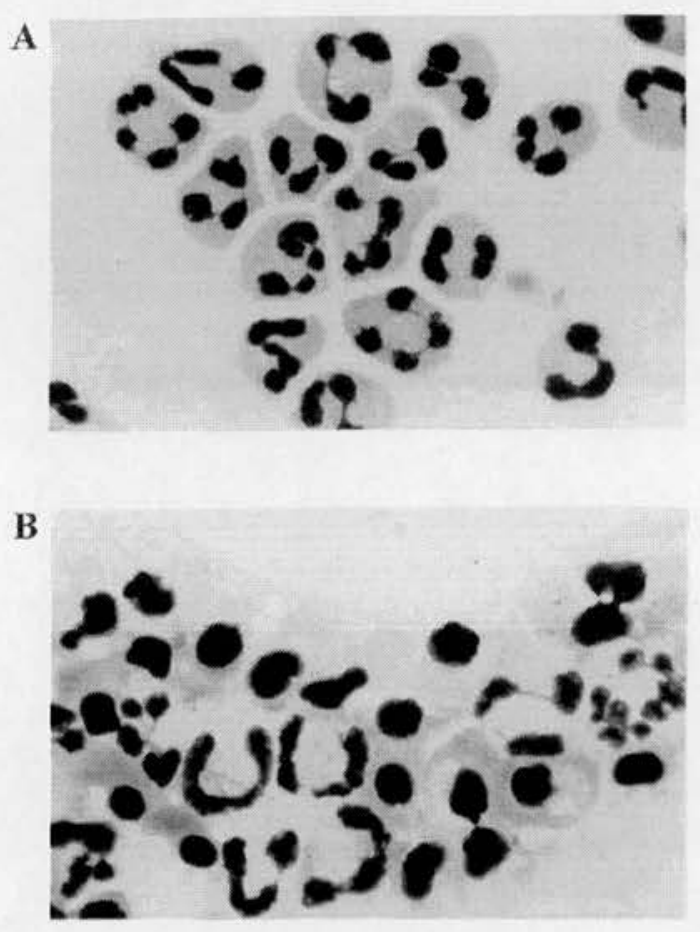

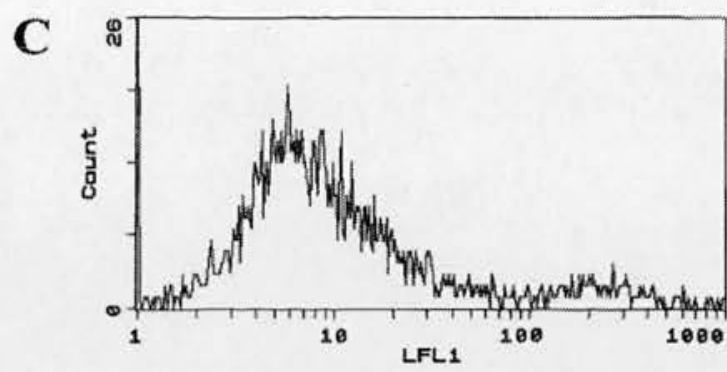

D

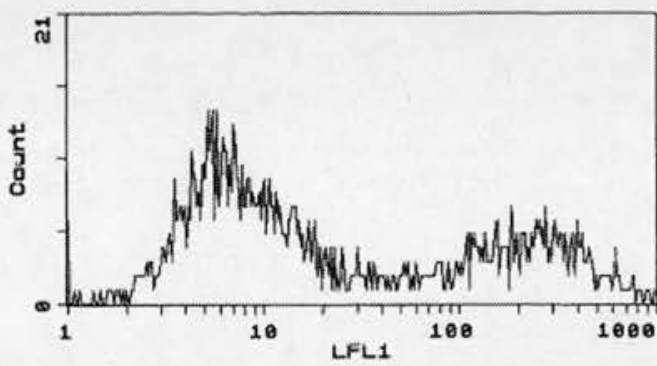

$\mathbf{E}$

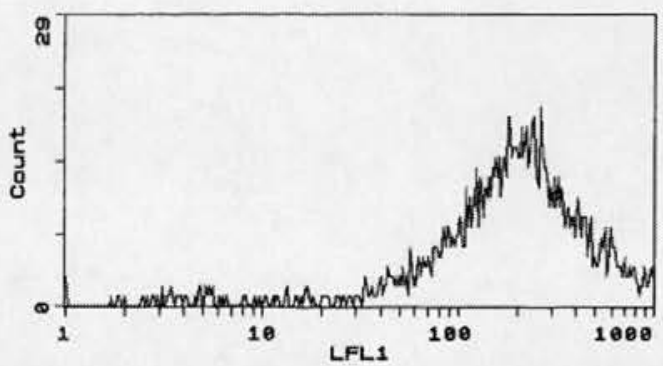

FIG. 1. Effect of the NO donor GEA 3162 on human neutrophil apoptosis. Neutrophils $\left(5 \times 10^{6} / \mathrm{mL}\right)$ were cultured with (A) vehicle control or (B) GEA $3162(100 \mu \mathrm{M})$ for $6 \mathrm{hr}$ and cytocentrifuge preparations made (magnification $\times 1000)$. Neutrophils were cultured for $6 \mathrm{hr}$ as above and treated with (C) vehicle control, (D) GEA $3162(30 \mu \mathrm{M})$, or (E) GEA $3162(100 \mu \mathrm{M})$, and samples were processed for annexin V binding to determine phosphatidylserine expression (LFL1, log fluorescence).

\section{Effects of NO Donors Potentially Mediated by the Generation of Oxygen free Radicals}

The capacity for certain $\mathrm{NO}$ donors to co-release $\mathrm{O}_{2}$ is well recognized. However, neither receptor-mediated generation of $\mathrm{O}_{2}$ nor the application of exogenous reactive oxygen species (e.g. hydrogen peroxide) is able to induce comparable apoptotic responses in neutrophils [32]. To examine whether the generation of $\mathrm{O}_{2}$ may be contributing to the pro-apoptotic effect of the NO donors, the effects of SOD $(6 \mu \mathrm{g} / \mathrm{mL})$ on SIN-1- and GEA 3162-induced apoptosis were assessed. Figure 4 demonstrates that SOD abolished the pro-apoptotic effect of SIN-1 (which releases more $\mathrm{O}_{2}$ than GEA 3162) and partially inhibited the effect of the latter, this being particularly evident at submaximal GEA 3162 concentrations. We investigated whether $\mathrm{ONOO}^{-}$, a major product of $\mathrm{O}_{2}$ and $\mathrm{NO}$ interaction, could trigger neutrophil apoptosis directly. Figure 5 shows that $\mathrm{ONOO}^{-}$ $(\geq 100 \mu \mathrm{M})$ caused a dramatic and concentration-dependent induction of neutrophil apoptosis at $4 \mathrm{hr}$ (e.g. $500 \mu \mathrm{M}$ gave approximately $40 \%$ apoptosis), although at higher concentrations and longer incubation periods evidence of necrosis is evident, as assessed by trypan blue exclusion (e.g. $855 \mu \mathrm{M}$ induced $>30 \%$ necrosis at $4 \mathrm{hr}$ ). Given the high rates of apoptosis and the onset of necrosis by $4 \mathrm{hr}$, the $6-\mathrm{hr}$ timepoint was not examined.

\section{Effects of the NO Donor GEA 3162 on Neutrophil Activation}

The rapid and dramatic pro-apoptotic effects of NO donors raised the possibility of initiation of signals within neutrophils that lead to engagement of the apoptotic machinery. We therefore examined whether these agents affected neutrophil polarization and directed migration as indices of neutrophil activation. After 45 -min pretreatment in media alone, the mean percentage shape change for control neutrophils was $14.2 \pm 1.8 \%(\mathrm{~N}=3)$, as determined by a flow cytometric method to assess shape change using forward and side scatter parameters (Fig. 6, A-D). Treatment with FMLP ( $10 \mathrm{nM}, 10 \mathrm{~min}$ ) increased shape change to $89.5 \pm 1.9 \%(\mathrm{~N}=3)$. Preincubation of neutrophils with GEA $3162(1-100 \mu \mathrm{M})$ caused a concentration-dependent inhibition $\left(\mathrm{IC}_{50}=9 \mu \mathrm{M}\right)$ of FMLP $(10 \mathrm{nM}, 10 \mathrm{~min})$ induced shape change (Fig. 6E). Note that these experiments were performed in PBS, since the presence of serum itself can induce cell polarisation. Optimal conditions for 

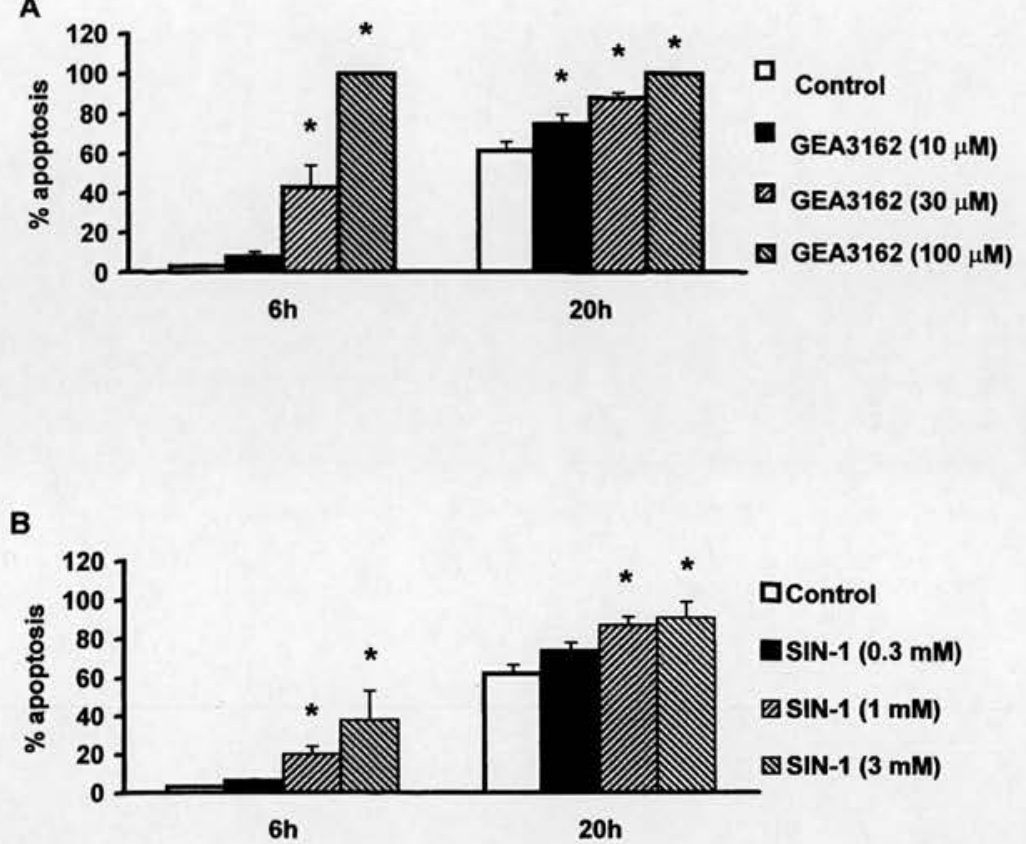

FIG. 2. Effect of the NO donors GEA 3162 and SIN-1 on human neutrophil apoptosis. Neutrophils $\left(5 \times 10^{6} / \mathrm{mL}\right)$ were cultured with (A) GEA 3162 $(10-100 \mu \mathrm{M})$ or (B) SIN-1 $(0.3-3 \mathrm{mM})$ for 6 or $20 \mathrm{hr}$ and apoptosis assessed morphologically. Results are expressed as the mean \pm SEM percent apoptosis of 3-4 separate experiments. Significant difference $(P<0.05)$ from the appropriate control (open bars) is indicated by *. chemotaxis were achieved using neutrophils suspended in Iscove's MDM containing $10 \%$ autologous serum. Mean O.D. values under control conditions (reflecting spontaneous chemokinesis) and maximal FMLP-stimulated conditions (directed chemotaxis) were $0.55 \pm 0.04$ and $1.17 \pm$ 0.14 , respectively $(\mathrm{N}=9)$. Preincubation of neutrophils with GEA $3162(1-100 \mu \mathrm{M})$ inhibited FMLP (100 nM). induced chemotaxis in a concentration-dependent fashion $\left(\mathrm{IC}_{50} \approx 7 \mu \mathrm{M}\right)$ (Fig. 7 ). SIN-1 $\left(\mathrm{IC}_{50} 1 \mathrm{mM}\right)$ caused a similar inhibition of FMLP $(100 \mathrm{nM})$-induced chemotaxis (data not shown).

\section{DISCUSSION}

Our results clearly show the capacity of structurally distinct NO donors (GEA 3162 and SIN-1) to induce apoptosis in human neutrophils. These data corroborate and extend our initial findings [23] and also those recently reported [23 25]. These results are of particular interest since neutrophils are relatively resistant to receptor-mediated or pharmacological induction of apoptosis and fail to undergo augmented apoptosis in response to stimuli such as glucocorticoids, agents that increase cAMP or cytosolic free $\mathrm{Ca}^{2+}$, and hypoxia [11-15, 32]. Although some variability occurred in the levels of induction of apoptosis between experiments, this was mainly due to donor variation and differences in activity between batches of GEA 3162 and SIN-1 (data not shown). To examine the mechanism of NO-induced neutrophil apoptosis, we initially investigated whether such effects were mediated via activation of sGC with the subsequent generation of cGMP. Although neutrophils possess a sGC [33], the mechanism of induction of apoptosis by NO does not appear to utilize the sGC-cGMP pathway, since the cyclic nucleotide analogue db-cGMP $(0.002-200 \mu \mathrm{M})$ did not induce apoptosis and the GEA 3162 and SIN-1 pro-apoptotic effect could not be blocked by the sGC inhibitor LY-83583. On the contrary, dbcGMP at a higher concentration of $2 \mathrm{mM}$ inhibited apoptosis. Preliminary data indicate that the ability of $\mathrm{db}-\mathrm{cGMP}$ to inhibit neutrophil apoptosis at higher concentrations may be mediated via cross-activation of protein kinase $\mathrm{A}$, since the protein kinase $\mathrm{A}$ inhibitor $\mathrm{H} 89$ blocks the inhibition of apoptosis by db-cGMP as well as the effect of agents that elevate cAMP [12 and *].

We next examined whether the pro-apoptotic effect of the NO donors involved, or was mediated by, the formation of oxygen-derived free radicals. Human neutrophils have the capacity to generate $\mathrm{NO}$ and $\mathrm{O}_{2}$, which may react to form $\mathrm{ONOO}^{-}[34,35]$ with the potential for the induction of apoptosis [36]. In the presence of NO donors, neutrophilderived extracellular $\mathrm{O}_{2}^{-}$-mediated generation of $\mathrm{ONOO}^{-}$ can be prevented by the presence of exogenous SOD. Furthermore, direct addition of $\mathrm{ONOO}^{-}$to neutrophils resulted in a rapid and marked induction of apoptosis, again suggesting that GEA 3162- and SIN-1-induced apoptosis may be mediated, at least in part, by $\mathrm{ONOO}^{-}$formation, particularly in the case of SIN-1, which releases equimolar concentrations of $\mathrm{NO}$ and $\mathrm{O}_{2}^{*}$. The resulting protection against SIN-1 - and GEA 3162-induced apoptosis provides strong evidence in support of this hypothesis, not least because SOD is not cell-permeable, its effects thus being restricted to protecting against extracellular $\mathrm{O}_{2}^{*}$. The finding that the pro-apoptotic effect of GEA 3162 at 100 $\mu \mathrm{M}$ was not blocked by SOD suggests that either GEA

\footnotetext{
* Wong TH and Rossi AG, unpublished observations.
} 

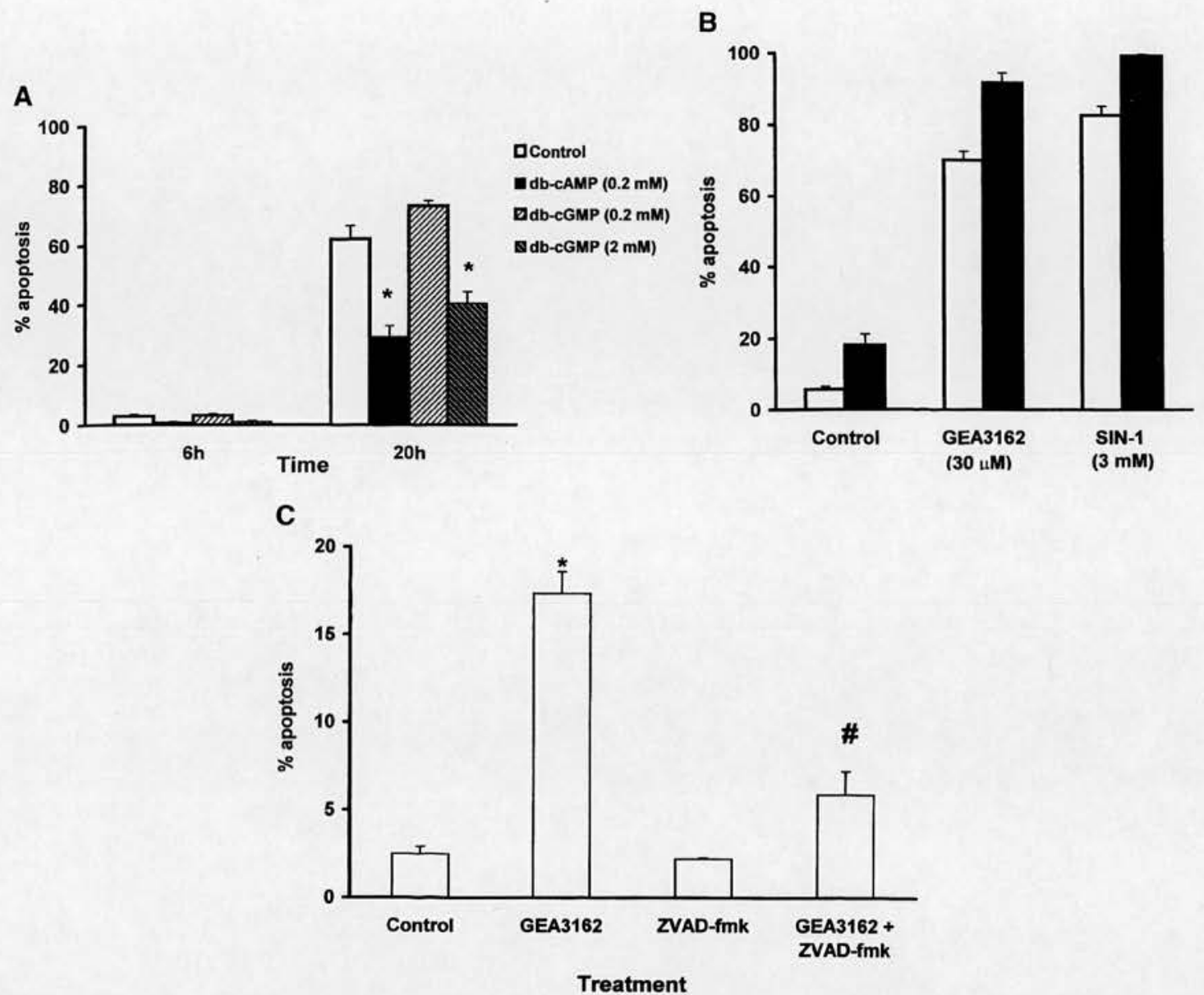

FIG. 3. (A) Effect of the cyclic nucleotide analogues db-cAMP and db-cGMP on human neutrophil apoptosis. Neutrophils $(5 \times$ $10 \% \mathrm{~mL})$ were cultured with db-cAMP $(0.2 \mathrm{mM})$ and db-cGMP $(0.2$ and $2 \mathrm{mM})$ for 6 or $20 \mathrm{hr}$ and apoptosis assessed morphologically. Results are expressed as the mean \pm SEM percent apoptosis of 6 separate experiments. Significant difference $(P<0.05)$ from the appropriate control (open bars) is indicated by *. (B) Effect of the sGC inhibitor LY-83583 on NO donor-induced human neutrophil apoptosis. Neutrophils $(5 \times 10 \% \mathrm{~mL})$ were cultured with GEA $3162(30 \mu \mathrm{M})$ or SIN-1 $(3 \mathrm{mM})$ in the absence (open bars) or presence of $100 \mu \mathrm{M}$ LY-83583 (filled bars) for $6 \mathrm{hr}$ and apoptosis assessed morphologically. Data represent means \pm SD of triplicate determinations from a single representative experiment. (C) Effect of the caspase inhibitor ZVAD-fmk on GEA 3162-induced human neutrophil apoptosis. Neutrophils $\left(5 \times 10^{6} / \mathrm{mL}\right)$ were cultured with GEA $3162(30 \mu \mathrm{M})$ in the presence or absence of $100 \mu \mathrm{M}$ ZVAD-fmk for $4 \mathrm{hr}$ and apoptosis thereafter assessed morphologically. Results are expressed as the mean \pm SEM percent apoptosis of 3 separate experiments. Significant difference $(P<0.05)$ from the control and from GEA 3162 is indicated by * and ${ }^{*}$, respectively.

3162 triggers apoptosis by both $\mathrm{ONOO}^{-}$-dependent and $\mathrm{ONOO}^{-}$-independent routes or that SOD is unable to provide complete protection against $\mathrm{ONOO}^{-}$formation at this concentration of $\mathrm{NO}$ donor. Whether $\mathrm{O}_{2}$ is produced as a byproduct of $\mathrm{NO}$ during its oxidation to $\mathrm{NO}_{2}$ by dioxygen or released after endogenous production by neutrophils themselves, the presence of SOD in these experiments should prevent the formation of oxygen-derived free radicals. In addition, further preliminary data suggest that the generation of endogenous NO by the neutrophil does not appear to play a critical role in regulating the constitutive rate of apoptosis in these cells since (i) depletion of the NO synthase substrate L-arginine had no significant effect on the rate of apoptosis at $20 \mathrm{hr}$ and (ii) incubation of neutrophils with the NO synthase inhibitors L-NMMA $\left(\mathrm{N}^{\mathrm{G}}\right.$-monomethyl-L-arginine) $(0.3 \mathrm{mM})$ and L-NAME
( $N^{\mathrm{G}}$-nitro-L-arginine methyl ester) $(0.3 \mathrm{mM})$ likewise did not affect the rate of apoptosis compared to control untreated cells (data not shown). Interestingly, the sGC inhibitor LY-83583 $(100 \mu \mathrm{M})$ reproducibly increased the rate of constitutive neutrophil apoptosis. The data shown (Fig. 3, A and B) suggest that cGMP, while not involved in NO-induced apoptosis, can clearly enhance the survival of neutrophils. This supports the view that activation of sGC may play a role in regulating inhibition of apoptosis, particularly since the inhibitor enhances the increase in the rate of apoptosis achieved by $\mathrm{NO}$ alone.

A number of possible molecular mechanisms may account for the pro-apoptotic effect of $\mathrm{ONOO}^{-}$and $\mathrm{NO}$ donors. Firstly, reactive oxygen and nitrogen radicals can lead to direct 'free radical-mediated' DNA damage, including deamination and DNA strand breaks [37, 38]. Sec- 


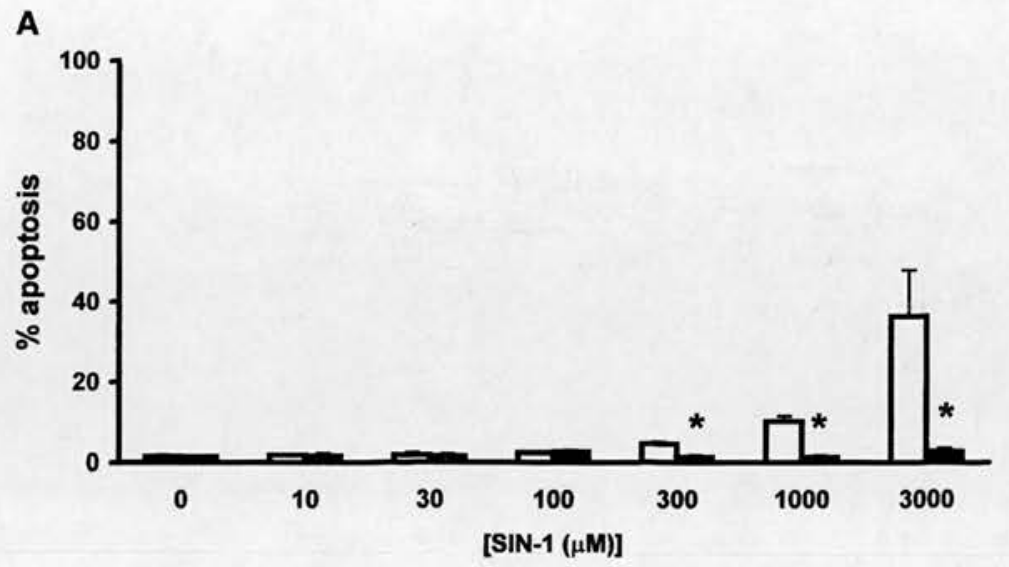

B

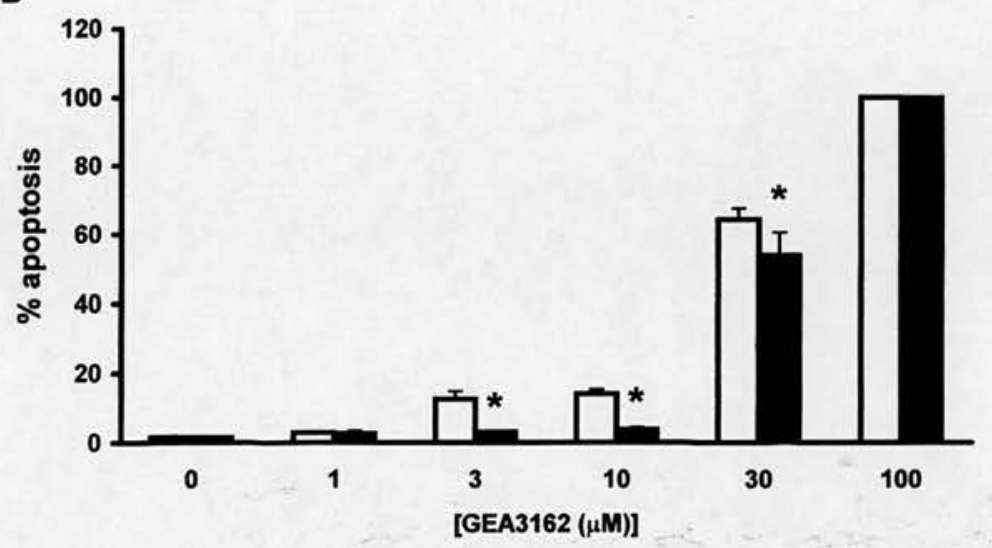

FIG. 4. Effect of SOD on GEA 3162- and SIN-1induced human neutrophil apoptosis. Neutrophils $\left(5 \times 10^{6} / \mathrm{mL}\right)$ were cultured with (A) SIN-1 $(10-3000 \mu \mathrm{M})$ and (B) GEA $3162(1-100 \mu \mathrm{M})$ in the absence (open bars) or in the presence (filled bars) of $6 \mu \mathrm{g} / \mathrm{mL}$ SOD for $6 \mathrm{hr}$ and apoptosis assessed morphologically. Similar results were obtained after 20-hr culture (data not shown). Results are expressed as the mean \pm SEM percent apoptosis of 3 separate experiments. Significant difference $(P<0.05)$ from the appropriate control (open bars) is indicated by *.

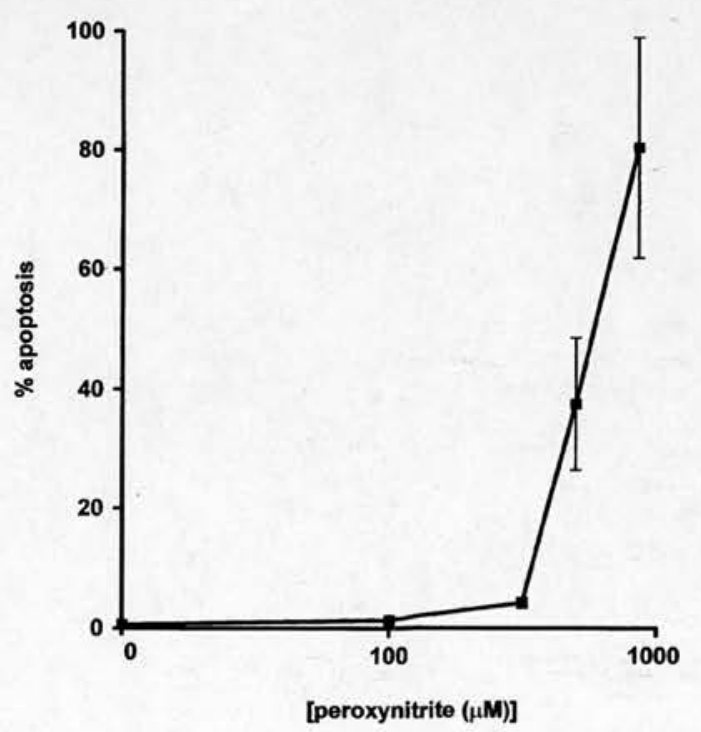

FIG. 5. Effect of $\mathrm{ONOO}^{-}$on human neutrophil apoptosis. Neutrophils $\left(5 \times 10^{6} / \mathrm{mL}\right.$ in PBS in the absence of serum) were cultured with $\mathrm{ONOO}^{-}$for $4 \mathrm{hr}$ and apoptosis assessed morphologically. Results are expressed as the mean \pm SEM percent apoptosis of 3 separate experiments. ondly, recent evidence indicates that NO effects could be mediated by inhibition of cytosolic ADP-ribosyltransferase, an enzyme that catalyzes the transfer of the ADP-ribose moiety of $\mathrm{NAD}^{+}$to other proteins [39]. Finally, conversion of $\mathrm{NO}$ to $\mathrm{ONOO}^{-}$may deplete intracellular $\mathrm{Ca}^{2+}$ stores [40]. This effect could play a crucial role in neutrophil apoptosis, since elevation of cytosolic free $\mathrm{Ca}^{2+}$ delays neutrophil apoptosis $[11,14]$ whereas depletion may induce apoptosis [11]. Our results suggest that the effects of NO donors are not due to direct DNA damage, since the caspase inhibitor ZVAD-fmk dramatically inhibited the pro-apoptotic effect of NO donors. NO has been reported to inhibit the activity of the transcription factor nuclear factor-kappaB (NF-kB) [41], and interestingly we have recently demonstrated that inhibition of this transcription factor results in the onset of neutrophil apoptosis [42]. We are currently investigating whether $\mathrm{NO}$ can inhibit NF- $\mathrm{kB}$ in neutrophils, thus providing another possible mechanism for NO-induced apoptosis in these cells. Results in Figs. 6 and 7 clearly demonstrate that GEA 3162 inhibits FMLP. induced shape change and chemotaxis consistent with inhibitory actions of NO upon neutrophil activation [20, 43-45]. Clearly, further work is required to ascertain the precise signaling pathways involved and whether the reported effects of $\mathrm{NO}$ on cytoskeletal elements via ADP. 
A

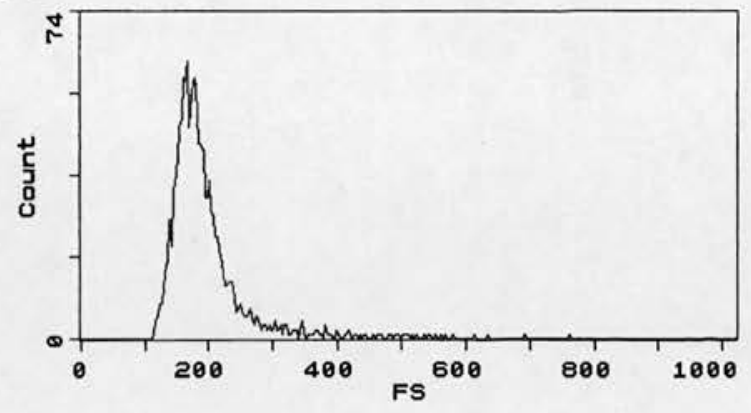

C

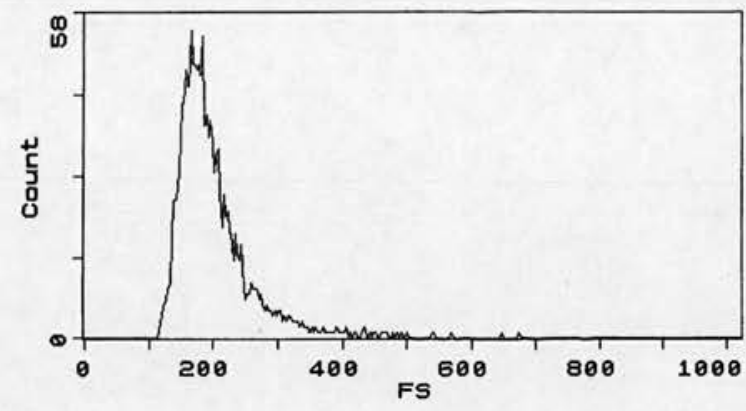

B

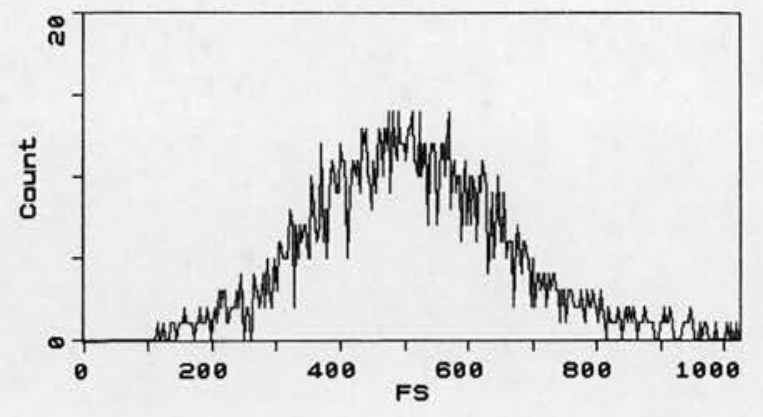

D

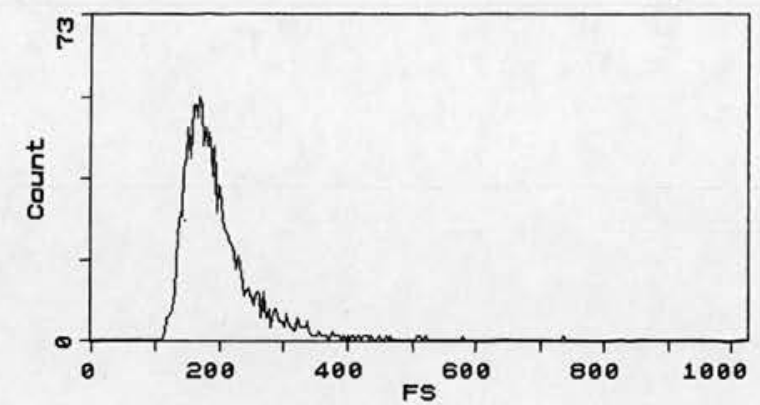

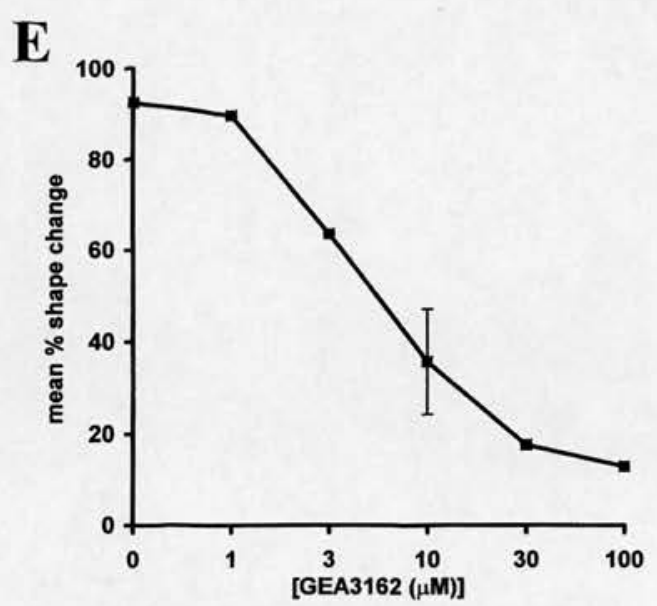

FIG. 6. Effect of GEA 3162 on FMLP-induced shape change. Neutrophils $\left(2 \times 10^{6} / \mathrm{mL}\right.$ in PBS in the absence of serum) were preincubated with GEA 3162 for $45 \mathrm{~min}$ at $37^{\circ}$ before the addition of $10 \mathrm{nM}$ FMLP for $10 \mathrm{~min}$. The incubations were terminated by the addition of $500 \mu \mathrm{L} 2.5 \%$ gluteraldehyde. Samples were assessed by an EPICS (Profile II) flow cytometer. Representative flow cytometry histograms of control cells (A), FMLP-stimulated cells (B), cells preincubated with GEA 3162 alone (C), and cells preincubated with GEA 3162 followed by FMLP stimulation (D) are shown, where the $x$-axis shows mean forward light scatter (FS) and the $y$-axis is the relative cell number. The mean percentage shape change (E) was calculated from the FS of each sample by gating out the population of non-shape-changed neutrophils. Results are expressed as the mean \pm SEM percentage shape change of 3 separate experiments.

ribosylation of actin [46] are responsible for the effects on neutrophil activation and apoptosis. Our results, however, show that NO donors can functionally uncouple neutrophil responsiveness to external stimuli by two potential mechanisms: by directly inhibiting neutrophil function (inhibition of shape change and chemotaxis) and by stimulating programmed cell death (inducing apoptosis).

In conclusion, we have demonstrated that structurally distinct NO donors have the unique ability to induce neutrophil apoptosis and inhibit neutrophil activation. The induction of apoptosis by NO donors appears to be mediated via a caspase-dependent mechanism as well as a cGMP-independent pathway, possibly involving the formation of $\mathrm{ONOO}^{-}$. Since the induction of neutrophil apoptosis and the functional uncoupling of these cells appear to be prerequisites for the successful resolution of inflamma- 


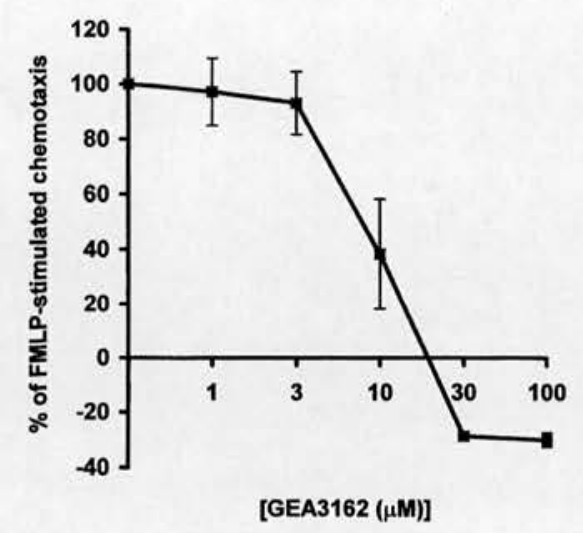

FIG. 7. Effect of GEA 3162 on FMLP-induced chemotaxis. Neutrophils $\left(3 \times 10^{6} / \mathrm{mL}\right)$ were preincubated with vehicle control or GEA $3162(1-100 \mu \mathrm{M})$ for $45 \mathrm{~min}$ at $37^{\circ}$ before chemotaxis to FMLP $\left(100 \mathrm{nM} ; 90 \mathrm{~min} ; 37^{\circ}\right)$ was assessed. Results are expressed as a mean \pm SEM percentage of control O.D. values obtained at $550 \mathrm{~nm}$ of 3 separate experiments. Similar results were obtained with SIN-1 (data not shown).

tion, the ability of NO donors to trigger both these events may provide a novel pharmacological approach to promote resolution of inflammation.

The Medical Research Council, U.K., Programme Grant Number G9016491 and The Wellcome Trust supported this work. E.R.C. is a Wellcome Senior Researcher in Clinical Science. A.G.R. and E.R.C. contributed equally to this manuscript. C.W. and T.H.W. are joint first authors. We thank Dr. Ian Dransfield for constructive criticism of this manuscript.

\section{References}

1. Kerr JFR, Wyllie AH and Currie AR, Apoptosis: A basic biological phenomenon with wide-ranging implications in tissue kinetics. Br J Cancer 26: 239-257, 1972.

2. Rossi AG and Haslett C, Inflammation, cell injury, and apoptosis. In: Lung Biology in Health and Disease (Ed. Said SI), Vol. 112, pp. 9-24. Marcel Dekker Inc., New York, 1998.

3. Savill JS, Wyllie AH, Henson JE, Walport MJ, Henson PM and Haslett C, Macrophage phagocytosis of aging neutrophils in inflammation. Programmed cell death in the neutrophil leads to its recognition by macrophages. $J$ Clin Invest 83: $865-875,1989$.

4. Lee A, Whyte MK and Haslett C, Inhibition of apoptosis and prolongation of neutrophil functional longevity by inflammatory mediators. J Leukoc Biol 54: 283-288, 1993.

5. Whyte MK, Meagher LC, MacDermot J and Haslett C, Impairment of function in aging neutrophils is associated with apoptosis. J Immunol 150: 5124-5134, 1993.

6. Dransfield I, Buckle AM, Savill JS, McDowall A, Haslett C and Hogg N, Neutrophil apoptosis is associated with a reduction in CD16 (FcyRIII) expression. J Immunol 153: 1254-1263, 1994.

7. Meagher LC, Savill JS, Baker A, Fuller RW and Haslett C, Phagocytosis of apoptotic neutrophils does not induce macrophage release of thromboxane $B_{2}$. J Leukoc Biol 52: 269 273, 1992.

8. Barnes PJ and Belvisi MG, Nitric oxide and lung disease. Thorax 48: 1034-1043, 1993.

9. Kubes P, Nitric oxide affects microvascular permeability in the intact and inflamed vasculature. Microcirculation 2: 235 $244,1993$.

10. Moilanen $\mathrm{E}$ and Vapaatalo $\mathrm{H}$, Nitric oxide in inflammation and immune response. Ann Med 27: 359-367, 1995.

11. Whyte MK, Hardwick SJ, Meagher LC, Savill JS and Haslett C, Transient elevations of cytosolic free calcium retard subsequent apoptosis in neutrophils in vitro. J Clin Invest 92: $446-455,1993$.

12. Rossi AG, Cousin JM, Dransfield I, Lawson MF, Chilvers ER and Haslett C, Agents that elevate cAMP inhibit human neutrophil apoptosis. Biochem Biophys Res Commun 217: 892-899, 1995.

13. Meagher LC, Cousin JM, Seckl JR and Haslett C, Opposing effects of glucocorticoids on the rate of apoptosis in neutrophilic and eosinophilic granulocytes. J Immunol 156: 4422 4428, 1996.

14. Cousin JM, Haslett C and Rossi AG, Regulation of granulocyte apoptosis by PKC inhibition and elevation of $\left[\mathrm{Ca}^{2+}\right]_{\text {. }}$. Biochem Soc Trans 25: 243S, 1997.

15. Cousin JM, Haslett C and Rossi AG, Effect of the protein phosphatase inhibitors, okadaic acid and calyculin A, on dexamethasone-mediated inhibition of neutrophil apoptosis. Biochem Soc Trans 25: 246S, 1997.

16. Wyllie AH, Glucocorticoid-induced thymocyte apoptosis is associated with endogenous endonuclease activation. Nature 284: 555-556, 1980.

17. McConkey DJ and Orrenius S, Signal transduction pathways in apoptosis. Stem Cells 14: 619-631, 1996.

18. Murray J, Barbara JA, Dunkley SA, Lopez AF, Van Ostade X, Condliffe AM, Dransfield I, Haslett C and Chilvers ER, Regulation of neutrophil apoptosis by tumor necrosis factoralpha: requirement for TNFR55 and TNFR75 for induction of apoptosis in vitro. Blood 90: 2772-2783, 1997.

19. Colotta F, Re F, Polentarutti N, Sozzani S and Mantovani A, Modulation of granulocyte survival and programmed cell death by cytokines and bacterial products. Blood 80: 20122020, 1992.

20. Moilanen E, Vuorinen P, Kankaanranta H, Metsa-Ketela T and Vapaatalo $\mathrm{H}$, Inhibition by nitric oxide-donors of human polymorphonuclear leukocyte functions. $\mathrm{Br} J$ Pharmacol 109: 852-858, 1993.

21. Feelisch M and Noack EA, Correlation between nitric oxide formation during degradation of organic nitrates and activation of guanylate cyclase. Eur J Pharmacol 139: 19-30, 1987.

22. Wyatt TA, Lincoln TM and Pryzwansky KB, Regulation of human neutrophil degranulation by LY- 83583 and L-arginine: role of cGMP-dependent protein kinase. Am J Physiol 265: C201-C211, 1993.

23. Wong TH, Rossi AG and Chilvers ER, Inhibition of neutrophil function and survival by nitric oxide donors. Clin Sci 92: 7P, 1997.

24. Blaylock MG, Cuthbertson BH, Galley HF, Ferguson NR and Webster NR, The effect of nitric oxide and peroxynitrite on apoptosis in human polymorphonuclear leukocytes. Free Radic Biol Med 25: 748-752, 1998.

25. Fortenberry JD, Owens ML, Brown MR, Atkinson D and Brown LA, Exogenous nitric oxide enhances neutrophil cell death and DNA fragmentation. Am J Respir Cell Mol Biol 18: 421-428, 1998.

26. Haslett C, Guthrie LA, Kopaniak MM, Johnston RB and Henson PM, Modulation of multiple neutrophil functions by preparative methods or trace concentrations of bacterial lipopolysaccharide. Am J Pathol 119: 101-110, 1985.

27. Koopman G, Reutelingsperger CP, Kuijten GA, Keehnen $\mathrm{RM}$, Pals ST and Van oers MH, Annexin V for flow cytometric detection of phosphatidylserine expression on B cells undergoing apoptosis. Blood 84: 1415-1420, 1994.

28. Kitchen E, Rossi AG, Condliffe AM, Haslett C and Chilvers 
ER, Demonstration of reversible priming of human neutrophils using platelet-activating factor. Blood 88: 4330-4337, 1996.

29. Uppu RM and Pryor WA, Synthesis of peroxynitrite in a two-phase system using isoamyl nitrite and hydrogen peroxide. Anal Biochem 236: 242-249, 1996.

30. Favre CJ, Ufret-Vincenty CA, Stone MR, Ma H and Gill DL, $\mathrm{Ca}^{2+}$ pool emptying stimulates $\mathrm{Ca}^{2+}$ entry activated by S-nitrosylation. J Biol Chem 273: 30855-30858, 1998.

31. Holm P, Kankaanranta $\mathrm{H}$, Metsa-Ketela $\mathrm{T}$ and Moilanen E, Radical releasing properties of nitric oxide donors GEA 3162, SIN-1 and S-nitroso-N-acetylpenicillamine. Eur J Pharmacol 346: 97-102, 1998.

32. Hannah S, Mecklenburgh K, Rahman I, Bellingan GJ, Greening $\mathrm{A}$, Haslett $\mathrm{C}$ and Chilvers ER, Hypoxia prolongs neutrophil survival in vitro. FEBS Lett 372: 233-237, 1995.

33. Lad PM, Glovsky MM, Richards JH, Smiley PA and Backstrom B, Regulation of human neutrophil guanylate cyclase by metal ions, free radicals and the muscarinic cholinergic receptor. Mol Immunol 22: 731-739, 1985.

34. Carreras MC, Pargament GA, Catz SD, Poderosos JJ and Boveris A, Kinetics of nitric oxide and hydrogen peroxide production and formation of peroxynitrite during the respiratory burst of human neutrophils. FEBS Lett 341: 65-68, 1994.

35. Fukuyama N, Ichimori K, Su Z, Ishida $H$ and Nakazawa $H$, Peroxynitrite formation from activated human leukocytes. Biochem Biophys Res Commun 224: 414-419, 1996.

36. Salgo MG, Squadrito GL and Pryor WA, Peroxynitrite causes apoptosis in rat thymocytes. Biochem Biophys Res Commun 215: 1111-1118, 1995.

37. Wink DA, Kasprzak KS, Maragos CM, Elespuru RK, Misra M, Dunams TM, Cebula TA, Koch WH, Andrews AW, Allen JS and Keefer LK, DNA deaminating ability and genotoxicity of nitric oxide and its progenitors. Science 254: 1001-1003, 1991.

38. Schwartzman RA and Cidlowski JA, Apoptosis: The biochemistry and molecular biology of programmed cell death. Endocr Rev 14: 133-151, 1993.

39. Kuo ML, Chau YP, Wang JH and Shiah SG, Inhibitors of poly (ADP-ribose) polymerase block nitric oxide-induced apoptosis but not differentiation in human leukemia HL-60 cells. Biochem Biophys Res Commun 219: 502-508, 1996.

40. Elliot SJ, Peroxynitrite modulates receptor-activated $\mathrm{Ca}^{2+}$ signaling in vascular endothelial cells. Am J Physiol 270: L954-L961, 1996.

41. Matthews JR, Botting CH, Panico M, Morris HR and Hay $\mathrm{RT}$, Inhibition of NF-kB DNA binding by nitric oxide. Nucleic Acids Res 24: 2236-2242, 1996.

42. Ward C, Chilvers ER, Lawson MF, Pryde JG, Fujihara S, Farrow SN, Haslett $\mathrm{C}$ and Rossi $\mathrm{AG}, \mathrm{NF}-\mathrm{kB}$ activation is a critical regulator of human granulocyte apoptosis in vitro. J Biol Chem 274: 4309-4318, 1999.

43. Kubes P, Suzuki M and Granger DN, Nitric oxide: An endogenous modulator of leukocyte adhesion. Proc Natl Acad Sci U S A 88: 4651-4655, 1991.

44. Clancy RM, Leszczynska-Piziak J and Abramson SB, Nitric oxide stimulates the ADP-ribosylation of actin in human neutrophils. Biochem Biophys Res Commun 191: 847-852, 1992.

45. Wanikiat $P$, Woodward DF and Armstrong RA, Investigation of the role of nitric oxide and cyclic GMP in both the activation and inhibition of human neutrophils. $\mathrm{Br} J$ Pharmacol 122: 1135-1145, 1997.

46. Clancy RM, Leszczynska-Piziak J and Abramson SB, Nitric oxide, an endothelial cell relaxation factor, inhibits neutrophil superoxide anion production via a direct action on the NADPH oxidase. J Clin Invest 90: 1116-1121, 1992. 


\title{
Temperature-dependent Arrest of Neutrophil Apoptosis
}

\author{
FAILURE OF Bax INSERTION INTO MITOCHONDRIA AT $15^{\circ} \mathrm{C}$ PREVENTS THE RELEASE OF CYTOCHROME $c^{*}$
}

Received for publication, February 8, 2000, and in revised form, July 12, 2000

Published, JBC Papers in Press, July 13, 2000, DOI 10.1074/jbc.M001008200

\author{
James G. Pryde $\ddagger$, Annemieke Walker§, Adriano G. Rossi, Sharon Hannah, \\ and Christopher Haslett \\ From the Rayne Laboratory, University of Edinburgh Medical School, Teviot Place, \\ Edinburgh EH8 9AG, United Kingdom
}

\begin{abstract}
Apoptosis is essential for the resolution of neutrophilic inflammation. To define the mechanisms triggering the execution phase of apoptosis we developed and utilized a model in which culture of human neutrophils at $15^{\circ} \mathrm{C}$ for $20 \mathrm{~h}$ arrested apoptosis and subsequent warming to $37^{\circ} \mathrm{C}$ triggered a synchronous burst of apoptosis. Treatment of $15{ }^{\circ} \mathrm{C}$ cultured neutrophils with the pan-caspase inhibitor zVAD-fmk just before warming to $37^{\circ} \mathrm{C}$ inhibited the morphological changes associated with apoptosis, but did not prevent the insertion of the proapoptotic protein Bax into mitochondria nor the inhibition of secretion and the externalization of phosphatidylserine, indices of neutrophil apoptosis. In both intact neutrophils and a cell-free extract, cytochrome $c$ released from mitochondria induced proteolytic cleavage of procaspase-3. At $15{ }^{\circ} \mathrm{C}$ the binding of Bax to mitochondria was uncoupled from Bax insertion into the mitochondrial membrane required for the release of cytochrome c. Apoptosis was also inhibited by low pH during warming to $37^{\circ} \mathrm{C}$, suggesting that changes to the conformation of Bax, necessary for membrane insertion, were being inhibited. Bax insertion was only sensitive to zVAD-fmk when added at the start of the $15^{\circ} \mathrm{C}$ culture period, suggesting that a cytoplasmic substrate of the effector caspases may mediate in the mechanism of Bax insertion into mitochondria.
\end{abstract}

Successful resolution of the inflammatory response requires that granulocytes, neutrophils and eosinophils, trigger an intracellular program for "silent" self-destruction called apoptosis $(1,2)$. If cell-death occurs by necrosis the cytotoxic cargo of granulocyte molecules is released, inducing tissue damage and chronic inflammation and stimulating the release of proinflammatory macrophage products to promote inflammation by other routes. The apoptotic program induces the morphological hallmarks of apoptosis, nuclear condensation and cell shrinkage, and shuts down the secretory potential of granulocytes (3). Changes to the molecular profile of the surface of apoptotic neutrophils target them for phagocytosis by macrophages (4,

* This work is supported by Program Grant G9016491 from the Medical Research Council, United Kingdom. The costs of publication of this article were defrayed in part by the payment of page charges. This article must therefore be hereby marked "advertisement" in accordance with 18 U.S.C. Section 1734 solely to indicate this fact.

$\doteqdot$ To whom correspondence should be addressed: Respiratory Medicine Unit, Dept. of Medicine (RIE), Rayne Laboratory, University of Edinburgh Medical School, Teviot Place, Edinburgh, EH8 9AG. Scotland, UK. Tel.: 44-131-650-6949; Fax: 44-131-650-4384; E-mail: j.pryde@ed.ac.uk.

$\$$ Supported by University of Edinburgh Faculty of Medicine, Vans Dunlop, and Shaw McFie Lang Postgraduate Research Scholarships.
5), without release of proinflammatory mediators from macrophages (6). Proinflammatory mediators and cytokines, such as granulocyte-macrophage colony-stimulating factor, lipopolysaccharide, C5a, or an hypoxic environment at the site of inflammation can prolong the functional life span of granulocytes by delaying apoptosis $(7,8)$ through increased expression of the anti-apoptotic proteins Bcl- $\mathrm{X}_{\mathrm{L}}(9)$ and Mcl-1 (10). The molecular mechanism triggering the execution phase of apoptosis in granulocytes is unknown but activation of tumor necrosis factor $\alpha$ and Fas (CD95) cell surface receptors increase expression of the proapoptotic proteins Bax and procaspase- 3 (9-14). Both the p38 mitogen-activated protein kinase and p42/p44 mitogen-activated protein kinase, and the transcription factor nuclear factor- $\kappa \mathrm{B}$, regulate the granulocyte apoptotic program $(15,16)$, the signals transduced by these pathways converging to induce activation of procaspase-3 (17-20).

In mammalian cells the execution phase of apoptosis involves either the direct activation of procaspase- 3 by caspase- 8 (21), or indirect activation of procaspase- 3 through the release of apoptosis-inducing factors, such as cytochrome $c$, from mitochondria (22-30). The proapoptotic Bcl-2 family member Bax is a soluble, monomeric, cytoplasmic protein (31) that inserts an hydrophobic C-terminal membrane-spanning domain into mitochondria $(32,33)$, inducing release of cytochrome $c$ (31-35), triggering the activation of caspase- $3(36-38)$ and the execution phase of apoptosis. Bax dimerization (39), the addition of recombinant Bax to isolated mitochondria (36) or overexpression of Bax (40) has also been shown to induce the release of cytochrome $c$. The mechanism by which cytochrome $c$ is translocated from mitochondria into the cytoplasm is controversial (41). However, once in the cytoplasm cytochrome $c$ complexes with apoptosis-protease-activating factor-1 (Apaf-1) and procaspase-9 (26) to form a protein complex the "apoptosome" (42). In the presence of dATP this complex induces the proteolytic cleavage and activation of procaspase- 3 that triggers a downstream cascade of caspase activity (43). It has been reported that after differentiation and maturation neutrophils have a reduced number of phenotypically atypical mitochondria, obtaining ATP predominantly by glycolysis (10). Thus, whether mitochondria play a role in triggering neutrophil apoptosis remains to be established.

Here we show that peripheral blood neutrophils cultured in vitro at $15^{\circ} \mathrm{C}$ for $20 \mathrm{~h}$ failed to induce the execution phase of apoptosis until warmed to $37^{\circ} \mathrm{C}$, when they showed a synchronous burst of apoptosis. In temperature-arrested neutrophils endogenous Bax showed peripheral binding to mitochondria but failed to induce activation of caspase- 3 and apoptosis. On warming to $37^{\circ} \mathrm{C}$ Bax inserted into the neutrophil membranes with concomitant proteolytic cleavage of procaspase-3 and induction of apoptosis. In both intact neutrophils and cell-free 
neutrophil extracts we show that the proteolytic cleavage of procaspase- 3 is induced by translocation of cytochrome $c$ into the cytoplasm. Analysis of plasma membrane events showed that externalization of phosphatidylserine and the inhibition of secretion were uncoupled from the activation of caspase-3, when the pan-caspase inhibitor benzyloxycarbonyl-Val-AlaAsp-fluoromethyl ketone (zVAD-fmk) ${ }^{1}$ was added to $15^{\circ} \mathrm{C}$ cultured neutrophils before warming them to $37^{\circ} \mathrm{C}$. Under these conditions the caspase inhibitor did not prevent Bax insertion into mitochondria when the cells were warmed from 15 to $37^{\circ} \mathrm{C}$. However, surprisingly, Bax insertion was inhibited if zVAD-fmk was added to neutrophils at the start of their incubation at $15^{\circ} \mathrm{C}$. This experimental model of apoptosis has provided insights into the molecular mechanisms that trigger the execution phase of neutrophil apoptosis.

\section{EXPERIMENTAL PROCFDURFS}

Granulocyte Isolation and Culture-Neutrophils were purified on gradients of Percoll from Amersham Pharmacia Biotech (Bucks, United Kingdom) (44). They were cultured in Tuf-Tainer Teflon pots from Pierce \& Warriner Ltd. (Chester, UK) at $5 \times 10^{6} \mathrm{cells} / \mathrm{ml}$ in growth medium containing: Iscove's modified Dulbecco's medium supplemented with $2 \mathrm{~mm}$ glutamine, $100 \mathrm{units} / \mathrm{ml}$ penicillin, $100 \mathrm{\mu g} / \mathrm{ml}$ strep tomycin, and $10 \%(\mathrm{v} / \mathrm{v})$ autologous serum. Culture at $15^{\circ} \mathrm{C}$ was in growth medium containing: $25 \mathrm{~mm}$ Hepes- $\mathrm{NaOH}, \mathrm{pH} 7,0.2 \%$ (w/y) endotoxin-free bovine serum albumin (BSA), and $20 \mu \mathrm{g} / \mathrm{ml}$ cycloheximide. BSA and all other chemicals were from Sigma. Neutrophil preparations were $98 \%$ pure with $<2 \%$ eosinophil contamination (16). Granulocytes from atopic donors were used to purify eosinophils by a negative selection procedure (16). Incubations with $\mathrm{zVAD}$-fmk from BACHEM Ltd. (Essex, UK), staurosporine from CN Biosciences (Nottingham, UK), and bongkrekic acid from BIOMOL Research Laboratories Inc. (Plymouth Meeting, PA) were in Iscove's medium with $0.2 \%$ (w/v) BSA and $20 \mu \mathrm{g} / \mathrm{ml}$ cycloheximide.

Assessment of Granulocyte Apoptosis-Nuclear morphology was assessed on cytocentrifuged slides stained with Diff-Quik ${ }^{\circledast a m i d o r ~ L t d . ~}$ (Abingdon, Oxon, UK) (16). Annexin V-FITC, from BenderMed Systems (Vienna, Austria), was used at 1:200 dilution $\left(5 \times 10^{5} \mathrm{cells} / \mathrm{ml}\right)$ to assay phosphatidylserine externalization and propidium iodide $(10 \mu \mathrm{g} / \mathrm{ml}$ in $\mathrm{Ca}^{2+} / \mathrm{Mg}^{2+}$-free phosphate-buffered saline) was used to monitor membrane integrity by flow cytometry on an EPICS Profile II from Coulter Electronics (Luton, UK) (16). DNA fragmentation was detected by the TUNEL in situ cell death detection kit from Roche Molecular Biochemicals (East Sussex, UK) and by sizing of DNA fragments on agarose gels, capturing images using a GS $1600 \mathrm{~m}$ gel documentation sys tem Ultra-Violet Products Ltd. (Cambridge, UK) $(16,45)$. The data is representative of at least three experiments unless indicated.

Measurement of Intracellular $p H$-Neutrophils $\left(5 \times 10^{4}\right.$ cells $)$ in 100 $\mu \mathrm{l}$ of Hank's buffered saline, supplemented with $20 \mathrm{~mm}$ Hepes-NaOH $\mathrm{pH} 7.4,5 \mathrm{~mm}$ glucose, $0.2 \%$ (w/v) BSA, and $10 \mu \mathrm{M}$ SNARF-1/AM from Molecular Probes (Eugene, OR) were incubated for $10 \mathrm{~min}$ at $37^{\circ} \mathrm{C}$. Intracellular SNARF-1 was excited at $488 \mathrm{~nm}$ and emission was meas ured at 575 and $670 \mathrm{~nm}$ using linear amplifiers and data plotted as forward scatter versus fluorescence ratio (FL2/FL3). The intracellular $\mathrm{pH}\left(\mathrm{pH}_{i}\right)$ was determined by comparing mean $575 / 670 \mathrm{~nm}$ fluorescence ratio values of histograms, to a calibration curve of histograms from fresh neutrophils. The $\mathrm{pH}$ was clamped between 5.6 and 7.8 , with overlapping $0.2 \mathrm{pH}$ unit intervals, in $20 \mathrm{~mm}$ Mes, Pipes, and Hepe buffers and $2 \mu \mathrm{g} / \mathrm{ml}$ nigericin, in a high-potassium medium containing: $110 \mathrm{~mm} \mathrm{KCl}, 20 \mathrm{~mm} \mathrm{NaCl}, 5 \mathrm{~mm}$ glucose, $1 \mathrm{~mm} \mathrm{MgCl}_{2}, 1.5 \mathrm{~mm} \mathrm{CaCl}$, $0.2 \%(\mathrm{w} / \mathrm{v}) \mathrm{BSA}$

Preparation of Cytosol from $15^{\circ} \mathrm{C}$ Neutrophils-Neutrophils $(5 \times$ $10^{8} ;<5 \%$ apoptotic after $20 \mathrm{~h}$ at $15^{\circ} \mathrm{C}$ ) were sedimented at $1,500 \times g_{\text {av }}$ at $4{ }^{\circ} \mathrm{C}$. They were washed twice in $50 \mathrm{ml}$ of homogenization buffer: 15 mм Pipes- $\mathrm{NaOH}, \mathrm{pH} 7.4,80 \mathrm{~mm} \mathrm{KCl}, 20 \mathrm{~mm} \mathrm{NaCl}, 0.25 \mathrm{M}$ sucrose, $1 \mathrm{~mm}$ dithiothreitol, and a 1:1000 dilution of protease inhibitor mixture: 87

${ }^{1}$ The abbreviations used are: zVAD-fmk, benzyloxycarbonyl-Val-AlaAsp-fluoromethylketone; Apaf-1, apoptosis protease activating factor PARP, poly(ADP-ribose) polymerase; FITC, fluorescein isothiocyanate; PAGE, polyacrylamide gel electrophoresis; Pipes, 1,4-piperazinediethane sulfonic acid; Mes, 2-(N-morpholino)ethanesulfonic acid; TUNEL, terminal deoxynucleotydyl transferase (tdt)-mediated dUTP FITC nick end labeling; BSA, bovine serum albumin; mtHSP, mitochondrial heat shock protein 70; SNARF-1, seminaphthorhodafluor-1. $\mathrm{mg}$ of phenylmethylsulfonyl fluoride, $160 \mathrm{mg}$ benzamidine, and $10 \mathrm{mg}$ of leupeptin and aprotinin, and $5 \mathrm{mg}$ each of bestatin, antipain, chymostatin, and pepstatin A solubilized in $1 \mathrm{ml}$ of $\mathrm{Me}_{2} \mathrm{SO}$. Neutrophils were resuspended in $1 \mathrm{ml}$ of homogenization buffer then broken with 20-30 strokes of a tight-fitting pestle in a glass Dounce homogenizer from Wheaton (Millville, NJ), until $80 \%$ of the nuclei stained with trypan blue. Intact cells and nuclei were removed by centrifugation at $350 \times$ $g_{a v}$ for 5 min at $4^{\circ} \mathrm{C}$. Post-nuclear supernatants were centrifuged at $17,000 \times g_{a v}$ in a TLA 100.3 rotor from Beckman (High Wycombe, Bucks, UK) for $10 \mathrm{~min}$ to remove secretory granules and mitochondria and the supernatant centrifuged at $541,000 \times g_{\max }$ for $15 \mathrm{~min}$ in a TLA 100.3 rotor to yield a membrane-free cytosol. Cytosol $(200 \mu \mathrm{l})$ was frozen and stored in liquid nitrogen. Protein concentrations were assayed by the bicinchonic acid protocol from Pierce \& Warriner Ltd. The immunoprecipitation procedure, with a monoclonal antibody to a native cytochrome $c$ epitope (6H2.B4, PharMingen), was as described previously (46).

Cell-free Proteolytic Cleavage of Procaspase-3-Cell-free mixtures for the proteolytic cleavage of procaspase- 3 contained: $10 \mu \mathrm{l}$ of $15^{\circ} \mathrm{C}$ cytosol (50 mg of protein/ml) and $10 \mu \mathrm{l}$ of an assay dilution buffer containing: $10 \mathrm{~mm}$ Hepes- $\mathrm{NaOH}$, pH 7.4, $40 \mathrm{~mm} \beta$-glycerophosphate, $50 \mathrm{~mm} \mathrm{NaCl}$, $2 \mathrm{mM} \mathrm{MgCl}, 5 \mathrm{mM} \mathrm{EGTA}, 1 \mathrm{~mm}$ dithiothreitol, supplemented with dATP $(20 \mu \mathrm{M})$ and inhibitors where appropriate. Incubations were stopped by transferring samples to ice and adding $20 \mu \mathrm{l}$ of $50 \mathrm{~mm}$ Tris- $\mathrm{HCl}, \mathrm{pH} 8,0.4 \mathrm{M} \mathrm{NaCl}, 1 \%(\mathrm{w} / \mathrm{v})$ deoxycholate, $1 \%(\mathrm{w} / \mathrm{v})$ Nonidet P-40, 5 mM EDTA, containing protease inhibitor mixture (lysis buffer) for $0.5 \mathrm{~h}$.

Isolation of Mitochondria-Rat liver was washed in $10 \mathrm{~mm}$ Pipes$\mathrm{NaOH}, \mathrm{pH} 7.2,0.25 \mathrm{~m}$ sucrose, $2 \mathrm{mM}$ EDTA and protease inhibitor mixture then forced through a stainless steel sieve $(150 \mu \mathrm{m}$ aperture; Endecotes Ltd., London) to break cells (46). The mitochondria were isolated from a post-nuclear supernatant as described previously and washed in the assay dilution buffer described above (47).

SDS-PAGE and Immunoblotting-Assay samples $(40 \mu \mathrm{l})$, treated with ice-cold lysis buffer for $0.5 \mathrm{~h}(40 \mu \mathrm{l})$ were solubilized with $80 \mu \mathrm{l}$ of $2 \times$ SDS-PAGE sample buffer $(46)$ at $95{ }^{\circ} \mathrm{C}$ for $10 \mathrm{~min}$, then treated with $1 \mathrm{~mm}$ dithiothreitol, cooled, and treated with $10 \mathrm{~mm}$ iodoacetamide. The proteins were separated on $12 \%(\mathrm{w} / \mathrm{v})$ polyacrylamide gels and electrophoretically transferred to nitrocellulose (46). The blots were probed with monoclonal antibodies to procaspase-3 (clone 19) and procaspase-7 (clone 51) from Transduction Laboratories (Lexington, KY) and monoclonal antibodies to procaspase-8 (clone B9-2), procaspase-9 (clone B40), cytochrome $c$ (clone 7H8.2C12), and polyclonal human Bax (13666E) all from PharMingen (San Diego, CA), at dilutions of 1:5001:1000. A hybridoma supernatant to poly(ADP-ribose)polymerase (PARP), used at 1:500 dilution, was a gift from Said Aoufuchi (Laboratory of Molecular Biology, Cambridge, UK). The horseradish peroxidase-conjugated anti-mouse and anti-rabbit IgGs from Kirkegaard \& Perry Labs (Gaithersburg, MA) were used at 1:4000 dilution for $0.5 \mathrm{~h}$ and detected by enhanced chemiluminescence (46). The level of protein loading and nonspecific proteolysis were monitored by Ponceau S staining (46). Tubulin and actin were assayed with anti-bovine $\alpha$-tubulin monoclonal antibody (236-10501) from Molecular Probes and a monoclonal antibody to actin; a gift from Simon Brown (Center for Inflammation Research, University of Edinburgh).

Confocal Microscopy-Neutrophils ( $10^{5}$ cells in $100 \mu \mathrm{l}$ of medium) were cytocentrifuged ( $300 \mathrm{rpm}$ for $3 \mathrm{~min}$ ) onto $1.5 \times 22 \times 22$-mm glass coverslips and fixed in methanol-free $3 \%$ (w/v) $p$-formaldehyde/phosphate-buffered saline and processed for immunofluorescence microscopy as described previously (46). The fixed cells were permeabilized with $0.1 \%(\mathrm{w} / \mathrm{v})$ Triton X-100 and nonspecific binding sites blocked for $1 \mathrm{~h}$ with $0.2 \%(\mathrm{w} / \mathrm{v})$ fish skin gelatin and $20 \%(\mathrm{v} / \mathrm{v})$ sheep serum in phosphate-buffered saline. A monoclonal antibody to mitochondrial heat shock protein 70 (mtHSP70) from Affinity Bioreagents Inc. (Golden, $\mathrm{CO}$ ) and a rabbit polyclonal antibody to ubiquinol-cytochrome $c$ oxidoreductase (complex III) produced by Herman Schagger (University of Frankfurt-am-Main), were used at 1:200 dilution to stain mitochondria. The polyclonal antibody to human Bax was used at 1:200 dilution. The secondary antibodies used at 1:400 dilution were Alexa 488 (green) goat anti-mouse IgG (highly cross-adsorbed) and Alexa 568 (red) goat anti-rabbit (highly cross-adsorbed) and the nucleic acid stain TOPO-3® $(8 \mu \mathrm{M})$, from Molecular Probes. Cells were observed using a $\times 63$ water immersion objective lens with a numerical aperture of 1.2 on a Leica TCS NT confocal laser scanning microscope system (Heidelberg, GMBH). Single optical sections of the images captured with Leica TCS software were digitally processed using Adobe Photoshop 5.02 and Paint Shop Pro 4. 


\section{RESULTS}

Neutrophil Apoptosis Is Arrested at $15{ }^{\circ} \mathrm{C}$-Neutrophils from peripheral blood can be maintained in culture at $37^{\circ} \mathrm{C}$ for several hours in autologous serum before asynchronously undergoing apoptosis (Fig. 1A, closed squares). Apoptosis was quantified using annexin V-FITC binding to externalized phosphatidylserine and morphological counting of pyknotic nuclei (16). A synchronous commitment to apoptosis has previously been induced in cells by using cell-free systems (28, 47-49). Dividing cells, for example, blocked at cell cycle checkpoints provide an homogeneous cytosol (49). Although neutrophils are terminally differentiated (post-replicative) cells, we synchronized their commitment to the execution phase of apoptosis by exposure to low temperature.

Low temperature blocks intracellular pathways that rely on membrane fission and fusion, as exemplified by vesicular transport $(50,51)$. In neutrophils (Fig. 1A, open circles) or eosinophils (data not shown) cultured at $15^{\circ} \mathrm{C}$, apoptosis was arrested suggesting that a membrane-associated event required for apoptosis was inhibited. In contrast, culture of $\mathrm{HL}-60$ promyelocytic leukemia cells at $15^{\circ} \mathrm{C}$ induced apoptosis (data not shown) as described previously for these and many other dividing mammalian cells (52), suggesting that there may be cell-type or differentiation state-dependent pathways for apoptosis. The rate of neutrophil apoptosis at $37^{\circ} \mathrm{C}$ was accelerated by treatment with cycloheximide (Fig. $1 \mathrm{~A}$, closed circles). In addition to preventing the translation of new proteins in the cytoplasm, cycloheximide can induce apoptosis through FADD-dependent mechanisms downstream of cell-surface Fas death receptors (53). However, when we cultured neutrophils at $15{ }^{\circ} \mathrm{C}$ with cycloheximide there was no increase in the rate of apoptosis (Fig. 1A, open circles). Treatment of $15^{\circ} \mathrm{C}$ cultured neutrophils with tumor necrosis factor $\alpha(16)$ did not induce apoptosis (data not shown), suggesting that the block to the induction of the execution phase of apoptosis was downstream of these plasma membrane-associated events.

Temperature Shift to $37{ }^{\circ} \mathrm{C}$ Triggers Synchronous Apoptosis-The low temperature arrest of neutrophil apoptosis was reversed by re-warming neutrophils, cultured for $20 \mathrm{~h}$ at $15^{\circ} \mathrm{C}$, to $37^{\circ} \mathrm{C}$. There was reorganization of the cytoskeleton associated with cell polarization (shape-change), monitored by flow cytometry, that showed cytoskeletal integrity had been maintained at $15{ }^{\circ} \mathrm{C}$ (data not shown). This polarization of the neutrophils was followed by a burst of synchronous apoptosis (Fig. $1 B$, closed circles). The initial rate of apoptosis was 10 -fold greater than the rate of constitutive apoptosis in neutrophils maintained at $37^{\circ} \mathrm{C}$ (Fig. $1 \mathrm{~A}$, closed squares), and by $2 \mathrm{~h}$ after warming $80-90 \%$ of the cells were apoptotic (Fig. 1B, closed circles). Neutrophils maintained for a further $2 \mathrm{~h}$ at $15^{\circ} \mathrm{C}$ showed no shape change and no increase in their rate of apoptosis (Fig. $1 B$, open circles). By culturing neutrophils in medium containing BSA and cycloheximide we removed experimental variables induced by serum factors and translation of mRNA into new protein.

The accelerated rate of neutrophil apoptosis at $37^{\circ} \mathrm{C}$, following preincubation at $15^{\circ} \mathrm{C}$, depended on the period of time neutrophils had been cultured at $15^{\circ} \mathrm{C}$. It was not a cold-shock response, as previously shown for lymphocytes (54). We demonstrated this by maintaining cells at $15^{\circ} \mathrm{C}$ for increasing periods of time before warming them to $37^{\circ} \mathrm{C}$ and estimating the initial rate of apoptosis over a 1.5 -h period as shown in Fig. $1 B$ (closed circles). For the first $6 \mathrm{~h}$ in culture there was no increase in the initial rate of apoptosis (Fig. 1C). However, as the cells were cultured for longer periods at $15^{\circ} \mathrm{C}$ there was an increase in the initial rate of apoptosis on warming (Fig. 1 C), These data suggested that there was a time- and temperature-
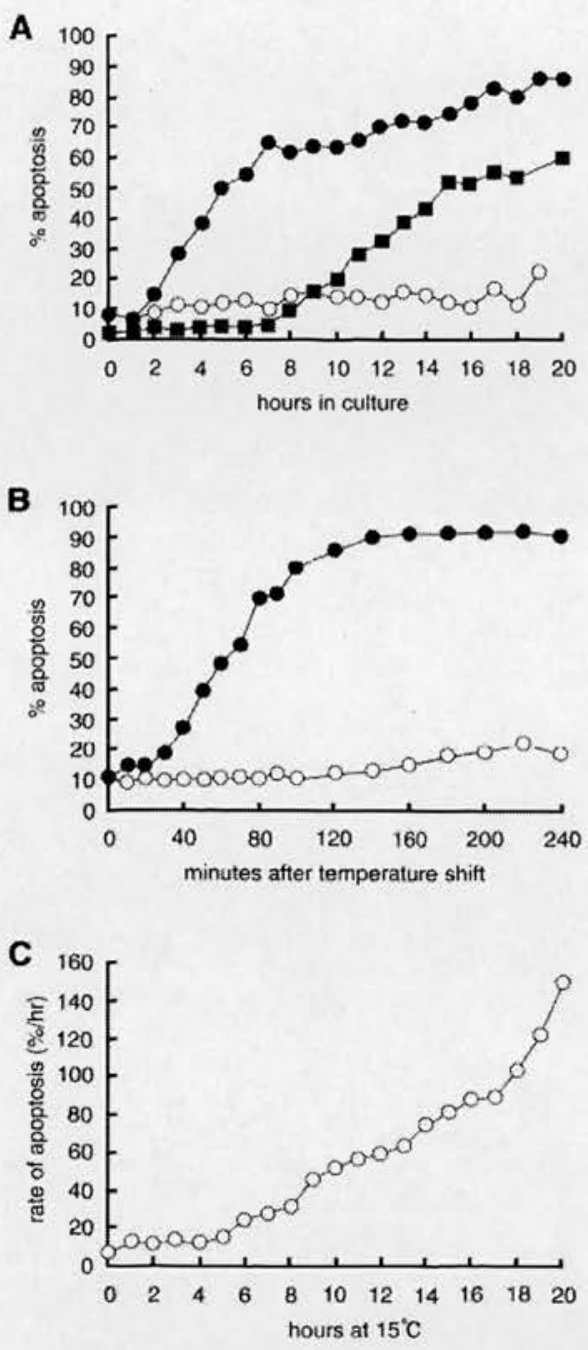

D

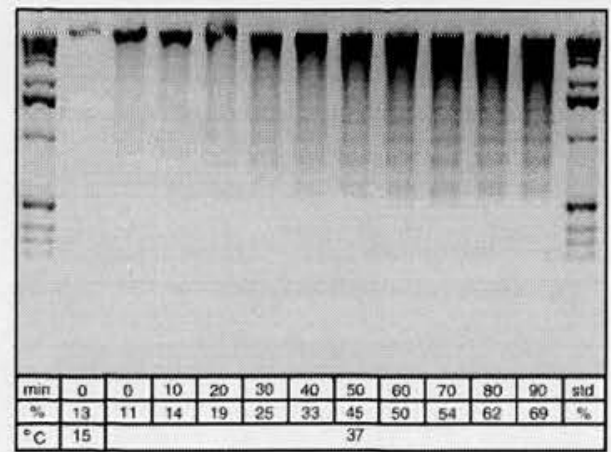

FIG. 1. Human neutrophil apoptosis was arrested at $15^{\circ} \mathrm{C}$ and warming to $37^{\circ} \mathrm{C}$ induced a rapid and synchronous apoptosis. Neutrophils were cultured in medium supplemented with: $A, 0.2 \%$ BSA and $20 \mu \mathrm{g} / \mathrm{ml}$ cycloheximide at $15^{\circ} \mathrm{C}(\mathrm{O})$ and $37^{\circ} \mathrm{C}(\bullet)$, an incubation with $10 \%(\mathrm{v} / \mathrm{v})$ autologous serum, without cycloheximide at $37^{\circ} \mathrm{C}$ is also shown (匹). Apoptosis was assessed by annexin V-FITC binding analyzed by flow cytometry. $B$, neutrophils cultured and analyzed as described in $A$ were held at $15^{\circ} \mathrm{C}$ for $20 \mathrm{~h}(O)$, and subsequently warmed to $37^{\circ} \mathrm{C},-C$, neutrophils were cultured at $15^{\circ} \mathrm{C}$ as described in $A$ and at 1-h intervals harvested and warmed to $37^{\circ} \mathrm{C}$ for $1.5 \mathrm{~h}$ as described in $B$ to assess the initial rate of apoptosis (single experiment). D, DNA, extracted from $5 \times 10^{6}$ neutrophils cultured at $15^{\circ} \mathrm{C}$ for $20 \mathrm{~h}$ and warmed to $37^{\circ} \mathrm{C}$ as described in $B$, was separated in agarose gels and stained with ethidium bromide. A 1-kilobase ladder of DNA standards (Std) is shown. Annexin V-FITC binding was used to assess apoptosis and the percentage (\%) apoptosis for each sample is shown. 
FIG. 2, zVAD-fmk inhibited morphological apoptosis but not the externalization of phosphatidylserine that is inhibited by staurosporine but not okadaic acid. Effects of the global caspase inhibitor zVAD-fmk on the triggering of neutrophil apoptosis. $A$, neutrophils were cultured, as described in the legend to Fig. $1 \mathrm{~A}$, at $15^{\circ} \mathrm{C}$ for $20 \mathrm{~h}(\mathrm{O}, \square)$ and warmed to $37^{\circ} \mathrm{C}(\mathbf{0})$ and apoptosis estimated by morphological counting. The neutrophils were treated with $100 \mu \mathrm{M}$ zVAD-fmk for 15 $\min (\square, \square)$ prior to warming to $37^{\circ} \mathrm{C}$ and control neutrophils were mock treated with $\mathrm{Me}_{2} \mathrm{SO}(\mathrm{O}, 0), B$, DNA fragmentation in the 15 and $37^{\circ} \mathrm{C}$ neutrophils shown in $A$ was analyzed by TUNEL and flow cytometry. $C$, the externalization of phosphatidylserine at the surface of the neutrophil plasma membrane at $15^{\circ} \mathrm{C}(\mathrm{O}, \square)$ and $37^{\circ} \mathrm{C}(\mathbf{\square})$ was estimated by annexin $\mathrm{V}$ FITC binding for the samples shown in $A$ above. $D$, staurosporine inhibited phosphatidylserine externalization. Apoptosis, assessed by morphological counting for neutrophils warmed from $15^{\circ} \mathrm{C}(\mathrm{O})$ to $37^{\circ} \mathrm{C}(\mathbf{)})$ was not inhibited by $2 \mu \mathrm{M}$ staurosporine treatment of $15^{\circ} \mathrm{C}$ cultured neutrophils ( $\square)$ for $1 \mathrm{~h}$ prior to warming to $37^{\circ} \mathrm{C}(\mathbf{\square}) . E$, however, the externalization of phosphatidylserine, assessed by annexin V-FITC-binding, at $15^{\circ} \mathrm{C}(\mathrm{O})$, and after warming to $37^{\circ} \mathrm{C}(0)$ was inhibited by treatment with staurosporine ( $\square, \mathbf{\square}) . F$, neutrophils were cultured at $15^{\circ} \mathrm{C}$ for $20 \mathrm{~h}(\mathrm{O})$ and before warming to $37^{\circ} \mathrm{C}(\mathbf{)})$ they were treated at $15^{\circ} \mathrm{C}(\square)$ for $1 \mathrm{~h}$ with the $1 \mu \mathrm{M}$ okadaic acid then warmed to $37^{\circ} \mathrm{C}(\mathbf{})$ and showed no inhibition of the triggering of apoptosis assessed by annexin V-FITC binding.
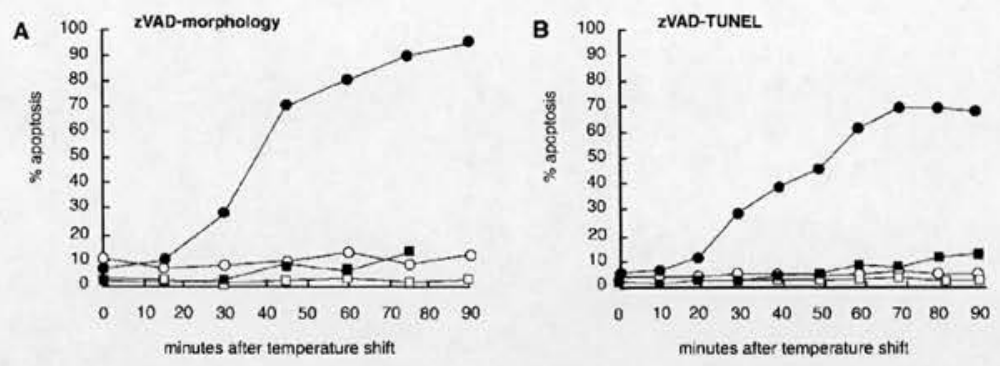

C
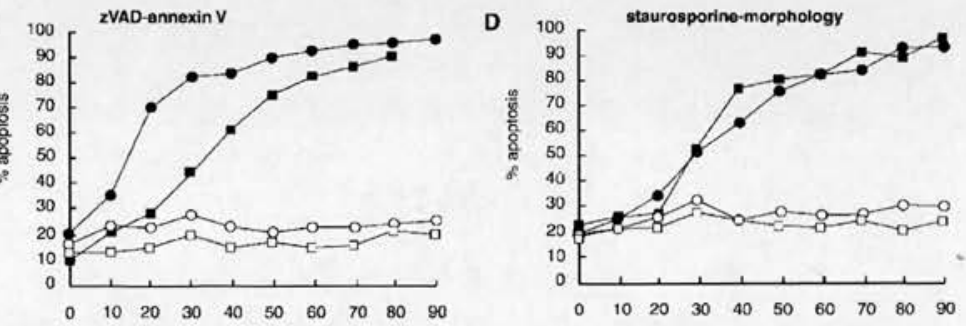

minutes atter temperature shift

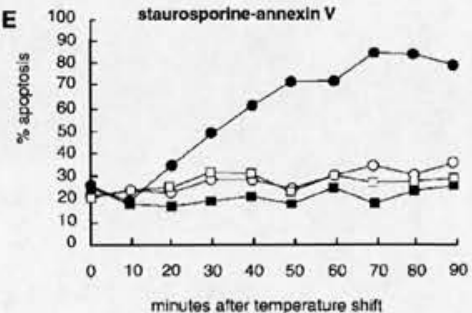

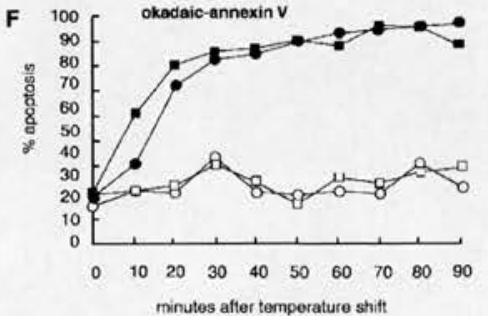

dependent accumulation of a proapoptotic factor at the site of the temperature arrest that led to a synchronization of apoptosis when the cells were subsequently warmed to $37^{\circ} \mathrm{C}$. DNA laddering (Fig. $1 D$ ), a downstream hallmark of activation of the execution phase of apoptosis, showed kinetics similar to the morphological and annexin V-FITC estimates of apoptosis when $15^{\circ} \mathrm{C}$ cultured neutrophils were warmed to $37^{\circ} \mathrm{C}$.

$15^{\circ} \mathrm{C}$ Arrest Is Proximal to Procaspase-3 Activation-To establish whether inhibition of neutrophil apoptosis at $15^{\circ} \mathrm{C}$ (Fig. 2A, open circles) was upstream of caspase-3 activation and apoptosis at $37^{\circ} \mathrm{C}$ (Fig. $2 A$, closed circles), $15^{\circ} \mathrm{C}$ cultured neutrophils were treated with $100 \mu \mathrm{M}$ zVAD-fmk (18) (Fig. 2A, open squares) for $15 \mathrm{~min}$ before warming to $37^{\circ} \mathrm{C}$. zVAD-fmk inhibited chromatin condensation and the formation of pyknotic nuclei when neutrophils were warmed from 15 to $37^{\circ} \mathrm{C}$ (Fig. $2 A$, closed squares). DNA fragmentation at $37^{\circ} \mathrm{C}$ (Fig. $2 B$, closed circles), indicative of caspase-3 activation, was also inhibited (Fig. $2 B$, closed squares) by zVAD-fmk. Phosphatidylserine was still translocated to the cell surface in the presence of zVAD-fmk (Fig. 2C, closed squares), although the kinetics of translocation were significantly different from the untreated neutrophils (Fig. 2C, closed circles). Phosphatidylserine externalization has been linked to caspase-3 activity $(19,55-57)$ so their uncoupling was surprising. However, two other plasma membrane hallmarks of apoptosis phagocytosis (57) and regulated secretion ${ }^{2}$ were also uncoupled from caspase-3 by zVADfmk. The protein kinase inhibitor staurosporine together with cycloheximide induces apoptosis in many cells (58). However, staurosporine did not stimulate apoptosis at $15^{\circ} \mathrm{C}$ (Fig. $2 D$, open squares) nor did it accelerate the induction of morpholog-

${ }^{2} \mathrm{~J}$. Pryde unpublished results. ical apoptosis when neutrophils were warmed to $37^{\circ} \mathrm{C}$ (Fig. $2 D$, closed squares). Phosphatidylserine, detected on the cell surface by annexin-V-FITC after warming to $37^{\circ} \mathrm{C}$ (Fig. $2 E$, closed circles), was not detected in the presence of staurosporine (Fig. $2 E$, closed squares), suggesting that translocation of this phospholipid to the cell surface may rely on a critical phosphorylation event. The inhibition of mitochondrial respiration also blocks phosphatidylserine externalization in apoptotic U937 and THP.1 cells, suggesting that this may be an energy-dependent event (57). Okadaic acid, an inhibitor of phosphatases 1 and 2A (46) had no effect on the induction of apoptosis in $15{ }^{\circ} \mathrm{C}$ cultured neutrophils warmed to $37^{\circ} \mathrm{C}$ (Fig. $2 F$, closed squares). This suggested that caspase activation might not be modulated by phosphorylation events in this experimental model. A number of other agents that induce neutrophil apoptosis, such as the nuclear factor- $\kappa \mathrm{B}$ inhibitor gliotoxin (16) and the phosphatidylinositol kinase inhibitor wortmannin, had no effect on the rate of apoptosis in $15^{\circ} \mathrm{C}$ cultured neutrophils (data not shown). Our results suggest that the temperaturedependent arrest of apoptosis in neutrophils was not due to signal transduction nor gene transcription events.

A Low Intracellular $p H$ Inhibits the Triggering of Apoptosis-Acid conditions at sites of inflammation inhibit neutrophil apoptosis (59), but conversely, low $\mathrm{pH}$ has also been implicated in triggering apoptosis in many cells $(60,61)$. The $\mathrm{pH}_{i}$ of freshly isolated neutrophils was 7.1, equivalent to the "set point" for resting cells in culture when measured by accumulation of carboxy-seminaphthorhodafluor-1 (SNARF-1), a fluorescent probe whose emission changes with $\mathrm{pH}(61-63)$. The $\mathrm{pH}_{i}$ of neutrophils incubated at $15^{\circ} \mathrm{C}$ for $20 \mathrm{~h}$ was $6.8-7.0$ (Fig. $3 A$, open circles). However, following the induction of apoptosis (assayed by annexin-V-binding and morphology, Fig. 3, B and 
FIG. 3. Acid pH arrested the triggering of apoptosis following a shift in temperature from 15 to $37^{\circ} \mathrm{C}$. $A$, neutrophils were cultured at $15^{\circ} \mathrm{C}$ for $20 \mathrm{~h}$ then either maintained at an external $\mathrm{pH}$ of 7.2 at $15^{\circ} \mathrm{C}(\mathrm{O})$ or $37^{\circ} \mathrm{C}(\bullet)$ for $1.5 \mathrm{~h}$, clamped at $\mathrm{pH} 6.2$ in a high-potassium buffer containing nigericin to maintain a $\mathrm{pH}_{i}$ of 6.2 when cultured at $15^{\circ} \mathrm{C}(\square)$ or warmed to $37^{\circ} \mathrm{C}(\mathbf{)})$ for $1.5 \mathrm{~h}$ or exposed to an external $\mathrm{pH}$ of 6.2 , in the absence of any added nigericin and potassium, at $15^{\circ} \mathrm{C}(\triangle)$ or $37^{\circ} \mathrm{C}(\Delta) . B$, apoptosis for the samples shown in $A$ was assessed by annexin V-FITC-binding at $15^{\circ} \mathrm{C}(\mathrm{O}, \square, \Delta)$ and $37{ }^{\circ} \mathrm{C}(\bullet, \mathbf{\bullet}, \mathbf{\Lambda})$ for neutrophils exposed to an external pH of $7.2(\mathrm{O}, \bullet)$ or 6.2 $(\triangle, \mathbf{\Lambda})$ or clamped at $\mathrm{pH} 6.2$ with nigericin in a high potassium buffer $(\square, \boldsymbol{\square})$. $C$, morphological estimates for neutrophil apoptosis of samples in $B$ above at $1.5 \mathrm{~h}$ after warming from 15 to $37^{\circ} \mathrm{C}$ and for neutrophils exposed to external $\mathrm{pH}$ of $6.2 \mathrm{(pH}$ 6.2 ) or clamped with nigericin and potassium ( $\mathrm{pH} 6.2 \mathrm{~N}) . D$, apoptosis estimated by annexin-V-FITC binding for neutrophils cultured at $15^{\circ} \mathrm{C}$ for $20 \mathrm{~h}$ and either held at $15^{\circ} \mathrm{C}(\mathrm{O})$ or warmed to $37^{\circ} \mathrm{C}($ and their $\mathrm{pH}_{i}$ clamped at either $7.2(\square, \boldsymbol{\square})$ or $6.2(\triangle, \Delta)$ in a high potassium buffer containing nigericin.
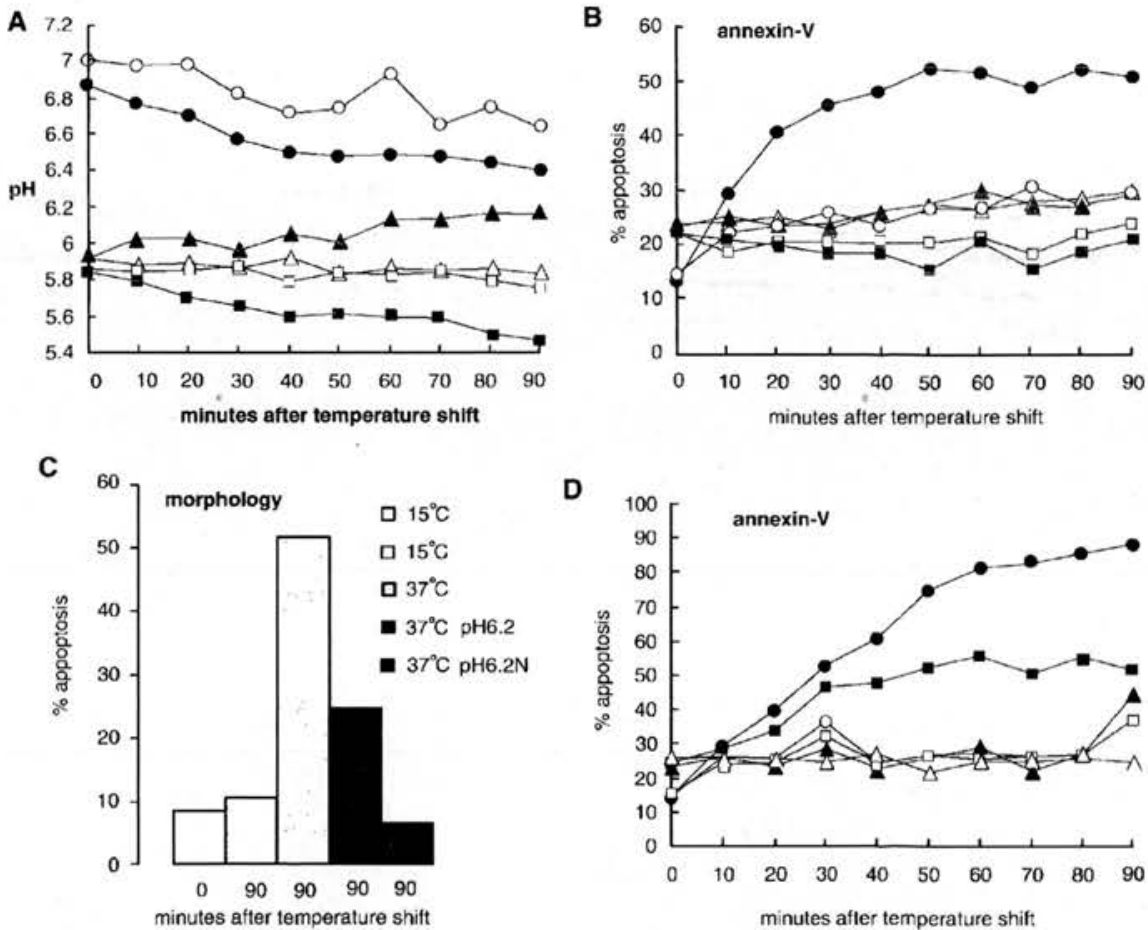

$C$ ), by increasing the temperature to $37^{\circ} \mathrm{C}$ the $\mathrm{pH}_{i}$ dropped to 6.4 (Fig. $3 A$, closed circles) as apoptosis progressed (Fig. $3 B$, closed circles), consistent with previous measurements of acidic $\mathrm{pH}_{i}$ during apoptosis $(60,61)$. However, clamping neutrophils at $\mathrm{pH} 6.2$, with nigericin and high $\mathrm{K}^{+}$(Fig. $3 A$, squares) did not trigger apoptosis at $15^{\circ} \mathrm{C}$ (Fig. $3, B$, open squares, and $C$ ) or at $37^{\circ} \mathrm{C}$ (Fig. 3, B, closed squares, and $C$ ). This suggested that acid $\mathrm{pH}$ alone was not a sufficient trigger for apoptosis as previously suggested $(60,61)$. Neutrophils cultured in growthmedium buffered at $\mathrm{pH} 6.2$, in the absence of nigericin (Fig. $3 A$, triangles), did not induce apoptosis at $15^{\circ} \mathrm{C}$ (Fig. $3, B$, open triangles, and $C$ ) nor on warming to $37^{\circ} \mathrm{C}$ (Fig. $3, B$, closed triangles, and $C$ ). This in vitro response to low $\mathrm{pH}$ appears to mimic the arrest of neutrophil apoptosis at inflammatory foci where the $\mathrm{pH}$ has dropped below 7 (59). When the $\mathrm{pH}_{i}$ of $15^{\circ} \mathrm{C}$ cultured neutrophils was clamped at 7.2, the rate of apoptosis was significantly greater when the cells were warmed to $37^{\circ} \mathrm{C}$ (Fig. $3 D$, squares) than in cells clamped at $\mathrm{pH} 6.2$ (Fig. $3 D$, closed triangles). However, apoptosis was still not as efficient as in the untreated cells (Fig. $3 D$, closed circles). This result is, however, consistent with reports that suggest an alkaline $\mathrm{pH}$ transient is necessary to trigger the execution phase of apoptosis (64).

Neutrophil Apoptosis Is Correlated with Procaspase-3 Cleavage-Procaspase-3 cleavage is required for neutrophil apoptosis (65), but was not detected by immunoblotting in freshly isolated human neutrophils (Fig. $4 A$, lane 2). However, treatment of neutrophils with diisopropyl fluorophosphate, a serine protease inhibitor, before solubilization at $0{ }^{\circ} \mathrm{C}$ in a nondenaturing lysis buffer and SDS sample buffer for PAGE, prevented nonspecific proteolysis of procaspase-3 (Fig. 4A, lane 3). Neutrophils were treated with diisopropyl fluorophosphate before isolating cytosols, but while this was not absolutely necessary for $15{ }^{\circ} \mathrm{C}$ cultured neutrophils (Fig. $4 A$, lanes 4 and 5 ), diisopropyl fluorophosphate treatment did allow detection of endogenous procaspase- 7 and procaspase- 8 by immunoblotting. We have been unable to detect procaspase-9 (data not shown).

Neutrophils cultured at $15{ }^{\circ} \mathrm{C}$ for $20 \mathrm{~h}$, when warmed to $37^{\circ} \mathrm{C}$ for $2 \mathrm{~h}$, proteolytically cleaved procaspase- 3 in a time-dependent manner (Fig. 4B, upper panel, lanes 1-11) that correlated with estimates of apoptosis by counting of pyknotic nuclei and annexin V-FITC binding (Fig. 2, $A$ and $C$ ). Neutrophils held for a further $2 \mathrm{~h}$ at $15^{\circ} \mathrm{C}$ showed no proteolytic processing of procaspase-3 (Fig. 4B, lower panel, lanes 1-11).

Proapoptotic Events Can be Detected in Neutrophil Cy. tosols-To identify the molecular events leading to the proteolytic cleavage and activation of neutrophil procaspase-3, cytosols were prepared from cultures of neutrophils maintained at $15^{\circ} \mathrm{C}$ for $20 \mathrm{~h}$. The neutrophils were homogenized in buffered sucrose and fractionated using a two-step ultracentrifugation procedure (see "Experimental Procedures") to minimize damage to organelles, particularly secretory granules. Elastase, a secretory granule marker, sedimented with membrane fractions (data not shown), while procaspase-3 remained in the cytosol (Fig. 5A, lane 1). Neutrophils cultured at $15^{\circ} \mathrm{C}$ and warmed to $37^{\circ} \mathrm{C}$ for $1.5 \mathrm{~h}$ before preparing the cytosols did not contain procaspase-3 (Fig. $5 \mathrm{~A}$, lane 2 ). The proteolytic cleavage of procaspase-3, observed in unbroken $15{ }^{\circ} \mathrm{C}$ cultured neutrophils warmed to $37^{\circ} \mathrm{C}$ (Fig. $4 B$, lanes 5-11), had presumably been triggered by apoptosis-inducing factors released from the membranes into the cytoplasm before the cells had been homogenized. Significantly, when cytosols from $15^{\circ} \mathrm{C}$ cultured neutrophils were warmed to $37^{\circ} \mathrm{C}$ in the presence of dATP, a cofactor involved in the activation of the apoptosome (42), procaspase- 3 was not proteolytically cleaved (Fig. $5 A$, lane 5 ) and was present in an amount comparable to the zVAD-fmk-treated controls (Fig. 5A, lane 6). These data suggested that apoptosisinducing factors were missing from the cytosol and had been removed with the membrane fraction. The fraction of cytosol from the small number of contaminating apoptotic cells did not catalyze a significant rate of proteolytic cleavage of procaspase-3.

Mitochondria have been shown to play a key role in the control and amplification of apoptotic signals $(23,42)$. Cytosols from Xenopus laevis eggs only trigger apoptosis when membrane fractions enriched in mitochondria are added $(25,27,48)$. When rat liver mitochondria were added to cytosols isolated 
Fig. 4. Temperature-dependent proteolytic cleavage of endogenous procaspase-3 in intact neutrophils. $A$, freshly isolated human neutrophils $(P M N)$ (lanes 2 and 3) and neutrophils cultured at $15{ }^{\circ} \mathrm{C}$ for $20 \mathrm{~h}$ (lanes 4 and 5 ) were either treated with $2.3 \mathrm{~mm}$ diisopropyl fluorophosphate for $15 \mathrm{~min}$ on ice (lanes 3 and 5) or left untreated (lanes 2 and 4). Each lane contains $80 \mu \mathrm{g}$ of protein solubilized in lysis buffer on ice then in SDS sample buffer before separation on a $12 \%(\mathrm{w} / \mathrm{v})$ polyacrylamide gel and electrophoretic transfer to nitrocellulose sheets and probing with a monoclonal antibody to procaspase-3. Lane 1 shows cytosol from $15^{\circ} \mathrm{C}$ cultured neutrophils. $B$, neutrophils cultured at $15^{\circ} \mathrm{C}$, as described in the legend to Fig. 1, and cell pellets $\left(5 \times 10^{6}\right.$ cells) were solubilized and analyzed as described in $A$. The $15^{\circ} \mathrm{C}$ cultured neutrophils were either warmed to $37^{\circ} \mathrm{C}$ (upper panel, lanes $1-11$ ) or held at $15^{\circ} \mathrm{C}$ (lower panel, lanes 1-11) for the times indirated and protenlytic cloavoge of procaspase- 3 assessed by immunoblotting. Re-probing the blots with antibodies to tubulin or actin (not shown) assessed protein loading and nonspecific proteolysis.
A

\begin{tabular}{|c|c|c|c|c|c|}
\cline { 2 - 6 } \multicolumn{1}{c|}{} & Cyt & \multicolumn{2}{|c|}{ Fresh PMN } & \multicolumn{2}{|c|}{15 C PMN } \\
\hline DFP & - & - & + & - & + \\
\hline $\begin{array}{l}\text { Procaspase-3 } \\
34-k D a\end{array}$ & \multicolumn{5}{|c|}{} \\
\hline
\end{tabular}

B

\begin{tabular}{|c|c|c|c|c|c|c|c|c|c|c|c|}
\hline Minutes & 0 & 10 & 20 & 30 & 40 & 50 & 60 & 70 & 80 & 90 & 120 \\
\hline $\begin{array}{l}\text { Procapase-3 } \\
34-\mathrm{kDa} \\
37 \mathrm{C}\end{array}$ & & & & $=$ & $m$ & 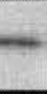 & - & & & & \\
\hline \multirow[t]{2}{*}{$\begin{array}{l}\text { Procapase-3 } \\
34 \mathrm{kDa} \\
15 \mathrm{C} \\
\end{array}$} & & & & & & & & & & & \\
\hline & 1 & 2 & 3 & 4 & 5 & 6 & 7 & 8 & 9 & 10 & 11 \\
\hline
\end{tabular}

from $15{ }^{\circ} \mathrm{C}$ cultured neutrophils, supplemented with dATP then incubated at $15{ }^{\circ} \mathrm{C}$ for $2 \mathrm{~h}$, immunoblotting revealed no significant proteolytic processing of procaspase-3 (Fig. $5 B$, lanes 1-5). The degree of proteolytic processing was compared with $15^{\circ} \mathrm{C}$ cytosols pretreated with zVAD-fmk (Fig. $5 B$, lane 6) and with $15{ }^{\circ} \mathrm{C}$ cytosols without added mitochondria (Fig. $5 B$, lane 8 ). In cytosols containing dATP and mitochondria permeabilized with Triton X-100, to release apoptosis-inducing factors, an efficient proteolytic cleavage of procaspase- 3 was observed by $2 \mathrm{~h}$ at either 15 or $37^{\circ} \mathrm{C}$ (Fig. $5 B$, lanes 7 and 14). Cytosol from $15{ }^{\circ} \mathrm{C}$ neutrophils warmed to $37^{\circ} \mathrm{C}$ with rat liver mitochondria, and dATP induced proteolytic cleavage of procaspase-3 in a time-dependent manner (Fig. 5B, lanes 9-12). The protease activity was inhibited by zVAD-fmk (Fig. $5 B$, lane 13) and was dependent upon the addition of mitochondria at $37^{\circ} \mathrm{C}$ (Fig. $5 B$, lane 15 ).

The involvement of membrane-associated events in the triggering of neutrophil apoptosis was also suggested by analysis of the rate of apoptosis as a function of temperature (Fig. $5 \mathrm{C}$ ). There was a sharp decline in the rate of apoptosis below $20^{\circ} \mathrm{C}$ and an Arrhenius plot of this data (not shown) (66) showed that the temperature dependence was biphasic. As the temperature drops to $15^{\circ} \mathrm{C}$ reduction in the fluidity of the membrane lipid may affect the behavior of membrane-associated proteins (50, 51,66 ). Mitochondrial anion channels have also been shown to respond to lowered temperature by changing their probability of being open, a parameter that is also affected by changes in $\mathrm{pH}$ (67). Thus, in our cell-free assay low temperature maintained the segregation of apoptosis-inducing factors within mitochondria, preventing apoptosome activation.

Cytochrome c Induces Procaspase-3 Cleavage-When horse heart cytochrome $c$ and dATP were added to cytosol from $15^{\circ} \mathrm{C}$ cultured neutrophils and incubated at 15 or $37^{\circ} \mathrm{C}$ for $1 \mathrm{~h}$, proteolytic cleavage of procaspase-3 was induced (Fig. $5 D$, lanes 2 and 4). The kinetics of procaspase-3 proteolytic cleavage were dependent on the concentration of cytochrome $c$, being complete between 10 and $100 \mathrm{ng}$ of cytochrome $c / 80 \mu \mathrm{g}$ of cytosol protein after $1 \mathrm{~h}$ at $37^{\circ} \mathrm{C}$. Treatment of the neutrophil cytosol with $100 \mu \mathrm{M} \mathrm{zVAD}$-fmk prior to the addition of cytochrome $c$ prevented proteolytic cleavage of procaspase-3 at 15 and $37^{\circ} \mathrm{C}$ (Fig. $5 D$, lanes 3 and 5). Since an efficient proteolytic cleavage of procaspase- 3 occurred at $15{ }^{\circ} \mathrm{C}$ the arrest of apo- ptosis was unlikely to be a consequence of the failure of the apoptosome proteins to undergo conformational changes at low temperature (68).

Poly(ADP-ribose)polymerase Is Cleaved by $15{ }^{\circ} \mathrm{C}$ CytosolsNeutrophil cytosols containing cytochrome $c$ and dATP produced active caspase-3 that processed HL-60 PARP (116 kDa), not present in mature neutrophils (17), to an $85-\mathrm{kDa}$ polypeptide fragment after a $1.5 \mathrm{~h}$ incubation at $15^{\circ} \mathrm{C}$ (Fig. 6, lower panel, lane 3 ). This proteolytic cleavage was inhibited by $\mathrm{zVAD}$ fmk (Fig. 6, lower panel, lane 4). There was no apparent proteolysis of procaspase-3 under these conditions (Fig. 6, upper panel, lane 3 ). There was variability between cytosol preparations, and under similar conditions procaspase- 3 was fully processed (see Fig. $5 \mathrm{D}$, lane 2). At $37^{\circ} \mathrm{C}$ there was complete proteolytic cleavage of procaspase-3 (Fig. 6, upper panel, lane 5) with concomitant proteolytic cleavage of PARP (Fig. 6, lower panel, lane 5); again this was inhibited by zVAD-fmk (Fig. 6 , lower panel, lane 6). No proteolytic processing of neutrophil procaspase-3 or HL-60-PARP by endogenous proteases was detected in the absence of cytochrome $c$ (Fig. 6, upper panel, lane 1 , and lower panel, lane 7 , respectively).

Release of Cytochrome $c$ from Neutrophil Mitochondria Occurs at 37 but not $15^{\circ} \mathrm{C}$-Cytochrome $c$ induced the proteolytic cleavage of endogenous neutrophil procaspase-3 but there was the possibility that this was only a property of the cell-free assay, particularly since we were unable to detect procaspase- 9 a component of the apoptosome (42). To monitor translocation of cytochrome $c$ from mitochondria into the cytoplasm of intact neutrophils, warmed from 15 to $37^{\circ} \mathrm{C}$, we separated postnuclear supernatants into membrane and cytosol fractions. Cytochrome $c$ was immunoprecipitated from the fractions with a monoclonal antibody to a native cytochrome $c$ epitope and identified by immunoblotting with an anti-cytochrome $c$ antibody that recognized SDS-denatured cytochrome $c$. Neutrophil cytochrome $c$ (Fig. 7, lane 3) co-migrated with cytochrome $c$ from both horse heart and HeLa cells (Fig. 7, lanes 1 and 2). In neutrophils held at $15^{\circ} \mathrm{C}$, cytochrome $c$ remained with the membrane fraction (Fig. 7, lanes 4 and 5), but on warming to $37^{\circ} \mathrm{C}$ cytochrome $c$ was translocated to the cytosol (Fig. 7, lanes 7 and 8 ). Thus neutrophils released endogenous cytochrome $c$ from mitochondria into the cytoplasm, an event that correlated with the onset of apoptosis. 
A

\begin{tabular}{|c|c|c|c|c|c|c|}
\hline Temperature ${ }^{\circ} \mathrm{C}$ & 0 & 0 & 15 & 15 & 37 & 37 \\
\hline $15^{\circ}$ C PMN cytosol $^{\text {A PMAD-fmk }}$ & + & - & + & + & + & + \\
\hline $\begin{array}{c}\text { zVAD } \\
\text { Procaspase-3 } \\
34-\mathrm{kDa}\end{array}$ & - & - & - & + & - & + \\
\hline & 1 & 2 & 3 & 4 & 5 & 6 \\
\hline
\end{tabular}

B

\begin{tabular}{|c|c|c|c|c|c|c|c|c|c|c|c|c|c|c|c|}
\hline Temperature $\mathrm{C}$ & 15 & 15 & 15 & 15 & 15 & 15 & 15 & 15 & 37 & 37 & 37 & 37 & 37 & 37 & 37 \\
\hline Mitochondria & + & + & + & + & + & + & + & - & + & + & + & + & + & + & - \\
\hline Triton & - & - & - & - & - & - & + & - & - & - & - & - & - & + & - \\
\hline zVAD-fmk & - & - & - & - & - & + & - & - & - & - & - & - & + & - & - \\
\hline Minutes & 0 & 30 & 60 & 90 & 120 & 120 & 120 & 120 & 30 & 60 & 90 & 120 & 120 & 120 & 120 \\
\hline $\begin{array}{l}\text { Procaspase-3 } \\
\text { 34-kDa }\end{array}$ & \multicolumn{10}{|c|}{} \\
\hline
\end{tabular}

C

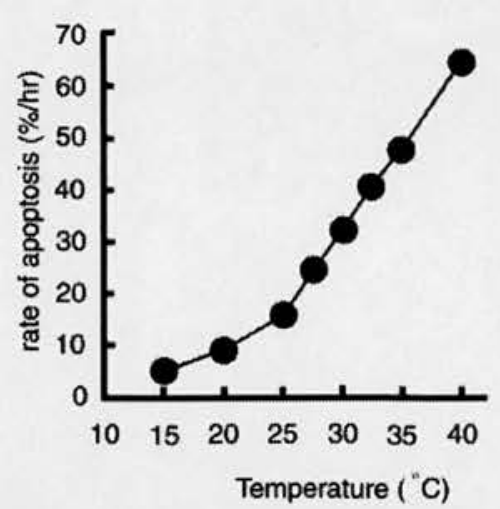

D

\begin{tabular}{|c|c|c|c|c|c|c|}
\hline Temperature $\mathrm{C}$ & 37 & 15 & 15 & 37 & 37 & 0 \\
\hline $15^{\circ} \mathrm{C}$ PMN cytosol & + & + & + & + & + & + \\
\hline zVAD-fmk & + & - & + & - & + & - \\
\hline Cytochrome c & - & + & + & + & + & - \\
\hline \multirow[t]{2}{*}{$\begin{array}{l}\text { Procaspase-3 } \\
34-\mathrm{kDa}\end{array}$} & & & & & & \\
\hline & 1 & 2 & 3 & 4 & 5 & 6 \\
\hline
\end{tabular}

Fig. 5. Cell-free proteolytic cleavage of procaspase-3. A, the proteolytic cleavage of endogenous neutrophil procaspase-3 was analyzed in cytosol supplemented with $20 \mu \mathrm{M}$ dATP (lanes $1,3-6$ ), and in $15^{\circ} \mathrm{C}$ cultured neutrophil cytosol warmed to $37^{\circ} \mathrm{C}$ (lane 2 ) by immunoblotting. Samples of the $15^{\circ} \mathrm{C}$ cytosol (lane 1) and cytosol from $15^{\circ} \mathrm{C}$ cultured cells warmed to $37^{\circ} \mathrm{C}$ (lane 2 ) were held at $0{ }^{\circ} \mathrm{C}$. The $15{ }^{\circ} \mathrm{C}$ cytosol was also held at $15{ }^{\circ} \mathrm{C}$ (lane 3 ) or warmed to $37^{\circ} \mathrm{C}$ (lane 5) in the presence (lanes 4 and 6 ) or absence (lanes 3 and 5 ) of $100 \mu \mathrm{M}$ zVAD-fmk. B, cell-free assays containing cytosol from $15{ }^{\circ} \mathrm{C}$ cultured neutrophils were supplemented with $5 \mu$ l of rat liver mitochondria fraction (47) and incubated at $15^{\circ} \mathrm{C}$ for the times indicated (lanes $1-5$, respectively). Control incubations at $15{ }^{\circ} \mathrm{C}$ contained: $100 \mu \mathrm{M}$ zVD-fmk (lane 6 ), mitochondria plus $0.1 \%$ (w/v) Triton X-100 (lane 7), or no mitochondria (lane 8). Lanes $9-12$ show incubations at $37^{\circ} \mathrm{C}$ for the times indicated, a $\mathrm{zVAD}$-fmk $2 \mathrm{~h}$ control (lane 13 ), a mitochondria plus $0.1 \%(\mathrm{w} / \mathrm{v})$ Triton X-100 control (lane 14), and a control lane with no added mitochondria (lane 15). $C$, the rate of neutrophil apoptosis is temperature dependent. Neutrophils were incubated for $22 \mathrm{~h}$ at $15^{\circ} \mathrm{C}$ and then warmed to the given temperatures (between 15 and $40^{\circ} \mathrm{C}$ ). The rate of apoptosis was estimated by annexin V-FITC binding as described in the legend for Fig. $1 B$. $D$, induction of the proteolytic cleavage of procaspase- 3 by horse heart cytochrome $c$. Incubations and analysis were as described above in $A$ and were for $1 \mathrm{~h}$ at the temperatures shown. Lane $1,37^{\circ} \mathrm{C}$ cytosol control. In lanes 2-6 the assays were supplemented with $200 \mathrm{ng}$ of cytochrome $c$. Incubations were at $15{ }^{\circ} \mathrm{C}$ (lanes 2 and 3 ), $37^{\circ} \mathrm{C}$ (lanes 4 and 5), and $0{ }^{\circ} \mathrm{C}$ (lane 6). Controls containing $100 \mu \mathrm{M} \mathrm{zVAD}$-fmk are shown in lanes 1,3 , and 5 .

The Translocation of Bax to Membrane Fractions-How the mitochondrial membrane is permeabilized to release cytochrome $c$ during apoptosis is not known $(68,69)$. However, while the opening or formation of the putative membrane channels may be temperature-sensitive, movement of cytochrome $c$ through the channels may not be sensitive to reduced temperature (30). Bax is highly expressed in neutrophils (9-14) and can form membrane pores (39) so we examined the subcellular distribution of Bax during neutrophil apoptosis. Immunoblotting showed that Bax was present in the postnuclear supernatants of $15^{\circ} \mathrm{C}$ cultured neutrophils (Fig. 8, upper panel, lane 1). Sedimentation of the membranes by ultracentrifugation (Fig. 8 , upper panel, lane 2) showed that Bax partitioned into the cytosol (Fig. 8, upper panel, lane 3) with procaspase-3 (Fig. 8, lower panel, lane 3 ). This is consistent with their localization in freshly isolated neutrophils (data not shown), murine thymo- cytes, splenocytes, and HL-60 cells $(33,35)$. In $15^{\circ} \mathrm{C}$ cultured neutrophils warmed to $37^{\circ} \mathrm{C}$ for $3 \mathrm{~h}$, procaspase- 3 was proteolytically cleaved (Fig. 8, lower panel, lanes 4-6) and Bax had translocated to the washed membrane fraction (Fig. 8, upper panel, lane 5) from the cytosol (Fig. 8, upper panel, lane 6). This location suggested that Bax had inserted its hydrophobic membrane-spanning domain into mitochondria (40). In some experiments membrane-associated Bax was processed to an $18-\mathrm{kDa}$ fragment (data not shown), a cleavage product identified in a number of other apoptotic cells $(70,71)$.

Bax insertion into mitochondria and the release of cytochrome $c$ in many experimental models is insensitive to zVADfmk (28). In subcellular fractions from neutrophils cultured for $18 \mathrm{~h}$ at $15^{\circ} \mathrm{C}$ and treated with $100 \mu \mathrm{M}$ zVAD-fmk for $2 \mathrm{~h}$, before warming to $37^{\circ} \mathrm{C}$, Bax had translocated to the membrane fraction (Fig. 8, upper panel, lane 11). Under these conditions 
FIG. 6. Proteolytic processing of HL-60 PARP in heterologous cell-free assays. HL-60 nuclei were incubated with $15^{\circ} \mathrm{C}$ neutrophil cytosol and dATP and proteolytic cleavage of procaspase- 3 triggered by the addition of cytochrome $c$ $(200 \mathrm{ng})$. Samples were taken at $0 \mathrm{~h}$ (lane 2) and at $1.5 \mathrm{~h}$ after incubation at $15^{\circ} \mathrm{C}$ (lanes 3 and 4), without zVAD-fmk treatment (lanes $1-3,5$ and 7 ), with $100 \mu \mathrm{M}$ zVAD-fmk treatment (lanes 4 and 6 ), and after incubation at $37^{\circ} \mathrm{C}$ (lanes 1 and 5-7). Samples were solubilized as described in the legend to Fig. $5 B$ and the immunoblots probed with monoclonal antibodies to procaspase-3 (upper panel) and PARP (lower panel)

\begin{tabular}{|c|c|c|c|c|c|c|c|}
\hline Temperature C & 37 & 0 & 15 & 15 & 37 & 37 & 37 \\
\hline Time (minutes) & 90 & 0 & 90 & 90 & 90 & 90 & 90 \\
\hline 15 C PMN cytosol & + & + & + & + & + & + & - \\
\hline HL60 nuclei & - & + & + & + & + & + & + \\
\hline Cytochrome c & - & + & + & + & + & + & - \\
\hline zVAD-fmk & - & $=$ & - & + & - & + & - \\
\hline $\begin{array}{l}\text { Procaspase-3 } \\
34-\mathrm{kDa}\end{array}$ & & & & & & & \\
\hline \multirow[t]{2}{*}{$\begin{array}{ll}\text { PARP } & 116-\mathrm{kDa} \\
& 85-\mathrm{kDa}\end{array}$} & & & & & & & \\
\hline & 1 & 2 & 3 & 4 & 5 & 6 & 7 \\
\hline
\end{tabular}

\begin{tabular}{|c|c|c|c|c|c|c|c|c|}
\hline Temperature C & 0 & 0 & 15 & 15 & 15 & 37 & 37 & 37 \\
\hline Fraction & $\mathrm{C}$ & $\mathrm{H}$ & $\mathrm{P}$ & $\mathrm{M}$ & $\mathrm{S}$ & $\mathrm{P}$ & $\mathrm{M}$ & $\mathrm{S}$ \\
\hline $\begin{array}{c}\text { Cytochrome c } \\
15 \mathrm{kDa}\end{array}$ & \multicolumn{10}{|c|}{} \\
\hline
\end{tabular}

Fig. 7. The release of endogenous neutrophil cytochrome $c$ from membrane fractions into the cytosol. Neutrophils were cultured at $15^{\circ} \mathrm{C}$ as described in the legend to Fig. $1 A$. Samples were solubilized in a nondenaturing lysis buffer for immunoprecipitation (46) with monoclonal antibodies to a native epitope on cytochrome $c$. After SDS-PAGE and electroblotting to nitrocellulose, cytochrome $c$ was identified with a monoclonal antibody to the SDS-denatured form of cytochrome $c$. Lane 1 contains $50 \mathrm{ng}$ of horse heart cytochrome $c(C)$. Lane 2 shows an immunoprecipitation of cytochrome $c$ from $0.5 \times 10^{6}$ HeLa cells $(H)$. Lanes $3-8$ show cytochrome $c$ immunoprecipitated from: in lane 3, a post-nuclear supernatants $(P)$ of $15^{\circ} \mathrm{C}$ cultured neutrophils $\left(7.5 \times 10^{7}\right.$ cells); lane $4,15^{\circ} \mathrm{C}$ cultured neutrophil membrane fraction $(M)$; lane 5 , $15^{\circ} \mathrm{C}$ cultured neutrophil supernatant $(S)$ fraction; lane 6 , post-nuclear supernatants $(S)$ of $15^{\circ} \mathrm{C}$ cultured neutrophils warmed to $37^{\circ} \mathrm{C}$ for $2 \mathrm{~h}$ (apoptosis by annexin V-FITC $85 \%$ ); lane $7,37^{\circ} \mathrm{C}$ membrane fraction $(\mathrm{M})$; lane $8,37^{\circ} \mathrm{C}$ supernatant $(S)$ fraction.

\begin{tabular}{|c|c|c|c|c|c|c|c|c|c|c|c|c|}
\hline Temperature $\mathrm{C}$ & & 15 & & & 37 & & \multicolumn{3}{|c|}{37} & \multicolumn{3}{|c|}{37} \\
\hline $100 \mu \mathrm{M}$ zVAD-fmk & & . & & & 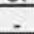 & & \multirow{2}{*}{\multicolumn{3}{|c|}{$\frac{+T_{0}}{12}$}} & \multicolumn{3}{|c|}{$+T 18$} \\
\hline$\%$ Apoptosis & \multicolumn{3}{|c|}{4} & & 94 & & & & & \multicolumn{3}{|c|}{11} \\
\hline Cell fraction & PNS & $M$ & C & PNS & $M$ & $\mathrm{C}$ & PNS & $M$ & C & PNS & $M$ & C \\
\hline \multicolumn{13}{|l|}{ Bax $21-\mathrm{kDa}$} \\
\hline \multicolumn{13}{|l|}{$\begin{array}{r}\text { Procaspase-3 } \\
34-\mathrm{kDa}\end{array}$} \\
\hline & 1 & 2 & 3 & 4 & 5 & 6 & 7 & 8 & 9 & 10 & 11 & 12 \\
\hline
\end{tabular}

Fig. 8. Subcellular localization of Bax. Neutrophils $\left(3 \times 10^{8}\right.$ cells) were held at $15^{\circ} \mathrm{C}$ for $18 \mathrm{~h}$. $10^{8}$ cells were incubated at $15{ }^{\circ} \mathrm{C}$ for $5 \mathrm{~h}($ lanes $1-3$ ); $10^{8}$ cells were cultured for a further $2 \mathrm{~h}$ at $15^{\circ} \mathrm{C}$ before warming to $37^{\circ} \mathrm{C}$ for $3 \mathrm{~h}$ (lanes $4-6$ ) and $10^{8}$ cells were treated for $2 \mathrm{~h}$ at $15^{\circ} \mathrm{C}$ with $100 \mu \mathrm{M}$ zVAD-fmk before warming to $37^{\circ} \mathrm{C}$ for $3 \mathrm{~h}$ (T18: lanes $10-12$ ). One sample of $10^{8}$ freshly isolated neutrophils was treated with $100 \mu \mathrm{M}$ zVAD-fmk at $15^{\circ} \mathrm{C}$ for $20 \mathrm{~h}$ and then warmed to $37^{\circ} \mathrm{C}$ for $3 \mathrm{~h}$ (lanes 7-9). Post-nuclear supernatants (post-nuclear supernatants: lanes 1 , 4, 7, and 10 ) were fractionated into membrane $(M$ : lanes $2,5,8$, and 11$)$ and cytosol $(C$ : lanes $3,6,9$, and 12$)$ by ultracentrifugation, the membrane fractions being re-homogenized to their original volume in buffer. The fractions were immunoblotted with antibodies to Bax (upper panel) and procaspase-3 (lower panel).

procaspase-3 was still present in the cytosol (Fig. 8, lower panel, lane 12). However, when freshly isolated neutrophils were treated with $100 \mu \mathrm{m} \mathrm{zVAD}$-fmk at $15^{\circ} \mathrm{C}$ for $20 \mathrm{~h}$ prior to warming to $37^{\circ} \mathrm{C}$, Bax remained in the cytosol fraction (Fig. 8, upper panel, lane 9) with procaspase-3 (Fig. 8, lower panel, lane 9). The Bax present in the membrane fraction (Fig. 8, upper panel, lane 8 ) can be accounted for by the apoptotic cells (12\%) present. These results suggest that during incubation at $15^{\circ} \mathrm{C}$, caspase activity may prepare Bax for translocation from the cytosol to mitochondria and insertion into the mitochondrial membrane on warming to $37^{\circ} \mathrm{C}$. Caspase-8, a potential candidate for the indirect activation of Bax, can be detected in neutrophil cytosols. However, procaspase- 8 was not proteolytically cleaved during either incubations at $15^{\circ} \mathrm{C}$ or during the warming of $15^{\circ} \mathrm{C}$ cultured neutrophils to $37^{\circ} \mathrm{C}$ in the presence of zVAD-fmk (data not shown).

The Translocation of Bax to Neutrophil Mitochondria-We followed the translocation of Bax from the cytoplasm to mito- chondria in apoptotic neutrophils by confocal microscopy. Immunofluorescence staining of neutrophils with rabbit preimmune sera and Alexa dye-tagged secondary antibodies showed nuclear staining in freshly isolated neutrophils (Fig. 9A) and no significant cytoplasmic staining. Anti-Bax polyclonal antibodies also showed nuclear staining (Fig. 9B), but similar staining in HeLa cells and Bax-deficient tumor cells suggested that this staining was nonspecific (72). In fresh preparations of neutrophils the few constitutively apoptotic neutrophils showed Bax staining of large cytoplasmic structures (Fig. 9B, arrow). These cytoplasmic structures were also stained with polyclonal antibodies to ubiquinol cytochrome $c$ oxidoreductase (complex III) that co-localized with mtHSP70 staining (Fig. 9C), whose expression was increased in many preparations of $15^{\circ} \mathrm{C}$ cultured neutrophils. Significantly, staining of $15^{\circ} \mathrm{C}$ cultured neutrophils with mtHSP70 (Fig. 9D) and Bax (Fig. 9E) showed colocalization (yellow staining) in merged images (Fig. $9 F$ ) of 
FIG. 9. Intracellular localization of Bax by confocal microseopy. Freshly isolated neutrophils showed no significant cytoplasmic staining with rabbit preimmune serum and anti-rabbit IgG-conjugated to Alex 568 (red) (A), with polyclonal antibodies against $\operatorname{Bax}(B)$ nor with monoclonal antibodies to the mitochondrial marker mtHSP70 detected with anti-mouse IgG-conjugated to Alexa $₫ 488$ (y,ern) (B). Nucles were stainod with TOPO- $3^{\pi}$ and the cells were analyzed by confocal microscopy and single optical sections are shown. Apoptotic cells present in the freshly isolated cells, however, showed Bax staining (red) of large cytoplasmic structures (arrow in $B$ ) that colocalized (yellow) with staining for both the mitochondrial markers mtHSP70 (red) and complex III (green) (C). After culture for $20 \mathrm{~h}$ at $15^{\circ} \mathrm{C}(D-F)$ neutrophils showed staining for cytoplasmic structures with mtHSP70 $(D)$ and Bax $(E)$ that co-localized (yellow staining) in merged micrographs $(F)$. When the $15^{\circ} \mathrm{C}$ cultured neutrophils were warmed to $37^{\circ} \mathrm{C}$ for $3 \mathrm{~b}$ $(G-D$, they showed staining of several discrete cytoplasmic structures. These were also stained for $\operatorname{mtHSP} 70(G)$ and $\operatorname{Bax}(H)$ and when the micrographs were merged (I) the Bax and mtHSP70 staining was mainly co-localized, although there were some structures that stained for only one or another of the antibodies. A control $15{ }^{\circ} \mathrm{C}$ culture was warmed to $37^{\circ} \mathrm{C}$ for $3 \mathrm{~h}$ and stained with rabbit preimmune $(J)$. Freshly isolated neutrophils were treated for $20 \mathrm{~h}$ with $100 \mu \mathrm{M} \mathrm{zVAD}$-fmk and then warmed to $37^{\circ} \mathrm{C}$ for 3 h. The micrograph $(K)$ shows the merged staining for Bax and mtHSP70. Neutrophils were cultured at $15{ }^{\circ} \mathrm{C}$ for $18 \mathrm{~h}$ and then treated at $15^{\circ} \mathrm{C}$ for $2 \mathrm{~h}$ with $100 \mu \mathrm{M}$ zVAD-fmk before warming to $37^{\circ} \mathrm{C}$ for $3 \mathrm{~h}$. The micrograph $(L)$ again shows the merged staining for Bax and mtHSP70. The bar represents $5 \mu \mathrm{M}$.
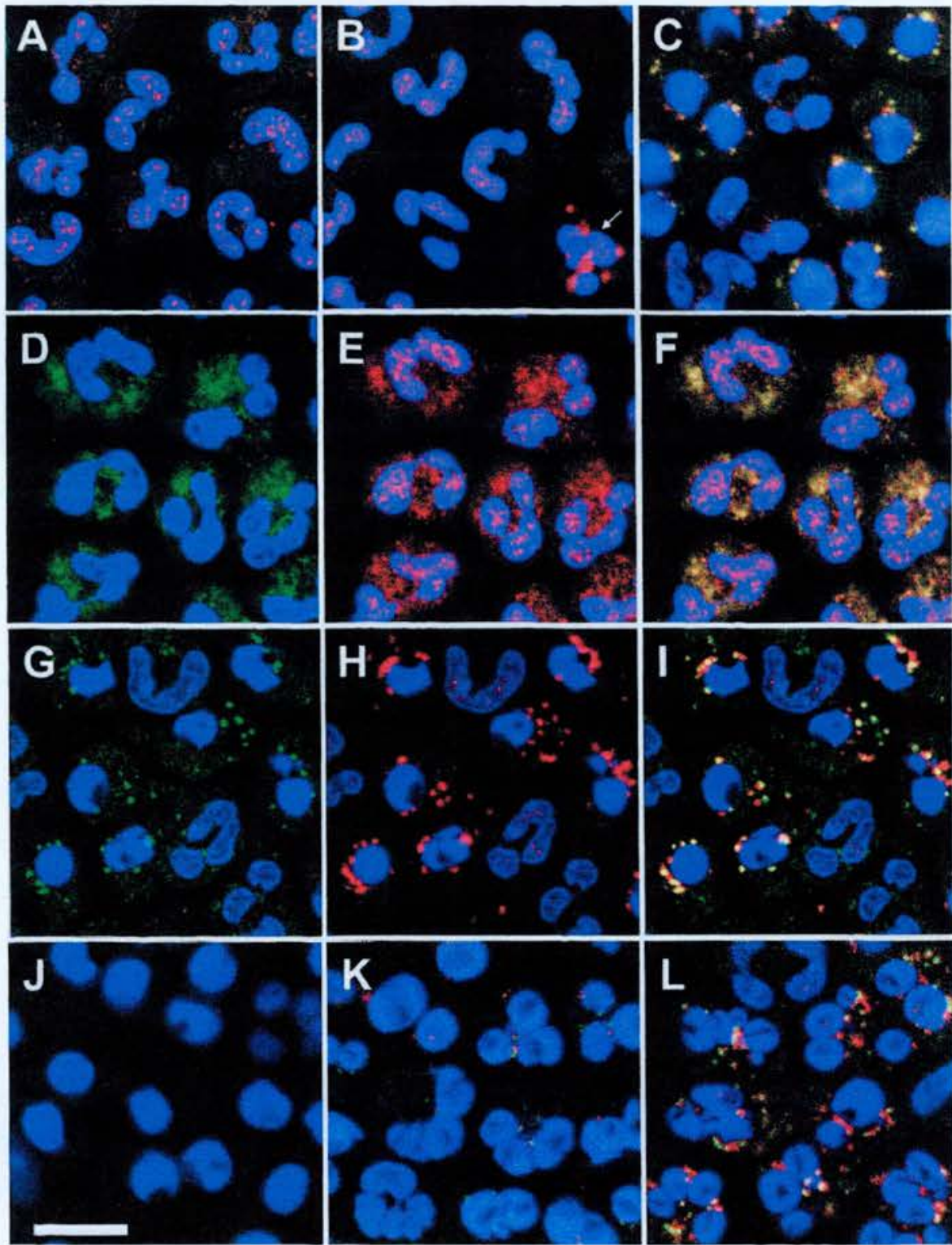

cytoplasmic structures that were present in cells that showed none of the pyknotic nuclear morphology associated with neutrophil apoptosis. Co-localization analysis, on single optical sections using Leica TCS software, confirmed the subcellular co-localization of Bax and mtHSP70 seen in the merged fluorescent micrographs (data not shown). Bax appeared therefore to translocate to mitochondria at $15^{\circ} \mathrm{C}$, without triggering apoptosis. The binding of Bax to mitochondria without insertion of its hydrophobic membrane-spanning domain would be consistent with Bax being peripherally associated with the mitochondrial membrane and redistributing to the cytosol fraction during homogenization and washing of the membrane fraction (Fig. 8, lane 3). After warming the $15^{\circ} \mathrm{C}$ cultured neutrophils to $37^{\circ} \mathrm{C}$ for $3 \mathrm{~h}$, mtHSP70 staining (Fig. $9 G$ ) and Bax staining (Fig. $9 H$ ) were co-localized in the merged micrograph (Fig. 9I) with a small number of large, but discrete, cytoplasmic structures. Similar cytoplasmic structures have been identified in HeLa cells treated with staurosporine to induce apoptosis (72) and in cells overexpressing Bax $(37,38)$ as aggregates of mitochondria. Not all mitochondria stained for Bax and some Bax-stained structures did not stain with
mtHSP70. There was therefore a differential response by mitochondria to apoptotic signals and Bax may also translocate to other membrane compartments. Neutrophils cultured at $15^{\circ} \mathrm{C}$ for $20 \mathrm{~h}$ then warmed to $37^{\circ} \mathrm{C}$ for $3 \mathrm{~h}$ showed no staining with rabbit preimmune sera (Fig. $9 J$ ).

After culture of neutrophils at $15^{\circ} \mathrm{C}$ for $20 \mathrm{~h}$ with zVAD-fmk and warming to $37^{\circ} \mathrm{C}$ for $3 \mathrm{~h}$ there was no significant Bax staining of neutrophil mitochondria that showed non-apoptotic nuclear morphology (Fig. $9 K$ ). This was consistent with the immunoblotting data (Fig. 8, upper panel, lanes 7-9) that showed no insertion of Bax into mitochondria. However, neutrophils cultured at $15^{\circ} \mathrm{C}$ for $18 \mathrm{~h}$, then treated for $2 \mathrm{~h}$ with zVAD-fmk before warming to $37^{\circ} \mathrm{C}$ for $3 \mathrm{~h}$, did show significant Bax and mtHSP70 staining (Fig. 9L). This was consistent with immunoblotting data that showed that Bax had translocated to the membrane fraction (Fig. 8, upper panel, lanes 10-12). While the nuclear morphology was significantly different from the nuclei of freshly isolated neutrophils under these conditions, the pyknotic nuclear morphology characteristic of apoptotic neutrophils was not detected and there was no fragmentation of DNA under these conditions (see Fig. 2, $A-C$ ). 


\section{DISCUSSION}

We have shown for the first time that culturing neutrophils at $15^{\circ} \mathrm{C}$ reversibly arrests the induction of apoptosis, with subsequent warming to $37^{\circ} \mathrm{C}$ triggering a burst of synchronous apoptosis. The molecular consequences of temperature reduction on cells are poorly understood. However, between 10 and $20^{\circ} \mathrm{C}$ there is a reduction in membrane lipid fluidity, a decrease in the rate of protein translation, and an inhibition of vesicular trafficking and neutrophil respiratory burst activity $(67,50,51)$. Our results suggest that the arrest of apoptosis in neutrophils cultured at $15^{\circ} \mathrm{C}$ may be due to the failure of the proapoptotic protein Bax to undergo the conformational changes necessary for it to insert into mitochondria. Once in the membrane Bax triggers the release of cytochrome $c$ and the subsequent activation of caspase- 3 that induces the execution phase of neutrophil apoptosis.

Bax is a soluble protein located in the cytoplasm of freshly isolated neutrophils (10-12). Our immunofluorescence studies on Bax localization in neutrophils cultured at $15^{\circ} \mathrm{C}$ have shown that in addition to its cytoplasmic localization, Bax was also associated with mitochondria that showed signs of aggregation; both observations reported for many cell lines cultured at $37^{\circ} \mathrm{C}$ (73). However, in neutrophils cultured at $15^{\circ} \mathrm{C}$ Bax failed to undergo the conformational changes necessary for insertion of its C-terminal membrane-spanning domain into mitochondria (33). Bax was readily washed from membranes isolated from neutrophils cultured at $15^{\circ} \mathrm{C}$ suggesting a peripheral association with the membrane fraction. The N-terminal region of the Bax molecule may bind to and mask its C-terminal domain in the cytoplasm and the removal of the $\mathrm{N}$-terminal domain leads to an autoactivation and constitutive insertion of the mutant protein into mitochondria (72). The cytoplasmic components that normally facilitate this unmasking of the C-terminal membrane-spanning domain and the conformational changes to neutrophil Bax are unknown. However, in HeLa cells, for example, the proapoptotic protein Bid, can induce the insertion of Bax into membranes $(22,72)$ and may be one of a number of cytoplasmic factors that play a role in the release of cytochrome $c$ from mitochondria (29).

In many models of apoptosis the insertion of Bax into mitochondria and the subsequent release of cytochrome $c$ are not inhibited by the pan-caspase inhibitor zVAD-fmk, while proteolytic cleavage of procaspase- 3 is inhibited (28). Treatment of neutrophils, cultured at $15^{\circ} \mathrm{C}$ for $18 \mathrm{~h}$, with zVAD-fmk prior to triggering apoptosis by warming to $37^{\circ} \mathrm{C}$, also failed to inhibit Bax translocation to neutrophil membranes under conditions where procaspase- 3 was not proteolytically cleaved, consistent with other models of apoptosis. However, when freshly isolated neutrophils were treated with zVAD-fmk at the beginning of the culture period at $15^{\circ} \mathrm{C}$, Bax failed to insert into mitochondria when the cells were warmed to $37^{\circ} \mathrm{C}$. Assuming the fidelity of zVAD-fmk for its caspase targets, these experiments suggested that caspase-mediated events were necessary for Bax insertion (74) but were not in themselves a sufficient trigger for the induction of Bax insertion into mitochondria at $15^{\circ} \mathrm{C}$. Whatever the role the caspases play in preparing Bax, or in activating a putative effector protein necessary for the activation of Bax binding and insertion once the neutrophils are warmed to $37^{\circ} \mathrm{C}$, we saw no evidence that Bax was proteolytically cleaved at $15^{\circ} \mathrm{C}$. Activation of an effector protein and Bax binding at $15{ }^{\circ} \mathrm{C}$ would provide an explanation for the synchronization of apoptosis we observe on warming the cells to $37^{\circ} \mathrm{C}$ after culture at $15^{\circ} \mathrm{C}$. Direct proteolytic cleavage of p21 Bax does not appear to be involved in the translocation of Bax to mitochondria $(70,71)$. However, caspases have been shown to activate the calcium-activated cysteine protease, calpain, that cleaves p21 Bax to p18 Bax at the mitochondrial membrane $(70,71)$. While this proteolytic processing by calpain augments the homodimerization of Bax and the release of cytochrome $c$ it is a relatively late apoptotic event concomitant temporally with the cleavage of many other caspase- 3 substrates and with DNA fragmentation $(75,76)$.

The conformational changes necessary for neutrophil Bax to insert into the mitochondrial membrane were not only inhibited by low temperature, but also by clamping neutrophils at an acidic $\mathrm{pH}_{i}$ during warming to $37^{\circ} \mathrm{C}$, a treatment that was dominant over the effect of temperature reduction. In FL5.12 cells and D1 thymocyte cell lines transient increases in cytoplasmic $\mathrm{pH}_{i}$ have been correlated with $\mathrm{pH}$-dependent conformational change in Bax, an effect that is also inhibited by acid $\mathrm{pH}(39,64,73)$. This observation may provide an explanation for the inhibition of neutrophil apoptosis observed at inflammatory foci where an acid environment has developed (59). Our data are also consistent with the observation that tumor necrosis factor/cycloheximide-induced cytochrome $c$ release is inhibited by $\mathrm{zVAD}-\mathrm{fmk}(30)$ and that caspase- 8 may trigger the release of cytochrome $c(77)$. However, our preliminary data (not shown) have suggested that procaspase-8, like procaspase-3 is not proteolytically cleaved at 15 or at $37^{\circ} \mathrm{C}$ in the presence of zVAD-fmk, under conditions where Bax insertion occurs.

How cytochrome $c$ is translocated across the outer membrane of mitochondria is not known $(41,68,78,79)$. Bax insertion may affect membrane channels by inducing permeability changes that result in the release of cytochrome $c(78,80-82)$. Many cells that obtain their ATP by oxidative phosphorylation can still release cytochrome $c$ from mitochondria at low temperature $(30,81)$. It was possible that extended culture at low temperature would lead to collapse of the inner mitochondrial membrane potential $\left(\Delta \Psi_{m}\right)$ and trigger the opening of the mitochondrial permeability transition pore in neutrophils, an event that correlates with the insertion of Bax and the induction of apoptosis $(27,41,68)$. In granulocytes, however, ATP is obtained predominantly by glycolysis and this may allow their mitochondria to use pyruvate to maintain their $\Delta \Psi_{m}$ at low temperature. Apoptosis triggered by warming $15^{\circ} \mathrm{C}$ cultured neutrophils to $37^{\circ} \mathrm{C}$ was not inhibited by preincubation with 50 $\mu \mathrm{M}$ bongkrekic acid (data not shown), an inhibitor that blocks the permeability transition pore $(37,77)$. This experiment suggested that the collapse of the membrane potential might be an event triggered downstream of caspase-3 activation (28).

The Bax-dependent release of cytochrome $c$ from mitochondria in $15^{\circ} \mathrm{C}$ cultured neutrophils, treated with zVAD-fmk just before warming to $37^{\circ} \mathrm{C}$ to inhibit caspase-3, triggered the activation of apoptosis inducing activities that led to the arrest of both secretion and phagocytosis (57). The externalization of phosphatidylserine on the cell surface was also induced and this may trigger recognition and phagocytosis of granulocytes by macrophages (55). We have established that mitochondria play a role in triggering neutrophil apoptosis. Our $15^{\circ} \mathrm{C}$ cytosols will now enable us to investigate not only the zVAD-fmk sensitive activity that is required for Bax insertion into mitochondria, but also the zVAD-fmk insensitive activities triggered during cytochrome $c$ release, that lead to the apoptotic changes associated with the neutrophil plasma membrane. Finally, the differential response of granulocytes and proliferating cells to temperature reduction suggests that therapeutic targets specific for triggering of neutrophil apoptosis may possibly be identified in this clinically relevant cellular model of apoptosis.

Acknowledgments-We thank Ian Dransfield for help with flow cytometry and Linda Sharp for help with confocal microscopy. Said 
Aoufuchi, Simon Brown, and David Apps are thanked for gifts of antibodies. We are grateful to Tim Allsopp, David Apps, Ian Dransfield,Jonathan Lamb, Mary McElroy, and Deborah Pryde for critical reading of the manuscript and discussions during the course of this work.

\section{REFERENCES}

1. Wyllie, A. H., Kerr, J. F. R, and Currie, A. R. (1980) Int. Rev, Cytol, 68, 251-306

2. Savill, J. S., Wyllie, A. H., Henson, J. E., Walport, M. J., Henson, P. M., and Haslett, C. (1989) J. Clin. Invest. 83, 865-875

3. Whyte, M. K. B. Meagher, L. C., MacDermot, J., and Haslett, C. (1993) J. Immunol. 150, 5124-5134

4. Savill, J., Dransfield, I. Hogg, N., and Haslett, C. (1990) Nature 343, 170-173

5. Savill, J., Hogg, N., Ren, Y., and Haslett, C. (1992) J. Clin. Invest. 90, 1513-1522

6. Meagher, L. C. Savill, J. S., Baker, A., Fuller, R. W., and Haslett, C. (1992) J. Leukocyte Biol. 52, 269-273

7. Lee, A., Whyte, M. K. B., and Haslett, C. (1993) J. Leukocyte Biol. 54, 283-288

8. Hannah, S., Mecklenburgh, K., Rahman, I, Bellingan, G. J., Greening, A. Haslett, C., and Chilvers, E. R. (1995) FEBS Lett. 372, 233-237

9. Weinmann, P., Gaehtgens, P., and Walzog, B. (1999) Blood 93, 3106-3115

10. Moulding, D. A., Quayle, J. A., Hart, C. A., and Edwards, S. W. (1998) Blood 92, 2495-2502

11. Lagasse, E., and Weissman, I. L. (1994) J. Exp. Med. 179, 1047-1052

12. Ohta, H., Yatomi, Y., Sweeney, E. A., Hakomori, S., and Igarashi, Y. (1994) FEBS Lett. 355, 267-270

13. Liles, W C Kiener, P. A Ledbetter, J. A Aruffo, A and Klebanoff, S, J. (1996) J. Exp. Med. 184, 429-440

14. Matsumoto, K., Schleimer, R. P., Saito, H., Iikura, Y., and Bochner, B. S. (1995) Blood 86, 1437-1443

15. Frasch, S. C., Nick, J. A., Fadok, V. A., Bratton, D. L., Worthen, G. S., and Henson, P. M. (1998) J. Biol. Chem. 273, 8389-8397

16. Ward, C., Chilvers, E. R., Lawson, M. F., Pryde, J. G., Fujihara, S., Farrow, S. N., Haslett, C., and Rossi, A. G. (1999) J. Biol. Chem. 274, 4309-4318

17. Sanghavi, D. M., Thelen, M., Thornberry, N. A., Casciola-Rosen, L., and Rosen, A. (1998) FEBS Lett. 422, 179-184

18. Korsmeyer, S. J. (1995) Trends Genet. 11, 101-105

19. Yamashita, K., Takahashi, A., Kobayashi, S. Hirata, H., Mesner, P. W., Kaufmann, S. H., Yonehara, S., Yamamoto, K., Uchiyama, T., and Sasada, M. (1999) Blood 93, 674-685

20. Yuan, J., and Horvitz, H. R. (1990) Dev. Biol. 138, 33-41

21. Stennicke, H. R., Jürgensmeier, J. M., Shin, H., Deveraux, Q., Wolf, B. B. Yang, X., Zhou, Q., Ellerby, H. M., Ellerby, L. M., Bredesen, D., Green, D. R., Reed, J. C., Froelich, C. J., and Salvesen, G. S. (1998) J. Biol. Chem. 273, 27084-27090

22. Kuwana, T., Smith, J. J., Muzio, M., Dixit, V., Newmeyer, D. D., and Kornbluth, S. (1998) J. Biol. Chem. 273, 16589-16594

23. Susin, S. A., Zamzami, N., Castedo, M., Hirsch, T., Marchetti, P., Macho, A. Daugas, E., Geuskens, M., and Kroemer, G. (1996) J. Exp. Med. 184, 1331-1341

24. Li, F., Srinivasan, A., Wang, Y., Armstrong, R. C., Tomaselli, K. J., and Fritz, L. C. (1997) J. Biol. Chem. 272, 30299-30305

25. Kluck, R. M., Martin, S. J., Hoffman, B. M., Zhou, J. S., Green, D. R., and Newmeyer, D. D. (1997) EMBO J. 16, 4639-4649

26. Zou, H., Henzel, W. J., Liu, X., Lutschg, A., and Wang, X. (1997) Cell 90, 405-413

27. Kluck, R. M., Bossy-Wetzel, E., Green, D. R., and Newmeyer, D. D. (1997) Science 275, 1132-1136

28. Bossy-Wetzel, E., Newmeyer, D. D., and Green D. R. (1998) EMBO J. 17, $37-49$

29. Bossy-Wetzel, E., and Green, D. R. (1999) J. Biol. Chem. 274, 17484-17490

30. Goldstein, J. C., Waterhouse, N. J., Juin, P., Evan, G. I., and Green, D. R. (2000) Nat. Cell Biol. 2, 156-162

31. Oltvai, Z. N., Milliman, C. L., and Korsmeyer, S. J. (1993) Cell 74, 609-619

32. Wolter, K. G., Hsu, Y-T., Smith, C. L., Nechushtan, A., Xi, X-G., and Youle, R. J. (1997) J. Cell Biol. 139, 1281-1292

33. Nechushtan, A., Smith, C. L., Hsu, Y-T., and Youle, R. J. (1999) EMBO J. 18 $2330-2341$

34. Hsu, Y-T., Wolter, K. G., and Youle, R. J. (1997) Proc. Natl. Acad. Sci. U. S. A. 94, 3668-3672

35. Hsu, Y-T., and Youle, R. J. (1997) J. Biol. Chem. 272, 13829-13834

36. Jürgensmeier, J. M., Xie, Z., Deveraux, Q., Ellerby, L., Bredesen, D., and Reed, J. C. (1998) Proc. Natl. Acad. Sci. U. S. A. 95, 4997-5002

37. Eskes, R., Antonsson, B., Osen-Sand, A., Montessuit, S., Richter, C., Sadoul, R., Mazzei, G., Nichols, A., and Martinou, J-C. (1998) J. Cell Biol. 143, 217-224

38. Narita, M., Shimizu, S., Ito, T., Chittenden, T., Lutz, R. J., Matsuda, H., and Tsujimoto, Y. (1998) Proc. Natl. Acad. Sci. U. S. A. 95, 14681-14686

39. Gross, A., Jockel, J., Wei, M. C., and Korsmeyer, S. J. (1998) EMBO J. 17, $3878-3885$

40. Rosse, T., Olivier, R., Monney, L., Rager, M., Conus, S., Fellay, I., Jansen, B., and Borner, C. (1998) Nature 391, 496-499

41. Shimizu, S., Narita, M., and Tsujimoto, Y. (1999) Nature 399, 483-487

42. Li, P., Nijhawan, D., Budihardjo, I., Srinivasula, S. M., Ahmad, M., Alnemri, E. S., and Wang, X. (1997) Cell 91, 479-489

43. Slee, E. A., Harte, M. T., Kluck, R. M., Wolf, B. B., Casiano, C. A., Newmeyer, D. D., Wang, H-G., Reed, J. C., Nicholson, D. W., Alnemri, E. S., Green, D. R., and Martin, S. J. (1999) J. Cell Biol. 144, 281-292

44. Haslett, C., Guthrie, L. A., Kopaniak, M. M., Johnston, R. B., and Henson, P. M. (1985) Am. J. Pathol. 119, 101-110

45. Eldadah, B. A., Yakovlev, A. G., and Faden, A. I. (1996) Nucleic Acids Res, 24, 4092-4093

46. Pryde, J. G. (1994) J. Cell Sci. 107, 3425-3436

47. Nicholson, D. W., and MeMurray, W. C. (1984) Can. J. Biochem. Cell Biol.62, 1205-1216

48. Newmeyer, D. D., Farschon, D. M., and Reed, J. C. (1994) Cell 79, 353-364

49. Earnshaw, W. C. (1995) Curr. Opin. Cell Biol. 7, 337-343

50. Tartakoff, A. M. (1986) EMBO J. 5, 1477-1482

51. Manara, F. S., and Schneider, D. L. (1985) Biochem. Biophys. Res. Commun 132, 696-701

52. Shimura, M., Ishizaka, Y., Yuo, A., Hatake, K., Oshima, M., Sasaki, T., and Takaku, F. (1997) FEBS Lett. 417, 379-384

53. Tang, D., Lahti, J. M., Grenet, J., and Kidd, V. J. (1999) J. Biol. Chem. 274, $7245-7252$

54. Gregory, C. D., and Milner, A. E. (1994) Int. J. Cancer. 57, 419-426

55. Martin, S. J., Finucane, D. M., Amarante-Mendes, G. P., O'Brien, G. A., and Green, D. R. (1996) J. Biol. Chem. 271, 28753-28756

56. Vanags, D. M., Pörn-Ares, M. I., Coppola, S., Burgess, D. H., and Orrenius, S. (1996) J. Biol. Chem. 271, 31075-31085

57. Zhuang, J., Ren, Y., Snowden, R. T., Zhu, H., Gogvadze, V., Savill, J. S., and Cohen, G. M. (1998) J. Biol. Chem. 273, 15628-15632

58. Jacobson, M. D., Weil, M., and Raff, M. C. (1997) Cell 88, 347-354

59. Trevani, A. S., Andonegui, G., Giordano, M., López, D. H., Gamberale, R. Minucci, F., and Geffner, J. R. (1999) J. Immunol. 162, 4849-4857

60. Li, J., and Eastman, A. (1995) J. Biol. Chem. 270, 3203-3211

61. Furlong, I. J., Ascaso, R., Rivas, A. L., and Collins, M. K. (1997) J. Cell Sci. 110 653-661

62. Musgrove, E. A., and Hedley, D. W. (1990) Methods Cell Biol. 33, 59-69

63. Benson, R. S. P., Dive, C., and Watson, A. J. M. (1999) J. Cell Sci. 112, $1755-1760$

64. Khaled, A. R., Kim, K., Hofmeister, R., Muegge, K., and Durum, S. K. (1999) Proc. Natl. Acad. Sci. U.S. A. 96, 14476-14481

65. Woo, M., Hakem, R., Soengas, M. S., Duncan, G. S., Shahinian, A., Kägi, D., Hakem, A., McCurrach, M., Khoo, W., Kaufman, S. A., Senaldi, G., Howard, T. Lowe, S. W and Mak, T. W. (1998) Genes Dev. 12, 806-819

66. Kumamoto, J., Raison, J. K., and Lyons, J. M. (1971) J. Theor. Biol. 31, 47-51

67. Liu, G., Hinch, B., Davatol-Hag, H., Lu, Y., Powers, M., and Beavis, A. D. (1996) J. Biol. Chem. 271, 19717-19723

68. Green, D. R., and Reed, J. C. (1998) Science 281, 1309-1312

69. Schlesinger, P. H., Gross, A., Yin, X-M., Yamamoto, K., Saito, M., Waksman, G., and Korsmeyer, S. J. (1997) Proc. Natl. Acad. Sci. U.S. A. 94, $11357-11362$

70. Wood, D. E., Thomas, A., Devi, L. A., Berman, Y., Beavis, R. C., Reed, J. C., and Newcomb, E. W. (1998) Oncogene 17, 1069-1078

71. Kirsch, D. G., Doseff, A. Chau, B. N Lim, D-S., De Souza-Pinto, N. C., Hansford, R., Kastan, M. B., Lazebnik, Y. A., and Hardwick, J. M. (1999) J. Biol. Chem. 274, 21155-21161

72. Desagher, S., Osen-Sand, A., Nichols, A., Eskes, R., Montessuit, S., Lauper, S. Maundrell, K., Antonsson, B., and Martinou, J-C. (1999) J. Cell Biol. 144, 891-901

73. Goping, I. S., Gross, A., Lavoie, J. N., Nguyen, M., Jemmerson, R., Roth, K. Korsmeyer, S. J., and Shore, G. C. (1998) J. Cell Biol. 143, 207-215

74. Murphy, K. M., Streips, U. N., and Lock, R. B. (1999) Oncogene 18, 5991-5999 75. Wood, D. E., and Newcomb, E. W. (1999) J. Biol. Chem. 274, 8309-8315

76. Wood, D. E., and Newcomb, E. W. (2000) Exp. Cell Res. 256, 375-382

77. Srinivasan, A., Li, F., Wong, A., Kodandapani, L., Smidt, R., Krebs, J. F., Fritz, L. C., Wu, J. C., and Tomaselli, K. J. (1998) J. Biol. Chem. 273, 4523-4529

78. Heidin, M. G., Chandel, N. S., Schumacker, P. T., and Thompson, C. B. (1999) Mol. Cell 3, 159-167

79. Marzo, I., Brenner, C., Zamzami, N., Susin, S. A., Beutner, G., Brdiczka, D. Remy, R., Xie, Z. H., Reed, J. C., and Kroemer, G. (1998) J. Exp. Med. 187, 1261-1271

80. Marchetti, P., Castedo, M., Susin, S. A., Zamzami, N., Hirsch, T., Macho, A. Haeffner, A., Hirsch, F., Geuskens, M., and Kroemer, G. (1996) J. Exp. Med. $184,1155-1160$

81. Heiskanen, K. M., Bhat, M. B., Wang, H. W., Ma, J., and Nieminen, A. L (1999) J. Biol. Chem. 274, 5654-5658

82. Basanez, G., Nechushtan, A., Drozhinin, O., Chanturitya, A., Choe, E., Tutt, S., Wood, K. A., Hsu, Y-T., Zimmerberg, J., and Youle, R. J. (1999) Proc. Natl. Acad. Sci. U. S. A. 96, 5492-5497 
receptor activation. We found high levels of nitric oxide associated with Fas receptor resistance in nasal polyp tissues. Therefore, inhibition of apoptosis of eosinophils can be achieved by increased expression of survival factors and disruption of death signals. Future studies will show whether additional mechanisms exist.

The intracellular regulation of apoptosis includes members of the Bcl-2 family. Normal eosinophils express significant levels of $\mathrm{Bcl}-\mathrm{xL}$ and $\mathrm{Bax}$, but no or little Bcl$\mathrm{xS}$ and $\mathrm{Bcl}-2$ (11). In allergic diseases, IL-5 upregulates Bcl$\mathrm{xL}$, but does not alter $\mathrm{Bax}, \mathrm{Bcl}-\mathrm{xS}$ or $\mathrm{Bcl}-2$ levels in eosinophils. In contrast, $\mathrm{Bcl}-2$ is expressed in eosinophils derived from a subgroup of patients with the hypereosinophilic syndrome (12). Overexpression of Bcl-2 in eosinophils delays eosinophil apoptosis in a cytokine-independent manner.

In conclusion, delayed eosinophil apoptosis is an important pathogenic event, which contributes to eosinophil accumulation in diseases associated with eosinophilia. Several mechanisms are described that may participate in the inhibition of the death process. The identification of molecules involved in the anti-apoptosis pathways in eosinophils offers hope for the development of new drugs that reduce inflammation in eosinophilic disorders.

\section{References}

1. Simon H-U. Novel therapeutic strategies via the apoptosis pathways to resolve eosinophilic inflammation associated with allergy. Cell Death Diff 1996; 3: 349-356.

2. Simon H-U, Grotzer M, Nikolaizik WH, Blaser K, Schöni MH. High altitude climate therapy reduces peripheral blood $\mathrm{T}$ lymphocyte activation, eosinophilia and bronchial obstruction in children with house-dust mite allergic asthma. Pediatr Pulmonol 1994; 17: 304-311.

3. Simon H-U, Yousefi, S, Dommann-Scherrer CC, Zimmermann DR, Bauer S, Barandun J, Blaser K, Expansion of cytokine producing $\mathrm{CD}^{-} \mathrm{CD} 8^{-} \mathrm{T}$ cells associated with abnormal Fas expression and hypereosinophilia. J Exp Med 1996; 183: 1071-1082.

4. Simon H-U, Plötz S G, Dummer R, Blaser K, Abnormal clones of $\mathrm{T}$ cells producing interleukin 5 in idiopathic eosinophilia. $N$ Engl J Med 1999; 341: 1112 1120.

5. Simon H-U Yousefi S, Schranz C, Schapowal A, Bachert C, Blaser K. Direct demonstration of delayed eosinophil apoptosis as a mechanism causing tissue eosinophilia. J Immunol 1997; 158: 3902-3908.

6. Simon H-U, Eosinophil apoptosis in allergic disease an emerging new new issue. Clin Exp Allergy 1998; 28: $1321-1324$

7. Dibbert B, Weber M, Nikolaizik W H, et al. Cytokinemediated Bax deficiency associated with delayed neutrophil apoptosis in inflammation. Proc Natl Acad Sci USA 1999; 96: 13330-13335.
8. Hebestreit H, Yousefi S, Balatti I et al. Expression and function of the Fas receptor on human blood and tissue eosinophils. Eur J Immunol 1996; 26: 1775-1780.

9. Simon H-U, Yousefi S, Dibbert B, et al. Role for tyrosine phosphorylation and Lyn tyrosine kinase in Fas receptor-mediated apoptosis in eosinophils. Blood 1998; 92: 547-557.

10. Hebestreit H, Dibbert B, Balatti I, et al. Disruption of Fas receptor signaling by nitric oxide in eosinophils. $J$ Exp Med 1998; 187: 415-425.

11. Dibbert B, Daigle I, Braun D, et al. Role for Bcl- $\mathrm{x}_{\mathrm{L}}$ in delayed eosinophil apoptosis mediated by granulocytemacrophage colony-stimulating factor and interleukin5. Blood 1998; 92: 778-783.

12. Plötz SG, Dibbert B, Abeck D, Ring J, Simon H-U. Bcl-2 expression by eosinophils in a patient with hypereosinophilia. J Allergy Clin Immunol 1998; 102: 1037-1040.

\section{Transcriptional regulation of eosinophil apoptosis}

doi: $10.1053 /$ rmed.2000.0980

\section{A. G. Rossi, C. Ward, J. Murray, M. C. Martin, S. FuJIHARA, I. Dransfield AND C. HASLETT}

Respiratory Medicine Unit, Department of Medicine, University of Edinburgh Medical School, Teviot Place, Edinburgh, EH8 9AG, U.K.

Eosinophilic granulocytes play a key role in the host's defence against invading organisms; for example, they play a particularly prominent role in the fight against parasitic infection. They are also involved in orchestrating the pathogenesis and propagation of certain allergic and inflammatory diseases such as allergic rhinitis and asthma (1). It has become abundantly clear that the development of chronic inflammatory diseases depend not only upon the recruitment and activation of inflammatory cells but also upon their subsequent removal from the inflammatory milieu (2). Apoptosis or programmed cell death is now regarded as a fundamental process regulating inflammatory cell survival and is critically involved in ensuring the successful resolution of an inflammatory response (2). This process therefore provides an efficient non-inflammatory mechanism for removal of potentially highly histotoxic cells from the inflamed site after the useful function of these cells has been served. Therefore, together with preventing excessive granulocyte invasion and activation, a parallel strategy of selectively inducing apoptosis and augmenting phagocytic removal of these cells should prove beneficial for the development of novel therapeutic targets $(2,3)$.

Eosinophils and neutrophils have a similar ontogeny, in that they are derived from a common myeloid precursor, and when activated these cells can exhibit similar functional 
responses. Comparison of functional responses in these cell types has proven to be a useful tool to understand granulocyte behaviour, including apoptosis. Several priming and pro-inflammatory cytokines present at an inflammatory site not only affect granulocyte responsiveness but can also delay the natural constitutive rate of apoptosis. For example, IL-5 and GM-CSF cause a very profound inhibition of eosinophil apoptosis in vitro and a similar effect is observed in neutrophils exposed to LPS or GMCSF $(2,3)$. Thus selective promotion of apoptosis by blocking the influence of these survival factors may be achievable. In addition, it has been convincingly demonstrated that the constitutive rate of neutrophil and eosinophil apoptosis can be regulated in a selective manner; glucocorticoids and agents that elevate cytosolic $\mathrm{Ca}^{2+}$ induce eosinophil apoptosis whereas such agents delay neutrophil apoptosis $(2,4-6)$. Furthermore, the recent observation that glucocorticoids dramatically up-regulate the capacity of macrophages to phagocytose apoptotic cells, including eosinophils and neutrophils, by a noninflammatory mechanism has underpinned the importance of studying and targeting this natural cell suicide process (7). In support of these in vitro observations, it has recently been demonstrated that amelioration of the clinical symptoms of asthma by treating asthmatic patients with oral corticosteroids causes a major increase in the number of phagocytosed apoptotic eosinophils appearing in the sputum. This strongly indicates the importance of these processes in clinical situations (8). Therefore, understanding the molecular mechanisms controlling granulocyte apoptosis in general and unravelling the reasons why eosinophil and neutrophil apoptosis is regulated differently may help in the search for novel therapeutic targets. It has become apparent that both granulocytes, despite being terminally differentiated, are transcriptionally active and consequently have the capacity to synthesize specific proteins. Neutrophils, for example, can synthesize and release a number of inflammatory mediators (e.g. IL-8, GM-CSF) which can themselves influence cell responsiveness and regulate apoptosis (9). Similarly, eosinophils are capable of synthesizing a plethora of inflammatory cytokines and chemokines (1). Recently, we have shown that eosinophils, in response to pharmacological stimuli (e.g. PMA) and physiological secretagogues (e.g. IL-5, $\mathrm{C} 5 \mathrm{a})$, release the pro-inflammatory cytokine macrophage migration inhibitory factor (MIF). PMA-induced MIF secretion is attenuated by cycloheximide and a protein kinase C inhibitor Ro 31-8220 indicating that protein synthesis and protein kinase $\mathrm{C}$ activation are involved in MIF secretion (10). Interestingly, BAL fluid obtained from asthmatic patients contained significantly higher levels of MIF than BAL fluid obtained from normal subjects (10). Despite the fact that granulocytes clearly have the capacity to synthesize and secrete proteins and that investigations into transcriptional regulation of granulocyte responsiveness and apoptosis is in its infancy, some transcription factors have been identified as playing a significant regulatory role. Many of the transcription factors identified so far have yet to be directly demonstrated in granulocytes and shown to be involved in their subsequent behaviour.
These transcription factors are proteins usually located in the cytoplasm that translocate to the nucleus when activated. They then bind to regulatory sequences, usually in the upstream $\left(5^{\prime}\right)$ promoter region of target genes that control the rate of gene transcription. This results in altered protein synthesis of important cytokines, receptors, adhesion molecules, etc, which orchestrate the inflammatory response.

The constitutive rate of neutrophil apoptosis can be enhanced by blockade of protein synthesis using agents such as actinomycin D and cycloheximide suggesting that synthesis of proteins limit induction of apoptosis in neutrophils (11). This is somewhat different in other cell systems, for example, thymocyte apoptosis is suppressed by protein synthesis inhibition. There is also evidence that eosinophil apoptosis, like the neutrophil, can be enhanced by inhibition of protein synthesis (12, Fig. 1). In addition, IL-5 and GM-CSF mediated survival of human eosinophils (13) and neutrophils (14) respectively are prevented by protein synthesis inhibition. Recent evidence indicates that the eosinophil survival agents IL-5, IL-3 and GM-CSF share common signal transducers and mediate their responses via the Lyn-Ras-Raf-1-MAP kinase pathway and the pathways involving the Janus kinase (JAK)/signal transduction and activation of transcription (STAT) family (see 1, 15, 16 for reviews).

We have recently shown that the ubiquitous transcription factor NF- $\kappa$ B plays a critical role in controlling apoptosis both in neutrophils and eosinophils (17). Due to the relatively short life of both cells in vitro and the consequent difficulty of performing long-term molecular biological techniques on these cells, we have relied heavily on a pharmacological approach to investigate the role of transcription factors in regulating granulocyte apoptosis. For this, we have used specific inhibitors of NF- $\kappa \mathrm{B}$ to probe the role of this transcription factor. We have shown that the

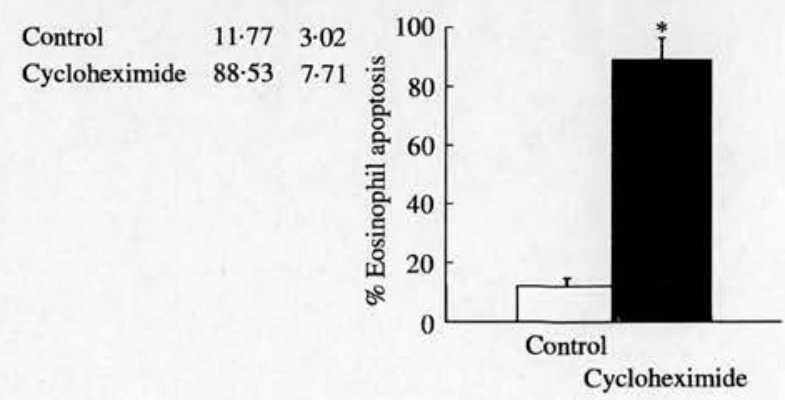

Fig. 1. The effect of cycloheximide on constitutive eosinophil apoptosis. Eosinophils $\left(2 \times 10^{6} / \mathrm{ml}\right)$, cultured in Iscoves DMEM supplemented with $10 \%$ autologous serum, were untreated (open bar) or treated with $50 \mu \mathrm{M}$ cycloheximide (filled bar) and apoptosis assessed morphologically after $20 \mathrm{~h}$ of culture (a minimum of 500 cells were examined per slide). Each treatment was performed in triplicate and the data represent the mean \pm SEM of 4 separate experiments. ${ }^{*} P<0.05$ compared with control. 
selective NF- $\kappa \mathrm{B}$ inhibitor, gliotoxin, but not its structurally similar inactive analogue, methylthiogliotoxin, greatly accelerated the onset of apoptosis of both neutrophils and eosinophils (17). The induction of neutrophil apoptosis was mimicked by other agents that interfere with the NF- $\kappa$ B pathway (e.g. curcumin, PDTC, MG-132 and the cell permeable inhibitory peptide SN50). In neutrophils, the pro-survival bacterial product LPS, and the cytokine TNF$\alpha$ which induces an early pro-apoptotic effect followed by a later anti-apoptotic effect (18), directly stimulated NF- $\kappa$ B translocation from the cytoplasm to the nucleus as assessed by electrophoretic mobility shift assays. Gliotoxin selectively inhibited the NF- $\kappa \mathrm{B}$ activation process and enhanced dramatically the pro-apoptotic effect of TNF- $\alpha$.

In addition, cycloheximide similarly enhanced TNF- $\alpha$ induced neutrophil apoptosis even at time points when cycloheximide alone exerted no pro-apoptotic effect. Taken together these data indicate that NF- $\kappa \mathrm{B}$ may regulate the production of protein(s) which protects neutrophils from the cytotoxic effect of TNF- $\alpha$. In the eosinophil, TNF- $\alpha$ alone failed to alter the rate of constitutive apoptosis, however, when these cells were cultured simultaneously with TNF- $\alpha$ and gliotoxin there was a dramatic synergistic enhancement of apoptosis indicating that blockade of NF$\kappa \mathrm{B}$ could unmask the ability of TNF- $\alpha$ to induce apoptosis in eosinophils (17). Although we have detected the presence of the inhibitory subunit of NF- $\kappa \mathrm{B}, \kappa \mathrm{I} \kappa \mathrm{B} \alpha$, in eosinophils (Fujihara, Ward and Rossi, unpublished observations) the precise mechanism of NF- $\kappa \mathrm{B}$ inhibition by gliotoxin and other inhibitors in granulocytes is still to be determined (see Fig. 2). Despite this, it is abundantly clear that the transcription factor NF- $\kappa \mathrm{B}$ plays a crucial role in regulating apoptosis in both neutrophils and eosinophils.

Glucocorticoids exert differential effects on granulocyte behaviour e.g. dexamethasone induces eosinophil apoptosis whereas neutrophil apoptosis it delayed. It is therefore of great scientific and clinical interest to understand the mechanisms controlling these differences particularly in view of the wide use in managing inflammatory diseases. Despite the fact that the effects of glucocorticoids on granulocyte apoptosis are mediated by ligation of glucocorticoid receptors and are dependent on protein synthesis, the precise intracellular signalling mechanism has not been elucidated. We are currently probing whether the differential effects of glucocorticoids are mediated by transactivation of the glucocorticoid response element (GRE), transrepression of other transcription factors [e.g. NF- $\kappa \mathrm{B}$, activator protein (AP-1)] and interactions with different transcription factors [cyclicAMP response element binding protein (CREB), STATs]. There is already good evidence that glucocorticoid receptors do indeed interact with other transcription factors in other cell systems $(19,20)$. Hence, it is possible that the differential effects of glucocorticoids in regulating neutrophil and eosinophil apoptosis may be due to divergent effects on transcriptional regulation of apoptosis in the two cell types. We therefore believe that understanding the molecular mechanisms controlling apoptosis, especially at the transcriptional level, will help in the search for therapeutic agents that can selectively drive granulocyte apoptosis.

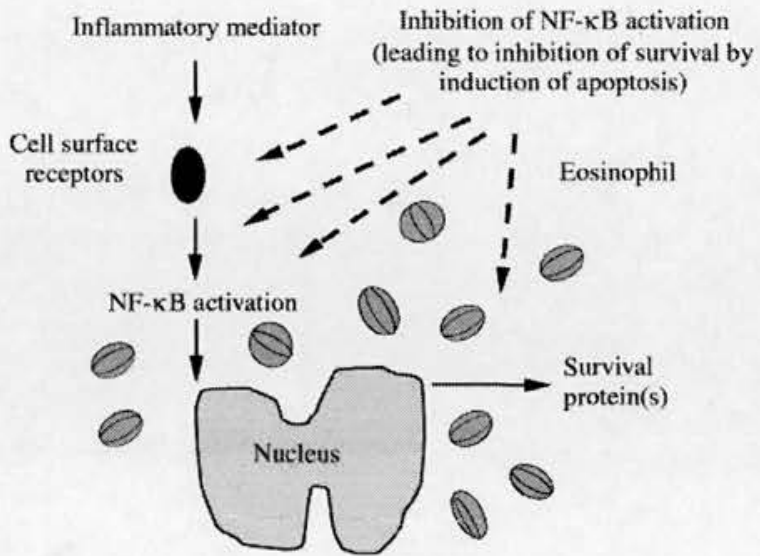

Fig. 2. Possible role of NF- $\kappa \mathrm{B}$ in eosinophil apoptosis Activation of cell surface receptors by cognate ligands may result in NF- $\kappa \mathrm{B}$ activation resulting in the production of survival proteins which inhibit apoptosis. Blockade of the NF- $\kappa$ B pathway by specific agents such as gliotoxin may lead to the inhibition of survival by the induction of apoptosis.

\section{Acknowledgements}

This work presented in this supplement was supported by a MRC programme grant (G9016491).

\section{References}

1. Giembycz MA, Lindsay MA, Pharmacology of the eosinophil. Pharmacol Rev 1999; 51: 213-340.

2. Rossi AG, Haslett, C Inflammation, cell injury and apoptosis. In: Lenfant C, ed. Lung Biology in Health and Disease. Vol 117, Chapt 2, pp 9-24. 1998.

3. Chilvers ER, Rossi AG, Murray J, Haslett C, Regulation of granulocyte apoptosis and implications for anti-inflammatory therapy. Thorax 1998; 53: 533-534.

4. Whyte MK, Hardwick SJ, Meagher LC, Savill JS, Haslett C, Transient elevations of cytosolic free calcium retard subsequent apoptosis in neutrophils in vitro. $J$ Clin Invest 1993; 92: 446-455.

5. Meagher LC, Cousin JM, Seckl JR, Haslett C, Opposing effects of glucocorticoids on the rate of apoptosis in neutrophilic and eosinophilic granulocytes. J Immunol 1996; 156: 4422-4428.

6. Cousin JM, Haslett C, Rossi AG, Regulation of granulocyte apoptosis by PKC inhibition and elevation of $\left[\mathrm{Ca}^{2+}\right]_{\mathrm{i}}$. Biochem Soc Trans 1997; 25: 243S.

7. Liu Y, Cousin JM, Hughes J, et al. Glucocorticoids promote nonphlogistic phagocytosis of apoptotic leukocytes. J Immunol 1999; 162: 3639-3646.

8. Woolley KL, Gibson PG, Carty K, Wilson AJ, Twaddell SH, Woolley MJ. Eosinophil apoptosis and 
the resolution of airway inflammation in asthma. $\mathrm{Am} \mathrm{J}$ Respir Crit Care Med 1996; 154: 237-243.

9. Cassatella MA, The production of cytokines by polymorphonuclear neutrophils. Immunol Today 1995; 16: $21-26$.

10. Rossi AG, Haslett C, Hirani $\mathrm{N}$, et al. Human circulating eosinophils secrete macrophage migration inhibitory factor (MIF). Potential role in asthma. $J$ Clin Invest 1998; 101: 2869-2874.

11. Whyte MK, Savill J, Meagher LC, Lee A, Haslett C, Coupling of neutrophil apoptosis to recognition by macrophages: coordinated acceleration by protein synthesis inhibitors. J Leukoc Biol 1997; 62: 195-202.

12. Ward C, Mechanisms regulating granulocyte apoptosis. $\mathrm{PhD}$ Thesis, 1998 University of Edinburgh.

13. Yamaguchi Y, Suda T, Ohta S, Tominaga K, Miura Y, Kasahara T, Analysis of the survival of mature human eosinophils: interleukin-5 prevents apoptosis in mature human eosinophils. Blood 1991; 78: 2542-2547.

14. Brach MA, deVos S, Gruss HJ, Herrmann F, Prolongation of survival of human polymorphonuclear neutrophils by granulocyte-macrophage colony-stimulating factor is caused by inhibition of programmed cell death. Blood 1992; 80: 2920-2924.

15. Adachi T, Alam R, The mechanism of IL-5 signal transduction. Am J Physiol 1998; 275: C623-633

16. Simon H, Alam R, Regulation of eosinophil apoptosis: transduction of survival and death signals. Int Arch Allergy Immunol 1999; 118: 7-14.

17. Murray J, Barbara JA, Dunkley SA, et al, Regulation of neutrophil apoptosis by tumor necrosis factor-alpha: requirement for TNFR55 and TNFR75 for induction of apoptosis in vitro. Blood 1997; 90: 2772-2783.

18. Ward C, Chilvers ER, Lawson MF, et al. NF?- $\kappa \mathrm{B}$ activation is a critical regulator of human granulocyte apoptosis in vitro. J Biol Chem 1999; 274: 4309-4318.

19. Barnes PJ, Anti-inflammatory actions of glucocorticoids: molecular mechanisms. Clin Sci (Colch) 1998; 94: 557-572.

20. Barnes PJ, Adcock IM, Transcription factors and asthma. Eur Respir J 1998; 12: 221-234.

\section{Role and mechanisms of eosinophil apoptosis in atopic dermatitis}

doi:10.1053/rmed.2000.0981

\section{B. WEDI, U. RAAP, J. StRaede AND A. KapP}

Department of Dermatology and Allergology, Hannover Medical University, Ricklinger Str. 4, 30449 Hannover, Germany.

In general, atopy is defined by specific sensitization to allergens. However, at present there is scant evidence that allergy is central to the development of atopic dermatitis. Despite their atopic constitution, many patients with mild to moderate atopic dermatitis do not have clinically relevant food or aeroallergen sensitivities; therefore, controversy has surrounded the contribution of IgE-mediated hypersensitivity to the pathogenesis of atopic dermatitis classically belonging to the atopic diseases. Elevated blood eosinophil counts are common in atopic dermatitis and immigration and degranulation of activated eosinophils is observed in lesional skin. Thus, the regulation of eosinophil survival may represent a major mechanism through which functional eosinophil are accumulated in inflammatory sites. It is well known that several cytokines, including GMCSF, IL-5 and IL-3, are able to support eosinophil survival and delay apoptosis. We compared eosinophil survival and apoptosis in non-atopic volunteers and in patients with inhalant allergy or acute exacerbation of atopic dermatitis. In eosinophils from subjects with inhalant allergy, and particularly with atopic dermatitis, eosinophil survival was prolonged due to delayed apoptosis when compared to eosinophil from non-atopic subjects (1). There was no difference in the occurrence of apoptosis between the extrinsic and the intrinsic type of atopic dermatitis, pointing to a secondary role of specific sensitization. IL-3 $>$ GMCSF $>$ IL-5 were able to increase viability of cultured eosinophil in non-atopics and subjects with inhalant allergy, but were hardly able to further increase eosinophil survival in subjects with atopic dermatitis which was increased per se. Eosinophil supernatants of patients with atopic dermatitis more than of patients with inhalant allergy dose-dependently inhibited apoptosis in non-atopic eosinophil, and it was shown by cytokine specific ELISA that this effect was possibly due to autocrine production of GM-CSF, probably IL-5, but not IL- 3 or TGF- $\beta 1$. The observed effects in atopic dermatitis were, in fact, mediated by an autocrine production of GM-CSF, since neutralizing anti-GM-CSF mAbs significantly reduced atopic dermatitis eosinophil survival. Moreover, analysing the effect of several other cytokines on eosinophil apoptosis, IL-1 $\beta$, IL-8, IL-12, platelet activating factor, TNF- $\alpha$ and eotaxin did not show any effect. However, IL-4 dose-dependently inhibited eosinophil survival by inducing apoptosis (2). This effect was abrogated by pre-incubation with neutralizing anti-IL-4 antibodies and was most evident in atopic dermatitis eosinophils. However, in co-incubation experiments IL-4 did not overcome the survival-prolonging effect of IL-3, IL-5 and GM-CSF. Moreover, IL-3, IL-5, GMCSF and IL-4 did not modulate eosinophil surface expression of APO-1/Fas antigen (CD95), and Fas antigen expression was similar between the groups studied. However, in inhalant allergy, and particularly in atopic dermatitis, eosinophils demonstrated a certain resistance to anti-Fas $\mathrm{mAb}$ induced apoptosis when compared to nonatopic eosinophils (3). The pathomechanism of this resistance to anti-Fas $\mathrm{mAb}$ as well as the mechanisms for induction of eosinophil apoptosis in general remain uncertain. The role of oxidative stress has not been investigated. Thus, we next analysed the role of reactive oxygen species and selective antioxidants in eosinophil apoptosis. Eosinophils were cultured with the heavy metal 


\title{
Glucocorticoid Augmentation of Macrophage Capacity for Phagocytosis of Apoptotic Cells Is Associated with Reduced p130Cas Expression, Loss of Paxillin/pyk2 Phosphorylation, and High Levels of Active Rac ${ }^{1}$
}

\author{
Katherine M. Giles, * Katherine Ross, * Adriano G. Rossi, * Neil A. Hotchin, ${ }^{\dagger}$ \\ Christopher Haslett,* and Ian Dransfield ${ }^{2 *}$
}

\begin{abstract}
Phagocytic clearance of apoptotic granulocytes has a pivotal role in determining an inflammatory outcome, resolution or progression to a chronic state associated with development of fibrotic repair mechanisms, and/or autoimmune responses. In this study, we describe reprogramming of monocyte to macrophage differentiation by glucocorticoids, resulting in a marked augmentation of their capacity for phagocytosis of apoptotic neutrophils. This monocyte/macrophage phenotype was characterized by decreased phosphorylation, and therefore recruitment of paxillin and pyk2 to focal contacts and a down-regulation of p130Cas, a key adaptor molecule in integrin adhesion signaling. Glucocorticoid-treated cells also displayed higher levels of active Rac and cytoskeletal activity, which were mirrored by increases in phagocytic capability for apoptotic neutrophils. We propose that changes in the capacity for reorganization of cytoskeletal elements induced by glucocorticoids are essential for efficient phagocytic uptake of apoptotic cells. The Journal of Immunology, 2001, 167: 976-986.
\end{abstract}

$\mathrm{T}$ he acute inflammatory response provides a defense mechanism against microbial infection or tissue injury involving the rapid and coordinated recruitment of granulocytes and other inflammatory cells in response to chemokines and other inflammatory mediators. This response is normally self-resolving, but the pathogenesis of a number of diseases such as asthma, emphysema, and rheumatoid arthritis is characterized by a persistent accumulation of inflammatory cells presumably as a result of failure of the natural resolution process (1-3). During normal inflammatory resolution, clearance of extravasated granulocytes requires the induction of apoptosis and concomitant uptake by macrophages (4). Apoptotic cell death, in contrast to necrosis, is associated with maintenance of cell membrane integrity (4) and downregulation of granulocyte secretory function (5), thereby inhibiting potential exacerbation of the inflammatory response through the release of cytotoxic granule contents and proinflammatory cytokines. Additionally, macrophage ingestion of apoptotic cells, unlike phagocytosis of necrotic cells or opsonized particles, does not induce proinflammatory mediator production (6) and can functionally down-regulate cytokine release induced by LPS or opsonized cells (7). Failure to clear apoptotic cells may result in secondary necrosis with predictable consequences in terms of tissue injury and/or initiation of autoimmune processes when phagocyte clearance processes in vivo are overwhelmed by excess apoptotic cell

*Medical Research Council Centre for Inflammation Research, University of Edinburgh Medical School, Edinburgh, United Kingdom; and 'School of Biosciences, The University of Birmingham, Edgbaston, Birmingham, United Kingdom

Received for publication December 11, 2000. Accepted for publication May 4, 2001.

The costs of publication of this article were defrayed in part by the payment of page charges. This article must therefore be hereby marked advertisement in accordance with 18 U.S.C. Section 1734 solely to indicate this fact.

' This work was supported by Medical Research Council Program Grant 9016491 (to C.H., A.G.R., K.R., and I.D.) and the Wellcome Trust (to K.M.G.).

${ }^{2}$ Address correspondence and reprint requests to Dr. I. Dransfield, MRC Centre for Inflammation Research, University of Edinburgh Medical School, Teviot Place, Edinburgh EH8 9AG, U.K. E-mail address: i.dransfield@ed.ac.uk load (8-10). Clearly, if induction of apoptotic pathways is to be considered as a potential therapy for cancer or inflammatory disease, a parallel strategy to maximize phagocytic clearance is likely to be required to avoid the deleterious consequences of necrotic cell death.

A number of cell surface molecules have been proposed to mediate the uptake of apoptotic cells; these include lectins (11), $\alpha_{v} \beta_{3}$ integrin/CD36/thrombospondin complex $(12,13)$, phosphatidylserine receptors (14), scavenger receptors (15), receptors for oxidized lipids (16), CD14 (17), CD29 (18), the ABC1 transporter (19), and receptors for complement components C3bi (CR3/CR4) (20) and Clq (21). The lack of complete inhibition of phagocytosis by soluble ligands or blocking $\mathrm{mAb}$ points to functional redundancy, and we have suggested that a more effective strategy for altering clearance of apoptotic cells would be manipulation of macrophage phagocytic potential (22). Although cytokines such as TNF, GM-CSF, TGF- $\beta$, or IL- 1 may be used to augment phagocytic clearance (23), the effects have been small and the pleiotropic consequences of some of these agents may restrict their potential therapeutic value. Our previous studies have revealed that disruption of cytoskeletal and adhesion contacts in monocyte-derived macrophages by elevation of intracellular cAMP inhibits phagocytosis of apoptotic cells (24). In contrast, ligation of CD44 (25) or adhesion to fibronectin (26) results in a rapid and dramatic augmentation of apoptotic cell uptake. For example, CD44 induces a $400 \%$ increase in phagocytic index within a 30 -min assay period. If similar augmentation of phagocytic activity were attainable in vivo, the potential for clearance of apoptotic cells over the course of an inflammatory response would be considerable. Recent genetic studies in Caenorhabditis elegans have further suggested a role for adhesion signaling in the control of phagocytosis. Ced-5, -2 , and -10 , members of a family of genes required for clearance of cellular corpses during development, are highly homologous to mammalian proteins DOCK180 (27) (myoblast city protein in Drosophila) (28), Crk, and Rac (29), involved in mediating integrin signaling in mammalian cells. Integrin ligation induces the 
formation of a multiprotein complex involving DOCK180 and $\mathrm{Crk}$, the adapter protein $\mathrm{p} 130 \mathrm{Cas}$, and the guanine nucleotide exchange factor $\mathrm{C} 3 \mathrm{G}(30-33)$. Assembly and membrane localization of this complex activates the GTPases Rac $(34,35)$ and Rho $(36)$, inducing membrane ruffling and lamellipodia formation (37) required for spreading, adhesion, and cell migration. Ced-5 and ced- 2 have been shown to interact and activate ced-10 GTPase activity in vitro (29), and ced-5-, -2-, and -10-deficient animals have defects in both cell migration and phagocytosis $(27,29)$.

Glucocorticoids represent a powerful antiinflammatory treatment due to their capacity for inhibition of inflammatory cell recruitment and down-regulation of production and responsiveness of cells to proinflammatory cytokines (38). We have recently described a novel glucocorticoid receptor-dependent promotion of macrophage capacity for phagocytosis of apoptotic cells following short-term exposure of macrophages to glucocorticoids (39). In the present study, we show that long-term exposure of monocytes to the synthetic glucocorticoid dexamethasone $(\mathrm{DX})^{3}$ reprograms monocyte differentiation toward a proresolution phenotype, exhibiting increased phagocytosis of apoptotic cells. Monocytes treated with DX represented a homogeneous cell population characterized by a more rounded appearance. Marked down-regulation of expression of p130Cas, which is required for integrin adhesion signaling through the DOCK $180 / \mathrm{Crk} / \mathrm{C} 3 \mathrm{G}$ complex, together with reduced phosphorylation and recruitment of paxillin and pyk2 to sites of adhesion, may account for this phenotypic alteration. We therefore propose that the dramatic increase in phagocytic potential in DX-treated monocyte-derived macrophages results from changes in the capacity for adhesion-dependent reorganization of cytoskeletal elements that are then available for coordinated phagocytic uptake of apoptotic cells.

\section{Materials and Methods}

\section{Abs and other reagents}

Reagents were obtained from Sigma (Poole, U.K.), unless otherwise stated. Iscove's DMEM (IDMEM) was from Life Technologies (Paisley, U.K.). Dextran and Percoll were from Amersham Pharmacia Biotech (Buckingham, U.K.). DX was obtained from David Bull Laboratories (Warwick, U.K.). Primary Abs were from the following sources: p130Cas, paxillin, Pyk2, Rac, and RC-20 (anti-phosphotyrosine) mAb were from Transduction Laboratories (supplied by Becton Dickinson, Oxford, U.K.); CrkL and C3G rabbit polyclonal $\mathrm{Ab}$ were from Santa Cruz (supplied by Insight Biotechnology, Wembley, U.K.). mAb specific for CD44v3 (3G5), $\beta 1$ (12G10), and class II (WR18) were from Serotec (Oxford, U.K.). Control mouse Igs (IgGland IgG2a), BerMac3 (CD163) and rabbit Igs, and F(ab') goat anti-mouse Ig FITC and HRP conjugates were from Dako (Ely, U.K.). The following monoclonals were generously provided as gifts: $5 \mathrm{~A} 4$ (CD44; G. Dougherty, University of California, San Francisco, CA), SM $\phi$ and 15.2 (CD36 and CD54, respectively; N. Hogg, Imperial Cancer Research Fund (London, U.K.), 23C6 (CD51/61; M. Horton, UCL, London, U.K.), PM6/13 (CD61; M. Wilkinson, Wellcome Trust, London, U.K.), 3G8 (CD16; J. Unkeless, Mount Sinai Medical School, New York, NY), UCHM1 (CD14; P. Beverley, UCL), 61 D3 (CD14; C. Gregory, Nottingham, U.K.). Secondary anti-rabbit HRP was from Amersham Pharmacia Biotech.

\section{Cell isolation and culture}

Mononuclear and polymorphonuclear leukocytes were isolated as previously described (25). In brief, erythrocytes were sedimented from freshly drawn peripheral blood, with $0.6 \%(\mathrm{w} / \mathrm{v})$ dextran $\mathrm{T} 500$, followed by fractionation of leukocytes on a discontinuous Percoll gradient (prepared in $\mathrm{Ca}^{2+} / \mathrm{Mg}^{2+}$-free PBS with final concentrations of Percoll of 50,63 , and $73 \%$ ) at $720 \times g$ for $20 \mathrm{~min}$. Mononuclear cells were aspirated from the $50 / 63$ interface, and neutrophils from the $63 / 73 \%$ interface, and washed three times in PBS (without $\mathrm{Ca}^{2+} / \mathrm{Mg}^{2+}$ ) before culture. Neutrophils (resuspended at $4 \times 10^{6} \mathrm{cells} / \mathrm{ml}$ in IDMEM containing $10 \%$ autologous se-

${ }^{3}$ Abbreviations used in this paper: DX, dexamethasone; IDMEM, Iscove's DMEM; PAK, p21-activated kinase. rum) were cultured at $37^{\circ} \mathrm{C}$ in a $5 \% \mathrm{CO}_{2}$ atmosphere for $20 \mathrm{~h}$ in Falcon tissue culture flasks. Cultured populations were $>50 \%$ apoptotic, as determined by morphological analysis and annexin $\mathrm{V}$ binding, and $<5 \%$ propidium iodide positive. Mononuclear cells were plated at $4 \times 10^{6} / \mathrm{ml}$ in IDMEM and incubated for $30-60 \mathrm{~min}$, at $37^{\circ} \mathrm{C}, 5 \% \mathrm{CO}_{2}$ after which nonadherent lymphocytes were removed by washing with HBSS (without $\left.\mathrm{Ca}^{2+} / \mathrm{Mg}^{2+}\right)$ and monocytes were cultured for a period of 5 days in IDMEM plus $10 \%$ autologous serum, $\pm 1 \mu \mathrm{M}$ DX.

\section{Macrophage phagocytosis assay}

Monocyte-derived macrophages cultared in 48-well tissue culture plates, as described above, were cultured in the presence or absence of DX, or $10 \mu \mathrm{M}$ RU38486 for varying periods of time. For experiments using inhibitors of phagocytosis, macrophages were washed once, then incubated with phagocytosis inhibitors (at the concentrations described in figure legends) for 15 min before the phagocytosis assay. The macrophage monolayer was then overlaid with apoptotic neutrophils (washed and resuspended at a final concentration of $4 \times 10^{6} / \mathrm{ml}$ in IDMEM) and incubated at $37^{\circ} \mathrm{C}, 5 \% \mathrm{CO}_{2}$ for $20 \mathrm{~min}$. Noningested neutrophils were removed by washing in IDMEM, and monolayers were then fixed in $2.5 \%$ glutaraldehyde. The percentage of phagocytosis of neutrophils stained for myeloperoxidase activity with 0.1 $\mathrm{mg} / \mathrm{ml}$ dimethoxybenzidine and $0.03 \%(\mathrm{v} / \mathrm{v})$ hydrogen peroxide was quantified microscopically by counting at least 500 cells in randomly selected fields per well, and an average between the duplicate wells was calculated. Phagocytic index was calculated as: (average number of neutrophils phagocytosed per macrophage $) \times(\%$ macrophages that had phagocytosed one or more neutrophil). Controls for inhibitors used were as follows: All mAb used have previously been shown to inhibit apoptotic cell phagocytosis and were used at concentrations that were deemed to be saturating by flow cytometry. The following inhibitors were found to be functionally active at concentrations used in this study. RGDS (integrin inhibition peptide) was shown to inhibit $\alpha_{5} \beta_{1}$ integrin-mediated adhesion of $\mathrm{T}$ lymphocytes to fibronectin. Phospho-L-serine was shown to inhibit binding of FITC annexin V to apoptotic neutrophils in flow cytometric analysis. Dextran sulfate inhibited uptake of acetylated low density lipoprotein by monocytederived macrophages. Other reagents (glyburide and glucosamine) were used at concentrations that have previously been shown to exert inhibitory effects.

\section{Flow cytometry}

Flow cytometry was performed essentially as described (25), with all incubations conducted on ice to prevent internalization of $\mathrm{Ab}$. Macrophages were detached from tissue culture plastic using PBS containing $2 \mathrm{mM}$ EDTA and $0.5 \%$ serum. After washing with ice-cold PBS containing $0.2 \%$ $(\mathrm{w} / \mathrm{v}) \mathrm{BSA}$ and $0.1 \%(\mathrm{w} / \mathrm{v})$ sodium azide cells $\left(10^{5} / \mathrm{assay}\right)$ were preincubated for $10 \mathrm{~min}$ with $20 \%(\mathrm{v} / \mathrm{v})$ normal rabbit serum to block nonspecific binding to $\mathrm{Fc} \gamma \mathrm{R}$. Cells were then incubated with saturating concentrations of $\mathrm{mAb}$ for $30 \mathrm{~min}$, and washed twice in PBS containing $0.2 \%$ BSA and $0.1 \%$ sodium azide before incubation with FITC-conjugated $\mathrm{F}\left(\mathrm{ab}^{\prime}\right)_{2}$ goat anti-mouse Ig (Dako) for $30 \mathrm{~min}$, and washed twice more before analysis using either an EPICS Profile II (Beckman-Coulter, High Wycombe, U.K.) or a FACSCalibur (Becton Dickinson) flow cytometer.

\section{Electron microscopy}

Macrophages cultured on glass coverslips in the presence or absence of 1 $\mu \mathrm{M}$ DX for 5 days were fixed with $3 \%$ gluteraldehyde in $0.1 \mathrm{M}$ sodium cacodylate buffer, $\mathrm{pH} 7.4$, for $3 \mathrm{~h}$, and postfixed with $1 \%$ osmium tetroxide in $0.1 \mathrm{M}$ cacodylate buffer for $2 \mathrm{~h}$. After dehydration in an ascending acetone series, and critical point drying with $\mathrm{CO}_{2}$, samples were sputter coated with $20 \mathrm{~nm}$ gold/palladium and examined using a Phillips 505 scanning electron microscope.

\section{Immunoprecipitation and Western blotting}

Adherent macrophage cultures were washed with PBS containing $0.1 \mathrm{mM}$ $\mathrm{NaVO}_{3}$ plus protease inhibitor cocktail (Boehringer Mannheim, Mannheim, Germany), and were lysed by incubation with PBS containing $1 \%$ Nonidet P-40, $0.1 \mathrm{mM} \mathrm{NaVO}_{3}$, and protease inhibitor cocktail, $10 \mathrm{~min}$ on ice. Membrane and nuclear material were removed by centrifugation at $14,000 \times \mathrm{g}, 4^{\circ} \mathrm{C}, 30 \mathrm{~min}$. Lysates were precleared by incubation with protein $\mathrm{A}$ agarose-coupled rabbit anti-mouse $\operatorname{IgG}, 4^{\circ} \mathrm{C}, 30 \mathrm{~min}$. The resulting lysates were tested for protein concentration using a detergentcompatible protein estimation kit (Pierce, Rockford, IL), and equilibrated to contain equivalent levels of protein. A total of $100 \mu$ l lysate $(100-150$ $\mu \mathrm{g}$ total protein) was incubated with $1 \mu \mathrm{g}$ of either mouse IgG control, anti-paxillin, or pyk $2 \mathrm{mAb}, 4^{\circ} \mathrm{C}, 30 \mathrm{~min}$, shaking. Immunoprecipitation was achieved by incubation for $30 \mathrm{~min}$ with protein A-coupled rabbit anti- 
mouse IgG (Sigma), and washed twice in TBS containing $0.1 \%$ Triton $\mathrm{X}-100$, and once in $25 \mathrm{mM}$ Tris, plus $0.05 \%$ SDS. Samples were resolved using a $9 \%$ reducing polyacrylamide gel and transferred electrophoretically (50 $\mathrm{V}$ for $1 \mathrm{~h}$ ) onto nitrocellulose (Amersham Pharmacia Biotech). For detection of phosphotyrosine, membranes were blocked with TBS plus $0.05 \%$ Tween 20 (TBS-T) and all other blots with TBS-T plus $10 \%$ nonfat dried milk powder $(w / v)$.

\section{Assay for detection of activated Rac}

Adherent macrophage cultures were lysed in radioimmunoprecipitation assay buffer containing protease inhibitor cocktail (Boehringer Mannheim) plus $1 \mathrm{mM}$ PMSF. Lysates were cleared of membrane and nuclear material by centrifugation, total protein was estimated, and levels were equilibrated as described for immunoprecipitation. A total of $20 \mu \mathrm{l}$ lysate was removed for estimation of total Rac protein, and the remaining $(\sim 300 \mu \mathrm{g})$ was incubated with GST-p21-activated kinase (PAK) (cdc42 or Rac interacting binding domain) fusion protein coupled to Sepharose beads, $4^{\circ} \mathrm{C}, 1 \mathrm{~h}$, shaking. Beads were washed four times in ice-cold Tris buffer $(50 \mathrm{mM}$ Tris (pH 7.2), $150 \mathrm{mM} \mathrm{NaCl}, 10 \mathrm{mM} \mathrm{MgCl}, 1 \%$ Triton, protease inhibitor mixture, $1 \mathrm{mM}$ PMSF), and the amount of active Rac bound to the PAK cdc or Rac interacting binding domain quantified by SDS PAGE and Western blotting, as described for immunoprecipitation.

\section{$R N A$ isolation and RT-PCR}

Macrophages were washed once in ice-cold PBS, lysed, and RNA extracted using TRIzol (Life Technologies; protocol as manufacturer's instructions). RNA was DNase treated to remove genomic DNA for $1 \mathrm{~h}$, $37^{\circ} \mathrm{C}$, and the resulting RNA was used in RT-PCR reaction using Life Technologies One Step RT-PCR kit (protocol as manufacturer's instructions). Primers used ( 35 cycles, annealing $\left.53^{\circ} \mathrm{C}\right)$ were as follows: DOCK 180, 5'-GAGGCAGAGGAGACGAACAG, 3'-AAGCCGATTCG GTGTAGTTA; M-DOCK, 5'-TGCTGAAGTGGCGTATGAAG, 3'CCTCGACCGAACAATGAACT; actin, 5'-CCACCAACTGGGACGA CATG, 3'-GTCTCAAACATGATCTGGGTCATC.

\section{Results \\ Glucocorticoids augment phagocytosis of apoptotic cells in a time-dependent manner}

Monocytes isolated from peripheral blood were cultured in vitro for up to 5 days in the presence of the synthetic glucocorticoid DX, and macrophage potential for phagocytosis of apoptotic cells was determined. Preliminary experiments revealed that macrophages treated with DX for $>24 \mathrm{~h}$ were highly phagocytic, leading to some cell detachment during our standard assay (data not shown). We therefore reduced the assay time from 30 to $20 \mathrm{~min}$ to minimize cell loss and reveal differences between treated cells. DX treatment consistently increased monocyte-derived macrophage phagocytosis of apoptotic neutrophils in a manner that was related to the duration of treatment (Fig. $1 A$ ), and blocked by addition of the steroid receptor antagonist RU38486 $(10 \mu \mathrm{M})$, indicating that a glucocorticoid receptor-dependent process mediated these effects (results not shown). In particular, we noted a striking effect of exposure of freshly isolated monocytes to DX for $120 \mathrm{~h}$ upon phagocytic potential. We therefore investigated whether a time window for steroid exposure early in maturation was sufficient to generate a highly phagocytic phenotype. Monocytes were incubated with DX for periods of $24 \mathrm{~h}(0-24,24-48,48-72,72-96$, and 96-120). To ensure that observed changes were the result of DX exposure exclusively during the stated time window, the steroid receptor antagonist RU38486 was added following removal of DX-containing media. The addition of the antagonist alone had no significant effect (data not shown). Augmentation of apoptotic cell phagocytosis was much less pronounced when DX was added later in the culture period, and that exposure of monocytes to DX within the first $24 \mathrm{~h}$ was sufficient to induce highly phagocytic macrophages (Fig. 1B). Comparison of phagocytic indices revealed that 5-day DX-treated monocytes/macrophages had a 9-fold higher capacity for apoptotic cell clearance in a 20-min assay (Fig. 1, C-E). Thus, glucocorticoids represent the most potent stimulus for aug- mentation of macrophage capacity for phagocytosis of apoptotic cells described to date. Moreover, glucocorticoid modulation of phagocytosis could have profound effects on clearance of apoptotic cells during the resolution of inflammation.

\section{Glucocorticoids reprogram macrophage phenotype}

Examination of macrophages by phase-contrast microscopy revealed that augmented phagocytic potential following 5-day treatment with DX was accompanied by distinct morphological changes consistent with the suggestion that DX influences in vitro differentiation of monocytes. Under our standard culture conditions (IDMEM containing 10\% autologous serum) at 5 days, macrophages were composed of a heterogeneous population of cells with different morphologies: multinucleated giant cells, spread apparently motile cells with evidence of membrane ruffling, rounded less spread cells, and occasional dendritic-like cells (Fig. $2 A$ ). Microscopy-based quantitation revealed that large macrophages $(>25$ $\mu \mathrm{m}$ diameter) represented $29.5 \pm 5.8 \%$ of cells present in 5-day monocyte/macrophage preparations ( $n=4$ separate experiments). Treatment of freshly isolated monocytes with DX for 5 days resulted in consistent morphological changes, with the absence of large multinucleated cell populations and reduced numbers of spread macrophages (large macrophages representing less than $2 \%$ of the cells present). In contrast, the proportion of rounded cells was increased (Fig. $2 B$ ). Further examination of macrophage morphology using scanning electron microscopy demonstrated that the rounded DX-treated cells were attached, with ruffled membranes and filopodial processes (Fig. 2D) when compared with untreated cells (Fig. 2C). An increase in the morphological homogeneity of DX-treated monocyte-derived macrophages was also reflected in the laser scatter properties of DX-treated cells and more uniform expression of a number of surface molecules, including CD14, when analyzed by flow cytometry (Fig. $2 E$ ). Analysis of the coefficient of variation for the fluorescence peaks (untreated vs DXtreated \pm SEM) for CD14 $(108 \pm 14 ; 60 \pm 5, n=13)$, CD16 $(96 \pm 8 ; 62 \pm 4, n=11)$, HLA-DR $(122 \pm 11 ; 96 \pm 6, n=10)$, CD44 $(99 \pm 15 ; 66 \pm 7, n=6)$, and CD51 $(81 \pm 5 ; 53 \pm 4, n=$ 11) confirmed this impression and would be consistent with reprogramming of monocyte differentiation by DX during in vitro culture,

\section{Increased phagocytosis of apoptotic cells involves multiple phagocytic receptors}

Flow cytometric analysis was further used to determine whether DX augmentation of phagocytosis was associated with increased expression of receptors previously implicated in the recognition process (see Ref. 22 for review and Table I). Although most receptors examined exhibited more uniform levels of surface expression, as described above, we did not observe changes in the percentages of positive cells following DX treatment. However, comparison of mean fluorescence intensity of binding revealed some consistent alterations in the levels of surface expression of certain receptors. HLA-DR and the macrophage differentiation markers Fc $\gamma$ RIII (CDI6) and BerMac (CD163) were expressed at slightly elevated levels on DX-treated macrophages (Table I). In contrast, we found significantly reduced expression of CD44, CD44v3, ICAM-1 (CD54), and integrin $\beta_{3}$ subunit (CD61) $(p<$ 0.05 using Student's $t$ test). Binding of the CD36 mAb SM $\phi$ was always found to be lower than that of the IVC7 CD36 mAb, even when both Abs are used at saturating concentrations, suggesting that SM $\phi$ may recognize an epitope that is not present on all CD36 molecules. Interestingly, although overall levels of CD36 expression (IVC7 staining) were decreased with DX treatment, binding of the $\mathrm{SM} \phi \mathrm{mAb}$ was not reduced. Together these results indicated 
FIGURE 1. Effects of DX on macrophage phagocytosis of apoptotic neutrophils. Adherent peripheral blood monocyte-derived macrophages were cultured in the presence or absence of $1 \mu \mathrm{M}$ DX for different times, as shown. The capacity for phagocytosis of apoptotic neutrophils was determined in a 20min assay by microscopic visualization of myeloperoxidase activity. $A$, Monocytederived macrophages were incubated with 1 $\mu \mathrm{M}$ DX for the time periods shown during 5 days of culture before assessment of phagocytosis. Data shown as mean phagocytosis \pm SEM for three separate experiments. $B$, Monocyte-derived macrophages were exposed to a 24-h pulse of $1 \mu \mathrm{M} \mathrm{DX}$ for the time periods shown during 5 days of culture, before assessment of phagocytosis. Data shown are one representative experiment of four that were performed. Representative photomicrographs showing untreated and 5-day DX-treated monocyte-derived macrophage capacity for phagocytosis are shown in $C$ and $D$, respectively. Phagocytic index (calculated as (average number of neutrophils phagocytosed/macrophage) $\times$ (percentage of macrophages phagocytosing one or more neutrophils) for untreated and 5-day DX-treated monocyte-derived macrophages (values taken from mean of duplicates of a minimum of 500 cells \pm SEM for five separate experiments) is shown in $E$.
A

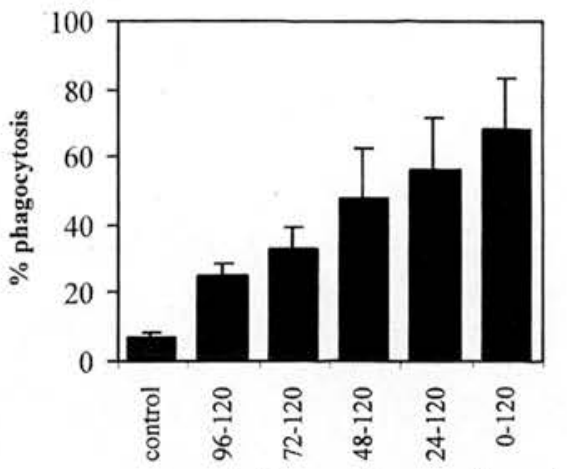

exposure to dexamethasone (hours)

C

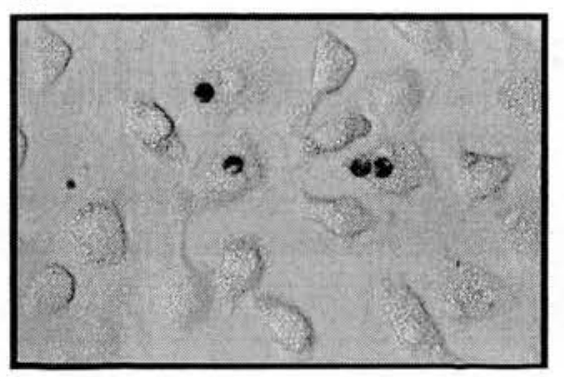

E

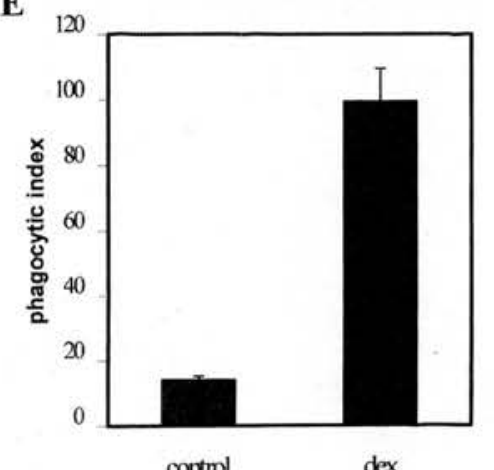

control

dex
B

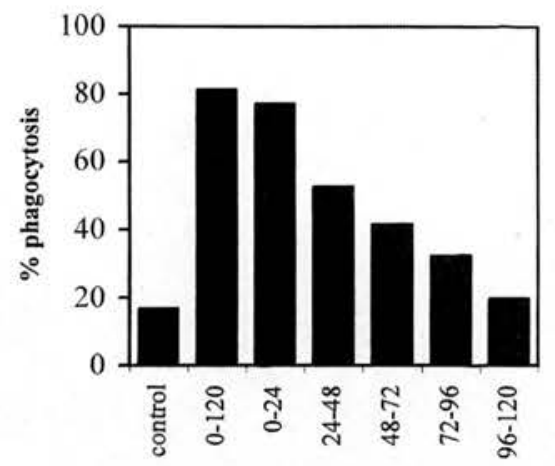

exposure to dexamethasone (hours)

D

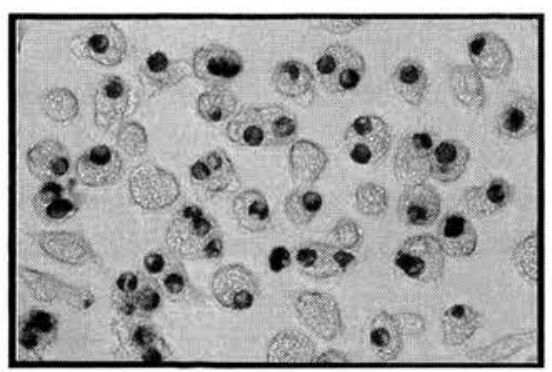

that augmented phagocytic capacity was not associated with increased expression of putative apoptotic cell recognition receptors. However, since surface expression does not necessarily indicate the presence of functionally active receptors, we used specific $\mathrm{mAb} /$ soluble ligand inhibitors of apoptotic recognition pathways to define their contribution to DX-treated macrophage phagocytic activity. Inhibitors of $\mathrm{CD} 36$ (SM $\phi \mathrm{mAb}, 1: 50$ ascites) and $\alpha_{\mathrm{v}} \beta_{3}$ ( $0.5 \mathrm{mM}$ RGDS peptide) did not prevent phagocytosis of apoptotic neutrophils by DX-treated macrophages (Table I), suggesting that this pathway does not play a major role in DX-augmented phagocytosis. In addition, $10 \mathrm{mM}$ glucosamine exhibited only partial inhibition of DX-augmented phagocytosis, further suggesting that integrin-mediated recognition is not the dominant pathway utilized. However, it should be noted that although we have validated that RGDS is functionally active in preventing $\alpha_{5} \beta_{1}$-mediated T cell adhesion to fibronectin, in our experimental system RGDS does not inhibit untreated macrophage phagocytosis either (percentage of phagocytosis control $28.5 \pm 4 \%$, RGDS $27.5 \pm 4 \%$; mean \pm SEM, $n=7$ ). Therefore, these data cannot be taken to indicate that treatment of monocytes with DX induces a switch to $\alpha_{v} \beta_{3}$-independent recognition of apoptotic cells. Inhibition of CD14 with the $61 \mathrm{D} 3 \mathrm{mAb}$ also failed to inhibit DX-treated mac- rophage phagocytosis of apoptotic neutrophils, suggesting that glucocorticoid up-regulated phagocytosis was not CD14 dependent (data not shown). We observed a small inhibitory effect of $2 \mathrm{mM}$ phospho-L-serine upon DX-induced phagocytosis of apoptotic neutrophils, implying that the phosphatidylserine receptor does not play a major role. However, it is possible that phospho-L-serine is not the most effective inhibitor of phosphatidylserine receptor function. Phagocytosis was markedly inhibited in the presence of $500 \mu \mathrm{g} / \mathrm{ml}$ fucoidan ( $31 \%$ of control, $p<0.01)$, implicating scavenger receptor involvement. However, other scavenger receptor ligands, including dextran sulfate (Table I), only partially inhibited phagocytosis. These data suggest broader effects of fucoidan, which may also inhibit other carbohydrate recognition molecules (for example, selectins). One interpretation of these data is that DX augmentation may involve multiple receptor pathways acting cooperatively. Alternatively, DX might promote engagement of a novel receptor pathway to provide more efficient apoptotic cell recognition.

\section{Glucocorticoids alter macrophage cytoskeletal organization}

The distinct morphological appearance of DX-treated macrophages shown in Fig. 2 suggested that control of adhesion was 
A

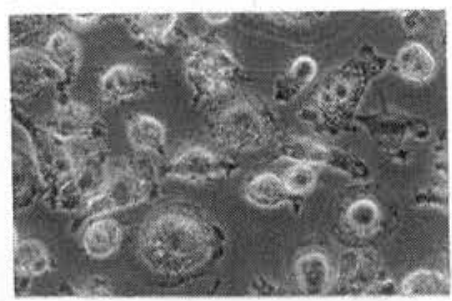

C

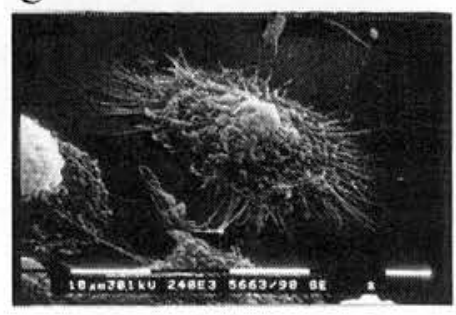

B

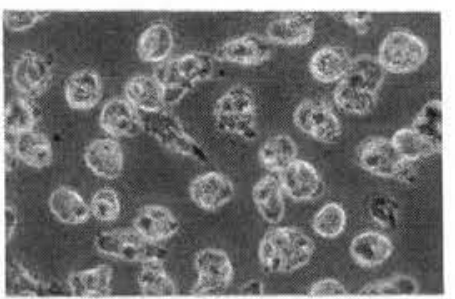

D

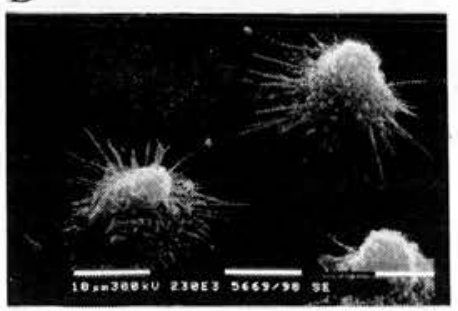
notype. Adherent peripheral blood monocytes cultured for 5 days in the presence or absence of $1 \mu \mathrm{M} \mathrm{DX}$ on either tissue culture-treated plastic ( $A$ and $B$ ) or glass coverslips $(C$ and $D$ ). Morphology of untreated $(A$ and $C)$, and DX-treated $(B$ and $D)$ was assessed by phase-contrast microscopy ( $A$ and $B)$ ( $\times 40$ objective), and scanning electron microscopy $(C$ and $D)$ (bar, $10 \mu \mathrm{m}$ ). Note the uniform appearance of DX-treated cells by phasecontrast microscopy, which is also observed in electron micrographs. $E$, Monocyte-derived macrophage surface phenotype was examined by indirect immunofluorescence using control IgG1 $\mathrm{mAb}$, CD44 mAb (5A4), or CD14 mAb (UCHM1) together with flow cytometry. The profiles (from one of at least four separate determinations) shown here illustrate the more homogeneous laser scatter properties of DX-treated macrophages. The fluorescence histograms (FL1-H) for cells within the gates shown illustrate the more uniform cell surface expression of CD14 and CD44 following DX treatment. Dotted lines show binding of relevant isotype controls; solid lines, $\mathrm{mAb}$ binding to untreated macrophages; and bold lines, $\mathrm{mAb}$ binding to DX-treated macrophages. FSC, Forward scatter; ssc, side scatter.
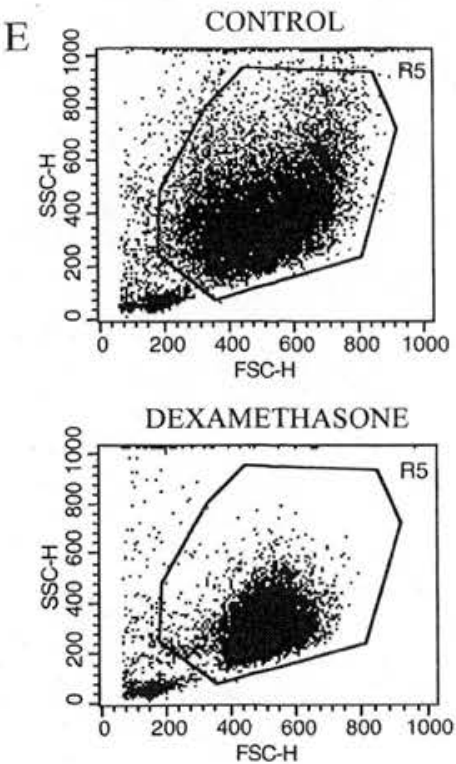
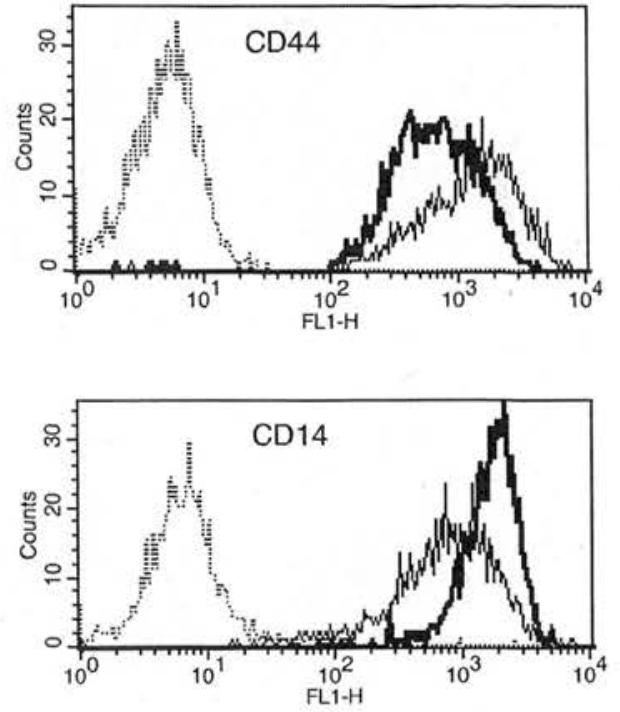

altered following DX treatment. In untreated cells, visualization of focal contacts within macrophages cultured for 5 days on glass slides showed punctate (podosome-like) staining of actin (Fig. 3C) with concentric association of talin (Ref. 24 and our unpublished data) and paxillin (Fig. 3D), probably representing sites of cellsubstratum contact. Paxillin and other proteins associated with adhesion (vinculin and tyrosine-phosphorylated proteins (data not shown)) were also observed toward the cell periphery (see Fig. $3 D$ ), consistent with the adherent phenotype of macrophages. In contrast, DX-treated cells showed an absence of actin- and paxillin-containing podosomes (Fig. 3, $E$ and $F$ ), suggesting an altered organization of adhesion structures.

\section{Altered cytoskeletal protein phosphorylation and organization in} DX-treated macrophages

Since paxillin recruitment to sites of adhesion is regulated by phosphorylation, we next examined levels of expression and the tyrosine phosphorylation status of paxillin and pyk 2 by Western blot analysis of immunoprecipitated proteins. Although paxillin and pyk2 are still expressed at equivalent levels in DX-treated macro- phages (not shown), phosphorylation was found to be reduced in adherent DX-treated macrophages when compared with untreated macrophages (Fig. 4). The observed decrease in phosphorylation of paxillin and pyk 2 was consistent with the altered distribution of paxillin in macrophages and raised the possibility that DX treatment disrupted adhesion-dependent signaling. The p130Cas/Crk/ DOCK 180 complex is a major mediator of adhesion signaling. We therefore examined the levels of expression of CrkL, CrkII, and p130Cas by Western blot analysis, and since we were unable to reproducibly immunoblot DOCK180 (data not shown), we used RT-PCR to test for mRNA for DOCK180 and M-DOCK. Although we did not see changes in levels of CrkL (Fig. $5 \mathrm{~A}$ ) or CrkII (data not shown), p130Cas expression was markedly reduced in DX-treated macrophages (Fig. $5 B$ ). No differences in the levels of mRNA for DOCK 180 or M-DOCK were noted in PCR analysis (Fig. 5C), although differences may be apparent at the level of protein. Despite these marked changes in cytoskeletal organization and phosphorylation, we were surprised to find that DX-treated cells were extremely active in formation of lamellipodia and cellular extensions necessary for phagocytosis in time lapse video 
Table I. Monocytes treated for 5 days with $I \mu M D X$, and the effects on surface receptor expression and apoptotic cell recognition pathways using previously defined inhibitors of phagocytosis

\begin{tabular}{|c|c|c|c|c|c|}
\hline & \multicolumn{2}{|c|}{ Expression } & \multicolumn{2}{|c|}{ Function } & \multirow[b]{2}{*}{ Ref. } \\
\hline & $\mathrm{mAb}$ & $\begin{array}{l}\% \text { relative to } \\
\text { control }^{\alpha}\end{array}$ & Inhibitor & $\begin{array}{l}\% \text { phagocytosis relative } \\
\text { to } \text { control }^{\alpha}\end{array}$ & \\
\hline \multicolumn{6}{|l|}{$\alpha_{\mathrm{v}} \beta_{3} / \mathrm{CD} 36 /$ thrombospondin } \\
\hline$\alpha_{\mathrm{y}}$ & $13 \mathrm{C} 2$ & $98.7 \pm 7.3$ & & & \\
\hline$\beta_{3}$ & $\operatorname{lm} 6 / 13$ & $82.7 \pm 7.7$ & & & \\
\hline \multirow[t]{2}{*}{$\alpha_{v} \beta_{3}$} & $23 \mathrm{C6}$ & $83.6 \pm 7.0$ & RGDS (active) & $85.7 \pm 5.7(n=7)$ & 12,13 \\
\hline & & & RADS (inactive) & $101.3 \pm 8.6(n=3)$ & \\
\hline \multirow{2}{*}{$\mathrm{CD} 36$} & ICV7 & $58.1 \pm 8.1$ & & & 12,13 \\
\hline & $\operatorname{Sm} \phi$ & $86.6 \pm 20.0$ & $\operatorname{Sm} \phi$ & $76.6 \pm 7.0(n=9)$ & \\
\hline Charge sensitive & & & Glucosamine & $63.8 \pm 10.0(n=7)$ & \\
\hline \multicolumn{6}{|l|}{ Pattern recognition receptors } \\
\hline Mannose receptor & & & Mannan & $81.5 \pm 5.8(n=8)$ & 57 \\
\hline CD14/ICAM-3 & UCHM1 & $94.9 \pm 14.4$ & & & 17 \\
\hline \multicolumn{6}{|l|}{ CD44 } \\
\hline CD44 (all isoforms) & $5 \mathrm{~A} 4$ & $71.6 \pm 9.0$ & & & 25 \\
\hline $\mathrm{CD} 44 y^{3}$ & $3 \mathrm{G} 5$ & $58.4 \pm 6.4$ & & & \\
\hline Phosphatidylserine receptor & & & Phospho-L-serine & $80.8 \pm 6.3(n=8)$ & 14 \\
\hline \multicolumn{6}{|l|}{ "Scavenger" receptors } \\
\hline SR-A & & & Dextran sulfate & $67.9 \pm 8.6(n=10)$ & 15 \\
\hline CD163 & BerMac3 & $189.9 \pm 43.3$ & & & \\
\hline \multicolumn{6}{|l|}{ Other molecules } \\
\hline HLA-DR & WR18 & $123.6 \pm 20.6$ & $\mathrm{NA}^{b}$ & NA & \\
\hline CD16 & $3 \mathrm{G} 8$ & $130.5 \pm 11.5$ & NA & NA & \\
\hline
\end{tabular}

a "Control" receptor expression represents expression on 5-day DX-treated monocyte-derived macrophages relative to untreated 5-day monocyte-derived macrophages. "Control" phagocytosis represents phagocytosis of apoptotic neutrophils by 5-day DX-treated monocyte-derived macrophages in the absence of inhibitor.

${ }^{b} \mathrm{NA}$, Not available.

microscopy analysis (data not shown). To test whether the actinregulatory machinery remained functional in DX-treated macrophages, we assessed the levels of activity of the Rho family GTPase Rac, which is involved in membrane ruffling and extension of cellular processes. Using pull-down assays with p21-activated kinase-GST agarose, we found that DX caused a marked increase in the amount of active Rac detectable within macrophage lysates (Fig. 5D). The pronounced morphological alteration observed in DX-treated cells may reflect reprogramming of the capacity for adhesion-dependent signal transduction via down-regulation of p130Cas, reduced paxillin, and pyk2 phosphorylation, and failure to form podosome-like adhesion structures. However, DXtreated macrophages were found to have high levels of active Rac that might contribute to the increased capacity for cytoskeletal reorganization necessary for phagocytosis.

\section{Discussion}

In this study, we present evidence that a macrophage phenotype with augmented phagocytic potential for clearance of apoptotic cells is induced following exposure to DX. We believe that DX exerts distinct regulatory mechanisms upon macrophage behavior, depending upon the stage of monocyte maturation. In our previously published work, monocytes/macrophages were treated with DX from 96 to $120 \mathrm{~h}$, leading to a relatively modest increase in phagocytic potential (39). In contrast with the morphological changes described in this work for cells treated with DX immediately following isolation, no gross changes in morphology are observed following exposure of 96-h monocytes/macrophages to DX (data not shown). Importantly, we demonstrate that treatment of monocytes with DX for the first $24 \mathrm{~h}$ of the 5-day culture period following isolation from peripheral blood is critical for induction of this phenotype, suggesting that glucocorticoids, acting via glucocorticoid receptors, have the potential to reprogram monocyte differentiation. In support of our suggestion, we observe that DXtreated monocytes mature into macrophages that exhibit a uniform morphological appearance consisting of smaller, more rounded cells with more homogeneous laser scatter properties in flow cytometric analysis. Although a number of macrophage surface receptors are expressed at slightly reduced levels in DX-treated cells when compared with untreated cells, the range of expression within the population was found to be less in DX-treated monocytes/macrophages, indicating that functional homogeneity is matched by cell surface phenotype. In addition, the specific increase in BerMac (CD163) expression following DX treatment lends further support to the suggestion of a reprogramming event. However, our analysis failed to reveal any single surface molecular change that would define a prophagocytic phenotype.

In preliminary experiments, we noted that highly phagocytic macrophages were prone to detach during washing following a 30 -min assay. One speculation would be that internalization of plasma membrane during phagocytosis of apoptotic cells may compromise cellular adhesion. In addition, the marked functional alterations that we observe following glucocorticoid treatment of monocytes further illustrate a close association between control of monocyte/macrophage adhesion and clearance of apoptotic cells. Many of the receptors implicated in phagocytosis also have key roles in macrophage adhesion and migration (22). In this study, we demonstrate that augmentation of phagocytic potential is associated with decreased tyrosine phosphorylation of paxillin and $p y k 2$, proteins that represent important components of adhesion contacts (for review, see Ref. 40), and decreased expression of p130Cas, a mediator of adhesion signaling (32). Immunofluorescence analysis revealed that adhesion structures containing localized paxillin and actin were altered in DX-treated macrophages. Reduced expression of p130Cas would be predicted to disrupt Crk/DOCK180 
FIGURE 3. Effects of DX on localization of actin and paxillin in macrophages. Adherent peripheral blood monocyte-derived macrophages were cultured for 5 days in the presence or absence of $1 \mu \mathrm{M} \mathrm{DX}$. Localization of actin and paxillin was determined using either rhodamine phalloidin $(C$ and $E$ ) or anti-paxillin $\mathrm{mAb}(D$ and $F)$ together with fluorescence microscopy. Staining observed with control mAb is shown in $A$ and $B$. These representative micrographs illustrate typical punctate actin staining of contact sites similar to podosomes in control macrophages $(C$ and $D)$ that are absent in DX-treated macrophages $(E$ and $F)$. Paxillin is also localized to smaller focal adhesion-like structures near the cell periphery in control macrophages that are less well defined in DX-treated macrophages.
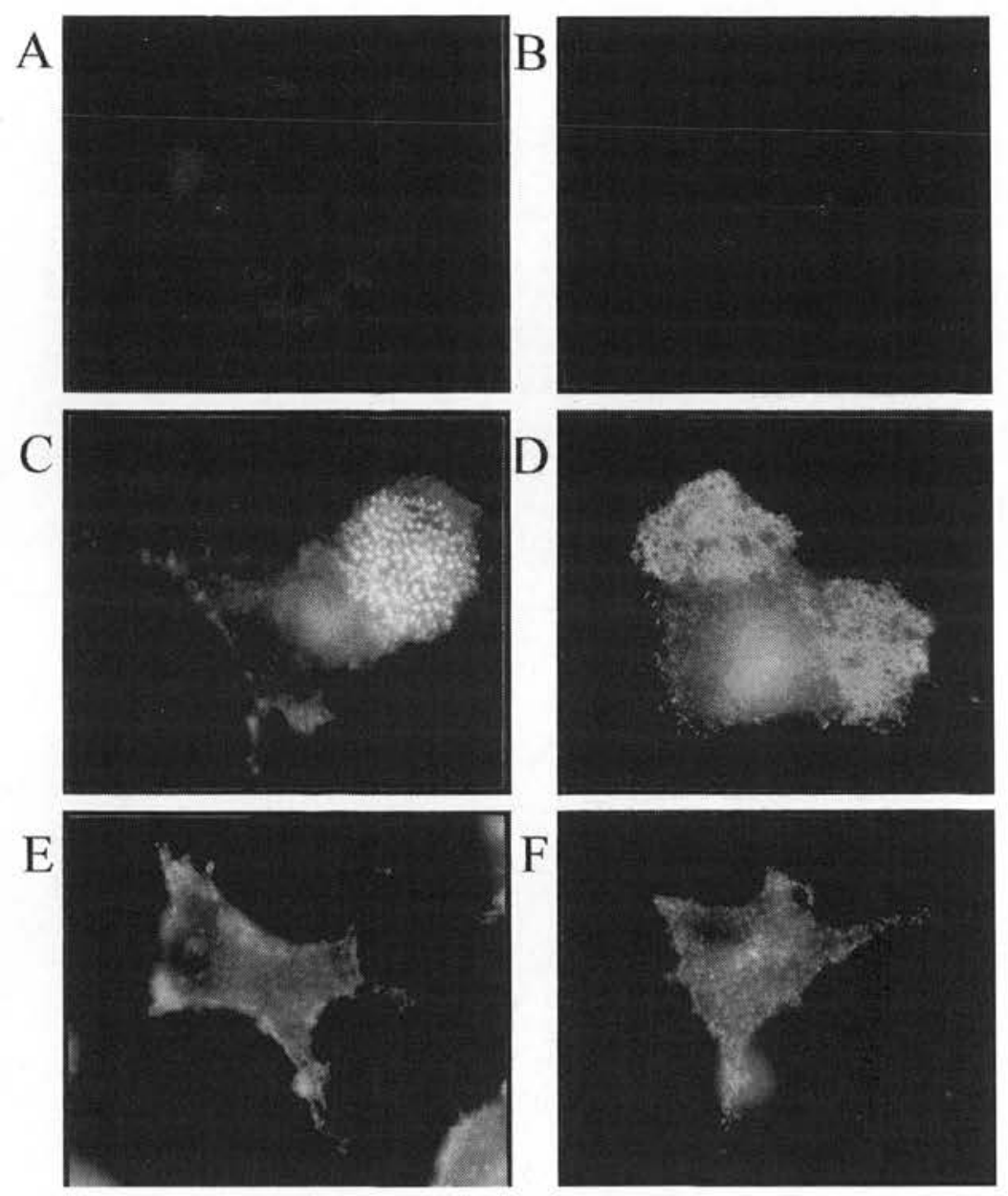

complexes, which together with reduced phosphorylation of paxillin and pyk 2 may have implications for control of the turnover of adhesion structures in macrophages (shown schematically in Fig. 6). Since decreased p130Cas expression in DX-treated macrophages is associated with augmentation of phagocytic capacity, specific recruitment of p130Cas to focal contacts following adhesion to matrix may mimic loss of p130Cas observed in DX-treated cells and influence the availability of other components to drive cytoskeletal reorganization necessary for phagocytosis. We have tried to define whether cytoskeletal components we have examined contribute directly to phagocytosis. However, these studies are made difficult by the morphological and functional heterogeneity of macrophage preparations. Furthermore, cytoskeletal elements present within internalized apoptotic neutrophils complicate interpretation of observed staining patterns. Both Crk and p130Cas have previously been shown to influence the capacity for actin reorganization in rat-1 fibroblast cells $(41,42)$; therefore, downregulation of the central signaling molecule p130Cas is likely to have important implications for the control of adhesion and migration in DX-treated monocytes. We propose that the repertoire of adhesion receptors that are engaged on the macrophage surface might control phagocytic potential indirectly by releasing or sequestering key regulatory molecules such as p130Cas from focal adhesion complexes.

Time-lapse video microscopy reveals that despite the small rounded appearance of DX monocytes/macrophages, these cells remain extremely membrane active, rapidly extending and retracting cellular processes (data not shown). Increased levels of active Rac in DX-treated macrophages lend support to the suggestion that although recruitment of proteins such as paxillin to podosome adhesion-signaling complexes does not occur in the absence of p130Cas, Rac may still drive the extension and retraction of processes observed in DX-treated cells. One possibility is that other p130Cas-like adapters such as HEF1 and Efts/Sin (Ref. 32 and

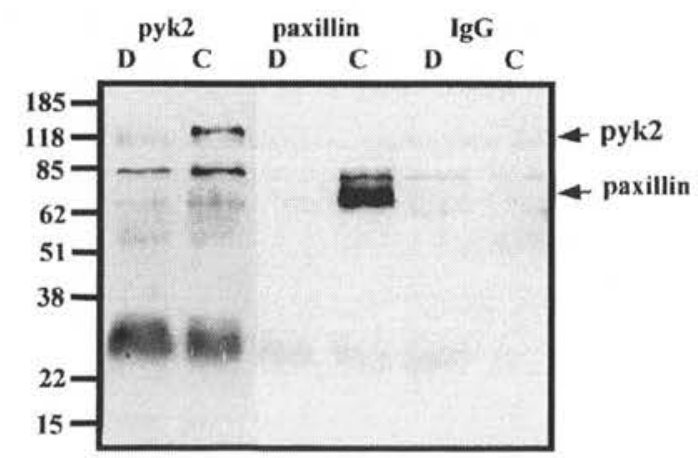

FIGURE 4. DX down-regulates phosphorylation of pyk2 and paxillin. Adherent peripheral blood monocyte-derived macrophages were cultured for 5 days in the presence or absence of $1 \mu \mathrm{M}$ DX. Pyk 2 and paxillin were immunoprecipitated from cell lysates, and protein phosphorylation of immunoprecipitated proteins was analyzed by SDS-PAGE together with Western blotting using the anti-phosphotyrosine mAb RC-20 and ECL detection. In this gel, $\operatorname{lgG}$ denotes protein phosphorylation patterns associated with a nonbinding IgGl control $\mathrm{mAb}$. The band at $\sim 85 \mathrm{kDa}$, present to some extent in all immunoprecipitates, probably represents a nonspecific component. C lanes are from untreated control macrophages, and D lanes are from DX-treated macrophages. 
A

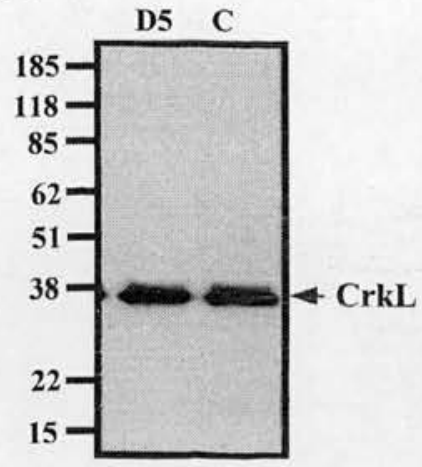

B

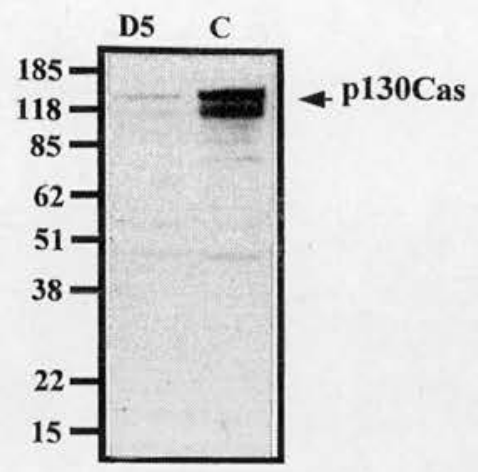

C

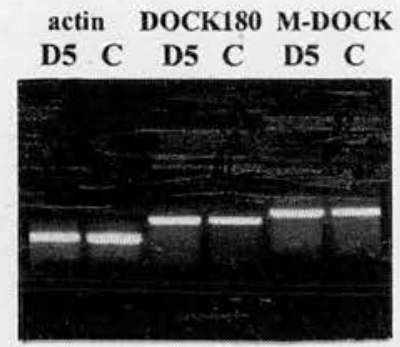

D

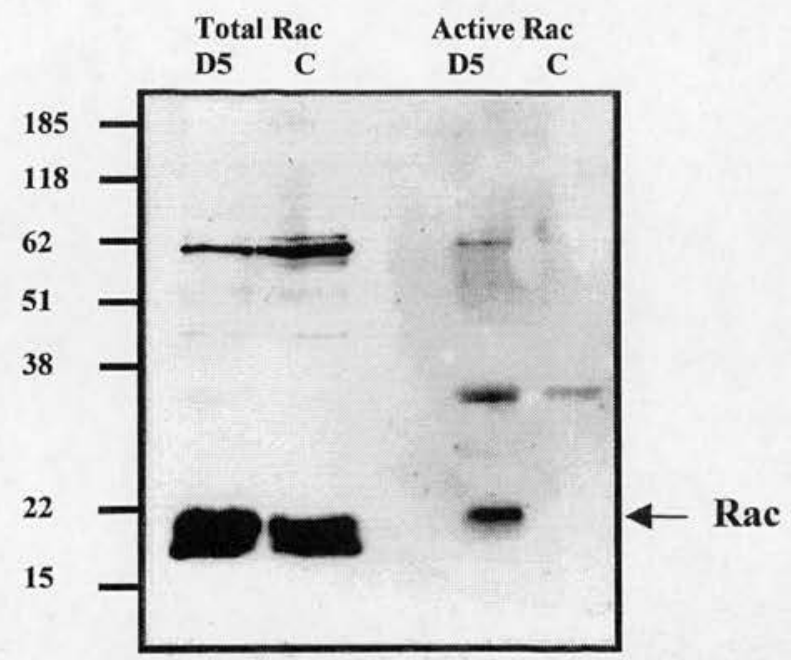

FIGURE 5. Specific down-regulation of p130Cas and increased activation of Rac following DX treatment of macrophages. Adherent peripheral blood monocyte-derived macrophages were cultured in the absence (C) or presence of $1 \mu \mathrm{M}$ DX for 5 days (D5). Macrophage cell lysates were assessed for expression of $A, \operatorname{CrkL}(36 \mathrm{kDa})$ and $B, \mathrm{p} 130 \mathrm{Cas}(130 \mathrm{kDa})$ by SDS-PAGE and Western blotting. $C$, Total RNA from untreated and DX-treated macrophages were assessed for expression of actin, DOCK180, and M-DOCK/DOCK2 transcripts using RT-PCR with primers described in Materials and Methods. D, Activity of the GTPase Rac in macrophage lysates was assessed by pull-down assays using Sepharose coupled with GST-PAK protein. SDS-PAGE and Western blotting with a Rac-specific mAb were used to determine whether Rac was present in pull-downs (Active Rac) or in whole cell lysates (Total Rac) to test whether Rac was present at similar levels in untreated and DX-treated cells.

references therein), present in macrophages, may allow the recruitment of Rac/Crk/DOCK 180 specifically to membranes in a manner that facilitates phagocytosis of apoptotic cells and possibly other particles (43). Indeed, in preliminary experiments using low density IgG-opsonized particles, we have found that DX-treated monocytes/macrophages are more efficient phagocytes (untreated, $24.5 \%$ phagocytosis; DX treated, $53.5 \%$ phagocytosis, $n=4$ ). Importantly, these data suggest that expression or phosphorylation of p130Cas may have a negative regulatory role upon macrophage phagocytic potential. We would speculate that decreased expression/phosphorylation of p130Cas together with augmented Rac activity defines a subpopulation of macrophages highly competent for phagocytosis of apoptotic cells.

Interestingly, recent data suggest that dendritic cell capacity for presentation of apoptotic cell-derived material via MHC class I or class II may depend on $\alpha_{v} \beta_{5}$-mediated internalization mechanisms (44). Our data suggest that in addition to differences in integrin usage, DX-treated macrophages lack p130Cas, a molecule that is recruited following apoptotic cell binding to dendritic cells (45). One possibility is that $\mathrm{p} 130 \mathrm{Cas}$ expression may determine the cel- lular consequences of apoptotic cell handling in macrophages and other cell types. Recent studies have shown that immature dendritic cells treated with glucocorticoids down-regulate the capacity for production of $\mathrm{IL}-12$, and consequently induce a regulatory phenotype (46-48). Changes in the adhesion status of macrophages described in this work may also have important consequences for other macrophage functions that influence the progression of inflammation. Loss of podosome adhesion structures observed in Wiskott-Aldrich syndrome macrophages leads to defective chemotaxis responses as a result of loss of the capacity for polarization (49). Whether DX-treated macrophages show increased potential for directed migration necessary for recruitment or emigration of cells to and from the inflamed site has not been assessed and may also be dependent on the effects of DX upon expression of chemokine receptors and activation of kinases such as extracellular signal-related kinase 1 and 2 . One speculation would be that in addition to augmented capacity for clearance of apoptotic cells, emigration of DX-treated macrophages from inflamed sites to draining lymph nodes might be altered. Formation of podosome structures in osteoclasts has also been shown to require p130Cas/ 
A

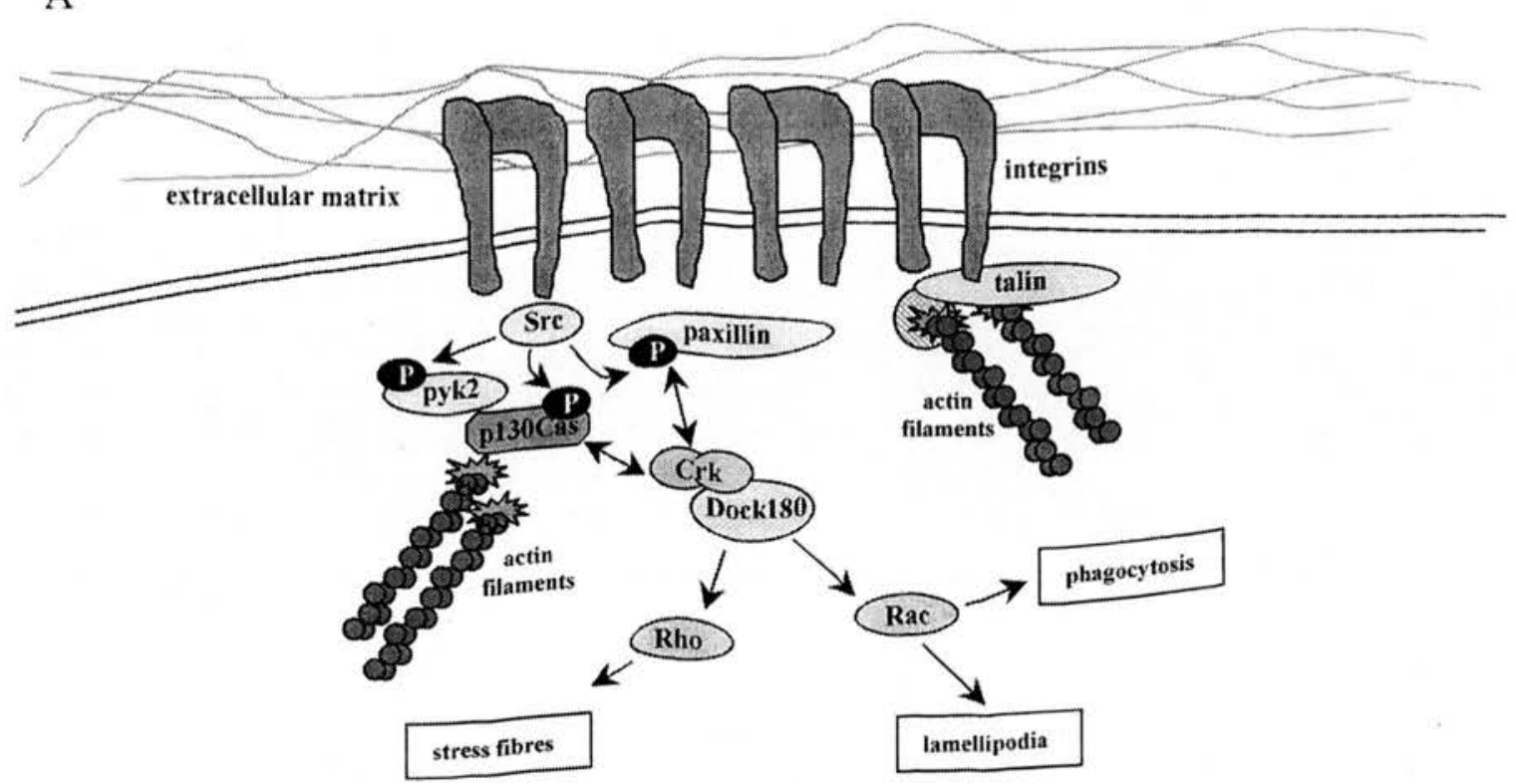

B

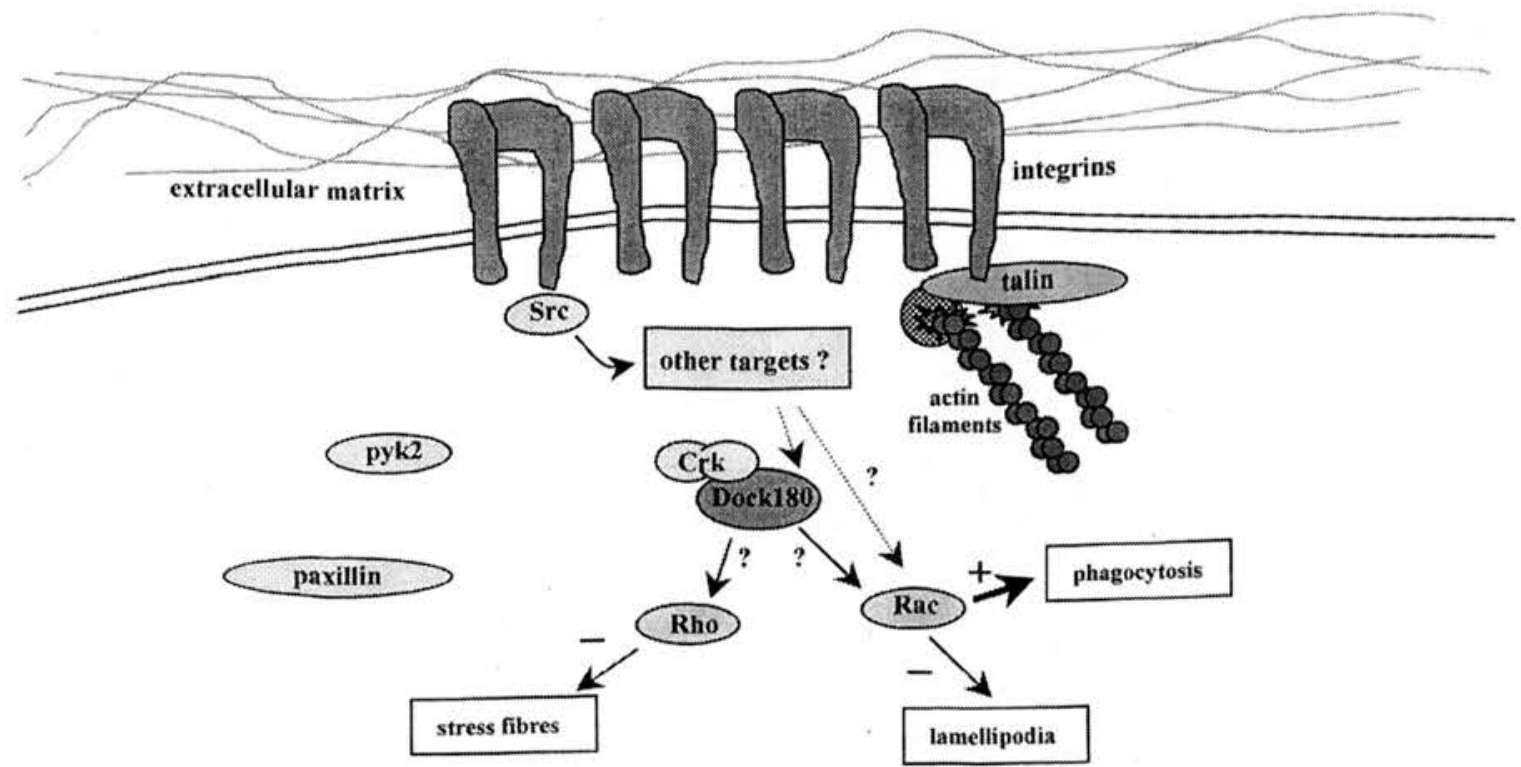

FIGURE 6. Schematic representation of the effects of DX upon cytoskeletal elements present in macrophages. $A$, Attachment to matrix activates a number of adhesion pathways. Integrin ligation induces Src activation and phosphorylation of pyk2 and the scaffolding protein paxillin and p130Cas. The resulting phosphotyrosine motifs recruit Crk and downstream Crk effectors, including DOCK180. The DOCK180/Crk/p130Cas complex may facilitate actin redistribution and podosome formation and may lead to activation of the small GTPases Rac and Rho. B, Following DX treatment, loss of phosphorylation of paxillin and pyk2 would be expected to disrupt interactions of these molecules with other effectors. Crk and DOCK1 80 complexes that are required for formation of adhesion structures may be altered by the reduced levels of p130Cas. Our data would suggest that activation of Rac is facilitated despite the reduced levels of p130Cas in DX-treated cells.

Crk/DOCK180 (50). One interesting possibility is that the detrimental effects of prolonged steroid treatment on bone homeostasis may be mediated though disruption of important adhesion events associated with bone homeostasis.

A striking feature of DX-treated macrophage phenotype was the lack of large multinucleated cells observed in culture when compared with untreated cells. Preliminary data indicate that formation of multinucleated macrophages promoted by treatment with IFN- $\gamma$ (51), or following stimulation with CD98 (52), is inhibited by DX, consistent with data on alveolar macrophages (53), and suggests a dominant regulatory effect of glucocorticoids upon macrophage differentiation. Although previous reports have suggested that glucocorticoids drive monocyte apoptosis (54), we believe that these apparently discrepant results are accounted for by differences in the culture media used. In this present study, monocytes were cultured in $10 \%$ autologous serum with no differences in the numbers of cells recovered with or without DX (control, $152 \pm 16$ cells/ field; DX, $140 \pm 16$ cells/field; average counts \pm SEM from 10 separate experiments). Moreover, the phenotype we observe is unlikely to represent selection of a subpopulation of monocytes that 
are resistant to glucocorticoid-induced death. The percentage of monocyte-derived macrophages that phagocytose apoptotic cells is increased at least 3-fold by DX treatment, requiring that most of the cells in the initial population be lost if selection of a phagocytic phenotype occurred. Although production of modulatory cytokines (e.g., TGF $\beta$ ) following DX treatment might influence monocyte differentiation and phagocytic potential, exposure of monocytes to TGF $\beta$ or IL-10 does not induce the prophagocytic functional phenotype described in this study (not shown).

In conclusion, we report the novel finding that early exposure of monocytes to glucocorticoids induces a proresolution phenotype. The proportion of monocytes/macrophages capable of phagocytosis of apoptotic cells and the phagocytic index are increased dramatically following DX treatment. The combined effect would give rise to a markedly enhanced potential for clearance of apoptotic cells from an inflammatory site following DX treatment, with considerable implications for therapeutic strategies for manipulation of inflammatory processes in vivo. The prophagocytic phenotype of DX-treated monocytes/macrophages was characterized by profound morphological changes, down-regulation of phosphorylation of paxillin and pyk2, and loss of p130Cas expression. We propose that the increased Rac activity we observe together with these changes in cytoskeletal changes may define a prophagocytic macrophage phenotype. These data further emphasize the importance of understanding the contribution of adhesionrelated signaling pathways in the regulation of macrophage phagocytosis.

\section{Acknowledgments}

We thank our colleagues in the Centre for Inflammation Research for their help with cell isolation and other aspects of this work; in particular, Dr. Graham Thomas for help with microscopy, Dr. Sandrine Prost for video microscopy, and Stephen Mitchell for electron microscopy.

\section{References}

1. Weiss, S. J. 1989. Mechanisms of disease: tissue destruction by neutrophils. N. Engl. J. Med. 320:365

2. Newman, S. L., J. E. Henson, and P. M. Henson. 1982. Phagocytosis of senescent neutrophils by human monocyte-derived macrophages and rabbit inflammatory macrophages. J. Exp. Med. 156:430.

3. Haslett, C. 1992. Resolution of acute inflammation and the role of apoptosis in the tissue fate of granulocytes. Clin. Sci. 83:639.

4. Savill, J. S., A. H. Wyllie, J. E. Henson, M. J. Walport, P. M. Henson, and C. Haslett. 1989. Macrophage phagocytosis of aging neutrophils in inflammation: programmed cell death in the neutrophil leads to its recognition by macrophages. J. Clin. Invest. 83:865.

5. Whyte, M. K., L. C. Meagher, J. MacDermot, and C. Haslett. 1993. Impairment of function in ageing neutrophils is associated with apoptosis. J. Immunol. 150: 5124 .

6. Meagher, L. C., J. S. Savill, A. Baker, R. W. Fuller, and C. Haslett. 1992. Phagocytosis of apoptotic neutrophils does not induce macrophage release of thromboxane B2. J. Leukocyte Biol. 52:269.

7. Fadok, V. A., D. L. Bratton, A. Konowal, P. W. Freed, J. Y. Westcott, and P. M. Henson. 1998. Macrophages that have ingested apoptotic cells in vitro inhibit proinflammatory cytokine production through autocrine/paracrine mechanisms involving TGF- $\beta, \mathrm{PGE}_{2}$, and PAF. J. Clin. Invest. 101:890.

8. Ogasawara, J., R. Watanabe-Fukunaga, M. Adachi, A. Matsuzawa, T. Kasugai, Y. Kitamura, N. Itoh, T. Suda, and S. Nagata. 1993. Lethal effect of the anti-Fas antibody in mice. Nature 364:806.

9. Kuwano, K., N. Hagimoto, M. Kawasaki, T. Yatomi, N. Nakamura, S. Nagata, T. Suda, R. Kunitake, T. Maeyama, H. Miyazaki, and N. Hara. 1999. Essentia roles of the Fas-Fas ligand pathway in the development of pulmonary fibrosis J. Clin. Invest. 104:13.

10. Ravirajan, C. T., V. Pittoni, and D. A. Isenberg. 1999. Apoptosis in human autoimmune diseases. Int. Rev. Immunol. 18:563.

11. Duvall, E., A. H. Wyllie, and R. G. Morris. 1985. Macrophage recognition of cells undergoing programmed cell death (apoptosis). Immunology 56:35I.

12. Savill, J. S., I. Dransfield, N. Hogg, and C. Haslett. 1990. Vitronectin receptormediated phagocytosis of cells undergoing apoptosis. Nature 342:170.

13. Savill, J. S., N. Hogg, Y. Ren, and C. Haslett. 1992. Thrombospondin cooperates with CD36 and the vitronectin receptor in macrophage recognition of neutrophils undergoing apoptosis. J. Clin. Invest. 90:1513.

14. Fadok, V. A., D. L. Bratton, D. M. Rose, A. Pearson, R. A. Ezekewitz, and P. M. Henson. 2000. A receptor for phosphatidylserine-specific clearance of apoptotic cells. Nature 405:85.
15. Platt, N., H. Suzuki, Y. Kurihara, T. Kodama, and S. Gordon. 1996. Role for the class A macrophage scavenger receptor in the phagocytosis of apoptotic thymocytes in vitro. Proc. Natl. Acad. Sci. USA 93:I2456.

16. Chang, M. K., C. Bergmark, A. Laurila, S. Horkko, K. H. Han, P. Friedman, E. A. Dennis, and J. L. Witztum. 1999. Monoclonal antibodies against oxidized low density lipoprotein bind to apoptotic cells and inhibit their phagocytosis by elicited macrophages: evidence that oxidation-specific epitopes mediate macrophage recognition. Proc. Natl. Acad. Sci. USA 96:6353.

17. Devitt, A., O. D. Moffatt, C. Raykundalia, J. D.Capra, D. L. Simmons, and C. D. Gregory. 1998. Human CD14 mediates recognition and phagocytosis of apoptotic cells. Nature 392:505.

18. Schwartz, B. R., A. Karsan, T. Bombeli, and J. M. Harlan. 1999. A novel $\beta$ integrin-dependent mechanism of leukocyte adherence to apoptotic cells. $J$. $I \mathrm{~m}$ munol. 162:4842.

19. Luciani, M. F., and G. Chimini. 1996. The ATP binding cassette transporter, $\mathrm{ABCl}$, is required for the engulfment of corpses generated by apoptotic cell death. $E M B O J .15: 226$

20. Mevorach, D., J. O. Mascarenhas, D. Gershov, and K. B. Elkon. 1998. Complement-dependent clearance of apoptotic cells by human macrophages. J. Exp. Med. 188:2313.

21. Botto, M., C. A. E. Dell'Agnola, E. M. Bygrave, H. T. Thompson, F. Cook, M. Petry, M. Loos, P. P. Pandolfi, and M. J. Walport. 1998. Homozygous Cld deficiency causes glomerulonephritis associated with multiple apoptotic bodies. Nat. Genet. 19:56.

22. Giles, K. M., S. P. Hart, C. Haslett, A. G. Rossi, and I. Dransfield. 2000. An appetite for apoptotic cells? Controversies and challenges. Br. J. Haematol. 109:1.

23. Ren, Y., and J. Savill. 1995. Proinflammatory cytokines potentiate thrombospondin-mediated phagocytosis of neutrophils undergoing apoptosis. J. Immunol. 154:2366.

24. Rossi, A. G., J. C. McCutcheon, N. Roy, E. R. Chilvers, C. Haslett, and I. Dransfield. 1998. Regulation of macrophage phagocytosis of apoptotic cells by cAMP. J. Immunol. 160:3562.

25. Hart, S. P., G. J. Dougherty, C. Haslett, and I. Dransfield. 1997. CD44 regulates phagocytosis of apoptotic neutrophil granulocytes, but not apoptotic lymphocytes, by human macrophages. J. Immunol. 159:919.

26. McCutcheon, J. C., S. P. Hart, M. Canning, K. Ross, M. J. Humphries, and I. Dransfield. 1998. Regulation of macrophage phagocytosis of apoptotic cells by adhesion to fibronectin. J. Leukocyte Biol. 64:L

27. Wu, Y. C., and H. R. Horvitz. 1998. C. elegans phagocytosis and cell-migration protein CED-5 is similar to human DOCK180. Nature 392:501.

28. Erickson, M. R., B. J. Galletta, and S. M. Abmayr. 1997. Drosophila myoblast city encodes a conserved protein that is essential for myoblast fusion, dorsal closure, and cytoskeletal organization. J. Cell Biol. 138:589.

29. Reddien, P. W., and H. R. Horvitz. 2000. CED-2/CrkII and CED-10/Rac control phagocytosis and cell migration in Caenorhabditis elegans. Nat. Cell Biol. 2:131.

30. Sakai, R., A. Iwamatsu, N. Hirano, S. Ogawa, T. Tanaka, H. Mano, Y. Yazaki, and H. Hirai. 1994. A novel signaling molecule, pl30, forms stable complexes in vivo with $\mathrm{v}$-Crk and $\mathrm{v}$-Src in a tyrosine phosphorylation-dependent manner. EMBO J. 13:3748

31. Kirsch, K. H., M. M. Georgescu, and H. Hanafusa. 1998. Direct binding of p130(Cas) to the guanine nucleotide exchange factor C3G. J. Biol. Chem. 273 . 25673.

32. O'Neill, G. M., S. J. Fashena, and E. A. Golemis. 2000. Integrin signalling: a new Cas $(\mathrm{t})$ of characters enters the stage. Trends Cell Biol. 10:111.

33. Hasegawa, H., E. Kiyokawa, S. Tanaka, K. Nagashima, N. Gotoh, M. Shibuya, T. Kurata, and M. Matsuda. 1996. DOCK180, a major CRK-binding protein, alters cell morphology upon translocation to the cell membrane. Mol. Cell. Biol. 16:1770.

34. Kiyokawa, E., Y. Hashimoto, S. Kobayashi, H. Sugimura, T. Kurata, and M. Matsuda. 1998. Activation of Rac1 by a Crk SH3-binding protein, DOCK180. Genes Dev. 12:3331.

35. Kiyokawa, E., Y. Hashimoto, T. Kurata, H. Sugimura, and M. Matsuda.1998. Evidence that DOCK180 up-regulates signals from the CrkII-p130(Cas) complex. J. Biol. Chem. 273:24479.

36. Altun-Gultekin, Z. F., S. Chandriani, C. Bougeret, T. Ishizaki, S. Nanumiya, P. de Graaf, P. Van Bergen en Henegouwen, H. Hanafusa, J. A. Wagner, and R. B. Birge. 1998. Activation of Rho-dependent cell spreading and focal adhesion biogenesis by the v-Crk adaptor protein. Mol. Cell. Biol. 18:3044.

37. Allen, W. E., G. E. Jones, J. W. Pollard, and A. J. Ridley. 1997. Rho, Rac and $\mathrm{Cdc} 42$ regulate actin organization and cell adhesion in macrophages. J. Cell $\mathrm{Sci}$. 110:707.

38. Schleimer, R. P. 1993. An overview of glucocorticoid anti-inflammatory actions. Eur. J. Clin. Pharmacol. 45(Suppl. 1):S43.

39. Liu, Y., J. M. Cousin, J. Hughes, J. Van Damme, J. R. Seckl, C. Haslett, I. Dransfield, J. Savill, and A. G. Rossi. 1999. Glucocorticoids promote nonphlogistic phagocytosis of apoptotic leukocytes. J. Immunol. 162:3639.

40. Aplin, A. E., A. Howe, S. K.Alahari, and R. L. Juliano. 1998. Signal transduction and signal modulation by cell adhesion receptors: the role of integrins, cadherins, immunoglobulin-cell adhesion molecules, and selectins. Pharmacol. Rev. 50:197.

41. Nakashima, N., D. W. Rose, S. Xiao, K. Egawa, S. S. Martin, T. Haruta, A. R. Saltiel, and J. M. Olefsky. 1999. The functional role of CrkII in actin cytoskeleton organization and mitogenesis. J. Biol. Chem. 274:3001.

42. Honda, H. H. Oda, T Nakamoto, Z. Honda, R. Sakai, T Suzuki, T Saito, K. Nakamura, K. Nakao, T. Ishikawa, et al. 1998. Cardiovascular anomaly, impaired actin bundling and resistance to Src-induced transformation in mice lacking p130Cas. Nat. Genet. 19:361. 
43. Van der Goes, A., K. Hoekstra, T. K. van den Berg, and C. D. Dijkstra. 2000 Dexamethasone promotes phagocytosis and bacterial killing by human monocytes/macrophages in vitro. J. Leukocyte Biol, 67:801.

44. Albert, M. L., N. Sauter, and N. Bhardwaj. 1998. Dendritic cells acquire antigen from apoptotic cells and induce class-I-restricted CTLs. Nature 392:86.

45. Albert, M. L., J. Kim, and R. B. Birge. 2000, $\alpha_{v} \beta_{5}$ integrin recruits the CrkIIDock180-Racl complex for phagocytosis of apoptotic cells. Nat. Cell Biol. 2:899.

46. Woltman, A. M., J. W. de Fijter, S. W. Kamerling, L. C. Paul, M. R. Daha, and C. van Kooten. 2000. The effect of calcineurin inhibitors and corticosteroids on the differentiation of human dendritic cells. Eur. J. Immumol. 30:1807.

47. Matyszak, M. K., S. Citterio, M. Rescigno, and P. Ricciardi-Castagnoli. 2000 Differential effects of corticosteroids during different stages of dendritic cell maturation. Eur. J. Immunol. 30:1233.

48. De Jong, E. C., P. L. Vieira, P. Kalinski, and M. L. Kapsenberg. 1999. Corticosteroids inhibit the production of inflammatory mediators in immature monocytederived DC and induce the development of tolerogenic DC3. J. Leukocyte Biol. 66:201.
49. Linder, S., H. Higgs, K. Hufner, K. Schwarz, U. Pannicke, and M. Aepfelbacher 2000. The polarization defect of Wiscott-Aldrich syndrome macrophages is linked to dislocalization of the Arp2/3 complex. J. Immunol. 165:221.

50. Nakamura, I., E. Jimi, L. T. Duong, T. Sasaki, N. Takahashi, G. A. Rodan, and T. Suda. 1998. Tyrosine phosphorylation of p130Cas is involved in actin organization in osteoclasts. J. Biol. Chem. 273:11144.

51. Weinberg, J. B., M. M. Hobbs, and M. A. Misukonis. 1985. Phenotypic charac terization of $\gamma$ interferon-induced human monocyte polykaryons. Blood 66:1241.

52. Ohgimoto, S., N. Tabata, S. Suga, M. Nishio, H. Ohta, M. Tsurudome, H. Komada, M. Kawano, N. Watanabe, and Y. Ito, 1995. Molecular characterization of fusion regulatory protein-1 (FRP-1) that induces multinucleated giant cell formation of monocytes and HIV gp 160-mediated cell fusion: FRP-1 and 4F2/CD98 are identical molecules. J. Immunol. 155:3585.

53. Nagasawa, H., C. Miyaura, E. Abe, T. Suda, M. Horiguchi, and T. Suda. 1987. Fusion and activation of human alveolar macrophages induced by recombinant interferon- $\gamma$ and their suppression by dexamethasone. Am. Rev. Respir. Dis. 136: 916

54. Schmidt, M., H. G. Pauels, N. Lugering, A. Lugering, W. Domschke, and T. Kucharzik. 1999. Glucocorticoids induce apoptosis in human monocytes: potential role of IL-1 B. J. Immunol. 163:3484. 


\title{
Cyclic AMP Regulation of Neutrophil Apoptosis Occurs via a Novel Protein Kinase A-independent Signaling Pathway*
}

Received for publication, June 6, 2001, and in revised form, August 29, 2001 Published, JBC Papers in Press, September 17, 2001, DOI 10.1074/jbc.M105197200

\author{
Morag C. Martin†, Ian Dransfield, Christopher Haslett, and Adriano G. Rossi \\ From the Medical Research Council Centre for Inflammation Research, Respiratory Medicine Unit, \\ University of Edinburgh Medical School, Edinburgh EH8 9AG, United Kingdom
}

\begin{abstract}
The second messenger molecule cyclic AMP dramatically modulates the apoptotic program in a wide variety of cells, accelerating apoptosis in some and delaying the rate of apoptosis in others. Human neutrophil apoptosis, a process that regulates the fate and numbers of these potentially histotoxic cells in inflammatory sites, is profoundly delayed by the cell-permeable analog of cyclic AMP, dibutyryl-cAMP. We have investigated the mechanisms underlying cyclic AMP-mediated delay of neutrophil apoptosis, and we show that cyclic AMP inhibits loss of mitochondrial potential occurring during constitutive neutrophil apoptosis. Furthermore, we demonstrate that cyclic AMP also suppresses caspase activation in these inflammatory cells. Despite increasing protein kinase $A$ activity, this kinase is unlikely to mediate the effect of cyclic AMP on apoptosis because blockade of protein kinase $A$ activation did not influence the survival effects of cyclic AMP. Further investigation of the signaling mechanism demonstrated that the delay of apoptosis is independent of phosphoinositide 3-kinase and MAPK activation. Our results suggest cyclic AMP delays neutrophil apoptosis via a novel, reversible, and transcriptionally independent mechanism. We show that proteasome activity in the neutrophil is vitally involved in this process, and we suggest that a balance of pro-apoptotic and anti-apoptotic proteins plays a key role in the powerful ability of cyclic AMP to delay neutrophil death.
\end{abstract}

The neutrophil is a terminally differentiated phagocytic cell that plays a key role in first line defense against invading bacteria. Neutrophils are rapidly recruited to inflamed sites in response to infection and, following phagocytosis of the invading organism, release a variety of toxic granule contents into the phagolysosome containing the engulfed microorganisms ( 1 , 2 ). The neutrophil normally has a short life span, and senescent neutrophils must be prevented from releasing their cytotoxic cell contents into the surrounding milieu because such liberation will lead to local tissue damage. To avoid this undesirable and inappropriate response, the neutrophil undergoes a regulated process of programmed cell death or apoptosis (3-5), allowing shutdown of secretory capacity (6) and phagocytic removal of the intact effete cell by a mechanism that does not incite an inflammatory response (7-9).

* This work was supported by Medical Research Council UK Program Grant G9016491 and a Medical Research Council UK research studentship. The costs of publication of this article were defrayed in part by the payment of page charges. This article must therefore be hereby marked "advertisement" in accordance with 18 U.S.C. Section 1734 solely to indicate this fact.

$\$$ To whom correspondence should be addressed. Tel.: 44-131-651 1323/1572; Fax: 44-131-651-4384; E-mail: moragmartin@hotmail.com.
The execution of the apoptotic program generally involves the activation of a family of cysteine proteases, collectively referred to as the caspases, that are ultimately responsible for the structural dismantling of the cell $(10,11)$. In addition, the mitochondria play a central role through their ability to integrate anti-apoptotic or pro-apoptotic signals from Bcl-2 family members with coordinated activation of downstream caspases and nucleases $(12,13)$. In many cell types it has been documented that apoptosis is accompanied by an early dissipation of the mitochondrial transmembrane potential $(\Delta \Psi m)$ with increased permeability of the outer mitochondrial membrane allowing release of apoptosis-inducing factors such as cytochrome $c(12-14)$. Neutrophils are thought to contain very few mitochondria, and it has not yet been fully established whether they have the capacity to play a functional role in regulation of neutrophil apoptosis $(15,16)$.

Neutrophils undergo constitutive apoptosis during in vitro culture and exhibit the classic changes associated with apoptosis including cytoplasmic condensation, internucleosomal cleavage of DNA by endogenous endonucleases, and exposure of phosphatidylserine on the outer leaflet of the plasmalemma (3). Although the apoptotic program in neutrophils is an intrinsic cell process, the rate of apoptosis can be altered dramatically by a number of agents (17). In particular, we and others (18-20) have shown that elevated levels of the second messenger cyclic AMP can prolong neutrophil longevity by delaying apoptosis.

The cyclic AMP-dependent signaling transduction pathway is a multienzyme cascade that regulates a diverse array of biological processes. Specific ligation of appropriate G-proteincoupled receptors followed by adenylate cyclase activation leads to the production of cyclic AMP. Cyclic AMP then binds to cytoplasmic protein kinase $\mathrm{A}$, a tetrameric structure composed of two regulatory $(\mathrm{R})$ and two catalytic $(\mathrm{C})$ subunits, resulting in dissociation of the $\mathrm{C}$ subunits and subsequent phosphorylation of target proteins (21). Although most components of this signaling cascade are well characterized, the molecular mechanisms underlying cyclic AMP-mediated modulation of apoptosis remain to be elucidated. The signaling mechanism(s) used by cyclic AMP to control these events is (are) likely to be complex and cell type-specific. For example, in contrast to the profound delay in the engagement of the apoptotic process in neutrophils (18-20), cyclic AMP elevation induces apoptosis in thymocytes (22) and leukemic cell lines $(23,24)$. The signaling mechanism determining this ability to differentially influence apoptosis in diverse cell types remains to be elucidated.

In the present study we show that elevated cyclic AMP inhibits activation of caspase-3 and loss in mitochondrial potential $(\Delta \Psi m)$ when neutrophils are aged in vitro, i.e. effects that appear to be associative rather that causative. Although 

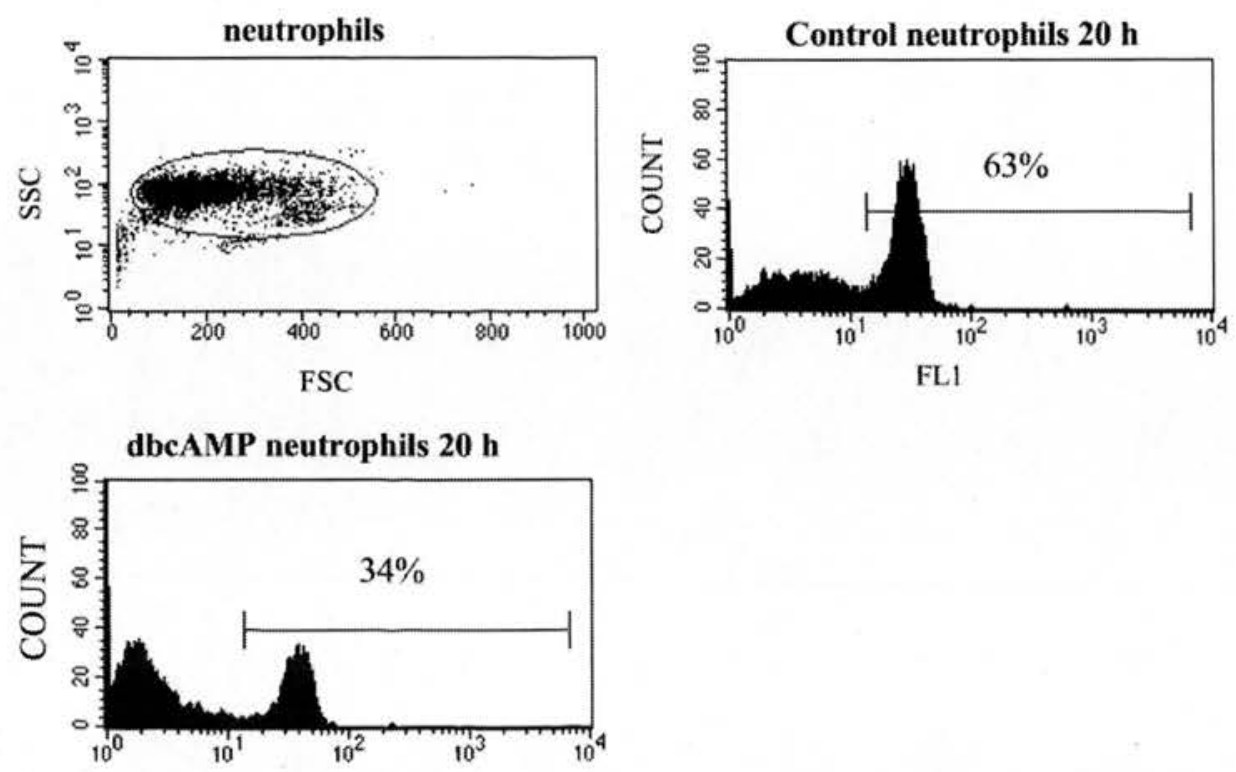

FL1

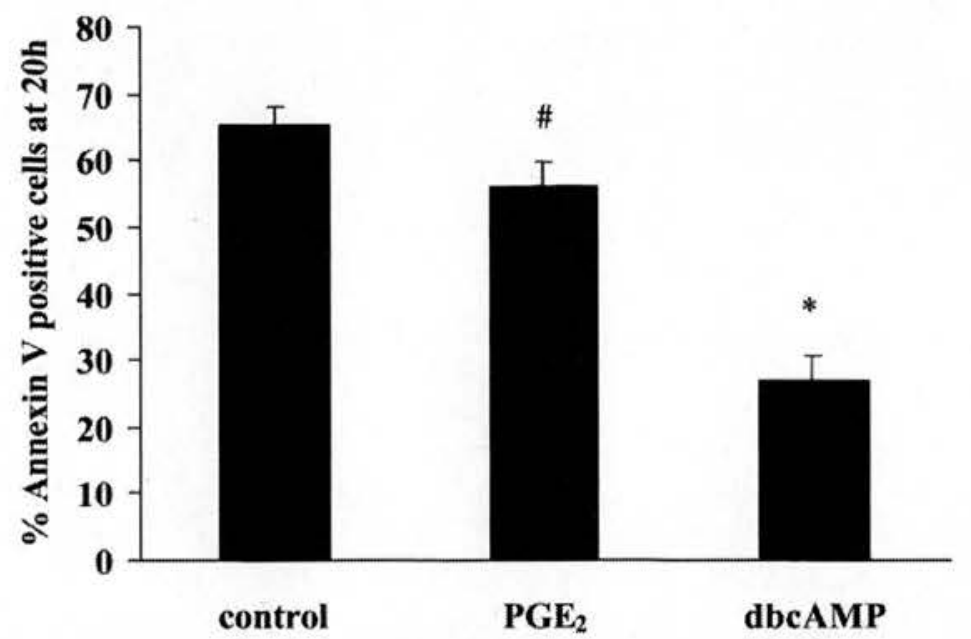

FIG. 1. Effect of cyclic AMP elevation on human neutrophil apoptosis. Human neutrophils $\left(5 \times 10^{6} / \mathrm{ml}\right)$ were cultured at $37^{\circ} \mathrm{C}$ in Iscove's DMEM containing $10 \%$ autologous serum and treated with Bt, $A M P(d b c A M P)(0.2 \mathrm{mM})$ or $\mathrm{PGE}_{2}(10 \mu \mathrm{M})$. After $20 \mathrm{~h}$, the cells were incubated with FITC-labeled recombinant human annexin $\mathrm{V}$ to determine phosphatidylserine expression. The cells were then assessed by flow cytometry on a FACSCalibur and analyzed on associated CellQuest software. Data from a minimum of 5000 cells were analyzed for each condition. All values represent mean \pm S.E. of $n=5$ - 8 experiments, each performed in duplicate where significant difference from control is represented by $*, p<0.001$, and \#, $p<0.05$. Similar results were found by morphological assessment of apoptosis (data not shown).

we could demonstrate that cyclic AMP rapidly elevates endogenous PKA ${ }^{1}$ activity in cultured neutrophils, blockade of PKA activation did not influence the observed delay in neutrophil apoptosis induced by cyclic AMP elevation. We also show that cyclic AMP elevation delays neutrophil apoptosis via a transcriptionally independent and reversible pathway, which does not require PI 3-kinase and MAPK activity. Together these data point to a novel mode of action for the major retardation of neutrophil apoptosis induced by cyclic AMP elevation.

${ }^{1}$ The abbreviations used are: PKA, protein kinase $\mathrm{A} ; \mathrm{Bt}_{2} \mathrm{cAMP}$, dibutyryl cyclic AMP; DMEM, Dulbecco's modified Eagle's medium; GM-CSF, granulocyte macrophage-colony-stimulating factor; JC-1 [5,5',6,6'-tetrachloro-1,1',3,3'-tetraethylbenzimidazocarbocyaniniodide; MAPK, mitogen-activated protein kinase; PI 3-kinase, phosphoinositide 3-kinase; PBS, phosphate-buffered saline; PGE $_{2}$, prostaglandin $\mathrm{E}_{2}$; FITC, fluorescein isothiocyanate.

\section{EXPERIMENTAL PROCEDURES \\ Granulocyte Isolation and Culture}

Neutrophils were purified from the peripheral blood of normal donors by dextran sedimentation (Sigma) followed by centrifugation on discontinuous Percoll ${ }^{\mathrm{TM}}$ (Amersham Pharmacia Biotech) gradients as described previously $(25,26)$. Only neutrophil preparations with a purity of $>98 \%$ were used. Cells were cultured in flat-bottomed Falcon flexible wells (Becton Dickinson, Oxford, UK) at $37^{\circ} \mathrm{C}$ in a $5 \% \mathrm{CO}_{2}$ atmosphere at a concentration of $5 \times 10^{6} / \mathrm{ml}$ in Iscove's modified Dulbecco's medium (Life Technologies, Inc.) supplemented with 100 units/ml penicillin/ streptomycin (Life Technologies, Inc.) and $10 \%(\mathrm{v} / \mathrm{v})$ autologous serum. As an index of necrosis, cell membrane integrity was assessed by the ability of cells to exclude the vital dye trypan blue (Sigma). Under all experimental conditions, greater than $99 \%$ of the cells consistently excluded trypan blue.

\section{Assessment of Granulocyte Apoptosis}

Morphology-Cells were cyto-centrifuged, fixed in methanol, stained with Diff-Quik ${ }^{\mathrm{TM}}$ Gamidor Ltd. (Abingdon, Oxon, UK), and counted 
FIG. 2. Time course for the effect of $\mathrm{Bt}_{2}$ CAMP (dbcAMP) on caspase-3 expression during human neutrophil apoptosis. A, Western blot of cytoplasmic extracts from neutrophils treated with control buffer or $\mathrm{Bt}_{2} \mathrm{cAMP}(0.2 \mathrm{mM})$ for the time points indicated. Cell lysates were prepared and immunoblotted as described under "Experimental Procedures." Lysates were prepared from equivalent numbers of cells and subjected to SDS-polyacrylamide gel electrophoresis/immunoblot analysis using a rabbit polyclonal antibody specific for caspase- 3 . The caspase- 3 antibody recognizes both the $32-\mathrm{kDa}$ pro-caspase-3 and the $17-\mathrm{kDa}$ subunit of active caspase- 3 . The $17-\mathrm{kDa}$ caspase- 3 cleavage product is faintly visible in control (con) lysates at $8 \mathrm{~h}$ becoming more apparent by $20 \mathrm{~h}$. There appears to be less active caspase- 3 in $\mathrm{Bt}_{2} \mathrm{cAMP}$ $(d b c A M P)$-treated cell lysates compared with control cell lysates. The gel is representative of three experiments. $B$, human neutrophils were treated with or without $\mathrm{Bt}_{2}$ cAMP $(0.2 \mathrm{mM})$ for the time points indicated under equivalent culture conditions as the cells used for caspase- 3 expression assessment above. Cells were assessed for apoptosis by measurement of phosphatidylserine expression using annexin V FITC. Data from a minimum of 5000 cels were analyzed for each condition. All values represent mean \pm S.E. of $n=3$ experiments, each performed in duplicate.

using oil immersion microscopy to determine the proportion of cells with distinctive apoptotic morphology $(3,26)$. At least 500 cells were counted per slide with the observer blinded to the experimental conditions. The results were expressed as the mean percent apoptosis \pm S.E

Annexin V Binding-A separate and independent assessment of apoptosis was performed by flow cytometry using annexin $\mathrm{V}$ binding (annexin V-FLUOS, Roche Molecular Biochemicals) to measure phosphatidylserine exposure on the surface of apoptotic cells. A working solution of annexin V-FLUOS was made from stock annexin V-FLUOS $(0.1$ $\mu \mathrm{g} / \mu \mathrm{l}$ ) diluted 1:3000 in Hanks' balanced salt solution (Sigma) supplemented with $2.5 \mathrm{mM} \mathrm{CaCl}$. Neutrophils $\left(20 \mu \mathrm{l}\right.$ of $\left.5 \times 10^{6} / \mathrm{ml}\right)$ were added to $200 \mu \mathrm{l}$ of the working solution of annexin V-FLUOS before being assessed by flow cytometry on a FACSCalibur (Becton Dickinson, Oxford, UK) and analyzed on associated CellQuest (Becton Dickinson) software. All experiments were performed at least three times unless otherwise indicated.

\section{Measurement of PKA Activity}

PKA activity was measured using Promega's SignaTECT TM ${ }^{\text {TMMP- }}$ dependent Protein Kinase (PKA) Assay System, which utilizes biotinylated Kemptide (LRRASLG), a peptide substrate derived from the in vivo substrate pyruvate kinase. Neutrophils $\left(5 \times 10^{6}\right.$ cells $)$ were preincubated with control buffer or $10 \mu \mathrm{M} \mathrm{H} 89$ (Calbiochem) for $1 \mathrm{~h}$ in PBS with $\mathrm{Ca}^{2+} / \mathrm{Mg}^{2+}$ (or for $19 \mathrm{~h}$ in DMEM Iscove's with $10 \%$ autologous serum) at $37^{\circ} \mathrm{C}$ before being stimulated with $0.2 \mathrm{mM} \mathrm{Bt}$ cAMP or $1 \mu \mathrm{M}$ $\mathrm{PGE}_{2}$ (both from Sigma) for $30 \mathrm{~min}$ at $37^{\circ} \mathrm{C}$. Following one wash in ice-cold PBS, neutrophils were resuspended in $0.5 \mathrm{ml}$ of cold extraction buffer (25 mM Tris- $\mathrm{HCl}$, pH 7.4, $0.5 \mathrm{~mm}$ EDTA, $0.5 \mathrm{~mm}$ EGTA, $10 \mathrm{~mm}$ $\beta$-mercaptoethanol, $1 \mu \mathrm{g} / \mathrm{ml}$ leupeptin, $1 \mu \mathrm{g} / \mathrm{ml}$ aprotinin, $1 \mathrm{~mm}$ phenylmethylsulfonyl fluoride, and $1 \%$ Triton X-100 (Sigma)). The lysates were centrifuged $\left(5 \mathrm{~min}\right.$ at $\left.4^{\circ} \mathrm{C} ; 14,000 \times g\right)$ and the supernatants retained. The PKA reaction mixture consisting of $5 \mu \mathrm{l}$ of $5 \times$ PKA Assay Buffer, $5 \mu \mathrm{l}$ of cyclic AMP $(0.025 \mathrm{~mm}), 5 \mu \mathrm{l}$ of PKA-biotinylated peptide substrate $(0.5 \mathrm{mM}), 5 \mu \mathrm{l}$ of $\left[\gamma^{33} \mathrm{P}\right] \mathrm{ATP}$ mixture $(5 \mu \mathrm{l}$ of $0.5 \mathrm{~mm}$ ATP and $0.05 \mu \mathrm{l}$ of $\left.\left[\gamma_{-}{ }^{33} \mathrm{P}\right] \mathrm{ATP}(3,000 \mathrm{Ci} / \mathrm{mmol}) 10 \mu \mathrm{Ci} / \mu \mathrm{l}\right)$ was mixed gently and preincubated at $30^{\circ} \mathrm{C}$ for $5 \mathrm{~min}$ (Promega, Southampton, UK). A control reaction without substrate was performed to determine background counts. The PKA activity reaction was initiated by adding $5 \mu \mathrm{l}$ of the lysates to the reactants and incubated at $30^{\circ} \mathrm{C}$ for $5 \mathrm{~min}$. The reaction was terminated by adding $12.5 \mu \mathrm{l}$ of Termination Buffer to each sample (Promega, Southampton, UK). Aliquots $(10 \mu \mathrm{l})$ from each terminated reaction sample were spotted onto prenumbered $\mathrm{SAM}^{2 \mathrm{TM}}$ membrane

$\begin{array}{ccccccc}\text { on con } & \text { dbeAMP } & \text { con } & \text { dbcaMP } & \text { con dbeAMP } \\ \text { th } & 1 \mathrm{~h} & 1 \mathrm{~h} & 8 \mathrm{~h} & 8 \mathrm{~h} & 20 \mathrm{~h} & 20 \mathrm{~h}\end{array}$

A

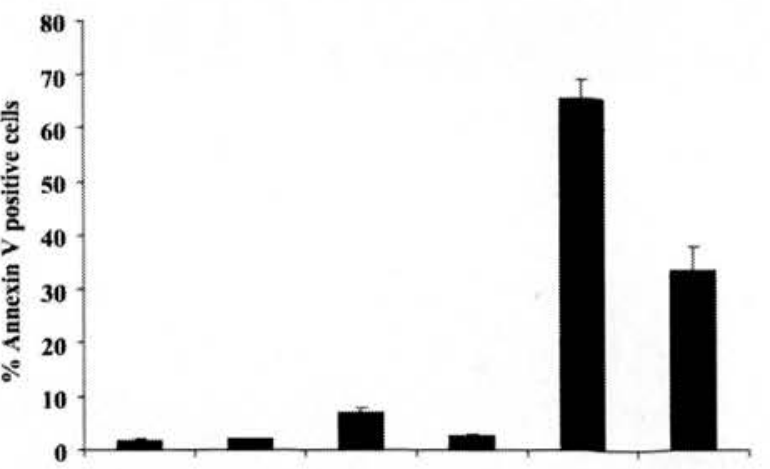

control dbcAMP control dbcAMP control dbcAMP $4 \mathrm{~h} \quad 8 \mathrm{~h} \longrightarrow 20 \mathrm{~h}$

squares (Promega, Southampton, UK). The SAM ${ }^{2 T M}$ membrane square containing the spotted samples were then washed 1 time for $30 \mathrm{~s}$ with $200 \mathrm{ml}$ of $2 \mathrm{M} \mathrm{NaCl}$ (Sigma) followed by 3 washes for 2 min with $200 \mathrm{ml}$ of $2 \mathrm{M} \mathrm{NaCl}$ and then 4 washes for 2 min with $200 \mathrm{ml}$ of $2 \mathrm{M} \mathrm{NaCl}$ in $1 \%$ $\mathrm{H}_{3} \mathrm{PO}_{4}$. Finally the Membrane squares were quickly washed in deionized water before being allowed to dry. PKA activity was measured by scintillation counting.

\section{Measurement of Mitochondrial Dissipation}

Changes in mitochondrial potential were measured in neutrophils following stimulation using JC-1 (5, $5^{\prime}, 6,6^{\prime}$ 'tetrachloro-1,1',3,3'-tetraethylbenzimidazocarbocyaniniodide (Molecular Probes), a cationic dye that exhibits potential dependent accumulation in mitochondria indicated by a fluorescence emission shift from green $(525 \mathrm{~nm})$ to red (590 $\mathrm{nm}$ ) (27). Mitochondrial depolarization is therefore indicated by a decrease in the red/green fluorescence intensity ratio. JC-1 $(10 \mu \mathrm{g} / \mathrm{ml})$ was diluted in PBS from stock $\mathrm{JC1}\left(5 \mathrm{mg} / \mathrm{ml}\right.$ in $\left.\mathrm{Me}_{2} \mathrm{SO}\right)$ and added to neutrophils $\left(1 \times 10^{6} / \mathrm{ml}\right)$ for $10 \mathrm{~min}$ at $37^{\circ} \mathrm{C}$. Neutrophil mitochondria labeled with $\mathrm{JC}-1$ were examined by confocal fluorescent microscopy together with TO-PRO-3 (1 $\mu \mathrm{M})$ (Molecular Probes) (28) to assess neutrophils with necrotic morphology. Alternatively, neutrophils labeled with JC-1 were assessed by flow cytometric analysis using FACSCalibur (Becton Dickinson, Oxford, UK) and analyzed on associated CellQuest (Becton Dickinson) software. Non-apoptotic neutrophils were removed using immunomagnetic separation with sheep anti-mouse IgG-Dynabeads (Dynabeads M-450, Dynal, Mersyside, UK) coated with the murine anti-neutrophil antibody 3G8 (anti-CD16; a gift from Dr. J. Unkeless, Mount Sinai Medical School, New York). Cells were mixed with washed antibody-coated magnetic beads on a rotary mixer at $4{ }^{\circ} \mathrm{C}$ for $20 \mathrm{~min}$, and the beads removed magnetically by two 3-min stationary magnetic contacts (Dynal Magnetic Particle Concentrator, MPC-1) to yield an apoptotic neutrophil preparation. After purification, the apoptotic neutrophils were labeled with JC-1 as described previously.

\section{Western Blotting}

Human neutrophils $\left(5 \times 10^{6} / \mathrm{ml}\right)$ were cultured with or without Bt ${ }_{2}$ AMP $(0.2 \mathrm{~mm})$ at $37^{\circ} \mathrm{C}$ for various time points as detailed under "Results." Cytoplasmic extracts were then prepared from equivalent numbers of cells $\left(10 \times 10^{6}\right.$ cells). To minimize problems with proteolysis, lysates were prepared using methods normally used for electrophoretic mobility shift assay preparations $(26,29)$ with the addition of $1 \mathrm{~mm}$ phenylmethylsulfonyl fluoride. Samples were loaded onto a $12.5 \%$ 

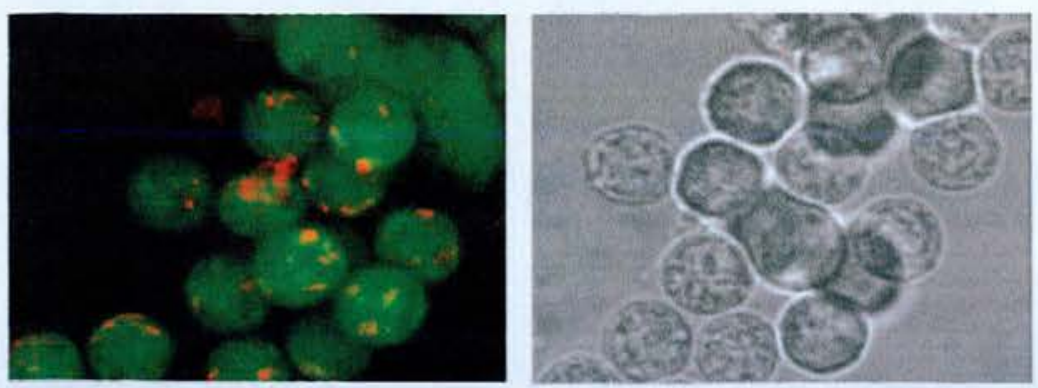

A
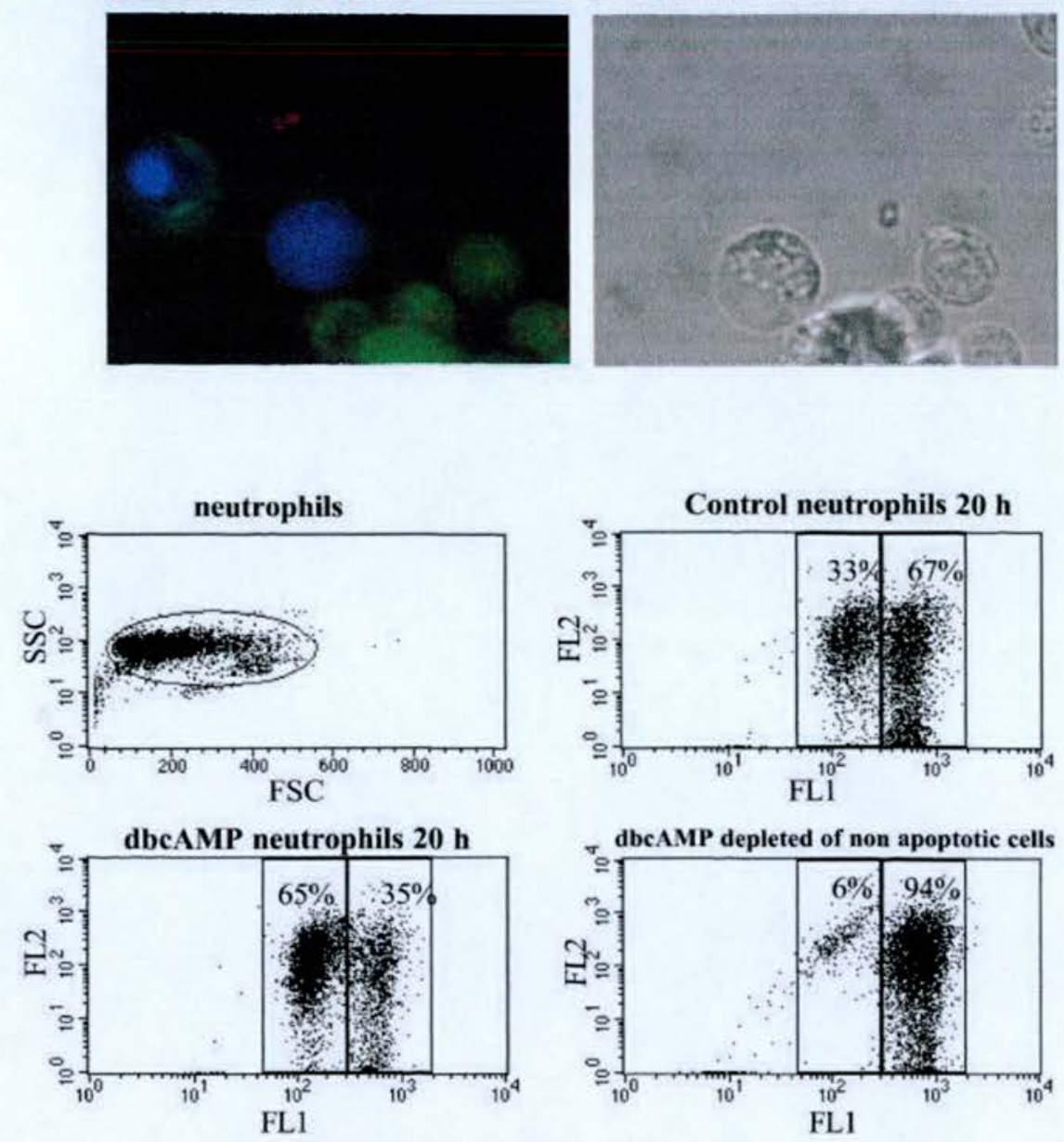

Fig. 3. A, determination if neutrophils contain mitochondria. Human neutrophils $\left(1 \times 10^{6} / \mathrm{ml}\right)$ were cultured at $37^{\circ} \mathrm{C}$ in Iscove's DMEM containing $10 \%$ autologous serum. Neutrophils were labeled with JC-1, a mitochondrial specific dye, and examined by confocal fluorescent microscopy as described under "Experimental Procedures." Bottom panels show TO-PRO-3 staining for neutrophils with necrotic morphology (blue). $B$, effect of $\mathrm{Bt}_{2} \mathrm{AMMP}$ (dbcAMP) on dissipation of mitochondrial transmembrane potential during human neutrophil apoptosis. Human neutrophils $\left(5 \times 10^{6} / \mathrm{ml}\right)$ were cultured for $20 \mathrm{~h}$ at $37^{\circ} \mathrm{C}$ in Iscove's DMEM containing $10 \%$ autologous serum with or without Bt $\mathrm{c}_{2} \mathrm{AMP}$ (dbcAMP) $(0.2 \mathrm{~mm})$. Cells were then labeled with the mitochondrial specific dye JC-1 as described under "Experimental Procedures" before flow cytometric analysis of mitochondrial membrane potential using a FACSCalibur and associated CellQuest software. Non-apoptotic neutrophils (bottom right panel) were removed by anti-CD16 immunodepletion before the remaining cells were labeled with JC-1. Shown is one representative experiment.

Tris- $\mathrm{HCl}$ polyacrylamide mini-gel under reducing conditions and transferred to nitrocellulose membrane (Amersham Pharmacia Biotech) at $60 \mathrm{~V}$ for $1 \mathrm{~h}$ before overnight incubation at $4{ }^{\circ} \mathrm{C}$ with an antibody specific to caspase-3 (catalog number 65906E, PharMingen). After washing, blots were incubated with donkey anti-rabbit horseradish peroxidase conjugate (Amersham Pharmacia Biotech) diluted 1:2000 and developed using a commercial chemiluminescence detection system (ECL, Amersham Pharmacia Biotech).

\section{Further Materials}

Further specific materials were obtained as follows: $\left(R_{\mathrm{p}}\right)-8-\mathrm{Br}$ cAMPS, PD98059, SB203580, and cycloheximide (Calbiochem); lactacystin and epoxomicin (Affiniti, Mamhead, UK); and LY294002 (New England Biolabs, Hertfordshire, UK).

\section{Statistical Analysis}

Statistical analysis was performed using the Student's $t$ test or by analysis of variance with comparisons between groups made using the Newman-Keuls procedure. Differences were considered significant when $p<0.05$.

\section{RESULTS}

Elevation of Cyclic AMP Delays Neutrophil Apoptosis-To examine the effects of cyclic AMP on apoptosis induction, neutrophils were exposed to $\mathrm{Bt}_{2} \mathrm{cAMP}$, a membrane-permeant cyclic $\mathrm{AMP}$ analog and the receptor-directed stimulus $\mathrm{PGE}_{2}$, for $20 \mathrm{~h}$. Both $\mathrm{Bt}_{2} \mathrm{cAMP}$ and $\mathrm{PGE}_{2}$ delay neutrophil apoptosis, determined by standard morphological criteria (data not 


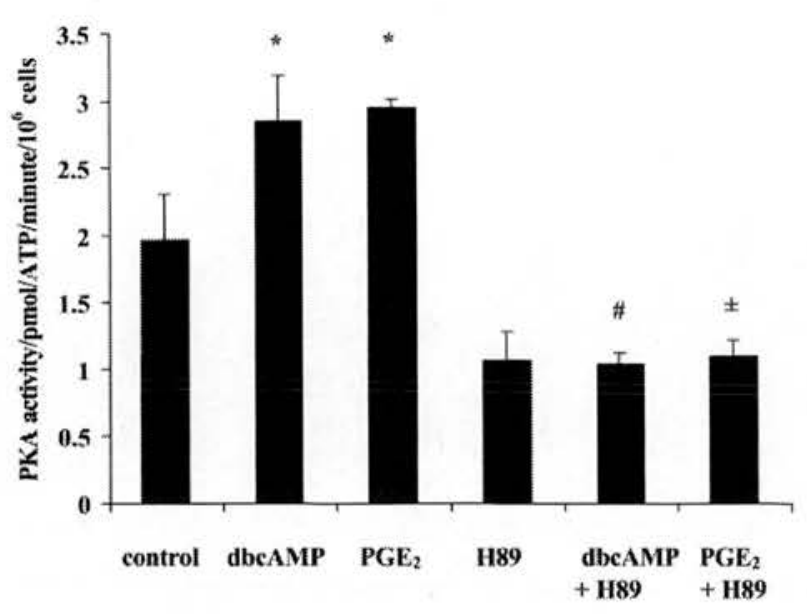

FIG. 4. Measurement of PKA activation by elevators of cyclic AMP in human neutrophils. Human neutrophils $\left(5 \times 10^{6} / \mathrm{ml}\right)$ were preincubated with $10 \mu \mathrm{M}$ H89 for $1 \mathrm{~h}$ before being stimulated with $\mathrm{Bt}, \mathrm{cAMP}(d b c A M P)(0.2 \mathrm{mM})$ or $\mathrm{PGE}_{2}(1 \mu \mathrm{M})$ for $30 \mathrm{~min}$ at $37^{\circ} \mathrm{C}$. PKA activity was measured as described under "Experimental Procedures." All values represent mean $\pm \mathrm{S}$.E. of $n=3$ experiments where significant difference from control values is represented by *, $p<0.05$. Significant difference from $\mathrm{Bt}_{2} \mathrm{cAMP}$ alone is represented by $\#, p<$ 0.001 , and significant difference from $\mathrm{PGE}_{2}$ alone is represented by \pm , $p<0.001$.

shown) and annexin $\mathrm{V}$ binding (Fig. 1). It is interesting to note that maximal concentrations of $\mathrm{Bt}_{2} \mathrm{cAMP}(0.2 \mathrm{~mm})$ were more effective at delaying neutrophil apoptosis compared with maximal concentrations of $\mathrm{PGE}_{2}(10 \mu \mathrm{M})$ (Fig. 1).

Bt ${ }_{2}$ cAMP Inhibits Caspase Activation but Is Unlikely to Act Directly as an Inhibitor of Caspases - It is widely believed that caspases act as the main executioners of apoptosis, with their activation resulting in chromatin condensation and DNA fragmentation. Whether cyclic AMP delays constitutive neutrophil apoptosis by directly suppressing caspase activation in neutrophils has not been examined. Therefore, we investigated the effect of cyclic AMP on activation of caspase-3 during constitutive apoptosis by immunoblotting. Neutrophils were found to express active caspase- $3(17 \mathrm{kDa})$ which closely correlates with the exposure of phosphatidylserine during constitutive neutrophil apoptosis (Fig. 2, $A$ and $B$ ). By $20 \mathrm{~h}$ there is significant caspase- 3 activity $(17 \mathrm{kDa})$ which can be inhibited by $\mathrm{Bt}_{2} \mathrm{cAMP}$. The appearance of some caspase- 3 activity in the presence of $\mathrm{Bt}_{2} \mathrm{cAMP}$ at $20 \mathrm{~h}$ probably reflects the presence of some apoptotic cells in the population (Fig. $2 B$ ).

Apoptosis in Neutrophils Is Accompanied by Dissipation of Mitochondrial Transmembrane Potential That Can Be Inhibited by $B t_{2} c A M P$ - In many cell models, apoptosis is accompanied by an early dissipation of the mitochondrial transmembrane potential $(\Delta \Psi m)$. Previous data (16) have indicated that neutrophils do not respire, and it was thought unlikely that they contained mitochondria. By using confocal microscopy and flow cytometry, we have been able to demonstrate that neutrophils do contain mitochondria (orange), which during overnight culture exhibit loss of mitochondrial potential as indicated by an increase in green fluorescence (Fig. $3 A$ ). Our studies also reveal that $\mathrm{Bt}_{2} \mathrm{cAMP}$ inhibits changes in mitochondrial potential occurring during constitutive neutrophil apoptosis (Fig. $3 B$ ). As the number of cells showing loss of mitochondrial potential appeared to correlate with the number of apoptotic cells measured by annexin $\mathrm{V}$ positivity in previous experiments (Fig. 1), we examined directly if loss in mitochondrial potential occurred in those neutrophils undergoing apoptosis. It is well established that neutrophils lose cell surface expression of CD16 during the process of apoptosis (30). Immunodepletion of
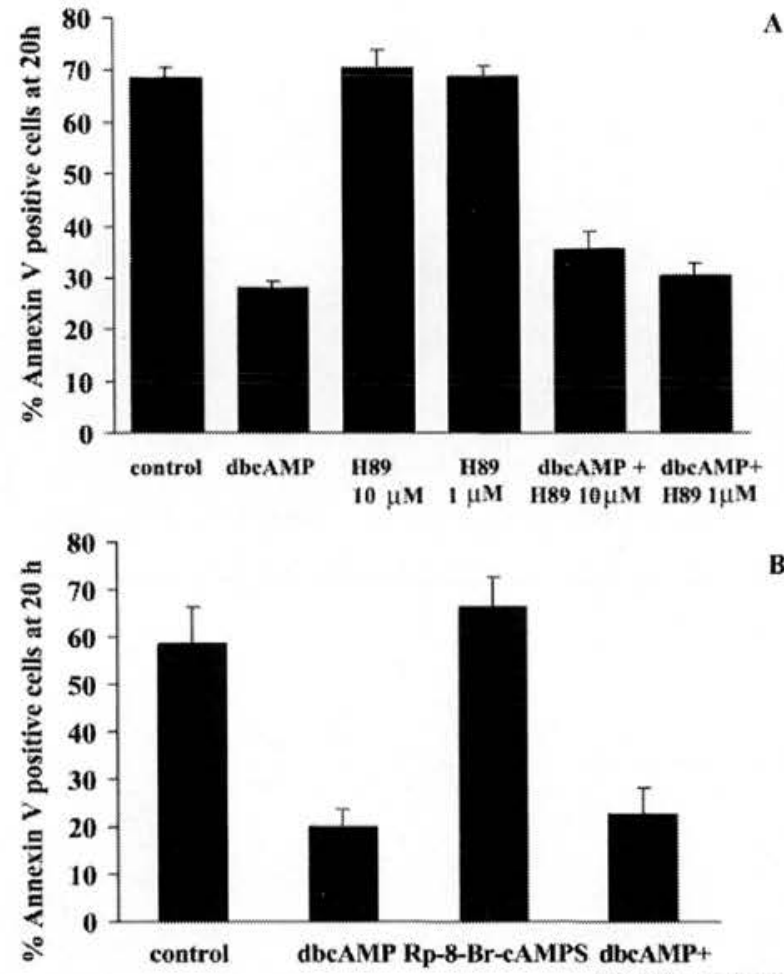

B

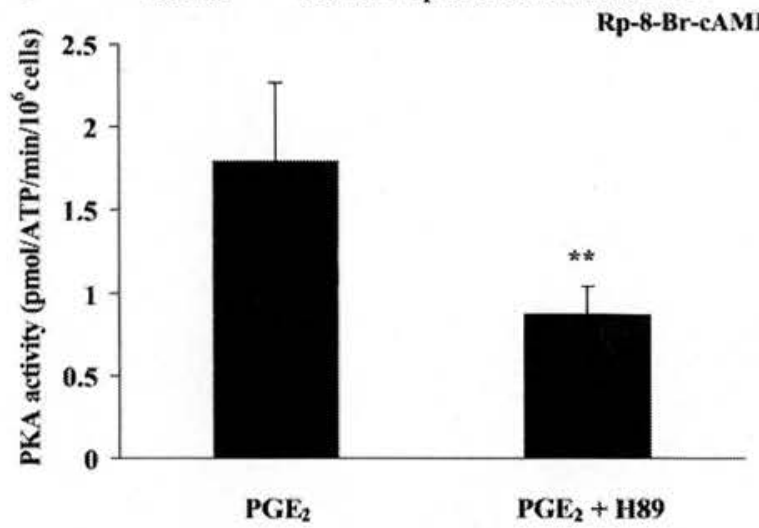

FIG. 5. The effect of pharmacological blockade of PKA activity on $\mathrm{Bt}_{2} \mathrm{cAMP}$-mediated delay of neutrophil apoptosis. $A$ and $B$, human neutrophils $\left(5 \times 10^{6} / \mathrm{ml}\right)$ cultured in Iscove's DMEM containing $10 \%$ autologous serum at $37^{\circ} \mathrm{C}$ were preincubated for 30 min with H89 $(10 \mu \mathrm{M})(A)$ or $\left(R_{\mathrm{p}}\right)-8$-Br-cAMPS $(100 \mu \mathrm{M})(B)$ before stimulation with $\mathrm{Bt}_{2} \mathrm{cAMP}(d b c A M P)(0.2 \mathrm{mM})$. After a further $20 \mathrm{~h}$ in culture, the cells were incubated with FITC-labeled recombinant human annexin V to determine phosphatidylserine expression. The cells were then analyzed by flow cytometry using a FACSCalibur and associated CellQuest software. Data from a minimum of 5000 cells were analyzed for each condition. All values represent mean \pm S.E. of $n=3$ experiments, each performed in duplicate. Similar results were found when cells were assessed for apoptosis by morphological examination (data not shown). $C$, human neutrophils $\left(5 \times 10^{6} / \mathrm{ml}\right)$ cultured in Iscove's DMEM containing $10 \%$ autologous serum at $37^{\circ} \mathrm{C}$ were preincubated with $10 \mu \mathrm{M} H 89$ for $19 \mathrm{~h}$ before being stimulated with $\mathrm{PGE}_{2}(1 \mu \mathrm{M})$ for $1 \mathrm{~h}$ at $37^{\circ} \mathrm{C}$. PKA activity was measured as described under "Experimental Procedures." All values represent mean \pm S.E. of $n=5$ experiments where significant difference from $\mathrm{PGE}_{2}$ alone is represented by ${ }^{* *}, p<0.01$.

non-apoptotic neutrophils using anti-CD16 magnetic beads demonstrated apoptotic neutrophils were indeed positive for loss of mitochondrial potential (Fig. $3 B$ ) indicating that dissipation of mitochondrial membrane potential occurs in neutrophils undergoing programmed cell death.

It has been shown previously (31) that inhibitors of the 
FIG. 6. Effect of PI 3-kinase and MAPK inhibition on $\mathrm{Bt}_{2} \mathrm{cAMP}$ (dbcAMP)-mediated delay of neutrophil apoptosis. Human neutrophils $\left(5 \times 10^{6} \%\right.$ ml) cultured in Iscove's DMEM containing $10 \%$ autologous serum at $37^{\circ} \mathrm{C}$ were treated with LY294002 $(L Y, 10 \mu \mathrm{M})$ or PD98059 (PD, $10 \mu \mathrm{M})$ for 30 min prior to stimulation by $\mathrm{Bt}_{2} \mathrm{cAMP}(0.2 \mathrm{mM})$. After a further $20 \mathrm{~h}$ in culture, the cells were incubated with FITC-labeled recombinant human annexin $\mathrm{V}$ to determine phosphatidylserine expression. The cells were then analyzed by flow cytometry using a FACSCalibur and associated CellQuest software. Data from a minimum of 5000 cells were analyzed for each condition. All values represent mean \pm S.E. of $n=3$ experiments, each performed in duplicate where significant difference from GM-CSF alone is represented by *, $p<0.01$. con, control.

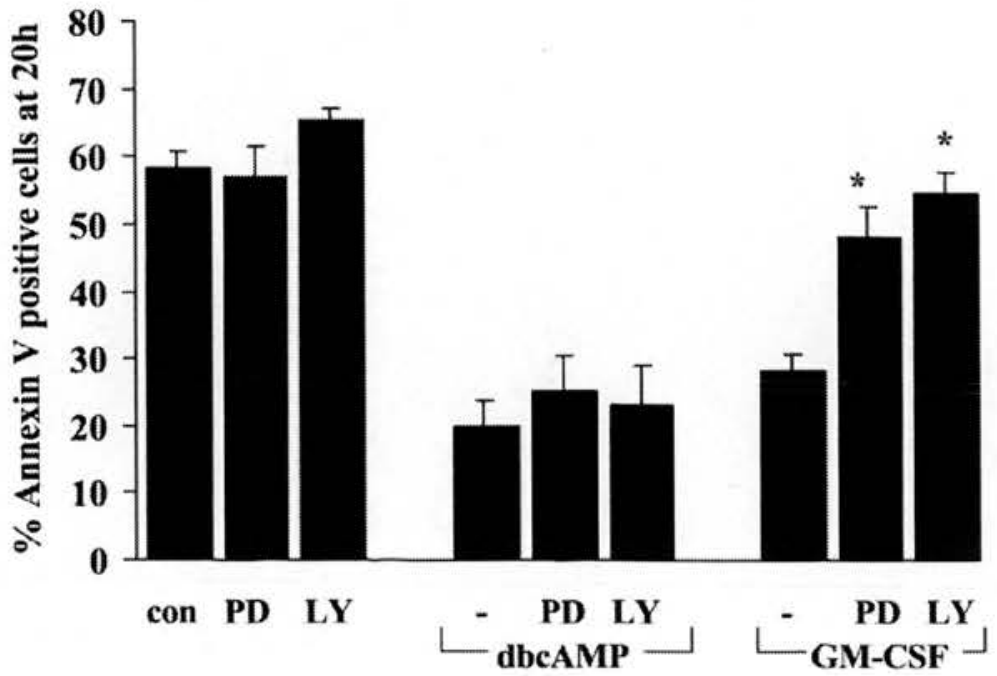

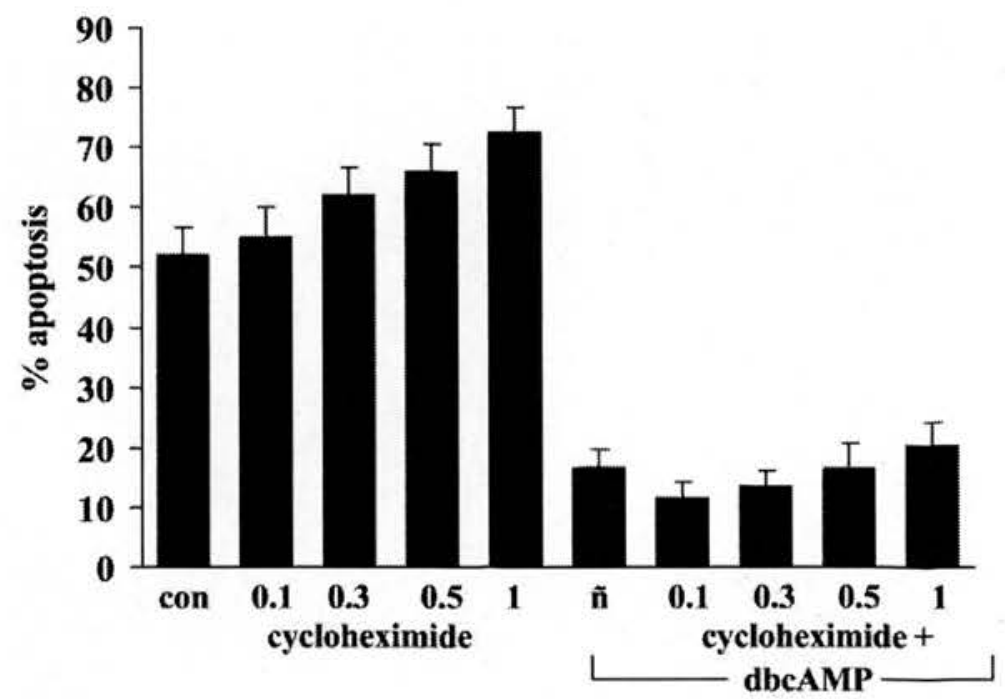

Fig. 7. Effect of protein synthesis inhibition by cycloheximide on $\mathrm{Bt}_{2} \mathrm{cAMP}$ (dbcAMP)-mediated delay of neutrophil apoptosis. Human neutrophils $\left(5 \times 10^{6} / \mathrm{ml}\right)$ were cultured in Iscove's DMEM containing $10 \%$ autologous serum at $37^{\circ} \mathrm{C}$ and treated with the indicated concentrations of cycloheximide $(\mu \mathrm{g} / \mathrm{ml})$ with or without $\mathrm{Bt}_{2} \mathrm{cAMP}(0.2$ $\mathrm{mM})$. After $20 \mathrm{~h}$, cells were harvested and assessed morphologically for apoptosis. All values represent mean \pm S.E. of $n=$ 10 experiments, each performed in triplicate. Similar results were found when cells were assessed for apoptosis by annexin $\mathrm{V}$ binding (data not shown). con, control.

mitochondrial respiratory chain do not affect constitutive neutrophil apoptosis, raising the question of the source of their $\Delta \Psi m$. It may be the case that the neutrophil maintains a transmembrane gradient by a functional $\mathrm{F}_{1}, \mathrm{~F}_{0}$-ATPase; however, this needs to be investigated in more detail.

Cyclic AMP Elevation Stimulates PKA Activation in Neutrophils, an Effect That Is Blocked by Pharmacological Inhibitors-To elucidate further the mechanism by which cyclic AMP regulates neutrophil apoptosis, we examined its downstream signaling pathway. The effects of cyclic AMP are thought to be mediated through binding of cyclic AMP to the intracellular kinase, PKA. This leads to the dissociation of PKA into regulatory and catalytic subunits, which can consequently lead to phosphorylation events of proteins such as the cyclic AMPresponse element-binding protein (21). To explore whether cyclic AMP suppresses apoptosis and apoptotic signaling via activating the PKA pathway in neutrophils, we examined the effects of $\mathrm{Bt}_{2}$ cAMP on endogenous PKA activation (Fig. 4). We found rapid activation of PKA when cells were treated with both $\mathrm{Bt}_{2}$ cAMP and $\mathrm{PGE}_{2}$ Furthermore, the activation of PKA upon stimulation of neutrophils with cyclic AMP elevators could be blocked by the pharmacological PKA inhibitor H89 (32) (Fig. 4).

Activation of the PKA Pathway Does Not Account for Cyclic
AMP-mediated Retardation of Apoptosis-To investigate whether PKA activation by cyclic AMP was necessary for cyclic AMP-mediated delay of apoptosis, neutrophils were incubated with the PKA inhibitor H89 before being stimulated with $\mathrm{Bt}_{2} \mathrm{cAMP}$ and assessed for apoptosis. Surprisingly, whereas pre-treatment with $\mathrm{H} 89$ prevented activation of PKA, it did not prevent the inhibition of apoptosis by $\mathrm{Bt}_{2} \mathrm{CAMP}$ (Fig. $5 \mathrm{~A}$ ). Additionally, the highly specific inhibitor of PKA, $\left(R_{\mathrm{p}}\right)-8-\mathrm{Br}$ cAMPS $(100 \mu \mathrm{M})$, also failed to block $\mathrm{Bt}_{2}$ cAMP inhibition of neutrophil apoptosis (Fig. $5 B$ ). This suggests that cyclic AMP elevation stimulates PKA activity but PKA does not play a major role in the anti-apoptotic effect of cyclic AMP elevation in neutrophils.

The ability of H89 to block PKA activity, as shown by direct measurement of kinase activity, suggests a lack of involvement of PKA in the anti-apoptotic effect of cyclic AMP in neutrophils. Most importantly, we investigated whether H89 could block PKA activity for the full overnight culture period and under identical culture conditions that we use for our apoptosis assay. Neutrophils were therefore cultured in serum-supplemented Iscove's DMEM for $19 \mathrm{~h}$ in the presence or absence of H89 before stimulation with $\mathrm{PGE}_{2}$ for $1 \mathrm{~h}$. PKA activity was then measured as described under "Experimental Procedures." We found that $\mathrm{H} 89$ could still block $\mathrm{PGE}_{2}$-stimulated PKA activity 


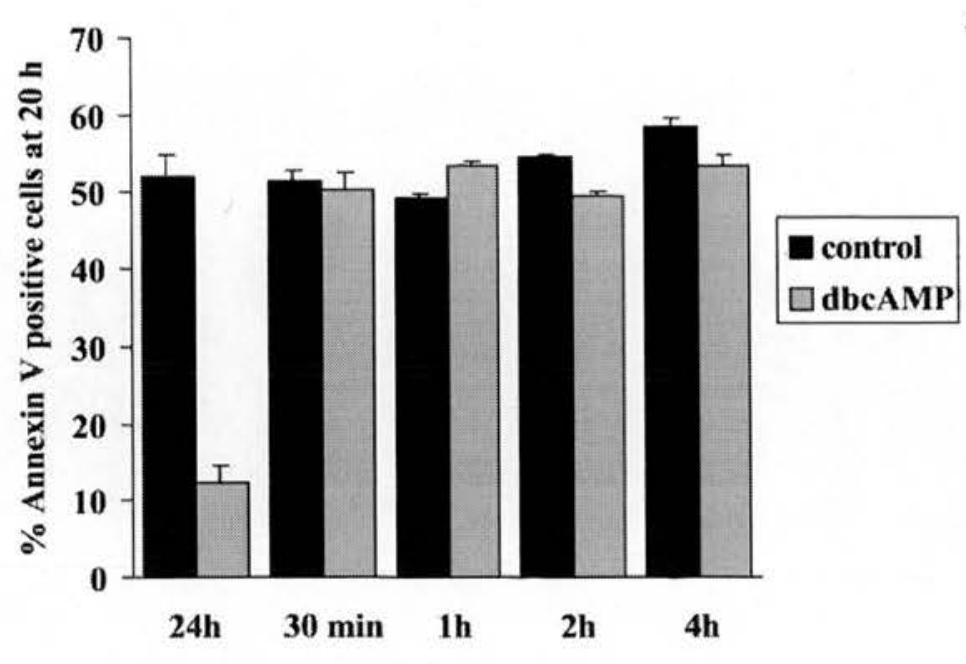

Period of cell stimulation before wash out
Fig. 8. Loss of $\mathrm{Bt}_{2} \mathrm{cAMP}$ (dbcAMP)mediated delay of neutrophil apoptosis by washing and rescue of cultured neutrophils from apoptosis by delayed addition of $\mathbf{B t}_{2} \mathbf{c A M P}$. $A$, human neutrophils $\left(5 \times 10^{6} / \mathrm{ml}\right)$ cultured in Iscove's DMEM containing $10 \%$ autologous serum at $37^{\circ} \mathrm{C}$ were treated with or without $\mathrm{Bt} \mathrm{t}_{2} \mathrm{cAMP}(d b c A M P)(0.2 \mathrm{mM})$ for the time points indicated before the cells were washed 2 times in PBS to remove $\mathrm{Bt}_{2} \mathrm{cAMP}$ and returned to culture. Cells were cultured in Iscove's DMEM containing $10 \%$ autologous serum until $20 \mathrm{~h}$ when the cells were resuspended and incubated with FITC-labeled recombinant human annexin $\mathrm{V}$ to determine phosphatidylserine expression. The cells were then assessed by flow cytometry on a FACSCalibur and analyzed on associated CellQuest software. Data from a minimum of 5000 cells were analyzed for each condition. All values represent mean \pm S.E. of $n=3$ experiments, each performed in duplicate. $B$, human neutrophils $(5 \times$ $10^{6} / \mathrm{ml}$ ) cultured in Iscove's DMEM containing $10 \%$ autologous serum at $37^{\circ} \mathrm{C}$ for the time points indicated before addition of $\mathrm{Bt}_{2}$ cAMP $(0.2 \mathrm{mM})$. At $20 \mathrm{~h}$, cells were resuspended and incubated with FITC-labeled recombinant human annexin $\mathrm{V}$ to determine phosphatidylserine expression. The cells were then assessed by flow cytometry on a FACSCalibur and analyzed on associated CellQuest software. Data from a minimum of 5000 cells were analyzed for each condition. All values represent mean \pm S.E. of $n=3$ ex periments, each performed in duplicate.

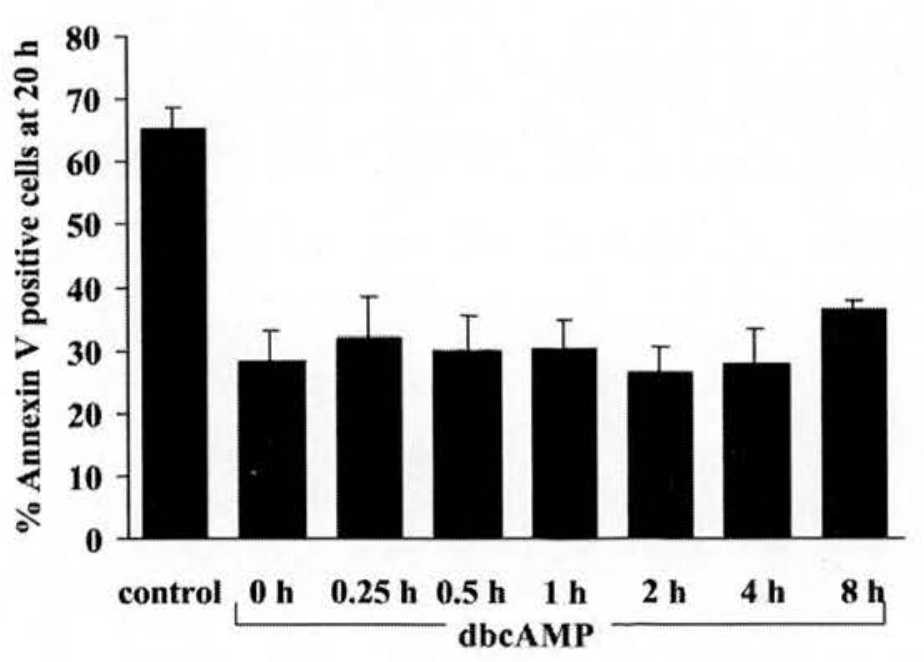

Period of cell culture before addition of dbcAMP

at $20 \mathrm{~h}$ (Fig. $5 \mathrm{C}$ ). This is very important because it demonstrates that the inability of $\mathrm{H} 89$ to reverse cyclic AMP-mediated delay of neutrophil apoptosis is not due to degradation of H89 during the overnight culture period. Furthermore, it also demonstrates that $\mathrm{H} 89$ is not inactivated by autologous serum that is used in our apoptosis assay.

Activation of Akt/PI 3-Kinase or Mitogen-activated Kinase Pathways Does Not Account for the Bt.cAMP-mediated Delay of Neutrophil Apoptosis-The phosphoinositide 3-kinase/Akt pathway plays an essential role in cell survival in various cell types (33) and may be involved in the cyclic AMP-signaling cascade. For example, it has been reported that cyclic AMP requires PI 3-kinase activation for DNA synthesis induced by insulin-like growth factor I in FRTL-5 cells (34) and is involved in the ability of cyclic AMP to attenuate chemoattractantinduced respiratory burst in neutrophils (35). Therefore, we examined whether PI 3-kinase is involved in the signaling pathway mediating the protective effect of cyclic AMP on neutrophil survival. Cells were preincubated with the specific PI 3-kinase inhibitor LY294002 (36) prior to exposing them to $\mathrm{Bt}_{2} \mathrm{cAMP}$ or GM-CSF (Fig. 6). We found that the PI 3-kinase inhibitor suppressed GM-CSF-mediated delay of neutrophil apoptosis, which has been reported previously (37), yet had no effect on suppression of apoptosis by $\mathrm{Bt}_{2} \mathrm{cAMP}$.

We also investigated whether cyclic AMP could be acting through the MAPK signaling pathway to delay neutrophil apoptosis. Activation of extracellular signal-regulated kinase has been implicated in a number of systems to contribute as a negative regulator of apoptosis $(38,39)$. Elevation of cyclic AMP levels is also known to either inhibit or activate MAPK in a cell typeand stimulus-specific manner $(40,41)$. The protective effect of cAMP-elevating agents does not appear to act through the MAPK pathway in our system because the p42/p44 MAPK kinase inhibitor PD98059 had no effect on the anti-apoptotic functions of cyclic AMP in neutrophils, yet reversed the anti-apoptotic functions of GM-CSF treatment in neutrophils (Fig. 6). Similarly the p38 MAPK inhibitor SB203580 (42) did not reverse $\mathrm{Bt}_{2} \mathrm{CAMP}$ mediated delay of neutrophil apoptosis (data not shown). 
Retardation of Neutrophil Apoptosis by Bt $t_{2}$ AMP Does Not Require New Protein Synthesis-Our results suggest $\mathrm{Bt}_{2} \mathrm{cAMP}$ suppresses neutrophil apoptosis via a previously uncharacterized signaling mechanism. We therefore determined whether $\mathrm{Bt}_{2} \mathrm{cAMP}$ stimulated a novel signaling pathway that would require transcriptional activation to suppress neutrophil apoptosis.

To block protein synthesis cycloheximide was titrated to low concentrations to minimize the induction of neutrophil apoptosis that has been reported by this compound (43). Apoptosis was assessed by morphology and annexin $\mathrm{V}$ binding following overnight culture of neutrophils with $\mathrm{Bt}_{2} \mathrm{cAMP}$ and cycloheximide (Fig. 7). Cycloheximide failed to reverse the suppression of apoptosis by cyclic AMP. It did however block glucocorticoidmediated suppression of neutrophil apoptosis at these concentrations (Ref. 44 and data not shown), suggesting that gene transcription is not necessary for the suppression of neutrophil apoptosis by $\mathrm{Bt}_{2}$ cAMP.

We next examined the possibility that $\mathrm{Bt}_{2} \mathrm{cAMP}$ might activate a rapid and reversible signaling pathway rather than stimulate new protein synthesis, which would occur over several hours. Cells were cultured in the presence of $\mathrm{Bt}_{2} \mathrm{cAMP}$ for the time points indicated before $\mathrm{Bt}_{2} \mathrm{CAMP}$ was removed from culture by gently washing in PBS and then returned to normal culture conditions. $\mathrm{Bt}_{2} \mathrm{cAMP}$ was required to be continually present in culture to suppress neutrophil apoptosis (Fig. 8A). However, $\mathrm{Bt}_{2} \mathrm{cAMP}$ rescued neutrophils from apoptosis when added at later time points (Fig. $8 B$ ). We are investigating whether the effects of cyclic AMP occur when the rate of apoptosis is high (between 8 and $20 \mathrm{~h}$ ). However, during constitutive apoptosis, cells at different stages of the apoptotic program are present in the population at any one time point. We are currently investigating whether synchronous apoptosis triggered by Fas ligation, tumor necrosis factor- $\alpha$ (26), or temperature shift (15) can be modulated by cyclic AMP. Taken together, our results suggest that $\mathrm{Bt}_{2} \mathrm{cAMP}$ exerts a powerful direct signaling mechanism, independent of new protein synthesis, to suppress neutrophil apoptosis, and this suppression is rapidly lost when $\mathrm{Bt}_{2} \mathrm{cAMP}$ is removed from culture.

Proteasome Inhibitors Are Able to Reverse Retardation of Neutrophil Apoptosis by $\mathrm{Bt}_{2} \mathrm{cAMP}$ - The ubiquitin/proteasome system plays an important role in the degradation of cellular proteins that regulate various cellular processes, including apoptosis. The observations above reveal $\mathrm{Bt}_{2} \mathrm{cAMP}$ delays neutrophil apoptosis independently of new protein synthesis, suggesting that $\mathrm{Bt}_{2} \mathrm{cAMP}$ is unlikely to stimulate the production of a survival protein. Thus we examined whether alternatively $\mathrm{Bt}_{2} \mathrm{cAMP}$ was accelerating the degradation or modification of pro-death proteins within the neutrophil to increase survival. Neutrophils were co-incubated with $\mathrm{Bt}_{2} \mathrm{CAMP}$ and the irreversible proteasome inhibitors lactacystin $(10 \mu \mathrm{M})(45)$ and epoxomicin $(10 \mu \mathrm{M})(46)$ for $20 \mathrm{~h}$ (Fig. 9, $A$ and $B$ ). Both proteasome inhibitors eliminated the delay of neutrophil apoptosis induced by $\mathrm{Bt}_{2} \mathrm{cAMP}$.

\section{DISCUSSION}

Human neutrophils undergo apoptosis, a process that is centrally important in the resolution of inflammation. It has been shown previously that cyclic AMP is an important regulator of neutrophil apoptosis (18-20), yet little is known of the signaling mechanism by which by cyclic AMP controls neutrophil cell death. The studies herein have established that cyclic AMP acts upstream of caspase-3 activation to inhibit the apoptotic pathway in neutrophils. For the first time, it was also demonstrated that neutrophils contain a small but significant number of mitochondria, which exhibit a loss of membrane potential during constitutive apoptosis, which can be delayed by cyclic
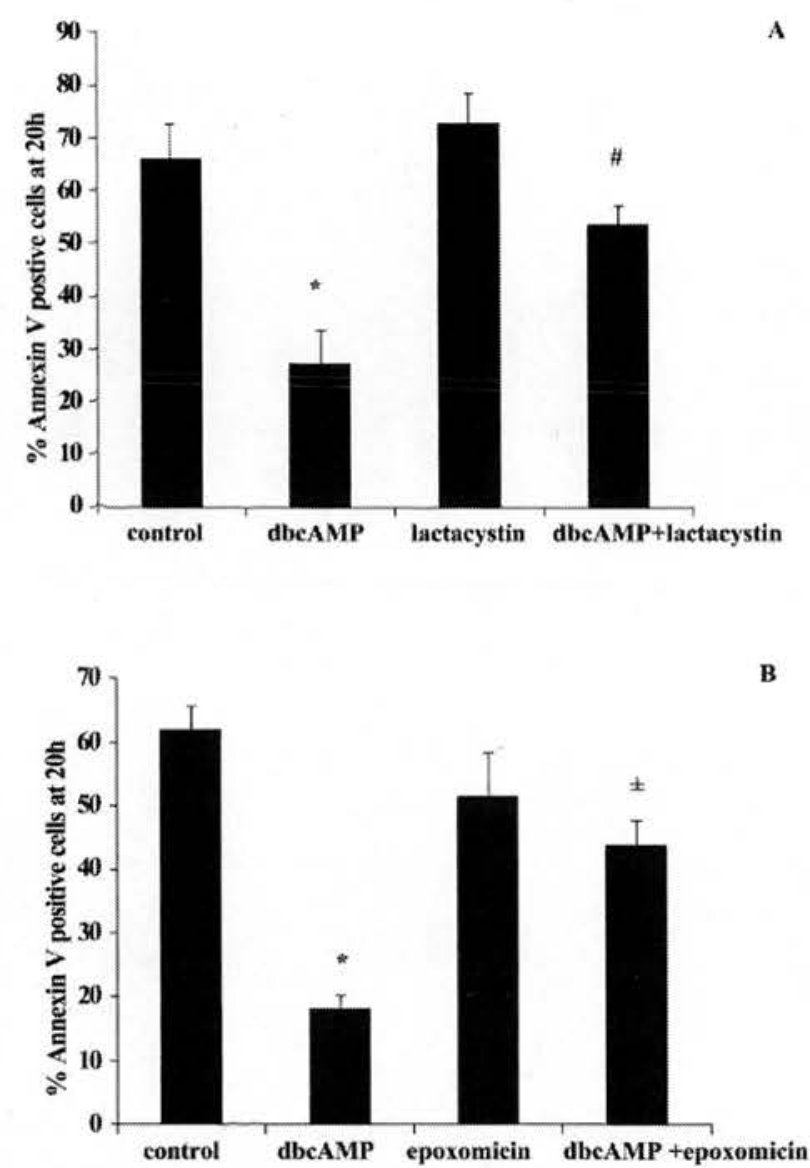

FIG. 9. Effects of proteasome inhibitors on Bt ${ }_{2} \mathbf{c A M P}$ (dbcAMP)-mediated delay of neutrophil apoptosis. Human neutrophils $\left(5 \times 10^{6} / \mathrm{ml}\right)$ were cultured in Iscove's DMEM containing $10 \%$ autologous serum at $37^{\circ} \mathrm{C}$ and treated with lactacystin $(10 \mu \mathrm{M}, A)$ or epoxomicin $(10 \mu \mathrm{M}, B)$ with or without Bt ${ }_{2} \operatorname{cAMP}(0.2 \mathrm{mM})$. After $20 \mathrm{~h}$ in culture, the cells were incubated with FITC-labeled recombinant human annexin $\mathrm{V}$ to determine phosphatidylserine expression. The cells were then analyzed by flow cytometry using a FACSCalibur and associated CellQuest software. Data from a minimum of 5000 cells were analyzed for each condition. All values represent mean \pm S.E. of $n=3$ experiments, each performed in duplicate where significant difference from control is represented by ${ }^{*} p<0.001$. Significant difference from $\mathrm{Bt}_{2} \mathrm{cAMP}$ alone represented by $\# p<0.001$ or $\pm p<0.01$.

AMP elevation. We are currently investigating whether loss of mitochondrial membrane potential occurs before other indices of apoptosis in neutrophils, such as phosphatidylserine exposure and nuclear condensation. This would help ascertain whether loss of mitochondrial potential during neutrophil apoptosis, shown to trigger apoptosis in other cell types, has a similar function in neutrophils and whether $\mathrm{Bt}_{2} \mathrm{cAMP}$ can directly affect loss of mitochondrial potential to delay neutrophil apoptosis.

It has been suggested that PKA plays an important role in cyclic AMP-mediated delay of neutrophil apoptosis (18-20). It is known that cyclic AMP analogs, which selectively activate type I PKA, attenuate neutrophil apoptosis, compared with analogs that preferentially activate type II PKA suggesting that that type I PKA is necessary and sufficient to mediate the cyclic AMP-induced delay in human neutrophil apoptosis (19). We suggest, alternatively, that PKA activation by cyclic AMP is not responsible for the major apoptosis-retarding influences of cyclic AMP in neutrophils. Indeed, we have demonstrated directly that cyclic AMP elevation in neutrophils stimulates an 
increase in PKA activity, which is blocked by pharmacological inhibitors. Importantly, however, blockade of PKA was not sufficient to reverse the anti-apoptotic effect of cyclic AMP, implying that this molecule has little or no role in the cyclic AMP signaling pathway responsible for delay of neutrophil apoptosis.

Previous publications $(18,19)$ have implicated a role for PKA in cyclic AMP regulation of neutrophil apoptosis using concentrations of $\mathrm{H} 89$ greater than $10 \mu \mathrm{M}$. The specificity of $\mathrm{H} 89$ at these concentrations is questionable, and it has been published (47) that H89 may inhibit several other kinases, some with potency similar to or greater than that for PKA. We propose that failure to directly measure PKA activity together with the use of high and possibly nonspecific concentrations of H89 could have led to misinterpretation of previous data. We have demonstrated that $10 \mu \mathrm{M} \mathrm{H} 89$ is sufficient to block PKA activity for extended culture periods and is active in the presence of autologous serum. The failure therefore of both $\mathrm{H} 89$ and $\left(R_{\mathrm{p}}\right)-$ 8-Br-cAMPS, a highly specific inhibitor of PKA, to reverse cyclic AMP-mediated delay of neutrophil apoptosis points to a novel signaling pathway used by cyclic AMP to inhibit neutrophil apoptosis, which is independent of PKA activation.

There have been a few studies reporting PKA-independent effects of cyclic AMP; however, little has been elucidated of the alternative signaling pathways downstream of cyclic AMP. Pharmacological blockade of the MAPK and PI 3-kinase signaling cascades in this study suggest that neither of these signaling pathways are likely to be important in the cyclic AMPmediated delay of neutrophil apoptosis. There has been interest in the discovery that cyclic AMP can bind specifically to and activate small guanine nucleotide exchange factors which, when bound by cyclic AMP, activate the small Ras-like GTPase, Rap1 $(48,49)$. The biological function of Rap1 is still unclear, but it has been proposed that activation of this small GTPase may feed into MAPK signaling pathways (50). As an approach to establishing if Rap1 has a role in cyclic AMPmediated delay of neutrophil apoptosis, we have blocked Rap1 activity using the Clostridium sordellii lethal toxin, which has been reported to inhibit specifically the small GTPases Rap1, Ras, and Rac (51). Furthermore, we have tested GGTI-286, a geranylgeranyltransferase inhibitor, which blocks geranylgeranylation required by Rap1 to achieve its mature, biologically active form (52). Thus our preliminary experiments suggest that Rap1 is not involved in cyclic AMP-mediated delay of neutrophil apoptosis (data not shown): however, this area of research is still under investigation. Our studies are in accord with a very recent publication that demonstrates that cyclic AMP-dependent inhibition of interleukin-5 from human T lymphocytes is not mediated by PKA or by the Rap1 signaling pathway (53).

Regulation of neutrophil apoptosis is thought to depend on the balance between pro-apoptotic and anti-apoptotic death factors expressed in the cell $(17,54)$. Neutrophils contain death regulator proteins, including Bax and Bad, and also express some members of the anti-apoptotic family such as Mcl-1 and Bcl- $x_{\mathrm{L}}$ but not Bcl-2 $(17,54)$. It has been proposed that neutrophil longevity may be prolonged by the synthesis of anti-apoptotic proteins such as Mcl-1 (55). However, it is unlikely that cyclic AMP effects are mediated by such a mechanism in the retardation of neutrophil apoptosis since we have demonstrated that cyclic AMP-mediated delay of neutrophil apoptosis does not require gene transcription. Furthermore, "wash out" experiments have revealed that retardation of neutrophil apoptosis is rapidly lost when $\mathrm{Bt}_{2} \mathrm{cAMP}$ is removed from culture, even after incubation periods that should permit new protein synthesis.
Together, these data suggest a mechanism whereby cyclic AMP does not stimulate production of a survival protein but may alternatively induce post-transitional modifications in the neutrophil to promote survival. One potential mechanism for cyclic AMP-mediated retardation of neutrophil apoptosis may involve cyclic AMP specifically targeting a death protein(s) to the proteasome for degradation. We have demonstrated that blockade of proteasome activity results in a dramatic loss of the pro-survival effect of cyclic AMP. We speculate that cyclic AMP may be involved in the post-translational modification of a death protein, which targets the neutrophil proteasome. If cyclic AMP stimulation is removed or proteasome activity is blocked, then the accumulation of a death protein(s) would be predicted to permit the constitutive death pathway of neutrophils to be reconstituted. Further characterization of proteasome activity in this signaling pathway and possible death protein targets of cyclic AMP are currently under investigation.

In conclusion, cyclic AMP delays neutrophil apoptosis via a novel, reversible, and transcriptionally independent mechanism. Our results contest the dogma that cyclic AMP exerts is physiological functions almost entirely through activation of PKA, and we are currently investigating the involvement of novel downstream signaling pathways in cyclic AMP regulation of neutrophil cell death

Acknowledgment-We thank Dr. M. Popoff for supplying the $C$. sordellii lethal toxin.

\section{REFERENCES}

1. Haslett, C., Savill, J. S., and Meagher, L. (1989) Curr. Opin. Immunol, 2, $10-18$

2. Rossi, A. G., and Hellewell, P. G. (1994) in Immunopharmacology of Neutro phils (Hellewell P. G., and Williams, T. J., eds) pp. 223-243, Academic Press, London

3. Savill, J. S., Wyllie, A. H., Henson, J. E., Walport, M. J., Henson, P. M., and Haslett, C. (1989) J. Clin. Invest. 83, 865-875

4. Rossi, A. G., and Haslett, C. (1998) in Lung Biology in Health and Disease (Lenfant, C., ed) Vol. 112, pp. 9-24, Marcel Dekker, Inc., New York

5. Haslett, C. (1999) Am. J. Respir. Crit. Care Med. 160, (suppl.) 5-11

6. Whyte, M. K. B., Meagher, L. C., MacDermot, J., and Haslett, C. (1993) J. Immunol. 150, 5124-5134

7. Meagher, L. C., Savill, J. S., Baker, A., Fuller, R. W., and Haslett, C. (1992 J. Leukocyte Biol. 52, 269-273

8. Fadok, V. A., Bratton, D. L., Konowal, A., Freed, P. W., Westcott, J. Y., and Henson, P. M. (1998) J. Clin. Invest. 101, 890-898

9. Liu, Y., Cousin, J. M., Hughes, J., Van Damme, J., Seckl, J. R., Haslett, C. Dransfield I., Savill, J., and Rossi, A. G. (1999) J. Immunol. 162, 3639-3646

10. Earnshaw, W. C., Martins, L. M., and Kaufmann, S. H. (1999) Annu. Rev. Biochem. 68, 383-424

11. Hengartner, M. O. (2000) Nature 407, 770-776

12. Green, D. R., and Reed, J. C. (1998) Science 281, 1309-1312

12. Green, D. R., and Reed, J. C. (1998) Science 281,

14. Kluck, R. M., Bossy-Wetzel, E., Green, D. R., and Newmeyer, D. D. (1997) Science 275, 1132-1136

5. Pryde, J. G., Walker, A., Rossi, A. G., Hannah, S., and Haslett, C. (2000) J. Biol. Chem. 275, 33574-33584

16. Simon, H. U. (2001) Immunol, Rev, 179, 156-162

17. Ward, C., Dransfield, I., Chilvers, E. R., Haslett, C., and Rossi, A. G. (1999) Trends Pharmacol. Sci. 20, 503-509

18. Rossi, A. G., Cousin, J. M., Dransfield, I., Lawson, M. F., Chilvers, E. R., and Haslett, C. (1995) Biochem. Biophys. Res. Commun. 217, 892-899

19. Parvathenani, L. K., Buescher, E. S., Chacon-Cruz, E., and Beebe, S. J. (1998), J. Biol. Chem. 273, 6736-6743

20. Ottonello, L., Gonella, R., Dapino, P., Sacchetti, C., and Dallegri, F. (1998) Exp. Hematol. 26, 895-902

21. Daniel, P. B., Walker, W. H., and Habener, J. F. (1998) Annu. Rev. Nutr. 18 353-383

22. McConkey, D. J., Orrenius, S., and Jondal, M. (1990) J. Immunol. 145, $1227-1230$

23. Lanotte, M., Riviere, J. B., Hermouet, S., Houge, G., Vintermyr, O. K., Gjertsen, B. T, and Doskeland, S. O. (1991) Cell. Physiol, 146, 73-80

24. Kiefer, J., Okret, S., Jondal, M., and McConkey, D. J. (1995) J. Immunol. 155 , $4525-4528$

25. Haslett, C., Guthrie, L. A., Kopaniak, M. M., Johnston, R. B., Jr., and Henson, P. M. (1995) Am. J. Pathol. 119, 101-110

26. Ward, C., Chilvers, E. R., Lawson, M. F., Pryde, J. G., Fujihara, S., Farrow, S. N., Haslett, C., and Rossi, A. G. (1999) J. Biol. Chem. 274, 4309-4318

27. Reers, M., Smith, T. W., and Chen, L. B. (1991) Biochemistry 30, 4480-4486 28. Van Hooijdonk, C. A, Glade, C. P., and Van Erp, P. E. (1994) Cytometry 17, 185-189

29. Dignam, J. D., Lebovitz, R. M., and Roeder, R. G. (1983) Nucleic Acids Res. 11 , 1475-1489

30. Dransfield, I., Buckle, A. M., Savill, J. S., McDowall, A., Haslett, C., and Hogg, 
N. (1994) J. Immunol. 153, 1254-1263

31. Hannah, S., Mecklenburgh, K., Rahman, I., Bellingan, G. J., Greening, A., Haslett, C., and Chilvers, E. R. (1995) FEBS Lett. 372, 233-237

32. Chijiwa, T., Mishima, A., Hagiwara, M., Sano, M., Hayashi, K., Inoue, T. Naito, K., Toshioka, T., and Hidaka, H. (1990) J. Biol. Chem. 265, 5267-5272

33. Datta, S. R., Brunet, A., and Greenberg, M. E. (1999) Genes Dev. 13, 2905-2927

34. Nedachi, T., Akahori, M., Ariga, M., Sakamoto, H., Umesaki, K., Hakuno, F., and Takahashi, S. I. (2000) Endocrinology 141, 2429-2438

35. Ahmed, M. U., Hazeki, K., Hazeki, O., Katada T., and Ui, M. (1996) Biochem. J. 318, 965-971

36. Vlahos, C. J., Matter, W. F., Hui, K. Y., and Brown, R. F. (1994) J. Biol. Chem. 269, 5241-5248

37. Klein, J. B., Rane, M. J., Scherzer, J. A., Coxon, P. Y., Kettritz, R., Mathiesen, J. M., Buridi, A., and McLeish, K. R. (2000) J. Immunol, 164, 4286-4291

38. Tran, S. E., Holmstrom, T. H., Ahonen, M., Kahari, V. M., and Eriksson, J. E. (2001) J. Biol. Chem. 276, 16484-16490

39. Xia, Z., Dickens, M., Raingeaud, J., Davis, R. J., and Greenberg, M. E. (1995) Science 270, 1326-133

40. Plevin, R., Malarkey, K., Aidulis, D., McLees, A., and Gould, G. W. (1997) Cell Signal. 9, 323-328

41. Erhardt, P., Troppmair, J., and Rapp, U. R., and Cooper, G. M. (1995) Mol. Cell. Biol. 15, 5524-5530

42. Cuenda, A., Rouse, J., Doza, Y. N., Meier, R., Cohen, P., Gallagher, T. F., Young, P. R., and Lee, J. C. (1995) FEBS Lett. 364, 229-233
43. Whyte, M. K. B., Savill, J., Meagher, L. C., Lee, A., and Haslett, C. (1997) J. Leukocyte Biol. 62, 195-202

44. Cox, G., and Austin, R. C. (1997) J. Leukocyte Biol. 61, 224-230

45. Imajoh-Ohmi, S., Kawaguchi, T., Sugiyama, S., Tanaka, K., Omura, S., and Kikuchi, H. (1995) Biochem. Biophys. Res. Commun. 217, 1070-1077

46. Meng, L., Mohan, R., Kwok, B. H., Elofsson, M., Sin, N., and Crews, C. M. (1999) Proc. Natl. Acad. Sci. U. S. A. 96, 10403-10408

47. Davies, S. P., Reddy, H., Caivano, M., and Cohen, P. (2000) Biochem. J. 351 , 95-105

48. DeRooij, J., Zwartkruis, F. J., Verheijen, M. H., Cool, R. H., Nijman, S. M., Wittinghofer, A., and Bos, J. L. (1998) Nature 396, 474-477

49. Kawasaki, H., Springett, G. M., Mochizuki, N., Toki, S., Nakaya, M., Matsuda, M., Housman, D. E., and Graybiel, A. M. (1998) Science 282, 2275-2279

50. Bos, J. L. (1998) EMBO J. 17, 6776-6782

51. Popoff, M. R., Chaves-Olarte, E., Lemichez, E, von Eichel-Streiber C., Thelestam, M., Chardin, P., Cussac, D., Antonny, B., Chavrier, P., Flatau, G., Giry, M., de Gunzburg, J., and Boquet, P. (1996) J. Biol. Chem. 271, 10217-10224

52. Lerner, E. C., Qian, Y., Hamilton, A. D., and Sebti, S. M. (1995) J. Biol. Chem. 270, 26770-26773

53. Staples, K. J., Bergmann, M., Tomita, K., Houslay, M. D., McPhee, I., Barnes, P. J., Giembycz, M. A., and Newton, R. (2001) J. Immunol. 167, 2074-2080 54. Akgul, C., Moulding, D. A., and Edwards, S. W. (2001) FEBS Lett. 487, $318-322$

55. Moulding, D. A., Quale, J. A., Hart, C. A., and Edwards, S. W. (1998) Blood 92, 2495-2502 


\title{
Dissociation of DNA Fragmentation from Other Hallmarks of Apoptosis in Nitric Oxide-Treated Neutrophils: Differences between Individual Nitric Oxide Donor Drugs
}

\author{
Emma L. Taylor,* Ian L. Megson, † Christopher Haslett, ${ }^{*}$ and Adriano G. Rossi*, \\ * Centre for Inflammation Research and $†$ Centre for Cardiovascular Science, \\ University of Edinburgh, Edinburgh EH8 9AG, United Kingdom
}

The events of apoptotic cell death can be experimentally dissociated from each other in certain cell types. Here we demonstrate the ability of structurally diverse nitric oxide (NO) donating compounds to delay or enhance neutrophil apoptosis and to differentially influence distinct parameters of programmed cell death. We provide evidence that high concentrations of the NO donors GEA 3162, SPER/NO, and DEA/NO induce morphological and biochemical markers of neutrophil apoptosis, but that only DEA/NO causes a concomitant increase in DNA fragmentation as evidenced by nuclear propidium iodide intercalation and the classical laddering pattern of electrophoresed DNA. In contrast, both GEA 3162 and SPER/NO inhibit DNA cleavage in a time- and concentration-dependent manner. We are the first to show that DNA fragmentation can be dissociated from other changes of apoptosis in NO-treated neutrophils and that it may therefore be inappropriate to assess NO-induced apoptosis solely by measuring DNA fragmentation in this cell type. 2001 Elsevier Science

Key Words: neutrophil; apoptosis; nitric oxide; DNA fragmentation.

Internucleosomal DNA fragmentation is a characteristic feature of apoptotic cell death in many cell types, and is frequently used as the sole marker of apoptosis, as assessed by gel electrophoresis yielding character-

Abbreviations used: NO, nitric oxide; GEA 3162, [1,2,3,4,oxatriazolium, 5-amino-3-(3,4-dichlorophenyl)-, chloride]; SPER/NO, spermine diazeniumdiolate (NONOate); DEA/NO, diethylamine diazeniumdiolate (NONOate); IMDM, Iscove's modified Dulbecco's medium; FITC, fluorescein isothiocyanate; PS, phosphatidylserine; HBSS, Hanks' balanced salt solution; mAb, monoclonal antibody; PBS, phosphate-buffered saline; PI, propidium iodide.

'To whom correspondence and reprint requests should be addressed at Centre for Inflammation Research, Rayne Laboratory, Medical School, Teviot Place, Edinburgh EH8 9AG, UK. Fax: +44 131650 4384. E-mail: a.g.rossi@ed.ac.uk. istic DNA ladders, by incorporation of propidium iodide (PI) into DNA of permeabilized cells, or by terminal deoxynucleotidyl transferase (TdT)-mediated d-UTP nick end-labeling (TUNEL) staining. However, it has been established that there is no absolute requirement for DNA degradation in order for this form of cell death to proceed (1). The process of DNA fragmentation may be dissociated from morphological evidence of apoptosis in certain cell types including thymocytes, lymphocytes, hematopoietic cells, cardiac myocytes, neurons, and various cell lines $(1-15)$. Nevertheless, this phenomenon has not previously been reported in neutrophils induced to undergo apoptosis by exogenous nitric oxide (NO).

NO has the ability to modulate the apoptotic process in a number of different cell types. Both inhibition and induction of cell death are seemingly dependent on the concentration and rate of release of NO, the cell type, and the cellular redox environment and defence mechanisms. It has been suggested that low concentrations of NO, such as those that are produced by the constitutive endothelial form of nitric oxide synthase (eNOS) tend to have a protective effect on cells $(16,17)$. In contrast, the high concentrations produced when the inducible form of NOS (iNOS) is activated are more likely to be injurious to cells $(16,17)$.

Granulocyte apoptosis is believed to be a key event for the successful resolution of inflammation as this process renders intact cells unresponsive to external stimuli and easily recognizable by phagocytes using a noninflammatory mechanism (18). Furthermore, isolated neutrophilic granulocytes readily undergo spontaneous apoptosis in culture, with approximately 50 $80 \%$ of cells dying within $20 \mathrm{~h}$ of culture (19). We have previously demonstrated that GEA 3162-derived NO can enhance the rate of apoptosis in this cell type, by a mechanism that is dependent on caspase enzymes, but is independent of the rise in cGMP through which most other effects of NO are mediated (20). A similar induc- 
tion of neutrophil apoptosis has been reported using a number of different NO donors, including sodium nitroprusside, $S$-nitrosoglutathione, GEA 3162 and SIN-1, plus solutions of NO gas $(20-24)$.

In the present study, we have compared the effects of three distinct NO-donor compounds with different mechanisms and rates of NO release; GEA 3162, diethylamine diazeniumdiolate (DEA/NO) and spermine diazeniumdiolate (SPER/NO) (25), on a number of parameters associated with human neutrophil apoptosis. GEA 3162 is a mesoionic 3-aryl substituted oxatriazole-5-imine derivative, while SPER/NO and DEA/NO belong to the diazeniumdiolate (NONOate) group of NO-releasing compounds. DEA/NO has a half-life of just $9 \mathrm{~min}$ at physiological temperature and $\mathrm{pH}$, while SPER/NO releases NO more slowly, having a half-life of $39 \mathrm{~min}(25,26)$. We wanted to test two hypotheses; firstly that different compounds, with varying rates and mechanisms of NO liberation, may have differential effects on apoptotic events, and secondly that any given drug may influence these events to varying degrees.

Our results confirm the hypothesis that individual classes of compounds may have differential effects on neutrophil apoptosis, possibly reflecting differences in the rate and mechanism of NO release. Although we anticipated variations in the extent to which the compounds influenced the different events of apoptosis, we unexpectedly found that certain NO-donors exerted opposing effects on independent markers of apoptosis. GEA 3162 and SPER/NO both inhibited DNA fragmentation (as assessed by DNA ladders and propidium iodide nuclear intercalation), despite a profound increase in morphological (cell shrinkage and the appearance of hyperchromatic pyknotic nuclei) and plasmalemmal changes (annexin V binding and loss of CD16 expression) that occur during neutrophil apoptosis. We therefore conclude that morphological and cell surface changes associated with human neutrophil apoptosis can be dissociated from internucleosomal DNA degradation when these cells are exposed to specific NO donor drugs.

\section{MATERIALS AND METHODS}

\section{Materials}

IMDM cell culture medium was purchased from Life Technologies (Paisley, UK) and cell culture plastic was from Becton Dickinson (Oxford, UK). Dextran T-500 and Percoll for neutrophil isolation were bought from Pharmacia (Milton Keynes, UK), and Diff-Quik for cytocentrifuge slide staining was from Baxter Healthcare (Glasgow, UK). For the DNA ladders, Wizard SV Miniprep DNA extraction kits were bought from Promega (Southampton, UK) and Sea Kem agarose was from Flowgen (Ashby de-la-Zouch, UK). The NO donating agents; GEA 3162, spermine NONOate and diethylamine NONOate were purchased from Alexis Biochemicals (Nottingham, UK). All other reagents were purchased from Sigma Chemical Co (Poole, UK).

\section{Neutrophil Isolation}

Neutrophils were isolated from the blood of healthy volunteers as previously described $(27,28)$. Briefly, whole, citrated blood was centrifuged $(200 \mathrm{~g}, 20 \mathrm{~min})$ and platelet-rich plasma aspirated. Leukocytes were separated from erythrocytes by dextran sedimentation, then further divided into mononuclear cell and granulocyte populations by centrifugation through a discontinuous Percoll gradient $(720 \mathrm{~g}, 20 \mathrm{~min})$. Granulocytes were harvested from the $79: 68 \%$ interface of the gradient, and only neutrophil preparations of $\geq 95 \%$ purity were used.

\section{In Vitro Culture of Neutrophils}

Neutrophils $\left(4.5 \times 10^{6} \mathrm{cells} / \mathrm{ml}\right)$ were suspended in IMDM containing penicillin $(100 \mathrm{U} / \mathrm{ml})$ and streptomycin $(100 \mu \mathrm{g} / \mathrm{ml})$, and supplemented with $10 \%(\mathrm{v} / \mathrm{v})$ autologous serum. They were cultured in flat-bottomed 96 -well polypropylene plates $\left(37^{\circ} \mathrm{C}, 5 \% \mathrm{CO}_{2}\right)$ for $1-20 \mathrm{~h}$ in the presence of either IMDM (controls) or NO donors at concentrations as shown in the figure legends.

\section{Assessment of Apoptosis}

Morphology. Neutrophils ( $100 \mu \mathrm{l}$ of recovered cells) were cytocentrifuged in duplicate, fixed in methanol and stained using Diff-Quik physiological stain, then observed by light microscopy $(\times 100$ objective) to determine the proportion of darkly stained cells with condensed nuclei. At least 500 cells per slide were counted, with the observer blinded to the experimental conditions (28).

Annexin $V$ binding. FITC-Annexin V $(180 \mu \mathrm{l})$ diluted $1: 3000$ in HBSS plus $5 \mathrm{mM} \mathrm{CaCl}$, was added to $20 \mu \mathrm{l}$ of cell samples and incubated $\left(4^{\circ} \mathrm{C}, 10 \mathrm{~min}\right)$ before flow cytometric analysis using an EPICS XL2, to measure phosphatidylserine (PS) exposure on the surface of apoptotic cells (20).

Cell surface CD16 expression. Neutrophils $(60 \mu \mathrm{l}$ of recovered cells) were centrifuged $(200 \mathrm{~g}, 3 \mathrm{~min})$, then FITC-conjugated $\mathrm{mAb}$ against human neutrophil CD16 added. Following incubation $\left(4^{\circ} \mathrm{C}\right.$, $30 \mathrm{~min}$ ), the cells were washed $3 \times$ in PBS without $\mathrm{Ca}^{2+} / \mathrm{Mg}^{2+}$, resuspended and analyzed using an EPICS XL2, with CD16 'low' expressing cells representing the apoptotic population (29).

\section{DNA Fragmentation Assays}

(i) Propidium iodide intercalation. Neutrophils $(100 \mu \mathrm{l}$ of recovered cells) were centrifuged $(200 \mathrm{~g}, 3 \mathrm{~min})$, then fixed and permeabilized in $70 \%$ ethanol $\left(4^{\circ} \mathrm{C}, 10 \mathrm{~min}\right)$. The cells were washed $3 \times$ in PBS without $\mathrm{Ca}^{2+} / \mathrm{Mg}^{2+}$ before addition of $60 \mu \mathrm{l}$ RNase $\mathrm{A}(0.5 \mathrm{mg} / \mathrm{ml})$ and $60 \mu \mathrm{l}$ propidium iodide (PI) $(0.1 \mathrm{mg} / \mathrm{ml})$, then assessed by flow cytometry using an EPICS XL2, to assess DNA fragmentation.

(ii) Gel electrophoresis of isolated DNA. Cells were lysed using 7 $M$ guanidine hydrochloride, then DNA was extracted using a commercially-available miniprep kit according to the manufacturer's instructions, from neutrophils treated with medium alone, gliotoxin $(0.1 \mu \mathrm{g} / \mathrm{ml})$, dibutyryl-cAMP $(0.2 \mathrm{mM})$ or NO donors for 8 or $20 \mathrm{~h}$. DNA was run on a $2 \%$ Sea Kem agarose gel containing ethidium bromide (100 V, $1 \mathrm{~h})$, and fragmentation (laddering) was visualized using a u.v. lamp.

\section{Statistic Analysis}

The results are expressed as the mean \pm SEM for $(n)$ separate experiments. Values were compared using repeated measures analysis of variance (ANOVA) followed by Student-Newman-Keuls analysis. Differences were considered significant when $P<0.05$.

\section{RESULTS AND DISCUSSION}

A number of studies have reported either a natural dissociation of apoptotic cell death and DNA fragmen- 
tation, or the ability to separate the two processes by experimental manipulation, in several cell types (115). It has been suggested that DNA fragmentation should not be used as the only marker of apoptosis in studies of programmed cell death $(9,30)$. It is therefore clear that although internucleosomal DNA cleavage is frequently observed alongside apoptosis, it is not necessarily an integral part of the death process, and as such is not essential for this form of cell death to occur. Such a dissociation has not previously been observed in neutrophils; indeed a number of studies have demonstrated an increase in the endonuclease-mediated cleavage of DNA in neutrophils treated with NO $(22$, 23). Here we report that neutrophil apoptosis may proceed alongside an inhibition of internucleosomal DNA cleavage, and that this phenomenon depends upon the specific characteristics of the NO-donating species used.

GEA 3162 is a mesoionic 3-aryl substituted oxatriazole-5-imine derivative with a structure similar to that of molsidomine, the precursor of the peroxynitritedonating compound SIN-1 (25). There is some controversy as to whether this compound is a pure NO donor, as reported by some groups $(31,32)$, or whether superoxide anion is simultaneously generated during NO release, effectively making GEA 3162 a peroxynitrite donor (33).

The diazeniumdiolate, or 'NONOate' group of NOdonating agents have an NO dimer linked to a nucleophilic molecule via a nitrogen atom. They predictably and spontaneously release up to two molecules of NO per molecule of compound in aqueous solution, in a reaction that is highly temperature- and $\mathrm{pH}$-dependent $(25,26)$. We used the fast-releasing drug diethylamine NONOate (DEA/NO) which has a half-life of just $9 \mathrm{~min}$ at physiological temperature and $\mathrm{pH}$, and the slower releasing drug spermine NONOate (SPER/NO), the half-life of which is $39 \mathrm{~min}(25,26)$.

Concentration-response curves for the effects of 0.1 $\mu \mathrm{M}$ to $1 \mathrm{mM}$ GEA 3162 , SPER/NO and DEA/NO on neutrophil apoptosis over a 20 -h culture period were constructed (Fig. 1). NO derived from the NONOates exerted a biphasic effect, in that low concentrations delayed, but high concentrations enhanced the rate of constitutive neutrophil apoptosis. In contrast, NO from GEA 3162 showed no reduction in the rate of apoptosis at equivalent concentrations to those at which the NONOates gave an inhibition. However, at higher concentrations $(\geq 30 \mu \mathrm{M})$, a concentration-dependent induction of apoptosis was observed. Therefore, there are obvious differences in the response of neutrophils to different types of NO donor. A concentration of NONOates that inhibits $(0.1 \mu \mathrm{M})$ and a concentration that induces $(1 \mathrm{mM})$ neutrophil apoptosis, together with two concentrations of GEA 3162 that induce neutrophil apoptosis (30 and $100 \mu \mathrm{M})$ were then selected for further studies.
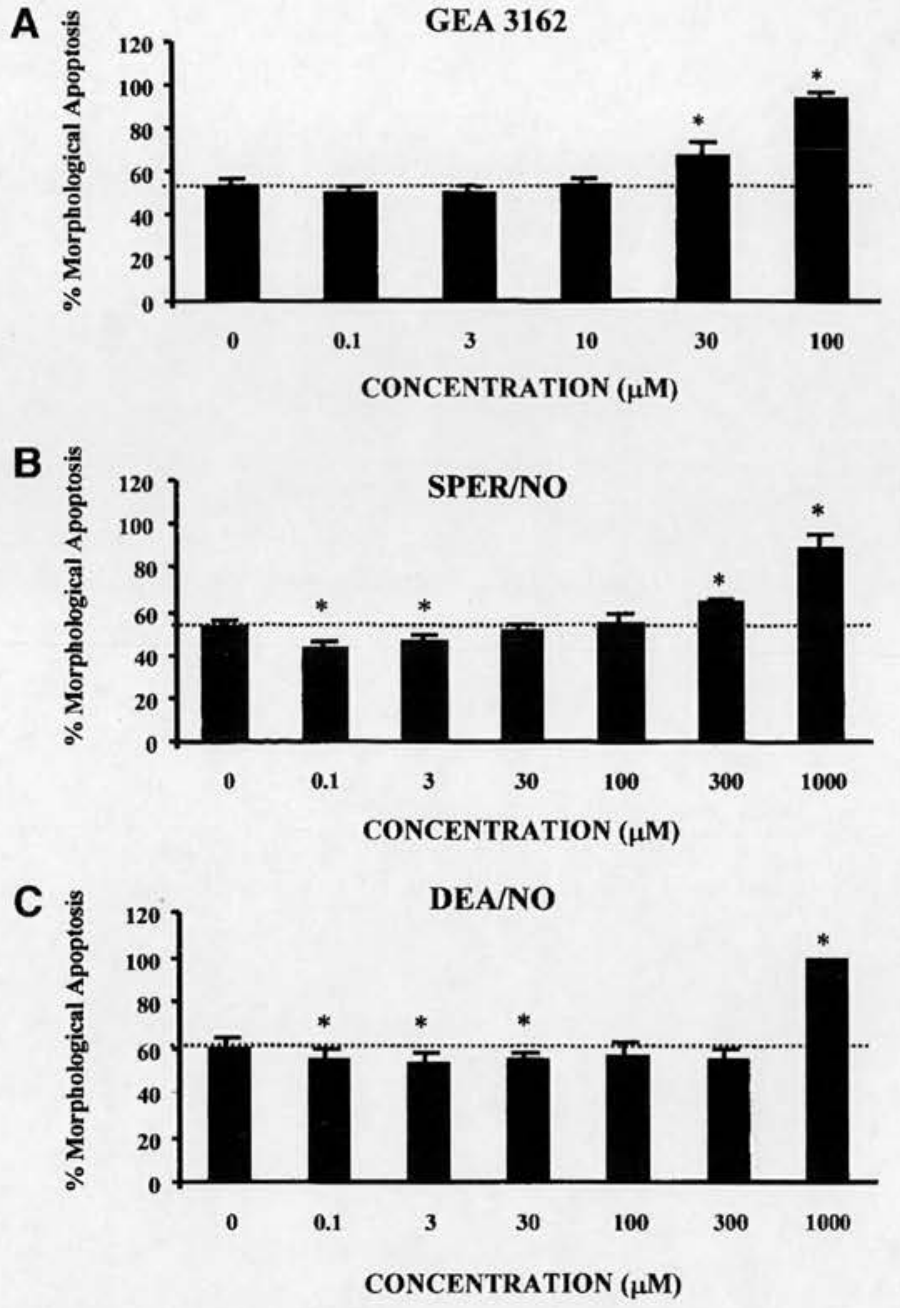

FIG. 1. Concentration-response graphs showing the effects of the NO donor drugs (A) GEA $3162(0.1-100 \mu \mathrm{M})$, (B) SPER/NO $(0.1 \mu \mathrm{M}$ to $1 \mathrm{mM})$ and $(\mathrm{C})$ DEA/NO $(0.1 \mu \mathrm{M}$ to $1 \mathrm{mM})$ on morphological neutrophil apoptosis over a 20-h culture period, as described under Materials and Methods. Values represent means \pm SEM from $n=6$ separate experiments. Dotted lines show the amount of spontaneous neutrophil apoptosis and asterisks denote a significant $(P<0.05)$ difference from control levels of apoptosis.

Figure 2 shows the rate of constitutive neutrophil apoptosis, as assessed by four techniques, namely morphology, annexin V binding, CD16 shedding and PI intercalation as described under Materials and Methods. Over a 20 -h culture period, neutrophils spontaneously underwent approximately $60 \%$ apoptosis as assessed by morphological changes, in agreement with previous observations $(19,28)$. There was good correlation between all assays used in this study, therefore all of these techniques are appropriate indices of constitutive neutrophil apoptosis.

Time course studies to compare the effects of GEA 3162 on the four different markers of apoptosis showed that this compound enhanced the rate of neutrophil apoptosis over the 20 -h time period, as assessed by 


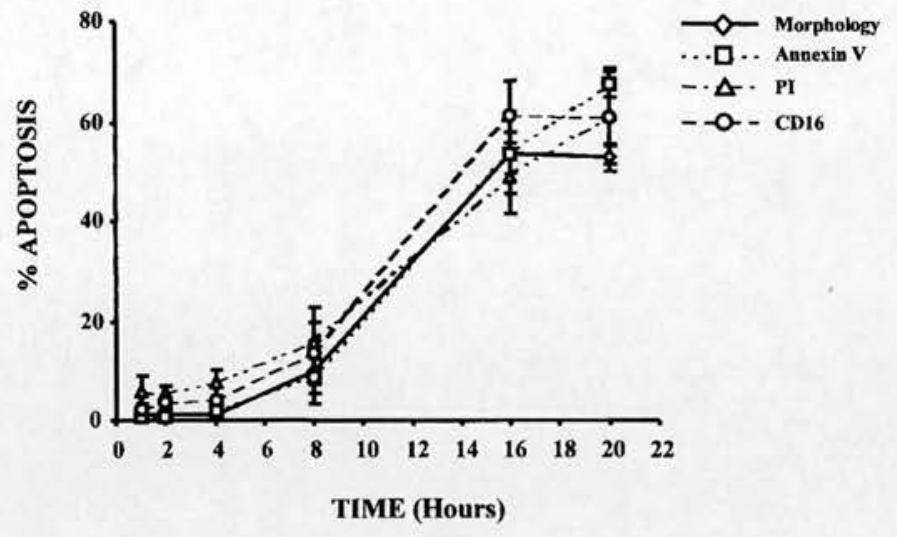

FIG. 2. Constitutive neutrophil apoptosis over a 20-h culture period, as assessed by morphology, annexin V binding, CD16 shedding and propidium iodide intercalation, as described under Materials and Methods. Values represent means \pm SEM from $n=3-6$ separate experiments.

morphology and CD16 shedding, in a concentrationand time-dependent manner (Figs. 3A and 3B). However, measurement of phosphatidylserine (PS) exposure using annexin $\mathrm{V}$ only showed an induction at earlier time points, up to $8 \mathrm{~h}$ for $30 \mu \mathrm{M}$ and $16 \mathrm{~h}$ for 100 $\mu \mathrm{M}$. At later time points, no significant difference from control was observed. As yet we are unable to speculate on the reason for the 'catch-up' of control cells in terms of PS exposure, we can only suggest that the annexin V assay may not be suitable for measuring the effects of certain NO donors at later time points in this cell type.

Paradoxically, assessment of DNA fragmentation by PI intercalation into the DNA of permeabilized cells showed a concentration- and time-dependent decrease in the hypodiploid population of GEA 3162-treated cells compared to controls, rather than the expected increase. Typical flow cytometry histograms are shown in Fig. 3C. This suggests that GEA 3162 is able to induce morphological and biochemical events of apoptosis, whilst exerting a concomitant inhibitory influence on the endonuclease-mediated DNA cleavage pathway.

In order to confirm this inhibition, DNA gels were run, to look for the distinctive laddering pattern derived from internucleosomal DNA degradation. Figures 3D and 3E show increased DNA laddering with the known inducer of neutrophil apoptosis, gliotoxin (lane 2) (28) and decreased laddering with the cell permeable analogue of cyclic-AMP, db-cAMP (lane 3$)(34,35)$, compared to control cells (lane 1) at 8 and $20 \mathrm{~h}$. However, there was complete inhibition of DNA fragmentation in $30 \mu \mathrm{M}$ GEA 3162-treated cells (lane 4) at $8 \mathrm{~h}$ and in $100 \mu \mathrm{M} \mathrm{GEA}$ 3162 -treated cells (lane 5) at 8 and $20 \mathrm{~h}$. Although a small degree of DNA fragmentation could be seen in $30 \mu \mathrm{M}$ GEA 3162-treated neutrophils at $20 \mathrm{~h}$, this was less evident than in control cells. These results offer further evidence that inhibition of DNA fragmentation by GEA 3162 is concentration-dependent.
Similar experiments using SPER/NO demonstrated that $0.1 \mu \mathrm{M}$ of this compound inhibits neutrophil apoptosis as measured by all four apoptotic markers (Fig. 4A). However, induction of neutrophil apoptosis by 1 mM SPER/NO was only detectable by morphology, CD16 shedding and PS exposure-which in this case correlated with morphology to the end of the 20-h culture period (Fig. 4B).

When PI intercalation was assessed, an inhibition of DNA fragmentation was again observed (Fig. 4C), although this was less pronounced than that observed with GEA 3162. Again, these findings were confirmed by the use of DNA gels, which showed a concentrationdependent inhibition of DNA laddering for $0.1 \mu \mathrm{M}$ (lane 4) and $1 \mathrm{mM}$ (lane 5) SPER/NO at 8 and $20 \mathrm{~h}$ (Figs. 4D and 4E).

The only compound for which all four assays gave concurring results was DEA/NO (Figs. 5A and 5B), as delayed neutrophil apoptosis by $0.1 \mu \mathrm{M}$ and the induction of apoptosis by $1 \mathrm{mM}$ DEA/NO over the 20 -h time period were both detected by all four techniques. This shows that NO derived from this compound induces morphological apoptosis with associated DNA fragmentation (Fig. 5C). The DNA ladders for this compound (Figs. 5D and 5E) showed a decrease in laddering in $0.1 \mu \mathrm{M}$-treated cells (lane 4 ), but an increase in $1 \mathrm{mM}$-treated cells (lane 5) at 8 and $20 \mathrm{~h}$, thus confirming the correlation between morphological apoptosis and DNA fragmentation. Thus, NO from some donors enhances neutrophil apoptosis independently of DNA fragmentation, whilst with others, the two processes occur simultaneously.

Overall, these studies demonstrate that different groups of NO donors have distinct effects on neutrophil apoptosis, which may potentially be exploited to determine the mechanism(s) through which such effects are mediated. Similar differential mechanistic effects of different NO donors have previously been reported for platelet aggregation, and are believed to depend partly on the site of NO release (36). A small but statistically significant inhibition of neutrophil apoptosis occurs to a similar degree and over a similar time course in cells treated with $0.1 \mu \mathrm{M}$ SPER/NO and DEA/NO, therefore this effect is unlikely to be governed by the rate of release of NO from NO donors. No inhibitory effect was observed with GEA 3162 suggesting that the mechanism or site of NO release, or even the amount of peroxynitrite generated, is crucial in determining whether or not a particular compound exerts an inhibitory effect.

The induction of neutrophil apoptosis by higher concentrations of NO donors, on the other hand, may be influenced by the rate of $\mathrm{NO}$ release. $1 \mathrm{mM}$ DEA/NO, which releases $\mathrm{NO}$ very quickly and therefore provides a rapid 'spike' of NO, caused increased morphological apoptosis which was evident by $2 \mathrm{~h}$, whereas with the slower-releasing drug SPER/NO, such an induction did 
A

30 $\mu$ M GEA 3162
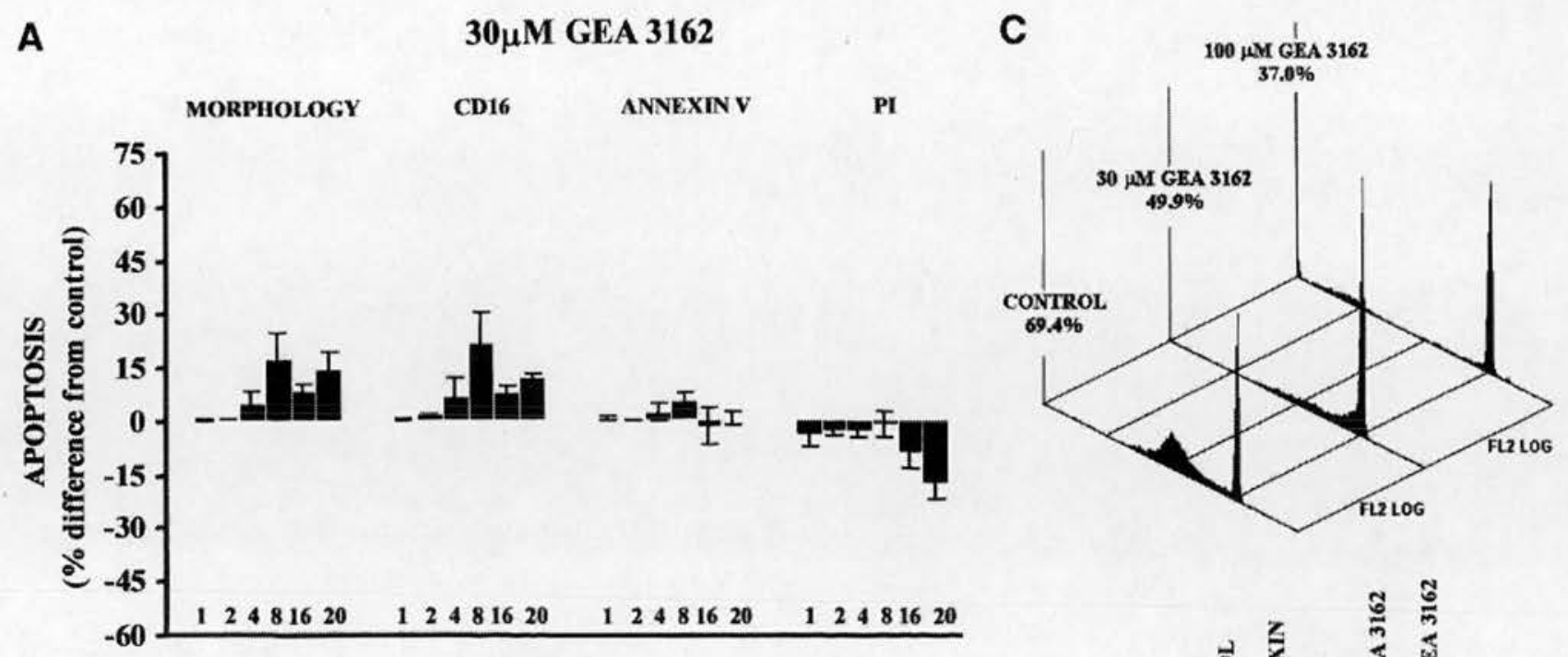

TIME (hours)

B

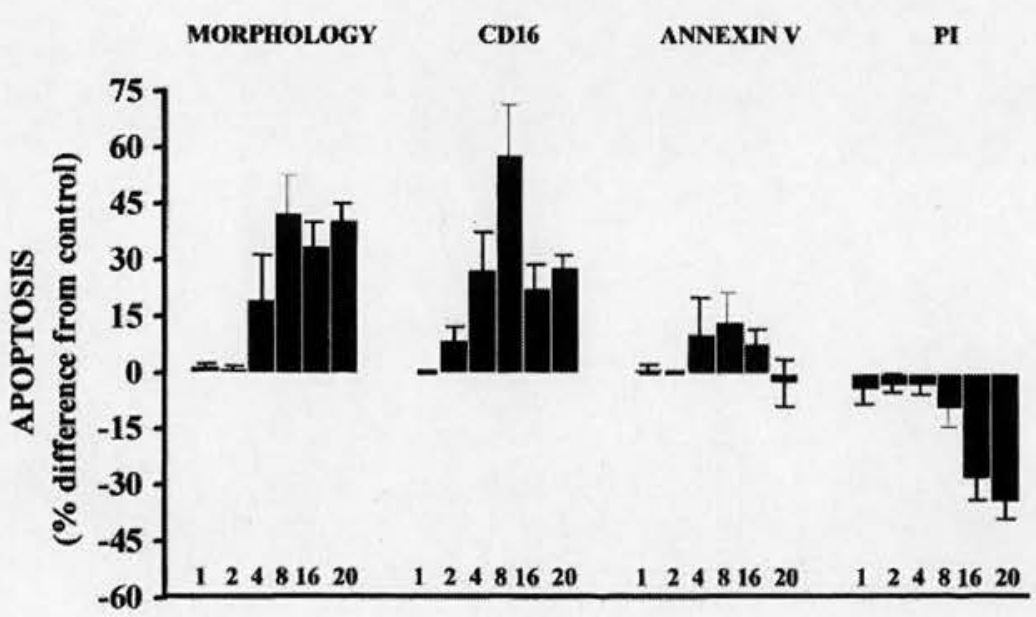

$100 \mu M$ GEA 3162

TIME (hours)

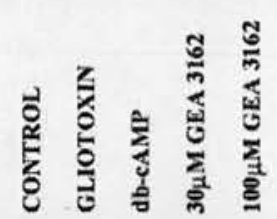

D

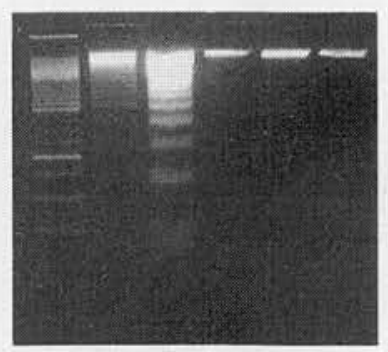

$\mathbf{E}$

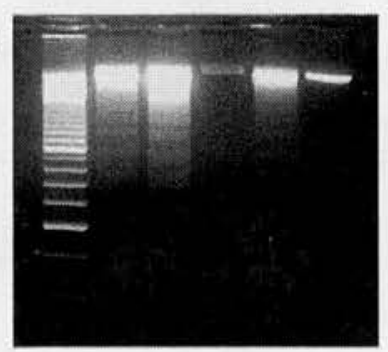

FIG. 3. Effects of (A) $30 \mu \mathrm{M}$ and (B) $100 \mu \mathrm{M}$ GEA 3162 on four independent markers of neutrophil apoptosis. Neutrophils were incubated for 1-20 h in the absence or presence of GEA 3162, with apoptosis assessed by morphology, CD16 shedding, annexin V binding and PI intercalation (DNA fragmentation), as described under Materials and Methods. Values represent the mean \pm SEM difference from levels of apoptosis in cells incubated without GEA 3162, from $n=3-6$ separate experiments. (C) Flow cytometry histograms from one representative experiment, showing the concentration-dependent inhibition of PI intercalation by GEA 3162. The broader peak on the histograms represents the population of hypodiploid apoptotic cells. DNA ladders show DNA fragmentation in neutrophils incubated with medium alone (control), the NF $\kappa$ B survival factor inhibitor gliotoxin $(0.1 \mu \mathrm{g} / \mathrm{ml}$, positive control $)$, dibutyryl cyclic-AMP $(0.2 \mathrm{mM}$, negative control), $30 \mu \mathrm{M}$ GEA 3162 or $100 \mu \mathrm{M}$ GEA 3162 for (D) $8 \mathrm{~h}$ or (E) $20 \mathrm{~h}$, as described under Materials and Methods.

not appear until $16 \mathrm{~h}$. This suggests that a rapid burst of higher concentrations of $\mathrm{NO}$ is more effective at inducing neutrophil apoptosis than prolonged exposure to lower concentrations. GEA 3162 enhanced apoptosis at lower concentrations than the NONOates, and this effect could be seen by $4 \mathrm{~h}$ with $100 \mu \mathrm{M}$ or $8 \mathrm{~h}$ with 30 $\mu \mathrm{M}$. Again, this possibly reflects differences in the mechanism of NO liberation from this compound compared to the NONOates. For all three compounds, shedding of CD16 was the first indication of neutrophil apoptosis, occurring a few hours before morphological evidence of cell death, which preceded DNA fragmentation by several hours, thus confirming previous reports that internucleosomal DNA breakdown is a late event in apoptosis $(1,37)$.

Another interesting concept that arises from these studies is that individual compounds may have opposing effects on different parameters of neutrophil apop- 
A

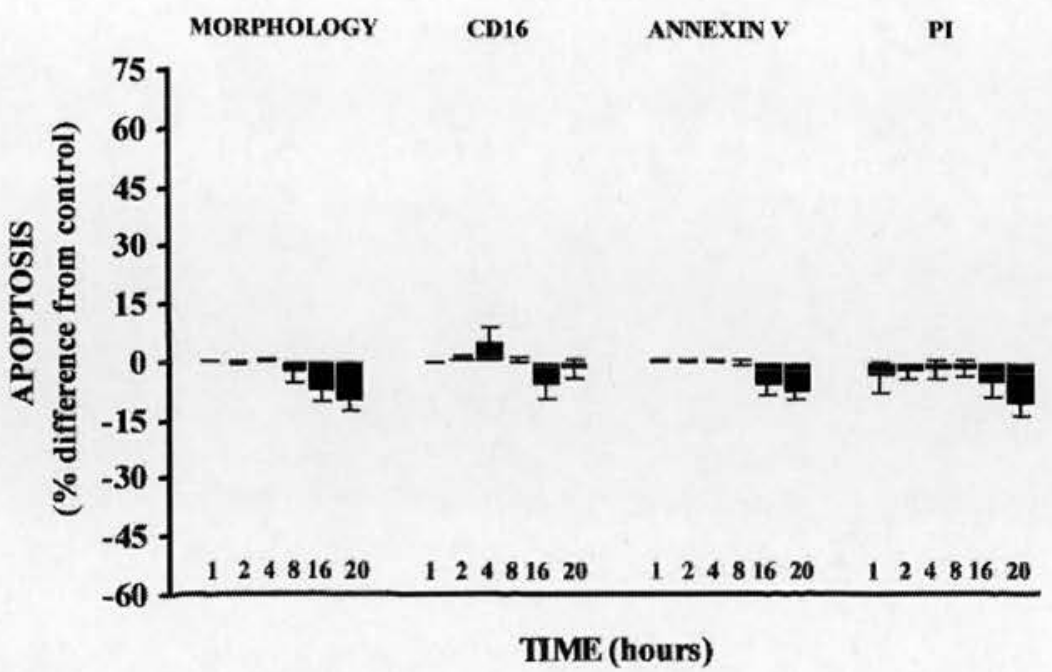

B

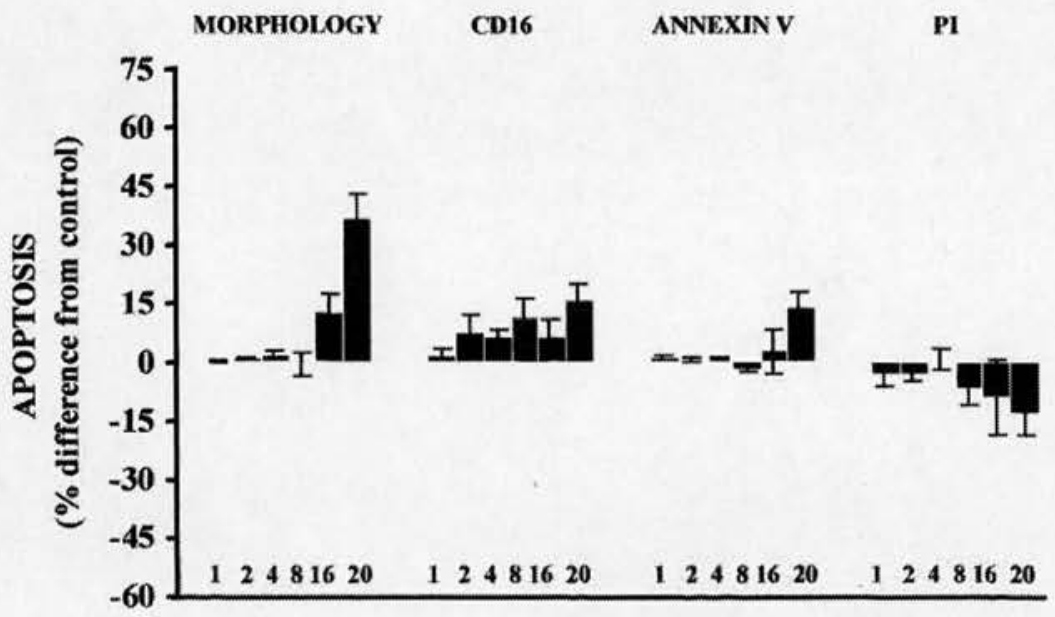

C
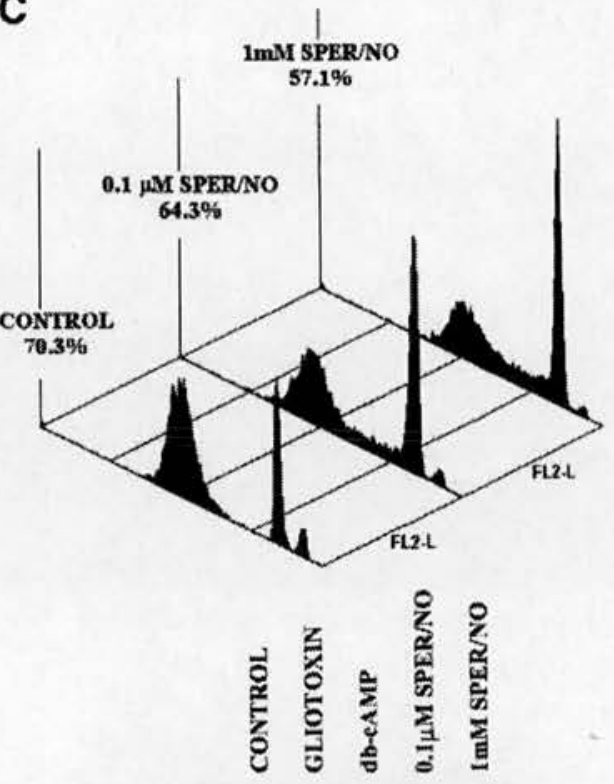

D

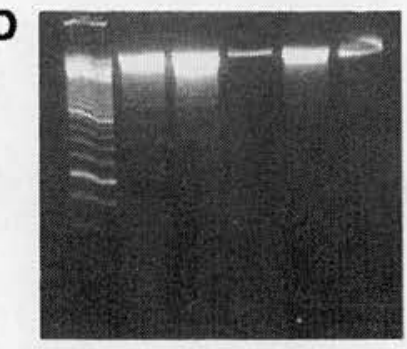

$\mathbf{E}$

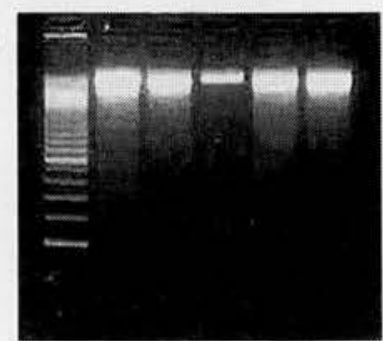

TIME (hours)

FIG. 4. Effects of (A) $0.1 \mu \mathrm{M}$ and (B) $1 \mathrm{mM}$ SPER/NO on four independent markers of neutrophil apoptosis. Neutrophils were incubated for 1-20 h in the absence or presence of SPER/NO, with apoptosis assessed by morphology, CD16 shedding, annexin V binding and PI intercalation (DNA fragmentation), as described under Materials and Methods. Values represent the mean \pm SEM difference from levels of apoptosis in cells incubated without SPER/NO, from $n=3-6$ separate experiments. (C) Flow cytometry histograms from one répresentative experiment, showing the concentration-dependent inhibition of PI intercalation by SPER/NO. The broader peak on the histograms represents the population of hypodiploid apoptotic cells. DNA ladders show DNA fragmentation in neutrophils incubated with medium alone (control), the NF $\kappa \mathrm{B}$ survival factor inhibitor gliotoxin $(0.1 \mu \mathrm{g} / \mathrm{ml}$, positive control), dibutyryl cyclic-AMP $(0.2 \mathrm{mM}$, negative control), $0.1 \mu \mathrm{M}$ SPER/NO or $1 \mathrm{mM}$ SPER/NO for (D) $8 \mathrm{~h}$ or (E) $20 \mathrm{~h}$, as described under Materials and Methods.

tosis. A clear dissociation of morphological and biochemical changes of apoptosis from DNA fragmentation was observed in neutrophils treated with GEA 3162 and SPER/NO, although no such discrepancy was seen with DEA/NO. This effect was most likely due to the NO-donors themselves, as a good correlation between individual apoptotic markers was observed in untreated cells undergoing spontaneous apoptosis. It has been suggested that DNA fragmentation, but not cell death itself, is dependent on caspase 3 activation $(5,8,10-13)$, and that NO is able to inhibit this enzyme through modification of free thiols $(38,39)$. Therefore it is conceivable that neutrophil cell death and DNA cleavage may be dissociated through inhibition of caspase 3 , which could prevent activation of the DNA degradation pathway but be insufficient to impede cell death, which may be effected through different caspase enzymes. Alternative mechanisms could be 

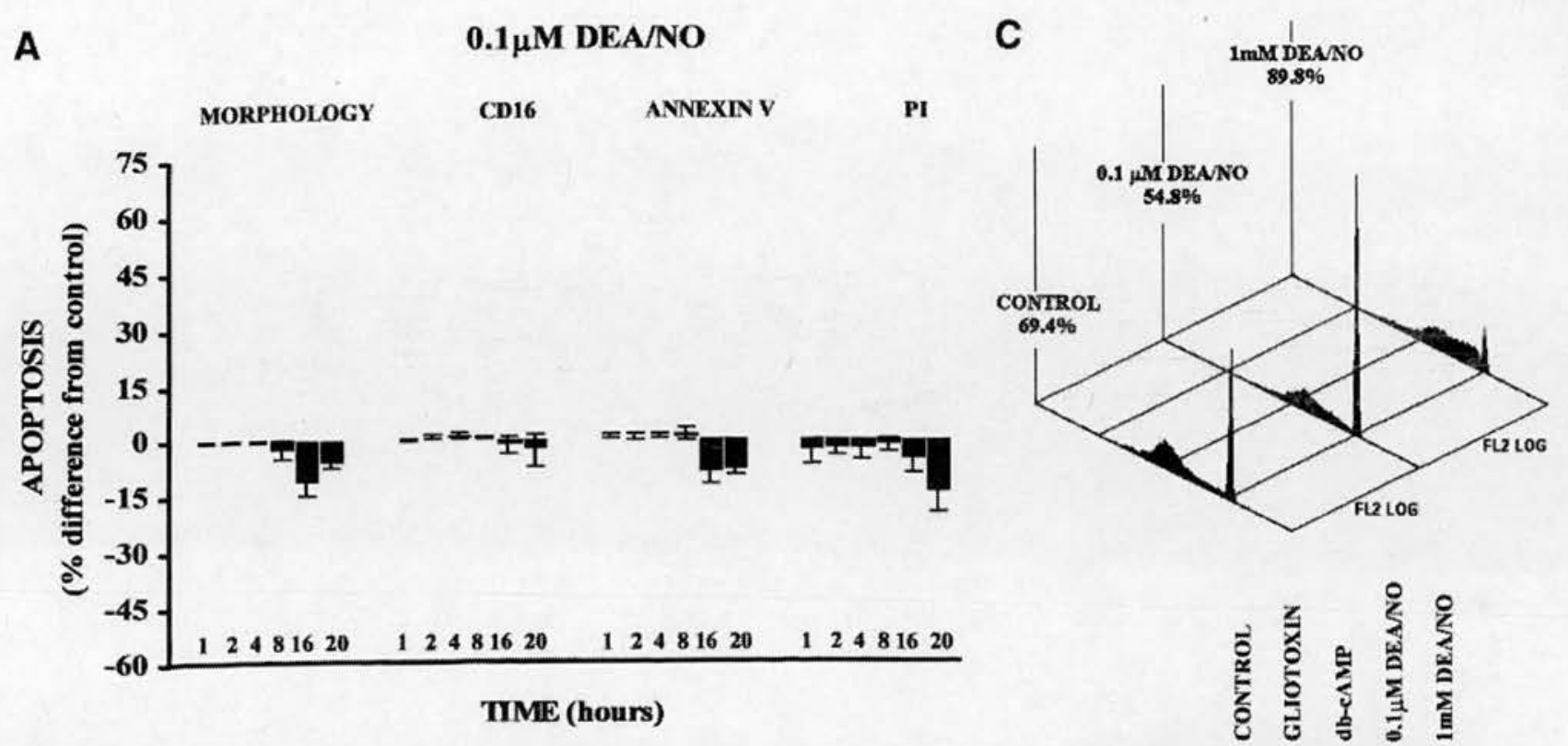

B

\section{$1 \mathrm{mM}$ DEA/NO}

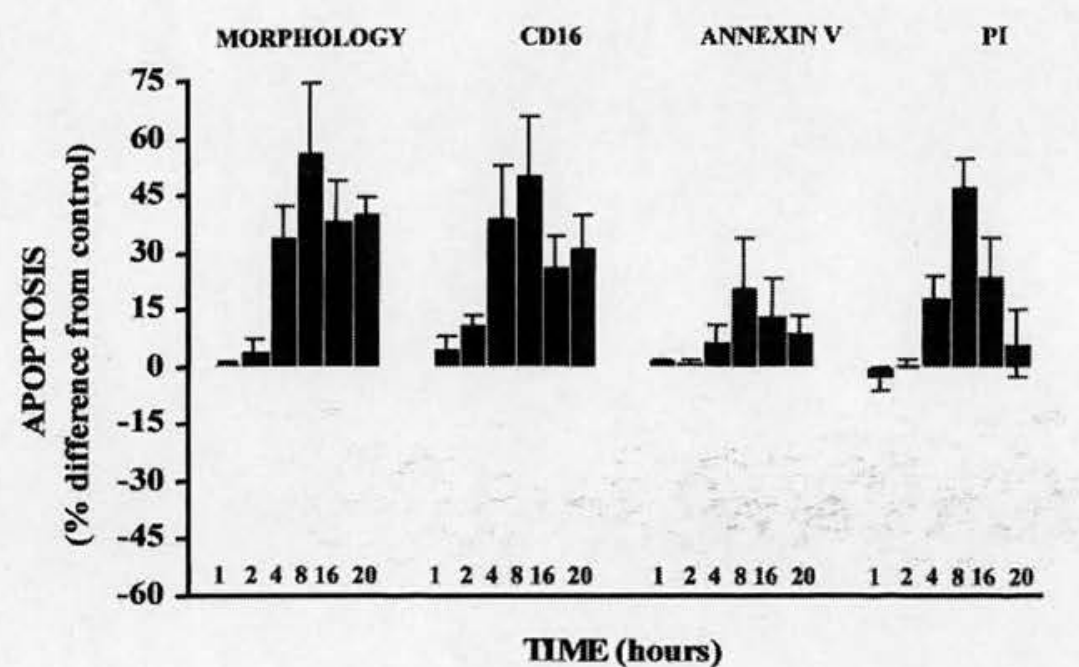

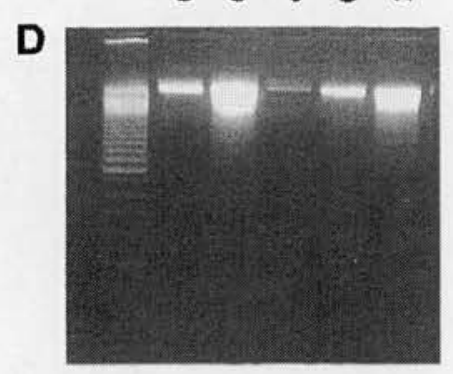

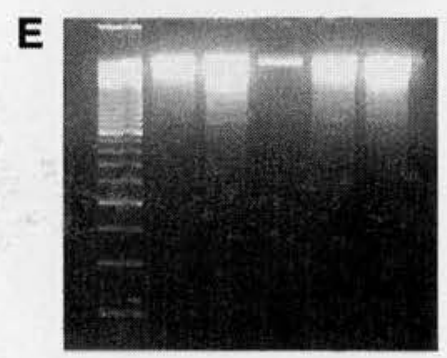

FIG. 5. Effects of (A) $0.1 \mu \mathrm{M}$ and (B) $1 \mathrm{mM}$ DEA/NO on four independent markers of neutrophil apoptosis. Neutrophils were incubated for $1-20 \mathrm{~h}$ in the absence or presence of DEA/NO, with apoptosis assessed by morphology, CD16 shedding, annexin V binding and PI intercalation (DNA fragmentation), as described under Materials and Methods. Values represent the mean \pm SEM difference from levels of apoptosis in cells incubated without DEA/NO, from $n=3-6$ separate experiments. (C) Flow cytometry histograms from one representative experiment, showing the biphasic effect of DEA/NO on PI intercalation. The broader peak on the histograms represents the population of hypodiploid apoptotic cells. DNA ladders show DNA fragmentation in neutrophils incubated with medium alone (control), the NF $\kappa B$ survival factor inhibitor gliotoxin $(0.1 \mu \mathrm{g} / \mathrm{ml}$; positive control), dibutyryl cyclic-AMP $(0.2 \mathrm{mM}$, negative control), $0.1 \mu \mathrm{M}$ DEA/NO or $1 \mathrm{mM}$ DEA/NO for (D) $8 \mathrm{~h}$ or (E) $20 \mathrm{~h}$, as described under Materials and Methods.

that NO directly modifies vital cysteine residues in enzymes involved in the DNA cleavage pathway, or indirectly modifies tyrosine residues via formation of peroxynitrite.

It is worth noting that the absence of internucleosomal DNA cleavage should not affect the clearance of neutrophils from inflammatory sites by macrophages, a vital event in resolution of inflammation (18). Cell surface events that allow macrophage recognition of apoptotic neutrophils utilize a different, caspase-independent, mechanism than the pathways that bring about cleavage of DNA $(40,41)$. The ability of macrophages to detect and remove the apoptotic cells would therefore not be diminished, as these cell surface events are still evident, despite the lack of DNA fragmentation. This is particularly relevant because it has been shown that NO generation by activated macrophages can induce apoptosis in neighboring mesangial cells that are subsequently removed by phagocytes (42). 
We have demonstrated a clear dissociation between some of the events of apoptotic cell death in neutrophils, whereby discrepancies between DNA breakdown and other parameters of apoptotic cell death may be seen during NO-induced apoptosis, depending on the NO-donating compound used. To the best of our knowledge, this lack of correlation has not previously been reported in this cell type, indeed most studies report a simultaneous increase in DNA fragmentation and other apoptotic markers $(22,23)$. We conclude from these studies that analysis of NO-induced neutrophil apoptosis by DNA fragmentation alone is not to be recommended, as this may cause vital consequences of the application of chemical agents to neutrophils to be overlooked. It is important to exploit two or preferably three independent markers of apoptosis before results can be treated with any confidence.

\section{ACKNOWLEDGMENTS}

This work was supported by the Wellcome Trust and an MRC programme grant (G9016491). E.L.T. is a Wellcome Trust 4-year scholar funded through the University of Edinburgh Cardiovascular Research Initiative.

\section{REFERENCES}

1. Cohen, G. M., Sun, X. M., Snowden, R. T., Dinsdale, D., and Skilleter, D. N. (1992) Biochem. J. 286(Pt. 2), 331-334.

2. Xu, J., Xu, Y., Nguyen, Q., Novikoff, P. M., and Czaja, M. J. (1996) Am. J. Physiol. 270, G60-G70.

3. Marini, M., and Musiani, D. (1998) Exp. Cell Res. 239, 393-398.

4. Gooch, J. L., and Yee, D. (1999) Cancer Lett. 144, 31-37.

5. McIlroy, D., Sakahira, H., Talanian, R. V., and Nagata, S. (1999) Oncogene 18, 4401-4408.

6. Rasola, A., Farahi, F. D., Hofman, P., and Rossi, B. (1999) FASEB J. 13, 1711-1723.

7. Sakahira, H., Enari, M., Ohsawa, Y., Uchiyama, Y., and Nagata, S. (1999) Curr. Biol. 9, 543-546.

8. Tanabe, K., Nakanishi, H., Maeda, H., Nishioku, T., Hashimoto, K., Liou, S. Y., Akamine, A., and Yamamoto, K. (1999) J. Biol. Chem. 274, 15725-15731.

9. Chiu, L. C., Wan, J. M., and Ooi, V. E. (2000) Int. J. Oncol. 17, 789-796.

10. D'Mello, S. R., Kuan, C. Y., Flavell, R. A., and Rakic, P. (2000) J. Neurosci. Res. 59, 24-31.

11. Kim, D. K., Cho, E. S., and Um, H. D. (2000) Exp. Cell Res. 257, 82-88.

12. Kouroku, Y., Fujita, E., Urase, K., Tsuru, T., Setsuie, R., Kikuchi, T., Yagi, Y., Momoi, M. Y., and Momoi, T. (2000) J. Neurosci. Res. 62, 547-556.

13. Jiang, D., Jha, N., Boonplueang, R., and Andersen, J. K. (2001) J. Neurochem. 76, 1745-1755.

14. Takemura, G., Kato, S., Aoyama, T., Hayakawa, Y., Kanoh, M., Maruyama, R., Arai, M., Nishigaki, K., Minatoguchi, S., Fukuda, K., Fujiwara, T., and Fujiwara, H. (2001) J. Pathol. 193, 546556.

15. Yuste, V. J., Bayascas, J. R., Llecha, N., Sanchez-Lopez, I., Boix, J., and Comella, J. X. (2001) J. Biol. Chem. 276, 22323-22331.

16. Kim, P. K. M., Zamora, R., Petrosko, P., and Billiar, T. R. (2001) Int. Immunopharmacol. 1, 1421-1441.
17. Dimmeler, S., and Zeiher, A. M. (1997) Nitric. Oxide. 1, 275-281.

18. Ward, C., Dransfield, I., Chilvers, E. R., Haslett, C., and Rossi, A. G. (1999) Trends Pharmacol. Sci. 20, 503-509.

19. Hannah, S., Nadra, I., Dransfield, I., Pryde, J. G., Rossi, A. G., and Haslett, C. (1998) FEBS Lett. 421, 141-146.

20. Ward, C., Wong, T. H., Murray, J., Rahman, I., Haslett, C., Chilvers, E. R., and Rossi, A. G. (2000) Biochem. Pharmacol. 59, 305-314.

21. Blaylock, M. G., Cuthbertson, B. H., Galley, H. F., Ferguson, N. R., and Webster, N. R. (1998) Free Radical Biol. Med. 25, 748-752.

22. Fortenberry, J. D., Owens, M. L., Brown, M. R., Atkinson, D., and Brown, L. A. (1998) Am. J. Respir. Cell Mol. Biol. 18, 421-428.

23. Fortenberry, J. D., Owens, M. L., and Brown, L. A. (1999) Am. J. Physiol. 276, L435-L442.

24. Singhal, P. C., Patel, P., Nahar, N., Franki, N., Kapasi, A., Reddy, K., Shah, N., Nwakoby, I. E., and Mehrotra, B. (1999) J. Leukocyte Biol. 66, 930-936.

25. Megson, I. L. (2000) Drugs Future 25, 701-715.

26. Maragos, C. M., Morley, D., Wink, D. A., Dunams, T. M., Saavedra, J. E., Hoffman, A., Bove, A. A., Isaac, L., Hrabie, J. A., and Keefer, L. K. (1991) J. Med. Chem. 34, 3242-3247.

27. Haslett, C., Worthen, G. S., Giclas, P. C., Morrison, D. C., Henson, J. E., and Henson, P. M. (1987) Am. Rev. Respir. Dis. 136, $9-18$.

28. Ward, C., Chilvers, E. R., Lawson, M. F., Pryde, J. G., Fujihara, S., Farrow, S. N., Haslett, C., and Rossi, A. G. (1999) J. Biol. Chem. 274, 4309-4318.

29. Dransfield, I., Buckle, A. M., Savill, J. S., McDowall, A., Haslett, C., and Hogg, N. (1994) J. Immunol. 153, 1254-1263.

30. Collins, R. J., Harmon, B. V., Gobe, G. C., and Kerr, J. F. (1992) Int. J. Radiat. Biol. 61, 451-453.

31. Malo-Ranta, U., Yla-Herttuala, S., Metsa-Ketela, T., Jaakkola, O., Moilanen, E., Vuorinen, P., and Nikkari, T. (1994) FEBS Lett. 337, 179-183.

32. Holm, P., Kankaanranta, H., Metsa-Ketela, T., and Moilanen, E. (1998) Eur. J. Pharmacol. 346, 97-102.

33. Schmidt, K., Schrammel, A., Gorren, A. C. F., Koesling, D., and Mayer, B. (2001) Naunyn-Schmiedebergs Arch. Pharmacol. 363, 161-161.

34. Rossi, A. G., Cousin, J. M., Dransfield, I., Lawson, M. F., Chilvers, E. R., and Haslett, C. (1995) Biochem. Biophys. Res. Commun. 217, 892-899.

35. Martin, M. C., Dransfield, I., Haslett, C., and Rossi, A. G. (2001) J. Biol. Chem., in press.

36. Sogo, N., Magid, K. S., Shaw, C. A., Webb, D. J., and Megson, I. L. (2000) Biochem. Biophys. Res. Commun. 279, 412-419.

37. Rucker-Martin, C., Henaff, M., Hatem, S. N., Delpy, E., and Mercadier, J. J. (1999) Basic Res. Cardiol. 94, 171-179.

38. Melino, G., Bernassola, F., Knight, R. A., Corasaniti, M. T., Nistico, G., and Finazzi-Agro, A. (1997) Nature 388, 432- 433.

39. Mohr, S., Zech, B., Lapetina, E. G., and Brune, B. (1997) Biochem. Biophys. Res. Commun. 238, 387-391.

40. Zhuang, J., Ren, Y., Snowden, R. T., Zhu, H., Gogvadze, V., Savill, J. S., and Cohen, G. M. (1998) J. Biol. Chem. 273, 15628 15632.

41. Pryde, J. G., Walker, A., Rossi, A. G., Hannah, S., and Haslett, C. (2000) J. Biol. Chem. 275, 33574-33584.

42. Duffield, J. S., Erwig, L. P., Wei, X., Liew, F. Y., Rees, A. J., and Savill, J. S. (2000) J. Immunol, 164, 2110-2119. 


\title{
Inhibition of nuclear factor-кB activation un-masks the ability of TNF- $\alpha$ to induce human eosinophil apoptosis
}

\author{
Satoko Fujihara', Carol Ward', Ian Dransfield', Ronald T. Hay ${ }^{2}$, lain J. Uings ${ }^{3}$, Brian \\ Hayes $^{3}$, Stuart N. Farrow ${ }^{3}$, Christopher Haslett' ${ }^{1}$ and Adriano G. Rossi ${ }^{1}$ \\ ${ }^{1}$ Rayne Laboratory, Respiratory Medicine Unit, Centre for Inflammation Research, Department \\ of Medicine, University of Edinburgh Medical School, Edinburgh, GB \\ ${ }^{2}$ School of Biomedical Sciences, University of St Andrews, North Haugh, St Andrews, GB \\ ${ }^{3}$ The Cell Biology Unit, GlaxoSmithKline, Stevenage, GB
}

Apoptosis renders eosinophils functionally effete and marks them for 'silent' removal from inflamed sites by macrophages. We show, for the first time, that eosinophils exposed to TNF- $\alpha$ rapidly lose their cytoplasmic levels of $\operatorname{lkB} \alpha$, the inhibitory subunit of NF-kB. Consequently, TNF- $\alpha$ triggers NF-kB mobilization from the cytoplasm to the nucleus, as determined by tracking the NF- $\mathrm{kB}$ subunit $\mathrm{p} 65$ by immunofluorescence and Western blot analysis. Inhibition of TNF- $\alpha$-mediated $\mathrm{I} \mathrm{KB} \alpha$ degradation and NF- $\mathrm{kB}$ activation by gliotoxin or the proteasome inhibitor MG-132 un-masks the caspase-dependent pro-apoptotic properties of TNF- $\alpha$. In addition, cycloheximide similarly renders TNF- $\alpha$ pro-apoptotic, suggesting that $\mathrm{NF}-\mathrm{kB}$ activation controls the production of a protein(s) that protects eosinophils from the cytotoxic effects of TNF- $\alpha$. Evidence is presented suggesting that TNF- $\alpha$ triggered apoptosis is more susceptible to NF- $\mathrm{kB}$ inhibition than constitutive apoptosis, leading to the possibility of the specific targeting of apoptosis in eosinophil sub-populations. Prior to morphological signs of apoptosis, TNF- $\alpha$-induced IL- 8 synthesis is abrogated by inhibition of $\mathrm{NF}-\mathrm{kB}$. We propose that NF- $\mathrm{KB}$ activation plays a critical role in controlling eosinophil responsiveness and apoptosis, and speculate that selective inhibitors of eosinophil NF- $\mathrm{KB}$ activation may ultimately provide alternative therapeutic agents for the treatment of eosinophilic diseases, including asthma and allergic rhinitis.

Key words: Eosinophil / NF-kB / Apoptosis / $\mathrm{l} \mathrm{kB} \alpha$ / Inflammation

\begin{tabular}{|lr|}
\hline Received & $18 / 6 / 01$ \\
Revised & $9 / 10 / 01$ \\
Accepted & $27 / 11 / 01$ \\
\hline
\end{tabular}

\section{Introduction}

Eosinophilic granulocytes are normally involved in orchestrating the destruction and removal of invading parasites. However, if these cells are inappropriately recruited or activated at inflammatory sites they can liberate excessive amounts of toxic products (e.g. free $\mathrm{O}_{2}$ radicals, major basic protein, etc.), which can cause damage to the surrounding tissue. Thus, eosinophilmediated epithelial cell damage in the respiratory tract is likely to contribute to the pathogenesis and propagation of asthma and allergic rhinitis [1, 2]. Ligation of specific cell surface receptors and the consequent triggering of diverse signal transduction pathways control eosinophil functional responsiveness [3] and physiological programmed cell death (apoptosis) [4]. During apoptosis

[1 22139] granulocytes undergo a series of dramatic morphological transformations (e.g. shrinkage and chromatin condensation) and surface molecule alterations (e.g. expression of phosphatidylserine) [5-8]. Apoptosis also causes a marked reduction in the cell's ability to respond to proinflammatory stimuli [5], and is critical for the efficient non-inflammatory $[5,6]$ removal of effete granulocytes from the inflammatory site by macrophages [7] and other cells including epithelial cells [9].

Eosinophils have the capacity to release inflammatory mediators derived from lipid metabolism and are capable of synthesizing pro-inflammatory cytokines [e.g. IL-8, TNF- $\alpha$, granulocyte/macrophage-colony stimulating factor (GM-CSF), IL-5, etc.] [3], many of which are regulated by the transcription factor nuclear factor- $\mathrm{kB}$ (NF-кB) [10-12]. Our recent demonstration that neutrophil apoptosis is critically regulated by the synthesis of NF- $\mathrm{kB}$ controlled survival protein(s) $[13,14]$ suggests that NFкВ may control gene transcription of proteins that influence granulocyte apoptosis as well the synthesis of pro- 
inflammatory agents. NF-kB1 (p50), NF-kB2 (p52), RelA (p65), relB and c-rel are a family of proteins which bind to one another to form NF-kB dimers (often p50/p65), that reside predominantly in the cytoplasm. NF- $\mathrm{KB}$ is held in the cytoplasm in an inactive state by being bound to an inhibitory protein from the $\mathrm{k} \mathrm{kB}$ family in which the $37-\mathrm{kDa}$ $\mathrm{l} \mathrm{kB} \alpha$ is the major form. When cells are appropriately stimulated, the $\mid \kappa B \alpha$ subunit is rapidly phosphorylated and ubiquinated before finally being degraded by the $26 \mathrm{~S}$ proteasome [10-12]. Translocation of NF- $\mathrm{kB}$ subunits to the nucleus, where they bind to regulatory DNA sequences in the upstream promoter region of target genes, controls rates of transcription $[10,11]$. Inflammatory cells have the capacity to synthesize and respond to exogenous NF- $\mathrm{KB}$ regulated pro-inflammatory cytokines [15]. For example, $\mathrm{TNF}-\alpha$ is capable of priming neutrophils, rendering them more susceptible to activation upon subsequent exposure to other neutrophil secretagogues $[16,17]$. Furthermore, TNF- $\alpha$ can influence apoptosis in many cells including granulocytes [13, 18-20]; effects that may be controlled by activation of NF-kB $[13,18,20]$. For example, apoptosis is promoted when neutrophils are exposed to TNF- $\alpha$ for short periods of culture (i.e. 2-8 h) in a concentrationdependent manner. When apoptosis is assessed after $20 \mathrm{~h}$ of TNF- $\alpha$ exposure apoptosis is delayed [18]. Interestingly, TNF- $\alpha$ has the ability to induce apoptosis in human neutrophils via a mechanism where TNF receptor 2 (TNFR2) facilitates the dominant TNF receptor 1 (TNFR1) death effect. In human eosinophils TNF- $\alpha$ has been shown to exert an anti-apoptotic effect that signals partly via the p38 MAP kinase pathway [19], and more recently Temkin and Levi-Schaffer [20] have shown that TNF- $\alpha$-mediated eosinophil survival occurs via ligation of both TNF receptor subtypes and through the generation of the eosinophil survival factor GM-CSF; an effect that seems to involve activation of NF-kB. Although we [13] and others $[20,21,22]$ have shown that NF- $\mathrm{kB}$ is present in granulocytes, the role of NF- $\mathrm{KB}$ in the regulation of eosinophil-derived chemokine production and apoptosis remains poorly understood. We used two compounds that inhibit NF-kB activation; MG-132, a widely used proteasome inhibitor that prevents $\mathrm{l} \mathrm{KB}$ degradation [23] and gliotoxin [13, 24]. Gliotoxin is an epipolythiodioxoperazine which exhibits immune suppressive activity both in vitro and in vivo $[24,25]$ and selectively inhibits NF-kB activation [24]. We demonstrate that both gliotoxin and MG-132 can un-mask the potential of TNF- $\alpha$ to induce eosinophil apoptosis. At the same concentration that affects apoptosis and inhibits TNF- $\alpha$-induced IL- 8 secretion, gliotoxin inhibits the translocation of p65 to the nucleus from the cytoplasm by inhibiting the degradation of $\mathrm{I}_{\mathrm{K}} \mathrm{B} \alpha$. From our data we therefore propose that the NF- $\mathrm{KB}$ pathway plays a key role in regulation of eosinophil responsiveness and apoptosis; a system that may be modulated for therapeutic gain.

\section{Results}

\subsection{NF-kB regulates eosinophil apoptosis}

We have shown that NF-KB plays a central role in eosinophil apoptosis by demonstrating that blockade of NF- $\mathrm{kB}$ activation by gliotoxin un-masks the ability of TNF- $\alpha$ to dramatically induce eosinophil apoptosis. This is clearly depicted in Fig. $1 \mathrm{~A}$, where TNF- $\alpha(10 \mathrm{ng} / \mathrm{ml})$ alone and gliotoxin $(100 \mathrm{ng} / \mathrm{ml})$ alone cultured with eosinophils for $4 \mathrm{~h}$ caused little morphological apoptosis; however, when eosinophils were cultured in the presence of TNF- $\alpha$ plus gliotoxin there was a dramatic induction of characteristic pyknotic nuclei typical of eosinophil apoptosis. Our preliminary data indicated that it requires a significant amount of time ( $>3 \mathrm{~h}$ ) to observe significant apoptosis and that the 4-h time point is optimal for observing the synergistic effects of TNF- $\alpha$ and gliotoxin (data not shown). As an additional independent assessment of eosinophil apoptosis, we performed experiments in which surface changes associated with the apoptotic program were also assessed. For this we measured FITC-labeled annexin $\mathrm{V}$ binding in the presence of $\mathrm{Ca}^{2+}$ to phosphatidylserine molecules exposed on the outer membrane of apoptotic cells, where the annexin V 'low peak' represents non-apoptotic cells and the annexin $V$ 'high peak' represents apoptotic cells. In agreement with morphological assessment of apoptosis, Fig. $1 \mathrm{C}$ shows that, although control and TNF- $\alpha$-treated eosinophils at $4 \mathrm{~h}$ exhibit low rates of apoptosis, the small increase in annexin $\mathrm{V}^{+}$cells observed with gliotoxin alone is again dramatically augmented when the cells are cultured in the presence of both reagents together. Furthermore, the marked synergism that was displayed by gliotoxin and $\mathrm{TNF}-\alpha$ is mediated by triggering of the caspase pathway since the broad-spectrum caspase inhibitor, zVAD-fmk $(100 \mu \mathrm{M})$ completely blocked the pro-apoptotic effects induced by the combination of TNF- $\alpha$ plus gliotoxin (Fig. 2). When eosinophils were co-cultured with the protein synthesis inhibitor cycloheximide $(5 \mu \mathrm{M})$, at a concentration and time point that had almost no direct effect on eosinophil apoptosis, a similar un-masking of the proapoptotic effect of TNF- $\alpha$ was observed; an effect that was also suppressed by zVAD-fmk (Fig. 2).

In addition, when eosinophils were cultured with the proteasome inhibitor MG-132 $(20 \mu \mathrm{M})$ a marked induction of apoptosis was also observed, although the proapoptotic effect required longer incubation times than gliotoxin. For example, no significant induction of apoptosis was observed above constitutive levels when eosinophils were cultured for $8 \mathrm{~h}$ with MG-132 (data not shown). Even with incubation periods of $30 \mathrm{~h}, \mathrm{MG}-132$ induced little apoptosis; however, in the presence of TNF- $\alpha$ there was a dramatic synergistic induction of 
A

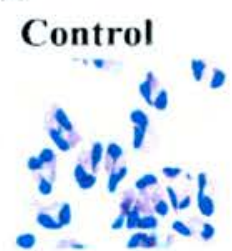

Gliotoxin

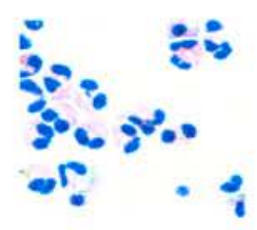

B
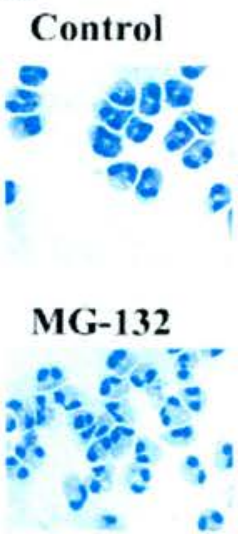

C

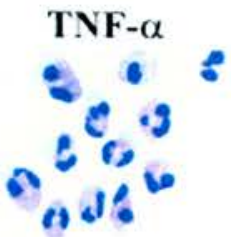

Gliotoxin/TNF- $\alpha$

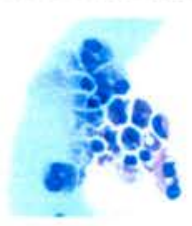

TNF- $\alpha$

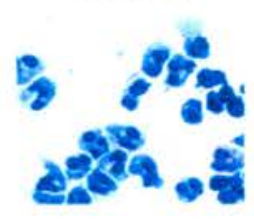

MG-132/TNF- $\alpha$
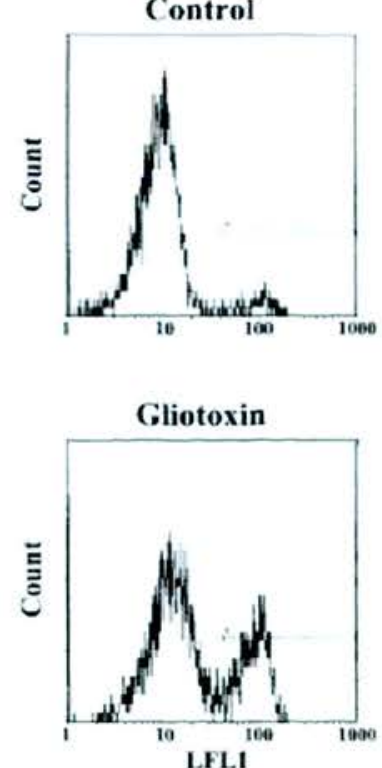

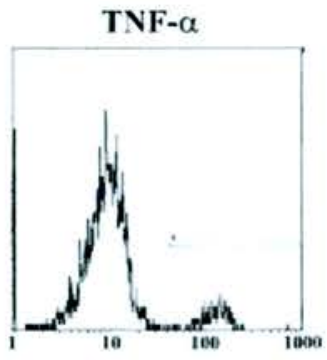

Gliotoxin/TNF- $\alpha$

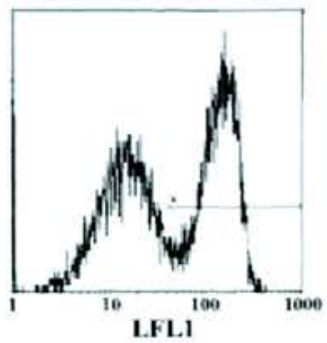

Fig. 1. The effect of gliotoxin, MG-132 and TNF- $\alpha$ on eosinophil apoptosis. (A) Cytocentrifuge preparations were made of human eosinophils incubated for $4 \mathrm{~h}$ at $37^{\circ} \mathrm{C}$ in medium alone (control), $10 \mathrm{ng} / \mathrm{ml} \mathrm{TNF}-\alpha, 100 \mathrm{ng} / \mathrm{ml}$ gliotoxin, and combination of these reagents. The rates of apoptosis in this experiment are control, $5 \%$; TNF- $\alpha, 5 \%$; gliotoxin, $26.5 \%$; and gliotoxin/TNF- $\alpha, 47.5 \%$. (B) Cytocentrifuge preparations of human eosinophils incubated for $30 \mathrm{~h}$ at $37^{\circ} \mathrm{C}$ in medium alone (control), $10 \mathrm{ng} / \mathrm{ml} \mathrm{TNF}-\alpha$, MG$132(20 \mu \mathrm{M})$, and combination of these reagents. The rates of apoptosis in this experiment are control, $14.6 \% ;$ TNF- $\alpha, 233 \%$; MG-132, 11.4\%; and MG-132/TNF- $\alpha, 75.7 \%$. (C) Aliquots $(20 \mu \mathrm{l})$ were taken from human eosinophils that had been incubated for $4 \mathrm{~h}$ at $37^{\circ} \mathrm{C}$ in medium alone (control), $10 \mathrm{ng} / \mathrm{ml} \mathrm{TNF- \alpha ,100} \mathrm{ng/ml} \mathrm{gliotoxin,} \mathrm{and} \mathrm{a} \mathrm{combination} \mathrm{of} \mathrm{these} \mathrm{reagents} \mathrm{and} \mathrm{incu-}$ bated with FITC-labeled recombinant human annexin $V$ to determine the phosphatidylserine expression on the cell surface. Flow cytometric traces from a typical experiment are depicted with the percentage of cells in the annexin- $\mathrm{V}$ high population being $7.0 \%$ in control, $9.8 \%$ in TNF- $\alpha, 28.5 \%$ in gliotoxin and $50.8 \%$ in TNF- $\alpha$ plus gliotoxin-treated samples.

apoptosis (Fig. 1B). When apoptosis was assessed after 40-h treatment with MG-132 there was a significant induction of apoptosis (control, 24.8 $\pm 8.7 \%$; MG-132, $72.5 \pm 15.8 \%$ apoptosis; $p<0.05, n=4$ ). The pro-apoptotic effects of MG-132 per se and the enhanced apoptosis observed with a combination of MG-132 and TNF- $\alpha$ were also suppressed by zVAD-fmk (data not shown).

Taken together the above results support the view that TNF- $\alpha$ treatment results in the NF- $\mathrm{KB}$ controlled generation of a survival protein(s) that protects eosinophils from the TNF- $\alpha$ receptor- and caspase-dependent induction of apoptosis. To substantiate this hypothesis we performed a detailed direct analysis of NF- $\mathrm{KB}$ activation.

\subsection{TNF- $\alpha$ induced cytoplasmic $1 \mathrm{kB} \alpha$ degradation and p65 disappearance in eosinophils}

Upon appropriate cell stimulation, the inhibitory subunit of $\mathrm{NF}-\mathrm{kB}$, $\mathrm{I} \mathrm{KB} \alpha$, is rapidly phosphorylated and undergoes proteolytic breakdown by the proteasome, thereby permitting NF- $\mathrm{kB}$ to translocate from the cytoplasm from 
the nucleus [10]. Since the phosphorylation and subsequent degradation of $\mathrm{I} \kappa \mathrm{B} \alpha$ is a prerequisite for NF- $\mathrm{KB}$ activation, the amount of $\mathrm{I} \mathrm{kB} \alpha$ in cytosolic extracts of eosinophils was examined by Western blotting to find evidence for the activation of the NF-kB pathway. In quiescent un-stimulated cells, $\mathrm{I}_{\mathrm{KB}} \alpha$ appeared as a single band of $37 \mathrm{kDa}$, which disappeared following TNF- $\alpha$ stimulation, indicating degradation of $\operatorname{lkB} \alpha$ (Fig. $3 \mathrm{~A}$ ) and translocation of NF-KB to the nucleus. Following TNF- $\alpha$ stimulation the amount of p65 in the cytoplasm was reduced (Fig. $3 \mathrm{~B}$ ), indicating that, following the degradation of $1 \mathrm{kB} \alpha, \mathrm{p} 65$ is released from $I \mathrm{~KB} \alpha$ in the cytoplasm and translocated into the nucleus. As shown in Fig. 3A, gliotoxin had no direct effect on IKB $\alpha$ levels but inhibited TNF- $\alpha$-induced disappearance of $1 \kappa B \alpha$. The latter observation is consistent with gliotoxin-mediated inhibition of TNF- $\alpha$-induced loss of cytoplasmic p65 (Fig. 3B). These results therefore suggest that TNF $\alpha$ stimulation results in $\mathrm{l} \mathrm{KB} \alpha$ degradation and translocation of NF- $\mathrm{kB}$ from the cytoplasm to the nucleus.

\subsection{Immunohistochemical analysis of NF- $\mathrm{kB}$ translocation in eosinophils}

To provide compelling evidence for subcellular movement of NF- $\mathrm{KB}$ in eosinophils we performed quantitative immunohistochemical analysis of TNF- $\alpha$-induced trans-

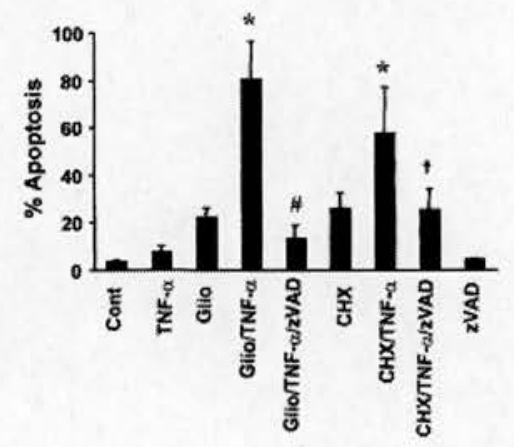

Fig. 2. Effects of the caspase inhibitor, ZVAD-fmk, and the protein synthesis inhibitor, cycloheximide, on eosinophil apoptosis induced by gliotoxin, TNF- $\alpha$ or on a combination of these agents. Human eosinophils were treated with the indicated reagents for $4 \mathrm{~h}$ at $37^{\circ} \mathrm{C}$ before cytocentrifuge preparations were made and apoptosis assessed morphologically. Concentrations are: $10 \mathrm{ng} / \mathrm{ml} \mathrm{TNF}-\alpha, 100 \mathrm{ng} / \mathrm{ml}$ gliotoxin, $100 \mu \mathrm{M} z V A D-f m k$, and $5 \mu \mathrm{M}$ cycloheximide (CHX). The data are expressed as the mean \pm SEM of four separate experiments using eosinophils isolated from different donors. *Represents significance differences $(p<0.05)$ from control, and \# represents significance difference from Glio/TNF- $\alpha$ and $\dagger$ represents significance difference form CHX/TNF- $\alpha$. location of p65. Immunofluorescent confocal images of p65 (FITC: green) reveal translocation into the nucleus (propidium iodide: red) by TNF- $\alpha$ stimulation (shown as yellow as the result of overlapping green p65-FITC, and red propidium iodide) (Fig. 4) that is effectively prevented by gliotoxin. These results are consistent with the Western blot analysis shown in Fig. $3 \mathrm{~A}$ and $\mathrm{B}$, and therefore provide evidence that gliotoxin acts by inhibition of NF$\mathbf{K B}$ activation. Quantification of translocation is depicted in Fig. 5A-C where the immunofluorescent images are analyzed on a Leica Q550IW image analyzer to detect p65 amount in the cytoplasm and nucleus and the results expressed as a ratio. Fig. $5 \mathrm{~A}$ and $\mathrm{B}$ clearly show the inhibition of TNF- $\alpha$-induced NF-KB translocation into the nucleus by gliotoxin and MG-132. In Fig. $5 \mathrm{C}$ the time course of translocation of p65 stimulated with TNF- $\alpha$ from a representative experiment is depicted. Maximum induction of TNF- $\alpha$-stimulated p65 translocation in eosinophils isolated from this particular donor occurred after 60 -min exposure to TNF- $\alpha$ as assessed by immunofluorescence. Following this time point, NF-кB levels in the nucleus rapidly decrease almost to basal levels, perhaps reflecting rapid re-synthesis of $\mathrm{I}_{\mathrm{K}} \mathrm{B} \alpha$ and consequent removal of NF-kB from the nucleus. Although different levels of basal and stimulated p65 distribution were observed between individual donors, substantial

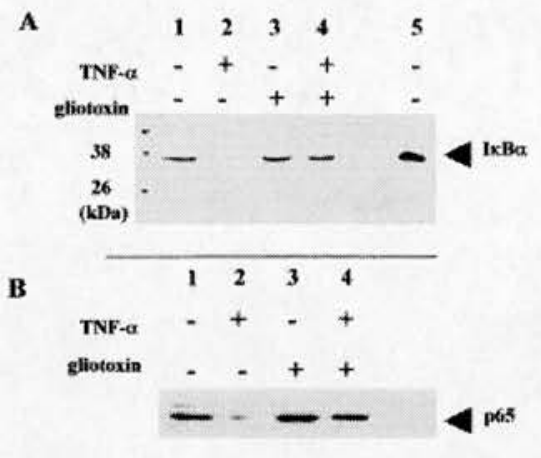

Fig. 3. Western blot analysis of cytoplasmic $1 \mathbf{k B} \alpha$ and $\mathrm{p} 65$. Eosinophils were stimulated with $10 \mathrm{ng} / \mathrm{ml}$ TNF- $\alpha, 100 \mathrm{ng} /$ $\mathrm{ml}$ gliotoxin for $15 \mathrm{~min}$ in Iscove's DMEM at $37^{\circ} \mathrm{C}$ and cytoplasmic extract prepared and analyzed by Western blotting with anti-|kB $\alpha$ antibody, and anti-p65 antibody. (A) Cytoplasmic $I_{\kappa} B \alpha$ expression. Upon TNF- $\alpha$ stimulation the $I_{\kappa} B \alpha$ band disappears, indicative of proteosomal degradation; treatment of the cells with gliotoxin stabilizes $\mid \mathbf{k B} \alpha$ in the cytoplasm both in the absence and presence of TNF- $\alpha$. For a control Jurkat cell cytoplasmic extracts were included (lane 5). (B) The amount cytoplasmic p65. The loss of p65 expression in the cytoplasm with TNF- $\alpha$ stimulation correlated with $1 \mathrm{kB} \alpha$ loss and suggests translocation of p65 into the nucleus, a process blocked by gliotoxin. The blots are representative of at least three separate experiments. 

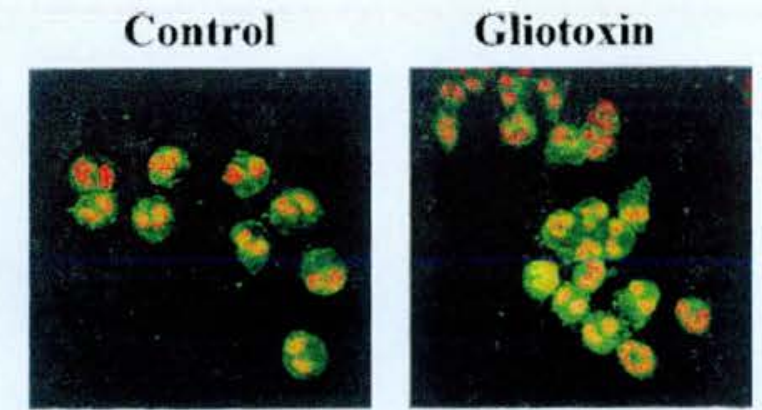

TNF- $\alpha$

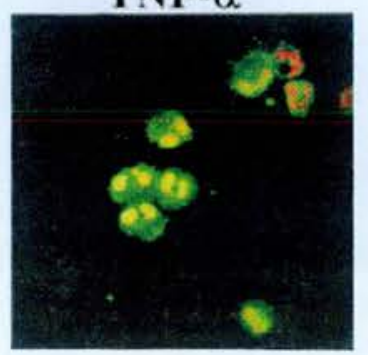

Glio/TNF- $\alpha$

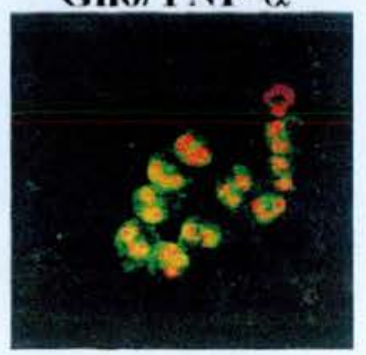

Fig. 4. Confocal microscope images of p65 expression in human eosinophils stimulated with TNF- $\alpha$ with and without gliotoxin. Eosinophils were stimulated for $\mathbf{4 5} \mathrm{min}$ in Iscove's DMEM with $10 \mathrm{ng} / \mathrm{ml} \mathrm{TNF-} \alpha$ and $100 \mathrm{ng} / \mathrm{ml}$ gliotoxin. Antip65 antibody was FITC labeled (shown as green) and the nucleus was stained with propidium iodine (shown as red). Upon TNF- $\alpha$ stimulation p65 migrates into the nucleus, resulting in an enhanced yellow color. Gliotoxin, with or without TNF- $\alpha$, dramatically inhibits p65 nuclear migration.

NF- $\mathrm{kB}$ activation by TNF- $\alpha$ stimulation was observed (data not shown).

\subsection{NF-kB activation mediates IL-8 production by human eosinophils}

Having clearly shown the presence and activation of the NF- $\mathrm{kB}$ pathway in eosinophils and demonstrated that this pathway regulates the eosinophil apoptotic program in response to TNF- $\alpha$, we next sought to investigate its role in regulating eosinophil responsiveness. It is established in many cell types $[11,26]$ that IL- 8 production is tightly controlled by NF-KB, and eosinophils have the capacity to synthesize this important pro-inflammatory cytokine $[3,21]$. TNF- $\alpha$ is a powerful stimulator of IL-8 production in eosinophils (Fig. 6) even at the early time point of $90 \mathrm{~min}$, a time point chosen since there is no gliotoxin-induced apoptosis that could influence IL-8 production (data not shown). The production of IL-8 was dramatically suppressed by gliotoxin at a concentration that effectively inhibits NF- $\mathrm{kB}$ activation at this time point where there is no significant apoptosis.
A

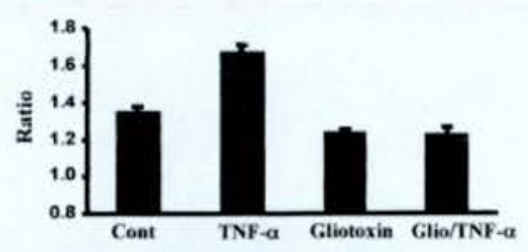

B

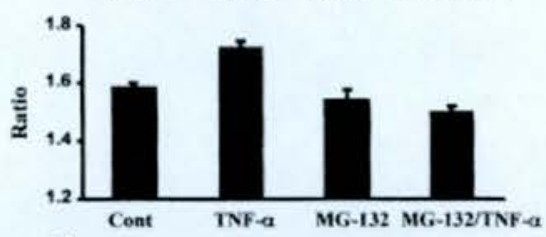

C

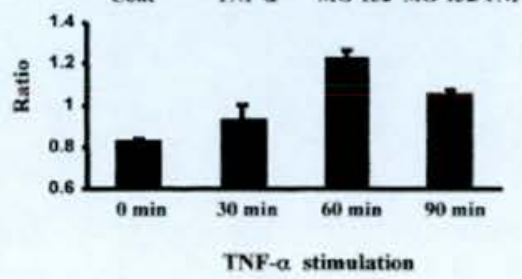

Fig. 5. Quantitative analysis of p65 translocation to the nucleus. Computer analysis of p65 expression in the nucleus was measured as the ratio of p65-FITC nuclear fluorescence versus cytoplasmic fluorescence in each cell. At least 300 cells were counted per condition and the data are represented as mean \pm SEM determined for each experiment independently. Eosinophils were incubated in Iscove's DMEM at $37^{\circ} \mathrm{C}$ with the indicated stimuli (A and $B$ ) for 45 min and with TNF- $\alpha$ for the indicated times (C). (A) The effect of $100 \mathrm{ng} / \mathrm{ml}$ gliotoxin on activation by $10 \mathrm{ng} / \mathrm{ml} \mathrm{TNF}-\alpha$. (B) The effect of $20 \mu \mathrm{M} M G-132$ on p65 translocation into the nucleus. (C) Time course of the effect of $10 \mathrm{ng} / \mathrm{ml} \mathrm{TNF- \alpha .}$ The data are representative of one experiment of at least three similar experiments.

\section{Discussion}

We have clearly demonstrated that human eosinophils possesses a fully functional NF- $\mathrm{KB}$ signaling system that plays an important role in regulating cellular responsiveness and apoptosis. Our data show that the eosinophil contains p65 and that NF-kB is presumably maintained in the cytoplasm by the inhibitor protein $1 \mathrm{kB} \alpha$. Upon exposure to the pro-inflammatory agent TNF- $\alpha, \mid \kappa B \alpha$ is rapidly lost from the cytoplasm by a process that involves phosphorylation and ubiquitination, followed by subsequent degradation by the proteasome. In eosinophils, Western blot analysis and immunofluorescence data demonstrate that $\mathrm{p} 65$ translocates from the cytoplasm to the nucleus in response to TNF- $\alpha$. One likely partner for p65 in eosinophils is p50, as identified by Western blot analysis ([21] and data not shown). Gliotoxin and MG-132 effectively prevents p65 translocation to the nucleus, which we suggest is important for the 


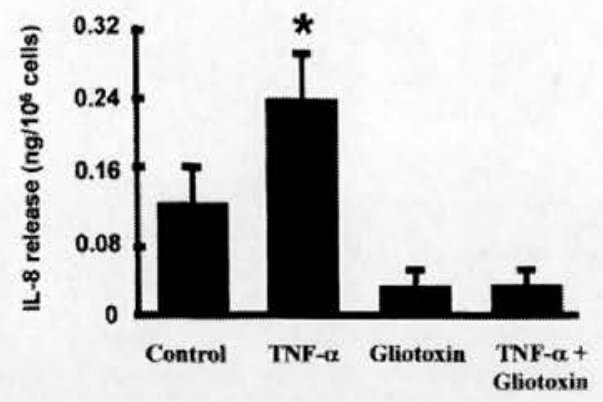

Fig. 6. Effect of gliotoxin on TNF- $\alpha$-induced IL- 8 release from human eosinophils. Eosinophils were stimulated with $10 \mathrm{ng} / \mathrm{ml} \mathrm{TNF}-\alpha$ for $90 \mathrm{~min}$ in the absence and presence of $100 \mathrm{ng} / \mathrm{ml}$ gliotoxin and the IL-8 concentrations in the cell supernatants determined by specific ELISA. The data are expressed as the mean \pm SEM of four separate experiments using eosinophils isolated from different donors. Significant increase $(p<0.05)$ from the control is indicated by *

direct effect of these compounds on apoptosis. TNF- $\alpha$ is a powerful activator of NF- $\mathrm{KB}$ and gliotoxin and MG-132 inhibit NF- $\mathrm{kB}$ activation upon TNF- $\alpha$ stimulation resulting in a dramatic synergistic induction of apoptosis. Interestingly, the following observations suggest that TNF- $\alpha$-triggered apoptosis is more susceptible to NF- $\mathrm{kB}$ inhibition than constitutive apoptosis; firstly, higher concentrations of gliotoxin are required to directly induce apoptosis than required to render the cells responsive to the pro-apoptotic effects of TNF- $\alpha$ [13] and secondly MG-132 augments TNF- $\alpha$-induced apoptosis at earlier time points than are required to induce apoptosis directly. These findings clearly require further investigation but provide the intriguing possibility of selectively targeting the removal of potentially histotoxic eosinophils from inflammatory sites by manipulating apoptosis of such eosinophil sub-populations without influencing constitutive tissue eosinophils.

The NF- $\mathrm{kB}$ complex binds to DNA regulatory sites on the upstream (5') promotor sequences of target genes that control the rate of gene transcription for many proinflammatory mediators, some of which can influence granulocyte function and apoptosis (e.g. TNF- $\alpha$ and IL-8). Eosinophils exposed to TNF- $\alpha$ (Fig. 6) and lipopolysaccharide [27] are capable of releasing IL-8. IL-8 itself can cause recruitment of inflammatory cells into sites of inflammation and can also prime and/or activate granulocytes [28, 29]. Thus activation of the NF-kB pathway can augment the inflammatory response by generating further pro-inflammatory mediators. In our studies, synthesis of IL-8 is inhibited by relatively low concentrations of gliotoxin even at a time point $(90 \mathrm{~min})$ at which there is no significant induction of apoptosis, consistent with the observation that TNF- $\alpha$ activates NF- $\mathrm{KB}$ to induce IL-8 production in human eosinophils (Fig. 6; [21]). Although we have shown previously that gliotoxin, but not its structurally similar analog, methylthiogliotoxin, can directly induce neutrophil apoptosis and enhance apoptosis in the presence of TNF- $\alpha$ [13] by selectively inhibiting NF- $\mathrm{KB}$ activation, the underlying mechanisms were not identified. We show here that gliotoxin not only prevents translocation of p65 from the cytoplasm to the nucleus but also prevents $\mathrm{l} \mathrm{B} \mathrm{B} \alpha$ degradation. Interestingly, MG-132, like gliotoxin, can also induce eosinophil apoptosis directly and also renders eosinophils responsive to the pro-apoptotic effects of TNF- $\alpha$. It is noteworthy that MG-132 required a longer incubation period in comparison to gliotoxin to un-mask TNF- $\alpha$-induced apoptosis. The precise reason for this is unknown but may reflect a difference in the mechanism of action or in the rates of cellular incorporation between the two inhibitors of NF-kB activation. We do, however, propose that NF- $\mathrm{KB}$ activation is a critical regulator of granulocyte apoptotic programs. Studies using cycloheximide suggests that granulocytes synthesize a survival protein(s) that inhibits apoptosis [30]. Our results showing that cycloheximide dramatically un-masks the pro-apoptotic effect of TNF- $\alpha$ in eosinophils are entirely consistent with our hypothesis that NF- $\mathrm{KB}$ activation leads to the synthesis of a survival protein that limits the ability of the cell to undergo apoptosis. Thus, TNF- $\alpha$ triggers a number of signaling pathways following ligation of TNF- $\alpha$ receptors. We have previously shown that neutrophils undergo an early induction of apoptosis (2-8 h) and a later $(>18 \mathrm{~h})$ inhibition of apoptosis when cultured in vitro in the presence of TNF- $\alpha$ [18]. The pro-apoptotic effect of TNF- $\alpha$ is thought to be mediated by ligation of the TNFR1 containing a death domain that interacts with the TNF-receptor-associated death domain protein (TRADD) allowing recruitment of Fas-associated death domain (FADD), leading to activation of the caspase enzyme pathway. This highly regulated sequence of events enables apoptosis to be triggered. TNFR 2, often in co-operation with TNFR1, results in the activation of many kinases including the $\mathrm{p} 38$-Jun $\mathrm{N}$-terminal kinase (JNK) pathway and IKK [31]. Prevention of the survival pathway by blocking the generation of NF-kB-mediated protein synthesis allows the death pathway to predominate and a massive induction of apoptosis is observed. It remains possible that blockade of NF- $\mathrm{kB}$ will prevent the synthesis of powerful NF-kB-regulated survival cytokines such as GM-CSF, IL-5 and IL-3. Indeed, it has very recently been published that TNF- $\alpha$ via ligation of both TNFRI and TNFR2 subtypes mediated eosinophil survival (as assessed by Trypan blue exclusion), and that this occurred through regulation of GM-CSF but not IL-3 and IL-5 production [20]. Furthermore, it was shown that GM-CSF is indeed synthesized when eosinophils are challenged with TNF- $\alpha$ and that TNF- $\alpha$ enhanced eosin- 
ophil survival is significantly inhibited by the proteasome inhibitor MG-132 [20]. It also remains likely that eosinophils like other cells can generate protein(s) that directly influence the apoptotic program. However, the precise identity of the potential survival protein(s) is unknown (see [14] for a review); however, several likely candidate proteins have been implicated including members of the oncogene Bcl2 family (e.g. A1 [32, 33] and $\mathrm{Mcl}-1$ [34]) and others such as C-Myc [35], A20 [36], $\operatorname{clAP}[37,38]$, and IEX-1L [39].

Although it is abundantly clear that NF- $\mathrm{KB}$ activation can play an important role in regulating the production of cytokines and adhesion molecules that are vital for orchestrating the inflammatory response, there is relatively little direct in vivo evidence indicating that this transcription factor can influence eosinophilic inflammation. Compelling evidence that NF- $\mathrm{kB}$ plays an essential role in the induction of eosinophilia in allergic airway inflammation, was obtained using mice deficient in p50, when compared with wild-type mice [40]. In addition to being incapable of mounting eosinophilic airway inflammation, p50-1- mice had a dramatically reduced capacity for the production NF- $\mathrm{KB}$ regulated inflammatory mediators such as the Thelper 2 cytokine IL- 5 and the chemokines eotaxin and macrophage inflammatory protein (MIP)- $1 \alpha$ and MIP-1 $\beta$. We believe that NF-kB plays a prominent role in controlling eosinophil responsiveness and longevity, and therefore could have far reaching implications for understanding not only the generation and propagation, but also the resolution, of eosinophilic inflammation. Further investigations may lead to the development of novel compounds that interfere with the NF- $\mathrm{KB}$ signaling pathway which may be applied to clinically relevant eosinophilic diseases such as asthma and allergic rhinitis.

\section{Materials and methods}

\subsection{Eosinophil isolation and culture}

Granulocytes were isolated from the peripheral blood of normal or mildly atopic donors by dextran sedimentation followed by centrifugation through discontinuous Percoll (Amersham/Pharmacia, Little Chalfont, GB) gradients [13, 41]. Eosinophils were separated from contaminating neutrophils by negative selection using an immunomagnetic separation step with sheep anti-mouse IgG-Dynabeads (Dynabeads M-450, Dynal, Merseyside, GB) coated with the murine anti-neutrophil antibody 3 G8 (anti-CD16; a gift from Dr. J. Unkeless, Mount Sinai Medical School, New York) as described [42]. Eosinophils were routinely $>98 \%$ pure and $>99 \%$ viable. After purification, eosinophils were washed twice in PBS without $\mathrm{Ca}^{2+}$ and $\mathrm{Mg}^{2+}$ and resuspended as indicated below. Eosinophils in Iscove's Dulbecco's modified Eagle's medium (DMEM) (Life Technologies, Paisley,
GB) supplemented with $10 \%$ autologous serum were cultured in Falcon flat-bottom flexible 96-well plates (Becton Dickinson, Oxford, $\mathrm{GB}$ ) at $37^{\circ} \mathrm{C}$ in a $5 \% \mathrm{CO}_{2}$ atmosphere at $2 \times 10^{6} / \mathrm{ml}$. Cells were cultured in the absence or presence of test agents as described in the figure legends.

\subsection{Assessment of eosinophil apoptosis}

\subsubsection{Morphology}

Cells $\left(120 \mu \mathrm{l}\right.$ of $\left.2 \times 10^{6} / \mathrm{ml}\right)$ were cyto-centrifuged, fixed in methanol, stained with Diff-Quik ${ }^{\mathrm{TM}}$ (Gamidor Ltd., Abingdon, GB) and counted using oil immersion microscopy $(\times 100$ objective) to determine the proportion of cells with distinctive apoptotic morphology $[4,7,13]$. At least 500 cells were counted per slide. All experiments were performed at least three times and each treatment done in duplicate and the results are expressed as the mean $\%$ apoptosis \pm SEM.

\subsubsection{Annexin V binding}

A separate and independent assessment of apoptosis was performed by flow cytometry using FITC-labeled recombinant human annexin $\mathrm{V}$ that binds to phosphatidylserine exposed on the surface of apoptotic cells. Stock annexin V (Bender MedSystems, Vienna, Austria) was diluted 1:200 with binding buffer and then added $(20 \mu \mathrm{l})$ to $120 \mu \mathrm{l}$ of the recovered cell samples. Following a 10 -min incubation at $4^{\circ} \mathrm{C}$, these samples were fixed by the addition of $100 \mu \mathrm{l}$ of $3 \%$ paraformaldehyde in PBS before analysis using an EPICS Profile II (Coulter Electronics, Luton, GB) [13].

\subsection{Preparation of cell cytoplasmic extracts}

Following incubation at $37^{\circ} \mathrm{C}$ with the indicated reagents (see figure legends for concentrations and times) eosinophils were washed with ice-cold PBS and the cells lysed at $4^{\circ} \mathrm{C}$ for 15 min with Nonidet $\mathrm{P}-40$ lysis buffer as described [43] (50 mM NaF, $5 \mathrm{mM} \mathrm{Na} \mathrm{P}_{2} \mathrm{O}_{7}, 1 \mathrm{mM} \mathrm{Na} \mathrm{VO}_{4}, 10 \mathrm{mM}$ $\beta$-glyceropyrophosphate, $0.5 \%$ (v/v) Nonidet P-40, $2 \mathrm{mM}$ EDTA, $16.8 \mathrm{mM} \mathrm{Na}_{2} \mathrm{HPO}_{4}, 3.2 \mathrm{mM} \mathrm{NaH} \mathrm{PO}_{4}$ ) containing complete protease inhibitor cocktail EDTA free (Roche Diagnostics, Mannheim, Germany). The cell fragments were then centrifuged $\left(13,000 \times \mathrm{g}, 10 \mathrm{~min}, 4^{\circ} \mathrm{C}\right)$ and the supernatants containing the cytoplasmic extracts collected.

\subsection{Western blot analysis}

The cell extracts were diluted with $3 x$ sample buffer $[5.7 \%$ (wt/v) SDS, $14 \%$ mercaptoethanol, $150 \mathrm{mM}$ Tris- $\mathrm{HCl}, \mathrm{pH}$ $6.7 ; 30 \%(\mathrm{v} / \mathrm{v})$ glycerol] and heated at $95^{\circ} \mathrm{C}$ for $3 \mathrm{~min}$ before running on $10 \%$ (wt/v) SDS-polyacrylamide gel. Electophoretically separated proteins were transferred on PVDF microporous membranes (Millipore Corporation). Non-specific 
binding was blocked with PBS containing $5 \%$ non-fat dry milk and $0.1 \%$ Tween 20 (Sigma Co., Poole, GB) and probed with the indicated antibodies (anti-lkB $\alpha$ from New England Biolab Ltd., Hertfordshire, GB; anti-p65 and -p50 antibodies from Santa Cruz Biotechnology, CA). As a positive control $10 \mu \mathrm{g}$ of protein extracted from un-treated Jurkat cell [maintained in RPMI 1640 (Life Technologies), 10\% FCS (Life Technologies)] extract was also run on the gels. Immunodetected proteins were visualized by $\mathrm{ECL}^{\mathrm{TM}}$ detection reagents (Amersham/Pharmacia).

\subsection{Immunohistochemistry}

Eosinophils $\left(2.5 \times 10^{6} / \mathrm{ml}\right)$ were incubated in Iscove's DMEM containing $5 \% \mathrm{FCS}$ for $2 \mathrm{~h}$ at $37^{\circ} \mathrm{C}$ before treatment with the indicated agents and time (see figure legends for details). The cells were cyto-centrifuged, air dried for $10 \mathrm{~min}$ and then fixed with $4 \%(\mathrm{w} / \mathrm{v}) \mathrm{p}$-formaldehyde/PBS for $10 \mathrm{~min}$ and washed three times with PBS. The cells were permeabilized and nonspecific binding was blocked in buffer containing $0.2 \%(\mathrm{w} / \mathrm{v})$ Triton $\mathrm{X}-100$ protein block serum-free buffer (DAKO Corp., Glostrup, Denmark) at room temperature for $30 \mathrm{~min}$. The cells were incubated with rabbit polyclonal p65 antibody (Santa Cruz Biotechnology; 1:100 dilution) in antibody diluent (DAKO) with $0.2 \%$ Triton $\mathrm{X}-100$ for $1 \mathrm{~h}$. The cells were washed three times in antibody diluent with $0.2 \%$ Triton X-100 and then incubated (1:100 dilution) with Alexa ${ }^{\mathrm{TM}}$ 488 (goat anti-rabbit) IgG antibody (Molecular Probes, Eugene, OR) for $1 \mathrm{~h}$ in antibody diluent with $0.2 \%$ Triton $\mathrm{X}$ 100 then washed three times in the same buffer. The nucleus was stained with $0.1 \%(\mathrm{v} / \mathrm{v})$ propidium iodide (Sigma) with $2 \%(\mathrm{w} / \mathrm{v})$ RNase in PBS at $37^{\circ} \mathrm{C}$ for $30 \mathrm{~min}$ followed by washing $(\times 3)$ with PBS before applying a glass coverslip.

\subsection{Confocal microscopy and image analysis}

Images are from single confocal sections, $0.3 \mu \mathrm{m}$ thick, taken through the center of the nucleus, on a Leica TSC 4D confocal microscope using a $63 \times$ objective lens. NF-kB nuclear translocation was determined with a Leica DMRB microscope using a 40x NA 0.7 objective lens and Kappa integrating monochrome CCD camera. Image analysis was integrated with image capture as described before [44]. The image analyzer captures an image of the green NF-кB fluorescence and using an automated macro-program developed for this application on a Leica Q550IW image analyzer and uses the binary images of the propidium iodide staining as a mask to measure only the NF-kB fluorescence of the nuclei. The image analyzer then detects the green fluorescence ( $488 \mathrm{~nm}$ excitation, $530 \mathrm{~nm}$ emission) and subtracts the binary image of the red fluorescence $(570 \mathrm{~nm}$ excitation, $620 \mathrm{~nm}$ emission) to give a binary mask for the cytoplasm. At least 300 cells were counted per condition and the data accumulated. Means \pm SEM are determined for each experiment independently.

\subsection{IL-8 measurement}

The ELISA was performed using IL- 8 recombinant protein as a standard and $\mathrm{IgG}$ anti-human IL-8 according to the manufacture's instructions (R\&D systems, Abingdon, GB).

\subsection{Materials}

Further specific materials were obtained as follows: recombinant human TNF- $\alpha$ (R\&D Systems), MG-132 (N-cbz-LeuLeu-leucinal) (Biomol, Affinity Research Products, Mamhead, GB) and zVAD-fmk (benzylocarbonyl-Val-Ala-Aspfluoromethylketone) (Bachem Ltd., Saffron Walden, GB).

\subsection{Statistical analysis}

The results are expressed as the mean \pm SEM of the number ( $n$ ) of independent experiments each using cells isolated from different donors with each treatment performed in duplicate or triplicate. Statistical analysis was performed by ANOVA with comparisons between groups made using the Newmans-Kuels procedure. Where appropriate, analysis was performed using the paired Student's $t$-test. Differences were considered significant when $p<0.05$, and are represented by the indicated symbol.

Acknowledgements: This work was supported by a Medical Research Council Program Grant G9016491. S. Fujihara is supported by a GlaxoWellcome studentship.

\section{References}

1 Weller, P. F., Human eosinophils. J. Allergy Clin. Immunol. 1997. 100: 283-287.

2 Gleich, G. J., Mechanisms of eosinophil-associated inflammation. J. Allergy Clin. Immunol. 2000. 105: 651-663.

3 Giembycz, M. A. and Lindsay, M. A., Pharmacology of the eosinophil. Pharmacol. Rev. 1999. 51: 213-340.

4 Stern, M., Meagher, L., Savill, J. and Haslett, C., Apoptosis in human eosinophils. Programmed cell death in the eosinophil leads to phagocytosis by macrophages and is modulated by IL-5. J. Immunol. 1992. 148: 3543-3449.

5 Stern, M., Savill, J. S. and Haslett, C., Human monocytederived macrophage phagocytosis of senescent eosinophils undergoing apoptosis. Mediation by $\alpha \mathrm{v} \beta 3 / \mathrm{CD} 36 /$ thrombospondin recognition mechanism and lack of phlogistic response. Am. J. Pathol. 1996. 149: 911-921.

6 Meagher, L. C., Savill, J. S., Baker, A., Fuller, R. W. and Haslett, C., Phagocytosis of apoptotic neutrophils does not induce macrophage release of thromboxane B2. J. Leukoc. Biol. 1992. 52: 269-273.

7 Savill, J. S., Wyllie, A. H., Henson, J. E., Walport, M. J., Henson, P. M. and Haslett, C., Macrophage phagocytosis of aging neutrophils in inflammation. Programmed cell death in the neutrophil leads to its recognition by macrophages. J. Clin. Invest. 1989. 83: 865-875. 
8 Colotta, F., Re, F., Polentarutti, N., Sozzani, S. and Mantovani, A., Modulation of granulocyte survival and programmed cell death by cytokines and bacterial products. Blood 1992. 80: 2012-2020.

9 Walsh, G. M., Sexton, D. W., Blaylock, M. G. and Convery, C. M., Resting and cytokine-stimulated human small airway epithelial cells recognize and engulf apoptotic eosinophils. Blood 1999. 94: $2827-2835$.

10 Matthews, J. R. and Hay, R. T., Regulation of the DNA binding activity of NF-kB. Int. J. Biochem. Cell Biol. 1995. 27: 865-879.

11 McKay, L. I. and Cidlowski, J. A., Molecular control of immune/ inflammatory responses: interactions between nuclear factor-kB and steroid receptor-signaling pathways. Endocr. Rev. 1999. 20: 435-459.

12 Karin, M., The beginning of the end: $I_{K} B$ kinase $(\mathrm{IKK})$ and NF-KB activation. J. Biol. Chem. 1999. 274: 27339-27342.

13 Ward, C., Chilvers, E. R., Lawson, M. F., Pryde, J. G., Fujihara, S., Farrow, S. N., Haslett, C. and Rossi, A. G., NF-кB activation is a critical regulator of human granulocyte apoptosis in vitro. J. Biol. Chem. 1999. 274: 4309-4318.

14 Ward, C., Dransfield, I., Chilvers, E. R., Haslett, C. and Rossi, A. G., Pharmacological manipulation of granulocyte apoptosis: potential therapeutic targets. Trends Pharmacol. Sci. 1999. 20: 503-509.

15 Cassatella, M. A., Neutrophil-derived proteins: selling cytokines by the pound. Adv. Immunol. 1999. 73: 369-509.

16 O'Flaherty, J. T., Rossi, A. G., Redman, J. F. and Jacobson, D. P., Tumor necrosis factor- $\alpha$ regulates expression of receptors for formyl-methionyl-leucyl-phenylalanine, leukotriene B4, and platelet-activating factor. Dissociation from priming in human polymorphonuclear neutrophils. J. Immunol. 1991. 147: 3842-3847

17 O'Flaherty, J. T. and Rossi, A. G., 5-hydroxyicosatetraenoate stimulates neutrophils by a stereospecific, G protein-linked mechanism. J. Biol. Chem. 1993. 268: 14708-14714.

18 Murray, J., Barbara, J. A. J., Dunkley, S. A., Lopez, A. F., Van Ostade, X., Condliffe, A. M., Dransfield, I., Haslett, C. and Chilvers, E. R., Regulation of neutrophil apoptosis by tumo necrosis factor-alpha: requirement for TNFR55 and TNFR75 for induction of apoptosis in vitro. Blood 1997. 90: 2772-2783.

19 Tsukahara, K., Nakao, A., Hiraguri, M., Miike, S., Mamura, M. Saito, Y. and Iwamoto, I., Tumor necrosis factor- $\alpha$ mediates antiapoptotic signals partially via p38 MAP kinase activation in human eosinophils. Int. Arch. Allergy Immunol. 1999. 120 (Suppl 1): 54-59.

20 Temkin, V. and Levi-Schaffer, F. Mechanism of tumour necrosis factor alpha mediated eosinophil survival. Cytokine 2001. 15: 20-26.

21 Yamashita, N., Koizumi, H., Murata, M., Mano, K. and Ohta, K., Nuclear factor $\mathrm{kB}$ mediates interleukin-8 production in eosinophils. Int. Arch. Allergy Immunol. 1999. 120: 230-236.

22 McDonald, P. P., Bald, A. and Cassatella, A. A., Activation of the NF- $\mathrm{kB}$ pathway by inflammatory stimuli in human neutrophils. Blood 1997. 89: 3421-3433.

23 Traenckner, E.-B., Wilk, S. and Baeuerle, P. A., A proteasome inhibitor prevents activation of NF-kB and stabilizes a newly phosphorylated form of $1 \mathrm{kB}-\alpha$ that is still bound to NF-kB. EMBO J. 1994. 13: 5433-5441.

24 Pahl, H. L., Krauß B., Schulze-Osthoff, K., Decker, T. Traeneckner, E. B., Vogt, M., Myers, C., Parks, T., Warring, P. Mühlbacher, A., Czernilofsky, A.-P. and Baeuerle, P. A., The immunosuppressive fungal metabolite gliotoxin specifically inhibits transcription factor NF-кB. J. Exp. Med. 1996. 183: 1829-1840.

25 Sutton, P., Newcombe, N. R., Waring, P. and Müllbacher, A., In vivo immunosuppressive activity of gliotoxin, a metabolite produced by human pathogenic fungi. Infect. Immun. 1994. 62: 1192-1198.

26 Roebuck, K. A., Regulation of interleukin-8 gene expression. J. Interferon Cytokine Res. 1999. 19: 429-438.

27 Takanaski, S., Nonaka, R., Xing, Z., O'Byrne, P., Dolovich, J. and Jordana, M., Interleukin 10 inhibits lipopolysaccharideinduced survival and cytokine production by human peripheral blood eosinophils. J. Exp. Med. 1994. 180: 711-715.

28 Baggiolini, M., Imboden, P. and Detmers, P., Neutrophil activation and the effects of interleukin-8/neutrophil-activating peptide 1 (IL-8/NAP-1). Cytokines 1992. 4: 1-17.

29 Harada, A., Sekido, N., Akahoshi, T., Wada, T., Mukaida, N. and Matsushima, K., Essential involvement of interleukin-8 (IL-8) in acute inflammation. J. Leukoc. Biol. 1994. 56: 559-564.

30 Whyte, M. K., Savill, J., Meagher, L. C., Lee, A. and Haslett, C., Coupling of neutrophil apoptosis to recognition by macrophages: coordinated acceleration by protein synthesis inhibitors. J. Leukoc. Biol. 1997. 62: 195-202.

31 Rath, P. C. and Aggarwal, B. B., TNF-induced signaling in apoptosis. J. Clin. Immunol. 1999. 19: 350-364.

32 Chuang, P. I., Yee, E., Karsan, A., Winn, R. K. and Harlan, J. M., A1 is a constitutive and inducible Bcl-2 homologue in mature human neutrophils. Biochem. Biophys. Res. Commun. 1998. 249: $361-365$.

33 Hamasaki, A., Sendo, F., Nakayama, K., Ishida, N., Negishi, I., Nakayama, K. and Hatakeyama, S., Accelerated neutrophil apoptosis in mice lacking A1-a, a subtype of the bcl-2-related A1 gene. J. Exp. Med. 1998. 188: 1985-1992.

34 Moulding, D. A., Quayle, J. A., Hart, C. A. and Edwards, S. W., Mcl-1 expression in human neutrophils: regulation by cytokines and correlation with cell survival. Blood 1998. 92: 2495-2502.

35 Evan, G. I., Wyllie, A. H., Gilbert, C. S., Littlewood, T. D., Land, H, Brooks, M., Waters, C. M., Penn, L. Z. and Hancock, D. C., Induction of apoptosis in fibroblasts by c-myc protein. Cell 1992. 69: $119-128$

36 Sarma, V., Lin, Z., Clark, L., Rust, B. M., Tewari, M., Noelle, R. $J$ and Dixit, V. M. Activation of the B cell surface receptor CD40 induces $A 20$, a novel zinc finger protein that inhibits apoptosis. J. Biol. Chem. 1995. 270: 12343-12346.

37 Chu, Z. L., McKinsey, T. A., Liu, L., Gentry, J. J., Malim, M. H. and Ballard, D. W., Suppression of tumor necrosis factorinduced cell death by inhibitor of apoptosis C-IAP2 is under NFкB control. Proc. Natl. Acad. Sci. USA 1997. 94: 10057-10062.

38 Wang, C. Y., Mayo, M. W., Korneluk, R. G., Goeddel, D. V. and Baldwin, A. S. Jr., NF- $\mathrm{kB}$ antiapoptosis: induction of TRAF1 and TRAF2 and C-IAP1 and C-IAP2 to suppress caspase-8 activation. Science 1998. 281: 1680-1683.

39 Wu, M. X., Ao, Z., Prasad, K. V., Wu, R. and Schlossman, S. F., IEX-1L, an apoptosis inhibitor involved in NF-kB-mediated cell survival. Science 1998. 281: 998-1001.

40 Yang, L., Cohn, L., Zhang, D. H., Homer, R., Ray, A. and Ray, P., Essential role of nuclear factor $\mathbf{k B}$ in the induction of eosinophilia in allergic airway inflammation. J. Exp. Med. 1998. 188: 1739-1750.

41 Haslett, C., Guthrie, L. A., Kopaniak, M. M., Johnston, R. B. Jr. and Henson, P. M., Modulation of multiple neutrophil functions 
by preparative methods or trace concentrations of bacterial lipopolysaccharide. Am. J. Pathol. 1985. 119: 101-110.

42 Rossi, A. G., Haslett, C., Hirani, N., Greening, A. P., Rahman, I. Metz, C. N., Bucala, R. and Donnelly, S. C., Human circulating eosinophils secrete macrophage migration inhibitory factor (MIF). Potential role in asthma. J. Clin. Invest. 1998. 101: 2869-2874.

43 Rodriguez, M. S., Thompson, J., Hay, R. T. and Dargemont, C., Nuclear retention of IKBalpha protects it from signal-induced degradation and inhibits nuclear factor $\mathrm{\kappa} B$ transcriptional activation. J. Biol. Chem. 1999. 274: 9108-9115.
44 Deptala, A., Bendner, E., Gorczyca, W. and Darzynkiewicz, Z., Activation of nuclear factor $\mathrm{\kappa} B(\mathrm{NF}-\mathrm{\kappa B})$ assayed by laser scanning cytometry (LSC). Cytometry 1998. 33: 376-382.

Correspondence: Adriano G. Rossi, Rayne Laboratory, Respiratory Medicine Unit, Centre for Inflammation Research, Department of Medicine, University of Edinburgh Medical School, Teviot Place, Edinburgh EH8 9AG, GB

Fax: +44-131-650-3484

e-mail: a.g.rossi@ed.ac.uk 


\title{
Prostaglandin $\mathrm{D}_{2}$ and Its Metabolites Induce Caspase-Dependent Granulocyte Apoptosis That Is Mediated Via Inhibition of $I \kappa B \alpha$ Degradation Using a Peroxisome Proliferator-Activated Receptor- $\gamma$-Independent Mechanism ${ }^{1}$
}

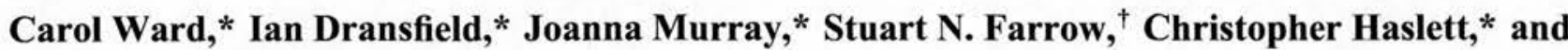 \\ Adriano G. Rossi ${ }^{2 *}$
}

\begin{abstract}
Many inflammatory mediators retard granulocyte apoptosis. Most natural PGs studied herein (e.g., PGE $_{2}$, PGA $_{2}$, PGA $_{1}$, PGF $_{2 \alpha}$ ) either delayed apoptosis or had no effect, whereas $\mathrm{PGD}_{2}$ and its metabolite $\mathrm{PGJ}_{2}$ selectively induced eosinophil, but not neutrophil apoptosis. This novel proapoptotic effect does not appear to be mediated via classical PG receptor ligation or by elevation of intracellular cAMP or $\mathrm{Ca}^{2+}$. Intriguingly, the sequential metabolites $\Delta^{12} \mathrm{PGJ}_{2}$ and 15-deoxy- $\Delta^{12,} \Delta^{14}-\mathrm{PGJ}_{2}\left(\mathbf{1 5 d P G J}_{2}\right)$ induced caspase-dependent apoptosis in both granulocytes, an effect that did not involve de novo protein synthesis. Despite the fact that $\Delta^{12}$ PGJ $_{2}$ and 15dPGJ $J_{2}$ are peroxisome proliferator-activated receptor- $\gamma$ (PPAR- $\gamma$ ) activators, apoptosis was not mimicked by synthetic PPAR- $\gamma$ and PPAR- $\alpha$ ligands or blocked by an irreversible PPAR- $\gamma$ antagonist. Furthermore, $\Delta^{12}$ PGJ $_{2}$ and $^{15 d P G J}{ }_{2}$ inhibited LPS-induced $I \kappa B \alpha$ degradation and subsequent inhibition of neutrophil apoptosis, suggesting that apoptosis is mediated

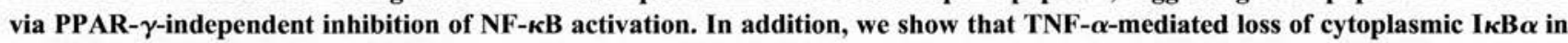
eosinophils is inhibited by $15 \mathrm{dPGJ}_{2}$ in a concentration-dependent manner. The selective induction of eosinophil apoptosis by PGD $_{2}$ and $\mathbf{P G J}_{2}$ may help define novel therapeutic pathways in diseases in which it would be desirable to specifically remove eosinophils but retain neutrophils for antibacterial host defense. The powerful proapoptotic effects of $\Delta^{12} \mathrm{PGJ}_{\mathbf{2}}$ and $15 \mathrm{dPGJ}_{\mathbf{2}}$ in both granulocyte types suggest that these natural products control the longevity of key inflammatory cells and may be relevant to understanding the control and resolution of inflammation. The Journal of Immunology, 2002, 168: 6232-6243.
\end{abstract}

$\mathrm{N}$ eutrophilic and eosinophilic granulocytes are key effector cells in host defense against bacteria and parasites, respectively; however, the over-recruitment, uncontrolled activation, or defective removal of these cells plays a prominent role in the initiation and propagation of chronic inflammatory diseases. Granulocyte apoptosis occurs as part of the normal resolution process, rendering these cells unresponsive to subsequent stimulation but allowing recognition by phagocytes (e.g., macrophages) by a silent mechanism that does not cause the release of phagocyte proinflammatory mediators (1-4). Indeed, Fadok et al. (5) have shown that anti-inflammatory agents such as TGF- $\beta$ are released. At sites of inflammation, granulocytes are exposed to a plethora of different inflammatory mediators, most of which not only influence granulocyte responsiveness but also prolong their functional longevity by delaying apoptosis (6). A notable exception to this rule is the acceleration of apoptosis by the proinflam-

*Rayne Laboratory, Respiratory Medicine Unit, Medical Research Council Center for Inflammation Research, University of Edinburgh Medical School, Edinburgh, United Kingdom; and ${ }^{\dagger}$ Cell Biology Unit, GlaxoSmithKline, Stevenage, United Kingdom

Received for publication December 26, 2001. Accepted for publication April 17, 2002.

The costs of publication of this article were defrayed in part by the payment of page charges. This article must therefore be hereby marked advertisement in accordance with 18 U.S.C. Section 1734 solely to indicate this fact.

${ }^{1}$ This work was supported in part by Medical Research Council Program Grant G9016491.

${ }^{2}$ Address correspondence and reprint requests to Dr. Adriano G. Rossi, Rayne Laboratory, Respiratory Medicine Unit, Medical Research Council Center for Inflammation Research, University of Edinburgh Medical School, Teviot Place, Edinburgh, EH8 9AG U.K. E-mail address: a.g.rossi@ed.ac.uk matory mediator TNF- $\alpha$ after exposure of neutrophils to this cytokine (7), particularly in the presence of NF- $\kappa \mathrm{B}$ inhibitors (8).

PGs, a group of $\mathrm{C}_{20}$ carboxylic acids containing a cyclopentane ring, have been unequivocally shown to play a prominent role in the inflammatory process; however, their effects on granulocyte apoptosis have not been fully examined. Prostanoid formation occurs when cyclooxygenase oxygenates arachidonate converting it to $\mathrm{PGG}_{2}$, which is then reduced to $\mathrm{PGH}_{2}$. $\mathrm{PGH}_{2}$, in turn, is converted to five primary active metabolites, $\mathrm{PGD}_{2}, \mathrm{PGE}_{2}, \mathrm{PGF}_{2} \alpha$, $\mathrm{PGI}_{2}$, or thromboxane $\mathrm{A}_{2}$ via distinct synthases (9-11). $\mathrm{PGE}_{1}$ and $\mathrm{PGE}_{2}$ can be metabolized to $\mathrm{PGA}_{1}$ and $\mathrm{PGA}_{2}(12)$, whereas $\mathrm{PGD}_{2}$ sequentially forms metabolites of the $\mathrm{J}$ series, 9-deoxy- $\Delta^{9} \mathrm{PGD}_{2}$ $\left(\mathrm{PGJ}_{2}\right), \Delta^{12} \mathrm{PGJ}_{2}$, and 15-deoxy- $\Delta^{12}, \Delta^{14} \mathrm{PGJ}_{2}\left(15 \mathrm{dPGJ}_{2}\right)^{3}$ (13). Both neutrophils and eosinophils synthesize, to varying degrees, some of these PGs and are capable of responding to specific PGs by interaction with their cognate classical seven-transmembrane prostanoid receptors $(14,15)$. Interestingly, $\mathrm{PGD}_{2}$, which is produced by both neutrophils and eosinophils $(16,17)$, is also generated by Ag-stimulated human Th2 cells (18) and is the major arachidonic acid metabolite released from activated human mast cells (19). Thus, this PG is considered to be an important mediator of allergic disorders such as allergic rhinitis and is present after $\mathrm{Ag}$ challenge in the airways of patients with asthma (20). Although $\mathrm{PGD}_{2}$ binds preferentially to the $\mathrm{PGD}_{2}$ receptor (DP receptor), it

\footnotetext{
${ }^{3}$ Abbreviations used in this paper: $15 \mathrm{dPGJ}_{2}, 15-$ deoxy- $\Delta^{12}, \Delta^{14}-\mathrm{PGJ}_{2} ;\left[\mathrm{Ca}^{2+}\right]_{i}$, cytosolic free $\mathrm{Ca}^{2+}$ concentration; IKK, I $\kappa \mathrm{B}$ kinase; $\mathrm{PGJ}_{2}$, 9-deoxy- $\Delta^{9}-\mathrm{PGD}_{2}$; DP receptor, $\mathrm{PGD}_{2}$ receptor; IP receptor, prostacyclin receptor; TP receptor, thromboxane $\mathrm{A}_{2}$ receptor; FP receptor, $\mathrm{PGF}_{2 \alpha}$ receptor; EP receptor, $\mathrm{PGE}$ receptor; PPAR, peroxisome proliferator-activated receptor; z-VAD-fmk, benzylocarbonylvalylalanylaspartyl fluoromethylketone; dbcAMP, dibutyryl cAMP.
} 
can also bind to other PG receptors, thereby triggering several different signaling pathways. For example, binding to the DP receptor increases intracellular cAMP and/or cytosolic free $\mathrm{Ca}^{2+}$ concentration $\left(\left[\mathrm{Ca}^{2+}\right]_{i}\right.$ ), whereas PGE receptor (EP receptor) 1 , $\mathrm{PGF}_{2 \alpha}$ receptor (FP receptor), or thromboxane $\mathrm{A}_{2}$ receptor (TP receptor) ligation increases $\left[\mathrm{Ca}^{2+}\right]_{i}$ but has no direct effect on cAMP levels. Prostacyclin receptor (IP receptor) activation also increases intracellular cAMP $(9,21-23)$. The metabolites of $\mathrm{PGD}_{2}$, $\Delta^{12} \mathrm{PGJ}_{2}$ and $15 \mathrm{dPGJ}_{2}$, have been shown to activate intracellular peroxisome proliferator-activated receptors (PPARs) which are transducer proteins belonging to the steroid/thyroid/retinoid receptor superfamily that regulate target genes by binding to PPAR response elements $(24,25)$. Three isoforms of PPAR (PPAR- $\gamma$, PPAR- $\delta$, and PPAR- $\alpha$ ) are present in human cells (26-28). PPAR- $\alpha$ is primarily expressed in tissues with high fatty acid catabolism; PPAR- $\gamma$ is expressed in adipose tissue, adrenal gland, spleen, and several myeloid cell lines; and PPAR- $\delta$ is highly expressed in heart, kidney, and intestine (29-31).

In this study, we demonstrate differential effects of $\mathrm{PGD}_{2}$ on granulocyte apoptosis; selectively inducing eosinophil but not neutrophil apoptosis. This intriguing result prompted us to examine the actions of this PG and its metabolites more closely. We show that the $\mathrm{PGD}_{2}$ metabolites, $\Delta^{12} \mathrm{PGJ}_{2}$ and $15 \mathrm{dPGJ}_{2}$, are powerful inducers of caspase-dependent granulocyte apoptosis. These data could not be mimicked using synthetic PPAR- $\gamma$ agonists such as the thiazolidinediones BRL49653 and ciglitazone (32), nor could the PPAR- $\gamma$ antagonist GW9662 (33) prevent induction of cell death by $15 \mathrm{dPGJ}_{2}$. PGs of the $\mathrm{J}$ series also activate PPAR- $\alpha$; however, a synthetic ligand to this isoform, pirinixic acid (WY-14643), had no effect on granulocyte apoptosis. We also rule out a significant proapoptotic role for the other classical cell surface PG receptors in both neutrophils and eosinophils. We have recently shown that NF- $\kappa \mathrm{B}$ plays a critical role in the regulation of granulocyte apoptosis where specific inhibition of NF- $\kappa$ B can directly induce granulocyte apoptosis and can enhance apoptosis induced by TNF- $\alpha$ and block the delayed apoptosis induced by LPS (8). In view of the suggestion that PPAR- $\gamma$ ligands may inhibit NF- $\kappa$ B activation, we examine whether the effects of $\Delta^{12} \mathrm{PGJ}_{2}$ and $15 \mathrm{dPGJ}_{2}$ are mediated via inhibition of this transcription factor. We demonstrate in the neutrophil that $\mathrm{PGD}_{2}$ metabolites inhibit LPS-induced degradation of $\mathrm{I} \kappa \mathrm{B} \alpha$ (the inhibitory subunit of NF$\kappa \mathrm{B})$ and block LPS-mediated inhibition of apoptosis. Furthermore, TNF- $\alpha$-induced I $\kappa \mathrm{B} \alpha$ breakdown in the eosinophil is also inhibited by $\mathrm{PGD}_{2}$ metabolites. We conclude that the PPAR- $\gamma$ ligands $\Delta^{12} \mathrm{PGJ}_{2}$ and $15 \mathrm{dPGJ}_{2}$ influence granulocyte apoptosis by interfering with the prosurvival NF- $\kappa \mathrm{B}$ pathway, an effect that is independent of PPAR- $\gamma$ ligation.

\section{Materials and Methods \\ Neutrophil and eosinophil isolation and culture}

Neutrophils and eosinophils were isolated from the peripheral blood of normal donors by dextran sedimentation followed by centrifugation through discontinuous Percoll gradients (Amersham Pharmacia Biotech, Little Chalfont, U.K.) $(34,35)$. Only neutrophil preparations with a cell purity of $>98 \%$ were used. Eosinophils were separated from contaminating neutrophils using immunomagnetic separation with sheep anti-mouse IgGDynabeads (Dynabeads M-450; Dynal, Merseyside, U.K.) coated with the murine anti-neutrophil Ab 3G8 (anti-CD16; a gift from Dr. J. Unkeless, Mount Sinai Medical School, New York, NY). Cells were mixed with washed $\mathrm{Ab}$-coated magnetic beads at a bead-neutrophil ratio of $3: 1$ on a rotary mixer at $4^{\circ} \mathrm{C}$ for $20 \mathrm{~min}$, and the beads were removed magnetically by two 3-min stationary magnetic contacts (Dynal Magnetic Particle Concentrator, MPC-1) to yield an eosinophil population of $>98 \%$ purity. After purification, cells were washed twice in PBS without $\mathrm{Ca}^{2+}$ and $\mathrm{Mg}^{2+}$ before resuspending in IMDM (Life Technologies, Paisley, U.K.) supplemented with $10 \%$ autologous serum (unless otherwise stated in figures)
Both cell types were cultured in flat-bottom Falcon flexible well plates (BD Biosciences, Oxford, U.K.) at $37^{\circ} \mathrm{C}$ in a $5 \% \mathrm{CO}_{2}$ atmosphere; or in $2 \mathrm{ml}$ round-bottom Eppendorf tubes in a shaking water bath at $37^{\circ} \mathrm{C}$, with neutrophils $5 \times 10^{6} / \mathrm{ml}$ and eosinophils $2 \times 10^{6} / \mathrm{ml}$. Cells were cultured in the absence or presence of test agents as described in the figures. All experiments were performed at least three times in triplicate.

\section{Assessment of granulocyte apoptosis}

Morphology. Cells were cytocentrifuged, fixed in methanol, stained with DiffQuik (Gamidor, Abingdon, U.K.) and counted using oil immersion microscopy ( $\times 100$ objective) to determine the proportion of cells with distinctive apoptotic morphology (8). At least 500 cells were counted per slide with the observer blinded to the experimental conditions. The results were expressed as the mean percent apoptosis \pm SEM

Annexin V binding and propidium iodide staining. A separate and independent assessment of apoptosis was performed by flow cytometry using FITC-labeled recombinant human annexin V that binds to phosphatidylserine exposed on the surface of apoptotic cells and propidium iodide as an index of loss of cell membrane integrity (8). Stock annexin V (Bender MedSystems, Vienna, Austria) was diluted 1/200 with binding buffer and then added $(25 \mu \mathrm{l})$ to $75 \mu \mathrm{l}$ of the recovered cell samples. After a 10-min incubation at $4^{\circ} \mathrm{C}$, these samples were treated with propidium iodide (final concentration, $10 \mu \mathrm{g} / \mathrm{ml}$ ) for $2 \mathrm{~min}$ before flow cytometric analysis using an EPICS XL2.

DNA fragmentation assay. Cells were lysed, DNA was extracted and run on an agarose gel containing ethidium bromide, and DNA fragmentation (laddering) was visualized as described (36).

\section{Assessment of membrane integrity and cell recovery}

To ensure that the cell death observed was due solely to apoptosis, the membrane integrity of treated and untreated cells was assessed using the vital dye trypan blue. In addition, cells were counted at the start of culture and at the end of the indicated period. Under all conditions and treatments used, there was no loss of cell membrane integrity, and cell loss was minimal.

\section{Measurement of $\left[\mathrm{Ca}^{2+}\right]_{i}$}

Freshly isolated granulocytes were washed (three times) in $\mathrm{HBSS}\left(\mathrm{Ca}^{2+}\right.$ and $\mathrm{Mg}^{2+}$ free) before being resuspended at $10^{7} / \mathrm{ml}$ in $\mathrm{HBSS}\left(\mathrm{Ca}^{2+}\right.$ and $\mathrm{Mg}^{2+}$ free), for incubation with fura 2-acetoxymethyl ester (final concentration, $2 \mu \mathrm{M})$ for $30 \mathrm{~min}$ at $37^{\circ} \mathrm{C}(37,38)$. The cells were then washed (twice) to remove fura 2-acetoxymethyl ester and left in $\mathrm{HBSS}\left(\mathrm{Ca}^{2+}\right.$ and $\mathrm{Mg}^{2+}$ free) for a further $10 \mathrm{~min}$ for optimal deesterification, before finally resuspending the granulocytes at $2 \times 10^{6} / \mathrm{ml}$ in HBSS (containing $\mathrm{Ca}^{2+}$ and $\left.\mathrm{Mg}^{2+}\right)$. Changes in fluorescence upon agonist addition were determined using a PerkinElmer (Wellesley, MA) LS50B fluorometer, with dual wavelength excitation ( 340 and $380 \mathrm{~nm}$ ) and emission at $510 \mathrm{~nm}$, fitted with a thermostated cuvette compartment and stirring attachment, to ensure complete mixing of reagents. $\left[\mathrm{Ca}^{2+}\right]_{\mathrm{i}}$ was calibrated as previously described $(37,38)$

\section{Western blotting for $I \kappa B \alpha$}

Cell samples $\left(5 \times 10^{6} / \mathrm{ml}\right)$ were incubated in a shaking water bath at $37^{\circ} \mathrm{C}$ with the agents of interest as described in the figure legends. After treatment, cells were immediately placed on ice, and all lysates were prepared at $4{ }^{\circ} \mathrm{C}$. To minimize problems with proteolysis, lysates were prepared by methods normally used for EMSA preparations (8). Lysates were run on a $9 \%$ SDS gel and, after transfer, blocked by $5 \%$ milk protein before an overnight incubation with primary $\mathrm{I} \kappa \mathrm{B} \alpha \mathrm{Ab}$ (New England Biolabs, Beverly, MA) diluted $1 / 500$. After washing, blots were incubated with HRPconjugated anti-biotin $\mathrm{Ab}$ diluted at $1 / 2500$ and developed using standard ECL reagents (Amersham, Arlington Heights, IL).

\section{Other materials}

Further specific materials were obtained as follows: LPS (Escherichia coli 0127:B8) (Sigma, Poole, U.K.); benzylocarbonylvalylalanylaspartyl fluoromethylketone (z-VAD-fmk; Bachem U.K., Saffron Walden, U.K.); $\Delta^{12} \mathrm{PGJ}_{2}, 15 \mathrm{dPGJ}_{2}, \mathrm{PGJ}_{2}, \mathrm{PGD}_{2}, \mathrm{PGA}_{2}, \mathrm{PGA}_{1}, \mathrm{U} 46619$, ciglitazone, and WY-14643 (Biomol; Affinity Research Products, Mamhead, U.K.); recombinant human TNF- $\alpha$ (R\&D Systems, Abingdon, U.K.). GW9662 was a gift from T. Willson (Glaxo Wellcome, Research Triangle Park, NC), and BRL49653 was a gift from K. Chaterjee (University of Cambridge, Cambridge, U.K.). All other reagents were obtained from Sigma U.K. and were of the highest purity. 


\section{Statistical analysis}

The results are expressed as mean \pm SEM of the number $(n)$ of independent experiments each using cells from separate donors with each treatment performed in triplicate. Statistical analysis was performed by ANOVA with comparisons between groups made using the Newman-Kuels procedure. Differences were considered significant when $p<0.05$.

\section{Results}

Most PG either inhibit or have no direct effect on granulocyte apoptosis

The effects of PGs on granulocyte apoptosis are shown in Table I. $\mathrm{PGE}_{2}$ and 11-deoxyPGE $\mathrm{PG}_{1}$ delayed neutrophil apoptosis as did the dehydration product of $\mathrm{PGE}_{1}, \mathrm{PGA}_{1}$. $\mathrm{PGA}_{2}$ had no significant effect on apoptosis in either cell type, whereas $\mathrm{PGF}_{2 \alpha}$ decreased the rate of constitutive apoptosis in both neutrophils and eosinophils. The thromboxane $A_{2}$ mimetic $U 46619$ had no direct effect on cell death. Only $\mathrm{PGD}_{2}$ increased the constitutive rate of esinophil apoptosis

\section{$\mathrm{PGD}_{2}$ has differential effects on neutrophil and eosinophil apoptosis}

$\mathrm{PGD}_{2}$ did not enhance the rate of constitutive neutrophil apoptosis at $20 \mathrm{~h}$ (Fig. $1 \mathrm{~A}$ ) or at earlier time points (e.g., 2, 3, 4, or $6 \mathrm{~h}$ ) when basal levels of apoptosis are much lower (data not shown). However, $\mathrm{PGD}_{2}$ significantly increased the rate of eosinophil apoptosis, after both 20 and $40 \mathrm{~h}$ in culture, with levels of apoptosis in treated cells being $\sim 4$ times higher than those in control untreated cells (Fig. 1B). As a further control in these experiments, when neutrophils were cultured at the same density as eosinophils $\left(2 \times 10^{6} \%\right.$ $\mathrm{ml}$ ), $\mathrm{PGD}_{2}$ still did not induce neutrophil apoptosis (control, $85.9 \pm 4.6 ; \mathrm{PGD}_{2}(10 \mu \mathrm{M}), 87.9 \pm 1.3(n=3$, each experiment performed in triplicate)). To directly demonstrate the efficacy of $\mathrm{PGD}_{2}$ on eosinophils, apoptosis was induced by $\mathrm{PGD}_{2}$ and compared with apoptosis induced by maximal concentrations of dexamethasone $(1 \mu \mathrm{M})$, an established accelerator of eosinophil apoptosis (39), during concurrent experiments on cells from the same donor. $\mathrm{PGD}_{2}(10 \mu \mathrm{M})$ induced apoptosis to a greater extent than dexamethasone at both time points $(20$ and $40 \mathrm{~h}$ ) tested (Fig. 1B). In addition, this increase in apoptosis appeared to be restricted to eosinophils because, as shown in Table II, further studies using other immune cells determined that $\mathrm{PGD}_{2}$ did not induce cell death in Jurkat T lymphocytes or human PBL. Only the J series metabolite $\Delta^{12} \mathrm{PGJ}_{2}$ substantially induced apoptosis in the Jurkat cell line. Furthermore, $\mathrm{PGD}_{2}$ did not influence the constitutive rates of apoptosis in either peripheral blood monocytes or monocyte-derived macrophages after $20 \mathrm{~h}$ of culture (data not shown).

\section{Role of the DP receptor in the regulation of gramulocyte apoptosis}

$\mathrm{PGD}_{2}$ can be metabolized to biologically active breakdown products. Therefore, to further investigate the possible mechanism of $\mathrm{PGD}_{2}$-mediated acceleration of eosinophil apoptosis, we used a stable $\mathrm{PGD}_{2}$ mimetic $(5 Z, 13 E)-(9 R, 11 R, 15 S)$-9-chloro-15-hydroxy16,17,18,19,20-pentano-3-oxa-5,13-prostadienoic acid (ZK 118.182)) (40). This compound allowed investigation of the consequences of activating the DP receptor without the production of active $\mathrm{PGD}_{2}$ metabolites. In contrast to $\mathrm{PGD}_{2}, \mathrm{ZK} 118.182$ significantly inhibited apoptosis in neutrophils (Fig. 1C) while increasing eosinophil apoptosis at $40 \mathrm{~h}$ to a small but significant extent; however, the effect was minimal in comparison to levels of cell death induced by $\mathrm{PGD}_{2}$ (Fig. $1 D$ ). Thus, these disparate results suggest that ligation of the DP receptor is unlikely to account for the proapoptotic effects observed with $\mathrm{PGD}_{2}$.

The increase in eosinophil apoptosis is not explained by binding to other surface $P G$ receptors

$\mathrm{PGD}_{2}$ at the concentrations used in this study, has the ability to bind other PG receptors, namely, the EP, FP, IP, and TP receptors (9). We therefore examined the effects of agonist binding to these receptors to determine whether the increase in apoptosis observed with $\mathrm{PGD}_{2}$ could be reproduced. The FP receptor binds $\mathrm{PGF}_{2 \alpha}$, which inhibits several functional activities in human neutrophils $(41,42)$; however, little is known of its effects on eosinophils. As shown in Table I, PGF $_{2 \infty}$, significantly increased neutrophil survival, and by $40 \mathrm{~h}$ of culture, eosinophil apoptosis was almost $50 \%$ of control values. Had $\mathrm{PGD}_{2}$ been acting via the FP receptor, apoptosis should have been inhibited in both eosinophils and neutrophils.

The use of U-46619, a thromboxane $A_{2}$ receptor agonist, demonstrated that activation of putative TP receptors on granulocytes had no significant effect on apoptosis in either neutrophils or eosinophils (Table $\mathrm{I}$ ), again suggesting that $\mathrm{PGD}_{2}$ is not acting via this receptor. Activation of the DP, IP, $\mathrm{EP}_{2}, \mathrm{EP}_{3}$, and $\mathrm{EP}_{4}$ receptors has been demonstrated to increase intracellular cAMP levels in many cell types including granulocytes (9). To mimic elevation of cAMP, we used the stable, cell-permeable analog of cAMP, dbcAMP (dbcAMP), and show that this compound powerfully delays apoptosis in both neutrophilic (Fig. 1E) and eosinophilic (Fig. 1F) granulocytes. Therefore, elevation of cAMP by ligation of certain PG receptors cannot explain the dramatic augmentation of apoptosis induced by $\mathrm{PGD}_{2}$.

Ligation of the $\mathrm{EP}_{1}$ receptor has no reported effects on intracellular cAMP levels, but it does increase $\left[\mathrm{Ca}^{2+}\right]_{i}$ in some cell systems. To investigate directly whether $\mathrm{PGD}_{2}$ can influence $\left[\mathrm{Ca}^{2+}\right]_{\mathrm{i}}$

Table I. Effect of PG on neutrophil and eosinophil apoptosis ${ }^{a}$

\begin{tabular}{|c|c|c|c|c|c|c|}
\hline \multirow[b]{2}{*}{ Treatment } & \multicolumn{2}{|c|}{ Neutrophils $(20 \mathrm{~h})$} & \multicolumn{2}{|c|}{ Eosinophils $(20 \mathrm{~h})$} & \multicolumn{2}{|c|}{ Eosinophils $(40 \mathrm{~h})$} \\
\hline & Control & Treated & Control & Treated & Control & Treated \\
\hline $\mathrm{PGE}_{2}$ & $65.9 \pm 6.6$ & $45.8 \pm 6.8^{b}$ & $7.7 \pm 1.4$ & $6.6 \pm 3.6$ & $12.8 \pm 1.0$ & $14.0 \pm 4.4$ \\
\hline 11-DeoxyPGE & $65.3 \pm 6.9$ & $50.3 \pm 7.0^{b}$ & $8.4 \pm 2.0$ & $5.4 \pm 1.9$ & $15.0 \pm 4.5$ & $5.6 \pm 1.7^{h}$ \\
\hline $\mathrm{PGA}_{1}$ & $67.3 \pm 1.1$ & $56.1 \pm 0.9^{b}$ & ND & & ND & \\
\hline $\mathrm{PGA}_{2}$ & $71.4 \pm 5.6$ & $64.6 \pm 9.4$ & $7.7 \pm 1.4$ & $10.2 \pm 6.1$ & $12.8 \pm 1.0$ & $18.7 \pm 3.4$ \\
\hline $\mathrm{PGF}_{2 \alpha}$ & $66.0 \pm 6.7$ & $57.7 \pm 8.0^{b}$ & $7.7 \pm 1.4$ & $5.1 \pm 1.9^{b}$ & $12.8 \pm 1.4$ & $6.9 \pm 0.7^{b}$ \\
\hline U 46619 & $70.2 \pm 5.1$ & $70.3 \pm 3.2$ & 11.5 & 9.71 & ND & \\
\hline $\mathrm{PGD}_{2}$ & $61.0 \pm 6.2$ & $65.1 \pm 2.3$ & $10.9 \pm 2.4$ & $41.1 \pm 7.9^{b}$ & $19.9 \pm 4.9$ & $83.7 \pm 5.5^{b}$ \\
\hline
\end{tabular}

${ }^{a}$ Neutrophils $\left(5 \times 10^{6} / \mathrm{ml}\right)$ or eosinophils $\left(2 \times 10^{6} / \mathrm{ml}\right)$ were incubated in IMDM supplemented with serum alone (control) or with PG $(10 \mu \mathrm{M})$ as detailed above and harvested at $20 \mathrm{~h}$ (neutrophils) and 20 and $40 \mathrm{~h}$ (eosinophils). Apoptosis was assessed morphologically. Data represent the mean \pm SEM of at least three separate experiments. All experiments were performed in triplicate.

${ }^{b}$ Significant differences $(p<0.05)$ from the appropriate control. 
A

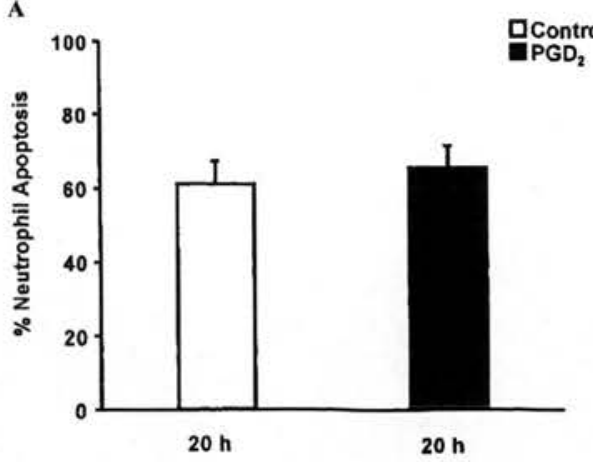

C

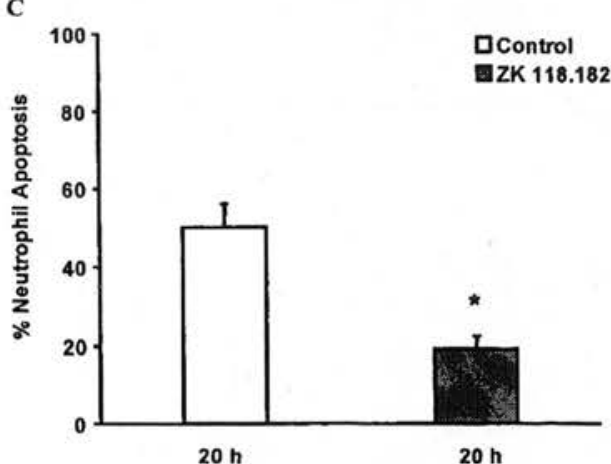

E

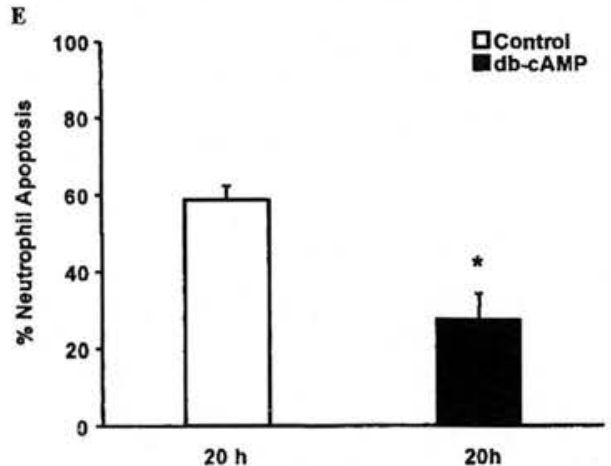

B

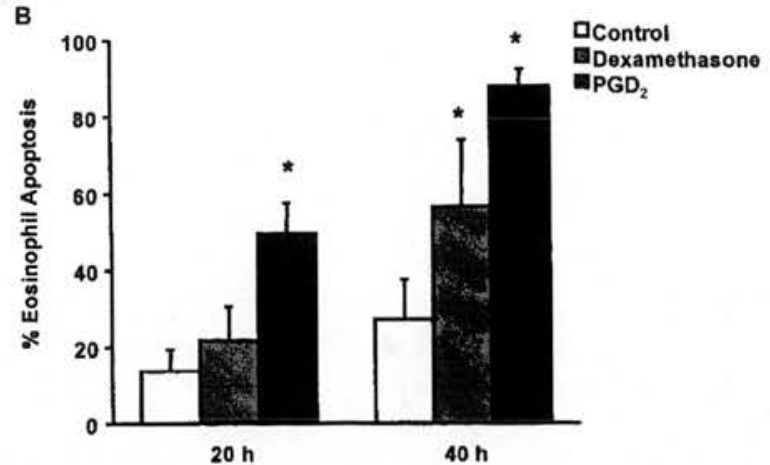

D

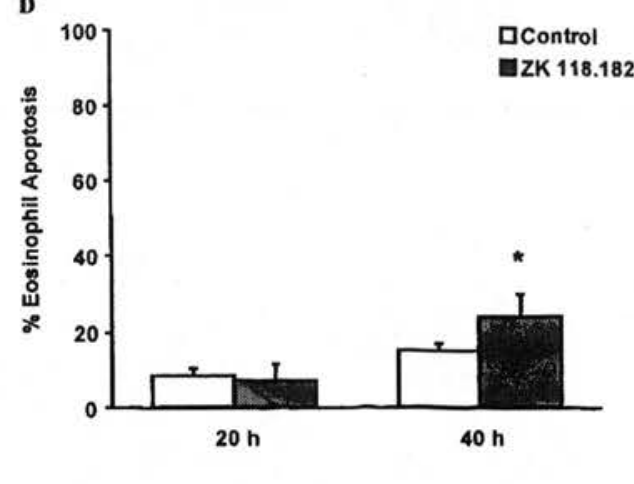

F

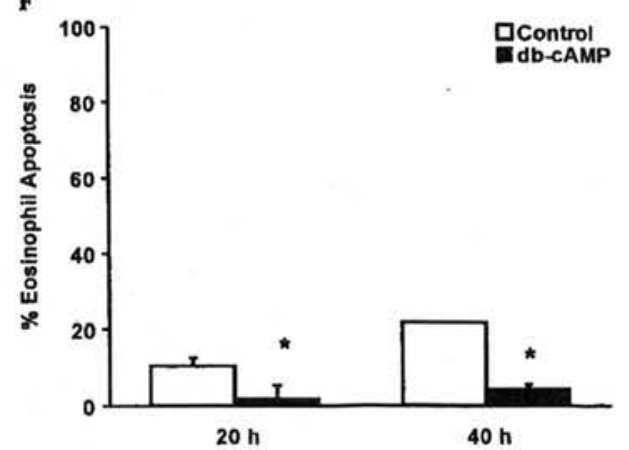

FIGURE 1. Effect of $\mathrm{PGD}_{2}, \mathrm{ZK} 118.182$, and dbcAMP on constitutive granulocyte apoptosis. Neutrophils $\left(A, C\right.$, and $\left.E ; 5 \times 10^{6} / \mathrm{ml}\right)$ and eosinophils $\left(B, D\right.$, and $\left.F ; 2 \times 10^{6} / \mathrm{ml}\right)$ were incubated in IMDM supplemented with serum alone (control) or with the indicated reagent and harvested at $20 \mathrm{~h}$ (neutrophils) and 20 and $40 \mathrm{~h}$ (eosinophils), and apoptosis was assessed morphologically. In all panels, $\square$ is vehicle control-treated cells. $A$, PGD $(10$ $\mu \mathrm{M})$-treated cells $(\mathbf{E}) ; B$, dexamethasone $(1 \mu \mathrm{M})(\square), \mathrm{PGD}_{2}(10 \mu \mathrm{M})(\mathbf{E}) ; C$ and $D, \mathrm{ZK} 118.182(30 \mu \mathrm{M})(\square) ; E$ and $F$, dbcAMP $(0.2 \mathrm{mM})$-treated cells (E). Data represent the mean $\pm \mathrm{SEM}$ of three separate experiments. All experiments were performed in triplicate. $*, p<0.05$ compared with control values.

in granulocytes, we performed studies using fura 2-loaded cells. Neutrophils (Fig. 2) and eosinophils (Fig. 3) respond to stimuli such as platelet-activating factor and leukotriene $\mathrm{B}_{4}$, which act on specific $\mathrm{G}$ protein-coupled receptors by a rapid and reversible elevation of $\left[\mathrm{Ca}^{2+}\right]_{1}$. We found that $\mathrm{PGD}_{2}$ at $10 \mu \mathrm{M}$ induced a rapid and reversible elevation of $\left[\mathrm{Ca}^{2+}\right]_{i}$ in eosinophils but not in neutrophils. These differential effects were mirrored by the $\mathrm{PGD}_{2}$ sequential metabolites $\mathrm{PGJ}_{2}, \Delta 12 \mathrm{PGJ}_{2}$, and $15 \mathrm{dPGJ}_{2}$. Interestingly, in eosinophils there was both homologous and heterologous desensitization between the different $\mathrm{PGD}_{2}$ metabolites and indeed $\mathrm{PGD}_{2}$ itself (Fig. 3 and data not shown). It has been recently reported that $\mathrm{PGD}_{2}$ metabolites are potent selective activators of human eosinophils inducing calcium mobilization, actin polymerization, and CD1 $1 \mathrm{~b}$ expression by interacting with $\mathrm{DP}_{2}(43)$. We have previously reported that increasing levels of $\left[\mathrm{Ca}^{2+}\right]_{i}$ inhibits neutrophil apoptosis while accelerating this process in eosinophils
$(44,45)$. However, use of calcium ionophores and other pharmacological agents (e.g., thapsigargin) that elevate $\left[\mathrm{Ca}^{2+}\right]_{\mathrm{i}}$ also cause degranulation in the eosinophil and cause necrosis rather than apoptosis by $40 \mathrm{~h}$. Neither degranulation nor necrosis was observed in either cell type when treated with $\mathrm{PGD}_{2}$ at any time point examined. This suggests that increases in $\left[\mathrm{Ca}^{2+}\right]_{i}$ were not responsible for the proapoptotic effect of this PG. Therefore, the proapoptotic effect of $\mathrm{PGD}_{2}$ on eosinophils does not appear to be mediated via any known classical cell surface PG receptor or by increasing intracellular cAMP or $\left[\mathrm{Ca}^{2+}\right]_{i}$.

The $P G D_{2}$ metabolites $P G J_{2}, \Delta^{\prime 2} P G J_{2}$, and $15 d P G J_{2}$ induce granulocyte apoptosis

$\mathrm{PGJ}_{2}$, an active $\mathrm{PGD}_{2}$ metabolite, is also capable of selectively triggering the DP receptor $(13,22)$. As demonstrated in Fig. $4 A$, $\mathrm{PGJ}_{2}$ produced results similar to those of $\mathrm{PGD}_{2}$ in neutrophils, 
Table II. Effects of $P G D_{2}$ and $P G D_{2}$ metabolites on lymphocyte apoptosis ${ }^{\circ}$

\begin{tabular}{|c|c|c|c|c|}
\hline \multirow[b]{2}{*}{ Treatment } & \multicolumn{2}{|c|}{$\begin{array}{c}\text { Jurkat } \mathrm{T} \\
\text { Lymphocytes }\end{array}$} & \multicolumn{2}{|c|}{$\begin{array}{l}\text { Human Peripheral } \\
\text { Lymphocytes }\end{array}$} \\
\hline & Mean & SEM & Mean & SEM \\
\hline Control & 2.6 & 0.4 & 2.8 & 0.7 \\
\hline Dexamethasone $(1 \mu \mathrm{M})$ & ND & & $6.8^{b}$ & 0.1 \\
\hline $\mathrm{PGD}_{2}(10 \mu \mathrm{M})$ & 2.2 & 1.0 & 2.9 & 0.4 \\
\hline $\mathrm{PGJ}_{2}(10 \mu \mathrm{M})$ & 2.2 & 0.8 & 3.8 & 0.8 \\
\hline$\Delta 12 \mathrm{PGJ}_{2}(10 \mu \mathrm{M})$ & $17.0^{b}$ & 5.2 & ND & \\
\hline $15 \mathrm{dPGJ}_{2}(10 \mu \mathrm{M})$ & 2.5 & 0.9 & ND & \\
\hline
\end{tabular}

${ }^{a}$ Jurkat T lymphocytes $\left(5 \times 10^{6} / \mathrm{ml}\right)$ in RPMI with $10 \%$ FBS and human peripheral lymphocytes $\left(5 \times 10^{6} / \mathrm{ml}\right)$ in IMDM with $10 \%$ autologous serum were treated with the reagents as indicated and cultured at $37^{\circ} \mathrm{C}$ for $20 \mathrm{~h}$. The cells were then fixed and stained, and apoptosis was determined by morphological assessment. All values are from $n=3-5$ separate experiments, each performed in triplicate.

${ }^{b}$ Represents significance differences $(p<0.05)$ from the appropriate control.

causing no significant change in the rate of constitutive apoptosis at $20 \mathrm{~h}$. However, $\mathrm{PGJ}_{2}$ significantly increased eosinophil apoptosis at 20 and $40 \mathrm{~h}$, but this increase was markedly less than that induced by the parent compound, $\mathrm{PGD}_{2}$, when used at equimolar concentrations (Fig. $4 B$ ).

These results suggested that the differential effect of $\mathrm{PGD}_{2}$ on eosinophil and neutrophil apoptosis might be the result of differences in the metabolic products produced by these two cells. We therefore investigated whether the $\mathrm{PGJ}_{2}$ metabolites $\Delta^{12} \mathrm{PGJ}_{2}$ and $15 \mathrm{dPGJ}_{2}$ could increase levels of constitutive granulocyte apoptosis. Fig. 4, $C$ and $D$, illustrates that these metabolites are efficacious inducers of programmed cell death in both cell types causing increases in eosinophil apoptosis comparable with those observed with $\mathrm{PGD}_{2}$. However, $\Delta^{12} \mathrm{PGJ}_{2}$ and $15 \mathrm{dPGJ}_{2}$ also had powerful proapoptotic effects on neutrophils, increasing control rates from 66 to $\sim 90 \%$ at $20 \mathrm{~h}$. These data support the possibility that neutrophils and eosinophils may metabolize $\mathrm{PGD}_{2}$ differently. The proportion of cells exhibiting classical pyknotic nuclei of apoptotic neutrophils and eosinophils increases markedly in cells treated with $15 \mathrm{dPGJ}_{2}$ and $\Delta^{12} \mathrm{PGJ}_{2}$ even at the early time points of 3-4 h (Fig. 6 and data not shown). When DNA was extracted from granulocytes treated with $\mathrm{PGD}_{2}$ metabolites for short period of culture (e.g. $4 \mathrm{~h}$ ) and run on an agarose gel, a typical DNA ladder pattern was evident whereas there was no DNA "laddering" observed in control cells (data not shown). Moreover, the proportion of granulocytes binding FITC-labeled annexin V (indicative of cell surface changes associated with apoptosis) was increased when cells were treated with $\mathrm{PGD}_{2}$ and its metabolites (Fig. 5). A smaller increase in propidium iodide staining was also observed with the PGs. We believe that this increase is likely due to secondary necrosis, in that failure of apoptotic eosinophil clearance results in these cells quickly undergoing necrosis and it is possible that actual processing of eosinophils during the double staining technique can contribute to an abnormally high level of propidium iodide staining. This latter point is further supported by the observation that we failed to observe any significant uptake of the vital dye trypan blue when the cells are treated with these PGs.

The induction of granulocyte apoptosis by $\Delta^{12} P G J_{2}$ and $15 \mathrm{dPGJ}_{2}$ is independent of synthesis of a death protein but dependent on activation of caspases

To investigate whether the proapoptotic effect of $\Delta^{12} \mathrm{PGJ}_{2}$ and $15 \mathrm{dPGJ}_{2}$ requires synthesis of protein(s) (e.g., a death-inducing protein), cells were cultured with a protein synthesis inhibitor, cycloheximide. Although cycloheximide itself is a potent accelerator

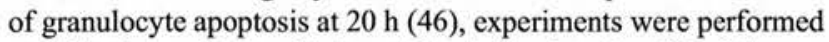
at a 3-h time point at which cycloheximide alone has little influence on this process. Interestingly, the proapoptotic effect of $15 \mathrm{dPGJ}_{2}$ was apparent even at this short incubation period (Fig. 6A). Cycloheximide did not inhibit the induction of cell death but rather produced an additive increase in the levels of apoptosis produced by this metabolite. The increase in apoptosis induced by $15 \mathrm{dPGJ}_{2}$ was attenuated when cells were cotreated with the pan-caspase inhibitor z-VAD-fmk but not by the vehicle control ( $0.02 \%$ DMSO) (Fig. $6 B)$. z-VAD-fmk
FIGURE 2. Effect of $\mathrm{PGD}_{2}, \mathrm{PGJ}_{2}, \Delta 12 \mathrm{PGJ}_{2}$, and $15 \mathrm{dPGJ}_{2}$ on neutrophil $\left[\mathrm{Ca}^{2+}\right]_{\mathrm{i}}$. Fura 2-loaded neutrophils $(2 \times 10 \% \mathrm{ml})$ were stimulated with platelet-activating factor (PAF; $100 \mathrm{nM}), \mathrm{PGD}_{2}(10 \mu \mathrm{M}), \mathrm{PGJ}_{2}(10$ $\mu \mathrm{M}), \Delta 12 \mathrm{PGJ}_{2}(10 \mu \mathrm{M})$, and $15 \mathrm{dPGJ}_{2}(10 \mu \mathrm{M})$ as indicated above. Changes in $\left[\mathrm{Ca}^{2+}\right]_{i}$ were determined as described in Materials and Methods, and data are representative of three separate experiments.
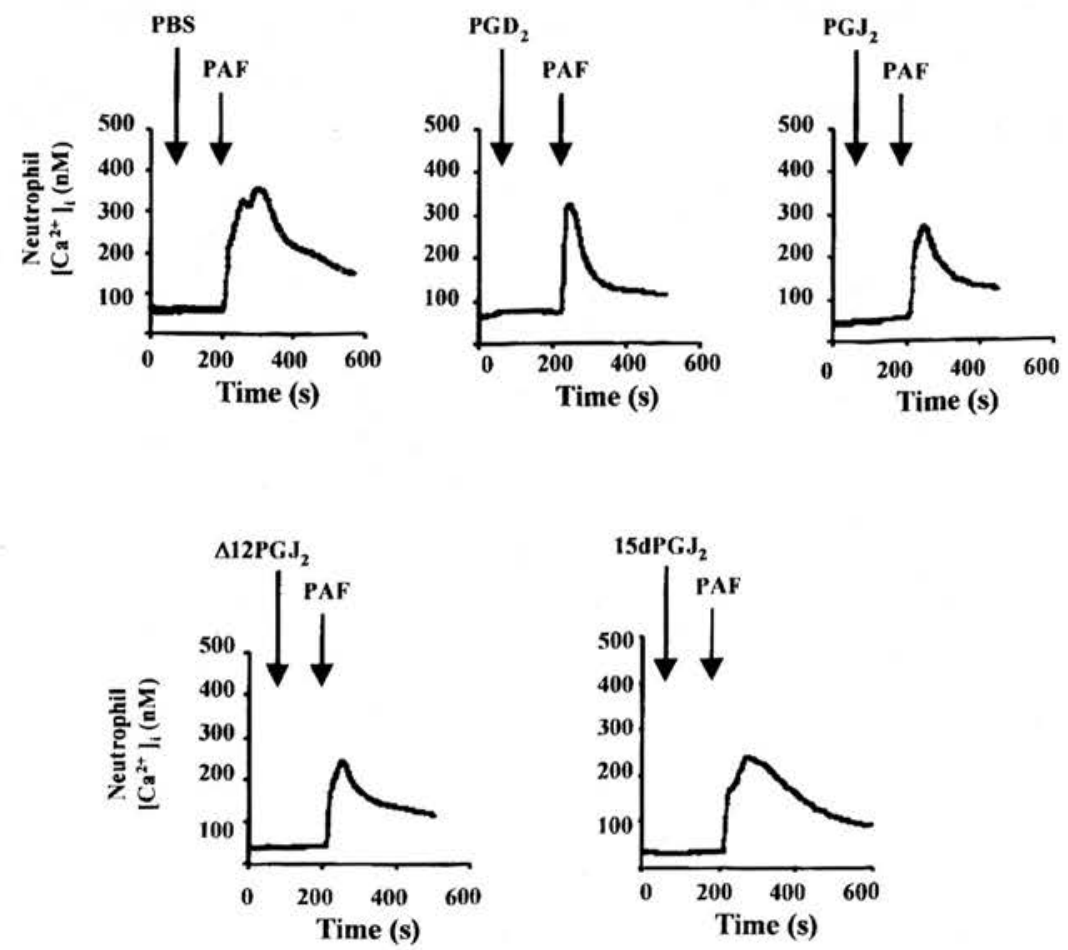
FIGURE 3. Effect of $\mathrm{PGD}_{2}, \mathrm{PGJ}_{2}, \Delta 12 \mathrm{PGJ}_{2}$, and $15 \mathrm{dPGJ}_{2}$ on eosinophil $\left[\mathrm{Ca}^{2+}\right]_{\mathrm{i}}$. Fura 2-loaded eosinophils $(2 \times 10 \% / \mathrm{ml})$ were stimulated with leukotriene $\mathrm{B}_{4}\left(\mathrm{LTB}_{4} ; 100 \mathrm{nM}\right), \mathrm{PGD}_{2}(10 \mu \mathrm{M}), \mathrm{PGJ}_{2}(10$ $\mu \mathrm{M}), \Delta 12 \mathrm{PGJ}_{2}(10 \mu \mathrm{M})$, and $15 \mathrm{dPGJ}_{2}(10 \mu \mathrm{M})$ as indicated above. Changes in $\left[\mathrm{Ca}^{2+}\right]_{i}$ were determined as described in Materials and Methods, and data are representative of three separate experiments.
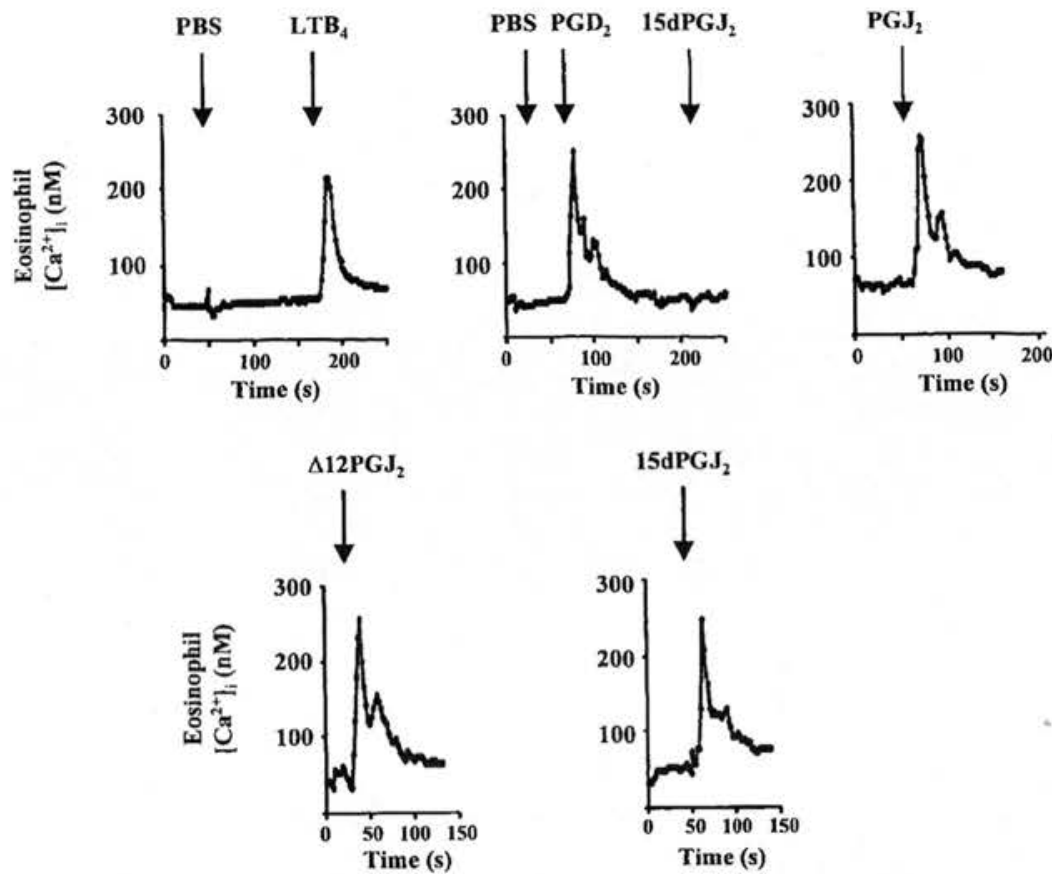

also blocked the induction of eosinophil apoptosis by both metabolites (data not shown). Taken together, these results clearly demonstrate that the proapoptotic nature of the $\mathrm{PGD}_{2}$ metabolites likely does not result from the synthesis of a death-inducing protein as has been suggested for the proapoptotic effect observed in other cells (47) but is, however, critically dependent on the activation of caspases.

Synthetic PPAR- $\gamma$ activators do not induce granulocyte apoptosis

Because $\Delta^{12} \mathrm{PGJ}_{2}$ and $15 \mathrm{dPGJ}_{2}$ are known ligands for PPAR- $\gamma$, we investigated the effects of other known PPAR- $\gamma$ activators on gran- ulocyte apoptosis. The synthetic PPAR- $\gamma$ ligands, BRL49653 and ciglitazone, used at concentrations ranging from $1 \mathrm{nM}$ to $100 \mu \mathrm{M}$, did not affect the rate of constitutive apoptosis in either cell type (Fig. 6C). Moreover, GW9662, an irreversible PPAR- $\gamma$ antagonist (33), did not prevent the induction of apoptosis induced by $15 \mathrm{dPGJ}_{2}$ (Fig. $6 D$ ). Because some reports indicate that $\mathrm{PGD}_{2}$ metabolites can also activate PPAR- $\alpha$ at higher concentrations (48), we also incubated granulocytes with pirinixic acid (WY-14643), a PPAR- $\alpha$ agonist. This compound did not influence apoptosis in either cell type over the wide range of concentrations examined $(1-300 \mu \mathrm{M})$ (Fig. 6C). Our data strongly suggest the powerful

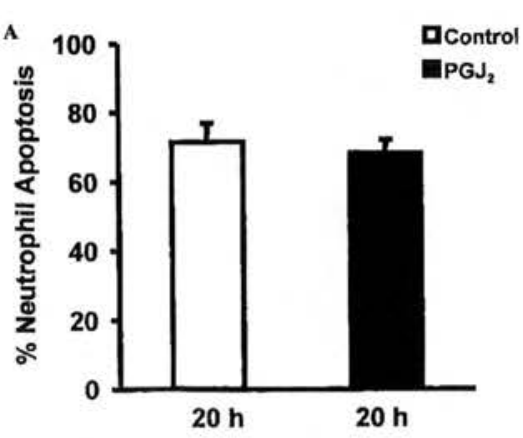

C

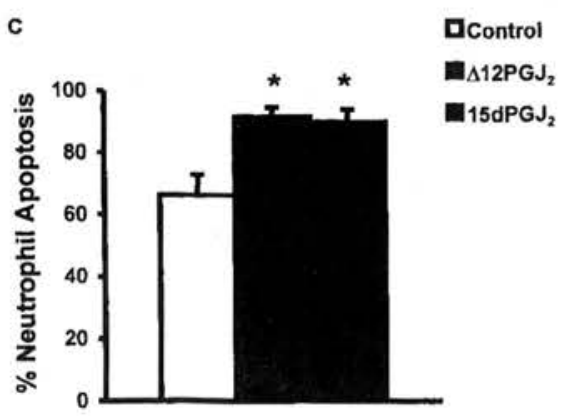

$20 \mathrm{~h}$

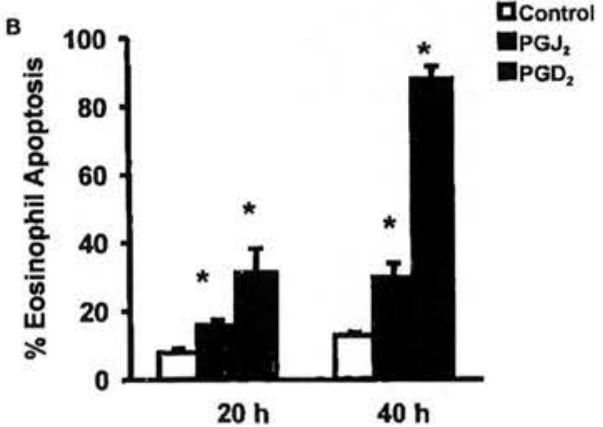

D

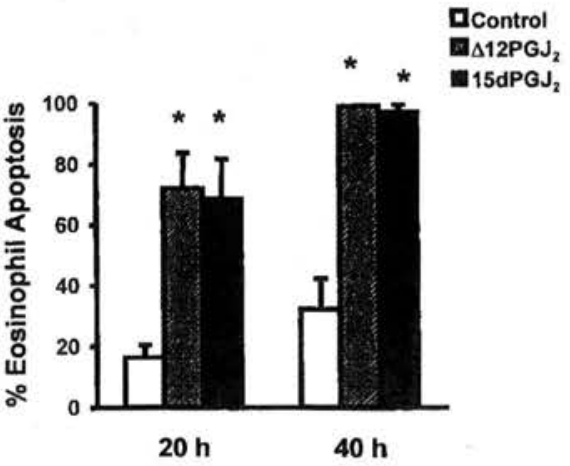

FIGURE 4. Effect of $\mathrm{PGJ}_{2}, \Delta 12 \mathrm{PGJ}_{2}$ and $15 \mathrm{dPGJ}_{2}$ on constitutive granulocyte apoptosis. Neutrophils ( $A$ and $C$ $\left.5 \times 10^{6} / \mathrm{ml}\right)$ and eosinophils $(B$ and $D$; $\left.2 \times 10^{6} / \mathrm{ml}\right)$ were incubated in IMDM supplemented with serum alone (control) or with the indicated reagent and harvested at $20 \mathrm{~h}$ (neutrophils) and 20 and $40 \mathrm{~h}$ (eosinophils) and apoptosis was assessed morphologically. In all panels $\square$ is vehicle control-treated cells. $A, \mathrm{PGJ}_{2}(10 \mu \mathrm{M})$-treated cells (ㅌ); $B, \mathrm{PGJ}_{2}(10 \mu \mathrm{M})(\mathbf{E}), \mathrm{PGD}_{2}(10$ $\mu \mathrm{M})(\square) ; C$ and $D, \Delta 12 \mathrm{PGJ}_{2}(10 \mu \mathrm{M})$ (口) and $15 \mathrm{dPGJ}_{2}(10 \mu \mathrm{M})$ (四). Data represent the mean \pm SEM of three separate experiments $(A$ and $B)$ and four separate experiments $(C$ and $D)$. All experiments were performed in triplicate. $*, p<0.05$ compared with control values. 


\section{Control}

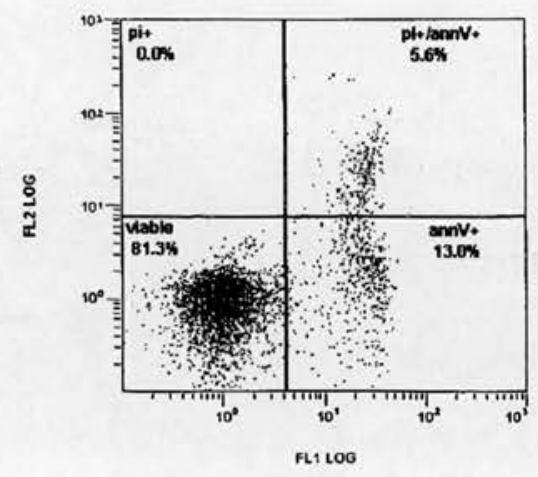

\section{$\mathrm{PGJ}_{2}$}

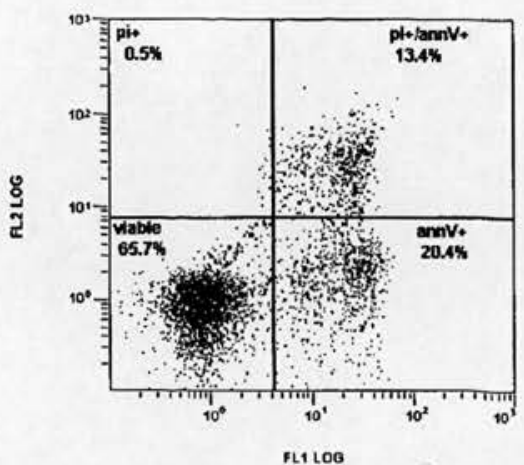

$\mathrm{PGD}_{2}$

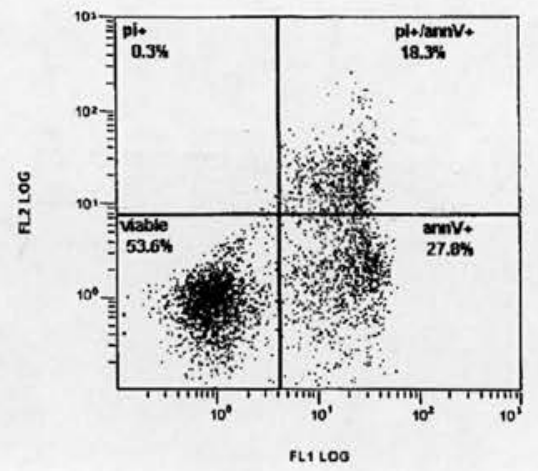

$\Delta 12 \mathrm{PGJ}_{2}$

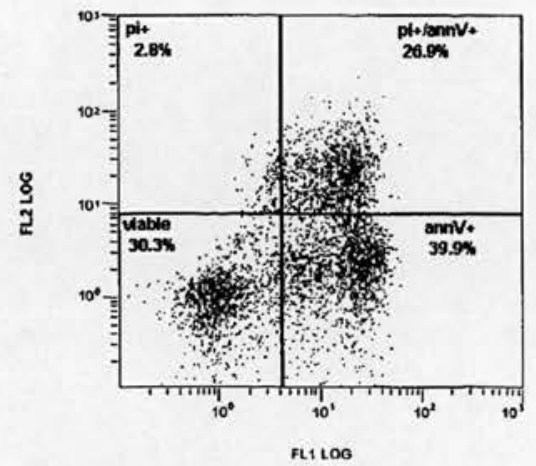

5. Effect of $\mathrm{PGD}_{2}, \mathrm{PGJ}$ $\mathrm{A}_{2}$, and $15 \mathrm{dPGJ}_{2}$ on eosinophil were incubated in IMDM supplemented with serum alone (control) or with $\mathrm{PGD}_{2}, \mathrm{PGJ}_{2}, \Delta 12 \mathrm{PGJ}_{2}$ and $15 \mathrm{dPGJ}_{2}$ (all at $10 \mu \mathrm{M}$ ); harvested at $20 \mathrm{~h}$; and incubated with FITClabeled recombinant human annexin $\mathrm{V}$ (annV) to determine phosphatidylserine expression and propidium iodide (pi) to determine loss of cell membrane integrity. Fluorescence was assessed using a EPICS XL2 flow cytometer. Data from a minimum of 5000 cells were analyzed for each condition, and the proportion of cells in each quadrant is indicated.

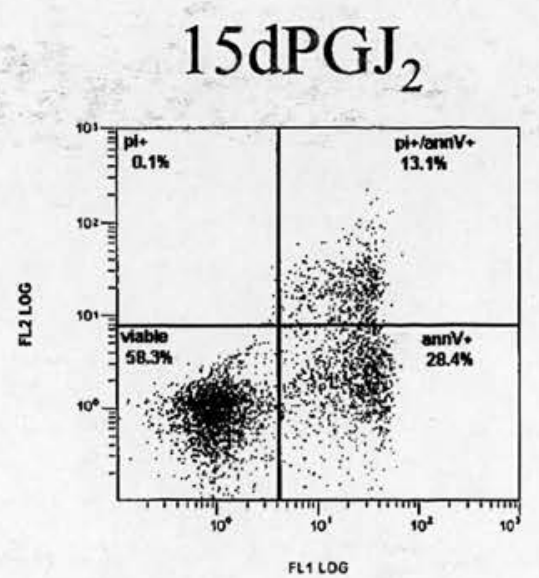

ing NF- $\kappa$ B activation. We therefore examined this possibility directly by Western blot analysis of $\mathrm{I} \kappa \mathrm{B}$ degradation. As shown in Fig. $7 B$, $15 \mathrm{dPGJ}_{2}$ and $\Delta^{12} \mathrm{PGJ}_{2}$ did not cause $\mathrm{I} \kappa \mathrm{B} \alpha$ degradation, but both metabolites inhibited the proteolytic breakdown of $\mathrm{I} \kappa \mathrm{B} \alpha$ in response to LPS stimulation. Further studies determined that neither $\mathrm{PGD}_{2}$ nor $\mathrm{PGJ}_{2}$, which do not induce apoptosis in neutrophils, could prevent $\mathrm{I} \kappa \mathrm{B} \alpha$ degradation in these cells (data not shown). It is therefore apparent that the proapoptotic effect of $\Delta^{12} \mathrm{PGJ}_{2}$ and $15 \mathrm{dPGJ}_{2}$ may be mediated by inhibition of NF- $\kappa \mathrm{B}$ activation. This is further supported by the demonstration that the inhibition of LPS by $\Delta^{12} \mathrm{PGJ}_{2}$ in neutrophils (Fig. $8 A$ ) and TNF- $\alpha$-mediated $\mathrm{I} \kappa \mathrm{B} \alpha$ breakdown by $15 \mathrm{dPGJ}_{2}$ in eosinophils (Fig. $8 B$ ) is concentration dependent. 

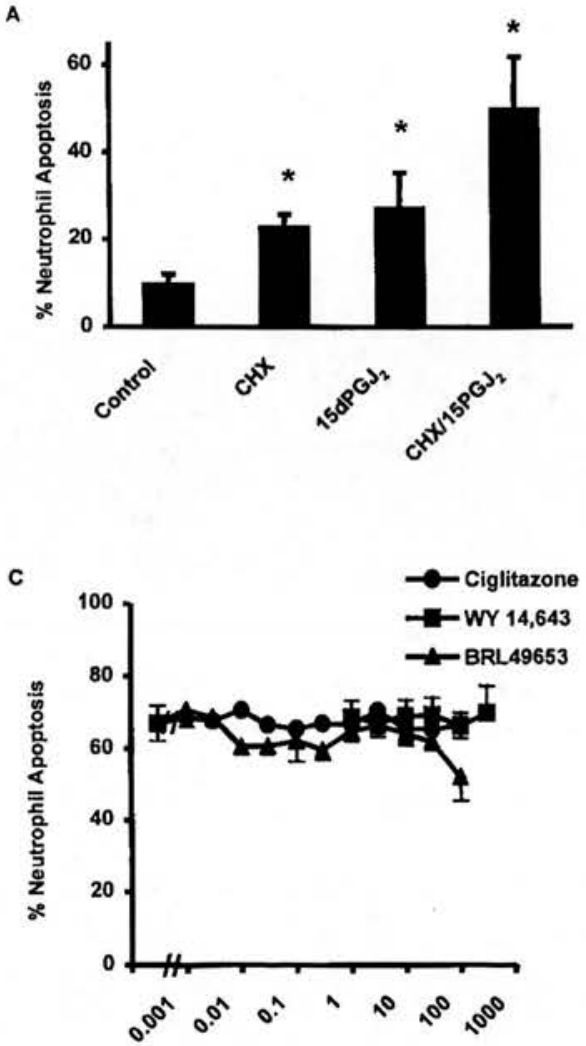

Synthetic PPAR ligand [uM]
B
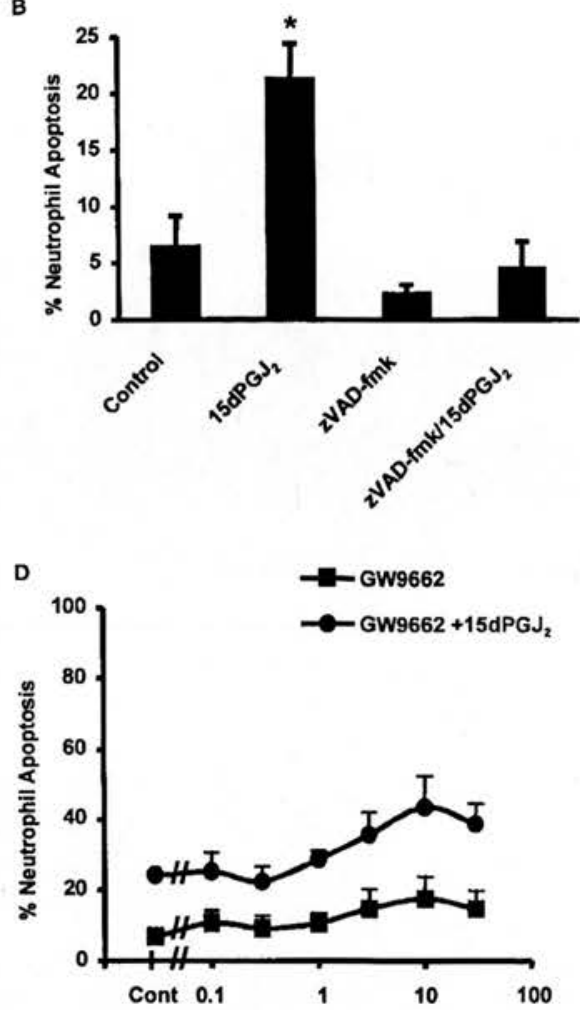

GW9662 [uM]

FIGURE 6. $A$ and $B$, Effect of cycloheximide (CHX) and $z-V A D$-fmk on $\Delta^{12}-\mathrm{PGJ}_{2^{-}}$and $15 \mathrm{dPGJ}$-induced neutrophil apoptosis. $A$, Neutrophils $(5 \times$ $10 \% \mathrm{ml}$ ) were incubated in PBS with calcium and magnesium alone (control) or with $\Delta^{12}-\mathrm{PGJ}_{2}$ or $15 \mathrm{dPGJ}_{2}(10 \mu \mathrm{M}) \pm$ cycloheximide $(5 \mu \mathrm{M})$. $B$, Neutrophils $\left(5 \times 10^{6} / \mathrm{ml}\right)$ were incubated in PBS with calcium and magnesium alone (control) or with $\Delta^{12}-\mathrm{PGJ}_{2}$ or $15 \mathrm{dPGJ}_{2}(10 \mu \mathrm{M}) \pm \mathrm{z}-\mathrm{VAD}$-fmk $(100$ $\mu \mathrm{M})$. Cells were harvested at $3 \mathrm{~h}$, and apoptosis was assessed morphologically. Data represent the mean \pm SEM of four separate experiments $(A)$ and three separate experiments $(B)$. All experiments were performed in triplicate. $*, p<0.05$ compared with control values. $C$, Effect of PPAR $\gamma$ ligands ciglitazone and BRL49653 and the PPAR- $\alpha$ agonist WY-14643 on neutrophil apoptosis. Neutrophils ( $\left.5 \times 10^{6} / \mathrm{ml}\right)$ were incubated in IMDM supplemented with serum alone (control) with WY-14643 (1-300 $\mu \mathrm{M})$, ciglitazone, and BRL49653 (0.001-100 $\mu \mathrm{M})$, and harvested at $20 \mathrm{~h}$. Apoptosis was assessed morphologically. Data represent the mean \pm SEM of three separate experiments. All experiments were performed in triplicate. $D$, Effect of the irreversible PPAR- $\gamma$ antagonist GW9662 on $15 \mathrm{dPGJ}_{2}$-induced neutrophil apoptosis. Neutrophils $\left(5 \times 10^{6} / \mathrm{ml}\right)$ were incubated in PBS with calcium and magnesium alone (control) or with $15 \mathrm{dPGJ}_{2}(10 \mu \mathrm{M}) \pm \mathrm{GW} 9662(0.1-30 \mu \mathrm{M})$. Cells were harvested at $3 \mathrm{~h}$. Apoptosis was assessed morphologically. Data represent the mean $\pm \mathrm{SEM}$ of three separate experiments. All experiments were performed in triplicate.

\section{Discussion}

This study demonstrates for the first time that $\mathrm{PGD}_{2}$ is a powerful selective inducer of eosinophil apoptosis while exerting no effect on survival in neutrophils, Jurkat T lymphocytes, monocytes, or macrophages. $\mathrm{PGD}_{2}$ has been shown to influence granulocyte responsiveness by binding to specific 7-transmembrane $\mathrm{G}$ proteincoupled DP receptors $(42,43,50)$. Despite convincing evidence that ligand binding increased cAMP production in COS-M6 and HEK 293 cells transfected with the cloned human DP receptor (51), it is highly unlikely that $\mathrm{PGD}_{2}$ induces cell death in eosinophils via elevation of cAMP because this would delay granulocyte apoptosis (see Fig. 1, $B$ and $C$ ) rather than induce apoptosis. $\mathrm{PGD}_{2}$ causes a rapid transient increase in $\left[\mathrm{Ca}^{2+}\right]_{\mathrm{i}}$ in human eosinophils (Ref. 23; see Fig. 3) and primes eosinophils for enhanced release of $\mathrm{LTC}_{4}$ in response to the calcium ionophore $\mathrm{A} 23187$ (23). Although fura 2-loaded eosinophils exposed to $\mathrm{PGD}_{2}$ and its metabolites exhibit a rapid transient elevation of $\left[\mathrm{Ca}^{2+}\right]_{i}$ (Fig. 3), we believe that an increase in $\left[\mathrm{Ca}^{2+}\right]_{\mathrm{i}}$ is not responsible for the $\mathrm{PGD}_{2-}$ mediated eosinophil proapoptotic effect because: 1) fura 2-loaded neutrophils do not exhibit a $\mathrm{Ca}^{2+}$ transient when exposed to the $\mathrm{PGD}_{2}$ metabolites although these metabolites induce apoptosis in neutrophils; 2) elevation of eosinophil $\left[\mathrm{Ca}^{2+}\right]_{i}$ by pharmacological agents such as A23187 and thapsigargin causes degranulation and necrosis at time points when $\mathrm{PGD}_{2}$ induces apoptosis; 3 ) receptordirected stimuli such as IL-5 that increase eosinophil $\left[\mathrm{Ca}^{2+}\right]_{i}$ inhibit rather than induce apoptosis $(52,53)$; 4) $\mathrm{PGD}_{2}$ and $\mathrm{PGJ}_{2}$ exhibit similar $\mathrm{Ca}^{2+}$ responses whereas $\mathrm{PGD}_{2}$ is a more powerful inducer of eosinophil apoptosis; and 5) chemotactic agents such as eotaxin that cause an increase in $\left[\mathrm{Ca}^{2+}\right]_{\mathrm{i}}(54)$ do not induce eosinophil apoptosis (C. Ward and A. G. Rossi, unpublished observations). A newly described receptor $\mathrm{CRTH} 2 / \mathrm{DP}_{2}$ has been found to mediate $\left[\mathrm{Ca}^{2+}\right]_{i}$ flux and responsiveness to $\operatorname{PGD}_{2}(55,56)$ and indeed $\mathrm{Ca}^{2+}$ mobilization in response to $\mathrm{PGD}_{2}$ metabolites (43) in eosinophils; however, it is unlikely that $\left[\mathrm{Ca}^{2+}\right]_{i}$ flux, and hence this receptor, are involved in the proapoptotic effect of the $\mathrm{PGD}_{2}$ metabolites. Furthermore, this receptor binds $\mathrm{PGE}_{2}$ and $\mathrm{PGF}_{2 \alpha}$ with similar affinities to $15 \mathrm{dPGJ}_{2}$, and as illustrated in Table I both these PGs inhibit rather than induce eosinophil apoptosis. This receptor is not present on neutrophils; therefore, it cannot be responsible for the proapoptotic effects of $\Delta^{12} \mathrm{PGJ}_{2}$ and $15 \mathrm{dPGJ}_{2}$ observed in these cells.

$\mathrm{PGD}_{2}$ shows a promiscuous PG receptor binding profile (57, 58 ); and in the absence of direct evidence for the expression of IP, FP, or TP receptors on granulocytes, it is possible that other 


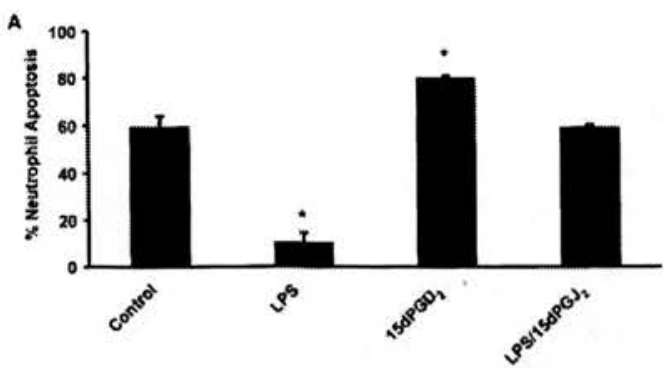

B

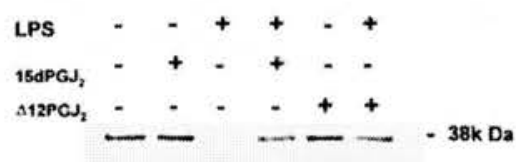

FIGURE 7. $A$, Effect of $15 \mathrm{dPGJ}_{2}$ on LPS-induced inhibition of neutrophil apoptosis. Neutrophils $\left(5 \times 10^{6} / \mathrm{ml}\right)$ were preincubated in PBS with calcium and magnesium alone (control) or with $15 \mathrm{dPGJ}_{2}(10 \mu \mathrm{M})$ for $1 \mathrm{~h}$ before the addition of LPS $(1 \mu \mathrm{g} / \mathrm{ml})$ and $10 \%$ autologous serum. Cells were harvested at $20 \mathrm{~h}$. Apoptosis was assessed morphologically. Data represent the mean \pm SD of a representative experiment (of six) performed in triplicate. All experiments were performed in triplicate. *, $p<0.05$ compared with control values. $B$, Western blot analysis of $15 \mathrm{dPGJ}_{2}$ and $\Delta^{12}-\mathrm{PGJ}_{2}$ inhibition of LPS-induced $\mathrm{I} \kappa \mathrm{B} \alpha$ degradation. Neutrophils $(5 \times$ $10^{6} / \mathrm{ml}$ ) were preincubated in PBS with calcium and magnesium alone (control) or with $15 \mathrm{dPGJ}_{2}(10 \mu \mathrm{M})$ for $1 \mathrm{~h}$ before the addition of LPS (1 $\mu \mathrm{g} / \mathrm{ml}$ ) and $10 \%$ autologous serum in a shaking water bath at $37^{\circ} \mathrm{C}$. After $15 \mathrm{~min}$, cells were lysed using procedures detailed in Materials and Meth$o d s$, and the resulting lysates were run on a $9 \%$ acrylamide gel. Data are representative of one experiment of at least five.

receptors (e.g., EP) are mediating the proapoptotic activities of $\mathrm{PGD}_{2}$. However, the results obtained using a wide variety of PGs or PG analogs demonstrate that the proapoptotic effect of $\mathrm{PGD}_{2}$ could not be reproduced through triggering of any of the classical cell surface PG receptors for which it has a known affinity (Table I; Figs. 1 and 2). It is unlikely that the DP receptor is involved in mediating the proapoptotic effect in eosinophils because the stable $\mathrm{PGD}_{2}$ mimetic ZK118.182 induced minimal increases in eosinophil apoptosis and inhibited this process in the neutrophil. A plausible explanation for the observed differential proapoptotic effects of $\mathrm{PGD}_{2}$ could be that eosinophil and neutrophil granulocytes metabolize $\mathrm{PGD}_{2}$ differently, resulting in distinct functional outcomes. It is possible that neutrophils fail to metabolize $\mathrm{PGJ}_{2}$ to the $\Delta^{12} \mathrm{PGJ}_{2}$ and $15 \mathrm{dPGJ}_{2}$ sequential metabolites and are thus protected from the proapoptotic effects of these products. It is also possible that eosinophils readily metabolize $\mathrm{PGD}_{2}$ into the active $\mathrm{PGD}_{2}$ proapoptotic metabolites or alternatively that eosinophil uptake of $\mathrm{PGD}_{2}$ and $\mathrm{PGJ}_{2}$ may differ from the process in neutrophils. These intriguing possibilities await further investigation. Interestingly, a very recent study has specifically and conclusively shown, using a newly described $\mathrm{mAb}$ raised against $15 \mathrm{dPGJ}_{2}$, that this metabolite is present in the cytoplasm of macrophages in human atherosclerotic plaques (59). Thus, these authors have set a precedent for the detection of $\mathrm{PGD}_{2}$ metabolites in vivo and specifically demonstrated that this metabolite can be generated during an inflammatory response in an important human disease. Although we and many others have used low micromolar concentrations of $\mathrm{PGD}_{2}$ and its metabolites, we believe that such levels could be
A
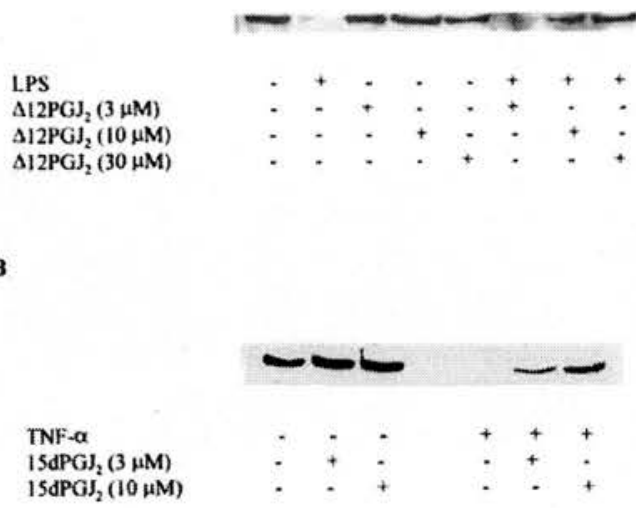

FIGURE 8. A. Western blot analysis of the concentration dependency of $\Delta^{12}-\mathrm{PGJ}_{2}$-mediated inhibition of LPS-induced $\mathrm{I} \kappa \mathrm{B} \alpha$ degradation in human neutrophils. Neutrophils $\left(5 \times 10^{6} / \mathrm{ml}\right)$ were preincubated in PBS with calcium and magnesium alone (control) or with $\Delta^{12}-\mathrm{PGJ}_{2}(3-30 \mu \mathrm{M})$ for $1 \mathrm{~h}$ before the addition of LPS $(1 \mu \mathrm{g} / \mathrm{ml})$ and $10 \%$ autologous serum in a shaking water bath at $37^{\circ} \mathrm{C}$. After $15 \mathrm{~min}$, cells were lysed using procedures detailed in Materials and Methods, and the resulting lysates were run on a $9 \%$ acrylamide gel. This is a representative experiment of at least three. $B$, Western blot analysis of the concentration dependency of $15 \mathrm{dPGJ}_{2}$-mediated inhibition of TNF- $\alpha$-induced $\mathrm{I} \kappa \mathrm{B} \alpha$ degradation in human eosinophils. Eosinophils $\left(5 \times 10^{6} / \mathrm{ml}\right)$ were preincubated in PBS with calcium and magnesium alone (control) or with $15 \mathrm{dPGJ}_{2}(10 \mu \mathrm{M})$ for $1.5 \mathrm{~h}$ before the addition of TNF- $\alpha(10 \mathrm{ng} / \mathrm{ml})$ and $10 \%$ autologous serum in a shaking water bath at $37^{\circ} \mathrm{C}$. After $15 \mathrm{~min}$, cells were lysed using procedures detailed in Materials and Methods, and the resulting lysates were run on a $9 \%$ acrylamide gel. These are results of a representative experiment of at least three.

achieved in vivo at their site of action. There is convincing evidence demonstrating that certain PGs can reach concentrations in the micromolar range at sites of acute inflammation (60) and in certain biological fluids, e.g., seminal fluid (61). Actual concentrations of $\mathrm{PGD}_{2}$ metabolites at relevant sites in vivo await confirmation. Because the metabolites can be produced intracellularly and extracellularly it would be difficult to estimate or assess actual concentrations at their target sites.

The mechanisms by which $\Delta^{12} \mathrm{PGJ}_{2}$ and $15 \mathrm{dPGJ}_{2}$ induce granulocyte apoptosis involve caspase activation because death was inhibited by $z$-VAD-fmk. Although $\Delta^{12} \mathrm{PGJ}_{2}$ and $15 \mathrm{dPGJ}_{2}$ may have their primary targets in the nucleus, where they regulate the expression of specific genes, e.g., via binding to PPAR- $\gamma$, we found that other synthetic agonists to PPAR- $\gamma$ and PPAR- $\alpha$ could not mimic the proapoptotic effects of $\Delta^{12} \mathrm{PGJ}_{2}$ and $15 \mathrm{dPGJ}_{2}$. Although the compound WY-14643 is an effective activator of PPAR- $\alpha$, PPAR- $\gamma$ is also activated by this agent at concentrations of $100 \mu \mathrm{M}$ (32). Despite the use of WY-14643 concentrations up to $300 \mu \mathrm{M}$ (Fig. $3 C$ ), apoptosis was not affected in either cell type. In addition, the thiazolidinediones BRL49653 and ciglitazone, potent activators of PPAR- $\gamma$, did not induce apoptosis in either neutrophils or eosinophils despite the use of concentrations as high as $100 \mu \mathrm{M}$. Moreover, when PPAR- $\gamma$ was blocked using the irreversible antagonist, GW9662 (32), the induction of apoptosis mediated by either of the PPAR- $\gamma$ ligands was unaffected despite the use of concentrations in excess of those known to block PPAR- $\gamma$ activation. Taken together, these data strongly indicate that the 
proapoptotic effects of $\Delta^{12} \mathrm{PGJ}_{2}$ and 15dPGJ on granulocytes signal independently of PPAR- $\gamma$ (or PPAR- $\alpha$ ) and point to the existence of another mechanism. Evidence supporting this possibility has been reported by Thieringer et al. (62), who showed that $15 \mathrm{dPGJ}_{2}$ but not other PPAR- $\gamma$ agonists could inhibit cytokine production in primary human monocyte-derived macrophages and RAW 264.7 cells. However, the signaling mechanisms of cyclopentenone PGs appear to be cell type specific (49). For example, in activated macrophages, $15 \mathrm{dPGJ}_{2}$ as well as BRL49653 antagonize AP-1, STAT, and NF- $\kappa$ B in a PPAR- $\gamma$-dependent manner (63), whereas in human monocytes there is no inhibitory effect of PPAR- $\gamma$ agonists on the induced expression of TNF- $\alpha$ and IL-6, products that are controlled by these transcription factors (64). In human monocyte-derived macrophages, both BRL49653 and $15 \mathrm{dPGJ}_{2}$ induced apoptosis (PPAR- $\gamma$-dependent) (65); and in endothelial cells, both $15 \mathrm{dPGJ}_{2}$ and ciglitazone caused cell death via a caspase and PPAR- $\gamma$-dependent mechanism (66). These putative PPAR- $\gamma$ ligands can therefore act in some cells in a PPAR- $\gamma$ independent manner. Studies in human neutrophils have shown that $15 \mathrm{dPGJ}_{2}$ inhibits the $\beta_{2}$ integrin-dependent respiratory burst via a PPAR- $\gamma$-independent pathway and also suggest the presence of an as yet unidentified receptor (67). Data obtained suggested that such a receptor may act via increases in cytosolic cAMP. Our data, however, indicate that such a mechanism could not be responsible for the proapoptotic effect because an elevation of cAMP strongly inhibits granulocyte apoptosis (Fig. 1, $E$ and $F$ and Refs. 68 and 69).

Because we have recently shown that activation of an inducible form of NF- $\kappa$ B is crucial to granulocyte survival (8), the reported inhibition of this transcription factor by these metabolites $(49,63$, 70) made an attractive hypothesis to explain the increases in cell death observed in our studies. Thus, we examined the effects of $\Delta^{12} \mathrm{PGJ}_{2}$ and $15 \mathrm{dPGJ}_{2}$ on the activation of NF- $\kappa \mathrm{B}$ in granulocytes and found that both metabolites could inhibit LPS-induced degradation of $\mathrm{I} \kappa \mathrm{B} \alpha$ in neutrophils with concentrations reported in other studies $(49,63,70)$. Interestingly, we show for the first time that TNF- $\alpha$-mediated loss of cytoplasmic I $\kappa \mathrm{B} \alpha$ in eosinophils is also inhibited, in a concentration-dependent manner, by $15 \mathrm{dPGJ}_{2}$. Thus, we have shown in granulocytes that this inhibition was concentration dependent and correlated well with the proapoptotic effects observed. Recently, it has been demonstrated that the cyclopentenone PG, $\mathrm{PGA}_{\mathrm{I}}$, inhibits $\mathrm{I} \kappa \mathrm{B}$ kinase (IKK) activity in Jurkat, $\mathrm{HeLa}$, and COS cells transfected with IKK $\alpha$. Moreover, in HeLa cells, which do not express PPAR- $\gamma, 15 \mathrm{dPGJ}_{2}$ inhibits IKK and $\mathrm{NF}-\kappa \mathrm{B}$ activation by TNF- $\alpha$ (70). This group shows that A- and J-type PGs inhibited IKK activity and thus that a reactive $\alpha \beta$ unsaturated carbonyl group in the cyclopentane ring was critical for IKK inhibition (70). However, as shown in Table I, neither $\mathrm{PGA}_{1}$ nor $\mathrm{PGA}_{2}$, both of which contain this reactive carbonyl group $(48,70)$, induced apoptosis in neutrophils; indeed by $20 \mathrm{~h}$, $\mathrm{PGA}_{1}$ had produced a small but significant decrease in neutrophil programmed cell death. Because we have previously shown that $\mathrm{NF}-\kappa \mathrm{B}$ activation is crucial for survival in these cells (8), these data also suggest a degree of specificity in the interaction of cells with these PGs and that the effects of PGs on NF- $\kappa$ B may be dependent on cell type.

Knowledge of the mechanisms by which these metabolites exert their proapoptotic effects may be central to understanding why such products influence inflammation. For example, $15 \mathrm{dPGJ}_{2}$ suppresses adjuvant-induced arthritis in the rat (71), whereas in a rat model of pleurisy, increased levels of $15 \mathrm{dPGJ}_{2}$ and $\mathrm{PGD}_{2}$ have been shown to correlate with the resolution of inflammation (72). PPAR- $\gamma$ ligands also affect the acquired immune response; e.g., ciglitazone and $15 \mathrm{dPGJ}_{2}$ inhibit helper $\mathrm{T}$ cell responses by inhib- iting IL-2 secretion (73). However, whether this phenomenon is concurrent with apoptosis and clearance of inflammatory cells from the respiratory system, is currently unknown. Our results depicting $\mathrm{PGD}_{2}$ as a powerful selective inducer of eosinophil apoptosis may have implications where induction of apoptosis of a specific inflammatory cell type may be of importance in the control of eosinophilic type inflammation. The mechanism involved is likely to depend on the metabolism of this parent prostanoid to products such as $\mathrm{PGJ}_{2}$ and the PPAR- $\gamma$ ligands $\Delta^{12} \mathrm{PGJ}_{2}$ and $15 \mathrm{dPGJ}_{2}$, which also induce eosinophil and neutrophil apoptosis. However, this proapoptotic effect is not mediated by activation of PPAR- $\gamma$ or PPAR- $\alpha$. Interestingly, cyclopentenone PGs have been shown to bind irreversibly to nascent proteins in the endoplasmic reticulum (74). In granulocytes, this mechanism could interfere with the function of survival proteins needed to prevent activation of the apoptotic pathway, a possibility that is currently under investigation.

In conclusion, we have shown for the first time that $\mathrm{PGD}_{2}$ is a powerful, selective accelerator of eosinophil apoptosis, the effects being more significant that those described previously for corticosteroids (50). A preliminary investigation of the underlying mechanisms have shown that its sequential metabolite $\mathrm{PGJ}_{2}$ also induces eosinophil cell death but that the sequential natural metabolites $\Delta^{12} \mathrm{PGJ}_{2}$ and $15 \mathrm{dPGJ}_{2}$, which inhibit the degradation of $\mathrm{I} \kappa \mathrm{B} \alpha$ in granulocytes, also accelerate apoptosis in both neutrophils and eosinophils. The proapoptotic effects of these cyclopentenone PGs are caspase dependent but do not involve ligation of PPAR- $\gamma$ or PPAR- $\alpha$ receptors. Our experiments suggest that the mechanism involves inhibition of NF- $\kappa \mathrm{B}$ activation, a central event in the control of granulocyte apoptosis (8), and thus these metabolites may fundamentally influence the resolution phase of inflammation.

\section{Acknowledgments}

We thank Professor Edwin Chilvers (Respiratory Medicine Unit, Department of Medicine, University of Cambridge, Cambridge, U.K.) for useful comments throughout this work.

\section{References}

1. Savill, J. S., A. H. Wyllie, J. E. Henson, M. J. Walport, P. H. Henson, and C. Haslett. 1989. Macrophage phagocytosis of aging neutrophils in inflammation: programmed cell death in the neutrophil leads to its recognition by macrophages. J. Clin. Invest. 83:865.

2. Whyte, M. K. B., L. C. Meagher, J. MacDermot, and C. Haslett. 1993. Impairment of function in aging neutrophils is associated with apoptosis. J. Immunol. 150:5124.

3. Meagher, L. C., J. S. Savill, A. Baker, R. W. Fuller, and C. Haslett. 1992. Phagocytosis of apoptotic neutrophils does not induce macrophage release of thromboxane $\mathrm{B}_{2}$. J. Leukocyte Biol. 52:269.

4. Liu, Y., J. M. Cousin, J. Hughes, J. Van Damme, J. R. Seckl, C. Haslett, I. Dransfield, J. Savill, and A. G. Rossi. 1999. Glucocorticoids promote nonphlogistic phagocytosis of apoptotic leukocytes. J. Immunol. 162:3639.

5. Fadok, V. A., D. L. Bratton, A. Konowal, P. W. Freed, J. Y. Westcott, and P. M. Henson. 1998. Macrophages that have ingested apoptotic cells in vitro inhibit proinflammatory cytokine production through autocrine/paracrine mechanisms involving TGF- $\beta, \mathrm{PGE}_{2}$, and PAF. J. Clin. Invest. 101:890.

6. Ward, C., I. Dransfield, E. R. Chilvers, C. Haslett, and A. G. Rossi. 1999. Pharmacological manipulation of granulocyte apoptosis: potential therapeutic targets. Trends Pharmacol. Sci. 20:503.

7. Murray, J., J. A. J. Barbara, S. A. Dunkley, A. F. Lopez, X. Van Ostade, A. M. Condliffe, I. Dransfield, C. Haslett, and E. R. Chilvers. 1997. Regulation of neutrophil apoptosis by tumor necrosis factor- $\alpha$ : requirement for TNFR 55 and TNFR75 for induction of apoptosis in vitro. Blood 90:2772.

8. Ward, C., E. R. Chilvers, M. F. Lawson, J. G. Pryde, S. Fujihara, S. N. Farrow, C. Haslett, and A. G. Rossi. 1999. NF- $\kappa$ B activation is a critical regulator of human granulocyte apoptosis in vitro. J. Biol. Chem. 274:4309.

9. Coleman, R. A., W. L. Smith, and S. Narumiya. 1994. International Union of Pharmacology classification of prostanoid receptors: properties, distribution, and structure of the receptors and their subtypes. Pharmacol. Rev. 46:205.

10. Versteeg, H. H., P. M. P. van Bergen en Henegouwen, S. J. H. van Deventer, and Peppelenbosch, M. P. 1999. Cyclooxygenase-dependent signalling: molecular events and consequences. FEBS Lett. 445:1.

11. Pierce, K. L., D. W. Gil, D. F. Woodward, and J. W. Regan. 1995. Cloning of human prostanoid receptors. Trends Pharmacol. Sci. 16:253. 
12. Ohno, K., M. Fukiwara, M. Fukushima, and S. Narumiya. 1986. Metabolic dehydration of prostaglandin $\mathrm{E}_{2}$ and cellular uptake of the dehydration product: correlation with prostaglandin $\mathrm{E}_{2}$-induced growth inhibition. Biochem. Biophys. Res. Commun. 39:808.

13. Fukushima, M. 1992. Biological activities and mechanisms of action of $\mathrm{PGJ}_{2}$ and related compounds: an update. Prostaglandins Leukotrienes Essent. Fatty Acids 47:1.

14. Hubscher, T. 1975. Role of the eosinophil in the allergic reactions. II. Release of prostaglandins from human eosinophilic leukocytes. J. Immunol. 114:1389.

15. Goldstein, I. M., C. L. Malmstem, B. Samuelsson, and G. Weissman. 1977. Prostaglandins, thromboxanes, and polymorphonuclear leukocytes: mediation and modulation of inflammation. Inflammation 2:309.

16. McGuire, J. C., and F. F. Sun. 1980. Metabolism of arachidonic acid and prostaglandin endoperoxide by assorted leukocytes. Adv. Prostaglandin Thromboxane Res, 8:1665.

17. Kroegel, C., and H. Matthys. 1993. Platelet-activating factor-induced human eosinophil activation: generation and release of cyclo-oxygenase metabolites in human blood eosinophils from asthmatics. Immunology 78:279.

18. Tanaka, K., K. Ogawa, K. Sugamura, M. Nakamura, S. Takano, and K. Nagata. 2000. Cutting edge: differential production of prostaglandin $D_{2}$ by human helper T cell subsets. $J$. Immunol. 164:2277.

19. Peters, S. P., D. W. MacGlashan, E S. Schulman, R. P. Schleimer, E. C. Hayes, J. Rakach, N. F. Adkinson, and L. Lichtenstein. 1984. Arachidonic acid metabolism in purified human lung mast cells. J. Immunol. 132:1972.

20. Murray, J. J., A. B. Tonnel, A. R. Brash, L. J. Roberts, P. Gosset, M. D. Workman, A. Capron, and J. A. Oates. 1986. Release of prostaglandin D into human airways during acute antigen challenge. N. Engl. J. Med. 315:800.

21. Ito, S., M. Negishi, K. Sugama, E. Okuda-Ashitaka, and O. Hayaishi. 1991. Signal transduction coupled to prostaglandin $\mathrm{D}_{2}$. Adv. Prostaglandin Thromboxane Leukotriene Res, 21:371.

22. Bundy, G. L., D. R. Morton, D. C. Peterson, E. E. Nishizawa, and W. L. Miller. 1983. Synthesis and platelet aggregation inhibiting activity of prostaglandin D analogues. J. Med. Chem. 26:790.

23. Raible, D. G., E. S. Schulman, J. DiMuzio, R. Cardillo, and T. J. Post. 1992. Mast cell mediators prostaglandin- $\mathrm{D}_{2}$ and histamine activate human eosinophils. J. Immunol. 148:3536.

24. Lemberger, T., B. Desvergne, and W. Wahli. 1996. Peroxisome proliferatoractivated receptors: a nuclear receptor signaling pathway in lipid physiology. Annu. Rev. Cell Dev. Biol. 12:335.

25. Issemann, I., and S. Green. 1990. Activation of a member of the steroid hormone receptor superfamily by peroxisome proliferators. Nature 347:645.

26. Schmidt, A., N. Endo, S. J. Rutledge, R. Vogel, D. Shinar, and G. A. Rodan 1992. Identification of a new member of the steroid hormone receptor superfamily that is activated by a peroxisome proliferator and fatty acids. Mol. Endocrinol. 6:1634.

27. Sher, Y., H. F. Yi, O. W. McBride, and F. J. Gonzalez. 1993. cDNA cloning, chromosomal mapping, and functional characterization of the human peroxisome proliferator activated receptor. Biochemistry 32:5598.

28. Greene, M. E., B. Blumberg, O. W. McBride, H. F. Yi, K. Kronquist, K. Kwan, L. Hsieh, G. Greene, and S. D. Nimer. 1995. Isolation of the human peroxisome proliferator activated receptor $\gamma \mathrm{cDNA}$ : expression in hematopoietic cells and chromosomal mapping. Gene Expression 4:281.

29. Kliewer, S. A., B. M. Forman, B. Blumberg, E. S. Ong, U. Borgmeyer, D. J. Manglesdorf, K. Umesono, and R. M. Evans. 1994. Differential expression and activation of a family of murine peroxisome proliferator-activated receptors. Proc. Natl. Acad. Sci. USA 91:7355.

30. Tontonoz, P., E. Hu, and B. M. Spiegelman. 1994. mPPAR $\gamma 2$ : tissue-specific regulator of an adipocyte enhancer. Genes Dev. 8:1224.

31. Braissant, O., F. Foufelle, C. Scotto, M. Dauca, and W. Wahli. 1996. Differential expression of peroxisome proliferator-activated receptors (PPARs): tissue distribution of PPAR- $\alpha,-\beta$, and $-\gamma$ in the adult rat. Endocrinology 137:354.

32. Lehmann, J. M., L. B. Moore, T. A. Smith-Oliver, W. O. Wilkinson, T. M. Willson, and S. A. Kliewer. 1995. An antidiabetic thiazolidinedione is a high affinity ligand for peroxisome proliferator-activated receptor $\gamma$ (PPAR $\gamma$ ). J. Biol. Chem, 270:12953.

33. Huang, J. T., J. S. Welch, M. Ricote, C. J. Binder, T. M. Willson, C. Kelly, J. L. Witztum, C. D. Funk, D. Conrad, and C. K. Glass. 1999. Interleukin-4 dependent production of PPAR- $\gamma$ ligands in macrophages by 12/15-lipoxygenase. Nature 400:378

34. Rossi, A. G., C Haslett, N. Hirani, A. P. Greening, I. Rahman, C. N. Metz, R. Bucala, and S. C. Donnelly. 1998. Human circulating eosinophils secrete macrophage migration inhibitory factor (MIF): potential role in asthma. J. Clin. Invest. 101:2869.

35. Haslett, C., L. A. Guthrie, M. M. Kopaniak, R. B. Johnston, Jr., and P. M. Henson. 1995. Modulation of multiple neutrophil functions by preparative methods or trace concentrations of bacterial lipopolysaccharide. Am. J. Pathol 119:101.

36. Taylor, E. L., I. L. Megson, C. Haslett, and A. G. Rossi. 2001. Dissociation of DNA fragmentation from other hallmarks of apoptosis in nitric oxide-treated neutrophils: differences between individual nitric oxide donor drugs. Biochem. Biophys, Res. Commun. 289:1229.

37. O'Flaherty, J. T., and A. G. Rossi. 1993. 5-Hydroxyicosatetraenoate stimulates neutrophils by a stereospecific, G protein-linked mechanism. J. Biol. Chem. 268 . 14708 .

38. Ruchaud-Sparagano, M. H., T. R. Walker, A. G. Rossi, C. Haslett, and I. Dransfield. 2000. Soluble E-selectin acts in synergy with platelet-activating factor to activate neutrophil $\beta_{2}$-integrins: role of tyrosine kinases and $\mathrm{Ca}^{2+}$ mobilization. J. Biol. Chem. 275:15758.

39. Meagher, L. C., J. M. Cousin, J. R. Seckl, and C. Haslett. 1996. Opposing effects of glucocorticoids on the rate of apoptosis in neutrophilic and eosinophilic granulocytes. J. Immunol. 156:4422.

40. Pons, F., T. J. Williams, S. A. Kirk, F. McDonald, and A. G. Rossi. 1994. Proinflammatory and anti-inflammatory effects of the stable prostaglandin $\mathrm{D}_{2}$ analogue, ZK 118.182. Eur. J. Pharmacol. 261:237.

41. Gryglewski, R. J., A. Szczeklik, and M. Wandzilak. 1987. The effect of six prostaglandins, prostacyclin and iloprost on generation of superoxide anions by human polymorphonuclear leukocytes stimulated by zymosan or formyl-methionyl-leucyl-phenylalanine. Biochem. Pharmacol. 36:4209.

42. Wheeldon, A., and C. J. Vardey. 1993. Characterization of the inhibitory prostanoid receptors on human neutrophils. Br. J. Pharmacol. 108:1051.

43. Monneret, G., H. Li, J. Vasilescu, J. Rokach, and W. S. Powell. 2002. 15-Deoxy$\Delta^{12,14}$-prostaglandins $\mathrm{D}_{2}$ and $\mathrm{J}_{2}$ are potent activators of human eosinophils. J. Immunol. 168:3563.

44. Whyte, M. K. B., S. J. Hardwick, L. Meagher, J. S. Savill, and C. Haslett. 1993. Transient elevations of cytosolic free calcium retard subsequent apoptosis in neutrophils in vitro. J. Clin. Invest. 92:446.

45. Cousin, J. M., C. Haslett, C, and A. G. Rossi. 1997. Regulation of granulocyte apoptosis by PKC inhibition and elevation of $\left[\mathrm{Ca}^{2+}\right]_{i}$. Biochem. Soc. Trans. 25: $243 S$.

46. Whyte, M. K. B., J. Savill, L. C. Meagher, A. Lee, and C. Haslett. 1997. Coupling of neutrophil apoptosis to recognition by macrophages: coordinated acceleration by protein synthesis inhibitors. J. Leukocyte Biol. 62:195.

47. Kim, I., J. Lee, H. Sohn, H. Kim, and S. Kim. 1993. Prostaglandin $A_{2}$ and $\Delta^{12}$-prostaglandin $\mathrm{J}_{2}$ induce apoptosis in L1210 cells. FEBS Lett. 321:209.

48. Yu, K., W. Bayona, C. B. Kallen, H. P. Harding, C. P. Ravera, G McMahon, M. Brown, and M. A. Lazar. 1995. Differential activation of peroxisome proliferator-activated receptors by eicosanoids. J. Biol. Chem. 270;23975.

49. Straus, D. S., G. Pascual, M. Li, J. S. Welch, M. Ricote, C. Hsiang, L. L. Sengchanthalangsy, G. Ghosh, and C. K. Glass. 2000. 15-deoxy- $\Delta^{12,14}$ prostaglandin $\mathrm{J}_{2}$ inhibits multiple steps in the NF- $\mathrm{KB}$ signaling pathway. Proc. Natl. Acad. Sci. USA 97:4844.

50. Rossi, A. G., and J. T. O'Flaherty. 1989. Prostaglandin binding sites in human polymorphonuclear neutrophils Prostaglandins 37:641.

51. Boie, Y., N. Sawyer, D. M. Slipetz, K. M. Metters, and M. Abramovitz. 1995. Molecular cloning and characterization of the human prostanoid DP receptor. J. Biol. Chem. 270:18910.

52. Coeffier, E., D. Joseph, and B. B. Vargaftig. 1991. Activation of guinea pig eosinophils by human recombinant IL-5: selective priming to platelet-activating factor-acether and interference of its antagonists. J. Immunol. 147:2595.

53. Stern M., L. Meagher, J. Savill, and C. Haslett. 1992. Apoptosis in human eosinophils: programmed cell death in the eosinophil leads to phagocytosis by macrophages and is modulated by IL-5. J. Immunol. 148:3543.

54. Griffiths-Johnson, D. A., P. D. Collins, A. G. Rossi, P. J. Jose, and T. J. Williams 1993. The chemokine, eotaxin, activates guinea-pig eosinophils in vitro and causes their accumulation into the lung in vivo. Biochem. Biophys. Res. Commun 197:1167.

55. Hirai, H., K. Tanaka, O. Yoshie, K. Ogawa, K. Kenmotsu, Y. Takamori, M. Ichimasa, K. Sugamura, M. Nakamura, S. Takano, and K. Nagata. 2001 Prostaglandin $\mathrm{D}_{2}$ selectively induces chemotaxis in $T$ helper type 2 cells, eosinophils, and basophils via seven-transmembrane receptor CRTH2. J. Exp. Med. 193:255.

56. Monneret, G., S. Gravel, M. Diamond, J. Rokach, and W. S. Powell. 2001. Prostaglandin $\mathrm{D}_{2}$ is a potent chemoattractant for human eosinophils that acts via a novel DP receptor. Blood 98:1942.

57. Narumiya, S., and N. Toda. 1985. Different responsiveness of prostaglandin D2 sensitive systems to prostaglandin $\mathrm{D}_{2}$ and its analogues. Br. J. Pharmacol. 85 , 367.

58. Hamid-Bloomfield, S., A. N. Payne, A. A. Petrovic, and B. J. Whittle. 1990. The role of prostanoid TP- and DP-receptors in the bronchoconstrictor effect of inhaled $\mathrm{PGD}_{2}$ in anaesthetized guinea-pigs: effect of the DP-antagonist BW A868C. Br. J. Pharmacol. 100:761.

59. Shibata, T., M. Kondo, T, Osawa, N. Shibata, M. Kobayashi, and K. Uchida 2002. 15-Deoxy- $\Delta^{12,14}$-prostaglandin $\mathrm{J}_{2}$ : a prostaglandin $\mathrm{D}_{2}$ metabolite generated during inflammatory processes. J. Biol. Chem. 277:10459.

60. Offenbacher, S., B. M. Odle, and T. E. Van Dyke. 1986. The use of crevicular fluid prostaglandin $E_{2}$ levels as a predictor of periodontal attachment loss. J. Peridont. Res. 21:101.

61. Cosentino, M. J., L. B. Emilson, and A. T. Cockett. 1984. Prostaglandins in semen and their relationship to male fertility: a study of 145 men. Fertil. Steril. 41:88.

62. Thieringer, R., J. E. Fenyk-Melody, C. B. Le Grand, B. A. Shelton, P. A. Detmers, E. P. Somers, L. Carbin, D. E. Moller, S. D. Wright, and J. Berger 2000. Activation of peroxisome proliferator-activated receptor $\gamma$ does not inhibit IL-6 or TNF- $\alpha$ responses of macrophages to lipopolysaccharide in vitro or in vivo. J. Immunol. 164:1046.

63. Ricote, M., A. C. Li, T. M. Willson, C. J. Kelly, and C. K. Glass. 1998. The peroxisome proliferator-activated receptor- $\gamma$ is a negative regulator of macrophage activation. Nature 391:79.

64. Shu, H., B. Wong, G. Zhou, Y. Li, J. Berger, J. W. Woods, S. D. Wright, and T. Q. Cai. 2000. Activation of PPAR $\alpha$ or $\gamma$ reduces secretion of matrix metalloproteinase 9 but not interleukin 8 from human monocytic THP-1 cells. Biochem. Biophys. Res. Commun. 267:345. 
65. Chinetti, G., S. Griglio, M. Antonucci, I. P. Torra, P. Delerive, Z. Majd, J. Fruchart, J. Chapman, J. Najib, and B. Staels. 1998. Activation of proliferatoractivated receptors $\alpha$ and $\gamma$ induces apoptosis of human monocyte-derived macrophages. J. Biol. Chem. 273:25573.

66. Bishop-Bailey, D., and T. Hla. 1999. Endothelial cell apoptosis induced by the peroxisome proliferator-activated receptor (PPAR) ligand 15-deoxy- $\Delta^{12.14}$-prostaglandin $\mathrm{J}_{2}$. J. Biol. Chem. 274:17042.

67. Vaidya, S., E. P. Somers, S. D. Wright, P. A. Detmers, and V. S. Bansal. 1990 15-Deoxy- $\Delta^{12,14}$-prostaglandin $\mathrm{J}_{2}$ inhibits the $\beta_{2}$ integrin-dependent oxidative burst: involvement of a mechanism distinct from peroxisome proliferator-activated receptor $\gamma$ ligation. J. Immunol. 163:6187.

68. Rossi, A. G., J. M. Cousin, I. Dransfield, M. F. Lawson, E. R. Chilvers, and C. Haslett. 1995. Agents that elevate cAMP inhibit human neutrophil apoptosis. Biochem. Biophys. Res. Commun. 217:892.

69. Martin, M. C., I. Dransfield, C. Haslett, and A. G. Rossi. 2001. Cyclic AMP regulation of neutrophil apoptosis occurs via a novel protein kinase A-independent signaling pathway. J. Biol. Chem. 276:45041.
70. Rossi, A., P. Kapahi, G. Natoli, T. Takahashi, Y. Chen, M. Karin, and M. G. Santoro. 2000. Anti-inflammatory cyclopentenone prostaglandins are direct inhibitors of I $\kappa$ B kinase. Nature 403:103.

71. Kawahito, Y., M. Kondo, Y. Tsubouchi, A. Hashiramoto, D. Bishop-Bailey, K. Inoue, M. Kohno, R. Yamada, T. Hla, and H. Sano. 2000. 15-Deoxy- $\Delta^{12,14}$ $\mathrm{PGJ}_{2}$ induces synoviocyte apoptosis and suppresses adjuvant-induced arthritis in rats. J. Clin. Invest. 106:189.

72. Gilroy, D. W., P. R. Colville-Nash, D. Willis, J. Chilvers, M. J. Paul-Clark, and D. A. Willoughby. 1999. Cyclopentenone prostaglandins: new allies in the war on inflammation. Nat. Med. 5:698.

73. Clark, R. B., D. Bishop-Bailey, T. Estrada-Hernandez, T. Hla, L. Puddington, and S. J. Padula. 2000. The nuclear receptor PPAR $\gamma$ and immunoregulation: PPAR $\gamma$ mediates inhibition of helper T cell responses. J. Immunol. 164:1364.

74. Takahashi, S., N. Odani, K. Tomokiyo, K. Furuta, M. Suzuki, A. Ichikawa, and M. Negishi. 1998. Localization of a cyclopentenone prostaglandin to the endoplasmic reticulum and induction of BiP mRNA. Biochem. J. 335:35. 


\title{
Cigarette Smoke Prevents Apoptosis through Inhibition of Caspase Activation and Induces Necrosis
}

\author{
Julie A. Wickenden, Murray C. H. Clarke, Adriano G. Rossi, Irfan Rahman, Stephen P. Faux, Kenneth Donaldson, \\ and William MacNee
}

ELEGI/Colt, Phagocyte, and MRC Laboratories, Centre for Inflammation Research, University of Edinburgh Medical School; and Institute of Occupational Medicine, Roxburgh Place, Edinburgh, Scotland, United Kingdom

\begin{abstract}
Emphysema is characterized by enlargement of the distal airspaces in the lungs due to destruction of alveolar walls. Alveolar endothelial and epithelial cell apoptosis induced by cigarette smoke is thought to be a possible mechanism for this cell loss. In contrast, our studies show that cigarette smoke condensate (CSC) induces necrosis in alveolar epithelial cells and human umbilical vein endothelial cells. Furthermore, study of the cell death pathway in a model system using Jurkat cells revealed that in addition to inducing necrosis, CSC inhibited apoptosis induced by staurosporine or Fas ligation, with both effects prevented by the antioxidants glutathione and dithiothreitol. Time course experiments revealed that CSC inhibited an early step in the caspase cascade, whereby caspase- 3 was not activated. Moreover, cell-free reconstitution of the apoptosome in cytoplasmic extracts from CSC-treated cells, by addition of cytochrome-c and dATP, did not result in activation of caspases-3 or $\mathbf{- 9}$. Thus, smoke treatment may alter the levels of pro- and antiapoptogenic factors downstream of the mitochondria to inhibit active apoptosome formation. Therefore, unlike previous studies, cell death in response to cigarette smoke by necrosis and not apoptosis may be responsible for the loss of alveolar walls and inflammation observed in emphysema.
\end{abstract}

Cigarette smoke is a complex mixture of chemicals containing high levels of oxidants and is the major etiologic factor in the development of chronic obstructive pulmonary disease (COPD), of which emphysema is a major component. Emphysema is characterized by enlargement of distal airspaces due to destruction of alveolar wall endothelial cells, epithelial cells, and connective tissue resulting from both protease/antiprotease and oxidant/antioxidant imbalances $(1,2)$. Recently, it has been proposed that apoptosis of alveolar wall cells occurs in response to cigarette smoking, resulting in progressive cell loss and emphysema (3-7).

The study of cell death, including apoptosis, has attracted intense interest as the physiologic program for deletion of harmful or unwanted cells in vivo. A variety of newly

(Received in original form November 6, 2002 and in revised form May 7, 2003)

Address correspondence to: Prof. W. MacNee, ELEGI/Colt Research Laboratories, Wilkie Building, University of Edinburgh Medical School, Teviot Place, Edinburgh EH8 9AG, UK. E-mail: w.macnee@ed.ac.uk

Abbreviations: calcium magnesium-free phosphate-buffered saline, CMFPBS; chronic obstructive pulmonary disease, COPD; cigarette smoke condensate, CSC; dithiothreitol, DTT; glutathione, GSH; glutathione disulphide, GSSG; lactate dehydrogenase, LDH; human umbilical vein endothelial cells, HUVECs; poly (ADP-ribose) polymerase, PARP; reactive oxygen species, ROS; staurosporine, SS.

Am. J. Respir. Cell Mol. Biol. Vol. 29, pp. 562-570, 2003

Originally Published in Press as DOI: 10.1165/rcmb.2002-0235OC on May 14, 2003 Internet address: www.atsjournals.org characterized forms of cell death have highlighted the complexity, diversity, and redundancy that exist in a cell's ability to die (8). In general, there are two main forms of cell death: apoptosis and necrosis. Apoptosis is a well-defined programmed response that results in characteristic morphologic and biochemical changes, such as cell shrinkage and the condensation and fragmentation of nuclear material (9). Inappropriate apoptosis has been implicated in many pathologic conditions, such as neurodegenerative disorders and cancer (10). Necrosis, however, is regarded as a passive response to extremes of environmental stimuli, such as heat and ultraviolet light, and is characterized by cytoplasmic swelling, the rapid loss of plasma membrane integrity, and eventually cell lysis (11). It has been documented that the mode of cell death may be dependent on the cell type, the concentration of stimulus employed, and its environmental setting (12).

The most characterized effectors of apoptotic cell death are the caspases, a family of cysteine proteases that interact with each other in a hierarchical manner (13). One pathway involves induction of apoptosis by ligation of surface death receptors such as Fas and tumor necrosis factor. This so-called "extrinsic" pathway results in auto-activation of caspase-8, and the subsequent cleavage of procaspase- 3 into its active subunits (14). The "intrinsic" mitochondrial pathway can be activated in response to stimuli such as ultraviolet light and oxidative stress, and results in the release of mitochondrial cytochrome-c, initiating formation of the apoptosome complex $(15,16)$. Consisting of APAF-1, cytochrome-c, and caspase- 9 , in the presence of dATP, formation of the apoptosome results in the autoactivation of caspase- 9 and again, activation of the effector caspase-3 (17). Through cleavage of a distinct subset of cellular substrates, caspase- 3 initiates many of the key changes witnessed during apoptosis, thus explaining how a diverse range of stimuli manifest identical phenotypic outcomes during cell death (13). It has been shown that interference with one or more of these stages may result in inhibition of the entire apoptotic process (18).

Reactive oxygen species (ROS) are molecules that have been implicated in mediating apoptotic processes. Depending on the concentration, ROS have been shown to both promote and inhibit apoptosis $(12,19)$. Studies have also shown that oxidants, including hydrogen peroxide $\left(\mathrm{H}_{2} \mathrm{O}_{2}\right)$, inhibit the apoptotic process initiated by other stimuli $(19,20-22)$. Inhibition of the caspase cascade (19-23) and activation of poly (ADP-ribose) polymerase (PARP) $(21,22,24)$ have been proposed as mechanisms for the oxidant-mediated inhibition of apoptosis. 
The aim of the present study was to characterize cigarette smoke-mediated induction of cell death in epithelial and endothelial cells. An alveolar epithelial type II cell line (A549) and primary human umbilical vein endothelial cells (HUVECs) were chosen as surrogate cells to represent alveolar epithelial type II cells and pulmonary microvascular endothelial cells. Here we demonstrate that, in contrast to previous studies $(3,4,25-28)$, cigarette smoke induced necrosis with no evidence of apoptosis, and in addition was able to inhibit apoptosis induced by staurosporine (SS). The cell death machinery is ubiquitous and highly conserved; thus, Jurkat $\mathrm{T}$ cells are commonly used to study the mechanisms of cell death, given that they undergo apoptosis readily and display classical apoptotic markers. Therefore, Jurkat cells were used as a model system to determine the effect of cigarette smoke condensate (CSC) on their well-characterized apoptotic pathway. Here we report that cigarette smoke exposure prevents caspase activation, thus inhibiting apoptosis, and instead promoting necrosis.

\section{Materials and Methods \\ Materials}

All chemicals were of analytical reagent grade and purchased from Sigma Chemical Co. (Poole, UK) unless stated otherwise.

\section{Cell Culture}

A549 cells (ECACC; Porton Down, UK) were grown in Dulbecco's modified Eagle's medium supplemented with $10 \%$ fetal calf serum (LabTech International, Ringmer, UK), $100 \mathrm{U} / \mathrm{ml}$ penicillin, 100 $\mu \mathrm{g} / \mathrm{ml}$ streptomycin (P/S; Invitrogen, Paisley, UK) and $2 \mathrm{mM}$ L-Glutamine (L-Glut; Invitrogen). A549 cells were seeded into 6 well plates at a density of $0.3 \times 10^{6}$ cells per well or 96 well plates at $9 \times 10^{4}$ cells per well for treatment. Cells were quiesced overnight in serum-free media and subsequently treated under serum-free conditions. HUVECs were grown in EBM-2 media (BioWhittaker, Verviers, Belgium) with supplements supplied by the manufacturer. HUVECs, between passages 4 and 6 , were seeded into 24well plates at a density of $5 \times 10^{4}$ cells per well or 96 -well plates at $1 \times 10^{4}$ cells per well, and cultured overnight before treatment. Cells were washed once in calcium magnesium-free phosphatebuffered saline (CMF-PBS) before being exposed to varying concentrations of CSC in full media. Jurkat cells (ECACC) were grown in RPMI supplemented with $10 \%$ fetal bovine serum, $\mathrm{P} / \mathrm{S}$, and L-Glut and treated at a cell density of $1 \times 10^{6} \mathrm{cells} / \mathrm{ml}$ with either normal media, $2 \mu$ M SS (Calbiochem, Nottingham, UK), 100 $\mathrm{ng} / \mathrm{ml}$ anti-Fas activating antibody (CH-11; Upstate Biotechnology, Lake Placid, NY), $10 \%$ CSC or a combination of $2 \mu \mathrm{M}$ SS and $10 \%$ CSC or $100 \mathrm{ng} / \mathrm{ml} \mathrm{CH}-11$ and $10 \%$ CSC.

\section{Preparation of CSC}

CSC was prepared fresh at a concentration of 1 cigarette $/ \mathrm{ml}$ in CMF-PBS. Whole smoke from a king-size medium tar filter-tipped cigarette was drawn into a glass syringe and passed over CMFPBS in a tonometer with agitation $(29,30)$. The condensate was sterile filtered through a 0.22 -mm syringe filter before use.

\section{Determination of Apoptosis and Necrosis}

Acridine orange/ethidium bromide staining was performed as previously described (31). Briefly, treated cells were stained with $4 \mu \mathrm{g} /$ $\mathrm{ml}$ acridine orange and $4 \mu \mathrm{g} / \mathrm{ml}$ ethidium bromide and visualized by epifluoresence microscopy. Viable (normal, green nuclei), early apoptotic (condensed, green nuclei), late apoptotic (condensed, red nuclei), and necrotic (normal, red nuclei) cells were counted. For assessment of DNA fragmentation, $2 \times 10^{6}$ cells were lysed in $500 \mathrm{ml} 7 \mathrm{M}$ guanidine hydrochloride and applied to Wizard SV miniprep columns (Promega, Madison, WI). Columns were centrifuged at $10,000 \times g$ for $2 \mathrm{~min}$, column wash solution $(9 \mathrm{mM}$ Tris.Cl pH 7.4, $90 \mathrm{mM} \mathrm{NaCl}, 2.25 \mathrm{mM}$ EDTA, 55\% ethanol) was applied and the columns were re-centrifuged. The wash was repeated before eluting DNA with $50 \mathrm{ml}$ TE/RNase and performing electrophoresis on a $1.8 \%$ agarose gel. The lactate dehydrogenase (LDH) assay (Roche, Lewes, UK) was performed on cells grown in 96-well plates. Treated cells were incubated at $37^{\circ} \mathrm{C}$ for times as indicated, plates centrifuged at $250 \times \mathrm{g}$ for $10 \mathrm{~min}$ and $100 \mu \mathrm{l}$ of supernatant transferred to a fresh 96-well plate. The LDH assay was performed as per manufacturer's instructions. Jurkat cells were prepared by Cytospin (Shandon, Pittsburgh, PA), stained with DiffQuick (Dade Behring, Marburg, Germany), and viable, apoptotic and necrotic cells counted by brightfield microscopy (Olympus, London, UK). Two Cytospins were prepared for each treatment and at least 300 cells counted per slide.

\section{Cell-Free Apoptosis Assay and Western Blotting}

The assay was performed as previously described (32). Briefly, Jurkat or A549 cells, were incubated in cell extract buffer (CEB, $20 \mathrm{mM}$ HEPES-KOH, $\mathrm{pH} 7.5,10 \mathrm{mM} \mathrm{KCl}, 1.5 \mathrm{mM} \mathrm{MgCl}, 1 \mathrm{mM}$ EDTA, $1 \mathrm{mM}$ EGTA, $1 \mathrm{mM}$ DTT, $100 \mu \mathrm{M}$ PMSF, $10 \mu \mathrm{g} / \mathrm{ml}$ leupeptin, $2 \mu \mathrm{g} / \mathrm{ml}$ aprotinin) on ice for $10 \mathrm{~min}$, before passing through a $25-\mathrm{G}$ needle ten times and centrifuged at $10,000 \times \mathrm{g}$ for $15 \mathrm{~min}$ at $4^{\circ} \mathrm{C}$. Supernatants were removed and stored at $-70^{\circ} \mathrm{C}$ until used. Cell-free apoptosis was initiated by the addition of 10 $\mu \mathrm{M}$ cytochrome-c and $1 \mathrm{mM}$ dATP to the extracts, followed by incubation at $37^{\circ} \mathrm{C}$. Aliquots were removed at the time points indicated, $4 \times$ Laemmli buffer added, and the sample heated to $96^{\circ} \mathrm{C}$ for $5 \mathrm{~min}$. Samples equivalent to $1 \times 10^{6}$ cells were analyzed by Western blot using a polyclonal anti-caspase- 3 antibody, or a polyclonal anti-caspase-9 antibody (Pharmingen, Oxford, UK) recognizing both pro- and active forms. The signal was detected using a horseradish peroxidase-conjugated secondary antibody (Santa Cruz, Wembley, UK) and enhanced chemiluminesence (ECL; Amersham, Little Chalfont, UK).

\section{Assessment of Recombinant Caspase Activity}

Caspase- 3 activity was determined using the caspase- 3 assay kit (Calbiochem), as per manufacturer's instructions. Briefly, active caspase-3 $(30 \mathrm{U})$ was placed into a half-volume 96 -well plate before addition of inhibitor, $1,5,10 \% \mathrm{CSC}$, or $1 \mathrm{mM} \mathrm{H}_{2} \mathrm{O}_{2}$. The plate was incubated at $37^{\circ} \mathrm{C}$ for $1 \mathrm{~h}$ before addition of colorimetric caspase-3 substrate, DEVD-pNA. Caspase-3 activity was determined by measuring the change in absorbance at $405 \mathrm{~nm}$ after 2.5 h at $37^{\circ} \mathrm{C}$.

\section{Measurement of Glutathione Levels}

Four sets of glutathione (GSH) standards were prepared in KPE buffer ( $0.1 \mathrm{M}$ phosphate buffer, $5 \mathrm{mM}$ EDTA, pH 7.4), CSC added to a final concentration of 1,5 , or $10 \%$, and solutions incubated with agitation for $1 \mathrm{~h}$ at $37^{\circ} \mathrm{C}$. GSH levels were measured using a microplate assay adapted from the enzymatic method developed by Tietze and coworkers (33) and Vandeputte and colleagues (34). 
In brief, $1.67 \mathrm{U} / \mathrm{ml} \mathrm{GSH}$ reductase and $0.2 \mathrm{mg} / \mathrm{ml}$ dithiobisnitrobenzoic acid were added to the GSH standards for $30 \mathrm{~s}$ to enable the conversion of GSSG to GSH before $0.2 \mathrm{mg} / \mathrm{ml}$ reduced $\beta$ nicotinamide adenoside diphosphate ( $\beta$-NADPH) was added. The change in absorbance was measured over $2 \mathrm{~min}$ at $405 \mathrm{~nm}$ in a microplate reader (MRX).

\section{Statistical Analysis}

ANOVA was performed using the MiniTab package with Tukey's post-testing to determine significance between treatments. A $P$ value $<0.05$ was deemed significant.

\section{Results}

CSC Induces Necrosis in Fpithelial and Endothclial Cells A significant increase in LDH release from A549 cells and HUVECs was seen in a dose-dependent manner after a 24-h exposure to CSC (Figure 1A). LDH release from A549 cells was also time-dependent (Figure 1B). In addition, acridine orange and ethidium bromide staining revealed that CSC induced necrosis, with no evidence of apoptosis in either A549 cells or HUVECs (Figures 2 and 3). The absence of apoptosis in response to CSC was further confirmed

A

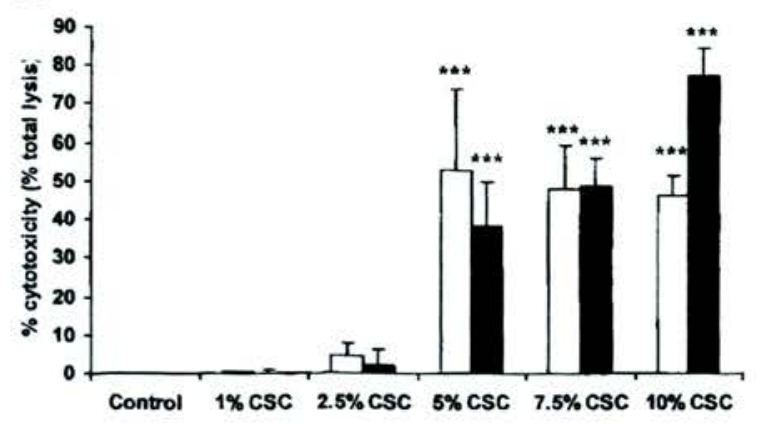

B

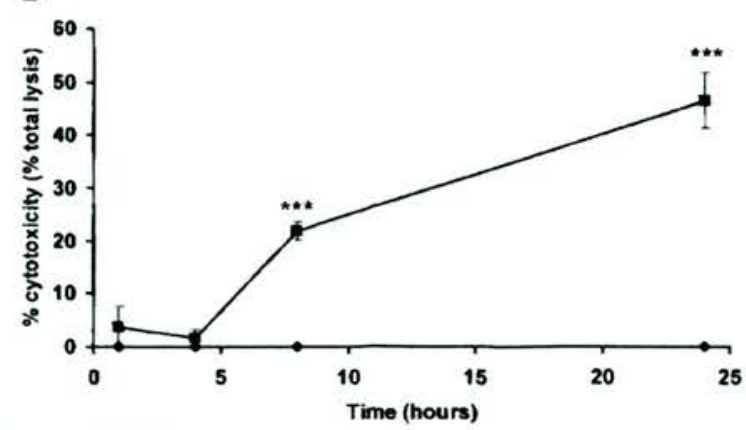

Figure 1. CSC induces necrosis in A549 alveolar epithelial cells and HUVECs. (A) A549 cells (open bars) and HUVECs (closed bars) were exposed to $1-10 \%$ CSC for $24 \mathrm{~h}$ and the percentage of $\mathrm{LDH}$ released into the culture media was determined, compared with a total lysis control ( $1 \%$ Triton X-100). (B) Cytotoxicity to A549 cells in response to $10 \%$ CSC (closed squares) was timedependent; control media (closed diamonds). Results are mean of three experiments \pm SEM. ${ }^{* * *} P<0.001$ compared with control. by a number of methods, including electron microscopy, oligonucleosomal DNA fragmentation, and chromatin condensation assessed by Hoechst 33342, over a range of doses and time points (data not shown). In contrast, however, apoptosis was induced by staurosporine in both cell types (Figures 2E and 3E). Interestingly, A549 cells co-cultured with SS and CSC together did not die by apoptosis, but instead underwent necrosis (Figure 4), thereby implicating a potential for a direct inhibitory effect of CSC on the apoptotic machinery. These results clearly demonstrate that epithelial and endothelial cells undergo necrosis, not apoptosis, in response to CSC.

\section{CSC Prevents SS- and CH-11-Induced Apoptosis, Resulting in Necrosis}

The Jurkat cell is ubiquitously used to study the "core" molecular machinery of cell death, thus this model was employed to elucidate the effect of CSC on the caspase pathway in particular. Jurkat cells treated simultaneously with SS or $\mathrm{CH}-11$ underwent the classical morphologic and biochemical changes indicative of apoptosis, such as chromosomal condensation and oligonucleosomal DNA fragmentation, or laddering (Figures 5A-5C). However, as ob-
A
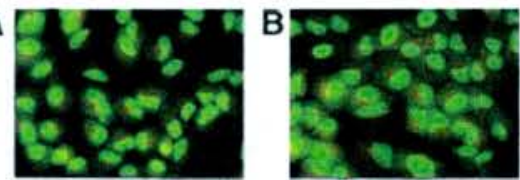

D 2
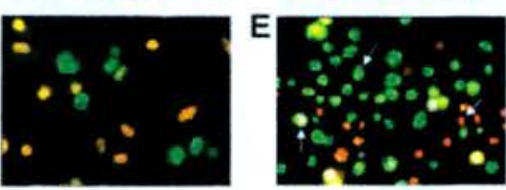

G

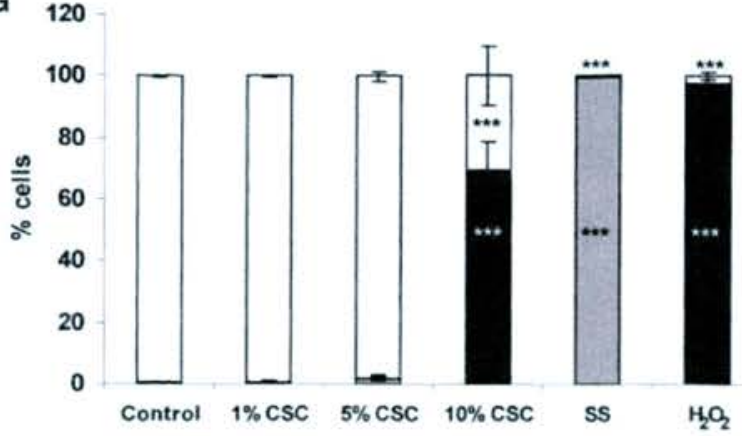

Figure 2. CSC induces necrosis in A549 alveolar epithelial cells with no evidence of apoptosis. A549 cells were exposed to media alone $(A), 1 \% \operatorname{CSC}(B), 5 \% \operatorname{CSC}(C), 10 \% \operatorname{CSC}(D), 2 \mu \mathrm{M}$ SS $(E)$, or $5 \mathrm{mM} \mathrm{H} \mathrm{O}_{2}(F)$ for up to $24 \mathrm{~h}$. Acridine orange and ethidium bromide staining was performed, and the percentage of viable (white bars), apoptotic (gray bars), and necrotic (black bars) cells was determined $(G, 24 \mathrm{~h}$ shown). Note classical apoptotic nuclei in $E$ (arrows) and absence of this morphology in $B, C$, and $D$. Results are mean of three experiments \pm SEM. ${ }^{* * *} P<0.001$ compared with control. Magnification: $\times 36$. 
A
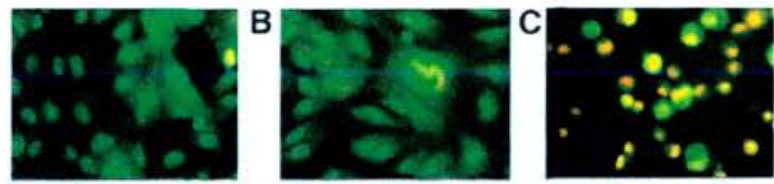

D
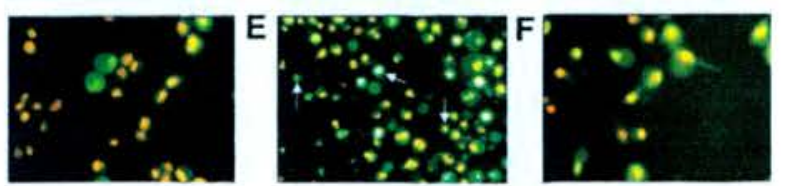

G

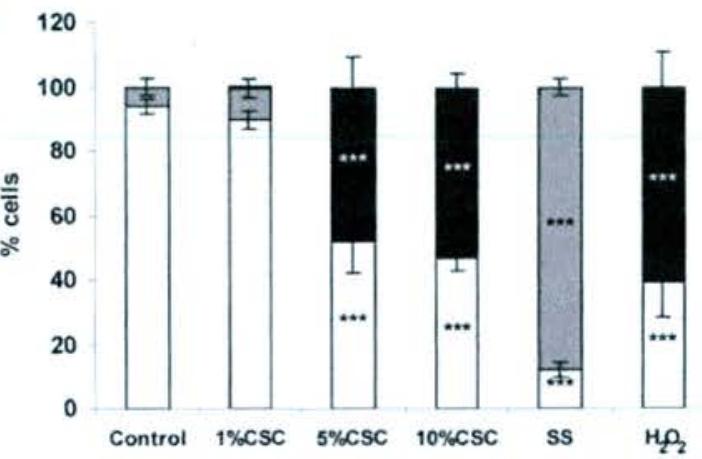

Figure 3. CSC induces necrosis in HUVECs with no evidence of apoptosis. HUVECs were exposed to media alone $(A), 1 \%$ CSC $(B), 5 \% \operatorname{CSC}(C), 10 \% \operatorname{CSC}(D), 2 \mu \mathrm{M} \mathrm{SS}(E)$, or $5 \mathrm{mM} \mathrm{H}_{2} \mathrm{O}_{2}$ $(F)$ for up to $24 \mathrm{~h}$. Acridine orange and ethidium bromide staining was performed and the percentage of viable (white bars), apoptotic (gray bars), and necrotic (black bars) cells was determined ( $G$, $24 \mathrm{~h}$ shown). Note classical apoptotic nuclei in $E$ (arrows) and absence of this morphology in $B, C$, and $D$. Results are mean of three experiments \pm SEM. ${ }^{* * *} P<0.001$ compared with control. Magnification: $\times 36$.

served with A549 cells, apoptosis did not occur when CSC was added to the SS- or $\mathrm{CH}$-11-treated cells or after CSC treatment alone (Figures 5D-5G). Morphologically, the predominant form of cell death in the presence of CSC was necrosis. This necrotic mode of cell death was underscored by the concurrent release of LDH from the cells treated with CSC or a combination of SS and CSC over $24 \mathrm{~h}$ (Figure 6). A low level of LDH release was observed from cells incubated with SS alone and this was attributed to secondary necrosis given the absence of phagocytic clearance. These results indicate that cigarette smoke prevents apoptosis induced by SS or CH-11, and instead promotes necrosis.

To ascertain more accurately the stage of apoptosis that was inhibited, Jurkat cells were treated with SS and $10 \%$ CSC was added at hourly intervals, after which the cells were coincubated for the remainder of the experiment. A higher ratio of apoptotic cells to necrotic cells was seen as the time between SS and CSC treatment increased (Figure 7). This suggests that CSC may impede an early phase in the apoptotic pathway, as CSC only affects apoptosis when present in the early stages of the process.

CSC-Induced Necrosis Is Not Mediated by Oxidative Stress Inhibition of apoptosis and induction of necrosis has been demonstrated in response to oxidants such as $\mathrm{H}_{2} \mathrm{O}_{2}(20-22)$, which is present in cigarette smoke (35). Therefore, to determine whether the oxidant component of cigarette smoke was mediating inhibition of apoptosis, SS-exposed Jurkat cells were treated with CSC in the presence of various antioxidants and evaluated by morphology and DNA laddering. No effect was seen on the necrosis-inducing ability of CSC in the presence of the antioxidant mannitol. However, GSH and dithiothreitol (DTT) prevented necrosis induced by CSC, with cells remaining viable, whereas cells cocultured with SS and CSC underwent apoptosis rather than necrosis (Table 1 and Figure 8).

An alternative role for GSH in vivo is in the detoxification of electrophilic compounds by direct conjugation via the thiol group. A dose-dependent decrease in measurable GSH was observed after incubation with CSC (results expressed as percentage of control: $1 \%$ CSC, $80.66 \pm 4.4 ; 5 \%$ CSC, $17.97 \pm 5.15, P<0.001 ; 10 \%$ CSC, $7.11 \pm 0.44, P<$ $0.001)$. The assay employed measures both reduced $(\mathrm{GSH})$ and oxidized (GSSG) glutathione. If oxidation had taken place, then no decrease in total GSH would have occurred.

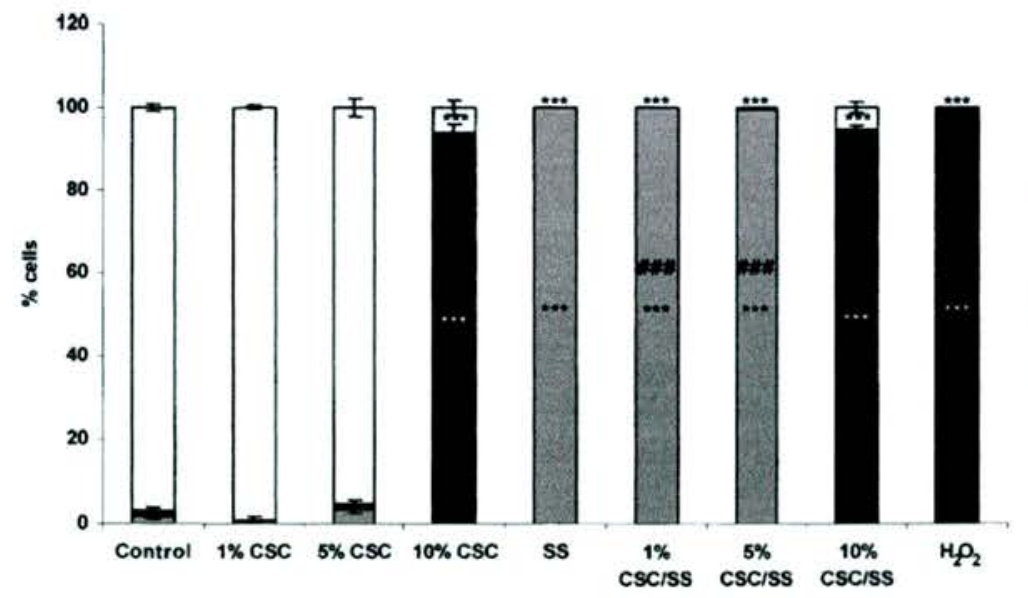

Figure 4. CSC prevents apoptosis and induces necrosis in A.549 cells. A 549 cells were incubated with either media alone, or media containing CSC, $2 \mu \mathrm{M} \mathrm{SS}, 5$ $\mathrm{mM} \mathrm{H}_{2} \mathrm{O}_{2}$, or a combination of SS and CSC for $24 \mathrm{~h}$. The percentage of viable (white bars), apoptotic (gray bars), and necrotic (black bars) cells was determined following acridine orange and ethidium bromide staining. Results expressed as the mean of three experiments \pm SEM. ${ }^{* * *} P<0.001$ compared with control. $1 \% \mathrm{CSC} / \mathrm{SS}$ and $5 \% \mathrm{CSC} / \mathrm{SS}$ were significantly different from smoke alone treatments $(P<0.001)$. 

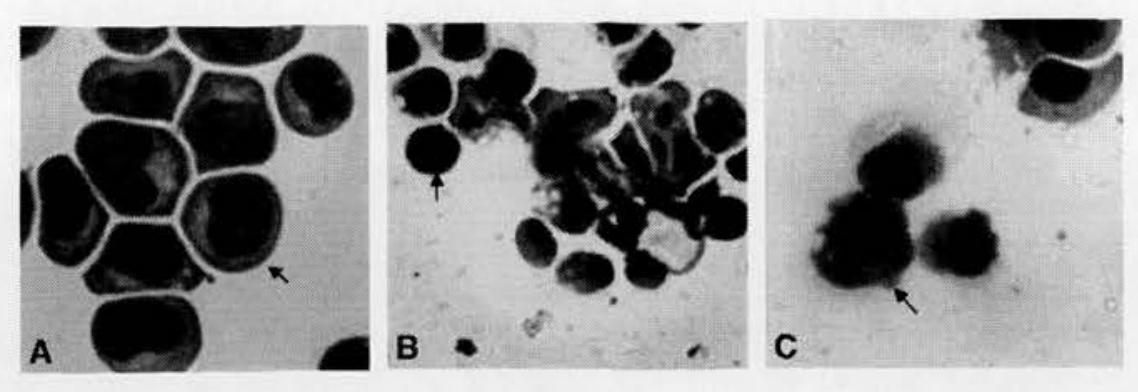

D

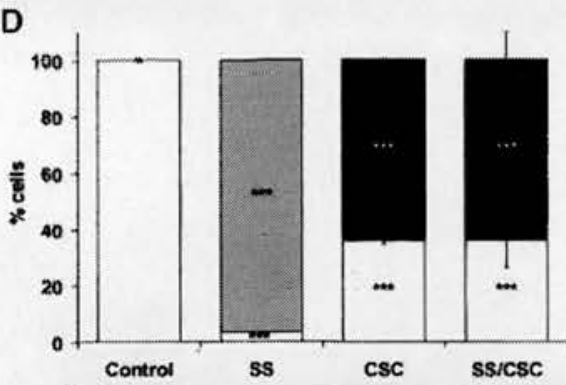

E

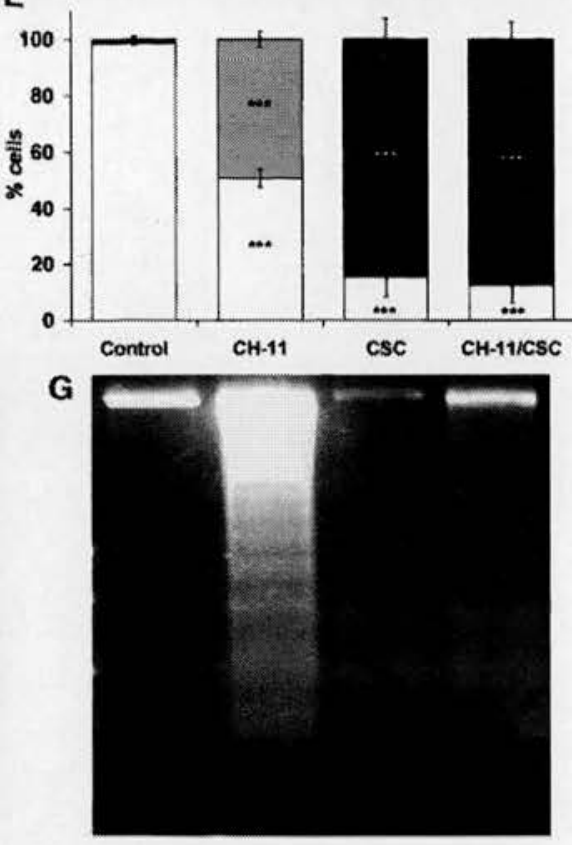

Figure 5. CSC switches apoptosis induced by $\mathrm{SS}$ or $\mathrm{CH}-11$ to necrosis. Jurkat cells were treated with SS, CH.11, $10 \%$ CSC, or a combination as indicated. After a 6-h exposure $(D)$ or 9-h exposure $(E)$, death was assessed by morphology and the percentage of normal ( $A$, white bars), apoptotic ( $B$, gray bars), and necrotic ( $C$, black bars) cells was determined. Results represent mean of three experiments \pm SEM, where at least 300 cells were counted per slide. ${ }^{* * *} P<0.001$ compared with control. Apoptosis was confirmed by the presence of oligonucleosomal DNA fragments, or so-called "ladders" $(F, G)$.
Thus, this data implies that cigarette smoke components may conjugate with GSH.

In addition, unlike in response to oxidants $(21,22,24)$, the presence of a PARP inhibitor, 3-aminobenzinamide, did not alter the relative levels of apoptosis and necrosis in response to SS and CSC (data not shown), implying that depletion of ATP as a result of PARP activation is not involved in CSC-mediated cell death. These data suggest that oxidative stress induced by CSC is not responsible for inhibition of apoptosis and induction of necrosis.

\section{CSC Inhibits Caspase-3 Cleavage but Not Activity}

Because it was established that CSC affected an early stage of apoptosis, caspase activation was investigated. Caspases can be inhibited by direct modification; however, CSC had no direct effect on recombinant caspase-3 activity (data not shown). Caspases exist as an inactive proform that is cleaved to yield active subunits; thus, activation can be monitored by Western blot. Assessment of caspase-3 activation in Jurkat cells revealed cleavage of the proform after SS treatment, but not after CSC or coculture with SS and CSC
(Figure 8), indicating that the caspase pathway may be halted in the presence of CSC. Furthermore, when cells were treated with SS and CSC in the presence of DTT or GSH, caspase- 3 cleavage occurred, whereas mannitol was again not effective (Figure 8). Thus, GSH and DTT are able to "quench" or antagonize components in CSC that prevent caspase-3 activation.

Cell-Free Reconstitution of the Apoptosome Reveals CSC-Mediated Inhibition of Apoptosome Formation

To ascertain how CSC affected the caspase pathway, an active apoptosome was reconstituted in a cell-free system with cytochrome-c and dATP, in either the presence of CSC, or in extracts prepared from cells treated with CSC. No effect on activation of caspase- 3 was observed when CSC was added to the lysates directly (data not shown). However, in extracts from Jurkats exposed to CSC for $2 \mathrm{~h}$ before preparation, neither caspase-3 nor caspase- 9 activation occurred, in contrast to the progressive processing to the active form seen in lysates from untreated cells (Figures 9A-9D). This caspase inhibition was not dependent on PI3-kinase activation, due 


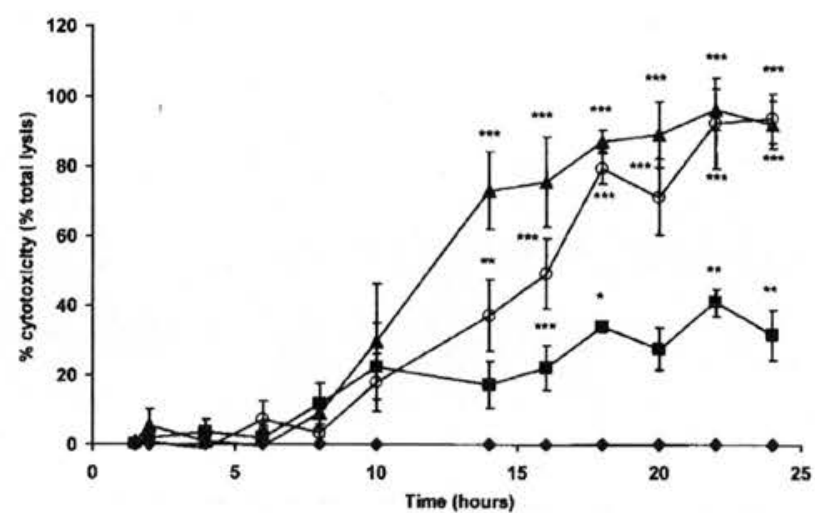

Figure 6. CSC induces LDH release from Jurkat cells in a timedependent manner. Cells were treated with control media (diamonds), $2 \mu \mathrm{M} \mathrm{SS}$ (squares), $10 \% \operatorname{CSC}$ (triangles), or a combination of SS and CSC (open circles), and the level of released LDH measured over $24 \mathrm{~h}$ was determined. Results are expressed as percentage LDH release compared with a total lysis control. Mean of three experiments performed in triplicate \pm SEM. $* P<0.05$, ${ }^{* *} P<0.01,{ }^{* * *} P<0.001$ compared with untreated cells.

to the lack of effect of the inhibitor LY294002 (data not shown). Moreover, caspase-3 was not cleaved after reconstitution of the apoptosome in lysates from A549 cells treated with CSC (Figure 9E). Thus, cigarette smoke prevents apoptosis by inhibiting the formation of an active apoptosome complex and the subsequent activation of caspase- 9 and -3 .

\section{Discussion}

Cigarette smoking is the major etiologic factor in the pathogenesis of emphysema. The gas phase of cigarette smoke contains many free radicals and oxidant molecules, with the potential to generate additional oxidant molecules via redox cycling, leading to an increased oxidative burden in the lungs of smokers (35). Therefore, cigarette smoke inhalation leads to a depletion of antioxidants, release of inflammatory mediators, and an increase in epithelial permeability (36-39). Recent evidence has suggested that apoptosis of lung cells may be a factor in cigarette smokeinduced emphysema $(3-5,7,40)$. In contrast, our studies show that CSC does not induce apoptosis and in fact induces necrosis in the alveolar epithelial type II cell line (A549) and primary endothelial cells (HUVECs). Interestingly, CSC also prevented apoptosis induced by SS in A549 cells.

Oxidative stress is responsible for many of the effects of cigarette smoking. Given that oxidants, such as hydrogen peroxide, have been shown to inhibit apoptosis and induce necrosis (19-22) we hypothesized that CSC may function in a similar manner. Jurkat cells, which undergo apoptosis readily and display easily identifiable markers, treated with CSC underwent necrosis. Moreover, Jurkat cells cocultured with a combination of SS or CH-11 and CSC also underwent necrosis, comparable to A549 cells, with no evidence of apoptosis. Therefore it appeared that CSC was inhibiting apoptosis and promoting necrosis.

An investigation into the role of oxidants demonstrated
A

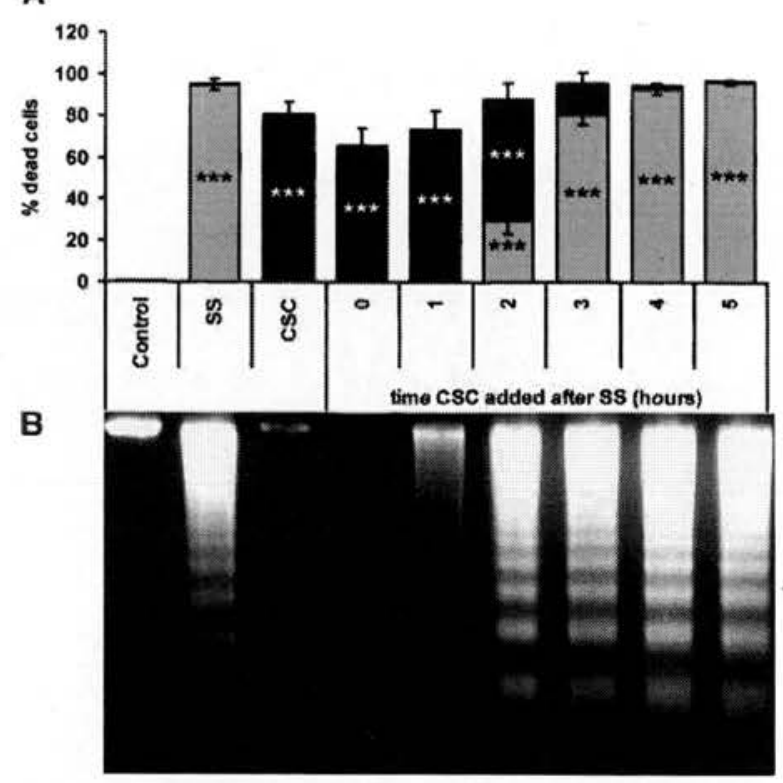

Figure 7. Cigarette smoke can switch SS-induced apoptosis to necrosis when added after SS treatment. Jurkat cells were treated with $2 \mu \mathrm{M}$ SS, followed by addition of $10 \%$ CSC at hourly intervals. Six hours after initial SS treatment cytospins were prepared and the percentage of normal (white bars), apoptotic (gray bars), and necrotic (black bars) cells was determined $(A)$. Apoptosis was confirmed by the presence of oligonucleosomal DNA "ladders" (B). Results are mean of three experiments \pm SEM, where at least 300 cells were counted per slide. $* * * P<0.001$ compared with control.

that GSH and DTT protected against CSC-induced necrosis, and prevented the inhibition of apoptosis usually seen during SS and CSC coculture. However, the antioxidant mannitol was ineffective. GSH is an important antioxidant in vivo; however, it also fulfils other vital roles such as regulation of immune function, signal transduction, metabolism, and the detoxification of electrophilic compounds $(41-45)$. Detoxification occurs via the thiol group under the control of GSH-S-transferases, although conjugation is also observed in the absence of the enzyme $(43,45)$. A dosedependent decrease in measurable GSH, in the absence of GSSG formation, after incubation with CSC was observed, indicating that GSH may form conjugates with the many electrophilic compounds present in cigarette smoke. Considering that the thiol compounds GSH and DTT were both able to prevent CSC-induced necrosis and CSC-mediated inhibition of apoptosis, whereas mannitol was not, indicates that electrophilic compounds, not oxidants, may be responsible for these effects.

To elucidate the mechanism of apoptosis inhibition, the caspase pathway was studied in more detail. Western blot analysis revealed that caspase- 3 activation did not occur in cells treated with CSC. Moreover, treatment with GSH and DTT, and not mannitol, prevented the inhibitory effect of CSC on caspase activation. Caspases contain a central thiol group that is essential for function and prone to oxidation, 
TABLE 1

The antioxidants DTT and GSH prevent CSC-induced necrosis and inhibition of apoptosis

\begin{tabular}{llcc}
\hline & $\%$ Apoptosis & $\%$ Necrosis \\
\hline Control & No antioxidants & $0.3 \pm 0.1$ & $0.1 \pm 0.1$ \\
& Mannitol & $0.3 \pm 0.2$ & $0.2 \pm 0.1$ \\
& DTT & $2.4 \pm 1.3$ & $0.7 \pm 0.4$ \\
& GSH & $0.4 \pm 0.2$ & $0.2 \pm 0.2$ \\
SS & No antioxidants & $94.9 \pm 2.6^{*}$ & $0.4 \pm 0.2$ \\
& Mannitol & $97.4 \pm 1.2^{*}$ & $0.9 \pm 0.7$ \\
& DTT & $90.3 \pm 4.2^{*}$ & $0.1 \pm 0.1$ \\
& GSH & $93.4 \pm 4.5^{*}$ & $0.2 \pm 0.1$ \\
CSC & No antioxidants & $0.0 \pm 0.0$ & $74.6 \pm 5.0^{*}$ \\
& Mannitol & $0.0 \pm 0.0$ & $75.4 \pm 11.8^{*}$ \\
& DTT & $0.4 \pm 0.3$ & $16.3 \pm 1.7^{\dagger}$ \\
SS/CSC & GSH & $12.9 \pm 4.7$ & $0.3 \pm 0.3^{\dagger}$ \\
& No antioxidants & $0.0 \pm 0.0$ & $69.5 \pm 4.9^{*}$ \\
& Mannitol & $23.2 \pm 18.4^{\dagger}$ & $61.4 \pm 18.5^{*}$ \\
& DTT & $79.5 \pm 11.4^{* \dagger}$ & $0.6 \pm 0.4^{\dagger}$ \\
& GSH & $98.5 \pm 0.5^{* \dagger}$ & $0.3 \pm 0.2^{\dagger}$ \\
\hline
\end{tabular}

$* P<0.001$ compared with antioxidant control.

'P $P<0.001$ compared with no antioxidant treatment.

Jurkat cells were treated as indicated with $5 \mathrm{mM}$ mannitol, $1 \mathrm{mM}$ dithiothreitol, or $1 \mathrm{mM}$ glutathione for $6 \mathrm{~h}$. Cytospins were prepared and the percentage of normal, apoptotic and necrotic cells were determined. Results are mean of three experiments \pm SEM where at least 300 cells were counted per slide.

alkylation, and s-nitrosylation (46). However, direct addition of CSC to recombinant caspase- 3 had no effect on the ability of the enzyme to cleave its substrate.

The effect of CSC on caspase activation was studied further by reconstitution of the apoptosome, in cytoplasmic extracts of Jurkat cells, on addition of cytochrome-c and dATP. The occurrence of caspase- 3 cleavage, as determined by Western blot, was used as a positive indicator of apoptosome formation and caspase-9 activation. Initially, CSC was incubated with the extracts before addition of cytochrome-c and dATP, whereby caspase- 3 cleavage was observed, indicating that CSC had no direct effect on formation of an active apoptosome. Interestingly, in extracts from Jurkat cells treated with CSC for $2 \mathrm{~h}$ before preparation, caspase- 3 activation was prevented. Moreover, caspase-9 activation did not occur, indicating CSC treatment prevented the formation of a functional apoptosome. Importantly, apoptosome formation and caspase-3 cleavage was also prevented by CSC treatment of A549 lysates, thus underscoring this effect on the cell death machinery to be more general and not Jurkat-specific. Numerous inducible regulators of apoptosis exist, presenting the possibility that CSC exposure may mediate an alteration of the intracellular balance between pro- and antiapoptogenic factors.

Although Jurkat cells were used as a model here, the involvement of $\mathrm{T}$ cells in the development of emphysema must not be overlooked. Increased numbers of $T$ cells are observed in the lungs of emphysema sufferers, and their presence is correlated with increased lung destruction (47). Necrosis of $\mathrm{T}$ cells present in the lung may also contribute to the progression of emphysema by increasing local tissue damage by release of intracellular contents.

The data presented here are in contradiction to some

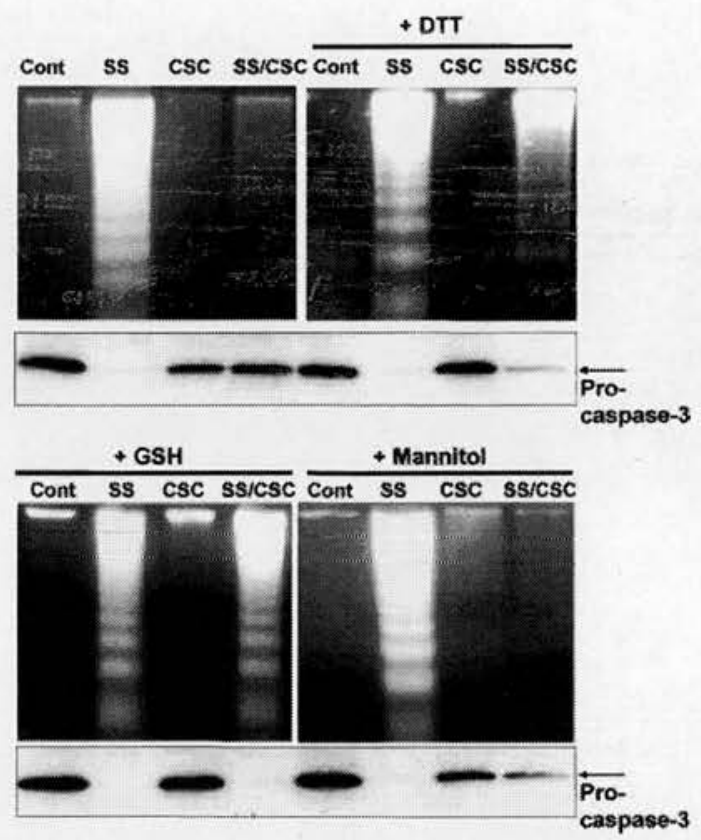

Figure 8. Activation of caspase-3 and subsequent DNA "laddering" does not occur in Jurkats treated with CSC or SS/CSC. Cells were treated with $2 \mu \mathrm{M} \mathrm{SS}$ and/or $10 \%$ CSC in the presence of the thiol antioxidants DTT or GSH, or mannitol. Activation of caspase- 3 was determined by Western blot for loss of the pro-form and the resultant DNA "laddering" was determined by agarose gel electrophoresis. Representative of three experiments.

previous studies $(3,4,25-28)$. However, a number of these have used TUNEL nick-end labeling to identify apoptotic cells $(26,28)$, a method that merely detects DNA strand breaks. Cigarette smoke exposure results in oxidant-induced DNA strand breaks (48), and so these studies may have inadvertently identified cells with oxidant-induced DNA damage as apoptotic. Moreover, caspase activation was either not involved (4), or not studied (27), in "cigarette smokeinduced apoptosis". Characterization of cell death is becoming increasingly complex. A number of alternative forms of cell death have been identified with many of the "classical" markers of apoptosis evident. However, in some cases cell death is independent of caspase activation (8). For this reason, it is becoming increasingly necessary to characterize cell death by a number of methods. This presents a plausible explanation as to why previous studies have purported to observe apoptosis in response to smoke exposure. Additionally, no standardized protocol for the production of CSC exists; each procedure can isolate a slightly different spectrum of components. Nevertheless, we believe that our method of exposing cells to CSC replicates the situation in a smoker's lung, whereby passing the smoke over the buffer in the tonometer system more accurately mimics smoke filling the airspace and exposing the lung lining fluid.

The key finding of this study is that cigarette smoke induces necrosis in alveolar type II cells, endothelial cells, and Jurkat cells. Moreover, CSC inhibited caspase activation and apoptosis in A549 and Jurkat cells. From these 


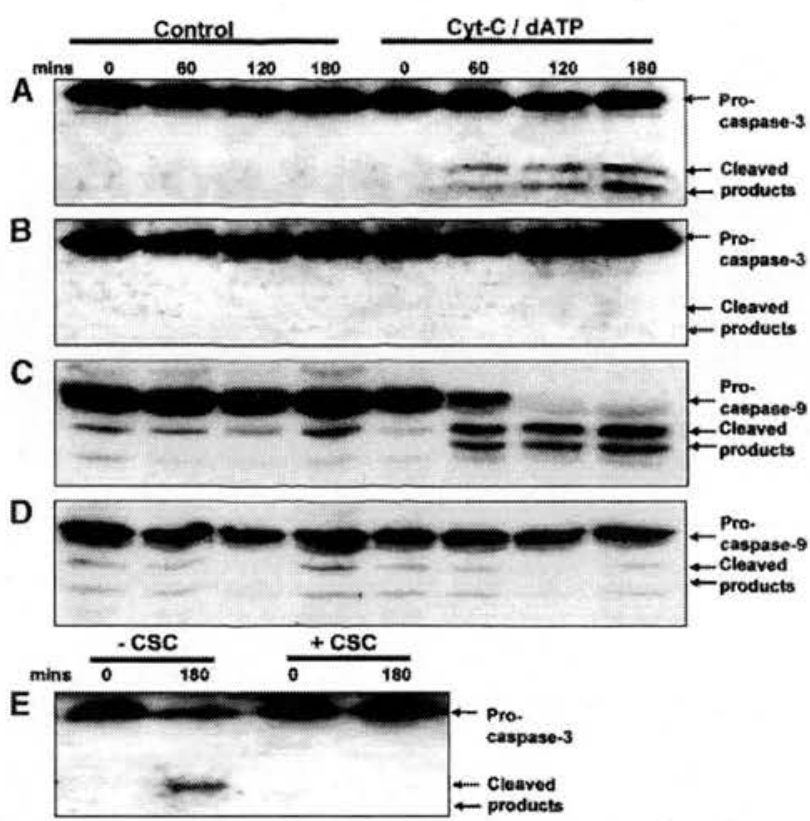

Figure 9. CSC treatment inhibits apoptosome formation and subsequent activation of the caspase pathway. Jurkat cells were incubated for $2 \mathrm{~h}$ with either control media $(A, C)$ or $10 \% \operatorname{CSC}(B$, $D)$. Cytoplasmic extracts were prepared and reconstitution of the apoptosome was initiated by addition of $10 \mathrm{mM}$ cytochrome-c and $1 \mathrm{mM}$ dATP. Western blotting for caspase-3 $(A, B)$, or caspase-9 $(C, D)$ indicated that apoptosome formation and activation of the caspase cascade did not occur in CSC-treated lysates. Similarly, CSC treatment inhibited apoptosome formation and caspase-3 activation in identically prepared A549 cell lysates $(E)$. Result is representative of three experiments.

findings, we suggest a mechanism whereby cigarette smoke may induce emphysema. Although initially thought to be due to apoptosis $(3-5,7,40)$, emphysema may in fact result from loss of alveolar tissue by necrosis of lung epithelial and endothelial cells. In these experiments, the effects of CSC could be prevented by the presence of extracellular thiol compounds such as GSH, which is native to the lung and forms one of the most important lung antioxidant defenses (49). Therefore, it could be deduced that the risk of necrosis in response to cigarette smoking is insignificant. However, in situations of acute smoking, GSH has been shown to be decreased in lung lining fluid with a subsequent rebound to levels higher than those of nonsmokers $(36,38$, $50,51)$. During this window of antioxidant depletion, the cells of the lung are likely to be susceptible to necrotic cell death as a consequence of additional cigarette smoke exposure. Moreover, local tissue damage may be amplified by the subsequent release of intracellular enzymes and lysosomal contents, resulting in recruitment of inflammatory cells to the site of injury and further necrosis of surrounding tissue. This scenario is supported by Retamales and coworkers (52), who observed increased inflammation in increased severity of emphysema. Thus, although much interest has been generated on the involvement of apoptosis, our studies emphasize the role of necrosis and the subsequent proin- flammatory responses as more likely candidates in the pathogenesis of emphysema.

Acknowledgments: The authors thank Drs. Peter Henriksen and Jean-Michel Sallenave for supply of HUVECs, Drs. Ellen Drost and Peter Gilmour for helpful advice and comments on the manuscript, and The Colt Foundation for financial support.

\section{References}

1. Riley, D. J., and J. S. Kerr. 1985. Oxidant injury of the extracellular matrix: potential role in the pathogenesis of pulmonary emphysema. Lung 163: 1-13.

2. Gadek, J. E., and E. R. Pacht. 1990. The protease-antiprotease balance within the human lung: implications for the pathogenesis of emphysema. Lung 168:552-564

3. Tuder, R. M., K. Wood, L. Tarasevicine, S. C. Flores, and N. F. Voelkel. 2000. Cigarette smoke extract decreases the expression of vascular endothelial growth factor by cultured cells and triggers apoptosis of pulmonary endothelial cells. Chest 117:241S-242S. (Abstr)

4. Hoshino, Y., T. Mio, S. Nagai, H. Miki, I. Ito, and T. Izumi. 2001. Cytotoxic effects of cigarette smoke extract on an alveolar type II cell-derived cell line. Am. J. Physiol. Lung Cell. Mol. Physiol. 281:L509-L516.

5. Kasahara, Y., R. M. Tuder, L. Taraseviciene-Stewart, T. D. Le Cras, S. Abman, P. K. Hirth, J. Waltenberger, and N. F. Voelkel. 2000. Inhibition of VEGF receptors causes lung cell apoptosis and emphysema. $J$. Clin. Invest. 106:1311-1319.

6. Lucey, E. C., J. Keane, P. P. Kuang, G. L. Snider, and R. H. Goldstein. 2002. Severity of elastase-induced emphysema is decreased in tumor necrosis factor-alpha and interleukin-1beta receptor-deficient mice. Lab. Invest. 82:79-85.

7. Kasahara, Y., R. M. Tuder, C. D. Cool, D. A. Lynch, S. C. Flores, and N. F. Voelkel. 2001. Endothelial cell death and decreased expression of vascular endothelial growth factor and vascular endothelial growth factor receptor 2 in emphysema. Am. J. Respir. Crit. Care Med. 163:737-744.

8. Leist, M., and M. Jaattela. 2001. Four deaths and a funeral: from caspases to alternative mechanisms. Nat. Rev. Mol. Cell Biol. 2:589-598.

9. Kerr, J. F., A. H. Wyllie, and A. R. Currie. 1972. Apoptosis: a basic biological phenomenon with wide-ranging implications in tissue kinetics. Br. J. Cancer 26:239-257.

10. Thompson, C. B. 1995. Apoptosis in the pathogenesis and treatment of disease. Science 267:1456-1462.

11. Majno, G., and I. Joris. 1995. Apoptosis, oncosis, and necrosis: an overview of cell death. Am. J. Pathol. 146:3-15.

12. Lennon, S. V., S. J. Martin, and T. G. Cotter. 1991. Dose-dependent induction of apoptosis in human tumour cell lines by widely diverging stimuli. Cell Prolif. 24:203-214.

13. Thornberry, N. A., and Y. Lazebnik. 1998. Caspases: enemies within. Science 281:1312-1316.

14. Budihardjo, I., H. Oliver, M. Lutter, X. Luo, and X. Wang. 1999. Biochemical pathways of caspase activation during apoptosis. Annu. Rev. Cell Dev. Biol. 15:269-290.

15. Kluck, R. M., E. Bossy-Wetzel, D. R. Green, and D. D. Newmeyer. 1997. The release of cytochrome $\mathrm{c}$ from mitochondria: a primary site for $\mathrm{Bcl}-2$ regulation of apoptosis. Science 275:1132-1136.

16. Liu, X., C. N. Kim, J. Yang, R. Jemmerson, and X. Wang. 1996. Induction of apoptotic program in cell-free extracts: requirement for dATP and cytochrome c. Cell 86:147-157.

17. Li, P., D. Nijhawan, I. Budihardjo, S. M. Srinivasula, M. Ahmad, E. S. Alnemri, and X. Wang. 1997. Cytochrome $\mathrm{c}$ and dATP-dependent formation of Apaf-1/caspase-9 complex initiates an apoptotic protease cascade. Cell $91: 479-489$

18. Concha, N. O., and S. S. Abdel-Meguid. 2002. Controlling apoptosis by inhibition of caspases. Curr. Med. Chem. 9:713-726.

19. Hampton, M. B., and S. Orrenius. 1997. Dual regulation of caspase activity by hydrogen peroxide: implications for apoptosis. FEBS Lett. 414:552-556.

20. Samali, A., H. Nordgren, B. Zhivotovsky, E. Peterson, and S. Orrenius. 1999. A comparative study of apoptosis and necrosis in HepG2 cells: oxidantinduced caspase inactivation leads to necrosis. Biochem. Biophys. Res. Commun. 255:6-11.

21. Lee, Y. J., and E. Shacter. 1999. Oxidative stress inhibits apoptosis in human lymphoma cells. J. Biol. Chem. 274:19792-19798.

22. Lee, Y. J., and E. Shacter. 2000. Hydrogen peroxide inhibits activation, not activity, of cellular caspase-3 in vivo. Free Radic. Biol. Med. 29:684-692.

23. Borutaite, V., and G. C. Brown. 2001. Caspases are reversibly inactivated by hydrogen peroxide. FEBS Lett. 500:114-118. 
24. Palomba, L., P. Sestili, F. Cattabeni, A. Azzi, and O. Cantoni. 1996. Prevention of necrosis and activation of apoptosis in oxidatively injured human myeloid leukemia U937 cells. FEBS Lett. 390:91-94.

25. Ishii, T., T. Matsuse, H. Igarashi, M. Masuda, S. Teramoto, and Y. Ouchi 2001. Tobacco smoke reduces viability in human lung fibroblasts: protective effect of glutathione S-transferase P1. Am. J. Physiol. Lung Cell. Mol. Physiol. 280:L1189-L1195.

26. D'Agostini, F., R. M. Balansky, A. Izzotti, R. A. Lubet, G. J. Kelloff, and S. De Flora. 2001. Modulation of apoptosis by cigarette smoke and cancer chemopreventive agents in the respiratory tract of rats. Carcinogenesis 22:375-380.

27. Vayssier, M., N. Banzet, D. Francois, K. Bellmann, and B. S. Polla. 1998. Tobacco smoke induces both apoptosis and necrosis in mammalian cells: differential effects of HSP70. Am. J. Physiol. 275:L771-L779.

28. Wang, J., D. E. Wilcken, and X. L. Wang. 2001. Cigarette smoke activates caspase- 3 to induce apoptosis of human umbilical venous endothelial cells. Mol. Genet. Metab. 72:82-88.

29. Rahman, I., X. Y. Li, K. Donaldson, D. J. Harrison, and W. MacNee. 1995. Glutathione homeostasis in alveolar epithelial cells in vitro and lung in vivo under oxidative stress. Am. J. Physiol. 269:L285-L292.

30. Rahman, I., C. A. Smith, M. F. Lawson, D. J. Harrison, and W. MacNee. 1996. Induction of gamma-glutamylcysteine synthetase by cigarette smoke is associated with AP-1 in human alveolar epithelial cells. FEBS Lett. 396:21-25.

31. Martin, D and Leonardo, M. 1998. Microscopic quantitation of apoptotic index and cell viability using vital and fluorescent dyes. Current Protocols in Immunology 1, 3.17.1-3.17.39.

32. Slee, E. A., M. T. Harte, R. M. Kluck, B. B. Wolf, C. A. Casiano, D. D. Newmeyer, H. G. Wang, J. C. Reed, D. W. Nicholson, E. S. Alnemri, D. R. Green, and S. J. Martin. 1999. Ordering the cytochrome c-initiated caspase cascade: hierarchical activation of caspases-2, $-3,-6,-7,-8$, and -10 in a caspase-9-dependent manner. J. Cell Biol. 144:281-292.

33. Tietze, F. 1969. Enzymic method for quantitative determination of nanogram amounts of total and oxidized glutathione: applications to mammalian blood and other tissues. Anal. Biochem. 27:502-522.

34. Vandeputte, C., I. Guizon, I. Genestie-Denis, B. Vannier, and G. Lorenzon. 1994. A microtiter plate assay for total glutathione and glutathione disulfide contents in cultured/isolated cells: performance study of a new miniaturized protocol. Cell Biol. Toxicol. 10:415-421.

35. Pryor, W. A., and K. Stone. 1993. Oxidants in cigarette smoke: radicals, hydrogen peroxide, peroxynitrate, and peroxynitrite. Ann. N. Y. Acad Sci. 686:12-27.

36. Li, X. Y., I. Rahman, K. Donaldson, and W. MacNee. 1996. Mechanisms of cigarette smoke induced increased airspace permeability. Thorax 51:465471.
37. Jones, J. G., B. D. Minty, P. Lawler, G. Hulands, J. C. Crawley, and N. Veall. 1980. Increased alveolar epithelial permeability in cigarette smokers. Lancet 1:66-68.

38. Rahman, I., D. Morrison, K. Donaldson, and W. MacNee. 1996. Systemic oxidative stress in asthma, COPD, and smokers. Am. J. Respir. Crit. Care Med. 154:1055-1060.

39. Morrison, D., I. Rahman, S. Lannan, and W. MacNee. 1999. Epithelial permeability, inflammation, and oxidant stress in the air spaces of smokers. Am. J. Respir. Crit. Care Med. 159:473-479.

40. Tuder, R. M., Y. Kasahara, and N. F. Voelkel. 2000. Inhibition of vascular endothelial growth factor receptors causes emphysema in rats. Chest 117:281S (Abstr)

41. Hudson, V. M. 2001. Rethinking cystic fibrosis pathology: the critical role of abnormal reduced glutathione (GSH) transport caused by CFTR mutation. Free Radic. Biol. Med. 30:1440-1461.

42. Brown, L. A. 1994. Glutathione protects signal transduction in type II cells under oxidant stress. Am. J. Physiol. 266:L172-L177.

43. Chasseaud, L. F. 1979. The role of glutathione and glutathione S-transferases in the metabolism of chemical carcinogens and other electrophilic agents. Adv. Cancer Res. 29:175-274.

44. Droge, W., K. Schulze-Osthoff, S. Mihm, D. Galter, H. Schenk, H. P. Eck, S. Roth, and H. Gmunder. 1994. Functions of glutathione and glutathione disulfide in immunology and immunopathology. FASEB J. 8:1131-1138.

45. Meister, A., and M. E. Anderson. 1983. Glutathione. Annu. Rev. Biochem. 52:711-760.

46. Melino, G., F. Bernassola, R. A. Knight, M. T. Corasaniti, G. Nistico, and A. Finazzi-Agro. 1997. S-nitrosylation regulates apoptosis. Nature 388: $432-433$.

47. Finkelstein, R., R. S. Fraser, H. Ghezzo, and M. G. Cosio. 1995. Alveolar inflammation and its relation to emphysema in smokers. Am. J. Respir. Crit. Care Med. 152:1666-1672.

48. Leanderson, P. 1993. Cigarette smoke-induced DNA damage in cultured human lung cells. Ann. N. Y. Acad. Sci. 686:249-259.

49. Kelly, F. J. 1999. Gluthathione: in defence of the lung. Food Chem. Toxicol. 37:963-966.

50. Li, X. Y., K. Donaldson, I. Rahman, and W. MacNee. 1994. An investigation of the role of glutathione in increased epithelial permeability induced by cigarette smoke in vivo and in vitro. Am. J. Respir. Crit. Care Med. cigarette smoke

51. Cantin, A. M., S. L. North, R. C. Hubbard, and R. G. Crystal. 1987. Normal alveolar epithelial lining fluid contains high levels of glutathione. J. AppL. Physiol. 63:152-157.

52. Retamales, I., W. M. Elliott, B. Meshi, H. O. Coxson, P. D. Pare, F. C. Sciurba, R. M. Rogers, S. Hayashi, and J. C. Hogg. 2001. Amplification of inflammation in emphysema and its association with latent adenoviral infection. Am. J. Respir. Crit. Care Med. 164:469-473. 


\title{
Role of leukotrienes in the regulation of human granulocyte behaviour: dissociation between agonist-induced activation and retardation of apoptosis
}

\author{
'Joanna Murray, 'Carol Ward, ' Joseph T. O'Flaherty, 'Ian Dransfield, 'Christopher Haslett, \\ ${ }^{3}$ Edwin R. Chilvers \& ${ }^{*, 1}$ Adriano G. Rossi \\ ${ }^{1}$ Rayne Laboratory, Respiratory Medicine Unit, Centre for Inflammation Research, University of Edinburgh Medical School, \\ Teviot Place, Edinburgh EH8 9AG; ${ }^{2}$ Department of Internal Medicine, Section on Infectious Diseases, Wake Forest University \\ School of Medicine, Medical Center Blvd., Winston-Salem, NC 27156, U.S.A. and ${ }^{3}$ Respiratory Medicine Division, Department \\ of Medicine, University of Cambridge School of Medicine, Addenbrooke's and Papworth Hospitals, Hills Road, Cambridge CB2 \\ 2QQ
}

1 Since most inflammatory mediators that stimulate granulocyte responsiveness also delay apoptosis, it is often assumed that activation and longevity are causally related. Using isolated human peripheral blood neutrophils and eosinophils, we examined this association by exploiting the proinflammatory lipid mediators, the leukotrienes (LTs), and investigated granulocyte function and apoptosis.

$2 \mathrm{LTB}_{4}$ induced elevation of intracellular free $\mathrm{Ca}^{2+}$ concentration $\left(\left[\mathrm{Ca}^{2+}\right]_{i}\right)$, cell polarisation and retardation of neutrophil apoptosis, although the antiapoptotic effect occurred only at concentrations $\geqslant 300 \mathrm{nM}$. $\mathrm{LTB}_{4}$-induced activation was attenuated by CP-105,696, a BLT1-specific antagonist suggesting classical $\mathrm{LTB}_{4}$ receptor BLT1 involvement.

3 Despite demonstrating the presence of the neutrophil intracellular $\mathrm{LTB}_{4}$ receptor peroxisomeproliferator activator receptor- $\alpha$ (PPAR $\alpha$ ) in neutrophils, the selective PPAR $\alpha$ agonist WY-14,643 did not mimic $\mathrm{LTB}_{4}$-induced prosurvival effects.

$4 \mathrm{LTB}_{4}$-induced survival, however, also appeared to be mediated by BLT1 since CP-105,696 inhibited the $\mathrm{LTB}_{4}$-mediated antiapoptotic effect. Furthermore, based on studies with CP-105,696 and 5-lipoxygenase inhibitors, lipopolysaccharide (LPS)-, granulocyte-macrophage colony-stimulating factor (GM-CSF)-, dexamethasone- and dibutyryl-cAMP (db-cAMP)-induced delay of neutrophil apoptosis did not involve autocrine production of $\mathrm{LTB}_{4}$.

5 Although $\mathrm{LTB}_{4}$ and $\mathrm{LTD}_{4}$ induced human eosinophil $\left[\mathrm{Ca}^{2+}\right]_{i}$ elevation and polarization, these $\mathrm{LTs}$ did not influence eosinophil apoptosis. Furthermore, $\mathrm{LTB}_{4}{ }^{-}$and $\mathrm{LTD}_{4}$-induced eosinophil activation was attenuated by $\mathrm{CP}-105,696$ and the Cys-LT ${ }_{1}$ receptor antagonist montelukast, respectively, highlighting specific receptor dependency.

6 Thus, mediator-triggered granulocyte activation and antiapoptotic pathways are distinct events that can be differentially regulated.

British Journal of Pharmacology (2003) 139, 388 - 398. doi:10.1038/sj.bjp.0705265

Keywords: Leukotrienes; neutrophils; eosinophils; apoptosis; calcium

Abbreviations: BLT, $\mathrm{LTB}_{4}$ receptor; $\left[\mathrm{Ca}^{2+}\right]_{\mathrm{l}}$, intracellular free $\mathrm{Ca}^{2+}$ concentration; Cys-LT, cysteinyl leukotriene db-cAMP, dibutyryl-cAMP; fMLP, formyl-leucyl-phenylalanine; GM-CSF, granulocyte/macrophage colonystimulating factor; LPS, lipopolysaccharide; PAF, platelet-activating factor; PPAR, peroxisome-proliferator activator receptor

\section{Introduction}

Granulocytes are key effector cells of the immune system, where neutrophils provide first-line protection against bacterial and fungal invasion and eosinophils play an important role in mediating antiparasitic defences. Over-recruitment, inappropriate activation or dysregulated clearance of these cells likely contribute to the pathogenesis and propagation of a wide variety of inflammatory disorders. While neutrophil

*Author for correspondence at: Rayne Laboratory, Respiratory Medicine Unit, Centre for Inflammation Research, University of Edinburgh Medical School, Teviot Place, Edinburgh, EH8 6AG, U.K.; E-mail: a.g.rossi@ed.ac.uk products can cause tissue damage in inflammatory diseases such as chronic obstructive pulmonary disease and rheumatoid arthritis, inappropriate release of eosinophil products is associated with allergic disorders such as allergic rhinitis and asthma (Rossi \& Haslett, 1998; Giembycz \& Lindsay, 1999; Gompertz \& Stockley, 2000). Apoptosis or programmed cell death is a critical process regulating the lifespan of inflammatory cells and the resolution phase of inflammation (Haslett, 1999; Ward et al., 1999b; Lawrence et al., 2002). Apoptosis is associated with the maintenance of plasma membrane integrity, downregulation of functional responsiveness (Whyte et al., 1993b), and triggers apoptotic cell recognition 
and engulfment by phagocytic cells (Savill et al., 1989; Stern et al., 1992; Walsh et al., 1999). Remarkably, the phagocytic clearance of these cells fails to excite an inflammatory response (Meagher et al., 1992); indeed, ingestion of apoptotic granulocytes in vitro induces an anti-inflammatory cytokine and mediator profile in the phagocyte (Stern et al., 1996; Fadok et al., 1998; Liu et al., 1999). Thus, this process provides an efficient and noninflammatory mechanism for the clearance of potentially toxic and unneeded cells from the inflamed site.

Neutrophils and eosinophils are derived from a common myeloid precursor, and when activated, exhibit similar responses (e.g., cell polarisation, chemotaxis, adhesion, degranulation and superoxide anion generation). Comparative studies of functional responses in these cells have contributed significantly to our understanding of granulocyte behaviour, including apoptosis. Of interest, despite their close haematopoietic origins, apoptosis of eosinophil and neutrophil granulocytes can be regulated in a selective manner. For example, glucocorticoids induce eosinophil apoptosis but retard this process in the neutrophil (Meagher et al., 1996). Many of the proinflammatory mediators present at an inflamed site not only influence granulocyte responsiveness but also delay the rate of constitutive apoptosis. For example, lipopolysaccharide (LPS) and granulocyte/macrophage colony-stimulating factor (GM-CSF) have been shown to upregulate neutrophil function by priming for enhanced functional responsiveness to secretagogue agonists and also prolong cell longevity by delaying apoptosis (Colotta et al., 1992; Lee et al., 1993). A similar dual effect has been observed in eosinophils stimulated with IL-5 and GM-CSF with both cytokines enhancing eosinophil function and delaying apoptosis (Stern et al., 1992; Walsh et al., 1996). Thus, it is often perceived that a close and inevitable relation exists between granulocyte activation status and longevity; indeed, a large family of inflammatory mediators promote granulocyte function by initially inducing cell priming and subsequently by delaying the onset of constitutive apoptosis (Mecklenburgh et al., 1999). In this study, we investigated this association by exploiting the archetypal proinflammatory lipid mediators, the leukotrienes (LTs).

The LTs are produced by the oxygenation of deacylated membrane-bound arachidonic acid through a reaction catalysed by 5-lipoxygenase (5-LO) and 5-lipoxygenase-activating protein (FLAP) to yield 5-hydroperoxyeisosatetraenoic acid (5-HPETE), which is converted to leukotriene $\mathrm{A}_{4}\left(\mathrm{LTA}_{4}\right)$, an unstable epoxide intermediate. In neutrophils, and other cells that contain $\mathrm{LTB}_{4}$ synthase (e.g., alveolar macrophages), $\mathrm{LTA}_{4}$ is converted to $\mathrm{LTB}_{4}$ (Samuelsson et al., 1987). Indeed, neutrophils when activated by a variety of stimuli (e.g., the calcium ionophore, A23187 and receptor-directed stimuli such as platelet-activating factor (PAF), formyl-leucyl-phenylalanine (fMLP) and GM-CSF) are capable of synthesising and releasing $\mathrm{LTB}_{4}$ (Lin et al., 1982; Samuelsson et al., 1987; Dahinden et al., 1988; Rossi et al., 1993). LTB 4 has also been shown to be a powerful activator of neutrophil responsiveness causing cell polarisation, chemotaxis, adhesion, degranulation and reactive oxygen species generation (Ford-Hutchinson et al., 1980; Rossi et al., 1993). In addition, $\mathrm{LTB}_{4}$ has been shown to influence neutrophil longevity by delaying apoptosis (Hebert et al., 1996). LTB ${ }_{4}$ is believed to elicit its effects by interacting with distinct seven transmembrane G-proteincoupled receptors, the classical BLT1 receptor (Yokomizo et al., 1997) and the recently identified BLT2 receptor (Kamohara et al., 2000; Serhan \& Prescott, 2000; Yokomizo et al., 2000). The other receptor type that binds $\mathrm{LTB}_{4}$ that has received much recent attention is the peroxisome-proliferator activator receptor- $\alpha(\operatorname{PPAR} \alpha)($ Devchand et al., 1996). This receptor is a member of the PPAR group of transducer proteins belonging to the steroid/thyroid/retinoid receptor family, which regulate the expression of target genes by binding to PPAR response elements. PPAR $\alpha$ is primarily expressed in tissues that have high fatty acid catabolism, including the liver and the immune system (Devchand et al., 1996).

In eosinophils, mast cells and other cells that contain $\mathrm{LTC}_{4}$ synthase, LTA $_{4}$ is converted mainly to $\mathrm{LTC}_{4}$, through conjugation with reduced glutathione. $\mathrm{LTC}_{4}$ is exported from the cytosol to the extracellular environment and sequentially cleaved to form $\mathrm{LTD}_{4}$ and the less potent $\mathrm{LTE}_{4}$. These cysteinyl LTs are now well established as mediators of immediate hypersensitivity reactions, and are potent agonists of bronchoconstriction and vascular permeability and play a role in the persistent tissue eosinophilia that is characteristic of asthma (Leff, 2000). Cysteinyl LTs may influence various stages of the eosinophil life cycle including bone marrow differentiation, adhesion and margination; however, the effects of the cysteinyl LTs on the activation status and functional longevity of these cells are ill defined. In this study, we have examined the relation between granulocyte activation and apoptosis by comparing the effects of the LTs on neutrophil and eosinophil function and lifespan in vitro.

\section{Methods}

\section{Neutrophil and eosinophil isolation}

Neutrophils and eosinophils were isolated from the peripheral venous blood of healthy adult donors by dextran sedimentation followed by centrifugation through discontinuous PBSPercoll gradients (Rossi et al., 1998; Ward et al., 1999a). Only neutrophil preparations with a purity of $>98 \%$ were used. Eosinophils were separated from contaminating neutrophils using an immunomagnetic separation step with sheep anti-mouse IgG-Dynabeads (Dynabeads M-450, Dynal, Merseyside, U.K.) coated with the murine antineutrophil antibody 3G8 (anti-CD16; a gift from Dr J. Unkeless, Mount Sinai Medical School, New York, U.S.A.). Cells were mixed with washed 3G8-coated Dynabeads at a bead: neutrophil ratio of $3: 1$ on a rotary mixer at $4{ }^{\circ} \mathrm{C}$ for $20 \mathrm{~min}$, and the beads removed magnetically by two $3 \mathrm{~min}$ stationary magnetic contacts (Dynal Magnetic Particle Concentrator, MPC-1) to yield an eosinophil population of $>98 \%$ purity. After harvesting and purification, cells were washed twice in PBS (without divalent cations) and once in PBS containing $\mathrm{MgCl}_{2}$ and $\mathrm{CaCl}_{2}$ before resuspending in appropriate buffer.

\section{Culture of gramulocytes}

Neutrophils $\left(5 \times 10^{6}\right.$ cells ml $\left.{ }^{-1}\right)$ and eosinophils $\left(2.5 \times 10^{6}\right.$ cells $\mathrm{ml}^{-1}$ ) were resuspended in Iscove's MDM (Life Technologies, Paisley, U.K.) containing $50 \mathrm{U} \mathrm{ml}^{-1}$ penicillin and $50 \mathrm{U} \mathrm{ml}^{-1}$ streptomycin and supplemented with $10 \%$ autologous serum (or $0.1 \%$ BSA) and cultured in flat-bottomed 96- 
well Falcon flexiwell plates (Becton-Dickinson, U.K.) at $37^{\circ} \mathrm{C}$ in a humidified $5 \% \mathrm{CO}_{2}$ atmosphere for the time periods indicated. The cells were cultured in the absence or presence of the reagents of interest as described in the figure legends and unless otherwise stated, all experiments were done at least three times with each treatment performed in triplicate.

\section{Morphological assessment of granulocyte apoptosis}

After culture, cells were gently resuspended and $100 \mu \mathrm{l}$ harvested from each well, cytocentrifuged in a Shandon Cytospin II (Shandon, U.K.) and the resulting slide preparations air dried, fixed in methanol and stained with Diff-Quik ${ }^{\mathrm{TM}}$. Cell morphology was examined by $\times 100$ objective oil immersion light microscopy to determine the proportion of cells with highly characteristic apoptotic morphology (Savill et al., 1989; Stern et al., 1992; Ward et al., 1999a). For each condition examined, slides were prepared from triplicate incubations and after coding, a total of at least 500 granulocytes were counted over a minimum of five high power fields with the observer blinded to the assay conditions. The data were expressed as the mean per cent apoptosis \pm s.e.m. Cell viability was determined in parallel by the ability of granulocytes to exclude the vital dye trypan blue assessed by light microscopy and, on occasion, by cellular incorporation of propidium iodide as assessed by flow cytometry (Ward et al., 1999a). Under all conditions and treatments used, there was no loss of cell membrane integrity and cell loss was minimal.

\section{Annexin $V$ binding}

A separate and independent assessment of apoptosis was performed by flow cytometry using FITC-labelled recombinant human annexin $\mathrm{V}$ that binds to phosphatidylserine exposed on the surface of apoptotic cells. Stock annexin V (Bender MedSystems, Vienna, Austria) was diluted 1:200 with binding buffer and then added $(25 \mu \mathrm{l})$ to $75 \mu \mathrm{l}$ of the recovered cell samples. Following a $10 \mathrm{~min}$ incubation at $4^{\circ} \mathrm{C}$, these samples were fixed by the addition of $100 \mu \mathrm{l}$ of $3 \%$ paraformaldehyde before analysis using an EPICS Profile II (Coulter Electronics, Luton, U.K.) or a FACSCalibur (Becton Dickinson, Oxford, U.K.) (Ward et al., 1999a). The fluorescence from a minimum of 5000 cells was recorded.

\section{Determination of cytosolic free calcium ion levels $\left(\left[\mathrm{Ca}^{2+}\right]_{i}\right)$}

Freshly isolated granulocytes were washed $(3 \times)$ in HBSS $\left(\mathrm{Ca}^{2+}\right.$ and $\mathrm{Mg}^{2+}$ free) before being resuspended at $10^{7} \mathrm{ml}^{-1}$ in HBSS $\left(\mathrm{Ca}^{2+}\right.$ and $\mathrm{Mg}^{2+}$ free) and incubated with fura-2/AM (final concentration $2 \mu \mathrm{M}$ ) for $30 \mathrm{~min}$ at $37^{\circ} \mathrm{C}$ (O'Flaherty \& Rossi, 1993). The cells were then washed $(2 \times)$ and left in HBSS $\left(\mathrm{Ca}^{2+}\right.$ and $\mathrm{Mg}^{2+}$ free) for a further $10 \mathrm{~min}$ for optimal de-esterification of the fura-2-acetoxymethyl ester, before finally resuspending the granulocytes at $2 \times 10^{6} \mathrm{cells} \mathrm{ml}^{-1}$ in HBSS (containing $\mathrm{Ca}^{2+}$ and $\mathrm{Mg}^{2+}$ ). Changes in fluorescence following agonist addition were determined using a PerkinElmer LS50B fluorimeter, with dual wavelength excitation ( 340 and $380 \mathrm{~nm}$ ) and emission at $510 \mathrm{~nm}$. The fluorimeter has a fitted thermostated cuvette compartment and stirring attachment to ensure complete mixing of reagents at $37^{\circ} \mathrm{C}$. Calibration of $\left[\mathrm{Ca}^{2+}\right]_{\mathrm{i}}$ was performed as described (O'Flaherty \& Rossi, 1993).

\section{Granulocyte shape change assay}

Freshly isolated granulocytes $\left(180 \mu \mathrm{l}, 2 \times 10^{6} \mathrm{cells} \mathrm{ml}^{-1}\right.$ in PBS containing $\mathrm{CaCl}_{2}$ and $\mathrm{MgCl}_{2}$ ) were equilibrated at $37^{\circ} \mathrm{C}$ in a shaking water bath for $15 \mathrm{~min}$ in the presence of buffer alone or the antagonist of interest. Agonists were then added to the required concentration and incubations continued for a further $15 \mathrm{~min}$ before the addition of $200 \mu \mathrm{l}$ of $2.5 \%$ glutaraldehyde. Samples were analysed for shape change by flow cytometry (Coulter EPICS Profile II; Coulter Electronics, Luton, U.K.) (Kitchen et al., 1996). Percentage shape change was calculated from the mean forward light scatter of each sample by gating on the nonshape-changed population.

\section{Western blotting for $P P A R \alpha$}

Cell samples $\left(5 \times 10^{6}\right.$ cells ml $\left.^{-1}\right)$ were lysed (Ward et al., 1999a, 2002; Fujihara et al., 2002) and the whole cell lysates were run on a $9 \%$ SDS gel and after transfer to nitrocellulose membranes (Amersham Pharmacia Biotech U.K. Ltd), blocked by $5 \%$ milk protein before an overnight incubation with the polyclonal PPAR $\alpha$ antibody, N/19 (SantaCruz Biotechnology Inc., Santa Cruz, CA, U.S.A.) diluted 1:500. After washing, blots were incubated with HRP-conjugated antibiotin antibody diluted at 1:2500 and developed using standard ECL reagents.

\section{Materials}

Further specific materials were obtained as follows: $\mathbf{L T B}_{4}$, LTC $_{4}$, LTD $_{4}$, and WY-14,643 (Biomol, Affinity Research Products, Mamhead, U.K.) and GM-CSF, IL-5 (R\&D Systems Europe Ltd, Oxon, U.K.) and MK-886 (Calbiochem Ltd, Beeston, Nottingham, U.K.). CP-105,696 was a gift from Henry J. Showell, Pfizer Inc., Connecticut, U.S.A. Montelukast (1-(((1(R)-(3-(2-(7-chloro-2-quinolinyl)-(E)-ethenyl)phenyl)(3-2-(1-hydroxy-1-methylethyl)phenyl)propyl)thio)methyl) cyclopropane) acetic acid sodium salt) was a gift from $\mathrm{Dr}$ Richard Tomiak, Merck, Sharp and Dohme Ltd. All other reagents were obtained from Sigma Chemical Co., Poole, U.K. and were of the highest purity.

\section{Statistical analysis}

The results are expressed as means \pm s.e.m. of $(n)$ the number of independent experiments each using cells isolated from separate donors with each treatment performed in triplicate, unless otherwise stated. Statistical analysis was performed by ANOVA with comparisons between groups made using the Newman-Kuels procedure. Differences were considered significant when $P<0.05$.

\section{Results}

\section{Effect of $\mathrm{LTB}_{4}$ on human neutrophil apoptosis}

Initially, we demonstrated that incubation of human neutrophils for $20 \mathrm{~h}$ with $\mathrm{LTB}_{4}(1 \mu \mathrm{M})$ delayed the rate of constitutive apoptosis as assessed by morphological criteria (Figure 1a). In contrast, the cysteinyl LTs $\mathrm{LTC}_{4}(1 \mu \mathrm{M})$ and $\mathrm{LTD}_{4}(1 \mu \mathrm{M})$ had no effect on the constitutive rate of apoptosis in these cells 

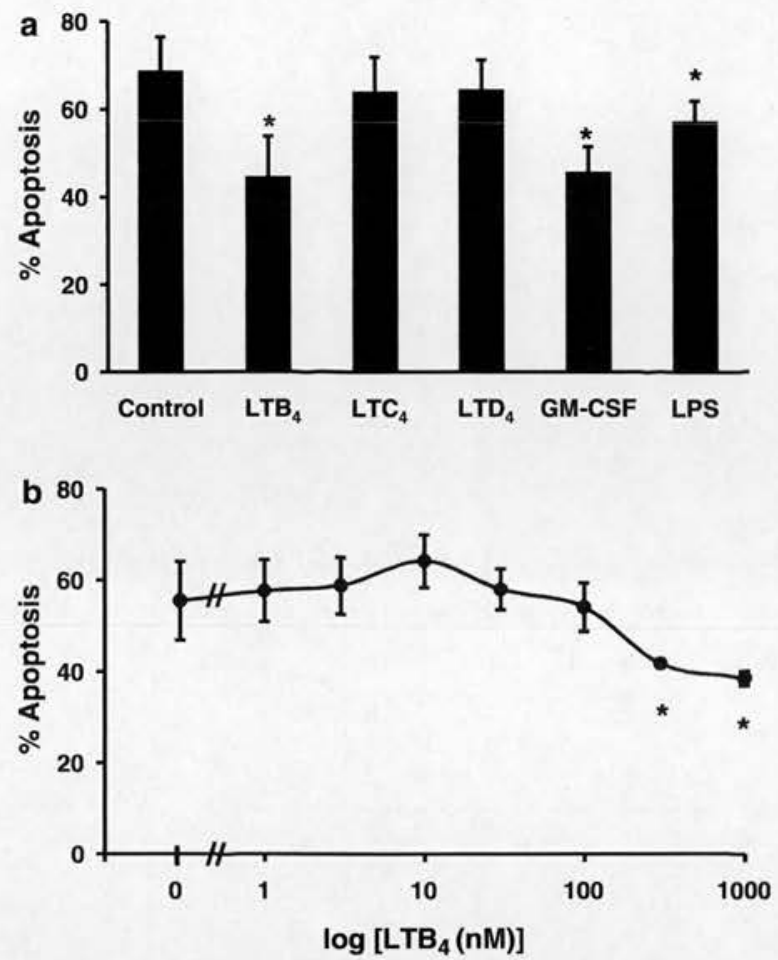

Figure 1 Effect of LTs on human neutrophil apoptosis. Neutrophils $\left(5 \times 10^{6}\right.$ cells $\left.\mathrm{ml}^{-1}\right)$ were cultured in serum-supplemented Iscove's MDM alone (control) or in identical medium containing stimuli (final concentration). (a) $\mathrm{LTB}_{4}(1 \mu \mathrm{M}), \mathrm{LTC}_{4}(1 \mu \mathrm{M}), \mathrm{LTD}_{4}$ $(1 \mu \mathrm{M})$, GM-CSF $\left(50 \mathrm{U} \mathrm{ml}^{-1}\right)$ or LPS $\left(1 \mu \mathrm{g} \mathrm{ml}^{-1}\right)$ and (b) $\mathrm{LTB}_{4}$ $(1 \mathrm{nM}-1 \mu \mathrm{M})$. Cells were harvested following $20 \mathrm{~h}$ in culture and assessed for morphological features of apoptosis. Data represent the means \pm s.e.m. of three separate experiments, each performed in triplicate, where * represents $P<0.05$ compared to control values.

(Figure 1a). The extent of the retardation of neutrophil apoptosis observed with $\mathrm{LTB}_{4}$ was similar to that observed with human recombinant GM-CSF $\left(50 \mathrm{Uml}^{-1}\right)$ and LPS $\left(1 \mu \mathrm{g} \mathrm{ml}^{-1}\right)$ (Figure la). Assessment of cell recovery (haemocytometer counts) and viability (trypan blue exclusion) demonstrated that the inflammatory mediators investigated had no effect on either of these parameters as compared to vehicle-treated controls (data not shown). Experiments performed to examine the concentration dependency of $\mathbf{L T B}_{4}$ mediated neutrophil survival revealed that this response was only observed with relatively high concentrations of $\mathrm{LTB}_{4}$ $\left(\geqslant 300 \mathrm{nM}\right.$; Figure 1b). Accordingly, an $\mathrm{LTB}_{4}$ concentration of $1 \mu \mathrm{M}$ was selected for use in all further apoptosis studies.

\section{Effect of $\mathrm{LTB}_{4}$ on neutrophil $\left[\mathrm{Ca}^{2+}\right]_{i}$ levels and cell} polarisation

Signalling mechanisms such as changes in $\left[\mathrm{Ca}^{2+}\right]_{\mathrm{i}}$ are fundamental in regulating neutrophil activation and may play a key role regulating granulocyte apoptosis (Whyte et al., 1993a). We therefore examined the effects of $\mathrm{LTB}_{4}$ on $\left[\mathrm{Ca}^{2+}\right]_{i}$ in human neutrophils and compared the responses to those induced by other agonists. $\left[\mathrm{Ca}^{2+}\right]_{\mathrm{i}}$ levels in freshly isolated neutrophils were measured using the $\mathrm{Ca}^{2+}$-sensitive dye fura-2 following the addition of PAF (100nM), $\mathrm{LTB}_{4}(100 \mathrm{nM})$ or fMLP $(100 \mathrm{nM})$ as indicated in Figure 2. The resting $\left[\mathrm{Ca}^{2+}\right]_{\mathrm{i}}$ levels recorded in these experiments $(<100 \mathrm{nM})$ were consistent with those reported in previous studies in neutrophils (O'Flaherty \& Rossi, 1993; Whyte et al., 1993a). Addition of PAF, $\mathrm{LTB}_{4}$ or fMLP induced a rapid rise in $\left[\mathrm{Ca}^{2+}\right]_{i}$ to a peak of approximately $600 \mathrm{nM}$, before returning to control values (Figure 2). To investigate the mechanism of $\mathrm{LTB}_{4}$-mediated elevation of $\left[\mathrm{Ca}^{2+}\right]_{i}$, neutrophils were preincubated in the presence or absence of the specific BLT1 receptor antagonist CP-105,696 ((+)-1-(3S,4R)-[3-(4-phenyl-benzyl)-4-hydroxychroman-7-yl]-cyclopentane carboxylic acid; Showell et al., 1995), prior to the addition of $\mathrm{LTB}_{4}$, PAF or fMLP (100 nM). CP-105,696 $(1 \mu \mathrm{M})$ had no effect on either the basal levels of $\left[\mathrm{Ca}^{2+}\right]_{i}$, or on PAF- or fMLP-induced responses, but abrogated $\mathrm{LTB}_{4}$-mediated elevation of $\left[\mathrm{Ca}^{2+}\right]_{i}$ (Figure 2) indicating that this response is a specific, BLT1 receptormediated event. Moreover, the data suggest that fMLP- and PAF-induced elevation of $\left[\mathrm{Ca}^{2+}\right]_{i}$ is not mediated by the autocrinic production of $\mathrm{LTB}_{4}$.

We next examined the effect of a range of $\mathrm{LTB}_{4}$ concentrations on cell polarisation. Incubation of freshly isolated neutrophils with $\mathrm{LTB}_{4}$ for $10 \mathrm{~min}$ induced a dramatic polarisation response as assessed by changes in forward scatter by flow cytometric analysis (compare Figure $3 a$ and $b$ ). This response exhibited a concentration-dependency and was sensitive to much lower $\mathrm{LTB}_{4}$ concentrations of $1 \mathrm{nM}$ and above (Figure 3e). The cysteinyl LTs $\mathrm{LTC}_{4}$ and $\mathrm{LTD}_{4}$ failed to elicit a response at $1 \mu \mathrm{M}$, a concentration that was maximal for $\mathrm{LTB}_{4}$-induced shape change (Figure $3 \mathrm{e}$ ). Preincubation of neutrophils $\left(15 \mathrm{~min}\right.$ at $\left.37^{\circ} \mathrm{C}\right)$ with the BLT1 receptor antagonist CP-105,696 $(3 \mu \mathrm{M})$ had no effect on basal shape change (compare Figure $3 \mathrm{a}$ and $\mathrm{c}$ ) but abrogated the maximal $(1 \mu \mathrm{M})$ $\mathrm{LTB}_{4}$-induced shape change (compare Figure $3 \mathrm{~b}$ and d) without influencing fMLP-induced polarisation (Figure 3f). Taken together, these data indicate that the rapid activation responses to $\mathrm{LTB}_{4}$ observed in human neutrophils are BLT1 receptor-mediated and support the use of CP-105,696 as a potent and specific BLT1 receptor antagonist.

\section{Effect of a BLTI receptor antagonist on $L T B_{4}$-mediated survival of human neutrophils}

As a separate assessment of apoptosis, we show that incubation of neutrophils with $\mathrm{LTB}_{4}(1 \mu \mathrm{M})$ for $20 \mathrm{~h}$ delayed apoptosis as assessed by annexin $\mathrm{V}$ binding (Figure $4 \mathrm{a}$ and $\mathrm{b}$ ). Initial preliminary experiments showed that the presence of autologous serum interfered with the ability of CP-105,696 to inhibit $\mathrm{LTB}_{4}$-mediated retardation of neutrophil apoptosis (data not shown). We therefore cultured our cells in the presence of $0.1 \%$ BSA since delayed apoptosis induced by $\mathrm{LTB}_{4}$ is not dependent on the presence of serum and neutrophils cultured for $20 \mathrm{~h}$ in the absence of any protein would invariably undergo secondary necrosis. Thus, to explore the receptor-dependency of $\mathrm{LTB}_{4}$-mediated delay of neutrophil apoptosis, cells were cocultured in Iscove's containing $0.1 \%$ BSA with the BLT1 receptor antagonist CP-105,696 at a concentration shown in this study to completely antagonise maximal $\mathrm{LTB}_{4}$-stimulated $\left[\mathrm{Ca}^{2+}\right]_{i}$ elevation and shape change (Figures 2 and $3 \mathrm{c}$ ). $\mathrm{LTB}_{4}$-delayed neutrophil apoptosis was now almost totally inhibited by CP-105,696 (Figure 4c). 

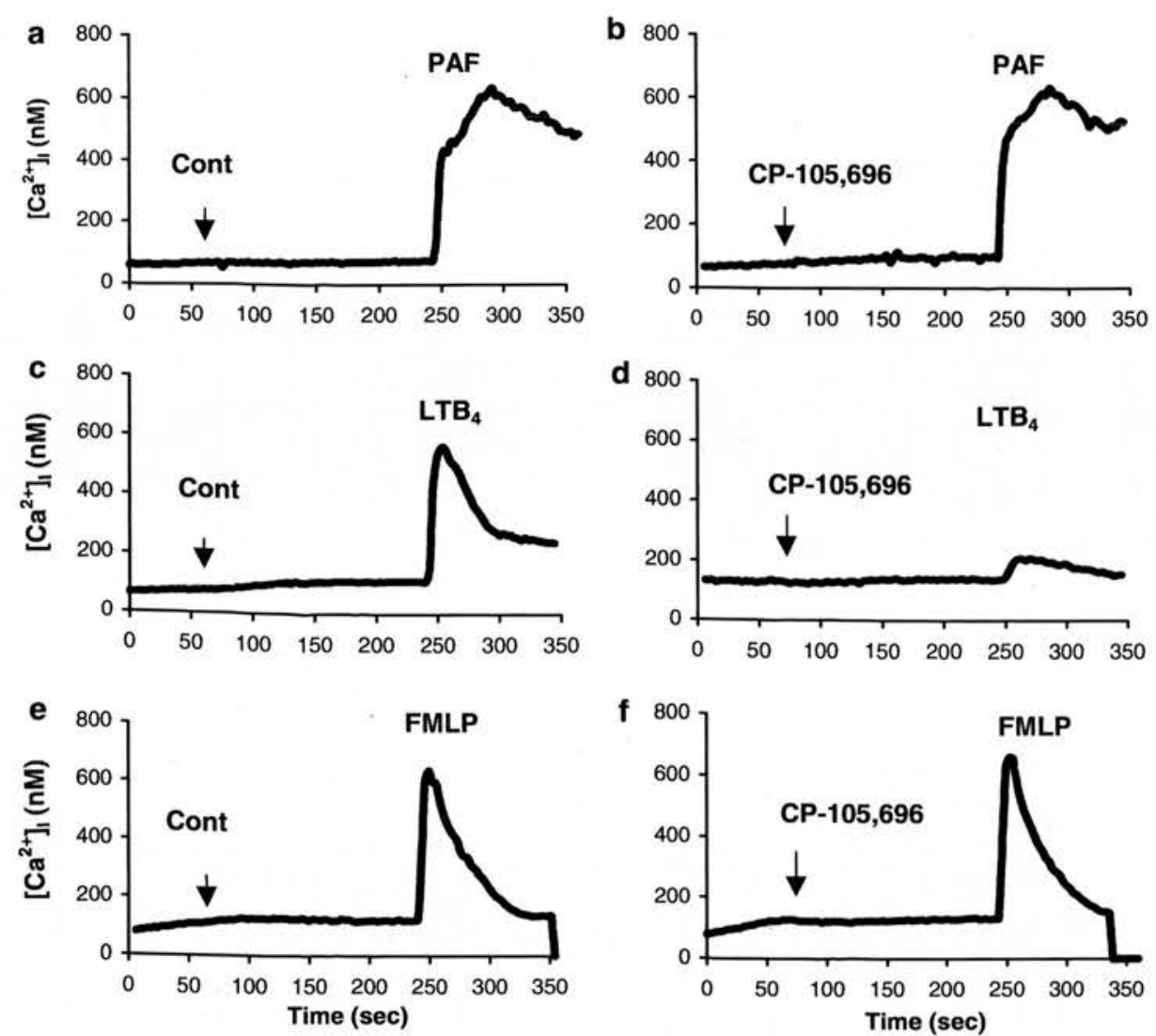

Figure 2 Measurement of cytosolic calcium levels in freshly isolated human neutrophils in response to agonist stimulation Cytosolic calcium measurements (nM) in fura-2-loaded, neutrophils $\left(2 \times 10^{6}\right.$ cells ml $\left.{ }^{-1}\right)$ over $350 \mathrm{~s}$. Buffer alone (control, a,c,e) or CP-105,696 $(1 \mu \mathrm{M} ; \mathrm{b}, \mathrm{d}, \mathrm{f})$ was added to the cell suspension followed by the sequential addition of PAF $(100 \mathrm{nM} ; \mathrm{a}, \mathrm{b}), \mathrm{LTB}_{4}(100 \mathrm{nM}$ c, d), or fMLP ( $100 \mathrm{nM} ; \mathrm{e}, \mathrm{f})$, after $245 \mathrm{~s}$. The data show traces obtained from a typical experiment performed at least three times.

Effect of a BLTI receptor antagonist and a FLAP inhibitor on GM-CSF-, LPS-, dexamethasone- and $d b-$ cAMP-mediated survival of human neutrophils

It has been suggested that the delay of neutrophil apoptosis by GM-CSF, LPS and dexamethasone is mediated by autocrine generation of $\mathrm{LTB}_{4}$ (Lee et al., 1999). To pursue this hypothesis in more detail, we examined: (i) whether the survival effects of $\mathrm{LTB}_{4}$ and GM-CSF/LPS were additive; (ii) the effect of FLAP and 5-LO inhibitors on GM-CSF and LPSmediated survival and (iii) whether the effects of these inflammatory mediators, dexamethasone and the cell-permeable analogue of cAMP, db-cAMP was modified by CP105,696 at a concentration shown to block $\mathrm{LTB}_{4}$-mediated shape change, $\left[\mathrm{Ca}^{2+}\right]_{i}$ transients and survival. Coculture experiments demonstrated that the antiapoptotic effects of $\mathrm{LTB}_{4}$ and GM-CSF, and $\mathrm{LTB}_{4}$ and LPS were in fact additive (Figure 5a), perhaps suggesting that these agents act via discrete rather than common signalling pathways. This latter observation however requires further investigation. Moreover, preincubation of neutrophils $\left(30 \mathrm{~min}, 37^{\circ} \mathrm{C}\right)$ with the specific FLAP inhibitor (MK-886; $1 \mu \mathrm{M}$ ), or the BLT1 antagonist CP-105,696 $(1$ and $3 \mu \mathrm{M})$, had no influence on basal apoptosis per se and did not inhibit GM-CSF-, LPS-, dexamethasone- or db-cAMP-mediated suppression of apoptosis (Table 1, Figure 5b). Taken together, these data demonstrate that
GM-CSF and LPS are unlikely to mediate directly their survival signal in neutrophils via the generation and autocrine action of $\mathrm{LTB}_{4}$.

\section{Role of PPAR $\alpha$ in $\mathrm{LTB}_{4}$-mediated survival of human neutrophils}

It is now well recognised that $\mathrm{LTB}_{4}$ is a potent activating ligand for PPAR $\alpha$ (Devchand et al., 1996). In these studies, we have detected PPAR $\alpha$ (molecular weight $55 \mathrm{kDa})$ in the cytosol of freshly isolated human neutrophils and eosinophils as well as monocytes and monocyte-derived macrophages by Western blotting (Figure 6a). To determine whether the $\mathrm{LTB}_{4}$-stimulated survival signal in human neutrophils is mediated via this receptor, we exploited the synthetic PPARactivating ligand pirinixic acid (WY-14643), which has been shown to be selective for PPAR $\alpha$ (Yu et al., 1995). Culture of neutrophils for $20 \mathrm{~h}$ with a wide range of concentrations of WY-14643 $(1-300 \mu \mathrm{M})$ had no influence on the rate of constitutive apoptosis in these cells (Figure 6b). Therefore, although PPAR $\alpha$ is present in human neutrophils, stimulation of this receptor does not mimic $\mathrm{LTB}_{4}$-mediated retardation of neutrophil death suggesting that the survival effect of $\mathrm{LTB}_{4}$ may not be mediated via activation of this receptor. 

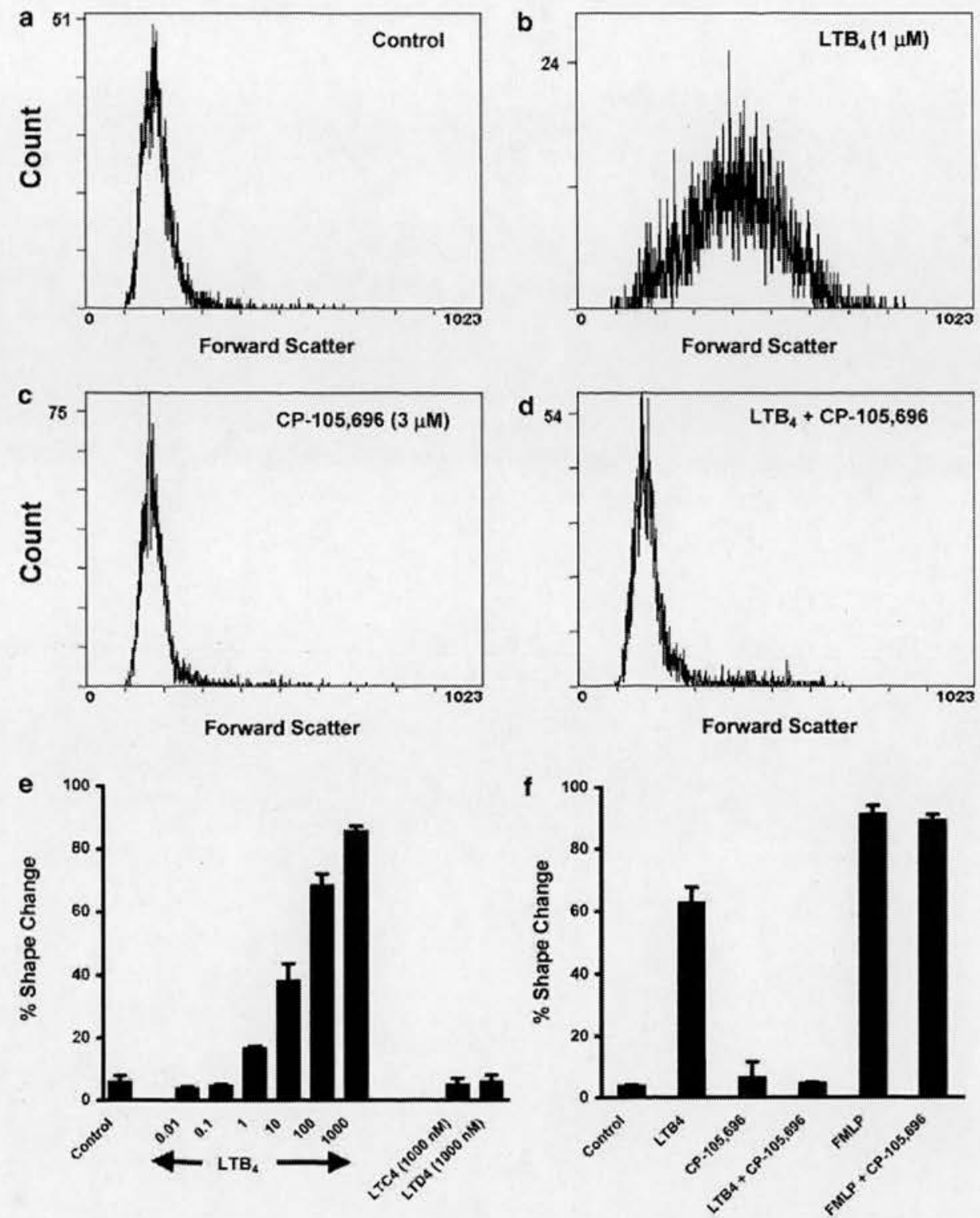

Figure 3 Effect of $\mathrm{LTB}_{4}$ on shape change in human neutrophils. Neutrophils $\left(2 \times 10^{6} \mathrm{cells} \mathrm{ml}^{-1}\right.$ in $180 \mu \mathrm{l}$ PBS with $\mathrm{CaCl}_{2}$ and $\left.\mathrm{MgCl}_{2}\right)$ were equilibrated for $15 \mathrm{~min}$ at $37^{\circ} \mathrm{C}$, then preincubated with $10 \mu \mathrm{l}$ CP-105,696 $(3 \mu \mathrm{M})$ or vehicle prior to stimulation for 10 min with $10 \mu \mathrm{LTB}_{4}(1 \mu \mathrm{M})$, or vehicle. (a) represents control, (b) LTB ${ }_{4}$, (c) CP-105,696 and (d) LTB 4 plus CP-105,696. (e) Shows the concentration - response for $\mathrm{LTB}_{4}$-induced shape change where neutrophils $\left(2 \times 10^{6} \mathrm{cells} \mathrm{ml}^{-1}\right.$ in $190 \mu \mathrm{l}$ PBS with $\mathrm{CaCl}_{2}$ and $\left.\mathrm{MgCl}_{2}\right)$ were equilibrated for $15 \mathrm{~min}$ at $37^{\circ} \mathrm{C}$, then incubated with $10 \mu \mathrm{l}$ buffer alone (control), $\mathrm{LTB}_{4}(0.01 \mathrm{nM}-1 \mu \mathrm{M}), \mathrm{LTC}_{4}(1 \mu \mathrm{M})$ or $\mathrm{LTD}_{4}(1 \mu \mathrm{M})$ for $10 \mathrm{~min}$. (f) Shows the specificity of CP-105,696 for BLT1-mediated shape change where neutrophils $\left(2 \times 10^{6}\right.$ cells ml ${ }^{-1}$ in $180 \mu \mathrm{l}$ PBS with $\mathrm{CaCl}_{2}$ and $\mathrm{MgCl}_{2}$ ) were equilibrated for $15 \mathrm{~min}$ at $37^{\circ} \mathrm{C}$, then incubated with $10 \mu \mathrm{l}$ buffer alone (control), or CP-105,696 $(3 \mu \mathrm{M})$ prior to stimulation for $10 \mathrm{~min}$ with $10 \mu \mathrm{l} \mathrm{LTB} 4(1 \mu \mathrm{M})$, fMLP $(100 \mathrm{nM})$, or buffer alone. All reactions were terminated by addition of $200 \mu 12.5 \%$ glutaraldehyde. (a-d) Show representative flow cytometry (EPICS Profile II), $x$-axis: mean forward light scatter (FS); $y$-axis: relative cell number. (e) and (f) show per cent shape change as analysed by flow cytometry as detailed in Methods. Data represent means \pm s.e.m. of one representative experiment of three, each performed in triplicate.

\section{Effects of LTs on eosinophil $\left[\mathrm{Ca}^{2+}\right]_{i}$ levels}

The effects of LTs on $\left[\mathrm{Ca}^{2+}\right]_{i}$ in freshly isolated human eosinophils were measured using fura- 2 in a similar set of experiments as described for neutrophils. The rise in eosinophil $\left[\mathrm{Ca}^{2+}\right]_{\mathrm{i}}$ in response to $\mathrm{LTB}_{4}(100 \mathrm{nM}$; Figure $7 \mathrm{a})$, was abrogated by preincubation with the BLT1 receptor antagonist CP-105,696 (data not shown). While $\mathrm{LTC}_{4}$ had no effect on
$\left[\mathrm{Ca}^{2+}\right]_{\mathrm{i}}$ (Figure 7a), incubation with $\mathrm{LTD}_{4}$ induced a rapid and transient rise in $\left[\mathrm{Ca}^{2+}\right]_{i}$ (Figure $7 \mathrm{a}$ and b). Moreover, $\mathrm{LTD}_{4^{-}}$ mediated flux in $\left[\mathrm{Ca}^{2+}\right]_{i}$ was blocked by preincubation with the Cys-LT $\mathrm{L}_{1}$ receptor antagonist montelukast $(1 \mu \mathrm{M})$ (Figure $\left.7 \mathrm{~b}\right)$, which had no influence on resting $\left[\mathrm{Ca}^{2+}\right]_{i}$ or on $\mathrm{LTB}_{4}$ or PAFmediated responses (Figure $7 \mathrm{~b}$ ). Interestingly, there was no evidence of heterologous desensitisation with regard to the $\left[\mathrm{Ca}^{2+}\right]_{\mathrm{i}}$ responses induced by $\mathrm{LTB}_{4}, \mathrm{LTD}_{4}$ and PAF (see 

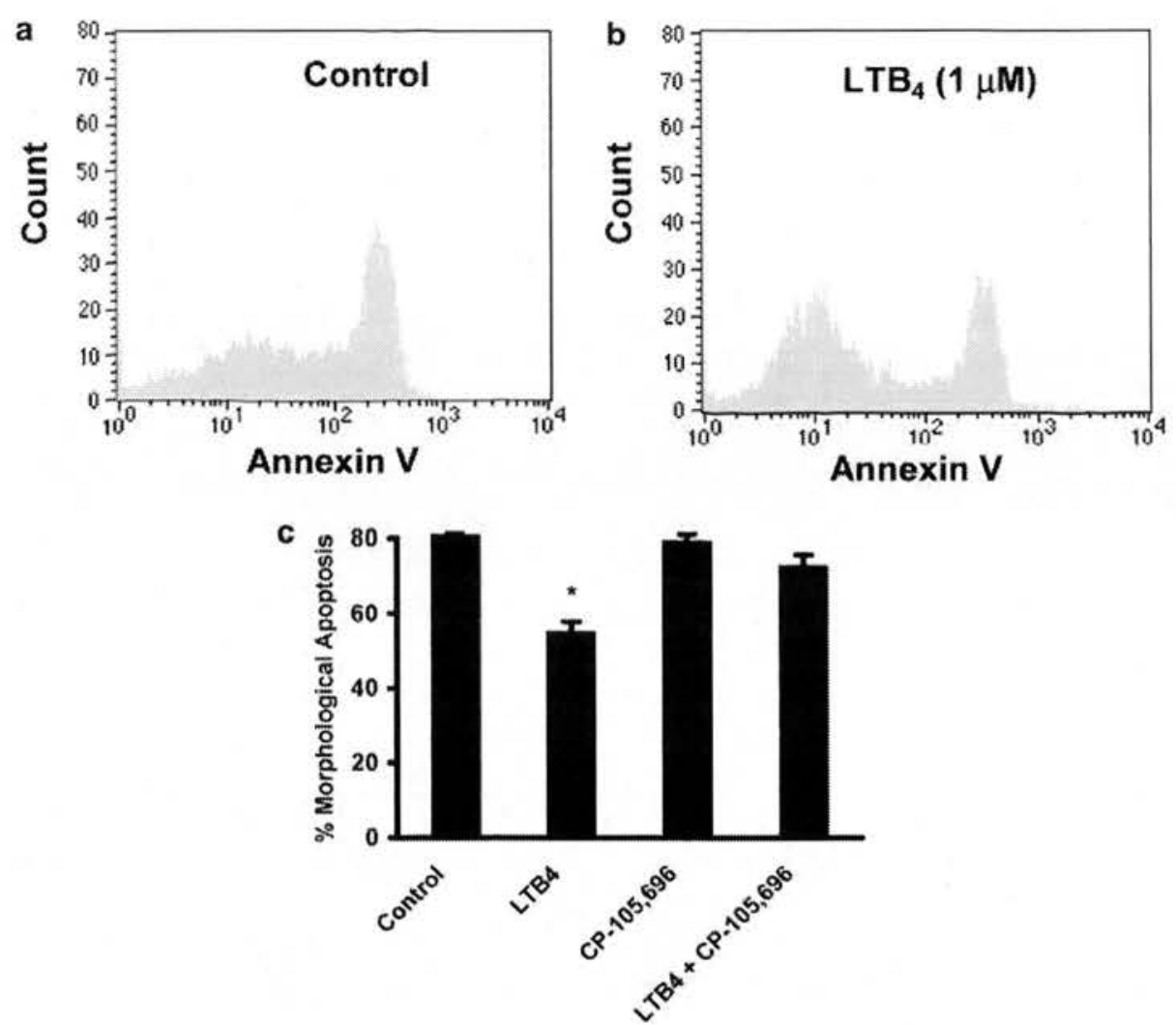

Figure 4 Effect of BLT1 receptor antagonist CP-105,696 on $\mathrm{LTB}_{4}$-mediated retardation of human neutrophil apoptosis. Neutrophils $\left(5 \times 10^{6} \mathrm{cells} \mathrm{ml}^{-1}\right)$ were cultured in serum-supplemented Iscove's MDM alone (control, a) or in the presence of LTB ${ }_{4}$ $(1 \mu \mathrm{M}, \mathrm{b})$. Neutrophils were resuspended following $20 \mathrm{~h}$ in culture and incubated with FITC-labelled recombinant human Annexin V to determine phosphatidylserine expression. The cells were then fixed and analysed using an EPICS Profile II. Mean fluorescence values are shown for a minimum of 5000 cells for each condition. (c) Neutrophils $\left(5 \times 10^{6} \mathrm{cells} \mathrm{ml}^{-1}\right)$ were preincubated $\left(37^{\circ} \mathrm{C}\right.$, $15 \mathrm{~min})$ in serum-supplemented Iscove's MDM containing BSA $(0.1 \%)$ and treated with $\mathrm{LTB}_{4}(1 \mu \mathrm{M})$ with or without CP-105,696 $(3 \mu \mathrm{M})$. Samples were harvested to assess for morphological features of apoptosis; data represent the means \pm s.e.m. of three independent experiments performed in triplicate, where * represents $P<0.05$ compared to control values.

Figure 7 and data not shown). This supports the existence of a specific Cys- $\mathrm{LT}_{1}$-receptor mediated $\mathrm{LTD}_{4}$-induced flux in $\left[\mathrm{Ca}^{2+}\right]_{i}$ in human eosinophils.

\section{Effect of LTs on eosinophil apoptosis}

In these studies, we sought to determine the potential role of $\mathrm{LTB}_{4}$ and the cysteinyl LTs $\left(\mathrm{LTC}_{4}\right.$ and $\left.\mathrm{LTD}_{4}\right)$ in modulating eosinophil apoptosis. In initial experiments, we examined the effects of $\mathrm{LTB}_{4}, \mathrm{LTC}_{4}$, and $\mathrm{LTD}_{4}(1 \mu \mathrm{M})$ on eosinophil longevity at $20 \mathrm{~h}$, when the constitutive rate of apoptosis in these cells was still relatively very low $(17.0 \pm 1.2 \%$; Figure 8$)$. At this time point, we were unable to detect any significant effects of the LTs on eosinophil apoptosis. The cys-LT receptor antagonist montelukast $(10 \mu \mathrm{M})$ was also unable to modulate the constitutive rate of eosinophil apoptosis, either on its own, or following coculture with the LTs (Figure 8). The responsiveness of these cells to stimuli that have been previously reported to modulate apoptosis was confirmed by the delay of apoptosis by IL-5 $\left(50 \mathrm{ng} \mathrm{ml}^{-1}\right)$ (Stern et al., 1992). In experiments where cells were harvested following longer periods in culture, where the rates of constitutive apoptosis recorded were much higher $(40 \mathrm{~h}: 30.3 \pm 1.9 \% ; 60 \mathrm{~h}$ : $70.7 \pm 1.2 \%$ ), we were still unable to detect any influence of $\mathrm{LTB}_{4}, \mathrm{LTC}_{4}, \mathrm{LTD}_{4}$ or montelukast on eosinophil apoptosis (data not shown). Similar results were also obtained at these time points using LTs at 10 and $300 \mathrm{nM}$, and montelukast at $3 \mu \mathrm{M}$ (data not shown). Thus, in these studies, neither $\mathrm{LTB}_{4}$ nor the cysteinyl LTs, $\mathrm{LTC}_{4}$ and $\mathrm{LTD}_{4}$ had any influence on the rate of eosinophil apoptosis at the various concentrations and time points investigated.

\section{Discussion}

In this study, we have examined the effects of the proinflammatory lipid mediators, the LTs, on granulocyte activation and survival. $\mathrm{LTB}_{4}$ is a potent stimulus of rapid activation events in neutrophils (i.e., $\left[\mathrm{Ca}^{2+}\right]_{\mathrm{i}}$ fluxes and shape change). For example, $\mathrm{LTB}_{4}$-stimulated cell polarisation occurred at concentrations as low as $1 \mathrm{nM}$, with an $\mathrm{EC}_{50}$ of $\cong 10 \mathrm{nM}$. In addition, $\mathrm{LTB}_{4}$-induced $\left[\mathrm{Ca}^{2+}\right]_{i}$ elevation and shape change were attenuated by the specific BLT1 receptor antagonist CP105,696 whereas these responses induced by PAF and fMLP appear to be mediated by non-LTB ${ }_{4}$ receptor-dependent mechanisms.

To investigate $\mathrm{LTB}_{4}$-mediated survival of human neutrophils, we examined the potential role of specific receptordependent mechanisms. Our initial studies using CP-105,696 also suggested that $\mathrm{LTB}_{4}$-induced retardation of this response 

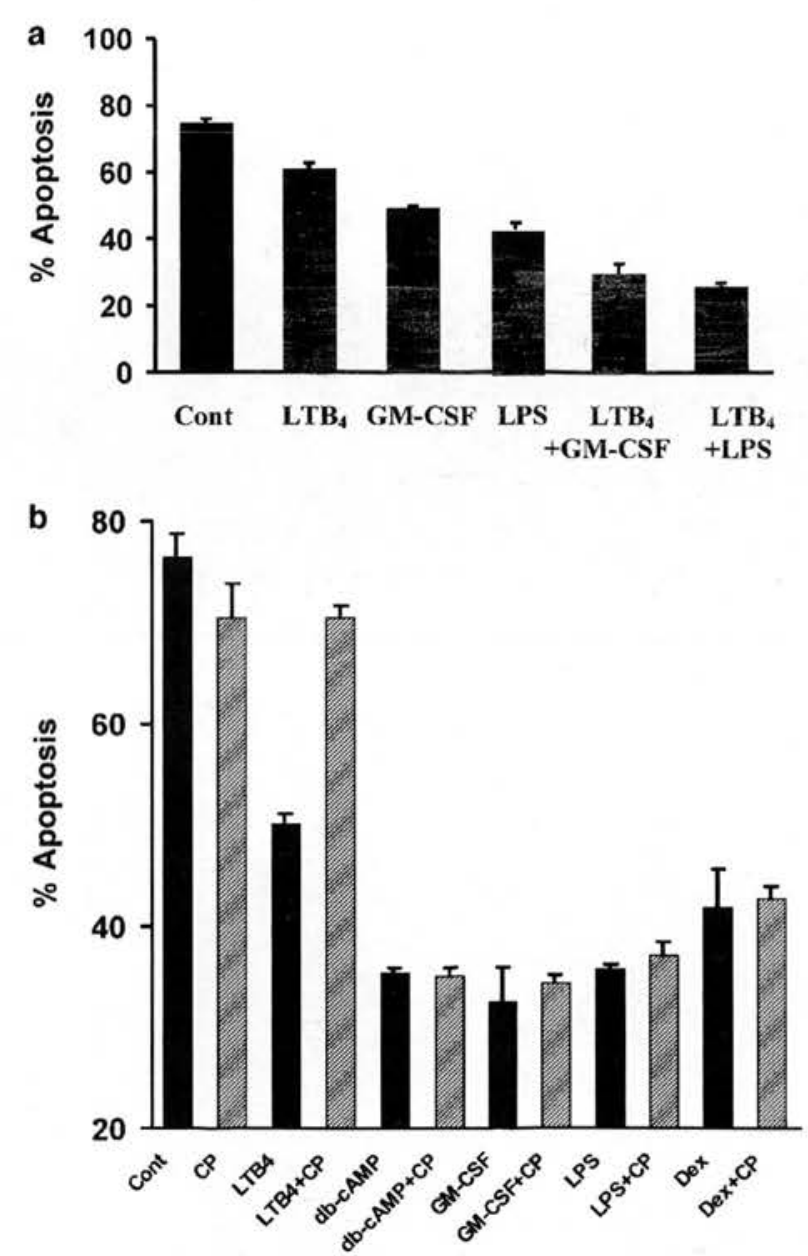

Figure 5 (a) Effect of coculture with GM-CSF and LPS on $\mathrm{LTB}_{4}$ mediated retardation of human neutrophil apoptosis. Neutrophils $\left(5 \times 10^{6}\right.$ cells $\left.\mathrm{ml}^{-1}\right)$ were incubated in serum-supplemented Iscove's MDM alone or in identical medium containing $\mathrm{LTB}_{4}(1 \mu \mathrm{M}), \mathrm{GM}$ CSF $\left(50 \mathrm{Uml}^{-1}\right)$, LPS $\left(1 \mu \mathrm{g} \mathrm{ml}^{-1}\right), \operatorname{LTB}_{4}(1 \mu \mathrm{M})$ plus GM-CSF $\left(50 \mathrm{U} \mathrm{ml}^{-1}\right)$ or $\mathrm{LTB}_{4}(1 \mu \mathrm{M})$ plus LPS $\left(1 \mu \mathrm{g} \mathrm{ml}^{-1}\right)$. Neutrophils were harvested following $20 \mathrm{~h}$ in culture and assessed for morphological features of apoptosis. Data represent the means \pm s.e.m. of one representative experiment of three, each performed in triplicate. (b) Effect of CP-105,696 on LTB $_{4^{-}}$, db-cAMP-, GM-CSF-, LPS- and dexamethasone-mediated retardation of human neutrophil apoptosis. Neutrophils $\left(5 \times 10^{6}\right.$ cells $\left.\mathrm{ml}^{-1}\right)$ were pre-incubated $\left(37^{\circ} \mathrm{C}\right.$, $15 \mathrm{~min})$ in BSA $(0.1 \%)$-supplemented Iscove's MDM alone (control; black bars) or in identical medium containing CP-105,696 at final concentrations of $3 \mu \mathrm{M}$ (hatched bars) prior to cell culture in the medium alone (control), $\mathrm{LTB}_{4}(1 \mu \mathrm{M})$, db-cAMP $(0.2 \mathrm{mM}), \mathrm{GM}-\mathrm{CSF}$ $\left(50 \mathrm{U} \mathrm{ml}^{-1}\right)$, LPS $\left(1 \mu \mathrm{g} \mathrm{ml}^{-1}\right)$ or dexamethasone $(1 \mu \mathrm{M})$. Neutrophils were harvested following $20 \mathrm{~h}$ in culture and assessed for morphological features of apoptosis. Data represent the means \pm s.e.m. of triplicate samples from an individual experiment from at least 3 separate experiments.

appears to be mediated via the BLT1 receptor. This conclusion is supported by our studies investigating the now recognised $\mathrm{LTB}_{4}$ receptor PPAR $\alpha$. For this, we initially probed for the presence of the receptor by Western blotting analysis and show for the first time that PPAR $\alpha$ (molecular weight $55 \mathrm{kDa}$ ) is present in whole-cell lysates of freshly isolated human neutrophils and eosinophils, as well as monocytes and
Table 1 Effect of the FLAP inhibitor MK-886 on $\mathrm{LTB}_{4}$, GM-CSF and LPS-mediated retardation of human neutrophil apoptosis

$\begin{array}{lcc}\text { Treatment } & \text { Control } & M K-866 \\ \text { Control } & 72.5 \pm 5.0 & 72.0 \pm 5.8 \\ \text { LTB }_{4} & 59.1 \pm 3.3 & 58.9 \pm 3.7 \\ \text { GM-CSF } & 50.9 \pm 2.7 & 51.8 \pm 3.7 \\ \text { LPS } & 51.1 \pm 9.2 & 49.3 \pm 8.0\end{array}$

Human neutrophils $\left(5 \times 10^{6}\right.$ cells $\left.\mathrm{ml}^{-1}\right)$ were preincubated $\left(37^{\circ} \mathrm{C}, 30 \mathrm{~min}\right)$ in serum supplemented Iscove's MDM alone or in identical medium containing MK $-886(1 \mu \mathrm{M})$ prior to cell culture in the medium alone (control), $\operatorname{LTB}_{4}(1 \mu \mathrm{M}), \mathrm{GM}-\mathrm{CSF}$ $\left(50 \mathrm{U} \mathrm{ml}^{-1}\right)$ or LPS $\left(1 \mu \mathrm{g} \mathrm{ml}^{-1}\right)$. Neutrophils were harvested following $20 \mathrm{~h}$ in culture and assessed for morphological features of apoptosis (expressed as percentage of apoptotic cells). Data represent the means \pm s.e.m. of three separate experiments, each performed in triplicate.

a
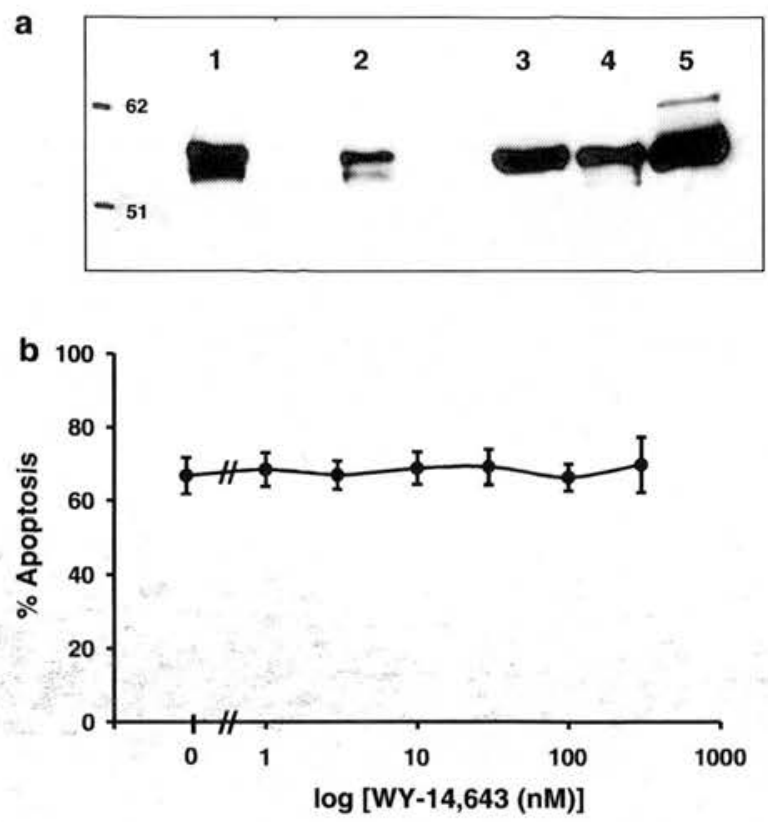

Figure 6 Detection of PPAR $\alpha$ in freshly isolated human leukocytes (a). Leukocyte cytosolic samples $\left(5-10 \times 10^{6}\right.$ cells in $100 \mu \mathrm{l}$ of lysis buffer as detailed in the Methods section) were run on a $9 \%$ acrylamide gel and probed for human $\operatorname{PPAR} \alpha$. Lanes represent: (1) fresh neutrophils $\left(10 \times 10^{6}\right)$, (2) fresh neutrophils $\left(5 \times 10^{6}\right),(3)$ fresh eosinophils $\left(10 \times 10^{6}\right)$, (4) monocytes $\left(10 \times 10^{6}\right)$, (5) monocytederived macrophages $\left(10 \times 10^{6}\right)$. Effect of WY-14,643, a synthetic PPAR $\alpha$-activating ligand, on human neutrophil apoptosis (b). Human neutrophils $\left(5 \times 10^{6} \mathrm{cells} \mathrm{ml}^{-1}\right)$ were incubated in serumsupplemented Iscove's MDM alone (control) or in identical medium containing a range of concentrations of WY-14643 $(1-300 \mu \mathrm{M})$. Cells were harvested following $20 \mathrm{~h}$ in culture and assessed for morphological features of apoptosis. Data represent the mean$\mathrm{s} \pm$ s.e.m. of three separate experiments, each performed in triplicate.

monocyte-derived macrophages. Despite the presence of $\operatorname{PPAR} \alpha$ protein in freshly isolated neutrophils, culture of these cells for $20 \mathrm{~h}$ with a wide range of concentrations of the synthetic and selective PPAR $\alpha$-activating ligand pirinixic acid (WY-14643) $(1-300 \mu \mathrm{M})$ had no influence on the rate of constitutive apoptosis in these cells. The higher concentrations of WY-14643 used in our study are by far in excess of that used $(10 \mu \mathrm{M})$ to enhance interferon- $\gamma$ plus TNF- $\alpha$-induced apoptosis 


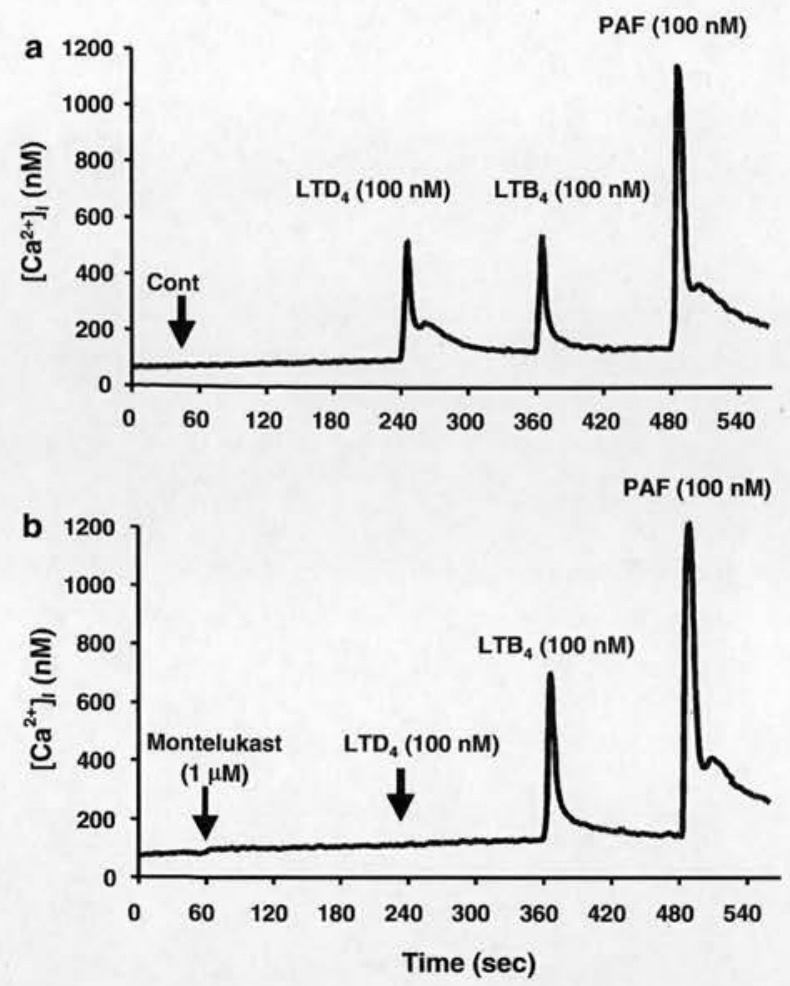

Figure 7 Effect of the Cys-LT, receptor antagonist montelukast on agonist-induced calcium fluxes in human eosinophils. Cytosolic calcium measurements $(\mathrm{nM})$ in fura-2-loaded eosinophils $\left(2 \times 10^{6}\right.$ cells ml ${ }^{-1}$ in HBSS without phenol red, with $\mathrm{CaCl}_{2}$ and $\mathrm{MgCl}_{2}$ ) over $540 \mathrm{~s}$. Buffer alone (control, a) or montelukast ( $\left.1 \mu \mathrm{M}, \mathrm{b}\right)$ was added to the cell suspension after $60 \mathrm{~s}$, followed by the sequential addition of $\mathrm{LTD}_{4}(100 \mathrm{nM}), \mathrm{LTB}_{4}(100 \mathrm{~nm})$ and PAF $(100 \mathrm{nM})$ after 245,365 and $485 \mathrm{~s}$, respectively. The data show traces obtained from a typical experiment performed at least three times.

in isolated human differentiated macrophages (Chinetti et al., 1998). Of particular relevance to our studies is the recent characterisation and cloning of a novel cell surface-bound G-protein-coupled receptor for $\mathrm{LTB}_{4}$, designated BLT2 (Yokomizo et al., 2000; Kamohara et al., 2000). This receptor binds LTB $_{4}$ with a $K_{\mathrm{d}}$ value of $23 \mathrm{nM}$ compared with $1.1 \mathrm{nM}$ for BLT1, but still efficiently transduces intracellular signalling. In contrast to BLT1, which is expressed predominantly in leucocytes, BLT2 is expressed more ubiquitously with a large amount of BLT2 mRNA detected in human spleen as well as in peripheral blood leucocytes (Kamohara et al., 2000; Yokomizo et al., 2000). Yokomizo et al. (2000) also revealed that several BLT1 antagonists failed to inhibit $\mathrm{LTB}_{4}$ binding to BLT2 demonstrating that BLT2 is pharmacologically distinct and may mediate cellular functions in tissues other than leucocytes. Further work is required to investigate the potential role of this receptor in $\mathrm{LTB}_{4}$-induced neutrophil function and, in particular, apoptosis. This is especially important given that there is evidence suggesting that $\mathrm{LTB}_{4}$ may mediate retardation of neutrophil apoptosis via cell surface receptor signalling events distinct from those involved in neutrophil degranulation and respiratory burst (Hebert et al., 1996). Taken together, however, our results suggest that the BLT1, and not the $\operatorname{PPAR} \alpha$ receptor, is the likely receptor responsible for the retardation of neutrophil apoptosis induced by $\mathrm{LTB}_{4}$.

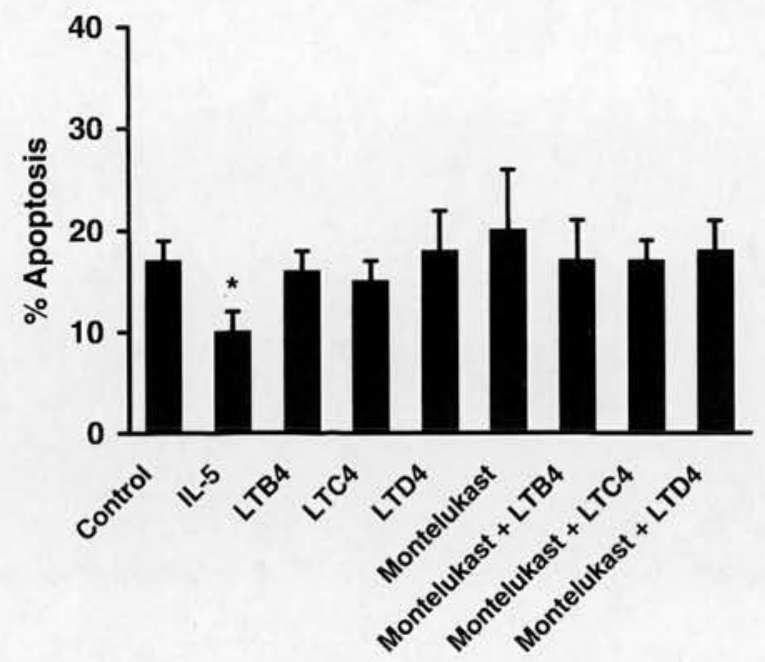

Figure 8 Effect of LTs $\left(\mathrm{LTB}_{4}, \mathrm{LTC}_{4}, \mathrm{LTD}_{4}\right)$ and montelukast on human eosinophil apoptosis at $20 \mathrm{~h}$ in vitro. Eosinophils $\left(2.5 \times 10^{6} \mathrm{cells} \mathrm{ml}^{-1}\right)$ were cultured in serum-supplemented Iscove's MDM alone (control) or in identical medium containing: final concentration $\mathrm{LTB}_{4}(1 \mu \mathrm{M}), \mathrm{LTC} 4(1 \mu \mathrm{M}), \mathrm{LTD}_{4}(1 \mu \mathrm{M})$, montelukast $(10 \mu \mathrm{M})$ or IL-5 $\left(50 \mathrm{ng} \mathrm{ml}^{-1}\right)$. Coculture conditions were also prepared with $\mathrm{LTB}_{4}, \mathrm{LTC}_{4} \mathrm{LTD}_{4}(1 \mu \mathrm{M})$ in the presence of montelukast $(10 \mu \mathrm{M})$. Eosinophils were harvested following $20 \mathrm{~h}$ in culture and assessed for morphological features of apoptosis. Data represent the means \pm s.e.m. of three independent experiments, each performed in triplicate, where ${ }^{*}$ represents $P<0.05$ compared to control values.

We have also demonstrated, using the specific FLAP antagonist MK-886, that GM-CSF- and LPS-mediated survival in neutrophils does not appear to be mediated via the generation and autocrine effects of $\mathrm{LTB}_{4}$. These data conflict with a recent report (Lee et al., 1999) that proposed that the delay of neutrophil apoptosis observed with GM-CSF, LPS and dexamethasone could be attributed to the generation of $\mathrm{LTB}_{4}$. These latter observations are supported by a recent report showing that BLT1 receptor expression is upregulated by dexamethasone and that this effect may be one of the mechanisms through which glucocorticoids can prolong neutrophil survival. Indeed, dexamethasone-induced survival may be mediated by $\mathrm{LTB}_{4}$ as determined by use of an $\mathrm{LTB}_{4}$ receptor antagonist (Stankova et al., 2002). Difference between these studies and our observations may be because of differences in isolation procedures, activation/priming status of the cells, culture conditions or reagents and inhibitors used. Our studies however indicate that, whatever the precise mechanism utilised by $\mathrm{LTB}_{4}$ to mediate its suppression of neutrophil apoptosis, it is unlikely that dexamethasone, LPS, GM-CSF or db-cAMP delay apoptosis by this mechanism since blockade of $\mathrm{LTB}_{4}$ action by CP-105,696 and/or $\mathrm{LTB}_{4}$ synthesis by the FLAP inhibitor do not abrogate the effects of these agents.

It has been assumed previously that a close relation exists between neutrophil activation and survival in that agents which serve to prime neutrophils for enhanced functional responsiveness to secretagogue signals or directly activate these cells also increase functional longevity by delaying the onset of constitutive apoptosis. We have shown that while $\mathrm{LTD}_{4}$, and to a lesser extent $\mathrm{LTB}_{4}$, were potent stimuli for elevation of $\left[\mathrm{Ca}^{2+}\right]_{\mathrm{i}}$ in freshly isolated human eosinophils, neither $\mathrm{LTB}_{4}$, 
nor the cysteinyl LTs $\mathrm{LTC}_{4}$ and $\mathrm{LTD}_{4}$ had any influence on the basal rate of apoptosis. Although it has been reported that cysteinyl LTs can delay apoptosis of eosinophils isolated from asthmatic patients (Lee et al., 2000), our data suggest that the LTs, despite elevating $\left[\mathrm{Ca}^{2+}\right]_{i}$, do not prolong survival of eosinophils isolated from normal or mildly atopic individuals. Furthermore, analyses of the documented effects of various agonists on granulocyte activation reveals that there may be no unifying hypothesis to correlate these two events, that is that the mechanisms leading to granulocyte activation do not appear to be critical for delaying granulocyte apoptosis. This is supported by the observation that $\mathrm{TNF} \alpha$, a potent neutrophil priming agent, induces neutrophil apoptosis at early time points $(<8 \mathrm{~h})$ while delaying this process at later times $(<20 \mathrm{~h})$ (Murray et al., 1997). Moreover, while nitric oxide (NO) donors induce neutrophil apoptosis (Blaylock et al., 1998; Fortenberry et al., 1998; Ward et al., 2000; Taylor et al., 2001), these reagents also have the capacity to downregulate other functions such as shape change and chemotaxis (Ward et al., 2000). Interestingly, the ultimate biological effect of NO on apoptosis and function may depend on the source and concentration of $\mathrm{NO}$, and the cell under investigation (Taylor et al., 2003). In addition, neutrophils exposed to agents that elevate cAMP are rendered less responsive to activation but exhibit a greater longevity (Rossi et al., 1995; Martin et al., 2001). This is further supported by our demonstration that preincubation of neutrophils with PAF $(1 \mu \mathrm{M})$ prior to stimulation with fMLP $(100 \mathrm{nM})$, a protocol that results in a dramatic upregulation of FMLP-induced superoxide anion release (Ward et al., 2000), has no effect on neutrophil apoptosis following 6 and $20 \mathrm{~h}$ in culture (data not shown). These data offer the possibility that more rational and selective anti-inflammatory therapies may be designed and further underline the importance of elucidating and understanding the mechanisms of activation and signalling in response to local LT generation and other inflammatory agents present at sites of inflammation.

This work was supported by The National Asthma Campaign (01 042), The Medical Research Council Program Grant (G9016491), The Wellcome Trust (035662) and by Merck Sharp and Dohme. JM and CW are joint first authors.

\section{References}

BLAYLOCK, M.G., CUTHBERTSON, B.H., GALLEY, H.F., FERGUSON, N.R. \& WEBSTER, N.R. (1998). The effect of nitric oxide and peroxynitrite on apoptosis in human polymorphonuclear leukocytes. Free Radic. Biol. Med., 25, $748-752$.

CHINETTI, G., GRIGLIO, S., ANTONUCCI, M., TORRA, I.P., DELERIVE, P., MAJD, Z., FRUCHART, J.C., CHAPMAN, J., NAJIB, J. \& STAELS, B. (1998). Activation of proliferator-activated receptors $\alpha$ and $\gamma$ induces apoptosis of human monocyte-derived macrophages. J. Biol. Chem., 273, 25573-25580.

COLOTTA, F., RE, F., POLENTARUTTI, N., SOZZANI, S. \& MANTOVANI, A. (1992). Modulation of granulocyte survival and programmed cell death by cytokines and bacterial products. Blood, $\mathbf{8 0}$, $2012-2020$.

DAHINDEN, C.A., ZINGG, J., MALY, F.E. \& DE WECK, A.L. (1988). Leukotriene production in human neutrophils primed by recombinant human granulocyte/macrophage colony-stimulating factor and stimulated with the complement component C5A and FMLP as second signals. J. Exp. Med., 167, 1281-1295.

DEVCHAND, P.R., KELLER, H., PETERS, J.M., VAZQUEZ, M., GONZALEZ, F.J. \& WAHLI, W. (1996). The PPAR $\alpha$-leukotriene $\mathrm{B}_{4}$ pathway to inflammation control. Nature, 384, 39-43.

FADOK, V.A., BRATTON, D.L., KONOWAL, A., FREED, P.W., WESTCOTT, J.Y. \& HENSON, P.M. (1998). Macrophages that have ingested apoptotic cells in vitro inhibit proinflammatory cytokine production through autocrine/paracrine mechanisms involving TGF- $\beta, \mathrm{PGE}_{2}$, and PAF. J. Clin. Invest., 101, $890-898$.

FORD-HUTCHINSON, A.W., BRAY, M.A., DOIG, M.V., SHIPLEY, M.E. \& SMITH, M.J. (1980). Leukotriene B, a potent chemokinetic and aggregating substance released from polymorphonuclear leukocytes. Nature, 286, $264-265$.

FORTENBERRY, J.D., OWENS, M.L., BROWN, M.R., ATKINSON, D. \& BROWN, L.A. (1998). Exogenous nitric oxide enhances neutrophil cell death and DNA fragmentation. Am. J. Respir. Cell. Mol. Biol., 18, $421-428$.

FUJIHARA, S., WARD, C., DRANSFIELD, I., HAY, R.T., UINGS, I.J., HAYES, B., FARROW, S.N., HASLETT, C. \& ROSSI, A.G. (2002). Inhibition of nuclear factor $-\kappa \mathrm{B}$ activation un-masks the ability of TNF- $\alpha$ to induce human eosinophil apoptosis. Eur. J. Immunol., 32, $457-466$.

GIEMBYCZ, M.A. \& LINDSAY M.A. (1999). Pharmacology of the eosinophil. Pharmacol. Rev., 51, 213-340.

GOMPERTZ, S. \& STOCKLEY, R.A. (2000). Inflammation-role of the neutrophil and the eosinophil. Semin. Respir. Infect., 15, $14-23$.
HASLETT, C. (1999). Granulocyte apoptosis and its role in the resolution and control of lung inflammation. Am. J. Respir. Crit. Care Med., 160, S5-S11.

HEBERT, M-J., TAKANO, T., HOLTHÖFER, H. \& BRADY, H.R. (1996). Sequential morphologic events during apoptosis of human neutrophils. Modulation by lipoxygenase-derived eicosanoids. $J$. Immunol., 157, 3105-3115.

KAMOHARA, M., TAKASAKI, J., MATSUMOTO, M., SAITO, T., OHISHI, T., ISHII, H. \& FURUICHI, K. (2000). Molecular cloning and characterization of another leukotriene $\mathrm{B}_{4}$ receptor. J. Biol. Chem., 275, $27000-27004$

KITCHEN, E., ROSSI, A.G., CONDLIFFE, A.M., HASLETT, C. \& CHILVERS, E.R. (1996). Demonstration of reversible priming of human neutrophils using platelet-activating factor. Blood, 88, $4330-4337$

LAWRENCE, T., WILLOUGHBY, D.A. \& GILROY, D.W. (2002) Antiinflammatory lipid mediators and insights into the resolution of inflammation. Nat. Rev. Immunol., 2, 787-795.

LEE, A., WHYTE, M.K.B. \& HASLETT, C. (1993). Inhibition of apoptosis and prolongation of neutrophil functional longevity by inflammatory mediators. J. Leukoc. Biol., 54, 283-288.

LEE, E., LINDO, T., JACKSON, N., MENG-CHOONG, L., REYNOLDS, P., HILL, A., HASWELL, M., JACKSON, S. \& KILFEATHER, S. (1999). Reversal of human neutrophil survival by leukotriene $B_{4}$ receptor blockade and 5-lipoxygenase and 5-lipoxygenase activating protein inhibitors. Am. J. Respir. Crit. Care Med., 160, 2079-2085.

LEE, E., ROBERTSON, T., SMITH, J. \& KILFEATHER, S. (2000). Leukotriene receptor antagonists and synthesis inhibitors reverse survival in eosinophils of asthmatic individuals. Am. J. Respir. Crit. Care Med., 161, 1881-1886.

LEFF, A.R. (2000). Role of leukotrienes in bronchial hyperresponsiveness and cellular responses in airways. Am. J. Respir. Crit. Care Med., 161, S125-S132.

LIN, A.H., MORTON, D.R. \& GORMAN, R.R. (1982). Acetyl glyceryl ether phosphorylcholine stimulates leukotriene B4 synthesis in human polymorphonuclear leukocytes. J. Clin. Invest., 70, $1058-$ 1065.

LIU, Y., COUSIN, J.M., HUGHES, J., VAN DAMME, J., SECKL, J.R., HASLETT, C., DRANSFIELD, I., SAVILL, J. \& ROSSI, A.G. (1999). Glucocorticoids promote nonphlogistic phagocytosis of apoptotic leukocytes. J. Immunol., 162, 3639-3646.

MARTIN, M.C., DRANSFIELD, I., HASLETT, C. \& ROSSI, A.G. (2001). Cyclic AMP regulation of neutrophil apoptosis occurs via a novel protein kinase A-independent signaling pathway. J. Biol. Chem., 276, $45041-45050$. 
MEAGHER, L.C., COUSIN, J.M., SECKL, J.R. \& HASLETT, C. (1996). Opposing effects of glucocorticoids on the rate of apoptosis in neutrophilic and eosinophilic granulocytes. J. Immunol., 156, $4422-4428$.

MEAGHER, L.C., SAVILL, J.S., BAKER, A., FULleR, R.W. \& HASLETT, C. (1992). Phagocytosis of apoptotic neutrophils does not induce macrophage release of thromboxane $\mathrm{B}_{2}$. J. Leukoc. Biol., 52, $269-273$.

MECKLENBURGH, K., MURRAY, J., BRAZIL, T., WARD, C., ROSSI, A.G. \& CHILVERS, E.R. (1999). Role of neutrophil apoptosis in the resolution of pulmonary inflammation. Monaldi Arch. Chest Dis., 54, 345-349.

MURRAY, J., BARBARA, J.A.J., DUNKLEY, S.A., LOPEZ, A.F., VAN OSTADE, X., CONDLIFFE, A.M., DRANSFIELD, I., HASLETT, C. \& CHILVERS, E.R. (1997). Regulation of neutrophil apoptosis by tumor necrosis factor- $\alpha$ : requirement for TNFR 55 and TNFR75 for induction of apoptosis in vitro. Blood, 90, 2772-2783.

O'FLAHERTY, J.T. \& ROSSI, A.G. (1993). 5-Hydroxyicosatetraenoate stimulates neutrophils by a stereospecific, $G$ protein-linked mechanism. J. Biol. Chem., 268, 14708-14714.

ROSSI, A.G., COUSIN, J.M., DRANSFIELD, I., LAWSON, M.F., CHILVERS, E.R. \& HASLETT, C. (1995). Agents that elevate cAMP inhibit human neutrophil apoptosis. Biochem. Biophys. Res. Commun., 217, $892-899$.

ROSSI, A.G. \& HASLETT, C. (1998). Inflammation, cell injury and apoptosis. In: Lung Biology in Health and Disease. ed. Lenfant, C. pp. 9-24. New York: Marcel-Dekker.

ROSSI, A.G., HASLETT, C., HIRANI, N., GREENING, A.P., RAHMAN, I., METZ, C.N., BUCALA, R. \& DONNELLY, S.C. (1998). Human circulating eosinophils secrete macrophage migration inhibitory factor (MIF). Potential role in asthma. J. Clin. Invest., 101, 28692974.

ROSSI, A.G., MACINTYRE, D.E., JONES, C.J.P. \& MCMILLAN, R.M (1993). Stimulation of human polymorphonuclear leukocytes by leukotriene $\mathrm{B}_{4}$ and platelet-activating factor: an ultrastructural and pharmacological study. J. Leukoc. Biol., 53, $117-125$.

SAMUELSSON, B., DAHLEN, S.E., LINDGREN, J.A., ROUZER, C.A. \& SERHAN, C.N. (1987). Leukotrienes and lipoxins: structures, biosynthesis, and biological effects. Science, 237, 1171-1176.

SAVILL, J.S., WYLLIE, A.H., HENSON, J.E., WALPORT, M.J., HEN SON, P.M. \& HASLETT, C. (1989). Macrophage phagocytosis of ageing neutrophils in inflammation. Programmed cell death in the neutrophil leads to recognition by macrophages. J. Clin. Invest. 83, $865-875$.

SERHAN, C.N. \& PRESCOTT, S.M. (2000). The scent of a phagocyte: advances on leukotriene $\mathrm{B}_{4}$ receptors. J. Exp. Med., 192 , F5.

SHOWELl, H.J., PETTIPHER, E.R., CHENG, J.B., BRESLOW, R., CONKLYN, M.J., FARRELL, C.A., HINGORANI, G.P., SALTER, E.D., HACKMAN, B.C., WIMBERLY, D.J., DOHERTY, N.S., MELVIN, L.S., REITER, L.A., BIGGERS, M.S. \& KOCK, K. (1995). The in vitro and in vivo pharmacologic activity of the potent and selective leukotriene $B_{4}$ receptor antagonist CP-105696. $J$. Pharmacol. Exp. Ther., 273, $176-184$.

STANKOVA, J., TURCOTTE, S., HARRIS, J. \& ROLA-PLESZCZYNSKI, M. (2002). Modulation of leukotriene $B_{4}$ receptor-1 expression by dexamethasone: potential mechanism for enhanced neutrophil survival. J. Immunol., 168, 3570 - 3576 .

STERN, M., MEAGHER, L., SAVILL, J. \& HASLETT, C. (1992). Apoptosis in human eosinophils. Programmed cell death in the eosinophil leads to phagocytosis by macrophages and is modulated by IL-5. J. Immunol., 148, $3543-3549$.

STERN, M., SAVILL, J. \& HASLETT, C. (1996). Human monocyte-derived macrophage phagocytosis of senescent eosinophils undergoing apoptosis. Mediation by $\alpha v \beta 3 / \mathrm{CD} 36 /$ thrombospondin recognition mechanism and lack of phlogistic response. Am. $J$. Pathol., 149, $911-921$

TAYLOR, E.L., MEGSON, I.L., HASLETT, C. \& ROSSI, A.G. (2001). Dissociation of DNA fragmentation from other hallmarks of apoptosis in nitric oxide-treated neutrophils: differences between individual nitric oxide donor drugs. Biochem. Biophys. Res. Commun., 289, $1229-1236$.

TAYLOR, E.L., MEGSON, I.L., HASLETT, C. \& ROSSI, A.G. (2003). Nitric oxide; a key regulator of inflammatory cell apoptosis. Cell Death Differ, 10, $418-430$.

WALSH, G.M., SEXTON, D.W., BLAYLOCK, M.G. \& CONVERY, C.M. (1999). Resting and cytokine-stimulated human small airway epithelial cells recognize and engulf apoptotic eosinophils. Blood, 94, $2827-2835$.

WALSH, G.M., WILLIAMSON, M.L., SYMON, F.A., WILLARS, G.B. \& WARDLAW, A.J. (1996). Ligation of CD69 induces apoptosis and cell death in human eosinophils cultured with granulocyte-macrophage colony-stimulating factor. Blood, 87, 2815-2821.

WARD, C., CHILVERS, E.R., LAWSON, M.F., PRYDE, J.G., FUJIHARA, S., FARROW, S.N., HASLETT, C. \& ROSSI, A.G. (1999a). $\mathrm{NF}-\kappa \mathrm{B}$ activation is a critical regulator of human granulocyte apoptosis in vitro. J. Biol. Chem., 274, 4309-4319.

WARD, C., DRANSFIELD, I., CHILVERS, E.R., HASLETT, C. \& ROSSI, A.G. (1999b). Pharmacological manipulation of granulocyte apoptosis: potential therapeutic targets. Trends Pharmacol. Sci., 20, $503-509$.

WARD, C., DRANSFIELD, I., MURRAY, J., FARROW, S.N., HASLETT, C. \&. ROSSI, A.G. (2002). Prostaglandin $\mathrm{D}_{2}$ and its metabolites induce caspase-dependent granulocyte apoptosis that is mediated via inhibition of $\mathrm{I} \kappa \mathrm{B} \alpha$ degradation using a peroxisome proliferator-activated receptor- $\gamma$-independent mechanism. J. Immunol., 168, $6232-6243$.

WARD, C., WONG, T.H., MURRAY, J., RAHMAN, I., HASLETT, C., CHILVERS, E.R. \& ROSSI, A.G. (2000). Induction of human neutrophil apoptosis by nitric oxide donors: evidence for a caspase-dependent, cyclic-GMP-independent, mechanism. Biochem. Pharmacol., 59, $305-314$.

WHYTE, M.K.B., MEAGHER, L.C., HARDWICK, S., SAVILL, J.S. \& HASLETT, C. (1993a). Transient elevations of cytosolic free calcium retard subsequent apoptosis in neutrophils in vitro. J. Clin. Invest., 92, $446-455$.

WHYTE, M.K.B., MEAGHER, L.C., MACDERMOT, J. \& HASLETT, C. (1993b). Impairment of function in aging neutrophils is associated with apoptosis. J. Immunol., 150, $5123-5134$

YOKOMIZO, T., IZUMI, T., CHANG, K., TAKUWA, Y. \& SHIMIZU, T. (1997). A G-protein-coupled receptor for leukotriene B4 that mediates chemotaxis. Nature, 387, 620-624.

YOKOMIZO, T., KATO, K., TERAWAKI, K., IZUMI, T. \& SHIMIZU, T. (2000). A second leukotriene $B_{4}$ receptor, BLT2: a new therapeutic target in inflammation and immunological disorders. J. Exp. Med., $192,421-432$.

YU, K., BAYONA, W., KALLEN, C.B., HARDING, H.P., RAVERA, C.P., MCMAHON, G., BROWN, M. \& LAZAR, M.A. (1995). Differential activation of peroxisome proliferator-activated receptors by eicosanoids. J. Biol. Chem., 270, 23975-23983.

(Received January 15, 2003

Revised February 17, 2003

Accepted February 26, 2003) 


\title{
Hypoxic Regulation of Neutrophil Apoptosis Role of Reactive Oxygen Intermediates in Constitutive
and Tumor Necrosis Factor $\alpha$-Induced Cell Death
}

\author{
J. MURRAY, S.R. WALMSLEY, K.I. MECKLENBURGH, A.S. COWBURN, \\ J.F. WHITE, A.G. ROSSI, AND E.R. CHILVERS \\ Respiratory Medicine Division, Deparlment of Medicine, \\ University of Cambridge School of Clinical Medicine, \\ Addenbrooke's and Papworth Hospitals, Cambridge CB2 2QQ, UK \\ Rayne Laboratory, Respiratory Medicine Unit, \\ MRC Center for Inflammation Research, University of Edinburgh Medical School, \\ Teviot Place, Edinburgh EH8 9AG, UK
}

\begin{abstract}
Activation of the NADPH oxidase system to generate reactive oxygen species (ROS) plays a key role in bacterial killing by human neutrophils. However, the involvement of such radicals in spontaneous and TNF $\alpha$-driven neutrophil apoptosis remains uncertain. While incubation of cells under anoxic conditions attenuated the pro-apoptotic effect of TNF $\alpha$, full activation of the respiratory burst using PAF followed by fMLP, or the addition of physiologically relevant concentrations of $\mathrm{H}_{2} \mathrm{O}_{2}$, had no effect on the rate of apoptosis. Furthermore, the phosphoinositide 3-kinase inhibitor, LY294002, which abolishes receptor-mediated activation of the NADPH oxidase, and five discrete anti-oxidants all failed to affect apoptotic thresholds. Thus ROS do not appear to modulate constitutive apoptosis in neutrophils or appear sufficient to mediate the pro-apoptotic effect of TNF $\alpha$.
\end{abstract}

KEYwORDS: apoptosis; neutrophils; NADPH oxidase; TNF $\alpha$

\section{INTRODUCTION}

Human neutrophils undergo constitutive apoptosis when aged in vitro and this results in hypo-responsiveness to external signals and the expression of cell surface motifs that initiate recognition by inflammatory macrophages. ${ }^{1}$ The speed and capacity of the macrophage phagocytic response towards apoptotic neutrophils, together with the observation that engulfment excites an anti-inflammatory rather than pro-inflammatory macrophage response, predicts that this process plays a pivotal role in the safe disposal of intact but effete neutrophils from an inflamed focus. This view is supported by in vivo data in animal models of acute lung injury and glomerulonephritis and in the neonatal respiratory distress syndrome. ${ }^{2}$

Address for correspondence: Professor Edwin Chilvers, Department of Medicine, Box 157 Addenbrooke's Hospital, University of Cambridge School of Clinical Medicine, Hills Road, Cambridge CB2 2QQ, UK. Voice and fax: (44) 1223762007.

erc24@cam.ac.uk

Ann. N.Y. Acad. Sci. 1010: 417-425 (2003). (C) 2003 New York Academy of Sciences. doi: 10.1196/annals.1299.075 
While the mechanism(s) regulating neutrophil survival and death are poorly understood, there is now considerable evidence to show that this process can be altered by physiological stimuli. For example the pro-inflammatory mediators G-CSF, GMCSF, lipopolysaccharide, C5a, IL-1 $\beta$, IFN $\gamma$, and $\mathrm{LTB}_{4}$ all increase the survival of these cells by delaying apoptosis. ${ }^{3}$ Moreover, compared to other myeloid cells, neutrophils are relatively resistant to agonist-stimulated apoptosis. We have previously reported that TNF $\alpha$ has the unique capacity to drive neutrophil apoptosis in a proportion of cells at early times $(<12 \mathrm{~h})$ while decreasing the overall extent of apoptosis at later times. Of note, TNF $\alpha$ has also been shown to "prime" neutrophils for apoptotic killing by agents such as C5a, IgG, fMLP and $E$. coli ${ }^{4}$ and to enhance the phagocytosis of apoptotic neutrophils by monocyte-derived macrophages. These data suggest a potentially synergistic or dual mechanism whereby TNF $\alpha$ may drive the removal of neutrophils from an inflamed site both by accelerating receptor-mediated apoptosis and by facilitating apoptotic cell clearance.

TNF $\alpha$ causes a rapid rise in the levels of intracellular $\operatorname{ROS}^{5}$ and this response has been proposed to underly the cytotoxic effects of this cytokine. Consistent with this, the anti-oxidants, thioredoxin and $\mathrm{N}$-acetylcysteine, have been reported to inhibit TNF $\alpha$-mediated apoptosis. TNF $\alpha$ cytotoxicity can also be affected by certain mitochondrial inhibitors, ${ }^{6}$ and alterations in mitochondrial ultrastructure after TNF $\alpha$. treatment are well documented. Furthermore, cellular sensitivity or resistance to $\mathrm{TNF} \alpha$ have been correlated with decreased or increased levels of mitochondrial superoxide dismutase, respectively. ${ }^{7}$

The aim of this study was to determine the role of ROS in constitutive and TNF $\alpha$ stimulated apoptosis in human neutrophils. Neutrophils were resistant to killing by $\mathrm{H}_{2} \mathrm{O}_{2}$ or maximal activation of the NADPH oxidase, and the mitochondrial complex III inhibitor actinomycin that increases ROS release inhibited rather than increased TNF $\alpha$-induced apoptosis. While TNF $\alpha$-mediated killing was inhibited under anoxic conditions, the above data suggest that neutrophil apoptosis is not influenced by ROS when generated under physiological conditions.

\section{MATERIALS AND METHODS}

\section{Neutrophil Preparation and Culture}

Human neutrophils were purified from the peripheral blood of healthy human volunteers as previously detailed. ${ }^{8}$ Cell purity was $>95 \%$ neutrophils with $<0.1 \%$ mononuclear cell contamination and $>99 \%$ cell viability. Freshly harvested neutrophils were suspended at $5 \times 10^{6} / \mathrm{mL}$ in Iscoves MDM supplemented with $10 \%$ autologous serum and $50 \mathrm{U} / \mathrm{mL}$ penicillin and $50 \mathrm{U} / \mathrm{mL}$ streptomycin and cultured at $37^{\circ} \mathrm{C}$ in flat-bottomed 96-well Falcon flexiwell plates (Becton-Dickinson, U.K.) in the presence or absence of test reagents (TNF $\alpha, 12.5 \mathrm{ng} / \mathrm{mL} ; \mathrm{PAF}, 1 \mu \mathrm{M}$; $\mathrm{PMLP}, 100$ $\mathrm{nM}$; LY 294002, $10 \mu \mathrm{M}$; Trolox, $10 \mathrm{mM}$; SOD, $200 \mu \mathrm{g} / \mathrm{mL}$; CAT, $250 \mu \mathrm{g} / \mathrm{mL}$; glutathione, $5 \mathrm{mM}$; NAC, $10 \mathrm{mM}$; rotenone, $0.1 \mu \mathrm{g} / \mathrm{mL}$; actinomycin A, $50 \mu \mathrm{M}$ ). Normoxic environments $\left(\mathrm{PO}_{2}\right.$ of media $19 \mathrm{kPa}$; radiometer, Copenhagen) were controlled using a humidified $5 \% \mathrm{CO}_{2} /$ air incubator, and anaerobic conditions $\left(\mathrm{PO}_{2}\right.$ $0 \mathrm{kPa}$ ) maintained with a MACS 500 Don Whitley $\mathrm{H} 2$ catalyst-dependent anaerobic incubator with a $5 \% \mathrm{CO}_{2} / 10 \% \mathrm{H}_{2} /$ balanced $\mathrm{N}_{2}$ gas mix. 


\section{Morphological Assessment of PMN Apoptosis}

Cell morphology was examined under oil immersion light microscopy $(\times 100$ objective) and apoptotic neutrophils were defined as cells containing darkly stained condensed pyknotic nuclei. ${ }^{1}$ A total of 300 neutrophils were counted over at least five high-power fields with the observer blinded to the assay conditions. Our previous studies have shown that morphologic assessment of apoptosis correlates closely to other apoptotic markers including annexin $\mathrm{V}$ binding, propidium iodide staining, CD16 shedding, and DNA cleavage, and remains the most reliable index of apoptosis in neutrophils. ${ }^{9,10}$

\section{Assessment of Superoxide Anion Generation}

Neutrophils were suspended at $10^{6}$ cells in $90 \mu \mathrm{L}$ PBS with $\mathrm{CaCl}_{2}$ and $\mathrm{MgCl}_{2}$ and equilibrated at $37^{\circ} \mathrm{C}$ in a thermomixer for $5 \mathrm{~min}$. Cells were primed with platelet-

A

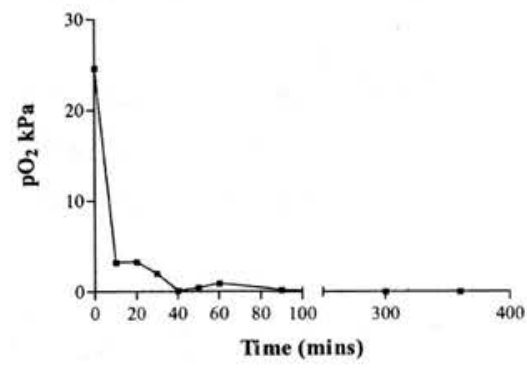

B

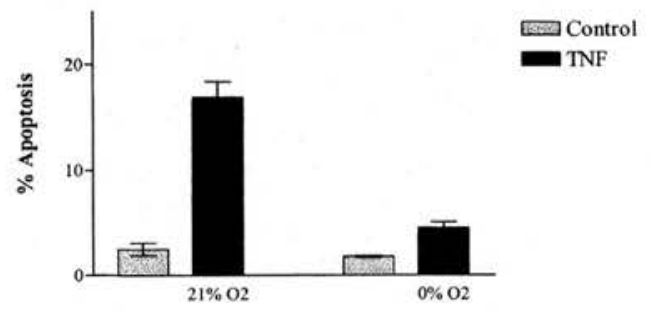

FIGURE 1. TNFa-induced apoptosis is inhibited under anoxic conditions. (A) Medium was pre-equilibrated to $0 \%$ by incubating for $40 \mathrm{~min}$ in a MACS 500 Don Whitley $\mathrm{H}_{2}$ catalyst-dependent anaerobic incubator using $5 \% \mathrm{CO}_{2} / 10 \% \mathrm{H}_{2} /$ balanced $\mathrm{N}_{2}$ gas mix. (B) Neutrophils were subsequently incubated in $21 \%$ oxygen or in $0 \%$ oxygen in the pre-equilibrated medium at $5 \times 10^{6}$ cells $/ \mathrm{mL}$. TNF $\alpha(12.5 \mathrm{ng} / \mathrm{mL})$, when present, was added at the start of each incubation, and cells harvested at $6 \mathrm{~h}$. Apoptosis was assessed morphologically from cytocentrifuge preparations. (B) Data represent mean \pm SEM of $n=3$ experiments each performed in triplicate. 
activating factor (PAF, $1 \mu \mathrm{M}$ ) or PBS for $5 \mathrm{~min}$ and then stimulated with fMLP $(100 \mathrm{nM})$ or PBS in the presence of pre-warmed cytochrome $c(1.2 \mathrm{mg} / \mathrm{ml}$ in PBS) or lucigenin $\left(0.25 \mathrm{mM}\right.$ in BSA $1 \mathrm{mg} / \mathrm{mL}$ PBS without $\mathrm{MgCl}_{2}$ or $\left.\mathrm{CaCl}_{2}\right)$. The superoxide dismutase-inhibitable reduction of cytochrome $c$ was determined as previously described and expressed as nmoles $\mathrm{O}_{2}^{-}$generated per $10^{6}$ neutrophils. Lucigenindependant chemiluminescence (LDCL) was recorded continuously at 9-s intervals for $7 \mathrm{~min}$; data were recorded on-line (Cellular Chemiluminescence, Dynatech Laboratories Ltd.) to produce mean LDCL values from triplicate wells.

\section{Statistical Analysis}

All values are presented as the mean $\pm \operatorname{SEM}$ of $(n)$ number of independent experiments. The data were evaluated using the paired or unpaired Students $t$-test: $P$ values $<0.05$ were considered to be statistically significant.

\section{RESULTS}

\section{TNF $\alpha$-Induced Apoptosis is Inhibited under Anoxic Conditions}

To address whether the pro-apoptotic effect of TNF $\alpha$ in neutrophils requires oxidative metabolism, the effect of absolute hypoxia was examined. Neutrophils were cultured in the presence or absence of TNF $\alpha$ under normoxic or anoxic conditions using medium that had been fully deoxygenated prior to the addition of TNF $\alpha$ (FIG. 1A). The pro-apoptotic effect of TNF $\alpha$ was greatly reduced under such conditions (FIG. 1B), indicating an essential role for molecular oxygen in the cytocidal effect of TNF $\alpha$. In subsequent experiments, neutrophils were suspended in normoxic rather than deoxygenated medium prior to anoxic incubation. In these experiments the extent of TNF $\alpha$-mediated apoptosis was not significantly different from that observed under normoxic conditions, indicating that the commitment of these cells to undergo apoptosis in response to TNF $\alpha$ is extremely rapid, occurring within $30 \mathrm{~min}$ of exposure to this cytokine (data not shown).

\section{Role of the NADPH Oxidase in TNF $\alpha$-Stimulated Neutrophil Apoptosis}

Oxygen radicals have been proposed as playing an essential role in TNF $\alpha$ and phagocytosis-induced apoptotic cell death in neutrophils. ${ }^{11}$ However it has yet to be determined whether activation of the NADPH oxidase under physiological conditions results in apoptosis. To address this we activated neutrophils by first incubating with a priming concentration of PAF $(1 \mu \mathrm{M}, 5 \mathrm{~min})$ followed by the addition of a maximally effective concentration of the bacterial tripeptide fMLP (100 nM, $10 \mathrm{~min}$ ). As shown in FIGURE $2 \mathrm{~A}$ and $\mathrm{B}$, this protocol resulted in a robust stimulation of superoxide anion release yet had no effect on the subsequent rate of constitutive apoptosis measured either at 6 or 20 hours (FIG. 2C).

Activation of the phosphoinositide 3-kinase (PI3-kinase)/Akt pathway is an important mechanism underlying growth factor-mediated survival in mesenchymal cells and cytokine-stimulated neutrophils. Gardai and colleagues ${ }^{12}$ proposed that NADPH oxidase-derived oxidants, acting through Lyn and the SH2-containing inositol-5-phosphatase (SHIP), serve to inhibit Akt and thereby trigger neutrophil apop- 
A

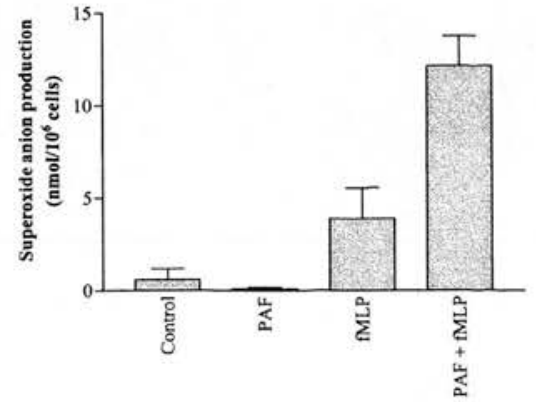

B

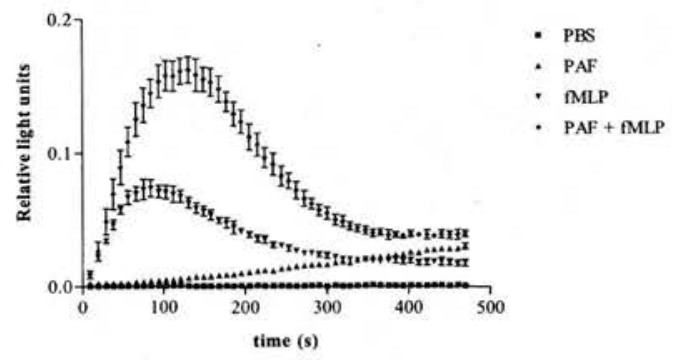

$\mathrm{C}$

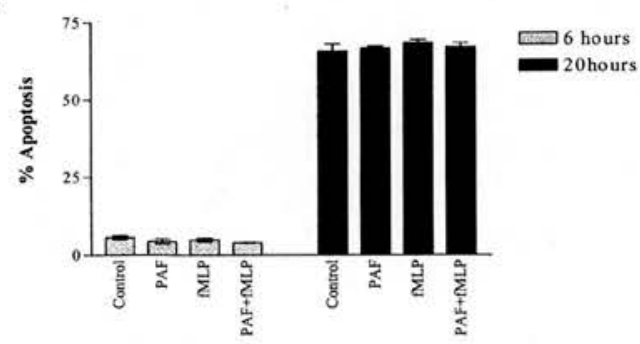

FIGURE 2. Role of NADPH oxidase in TNF $\alpha$-stimulated neutrophil apoptosis. (A) Neutrophils were incubated at $5 \times 10^{6}$ cells $/ \mathrm{ml}$ with test reagents PAF $(1 \mu \mathrm{M})$ and/or fMLP $(100 \mathrm{nM})$. Superoxide dismutase-inhibitable reduction of cytochrome $c$ was measured at $35-565 \mathrm{~nm}$ and expressed as nmoles $\mathrm{O}_{2}{ }^{-}$generated per $10^{6}$ neutrophils. (B) Kinetics of ROS release was measured by lucigenin-dependent chemiluminescence with mean data produced from triplicate wells. (C) At the time points indicated, cells were recovered and apoptosis assessed by morphologic examination of cytocentrifuge preparations. Data in $\mathbf{A}$ and $\mathbf{C}$ represent mean \pm SEM of three separate experiments, each performed in triplicate. Data in $\mathbf{B}$ are from triplicate determinations from a single experiment and representative of two further experiments. 
tosis. Despite this, LY29008, a PI3-kinase inhibitor that we have shown abolishes agonist-stimulated NADPH oxidase and the survival effect of GM-CSF, had no effect on the extent of apoptosis under normoxic conditions (\% apoptosis $20 \mathrm{~h}$ : normoxia $63 \pm 3$, normoxia + LY29008 $62 \pm 2, n=3$ ). These data concur with our previous observation that the PI3-kinase inhibitor wortmannin fails to block TNF $\alpha$ induced neutrophil apoptosis ${ }^{13}$ and would support our hypothesis that activation of the NADPH oxidase does not mediate the pro-apoptotic effect of TNF $\alpha$.

A

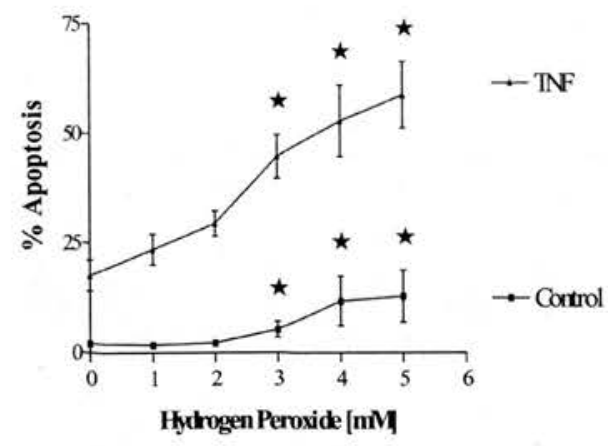

B

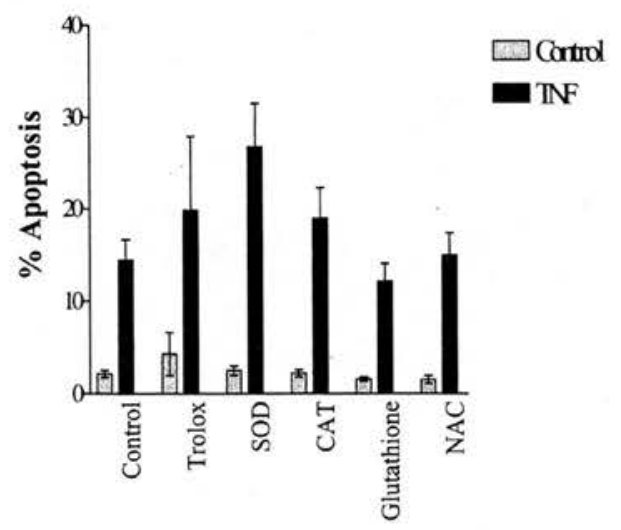

FIGURE 3. Role of reactive oxygen species and anti-oxidants on TNF $\alpha$-stimulated neutrophil apoptosis. (A) The effect of exogenous $\mathrm{H}_{2} \mathrm{O}_{2}$ on neutrophil apoptosis was assessed by incubating cells at $5 \times 10^{6} / \mathrm{mL}$ with a range of $\mathrm{H}_{2} \mathrm{O}_{2}$ concentrations from $1-5 \mathrm{mM}$. Cells were harvested at $6 \mathrm{~h}$ and apoptosis scored morphologically. (B) Neutrophils were incubated at $5 \times 10^{6}$ cells $/ \mathrm{ml}$ with or without TNF $\alpha(12.5 \mathrm{ng} / \mathrm{mL})$ in the presence of the antioxidants trolox $(10 \mathrm{mM})$, SOD $(200 \mu \mathrm{g} / \mathrm{mL})$, CAT $(250 \mu \mathrm{g} / \mathrm{mL})$, glutathione $(5 \mathrm{mM})$ or NAC $(10 \mathrm{mM})$. Cells were harvested at $6 \mathrm{~h}$ and apoptosis assessed morphologically. Data represent mean \pm SEM of $n=3$ experiments each performed in triplicate with significant differences to control values $(P<0.05)$ marked *. 


\section{Role of Reactive Oxygen Species in TNF $\alpha$-Stimulated Neutrophil Apoptosis}

To explore further the capacity of ROS to influence apoptotic thresholds in neutrophils these cells were incubated for 6 hours in the presence or absence of TNF $\alpha$ with increasing concentrations of $\mathrm{H}_{2} \mathrm{O}_{2}$. Detailed concentration-response studies demonstrated that $\mathrm{H}_{2} \mathrm{O}_{2}$ (up to $1 \mathrm{mM}$ ) had no effect on the rate of constitutive or TNF $\alpha$-stimulated apoptosis (data not shown). While the use of much higher (supraphysiological) concentrations of $\mathrm{H}_{2} \mathrm{O}_{2}$ increased the extent of basal and TNF $\alpha$-stimulated apoptosis at $6 \mathrm{~h}$ (FIG. 3A), the overall extent of apoptosis remained less than $20 \%$, even in the presence of $5 \mathrm{mM} \mathrm{H}_{2} \mathrm{O}_{2}$. These data again suggest that human neutrophils are highly resistant to oxidant-induced apoptosis.

To assess whether the inhibition of TNF $\alpha$-induced neutrophil apoptosis observed under hypoxic conditions reflected changes in the intracellular generation of ROS the effects of a panel of structurally discrete antioxidants were examined. In agreement with the above data, trolox $(10 \mu \mathrm{M})$, superoxide dismutase (SOD, $200 \mu \mathrm{g} / \mathrm{mL}$ ), catalase $(\mathrm{CAT}, 250 \mu \mathrm{g} / \mathrm{mL})$, reduced glutathione $(5 \mathrm{mM})$ and $N$-acetyl-L-cysteine (NAC, $10 \mathrm{mM}$ ) were all unable to inhibit the pro-apoptotic effect of TNF $\alpha$ (FIG. 3B).

The mitochondria are an important source of ROS formation through auto-oxidation of Complex I flavin mononucleotide and Complex III ubisemiquinone, and have been previously shown to modulate TNF $\alpha$-induced cytotoxicity in L929 and WEH1 cells. To investigate the putative role of these organelles in TNF $\alpha$-mediated neutrophil apoptosis we preincubated cells with the mitochondrial inhibitors rotenone $(0.1 \mu \mathrm{g} / \mathrm{mL})$, a Complex I inhibitor, or actinomycin A $(50 \mu \mathrm{M})$, a Complex III inhibitor, prior to cytokine stimulation. While neither of these agents influenced the rate of constitutive apoptosis at $6 \mathrm{~h}$ (data not shown), actinomycin A caused a significant inhibition TNF $\alpha$-stimulated apoptosis (\% apoptosis $6 \mathrm{~h}$ : TNF $\alpha 17 \pm 3, \mathrm{TNF} \alpha+$ actinomycin A $8 \pm 1, n=3, P<0.05$ ).

\section{DISCUSSION}

We have previously reported that TNF $\alpha$ has a bimodal effect on the survival of human neutrophils in vitro with the capacity to induce early apoptosis but decrease the extent of cell death at later times. This early killing effect of TNF $\alpha$ requires activation of the high-affinity TNFR75 in addition to TNFR55. ${ }^{14}$ Despite many attempts to elucidate the mechanisms of TNF $\alpha$-mediated cytotoxicity in hematopoietic and other cell types, there is currently no unifying theory to account for the pro-apoptotic effect of TNF $\alpha$ in neutrophils. In this study we have sought to identify the intracellular mechanisms underlying the induction of neutrophil apoptosis by TNF $\alpha$ and, in particular, assess the involvement of ROS in both constitutive and TNF $\alpha$-induced apoptosis.

Gardai and co-workers ${ }^{12}$ demonstrated that TNF $\alpha$ in combination with either the $\beta 2$-integrin-activating antibody VIM12, GM-CSF, or LPS, promoted apoptosis via the phosphorylation and activation of the tyrosine kinase Lyn with subsequent recruitment and activation of SHIP and downregulation of Akt. This work also proposed a role for oxidants in the apoptotic response since the initial activation of Lyn was dependent on the NADPH oxidase. Oxidant-dependent induction of neutrophil apoptosis has also been proposed in models of neutrophil phagocytosis of E. coli or 
opsonized particles. ${ }^{15}$ By contrast, in our experimental system we have been unable to modulate neutrophil apoptosis with maximal physiological stimulation of the NADPH oxidase or by the addition of exogenous $\mathrm{H}_{2} \mathrm{O}_{2}$ (up to a concentration of $1 \mathrm{mM}$ ).

Studies in L929 cells have identified the mitochondria as the first site of damage in TNF $\alpha$-mediated cytolysis, ${ }^{16}$ and mitochondrial inhibitors have been shown to influence TNF $\alpha$-induced cytotoxicity in several cell types. It has long been recognized that the mitochondria are an important source of ROS under certain conditions and according to Boveris and Chance ${ }^{17}(1973)$, this pathway accounts for about $1-2 \%$ of the oxygen consumed by cells during resting respiration. Two sites within the mitochondrial respiratory chain have been identified as responsible for ROS formation; the first is dependent on the auto-oxidation of the flavin mononucleotide from the NADH-dehydrogenase (Complex I), whereas the second and probably more relevant site depends on the auto-oxidation of the unstable ubisemiquinone (Complex III), which is an intermediate of the Q-cycle reaction. It is important to note that while the production of oxygen radicals at the ubiquinone site is diminished by complex I and II inhibitors the formation of ROS actually increases several-fold in the presence of actinomycin A, implicating ubisemiquinone as the main reductant site of oxygen. ${ }^{18}$ Indeed this ability of actinomycin A to enhance mitochondrial ROS generation appears to underly its capacity to augment TNF $\alpha$-stimulated apoptosis in both L929 and WEHI mouse fibrosarcoma cells. ${ }^{6}$ Hence our finding that this compound inhibits rather than increases the pro-apoptotic effect of TNF $\alpha$ further questions the role of ROS in TNF $\alpha$-induced killing in neutrophils. This conclusion is supported by the lack of effect of the antioxidants trolox, SOD, catalase, reduced glutathione, and NAC on the TNF $\alpha$ response.

While ROS generation either via activation of the NADPH oxidase or the mitochondrial respiratory burst does not appear to be involved in TNF $\alpha$-killing, our data indicate that the ability of TNF $\alpha$ to induce neutrophil apoptosis still requires the presence of molecular oxygen. Two models exist regarding the effect of oxygen deprivation on the mitochondrial respiratory chain. Firstly, a reduction in $\mathrm{O}_{2}$ can be associated with a decline in ROS. ${ }^{19}$ Secondly, under hypoxic conditions the reduction of $\mathrm{O}_{2}$ to $\mathrm{H}_{2} \mathrm{O}$ by cytochrome $c$ oxidase (Complex IV) is inhibited, resulting in release of $\mathrm{O}_{2}^{-}$and electrons at Complex III. ${ }^{20}$ In neutrophils, while it is evident that the absence of environmental oxygen, or the presence of mitochondrial complex III inhibitors, blocks TNF $\alpha$ killing, the lack of effect of exogenous $\mathrm{H}_{2} \mathrm{O}_{2}$, antioxidants, and complex I inhibitors indicates a regulatory step independent of ROS.

Together, our data indicate that the generation of ROS under physiological conditions does not affect the rate of constitutive apoptosis in neutrophils or underlie the pro-apoptotic effect of TNF $\alpha$. This conclusion would be entirely consistent with the fact that these cells are required to produce large quantities of such intermediates to effect efficient bacterial killing.

\section{ACKNOWLEDGMENTS}

This work was supported by the Wellcome Trust (035662), the MRC (G9016491), and the National Asthma Campaign (01/042). S.R.W. and J.A.W. hold MRC Clinical Research Training Fellowships. 


\section{REFERENCES}

1. SAVILl, J., J.E. Henson, A.H. Wyllie, et al. 1989. Macrophage phagocytosis of aging neutrophils in inflammation. Programmed cell death in the neutrophil leads to recognition by macrophages. J. Clin. Invest. 83: 865-875.

2. GrigG, J.M., J. SAVILL, C. SARRAF, et al. 1991. Neutrophil apoptosis and clearance by macrophages in the lungs of neonates with pulmonary inflammation Lancet 338: $720-722$.

3. LeE, A., M.K. Whyte \& C. Haslett. 1993. Inhibition of apoptosis and prolongation of neutrophil functional longevity by inflammatory mediators. J. Leukoc. Biol. 54: $283-288$.

4. Salamone, G., M. Giordano, A.S. Trevani, et al. 2001. Promotion of neutrophil apoptosis by TNF-alpha. J. Immunol. 166: 3476-3483.

5. Matthews, N., M.L. Neale, S.K. Jackson \& J. Stark. 1987. Tumour cell killing by tumour necrosis factor: inhibition by anaerobic conditions, free-radical scavengers and inhibitors of arachidonate metabolism. Immunology 62: 153-155.

6. Schulze-Osthoff, K., A.C. BAKker, B. VAN Haesebroeck, et al. 1992. Cytotoxic activity of tumour necrosis factor is mediated by early damage of mitochondrial function: evidence for the involvement of mitochondrial radical generation. J. Biol. Chem. 267: 5317-5323.

7. Hirose, K.D., D.L. Longo, J.J. Oppenheim \& K. Matsushima, 1993, Over expression of mitochondrial manganese superoxide dismutase promotes the survival of tumour cells exposed to interleukin-1, tumour necrosis factor, selected anticancer drugs, and ionizing radiation. FASEB J. 7: 361-368.

8. Haslett, C., L.A. Guthrie, M.M. Kopamiak, et al. 1985. Modulation of multiple neutrophil functions by preparative methods or trace concentrations of bacterial lipopolysaccaride. Am. J. Path. 119: 101-110.

9. Howburn, A., K.A. Cadwallader, B.J. Reed, et al 2002. Role of PI3-kinasedependent Bad phosphorylation and altered transcription in cytokine-mediated neutrophil survival. Blood 100: 2607-2616.

10. Dransfield, I., A.M. Buckle, J. SAvill, et al. 1994. Neutrophil apoptosis is associated with a reduction in CD16 (Fc gamma RIII) expression. J. Immunol. 153: 1254-1263.

11. Coxon, A., P. RIEU, F.J. BARKLow, et al. 1996. A novel role for the $\beta 2$ integrin CD11b/ CD18 in neutrophil apoptosis: a homeostatic mechanism in inflammation. Immunity 5: 653-666.

12. Gardai, S., B.B. Whitlock, C. Helgason, et al. 2002. Activation of SHIP by NADPH oxidase-stimulated Lyn leads to enhanced apoptosis in neutrophils. J. Biol. Chem. 277: 5236-5246.

13. Murray, J., A.M. Condliffe, C. Haslett \& E.R. Chilvers. 1996. Wortmannin enhances tumour necrosis factor alpha-stimulated neutrophil apoptosis. Biochem. Soc. Trans. 24: $80 \mathrm{~s}$

14. Murray, J., J.A. Barbara, S.A. Dunkley, et al. 1997. Regulation of neutrophil apoptosis by tumor necrosis factor-alpha: requirement for TNFR55 and TNFR75 for induction of apoptosis in vitro. Blood 90: 2772-2783.

15. Lock, R., C. DAHLGRen, M. Linden, et al. 1990. Neutrophil killing of two type 1 fimbria-bearing Escherichia coli strains: dependence on respiratory burst activation. Infect. Immun. 58: 37-42.

16. MatThEws, N. 1983. Anti-tumour cytotoxin produced by human monocytes: studies on its mode of action. Br. J. Cancer 48: $405-410$.

17. BOVERIS, A. \& B. CHANCE. 1973. The mitochondrial generation of hydrogen peroxide: general properties and effect of hyperbaric oxygen. Biochem. J. 134: 707-716.

18. Boveris, A. 1976. Mitochondrial production of hydrogen peroxide in Saccharomyces cerevisiae. Acta Physiol. Lat. Am. 26: 303-309.

19. Ehleben, W., B. Bolling, E. Merten, et al. 1998. Cytochromes and oxygen radicals as putative members of the oxygen sensing pathway. Respir. Physiol. 114: 25-36.

20. Chandel, NS., E. Maltepe, E. Goldwasser, et al. 1998. Mitochondrial reactive oxygen species trigger hypoxia-induced transcription. Proc. Natl. Acad. Sci. USA 95: $11715-11720$ 


\title{
Golgi fragmentation during Fas-mediated apoptosis is associated with the rapid loss of GM130
}

\author{
Annemieke Walker, ${ }^{*}$ Carol Ward, Tara A. Sheldrake, Ian Dransfield, Adriano G. Rossi, \\ James G. Pryde, and Christopher Haslett
}

Centre for Inflammation Research, The University of Edinburgh, Medical School, Teviot Place, Edinburgh EH8 $9 A G$, UK

Received 2 February 2004

\begin{abstract}
During apoptosis, the Golgi complex becomes fragmented and key proteins (e.g., GRASP65 and p115) are targets for caspase cleavage. GM130, an integral membrane protein, contributes to the maintenance of Golgi structure and facilitates membrane fusion with secretory vesicles. We show that GM130 levels decrease during Fas-induced apoptosis but not during staurosporine-induced apoptosis while in both models p115 levels remain unaffected. We conclude that GM130 is rapidly diminished during Fas-mediated apoptosis associated with Golgi fragmentation in contrast to previous studies which have suggested that loss of GM130 during apoptosis is a late event.
\end{abstract}

(c) 2004 Elsevier Inc. All rights reserved.

Keywords: Golgi; Apoptosis; p115; GM130; Staurosporine; Fas

The Golgi complex is the 'organising centre' for protein cargo en route from the endoplasmic reticulum to the plasma membrane. During mitosis, this structure becomes fragmented with a concomitant arrest in vesicle trafficking [1]. During apoptosis the Golgi complex also fragments [2-4] and it is possible that the secretory pathway is similarly inhibited, which would in part explain why apoptotic cells fail to respond to various external stimuli [5]. Mitotic Golgi fragmentation has been demonstrated to be dependent on cyclin-dependent-kinase-1 (CDK1) and later GM130 was identified as a CDK1 substrate [6]. GM130, a cis-Golgi matrix protein, is associated with the cytoplasmic face of the Golgi via GRASP65 [7], a N-myristoylated peripheral membrane protein [8], and binds the p115 tethering protein present on incoming vesicles $[9,10]$. Following phosphorylation, GM130 is no longer able to bind to p115 and dissociation of p115-GM130 is thought to contribute to fragmentation of the Golgi complex

Abbreviations: z-VAD-fmk, benzylocarbonyl-Val-Ala-Asp-flouromethylketone; PI, propidium iodide; SSP, staurosporine.

"Corresponding author. Fax: +44-131-650-4384.

E-mail address: awalker4@staffmail.ed.ac.uk (A. Walker). during mitosis [9]. GM130, GRASP65, and p115 have already been shown to be cleaved during apoptosis in a caspase-dependent manner facilitating Golgi fragmentation during apoptosis [2,3]. These studies concluded that GM130 cleavage during apoptosis is secondary to GRASP65 cleavage and a relatively late event [2,3]. However, these conclusions were based on staurosporine (broad-range kinase inhibitor) -induced apoptosis and work by Cha et al. [11] demonstrates phosphorylation of Golgi-associated targets may modulate cleavage. To address this issue, we followed the fate of GM130 during apoptosis in human J.CaM1.6 cells, a derivative of Jurkat T lymphocyte cells by triggering the physiologically relevant Fas death pathway [12] and for comparison by the broad spectrum kinase inhibitor staurosporine.

\section{Materials and methods}

Cell line. J.CaM1.6 cells were obtained from the European Collection of Animal Cell Cultures (Porton Down, UK) and routinely maintained in RPMI-1640 containing $10 \%$ FCS, $100 \mathrm{U} / \mathrm{ml}$ penicillin/ streptomycin, $2 \mathrm{mM}$ L-glutamine, and $1 \%$ non-essential amino acids. Cells were grown in a $5 \% \mathrm{CO}_{2}$ humidified incubator at $37^{\circ} \mathrm{C}$. 
Induction of apoptosis. J.CaM1.6 cells were incubated in 96-well plates at $5 \times 10^{6}$ cells $/ \mathrm{ml}$. Cells were incubated with either the broadrange kinase inhibitor staurosporine $(2 \mu \mathrm{M})$ (Calbiochem-Novabiochem, UK) or the anti-Fas monoclonal antibody $\mathrm{CH} 11(500 \mathrm{ng} / \mathrm{ml})$ (TCS Biologicals, UK) to induce apoptosis. One hundred micromolar of the pan-caspase inhibitor z-VAD-fmk (Bachem, UK) was added to appropriate wells in both staurosporine and $\mathrm{CH} 11$ assays and ZB4 (Fas blocking monoclonal antibody, $500 \mathrm{ng} / \mathrm{ml}$, TCS Biologicals, UK) was added to appropriate wells in $\mathrm{CH} 11$ assays.

Flow cytometry. Externalisation of phosphatidylserine was monitored by FITC-labelled recombinant human Annexin-V binding. Stock Annexin-V-FITC (Bender MedSystems, Austria) was diluted 1/3000 with Annexin-V-binding buffer $(500 \mathrm{ml}$ Hanks' Balanced Salt Solution containing $5 \mu \mathrm{M} \mathrm{CaCl}$ ). Cells were incubated on ice for $10 \mathrm{~min}$ with Annexin-V-FITC and $1 \mathrm{~min}$ with propidium iodide (PI). Annexin-VFITC/PI binding was assessed by flow cytometry on a Coulter EPICS (Beckman Coulter, UK) and analysed on associated EXPO software. All experiments were carried out in triplicate $(\mathrm{n}=3)$.

Immunofluorescence and image acquisition. The indirect immunofluorescence protocol was as described [13]. Cells were cytocentrifuged ( $300 \mathrm{rpm}$ for $3 \mathrm{~min}$ ) onto $1.5 \times 22 \times 22$-mm glass coverslips and fixed in methanol-free $3 \%(\mathrm{w} / \mathrm{v})$ para-formaldehyde/PBS containing $1 \mathrm{mM}$ $\mathrm{CaCl}_{2}$ and $1 \mathrm{mM} \mathrm{MgCl}$ for $20 \mathrm{~min}$ at $20^{\circ} \mathrm{C}$. Cells were permeabilised with $0.1 \%(\mathrm{w} / \mathrm{v})$ Triton $\mathrm{X}-100$ and non-specific binding was blocked with $10 \%(\mathrm{v} / \mathrm{v})$ sheep serum in $0.2 \%(\mathrm{w} / \mathrm{v})$ fish skin gelatin (Sigma, UK). Primary antibodies used were: anti-active-caspase-3 rabbit polyclonal antibody (BD Pharmingen, UK) and mouse anti-rat GM130 monoclonal antibody (BD Transduction Laboratories, UK). Secondary antibodies used were Alexa 488 (green) goat anti-mouse IgG (highly cross-adsorbed) and Alexa 568 (red) goat anti-rabbit (highly cross-adsorbed) (Molecular Probes, Netherlands). Nuclei were stained with TO-PRO3 (Molecular Probes). Images for each channel were captured separately and assembled into a single file with TCSMERGE software (Leica Microsystems, Heidelberg, Germany) prior to analysis. All confocal images were processed digitally using Adobe Photoshop 5.02 and Paint Shop Pro 4.

Western blotting. J.CaM1.6 cells $\left(2.5 \times 10^{6}\right)$ were harvested per sample and lysed as described [13]. Each sample was loaded onto a $10 \%$ SDS - polyacrylamide gel and proteins were separated and electrophoretically transferred to nitrocellulose. The nitrocellulose membranes were probed with mouse monoclonal antibodies to procaspase-3 (BD Pharmingen, UK), GM130, p115 (BD Transduction Laboratories, UK), and actin (Sigma, UK) followed by HRP-Goat anti mouse IgG (Dako, UK). The membranes were then incubated with ECL reagent (Amersham Biosciences, UK), placed under BioMax MS-1 X-ray sensitive film, and processed through an X-ray developer (X-Ograph Imaging Systems, Wilts, UK).

Statistical analysis. Results are reported either as pooled data from a series of $n$ separate experiments (mean \pm SEM) or as individual representative experiments (mean \pm SD). Statistical significance was assessed by one-way analysis of variance with comparisons between groups made using the Tukey procedure.

\section{Results}

\section{Induction of apoptosis in J.CaM1.6 cells}

To examine whether GM130 was affected during Fasmediated apoptosis, we used J.CaM1.6 cells, a derivative of Jurkat cells, which constitutively expresses Fas. J.CaM1.6 cells were treated with $500 \mathrm{ng} / \mathrm{ml} \mathrm{CH} 11$ in the presence of FCS, over $6 \mathrm{~h}$ and the proportion of apoptotic cells, as assessed by Annexin-V-FITC binding, was monitored at 1, 2, 4, and $6 \mathrm{~h}$ (Fig. 1A). At $1 \mathrm{~h}$, the percentage of apoptotic cells was $\sim 12 \%$, significantly $(p<0.05)$ higher than present in control populations ( $\sim 2 \%$ ), increasing up to $4 \mathrm{~h}$, where the amount of apoptosis was $\sim 60 \%$, after which the rate of apoptosis began to decline. To ensure that induction of apoptosis occurred via the Fas pathway, control samples were preincubated with $500 \mathrm{ng} / \mathrm{ml}$ of antagonist Fas antibody ZB4 for $30 \mathrm{~min}$, prior to addition of CH11. ZB4 abolished the apoptotic effect of $\mathrm{CH} 11$ upon J.CaM1.6 cells, decreasing the proportion of cells which bound Annexin-V-FITC $(\sim 5 \%$ not significant $(p<0.05)$ from untreated control levels). In addition, z-VAD-fmk completely inhibited Annexin-V-FITC binding, which was not significantly different from control levels. Fig. 1B shows representative flow cytometry profiles demonstrating that the amount of PI positive cells never increases significantly above control levels.

To follow the kinetics of apoptosis induction in J.CaM1.6 cells by staurosporine, cells were incubated in the presence of $2 \mu \mathrm{M}$ staurosporine over $4 \mathrm{~h}$ (Fig. 2A) and apoptosis was assessed by Annexin-V-FITC binding and exclusion of PI. The results from three separate experiments show that at $1 \mathrm{~h}$, the number of apoptotic cells present, $\sim 14 \%$, was already significantly $(p<0.05)$ increased from control cells $\sim 2 \%$. This trend continued up to $4 \mathrm{~h}$, where $\sim 80 \%$ of cells bound Annexin-V-FITC and excluded PI. Apoptosis was inhibited by z-VADfmk, demonstrating that the effects of staurosporine were due to caspase activity. Fig. 2B shows representative flow cytometry profiles revealing that staurosporine does not induce significant amounts of necrosis over this time frame.

\section{Immunofluorescence of Golgi fragmentation in apoptotic J. CaM1.6 cells}

To examine the fragmentation of the Golgi in apoptotic J.CaM1.6 cells, cells were treated with $500 \mathrm{ng} / \mathrm{ml}$ $\mathrm{CH} 11$ or $2 \mu \mathrm{M}$ staurosporine over $4 \mathrm{~h}$ to induce apoptosis. Apoptotic cells were identified by their fragmented nuclei and by positive staining with the polyclonal antibody raised against active-caspase-3 (Fig. 3). Many of the cells treated with $500 \mathrm{ng} / \mathrm{ml} \mathrm{CH} 11$ had caspase- 3 activity throughout the cytoplasm and in those cells the nuclei and Golgi were fragmented. In cells that had been pre-treated with the neutralising antibody ZB4 against Fas or the caspase inhibitor z-VAD-fmk, caspase-3 activation, and Golgi fragmentation were prevented. This demonstrated that Golgi fragmentation and nuclear "pebbling" were a direct consequence of signalling through the Fas death pathway in CH11-treated cells and that the morphological changes observed were caspase-dependent. To compare the fragmentation of the Golgi complex during staurosporine-induced apoptosis, J.CaM1.6 cells were treated with $2 \mu \mathrm{M}$ 

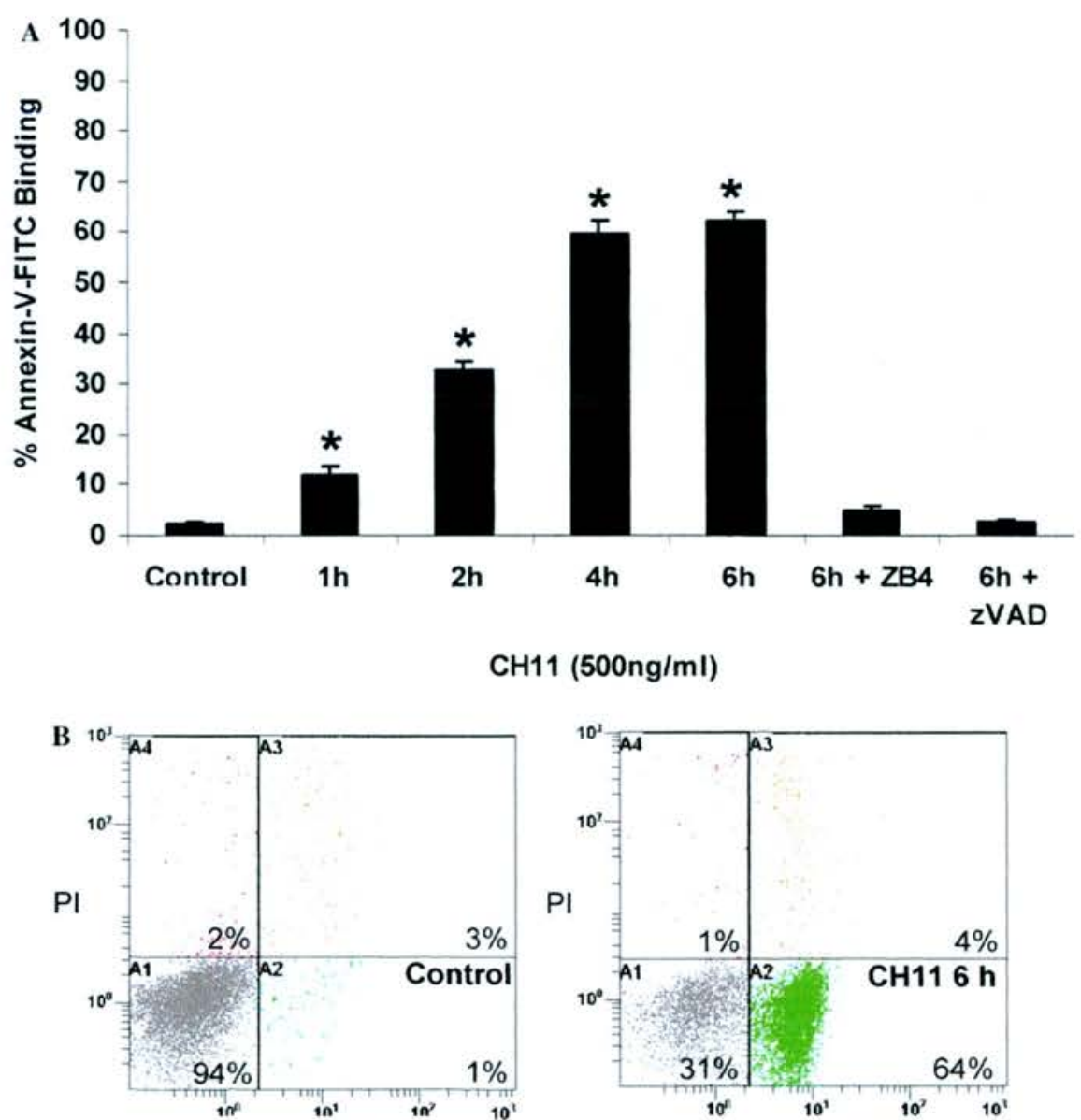

Annexin-V-FITC
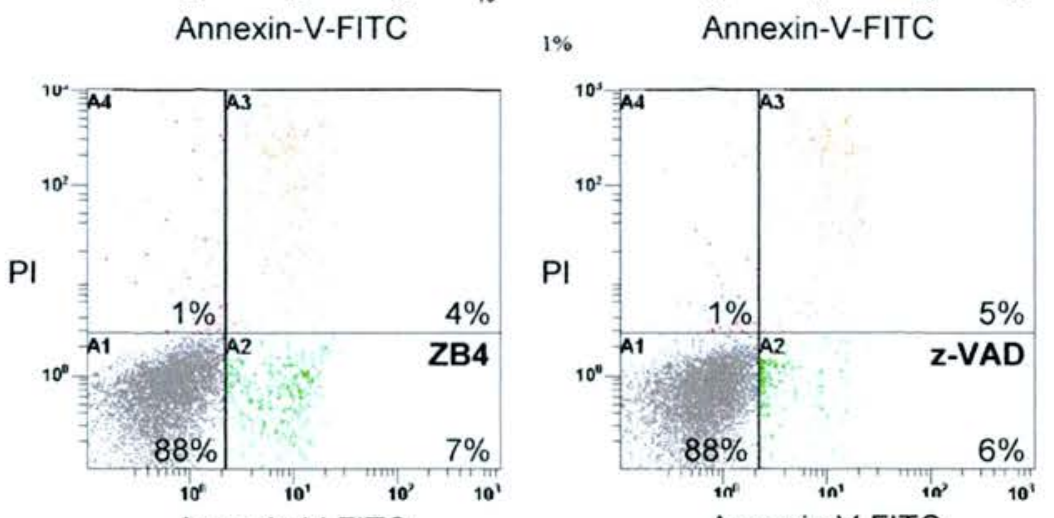

Annexin-V-FITC

Fig. 1. Fas-induced apoptosis in J.CaM1.6 cells. (A) J.CaM1.6 cells were incubated with the anti-Fas monoclonal antibody CH11 (500 ng/ml) over $6 \mathrm{~h}$. Samples were taken at the time points indicated and the rates of apoptosis and necrosis were assessed by Annexin-V binding and PI staining. The amount of apoptosis increased significantly over $6 \mathrm{~h}$ and this was blocked by the Fas neutralising antibody $\mathrm{ZB} 4$ ( $500 \mathrm{ng} / \mathrm{ml})$ and the pan-caspase inhibitor z-VAD-fmk $(100 \mu \mathrm{M})$. Necrosis as measured by PI staining did not increase significantly during any of the treatments. Data represent means \pm SEM of three separate experiments. All experiments were performed in triplicate $\left({ }^{*} p<0.05\right.$ compared with control values). (B) Representative scatter plots demonstrating little PI staining (upper quadrants) in control cell populations and CH11-treated cell populations where Annexin-FITC binding is increased.

staurosporine for $4 \mathrm{~h}$. The proportion of apoptotic cells was higher in staurosporine-treated cells than $\mathrm{CH} 11$ treated cells after $4 \mathrm{~h}$ with the majority of cells being apoptotic. From the kinetic studies, it can be seen that the majority ( $\sim 80 \%$ ) of staurosporine-treated J.CaM1.6 cells are apoptotic at $4 \mathrm{~h}$ (Fig. 3). All cells displaying nuclear fragmentation also had caspase- 3 activity yet strikingly, a large proportion of these cells still had intact Golgi. 


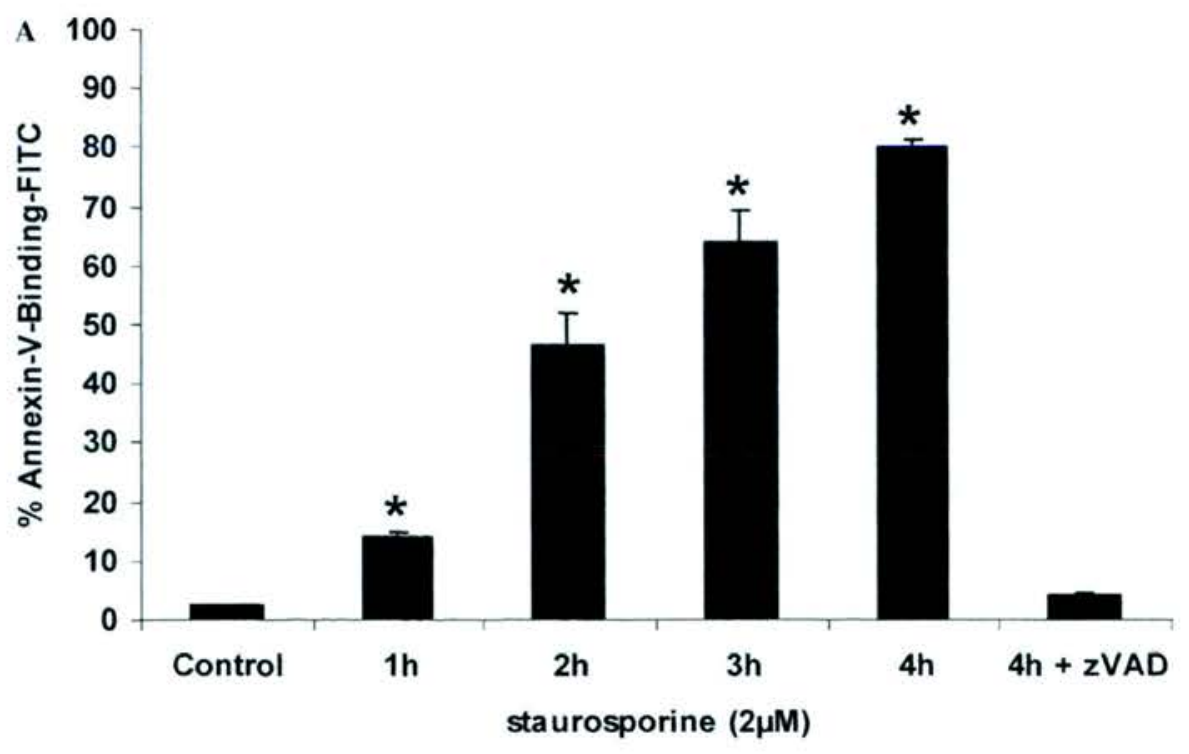

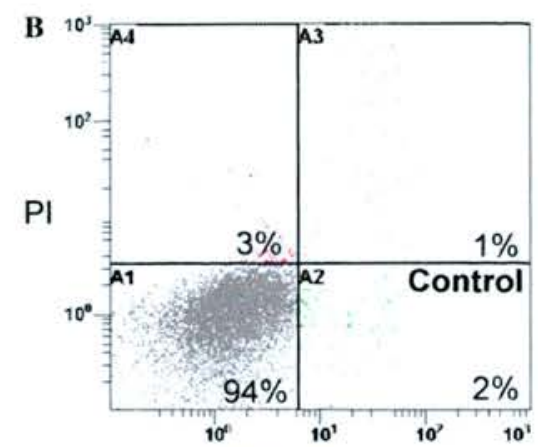

Annexin-V-FITC

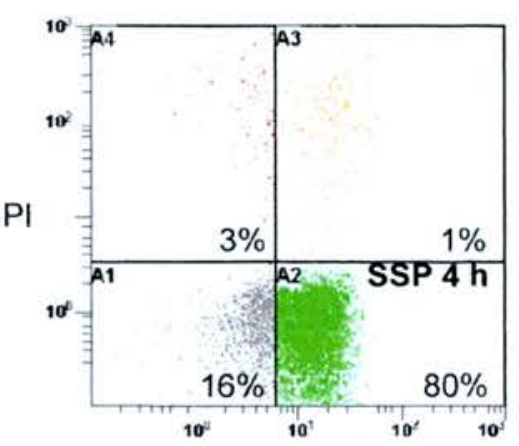

Annexin-V-FITC

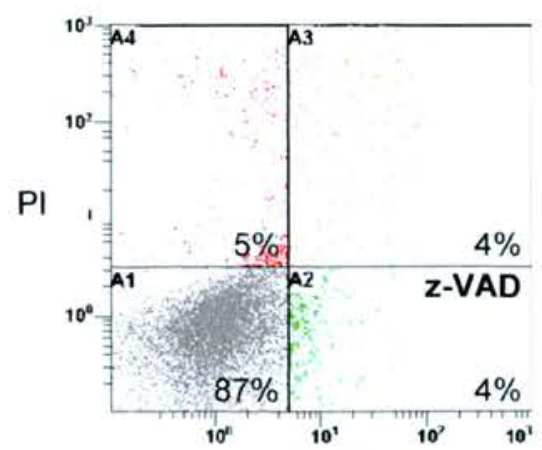

Annexin-V-FITC

Fig. 2. Staurosporine-induced apoptosis in J.CaM1.6 cells. (A) J.CaM1.6 cells were incubated with $2 \mu \mathrm{M}$ staurosporine over 4 h. Samples were taken at the time points indicated and the rates of apoptosis and necrosis were assessed by Annexin-V binding and PI staining. The amount of apoptosis increased significantly over $4 \mathrm{~h}$ and this was blocked by $\mathrm{z}-\mathrm{VAD}$-fmk $(100 \mu \mathrm{M})$. Necrosis as measured by PI staining did not increase significantly during any of the treatments. Data represent means \pm SEM of three separate experiments. All experiments were performed in triplicate $\left({ }^{*} p<0.05\right.$ compared with control values). (B) Representative scatter plot demonstrating little PI staining (upper quadrants) in control cell populations and staurosporine-treated cell populations where Annexin-FITC binding is increased.

\section{GM130 levels decrease during CH11-, but not stauro-} sporine-, induced apoptosis

Immunofluorescence studies suggested that the kinetics of Golgi fragmentation in apoptotic cells treated with staurosporine were different to the kinetics of Golgi fragmentation in cells treated with $\mathrm{CH} 11$ (Fig. 3). Of particular interest was the fate of the Golgi matrix protein GM130 and p115 during apoptosis. J.CaM1.6 cells were incubated with $500 \mathrm{ng} / \mathrm{ml} \mathrm{CH11} \mathrm{over} 6 \mathrm{~h}$ and the levels of GM130 and p115 were assessed by Western blotting together with procaspase- 3 and actin. After $4 \mathrm{~h}$, procaspase- 3 was completely cleaved and there was a concomitant decrease in GM130 compared to control levels, although p115 and actin levels were similar to control (Fig. 4). At 6h, GM130 was barely detectable while p115 and actin levels remained comparable to control levels. Procaspase-3 processing and apoptosis was inhibited by ZB4, demonstrating that apoptosis was specific to the induction of the Fas-mediated death pathway. Procaspase-3 cleavage was also completely inhibited by z-VAD-fmk and the rate of apoptosis was similar to control levels. In both cases, pre-incubation with ZB4 and z-VAD-fmk, GM130 levels were similar to control demonstrating that the reduced levels of GM130 were specific to Fas-mediated apoptosis and were caspase-dependent.

To assess whether GM130 levels also diminished during staurosporine-induced apoptosis we treated J.CaM1. 6 cells with $2 \mu \mathrm{M}$ staurosporine over $4 \mathrm{~h}$ and the levels of GM130, p115, procaspase-3, and actin were monitored again by Western blot analysis. By $2 \mathrm{~h}$, procaspase- 3 was no longer detected but GM130, p115, and actin were not diminished. After 3 and $4 \mathrm{~h}$, despite 

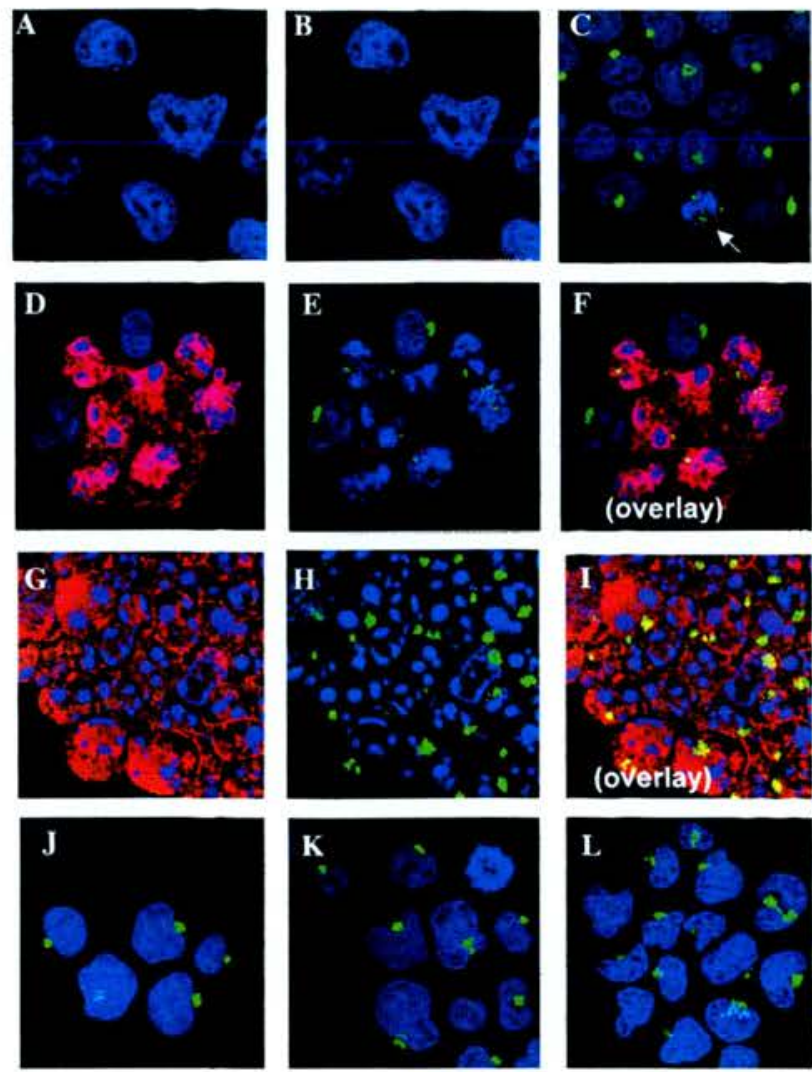

Fig. 3. Golgi morphology in J.CaM1.6 cells treated with $500 \mathrm{ng} / \mathrm{ml}$ $\mathrm{CH} 11$ or $2 \mu \mathrm{M}$ staurosporine for $4 \mathrm{~h}$. Cells were stained with anti-active-caspase- 3 antibody followed by goat-anti-rabbit-Alexa 568 (red) and anti-GM130 followed by goat-anti-mouse-Alexa 488 (green) and the nuclei with TO-PRO-3 (blue). J.CaM1.6 cells stained with (A) rabbit IgG, goat-anti-rabbit-Alexa 568 and (B) mouse IgG, goat-antimouse-Alexa 488. (C) Untreated cells stained with anti-GM130 and anti-active-caspase- 3 (arrow indicates mitotic cell). Cells treated with CH11 ( $500 \mathrm{ng} / \mathrm{ml})$ over $4 \mathrm{~h}(\mathrm{D}-\mathrm{F})$, where (D) shows anti-active-caspase-3, (E) shows anti-GM130, and (F) is the overlay of (D,E). Cells treated with staurosporine $(2 \mu \mathrm{M})$ over $4 \mathrm{~h}(\mathrm{G}-\mathrm{I})$, where $(\mathrm{G})$ shows active-caspase- 3 staining, $(\mathrm{H})$ shows GM130 staining, and $(\mathrm{I})$ is the overlay of $(\mathrm{G}, \mathrm{H})$. Composite micrograph of cells treated with $\mathrm{CH} 11$ in the presence of (J) ZB4 $(500 \mathrm{ng} / \mathrm{ml})$, (K) z-VAD-fmk $(100 \mu \mathrm{M})$. Cells treated with staurosporine in the presence of z-VAD-fmk (L). Data are representative of three experiments.

procaspase- 3 processing and $\sim 80 \%$ apoptosis (Fig. 2), GM130 was present at levels comparable to untreated cells. Procaspase- 3 cleavage was completely inhibited by z-VAD-fmk and similar to control levels. GM130 displays increased resistance to caspase-dependent cleavage in the presence of staurosporine, perhaps reflecting the apparent inhibition of Golgi fragmentation in staurosporine-treated J.CaM1.6 cells (Fig. 3).

\section{Discussion}

GM130 is centrally involved in mediating incoming vesicle fusion and is rapidly phosphorylated during
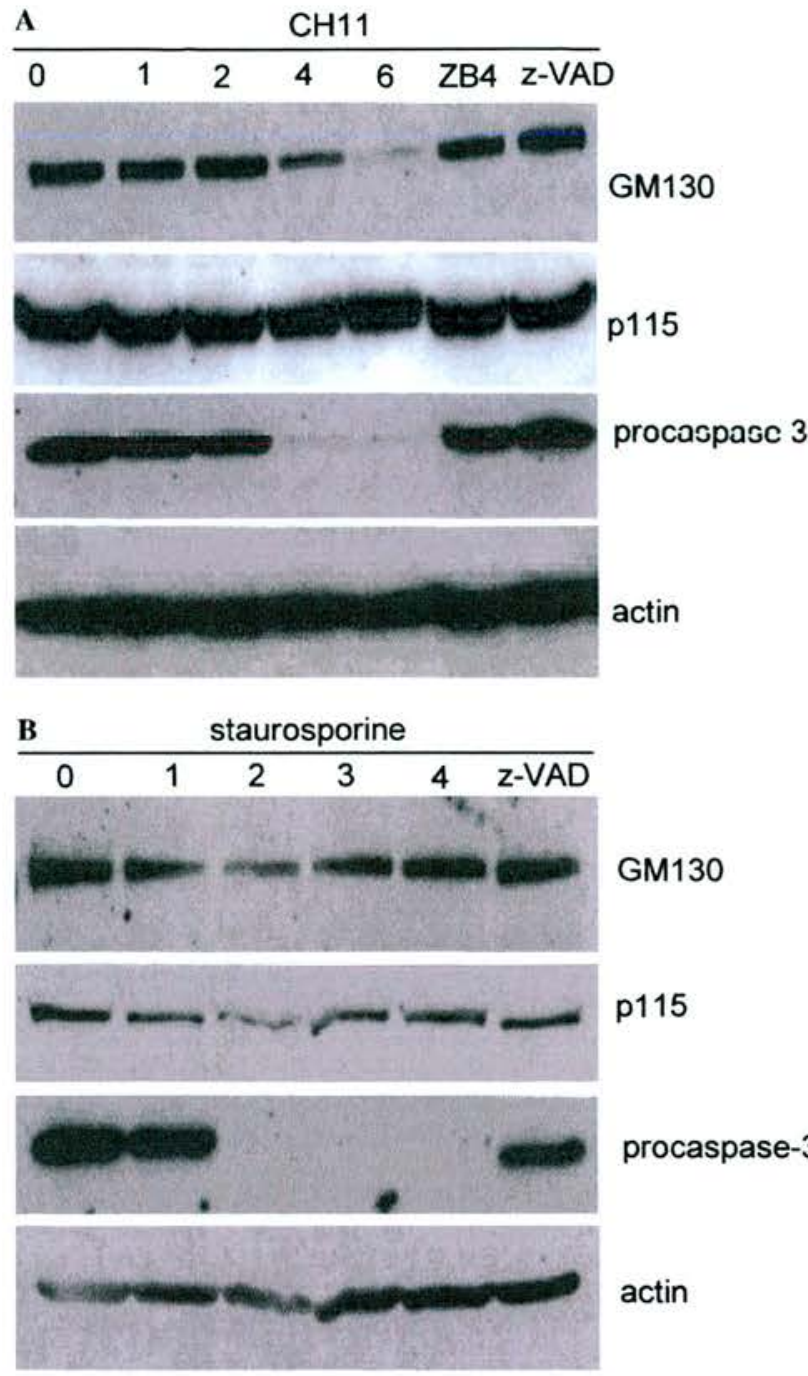

Fig. 4. GM130 levels decrease during $\mathrm{CH} 1 \mathrm{l}$-induced apoptosis in J.CaM1.6 cells but not in staurosporine-induced apoptosis. (A) J.CaMl 6 cells were incubated with or without $\mathrm{CH} 11(500 \mathrm{ng} / \mathrm{ml})$ over $6 \mathrm{~h}$. At the indicated times, cell lysates were prepared for Western blotting using antibodies to GM130, p115, procaspase-3, and actin. After $4 \mathrm{~h}$ procaspase- 3 was no longer detected and GMI30 is diminished as compared to control. At 6 h GMI30 is barely detected and this effect is inhibited by ZB4 $(500 \mathrm{ng} / \mathrm{ml})$ and by z-VAD-fmk $(100 \mu \mathrm{M})$. (B) J.CaM1.6 cells were incubated with or without staurosporine $(2 \mu \mathrm{M})$ over $4 \mathrm{~h}$. At the indicated times, cell lysates were prepared for Western blotting using antibodies to GM130, p115, procaspase-3, and actin. After $2 \mathrm{~h}$ procaspase- 3 was no longer detected and yet at $4 \mathrm{~h}$ GM130 levels were comparable to control. In cells pre-treated with z-VAD-fmk $(100 \mu \mathrm{M})$, procaspase-3 levels were as in control. Data are representative of three separate experiments.

mitosis, contributing to the fragmentation of the Golgi complex $[6,14]$. It therefore seems likely that GM130 would also be an important early target during apoptosis to facilitate Golgi fragmentation. However, studies so far have not borne this out. Most recently, GM130 detection was shown to be diminished by Western blot analysis at the same time as p115 in COS- 
7 cells treated with etoposide over $72 \mathrm{~h}$ and staurosporine over $48 \mathrm{~h}$ [2]. COS-7 cells have an impaired Fas-receptor-mediated signalling pathway [15] and seem to be particularly recalcitrant to the induction of apoptosis via a number of stimuli (personal observation). In contrast, using Fas-sensitive Jurkat cells (J.CaM1.6) we have shown that GM130 levels diminish rapidly following induction of Fas-mediated apoptosis, while p115 levels remained unchanged. One possible explanation for these differing observations would be that the missing components of the apoptotic pathways in COS-7 cells may normally be responsible for the rapid breakdown of GM130 during apoptosis. Although Golgi fragmentation and GRASP65 cleavage during apoptosis have been reported, These studies failed to show early GM130 cleavage [3]. However, this group [3] used the non-selective kinase inhibitor staurosporine extensively, which our data suggest may mask early GM130 cleavage. GM130 remained unaffected at $4 \mathrm{~h}$ when treated with staurosporine (Fig. 4B). GM130 levels might decrease at later time point during staurosporine-induced apoptosis, allowing efficient Golgi fragmentation. Interestingly, Nozawa et al. [16] demonstrated that necrosis also diminishes GM130 levels, highlighting the importance of carefully monitoring necrosis levels. Our results clearly show that despite higher levels of apoptosis induced by staurosporine, GM130 remained unaffected, whereas triggering the physiologically relevant Fas death pathway with $\mathrm{CH} 11$ resulted in decreasing GM130 levels. In addition, there was also a striking morphological difference in the appearance of the Golgi in staurosporine- and CH11-treated cells. For the staurosporinetreated cells Golgi fragmentation appeared to be incomplete, despite caspase-3 activation (Fig. 3). It is possible that the kinetics of GM130 cleavage and other key Golgi-associated proteins may be cell-type-dependent or dependent on whether the stress pathway or the receptor-mediated pathway of apoptosis is activated. Alternatively, we would speculate that the rapid cleavage of GM130 may involve a phosphorylation step to allow for efficient caspase recognition of the cleavage site as is the case with golgin-160 [11]. The precise kinetics of GM130 depletion during apoptosis in relation to other key Golgi proteins remains to be fully determined. The manner in which apoptosis is induced is important in determining whether key proteins such as GM130 are targeted, for example, we show that apoptosis triggered by the Fas pathway, but not induced by staurosporine, results in rapidly reduced GM130 levels. This is critical since staurosporine-induced apoptosis is a widely used model in the study of Golgi fragmentation during apoptosis.

\section{Acknowledgments}

We thank Linda Wilson for help with confocal microscopy. This work was supported by the Norman Salvesen Emphysema Research Trust and the National Asthma Campaign (D1/D42).

\section{References}

[1] C. Featherstone, G. Griffiths, G. Warren, Newly synthesized G protein of vesicular stomatitis virus is not transported to the Golgi complex in mitotic cells, J. Cell Biol. 101 (1985) 2036-2046.

[2] R. Chiu, L. Novikov, S. Mukherjee, D. Shields, A caspase cleavage fragment of p115 induces fragmentation of the Golgi apparatus and apoptosis, J. Cell Biol. 159 (2002) 637-648.

[3] J.D. Lane, J. Lucocq, J. Pryde, F.A. Barr, P.G. Woodman, V.J. Allan, M. Lowe, Caspase-mediated cleavage of the stacking protein GRASP65 is required for Golgi fragmentation during apoptosis, J. Cell Biol. 156 (2002) 495-509.

[4] M. Mancini, C.E. Machamer, S. Roy, D.W. Nicholson, N.A. Thornberry, L.A. Casciola-Rosen, A. Rosen, Caspase-2 is localized at the Golgi complex and cleaves golgin-160 during apoptosis, J. Cell Biol. 149 (2000) 603-612.

[5] M.K. Whyte, L.C. Meagher, J. MacDermot, C. Haslett, Impairment of function in aging neutrophils is associated with apoptosis, J. Immunol. 150 (1993) 5124-5134.

[6] M. Lowe, C. Rabouille, N. Nakamura, R. Watson, M. Jackman, E. Jamsa, D. Rahman, D.J. Pappin, G. Warren, Cdc2 kinase directly phosphorylates the cis-Golgi matrix protein GM130 and is required for Golgi fragmentation in mitosis, Cell 94 (1998) 783-793.

[7] F.A. Barr, N. Nakamura, G. Warren, Mapping the interaction between GRASP65 and GM130, components of a protein complex involved in the stacking of Golgi cisternae, EMBO J. 17 (1998) 3258-3268.

[8] F.A. Barr, M. Puype, J. Vandekerckhove, G. Warren, GRASP65, a protein involved in the stacking of Golgi cisternae, Cell 91 (1997) 253-262.

[9] N. Nakamura, M. Lowe, T.P. Levine, C. Rabouille, G. Warren, The vesicle docking protein p115 binds GM130, a cis-Golgi matrix protein, in a mitotically regulated manner, Cell 89 (1997) 445-455.

[10] B. Sonnichsen, M. Lowe, T. Levine, E. Jamsa, B. Dirac-Svejstrup, G. Warren, A role for giantin in docking COPI vesicles to Golgi membranes, J. Cell Biol. 140 (1998) 1013-1021.

[11] H. Cha, B.L. Smith, K. Gallo, C.E. Machamer, P. Shapiro, Phosphorylation of golgin-160 by mixed lineage kinase 3, J. Cell Sci. 117 (2004) 751-760.

[12] M.E. Peter, P.H. Krammer, The CD95(APO-1/Fas) DISC and beyond, Cell Death Differ. 10 (2003) 26-35.

[13] J.G. Pryde, A. Walker, A.G. Rossi, S. Hannah, C. Haslett, Temperature-dependent arrest of neutrophil apoptosis. Failure of Bax insertion into mitochondria at $15^{\circ} \mathrm{C}$ prevents the release of cytochrome $c$, J. Biol. Chem. 275 (2000) 33574-33584.

[14] M. Lowe, N.K. Gonatas, G. Warren, The mitotic phosphorylation cycle of the cis-Golgi matrix protein GM130, J. Cell Biol. 149 (2000) 341-356.

[15] S.A. Memon, J. Hou, M.B. Moreno, C.M. Zacharchuk, Apoptosis induced by a chimeric Fas/FLICE receptor: lack of requirement for Fas- or FADD-binding proteins, J. Immunol. 160 (1998) 2046-2049.

[16] K. Nozawa, C.A. Casiano, J.C. Hamel, C. Molinaro, M.J. Fritzler, E.K. Chan, Fragmentation of Golgi complex and Golgi autoantigens during apoptosis and necrosis, Arthritis Res. 4 (2002) R3. 


\title{
Macrophage phagocytosis of apoptotic neutrophils is compromised by matrix proteins modified by cigarette smoke and lipid peroxidation products
}

\author{
Paul A. Kirkham, ${ }^{\text {a,* }}$ Gillian Spooner, ${ }^{a}$ Irfan Rahman, ${ }^{\mathrm{b}}$ and Adriano G. Rossi ${ }^{\mathrm{b}}$ \\ ${ }^{a}$ Novartis Institute for Biomedical Research (Horsham), West Sussex, UK \\ ${ }^{\mathrm{b}}$ Respiratory Medicine Unit, MRC Centre for Inflammation Research, University of Edinburgh Medical School, Edinburgh, UK
}

Received 30 March 2004

\begin{abstract}
Clearance of apoptotic cells by phagocytosis plays an important role in the resolution of an inflammatory response. Macrophages interacting with extracellular matrix (ECM) proteins upregulate their phagocytic capacity. Cigarette smoke contains highly reactive carbonyls that modify proteins which directly/indirectly affects cellular function. We observed, in vitro, that human macrophages interacting with carbonyl or cigarette smoke modified ECM proteins dramatically down regulated their ability to phagocytose apoptotic neutrophils. We also show that this interaction with carbonyl-adduct modified ECM proteins led to increased macrophage adhesion in vitro. We hypothesise that changes in the ECM environment as a result of cigarette smoking affect the ability of macrophages to remove apoptotic cells. Moreover, we postulate that this decreased phagocytic activity was as a result of sequestration of receptors involved in the uptake of apoptotic cells towards that of recognition of carbonyl adducts on the modified ECM proteins leading to increased macrophage adhesion.

(c) 2004 Elsevier Inc. All rights reserved.
\end{abstract}

Keywords: Macrophages; Neutrophils; Phagocytosis; Inflammation

Phagocytosis of apoptotic cells plays a major role in the resolution of an inflammatory response [1]. An impairment of this process can therefore lead to chronic inflammatory conditions. The presence of such a chronic inflammatory state within the airways is one major characteristic of chronic obstructive pulmonary disease (COPD) [2]. There is a pronounced general increase in inflammatory cells, with profound increases in neutrophils [3], macrophages [4], and $\mathrm{CD}^{+} \mathrm{T}$ cells [5] being observed. Although their lifespan and recruitment dynamics may differ, they nonetheless all play a role in the ongoing inflammatory response within COPD. Moreover, it is the severity of the inflammatory response that can lead to airway obstruction and emphysema in COPD [6]. The inflammatory response can also be viewed as part of an innate tissue repair process fol-

'Corresponding author. Fax: +44-1403-323837.

E-mail address: paul.kirkham@pharma.novartis.com (P.A. Kirkham). lowing an insult, such as invading pathogens or damaged cells as a result of cigarette smoking. The ultimate fate of many recruited inflammatory cells is death, be it apoptotic or necrotic. It is the balance between inflammatory cell recruitment and the removal of apoptotic cells by phagocytosis that ultimately determines whether the inflammatory response will resolve [1,7]. Failure to remove inflammatory cells, such as apoptotic neutrophils, can result in secondary necrosis, releasing their toxic granule contents causing further tissue damage thereby exacerbating the inflammatory response $[1,7]$ and ultimately leading to a chronic inflammatory state [8]. In contrast, phagocytic removal of apoptotic cells by macrophages is an immunologically silent process that does not provoke release of pro-inflammatory mediators [9]. Consequently, this limits further tissue damage promoting a resolution of the inflammatory response.

Regulating the clearance of apoptotic cells can be viewed from two perspectives, first influencing the rate at which cells enter apoptosis and second controlling the 
phagocytic removal of apoptotic cells [7,10,11]. The former is known to be affected by inflammatory mediators and growth factors $[12,13]$. In addition, phagocytosis can also be controlled by a number of factors such as pro-inflammatory cytokines [14], soluble factors such as cAMP [10], glucocorticoids [15,16], extracellular matrix proteins, for example fibronectin [11] and collagen [17], or cell surface molecules such as CD44 [18]. In a disease such as COPD, where cigarette smoking can cause rises in both neutrophil and macrophage numbers in lung tissues, any factor or mechanism that can upset the balance between the number of cells entering apoptosis and the ability of macrophages to remove them could profoundly affect the outcome of the inflammatory response. Indeed, it has been shown that in COPD alveolar macrophages have a decreased propensity for phagocytosis [19] that could potentially exacerbate the inflammatory response.

Cigarette smoke, a complex mixture of chemicals and oxidants, is a major aetiological factor in the development of COPD [2]. Examples of such chemicals include the highly reactive aldehydes acrolein and 4-hydroxynonenal (4-HNE) [20], which can also be formed in vivo as a result of lipid peroxidation. These highly reactive water-soluble carbonyls can in turn, by attacking residues such as lysine, arginine, cysteine or histidine result in covalent post-translational protein modifications. Within this context, both 4-HNE and acrolein derived protein modifications have been shown to be markers for oxidative stress derived tissue damage in chronic diseases [20,21]. Moreover, 4-HNE modifications have been shown to inversely correlate with a loss of lung function including a decline in the forced expiratory volume in one second $\left(\mathrm{FEV}_{1}\right)$ in COPD [22]. It has previously been shown that macrophages can adhere to these carbonyl modified proteins through the class A macrophage scavenger receptor (SR-A) [23], a receptor that also plays a functional role in the phagocytosis and uptake of apoptotic cells [24]. However, the impact of ECM modifications by specific carbonyls found in cigarette smoke, such as 4-HNE and acrolein, on macrophage phagocytosis has not been studied. We therefore determined what effect modification of ECM proteins by reactive carbonyls could have on the clearance of apoptotic neutrophils by macrophages in vitro. Moreover, we also show that the macrophage interaction with modified ECM proteins is not solely through SR-A.

\section{Materials and methods}

Preparation and culture of human monocyte derived macrophages from healthy non-smokers. Human peripheral blood cells were separated into mononuclear cells and granulocytes from fresh, citrated blood of healthy volunteers by dextran sedimentation and discontinuous PBS-Percoll density gradient centrifugation as described [13]. Human monocytes were isolated from the mononuclear cells by plating out the mononuclear cell fraction (PBS-Percoll interphase fraction) into 48-well tissue culture plates as described previously [23]. After removing non-adherent cells (essentially the lymphocytes), the remaining adherent monocytes were cultured for six days in Iscove's MDM containing $10 \%$ autologous serum with fresh medium being added on the third day. The resulting monocyte derived macrophages were harvested by leaving in calcium/magnesium free PBS containing $2 \mathrm{mM}$ EDTA for $45 \mathrm{~min}$ on ice followed by vigorous aspiration. The macrophages were then resuspended in fresh Iscove's MDM in a polypropylene falcon tube at a cell density of $1 \times 10^{6} / \mathrm{ml}$.

Isolation and preparation of fluorescently labelled apoptotic neutrophils. Human peripheral blood neutrophils isolated as described above were then resuspended at $20 \times 10^{6} / \mathrm{ml}$ in Iscove's MDM and fluorescently labelled as described $[25,26]$ with the following modifications. The neutrophils were incubated for $30 \mathrm{~min}$ at $37^{\circ} \mathrm{C}$ in a $40 \mathrm{ml}$ falcon tube with $10 \mu \mathrm{l} \mathrm{CM-Orange} \mathrm{(Catalog} \mathrm{No.} \mathrm{C2927;} \mathrm{Molecular} \mathrm{Probes,}$ Leiden, The Netherlands).

The cell suspension was then diluted with Iscove's MDM to give a cell density of $4 \times 10^{6}$ neutrophils $/ \mathrm{ml}$ and the cells aged $\left(20 \mathrm{~h}, 37^{\circ} \mathrm{C}\right)$ in tissue culture flasks in the presence of autologous donor serum $(10 \%)$. Prior to use in the phagocytosis assay the neutrophils were washed $(2 \times)$ in Iscove's MDM and the percentage of neutrophils that had undergone apoptosis was determined by typical morphology $[13,27]$. Only aged neutrophils with a viability (assessed by trypan blue dye exclusion) $>98 \%$ were used.

Adhesion assay. Tissue culture plates (96 well) were coated with collagen IV by drying $100 \mu \mathrm{l}$ of $100 \mu \mathrm{g} / \mathrm{ml}$ protein solution onto the surface or each well. The wells were washed with distilled water and then left untreated or modified with $100 \mathrm{mM}$ acrolein or $5 \mathrm{mM} 4-\mathrm{HNE}$ in PBS for $24 \mathrm{~h}$ at $37^{\circ} \mathrm{C}$, after which the plates were extensively washed with PBS. PMA treated U937 cells [23] were preloaded with BCECF$\mathrm{AM}$ at $10 \mu \mathrm{g} / 10^{7}$ cells $/ \mathrm{ml}$ for $30 \mathrm{~min}$ in PBS supplemented with $1 \%$ BSA, and then washed with $\mathrm{Ca}^{2+} / \mathrm{Mg}^{2+}$ free PBS supplemented with $0.1 \%$ BSA and $5 \mathrm{mM}$ EDTA. The cells were then left untreated or pretreated for $30 \mathrm{~min}$ with $100 \mu \mathrm{g} / \mathrm{ml}$ fucoidan before addition to each well at $2 \times 10^{5}$ cells/well. The cells were left to adhere for $60 \mathrm{~min}$ at $37^{\circ} \mathrm{C}$ and then non-adherent cells were washed off by gentle washing with PBS supplemented with $0.1 \%$ BSA, $5 \mathrm{mM}$ EDTA and the level of adherent cells remaining quantified by measuring fluorescence (Ex $485 \mathrm{~nm}$; Em $538 \mathrm{~nm}$ ).

Phagocytosis assay. Tissue culture plates ( 24 well) were precoated with $10 \mu \mathrm{g} / \mathrm{ml}$ fibronectin in PBS and left to incubate for $16 \mathrm{~h}$ at $37^{\circ} \mathrm{C}$. Collagen IV precoated 24-well plates were obtained from BectonDickinson. Plate wells were then left untreated or treated with either cigarette smoke condensate [28], $100 \mathrm{mM}$ acrolein, $5 \mathrm{mM} 4-\mathrm{HNE}$ or $10 \mathrm{mM} \mathrm{HOCl}$ as described above. Human monocyte derived macrophages were then plated out at $1 \times 10^{6} /$ well and left to incubate for $45 \mathrm{~min}$ at $37^{\circ} \mathrm{C}$. As positive and negative controls for regulation of phagocytic uptake anti-CD44 (approx. $10 \mu \mathrm{g} / \mathrm{ml}$ of Ab from $5 \mathrm{~A} 4$ hybridoma) and $2 \mathrm{mM}$ dibutyryl-cAMP, respectively, were added to macrophages plated on either unmodified collagen IV or fibronectin and left for a further $15 \mathrm{~min}$ at $37^{\circ} \mathrm{C}$. Apoptotic CM-Orange loaded neutrophils $\left(4 \times 10^{6}\right)$ were then added to each well and left to incubate with the macrophages for $1 \mathrm{~h}$ at $37^{\circ} \mathrm{C}$. The macrophages were then harvested with trypsin/EDTA and kept on ice in the dark prior to analysis by FACS

Flow cytometric analysis. Samples were analysed by flow cytometry in a FACSCalibur with CellQUEST software (Becton-Dickinson, UK). Neutrophil and macrophage populations were identified and separated by forward and side scatter profiles. Data acquisition was terminated when 3000 events in the macrophage gate had been collected. During analysis the macrophage population was then plotted against forward scatter and FL-2 and separated into red fluorescent positive and negative populations. The number of red fluorescent positive events was then divided by the total sum of events in the macrophage gate to determine the percentage of macrophages that had ingested apoptotic neutrophils. 


\section{Results and discussion}

Many factors have been shown to upregulate the nonphlogistic clearance of apoptotic leukocytes by phagocytosis, such as lipoxins, cytokines, and corticosteroids $[14,15,29]$. Interaction of macrophages with ECM components such as fibronectin through the $\beta 1$ integrin receptors [11] and collagen through Fc receptors can also upregulate phagocytosis. In this study we have investigated the impact of modification of ECM proteins by cigarette smoke and its constituents, namely reactive aldehydes and oxidants, on macrophage function

Table 1

Percentage of macrophages phagocytosing apoptotic neutrophils on unmodified ECM proteins

\begin{tabular}{lll}
\hline & Mean (\%) & SEM \\
\hline Collagen IV & 34.5 & 9.6 \\
Fibronectin & 32.4 & 8.4 \\
\hline
\end{tabular}

Macrophages plated onto surfaces coated with either collagen IV or fibronectin were allowed to engulf dye labelled apoptotic neutrophils for a period of $1 \mathrm{~h}$. Three thousand macrophages were then analysed by flow cytometry and the number of macrophages engulfing dye labelled apoptotic neutrophils in this population is represented as a percentage of the total population counted. Data are means \pm SEM from three experiments, each experiment using human monocyte derived macrophages from a different donor. through their ability to phagocytose apoptotic neutrophils.

\section{Cigarette smoke and carbonyl modified ECM proteins decrease nonphlogistic phagocytosis of apoptotic PMN}

Two ECM proteins, collagen IV and fibronectin, were studied for their effect on macrophage phagocytic activity when modified with a variety of agents. In Table 1, the mean basal levels of phagocytosis from three separate experiments are shown when macrophages are plated onto either collagen IV or fibonectin. Each experiment used monocyte derived macrophages from a different donor. This table also shows that both ECM proteins, collagen IV or fibronectin, triggered similar levels of phagocytic activity towards apoptotic neutrophils. Fig. 1A illustrates a representative FL-2 histogram profile for these gated macrophages engaged in phagocytosis when plated out onto unmodified collagen IV compared to that for acrolein modified collagen IV (Fig. 1B). A reduction in FL2 fluorescence for the macrophage population is observed as less dye labelled apoptotic neutrophils are engulfed. In contrast, Fig. 1C demonstrates an increase in FL-2 fluorescence as antiCD44 stimulation of macrophages upregulates phagocytic uptake of dye labelled apoptotic neutrophils.
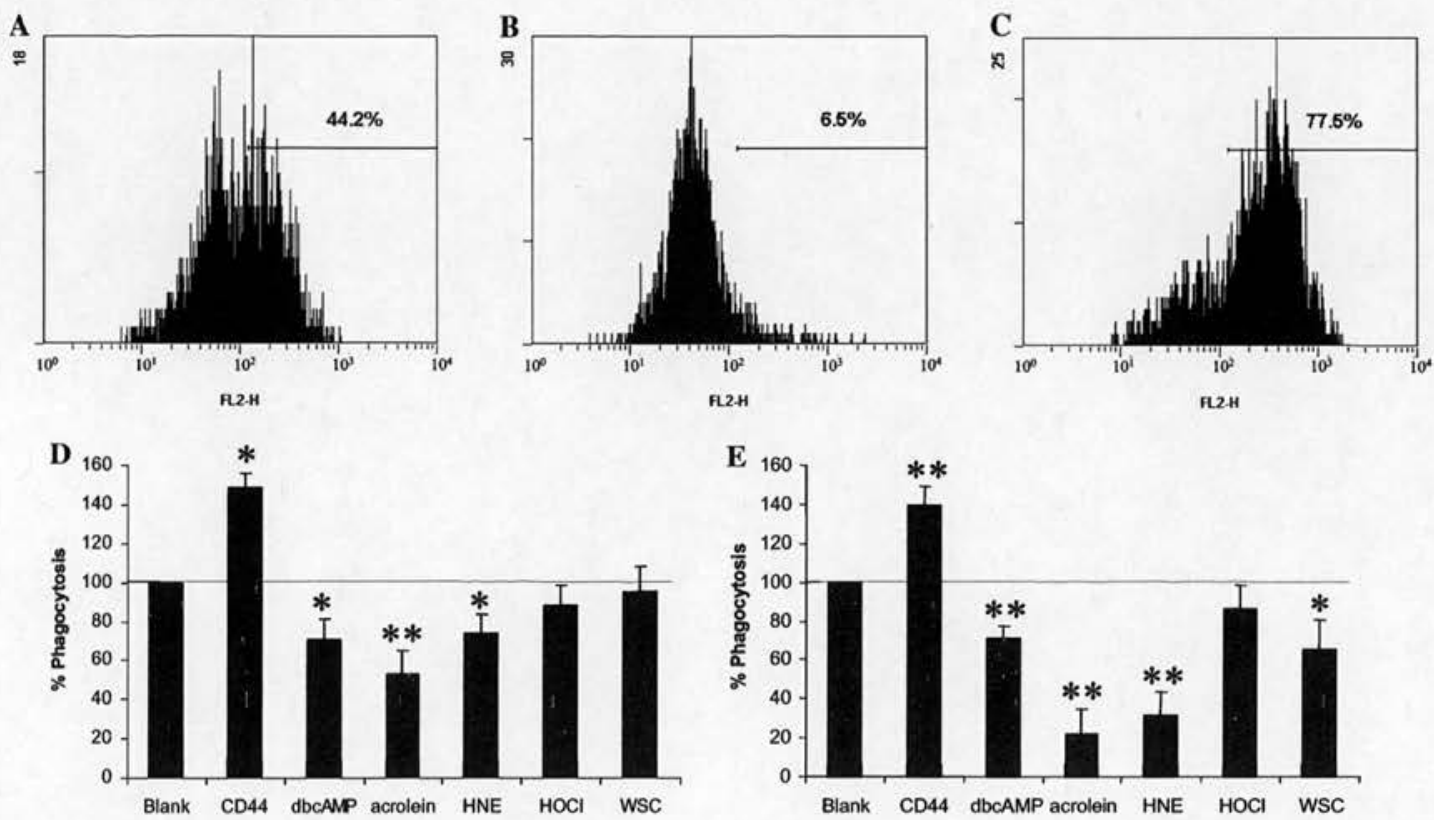

Fig. 1. Extracellular matrix proteins modified by 4-HNE, acrolein or cigarette smoke down regulate macrophage phagocytosis of apoptotic neutrophils. (A-C) Representative laser flow cytometry profiles for FL2 versus cell number demonstrating phagocytic potential of gated macrophages when plated onto unmodified collagen IV (A), acrolein modified collagen IV (B) or pre-treatment with anti-CD44 prior to plating onto unmodified collagen IV (C). Tissue culture plates precoated with either (D) Fibronectin or (E) collagen IV were left untreated or treated with either acrolein, 4-HNE, $\mathrm{HOCl}$ or cigarette smoke condensate (WSC). Gated macrophages were analysed by flow cytometry for FL-2 versus cell number as described in Materials and methods. The number of FL-2 positive events under each condition is then normalised and expressed as \% phagocytosis, relative to macrophage phagocytosis on unmodified matrix protein. Data are means \pm SEM from three experiments, ${ }^{*} p<0.05,{ }^{* *} p<0.01$ as determined by a two-tailed Student's $t$ test. 
Therefore, normalising basal phagocytosis levels to $100 \%$ for macrophages plated onto unmodified fibronectin (Fig. 1D) and collagen IV (Fig. 1E), the effect of various modifying agents on the ECM proteins, and the resultant impact on macrophage phagocytosis are shown. When cigarette smoke condensate was used to modify ECM proteins, only modified collagen IV (Fig. 1E) showed any significant reduction in phagocytic activity $(p<0.05)$ by as much as $40 \%$. Highly reactive aldehydes, such as acrolein and 4-HNE, are constituents of cigarette smoke. Therefore, ECM proteins were also treated in vitro with these aldehydes in order to determine whether they could account for the effect of cigarette smoke exposed ECM proteins on reducing macrophage phagocytic activity. When collagen IV (Fig. 1E) was modified with either 4-HNE or acrolein, there was an $80 \%$ reduction $(p<0.01)$ in phagocytic activity. Fibronectin (Fig. 1D) on the other hand only gave a $50 \%(p<0.01)$ and $30 \%(p<0.05)$ reduction in phagocytic activity when modified with acrolein and 4-HNE, respectively. For positive and negative controls in this experiment, CD44 and dbcAMP, as has previously been shown $[10,18]$, were able to increase and decrease macrophage phagocytic activity respectively. These results would therefore imply that the reactive aldehyde content of cigarette smoke may, in part, be responsible for the observed decrease in phagocytic activity when the macrophages were plated onto collagen IV that had been previously exposed to cigarette smoke condensate. Interestingly, unlike collagen IV, fibronectin when exposed to cigarette smoke did not affect phagocytosis. This could be explained by collagen IV having more susceptible amino acid residues exposed to attack by reactive aldehydes, leading to greater levels of post-translational modification. Indeed, when ECM proteins are exposed to modification by reactive carbonyls alone, be it acrolein or 4-HNE, there was a greater reduction in phagocytic activity of macrophages in contact with modified collagen IV as compared to modified fibronectin.

ECM proteins exposed to oxidative stress do not impact on nonphlogistic phagocytosis of apoptotic PMN

Cigarette smoke is also a direct source of oxidants with high levels being present $\left(10^{17}\right.$ oxidants/puff). Therefore in order to determine the impact of oxidative stress itself on ECM protein environment and how this could impact on macrophage function, ECM proteins were pre-treated with the powerful oxidant $\mathrm{HOCl}$. This oxidant can also be produced in vivo under conditions of oxidative stress [30]. Our results showed that ECM protein pre-treatment with $\mathrm{HOCl}$ had no significant effect on phagocytic activity towards apoptotic neutrophils (Figs. 1D and E). Whilst $\mathrm{HOCl}$ has been shown to destroy or modify the arginine residues in the integrin related RGD binding motif in ECM proteins, thereby affecting cell adhesion and survival [31], removal of the RGD motif within fibronectin failed to modulate phagocytic activity [11]. Consequently, our data would agree with these previous findings.

\section{Receptors for phagocytosis and macrophage adhesion}

Numerous receptors, including type A macrophage scavenger receptor (MSR-A) and CD36, have been shown to be involved in the uptake and recognition of apoptotic cells [24,32,33]. Indeed, there have been at least 10 molecules identified so far that have been implicated in macrophage uptake of apoptotic cells [34]. Some of these receptors, such as MSR-A and CD36, have also been shown to be involved in macrophage adhesion to ECM proteins post-translationally modified by lipid peroxidation products or cigarette smoke $[23,35,36]$. In Fig. 2 we show that both 4 -HNE and acrolein adducts on collagen IV can facilitate macrophage adhesion in a non-integrin related manner, as these experiments were performed in the presence of $\mathrm{Ca}^{2+} / \mathrm{Mg}^{2+}$ free media containing $5 \mathrm{mM}$ EDTA, thereby preventing any classical integrin related adhesion events. Interestingly, the adhesion to 4-HNE adducts is greater than that seen with acrolein by a factor of 2 . Moreover, this increased adhesion occurred when using a 20-fold lower concentration of 4-HNE compared to acrolein for modifying the matrix protein. In a similar context, macrophage adhesion to cigarette smoke condensate modified matrix protein has also been demonstrated by others, but occurred to a much lesser extent than that observed for acrolein alone [23], and may account for the smaller impact of cigarette smoke modified matrix

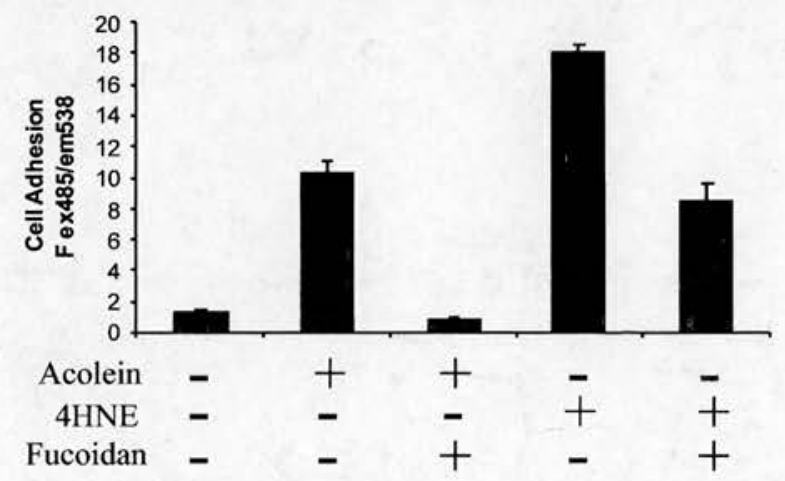

Fig. 2. 4-HNE and acrolein modified collagen IV promotes macrophage adhesion. Tissue culture plates precoated with human collagen IV were modified with either acrolein or 4-HNE. PMA differentiated U937 cells loaded with BCECF were plated out onto the modified collagen coated plates in the presence or absence of $100 \mu \mathrm{g} / \mathrm{ml}$ fucoidan. Adherent cells were detected by measuring fluorescence. Data are presented as means \pm SEM for quadruplicate determinations. Further details are as described in Materials and methods. 
proteins in down regulating phagocytosis as observed here (Fig. 1). Prevention of MSR-A mediated binding by use of fucoidan, whilst completely blocking acrolein adduct based adhesion, only blocked 4-HNE adduct related adhesion by $50 \%$. Therefore, unlike acrolein modified protein, adhesion to 4-HNE modified protein is not completely mediated through MSR-A, as the MSR-A inhibitor fucoidan failed to block all macrophage adhesion. This would suggest that another as yet unidentified receptor is involved in recognition of 4-HNE modified ECM protein.

The number of macrophages adhering to acrolein or 4-HNE modified collagen IV has been shown to be dependent on the extent of modification present [23]. Moreover, adhesion to 4-HNE adducts could still be achieved after modifying protein with a single exposure to low micromolar concentrations of 4-HNE compared to that for acrolein [23]. However, frequency of exposure to carbonyls, as a result of cigarette smoking for example, will also have a big impact on the level of protein modifications attained [23]. Consequently, because adhesion to carbonyl modified collagen IV was not only greater but occurred using lower concentrations of 4-HNE than that for acrolein (Fig. 2), it is possible that 4-HNE modifications may be more pathophysiologically important than acrolein. Indeed, Rahman et al. [22] have recently shown that there are increasing levels of 4-HNE adducts within lung tissue with increasing severity of COPD status as defined by a worsening $\mathrm{FEV}_{1}$.

Besides MSR-A, another receptor involved in recognition of carbonyl-modified proteins is CD36 and may well account for the fucoidan insensitive binding to 4 HNE modified ECM protein. Nevertheless, we propose that it is the sequestration of receptors towards adhesion of the macrophages to the post-translationally modified ECM proteins (seen in Fig. 2) that prevents recognition of and consequently reduces phagocytosis of apoptotic neutrophils as observed here (Fig. 1). This hypothesis is similar to that described by Maxeiner et al. [35] and El Khoury et al. [36] where MSR-A and/or CD36 receptors were shown to mediate macrophage adherence to surfaces coated with either carbonyl modified collagen IV or oxLDL, the latter being a rich source of carbonyl modified proteins. Moreover, this adhesion prevented scavenger receptor phagocytosis of modified LDL particles but not Fc mediated phagocytosis of IgG coated red blood cells. Although MSR-A and CD36 are involved in the clearance of apoptotic cells, other receptors are just as important. This was demonstrated by Teder et al. [8] using mice deficient for the hyaluron receptor CD44. These mice exhibited an impaired clearance of apoptotic neutrophils and a persistent inflammatory state following non-infectious lung injury. Consequently, the clearance of apoptotic cells most likely involves the coordinated response of numerous receptors and a dysfunction in any one can have a detrimental effect.

In conclusion, we have shown that surfaces coated with ECM proteins modified by carbonyls in cigarette smoke or products of lipid peroxidation, acrolein and 4-HNE, can trigger macrophage adhesion. We postulate this may account, in part, to the increased macrophage retention and adhesion observed by others in the lungs of both COPD and cigarette smoke exposed rats. This in turn results in a down regulation in the ability of these adherent macrophages to phagocytose apoptotic neutrophils. Our in vitro findings may therefore provide another mechanism by which post-translational alterations in the extracellular environment as seen in COPD [22] could affect cellular function. Such a sequence of events in vivo could lead to an imbalance in the effective clearance of apoptotic neutrophils resulting in secondary necrosis, exacerbating the inflammatory response $[1,7]$ and establishment of a chronic inflammatory state.

\section{References}

[1] C. Haslett, Granulocyte apoptosis and its role in the resolution and control of lung inflammation, Am. J. Respir. Crit. Care Med. 160 (1999) S5-S11.

[2] P. Barnes, Mechanisms in COPD: differences from asthma, Chest 117 (2000) 10S-14S.

[3] A. Pesci, B. Balbi, M. Majori, G. Cacciani, S. Bertacco, P. Alciato, C.F. Donner, Inflammatory cells and mediators in bronchial lavage of patients with chronic obstructive pulmonary disease, Eur. Respir. J. 12 (1998) 380-386.

[4] S.D. Shapiro, The macrophage in chronic obstructive pulmonary disease, Am. J. Respir. Crit. Care Med. 160 (1999) S29-S32.

[5] M. Saetta, A.D. Stefano, G. Turato, F.M. Facchini, L. Corbino, C.E. Mapp, P. Maestrelli, A. Ciaccia, L.M. Fabbri, CD8 ${ }^{+}$Tlymphocytes in peripheral airways of smokers with chronic obstructive pulmonary disease, Am. J. Respir. Crit. Care Med. 157 (1998) 822-826.

[6] M. Seatta, G. Turato, P. Maestrelli, C.E. Mapp, L.M. Fabbri, Cellular and structural bases of chronic obstructive pulmonary disease, Am. J. Respir. Crit. Care Med. 163 (2001) $1304-1309$.

[7] C. Ward, I. Dransfield, E.R. Chilvers, C. Haslett, A.G. Rossi, Pharmacological manipulation of granulocyte apoptosis: potential therapeutic targets, Trends Pharmacol. Sci. 20 (1999) 503-509.

[8] P. Teder, R.W. Vandivier, D. Jiang, J. Liang, L. Cohn, E. Puré, P.M. Henson, P.W. Noble, Resolution of lung inflammation by CD44, Science 296 (2002) 155-158.

[9] L.C. Meagher, J.S. Savill, A. Baker, C. Haslett, Phagocytosis of apoptotic neutrophils does not induce release of thromboxane B2, J. Leukocyte Biol. 52 (1992) 269-273.

[10] A.G. Rossi, J.C. McCutcheon, N. Roy, E.R. Chilvers, C. Haslett, I. Dransfield, Regulation of macrophage phagocytosis of apoptotic cells by cAMP, J. Immunol. 160 (1998) 3562-3568.

[11] J.C. McCutcheon, S.P. Hart, M. Canning, K. Ross, M.J. Humphries, I. Dransfield, Regulation of macrophage phagocytosis of apoptotic neutrophils by adhesion to fibronectin, J. Leukocyte Biol. 64 (1998) 600-607.

[12] A. Walker, C. Ward, I. Dransfield, C. Haslett, A.G. Rossi, Regulation of granulocyte apoptosis by hemopoietic growth factors, cytokines and drugs: potential relevance to allergic 
inflammation, Curr. Drug Targets-Inflam. Allergy 2 (2003) 339347.

[13] C. Ward, E.R. Chilvers, M.F. Lawson, J.G. Pryde, S. Fujihara, S.N. Farrow, C. Haslett, A.G. Rossi, NF-кB activation is a critical regulator of human granulocyte apoptosis in vitro, J. Biol. Chem. 274 (1999) 4309-4318.

[14] Y. Ren, J. Savill, Proinflammatory cytokines potentiate thrombospondin mediated phagocytosis of neutrophils undergoing apoptosis, J. Immunol. 154 (1995) 2366-2374.

[15] Y. Liu, J.M. Cousin, J. Hughes, J. Van Damme, J.R. Seckl, C. Haslett, I. Dransfield, J. Savill, A.G. Rossi, Glucocorticoids promote nonphlogistic phagocytosis of apoptotic leukocytes, J. Immunol. 162 (1999) 3639-3646.

[16] K.M. Giles, K. Ross, A.G. Rossi, N.A. Hotchin, C. Haslett, I Dransfield, Glucocorticoid augmentation of macrophage capacity for phagocytosis of apoptotic cells is associated with reduced p130Cas expression, loss of paxillin/pyk2 phosphorylation and high levels of active rac, J. Immunol. 167 (2001) 976-986.

[17] S.L. Newman, M.A. Tucci, Regulation of human monocyte/ macrophage function by extracellular matrix. Adherence of monocytes to collagen matrices enhances phagocytosis of opsonised bacteria by activation of complement receptors and enhancement of Fc receptor function, J. Clin. Invest. 86 (1990) 703-714.

[18] S.P. Hart, G.J. Dougherty, C. Haslett, I. Dransfield, CD44 regulates phagocytosis of apoptotic neutrophil granulocytes, but not apoptotic lymphocytes, by human macrophages, J. Immunol. 159 (1997) 919-925.

[19] F. Ferrara, D. D'Adda, M. Falchi, L. Dall'Astra, The macrophage activity of patients affected by pneumonia and chronic obstructive pulmonary disease, Int. J. Tissue React. 18 (1996) 109 114.

[20] H. Esterbauer, R.J. Schaur, H. Zollner, Chemistry and biochemistry of 4-hydroxynonenal, malonaldehyde, and related aldehydes, Free Radic. Biol. Med. 11 (1991) 81-128.

[21] K. Uchida, Current status of acrolein as a lipid peroxidation product, Trends Cardiovasc. Med. 9 (1999) 109-113.

[22] I. Rahman, A.A. Van Schadewijk, A.J. Crowther, P.S. Heimstra, J. Stolk, W. MacNee, W.I. De Boer, 4-Hydrox-2-nonenal, a specific lipid peroxidation product, is elevated in lungs of patients with chronic obstructive pulmonary disease, Am. J. Respir. Crit. Care Med. 166 (2002) 490-495.

[23] P.A. Kirkham, G. Spooner, C. Ffoulkes-Jones, R. Calvez, Cigarette smoke triggers macrophage adhesion and activation: role of lipid peroxidation products and scavenger receptor, Free Radic. Biol. Med. 35 (2003) 697-710.

[24] N. Platt, H. Suzuki, Y. Kurihara, T. Kodama, S. Gordon, Role for the class A scavenger receptor in the phagocytosis of apoptotic thymocytes in vitro, Proc. Natl. Acad. Sci. USA 93 (1996) 12456 12460 .
[25] H.P. Jersmann, K.A. Ross, S. Vivers, S.B. Brown, C. Haslett, I. Dransfield, Phagocytosis of apoptotic cells by human macrophages: analysis by multiparameter flow cytometry, Cytometry A 51 (2002) 7-15.

[26] S.B. Brown, M.C.H. Clarke, L. Magowan, H. Sanderson, J. Savill, Constitutive death of platelets leading to scavenger receptor-mediated phagocytosis. A caspase-independent cell clearance program, J. Biol. Chem. 275 (2000) 5987-5996.

[27] J.S. Savill, A.H. Wyllie, J.E. Henson, M.J. Walport, P.M. Henson, C. Haslett, Macrophage phagocytosis of aging neutrophils in inflammation: programmed cell death in the neutrophil leads to its recognition by macrophages, J. Clin. Invest. 83 (1989) 865-875.

[28] I. Rahman, X. Li, K. Donaldson, D.J. Harrison, W. MacNee, Glutathione homeostasis in alveolar epithelial cells in vitro and lung in vivo under oxidative stress, Am. J. Physiol. 269 (1995) L285-L292.

[29] C. Godson, S. Mitchell, K. Harvey, N.A. Petasis, N. Hogg, H.R. Brady, Lipoxins rapidly stimulate nonphlogistic phagocytosis of apoptotic neutrophils by monocyte derived macrophages, J. Immunol. 164 (2000) 1663-1667.

[30] M.M. Anderson, S.L. Hazen, F.F. Hsu, J.W. Heinecke, Human neutrophils employ the myeloperoxidase-hydrogen peroxidechloride system to convert hydroxyl amino acids into glycolaldehyde, 2-hydoxypropanal and acrolein. A mechanism for the generation of highly reactive $\alpha$-hydoxy and $\alpha, \beta$-unsaturated aldehydes by phagocytes at sites of inflammation, J. Clin. Invest. 99 (1997) $424-432$.

[31] H. Vissers, C. Thomas, Hypochlorous acid disrupts the adhesive properties of subendothelial matrix, Free Radic. Biol. Med. 23 (1997) 401-411.

[32] J. Savill, V. Fadok, P. Henson, C. Haslett, Phagocyte recognition of cells undergoing apoptosis, Immunol. Today 14 (1993) 131136.

[33] S.P. Hart, C. Haslett, I. Dransfield, Recognition of apoptotic cells by phagocytes, Experientia 52 (1996) 950-956.

[34] J. Savill, I. Dransfield, C. Gregory, C. Haslett, A blast from the past: clearance of apoptotic cells regulates immune responses, Nat. Rev. Immunol. 2 (2002) 965-975.

[35] H. Maxeiner, J. Husemann, C.A. Thomas, J.D. Loike, J. ElKhoury, S.C. Silverstein, Complementary roles for scavenger receptor $\mathrm{A}$ and $\mathrm{CD} 36$ of human monocyte derived macrophages in adhesion to surfaces coated with oxidised low-density lipoproteins and in secretion of $\mathrm{H}_{2} \mathrm{O}_{2}$, J. Exp. Med. 188 (1998) 22572265.

[36] J. El-Khoury, C.A. Thomas, J.D. Loike, S.E. Hickman, L. Cao, S.C. Silverstein, Macrophages adhere to glucose modified basement membrane collagen IV via their scavenger receptors, J. Biol. Chem. 269 (1994) 10197-10200. 


\title{
Interferon $\gamma$ suppresses glucocorticoid augmentation of macrophage clearance of apoptotic cells
}

\author{
Sarah J. Heasman, Katherine M. Giles, Adriano G. Rossi, Judith E. Allen, Christopher \\ Haslett and lan Dransfield
}

MRC Centre for Inflammation Research, University of Edinburgh Medical School, Edinburgh, GB

\begin{abstract}
One of beneficial effects of glucocorticoids (GC) in inflammation may be the augmentation of macrophages' capacity for phagocytosis of apoptotic cells, a process that has a central role in resolution of inflammation. Here we define the phenotype of GC-treated monocytederived macrophages, comparing to IFN- $\boldsymbol{\gamma}$-treated and IL-4-treated monocyte-derived macrophages and combinatorial treatment. Our data indicate that the cytokine microenvironment at an inflammatory site will critically determine monocyte functional capacity following treatment with GC. In particular, whilst GC exert dominant regulatory effects over IFN- $\gamma$ in terms of cell surface receptor repertoire and morphology, the acquisition of a macrophage capacity for clearance of apoptotic cells is prevented by combined treatment. In terms of mechanism, GC augmentation of phagocytosis was reversed even when monocytes were pre-incubated with GC for the first $24 \mathrm{~h}$ of culture, a period that is critical for induction of a highly phagocytic macrophage phenotype. These findings have important implications for the effectiveness of GC in promoting acquisition of a pro-phagocytic macrophage phenotype in inflammatory diseases associated with high levels of IFN- $\gamma$
\end{abstract}

Key words: Monocyte/macrophage / Inflammation / Apoptosis / Cytokine / Adhesion

\begin{tabular}{|lr|}
\hline Received & $27 / 10 / 03$ \\
Revised & $19 / 3 / 04$ \\
Accepted & $8 / 4 / 04$ \\
\hline
\end{tabular}

\section{Introduction}

Macrophages play a central role in the immune system, initiating and co-ordinating both innate immunity and the adaptive immune response $[1,2]$. Macrophage production of matrix, enzymes and the cytokines that regulate fibroblast migration and proliferation modulates the resolution phase of inflammation [3, 4]. In addition, the efficient clearance of extravasated leukocytes that have been induced to undergo apoptosis prevents further injury through the release of toxic or immunostimulatory intracellular contents [5]. Importantly, phagocytic clearance of apoptotic leukocytes by macrophages induces release of anti-inflammatory cytokines that promote resolution of inflammation [6] and triggers changes in macrophage behavior that promote tolerogenic responses. However, in situations where prolonged or chronic inflammatory responses occur, leukocytes may not be efficiently cleared by macrophages and consequently

[DOI 10.1002/eji.200324698]

The first two authors contributed equally to this manuscript.

Abbreviations: GC: Glucocorticoid IDMEM: Iscove's modification of Dulbecco's modified Eagles medium MDMФ: Monocyte-derived macrophage undergo secondary necrosis, releasing cytotoxic granule contents and further exacerbating the inflammatory response, potentially leading to the development of autoimmunity [7]. For example, the defective phagocytosis of apoptotic cells that is observed in $\mathrm{C} 1 \mathrm{q}$ deficiency contributes to the development of systemic lupus erythematosus [8]. Thus, defining the mechanisms that regulate this important clearance process is essential for understanding the pathogenesis of many inflammatory diseases.

A number of studies indicate that micro-environmental cues, e.g. extracellular matrix components and the cytokine repertoire, have a critical role in determining macrophage behavior [9]. It is well established that pro- and anti-inflammatory cytokines differentially activate macrophages. LPS or pro-inflammatory cytokines such as IFN$\gamma$ and TNF- $\alpha$ induce "classically activated" macrophages, which can be defined by production of nitric oxide and reactive oxygen species $[10,11]$. These macrophages show increased potential for the engulfment and destruction of pathogenic organisms which is reflected in the repertoire of receptors expressed; these receptors include Fc and complement receptors that enable recognition and internalization of immunoglobulin- and complement-opsonized particles [12]. Conversely, in response to cytokines with anti-in- 
flammatory potential such as IL-4 and IL-13, macrophage release of pro-inflammatory cytokines is inhibited, inducing an immunoregulatory phenotype. These macrophages are characterized by increased expression of specific pattern-recognition molecules, such as the mannose receptor $[13,14]$ and enhanced capacity for endocytosis and antigen presentation [15]. Arginase-1 expression is also enhanced and competes with NO synthases, increasing the metabolism of arginine to ornithine and urea, resulting in a decreased capacity for the production of reactive nitrogen species [16] and a reduced ability for pathogen clearance.

We have recently demonstrated that exposure of peripheral blood monocytes to glucocorticoids (GC), e.g. the synthetic glucocorticoid dexamethasone, results in differentiation towards a macrophage phenotype that exhibits a markedly increased phagocytic capacity for apoptotic cells; exposure to mineralocorticoids or sex steroids does not do this [17]. Prolonged exposure of monocytes to GC induced a homogeneous monocytederived macrophage (MDM $\Phi$ ) phenotype consisting of small "rounded" cells with profound changes in their cytoskeletal organization and a loss of actin-containing podosomes [18].

We sought to define the cell surface phenotype and functional repertoires of human monocytes treated with GC and the cytokines IL- 4 and IFN- $\gamma$. In this manuscript we present evidence that MDM $\Phi$ that have differentiated in the presence of dexamethasone exhibit distinct receptor expression patterns and functional status from either IFN- $\gamma$ - or IL-4-activated MDM $\Phi$. GC exert a dominant effect upon the morphology and adhesive status of MDM $\Phi$ following combination treatment with IFN- $\gamma$ or IL4. However, we demonstrate that IFN- $\gamma$ profoundly inhibits the GC-induced capacity to clear apoptotic cells. Indeed, even after exposure of MDM $\Phi$ to GC for $24 \mathrm{~h}$, subsequent exposure to IFN- $\gamma$ inhibited the acquisition of a phagocytic phenotype, indicating that GC-induced alterations in MDM $\Phi$ function are reversible. Our data suggest that the cytokine milieu at an inflammatory site will critically determine whether GC induce an MDM $\Phi$ phenotype with high capacity to clear apoptotic cells; a phenotype that would favor the resolution of inflammation.

\section{Results}

\subsection{Dexamethasone-augmented MDM $\Phi$ phagocytosis of apoptotic cells is inhibited in the presence of IFN- $\gamma$}

We examined the effects of IFN- $\gamma$ or IL- 4 , in combination with dexamethasone, on the phagocytosis of apoptotic neutrophils by MDM $\Phi$. Dexamethasone, or dexametha- sone in combination with IL-4, strongly increased the percentage of MDM $\Phi$ that phagocytosed apoptotic neutrophils compared with phagocytosis by untreated MDM $\Phi$ (Fig. 1). We did not observe a difference in the percentage of MDM $\Phi$ that phagocytosed apoptotic cells when comparing IFN- $\gamma$-treated and control MDM $\Phi$. However, in the presence of IFN- $\gamma$, dexamethasone failed to augment this phagocytosis (Fig. 1).

We assessed the mean fluorescence of phagocytic MDM $\Phi$ populations to provide further information about the effects of these treatments on the number of apoptotic cells phagocytosed per MDM $\Phi$ (expressed as mean fluorescence of phagocytic cells after subtraction of the mean fluorescence of non-phagocytic cells). Dexamethasone-treated MDM $\Phi$ showed increased fluorescence when compared with untreated MDM $\Phi$ (the mean \pm SE was $655 \pm 84$ compared with $407 \pm 111$ ). Phagocytic MDM $\Phi$ from IL-4- or IFN- $\gamma$-treated populations also showed slightly higher mean fluorescences $(510 \pm 163$ and $650 \pm 142$, respectively). Interestingly, phagocytic MDM $\Phi$ treated with dexamethasone plus IL4 or dexamethasone plus IFN- $\gamma$ had lower mean fluorescences of $455 \pm 101$ and $456 \pm 106$, respectively.

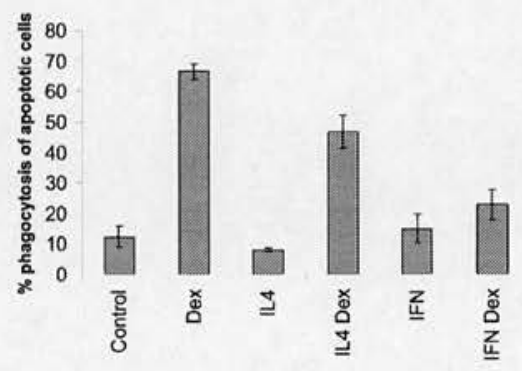

Fig. 1. Dexamethasone-induced phagocytosis of apoptotic neutrophils is reduced in the presence of IFN- $\gamma$. The phagocytic ability of MDM $\Phi$ cultured in IDMEM containing $10 \%$ autologous serum alone (control) or with combinations of dexamethasone, IL- 4 or IFN- $\gamma$ as described in Sect. 4.2 was determined on day 5 by flow cytometric quantification of the percentage of fluorescent MDM $\Phi$ following a $60-\mathrm{min}$ incubation with CMFDA-labeled apoptotic neutrophils. Dexamethasone treatment results in a highly phagocytic population of macrophages when compared with untreated macrophages. MDM $\Phi$ treated with IFN- $\gamma$ plus dexamethasone have a phagocytic ability similar to MDM $\Phi$ treated with IFN$\gamma$ alone, demonstrating that IFN- $\gamma$ is dominant in determining phagocytic capacity. The results shown here represent the mean \pm S.E of five separate experiments. 


\subsection{Suppression of pro-inflammatory cytokine release by dexamethasone}

In view of the dominant effects of IFN- $\gamma$ on dexamethasone-induced augmentation of phagocytic function, we next examined the release of pro-inflammatory cytokines by treated MDM $\phi$. As would be expected, IFN$\gamma$-treated MDMФ strongly up-regulated production of TNF- $\alpha$, IL- 1 and IL- 6 when compared with untreated MDM $\Phi$ or IL-4-treated MDM $\Phi$ (Fig. 2). Although production of most cytokines was effectively inhibited by dexamethasone alone, co-culture of MDM $\Phi$ in the presence of IL-4 and dexamethasone together produced further inhibitory effects on the production of IL-6.

In contrast to the dominant effects of IFN- $\gamma$ on phagocytosis by dexamethasone-treated MDM $\Phi$, reduced levels of TNF- $\alpha, \mathrm{IL}-6$ and $\mathrm{IL}-1$ production were found for MDM $\Phi$ treated with dexamethasone in combination with IFN- $\gamma$, suggesting that dexamethasone remained able to suppress the inflammatory cytokine production that was induced by IFN- $\gamma$.

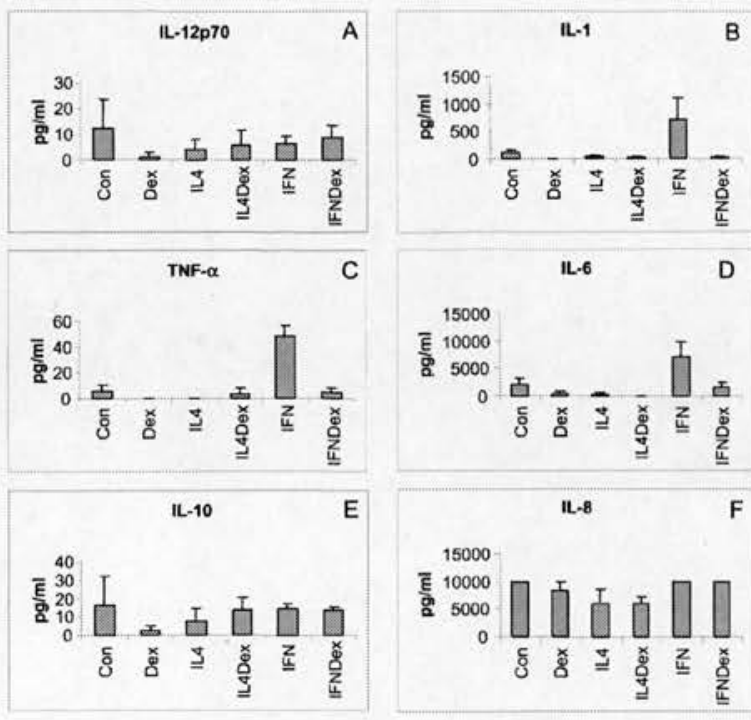

Fig. 2. Cytokine profiles of MDM $\Phi$ treated with dexamethasone $\pm \mathrm{IL}-4$ and IFN- $\gamma$ for 5 days. Adherent monocytes were cultured for 5 days either in $10 \%$ autologous serum alone or with combinations of dexamethasone, IL-4 and IFN- $\gamma$. MDM $\Phi$ supernatants were collected after 5 days and analyzed using an inflammation cytokine bead array kit (Becton Dickinson). Cytokine levels were determined by extrapolation from standard curves using cytokine bead array analysis software. Results shown are the cytokine levels (mean \pm S.E) from three separate experiments using $M D M \Phi$ from three different, normal donors.

\subsection{MDMФ cultured in dexamethasone exhibit distinct surface receptor profiles}

We next compared cell surface receptor profiles of MDM $\Phi$ cultured with dexamethasone, IFN- $\gamma$ or IL-4 alone, or with combinations of treatments. As would be expected, the high affinity receptor for immunoglobulin, CD64 (FcyRI), was strongly up-regulated by IFN- $\gamma$ treated MDM $\Phi$ when compared with untreated MDM $\Phi$ (Fig. $3 \mathrm{~A}$ ) and was also augmented when MDMФ were exposed to IFN- $\gamma$ in combination with dexamethasone. In contrast, CD64 was down-regulated by dexamethasone when used alone (35\% reduction in levels of expression) and markedly down-regulated by IL-4 (70\% reduction in expression).

Dexamethasone-treated MDM $\Phi$ expressed slightly reduced levels of HLA-DR when compared with untreated cells; MDM $\Phi$ exposed to IL- 4 or IFN- $\gamma$ showed increased expression compared with untreated cells (Fig. 3B). Interestingly, up-regulation of expression of HLA-DR was not observed when MDM $\Phi$ were treated with IFN- $\gamma$ or IL- 4 in combination with dexamethasone, suggesting a suppressive effect of dexamethasone
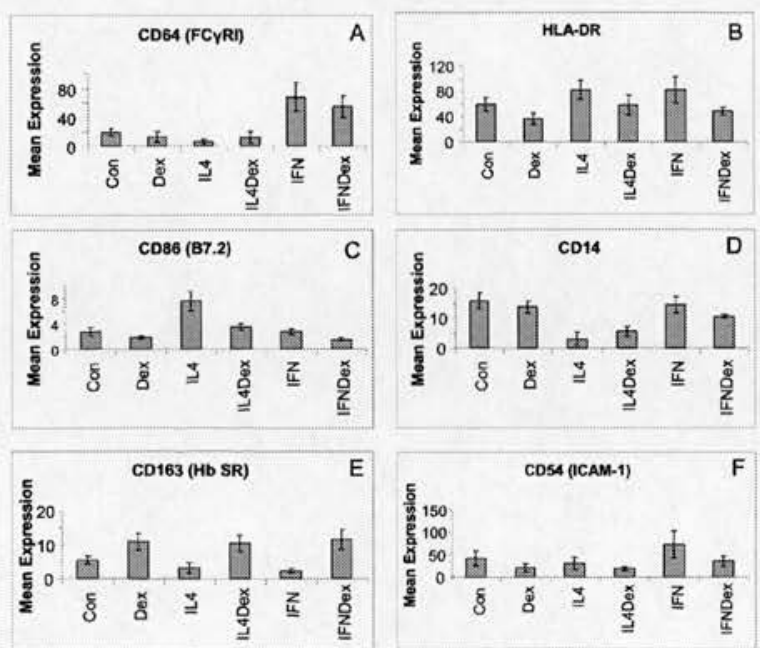

Fig. 3. Effects of dexamethasone, IL-4 and IFN- $\gamma$ on macrophage cell surface receptor expression. Adherent monocytes were cultured for 5 days either in $10 \%$ autologous serum or with combinations of dexamethasone, IL-4 and IFN- $\gamma$. The surface phenotype was assessed by indirect immunofluorescence and flow cytometry using control lgG1 $m A b, C D 64$ mAb (A), HLA-DR mAb (B), CD86 mAb (C), CD14 mAb (D), CD163 mAb (E) and ICAM-1 mAb (F). Results, corrected for the mean fluorescence intensity recorded for the IgG1 control, are expressed as the average mean fluorescence $( \pm$ S.E.) recorded for each antibody from a minimum of three separate donors. 
(Fig. 3B). IL-4-treated MDMФ expressed approximately 3-times the levels of CD86 when compared with IFN- $\gamma$ treated or dexamethasone-treated MDMФ (Fig. 3C). Expression of the adhesion molecule ICAM-1 on MDM $\Phi$ was increased following IFN- $\gamma$ treatment, consistent with published data [19]. In contrast, both IL-4 and dexamethasone reduced ICAM-1 expression and IFN- $\gamma$ induced up-regulation of ICAM-1 was suppressed following combined IFN- $\gamma$ and dexamethasone treatment (Fig. 3F).

MDMФ expression of CD14 was not affected by dexamethasone treatment alone. In contrast, IL-4-treated macrophages exhibit markedly reduced CD14 expression, consistent with the induction of an immature "dendritic-like" cell phenotype (Fig. 3D). Reduced expression of CD14 expression was also seen following treatment with IL-4 and dexamethasone in combination, suggesting that the effects of IL-4 predominate (Fig. 3D). The divergent effects of IL- 4 and dexamethasone were also found when expression of the mannose receptor was examined. Dexamethasone and IFN- $\gamma$ both downregulated expression of the mannose receptor, whereas IL-4 up-regulated expression (mean fluorescence intensities: control=58.7, IL-4=85.8, dexamethasone $=18.4$, IFN- $\gamma=4.7) \quad(n=2)$. Expression of the GC-responsive hemoglobin scavenger receptor CD163 [20] was downregulated following culture in the presence of IL- 4 or IFN$\gamma$ alone, but increased in dexamethasone-treated MDMФ (Fig. 3E). Increased CD163 expression levels were also observed following combined treatment with dexamethasone and IFN- $\gamma$, again suggesting a dominant effect of dexamethasone.

\subsection{Effects of dexamethasone and cytokines on MDMФ morphology}

Our analysis suggests that suppression of dexamethasone-augmented phagocytosis by IFN- $\gamma$ is not reflected by surface phenotype alterations, with dexamethasone exerting a dominant effect. Morphological examination demonstrated that a homogeneous population of smaller, less-well-spread MDM $\Phi$ was induced by GC when compared with the heterogeneous untreated MDM $\Phi$ population (Fig. 4). When cultured in the presence of IL-4 alone, MDM $\Phi$ exhibited a highly spread, polarized morphology with pronounced cellular processes and evidence of the formation of homotypic cell aggregates (Fig. 4). A heterogeneous MDMФ population comprising multinucleated "giant" cells together with smaller macrophages was observed in the presence of IFN- $\gamma$ (Fig. 4). Culture of MDM $\Phi$ in the presence of IFN- $\gamma$ plus dexamethasone, or IL-4 plus dexamethasone, yielded a population of smaller "rounded" cells similar to
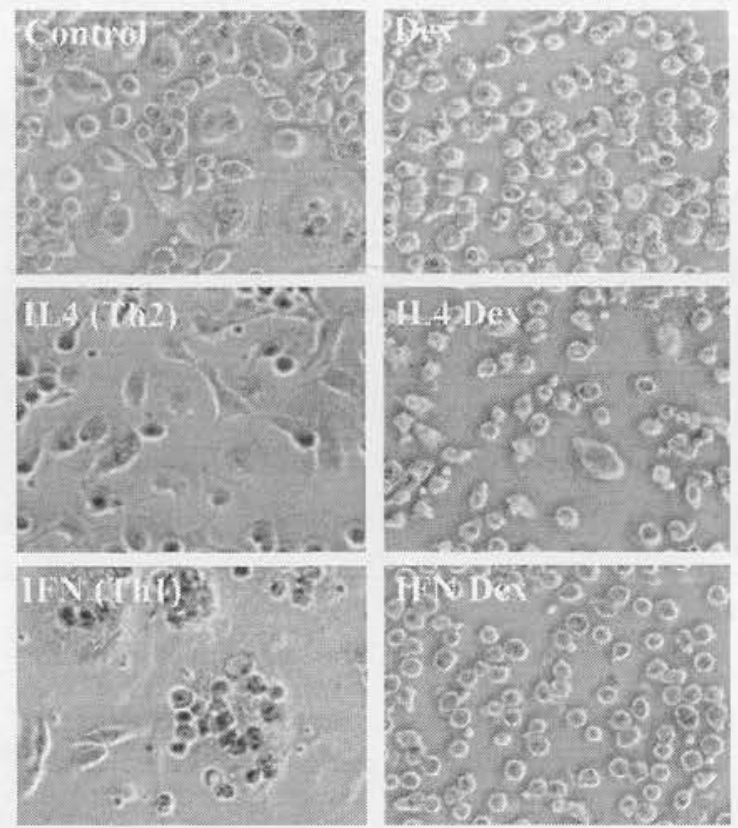

Fig. 4. Dexamethasone is dominant over IFN- $\gamma$ and IL-4 cytokines in inducing cellular morphology. Adherent monocytes were cultured for 5 days on glass coverslips either in $10 \%$ autologous serum or with combinations of dexamethasone, IL-4 and IFN- $\gamma$. Representative photomicrographs depict cellular morphology using phase-contrast microscopy with a $\times 63$ objective. Control MDM $\Phi$ represent a heterogeneous population of cells containing giant, multinucleated cells as well as smaller mononuclear cells. Dexamethasone-differentiated MDM $\Phi$ form a homogeneous population of small, rounded cells. Combined treatment with either IL- 4 or IFN- $\gamma$ plus dexamethasone also yields a homogeneous population of small rounded cells, similar to the dexamethasone-treated population, implying that dexamethasone is dominant over both cytokines in inducing cellular morphological changes.

those seen with dexamethasone alone. Together these data suggest that dexamethasone exerts dominant effects upon MDM $\phi$ morphology.

\subsection{Dexamethasone treatment alters adhesion structures in MDM $\Phi$}

We next examined the characteristic "podosome-like" adhesion signaling complexes consisting of a punctate actin foci surrounded by a ring of paxillin, vinculin and other cytoskeletal proteins [21] that are present in MDM $\Phi$. Untreated or IFN- $\gamma$-treated MDM $\Phi$ contain abundant podosomes, whereas these structures were absent from dexamethasone-treated MDMФ (Fig. 5). IFN- $\gamma$-treatment appears to drive differentiation towards 

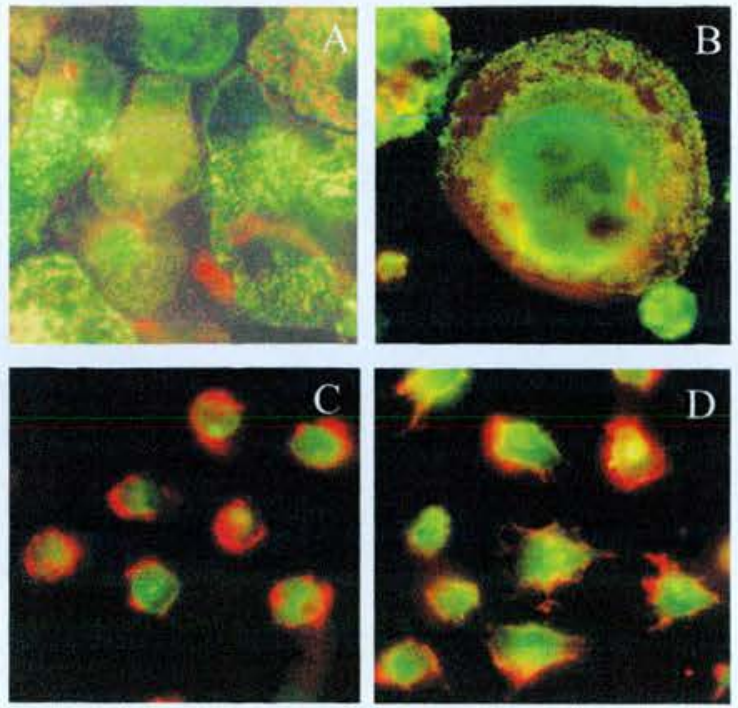

Fig. 5. Dexamethasone is dominant over IFN- $\gamma$ and IL-4 cytokines in determining the adhesion status of the macrophages. Adherent monocytes were cultured for 5 days on glass coverslips either in $10 \%$ autologous serum or with combinations of dexamethasone, IL-4 and IFN- $\gamma$. Fixed and permeabilized cells were stained for F-actin using rhodamine phalloidan and paxillin (paxillin mAb and FITCconjugated anti-mouse-lgG), to visualize cytoskeletal organization. In the representative photomicrographs shown, podosomes are characterized by a foci of actin (red) which is surrounded by a ring of paxillin (green). Untreated control MDM $\Phi$ contain abundant podosomes (A). Podosomes are also present in IFN- $\gamma$-treated MDM $\Phi$ (B). By contrast, MDM $\Phi$ differentiated in the presence of dexamethasone alone or dexamethasone plus IFN- $\gamma$ (C and D, respectively) do not contain podosomes. These data demonstrate the dominance of dexamethasone over IFN- $\gamma$ in determining the adherence status of the macrophages.

a multinucleated phenotype and the podosomes in these "giant" cells were particularly striking, where a cortical ring of podosomes were formed, similar to the resorption zone described for osteoclasts (Fig. 5B) [22]. Interestingly, IL-4 induces a polarized morphology with podosomes localized at the leading edge of the cell (data not shown). Podosomes were absent from MDMФ cultured in the presence of dexamethasone (Fig. $5 \mathrm{C}$ ) or IFN- $\gamma$ plus dexamethasone (Fig. 5D), in keeping with morphological observations. Podosomes were also absent from MDM $\Phi$ treated with IL-4 plus dexamethasone (data not shown). These data confirm the dominant effect of dexamethasone in terms of both morphology and cytoskeletal organization in MDM $\Phi$.

\subsection{The mechanism of IFN- $\gamma$ suppression of phagocytosis}

In view of the finding that the first $24 \mathrm{~h}$ of culture was critical for GC-mediated augmentation of MDM $\Phi$ phagocytosis [18], we sought to investigate whether IFN- $\gamma$ could over-ride the effects of dexamethasone after this initial 24-h culture period. MDM $\Phi$ were exposed to either dexamethasone or IFN- $\gamma$ for the first $24 \mathrm{~h}$ of culture, followed by culture for 4 days in medium alone, dexamethasone or IFN- $\gamma$. MDMФ phagocytosis of apoptotic cells was augmented when monocytes were cultured in medium for $24 \mathrm{~h}$ followed by the addition of dexamethasone although less than when dexamethasone was present during the first $24 \mathrm{~h}$ (Fig. 6A). Addition of IFN- $\gamma$ alone failed to alter the phagocytic capacity of MDMФ when compared to untreated control MDMФ. However, if monocytes were incubated with IFN- $\gamma$ for $24 \mathrm{~h}$ prior to the addition of dexamethasone, augmentation of MDM $\Phi$ phagocytosis of apoptotic cells was not observed. Surprisingly, if monocytes were incubated with dexamethasone for the first $24 \mathrm{~h}$ of culture followed by addition of IFN- $\gamma$, the phagocytic capacity was also not augmented.

In view of the "switch off" of phagocytosis following IFN$\gamma$ treatment $24 \mathrm{~h}$ after dexamethasone treatment of monocytes, we next sought to test whether engagement of IFN- $\gamma$ signaling in GC-treated MDM $\Phi$ would also inhibit phagocytosis. MDM $\Phi$ were treated with dexamethasone at day 0 and then IFN- $\gamma$ was added on day 1 , day 3 or day 6 (on day 6 , it was added either $1 \mathrm{~h}$ or $3 \mathrm{~h}$ prior to the phagocytosis assay) (Fig. 6B). The results show that treatment with IFN $-\gamma$ for a few hours prior to phagocytosis did not inhibit phagocytic ability when compared with MDM $\Phi$ treated with dexamethasone alone. Indeed, the suppressive effect of IFN- $\gamma$ upon GCaugmented phagocytosis was less pronounced the longer the macrophages were exposed to dexamethasone before IFN- $\gamma$ was added. These results demonstrate that the IFN- $\gamma$-mediated suppression of augmented phagocytosis most likely induces changes in MDM $\Phi$ phenotype rather than being a direct consequence of engagement of IFN- $\gamma$-mediated signaling pathways.

\section{Discussion}

One of the most important observations in our study is that IFN- $\gamma$ strongly suppressed the dexamethasoneinduced augmentation of apoptotic cell phagocytosis by macrophages (Fig. 1, 6A and 6B). Indeed, IFN- $\gamma$ retained the ability to inhibit GC-augmented phagocytosis even when monocytes had been pre-treated with dexamethasone for $24 \mathrm{~h}$. Exposure of MDM $\Phi$ to IFN- $\gamma$ at later time points following dexamethasone treatment failed to 

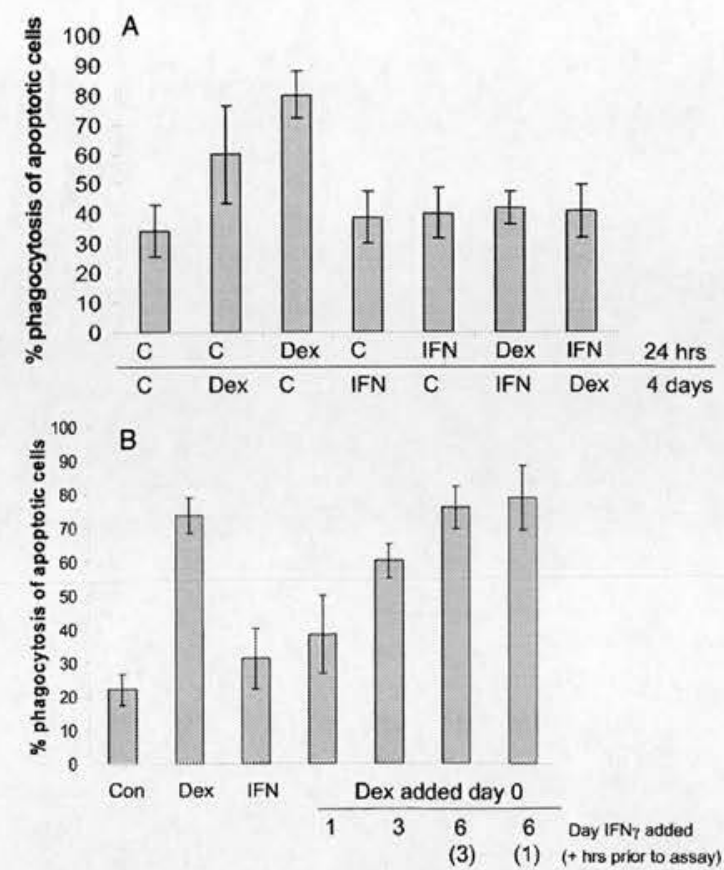

Fig. 6. IFN- $\gamma$ reverses and blocks dexamethasone augmentation of MDM $\Phi$ phagocytosis of apoptotic cells. (A) Adherent monocytes were cultured for the first $24 \mathrm{~h}$ in IDMEM containing $10 \%$ autologous serum alone, dexamethasone or IFN- $\gamma$. Cells were washed and then treated for the remaining 4 days in IDMEM containing $10 \%$ autologous serum, dexamethasone or IFN- $\gamma$ to give a checkerboard of combination treatments ( $24 \mathrm{~h} \mathrm{/} 4$ days). The phagocytic ability of the MDM $\Phi$ populations was then determined by flow cytometry as described in Sect. 4.5. Results are shown as the mean percentage of macrophage phagocytosis \pm S.E. from at least three separate experiments. Augmentation of MDM $\Phi$ phagocytic ability was observed following exposure to dexamethasone for the first $24 \mathrm{~h}$ only, or for the final 4 days of culture when compared with untreated $М D M \Phi$. In contrast, exposure to dexamethasone followed by IFN- $\gamma$, or to IFN- $\gamma$ prior to dexamethasone, resulted in a significant down-regulation in MDM $\Phi$ phagocytic ability, demonstrating that IFN- $\gamma$ can both reverse and block the dexamethasone-augmented phagocytosis of apoptotic neutrophils. (B) Adherent monocytes were cultured in IDMEM containing $10 \%$ autologous serum and dexamethasone, with the subsequent addition of IFN- $\gamma$ on day 1 , or day 3 , or $1 \mathrm{~h}$ or $3 \mathrm{~h}$ prior to the phagocytosis assay on day 6 . The phagocytic ability of the MDM $\Phi$ populations was then determined by flow cytometry. Results are shown as mean percentage of macrophage phagocytosis \pm S.E. from at least three separate experiments. MDM $\Phi$ that were cultured in dexamethasone and exposed to IFN- $\gamma$ on day 1 showed a suppression in phagocytic ability compared with MDM $\Phi$ treated only with dexamethasone. The addition of IFN- $\gamma$ on day 3 led to only a slight reduction in phagocytic ability and MDM $\Phi$ exposed to IFN- $\gamma$ for $1 \mathrm{~h}$ or $3 \mathrm{~h}$ only showed no inhibition in phagocytic ability. reverse the augmentation of phagocytosis, suggesting that the inhibition was not due to a direct effect of IFN- $\gamma$ mediated signaling. Previous studies have also suggested that the cytokine environment may regulate macrophage phagocytosis of apoptotic cells. When human MDM $\phi$ were given a 4-h exposure of IFN- $\gamma$ after 4 days of culture, they displayed augmented phagocytosis of apoptotic cells [23]. In contrast, phagocytosis of apoptotic cells by rat bone-marrow-derived macrophages was inhibited by treatment for $48 \mathrm{~h}$ with IFN- $\gamma$ [24]. Our data suggest that although IFN- $\gamma$-treated MDM $\Phi$ show some augmentation of phagocytosis of apoptotic cells, acquisition of a pro-phagocytic macrophage phenotype in response to $\mathrm{GC}$ is lost in the presence of this cytokine.

One important implication of this work is that GC may fail to stimulate macrophage phagocytosis in a Th1 environment. Thus, one of the potential beneficial effects of GCtreatment, i.e. facilitation of apoptotic cell clearance, may be inhibited by IFN- $\gamma$. Interestingly, a Th 1 cytokine environment has been suggested to contribute to corticosteroid resistance in diseases like rheumatoid arthritis [25]. This may also impact on the use of IFN- $\gamma$ to boost host defense against infection via macrophage activation following severe injury. Many trials of IFN- $\gamma$ therapy have failed to show a clear improvement in patient outcome [26]. Whether IFN- $\gamma$ inhibits the ability of endogenous GC to regulate macrophage phagocytosis is not known. Interestingly, expression of $11 \beta$ hydroxysterone dehydrogenase is rapidly induced during monocyte differentiation [27] concomitantly with acquisition of capacity for phagocytosis of apoptotic cells [28].

We have previously suggested that the altered adhesion observed in dexamethasone-treated MDM $\Phi$ may be linked to augmentation of phagocytic capacity. On the basis of morphological appearance, we would have predicted that co-incubation with either dexamethasone plus IFN- $\gamma$ or dexamethasone plus IL- 4 would give rise to a highly phagocytic phenotype. However, our data provide the first evidence for dissociation between morphology/adhesion status and phagocytic capacity. Thus, although cells treated with IFN- $\gamma$ plus dexamethasone exhibit a small and rounded cell morphology without distinct podosome adhesion structures, the augmented phagocytic capacity of dexamethasone-treated macrophages is not shared by MDM $\Phi$ treated with IFN- $\gamma$ plus dexamethasone.

For other characteristics of MDM $\Phi$ that we examined, dexamethasone exerted dominant effects over IFN- $\gamma$, inhibiting IFN- $\gamma$-driven pro-inflammatory cytokine production and morphological appearance. Expression of CD163 was up-regulated by dexamethasone even in the presence of IFN- $\gamma$ and the IFN- $\gamma$-dependent up- 
regulation of ICAM-1 and HLA-DR was attenuated by dexamethasone. Our phenotype analysis also revealed that the expression levels of the mannose receptor and CD14, both previously implicated as phagocytic receptors $[29,30]$, show no correlation with the increased phagocytic ability of dexamethasone-treated MDMФ. The mannose receptor was down-regulated following GC treatment and CD14 showed no change in expression when compared with untreated MDM $\Phi$, potentially excluding the involvement of these receptors in the augmented phagocytosis seen following GC treatment of MDM $\Phi$.

Our data support the suggestion that monocytes treated with the GC differentiate to a phenotype distinct from the classically activated IFN- $\gamma$ - treated MDM $\Phi$ or an "alternatively" activated IL-4-treated MDM $\Phi$. We found that the mannose receptor, a well-defined marker of alternative activation [13], was expressed at higher levels on IL4-treated MDM $\Phi$, but was reduced on MDM $\Phi$ cultured in dexamethasone. Several other receptors exhibit differential expression on IL-4- and GC-treated MDMФ, including HLA-DR, CD86 and CD163. MDM $\Phi$ that have differentiated in GC or IL-4 exhibit distinct phenotypes, the latter inducing a polarized appearance with many cellular processes that is very different from the "rounded" appearance of dexamethasone-treated cells. Most importantly in terms of capacity for clearance of apoptotic cells, IL-4 fails to induce a MDM $\Phi$ phenotype that is capable of efficient phagocytosis of apoptotic cells. IL-4-treated monocytes would have the potential for antigen presentation and immunomodulation (high HLA-DR and CD86), whereas dexamethasone-treated MDMФ exhibit "anti-inflammatory" characteristics with reduced capacity for immunostimulation because of down-regulation of HLA-DR and CD86.

Although both IL- 4 and GC may be involved in countering pro-inflammatory factors, their impact on macrophage function would have distinct consequences in the outcome of an inflammatory response. For example, discordant regulation of the capacity of macrophages for antigen presentation and apoptotic cell clearance may be critical for "safe" disposal of apoptotic and necrotic cells without the potential for "cross-presentation" of autoantigen and the induction of an autoimmune response. Together, these data suggest that the functional repertoire of $\mathrm{IL}-4$ - or dexamethasone-treated MDM $\phi$ is distinct. GC promote a "tolerogenic" phenotype similar to that reported to be induced by IL- $10[31$, 32]. Interestingly, like GC, IL-10 up-regulates expression of CD163, although the mechanism appears to be distinct [33]. Preliminary experiments indicate that treatment of monocytes with anti-IL-10 antibodies fails to block the development of the phenotype we observe fol- lowing treatment with GC (S. J. Heasman, unpublished observations). However, IL-10-cultured MDMФ show some augmentation of phagocytic capacity for apoptotic cells (data not shown), suggesting that there may be parallels in the mechanism of action of GC and IL- 10 .

In summary, the role that particular cytokines, steroids and lipid mediators play individually, and in concert, will critically determine the balance between pathogen clearance, resolution of inflammation, tolerance induction and wound healing. In particular, data presented here suggest that defining the interplay between GC and IFN- $\gamma$ in the regulation of macrophage function may unveil novel therapeutic targets for treatment of inflammatory disease.

\section{Materials and methods}

\subsection{Antibodies and other reagents}

All chemicals were from Sigma (Poole, Dorset, GB) unless otherwise stated. Antibodies were used at saturating concentrations as determined by titration in indirect immunoassays and flow cytometry as follows: HLA-DR (clone WR18, IgG2a, used at 1/100, Serotec, Oxford, GB), CD14 (clone UCHM1, IgG2a, provided by Dr. Peter Beverley, Edward Jenner Institute for Vaccine Research, Compton, GB), C54 (clone 15.2, provided by Dr. Nancy Hogg, Cancer Research UK, London, GB), CD64 (clone 10.1, IgG1, used at 1/100, provided by Dr.Nancy Hogg), CD86 (clone BU63, IgG1, used at 1/50, Caltag, B-D Biosciences, GB), CD163 (clone Bermac, IgG1, used at $1 / 35$, Dako, Oxford, GB), mannose receptor (clone 19.2, IgG1, Serotec), and IgG1 control (MOPC, mouse IgG1 plasmacytoma, obtained from ECACC, GB).

\subsection{Cell isolation and culture}

Mononuclear cells (MNC) and polymorphonuclear cells were isolated from human blood by dextran sedimentation and centrifugation over discontinuous Percoll ${ }^{\mathrm{TM}}$ (Amersham Pharmacia Biotech, Buckingham, GB) gradients as previously described [34]. The MNC (typically 15-20\% monocytes by morphological analysis of cytocentrifuge preparations or flow cytometric determination on the basis of laser scatter properties and CD14 reactivity) were resuspended at $4 \times 10^{6} / \mathrm{ml}$ in Iscove's modification of Dulbecco's modified Eagles medium (IDMEM; Life Technologies, Paisley, GB) and enriched for monocytes by selective adherence to 48 -well ( $0.5 \mathrm{ml} /$ well) or 6 -well $(4.0 \mathrm{ml} /$ well) tissue culture plates for $1 \mathrm{~h}$ at $37^{\circ} \mathrm{C}$ in $5 \% \mathrm{CO}_{2}$. Adherent monocytes were washed three times and then allowed to differentiate for 5 days in IDMEM containing penicillin/streptomycin and $10 \%$ autologous serum prepared by recalcification of platelet-rich plasma. Washed adherent MDM $\Phi$ consisted of $>90 \%$ 
CD14-positive cells at day 5 as assessed by flow cytometry. In our hands GC did not induce monocyte apoptosis as reported by Schmidt et al. [35]. Our unpublished data suggest that autologous serum over-rides the pro-apoptotic effects of GC on monocytes. As detailed in the text, dexamethasone was added to a final concentration of up to $1 \mu \mathrm{m}$, whereas recombinant IL- 4 and recombinant human IFN- $\gamma$ (both obtained from R\&D Systems, Minneapolis, USA) were used at $10 \mathrm{ng} / \mathrm{ml}$.

Isolated polymorphonuclear cells (typically $95-98 \%$ neutrophils by morphological examination of cytocentrifuge preparations) were resuspended at $20 \times 10^{6}$ cells $/ \mathrm{ml}$ cells in IDMEM and labeled with the fluorescent cell tracker dye carboxymethylfluorescein diacetate (CMFDA; Molecular Probes, Leiden, The Netherlands), $2 \mu \mathrm{g} / \mathrm{ml}$ for $15 \mathrm{~min}$ at $37^{\circ} \mathrm{C}$ in $5 \%$ $\mathrm{CO}_{2}$. Cells were then washed and cultured in $75 \mathrm{~mm}$ tissue culture flasks (Nunc, Fisher Scientific, Leicestershire, GB) for $18-24 \mathrm{~h}$ at $4 \times 10^{6} / \mathrm{ml}$ in IDMEM containing $10 \%$ autologous serum to induce spontaneous apoptosis. Microscopic examination of nuclear morphology of cytocentrifuge preparations of cultured cells was used to assess levels of apoptosis and neutrophils were typically $50-60 \%$ apoptotic with $15-20 \%$ necrotic cells present, as determined by dual annexin $\mathrm{V} /$ propidium iodide staining and flow cytometry.

\subsection{Flow cytometric determination of cytokine release}

Supernatants from MDM $\Phi$ that had been cultured for 5 days were analyzed for the presence of IL-8, IL-6, IL-1 1 , IL-10, TNF- $\alpha$ and IL-12p70 using the Human Inflammation BD Cytometric Bead Assay (Becton Dickinson, Oxford, GB) as described by the manufacturer's assay protocol. Briefly, $50 \mu \mathrm{l}$ samples of cell supernatant were incubated with capture beads to the six cytokines and a PE cytokine detection reagent for $3 \mathrm{~h}$ at room temperature. Samples were then washed once and 6000 events were acquired using a FACSCalibur flow cytometer (Becton Dickinson). Analysis of the median FL-2 fluorescence associated with bead populations defined by FL-3 labeling was made using Cellquest software (Becton Dickinson).

\subsection{Flow cytometric analysis of surface receptor expression}

MDM $\Phi$ obtained after 5 days of culture were washed in Hanks' balanced salt solution (HBSS) and incubated with $5 \mathrm{mM}$ EDTA in $\mathrm{Ca}^{2+} / \mathrm{Mg}^{2+}$-free PBS on ice for $15 \mathrm{~min}$ to detach the cells from the wells. The detached MDM $\Phi$ were centrifuged at $220 \times \mathrm{g}$ and washed in $\mathrm{Ca}^{2+} / \mathrm{Mg}^{2+}$-free PBS containing $0.2 \%$ BSA and $0.1 \%$ sodium azide (flow buffer). The pelleted cells were then incubated on ice for $30 \mathrm{~min}$ with saturating concentrations of monoclonal antibodies to cell surface determinants. The cells were then washed twice in flow buffer and incubated for a further $30 \mathrm{~min}$ on ice with FITC-labeled $F\left(a b^{\prime}\right)_{2}$ fragments of goat anti-mouse- immunoglobulin (Dako; used at 1/50). Following two further washes in flow buffer, the labeled cells were analyzed using either an EPICS XL (Beckman Coulter, High Wycombe, GB) or FACSCalibur flow cytometer (Becton Dickinson) after acquiring $5000-8000$ events per sample. Post-acquisition analysis was performed either using Cellquest (Becton Dickinson) or EXPO32 (Beckman Coulter).

\subsection{Quantitation of phagocytosis of apoptotic cells}

Monocytes were cultured for 5 days in 48 -well plates as described above either in the presence of $10 \%$ autologous serum alone (control), or in the presence of dexamethasone, IL- 4 or IFN- $\gamma$, or combinatorial treatments at the concentrations detailed in the text. MDM $\Phi$ were gently washed in $\mathrm{Ca}^{2+} / \mathrm{Mg}^{2+}$-free HBSS and phagocytosis of apoptotic neutrophils was then performed as described previously [36]. Briefly, MDM $\Phi$ were co-incubated with $0.5 \mathrm{ml}$ of CMFDAlabeled apoptotic neutrophils at $4 \times 10^{6} / \mathrm{ml}$ for $1 \mathrm{~h}$ at $37^{\circ} \mathrm{C}$ in $5 \% \mathrm{CO}_{2}$ after which the neutrophils were gently aspirated and $0.25 \mathrm{ml}$ of trypsin/EDTA added. Following incubation at $37^{\circ} \mathrm{C}$ in $5 \% \mathrm{CO}_{2}$ for $15 \mathrm{~min}$ and then at $4^{\circ} \mathrm{C}$ for $15 \mathrm{~min}$, MDM $\Phi$ were detached by vigorous pipetting. Flow cytometry was then used to determine the proportion of phagocytic macrophages (gated on the basis of forward- and sidescatter properties) and the percentage of FL-1-labeled MDM $\Phi$ was determined.

\subsection{Indirect immunofluorescence analysis of macrophage cytoskeleton}

Monocytes were plated on sterile coverslips in 24-well plates at $4 \times 10^{6} / \mathrm{ml}$. After 5 days of differentiation, adherent MDM $\Phi$ were fixed in $2 \mathrm{ml}$ of $3 \%(\mathrm{w} / \mathrm{v})$ para-formaldehyde at room temperature for $20 \mathrm{~min}$. Coverslips were then washed three times in $\mathrm{Ca}^{2+} / \mathrm{Mg}^{2+}$-free PBS and free aldehyde groups were then quenched with $50 \mathrm{mM} \mathrm{NH}_{4} \mathrm{Cl} / \mathrm{PBS}$ at room temperature for $15 \mathrm{~min}$. After three further washes in PBS, cells were permeabilized using $0.1 \%$ Triton $X-100$ for $4 \mathrm{~min}$. Following a further three washes in PBS the coverslips were incubated for $10 \mathrm{~min}$ in heat-inactivated $A B$ serum $(1 / 10)$ to block non-specific antibody binding to Fc receptors. To visualize podosomes within $\mathrm{MDM} \Phi$, cells were then incubated on ice for 30 min with mAb specific for paxillin (BD Transduction Labs, Belgium). The cells were washed and then labeled by incubating for $30 \mathrm{~min}$ with an Alexa-488 goat anti-mouse-immunoglobulin antibody (1/400 in PBS; Molecular Probes). After a further three washes cells were incubated with rhodamine phalloidin (1/800 in PBS; Molecular Probes). The coverslips were then mounted onto slides using an antifadent mounting medium (Molecular Probes) and examined under oil immersion microscopy using a $\times 63$ objective using an Axiovert $\mathbf{S} 100$ immunofluorescence microscope with Coolsnap LCD camera and Openlab image acquisition software. 
Acknowledgements: We would like to thank colleagues in the Centre for Inflammation Research for help in the preparation of leukocytes, and Paul Hartley for help with microscopy. This work was supported by the Medical Research Council (Program grant 9016491) and the Wellcome Trust (S. J. H. and K. M. G.).

\section{References}

1 Nathan, C. F., Murray, H. W. and Cohn, Z. A., The macrophage as an effector cell. N. Engl. J. Med. 1980. 303: 622-626.

2 Gordon, S., Keshav, S. and Chung, L. P., Mononuclear phagocytes: tissue distribution and functional heterogeneity. Curr. Opin. Immunol. 1988, 1: 26-35.

3 Scott, W. A., Pawlowski, N. A., Cramer, E. B. and Cohn, Z. A., Secretory functions of the mononuclear phagocyte. Adv. Exp. Med. Biol. 1985. 183: 17-25.

4 Keshav, S., Chung, L. P. and Gordon, S., Macrophage products in inflammation. Diagn. Microbiol. Infect. Dis. 1990. 13: 439-447.

5 Savill, J., Dransfield, I., Gregory, C. and Haslett, C., A blast from the past: clearance of apoptotic cells regulates immune responses. Nat. Rev. Immunol. 2002. 2: 965-975.

6 Fadok, V. A., Bratton, D. L., Konowal, A., Freed, P. W., Westcott, J. Y. and Henson, P. M., Macrophages that have ingested apoptotic cells in vitro inhibit proinflammatory cytokine production through autocrine/paracrine mechanisms involving TGFbeta, PGE2, and PAF. J. Clin. Invest. 1998. 101: 890-898.

7 Haslett, C., Savill, J. S., Whyte, M. K., Stern, M., Dransfield, I. and Meagher, L. C., Granulocyte apoptosis and the control of inflammation. Philos. Trans. R. Soc. Lond. B Biol. Sci. 1994. 345: $327-333$

8 Botto, M., Dell'Agnola, C., Bygrave, A. E., Thompson, E. M., Cook, H. T., Petry, F., Loos, M., Pandolfi, P. P. and Walport, M. J., Homozygous C1q deficiency causes glomerulonephritis associated with multiple apoptotic bodies. Nat. Genet. 1998. 19: 56-59.

9 Gratchev, A., Schledzewski, K., Guillot, P. and Goerdt, S., Alternatively activated antigen-presenting cells: molecular repertoire, immune regulation, and healing. Skin Pharmacol. Appl. Skin Physiol. 2001. 14: 272-279.

10 Nathan, C. F., Murray, H. W., Wiebe, M. E. and Rubin, B. Y., Identification of interferon-gamma as the lymphokine that activates human macrophage oxidative metabolism and antimicrobial activity. J. Exp. Med. 1983. 158: 670-689.

11 Pace, J. L., Russell, S. W., Schreiber, R. D., Altman, A. and Katz, D. H., Macrophage activation: priming activity from a T-cell hybridoma is attributable to interferon-gamma. Proc. Natl. Acad. Sci. U. S. A. 1983. 80: 3782-3786.

12 Rabinovitch, M., Hamburg, S. I. and Fleit, H. B., Interferoninduced enhancement of $\mathrm{FC}$ receptor-mediated macrophage phagocytosis. J. Reticuloendothel. Soc. 1980. 28: 27s-28s.

13 Stein, M., Keshav, S., Harris, N. and Gordon, S., Interleukin 4 potently enhances murine macrophage mannose receptor activity: a marker of alternative immunologic macrophage activation. J. Exp. Med. 1992. 176: 287-292.

14 Doyle, A. G., Herbein, G., Montaner, L. J., Minty, A. J., Caput, D., Ferrara, P. and Gordon, , S., Interleukin-13 alters the activation state of murine macrophages in vitro: comparison with interleukin-4 and interferon-gamma. Eur. J. Immunol. 1994. 24: $1441-1445$.
15 te Velde, A. A., Klomp, J. P., Yard, B. A., de Vries, J. E. and Figdor, C. G., Modulation of phenotypic and functional properties of human peripheral blood monocytes by IL-4. J. Immunol. 1988. 140: $1548-1554$

16 Munder, M., Eichmann, K. and Modolell, M., Alternative metabolic states in murine macrophages reflected by the nitric oxide synthase/arginase balance: competitive regulation by $C D 4+T$ cells correlates with Th1/Th2 phenotype. J. Immunol. 1998. 160: 5347-5354.

17 Liu, Y., Cousin, J. M., Hughes, J., Van Damme, J., Seckl, J. R., Haslett, C., Dransfield, I., Savill, J. and Rossi, A. G., Glucocorticoids promote nonphlogistic phagocytosis of apoptotic leukocytes. J. Immunol. 1999. 162: 3639-3646.

18 Giles, K. M., Ross, K., Rossi, A. G., Hotchin, N. A., Haslett, C. and Dransfield, I., Glucocorticoid augmentation of macrophage capacity for phagocytosis of apoptotic cells is associated with reduced $\mathrm{p} 130 \mathrm{Cas}$ expression, loss of paxillin/pyk2 phosphorylation, and high levels of active Rac. J. Immunol. 2001. 167: 976-986.

19 Dustin, M. L., Rothlein, R., Bhan, A. K., Dinarello, C. A. and Springer, T. A., Induction by IL 1 and interferon-gamma: tissue distribution, biochemistry, and function of a natural adherence molecule (ICAM-1). J. Immunol. 1986. 137: 245-254

20 Hogger, P., Dreier, J., Droste, A., Buck, F. and Sorg, C., Identification of the integral membrane protein $\mathrm{RM} 3 / 1$ on human monocytes as a glucocorticoid-inducible member of the scavenger receptor cysteine-rich family (CD163). J. Immunol. 1998. 161: 1883-1890.

21 Linder, S., Nelson, D., Weiss, M. and Aepfelbacher, M. Wiskott-Aldrich syndrome protein regulates podosomes in primary human macrophages. Proc. Natl. Acad. Sci. U. S. A. 1999. 96: 9648-9653.

22 Chellaiah, M. A., Soga, N., Swanson, S., McAllister, S. Alvarez, U., Wang, D., Dowdy, S. F. and Hruska, K. A., Rho-A is critical for osteoclast podosome organization, motility, and bone resorption. J. Biol. Chem. 2000. 275: 11993-12002.

23 Ren, Y. and Savill, J., Proinflammatory cytokines potentiate thrombospondin-mediated phagocytosis of neutrophils undergoing apoptosis. J. Immunol. 1995. 154: 2366-2374.

24 Erwig, L. P., Gordon, S., Walsh, G. M. and Rees, A. J., Previous uptake of apoptotic neutrophils or ligation of integrin receptors downmodulates the ability of macrophages to ingest apoptotic neutrophils. Blood 1999. 93: 1406-1412.

25 De, A., Blotta, H. M., Mamoni, R. L., Louzada, P., Bertolo, M. B., Foss, N. T., Moreira, A. C. and Castro, M., Effects of dexamethasone on lymphocyte proliferation and cytokine production in rheumatoid arthritis. J. Rheumatol. 2002. 29: 46-51.

26 Dries, D. J. and Perry, J. F. Jr., Interferon-gamma: titration of inflammation. Crit. Care Med. 2002. 30: 1663-1664.

27 Thieringer, R., Le Grand, C. B., Carbin, L., Cai, T. Q., Wong, B. Wright, S. D. and Hermanowski-Vosatka, A., 11 Betahydroxysteroid dehydrogenase type 1 is induced in human monocytes upon differentiation to macrophages. J. Immunol. 2001. 167: $30-35$

28 Savill, J., Dransfield, I., Hogg, N. and Haslett, C., Vitronectin receptor-mediated phagocytosis of cells undergoing apoptosis. Nature 1990. 343: 170-173.

29 Devitt, A., Moffatt, O. D., Raykundalia, C., Capra, J. D. Simmons, D. L. and Gregory, C. D., Human CD14 mediates recognition and phagocytosis of apoptotic cells. Nature 1998. 392: 505-509.

30 Hall, S. E., Savill, J. S., Henson, P. M. and Haslett, C., Apoptotic neutrophils are phagocytosed by fibroblasts with participa- 
tion of the fibroblast vitronectin receptor and involvement of a mannose/fucose-specific lectin. J.Immunol. 1994. 153: 3218-3227.

31 Capsoni, F., Minonzio, F., Mariani, C., Ongari, A. M., Bonara, P. and Fiorelli, G., Development of phagocytic function of cultured human monocytes is regulated by cell surface IL-10. Cell. Immunol. 1998. 189: 51-59.

32 Stumpo, R., Kauer, M., Martin, S. and Kolb, H., Alternative activation of macrophage by IL-10. Pathobiology 1999. 67: 245-248.

33 Buechler, C., Ritter, M., Orso, E., Langmann, T., Klucken, J. and Schmitz, G., Regulation of scavenger receptor CD163 expression in human monocytes and macrophages by pro- and antiinflammatory stimuli. J. Leukoc. Biol. 2000. 67: 97-103.

34 Hart, S. P., Ross, J. A., Ross, K., Haslett, C. and Dransfield, I. Molecular characterization of the surface of apoptotic neutrophils: implications for functional downregulation and recognition by phagocytes. Cell Death Differ. 2000. 7: 493-503.
35 Schmidt, M., Pauels, H. G., Lugering, N., Lugering, A., Domschke, W. and Kucharzik, T., Glucocorticoids induce apoptosis in human monocytes: potential role of IL-1 beta. J. Immunol. 1999. 163: 3484-3490.

36 Jersmann, H. P., Ross, K. A., Vivers, S., Brown, S. B., Haslett, C. and Dransfield, I., Phagocytosis of apoptotic cells by human macrophages: analysis by multiparameter flow cytometry. Cytometry 2003. 51A: 7-15.

Correspondence: Ian Dransfield, MRC Centre for Inflammation Research, University of Edinburgh Medical School, Teviot Place, Edinburgh EH8 9AG, GB

Fax: +44-131-6504384

e-mail: i.dransfield@ed.ac.uk 


\title{
Heme Inhibits Human Neutrophil Apoptosis: Involvement of Phosphoinositide 3-Kinase, MAPK, and NF- $\kappa$ B
}

\author{
Maria Augusta Arruda, ${ }^{*}$ Adriano G. Rossi, ${ }^{\ddagger}$ Marta S. de Freitas, ${ }^{*}$ Christina Barja-Fidalgo, ${ }^{1}$ * \\ and Aurélio V. Graça-Souza ${ }^{\dagger}$
}

High levels of free heme are found in pathological states of increased hemolysis, such as sickle cell disease, malaria, and ischemia reperfusion. The hemolytic events are often associated with an inflammatory response that usually turns into chronic inflammation. We recently reported that heme is a proinflammatory molecule, able to induce neutrophil migration, reactive oxygen species generation, and IL-8 expression. In this study, we show that heme (1-50 $\mu \mathrm{M})$ delays human neutrophil spontaneous apoptosis in vitro. This effect requires heme oxygenase activity, and depends on reactive oxygen species production and on de novo protein synthesis. Inhibition of ERK and PI3K pathways abolished heme-protective effects upon human neutrophils, suggesting the involvement of the Ras/Raf/MAPK and PI3K pathway on this effect. Confirming the involvement of these pathways in the modulation of the antiapoptotic effect, heme induces Akt phosphorylation and ERK-2 nuclear translocation in neutrophils. Fu-

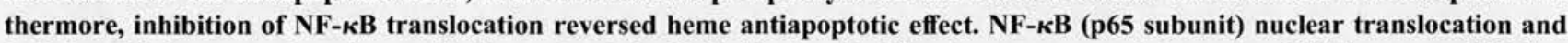
IкB degradation were also observed in heme-treated cells, indicating that free heme may regulate neutrophil life span modulating signaling pathways involved in cell survival. Our data suggest that free heme associated with hemolytic episodes might play an important role in the development of chronic inflammation by interfering with the longevity of neutrophils. The Journal of Immunology, 2004, 173: 2023-2030.

$\mathrm{S}$ evere hemolysis occurring during pathological states such as sickle cell disease, ischemia reperfusion, and malaria results in high levels of free heme (up to $20 \mu \mathrm{M}$ ). Under these conditions, the physiological mechanisms of removing free heme from the circulation, especially its binding to hemopexin, collapse, allowing nonspecific heme uptake and heme-catalyzed oxidation reactions $(1,2)$. We have recently reported that heme is a proinflammatory molecule able to induce neutrophil migration in vivo and in vitro (3). Interaction of free heme with human neutrophils leads to actin cytoskeleton reorganization and reactive oxygen species (ROS) $)^{2}$ generation through the induction of protein kinase C (PKC) activity, and also increases IL-8 expression (3). These findings attested to a prominent role for free heme in the development of inflammation associated with hemolytic diseases. In agreement, it has been shown that increased levels of heme in plasma are accompanied by a rise in cytokine and chemokine concentrations, as well as enhanced leukocyte function (4-8), events often associated with an inflammatory response that usually develops into chronic inflammation $(9,10)$.

\footnotetext{
*Departamento de Farmacologia, Instituto de Biologia, Universidade do Estado do Rio de Janeiro, and 'Departamento de Bioquímica Médica, Instituto de Ciências Biomédicas/Centro de Ciências da Saúde, Universidade Federal do Rio de Janeiro, Rio de Janeiro, Brazil; and ${ }^{\ddagger}$ Centre for Inflammation Research, Respiratory Medicine Unit, University of Edinburgh Medical School, Edinburgh, United Kingdom.

Received for publication January 20, 2004. Accepted for publication May 19, 2004.

The costs of publication of this article were defrayed in part by the payment of page charges. This article must therefore be hereby marked advertisement in accordance with 18 U.S.C. Section 1734 solely to indicate this fact.

${ }^{1}$ Address correspondence and reprint requests to Dr. Christina Barja-Fidalgo, Departamento de Farmacologia, Instituto de Biologia, Universidade do Estado do Rio de Janeiro, Av 28 de setembro 87-Vila Izabel, Rio de Janeiro, RJ, 20551-030 Brazil. E-mail address: barja-fidalgo@uerj.br

${ }^{2}$ Abbreviations used in this paper: ROS, reactive oxygen species; BIM, bis-indoylmaleimide IV; CO, carbon monoxide; DPI, diphenyleneiodonium; HO, heme oxygenase; PDTC, pyrrolidine dithiocarbamate; PKC, protein kinase C; SnPPIX, tin protoporphyrin IX.
}

Neutrophil apoptosis and subsequent clearance by phagocytes are critical to the resolution of acute inflammation $(11,12)$. These terminally differentiated cells constitute the first line of host defense against invading microorganism, being promptly recruited to inflamed loci in response to infection or tissue injury. Once activated, neutrophils are able to phagocytose, to release granular lytic enzymes and antimicrobial polypeptides into the phagolysosome, and to generate large amounts of ROS as well as reactive nitrogen species (13). Under normal conditions, neutrophils have a very short $t_{1 / 2}$, being committed to programmed cell death (apoptosis). During this process, cell membrane integrity is maintained, avoiding the release of proinflammatory and potentially cytotoxic agents and the subsequent amplification of the inflammatory response. Apoptotic neutrophils also express surface markers that allow their recognition and nonphlogistic ingestion by professional phagocytes such as macrophages, or potential phagocytes such as fibroblasts and mesangial cells (14).

Culturing peripheral blood neutrophils in vitro reproducibly results in spontaneous apoptosis of $>50 \%$ of the cells within $24 \mathrm{~h}$. These apoptotic cells exhibit the classical features associated with this phenomenon, such as cytoplasmic condensation, phosphatidylserine exposure on the outer leaflet of the plasma membrane, and internucleosomal DNA cleavage, followed by chromatin condensation. Ultimately, it is the activation of caspases, a family of redox-sensitive cysteine proteases, that coordinates the structural dismantling of the cell (15).

Agents that promote neutrophil responsiveness, such as IL-8, GM-CSF, LPS, and leukotriene $\mathrm{B}_{4}$, also delay human neutrophil apoptosis (16-19). These stimuli promote neutrophil survival by modulating intracellular signaling pathways, including the MAPK, especially ERK and PI3K/Akt pathways $(20,21)$. Evidence has shown that activation of NF- $\kappa \mathrm{B}$ pathway has a protective effect in several cell types, regulating the expression of antiapoptotic genes (22). In human neutrophils, NF- $\kappa \mathrm{B}$ activation seems to regulate spontaneous apoptosis and the antiapoptotic effect of TNF- $\alpha$, a 
cytokine that exerts dual effects upon these cells. The modulation of all those pathways most likely regulates the balance between pro- and antiapoptotic proteins to influence neutrophil survival, especially the members of Bcl-2 family, which comprises both pro- and antiapoptotic members (23).

In the present study, we demonstrate that heme is able to prolong neutrophil life span by inhibiting apoptosis by a mechanism dependent on heme oxygenase (HO) activity and ROS generation. This effect depends on de novo protein synthesis and seems to be mediated by MAPK and PI3K/Akt pathways and involves NF- $\kappa$ B activation, indicating that heme may control neutrophil apoptosis through activation of these survival pathways. Our data support a role for heme as a proinflammatory mediator during hemolytic states, suggesting that this molecule is important in the development of chronic inflammation associated with hemolysis and hemoglobinemia.

\section{Materials and Methods \\ Reagents}

Cycloheximide, diphenyleneiodonium (DPI), pyrrolidine dithiocarbamate (PDTC), and apocynin (acetovanillone) were purchased from SigmaAldrich (St. Louis, MO). LY294002, bis-indoylmaleimide IV (BIM), and PD98059 were from Calbiochem (San Diego, CA). Biliverdin and bilirubin were from Valeant Pharmaceuticals (Costa Mesa, CA). Tin protoporfirin IX (SnPPIX) was from Porphyrin Products (Logan, UT). Human rIL-8 was a gift from F. Cunha (Faculdade de Medicina de Ribeirão Preto-Universidade de São Paulo, São Paulo, Brazil), and anti-IL-8 mAb was donated by P. Bozza (Fundaçao Oswaldo Cruz, Rio de Janeiro, Brazil).

\section{Heme}

Hemin (cell culture grade; Sigma-Aldrich) stock solutions were made in DMSO (culture grade; Sigma-Aldrich) and diluted in sterile PBS immediately before use. The final concentration of DMSO was kept lower than $0.01 \%$ for all assays.

\section{Neutrophil isolation and culture}

Neutrophils were isolated from EDTA $(0.5 \%)$-treated peripheral venous blood of healthy human volunteers by a method of dextran sedimentation and density gradient centrifugation, as previously described (24). Residual erythrocytes were removed by hypotonic lysis. Isolated neutrophils $(5 \times$ $10 \% \mathrm{ml}$ ) were incubated in DMEM supplemented with $10 \%$ heat-inactivated $\mathrm{FCS}, 100 \mathrm{U} / \mathrm{ml}$ penicillin, and $100 \mathrm{mg} / \mathrm{ml}$ streptomycin at $37^{\circ} \mathrm{C}$ in a humidified atmosphere containing $5 \% \mathrm{CO}_{2}$ for $20 \mathrm{~h}$, unless otherwise indicated. Under all experimental conditions, $>99 \%$ of cells were viable, as assessed by trypan blue dye exclusion.

\section{Assessment of neutrophil apoptosis}

Morphology. Cells were cytocentrifuged, stained with Diff-Quik, and counted under light microscopy $(\times 1000)$ to determine the proportion of cells showing characteristic apoptotic morphology. At least 400 cells were counted per slide. The results were expressed as mean $\pm \mathrm{SD}$.

\section{Annexin $V$-binding assay}

To measure phosphatidylserine exposure on apoptotic cell surface, a flow cytometric assay using annexin V binding (annexin V-FLUOS; Roche Molecular Biochemicals, Mannheim, Germany) was performed. A working solution of annexin V-FLUOS was made from stock annexin V-FLUOS $(0.1 \mu \mathrm{g} / \mathrm{ml})$ diluted $1 / 3000$ in HBSS supplemented with $2.5 \mathrm{mM} \mathrm{CaCl}_{2}$. Neutrophils $\left(20 \mu \mathrm{l}\right.$ of $\left.5 \times 10^{6} / \mathrm{ml}\right)$ were added to $200 \mu \mathrm{l}$ of a working solution of annexin V before being assessed on a FACSCalibur flow cytometer (BD Biosciences, San Jose, CA) and analyzed on associated CellQuest (BD Biosciences) software. All experiments were performed at least three times.

\section{DNA electrophoresis}

DNA fragmentation was analyzed, as previously described (25). Briefly, neutrophils $\left(5 \times 10^{6}\right.$ cells $\left./ \mathrm{ml}\right)$ were taken after $20 \mathrm{~h}$ and lysed with $500 \mu \mathrm{l}$ of lysing buffer $(0.2 \%$ Triton X-100; 100 and $1 \mathrm{mM}$ EDTA, pH 7.4). Cell lysates were then centrifuged at $13,000 \times \mathrm{g}$, and the supernatants (containing fragmented DNA) were separated from the pellet. The supernatants obtained were treated with $50 \mu \mathrm{l}$ of $5 \mathrm{M} \mathrm{NaCl}$ and $500 \mu \mathrm{l}$ of isopropanol and left for $12 \mathrm{~h}$ at $-70^{\circ} \mathrm{C}$. DNA pellets were washed with $70 \%$ ethanol, air dried, and resuspended in TE buffer (10 mM Tris, $1 \mathrm{mM}$ EDTA, pH 7.4). Fragmented DNA was separated on a $1 \%$ agarose gel electrophoresis containing $1 \mu \mathrm{g} / \mathrm{ml}$ ethidium bromide. The products of DNA fragmentation were visualized and documented under UV light.

\section{Subcellular localization}

Cells were cytocentrifuged and fixed with paraformaldehyde $(4 \%)$, and then permeabilized with $0.5 \%$ Triton X-100 in PBS for 20 min. The slides were incubated with rabbit polyclonal anti-ERK-2 Ab (Santa Cruz Biotechnology, Santa Cruz, CA; $1 / 200$ ) at $4^{\circ} \mathrm{C}$ overnight, incubated at room temperature for $1 \mathrm{~h}$ with biotin-conjugated goat anti-rabbit IgG (Santa Cruz Biotechnology; 1/1000), and finally incubated with FITC-conjugated streptavidin for $1 \mathrm{~h}$ at room temperature. Slides were then mounted using $\mathrm{N}$-propylgalate solution before examination under an Olympus BX40F4 microscope (Melville, NY) equipped for epifluorescence. Images were analyzed using Adobe Photoshop software (Adobe Systems, San Jose, CA).

\section{Preparation of cell extracts}

To obtain the whole cell extracts to analyze $\mathrm{I} \kappa \mathrm{B} \alpha$ degradation, an indicator of NF- $\kappa$ B pathway activation, neutrophils $\left(5 \times 10^{6}\right.$ cells $\left./ \mathrm{ml}\right)$ were resuspended in lysis buffer ( $50 \mathrm{mM}$ HEPES, pH $6.4,1 \mathrm{mM} \mathrm{MgCl}, 10 \mathrm{mM}$ EDTA, $1 \%$ Triton X-100, $1 \mu \mathrm{g} / \mathrm{ml}$ DNase, $0.5 \mu \mathrm{g} / \mathrm{ml}$ RNase) containing the following protease inhibitors: $1 \mathrm{mM}$ PMSF, $1 \mathrm{mM}$ benzamidine, $1 \mu \mathrm{M}$ leupeptin, and $1 \mu \mathrm{M}$ soybean trypsin inhibitor (Sigma-Aldrich).

\section{Preparation of nuclear extracts}

For the analysis of NF- $\kappa \mathrm{B}$ nuclear translocation, neutrophils $\left(5 \times 10^{6}\right.$ cells $/ \mathrm{ml})$ were incubated with heme $(3 \mu \mathrm{M})$ for 1 or $2 \mathrm{~h}$ at $37^{\circ} \mathrm{C}$ in a $5 \%$ $\mathrm{CO}_{2}$ atmosphere. Nuclear extracts were obtained, as described earlier (26). Briefly, cells were lysed in ice-cold buffer A (10 mM HEPES, pH 7.9, 10 $\mathrm{mM} \mathrm{KCl}, 0.1 \mathrm{mM}$ EDTA, $0.1 \mathrm{mM}$ EGTA, $1 \mathrm{mM}$ DTT, and $0.5 \mathrm{mM}$ PMSF), and after a 15 -min incubation on ice, Nonidet $\mathrm{P}-40$ was added to a final concentration of $0.5 \%(\mathrm{v} / \mathrm{v})$. Nuclei were collected by centrifugation $\left(1,810 \times g ; 5\right.$ min at $\left.4^{\circ} \mathrm{C}\right)$. The nuclear pellet was suspended in ice-cold buffer C (20 mM HEPES, pH 7.9, $400 \mathrm{mM} \mathrm{NaCl}, 1 \mathrm{mM}$ EDTA, $1 \mathrm{mM}$ EGTA, $1 \mathrm{mM}$ DTT, $1 \mathrm{mM}$ PMSF, $1 \mu \mathrm{g} / \mathrm{ml}$ pepstatin, $1 \mu \mathrm{g} / \mathrm{ml}$ leupeptin, and $20 \%(\mathrm{v} / \mathrm{v})$ glycerol) and incubated for $30 \mathrm{~min}$. Nuclear proteins were collected in the supernatant after centrifugation $(12,000 \times g ; 10 \mathrm{~min}$ at $4^{\circ} \mathrm{C}$ ), and the immunoblotting for nuclear NF- $\kappa \mathrm{B}$ and histone $(\mathrm{H} 3)$ content was performed, as described below.

\section{Immunoprecipitation}

Neutrophils $\left(5 \times 10^{6}\right.$ cells $\left./ \mathrm{ml}\right)$ were incubated with heme $(3 \mu \mathrm{M})$ for 5,15 , and $30 \mathrm{~min}$ at $37^{\circ} \mathrm{C}$ in a $5 \% \mathrm{CO}_{2}$ atmosphere in the absence or the presence of LY290042 $(3 \mu \mathrm{M})$ or BIM $(10 \mathrm{nM})$. Cells were lysed in $50 \mathrm{mM}$ Tris$\mathrm{HCl}, \mathrm{pH} 7.4,150 \mathrm{mM} \mathrm{NaCl}, 1.5 \mathrm{mM} \mathrm{MgCl}_{2}, 1.5 \mathrm{mM}$ EDTA, Triton X-100 $(1 \%, \mathrm{v} / \mathrm{v})$, glycerol $(10 \%, \mathrm{v} / \mathrm{v})$, aprotinin $(10 \mu \mathrm{g} / \mu \mathrm{l})$, leupeptin $(10 \mu \mathrm{g} / \mu \mathrm{l})$, pepstatin $(2 \mu \mathrm{g} / \mu \mathrm{l})$, and $1 \mathrm{mM}$ PMSF. Lysates $(2 \mu \mathrm{g}$ of protein $/ \mu \mathrm{l})$ were incubated overnight at $4^{\circ} \mathrm{C}$ with polyclonal anti-Akt1 (1/200; Santa Cruz Biotechnology) Ab. After this time, protein $\mathrm{A} / \mathrm{G}$-agarose $(20 \mu \mathrm{l} / \mathrm{mg}$ protein; Santa Cruz Biotechnology) was added, and samples were incubated at $4^{\circ} \mathrm{C}$ in a rotatory shaker for $2 \mathrm{~h}$. The content of total and phosphorylated Akt on serine residues was analyzed by Western blot, as described below.

\section{Western blot analysis}

The total protein content in the cell extracts was determined by Bradford's method (27). Cell lysates were denatured in sample buffer $(50 \mathrm{mM}$ Tris$\mathrm{HCl}, \mathrm{pH} 6.8,1 \% \mathrm{SDS}, 5 \% 2-\mathrm{ME}, 10 \%$ glycerol, and $0.001 \%$ bromphenol blue) and heated in a boiling water bath for $3 \mathrm{~min}$. Samples ( $30 \mu \mathrm{g}$ of total protein) were resolved by $12 \%$ SDS-PAGE, and proteins were transferred to polyvinylidene difluoride membranes (Hybond-P; Amersham Biosciences). Rainbow markers (Amersham Biosciences, Uppsala, Sweden) were run in parallel to estimate molecular weights. Membranes were blocked with Tween TBS $(20 \mathrm{mM}$ Tris- $\mathrm{HCl}$, pH 7.5, $500 \mathrm{mM} \mathrm{NaCl}, 0.1 \%$ Tween 20 ) containing $1 \% \mathrm{BSA}$ and probed with polyclonal anti-Bcl- $\mathrm{x}_{\mathrm{L}}$ (Santa Cruz Biotechnology; 1/500), polyclonal anti-Bad (Santa Cruz Biotechnology; 1/500), monoclonal anti-phosphoserine (Sigma-Aldrich; 1/1000), polyclonal anti-Akt1 Ab (Santa Cruz Biotechnology; 1/1000), polyclonal anti-NF- $\kappa B$ Ab (Santa Cruz Biotechnology; 1/1000), polyclonal anti-I $\kappa \mathrm{B}$ Ab (Santa Cruz Biotechnology; 1/1000), or monoclonal antihistone H3 Ab (Cell Signaling Technology, Beverly, MA; 1/1000). After extensive washing in Tween TBS, polyvinylidene difluoride sheets were incubated with biotin-conjugated anti-rabbit or anti-mice IgG (Santa Cruz 
Biotechnology; 1:1000) $\mathrm{Ab}$ for $1 \mathrm{~h}$ and then incubated with HRP-conjugated streptavidin (Caltag Laboratories, Burlingame, CA; 1/1000). Immunoreactive proteins were visualized by $3,3^{\prime}$-diaminobenzidine (Sigma-Aldrich) staining. The bands were also quantified by densitometry using Scion Image Software (Scion, Frederick, MD).

\section{Statistical analysis}

Statistical significance was assessed by ANOVA, followed by Bonferroni's $t$ test, and $p<0.05$ was taken as statistically significant.

\section{Results}

Heme delays human neutrophil apoptosis in vitro

The effect of heme in the modulation of spontaneous neutrophil apoptosis was simultaneously assessed by morphology (Fig. 1A), annexin $\mathrm{V}$ binding (Fig. $1 B$ ), and DNA electrophoresis (Fig. 1C). We have observed that incubation of human neutrophils with heme (3 $\mu \mathrm{M})$ significantly delayed the apoptotic rate of these cells. Heme-mediated delay of apoptosis at $1-3 \mu \mathrm{M}$ was similar to that observed with IL-8 $(100 \mathrm{nM})$, a cytokine with a known ability to protect neutrophil from apoptosis. Heme was able to delay neutrophil apoptosis in vitro at all concentrations studied (1-50 $\mu \mathrm{M}$; Fig. $2 A$ ), although its protective effects were less pronounced at the highest concentration used $(50 \mu \mathrm{M})$. Because heme is able to evoke IL-8 synthesis in human neutrophils (3), we speculated whether the effect of free heme on neutrophil apoptosis would be mediated by an autocrine production of this antiapoptotic chemokine. Pretreatment of neutrophils with anti-IL- $8 \mathrm{Ab}$ did not inhibit the heme effect, indicating that heme inhibits human neutrophil apoptosis per se (Fig. 2B).

\section{A}

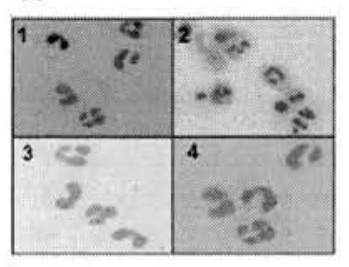

C

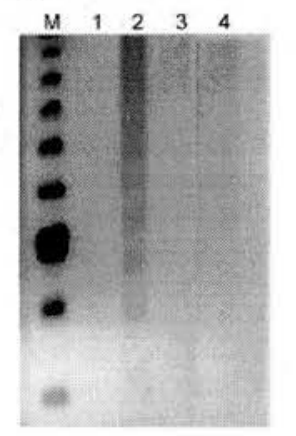

FIGURE 1. Inhibition of spontaneous apoptosis in human neutrophils by heme. $A$, Morphological analysis of apoptotic neutrophils: 1) medium alone, $0 \mathrm{~h}$; 2) medium alone, $20 \mathrm{~h}$; 3) $3 \mu \mathrm{M}$ heme, $20 \mathrm{~h}$; 4) $100 \mathrm{nM} \mathrm{IL}-8$, $20 \mathrm{~h} . B$, Flow cytometric analysis of annexin $\mathrm{V}$ binding in fresh neutrophils (1), neutrophils incubated for $20 \mathrm{~h}$ in the absence (2) or the presence of 3 $\mu \mathrm{M}$ heme (3), or $100 \mathrm{nM} \mathrm{IL-8} \mathrm{(4).} \mathrm{The} \mathrm{percentage} \mathrm{of} \mathrm{annexin} \mathrm{V-positive}$ cells in each sample is indicated. This representative experiment was repeated three times, showing similar results each time. $C$, Agarose gel electrophoresis of DNA showing an increase in the formation of oligonucleosomal DNA fragments in spontaneous apoptosis after $20 \mathrm{~h}$ (lane $1,0 \mathrm{~h}$; lane $2,20 \mathrm{~h}$ ), which is inhibited by incubation with $3 \mu \mathrm{M}$ heme (lane 3 ) and $100 \mathrm{nM} \mathrm{IL-8} \mathrm{(lane} \mathrm{4).} \mathrm{Lane} M$ represents the m.w. marker.
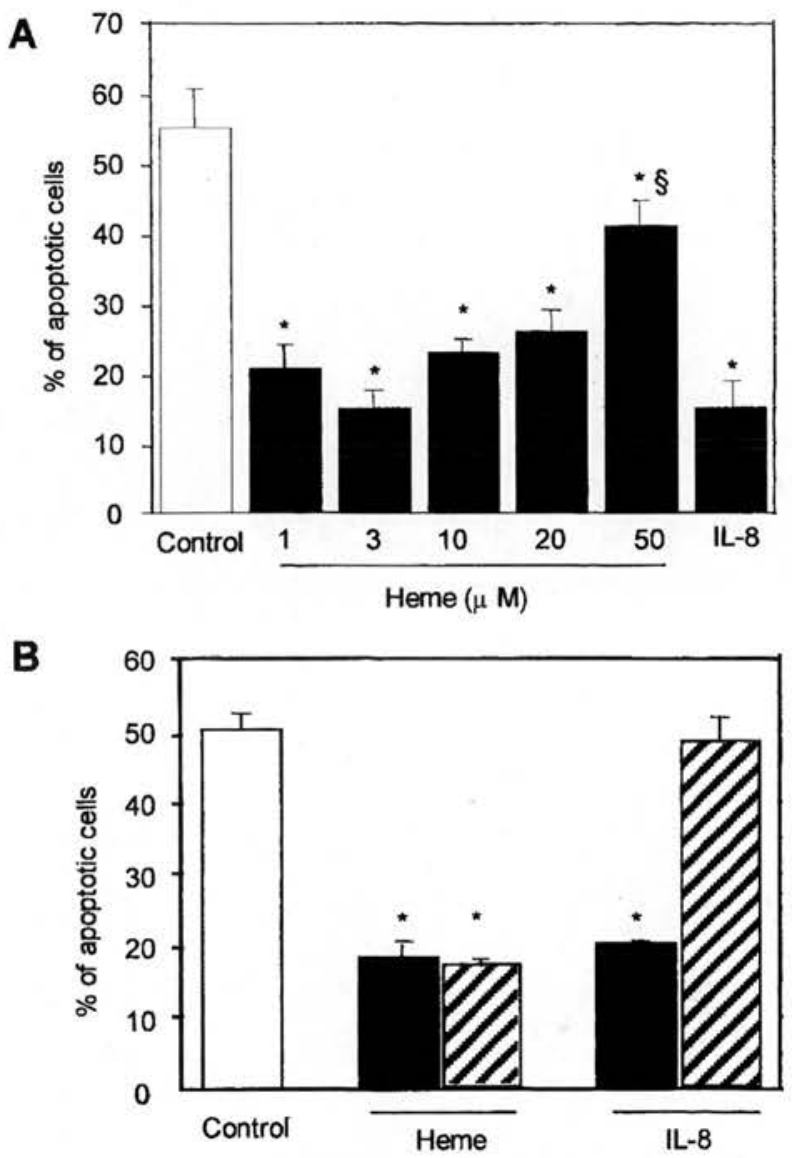

FIGURE 2. Concentration-dependent effect of heme on neutrophil apoptosis. $A$, Neutrophils $\left(5 \times 10^{6} / \mathrm{ml}\right)$ were incubated in the absence or in the presence of varying concentrations of heme (1-50 $\mu \mathrm{M})$ or IL-8 (100 $\mathrm{nM}$ ). After $20 \mathrm{~h}$, cells were cytocentrifuged, and the number of apoptotic cells was determined microscopically. $B$, The same protocol was performed with a single heme $(3 \mu \mathrm{M})$ or IL- $8(100 \mathrm{nM})$ concentration in the absence or the presence of anti-IL- $8 \mathrm{mAb}(7.2 \mu \mathrm{g} / \mathrm{ml}$; 2$)$. Data shown are the results (mean $+\mathrm{SD}$ ) of three experiments, each performed in triplicate. *, Indicates a significant difference $(p<0.001)$ between heme- or IL-8treated and control neutrophils. $\S$, Indicates that this group is significantly different from other treatments $(p<0.05)$.

\section{SnPPIX reverses heme effects on neutrophil apoptosis}

We have previously observed that heme enhances HO-1 protein expression on neutrophils (A.V.G.-S., C.B.-F., and M.A.A., unpublished data). To investigate the involvement of $\mathrm{HO}$ in the inhibition induced by heme on neutrophil apoptosis, cells were coincubated with SnPPIX $(50 \mu \mathrm{M})$, a competitive inhibitor of this enzyme (Fig. 3). HO inhibition partially reversed heme effects on neutrophil apoptosis, suggesting that heme metabolites may play an important role on neutrophil survival under these conditions. However, neither biliverdin nor bilirubin $(3 \mu \mathrm{M})$ protected human neutrophils from spontaneous apoptosis (51 and $53 \%$ of apoptotic cells, repectively), suggesting that other $\mathrm{HO}$ metabolites might be involved in heme effect.

\section{Delaying of neutrophil apoptosis by heme requires ROS production}

We have previously reported that heme evokes an oxidative burst in human neutrophils (3), which may lead to profound changes in the redox status of the cells. These alterations are known to modulate the activity of redox-sensitive proteins, including caspases 


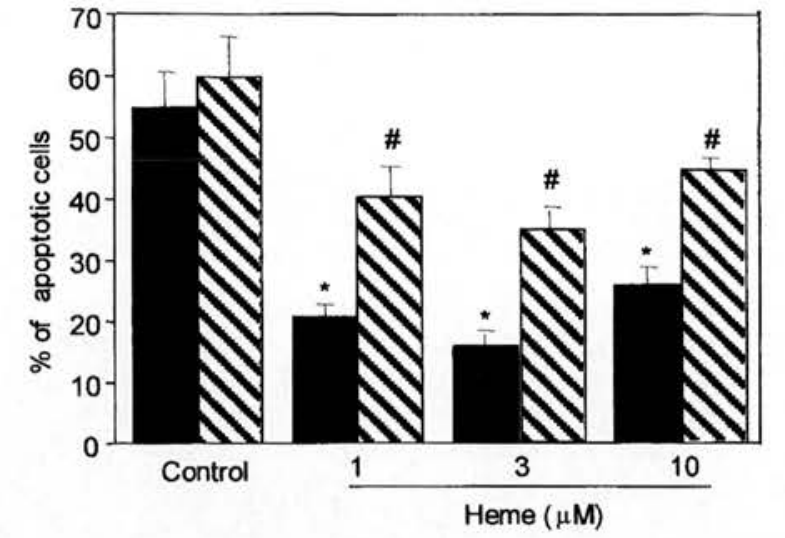

FIGURE 3. Inhibition of $\mathrm{HO}$ activity by SnPPIX reverses the antiapoptotic effect of heme on human neutrophils. Neutrophils $\left(5 \times 10^{6} / \mathrm{ml}\right)$ were incubated with indicated concentrations of heme in the absence (- or in the presence of SnPPIX $(50 \mu \mathrm{M} ; \mathbb{Q})$. After $20 \mathrm{~h}$, cells were cytocentrifuged, and the number of apoptotic cells was determined microscopically. Data shown are the results (mean $+\mathrm{SD})$ of three experiments, each performed in triplicate. $*$, Indicates a significant difference $(p<0.001)$ between heme-treated and control neutrophils. \#, Indicates that SnPPIX significantly inhibited the delay of apoptosis induced by heme $(p<0.05)$.

(28). In Fig. 4, we show that the incubation of cells with DPI and apocynin, two different NADPH oxidase inhibitors, reversed the inhibition of apoptosis in neutrophils treated with heme. These results suggest that alterations in the redox potential of these cells, mediated by NADPH oxidase activity, might be critical for the heme-induced antiapoptotic effect.

\section{Heme-induced delay of neutrophil apoptosis requires protein synthesis}

The balance between levels of pro- and antiapoptotic proteins has a pivotal role in the modulation of apoptosis. To determine the requirement of de novo protein synthesis on the heme-induced effect on neutrophil apoptosis, cells were pretreated with cyclo-

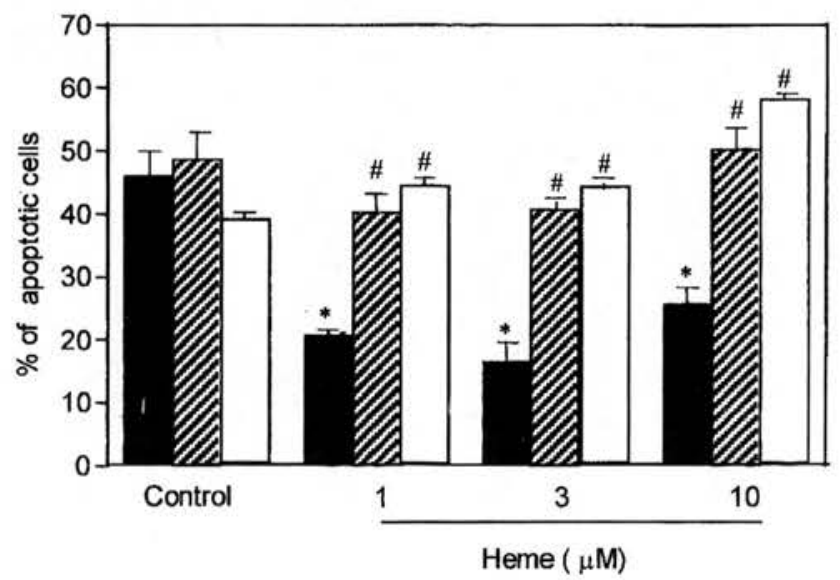

FIGURE 4. Heme inhibition of neutrophil apoptosis requires NADPH oxidase activity. Neutrophils $\left(5 \times 10^{6} / \mathrm{ml}\right)$ were cultured with or without heme $(1-10 \mu \mathrm{M})$ in the absence (E) or in the presence of DPI $(10 \mu \mathrm{M}$; or apocynin $(10 \mu \mathrm{M} ; \square)$. After $20 \mathrm{~h}$, cells were cytocentrifuged, and the number of apoptotic cells was determined microscopically. Data shown are the results (mean $+\mathrm{SD}$ ) of three experiments, each performed in triplicate. $*$, Indicates a significant difference $(p<0.01)$ between heme-treated and control neutrophils. \#, Indicates that DPI and apocynin significantly inhibited the delay of apoptosis induced by heme $(p<0.05)$.

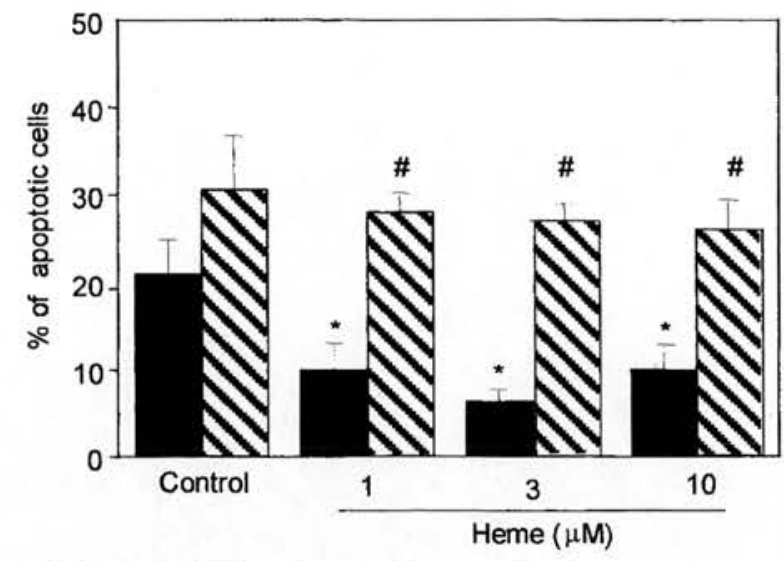

FIGURE 5. Inhibition of neutrophil apoptosis by heme requires protein synthesis. Neutrophils $\left(5 \times 10^{6} / \mathrm{ml}\right)$ were incubated in the absence or in the presence of heme $(1-10 \mu \mathrm{M})$, with $(\mathbb{\mathbb { N }})$ or without $(\square)$ cycloheximide (3.6 $\mu \mathrm{M})$. After $5 \mathrm{~h}$, cells were cytocentrifuged, and the number of apoptotic cells was determined microscopically. Data shown are the results (mean + SD) of three experiments, each performed in triplicate. *, Indicates a significant difference $(p<0.05)$ between heme-treated and control neutrophils. \#, Indicates that cycloheximide significantly inhibited the delay of apoptosis induced by heme $(p<0.05)$. The results are expressed as mean $\pm \mathrm{SD}$

heximide $(3.6 \mu \mathrm{M})$ and incubated with heme (1-10 $\mu \mathrm{M}$; Fig. 5). As we have previously observed that cycloheximide is cytotoxic at later time points (e.g., $20 \mathrm{~h}$ ) (data not shown), we evaluated apoptosis after 5 -h incubation in which there is no detectable cytotoxicity. Cycloheximide completely abolished the survival effect induced by heme on human neutrophil apoptosis, suggesting that the effects of heme rely on newly synthesized antiapoptotic proteins.

Heme induces Bad degradation and Bcl- $x_{L}$ expression on human neutrophils

The balance between the expression of anti- and proapoptotic proteins of Bcl-2 family has been shown to be a prominent feature on the control of apoptosis. As mature neutrophils exhibit a very short life span, the expression of proapoptotic Bcl-2 members is constitutively high, whereas antiapoptotic members' levels are very low or not detectable (23). Fig. $6 A$ shows that heme induced the degradation of Bad, a proapoptotic Bcl-2 member, which reached lower levels $30 \mathrm{~min}$ after heme treatment. In contrast, heme induced the synthesis of the antiapoptotic protein $\mathrm{Bcl}-\mathrm{x}_{\mathrm{L}}$ (Fig. $6 B$ ), reinforcing the regulation of prosurvival signaling by heme.

Heme delays neutrophil apoptosis via PI3K- and ERKdependent pathways

Because the Ras/Raf/MAPK and PI3K pathways have been reported to be actively involved in regulating the antiapoptotic effect of other proinflammatory agents, we investigated the participation of these signaling pathways in heme-mediated delay of neutrophil apoptosis in vitro. Pretreatment of cells with the PI3K inhibitor LY294002 $(3 \mu \mathrm{M})$ or the MEK1/2 inhibitor PD98059 $(10 \mu \mathrm{M})$ totally reversed the delay of apoptosis promoted by heme $(3 \mu \mathrm{M})$ and IL-8 (100 nM; Fig. 7), suggesting a pivotal role of these signaling pathways in the modulation of heme effect on neutrophils.

\section{Heme activates ERK-2 nuclear translocation}

The phosphorylation of ERK-1/2 regulatory sites can drive its translocation to the nucleus, where ERK exerts part of its biological activity. Confirming the involvement of the ERK pathway in 
A

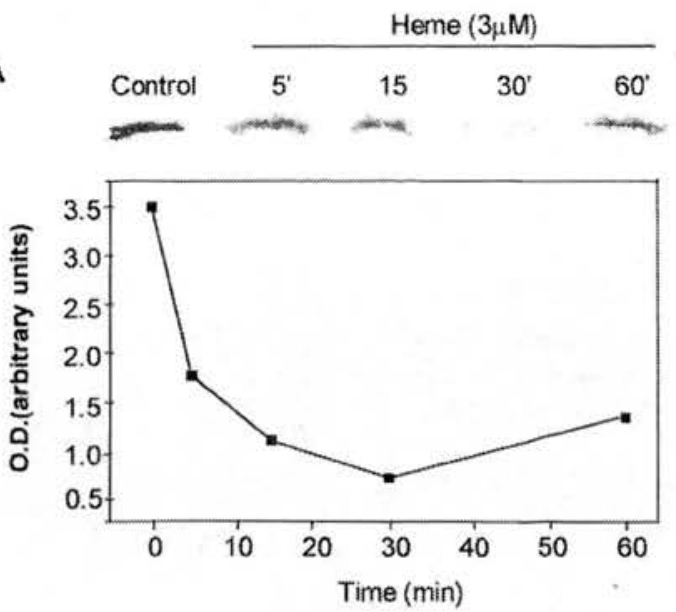

B
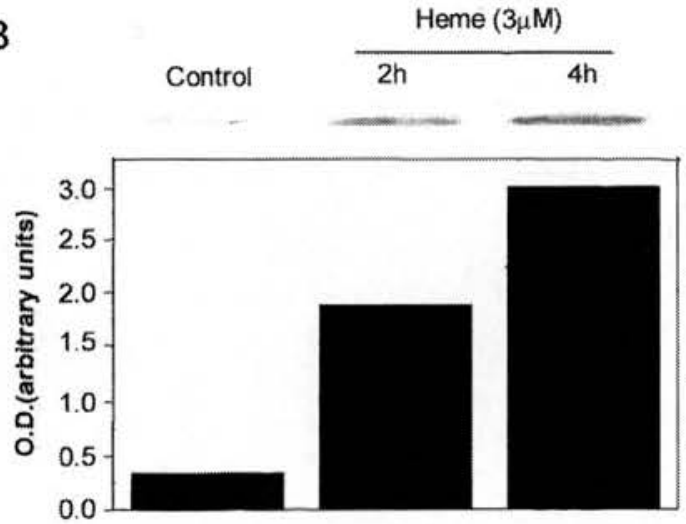

FIGURE 6. Heme induces Bad degradation and $\mathrm{Bcl}-\mathrm{x}_{\mathrm{L}}$ synthesis. Neutrophils $\left(5 \times 10^{6} / \mathrm{ml}\right)$ were cultured in the absence or the presence of heme $(3 \mu \mathrm{M})$. At the indicated time points, $\mathrm{Bad}(A)$ or $\mathrm{Bcl}-\mathrm{x}_{\mathrm{L}}(B)$ protein expression was accessed by Western blot analysis, as described in Materials and Methods. Quantification of band OD is expressed in arbitrary units.

heme antiapoptotic effects, Fig. 8 shows that heme ( $3 \mu \mathrm{M})$, as well as IL-8 (100 nM), promoled ERK-2 translocation to the nucleus in human neutrophils, after $1 \mathrm{~h}$ of incubation, as detected by immunofluorescence microscopic analysis.

\section{Heme induces Akt activation}

Akt phosphorylation on serine residues is a kcy event in PI3K/Akt signaling cascade. Once activated, Akt is able to promote cell survival phosphorylating proapoptotic Bcl-2 family members, especially Bad, inducing their degradation by the proteasome (20). Fig. 9 shows that heme $(3 \mu \mathrm{M})$ induced Akt phosphorylation on serine residues. This effect was highly significant after $5 \mathrm{~min}$ of incubation, peaking after $15 \mathrm{~min}$ and decreasing thereafter. The time course of Akt phosphorylation seems to be modulated hierarchically by PI3K and PKC. Although LY294002 $(3 \mu \mathrm{M} ; \mathbb{Q})$ inhibited only the early effect observed at $5 \mathrm{~min}$ after incubation with heme, $\mathrm{BIM}$, a PKC inhibitor (10 nM; $\square$ ), exclusively inhibited hemeinduced Akt phosphorylation on later time points (15-30 min). These results strongly suggest that heme triggers Akt signaling in human neutrophils in a PI3K-dependent manner, but requires PKC activity to sustain this effect.

\section{Heme induces a redox-sensitive $N F-\kappa B$ activation}

It is well established that NF- $\kappa \mathrm{B}$ modulates prosurvival signaling pathways, inhibiting apoptosis of several cell types (29-31).

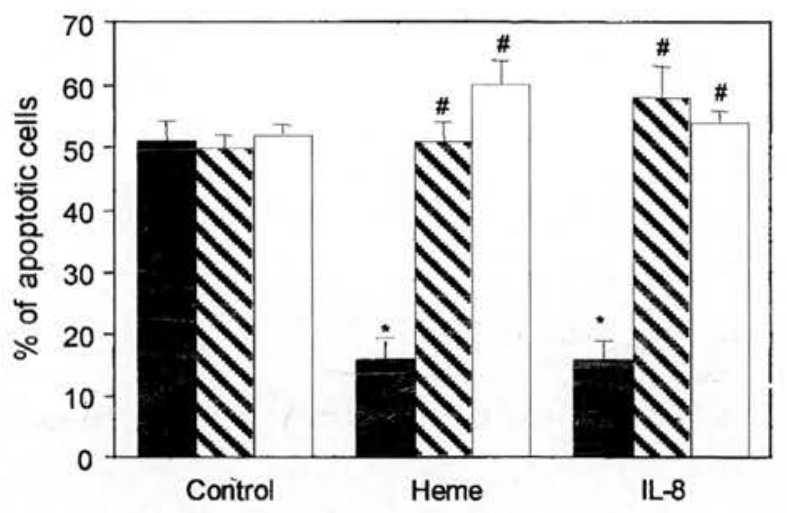

FIGURE 7. Inhibition of PI3K and ERK pathway abrogates the antiapoptotic effect of heme on neutrophils. Neutrophils $\left(5 \times 10^{6} / \mathrm{ml}\right)$ were cultured with heme ( $3 \mu \mathrm{M})$ or IL- $8(100 \mathrm{nM})$, in the absence (I) or in the presence of LY294002 (3 $\mu \mathrm{M} ; \mathbb{\$})$ or PD98059 $(10 \mu \mathrm{M} ; \square)$. After $20 \mathrm{~h}$, cells were cytocentrifuged, and the number of apoptotic cells was determined microscopically. Data shown are the results (mean + SD) of three experiments, each performed in triplicate. $*$, Indicates a significant difference $(p<0.01)$ between heme- or IL-8-treated and control neutrophils. \#, Indicates that PD98059 and LY294002 significantly inhibited the delay of apoptosis induced by IL- 8 and heme $(p<0.05)$. The results are expressed as mean $\pm \mathrm{SD}$.

$\mathrm{NF}-\kappa \mathrm{B}$ activation requires $\mathrm{I} \kappa \mathrm{B}$ phosphorylation and degradation in the cytoplasm and subsequent translocation of NF- $\kappa \mathrm{B}$ to the nucleus. This process can be regulated by redox-sensitive mechanisms. In Fig. 10A, we show that PDTC (100 nM), an antioxidant able to inhibit NF- $\kappa \mathrm{B}$ activation, abrogated the delay of neutrophil apoptosis induced by heme, suggesting that NF- $\kappa \mathrm{B}$ activation modulates heme-mediated cell survival.

To confirm that NF- $\kappa \mathrm{B}$ activation occurs in neutrophils stimulated with heme, we evaluated the degradation of cytoplasmic $I \kappa B$ and the translocation of NF- $\kappa \mathrm{B}$ p 65 subunit to the nucleus in these
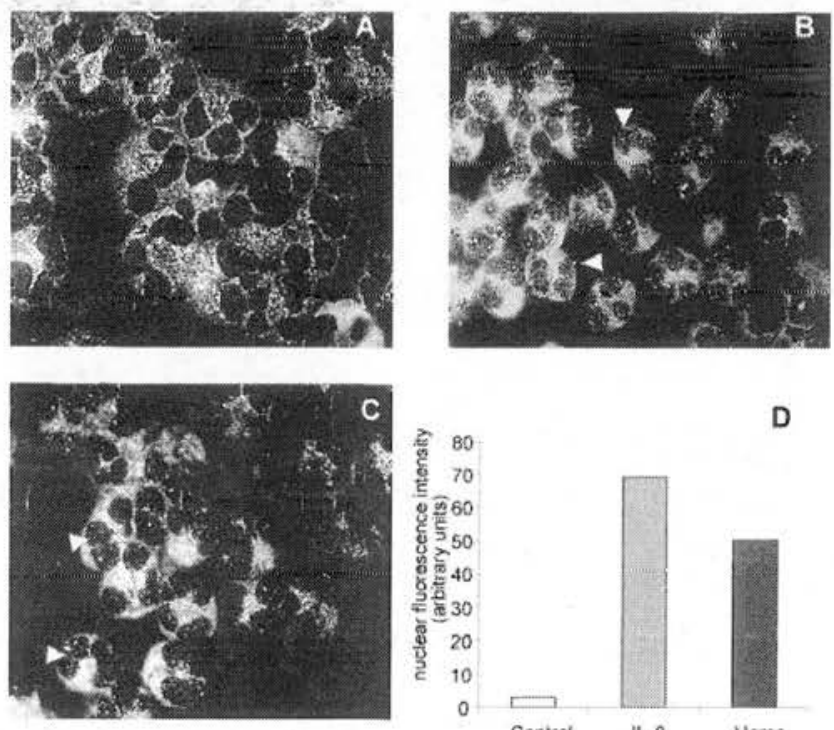

D

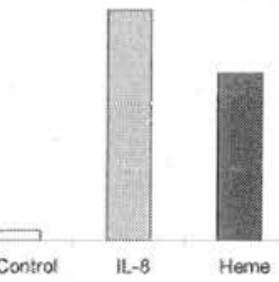

FIGURE 8. Heme induces ERK nuclear translocation. Neutrophils $(5 \times$ $\left.10^{6} / \mathrm{ml}\right)$ were incubated for $1 \mathrm{~h}$ with medium alone (control; $\left.A\right)$, IL-8 (100 $\mathrm{nM} ; B)$, or heme ( $3 \mu \mathrm{M} ; C$ ). Cytocentrifuge preparations were fixed, and subcellular localization of endogenous ERK-2 was detected by staining with anti-ERK-2 Ab. Quantification of nuclear fluorescence intensity (arrowheads) is shown in $D$. Experiments were performed three times with similar results. 


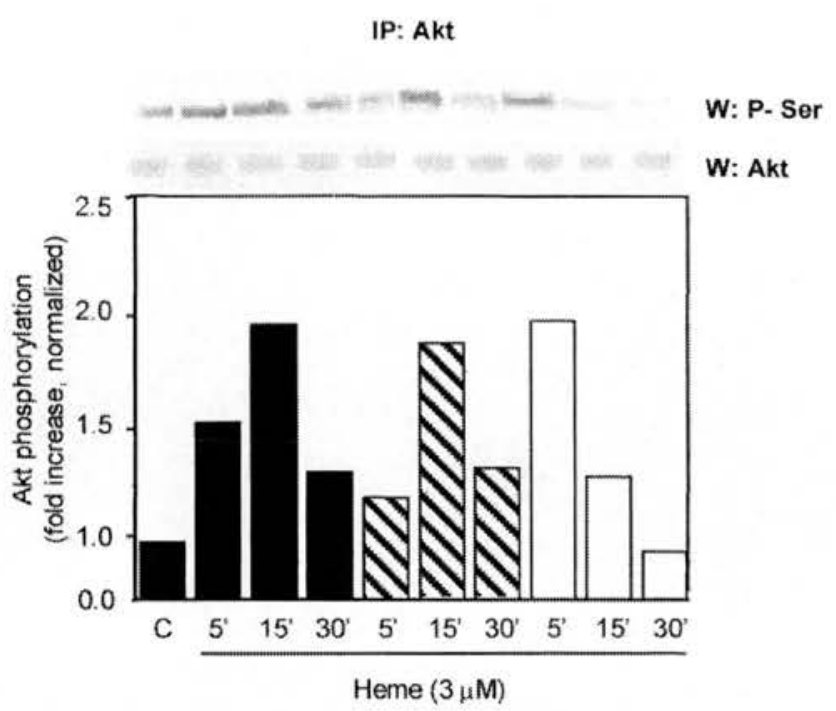

FIGURE 9. Heme induces Akt phosphorylation via Pl3K and PKC. Neutrophils $\left(5 \times 10^{6} / \mathrm{ml}\right)$ were incubated with heme $(3 \mu \mathrm{M})$ for the indicated time points in the absence or in the presence of LY294002 $(3 \mu \mathrm{M} ; \mathbb{\mathbb { N }})$ or BIM (10 nM; $\square)$. Cells were then harvested, cell extracts were immunoprecipitated with anti-Akt, and Western blots were performed for phosphoserine detection. Quantification of band OD is expressed in arbitrary units.

cells. After $1 \mathrm{~h}$ of incubation with $3 \mu \mathrm{M}$ heme, the levels of $\mathrm{I} \kappa \mathrm{B}$ significantly decreased in neutrophils treated with heme (Fig. $10 B$ ). This is followed by a significant increase of the NF- $\kappa \mathrm{B}$ p65 subunit in nuclear extracts of neutrophils, which was more prominent after $2 \mathrm{~h}$ of incubation with heme and comparable to that evoked by LPS $(1 \mu \mathrm{g} / \mathrm{ml}$; Fig. $10 \mathrm{C})$. The translocation of NF- $\kappa B$ evoked by heme and LPS was inhibited when cells were treated with the NADPH oxidase inhibitor DPI, providing an additional evidence for the role of ROS on NF- $\kappa \mathrm{B}$ activation.

\section{Discussion}

Chronic inflammation, an important feature in hemolytic diseases, involves an intense neutrophil activation accompanied by increased survival rate of these cells (12), events that may lead to an undesirable persistence of inflammation. In this study, we show, for the first time, that free heme increases neutrophil survival, providing evidence that this effect may contribute to the inflammatory process often associated with hemolytic episodes.

It was previously reported that erythrocytes are able to inhibit apoptosis of human neutrophils even when these cells are physically separated (32), suggesting the existence of a diffusible molecule able to retard neutrophil apoptosis. Our findings strongly suggest that the ability of erythrocytes to prevent neutrophil apoptosis may be directly linked to an increase in free heme concentrations released by lysed RBC.

A putative mechanism by which the organism can control the deleterious effect of large amounts of heme is through the HO activity. $\mathrm{HO}$ are ubiquitous enzymes able to catalyze the initial and rate-limiting step in the oxidative degradation of heme to bilirubin, producing equimolar amounts of biliverdin, free iron $(\mathrm{Fe})$, and carbon monoxide (CO) (33). The expression of the inducible HO isoform (HO-1) is positively modulated by several inflammatory mediators and oxidative stress and also by heme itself. HO-1 has also been highlighted as one of the major inducible enzymes during the inflammatory response, especially during the resolution phase of inflammation (34). Its expression is associated with the
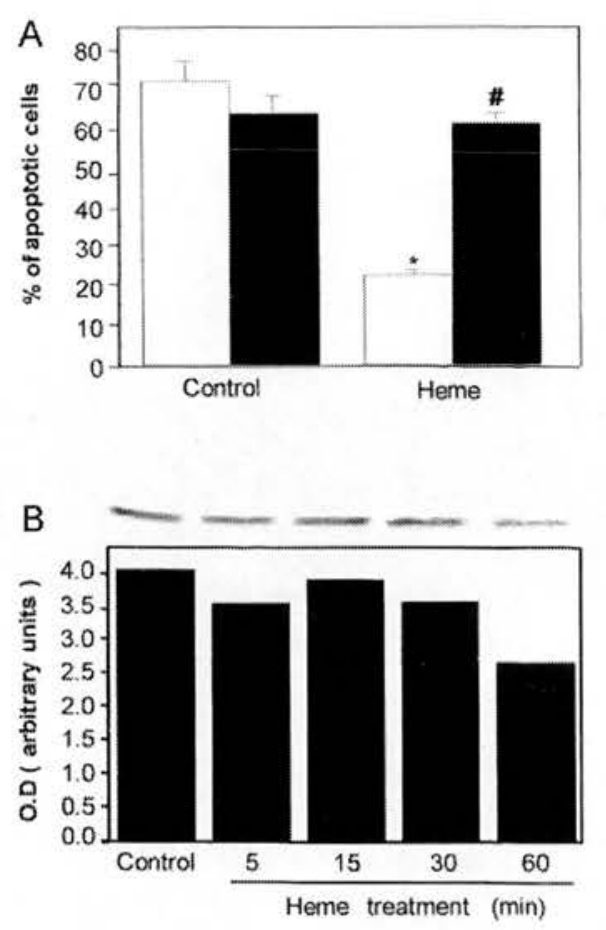

IKB- $\alpha$

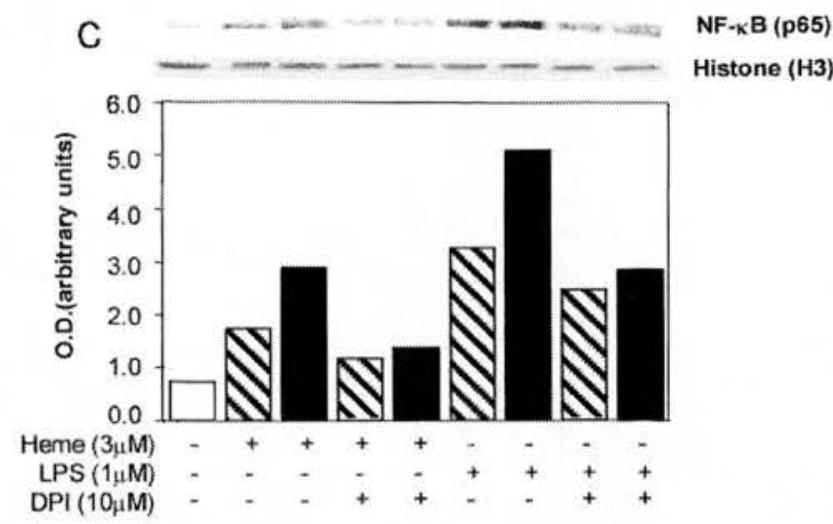

FIGURE 10. Heme-mediated delay of apoptosis requires NF- $\kappa \mathrm{B}$ activation. $A$, Neutrophils $\left(5 \times 10^{6} / \mathrm{ml}\right)$ were incubated with heme $(3 \mu \mathrm{M})$ in the absence ( $\square$ ) or in the presence (四) of PDTC ( $100 \mathrm{nM})$. After $20 \mathrm{~h}$, cells were cytocentrifuged, and the number of apoptotic cells was determined microscopically. Data shown are the results (mean $+\mathrm{SD}$ ) of three experiments, each performed in triplicate. $*$, Indicates a significant difference $(p<0.01)$ between heme-treated and control neutrophils. \#, Indicates that PDTC significantly inhibited the delay of apoptosis induced by heme $(p<$ $0.05)$. $B$, Western blot analysis of whole cell extracts prepared from nonstimulated or heme-stimulated $(3 \mu \mathrm{M})$ neutrophils for the indicated time points. $C$, Western blot analysis of nuclear extracts prepared from neutrophils stimulated or not with heme $(3 \mu \mathrm{M})$ or LPS $(1 \mu \mathrm{g} / \mathrm{ml})$ for 1 or $2 \mathrm{~h}$ in

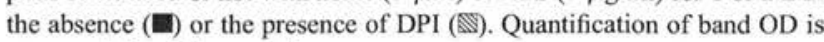
expressed in arbitrary units.

inhibition of apoptosis of several cell types such as fibroblasts, endothelial cells, pancreatic $\beta$ cells, and hepatocytes (35), and could be related to the action of one or more catalytic by-products generated by HO-1 (36). Recent reports have shown that the induction of HO-1 activity inhibited TNF- $\alpha$-induced apoptosis in endothelial cell, via $\mathrm{CO}$ generation (37), whereas E-selectin and VCAM expression promoted by this stimulus is negatively modulated by $\mathrm{HO}-1$ activity via bilirubin and $\mathrm{Fe}(38)$. In agreement 
with these findings, we showed that $\mathrm{HO}$ activity is, at least in part, required for heme effects upon human neutrophils because the delay of neutrophil apoptosis was partially reversed by a competitive inhibitor of this enzyme. However, neither bilverdin nor its metabolite, bilirubin, was able to influence on neutrophil life span, suggesting that probably $\mathrm{CO}$ and/or Fe might be the effector molecules generated by HO-1. The role of heme and HO-1 in modulating neutrophil spontaneous apoptosis points to a new and intriguing role of this system during the inflammatory response.

We have shown that heme is also able to trigger oxidative burst in neutrophils (3). This effect appears to be closely related to the antiapoptotic effect of heme because the inhibition of neutrophil apoptosis is reversed by DPI and apocynin, two NADPH oxidase inhibitors, providing evidence that alterations in redox potential mediate heme effects on human neutrophils. Data concerning the relationship between ROS generation, and the subsequent alterations in the redox potential, and the apoptotic process of different cells have been conflicting. Several reports have shown that antioxidants could both elicit or delay apoptosis $(39,40)$. As neutrophils can change their redox potential through their primary function of killing invading microorganisms, ROS generation as well as the presence of intracellular antioxidant molecules may interfere with the regulation of apoptosis in these cells. Although ROS and glutathione were shown to block caspase activity, both can inhibit, as well as trigger, apoptosis in human neutrophils, depending on the experimental conditions $(41,42)$. These data indicate that the regulation of neutrophil survival most likely involves a delicate balance in the redox status of the cell rather than the prevalence of intracellular oxidants or antioxidants. This may probably explain the reduced inhibition of neutrophil apoptosis when the cells were incubated with a higher concentration of heme ( $50 \mu \mathrm{M}$; Fig. 2), which was able to generate higher superoxide anion production (3).

Evidence has shown that the antiapoptotic effect of some agents require de novo protein synthesis, although it was recently reported that cAMP inhibits neutrophil apoptosis via a novel, reversible, and transcriptionally independent mechanism (43). Heme inhibition of neutrophil apoptosis was blocked by cycloheximide, suggesting that newly synthesized antiapoptotic proteins are likely to be involved in this effect. Neutrophils constitutively express proapoptotic proteins, including $\mathrm{Bax}, \mathrm{Bid}, \mathrm{Bak}$, and $\mathrm{Bad}$, while the expression of antiapoptotic $\mathrm{Bcl}$ members $\left(\mathrm{Bcl}-\mathrm{x}_{\mathrm{L}}, \mathrm{A}_{1}\right.$, and $\left.\mathrm{Mcl}-1\right)$ is very low or undetectable in resting cells (23). These antiapoptotic proteins are highly and transiently expressed when neutrophils are exposed to survival factors, such as IL- 8 and GM-CSF, which act through the activation of MAPK, PI3K, NF- $\kappa$ B, and other distinct signaling pathways $(20,21,23)$. We have shown that exposition of neutrophils to heme induced the degradation of Bad reducing after to $30 \mathrm{~min}$ to very low levels. In contrast, heme treatment increased the levels of Bcl- $\mathrm{x}_{\mathrm{L}}$, corroborating to heme antiapoptotic effects. In parallel, we have also shown that the inhibition of ERK and PI3K pathways, which have been directly correlated to the expression of those proteins, successfully reversed heme-induced delay of neutrophil apoptosis. Involvement of ERK activation in the heme-mediated antiapoptotic effects was supported by the observation that heme induces ERK-2 nuclear translocation, a prominent feature of ERK activation.

As an additional parameter to evaluate the involvement of PI3K on heme antiapoptotic effect, we analyzed the phosphorylation of Akt on serine residues. Akt is the main downstream target of PI3K, which induce Akt translocation to the plasma membrane and subsequent phosphorylation by the PI3K-dependent kinase. Heme-induced Akt phosphorylation was dependent on PI3K and PKC activation. However, while PI3K activation is an essential and early event for Akt phosphorylation induced by heme, PKC appears to be involved in the late and sustained activation of Akt, which is probably associated with the modulation of antiapoptotic effect. A great deal of effort has been directed toward defining which PKC isoforms are involved in the regulation of the apoptotic process. It is now believed that classical $\left(\alpha, \beta_{\mathrm{l}}, \beta_{\mathrm{II}}\right.$, and $\left.\gamma\right)$ and atypical $(\zeta, \mu$, and $\lambda$ ) isoforms are associated with cell survival, whereas novel PKC isoenzymes $(\delta, \epsilon, \eta$, and $\theta$ ) are considered proapoptotic (44, 45). Although the precise participation of different PKC member on heme antiapoptotic effect requires further investigation, our data suggest a prominent role for PI3K/Akt pathway on heme antiapoptotic effect, which is probably up-regulated by PKC.

Many inflammatory mediators regulate gene expression in target cells by influencing the activity of transcription factors, especially $\mathrm{NF}-\kappa \mathrm{B}$, to evoke their proinflammatory response (46). NF- $\kappa \mathrm{B}$ comprises a family of transcription factors that act to regulate genes involved in a variety of events such as inflammatory and immune responses, apoptosis, proliferation, and differentiation $(47-50)$. NF- $\kappa \mathrm{B}$ regulation seems to be highly cell specific and redox sensitive (51-53). Moreover, Asehnoune et al. (54) have recently shown that the antioxidants $N$-acetylcysteine and $\alpha$-tocopherol prevented LPS-induced nuclear translocation of NF- $\kappa \mathrm{B}$ on early events in TLR4 signaling. We have observed that heme activates NF- $\kappa \mathrm{B}$ in polymorphonuclear cells, inducing its translocation to the nucleus. The inhibition of the heme-induced antiapoptotic effect by PDTC suggests that heme induces redox-sensitive NF- $\kappa \mathrm{B}$ activation. The evidence that the inhibitor DPI impairs both heme- and LPS-induced NF- $\kappa \mathrm{B}$ nuclear translocation points to a prominent role of ROS generated by NADPH oxidase on this phenomenon. Furthermore, this is the first report showing the importance of NADPH oxidase-derived ROS in NF- $\kappa$ B activation on human neutrophils. The involvement of NF- $\kappa$ B on heme cytoprotective effects reinforces the close relationship between activation status and enhanced granulocyte life span already observed for other antiapoptotic stimuli (52).

Taken together, our data strongly suggest that the protective effect of heme on human neutrophil apoptosis is modulated by $\mathrm{PI} 3 \mathrm{~K}$ and MAPK pathways and involves NF- $\kappa \mathrm{B}$ activation in an NADPH oxidase-dependent manner. The activation of those pathways might lead to the transcription of HO-1 (55), whose activity could modulate the heme-induced antiapoptotic effect, among other antiapoptotic proteins, as previously discussed.

In summary, we are presenting novel data showing that free heme is able to delay human neutrophil apoptosis in vitro at concentrations found during hemolytic events in vivo. This effect is dependent on $\mathrm{HO}$ activity and ROS generation, requires de novo protein synthesis, and is modulated through PI3K, ERK, and $\mathrm{NF}-\kappa \mathrm{B}$ pathways. Furthermore, the data suggest that heme may contribute to the development of chronic inflammation associated with hemolytic episodes by delaying apoptosis and promoting functional longevity of neutrophils. The understanding of this process can lead to the establishment of new strategies to ameliorate tissue damage associated with severe hemolysis.

\section{Acknowledgments}

We thank Dr. I. M. Fierro for the critical reading of this manuscript. We also thank Dr. F. Q. Cunha for supplying human rIL-8, and Dr. P. Bozza for anti-IL-8 mAb, as well as Carla Gregório and Pedro Barcellos de Souza for excellent technical assistance.

\section{References}

1. Muller-Eberhard, U., and M. Fraig. 1993. Bioactivity of heme and its containment. Am. J. Hematol. 42:59.

2. Reiter, C. D., X. Wang, J. E. Tanus-Santos, N. Hogg, R. O. Cannon III, A. N. Schechter, and M. T. Gladwin. 2002. Cell-free hemoglobin limits nitric oxide bioavailability in sickle-cell disease. Nat. Med. $8: 1383$. 
3. Graça-Souza, A. V., M. A. Arruda, M. S. de Freitas, C. Barja-Fidalgo, and P. L. Oliveira. 2002. Neutrophil activation by heme: implications for inflammatory processes. Blood 99:4160.

4. Haeger, M., M. Unander, B. Andersson, A. Tarkowski, J. P. Arnestad, and A. Bengtsson. 1996. Increased release of tumor necrosis factor- $\alpha$ and interleukin- 6 in women with the syndrome of hemolysis, elevated liver enzymes, and low platelet count. Acta Obstet. Gynecol. Scand. 75:695.

5. Davenport, R. D., R. M. Strieter, T. J. Standiford, and S. L. Kunkel. 1990. Interleukin-8 production in red blood cell incompatibility. Blood 76:2439.

6. Davenport, R. D., and S. L. Kunkel. 1994. Cytokine roles in hemolytic and nonhemolytic transfusion reactions. Transfis. Med. Rev. 8:157.

7. Mollapour, E., J. B. Porter, R. Kaczmarski, D. C. Linch, and P. J. Roberts. 1998. Raised neutrophil phospholipase $A_{2}$ activity and defective priming of NADPH oxidase and phospholipase $\mathrm{A}_{2}$ in sickle cell disease. Blood 91:3423.

8. Gonçalves, M. S., I. L. Queiroz, S. A. Cardoso, A. Zanetti, A. C. Strapazoni, E. Adorno, A. Albuquerque, A. Sant'Ana, M. G. dos Reis, A. Barral, and M. Barral Netto. 2001. Interleukin 8 as a vaso-occlusive marker in Brazilian patients with sickle cell disease. Braz. J. Med. Biol. Res. 34:1309.

9. Wun, T. 2001. The role of inflammation and leukocytes in the pathogenesis of sickle cell disease; haemoglobinopathy. Hematology 5:403.

10. Kaul, D. K., and R. P. Hebbel. 2000. Hypoxia/reoxygenation causes inflammatory response in transgenic sickle mice but not in normal mice. J. Clin. Invest 106:411.

11. Savill, J. 1997. Apoptosis in resolution of inflammation. J. Leukocyte Biol. $61: 375$.

12. Ward, C., I. Dransfield, E. R. Chilvers, C. Haslett, and A. G. Rossi. 1999. Pharmacological manipulation of granulocyte apoptosis: potential therapeutic targets. Trends Pharmacol. Sci. 20:503.

13. F.dwards, S. W. 2000. Biochemistry and Physiology of the Neutrophil. Cambridge University Press, New York.

14. Savill, J., I. Dransfield, C. Gregory, and C. Haslett. 2002. A blast from the past: clearance of apoptotic cells regulates immune responses. Nat. Rev. Immuno. 2:965.

15. Hengartner, M. O. 2000. The biochemistry of apoptosis. Nature 407:770.

16. Kettritz, R., M. L. Gaido, H. Haller, F. C. Luft, C. J. Jennette, and R. J. Falk. 1998. Interleukin- 8 delays spontaneous and tumor necrosis factor- $\alpha$-mediated apoptosis of human neutrophils. Kidney Int. 53:84.

17. Lee, A., M. K. Whyte, and C. Haslett. 1993. Inhibition of apoptosis and prolongation of neutrophil functional longevity by inflammatory mediators. J. Leukocyte Biol. 54:283.

18. Colotta, F., F. Re, N. Polentarutti, S. Sozzani, and A. Mantovani. 1992. Modulation of granulocyte survival and programmed cell death by cytokines and bacterial products. Blood 80:2012.

19. Hebert, M. J., T. Takano, H. Holthofer, and H. R. Brady. 1996. Sequential morphologic events during apoptosis of human neutrophils: modulation by lipoxygenase-derived eicosanoids. J. Immunol. 157:3105.

20. Klein, J. B., M. J. Rane, J. A. Scherzer, P. Y. Coxon, R. Kettritz, J. M. Mathiesen, A. Buridi, and K. R. McLeish. 2000. Granulocyte-macrophage colony-stimulating factor delays neutrophil constitutive apoptosis through phosphoinositide 3-kinase and extracellular signal-regulated kinase pathways. J. Immunol. 16:4286.

21. Klein, J. B., A. Buridi, P. Y. Coxon, M. J. Rane, T. Manning, R. Kettritz, and K. R. McLeish. 2001. Role of extracellular signal-regulated kinase and phosphatidylinositol-3 kinase in chemoattractant and LPS delay of constitutive neutrophil apoptosis. Cell. Signal. 13:335.

22. Ward, C., E. R. Chilvers, M. F. Lawson, J. G. Pryde, S. Fujihara, S. N. Farrow, C. Haslett, and A. G. Rossi. 1999. NF- $-\mathrm{B}$ activation is a critical regulator of human granulocyte apoptosis in vitro. J. Biol. Chem. 274:4309.

23. Akgul, C., D. A. Moulding, and S. W. Edwards. 2001. Molecular control of neutrophil apoptosis. FEBS Lett. 487:318.

24. Boyum, A. 1976. Isolation of lymphocytes, granulocytes and macrophages Scand. J. Immunol. (Suppl. 5):9.

25. Fierro, I. M., C. Barja-Fidalgo, R. M. Canedo, F. Q. Cunha, and S. H. Ferreira, 1995. An increase in nitric oxide produced by rat peritoneal neutrophils is not involved in cell apoptosis. Mediat. Inflamm. 4:222.

26. Coelho, A. L., M. S. De Freitas, A. Mariano-Oliveira, A. L. Oliveira-Carvalho, R. B. Zingali, and C. Barja-Fidalgo. 2001. Interaction of disintegrins with human neutrophils induces cytoskeleton reorganization, focal adhesion kinase activation, and extracellular-regulated kinase-2 nuclear translocation, interfering with the chemotactic function. FASEB J. 15:1643.

27. Bradford, M. 1976. A rapid and sensitive method for quantification of microgram quantities of protein utilizing the principle of protein-dye binding. Anal. Biochem. $72: 248$.

28. Fadeel, B., A. Ahlin, J. I. Henter, S. Orrenius, and M. B. Hampton. 1998. Involvement of caspases in neutrophil apoptosis: regulation by reactive oxygen species. Blood 92:4808.

29. Ferran, C., D. M. Stroka, A. Z. Badrichani, J. T. Cooper, C. J. Wrighton, M. Soares, S. T. Grey, and F. H. Bach. 1998. A20 inhibits NF- $\kappa$ B activation in endothelial cells without sensitizing to tumor necrosis factor-mediated apoptosis. Blood 91:2249.

30. Caamano, J., and C. A. Hunter. 2002. NF- $\kappa$ B family of transcription factors: central regulators of innate and adaptive immune functions. Clin. Microbiol. Rev. 15:414.

31. Zheng, X., A. Karsan, V. Duronio, F. Chu, D. C. Walker, T. R. Bai, and R. R. Schellenberg. 2002. Interleukin-3, but not granulocyte-macrophage colonystimulating factor and interleukin-5, inhibits apoptosis of human basophils through phosphatidylinositol 3-kinase: requirement of NF-kB-dependent and -independent pathways. Immunology 107:306.

32. Aoshiba, K., Y. Nakajima, S. Yasui, J. Tamaoki, and A. Nagai. 1999. Red blood cells inhibit apoptosis of human neutrophils. Blood 93:4006.

33. Choi, A. M., and J. Alam. 1996. Heme oxygenase-1: function, regulation, and implication of a novel stress-inducible protein in oxidant-induced lung injury. Am. J. Respir. Cell Mol. Biol. 15:9.

34. Willoughby, D. A., A. R. Moore, P. R. Colville-Nash, and D. Gilroy. 2000. Resolution of inflammation. Int. J. Immunopharmacol. 22:1131.

35. Wagener, F. A. D. T. G., H. Volk, D. Willis, N. G. Abraham, M. P. Soares, G. J. Adema, and C. G. Figdor. 2003. Different faces of the heme-heme oxygenase system in inflammation. Pharmacol. Rev. 55:551.

36. Otterbein, L. E., L. L. Mantell, and A. M. Choi 1999. Carbon monoxide provides protection against hyperoxic lung injury. Am. J. Physiol. 276:L688.

37. Brouard, S., P. O. Berberat, E. Tobiasch, M. P. Seldon, F. H. Bach, and M. P. Soares. 2002. Heme oxygenase-1-derived carbon monoxide requires the activation of transcription factor NF- $\kappa \mathrm{B}$ to protect endothelial cells from tumor necrosis factor- $\alpha$-mediated apoptosis. J. Biol. Chem. 277:17950.

38. Soares M. P., M. P. Seldon, I. P. Gregoire, T. Vassilevskaia, P. O. Berberat, J. Yu, T. Y. Tsui, and F. H. Bach. 2004. Heme oxygenase-1 modulates the expression of adhesion molecules associated with endothelial cell activation. $J$. Immunol. 172:3553.

39. Hockenbery, D. M., Z. N. Oltvai, X. M. Yin, C. L. Milliman, and S. J. Korsmeyer. 1993. Bcl-2 functions in an antioxidant pathway to prevent apoptosis. Cell 75:241.

40. Hampton, M. B., and S. Orrenius. 1997. Dual regulation of caspase activity by hydrogen peroxide: implications for apoptosis. FEBS Lett. 414:552.

41. Hsu, M. J., S. S. Lee, and W. W. Lin. 2002. Polysaccharide purified from $\mathrm{Ga}$ noderma lucidum inhibits spontaneous and Fas-mediated apoptosis in human neutrophils through activation of the phosphatidylinositol 3 kinase/Akt signaling pathway. J. Leukocyte Biol. 72:207.

42. Watson, R. W. 2002. Redox regulation of neutrophil apoptosis. Antioxid. Redox Signal. 4:97.

43. Martin, M. C., I. Dransfield, C. Haslett, and A. G. Rossi. 2001. Cyclic AMP regulation of neutrophil apoptosis occurs via a novel protein kinase A-independent signaling pathway. J. Biol. Chem. 276:45041.

44. Pongracz, J., P. Webb, K. Wang, E. Deacon, O. J. Lunn, and J. M. Lord. 1999. Spontaneous neutrophil apoptosis involves caspase 3-mediated activation of protein kinase C- $\delta$. J. Biol. Chem. 274:37329.

45. Cross, T. G., D. Schell-Toellner, N. V. Henriquez, E. Deacon, M. Salmon, and J. M. Lord. 2000. Serine/threonine protein kinases and apoptosis. Exp. Cell Res. 256:34.

46. Lentsch, A. B., and P. A. Ward. 1999. Activation and regulation of NF- $\kappa$ B during acute inflammation. Clin. Chem. Lab. Med. 37:205.

47. Baldwin, A. S., Jr. 2001. Series introduction: the transcription factor NF- $\kappa$ B and human disease. J. Clin. Invest. 107:3.

48. Yamamoto, Y., and R. B. Gaynor. 2001. Therapeutic potential of inhibition of the $\mathrm{NF}-\kappa \mathrm{B}$ pathway in the treatment of inflammation and cancer. $J$. Clin. Invest. 107:135.

49. Barkett, M., and T. D. Gilmore. 1999. Control of apoptosis by Rel/NF- $\kappa$ B transcription factors. Oncogene 18:6910.

50. Aggarwal, B. B. 2000. Apoptosis and NF- $\kappa$ B: a tale of association and dissociation. Biochem. Pharmacol. 60:1033.

51. Vancurova, L, V. Miskolci, and D. Davidson. 2001. NF- $\mathrm{kB}$ activation in tumor necrosis factor $\alpha$-stimulated neutrophils is mediated by protein kinase $\mathrm{C} \delta$ : correlation to nuclear I $\mathrm{KB} \alpha$. J. Biol. Chem. 276:19746.

52. Castro-Alcaraz, S., V. Miskolci, B. Kalasapudi, D. Davidson, and I. Vancurova. 2002. NF- $\kappa \mathrm{B}$ regulation in human neutrophils by nuclear $\mathrm{I} \kappa \mathrm{B} \alpha$; correlation to apoptosis. J. Immunol. 1693947

53. Haddad, J. J. 2002. Science review: redox and oxygen-sensitive transcription factors in the regulation of oxidant-mediated lung injury: role for nuclear factor$\kappa$ B. Crit. Care 6:481.

54. Asehnoune, K., D. Strassheim, S. Mitra, J. Y. Kim, and E. Abraham. 2004. Involvement of reactive oxygen species in Toll-like receptor 4-dependent activation of NF- $\kappa$ B. J. Immunol. 172:2522.

55. Zhang, X., E. L. Bedard, R. Potter, R. Zhong, J. Alam, A. M. Choi, and P. J. Lee. 2002. Mitogen-activated protein kinases regulate $\mathrm{HO}^{-1}$ gene transcription after ischemia-reperfusion lung injury. Am. J. Physiol. 283:L815. 


\title{
GEA 3162 decomposes to co-generate nitric oxide and superoxide and induces apoptosis in human neutrophils via a peroxynitrite-dependent mechanism
}

\author{
${ }^{1}$ Emma L. Taylor, ${ }^{*, 1}$ Adriano G. Rossi, ${ }^{2}$ Catherine A. Shaw, ${ }^{2}$ Francesco P. Dal Rio, \\ ${ }^{1}$ Christopher Haslett \& ${ }^{2}$ Ian L. Megson
}

${ }^{1}$ Centre for Inflammation Research, Rayne Laboratory, Medical School, University of Edinburgh, Teviot Place, Edinburgh EH8 9AG and ${ }^{2}$ Centre for Cardiovascular Science, Hugh Robson Building, University of Edinburgh, George Square, Edinburgh EH8 9XD

1 GEA 3162 (1,2,3,4,-oxatriazolium, 5-amino-3-(3,4-dichlorophenyl)-chloride), has powerful effects on neutrophil function and apoptosis, but the underlying mechanisms are unclear, particularly with respect to the possible roles of nitric oxide $(\mathrm{NO})$ and/or peroxynitrite $\left(\mathrm{ONOO}^{-}\right)$.

2 Our hypothesis was that GEA 3162 is a generator of $\mathrm{ONOO}^{-}$and that its biological effects on neutrophil apoptosis differ from those of a conventional NO donor. The effects of GEA 3162 were compared to those of the established $\mathrm{ONOO}^{-}$donor, 3-morpholinosydnonimine (SIN-1), and the NO donor, diethylamine diazeniumdiolate $(\mathrm{DEA} / \mathrm{NO})$ in neutrophils from healthy volunteers. Electrochemical detection and electron paramagnetic resonance were used to define the NO-related species generated from these agents.

3 GEA 3162 and SIN-1 influence neutrophil apoptosis differently from DEA/NO. All three compounds induced morphological neutrophil apoptosis. However, both GEA 3162 and SIN-1 paradoxically inhibited internucleosomal DNA fragmentation, whereas DEA/NO induced fragmentation compared to control.

4 In contrast to DEA/NO, generation of free NO was not detectable in solutions of GEA 3162 or SIN-1 $(100 \mu \mathrm{M})$. However, $\mathrm{Cu} / \mathrm{Zn}$ superoxide dismutase (SOD; $50-750 \mathrm{U} \mathrm{ml}^{-1}$ ) unmasked NO generated from these compounds in a concentration-dependent manner. GEA 3162 and SIN-1 oxidised the $\mathrm{O}_{2}^{-}$- and $\mathrm{ONOO}^{-}$-sensitive dye, dihydrorhodamine 123 (DHR $123 ; 1 \mu \mathrm{M}$ ), suggesting that $\mathrm{ONOO}^{-}$released from these compounds is responsible for oxidation of DHR 123.

5 We conclude that GEA 3162 is an $\mathrm{ONOO}^{-}$donor with pro-apoptotic properties that more closely resemble SIN-1 than the NO donor, DEA/NO. Moreover, unlike NO, ONOO- induces apoptosis in neutrophils via a mechanism that does not require DNA fragmentation.

British Journal of Pharmacology (2004) 143, 179-185. doi:10.1038/sj.bjp.0705909

Keywords: Nitric oxide; peroxynitrite; GEA 3162; neutrophil; apoptosis

Abbreviations: DEA/NO, diethylamine diazeniumdiolate; DHR 123, dihydrorhodamine 123; EPR, electron paramagnetic resonance; GEA 3162, 1,2,3,4-oxatriazolium, 5-amino-3-(3,4-dichlorophenyl)-chloride; $\mathrm{H}_{2} \mathrm{O}_{2}$, hydrogen peroxide; $\mathrm{HOCl}$, hypochlorous acid; IMDM, Iscove's modified Dulbecco's medium; NO, nitric oxide; NOS, nitric oxide synthase; $\mathrm{O}_{2}^{-}$, superoxide anion; $\mathrm{ONOO}^{-}$, peroxynitrite; PBS, phosphate-buffered saline; PI, propidium iodide; PMA, phorbol 12-myristate 13-acetate; SIN-1, 3-morpholinosydnonimine; SOD, superoxide dismutase

\section{Introduction}

Nitric oxide (NO) is a free radical that was originally identified as an endogenous endothelium-derived vasodilator (Furchgott \& Zawadzki, 1980; Palmer et al., 1987), but is now recognised to have a role in a large number of other physiological and pathophysiological processes, including haemostasis, neurotransmission and inflammation (Quinn et al., 1995). The role of NO in inflammation is particularly complex, with macrophages in particular capable of generating high levels of NO through promotion of transcription factor activity and consequent expression of the unregulated, inducible isoform of NO synthase (iNOS) in response to inflammatory stimuli

*Author for correspondence; E-mail: a.g.rossi@ed.ac.uk

Advance online publication: 2 August 2004
(Hecker et al., 1996). The primary role of iNOS-derived NO is accepted to be that of a powerful antipathogenic agent, but it is also clear that NO has a complex impact on apoptosis (Dimmeler \& Zeiher, 1997; Kim et al., 1999; Taylor et al., 2003), a physiological form of cell death which eliminates effete or unhealthy cells. The issue is further complicated by the rapid reaction of $\mathrm{NO}$ with superoxide anion $\left(\mathrm{O}_{2}^{-}\right)$to form the powerful oxidising agent, peroxynitrite $\left(\mathrm{ONOO}^{-} ; \mathrm{Kelm}\right.$ et al., 1997).

Despite $\mathrm{NO}$ and $\mathrm{ONOO}^{-}$sharing several biological properties (Ronson et al., 1999; Low et al., 2002), including an ability to induce apoptosis (Taylor et al., 2003), key differences have been noted in their biological effects and mechanisms of action. For example, although both $\mathrm{NO}$ and $\mathrm{ONOO}^{-}$are 
capable of blocking mitochondrial respiration, they do so through inhibition of different complexes in the respiratory chain and their effects are differentially susceptible to reversal by thiols and carbohydrates (Lizasoain et al., 1996). These differences highlight the importance of the accurate identification of the nature of the species generated by the so-called 'NO donors'; $\mathrm{ONOO}^{-}$generated as a result of concomitant release of $\mathrm{NO}$ and $\mathrm{O}_{2}^{-}$may exert effects that are similar but mechanistically distinct from pure $\mathrm{NO}$ in biological systems. To date, however, little is known about potential differential effects of $\mathrm{NO}$ and $\mathrm{ONOO}^{-}$on inflammatory cell apoptosis.

GEA 3162 is an oxatriazole-5-imine derivative with vasodilator (Nurminen \& Vapaatalo, 1996) and pro-apoptotic (Ward et al., 2000; Taylor et al., 2001) properties. However, its identity as a 'pure' NO donor is controversial (Kankaanranta et al., 1996; Holm et al., 1998; Schmidt et al., 2001). In this study, we set out to establish the identity of the NO-related species generated from GEA 3162, and to compare its biological properties to an established NO donor (diethylamine diazeniumdiolate; DEA/NO) and an ONOO donor (SIN-1) with respect to neutrophil apoptosis. All the three compounds have previously been shown to accelerate the rate of programmed cell death in neutrophils in vitro (Blaylock et al., 1998; Ward et al., 2000; Taylor et al., 2001); however, the precise mechanisms have not been elucidated.

\section{Experimental procedures}

\section{Isolation of neutrophils}

Neutrophils were isolated from the blood of healthy volunteers as described previously (Ward et al., 1999a). Briefly, whole, citrated blood was centrifuged $(200 \times g, 20 \mathrm{~min})$ and plateletrich plasma aspirated. Leukocytes were separated from erythrocytes by dextran sedimentation, then further divided into mononuclear cell and granulocyte populations by centrifugation through a discontinuous Percoll gradient $(720 \times \mathrm{g}, 20 \mathrm{~min})$. Granulocytes were harvested from the $79: 68 \%$ interface of the gradient, and only neutrophil preparations of $\geqslant 95 \%$ purity were used. Neutrophil preparations were tested for cellular homogeneity by preparation of a cytospin slide ( $100 \mu \mathrm{l}$ of cell suspension, 300 r.p.m., $3 \mathrm{~min})$, which was then stained and examined by oil-immersion light microscopy, with at least 500 cells counted. The percentage of contaminating leukocytes (eosinophils, monocytes and lymphocytes) was then calculated and the cell preparation was discarded if levels of contamination reached or exceeded $5 \%$ of the total cell population.

\section{Apoptosis studies}

Neutrophils $\left(4.5 \times 10^{6}\right.$ cells ml $\left.{ }^{-1}\right)$ were suspended in Iscove's modified Dulbecco's medium (IMDM; Life Technologies, Paisley, U.K.) containing penicillin $\left(100 \mathrm{U} \mathrm{ml}^{-1}\right)$ and streptomycin $\left(100 \mu \mathrm{g} \mathrm{ml}^{-1}\right)$, and supplemented with $10 \%\left(\mathrm{vv}^{-1}\right)$ autologous serum. They were cultured in flat-bottomed $96-$ well Falcon polypropylene plates $\left(37^{\circ} \mathrm{C}, 5 \% \mathrm{CO}_{2}\right)$ for $1-20 \mathrm{~h}$ in the presence of either phosphate-buffered saline (PBS) at $\mathrm{pH}$ 7.4 (controls) or NO donors $(100 \mu \mathrm{M}-3 \mathrm{mM})$. The concentra- tions of NO donors used in these studies were selected on the basis of previously published data (Taylor et al., 2001), which have been shown to modulate neutrophil apoptosis.

Following incubation, $100 \mu \mathrm{l}$ of recovered cells were cytocentrifuged in duplicate, fixed in methanol and stained using Diff-Quik ${ }^{\mathrm{TM}}$ physiological stain, then observed by light microscopy $(\times 100$ objective $)$ to determine the proportion of darkly stained cells with condensed nuclei. At least 500 cells per slide were counted, with the observer blinded to the experimental conditions.

A further $100 \mu \mathrm{l}$ of recovered cells were centrifuged $(200 \times g$, $3 \mathrm{~min})$, then fixed and permeabilised in $70 \%$ ethanol $\left(4^{\circ} \mathrm{C}\right.$, $10 \mathrm{~min})$. The cells were washed $(\times 3)$ in PBS without $\mathrm{Ca}^{2+}$ $\mathrm{Mg}^{2+}$ before addition of $60 \mu \mathrm{l} \mathrm{RNase} \mathrm{A}\left(0.5 \mathrm{mg} \mathrm{ml}^{-1}\right)$ and $60 \mu \mathrm{l}$ propidium iodide (PI; $0.1 \mathrm{mg} \mathrm{ml}^{-1}$ ), then assessed by flow cytometry using an EPICS XL2 (Coulter Electronics, Luton, U.K.), to measure DNA fragmentation.

\section{Dihydrorhodamine 123 studies}

Dihydrorhodamine 123 (DHR 123) is a fluorescent dye which is activated by various reactive oxygen species, including $\mathrm{ONOO}^{-}, \mathrm{O}_{2}^{-}$, hydrogen peroxide $\left(\mathrm{H}_{2} \mathrm{O}_{2}\right)$ and $\mathrm{HOCl}$, but not by NO (Crow, 1997). This compound can therefore be used to discriminate between agents that release NO only and those that generate $\mathrm{NO}$ and $\mathrm{O}_{2}^{-}$simultaneously.

PBS $(100 \mu \mathrm{l})$, the neutrophil-activating agent, phorbol 12myristate 13-acetate (PMA; final concentration $10 \mathrm{nM}$ ), SIN-1 $(1 \mathrm{mM})$, GEA $3162(100 \mu \mathrm{M})$ or DEA/NO $(1 \mathrm{mM})$ was added to $900 \mu \mathrm{l}$ PBS in $2 \mathrm{ml}$ Eppendorf tubes. DHR 123 was added to each tube to a final concentration of $1 \mu \mathrm{M}$. Tubes were incubated for $60 \mathrm{~min}\left(37^{\circ} \mathrm{C}, 5 \% \mathrm{CO}_{2}\right)$. A volume of $450 \mu \mathrm{l}$ was then transferred to a $0.5 \mathrm{ml}$ cuvette, excited at $480 \mathrm{~nm}$ and the fluorescence emitted at $500 \mathrm{~nm}$ was read using a spectrofluorimeter (Perkin Elmer, U.K.).

\section{Electrochemical detection of $\mathrm{NO}$}

Free NO from GEA 3162 or SIN-1 (both $100 \mu \mathrm{M}$ ) and DEA/ NO $(5 \mu \mathrm{M})$ was measured in Iscove's MDM cell culture medium using an NO electrode (Iso-NO II, World Precision Instruments) calibrated daily with DEA/NO $(0.1-1.6 \mu \mathrm{M})$ in pH 4 buffer. Superoxide dismutase (SOD; $50-750 \mathrm{U} \mathrm{ml}^{-1}$ ) was added cumulatively in an effort to unmask NO generated from these agents (Lizasoain et al., 1996), and the concentration of NO was measured at plateau approx. $5 \mathrm{~min}$ following SOD addition.

\section{Electron paramagnetic detection of $\mathrm{O}_{2}^{-}$and $\mathrm{ONOO}^{-}$}

Spin-trapping experiments were performed in Iscove's MDM cell culture medium containing $100 \mu \mathrm{M}$ SIN-1, GEA 3162 or DEA/NO in the presence or absence of SOD $\left(500 \mathrm{U} \mathrm{ml}^{-1}\right)$, and the spin trap Tempone-H (1 mM) (Dikalov et al., 1997) prepared in water containing EDTA $(10 \mathrm{mM})$. Reaction mixtures were incubated at $37^{\circ} \mathrm{C}$ throughout the experiments and the intensity of the electron paramagnetic resonance (EPR) signal corresponding to the formation of 4-oxo-Tempo (triplet centred at $3364 \mathrm{G}$ ) was measured (arbitrary units) at timed intervals for each of the donor drugs (Magnettech ${ }^{\text {to }}$ miniscope MS100 with the following parameter settings: field 
sweep $51.2 \mathrm{G}$, microwave frequency $9.5 \mathrm{GHz}$, microwave power $20 \mathrm{~mW}$, modulation amplitude $1500 \mathrm{mG}$ ). In control experiments without any NO-generating compounds present, there was a slow increase in EPR signal corresponding to the auto-oxidation of Tempone-H to 4-Oxo-Tempo; these signals have been subtracted from the data shown.

\section{Statistical analysis}

Data were assessed for statistical significance using two-way analysis of variance (ANOVA) with a Student-Neuman-Keuls post-test. Probability values of $P<0.05$ were considered statistically significant.

\section{Results}

Electrochemical detection of DEA/NO-derived NO revealed that this compound liberated free NO in a spontaneous and predictable manner. A bolus addition of DEA/NO $(5 \mu \mathrm{M})$ to culture medium caused a rapid transient rise in NO concentration, reaching a maximum of $\sim 5 \mu \mathrm{M}$, before subsiding over the subsequent $30 \mathrm{~min}$ (Figure 1a). The signal was not altered by incubation with SOD $\left(150 \mathrm{U} \mathrm{ml}^{-1}\right)$, but the rate of decay of the NO signal was markedly enhanced in the presence of neutrophils $\left(5 \times 10^{6} \mathrm{ml}^{-1}\right.$; Figure 1a). SOD $\left(150 \mathrm{U} \mathrm{ml}^{-1}\right)$ failed to increase the signal seen with neutrophils; instead, it caused a significant and surprising inhibition of NO generation.

In contrast to DEA/NO, NO was not detectable from GEA $3162(100 \mu \mathrm{M})$ in medium. However, NO generation from this agent was detected in the presence of SOD $\left(150 \mathrm{U} \mathrm{ml}^{-1}\right)$, with maximal NO concentrations reaching $\sim 3 \mu \mathrm{M}$ after $30 \mathrm{~min}$ incubations (Figure $1 \mathrm{~b}$ ). In the presence of neutrophils $\left(5 \times 10^{6} \mathrm{ml}^{-1}\right)$ and SOD $\left(150 \mathrm{U} \mathrm{ml}^{-1}\right)$, NO generation was markedly attenuated for incubation periods of $>10 \mathrm{~min}$. NO generation bore a nonlinear relationship to SOD concentration for both GEA 3162 and SIN-1 (Figure 1c), and there was no significant difference between the two agents under these conditions $(P>0.05)$.

Results from EPR spectroscopy identified a signal corresponding to 4-oxo-Tempo formation from the reduced form of the spin-trap, Tempone- $\mathrm{H}$, after incubation with GEA 3162 or SIN-1 (Figure 2a). The intensity of the signal obtained with both GEA-3162 and SIN-1 was dependent on the incubation time and was severely attenuated in the presence of SOD $\left(500 \mathrm{U} \mathrm{ml}^{-1}\right)$. DEA/NO failed to generate a significant signal under the same conditions (Figure 2b).

Assessment of the oxidation of DHR 123 to fluorescent rhodamine 123 using a spectrofluorimeter showed that both SIN-1 $(1 \mathrm{mM})$ and GEA $3162(100 \mu \mathrm{M})$ produced high levels of fluorescence ( $803 \pm 74$ and $757 \pm 100 \mathrm{U}$, respectively; $P<0.05$; $n=3$ ) compared to control (PBS alone, $23 \pm 5 \mathrm{U}$; Figure 3 ). In contrast, DEA/NO failed to significantly oxidise DHR 123 $(53 \pm 9 \mathrm{U} ; n=3)$. None of the compounds alone (in the absence of DHR 123) produced a fluorescent signal that exceeded 1.0 arbitrary units (data not shown), thus excluding autofluorescence as a possible explanation for the differential fluorescence observed.

High concentrations of DEA/NO (1 mM) and GEA 3162 $(100 \mu \mathrm{M})$ accelerated morphological changes characteristic of

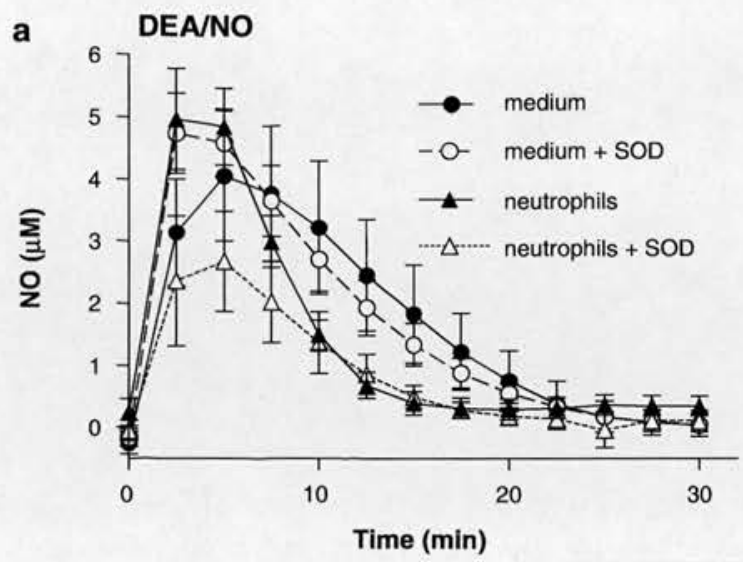

b GEA 3162

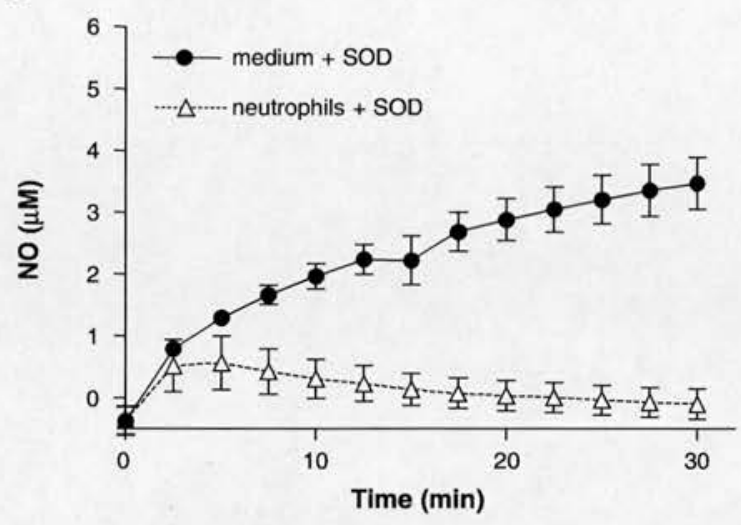

C SIN1/GEA $3162+$ SOD

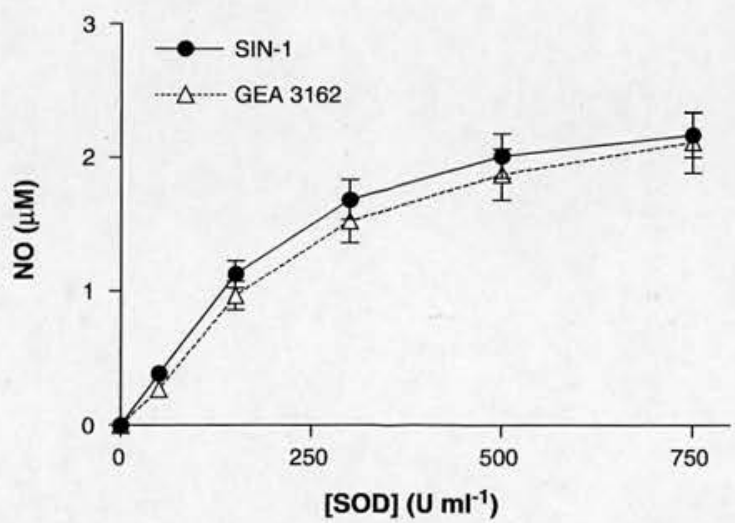

Figure 1 Measurement of NO release from DEA/NO, SIN-1 and GEA 3162 in the absence and presence of neutrophils and/or SOD. Mean NO electrode recordings for (a) DEA/NO $(5 \mu \mathrm{M})$ and (b) GEA-3162 $(100 \mu \mathrm{M})$ in Iscove's medium, in the presence and absence of human neutrophils $\left(4.5 \times 10^{6} \mathrm{cells} \mathrm{ml}^{-1}\right)$. NO release from GEA 3162 was not detectable in the absence of SOD $\left(150 \mathrm{U} \mathrm{ml}^{-1}\right)$. Concentrations of NO were calculated using a calibration curve generated using DEA/NO $(100 \mathrm{nM} 1.6 \mu \mathrm{M})$ in $\mathrm{pH} 4$ buffer. Results are mean + s.e.m. $(n=6)$. (c) Generation of NO by SIN-1 and GEA 3162 (both $100 \mu \mathrm{M})$ in the presence of SOD $\left(50-750 \mathrm{U} \mathrm{ml}^{-1}\right)$. Data represent NO concentrations at plateau approx. $5 \mathrm{~min}$ following addition of SOD. Results are mean +s.e.m. $(n=6)$.

neutrophil apoptosis over a 20 -h time course compared to untreated controls (Figure 4a). Approximately $50-60 \%$ of control cells underwent spontaneous apoptosis during the 
a

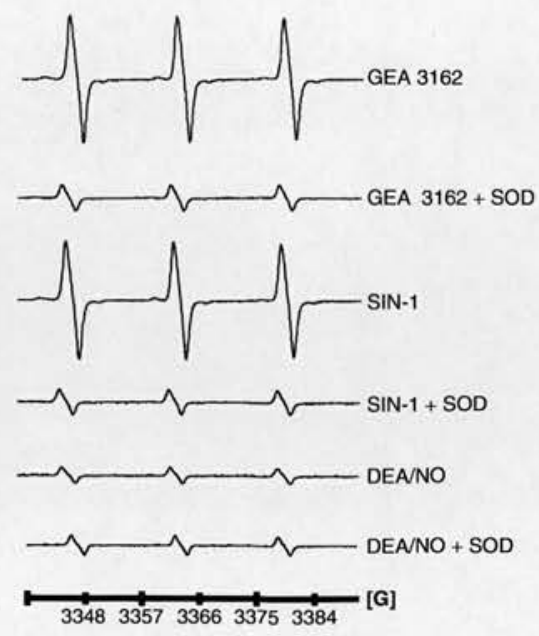

b

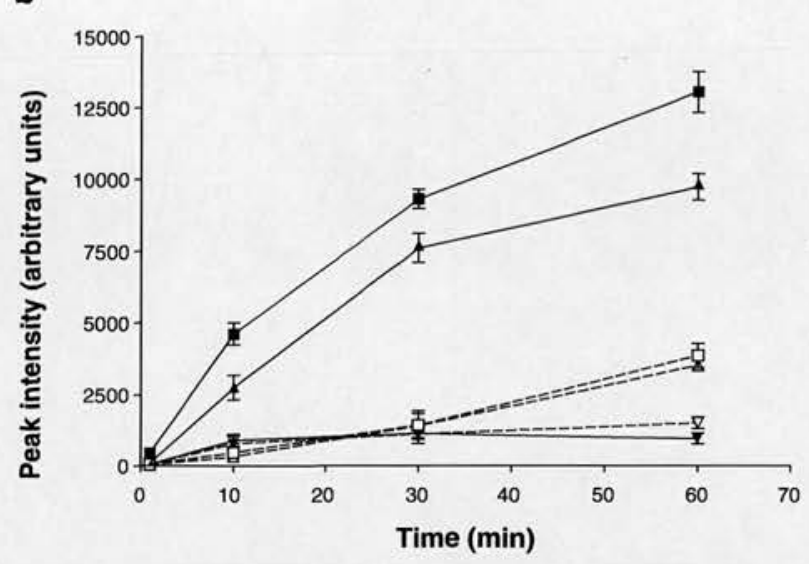

- - GEA $3162-$ - - GEA $3162+$ SOD $\leftarrow$ SIN-1

$-\triangle-$ SIN-1 + SOD $\rightarrow$ DEANO $-\nabla-$ DEANO + SOD

Figure 2 Generation of $\mathrm{O}_{2}^{-} / \mathrm{ONOO}^{-}$by donor drugs. (a) Representative EPR spectra showing the signal generated through oxidation of Tempone- $\mathrm{H}(1 \mathrm{mM})$ by $\mathrm{O}_{2}^{-} / \mathrm{ONOO}^{-}$after incubation $\left(30 \mathrm{~min}, 37^{\circ} \mathrm{C}\right.$ ) with SIN-1, GEA 3162 and DEA/NO (all $100 \mu \mathrm{M}$ ) in the presence or absence of SOD $\left(500 \mathrm{U} \mathrm{ml}^{-1}\right)$. Control spectra of Iscove's medium and $1 \mathrm{mM}$ Tempone- $\mathrm{H}$ have been subtracted from each trace. (b) Relative intensities (arbitrary units) for EPR signals generated over time in the presence of SIN-1, GEA-3162 or DEA/ NO $(100 \mu \mathrm{M})$ in the presence or absence of SOD $\left(500 \mathrm{U} \mathrm{ml}^{-1}\right)$. Results are mean \pm s.e.m. $(n=6)$.

culture period, whereas those treated with either compound were virtually all apoptotic. However, when internucleosomal DNA fragmentation was assessed by flow cytometric measurement of PI intercalation, key differences were observed between the two compounds. Whereas DEA/NO increased DNA fragmentation compared to control, GEA 3162 produced an unexpected inhibition of this process (Figure $4 \mathrm{~b}$ ). The effects of $1-3 \mathrm{~mm}$ SIN-1 on morphology and DNA fragmentation at $20 \mathrm{~h}$ were then studied in order to determine how $\mathrm{ONOO}^{-}$influences these apoptotic events in neutrophils. This compound had effects similar to GEA 3162 but opposing effects to DEA/NO, in that a concentration-dependent inhibition of DNA fragmentation occurred alongside an induction of apoptotic morphology (Figure 4c).

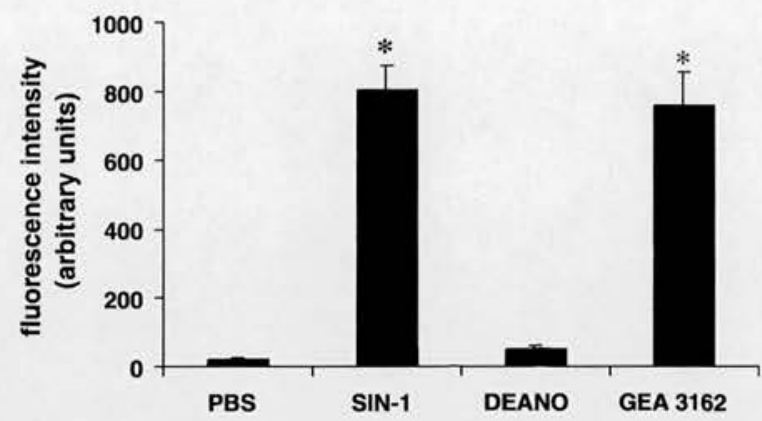

Figure 3 Spectrofluorimetric assessment of DHR 123 oxidation. DHR $123(1 \mu \mathrm{M})$ was added to solutions of PBS (control), SIN-1 $(1 \mathrm{mM}), \mathrm{DEA} / \mathrm{NO}(1 \mathrm{mM})$ or GEA $3162(100 \mu \mathrm{M})$ and incubated for $60 \mathrm{~min}$ at $37^{\circ} \mathrm{C}$. The extent of DHR 123 oxidation to rhodamine 123 was assessed using a spectrofluorimeter at an emission wavelength of $500 \mathrm{~nm}$. Results are mean \pm s.e.m. $(n=3)$. Asterisks represent significant difference $(P<0.05)$ from control fluorescence.

\section{Discussion}

Our results show that the oxatriazole-5-imine-derived compound, GEA 3162 , generated an oxidising species that induced apoptosis in human neutrophils via a mechanism that was independent of DNA fragmentation. Similar results were obtained with the known $\mathrm{ONOO}^{-}$generator, SIN-1, but the NO donor, DEA/NO, failed to generate an oxidising species, and apoptosis induction in response to this agent was associated with increased DNA fragmentation. Further experiments to determine the NO-related species generated from these agents established that GEA 3162 and SIN-1 concomitantly generate $\mathrm{NO}$ with $\mathrm{O}_{2}^{-}$, resulting in rapid formation of $\mathrm{ONOO}^{-}$. The $\mathrm{NO}$ component was unmasked by removal of $\mathrm{O}_{2}^{-}$by high concentrations of the enzyme, SOD. These results were in common with those of the recognised ONOO $^{-}$generator, SIN-1 (Hogg et al., 1992), but were at odds with the NO donor drug, DEA/NO (Maragos et al., 1991), which was found to generate $\mathrm{NO}$ in the absence of $\mathrm{O}_{2}^{-}$ scavenger systems, and did not oxidise Tempone- $\mathrm{H}$ to generate an EPR signal.

Previous studies have suggested that GEA 3162 does not liberate $\mathrm{O}_{2}^{-}$alongside $\mathrm{NO}$; therefore it was suggested to be a 'pure' NO donor (Holm et al., 1998). However, in contrast, recent preliminary data have indicated that GEA 3162 resembles $\mathrm{SIN}-1$, in that both compounds simultaneously generate $\mathrm{NO}$ and $\mathrm{O}_{2}^{-}$and are therefore both $\mathrm{ONOO}^{-}$donors (Schmidt et al., 2001). Given that SIN-1 is accepted to be an $\mathrm{ONOO}^{-}$donor (Hogg et al., 1992), while DEA/NO generates pure NO (Maragos et al., 1991), our results suggest that $\mathrm{ONOO}^{-}$is a requirement for the inhibition of DNA fragmentation and implies that GEA 3162 is also an $\mathrm{ONOO}^{-}$generating agent. We therefore carried out a number of experiments to determine the nature of the NO-related species liberated from GEA 3162.

An NO-specific electrode detected NO release from DEA/ $\mathrm{NO}$ in culture medium, as has been shown previously (Crane et al., 2002). Release of NO from this compound $(5 \mu \mathrm{M})$ occurred spontaneously at physiological temperature and $\mathrm{pH}$. The signal was not significantly affected by SOD alone, suggesting that there was no concurrent release of $\mathrm{O}_{2}^{-}$under these conditions. In the presence of neutrophils, there was a mild acceleration of the decay of NO signal from DEA/NO, 

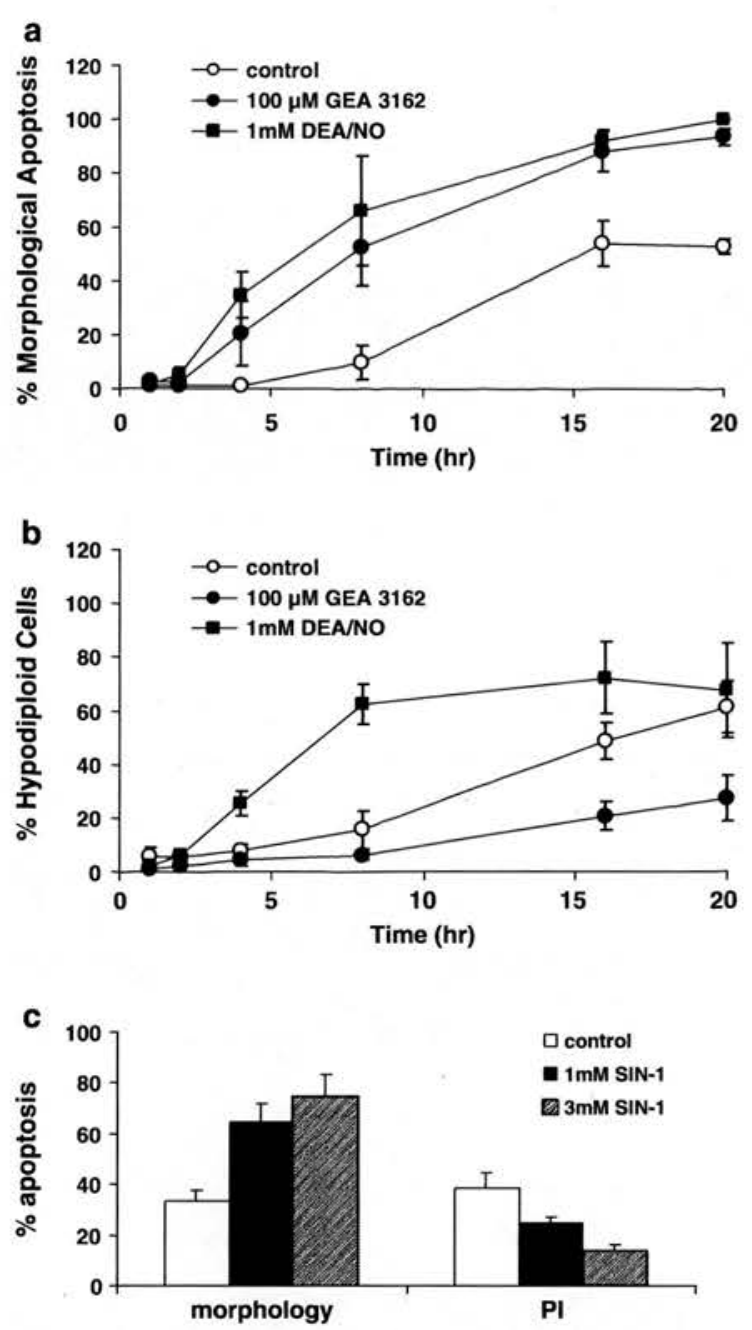

Figure 4 Differential effects of compounds on independent parameters of neutrophil apoptosis. Human neutrophils were incubated at $37^{\circ} \mathrm{C}$ for $1-20 \mathrm{~h}$ with PBS (control), DEA/NO (1 mM) or GEA $3162(100 \mu \mathrm{M})$. Apoptosis was then assessed by (a) morphological changes using oil-immersion light microscopy and (b) PI intercalation to show hypodiploid DNA content characteristic of apoptotic cells. Results are mean \pm s.e.m. $(n=3-6)$. (c) Neutrophils were incubated for $20 \mathrm{~h}$ with SIN-1 (1-3 mM) and apoptosis was assessed by morphology and PI intercalation. Results are mean \pm s.e.m. $(n=5)$.

suggesting a scavenging effect of the cells. However, NO detection in the presence of neutrophils and SOD was not enhanced; indeed, it was surprisingly blunted, perhaps indicating a scavenging effect of the enzyme under these conditions. On the other hand, SIN-1 and GEA 3162 failed to produce measurable free NO under the same conditions, despite the relatively high concentrations of these drugs used $(100 \mu \mathrm{M})$. The limit for NO detection of the electrode used in these studies is $\sim 50 \mathrm{nM}$. Rapid scavenging of NO through reaction with $\mathrm{O}_{2}^{-}$generated simultaneously might account for the lack of detectable NO under these conditions. It is well established that this is the case for SIN-1, which is recognised to be an $\mathrm{ONOO}^{-}$donor rather than a pure NO donor (Hogg et al., 1992), but it was a matter of debate whether NO release from GEA 3162 also involves the concomitant liberation of
$\mathrm{O}_{2}^{-}$. In order to test this, the buffer into which the electrode was immersed was preincubated with varying concentrations of $\mathrm{Cu} / \mathrm{Zn} \mathrm{SOD}$, which rapidly converts $\mathrm{O}_{2}$ to $\mathrm{H}_{2} \mathrm{O}_{2}$, in an effort to 'unmask' $\mathrm{NO}$ by removal of $\mathrm{O}_{2}^{-}$. If SIN-1 and GEA 3162 both liberate a combination of $\mathrm{NO}$ and $\mathrm{O}_{2}^{-}$, then free $\mathrm{NO}$ might be detectable in the presence of SOD, which would prevent at least some of the $\mathrm{NO}$ being oxidised to $\mathrm{ONOO}^{-}$ (Lizasoain et al., 1996). With reference to the rate constants, the relative rates of reaction can be estimated as follows (for a concentration of $1 \mu \mathrm{M} \mathrm{NO}$ and assuming the amount of NO generated is matched by $\mathrm{O}_{2}^{-}$):

$$
\begin{aligned}
& 2 \mathrm{O}_{2}^{-}+\mathrm{H}^{+} \stackrel{\text { SOD }}{\longrightarrow} \mathrm{H}_{2} \mathrm{O}_{2}+\mathrm{O}_{2} \mathrm{k}=2 \times 10^{9} \mathrm{M}^{-1} \mathrm{~s}^{-1} \text { Rate }=2 \mu \mathrm{Ms}^{-1} \\
& \mathrm{O}_{2}^{-}+\mathrm{NO} \longrightarrow \mathrm{ONOO}^{-} \mathrm{k}=6 \times 10^{9} \mathrm{M}^{-1} \mathrm{~s}^{-1} \text { Rate }=6 \mu \mathrm{Ms}^{-1}
\end{aligned}
$$

From these reaction rales, it is reasonable to predict that some NO might be unmasked in the presence of SOD, but that the amount observed would be a relatively small percentage of the total amount generated, on account of the fact that the rate of reaction of $\mathrm{NO}$ with $\mathrm{O}_{2}^{-}$is three times faster than that catalysed by SOD. In the event, NO was detected from both SIN-1 and GEA 3162; indeed, the profile of NO release from the two compounds in the presence of SOD was virtually identical, and correlated well with previously published data for SIN-1 (Lizasoain et al., 1996). The role of oxygen-derived free radicals in quenching of NO was confirmed by EPR experiments using the $\mathrm{O}_{2}^{-}$and $\mathrm{ONOO}^{-}$-specific spin-trap, Tempone-H. A signal corresponding to 4-oxo-Tempo increased in intensity during incubations with both SIN-1 and GEA-3162, but was not seen with DEA/NO. The 4-oxoTempo signal was quenched by SOD, indicating that the species responsible for oxidation of Tempone- $\mathrm{H}$ was $\mathrm{O}_{2}^{-}$, or a downstream product of $\mathrm{O}_{2}^{-}$(e.g. $\mathrm{ONOO}^{-}$). Taken together, these results indicate that GEA-3162 is indeed an $\mathrm{ONOO}^{-}$ generator, with characteristics that are very similar to SIN-1 and quite distinct from the NO donor, DEA/NO.

DHR 123 is oxidised to the fluorochrome, rhodamine 123, by a number of oxidative and nitrosative species, including $\mathrm{ONOO}^{-}, \mathrm{O}_{2}^{-}, \mathrm{H}_{2} \mathrm{O}_{2}$ and $\mathrm{HOCl}$, and is widely used in flow cytometric assessment of respiratory burst in neutrophils (Smith \& Weidemann, 1993; Ruchaud-Sparagano et al., 1997). However, it has been demonstrated that NO does not have the capacity to oxidise this molecule (Crow, 1997). This makes DHR 123 an ideal tool for discriminating between agents that release $\mathrm{NO}$ and those that liberate $\mathrm{ONOO}^{-}$. Addition of DHR 123 to solutions of SIN-1 (1 mM) and GEA $3162(100 \mu \mathrm{M})$ led to the generation of fluorescent rhodamine 123 , whereas with PBS and DEA/NO (1 mM), no fluorescence could be detected. This suggests that both SIN-1 and GEA 3162 generate species distinct from $\mathrm{NO}$, such as $\mathrm{ONOO}^{-}$, which does react with DHR 123.

Functionally, SIN-1 and GEA 3162 share properties that differ from DEA/NO. Apoptosis studies showed that all the three compounds accelerated morphological neutrophil apoptosis at high concentrations, as has previously been demonstrated by this group (Ward et al., 2000; Taylor et al., 2001). Only extremely high concentrations of DEA/NO were able to induce neutrophil apoptosis (Taylor et al., 2001), which are likely to be suprapathophysiological, thereby suggesting that even high concentrations of NO generated from iNOS during excessive inflammation are unable to promote neutrophil apoptosis by themselves. Therefore, endogenously formed NO, 
whatever its source, is unlikely to induce apoptosis in this cell type if it is not converted to $\mathrm{ONOO}^{-}$.

Only DEA/NO produced the expected rise in the level of internucleosomal DNA fragmentation that mirrored the morphological changes observed. In the presence of both SIN1 and GEA 3162, this process was inhibited compared to control, indicating an uncoupling of DNA fragmentation from other apoptotic events with these two compounds, and provides further evidence that the species generated by GEA 3162 more closely resembles that from SIN-1 than from DEA/ NO. Although this study did not investigate the potential underlying mechanism, this phenomenon could potentially be due to the inhibitory modification of tyrosine (nitration) or reduced cysteine ( $S$-nitrosation) residues (Kuo \& Kocis, 2002) in critical proteins of the DNA fragmentation pathway such as caspase 3 (Kim et al., 1997) or DFF40 (Widlak, 2000), thus affecting the activity of these proteins and therefore the DNA fragmentation process.

Overall, we have demonstrated that GEA 3162 is an $\mathrm{ONOO}^{-}$donor that induces neutrophil apoptosis via a mechanism that is not dependent on DNA fragmentation. Chemically and biologically, therefore, GEA 3162 resembles SIN-1 and is clearly distinct from DEA/NO. The induction of neutrophil apoptosis may be of therapeutic benefit in a number of inflammatory conditions in which resolution of inflammation is impaired (such as arthritis, pancreatitis, pneumonia and asthma), which causes neutrophils to persist in the tissue and then potentially subsequently die by necrosis (Ward et al., 1999b; Taylor et al., 2003). $\mathrm{ONOO}^{-}$is an important molecule in inflammation as iNOS-derived NO and $\mathrm{O}_{2}^{-}$are both produced by inflammatory cells and the resulting $\mathrm{ONOO}^{-}$may contribute to the pathophysiology of a number of chronic inflammatory conditions (Szabo, 1996).

E.L.T and C.A.S. are funded by the Wellcome Trust, as part of a 4-year Ph.D. programme in Cardiovascular Biology within the Cardiovascular Research Initiative at the University of Edinburgh, and FPDR was funded by a Wellcome Trust vacation scholarship (VS/02/EDI/3). The EPR miniscope was supported by a BHF core facility grant (CUI/99010).

\section{References}

BLAYLOCK, M.G., CUTHBERTSON, B.H., GALLEY, H.F., FERGUSON, N.R. \& WEBSTER, N.R. (1998). The effect of nitric oxide and peroxynitrite on apoptosis in human polymorphonuclear leukocytes. Free Radic. Biol. Med., 25, 748-752.

CRANE, M.S., OLLOSSON, R., MOORE, K.P., ROSSI, A.G. \& MEGSON, I.L. (2002). Novel role for low molecular weight plasma thiols in nitric oxide-mediated control of platelet function. J. Biol. Chem. 277, 46858-46863.

CROW, J.P. (1997). Dichlorodihydrofluorescein and dihydrorhodamine 123 are sensitive indicators of peroxynitrite in vitro: implications for intracellular measurement of reactive nitrogen and oxygen species. Nitric Oxide, 1, 145-157.

DIKALOV, S., SKATCHKOV, M. \& BASSENGE, E. (1997). Spin trapping of superoxide radicals and peroxynitrite by 1-hydroxy-3carboxy-pyrrolidine and 1-hydroxy-2,2,6,6-tetramethyl-4-oxopiperidine and the stability of corresponding nitroxyl radicals towards biological reductants. Biochem. Biophys. Res. Commun. 231, 701-704

DIMMELER, S. \& ZEIHER, A.M. (1997). Nitric oxide and apoptosis: another paradigm for the double-edged role of nitric oxide. Nitric Oxide: Biol. Chem., 1, 275-281.

FURCHGOTT, R.F. \& ZAWADZKI, J.V. (1980). The obligatory role of endothelial cells in the relaxation of arterial smooth muscle by acetylcholine. Nature, 288, 373-376.

HECKER, M., PREISS, C., KLEMM, P. \& BUSSE, R. (1996). Inhibition by antioxidants of nitric oxide synthase expression in murine macrophages: role of nuclear factor kappa B and interferon regulatory factor $1 . B r$. J. Pharmacol., 118 , 2178-2184

HOGG, N., DARLEY-USMAR, V.M., WILSON, M.T. \& MONCADA, S (1992). Production of hydroxyl radicals from the simultaneous generation of superoxide and nitric oxide. Biochem. J., 281 (Part 2), 419-424.

HOLM, P., KANKAANRANTA, H., METSA-KETELA, T. \& MOILANEN, E. (1998). Radical releasing properties of nitric oxide donors GEA 3162, SIN-1 and $S$-nitroso- $N$-acetylpenicillamine. Eur. J. Pharmacol., 346, 97-102.

KANKAANRANTA, H., RYDELl, E., PETERSSON, A.S., HOLM, P., MOILANEN, E., CORELL, T., KARUP, G., VUORINEN, P., PEDERSEN, S.B., WENNMALM, A. \& METSA-KETELA, T (1996). Nitric oxide-donating properties of mesoionic 3-aryl substituted oxatriazole-5-imine derivatives. Br. J. Pharmacol., 117, 401-406.

KELM, M., DAHMANN, R., WINK, D. \& FEELISCH, M. (1997). The nitric oxide/superoxide assay. Insights into the biological chemistry of the NO/O-2 interaction. J. Biol. Chem., 272, 9922-9932.

KIM, Y.M., BOMBECK, C.A. \& BILLIAR, T.R. (1999). Nitric oxide as a bifunctional regulator of apoptosis. Circ. Res., 84, 253-256.

KIM, Y.M. TALANIAN, R.V. \& BILLIAR, T.R. (1997). Nitric oxide inhibits apoptosis by preventing increases in caspase-3-like activity via two distinct mechanisms. J. Biol. Chem., 272, 31138-31148.

KUO, W.N. \& KOCIS, J.M. (2002). Nitration/S-nitrosation of proteins by peroxynitrite-treatment and subsequent modification by glutathione $S$-transferase and glutathione peroxidase. $\mathrm{Mol}$. Cell Biochem., 233, 57-63.

LIZASOAIN, I., MORO, M.A., KNOWLES, R.G., DARLEY-USMAR, V \& MONCADA, S. (1996). Nitric oxide and peroxynitrite exert distinct effects on mitochondrial respiration which are differentially blocked by glutathione or glucose. Biochem. J., 314 (Part 3), $877-880$

LOW, S.Y., SABETKAR, M., BRUCKDORFER, K.R. \& NASEEM, K.M (2002). The role of protein nitration in the inhibition of platele activation by peroxynitrite. FEBS Lett., 511, 59-64.

MARAGOS, C.M., MORLEY, D., WINK, D.A., DUNAMS, T.M SAAVEDRA, J.E., HOFFMAN, A., BOVE, A.A., ISAAC, L., HRABIE J.A. \& KEEFER, L.K. (1991). Complexes of NO with nucleophiles as agents for the controlled biological release of nitric oxide. Vasorelaxant effects. J. Med. Chem., 34, 3242-3247.

NURMINEN, M.L. \& VAPAATALO, H. (1996). Effect of intracerebroventricular and intravenous administration of nitric oxide donors on blood pressure and heart rate in anaesthetized rats. $\mathrm{Br} . \mathrm{J}$. Pharmacol., 119, 1422-1426.

PALMER, R.M., FERRIGE, A.G. \& MONCADA, S. (1987). Nitric oxide release accounts for the biological activity of endothelium-derived relaxing factor. Nature, 327, 524-526.

QUINN, A.C., PETROS, A.J. \& VALlANCE, P. (1995). Nitric oxide: an endogenous gas. Br.J. Anaesth., 74, 443-451

RONSON, R.S., NAKAMURA, M \& VINTEN-JOHANSEN, J. (1999). The cardiovascular effects and implications of peroxynitrite. Cardiovasc. Res., 44, 47-59.

RUCHAUD-SPARAGANO, MH, STOCKS, SC, TURLEY, H \& DRANSFIELD, I. (1997). Activation of neutrophil function via CD66: differential effects upon beta 2 integrin mediated adhesion. Br. J. Haematol., 98, 612-620.

SCHMIDT, K., SCHRAMMEL, A., GORREN, A.C.F., KOESLING, D. \& MAYER, B. (2001). Decomposition of the 'NO donor' GEA 3162 results in the formation of peroxynitrite. Naunyn-Schmiedeberg's Arch. Pharmacol., 363, S161.

SMITH, J.A. \& WEIDEMANN, M.J. (1993). Further characterization of the neutrophil oxidative burst by flow cytometry. J. Immmol. Methods, 162, 261-268. 
SZABO, C. (1996). The pathophysiological role of peroxynitrite in shock, inflammation, and ischemia-reperfusion injury. Shock, 6, $79-88$.

TAYLOR, E.L., MEGSON, I.L., HASLETT, C. \& ROSSI, A.G. (2001) Dissociation of DNA fragmentation from other hallmarks of apoptosis in nitric oxide-treated neutrophils: differences between individual nitric oxide donor drugs. Biochem. Biophys. Res. Commun., 289, 1229-1236.

TAYLOR, E.L., MEGSON, I.L., HASLETT, C. \& ROSSI, A.G. (2003) Nitric oxide: a key regulator of myeloid inflammatory cell apoptosis. Cell Death Differ., 10, 418-430.

WARD, C., CHILVERS, E.R., LAWSON, M.F., PRYDE, J.G., FUJIHARA, S., FARROW, S.N., HASLETT, C. \& ROSSI, A.G. (1999a). NF-kappaB activation is a critical regulator of human granulocyte apoptosis in vitro. J. Biol. Chem., 274, 4309-4318.
WARD, C., DRANSFIELD, I., CHILVERS, E.R., HASLETT, C. \& ROSSI, A.G. (1999b). Pharmacological manipulation of granulocyte apoptosis: potential therapeutic targets. Trends Pharmacol. Sci., 20, 503-509.

WARD, C., WONG, T.H., MURRAY, J., RAHMAN, I., HASLETT, C., CHILVERS, E.R. \& ROSSI, A.G. (2000). Induction of human neutrophil apoptosis by nitric oxide donors: evidence for a caspase-dependent, cyclic-GMP-independent, mechanism. Biochem. Pharmacol., 59, 305-314.

WIDLAK, P. (2000). The DFF40/CAD endonuclease and its role in apoptosis. Acta Biochim. Pol., 47, 1037-1044.

(Received March 24, 2004 Revised May 24, 2004 Accepted June 1, 2004) 


\title{
Inhibition of NF-кB by a cell permeable form of IкB $\alpha$ induces apoptosis in eosinophils
}

\author{
Satoko Fujihara ${ }^{\mathrm{a}, *}$, Ellis Jaffray ${ }^{\mathrm{b}}$, Stuart N. Farrow ${ }^{\mathrm{c}}$, Adriano G. Rossi ${ }^{\mathrm{a}}$, \\ Christopher Haslett ${ }^{\mathrm{a}}$, Ronald T. Hay ${ }^{\mathrm{b}}$ \\ a Department of Medicine, Centre for Inflammation Research, University of Edinburgh Medical School, Teviot Place, Edinburgh EH8 $9 A G$, UK \\ ${ }^{\mathrm{b}}$ Centre for Biomolecular Sciences, School of Biology, University of St. Andrews, North Haugh, St. Andrews, Fife KY16 9ST, UK \\ ${ }^{\mathrm{c}}$ The Asthma Biology Department, GlaxoSmithKline, Stevenage, Herts SGI 2NT, UK
}

Received 15 November 2004

Available online 7 December 2004

\begin{abstract}
An 11 amino acid HIV-TAT peptide can deliver target proteins into a variety of cells in a receptor-independent manner. To generate a highly specific inhibitor of the transcription factor NF- $\kappa \mathrm{B}$, we have fused the TAT-peptide to a version of IкB $\alpha$ that is resis-

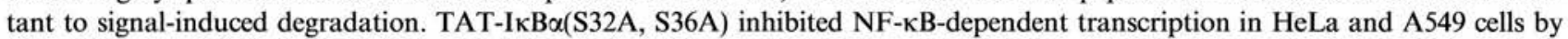

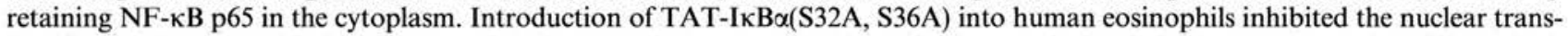

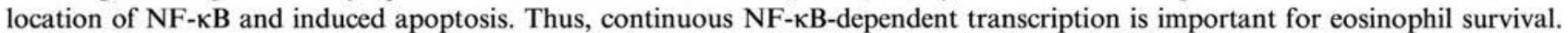
While eosinophils are normally refractive to standard methods of gene delivery, the ability of TAT fusion proteins to be taken up by these cells should enable a detailed molecular analysis of survival pathways in these cells.
\end{abstract}

(c) 2004 Elsevier Inc. All rights reserved.

Keywords: HIV-TAT; NF-кB; IкB $\alpha$; TAT-IкB $\alpha$; Eosinophils; Apoptosis

Excessive eosinophil recruitment and activation at inflammatory sites are likely to cause surrounding tissue damage by liberation of their toxic granule contents. Toxic granule proteins are important for defence against parasites, but if they are released in the host tissue when eosinophils are inappropriately activated, they will contribute to the progression of inflammatory diseases such as asthma. Thus, levels of eosinophilic toxic granule components such as major basic protein, eosinophil cationic protein, eosinophil peroxidase, and cysteinyl leukotrienes have been found in significantly increased amounts in the airways of asthmatic patients and animal models, suggesting that they may be responsible for the

\footnotetext{
* Corresponding author. Present address: Medicine and Therapeutic, University of Dundee, Ninewells Hospital, Dundee, Scotland UKDD1 9SY, UK. Fax: +44 138264972.

E-mail address: s.fujihara@dundee.ac.uk (S. Fujihara).
}

airway tissue damage and remodelling [1-3]. An additional function of eosinophils is the production of pro-inflammatory cytokines including IL-1, IL-4, IL-6, IL-8, TNF $\alpha$, GM-CSF, and IL-5 [4]. Synthesis of these proteins in eosinophils is dependent on the activation of intracellular signalling cascades and key transcription factors. Some of these proteins trigger the activation of inflammatory cells, including eosinophils, thereby contributing to an amplification of the inflammatory response and disease progression. Therefore, effective removal of eosinophils from inflammatory sites may be a key factor for successful resolution of the inflammatory process. As previously shown from our study $[5,6]$ and others $[7,8]$ NF- $\mathrm{\kappa B}$ is a key regulator of eosinophil activation and survival.

$\mathrm{NF}-\mathrm{KB}$ is a family of transcription factors that regulates survival and inflammatory responses $[9,10]$. The NF-kB family contains the proteins p65/Rel A, c-Rel, 
$\operatorname{Rel} \mathrm{B}, \mathrm{p} 50 / \mathrm{NF}-\kappa \mathrm{B} 1$, and $\mathrm{p} 52 / \mathrm{NF}-\kappa \mathrm{B} 2$ in various combinations to form the active transcriptional dimer. NF- $\mathrm{\kappa B}$ transcriptional activity is controlled by the $\mathrm{I} \kappa \mathrm{B}$ family of inhibitory proteins which contain ankyrin repeat domains (ARD) and share the function of retaining NF$\kappa \mathrm{B}$ in the cytoplasm. $\mathrm{I} \kappa \mathrm{B} \alpha$ is a $37 \mathrm{kDa}$ protein that binds to NF- $\kappa \mathrm{B}$, retaining it in the cytoplasm and inhibiting DNA binding. The pro-inflammatory cytokines TNF $\alpha$ and IL- $1 \beta$ initiate a signalling cascade via their receptors on the cell surface and activate the I $\kappa \mathrm{B}$ kinases consisting of subunits, IKK-1 (or IKK- $\alpha$ ) and IKK-2 (or IKK- $\beta$ ), and a structural subunit IKK- $\gamma$ or NEMO. The activated kinase phosphorylates I $\mathrm{K} \mathrm{B} \alpha$ on serines 32 and 36 , and phosphorylated $\mathrm{I} \kappa \mathrm{B} \alpha$ is selectively ubiquitinated on lysine 21 and 22 by an SCF E3 ubiquitin ligase containing $\beta \operatorname{TrCP}$. Poly-ubiquitinated $\operatorname{I\kappa B} \alpha$ is degraded by the $26 \mathrm{~S}$ proteasome [11] and the released $\mathrm{NF}-\mathrm{\kappa B}$ translocates to the nucleus where it activates transcriptionally responsive genes. The pro-inflammatory cytokine TNF $\alpha$ can trigger at least two antagonistic pathways: a survival pathway via NF- $\kappa \mathrm{B}$ transcriptional activation and a pro-apoptotic pathway via activation of caspase cascades. Thus, inhibition of NF-KB unmasks the caspase-dependent pro-apoptotic properties of $\mathrm{TNF} \alpha$ in many cells [12-14] including granulocytes [6].

The study of specific intracellular signalling pathways in eosinophils is technically challenging because they are refractory to many methods of direct molecular manipulation such as transfection, viral infection or microinjection. Recently, an 11 amino acid HIV-TAT has been highlighted for its ability to deliver target proteins into a variety of cells in a receptor-independent manner. It has been demonstrated that the arginine rich motif (ARM), corresponding to amino acids 47-57 of HIV-1 TAT (YGRKKRRQRRR), could transduce materials such as antibodies and enzymes, nucleic acids, and beads into cells in a receptor- and transporter-independent manner [15]. Furthermore, linkage of TAT to proteins such as p27 [15], caspase3 [16], GTPase [17], and $\beta$-galactosidase [18] resulted in almost $100 \%$ transduction efficiency in $30 \mathrm{~min}$ of many cell types including granulocytes [19-22].

Here, we generated a TAT-I $\kappa \mathrm{B} \alpha$ fusion protein that is resistant to signal-induced degradation and demonstrate that it is capable of inhibiting NF- $\kappa \mathrm{B}-$ dependent transcription in a variety of cell types. Introduction of TAT-IкB $\alpha(\mathrm{S} 32 \mathrm{~A}, \mathrm{~S} 36 \mathrm{~A})$ into human eosinophils inhibited the nuclear translocation of NF- $\mathrm{KB}$ and induced apoptosis, thus demonstrating the utility of this approach.

\section{Materials and methods}

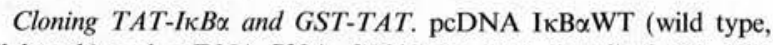
full length) and pcDNA S32A, S36AIкB $\alpha$ were described [23]. The sequence encoding an 11 amino acid TAT peptide (YGRKKRR QRRR) [15] inserted into pGEX2T (Pharmacia, New Jersey, USA).

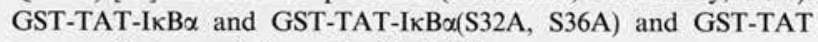
were purified essentially as described [24].

Labelling TAT with FITC. Fluorescein 5-isothiocyanate (FITC) was incubated with TAT-I $\kappa \mathrm{B} \alpha$, recombinant I $\mathrm{B} \boldsymbol{\alpha} \alpha$ or GST-TAT (40$80 \mu \mathrm{g} / \mathrm{mg}$ protein) for $1 \mathrm{~h}$ at room temperature then, overnight at $4{ }^{\circ} \mathrm{C}$ in the dark. After coupling, protein was dialysed against buffer $(10 \mathrm{mM}$ Tris, $150 \mathrm{mM} \mathrm{NaCl}, \mathrm{pH} 8.2$ ), at $4{ }^{\circ} \mathrm{C}$ with four changes of the same buffer and the protein concentration was determined as described [24].

Luciferase assay. HeLa cells, 57A [25] containing an integrated NF$\kappa \mathrm{B}$-dependent luciferase reporter, were maintained in $10 \% \mathrm{FCS}$ in DMEM. Following treatment with TAT-I $\kappa \mathrm{B} \alpha$ or GST-TAT cells were exposed to TNF $\alpha$ to activate NF- $\mathrm{kB}$ and $6 \mathrm{~h}$ later luciferase specific activity (RLU/mg) was determined as described [25].

Other experimental methods. Human cosinophil isolation, assessment of eosinophil apoptosis, statistical analysis, isolation of cytoplasmic, and nuclear extracts for Western blotting, and fluorescence microscopy were as described [5], with the following modifications. The images of fluorescence microscopy were captured using Open Lab software (Improvision, Coventry, UK) and CoolSnap digital camera (Media Cybernetics, Silver Spring, MD, USA). To prepare cytoplasmic and nuclear extracts for Western blotting, $5 \times 10^{6}$ eosinophils and $1 \times 10^{6}$ A549 cells were used for each condition, and were analysed with anti-IкB $\alpha$ (Cell Signalling Technology, Manchester, UK) and anti-p65 antibodies (Santa Cruz Biotechnology, California, USA).

\section{Results}

\section{Inhibition of $N F-\kappa B$ activation by $T A T-I \kappa B \alpha$}

The 11 amino acid TAT peptide (YGRKKRRQ RRR) can mediate uptake of coupled molecules into cells. Therefore, to generate a cell permeable molecule capable of specifically inhibiting NF-кB the TAT peptide was fused to $I \kappa B \alpha$, the natural inhibitor of NF$\kappa \mathrm{B}$, and $\mathrm{I} \kappa \mathrm{B} \alpha(\mathrm{S} 32 \mathrm{~A}, \mathrm{~S} 36 \mathrm{~A})$ (Fig. 1A) that is resistant to signal-induced degradation [26]. As a negative control the TAT peptide was also fused to GST. These proteins and recombinant $\mathrm{I} \kappa \mathrm{B} \alpha$ lacking the TAT peptide were expressed in bacteria and purified as described [24]. MALDI-TOF mass spectrometry confirmed that the proteins were of the predicted molecular mass (TAT-IкB $\alpha(\mathrm{S} 32 \mathrm{~A}, \mathrm{~S} 36 \mathrm{~A}) ; 37,570$ a.m.u. and recombinant $\mathrm{I} \kappa \mathrm{B} \alpha(\mathrm{rI} \kappa \mathrm{B} \alpha) ; 35,471$ a.m.u.) and SDS-PAGE followed by Coomassie blue staining indicated that they were essentially homogeneous (Fig. 1B).

To determine the ability of the TAT-IкB $\alpha$ fusion to block NF- $\mathrm{KB}$ activation, recombinant proteins were applied to a HeLa cell line (57A) containing an integrated NF- $\mathrm{KB}$ luciferase reporter gene [25] and preincubated for $30 \mathrm{~min}$ prior to addition of TNF $\alpha$. After a further $6 \mathrm{~h}$ cells were harvested and luciferase activity was determined. TNF $\alpha$ alone induces a large (approximately 100-

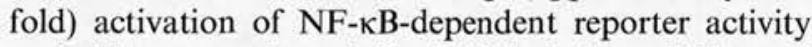
and this was substantially inhibited by addition of TAT-I $\kappa \mathrm{B} \alpha(\mathrm{S} 32 \mathrm{~A}, \mathrm{~S} 36 \mathrm{~A})$, but not by GST-TAT or rI $\kappa \mathrm{B} \alpha$ (Fig. 1C). To compare the efficiency with which

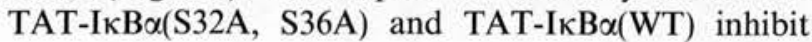


A

lacBa.
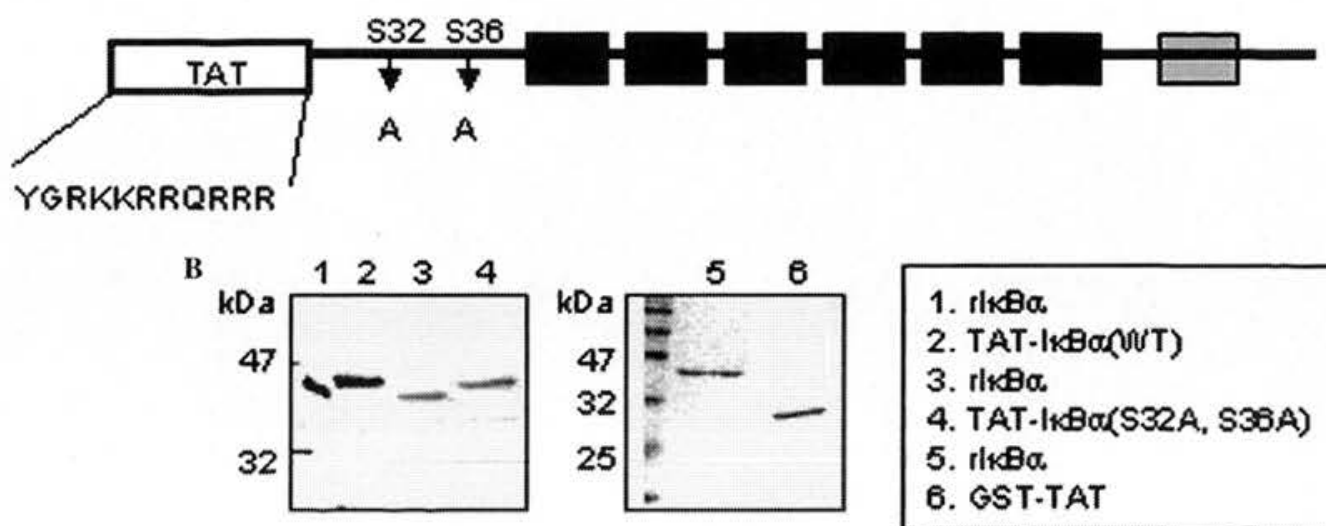

1. $\operatorname{rik} B a$

2. TAT- IKBa(N)

3. $r \mid \kappa B a$.

4. TAT- IKB $\alpha(S 32 A, S 36$ A)

5. $\|_{1} \theta a \alpha$

6. GST-TAT
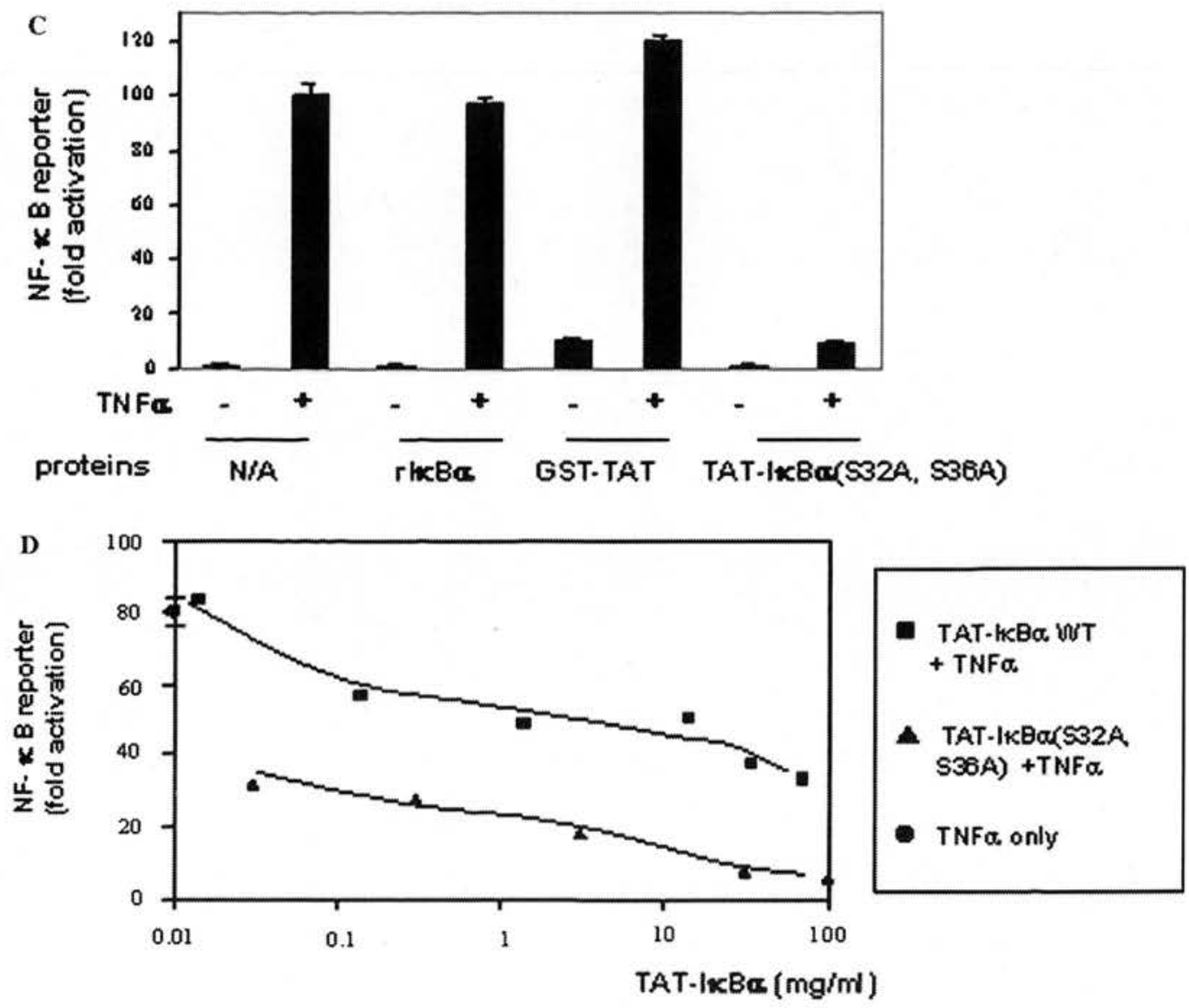

Fig. 1. (A) Schematic diagram representing the structure of TAT-IкB $\alpha(\mathrm{S} 32 \mathrm{~A}, \mathrm{~S} 36 \mathrm{~A})$. (B) The indicated purified proteins were analysed by SDSPAGE followed by Coomassie blue staining. (C) HeLa 57A cells containing an NF-KB-dependent luciferase reporter were incubated with

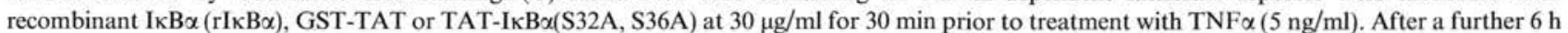
luciferase activity was determined and expressed as fold activation relative to the activity in cells that were not exposed to TNF $\alpha$. (D) The effect of

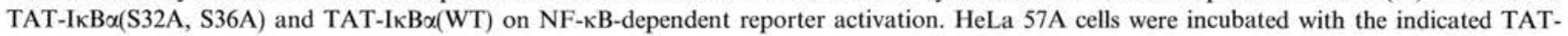
$\mathrm{I} \kappa \mathrm{B} \alpha(\mathrm{S} 32 \mathrm{~A}, \mathrm{~S} 36 \mathrm{~A})$ or TAT-IкB $\alpha(\mathrm{WT})$ for $30 \mathrm{~min}$ at $37^{\circ} \mathrm{C}$ and exposed to $\mathrm{TNF} \alpha(5 \mathrm{ng} / \mathrm{ml})$ for further $30 \mathrm{~min}$. Errors were not shown as they were within the range of the symbols.

$\mathrm{NF}-\kappa \mathrm{B}$ activation, a range of concentrations of the proteins were preincubated with HeLa $57 \mathrm{~A}$ prior to addition of $\mathrm{TNF} \alpha$ and reporter activities were determined.
While TAT-IкB $\alpha(\mathrm{WT})$ displays some inhibitory potential, it is clear that TAT-I $\kappa \mathrm{B} \alpha(\mathrm{S} 32 \mathrm{~A}, \mathrm{~S} 36 \mathrm{~A})$ is considerably more efficient at inhibiting $\mathrm{NF}-\kappa \mathrm{B}-d e p e n d e n t$ 
transcription (Fig. 1D). Even at a low dose $(0.03 \mu \mathrm{g} / \mathrm{ml})$ TAT-IкB $\alpha(\mathrm{S} 32 \mathrm{~A}, \mathrm{~S} 36 \mathrm{~A})$ reduces $\mathrm{TNF} \alpha$-activated luciferase activity by $61 \%$. Therefore, TAT-I $\kappa \mathrm{B} \alpha(\mathrm{S} 32 \mathrm{~A}$, S36A) was chosen for further study.

\section{Mechanism of inhibition of $N F-\kappa B$ by $T A T-I \kappa B \alpha$}

To investigate the mechanism by which TAT-ІкB $\alpha$ inhibits signal-induced activation of $N F-\kappa B$, the alveolar epithelial cell line A549 was preincubated with TAT-I $\kappa \mathrm{B} \alpha(\mathrm{S} 32 \mathrm{~A}, \mathrm{~S} 36 \mathrm{~A})$ prior to addition of $\mathrm{TNF} \alpha$, cells were lysed, and $\mathrm{I} \kappa \mathrm{B} \alpha$ levels were determined by Western blotting (WB) analysis. In the absence of TAT-I $\kappa \mathrm{B} \alpha(\mathrm{S} 32 \mathrm{~A}, \mathrm{~S} 36 \mathrm{~A}), \mathrm{TNF} \alpha$ induces the degradation of endogenous $I \kappa B \alpha$ (Fig. 2A, lanes 1 and 2). Exogenous TAT-I $\kappa \mathrm{B} \alpha(\mathrm{S} 32 \mathrm{~A}, \mathrm{~S} 36 \mathrm{~A})$ is resistant to $\mathrm{TNF} \alpha$-induced degradation, but does not protect endogenous $\mathrm{I} \kappa \mathrm{B} \alpha$ from signal-induced degradation (Fig. 2A, lanes 3 and 4). GST-TAT does not influence $\mathrm{TNF} \alpha$-induced degradation of $\mathrm{I} \kappa \mathrm{B} \alpha$ (Fig. $2 \mathrm{~A}$, lanes 5 and 6).
To determine the influence of TAT-I $\kappa \mathrm{B} \alpha(\mathrm{S} 32 \mathrm{~A}$, $\mathrm{S} 36 \mathrm{~A}$ ) on $\mathrm{TNF} \alpha$-induced nuclear translocation of NF$\kappa \mathrm{B}$, cells were pretreated with TAT fusion protein and the subcellular localisation of $\mathrm{NF}-\kappa \mathrm{B}$ was determined by immunofluorescence microscopy.

In untreated cells, p65 is predominantly cytoplasmic but after treatment with TNF $\alpha$ translocates to the nucleus (Fig. 2B, left panels). However, pre-treatment of cells with TAT-IкB $\alpha(\mathrm{S} 32 \mathrm{~A}, \mathrm{~S} 36 \mathrm{~A})$ blocks TNF $\alpha$ induced nuclear translocation of NF- $\mathrm{B}$ p 65 . Pre-treatment of cells with GST-TAT did not influence $\mathrm{TNF} \alpha$-induced nuclear translocation of NF- $\kappa \mathrm{B}$ p65 (Fig. 2B, right panels). Thus, TAT-IкB $\alpha(\mathrm{S} 32 \mathrm{~A}, \mathrm{~S} 36 \mathrm{~A})$ does not inhibit $\mathrm{TNF} \alpha$-induced degradation of endogenous $I \kappa \mathrm{B} \alpha$ but blocks nuclear translocation of the released NF- $\mathrm{NB}$.

\section{$T A T-I \kappa B \alpha$ transduction into eosinophils}

Unlike established cell lines, eosinophils are refractory to most forms of transfection or viral infection.

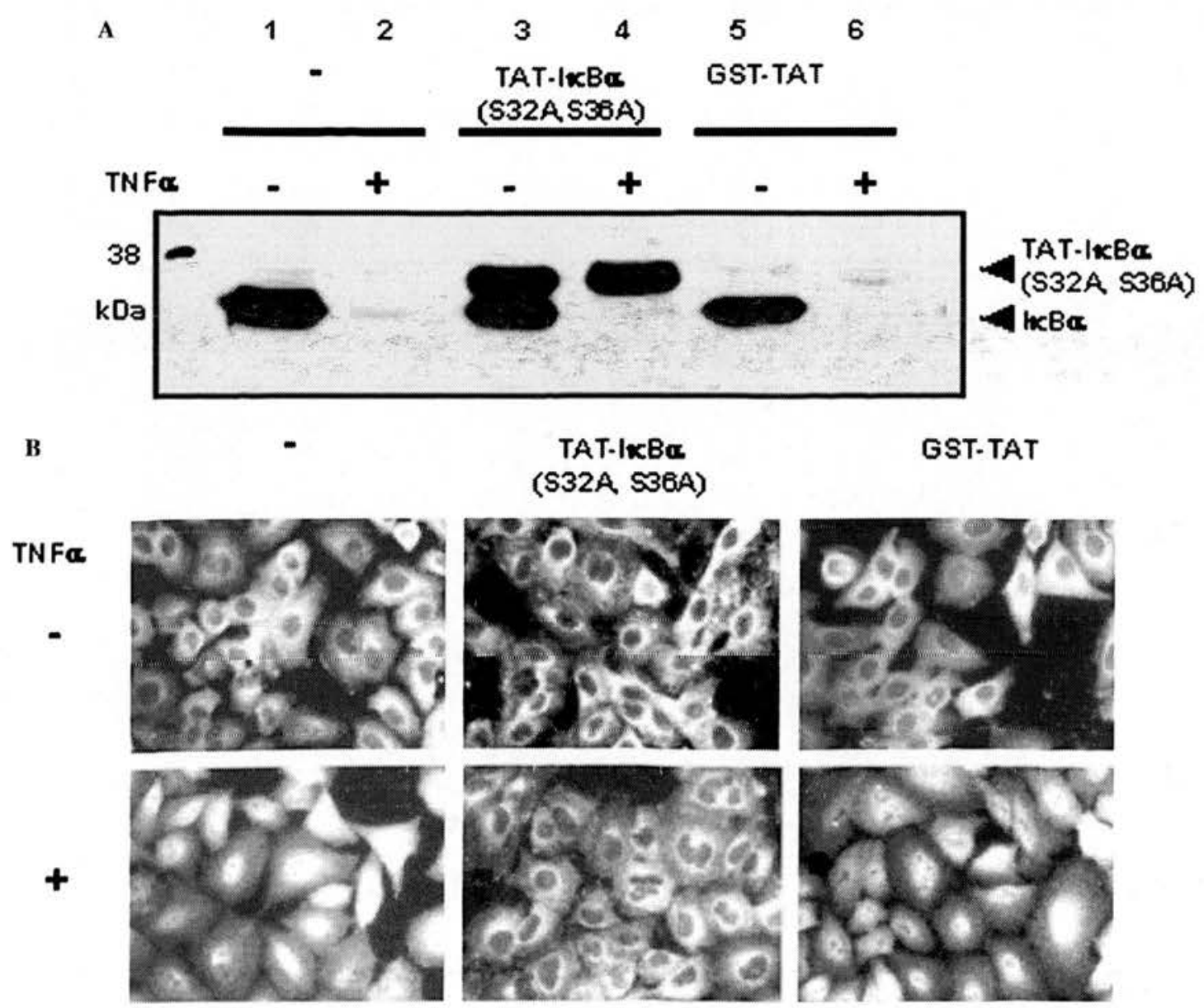

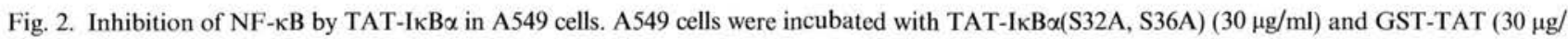
$\mathrm{ml}$ ) for $30 \mathrm{~min}$ at $37^{\circ} \mathrm{C}$ and then treated with $\mathrm{TNF} \alpha(5 \mathrm{ng} / \mathrm{ml})$ for $30 \mathrm{~min}$. (A) Cytoplasmic extract was analysed by WB with anti-I $\mathrm{B} \alpha$ antibody. (B) Cells were stained with anti-p65 antibody and viewed by fluorescence microscopy as indicated in Materials and methods. 


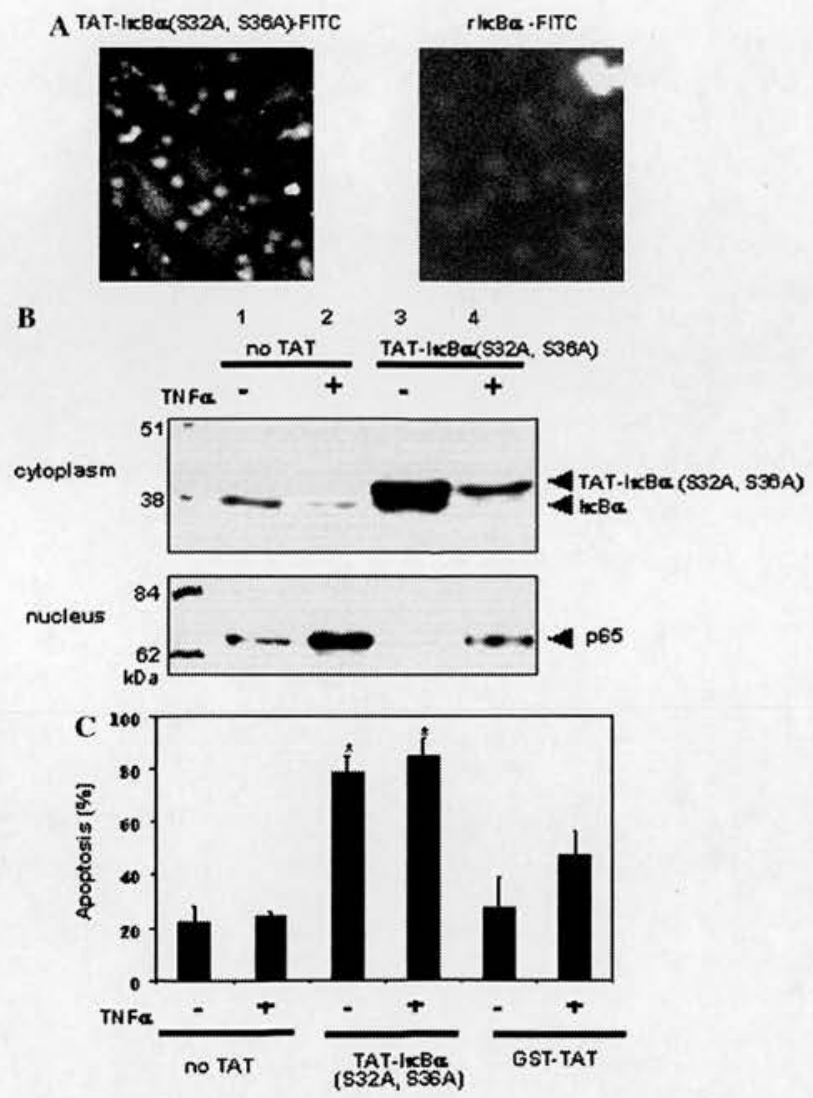

Fig. 3. (A) TAT-IкB $\alpha$ transduction into eosinophils. FITC conjugated TAT-I $\kappa \mathrm{B} \alpha$ or $\mathrm{rI} \kappa \mathrm{B} \alpha$ (both at $30 \mu \mathrm{g} / \mathrm{ml}$ ) was incubated with purified human eosinophils for $30 \mathrm{~min}$ at $37^{\circ} \mathrm{C}$ in the dark. After extensive washing to remove unbound material, FITC conjugated proteins were visualised by fluorescence microscopy. (B) Eosinophils were incubated with TAT-I $\kappa$ B $\alpha(\mathrm{S} 32 \mathrm{~A}, \mathrm{~S} 36 \mathrm{~A})(30 \mu \mathrm{g} / \mathrm{ml})$ for $30 \mathrm{~min}$ at $37^{\circ} \mathrm{C}$ and further treated with $\mathrm{TNF} \alpha(5 \mathrm{ng} / \mathrm{ml})$ for $30 \mathrm{~min}$, prior to preparation of nuclear and cytoplasmic extracts. I $\mathrm{B} \alpha$ levels in cytoplasmic extracts and p65 levels in nuclear extracts were determined by WB. (C) Eosinophils were treated with TAT-IкB $\alpha(\mathrm{S} 32 \mathrm{~A}, \mathrm{~S} 36 \mathrm{~A})(30 \mu \mathrm{g} / \mathrm{ml})$ and GST-TAT $(30 \mu \mathrm{g} / \mathrm{ml})$ for $30 \mathrm{~min}$ at $37^{\circ} \mathrm{C}$ and further treated with $\mathrm{TNF} \alpha(10 \mathrm{ng} / \mathrm{ml})$ as indicated. At $24 \mathrm{~h}$, apoptosis was determined morphologically. All values represent means $\pm \mathrm{SE}$ of $n=3$ experiments. *Represents significant difference from appropriate controls $(p<0.05)$.

To investigate the possibility that eosinophils could take up exogenously added protein TAT-I $\kappa \mathrm{B} \alpha(\mathrm{S} 32 \mathrm{~A}, \mathrm{~S} 36 \mathrm{~A})$ and $\mathrm{rI} \kappa \mathrm{B} \alpha$ were conjugated to FITC to allow their direct visualisation. TAT-I $\kappa \mathrm{B} \alpha(\mathrm{S} 32 \mathrm{~A}, \mathrm{~S} 36 \mathrm{~A})$-FITC was efficiently taken up by eosinophils whereas rI $\kappa \mathrm{B} \alpha$-FITC showed no evidence of uptake into eosinophils. To establish the ability of TAT-I $\kappa \mathrm{B} \alpha$ to inhibit NF- $\kappa \mathrm{B}$ in eosinophils, TAT-I $\kappa \mathrm{B} \alpha(\mathrm{S} 32 \mathrm{~A}, \mathrm{~S} 36 \mathrm{~A})$ was preincubated with eosinophils prior to treatment with $\mathrm{TNF} \alpha$. Cells were fractionated into nucleus and cytoplasm, and analysed by WB with IкB $\alpha$ and p 65 antibodies, respectively. As in A549 cells, TNF $\alpha$ induced degradation of endogenous $\mathrm{I} \kappa \mathrm{B} \alpha$ in eosinophils, irrespective of the presence of TAT-I $\kappa \mathrm{B} \alpha(\mathrm{S} 32 \mathrm{~A}, \mathrm{~S} 36 \mathrm{~A})$ (Fig. 3B, upper panel).
In the absence of TAT-I $\kappa \mathrm{B} \alpha(\mathrm{S} 32 \mathrm{~A}, \mathrm{~S} 36 \mathrm{~A})$ a significant amount of NF- $\kappa \mathrm{B}$ p65 is detected in the nuclei of eosinophils, suggesting that a fraction of NF- $\kappa \mathrm{B}$ is constitutively active in these cells. However, the nuclear content of NF- $\kappa \mathrm{B}$ p 65 is dramatically increased after treatment with $\mathrm{TNF} \alpha$ (Fig. 3B, lower panel). In the presence of exogenous TAT-I $\kappa \mathrm{B} \alpha(\mathrm{S} 32 \mathrm{~A}, \mathrm{~S} 36 \mathrm{~A})$, the eosinophil nuclear extract is cleared of constitutively active $\mathrm{NF}-\kappa \mathrm{B}$ p 65 and $\mathrm{TNF} \alpha$ induced nuclear accumulation of NF- $\mathrm{NB}$ p65 is substantially reduced (Fig. 3B, lower panel).

To establish the biological consequence of inhibiting $\mathrm{NF}-\kappa \mathrm{B}$ in human eosinophils TAT-I $\kappa \mathrm{B} \alpha(\mathrm{S} 32 \mathrm{~A}, \mathrm{~S} 36 \mathrm{~A})$ was transduced into these cells and half of the cells were treated with TNF $\alpha$. After $24 \mathrm{~h}$ the proportion of cells exhibiting characteristic apoptotic morphology was determined. In untreated or GST-TAT treated eosinophils $20 \%$ of cells became apoptotic after $24 \mathrm{~h}$, regardless of the presence of $\mathrm{TNF} \alpha$. In the presence of TAT-I $\kappa \mathrm{B} \alpha(\mathrm{S} 32 \mathrm{~A}, \mathrm{~S} 36 \mathrm{~A})$, there is a dramatic increase in the proportion of apoptotic cells $(78 \%)$ that is independent of TNF $\alpha$ treatment (Fig. 3C). These results suggest that the presence of constitutively active NF- $\kappa \mathrm{B}$ is required for eosinophil survival.

\section{Discussion}

Eosinophils are notoriously difficult cells in which to carry out molecular analysis as they are refractive to transfection and viral infection. Here, we establish the principle of HIV TAT arginine rich motif peptide (YGRKKRRQRRR) mediated uptake of proteins into eosinophils and demonstrate that TAT fused to the complete I $\mathrm{B} \boldsymbol{\alpha} \alpha$ protein is efficiently internalised in these cells. TAT-I $\kappa \mathrm{B} \alpha(\mathrm{S} 32 \mathrm{~A}, \mathrm{~S} 36 \mathrm{~A})$ efficiently enters HeLa cells and blocks signal-induced activation of NF- $\kappa$ Bdependent transcription. In A549 cells exogenous TAT-IкB $\alpha(\mathrm{S} 32 \mathrm{~A}, \mathrm{~S} 36 \mathrm{~A})$ does not stop TNF $\alpha$-induced degradation of endogenous $I \kappa \mathrm{B} \alpha$, but it does inhibit nuclear translocation of NF-кB p65. Thus, it is likely that $\mathrm{NF}-\kappa \mathrm{B}$ heterodimers released from endogenous $\mathrm{I} \kappa \mathrm{B} \alpha$ are bound by TAT-IкB $\alpha(\mathrm{S} 32 \mathrm{~A}, \mathrm{~S} 36 \mathrm{~A})$ and retained in a transcriptionally inert state in the cytoplasm. This is coincident with recently published studies employing various versions of TAT-I $\kappa \mathrm{B} \alpha$ carried out in HeLa cells, Jurkat cells, and bone marrow macrophages [27,28]. The biological consequences of introducing TAT-IкB $\alpha$ (S32A, S36A) into eosinophils are striking and a dramatic increase in apoptosis is observed, even in the absence of TNF $\alpha$ (Fig. 3C) although this is somewhat donor dependent. This suggests that in eosinophils constitutively active NF- $\mathrm{NB}$ is required to maintain a survival response and inhibition of $\mathrm{NF}-\kappa \mathrm{B}$ by ТАT-IкB $\alpha(\mathrm{S} 32 \mathrm{~A}, \mathrm{~S} 36 \mathrm{~A})$ unmasks a potent pro-apoptotic signal. This is consistent with the observation that 
NF- $\kappa \mathrm{B}$ p65 is detected in the nuclei of unstimulated eosinophils and this nuclear p65 is cleared by exogenously added TAT-I $\mathrm{B} \alpha(\mathrm{S} 32 \mathrm{~A}, \mathrm{~S} 36 \mathrm{~A})$ (Fig. 3B). Given that free $\mathrm{I} \kappa \mathrm{B} \alpha$ has the ability to translocate to the nucleus, disengage bound NF- $\mathrm{KB}$ from DNA, and export the $N F-\kappa B / I \kappa B \alpha$ complex back to the cytoplasm [29], it is likely that exogenously added TAT-I $\kappa \mathrm{B} \alpha(\mathrm{S} 32 \mathrm{~A}$, S36A) inhibits NF- $\kappa$ B-dependent transcription in eosinophils by this mechanism. A requirement for constitutive activation of NF- $\mathrm{KB}$ to provide a survival response was also evident from previous studies in eosinophils where pharmacological inhibitors of $\mathrm{NF}-\kappa \mathrm{B}$ activity such as gliotoxin and $\mathrm{Mg}$ 132-induced apoptosis [5].

\section{Acknowledgments}

Satoko Fujihara was sponsored by a GSK PhD studentship. This work was supported by BBSRC. We thank Dr. Jean-Michel Sallenave for reviewing the manuscript. 3G8 was a kind gift from Dr. J. Unkeless (Mount Sinai Medical School, New York).

\section{References}

[1] W.W. Busse, R. Lemanske, Asthma New Engl. J. Med. 344 (2001) 350-362.

[2] K. Blease, N.W. Lukacs, C.M. Hogaboam, S.L. Kunkel, Respir. Res. 1 (2000) 54-61.

[3] J. Bousquet, P.K. Jeffery, W.W. Busse, M. Johnson, A.M. Vignola, Am. J. Respir. Crit. Care Med. 161 (2000) 1720 1745.

[4] M.A. Giembycz, M.A. Lindsay, Pharmacol. Rev. 51 (1999) 2.

[5] S. Fujihara, C. Ward, I. Dransfield, R.T. Hay, I.J. Uings, B. Hayes, S.N. Farrow, C. Haslett, A.G. Rossi, Eur. J. Immunol. 32 (2002) 457-466.

[6] C. Ward, E.R. Chilvers, M.F. Lawson, J.G. Pryde, S. Fujihara, S.N. Farrow, C. Haslett, A.G. Rossi, J. Biol. Chem. 274 (1999) 4309-4318.
[7] N. Yamashita, H. Koizumi, M. Murata, K. Mano, K. Ohta, Int. Arch. Allergy. Immunol 120 (1999) 230-236.

[8] M. Choi, S. Rolle, M. Wellner, M.C. Cardoso, C. Scheidereit, F.C. Luft, R. Kettritz, Blood 102 (2003) 2259-2267.

[9] P.J. Barnes, I.M. Adcock, Eur. Respir. J. 12 (1998) 221-234.

[10] P.J. Barnes, M. Karin, N. Engl. J. Med. 336 (1997) 1066-1071.

[11] S. Gosh, M. Karin, Cell 109 (2002) 81-96.

[12] A.A. Beg, W.C. Shar, R.T. Bronson, S. Ghosh, D. Baltimore, Nature 376 (1995) 167-170.

[13] D.J. Van Antwerp, S.J. Martin, T. Kafri, D.R. Green, I.M. Verma, Science 274 (1996) 787-789.

[14] C.Y. Wang, M.W. Mayo, A.S. Baldwin Jr., Science 274 (1996) $784-787$.

[15] H. Nagahara, A.M. Vocero-Akbani, E.L. Snyder, A. Ho, D.G. Latham, N.A. Lissy, M. Becker-Hapak, S.A. Exhevsky, S.F. Dowdy, Nat. Med. 4 (1998) 1449-1452.

[16] A. Vocero-Akbani, N.V. Heyden, N.A. Lissy, L. Rantner, S.F. Dowdy, Nat. Med. 5 (1999) 29-33.

[17] A. Vocero-Akbani, N.A. Lissy, S.F. Dowdy, Methods Enzymol. 332 (2000) 36-49.

[18] S.R. Schwarze, K.A. Hruska, S.F. Dowdy, Trends Cell Biol. 10 (2000) 290-295.

[19] D.J. Hall, J. Cui, M.E. Bates, B.A. Stout, L. Koendeman, P.J. Coffer, P. Bertics, Blood 98 (2001) 2014-2021.

[20] J. Albals, L. Ulfman, P. Hordijk, L. Koenderman, Mol. Biol. Cell 12 (2001) 2137-2145.

[21] W.J. Bruynickx, K.M. Comerford, D.W. Lawrence, S.P. Colgan, Blood 97 (2001) 3245-3251.

[22] S. Myou, X. Zhu, E. Boetticher, S. Myo, A. Meliton, A. Lamberino, N.M. Munoz, A.R. Leff, J. Immunol. 169 (2002) 2670-2676.

[23] M.S. Rodriguez, I. Michalopoulos, F. Arenzana-Seisdedos, R.T. Hay, Mol. Cell. Biol. 15 (1995) 2413-2419.

[24] E. Jaffray, K.M. Wood, R.T. Hay, Mol. Cell. Biol. 15 (1995) 2166-2172.

[25] M.S. Rodriguez, J. Thompson, R.T. Hay, C. Dargemont, J. Biol. Chem. 274 (1999) 9108-9115.

[26] M. Roff, J. Thomson, M.S. Rodriguez, J.-M. Jacque, F. Baleux, F. Arenzana-Seisdedos, R.T. Hay, J. Biol. Chem. 271 (1996) $7844-7850$.

[27] Y. Abu-Amer, S.F. Dowdy, P.F. Ross, J.C. Clohisy, S.L. Teitelbaum, J. Biol. Chem. 276 (2001) 30499-30503.

[28] P.S. Kabouridis, M. Hasan, J. Newson, D.W. Gilroy, T. Lawrence, J. Immunol. 169 (2002) 2587-2593.

[29] F. Arenzana-Seisdedos, J. Thompson, M.S. Rodriguez, F. Bachelerie, D. Thomas, R.T. Hay, Mol. Cell. Biol. 15 (1995) 2689-2696. 


\title{
Phosphoinositide-3 kinases critically regulate the recruitment and survival of eosinophils in vivo: importance for the resolution of allergic inflammation
}

\author{
Vanessa Pinho, ${ }^{\star}$ Danielle G. Souza, ${ }^{\star}$ Michele M. Barsante, ${ }^{\star}$ Fabiana P. Hamer, ${ }^{\star}$ \\ Marta S. De Freitas, ${ }^{\dagger}$ Adriano G. Rossi, ${ }^{\star}$ and Mauro M. Teixeira ${ }^{\star, 1}$ \\ *Immunopharmacology, Departamento de Bioquímica e Imunologia, ICB, Universidade Federal de Minas Gerais, \\ Belo Horizonte, Brazil; ' Departamento de Farmacologia, Instituto de Biologia, Universidade do Estado do Rio de \\ Janeiro, Brazil; and ${ }^{\ddagger}$ Respiratory Medicine Unit, MRC, Centre for Inflammation Research, University of Edinburgh \\ Medical School, Scotland
}

\begin{abstract}
The phosphatidylinositol-3 kinase (PI3K) family of signaling enzymes plays a crucial role in leukocyte recruitment and activation and hence, likely regulates the induction and propagation phases of inflammation. However, little data have emerged showing a role for these processes in the resolution phase in models of in vivo inflammation. Here, we have evaluated the role of PI3K for the migration and survival of eosinophils in a model of allergic pleurisy in mice. Eosinophil accumulation in PI3K $\gamma$-deficient mice was inhibited at $48 \mathrm{~h}$, as compared with wild-type mice but not at earlier time-points (6 and $24 \mathrm{~h}$ ). Experiments with adoptive transfer of bone marrow showed that PI3K $\gamma$ in eosinophils but not in non-bone marrow-derived cells was required for their accumulation. Systemic treatment with PI3K inhibitors before antigen challenge prevented the recruitment of eosinophils. This was associated with decreased Akt phosphorylation, interleukin-5 production, and eosinophil release from the bone marrow. Treatment with PI3K inhibitors $24 \mathrm{~h}$ after antigen challenge markedly cleared the accumulated eosinophils, an effect associated with inhibition of Akt phosphorylation and an increased number of apoptotic events. Altogether, our data demonstrate an important role of PI3K $\gamma$ for the maintenance of eosinophilic inflammation in vivo, whereas other isoforms of PI3K may be relevant for the recruitment process. J. Leukoc. Biol. 77: 800-810; 2005.
\end{abstract}

Key Words: eosinophilia $\cdot$ signal transduction $\cdot$ apoptosis $\cdot$ allergy

\section{INTRODUCTION}

Phosphatidylinositol-3 kinases (PI3Ks) are a family of proteins that catalyze the phosphorylation of the $3^{\prime}-\mathrm{OH}$ position of the inositol ring of PIs, resulting in the formation of $\mathrm{PI}(3) \mathrm{P}$, $\mathrm{PI}(3,4) \mathrm{P} 2$, and $\mathrm{PI}(3,4,5) \mathrm{P} 3$, collectively termed 3 '-PI lipids [1, $2]$. These molecules work as second messengers and are im- portant in receptor signaling by growth factors, cytokines, and hormones [1-4]. There are different isoforms of PI3Ks, which can be divided in three main classes. The class I PI3Ks are heterodimers consisting of a catalytic subunit and an adaptor/ regulatory subunit present mainly in the cytosol of cells and can be subdivided in two subclasses. Class IA enzymes may have one of three catalytic isoforms (p110 $\alpha$, p110 $\beta$, or $\mathrm{p} 110 \delta)$ associated to five regulatory isoforms and signal downstream of tyrosine kinases. Class IB enzymes are constituted of a unique catalytic subunit $(\mathrm{pl} 10 \gamma)$ associated with a regulatory subunit pl01 and signal downstream of heterotrimeric G-protein-coupled receptors (GPCRs). There is also a class II PI3K, characterized by the presence of a C2 domain, and a class III PI3K, which uses only PI as a substrate $[1,2]$.

Recently, several studies have demonstrated the importance of PI3K in cellular migration in vitro and in vivo [5-9]. Early evidence for the role of PI3K in chemotaxis was the demonstration that human $\mathrm{T}$ cell migration induced by regulated on activation, normal $\mathrm{T}$ expressed and secreted was PI3K activation-dependent [10]. In the immune system, PI3K may also be activated by antigen receptors, such as the B cell, the $\mathrm{T}$ cell, and Fc receptors (FcRs)-receptors for mitogenic and inflammatory cytokines-and chemokine receptors [11].

Eosinophils are effector cells that play an important role in the pathophysiology of allergic disease [12-14]. In allergic diseases, such as asthma, eosinophils are a crucial source of cytotoxic proteins, lipid mediators, oxygen metabolites, and cytokines, which may contribute to the severity of disease [15]. Thus, there is a great interest in the understanding of mechanisms involved in the recruitment, activation, and survival of eosinophils in inflammatory sites. Eosinophil recruitment to sites of allergic inflammation depends on the concerted action of a variety of molecules, including chemokines, which act through GPCRs, and may result in the activation of PI3K

\footnotetext{
${ }^{1}$ Correspondence: Imunofarmacologia, Departamento de Bioquímica e Imunologia, Instituto de Ciências Biológicas, Universidade Federal de Minas Gerais, Av. Antonio Carlos, 6627-Pampulha, 31270-901-Belo Horizonte, MG, Brazil. E-mail: mmtex@icb.ufmg.br

Received July 6, 2004; accepted December 23, 2004; doi: 10.1189/ jlb.0704386.
} 
isoforms, especially PI3K $\gamma[4,11,16]$. Several studies have now shown that the deficiency of PI3K $\gamma$ impairs the migration of neutrophils and macrophages in vitro and in a septic peritonitis model $[6,8]$. PI3K activation may also be important for the induction of survival in cells via the phosphorylation of Akt/protein kinase B (PKB) $[2,11,17,18]$. However, little is known about the role of PI3K in the recruitment and/or clearance of eosinophils in sites of allergic reactions. Moreover, the role of PI3K $\gamma$ isoforms for eosinophil migration has not been determined.

In this study, we have investigated the role of PI3K for the recruitment and survival of eosinophils into the pleural cavity of antigen-challenged and -sensitized mice. Initial experiments investigated the role of the PI3K $\gamma$ isoform by using PI3K $\gamma$-deficient mice (PI3K $\left.\gamma^{-1-}\right)$. In addition, we investigated the role of PI3K in the recruitment and survival of eosinophils in the allergic pleurisy model by using wortmannin and LY294002, inhibitors of all isoforms of PI3K [19].

\section{MATERIALS AND METHODS}

\section{Animals}

$\mathrm{PI} 3 \mathrm{~K}^{-1-}$ male $\mathrm{C} 57 \mathrm{Bl} / 6 \times \mathrm{sv} 129$ mice and their wild-type (WT) littermate control mice $(+/+; 18-22 \mathrm{~g})$ were used throughout these experiments, and those with drugs were conducted in BALB/c mice (18-22 g). PI3K $\gamma^{-1-}$ mice (in a C57Bl/ $6 \times$ sv1 29 background) were generated as described previously [8]. The mice were a kind gift of Dr. Josef M. Penninger and supplied by Taconic Farms, Inc. (Germatown, NY). PI3K $\gamma$ gene deletion was confirmed by polymerase chain reaction using specific primers (WT primers: sense, 5-TCAGGCTCGGAGATTAGGTA-3; antisense, 5-GCCCAATCGGTGGTAGAACT-3; $P I 3 K^{-1-}$ primers: sense, 5-GGACACGGCTTTGATTACAATC-3; antisense, 5-GGGGTGGGATTAGATAAATG-3, as described previously; ref. [8]). Animals were bred and housed in a temperature-controlled room with free access to water and food. All experimental protocols have been subjected to evaluation and were approved by the local animal ethics committee.

\section{Drugs and reagents}

Recombinant murine eotaxin was purchased from Peprotech (London, UK). Eotaxin was dissolved in water, diluted further in phosphate-buffered saline (PBS; pH 7.4) containing $0.01 \%$ bovine serum albumin (BS $\Lambda$ ), and stored at $-20^{\circ} \mathrm{C}$ until use. Wortmannin was purchased from Calbiochem (San Diego, CA). Wortmannin was diluted in dimethyl sulfoxide (DMSO) and stored at $-70^{\circ} \mathrm{C}$ until use. LY294002 was purchased from Alamone Laboratories (Jerusalem, Israel), diluted in DMSO, and stored at $-70^{\circ} \mathrm{C}$ until use. Ovalbumin (OVA) was purchased from Sigma Chemical Co. (St. Louis, MO). Annexin-V was a gift of Dr. Gustavo Amarantes-Mendes (Universidade de São Paulo, Brazil).

\section{Sensitization}

Animals were immunized with OVA, adsorbed to an aluminium hydroxide gel, as described previously [20]. Briefly, mice were injected subcutaneously on days 1 and 8 with $0.2 \mathrm{ml}$ solution containing $100 \mu \mathrm{g}$ OVA and $70 \mu \mathrm{g}$ aluminum hydroxide (Reheiss, Dublin, Ireland).

\section{Leukocyte migration into the pleural cavity induced by antigen}

Sensitized WT or PI3K $\gamma^{-1-}$ mice were challenged by intrapleural (i.pl.) administration of antigen (OVA) or PBS. The cells present in the pleural cavity were harvested 6,24 , or $48 \mathrm{~h}$ after antigen challenge by injecting $2 \mathrm{ml}$ PBS and total cell counts performed in a modified Neubauer chamber using Turk's stain. For the experiments evaluating leukocyte apoptosis, infiltrating leukocytes were examined 2, 6, and $48 \mathrm{~h}$ after antigen challenge. Differential cell counts were performed on cytospin preparations (Shandon III) stained with MayGrumwald-Giemsa using standard morphologic criteria to identify cell types. The results are presented as the number of cells per cavity.

\section{Production of chimeric mice}

Chimeric mice, which lacked PI3K $\gamma$ on bone marrow-derived or on non-bone marrow-derived cells, were produced by transplanting bone marrow cells into irradiated recipient animals. Femurs and tibias of WT or PI3K $\gamma^{-1-}$ donor mice were dissected and flushed with $2 \mathrm{ml}$ incomplete RPMI medium. The resultant cell suspension was washed twice and resuspended in PBS. Recipient WT or PI3K $\gamma^{-1-}$ mice were irradiated with $900 \mathrm{rad}$ and then injected intravenously with $3 \times 10^{7}$ bone marrow cells in $0.2 \mathrm{ml}$ PBS from WT or PI3K $\gamma^{-1-}$ donor mice. Blood of bone marrow-chimeric mice was tested for the total number of circulating leukocytes at 7,14 , and 21 days after reconstitution. All mice were treated intraperitoneally (i.p.) with antibiotic (ciprofloxacin, $100 \mu \mathrm{g} / \mathrm{animal} /$ day) for 10 days. After 21 days, the total and differential number of leukocytes had normalized (data not shown), and mice were submitted to protocols of sensitization and challenge with OVA or PBS.

\section{Collection of bone marrow and peripheral blood cells}

Bone marrow cells were isolated from the left femur. The femoral head and condyles were removed, and the displaceable cells were recovered by flushing the marrow cavity of the femur shaft with $1 \mathrm{~mL}$ PBS containing heparin (10 $\mathrm{U} / \mathrm{ml}$ ). Blood samples were collected from the brachial plexus. Total cell counts were performed in a modified Neubauer chamber using Turk's stain. Differential cell counts were performed on cytospin preparations (Shandon III) stained with May-Grumwald-Giemsa using standard morphologic criteria to identify cell types. The results are presented as the number of cells/femur or cells/ml blood.

\section{Treatment with inhibitors of PI3K}

The role of PI3K on eosinophil recruitment in the allergic pleurisy was also investigated by using selective inhibitors of PI3K, wortmannin, and LY294002. Wortmannin was administered systemically (i.p.) or locally (i.pl. injection) at the dose of $1.0 \mathrm{mg} / \mathrm{kg}, 60 \mathrm{~min}$ prior to or $24 \mathrm{~h}$ after the i.pl. administration of OVA. This dose was shown to be effective in other experimental systems [21]. Moreover, preliminary experiments showed the dose of $1.0 \mathrm{mg} / \mathrm{kg}$ to be maximally effective at inhibiting eosinophil recruitment in the model (data not shown). LY294002 was administered systemically or locally at the dose of $1.0 \mathrm{mg} / \mathrm{kg}, 60 \mathrm{~min}$ prior to or $24 \mathrm{~h}$ after antigen challenge. Drugs were dissolved in DMSO and further diluted in PBS. Control animals received drug vehicle.

\section{Assessment of eosinophil apoptosis}

\section{Morphology}

Apoptosis was assessed as deseribed previously [22]. Briefly, cells $\left(5 \times 10^{4}\right)$ collected $48 \mathrm{~h}$ after antigen challenge were cylocentrifuged, fixed, and stained with May-Grunwald-Giemsa and counted using oil immersion microscopy ( $\times 100$ objective) to determine the proportion of cells with distinctive apoptotic morphology. Twenty-five fields were counted per slide, and results are expressed as the mean \pm SEM of the number of apoptotic cells in 25 fields.

\section{Annexin-V binding and propidium staining}

Assessment of apoptosis was also performed by flow cytometry using fluorescein isothiocyanate (FITC)-labeled annexin-V, which binds to phosphatidylserine exposed on the surface of apoptotic cells and propidium iodide as an index of loss of cell membrane integrity. Stock annexin-V was diluted 1:1000 with binding buffer and then added to $100 \mu \mathrm{l} 2.5 \times 10^{5}$ cells collected after 2 and $6 \mathrm{~h}$ after wortmannin treatment. Following $10 \mathrm{~min}$ incubation at room temperature, these samples were treated with $5 \mu \mathrm{l}$ propidium iodide (concentration, $50 \mu \mathrm{g} / \mathrm{ml}$ ) and analyzed using Becton Dickinson (San Jose, CA) FACScan and CELLQuest software. Results are expressed as cells undergoing the early stage of apoptosis, quantified by staining with annexin-V but not propidium iodide. 


\section{Measurement of eotaxin and interleukin (IL)-5}

Frozen supernatants obtained from pleural cavity washes after $6 \mathrm{~h}$ of the challenge with OVA ( $1 \mu \mathrm{g} /$ cavity) were used for eotaxin and IL-5 detection. The concentration of eotaxin and $\mathrm{IL}-5$ protein in pleural effluents was measured by enzyme-linked immunosorbent assay (ELISA), using commercially available antibody pairs and as specified by the supplier (R\&D Systems, Minneapolis, MN).

\section{Measurement of anti-OVA serum antibodies}

Ninety-six-well ELISA plates (Nunc, Roshilde, Denmark) were coated overnight at $4^{\circ} \mathrm{C}$ with $2 \mu \mathrm{g}$ per well OVA in coating buffer. Plates were blocked with $200 \mu \mathrm{l} /$ well PBS containing $0.25 \%$ casein for $1 \mathrm{~h}$ at room temperature. After washing with PBS containing $0.1 \%$ Tween-20, serum samples were diluted in PBS/0.25\% casein (starting at 1:100). Plates were incubated for $1 \mathrm{~h}$ at $37^{\circ} \mathrm{C}$, washed six times, and incubated with peroxidase-conjugated goat anti-mouse antibody for $1 \mathrm{~h}$ at $37^{\circ} \mathrm{C}$. After six washes, the reaction was developed at room temperature with $100 \mu \mathrm{l} /$ well o-phenylenediamine $(1 \mathrm{mg} /$ $\mathrm{ml}), 0.04 \% \mathrm{H}_{2} \mathrm{O}_{2}$ in sodium citrate buffer. The reaction was interrupted by the addition of $20 \mu \mathrm{l}$ per well of $2 \mathrm{~N} \mathrm{H}_{2} \mathrm{SO}_{4}$. Absorbance was measured at $492 \mathrm{~nm}$ by an ELISA reader (Bio-Rad, Hercules, CA). ELISA was computed by optical densities between 1:100 and 1:6400 of serum dilutions in individual mice.

\section{Immunoprecipitation}

Cells $\left(5 \times 10^{6}\right.$ cells $\left./ \mathrm{ml}\right)$ obtained from the pleural cavity of challenged mice were lysed in $50 \mathrm{mM}$ Tris- $\mathrm{HCl}$, pH 7.4, $150 \mathrm{mM} \mathrm{NaCl}, 1.5 \mathrm{mM} \mathrm{MgCl}, 1.5 \mathrm{mM}$ EDTA, $1 \%$ Triton X-100, $10 \%$ glycerol, $10 \mu \mathrm{g} / \mu \mathrm{l}$ aprotinin, $10 \mu \mathrm{g} / \mu \mathrm{l}$ leupeptin, $2 \mu \mathrm{g} / \mu \mathrm{l}$ pepstatin. and $1 \mathrm{mM}$ phenylmethylsulfonyl fluoride. Lysates (2 $\mu \mathrm{g} / \mu \mathrm{l})$ were incubated overnight at $4^{\circ} \mathrm{C}$ with anti-Akt antibody $(1: 200$, Santa Cruz Biotechnology, CA). Blocking peptide (Santa Cruz Biotechnology) was used as a negative control for anti-Akt antibody. Then, protein A/G-agarose (20 $\mu \mathrm{l} / \mathrm{mg}$ protein, Santa Cruz Biotechnology) was added, and samples were incubated at $4^{\circ} \mathrm{C}$ under rotation for $2 \mathrm{~h}$. The contents of Akt and phosphorylated Akt were analyzed by Western blotting, as described subsequently.

\section{Immunoblotting analysis for Akt}

Lysates $(30 \mu \mathrm{g})$ were resolved by $12 \%$ sodium dodecyl sulfate-polyacrylamide gel electrophoresis, and proteins were transferred to polyvinylidene difluoride (PVDF) filters (Hybond-P, Amersham Bioseiences, I.ittle Chalfont, UK). Rainbow markers (Amersham Biosciences) were run in parallel to estimate molec- ular weights. Membranes were blocked with Tween-Tris-buffered saline (TBS; $20 \mathrm{mM}$ Tris- $\mathrm{HCl}, \mathrm{pH} 7.5,500 \mathrm{mM} \mathrm{NaCl}, 0.1 \%$ Tween-20) containing $1 \%$ BSA. Primary antibodies used in Western analysis were monoclonal antiphosphoserine (1:1000, Sigma Chemical Co.) and polyclonal anti-Akt (1:1000, Santa Cruz Biotechnology). The PVDF filters were next washed three times with Tween-TBS, followed by $1 \mathrm{~h}$ incubation with appropriate secondary antibody conjugated to biotin (Santa Cruz Biotechnology). Then, the filters were incubated with streptavidin-conjugated horseradish peroxidase (1:1000, Caltag Laboratories, South San Francisco, CA). Immunoreactive proteins were visualized by $3,3^{\prime}$-diaminobenzidine (Sigma Chemical Co.) staining. The bands were quantified by densitometry, using Scion image software (Scion Co., Frederick, MD).

\section{Statistical analysis}

All results are presented as the mean \pm SEM. Normalized data were analyzed by one-way ANOVA, and differences between groups were assessed using the Student-Newman-Keuls post-test. A $P$ value $<0.05$ was considered significant.

\section{RESULTS}

\section{Role of PI3K $\gamma$ for eosinophil accumulation in a model allergic pleurisy}

To investigate the importance of PI3K for eosinophil recruitment, sensitized WT and PI3K $\gamma^{-1-}$ mice were challenged (i.pl.) with OVA. The i.pl. injection of OVA in sensitized WT and PI3K $\gamma^{-1-}$ mice $(1 \mu \mathrm{g} /$ cavity $)$ induced a significant recruitment of eosinophils at 6 and $24 \mathrm{~h}$ after antigen challenge $(\mathbf{F i g}$. 1A). However, the number of eosinophils in PI3K $\gamma^{-1-}$ mice was diminished by $82 \%$ after $48 \mathrm{~h}$, as compared with WT mice. At this time-point, a significant accumulation of mononuclear cells was also observed in WT but not in PI3K $\gamma^{-1-}$ mice (Table 1). The accumulation of macrophages and neutrophils at 6 and $24 \mathrm{~h}$ after challenge was not altered in PI3K $\gamma^{-1-}$ mice when compared with WT mice (data not shown). The baseline levels of leukocytes in blood and bone marrow of WT and
A

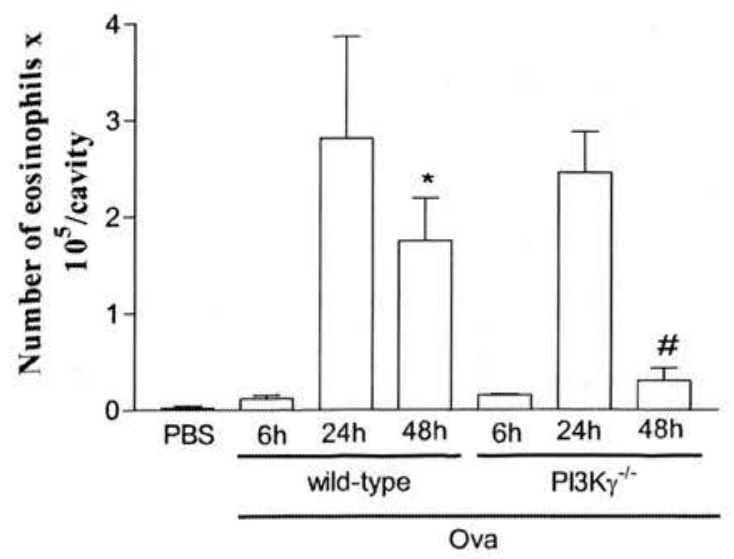

B

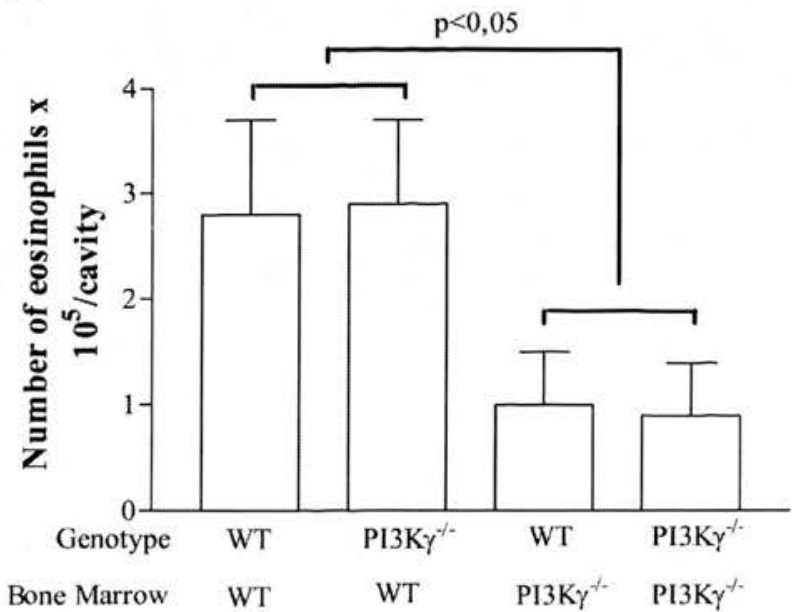

Fig. 1. Eosinophil accumulation induced by antigen challenge of sensitized PI3K $\gamma^{-1-}$ and WT mice. (A) Immunized WT or PI3K $\gamma^{-1-}$ mice or (B) immunized chimeric mice were challenged with an i.pl. injection of the OVA ( $1 \mu \mathrm{g} /$ cavity) or PBS and eosinophil accumulation assessed at the time-point described in the figure. Chimeric mice, which lacked PI $3 \mathrm{~K} \gamma$ on bone marrow-derived or on non-bone marrow-derived cells, were produced by transplanting bone marrow cells into irradiated recipient animals. Results are expressed as the means \pm SEM of five mice in each group. $*, P<0.01$, when compared with PBS-injected mice and \#, $P<0.01$, when compared with OVA-injected WT mice. 
TABLE 1. Recruitment of Leukocytes $\left(\times 10^{5}\right.$ Cells/Cavity) Induced by Antigen Challenge of Sensitized PI3K $\gamma^{-1-}$ and WT Mice

\begin{tabular}{lccccc}
\hline \hline & \multicolumn{2}{c}{ WT } & & \multicolumn{2}{c}{ PI3K $\gamma^{-1-}$} \\
\cline { 2 - 3 } \cline { 5 - 6 } & PBS & OVA & & PBS & OVA \\
\hline Total & $5.3 \pm 1.2$ & $25.6 \pm 2.4^{*}$ & & $8.3 \pm 1.7$ & $8.8 \pm 0.9^{n}$ \\
Mononuclear & $5.2 \pm 1.2$ & $23.9 \pm 2.1^{*}$ & & $8.2 \pm 1.7$ & $8.5 \pm 0.8^{n}$ \\
Neutrophils & 0 & 0 & & 0 & 0 \\
Eosinophils & $0.07 \pm 0.03$ & $1.8 \pm 0.4^{*}$ & & $0.03 \pm 0.02$ & $0.3 \pm 0.1^{\#}$ \\
\hline
\end{tabular}

Immunized WT or PI3K $\gamma^{-1-}$ mice were challenged with an i.pl. injection of OVA ( $1 \mu \mathrm{g} /$ cavity) or PBS, and eosinophil recruitment was assessed after $48 \mathrm{~h}$. Results are expressed as the means \pm SEM of five mice in each group. $* P<$ 0.01 when compared with PBS-injected mice and ${ }^{*} P<0.01$ when compared with OVA-infected WT mice.

PI3K $\gamma^{-1-}$ mice were not different (Table 2). Furthermore, analysis of the cellular infiltrate in bone marrow of WT and $\mathrm{PI} 3 \mathrm{~K} \gamma^{-1-}$ mice at 6,24 , and $48 \mathrm{~h}$ after antigen challenge demonstrated that there were no differences in the number of eosinophils between the two groups in the various time-points (Table 2). There were no morphological figures typical of apoptosis in the pleural cavity of immunized and challenged WT and PI3K $\gamma^{-1-}$ mice at 24,36 , and $48 \mathrm{~h}$ (data not shown). There were few apoptotic figures of eosinophils in bone marrow of WT or PI3K $\gamma^{-1-}$ mice (data not shown).

Although PI3K $\gamma$ is mainly expressed in leukocytes, studies have demonstrated that this PI3K isoform may also be expressed by endothelial cell [23]. Thus, to verify the relevance of PI3K $\gamma$ expressed on leukocytes and on non-bone marrowderived cells for the process of eosinophil accumulation in the pleural cavity, we made use of bone marrow transplantation. PI3K $\gamma^{-1-}$ mice were reconstituted with bone marrow from PI3K $\gamma^{+1+}$ (WT mice) donors so that they had PI3K $\gamma$-positive leukocytes but PI3K $\gamma$-negative non-bone marrow cells, and irradiated WT mice were reconstituted with bone marrow from $\mathrm{PI} 3 \mathrm{~K} \gamma^{-1-}$ donors so that they had PI3K $\gamma$-positive non-bone marrow cells but PI3K $\gamma$-negative leukocytes. In control groups, irradiated PI3K $\gamma^{-1-}$ mice were reconstituted with bone marrow from PI3K $\gamma^{-1-}$ donors, and irradiated WT mice were reconstituted with bone marrow from WT donors. The i.pl. injection of OVA in sensitized WT and PI3K $\gamma^{-1-}$ mice whose bone marrow had been reconstituted with WT bone marrow induced a significant recruitment of eosinophils after $48 \mathrm{~h}$ (Fig. 1B). In contrast, the eosinophil accumulation induced by i.pl. injection of OVA in sensitized WT and PI3K $\gamma^{-1-}$ mice whose bone marrow had been reconstituted with PI3K $\gamma^{-1-}$ bone marrow was significantly reduced, suggesting that PI3K $\gamma$ on bone marrow-derived cells was required for eosinophil accumulation into the pleural cavity $48 \mathrm{~h}$ after antigen challenge (Fig. 1B). After bone marrow transfer, the number of circulating leukocytes in blood was analyzed every 7 days, and we failed to observe any differences between the groups (data not shown).

Previous studies have shown that PI3K $\gamma^{-1-}$ mice exhibited low titers of immunoglobulin $\mathrm{G}_{1}\left(\mathrm{IgG}_{1}\right)$ antibodies to haptenconjugated OVA, suggesting that PI $3 \mathrm{~K} \gamma$ is required to generate functional $\mathrm{T}$ helper cell-dependent responses to antigen in vivo
[8]. In our model, the titers of total anti-OVA $\mathrm{Ig}$ and $\mathrm{IgG}_{1}$ are shown in Figure 2, $\mathbf{A}$ and $\mathbf{B}$, respectively. Thus, whereas WT mice exhibited high, total anti-OVA-specific Ig and $\mathrm{IgG}_{1}$ titers, these antibodies were reduced in PI3K $\gamma^{-1-}$ mice.

Next, we evaluated the production of eotaxin and IL-5, important mediators of eosinophil accumulation, in the allergic pleurisy model $[24,25]$. At $6 \mathrm{~h}$ after antigen challenge, there was no difference in the production of eotaxin (OVA-WT mice, $252 \pm 40 \mathrm{pg} /$ pleural cavity; OVA-PI3K $\gamma^{-1-}, 247 \pm 58 \mathrm{pg} / \mathrm{pleu}-$ ral cavity; $n=5$ ) or IL-5 (OVA-WT mice, $27.9 \pm 10.2 \mathrm{pg} /$ pleural cavity; OVA-PI3K $\gamma^{-1-}, 38.0 \pm 14.6 \mathrm{pg} /$ pleural cavity; $\mathrm{n}=5$ ) in the pleural cavity of WT and PI3K $\gamma^{-1-}$ mice. There was no difference in basal production of eotaxin or IL-5 between WT or PI3K $\gamma^{-1-}$ mice (data not shown).

\section{Effects of treatment with PI3K inhibitors (wortmannin and LY294002) on recruitment of eosinophils}

The next experiments were designed to investigate whether a pharmacological strategy, i.e., inhibitor of PI3Ks, could mimic the phenotype observed in PI3K $\gamma^{-1-}$ mice. Preliminary doseresponse studies demonstrated that the best dose of systemically administered wortmannin to affect eosinophil accumulation was $1 \mathrm{mg} / \mathrm{kg}$ (OVA+vehicle, $4.5 \pm 0.2 \times 10^{5}$ eosinophil/ cavity; OVA + wortmannin, $0.1 \mathrm{mg} / \mathrm{kg}: 4.9 \pm 0.9 \times 10^{5}$ eosinophil/ cavity; OVA + wortmannin, $0.3 \mathrm{mg} / \mathrm{kg}: 4.2 \pm 0.6 \times 10^{5}$ eosinophil/ cavity; OVA + wortmannin, $1 \mathrm{mg} / \mathrm{kg}: 1.4 \pm 0.3 \times 10^{5}$ eosinophil/ cavity). Thus, as seen in Figure 3A, sensitized mice that had been treated systemically with the PI3K inhibitor wortmannin ( $1 \mathrm{mg} / \mathrm{kg}), \mathrm{l}$ h before OVA challenge, had a significant reduction in the recruitment of eosinophils into the pleural cavity when compared with vehicle-treated controls. A significant reduction in the recruitment of total cells, mononuclear cells, and neutrophils was also observed with the wortmannin treatment (Table 3). In contrast to its effects when given systemically, wortmannin failed to affect the allergen-induced recruitment of eosinophils when given into the pleural cavity (locally) $1 \mathrm{~h}$ before the challenge (Fig. $3 \mathrm{~B}$ ). The reduction in the number of eosinophils was also seen when LY294002 $(1.0 \mathrm{mg} / \mathrm{kg})$, which is a structurally distinct PI3K inhibitor, was injected systemically but not when injected locally $1 \mathrm{~h}$ before challenge in sensitized mice (Fig. 3).

TABLE 2. Number of Eosinophils in Bone Marrow and Blood at 6,24, and $48 \mathrm{~h}$ after Antigen Challenge of Sensitized PI3K $\gamma^{-1-}$ and WT Mice

\begin{tabular}{lrrrrrr}
\hline \hline & \multicolumn{2}{c}{ Bone marrow } & & \multicolumn{2}{c}{ Blood } \\
\cline { 3 - 4 } \cline { 6 - 7 } \cline { 6 - 7 } & & WT & PI3K $\gamma^{-1-}$ & & WT & PI3K $\gamma^{-1-}$ \\
\hline PBS & & $5.5 \pm 0.7$ & $5.1 \pm 1.3$ & & $0.4 \pm 0.3$ & $1.5 \pm 0.7$ \\
OVA & $6 \mathrm{~h}$ & $4.9 \pm 1.1$ & $5.8 \pm 1.1$ & & $2.2 \pm 0.7$ & $2.0 \pm 0.7$ \\
& $24 \mathrm{~h}$ & $7.8 \pm 2.5$ & $5.4 \pm 1.6$ & & $1.2 \pm 0.4$ & $2.6 \pm 1.9$ \\
& $48 \mathrm{~h}$ & $3.2 \pm 1.5$ & $4.5 \pm 0.9$ & & $0.3 \pm 0.1$ & $1.2 \pm 0.5$ \\
\hline
\end{tabular}

Immunized WT or PI3K $\gamma^{-1-}$ mice were challenged with an i.pl. injection of OVA ( $1 \mu \mathrm{g} /$ cavity) or PBS, and number of eosinophils in bone marrow and blood was assessed after 6,24 , and $48 \mathrm{~h}$. Results are expressed as the number of cells $\times 10^{5}$ per femur (bone marrow) or $\mathrm{ml}$ (blood) and are the means \pm SEM of five mice in each group. $P<0.01$ when compared with WT mice. 
A

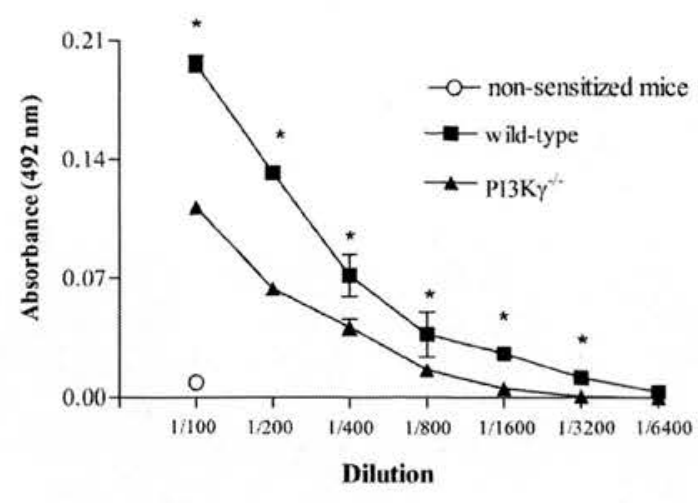

B

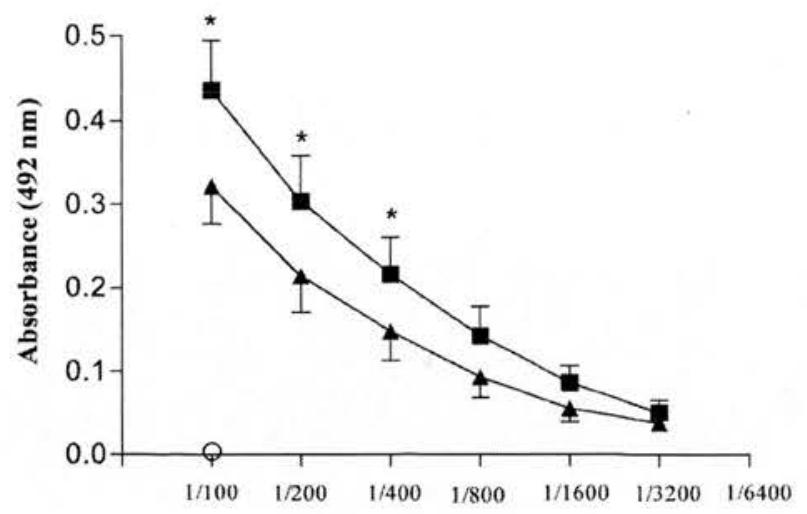

Dilution

Fig. 2. Titers of anti-OVA Ig in nonsensitized and in sensitized PI3K $\gamma^{-1-}$ and WT mice. PI3K $\gamma^{-1-}$ or WT mice were immunized with OVA and blood serum. samples obtained 7 days after the last immunization. The titers of (A) total and (B) $\mathrm{IgG}_{1}$ anti-OVA Ig were assessed by ELISA. Results are expressed as means \pm SEM absorhance of five mice in eight serial dilutions. ${ }^{*}, P<0.01$, when compared with WT mice.

As eosinophil accumulation in PI3K $\gamma^{-1-}$ mice was inhibited at $48 \mathrm{~h}$ as compared with WT mice but not at earlier time-points ( 6 and $24 \mathrm{~h}$ ), we evaluated whether administration of wortmannin $1 \mathrm{~h}$ before antigen challenge could also affect responses in PI3K $\gamma^{-1-}$ mice. The recruitment of eosinophils into the pleural cavity $24 \mathrm{~h}$ after antigen challenge was reduced significantly in sensitized $\mathrm{PI} 3 \mathrm{~K} \gamma^{-1-}$ mice that had been pretreated systemically with the PI3K inhibitor wortmannin (vehicle+OVA: $2.5 \pm 0.4 \times 10^{5}$ eosinophil/cavity; wortamannin + OVA: $0.2 \pm 0.2 \times 10^{5}$ eosinophil/ cavity; $n=4)$.

The serine-threonine PKB/Akt is a major target of PI3K activation $[2,11,17,18]$. Thus, a series of experiments analyzing Akt phosphorylation was carried out to verify whether OVA challenge was accompanied by PI3K activation and whether the dose of wortmannin had been effective for enzyme inhibition. When compared with PBS controls, OVA challenge of sensitized mice triggered significant Akt phosphorylation in pleural cell extracts at $2 \mathrm{~h}$ after challenge (Fig. 4). Systemic treatment with wortmannin $1 \mathrm{~h}$ before OVA challenge inhibited Akt phosphorylation to baseline levels (Fig. 4).

We then evaluated whether systemic treatment with wortmannin $1 \mathrm{~h}$ before OVA challenge could affect the antigenassociated increase of eosinophils in the bone marrow. Sensitized mice were challenged with OVA, and the number of eosinophils in bone marrow was examined. Antigen challenge resulted in a decrease in the number of eosinophils in the bone marrow that was first noticed at $6 \mathrm{~h}$ and was marked at $24 \mathrm{~h}$ after challenge when compared with sensitized mice challenged with PBS (Fig. 5). Systemic treatment with wortmannin
A

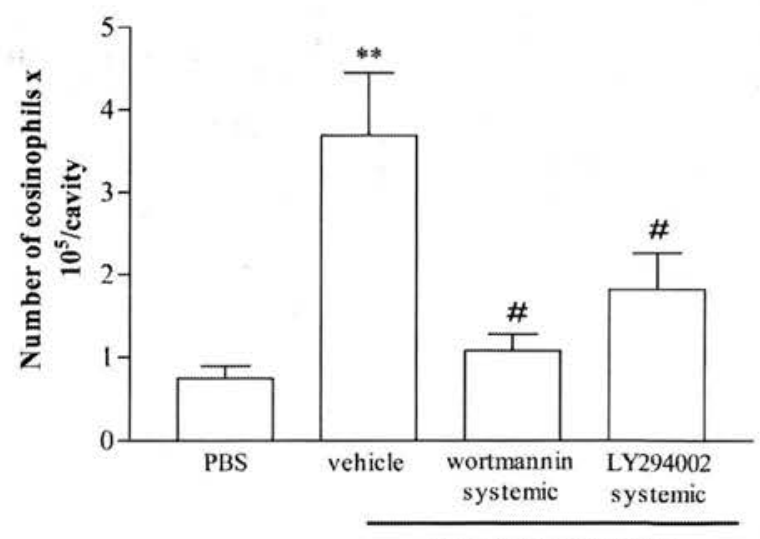

OVA (1 $\mu \mathrm{g}$ per cavity)
B

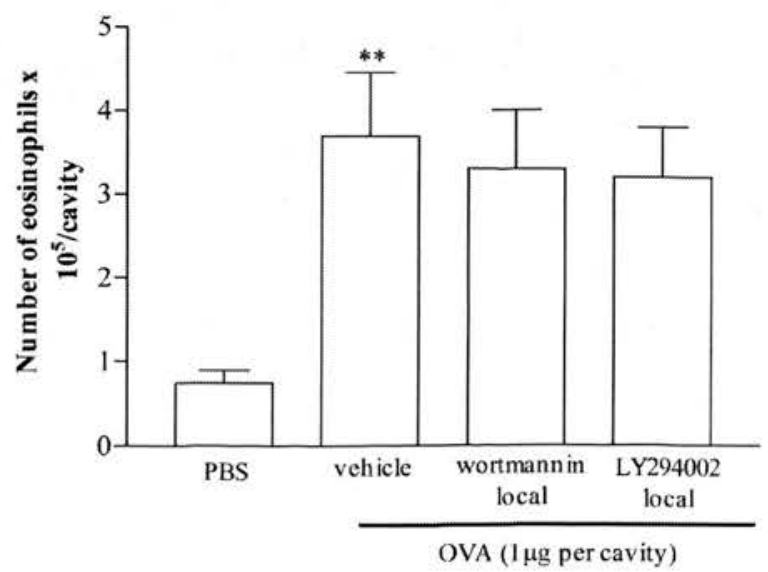

Fig. 3. Effects of the pretreatment with the PI3K inhibitors wortmannin and LY294002 on the recruitment of eosinophils induced by antigen challenge of sensitized mice. Wortmannin or LY294002 was administered (A) systemically (i.p.) or (B) locally (i.pl.) at the dose of $30 \mu \mathrm{g} / \mathrm{mouse}$ ( $1.0 \mathrm{mg} / \mathrm{kg}$ ), $60 \mathrm{~min}$ prior to the challenge with OVA (1 $\mu \mathrm{g} /$ cavity) or PBS. Eosinophil recruitment was assessed $48 \mathrm{~h}$ after antigen challenge. Results are expressed as the means \pm SEM of five mice in each group. ${ }^{* *}, P<0.01$, when compared with PBS-injected mice and $\#, P<0.01$, when compared with vehicle-treated, OVA-challenged mice. 
TABLE 3. Effects of the Systemic Pretreatment with the PI3K Inhibitor, Wortmannin, on the Recruitment of Leukocytes Induced by Antigen Challenge of Sensitized Mice

\begin{tabular}{lllll}
\hline \hline & \multicolumn{1}{c}{ Total } & Mononuclear & Neutrophils & Eosinophils \\
\hline PBS & $12.8 \pm 1.6$ & $12.2 \pm 1.5$ & $0.05 \pm 0.03$ & $0.6 \pm 0.2$ \\
OVA & $26.2 \pm 4.4^{*}$ & $22.8 \pm 5.5^{*}$ & $0.15 \pm 0.03^{*}$ & $3.2 \pm 0.9^{*}$ \\
OVA + & & & & \\
$\quad$ wortmannin & $11.4 \pm 1.9^{\#}$ & $10.3 \pm 1.8^{\#}$ & $0.05 \pm 0.03^{\#}$ & $1.1 \pm 0.2^{\#}$ \\
\hline
\end{tabular}

Wortmannin was administered systemically (i.p.) at the dose of $30 \mu \mathrm{g} /$ mouse (1.0 mg/kg), $60 \mathrm{~min}$ prior to the challenge with OVA (1 $\mu \mathrm{g} /$ cavity) or PBS. Leukocyte recruitment was assessed $48 \mathrm{~h}$ after antigen challenge. Results are expressed as the means \pm SEM of five mice in each group. ${ }^{*} P<0.01$ when compared with PBS-injected mice and "P $<0.01$ when compared with vehicle-treated, OVA-challenged mice.

prevented the fall in bone marrow eosinophil numbers at $6 \mathrm{~h}$ after antigen challenge but had no significant effects at 24. (Fig. 5) or $48 \mathrm{~h}$ (data not shown). Consistently, a lower number of circulating eosinophils was detected $24 \mathrm{~h}$ after antigen challenge in wortmannin-treated mice $\left(\mathrm{OVA}, 2.1 \pm 0.3 \times 10^{5}\right.$ eosinophils/ml blood; wortmannin+OVA, $1.2 \pm 0.2 \times 10^{5}$ eosinophils/ml blood; $\mathrm{n}=5, P<0.001)$. It is interesting that the antigen-induced increase in concentrations of IL-5 in the pleural cavity at $6 \mathrm{~h}$ after challenge was prevented by systemic treatment with wortmannin (Fig. 6).

\section{Effects of treatment with $\mathrm{PI} 3 \mathrm{~K}$ inhibitors (wortmannin and LY294002) on survival of eosinophils}

Next, we examined whether in addition to suppressing eosinophil recruitment, wortmannin could affect eosinophil survival in the allergic pleurisy model. As seen in Figure 4, there was significant Akt phosphorylation (and hence, PI3K activation) at $26 \mathrm{~h}$ after challenge. To this end, wortmannin or drug vehicle was administered $24 \mathrm{~h}$ after challenge, and the number of eosinophils accumulated in the cavity was examined after a further $24 \mathrm{~h}$. Our previous studies have shown that the number of eosinophils in the pleural cavity of allergen-challenged, sensitized mice is stable between 24 and $48 \mathrm{~h}$ after challenge [26]. As seen in Figure 7, systemic treatment with wortmannin $24 \mathrm{~h}$ after OVA challenge failed to affect the accumulation of eosinophils in the pleural cavity observed after a further $24 \mathrm{~h}$ (Fig. 7A). In contrast, local treatment with the drug markedly diminished the accumulation of these cells (Fig. 7B). Again, the reduction in the number of eosinophils was also seen when LY294002 (1.0 mg/kg) was injected only locally $24 \mathrm{~h}$ after challenge in sensitized mice (OVA, $6.9 \pm 1.1 \times 10^{5}$ eosinophils/ cavity; OVA+LY294002, $2.8 \pm 0.3 \times 10^{5}$ eosinophils/cavity; $\mathrm{n}=5, P<0.05)$. In addition, local treatment with wortmannin $24 \mathrm{~h}$ after the challenge inhibited the late antigen-associated Akt phosphorylation to background levels (Fig. 4).

As few eosinophils were recruited from 24 to $48 \mathrm{~h}$ after antigen challenge, and local wortmannin treatment markedly inhibited the accumulation of these cells and Akt phosphorylation in the pleural cavity, we tested whether wortmannininduced apoptosis could account for the observed results. The number of apoptotic eosinophils, as assessed by morphologic criteria, was markedly elevated in the pleural cavity of antigen- challenged and then wortmannin-treated mice (Fig. 8A). Similarly, local administration of LY294002 $24 \mathrm{~h}$ after antigen challenge significantly increased the number of apoptotic cells (Fig. 8A). In both cases, the majority of apoptotic cells had typical eosinophil granules and was located within macrophages, presumably having been engulfed by the latter cells (data not shown). To confirm the morphological findings, we performed additional experiments in which surface changes associated with apoptosis were assessed at earlier time-points, as the exposure of phosphatidylserine precedes the recognition of the apoptotic cells by phagocytes [27]. To this end, we used FITC-labeled annexin- $\mathrm{V}$ binding in the presence of $\mathrm{Ca}^{+2}$ to label phosphatidylserine molecules exposed on the outer membrane of apoptotic cells [27, 28]. In agreement with the morphological assessment, there was a rapid increase in the number of apoptotic events $2 \mathrm{~h}$ after treatment with wortmannin when compared with untreated mice (Fig. 8B).
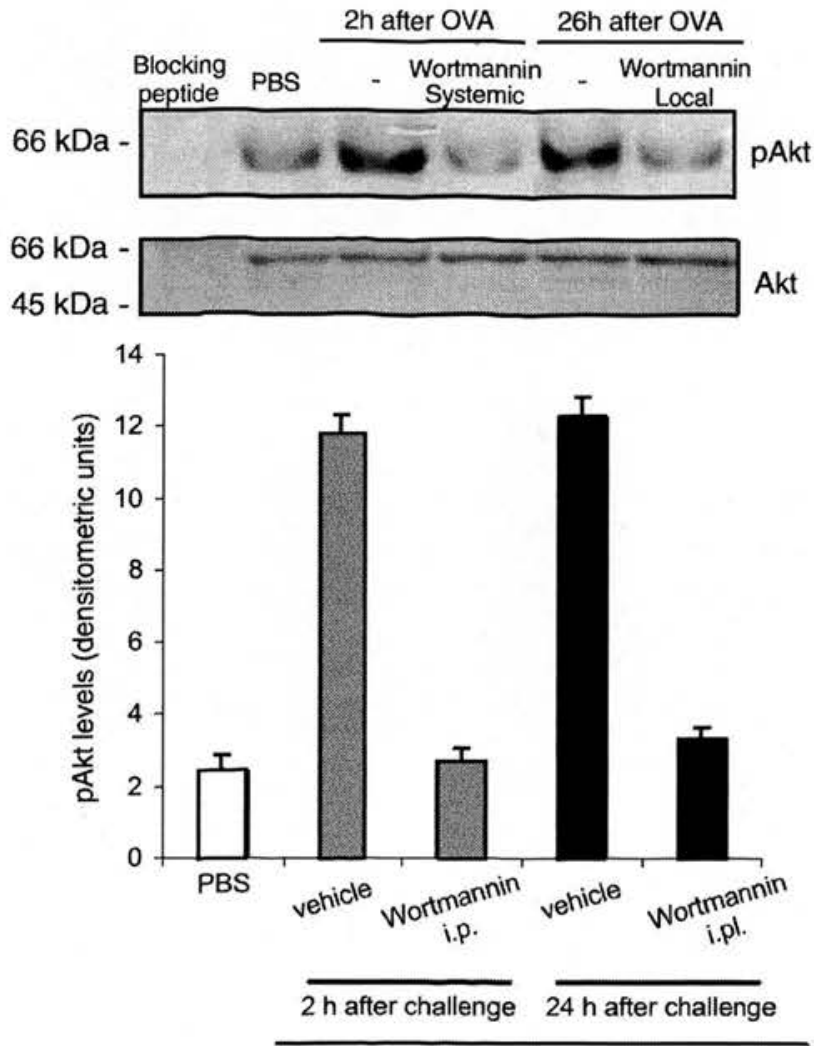

OVA

Fig. 4. Effects of pre- and post-treatment with wortmannin on OVA-induced Akt phosphorylation. Wortmannin or drug vehicle was administered systemically (i.p.), $60 \mathrm{~min}$ prior to or locally (i.pl.), $24 \mathrm{~h}$ after the challenge with OVA ( $1 \mu \mathrm{g} /$ cavity) in sensitized mice. Controls received PBS in challenge. Cell lysates harvested from the pleural cavity 2 or $26 \mathrm{~h}$ after the challenge were then immunoprecipitated with anti- $\mathrm{\Lambda kt}$ antibody and immunoblotted with anti- $\mathrm{\Lambda kt}$ and antiphosphoserine antibodies. The bands were quantified by densitometry, using Scion image software. Negative control is shown by using blocking peptide. The position to which the molecular mass standards migrated is marked: BSA (66 kDa); OVA (45 kDa). Results are expressed as the means 士 SEM of three mice in each group. 


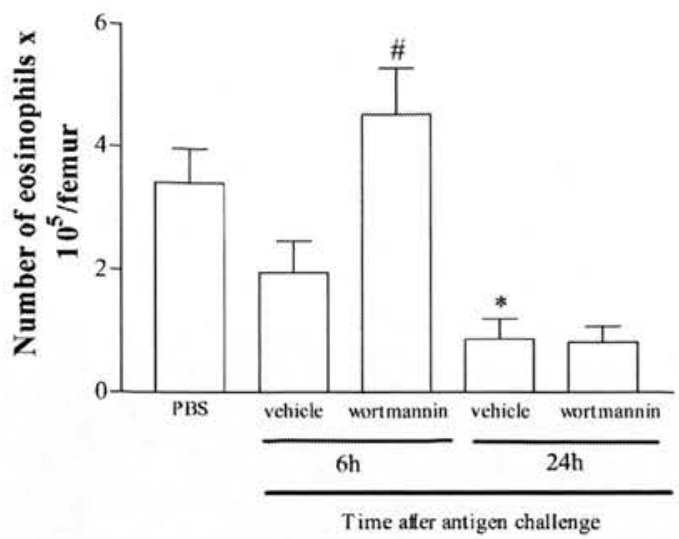

Fig. 5. Effects of the pretreatment with the PI3K inhibitor wortmannin on the number of eosinophils in the bone marrow after antigen challenge of sensitized mice. Wortmannin was administered systemically (i.p.) at the dose of 30 $\mu \mathrm{g} /$ mouse $(1.0 \mathrm{mg} / \mathrm{kg}), 60 \mathrm{~min}$ prior to the challenge with OVA ( $1 \mu \mathrm{g} /$ cavity) or PBS. Eosinophil number in the bone marrow was assessed at 6 and $24 \mathrm{~h}$ after antigen injection. Results are expressed as the means \pm SEM of five mice in each group. *, $P<0.01$, when compared with PBS-injected mice and \#, $P<0.01$, when compared with vehicle-treated, OVA-challenged mice.

\section{DISCUSSION}

In allergic diseases, such as asthma and atopic dermatitis, there is a characteristic, T cell-mediated recruitment of eosinophils into the affected tissues [12]. Although the role of eosinophils in asthma and other allergic diseases is not completely understood, it is thought that the recruitment and action of eosinophils may underlie several of the pathophysiological manifestations of allergic diseases $[12,13,15]$. Thus, an understanding of the mechanisms involved in eosinophil recruitment, activation, and survival in sites of allergic inflammation may be useful for the development of novel pharmacological therapies to control allergic diseases. More recently, there has been a great interest in the understanding of the signal transduction pathways following activation of serpentine receptors and especially following activation of chemokine receptors, which appear to play a major role in the recruitment of leukocytes in vivo [29]. In the present study, we have used pharmacological and genetic approaches to evaluate the role of PI3K for the migration of eosinophils in allergic reactions in the pleural cavity of mice. In addition, we investigated the importance of signaling through the PI3K pathway for eosinophil survival in these inflammatory sites.

Recent studies have demonstrated the importance of the class IB of PI3K (PI3K $\gamma$ ) for leukocyte recruitment during acute inflammation [6, 8]. Indeed, the recruitment of neutrophils and macrophages was reduced in $\mathrm{PI} 3 \mathrm{~K}^{-1-}$ mice after stimulation with various chemoattractants and in a septic peritonitis model $[6,8,30]$. Here, we demonstrate that eosinophil accumulation was similar in PI3K $\gamma^{-1-}$ and W'T mice in an allergic pleurisy model when evaluated 6 and $24 \mathrm{~h}$ after antigen challenge. However, at $48 \mathrm{~h}$, the accumulation of eosinophils at sites of allergic inflammation was markedly reduced in PI3K $\gamma^{-1-}$ mice when compared with their WT counterparts. We made chimerical mice lacking PI3K $\gamma$ on bone marrow-derived cells or on non-bone marrow-derived cells. Our results demonstrate that the lack of PI3K $\gamma$ in bone marrow-derived leukocytes but not on non-bone marrow cells is responsible for the reduction in the accumulation of eosinophils at $48 \mathrm{~h}$ after antigen challenge. Thus, it is clear from the data above that the expression of PI3K $\gamma$ by bone marrowderived leukocytes, presumably by the eosinophil itself, is not essential for the early recruitment (arrival) of eosinophils but is necessary for their persistence in the pleural cavity $48 \mathrm{~h}$ after allergen challenge of sensitized mice. Although we failed to detect enhanced numbers of apoptotic figures in PI3K $\gamma^{-1-}$ mice, the clearance of eosinophils observed at $48 \mathrm{~h}$ after challenge in PI3K $\gamma^{-1-}$ mice may be related to the relevance of this enzyme in preventing eosinophil apoptosis and the resolution of the inflammatory process.

It is interesting that PI3K $\gamma^{-1-}$ mice had lower titers of total serum $\mathrm{Ig}$ and $\operatorname{IgG}_{1}$. These results are consistent with studies demonstrating that $\mathrm{PI} 3 \mathrm{~K} \gamma^{+1-}$ mice exhibited high titers of nitroiodophenylacetic acid conjugated to OVA-specific IgG antibodies but lower levels of these antibodies in PI3K $\gamma^{-1-}$ mice [8]. Initial events during an allergic reaction involve the release of proinflammatory mediators by mast cells triggered by the binding of the allergen to $\mathrm{IgE}$ antibodies bound to mast cells FceRI. Murine $\mathrm{IgG}_{1}$ is also capable of sensitizing murine mast cells [31]. In addition, it has recently been demonstrated that mast cells from PI3K $\gamma^{-1-}$ mice had defective degranulation in vitro and responded poorly to adenosine or a passive systemic anaphylaxis in vivo [32]. Thus, although the differences in titers between WT and PI3K $\gamma^{-1-}$ mice were small, the diminished eosinophil accumulation observed in our studies could be secondary not only to a deficient production of $\mathrm{Ig}$ and insufficient mast cell sensitization but also to deficient mast cell function. Nevertheless, it is worth noting that the production of eotaxin and IL-5 in the pleural cavity was similar in PI3K $\gamma^{-1-}$ and WT mice. We have previously shown that eotaxin is released in the allergic pleurisy model and contrib-

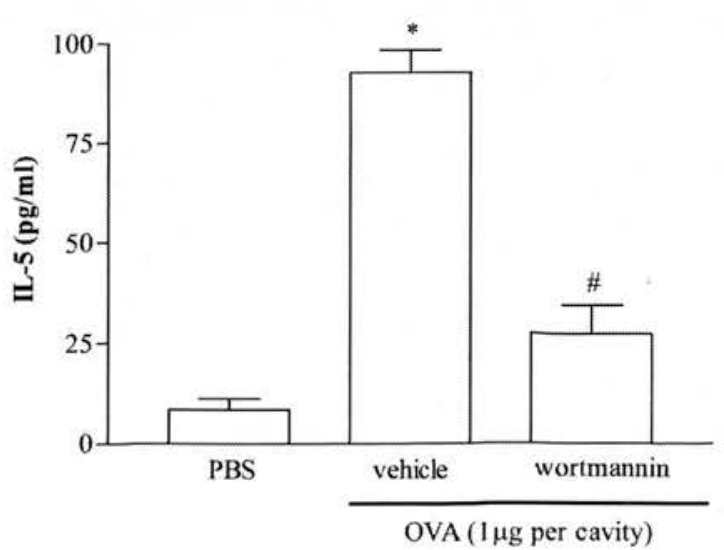

Fig. 6. Effects of the pretreatment with the PI3K inhibitor wortmannin on the production of IL-5 in the pleural cavity after antigen challenge of sensitized mice. Wortmannin was administered systemically (i.p.) at the dose of 30 $\mu \mathrm{g} /$ mouse $(1.0 \mathrm{mg} / \mathrm{kg}), 60 \mathrm{~min}$ prior to the challenge with OVA $(1 \mu \mathrm{g} /$ cavity) or PBS. IL-5 concentrations were assessed $6 \mathrm{~h}$ after antigen challenge. Results are expressed as the means \pm SEM of five mice in each group. ${ }^{*}, P<0.01$, when compared with PBS-injected mice and \#, $P<0.01$, when compared with vehicle-treated, OVA-challenged mice. 
A

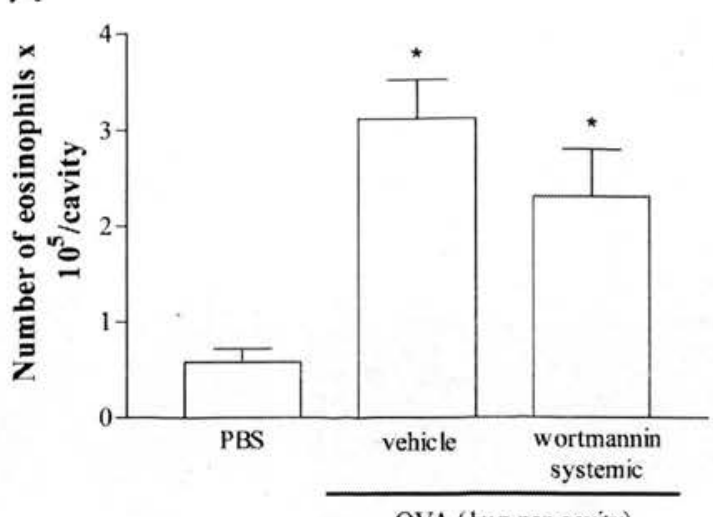

B

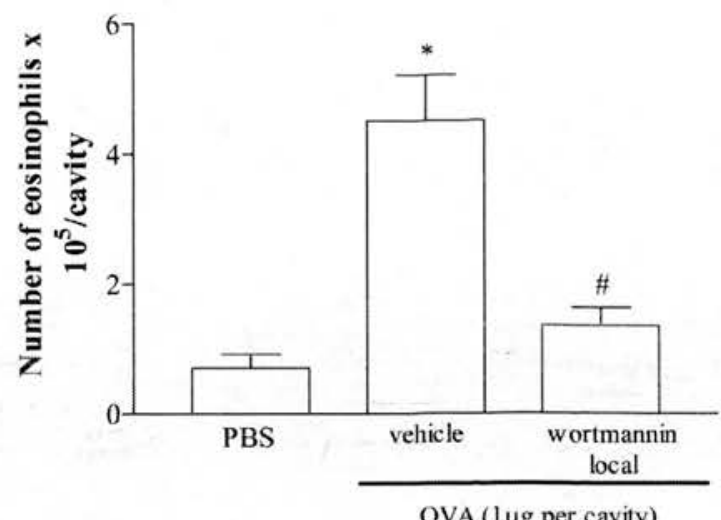

Fig. 7. Effects of therapeutic treatment with the PI3K inhibitor wortmannin on the accumulation of eosinophils in the pleural cavity of allergic mice. Immunized animals were challenged with OVA ( $1 \mu \mathrm{g} /$ cavity) or PBS and $24 \mathrm{~h}$ later, received a (A) systemic (i.p.) or (B) local (i.pl.) injection of wortmannin at the dose of 30 $\mu \mathrm{g} /$ mouse $(1.0 \mathrm{mg} / \mathrm{kg})$. The number of eosinophils accumulating in the pleural cavity was assessed after a further $24 \mathrm{~h}$. Results are expressed as the means \pm SEM of five mice in each group. ${ }^{*}, P<0.01$, when compared with PBS-injected mice and \#, $P<0.01$, when compared with vehicle-treated, OVA-challenged mice.

utes markedly to eosinophil recruitment in response to antigen challenge [25]. Similarly, many studies have demonstrated an important role of IL-5 for the recruitment of eosinophils in vivo [33, 34]. Although IL-5 and eotaxin appear to be produced by T cells [35] and macrophages [25], respectively, the results suggest that the initial mast cell-triggered events occur adequately in PI3K $\gamma^{-1-}$ mice. Thus, it appears that diminished sensitization (as seen by the decrease in Ig titers) and defective mast cell function may not be functionally relevant phenomena suppressed in PI3K $\gamma^{-1-}$ mice, as the most relevant mediators of eosinophil recruitment were being produced in adequate concentrations. This is consistent with the findings that the number of eosinophils that were recruited into the pleural cavity in the initial phase $(6-24 \mathrm{~h})$ of the allergic response was similar in $\mathrm{PI} 3 \mathrm{~K} \gamma^{-1-}$ and WT mice.

Next, we evaluated whether modulation of eosinophil recruitment was also observed when a pharmacological strategy was used to prevent PI3K function. As selective PI3K $\gamma$ inhibitors are not commercially available, we tested the effects of the isoform-nonselective PI3K inhibitors wortmannin and LY294002 in the allergic pleurisy reaction. In our experiments, no inhibition of eosinophil recruitment was observed when wortmannin or LY294002 was administrated locally $1 \mathrm{~h}$ before antigen challenge. This is in agreement with another study demonstrating that intranasal-administered wortmannin could inhibit allergen-induced airway hyper-responsiveness in vivo, probably by inhibiting eosinophil degranulation, but not their accumulation in bronchoalveolar lavage fluid [21]. However, the intrapulmonary administration of wortmannin before allergen challenge was an efficient inhibitor of the immediatetype allergic response and the late-phase pulmonary inflammation in a model of experimental asthma in Brown Norway rats [36]. Why shouldn't the drug then function when given locally? Two major possibilities may be raised to explain these apparent contradictory results. First, wortmannin may have already been cleared when the relevant chemoattractant molecules are produced. For example, eotaxin production peaks $6 \mathrm{~h}$ after antigen challenge [25] and hence, at least $6 \mathrm{~h}$ after local administration of wortmannin. Second, the main site of action of the drug may be the eosinophil itself. The latter possibility entails that the leukocyte has to be inhibited by the drug before it is recruited to the site of allergic inflammation. However, as discussed above, the lack of PI3K $\gamma$ on eosinophils was not associated with defective recruitment (arrival) but only with defective late accumulation of these cells.

In contrast to its lack of effect when administered locally, wortmannin markedly prevented eosinophil migration in the pleurisy model when given systemically. These results were confirmed by use of LY294002, which is a structurally distinct PI3K inhibitor [37]. The inhibition of PI3K on circulating eosinophils or at a site relevant for the recruitment of eosinophils but distinct from the site of allergic inflammation could provide an alternative possibility to explain the latter results. The bone marrow is a possible site for the action of the PI3K inhibitors, as several studies have pointed to the major role of this organ for the recruitment of eosinophils in vivo [38, 39]. In allergen-challenged mice, wortmannin prevented the antigeninduced fall in bone marrow eosinophils at $6 \mathrm{~h}$ after challenge. The effect of systemic wortmannin on bone marrow eosinophils was consistent with the finding that antigen-induced IL-5 production and a circulating number of eosinophils were also prevented by the drug. It has been shown previously that the mobilization of bone marrow eosinophils stimulated by IL- 5 involves signaling through PI3K [40]. It is worth noting the contrasting effects of wortmannin treatment (inhibition) and the genetic strategy (no effect) on the concentrations of IL-5 after allergen challenge, suggesting that a PI3K isoform other than $\mathrm{PI} 3 \mathrm{~K} \gamma$ is involved in the inhibitory effects of wortmannin on IL-5 production. This result supports previous reports in which production of IL-4 and IL- 5 by mesenteric lymphocyte from $\mathrm{PI} 3 \mathrm{~K} / \mathrm{p} 85^{-1-}$ mice (deficient in class IA PI3K isoforms) infected with Strongyloides venezuelensis was reduced [41]. Therefore, one possibility to explain the effects of systemic but not local treatment with the PI3K inhibitors on the recruitment of eosinophils in the allergic pleurisy model could be that the inhibitors are preventing eosinophil release from bone marrow 


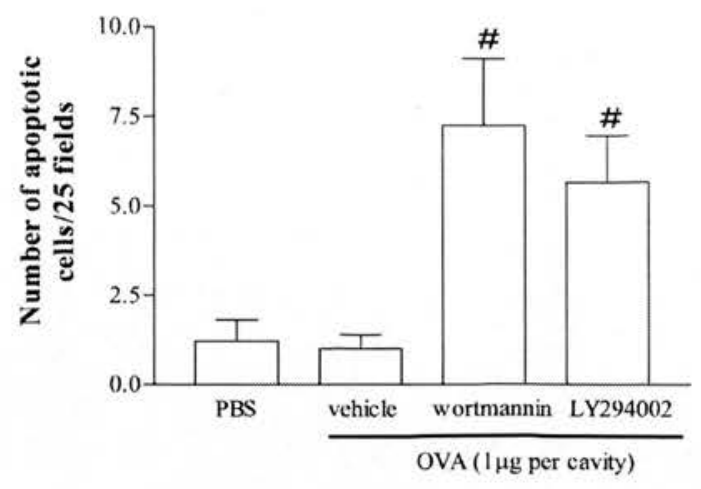

B

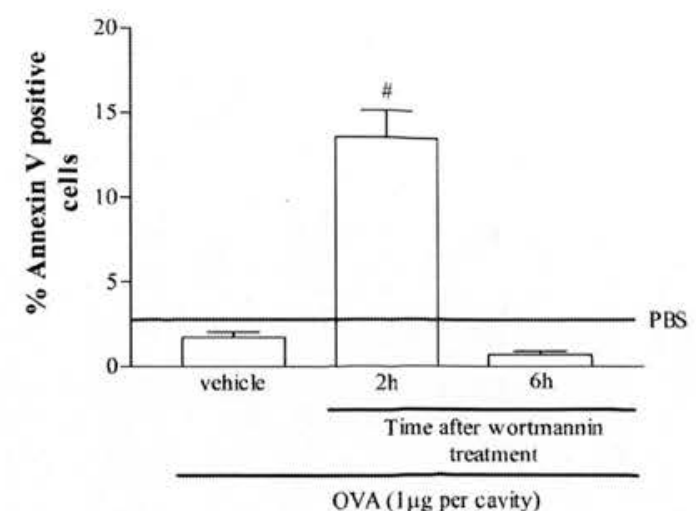

C
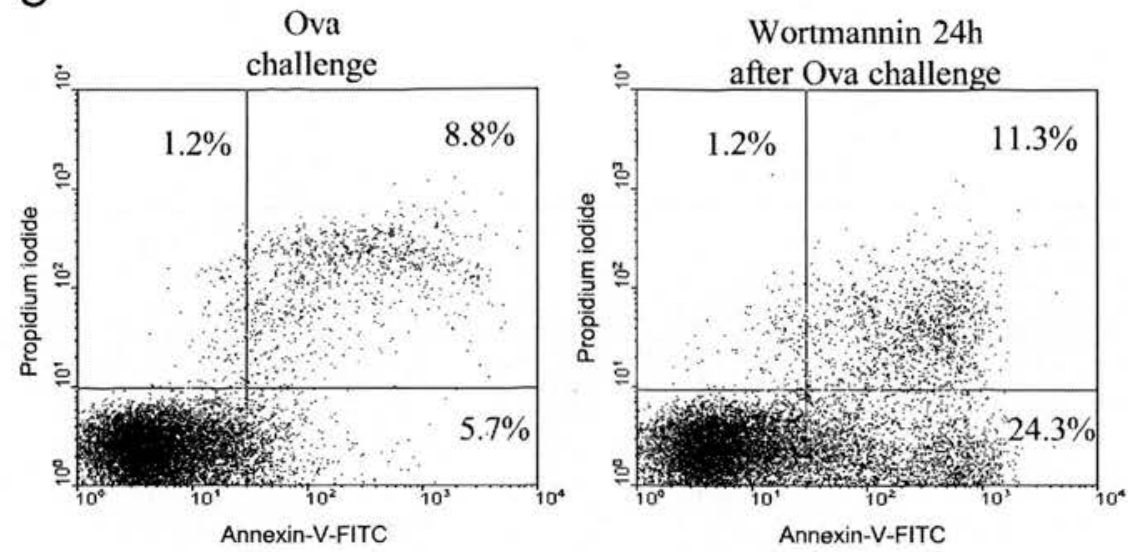

Fig. 8. Effects of therapeutic treatment with the PI3K inhibitors wortmannin or LY294002 on the number of apoptotic cells in the pleural cavity of allergic mice. Immunized animals were challenged with OVA ( $1 \mu \mathrm{g} /$ cavity) or PBS and $24 \mathrm{~h}$ later, received a local (i.pl.) injection of wortmannin or LY294002 at the dose of $30 \mu \mathrm{g} /$ mouse $(1.0 \mathrm{mg} / \mathrm{kg})$. The number of cells undergoing apoptosis was defined (A) morphologically or (B and C) by flow cytometry at the indicated times. (A) Cells $\left(5 \times 10^{4}\right)$ were collected $48 \mathrm{~h}$ after antigen challenge and were cytocentrifuged, fixed, and stained with May-Grunwald-Giemsa. The proportion of cells with distinctive apoptotic morphology was evaluated in 25 fields. Results are expressed as the mean \pm SEM of the number of apoptotic cells in 25 fields. (B and C) Cells $\left(2.5 \times 10^{5}\right)$ were collected $2 \mathrm{~h}(\mathrm{~B}$ and $\mathrm{C})$ and $6 \mathrm{~h}(\mathrm{~B})$ after wortmannin or vehicle treatment and incubated with FITC-labeled annexin-V and propidium iodide. Results are expressed as the mean \pm SEM of the percentage of cells staining with annexin-V-FITC alone. There were at least five mice in each group. \#, $P<0.01$, when compared with vehicle-treated, OVA-challenged mice.

when given systemically. Alternatively (or in addition to the mechanism proposed above), it is possible that the released eosinophils could have their PI3K enzymes inhibited by the systemic treatment with the inhibitors in blood, thus, prior to being stimulated to migrate to tissue. As systemic PI3K inhibitors prevented eosinophil recruitment, and no difference was found in eosinophil recruitment into the pleural cavity 6 and $24 \mathrm{~h}$ after antigen challenge in $\mathrm{PI} 3 \mathrm{~K} \gamma^{-1-}$ mice, we suggest that a PI3K isoform other than PI3K $\gamma$ may be contributing to eosinophil recruitment in our model. In keeping with the latter suggestion, the migration of neutrophils and macrophage in response to some chemokines was only partially reduced in $\mathrm{PI} 3 \mathrm{~K} \gamma^{-1-}$ mice, although these cells were unable to produce $\mathrm{PI}(3,4,5) \mathrm{P}_{3}$ and activate Akt/PKB $[6,8]$. Furthermore, this hypothesis is supported by the observation that the pretreatment of PI3K $\gamma^{-1-}$ mice with wortmannin, $1 \mathrm{~h}$ before OVA challenge, prevented the recruitment of eosinophils to the pleural cavity of drug-treated mice. Moreover, it has recently been demonstrated that antigen-challenged mice treated with a dominant-negative form of the class IA PI3K regulatory subunit had reduced airway eosinophilia and airway hyper-reactivity [42]. In this regard, a role for PI3K $\delta$ to polymorphonuclear cell polarization and directional migration has been documented previously [43]. Therefore, in future studies, it will be important to verify the role of other classes of PI3K in our model.

The eosinophil possesses a considerable variety of histotoxic molecules, which contribute to the initiation and maintenance of the allergic inflammatory response $[12,13,44]$. Thus, the clearance of eosinophils from inflammatory sites may have therapeutic implications [45]. In addition to their central role in cell proliferation and migration, class I PI3K has also been implicated in the prevention of apoptotic cell death [46, 47]. Thus, studies have demonstrated that the PI3K/Akt pathway is constitutively activated in the majority of human pancreatic cancer cell lines [48]. Moreover, use of selective inhibitors of PI3K could inhibit growth and survival of tumors [49]. Other studies have shown that PI3K is an important factor of survival 
in monocytes [50], neutrophils [51, 52], and eosinophils [53] when stimulated with growth factors in vitro. In our study, we demonstrate that when wortmannin or LY294002 was injected locally $24 \mathrm{~h}$ after challenge, there was a decrease of the number of eosinophils in the pleural cavity. It is interesting that intratracheal wortmannin also suppressed pulmonary eosinophil influx when administered before and after antigen challenge in mice [54]. The inhibition of eosinophil influx after post-treatment with wortmannin correlated with inhibition of Akt phophorylation and an increase in the number of apoptotic cells. Thus, local inhibition of PI3K after antigen challenge may be blocking an important signaling pathway induced by eosinophil survival factors. The nature of these PI3K-dependent eosinophil survival factors needs to be investigated. The increase of apoptotic cells and consequent decrease in number of eosinophils in the pleural cavity were not seen when wortmannin was administrated systemically, possibly as a result of limited access of the drug into the pleural cavity. Together, these results demonstrate that wortmannin may prevent the accumulation of eosinophils in an allergic pleurisy model by inhibiting eosinophil recruitment when given systemically before antigen challenge and by inducing eosinophil apoptosis when given locally after antigen challenge. The ability of PI3K inhibitors to induce eosinophil apoptosis may provide a good explanation for the absence of eosinophils $48 \mathrm{~h}$ after antigen challenge of immunized PI3K $\gamma^{-1-}$ mice.

In conclusion, our data demonstrate an important role of PI3K for the recruitment and survival of eosinophils in a model of allergic pleurisy. Eosinophil accumulation in the allergic pleurisy model is sensitive to wortmannin and LY294002, thus suggesting a role of PI3K enzymes. However, the PI3K $\gamma$ isoform appears not to be the most relevant for the recruitment process, and other PI3K isoforms could be involved. Conversely, eosinophil persistence in the pleural cavity is wortmannin- and LY294002-sensitive and depends on the expression of PI3K $\gamma$ by bone marrow-derived cells, presumably by eosinophils themselves. Strategies modulating the resolution of the inflammatory process may yield novel therapies for the treatment of inflammatory and autoimmune diseases [45, 55, 56]. In this regard, manipulation of PI3K activation might be useful therapeutically, not only for the prevention but also for the resolution (by influencing apoptosis of eosinophils) of allergic inflammation.

\section{ACKNOWLEDGMENTS}

We are grateful to Conselho Nacional de Desenvolvimento Científico e Tecnológico (CNPq), Fundação de Amparo a Pesquisas do Estado de Minas Gerais (FAPEMIG), and the Wellcome Trust for financial support for this work.

\section{REFERENCES}

1. Kapeller, R., Cantley, L. C. (1994) Phosphatidylinositol 3-kinase. Bioessays 16, 565-576.
2. Vanhaesebroeck, B., Waterfield, M. D. (1999) Signaling by distinct classes of phosphoinositide 3-kinases. Exp. Cell Res. 253, 239-254.

3. Ward, S. G., June, C. H., Olive, D. (1996) PI 3-kinase: a pivotal pathway in T-cell activation? Immunol. Today 17, 187-197.

4. Curnock, A. P., Logan, M. K., Ward, S. G. (2002) Chemokine signalling: pivoting around multiple phosphoinositide 3-kinases. Immunology 105 , 125-136.

5. Vicente-Manzanares, M., Rey, M., Jones, D. R., Sancho, D., Mellado, M., Rodriguez-Frade, J. M., Del Pozo, M. A., Yanez-Mo, M., De Ana, A. M., Martinez-A, C., Merida, I., Sanchez-Madrid, F. (1999) Involvement of phosphatidylinositol 3-kinase in stromal cell-derived factor-1 $\alpha$-induced lymphocyte polarization and chemotaxis. J. Immunol. 163, 4001-4012.

6. Hirsch, E., Katanaev, V. L., Garlanda, C., Azzolino, O., Pirola, L., Silengo, L., Sozzani, S., Mantovani, A., Altruda, F., Wymann, M. P. (2000) Central role for $\mathrm{G}$ protein-coupled phosphoinositide 3-kinase $\gamma$ in inflammation. Science 287, 1049-1053.

7. Rickert, P., Weiner, O. D., Wang, F., Bourne, H. R., Servant, G. (2000) Leukocytes navigate by compass: roles of PI3K $\gamma$ and its lipid products. Trends Cell Biol. 10, 466-473.

8. Sasaki, T., Irie-Sasaki, J., Jones, R. G., Oliveira-Dos-Santos, A. J., Stanford, W. L., Bolon, B., Wakeham, A., Itie, A., Bouchard, D., Kozieradzki, I., Joza, N., Mak, T. W., Ohashi, P. S., Suzuki, A., Penninger, J. M. (2000) Function of PI3K $\gamma$ in thymocyte development, T cell activation, and neutrophil migration. Science 287, 1040-1046.

9. Servant, G., Weiner, O. D., Herzmark, P., Balla, T., Sedat, J. W., Bourne, H. R. (2000) Polarization of chemoattractant receptor signaling during neutrophil chemotaxis. Science 287, 1037-1040.

10. Turner, S. J., Ward, S. G., Westwick, J. (1995) RANTES-activated human T lymphocyte. A role for phosphoinositide3-kınase. J. Immunol. 155, 2437-2444.

11. Fruman, D. A., Cantley, L. C. (2002) Phosphoinositide 3-kinase in immunological systems. Semin. Immunol. 14, 7-18.

12. Cara, D. C., Negrao-Correa, D., Teixeira, M. M. (2000) Mechanisms underlying eosinophil trafficking and their relevance in vivo. Histol. Histopathol. 15, 899-920.

13. Lacy, P., Moqbel, R. (2001) Immune effector functions of eosinophils in allergic airway inflammation. Curr. Opin. Allergy Clin. Immunol. 1, 7984.

14. Leiferman, K. M. (2001) A role for eosinophils in atopic dermatitis. J. Am. Acad. Dermatol. 45, S21-S24.

15. Kita, H., Gleich, G. J. (1996) Chemokines active on eosinophils: potential roles in allergic inflammation. J. Exp. Med. 183, 2421-2426.

16. Sotsios, Y., Ward, S. G. (2000) Phosphoinositide 3-kinase: a key biochemical signal for cell migration in response to chemokines. Immunol. Rev. 177, 217-235.

17. Datta, S. R., Dudek, H., Tao, X., Masters, S., Fu, H., Gotoh, Y., Greenberg, M. E. (1997) Akt phosphorylation of BAD couples survival signals to the cell-intrinsic death machinery. Cell 91, 231-241.

18. Franke, T. F., Kaplan, D. R., Cantley, L. C. (1997) PI3K: downstream AKTion blocks apoptosis. Cell $\mathbf{8 8}, 435-437$

19. Wymann, M. P., Bulgarelli-Leva, G., Zvelebil, M. J., Pirola, L., Vanhaesebroeck, B., Waterfield, M. D., Panayotou, G. (1996) Wortmannin inactvates phosphoinositide 3-kinase by covalent modification of Lys-802, a residue involved in the phosphate transfer reaction. Mol. Cell. Biol. 16, 1722-1733.

20. Das, A. M., Flower, R. J., Hellewell, P. G., Teixeira, M. M., Perretti, M. (1997) A novel murine model of allergic inflammation to study the effect of dexamethasone on eosinophil recruitment. Br. J. Pharmacol. 121, 97-104.

21. Ezeamuzie, C. I., Sukumaran, J., Philips, E. (2001) Effects of wortmannin on human eosinophil response in vitro and on bronchial inflammation and airway hyperresponsiveness in Guinea pigs in vivo. Am. J. Respir. Crit. Care Med. 164, 1633-1639.

22. Fujihara, S., Ward, C., Dransfield, I., Hay, R. T., Uings, I. J., Hayes, B., Farrow, S. N., Haslett, C., Rossi, A. G. (2002) Inhibition of nuclear factor- $\mathrm{B}$ activation un-masks the ability of TNF- $\alpha$ to induce human eosinophil apoptosis. Eur. J. Immunol. 32, 457-466.

23. Cieslik, K., Abrams, C. S., Wu, K. K. (2001) Up-regulation of endothelial nitric-oxide synthase promoter by the phosphatidylinositol 3-kinase $\gamma / \mathrm{Ja}$ nus kinase 2/MEK-1-dependent pathway. J. Biol. Chem. 276, 12111219.

24. Bozza, P. T., Castro-Faria-Neto, H. C., Penido, C., Larangeira, A. P., Silva, P. M., Martins, M. A., Cordeiro, R. S. (1994) IL-5 accounts for the mouse pleural eosinophil accumulation triggered by antigen but not by LPS. Immunopharmacology 27, 131-136.

25. Klein, A., Talvani, A., Silva, P. M., Martins, M. A., Wells, T. N., Proudfoot, A., Luckacs, N. W., Teixeira, M. M. (2001) Stem cell factor-induced leukotriene B4 production cooperates with eotaxin to mediate the recruit- 
ment of eosinophils during allergic pleurisy in mice. J. Immunol. 167, $524-531$.

26. Klein, A., Talvani, A., Cara, D. C., Gomes, K. L., Lukacs, N. W., Teixeira, M. M. (2000) Stem cell factor plays a major role in the recruitment of eosinophils in allergic pleurisy in mice via the production of leukotriene B4. J. Immunol. 164, 4271-4276.

27. Fadok, V. A., Savill, J. S., Haslett, C., Bratton, D. L., Doherty, D. E., Campbell, P. A., Henson, P. M. (1992) Different populations of macrophages use either the vitronectin receptor or the phosphatidylserine receptor to recognize and remove apoptotic cells. J. Immunol. 149, 40294035.

28. Koopman, G., Reutelingsperger, C. P., Kuijten, G. A., Keehnen, R. M., Pals, S. T., Van Oers, M. H. (1994) Annexin V for flow eytometric detection of phosphatidylserine expression on B cells undergoing apoptosis. Blood 84, 1415-1420.

29. Thelen, M. (2001) Dancing to the tune of chemokines. Nat. Immunol. 2, 129-134.

30. Li, Z., Jiang, H., Xie, W., Zhang, Z., Smrcka, A. V., Wu, D. (2000) Roles of PLC- $\beta 2$ and $-\beta 3$ and PI3K $\gamma$ in chemoattractant-mediated signal transduction. Science 287, 1046-1049.

31. Tigelaar, R. E., Vaz, N. M., Ovary, Z. (1971) Immunoglobulin receptors on mouse mast cells. J. Immunol. 106, 661-672.

32. Laffargue, M., Calvez, R., Finan, P., Trifilieff, A., Barbıer, M., Altruda, F., Hirsch, E., Wymann, M. P. (2002) Phosphoinositide 3-kinase $\gamma$ is an essential amplifier of mast cell function. Immunity 16, 441-451.

33. Foster, P. S., Hogan, S. P., Ramsay, A. J., Matthaei, K. I., Young, I. G. (1996) Interleukin 5 deficiency abolishes eosinophilia, airways hyperreactivity, and lung damage in a mouse asthma model. J. Exp. Med. 183, 195-201.

34. Kopf, M., Brombacher, F., Hodgkin, P. D., Ramsay, A. J., Milbourne, E. A., Dai, W. J., Ovington, K. S., Behm, C. A., Kohler, G., Young, I. G., Matthaei, K. I. (1996) IL-5-deficient mice have a developmental defect in CD5 + B-1 cells and lack eosinophilia but have normal antibody and cytotoxic T cell responses. Immunity 4, 15-24.

35. Ying, S., Humbert, M., Barkans, J., Corrigan, C. J., Pfister, R., Menz, G.. Larche, M., Robinson, D. S., Durham, S. R., Kay, A. B. (1997) Expression of IL-4 and IL-5 mRNA and protein product by CD4+ and CD8+ T cells, eosinophils, and mast cells in bronchial biopsies obtained from atopic and nonatopic (intrinsic) asthmatics. J. Immunol. 158, 3539-3544.

36. Tigani, B., Hannon, J. P., Mazzoni, L., Fozard, J. R. (2001) Effects of wortmannin on airways inflammation induced by allergen in actively sensitized Brown Norway rats. Eur. J. Pharmacol. 433, 217-223.

37. Ward, S., Sotsios, Y., Dowden, J., Bruce, I., Finan, P. (2003) Therapeutic potential of phosphoinositide 3-kinase inhibitors. Chem. Biol. 10, 207213.

38. Hogan, M. B., Piktel, D., Landreth, K. S. (2000) IL-5 production by bone marrow stromal cells: implications for eosinophilia associated with asthma. J. Allergy Clin. Immunol. 106, 329-336.

39. Larangeira, A. P., Silva, A. R., Gomes, R. N., Penido, C., Henriques, M. G., Castro-Faria-Neto, H. C., Bozza, P. T. (2001) Mechanisms of allergen- and LPS-induced bone marrow eosinophil mobilization and eosinophil accumulation into the pleural cavity: a role for CD11b/CD18 complex. Inflamm. Res. 50, 309-316.

40. Palframan, R. T., Collins, P. D., Severs, N. J., Rothery, S., Williams, T. J., Rankin, S. M. (1998) Mechanisms of acute eosinophil mobilization from the bone marrow stimulated by interleukin 5 : the role of specific adhesion molecules and phosphatidylinositol 3-kinase. J. Exp. Med. 188, 16211632.
41. Fukao, T., Yamada, T., Tanabe, M., Terauchi, Y., Ota, T., Takayama, T., Asano, T., Takeuchi, T., Kadowaki, T., Hata Ji, J., Koyasu, S. (2002) Selective loss of gastrointestinal mast cells and impaired immunity in PI3K-deficient mice. Nat. Immunol. 3, 295-304.

42. Myou, S., Leff, A. R., Myo, S., Boetticher, E., Tong, J., Meliton, A. Y., Liu, J.. Munoz. N. M., 7hu, X. (2003) Blockade of inflammation and airway hyperresponsiveness in immune-sensitized mice by dominant-negative phosphoinositide 3-kinase-TAT. J. Exp. Med. 198, 1573-1582.

43. Sadhu, C., Masinovsky, B., Dick, K., Sowell, C. G., Staunton, D. E. (2003) Essential role of phosphoinositide 3-kinase $\delta$ in neutrophil directional movement. J. Immunol. 170, 2647-2654.

44. Giembycz, M. A., Lindsay, M. A. (1999) Pharmacology of the eosinophil Pharmacol. Rev. 51, 213-340.

45. Ward, C., Dransfield, I., Chilvers, E. R., Haslett, I., Rossi, A. G. (1999) Pharmacological manipulation of granulocyte apoptosis: potential therapeutic targets. Trends Pharmacol. Sci. 20, 503-509.

46. Condliffe, A. M., Cadwallader, K. A., Walker, T. R., Rintoul, R. C., Cowburn, A. S., Chilvers, E. R. (2000) Phosphoinositide 3-kinase: a critical signaling event in pulmonary cells. Respir. Res. 1, 24-29.

47. Krasilnikov, M. A. (2000) Phosphatidylinositol-3 kinase dependent pathways: the role in control of cell growth, survival, and malignant transformation. Biochemistry 65, 59-67.

48. Bondar, V. M., Sweeney-Gotsch, B., Andreeff, M., Mills, G. B., MeConkey, D. J. (2002) Inhibition of the phosphatidylinositol 3'-kinase-AKT pathway induces apoptosis in pancreatic carcinoma cells in vitro and in vivo. Mol. Cancer Ther. 1, 989-997.

49. Krystal, G. W., Sulanke, G., Litz, J. (2002) Inhibition of phosphatidylinositol 3-kinase-Akt signaling blocks growth, promotes apoptosis, and enhances sensitivity of small cell lung cancer cells to chemotherapy. Mol. Cancer Ther. 1, 913-922.

50. Kelley, T. W., Graham, M. M., Doseff, A. I., Pomerant», R. W., Lau, S. M., Ostrowski, M. C., Franke, T. F., Marsh, C. B. (1999) Macrophage colonystimulating factor promotes cell survival through $\mathrm{Akt} /$ protein kinase B. J. Biol. Chem. 274, 26393-26398.

51. Klein, J. B., Rane, M. J., Scherzer, J. A., Coxon, P. Y., Kettritz, R., Mathiesen, J. M., Buridi, A., Meleish, K. R. (2000) Granulocyte-macrophage colony-stimulating factor delays neutrophil constitutive apoptosis through phosphoinositide 3-kinase and extracellular signal-regulated kinase pathways. J. Immunol. 164, 4286-4291.

52. Cowburn, A. S., Cadwallader, K. A., Reed, B. J., Farahi, N., Chilvers, E. R. (2002) Role of PI3-kinase-dependent Bad phosphorylation and altered transcription in cytokine-mediated neutrophil survival. Blood 100, 2607-2616.

53. Dijkers, P. F., Medema, R. H., Pals, C., Banerji, L., Thomas, N. S., Lam, E. W., Burgering, B. M., Raaijmakers, J. A., Lammers, J. W., Koenderman, L., Coffer, P. J. (2000) Forkhead transcription factor FKHR-LI modulates cytokine-dependent transcriptional regulation of p27(KIP1). Mol. Cell. Biol. 20, 9138-9148.

54. Kwak, Y. G., Song, C. H., Yi, H. K., Hwang, P. H., Kim, J. S., Lee, K. S., Lee, Y. C. (2003) Involvement of PTEN in airway hyperresponsiveness and inflammation in bronchial asthma. J. Clin. Invest. 111, 1083-1092.

55. Serhan, C. N., Levy, B. (2003) Novel pathways and endogenous mediators in anti-inflammation and resolution. Chem. Immunol. Allergy 83, 115 145 .

56. Gilroy, D. W., Lawrence, T., Perretti, M., Rossi, A. G. (2004) Inflammatory resolution: new opportunities for drug discovery. Nat. Rev. Drug Discov. 3, 401-416. 


\title{
Interleukin-10 inhibits lipopolysaccharide-induced survival and extracellular signal-regulated kinase activation in human neutrophils
}

\author{
Carol Ward, Joanna Murray, April Clugston, Ian Dransfield, \\ Christopher Haslett and Adriano G. Rossi
}

MRC Centre for Inflammation Research, Queen's Medical Research Institute, University of Edinburgh, Edinburgh, UK

\begin{abstract}
Lipopolysaccharide (LPS) induces a marked delay in human neutrophil apoptosis that is reversed by the anti-inflammatory cytokine IL-10. The effect of IL-10 is specific since other agents that delay neutrophil apoptosis are not affected. To investigate mechanisms underlying the actions of IL-10, we examined signaling pathways activated by LPS per se and in response to IL-10. The MAPK kinase (MEK) 1 inhibitor PD098059, the protein kinase C (PKC) inhibitor Ro31,8220, and the phosphatidylinositol-3 kinase (PI3-K) inhibitor LY294002 all partially reversed LPS-mediated retardation of neutrophil apoptosis, but the p38 MAPK inhibitor SB203850 did not. LPS activates the transcription factor NF- $\mathrm{KB}$, however, IL-10 did not affect the ability of LPS to activate

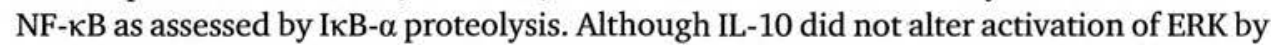
GM-CSF or TNF- $\alpha$, it did inhibit activation induced by LPS. Thus our data illustrate that LPS-induced neutrophil survival is regulated by the MAPK, PKC and PI3-K pathways as well as NF- $\mathrm{kB}$, and can be reversed by IL-10, through a mechanism involving inhibition of ERK activation. Because of the specific nature of this inhibition, we conclude that IL10 interferes with an ERK activation pathway, which is not involved in GM-CSF or TNF- $\alpha$ signaling.
\end{abstract}

Received $17 / 8 / 04$

Revised 16/5/05

Accepted 25/7/05

[DOI 10.1002/eji.200425561]

Key words:

Neutrophils

Apoptosis - IL-10

- Lipopolysaccharide

Extracellular signal-

regulated kinase

\section{Introduction}

Although neutrophils play a crucial role in innate immune defenses, dysregulated recruitment, activation, or clearance of these cells contributes to the development and persistence of many chronic inflammatory diseases. During resolution of inflammation, apoptosis

Correspondence: Dr. Adriano G. Rossi, MRC Centre for Inflammation Research, Queen's Medical Research Institute, University of Edinburgh, 47 Little France Crescent Edinburgh, EH16 4TJ, UK

Fax: +44-131-242-6554

e-mail: a.g.rossi@ed.ac.uk

Abbreviations: db-cAMP: Dibutyryl-cAMP - ERK: Extracellular signal-regulated kinase - IKB- $\alpha$ : Inhibitor of nuclear factor- $\kappa$ B- $\alpha$. JNK: C-Jun N-terminal kinase - LBP: LPS-binding protein MEK: MAPK/ERK kinase - DMEM: Dulbecco's modified Eagle's medium - PI3-K: Phosphatidylinositol 3-kinase of neutrophils results in their functional down-regulation and non-inflammatory removal by phagocytes [1-3]. Many factors encountered by neutrophils in the inflammatory milieu influence their activation status and life-span by altering the onset of apoptosis. For example, certain lipids (e.g., platelet-activating factor, leukotrienes, prostaglandins), cytokines (e.g., GM-CSF, IL-8), and hormones (e.g., glucocorticoids) can delay neutrophil apoptosis whereas other mediators promote apoptosis (e.g., TNF- $\alpha$ and nitric oxide) [4-7].

One powerful inhibitor of neutrophil apoptosis is LPS [8], the principle component of the outer membrane of Gram-negative bacteria, which activates innate immune responses. LPS affects inflammatory cell recruitment and activation and also influences the physiological cell death program. Inflammatory cells respond to LPS using a complex system involving the interaction of LPSbinding protein (LBP)/CD14/MD-2 and TLR. Neutro- 
phils express mRNA for CD14, MD-2 and TLR2 and 4 [9]. These receptors trigger inter-related signal transduction pathways, including MAPK [extracellular signal-regulated kinase (ERK) $1 / 2$, c-Jun N-terminal kinase (JNK) and p38 MAPK], phosphatidylinositol-3 kinase (PI3-K), $\mathrm{PKC}$ and $\mathrm{NF}-\kappa \mathrm{B}$ to orchestrate the innate immune response to this bacterial product. However, the precise pathway(s) responsible for prolongation of neutrophil survival and functional activity by LPS remain to be established. An inappropriate or over-reaction to LPS can lead to sepsis, septic shock, or acute respiratory distress syndrome [10] affecting over 18 million people a year. Understanding LPS signaling and elucidating possible inhibitory mechanisms are required for development of therapeutic strategies. IL-10 is reported as a powerful physiological inhibitor of LPS actions in various cell types.

IL-10 is synthesized by $\mathrm{T}$ cells, macrophages, monocytes and B cells and dampens many inflammatory responses [11], e.g., attenuating TNF- $\alpha$ production from LPS-activated monocytes and macrophages [12]. There is minimal understanding of how IL-10 achieves its effects and several signaling pathways have been implicated. IL-10 has been reported to inhibit activation of NF-кB [13], to activate Stat 3 [11], to induce expression of suppressor of cytokine signaling 3 (SOCS3) protein [14], to down-regulate expression of TLR4 [15] and to activate PI3-K [16]. However the data are controversial since IL-10 actions appear to be cell-type and/or species-specific and mainly deal with the effect of IL-10 on proliferation/activation rather than apoptosis.

There are small numbers of IL-10 receptors on the surface of freshly isolated neutrophils, which increase with exposure to LPS [14]. Human neutrophils store IL10 receptors in specific granules which are mobilized to the cell surface by pro-inflammatory mediators such as LPS, GM-CSF and TNF- $\alpha$ [17]. IL-10 suppresses the oxidative burst induced by various mediators [18] and inhibits secretion of TNF- $\alpha$, IL- $1 \beta$ and IL- 8 from LPSstimulated neutrophils [19]. We therefore examined the effect of IL-10 on LPS-mediated retardation of neutrophil apoptosis.

We show that IL-10, despite not influencing neutrophil apoptosis per se, inhibits LPS-induced neutrophil longevity in a concentration-dependent and specific manner, having no effect on the delay of neutrophil apoptosis induced by other agents [namely, glucocorticoids, GM-CSF, TNF- $\alpha$ and dibutyryl-cAMP (db-cAMP)]. We investigated the role of NF- $\mathrm{B}$ activation and the effects of specific pharmacological inhibitors of relevant kinase pathways to determine a possible mechanism for the actions of IL-10 on LPS-induced survival. This revealed that ERK, PI3-K, PKC and NF- $\mathrm{B}$ all are involved in LPS mediated delay of neutrophil apoptosis. However, p38 was not involved in the regulation of constitutive apoptosis or the prolongation of functional longevity by LPS. Importantly our data indicate that ERK signaling is a likely target of IL-10 action, and further study determined that IL-10 inhibited ERK activation by LPS in a specific manner, suggesting that IL-10 inhibits LPS induced neutrophil survival by influencing a component of the signaling pathway between TLR4 and MAPK [42, 44].

\section{Results}

IL-10 (1-300 ng/mL; equivalent to $14.5-4350 \mathrm{U} / \mathrm{mL}$ or 0.056-16.7 $\mathrm{nM}$ ) did not affect constitutive neutrophil apoptosis after 6 or 20 h of culture (Fig. 1), in agreement with our preliminary work [20]. To ensure that the cytokine was biologically active, ELISA were performed to determine the effect of IL-10 on inhibition of TNF- $\alpha$ production from LPS-treated monocytes [i.e., LPS $(1 \mu \mathrm{g} / \mathrm{mL})$ stimulated $555.1 \pm 27.1 \mathrm{pg} / \mathrm{mL}$ TNF- $\alpha$ release; LPS + IL-10 ( $1 \mathrm{ng} / \mathrm{mL}) 222.8 \pm 1.4$; LPS + IL-10 (10 ng/mL) 127.3 $\pm 5.9 ; \mathrm{LPS}+\mathrm{IL}-10(100 \mathrm{ng} / \mathrm{mL})$ $132.1 \pm 3.2$; all control and IL-10 alone samples produced negligible levels of TNF- $\alpha ; n=3$ ] and macrophages [i.e., LPS $(1 \mu \mathrm{g} / \mathrm{mL})$-stimulated $210.2 \pm 4.8 \mathrm{pg} / \mathrm{mL}$ TNF- $\alpha$ release; LPS + IL-10 ( $1 \mathrm{ng} / \mathrm{mL}) 98.2 \pm 2.1 ; \mathrm{LPS}+\mathrm{IL}-10$ $(10 \mathrm{ng} / \mathrm{mL}) 76.3 \pm 3.0 ; \mathrm{LPS}+\mathrm{IL}-10$ (100 ng/mL) 31.0 \pm 2.1 ; all control and IL-10 alone samples produced negligible levels of TNF- $\alpha ; n=3$ ].

Interestingly, however, further experimentation demonstrated that IL-10 could inhibit the retardation of neutrophil apoptosis by LPS at $20 \mathrm{~h}$ (Fig. 1B and C). This effect was concentration dependent with significant effects seen at concentrations as low as $3 \mathrm{ng} / \mathrm{mL}$ and maximal effects observed at $100 \mathrm{ng} / \mathrm{mL}$. We therefore assessed whether IL-10 could directly interfere with pivotal neutrophil survival pathways using a concentration of $100 \mathrm{ng} / \mathrm{mL}$. A similar approach has been taken by Clarke et al. [21], who investigated the signaling pathways responsible for IL-10-mediated suppression of TNF- $\alpha$ production. In this study the authors demonstrated concentration-dependent effects of IL-10 on functional responsiveness, and subsequently successfully used the concentration of $100 \mathrm{ng} / \mathrm{mL}$ for investigations of signaling mechanisms [21]. Thus, neutrophils were cultured with factors known to delay apoptosis using different signaling pathways, with or without IL$10(100 \mathrm{ng} / \mathrm{mL})$. Dexamethasone [4], db-cAMP [22, 23], and GM-CSF [8] significantly increased neutrophil survival, but only LPS-induced survival was significantly reversed by IL-10 (Fig. 2A). TNF- $\alpha$ is pro-apoptotic at early culture periods $(6 \mathrm{~h})$ while enhancing survival at $20 \mathrm{~h}$ [5]; IL-10 did not influence either the pro- or antiapoptotic effect (Fig. 2B). These data demonstrate that 
A

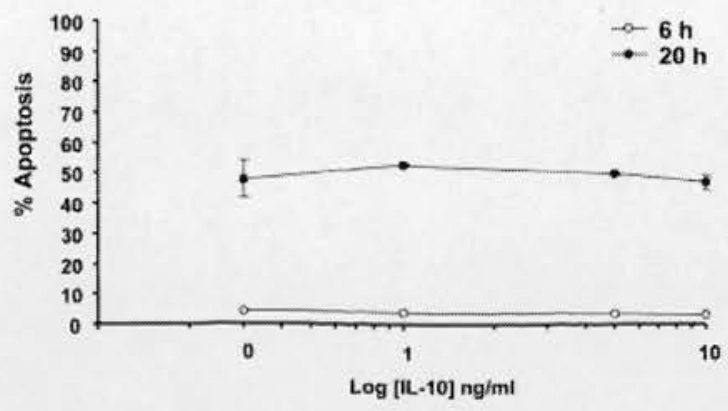

B

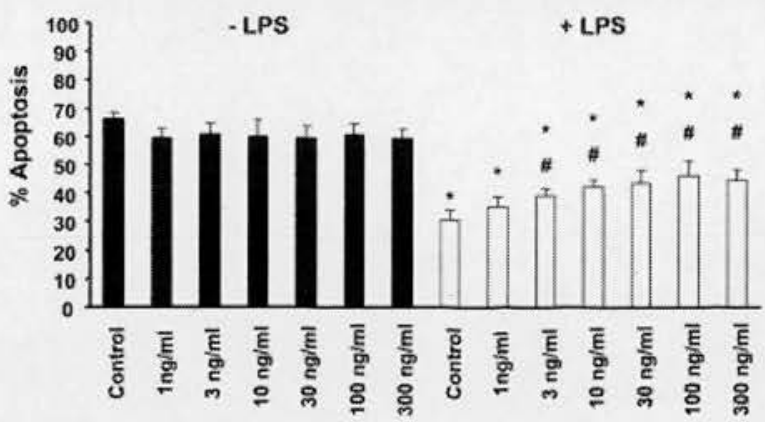

C
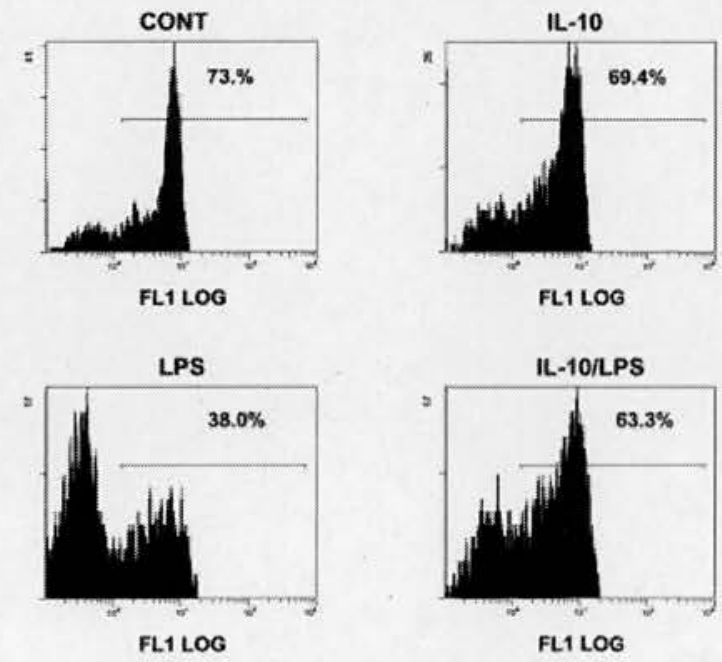

with regard to apoptosis, IL-10 appears to act on a mechanism that is particular to LPS signaling in neutrophils. Because IL-10 selectively reversed neutrophil survival induced by LPS, we examined LPSactivated signaling pathways to determine the possible mechanisms involved.

$\mathrm{NF}-\kappa \mathrm{B}$ is critically involved in neutrophil survival by a mechanism that involves the de novo synthesis of survival factors [24]. LPS activates NF- $\kappa$ B in neutrophils and protein synthesis inhibitors block the survival effect of LPS ([24] and our unpublished data), suggesting that LPS enhances neutrophil survival by a mechanism that

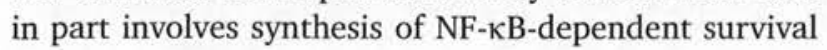

4 Fig. 1. (A) Effect of IL-10 on constitutive neutrophil apoptosis. Neutrophils $\left(5 \times 10^{6} / \mathrm{mL}\right)$ were cultured in Iscove's DMEM containing $10 \%$ autologous serum and cultured with the indicated concentrations of IL-10 for 6 or $20 \mathrm{~h}$. Apoptosis was assessed morphologically. All values represent mean \pm S.E. of $n=3$ experiments; each performed in triplicate. (B) Effect of IL-10 on LPS-mediated inhibition of constitutive neutrophil apoptosis. Neutrophils $\left(5 \times 10^{6} / \mathrm{mL}\right)$ were cultured in Iscove's DMEM and treated with the indicated concentrations of IL-10 for $1 \mathrm{~h}$ before the addition of LPS $(1 \mu \mathrm{g} / \mathrm{mL})$ or control medium in the presence of autologous serum $(10 \%)$, and harvested at $20 \mathrm{~h}$. Apoptosis was assessed both morphologically and by FITC-annexin V binding. All values represent mean \pm S.E of $n=$ 4 experiments; each performed in triplicate. * $^{*} 0.05$ compared with control values, \# $<0.05$ compared with LPS treatment alone. Similar results were obtained when $10 \%$ autologous serum was present at the beginning of the experiment. (C) Flow cytometric profiles of FITC-annexin V binding. Neutrophils $(5 \times$ $10^{6} / \mathrm{mL}$ ) were cultured as indicated in the legend to (B) and treated with control buffer (CONT), $100 \mathrm{ng} / \mathrm{mL}$ IL-10 (IL-10), $1 \mu \mathrm{g} / \mathrm{mL}$ LPS (LPS) or IL-10 plus LPS (IL-10/LPS). Cells were harvested at $20 \mathrm{~h}$. Percentages of annexin V-high neutrophils are indicated on the profiles. Data shown are representative of four different experiments. Similar results were obtained when $10 \%$ autologous serum was present at the beginning of the experiment.

proteins. In monocytes, IL-10 inhibits NF- $\kappa B$ activation by blocking I $\mathrm{B}$ kinase (IKK) activity [13] and in both 70Z/3 pre-B cells and RAW 264.7 macrophages IL-10 (100 $\mathrm{ng} / \mathrm{mL}$ ) was found to inhibit NF- $\mathrm{KB}$ activity by approximately $70 \%$ [21]. We examined whether IL-10 could inhibit LPS-induced activation of NF- $\mathrm{BB}$ using Western Blotting to detect the breakdown of $\mathrm{I \kappa B}-\alpha$ and I $\kappa \mathrm{B}-\beta$, the inhibitory subunits that prevent NF- $\kappa \mathrm{B}$ nuclear translocation. LPS caused a rapid and almost total disappearance of I $\mathrm{KB}-\alpha$ from cytoplasmic extracts within 15 min. Despite a 1 -h pre-incubation with IL-10 $(100 \mathrm{ng} / \mathrm{mL})$ before the addition of LPS $(1 \mu \mathrm{g} / \mathrm{mL})$, there was no inhibitory effect on LPS-mediated I $\mathrm{KB}-\alpha$ proteolysis (Fig. 3), even with pre-incubations of up to $3 \mathrm{~h}$ (data not shown). Breakdown of IкB- $\beta$ was not observed using LPS as a stimulus (data not shown) although this I $\kappa$ B isoform has been implicated in LPS signaling in other cells [25]. Since IL-10 had no effect on LPS-induced I $\kappa \mathrm{B}-\alpha$ proteolysis we examined other pathways activated by this bacterial component.

Because human neutrophils have a short lifespan and transfection methods have been largely unsuccessful, we examined pathways activated by LPS using pharmacological inhibitors to determine possible mechanisms for the effect of IL-10. Neutrophils were pre-incubated with the p38 inhibitor SB203580 [26, 27] before the addition of LPS or other cytokines for comparison. SB203580 did not affect the rate of constitutive neutrophil apoptosis or levels of apoptosis in cells treated with either LPS or TNF- $\alpha$ (Fig. 4A) or GM-CSF (data not shown). Several 


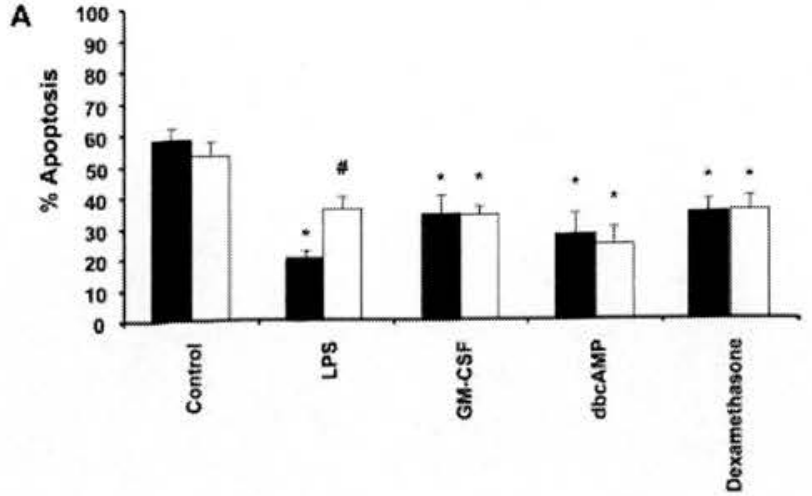

B

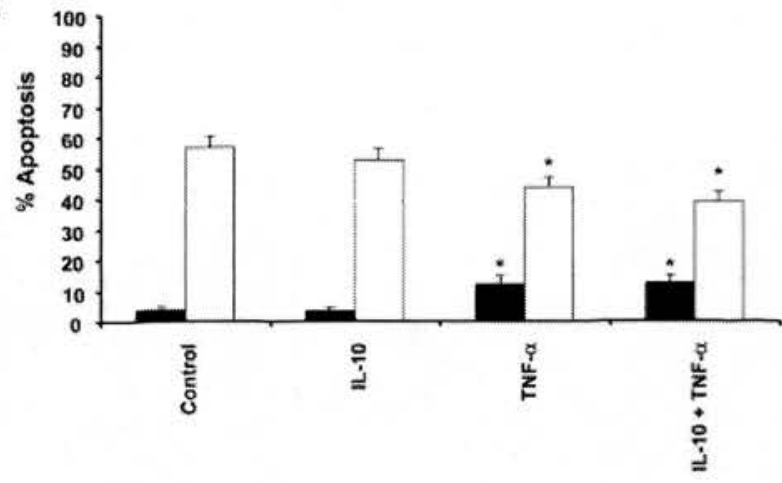

Fig. 2. (A) Effect of IL-10 on stimulus induced inhibition of neutrophil apoptosis. Neutrophils $\left(5 \times 10^{6} / \mathrm{mL}\right)$ were cultured in Iscove's DMEM containing $10 \%$ autologous serum and treated with control medium, dexamethasone $(1 \mu \mathrm{M})$, LPS $(1 \mu \mathrm{g} / \mathrm{mL})$, db-CAMP $(0.2 \mathrm{mM})$ or GM-CSF $(50 \mathrm{U} / \mathrm{mL})$ in the absence or presence of IL-10 (100 ng/mL). Apoptosis was assessed morphologically after $20 \mathrm{~h}$. Filled bars represent treatment, open bars represent IL-10 plus treatment. All values represent mean $\pm \mathrm{SE}$ of $n=3$ experiments; each performed in triplicate. ${ }^{*}<0.05$ compared with control values, \#< 0.05 compared with treatment alone. Similar results were obtained when neutrophils were cultured for $1 \mathrm{~h}$ with IL-10 prior to addition of the survival factors. (B) Effect of IL-10 on TNF- $\alpha$ induced neutrophil apoptosis and survival. Neutrophils $\left(5 \times 10^{6} / \mathrm{mL}\right)$ were cultured in Iscove's DMEM containing $10 \%$ autologous serum and treated with control medium, TNF- $\alpha(10 \mathrm{ng} / \mathrm{mL})$, IL-10 $(100 \mathrm{ng} / \mathrm{mL})$ or a combination of both cytokines before harvesting at 6 or $20 \mathrm{~h}$. Apoptosis was assessed morphologically at $6 \mathrm{~h}$ (filled bars) and $20 \mathrm{~h}$ (open bars). All values represent mean \pm SE of $n=3$ experiments; each performed in triplicate. ${ }^{*}<0.05$ compared with control values. Similar results were obtained when neutrophils were cultured for $1 \mathrm{~h}$ with IL-10 prior to addition of TNF- $\alpha$.

groups suggest that $20 \mu \mathrm{M}$ SB203580 is not specific for inhibition of p38, but can also inhibit JNK. Therefore these experiments were repeated using $5 \mu \mathrm{M}$ SB203580, a concentration shown to be specific for p38 [26], but results were similar (data not shown), suggesting that neither JNK nor p38 activation is required for the inhibitory action of LPS.

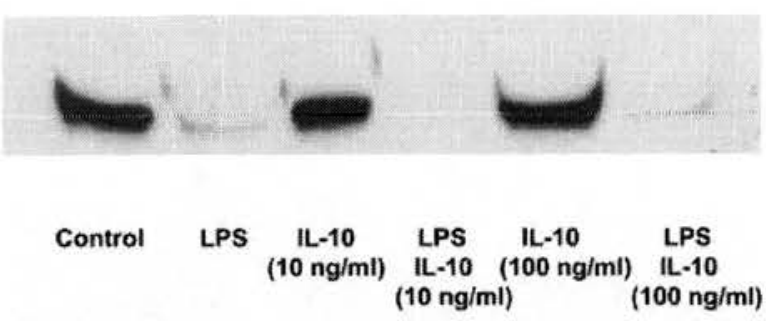

Fig. 3. Effect of IL-10 on I $1 \kappa \mathrm{B}-\alpha$ breakdown in response to LPS. Neutrophils $\left(5 \times 10^{6} / \mathrm{mL}\right)$ were pre-incubated with IL-10 (10 or $100 \mathrm{ng} / \mathrm{mL})$ for $1 \mathrm{~h}$ before the addition of LPS $(1 \mu \mathrm{g} / \mathrm{mL})$ or control medium in the presence of $10 \%$ autologous serum for $15 \mathrm{~min}$. Blot was probed for I $\mathrm{B}$ - $\alpha$. Data shown are representative of four different experiments.

Inhibition of PKC signaling pathways using the broad spectrum PKC inhibitor Ro31,8220 [28] determined that $\mathrm{PKC}$ is involved in the inhibition of neutrophil apoptosis by both LPS and TNF- $\alpha$. The data suggest that while PKC is important in LPS-mediated survival, it may also be part of common survival pathways rather than specific to LPS (Fig. 4B). This is further supported by the small, significant increase in constitutive apoptotic rates in control cells treated with Ro31,8220 alone.

The PI3-K inhibitor LY294002 [29] did not significantly affect rates of constitutive neutrophil apoptosis at $20 \mathrm{~h}$, but successfully reversed the delay of neutrophil apoptosis induced by GM-CSF, TNF- $\alpha$ and LPS (Fig. 4C). These data demonstrate that PI3-K is activated by many survival stimuli in neutrophils; therefore its effects are unlikely to be specific to LPS.

The MEK inhibitor PD098059 [30] had no effect on constitutive neutrophil apoptosis, nor did it significantly alter the decrease in neutrophil apoptosis by TNF- $\alpha$, dexamethasone, or dbcAMP (Fig. 4D). However, this compound almost completely reversed the delay of neutrophil apoptosis induced by LPS and GM-CSF, suggesting that the ERK kinases are involved in neutrophil survival mediated by both LPS and GMCSF (Fig. 4D).

Because we previously determined that inactivation of NF-кB $[24,31]$ reverses the survival effect of LPS, we examined whether PD098059 or LY294002 could block activation of NF- $\kappa \mathrm{B}$ by LPS, indicating a secondary mode of activation. Fig. $5 \mathrm{~A}$ and $\mathrm{B}$ demonstrates that these inhibitors did not block I $\kappa \mathrm{B}-\alpha$ proteolysis indicating that a link between these kinases and NF- $\kappa \mathrm{B}$ translocation is not involved in LPS-or TNF- $\alpha$-mediated survival.

ERK activation is clearly involved in the enhancement of neutrophil survival by LPS and GM-CSF (Fig. 4D), however IL-10 alone did not alter ERK activation regardless of time point examined (Fig. 6 and data not shown). Maximal activation of ERK occurred after 15 min of LPS treatment. GM-CSF demonstrated high ERK activity between 5 and $15 \mathrm{~min}$, while TNF- $\alpha$ 
A

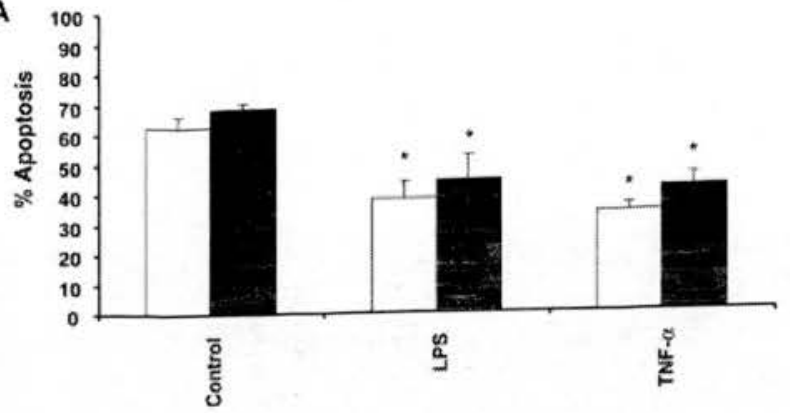

B

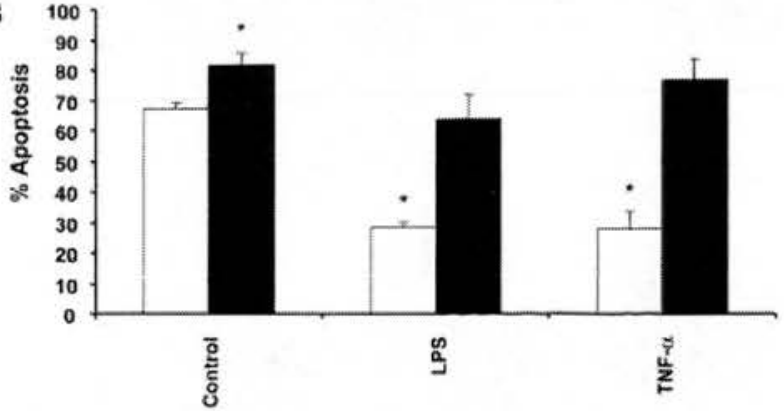

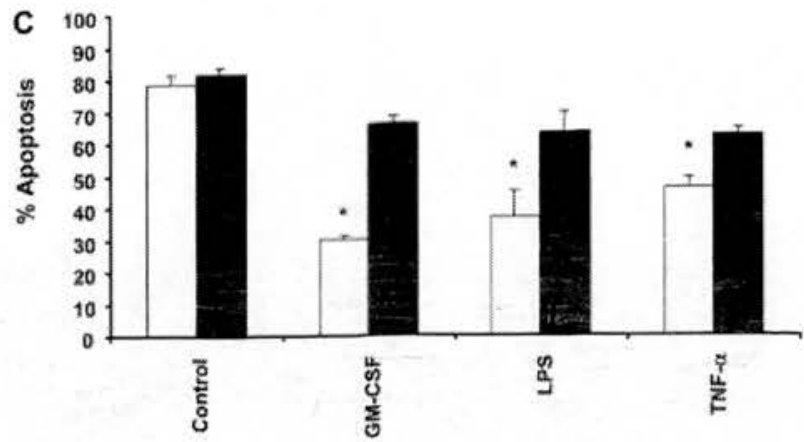

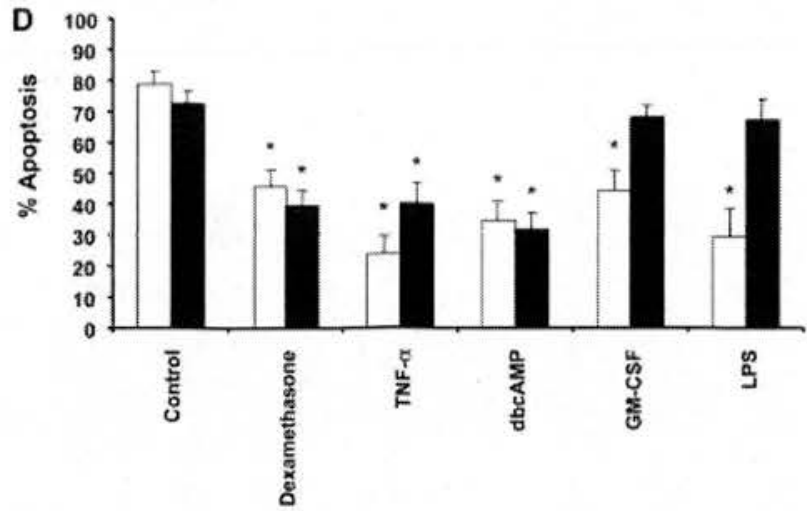

Fig. 4. (A) Effect of the p38 inhibitor SB203580 on stimulus-induced neutrophil survival. Neutrophils $\left(5 \times 10^{6} / \mathrm{mL}\right)$ were cultured in Iscove's DMEM for 30 min with SB203580 $(20 \mu \mathrm{M})$ before the addition of control medium, LPS $(1 \mu \mathrm{g} / \mathrm{mL})$ or TNF- $\alpha(10 \mathrm{ng} / \mathrm{mL})$ in the presence of $10 \%$ autologous serum. Apoptosis was assessed by flow cytometry after $20 \mathrm{~h}$. Open bars represent stimulus alone and filled bars indicate stimulus plus SB203580. All values represent mean \pm SE of $n=4$ experiments; each performed in triplicate. *< 0.05 compared with control values. (B) Effect of the PKC inhibitor Ro31,8220 on stimulus-induced neutrophil survival. Neutrophils $\left(5 \times 10^{6} / \mathrm{mL}\right)$ were cultured in Iscove's DMEM for $30 \mathrm{~min}$ with Ro31,8220 $(1 \mu \mathrm{M})$ before the addition of control medium, LPS $(1 \mu \mathrm{g} / \mathrm{mL})$ or TNF- $\alpha(10 \mathrm{ng} / \mathrm{mL})$ in the presence of $10 \%$ autologous serum. Apoptosis was assessed by flow cytometry after $20 \mathrm{~h}$. Open bars represent stimulus alone and filled bars indicate stimulus plus Ro31,8220. All values represent mean $\pm \mathrm{SE}$ of $n=3$ experiments; each performed in triplicate. * $<0.05$ compared with control values. (C) Effect of the PI3-K inhibitor LY294002 on stimulus-induced neutrophil survival. Neutrophils $\left(5 \times 10^{6} / \mathrm{mL}\right)$ were cultured in Iscove's DMEM for $30 \mathrm{~min}$ with LY294002 $(10 \mu \mathrm{M})$ before the addition of control medium, LPS $(1 \mu \mathrm{g} / \mathrm{mL})$, TNF- $\alpha(10 \mathrm{ng} / \mathrm{mL})$ or GM-CSF $(50 \mathrm{U} / \mathrm{mL})$ in the presence of $10 \%$ autologous serum. Apoptosis was assessed morphologically after $20 \mathrm{~h}$. Open bars represent stimulus alone and filled bars indicate stimulus plus LY294002. All values represent mean $\pm \mathrm{SE}$ of $n=3$ experiments; each performed in triplicate. * $<0.05$ compared with control values. (D) Effect of PD098059 on stimulus induced inhibition of neutrophil apoptosis. Neutrophils $\left(5 \times 10^{6} / \mathrm{mL}\right)$ were cultured in Iscove's DMEM for $30 \mathrm{~min}$ with PD098059 $(50 \mu \mathrm{M})$ before the addition of control medium, LPS $(1 \mu \mathrm{g} / \mathrm{mL}), \mathrm{TNF}-\alpha(10 \mathrm{ng} / \mathrm{mL}), \mathrm{GM}-\mathrm{CSF}(50 \mathrm{U} / \mathrm{mL})$, dexamethasone $(1 \mu \mathrm{M})$, or db-cAMP $(0.2 \mathrm{mM})$ in the presence of $10 \%$ autologous serum. Apoptosis was assessed by flow cytometry after $20 \mathrm{~h}$. Open bars represent stimulus alone and filled bars indicate stimulus plus PD098059. All values represent mean \pm S.E of $n$ $=4$ experiments; each performed in triplicate. ${ }^{*}<0.05$ compared with control values.

stimulation was maximal at $5 \mathrm{~min}$. Fig. $6 \mathrm{~A}$ clearly demonstrates that IL-10 inhibits activation of ERK by LPS, suggesting that this mechanism could account for the reversal of LPS-mediated neutrophil survival by this cytokine. However, there is no significant difference in the activity of ERK between cells treated with both IL-10 and GM-CSF or GM-CSF alone (Fig. 6A and B). IL-10 did not decrease ERK activation by TNF- $\alpha$ (Fig. $6 \mathrm{C}$ ); there was either no effect or in some experiments a slight increase in ERK phosphorylation was observed. These data demonstrate that IL-10 specifically inhibited LPSinduced ERK activation and suggests that this effect is not due to a nonspecific mechanism such as activation of phosphatases. Further, these data indicate that regulation of ERK activation by LPS and GM-CSF or TNF- $\alpha$ involves different mechanisms.

\section{Discussion}

We have demonstrated that IL-10 specifically inhibits LPS-induced neutrophil survival, having no effect on survival induced by GM-CSF, dexamethasone, dbcAMP or TNF- $\alpha$ (Fig. 2A and B). However no reported actions 
A

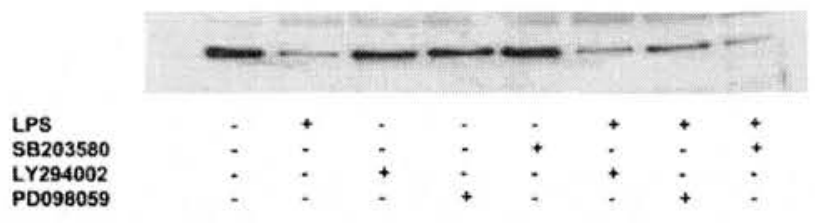

B

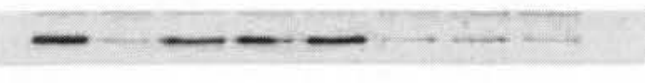

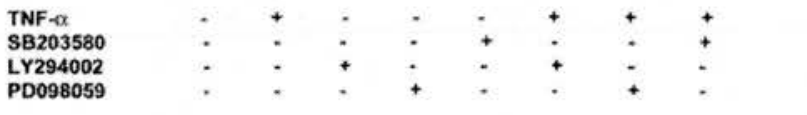

Fig. 5. (A) Effect of MAPK inhibitors on LPS-mediated proteolysis of IKB- $\alpha$. Neutrophils $\left(5 \times 10^{6}\right)$ were pre-incubated with SB203580 $(20 \mu \mathrm{M})$, LY294002 $(10 \mu \mathrm{M})$, or PD098059 $(50 \mu \mathrm{M})$ for $1 \mathrm{~h}$ before the addition of control medium or LPS $(1 \mu \mathrm{g} / \mathrm{mL})$ in the presence of $10 \%$ autologous serum for $15 \mathrm{~min}$. Western blot was probed for IкB- $\alpha$. Figure is a representative of three similar experiments. (B) Effect of MAPK inhibitors on TNF- $\alpha$ mediated proteolysis of IкB- $\alpha$. Neutrophils $\left(5 \times 10^{6}\right)$ were preincubated with SB203580 $(20 \mu \mathrm{M})$, LY294002 $(10 \mu \mathrm{M})$, or PD098059 $(50 \mu \mathrm{M})$ for $1 \mathrm{~h}$ before the addition of control medium or TNF- $\alpha(10 \mathrm{ng} / \mathrm{ml})$ in the presence of $10 \%$ autologous serum for $15 \mathrm{~min}$. Western blot was probed for IкB- $\alpha$. Figure is a representative of three similar experiments.

of IL-10 account for this specificity. IL-10 inhibits tyrosine phosphorylation in LPS-stimulated neutrophils [32], but had no effect on GM-CSF-induced neutrophil or eosinophil survival [33] (and our unpublished observations), although tyrosine phosphorylation has a crucial role in granulocyte survival [34]. IL-10 also blocks the increase in TLR4 expression induced by LPS in neutrophils [15]. However, IL-10 mediated suppression of the LPS effect was similar whether both mediators were cocultured or if IL-10 was added $1 \mathrm{~h}$ before LPS addition, suggesting that the suppressive effect of IL-10 is unlikely to be due to changes in TLR4 expression. IL-10 signals via Jak1 and Tyk2 that activate Stat1, Stat3 and in some cells Stat5, and also induces suppressor of cytokine signaling 3 (SOCS-3) protein. GM-CSF is another powerful inducer of SOCS-3 in neutrophils [14] and also activates Stat3 [35]. Since GM-CSF is a neutrophil survival factor, such a mechanism is unlikely to account for the specific decrease in LPS-induced survival by IL-10.

Because these actions could not account for the specific effects in our studies, we examined LPSactivated signaling pathways. NF- $\mathrm{KB}$ plays an important role in LPS-mediated neutrophil survival $[24,31]$. Although LPS induced the proteolytic breakdown of
A
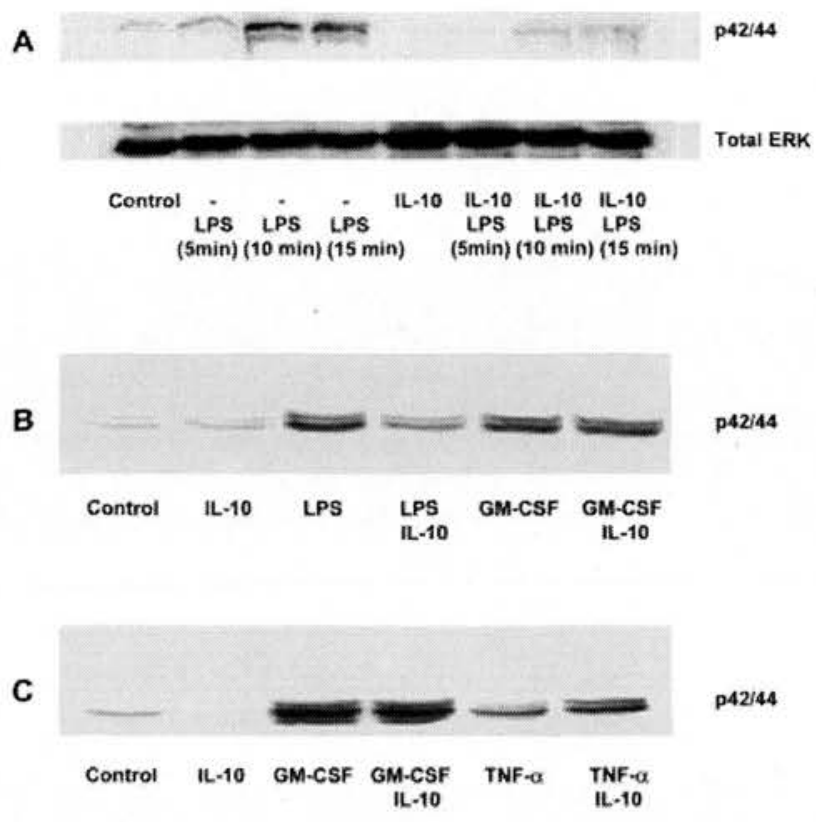

Fig. 6. (A and B) IL-10 inhibits ERK activation in response to LPS stimulation. Neutrophils $\left(5 \times 10^{6}\right)$ were pre-incubated with IL$10(100 \mathrm{ng} / \mathrm{mL})$ for $1 \mathrm{~h}$ before the addition of control medium, LPS $(1 \mu \mathrm{g} / \mathrm{ml})$ or GM-CSF $(50 \mathrm{U} / \mathrm{ml})$ in the presence of $10 \%$ autologous serum for $15 \mathrm{~min}$. Western blot was probed for phosphorylated ERK. Figure is a representative of five similar experiments. Equal protein loading was determined by Western blotting for total ERK. (C) IL-10 does not inhibit ERK activation in response to GM-CSF or TNF- $\alpha$ stimulation. Neutrophils $\left(5 \times 10^{6}\right)$ were pre-incubated with IL-10 (100 ng/ $\mathrm{ml}$ ) for $1 \mathrm{~h}$ before the addition of control medium, TNF- $\alpha$ $(10 \mathrm{ng} / \mathrm{ml})$ for $5 \mathrm{~min}$ or $\mathrm{GM}-\mathrm{CSF}(50 \mathrm{U} / \mathrm{ml})$ for $10 \mathrm{~min}$ in the presence of $10 \%$ autologous serum. Western blot was probed for phosphorylated ERK. Figure is a representative of five similar experiments.

I $\mathrm{B}-\alpha$, this was not prevented by IL-10 even with incubation periods of up to $3 \mathrm{~h}$ before LPS addition (Fig. 3). Inactivation of NF-KB causes massive cell death in TNF- $\alpha$-treated neutrophils within $2 \mathrm{~h}$ of culture [24]. IL-10 had no effect on TNF- $\alpha$-induced cell death, again suggesting that IL-10 does not directly interfere with NF$\kappa \mathrm{B}$ activation in neutrophils, in agreement with other studies $[14,36]$. Further, our data indicate that the inhibitors used in these studies did not block LPSactivation of NF-KB.

Inhibition of $\mathrm{p} 38$ or $\mathrm{p} 38 / \mathrm{JNK}$ pathways using SB203580 at concentrations known to inhibit either one or both kinases simultaneously [27] did not influence either constitutive neutrophil apoptosis or survival induced by any of the factors examined. This makes it unlikely that IL-10 is affecting these signaling pathways or that p38 is involved in constitutive neutrophil apoptosis or involved in LPS or TNF- $\alpha$ induced survival. This is in agreement with other work [37, 38]. In contrast, LY 294002, an inhibitor of PI3-K 
[29] reversed the inhibition of neutrophil apoptosis by GM-CSF, TNF- $\alpha$ and LPS. This suggests that PI3-K, forms part of a pivotal survival pathway in neutrophils, since inactivation of this kinase can also reverse the effects of other survival factors such as C5a [39]. Because IL-10 had no effect on GM-CSF- or TNF- $\alpha$-induced survival, it is doubtful that PI3-K activity is directly influenced by IL-10. The broad spectrum PKC inhibitor Ro31,8220 [28] reversed both LPS- and TNF- $\alpha$-induced survival. Interestingly, Ro31,8220 significantly induced neutrophil apoptosis, suggesting that PKC activation is an important pro-survival pathway in neutrophils, and is therefore unlikely to be the site of action of a specific inhibitor. The MAPK [42, 44] inhibitor PD098059 [30] blocked the inhibition of neutrophil apoptosis by GMCSF and LPS, in accordance with results of Klein et al. [40]. Because IL-10 did not affect GM-CSF-induced survival, these data at first appeared to rule out this pathway. Therefore, the inhibitor data gave no clear explanation for the action of IL-10. However, the most attractive target appeared to be ERK since there was no effect on constitutive apoptosis and only LPS and GMCSF induced survival was significantly reversed by the inhibitor.

Studies of the LPS signaling pathways in neutrophils reveal controversial results. For example one group found no effect of IL-10 on LPS induced neutrophil survival [41] whereas others [32, 42], in agreement with our study, found that IL-10 reverses LPS-mediated neutrophil apoptosis. The reasons for these differences are unclear but it is possible that differences in neutrophil isolation techniques, cell density, culture medium and tissue culture, serum concentration and type, and LPS serotype and concentration may account for the discrepancies (see [43] for a discussion on problems encountered when working with neutrophils). Previous reports [44, 45] suggest that LPS does not activate ERK in neutrophils, however, other studies support our results [46]. It has also been suggested that some, but not all, of the observed effects of LPS on neutrophil apoptosis are due to monocyte contamination [47]. However, other studies have shown direct LPS effects in these cells, even at the level of gene expression, when factors such as this are controlled for [48-50]. We show clear activation of ERK by LPS (Fig. 6). However, phosphorylation only occurred in the presence of serum, indicating that this effect is CD14 dependent, in agreement with Bonner et al. [51], implying that LPS activates ERK via TLR4-signaling pathways. However, IL-10 did not reverse GM-CSF- or TNF- $\alpha$-induced ERK activation, inferring that there is no direct interference with activation of this kinase by IL-10.

GM-CSF and LPS may activate ERK using different pathways. For example, GM-CSF has been shown to activate ERK via p21 ${ }^{\text {ras }}$ [52] and Swantek et al. [53] have demonstrated a link between IRAK activation by LPS and subsequent ERK stimulation in murine peritoneal macrophages, indicating that LPS can indeed signal ERK activation through a non-classical pathway. Our data strongly support the hypothesis that IL-10 can reverse LPS-mediated neutrophil survival by inhibiting a component of the ERK-signaling pathway, which is not involved in that used by TNF- $\alpha$ or GM-CSF. Inhibition of ERK activation by IL-10 is not confined to neutrophils; e.g., it has also been demonstrated in monocytes that IL10 can inhibit CD40 [54] and LPS [55] mediated ERK activation. The precise mechanism that IL-10 targets in the LPS signaling pathways is unknown; however, it is possible that the recently described adaptor protein ECSIT (evolutionary conserved signaling intermediate in Toll pathway) [56] may be targeted. Other negative regulators of LPS signaling, Dok-1 and Dok- 2 have been demonstrated to inhibit ERK activation [57], but whether $\mathrm{LL}-10$ can affect these proteins is currently unknown.

Interestingly, phagocytic ingestion of apoptotic cells is accompanied by the release of IL-10 [58] and IL-10 itself has recently been shown to up-regulate phagocytosis of apoptotic cells [59]. Our studies demonstrate IL10 could also prevent the increased functional longevity of neutrophils in response to LPS, allowing apoptosis and clearance of effete cells to occur and inflammation to resolve. Further, in an in vivo rat model of pulmonary inflammation induced by LPS, IL-10 has been shown to enhance resolution [42] and it may also dampen inflammation by generating uncoupled decoy receptors on activated human cells which can scavenge inflammatory chemokines [60]. Therefore the specific mechanisms involved in IL-10 inhibition of LPS-induced neutrophil survival may reveal possible targets for antiinflammatory therapies especially since modulation of resolution mechanisms controlling inflammation offers new opportunities for drug discovery [61].

\section{Materials and methods}

\section{Materials}

rhTNF- $\alpha$ and hIL-10 were obtained from R \& D Systems (Abingdon, UK), rhGM-CSF from Genzyme Diagnostics (Kent, UK), dexamethasone from David Bull Laboratories (Warwick, UK), Ro31,8220, PD098059, SB203850, and LY294002 from Calbiochem, and IкB- $\alpha$, and phosphor-ERK antibodies from New England BioLabs. All other reagents were obtained from Sigma Co, Poole, UK, including LPS (E. Coli 0127:B8). Note that $\mathrm{IL}-10$ at $100 \mathrm{ng} / \mathrm{mL}$ is equivalent to $1450 \times 10^{3} \mathrm{U} / \mathrm{mL}$ or 5. $6 \mathrm{nM}$. 


\section{Cell isolation and culture}

Neutrophils, monocytes and macrophages were prepared as previously detailed $[23,62]$. Purified cells were suspended in Iscove's DMEM (Life Technologies, Paisley, UK) supplemented with $10 \%$ autologous serum (unless otherwise stated) at $5 \mathrm{x}$ $10^{6} / \mathrm{mL}$ before culturing in flat-bottom Falcon flexible well plates (Becton Dickinson, Oxford, UK) at $37^{\circ} \mathrm{C}$ in a $5 \% \mathrm{CO}_{2}$ atmosphere; or in Eppendorf tubes in a shaking water bath.

\section{Assessment of granulocyte apoptosis}

\section{Morphology}

Cells (100 $\mu \mathrm{L} /$ sample) were cyto-centrifuged, fixed in methanol, stained with Diff-Quik ${ }^{\mathrm{TM}}$, and counted using oil immersion microscopy (x100 objective) to determine the proportion of cells with distinctive apoptotic morphology [24]. At least 500 cells were counted per slide, three slides per treatment.

\section{Annexin $\mathrm{V}$ binding}

Apoptosis was also assessed by flow cytometry using FITClabeled rh-annexin V (Bender MedSystems, Vienna, Austria) diluted 1:2500 with binding buffer and then added $(180 \mu \mathrm{L})$ to $20 \mu \mathrm{L}$ of the recovered cell samples. Following 10-min incubation at $4^{\circ} \mathrm{C}$, the samples were analyzed using a FACSCaliber (Becton Dickenson).

\section{Assessment of membrane integrity and cell recovery}

The membrane integrity of both treated and untreated cells was assessed using the vital dye trypan blue or alternatively determined by adding propidium iodide at a final concentration of $10 \mu \mathrm{g} / \mathrm{mL}$ to annexin $\mathrm{V}$ samples and assessing necrosis by flow cytometry.

\section{Western blotting}

Cell samples $\left(5 \times 10^{6} / \mathrm{mL}\right)$ were incubated in a shaking water bath at $37^{\circ} \mathrm{C}$ with the agents of interest and for times as described in figure legends. After treatment, cells were immediately placed on ice and all lysates were prepared at $4^{\circ} \mathrm{C}$.

\section{Cytoplasmic extracts}

Samples were centrifuged and $100 \mu \mathrm{L}$ of hypotonic buffer [10 mM Tris- $\mathrm{HCl}$, pH 7.8, 1.5 mM NaEDTA, $10 \mathrm{mM} \mathrm{KCl}, 10 \mu \mathrm{g}$ ] $\mathrm{mL}$ aprotinin, leupeptin and pepstatin A, $10 \mu \mathrm{M} 4-(2-$ aminoethyl)benzenesulfonyl fluoride, $10 \mathrm{mM}$ sodium orthovanadate, $5 \mathrm{mM}$ benzamidine and $20 \mathrm{mM}$ levamisole] added and incubated on ice for $10 \mathrm{~min}$. Following the addition of 0.1 volumes of $10 \% \mathrm{NP}-40(\mathrm{v} / \mathrm{v})$ the cells were vortexed briefly and centrifuged at $12000 \times \mathrm{g}$ for $2 \mathrm{~min}$ at $4^{\circ} \mathrm{C}$. The supernatant was added to loading buffer, boiled for $5 \mathrm{~min}$ and stored at $4^{\circ} \mathrm{C}$ until use.

\section{Whole-cell extracts}

Whole-cell extracts were prepared by adding $100 \mu \mathrm{L}$ of buffer (Sigma Protease Cocktail 1:100 dilution in $1 \mathrm{X}$ Tris-buffered saline solution, containing protease inhibitors as detailed above, and $0.1 \% \mathrm{NP}-40$ ) to pelleted cell samples. Cells were incubated on ice for $15 \mathrm{~min}$ before centrifugation at $12000 \times$ $\mathrm{g}$ for $20 \mathrm{~min}$ at $4^{\circ} \mathrm{C}$. The supernatant was added to loading buffer, boiled for $5 \mathrm{~min}$ and stored at $4^{\circ} \mathrm{C}$ until use.

Lysates were run on a $9 \%$ SDS gel and after transfer, blocked by $5 \%$ milk protein before an overnight incubation with primary antibody at $4^{\circ} \mathrm{C}$ diluted $1: 500$. After washing, blots were incubated with HRP-conjugated anti-biotin secondary antibody diluted at 1:2500 and developed using standard ECL reagents (Amersham). Ponceau S staining was used to determine equal protein loading on each gel run.

\section{TNF- $\alpha$ measurement}

Monocytes and macrophages were cultured $\left(2 \times 10^{6} / \mathrm{mL}\right.$ in Iscove's containing $10 \%$ autologous serum) plus or minus LPS $(1 \mu \mathrm{g} / \mathrm{mL})$ and $\mathrm{IL}-10(1-100 \mathrm{ng} / \mathrm{mL})$. Supernatants were harvested after 4 -h culture and TNF- $\alpha$ was measured by ELISA using paired monoclonal antibodies from Boehringer Mannheim.

\section{Statistical analysis}

Results are expressed as mean \pm SE of the number $(n)$ of independent experiments each using cells from separate donors with each treatment performed in triplicate. Statistical analysis was performed by ANOVA with comparisons between groups made using the Newman-Kuels procedure. Differences were considered significant with $p<0.05$.

Acknowledgements: This work was supported by Asthma UK (01/042). We thank Dr Lorna Bruce for running ELISA samples to ensure IL-10 activity and Dr Joanna Cousin for preliminary studies with SB203580 and PD098059.

\section{References}

1 Whyte, M. K. B., Meagher, L. C., MacDermot, J. and Haslett, C., Impairment of function in ageing neutrophils is associated with apoptosis. $J$. Immunol. 1993. 150: 5124-5134.

2 Savill, J. S., Wyllie, A. H., Henson, J. E., Walport, M. J., Henson, P. M. and Haslett, C., Macrophage phagocytosis of aging neutrophils in inflammation. Programmed cell death in the neutrophil leads to its recognition by macrophages. J. Clin. Invest. 1989. 83: 865-875.

3 Liu, Y., Cousin, J. M., Hughes, J., VanDamme, J., Seckl, J. R., Haslett, C., Dransfield, I. et al., Glucocorticoids promote nonphlogistic phagocytosis of apoptotic leukocytes. J. Immunol. 1999. 162: 3639-3646.

4 Meagher, L. C., Cousin, J. M., Seckl, J. R. and Haslett, C., Opposing effects of glucocorticoids on the rate of apoptosis in neutrophilic and eosinophilic granulocytes. J. Immunol. 1996. 156: 4422-4428.

5 Murray, J., Barbara, J. A., Dunkley, S. A., Lopez, A. F., Van Ostade, X., Condliffe, A. M., Dransfield, I. et al., Regulation of neutrophil apoptosis by tumor necrosis factor- $\alpha$ : requirement for TNFR55 and TNFR75 for induction of apoptosis in vitro. Blood 1997. 90: 2772-2783. 
6 Colotta, F., Re, F., Polentarutti, N., Sozzani, S. and Mantovani, A., Modulation of granulocyte survival and programmed cell death by cytokines and bacterial products. Blood 1992. 80: 2012-2020.

7 Ward, C., Dransfield, I., Chilvers, E. R., Haslett, C. and Rossi, A. G.Pharmacological manipulation of granulocyte apoptosis: potential therapeutic targets. Trends Pharmacol. Sci. 1999. 20: 503-509.

8 Lee, A., Whyte, M. K. and Haslett, C., Inhibition of apoptosis and prolongation of neutrophil functional longevity by inflammatory mediators. J. Leukoc. Biol 1993. 54: 283-288.

9 Zarember, K. A. and Godowski, P. J., Tissue expression of human Toll-like receptors and differential regulation of Toll-like receptor mRNAs in leukocytes in response to microbes, their products, and cytokines. $J$. Immunol. 2002. 168: 554-563.

10 Ulloa, L. and Tracey, K. J., The 'cytokine profile': a code for sepsis. Trends Mol. Med 2005. 11: 56-63.

11 Moore, K. W., deWaal Malefyt, R., Coffman, R. L. and O'Garra, A., Interleukin-10 and the interleukin-10 receptor. Annu. Rev. Immunol 2001. 19: $683-765$

12 Bogdan, C., Vodovotz, Y. and Nathan, C., Macrophage deactivation by interleukin 10. J. Exp. Med. 1991. 174: 1549-1555.

13 Schottelius, A. J., Mayo, M. W., Sartor, R. B. and Baldwin, A. S. Jr., Interleukin-10 signaling blocks inhibitor of kappaB kinase activity and nuclear factor кB DNA binding J. Biol. Chem. 1999. 274: 31868-31874.

14 Crepaldi, L., Gasperini, S., Lapinet, J. A., Calzetti, F., Pinardi, C., Lui, Y. Zurawski, S. et al., Up-regulation of $\mathrm{IL}-10 \mathrm{R} 1$ expression is required to render human neutrophils fully responsive to IL-10. J. Immunol 2001. 167: 2312-2322.

15 Muzio, M., Bosisio, D., Polentarutti, N., D'Amico, G., Stoppacciaro, A. Mancinelli, R., Van't Veer, C. et al., Differential expression and regulation of toll-like receptors (TLR) in human leukocytes: selective expression of TLR3 in dendritic cells. J. Immunol 2000. 164: 5998-6004.

16 Crawley, J. B., Williams, L. M., Mander, T., Brennan, F. M. and Foxwell, B. M. J., Interleukin-10 stimulation of phosphatidylinositol 3-kinase and p70 S6 kinase is required for the proliferative but not the antiinflammatory effects of the cytokine. J. Biol. Chem. 1996. 271: 16357-16362.

17 Elbim, C., Reglier, H., Fay, M., Delarche, C., Andrieu, V., El Benna, J. and Gougerot-Pocidalo, M. A., Intracellular pool of IL-10 receptors in specific granules of human neutrophils: differential mobilization by proinflammatory mediators. J. Immunol. 2001. 166: 5201-5207.

18 Capsoni, F., Minonzio, F., Ongari, A. M., Carbonelli, V., Galli, A. and Zanussi, C., Interleukin-10 down-regulates oxidative metabolism and antibody-dependent cellular cytotoxicity of human neutrophils. Scand. J. Immunol. 1997, 45: 269-275.

19 Cassatella, M. A., Meda, L., Bonora, S., Ceska, M. and Constantin, G. Interleukin 10 (IL-10) inhibits the release of proinflammatory cytokines from human polymorphonuclear leukocytes. Evidence for an autocrine role of tumor necrosis factor and $\Pi-1 \beta$ in mediating the production of $\mathrm{L}-8$ triggered by lipopolysaccharide. J. Exp. Med 1993. 178: 2207-2211.

20 Ward, C., Murray, J., Bruce, L., Farrow, S., Chilvers, E. R., Hannah, S., Haslett, C. and Rossi, A. G., Interleukin-10 does not directly affect the constitutive rate of human neutrophil or eosinophil apoptosis. Biochem. Soc. Trans 1997. 25:245S

21 Clarke, C. J., Hales, A., Hunt, A. and Foxwell, B. M.IL-10-mediated suppression of TNF- $\alpha$ production is independent of its ability to inhibit NFКB activity. Eur. J. Immunol. 1998. 28: 1719-1726.

22 Rossi, A. G., Cousin, J. M., Dransfield, I., Lawson, M. F., Chilvers, E. R. and Haslett, C., Agents that elevate CAMP inhibit neutrophil apoptosis Biochem. Biophys. Res. Comm. 1995. 217: 892-899.

23 Martin, M. C., Dransfield, I., Haslett, C. and Rossi, A. G.Cyclic AMP regulation of neutrophil apoptosis occurs via a novel protein kinase A independent signaling pathway. J. Biol. Chem 2001. 276: 45041-45050.

24 Ward, C., Chilvers, E. R., Lawson, M. F., Pryde, J. G., Fujihara, S., Farrow, S. N., Haslett, C. and Rossi, A. G., NF-KB activation is a critical regulator of human granulocyte apoptosis in vitro. J. Biol. Chem. 1999. 274: 4309-4318.

25 Thompson, J. E., Phillips, R. J., Erdjument-Bromage, H., Tempst, P. and Ghosh, S., IкB- $\beta$ regulates the persistent response in a biphasic activation of NF-кB. Cell 1995. 80: 573-582
26 Cuenda, A., Rouse, J., Doza, Y. N., Meier, R., Cohen, P., Gallagher, T. F., Young, P. R. and Lee, J. C., SB 203580 is a specific inhibitor of a MAP kinase homologue which is stimulated by cellular stresses and interleukin-1. FEBS Lett. 1995. 364: 229-233.

27 Whitmarsh, A. J., Yang, S., Su, M. S., Sharrocks, A. D. and Davis, R. J., Role of p38 and JNK mitogen-activated protein kinases in the activation of ternary complex factors. Mol. Cell. Biol. 1997. 17: 2360-2371.

28 Davis, P. D., Hill, C. H., Lawton, G., Nixon, J. S., Wilkinson, S. E., Hurst, S. A., Keech, E. and Turner, S. E., Inhibitors of protein kinase C. 1.2,3Bisarylmaleimides. J. Med. Chem 1992. 35: 177-184.

29 Vlahos, C. J., Matter, W. F., Hui, K. Y. and Brown, R. F., A specific inhibitor of phosphatidylinositol 3-kinase, 2-(4-morpholinyl)-8-phenyl-4H-1-benzopyran-4-one (LY294002). J. Biol. Chem. 1994. 269: 5241-5248.

30 Pang, L., Sawada, T., Decker, S. J. and Saltiel, A. R.Inhibition of MAP kinase kinase blocks the differentiation of PC-12 cells induced by nerve growth factor. J. Biol. Chem. 1995. 270: 13585-13588.

31 Ward, C., Dransfield, I., Murray, J., Farrow, S. N., Haslett, C and Rossi, A. G.Prostaglandin $\mathrm{D}_{2}$ and its metabolites induce caspase-dependent granulocyte apoptosis that is mediated via inhibition of $\mathrm{I \kappa} \mathrm{B} \alpha$ degradation using a peroxisome proliferator-activated receptor- $\gamma$-independent mechanism. $J$. Immunol. 2002. 168: 6232-6243.

32 Keel, M., Ungethum, U., Steckholzer, U., Niederer, E., Hartung, T., Trentz, O. and Ertel, W., Interleukin-10 counterregulates proinflammatory cytokine-induced inhibition of neutrophil apoptosis during severe sepsis. Blood 1997. 90: 3356-3363.

33 Takanaski, S., Nonaka, R., Xing, Z., O'Byrne, P., Dolovich, J. and Jordana, M., Interleukin 10 inhibits lipopolysaccharide-induced survival and cytokine production by human peripheral blood eosinophils. J. Exp. Med 1994. 180: 711-715.

34 Yousefi, S., Green, D. R., Blaser, K. and Simon, H. U.Protein-tyrosine phosphorylation regulates apoptosis in human eosinophils and neutrophils. Proc. Natl. Acad. Sci. USA 1994. 91: 10868-10872.

35 Kuroki, M. and O'Flaherty, J. T.Extracellular signal-regulated protein kinase (ERK)-dependent and ERK-independent pathways target STAT3 on serine-727 in human neutrophils stimulated by chemotactic factors and cytokines. Biochem. J. 1999. 341: 691-696.

36 Denys, A., Udalova, I. A. Smith, C. Williams, L. M., Ciesielski, C. J., Campbell, J., Andrews, C. et al., Evidence for a dual mechanism for IL-10 suppression TNF- $\alpha$ production that does not involve inhibition of p38 mitogen-activated protein kinase or NF- $\mathrm{kB}$ in primary human macrophages. J. Immunol. 2002. 168: 4837-4845.

37 Pongracz, J., Webb, P., Wang, K., Deacon, E., Lunn, O. J. and Lord, J. M., Spontaneous neutrophil apoptosis involves caspase 3-mediated activation of protein kinase C-delta. J. Biol. Chem. 1999. 274: 37329-37334.

38 Frasch, S. C., Nick, J. A., Fadok, V. A., Bratton, D. L., Worthen, G. S. and Henson, P. M., p38 mitogen-activated protein kinase-dependent and-independent intracellular signal transduction pathways leading to apoptosis in human neutrophils. J. Biol. Chem. 1998. 273: 8389-8397.

39 Perianayagam, M. C., Balakrishnan, V. S., King, A. J., Pereira, B. J. and Jaber, B. L.C5a delays apoptosis of human neutrophils by a phosphatidylinositol 3-kinase-signaling pathway. Kidney Int. 2002. 61: 456-463.

40 Klein, J. B., Rane, M. J., Scherzer, J. A., Coxon, P. Y., Kettritz, R., Mathiesen, J. M., Buridi, A. and McLeish, K. R., Granulocyte-macrophage colony-stimulating factor delays neutrophil constitutive apoptosis through phosphoinositide 3-kinase and extracellular signal-regulated kinase pathways. J. Immunol. 2000. 164: 4286-4291.

41 Gasparini, S., Marchi, M., Calzetti, F., Laudanna, C., Vicentini, L., Olsen, H., Murphy, M., Liao, F. et al., Gene expression and production of the monokine induced by INF- $\gamma$ (MIG), IFN-inducible T cell $\alpha$ chemoattractant (I-TAC), and IFN- $\gamma$-protein-10 (IP-10) chemokines by human neutrophils. $J$. Immunol. 1999. 162: 4928-4937.

42 Cox, G.IL-10 enhances resolution of pulmonary inflammation in vivo by promoting apoptosis of neutrophils. Am. J. Physiol. 1996,. 271: L566-L.571.

43 Dransfield, I, and Rossi, A. G., Granulocyte apoptosis: who would work with a 'real' inflammatory cell? Biochem. Soc. Trans 2004. 32: 447-451.

44 Nahas, N., Molski, T. F. P., Fernandez, G. A. and Sha'afi, R. I., Tyrosine phosphorylation and activation of a new mitogen-activated protein (MAP)- 
kinase cascade in human neutrophils stimulated with various agonists. Biochem. J. 1996. 318: 247-253.

45 Nick, J. A., Avdi, N. J., Gerwins, P., Johnson, G. L. and Worthen, G. S. Activation of a p38 mitogen-activated protein kinase in human neutrophils by lipopolysaccharide. J. Immunol 1996. 156: 4867-4875.

46 Nolan, B., Duffy, A., Paquin, L., De, M., Collette, H., Graziano, C. M. and Bankey, P., Mitogen-activated protein kinases signal inhibition of apoptosis in lipopolysaccharide-stimulated neutrophils. Surgery 1999. 126: 406-412.

47 Sabroe, I., Jones, E. C., Usher, L. R. , Whyte, M. K. B. and Dower, S. K., Toll-like receptor (TLR) 2 and TLR4 in human peripheral blood granulocytes: a critical role for monocytes in leukocyte lipopolysaccharide responses. $J$. Immunol. 2002. 168: 4701-4710.

48 Fessler, M. B., Malcolm, K. C., Duncan, M. W. and Worthen, G. S., A genomic and proteomic analysis of activation of the human neutrophil by lipopolysaccharide and its mediation by p38 mitogen-activated protein kinase. J. Biol. Chem. 2002. 277: 31291-31302.

49 Haslett, C., Guthrie, L. A., Kopaniak, M. M., Johnston, R. B. Jr. and Henson, P. M., Modulation of multiple neutrophil functions by preparative methods or trace concentrations of bacterial lipopolysaccharide. Am. J. Pathol 1985. 119: 101-110.

50 Malcolm, K. C., Arndt, P. G., Manos, E. J., Jones, D. A. and Worthen, G. S. Microarray analysis of lipopolysaccharide-treated human neutrophils. Am.J. Physiol. Lung Cell. Mol. Physiol. 2003. 284: L663-L670.

51 Bonner, S., Yan, S. R., Byers, D. M. and Bortolussi, R., Activation of extracellular signal-related protein kinases 1 and 2 of the mitogen-activated protein kinase family by lipopolysaccharide requires plasma in neutrophils from adults and newborns. Infect. Immun 2001. 69: 3143-3149.

52 Coffer, P. J., Geijsen, N., M'Rabet, L., Schweizer, R. C., Maikow, T., Raaijmakers, J. A. M., Lammers, J. J. and Koenderman, L., Comparison of the roles of mitogen-activated protein kinase and phosphatidylinositol 3 . kinase signal transduction in neutrophil effector function. Biochem. J. 1998. 329: $121-130$.
53 Swantek, J. L., Tsen, M. F., Cobb, M. H. and Thomas, J. A., IL-1 receptorassociated kinase modulates host responsiveness to endotoxin. J. Immunol. 2000. 164: 4301-4306.

54 Suttles, J., Milhorn, D. M., Miller, R. W., Poe, J. C., Wahl, L. M. and Stout, R. D., CD 40 signaling of monocyte inflammatory cytokine synthesis through an ERK1/2-dependent pathway. A target of interleukin (il)-4 and IL-10 antiinflammatory action. J. Biol. Chem. 1999. 274: 5835-5842.

55 Niiro, H., Otsuka, T., Ogami, E., Yamaoka, K., Nagano, S., Akahoshi, M., Nakashima, $\mathrm{H}$. et al., MAP kinase pathways as a route for regulatory mechanisms of IL-10 and IL-4 which inhibit COX-2 expression in human monocytes. Biochem. Biophys. Res. Comm 1998. 250: 200-205.

56 Kopp, E., Medzhitoz, R., Carothers, J., Xiao, C., Douglas, I., Janeway, C. A. and Ghosh, S., ECSIT is an evolutionarily conserved intermediate in the Toll/IL-1 signal transduction pathway. Genes Dev 1999. 13: 2059-2071.

57 Shinohara, H., Inoue, A., Toyama-Sorimachi, N., Nagai, Y., Yasuda, T., Suzuki, H., Horai, R. et al., Dok-1 and Dok- 2 are negative regulators of lipopolysaccharide-induced signaling. J. Exp. Med. 2005. 201: 333-339.

58 Voll, R. E., Herrmann, M., Roth, E. A., Stach, C., Kalden, J. R. and Girkontaite, I., Immunosuppressive effects of apoptotic cells. Nature 1997. 390: $350-351$.

59 Ogden, C. A., Pound, J. D., Batth, B. K., Owens,, S., Johannessen, I. Wood, K. and Gregory, C. D., Enhanced apoptotic cell clearance capacity and B cell survival factor production by $\mathrm{IL}-10$-activated macrophages: implications for Burkitt's lymphoma. J. Immunol. 2005, . 174: 3015-3023.

60 D'Amico, G., Frascaroli, G., Bianchi, G., Transidico, P., Doni, A., Vecchi, A., Sozzani, S. et al., Uncoupling of inflammatory chemokine receptors by IL-10: generation of functional decoys. Nat. Immunol 2000. 1: 387-391

61 Gilroy, D. W., Lawrence, T., Perretti, M. and Rossi, A. G.Inflammatory resolution: new opportunities for drug discovery. Nat. Rev. Drug Discov. 2004. 3: 401-416.

62 Rossi, A. G., McCutcheon, J. C., Roy, N., Chilvers, E. R., Haslett, C. and Dransfield, I., Regulation of macrophage phagocytosis of apoptotic cells by CAMP. J. Immunol. 1998. 160: 3562-3568. 


\section{The Involvement of the Apoptosis-Modulating Proteins ERK 1/2, Bcl- $x_{L}$ and Bax in the Resolution of Acute Inflammation in Vivo}

\author{
Deborah A. Sawatzky, ${ }^{\star \dagger}$ Derek A. Willoughby, ${ }^{\star}$ \\ Paul R. Colville-Nash, ${ }^{*}$ and Adriano G. Rossi ${ }^{\text {* }}$ \\ From the Department of Experimental Pathology,* \\ St. Bartholomew's, and the Royal London Medical School, \\ London; and the Medical Research Council Centre for \\ Inflammation Research, ${ }^{\dagger}$ The Queen's Medical Research Institute, \\ University of Edinburgh Medical School, Edinburgh, United \\ Kingdom
}

Inflammatory cell recruitment, activation, and apoptosis are highly regulated processes involving several checkpoints controlling the resolution of inflammation. We investigated the role of the mitogenactivated protein kinase (MAPK) signaling pathway (ie, ERK1/2) and apoptosis-regulating Bcl-2 family members (ie, Bcl- $x_{\mathrm{L}}$ and Bax) in the resolution of a rat carrageenan-induced pleurisy model. The specific ERK1/2 inhibitor PD 98059 enhanced the resolution of inflammation, whereas the MEK1/2 inhibitor U0126 had no effect and the flavonoid apigenin, a nonspecific inhibitor of ERK1/2 and COX-2, augmented inflammation. Specifically, PD98059 significantly decreased the total number of macrophages and neutrophils in the pleural cavity, mainly by increasing the rate of neutrophil apoptosis, as measured by Annexin $\mathrm{V}$ labeling and morphological analysis. Conversely, a specific inhibitor of proapoptotic Bax (V5) increased inflammation, indicating that by preventing apoptosis in vivo, resolution of inflammation is delayed. This was associated with a decrease in neutrophil apoptosis and an increase in macrophage and neutrophil numbers perpetuating the inflammatory response. In conclusion, this study shows that ERK1/2, Bax, and Bcl- $x_{\mathrm{L}}$ play important functional roles in the resolution phase of the acute inflammatory response in vivo by influencing apoptosis. Importantly, these data may provide novel therapeutic targets for the treatment of inflammatory diseases. (Am J Pathol 2006, 168:33-41; DOI: 10.2353/ajpath.2006. 050058)
The inflammatory response is a highly regulated process in which the balance between cell survival and apoptosis is orchestrated to ultimately drive and resolve inflammation. In addition, inflammation is controlled by a series of checkpoints, in which an array of endogenous mediators act to either elicit and/or to suppress the inflammatory response, depending on chronology and circumstance. ${ }^{1}$ Classically, the onset of acute inflammation is characterized by the recruitment of neutrophils and eosinophils from the bone marrow to the local site of inflammation, regulated by several chemokines and cell adhesion molecules. ${ }^{2}$ Here, they are activated by various cytokines [for example, tumor necrosis factor- $\alpha$, interleukin (IL) $-1 \beta$, and IL-8], growth factors (GM-CSF), and several other endogenous mediators (for example, PAF and $\mathrm{LTB}_{4}$ ). Resultant degranulation of cytotoxic granules and generation of toxic oxygen radicals contribute to surrounding tissue damage. At the peak of the inflammatory response, with regard to leukocyte recruitment, there is an influx of monocytes that differentiate into macrophages in situ. It is thought that these professional phagocytes clear apoptotic cells and contribute to resolution by generation of anti-inflammatory cytokines, such as transforming growth factor (TGF)- $\beta_{1}$ and IL-10. On the contrary, phagocytosis of necrotic cells leads to the generation of proinflammatory signals that fuels persistent inflammation and tissue damage. ${ }^{3}$ It is therefore thought that the promotion of apoptosis of inflammatory cells, hence efficient clearance by phagocytes, would be a beneficial therapeutic strat-

Supported in part by Asthma UK (grant 01/042); the Arthritis Research Campaign (grants C0594 and C0635 to P.C.-N.); the Joint Research Board, St. Bartholomew's; and the Royal London School of Medicine and Dentistry (to D.A.S.)

Accepted for publication September 15, 2005.

Professor Derek Willoughby died March 13, 2004.

Current address of P.C.-N.: SW Thames Renal Unit, St Helier Hospital, Carshalton, Surrey, UK.

Address reprint requests to D.A. Sawatzky, MRC Centre for Inflammation Research, The Queen's Medical Research Institute, University of Edinburgh Medical School, 47 Little France Crescent, Edinburgh, Scotland, UK EH16 4TJ. E-mail: d.a.sawatzky@ed.ac.uk. 
egy for inflammatory diseases such as asthma, rheumatoid arthritis, and inflammatory bowel disease.

Recently, we have shown the cellular profile of apoptosis in an acute pleurisy model of inflammation in vivo. ${ }^{4}$ One of the key events that initiate the resolution of inflammation (24 to 36 hours) is the induction of neutrophil apoptosis, which allows for safe clearance by phagocytes, such as macrophages. During resolution the majority of macrophages migrate to the local lymph node, ${ }^{5}$ however we have shown that some macrophages undergo apoptosis in situ at 72 hours, when inflammation has almost fully resolved. The signaling molecules and mechanisms that govern inflammatory cell survival and apoptosis have not yet been fully elucidated and more importantly, whether modulating these molecules can prevent or promote the resolution of the inflammatory response in an in vivo scenario has not been evaluated. There are numerous signal transduction molecules that play a role in determining the fate of the cell, which are triggered by extracellular signals before committing to caspase-dependent or -independent cell death. ${ }^{6}$ The $\mathrm{Bcl}-2$ and mitogen-activated protein kinase (MAPK) pathways have been well characterized in terms of cell apoptosis and cell survival regulation in vitro, however their effects on the resolution of inflammation in vivo is still unclear. Bax and Bcl- $x_{L}$, proapoptotic and anti-apoptotic $\mathrm{Bcl}-2$ family members, respectively, play key roles in the regulation of apoptosis. ${ }^{7}$ For example, it has been shown that eosinophils isolated from children with acute asthma had an increased expression of $\mathrm{Bcl}-2$, which was inversely correlated with the expiratory flow rate. ${ }^{8}$ In addition, Bax expression is attenuated and $\mathrm{BCl}-\mathrm{x}_{\mathrm{L}}$ expression is increased in T cells isolated from the lamina propria from patients with Crohn's disease. ${ }^{9}$ This shows that inflammatory cell survival, by means of prosurvival and anti-apoptotic signaling mechanisms are important in the pathogenesis of inflammatory diseases. It has yet to be determined if modifying proteins from the Bcl-2 family can modulate inflammation per se.

The three main cascades by which MAPK homologues are released via MAPK kinase (MEK 1-7) are the extracellular signal-regulated kinase (ERK) (p42, p44, MAPK), c-Jun amino-terminal kinases (JNKs), and p38 MAPK pathways. ${ }^{10}$ The endogenous homologues of MAPK all play roles in cell differentiation, apoptosis, stress responses, and inflammation. ${ }^{11}$ In inflammatory diseases, such as asthma and rheumatoid arthritis, MAPK expression is increased. ${ }^{12}$ ERK $1 / 2$ can be activated for a relatively prolonged period of time under certain inflammatory conditions, in which it has been shown that macrophage inhibitory factor (MIF) induces ERK $1 / 2$ via $\mathrm{CPLA}_{2}$-induced arachidonic acid generation for a period of 24 hours in culture, which may partially account for the mechanism of ERK-induced cellular proliferation. ${ }^{13}$ In addition, macrophage ingestion of apoptotic cells liberate mediators $^{3}$ that can influence inflammatory cell survival via MAPK pathways.

Studies have also shown that inhibitors of p38 MAPK, such as RWJ-67657, SB203580, and SB242235, are effective anti-inflammatory agents in suppressing cytokine release in human clinical trials ex vivo ${ }^{14}$ and also in the rat adjuvant-induced arthritis and in murine collageninduced arthritis models in vivo. ${ }^{15,16}$ Inhibitors of ERK 1/2, such as PD98059, have also been shown to inhibit eosinophilic proinflammatory cytokine release, differentiation, and degranulation in vitro. ${ }^{17}$ Interestingly, flavonoids, such as apigenin, also possess anti-inflammatory properties in vitro through antioxidant mechanisms; ${ }^{18}$ inhibition of cell adhesion molecule expression, such as ICAM$1,{ }^{19,20}$ and by attenuating the release of inflammatory mediators such as IL- $1 \beta$-induced $\mathrm{PGE}_{2}$ via the inhibition of MAPK. ${ }^{21}$ In vivo studies have shown that flavonoids attenuate the onset of acute inflammation in murine models of carrageenan-induced paw edema and peritonitis, which is attributable to $\mathrm{PGE}_{2}$ and $\mathrm{LTB}_{4}$ inhibition. ${ }^{22} \mathrm{Al}-$ though flavonoids, such as apigenin, are effective at preventing the onset of inflammation, it is unclear whether flavonoids exert anti-inflammatory effects once inflammation is established and whether they can promote the resolution of inflammation. We therefore hypothesize that inhibiting prosurvival factors, such as ERK $1 / 2$ and $\mathrm{Bcl}-\mathrm{x}_{\mathrm{L}}$, or inducing proapoptotic proteins, such as Bax, may lead to the safe clearance of inflammatory cells and aid the resolution of acute inflammation. In this study, we examined the expression profile of apoptosis-modulating proteins during the inflammatory response and investigated whether inhibition of these specific proteins can result in the advancement or delay of the resolution of inflammation.

\section{Materials and Methods}

All materials were purchased from Sigma-Aldrich Ltd. (Poole, UK) unless otherwise stated. Bax-inhibiting peptide (V5), PD98059, and U0126 were purchased from Calbiochem (Nottingham, UK). Viscarin ( $\lambda$-carrageenan) was a kind gift from Marine Colloids Inc. (Philadelphia, PA). Antibodies to Bax, Bcl- $x_{1}$, and pERK $1 / 2$ were purchased from Autogen Bioclear (Wiltshire, UK).

\section{Carrageenan-Induced Pleurisy}

Male Lewis rats (250 to $300 \mathrm{~g}$ ) (Tuck and Sons, Battlebridge, UK) were housed in the University of Edinburgh animal facilities in accordance with local guidelines. Animals were fed on a normal diet with tap water ad libitum and were housed in a 12-hour light/dark cycle, at 20 to $21^{\circ} \mathrm{C}$ and with $50 \%$ humidity levels. The first described model of carrageenan-induced inflammation was by Winter and co-workers ${ }^{23}$ in the paw edema model. Since then, the carrageenan-induced pleurisy model has been further developed and widely used as an acute resolving model of inflammation. ${ }^{24}$ Briefly, the animals were anesthetized with halothane, a small incision was made to expose the musculature and $0.15 \mathrm{ml}$ of $1 \%$ viscarin $(\lambda$ carrageenan) was injected intrapleurally with a blunted 21 -gauge needle. Animals were killed by a rising concentration of $\mathrm{CO}_{2}$ at various time points after carrageenan administration, from the onset ( 6 hours), the peak (24 hours), and during the resolution of inflammation (36, 48 , and 72 hours). Pleural cavities were washed with $1 \mathrm{ml}$ 
of $3.15 \%(w / v)$ sodium citrate in physiological saline. Total inflammatory cell pellets were harvested for analysis by Western blotting.

\section{In Vivo Treatment with 2'-Amino-3'- Methoxyflavone (PD98059), 1,4-Diamino-2,3- Dicyano-1,4-Bis(O-Aminophenylmercapto) Butadiene (U0126), or Bax-Inhibiting Peptide V5}

Rats were treated with $0.15 \mathrm{ml}$ of saline control, $0.15 \mathrm{ml}$ of $0.1 \%$ or $0.8 \%$ dimethyl sulfoxide vehicle control, or with $300 \mu \mathrm{mol} / \mathrm{L}$ PD98059, $300 \mu \mathrm{mol} / \mathrm{L}$ U0126, or $200 \mu \mathrm{mol} / \mathrm{L}$ Bax-inhibiting peptide (V5) intrapleurally 36 hours after injection with $1 \%$ carrageenan. Animals were culled by a rising concentration of $\mathrm{CO}_{2} 48$ hours after injection with carrageenan (12 hours after drug treatment). Pleural cavities were washed with $1 \mathrm{ml}$ of $3.15 \%(\mathrm{w} / \mathrm{v})$ sodium citrate in physiological saline. Edema formation was measured by weighing the total pleural exudates, and total cell counts were measured with a Coulter Counter (model DN; Coulter Electronics, Luton, UK). An aliquot of $1 \times 10^{6}$ inflammatory cells were washed in PBS three times and labeled with fluorescein isothiocyanate-conjugated Annexin $\mathrm{V}$ according to the manufacturer's instructions. Total cell apoptosis was measured by Annexin $\mathrm{V}$ labeling according to the manufacturer's instructions and analyzed by the BD FacsCalibur (Becton-Dickinson, Mountain View, CA). Lymphocyte, polymorphonuclear leukocyte (PMNs), and monocyte/macrophage total numbers were counted by light microscopy from hematoxylin and eosin (H\&E)-stained cytospin preparations and confirmed by flow cytometry as described. ${ }^{4}$ Total neutrophil apoptosis was also measured by morphological analysis by light microscopy from H\&E-stained cytospin preparations of pleural inflammatory cells with data presented as the percentage of apoptotic neutrophils from the total number of neutrophils present.

\section{Western Blot Analysis}

Inflammatory cell pellets were lysed in the presence of protease inhibitory buffer containing $1 \mu \mathrm{mol} / \mathrm{L}$ phenylmethyl sulfonyl fluoride, $1.5 \mu \mathrm{mol} / \mathrm{L}$ pepstatin $\mathrm{A}$, and 0.2 $\mu \mathrm{mol} / \mathrm{L}$ leupeptin. Protein concentrations were determined by the Bradford assay, and equal protein loading was confirmed by $\beta$-actin blotting (data not shown). Samples were boiled for 5 minutes with equal volumes of Laemmli gel loading buffer ( $5 \mathrm{mmol} / \mathrm{L}$ Tris, $10 \%$ sodium dodecyl sulfate, $10 \%$ glycerol, $10 \% \beta$-mercaptoethanol, and $2 \mathrm{mg} / \mathrm{ml}$ bromophenol blue). Total protein equivalents of $10 \mu \mathrm{g}$ for each sample were run on $10 \%$ sodium dodecyl sulfate-polyacrylamide gel electrophoresis and were transferred to nitrocellulose paper (Amersham, UK) using the Bio-Rad minigel system (Bio-Rad, Hemel Hempstead, UK). Nonspecific antibody binding was blocked with $5 \%$ dried milk protein, and blots were incubated with either polyclonal goat or rabbit antibodies against pERK 1/2 (1:1000 dilution), Bax (1:500 dilution), or $\mathrm{BCl}-\mathrm{x}_{\mathrm{L}}$ (1:1000 dilution).

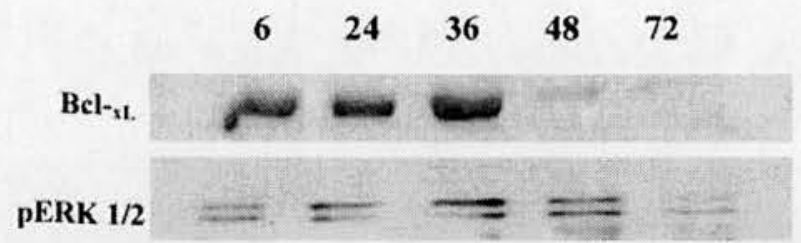

Bax

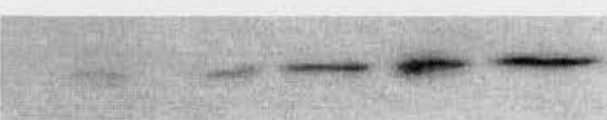

Figure 1. Expression of the prosurvival proteins, pERK $1 / 2$ and $\mathrm{BCl}-\mathrm{x}_{1}$, and the proapoptotic signaling protein, Bax, during the onset and resolving phase of inflammation. Male Lewis rats were injected intrapleurally with $1 \%$ carrageenan and inflammatory cells were harvested at various time points during the onset ( 6 to 24 hours) and resulving phase of acute inflammation ( 36 to 72 hours). Equal protein equivalent was determined by Bradford assay and 10 $\mu \mathrm{g}$ was loaded for each sample. Protein expression of $\mathrm{pERK} 1 / 2, \mathrm{BCl}-\mathrm{x}_{\mathrm{t}}$, and Bax were measured by electrophoresis and Western blotting. Data are representative of three separate experiments.

\section{Statistics}

All experiments were performed with six to eight rats per group, with experiments repeated to verify the original findings. Statistical analysis was performed by a one-way analysis of variance with Bonferroni multiple comparison post hoc test with a $95 \%$ confidence interval or by a Student's $t$-test as appropriate. Data are expressed as mean \pm SEM.

\section{Results}

\section{Expression Profiles of Signaling Molecules Involved in Apoptosis}

Inflammatory cells from the onset of inflammation in a rat carrageenan-induced pleurisy model ( 6 to 36 hours after carrageenan) expressed the prosurvival molecules pERK $1 / 2$ and $\mathrm{Bcl}-\mathrm{x}_{\mathrm{L}}$ with very low levels of Bax expression found (Figure 1). Conversely during the resolution of inflammation (48 hours after carrageenan), Bax protein expression was increased with negligible levels of pERK $1 / 2$ and $\mathrm{BCl}-\mathrm{x}_{\mathrm{L}}$ expression.

\section{Modulation of Proapoptotic or Prosurvival Proteins Can Enhance or Prevent the Resolution of Inflammation in Vivo}

Functional studies were conducted to assess whether inhibition of the prosurvival ERK $1 / 2$ could alter the resolution of inflammation in the carrageenan-induced pleurisy model. Once inflammation was established in the pleural cavity 36 hours after carrageenan, the flavonoid apigenin, a nonspecific ERK1/2 inhibitor, was given locally by intrapleural injection. Although apigenin did not affect edema, it was found to exacerbate inflammation with an increased total amount of pleural cell numbers of $112.6 \times 10^{6} \pm 6.4$ cells compared with vehicle-treated control of $71.7 \times 10^{6} \pm 7.3$ cells $\left({ }^{\star} P<0.05\right.$; Figure 2$)$.

The specific ERK1/2 inhibitor PD98059 augmented the resolution of inflammation by reducing cell numbers in 
A

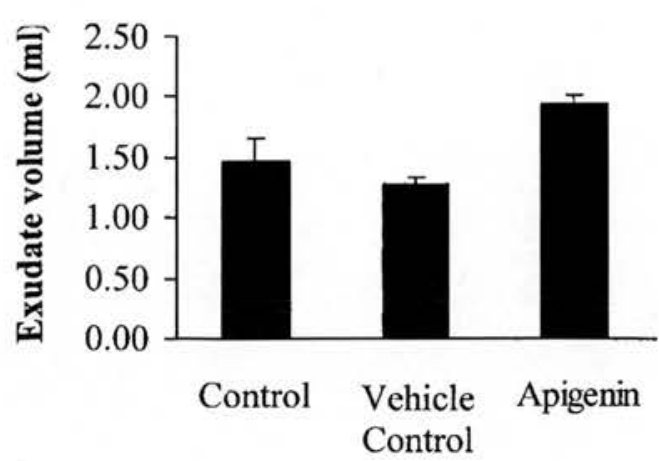

B

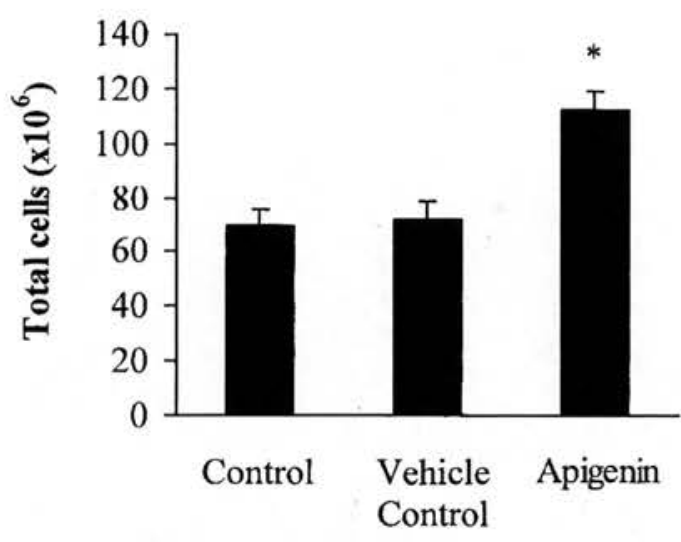

Figure 2. Apigenin, a nonspecific inhibitor of ERK1/2 and COX-2, inhibited the resolution of inflammation. Male Lewis rats were injected intrapleurally with $1 \%$ carrageenan and after 36 hours were treated intrapleurally with saline, vehicle-treated control, or $30 \mu \mathrm{mol} / \mathrm{L}$ apigenin. Twelve hours later edema formation $(\mathbf{A})$ and total inflammatory cell numbers $(\mathbf{B})$ were measured ( 48 hours after the onset of the carrageenan-induced pleurisy). Data are the mean of six animals per group $\pm \mathrm{SEM}, \cdot P<0.05$.

the pleural cavity to $50 \times 10^{6} \pm 5.2 \times 10^{6}$ cells $(n=8$; Figure 3B) compared with vehicle control of $81 \times 10^{6} \pm$ $5.6 \times 10^{6}$ cells $\left({ }^{* *} P<0.01\right)$ when given 36 hours after carrageenan. PD98059 pretreatment halved the total cell number of PMNs $\left({ }^{\star} P<0.05\right)$ and also monocytes/macrophages $\left({ }^{* * *} P<0.001\right)$ compared with vehicle-treated control (Figure 5A). Edema formation decreased with PD98059 treatment by $30 \%$ when compared with saline control; however, PD98059 treatment was not significantly different from vehicle-treated control (Figure 3A). Inflammatory cell apoptosis, measured by Annexin V binding and analyzed by flow cytometry, was significantly enhanced with PD98059 resulting in a $25 \%$ increase in binding when compared with vehicle control $\left(n=8\right.$, ${ }^{\star} P<$ 0.05 ; Figure $3 \mathrm{C}$ ). Specifically, there was as increase of apoptotic neutrophils with PD98059 pretreatment by $50 \pm 6 \%\left(n=6,{ }^{\star \star} P<0.01\right.$; Figure $\left.5 \mathrm{~B}\right)$, which was approximately double the amount of constitutive apoptosis found in the saline and vehicle-treated control groups. Neutrophil apoptosis was further illustrated with H\&Estained inflammatory cells (Figure $5 \mathrm{C}$ ). In contrast, the MEK $1 / 2$ inhibitor U0126 had no effect on the exudate volume, total cell number, or cell apoptosis levels (Figure 3, D-F).

We have shown that by enhancing apoptosis, via inhibition of ERK1/2, there is a significant attenuation of in-
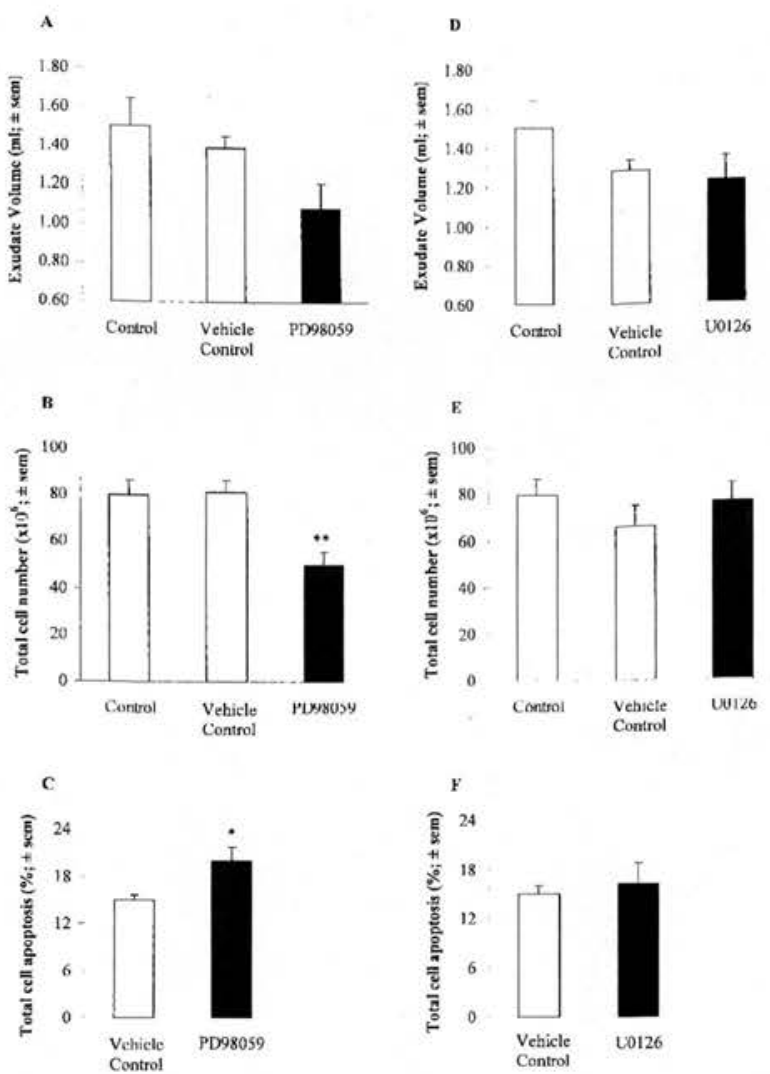

Figure 3. The specific ERK $1 / 2$ inhibitor, PD98059, enhanced the resolution of inflammation, whereas the MEK inhibitor U0126 had no effect. Male Lewis rats were injected intrapleurally with $1 \%$ carrageenan and after 36 hours were treated intrapleurally with saline, vehicle-treated control, $300 \mu \mathrm{mol} / \mathrm{L}$ PD98059, or $300 \mu \mathrm{mol} / \mathrm{L}$ U0126. Twelve hours later edema formation (A and D) and total inflammatory cell numbers (B and $\mathbf{E}$ ) were measured ( 48 hours after the onset of the carrageenan-induced pleurisy). Levels of apoptosis (C and $\mathbf{F}$ ) were measured by Annexin V binding and analyzed by flow cytometry. Data are the mean of eight animals per group \pm SEM, $\cdot P<0.05$ and $* P<0.01$.

flammation. On the contrary, it was also important to assess whether direct inhibition of apoptosis can alter the resolution of inflammation. A specific inhibitor of Bax prevented the safe resolution of inflammation with an increase in numbers of inflammatory cells to $121.9 \times$ $10^{6} \pm 16.7 \times 10^{6}$ cells $(n=8$; Figure 4) compared with saline alone to $78.3 \times 10^{6} \pm 9.5 \times 10^{6}$ cells $(n=8)$ when administered locally at the peak of inflammation. Specifically, the total amount of PMNs and monocytes/macrophages increased by $42 \%$ and $33 \%$, respectively, compared with saline control $\left({ }^{\star} P<0.05,{ }^{\star \star \star} P<0.001\right.$; Figure $5 A)$. There was also a significant decrease $\left(35 \%,{ }^{\star} P<\right.$ $0.05, n=8$ ) in Annexin V binding compared with control, which further supports the hypothesis that the augmented inflammation was due to the prevention of apoptosis, which would normally occur during the resolution phase of inflammation. In particular, Bax inhibition caused a decrease in the number of apoptotic neutrophils, in which only $7.2 \%$ of neutrophils were apoptotic within the total number of neutrophils present (Figure 5, B and $C$; ${ }^{*} P<0.05$ ). Interestingly, although $T G F-\beta_{1}$ was prevalent during resolution ( 36 hours after carrageenan), 


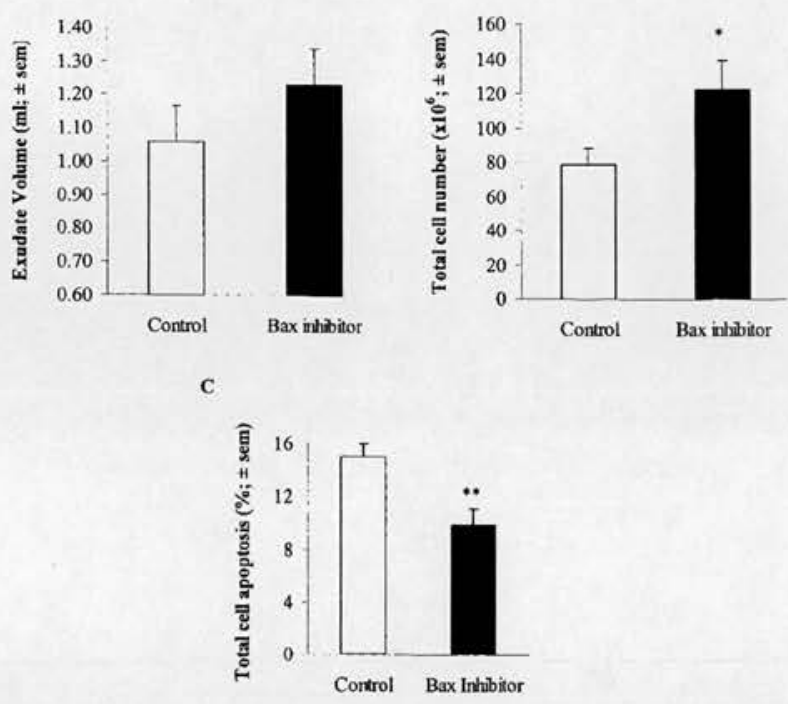

Figure 4. The Bax-inhibiting peptide V5 prevented the safe resolution of inflammation. Male Lewis rats were injected intrapleurally with $1 \%$ carrageenan and after 36 hours were treated intrapleurally with saline or 200 $\mu \mathrm{mol} / \mathrm{L}$ Bax-inhibiting peptide V5. Twelve hours later edema formation (A) and total inflammatory cell numbers (B) were measured ( 48 hours after the onset of the carrageenan-induced pleurisy). Levels of apoptosis (C) were measured by Annexin V binding and analyzed by flow cytometry. Data are the mean of 10 animals per group \pm SEM, $* P<0.05, * P=0.01$.

there was no significant difference in TGF- $\beta_{1}$ or IL-10 levels in any of the treatment groups (data not shown).

\section{Discussion}

The balance between prosurvival and proapoptotic signals is a highly regulated process involving many checkpoints that regulate cell survival and death. ${ }^{1,25}$ Several proinflammatory mediators, such as fMLP, ${ }^{26}$ tumor necrosis factor- $\alpha,{ }^{27} \mathrm{GM}-\mathrm{CSF},{ }^{28} \mathrm{PAF}^{29} \mathrm{LTB}_{4},{ }^{30}$ influence apoptosis of inflammatory cells, ${ }^{31}$ and trigger MAPK pathways. This study has shown that during the initiation of acute inflammation, expression of prosurvival molecules, such as ERK $1 / 2$ and $\mathrm{Bcl}-\mathrm{x}_{\mathrm{L}}$, are increased. Furthermore, we also show that the expression of these survival factors is down-regulated during the resolution phase. Conversely, the proapoptotic signaling molecule Bax is up-regulated when the inflammation is resolving with low expression levels found during the onset of inflammation. The functional role of cell signaling proteins, such as Bax, MEK 1/2, and ERK $1 / 2$, in inflammation in vivo was previously undetermined.

This study demonstrates for the first time that apoptosis-modulating pharmacological inhibitors can promote or delay the resolution of acute inflammation. Importantly, the specific ERK1/2 inhibitor, PD98059, accelerates resolution of inflammation even after inflammation was established. It is difficult to predict in patients when inflammation will occur, even in chronic inflammatory diseases, and therefore, treatment during flare-ups is more beneficial than a prolonged prophylactic drug treatment re- gime. Furthermore, this is an advantageous therapeutic strategy that will allow neutrophil infiltration to combat invading pathogens but will limit persistent inflammation and excessive tissue damage. Premature neutrophil apoptosis during infection is likely to be detrimental to host defense and lead to bacterial infection and sepsis. This is a known complication in cystic fibrosis patients in which the Pseudomonas aeruginosa-derived exotoxin pyocyanin induces neutrophil apoptosis, which allows inflammatory cells and bacteria to persist contributing to pulmonary damage and patient mortality. ${ }^{32}$

The effects of endogenous or exogenous mediators depend on the kinetics of the synthesis or release or the timing of the administration of the inflammatory process. Studies have shown that in the carrageenan-induced paw edema model, apigenin had an anti-inflammatory effect when given at the onset of inflammation. ${ }^{33}$ This effect would be consistent with the suppression of COX-2 inhibiting inflammation when given prophylactically. In the present study, the nonselective ERK $1 / 2$ inhibitor apigenin exacerbated inflammation, which in part is likely due to an inhibition of COX-2 because it has been shown that selective COX-2 inhibition by NS-398 and traditional NSAIDs, when given during the peak of inflammation, causes a prolonged inflammation. ${ }^{34}$ This is likely due to inhibition of the anti-inflammatory cyclopentenone prostaglandins, 15 deoxy $\delta,{ }^{12.14} \mathrm{PGJ}_{2}$, and $P \mathrm{PD}_{2}$, which directly promote the apoptosis of neutrophils and macrophages in situ. ${ }^{4}$ Further evidence of the anti-inflammatory role of COX-2 was also shown by Wallace and co-work$\mathrm{ers}^{35}$ in which inflammation failed to resolve in COX-2 knockout mice in a model of carrageenan-induced paw inflammation.

Here, we show that pretreatment with the specific ERK1/2 inhibitor PD98059 enhanced the resolution of acute inflammation, with attenuated total cell numbers of monocytes/macrophages and PMNs in the pleural cavity. This correlated with an increase in the number of free apoptotic neutrophils found after pretreatment with PD98059. The increased amount of free apoptotic cells was probably not due to a defective phagocytosis mechanism because previous studies have shown that PD98059 does not affect phagocytosis in many different in vitro assays. ${ }^{36-38}$ In the present study, we also qualitatively show that the macrophage can still ingest apoptotic cells, as indicated in Figure $5 \mathrm{C}$. The ability to visualize the free apoptotic neutrophils may be a result of a pronounced increase in apoptosis levels and a reduced number of the professional phagocytes (macrophages) in the pleural cavity. The persistence of free apoptotic cells has recently been suggested to be antiinflammatory dependent on the environment that the macrophage is exposed to. ${ }^{39,40}$ Interestingly, autologous administration of apoptotic cells has been clinically proven to help prevent heart, lung, and renal allograft transplant rejection. ${ }^{41-44}$ This is thought to occur as a result of tolerogenic changes of immature dendritic cells after the engulfment of the apoptotic cells, which then stimulates inhibitory T-regulatory cells that suppress immune rejection. ${ }^{45}$ This clearly shows that the administration of apoptotic cells and their safe engulfment in hu- 

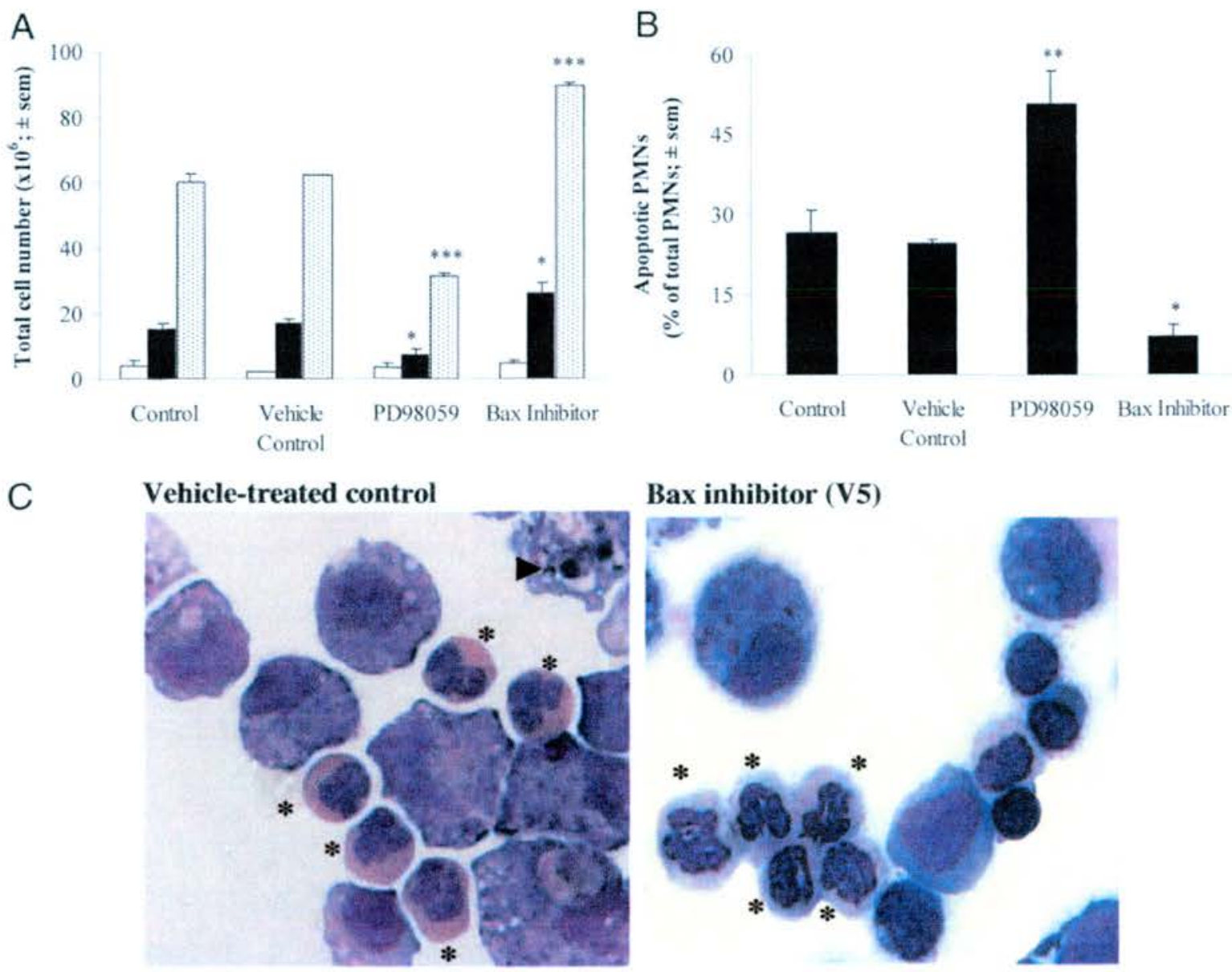

\section{Bax inhibitor (V5)}

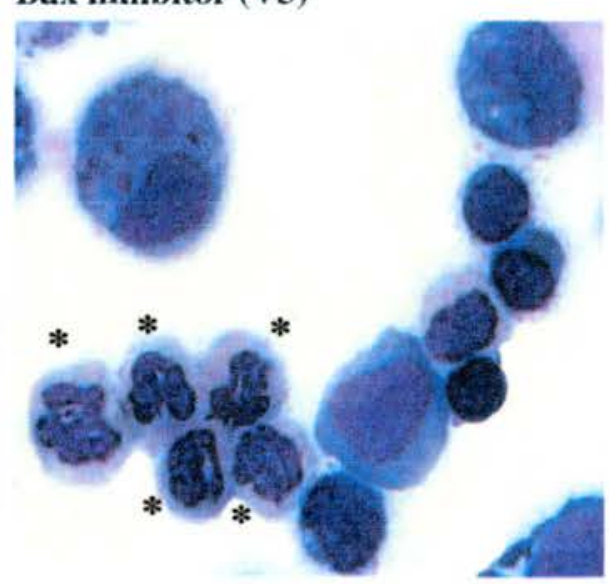

\section{Specific ERK1/2 inhibitor PD98059}

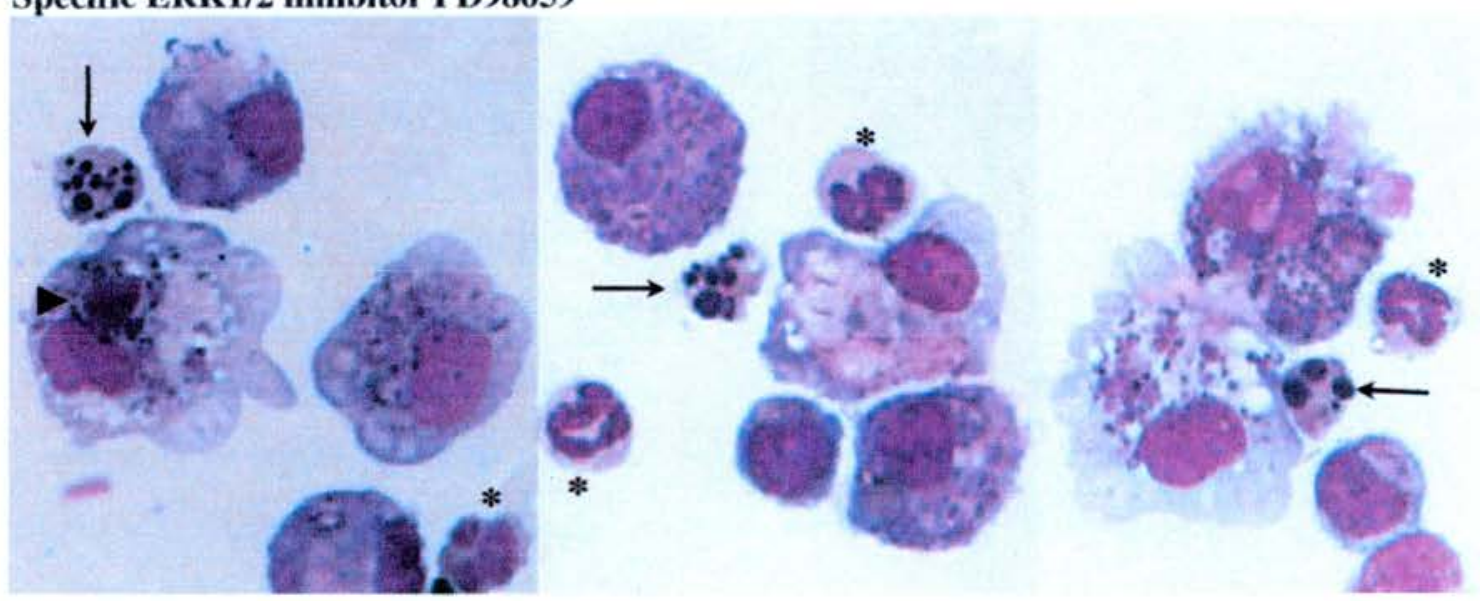

Figure 5. The specific ERK1/2 inhibitor PD98059 augments neutrophil apoptosis, whereas the Bax-inhibiting peptide promoted neutrophil survival. Male Lewis rats were injected intrapleurally with $1 \%$ carrageenan and after 36 hours were treated intrapleurally with saline, vehicle-treated control, $300 \mu$ mol/L PD 98059 , or $200 \mu \mathrm{mol} / \mathrm{L}$ Bax-inhibiting peptide V5. A: Twelve hours later, the total number of lymphocytes (open bars), neutrophils (filled bars), and monocytes, macrophages (dotted bars) was counted by flow cytometric analysis and differential cell counts by light microscopy. B: Neutrophil apoptosis was measured by morphological analysis by light microscopy after H\&E staining with data presented as the percentage of apoptotic neutrophils from the total number of neutrophils present. Examples of this in a typical field are illustrated showing the clistinct nuclei of apoptotic neutrophils (arrows), phagocytosed apoptotic neutrophils (arrowhead), and viable neutrophils (asterisk) after vehicle, PD98059, or Bax inhibitor (V5) pretreatment. Original magnifications, $\times 60$.

mans has a role in the adaptive and the innate immune system by stimulating tolerance and promoting the resolution of inflammation. In this study, there is an overall diminished inflammatory response with PD98059 treat- ment in which increased apoptosis of neutrophils leads to an enhanced resolution of inflammation.

The accelerated resolution of inflammation was a specific effect for PD98059 because the MEK1/2 inhibitor 
U0126 had no anti-inflammatory effect in terms of inflammatory cell numbers and apoptosis levels in vivo. U0126 has been shown to be specific for MEK $1 / 2$ and is noncompetitive with ERK and ATP. ${ }^{46}$ This indicates that MEK $1 / 2$ may be disassociated from the ERK $1 / 2$ signaling pathway, which may provide a novel pathway for cell survival in inflammation. Alternatively U0126 could be ineffective in this model. Recently however, administration of U0126 at the onset of inflammation in a murine model of ovalbumin-induced asthma has been shown to be effective in reducing eosinophilia, proinflammatory cytokine release, and airway hyperresponsiveness. ${ }^{47}$ In an in vitro study, there was a suppression of the release of eotaxin, RANTES, and GM-CSF from human airway smooth muscle cells with the MEK $1 / 2$ inhibitor U0126; however, no effect was observed with a range of concentrations of the ERK1/2 inhibitor PD98059. ${ }^{48}$ The authors argue that PD98059 has poor efficacy, which is different from the findings proposed here, in which this compound augmented the resolution of inflammation. The local concentrations of the PD98059 and U0126 compounds were consistent with $\mathrm{EC}_{50}$ values shown in other in vitro studies. $^{21}$

The Bax-inhibiting peptide V5 is a cell-permeable pentapeptide that blocks the ku70 binding domain and prevents Bax conformational change and mitochondrial translocation. ${ }^{49}$ This peptide has been shown to inhibit staurosporine-, UV-, and anti-cancer drug-induced apoptosis in vitro. ${ }^{50}$ In the present study, inhibition of Bax during the resolving phase of acute inflammation exacerbated the inflammatory response in vivo. Specifically, total pleural cell numbers of monocytes/macrophages and PMNs were increased, with a corresponding decrease in the amount of neutrophil apoptosis, after pretreatment with the specific Bax inhibitor. Other studies by Maiuri and co-workers ${ }^{51}$ show that induction of Bax via inhibition of nuclear factor $-\kappa \mathrm{B}$ in a carrageenan sponge model of chronic inflammation is associated with the apoptosis of neutrophils

A decrease in total cell apoptosis, which could be attributed mainly to neutrophil apoptosis, was observed with the Bax peptide inhibitor. In contrast, an increase in neutrophil apoptosis contributed to an enhanced resolution with the ERK $1 / 2$ inhibitor PD98059. The percent total apoptosis of pleural cells in control and drug-treated animals were relatively small. This likely reflects the difficulty in visualizing and measuring apoptosis due to efficient clearance by phagocytosis. There is also a very limited window of opportunity to visualize free apoptotic cells in vivo, in which the estimated duration of detectable apoptosis due to rapid recognition and efficient engulfment by phagocytes is only 1 to 2 hours. ${ }^{52,53}$ However. even the levels of apoptosis that we have observed may have significant biological effects because studies have shown that small changes in apoptosis levels can drastically affect the overall total cell number. For example, in the rat liver an increase of $2 \%$ apoptosis by histological examination results in an overall $25 \%$ reduction in cell number. ${ }^{54}$ Furthermore, our previous work shows that an approximate $3 \%$ induction of apoptosis contributes to an

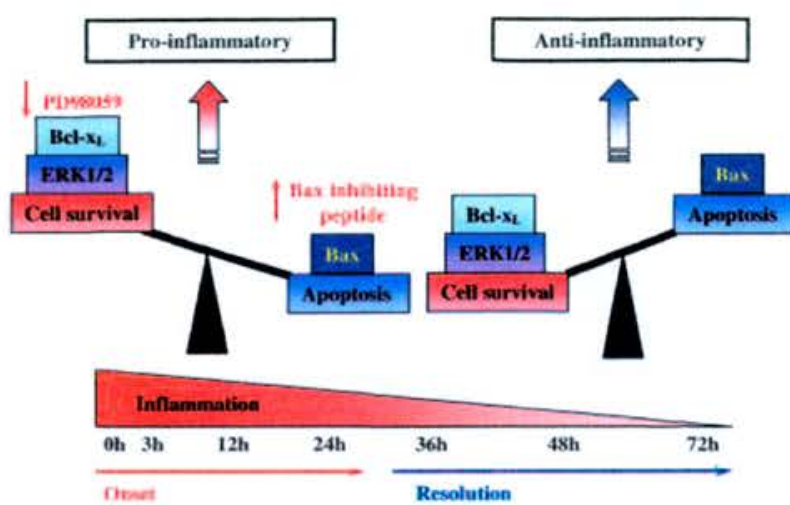

Figure 6. A schematic diagram showing the balance between prosurvival and proapoptotic signaling molecules from the onset to the resolution of acute inflammation. This figure shows that prosurvival proteins such as ERK $1 / 2$ and $\mathrm{Bcl}-\mathrm{x}_{\mathrm{L}}$ predominate during the onset of inflammation, which when inhibited promotes apoptosis and aids resolution. The expression of proapoptotic molecules such as Bax are relatively low at this time point. Conversely, during resolution of inflammation, the balance switches toward the expression of proapoptotic signaling molecules, Bax, which ultimately promotes cell death and safe clearance by phagocytosis.

$80 \%$ reduction in the number of neutrophils in the carrageenan-induced pleurisy model of inflammation in vivo. ${ }^{4}$

Results of previous studies suggest that phagocytosis of apoptotic cells results in release of anti-inflammatory cytokines, such as TGF- $\beta_{1}{ }^{3}$ and IL- $10 .{ }^{55}$ Furthermore, the released TGF- $\beta_{1}$ is capable of inhibiting the release of proinflammatory cytokines modulated via p38 MAPK whereby the inhibitor, SB 203580, prevented TGF- $\beta_{1}-$ mediated inhibition of lipopolysaccharide-induced tumor necrosis factor- $\alpha$ and MIP-2 release. ${ }^{56}$ In this study, however, there was no significant difference in TGF- $\beta_{1}$ or IL-10 levels found using the Bax inhibitor-, PD98059-, or the U0126-treated groups (data not shown). However, silent phagocytosis of early apoptotic cells can occur without affecting cytokine levels. ${ }^{57}$ The levels of proinflammatory cytokines, such as tumor necrosis factor- $\alpha$ and $\mathrm{IL}-1 \beta$, are insignificant during the resolution of inflammation; therefore, we were unable to establish whether inducing or preventing apoptosis in vivo affects these cytokines in particular.

Although we have clearly shown in this study that manipulation of pathways that influence apoptosis (namely ERK $1 / 2$ and $\mathrm{Bax}$ ) can accelerate the resolution of an acute model of inflammation, the question remains whether our observations could be extended to a more chronic inflammatory scenario. Many of the pathways that we have investigated here can affect cell recruitment and activation, in addition to influencing cell survival and apoptosis. Furthermore, it is now becoming clear that MAPK signaling pathways and proteins involved in the regulation of apoptosis are dysregulated in a number of pathological inflammatory conditions, such as asthma. ${ }^{12}$ The challenge now is to investigate whether such manipulation of these pathways influences the resolution phase of inflammation in animal models of chronic inflammation or, more importantly, whether chronic inflammatory diseases in humans can be beneficially influenced. The clinical trials currently underway with compounds that directly influence apoptosis (eg, the caspase inhibitors ${ }^{58}$ ) 
and compounds that have an indirect effect on cell survival (eg, JNK, ERK, and p38 kinase inhibitors ${ }^{59}$ ) will identify whether manipulation of apoptosis would be clinically beneficial for chronic inflammatory diseases.

Overall this study shows that direct modulation of apoptosis through interfering with the signaling of important molecules (ERK 1/2, Bcl- $\mathrm{x}_{L}, \mathrm{Bax}$ ) can exacerbate or attenuate cell infiltrate and edema formation. Thus evidence from in vitro data and from the evidence presented here suggest that promoting apoptosis and safe clearance of effete cells may be a valuable new therapeutic target for inflammatory diseases. ${ }^{60}$ There is also a greater need to elucidate effective mechanisms of inducing apoptosis of inflammatory cells in vivo and whether this affects the outcome of the disease process once inflammation is established. This study emphasizes that there is an intricate balance of survival and apoptotic signaling pathways that mediate the kinetics of the acute inflammatory response, as illustrated in Figure 6 . The enhanced resolution of inflammation by the inhibition of the prosurvival mediators, such as ERK 1/2 with PD98059 may prove to be an important advancement for the development of new treatments of inflammatory diseases.

\section{Acknowledgment}

This manuscript is dedicated to the late Professor Derek Willoughby for his great contribution to inflammation research.

We thank Rita Jones for technical support.

\section{References}

1. Nathan C: Points of control in inflammation. Nature $2002,420: 846-852$

2. Ley $\mathrm{K}$ : Integration of inflammatory signals by rolling neutrophils. Immunol Rev 2002, 186:8-18

3. Fadok VA, Bratton DL, Konowal A, Freed PW, Westcott JY, Henson PM: Macrophages that have ingested apoptotic cells in vitro inhibit proinflammatory cytokine production through autocrine/paracrine mechanisms involving TGF- $\beta, \mathrm{PGE}_{2}$, and PAF. J Clin Invest 1998, 101:890-898

4. Gilroy DW, Colville-Nash PR, McMaster S, Sawatzky DA, Willoughby DA, Lawrence $T$ : Inducible cyclooxygenase-derived 15-deoxy $(\Delta)^{12-14} \mathrm{PGJ}_{2}$ brings about acute inflammatory resolution in rat pleurisy by inducing neutrophil and macrophage apoptosis. FASEB J 2003, 17:2269-2271

5. Bellingan GJ, Caldwell $\mathrm{H}$, Howie SE, Dransfield I, Haslett C: In vivo fate of the inflammatory macrophage during the resolution of inflammation: inflammatory macrophages do not die locally, but emigrate to the draining lymph nodes. J Immunol 1996, 157:2577-2585

6. Jarpe MB, Widmann C, Knall C, Schlesinger TK, Gibson S, Yujiri T, Fanger GR, Gelfand EW, Johnson GL: Anti-apoptotic versus proapoptotic signal transduction: checkpoints and stop signs along the road to death. Oncogene 1998, 17:1475-1482

7. Reed JC: Double identity for proteins of the Bcl-2 family. Nature 1997. 387:773-776

8. El-Gamal Y, Heshmat N, Mahran M, El-Gabbas Z: Expression of the apoptosis inhibitor $\mathrm{Bcl}-2$ in sputum eosinophils from children with acute asthma. Clin Exp Allergy 2004, 34:1701-1706

9. Itoh J, de La Motte C, Strong SA, Levine AD, Fiocchi C: Decreased Bax expression by mucosal T cells favours resistance to apoptosis in Crohn's disease. Gut 2001, 49:35-41

10. Pearson G, Robinson F, Beers Gibson T, Xu BE, Karandikar M, Berman $\mathrm{K}$, Cobb $\mathrm{MH}$ : Mitogen-activated protein (MAP) kinase pathways: regulation and physiological functions. Endocr Rev 2001 22:153-183

11. Kumar S, Boehm J, Lee JC: p38 MAP kinases: key signalling molecules as therapeutic targets for inflammatory diseases. Nat Rev Drug Discov 2003, 2:717-726

12. Pelaia G, Cuda G, Vatrella A, Gallelli L, Caraglia M, Marra M, Abbruzzese A, Caputi M, Maselli R, Costanzo FS, Marsico SA: Mitogenactivated protein kinases and asthma. J Cell Physiol 2005 202:642-653

13. Mitchell RA, Metz CN, Peng T, Bucala R: Sustained mitogen-activated protein kinase (MAPK) and cytoplasmic phospholipase $A_{2}$ activation by macrophage migration inhibitory factor (MIF). Regulatory role in cell proliferation and glucocorticoid action. J Biol Chem 1999 274:18100-18106

14. Fijen JW, Zijlstra JG, De Boer P, Spanjersberg R, Tervaert JW, Van Der Werf TS, Ligtenberg JJ, Tulleken JE: Suppression of the clinica and cytokine response to endotoxin by RWJ-67657, a p38 mitogenactivated protein-kinase inhibitor, in healthy human volunteers. Clin Exp Immunol 2001, 124:16-20

15. Badger AM, Bradbeer JN, Votta B, Lee JC, Adams JL, Griswold DE: Pharmacological profile of SB 203580, a selective inhibitor of cytokine suppressive binding protein/p38 kinase, in animal models of arthritis, bone resorption, endotoxin shock and immune function. J Pharmaco Exp Ther 1996, 279:1453-1461

16. Badger AM, Griswold DE, Kapadia R, Blake S, Swift BA, Hoffman SJ, Stroup GB, Webb E, Rieman DJ, Gowen M, Boehm JC, Adams JL, Lee JC: Disease-modifying activity of SB 242235, a selective inhibitor of p38 mitogen-activated protein kinase, in rat adjuvant-induced arthritis. Arthritis Rheum 2000, 43:175-183

17. Adachi T, Choudhury BK, Stafford S, Sur S, Alam R: The differentia role of extracellular signal-regulated kinases and p38 mitogen-activated protein kinase in eosinophil functions. J Immunol 2000 $165: 2198-2204$

18. Naderi GA, Asgary S, Sarraf-Zadegan N, Shirvany H: Anti-oxidan effect of flavonoids on the susceptibility of LDL oxidation. Mol Cell Biochem 2003, 246:193-196

19. Bito T, Roy S, Sen CK, Shirakawa T, Gotoh A, Ueda M, Ichihashi M Packer L: Flavonoids differentially regulate IFN- $\gamma$-induced ICAM-1 expression in human keratinocytes: molecular mechanisms of action. FEBS Lett 2002, 520:145-152

20. Chen CC, Chow MP, Huang WC, Lin YC, Chang YJ: Flavonoids inhibit tumor necrosis factor- $\alpha$-induced up-regulation of intercellular adhesion molecule-1 (ICAM-1) in respiratory epithelial cells through activator protein-1 and nuclear factor- $\kappa \mathrm{B}$ : structure-activity relationships. Mol Pharmacol 2004, 66:683-693

21. Choudhury QG, McKay DT, Flower RJ, Croxtall JD: Investigation into the involvement of phospholipases $\mathrm{A}_{2}$ and map kinases in modulation of AA release and cell growth in A549 cells. Adv Exp Med Biol 2002 507:33-40

22. Ferrandiz ML, Alcaraz MJ: Anti-inflammatory activity and inhibition of arachidonic acid metabolism by flavonoids. Agents Actions 1991 32:283-288

23. Winter CA, Risley EA, Nuss GW: Carrageenin-induced edema in hind paw of the rat as an assay for antiinflammatory drugs. Proc Soc Exp Biol Med 1962, 111:544-547

24. Tomlinson A, Appleton I, Moore AR, Gilroy DW, Willis D, Mitchell JA Willoughby DA: Cyclo-oxygenase and nitric oxide synthase isoforms in rat carrageenin-induced pleurisy. Br J Pharmacol 1994 113:693-698

25. Lawrence T, Willoughby DA, Gilroy DW: Anti-inflammatory lipid mediators and insights into the resolution of inflammation. Nat Rev Immunol 2002, 2:787-795

26. Rane MJ, Carrithers SL, Arthur JM, Klein JB, McLeish KR: Formy peptide receptors are coupled to multiple mitogen-activated protein kinase cascades by distinct signal transduction pathways: role in activation of reduced nicotinamide adenine dinucleotide oxidase. $\mathrm{J}$ Immunol 1997, 159:5070-5078

27. Nick JA, Avdi NJ, Young SK, Lehman LA, McDonald PP, Frasch SC Billstrom MA, Henson PM, Johnson GL, Worthen GS: Selective activation and functional significance of $\mathrm{p} 38 \alpha$ mitogen-activated protein kinase in lipopolysaccharide-stimulated neutrophils. J Clin Invest 1999, 103:851-858

28. McLeish KR, Knall C, Ward RA, Gerwins P, Coxon PY, Klein JB, Johnson GL: Activation of mitogen-activated protein kinase cascades 
during priming of human neutrophils by TNF- $\alpha$ and GM-CSF. J Leukoc Biol 1998, 64:537-545

29. Khreiss T, Jozsef L, Chan JS, Filep JG: Activation of extracellular signal-regulated kinase couples platelet-activating factor-induced adhesion and delayed apoptosis of human neutrophils. Cell Signal 2004, 16:801-810

30. Lindsay MA, Haddad EB, Rousell J, Teixeira MM, Hellewell PG, Barnes PJ, Giembycz MA: Role of the mitogen-activated protein kinases and tyrosine kinases during leukotriene $\mathrm{B}_{4}$-induced eosinophil activation. J Leukoc Biol 1998, 64:555-562

31. Klein JB, Rane MJ, Scherzer JA, Coxon PY, Kettritz R, Mathiesen JM, Buridi A, McLeish KR: Granulocyte-macrophage colony-stimulating factor delays neutrophil constitutive apoptosis through phosphoinositide 3-kinase and extracellular signal-regulated kinase pathways. J Immunol 2000, 164:4286-4291

32. Usher LR, Lawson RA, Geary I, Taylor CJ, Bingle CD, Taylor GW, Whyte MK: Induction of neutrophil apoptosis by the Pseudomonas aeruginosa exotoxin pyocyanin: a potential mechanism of persistent infection. J Immunol 2002, 168:1861-1868

33. Gerritsen ME, Carley WW, Ranges GE, Shen CP, Phan SA, Ligon GF, Perry CA: Flavonoids inhibit cytokine-induced endothelial cell adhesion protein gene expression. Am J Pathol 1995, 147:278-292

34. Gilroy DW, Colville-Nash PR, Willis D, Chivers J, Paul-Clark MJ, Willoughby DA: Inducible cyclooxygenase may have anti-intlammatory properties. Nat Med 1999, 5:698-701

35. Wallace JL, Bak A, McKnight W, Asfaha S, Sharkey KA, MacNaughton WK: Cyclooxygenase 1 contributes to inflammatory responses in rats and mice: implications for gastrointestinal toxicity. Gastroenterology 1998, 115:101-109

36. Karimi K, Lennartz MR: Mitogen-activated protein kinase is activated during IgG-mediated phagocytosis, but is not required for target ingestion. Inflammation 1998, 22:67-82

37. Garcia-Garcia E, Sanchez-Mejorada G, Rosales C: Phosphatidylinositol 3-kinase and ERK are required for NF- $\mathrm{kB}$ activation but not for phagocytosis. J Leukoc Biol 2001, 70:649-658

38. Kurosaka K, Takahashi M, Kobayashi Y: Activation of extracellular signal-regulated kinase $1 / 2$ is involved in production of CXC-chemokine by macrophages during phagocytosis of late apoptotic cells. Biochem Biophys Res Commun 2003, 306:1070-1074

39. Gregory CD, Devitt A: The macrophage and the apoptotic cell: an innate immune interaction viewed simplistically? Immunology 2004, 113:1-14

40. Devitt A, Parker KG, Ogden CA, Oldreive C, Clay MF, Melville LA, Bellamy CO, Lacy-Hulbert A, Gangloff SC, Goyert SM, Gregory CD Persistence of apoptotic cells without autoimmune disease or inflammation in CD14-/- mice. J Cell Biol 2004, 167:1161-1170

41. Costanzo-Nordin MR, Hubbell EA, O'Sullivan EJ, Johnson MR, Mullen GM, Heroux AL, Kao WG, McManus BM, Pifarre R, Robinson JA Successful treatment of heart transplant rejection with photopheresis. Transplantation 1992, 53:808-815

42. Meiser BM, Kur $F$, Reichenspurner $H$, Wagner $F$, Boos KS, Vielhauer $S$, Weiss $M$, Rohrbach $H$, Kreuzer $E$, Uberfuhr $P$, Reichart B: Reduction of the incidence of rejection by adjunct immunosuppression with photochemotherapy after heart transplantation. Transplantation 1994, 57:563-568

43. Sunder-Plassman G, Druml W, Steininger R, Honigsmann $H$, Knobler R: Renal allograft rejection controlled by photopheresis. Lancet 1995, 346:506
44. Slovis BS, Loyd JE, King Jr LE: Photopheresis for chronic rejection of lung allografts. N Engl J Med 1995, 332:962

45. Lamioni A, Parisi F, Isacchi G, Giorda E, Di Cesare S, Landolfo A, Cenci F, Bottazzo GF, Carsetti R: The immunological effects of extracorporeal photopheresis unraveled: induction of tolerogenic dendritic cells in vitro and regulatory $T$ cells in vivo. Transplantation 2005 $79: 846-850$

46. Favata MF, Horiuchi KY, Manos EJ, Daulerio AJ, Stradley DA, Feese WS, Van Dyk DE, Pitts WJ, Earl RA, Hobbs F, Copeland RA, Magolda RL, Scherle PA, Trzaskos JM: Identification of a novel inhibitor of mitogen-activated protein kinase kinase. J Biol Chem 1998 273:18623-18632

47. Duan W, Chan JH, Wong CH, Leung BP, Wong WS: Anti-inflammatory effects of mitogen-activated protein kinase kinase inhibitor U0126 in an asthma mouse model. J Immunol 2004, 172:7053-7059

48. Hallsworth MP, Moir LM, Lai D, Hirst SJ: Inhibitors of mitogen-activated protein kinases differentially regulate eosinophil-activating cytokine release from human airway smooth muscle. Am J Respir Crit Care Med 2001, 164:688-697

49. Yoshida T, Tomioka I, Nagahara T, Holyst T, Sawada M, Hayes $P$, Gama V, Okuno M, Chen Y, Abe Y, Kanouchi T, Sasada H, Wang D, Yokota T, Sato E, Matsuyama S: Bax-inhibiting peptide derived from mouse and rat Ku70. Biochem Biophys Res Commun 2004. 321:961-966

50. Sawada M, Hayes P, Matsuyama S: Cytoprotective membranepermeable peptides designed from the Bax-binding domain of Ku70. Nat Cell Biol 2003, 5:352-357

51. Maiuri MC, Tajana G, luvone T, De Stefano D, Mele G, Ribecco MT, Cinelli MP, Romano MF, Turco MC, Carnuccio R: Nuclear factor- $k B$ regulates inflammatory cell apoptosis and phagocytosis in rat carrageenin-sponge implant model. Am J Pathol 2004, 165:115-126

52. Kerr JF, Wyllie AH, Currie AR: Apoptosis: a basic biological phenomenon with wide-ranging implications in tissue kinetics. $\mathrm{Br} \mathrm{J}$ Cancer 1972, 26:239-257

53. Wyllie AH, Kerr JF, Currie AR: Cell death: the significance of apoptosis. Int Rev Cytol 1980, 68:251-306

54. Bursch W, Paffe S, Putz B, Barthel G, Schulte-Hermann R: Determination of the length of the histological stages of apoptosis in normal liver and in altered hepatic foci of rats. Carcinogenesis 1990 $11: 847-853$

55. Voll RE, Herrmann M, Roth EA, Stach C, Kalden JR, Girkontaite I: Immunosuppressive effects of apoptotic cells. Nature 1997. 390:350-351

56. Xiao YQ, Malcolm K, Worthen GS, Gardai S, Schiemann WP, Fadok VA, Bratton DL. Henson PM: Cross-talk between ERK and p38 MAPK mediates selective suppression of pro-inflammatory cytokines by transforming growth factor- $\beta$. J Biol Chem 2002, 277:14884-14893

57. Kurosaka K, Takahashi M, Watanabe N, Kobayashi Y: Silent cleanup of very early apoptotic cells by macrophages. J Immunol 2003 , 171:4672-4679

58. Murphy FJ, Hayes I, Cotter TG: Targeting inflammatory diseases via apoptotic mechanisms. Curr Opin Pharmacol 2003, 3:412-419

59. Johnson GL, Lapadat R: Mitogen-activated protein kinase pathways mediated by ERK, JNK, and p38 protein kinases. Science 2002, 298:1911-1912

60. Gilroy DW, Lawrence T, Perretti M, Rossi AG: Inflammatory resolution: new opportunities for drug discovery. Nat Rev Drug Discov 2004. 3:401-416 


\title{
Cyclin-dependent kinase inhibitors enhance the resolution of inflammation by promoting inflammatory cell apoptosis
}

\author{
Adriano G Rossi ${ }^{1}$, Deborah A Sawatzky ${ }^{1,3}$, Annemieke Walker ${ }^{1,3}$, Carol Ward ${ }^{1}$, Tara A Sheldrake ${ }^{1}$, \\ Nicola A Riley ${ }^{1}$, Alison Caldicott ${ }^{1}$, Magdalena Martinez-Losa ${ }^{1}$, Trevor R Walker ${ }^{1}$, Rodger Duffin ${ }^{1}$, \\ Mohini Gray ${ }^{1}$, Elvira Crescenzi ${ }^{2}$, Morag C Martin ${ }^{1}$, Hugh J Brady ${ }^{2}$, John S Savill ${ }^{1}$, Ian Dransfield ${ }^{1}$ \& \\ Christopher Haslett ${ }^{1}$
}

\begin{abstract}
Apoptosis is essential for clearance of potentially injurious inflammatory cells and subsequent efficient resolution of inflammation. Here we report that human neutrophils contain functionally active cyclin-dependent kinases (CDKs), and that structurally diverse CDK inhibitors induce caspase-dependent apoptosis and override powerful anti-apoptosis signals from survival factors such as granulocyte-macrophage colony-stimulating factor (GM-CSF). We show that the CDK inhibitor $R$-roscovitine (Seliciclib or CYC202) markedly enhances resolution of established neutrophil-dependent inflammation in carrageenan-elicited acute pleurisy, bleomycininduced lung injury, and passively induced arthritis in mice. In the pleurisy model, the caspase inhibitor zVAD-fmk prevents $R$-roscovitine-enhanced resolution of inflammation, indicating that this CDK inhibitor augments inflammatory cell apoptosis. We also provide evidence that $R$-roscovitine promotes apoptosis by reducing concentrations of the anti-apoptotic protein Mcl-1. Thus, CDK inhibitors enhance the resolution of established inflammation by promoting apoptosis of inflammatory cells, thereby demonstrating a hitherto unrecognized potential for the treatment of inflammatory disorders.
\end{abstract}

Neutrophils have a central role in innate immunity and are rapidly recruited to sites of infection and injury; however, their many defense mechanisms that destroy and digest invading microorganisms are potentially deleterious to tissues ${ }^{1}$. Thus, it is vital that, once their physiological function has been achieved, these inflammatory cells and their potentially histotoxic contents are cleared rapidly. During spontaneous resolution of inflammation, neutrophils undergo apoptosis: a pre-programmed and highly regulated cell death process that results in shutdown of secretory capacity and allows recognition and removal by macrophages ${ }^{2,3}$. Neutrophil survival and apoptosis are profoundly influenced by the inflammatory milieu: inflammatory mediators such as GM-CSF or lipopolysaccharide (LPS), environmental conditions such as hypoxia, and the presence of pro-apoptotic stimuli such as tumor necrosis factor- $\alpha$ or Fas ligand markedly alter neutrophil longevity 4,5 .

Neutrophil apoptosis is controlled by a complex network of signaling pathways that regulate both the turnover of key molecules, including the anti-apoptotic protein myeloid cell leukemia 1 (Mcl-1) and the pro-apoptotic Bcl-2 family member Bax, and activation of the caspase family of proteases 5 . Once apoptosis has been engaged, the neutrophil secretory activity is shutdown; the cells remain intact and are phagocytosed by macrophages using recognition mechanisms that fail to elicit a pro-inflammatory response ${ }^{2,6}$. If macrophage phagocytosis or neutrophil apoptosis is impaired, however, chronic inflammation may ensue ${ }^{4,5,7}$. Consequently, the mechanisms involved in regulating inflammatory cell survival and apoptosis are the subject of considerable research endeavor.

Cell division of eukaryotic cells occurs in four phases (G1, S, G2, M) and in some circumstances, for example where growth factors are withdrawn or the cell is terminally differentiated, the cell will rest in G0 phase. The CDKs have been traditionally described as key regulators of the cell cycle, whereby different CDKs become activated during cell-cycle progression when complexed with their associated cyclin partners ${ }^{8}$. For this reason, targeting CDKs by specific inhibitors may prevent or limit tumor progression. Indeed, $\mathrm{CDK}$ inhibitors are under clinical trial for esophageal, lung, prostate and non-small-cell lung cancers ${ }^{9}$. In contrast, inhibition of CDKs attenuates apoptosis of terminally differentiated neurons ${ }^{10}$. The precise mechanisms that determine the effects of CDK inhibitors on apoptosis remain unclear, although these inhibitors downregulate Mcl-1 (refs. 11,12), a key protein regulating apoptosis ${ }^{13}$ including neutrophil apoptosis ${ }^{14}$. Neutrophils are terminally differentiated cells and therefore CDK inhibitors such as $R$-roscovitine would be predicted either to have no effect or, as in neurons, to inhibit apoptosis ${ }^{10}$.

${ }^{1}$ MRC Centre for Inflammation Research, Queen's Medical Research Institute, University of Edinburgh, 47 Little France Crescent, Edinburgh, EH16 4TJ, UK. ${ }^{2}$ Molecular Haematology and Cancer Biology Unit, Institute of Child Health, Great Ormond Street Hospital for Children NHS Trust, University College London, 30 Guilford Street, London WC1N 1EH, UK. ${ }^{3}$ These authors contributed equally to this article. Correspondence should be addressed to A.G.R. (a.g.rossi@ed.ac.uk).

Received 26 October 2005; accepted 24 July 2006; published online 3 September 2006; corrected after print 10 November 2006; doi:10.1038/nm1468 
a

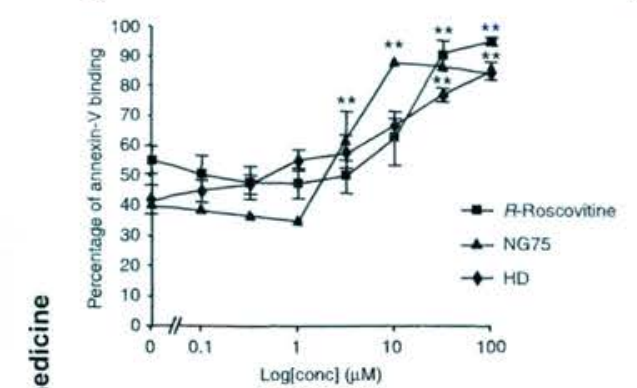

e

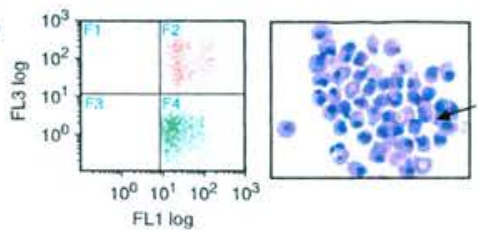

b
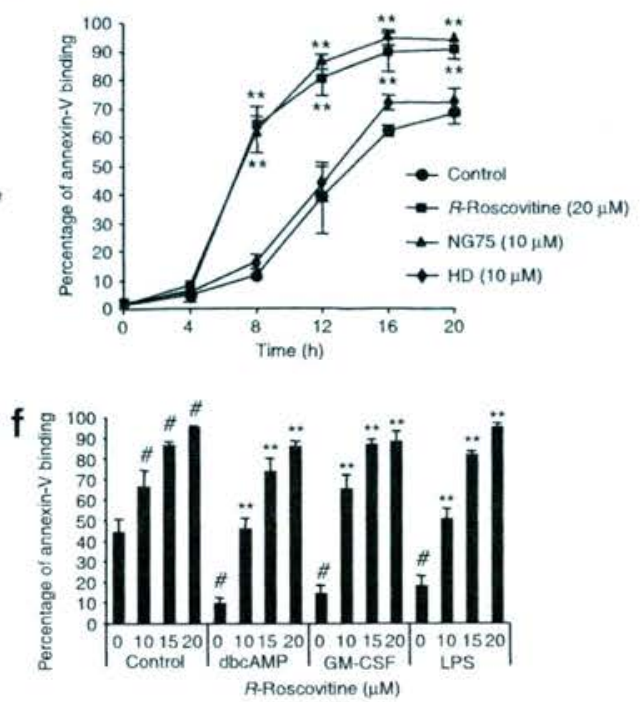

Figure 1 Effects of CDK inhibitors on human neutrophil apoptosis. (a) Different CDK inhibitors induce concentration-dependent apoptosis of neutrophils. Human neutrophils $\left(5 \times 10^{6} \mathrm{cells} / \mathrm{ml}\right)$ were incubated for $20 \mathrm{~h}$ with increasing concentrations of $R$-roscovitine, NG75 or hymenialdisine (HD). Apoptosis was assessed by annexin-V-FITC binding and confirmed by morphology. Data represent the mean \pm s.e.m. $(n \geq 3)$. ${ }^{* *} P<0.01$ versus control. (b) Different CDK inhibitors induce timedependent apoptosis of neutrophils. Human neutrophils $\left(5 \times 10^{6} \mathrm{cell} / \mathrm{s} / \mathrm{ml}\right)$ were incubated over $20 \mathrm{~h}$ with $20 \mu \mathrm{M} R$-roscovitine, $10 \mu \mathrm{M} \mathrm{NG75}$ or $10 \mu \mathrm{M}$ hymenialdisine. Apoptosis was assessed as in a $(n \geq 3) .{ }^{*} P<0.05,{ }^{\star *} P<0.01$ versus control. (c-e) The CDK inhibitor NG75 enhances annexin- $\mathrm{V}$ binding and induces morphological changes indicative of neutrophil apoptosis. Left,

flow cytometry dot plots of NG75-treated neutrophils, taken at $0 \mathrm{~h} \mathrm{(c),} 8 \mathrm{~h}$ (d) and $20 \mathrm{~h} \mathrm{(e).}$

Non-apoptotic cells are shown in gray, annexin- $\mathrm{V}-\mathrm{FITC}^{+}$cells in green, and annexin- $\mathrm{V}^{+} / \mathrm{PI}^{+}$cells in

red. Right, corresponding micrographs. In c, typical neutrophil morphology of multi-lobed nuclei is

seen. In d, arrow indicates typically apoptotic neutrophil morphology, showing condensed nuclei. In e, arrow indicates apoptotic neutrophil without nuclear staining and the cell membrane appears 'leaky'. This type of morphology may account for the annexin- $\mathrm{V}^{+} / \mathrm{PI}^{+}$cells seen by flow cytometry at $20 \mathrm{~h}$. ( $f-\mathrm{h}$ ) CDK inhibitors reverse dbcAMP-, GM-CSF- and LPS-mediated survival of neutrophils. Human neutrophils $\left(5 \times 10^{6} \mathrm{cells} / \mathrm{ml}\right)$ were pre-incubated with $0.2 \mathrm{mM}$ dbcAMP, $50 \mathrm{U} / \mathrm{ml}$ of GM-CSF or $1 \mu \mathrm{g} / \mathrm{ml}$ of LPS as indicated for $30 \mathrm{~min}$. Appropriate concentrations of $R$-roscovitine (f), NG75 (g) or hymenialdisine (h) were then added and the cells were incubated for a further $20 \mathrm{~h}$. Apoptosis was assessed as in a $(n \geq 3)$. ${ }^{\#} P<0.05$ versus control. ${ }^{*} P<0.05,{ }^{* \star} P<$ 0.01 versus control of relevant survival agent.

Here we have investigated whether CDK inhibitors can influence neutrophil apoptosis in vitro and consequently the resolution of neutrophilic inflammation in vivo. We show that human neutrophils express functional CDKs and that different CDK inhibitors directly induce caspase-dependent neutrophil apoptosis and inhibit cell survival induced by several biologically important powerful antiapoptotic agents. We also show that the CDK inhibitor $R$-roscovitine downregulates $\mathrm{Mcl}-1$ expression induced by survival factors in neutrophils. In addition, we demonstrate in vivo that $R$-roscovitine markedly enhances resolution of inflammation in mouse models of carrageenan-induced acute pleurisy, bleomycin-induced lung inflammation and passively induced arthritis. The $R$-roscovitineenhanced resolution of established pleurisy is driven by a caspasemediated pro-apoptotic effect. These findings suggest that CDK inhibitors may provide a therapeutic strategy to promote resolution of inflammatory diseases.

\section{RESULTS}

\section{CDK inhibitors induce human neutrophil apoptosis}

To investigate whether CDK inhibitors can affect apoptosis directly, human neutrophils were incubated over a 20 -h period with the structurally diverse CDK inhibitors $R$-roscovitine ${ }^{15-17}$, NG75 (refs.
C
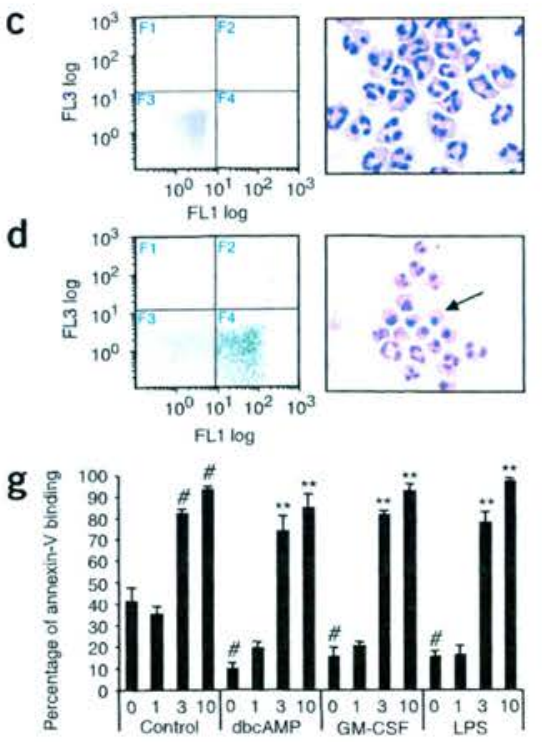

h

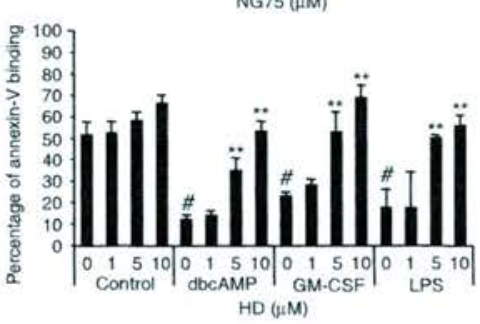

$18,19)$ and hymenialdisine $e^{20,21}$ at a range of concentrations similar to those known to inhibit CDKs specifically ${ }^{15-21}$. The CDK inhibitors markedly increased neutrophil apoptosis in a concentrationdependent (Fig. 1a) and time-dependent (Fig. 1b) manner. After $8 \mathrm{~h}$ of NG75 treatment, a marked increase in annexin-V-positive cells was observed (Fig. 1c-e); after prolonged treatment $(20 \mathrm{~h})$, an increased number of annexin- $\mathrm{V}$ - and propidium iodide (PI)-positive cells (indicating secondary necrosis) were present and these cells could be identified morphologically by their nuclear loss and ruffled plasma membrane (Fig. 1e). Similar profiles were seen with $R$-roscovitine and hymenialdisine treatment, and all annexin- $\mathrm{V}$ binding data was confirmed by morphological assessment of apoptosis (data not shown).

In a time-course study, $R$-roscovitine $(20 \mu \mathrm{M})$ and NG75 $(10 \mu \mathrm{M})$ increased the rate of apoptosis after $8 \mathrm{~h}$, whereas $10 \mu \mathrm{M}$ hymenialdisine did not increase apoptosis above the control rate (Fig. 1b). We deliberately used hymenialdisine at $10 \mu \mathrm{M}$, a concentration that did not affect apoptosis per se, because subsequently we wanted to test the effect of the CDK inhibitor on delayed apoptosis induced by survival factors (see below). Systematic study of its cellular targets has shown that $R$-roscovitine has high specificity for CDKl, CDK2 and CDK5, but not for other kinases including CDK4 and CDK6 (refs. 15-17); in addition, both NG75 (refs. 18,19) and 
Figure 2 CDK protein and activity in neutrophils and mechanisms governing the pro-apoptotic effect of CDK inhibitors. (a) The caspase inhibitor zVAD-fmk inhibits $R$-roscovitine-induced apoptosis of neutrophils. Human neutrophils $\left(5 \times 10^{6}\right.$ cells $\left./ \mathrm{ml}\right)$ were incubated for $6 \mathrm{~h}$ with $R$-roscovitine $(20 \mu \mathrm{M})$ with or without zVAD-fmk $(100 \mu \mathrm{M})$. Apoptosis was assessed as in Fig. 1a $(n=4) .{ }^{*} P<0.05$ versus control. (b) CDK 1 and CDK2 proteins are expressed in neutrophils and $R$-roscovitine induces caspase cleavage in

neutrophils. Human neutrophils $\left(5 \times 10^{6}\right.$ cells/ $\mathrm{ml})$ were lysed after isolation $(\mathrm{O} \mathrm{h})$ or after treatment $(4 \mathrm{~h})$ with buffer, GM-CSF $(50 \mathrm{ng} / \mathrm{ml})$, $R$-roscovitine $(20 \mu \mathrm{M})$, or GM-CSF $(50 \mathrm{ng} / \mathrm{ml})$ plus $R$-roscovitine $(20 \mu \mathrm{M})$. Proteins were subjected to western blotting with antibodies to caspase $3, \mathrm{CDK} 1, \mathrm{CDK} 2$ or $\beta$-actin. Blots are representative of at least three separate experiments. (c) CDK1 and CDK2 protein expression does not change during neutrophil apoptosis. Human neutrophils $\left(5 \times 10^{6} \mathrm{cells} / \mathrm{ml}\right)$ were lysed after isolation $(\mathrm{O} h)$ or after treatment $(20 \mathrm{~h})$ with buffer, GM-CSF $(50 \mathrm{U} / \mathrm{ml})$ or gliotoxin $(0.1 \mu \mathrm{g} / \mathrm{ml})$. Proteins were subjected to western blotting with antibodies to CDK1 or CDK2. Blots are representative of at least three separate experiments. (d,e) CDK1 activity is present in neutrophils and decreases after apoptosis induced by the activating antibody to

Fas $(\mathrm{CH} 11)$. Neutrophils $\left(5 \times 10^{6}\right.$ cells $\left./ \mathrm{ml}\right)$ were treated with $\mathrm{CH} 11(500 \mathrm{ng} / \mathrm{ml})$ for the indicated times. Subsequently, $10^{7}$ cells were lysed for each sample, the lysate was incubated with antibody to CDK1, and CDK1 activity was measured by ${ }^{32} \mathrm{P}$ transfer to histone $\mathrm{H} 1$ (d,e). Apoptosis $\left(\right.$ annexin- $\left.\mathrm{V}^{+} / \mathrm{PI}^{+}\right)(\mathrm{e})$ and CDK1 activity $(\mathrm{d}, \mathrm{e})$ were measured every $2 \mathrm{~h}$ over $8 \mathrm{~h}$. CDK1 activity was detected at time 0 and at $2 \mathrm{~h}$, but not at later time points as apoptosis increased (d,e). Again, no difference in CDK1 expression was detected over time (d). Results are representative of at least three separate experiments. (f) $R$-Roscovitine reduces survival factor-induced $\mathrm{Mcl}-1$ protein expression in neutrophils. Human neutrophils $\left(5 \times 10^{6}\right.$ cells/ml) were treated with buffer, GM-CSF $(50 \mathrm{ng} / \mathrm{ml}), R$-roscovitine $(20 \mu \mathrm{M})$, or GM-CSF ( $50 \mathrm{ng} / \mathrm{ml})$ plus $R$-roscovitine $(20 \mu \mathrm{M})$ for $2 \mathrm{~h}$. After cell lysis, proteins were subjected to western blotting with antibody to $\mathrm{Mcl}-1$. Blots are representative of at least three separate experiments. hymenialdisine $\mathrm{e}^{20,21}$ have high specificity for the same CDKs. The crystal structure of human CDK2 complexed with $R$-roscovitine has been described, together with evidence showing that the $R$ stereoisomer of roscovitine is slightly more potent at inhibiting purified CDK1/cyclin B activity than is the $S$ stereoisomer (half-maximal inhibitory concentrations of $0.45 \mu \mathrm{M}$ and $0.95 \mu \mathrm{M}$, respectively ${ }^{15}$ ). Our own studies with the stereoisomers used at $20 \mu \mathrm{M}$ showed that apoptosis after $6 \mathrm{~h}$ was $12.4 \pm 0.4 \%$ for the control, $12.8 \pm 1.3 \%$ for the dimethyl sulfoxide (DMSO; $0.04 \%$ ) control, $75.9 \pm 3.5 \%$ for $R$ roscovitine and $75.6 \pm 3.3 \%$ for $S$-roscovitine (mean \pm s.e.m.; $n=4$ separate donors, done in triplicate).

\section{CDK inhibitors overcome survival factor delay of apoptosis}

Because these CDK inhibitors accelerated apoptosis, we investigated whether they reversed the effects of agents that delay neutrophil apoptosis through different signaling pathways ${ }^{5}$. For example, dbcAMP penetrates the cell membrane to mimic endogenous CAMP22; GM-CSF acts through the GM-CSF receptor to activate phosphatidylinositol 3-kinase (PI3K), Janus kinase 2 and the transcription factor STAT1 (ref. 5); and LPS binds to Toll-like receptor-4 to activate mitogenactivation kinase (MAPK), PI3K and NF- $\mathrm{KB}$ signaling to inhibit apoptosis ${ }^{5,23-26}$. In addition, LPS-induced delay of neutrophil apoptosis is also dependent on contaminating monocytes ${ }^{25,26}$. The CDK inhibitors overrode all of these survival signals in a concentrationdependent manner (Fig. 1f-h) without directly inducing apoptosis per se (see $10 \mu \mathrm{M}$ hymenialdisine data). Thus, overriding of survival

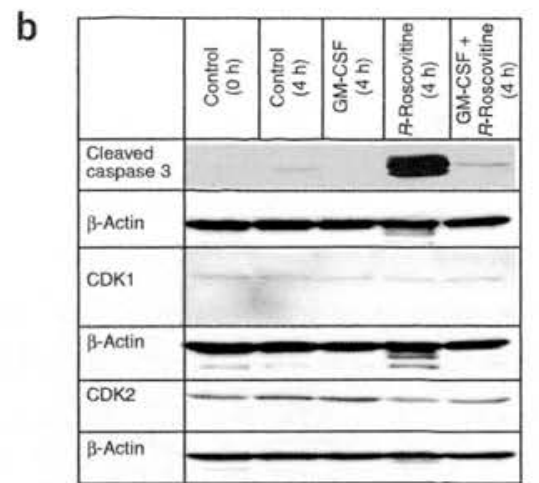

d

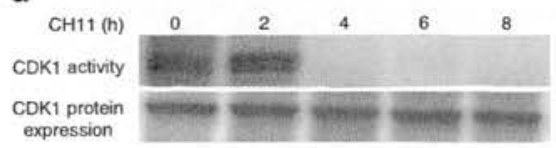

f

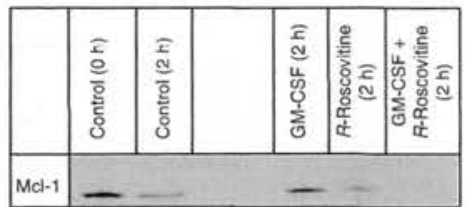

signals by CDK inhibitors occurs independently of, or is more sensitive to, direct effects of the inhibitors on apoptosis and takes place irrespective of the signaling pathways triggered in neutrophils.

\section{CDK inhibitors induce caspase-dependent apoptosis}

We found that CDK inhibitor-induced apoptosis was caspase dependent because pre-incubating neutrophils with the broadrange caspase inhibitor $\mathrm{zVAD}$-fmk prevented $R$-roscovitine-induced apoptosis (Fig. 2a). R-Roscovitine treatment resulted in caspase-3 cleavage, directly verifying that this $\mathrm{CDK}$ inhibitor activates caspases in neutrophils (Fig. 2b). In addition, we found that $R$-roscovitine-induced caspase- 3 cleavage was attenuated by the pro-survival factor GM-CSF (Fig. 2b), probably because of competing pro- and anti-apoptotic pathways. At the early time point of $4 \mathrm{~h}$, the rate of basal apoptosis was low $(<5 \%$; Fig. 1b) and consequently caspase-3 cleavage was minimal (Fig. 2b); however, caspase-3 cleavage was already very evident at $4 \mathrm{~h}$ when the neutrophils were treated with $R$-roscovitine alone. At $20 \mathrm{~h}$, the pro-apoptotic effect of $R$-roscovitine was dominant over survival factor-mediated effects (Fig. 1f).

\section{Human neutrophils express functionally active CDKs}

Because neutrophils are terminally differentiated and do not undergo cell division, little is known about CDKs and their associated partners in these cells. CDK1 and CDK2 were found to be present in neutrophils (Fig. $\mathbf{2 b}, \mathbf{c}$ ) and there was no difference in CDK protein 


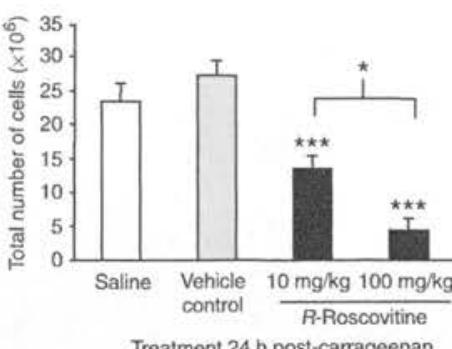

d

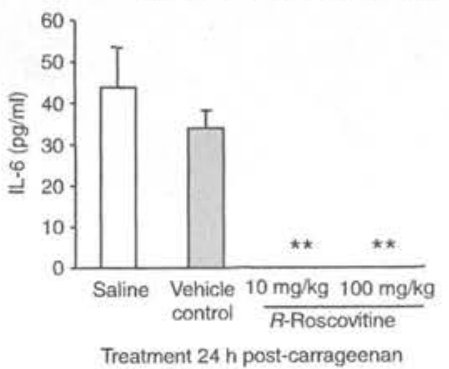

b

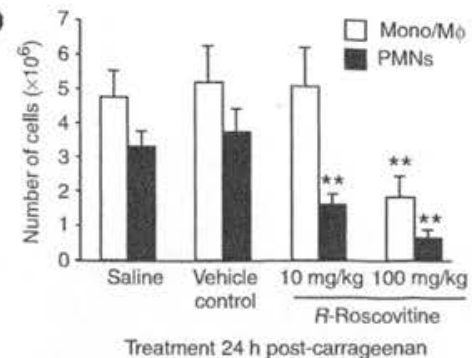

e

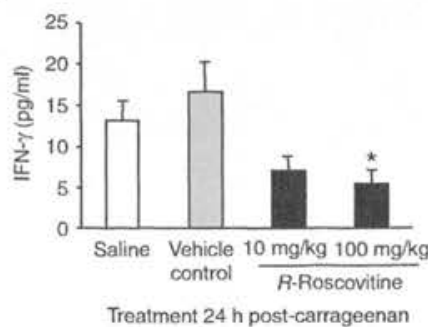

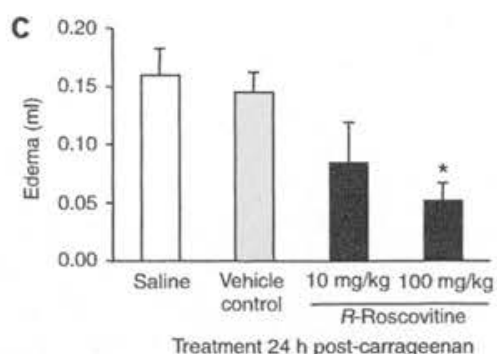

f

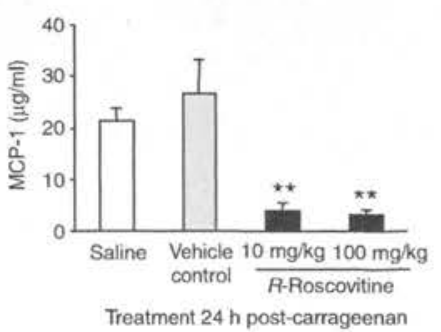

Figure 3 Effect of the CDK inhibitor $R$-roscovitine on resolution of carrageenaninduced pleurisy. (a-c) $R$-Roscovitine dose-dependently promotes resolution of inflammation in vivo. Twenty-four hours after intrapleural injection of $1 \%$ carrageenan, male C57/bl6 mice were treated with saline, DMSO, or 10 or $100 \mathrm{mg}$ per $\mathrm{kg}$ body weight $(\mathrm{mg} / \mathrm{kg}$ ) of $R$-roscovitine (i.p.). Mice were killed $36 \mathrm{~h}$ after carrageenan injection and the total pleural inflammatory cell numbers (a), mononuclear (Mono/M $\phi$ ) and polymorphonuclear cell (PMNs) numbers (b) and exudate volumes (c) were measured. Data represent the mean \pm s.e.m. ( $n=8-10$ mice per group). ${ }^{* \star} P<0.01$ and ${ }^{* * *} P<0.001$ versus DMSO control. (d-f) $R$-Roscovitine reduces pro-inflammatory cytokines in vivo. Mice were treated as in a-c, and IL-6 (d), IFN- $\gamma$ (e) and MCP-1 (f) were measured in pleural exudates with a cytokine bead assay $(n=8-10$ mice per group). ${ }^{* *} P<0.01$ and ${ }^{* * *} P<0.001$ versus DMSO control. (g) $R$ Roscovitine promotes resolution of inflammation in vivo in a time-dependent

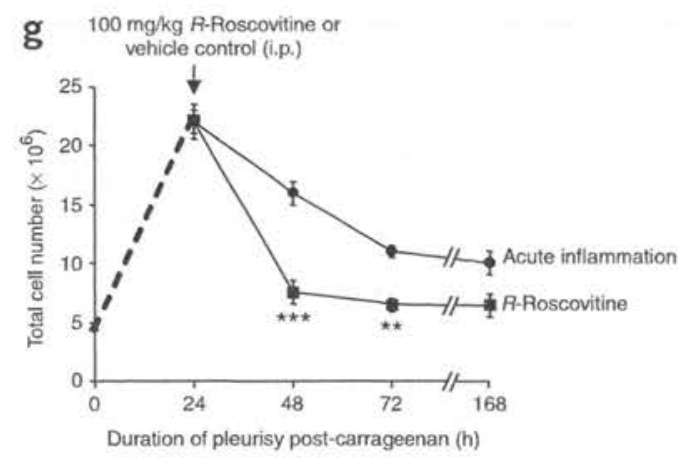
manner. Twenty-four hours after intrapleural injection of $1 \%$ carrageenan, male C57/bl6 mice were treated with vehicle control (DMSO) or 100 mg per $\mathrm{kg}$ body weight of $R$-roscovitine (i.p.). Mice were killed $24 \mathrm{~h}, 48 \mathrm{~h}$ or $168 \mathrm{~h}$ after carrageenan injection and the total pleural inflammatory cell numbers were determined ( $n=5-6$ mice per group). ${ }^{* *} P<0.01$ and ${ }^{* * *} P<0.001$ versus DMSO control.

quantities in freshly isolated neutrophils, aged neutrophils $(4 \mathrm{~h})$, or neutrophils treated with GM-CSF, $R$-roscovitine or both $(4 \mathrm{~h})$. Even when neutrophils were incubated for $20 \mathrm{~h}$ with buffer or GM-CSF, or when apoptosis was induced with gliotoxin ${ }^{23}$, the quantities of CDKs did not change (Fig. 2c). Thus, although CDKs seem to be important for inducing apoptosis, $\mathrm{CDK} 1$ and $\mathrm{CDK} 2$ are not targets for degradation during apoptosis.

In other cell systems, CDK1 and CDK2 have been shown to bind to various cyclin subtypes, whereas CDK5 does not seem to bind to cyclins but is activated by the non-cyclin p35 and p39 regulatory proteins. Because $R$-roscovitine can also inhibit CDK5 (ref. 16), we verified that CDK5 is expressed in human neutrophils (data not shown), in agreement with a previous study ${ }^{27}$ that demonstrated the presence of CDK5 mRNA and protein. CDK1 (Fig. 2d,e), CDK2 (not shown) and CDK5 (ref. 27) activity has been shown in neutrophils. We found that $\mathrm{CDKl}$ activity in neutrophils decreased rapidly during apoptosis induced by the activating Fas antibody $\mathrm{CH} 11$ (Fig. 2e). The CDK-binding partners and regulatory proteins in neutrophils remain to be identified.

\section{CDK inhibitors reduce neutrophil Mcl-1 expression}

We further probed the effect of $R$-roscovitine on the expression of a key regulator of apoptosis, Mcl-1(refs. 13,14), and found that Mcl-1 quantities in isolated cells fell rapidly within $2 \mathrm{~h}$ of culture, an effect that was prevented by GM-CSF treatment. $R$-Roscovitine inhibited GM-CSF-mediated upregulation of Mcl-1 (Fig. 2f).

Roscovitine-induced apoptosis enhances inflammatory resolution Having shown in vitro that neutrophil apoptosis was markedly influenced by CDK inhibitors, we determined the effects of $R$-roscovitine on the resolution of neutrophil-dominant inflammation in vivo. We used a well-established acute resolving mouse model of carrageenan-induced pleural inflammation ${ }^{28-31}$ to assess the effects of the CDK inhibitor on the kinetics of inflammatory cell recruitment and to investigate inflammatory mechanisms. $R$-Roscovitine accelerated the resolution of established inflammation when administered intraperitoneally (i.p.) $24 \mathrm{~h}$ after intrapleural injection of $1 \%$ carrageenan (Fig. 3). Thus, treatment with $10 \mathrm{mg}$ per $\mathrm{kg}$ body weight of $R$-roscovitine (i.p.) inhibited the total inflammatory cell number by more than $50 \%$ as compared with vehicle control (Fig. 3a), and also reduced the number of monocytes/ macrophages and neutrophils (Fig. 3b). Of note, $R$-roscovitine at $100 \mathrm{mg}$ per $\mathrm{kg}$ reduced the inflammatory cells to near basal numbers normally found in the naive mouse pleural cavity. Anti-inflammatory effects of $R$-roscovitine were also evident because edema formation (total exudate volume obtained by pleural 
a
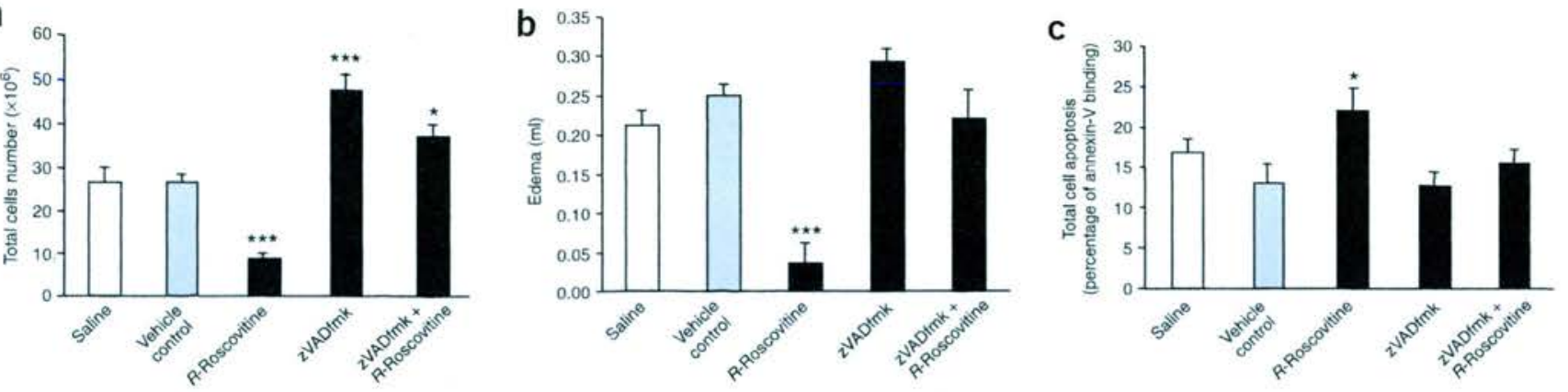

d

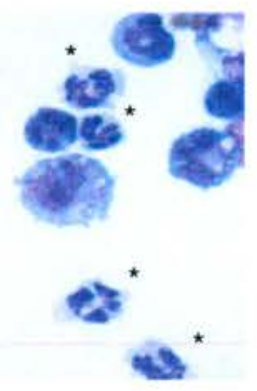

e
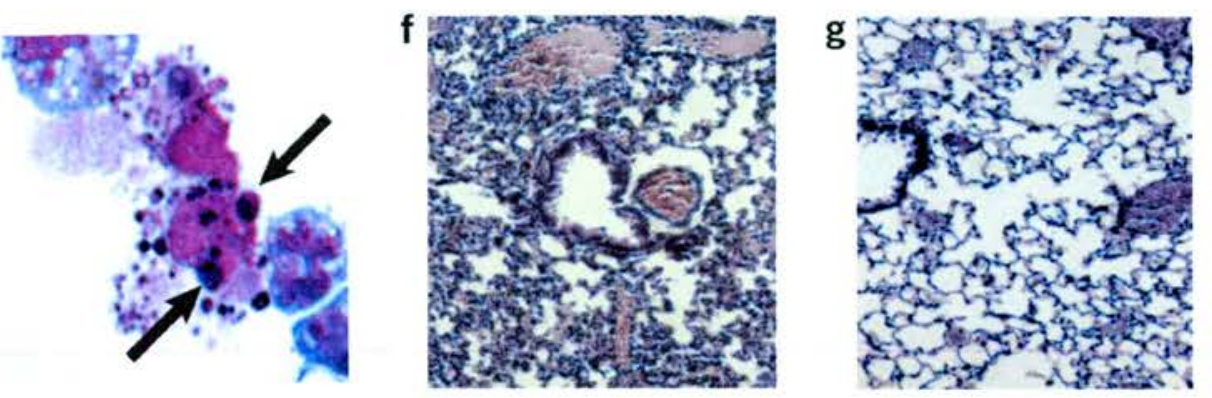

Figure 4 Role of caspase-dependent apoptosis in R-roscovitine-enhanced resolution of carrageenan-induced pleurisy. (a,b) The caspase inhibitor ZVAD-fmk prevents $R$-roscovitine-induced resolution of carrageenan-induced inflammation. Twenty-four hours after intrapleural injection of $1 \%$ carrageenan, male C57/bl6 mice were treated with $10 \mathrm{mg}$ per $\mathrm{kg}$ of $R$-roscovitine (i.p.) and/or $5 \mathrm{mg}$ per $\mathrm{kg}$ of $\mathrm{zVAD}$-fmk (i.p. at 4 -h intervals). Mice were killed $36 \mathrm{~h}$ after carrageenan injection and total pleural inflammatory cell numbers (a) and exudate volumes (b) were measured. Data represent the mean $\pm s . e . m$.

(8-10 mice per group). ${ }^{\star} P<0.05,{ }^{* \star} P<0.01$ and ${ }^{* * *} P<0.001$ versus DMSO control. (c) zVAD-fmk prevents apoptosis during $R$-roscovitine-induced resolution of carrageenan-induced inflammation. Mice were treated as in $\mathbf{a}, \mathbf{b}$, and apoptosis was analyzed in pleural inflammatory cells by annexin- $V$ labeling and flow cytometry ( $n=8-10$ mice per group). ${ }^{\star} P<0.05$ versus DMSO control. Inflammatory cell apoptosis was also assessed by morphology using cyto-centrifuge preparations stained with hematoxylin and eosin. (d-g) $R$-Roscovitine reduces inflammation in pleural lavage exudates and lung tissue, and ๑ decreases the numbers of macrophages containing apoptotic bodies in pleural lavage. Twenty four hours after intrapleural injection of $1 \%$ carrageenan, male C57/bl6 mice were treated i.p. with vehicle control (d,f) or $100 \mathrm{mg}$ per $\mathrm{kg}$ of $R$-roscovitine (e,g). Mice were killed $36 \mathrm{~h}$ after carrageenan injection.

Cyto-centrifuge preparations stained with hematoxylin and eosin (d,e) and tissue sections of pleural lavage and lungs (f,g) were assessed morphologically by microscopy (original magnification $\times 40$ ). Asterisks indicate viable neutrophils (d); arrows indicate phagocytosed apoptotic neutrophils (e).

washouts) decreased markedly with $R$-roscovitine treatment as compared with saline control $\left({ }^{*} P<0.05\right.$; Fig. 3c). In parallel, release of the pro-inflammatory mediators IL-6 (Fig. 3d), IFN- $\gamma$ (Fig. 3e) and MCP-1 (Fig. 3f) in the exudate was also diminished by $R$-roscovitine.

We next determined the effect, over a period of $7 \mathrm{~d}$, of administering $R$-roscovitine ( $100 \mathrm{mg}$ per kg i.p.) at the peak of inflammation $(24 \mathrm{~h})$. We observed a reduction in total cell numbers in the pleural cavity at all time points (Fig. $\mathbf{3 g}$ ) and in neutrophil and monocytes/ macrophages numbers at $36 \mathrm{~h}$ after carrageenan injection (Fig. 3a,b), providing further evidence that this $\mathrm{CDK}$ inhibitor enhances inflammatory resolution. Because $R$-roscovitine induced caspase-dependent apoptosis of human neutrophils in vitro (Fig. 2a,b), we established whether the enhanced resolution of inflammation by $R$-roscovitine was mediated by a similar induction of caspase-dependent inflammatory cell apoptosis in vivo. Systemic administration of the broad-spectrum caspase inhibitor $\mathrm{zVAD}$-fmk prevented the $R$-roscovitine-induced decrease in inflammatory cells and edema formation in the pleural cavity in carrageenan-induced pleural inflammation (Fig. 4). Administration of $\mathrm{zVAD}-\mathrm{fmk}$ prevented the resolution of inflammation in vivo by increasing the total inflammatory cell number (Fig. 4a) and edema formation (Fig. 4b) as compared with saline or vehicle controls. In addition, the increase in inflammatory cell apoptosis (Fig. 4c) and macrophages containing apoptotic bodies (see Fig. 4d,e) observed with $R$-roscovitine treatment in the pleural cavity was inhibited markedly by treatment with zVAD- fmk. We also noted that lungs of mice treated intrapleurally with carrageenan had a consequent marked inflammatory infiltrate (Fig. 4f), which was reduced by $R$-roscovitine treatment (Fig. $\mathbf{4 g}$ ).

\section{Roscovitine enhances inflammatory resolution in other models}

The anti-inflammatory and pro-resolving effects of $R$-roscovitine were further confirmed in another two inflammatory models. In the bleomycin-induced lung injury model, $R$-roscovitine $(100 \mathrm{mg}$ per $\mathrm{kg}$ i.p.), given after inflammation was established, reduced the total number of neutrophils in the bronchoalveolar fluid $3 \mathrm{~d}$ after bleomycin administration $\left({ }^{* *} P<0.01\right.$; Fig. 5a). Histological examination showed that, when bleomycin-induced lung injury was allowed to persist for $7 \mathrm{~d}, R$-roscovitine reduced bleomycin-induced inflammation (Fig. 5b-d). $R$-Roscovitine, when administered with the sham intratracheal saline control, had no obvious detrimental effects on the lung pathology (Fig. 5b). R-Roscovitine also reduced bleomycininduced lethality ( 3 out of 8 mice treated with bleomycin alone died by day 12 , as compared with none of the $R$-roscovitine- and bleomycin-treated mice). In addition, in a model of passively induced arthritis, we observed that arthritis, as assessed by clinical scores, resolved more quickly in $R$-roscovitine-treated than in control mice (Fig. 5e).

\section{DISCUSSION}

Specific induction of inflammatory cell apoptosis represents a new approach for future treatment of inflammatory diseases. The 


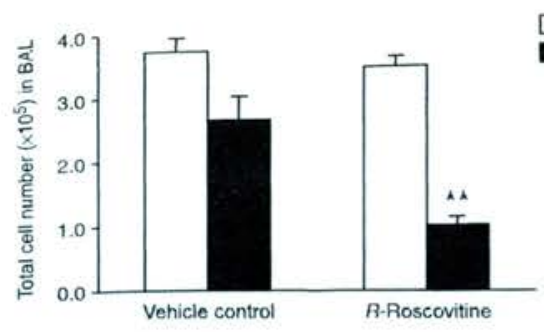

$\square$ Mono/Mro

Neutrophils

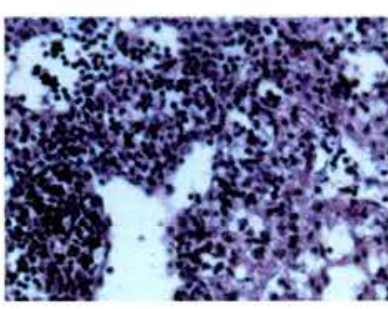

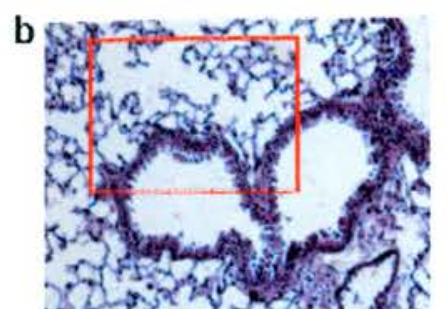
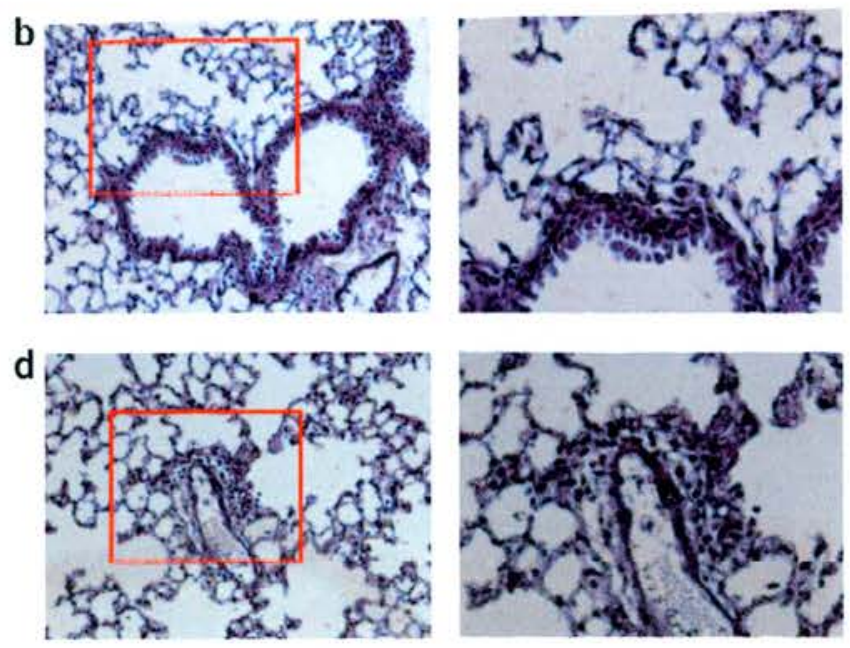

e

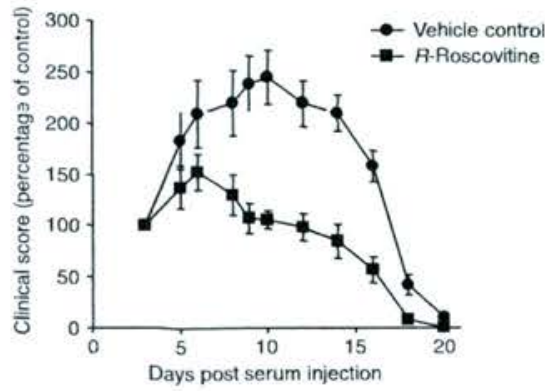

Figure 5 Effect of $R$-roscovitine on resolution of bleomycin-induced lung inflammation and serum-induced arthritis. (a) The total number of neutrophils in the lung is reduced by $R$ roscovitine after bleomycin-induced lung inflammation. Mice were givell 100 my per kg of $R$ roscovitine (i.p.) $48 \mathrm{~h}$ after intratracheal administration of bleomycin. Neutrophil and monocyte/ macrophage $(M o n o / M \phi)$ numbers were established by differential cell counts obtained from BAL

을 fluid $3 \mathrm{~d}$ after bleomycin administration. Data represent the mean \pm s.e.m. $(n=6$ mice per group). ${ }^{* *} P<0.01$ versus DMSO control. (b-d) $R$-Roscovitine inhibits inflammation and tissue damage in a chronic model of bleomycin-induced lung injury. Mice were given $100 \mathrm{mg}$ per $\mathrm{kg}$ of $R$-roscovitine (i.p.; d) or vehicle control (0.5\% DMSO, i.p.; c) $48 \mathrm{~h}$ after intratracheal administration of bleomycin or saline (sham control; b). Seven days after bleomycin treatment, lung inflammation and histological tissue damage were assessed by morphological examination (original magnification $\times 40$ ) of formaldehyde-fixed lung sections stained with hematoxylin and

eosin. Images are representative of six mice per group. (e) $R$-Roscovitine enhances the resolution of passively induced arthritis. Mice ( $n=10$ in each group) were injected twice (days 0 and 2) with $\mathrm{K} / \mathrm{BxN}$ serum derived from arthritic (day 60 ) $\mathrm{K} / \mathrm{B} \times \mathrm{N}$ transgenic mice. Clinical scores were assessed on the days indicated. Control mice received vehicle $(0.5 \mathrm{ml}$, i.p.) and treated mice received $R$-roscovitine $(0.5 \mathrm{ml}, 10 \mathrm{mg}$ per $\mathrm{kg}$, i.p.) on days $3,5,7,9,11,13,15,17$ and 19. Data represent the mean \pm s.e.m of the clinical score obtained on day 3 before the first injection of $R$-roscovitine, ${ }^{* * *} P<0.001$ by two-way ANOVA.

coordinated cascade of events that follows inflammatory insult is highly regulated from onset to resolution through checkpoints that control cell migration, infiltration and survival and perpetuation of the inflammatory response. Migratory cells either die in a pre-programmed manner through apoptosis or may persist in situ in a potentially detrimental manner to promote tissue destruction by continued excessive secretory activity or through the consequences of cell death by secondary necrosis. The ingestion of apoptotic cells by inflammatory macrophages also promotes the synthesis and release of mediators with anti-inflammatory properties (such as TGF- $\beta 1$ and $\mathrm{IL}-10)^{4,5}$. CDKs are essential cell signaling proteins that traditionally have been thought to control exclusively the fate of proliferating cells, in which CDK dysfunction is probably involved in increased cell turnover and tumor progression. Current therapies aimed at inhibiting CDKs are being developed for the treatment of various cancers.

Our study identifies a mechanism for both accelerating apoptosis of human neutrophils by CDK inhibitors in vitro and facilitating resolution of neutrophil-dependent inflammation in vivo. We have shown that human neutrophils possess CDK1, CDK2 and CDK5, and that specific inhibitors of CDKs (namely, $R$-roscovitine, NG75 and hymenialdisine) induce caspase-dependent apoptosis in a time- and concentration-dependent manner. The $\mathrm{CDK}$ inhibitors induced apoptosis even in the presence of diverse powerful pro-survival agents (dbcAMP, GM-CSF and LPS) that retard human neutrophil apoptosis through distinct molecular mechanisms. Compelling evidence suggests that contaminating monocytes contribute to LPS-mediated neutrophil survival 25,26 , triggering monocyte synthesis and release of neutrophil survival factors. Regardless of the precise mechanisms of LPS action, our data clearly show that the CDK inhibitors can overcome them. Thus, the ability of CDK inhibitors to override endogenous pro-survival factors further suggests their potential use in inflammatory diseases where concentrations of such mediators are increased.

We found that the expression of CDK1 and CDK2 proteins did not change during apoptosis, but that treatment of neutrophils with the pro-apoptotic activating antibody to Fas (CH11) resulted in loss of CDK1 activity before the onset of apoptosis. By contrast, a previous study has shown that non-cycling neuronal cells require activation of $\mathrm{CDK}$ for the induction of apoptosis and that CDK inhibitors can prevent cell death ${ }^{10}$. Our results suggest that CDK activity is necessary and fundamental to neutrophil survival. We have also shown that $R$-roscovitine-induced apoptosis is caspase dependent and is associated with a loss of expression of the $\mathrm{Bcl}-2$ family protein Mcl-1 (ref. 14). The loss of Mcl-1 caused by CDK inhibitors is probably an important mechanism controlling apoptosis ${ }^{11-13}$. In addition, because CDK5 is also a target for $R$-roscovitine $e^{16}$ and this $\mathrm{CDK}$ has been reported in human neutrophils ${ }^{27}$, we confirmed that CDK5 protein is expressed in human neutrophils (data not shown). Gene array experiments have reported the expression of several CDK mRNAs, endogenous CDK inhibitors and associated proteins in neutrophils ${ }^{32-35}$, some of which are altered during apoptosis ${ }^{36}$. 


\section{ARTICLES}

We previously showed that inhibiting pro-survival molecules from the MAPK and Bcl-2 families (namely, ERK1/2 and $\mathrm{Bcl}-\mathrm{x}_{\mathrm{L}}$ ) promotes resolution of inflammation by inducing inflammatory cell apoptosis in an acute rat model of carrageenan-induced pleurisy, whereas inhibition of the pro-apoptotic molecule Bax prevents resolution of inflammation ${ }^{30}$. We have now shown that $R$-roscovitine promotes resolution of inflammation in carrageenan-induced pleurisy in a dose-dependent manner. $R$-Roscovitine induced apoptosis in vivo, as assessed by annexin- $\mathrm{V}$ labeling and morphological analysis. $R$-Roscovitine also inhibited the release of pro-inflammatory cytokines, including IL-6, MCP-1 and IFN- $\gamma$, further supporting its pro-resolution role. In addition, $R$-roscovitine, administered at the peak of inflammation, enhanced the resolution of bleomycininduced lung inflammation. This model was used for its clinical relevance $\mathrm{e}^{37}$ and because progression of the marked acute inflammatory response induced by bleomycin leads to chronic inflammation and fibrosis ${ }^{38,39}$. Early accumulation of neutrophils in the BAL fluid and subsequent lung inflammation and injury were attenuated by $R$-roscovitine treatment. $R$-Roscovitine also reduced bleomycininduced lethality, indicating that $\mathrm{CDK}$ inhibition attenuates prolonged inflammation, leading to lung damage and consequent death. $R$-Roscovitine also caused enhanced inflammatory resolution in serum-induced arthritis as assessed by improved clinical scores.

Our in vitro work showing that the $\mathrm{CDK}$ inhibitors promote neutrophil apoptosis adds to the evidence indicating that neutrophils are essential in regulating the inflammatory responses in vivo, including models of carrageenan-induced pleurisy ${ }^{30}$, bleomycin induced-lung injury ${ }^{38,39}$ and arthritis ${ }^{7}$. We also showed that inhibition of apoptosis with zVAD-fmk prevents the enhanced resolution of inflammation by $R$-roscovitine in carrageenan-induced pleurisy. The dosing regime for $\mathrm{zVAD}$-fmk administration is paramount to elicit an effect in vivo and was based on studies where injections were administered every $3 \mathrm{~h}$ (ref. 40). Notably, R-roscovitine-induced apoptosis and its anti-inflammatory effects were reversed in vivo by the caspase inhibitor $\mathrm{zVAD}-\mathrm{fmk}$, providing further evidence that the anti-inflammatory mechanism of the CDK inhibitor is due to the induction of caspase-dependent inflammatory cell apoptosis.

Although neutrophils are terminally differentiated, evidence indicates that during the early stages of resolution of inflammation they are capable of phenotypic alteration, switching from a phenotype that generates pro-inflammatory mediators to one generating mediators that are anti-inflammatory and/or pro-resolving. For example, neutrophils can produce and respond to agents, such as lipoxins and resolvins, that have anti-inflammatory and pro-resolution properties $^{41-43}$. In addition, accelerated resolution of inflammation has been reported for endogenous lipid mediators such as protectin D1 (ref. 44), suggesting that control of inflammation can be achieved physiologically through production of endogenous mediators. Our hypothesis that manipulation of pro-resolution mechanisms can have profound effects on established inflammation is strongly supported by data showing that pharmacological manipulation of apoptosis and/or macrophage clearance can be achieved ${ }^{30,45}$ and our present finding that modulation of granulocyte apoptosis with CDK inhibitors promotes resolution of inflammation.

Thus, systemic administration of a specific inhibitor of CDKs induces apoptosis of inflammatory cells in situ and promotes inflammatory resolution. In vivo studies investigating the effect of $C D K$ inhibition in cancer have shown that systemic administration of $R$-roscovitine reduces tumor size ${ }^{46}$ and that $R$-roscovitine (even at high doses of $2 \mathrm{~g}$ per $\mathrm{kg}$ ) is well tolerated. It is widely acknowledged that tumor cells have defective $\mathrm{CDK}$ pathways that promote cell proliferation and prevent cell apoptosis ${ }^{8,9}$, suggesting that CDK inhibitors specifically target these pathways to elicit their therapeutic action. Our study has shown that $\mathrm{CDK}$ inhibitors also promote apoptosis in non-proliferating inflammatory cells in vitro and accelerate inflammatory resolution by promoting apoptosis and subsequent safe clearance of neutrophils by macrophages in vivo. In conclusion, given the renewed interest in the close association between inflammatory processes and cancer ${ }^{47,48}$ and the potential problems with using monoclonal antibodies and other biological agents, this newly identified role of the small-molecule anti-cancer $\mathrm{CDK}$ inhibitors may have potential for the treatment of diseases associated with increased or persistent inflammatory responses.

\section{METHODS}

Neutrophil isolation and assessment of apoptosis. We isolated human blood neutrophils ${ }^{23,49}$, a process that takes $\sim 2 \mathrm{~h}$, and cultured the cells $\left(5 \times 10^{6} / \mathrm{ml}\right.$, $37{ }^{\circ} \mathrm{C}$ ) in Iscove's modified Dulbecco's medium (IMDM) containing $10 \%$ autologous serum. The cells were incubated with the following reagents: $R$-roscovitine, $(R)$-2-[[9-(1-methylethyl)-6-[(phenylmethyl)amino]-9H-purin2-yl]amino]-1-butanol (A.G. Scientific); hymenialdisine, 4-(2-amino-4-oxo-2imidazolidin-5-ylidene)-2-bromo-4,5,6,7-tetrahydropyrrolo[2,3-c]azepin-8-one (from L. Meijer, Roscoff, France); NG75, 2-(1R-isopropyl-2-hydroxyethylamino)-6-(4-metoxy-benzylamino)-9-isopropylpurine (from N. Gray, Universiry of California, Berkeley); db-cAMP (Sigma); GM-CSF (R\&D Systems); and LPS (Escherichia coli 0127:B8, Sigma). We assessed apoptosis by flow cytometry using annexin-V-conjugated fluorescein isothiocynate (FITC; Roche) in combination with PI (Sigma) and used light microscopy to confirm the presence of morphological changes characteristic of apoptosis ${ }^{2,23}$.

Western blotting. We lysed cells $\left(5 \times 10^{6}\right)$ for 15 min with $0.1 \%$ Nonidet P40 in $1 \mathrm{ml}$ of TBS containing a protease inhibitor cocktail ${ }^{23}$ before centrifugation $\left(23,100 \mathrm{~g}, 4^{\circ} \mathrm{C}, 20 \mathrm{~min}\right)$. Protein samples $\left(40 \mu \mathrm{g}\right.$ or the equivalent of $1.5 \times 10^{6}$ cells per lane) were resolved by SDS-PAGE and then transferred to polyvinylidene difluoride (PVDF) membranes. Blots were blocked with 5\% skimmed milk powder in TBS plus Tween before probing with antibodies to CDK1 (Transduction Laboratories), CDK2 (Transduction Laboratories), CDK5 (Santa-Cruz Biotechnology), caspase-3 (Cell Signaling Technologies), Mcl-1 (SantaCruz Biotechnology) or $\beta$-actin (Sigma).

Kinase assays. We assayed kinase activity by immunoprecipitation of CDK1 (A17 antibody; Pharmingen) or CDK2 (M2 antibody; Santa Cruz Biotechnology), followed by incubation with histone $\mathrm{Hl}$ and $\left[\gamma_{-}{ }^{32} \mathrm{P}\right]$ ATP (ref. 50), Immunoprecipitates were resolved by SDS-PAGE, stained with Coomassie blue to visualize the histone $\mathrm{H} 1$ bands, dried and exposed for autoradiography.

Carrageenan-induced pleurisy. Male C57BL/6J mice (aged 8-12 weeks) were injected intrapleurally with $0.1 \mathrm{ml}$ of $1 \% \lambda$-carrageenan (Marine Colloids), followed $24 \mathrm{~h}$ later by $0.5 \mathrm{ml}$ of saline control, $0.5 \mathrm{ml}$ of $0.5 \%$ DMSO vehicle control, or 10 or $100 \mathrm{mg}$ per $\mathrm{kg}$ of $R$-roscovitine (i.p.). Mice were killed and pleural cavities were lavaged with $1 \mathrm{ml}$ of $3.15 \%$ (w/v) sodium citrate in PBS. Edema formation was measured by pleural exudate weight. Cell counts, apoptosis and macrophage phagocytosis in pleural cell samples were assessed microscopically.

Administration of zVAD-fmk. Twenty-four hours after intrapleural injection of carrageenan, mice were injected i.p. with $10 \mathrm{mg}$ per $\mathrm{kg}$ of $R$-roscovitine and/or $5 \mathrm{mg}$ per $\mathrm{kg}$ of $\mathrm{zVAD}$-fmk (z-Val-Ala-DL-Aspfluoromethylketone; Bachem) at a final concentration of $1 \mathrm{mg} / \mathrm{ml}$ in saline, or with appropriate amounts of DMSO vehicle control. Two additional doses of zVAD-fmk were given i.p. 4 and $8 \mathrm{~h}$ later, and all mice were killed after $12 \mathrm{~h}$.

Cytokine analysis. We measured cytokine levels by using a mouse inflammation cytokine bead kit (BD Biosciences) according to the manufacturer's instructions.

Bleomycin-induced lung injury. Male C57BL/6] mice (aged 8-12 weeks) were given either $0.05 \mathrm{ml}$ of $0.1 \mathrm{U}$ of bleomycin (Apollo Scientific) or saline intratracheally (sham control), and then treated $48 \mathrm{~h}$ later with either $0.5 \mathrm{ml}$ of $0.5 \%$ DMSO vehicle control or $100 \mathrm{mg}$ per $\mathrm{kg}$ of $R$-roscovitine. The mice were killed $72 \mathrm{~h}$ or $7 \mathrm{~d}$ after bleomycin or saline administration. In the acute 
72-h experiments, bronchoalveolar lavage were performed with three sequential washes with $0.8 \mathrm{ml}$ of ice-cold saline before perfusion with $4 \%$ formaldehyde for tissue histological analysis. Differential cell counts were done on cytocentrifuge preparations with eosin and hemotoxylin staining. Histological analysis of the 7-d experiments was done without bronchoalveolar lavage to maintain tissue integrity. Experiments were done on untreated control, sham control (saline and DMSO treatment), $R$-roscovitine control (saline and $100 \mathrm{mg}$ per $\mathrm{kg}$ of $R$-roscovitine), bleomycin plus vehicle (DMSO) control, and bleomycin plus $R$-roscovitine treatment groups, with six mice per group. Lungs were inflated, fixed with $1 \mathrm{ml}$ of $4 \%$ formaldehyde and then decalcified with $5 \%$ nitric acid for $3 \mathrm{~h}$, and lung injury was assessed by histological examination of paraffinembedded lung sections stained with hematoxylin and eosin.

Induction and assessment of arthritis. Female C57BL/6J mice (aged 6-8 weeks) received two i.p. injections at 48 -h intervals of sera $(100 \mu \mathrm{l})$ from arthritic $\mathrm{K} / \mathrm{BxN}$ mice (aged $60 \mathrm{~d}$ ) and $R$-roscovitine or vehicle was administered i.p. every $48 \mathrm{~h}$. Arthritis was scored by clinical examination: a score of 1 represents erythema alone or swelling of 1 digit; 2 represents erythema plus swelling of the tarsal joints, or swelling of the hock or wrist joint alone; 3 represents swelling in both tarsal and hock joints, both wrist and digits, or more than two digits and the tarsal joints. The mean cumulative limb scores for each mouse were calculated for each group, and data are expressed as the percentage of the clinical score obtained on day 3 before the first injection of $R$-roscovitine.

Statistical analysis. We analyzed in vitro experiments by analysis of variance (ANOVA) and Student-Newman-Keuls comparison tests. All in vivo experiments included at least six mice per group and experiments were repeated to verify the original findings. Statistical analysis was done by a one-way ANOVA with a Bonferroni multiple comparison post hoc test with a $95 \%$ confidence interval. Data are expressed as the mean \pm s.e.m. and values of $P<0.05$ were considered significant.

\section{ACKNOWLEDGMENTS}

We thank M. Clay and K. Miles for help with the in vivo models. We thank the Arthritis Research Campaign, the Medical Research Council, UK, the Norman Salvesen Emphysema Trust, Asthma UK, and the Juan Esplugues Foundation for financial support.

\section{AUTHOR CONTRIBUTIONS}

A.G.R. initiated, designed, directed and performed experiments and took overall responsibility for planning and writing the manuscript. D.A.S. helped design, perform and analyze the in vivo experiments and contributed to the writing of the in vivo component. A.W. helped design, perform and analyze the in vitro experiments and contributed to the writing of the in vitro component. C.W. contributed intellectually to the manuscript and helped with the in vitro work. T.A.S. performed some in vitro apoptosis and western blotting experiments N.A.R. performed the Mcl-1 western blot experiments. A.C. and M.M.-L performed some in vitro apoptosis experiments. T.R.W. helped in the design of the western blotting and kinase experiments. R.D. helped in the bleomycin experiments. M.G. helped in the design and execution of the arthritis experiments. E.C. helped in the design and execution of the kinase experiments. M.C.M. performed preliminary apoptosis experiments with the CDK inhibitors. H.J.B. provided intellectual input and provided significant input to the design and execution of the kinase experiments. J.S.S. provided intellectual input. I.D. provided intellectual input and contributed to the design of the experiments and the writing of the manuscript. C.H. provided intellectual input and helped in the design and coordination of the project.

\section{COMPETING INTERESTS STATEMENT}

The authors dedare that they have no competing financial interests.

Published online at http://www.nature.com/naturemedicine/

Reprints and permissions information is available online at http://npg.nature.com/ reprintsandpermissions/

1. Nathan, C. Points of control in inflammation. Nature 420, 846-852 (2002).

2. Savill, J.S. et al. Macrophage phagocytosis of aging neutrophils in inflammation. Programmed cell death in the neutrophil leads to its recognition by macrophages. J. Clin. Invest. 83, 865-875 (1989).

3. Savill, J., Dransfield, I., Hogg, N. \& Haslett, C. Vitronectin receptor-mediated phagocytosis of cells undergoing apoptosis. Nature $343,170-173$ (1990).
4. Gilroy, D.W., Lawrence, T., Perretti, M. \& Rossi, A.G. Inflammatory resolution: new opportunities for drug discovery. Nat. Rev. Drug Discov. 3, 401-416 (2004).

5. Riley, N.A. et al. Granulocyte apoptosis and macrophage clearance of apoptotic cells as targets for pharmacological intervention in inflammatory diseases. Anti-Inflamm. AntiAllergy Agents Medicinal Chem. 5, 3-12 (2006).

6. Whyte, M.K., Meagher, L.C., MacDermot, J. \& Haslett, C. Impairment of function in aging neutrophils is associated with apoptosis. J. Immunol. 150, 5124-5134 (1993).

7. Jonsson, H., Allen, P. \& Peng, S.L. Inflammatory arthritis requires Foxo3a to prevent Fas ligand-induced neutrophil apoptosis. Nat. Med. 11, 666-671 (2005).

8. Vermeulen, K., Van Bockstaele, D.R. \& Berneman, Z.N. The cell cycle: a review of reguIation, deregulation and therapeutic targets in cancer. Cell Prolif. 36, 131-149 (2003).

9. Senderowicz, A.M. Small-molecule cyclin-dependent kinase modulators. Oncogene 22, $6609-6620$ (2003)

10. Monaco, E.A., III. \& Vallano, M.L. Cyclin-dependent kinase inhibitors: cancer killers to neuronal guardians. Curr. Med. Chem. 10, 367-379 (2003).

11. MacCallum, D.E. et al. Seliciclib (CYC202, R-Roscovitine) induces cell death in multiple myeloma cells by inhibition of RNA polymerase II-dependent transcription and down-regulation of MCl-1. Cancer Res. 65, 5399-5407 (2005).

12. Raje, N. et al. Seliciclib (CYC2O2 or R-roscovitine), a small-molecule cyclin-dependent kinase inhibitor, mediates activity via down-regulation of $\mathrm{Mcl}-1$ in multiple myeloma. Blood 106, 1042-1047 (2005).

13. Michels, J., Johnson, P.W. \& Packham, G. Mcl-1. Int. J. Biochem. Cell Biol. 37, 267-271 (2005)

14. Moulding, D.A., Quayle, J.A., Hart, C.A. \& Edwards, S.W. Mcl-1 expression in human neutrophils: regulation by cytokines and correlation with cell survival. Blood 92, 2495-2502 (1998).

15. De Azevedo, W.F. et al. Inhibition of cyclin-dependent kinases by purine analogues: crystal structure of human cdk2 complexed with roscovitine. Eur. J. Biochem. 243, 518-526 (1997).

16. Meijer, L. et al. Biochemical and cellular effects of roscovitine, a potent and selective inhibitor of the cyclin-dependent kinases cdc2, cdk2 and cdk5. Eur. J. Biochem. 243, 527-536 (1997)

17. Bach, S. et al. Roscovitine targets, protein kinases and pyridoxal kinase. J. Biol. Chem. 280, 31208-31219 (2005).

18. Gray, N.S. et al. Exploiting chemical libraries, structure, and genomics in the search for kinase inhibitors. Science 281, 533-538 (1998).

19. Chang, Y.T. et al. Synthesis and application of functionally diverse 2,6,9-trisubstituted purine libraries as CDK inhibitors. Chem. Biol. 6, 361-375 (1999).

20. Meijer, L. et al. Inhibition of cyclin-dependent kinases, GSK-3 $\beta$ and CK1 by hymenialdisine, a marine sponge constituent. Chem. Biol. 7, 51-63 (2000).

21. Williams, O., Gil-Gomez, G., Norton, T., Kioussis, D. \& Brady, H.J. Activation of Cdk2 is a requirement for antigen-mediated thymic negative selection. Eur. J. Immunol. 30, 709-713 (2000)

22. Martin, M.C., Dransfield, I., Haslett, C. \& Rossi, A.G. Cyclic AMP regulation of neutrophil apoptosis occurs via a novel protein kinase $A$-independent signaling pathway. J. Biol. Chem. 276, 45041-45050 (2001)

23. Ward, C. et al. NF- $\mathrm{KB}$ activation is a critical regulator of human granulocyte apoptosis in vitro. J. Biol. Chem. 274, 4309-4318 (1999).

24. Ward, C. et al. Interleukin-10 inhibits lipopolysaccharide-induced survival and extracellular signal-regulated kinase activation in human neutrophils. Eur. J. Immunol. 35, 2728-2737 (2005).

25. Sabroe, I., Jones, E.C., Usher, L.R., Whyte, M.K. \& Dower, S.K. Toll-like receptor (TLR)2 and TLR4 in human peripheral blood granulocytes: a critical role for monocytes in leukocyte lipopolysaccharide responses. J. Immunol. 168, 4701-4710 (2002).

26. Sabroe, I. et al. Selective roles for Toll-like receptor (TLR)2 and TLR4 in the regulation of neutrophil activation and life span. J. Immunol. 170, 5268-5275 (2003).

27. Rosales, J.L., Ernst, J.D., Hallows, J. \& Lee, K.Y. GTP-dependent secretion from neutrophils is regulated by Cdk5. J. Biol. Chem. 279, 53932-53936 (2004).

28. Gilroy, D.W. et al. Inducible cyclooxygenase may have anti-inflammatory properties. Nat. Med. 5, 698-701 (1999).

29. Gilroy, D.W. et al. Inducible cyclooxygenase-derived 15-deoxy( $\Delta) 12-14 \mathrm{PGJ} 2$ brings about acute inflammatory resolution in rat pleurisy by inducing neutrophil and macrophage apoptosis. FASEB J. 17, 2269-2271 (2003).

30. Sawatzky, D.A., Willoughby, D.A., Colville-Nash, P.R. \& Rossi, A.G. The involvement of the apoptosis-modulating proteins ERK 1/2, Bcl- $\mathrm{x}_{\mathrm{L}}$ and $\mathrm{Bax}$ in the resolution of acute inflammation in vivo. Am. J. Pathol. 168, 33-41 (2006)

31. Cailhier, J.F. et al. Resident pleural macrophages are key orchestrators of neutrophil recruitment in pleural inflammation. Am. J. Respir. Crit. Care Med. 173, 540-547 (2006).

32. Klausen, P., Bjerregaard, M.D., Borregaard, N. \& Cowland, J.B. End-stage differentiation of neutrophil granulocytes in vivo is accompanied by up-regulation of p27kip1 and down-regulation of CDK2, CDK4, and CDK6. J. Leukoc. Biol. 75, 569-578 (2004).

33. Tsukahara, $Y$. et al. Gene expression in human neutrophils during activation and priming by bacterial lipopolysaccharide. J. Cell. Biochem. 89, 848-861 (2003).

34. Zhang, X. et al. Gene expression in mature neutrophils: early responses to inflammatory stimuli. J. Leukoc. Biol. 75, 358-372 (2004).

35. Subrahmanyam, Y.V. et al. RNA expression patterns change dramatically in human neutrophils exposed to bacteria. Blood 97, 2457-2468 (2001).

36. Kobayashi, S.D., Voyich, J.M., Whitney, A.R. \& DeLeo, F.R. Spontaneous neutrophi apoptosis and regulation of cell survival by granulocyte macrophage-colony stimulating factor. J. Leukoc. Biol. 78, 1408-1418 (2005). 
ARTICLES

37. Azambuja, E., Fleck, J.F., Batista, R.G. \& Menna Barreto, S.S. Bleomycin lung toxicity: who are the patients with increased risk? Pulm. Pharmacol. Ther. 18, 363-366 (2005)

38. Nagase, T. et al. A pivotal role of cytosolic phospholipase $A_{2}$ in bleomycin-induced pulmonary fibrosis. Nat. Med. 8, 480-484 (2002).

39. Teder, P. et al. Resolution of lung inflammation by CD44. Science 296, 155-158 (2002)

40. De Paepe, M.E., Mao, Q., Embree-Ku, M., Rubin, L.P. \& Luks, F.I. Fas/FasL-mediated apoptosis in perinatal murine lungs. Am. J. Physiol. Lung Cell. Mol. Physiol. 287, L730-L742 (2004).

41. Levy, B.D., Clish, C.B., Schmidt, B., Gronert, K. \& Serhan, C.N. Lipid mediator class switching during acute inflammation: signals in resolution. Nat. Immunol. 2, 612-619 (2001).

42. Serhan, C.N. et al. Resolvins: a family of bioactive products of omega-3 fatty acid transformation circuits initiated by aspirin treatment that counter proinflammation signals. J. Exp. Med. 196, 1025-1037 (2002)

43. Serhan, C.N. \& Savill, J. Resolution of inflammation: the beginning programs the end. Nat. Immunol. 6, 1191-1197 (2005).
44. Serhan, C.N. et al. Anti-inflammatory actions of neuroprotectin D1/protectin D1 and its natural stereoisomers: assignments of dihydroxy-containing docosatrienes. J. Immunol. 176, 1848-1859 (2006).

45. Pinho, V. et al. Phosphoinositide-3 kinases critically regulate the recruitment and survival of eosinophils in vivo: importance for the resolution of allergic inflammation. J. Leukoc. Biol. 77, 800-810 (2005).

46. McClue, S.J. et al. In vitro and in vivo antitumor properties of the cyclin dependent kinase inhibitor CYC202 ( $R$-roscovitine). Int. J. Cancer 102, 463-468 (2002).

47. Coussens, L.M. \& Werb, Z. Inflammation and cancer. Nature 420, 860-867 (2002).

48. Karin, M. \& Greten, F.R. NF-KB: linking inflammation and immunity to cancer development and progression. Nat. Rev. Immunol. 5, 749-759 (2005).

49. Haslett, C. Guthrie, L.A., Kopaniak, M.M. Johnston, R.B. Jr. \& Henson, P.M. Modulation of multiple neutrophil functions by preparative methods or trace concentrations of bacterial lipopolysaccharide. Am. J. Pathol. 119, 101-110 (1985).

50. Gil-Gomez, G., Berns, A. \& Brady, H.J. A link between cell cycle and cell death: $\mathrm{Bax}$ and $\mathrm{Bcl}-2$ modulate $\mathrm{Cdk2}$ activation during thymocyte apoptosis. EMBO J. 17. 7209-7218 (1998). 


\title{
Corrigendum: Cyclin-dependent kinase inhibitors enhance the resolution of inflammation by promoting inflammatory cell apoptosis
}

\author{
Adriano G Rossi, Deborah A Sawatzky, Annemieke Walker, Carol Ward, Tara A Sheldrake, Nicola A Riley, Alison Caldicott, \\ Magdalena Martinez-Losa, Trevor R Walker, Rodger Duffin, Mohini Gray, Elvira Crescenzi, Morag C Martin, Hugh J Brady, John S Savi l, \\ Ian Dransfield \& Christopher Haslett \\ Nature Medicine 12, 1056-1064 (2006); published online 3 September 2006; corrected after print 10 November 2006
}

In the version of this article initially published, the dose stated for $\mathrm{zVAD}$-fmk administration was incorrect. The methods reported on page 1062 should read, "Twenty-four hours after intrapleural injection of carrageenan, mice were injected i.p. with $10 \mathrm{mg}$ per kg of R-roscovitine and/or 5 mg per kg of zVAD-fmk (z-Val-Ala-DL-Asp-fluoromethylketone; Bachem)." Similarly, the legend to Figure 4, line 3, should read "C57/bl6 mice were treated with $10 \mathrm{mg}$ per $\mathrm{kg}$ of R-roscovitine (i.p.) and/or $5 \mathrm{mg}$ per $\mathrm{kg}$ of zVAD-fmk (i.p. at 4-h intervals)." The authors also made an error reporting the time of administration of $\mathrm{K} / \mathrm{Bxn}$ serum in the legend to Figure 5, line 14 . This should read "Mice ( $n=10$ in each group) were injected twice (days 0 and 2) with $\mathrm{K} / \mathrm{BxN}$ serum derived from arthritic (day 60) $\mathrm{K} / \mathrm{BxN}$ transgenic mice." The error has been corrected in the HTML and PDF versions of the article. 


\title{
NADPH oxidase-derived ROS: Key modulators of heme-induced mitochondrial stability in human neutrophils
}

\author{
Maria Augusta Arruda ${ }^{a}$, Pedro Barcellos-de-Souza $a^{a}$, André Luiz Franco Sampaio ${ }^{b}$, \\ Adriano G. Rossi ${ }^{c}$, Aurélio V. Graça-Souza ${ }^{d}$, Christina Barja-Fidalgo ${ }^{a, *}$ \\ aDepartamento de Farmacologia, Instituto de Biologia, Universidade do Estado do Rio de Janeiro, Av. 28 de setembro 87 -Vila Izabel, \\ Rio de Janeiro, RJ, 20551-030 Brazil \\ bepartamento de Farmacologia Aplicada, FarManguinhos-FIOCRUZ, Rio do Janeiro, Brazil \\ 'MRC Centre for Inflammation Research, University of Edinburgh Medical School, Edinburgh EH8 9AG, UK \\ dinstituto de Bioquímica Médica, Universidade Federal do Rio de Janeiro, Rio do Janeiro, Brazil
}

\section{ARTICLE INFORMATION}

\section{Article Chronology:}

Received 21 March 2006

Revised version received

24 August 2006

Accepted 26 August 2006

Available online 30 August 2006

\section{Keywords:}

NADPH oxidase

Neutrophil

Heme

Oxidative stress

Apoptosis

Signal transduction

Bcl-2 family proteins

Mitochondria

\begin{abstract}
A B S T R A C T
Heme is a proinflammatory molecule able to cause a profound delay of constitutive apoptosis of human neutrophils, an effect that likely contributes to chronic inflammation associated with hemolytic diseases. Herein we show that heme-induced delay of neutrophil apoptosis correlates with the prevention of mitochondrial potential $\left(\Delta \psi_{m}\right)$ dissipation by a mechanism dependent on NADPH oxidase (NADPHox)-generated reactive oxygen species (ROS) and NF- $\mathrm{kB} . \Delta \psi_{m}$ maintenance is accompanied by inhibition of Bax insertion into mitochondria and by a decrease in the $\mathrm{Bad} / \mathrm{Bcl}-\mathrm{X}_{\mathrm{L}}$ ratio. Heme induces Bad degradation in a completely ROS-dependent manner, as well as Bcl- $\mathrm{X}_{\mathrm{L}}$ synthesis, a phenomenon that also requires NF- $\kappa B$ activation. These data indicate that heme-induced preservation of mitochondrial integrity is a critical checkpoint controlled by NADPH oxidase generatedROS and redox-sensitive NF- $\mathrm{kB}$ activation.
\end{abstract}

(C) 2006 Elsevier Inc. All rights reserved.

\footnotetext{
- Corresponding author. Fax: 55+21+2587+6808.

E-mail address: barja-fidalgo@uerj.br (C. Barja-Fidalgo).
} 


\author{
Abbreviations: \\ NADPHox, NADPH oxidase \\ ROS, reactive oxygen species \\ $\Delta \psi_{m}$, mitochondria transmembrane \\ potential \\ MAPK, mitogen-activated protein \\ kinase \\ ERK, extracellular signal-regulated \\ protein kinase \\ PI3K, phosphatidylinositol 3 kinase \\ NF, nuclear factor \\ PKC, protein kinase C \\ IL, interleukin \\ GM-CSF, granulocyte macrophage \\ colony stimulation factor \\ DPI, diphenyleneiodonium \\ PDTC, pyrrolidine dithiocarbamate \\ COx, cytochrome $c$ oxidase \\ subunit IV \\ PICD, phagocytosis-induced cell \\ death \\ PMSF, phenylmethylsulfonyl \\ fluoride
}

\section{Introduction}

Phagocytic NADPH oxidase (NADPHox) is a multi-enzymatic complex responsible for the generation of high amounts of $\mathrm{O}_{2}^{-}$ through the reduction of molecular oxygen. The $\mathrm{O}_{2}^{-}$and $\mathrm{O}_{2}^{-}-$ derived ROS so generated have critical implications on the innate immune response, being involved in the killing of engulfed and/or surrounding pathogens [1]. In resting cells, NADPHox is inactive, once its subunits are found disassembled, distributed among cytosol and membrane. However, proper activation of these cells results in phosphorylation of NADPHox cytosolic subunit by protein kinase C (PKC) and other kinases, translocation to plasma membrane and association to the membrane-bound subunits, comprising the catalytic active NADPHox [1].

Beside the well-described cytotoxic properties, ROS have gained acceptance as important mediators of signal transduction, acting as second messengers. More specifically, it has been described that NADPHox-derived ROS mediate a number of prominent cellular processes such as proliferation, adhesion, motility, survival and apoptosis [reviewed in 2]. Among the ROS-activated molecular targets are the mitogen-activated protein kinases (MAPKs), the phosphoinositol PI3K/Akt pathway molecules and NF- $\mathrm{kB}$ [2].

Several proinflammatory mediators can trigger NADPHox in neutrophils, such as leukotriene $\mathrm{B}_{4}\left(\mathrm{LTB}_{4}\right)$, formylated peptides and interleukin (IL)-8 [3,4]. Our group has characterized heme (ferriprotoporphyrin IX), at a concentration range found during hemolytic episodes, as a potent proinflammatory molecule, triggering NADPHox-dependent ROS generation in a PKC-dependent manner [5]. More recently, we observed that free heme, as other proinflammatory agents, prolongs neutrophil longevity by delaying the onset of apoptosis, a critical event that likely leads to a chronic inflammatory state [6]. The anti-apoptotic effect of heme depends on ROS generation resulting in an up-regulation of NF- $\mathrm{\kappa B}$ activity, a transcription factor shown to critically regulate granulocyte apoptosis [7]. It also appears that PI3K/AKT and ERK signaling pathways are also required for the heme anti-apoptotic effect, as well as heme oxygenase activity. Levels of key Bcl-2 family members are also altered on exposure to heme, for example anti-apoptotic $\mathrm{Bcl}-\mathrm{X}_{\mathrm{L}}$ synthesis was increased whereas enhanced proapoptotic Bad degradation occurred in response to heme [6].

However, there is no consensus about the role of ROS on neutrophil lifespan. Most of reports affirm that NADPHoxderived ROS are directly linked to neutrophil induction of apoptosis, especially in phagocytosis-induced cell death (PICD) [8]. This way, a better understanding of the molecular and subcellular events regulating pro-survival signaling evoked by NADPHox-derived ROS becomes urgent.

Mitochondria are classically defined as organelles highly specialized in the generation of large amounts of ATP and thus essential for the maintenance of the bioenergetic machinery of virtually all cell types. However, studies performed during the last decade have conclusively shown that mitochondria also play a major role in the regulation of apoptosis [9]. The intermembrane spaces of mitochondria contain a number of proteins (e.g., cytochrome c, Smac/ DIABLO, Omi/HtrA2) that, once released into the cytosol, trigger the activation of the apoptotic cascade. Cytochrome $c$, the most prominent of these pro-apoptotic proteins, when associated with Apaf and pro-caspase 9 constitute the apoptosome, the initiator of the intrinsic apoptotic pathway [9].

The role of the mitochondria in neutrophil function had been regarded as trivial because these cells contain very poorly detectable mitochondria, mitochondrial poisons have no major effect on neutrophil function and neutrophils derive 
much of their energy from anaerobic glycolysis [10]. More recently, the development of specific fluorescent dyes has helped to show that neutrophils do indeed possess functionally important mitochondria [11]. The appearance and distribution of neutrophil mitochondria are different from the mitochondria of most cell types and appear to play an important role in regulating apoptosis in these terminally differentiated cells. In viable cells, these organelles are organized as a diffuse tubular network that clusters during apoptosis, colocalizing with the pro-apoptotic Bcl-2 member Bax. Bax is believed to be involved in pore formation on the outer mitochondria membrane leading to the release of proapoptotic proteins [11]. Recently, Maianski et al. characterized the neutrophil mitochondria, showing that when these organelles have lost their ability to produce ATP they are still able to maintain mitochondria transmembrane potential $\left(\Delta \psi_{m}\right)$. It is believed that when $\Delta \psi_{m}$ is disrupted, cells undergo apoptosis through the release of the pro-apoptotic factors locked in mitochondria [12].

The modulation of mitochondria outer membrane integrity is a key event in the switch between survival and apoptotic death. The control of this phenomenon ultimately depends upon the balance of pro- and anti-apoptotic members of the Bcl-2 family. The anti-apoptotic members (e.g., Bcl-2, Bcl- $\mathrm{X}_{\mathrm{L}}$ Mcl-1 and A1) act by promoting the stability of mitochondrial outer membrane and/or impairing the oligomerization and consequent insertion of pro-apoptotic Bax-like proteins [13]. A second group of pro-apoptotic Bcl-2 members (e.g., Bad, Bid) comprises proteins that modulate negatively the anti-apoptotic $\mathrm{Bcl}-2$ proteins and positively the pro-apoptotic ones [13]. In most cell types, the expression and activity of protective $\mathrm{Bcl}-2$ members is higher than pro-apoptotic members. However, mature neutrophils display the opposite, perhaps reflecting the fact that these cells are committed to programmed cell death. Neutrophils constitutively express pro-apoptotic proteins, whereas the expression of anti-apoptotic Bcl-2 members is very low or undetectable in resting cells. These antiapoptotic proteins are highly and transiently expressed when neutrophils are exposed to survival factors, such as heme, interleukin (IL)-8 and granulocyte macrophage colony stimulation factor (GM-CSF) $[4,14-16]$.

In this study, we aimed to determine the role of hemeinduced NADPHox activity on mitochondria stability and key components of the apoptotic machinery. We observed that heme preserves $\Delta \psi_{m}$ in aged neutrophils. We also show that ROS generation and NF- $\mathrm{kB}$ activation, which are critical to heme-induced delay of neutrophil apoptosis, mediate the effect on $\Delta \psi_{m}$. The impairment of $\Delta \psi_{m}$ dissipation promoted by heme correlates with inhibition of Bax insertion into neutrophil mitochondria, an effect that may be due to the reduction of $\mathrm{Bad} / \mathrm{Bcl}-\mathrm{X}_{\mathrm{L}}$ ratio. In addition, heme induces Bad degradation through a ROS-dependent manner. Bcl- $\mathrm{X}_{\mathrm{L}}$ protein synthesis also requires NADPHox-derived ROS as well as NF- $\mathrm{kB}$ activation. These data indicate that heme down-modulates neutrophil apoptosis by maintaining neutrophil $\Delta \psi_{m}$ through the regulation of the expression and activity of $\mathrm{Bcl}-2$ pro- and antiapoptotic proteins. These findings attest to a controversial, but intriguing role of ROS generated by NADPHox on the control of neutrophil physiology, and help to elucidate the mechanism of action of free heme on the delay of neutrophil apoptosis.

\section{Materials and methods}

\section{Reagents}

Diphenyleneiodonium (DPI), apocynin (acetovanillone) and pyrrolidine dithiocarbamate (PDTC) were purchased from Sigma (St. Louis, MO). JC-1 and MitoTracker FM were purchased from Molecular Probes (Eugene, OR).

\section{Heme}

Hemin (cell culture grade, Sigma) stock solutions were made in DMSO (culture grade, Sigma) and diluted in sterile PBS immediately before use. The final concentration of DMSO was kept lower than $0.01 \%$ for all assays.

\section{Neutrophil isolation and culture}

Neutrophils were isolated from EDTA (0.5\%)-treated peripheral venous blood of healthy human volunteers by a method of dextran sedimentation and density gradient centrifugation as previously described [17]. Residual erythrocytes were removed by hypotonic lysis. Isolated neutrophils $\left(5 \times 10^{6} / \mathrm{mL}\right)$ were incubated in DMEM supplemented with $10 \%$ heat-inactivated fetal calf serum, $100 \mathrm{U} / \mathrm{mL}$ penicillin and $100 \mathrm{mg} / \mathrm{mL}$ streptomycin at $37^{\circ} \mathrm{C}$ in a humidified atmosphere containing $5 \% \mathrm{CO}_{2}$. Under all experimental conditions, $>99 \%$ of cells were viable, as assessed by trypan blue dye exclusion.

\section{Assessment of neutrophil apoptosis}

Neutrophil apoptosis was assessed as previously described [7]. Briefly, cells were cytocentrifuged, stained with Diff-Quik ${ }^{\mathrm{TM}}$ and counted under light microscopy $(\times 1000)$ to determine the proportion of cells showing characteristic apoptotic morphology. At least 400 cells were counted per slide. The results were expressed as mean $\pm \mathrm{SD}$. Annexin V binding assay, showing similar results, confirmed this technique.

\section{Assessment of $\Delta \boldsymbol{\psi}_{m}$}

$\Delta \psi_{m}$ alteration was assessed as previously described [18]. Briefly, neutrophils $\left(5 \times 10^{6}\right.$ cells $\left./ \mathrm{mL}\right)$ were resuspended in $1 \mathrm{~mL}$ of HBSS containing $1 \mu \mathrm{g} / \mathrm{mL} \mathrm{JC}-1$ and incubated for $30 \mathrm{~min}$ at $37^{\circ} \mathrm{C}$. Cells were then cytocentrifuged and slides were mounted using $\mathrm{N}$-propylgalate solution before examination under an Olympus Fluoview version 3.34 confocal microscope (Ex 485 nm; Em JC-1 Red 546, Em JC-1 Green $532 \mathrm{~nm}$ ). Field mean fluorescence was assessed using Olympus Fluoview version 3.34 confocal microscope software and the red/green fluorescence ratio was obtained. At least fifteen fields were captured for each treatment, from three independent experiments.

\section{Subcellular localization (Confocal Microscopy)}

In order to determine Bax insertion into mitochondria, neutrophils $\left(5 \times 10^{6}\right.$ cells $\left./ \mathrm{mL}\right)$ were incubated with MitoTracker $\mathrm{FM}(100 \mathrm{nM})$ for $30 \mathrm{~min}$ at $37^{\circ} \mathrm{C}$. Cells were then cytocentrifuged, 
fixed with paraformaldehyde (4\%) and then permeabilized with $0.5 \%$ Triton X-100 in PBS for 20 min The slides were incubated with rabbit polyclonal anti-Bax antibody (Santa Cruz Biotechnology, $1: 200$ ) at $4^{\circ} \mathrm{C}$ overnight, incubated at room temperature for $1 \mathrm{~h}$ with biotin-conjugated goat anti-rabbit IgG (Santa Cruz Biotechnology, 1:1000), and finally incubated with Cy3-conjugated streptavidin for $1 \mathrm{~h}$ at room temperature. Slides were then mounted using $\mathrm{N}$-propylgalate solution before examination under an Olympus Fluoview version 3.34 confocal microscope.

\section{Preparation of cell extracts}

For obtaining whole cell lysates, neutrophils $\left(5 \times 10^{6}\right.$ cells $\left./ \mathrm{mL}\right)$ were resuspended in lysis buffer (50 mM HEPES, pH 6.4, $1 \mathrm{mM}$ $\mathrm{MgCl}_{2}, 10 \mathrm{mM}$ EDTA, $1 \%$ Triton X-100, $1 \mu \mathrm{g} / \mathrm{mL}$ DNAse, $0.5 \mu \mathrm{g} /$ $\mathrm{mL}$ RNAse) containing the following protease inhibitors cocktail: $1 \mathrm{mM}$ phenylmethylsulfonyl fluoride (PMSF), $1 \mathrm{mM}$ benzamidine, $1 \mu \mathrm{M}$ leupeptin and $1 \mu \mathrm{M}$ soybean trypsin inhibitor (Sigma).

Subcellular fractions were obtained as previously described [12]. Briefly, neutrophils were washed and resuspended in icecold cytosol extraction buffer $(250 \mathrm{nM}$ sucrose, $70 \mathrm{mM} \mathrm{KCl}$, $250 \mu \mathrm{g} / \mathrm{mL}$ digitonin and protease inhibitor cocktail in PBS). After $15 \mathrm{~min}$ incubation on ice, it was centrifuged at $1000 \times \mathrm{g}$ for $5 \mathrm{~min}$ and supernatant was collected (cytosolic fraction). Pellets were then resuspended in mitochondria lysis buffer (100 mM Tris- $\mathrm{HCl}, 10 \mathrm{mM} \mathrm{MgCl}$, 2 mM EGTA, 2 mM EDTA, 1\% Nonident P-40 (v/v), $10 \%$ glycerol $(\mathrm{v} / \mathrm{v})$, protease inhibitor cocktail in $50 \mathrm{mM}$ Tris, $\mathrm{pH} 7,5$ ), incubated for $10 \mathrm{~min}$ on ice and centrifuged at $10000 \times \mathrm{g}$ for $10 \mathrm{~min}$. The supernatant was taken as mitochondria fraction.

\section{Western blot analysis}

The total protein content in the cell extracts was determined by the Bradford's method [19]. Cell lysates were denatured in sample buffer (50 mM Tris- $\mathrm{HCl}, \mathrm{pH} 6.8,1 \%$ SDS, $5 \%$ 2-mercaptoethanol, $10 \%$ glycerol, $0.001 \%$ bromophenol blue) and heated in a boiling water bath for $3 \mathrm{~min}$. Samples ( $30 \mu \mathrm{g}$ total protein) were resolved by $12 \%$ SDS-PAGE and proteins were transferred to PVDF membranes (Hybond-P, Amersham Pharmacia Biotech). Rainbow markers (Amersham Pharmacia Biotech) were run in parallel to estimate molecular weights. Membranes were blocked with TweenTBS (20 mM Tris- $\mathrm{HCl}, \mathrm{pH} 7.5,500 \mathrm{mM} \mathrm{NaCl}, 0.1 \%$ Tween-20) containing $1 \%$ bovine serum albumin and probed with polyclonal anti-Bcl- $\mathrm{X}_{\mathrm{L}}$ (Santa Cruz Biotechnology, 1:500), polyclonal anti-Bad (Santa Cruz Biotechnology, 1:500), polyclonal anti-Bax (Santa Cruz Biotechnology, 1:500) or mouse monoclonal anti-cytochrome $c$ oxidase (COx) subunit IV (Molecular Probes, 1:1000). After extensive washing in Tween-TBS, PVDF sheets were incubated with biotin-conjugated anti-rabbit IgG (1:1000; Santa Cruz Biotechnology) antibody for $1 \mathrm{~h}$ and then incubated with horseradish peroxidase-conjugated streptavidin (1:1000; Caltag Laboratories, Burlingame, CA). Immunoreactive proteins were visualized by $3,3^{\prime}$-diaminobenzidine (Sigma) staining. The bands were also quantified by densitometry using Scion Image Software (Scion Co., MD, USA).

\section{Statistical analysis}

Statistical significance was assessed by ANOVA followed by Bonferroni's $t$ test, and $p<0.05$ was taken as statistically significant.

\section{Results}

Heme-induced delay of human neutrophil apoptosis in vitro is associated with maintenance of $\Delta \boldsymbol{\psi}_{m}$

We have previously shown that heme inhibits human neutrophil apoptosis in a concentration range found during hemolytic episodes [6]. Here we confirm that heme $(1-10 \mu \mathrm{M})$ delays neutrophil apoptosis and this effect is completely abolished when cells are pre-treated with two structural and functional uncorrelated inhibitors, DPI $(10 \mu \mathrm{M})$ and apocynin $(10 \mu \mathrm{M})$ (Fig. 1). The maintenance of $\Delta \psi_{m}$ is regarded as a critical regulator of the apoptotic program in virtually all cell types and can be measured using the cationic dye JC- 1 that exhibits a membrane potential-dependent accumulation in mitochondria [18]. When a loss of $\Delta \psi_{m}$ occurs during apoptosis, there is a characteristic fluorescence emission shift from red to green. The observed potential-dependent color shift is due to concentration-dependent formation of red fluorescent J aggregates. Consequently, $\Delta \psi_{m}$ dissipation can be assessed by a decrease in the JC-1-emited red/green fluorescence intensity ratio. Fresh, non apoptotic, neutrophil mitochondria exhibit preserved $\Delta \psi_{m}$, as determined by confocal microscopic analysis of JC-1 stained cells; they show a higher red $(546 \mathrm{~nm})$ fluorescence in relation to green $(536 \mathrm{~nm}$ ) fluorescence (Fig. 2). After $20 \mathrm{~h}$, cultured neutrophils undergo constitutive apoptosis and display an opposite staining profile where the ratio between the two emission wavelengths (red/green) is significantly lower. Heme treatment $(3 \mu \mathrm{M})$ inhibited $\Delta \psi_{m}$ dissipation and therefore preserved mitochondria integrity (Fig. 2). Inhibition of NADPHox by DPI reversed the heme-induced changes in $\Delta \psi_{m}$ indicating an involvement of ROS. We have previously shown the direct involvement of NF- $\kappa$ B signaling pathway in mediating the anti-apoptotic effect of heme using a number of different techniques (6). Now we show that this pathway is also involved in maintaining heme-induced $\Delta \psi_{m}$ because pharmacological inhibition of this signaling route with PDTC abolished heme protective effects (Fig. 2).

\section{Heme inhibits Bax insertion into the mitochondria}

Two events are closely related to $\Delta \psi_{m}$ dissipation and consequent progression of the apoptotic cascade in neutrophils: the clustering of mitochondrial tubular network and the formation of pores in the outer mitochondrial membrane by pro-apoptotic Bcl-2 members [13]. As observed in Fig. 3a, heme treatment $(3 \mu \mathrm{M})$ was able to prevent mitochondrial clustering in aged neutrophils since the mitochondria of heme-treated neutrophils are organized in a tubular network, similar to fresh neutrophils. Heme also inhibits Bax insertion into the mitochondria as assessed by confocal microscopy, an effect likely responsible 

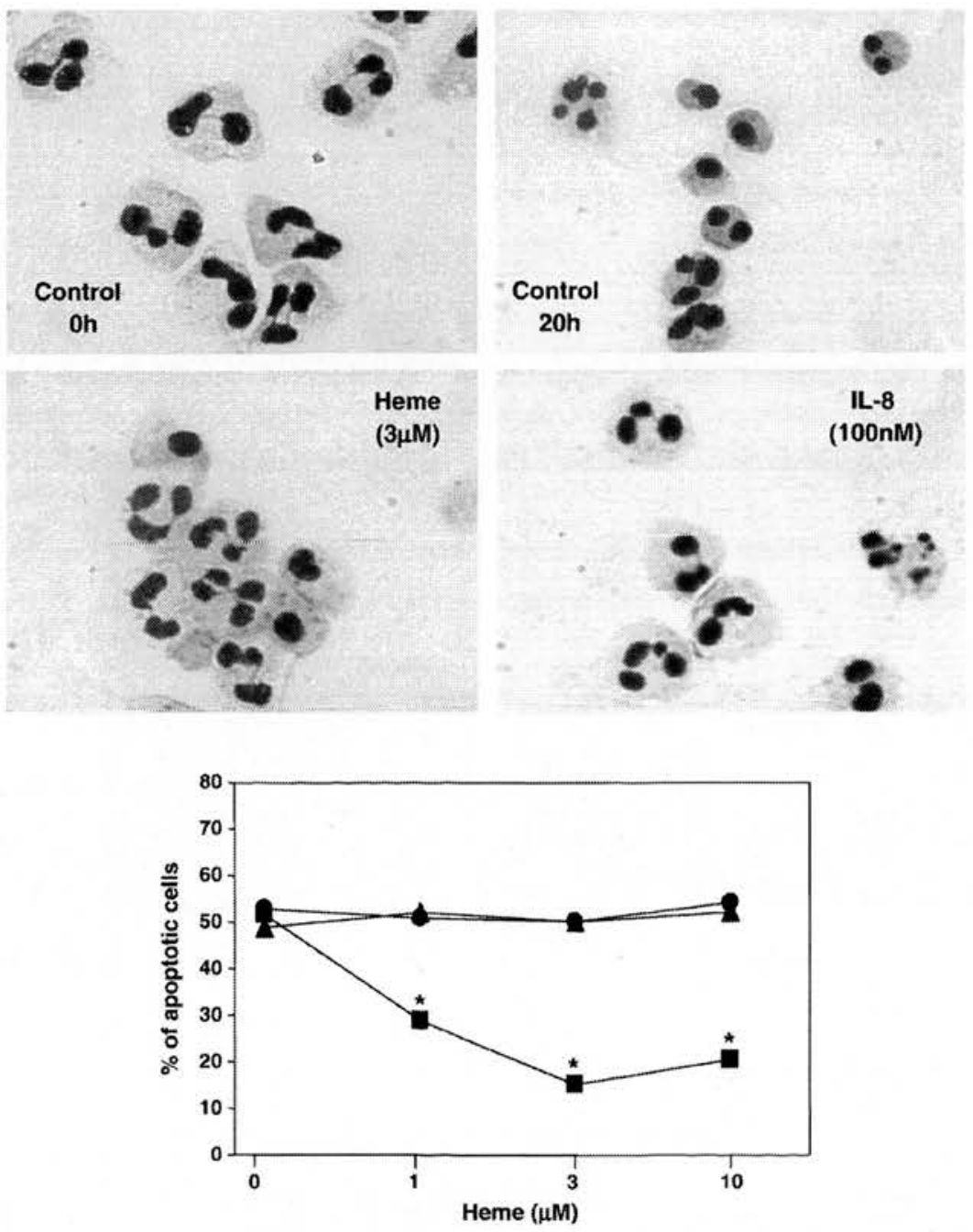

Fig. 1 - Effect of heme on neutrophil apoptosis. Neutrophils $\left(5 \times 10^{6} / \mathrm{mL}\right)$ were incubated in the absence or in the presence of varying concentrations of heme $(1-10 \mu \mathrm{M})$ in the absence (squares) or in the presence of DPI $(10 \mu \mathrm{M}$, circles) or apocynin (10 $\mu \mathrm{M}$, triangles). After $20 \mathrm{~h}$, cells were cytocentrifuged and the number of apoptotic cells was determined. Data shown are the results (mean \pm SD) of 3 experiments, each performed in triplicate. The asterisk (") indicates a significant difference $(p<0.001)$ between heme-treated and control neutrophils.

for maintaining the integrity of this organelle (Fig. 3b). Treatment with heme $(3 \mu \mathrm{M})$ significantly reduced the occurrence of Bax and mitochondria co-localization after $20 \mathrm{~h}$ of incubation, identified in merged images as false yellow points. The heme-induced inhibition of Bax insertion was also observed by Westem blot analysis of mitochondrial extracts (Fig. 4).

Heme-induced Bad degradation requires NADPHox-derived ROS

The balance of Bcl-2 member expression is likely to regulate the apoptotic process. It has been observed that heme promotes differential expression of pro- and anti-apoptotic $\mathrm{Bcl}-2$ proteins resulting in a decrease of the $\mathrm{Bad} / \mathrm{Bcl}-\mathrm{X}_{\mathrm{L}}$ ratio [6]. Heme-induced Bad degradation is delayed by the treat- ment with the NADPHox inhibitor DPI, suggesting the involvement of ROS in this event (Fig. 5).

Heme induces Bcl- $\mathrm{X}_{\mathrm{L}}$ expression-involvement of NADPHox and NF- $\kappa$ B activity

In fresh non-apoptotic neutrophils, the expression of antiapoptotic bcl-2 genes is very low or absent [16]. However, the presence of pro-inflammatory/anti-apoptotic mediators triggers their transcription and consequent protein synthesis [6,14-16]. Heme, like other anti-apoptotic agents, mediates its anti-apoptotic effect on neutrophil through de novo protein synthesis. It has also been shown that heme promotes $\mathrm{Bcl}-\mathrm{X}_{\mathrm{L}}$ protein synthesis [6], an effect indicating that this Bcl-2 member, among other inducible proteins, may play an important role in the control of neutrophil 

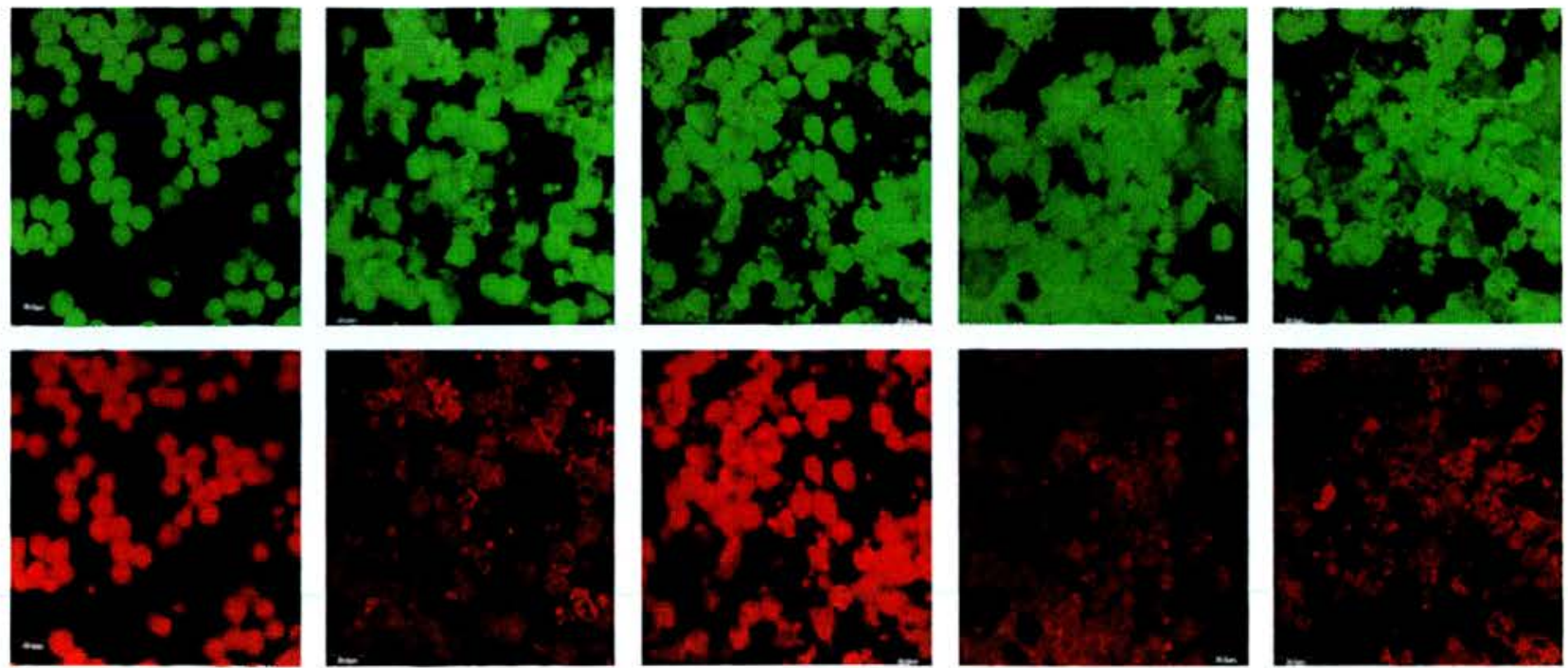

Control

Oh

Control

$-$

+PDTC

$+\mathrm{DPI}$

Heme $(3 \mu \mathrm{M})$

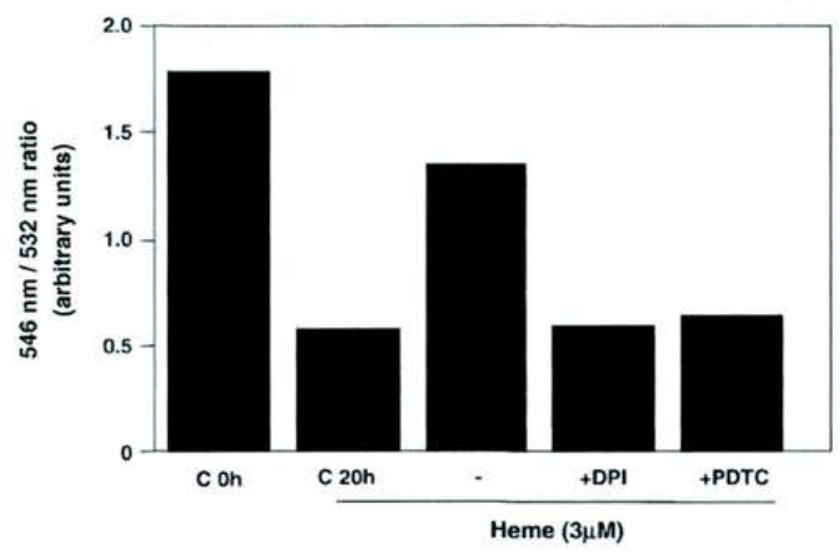

Fig. 2 - Heme inhibits $\Delta \boldsymbol{\psi}_{m}$ dissipation. Neutrophil were incubated for $20 \mathrm{~h}$ in the absence or in the presence of heme (3 $\left.\mu \mathrm{M}\right)$ with or without DPI $(10 \mu \mathrm{M})$ or PDTC $(100 \mathrm{nM})$, and $\Delta \psi_{m}$ was measured by confocal analysis of JC-1 staining. Freshly obtained neutrophils $(\mathrm{C} \mathrm{O} \mathrm{h})$ were used as negative control (non-apoptotic cells). Results represent the ratio between red ( $546 \mathrm{~nm}$ ) and green (532 nm) JC-1 emitted fluorescence. Using this dye, viable mitochondria usually display higher red fluorescence. These data are representative of three identical experiments with similar results. (For interpretation of the references to colour in this figure legend, the reader is referred to the web version of this article.)

lifespan. Heme-evoked Bcl- $\mathrm{X}_{\mathrm{L}}$ expression is shown to be a highly controlled event, which requires NADPHox-dependent ROS generation and redox-sensitive NF- $\kappa$ B activity (Fig. 6).

\section{Discussion}

Chronic inflammatory states are maintained by three major factors: sustained imbalance between the activity/production of pro-inflammatory (anti-apoptotic) versus anti-inflammatory (pro-apoptotic) stimuli, delay of neutrophil apoptosis and impairment of apoptotic neutrophil non-phlogistic clearance by professional phagocytes such as macrophages, or potential phagocytes such as fibroblasts and mesangial cells. This way, the machinery that controls neutrophil apoptosis seems to play a central role on this process, once it integrates the two other factors that corroborate to the establishment of chronic inflammation.

Hemolytic events, which can be triggered by a number of etiological agents, share the occurrence of high levels of free heme in circulation $[20,21]$. It is frequently accompanied by an acute inflammatory condition that can be both local and systemic and often develops to a chronic inflammatory response [22-27]. It has been described that heme itself is able to promote the expression of adhesion molecules on endothelial cells [28] providing an additional explanation to the vaso-occlusive events associated to systemic hemolytic episodes, and results from our group show that heme acts as a prototypical proinflammatory 


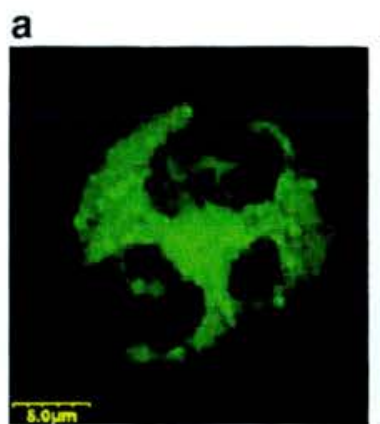

Control Oh

b
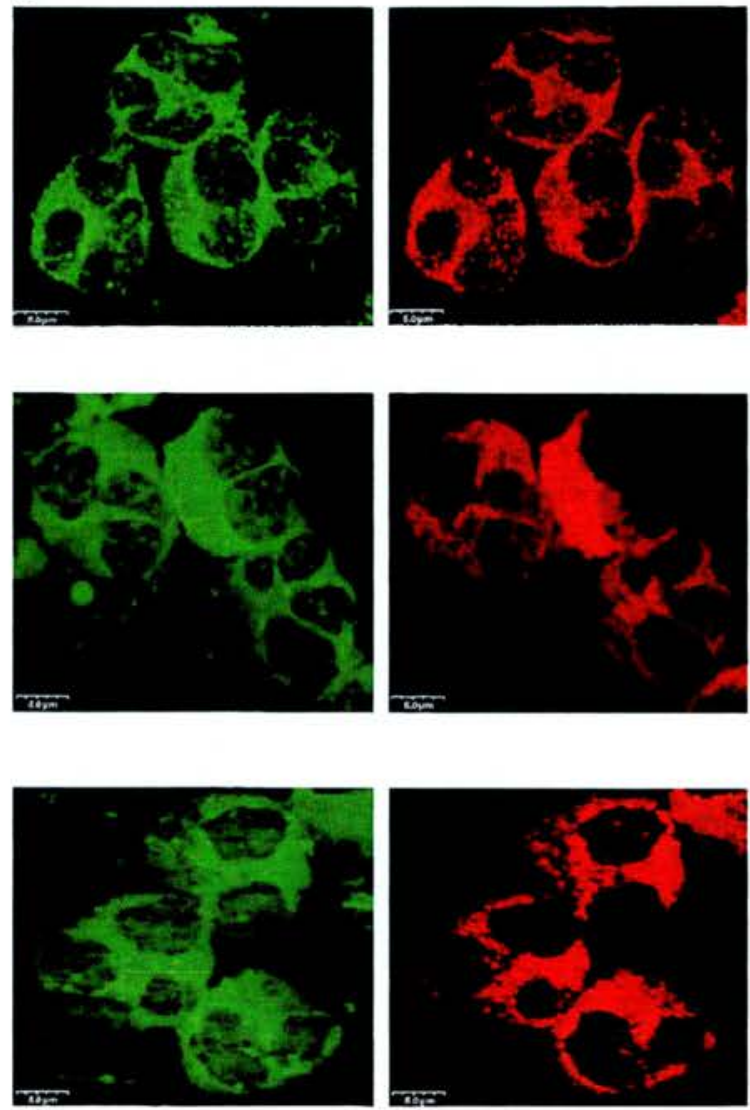

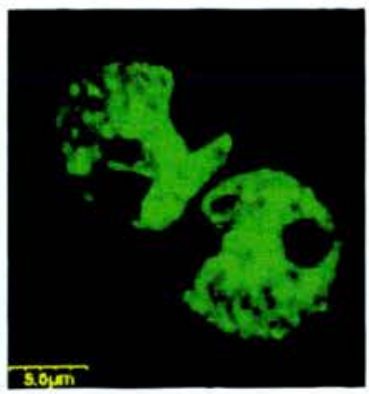

Control 20h
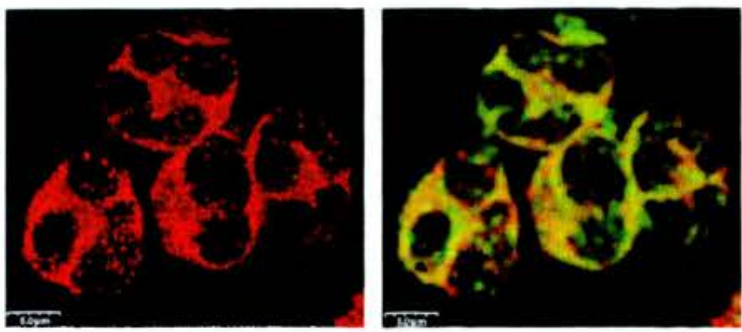

Control Oh

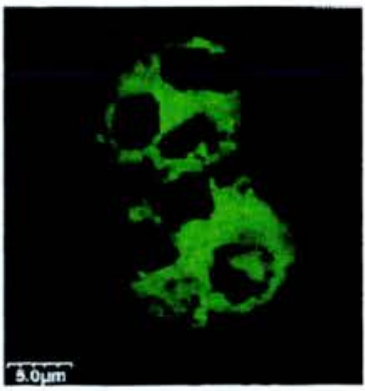

Heme 20h

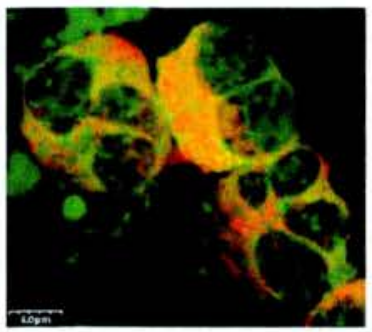

Control 20h

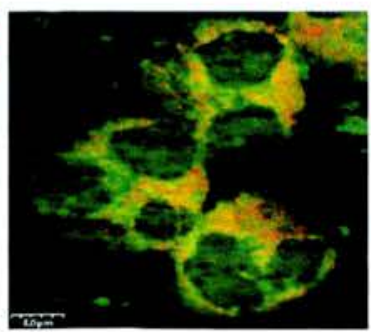

Heme 20h

Fig. 3 - Heme prevents Bax insertion into mitochondria: Confocal microscopy. (a) Mitochondrial morphology was assessed by confocal microscopy analysis of MitoTracker Green FM-stained cells. (b) Subcellular redistribution of Bax protein in neutrophils was assessed by confocal microscopy. Neutrophils were cultured for 0 or $20 \mathrm{~h}$ without additions or in the presence of heme $(3 \mu \mathrm{M})$. The cells were then stained with MitoTracker Green FM, fixed and permeabilized before addition of polyclonal antibody specific for Bax, and then analyzed by confocal microscopy. Co-localization regions are visualized as yellow dots. This figure is

molecule, potently activating neutrophils [5]. More recently we have reported that free heme delays human neutrophil apoptosis on a mechanism dependent on de novo protein synthesis via the activation of the MAPK, PI3K and NF- $\kappa B$ pathways [6]. The present study shows that heme alters neutrophil lifespan defined by the differentiation program of this cell, maintaining mitochondria stability through the alteration of expression/activity of pro- and anti-apoptotic Bcl-2 family proteins.

The sui generis neutrophil mitochondria, as elegantly demonstrated by Maianski et al. [12], lack life-supporting activities (e.g., ATP synthesis) being almost exclusively committed to the control of neutrophil apoptosis. We observed that free heme, in a concentration found during 


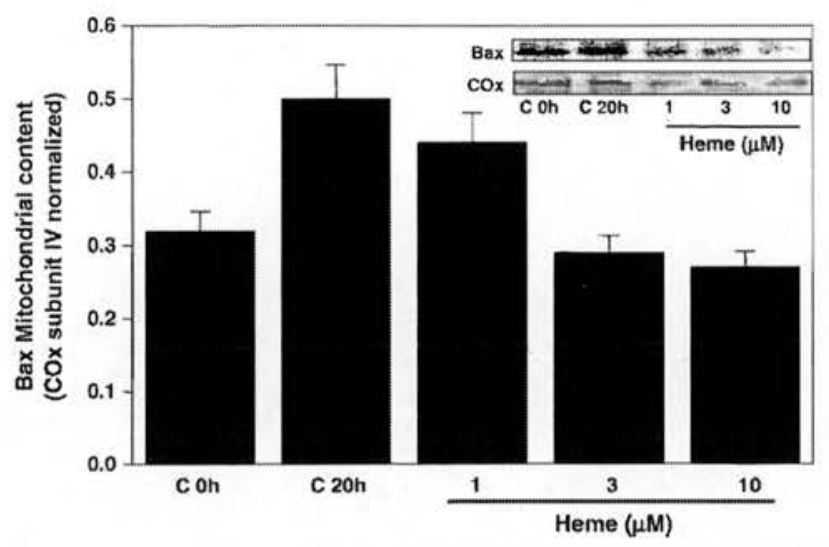

Fig. 4 - Heme prevents Bax insertion into mitochondria: Western blot. Detection of Bax insertion into the mitochondria was determined by Western blot. Digitonin-based cell fractionation was performed in fresh neutrophils and in neutrophils incubated for $20 \mathrm{~h}$ in the absence or in the presence of increasing concentrations of heme (1-10 $\mu \mathrm{M})$. The mitochondrial fractions were subjected to SDS-PAGE and Western blot was developed using specific antibody against Bax or COx (loading control). Data shown are the results (mean $\pm S D$ ) of 3 experiments. The asterisk (") indicates a significant difference $(p<0.05)$ when compared to fresh $(\mathrm{C} O \mathrm{~h})$ neutrophils.

hemolytic episodes $(3 \mu \mathrm{M})$, was able to maintain mitochondria stability, as observed by confocal microscopy analysis of JC-1 stained cells. Interestingly, pharmacological inhibition of $\mathrm{NF}-\kappa \mathrm{B}$ activation as well as NADPHox generation of ROS is once more required.

Heme protective effect is also associated to the inhibition of Bax insertion into the mitochondria (Figs. 3 and 4). Insertion of Bax into the mitochondria is a prominent feature of the apoptotic process. The formation of pores in the mitochondria outer membrane catalyzed by Bax oligomers promotes the leakage of pro-apoptotic proteins, which therefore lead to the activation of caspases and culminate in apoptotic cell death
[13]. We hypothesized that the control of Bax activity by heme relies on the integration of two distinct signals triggered by this molecule, the first one being the degradation of the proapoptotic protein Bad and the second one represented by the induction of the antiapoptotic protein $\mathrm{Bcl}-\mathrm{X}_{\mathrm{L}}$ expression [6]. Actually, we have observed that heme induces Bad degradation and Bcl- $\mathrm{X}_{\mathrm{L}}$ synthesis, and both effects require NADPHox activity. Moreover, NF- $\kappa B$ activity is also involved in Bcl- $\mathrm{X}_{\mathrm{L}}$ accumulation. These data suggest that the differential expression of these two proteins evoked by heme is crucial for the cytoprotective feature of this molecule. Bad, that is constitutively expressed in mature neutrophils, is rapidly and
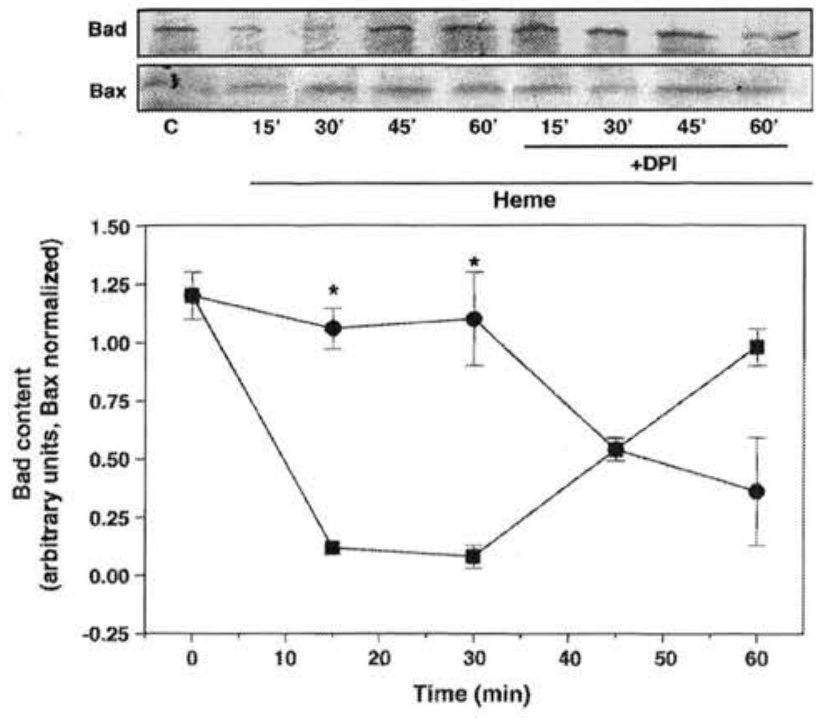

Fig. 5 - Heme-induced Bad degradation requires ROS. Neutrophils $\left(5 \times 10^{6} / \mathrm{mL}\right)$ were cultured with heme $(3 \mu \mathrm{M})$ in the absence (squares) or in the presence of DPI (10 $\mu \mathrm{M}$, circles). At the indicated time points, Bad protein expression was assessed by Western blot analysis. Data shown are the results (mean \pm SD) of 3 experiments. The asterisk (") indicates a significant difference $(p<0.05)$ between heme- and DPI-treated neutrophils. 


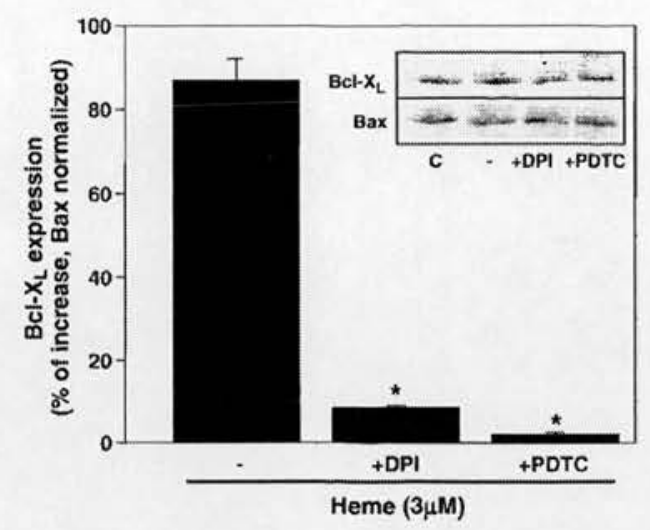

Fig. 6 - Heme induces Bcl-XL expression: role of NADPHox and NF- $\kappa$ B. Neutrophils $\left(5 \pm 10^{6} / \mathrm{mL}\right)$ were cultured with heme $(3 \mu \mathrm{M})$ in the absence or in the presence of DPI $(10 \mu \mathrm{M})$ or PDTC (100 nM). After $4 \mathrm{~h}, \mathrm{Bcl}-\mathrm{X}_{\mathrm{L}}$ protein expression was assessed by Western blot analysis. Data shown are the results (mean $\pm \mathrm{SD}$ ) of 3 experiments. The asterisk (") indicates a significant difference $(p<0.05)$ when compared to heme-treated neutrophils.

transiently reduced to very low levels after heme treatment. In contrast $\mathrm{Bcl}-\mathrm{X}_{\mathrm{L}}$, which content is very low in resting neutrophils, is highly induced after few hours. This condition facilitates the formation of $\mathrm{Bax} / \mathrm{Bcl}-\mathrm{X}_{\mathrm{L}}$ heterodimers, impairing Bax insertion into mitochondria (Arruda MA, unpublished data).

Noteworthy, the achievement that pharmacological inhibition of NADPHox as well as NF- $\kappa B$ leads to complete abolishment of heme protective effects [6], indicates an intense cross-talk between these signaling components, probably by changes in the redox status of the cell evoked by NADPHox activation. ROS, who have been primarily associated to life threatening events, have been shown to possess dual role on cellular physiology, being also associated to the activation of pro-survival signaling routs and inhibition of caspase 3, the main executioner of apoptotic program [27]. The apparent contradictory effects of ROS on neutrophil lifespan could be explained regarding the activation status of the cell. Some studies have shown the deleterious effect of exogenous ROS on resting neutrophils survival [29] and others showing PICD, when neutrophils generate large amounts of ROS intracellularly, it means, in the phagolysosome, in order to annihilate the engulfed pathogen [30]. After pathogen killing, neutrophil mission is theoretically accomplished, and it is reasonable to assume that rapid neutrophil apoptosis is beneficial in order to promote the resolution of inflammation. However, when neutrophils are activated by an inflammatory mediator, the NADPHox assembly and activation, which occurs few minutes after exposure to the stimulus, precedes other effector functions of activated neutrophils. We previously observed that heme induces NF- $\mathrm{KB}$ activation through ROS generated by NADPHox complex [6], which may act as second messengers, activating proinflammatory/anti-apoptotic signaling on neutrophils, and supporting this hypothesis, it has been shown that ROS are essential to LPS-induced TLR-4 signaling on neutrophils [31].
NF- $\nprec$ B comprises a family of transcription factors that act regulating genes involved in a variety of events such as apoptosis, proliferation and differentiation [32]. The regulation of NF- $\mathrm{kB}$ activation, which seems to be highly cell specific and redox sensitive [33,34], has a dual role on the amplification of the inflammatory response. It is involved on the transcription of both pro-inflammatory and anti-apoptotic genes not only on neutrophils, but also on a variety of cell types, revealing that the control of the activity of this transcription factor may have a major role on the control of acute and chronic inflammation $[7,35]$. However, studies using $\mathrm{NF}-\kappa \mathrm{B}$ inhibitors failed to control the inflammatory response [36]. Recently, it has been postulated that NF- $\kappa$ B is also involved in the transcription of a variety of genes that encode pivotal proteins in the resolution phase of inflammation. This way, the accurate knowledge of the NF- $\kappa B$ kinetic and modulation may be a powerful tool in the development of new strategies for the control of chronic inflammatory status.

In summary, we propose a mechanism for heme control of neutrophil apoptosis, on which it promotes the maintenance of $\Delta \psi_{\mathrm{m}}$, impairing Bax insertion into mitochondria through the reduction of $\mathrm{Bad} / \mathrm{Bcl}-\mathrm{X}_{\mathrm{L}}$ ratio. This phenomenon is likely to be controlled by NADPHox-derived ROS, via the activation of NF- $\kappa$ B pathway. Taken together, these data help the understanding of the molecular events involved on heme anti-apoptotic effect as well as corroborate to the statement of a new role for ROS generated by NADPHox on neutrophil physiology.

\section{Acknowledgments}

We would like to thank Carlos Bizarro Rodrigues for the confocal microscopy analysis and Dr. Iolanda M. Fierro for helpful comments. This work was supported by CNPq, FAPERJ and SR-2/UERJ (Brazil).

\section{REFERENCES}

[1] S.J. Chanock, J. el Benna, R.M. Smith, B.M. Babior, The respiratory burst oxidase, J. Biol. Chem. 269 (1994) 24519-24522.

[2] P. Chiarugi, PTPs versus PTKs: the redox side of the coin, Free Radical Res. 39 (2005) 353-364.

[3] B. Dewald, M. Baggiolini, Activation of NADPH oxidase in human neutrophils. Synergism between fMLP and the neutrophil products PAF and LTB4, Biochem. Biophys. Res. Commun. 128 (1985) 297-304.

[4] S.A. Jones, M. Wolf, S. Qin, C.R. Mackay, M. Baggiolini, Different functions for the interleukin 8 receptors (IL-8R) of human neutrophil leukocytes: NADPH oxidase and phospholipase D are activated through IL-8R1 but not IL-8R2, Proc. Natl. Acad. Sci. U. S. A. 93 (1996) 6682-6686.

[5] A.V. Graça-Souza, M.A. Arruda, M.S. de Freitas, C. Barja-Fidalgo, P.L. Oliveira, Neutrophil activation by heme: implications for inflammatory processes, Blood 99 (2002) 4160-4165.

[6] M.A. Arruda, A.G. Rossi, M.S. de Freitas, C. Barja-Fidalgo, A.V. Graça-Souza, Heme inhibits human neutrophil apoptosis: involvement of phosphoinositide 3-kinase, MAPK, and NF-kappaB, J. Immunol. 173 (2004) 2023-2030. 
[7] C. Ward, E.R. Chilvers, M.F. Lawson, J.G. Pryde, S. Fujihara, S.N. Farrow, C. Haslett, A.G. Rossi, NF-kappaB activation is a critical regulator of human granulocyte apoptosis in vitro, J. Biol. Chem. 274 (1999) 4309-4318.

[8] T.N. Mayadas, X. Cullere, Neutrophil beta2 integrins: moderators of life or death decisions, Trends Immunol. 26 (2005) 388-395.

[9] D.D. Newmeyer, S. Fregusson-Miller, Mitochondria: releasing power for life and unleashing the machineries of death, Cell 112 (2003) 481-490.

[10] N. Borregaard, T. Herlin, Energy metabolism of human neutrophils during phagocytosis, J. Clin. Invest. 70 (1982) 550-557.

[11] N.A. Maianski, D.A. Roos, T.W. Kuijpers, Tumor necrosis factor alpha induces a caspase-independent death pathway in human neutrophils, Blood 101 (2003) 1987-1995.

[12] N.A. Maianski, J. Geissler, S.M. Srinivasula, L.S. Alnemri, D. Roos, T.W. Kuijpers, Functional characterization of neutrophil mitochondria in neutrophils: a role restricted to apoptosis, Cell Death Differ. 11 (2004) 143-153.

[13] U.R. Rapp, U. Rennefahrt, J. Troppmair, Bcl-2 proteins: master switches at the intersection of death signaling and the survival control by Raf kinases, Biochim. Biphys. Acta 1644 (2004) 149-158.

[14] J.B. Klein, M.J. Rane, J.A. Scherzer, P.Y. Coxon, R. Kettritz, J.M. Mathiesen, A. Buridi, K.R. McLeish, Granulocyte-macrophage colony-stimulating factor delays neutrophil constitutive apoptosis through phosphoinositide 3-kinase and extracellular signal-regulated kinase pathways, J. Immunol. 164 (2000) 4286-4291.

[15] J.B. Klein, A. Buridi, P.Y. Coxon, M.J. Rane, T. Manning, R. Kettritz, K.R. McLeish, Role of extracellular signal-regulated kinase and phosphatidylinositol-3 kinase in chemoattractant and LPS delay of constitutive neutrophil apoptosis, Cell Signalling 13 (2001) 335-343.

[16] C. Akgul, D.A. Moulding, S.W. Edwards, Molecular control of neutrophil apoptosis, FEBS Lett. 487 (2001) 318-322.

[17] A. Boyum, Isolation of lymphocytes, granulocytes and macrophages, Scand. J. Immunol. 5 (1976) 9-15.

[18] M. Ankarcrona, J.M. Dypbukt, E. Bonfoco, B. Zhivotovsky, S. Orrenius, S.A. Lipton, P. Nicotera, Glutamate-induced neuronal death: a succession of necrosis or apoptosis depending on mitochondrial function, Neuron 15 (1995) 961-973.

[19] M. Bradford, Rapid and sensitive method for quantification of microgram quantities of protein utilising the principle of protein-dye binding, Anal. Biochem. 72 (1976) 248-254.

[20] U. Muller-Eberhard, M. Fraig, Bioactivity of heme and its containment, Am. J. Hematol. 42 (1993) 59-62.

[21] C.D. Reiter, X. Wang, J.E. Tanus-Santos, N. Hogg, R.O. CannonIII, A.N. Schechter, M.T. Gladwin, Cell-free hemoglobin limits nitric oxide bioavailability in sickle-cell disease, Nat. Med. 8 (2002) 1383-1389.

[22] R.D. Davenport, R.M. Strieter, T.J. Standiford, S.L. Kunkel, Interleukin-8 production in red blood cell incompatibility, Blood 76 (1990) 2439-2442.

[23] R.D. Davenport, S.L. Kunkel, Cytokine roles in hemolytic and nonhemolytic transfusion reactions, Transfus. Med. Rev. 8 (1994) 157-168.

[24] E. Mollapour, J.B. Porter, R. Kaczmarski, D.C. Linch, P.J. Roberts, Raised neutrophil phospholipase A2 activity and defective priming of NADPH oxidase and phospholipase A2 in sickle cell disease, Blood 91 (1998) 3423-3429.

[25] M.S. Gonçalves, I.L. Queiroz, S.A. Cardoso, A. Zanetti, A.C. Strapazoni, E. Adorno, A. Albuquerque, A. Sant'Ana, M.G. dos Reis, A. Barral, M. Barral Netto, Interleukin 8 as a vaso-occlusive marker in Brazilian patients with sickle cell disease, Braz. J. Med. Biol. Res. 34 (2001) 1309-1313.

[26] T. Wun, The role of inflammation and leukocytes in the pathogenesis of sickle cell disease; haemoglobinopathy, Hematol. 5 (2001) 403-412.

[27] D.K. Kaul, R.P. Hebbel, Hypoxia/reoxygenation causes inflammatory response in transgenic sickle mice but not in normal mice, J. Clin. Invest. 106 (2000) 411-420.

[28] F.A. Wagener, N.G. Abraham, Y. van Kooyk, T. de Witte, C.G. Figdor, Heme-induced cell adhesion in the pathogenesis of sickle-cell disease and inflammation, Trends Pharmacol. Sci. 22 (2001) 52-54.

[29] R.W. Watson, Redox regulation of neutrophil apoptosis, Antioxid. Redox Signal. 4 (2002) 97-104.

[30] B. Zhang, J. Hirahashi, X. Cullere, T.N. Mayadas, Elucidation of molecular events leading to neutrophil apoptosis following phagocytosis: cross-talk between caspase 8 , reactive oxygen species, and MAPK/ERK activation, J. Biol. Chem. 278 (2003) 28443-28454.

[31] K. Asehnoune, D. Strassheim, S. Mitra, J.Y. Kim, E. Abraham, Involvement of reactive oxygen species in Toll-like receptor 4-dependent activation of NF-kappa B, J. Immunol. 172 (2004) 2522-2529.

[32] M. Karin, A. Lin, NF-kappaB at the crossroads of life and death, Nat. Immunol. 3 (2002) 221-227.

[33] B.B. Aggarwal, Apoptosis and nuclear factor-kappa B: a tale of association and dissociation, Biochem. Pharmacol. 60 (2000) 1033-1039.

[34] J.J. Haddad, Redox and oxygen-sensitive transcription factors in the regulation of oxidant-mediated lung injury: role for nuclear factor-kappaB, Crit. Care 6 (2002) 481-490.

[35] D.W. Gilroy, T. Lawrence, M. Perretti, A.G. Rossi, Inflammatory resolution: new opportunities for drug discovery, Nat. Rev., Drug Discov. 3 (2004) 401-416.

[36] T. Lawrence, D.W. Gilroy, P.R. Colville-Nash, D.A. Willoughby, Possible new role for NF-kappaB in the resolution of inflammation, Nat. Med. 7 (2001) 1291-1297. 


\title{
GEA 3162, a peroxynitrite donor, induces Bcl-2-sensitive, p53-independent apoptosis in murine bone marrow cells
}

\author{
Emma L. Taylor ${ }^{a, *}$, John T. Li ${ }^{b}$, Joan C. Tupper ${ }^{c}$, Adriano G. Rossi ${ }^{d}$, Robert K. Winn ${ }^{e}$, \\ John M. Harlan ${ }^{c}$ \\ ${ }^{a}$ Institute of Biomedical and Clinical Science, Peninsula Medical School, Universities of Exeter and Plymouth, St Luke's Campus, \\ Heavitree Rd, Exeter, Devon EX1 2LU, UK \\ ${ }^{b}$ Department of Anesthesia/Critical Care Medicine, Children's Hospital Los Angeles, University of Southern California Keck School of Medicine, \\ Los Angeles, CA 90027, USA \\ ${ }^{\mathrm{C}}$ Department of Medicine, Research and Training Building, University of Washington, Harborview Medical Center, 300 Ninth Ave, Seattle, \\ WA 98104, USA \\ ${ }^{\mathrm{d} C e n t r e ~ f o r ~ I n f l a m m a t i o n ~ R e s e a r c h, ~ Q u e e n s ~ M e d i c a l ~ R e s e a r c h ~ I n s t i t u t e, ~ U n i v e r s i t y ~ o f ~ E d i n b u r g h, ~} 47$ Little France Crescent, \\ Edinburgh, EH16 4TJ, UK \\ ${ }^{e}$ Department of Surgery, Research and Training Building, University of Washington, Harborview Medical Center, 300 Ninth Ave, \\ Seattle, WA 98104, USA
}

\section{A R T I C L E I N F O}

Article history:

Received 22 April 2007

Accepted 7 June 2007

Keywords:

Peroxynitrite

Apoptosis

Caspase

p53

Bcl-2

Myeloid

\begin{abstract}
A B S T R A C T
Apoptosis may be regulated by oxidants such as peroxynitrite (ONOO- $)$. The tumour suppressor, p53, has been reported to play a crucial role in apoptosis induced by oxidants, therefore we assessed the ability of a ONOO- donor, GEA 3162, to activate caspases and induce mitochondrial permeability in a p53-deficient murine bone marrow cell line, Jaws II. Furthermore, these cells were stably transfected with $\mathrm{Bcl}-2$, in order to investigate the impact of this survival protein on $\mathrm{ONOO}^{-}$-induced apoptosis. GEA 3162 activated caspases and induced loss of mitochondrial membrane potential in Jaws II cells. In particular, caspases 3 and 2 were activated, alongside minor activation of caspases 8 and 9, and apoptosis was partially dependent upon p38 MAP kinase activation, with little or no role for JNK. Overexpression of Bcl-2 abolished activation of all caspases and reduced the change in mitochondrial membrane potential. Thus, we have demonstrated that the $\mathrm{ONOO}^{-}$donor, GEA 3162, induces apoptosis in Jaws II murine myeloid cells despite lacking functional p53, via a pathway that principally involves caspases 2 and 3 and mitochondrial changes. This is blocked by overexpression of Bcl-2 via a mechanism that does not appear to merely reflect stabilisation of the mitochondrial membrane.
\end{abstract}

(C) 2007 Elsevier Inc. All rights reserved

\footnotetext{
- Corresponding author at: Institute of Biomedical and Clinical Science, Peninsula Medical School, Universities of Exeter and Plymouth, St Luke's Campus, Heavitree Rd, Exeter, Devon EX1 2LU, UK. Tel.: +44 1392 262976; fax; +44 1392262926.

E-mail address: emma.taylor@pms.ac.uk (E.L. Taylor).

Abbreviations: GEA 3162, 1,2,3,4-oxatriazolium,5-amino-3-(3,4-dichlorophenyl)-chloride; STSP, staurosporine; GFP, green fluorescent protein; Jaws II-GFP, p53-deficient murine bone marrow cells stably transfected with a vector encoding GFP; Jaws II-Bcl-2, p53-deficient murine bone marrow cells stably transfected with a vector encoding GFP and the survival protein Bcl-2; JNK, c-Jun N-terminal kinase; MAP kinase, mitogen activated protein kinase; $\mathrm{NO}$, nitric oxide; $\mathrm{ONOO}^{-}$, peroxynitrite 0006-2952/\$ - see front matter (c) 2007 Elsevier Inc. All rights reserved.
}

doi:10.1016/j.bcp.2007.06.028 


\section{Introduction}

Apoptosis is a physiological form of programmed cell death that plays important roles in embryogenesis, inflammation and the safe and efficient clearance of damaged or unwanted cells. This process is characterised by cellular and nuclear condensation, maintenance of cell membrane integrity, the exposure of recognition molecules for phagocytic removal and internucleosomal DNA fragmentation [1]. During apoptotic cell death, a range of cysteine proteases called caspases are necessarily activated, which cleave proteins after aspartic acid residues following activation from their zymogen forms [2].

There are two principal pathways for the execution of apoptosis; a death receptor pathway and a stress pathway, as reviewed by Wyllie [1], and Zimmermann et al. [3]. The extrinsic death receptor-mediated pathway involves engagement of ligands such as tumour necrosis factor- $\alpha$ (TNF- $\alpha$ ) or Fas ligand (FasL) with the appropriate receptor and proceeds through activation of caspase 8 (initiator caspase) and caspase 3 , which is critical in cleaving cellular components to drive the irreversible dismantling of the cell. Additionally, the mitochondrial pathway described below can be activated through death receptor ligation via translocation of the truncated (active) form of Bid (tBid) to the mitochondria following cleavage by caspase 8 , as an amplification mechanism.

The intrinsic pathway occurs in response to cellular damage or external stresses, and mitochondria play a central role in this pathway. Activation of the stress pathway leads to mitochondrial changes, in which the membrane potential is lost, causing mitochondrial permeability. This allows the loss of pro-apoptotic factors from the mitochondria, formation of an 'apoptosome', and activation of caspase 9 (initiator caspase) and ultimately caspase 3. These mitochondrial changes are tightly regulated by the balance of pro- and anti-apoptotic members of the Bcl-2 proto-oncogene family [4], which interact with each other and the mitochondrial membrane to either promote or prevent mitochondrial permeabilisation. Pro-apoptotic members of the Bcl-2 family include Bax, Bad, Bid and Bak and tend to be located within the cytosol, whereas anti-apoptotic members such as Bcl-2, Bcl- $\mathrm{xL}$ and A1 are predominantly found on the mitochondrial membrane itself.

Mitochondrial changes in response to cellular damage or stress are often preceded by activation of the transcription factor, p53. This tumour suppressor induces apoptotic cell death if DNA damage is severe or irreparable. It controls transcription levels of several proteins involved in apoptosis in order to push the balance towards cell death, notably by increasing the pro-apoptotic proteins Fas (CD95), Bax, Bid, Noxa and Puma $[4,5]$ and down-regulating expression of the anti-apoptotic protein, Bcl-2 [6].

Further molecules with a role in the regulation of apoptosis are the mitogen activated protein (MAP) kinases. The JNK and p38 MAP kinase families are involved in the cellular response to stress and are important in apoptosis [7]. However, the relationship between pro- and anti-apoptotic effects of these kinases is complex, and may be cell type or stimulus-specific [8].

Peroxynitrite $\left(\mathrm{ONOO}^{-}\right)$is a powerful oxidant species formed through the combination of NO and superoxide anion
$\left(\mathrm{O}_{2}{ }^{-}\right)$. Inflammatory cells are able to produce both species when activated [9], and $\mathrm{ONOO}^{-}$is thought to have bactericidal effects in acute inflammation, and has additionally been shown to promote apoptosis in several cell types $[10,11]$.

Previous studies have demonstrated that the $\mathrm{ONOO}^{-}$ donor, GEA 3162, is able to promote apoptotic cell death in neutrophils [12-14] in a caspase-dependent manner. However, the effects of this compound on apoptosis have not been investigated in other cells of the myeloid lineage. We set out to investigate the effects of the $\mathrm{ONOO}^{-}$donor, GEA 3162, on apoptosis in the Jaws II murine immature bone marrow cell line, which is derived from the p53-/- mouse. We hypothesised that GEA 3162 would initiate apoptosis in these cells despite their lack of $\mathrm{p} 53$, and that overexpression of the survival protein, Bcl-2, would abolish this response through stabilisation of the mitochondrial membrane.

\section{Materials and methods}

\subsection{Cell culture}

Immature mouse bone marrow cells (Jaws II; ATCC, Manassas, VA) [15] were cultured in alpha minimum essential medium with ribonucleosides, deoxyribonucleosides enriched with $20 \%$ heat-inactivated fetal bovine serum (Hyclone Laboratories, Logan, UT), L-glutamine $4 \mathrm{mM}$, and sodium pyruvate $1 \mathrm{mM}$ (all purchased from BioWhittaker, Inc., Walkersville, $\mathrm{MD}$ ) in the presence of $5 \mathrm{ng} / \mathrm{ml}$ GM-CSF (R\&D Systems, Inc., Minneapolis, MN). Cells were seeded into T75 culture flasks. Flasks were incubated at $37^{\circ} \mathrm{C}$ in a humidified atmosphere containing $5 \% \mathrm{CO}_{2}$ until approximately $70-80 \%$ confluence was achieved. Cells were passaged 1 in 4 every 3-4 days.

In order to harvest the cells, suspension cells were removed into $50 \mathrm{ml}$ centrifuge tubes before addition of trypsin-EDTA to the flasks. Cells were incubated until the adherent cells detached and were removed into the appropriate centrifuge tube, and cell numbers counted using a haemacytometer. The tubes were centrifuged (1000 rpm, $10 \mathrm{~min}$ ) to form a cell pellet, then supernatant was aspirated and the cells resuspended in Jaws II medium at a density of $5 \times 10^{5}$ cells $/ \mathrm{ml}$ before being plated out as required.

\subsection{Construction and stable expression of Bcl-2 cDNA}

cDNA encoding the intronless open reading frame of the 717 base pair human Bcl-2 $\alpha$ (pORF-hBcl-2) (InvivoGen, San Diego, CA) was cloned into shuttle plasmid SL1180 (Amersham Pharmacia Biotech, Piscataway, NJ) utilizing the Nco I and Nhe I (New England BioLabs, Inc., Beverly, MA) restriction enzyme sites. The pORF-hBcl-2 was subsequently cloned into the EcoR I and Xho I (New England BioLabs, Inc., Beverly, MA) sites on the multiple cloning region of the bicistronic retroviral expression plasmid, pBMN-IRES-enhanced green fluorescent protein (EGFP) (kindly provided by Dr. Gary Nolan, Stanford University, Palo Alto, CA) [16]. High titer second-generation helper free retrovirus was produced by calcium phosphate mediated transfection of the Phoenix ecotropic packaging cell line (ATCC, Manassas, VA) with either $24 \mu \mathrm{g}$ of the hBcl-2 expression plasmid or pBMN-IRES-EGFP control plasmid. 
Recombinant retroviral supernatant was collected $48 \mathrm{~h}$ after transfection and filtered through a Millex-HV $0.45 \mu \mathrm{m}$ filter (Millipore Corp., Bedford, MA). For transduction, cell culture media from $\sim 70 \%$ confluent JAWS II cells in 6-well plates (Corning Inc., Corning, NY) were replaced with $2.5 \mathrm{ml}$ of retrovirus supernatant and centrifuged for $2 \mathrm{~h}(1430 \times \mathrm{g}$ at $\left.32{ }^{\circ} \mathrm{C}\right)$ and then incubated for $10 \mathrm{~h}\left(5 \% \mathrm{CO}_{2}, 37^{\circ} \mathrm{C}\right)$. Upon completion of the incubation period, retroviral supernatant was replaced by appropriate normal growth medium for each cell type. Cells were sorted for stable retrovirus transfection based on EGFP expression using a FACVantage SE cell sorter (Beckton Dickinson Corp., Franklin Lakes, NJ). High-expressing cells were collected and used in subsequent experiments, as previously published [17].

\subsection{Bcl-2 western blot}

JAWS II-Bcl-2 or JAWS II-GFP cells were lysed in $60 \mathrm{~mm}$ tissue culture dishes (Becton Dickinson, Franklin Lakes, NJ) using icecold modified radioimmunoprecipitation (RIPA) buffer (Tris$\mathrm{HCl} 50 \mathrm{mM}, \mathrm{NP}-401 \%$, Na-deoxycholate $0.25 \%, \mathrm{NaCl} 150 \mathrm{nM}$, $\mathrm{Na}_{3} \mathrm{VO}_{4} 1 \mathrm{mM}$, NaF $50 \mathrm{mM}$ ) (all purchased from Sigma, St. Louis, MO) with one Complete Mini ${ }^{\mathrm{TM}}$ tablet (Roche, Indianapolis, IN) per $10 \mathrm{ml}$ of modified RIPA buffer. Cell debris was removed by centrifugation at $14,000 \times g$ for $15 \mathrm{~min}$ at $4^{\circ} \mathrm{C}$. Supernatant was collected and stored at $-20^{\circ} \mathrm{C}$. BCA Protein Assay kit (Pierce, Rockford, IL) was used to measure protein concentrations of cell lysates. Cell lysates, $20 \mu \mathrm{g}$ total protein per lane, were resolved on Novex ${ }^{\mathbb{B}} 4-20 \%$ Tris-glycine gels (Invitrogen, Carlsbad, CA) and transferred onto methanol activated Immobilon ${ }^{\mathrm{TM}}$ PVDF membrane (Millipore Co., Bedford, MA). Membrane was blocked with $5 \%$ milk (Western Family Foods, Portland, OR) in TBS (Sigma, St. Louis, MO) at $4{ }^{\circ} \mathrm{C}$ overnight. After blocking, membrane was probed with mouse anti-human Bcl-2 monoclonal antibody (\#610539 BD Transduction Labs, San Diego, CA) at a 1:1000 dilution in TBS with $1 \%$ tween-20 (TBST) (Sigma, St. Louis, MO) for $1 \mathrm{~h}$ at room temperature. Membrane was washed three times in TBST. Goat anti-mouse IgG HRP conjugated secondary antibody (\#610094 BD Transduction Labs, San Diego, CA) was applied at 1:10,000 dilution in TBST and incubated for $1 \mathrm{~h}$ at room temperature. Membrane was washed four times in TBST followed by two washes in TBS. Enhanced chemiluminescence (ECL) plus (Amersham, Buckinhamshire, England) was applied for $5 \mathrm{~min}$ and chemiluminescence was detected on radiographic film (Eastman Kodak Co., Rochester, NY).

\subsection{MTT assay}

A colorimetric assay using (3-(4,5-dimethylthiazol-2-yl)-2,5diphenyltetrazolium bromide (MTT) provides a measure of the cytotoxicity of agents. The mitochondria of viable cells metabolise MTT to turn the solution from yellow to purple, which can be measured using a spectrophotometer at a wavelength of $500-600 \mathrm{~nm}$.

In order to measure cell viability following exposure to GEA $3162(30-100 \mu \mathrm{M}), 20,000$ cells per well $(40 \mu \mathrm{l})$ were plated into a 96-well plate, which was then incubated at $37^{\circ} \mathrm{C}$ overnight, before addition of $50 \mu \mathrm{l}$ medium. GEA $3162(10 \mu \mathrm{l}$ of $10 \times$ stock) was added to final concentrations as shown in the figure legends (concentrations that have previously been shown to promote apoptosis in human neutrophils [18]) and the plate incubated for $4 \mathrm{~h}$. The MTT assay was then carried out according to the manufacturers' instructions. Briefly, $10 \mu \mathrm{l}$ of MTT solution was added to each well and the plate incubated at $37^{\circ} \mathrm{C}$ for $4 \mathrm{~h}$ before addition of $100 \mu \mathrm{l}$ of solubilisation solution. The plate was then read with a test wavelength of $570 \mathrm{~nm}$ and a reference wavelength of $630 \mathrm{~nm}$.

\subsection{Homogeneous caspase activation}

Caspase proteases are activated during apoptotic cell death. Fluorogenic substrates can be cleaved by caspases, giving a quantitative indication of caspase enzyme activity which can be measured by fluorescent plate reader.

In order to measure levels of caspase activation in Jaws II cells treated with GEA 3162 (Alexis Biochemicals, San Diego, CA) or the pan-kinase inhibitor, staurosporine (STSP; Sigma, St. Louis, MO), 20,000 cells per well $(40 \mu \mathrm{l})$ were plated into an opaque 96-well plate, which was then incubated at $37^{\circ} \mathrm{C}$ overnight. GEA 3162 or STSP $(10 \mu$ l of $10 \times$ stock) were added to final concentrations as shown in the figure legends (concentrations that have previously been shown to promote apoptosis in human neutrophils [18]) in the absence or presence of the pan-caspase inhibitor, $\mathrm{zVAD}(100 \mu \mathrm{M})$. The volume in each well was made up to $100 \mu \mathrm{l}$ with $50 \mu \mathrm{l}$ culture medium. Following $4 \mathrm{~h}$ incubation at $37^{\circ} \mathrm{C}$ (time point determined by preliminary time course studies; data not shown), caspase activation was measured using a homogeneous caspase detection kit (Roche, Indianapolis, IN) according to the manufacturer's instructions. Briefly, $100 \mu \mathrm{l}$ of caspase substrate was added to each well, plus two wells containing positive control lysates provided in the kit, and two wells containing medium alone as 'blank' wells. The plate was then incubated at $37^{\circ} \mathrm{C}$ for at least $1 \mathrm{~h}$ before fluorescence was read using a fluorescent plate reader with excitation at $485 \mathrm{~nm}$ and emission measured at $530 \mathrm{~nm}$. Background fluorescence from 'blank' wells was then subtracted from all values obtained.

The same protocol was followed for other experiments using the pharmacological inhibitors, SP600125 (JNK inhibitor) and SB203580 (p38 inhibitor). For these experiments, the inhibitor was diluted in Jaws II culture medium at $2 \times$ desired concentration before addition of $50 \mu$ lo the wells at a final concentration of $10-50 \mu \mathrm{M}$.

\subsection{Specific caspase activation}

Individual caspases 2, 3, 8 and 9 were also assessed for activity using a fluorescent plate assay, in order to investigate possible pathways through which GEA 3162-induced apoptosis may proceed.

Jaws II-GFP and Jaws II-Bcl-2 cells were harvested as described above. 2 million cells were seeded into T25 flasks in $4.5 \mathrm{ml}$ culture medium and incubated overnight at $37^{\circ} \mathrm{C}$. The following day, $500 \mu \mathrm{l}$ of medium (controls) or GEA 3162 (final concentration $30-100 \mu \mathrm{M}$ ) were added, and flasks were incubated for $4 \mathrm{~h}$ at $37^{\circ} \mathrm{C}$. Cells were then harvested and centrifuged (1000 rpm, $5 \mathrm{~min}$ ). Activity of caspases 2, 3, 8 and 9 was then assessed using the ApoAlert Caspase Profiling kit 
(Clontech, Mountain View, CA) according to the manufacturers' instructions. Briefly, cell pellets were resuspended in $400 \mu \mathrm{l}$ of ice-cold cell lysis buffer and incubated on ice for $10 \mathrm{~min}$. Following lysis, tubes were vortexed and $50 \mu \mathrm{l}$ of cell lysate added in duplicate to wells containing immobilised substrate for individual caspases plus $50 \mu \mathrm{l}$ of $2 \times$ reaction buffer. The plate was then covered and incubated at $37^{\circ} \mathrm{C}$ for $2 \mathrm{~h}$. Cleavage of caspase substrates was then measured by fluorescent plate reader, with excitation at $360 \mathrm{~nm}$ and emission measured at $460 \mathrm{~nm}$.

\subsection{Mitochondrial membrane permeability assay}

A potential difference exists across the mitochondrial membrane in viable cells. However, during apoptosis that proceeds through the stress or mitochondrial pathway, the potential difference is lost and the mitochondria become permeable. This loss of potential difference can be measured by the use of a fluorescent dye that accumulates in the mitochondria of viable cells and fluoresces red, but is unable to enter the mitochondria of apoptotic cells and fluoresces green in the cytosol. The ratio of red:green fluorescence gives an indication of the proportion of viable:apoptotic cells, and can be measured using a fluorescent plate reader.

The change in mitochondrial membrane potential during apoptosis in Jaws II cells treated with GEA 3162 or STSP was measured using the Mit-E- $\psi$ apoptosis detection kit (Biomol International, Plymouth Meeting, PA) according to the manufacturer's instructions. Cells were harvested as described above and 10,00,000 cells added per well of a 6 -well plate in a total volume of $2 \mathrm{ml}$. Following overnight incubation at $37^{\circ} \mathrm{C}$ to allow cells to adhere, culture medium (control), STSP or GEA 3162 were added to the wells to final concentrations shown in the figure legends, then cells were incubated for a further $4 \mathrm{~h}$. The cells were then harvested and tubes were centrifuged (1000 rpm, $5 \mathrm{~min}$ ) to pellet the cells. Supernatant was then aspirated and cells were resuspended in $1 \mathrm{ml}$ Mit-E- $\psi$ solution and incubated at $37{ }^{\circ} \mathrm{C}$ for $15 \mathrm{~min}$. Assay buffer $(2 \mathrm{ml})$ was then added and the tubes centrifuged (1000 rpm, $5 \mathrm{~min}$ ) before resuspension of the cell pellet in $1 \mathrm{ml}$ assay buffer and further centrifugation ( $1000 \mathrm{rpm}, 5 \mathrm{~min}$ ) to wash the cells. Cells were resuspended once more in $1 \mathrm{ml}$ assay buffer before duplicate aliquots of $100 \mu \mathrm{l}$ were transferred from each tube to an opaque 96 -well plate, which was then analysed by fluorescent plate reader with excitation at $485 \mathrm{~nm}$ and emission measured at $590 \mathrm{~nm}$ (red fluorescence) and $530 \mathrm{~nm}$ (green fluorescence). The ratio of red to green fluorescence was then calculated.

\subsection{Statistical analysis}

Each experiment was carried out using duplicate samples for a minimum of three replicates, and the mean and standard error of the combined data was calculated. Significance between results was analysed using repeated measures ANOVA. Student-Newman-Keuls post-test was used to determine significance differences between treatments. A $p$ value $<0.05$ was considered statistically significant.

\section{Results}

3.1. Characterisation of the Bcl-2 overexpressing Jaws II cell line

Jaws II cells transfected with a vector encoding GFP/Bcl-2 were sorted for high-expressing cells on the basis of their green
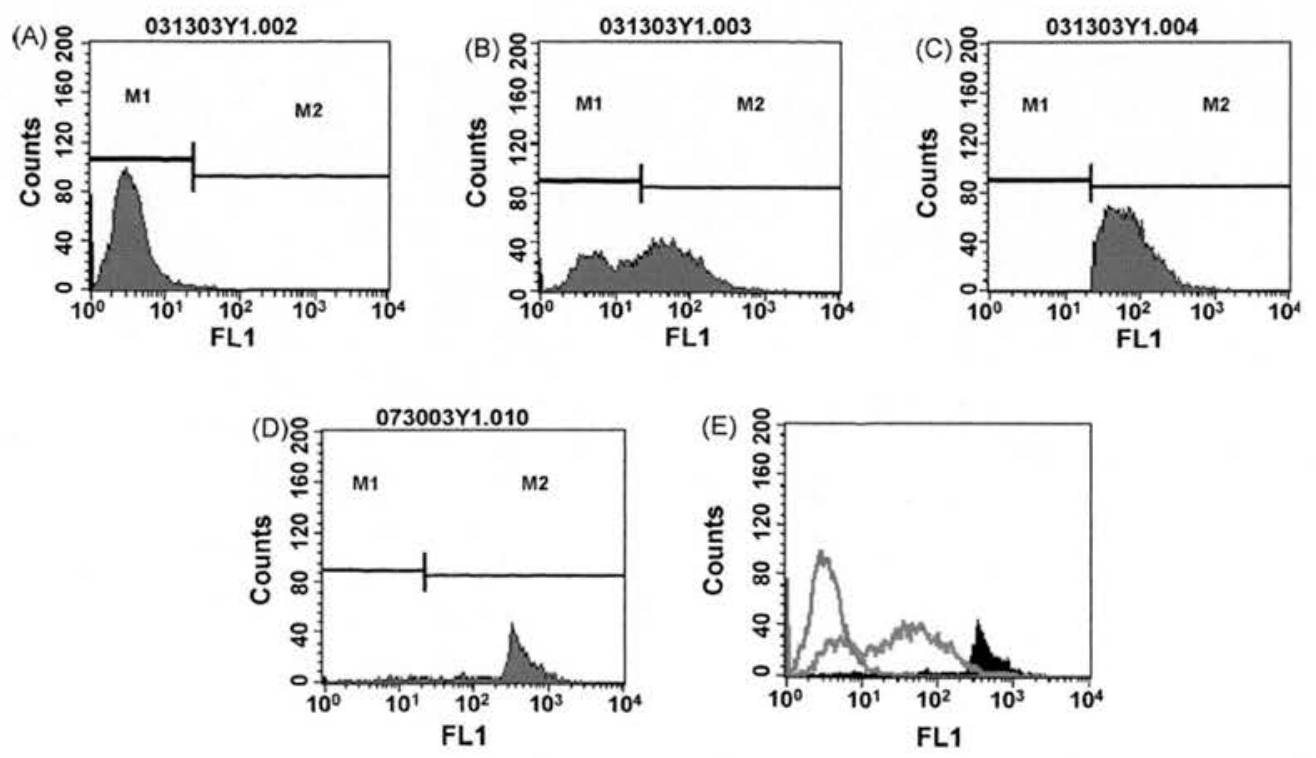

Fig. 1 - FACS analysis for transfection of JAWSII cells with retrovirus containing bcl-2-IRES-GFP. Cells successfully transfected to overexpress Bcl-2 were selected based on linked GFP expression in FL1. In panel (A) wild type cells demonstrated minimal fluorescence. (B) Cells transfected with Bcl-2-IRES-GFP retrovirus showed bimodal fluorescence and GFP positive cells were sorted based on gate M2. Post-sort analysis (C) showed a wide range in fluorescent intensity and these cells were sorted again for the highest GFP expressors (D). Panel (E) shows the population of cells after several passages: wild type (black line), transfected cells (grey line), high-expressing cells (black area). 
fluorescence (FL1). Non-transfected cells exhibited low fluorescence (Fig. 1A), which was shifted to a bimodal fluorescence profile following retroviral transfection (Fig. 1B). The higher expressing cells, corresponding to gate M2, were collected. Analysis of these cells showed a wide spread of fluorescence (Fig. 1C), so they were once again sorted for the highest expressing cells (Fig. 1D) which were collected and cultured for use in these studies.

Western blot analysis was carried out to demonstrate the effectiveness of the transfection of Bcl-2 into the Jaws II cell line. These blots clearly show that Jaws II-Bcl-2 cells express large quantities of $\mathrm{Bcl}-2$ protein, that are not detectable in cells containing GFP vector alone (Fig. 2A, lanes 1 and 2). The blot was stripped and reprobed for actin to demonstrate that lane loading was equal for both Jaws II-GFP and Jaws II-Bcl-2 cells. Expression was stable and did not diminish over time (Fig. 2B), as cell lysates generated at both passage 4 (lanes 1 and 2) and at passage 12 show marked Bcl-2 expression in Jaws II-Bcl-2 cells but not in Jaws II-GFP cells (lanes 3 and 4).

3.2. Effects of GEA 3162 on cell viability in Jaws II-GFP and Jaws II-Bcl-2 cells

Assessment of Jaws II cell viability by MTT assay following exposure to GEA $3162(30$ or $100 \mu \mathrm{M}$ ) for $4 \mathrm{~h}$ showed a decrease in viability in Jaws II-GFP cells (Fig. 3), with a significant $(p<0.05)$ effect seen with the higher concentration. Jaws II$\mathrm{Bcl}-2$ cells also showed a slight decrease in viability, although

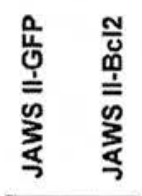

Anti-Bcl2

Anti-actin

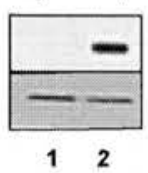

(B)

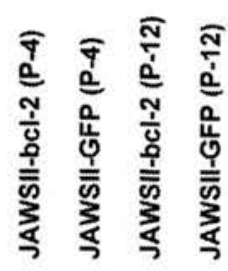

$26 \mathrm{kDa}$

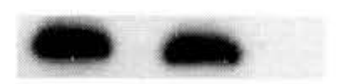

Fig. 2 - Transfection of Jaws II cells with human Bcl-2. (A) Western blot of cell lysates prepared from Jaws II cells transfected to overexpress Bcl-2 (Jaws II-Bcl-2) or vector alone (Jaws II-GFP), showing expression of Bcl-2 in Jaws IIBcl-2 but not Jaws II-GFP cells, and equal lane loading using actin. (B) Bcl-2 overexpression was stable over multiple passages. Westem blot of different passages of JAWS II cells stably transfected to overexpress human Bcl2 or GFP. Human Bcl-2 protein levels were unchanged from passage 4 to passage 12 .

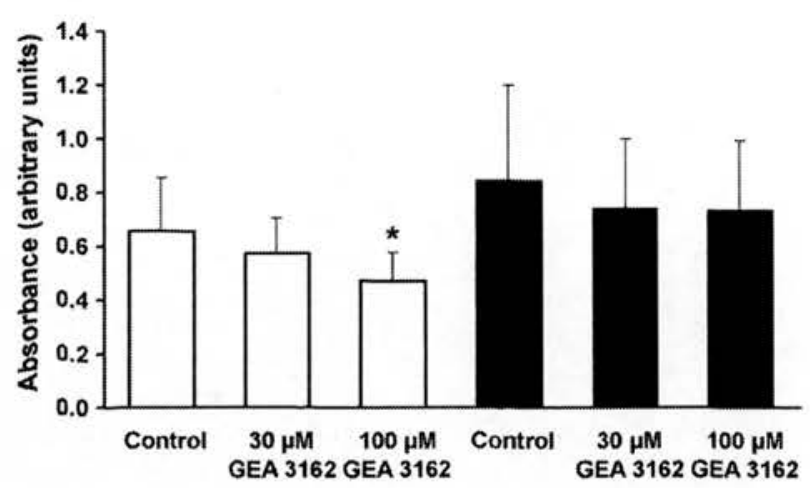

Jaws \|-GFP

Jaws $\|-B c l 2$

Fig. 3 - Effect of GEA 3162 on Jaws II cell viability. GFP or Bcl2-overexpressing Jaws II cells were exposed to GEA 3162 (30-100 $\mu \mathrm{M})$ for $4 \mathrm{~h}$ before assessment of cell viability by MTT assay. Data represents mean \pm S.D. following $\boldsymbol{n}=\mathbf{2}$ experiments. Asterisks represent significant $(p<0.05)$ difference from control (untreated) cells for each cell type (repeated measures ANOVA with Student-Newman-Keuls post-test).

this effect was reduced compared to the control cells and was associated with more variability in absorbance measured. Both sets of cells continued to exclude Trypan Blue following treatment with GEA 3162 (data not shown).

3.3. Total caspase activation in response to GEA 3162 in Jaws II-GFP and Jaws II-Bcl-2 cells

Measurement of caspase activation in Jaws II cells showed enhanced activation in cells containing the GFP vector in response to GEA 3162 . Following $4 \mathrm{~h}$ incubation in medium alone, Jaws II-GFP cells and the Bcl-2-overexpressing cells showed no significant difference in the basal level of fluorescence. In the presence of STSP $(200 \mathrm{nM})$, however, fluorescence was increased ( $p>0.05$ compared with GFP control) in Jaws II-GFP cells (Fig. 4A). Overexpression of Bcl-2 reduced the increase in fluorescence in response to STSP $(p>0.05)$, although fluorescence did not return to control levels. In the presence of the pan-caspase inhibitor, zVAD $(100 \mu \mathrm{M})$, fluorescence levels were similar in GFP cells, GFP cells plus STSP $(p>0.05)$, Bcl-2 overexpressing cells $(p>0.05)$ and Bcl-2 cells plus STSP $(p>0.05)$. Although the presence of $\mathrm{zVAD}$ was insufficient to return fluorescence back to control levels, it did markedly reduce the level of caspase activation in response to STSP in both Jaws II-GFP and Jaws II-Bcl-2 cells, although this was not significantly different from STSP-treated cells in the absence of zVAD $(p>0.05)$.

When Jaws II cells were exposed to the $\mathrm{ONOO}^{-}$donor, GEA $3162(30-100 \mu \mathrm{M})$, for $4 \mathrm{~h}$, caspase activation increased in a concentration-dependent manner in Jaws II-GFP cells (Fig. 4B), with those treated with $100 \mu \mathrm{M}$ GEA 3162 producing a statistically significant $(p<0.05)$ degree of activation. In contrast, in the presence of $100 \mu \mathrm{M} z V A D$, 

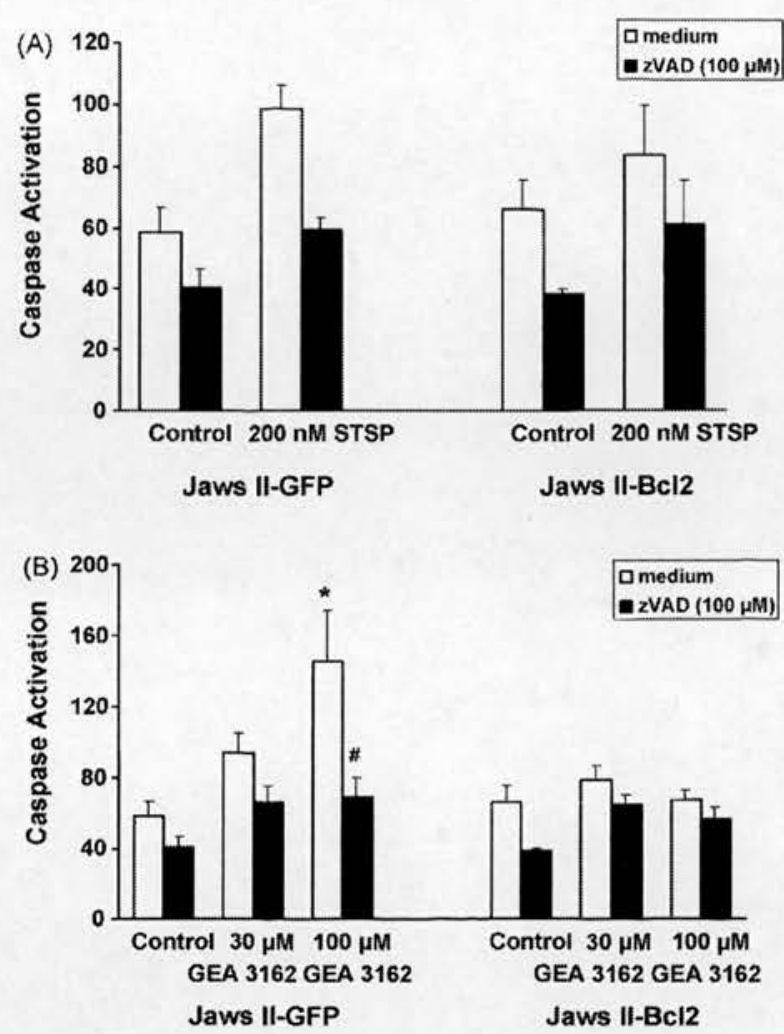

Fig. 4 - GEA 3162 induces caspase activation in Jaws II-GFP but not Jaws II-Bcl-2 cells. GFP or Bcl-2-overexpressing Jaws II cells were exposed to (A) STSP (200 nM) or (B) GEA $3162(30-100 \mu \mathrm{M})$ for $4 \mathrm{~h}$ before assessment of homogeneous caspase substrate cleavage (white bars). As an internal control for the assay, cells were also incubated with the pan-caspase inhibitor, zVAD (100 $\mu \mathrm{M}$; black bars). Data represents mean \pm S.E.M. following $n=4$ experiments performed in duplicate. Asterisks represent significant $(p<0.05$ ) difference from control (untreated) cells for each cell type, and hashes represent significant differences in fluorescence in the absence and presence of zVAD for a given treatment (repeated measures ANOVA with Student-Newman-Keuls post-test).

the increase in fluorescence was abrogated, and no significant difference was seen between treated and untreated cells $(p>0.05)$.

Overexpression of $\mathrm{Bcl}-2$ reduced the caspase activation seen in Jaws II cells on exposure to GEA 3162. Treatment of these cells with GEA 3162 produced no significant difference in fluorescence from control (untreated) cells $(p>0.05)$. There was, however, a significant decrease in caspase activation levels in Bcl-2-overexpressing cells exposed to $100 \mu \mathrm{M}$ GEA 3162 , compared to cells containing the GFP vector alone under the same conditions $(p<0.05)$. Addition of $\mathrm{zVAD}(100 \mu \mathrm{M})$ had little effect in Jaws II-Bcl-2 cells, but reduced fluorescence levels very slightly $(p>0.05)$, demonstrating that the vast majority of caspase activity was already inhibited by the overexpression of $\mathrm{Bcl}-2$.
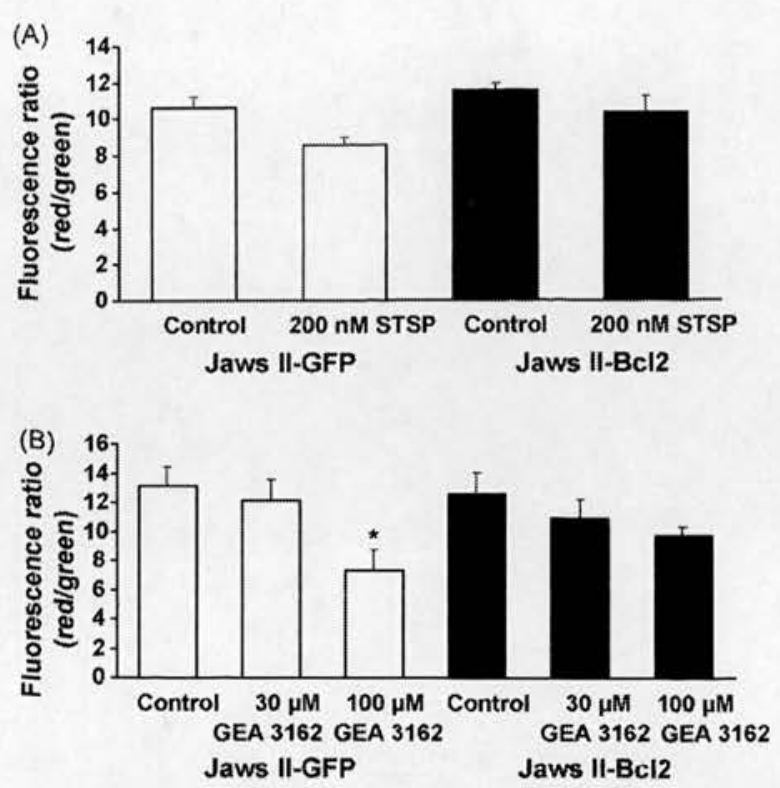

Fig. 5 - GEA 3162 induces loss of mitochondrial membrane potential that is reduced by Bcl-2 overexpression. GFP or Bcl-2-overexpressing Jaws II cells were exposed to (A) STSP (200 nM) or (B) GEA $3162(30-100 \mu \mathrm{M})$ for $4 \mathrm{~h}$ before assessment of mitochondrial permeability. Cells were harvested and centrifuged and cell pellets resuspended and incubated in a solution of fluorescent dye before washing, then red and green fluorescence was measured. The ratio of red (intact mitochondrial membrane potential) to green (mitochondrial membrane potential lost) cells is indicative of the extent of mitochondrial-dependent apoptosis. Data represents mean \pm S.E.M. following $n=4$ experiments performed in duplicate. Asterisks represent significant $(p<0.05)$ difference from control (untreated) cells for each cell type (repeated measures ANOVA with Student-Newman-Keuls post-test).

\subsection{Involvement of mitochondrial permeabilisation in apoptosis}

The loss of mitochondrial membrane potential $\left(\psi_{\mathrm{M}}\right)$ that is characteristic of the stress pathway of apoptosis was measured through the use of a fluorescent dye. Viable cells fluoresce red and apoptotic cells fluoresce green, therefore the red:green fluorescence ratio decreases with increasing levels of apoptotic cell death. STSP ( $200 \mathrm{nM}, 4 \mathrm{~h}$ ) promoted the loss of $\psi_{\mathrm{M}}$ in Jaws II cells containing the GFP vector alone, while overexpression of $\mathrm{Bcl}-2$ caused a reduction in the change of mitochondrial potential (Fig. 5A), thereby providing a protective effect against mitochondrial permeabilisation.

In Jaws II-GFP cells exposed to GEA $3162(30-100 \mu \mathrm{M})$ for $4 \mathrm{~h}$, there was a concentration-dependent reduction in the fluorescence ratio compared to control cells (Fig. 5B), with exposure to $100 \mu \mathrm{M}$ GEA 3162 producing a significant level of mitochondrial permeability $(p<0.05)$. Again, the overexpression of Bcl-2 in Jaws II cells conferred some protection against the loss of $\psi_{\mathrm{M}}$, although this was not complete. Cells exposed 
(A)

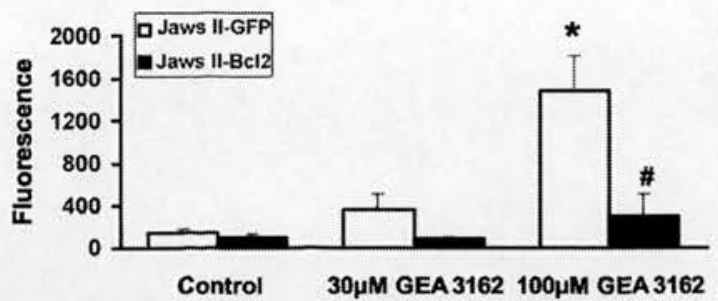

(B)

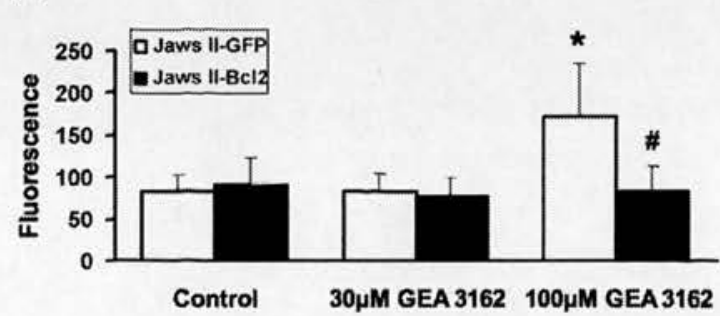

(C)

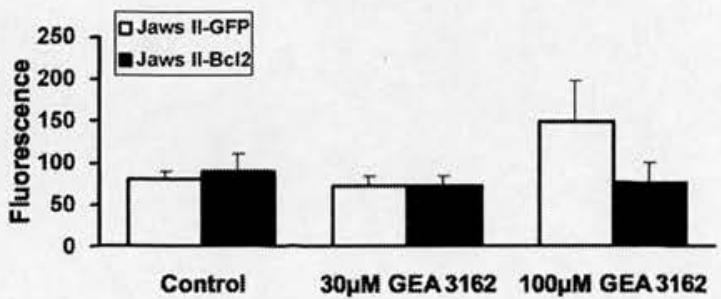

(D)

Caspase 2

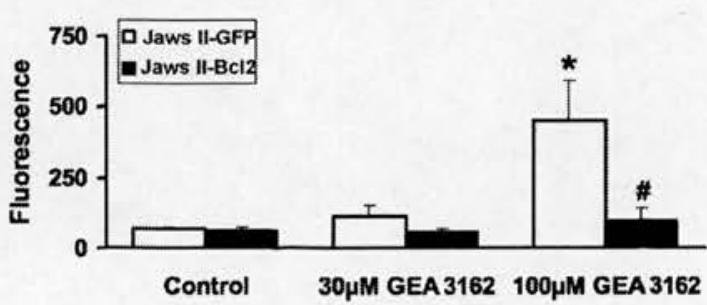

Fig. 6 - Specific caspases activated by GEA 3162. GFP or Bcl2-overexpressing Jaws II cells were exposed to GEA 3162 (30-100 $\mu \mathrm{M})$ for $4 \mathrm{~h}$ before assessment of the cleavage of specific fluorogenic substrates for (A) caspase 3 , (B) caspase 8 , (C) caspase 9 and (D) caspase 2. Cells were harvested, centrifuged and lysed, then lysates incubated with specific caspase substrates immobilised on a plate, with fluorescence indicating the extent of caspase activation. Data represents mean \pm S.E.M. following $n=4$ experiments performed in duplicate. Asterisks represent significant $(p<0.05)$ difference from control (untreated) cells for each cell type, and hashes represent significant differences between fluorescence in GFP and Bcl-2 cells for a given treatment (repeated measures ANOVA with Student-Newman-Keuls post-test). to 30 or $100 \mu \mathrm{M}$ GEA 3162 were not significantly different from control $(p>0.05)$.

\subsection{Activation of specific caspases}

Analysis of specific caspase activation showed that exposure of Jaws II-GFP cells to GEA 3162 leads to a large increase in the activity of caspases 2 and 3 , and a smaller increase in caspases 8 and 9 (Fig. 6). Treatment with 30 or $100 \mu$ M GEA 3162 produced a concentration-dependent increase in caspase 3 substrate cleavage in cells transfected with the GFP vector alone, with fluorescence levels reaching approximately $230 \%$ $(p>0.05)$ and $950 \%(p<0.05)$ of control levels respectively. Similarly, relatively high levels of activation were seen with caspase 2, with $30 \mu \mathrm{M}$ GEA 3162 producing around $160 \%$ $(p>0.05)$ and $100 \mu \mathrm{M}$ producing $650 \%(p<0.05)$ of control fluorescence. However, caspase 8 activation was less pronounced, with fluorescence in response to $30 \mu \mathrm{M}$ GEA 3162 being virtually identical to control $(p>0.05)$, and $100 \mu \mathrm{M}$ measured at $200 \%(p<0.05)$ of control. Caspase 9 levels were virtually identical in control and $30 \mu \mathrm{M}$ GEA 3162-treated cells $(p>0.05)$ while $100 \mu \mathrm{M}$-treated cells showed $185 \%(p>0.05)$ of control fluorescence. In contrast, overexpression of Bcl-2 greatly reduced or abolished all GEA 3162-induced increases in fluorescence, and there was no significant difference between any treatments in these cells for any of the caspases measured, indicating that $\mathrm{Bcl}-2$ overexpression prevents upregulation of the activity of multiple caspases in response to $\mathrm{ONOO}^{-}$.

\subsection{Effects of MAP kinase inhibitors on total caspase activity in Jaws II-GFP cells}

Homogeneous caspase activity in the presence of JNK (SP600125; 10-50 $\mu \mathrm{M}$ ) and p38 (SB203580; 10-50 $\mu \mathrm{M}$ ) MAP kinase inhibitors was also measured in Jaws II-GFP cells exposed to GEA $3162(30-100 \mu \mathrm{M})$ for $4 \mathrm{~h}$. SP600125 afforded slight protection against GEA 3162-induced apoptosis at the highest concentration (Fig. 7B). The level of fluorescence in untreated (control) cells was unaffected by the presence of $10 \mu \mathrm{M}(p>0.05)$ or $50 \mu \mathrm{M}(p>0.05)$ of the JNK inhibitor. The lower $(30 \mu \mathrm{M})$ concentration of GEA 3162 caused a small increase in caspase activity although this was not statistically significant by repeated measures ANOVA $(p>0.05)$. In the presence of SP600125, fluorescence was not significantly altered. Exposure to $100 \mu \mathrm{M}$ GEA 3162 for $4 \mathrm{~h}$ caused a significant increase in caspase activity $(p<0.05)$. Addition of $10 \mu \mathrm{M}$ SP600125 caused a small increase in caspase activity ( $p<0.05$ compared to untreated cells), but addition of $50 \mu \mathrm{M}$ SP600125 caused a small but statistically significant decrease. While this was still significantly different from untreated control cells, it was also a significant decrease compared to cells exposed to GEA 3162 in the absence of the JNK inhibitor $(p<0.05)$.

In contrast, SP600125 failed to affect caspase activation in response to $200 \mathrm{nM}$ STSP (Fig. 7A). STSP-treated cells exhibited a significant increase in fluorescence compared to control $(p<0.05)$ which was not abrogated by the presence of SP600125, as fluorescence remained significantly different from control. 

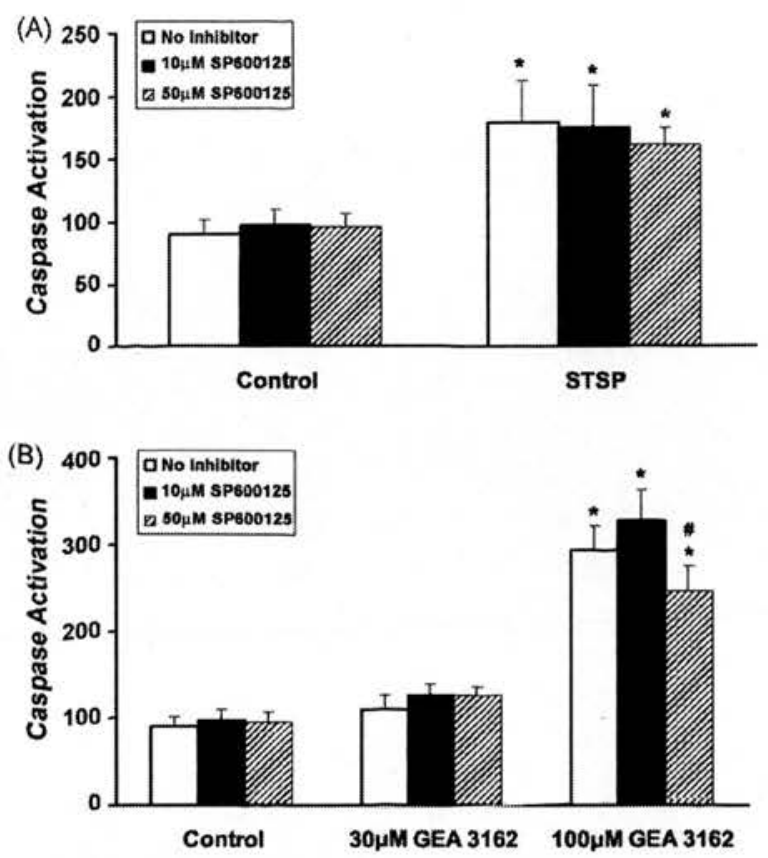

Fig. 7 - Effect of JNK inhibition on GEA 3162-induced apoptosis in Jaws II-GFP cells. Cells were exposed to (A) STSP $(200 \mathrm{nM})$ or (B) GEA $3162(30-100 \mu \mathrm{M})$ in the absence or presence of the JNK inhibitor, SP600125 (10-50 $\mu \mathrm{M})$. Total caspase activity was then measured by incubation with fluorogenic caspase substrate for $1 \mathrm{~h}$. Data represents mean \pm S.E.M. following $n=5$ experiments performed in duplicate. Asterisks represent significant $(p<0.05)$ difference from control (untreated) cells, and hashes represent significant differences in fluorescence in the absence and presence of SP600125 (repeated measures ANOVA with Student-Newman-Keuls post-test).

Analysis of total caspase activation in the presence of the p38 inhibitor, SB203580 (10-50 $\mu \mathrm{M})$ showed protection of Jaws II-GFP cells from GEA 3162-induced apoptosis (Fig. 8B). The inhibitor had no effect on apoptosis in untreated (control) cells, with fluorescence at similar levels in the presence of 0,10 and $50 \mu \mathrm{M}$ SB203580 ( $p>0.05$ between conditions). Caspases were activated in response to $30 \mu \mathrm{M}$ GEA $3162(p>0.05$ compared to untreated cells), and addition of 10 or $50 \mu \mathrm{M}$ of the p38 inhibitor had no effect on caspase activity $(p>0.05$ versus no inhibitor). Increased caspase activity was also detected on exposure to $100 \mu \mathrm{M}$ GEA, However there was a concentration-dependent reduction in fluorescence on addition of SB203580, with $50 \mu \mathrm{M}$ producing a statistically significant effect, bringing caspase activity down to a level that is significantly different to $100 \mu \mathrm{M}$ GEA 3162-treated cells in the absence of inhibitor, and not significantly different from untreated cells.

In contrast, inhibition of p38 enhanced STSP-induced apoptosis in Jaw II-GFP cells (Fig. 8A), with specific inhibition of 38 MAP kinase providing an additive apoptotic effect to the general kinase inhibition provided by STSP. Fluorescence levels were elevated in response to $200 \mathrm{nM}$ STSP. Addition of
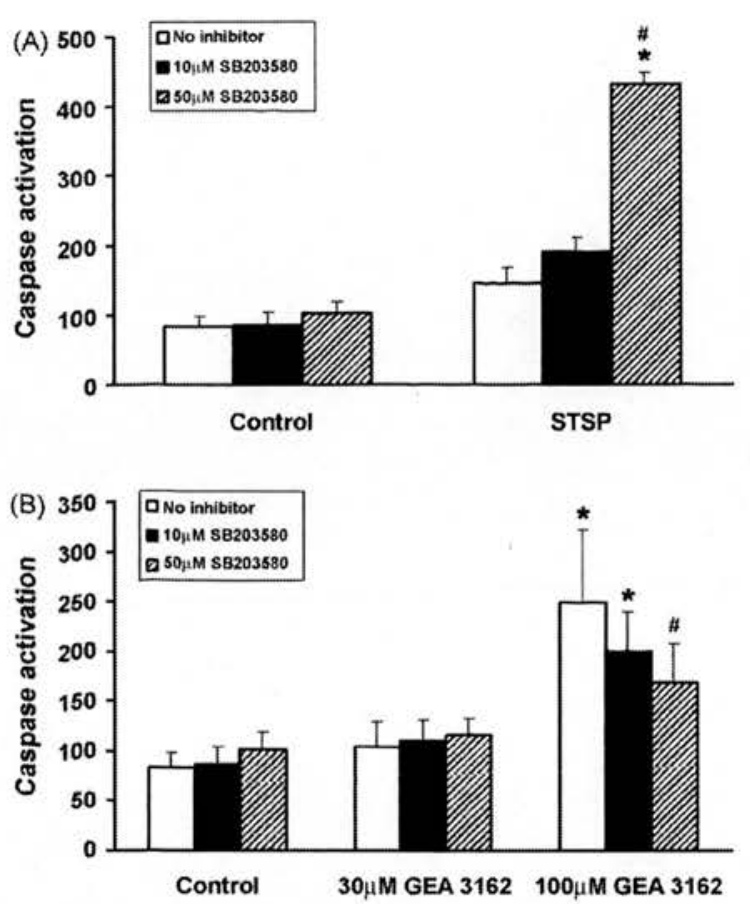

Fig. 8 - Effect of p38 inhibition on GEA 3162-induced apoptosis in Jaws II-GFP cells. Cells were exposed to (A) STSP $(200 \mathrm{nM})$ or (B) GEA $3162(30-100 \mu \mathrm{M})$ in the absence or presence of the p38 inhibitor, SB203580 (10-50 $\mu \mathrm{M})$. Total caspase activity was then measured by incubation with fluorogenic caspase substrate for $1 \mathrm{~h}$. Data represents mean \pm S.E.M. following $n=3$ experiments performed in duplicate. Asterisks represent significant $(p<0.05)$ difference from control (untreated) cells, and hashes represent significant differences in fluorescence in the absence and presence of SB203580 (repeated measures ANOVA with Student-Newman-Keuls post-test).

$10 \mu \mathrm{M}$ SB203580 in the presence of STSP increased fluorescence in a concentration-dependent manner, with $50 \mu \mathrm{M}$ having a significant effect ( $p<0.05$ compared to STSP alone).

\section{Discussion}

Apoptotic cell death is regulated by NO and related species, such as $\mathrm{ONOO}^{-}$, in several cell types including myeloidderived leukocytes such as neutrophils, eosinophils and monocytes/macrophages [10]. The biological effects of NO and $\mathrm{ONOO}^{-}$, including their effects on apoptosis, have recently been comprehensively reviewed [19]. Some studies have reported that $\mathrm{p} 53$ is required for apoptosis induced by NO and/or $\mathrm{ONOO}^{-}$in some cell types [20-22]. Other studies suggest that $\mathrm{p} 53$ may be inactivated by $\mathrm{ONOO}^{-}[23]$ or that apoptosis may proceed despite the absence of p53 [24] Alternatively, lack of p53 may confer a degree of resistance to $\mathrm{NO} / \mathrm{ONOO}^{-}$-stimulated apoptosis but other pathways may contribute to cell death or compensate when p53 is lacking [25-28]. In order to elucidate the dependence or otherwise of apoptosis on p53, we investigated the effects of GEA 3162, a 
$\mathrm{ONOO}^{-}$donor similar to SIN-1 [14], on apoptosis in a murine bone marrow cell line, Jaws II. This cell line is devoid of p53, and was studied containing either a vector encoding GFP alone (control) or a vector coding for both GFP and the anti-apoptotic protein, Bcl-2.

Although we have previously demonstrated that GEA 3162 simultaneously releases $\mathrm{NO}$ and $\mathrm{O}_{2}^{-}$in our system, and produces the same profile of apoptotic events as SIN-1 [14], other studies have found GEA 3162 to be a pure NO donor [29]. Furthermore, in culture conditions, factors such as $\mathrm{CO}_{2}$ may cause significant decay of $\mathrm{ONOO}^{-}$formed [30]. Thus, although in this study we refer to GEA 3162 as a $\mathrm{ONOO}^{-}$donor, the exact nature of the apoptogenic species is not known, and further studies (e.g. using SOD or NO scavengers) would have to be carried out to determine which species is responsible for these effects.

Apoptosis is a process that is dependent on the action of caspase proteases [1]. Therefore, total caspase activity was measured in Jaws II cells to determine whether these proteases are activated on exposure to GEA 3162, or STSP as a control apoptosis-initiating stimulus STSP increased caspase activation in Jaws II-GFP cells, although this failed to reach statistical significance. GEA 3162 also increased caspase activity; the effect was clearly concentration-dependent and there was a significant effect with the higher concentration. When the pan-caspase inhibitor, zVAD, was added as an internal control for the assay, fluorescence was reduced in all conditions, demonstrating that fluorescence measured was due to caspase activation. A significant difference was seen between control cells exposed to $100 \mu \mathrm{M}$ GEA 3162 in the absence and presence of zVAD, therefore GEA 3162 specifically enhances caspase activation. Thus, apoptosis proceeded despite the absence of p53 in these cells, and correlates with existing data that suggests that $\mathrm{p} 53$ is not essential for apoptosis, but alternative and/or compensatory pathways are involved.

Overexpression of Bcl-2 was protective against GEA 3162induced caspase activation, as no significant increase in caspase activity was seen in Jaws II-Bcl-2 cells compared to untreated cells of the same kind. However, a significant difference was observed between treatment with $100 \mu \mathrm{M}$ GEA 3162 in Jaws II-GFP compared with Jaws II-Bcl-2. Thus, it is the overexpressed $\mathrm{Bcl}-2$ that abrogates caspase activity. Similar findings have previously been reported in macrophage cell lines, which were protected from NO-induced apoptosis by overexpression of $\mathrm{Bcl}-2$ [31]. In that study, $\mathrm{Bcl}-2$ was proposed to neutralise the p53-dependent increase in Bax expression. The absence of p53 in Jaws II cells implies that an alternative mechanism of protection is also involved. One possibility is the antioxidant activity of $\mathrm{Bcl}-2$, which has been previously described [32]. Alternatively, Bcl-2 may have a protective role similar to that observed in C. elegans by its ortholog, CED-9. CED-9 acts to sequester CED-4 (the worm equivalent of Apaf1), and prevent activation of CED-3 (caspase 3 in humans). Therefore, it is possible that human Bcl-2 may have a similar, more direct, role in caspase regulation, and there is some data to support this proposal, as discussed by Cory and Adams [4].

Mitochondrial permeability was enhanced in Jaws II-GFP cells on exposure to GEA 3162, suggesting that the mitochondrial pathway is involved in $\mathrm{ONOO}^{-}$induced apoptosis in these cells. Bcl-2 overexpression reduced the change in $\Psi_{\mathrm{M}}$ so that it was no longer significantly different from control, but this protection was incomplete. This is in agreement with previous studies in $\mathrm{ONOO}^{-}$treated thymocytes, in which Bcl-2 offered complete protection against caspase activation and DNA fragmentation, but only partial inhibition of loss of $\Psi_{\mathrm{M}}$ [33].

Measurement of specific caspases showed that GEA 3162 strongly activated caspases 2 and 3 in Jaws II-GFP cells, while a lesser activity of caspases 8 and 9 was detectable. Such data shows remarkable similarity to that obtained in HL-60 cells on exposure to $\mathrm{ONOO}^{-}$, in which activity of caspases 2 and 3 was approximately $500 \%$ of control (untreated) levels, whereas caspases 8 and 9 had a maximal activation of $\sim 200-210 \%$ of control [34]. Interestingly, HL60 cells are also p53 null, suggesting that this profile of caspase activity may occur in response to $\mathrm{ONOO}^{-}$in cells lacking this gene. The lack of discernible phenotype in the caspase $2-1-$ mouse points towards a potential role for caspase 2 as a compensatory molecule activated in the absence of other pro-apoptotic proteins, such as p53. However, although it is possible that this is a cell-specific apoptotic pathway seen in cells lacking functional p53, a stimulus-specific effect in p53-replete cells in response to $\mathrm{ONOO}^{-}$cannot be ruled out. Further experiments in other p53-1- and p53+/+ cells, plus siRNA and repletion studies would need to be carried out to distinguish cell-specific and stimulus-specific effects of $\mathrm{ONOO}^{-}$.

Caspase 3 is the principal effector caspase involved in the apoptotic cascade, and cleaves specific target proteins within the cell, such as PARP, PKC- $\delta$, protein kinases and structural proteins [35]. However, little is known about the role of caspase 2 in apoptosis, as it has largely been ignored in investigations of the apoptotic process, due to the lack of phenotype in the knockout mouse. However, there have been several suggestions regarding its function (particularly that it acts just upstream of mitochondrial permeabilisation) and these proposed functions are reviewed by Troy and Shelanski [18]. A recent study also attributed caspase 2 function to an ER stress pathway of apoptosis, functioning as both an initiator and effector caspase [36]. As ER stress has been implicated in the apoptotic response to $\mathrm{NO} / \mathrm{ONOO}^{-}$in $\mathrm{p} 53$-deficient murine microglial cells [24], this is a feasible suggestion for the mechanism by which $\mathrm{ONOO}^{-}$induces apoptosis in Jaws II cells.

Caspase 9 activation measured by the profiling assay confirmed the involvement of mitochondria in apoptosis elicited by GEA 3162 in Jaws II-GFP cells. Cleavage of the caspase 8 substrate at low levels suggests either non-specific cleavage by high levels of caspases 2 and 3 , or a potential role for death receptor clustering that is amplified via the caspase cascade. It has been proposed that reactive oxygen species may induce ligand-independent death receptor clustering in lipid rafts and subsequent caspase activation [37]. It remains to be seen whether this mechanism has a role to play in GEA 3162-provoked apoptosis.

It is interesting to note that exogenous expression of human Bcl-2 does not simply reduce caspase activity, but abolishes it in both untreated and GEA 3162-exposed cells. This is similar to the 'complete' inhibition of apoptosis previously reported in various cell types on overexpression 
of $\mathrm{Bcl}-2[33,38]$, and suggests that $\mathrm{Bcl}-2$ may have a tighter regulatory role on $\mathrm{NO} / \mathrm{ONOO}^{-}$-induced caspase activation than mere mitochondrial stability. Additionally, all caspases are equally affected by Bcl-2, rather than just those downstream of mitochondria (caspases 9 and 3) pointing to a more general inhibitory effect than insertion of Bcl-2 into mitochondrial membranes. If only mitochondrial stability was altered, it would be expected that caspases 2 and 8 would still be elevated in the Jaws II-Bcl-2 cells. These data therefore support the hypothesis that cytoprotection afforded by Bcl-2 occurs via a similar pathway to that observed in C. elegans by CED-9.

The role of MAP kinases in cells is complex and somewhat variable, with both pro- and anti-apoptotic effects being reported, and is seemingly dependent upon the cell type being investigated $[7,8]$. However, there is evidence to suggest that both p38 and JNK MAP kinases may play a role in $\mathrm{NO}^{-} \mathrm{ONOO}^{-}$ mediated cell death $[39,40]$ as well as other apoptotic stimuli [41] and Bcl-2 has been shown to suppress p38 activation and subsequent NO-induced apoptosis [42], therefore it was hypothesised that these kinases may be involved in GEA 3162-induced apoptosis of Jaws II cells. Studies using pharmacological inhibitors of p38 and JNK demonstrated a pro-apoptotic role for $\mathrm{p} 38$ when Jaws II-GFP cells were exposed to $\mathrm{ONOO}^{-}$from GEA 3162, but little or no role for JNK. However the role of p38 is stimulus-specific, as this kinase has an antiapoptotic role when cells are exposed to STSP. Additional studies examining the expression and phosphorylation status of these MAP kinases would be useful in order to confirm and further investigate the involvement of these proteins and their role in $\mathrm{ONOO}^{-}$-induced apoptosis.

Protein modification may occur in response to reactive oxygen and nitrogen species, which may subsequently alter protein function and cause cellular effects including apoptosis [43]. One such modification, which may occur on exposure to $\mathrm{ONOO}^{-}$is formation of 3-nitrotyrosine (3-NT), in which susceptible protein tyrosine residues may be nitrated [44]. Additional studies are required to assess formation of 3-NT, and identification of target proteins, in Jaws II cells exposed to GEA 3162, to determine whether this mechanism may have a role in GEA 3162-induced apoptosis in these cells.

We have demonstrated an apoptotic response to $\mathrm{ONOO}^{-}$in a murine bone marrow cell line. To the best of our knowledge, this is the first time that apoptosis has been reported using this cell line, and demonstrates that functional p53 is not a requirement for $\mathrm{ONOO}^{-}$mediated cell death in this cell type. Furthermore, we have shown that caspases 2 and 3 are important for apoptosis to proceed, with roles for caspases 8 and 9, and p38 MAP kinase. The anti-apoptotic protein, Bcl-2, abolishes caspase activation involved in both intrinsic and extrinsic pathways, suggesting a survival mechanism that is not restricted solely to the mitochondria, and may represent a novel or previously only speculative mechanism by which Bcl2 protects against apoptosis.

\section{Acknowledgements}

This work was funded by the Wellcome Trust as part of an International Travelling Research Fellowship offered by the
Cardiovascular Research Initiative at the University of Edinburgh (ELT). Additionally, this work was supported by USPHS grant HL080623 (JMH).

REFERE N C E S

[1] Wyllie AH. Apoptosis: an overview. Br Med Bull 1997;53:451-65.

[2] Kidd VJ. Proteolytic activities that mediate apoptosis. Annu Rev Physiol 1998;60:533-73.

[3] Zimmermann KC, Bonzon C, Green DR. The machinery of programmed cell death. Pharmacol Ther 2001;92:57-70.

[4] Cory S, Adams JM. The Bcl2 family: regulators of the cellular life-or-death switch. Nat Rev Cancer 2002;2:647-56.

[5] Haupt S, Berger M, Goldberg Z, Haupt Y. Apoptosis - the p53 network. J Cell Sci 2003;116:4077-85.

[6] Weller M. Predicting response to cancer chemotherapy: the role of p53. Cell Tissue Res 1998;292:435-45.

[7] Wada T, Penninger JM. Mitogen-activated protein kinașes in apoptosis regulation. Oncogene 2004;23:2838-49.

[8] Liu J, Lin A. Role of JNK activation in apoptosis: a doubleedged sword. Cell Res 2005;15:36-42.

[9] Bosca L, Zeini M, Traves PG, Hortelano S. Nitric oxide and cell viability in inflammatory cells: a role for NO in macrophage function and fate. Toxicology 2005;208: 249-58.

[10] Taylor EL, Megson IL, Haslett C, Rossi AG. Nitric oxide: a key regulator of myeloid inflammatory cell apoptosis. Cell Death Differ 2003;10:418-30.

[11] Virag L, Szabo E, Gergely P, Szabo C. Peroxynitrite-induced cytotoxicity: mechanism and opportunities for intervention. Toxicol Lett 2003;140-141:113-24.

[12] Ward C, Wong TH, Murray J, Rahman I, Haslett C, Chilvers $\mathrm{ER}$, et al. Induction of human neutrophil apoptosis by nitric oxide donors: evidence for a caspase-dependent, cyclicGMP-independent, mechanism. Biochem Pharmacol 2000;59:305-14.

[13] Taylor EL, Megson IL, Haslett C, Rossi AG. Dissociation of DNA fragmentation from other hallmarks of apoptosis in nitric oxide-treated neutrophils: differences between individual nitric oxide donor drugs. Biochem Biophys Res Commun 2001;289:1229-36.

[14] Taylor EL, Rossi AG, Shaw CA, Dal Rio FP, Haslett C, Megson IL. GEA 3162 decomposes to co-generate nitric oxide and superoxide and induces apoptosis in human neutrophils via a peroxynitrite-dependent mechanism. Br J Pharmacol 2004;143:179-85.

[15] MacKay V, Moore EE. Immortalized dendritic cells. US Patent and Trademark Office. USA: ZymoGenetics Inc.; 1997. Ref Type: Generic

[16] Kinoshita S, Su L, Amano M, Timmerman LA, Kaneshima H, Nolan GP. The T cell activation factor NF-ATc positively regulates HIV-1 replication and gene expression in T cells Immunity 1997;6:235-44.

[17] Vanasse GJ, Winn RK, Rodov S, Zieske AW, Li JT, Tupper JC, et al. Bcl-2 overexpression leads to increases in suppressor of cytokine signaling-3 expression in B cells and de novo follicular lymphoma. Mol Cancer Res 2004;2:620-31.

[18] Troy CM, Shelanski ML. Caspase-2 redux. Cell Death Differ 2003;10:101-7.

[19] Pacher P, Beckman JS, Liaudet L. Nitric oxide and peroxynitrite in health and disease. Physiol Rey 2007;87:315-424.

[20] Messmer UK, Ankarcrona M, Nicotera P, Brune B. p53 expression in nitric oxide-induced apoptosis. FEBS Lett $1994 ; 355: 23-6$. 
[21] Kaji T, Kaieda I, Hisatsune T, Kaminogawa S. 3Morpholinosydnonimine hydrochloride induces $\mathrm{p} 53$ dependent apoptosis in murine primary neural cells: a critical role for p21(ras)-MAPK-p19(ARF) pathway. Nitric Oxide 2002;6:125-34.

[22] Martin LJ, Chen K, Liu Z. Adult motor neuron apoptosis is mediated by nitric oxide and Fas death receptor linked by DNA damage and p53 activation. J Neurosci 2005;25:6449-59.

[23] Cobbs CS, Whisenhunt TR, Wesemann DR, Harkins LE, Van Meir EG, Samanta M. Inactivation of wild-type $p 53$ protein function by reactive oxygen and nitrogen species in malignant glioma cells. Cancer Res 2003;63:8670-3.

[24] Kawahara K, Oyadomari S, Gotoh T, Kohsaka S, Nakayama $\mathrm{H}$, Mori M. Induction of CHOP and apoptosis by nitric oxide in p53-deficient microglial cells. FEBS Lett 2001;506:135-9.

[25] Messmer UK, Brune B. Nitric oxide-induced apoptosis: p53dependent and $\mathrm{p} 53$-independent signalling pathways. Biochem J 1996;319(Pt 1):299-305.

[26] Weller R, Billiar T, Vodovotz Y. Pro- and anti-apoptotic effects of nitric oxide in irradiated keratinocytes: the role of superoxide. Skin Pharmacol Appl Skin Physiol 2002;15: $348-52$.

[27] Jeon HK, Choi SU, Jung NP. Association of the ERK1/2 and p38 kinase pathways with nitric oxide-induced apoptosis and cell cycle arrest in colon cancer cells. Cell Biol Toxicol 2005;21:115-25.

[28] Li CQ Pang B, Kiziltepe T, Trudel LJ, Engelward BP, Dedon PC, et al. Threshold effects of nitric oxide-induced toxicity and cellular responses in wild-type and p53-null human lymphoblastoid cells. Chem Res Toxicol 2006;19:399-406.

[29] Kankaanranta H, Rydell E, Petersson AS, Holm P, Moilanen E, Corell T, et al. Nitric oxide-donating properties of mesoionic 3-aryl substituted oxatriazole-5-imine derivatives. Br J Pharmacol 1996;117:401-6.

[30] Romero N, Denicola A, Souza JM, Radi R. Diffusion of peroxynitrite in the presence of carbon dioxide. Arch Biochem Biophys 1999;368:23-30.

[31] Messmer UK, Reed UK, Brune B. Bcl-2 protects macrophages from nitric oxide-induced apoptosis. J Biol Chem 1996;271:20192-7.

[32] Hockenbery DM, Oltvai ZN, Yin XM, Milliman CL, Korsmeyer SJ. Bcl-2 functions in an antioxidant pathway to prevent apoptosis. Cell 1993;75:241-51.
[33] Virag L, Szabo C. BCL-2 protects peroxynitrite-treated thymocytes from poly(ADP-ribose) synthase (PARS)independent apoptotic but not from PARS-mediated necrotic cell death. Free Radic Biol Med 2000;29: 704-13.

[34] Zhuang S, Simon G. Peroxynitrite-induced apoptosis involves activation of multiple caspases in HL-60 cells. Am J Physiol Cell Physiol 2000;279:C341-51.

[35] Thornberry NA. The caspase family of cysteine proteases. Br Med Bull 1997;53:478-90.

[36] Cheung HH, Lynn KN, Liston P, Korneluk RG. Involvement of caspase-2 and caspase- 9 in endoplasmic reticulum stress-induced apoptosis: a role for the IAPs. Exp Cell Res 2006;312:2347-57.

[37] Scheel-Toellner D, Wang K, Assi LK, Webb PR, Craddock $\mathrm{RM}$, Salmon M, et al. Clustering of death receptors in lipid rafts initiates neutrophil spontaneous apoptosis. Biochem Soc Trans 2004;32:679-81.

[38] Melkova Z, Lee SB, Rodriguez D, Esteban M. Bcl-2 prevents nitric oxide-mediated apoptosis and poly(ADP-ribose) polymerase cleavage. FEBS Lett 1997;403:273-8.

[39] Ghatan S, Larner S, Kinoshita Y, Hetman M, Patel L, Xia Z, et al. p38 MAP kinase mediates bax translocation in nitric oxide-induced apoptosis in neurons. J Cell Biol 2000;150:335-47.

[40] Shacka JJ, Sahawneh MA, Gonzalez JD, Ye YZ, D'alessandro TL, Estevez AG. Two distinct signaling pathways regulate peroxynitrite-induced apoptosis in PC12 cells. Cell Death Differ 2006

[41] Derouet M, Thomas L, Moulding DA, Akgul C, Cross A, Moots RJ, et al. Sodium salicylate promotes neutrophil apoptosis by stimulating caspase-dependent turnover of Mcl-1. J Immunol 2006;176:957-65.

[42] Cheng A, Chan SL, Milhavet O, Wang S, Mattson MP. p38 MAP kinase mediates nitric oxide-induced apoptosis of neural progenitor cells. J Biol Chem 2001;276: 43320-7.

[43] Naoi M, Maruyama W, Shamoto-Nagai M, Yi H, Akao Y, Tanaka M. Oxidative stress in mitochondria: decision to survival and death of neurons in neurodegenerative disorders. Mol Neurobiol 2005;31:81-93.

[44] Kamat JP. Peroxynitrite: a potent oxidizing and nitrating agent. Indian J Exp Biol 2006;44:436-47. 


\title{
Tissue- and Stimulus-Dependent Role of Phosphatidylinositol 3-Kinase Isoforms for Neutrophil Recruitment Induced by Chemoattractants In Vivo ${ }^{1}$
}

\author{
Vanessa Pinho, ${ }^{2 \dagger}$ Remo de Castro Russo, * Flávio A. Amaral,* Lirlândia P. de Sousa,* \\ Michele M. Barsante,* Danielle G. de Souza, ${ }^{\S}$ José C. Alves-Filho, ${ }^{\text {ๆ }}$ Denise C. Cara, \\ Joel S. Hayflick, Christian Rommel, ${ }^{\#}$ Thomas Ruckle, ${ }^{\#}$ Adriano G. Rossi, ** \\ and Mauro M. Teixeira*
}

PI3K plays a fundamental role in regulating neutrophil recruitment into sites of inflammation but the role of the different isoforms of PI3K remains unclear. In this study, we evaluated the role of PI3K $\gamma$ and PI3K $\delta$ for neutrophil influx induced by the exogenous administration or the endogenous generation of the chemokine CXCL1. Administration of CXCL1 in PI3K $\gamma^{-1-}$ or wild-type (WT) mice induced similar increases in leukocyte rolling, adhesion, and emigration in the cremaster muscle when examined by intravital microscopy. The induction of neutrophil recruitment into the pleural cavity or the tibia-femoral joint induced by the injection of CXCL1 was not significantly different in PI3K $\gamma^{-1-}$ or WT mice. Neutrophil influx was not altered by treatment of W' $\mathrm{I}$ mice with a specific PI3K $\delta$ inhibitor, IC87114, or a specific PI3K $\gamma$ inhibitor, AS605240. The administration of IC87114 prevented CXCL1induced neutrophil recruitment only in presence of the PI3K $\gamma$ inhibitor or in PI3K $\gamma^{-1-}$ mice. Ag challenge of immunized mice induced CXCR2-dependent neutrophil recruitment that was inhibited by wortmannin or by blockade of and PI3K $\delta$ in PI3K $\gamma^{-1-}$ mice. Neutrophil recruitment to bronchoalveolar lavage induced by exogenously added or endogenous production of CXCL1 was prevented in PI3K $\gamma^{-1-}$ mice. The accumulation of the neutrophils in lung tissues was significantly inhibited only in PI3K $\gamma^{-1-}$ mice treated with IC87114. Neutrophil recruitment induced by exogenous administration of C5a or fMLP appeared to rely solely on PI3K $\gamma$. Altogether, our data demonstrate that there is a tissue- and stimulus-dependent role of PI3K $\gamma$ and PI3K $\delta$ for neutrophil recruitment induced by different chemoattractants in vivo. The Journal of Immunology, 2007, 179: 7891-7898.

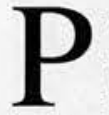

hosphatidylinositol 3-kinases are a family of proteins that catalyze the phosphorylation of the $3^{\prime}-\mathrm{OH}$ position of the inositol ring of phosphatidylinositols (PtdIns) ${ }^{3}$ resulting in the formation of PtdIns(3)P, PtdIns(3,4)P2, and PtdIns(3,4,5)P3, collectively termed $3^{\prime}$-PtdIns lipids $(1,2)$. These molecules work as second messengers and are important in regulation of various cellular responses, including proliferation, transformation, survival, superoxide production, adhesion, and cell migration (3).

*Departamento de Bioquímica e Imunologia, ${ }^{\dagger}$ Departamento de Morfologia, ${ }^{+}$Departamento de Patologia, and 'Departamento de Microbiologia, Instituto de Ciências Biológicas, Universidade Federal de Minas Gerais, Belo Horizonte, Brazil; " Departmento de Farmacologia, Escola de Medicina de Ribeirão Preto, Universidade de São Paulo, São Paulo, Brazil IICOS Corporation, Bothell, WA 98021; "Merck Serono International, Geneva, Switzerland and $* *$ Medical Research Council Centre for Inflammation Research, Queen's Medical Research Institute, University of Edinburgh, Edinburgh, United Kingdom

Received for publication March 27, 2007. Accepted for publication September 20, 2007.

The costs of publication of this article were defrayed in part by the payment of page charges. This article must therefore be hereby marked advertisement in accordance with 18 U.S.C. Section 1734 solely to indicate this fact.

'This work was supported by Conselho Nacional de Desenvolvimento Cientifico e Tecnológico (Brazil), Fundação de Amparo a Pesquisas do Estado de Minas Gerais (Brazil).

${ }^{2}$ Address correspondence and reprint requests to Dr. Vanessa Pinho, Imunofarmacologia, Departamento de Bioquímica e Imunologia, Departamento de Morfologia, Instituto de Ciências Biológicas, Universidade Federal de Minas Gerais, Avenida Antonio Carlos, 6627 Pampulha, 31270-901, Belo Horizonte, MG, Brazil. E-mail address: vpinho@icb.ufmg.br

${ }^{3}$ Abbreviations used in this paper: PtdIns, phosphatidylinositol; GPCR, G proteincoupled receptor; WT, wild type; i.pl., intrapleural; BAL, bronchoalveolar lavage; $\mathrm{MPO}$, myeloperoxidase.

Copyright $\odot 2007$ by The American Association of Immunologists, Inc. 0022-1767/07/\$2.00
There are different isoforms of PI3Ks that can be divided into three main classes. The class I PI3Ks are heterodimers consisting of a catalytic subunit and an adaptor/regulatory subunit present mainly in the cytosol of cells and can be subdivided into two subclasses: class IA enzymes may have one of three catalytic isoforms (p110 $\alpha$, $\mathrm{p} 110 \beta$, or $\mathrm{p} 110 \delta$ ) associated to five regulatory isoforms and signal downstream of tyrosine kinases; class IB enzymes are constituted by a unique catalytic subunit $(\mathrm{p} 110 \gamma)$ associated with a regulatory subunit p101 and signal downstream of heterotrimeric G proteincoupled receptors (GPCRs) $(1,2)$. There is also a class II PI3K, characterized by the presence of a C2 domain, and a class III PI3K, which uses only PtdIns as a substrate $(1,2)$.

Neutrophils, a major type of blood leukocytes, are indispensable for host defense of bacterial and fungal infections (4). This activity depends on the stimulation of various functions after activation of specific receptors, including the formyl methionyl leucyl peptide, chemotactic factor, and TLRs (5-10). Neutrophil migration is dependent on their activation by chemotactic stimuli, including chemokines, complement fragments, and lipid mediators, which enable these cells to enter the site of infection and destroy the invading pathogens. Chemotactic factors bind to seven-transmembrane domain receptors and activate heterotrimeric $\mathrm{G}$ proteins $(5$, $6,11,12)$. Downstream of these proteins a complex interrelated signaling network is activated and may result in the activation of PI3K isoforms (12-15). Involvement of PI3Ks in neutrophil activation has been demonstrated using the inhibitors of class I PI3K, wortmannin and LY294002, which block neutrophil activation and PtdIns $(3,4,5) \mathrm{P} 3$ generation, a main product of PI3K activation (16, 17). Studies using mice lacking the p110 catalytic subunit of PI3K $\gamma$ have demonstrated the importance of this PI3K isoform for 
chemokine-regulated recruitment of neutrophils in vitro $(18,19)$. Furthermore, PI3K $\gamma$-deficient neutrophils exhibited severe defects in GPCR-induced respiratory burst and produced less PtdIns(3,4,5)P3 after stimulation of cells with C5a, fMLP, and CXCL8 (IL-8) (19). It has been recently suggested that $\mathrm{PI} 3 \mathrm{~K} \delta$ may also modulate the activity and recruitment of neutrophils in vivo $(20,21)$. However, the exact extent to which PI $3 \mathrm{~K} \gamma$ participates in the process of neutrophil recruitment in vivo is not known.

The present study was conducted initially to investigate the role of PI3K $\gamma$ in modulating neutrophil rolling, adhesion, and transmigration induced by the chemokine CXCL1 in the mouse cremaster. However, as $\mathrm{PI} 3 \mathrm{~K} \gamma$ appeared not to be directly involved with CXCL1-induced neutrophil influx, further studies were conducted to investigate whether the enzyme was relevant for the neutrophil recruitment in other sites of inflammation and that induced by more complex inflammatory stimuli, including $\mathrm{Ag}$ challenge or bleomycin administration. We also evaluated the relevance of $\mathrm{PI} 3 \mathrm{~K} \gamma$ for neutrophil influx induced by other chemoattractant molecules and whether PI3K $\delta$ played a role in CXCL1-induced neutrophil influx. We demonstrate that the involvement of PI3K $\gamma$ on neutrophil recruitment depends on the chemotactic stimulus and on the tissue in which the inflammatory stimulation is occurring. $\mathrm{PI} 3 \mathrm{~K} \delta$ and $\mathrm{PI} 3 \mathrm{~K} \gamma$ have a redundant role in mediating exogenously added or endogenously generated CXCL1-induced neutrophil recruitment.

\section{Materials and Methods \\ Animals}

PI3K $\gamma$-deficient male C57BL/6 $\times$ sv129 mice and their wild-type (WT) littermate control mice $(+/+)(18-22 \mathrm{~g})$ were used throughout these experiments. PI3K $\gamma^{-1-}$ mice (in a C57BL $6 \times$ sv129 background) were generated as previously described (18). The mice were a gift of Dr. J. M. Penninger (Institute of Molecular Biotechnology of the Austrian Academy of Sciences, Vienna, Austria) and supplied by Taconic Farms. $P I 3 K \gamma$ gene deletion was confirmed by PCR using specific primers (WT primers: sense, 5-TCAGGC TCGGAGATTAGGTA-3; antisense, 5-GCCCAATCGGTGGTAGAACT-3; $P I 3 \mathrm{~K}^{-1-}$ primers: sense, 5-GGACACGGCTTTGATTACAATC-3; antisense primer, 5-GGGGTGGGATTAGATAAATG-3 as previously described (18). Animals were bred and housed in a temperature-controlled room with free access to water and food. All experimental protocols have been subjected to evaluation and were approved by the local animal ethics committee.

\section{Drugs and reagents}

Recombinant murine CXCL1 was purchased from PeproTech. CXCL1 was dissolved in water, diluted further in PBS (pH 7.4) containing $0.01 \%$ BSA and stored at $-70^{\circ} \mathrm{C}$ until use. OVA was purchased from Sigma-Aldrich and $\mathrm{C} 5 \mathrm{a}$ and wortmannin were purchased from Calbiochem. Wortmannin was diluted in DMSO and stored at $-70^{\circ} \mathrm{C}$ until use. LY294002 was purchased from Alamone Laboratories, diluted in DMSO, and stored at $-70^{\circ} \mathrm{C}$ until use. Bleomycin (blenoxane; Bristol-Meyers) was commercially available. The PI $3 K \delta$ inhibitor IC 87114 was a gift of the ICOS Corporation and stored at $-20^{\circ} \mathrm{C}$ until use. The PI3K $\gamma$ inhibitor AS605240 was a gift of Serono and was stored at $-20^{\circ} \mathrm{C}$ until use.

\section{Intravital microscopy}

The mouse cremaster preparation was used to study the behavior of leukocytes in the microcirculation and adjacent connective tissue, as previously described (22). Briefly, an incision was made in the scrotal skin to expose the left cremaster muscle, which was then carefully removed from the associated fascia. A lengthwise incision was made on the ventral surface of the cremaster muscle using a cautery. The testicle and the epididymis were separated from the underlying muscle and were moved into the abdominal cavity. The muscle was then spread out over an optically clear viewing pedestal and was secured along the edges with a 4-0 suture. The exposed tissue was superfused with warm bicarbonate-buffered saline $(\mathrm{pH}$ 7.4). An intravital microscope (Olympus $\mathrm{BX50F4)}$ with a $\times 20$ objective lens and $\mathrm{a} \times 10$ eyepiece was used to examine the cremasteric microcirculation. A video camera (5100 HS; Panasonic) was used to project the images onto a monitor, and the images were recorded for playback analysis using a conventional videocassette recorder.
Single, unbranched cremasteric venules (25-40 $\mu \mathrm{m}$ in diameter) were selected and, to minimize variability, the same section of cremasteric venule was observed throughout the experiment. The number of rolling, adherent, and emigrated leukocytes was determined offline during video playback analysis. Rolling leukocytes were defined as those cells moving at a velocity less than that of erythrocytes within a given vessel. The flux of rolling cells was measured as the number of rolling cells passing by a given point in the venule per minute. A leukocyte was considered to be adherent if it remained stationary for at least $30 \mathrm{~s}$, and total leukocyte adhesion was quantified as the number of adherent cells within a $100-\mu \mathrm{m}$ length of venule. Leukocyte emigration was defined as the number of cells in the extravascular space within an area of 50 $\mu \mathrm{m}$ of distance from the venule. Only cells adjacent to and clearly outside the vessel under study were counted as emigrated.

\section{Experimental protocols}

For each experiment, $500 \mathrm{ng}$ of murine rCXCL1 in $0.2 \mathrm{ml}$ of saline was administered locally by s.c. injection beneath the right scrotal skin using a 30-G needle, $2 \mathrm{~h}$ before exteriorization. The left cremaster was then prepared for intravital microscopy. Leukocyte kinetics was investigated, as described above, and at the end of each experiment whole blood was drawn by cardiac puncture. Total cell counts were performed in a modified Neubauer chamber using Turk's stain.

\section{Histology}

At the end of each intravital microscopy experiment, the cremaster muscles were removed and fixed in $10 \%$ neutral-buffered formalin. The tissues were dehydrated gradually in ethanol, embedded in paraffin, cut into $4-\mu \mathrm{m} \mathrm{sec}-$ tions, stained with H\&E, and examined under direct light microscopy.

\section{Sensitization}

Animals were immunized with OVA adsorbed to aluminum hydroxide gel as previously described (23). Briefly, mice were injected s.c. on days 1 and 8 with $0.2 \mathrm{ml}$ of a solution containing $100 \mu \mathrm{g}$ of OVA and $70 \mu \mathrm{g}$ of aluminum hydroxide (Reheiss).

\section{Leukocyte migration into the pleural cavity induced by Ag and chemoattractant agent}

Sensitized WT or PI3K $\gamma^{-1-}$ mice were challenged by intrapleural (i.pl.) administration of Ag (OVA) or PBS. In addition, to investigate the leukocyte migration into the pleural cavity induced by a chemoattractant agent, WT or PI3 $\mathrm{K}^{-1-}$ mice were injected into the pleural cavity with CXCL1 (30-100 ng/cavity), C5a (100 ng/cavity), and fMLP (200 ng/cavity). These were optimal doses to induce leukocyte recruitment in preliminary experiments (data not shown). The cells present in the pleural cavity were harvested $6 \mathrm{~h}$ after Ag challenge or chemoattractant agent administration by injecting $2 \mathrm{ml}$ of PBS and total cell counts performed in a modified Neubauer chamber using Turk's stain. This time was shown to be optimal for neutrophil recruitment in preliminary kinetics experiments. Differential cell counts were performed on cytospin preparations (Shandon III) stained with May-Grünwald-Giemsa using standard morphologic criteria to identify cell types. The results are presented as the number of cells per cavity

\section{Leukocyte migration into the lung induced by chemoattractant agent or bleomycin}

WT or PI3 $\mathrm{K}^{-1-}$ mice were injected intratracheally with $500 \mathrm{ng}$ of murine rCXCL1 or $0.125 \mathrm{U}$ of bleomycin. The stimulus was given in $50 \mu \mathrm{l}$ of sterile PBS. The control mice received PBS alone. The cells present in the alveolar space and the lung were harvested $6 \mathrm{~h}$ after CXCL1 administration or $48 \mathrm{~h}$ after bleomycin administration.

\section{Bronchoalveolar lavage (BAL)}

BAL was performed to obtain leukocytes in the alveolar spaces. The trachea was exposed and a $1.7-\mathrm{mm}$ outside-diameter polyethylene catheter was inserted. BAL was performed by instilling three 1-ml aliquots of PBS and $\sim 2 \mathrm{ml}$ of fluid was retrieved per mouse. The number of total leukocytes was determined by counting leukocytes in a modified Neubauer chamber after staining with Turk's solution. Differential counts were obtained from cytospin preparations by evaluating the percentage of each leukocyte on a slide stained with May-Grünwald-Giemsa.

\section{Quantification of neutrophil accumulation in lung tissue by myeloperoxidase (MPO) activity measurement}

The extent of neutrophil accumulation in the lung tissue was measured by assaying MPO activity as previously described (24). Using the conditions 
described below, this methodology is very selective for the determination of neutrophils over macrophages (data not shown). Before lung removal, the pulmonary vasculature was perfused with $3 \mathrm{ml}$ of PBS via the right ventricle. Upon thawing, the tissue $(0.1 \mathrm{~g}$ of tissue per $1.9 \mathrm{ml}$ of buffer) was homogenized in $\mathrm{pH} 4.7$ buffer $\left(0.1 \mathrm{M} \mathrm{NaCl}, 0.02 \mathrm{M} \mathrm{NaPO}_{4}, 0.015 \mathrm{M}\right.$ sodium EDTA), centrifuged at $3000 \times \mathrm{g}$ for $10 \mathrm{~min}$ and the pellet was subjected to hypotonic lyses $(1.5 \mathrm{ml}$ of $0.2 \% \mathrm{NaCl}$ solution followed $30 \mathrm{~s}$ later by addition of an equal volume of a solution containing $1.6 \% \mathrm{NaCl}$ and $5 \%$ glucose). After a further centrifugation, the pellet was resuspended in $0.05 \mathrm{M} \mathrm{NaPO}_{4}$ buffer (pH 5.4) containing $0.5 \%$ hexadecyl-trimethylammonium bromide and rehomogenized. One-milliliter aliquots of the suspension were transferred into $1.5-\mathrm{ml}$ Eppendorf tubes followed by three freeze-thaw cycles using liquid nitrogen. The aliquots were then centrifuged for $15 \mathrm{~min}$ at $3000 \times g$ to perform the assay. MPO activity in the resuspended pellet was assayed by measuring the change in $\mathrm{OD}_{450 \mathrm{~nm}} \mathrm{using}$ tetramethylbenzidine $(1.6 \mathrm{mM})$ and $\mathrm{H}_{2} \mathrm{O}_{2}(0.5 \mathrm{mM})$. The reaction was stopped by adding $100 \mu \mathrm{l}$ of $4 \mathrm{M} \mathrm{H}_{2} \mathrm{SO}_{4}$ and was quantified at $450 \mathrm{~nm}$ in a spectrophotometer (Emax; Molecular Devices). The neutrophil content was calculated from a standard curve based on MPO activity expressed as absorbance increase at $450 \mathrm{~nm}$ from $5 \%$ casein peritoneal-induced neutrophils assayed in parallel. The results were expressed in relative number of neutrophils per milligram of wet tissue.

\section{Leukocyte migration into an articular cavity}

The role of $\mathrm{PI} 3 \mathrm{~K} \gamma$ for neutrophil recruitment was also investigated after administration of CXCL1 in articular cavity. WT or PI3K $\gamma^{-1-}$ mice were injected into the tibia-femoral articulation with an optimal dose of murine rCXCL1 ( $30 \mathrm{ng} /$ cavity). The stimulus was given in $5 \mu \mathrm{l}$ of sterile PBS and control mice received PBS alone. The cells present in the tibia-femoral articulation were harvested $6 \mathrm{~h}$ after chemoattractant agent administration by washing the cavity with $30 \mu \mathrm{l}$ of PBS. The number of total leukocytes was determined by counting leukocytes in a modified Neubauer chamber after staining with Turk's solution. Differential counts were obtained from cytospin preparations by evaluating the percentage of each leukocyte on a slide stained with May-Grünwald-Giemsa.

\section{Treatment with inhibitors of $P I 3 K$}

The role of PI3K on neutrophils recruitment was also investigated by using selective inhibitors of PI3K, wortmannin and LY294002; the selective inhibitor of PI3K $\delta$, IC87114; and selective inhibitor of PI3K $\gamma$, AS605240. Wortmannin or LY294002 were administered systemically (i.p.) at the dose of 1.0 $\mathrm{mg} / \mathrm{kg} 60 \mathrm{~min}$ before the i.pl, administration of OVA or chemoattractant agent. This dose was shown to be effective in other experimental systems (25). Moreover, preliminary experiments showed the dose of $1.0 \mathrm{mg} / \mathrm{kg}$ to be maximally effective at inhibiting eosinophil recruitment in a similar model (26). IC87114, a highly selective inhibitor of $\mathrm{PI} 3 \mathrm{~K} \delta(20)$, was administered orally at the dose of $25 \mathrm{mg} / \mathrm{kg} 60 \mathrm{~min}$ before administration of chemoattractant agents. This dose was shown to be effective and selective in another model of leukocyte influx (21). AS605240, a highly selective inhibitor of $\mathrm{PI} 3 \mathrm{~K} \gamma(27)$, was administered orally at the dose of $50 \mathrm{mg} / \mathrm{kg} 60 \mathrm{~min}$ before administration of chemoattractant agents. This dose was shown to be effective and selective in vivo (27). Wortmannin and LY294002 were dissolved in DMSO and further diluted in PBS. IC87114 and AS605240 was suspended in $0.1 \%$ methylcellulose solution and ground in a homogenizer to ensure a uniform suspension. Control animals received drug vehicle.

\section{Preparation of bone marrow neutrophils}

The femurs and tibias from both hind limbs were removed of mice and freed of soft tissue attachments, and the extreme distal tip of each extremity was cut off. HBSS (without $\mathrm{Ca}^{2+}$ and $\mathrm{Mg}^{2+}$ ) was forced through the bone by using a 5 -ml syringe with a 22-gauge needle. After dispersing cell clumps and removing the debris, the bone marrow cells were suspended in $2 \mathrm{ml}$ of HBSS and laid on top of a two-layer Percoll gradient of 72 and $65 \%$ Percoll (Sigma-Aldrich) diluted in HBSS $(100 \%$ Percoll $=$ nine parts Percoll and one part 10× HBSS) and centrifuged $(1200 \times g, 30 \mathrm{~min}$, room temperature) without braking. The enriched neutrophil fraction was recovered at the interface between 65 and $72 \%$ Percoll. After washing twice with HBSS, $5.0 \pm 1.0 \times 10^{6}$ cells were obtained per mouse, $95 \%$ neutrophils, identified by staining the nuclei with Turk reagent (Merck).
FIGURE 1. Effects of the pretreatment with the nonselective PI3K inhibitor $(A)$, wortmannin, and PI3K $\delta$-selective inhibitor (B), IC87114, and PI3K $\gamma$-selective inhibitor, AS605240, on the recruitment of neutrophils induced by CXCL1 in WT and PI3 $\mathrm{K}^{-1-}$ mice. Wortmannin $(1.0 \mathrm{mg} /$ $\mathrm{kg}), \mathrm{IC} 87114(25 \mathrm{mg} / \mathrm{kg})$, and AS605240 $(50 \mathrm{mg} / \mathrm{kg})$ was administered $60 \mathrm{~min}$ before CXCL1 (100 ng/cavity) i.pl. administration. Neutrophil recruitment was assessed $6 \mathrm{~h}$ after chemokine injection. Results are expressed as the means \pm SEM of five mice in each group. $*, p<$ 0.01 when compared with PBS-injected mice; \#, $p<0.01$ when compared with vehicle-treated CXCL1-injected mice.

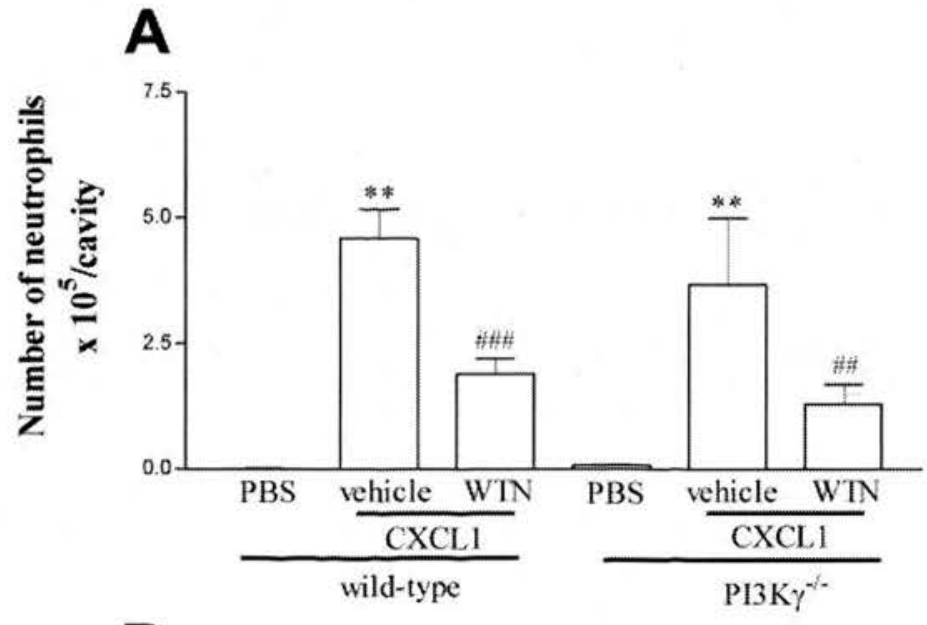

B

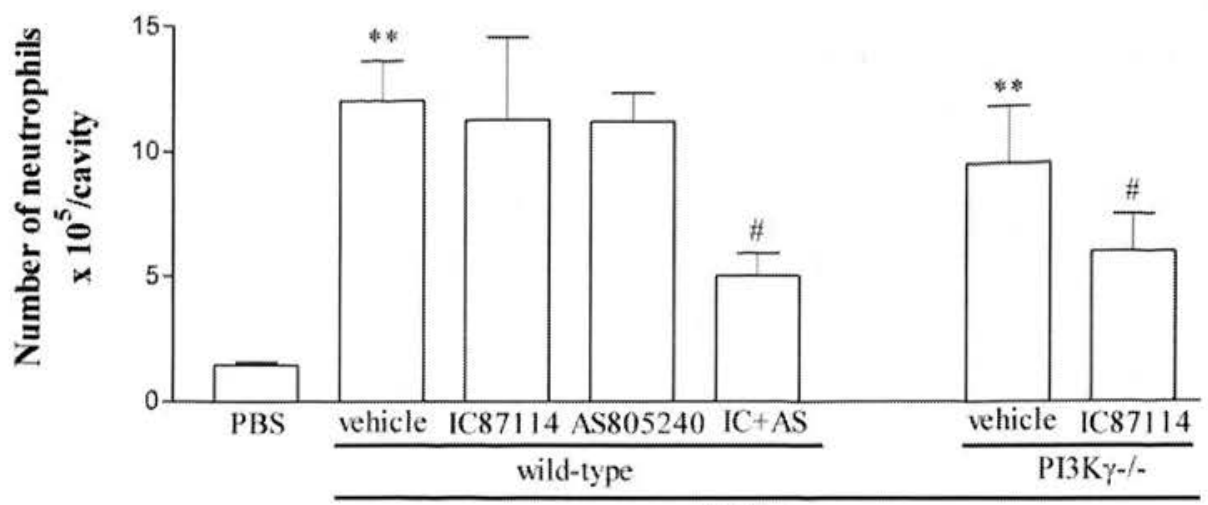

CXCL1 

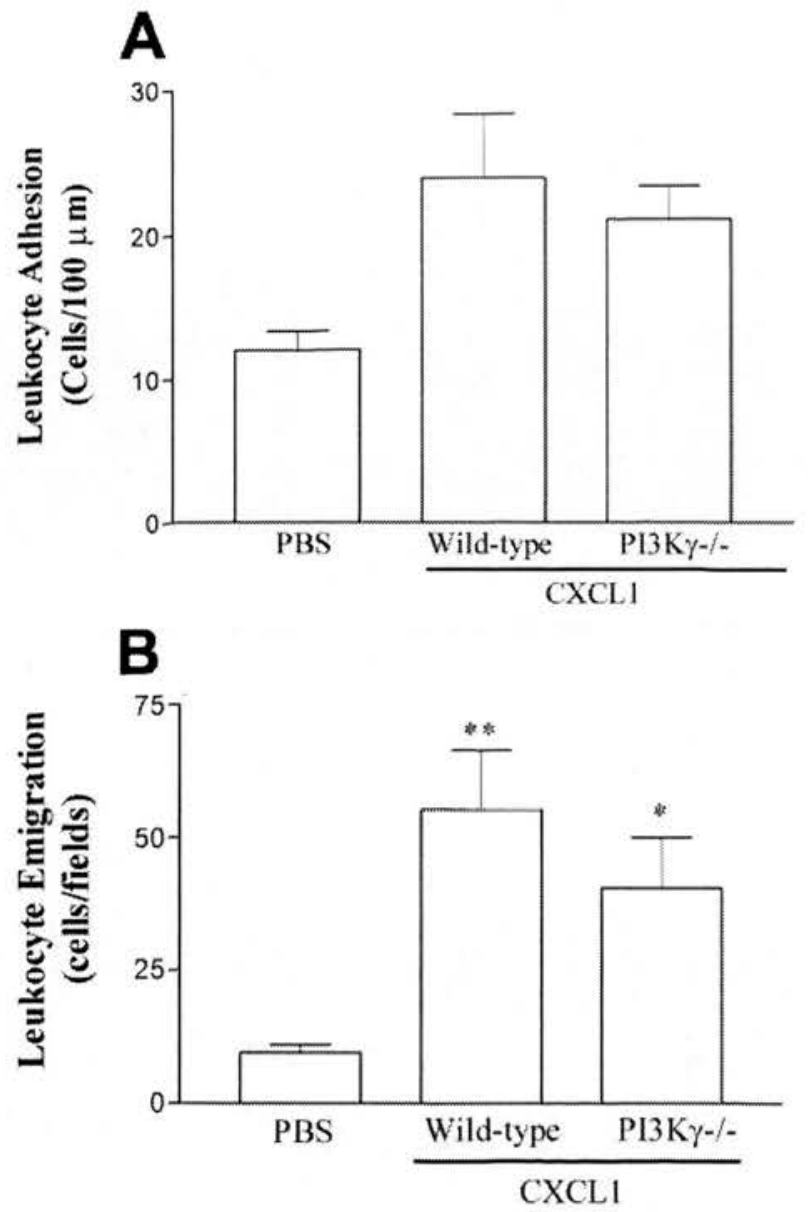

FIGURE 2. Neutrophil adhesion $(A)$ and emigration $(B)$ in the cremaster muscle of WT and PI3K $\gamma^{-1-}$ mice $2 \mathrm{~h}$ after intrascrotal administration of CXCL1 (500 ng/animal). Control mice were injected with PBS. Results are expressed as the means $\pm \mathrm{SEM}$ of four to five mice in each group. $*, p<$ 0.01 when compared with PBS-injected mice.

\section{Neutrophil chemotaxis}

A modified Boyden chamber assay to examine the neutrophil chemoattractant response to CXCL-1 and C5a was performed using a 48-well microchamber (Neuro Probe). Murine bone marrow neutrophils were isolated as above described and resuspended in running buffer $(1 \times$ HBSS, $2 \mathrm{mg} / \mathrm{ml} \mathrm{BSA}, 10 \mathrm{mM}$ HEPES, 1 $\mathrm{mM} \mathrm{CaCl}_{2}$, and $\left.1 \mathrm{mM} \mathrm{MgCl}\right)$. C5a (10 $\left.\mathrm{nM}\right)$ and recombinant mouse CXCL-1 $(20 \mathrm{ng} / \mathrm{ml})$ diluted in running buffer (for wells containing neutrophils) or appropriate buffer control was added to the lower chambers of the apparatus. A 5- $\mu \mathrm{m}$ pore polycarbonate membrane (Neuro Probe) was placed between the upper and lower chambers, and $5 \times 10^{4}$ cells in a volume of $50 \mu \mathrm{l}$ were added to the top chambers of the apparatus. Cells were allowed to migrate into the membrane for

Table I. Circulating leukocyte counts and venular diameter in untreated mice and mice treated intrascrotally with CXCLI (500 ng ${ }^{a}$

\begin{tabular}{llcc}
\hline \multicolumn{1}{c}{ Animal } & Treatment & $\begin{array}{c}\text { Circulating } \\
\text { Leukocyte } \\
\left(10^{\circ} / \mathrm{ml}\right)\end{array}$ & $\begin{array}{c}\text { Venular } \\
\text { Diameter } \\
(\mu \mathrm{m})\end{array}$ \\
\hline $\mathrm{WT}+\mathrm{PI} 3 \mathrm{~K} \gamma^{-1-}$ & PBS & $6.3 \pm 2.2$ & $30.9 \pm 2.3$ \\
WT & CXCL1 (500 ng) & $8.5 \pm 2.1$ & $33.4 \pm 1.7$ \\
$\mathrm{P} 3 \mathrm{~K} \gamma^{-1-}$ & CXCL1 (500 ng) & $6.8 \pm 1.3$ & $33.5 \pm 3.0$ \\
\hline
\end{tabular}

${ }^{a}$ Murine $\mathrm{rCXCL} 1$ in $0.2 \mathrm{ml}$ of saline was administered locally by s.c. injection beneath the right scrotal skin $2 \mathrm{~h}$ before exteriorization of cremaster muscle. The left cremaster was then prepared for intravital microscopy. Venular diameter was verified and circulating leukocyte kinetics were assessed in whole blood drawn by cardiac puncture. Total cell counts were performed in a modified Neubauer chamber using Turk's stain. Results are expressed as the means \pm SEM of five mice in each group.

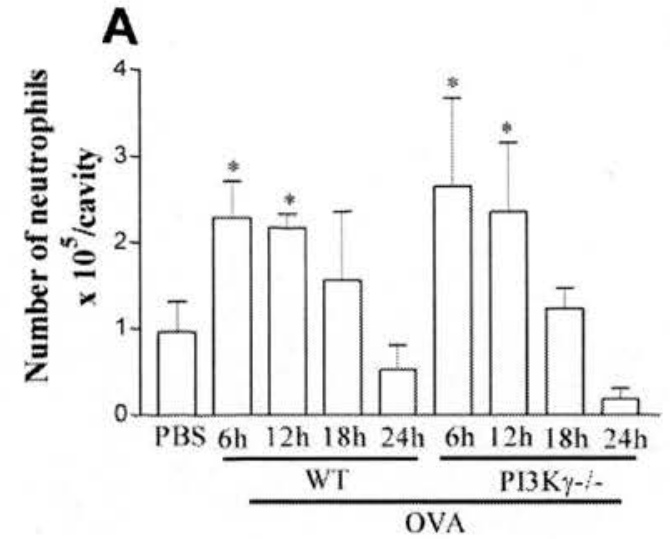

B
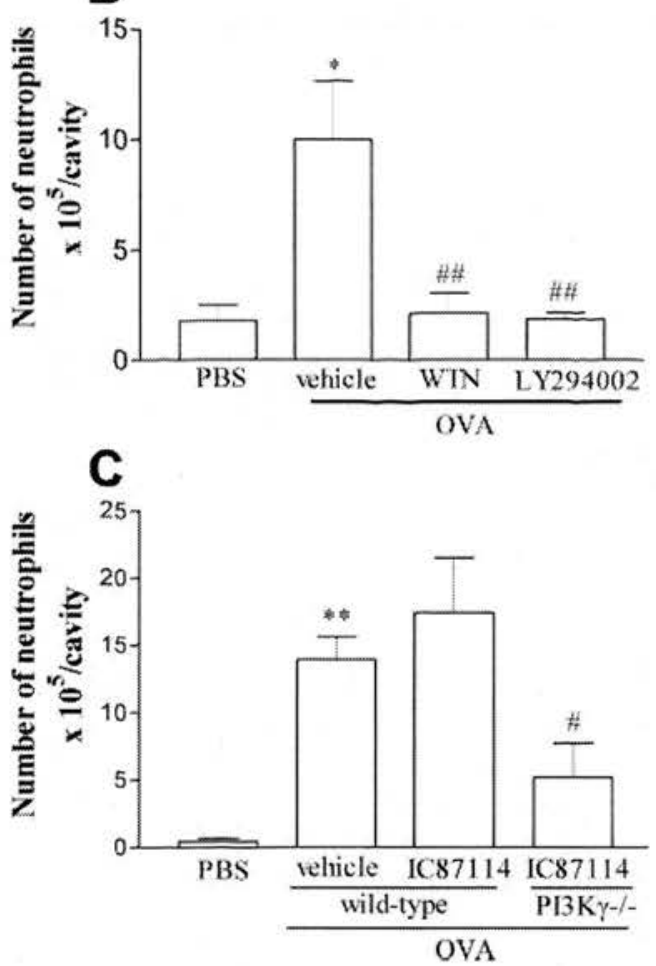

FIGURE 3. Role of PI3K in neutrophil accumulation induced by $\mathrm{Ag}$ challenge of sensitized PI3K $\gamma^{-1-}$ and WT mice. Neutrophil accumulation was assessed after $6 \mathrm{~h}$ in immunized WT or PI3K $\gamma^{-1-}$ mice and challenged with an i.pl. injection of OVA ( $1 \mu \mathrm{g} /$ cavity $)$ or PBS at the time point described in the figure $(A)$. Effects of the pretreatment with the nonselective PI3K inhibitors $(B)$, wortmannin $(1.0 \mathrm{mg} / \mathrm{kg})$, and LY294002 $(1.0 \mathrm{mg} / \mathrm{kg})$, and PI3K $\delta$-selective inhibitor $(C)$, IC $87114(25 \mathrm{mg} / \mathrm{kg})$, in the neutrophil accumulation induced by $\mathrm{Ag}$ were also verified. Results are expressed as the means \pm SEM of five mice in each group. $*, p<0.05$ when compared with PBS-injected mice; \#, $p<0.05$ when compared with vehicle-treated OVA-injected WT mice.

$1 \mathrm{~h}$ per treatment at $37^{\circ} \mathrm{C}$ with $5 \% \mathrm{CO}_{2}$. Following incubation, the chamber was disassembled and the membrane was scraped and washed three times in PBS to remove nonadherent cells before being fixed in methanol and stained using the Diff-Quik system (Dade Behring). Each well-associated membrane area was scored using light microscopy to count the intact cells present in five random fields. The results are expressed as the number of neutrophils per field.

\section{Statistical analysis}

All results are presented as the mean \pm SEM. Normalized data were analyzed by one-way ANOVA, and differences between groups were assessed using the Student-Newman-Keuls posttest. A $p$ value $<0.05$ was considered significant. 
FIGURE 4. Role of PI3K in neutrophil accumulation induced by C5a and fMLP in PI3K $\gamma^{-1-}$ and WT mice. $A$, C5a (100 ng/cavity) or, $B$, fMLP (200 $\mathrm{ng} /$ cavity) were injected in pleural cavity and neutrophil accumulation was assessed $6 \mathrm{~h}$ later. Results are expressed as the means \pm SEM of five mice in each group. $*, p<0.01$ when compared with PBSinjected mice; $\#, p<0.01$ when compared with OVA-injected WT mice.
A

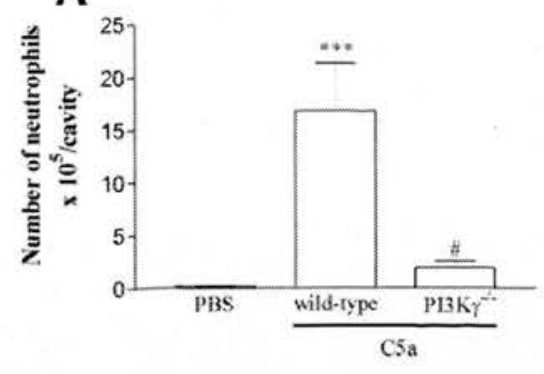

B

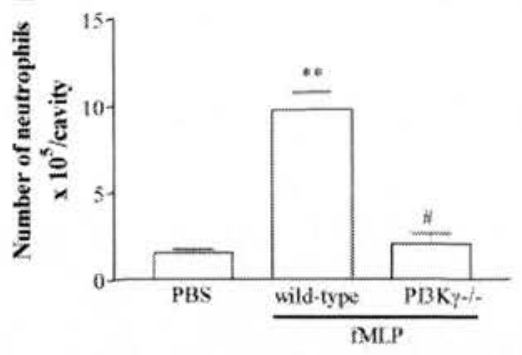

\section{Results}

Neutrophil recruitment induced by the i.pl. administration of 100 $\mathrm{ng}$ of CXCL1 was similar in PI3 $\mathrm{K}^{-1-}$ and WT mice (Fig. 1A). Neutrophil recruitment induced by lower doses (30 ng/cavity) of CXCL1 was also similar in PI3K $\gamma^{-1-}$ and WT mice (WT mice: $3.1 \pm 0.6 \times 10^{5}$ neutrophils/cavity; PI3K $\gamma^{-1-}$ mice: $2.1 \pm 0.4 \times$ $10^{5}$ neutrophils/cavity). Similar results were obtained using intravital microscopy in the CXCL1-treated cremaster muscle of $\mathrm{PI} 3 \mathrm{~K}^{-1-}$ or WT mice. The dose of CXCL1 used in these experiments was based in preliminary experiments and previous studies (28). Intrascrotal administration of $500 \mathrm{ng}$ of CXCL1 induced similar increases in leukocyte rolling (WT mice: $50.6 \pm 4.3 \mathrm{cell} / \mathrm{min}$; $\mathrm{PI} 3 \mathrm{~K}^{-1-}$ mice: $75.6 \pm 8.0 \mathrm{cell} / \mathrm{min}$ ), adhesion, and emigration in PI3 $\mathrm{K}^{-1-}$ or WT mice (Fig. 2). To identify the type of cells that had been recruited to the site of inflammation at this time point, $\mathrm{H} \& \mathrm{E}$ staining was performed on the tissues after the experiment. Neutrophils were the majority of the cells attached to endothelial cells or recruited to the cremaster muscle sections of both PI3K $\gamma^{-1-}$ or WT mice (data not shown). The circulating leukocyte counts were not different in these mice after administration of PBS or CXCL1 (Table I).

The next series of experiments were conducted in the pleural cavity for ease of performance. Despite the similar neutrophil recruitment in PI3K $\gamma^{-1-}$ and WT mice, treatment with the isoform nonselective PI3K inhibitor wortmannin $(1.0 \mathrm{mg} / \mathrm{kg})$ inhibited CXCL1-induced neutrophil recruitment both in PI3K $\gamma^{-1-}$ and WT mice (Fig. 1A). The latter results suggested that a PI3K was indeed relevant for chemokine-induced neutrophil influx. Treatment of WT mice with IC87114, a PI3K $\delta$ inhibitor, or AS605240, a PI3K $\gamma$ inhibitor, alone failed to affect the neutrophil recruitment induced by CXCL1 (Fig. 1B). However, the treatment of PI3K $\gamma^{-1-}$ mice with IC87114 and the concomitant treatment with both IC87114 and AS605240 significantly inhibited CXCL1-induced neutrophil recruitment (Fig. $1 B$ ). In vitro, the chemotaxis of bone marrowderived neutrophils obtained from PI3K $\gamma^{-1-}$ mice was decreased in comparison to their WT control (basal chemotaxis: WT, $5.0 \pm$ 0.4 neutrophils/field; PI3K $\gamma^{-1-}, 4.3 \pm 0.5$ neutrophils/field; CXCL1-induced chemotaxis: WT, $18.5 \pm 0.2$ neutrophils/field; $\mathrm{PI} 3 \mathrm{~K}^{-1-}, 10.5 \pm 0.4$ neutrophils/field, $p<0.05$ ).

We then examined whether PI $3 \mathrm{~K} \gamma$ deficiency might alter the neutrophil recruitment induced by endogenously generated CXCL1, i.e., after Ag challenge (OVA, $1 \mu \mathrm{g} /$ cavity) of sensitized mice. The i.pl. injection of OVA in sensitized WT mice induced a maximal neutrophil recruitment that occurred between 6 and $12 \mathrm{~h}$ and had dropped to baseline levels $24 \mathrm{~h}$ later (Fig. 3A). The neutrophil accumulation after $\mathrm{Ag}$ challenge was not altered in PI $3 \mathrm{~K} \gamma$ deficient mice (Fig. 3A). Treatment of sensitized WT mice with a CXCR2 antagonist greatly reduced OVA-induced neutrophil accumulation (PBS, $1.8 \pm 0.7 \times 10^{5}$ neutrophil/cavity; OVA, $10.0 \pm$ $2.6 \times 10^{5}$ neutrophils/cavity; OVA + CXCR2 antagonist, $2.9 \pm$ $1.6 \times 10^{5}$ neutrophils/cavity, $n=5, p<0.05$ ), demonstrating that the
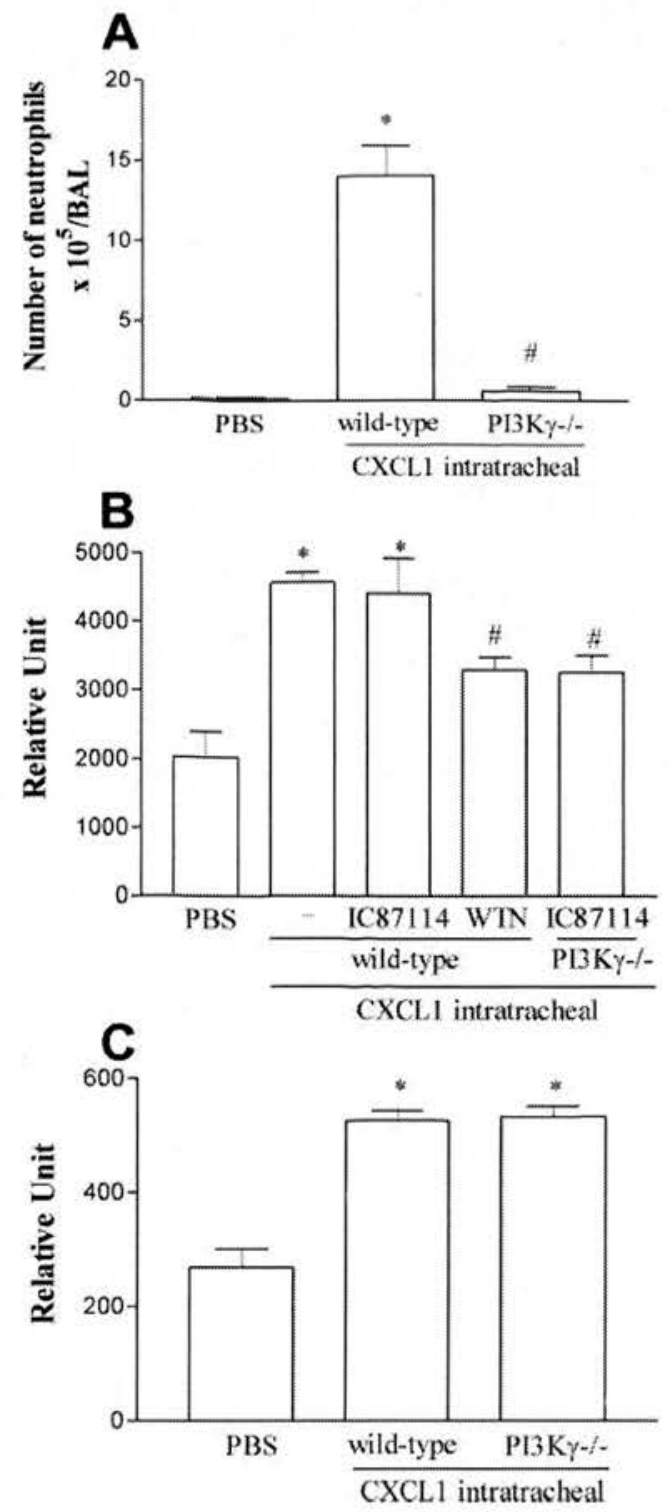

FIGURE 5. Role of PI3K in neutrophil recruitment to lung after intratracheal administration of CXCL1. CXCL1 (500 ng/animal) was administered intratracheally in WT and PI3K $\gamma^{-1-}$ mice. The BAL and lung removal was performed $6 \mathrm{~h}$ after chemokine administration. $A$, Neutrophil influx to alveolar spaces; $B, C X C L 1$-induced recruitment of neutrophil to lung tissues assessed by measuring tissue MPO content; and $C$, CXCL1induced recruitment of neutrophil to lung tissues assessed by measuring tissue MPO content after IC87114 treatment. Results are expressed as the means \pm SEM of five mice in each group. $*, p<0.01$ when compared with PBS-injected mice; \#, $p<0.01$ when compared with CXCL1-injected WT mice. 

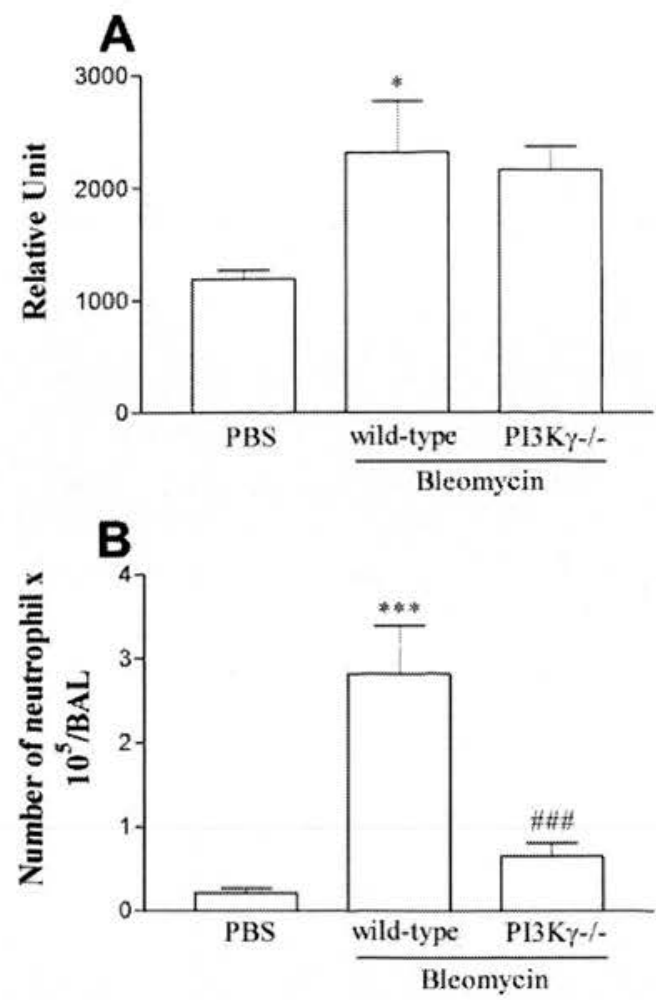

FIGURE 6. Role of PI3K in neutrophil recruitment to lung after intratracheal administration of bleomycin. Bleomycin $(0.125 \mathrm{U} / \mathrm{animal})$ was administered intratracheally in WT and PI $3 \mathrm{~K}^{-1-}$ mice. Lung removal was performed $48 \mathrm{~h}$ after bleomycin administration. $A$, Neutrophil influx to alveolar spaces and, $B$, bleomycin-induced recruitment of neutrophil to lung tissues assessed by measuring tissue MPO content. Results are expressed as the means \pm SEM of five mice in each group. $*, p<0.01$ when compared with PBS-injected mice; \#, $p<0.01$ when compared with OVAinjected WT mice.

neutrophil recruitment induced by $\mathrm{Ag}$ challenge of sensitized mice depended mainly on CXC chemokine production and CXCR2 activation. Treatment with wortmannin or LY294002, two structurally distinct PI3K inhibitors, diminished the accumulation of neutrophils induced by Ag challenge (Fig. 3B). Treatment of WT mice with IC 87114 did not affect the neutrophil recruitment induced by $\mathrm{Ag}$ challenge of sensitized mice. However, the treatment of PI3 $\mathrm{K}^{-1-}$ mice with IC87114 diminished the influx of neutrophils into the pleural cavity (Fig. 3C).

Using an in vitro chemotaxis assay, it has been previously shown that C5a-induced chemotaxis is impaired in neutrophils from PI3K $\gamma^{-1-}$ mice (19). In experiments with bone marrow-derived neutrophils, C5a-induced chemotaxis was partially diminished in PI3K $\gamma^{-1-}$ mice vs WT (basal chemotaxis: WT, $5.0 \pm 0.4$ neutrophils/field; $\mathrm{PI} 3 \mathrm{~K}^{-\prime-}, 4.3 \pm 0.5$ neutrophils/field; $\mathrm{C} 5 \mathrm{a}$-induced chemotaxis: WT, $17.8 \pm 1.2$ neutrophils/field; PI3K $\gamma^{-1-}$, $11.4 \pm 1.2$ neutrophils/field, $p<0.05$ ). In our in vivo experiments, the recruitment of neutrophils induced by i.pl. administration of C5a was abrogated in PI3K $\gamma^{-1-}$ mice (Fig. 4A). Similarly, neutrophil recruitment induced by the administration of the chemoattractant molecule fMLP was abrogated in $\mathrm{PI} 3 \mathrm{~K}^{-1-}$ mice as compared with their WT controls (Fig. 4B).

The recruitment of neutrophils to lung tissue and BAL fluid was evaluated after instillation of CXCL1 or bleomycin. Neutrophil influx to alveolar spaces was totally prevented in PI3K $\gamma$-deficient mice (Fig. 5A). In contrast, the deficiency of PI3K $\gamma$ had no effect on CXCL1-induced recruitment of neutrophils to lung tissues of mice, as assessed by measuring tissue MPO content (Fig. 5B). Treatment of WT mice with IC87114 failed to affect CXCL1-induced recruitment of neutrophils to lung tissues (Fig. 5C). However, the amount of neutrophils in lung tissues was significantly inhibited in PI3K $\gamma^{-1-}$ mice treated with IC87114 (Fig. 5C).

Bleomycin-induced neutrophil influx depends of the release of CXCL1 into the alveolar space and activation of CXCR2 receptors (R. de Castro Russo and M. M. Teixeira, unpublished data). In agreement with the findings after CXCL1 administration, neutrophil influx to alveolar space after bleomycin administration was markedly diminished in PI3K $\gamma^{-1-}$ as compared with WT mice (Fig. 6A) and the recruitment of neutrophils to lung tissues was unaltered in PI3K $\gamma^{-1-}$ mice given bleomycin (Fig. $6 B$ ).

Furthermore, the role of PI3K $\gamma$ in neutrophil recruitment induced by CXCL1 was further confirmed in another model of neutrophil recruitment using the tibia-femoral joint. Neutrophil influx into the tibia-femoral joint was not significantly affected in PI3K $\gamma$-deficient mice (number of neutrophils $\times 10^{6}$ cavity: WT mice: PBS, $1.2 \pm 0.9$; CXCL1, $12.8 \pm 5.9$; PI3K $\gamma^{-1-}$ mice: PBS, $0.2 \pm 0.1$; CXCL1, $12.4 \pm 6.4, n=5, p>0.05$ when comparing WT vs PI3K $\gamma^{-1-}$ mice).

\section{Discussion}

The present study investigated the role of $\mathrm{PI} 3 \mathrm{~K} \gamma$ and $\mathrm{PI} 3 \mathrm{~K} \delta$ for neutrophil influx induced by the exogenous administration or the endogenous generation of the chemokine CXCL1. Overall, administration of CXCL1 induced leukocyte migration that was similar in PI3K $\gamma^{-1-}$ or WT mice, or in mice treated with a specific $\mathrm{PI} 3 \mathrm{~K} \delta$ inhibitor, IC87114, or a specific PI3K $\gamma$ inhibitor, AS605240. CXCR2-dependent neutrophil recruitment was only inhibited when there was concomitant inhibition of both $\mathrm{PI} 3 \mathrm{~K} \delta$ and $\mathrm{PI} 3 \mathrm{~K} \gamma$. In contrast, neutrophil recruitment into alveolar space or in response to exogenous administration of C5a or fMLP appeared to rely solely on PI3K $\gamma$.

We demonstrated that PI $3 \mathrm{~K} \gamma$ deficiency did not interfere significantly with CXCL1-induced rolling, adhesion, and transmigration of neutrophil in cremaster muscle. Recently, it was shown that PI3K $\gamma$-deficient mice presented a decrease in CXCL1-induced leukocyte adhesion in venules of the exteriorized mouse cremaster muscle (29). However, the experimental conditions used by the authors differ from ours in an important point. Although the chemotactic stimulus was injected i.v. and leukocyte adhesion was counted $1 \mathrm{~min}$ after the administration of CXCL1, CXCL1 was given locally (i.e., into the tissue) in our experiments and rolling, adhesion, and emigration were evaluated $2 \mathrm{~h}$ later. Thus, whereas in our experiments the inflammatory stimulus was present in the tissues where they are usually generated, in the previous study (29), the chemokine was given i.v. In the latter case, there may be stimulation (activation) of leukocytes before they migrate into tissue permitting leukocyte adherence in vessels but not their migration into tissue. Leukocyte activation may mimic the situation of chemotaxis in vitro and neutrophil chemotaxis induced by chemotactic agents is dependent on PI3K $\gamma$ (Ref. 30 and our results). In contrast, injection of stimulus in the tissue was PI3K $\gamma$ independent and correlated better with the PI $3 \mathrm{~K} \gamma$-independent chemokinetic activity of neutrophils $(30,31)$. More recently, Liu et al. (38) showed that neutrophil emigration in response to CXCL1 was PI3K $\gamma$ dependent at least up to $90 \mathrm{~min}$ after the addition of stimulus and was PI $3 \mathrm{~K} \gamma$ independent thereafter. The results in later time points are consistent with the PI $3 \mathrm{~K} \gamma$-independent neutrophil influx in response to $\mathrm{Ag}$ and CXCL1 in our systems. Further studies are certainly needed to understand in greater detail the relevance of these in vitro phenomena (chemotaxis vs chemokinesis) for neutrophil recruitment in vivo and how the whole process is modulated by $\mathrm{PI} 3 \mathrm{~K} \gamma$. 
Although CXCL1-induced accumulation of neutrophils into the pleural cavity was similar in PI3K $\gamma$-deficient and WT mice, treatment with an isoform nonselective PI3K inhibitor, wortmannin, inhibited neutrophil accumulation induced by CXCL1. Wortmannin was capable of preventing neutrophil influx both in WT and $\mathrm{PI} 3 \mathrm{~K} \gamma^{-1-}$ mice. Previous studies have demonstrated that activation of $\mathrm{PI} 3 \mathrm{~K} / \mathrm{Akt}$ is required for the induction of human neutrophil migration in response to CXCL8 (a homolog of murine CXCL1) $(6,32)$. Thus, although CXC chemokine-induced neutrophil recruitment appears to be PI3K dependent, it appears that sole blockade (or absence) of PI3K $\gamma$ is not sufficient to prevent neutrophil influx induced by CXCL1. In contrast to our findings, Hirsch et al. (19) demonstrated that neutrophil influx induced by IL-8 was partially inhibited in $\mathrm{PI} 3 \mathrm{~K}^{-1-}$ mice. One possibility to explain the discrepancies between those and our findings could be the need for the stimulation of intermediate cells and molecules for human IL-8 to induce recruitment of neutrophils in rodents $(33,34)$.

Recent reports have also suggested a role for $\mathrm{PI} 3 \mathrm{~K} \delta$ in promoting neutrophil trafficking in a mouse model of TNF- $\alpha$-induced inflammation and in a model of acute pulmonary inflammation induced by LPS in rats $(20,21)$. Treatment with a PI3K $\delta$ inhibitor, IC87114, did not affect CXCL1-induced neutrophil recruitment into the pleural cavity of WT mice at a dose previously shown to be effective in vivo (21). However, the data with wortmannin still suggested that the recruitment of neutrophils induced by CXCL1 would still depend on the activation of other PI3K isoforms or of the joint action of diverse isoforms. Sadhu et al. (15) have proposed that different PI3K isoforms may be activated in response to in vitro stimulation of GPCRs leading to human neutrophil migration. In our experiments, we demonstrated that inhibition of PI3K $\delta$ in PI3K $\gamma^{-1-}$ mice or treatment with inhibitors of both enzymes was accompanied by significant inhibition of neutrophil migration induced by exogenous administration of CXCL1. This result suggests that neutrophil recruitment following triggering of CXCR2 depends on the function of both PI3K $\gamma$ and PI3K $\delta$. In human neutrophils, $\mathrm{PI} 3 \mathrm{~K} \gamma$ and $\mathrm{PI} 3 \mathrm{~K} \delta$ act together to amplify PIP3 production leading to cell polarization and chemotaxis (15). In accordance with the latter finding, $\mathrm{PI} 3 \mathrm{~K} \gamma / \mathrm{PI} 3 \mathrm{~K} \delta^{-1-}$ mice have a profound reduction of $\mathrm{T}$ cell development (35).

It has been shown that PI3K $\gamma$ deficiency affected the ability of the endothelium to adhere neutrophils, most likely due to a reduction in E-selectin-mediated rolling (36). However, deficiency in p $110 \gamma$ and p1 $10 \delta$ catalytic subunits of PI3K in the venular endothelium had an additive effect in the ability of this cell type to recruit neutrophils in response to TNF- $\alpha$ stimulation (36). More recent studies from the same group show that PI $3 \mathrm{~K} \gamma$ and $\mathrm{PI} 3 \mathrm{~K} \delta$ have temporally distinct roles in mediating leukocyte recruitment in vivo (31). Thus, $\mathrm{PI} 3 \mathrm{~K} \gamma$ and $\mathrm{PI} 3 \mathrm{~K} \delta$ mediate neutrophil influx in a kinetic- (31), tissue-, and stimulus- (this study) dependent manner.

A series of experiments were then conducted to examine the role of PI $3 \mathrm{~K} \gamma$ and PI $3 \mathrm{~K} \delta$ for the recruitment of neutrophils induced by administration of $\mathrm{Ag}$ to immunized mice. In this model, we show that neutrophil recruitment is totally dependent on the endogenous release of CXCR2-acting chemokines. As such, Ag-induced neutrophil recruitment was unchanged in $\mathrm{PI} 3 \mathrm{~K} \gamma$-deficient mice but was abrogated by treatment with PI3K-nonselective inhibitors. Administration of the PI $3 \mathrm{~K} \delta$ inhibitor failed to alter neutrophil recruitment in WT mice. In contrast, the PI $3 \mathrm{~K} \delta$ inhibitor greatly prevented neutrophil recruitment in Ag-challenged PI3K $\gamma$-deficient mice. Altogether, the latter results demonstrate that endogenous CXCR2-acting chemokines, such as CXCL1, are necessary for Ag-induced neutrophil influx and work via activation of both $\mathrm{PI} 3 \mathrm{~K} \gamma$ and $\mathrm{PI} 3 \mathrm{~K} \delta$.
Neutrophil infiltration into BAL fluid after intranasal administration of CXCL1 was greatly diminished in PI3K $\gamma$-deficient mice, an effect consistent with other studies (37). However, neutrophil influx into pulmonary tissue was not dependent on $\mathrm{PI} 3 \mathrm{~K} \gamma$. It has been demonstrated that intravascular, interstitial, and intra-alveolar accumulation of neutrophils may be differentially regulated; indeed, polymorphonuclear neutrophils can enter the pulmonary interstitium without advancing to the alveolar airway space and crossing the epithelial barrier (38). Thus, the differential mechanisms used by neutrophils to cross endothelial and epithelial cells may explain the differential use of PI3K $\gamma$ for neutrophil influx into lung tissues and alveolar spaces. However, the latter possibility needs to be investigated in more detail.

We also demonstrated in a model of bleomycin-induced pulmonary injury that deficiency of PI $3 \mathrm{~K} \gamma$ inhibited neutrophil influx into the alveolar space but did not interfere with neutrophil recruitment to lung tissue. In this model of pulmonary inflammation, neutrophil recruitment is associated with endogenous production and action of CXCL1 on CXCR2 receptors (R. de Castro Russo and M. M. Teixeira, unpublished data). Therefore, CXCL1-dependent neutrophil transmigration through lung endothelial cells depends on the cooperative action of both PI $3 \mathrm{~K} \gamma$ and PI $3 \mathrm{~K} \delta$. In contrast, migration through epithelial cells is totally dependent on PI3K $\gamma$.

There is evidence that GPCRs are able to activated class IA PI3K $(39,40)$. How might PI3K $\delta$ be activated by GPCRs? It is proposed that an initial burst of PIP 3 induced by PI $3 \mathrm{~K} \gamma$ activation could activate Rac and Src family tyrosine kinases that could then activate PI $3 \mathrm{~K} \delta(15)$. It is not clear whether the latter hypothesis would be functional in our experiments, as one would expect complete blockade of migration once $\mathrm{PI} 3 \mathrm{~K} \gamma$ or $\mathrm{PI} 3 \mathrm{~K} \delta$ were blocked. Additional experiments are necessary to understand the interplay (or functional relationships) between these two isoforms in vivo.

We also evaluated the importance of $\mathrm{PI} 3 \mathrm{~K} \gamma$ for neutrophil recruitment to the pleural cavity induced by the complement fragment C5a and the bacterial-derived chemotactic peptide fMLP. C5a is a potent chemoattractant for neutrophils, basophils, eosinophils, monocytes, and macrophages and is released after complement activation at sites of inflammation $(41,42)$. The role of PI $3 \mathrm{~K} \gamma$ to C5a-induced neutrophil migration is supported by previous studies demonstrating the inhibition of $\operatorname{PtdIns}(3,4,5) \mathrm{P} 3$ production and chemotaxis after in vitro stimulation of neutrophil with C5a in PI3K $\gamma^{-1-}$ neutrophils (19). It is well-known also that neutrophil recruitment in response to Gram-negative infection involves bacterial-derived chemotactic factors such as $N$-formyl peptides (fMLP) and that, in the absence of PI3K neutrophils, cannot migrate toward fMLP $(5,19,43,44)$. In agreement with these previous data, the recruitment of neutrophils induced by C5a and fMLP was prevented in PI3K $\gamma^{-1-}$ mice.

In conclusion, we demonstrate there is differential usage of $\mathrm{PI} 3 \mathrm{~K} \gamma$ when neutrophil recruitment is induced by different chemoattractant molecules (CXCL1, C5a, and FMLP) and whether recruitment occurs across endothelial or epithelial barriers. Whereas C5a and fMLP appear to rely entirely on PI3K $\gamma$ for induction of neutrophil influx, CXCL1-mediated effects largely depend on the redundant role of PI $3 \mathrm{~K} \gamma$ and PI $3 \mathrm{~K} \delta$. Neutrophil emigration across the epithelium also relies on PI $3 \mathrm{~K} \gamma$ expression. The redundant role of and cooperation between signaling molecules and enzymes may be fundamental in providing additional specificity and complexity to signals initiated after seven-transmembrane receptor activation.

\section{Acknowledgments}

We thank Valdinéria Borges and Ilma Marçal for technical assistance. 


\section{Disclosures}

Joel S. Hayflick is an employee of ICOS Corporation and Christian Rommel and Thomas Ruckle are employees of Merck Serono. These companies have commercial interest in developing PI3K-selective inhibitors for the treatment of inflammatory disease. The other authors declare no financial conflict of interest.

\section{References}

1. Kapeller, R., and L. C. Cantley. 1994. Phosphatidylinositol 3-kinase. BioEssays 16: $565-576$.

2. Vanhaesebroeck, B., and M. D. Waterfield. 1999. Signaling by distinct classes of phosphoinositide 3-kinases. Exp. Cell Res. 253: 239-254.

3. Toker, A., and L. C. Cantley. 1997. Signalling through the lipid products of phosphoinositide-3-OH kinase. Nature 387: 673-676.

4. Lehrer, R. I., T. Ganz, M. E. Selsted, B. M. Babior, and J. T. Curnutte. 1988. Neutrophils and host defense. Ann. Intern. Med. 109: 127-142.

5. Le, Y., P. M. Murphy, and J. M. Wang. 2002. Formyl-peptide receptors revisited. Trends Immunol. 23: 541-548.

6. Heit, B., S. Tavener, E. Raharjo, and P. Kubes. 2002. An intracellular signaling hierarchy determines direction of migration in opposing chemotactic gradients. J. Cell Biol. 159: 91-102,

7. Godaly, G., G. Bergsten, L. Hang, H. Fischer, B. Frendeus, A. C. Lundstedt, M. Samuelsson, P. Samuelsson, and C. Svanborg. 2001. Neutrophil recruitment, chemokine receptors, and resistance to mucosal infection. J. Leukocyte Biol. 69 : 899-906.

8. Jeyaseelan, S., H. W. Chu, S. K. Young, M. W. Freeman, and G. S. Worthen 2005. Distinct roles of pattern recognition receptors CD14 and Toll-like receptor 4 in acute lung injury. Infect. Immun. 73: 1754-1763.

9. Netea, M. G., C. A. Van Der Graaf, A. G. Vonk, I. Verschueren, J. W. Van Der Meer, and B. J. Kullberg. 2002. The role of Toll-like receptor (TLR) 2 and TLR4 in the host defense against disseminated candidiasis. J. Infect. Dis. 185: 1483-1489.

10. Baggiolini, M., F. Boulay, J. A. Badwey, and J. T. Curnutte. 1993. Activation of neutrophil leukocytes: chemoattractant receptors and respiratory burst. FASEB J. 11: $1004-1010$

11. Cicchetti, G., P. G. Allen, and M. Glogauer. 2002. Chemotactic signaling pathways in neutrophils: from receptor to actin assembly. Crit. Rev. Oral Biol. Med. 13: $220-228$

12. Niggli, V. 2003. Signaling to migration in neutrophils: importance of localized pathways. Int. J. Biochem. Cell Biol. 35: 1619-1638.

13. Curnock, A. P., M. K. Logan, and S. G. Ward. 2002. Chemokine signalling: pivoting around multiple phosphoinositide 3-kinases. Immunology 105: 125-136.

14. Fruman, D. A., and L. C. Cantley. 2002. Phosphoinositide 3-kinase in immunological systems. Semin. Immunol. 14: 7-18.

15. Sadhu, C., B. Masinovsky, K. Dick, C. G. Sowell, and D. E. Staunton. 2003. Essential role of phosphoinositide 3-kinase $\delta$ in neutrophil directional movement. J. Immunol. 170: 2647-2654.

16. Stephens, L., A. Smrcka, F. T. Cooke, T. R. Jackson, P. C. Sternweis, and P. T. Hawkins. 1994. A novel phosphoinositide 3 kinase activity in myeloidderived cells is activated by $\mathrm{G}$ protein $\beta \gamma$ subunits. Cell 77: 83-93.

17. Yum, H. K., J. Arcaroli, J. Kupfner, R. Shenkar, J. M. Penninger, T. Sasaki, K. Y. Yang, J. S. Park, and E. Abraham. 2001. Involvement of phosphoinositide 3-kinases in neutrophil activation and the development of acute lung injury. J. Immunol. 167: 6601-6608.

18. Sasaki, T., J. Irie-Sasaki, R. G. Jones, A. J. Oliveira-Dos-Santos, W. L. Stanford, B. Bolon, A. Wakeham, A. Itie, D. Bouchard, I. Kozieradzki, et al. 2000. Function of PI3K $\gamma$ in thymocyte development, T cell activation, and neutrophil migration. Science 287: 1040-1046.

19. Hirsch, E., V. L. Katanaev, C. Garlanda, O. Azzolino, L. Pirola, L. Silengo, S. Sozzani, A. Mantovani, F. Altruda, and M. P. Wymann. 2000. Central role for $\mathrm{G}$ protein-coupled phosphoinositide 3-kinase $\gamma$ in inflammation. Science 287: 1049-1053.

20. Sadhu, C., K. Dick, W. T. Tino, and D. E. Staunton. 2003. Selective role of PI3K $\delta$ in neutrophil inflammatory responses. Biochem. Biophys. Res. Commun. 308 : 764-769.

21. Puri, K. D., T. A. Doggett, J. Douangpanya, Y. Hou, W. T. Tino, T. Wilson, T. Graf, E. Clayton, M. Turner, J. S. Hayflick, and T. G. Diacovo. 2004. Mechanisms and implications of phosphoinositide 3-kinase $\delta$ in promoting neutrophil trafficking into inflamed tissue. Blood 103: 3448-3456.

22. Kanwar, S., D. C. Bullard, M. J. Hickey, C. W. Smith, A. L. Beaudet, B. A. Wolitzky, and P. Kubes. 1997. The association between $\alpha_{4}$-integrin, Pselectin, and E-selectin in an allergic model of inflammation. J. Exp. Med. 185 1077-1087.
23. Klein, A., A. Talvani, D. C. Cara, K. L. Gomes, N. W. Lukacs, and M. M. Teixeira 2000. Stem cell factor plays a major role in the recruitment of eosinophils in allergic pleurisy in mice via the production of leukotriene B4. J. Immunol. 164: 4271-4276.

24. De Matos, I. M., D. G. Souza, D. G. Seabra, L. Freire-Maia, and M. M. Teixeira. 1999. Effects of tachykinin NK1- or PAF-receptor blockade on the lung injury induced by scorpion venom. Eur. J. Pharmacol. 376: 293-300.

25. Ezeamuzie, C. I., J. Sukumaran, and E. Philips. 2001. Effects of wortmannin on human eosinophil response in vitro and on bronchial inflammation and airway hyperresponsiveness in guinea pigs in vivo. Am. J. Respir. Crit. Care Med. 164: 1633-1639.

26. Pinho, V., D. G. Souza, M. M. Barsante, F. P. Hamer, M. S. De Freitas, A. G. Rossi, and M. M. Teixeira. 2005. Phosphoinositide-3 kinases critically regulate the recruitment and survival of eosinophils in vivo: importance for the resolution of allergic inflammation. J. Leukocyte Biol. 77: 800-810,

27. Camps, M., T. Ruckle, H. Ji, V. Ardissone, F. Rintelen, J. Shaw, C. Ferrandi, C. Chabert, C. Gillieron, B. Francon, et al. 2005. Blockade of PI3K $\gamma$ suppresses joint inflammation and damage in mouse models of rheumatoid arthritis. Nat. Med. 11: 936-943.

28. Zhang, X. W., Q. Liu, Y. Wang, and H. Thorlacius. 2001. CXC chemokines, MIP-2 and KC, induce P-selectin-dependent neutrophil rolling and extravascular migration in vivo. Br. J. Pharmacol. 133: 413-421.

29. Smith, D. F., T. L. Deem, A. C. Bruce, J. Reutershan, D. Wu, and K. Ley. 2006. Leukocyte phosphoinositide-3 kinase $\gamma$ is required for chemokine-induced, sustained adhesion under flow in vivo. J. Leukocyte Biol. 80: 1491-1499.

30. Ferguson, G. J., L. Milne, S. Kulkarni, T. Sasaki, S. Walker, S. Andrews, T. Crabbe, P. Finan, G. Jones, S. Jackson, et al. 2007. PI3K $\gamma$ has an important context-dependent role in neutrophil chemokinesis. Nat. Cell. Biol. 9: 86-91.

31. Liu, L., K. D. Puri, J. M. Penninger, and P. Kubes. 2007. Leukocyte PI3K $\gamma$ and PI3K $\delta$ have temporally distinct roles for leukocyte recruitment in vivo. Blood 110: $1191-1198$.

32. Knall, C., G. S. Worthen, and G. L. Johnson. 1997. Interleukin 8-stimulated phosphatidylinositol-3-kinase activity regulates the migration of human neutrophils independent of extracellular signal-regulated kinase and p38 mitogen-activated protein kinases. Proc. Natl. Acad. Sci. USA. 94: 3052-3057.

33. Ribeiro, R. A., C. A. Flores, F. Q. Cunha, and S. H. Ferreira. 1991. IL-8 causes in vivo neutrophil migration by a cell-dependent mechanism. Immunology 73 : $472-477$.

34. Ramos, C. D., N. E. Heluy-Neto, R. A. Ribeiro, S. H. Ferreira, and F. Q. Cunha. 2003. Neutrophil migration induced by IL-8-activated mast cells is mediated by CINC-1. Cytokine 7: 214-223.

35. Webb, L. M., E. Vigorito, M. P. Wymann, E. Hirsch, and M. Turner. 2005. Cutting edge: $\mathrm{T}$ cell development requires the combined activities of the p110 $\gamma$ and pl $10 \delta$ catalytic isoforms of phosphatidylinositol 3-kinase. J. Immunol. 175: 2783-2787.

36. Puri, K. D., T. A. Doggett, C. Y. Huang, J. Douangpanya, J. S. Hayflick, M. Turner, J. Penninger, and T. G. Diacovo. 2005. The role of endothelial PI3K $\gamma$ activity in neutrophil trafficking. Blood 106: 150-157.

37. Thomas, M. J. A. Smith, D. H. Head, L. Milne, A. Nicholls, W. Pearce, B. Vanhaesebroeck, M. P. Wymann, E. Hirsch, A. Trifilieff, et al. 2005. Airway inflammation: chemokine-induced neutrophilia and the class I phosphoinositide 3-kinases. Eur. J. Immunol. 35: 1283-1291.

38. Li, Q. P. W. Park, C. L. Wilson, and W. C. Parks. 2002. Matrilysin shedding of syndecan-1 regulates chemokine mobilization and transepithelial efflux of neutrophils in acute lung injury. Cell 111: 635-646.

39. Curnock, A. P., Y. Sotsios, K. L. Wright, and S. G. Ward. 2003. Optimal chemotactic responses of leukemic $\mathrm{T}$ cells to stromal cell-derived factor-1 requires the activation of both class IA and IB phosphoinositide 3-kinases. J. Immunol. 170: $4021-4030$

40. Vicente-Manzanares, M., M. Rey, D. R. Jones, D. Sancho, M. Mellado, J. M. Rodriguez-Frade, M. A. del Pozo, M. Yanez-Mo, A. M. de Ana, C. Martinez-A, et al. 1999. Involvement of phosphatidylinositol 3-kinase in stromal cell-derived factor-1 $\alpha$-induced lymphocyte polarization and chemotaxis. J. Immunol. 163: 4001-4012.

41. Allegretti, M., A. Moriconi, A. R. Beccari, R. Di Bitondo, C. Bizzarri, R. Bertini, and F. Colotta. 2005. Targeting C5a: recent advances in drug discovery. Curr. Med. Chem. 12: 217-236.

42. Guo, R. F., and P. A. Ward. 2005. Role of C5a in inflammatory responses. Annu. Rev. Immunol. 23: 821-852

43. Marasco, W. A., S. H. Phan, H. Krutzsch, H. J. Showell, D. E. Feltner, R. Nairn, E. L. Becker, and P. A. Ward. 1984. Purification and identification of formylmethionyl-leucyl-phenylalanine as the major peptide neutrophil chemotactic factor produced by Escherichia coli. J. Biol. Chem. 259: 5430-5439.

44. Hannigan, M., L. Zhan, Z. Li, Y. Ai, D. Wu, and C. K. Huang. 2002. Neutrophils lacking phosphoinositide 3-kinase $\gamma$ show loss of directionality during $N$-formylMet-Leu-Phe-induced chemotaxis, Proc. Natl. Acad. Sci. USA 19; 3603-3608. 


\section{Galectin-3 Reduces the Severity of Pneumococcal Pneumonia by Augmenting Neutrophil Function}

\author{
Sarah L. Farnworth, ${ }^{\star}$ Neil C. Henderson, ${ }^{*}$ \\ Alison C. MacKinnon, ${ }^{*}$ Kirsten M. Atkinson, ${ }^{\star}$ \\ Tom Wilkinson, ${ }^{*}$ Kevin Dhaliwal, ${ }^{*}$ \\ Katsutoshi Hayashi, ${ }^{\dagger}$ A. John Simpson, ${ }^{\star}$ \\ Adriano G. Rossi, ${ }^{\star}$ Christopher Haslett, ${ }^{*}$ \\ and Tariq Sethi ${ }^{*}$
}

From the Centre for Inflammation Research, The Queen's Medical Research Institute, University of Edinburgh, Edinburgh, United Kingdom; and the Department of Respiratory Medicine, ${ }^{\dagger}$ Sendai City Medical Centre, Sendai City, Japan

The Gram-positive Streptococcus pneumoniae is the leading cause of community-acquired pneumonia worldwide, resulting in high mortality. Our in vivo studies show that galectin- $3^{-1-}$ mice develop more severe pneumonia after infection with $S$. pneumoniae, as demonstrated by increased bacteremia and lung damage compared to wild-type mice and that galectin-3 reduces the severity of pneumococcal pneumonia in part by augmenting neutrophil function. Specifically, we show that 1) galectin-3 directly acts as a neutrophil-activating agent and potentiates the effect of fMLP, 2) exogenous galectin-3 augments neutrophil phagocytosis of bacteria and delays neutrophil apoptosis, 3) phagocytosis of apoptotic neutrophils by galectin- $3^{-/-}$macrophages is less efficient compared to wild type, and 4) galectin-3 demonstrates bacteriostatic properties against $S$. pneumoniae in vitro. Furthermore, ad-back of recombinant galectin-3 in vivo protects galectin-3-deficient mice from developing severe pneumonia. Together, these results demonstrate that galectin-3 is a key molecule in the host defense against pneumococcal infection. Therapeutic strategies designed to augment galectin-3 activity may both enhance inflammatory cell function (by directly affecting neutrophil responsiveness and prolonging neutrophil longevity) and have direct bacteriostatic activity, improving clinical outcomes after severe pneumococcal infection. (Am J Pathol 2008, 172:395-405; DOI: 10.2353/ajpath.2008.070870)

Despite improvements in therapy and intensive-care support, mortality attributable to respiratory tract infections is ever increasing. The Gram-positive bacterium Streptococcus pneumoniae is the most common cause of communityacquired pneumonia. Mortality is high, especially in developing countries. ${ }^{1}$ Limitations of conventional therapies and emerging drug resistance among strains of $S$. pneumoniae to antibiotics, such as penicillin ${ }^{2}$ and vancomycin, ${ }^{3}$ necessitates continued study into the mechanisms involved in the pathogenesis of pneumococcal disease and the host immune response against pneumococcal invasion.

Alveolar macrophages and recruited macrophages and neutrophils play a key role in the clearance and killing of invading pathogens. In the lung, resident alveolar macrophages are the first line of cellular defense and play a phagocytic role during the early stages of infection. Interaction of these cells with pneumococcus provokes an inflammatory response inducing recruitment of the more efficient polymorphonuclear leukocytes (or neutrophils) and increased concentrations of serum opsonins at the site of infection. Polymorphonuclear leukocytes are major effector cells in host defense against pneumococcal pneumonia as they phagocytose pneumococci and synthesize a variety of cytotoxic products, including active oxygen metabolites and defensins. ${ }^{4} \mathrm{Al}-$ though these products kill the invading pathogens, they can also severely damage the lung tissue itself. ${ }^{4}$ Nonresident macrophages are subsequently recruited and contribute to the resolution of inflammation by phagocytosing cell debris and apoptotic neutrophils. Therefore, the mechanisms that orchestrate neutrophil and macrophage function are important for the clearance of bacteria and resolution of pneumonic infection.

Galectin-3 is a unique member of the growing family of $\beta$-galactoside-binding lectins. ${ }^{5-7}$ Galectin-3 contains a single carboxy-terminal domain and a glycine-rich amino-terminal domain through which it forms oligomers and

Supported by the Medical Research Council, UK (Ph.D. studentship to S.L.F.); and the Wellcome Trust, UK (clinical training fellowship to N.C.H. and senior research leave fellowship to T.S.)

S.L.F. and N.C.H. contributed equally.

Accepted for publication November 12, 2007

Address reprint requests to Tariq Sethi, Centre for Inflammation Research, The Queen's Medical Research Institute, University of Edinburgh, 47 Little France Crescent, Edinburgh EH16 4TJ, UK. E-mail: t.sethi@ ed.ac.uk. 
functions to cross-link both carbohydrate and noncarbohydrate ligands. Galectin-3 has been implicated in many facets of the inflammatory response including neutrophil adhesion and activation, ${ }^{8}$ chemoattraction of monocytes/ macrophages, ${ }^{9}$ and activation of mast cells ${ }^{10}$ and lymphocytes. ${ }^{11}$ Galectin-3 is abundantly expressed and secreted by macrophages. ${ }^{12}$ However, mouse neutrophils do not express galectin- $3 .^{13}$ Secreted galectin-3 can cross-link surface glycoproteins and activate pathways involved in several innate immune responses such as the oxidative burst in neutrophils ${ }^{11,14}$ and degranulation in mast cells. ${ }^{10}$ Galectin-3 also contributes to chemotaxis by mediating cell-cell and cell-substratum adhesion. ${ }^{15,16}$

Galectin-3 has been shown to play a role in apoptosis. Extracellular galectin-3 induces T-cell apoptosis ${ }^{17}$ whereas intracellular galectin-3 results in an inhibition of apoptosis. ${ }^{18}$ Furthermore, peritoneal macrophages taken from galectin$3^{-1-}$ mice are more prone to undergo apoptosis than wildtype (WT) macrophages. ${ }^{19}$ However, minimal data exist regarding the role of galectin-3 in neutrophil apoptosis.

Galectin-3 has been shown to play a critical role in phagocytosis of opsonized red blood cells ${ }^{20}$; however, its role in the phagocytic clearance of microorganisms and apoptotic neutrophils has not been elucidated. Clearance of apoptotic neutrophils by macrophages is a key step in the resolution of inflammation. Without this step, apoptotic neutrophils will undergo secondary necrosis resulting in the release of damaging toxic products. Removal of these potentially toxic apoptotic neutrophils results in the release of anti-inflammatory and reparative cytokines such as transforming growth factor- $\beta 1$. These clearance and resolution phases help to limit the degree of tissue injury.

Galectin-3 has recently been demonstrated to have antimicrobial activity toward the pathogenic fungus Candida albicans. ${ }^{21}$ This antimicrobial activity may also be relevant for other pathogens, thus revealing an interesting therapeutic use of this galectin. Sato and colleagues ${ }^{13}$ demonstrated that after pneumococcal infection of the lungs, galectin-3 accumulates in the alveolar space, and this correlates with the onset of neutrophil extravasation. However, although neutrophils were actively recruited into Escherichia coli pneumonia-infected lungs, there was no increase in galectin-3 expression. Furthermore galectin-3 was released by alveolar macrophages on incubation with $S$. pneumoniae membrane fraction. ${ }^{13}$ In addition, lipopolysaccharide expressed on $E$. coli has been shown to down-regulate galectin-3 expression. ${ }^{12,22}$ The relevance of these observations to the mechanistic role of galectin-3 in the host immune response to $S$. pneumoniae infection has not been examined. We therefore studied pneumococcal pneumonia in mutant mice lacking the galectin- 3 gene and demonstrate that reconstituting galectin-3 in a deficient mouse can reduce the severity of infection.

\section{Materials and Methods}

\section{Materials}

Tissue culture reagents were purchased from PAA (The Cell Culture Company, Somerset, UK). Recombinant mouse and human galectin-3 were prepared using the baculovirus expression system (Invitrogen, Paisley, UK). All other reagents were purchased from Sigma-Aldrich (Poole, UK) unless otherwise stated.

\section{Animals}

Generation of galectin- $3^{-1-}$ mice in a 129sv and C57/B6 background by gene targeting technology has been described. ${ }^{23}$ As controls, age- and sex-matched WT littermate mice were used. All procedures were undertaken with approved license from the Animal Scientific Procedure Division of the Home Office (London, UK).

\section{Bacteria}

S. pneumoniae type 3 was used in this study (American Type Culture Collection, Rockville, MD). After overnight incubation on $5 \%$ sheep blood agar plates (BD Biosciences, Oxford, UK), freshly grown colonies were suspended in heart infusion broth (BD Biosciences) with $10 \%$ heat-treated horse serum (HIB-S) (Invitrogen) at an $\mathrm{OD}_{550}$ of 0.17 . The bacterial suspension was diluted 100 fold with fresh HIB-S and was incubated for 4 hours at $37^{\circ} \mathrm{C}$ with shaking, corresponding to a point compatible with logarithmic growth. The suspension was centrifuged at $3000 \mathrm{rpm}$ for 10 minutes at room temperature. The supernatant was discarded, and the pellet was resuspended with the same volume of phosphate-buffered saline (PBS). Serial dilutions were used for determination of exact bacterial concentration. Fluorescein isothiocyanate (FITC)-labeled S. pneumoniae were prepared as described previously. ${ }^{24}$

\section{In Vivo Pneumonia Model}

Under anesthesia (avertin $10 \mu \mathrm{l} / \mathrm{g}$ body weight), WT or galectin- $3^{-1-}$ mice were intratracheally inoculated with $1 \times 10^{5}$ colony-forming units (CFU) of $S$. pneumoniae. Fifteen hours after bacterial administration, mice were sacrificed, blood was collected aseptically, and bronchoalveolar lavage (BAL) was performed with two separate aliquots of $400 \mu \mathrm{l}$ of sterile PBS. Half of the lung was stored in $2 \mathrm{ml}$ of PBS for bacterial counts. The other half was fixed in 10\% formalin (Sigma-Aldrich), paraffin-embedded, cut into $3-\mu \mathrm{m}$-thick sections, and stained with hematoxylin and eosin (H\&E). Blood was inoculated on a $5 \%$ sheep blood agar plate (BD Biosciences) and incubated at $37^{\circ} \mathrm{C}$ overnight. The BAL fluid was centrifuged at $3000 \mathrm{rpm}$ for 10 minutes at $4^{\circ} \mathrm{C}$. Protein concentration in BAL was determined by the BCA protein assay reagent (Pierce Biotechnology, Northumberland, UK) according to the manufacturer's instructions, and cytokines [interleukin (IL)-6 and tumor necrosis factor (TNF)- $\alpha$ ] were measured using the Mouse Inflammation Cytometric Bead Array kit (BD Biosciences) according to the manufacturer's instructions. The supernatant was stored at $-20^{\circ} \mathrm{C}$ until further use. The pellet was resuspended in $300 \mu \mathrm{l}$ of PBS, and total cell number was determined with a hemocytometer. For neutrophil and macrophage cell 
counts, cytospins were prepared $(280 \mathrm{rpm}, 3$ minutes at room temperature), and cells were stained with Diff-Quik to enable the morphological discrimination between mononuclear cells and polymorphonuclear cells. The lung was homogenized in $2 \mathrm{ml}$ of PBS, and the homogenates were serially diluted 10 -fold with PBS, inoculated on $5 \%$ sheep blood agar plates, and incubated at $37^{\circ} \mathrm{C}$ overnight. Colonies formed from lung homogenates were counted, and blood plates were examined for the presence (sepsis) or absence of colonies. Myeloperoxidase activity in lung homogenates was assayed using $3,3^{\prime}, 5,5^{\prime}$ tetramethylbenzidine (Sigma-Aldrich) as a substrate. Myeloperoxidase activity/mg lung homogenate was determined. Western blot analysis for galectin- 3 was performed on neat BAL and 1:10 dilution of lung homogenate using the antibody anti-galectin-3 clone A3A12 (Alexis Biochemicals, San Diego, CA). The galectin-3 ad-back experiment was performed by administration of $5 \mu \mathrm{g}$ of recombinant mouse galectin-3 intratracheally at the time of infection.

\section{Tissue Culture}

Bone marrow-derived macrophages (BMDMs) and bone marrow-derived neutrophils were prepared from WT and galectin- $3^{-1-}$ mouse femurs and tibias. BMDMs were prepared by maturing bone marrow cells in Dulbecco's modified Eagle's medium-F12 containing $10 \%$ heat inactivated fetal bovine serum (FBS), $1 \%$ penicillin and streptomycin, and 10\% L929 conditioned media as a source of granulocyte-macrophage colony-stimulating factor (GMCSF), for 7 to 9 days. L929-conditioned media was prepared by growing L929 cells to confluency and sterilefiltering the conditioned media before adding to the Dulbecco's modified Eagle's medium-F12.

Bone marrow-derived neutrophils were prepared from WT and galectin- $3^{-1-}$ mouse femurs and tibias. This technique has previously been shown to yield greater than $90 \%$ neutrophil purity. ${ }^{25}$ Furthermore, studies have reported that mouse bone marrow-derived neutrophils show very similar morphology and function to neutrophils isolated from mouse whole blood. ${ }^{26}$ Bone marrow-derived neutrophils were prepared by Percoll gradient centrifugation as described previously. ${ }^{25}$ To assess neutrophil purity, bone marrow-derived neutrophils were incubated with 1:200 phycoerythrin (PE)-conjugated LY-6G and LY-6C (GR-1, clone RB6-8C5) monoclonal antibody (BD Pharmingen, Oxford, UK) for 30 minutes at $4^{\circ} \mathrm{C}$ and analyzed by fluorescence-activated cell sorting (FACS) analysis using a BD FACSCalibur flow cytometer. When needed, mouse neutrophils were made apoptotic by culturing in suspension at $5 \times 10^{6} / \mathrm{ml}$ in RPMI supplemented with $10 \%$ heat-inactivated FBS, $1 \%$ penicillin and streptomycin, and $1 \%$ L-glutamine for the indicated duration. To measure neutrophil apoptosis, neutrophils were incubated with 1:200 PE-conjugated LY-6G and LY-6C (GR-1, clone RB6-8C5) monoclonal antibody for 30 minutes at $4^{\circ} \mathrm{C}$ followed by incubation with 1:500 Annexin-V-FITC (Roche, Hertfordshire, UK) in Annexin-V bind buffer (Hanks plus $5 \mathrm{mmol} / \mathrm{L} \mathrm{CaCl}_{2}$ ) for 10 minutes at $4^{\circ} \mathrm{C}$.
Samples were treated with 1:10,000 ToPro-3 (Invitrogen) and immediately analyzed by FACS analysis using a BD FACSCalibur flow cytometer.

Human peripheral blood neutrophils were prepared as described previously. ${ }^{27}$ Apoptotic human neutrophils were prepared by culturing neutrophils for up to 24 hours in Iscove's modified Dulbecco's medium containing $10 \%$ FBS and treated as indicated. Human neutrophil apoptosis was assessed after 18 hours by identifying morphological changes of Diff-Quik-stained cells followed by cell counting. Binding of Annexin $\mathrm{V}$ was also assessed by incubating neutrophils with 1:500 Annexin-V-FITC (Roche) in Annexin-V bind buffer (HANKS plus $5 \mathrm{mmol} / \mathrm{L} \mathrm{CaCl}$ ) for 10 minutes at $4^{\circ} \mathrm{C}$. Samples were treated with $1: 10,000$ ToPro-3 (Invitrogen) and immediately analyzed by FACS analysis using a BD FACSCalibur flow cytometer.

\section{Baculovirus Expression System}

The Invitrogen baculovirus expression system was used to produce endotoxin-free recombinant human or mouse galectin-3. Detailed protocols are given in the Bac-to-Bac Baculovirus Expression System manual (Invitrogen 10359016). Briefly, mouse or human galectin-3 was cloned out of pcDNA 3.1 or pGEM-T Easy vectors, respectively, and cloned into a pFastBac HT donor plasmid. Recombinant pFastBac vectors were amplified and transformed into $\mathrm{DH} 10 \mathrm{Bac} E$. coli containing a baculovirus shuttle vector (bacmid). Recombinant bacmid containing the galectin-3 sequence was produced on transposition of the pFastBac construct and purified using the S.N.A.P midi-prep kit (Invitrogen). Recombinant bacmid was transfected into Sf9 insect cells and amplified using the method given in the manual. Galectin-3 protein was purified using the Ni-NTA His bind resin (Novagen, San Diego, CA) according to the manufacturer's instructions. Galectin-3 was verified using sodium dodecyl sulfate-polyacrylamide gel electrophoresis and Western blotting using 1:2000 antigalectin-3 antibody (clone A3A12; Affinity Bioreagents, Nottingham, UK) (data not shown).

\section{Phagocytosis Assays}

Phagocytosis of $S$. pneumoniae by bone marrow-derived macrophages or neutrophils was performed as follows. FITC-S. pneumoniae were opsonized with $10 \%$ mouse serum for 1 hour at $37^{\circ} \mathrm{C}$. Bone marrow neutrophils were incubated with a 10:1 ratio of FITC-S. pneumoniae to neutrophils for the indicated time points. Excess bacteria were washed off, and neutrophils were incubated with 1:200 PE-conjugated LY-6G and LY-6C (GR-1, clone RB6-8C5) antibody for 30 minutes. Samples were treated with 1:10,000 ToPro-3 (Invitrogen) and immediately analyzed by FACS analysis using a BD FACSCalibur flow cytometer. In some experiments, bone marrow neutrophils were treated with $10 \mu \mathrm{g} / \mathrm{ml}$ of recombinant mouse galectin-3 for 1 hour at $37^{\circ} \mathrm{C}$ before phagocytosis. Bone marrow-derived macrophages were incubated with a 10:1 ratio of FITC-S. pneumoniae to macrophages for the 
indicated duration and analyzed by FACS analysis using a BD FACSCalibur flow cytometer.

Phagocytosis of apoptotic human neutrophils by BMDMs was performed as follows. BMDMs were seeded in 48well plates, stained with $1 \mu \mathrm{g} / \mathrm{ml}$ Cell Tracker Orange (CMTMR, Invitrogen) and incubated with 10:1 ratio of 1 $\mu \mathrm{g} / \mathrm{ml}$ Cell Tracker Green (CMFDA, Invitrogen) labeled day 1 apoptotic human neutrophils for the indicated time points at $37^{\circ} \mathrm{C}$. Excess neutrophils were washed off, and BMDMs were removed using $5 \mathrm{mmol} / \mathrm{L}$ ice-cold ethylenediaminetetraacetic acid with gentle scraping. Percentage of phagocytosis was determined by FACS analysis using a BD FACSCalibur flow cytometer.

\section{Neutrophil Activation Assays}

Three assays were used to examine neutrophil activation in response to galectin-3. On activation, neutrophils release reactive oxygen species (ROS) and up-regulate CD11b expression and shed L-selectin (CD62L). Priming of neutrophils induces shape change without a noticeable increase in activation. ROS release was assessed by two methods, cytochrome $c$ reduction, which measures the $\mathrm{O}_{2}^{-}$metabolite, ${ }^{28}$ and dihydrorhodamine (DHR) fluorescence, which measures the $\mathrm{H}_{2} \mathrm{O}_{2}$ metabolite. ${ }^{29}$ 1) Neutrophils isolated from human peripheral blood were incubated with varying concentrations of recombinant human galectin-3 $(0.1$ to $10 \mu \mathrm{g} / \mathrm{ml})$ or 1 $\mu \mathrm{mol} / \mathrm{L}$ PAF for 15 minutes followed by 15 minutes of incubation with $0.1 \mu \mathrm{mol} / \mathrm{L} \mathrm{fMLP}$ in $1 \mathrm{mg} / \mathrm{ml}$ cytochrome $c$ in Hanks' media. Color change in supernatants (an indication of $\mathrm{O}_{2}{ }^{-}$release) was detected using a scanning spectrophotometer 500 to $600 \mathrm{~nm}$. Absorbance was converted to concentration using the calculation $A=\Sigma$.I.c. 2) Neutrophils isolated from human peripheral blood were incubated with $0.1 \mathrm{mmol} / \mathrm{L} \mathrm{DHR}$ for 5 minutes at $37^{\circ} \mathrm{C}$ and incubated with $1 \mu \mathrm{mol} / \mathrm{L}$ PAF or $10 \mu \mathrm{g} / \mathrm{ml}$ of recombinant human $(\mathrm{Hu})$ or mouse $(\mathrm{Ms})$ galectin-3 for 15 minutes at $37^{\circ} \mathrm{C}$. Cells were incubated with $0.1 \mu \mathrm{mol} / \mathrm{L}$ fMLP or PBS for 15 minutes and DHR fluorescence was measured as mean FL-1 fluorescence on the BD FACSCalibur flow cytometer. 3) Shape change of isolated human neutrophils in response to varying concentrations of galectin-3 $(0.25,2.5,10$, and $20 \mu \mathrm{g} / \mathrm{ml})$ or $1 \mu \mathrm{mol} / \mathrm{L}$ PAF alone was performed by measuring change in forward scatter by FACS analysis. 4) CD11b and L-selectin expression on neutrophils from human whole blood and CD11b expression on neutrophils from WT and galectin- $3^{-1-}$ mouse whole blood were measured by FACS analysis. Heparinized human whole blood was incubated with $0.1 \mu \mathrm{mol} / \mathrm{L}$ $\mathrm{fMLP}, 100 \mathrm{ng} / \mathrm{ml}$ lipopolysaccharide, or varying concentrations of galectin-3 $(0.25$ to $25 \mu \mathrm{g} / \mathrm{ml})$ for 30 minutes. Blood was incubated with anti-human CD11b-APC (clone ICRF44) and anti-human CD62L-PE (clone DREG-56) for 30 minutes at $4^{\circ} \mathrm{C}$. Samples were treated with $1 \mathrm{ml}$ of $1 \times$ FACS lysing solution (BD Biosciences) for 5 minutes at room temperature to lyse the red blood cells. The cell pellet was washed and resuspended in $300 \mu$ l of $1 \%$ PFA in PBS containing $0.1 \%$ bovine serum albumin. Samples were analyzed using a BD FACSCalibur flow cytometer, and neutrophils were identified by forward and side scatter properties.

Mouse whole blood was collected from the vena cava of mice anesthetized with avertin. Whole blood was incubated with 1:200 PE-conjugated LY-6G and LY-6C (GR-1, clone RB6-8C5) antibody and analyzed by FACS analysis to confirm the neutrophil population in the mouse whole blood. Mouse whole blood was incubated with $30 \mu \mathrm{g} / \mathrm{ml}$ of recombinant mouse galectin-3 followed by anti-mouse CD11b-APC (clone M1/70) (1:66.7) antibody. Samples (gated on the GR-1-positive population) were analyzed using a BD FACSCalibur flow cytometer.

\section{Antibacterial Assay}

S. pneumoniae was incubated overnight on a blood agar plate at $37^{\circ} \mathrm{C}$. Ten $\mathrm{ml}$ of $100 \%$ heart infusion broth (BD Biosciences) was inoculated with one colony of S. pneumoniae and incubated overnight at $37^{\circ} \mathrm{C}$ with gentle shaking. Cultures were centrifuged at 3000 rpm for 20 minutes, washed with broth, and centrifuged again. The culture was resuspended in broth to an $\mathrm{OD}_{600}=0.1$ and diluted 1:100. Seventy-five $\mu$ l of the bacterial reaction mix was incubated with $75 \mu \mathrm{l}$ of antibiotic or galectin-3 at $37^{\circ} \mathrm{C}$ for 2 hours at $800 \mathrm{rpm}$ on the thermomixer. Antibiotics used included recombinant mouse galectin-3 (0.3 and $15 \mu \mathrm{g})$ and ampicillin $(20 \mu \mathrm{g} / \mathrm{ml})$. A reaction was prepared for $t=0$, which was plated before the 2-hour incubation. At time 0 and after 2 hours, the reactions were serially diluted into PBS, and $100 \mu$ l of each dilution was plated onto blood agar plates. Plates were incubated overnight and colonies counted the next day.

\section{Statistical Analysis}

Results are presented as means \pm SEM. Significance of the differences between means was assessed using oneway analysis of variance or two-tailed Student's $t$-test. Values of $P<0.05$ were considered significant.

\section{Results}

\section{Galectin-3 Is a Critical Regulator of the Severity of an Acute Pneumococcal Infection in the Lung in Vivo}

WT and galectin- $3^{-1-}$ mice were intratracheally inoculated with $1 \times 10^{5} \mathrm{CFU} S$. pneumoniae, and animals were sacrificed after 15 hours (Figure 1). Initial dosing experiments performed throughout a 24 -hour period resulted in high mortality of the galectin-3-null mice. This was most likely attributable to the inadequate host response to pneumococcal infection in the galectin-3-null mouse. A shorter duration of 6 hours did not prove long enough to show a prominent difference in the severity of disease outcome in WT and galectin- $3^{-1-}$ mice. We therefore monitored pneumonia for 15 hours with $1 \times 10^{5} \mathrm{CFU}$ because this time point and dose achieve measurable disease with no mortality. 

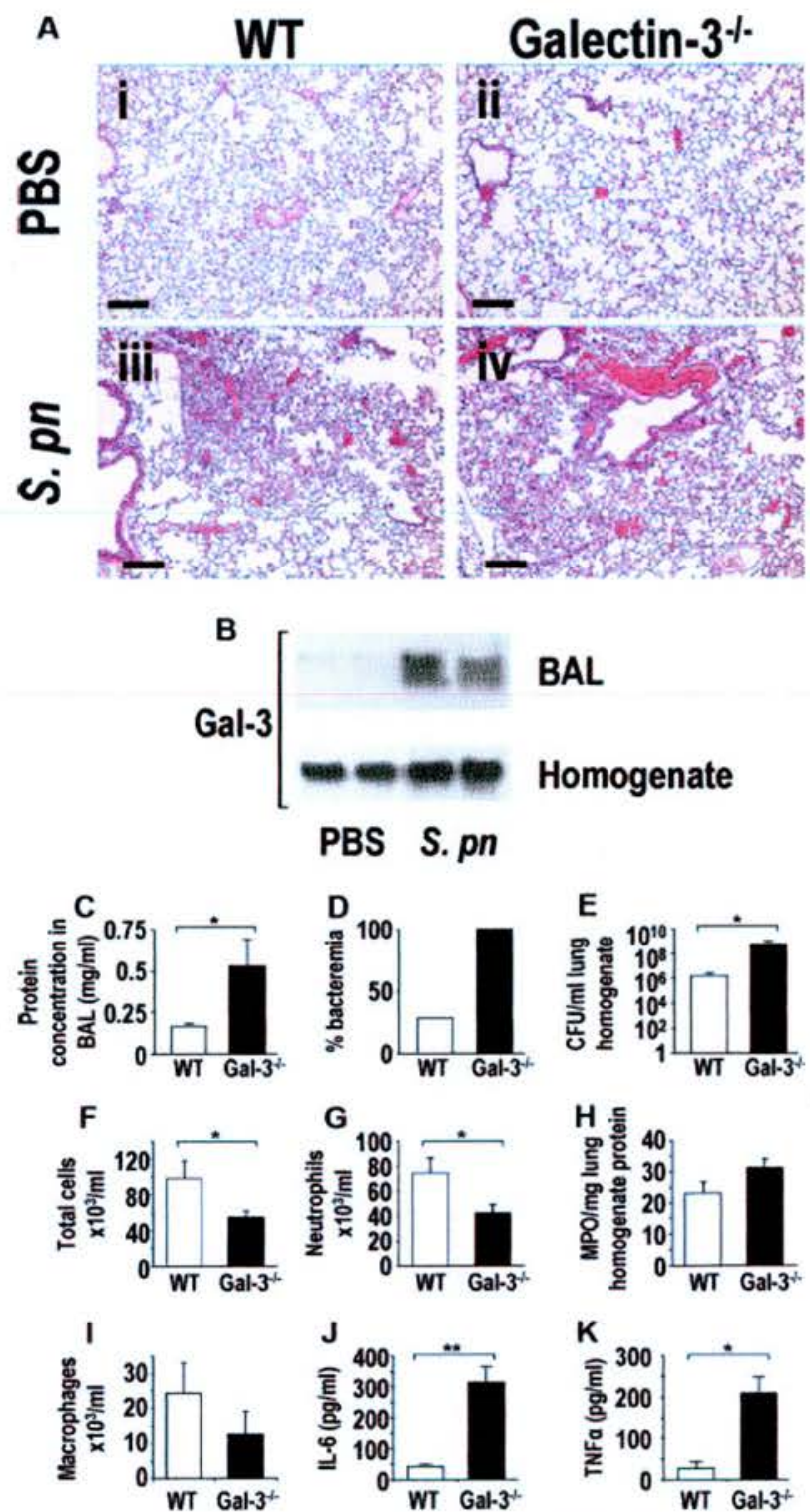

Figure 1. Galectin-3 plays a critical role in the clearance of an acute pneumococcal infection. Mice were inoculated intratracheally with $1 \times 10^{5} \mathrm{CFU} S$. pneumoniae for 15 hours ( $n=10$ mice in each group). A: H\&E staining of WT and galectin- $3^{-1-}$ lung tissue from control (i and ii) (PBS instilled intratracheally) and $S$. pneumoniae pneumonia (iii and iv) (S. pneumoniae inoculated intratracheally) at 15 hours after instillation. B: Representative galectin-3 Western blots showing galectin-3 levels in BAL (neat) and homog enates (1-10) of WT mice inoculated with $S$ pneumoniae compared to PBS control C-K: WT (white bars) and galectin- $3^{-1-}$ (black bars). C: Protein concentration was significantly higher in lavage fluid from galectin-3mice compared with WT $\left({ }^{*} P<0.05\right.$ compared to WT). D: Blood from galectin- $3^{-1}$ and WT mice was plated on blood agar plates and the percentage of plates with bacterial growth is expressed as percent bacteremia. $\mathbf{E}$. Lungs from galectin- $3^{-1-}$ and WT mice were homogenized, serially diluted plated on blood agar plates, and bacterial counts determined. Galectin-3 lung homogenate demonstrated higher bacterial counts compared to WT $\left({ }^{*} P<0.05\right.$ compared to WT). Total cell recruitment $(\mathbf{F})$ and neutrophil recruitment $(\mathbf{G})$ into the alveolar space was reduced in galectin- $3^{-1-}$ mice compared to WT mice after $S$. pnetumoniae infection $(* P<0.05$ compared to WT) H: Myeloperoxidase activity in lung homogenate was similar between the two groups. I: Macrophage recruitment into the alveolar space of WT and galectin- $3^{--}$mice was similar. IL-6 (J) and TNF- $\alpha(\mathbf{K})$ concentration in BAI was determined by CBA and demonstrated to be higher in galectin-3-deficient animals $(* P<0.01$ compared to WT and $* P<0.05$ compared to WT $)$. Scale bars $=100 \mu \mathrm{m}$.
Figure $1 \mathrm{~A}$ depicts representative $\mathrm{H} \& \mathrm{E}$ staining of lungs from WT and galectin- $3^{-1-}$ mice inoculated with S. pneumoniae or PBS as a control demonstrating increased cell infiltrate in the lungs of infected mice. Galectin- $3^{-1-}$ mice demonstrated more severe pneumonia with increased lung injury and septicemia. Expression of galectin-3 in lung homogenates and BAL was increased in WT mice inoculated with S. pneumoniae compared to PBS controls (Figure 1B). The concentration of galectin-3 in the BAL after infection was $\sim 50 \mu \mathrm{g} / \mathrm{ml}$. Overall protein concentration was significantly higher in BAL fluid from galectin$3^{-1-}$ mice compared to WT mice (Figure $1 \mathrm{C}$ ), indicating a greater amount of leakage from the vasculature and therefore a higher degree of tissue injury. One hundred percent of culture plates inoculated with galectin-3 $3^{-1-}$ blood produced colonies indicating that all galectin-3 $3^{-1-}$ mice were bacteremic after S. pneumoniae infection compared to $30 \%$ of WT mice (Figure 1D). Culture plates of lung homogenate from galectin- $3^{-1-}$ mice demonstrated $\sim 450$-fold greater bacterial load than culture plates inoculated with WT lung homogenate $\left(8.6 \times 10^{8} \mathrm{CFU} / \mathrm{ml}\right.$ in galectin-3 $3^{-1-}$ compared to $1.9 \times 10^{6} \mathrm{CFU} / \mathrm{ml}$ in WT) (Figure $1 \mathrm{E}$ ) indicating a clearance defect of $S$. pneumoniae in galectin- $3^{-1-}$ mice. Total cell counts were significantly reduced in galectin- $3^{-1-}$ mice after pneumococcal pneumonia infection compared to WT (Figure 1F). In addition, differential cell counts revealed reduced neutrophil recruitment in galectin- $3^{-1-}$ mice compared to WT (Figure 1G). However, myeloperoxidase activity was similar in lung homogenates from WT and galectin- $3^{-1-}$ mice after S. pneumoniae infection (Figure $1 \mathrm{H}$ ). This would suggest that neutrophils accumulate in the interstitial lung tissue during pneumonia in galectin- $3^{-1-}$ mice but are hindered from transmigrating into the alveolar space in the absence of galectin-3. Although galectin- $3^{-1-}$ mice demonstrated reduced macrophage numbers in lavage fluid compared to WT, this was not significant (Figure 1I). Increased severity of pneumococcal infection in galectin$3^{-1-}$ mice correlates with greater concentrations of IL-6 and TNF- $\alpha$ in BAL compared to WT mice (Figure 1, J and K). These proinflammatory cytokines can have pathological consequences on host tissue and may contribute to increased leakage from the vasculature and sepsis in the galectin-3-deficient mouse. These results demonstrate that galectin- 3 is a critical molecule regulating the severity of a pneumococcal pneumonia infection in vivo through its ability to enhance the clearance of pneumococcal pneumonia and to resolve inflammation.

\section{Expression of Galectin-3 in Macrophages Does Not Enhance Phagocytosis of S. pneumoniae but Does Augment Phagocytosis of Apoptotic Neutrophils}

Previous studies have shown that galectin- $3^{-1-}$ macrophages exhibit reduced phagocytosis of IgG-opsonized erythrocytes and apoptotic thymocytes. ${ }^{20}$ Galectin-3-deficient macrophages demonstrated no phagocytic defect toward S. pneumoniae compared to WT (Figure 2, A and 
A

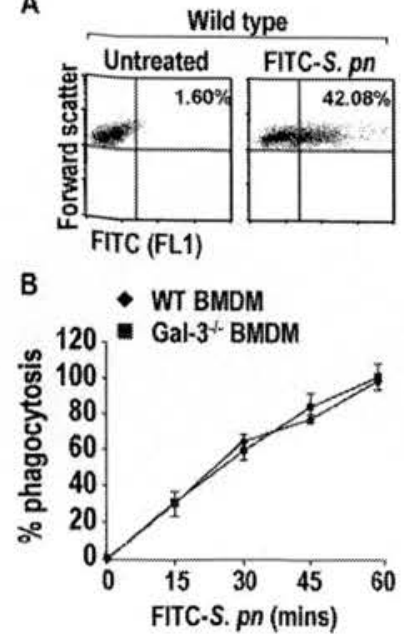

Galectin-3:

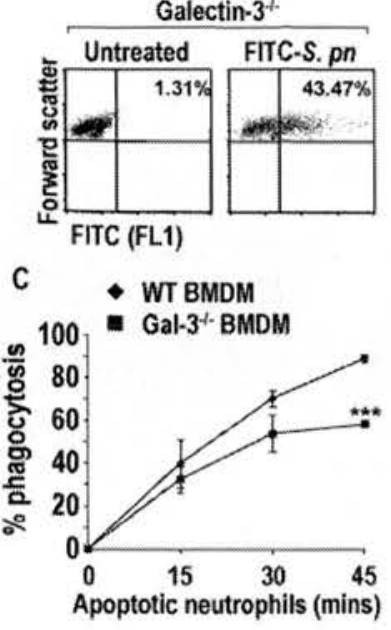

Figure 2. Expression of galectin-3 in macrophages does not enhance phagocytosis of $S$. pneumoniae but endogenous galectin-3 augments macrophage phagocytosis of apoptotic human neutrophils. A: Representative forward scatter versus FL1 dot blots of WT and galectin- $3^{-1}$ BMDMs treated for 60 minutes with 10:1 ratio of FITC-S. pneumoniae. Gated on macrophages according to forward and side scatter properties. Percent in upper right quadrant indicates macrophages that have phagocytosed FITC-labeled bacteria. B: Phagocytosis of a 10:1 ratio of opsonized FITC-S. pneumoniae for up to 1 hour by WT $(\bullet)$ and galectin- $3^{-1-}$ (四) BMDMs measured using FACS analysis. Results represent the mean percentage phagocytosis (compared to WT 60 minutes $)(n=3)$. C. Phagocytosis of a 10:1 ratio of apoptotic human neutrophils for up to 45 minutes by WT $(\bullet)$ and galectin- $3^{-1-}$ (国) BMDMs measured using FACS analysis. Results represent the mean percent phagocytosis $(n=3)(\cdots P<0.0001$ compared to WT 45 minutes $)$.

B). However, galectin-3 $3^{-1-}$ BMDMs demonstrated reduced phagocytosis of apoptotic human neutrophils (Figure $2 \mathrm{C}$ ). Phagocytosis of apoptotic cells is crucial for the resolution of inflammation by protecting tissues from excess exposure to inflammatory and immunogenic components of dying cells. ${ }^{30}$ This result may partially explain our in vivo findings of increased lung damage leading to septicemia in the galectin-3-deficient mouse. The different phagocytic responses toward pneumococcus and apoptotic cells may be attributable to alternate mechanisms regulating pathogen recognition by macrophages.

\section{Exogenously Added Recombinant Galectin-3 Causes Human and Mouse Neutrophil Activation}

Despite reduced neutrophil transmigration from the lung interstitia into the alveolar space after $S$. pneumoniae infection in galectin- $3^{-1-}$ mice compared to WT, recruitment is not completely abolished. We therefore examined the effect of galectin-3 on neutrophil function because neutrophils that are successfully recruited in the galectin3-deficient mouse may demonstrate reduced activity. Mouse neutrophils do not express galectin-3 (our own observations, data not shown), ${ }^{13}$ and it is widely believed that the action of galectin-3 on neutrophils is extracellular where it oligomerizes on the cell surface to exert its effects. ${ }^{31}$

On activation, neutrophils release ROS and up-regulate CD11b expression and shed L-selectin (CD62L). Priming of neutrophils induces shape change without a
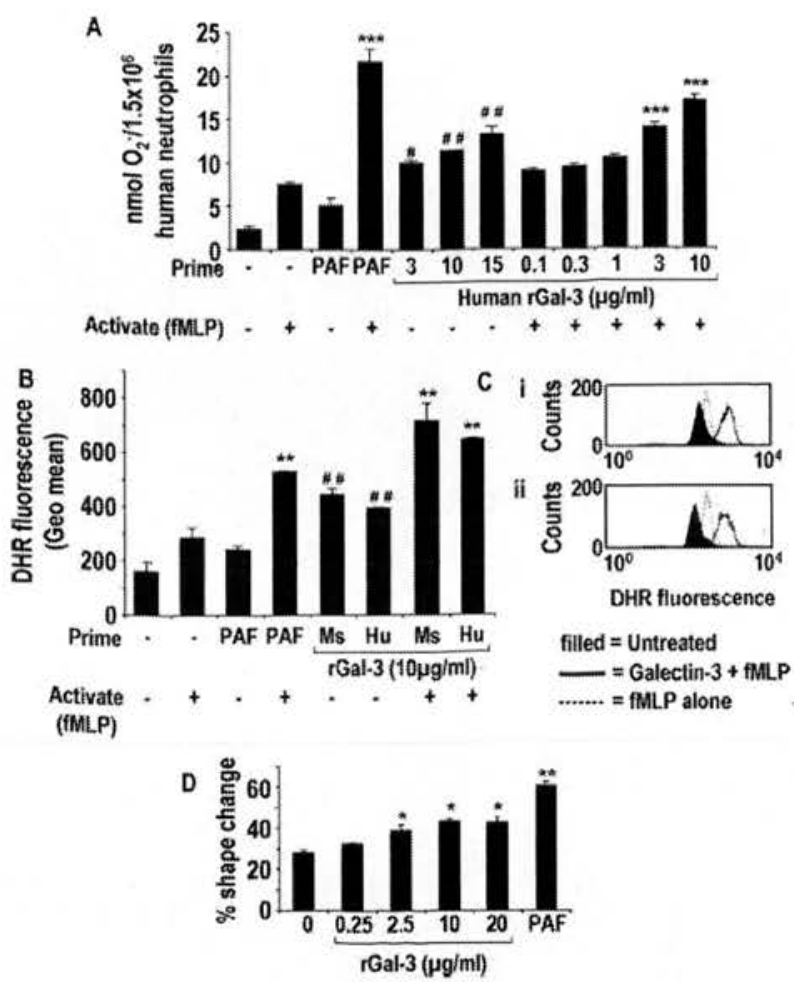

Figure 3. Exogenously added recombinant galectin-3 causes human neutrophil activation and the generation of ROS. Free radical generation from human neutrophils treated with PAF $(1 \mu \mathrm{mol} / \mathrm{L})$ or human galectin-3 $(0.1$ to $15 \mu \mathrm{g} / \mathrm{ml})$ for 15 minutes followed by fMLP $(0.1 \mu \mathrm{mol} / \mathrm{L})$ or PBS for 15 minutes was measured by cytochrome $c$ reduction $(\mathbf{A})(n=6)(\cdots p<0.0001$ compared to treatment with fML.P alone, "P<0.05 and ${ }^{m "} P<0.01$ compared to treatment with PAF alone) and DHR fluorescence using FACS analysis (B and $\mathbf{C})(n=3)\left(* P<0.01\right.$ compared to treatment with fMLP alone and ${ }^{* *} P<$ 0.01 compared to treatment with PAF alone). C: Representative histogram showing DHR fluorescence in response to PMLP pretreated with either 10 $\mu \mathrm{g} / \mathrm{ml}$ mouse (i) or human recombinant galectin-3 (ii). D: Percentage of shape change of human neutrophils treated with galectin-3 $(0.25$ to 20 $\mu \mathrm{g} / \mathrm{ml})$ or $1 \mu \mathrm{mol} / \mathrm{L}$ PAF for 15 minutes $(n=3)(\cdot P<0.05$ and $* P<0.01$ compared with untreated).

noticeable increase in activation. Isolated human neutrophils preincubated with recombinant human galectin- 3 at 3 and $10 \mu \mathrm{g} / \mathrm{ml}$ followed by fMLP produced significantly more superoxide as measured by cytochrome $c$ reduction compared with neutrophils treated with fMLP alone (Figure 3A). Incubation of neutrophils with galectin-3 alone $(3,10$, and $15 \mu \mathrm{g} / \mathrm{ml})$ also resulted in increased superoxide release compared to untreated or cells treated only with PAF (Figure 3A). When treated with either recombinant mouse (Ms) or human ( $\mathrm{Hu}$ ) galectin-3 $(10 \mu \mathrm{g} / \mathrm{ml}$ ) followed by $\mathrm{fMLP}$, the level of free radical generation, measured by DHR activation also exceeded that of neutrophils treated with fMLP alone (Figure 3B). This assay also demonstrated that galectin- 3 alone (10 $\mu \mathrm{g} / \mathrm{ml}$ ) is sufficient to elicit ROS release from neutrophils (Figure 3B). Figure $3 \mathrm{C}$ shows representative histogram overlays of neutrophils. Human neutrophils pretreated with mouse or human recombinant galectin-3 (solid line) before fMLP treatment produced more $\mathrm{H}_{2} \mathrm{O}_{2}$ compared to neutrophils treated with fMLP alone (dashed line). At concentrations less than those that directly activate isolated human neutrophils, galectin-3 alone does not significantly alter shape change. Higher concentrations of 


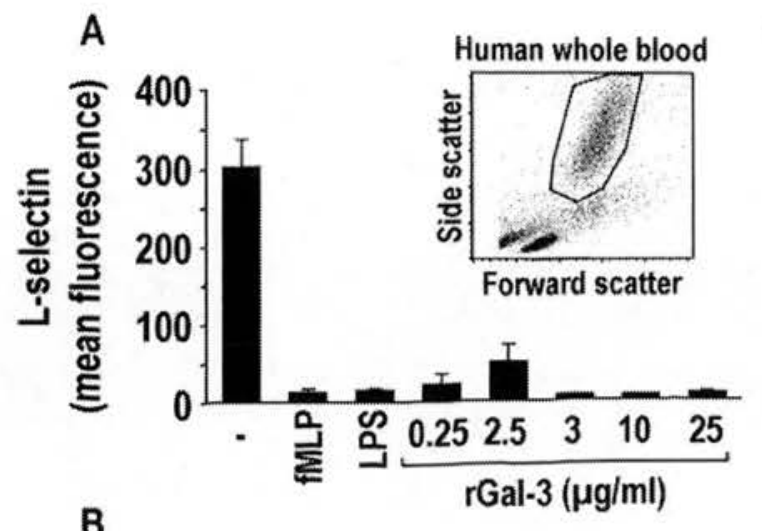

C
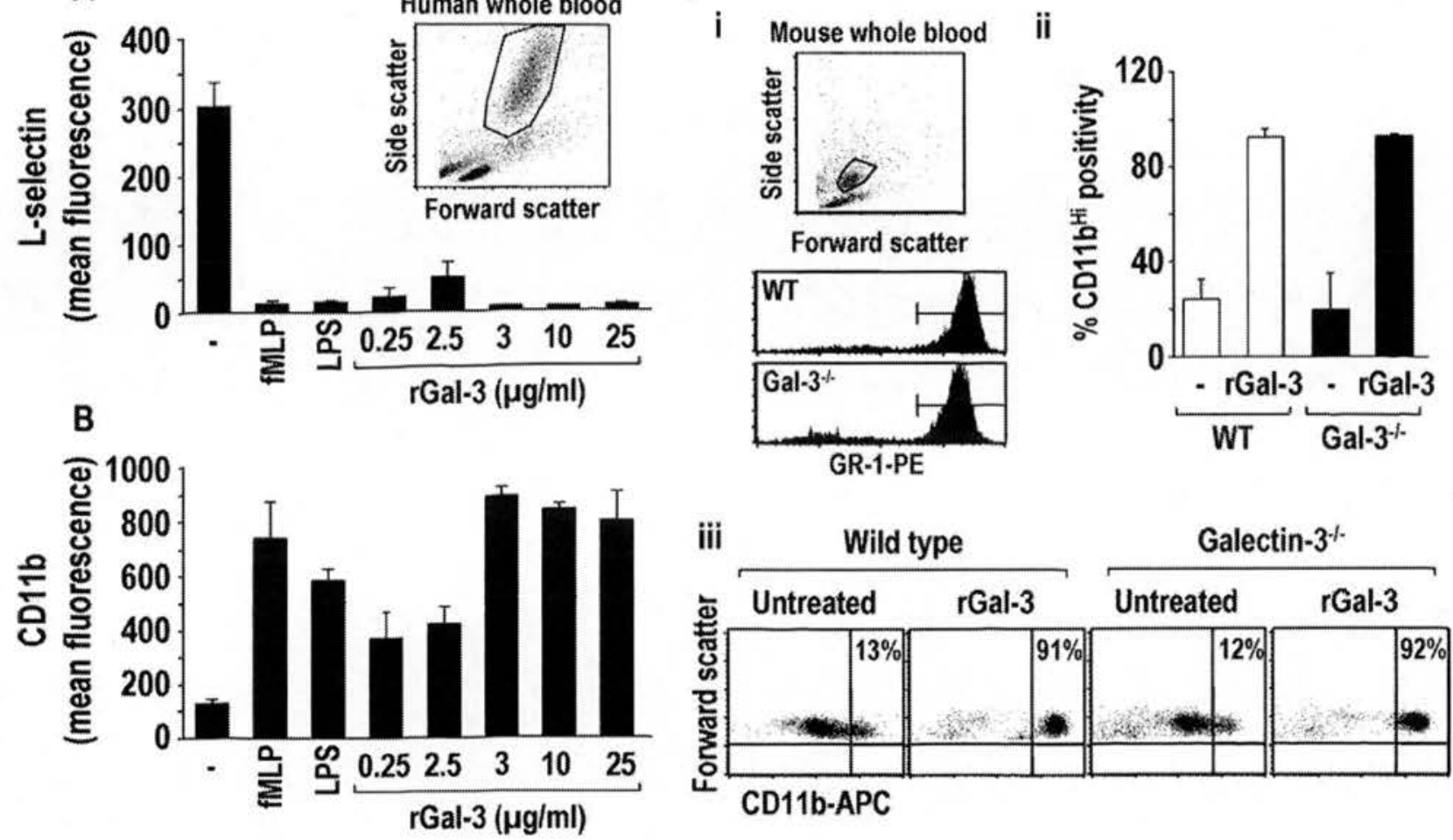

Figure 4. Exogenously added recombinant galectin-3 causes t-selectin shedding and CD11b up-regulation on human whole blood neutrophils and CD11b up-regulation on both WT and galectin- $3^{-/-}$mouse neutrophils. L-selectin shedding (A) and CD11b up-regulation (B) on human neutrophils after a 30-minute incubation with fMLP $(0.1 \mu \mathrm{mol} / \mathrm{L})$, lipopolysaccharide $(100 \mathrm{ng} / \mathrm{ml})$, or human galectin-3 $(0.25$ to $25 \mu \mathrm{g} / \mathrm{ml})$ was assessed by FACS analysis $(n=6)$ (for all treatments $P<0.01$ compared to untreated). A, inset: Representative dot blot showing forward and side scatter properties of human whole blood, gated on neutrophils. C: i: Representative dot blot showing forward and side scatter properties of WT mouse whole blood gated on GR-1-positive neutrophils. ii and iii: Mouse whole blood was treated with $30 \mu \mathrm{g} / \mathrm{ml}$ of mouse galectin-3 for 30 minutes and incubated with anti-CD11b-APC and anti-GR-1-PE. Galectin-3-treated WT (white bars) and galectin- $3^{-1-}$ (black bars) whole blood demonstrated increased CD11b expression (CD11 ${ }^{\text {Hit)}}$ ) on GR-1-positive neutrophils as measured by FACS analysis $(n=3)$.

galectin-3 $(>2.5 \mu \mathrm{g} / \mathrm{ml})$ produced a small increase in shape change but not to the magnitude as that observed with PAF (Figure 3D), suggesting that the predominant effect of galectin-3 is on neutrophil activation.

Activation of whole blood human neutrophils with fMLP, lipopolysaccharide, and galectin-3 caused L-selectin shedding and CD11b up-regulation (Figure 4, A and $B$, respectively). A dot blot (Figure $4 \mathrm{~A}$, inset) shows forward and side scatter of human peripheral blood neutrophils. Together these experiments show that galectin-3 can cause direct activation of neutrophils in whole blood and can both directly activate isolated neutrophils and prime neutrophils to subsequent activation by fMLP.

Galectin- $3^{-1-}$ mouse whole blood shows the same forward and side scatter properties as WT (data not shown). WT and galectin- $3^{-1-}$ mouse whole blood was treated with $30 \mu \mathrm{g} / \mathrm{ml}$ of recombinant mouse galectin-3 and high CD11b expression (CD11 $\mathrm{b}^{\mathrm{HI}}$ ) on neutrophils was assessed by FACS analysis. Samples were gated on GR-1-positive neutrophils (Figure 4Ci). CD11b ${ }^{H 1}$ expression on neutrophils in galectin-3-treated mouse whole blood was compared to those of untreated controls. Both WT and galectin- $3^{-1-}$ neutrophils demonstrated more than $90 \% \mathrm{CD} 11 \mathrm{~b}^{\mathrm{HI}}$ expression when treated with galectin-3 compared to just $20 \%$ of untreated neutrophils demonstrating $\mathrm{CD}_{11} \mathrm{~b}^{\mathrm{HI}}$ expression (Figure $4 \mathrm{C}$, ii and iii).
Together these data demonstrate that exogenously added galectin-3 can activate both human and mouse neutrophils. Furthermore, exogenous galectin- 3 increases CD11b expression on both WT and galectin- $3^{-1-}$ neutrophils to similar levels.

\section{Exogenous Galectin-3 Enhances Phagocytosis of Bacteria by Both WT and Galectin- $3^{-1-}$ Neutrophils}

WT and galectin- $3^{-1-}$ mouse bone marrow neutrophils were isolated as described previously. ${ }^{25}$ After an infection, this fully competent neutrophil reservoir in the bone marrow is required to rapidly supplement peripheral neutrophils at times of increased demand. ${ }^{26}$ Figure $5 \mathrm{~A}$ illustrates forward and side scatter properties of a bone marrow neutrophil preparation from WT and galectin- $3^{-1-}$ mice. The various cell populations observed most likely demonstrate neutrophils that are matured to different extents. However, when all cell populations are taken into account (gate R1), the percentage of GR-1-positive cells is $\sim 80 \%$.

Untreated WT and galectin-3-1- GR-1-positive mouse bone marrow neutrophils incubated with opsonized FITClabeled S. pneumoniae showed similar levels of phago- 
A
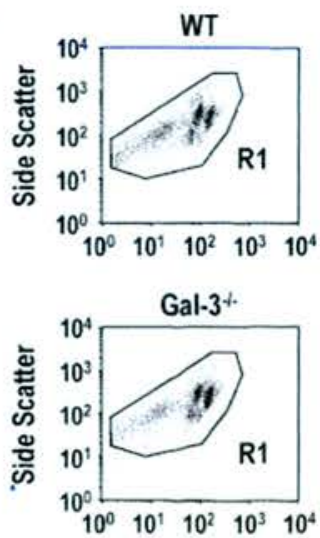

Forward Scatter

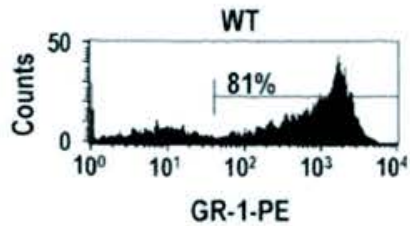

Gal-3+

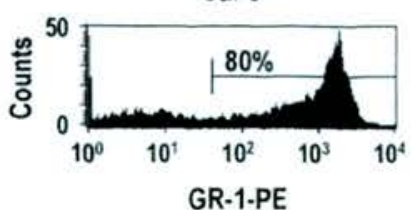

B

$\square$ WT neutrophil

Gal-3* neutrophil

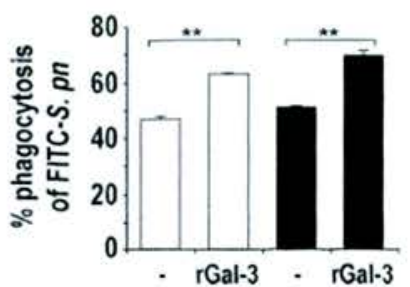

Figure 5. Exogenous galectin-3 enhances phagocytosis of $S$. pneumoniae by both WT and malectin-3-- neutrophils. A: Representative dot blots from WT and galectin- $3^{-\gamma-}$ mouse bone marrow neutrophil preparations gated on all populations (R1). Representative histograms demonstrate $-80 \%$ GR-1 positivity within the R1 gate. B: WT (white bars) and galectin-3-j- (black bars) bone marrow neutrophils were prepared as described in the Materials and Methods. Neutrophils were incubated with $30 \mu \mathrm{g} / \mathrm{ml}$ of recombinant mouse galectin-3 or PBS (control) for 1 hour at $37^{\circ} \mathrm{C}$ then incubated with a $10: 1$ ratio of opsonized FITC-S. pneumoniae for 1 hour at $37^{\circ} \mathrm{C}$. Samples were gated on GR-1-positive cells and analyzed using a BD FACSCalibur $(n=3)(* P<0.01$ compared to untreated).

cytosis. However, after a 1-hour incubation with $10 \mu \mathrm{g} / \mathrm{ml}$ of recombinant mouse galectin-3, both WT and galectin$3^{-1-}$ bone marrow neutrophils enhanced their phagocytic capability (Figure 5B). This indicates that exogenous galectin-3 can stimulate phagocytosis of $S$. pneumoniae by neutrophils from WT and galectin- $3^{-1-}$ mice.

\section{Neutrophils from WT and Galectin-3-1- Mice} Undergo Apoptosis at the Same Rate in Vitro, but Exogenous Galectin-3 Prolongs Neutrophil

\section{Survival}

Previous reports have demonstrated an important role for galectin-3 during apoptosis. ${ }^{17-19}$ We therefore examined the rate of spontaneous apoptosis of neutrophils from WT or galectin- $3^{-1-}$ mice in vitro. Figure $6 \mathrm{~A}$ shows that neutrophils from galectin- $3^{-1-}$ mice aged in vitro demonstrate a similar rate of cell death compared to WT neutrophils. However, in keeping with its ability to activate neutrophils, incubation with galectin- 3 delays spontaneous apoptosis in human peripheral blood neutrophils aged for 18 hours in culture assessed by both nuclear morphology and cell surface changes as measured by

A

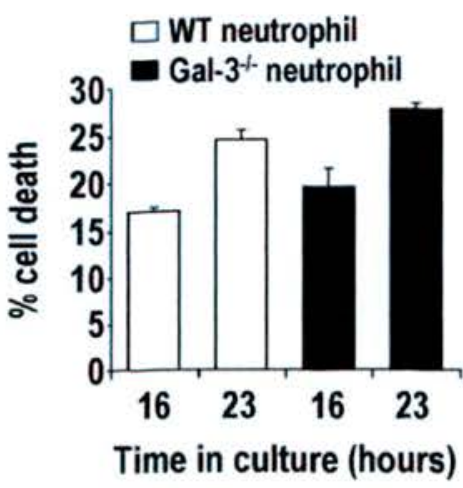

B

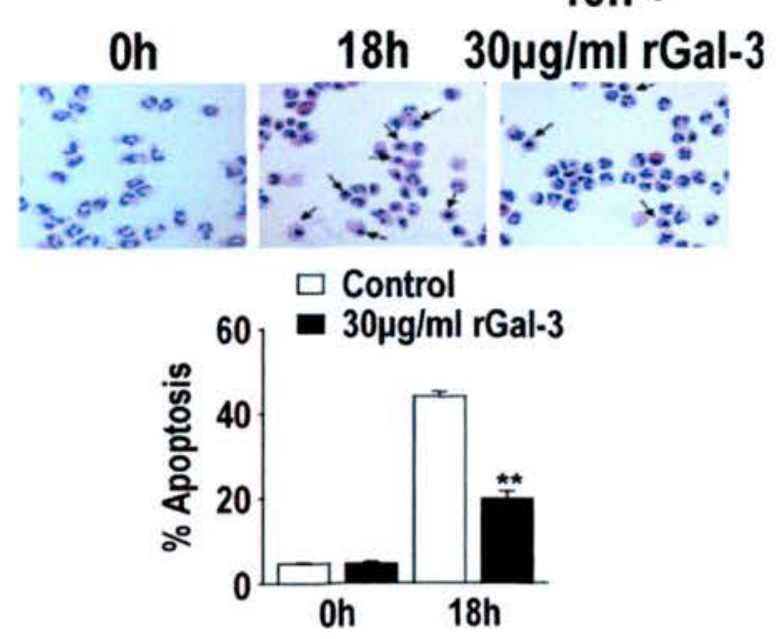

Time in culture (hours)

C

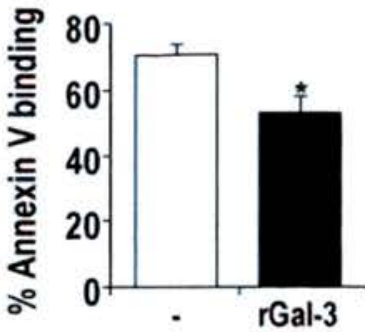

$18 \mathrm{~h}$ in culture

Figure 6. WT and galectin-3-1- mouse neutrophils undergo apoptosis at similar rates in culture and galectin-3 protects human neutrophils from apoptosis. A: Mouse bone marrow neutrophils were cultured for the indicated time points and incubated with PE-conjugated anti-GR-1 antibody followed by annexin-V-FITC. ToPro-3 was added to each sample before FACS analysis. Apoptotic and necrotic neutrophils were identified as those demonstrating GR-1 and annexin-V/ToPro-3 positivity $(n=3)$ B: Human peripheral blood neutrophils were cultured for 18 hours in Iscove's modified Dulbecco's medium containing $10 \%$ FBS and $30 \mu \mathrm{g} / \mathrm{ml}$ of human recombinant galectin-3 or PBS. Cytospin preparations were prepared, and neutrophil apoptosis was determined by morphology. Arrows show apoptotic humar neutrophils. Percent apoptosis was calculated from total cell number of five fields of each slide $(n=3)(* P<0.01$ compared to untreated $)$. C: Human peripheral blood neutrophils were cultured for 18 hours in Iscove's modified Dulbecen's medium containing $10 \%$ FBS and $20 \mu \mathrm{g} \mathrm{ml}$ of human recombinant galectin-3. Neutrophils were incubated with annexin-V-FITC, and ToPro-3 was added to each sample before FACS analysis. Annexin-V-positive and ToPro-3-negative neutrophils were classed as apoptotic $(n=3)\left({ }^{*} P<\right.$ 0.05 compared to untreated) 
annexin $\mathrm{V}$ binding (Figure $6, \mathrm{~B}$ and $\mathrm{C}$ ). The different rates of apoptosis demonstrated in Figure 6, B and C, reflect the techniques used to assess neutrophil apoptosis.

In addition to the above findings suggesting that galectin-3-deficient macrophages are less able to phagocytose apoptotic neutrophils than their WT counterparts (Figure 2C), galectin-3 delays spontaneous human neutrophil apoptosis. Thus after $S$. pneumoniae infection in vivo, the greater accumulation of apoptotic neutrophils in the galectin- $3^{-1-}$ mouse may contribute to tissue damage and subsequent bacteremia. These results suggest that the increased vasculature damage and bacteremia observed in galectin- $3^{-1-}$ mice is in part attributable to ineffective clearance of apoptotic cells by galectin- $3^{-1-}$ macrophages.

\section{Galectin-3 Is Bacteriostatic Toward \\ S. pneumoniae}

Galectin-3 has recently been shown to have antimicrobial activity toward the pathogenic fungus Candida albicans. ${ }^{21}$ We therefore investigated whether galectin-3 had a direct antimicrobial effect on S. pneumoniae. The same strain of S. pneumoniae used in the in vivo experiments was incubated with varying concentrations of galectin- 3 or ampicillin as a positive control as described in the Materials and Methods. Incubation of $15 \mu \mathrm{g}$ of galectin-3 with $S$. pneumoniae significantly inhibited bacterial growth (Figure 7A), suggesting that galectin-3 is bacteriostatic toward S. pneumoniae. Growth inhibition of S. pneumoniae by galectin-3 may further explain our in vivo findings that galectin- $3^{-1-}$ mice demonstrate more severe $S$. pneumoniae infection. This may have important implications as to the function of galectin-3 in the lungs of infected mice.

\section{Addition of Recombinant Galectin-3 into the Lungs of Galectin-3 ${ }^{-1-}$ Mice Reduces Severity of Pneumonia}

To expand our in vivo work, we inoculated galectin- $3^{-1-}$ mice with S. pneumoniae in the presence or absence of 5 $\mu \mathrm{g}$ of galectin-3. Sepsis and protein content in the BAL were assessed as indicators of lung injury and degree of pneumonic severity. Inoculation of galectin-3-deficient mice with $S$. pneumoniae and galectin-3 reduced the protein content in lavage indicating reduced lung injury (Figure 7B). Furthermore, severity of bacteremia was reduced in galectin-3-treated mice (Figure 7C) and IL-6 and TNF- $\alpha$ levels in the BAL were reduced in mice treated with galectin-3 (Figure 7, D and E). These results reveal the potential therapeutic use of galectin- 3 toward infection.

\section{Discussion}

The Gram-positive Streptococcus pneumoniae is the leading cause of community-acquired pneumonia worldwide, resulting in high mortality. The increasing prevalence of antibiotic resistance means that novel treatment strate-
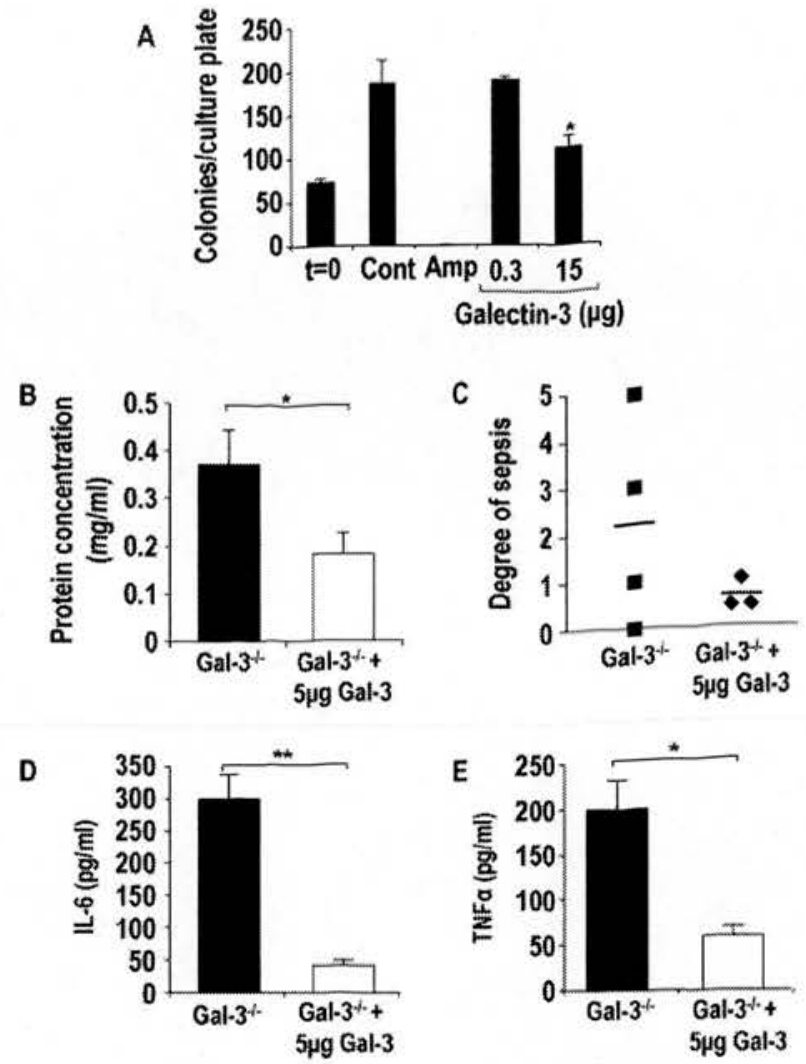

Figure 7. Galectin-3 is bacteriostatic, and delivery of recombinant galectin-3 into the lungs of galectin- $3^{--}$mice reduces severity of pneumonia. A: $S$. pneumoniae cultures were incubated with galectin-3 $(0.3$ and $15 \mu \mathrm{g})$ or ampicillin $(20 \mu \mathrm{g} / \mathrm{ml})$ for 2 hours, diluted $1: 10$, and plated onto blood agar plates. Colonies were counted the following day $(n=3)(* P<0.05 \mathrm{com}$ pared to control). Galectin-3-deficient mice were intratracheally inoculated with $1 \times 10^{5} \mathrm{CFU} S$, pneumoniae in the presence or absence of $5 \mu \mathrm{g}$ of recombinant mouse galectin- 3 for 15 hours. B: Protein concentration was significantly higher in lavage fluid from galectin $-3^{-1-}$ (black bars) mice compared with galectin- $3^{--}$mice treated with galectin-3 (white bars) $\left({ }^{*} P<\right.$ 0.05 compared to galectin- $3^{-i-}$ ). C: Blood from galectin- $3^{-/-}$and WT mice was plated on blood agar plates, and the degree of bacterial presence per plate was scored according to the arbitrary scale 0 to $5(0$, no bacterial growth; 5 , significant bacterial growth). Galectin- $3^{-1-}$ mice treated with galectin-3 demonstrated reduced sepsis compared to untreated galectin- $3^{-/-}$ mice. IL- 6 (D) and TNF- $\alpha$ (E) concentration in BAL was determined by CBA and demonstrated to be lower in galectin-3-treated galectin- $3^{-1-}$ mice $(*-P<$ 0.01 compared to galectin $-3^{-1-}$ and $* P<0.05$ compared to galectin- $3^{-1-}$ ).

gies to combat pneumonia are urgently required. Previous studies have demonstrated that galectin-3 is increased in the lung after infection with S. pneumoniae, and this correlates with the onset of neutrophil extravasation. ${ }^{13}$ However, the relevance of these observations to the mechanistic role of galectin- 3 in the host immune response to $S$. pneumoniae infection has not been examined. We therefore studied pneumococcal pneumonia in mutant mice lacking the galectin- 3 gene. Our in vivo studies show that galectin- $3^{-1-}$ mice develop more severe pneumonia after infection with S. pneumoniae, as demonstrated by increased septicemia and lung damage compared to WT mice. Neutrophil recruitment to the alveolar space was reduced in galectin- $3^{-1-}$ mice; however, myeloperoxidase activity in lung homogenates was not reduced in these mice compared to WT. This would suggest that neutrophils accumulate in the interstitial lung 
tissue during pneumonia in galectin- $3^{-1-}$ mice but are hindered from transmigrating into the alveolar space in the absence of galectin-3.

Galectin-3 is highly expressed and secreted from activated macrophages and acts as a powerful proinflammatory signal. ${ }^{12}$ Previous studies have shown that galectin-3 promotes the respiratory burst in neutrophils (and our observations), ${ }^{11,32}$ increases CD66 expression on the surface of neutrophils, ${ }^{33}$ and binds to CD66a and $\mathrm{CD} 66 \mathrm{~b},{ }^{34}$ the receptors most likely responsible for inducing neutrophil NADPH oxidase activation. Galectin-3 promotes the adhesion of neutrophils to $\operatorname{laminin}^{8}$ and enhances phagocytic activity of opsonized erythrocytes. ${ }^{33}$ However, the role of galectin-3 with regard to neutrophil function in response to infection is not well characterized.

Neutrophils play a critical role in the host immune defense against infection. Neutrophils are recruited early after infection, and their ability to phagocytose bacteria and secrete cytotoxic mediators makes them an important innate defense mechanism. Therefore, augmenting their function is critical to their antibacterial properties after infection. However, their removal from the site of infection during resolution is equally important because this limits host cell damage. Neutrophil apoptosis occurs as part of the normal resolution process, rendering neutrophils unresponsive to further stimulation and allowing noninflammatory recognition by tissue macrophages and their removal by phagocytosis. Factors that increase neutrophil activation and prolong neutrophil survival at sites of infection will have an important bearing on the host response to infection.

Here we demonstrate that galectin- 3 can directly activate both human and mouse neutrophils and potentiate the effect of fMLP. We have revealed that whole blood neutrophils can be directly activated by concentrations of galectin-3 lower than those that directly activate isolated neutrophils. This suggests that in whole blood there may be factors present in the serum (eg, GM-CSF) functioning to prime circulating neutrophils.

Although mouse neutrophils express very low levels of endogenous galectin-3 (our own observations), ${ }^{13}$ they can be activated by extracellular galectin-3, which is up-regulated in the surrounding tissue environment after infection. However, in galectin-3-null mice this galectin-3 up-regulation does not occur, resulting in reduced neutrophil recruitment into the alveolar spaces, activation, and phagocytosis.

Galectin-3 expression is up-regulated after pneumococcal infection (our own observations), ${ }^{13}$ and macrophages are capable of secreting large amounts of galectin-3. ${ }^{12,13}$ We show that longevity of human neutrophils is increased after incubation with exogenous galectin-3, thus delaying spontaneous apoptosis in vitro. Furthermore, galectin- $3^{-1-}$ macrophages displayed reduced phagocytosis of apoptotic human neutrophils compared to WT. The resultant accumulation of apoptotic neutrophils in the lungs of galectin- $3^{-1-}$ mice after infection would cause considerable damage to lung tissue, thus allowing bacteria to traverse the lung epithelia and enter the blood stream resulting in septicemia.
During S. pneumoniae infection the galectin-3 $3^{-1-}$ mouse mounts a greater Th1 response demonstrated by increased IL- 6 and TNF- $\alpha$ cytokine levels compared to WT mice or galectin- $3^{-1-}$ mice treated with recombinant galectin-3. This increased Th1 response may contribute to lung damage and subsequent septicemia. We have shown that galectin- $3^{-/-}$macrophages demonstrate a deficit in their ability to adopt an anti-inflammatory alternative (M2) phenotype. ${ }^{35}$ We therefore propose that, in addition to reduced neutrophil activation and apoptotic neutrophil clearance by macrophages in the galectin- $3^{-1-}$ mice, these mice are less able to dampen down the excessive inflammation and destructive potential of pneumococcal infection.

We propose that galectin-3 released from resident alveolar macrophages in response to pneumococcal pneumonia infection can activate neutrophils and enhance their bacterial killing and phagocytic capability, aiding pathogen clearance. In addition, we show that galectin-3 may play a further role in host defense by a direct bacteriostatic effect on S. pneumoniae. Galectin-3 $(15 \mu \mathrm{g})$ dramatically reduced $S$. pneumoniae growth in vitro. Sato and colleagues ${ }^{13}$ demonstrated that the galectin-3 concentration in the lung is increased in mice after $S$. pneumoniae infection. We observed very high concentrations of galectin-3 in lung homogenate and BAL after $S$. pneumoniae infection (concentration in BAL $>50 \mu \mathrm{g} / \mathrm{ml}$ ). This suggests that bacteriostatic concentrations of galectin-3 are achieved in the lung and may play an important role in the defense against $S$. pneumoniae infection.

Our study has shown that galectin- 3 protects against pneumococcal pneumonia through a variety of mechanisms including augmentation of neutrophil function and a direct bacteriostatic role. Strategies designed to augment galectin-3 expression in the lung may result in the development of novel treatments for pneumococcal pneumonia.

\section{Acknowledgment}

We thank Dr. Simon Hart for helpful discussions.

\section{References}

1. Paterson GK, Blue CE, Mitchell TJ: Role of interleukin-18 in experimental infections with Streptococcus pneumoniae. J Med Microbiol 2005, 54:323-326

2. Feldman C: Clinical relevance of antimicrobial resistance in the management of pneumococcal community-acquired pneumonia. J Lab Clin Med 2004, 143:269-283

3. Novak R, Henriques B, Charpentier E, Normark S, Tuomanen E: Emergence of vancomycin tolerance in Streptococcus pneumoniae. Nature 1999, 399:590-593

4. Sibille Y, Reynolds HY: Macrophages and polymorphonuclear neutrophils in lung defense and injury. Am Rev Respir Dis 1990. 141:471-501

5. Barondes $\mathrm{SH}$, Cooper DN, Gitt MA, Leffler H: Galectins. Structure and function of a large family of animal lectins. J Biol Chem 1994, 269:20807-20810

6. Hughes RC: The galectin family of mammalian carbohydrate-binding molecules. Biochem Soc Trans 1997, 25:1194-1198 
7. Cooper DN: Galectinomics: finding themes in complexity. Biochim Biophys Acta 2002, 1572:209-231

8. Kuwabara I, Liu FT: Galectin-3 promotes adhesion of human neutrophils to laminin. J Immunol 1996, 156:3939-3944

9. Sano H. Hsu DK, Yu L, Apgar JR, Kuwabara I, Yamanaka T, Hirashima M, Liu FT: Human galectin-3 is a novel chemoattractant for monocytes and macrophages. J Immunol 2000, 165:2156-2164

10. Frigeri LG, Zuberi RI, Liu FT: Epsilon BP, a beta-galactoside-binding animal lectin, recognizes IgE receptor (Fc epsilon RI) and activates mast cells. Biochemistry 1993, 32:7644-7649

11. Yamaoka A, Kuwabara I, Frigeri LG, Liu FT: A human lectin, galectin-3 (epsilon bp/Mac-2), stimulates superoxide production by neutrophils. J Immunol 1995, 154:3479-3487

12. Liu FT, Hsu DK, Zuberi RI, Kuwabara I, Chi EY, Henderson WR: Jr Expression and function of galectin-3, a beta-galactoside-binding lectin, in human monocytes and macrophages. Am J Pathol 1995, 147: $1016-1028$

13. Sato S, Ouellet N, Pelletier I, Simard M, Rancourt A, Bergeron MG: Role of galectin-3 as an adhesion molecule for neutrophil extravasation during streptococcal pneumonia. J Immunol 2002, 168:18131822

14. Nieminen J, St Pierre C, Sato S: Galectin-3 interacts with naive and primed neutrophils, inducing innate immune responses. J Leukoc Biol 2005, 78:1127-1135

15. Lotan R, Belloni PN, Tressler RJ, Lotan D, Xu XC, Nicolson GL: Expression of galectins on microvessel endothelial cells and their involvement in tumour cell adhesion. Glycoconj J 1994, 11:462-468

16. Dumic J, Dabelic S, Flogel M: Galectin-3: An open-ended story. Biochim Biophys Acta 2006, 1760:616-635

17. Fukumori T, Takenaka Y, Yoshii T, Kim HR, Hogan V, Inohara H, Kagawa S, Raz A: CD29 and CD7 mediate galectin-3-induced type II T-cell apoptosis. Cancer Res 2003, 63:8302-8311

18. Yang RY, Hsu DK, Liu FT: Expression of galectin-3 modulates T-cell growth and apoptosis. Proc Natl Acad Sci USA 1996, 93:6737-6742

19. Hsu DK, Yang RY, Pan Z, Yu L, Salomon DR, Fung-Leung WP, Liu FT: Targeted disruption of the galectin-3 gene results in attenuated peritoneal inflammatory responses. Am J Pathol 2000, 156:1073-1083

20. Sano H, Hsu DK, Apgar JR, Yu L, Sharma BB, Kuwabara I, Izui S, Liu FT: Critical role of galectin-3 in phagocytosis by macrophages. J Clin Invest 2003, 112:389-397

21. Kohatsu L, Hsu DK, Jegalian AG, Liu FT, Baum LG: Galectin-3 induces death of Candida species expressing specific beta-1,2-linked mannans. J Immunol 2006, 177:4718-4726

22. Sato $S$, Hughes RC: Regulation of secretion and surface expression of Mac-2, a galactoside-binding protein of macrophages. J Biol Chem 1994, 269:4424-4430
23. Coinot C, Ripoche MA, Milon G, Montagutelli X, Crocker PR, Poirier F: Maintenance of granulocyte numbers during acute peritonitis is defective in galectin-3-null mutant mice. Immunology 1998, 94:290-296

24. Lanoue A, Clatworthy MR, Smith P, Green S, Townsend MJ, Jolin HE, Smith KG, Fallon PG, McKenzie AN: SIGN-R1 contributes to protection against lethal pneumococcal infection in mice. J Exp Med 2004. 200:1383-1393

25. Looney MR, Su X, Van Ziffle JA, Lowell CA, Matthay MA: Neutrophils and their FC gamma receptors are essential in a mouse model of transfusion-related acute lung injury. J Clin Invest 2006, 116:16151623

26. Boxio R, Bossenmeyer-Pourie C, Steinckwich N, Dournon C, Nusse $\mathrm{O}$ : Mouse bone marrow contains large numbers of functionally competent neutrophils. J Leukoc Biol 2004, 75:604-611

27. Dransfield I, Stocks SC, Haslett C: Regulation of cell adhesion molecule expression and function associated with neutrophil apoptosis. Blood 1995, 85:3264-3273

28. Dahigren $C$, Karlsson A: Respiratory burst in human neutrophils. $\mathrm{J}$ Immunol Methods 1999, 232:3-14

29. Walrand S, Valeix S, Rodriguez C, Ligot P, Chassagne J, Vasson MP: Flow cytometry study of polymorphonuclear neutrophil oxidative burst: a comparison of three fluorescent probes. Clin Chim Acta 2003, 331:103-110

30. Savill J, Dransfield I, Gregory C, Haslett C: A blast from the past: clearance of apoptotic cells regulates immune responses. Nat Rev Immunol 2002, 2:965-975

31. Funakoshi N, Onizuka M, Yanagi K, Ohshima N, Tomoyasu M, Sato Y Yamamoto T, Ishikawa S, Mitsui T: A new model of lung metastasis for intravital studies. Microvasc Res 2000, 59:361-367

32. Karlsson A, Follin $P$, Leffler $H$, Dahlgren $C$ : Galectin-3 activates the NADPH-oxidase in exudated but not peripheral blood neutrophils. Blood 1998, 91:3430-3438

33. Fernández GC, llarregui JM, Rubel CJ, Toscano MA, Gomez SA, Beigier BM, Isturiz MA, Rabinovich GA, Palermo MS: Galectin-3 and soluble fibrinogen act in concert to modulate neutrophil activation and survival: involvement of alternative MAPK pathways. Glycobiology $2005,15: 519-527$

34. Feuk-Lagerstedt $E$, Jordan ET, Leffler $H$, Dahigren $C$, Karisson A: Identification of CD66a and CD66b as the major galectin-3 receptor candidates in human neutrophils. J Immunol 1999, 163:5592-5598

35. MacKinnon AC, Farnworth SL, Hodkinson PS, Henderson NC, Atkinson KM, Leffler H, Nilsson UJ, Haslett C, Forbes SJ, Sethi T: ReguIation of alternative macrophage activation by galectin-3. J Immunol 2008 (in press). 


\title{
Apoptotic human cells inhibit migration of granulocytes via release of lactoferrin
}

\author{
Irini Bournazou, ${ }^{1}$ John D. Pound, ${ }^{1}$ Rodger Duffin, ${ }^{1}$ Stylianos Bournazos, ${ }^{1}$ Lynsey A. Melville, ${ }^{1}$ \\ Simon B. Brown, ${ }^{1}$ Adriano G. Rossi, ${ }^{1}$ and Christopher D. Gregory, ${ }^{1,2}$
}

1The University of Edinburgh/Medical Research Council (MRC) Centre for Inflammation Research,

Queen's Medical Research Institute, Edinburgh, United Kingdom. ${ }^{2}$ ImmunoSolv Ltd., Edinburgh, United Kingdom.

\begin{abstract}
Apoptosis is a noninflammatory, programmed form of cell death. One mechanism underlying the non-phlogistic nature of the apoptosis program is the swift phagocytosis of the dying cells. How apoptotic cells attract mononuclear phagocytes and not granulocytes, the professional phagocytes that accumulate at sites of inflammation, has not been determined. Here, we show that apoptotic human cell lines of diverse lineages synthesize and secrete lactoferrin, a pleiotropic glycoprotein with known antiinflammatory properties. We further demonstrated that lactoferrin selectively inhibited migration of granulocytes but not mononuclear phagocytes, both in vitro and in vivo. Finally, we were able to attribute this antiinflammatory function of lactoferrin to its effects on granulocyte signaling pathways that regulate cell adhesion and motility. Together, our results identify lactoferrin as an antiinflammatory component of the apoptosis milieu and define what we believe to be a novel antiinflammatory property of lactoferrin: the ability to function as a negative regulator of granulocyte migration.
\end{abstract}

\section{Introduction}

Apoptosis is a programmed, physiological form of cell death that, in inflammatory terms, is quiet: apoptotic cells are rapidly phagocytosed by their neighbors or by mononuclear phagocytes that are attracted by chemotactic factors such as lysophosphatidylcholine (1) and fractalkine (2) released by apoptotic cells. The rapid engulfment of apoptotic cells militates against the potential tissue-injuring and proinflammatory features of dead cells as exemplified by necrosis (3-6). Indeed, failed or delayed clearance of apoptotic cells can have detrimental inflammatory consequences, including the development of autoimmune pathologies (7-9). The mechanisms underlying the non- or antiinflammatory nature of the apoptosis program are not understood in detail. Antiinflammatory mediators such as TGF- $\beta 1$ and IL- 10 are known to be produced at sites of apoptosis, both directly by apoptotic cells themselves and indirectly through interaction of apoptotic cells with phagocytes $(10$, 11). Apoptotic cells selectively attract mononuclear phagocytes $(1,12)$, but, curiously, the other class of professional phagocytes, granulocytes or polymorphonuclear phagocytes, do not migrate toward apoptotic cells in vitro (12) and do not normally engage in apoptotic cell engulfment. The absence of granulocytes from sites of homeostatic and developmental apoptosis suggests that apoptotic cells have the potential to selectively regulate the recruitment of mononuclear leukocytes.

In marked contrast to sites of apoptosis, sites of acute infection are characterized by the presence of granulocytes, most commonly neutrophils, as a means to protect the host by engulfing, killing, and digesting invading infectious agents. Neutrophils, as a first line of immune defense, are rapidly recruited to the site of infection in response to a variety of inflammatory stimuli, including

Conflict of interest: C.D. Gregory and J.D. Pound are founders of the company, ImmunoSolv Ltd., that supplied 2 of the monoclonal anti-lactoferrin antibodies used in Supplemental Figure 1.

Nonstandard abbreviations used: $\mathrm{BL}$, Burkitt lymphoma; $\left[\mathrm{Ca}^{2}\right]_{\mathrm{i}}$, intracellular calcium concentration; $\mathrm{ALLP}$, formyl-methionyl-leucyl-phenylalanine; $\mathrm{LTB}_{4}$, leukotriene $\mathrm{B}_{4}$. Citation for this article: J. Clin. Invest. doi: $10.1172 / \mathrm{JCl} 36226$. chemokines, cytokines, leukotrienes, and bacterial components such as LPS and $N$-formylated peptides $(13,14)$. Comparative studies on the slime mold Dictyostelium discoideum have shown that in response to chemoattractants, neutrophils orient themselves and migrate in an "ameboid motion" by anterior pseudopod extension accompanied by simultaneous posterior contraction and retraction. Such polarized morphology is characterized by the formation of a lamellipodium at the leading edge and a uropod at the trailing edge of the neutrophil (15-18). This process is tightly controlled not only to ensure the efficient migration of neutrophils to inflammatory sites, but also to prevent their aberrant infiltration and consequent tissue-damaging activities. Detrimental effects of neutrophils - caused, for example, by release of their proteolytic enzymes - contribute to many pathological inflammatory conditions, ranging from vasculitis and ischemia/reperfusion injury to glomerulonephritis, rheumatoid arthritis, and acute graft rejection (19). Therefore, at inflammatory sites, negative signals exist that prevent neutrophil recruitment, dampen neutrophil responsiveness, and counterbalance or terminate the inflammatory response. This antiinflammatory program is characterized by cessation of neutrophil infiltration, as arachidonic acid-derived prostaglandins and leukotrienes are switched to lipoxins, resolvins, and protectins $(20,21)$. Resolution of inflammation is ultimately achieved through neutrophil apoptosis, and apoptotic neutrophils are subsequently phagocytosed by macrophages, a process that leads to the release not only of antiinflammatory cytokines but also of such antiinflammatory and proresolving lipid mediators as lipoxin A4, resolvin E1, and protectin D1 (22-25).

Given (a) the production of negative signaling molecules at inflammatory sites to limit neutrophil recruitment and function and (b) failure of neutrophil migration to sites of apoptosis, we sought to determine whether apoptotic cells actively produce negative regulators of neutrophil chemotaxis. We postulated that the production by apoptotic cells of factors that inhibit neutrophil migration contribute to the non-phlogistic nature of the apoptosis program. Here, we present evidence that apoptotic cells actively inhibit neutrophil migration through the production of 
A

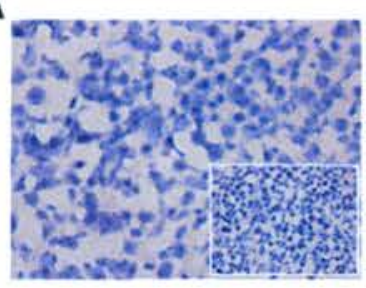

C

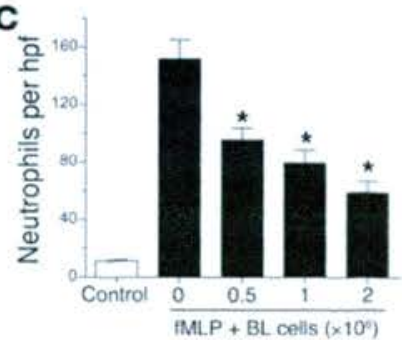

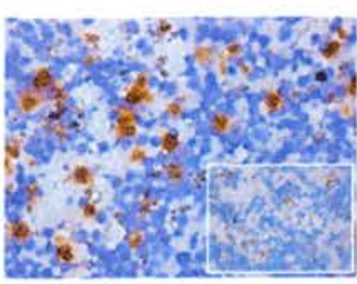

D

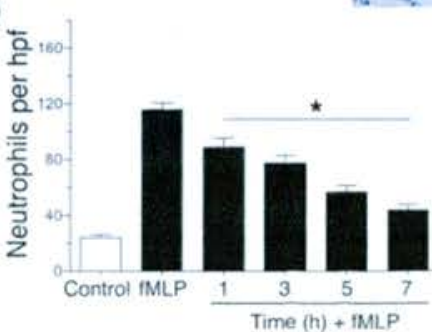

B fMLP fMLP + BL cells

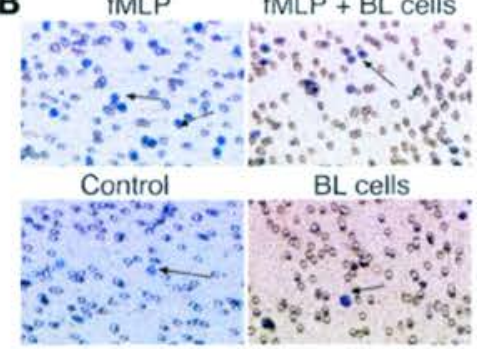

E

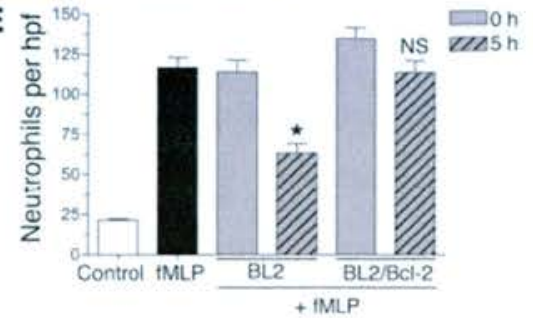

Figure 1

Apoptotic cells release factor(s) that inhibit neutrophil migration. (A) Immunohistochemical analysis of neutrophils in BL (left) and spleen (positive control; right) sections. Inset images represent isotype control. (B) Representative images of stained Transwell filters. (C) Neutrophil chemotaxis toward increasing concentrations of BL cells was assessed in the presence of fMLP $(100 \mathrm{nM}) . n=3 ;{ }^{*} P<0.05$ vs. time 0 . (D) BL cell-conditioned media obtained at the indicated time points were used to analyze fMLP-induced neutrophil chemotaxis. $n=3 ;{ }^{*} P<0.05$ vs. fMLP. (E) Neutrophil chemotaxis toward fMLP was analyzed in the presence of control or Bcl-2-transfected BL2 cells obtained following a 0- and 5-hour incubation at $37^{\circ} \mathrm{C} . n=3 ;{ }^{*} P<0.05$ vs. BL2 $0 \mathrm{~h} ; \mathrm{NS}$ vs. BL2/Bcl-2 $0 \mathrm{~h}$. Apoptosis levels were assessed by flow cytometry following staining with annexin $\mathrm{V} /$ propidium iodide. Error bars indicate SEM. Original magnification; $\times 400(\mathbf{A} ; \mathbf{A}$, insets; B). hpf, high-power field.

lactoferrin, an $80 \mathrm{kDa}$ antiinflammatory glycoprotein that specifically inhibits chemotaxis of neutrophils but not mononuclear phagocytes. Lactoferrin was found to be synthesized de novo following triggering of apoptosis and released by apoptotic cells of diverse lineages. Lactoferrin impaired neutrophil activation and prevented mobilization of the cells by inhibiting migratory polarization of the cell body. These results demonstrate that the non-phlogistic constitution of apoptotic cells includes an antiinflammatory molecule, lactoferrin, that we show, for the first time to our knowledge, has potent negative regulatory effects on neutrophil migration. These findings provide a rationale for the absence of neutrophils from apoptotic sites and have important implications for understanding the mechanisms involved in the resolution phase of inflammation.

\section{Results}

Apoptotic cells actively produce factor(s) that inhibit neutrophil chemotaxis. To address whether apoptotic cells influence migratory activity of neutrophils, we carried out a series of in vitro Boyden-type chemotaxis assays to investigate neutrophil migration toward Burkitt lymphoma (BL) cells. We initially employed BL as a model tissue as these tumor cell populations display high levels of apoptosis, a property that is retained constitutively in the tumor-derived cell lines. As at all sites of apoptosis, there is marked infiltration of macrophages that engulf the apoptotic cells, giving rise to the typical "starry sky" histological appearance of this tumor. As shown in Figure 1A (left), while macrophages were abundant in histological sections of BL, neutrophils were absent. We assessed the effects of BL cells on the migratory activity of neutrophils in vitro by adding neutrophils to the top compartment of a Transwell filter and inducing them to migrate toward the lower chamber containing BL cells in the presence of the powerful neutrophil chemoattractant formyl-methionyl-leucyl-phenylalanine (fMLP) (Figure 1B). As shown in Figure 1C, neutrophil migration was significantly inhibited in BL cells in a concentration-dependent manner. We observed a similar effect irrespective of the chemoattractant used (inhibition of neutrophil migration induced by $\mathrm{C} 5 \mathrm{a}, \mathrm{IL}-8$, and leukotriene $\mathrm{B}_{4}$ [ $\left.\mathrm{LTB}_{4}\right]$; data not shown, but see below). We carried out subsequent chemotaxis assays using BL-conditioned medium obtained over a 7-hour time course and found that BL cells actively released an inhibitory factor(s) (Figure 1D). The release of the inhibitory factor appeared to be linked to the levels of apoptosis in the BL cell populations, since the inhibitory activity was significantly lower in further chemotaxis assays using $\mathrm{BL}$-conditioned medium derived from cells overexpressing the apoptosis inhibitor $\mathrm{Bcl}-2$, as compared with that of parental cells (Figure 1E).

Biochemical characterization of neutrophil migration-inbibitory factor(s). In an attempt to gain further insight into the biochemical nature of the factor(s) that BL cells secrete in order to exclude neutrophils from their environment, we initially estimated the molecular weight range of the inhibitory factor(s) by using filters with molecular weight cutoff points of approximately $3,10,30$, 50 , and $100 \mathrm{kDa}$. BL-conditioned media obtained after 24 -hour incubation were fractionated, and each fraction was examined in vitro using the neutrophil chemotaxis assay described above. The results revealed that fractions containing molecules of less than $50 \mathrm{kDa}$ failed to display any inhibitory effect on neutrophil migration (Figure 2A). However, the use of $100 \mathrm{kDa}$ filters revealed that both fractions ( $>100 \mathrm{kDa}$ and $<100 \mathrm{kDa}$ ) displayed an inhibitory effect on neutrophil migration, indicating that at least 1 factor has a molecular weight that ranges between 50 and $100 \mathrm{kDa}$. The presence of inhibitory activity in the filtrate of the $100 \mathrm{kDa}$ 

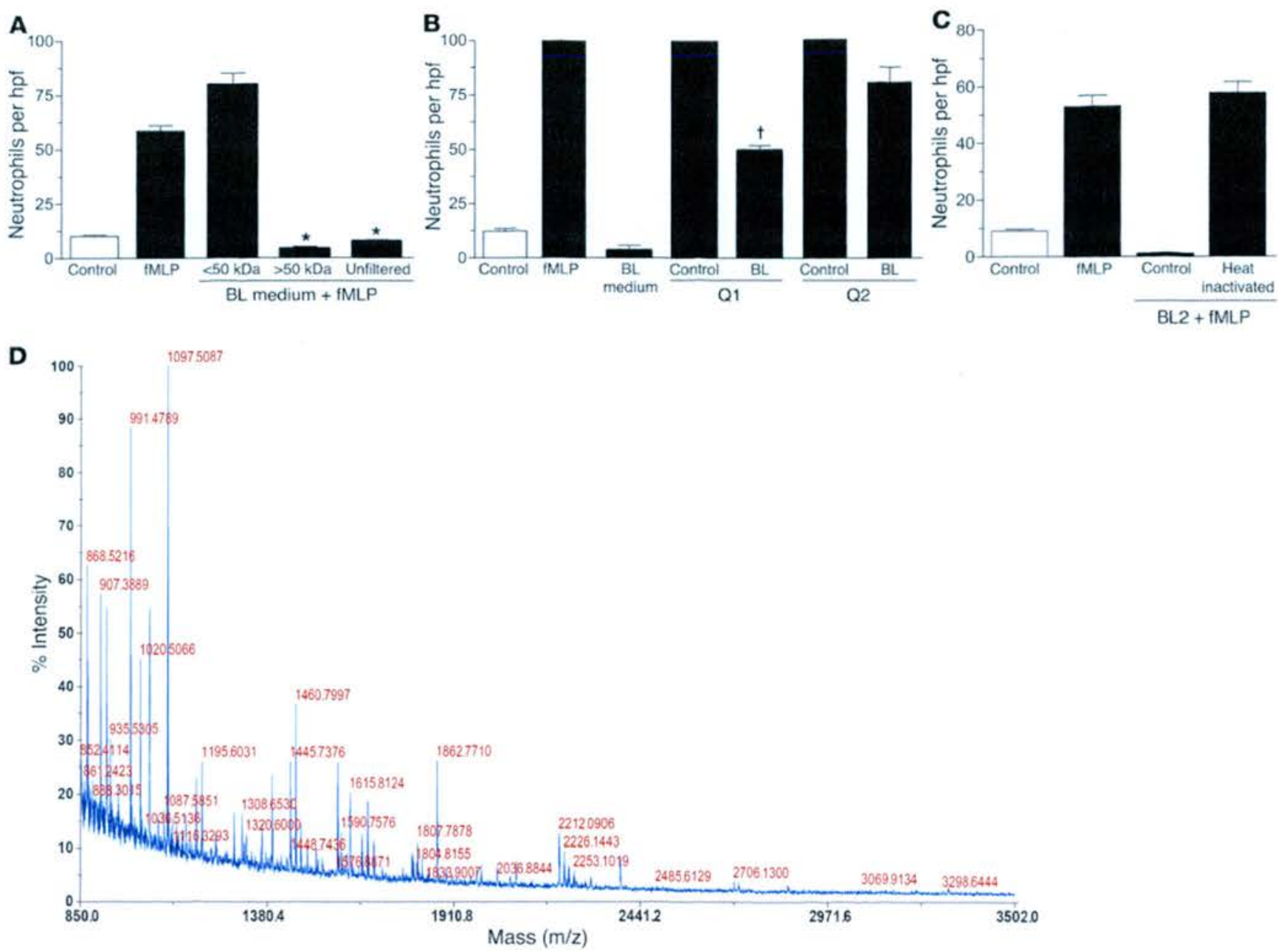

Figure 2

Biochemical characterization of the inhibitory factor. Conditioned media from BL2 cells cultured for 24 hours were size fractionated using filters with $50 \mathrm{kDa}(\mathbf{A})$ molecular weight cutoff sizes. Unfiltered medium was included as control. ${ }^{*} P<0.001$ compared with the corresponding positive control. Error bars indicate SEM. Ion exchange analysis included the use of Q Sepharose beads (positively charged) in order to distinguish positively and negatively charged molecules in the $<100 \mathrm{kDa}$ fraction $(\mathrm{B})$ of the BL medium. Unbound molecules (Q1 fraction) were collected, whereas bound molecules were eluted from the beads (Q2 fraction). Neutrophil migration toward these fractions in the presence of fMLP (100 nM) was assessed. Q1 and Q2 fractions (unbound and eluant fraction) of serum-free medium (no BL) were included as control. ${ }^{\dagger} P<0.05$ compared with the corresponding control. Error bars indicate SEM. (C) Chemotaxis assay of neutrophils toward BL-conditioned medium that was heat inactivated $\left(90^{\circ} \mathrm{C}\right.$ for 10 minutes). (D) MALDI-TOF mass spectrum for the tryptic digest of the peptide band identified as lactoferrin.

cutoff membrane is likely to result from (a) imprecise molecular weight cutoff of molecules in the 50-100 kDa range, (b) complex formation through multimerization of the 50-100 kDa factor or through interaction with other molecules, or (c) the existence of a distinct inhibitory activity of greater than $100 \mathrm{kDa}$. It should be noted that the selected isolation approach is skewed in favor of proteins and that additional low-molecular-weight (for example, lipid) mediators of neutrophil migration inhibition would not be identified by these procedures.

To investigate further the biochemical properties of the retentate and filtrate of the $100 \mathrm{kDa}$ cutoff membrane, we first determined the charge ( $\mathrm{pl}$ value) of the migration-inhibitory activity by means of an ion exchange analysis of BL-conditioned media. Using positively charged ion exchange beads (Q Sepharose), the $100 \mathrm{kDa}$ membrane retentate and filtrate were separated into posi- tively charged (Q-supernatant) and negatively charged (Q-eluant) fractions. As shown in Figure 2B, the filtrate of the $100 \mathrm{kDa}$ membrane ( $<100 \mathrm{kDa}$ fraction) contained migration-inhibitory activity in both the supernatant (positive charge) and the eluant (negative charge) of the $\mathrm{Q}$ beads. By contrast, analyses of the retentate ( $>100 \mathrm{kDa}$ fraction) revealed that only the negatively charged eluant displayed significant activity in inhibiting neutrophil migration. These results indicate that at least 2 moieties with neutrophil migration-inhibitory activity were present in $\mathrm{BL}$ cell-conditioned medium: one of 50-100 kDa with positive $\mathrm{PI}$ and a second of $100 \mathrm{kDa}$ or more and negatively charged.

We subsequently determined whether the neutrophil migrationinhibitory activity was heat labile. As shown in Figure 2C, heat inactivation completely abrogared all chemotaxis inhibitory activity in BL cell-conditioned medium, suggesting that the inhibitory 

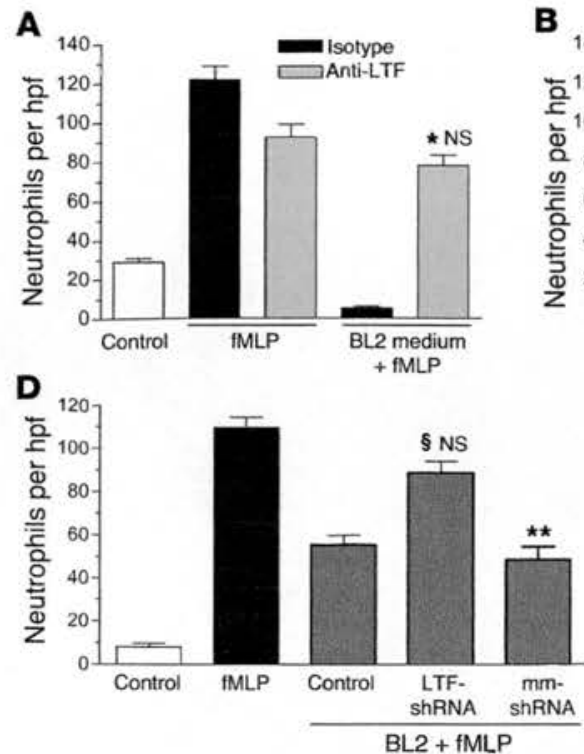

B

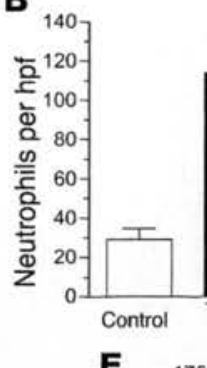

$\mathbf{E}$
C

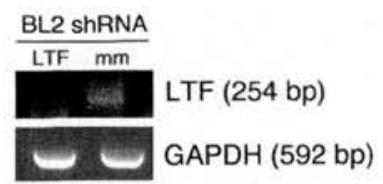

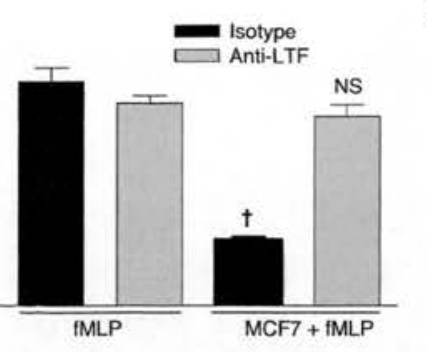

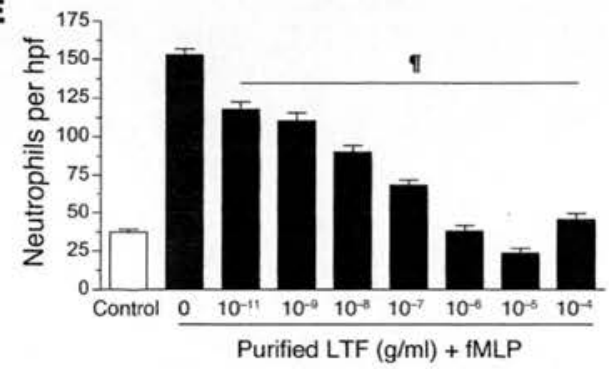

\section{Figure 3}

Lactoferrin specifically inhibits neutrophil chemotaxis. Neutrophil chemotaxis in the presence of human anti-lactoferrin (anti-LTF) polyclonal antibody (gray) or isotype control (black) using conditioned media from BL (A) and MCF7 (B) cells (A: $n=3,{ }^{*} P<0.05$ vs. isotype control, NS vs. $\mathrm{fMLP}$ anti-lactoferrin control; $\mathrm{B} ; n=3,{ }^{\dagger} P<0.001$ vs. fMLP/isotype; NS vs. fMLP/anti-LTF. (C) RT-PCR analysis to assess lactoferrin expression in BL cells stably expressing LTF shRNA (LTF) cells and mock-transfected $(\mathrm{mm})$ cells induced to become apoptotic $\left(1 \mu \mathrm{M} \mathrm{staurosporine;} 37^{\circ} \mathrm{C}\right)$. (D) Chemotaxis assay to determine neutrophil migration toward supernatants obtained from control, LTF shRNA, and mock-transfected BL cells $\left(n=5 ; \Im P<0.05\right.$ compared with mm shRNA control; ${ }^{\star *} P<0.05$ compared with $\mathrm{fMLP}$; NS compared with $\mathrm{fMLP}$ control). (E) Dose-response analysis of purified human lactoferrin. $n=3 ; \uparrow P<0.05 \mathrm{vs} .0 \mathrm{~g} / \mathrm{ml}$ purified LTF + fMLP. Error bars indicate SEM.

factor(s) were most likely protein in nature. We then analyzed the proteins released from BL cells in viable and apoptotic states by protein fingerprinting. Polypeptide bands of greater than $50 \mathrm{kDa}$ were excised from a $10 \%$ SDS polyacrylamide gel. Tryptic peptides were gel extracted, and matrix-assisted laser desorption/ionization-time of flight (MALDI-TOF) mass spectrometric analysis was carried out (Figure 2D). Also, given the crude biochemical characteristics described above, we undertook a candidate approach based on the proteins released from apoptotic BL cells. We identified the factor released by BL cells that prevented neutrophil chemotaxis as lactoferrin.

Lactoferrin specifically inbibits neutrophil chemotaxis toward a range of chemoattractants. Lactoferrin is a glycoprotein of approximately 75-80 kDa that belongs to the transferrin family of proteins due to its iron-binding properties. It is a well-characterized component of neutrophil secondary granules, lacrimal fluid, colostrum, saliva, and mucosal secretions, in which it confers antibacterial activity. We observed that addition of anti-lactoferrin antibody to BL-conditioned medium neutralized its neutrophil migration-inhibitory activity using either polyclonal or monoclonal antibodies (Figure 3A and Supplemental Figure 1; supplemental material available online with this article; doi:10.1172/JCI36226DS1). Similar results were observed with supernatants obtained from the mammary carcinoma line MCF-7, indicating that the neutrophil migration-inhibitory activity is not restricted to BL cell-derived lactoferrin (Figure 3B). Furthermore, lactoferrin purified from human milk displayed dose-dependent inhibitory activity toward neutrophil migration in response to AMLP (Figure 3E) and also inhibited migration toward $\mathrm{C} 5 \mathrm{a}, \mathrm{IL}-8$, and $\mathrm{LTB}_{4}$ to similar levels (Figure 4A). The neutrophil migration-inhibitory effect was also displayed by lactoferrin purified from human neutrophils (Figure 4D). It should be noted that both types of purified lactoferrin used in this study were free of endotoxin contamination, and the observed inhibitory effect did not appear to be due to any lactoferrin-associated molecules such as LPS. Furthermore, inhibition of lactoferrin expression by shRNA in BL cells provides additional support for the specificity of the observed lactoferrin effect. Thus, we found that neutrophil chemotaxis toward supernatants obtained from BL2 cells transfected with shRNA vectors targeted against lactoferrin was higher compared with supernatants from BL control or mock-transfected cells (Figures 3, C and D). Lactoferrin exerted no toxic effects on neutrophils, as assessed by annexin V/propidium iodide staining of control and lactoferrintreated neutrophils ( $>98 \%$ cell viability). These results suggested that lactoferrin binds to neutrophils and inhibits their ability to undergo chemotaxis. To exclude the possibility that the observed inhibitory activity of lactoferrin was due instead to its ability to bind and functionally neutralize the chemoattractants, additional chemotaxis assays were performed using chemoattractants (fMLP, $\mathrm{C} 5 \mathrm{a}, \mathrm{IL}-8$ ) that were preabsorbed with lactoferrin. To achieve this, we preincubated chemoattractants with lactoferrin. Subsequently, anti-lactoferrin antibody was used to remove the lactoferrin with the aid of magnetic beads. As shown in Figure 4B, no difference in neutrophil chemotactic activity was observed between the control and lactoferrin-absorbed chemoattractants, which excludes the possibility that lactoferrin binds to, and alters the activity of, the chemoattractants. Further supporting our conclusion that lactoferrin exerts its inhibitory effects through binding to neutrophils, we also observed that purified lactoferrin can directly associate with neutrophils (Figure 4C). In addition, Scatchard binding 

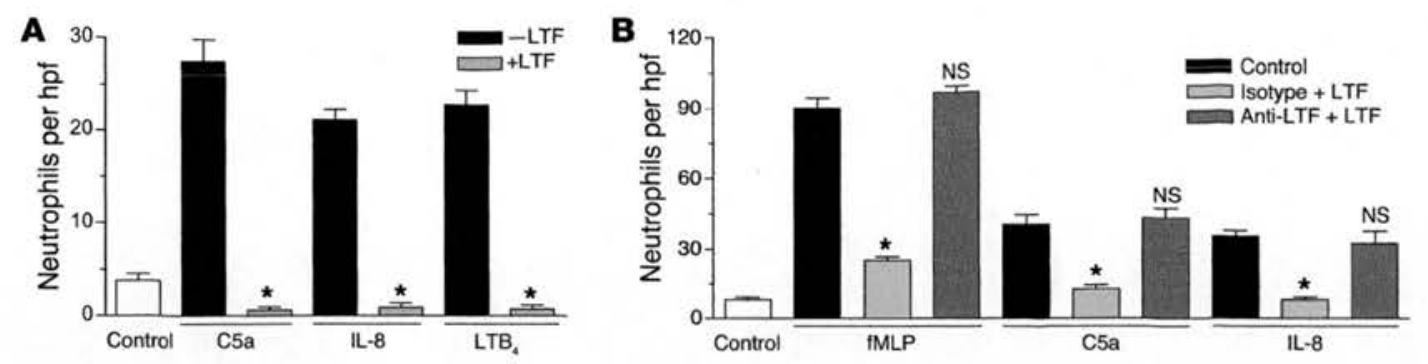

C
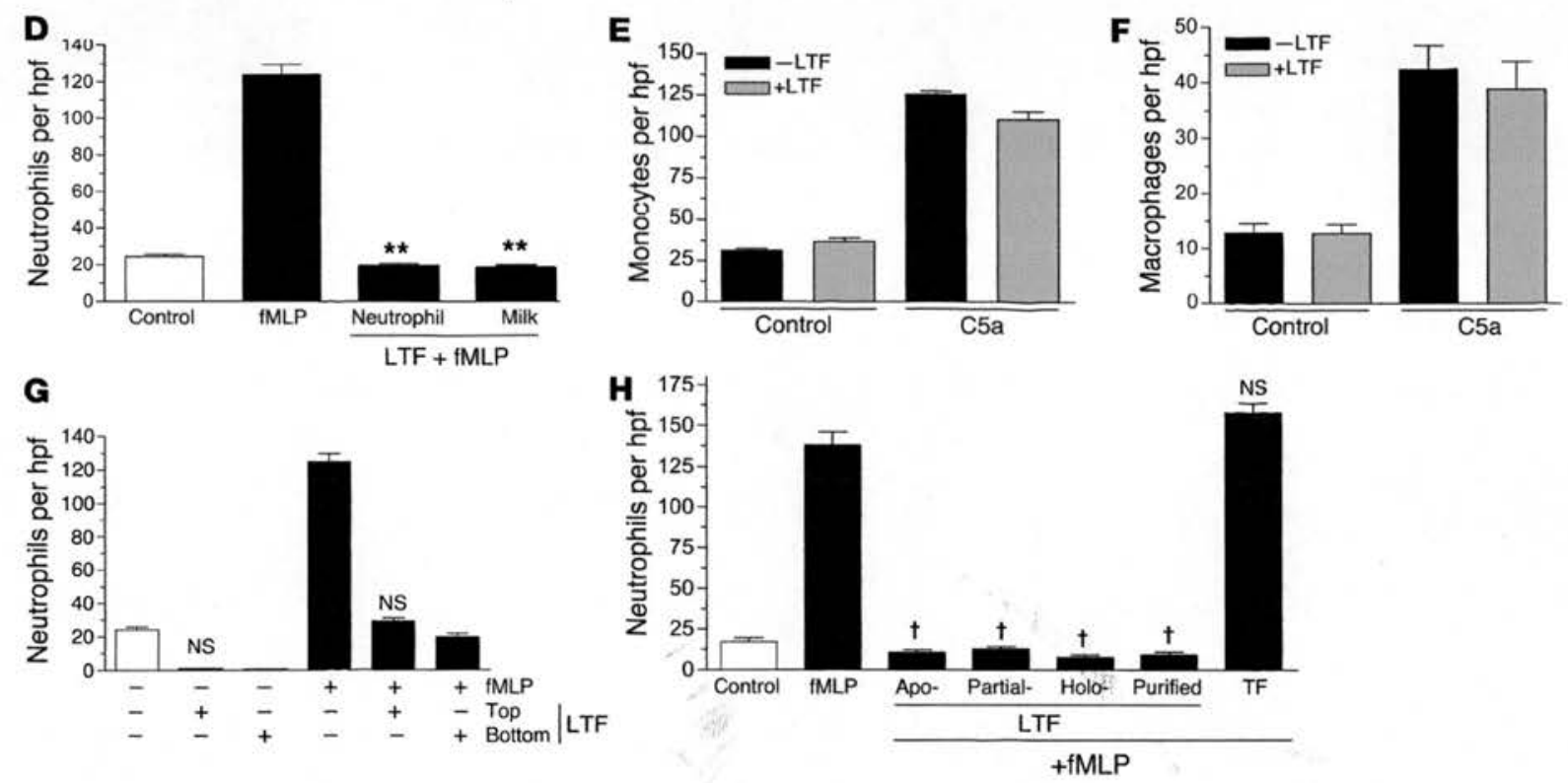

Figure 4

Neutrophil chemotaxis toward lactoferrin is irrespective of the chemoattractant used and its iron saturation status. (A) Neutrophil chemotaxis toward different chemoattractants. $n=3 ;{ }^{\star} P<0.05$. (B) Neutrophil chemotaxis toward chemoattractants (control) or chemoattractants that were incubated with lactoferrin $(10 \mu \mathrm{g} / \mathrm{ml})$ followed by the addition of isotype or anti-lactoferrin monoclonal antibody $(10 \mu \mathrm{g} / \mathrm{ml})$. Antibodies were removed using magnetic IgG beads. $n=3 ;{ }^{\star} P<0.05$, NS compared with chemoattractant control. (C) Immunoblot analysis of lysates of neutrophils incubated with or without biotinylated lactoferrin $(10 \mu \mathrm{g} / \mathrm{ml})$ at $37^{\circ} \mathrm{C}$ for 1 hour. (D) Neutrophil chemotaxis toward lactoferrin $(10 \mu \mathrm{g} / \mathrm{ml})$ purified from human neutrophils or human milk. ${ }^{* *} P<0.001$ vs. fMLP. C5a-induced monocyte (E) or macrophage (F) chemotaxis. (G) Neutrophil migration in the presence of lactoferrin $(10 \mu \mathrm{g} / \mathrm{ml})$ in the top or bottom compartment of the Transwell insert $(n=3$; NS vs. corresponding $+\mathrm{LTF}$ controls). (H) Chemotaxis assay to determine neutrophil migration toward purified recombinant iron-depleted (Apo-), partially iron-saturated, and fully iron-saturated (Holo-) recombinant lactoferrin $(10 \mu \mathrm{g} / \mathrm{ml})$. Milk-purified lactoferrin and partially iron-saturated transferrin (TF; $10 \mu \mathrm{g} / \mathrm{ml}) \mathrm{Were}$ added as control. $n=4 ;{ }^{\dagger} P<0.001$ compared with fMLP control. Error bars indicate SEM.

analysis of ${ }^{125} \mathrm{I}$-labeled apolactoferrin indicated, in accordance with earlier findings $(26,27)$, that lactoferrin bound to neutrophils via 2 classes of receptor that differ in affinity and number of binding sites per cell. We determined the higher-affinity receptors to be expressed at a density of $9,100 \pm 2,500$ binding sites per cell, with an affinity of $350 \pm 65 \mathrm{nM}$, and the lower-affinity receptors to be expressed at a density of $2.5 \times 10^{6} \pm 0.7 \times 10^{6}$ per cell, with an affinity of $20 \pm 10 \mu \mathrm{M}$ (Supplemental Figure 2).

To determine whether the migration-inhibitory effects of lactoferrin were specific to neutrophils among professional phagocytes, we analyzed its effects on monocyte and macrophage migration in vitro. As shown in Figure 4, E and F, C5a-induced chemotaxis of mononuclear phagocytes was unimpaired by lactoferrin. We further assessed whether lactoferrin acted by inhibiting neutrophil migration or promoting neutrophil repulsion. In chemotaxis assays, in which we added lactoferrin to the upper chamber along with neutrophils, we observed inhibition of neutrophil migration toward AMLP and control medium (Figure 4G), suggesting that lactoferrin exerts a direct effect on neutrophils by inhibiting their migratory ability and not by forcing them to migrate in all directions away from the chemoattractant.

Neutrophil migration-inhibitory effects of lactoferrin are not related to its iron-binding properties and are demonstrable in vitro and in vivo. Iron and iron-associated molecules have been previously shown to play an important role in many immunomodulatory functions. Indeed, suppression of IL-1 release by monocytes is observed by purified iron-saturated lactoferrin, whereas an inhibition of GM colony-stimulating activity production by monocytes and macrophages correlated with the iron saturation status of lactoferrin (28-30). Therefore, we further examined whether differences in the iron saturation profile of lactoferrin affect its ability to inhibit neutrophil migration. Chemotaxis assays to determine neutrophil migration toward iron-depleted (apo-lactoferrin), partially iron-saturated, or fully iron-saturated (holo-lactoferrin) lactofer- 

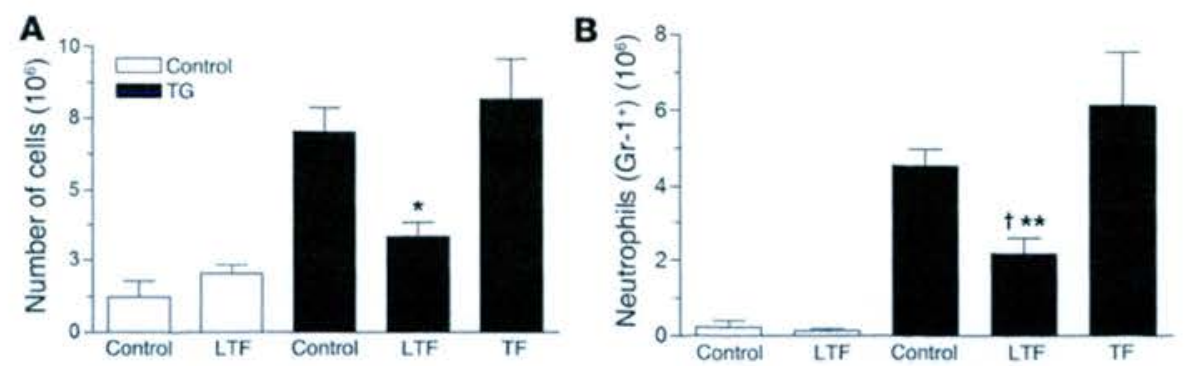

\section{Figure 5}

Lactoferrin inhibits neutrophil migration in vivo. Total cell (A) or neutrophil number (Gr-1+; B) obtained from peritoneal lavage. ${ }^{*} P<0.05$ vs. transferrin; ${ }^{\dagger} P<0.05$ vs. thioglycollate (TG) control, ${ }^{* *} P<0.01$ vs. transferrin control. Error bars indicate SEM. (C) Characteristic cytospin images. Original magnification, $\times 400$, top; $\times 200$, bottom.

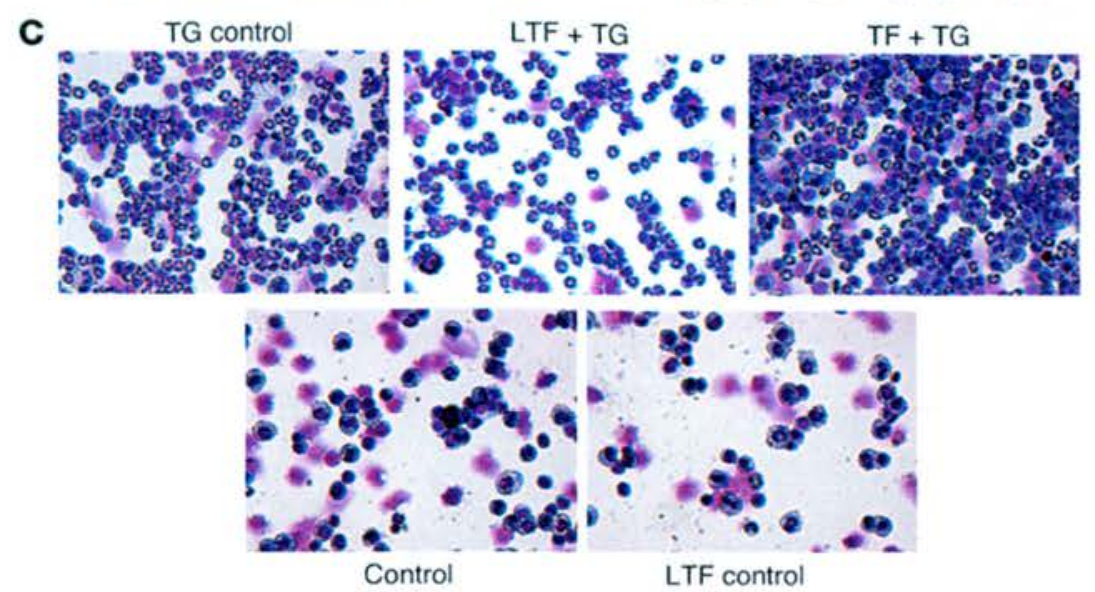

rin revealed that the level of iron saturation was not responsible for the observed inhibition in neutrophil migration (Figure $4 \mathrm{H}$ ). Also, as lactoferrin belongs to the transferrin family of proteins sharing $74 \%$ sequence homology with transferrin (both of them are $80 \mathrm{kDa}$ cationic iron-binding glycoproteins), we reasoned that, if the underlying neutrophil migration-inhibitory mechanism of lactoferrin was rooted in its ability to chelate iron, transferrin might show similar effects on neutrophil migration. To explore this possibility, we performed chemotaxis assays in which neutrophils were induced to migrate toward FMLP in the presence of partially iron-saturated transferrin. Our results showed that transferrin, unlike lactoferrin, had no effect on neutrophil chemotaxis, further supporting the conclusion that the observed neutrophil migration-inhibitory effect is lactoferrin specific and is unlikely to require iron-chelating activity (Figure $4 \mathrm{H}$ ).

Having established the inhibitory effects of lactoferrin on neutrophil chemotaxis in vitro, we then used a murine peritonitis model to assess the effect of lactoferrin on leukocyte recruitment in vivo. Lactoferrin and transferrin were tested for their ability to affect thioglycollate-induced leukocyte recruitment to the peritoneal cavity. As shown in Figure 5, A and B, thioglycollate caused rapid recruitment of leukocytes compared with vehicle alone, and the recruited leukocytes were predominantly neutrophils (88\%). In the presence of lactoferrin, the total number of neutrophils recruited to the peritoneal cavity was reduced by $52 \%$ compared with control, whereas transferrin had no effect. Lactoferrin reduced specifically the proportion and number of neutrophils migrating into the cavity but did not affect recruitment of other types of leukocytes in response to thioglycollate (Figure $5 \mathrm{C}$ ). These results demonstrate that, similar to its effect on neutrophil chemoattraction in vitro, lactoferrin is a potent inhibitor of neutrophil migration in vivo.

Impairment of neutrophil activation profile by lactoferrin. Neutrophil migration involves activation, adhesion, and extravasation pro- cesses that are accompanied by gross changes in cell morphology: whereas nonactivated neutrophils are rounded, activated neutrophils acquire a polarized morphology with spreading and adhesion to the available substratum (15). In order to initially assess the effects of lactoferrin on neutrophil activation, we performed time-lapse video microscopy of neutrophils and recorded directly their activation morphology, cell spreading, and locomotion. During a 1-hour time course, lactoferrin-pretreated neutrophil populations stimulated with $\mathrm{MLP}$ displayed a greater proportion of nonadherent cells as well as cells presenting a rounded, nonactivated morphology as compared with neutrophils treated with $\mathrm{fMLP}$ alone (Figure $6, \mathrm{~A}$ and $\mathrm{B}$ ). These quantitative differences between lactoferrin-treated and untreated neutrophils stimulated with AMLP were reflected in the locomotion of the cells around the substratum, with lactoferrin-treated cells displaying markedly reduced movement.

Changes in cell morphology following stimulation with AMLP or other neutrophil agonists are characterized by a rapid increase in intracellular cytoplasmic calcium levels through mobilization of calcium from ER stores and activation of calcium influx channels of the plasma membrane, mediated by the inositol triphosphate $\left(\mathrm{IP}_{3}\right)$ and diacylglycerol/PLC (DAG/PLC) pathways (31-33). In order to determine whether the observed cell shape alterations following lactoferrin treatment are related to changes in intracellular calcium concentrations $\left(\left[\mathrm{Ca}^{2+}\right]_{i}\right)$, we measured the levels of $\left[\mathrm{Ca}^{2+}\right]_{i}$ in control and lactoferrin-treated cells in response to fMLP stimulation ( $1 \mathrm{nM}$ or $10 \mathrm{nM})$. No changes were observed in the fMLP-mediated $\left[\mathrm{Ca}^{2+}\right]_{\mathrm{i}}$ response between control and lactoferrin-treated neutrophils, suggesting that lactoferrin acts downstream or independently of the mechanisms involved in intracellular calcium flux (Figure 6C).

As lactoferrin was shown to prevent neutrophil migration, we next explored whether it could also affect the neutrophil activation state. To this end, we chose to measure the expression of 2 
A

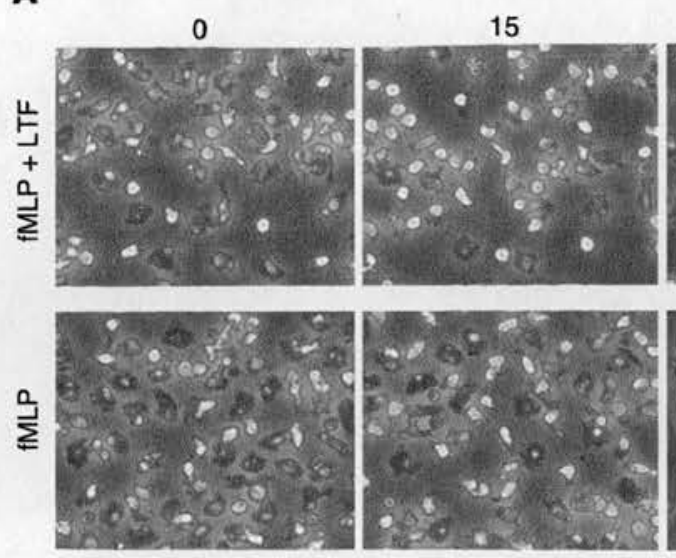

B

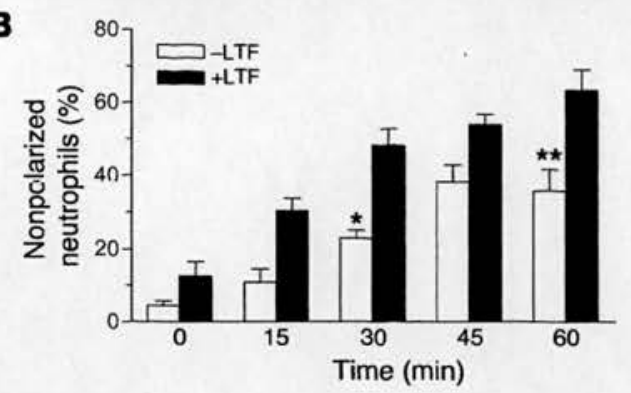

Time (min)
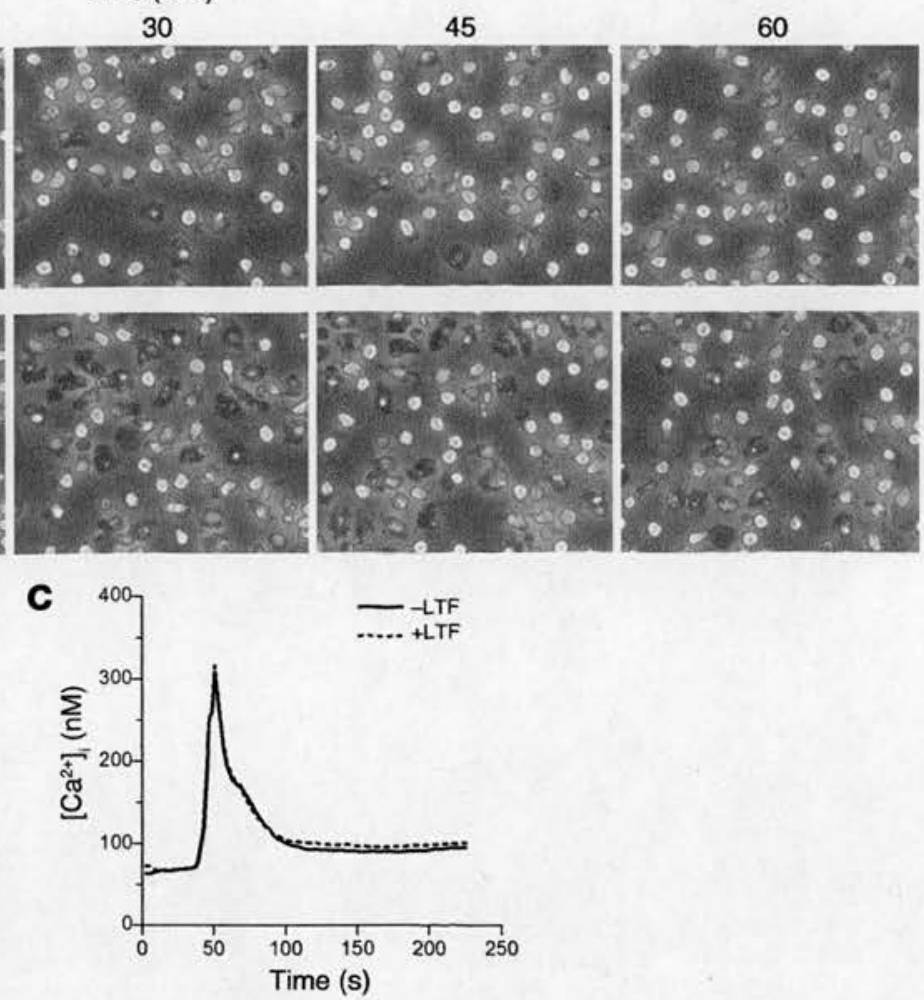

\section{Figure 6}

Effect of lactoferrin on neutrophil polarization morphology and spreading. (A) Time-lapse video microscopy frames of control or lactoferrin-pretreated neutrophils $\left(10 \mu \mathrm{g} / \mathrm{ml} ; 40\right.$ minutes at $\left.37^{\circ} \mathrm{C}\right)$ stimulated with $1 \mu \mathrm{M} \mathrm{fMLP}$ over a 1 -hour incubation time course. Original magnification, $\times 400$. (B) Quantification of neutrophils (nonpolarized) counted from 5 different fields; ${ }^{*} P<0.05,{ }^{* \star} P<0.01$ vs. corresponding $+\mathrm{LTF}$ control. Error bars

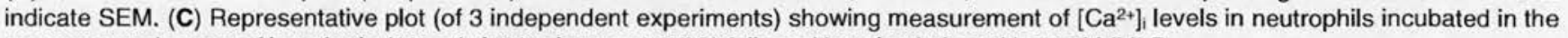
presence or absence of lactoferrin $\left(10 \mu \mathrm{g} / \mathrm{ml} ; 30\right.$ minutes at $\left.37^{\circ} \mathrm{C}\right)$ followed by stimulation with $10 \mathrm{nM} \mathrm{fMLP}$.

known neutrophil activation-associated markers, CD62L (L-selectin) and CD11b, using 2-color flow cytometry. Upon activation, $\mathrm{CD} 62 \mathrm{~L}$ is cleaved from the neutrophil surface, whereas $\mathrm{CD} 11 \mathrm{~b}$ expression is upregulated following translocation from cytoplasmic granules to the cell membrane. Freshly isolated neutrophils were pretreated with lactoferrin and then exposed to the activation stimuli PMLP, TNF- $\alpha$, and PMA. As shown in Figure 7, A-D, we found that, in lactoferrin-treated neutrophils compared with control cells, CD62L expression was significantly higher, whereas CD11b levels were lower. These effects were common to all activation stimuli used. Transferrin-treated neutrophils were also included but showed no significant differences compared with control cells. It is noteworthy that the lactoferrin effect was also evident when PMA, a specific PKC activator, was used as an agonist, indicating that lactoferrin acts downstream of PKC and not on pathways involved in $\mathrm{PKC}$ activation and $\mathrm{Ca}^{2+}{ }_{\mathrm{i}}$ responses, such as the $\mathrm{IP}_{3}$ and $\mathrm{DAG} / \mathrm{PLC}$ pathways. This finding prompted us to investigate putative downstream targets of PKC involved in the late signaling cascades following neutrophil activation that also regulate cell motility and actin reorganization. Such cascades involve the activation of MAP family kinases (34), and we therefore examined the phosphorylation of p44/42 (ERK1 and ERK2) MAPKs. Whereas in untreated neutrophils, ERK1 and ERK2 were phosphorylated following AMLP stimulation ( $100 \mathrm{nM})$, lower levels of phosphorylated ERK1/2 were observed in neutrophils that had been pretreated with lactoferrin prior to stimulation with fMLP (Figure 7E). Collectively, these data suggest that lactoferrin has a clear impact on neutrophil activation, including impairment of neutrophil degranulation, inhibition of expression of $\beta_{2}$ integrins, and reduction of activation of intracellular kinases, with profound effects on cell migration and motility.

Induction of apoptosis upregulates lactoferrin expression and release in diverse cell types. Pursuing our initial hypothesis, which was strengthened by early observations that inhibition of neutrophil migration by BL cells appeared to be correlated with BL cell apoptosis (Figure 1E), we assessed lactoferrin expression following induction of apoptosis in a panel of cells of diverse lineages. By transcriptional analysis using RT-PCR, we found that lactoferrin was expressed, as reported previously (35), by MCF-7 mammary epithelial cells in their viable state but not by Jurkat, BL2, or A549 cells. Upon apoptosis induction, lactoferrin expression was upregulated in MCF-7 cells and expressed de novo in Jurkat, BL2, and A549 cells (Figure 8). More specifically, lactoferrin was transcribed de novo early after induction of apoptosis in A549 cells by either $100 \mathrm{nM}$ etoposide or $1 \mu \mathrm{M}$ staurosporine (Figure $8 \mathrm{~B}$ ). Levels of lactoferrin induced by etoposide were reduced in cells treated in the presence of the broad-spectrum caspase inhibitor zVAD-fmk, which prevented apoptosis induction (Figure 8C). The link between lactoferrin expression and apoptosis induction was further supported by the effects of the apoptosis inhibitor Bcl-2. 
A
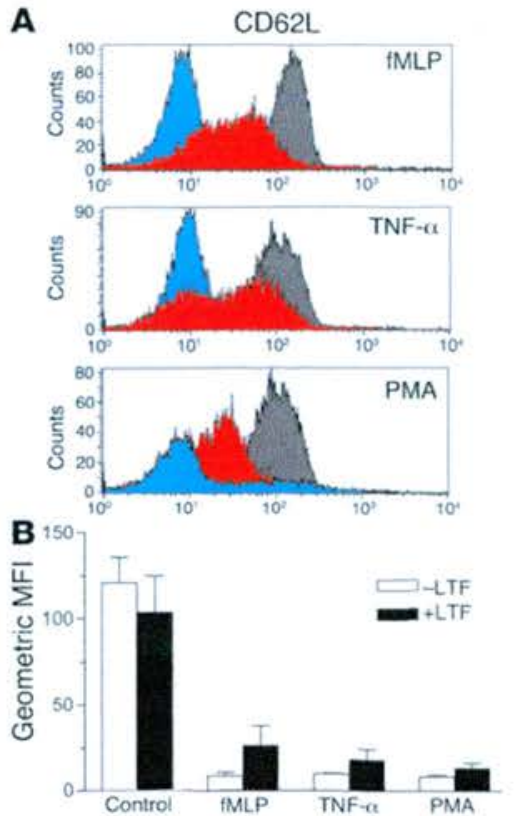

C
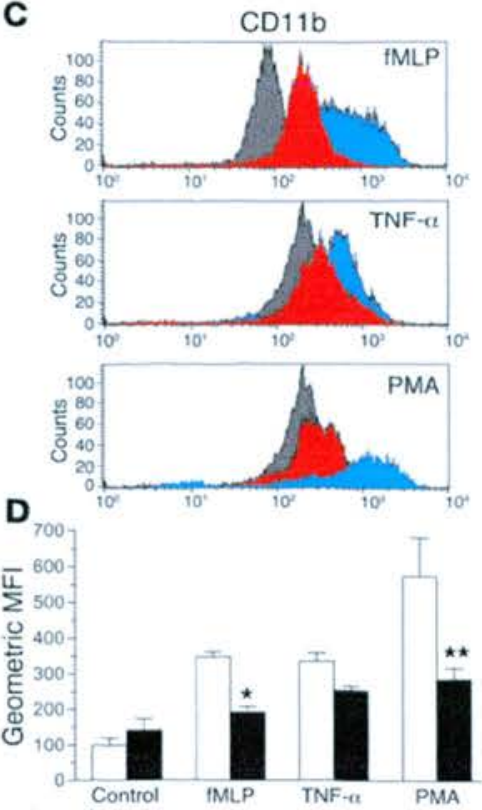

fMLP

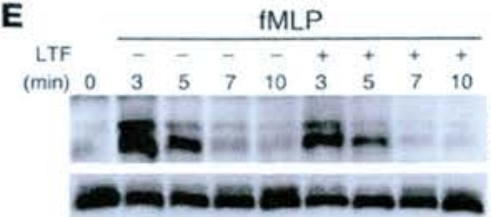

Figure 7

Effect of lactoferrin on neutrophil activation status. The expression of CD62L (A and B) and CD11b (C and D) was assessed in fMLP- $(100 \mathrm{nM}), \mathrm{TNF}-\alpha-(1 \mathrm{ng} \mathrm{ml})$, or PMA-stimulated $(100 \mathrm{nM})$ neutrophils (30 minutes at $\left.37^{\circ} \mathrm{C}\right)$ that were preincubated $\left(40\right.$ minutes at $\left.37^{\circ} \mathrm{C}\right)$ in the presence or absence of lactoferrin $(10 \mu \mathrm{g} / \mathrm{ml})$. Representative flow cytometry overlays of CD62L (A) and CD11b (C) expression in control (gray) and stimulated neutrophils (lactoferrin-treated: red; untreated: blue). $n=3 ;{ }^{*} P<0.05,{ }^{*} P<0.01$. Error bars indicate SEM. (E) Western blot analysis to determine levels of ERK $1 / 2$ phosphorylation. Neutrophils were incubated with lactoferrin $\left(10 \mu \mathrm{g} / \mathrm{ml} ; 40\right.$ minutes at $\left.37^{\circ} \mathrm{C}\right)$, followed by stimulation with $\mathrm{FMLP}(100 \mathrm{nM})$ for the indicated times. Membrane was stripped and reprobed for total ERK2. Results are representative of 3 independent experiments.
BL cells expressing exogenous $\mathrm{Bcl}-2$ that provided protection from apoptosis expressed lower levels of lactoferrin upon exposure to staurosporine than did their parental counterparts (Figure 8, A and D). Not only was apoptosis-related lactoferrin expression demonstrated at the transcriptional level, lactoferrin protein was also recovered from supernatants of cells undergoing apoptosis (Figure 8D). Treatment of A549 cells with brefeldin A, which interferes with intracellular transport of newly synthesized proteins, resulted in inhibition of apoptosis-induced lactoferrin release, providing further evidence for de novo synthesis and secretion of lactoferrin by cells undergoing apoptosis (Figure $8 \mathrm{E}$ ). An analogous effect was also evident from supernatants obtained from primary lymphocytes induced to become apoptotic in the presence of $1 \mu \mathrm{M}$ staurosporine. Finally, immunoblotting analyses and chemotaxis assays using supernatants of BL cells undergoing primary necrosis further revealed that the release of lactoferrin is not linked to necrosis but that lactoferrin is expressed and actively released from cells as a consequence of activation of their apoptosis program (Figure 8F and Supplemental Figure 3).

\section{Discussion}

Apoptosis plays a key role in regulating cell populations through programmed cell death, an intrinsically non-phlogistic process that contrasts with accidental, necrotic cell death, which has proinflammatory consequences. Defining the properties of apoptotic cells that contribute to the noninflammatory or antiinflammatory nature of the apoptosis program is critical to our understanding of this fundamental biological process. We have now identified lactoferrin as a cell-autonomous antiinflammatory mediator that is produced by cells of diverse lineages undergoing apoptosis. Furthermore, these studies demonstrate a hitherto unrecognized immunomodulatory function of lactoferrin: to inhibit directly the migration of neutrophil granulocytes. Lactoferrin is therefore a constitutive antiinflammatory component of apoptotic cells that, in addition to its known antiinflammatory properties, militates against the proinflammatory recruitment of granulocytes to sites of apoptosis.

First discovered in milk almost 70 years ago, lactoferrin is a highly pleiotropic iron-binding glycoprotein closely related to transferrin that is a well-known constituent of exocrine secretions and secondary granules of neutrophils. It has many antiinflammatory and immunoregulatory properties, and the majority of these are not related to its ability to chelate iron, although some of its bacteriostatic features are dependent on iron binding $(36,37)$. Its ability to inhibit neutrophil migration, as described here, is an additional antiinflammatory feature of the molecule that is independent of its iron-chelating activity. In addition to its well-established antimicrobial properties, lactoferrin can inhibit proinflammatory responses not only through its ability to bind to key components of bacteria and viruses, but also through its direct activity on innate immune cells and molecules $(36,37)$. Thus, lactoferrin can inhibit production of proinflammatory mediators such as TNF- $\alpha$ and IL-6 $(38,39)$ and can promote production of antiinflammatory mediators including IL- $10, \mathrm{IL}-4$, and TGF- $\beta 1(40,41)$.

The identification of specialized high- or low-affinity lactoferrin receptors on human neutrophils provides an initial mechanistic insight into how lactoferrin exerts its inhibitory effect on the neutrophil migration machinery. This is in accordance with our 


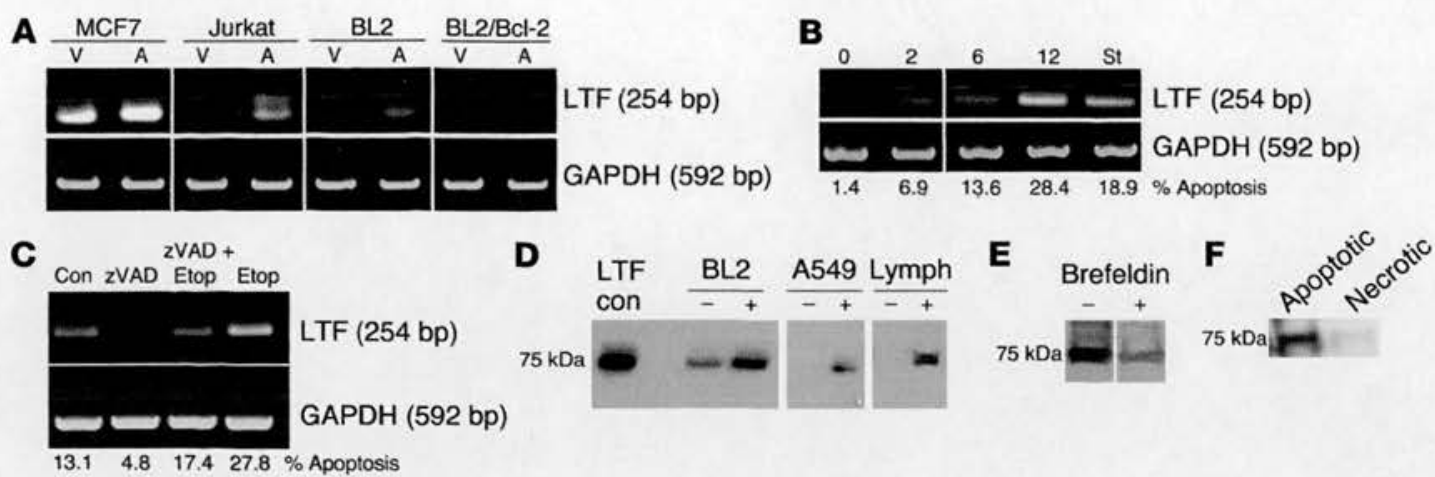

\section{Figure 8}

Induction of apoptosis upregulates lactoferrin expression and release. (A) RT-PCR analysis in cell lines stimulated to undergo apoptosis (A) and unstimulated controls (V). MCF7 cells transfected with caspase-3 (25.4\% apoptosis; $100 \mu \mathrm{M}$ etoposide, 20 hours), Jurkat (18.4\% apoptosis; $1 \mu \mathrm{M}$ staurosporine, 3 hours), BL2 (12.46\% apoptosis), and BL2/Bcl-2 (7.42\% apoptosis; $1 \mu \mathrm{M}$ staurosporine, 1 hour). The lanes were run on the same gel but, where indicated by the vertical lines, were noncontiguous. (B) Lactoferrin expression in A549 cells at defined time points (hours) following stimulation with $100 \mu \mathrm{M}$ etoposide or $1 \mu \mathrm{M}$ staurosporine. (C) Addition of pan-caspase inhibitor ZVAD-fmk (100 $\mu \mathrm{g} / \mathrm{ml})$ for $12 \mathrm{hours}$ in order to prevent etoposide-induced apoptosis in A549 cells. (D) Immunoblot analysis of cell supernatants from: BL2 and primary lymphocytes in the presence $(+)$ or absence $(-)$ of staurosporine $(1 \mu \mathrm{M})$ in serum-free conditions for 1 hour. A549 cells were stimulated with $(+)$ or without $(-) 100 \mu \mathrm{M}$ etopuside for 5 hours. All samples were run on the same gel. Noncontiguous samples of A549 cells and lymphocytes (Lymph) are indicated by the vertical lines. (E) A549 cells were induced to become apoptotic ( $100 \mu \mathrm{M}$ etoposide; 20 hours) in the presence or absence of brefeldin A $(1 \mu \mathrm{g} / \mathrm{ml})$, a protein release inhibitor. (F) Immunoblot analysis of cell supernatants from control BL2 cells $\left(1 \times 10^{6} / \mathrm{ml}\right)$ induced to undergo apoptosis ( $1 \mu \mathrm{M}$ staurosporine, 1 hour) or primary necrosis $\left(56^{\circ} \mathrm{C}, 1\right.$ hour) in serum-free conditions. St, staurosporine; con; control; Etop, etoposide.

results demonstrating that lactoferrin binds directly to neutrophils and impinges on intracellular signaling events coupled to neutrophil activation, rather than interacting with chemoattractants and modulating their activity. Moreover, the latter possibility was further excluded by the fact that the observed inhibitory effect of lactoferrin was (a) specific to neutrophil, not monocyte, migration, (b) observed with a diversity of chemoattractants, and (c) not directly neutralized by chemoattractants. Consistent with other studies $(26,27)$, our results demonstrated 2 classes of lactoferrin receptor on the surface of human neutrophils. Our results indicate that a relatively high-affinity receptor is expressed at relatively low density and that a relatively low-affinity receptor is expressed at relatively high density. However, the detailed characterization of the lactoferrin receptor(s) and dissection of downstream signaling pathways remain a significant challenge, as lactoferrin has been extensively reported to interact with a range of molecules and receptors in a cell-specific manner and therefore has the potential to elicit different signaling cascades in different cell types. Indeed, many researchers over many years have attempted the derailed characterization of lactoferrin receptors from various cell types with only partial success, and many candidate receptors have been proposed for different cell types. Importantly, as has been suggested elsewhere (42), critical difficulties in the identification of specific lactoferrin receptors stem from the cationic nature of the lactoferrin protein, allowing it to bind to anionic molecules found in virtually all cell types.

Here, we have observed several effects of lactoferrin on cell activation with clear implications for migration, adhesion, and motility of neutrophils. The initial phase of neutrophil activation following agonist stimulation is characterized by a rapid increase in $\left[\mathrm{Ca}^{2+}\right]_{i}$, which in turn triggers the activation of PKC as well as a range of calcium-dependent signaling pathways that control key neutrophil effector functions, including degranulation, cytoskeletal rearrangements, and cell migration (32, 43-45). Our data clearly suggest that the observed lactoferrin-mediated inhibitory effects on neutrophil chemotaxis could be attributed to modulation of molecules other than those involved in the $\mathrm{Ca}^{2+}{ }_{i}$ response following agonist stimulation. In addition, analysis of the expression of early activation markers such as CD62L and CD11b following stimulation with diverse neutrophil agonists, including PMA, a specific synthetic intracellular activator of PKC, further suggests that lactoferrin acts downstream or independently of the PKC pathway, affecting signaling cascade components of the later phases of neutrophil activation. This notion was strengthened by our observation that lactoferrin inhibited the phosphorylation of $\mathrm{p} 44 / 42$ (ERK1 and ERK2) MAPKs, indicating that its action is mediated, at least in part, via the MAPK pathway, a key pathway downstream of PKC with a crucial role in the regulation of cytoskeletal rearrangement and cell adhesion (46-48). The previously reported specific interaction of lactoferrin with the calciumcalmodulin complex $\left(\mathrm{Ca}^{2+} / \mathrm{CaM}\right)$ provides further insight into the potential intracellular signaling targets of lactoferrin (49). The $\mathrm{Ca}^{2+} / \mathrm{CaM}$ complex is formed following the increase in $\left[\mathrm{Ca}^{2+}\right]_{\mathrm{i}}$ and PKC activation and regulates the activity of a number of pathways involved in cell adhesion and migration, including the ERK $1 / 2$ kinases (50). Indeed, the use of specific inhibitors of calmodulin activity has been demonstrated to mimic the effect of lactoferrin - inhibition of neutrophil migration - reported here (51-53).

Lactoferrin has been shown previously to bind to receptors on mononuclear phagocytes and to inhibit proinflammatory responses via NF- $\mathrm{KB}(39,54,55)$. Taken together with the evidence that apoptotic cells can suppress proinflammatory responses of mononuclear phagocytes and can elicit antiinflammatory responses by these cells $(10,11)$, our results indicate that it is now reasonable to suspect that these effects are mediated, at least in part, by lactoferrin produced by apoptotic cells. However, the involvement of the NF- $\kappa B$ pathway in the inhibition of neutrophil migration seems unlikely, since lactoferrin-mediated inhibition of chemotaxis of 
neutrophils treated with gliotoxin, a specific NF- $\mathrm{B}$ inhibitor, was found to be identical to that of untreated cells (data not shown). In addition, no changes in the levels of $I \kappa B$, an inhibitor of NF- $\kappa B$ that is degraded following NF- $\kappa$ B activation, were observed in Western blot analysis of AMLP-stimulated neutrophils treated with or without lactoferrin (data not shown).

In general terms, in professional phagocytes, sensing mechanisms that are not yet well defined allow these cells to navigate to apoptotic cells through chemotactic processes involving lipid signaling molecules and classical chemokine mechanisms $(1,2)$. It is noteworthy that of the 2 categories of professional phagocytes - mononuclear (monocytes and macrophages) and polymorphonuclear (granulocytes) - apoptotic cells attract mononuclear phagocytes selectively (12). Indeed, the lack of recruitment of granulocytes to apoptotic zones is a hallmark of the non-phlogistic apoptosis program, distinguishing normal areas of cell death from sites of accidental or pathological tissue damage or infection. Recent evidence suggests that certain recombinant preparations of lactoferrin have the potential to chemoattract monocytes (56). Although we failed to observe monocyte attraction to lactoferrin in our studies with milk-derived lactoferrin, these results indicate that lactoferrin has the potential to function in the selective attraction of monocytes but exclusion of granulocytes from sites of apoptosis. Our observation that lactoferrin, released into the supernatants of apoptotic cells, is able to inhibit the migration of neutrophils, demonstrates not only that physiological concentrations of lactoferrin are functional in mediating this effect but also that apoptotic cells are active in producing a factor that can potently suppress migration of neutrophils, the major subset of professional phagocytes among circulating leukocytes, to sites of apoptosis. Although it is clear that the secretory events inhibited by brefeldin A treatment (transport from the ER and Golgi network) are important for the release of lactoferrin from apoptotic cells, further work will be required to elucidate the details of this secretory process - including whether lactoferrin is transported directly from Golgi membranes for release from the cell surface.

While neutrophils may lack the sensing mechanisms to guide them to sites of apoptosis, the constitutive production of a factor that blocks neutrophil migration to such sites is likely to be important when the apoptosis program is activated in the presence of signals that promote neutrophil recruitment during inflammatory responses. In this context, it is noteworthy that lactoferrin is active in inhibiting IL-8 production by endothelial cells (57). Furthermore, lactoferrin's ability to inhibit neutrophil migration is likely to be important in the resolution of acute inflammation. Thus, our results suggest that lactoferrin arising from the secondary granules of neutrophils and from apoptotic cells constitutes a negative feedback component that limits the influx of neutrophils to inflammatory sites. We would speculate that neutrophils undergoing apoptosis en route to resolution of inflammation would release lactoferrin as do other apoptotic cells but would be unlikely to be required to synthesize the protein de novo. The negative regulatory activity of lactoferrin described here places it as one of the few molecules, alongside lipoxins, netrin-1, and annexin-1, that negatively regulate neutrophil migration $(21,58,59)$. More importantly, based on the high specificity of its migration-inhibitory properties to neutrophils, lactoferrin is identified here as a promising therapeutic target for a range of chronic inflammatory conditions, including vasculitis, pulmonary fibrosis, and ischemia/reperfusion injury, that are characterized by excessive neutrophil infiltration leading to neutrophil-mediated host tissue damage and remodeling (19).

Since the properties of lactoferrin extend well beyond its ability to regulate infection, immune responses, and inflammation, the link established here between lactoferrin production and apoptosis has many additional implications. First, lactoferrin's ability to act as a growth factor $(60,61)$ extends evidence coupling the apoptosis program with tissue repair responses $(62,63)$. Second, additional recent evidence suggests that its protease activity may be instrumental in activating the caspase cascade. In particular, endogenous lactoferrin has been reported by 2 groups to induce apoptosis through activation of caspase- $3(64,65)$. It is tempting to speculate that the de novo synthesis of lactoferrin by cells triggered to undergo apoptosis in our studies is linked to the initiation of the apoptosis program as well as to its antiinflammatory features. Therefore, lactoferrin may have multiple properties in apoptosis: (a) regulating the initiation of the program, (b) influencing repair in the tissue microenvironment, and (c) promoting the non-phlogistic nature of the process.

Lactoferrin's functions in relation to tumor biology are currently unclear. Proteolyzed forms of exogenous lactoferrin have been reported to have proapoptotic and antitumor activities. In addition, lactoferrin appears to promote antitumor host immune responses (37), and activation of antigen-presenting cells has recently been reported using high doses of recombinant lactoferrin produced in Aspergillus (56). Its observed effects on cell growth are inconsistent, and both growth-promoting and growth-inhibiting effects have been reported $(60,61,66,67)$, suggesting the possible importance of tissue context. Since lactoferrin has potent ability to inhibit neutrophil migration, it seems likely that in tumors in which neutrophils play a supportive role, limitation of neutrophil infiltration through lactoferrin administration could be therapeutically beneficial. However, in tumors in which neutrophils are absent, we propose that, given the known antitumor effects of neutrophils (68-71), encouragement of neutrophil infiltration through inhibition of lactoferrin may effect tumor destruction. Our results might predict that tumors in which apoptosis is prominent would produce lactoferrin in situ. While this suggestion requires proper investigation, at least in one category, BL, a high-grade malignancy in which high-rate apoptosis occurs, in situ production of lactoferrin protein has been known for many years (72).

In conclusion, we describe a novel homeostatic function for the highly pleiotropic immunomodulatory glycoprotein lactoferrin. We demonstrate that lactoferrin production is closely coupled to the fundamental cell death program, apoptosis. In this respect, lactoferrin is much more generally expressed than previously realized. Our results show that lactoferrin endows apoptotic cells with antiinflammatory properties, including the capacity to inhibit neutrophil migration. Given the multifunctional abilities of lactoferrin, these results have broad implications for the influence of apoptotic cells on multiple physiological processes, including cell growth, differentiation, and innate and adaptive immune responses, as well as the pathological processes of inflammatory and malignant diseases.

\section{Methods}

Antibodies and reagents. The following antibodies and reagents were used in this study: rabbit polyclonal anti-human lactoferrin IgG antibody (SigmaAldrich), mouse monoclonal anti-human lactoferrin antibodies (LF-2B8, AbD Serotec; imab75 and imab77, ImmunoSolv), rabbit polyclonal immu- 
noglobulin (IgG) negative control (Dako), IgG1 isotype control (SigmaAldrich), allophycocyanin-conjugated (APC-conjugated) anti-CD11b (mIgG1; BD), FITC-conjugated anti-CD62L (mIgG1; AbD Serotec), purified human lactoferrin from milk (Sigma-Aldrich), purified human lactoferrin from neutrophils (Athens Research and Technology), recombinant human lactoferrin (Sigma-Aldrich), human recombinant apolactoferrin/ hololactoferrin (ProSpec), purified human transferrin (Sigma-Aldrich), recombinant human TNF- $\alpha$ (R\&D Bioscience), PMA (Sigma-Aldrich), fMLP (Sigma-Aldrich), C5a (Sigma-Aldrich), IL-8 (Sigma-Aldrich), LTB (Sigma-Aldrich), brefeldin A (Sigma-Aldrich), staurosporine (SigmaAldrich), and gliotoxin (Sigma-Aldrich). For lactoferrin absorption chemoattractants, each chemoattractant was incubated with purified human milk lactoferrin $(10 \mu \mathrm{g} / \mathrm{ml})$, and a mouse monoclonal anti-human lactoferrin antibody (LF-2B8; $10 \mu \mathrm{g} / \mathrm{ml}$ ) or isotype control was added. Antibodies were completely removed by BioMag goat anti-mouse IgG (QIAGEN) magnetic beads, and the efficiency of antibody removal was assessed by analysis of the preabsorbed chemoattractants by anti-mouse IgG ELISA (data not shown). Furthermore, the depleted antibody was readily recovered from the beads (data not shown).

Cell isolation. Fresh human venous blood was collected from volunteers according to the Royal Infirmary of Edinburgh (Scotland) Research Ethics Committee (approval 1702/95/3/11), and mononuclear and polymorphonuclear (PMN) leukocytes were isolated as previously described (73). Neutrophils represented more than $95 \%$ of isolated PMN cells. Monocytes ( $>90 \% \mathrm{CD} 4^{+}$cells) were positively selected from isolated mononuclear leukocytes using CD14 magnetic beads (Miltenyi Biotec). Human monocyte-derived macrophages were obtained following culture of monocytes for 6 days in Iscove's modified Dulbecco's medium (IMDM) containing $10 \%$ autologous serum.

Chemotaxis assay. In vitro leukocyte chemotaxis was measured following a well-established transfilter cell migration assay, as previously described (12) using polyvinyl uncoated Transwell inserts $(5 \mu \mathrm{m}$ pore size; Costar, Corning). Time of incubation $\left(37^{\circ} \mathrm{C} ; 5 \% \mathrm{CO}_{2}\right)$ varied for cell type (neutrophils: 60 minutes; monocytes: 90 minutes; macrophages: 4 hours). Unless otherwise stated, lactoferrin was used at $10 \mu \mathrm{g} / \mathrm{ml}$. Chemotactic agents included fMLP (100 nM; Sigma Aldrich), C5a (6.25 ng/ml; Sigma Aldrich), IL-8 (50 nM; R\&D Systems), and $\mathrm{LTB}_{4}(100 \mathrm{nM}$; Sigma Aldrich). For neutralization experiments, rabbit polyclonal anti-human lactoferrin antibody (Sigma Aldrich) or negative control (Dako) were used. Filters were observed using an inverted microscope (Zeiss Axiovert 25), and relative cell migration was determined by enumerating the number of migrated cells in 10 random high-power $(\times 400)$ fields.

shRNA lentiviral transduction of BL cells. Lactoferrin expression was downregulated using shRNA lentiviral vectors (MISSION; Sigma-Aldrich). Briefly, lactoferrin-targeted shRNA lentiviral plasmids (pLKO.1-puro) were cotransfected with ViraPower Lentiviral packaging mix (pLP1, pLP2, pLP-VSV-G; Invitrogen) to 293FT cells using Lipofectamine LTX (Invitrogen). BL2 cells were transduced with the shRNA-expressing lentivirus, and stable cell lines were generated by selection with puromycin ( $2 \mu \mathrm{g} / \mathrm{ml}$; Sigma-Aldrich).

RT.PCR analysis. Total RNA was extracted (RNeasy kit; QIAGEN) and reverse transcribed ( $2 \mu \mathrm{g}$ ) using SuperScript III RT (Invitrogen). Resulting CDNAs were used as template in PCR experiments at a concentration of $1 \mathrm{ng} / 50 \mu \mathrm{l}$ of PCR mixture. The primers used were: forward lactoferrin (5'-TGTCTTCCTCGTCCTGCTGTTCCTCG-3') and reverse lactoferrin (5'-CTGCCTCGTATATGAAACCACCATCAA- $3^{\prime}$ ), forward GAPDH primer (5'-CGACAGTCAGCCGCATCTTCTTTTGCGTCG-3') and reverse GAPDH primer (5'-GGACTGTGGTCATGAGTCCTTCCACGATAC-3'). Cycle parameters (lactoferrin: 40 cycles; GAPDH: 28 cycles) were: denaturation $94^{\circ} \mathrm{C}$ for 1 minute, primer annealing at $67^{\circ} \mathrm{C}$ (lactoferrin) or $50^{\circ} \mathrm{C}(\mathrm{GAPDH})$ for $1 \mathrm{~min}$ ute, and extension at $72^{\circ} \mathrm{C}$ for 45 seconds, with a first denaturation step at $94^{\circ} \mathrm{C}$ for 7 minutes and a final extension at $72^{\circ} \mathrm{C}$ for 5 minutes. Purified PCR products (QIAquick gel extraction kit; QIAGEN) were sequenced to confirm validity by the Sequencing Service of the School of Life Sciences, University of Dundee, using Applied Biosystems BigDye 3.1 chemistry on an Applied Biosystems model 3730 automated capillary DNA sequence analyzer.

Peritonitis model. All animal procedures were carried out under a UK Home Office Animals (Scientific Procedures) Act 1986 project licence. Mice (8- to 12 -week-old female $\mathrm{C} 57 \mathrm{BL} / 6$ mice; $n=7$ per group) were injected i.p. with purified human lactoferrin or transferrin ( $500 \mathrm{ng}$ in saline $/ 0.1 \%$; BSA Sigma Aldrich) or saline $/ 0.1 \% \mathrm{BSA}$ alone followed by a second i.p. injection with $1 \%$ thioglycollate $(500 \mu \mathrm{l})$ or saline $/ 0.1 \%$ BSA after 20 minutes. Recruited leukocytes were harvested after 4 hours by peritoneal lavage with ice-cold saline containing $2 \mathrm{mM}$ EDTA. Harvested cells were counted using a NucleoCounter (ChemoMetec), which excluded nonnucleated cells. To determine the number of neutrophils $\left(\mathrm{Gr}-1^{*}\right)$, cells were immunolabeled with PE-conjugated anti-mouse Ly6-Gr-1 and counted using Flow-Count beads (Beckman Coulter).

Histology and immunohistochemistry. Six- to 10-week-old BALB/c SCID mice were injected i.p. with $10^{7} \mathrm{BL} 2$ cells. Tumors developed i.p. within 2 months of injection. Mice were sacrificed and tumors excised. For positive control, $\mathrm{BALB} / \mathrm{c}$ mice were immunized with sheep red blood cells and spleens harvested and frozen 7 days after i.p. injection. Immunohistochemistry was performed on frozen acetone-fixed sections $(5 \mu \mathrm{m})$ of BL or spleen tissues using biotinylated anti-mouse $\mathrm{Gr}-1$ antibody ( $10 \mu \mathrm{g} / \mathrm{ml}$; BioLegend) or isotype control ( $\mathrm{AbD}$ Serotec). Nonspecific adsorption of antibodies was blocked using serum-free Protein Block (Dako). Reactions were amplified using VECTASTAIN Elite ABC avidin-biotinylated peroxidase complexes (Vector Laboratories). Hematoxylin was used as counterstain.

Flow cytometry. Unless otherwise stated, cells were suspended in PBS containing $5 \%$ normal mouse serum or $0.1 \% \mathrm{BSA}$, and all antibody incubations were performed for 20 minutes on ice. Mouse neutrophils were defined based on the expression of Gr-1 epitope using PE-conjugated rat anti-mouse Ly6G (Gr-1; eBioscience). For the assessment of neutrophil activation, the following antibodies were used: FITC-conjugated antiCD62L (FMC46, mIgG2b; AbD Serotec) and APC-conjugated anti-CD11b (ICRF44, mIgG1; BD). Isotype controls included mouse IgG1:FITC (AbD Serotec), mouse IgG1:APC (BD), and rat IgG2b:PE (eBioscience). Cell apoptosis was determined by labeling with annexin $\mathrm{V}$ and propidium iodide. Samples were analyzed using a BD FACSCalibur or FACScan cytometer, and data were analyzed using BD CellQuest software.

Size fractionation and ion exchange chromatography. Size fractionation of BL2 cell conditioned media was performed using filters with specific molecular weight cutoff sizes (Amicon Centrifugal filters YM-50 and YM-100; Millipore), following the manufacturer's instructions. Ion exchange chromatography was carried out using Q Sepharose Fast Flow beads (Sigma-Aldrich). Beads were washed with neutralizing buffer, and bound proteins were then eluted by addition of $10 \mathrm{mM} \mathrm{NaAc}, 0.5 \mathrm{M} \mathrm{NaCl}, \mathrm{pH} 4$. BL2 cell-conditioned medium or control medium (RPMI 1640) was mixed with the beads and incubated at room temperature for 5 minutes. Samples were centrifuged $(300 \mathrm{~g}, 5$ minutes) and supernatants stored. Beads were washed with neutralizing buffer, and bound proteins were then eluted by addition of the corresponding elution buffer (for S beads: $10 \mathrm{mM}$ Tris, $0.5 \mathrm{M} \mathrm{NaCl}$, $\mathrm{pH} 10$; for Q beads: $10 \mathrm{mM} \mathrm{NaAc}, 0.5 \mathrm{M} \mathrm{NaCl}, \mathrm{pH}$ 4). After a 5-minute incubation at room temperature, beads were centrifuged $(300 \mathrm{~g}, 5$ minutes) and supernatants collected and analyzed. Prior to chemotaxis analysis, the supernatants were diluted (1:100), and $\mathrm{pH}$ was adjusted to 7.0. Proteins were identified by peptide mass fingerprinting using MALDI-TOF mass spectrometry. The procedure was carried out by the Scottish Instrumentation and Resource Centre for Advanced Mass Spectrometry (SIRCAMS), School of Chemistry, University of Edinburgh. 
Immunoblotting. Conditioned media from viable and apoptotic BL2 and A549 cells were collected, and their protein content was TCA precipitated. Briefly, $100 \mu \mathrm{l} \mathrm{TCA}$ was added in $1 \mathrm{ml}$ conditioned medium at $4^{\circ} \mathrm{C}$. Samples were centrifuged at $18,000 \mathrm{~g}$ and the pellets washed in ice-cold acetone before resuspension in sample buffer (NuPAGE; Invitrogen). Neutrophils $\left(5 \times 10^{6}\right.$ cells $\left./ \mathrm{ml}\right)$ were lysed for 15 minutes with $1 \%$ NP-40 in TBS containing protease inhibitor cocktail (Sigma Aldrich), aprotinin, leupeptin, pepstatin A, 4-(2-aminoethyl)benzenesulfonyl fluoride, sodium orthovanadate, benzamidine, levamisole, and $\beta$-glycerophosphate. Samples were centrifuged $\left(20,000 \mathrm{~g}, 4^{\circ} \mathrm{C}, 15\right.$ minutes $)$ and resolved by SDS-PAGE using 4\%-12\% Bis-Tris gels (NuPAGE; Invitrogen). Proteins were then electroblotted onto a nitrocellulose membrane (NuPAGE; Invitrogen), blocked with $0.5 \%$ (for lactoferrin) or $3 \%$ BSA (for ERK), and probed with monoclonal mouse anti-human lactoferrin (1:100; LF.2B8; AbD Serotec) or mouse monoclonal anti-MAPK activated (diphosphorylated ERK1 and ERK2) antibody (1:1,000; Sigma-Aldrich) or polyclonal mouse ERK2 (1:1,000; Santa Cruz Biotechnology Inc.) or rabbit monoclonal anti-IкB $\alpha(74)$ $(1: 2,500 ; \mathrm{E} 130 ; \mathrm{Abcam})$ followed by HRP-conjugated goat anti-mouse IgG (1:2,000; Amersham) or HRP-conjugated goat anti-rabbit IgG $(1: 2,500$ Dako) and visualized using ECL (GE Healthcare).

Binding studies. Biotinylation of human milk-derived lactoferrin was performed using EZ-Link Sulfo-NHS-LC-Biotin (Pierce, Thermo Scientific). Fresh neutrophils were exposed to biotinylated lactoferrin $(10 \mu \mathrm{g} / \mathrm{ml})$ at $37^{\circ} \mathrm{C}$ for 1 hour, after which the cells were washed and lysates prepared, resolved by SDS-PAGE, electroblotted, blocked with $0.1 \%$ PBS-Tween, and probed with HRP-conjugated streptavidin (Dako). For Scatchard analyses, ${ }^{125}$ I-labeled human milk-derived lactoferrin, prepared using Pierce Iodination Reagent (Thermo Scientific), was added at a constant amount to fresh human neutrophils in the presence of increasing amounts of cold, unlabeled lactoferrin. After 30 minutes at $4^{\circ} \mathrm{C}$, cells were washed 3 times before gamma counting.
Measurement of $\left[\mathrm{Ca}^{2+}\right]_{i}$. Measurement of $\left[\mathrm{Ca}^{2+}\right]_{i}$ was performed as previously described (75). Briefly, freshly isolated neutrophils were resuspended $\left(10^{7} / \mathrm{ml}\right)$ in HBSS (without $\left.\mathrm{Ca}^{2+} / \mathrm{Mg}^{2+}\right)$ and were incubated with $2 \mu \mathrm{M}$ FURA 2/AM (Calbiochem) at $37^{\circ} \mathrm{C}$ for 30 minutes. The cells were washed twice, resuspended at $2 \times 10^{6} / \mathrm{ml}$ in $\mathrm{HBSS}$ (with $\mathrm{Ca}^{2+} / \mathrm{Mg}^{2+}$ ), incubated for an additional 30 minutes at $37^{\circ} \mathrm{C}$ in the presence of lactoferrin $(10 \mu \mathrm{g} / \mathrm{ml})$, and then stimulated with $\mathrm{fMLP}(1 \mathrm{nM}$ or $10 \mathrm{nM}) .\left[\mathrm{Ca}^{2+}\right] \mathrm{l}$ levels were determined based on $340: 380 \mathrm{~nm}$ dual wavelength excitation in a PerkinElmer luminescence spectrometer at $37^{\circ} \mathrm{C}$ with constant stirring. Calibration was performed after each experiment using Triton $\mathrm{X}\left(R_{\max }\right)$ and EGTA $\left(R_{\min }\right)\left(R_{\max }\right.$ and $R_{\min }$ are the maximum and minimum 340:380 fluorescence ratios, respectively). $[\mathrm{Ca} 2+]$, was calculated based on the $340: 380$ $\mathrm{nm}$ fluorescence ratio.

Statistics. Results from multiple experiments are presented as mean $\pm \mathrm{SEM}$. One-way ANOVA was performed, followed by Bonferroni's post-hoc test. In all cases, $P$ values of 0.05 or less were considered significant.

\section{Acknowledgments}

The authors thank Jason King for help in the lentiviral transduction of cells and the MRC and Leukaemia Research (United Kingdom) for supporting this work.

Received for publication May 16, 2008, and accepted in revised form October 15, 2008.

Address correspondence to: Christopher D. Gregory, MRC Centre for Inflammation Research, University of Edinburgh, Queen's Medical Research Institute, 47 Little France Crescent, Edinburgh EH16 4TJ, United Kingdom. Phone: 44-131-242-9170; Fax: 44131-242-9171; E-mail: chris.gregory@ed.ac.uk.
1. Lauber, K., et al. 2003, Apoptotic cells induce migration of phagocytes via caspase-3-mediated release of a lipid attraction signal. Cell. 113:717-730.

2. Truman, L.A., et al. 2008. $\mathrm{CX}_{3} \mathrm{CL} 1 /$ fractalkine is released from apoptotic lymphocytes to stimulate macrophage chemotaxis. Blood. 10.1182 /blood2008-06-162404.

3. Henson, P.M., and Hume, D.A. 2006. Apoptotic cell removal in development and tissue homeostasis. Trends Immunol. 27:244-250.

4. Ravichandran, K.S., and Lorenz, U. 2007. Engulfment of apoptotic cells: signals for a good meal. Nat. Rev. Immunol. 7:964-974.

5. Savill, J., Dransfield, I., Gregory, C., and Haslett, C. 2002. A blast from the past: clearance of apoptotic cells regulates immune responses. Nat. Rev. Immu nol. 2:965-975

6. Wyllie, A.H., Kerr, J.F., and Currie, A.R. 1980. Cell death: the significance of apoptosis. Int. Rev. Cytol. 68:251-306

7. Cohen, P.L., et al. 2002. Delayed apoptotic cell clearance and lupus-like autoimmunity in mice lacking the c-mer membrane tyrosine kinase. J. Exp. Med. 196:135-140.

8. Hanayama, R., et al, 2004. Autoimmune disease and impaired uptake of apoptotic cells in MFG E8-deficient mice. Science. 304:1147-1150.

9. Huynh, M.L., Fadok, V.A., and Henson, P.M. 2002 Phosphatidylserine-dependent ingestion of apop totic cells promotes TGF- $\beta 1$ secretion and the resolution of inflammation. J. Clin. Invest. 109:41-50.

10. Fadok, V.A., Bratton, D.L., Frasch, S.C., Warner, M.L., and Henson, P.M. 1998. The role of phosphatidylserine in recognition of apoptotic cells by phagocytes. Cell Death Differ. 5:551-562.

11. Voll, R.E., et al. 1997. Immunosuppressive effects of apoptotic cells. Nature, 390:350-351.

12. Truman, L.A., Ogden, C.A., Howie, S.E., and Grego- ry, C.D. 2004. Macrophage chemotaxis to apoptotic Burkitt's lymphoma cells in vitro: role of CD14 and CD36. Immunobiology. 209:21-30.

13. Huttenlocher, A., Sandborg, R.R., and Horwitz, A.F. 1995. Adhesion in cell migration. Curr. Opin. Cell Biol. 7:697-706.

14. Iijima, M., and Devreotes, P. 2002. Tumor suppressor PTEN mediates sensing of chemoattractant gradients. Cell. 109:599-610.

15. Parent, C.A., and Devreotes, P.N. 1999. A cell's sense of direction. Science. 284:765-770.

16. Servant, G., et al. 2000. Polarization of chemoattractant receptor signaling during neutrophil chemotaxis. Science. 287:1037-1040,

17. Drubin, D.G., and Nelson, W.J. 1996. Origins of cell polarity. Cell. 84:335-344.

18. Hogg, N., and Leitinger, B. 2001. Shape and shift changes related to the function of leukocyte integrins LFA-1 and Mac-1. J. Leukoc. Biol. 69:893-898.

19. Weiss, S.J. 1989. Tissue destruction by neutrophils. N. Engl. J. Med. 320:365-376.

20. Serhan, C.N., and Savill, J. 2005. Resolution of inflammation: the beginning programs the end. Nat. Immunol. 6:1191-1197.

21. Takata, S., et al. 1994. Remodeling of neutrophil phospholipids with $15(\mathrm{~S})$-hydroxyeicosatetraenoic acid inhibits leukotriene B4-induced neutrophil migration across endothelium. J. Clin. Invest. 93:499-508

22. Haslett, C. 1992. Resolution of acute inflammation and the role of apoptosis in the tissue fate of granulocytes. Clin. Sci. (Lond.). 83:639-648

23. Serhan, C.N. 2005. Novel omega-3-derived local mediators in anti-inflammation and resolution. Pharmacol. Ther: 105:7-21.

24. Rossi, A.G., et al. 2006. Cyclin-dependent kinase inhibitors enhance the resolution of inflammation by promoting inflammatory cell apoptosis.
Nat. Med. 12:1056-1064

25. Schwab, J.M., Chiang, N., Arita, M., and Serhan C.N. 2007. Resolvin E1 and protectin D1 activate inflammation-resolution programmes. Nature. 447:869-874

26. Maneva, A.I., Sirakov, L.M., and Manev, V.V. 1983. Lactoferrin binding to neutrophilic polymorphonuclear leucocytes. Int. J. Biochem. 15:981-984.

27. Spik, G., et al. 1994. Characterization of two kinds of lactotransferrin (lactoferrin) receptors on different target cells. Adv. Exp. Med. Biol. 357:13-19.

28. Zucali, J.R., Broxmeyer, H.E., Levy, D., and Morse, C. 1989. Lactoferrin decreases monocyte-induced fibroblast production of myeloid colony-stimulating activity by suppressing monocyte release of interleukin-1. Blood. 74:1531-1536.

29. Broxmeyer, H.E., Smithyman, A., Eger, R.R., Meyers, P.A., and de Sousa, M. 1978. Identification of lactoferrin as the granulocyte-derived inhibitor of colony-stimulating activity production. J. Exp. Med. 148:1052-1067.

30. Broxmeyer, H.E., et al. 1986. The effects in vivo of purified preparations of murine macrophage colony stimulating factor- 1 , recombinant murine granulocyte-macrophage colony stimulating factor and natural and recombinant murine interleukin 3 without and with pretreatment of mice with purified iron-saturated human lactoferrin. Immunobiol ogy. 172:168-174

31. Pozzan, T., Lew, D.P., Wollheim, C.B., and Tsien R.Y. 1983. Is cytosolic ionized calcium regulating neutrophil activation? Science. 221:1413-1415

32. Lew, P.D., Dayer, J.M., Wollheim, C.B., and Pozzan, T. 1984. Effect of leukotriene B4, prostaglandin E2 and arachidonic acid on cytosolic-free calcium in human neutrophils. FEBS Lett. 166:44-48.

33. Nigam, S., Muller, S., and Walzog, B. 1992. Effect of staurosporine on $\mathrm{PMet}$-Leu-Phe-stimulated human 
neutrophils: dissociated release of inositol 1, 4, 5trisphosphate, diacylglycerol and intracellular calcium. Biochim. Biophys. Acta. 1135:301-308.

34. Szczur, K., Xu, H., Atkinson, S., Zheng, Y., and Filippi, M.D. 2006. Rho GTPase CDC42 regulates directionality and random movement via distinct MAPK pathways in neutrophils. Blood. 108:4205-4213.

35. Siebert, P.D., and Huang, B.C. 1997. Identification of an alternative form of human lactoferrin mRNA that is expressed differentially in normal and tumor-derived cell lines. Proc. Natl. Acad. Sci. U. S. A. 94:2198-2203.

36. Legrand, D., Elass, E., Carpentier, M., and Mazurier, J. 2005. Lactoferrin: a modulator of immune and inflammatory responses. Cell. Mol. Life Sci. 62:2549-2559.

37. Ward, P.P., Paz, E., and Conneely, O.M. 2005. Multifunctional roles of lactoferrin: a critical overview. Cell. Mol. Life Sci. 62:2540-2548.

38. Crouch, S.P., Slater, K.J., and Fletcher, J. 1992. Regulation of cytokine release from mononuclear cells by the iron-binding protein lactoferrin. Blood. 80:235-240.

39. Haversen, L, et al. 2002. Lactoferrin down-regulates the LPS-induced cytokine production in monocytic cells via NF-kappa B. Cell Immunol. 220:83-95

40. Togawa, J., et al. 2002. Oral administration of lactoferrin reduces colitis in rats via modulation of the immune system and correction of cytokine imbalance. J. Gastroenterol. Hepatol. 17:1291-1298.

41. Zimecki, M., Artym, J., Chodaczek, G., Kocieba, M. and Kruzel, M. 2005. Effects of lactoferrin on the immune response modified by the immobilization stress. Pharmacol. Rep. 57:811-817.

42. Legrand, D., Elass, E., Carpentier, M., and Mazurier, J. 2006. Interactions of lactoferrin with cells involved in immune function. Biochem. Cell Biol. 84:282-290.

43. Anderson, R., Steel, H.C., and Tintinger, G.R 2005. Inositol 1, 4, 5-triphosphate-mediated shuttling between intracellular stores and the cytosol contributes to the sustained elevation in cytosolic calcium in FMLP-activated human neutrophils. Biochem. Pharmacol. 69:1567-1575.

44. Takenawa, T., Homma, Y., and Nagai, Y. 1983. Role of $\mathrm{Ca} 2+$ in phosphatidylinositol response and arachidonic acid release in formylated tripeptideor Ca2+ ionophore A23187-stimulated guinea pig neutrophils. J. Immunol. 130:2849-2855.

45. O'Flaherty, J.T., Rossi, A.G., Jacobson, D.P., and Redman, J.F. 1991. Roles of $\mathrm{Ca} 2+$ in human neutrophil responses to receptor agonists. Biochem.J. 277:705-711.

46. Thompson, H.L., Shiroo, M., and Saklatvala, J. 1993. The chemotactic factor $\mathrm{N}$-formylmethionylleucyl-phenylalanine activates microtubule-associated protein 2 (MAP) kinase and a MAP kinase kinase in polymorphonuclear leucocytes. Biochem.J. 290:483-488.
47. Thompson, H.L., Marshall, C.J., and Saklatvala, J. 1994. Characterization of two different forms of mitogen-activated protein kinase kinase induced in polymorphonuclear leukocytes following stimulation by $\mathrm{N}$-formylmethionyl-leucyl-phenylalanine or granulocyte-macrophage colony-stimulating factor. J. Biol. Chem. 269:9486-9492.

48. Van Lint, J., Van Damme, J., Billiau, A., Merlevede, W., and Vandenheede, J.R. 1993. Interleukin-8 activates microtubule-associated protein 2 kinase (ERK1) in human neutrophils. Mol. Cell. Biocbem. 127-128:171-177.

49. de Lillo, A., Tejerina, J.M., and Fierro, J.F. 1992. Interaction of calmodulin with lactoferrin. FEBS Lett. 298:195-198.

50. Verploegen, S., ex al. 2002. Role of Ca2+/calmodulin regulated signaling pathways in chemoattractant induced neutrophil effector functions. Comparison with the role of phosphotidylinositol-3 kinase. Eur. I. Biochem. 269:4625-4634.

51. Lian, J.P., et al. 2001. Antagonists of calcium fluxes and calmodulin block activation of the p21-activated protein kinases in neutrophils. J. Immunol. 166:2643-2650

52. Downey, G.P., et al. 1996. Chemotactic peptideinduced activation of MEK-2, the predominant isotorm in human neutrophtls. Inhibition by wortmannin. J. Biol. Chem. 271:21005-21011.

53. Naccache, P.H., et al. 1980. Calmodulin inhibitors block neutrophil degranulation at a step distal from the mobilization of calcium. Biochem. Biophys. Res. Commun, 97:62-68.

54. Birgens, H.S., Hansen, N.E., Karle, H., and Kristensen, L.O. 1983. Receptor binding of lactoferrin by human monocytes. Br. J. Haematol. 54:383-391.

55. Van Snick, J.L., and Masson, P.L. 1976. The binding of human lactoferrin to mouse peritoneal cells. J. Exp. Med. 144:1568-1580.

56. de la Rosa, G., Yang, D., Tewary, P., Varadhachary, A., and Oppenheim, J.J. 2008. Lactoferrin acts as an alarmin to promote the recruitment and activation of APCs and antigen-specific immune responses. J. Immunol. 180:6868-6876.

57. Elass, E., Masson, M., Mazurier, J., and Legrand, D. 2002. Lactoferrin inhibits the lipopolysaccharide-induced expression and proteoglycan-binding ability of interleukin-8 in human endothelial cells. Infect. Immun. 70:1860-1866.

58. Hayhoe, R.P., et al. 2006. Annexin 1 and its bioactive peptide inhibit neutrophil-endothelium interactions under flow: indication of distinct receptor involvement. Blood. 107:2123-2130.

59. Ly, N.P., et al. 2005. Netrin-1 inhibits leukocyte migration in vitro and in vivo. Proc. Natl. Acad. Sci. U.S. A. 102:14729-14734.

60. Cornish, J., et al. 2004. Lactoferrin is a potent regulator of bone cell activity and increases bone formation in vivo. Endocrinology. 145:4366-4374.

61. Ishii, T., et al. 2007. Bovine lactoferrin stimulates anchorage-independent cell growth via membrane-associated chondroitin sulfate and heparan sulfate proteoglycans in PC12 cells. J. Pharmacol. Sci. 104:366-373.

62. Golpon, H.A., et al. 2004. Life after corpse engulfment: phagocytosis of apoptotic cells leads to VEGF secretion and cell growth. FASEB J. 18:1716-1718.

63. Morimoto, K., et al. 2001. Alveolar macrophages that phagocytose apoptotic neutrophils produce hepatocyte growth factor during bacterial pneumonia in mice. Am. J. Respir. Cell Mol, Biol. 24:608-615.

64. Gorria, M., et al. 2008. A new lactoferrin- and irondependent lysosomal death pathway is induced by benzo[a]pyrene in hepatic epithelial cells. Toxicol. Appl. Pharmacol. 228:212-224.

65. Katunuma, N., et al. 2006. A novel apoptosis cascade mediated by lysosomal lactoferrin and its participation in hepatocyte apoptosis induced by D-galactosamine. FEBS Lett. 580:3699-3705.

66. Damiens, E., et al. 1999. Lactoferrin inhibits G1 cyclin-dependent kinases during growth arrest of human breast carcinoma cells. J. Cell. Biochem. 74:486-498.

67. Xiao, Y., Monitto, C.L., Minhas, K.M., and Sidransky, D. 2004. Lactoferrin down-regulates G1 cyclindependent kinases during growth arrest of head and neck cancer cells. Clin. Cancer Res. 10:8683-8686.

68. Lozupone, F., et al. 2000. Murine granulocytes control human tumor growth in SCID mice. Int. J. Cancer, 87:569-573.

69. Di Carlo, E., et al. 2001. The intriguing role of polymorphonuclear neutrophils in antitumor reactions. Blood. 97:339-345

70. Grote, D., Cattaneo, R., and Fielding, A.K. 2003. Neutrophils contribute to the measles virusinduced antitumor effect: enhancement by granulocyte macrophage colony-stimulating factor expression. Cancer Res. 63:6463-6468.

71. Dallegri, F, et al. 1991. Tumor cell lysis by activated human neutrophils: analysis of neutrophil-delivered oxidative attack and role of leukocyte function-associated antigen 1. Inflammation. 15:15-30.

72. Hoffer, P.B., Miller-Catchpole, R., and Turner, D.A. 1979. Demonstration of lactoferrin in tumor tissue from two patients with positive gallium scans. J. Nucl. Med. 20:424-427.

73. Dransfield, I., Stocks, S.C., and Haslett, C. 1995. Regulation of cell adhesion molecule expression and function associated with neutrophil apoptosis. Blood. 85:3264-3273.

74. Bournazos, S., Rennie, J., Hart, S.P., Fox, K.A., and Dransfield, I. 2008. Monocyte functional responsiveness after PSGL-1-mediated platelet adhesion is dependent on platelet activation status. Arterioscler. Thromb. Vasc. Biol. 28:1491-1498.

75. McMeekin, S.R., Dransfield, I., Rossi, A.G., Haslett, C., and Walker, T.R. 2006. E-selectin permits communication between PAF receptors and TRPC channels in human neutrophils. Blood. 107:4938-4945. 
A

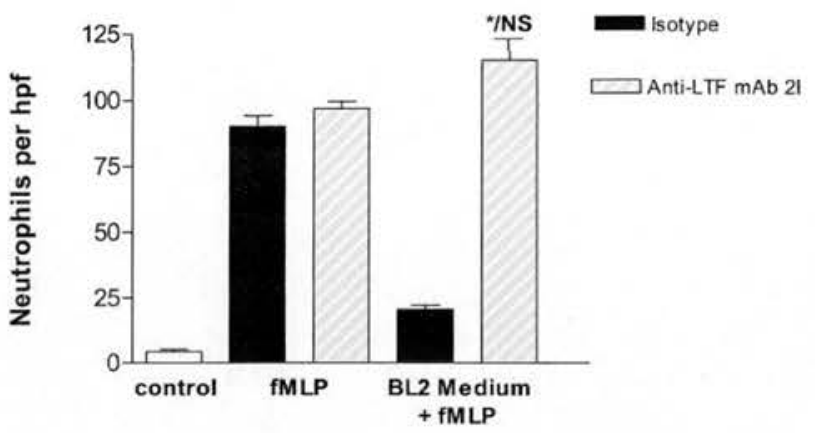

C

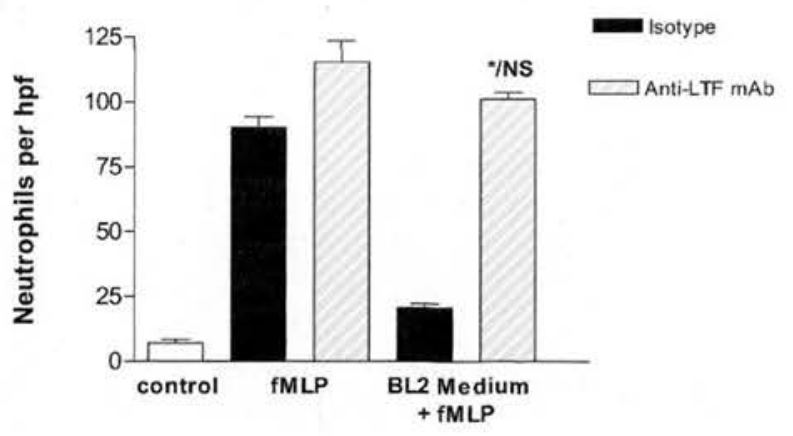

B

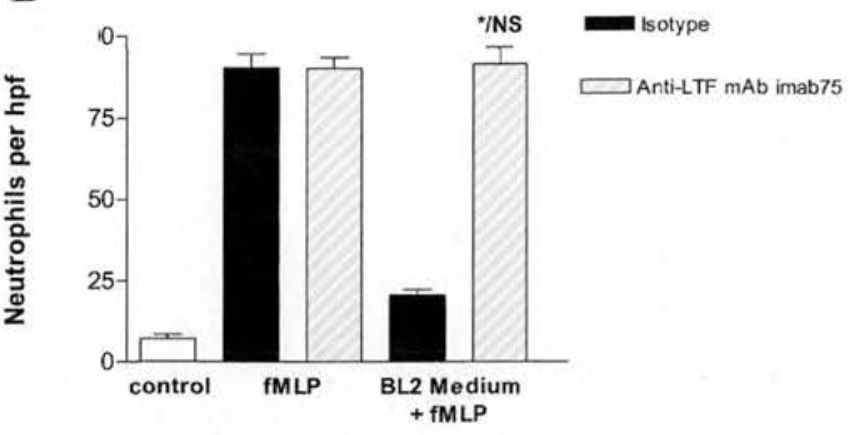

D

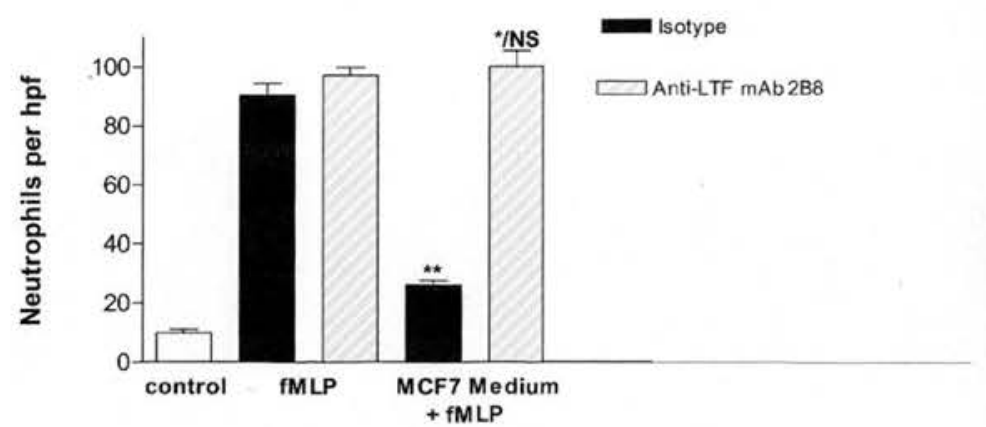

Supplemental Figure 1: Neutralisation experiments using monoclonal antibodies against human lactoferrin.

Neutrophil chemotaxis assays in the presence of three independent anti-lactoferrin

monoclonal antibodies (grey) or isotype control (black) using conditioned media from BL (A-C)

and MCF-7 (D) cells $\left(n=3 ;{ }^{*} p<0.05\right.$ vs isotype control, NS=non significant vs fMLP antilactoferrin control, ${ }^{\star *} \mathrm{p}<0.001$ compared to fMLP control.) Error bars indicate SEM. 


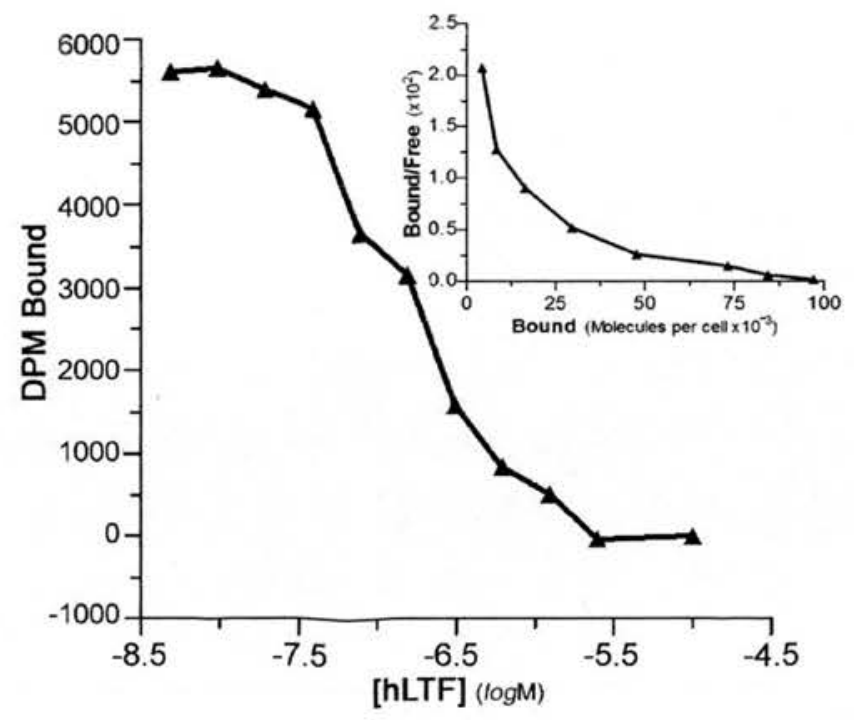

\section{Supplemental Figure 2: Analysis of ${ }^{125}$-labelled-lactoferrin binding to human} neutrophils.

Neutrophils $\left(2.5 \times 10^{6} \mathrm{ml}^{-1}\right)$ were incubated with $10 \mathrm{nM}^{125}$-labelled human milk-derived

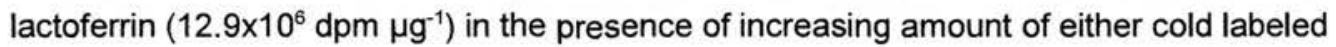
human lactoferrin (10 nM - 20 $\mu \mathrm{M})$ (specific competitor) or cold BSA (10 nM - $20 \mu \mathrm{M})$ (nonspecific competitor) for $30 \mathrm{~min}$ at $4^{\circ} \mathrm{C}$. Cells were washed three times prior to y measurement and all data were corrected for non-specific binding. Results are reported as dpm bound at the indicated ligand (cold) concentrations ( $\log \mathrm{M})$ and Scatchard analysis plot is shown as inset. Each point represents the mean of three experiments. 


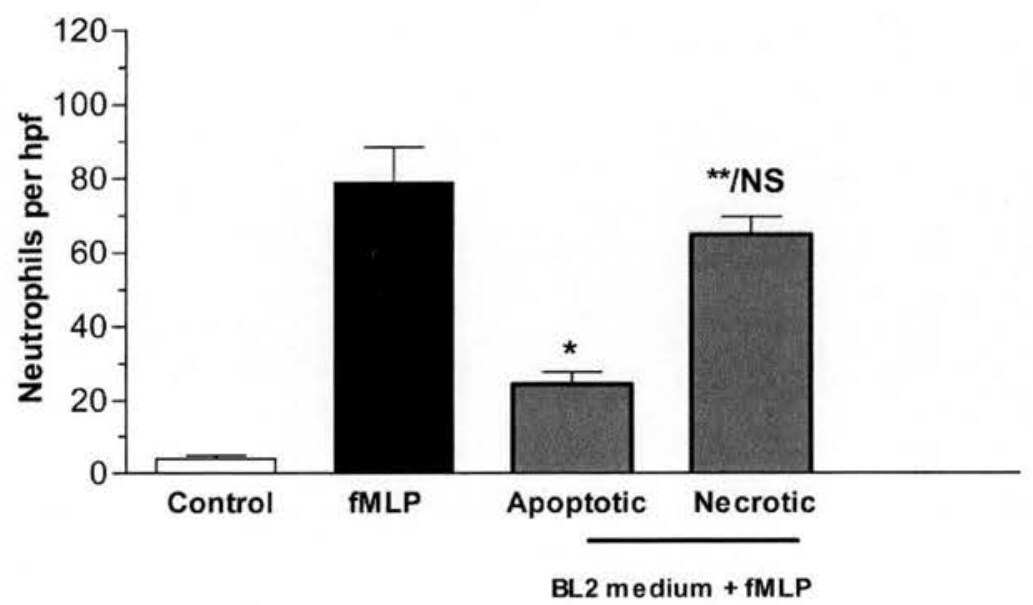

Supplemental Figure 3: Necrotic BL cells do not produce mediators of neutrophil migration inhibition.

Neutrophil chemotaxis assay to determine neutrophil migration towards supernatants from BL2 cells stimulated to undergo apoptosis (staurosporine-triggered) or primary necrosis (incubation at $56^{\circ} \mathrm{C}$ for $1 \mathrm{~h}$ ) in serum-free conditions $\left(n=3\right.$; ${ }^{*} p<0.001$ compared to $\mathrm{PMLP}$ control; ${ }^{\star \star} p<0.05$ vs apoptotic control; NS=non significant vs fMLP control). Error bars indicate SEM. 


\title{
Macrophage phagocytosis of apoptotic neutrophils is critically regulated by the opposing actions of pro-inflammatory and anti-inflammatory agents: key role for TNF- $\alpha$
}

\author{
Sylwia Michlewska, ${ }^{1}$ Ian Dransfield, Ian L. Megson, ${ }^{2}$ and Adriano G. Rossi \\ MRC Centre for Inflammation Research, The Queen's Medical Research Institute, University of \\ Edinburgh Medical School, Edinburgh, Scotland, UK
}

\begin{abstract}
Apoptosis of inflammatory cells and their subsequent clearance (efferocytosis) by macrophages $(\mathrm{M} \varphi \mathrm{s})$ are key mechanisms orchestrating successful resolution of inflammation. Although the powerful proinflammatory agents lipopolysaccharide (LPS) and tumor necrosis factor $\alpha$ (TNF- $\alpha$ ) influence rates of inflammatory cell apoptosis, little is known about their effects on efferocytosis. We have demonstrated that LPS and TNF- $\alpha$ potently inhibit efferocytosis of neutrophils by monocyte-derived M $\varphi$ s. Inhibition was both concentration and time dependent, although the effect of TNF- $\alpha$ was more rapid. We have found that soluble TNF receptor-I attenuated LPS inhibition of phagocytosis, indicating that TNF- $\alpha$ production is critical. Inhibition of efferocytosis by LPS was found to be positively associated with $\mathrm{M} \varphi$ production of TNF- $\alpha$, but negatively with interleukin-10 (IL-10) release. A critical role for $\mathrm{IL}-10$ in the regulation of phagocytosis was suggested by 2 important findings: LPS inhibition was observed more rapidly in the presence of an antihuman IL-10 receptor- $\alpha$ antibody, and efferocytosis by IL-10-deficient M $\varphi$ s was markedly reduced compared to wild-type M $\varphi$ s. Furthermore, exogenous IL-10 and glucocorticoids reversed inhibitory effects of LPS on efferocytosis via suppression of TNF- $\alpha$ production. We suggest that efferocytosis is regulated in an autocrine manner by pro- and anti-inflammatory mediators, and the inflammatory milieu determines whether inflammation successfully resolves.-Michlewska, S., Dransfield, I., Megson, I. L., Rossi, A. G. Macrophage phagocytosis of apoptotic neutrophils is critically regulated by the opposing actions of proinflammatory and anti-inflammatory agents: key role for TNF- $\alpha$. FASEB $J$. 23, 000-000 (2009)
\end{abstract}

Key Words: efferocytosis · interleukin- $10 \cdot$ glucocorticoids $\cdot$ soluble TNF-receptors

INFLAMMATION IS NORMALLY a tightly regulated physiological process playing a critical role in defense against pathogens and other injurious agents (1). However, loss of control and consequent exaggeration and prolongation of the inflammatory response can lead to tissue damage associated with chronic inflammatory conditions, such as rheumatoid arthritis, atherosclerosis, and chronic obstructive pulmonary disease. Evidence indicates that defects in the resolution of inflammation are likely to be major contributory factors to the pathogenesis of chronic inflammation $(2,3)$. In particular, the process of inflammatory cell apoptosis results in secretory shutdown (4) and exposure of surface molecules that allow their recognition by macrophages $(\mathrm{M} \varphi s)(5,6)$. These processes prevent the apoptotic cells from undergoing secondary necrosis and disgorgement of their histotoxic contents and, importantly, signals to the phagocytes to inhibit secretion of proinflammatory mediators while actively promoting antiinflammatory mediator release (7).

Sites of chronic inflammation (such as the rheumatoid synovium) are characterized by overexpression of various proinflammatory cytokines (8), and ligation of Toll-like receptors (TLRs) on leukocytes is an effective inducer of proinflammatory cytokine production (9). The proinflammatory cytokine tumor necrosis factor $\alpha$ (TNF- $\alpha$ ) is one of the most rapidly released cytokines after leukocyte exposure to the bacterial product and TLR4 ligand lipopolysaccharide (LPS). This cytokine plays a pivotal role in orchestrating inflammatory processes by switching on a TNF- $\alpha$-dependent cytokine cascade. Consequently, blockade of the TNF- $\alpha$ pathway reduces production of cytokines such as IL-1, GM-CMF, IL-6, or IL-8 (10). These findings suggested that targeting the overexpression of proinflammatory cytokines via blockade of one particular mediator (e.g., TNF- $\alpha$ ) is a viable option for anti-inflammatory therapy. In order for TNF- $\alpha$ to exert its biological function it has to be

\footnotetext{
${ }^{1}$ Correspondence: MRC Centre for Inflammation Research, The Queen's Medical Research Institute, University of Edinburgh Medical School, 47 Little France Crescent, Edinburgh, Scotland, EH16 4TJ UK. E-mail: s.michlewska@ sms.ed.ac.uk

${ }^{2}$ Current address: Department of Diabetes, UHI Millennium Institute, The Green House, Inverness, Scotland, IV2 3BL UK doi: $10.1096 /$ fj.08-121228
} 
released and bind to specific cell surface receptors. Therefore a reasonable strategy for therapy involves specific anti-TNF- $\alpha$ antibodies or soluble TNF receptors to reduce effective concentrations of TNF- $\alpha(11,12)$. Although the regulatory effects of TNF- $\alpha$ on proinflammatory cytokine production and its contribution to development of chronic inflammation are well established, the effects induced by TNF- $\alpha$ that lead to prolonged inflammatory responses and its harmful consequences remain to be fully defined. Two key processes involved in efficient resolution of inflammatory responses are apoptosis of inflammatory cells and their efferocytosis by phagocytes. Several reports describe the influence of proinflammatory mediators on apoptosis of inflammatory cells. For example, LPS markedly delays neutrophil apoptosis (13-15), whereas TNF- $\alpha$ may both inhibit or enhance neutrophil apoptosis depending on the time when apoptosis is assessed following TNF- $\alpha$ treatment (16). Data concerning the impact of proinflammatory cytokines on $\mathrm{M} \varphi$ uptake of apoptotic targets are limited and not consistent throughout the literature. Ren and Savill reported that treatment of human monocytc-derived M $\varphi s$ (MDM $\varphi s$ ) with granulocyte-macrophage colony-stimulating factor, IFN- $\gamma$, interleukin (IL) $-1 \beta$, or TNF- $\alpha$ for $6 \mathrm{~h}$ significantly increased uptake of apoptotic neutrophils (17). On the other hand, more recent findings demonstrate that both human MDM $\varphi$ s and murine J774 M $\varphi$ s preincubated for 20 min with TNF- $\alpha$, but not with LPS, have a reduced capacity for efferocytosis (18).

In this study we sought to thoroughly investigate the effect of proinflammatory agents (LPS and TNF- $\alpha$ ) on efferocytosis of neutrophils by human MDM $\varphi$ s and determine whether TNF- $\alpha$ plays a central role in any LPS-mediated effects. In addition, we investigated whether observed effects were regulated by the antiinflammatory cytokine IL-10 or by the powerful antiinflammatory drugs, the glucocorticoids.

\section{MATERIALS AND METHODS}

\section{Reagents}

All reagents were obtained from Sigma-Aldrich Co. (Poole, UK) unless otherwise stated. Iscove's Dulbecco modified Eagle medium (DMEM), HBSS, PBS, and culture supplements were purchased from PAA Laboratories (Pasching, Austria). DMEM F-12 GlutaMAX was obtained from Life Technologies, Inc. (Paisley, UK). Dextran T500 was obtained from Amersham Pharmacia Biotech (Buckingham, UK) and Percoll $^{\mathrm{TM}}$ from GE Healthcare Bio-Sciences AB (Uppsala, Sweden). LPS from E. coli, serotype 0127:B8, was purchased from Sigma-Chemicals Co. (St. Louis MO, USA). rhTNF- $\alpha$, soluble rhTNF receptor I/Fc Chimera (sTNF-RI), rhIL-10, anti-human IL-10 R $\alpha$, and DuoSet ${ }^{\otimes}$ ELISA (human/mouse TNF- $\alpha$ ) were obtained from R\&D Systems (Minneapolis, MN, USA). Roscovitine was purchased from Merck Chemicals Ltd (Nottingham, UK). BD ${ }^{\mathrm{TM}}$-Cytometric Bead Array (CBA) Human Inflammation Kit was obtained from BD Biosciences Pharmingen (San Diego, CA, USA).

\section{Cell isolation and culture}

Human blood cells were isolated from volunteers (15). Mononuclear cells were suspended in Iscove's DMEM at the concentration $8 \times 10^{5}$ monocytes $/ \mathrm{ml}$ (monocyte/lymphocyte ratio was assessed by flow cytometry) and plated at $0.5 \mathrm{ml} /$ well in 48-well plates. Following $1 \mathrm{~h}$ incubation at $37^{\circ} \mathrm{C}$, nonadherent cells were removed, and adherent monocytes were cultured in Iscove's DMEM with $10 \%$ autologous serum \pm treatment (all the treatments were added only once apart from anti-human IL-10 R $\alpha$ antibody, which was added every $48 \mathrm{~h}$ ) for 6 days at $37^{\circ} \mathrm{C}$ and $5 \% \mathrm{CO}_{2}$ atmosphere to allow their maturation into $\mathrm{MDM} \varphi \mathrm{s}$. Human neutrophils ( $>98 \%$ pure) were cultured at $37^{\circ} \mathrm{C}$ and $5 \% \mathrm{CO}_{2}$ atmosphere at a concentration of $5 \times 10^{6} / \mathrm{ml}$ in Iscove's DMEM with $10 \%$ autologous serum either for $20 \mathrm{~h}$ to undergo apoptosis or alternatively for $8 \mathrm{~h}$, when apoptosis $(>90 \%)$ was induced with $20 \mu \mathrm{M}$ R-roscovitine (15). These neutrophils are termed "apoptotic neutrophils." Apoptosis of neutrophils was assessed by oil-immersion light comparative microscopy of DiffQuik $^{\mathrm{TM}}$ stained cytocentrifuge preparations or alternatively by an annexin-V binding assay (15). Only neutrophils with $<5 \%$ necrosis (assessed by propidium iodide uptake) were used.

IL-10-deficient (IL-10 ${ }^{-1-}$ ) C57BL/6 (19) and wild-type (wt) $\mathrm{C} 57 \mathrm{BL} / 6$ mice were bred and maintained under specific pathogen-free conditions in the School of Biological Sciences Animal facility, University of Edinburgh. Bone marrow cells were flushed from dissected femurs of wt and IL $-10^{-1-}$ mice and cultured for 6 days in Teflon ${ }^{\text {TM }}$ pots in DMEM F-12 GlutaMAX medium supplemented with $10 \%$ FBS and $10 \%$ $\mathrm{L}-929$ conditioned medium at $37^{\circ} \mathrm{C}$ and $5 \% \mathrm{CO}_{2}$ atmosphere to allow their maturation into BMDM $\varphi$ s. On day 6 cells were harvested, resuspended at $1 \times 10^{6} \mathrm{cells} / \mathrm{ml}$, and plated at $0.5 \mathrm{ml} /$ well in 48 -well plates and cultured at $37^{\circ} \mathrm{C}$ and $5 \% \mathrm{CO}_{2}$ (at this point treatment was added).

\section{Assays for $\mathrm{M} \varphi$ phagocytosis of apoptotic neutrophils}

Following 6 days differentiation of MDM 4 s, culture supernatants were removed, and adherent MDM $\varphi$ s were washed with HBSS with $\mathrm{Ca}^{2+} / \mathrm{Mg}^{2+}$ and incubated with apoptotic neutrophils suspended at $5 \times 10^{6} / \mathrm{ml}$ for $30 \mathrm{~min}$ at $37^{\circ} \mathrm{C}$, providing a phagocyte-to-target ratio of $\sim 1: 10$. For the flow cytometric phagocytosis assay (20), neutrophils were stained with CellTracker Green ${ }^{\mathrm{TM}}$ (Molecular Probes, Leiden, The Netherlands) for $15 \mathrm{~min}$ and resuspended, prior to the assay. After incubation of $\mathrm{MDM} \varphi \mathrm{s}$ with apoptotic neutrophils, cells were incubated with trypsin/EDTA for $15 \mathrm{~min}$ at $37^{\circ} \mathrm{C}$, followed by $15 \mathrm{~min}$ on ice. Harvested cells were analyzed by flow cytometry in a FACSCalibur flow cytometer with CellQuest software (Becton-Dickinson, Oxford, UK). Neutrophil and $\mathrm{MDM}_{\varphi}$ populations were separated by forward and side scatter profiles, and a minimum of 6000 events within the $\mathrm{MDM} \varphi$ gate were acquired. The number of $\mathrm{FL1}^{+}$events in the MDM $\varphi$ gate was divided by the total number of MDM $\varphi$ s to obtain the percentage of MDM $\varphi$ s that had internalized neutrophils. For the microscopic phagocytosis assay, $\mathrm{MDM} \varphi \mathrm{s}$ were incubated with unstained apoptotic neutrophils and stained as described (21).

For $\mathrm{BMDM} \varphi$ phagocytosis assays, culture supernatants were removed, and the cells were labeled with $150 \mu \mathrm{l} /$ well of 2 $\mu \mathrm{g} / \mathrm{ml} \mathrm{FL}^{+}{ }^{+}$Cell Tracker Far Red ${ }^{\mathrm{TM}}$ (Invitrogen, Paisley, UK) for $30 \mathrm{~min}$, followed by addition of $100 \mu \mathrm{FBS}$ for $15 \mathrm{~min}$ and washing with $\mathrm{HBSS}$ with $\mathrm{Ca}^{2+} / \mathrm{Mg}^{2+}$. Then human apoptotic neutrophils (CellTracker Green labeled) were added and assay was performed as described above. The number of $\mathrm{FL}^{+}$ events in the $\mathrm{BMDM}_{\varphi} \mathrm{FL}_{4}^{+}$gate was divided by the total 
number of BMDM $\varphi$ s to obtain the percentage of BMDM 4 s that had internalized neutrophils.

\section{M $\varphi$ phagocytosis of immunoglobulin $G$ (IgG) -opsonized erythrocytes}

Following discontinuous Percoll density gradient centrifugation, erythrocytes were harvested, washed, and resuspended at $10^{7} / \mathrm{ml}$ in Iscove's DMEM, stained with CellTracker Green for $3 \mathrm{~h}$ and then incubated at $4^{\circ} \mathrm{C}$ with rabbit polyclonal anti-human erythrocyte $\mathrm{Ab}(1: 1000)$ for $30 \mathrm{~min}$. Opsonized erythrocytes were then washed and resuspended at $4 \times$ $10^{6} / \mathrm{ml}$, and $2 \times 10^{6}$ erythrocytes were added to each well with adherent $\mathrm{MDM} \varphi \mathrm{s}$ and incubated at $37^{\circ} \mathrm{C}$ for $30 \mathrm{~min}$ (21). The percentage phagocytosis was assessed by flow cytometry following the same protocol as for efferocytosis of neutrophils.

\section{Cytometric bead array}

Culture supernatants were collected at $120 \mathrm{~h}$ after treatment with $10 \mathrm{ng} / \mathrm{ml} \mathrm{LPS} \pm 1 \mu \mathrm{M}$ dexamethasone. All supernatants were centrifuged $(600 \mathrm{~g}, 6 \mathrm{~min})$ and stored in aliquots at $-70^{\circ} \mathrm{C}$. Cytokine concentrations in the culture supernatants were determined by CBA, performed according to the manufacturer's instructions.

\section{Enzyme-linked immunosorbent assay for measurement of} TNF- $\alpha$ and $\mathrm{IL}-10$ concentrations

MDM $\varphi$ s were treated for $1,6,12,24,48,72,96$, and $120 \mathrm{~h}$ with $10 \mathrm{ng} / \mathrm{ml} \mathrm{LPS}$ or for $96 \mathrm{~h}$ with increasing concentrations of LPS $(0.01,0.1,1,10,100 \mathrm{ng} / \mathrm{ml})$. Culture supernatants were collected (day 6 of the culture), centrifuged ( $600 \mathrm{~g} ; 6$ $\min )$, and stored in aliquots at $-70^{\circ} \mathrm{C}$. Concentrations of TNF- $\alpha$ and IL-10 were assessed by the DuoSet ELISA Development kit (R\&D Systems) according to the manufacturer's instructions.

\section{sTNF-RI binding assay}

Neutrophils, isolated from human blood (as described above), were cultured at $37^{\circ} \mathrm{C}$ and $5 \% \mathrm{CO}_{2}$ atmosphere at a concentration of $4 \times 10^{6} / \mathrm{ml}$ in Iscove's DMEM with $10 \%$ FBS (human IgG free) for $20 \mathrm{~h}$, then washed twice $(300 \mathrm{~g}, 5 \mathrm{~min}$, $\left.4^{\circ} \mathrm{C}\right)$, resuspended at the concentration of $0.8 \times 10^{6} / \mathrm{ml}$ in flow buffer (PBS without $\mathrm{Mg}^{2+} / \mathrm{Ca}^{2+}$ with $2 \% \mathrm{FBS}$ ), and plated at $100 \mu \mathrm{l} /$ well in 96-well FlexiPlate. Cells were incubated on ice for $20 \mathrm{~min}$ in flow buffer only or in the presence of $500 \mathrm{ng} / \mathrm{ml}$ sTNF-RI or $100 \mu \mathrm{g} / \mathrm{ml}$ heat-aggregated human recombinant IgG. Cells were washed twice ( $300 \mathrm{~g}, 5 \mathrm{~min}$, $\left.4^{\circ} \mathrm{C}\right)$, then incubated for $20 \mathrm{~min}$ on ice with goat $\mathrm{F}\left(\mathrm{ab}^{\prime}\right)_{2}$ anti-human IgG, fluorescein isothiocyanate conjugated antibody (1:50; Caltag Laboratories, Burlingame, CA, USA), washed twice $\left(300 \mathrm{~g}, 5 \mathrm{~min}, 4^{\circ} \mathrm{C}\right)$, and resuspended in annexin-V (1:40, APC conjugate) binding buffer. Assessment of binding was performed by flow cytometry analysis (22). The percentage of necrotic cells was assessed flow cytometrically by propidium iodide uptake.

\section{Statistical analysis}

Results are expressed as the mean \pm SE of $n=$ number of independent experiments using $\mathrm{MDM} \varphi$ s from different donors. Statistical significance (defined as $P<0.05$ ) was evaluated by ANOVA with comparisons between groups using the
Student-Newman-Keuls procedure when $n \geq 5$ or the Dunn's test when $n=4$.

\section{RESULTS}

LPS inhibits MDM $\varphi$ efferocytosis of neutrophils in a concentration- and time-dependent manner

To examine effects of LPS on MDM $\varphi$ efferocytosis, monocytes were treated with LPS $(10 \mathrm{ng} / \mathrm{ml})$ for the duration of $96-144 \mathrm{~h}$ and then cocultured with apoptotic neutrophils to determine phagocytosis. The percentage of MDM $\varphi$-ingesting apoptotic neutrophils, assessed by flow cytometric analysis, was significantly decreased by LPS treatment from control levels (Fig. 1A). MDM $\varphi$ s cultured for the same period in the presence of dexamethasone $(1 \mu \mathrm{M})$, reported previously to augment efferocytosis of neutrophils $(23,24)$, showed increased uptake of apoptotic neutrophils (Fig. 1A). Treatment of human blood monocytes with increasing concentrations of LPS for $120 \mathrm{~h}$ or with $10 \mathrm{ng} / \mathrm{ml}$ LPS for different durations indicated that the observed inhibitory effect is concentration and time dependent (Fig. $1 B)$. The approximate inhibitory concentration 50 $\left(\mathrm{IC}_{50}\right)$ value for LPS was $0.6 \mathrm{ng} / \mathrm{ml}$, and interestingly, LPS required at least $96 \mathrm{~h}$ to induce significant inhibition (96 h, $P<0.05$; or $144 \mathrm{~h}, P<0.001)$. Typical flow cytometric profiles of $\mathrm{MDM} \varphi$ phagocytosis under different treatments are shown in Fig. $1 C$, and confirmation of the different levels of phagocytosis by microscopy is depicted in Fig. 2C.

TNF- $\alpha$ inhibits MDM $\varphi$ efferocytosis of neutrophils in a concentration- and time-dependent manner

Since LPS is a powerful inducer of TNF- $\alpha$ production by M $\varphi s$ (25), we investigated whether TNF- $\alpha$ treatment of MDM $\varphi$ s would also decrease efferocytosis. Thus human blood monocytes were treated first with increasing concentrations of TNF- $\alpha$ for $120 \mathrm{~h}$ and, as shown in Fig. 2A1, TNF- $\alpha$ inhibited phagocytosis of apoptotic neutrophils in a concentration-dependent fashion. The approximate $\mathrm{IC}_{50}$ for $\mathrm{TNF}-\alpha$ was $8 \mathrm{ng} / \mathrm{ml}$. However, TNF- $\alpha$ requires a shorter time to induce significant inhibition ( $6 \mathrm{~h}, P<0.05$; or $48 \mathrm{~h}, P<0.001, n=6)$ in comparison to LPS (Fig. $2 A 2$ ). Figure $2 B$ represents a typical flow cytometric profile of $\mathrm{MDM} \varphi$ phagocytosis with TNF- $\alpha$ treatment, and its confirmation by microscopy is shown in Fig. $2 C$.

\section{LPS and TNF- $\alpha$ inhibit phagocytosis of \\ R-roscovitine-induced apoptotic neutrophils but not human IgG-opsonized erythrocytes}

Cultured neutrophils undergo spontaneous apoptosis at different rates, and therefore after $20 \mathrm{~h}$ of culture apoptotic cell populations contain a small number of viable and necrotic cells. Therefore we next investigated the effects of TNF- $\alpha(10 \mathrm{ng} / \mathrm{ml})$ and LPS (10 
A

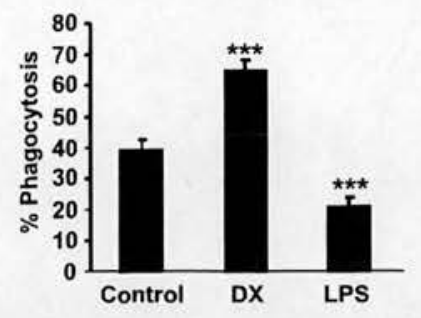

C

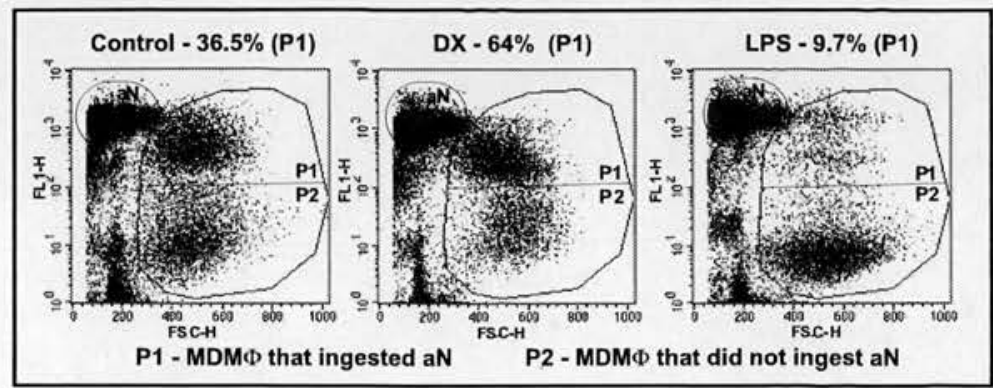

B2

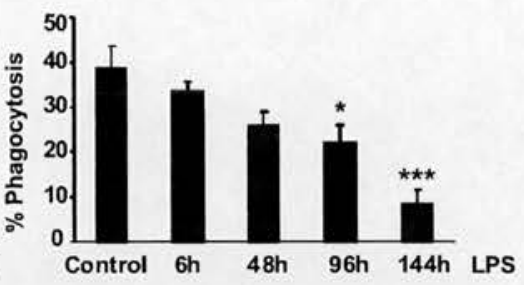

Figure 1. Effects of dexamethasone and LPS on $\mathrm{MDM} \varphi$ efferocytosis of neutrophils. A) Human MDM $\varphi$ s cultured in medium (control) for 6 days and treated on average for 96-144 $\mathrm{h}$ with either $1 \mu \mathrm{M}$ dexamethasone or 10 $\mathrm{ng} / \mathrm{ml}$ LPS were assessed for their ability to phagocytose apoptotic neutrophils. Percentage efferocytosis assessed on day 6 by flow cytometry is expressed as mean $\pm \mathrm{SE} ; n=19$ experiments. $B 1$, B2) Concentration response and time dependence of LPS treatment on $\mathrm{MDM} \varphi$ efferocytosis of neutrophils. Human MDM $\varphi s$ werc cultured with either increasing concentrations of LPS for $120 \mathrm{~h}(B 1)$ or with $10 \mathrm{ng} / \mathrm{ml}$ LPS for increasing durations of time (B2) and then assessed for their ability to phagocytose apoptotic neutrophils. Percentage efferocytosis assessed on day 6 by flow cytometry is expressed as mean \pm sE; $n=4$ separate experiments. $C$ ) Representative flow cytometric dot plots demonstrating changes in efferocytosis for treatments with either $1 \mu \mathrm{M}$ dexamethasone or 10 $\mathrm{ng} / \mathrm{ml}$ LPS for $120 \mathrm{~h}$ in comparison to medium control. aN, apoptotic (20 h culture) neutrophils; DX, dexamethasone. $* P<0.05, * * P<0.01, * * * P<0.001$ vs. medium control.

$\mathrm{ng} / \mathrm{ml}$ ) on efferocytosis of neutrophils undergoing early apoptosis without significant contaminating necrotic cells. For this purpose we induced apoptosis in neutrophils with the cyclin-dependent kinase inhibitor R-roscovitine, shown by our group to augment neutrophil apoptosis (15). TNF- $\alpha$ and LPS markedly inhibited uptake of R-roscovitine-induced apoptotic neutrophils, whereas dexamethasone-treated $\operatorname{MDM} \varphi s$ increased phagocytosis (Fig. 2D1). On the contrary, $\mathrm{MDM} \varphi$ phagocytosis of IgG-opsonized erythrocytes was not inhibited by TNF- $\alpha$ and LPS in comparison to control, whereas dexamethasone $(1 \mu \mathrm{M})$ significantly increased phagocytosis (Fig. 2D2).

\section{LPS inhibits MDM $\varphi$ efferocytosis of neutrophils via stimulation of TNF- $\alpha$ production}

As TNF- $\alpha$ inhibits MDM $\varphi$ efferocytosis (Fig. 2), we investigated whether the LPS-mediated effects were dependent on production of TNF- $\alpha$. We cotreated human monocytes for $96-144 \mathrm{~h}$ with $10 \mathrm{ng} / \mathrm{ml}$ LPS or $10 \mathrm{ng} / \mathrm{ml}$ TNF- $\alpha$ and increasing concentrations of sTNF-RI, which binds TNF- $\alpha$ present in the culture medium. Addition of $500 \mathrm{ng} / \mathrm{ml}$ sTNF-RI reversed both exogenous TNF- $\alpha$ and LPS-mediated inhibition of phagocytosis back to control levels (Fig. 3A).

sTNF-RI used in our study is a chimeric protein containing a fragment crystallizable (Fc) region of human $\operatorname{IgG}_{1}$. In its mature form, this protein forms homodimers, which were shown to be $\sim 45$-fold more potent inhibitors of TNF- $\alpha$ than the monomeric soluble
TNF-RI (26). However, presence of an Fc region in sTNF-RI raises the possibility that in our experiments reversal of the inhibitory effect of LPS and TNF- $\alpha$ is accomplished not only by a decrease in TNF- $\alpha$ activity but also by induction of Fc-mediated phagocytosis, similar to augmentation of phagocytosis by immune complexes (ICs; heat-aggregated IgG) (22). Binding of ICs to Fc receptors is a rapid process, and $30 \mathrm{~min}$ is sufficient to obtain optimal opsonization (22). Thus a series of control experiments were performed comparing the effectiveness of sTNF-RI on reversal of LPS- or TNF- $\alpha$-induced inhibition of phagocytosis. In all the experiments monocytes were cultured for 6 days in culture medium alone or treated for $96 \mathrm{~h}$ with either TNF- $\alpha(10 \mathrm{ng} / \mathrm{ml})$ or LPS $(10 \mathrm{ng} / \mathrm{ml})$. sTNF-RI was added either synchronically with LPS or TNF- $\alpha$ for $96 \mathrm{~h}$ to the monocyte culture or for $30 \mathrm{~min}$ to the $\mathrm{MDM} \varphi$ or neutrophil cultures, just prior to the phagocytosis assay. Alternatively, sTNF-RI was added directly to the MDM $\varphi$ s and apoptotic neutrophils during the phagocytosis assay $(1 \mathrm{~h})$. Presence of sTNF-RI in monocyte culture for $96 \mathrm{~h}$ significantly reversed the inhibitory effects of LPS and TNF- $\alpha$, while short durations of sTNF-RI treatment (30 $\mathrm{min}$ and $1 \mathrm{~h}$ ), sufficient for binding to the cell surface receptors, did not have any effect on phagocytosis in comparison to appropriate controls (Fig. $3 B$ ). Furthermore, we compared binding of sTNF-RI and IC to the apoptotic ( $20 \mathrm{~h}$ culture) neutrophils. As shown in Fig. $3 C$, following $30 \mathrm{~min}$ incubation, $>98 \%$ of neutrophils (viable and apoptotic) were opsonized with ICs, whereas $>98 \%$ did not bind sTNF-RI, excluding 

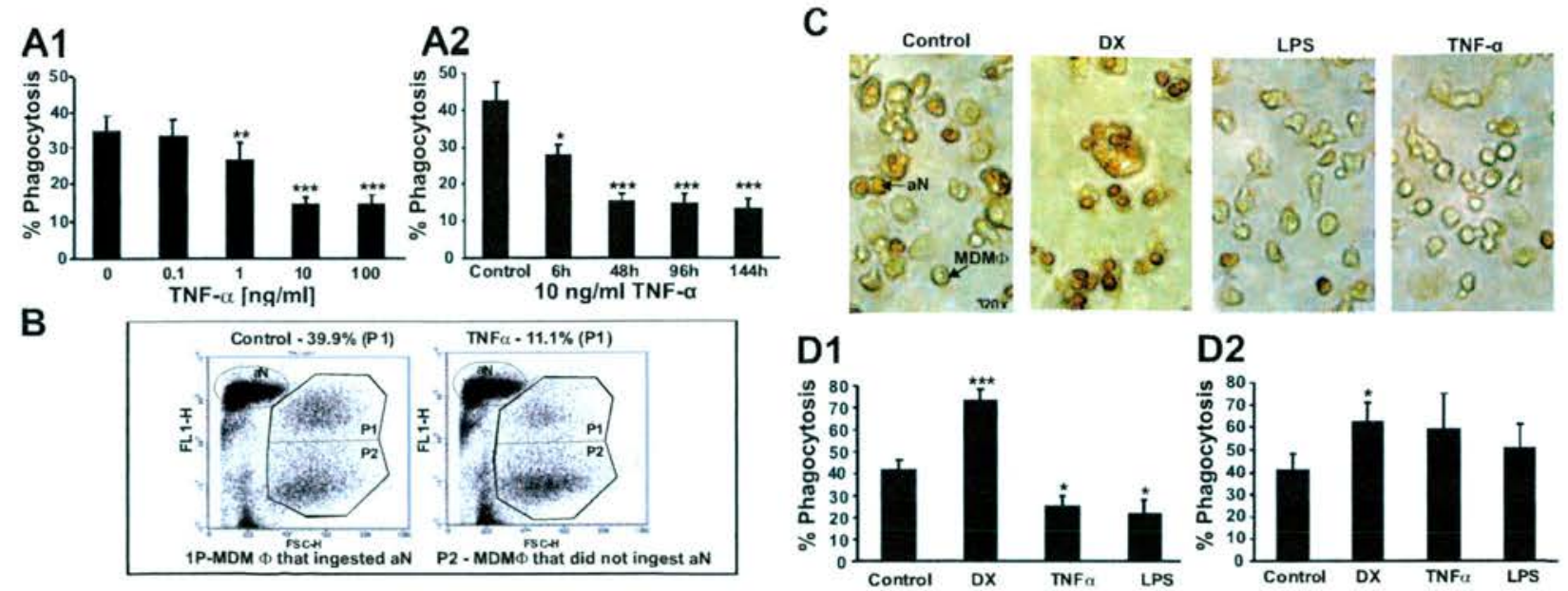

Figure 2. Concentration- and time-dependent effect of TNF- $\alpha$ on MDM $\varphi$ efferocytosis of neutrophils. $A 1, A 2) \mathrm{Human}$ MDM $\varphi s$ for concentration-response experiments were cultured for 6 days and treated for $120 \mathrm{~h}$ with increasing concentrations of TNF- $\alpha$ $(A 1)$, whereas for time course experiments, MDM $\varphi$ s were treated for increasing durations of time with $10 \mathrm{ng} / \mathrm{ml} \mathrm{TNF}-\alpha$ (A2). On day $6, \mathrm{MDM} \varphi$ efferocytosis of neutrophils was assessed by flow cytometry. Percentage efferocytosis is expressed as the mean \pm SE of $n=13(A 1)$ or $n=6(A 2)$ experiments. $B)$ Representative flow cytometric dot plots demonstrating changes in efferocytosis of neutrophils resulting from $96 \mathrm{~h}$ treatment with $10 \mathrm{ng} / \mathrm{ml}$ TNF- $\alpha$ in comparison to medium control. $C$ ) Representative photomicrographs showing $\mathrm{MDM}_{\varphi}$ efferocytosis of neutrophils (treatments the same as in Figs. $1 C$ and $2 B$ ). Images were obtained using an Axiovert S100 inverted microscope (Carl Zeiss), RS Photometrix camera, and analyzed with Open lab software, 3.1 .5 version. Original view $\times 320 . D 1, D 2)$ MDM $\varphi$ s were cultured for 6 days and treated for $120 \mathrm{~h}$ with either $1 \mu \mathrm{M}$ dexamethasone, $10 \mathrm{ng} / \mathrm{ml}$ TNF- $\alpha$, or $10 \mathrm{ng} / \mathrm{ml}$ LPS. On day 6 , ability of MDM $\varphi$ s to phagocytose either apoptotic neutrophils (apoptosis was induced by $20 \mu \mathrm{M}$ R-roscovitine, $8 \mathrm{~h}$ treatment) (D1) or human IgG opsonized erythrocytes (D2) was assessed by laser flow cytometric analysis. Data are expressed as mean \pm SE phagocytosis; $n=4$ experiments. $a N$, apoptotic ( $20 \mathrm{~h}$ culture) neutrophils; $D X$, dexamethasone. $* P<0.05$, $* * P<0.01$, $* * * P<0.001$ vs. medium control.

the possibility of Fc-induced augmentation of phagocytosis in our study (Fig. $3 \mathrm{C}$ ). Since some nonspecific binding of anti-human $\operatorname{IgG}$ antibodies to the low levels of necrotic cells in our cultures were detected (no difference between control, sTNF-RI and IC, samples), these cells were excluded from analysis.

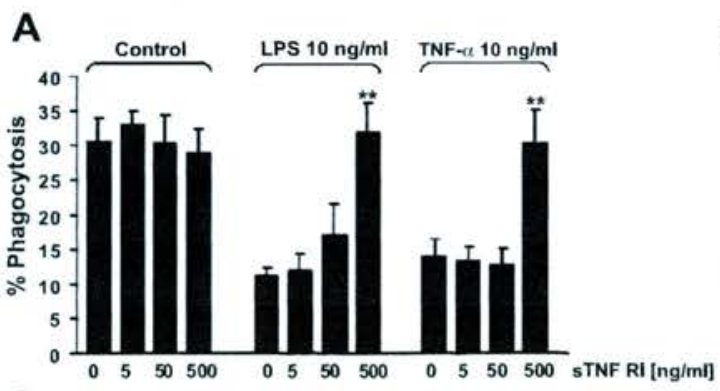

C
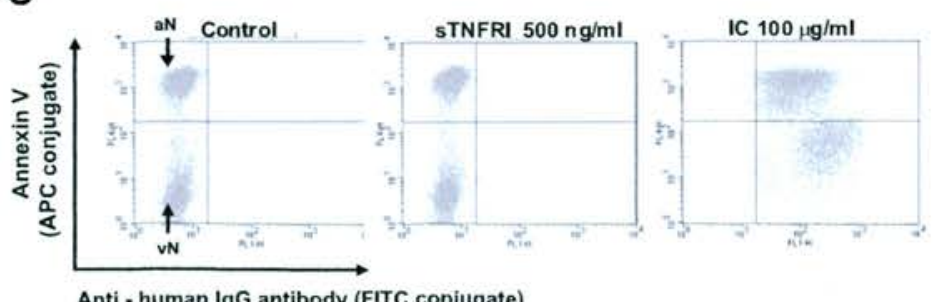

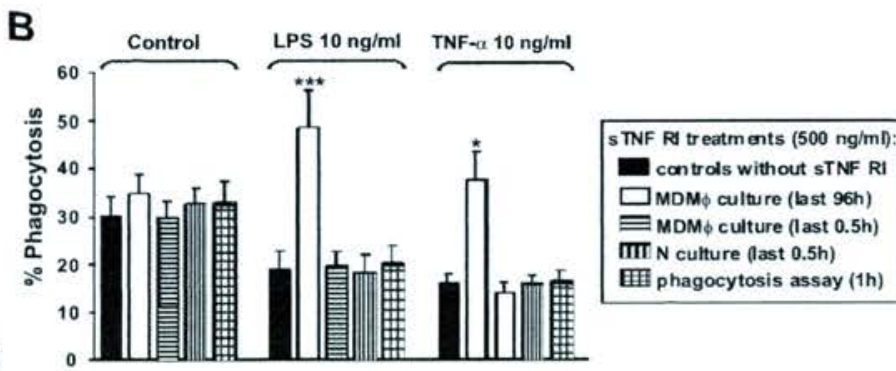

Figure 3. Neutralization of TNF- $\alpha$ released by LPS-stimulated $\mathrm{MDM} \varphi$ rescue efferocytosis. A) Human MDM $\varphi$ s were cultured for 6 days in culture medium alone or treated on average for the duration of $96-144 \mathrm{~h}$ with $10 \mathrm{ng} / \mathrm{ml} \mathrm{LPS}$ or $10 \mathrm{ng} / \mathrm{ml} \mathrm{TNF}-\alpha \pm$ increasing concentrations of sTNF-RI. On day $6, \mathrm{MDM} \varphi$ efferocytosis was assessed by flow cytometric analysis. Data are means $\pm \mathrm{SE} ; n=4$ separate experiments. ${ }^{*} * P<$ 0.01 vs. appropriate control. B) Human MDM $\varphi s$ were cultured for 6 davs in culture medium or treated for the duration of $96 \mathrm{~h}$ with either $10 \mathrm{ng} / \mathrm{ml}$ LPS or $10 \mathrm{ng} / \mathrm{ml}$ TNF- $\alpha$ alone. $s$ TNF-RI was added to MDM $\varphi$ s or alternatively to apoptotic (20 h culture) neutrophils at various times or was present in the phagocytosis assay, as indicated in $B$. On day $6, \mathrm{MDM} \varphi$ efferocytosis was assessed by flow cytometric analysis. Data are means \pm SE; $n=4$ separate experiments. $* P<0.05, * * * P<0.001$ vs. LPS or TNF- $\alpha$ alone, respectively. C) Apoptotic neutrophils, cultured for $20 \mathrm{~h}$ in Iscove's DMEM with $10 \% \mathrm{FCS}$, were incubated for $20 \mathrm{~min}$ in flow buffer alone (control) or with $500 \mathrm{ng} / \mathrm{ml}$ sTNF-RI or $100 \mu \mathrm{g} / \mathrm{ml}$ heat-aggregated IgG immune complexes, washed twice, and incubated with anti-human IgG antibody. Percentage of apoptotic neutrophils was assessed by annexin-V binding assay. $\mathrm{N}$, neutrophils; aN, apoptotic neutrophils; vN, viable neutrophils; IC, immune complexes. 
The ability of MDM $\varphi$ s to phagocytose apoptotic neutrophils depends on the levels of TNF- $\alpha$ in culture medium

Figure $\mathbf{4 A}$ depicts a scatter diagram in which paired data showing percentage phagocytosis on treatment with $10 \mathrm{ng} / \mathrm{ml}$ LPS (96-144h) vs. percentage phagocytosis in control conditions are plotted $(n=40)$. Even though the inhibitory effect of LPS is highly significant, we found some variation in monocyte response to LPS between donors. We hypothesized that the extent of inhibition of phagocytosis induced by LPS was dependent on endogenous TNF- $\alpha$ produced by cultured $\operatorname{MDM} \varphi s$ and consequently on the concentration of TNF- $\alpha$ in culture medium prior to the phagocytosis assay. To test this, we isolated monocytes from 6 selected donors, where we observed variability in response to LPS. Monocytes were cultured for 6 days, treated with $10 \mathrm{ng} / \mathrm{ml}$ LPS for $120 \mathrm{~h}$. On day 6 we performed paired experiments that involved collection of culture supernatants, measurement of cytokines by $\mathrm{CBA}$, and, in parallel, assessment of $\mathrm{MDM} \varphi$ efferocytosis by flow cytometry. We observed that the percentage of MDM $\varphi$ s ingesting apoptotic neutrophils was negatively correlated with the concentration of TNF- $\alpha$ in the supernatant $\left(R^{2}=0.84\right.$; Fig. $\left.4 B\right)$. Interestingly, we also noted that culture supernatants containing low concentrations of TNF- $\alpha$ showed increased levels of IL-10 (data not shown), which was reported to augment uptake of apoptotic cells by M $\varphi$ s (27). Therefore we examined the production of TNF- $\alpha$ and IL-10 in response to LPS treatment. Figure $4 C 1$ shows changes in concentrations of TNF- $\alpha$ and IL-10 at different time points after addition of $10 \mathrm{ng} / \mathrm{ml}$ LPS to culture medium. The first significant increase in TNF- $\alpha$ levels appears at the $6 \mathrm{~h}$ time point, followed by a decrease and then by a marked rise at the $96 \mathrm{~h}$ time point. We also found significant elevation in IL-10 levels at the $12 \mathrm{~h}$ time point with maximum concentration at $24 \mathrm{~h}$, followed by a continuous decline. As shown in Fig. 4C1, the increase in the IL-10 levels is associated with a decrease in TNF- $\alpha$ levels and vice versa. In addition, we measured concentrations of TNF- $\alpha$ and IL-10 following $96 \mathrm{~h}$ treatment with increasing concentrations of LPS. As indicated in Fig. $4 C 2$ the increase in TNF- $\alpha$ concentration is correlated with the concentration of LPS and is associated with low levels of IL-10. In the latter experiment the MDM $\varphi$ efferocytosis following LPS treatment $(1-100 \mathrm{ng} / \mathrm{ml})$ was significantly inhibited in comparison to control (data not shown).

\section{Presence of exogenous IL-10 blocks TNF- $\alpha$ production and inhibition of $\mathrm{MDM} \varphi$ efferocytosis induced by LPS}

As lower levels of TNF- $\alpha$ in culture supernatants were associated with elevated concentrations of IL-10 (Fig. $4 C 1$ ), we sought to investigate if, in our system, IL-10 is a negative regulator of TNF- $\alpha$ production by $\mathrm{MDM} \varphi \mathrm{s}$ exposed to LPS. For this purpose increasing concentra-
A

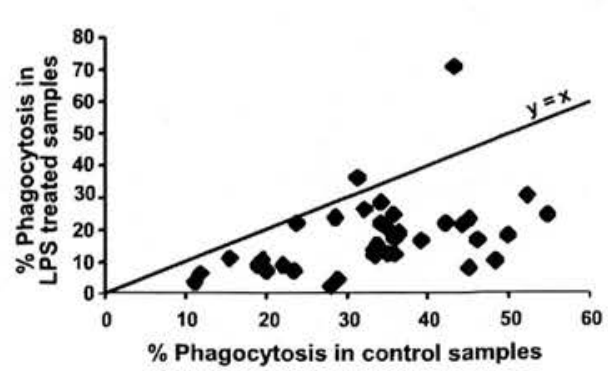

B

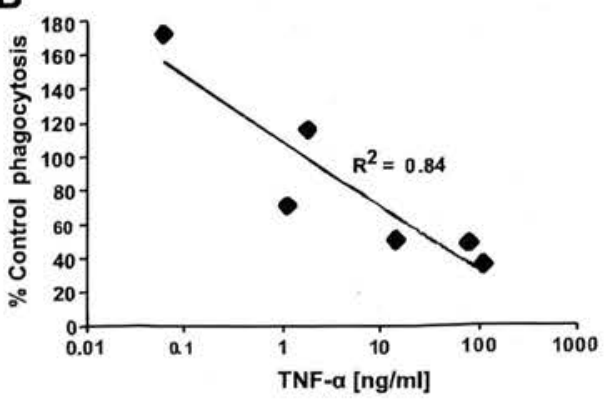

C1

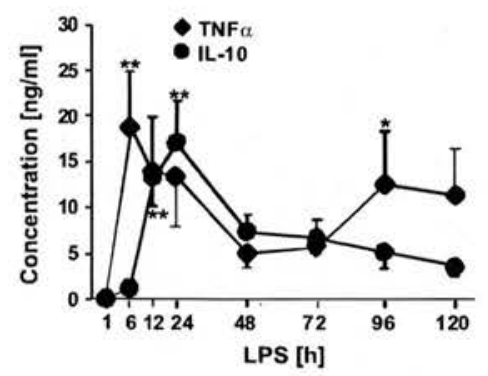

Figure 4. Effect of LPS on MDM $\varphi$ efferocytosis depends on concentration of TNF- $\alpha$ in the culture medium. A) Human MDM $\varphi$ s were cultured for 6 days in culture medium (control) or treated on average for the duration of 96-144 h with $10 \mathrm{ng} / \mathrm{ml}$ LPS. On day $6, \mathrm{MDM} \varphi$ efferocytosis was assessed by flow cytometry. Scatter diagram demonstrates paired data (control efferocytosis vs. efferocytosis on LPS treatment) from 40 experiments. Note variation in LPS inhibitory effect on the efferocytosis process. B) Monocytes isolated from blood of 6 donors were cultured for 6 days and treated with $10 \mathrm{ng} / \mathrm{ml}$ LPS for $120 \mathrm{~h}$. On day 6 , culture supernatants were collected, and TNF- $\alpha$ levels were measured by CBA, while efferocytosis was assessed by flow cytometry. Negative correlation $\left(R^{2}=0.84\right)$ was identified between efferocytosis levels and TNF- $\alpha$ concentrations. For comparisons, efferocytosis levels were normalized (medium control levels represented by $100 \%)$. C1, C2) Human MDM $\varphi$ s were cultured for 6 days and treated for increasing durations of time with $10 \mathrm{ng} / \mathrm{ml} \mathrm{LPS} \mathrm{(CI)} \mathrm{or} \mathrm{treated} \mathrm{for} 96 \mathrm{~h}$ with increasing concentrations of LPS (C2). On day 6, culture supernatants were collected, and TNF- $\alpha$ and IL-10 concentrations were measured by ELISA. Concentrations of TNF- $\alpha$ and IL-10 are expressed as means \pm SE for $n=7(C 1)$ or $n=4(C 2)$ experiments, $* P<0.05$, $* * P<0.01$ vs. respective concentrations at $1 \mathrm{~h}$ time point $(C 1)$ or samples not treated with LPS (C2). 
tions of IL-10 $(0.1-100 \mathrm{ng} / \mathrm{ml})$ were added to monocytes treated without (control) and with LPS or TNF- $\alpha$. Treatment was performed for the duration of $120 \mathrm{~h}$, and phagocytosis was assessed by flow cytometry. As shown in Fig. 5A, IL-10 significantly increased MDM $\varphi$ uptake of apoptotic cells. Furthermore, IL-10 reversed the inhibition of phagocytosis induced by LPS- but not by $\mathrm{TNF}-\alpha$-treated MDM $\varphi s$ in a concentration-dependent manner. On LPS treatment, efferocytosis was decreased, but addition of IL-10 at concentrations 10 $\mathrm{ng} / \mathrm{ml}$ and $100 \mathrm{ng} / \mathrm{ml}$ increased phagocytosis. It is noteworthy that these levels of efferocytosis are similar to those represented by MDM $\varphi$ s treated with 10 and $100 \mathrm{ng} / \mathrm{ml} \mathrm{IL-10}$ alone. To prove that IL-10 inhibited production of TNF- $\alpha$ in LPS-treated $(120 \mathrm{~h}) \mathrm{MDM} \varphi \mathrm{s}$, we measured TNF- $\alpha$ concentrations in $\operatorname{MDM} \varphi$ culture supernatants. As demonstrated in Fig. 5B, IL-10 reduced production of TNF- $\alpha$ in LPS-treated MDM $\varphi$ s in a concentration-dependent manner. IL-10 $(0.1$ and 1 $\mathrm{ng} / \mathrm{ml})$ significantly decreased TNF- $\alpha$ production by $\mathrm{MDM} \varphi s$, although the remaining levels of TNF- $\alpha(\sim 11$ and $\sim 4 \mathrm{ng} / \mathrm{ml}$ ) were still capable of inhibiting phagocytosis (Fig. 2A1). Higher concentrations of IL-10 (10 and $100 \mathrm{ng} / \mathrm{ml}$ ) further decreased TNF- $\alpha$ concentrations $(\sim 1 \mathrm{ng} / \mathrm{ml})$ and effectively reversed LPS-mediated inhibition of efferocytosis. Since the concentration-response curve for TNF- $\alpha$ (Fig. 2Al) indicates that $\mathrm{TNF}-\alpha$ at $1 \mathrm{ng} / \mathrm{ml}$ significantly inhibits efferocytosis, we examined if the presence of high concentrations of IL-10 may mask the inhibitory action of lower concentration of TNF- $\alpha$. For this purpose human monocytes were cultured for 6 days and treated for $72 \mathrm{~h}$ with increasing concentrations of TNF- $\alpha$ alone or with addition of IL-10 $(100 \mathrm{ng} / \mathrm{ml})$. In Fig. $5 C$ we show clearly that phagocytosis levels on combined treatment with TNF- $\alpha$ ( 1 or $10 \mathrm{ng} / \mathrm{ml}$ ) and $100 \mathrm{ng} / \mathrm{ml} \mathrm{IL-10} \mathrm{are}$ undistinguishable from medium control levels, whereas treatment with TNF- $\alpha$ alone causes significant inhibition of efferocytosis.

\section{IL-10 produced by LPS stimulated MDM $\varphi$ s delays the LPS inhibitory effect on efferocytosis}

Having found that LPS-induced inhibition of phagocytosis is blocked by the presence of exogenous IL-10, we next examined whether endogenous production of IL-10 delays the LPS inhibitory effect. We differentiated human monocytes for 6 days and cultured them with 10 $\mathrm{ng} / \mathrm{ml}$ LPS either alone or in the presence of $10 \mu \mathrm{g} / \mathrm{ml}$ anti-human IL-10 R $\alpha$ antibody for different durations of time: $24,48,72$, and $96 \mathrm{~h}$ prior to phagocytosis assessed by flow cytometry. Samples treated with LPS alone showed significant decrease in efferocytosis only after $96 \mathrm{~h}$ of treatment, concurring with our earlier observation (Fig. $1 \mathrm{Bl}$ and Fig. 6). Interestingly, in the presence of anti-human IL-10 R $\alpha$ antibody, LPS treatment caused significant inhibition at $24 \mathrm{~h}$, and this effect was maintained at 48,72 , and $96 \mathrm{~h}$ after treatment. Moreover, at $96 \mathrm{~h}$, LPS induced much stronger inhibition in the presence of anti-human IL-10 R $\alpha$ antibody than when used alone (Fig. 6).
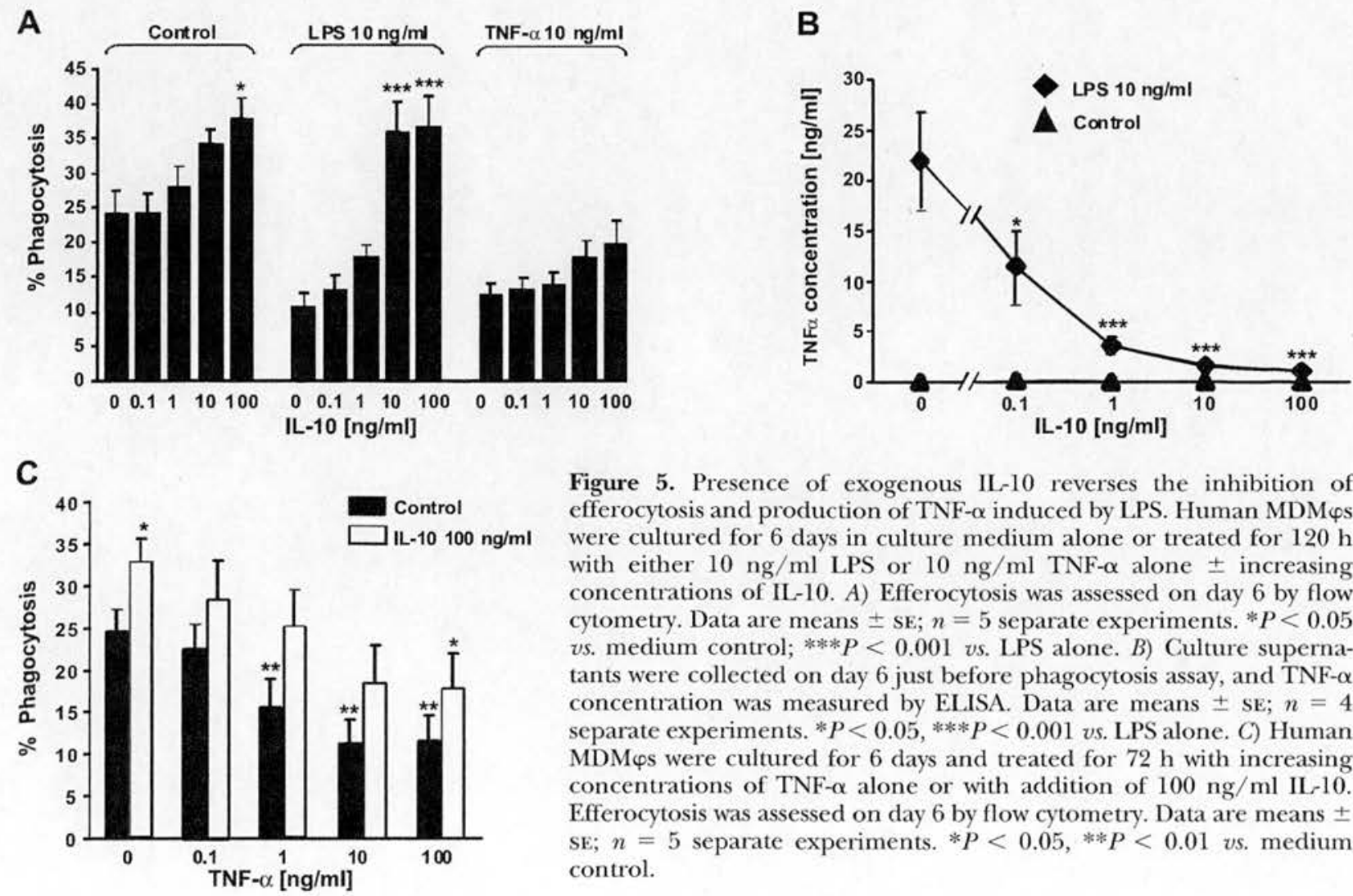

Figure 5. Presence of exogenous IL-10 reverses the inhibition of efferocytosis and production of TNF- $\alpha$ induced by LPS. Human MDM $\varphi s$ were cultured for 6 days in culture medium alone or treated for $120 \mathrm{~h}$ with either $10 \mathrm{ng} / \mathrm{ml}$ LPS or $10 \mathrm{ng} / \mathrm{ml} \mathrm{TNF-} \alpha$ alone \pm increasing concentrations of IL-10. A) Efferocytosis was assessed on day 6 by flow cytometry. Data are means $\pm \mathrm{SE} ; n=5$ separate experiments. $* P<0.05$ vs. medium control; $* * * P<0.001$ vs. LPS alone. $B$ ) Culture supernatants were collected on day 6 just before phagocytosis assay, and TNF- $\alpha$ concentration was measured by ELISA. Data are means $\pm \mathrm{SE} ; n=4$ separate experiments. $* P<0.05$, ${ }^{* * *} P<0.001$ vs. LPS alone. $C$ ) Human MDM $\varphi$ s were cultured for 6 days and treated for $72 \mathrm{~h}$ with increasing concentrations of TNF- $\alpha$ alone or with addition of $100 \mathrm{ng} / \mathrm{ml} \mathrm{IL}-10$. Efferocytosis was assessed on day 6 by flow cytometry. Data are means \pm SE; $n=5$ separate experiments. $* P<0.05$, $* * P<0.01$ vs. medium control. 


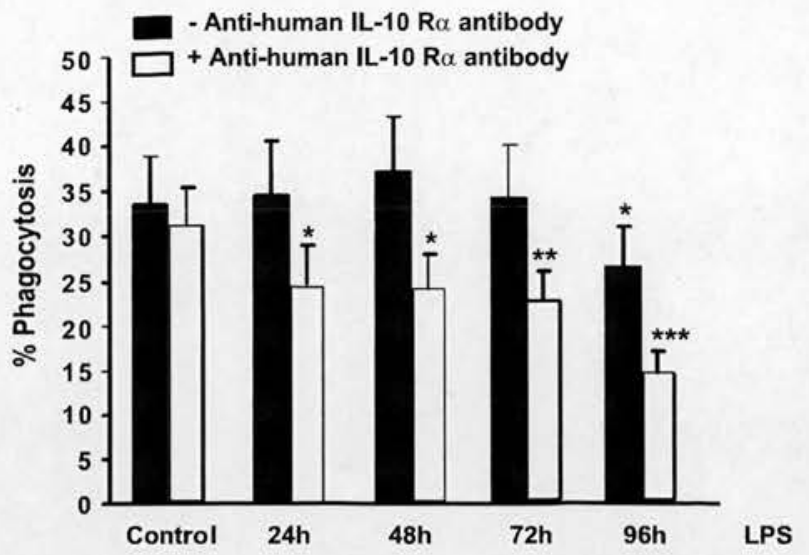

Figure 6. IL-10 produced by LPS-stimulated MDM $\varphi$ s delays LPS-induced inhibition of efferocytosis. Human $M D M \varphi s$ were cultured for 6 days and treated for $24,48,72$, and $96 \mathrm{~h}$ with $10 \mathrm{ng} / \mathrm{ml} \mathrm{LPS}$ alone or with addition of $10 \mu \mathrm{g} / \mathrm{ml}$ anti-human IL-10 R $\alpha$ antibody. Efferocytosis was assessed on day 6 by flow cytometry. Data are means \pm SE; $n=8$ separate experiments. $* P<0.05, * * P<0.01, * * * P<0.001$ vs. medium control.

To further investigate the role of endogenous IL-10 in maintaining high levels of efferocytosis under proinflammatory conditions we compared efferocytosis of neutrophils by $\mathrm{IL}-10^{-/-}$and wt $\mathrm{BMDM} \varphi \mathrm{s}$. As presented in Table 1 BMDM $\varphi$ s from IL-10-deficient mice have a much lower ability for phagocytosis in comparison to wt $\operatorname{BMDM} \varphi s$ in control conditions and when treated with LPS or TNF- $\alpha$.

\section{Dexamethasone rescues the ability of LPS-treated MDM $\varphi$ s to phagocytose apoptotic neutrophils}

Glucocorticoids, including the synthetic glucocorticoid dexamethasone, are recognized for their anti-inflammatory properties and ability to inhibit production of proinflammatory cytokines such as TNF- $\alpha$ (28). Furthermore, they are reported to increase nonphlogistic phagocytosis of apoptotic cells $(23,24)$. We therefore aimed to determine whether dexamethasone was able

TABLE 1. Comparison of efferocytosis by wt and

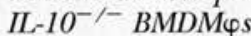

\begin{tabular}{lrc}
\hline \hline Treatment and genotype & Phagocytosis (\%) & Inhibition (\%) \\
\hline Control wt & $36.3 \pm 1.4$ & \\
Control IL-10-1- & $17.3 \pm 3.3$ & $52.6 \pm 8.4$ \\
LPS 24 wt & $24.4 \pm 4.3$ & \\
LPS24 IL $-10^{-1-}$ & $15.0 \pm 2.2$ & $37.3 \pm 5.9$ \\
LPS 48 wt & $4.5 \pm 2.1$ & \\
LPS 48 IL-10 $-1-$ & $2.1 \pm 0.9$ & $50.2 \pm 3.0$ \\
TNF- $\alpha$ 24 wt & $23.3 \pm 2.4$ & \\
TNF- $\alpha$ 24 IL-10 $-1-$ & $12.0 \pm 0.7$ & $47.4 \pm 5.5$ \\
TNF- $\alpha$ 48 wt & $7.5 \pm 3.0$ & \\
TNF- $\alpha$ 48 IL-10 $-1-$ & $1.9 \pm 0.5$ & $75.7 \pm 9.4$ \\
\hline
\end{tabular}

Phagocytosis by BMDM $\varphi$ s was assessed by flow cytometry. Data are expressed as mean \pm SE percentage of efferocytosis and percentage inhibition of efferocytosis, where corresponding wt and $\mathrm{IL}-10^{-1-}$ treatments were compared. Three animals/group were used. to exert similar effects to IL-10 and rescue LPS-inhibited efferocytosis through inhibition of TNF- $\alpha$ production or reversal of TNF- $\alpha$ action. As shown in Fig. 7A, dexamethasone augmented phagocytosis in a concentration-dependent manner with the most prominent effect at $0.1 \mu \mathrm{M}$ concentration or higher. Pretreatment of MDM $\varphi$ s with dexamethasone markedly reversed the inhibitory effects of LPS on efferocytosis. Therefore we next sought to investigate whether dexamethasone blocks TNF- $\alpha$ or enhances IL-10 production on LPS treatment. MDM $\varphi$ s were cultured in medium (control) or treated with either $1 \mu \mathrm{M}$ dexamethasone alone (dexamethasone control) or $10 \mathrm{ng} / \mathrm{ml}$ LPS alone or with both LPS and dexamethasone and supernatants were collected at the $120 \mathrm{~h}$ time point, prior to the phagocytosis assay. We found that dexamethasone partially decreased production of TNF- $\alpha$ but did not significantly change production of IL-10 in LPS-stimulated MDM $\varphi$ s (Fig. $7 B$ ), and this effect was accompanied by increases in phagocytosis. TNF- $\alpha$ and IL-10 levels in both controls were barely detectable (data not

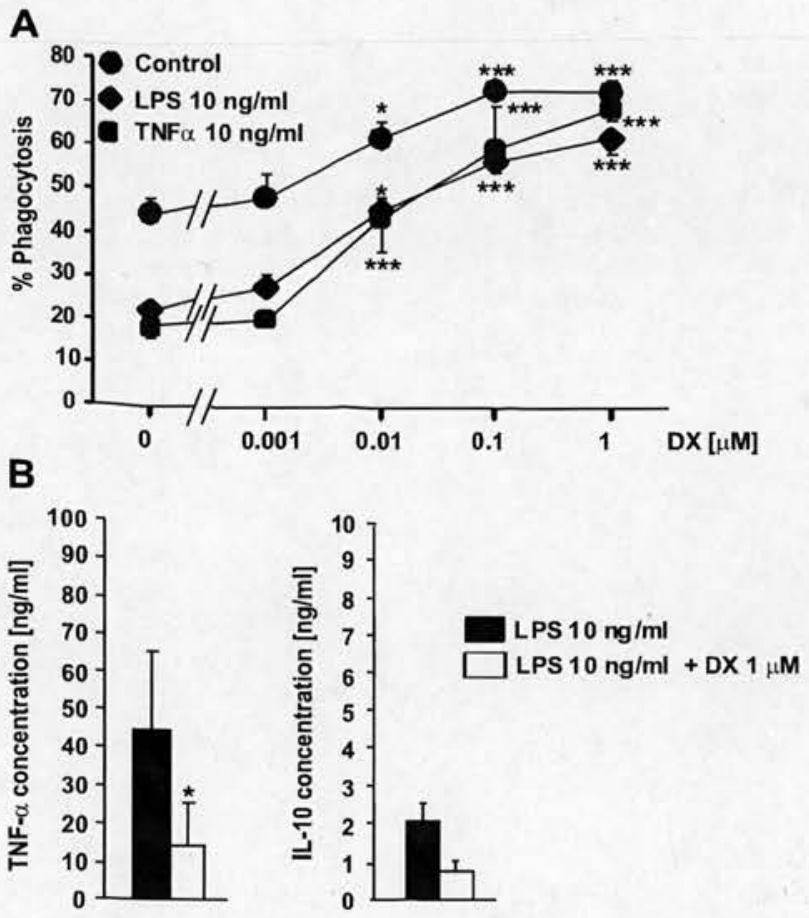

Figure 7. Dexamethasone reverses the inhibitory effects of TNF- $\alpha$ and LPS on efferocytosis. A) Human MDM $\varphi$ s were cultured for 6 days and treated for $120 \mathrm{~h}$ with increasing concentrations of dexamethasone alone or with addition of either $10 \mathrm{ng} / \mathrm{ml}$ LPS or $10 \mathrm{ng} / \mathrm{ml}$ TNF- $\alpha$. Efferocytosis was assessed on day 6 by flow cytometry. Data are means $\pm \mathrm{SE} ; n=$ 4-8 separate experiments. ${ }^{*} P<0.05, * * P<0.01$, $* * * P<$ 0.001 vs. medium, TNF- $\alpha$, or LPS control, respectively. $B$ ) Human MDM $\varphi$ s were cultured for $120 \mathrm{~h}$ in the presence of 10 $\mathrm{ng} / \mathrm{ml}$ LPS alone or with addition of $1 \mu \mathrm{M}$ dexamethasone. After $120 \mathrm{~h}$, supernatants were collected, and TNF- $\alpha$ and IL-10 concentrations were measured by CBA. Data are means $\pm \mathrm{SE} ; n=5$ separate experiments. Combined LPS and dexamethasone treatment at $120 \mathrm{~h}$ significantly changed TNF- $\alpha(* P<0.05)$ but not IL-10 levels as compared to treatment with LPS alone. DX, dexamethasone. 
shown). As indicated in Fig. $7 A$, dexamethasone also markedly reversed the inhibitory effect of TNF- $\alpha$ on phagocytosis in a concentration-dependent fashion.

\section{DISCUSSION}

The precise role of individual proinflammatory cytokines such as TNF- $\alpha$ at inflamed tissues and their involvement in the development of chronic inflammation is not fully understood. Because chronic inflammation has been shown to be related to defects in resolution of inflammation $(2,3)$, we hypothesized that an excess of proinflammatory mediators may affect this process. There are at least 2 main mechanisms involved in resolution of inflammation: inflammatory cell apoptosis and their subsequent clearance by $M \varphi s(2,6)$. It has been shown that key proinflammatory mediators such as LPS and TNF- $\alpha$ can influence the rate of inflammatory cell apoptosis $(13,16)$. In this study we focus on effects of proinflammatory mediators such as LPS and TNF- $\alpha$ on $\mathrm{M} \varphi$ efferocytosis.

We show for the first time that LPS during $\operatorname{MDM} \varphi$ differentiation decreased their ability to ingest apoptotic neutrophils that had undergone either constitutive apoptosis or R-roscovitine-induced apoptosis. We also provided convincing evidence that TNF- $\alpha$ is necessary and sufficient for LPS-mediated inhibition of efferocytosis because TNF- $\alpha$ depletion by sTNF-RI completely reverses the effect of LPS. Interestingly, phagocytosis of human IgG-opsonized erythrocytes was not attenuated by LPS or TNF- $\alpha$, indicating that proinflammatory stimuli do not block all phagocytic pathways in MDM $\varphi$ s and that there are likely to be differential mechanisms governing phagocytosis of apoptotic cells and IgGopsonized cells. These findings agree with other studies (21) indicating that $\mathrm{M} \varphi$ efferocytosis is likely regulated by mechanisms distinct from those modulating phagocytosis of cells coated with IgG and complement (29).

TNF- $\alpha$ is a rapidly released cytokine after trauma or infection (e.g., exposure to bacterial-derived LPS) and is one of the most abundant mediators in inflamed tissues (30). TNF- $\alpha$ is not only a "master regulator" of proinflammatory cytokine production (31) but also regulates many inflammatory processes such as inflammatory cell activation and recruitment and therefore is a key molecule involved in development of chronic inflammation (32). Consequently, TNF- $\alpha$ inhibitors such as infliximab (mouse-human chimeric antibody), adalimumab (human monoclonal antibody), or etanercept (TNF receptor II-IgG fusion protein) have been shown to have therapeutic benefit in the treatment of chronic inflammatory conditions (33). Etanercept is a bivalent receptor that binds TNF- $\alpha$ with 50-1000 times higher affinity to TNF- $\alpha$ than monomeric soluble TNF receptors and linkage of Fc portion of IgG significantly prolongs its half-life (34). Therefore in our study we used chimeric sTNF-RI fused to the portion of human $\operatorname{IgG}_{1}$, which we found to be much more efficient in decreasing TNF- $\alpha$ activity than monomeric receptor (our unpublished observation). Lack of binding of chimeric sTNF-RI to the surface of neutrophils clearly indicates that observed increase in efferocytosis does not involve Fc-mediated phagocytosis and that binding of TNF- $\alpha$ in the culture supernatants is primarily responsible for reversal of inhibitory effects of LPS and TNF- $\alpha$. Our data show that a TNF- $\alpha$ concentration as low as $1 \mathrm{ng} / \mathrm{ml}$ can have a significant effect on efferocytosis (Fig. 2A1). Therefore, to achieve significant reversal of the LPS and TNF- $\alpha$ effects, TNF- $\alpha$ concentrations must be reduced below this threshold level, for example, by excess sTNF-RI (i.e., $500 \mathrm{ng} / \mathrm{ml}$ ). So far, it has not been shown why binding of TNF- $\alpha$ at inflamed tissues leads to resolution of inflammation. Here we give evidence that excess TNF- $\alpha$ in inflamed tissue may result in insufficient efferocytosis of neutrophils and potentially other apoptotic cells. Consequently, higher rates of secondary necrosis and damage of the surrounding tissue will delay the resolution phase of inflammation and exacerbate chronic inflammatory conditions such as rheumatoid arthritis. As shown in Fig. 8, sTNF-RI reduces the biological activity of TNF- $\alpha$ and, therefore, reverses the influence of proinflammatory stimuli such as LPS on efferocytosis. Our data complement a recent study by McPhillips and colleagues indicating that production of oxidants through cPLA $A_{2}$ mediated arachidonic acid release, and Rho activation is involved in the inhibition of phagocytosis of apoptotic Jurkat cells by short-term incubation (20 min) of mature M $\varphi s$ with TNF- $\alpha$ (18). However, work by Ren and Savill suggests that not fully differentiated $\mathrm{MDM} \varphi s$ have increased ability to phagocytose apoptotic neutrophils when treated for $6 \mathrm{~h}$ with TNF- $\alpha$ (17). This may result from the fact that we used more mature macrophages ( 6 days culture) and assessed efferocytosis using a flow cytometric-based assay. We also focused on a more prolonged treatment with TNF- $\alpha$.

Interestingly, we were able to identify significant latency of the LPS inhibitory effect on phagocytosis in comparison to TNF- $\alpha$. Previously published data demonstrated that in monocytes maximal production of proinflammatory TNF- $\alpha$, IL- $1 \alpha$, IL- $1 \beta$, IL- 6 , or IL- 8 appears $4-8 \mathrm{~h}$ after proinflammatory challenge (i.e., LPS or interferon- $\gamma$ ) and is followed by maximal IL-10 production $24-48 \mathrm{~h}$ after activation (35). Since convincing evidence indicates that IL-10 can up-regulate efferocytosis (36), we sought to investigate if IL-10 was produced by LPS-activated monocytes and if its presence contributed to the observed latency. Following treatment with LPS we identified an increase in TNF- $\alpha$ concentration at the $6 \mathrm{~h}$ time point, followed by an increase in $\mathrm{IL}-10$ concentrations at $12-24 \mathrm{~h}$ time points (the latter effect is associated with a decrease in TNF- $\alpha$ levels). We also demonstrated that although the IL-10 concentration continuously declines, TNF- $\alpha$ levels rise again during the 72-96 h time points, consistent with the time when LPS treatment markedly inhibits efferocytosis. It is possible that LPS at later time points may still be present and active in culture media and/or LPS could be stimulating the production of secondary mediators that trigger production of TNF- $\alpha$ once IL -10 levels subside. Importantly, we demonstrated that endogenous IL-10 plays a protective role on 


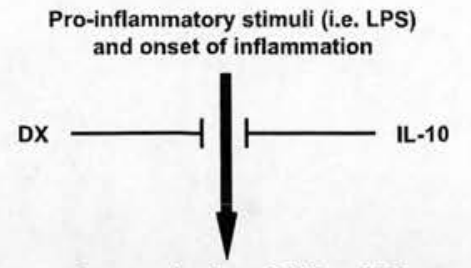

Overproduction of TNF- $\alpha$ shifting cytokine balance towards pro-inflammatory mediators

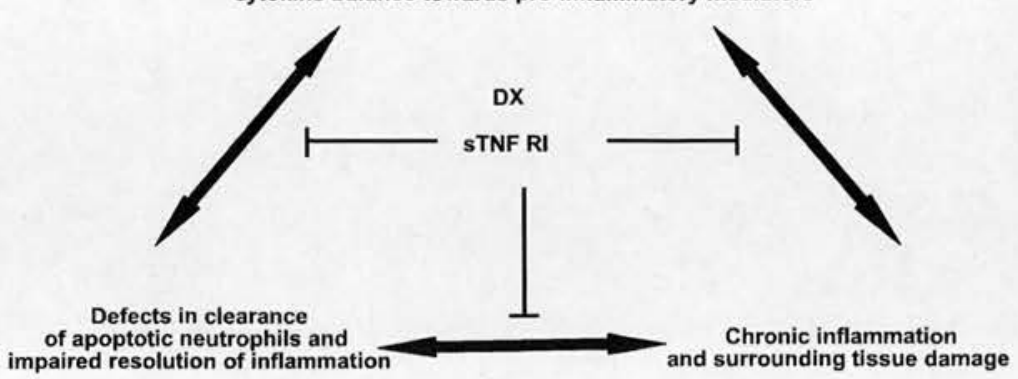

Figure 8. Glucocorticoid steroids, $\Pi-10$, and TNF- $\alpha$ inhibitors exert their powerful anti-inflammatory properties in part by influencing the TNF- $\alpha$ pathway. Overproduction of TNF- $\alpha$ at inflamed sites and subsequent inhibition of efferocytosis of neutrophils may have harmful consequences such as a higher rate of secondary necrosis, damage of surrounding tissues, and development of chronic inflammation. A decrease in local TNF- $\alpha$ concentrations or inhibition of its activity may prevent development of chronic inflammatory conditions such as rheumatoid arthritis. In our study, dexamethasone mimics the action of the anti-inflammatory cytokine $\mathrm{IL}-10$ by inhibiting the production of TNF- $\alpha$ in MDM $\varphi$ s challenged with proinflammatory stimuli. In addition, dexamethasone augments phagocytosis in MDM 4 exposed to TNF- $\alpha$. sTNF-RI and possibly other TNF- $\alpha$ inhibitors rescue efferocytosis by binding extracellular TNF- $\alpha$ and thus decreasing its biological activity. phagocytosis by blocking the biological action of IL-10 using the anti-human IL-10 R $\alpha$ antibody and achieving the LPS inhibitory effect much earlier. Experiments with $\mathrm{IL}-10^{-/-}$ animals further support the importance of endogenous IL-10 in maintaining high levels of phagocytosis in control and proinflammatory conditions. There are a number of other reports showing that IL-10 can exert opposite effects to LPS. For example, LPS-induced survival of neutrophils is reversed by $\mathrm{IL}-10$ through a mechanism that involves inhibition of extracellular regulated kinase activation (37). We also report that $\mathrm{IL}-10$ enhances $\mathrm{MDM} \varphi$ efferocytosis and, to a smaller extent, reverses inhibitory effects of TNF- $\alpha$ on phagocytosis. Therefore, we conclude that the presence of IL-10 in culture medium (especially in view of its inhibitory effects on TNF- $\alpha$ production) may at least in part explain the significant delay of LPS-induced inhibition.

We therefore conclude that the MDM $\varphi$ efferocytosis is dramatically regulated by their environment. After exposure to proinflammatory stimuli, at the onset of inflammation, the balance between cytokines shifts toward proinflammatory mediators (e.g., TNF- $\alpha$ ). However, during the resolution phase the balance of cytokines shifts toward anti-inflammatory mediators (e.g., IL-10), thereby augmenting efferocytosis and facilitating resolution of inflammation. Because $\mathrm{IL}-10$ is a very powerful inhibitor of proinflammatory cytokine production $(38,39)$, defects in either IL-10 secretion or function may represent a critical factor in development of inflammatory diseases. It is also noteworthy that uptake of various phagocytic targets may alter cytokine production in $\mathrm{M} \varphi s$ and consequently influence their environment in an autocrine fashion. Phagocytosis of apoptotic cells actively inhibits, whereas phagocytosis of IgG-opsonized cells induces production of proinflammatory cytokines such as TNF- $\alpha$, IL-1 $1 \beta$, or IL- 8 in both control or LPS-treated M $\varphi s(40)$. It has been also reported that apoptotic cells may have therapeutic potential in LPS-induced septic shock by reducing release of proinflammatory cytokines and enhancing II-10 production (41).

The other successful approach that we used to rescue efferocytosis of neutrophils by $\mathrm{MDM} \varphi \mathrm{s}$ exposed to proin- flammatory stimuli was treatment with the synthetic glucocorticosteroid dexamethasone. This anti-inflammatory agent was reported to augment, in a concentration- and time-dependent manner, $\mathrm{M} \varphi$ efferocytosis (23) as well as inhibit production of proinflammatory cytokines in $\mathrm{M} \varphi s$ (28). Dexamethasone, like IL-10, may regulate the production of TNF- $\alpha$ by influencing key signaling pathways such as NF-кB and MAPK kinase activation. However, in comparison to $\mathrm{IL}-10$, dexamethasone is a much more powerful suppressor of TNF- $\alpha$-induced inhibition of phagocytosis. Because glucocorticoid treatment neither completely inhibits TNF- $\alpha$ production nor elevates IL-10 release in LPS-treated MDM $\varphi$ s (see Fig. $7 B$ ), its effect seems to be more complex and likely involves modulation of other pathways. Our findings provide further insight into anti-inflammatory properties of glucocorticoids and may explain, in part, their efficacy in treatment of chronic inflammatory diseases.

In summary, we have determined that prolonged proinflammatory challenge may affect resolution of inflammatory responses via inhibition of $\mathrm{M} \varphi$ efferocytosis. We have shown that TNF- $\alpha$ is a key mediator in this process and that IL-10 exerts an important regulatory effect on TNF- $\alpha$ production and consequently on efferocytosis. As summarized in Fig. 8, data presented here indicate the significance of cytokine regulation in the resolution of inflammation as well as TNF- $\alpha$ in the development of chronic inflammation. Furthermore, we found several approaches to successfully decrease TNF- $\alpha$ production and activity, and consequently reverse LPS-mediated inhibition of efferocytosis. These findings provide important mechanistic information into the mode of action of steroids and anti-TNF- $\alpha$ agents and may help to explain their clinical success in treatment of chronic inflammatory diseases.

We thank Stelios Bournazos (University of Edinburgh, Edinburgh, UK) for providing immune complexes, anti-human IgG antibody, and useful comments, and Prof. David Gray (University of Edinburgh) for supplying the $\mathrm{IL}-10^{-1-}$ and wt mouse femurs. 


\section{REFERENCES}

1. Nathan, C. (2002) Points of control in inflammation. Nature 420, $846-852$

2. Serhan, C. N., Brain, S. D., Buckley, C. D., Gilroy, D. W., Haslett, C., O'Neill, L. A., Perretti, M., Rossi, A. G., and Wallace, J. L. (2007) Resolution of inflammation: state of the art, definitions and terms. FASEB J. 21, 325-332

3. Gilroy, D. W., Lawrence, T., Perretti, M., and Rossi, A. G. (2004) Inflammatory resolution: new opportunities for drug discovery. Nat. Rev. Drug Discov. 3, 401-416

4. Whyte, M. K., Meagher, L. C., MacDermot, J., and Haslett, C. (1993) Impairment of function in aging neutrophils is associated with apoptosis. J. Immunol. 150, 5124-5134

5. Savill, J. S., Wyllie, A. H., Henson, J. E., Walport, M. J., Henson, P. M., and Haslett, C. (1989) Macrophage phagocytosis of aging neutrophils in inflammation. Programmed cell death in the neutrophil leads to its recognition by macrophages. J. Clin. Invest. 83, 865-875

6. Serhan, C. N., and Savill, J. (2005) Resolution of inflammation: the beginning programs the end. Nat. Immunol. 6, 1191-1197

7. Fadok, V. A., Bratton, D. L., Konowal, A., Freed, P. W., Westcott, J. Y., and Henson, P. M. (1998) Macrophages that have ingested apoptotic cells in vitro inhibit proinflammatory cytokine production through autocrine/paracrine mechanisms involving TGF-beta, PGE2, and PAF. J. Clin. Invest. 101, 890-898

8. Feldmann, M., Brennan, F. M., Williams, R. O., Elliott, M. J., and Maini, R. N. (1995) Cytokine expression and networks in rheumatoid arthritis: rationale for anti-TNF alpha antibody therapy and its mechanism of action. J. Inflamm. 47, 90-96

9. Muller, J. M., Ziegler-Heitbrock, H. W., and Baeuerle, P. A. (1993) Nuclear factor kappa B, a mediator of lipopolysaccharide effects. Immunobiology 187, 233-256

10. Brennan, F. M., Chantry, D., Jackson, A., Maini, R., and Feldmann, M. (1989) Inhibitory effect of TNF alpha antibodies on synovial cell interleukin-1 production in rheumatoid arthritis. Lancet 2, 244-247

11. Feldmann, M., Brennan, F. M., Foxwell, B. M., Taylor, P. C., Williams, R. O., and Maini, R. N. (2005) Anti-TNF therapy: where have we got to in 2005? J. Autoimmun. 25(Suppl.), 26-28

12. Charles, P., Elliott, M. J., Davis, D., Potter, A., Kalden, J. R., Antoni, C., Breedveld, F. C., Smolen, J. S., Eberl, G., deWoody, K., Feldmann, M., and Maini, R. N. (1999) Regulation of cytokines, cytokine inhibitors, and acute-phase proteins following anti-TNFalpha therapy in rheumatoid arthritis. J. Immunol. 163, 1521-1528

13. Lee, A., Whyte, M. K., and Haslett, C. (1993) Inhibition of apoptosis and prolongation of neutrophil functional longevity by inflammatory mediators. J. Leukoc. Biol. 54, 283-288

14. Ward, C., Chilvers, E. R., Lawson, M. F., Pryde, J. G., Fujihara, S., Farrow, S. N., Haslett, C., and Rossi, A. G. (1999) NF-kappaB activation is a critical regulator of human granulocyte apoptosis in vitro. J. Biol. Chem. 274, 4309-4318

15. Rossi, A. G., Sawatzky, D. A., Walker, A., Ward, C., Sheldrake, T. A., Riley, N. A., Caldicott, A., Martinez-Losa, M., Walker, T. R., Duffin, R., Gray, M., Crescenzi, E., Martin, M. C., Brady, H. J., Savill, J. S., Dransfield, I., and Haslett, C. (2006) Cyclin-dependent kinase inhibitors enhance the resolution of inflammation by promoting inflammatory cell apoptosis. Nat. Med. 12, 1056-1064

16. Murray, J., Barbara, J. A., Dunkley, S. A., Lopez, A. F., VanOstade, X., Condliffe, A. M., Dransfield, I., Haslett, C., and Chilvers, E. R. (1997) Regulation of neutrophil apoptosis by tumor necrosis factor-alpha: requirement for TNFR55 and TNFR75 for induction of apoptosis in vitro. Blood $\mathbf{9 0 , 2 7 7 2 - 2 7 8 3}$

17. Ren, Y., and Savill, J. (1995) Proinflammatory cytokines potentiate thrombospondin-mediated phagocytosis of neutrophils undergoing apoptosis. J. Immunol. 154, 2366-2374

18. McPhillips, K., Janssen, W. J., Ghosh, M., Byrne, A., Gardai, S., Remigio, L., Bratton, D. L., Kang, J. L., and Henson, P. (2007) TNF-alpha inhibits macrophage clearance of apoptotic cells via cytosolic phospholipase A2 and oxidant-dependent mechanisms. J. Immunol. 178, 8117-8126

19. Kuhn, R., Lohler, J., Rennick, D., Rajewsky, K., and Muller, W. (1993) Interleukin-10-deficient mice develop chronic enterocolitis. Cell 75, 263-274

20. Jersmann, H. P., Ross, K. A., Vivers, S., Brown, S. B., Haslett, C., and Dransfield, I. (2003) Phagocytosis of apoptotic cells by human macrophages: analysis by multiparameter flow cytometry. Cytometry A 51, 7-15
21. Rossi, A. G., McCutcheon, J. C., Roy, N., Chilvers, E. R., Haslett, C., and Dransfield, I. (1998) Regulation of macrophage phagocytosis of apoptotic cells by cAMP. J. Immunol. 160, 3562-3568

22. Hart, S. P., Alexander, K. M., and Dransfield, I. (2004) Immune complexes bind preferentially to Fc gamma RIIA (CD32) on apoptotic neutrophils, leading to augmented phagocytosis by macrophages and release of proinflammatory cytokines. J. Immunol. 172, 1882-1887

23. Liu, Y., Cousin, J. M., Hughes, J., Van, D. J., Seckl, J. R., Haslett, C., Dransfield, I., Savill, J., and Rossi, A. G. (1999) Glucocorticoids promote nonphlogistic phagocytosis of apoptotic leukocytes. J. Immunol. 162, 3639-3646

24. McColl, A., Michlewska, S., Dransfield, I., and Rossi, A. G. (2007) Effects of glucocorticoids on apoptosis and clearance of apoptotic cells. Sci. World J. 7, 1165-1181

25. Fan, H., Teti, G., Ashton, S., Guyton, K., Tempel, G. E., Halushka, P. V., and Cook, J. A. (2003) Involvement of G(i) proteins and Src tyrosine kinase in TNFalpha production induced by lipopolysaccharide, group B streptococci and Staphylococcus aureus. Cytokine 22, 126-133

26. Aggarwal, B. B., and Natarajan, K. (1996) Tumor necrosis factors: developments during the last decade. Eur. Cytokine Netw. 7, 93-124

27. Xu, W., Roos, A., Schlagwein, N., Woltman, A. M., Daha, M. R., and van Kooten, C. (2006) IL-10-producing macrophages preferentially clear early apoptotic cells. Blood 107, 4930-4937

28. Barnes, P. J. (1998) Anti-inflammatory actions of glucocorticoids: molecular mechanisms. Clin. Sci. (Lond.) 94, 557-572

29. Michlewska, S., McColl, A., Rossi, A. G., Megson, I. L., and Dransfield, I. (2007) Clearance of dying cells and autoimmunity. Autoimmunity 40, 267-273

30. Feldmann, M., Brennan, F. M., Elliott, M., Katsikis, P., and Maini, R. N. (1994) TNF alpha as a therapeutic target in rheumatoid arthritis. Circ. Shock 43, 179-184

31. Maini, R. N., Elliott, M. J., Brennan, F. M., and Feldmann, M. (1995) Beneficial effects of tumour necrosis factor-alpha (TNF-alpha) blockade in rheumatoid arthritis (RA). Clin. Exp. Immunol. 101, 207-212

32. Clark, I. A. (2007) How TNF was recognized as a key mechanism of disease. Cytokine Growth Factor Rev. 18, 335-343

33. Feldmann, M., and Maini, R. N. (2003) Lasker Clinical Medical Research Award. TNF defined as a therapeutic target for rheumatoid arthritis and other autoimmune diseases. Nat. Med. 9, 1245-1250

34. Mazzon, E., and Cuzzocrea, S. (2008) Role of TNF-\{alpha\} in ileum tight junction alteration in mouse model of restraint stress. Am. J. Physiol. Gastrointest. Liver Physiol. 294, 1268-1280

35. De Waal, M. R., Abrams, J., Bennett, B., Figdor, C. G., and de Vries, J. E. (1991) Interleukin 10 (IL-10) inhibits cytokine synthesis by human monocytes: an autoregulatory role of IL-10 produced by monocytes. J. Exp. Med. 174, 1209-1220

36. Ogden, C. A., Pound, J. D., Batth, B. K., Owens, S., Johannessen, I., Wood, K., and Gregory, C. D. (2005) Enhanced apoptotic cell clearance capacity and B cell survival factor production by IL-10-activated macrophages: implications for Burkitt's lymphoma. J. Immunol. 174, 3015-3023

37. Ward, C., Murray, J., Clugston, A., Dransfield, I., Haslett, C., and Rossi, A. G. (2005) Interleukin-10 inhibits lipopolysaccharideinduced survival and extracellular signal-regulated kinase activation in human neutrophils. Eur. J. Immunol. 35, 2728-2737

38. Moore, K. W., Vieira, P., Fiorentino, D. F., Trounstine, M. L., Khan, T. A., and Mosmann, T. R. (1990) Homology of cytokine synthesis inhibitory factor (IL-10) to the Epstein-Barr virus gene BCRFI. Science 248, 1230-1234

39. Denys, A., Udalova, I. A., Smith, C., Williams, L. M., Ciesielski, C. J., Campbell, J., Andrews, C., Kwaitkowski, D., and Foxwell, B. M. (2002) Evidence for a dual mechanism for IL-10 suppression of TNF-alpha production that does not involve inhibition of p38 mitogen-activated protein kinase or NF-kappa B in primary human macrophages. J. Immunol. 168, 4837-4845

40. Voll, R. E., Herrmann, M., Roth, E. A., Stach, C., Kalden, J. R., and Girkontaite, I. (1997) Immunosuppressive effects of apoptotic cells. Nature 390, 350-351

41. Ren, Y., Xie, Y., Jiang, G., Fan, J., Yeung, J., Li, W., Tam, P. K., and Savill, J. (2008) Apoptotic cells protect mice against lipopolysaccharide-induced shock. J. Immunol. 180, 4978-4985

Received for publication September 11, 2008. Accepted for publication October 9, 2008. 


\title{
Journal of Inflammation
}

Research

\section{Cyclic GMP protects human macrophages against peroxynitrite-induced apoptosis Catherine A Shaw*1, David J Webb ${ }^{1}$, Adriano G Rossi² and Ian L Megson ${ }^{3}$}

\author{
Address: ${ }^{1}$ Centre for Cardiovascular Science, The Queen's Medical Research Institute, University of Edinburgh, Edinburgh, UK, ${ }^{2} \mathrm{MRC}$ Centre for \\ Inflammation Research, The Queen's Medical Research Institute, University of Edinburgh, Edinburgh, UK and ${ }^{3}$ Free Radical Research Facility, \\ Department of Diabetes and Cardiovascular Science, UHI Millennium Institute, Inverness, UK \\ Email: Catherine A Shaw* - catherine.shaw@ed.ac.uk; David J Webb - D.J.Webb@ed.ac.uk; Adriano G Rossi - a.g.rossi@ed.ac.uk; \\ Ian L Megson - Ian.Megson@uhi.ac.uk \\ - Corresponding author
}

This article is available from: http://www.journal-inflammation.com/content/6/1/14

(ㄷ) 2009 Shaw et al; licensee BioMed Central Ltd.

This is an Open Access article distributed under the terms of the Creative Commons Attribution License (http://creativecommons.org/licenses/by/2.0), which permits unrestricted use, distribution, and reproduction in any medium, provided the original work is properly cited.

\begin{abstract}
Background: Nitric oxide (NO) can be both pro- and anti-apoptotic in various cell types, including macrophages. This apparent paradox may result from the actions of NO-related species generated in the microenvironment of the cell, for example the formation of peroxynitrite (ONOO-). In this study we have examined the ability of $\mathrm{NO}$ and $\mathrm{ONOO}$ - to evoke apoptosis in human monocyte-derived macrophages (MDM $\varphi$ ), and investigated whether preconditioning by cyclic guanosine monophosphate (cGMP) is able to limit apoptosis in this cell type.
\end{abstract}

Methods: Characterisation of the NO-related species generated by (Z)-I- [2-(2-aminoethyl)-N(2-ammonioethyl)amino]diazen-1-ium-1,2-diolate (DETA/NO) and 1,2,3,4-oxatriazolium, 5-amino3-(3,4-dichlorophenyl)-, chloride (GEA-3|62) was performed by electrochemistry using an isolated NO electrode and electron paramagnetic resonance (EPR) spectrometry. Mononuclear cells were isolated from peripheral blood of healthy volunteers and cultured to allow differentiation into MDM $\varphi$. Resultant MDM $\varphi$ were treated for $24 \mathrm{~h}$ with DETA/NO $(100-1000 \mu \mathrm{M})$ or GEA-3162 $(10-300 \mu \mathrm{M})$ in the presence or absence of BAY 4I-2272 $(I \mu \mathrm{M})$, isobutylmethylxanthine (IBMX; I $\mu \mathrm{M})$, IH- [1,2,4]oxadiazolo [4,3-a]quinoxalin-I-one (ODQ; $20 \mu \mathrm{M})$ or 8-bromo-cGMP (I mM). Apoptosis in MDM $\varphi$ was assessed by flow cytometric analysis of annexin $\mathrm{V}$ binding in combination with propidium iodide staining.

Results: Electrochemistry and EPR revealed that DETA/NO liberated free NO radical, whilst GEA-3162 concomitantly released $\mathrm{NO}$ and $\mathrm{O}_{2}{ }^{\circ}$, and is therefore a ONOO-generator. $\mathrm{NO}$ (DETA/ NO) had no effect on cell viability, but ONOO- (GEA-3I62) caused a concentration-dependent induction of apoptosis in MDM $\varphi$. Preconditioning of MDM $\varphi$ with NO in combination with the phosphodiesterase inhibitor, 3-Isobutyl-I-methylxanthine (IBMX), or the NO-independent stimulator of soluble guanylate cyclase, BAY 4I-2272, significantly attenuated ONOO-induced apoptosis in a CGMP-dependent manner.

Conclusion: These results demonstrate disparities between the ability of NO and ONOO- to induce apoptosis in human MDM $\varphi$. Furthermore, this study provides evidence for a novel cGMPdependent pre-conditioning mechanism to limit ONOO-induced apoptosis in human MDM $\varphi$. 


\section{Background}

Apoptosis is a highly regulated and fundamental biological process governing cell survival. During apoptosis, the integrity of the cell membrane is maintained, therefore preventing release of the histotoxic cell contents. Because apoptotic cells are instantly recognised by phagocytes and removed from the inflammatory site, successful apoptosis is now recognised to be crucial to the resolution of inflammation. Failure of inflammatory cells to undergo apoptosis, or failure of subsequent phagocytic removal of apoptotic cells is believed to result in incomplete resolution and an exacerbation of the inflammatory response [1-4]. Thus, apoptosis is a non-inflammatory mechanism for the removal of inflammatory cells from a site of tissue damage.

The propensity of a cell to undergo apoptosis is determined by the net balance of many pro- and anti-apoptotic exogenous and endogenous factors [5-7]. The signalling molecule, nitric oxide (NO), has previously been reported to induce apoptosis in various cell types, including macrophages [8-12]. However, the role of NO in apoptosis is complicated by a number of reports indicating that it can be both pro- and anti-apoptotic [13-15]. The generally accepted paradigm is that lower NO concentrations produced constitutively by endothelial NO synthase (eNOS) and neuronal NO synthase ( $\mathrm{nNOS}$ ) are cytoprotective via primarily cGMP-dependent mechanisms, whilst higher, supraphysiological concentrations generated in some pathologies by the inducible form of NOS (iNOS) mediate apoptosis via mechanisms independent of cGMP signalling [16].

This apparent paradox may be explained, at least in part, by the production of intermediary NO-related species, with the ultimate outcome of any NO-mediated response being dependent on the precise NO-related species formed in the microenvironment, as well as the cell type in question. In biological systems NO often reacts with superoxide anions $\left(\mathrm{O}_{2}{ }^{-}\right)$, resulting in the formation of the powerful oxidising agent, peroxynitrite (ONOO-) [17-20]. ONOO- has been shown to induce apoptosis in human inflammatory cells, such as neutrophils $[21,22]$.

Disparities between the sensitivity of different cell types to apoptosis induced by NO (or NO-related species), suggests the presence of protective mechanisms in those cell types resistant to NO-evoked apoptosis. Such protective mechanisms may depend on the anti-apoptotic qualities of NO itself. Indeed, non-toxic concentrations of NO, and agents that act to elevate CGMP independently of NO, have been demonstrated to protect rodent macrophages and vascular smooth muscle cells (VSMC) against subsequent NO-induced cell death [23-26].
Here, we test the hypothesis that the NO-related species, ONOO, but not NO, induces apoptosis in human macrophages derived from the monocyte population of peripheral blood. Furthermore, we investigate whether low concentrations of NO, and the novel NO-independent stimulator of soluble guanylate cyclase, BAY 41-2272, are able to precondition human macrophages against subsequent $\mathrm{ONOO}$-induced apoptosis.

\section{Methods}

\section{Electrochemical Detection of NO}

NO radical released in Iscove's modified Dulbecco's tissue culture medium (IMDM; Gibco Life Technologies, UK) from (Z)-1- [2-(2-aminoethyl)- $\mathrm{N}$-(2-ammonioethyl)amino]diazen-1-ium-1,2-diolate (DETA/NO; Axxora Ltd, UK) and 1,2,3,4-oxatriazolium, 5-amino-3-(3,4dichlorophenyl)-, chloride (GEA-3162; Axxora Ltd, UK) was measured by an isolated NO electrode (Iso-NO II, World Precision Instruments, UK). NO production by DETA/NO $(300 \mu \mathrm{M})$ was recorded for $30 \mathrm{~min}$.

Superoxide dismutase (SOD; 50-500 U.ml-1; SigmaAldrich, UK) was added cumulatively in stepwise increments to unmask NO produced by GEA-3162 $(300 \mu \mathrm{M})$ [21]. Each bolus addition of SOD was added to the electrode chamber once the signal from the previous addition of SOD had reached a plateau, usually after approximately 3-5 min. Alternatively, GEA-3162 and $500 \mathrm{U} \cdot \mathrm{ml}^{-1}$ SOD were introduced simultaneously to the electrode chamber and the signal recorded until it decayed to baseline. Finally, because the structurally similar compound, SIN-1, can generate $\mathrm{ONOO}^{-}$in vitro, but NO in the presence of biological tissues [27], we investigated whether GEA-3162 could generate NO in the presence of a suspension of monocyte-derived macrophages $\left(1 \times 10^{6}\right.$ cells $\left./ \mathrm{ml}\right)$.

The NO scavenger, haemoglobin ( $\mathrm{Hb} ; 5 \mu \mathrm{M})$ [28], was introduced to the electrode chamber to confirm NO generation in experiments where the signal had not decayed to baseline at the end of the incubation period.

\section{Electron Paramagnetic Resonance Studies}

The concentration of oxidising free radical species generated by GEA-3162 in the presence or absence of SOD was assessed in IMDM tissue culture medium by electron paramagnetic resonance (EPR) spectrometry (electron spin resonance). GEA-3162 (10-300 $\mu \mathrm{M})$ was incubated for 30 $\mathrm{min}$ at $37^{\circ} \mathrm{C}$ in the presence or absence of SOD (500 U. $\mathrm{ml}^{-1}$ ) plus the chemical spin trap Tempone-H hydrochloride ( $1 \mathrm{mM}$; Axxora, UK; prepared in water containing EDTA $(10 \mathrm{mM})[29,30]$. The intensity of the EPR signals corresponding to the formation of the radical adduct, 4oxo-tempo (triplet centred around $3360 \mathrm{G}$ ), were recorded using a Miniscope MS100 X-band spectrometer 
(Magnettech, Germany) with the following parameter settings: field sweep $51.2 \mathrm{G}$, microwave frequency $9.5 \mathrm{GHz}$, microwave power $20 \mathrm{~mW}$, modulation amplitude 1500 $\mathrm{mG}$. Control experiments, consisting of tempone-H in IMDM in the absence of radical-generating compounds, were found to generate small signals due to the auto-oxidation of tempone-H to 4-oxo-tempo; these control signals were subtracted from the corresponding experimental signals. There experiments were repeated with GEA-3162 which had undergone spontaneous decomposition at $37^{\circ} \mathrm{C}$ for 7 days.

\section{Cell Isolation and Culture}

Mononuclear cells (MNC) and polymorphonuclear cells were isolated from human blood as previously described $[31,32]$. Briefly, whole, citrated blood was centrifuged $(200 \times g ; 20 \mathrm{~min})$ and platelet-rich plasma (PRP) aspirated. Leukocytes were separated from erythrocytes by dextran sedimentation, then further divided into MNC and granulocyte populations by centrifugation through a discontinuous Percoll ${ }^{\star}$ (Pharamcia, UK) gradient $(720 \times 8$; $20 \mathrm{~min}$ ). MNC were harvested at the 55:68\% interface and re-suspended at a density of $4 \times 10^{6}$ cells/ $\mathrm{ml}$ in IMDM supplemented with penicillin and streptomycin (both 100 U. $\mathrm{ml}^{-1}$ ), prior to enrichment for monocytes by selective adherence to 48 -well $\left(2 \times 10^{6}\right.$ cells/well $)$ tissue culture plates for $1 \mathrm{~h}$ at $37^{\circ} \mathrm{C}$ in $5 \% \mathrm{CO}_{2}$. Adherent monocytes were washed three times in phosphate buffered saline (PBS) and then allowed to differentiate into MDM $\varphi$ for 5 days $\left(37^{\circ} \mathrm{C} ; 5 \% \mathrm{CO}_{2}\right)$ in IMDM containing penicillin/ streptomycin and $10 \%$ autologous serum prepared by recalcification of PRP. Medium was replaced every 2-3 days throughout the differentiation period.

\section{Experimental Protocols}

In order to investigate the effect of NO radical and ONOO on cell viability, MDM $\varphi$ were treated for $24 \mathrm{~h}$ with the NO donor, DETA/NO (100-300 $\mu \mathrm{M})$, the ONOO-generator, GEA-3162 (10-300 $\mu \mathrm{M})$, or vehicle control (DMSO; $1 \%$ ) prior to assessment of apoptosis and necrosis by flow cytometry as described below. The experiments were repeated for GEA-3162 in the presence of the anti-oxidant enzymes SOD, or a combination of SOD plus catalase (both $500 \mathrm{U} . \mathrm{ml}^{-1}$ ). In order to investigate the actions of the breakdown products of the compound, additional experiments were conducted with GEA-3162 which had been allowed to decompose at $37^{\circ} \mathrm{C}$ for 7 days. The known pro-apoptotic agent, gliotoxin $\left(1 \mu \mathrm{g} \cdot \mathrm{ml}^{-1}\right.$; Sigma Aldrich, UK) was used as a positive control for the assay [33-35].

To investigate the role of NO:cGMP signalling in ONOO-induced apoptosis, MDM $\varphi$ were pre-treated for $24 \mathrm{~h}$ with DETA/NO $(10 \mu \mathrm{M})$ plus either the NO-independent sGC stimulator, BAY 41-2272 (1 $\mu \mathrm{M}$; Bayer, Germany; [36], or the non-specific phosphodiesterase (PDE) inhibitor, 3- isobutyl-1-methylxanthine (IBMX; $1 \mu \mathrm{M}$; Axxora, UK). BAY 41-2272 was used in combination with DETA/NO $(10 \mu \mathrm{M})$ because recent evidence has suggested that, rather than acting via direct stimulation of sGC, the actions of this compound are a result of the synergistic effects of inhibition of PDE V coupled with sensitisation of sGC toward endogenous NO $[37,38]$. As IBMX also raises intracellular cGMP by inhibiting its breakdown, DETA/NO is used to provide the initial stimulus for CGMP production because human macrophages are unlikely to generate enough endogenous NO to result in cellular cGMP production. The ability of the NO:cGMP pathway to inhibit ONOO-dependent apoptosis was further investigated by pre-treating $\mathrm{MDM} \varphi$ for $24 \mathrm{~h}$ with the cell permeable cGMP analogue, 8-bromo-cGMP (1 $\mathrm{mM})$, or a higher concentration of DETA/NO $(300 \mu \mathrm{M})$ in the presence or absence of the SGC inhibitor, $1-H-[1,2,4]$ oxadiazolo [4,3-a]quinoxalin-1-one (ODQ; $20 \mu \mathrm{M}$ ).

Following this pre-treatment period, cells were washed twice in PBS to remove pre-treatment compounds prior to incubation for a further $24 \mathrm{~h}$ in the presence of the ONOO-generator, GEA-3162 $(100 \mu \mathrm{M})$. At this concentration, GEA-3162 induces significant apoptosis in $\operatorname{MDM} \varphi$ without causing necrosis. Finally, a set of cells were treated for the final $24 \mathrm{~h}$ period only with the apoptotic agent, gliotoxin $\left(1 \mu \mathrm{g} \cdot \mathrm{ml}^{-1}\right)$, which serves as a positive control for the assay.

\section{Flow Cytometric Analysis of Annexin V Binding and Propidium lodide Staining}

Cell death was assessed by flow cytometric detection of fluorescein isothiocyanate (FITC)-conjugated annexin V binding to phosphatiylserine (PS) exposed on the surface of apoptotic cells, or propidium iodide (PI) staining of late apoptotic/necrotic cells. Following the experimental protocol detailed above, cells were removed from tissue culture plates ( $0.25 \%$ trypsin with EDTA) and recovered cell suspensions incubated for $10 \mathrm{~min}$ on ice in the presence of FITC-annexin V in annexin V-binding buffer (prepared as a 1:500 dilution of annexin V in Hank's balanced salt solution (HBSS) containing $5 \mathrm{mM} \mathrm{CaCl}_{2}$ ). Following this incubation period, PI (final concentration $2 \mu \mathrm{g} \cdot \mathrm{ml}^{-1}$ ) was added to the cell suspension/annexin $\mathrm{V}$ binding buffer mix for $1 \mathrm{~min}$ prior to analysis by Coulter EPICS XI flow cytometer (Beckman Coulter, USA) equipped with EXPO $^{\text {TM }} 32$ data analysis software.

\section{Quantification of cGMP}

Levels of CGMP produced by MDM $\varphi$ following incubation with DETA/NO in the absence and presence of BAY 412272 or IBMX were measured by enzyme-linked immunosorbant assay (ELISA; R\&D Systems, UK) according to the manufacturer's instructions. Briefly, cell culture supernatants were aspirated and adherent cells lysed ( $2 \%$ Triton X $100)$. Recovered solutions were acidified $(0.1 \mathrm{M} \mathrm{HCl})$ and 
acetylated prior to performing the assay. Optical density $(405 \mathrm{~nm})$ was measured by a Multiskan Ascent plate reader and the concentration of CGMP was calculated from a standard curved produced from serial dilutions of acetylated cGMP solutions $(0.08-50$ pmol.ml-1). All standards and samples were assayed in duplicate.

\section{Statistics}

Data are expressed throughout as mean \pm SEM, with $n$ values as indicated. Statistical tests are described in figure legends and were performed using GraphPad Prism software version 3.03 (GraphPad Software Inc., San Diego, USA). Results were considered to be statistically significant when $P<0.05$.

\section{Results}

\section{NO Release from DETA/NO and GEA-3162}

DETA/NO $(300 \mu \mathrm{M})$ generated a slow and prolonged NO release in IMDM that remained steady throughout a 30 min recording period. Introduction of the NO scavenger, haemoglobin $(\mathrm{Hb} ; 5 \mu \mathrm{M})$, to the electrode chamber successfully quenched the signal from the electrode, confirming NO release from DETA/NO (figure 1A).

In common with previous findings [21], GEA-3162 (300 $\mu \mathrm{M}$ ) failed to generate a detectable NO signal except in the presence of the superoxide scavenger, SOD (50 - 500 U. $\mathrm{ml}^{-1}$ ), when stepwise, incremental concentrations of NO were detectable. MDM $\varphi$ did not alter the inability of GEA-3162 to liberate NO in the absence of SOD, and did not alter concentration of NO released from GEA-3162 in the presence of SOD. Hb quenched the signal from GEA3162 (figure $1 B$ ). In the presence of SOD to unmask the NO released by GEA-3162, NO decayed from a max value of $2.2 \mu \mathrm{M}$ over a period of $\sim 4 \mathrm{~h}$ (figure $1 \mathrm{C}$ ).

\section{ONOO-Generation by GEA-3162}

GEA-3162 generated an EPR signal consistent with the production of the radical adduct, 4-oxo-tempo. SOD significantly attenuated the intensity of this signal by a maximum of $61 \%$ (figure 2). Incubation of GEA-3162 at $37^{\circ} \mathrm{C}$ for 7 days resulted in partial decomposition of the compound. The EPR signal from GEA-3162 treated in this way was diminished, but not completely abolished; the signal was reduced from an intensity of $6894 \pm 810(n=6)$ to $2939 \pm 650(n=3)$. This remaining signal was also quenched by SOD to $1443 \pm 290$.

\section{Quantification of cGMP}

Levels of cGMP were undetectable $\left(<0.08\right.$ pmol.ml-1 $\left.{ }^{-1}\right)$ in vehicle treated control MDM $\varphi$. Those cells that had been treated with DETA/NO plus BAY 41-2272 or IBMX generated 0.325 and 0.40 pmol.ml ${ }^{-1}$ cGMP per $1 \times 10^{6}$ cells respectively. Levels of CGMP generated by DETA/NO alone were below the limit of detection of the assay.

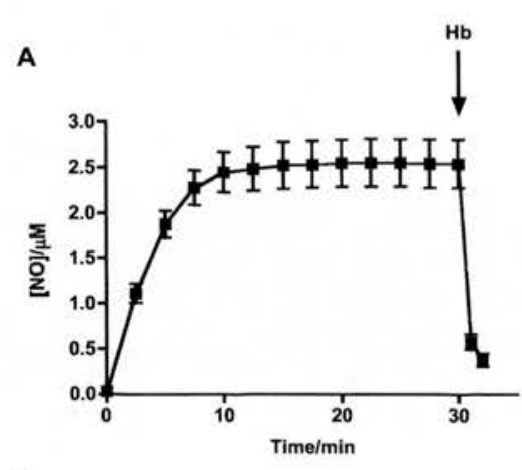

B
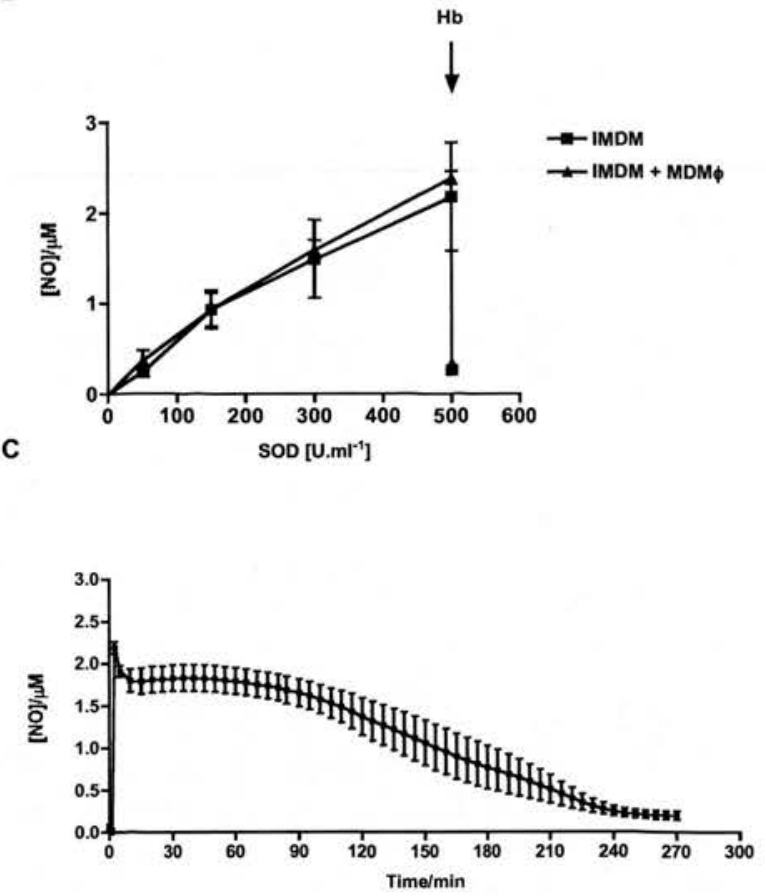

Figure I

NO generation by DETA/NO and GEA-3 162 measured by isolated NO electrode $A-N O$ liberated by DETANO $(300 \mu \mathrm{M})$ recorded for $30 \mathrm{~min}(n=6)$. B - GEA$3162(300 \mu \mathrm{M})$ failed to liberate free NO in solution except in the presence of SOD. Each addition of SOD is added approximately $3-5 \mathrm{~min}$ following the previous addition. The concentration of NO liberated in the presence of SOD was unchanged by $\operatorname{MDM} \varphi(n=3)$. Arrows indicate addition of $\mathrm{Hb}$. C - In the presence of $500 \mathrm{U} \cdot \mathrm{ml}^{-1}$ SOD, GEA-3I62 (300 $\mu \mathrm{M})$ liberated NO over a period of approximately $4 \mathrm{~h}(\mathrm{n}=$ 6).

Peroxynitrite, But Not NO, Induces Apoptosis in Human $\operatorname{MDM} \varphi$

The NO donor, DETA/NO, at concentrations of up to 1 $\mathrm{mM}$, had no affect on the levels of annexin $\mathrm{V}$ binding or PI staining in human $\mathrm{MDM} \varphi$. In contrast, the ONOO- 
A
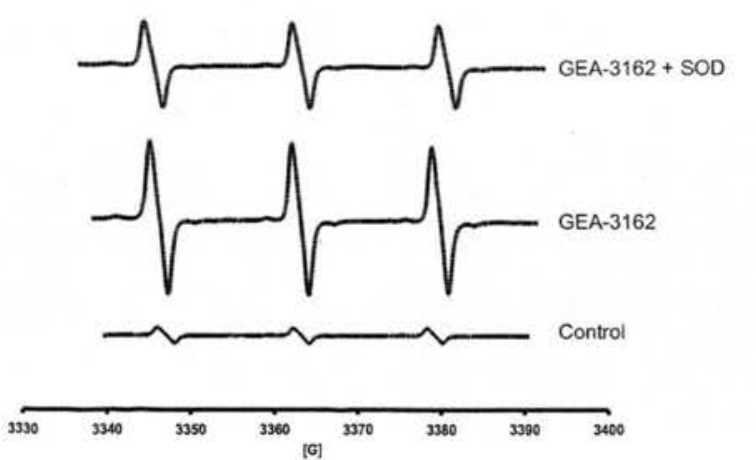

B

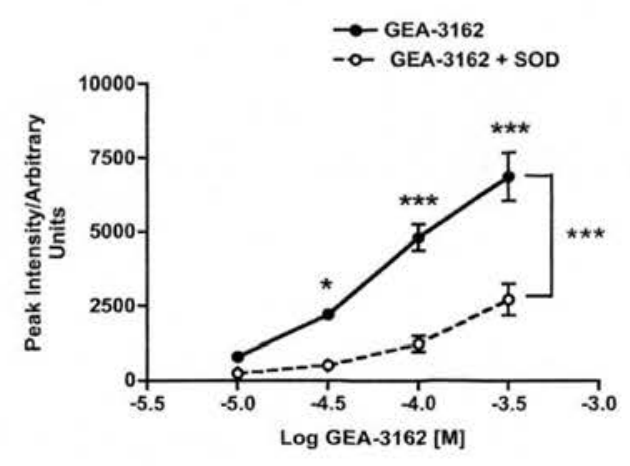

Figure 2

Oxidising radical generation by GEA-3 I62. A - representative EPR spectra generated by GEA-3162 (300 $\mu \mathrm{M})$ in the presence and absence of SOD (500 U. $\left.\mathrm{ml}^{-1}\right)$. B - SOD reduced the intensity of the GEA-3 I 62 EPR signal by a maximum of $61 \%$. $* p<0.05$, **k $P<0.001$ (Two-way ANOVA followed by post hoc Bonferroni's test; $n=6$ ).

generator, GEA-3162 (10-300 $\mu \mathrm{M})[21]$, caused a concentration-dependent induction of apoptosis in human $\operatorname{MDM} \varphi$, as demonstrated by an increase in annexin $\mathrm{V}$ binding (figure 3). At concentrations up to $100 \mu \mathrm{M}$, the increase in annexin $\mathrm{V}$ binding occurred in the absence of significant PI staining. However, at higher concentrations $(300 \mu \mathrm{M})$ a significant level of PI staining was also observed. Therefore, in order to specifically induce apoptosis, a concentration of $100 \mu \mathrm{M}$ GEA-3162 was selected for subsequent experiments. Whereas fresh GEA-3162 $(100 \mu \mathrm{M})$ induced a maximum of $61 \pm 7 \%(n=9)$ apoptosis, this was reduced to $39 \pm 9 \%(n=3)$ following treatment with partially decomposed GEA-3162.
The level of apoptosis (annexin V binding) induced by GEA-3162 (10-300 $\mu \mathrm{M})$ was further enhanced when $\operatorname{MDM} \varphi$ were treated with GEA-3162 in the presence of SOD (figure 3C). The additional elevation of apoptosis induced by SOD was suppressed when MDM $\varphi$ were treated with GEA-3162 in the presence of a combination of SOD plus catalase (figure $3 \mathrm{C}$ ). This combination did not, however, reduce overall levels of apoptosis induced by GEA-3162. Neither SOD nor catalase had any impact on the level of PI staining.

\section{cGMP Signalling Protects MDM $\varphi$ Against Peroxynitrite Induced Apoptosis}

Control sets of cells demonstrated that $24 \mathrm{~h}$ pre-treatment of MDM $\varphi$ with DETA/NO $(10 \mu \mathrm{M})$ in combination with BAY 41-2272 or IBMX (both $1 \mu \mathrm{M}$ ) had no effect on cell viability compared to untreated cells. Similarly, higher concentrations of DETA/NO $(300 \mu \mathrm{M})$ alone or in combination with the sGC inhibitor, ODQ $(20 \mu \mathrm{M})$, did not affect cell viability (figure 4A).

Treatment with GEA-3162 $(100 \mu \mathrm{M})$ alone induced apoptotic cell death (annexin V binding) in MDM $\varphi$. However, levels of apoptosis were significantly attenuated in those cells that had been subjected to previous $24 \mathrm{~h}$ pre-treatment with DETA/NO $(10 \mu \mathrm{M})$ in combination with BAY 41-2272 or IBMX ( $P<0.05$ for both treatments compared to GEA-3162 treated cells; figure 4). Pre-treatment with the cell permeable cGMP analogue, 8-bromo-cGMP, also significantly attenuated subsequent GEA-3162 induced apoptosis. Similarly, DETA/NO $(300 \mu \mathrm{M})$ afforded protection against GEA-3162 induced apoptosis and this protection was reversed in the presence of ODQ (figure 4).

\section{Discussion}

This study reveals disparities between the ability of NO radical and NO-related species to induce apoptosis in human macrophages. Our study highlights the opposing effects of ONOO- and NO is this setting and demonstrates that $\mathrm{ONOO}^{-}$, but not $\mathrm{NO}$ radical, induces apoptosis in human MDM $\varphi$. Furthermore, our data demonstrate for the first time that the pro-apoptotic effects of ONOO- are limited in human macrophages by pre-conditioning the cells with agents that act to elevate intracellular concentrations of cGMP.

During inflammation, neutrophilic leukocytes are recruited and activated during an initial wave of inflammatory cell recruitment. These inflammatory cells generate reactive oxygen and nitrogen species, which can combine to form ONOO- [39], thus resulting in a pro-oxidising environment within the inflammatory site. A second wave of cellular recruitment then results in an influx of monocytes that differentiate within the inflammatory 
A

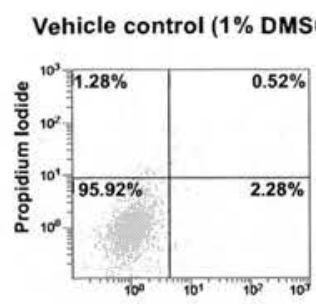

GEA-3162 (300 $\mu \mathrm{M})$

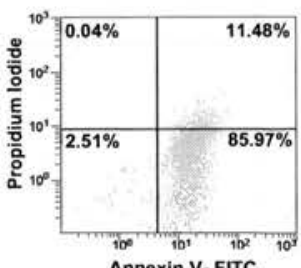

Annexin V. FITC
Gliotoxin $\left(1 \mu \mathrm{g} \cdot \mathrm{ml}^{-1}\right)$

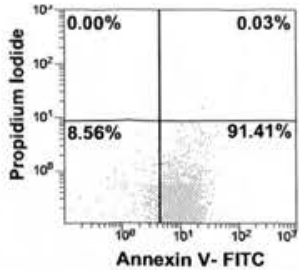

DETA/NO $(300 \mu \mathrm{M})$

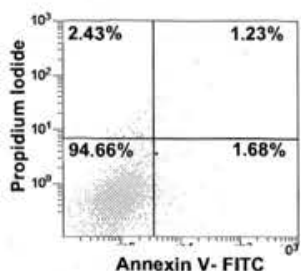

B

EOAnnexin V +ive

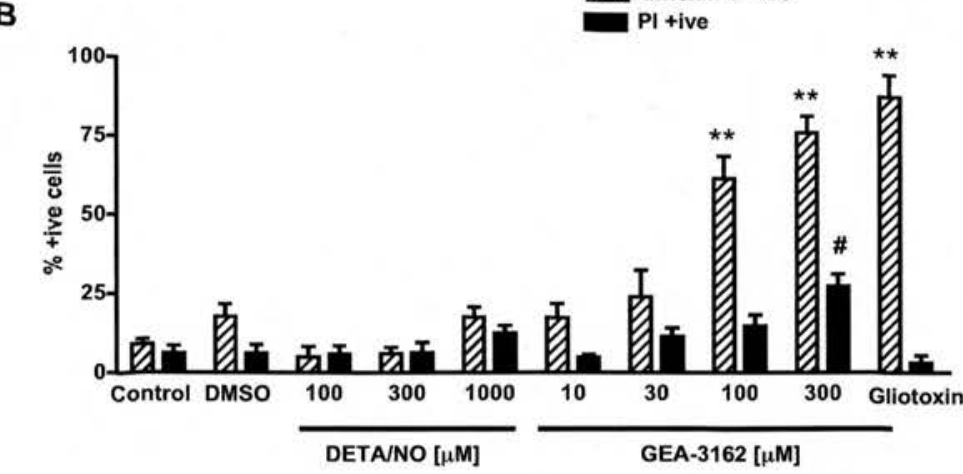

C
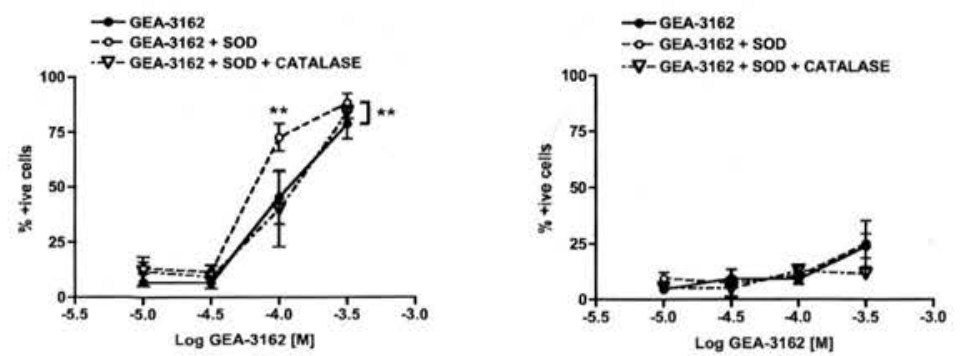

Figure 3

GEA-3162 induces apoptosis in human MDM $\varphi$. A - representative flow cytometry plots. B - induction of apoptosis by GEA-3162 but not DETA/NO. **P $<0.01$ significantly greater annexin $V$ binding compared to control; \#P $<0.05$ significantly greater PI staining compared to control (unpaired, one-way ANOVA followed by post hoc Dunnett's test; $n=6-9$ ). $C-$ apoptosis (annexin $V$ binding; left panel) induced by GEA-3162 was elevated by SOD $\left(500 \mathrm{U}^{-\mathrm{ml}^{-1}}\right)$. A combination of SOD and catalase (both $500 \mathrm{U} \cdot \mathrm{ml}^{-1}$ ) blunted the SOD-induced elevation of apoptosis but not reduce GEA-3162-induced apoptosis. PI staining (right panel) was unaffected by SOD or catalase. ${ }^{* * P}<0.01$; matched two ANOVA followed by post hoc Bonferroni's test; $\mathrm{n}=6$. 
A

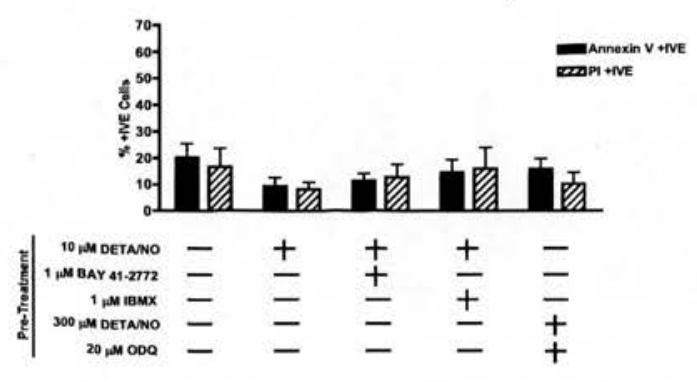

B

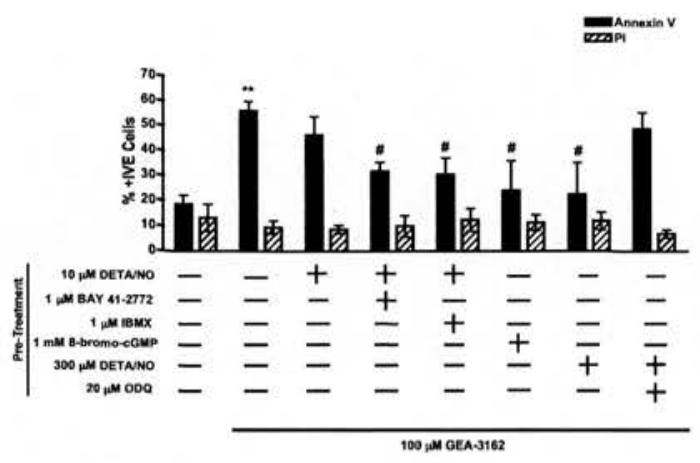

\section{Figure 4}

cGMP signalling inhibits apoptosis. MDM $\varphi$ were subjected to $24 \mathrm{~h}$ pre-treatment as shown below each column. A - The pre-treatments had no effect on cell viability after $24 \mathrm{~h}$ compared to vehicle ( $1 \% \mathrm{DMSO}$ ) treated cells (first column). B - The groups indicated were then treated for a further 24 $h$ with GEA-3162 (100 $\mu \mathrm{M})$. Pre-treatment with 8-bromocGMP, $300 \mu M$ DETA/NO, and $10 \mu M$ DETA/NO in combination with BAY 4I-2272 or IBMX, all significantly attenuated GEA-3162-induced apoptosis. ODQ prevented the attenuation of apoptosis induced by $300 \mu \mathrm{M}$ DETA/NO. **P $<0.01$ significantly different from vehicle (I\% DMSO) treated control cells (first column). \#P $<0.01$ significantly different from GEA-3162 treated cells (second column; one-way ANOVA followed by post hoc Dunnet's test; $n=3-9$ ).

site into macrophages. These macrophages play a crucial role in phagocytosing and removing apoptotic cells during the resolution of inflammation and tissue healing $[40,41]$. Macrophages resident in an inflammatory site will therefore be exposed to pro-apoptotic oxidising species, including ONOO-, generated during the inflammatory process.

In agreement with previous studies [21], our electrochemistry and EPR studies confirm that GEA-3162 decomposes to co-generate $\mathrm{NO}$ and $\mathrm{O}_{2}{ }^{\circ}$, and is therefore a ONOOgenerator, whilst DETA/NO liberates NO radicals in solution. In subsequent experiments, only $\mathrm{ONOO}^{-}$induced apoptosis, and the level of apoptosis induced was related to the activity of the compound. ONOO- is well documented to be cytotoxic in many cell types $[42,43]$. However, although ONOO- has previously been demonstrated to be pro-apoptotic in several cell types, including murine macrophages $[44,45]$, this is the first report of ONOOinducing apoptosis in human macrophages. Interestingly, in contrast to the actions of ONOO-, NO radical had no affect on cell viability. It is particularly surprising that NO radical was so ineffectual at causing either apoptosis or necrosis, even at these supraphysiological concentrations; this is in direct contrast to the observations of others who have demonstrated NO-induced apoptosis in a variety of inflammatory cells, including neutrophils [46] and murine, but not human, macrophages $[9,47]$. This disparity between the ability of ONOO- and NO to induce apoptosis may go some way to rationalise the apparently paradoxical reports of NO being both pro- and anti-apoptotic. Our results suggest that it is NO-related species, and $\mathrm{ONOO}^{-}$in particular, which might be formed in the microenvironment of the cell, that are responsible for proapoptotic signalling in human macrophages.

In rat thymocytes ONOO-induced apoptosis was inhibited by the antioxidant, Trolox, indicating that oxidising species are the apoptotic trigger [48]. We observed that the anti-oxidant and $\mathrm{O}_{2}{ }^{-}$- scavenger, SOD, actually elevated, rather than inhibited, ONOO-mediated apoptosis. As our electrochemical data demonstrate, in both the absence and presence of cells, SOD effectively 'unmasks' NO production from GEA-3162 by removing $\mathrm{O}_{2}$-from the system. Therefore, the NO liberated from GEA-3162 in the presence of SOD could itself be pro-apoptotic. However, given that we found that far higher NO concentrations released from DETA/NO failed to induce apoptosis, perhaps a more likely explanation is that hydrogen peroxide $\left(\mathrm{H}_{2} \mathrm{O}_{2}\right)$, formed by SOD [49] during the enzymatic conversion of $\mathrm{O}_{2}{ }^{-}$, is a more effective mediator of apoptosis than $\mathrm{ONOO}^{-}$per se. Indeed, $\mathrm{H}_{2} \mathrm{O}_{2}$ has previously been shown to be responsible for NO-induced apoptosis in murine macrophages by activating pro-apoptotic caspase enzymes [50]. Although $\mathrm{H}_{2} \mathrm{O}_{2}$ can inhibit caspase enzymes under certain circumstances, this usually occurs at very high $\mathrm{H}_{2} \mathrm{O}_{2}$ concentrations (higher that those likely to be generated in our experiments), and only once apoptosis has already been initiated [51]. Furthermore, inhibition of caspases once apoptosis has been initiated results in a switch from apoptosis to necrosis, which did not occur in this study. It is therefore possible that $\mathrm{H}_{2} \mathrm{O}_{2}$ would activate, rather than inhibit, caspase enzymes in our study. If $\mathrm{H}_{2} \mathrm{O}_{2}$ were formed by SOD in our experimental system, it would be predicted that a combination of 
SOD plus catalase (to eliminate $\mathrm{H}_{2} \mathrm{O}_{2}$ ) would inhibit overall levels of apoptosis. However, although the SODinduced elevation of apoptosis was blunted by catalase, overall levels of GEA-3162-induced apoptosis were not inhibited by a combination of SOD plus catalase. The reasons for this are not entirely clear, however, it has been reported that the enzymatic activity of catalase can be inhibited by $\mathrm{NO}$ via interactions with the ferric group of the enzyme $[52,53]$. It is therefore possible that catalase becomes inhibited by the NO unmasked from GEA-3162 in the presence of SOD and so cannot breakdown $\mathrm{H}_{2} \mathrm{O}_{2}$, thus apoptosis is not inhibited.

Earlier reports on the anti-apoptotic actions of NO in murine cells suggested that resistance to pro-apoptotic signals from NO, and/or NO-related species, may be a preconditioning response dependent on the anti-apoptotic properties of cGMP produced in response to the NO itself [23-25]. In common with these observations, our findings demonstrate that ONOO-induced apoptosis is attenuated in human MDM $\varphi$ following prior incubation with a low concentration of NO radical in combination with BAY 41-2272 (a sGC stimulator) to augment cGMP production, or with IBMX (a PDE inhibitor) to prevent cGMP breakdown. Similarly, the cell permeable cGMP analogue, 8-bromo-cGMP, and a higher concentration of the NO donor compound, DETA/NO, also reduced apoptosis following subsequent exposure to ONOO- As the cGMP-elevating agents were washed out of the cells in advance of ONOO- treatment, we believe this to be a cGMP-dependent pre-conditioning mechanism that protects human $\operatorname{MDM} \varphi$ from subsequent apoptosis. That this pre-conditioning response is dependent on cGMP signalling, rather than NO radicals per se, is further underlined by the observation that the sGC inhibitor, ODQ, reversed the protection against ONOO-induced apoptosis afforded by higher concentrations of DETA/NO.

In rodent macrophages, the observation that cGMP primes cells against subsequent pro-apoptotic signals has been proposed as a self-defence mechanism against high iNOS-derived NO concentrations generated by macrophages during host defence against invading pathogens $[24,25]$. Recently, it was reported that eNOS-derived NO plays a critical role in promoting iNOS induction and function in response to lipopolysaccharide (LPS) treatment in rodent macrophages and blood vessels [54,55]. Thus, it appears that during host defence, auto-regulation between NOS isoforms allows lower concentrations of $\mathrm{NO}$ to induce the production of supraphysiological NO concentrations whilst simultaneously affording cGMPdependent cellular protection against these high NO concentrations. Therefore it seems that NO is able to provide the cell with a means to finely balance pro- and antiapoptotic NO signals. However, because NO production by human macrophages is either absent or exceedingly low [56-58], self-defence is unlikely to account for the phenomenon in human cells. The studies conducted in murine cells used relatively high concentrations $(\sim 100$ $\mu \mathrm{M}$ ) of NO donor compounds to induce cytoprotection and crucially, the concentration of NO radical generated by the NO donor compounds was not measured in such studies. From our electrochemistry data we have estimated that the concentration of $\mathrm{NO}$ released from $10 \mu \mathrm{M}$ DETA/NO is likely to be in the $\mathrm{nM}$ range and arguably a more realistically physiological model of endogenous endothelial-derived NO. Our observations in human cells could, therefore, represent a cGMP-dependent mechanism by which, in the disease-free state, eNOS-derived NO from the healthy endothelium is able to limit apoptosis in macrophages. Protecting macrophages against the pro-apoptotic oxidising milieu of an inflammatory site would ensure maintenance of a sufficient population of phagocytes to effectively remove those cells that have undergone apoptosis, thus promoting the successful resolution of inflammation and preventing the tissue damage that can occur when apoptotic cells remaining in situ eventually rupture their membranes and release their histotoxic contents causing widespread necrosis [59].

The downstream signalling events responsible for cGMPdependent inhibition of apoptosis in human MDM $\varphi$ remain to be elucidated. Apoptosis involves multiple pathways and signalling cascades and is controlled by the regulation of various pro- and anti-apoptotic factors within the cell. It is likely that CGMP, possibly via activation of protein kinase $\mathrm{G}$ (PKG; [24]) ultimately results in a shift in the pro-/anti- apoptotic balance of the cell in favour of the later.

In summary, we have demonstrated the differential ability of NO and NO-related species to induce apoptosis in human MDM $\varphi$, and have established that ONOO; but not NO radical, is pro-apoptotic in this cell type (figure 5). Furthermore, our results demonstrate the presence of a cGMP-dependent pre-conditioning mechanism to limit ONOO-induced apoptosis in human macrophages.

\section{Abbreviations}

cGMP: cyclic guanosine monophosphate; DETA/NO: (Z)1- [2-(2-aminoethyl)-N-(2-ammonioethyl)amino]diazen-1-ium-1,2-diolate; DMSO: dimethylsulphoxide; EPR: electron paramagnetic resonance spectrometry; GEA3162: 1,2,3,4-oxatriazolium, 5-amino-3-(3,4-dichlorophenyl)-, chloride; Hb: haemoglobin; IBMX: isobutylmethylxanthine; IMDM: Iscove's modified Dulbecco's tissue culture medium; MDM $\varphi$ : monocyte-derived macrophages; MNC: Mononuclear cells; NO: nitric oxide; $\mathrm{O}_{2} \cdots$ superoxide; ONOO: peroxynitrite; PBS: phosphate buffered saline; PDE: phosphodiesterase; PI: propidium 


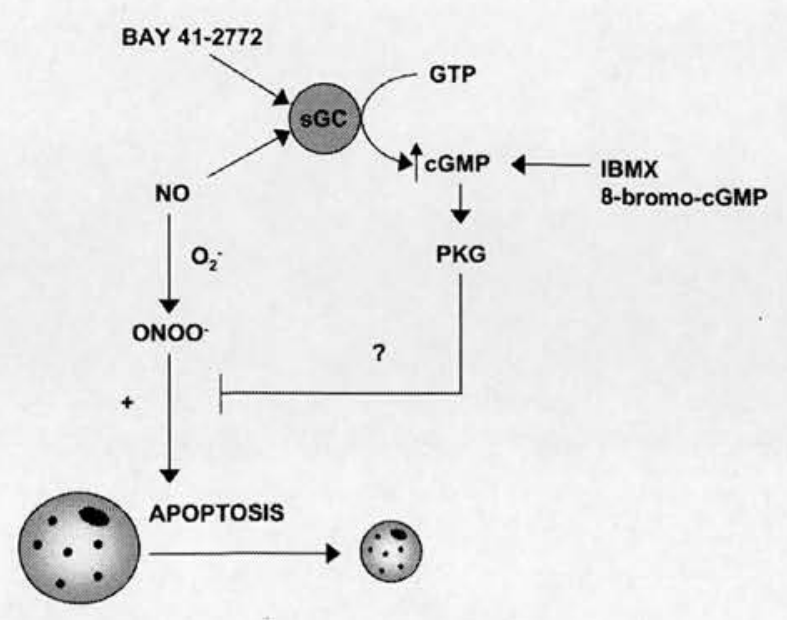

Figure 5

Schematic representation of the roles of NO, ONOO-, \& cGMP in macrophage apoptosis. NO and $\mathrm{O}_{2}$ - combine to form ONOO- which promotes macrophage apoptosis. Agents that act to elevate cGMP are able to limit ONOO-induced apoptosis.

iodide; PRP: platelet-rich plasma; SOD: superoxide dismutase.

\section{Competing interests}

The authors declare that they have no competing interests.

\section{Authors' contributions}

The manuscript was written and experiments designed by CAS, AGR, and ILM. All experiments were performed by CAS and supervised by ARG and ILM who also oversaw manuscript construction together with DJW. All authors have given final approval of the version to be published.

\section{Acknowledgements}

C.A.S is funded by the Wellcome Trust, as part of a 4-year Ph.D programme in Cardiovascular Biology within the Cardiovascular Research Initiative at the University of Edinburgh. The authors are grateful to Bayer (Leverkusen, Germany) for the kind gift of BAY 4I-2272.

\section{References}

I. Hart SP, Dransfield I, Rossi AG: Phagocytosis of apoptotic cells. Methods 2008, 44:280-285.

2. Gilroy DW, Lawrence T, Perretti M, Rossi AG: Inflammatory resolution: new opportunities for drug discovery. Nat Rev Drug Discov 2004, 3:40|-416.

3. Hallett JM, Leitch AE, Riley NA, Duffin R, Haslett C, Rossi AG: Novel pharmacological strategies for driving inflammatory cell apoptosis and enhancing the resolution of inflammation. Trends Pharmacol Sci 2008, 29:250-257.

4. Serhan CN, Brain SD, Buckley CD, Gilroy DW, Haslett C, O'Neill LA, Perretti M, Rossi AG, Wallace JL: Resolution of inflammation: state of the art, definitions and terms. Faseb J 2007, 21:325-332.

5. Shaw CA, Megson IL, Rossi AG: Apoptosis and Atherosclerosis: The Role of Nitric Oxide. Anti-Inflammatory \& Anti-Allergy Agents in Medicinal Chemsitry 2006, 5:27-33.
6. Shaw CA, Taylor EL, Megson IL, Rossi AG: Nitric oxide and the resolution of inflammation: implications for atherosclerosis. Mem Inst Oswaldo Cruz 2005, I00(SuppI I):67-7I.

7. Taylor EL, Megson IL, Haslett C, Rossi AG: Nitric oxide: a key regulator of myeloid inflammatory cell apoptosis. Cell Death Differ 2003, 10:418-430.

8. Muhl H, Sandau K, Brune B, Briner VA, Pfeilschifter J: Nitric oxide donors induce apoptosis in glomerular mesangial cells, epithelial cells and endothelial cells. Eur J Pharmacol 1996, 317:137-149.

9. Sarih M, Souvannavong V, Adam A: Nitric oxide synthase induces macrophage death by apoptosis. Biochem Biophys Res Commun 1993, 191:503-508.

10. Fehsel K, Kroncke KD, Meyer KL, Huber H, Wahn V, Kolb-Bachofen $\mathrm{V}$ : Nitric oxide induces apoptosis in mouse thymocytes. J Immunol 1995, 155:2858-2865.

II. Lee MH, Jang MH, Kim EK, Han SW, Cho SY, Kim CJ: Nitric oxide induces apoptosis in mouse $\mathrm{C} 2 \mathrm{C} 12$ myoblast cells. J Pharmacol Sci 2005, 97:369-376.

12. Battinelli E, Loscalzo J: Nitric oxide induces apoptosis in megakaryocytic cell lines. Blood 2000, 95:3451-3459.

13. Fiscus RR, Yuen JP, Chan SL, Kwong JH, Chew SB: Nitric oxide and cyclic GMP as pro- and anti-apoptotic agents. J Card Surg 2002, 17:336-339.

14. Kim PK, Zamora R, Petrosko P, Billiar TR: The regulatory role of nitric oxide in apoptosis. Int Immunopharmacol 2001, I:| $142|-| 44 \mid$.

15. Ward C, Wong TH, Murray J, Rahman I, Haslett C, Chilvers ER, Rossi AG: Induction of human neutrophil apoptosis by nitric oxide donors: evidence for a caspase-dependent, cyclic-GMP-independent, mechanism. Biochem Pharmacol 2000, 59:305-314.

16. Nicotera P, Brune B, Bagetta G: Nitric oxide: inducer or suppressor of apoptosis? Trends Pharmacol Sci 1997, 18:189-190.

17. Czapski G, Goldstein S: The role of the reactions of .NO with superoxide and oxygen in biological systems: a kinetic approach. Free Radic Biol Med 1995, 19:785-794.

18. Goldstein S, Czapski G: The reaction of NO. with O2.- and HO2.: a pulse radiolysis study. Free Radic Biol Med 1995, 19:505-510.

19. Reiter CD, Teng RJ, Beckman JS: Superoxide reacts with nitric oxide to nitrate tyrosine at physiological $\mathrm{pH}$ via peroxynitrite. J Biol Chem 2000, 275:32460-32466.

20. Saran M, Michel C, Bors W: Reaction of NO with O2-. implications for the action of endothelium-derived relaxing factor (EDRF). Free Radic Res Commun 1990, 10:221-226.

21. Taylor EL, Rossi AG, Shaw CA, Dal Rio FP, Haslett C, Megson IL: GEA 3162 decomposes to co-generate nitric oxide and superoxide and induces apoptosis in human neutrophils via a peroxynitrite-dependent mechanism. Br J Pharmacol 2004; 143:179-185.

22. Blaylock MG, Cuthbertson BH, Galley HF, Ferguson NR, Webster NR: The effect of nitric oxide and peroxynitrite on apoptosis in human polymorphonuclear leukocytes. Free Radic Biol Med 1998, 25:748-752.

23. von Knethen A, Callsen D, Brune B: NF-kappaB and AP-I activation by nitric oxide attenuated apoptotic cell death in RAW 264.7 macrophages. Mol Biol Cell 1999, 10:361-372.

24. Yoshioka Y, Yamamuro A, Maeda S: Nitric oxide at a low concentration protects murine macrophage RAW264 cells against nitric oxide-induced death via cGMP signaling pathway. $\mathrm{Br}$ J Pharmacol 2003, 139:28-34.

25. Yoshioka $Y$, Yamamuro A, Maeda S: Nitric oxide/cGMP signaling pathway protects RAW264 cells against nitric oxide-induced apoptosis by inhibiting the activation of p38 mitogen-activated protein kinase. JPharmacol Sci 2006, 10 I: 126-134.

26. Pan SL, Guh JH, Chang YL, Kuo SC, Lee FY, Teng CM: YC-I prevents sodium nitroprusside-mediated apoptosis in vascular smooth muscle cells. Cardiovasc Res 2004, 61:152-158.

27. Singh RJ, Hogg N, Joseph J, Konorev E, Kalyanaraman B: The peroxynitrite generator, $\mathrm{SIN}-\mathrm{I}$, becomes a nitric oxide donor in the presence of electron acceptors. Arch Biochem Biophys 1999, 361:331-339.

28. Martin W, Villani GM, Jothianandan D, Furchgott RF: Selective blockade of endothelium-dependent and glyceryl trinitrateinduced relaxation by hemoglobin and by methylene blue in the rabbit aorta. J Pharmacol Exp Ther 1985, 232:708-716. 
29. Dikalov S, Skatchkov M, Bassenge E: Quantification of peroxynitrite, superoxide, and peroxyl radicals by a new spin trap hydroxylamine I-hydroxy-2,2,6,6-tetramethyl-4-oxo-piperidine. Biochem Biophys Res Commun 1997, 230:54-57.

30. Dikalov S, Skatchkov M. Fink B, Bassenge E: Quantification of superoxide radicals and peroxynitrite in vascular cells using oxidation of sterically hindered hydroxylamines and electron spin resonance. Nitric Oxide 1997, 1:423-431.

31. Hart SP, Ross JA, Ross K, Haslett C, Dransfield I: Molecular characterization of the surface of apoptotic neutrophils: implications for functional downregulation and recognition by phagocytes. Cell Death Differ 2000, 7:493-503.

32. Rossi AG, McCutcheon JC, Roy N, Chilvers ER, Haslett C, Dransfield I: Regulation of macrophage phagocytosis of apoptotic cells by CAMP. J Immunol 1998, 160:3562-3568.

33. Pahl HL, Krauss B, Schulze-Osthoff K, Decker T, Traenckner EB, Vogt M, Myers C, Parks T, Warring P, Muhlbacher A, et al:: The immunosuppressive fungal metabolite gliotoxin specifically inhibits transcription factor NF-kappaB. J Exp Med 1996 183:1829-1840

34. Suen YK, Fung KP, Lee CY, Kong SK: Gliotoxin induces apoptosis in cultured macrophages via production of reactive oxygen species and cytochrome c release without mitochondrial depolarization. Free Radic Res 2001, 35:1-10.

35. Ward C. Dransfield I, Chilvers ER, Haslett C, Rossi AG: Pharmacological manipulation of granulocyte apoptosis: potential therapeutic targets. Trends Pharmacol Sci 1999, 20:503-509.

36. Stasch JP, Becker EM, Alonso-Alija C, Apeler H, Dembowsky K Feurer A, Gerzer R, Minuth T, Perzborn E, Pleiss U, et al:: NO-independent regulatory site on soluble guanylate cyclase. Nature 2001, 410:212-215.

37. Mullershausen F, Russwurm M, Friebe A, Koesling D: Inhibition of phosphodiesterase type 5 by the activator of nitric oxidesensitive guanylyl cyclase BAY 4/-2272. Circulation 2004, 109:17|1-17|3.

38. Friebe A, Mullershausen F, Smolenski A, Walter U, Schultz G, Koesling D: YC-I potentiates nitric oxide- and carbon monoxideinduced cyclic GMP effects in human platelets. Mol Pharmacol 1998, 54:962-967.

39. Fialkow L, Wang $Y$, Downey GP: Reactive oxygen and nitrogen species as signaling molecules regulating neutrophil function. Free Radic Biol Med 2007, 42:153-164.

40. Haslett C: Granulocyte apoptosis and inflammatory disease. BrMed Bull 1 997, 53:669-683.

41. Walker A, Ward C, Taylor EL, Dransfield I, Hart SP, Haslett C, Ross AG: Regulation of neutrophil apoptosis and removal of apoptotic cells. Curr Drug Targets Inflamm Allergy 2005, 4:447-454.

42. Szabo C: Multiple pathways of peroxynitrite cytotoxicity. Toxicol Lett 2003, 140-141: 105-112.

43. Pacher P, Beckman JS, Liaudet L: Nitric oxide and peroxynitrite in health and disease. Physiol Rev 2007, 87:315-424.

44. Sandoval M, Ronzio RA, Muanza DN, Clark DA, Miller MJ: Peroxynitrite-induced apoptosis in epithelial (T84) and macrophage (RAW 264.7) cell lines: effect of legume-derived polyphenols (phytolens). Nitric Oxide 1997, I:476-483.

45. Sandoval M, Zhang XJ, Liu X, Mannick EE, Clark DA, Miller MJ: Peroxynitrite-induced apoptosis in T84 and RAW 264.7 cells: attenuation by L-ascorbic acid. Free Radic Biol Med 1997, 22:489-495.

46. Taylor EL, Megson IL, Haslett C, Rossi AG: Dissociation of DNA fragmentation from other hallmarks of apoptosis in nitric oxide-treated neutrophils: differences between individual nitric oxide donor drugs. Biochem Biophys Res Commun 200I, 289:1229-1236.

47. Albina JE, Cui S, Mateo RB, Reichner JS: Nitric oxide-mediated apoptosis in murine peritoneal macrophages. J Immunol 1993. I 50:5080-5085.

48. Salgo MG, Squadrito GL, Pryor WA: Peroxynitrite causes apoptosis in rat thymocytes. Biochem Biophys Res Commun 1995, 215: $1111-1118$.

49. Beckman JS, Koppenol WH: Nitric oxide, superoxide, and peroxynitrite: the good, the bad, and ugly. Am J Physiol 1996. 271:C1424-1437.

50. Borutaite V, Brown GC: Nitric oxide induces apoptosis via hydrogen peroxide, but necrosis via energy and thiol depletion. Free Radic Biol Med 2003, 35:1457-1468.
51. Borutaite V, Brown GC: Caspases are reversibly inactivated by hydrogen peroxide. FEBS Lett 2001, 500:114-118.

52. Machiavelli LI, Poliandri AH, Quinteros FA, Cabilla JP, Duvilanski BH: Reactive oxygen species are key mediators of the nitric oxide apoptotic pathway in anterior pituitary cells. Nitric Oxide 2007, 16:237-246.

53. Cooper CE: Nitric oxide and iron proteins. Biochim Biophys Acto 1999, I4II:290-309.

54. Connelly L, Jacobs AT, Palacios-Callender M, Moncada S, Hobbs AJ: Macrophage endothelial nitric-oxide synthase autoregulates cellular activation and pro-inflammatory protein expression. J Biol Chem 2003, 278:26480-26487.

55. Vo PA, Lad B, Tomlinson JA, Francis S, Ahluwalia A: autoregulatory role of endothelium-derived nitric oxide (NO) on Lipopolysaccharide-induced vascular inducible NO synthase expression and function. J Biol Chem 2005, 280:7236-7243.

56. Albina JE: On the expression of nitric oxide synthase by human macrophages. Why no NO? J Leukoc Biol 1995, 58:643-649.

57. Schneemann M, Schoedon G: Species differences in macrophage NO production are important. Nat Immunol 2002, 3:102.

58. Thomassen MJ, Kavuru MS: Human alveolar macrophages and monocytes as a source and target for nitric oxide. Int Immunopharmacol 2001, I:1479-1490.

59. Maderna P, Godson C: Phagocytosis of apoptotic cells and the resolution of inflammation. Biochim Biophys Acta 2003, 1639:|4|-|5|.
Publish with BioMed Central and every scientist can read your work free of charge

"BioMed Central will be the most significant development for disseminating the results of biomedical research in our lifetime. "

Sir Paul Nurse, Cancer Research UK

Your research papers will be:

- available free of charge to the entire biomedical community

- peer reviewed and published immediately upon acceptance

- cited in PubMed and archived on PubMed Central

- yours - you keep the copyright
BioMedcentral 


\title{
Cyclic AMP enhances resolution of allergic pleurisy by promoting inflammatory cell apoptosis via inhibition of PI3K/Akt and NF- $\mathrm{B}$
}

\author{
Lirlândia P. Sousa ${ }^{a, b}$, Aline F. Carmo ${ }^{b}$, Bárbara M. Rezende ${ }^{b}$, Fernando Lopes ${ }^{\text {b,c }}$, Douglas M. Silva ${ }^{\mathrm{b}}$, \\ Ana L. Alessandri ${ }^{b}$, Cláudio A. Bonjardim ${ }^{\text {d }}$, Adriano G. Rossi ${ }^{\text {e }}$, Mauro M. Teixeira ${ }^{\text {b.*, }}$, Vanessa Pinho ${ }^{\text {b,c }}$ \\ ${ }^{a}$ Setor de Patologia Clínica, Colégio Técnico, Universidade Federal de Minas Gerais, Belo Horizonte, Brazil \\ bImunofarmacologia, Departamento de Bioquímica e Imunologia, ICB, Universidade Federal de Minas Gerais, Belo Horizonte, Brazil \\ ${ }^{\text {'}}$ Departamento de Morfologia, Universidade Federal de Minas Gerais, ICB, Belo Horizonte, Brazil \\ dDepartamento de Microbiologia, Universidade Federal de Minas Gerais, ICB, Belo Horizonte, Brazil \\ 'Medical Research Council Centre for Inflammation Research, Queen's Medical Research Institute, University of Edinburgh, Edinburgh, United Kingdom
}

\section{A RTICLE INFO}

\section{Article history:}

Received 13 March 2009

Accepted 27 April 2009

\section{Keywords:}

CAMP

Eosinophil

Apoptosis

Inflammation

Akt

$\mathrm{NF}-\mathrm{kB}$

\begin{abstract}
A B S T R A C T
Selective and timely induction of apoptosis is an effective means of resolving inflammation. The effects and putative mechanisms by which cyclic AMP (CAMP) modulates leukocyte apoptosis in vivo are still unclear. The present study aims at identifying intracellular pathways underlying the ability of cAMP elevating agents to resolve eosinophilic inflammation in a model of allergic pleurisy in mice. Ovalbumin (OVA) challenge of immunized mice induced eosinophil recruitment that peaked at $24 \mathrm{~h}$ and persisted till $48 \mathrm{~h}$. Treatment with the PDE4 inhibitor rolipram, cAMP mimetic db-cAMP or adenylate cyclase activator forskolin, at $24 \mathrm{~h}$ after antigen-challenge resulted in profound resolution of eosinophilic inflammation, without a decrease of mononuclear cell numbers. There was a concomitant increase in number of apoptotic cells in the pleural cavity. The effects of rolipram and db-cAMP were inhibited by the PKA inhibitor H89. Inhibition of PI3K/Akt or NF- $\mathrm{BB}$ induced resolution of inflammation that was associated with increased apoptosis. OVA-challenge resulted in a time-dependent activation of Akt and NF- $\kappa \mathrm{B}$, which was blocked by treatment with rolipram or PI3K/Akt pathway inhibitors. Thus, cAMP elevating agents resolve established eosinophilic inflammation by inducing leukocyte apoptosis. Mechanistically, the actions of cAMP are dependent on PKA and target a PI3K/Akt-dependent NF-KB survival pathway.
\end{abstract}

(c) 2009 Elsevier Inc. All rights reserved.

\section{Introduction}

Eosinophils are effectors cells that play an important role in the pathophysiology of allergic diseases [1,2]. In allergic diseases, such as asthma, eosinophils are a crucial source of cytotoxic proteins, lipid mediators, oxygen metabolites, and cytokines, which may contribute to the severity of disease [3]. The accumulation of eosinophils in tissue depends not only on the number of cells being recruited at any particular time, but also on the number of cells that are cleared or leave the tissue [4]. Thus, defective removal of these cells may play an important role in the initiation and propagation of chronic inflammatory diseases. There are two main

Abbreviations: cAMP, cyclic adenosine monophosphate; db-cAMP, dibutyryl-cAMP: i.pl., intrapleural; PDE4, phosphodiesterase 4; PI3K, phosphatidylinositol 3-kinase: $\mathrm{NF}-\mathrm{kB}$, nuclear factor kappa B; Akt/PKB, protein kinase B; EMSA, electrophoretic mobility shift assay.

- Corresponding author at: Av. Antonio Carlos, 6627. Pampulha, 31270-901, Belo Horizonte, MG, Brazil. Tel.: +55 313409 2651; fax: +55 3134092651.

E-mail address: mmtex@icb.ufmg.br (M.M. Teixeira). mechanisms that underlie the clearance of inflammatory cells from tissues, namely apoptosis followed by their subsequent removal by phagocytes and necrosis. Whereas the latter is undoubtedly associated with enhanced inflammation and tissue injury, the former is accompanied by shut down of cellular activity and inhibition of the inflammatory response [4]. Apoptosis is characterized by specific morphologic and biochemical events including cell shrinkage, cytoplasmic vacuolation, membrane blebbing, chromatin condensation and nuclear fragmentation associated with endonucleolytic DNA cleavage [5].

More recently, there has been great interest in understanding of the signal transduction pathways relevant for induction of the apoptosis or survival of leukocytes in vivo [6-8]. Cyclic adenosine $3^{\prime}, 5^{\prime}$ monophosphate (cAMP) is an important intracellular second messenger produced after adenylate cyclase activation that regulates different cellular processes by cAMP effectors $[9,10]$. Phosphodiesterases (PDEs) controls the intracellular cAMP levels by catalyzing its hydrolysis and inactivating these second messengers [10]. PDE isoenzymes have been classified into eleven distinct families [10]. Of these, PDE3, PDE4 and PDE7 are the most 
important for the regulation of cAMP in various types of cells. In neutrophils, eosinophils, mast cell and basophils, PDE4 isoenzymes appear to play a more important function in the regulation of cAMP in leukocyte [10]. Indeed, PDE4 inhibitors induce an increase in the intracellular levels of CAMP in leukocytes and have potent antiinflammatory activity [11-14]. Intracellular actions of cAMP can be mimicked by administration of the cell permeable analogue dibutyryl-cAMP (db-cAMP) or the adenylate cyclase activator forskolin. Similarly to rolipram, these compounds also inhibit leukocyte function and possess significant anti-inflammatory effects in vivo [15]. In vitro, inhibition of PDE4 enzymes and increase of intracellular levels of cyclic AMP may modify the survival of eosinophils. Indeed, cAMP elevating agents may enhance or prevent apoptosis of eosinophils depending on their activation status [16]. The effects of cAMP elevating/mimetics on leukocyte apoptosis and survival in vivo are not well-established.

The PI3K/Akt pathway has been also shown to mediate survival in many cell types [17]. Recently, we have demonstrated [8] that the PI3K/Akt pathway was important for the survival of eosinophils in vivo. It has been reported that there is a cross-talk between the cAMP-dependent and phosphatidylinositol 3-kinase (PI3K) pathways, but the effects of cAMP on PI3K/Akt activity are quite varied $[18,19]$ and cAMP can either stimulate or inhibit Akt activity. For example, cAMP activates PI3K/Akt in thyroid cells and hepatocytes $[20,21]$, whereas inhibition of PI3K/Akt pathway by cAMP has been reported in fibroblast and leukemia cells $[22,23]$. The transcription factor nuclear factor kappa B (NF- $\mathrm{KB}$ ) is a key regulator of several cellular functions, including leukocyte activation and survival [2426]. The pro-survival/anti-apoptotic effects of Akt can be mediated by NF-кB. For example, Akt may phosphorylate IкB kinase (IKK) leading to NF- $\mathrm{KB}$ activation $[27,28]$. It is not known whether the pro-survival effect of the PI3K/Akt pathway during allergic inflammation is mediated via modification of NF- $\mathrm{KB}$ function. Hence, it is of interest to examine whether any resolving effect of cAMP on allergic inflammation is mediated by prevention of the function of PI3K/Akt and consequent change in NF- $\mathrm{KB}$ function.

In the present study, we examined the ability of the PDE4 inhibitor rolipram and of cAMP-inducers/mimetics, forskolin and db-cAMP, to resolve eosinophilic inflammation in a model of allergic pleurisy in mice $[1,8,29,30]$. We show that rolipram, dbcAMP and forskolin resolve established eosinophilic inflammation by promoting apoptosis of inflammatory cells and by inhibiting a PI3K/Akt-dependent NF- $\kappa B$ survival pathway.

\section{Materials and methods}

\subsection{Animals}

All procedures described here had prior approval from the Animal Ethics Committee of Universidade Federal de Minas Gerais. Male C57/BL6 mice ( $8-10$ weeks) obtained from the Bioscience Unit of Instituto de Ciências Biológicas were housed under standard conditions and had free access to commercial chow and water.

\subsection{Drugs, reagents and antibodies}

Rolipram (Biomol ${ }^{\circledR}$, Plymouth Meeting, PA), forskolin and Akt inhibitor-IV (both from Calbiochem, San Diego, CA), gliotoxin (Glio) (Fluka Biochemika, Switzerland), LY294002 (Alamone labs, Jerusalem, Israel), and pyrrolidine dithiocarbamate (PDTC, SigmaAldrich, St Louis, MO) were diluted in DMSO and further in PBS. Dibutyryl-cAMP was from Sigma and was diluted in PBS. AnnexinV Detection Kit was from Caltag Laboratories (Burlingame, CA) Rabbit anti-P-Akt (Ser 473), anti-Akt, anti cleaved caspase-3 and mouse anti-phospho-IкB- $\alpha$ were from Cell Signaling Technology (Beverly MA, USA). Rabbit anti-IкB- $\alpha$ (C-21/sc-371), anti p65/RelA
(C-20/sc-372), anti p50/NF-кB1 (H-119/sc-7178) and anti Bax (P$19 /$ sc-526) or secondary anti-rabbit peroxidase conjugate antibodies were purchased from Santa Cruz Biotechnology (Santa Cruz, (A, EUA). Anti $\beta$-actin and anti-mouse peroxidase conjugate antibodies were from Sigma.

\subsection{Induction of pleurisy}

Animals were immunized with OVA (Sigma) adsorbed to aluminium hydroxide gel as described $[1,8,29,30]$. Briefly, mice were injected s.c. (subcutaneous) on days 1 and 8 with $0.2 \mathrm{ml}$ of a solution containing $100 \mu \mathrm{g}$ of OVA and $70 \mu \mathrm{g}$ of aluminium hydroxide (Reheiss, Dublin, Ireland). Sensitized mice were challenged by i.pl. (intrapleural) administration of antigen (OVA) or PBS. The cells present in the pleural cavity were harvested at different times after antigen-challenge by washing the cavity with $2 \mathrm{ml}$ of PBS and total cell counts performed in a modified Neubauer chamber using Turk's stain. For the experiments evaluating leukocyte apoptosis, infiltrating leukocytes were examined $2 \mathrm{~h}$ (annexin-V, DNA fragmentation assays, caspase-3 cleavage) and $24 \mathrm{~h}$ (morphologic apoptosis) after drug treatment. Differential cell counts were performed on cyto-centrifuge preparations (Shandon III) stained with May-Grunwald-Giemsa using standard morphological criteria to identify cell types. The results are presented as the number of cells per cavity.

\subsection{Treatment with drugs}

The role of CAMP on eosinophil accumulation into pleural cavity was investigated by using rolipram (a specific PDE4 inhibitor), forskolin (an adenylate cyclase activator), and db-cAMP (a cell permeable cAMP analogue). Rolipram was administered systemically (i.p.) at dose of $150 \mu \mathrm{g} / \mathrm{mouse}(6.0 \mathrm{mg} / \mathrm{kg}), 24 \mathrm{~h}$ after i.pl. OVAchallenge. This dose was shown to be effective in other experimental system [14]. Forskolin $10 \mu \mathrm{g} /$ mouse $(0.4 \mathrm{mg} / \mathrm{kg})$, Db-cAMP $100 \mu \mathrm{g} /$ mouse ( $4 \mathrm{mg} / \mathrm{kg}$ ), LY294002 (30 $\mu \mathrm{g} / \mathrm{mouse}, 1.0 \mathrm{mg} / \mathrm{kg})$, AKT inhibitor-IV $10 \mu \mathrm{g} / \mathrm{mouse}(0.4 \mathrm{mg} / \mathrm{kg})$ and gliotoxin $20 \mu \mathrm{g} / \mathrm{mouse}$ $(0.8 \mathrm{mg} / \mathrm{kg})$ were gived i.pl. at a volume of the $100 \mu \mathrm{l}, 24 \mathrm{~h}$ after OVA-challenge. PDTC was administered systemically (i.p.) at a dose of $100 \mathrm{mg} / \mathrm{kg}, 24 \mathrm{~h}$ after the i.pl. administration of OVA. As a positive control for anti-inflammatory activity, we used the synthetic glucocorticoid dexamethasone at dose of $2.0 \mathrm{mg} / \mathrm{kg}$ in PBS buffer. Glucocorticoids have been shown to induce eosinophil apoptosis and to enhance macrophage phagocytosis of apoptotic bodies [31]. Drugs were dissolved in DMSO and further diluted in PBS. Control mice received drug vehicle only.

\subsection{Assessment of leukocyte apoptosis}

\subsubsection{Morphology}

Apoptosis was assessed as previously described by us $[8,24]$. Briefly, cells $\left(5 \times 10^{4}\right)$ collected $48 \mathrm{~h}$ after antigen-challenge were cyto-centrifuged, fixed and stained with May-Grunwald-Giemsa and counted using oil immersion microscopy ( $\times 100$ objective) to determine the proportion of cells with distinctive apoptotic morphology (cells presented chromatin condensation, nuclear fragmentation and formation of apoptotic bodies out or inside macrophages). Twenty-five fields were counted per slide and results are expressed as the mean \pm S.E.M of number of apoptotic cells in 25 fields.

\subsubsection{Annexin- $V$ binding and propidium staining}

Assessment of apoptosis was also performed by flow cytometry using FITC-labeled annexin-V (Caltag laboratories), which binds to phosphatidylserine exposed on the surface of apoptotic cells, and propidium iodide, as an index of loss of cell membrane integrity. 
Annexin- $V$ was added to $100 \mu \mathrm{l}$ of $2.5 \times 10^{5}$ cells collected $2 \mathrm{~h}$ and $6 \mathrm{~h}$ after drugs treatment, in binding buffer. Following $20 \mathrm{~min}$ incubation at room temperature, these samples were treated with $5 \mu \mathrm{l}$ of propidium iodide $(50 \mu \mathrm{g} / \mathrm{ml})$ and analyzed using a Becton Dickenson FACScan (San Jose, CA) and FlowJo 7.2.2 software (Tree Star Company). Results are expressed as cells undergoing the early stage of apoptosis quantified by staining with annexin- $V$ but not propidium iodide. The cells were selected based on size and granularity, allowing separate analysis of granulocyte population. At the time point evaluated ( $26 \mathrm{~h}$ after OVA-challenge), morphological analysis showed that granulocytes were eosinophils (>95\%, data not shown).

\subsection{Lysate preparation and Western blot analysis}

Inflammatory cells harvested from the pleural cavity were washed with PBS and total cell extracts or nuclear and cytoplasmic cell extracts were prepared, as described $[32,33]$. Protein amounts were quantified with the Bradford assay reagent from Bio-Rad (Bio-Rad, USA). Total cell extracts $(40 \mu \mathrm{g})$, Nuclear $(15 \mu \mathrm{g})$ and cytoplasmic $(40 \mu \mathrm{g})$ extracts were separated by electrophoresis on a denaturing $10-15 \%$ polyacrylamide-SDS gel and transferred onto nitrocellulose membranes, as described [32]. Membranes were blocked overnight at $4{ }^{\circ} \mathrm{C}$ with PBS containing $5 \%(w / v)$ nonfat dry milk and $0.1 \%$ Tween-20, washed three times with PBS containing $0.1 \%$ Tween-20 and then incubated with specifics antibodies (1:1000) in phosphate-buffered saline containing $5 \%(\mathrm{w} / \mathrm{v}) \mathrm{BSA}$ and $0.1 \%$ Tween-20. After washing, membranes were incubated with appropriated horseradish peroxidase-conjugated secondary antibody $(1: 3000)$. Immunoreactive bands were visualized by using ECL detection system, as described by the manufacturer (GE Healthcare, Piscataway, NJ).

\subsection{Electrophoretic mobility shift assay (EMSA)}

Band shift assay was carried out of $10 \mu \mathrm{g}$ nuclear extracts essentially as described $[32,33]$, using a $5^{\prime} \quad\left[{ }^{32} \mathrm{P}\right]$-end-labeled double-stranded probe (only one strand is shown) corresponding to the consensus-binding site of NF- $\mathrm{KB}\left(5^{\prime}\right.$-AGT TGA GGG GAC TTT CCC AGG C $\left.-3^{\prime}\right)$. Heterologous competition assays were performed with a 100 -fold molar excess of cold oligonucleotide corresponding to c-fos SRE.

\subsection{Statistical analysis}

All results are presented as the mean \pm S.E.M. Normalized data were analyzed by one-way ANOVA, and differences between groups were assessed using the Student-Newman-Keuls post-test. A $P$-value
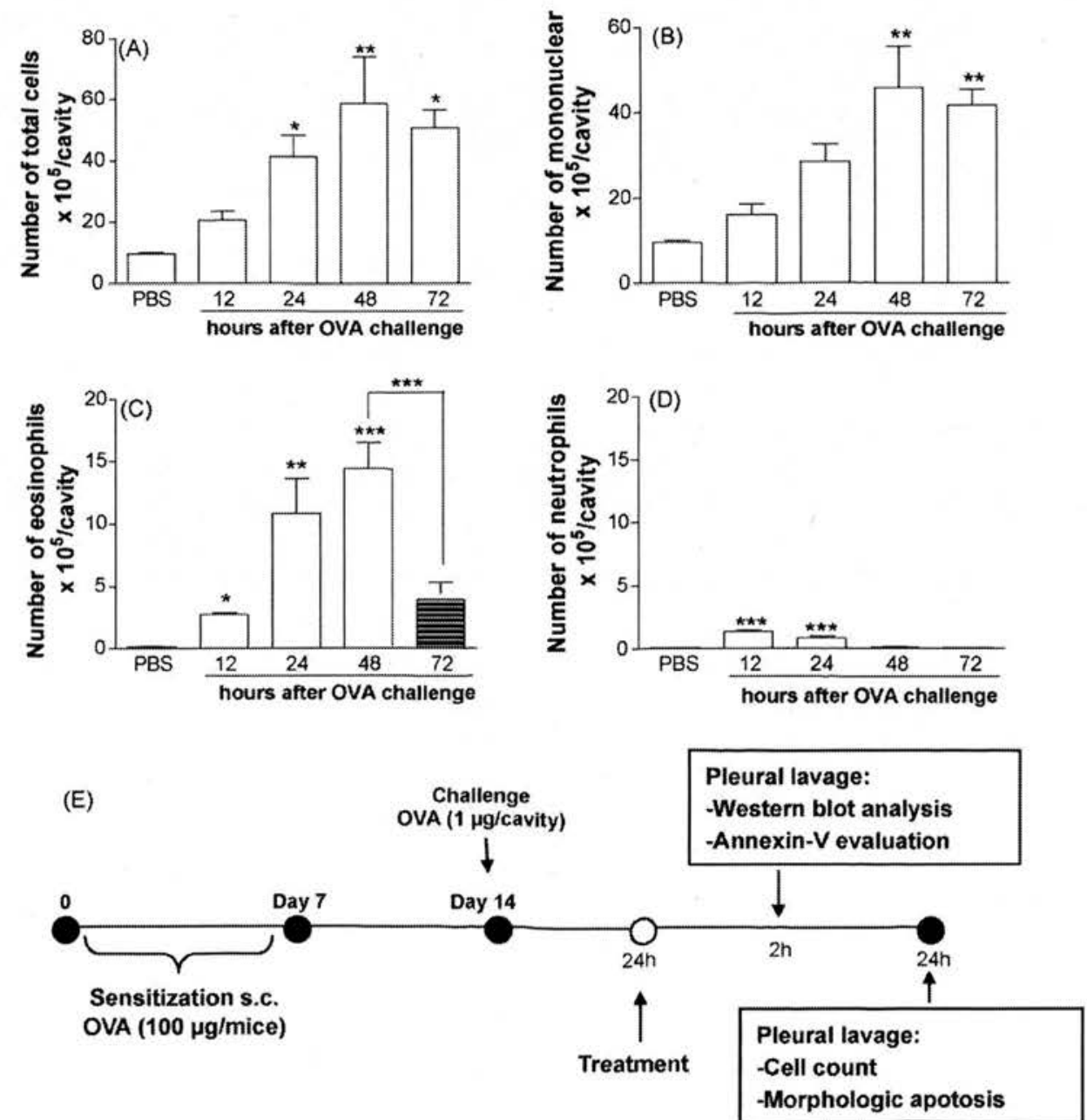

Fig. 1. Kinetics of leukocyte influx in allergic inflammation. Immunized mice were challenged with an i.pl. injection of OVA (1 $\mu \mathrm{g} / \mathrm{cavity}$ ) or PBS. The cells that migrated into the pleural cavity were collected at indicated times and processed for counting of leukocytes (A), mononuclear cells (B), eosinophils (C), and neutrophils (D). Results are expressed as the means \pm S.E.M of five mice in each group. ${ }^{*} P<0.05 ;{ }^{* *} P<0.01$ and ${ }^{* *} P<0.001$ when compared with PBS-injected mice. (E), schematic representation of the protocol of the induction of pleurisy and of the treatments used in this paper. 
$<0.05$ was considered significant. Calculations were performed using the prism 4.0 software program for Windows (GraphPad software, San Diego, CA).

\section{Results}

3.1. Cyclic AMP elevating agents promote resolution of allergic pleurisy by inducing leukocyte apoptosis

The model of allergic pleurisy used in the present experiment is a well-established model of acute eosinophilic inflammation
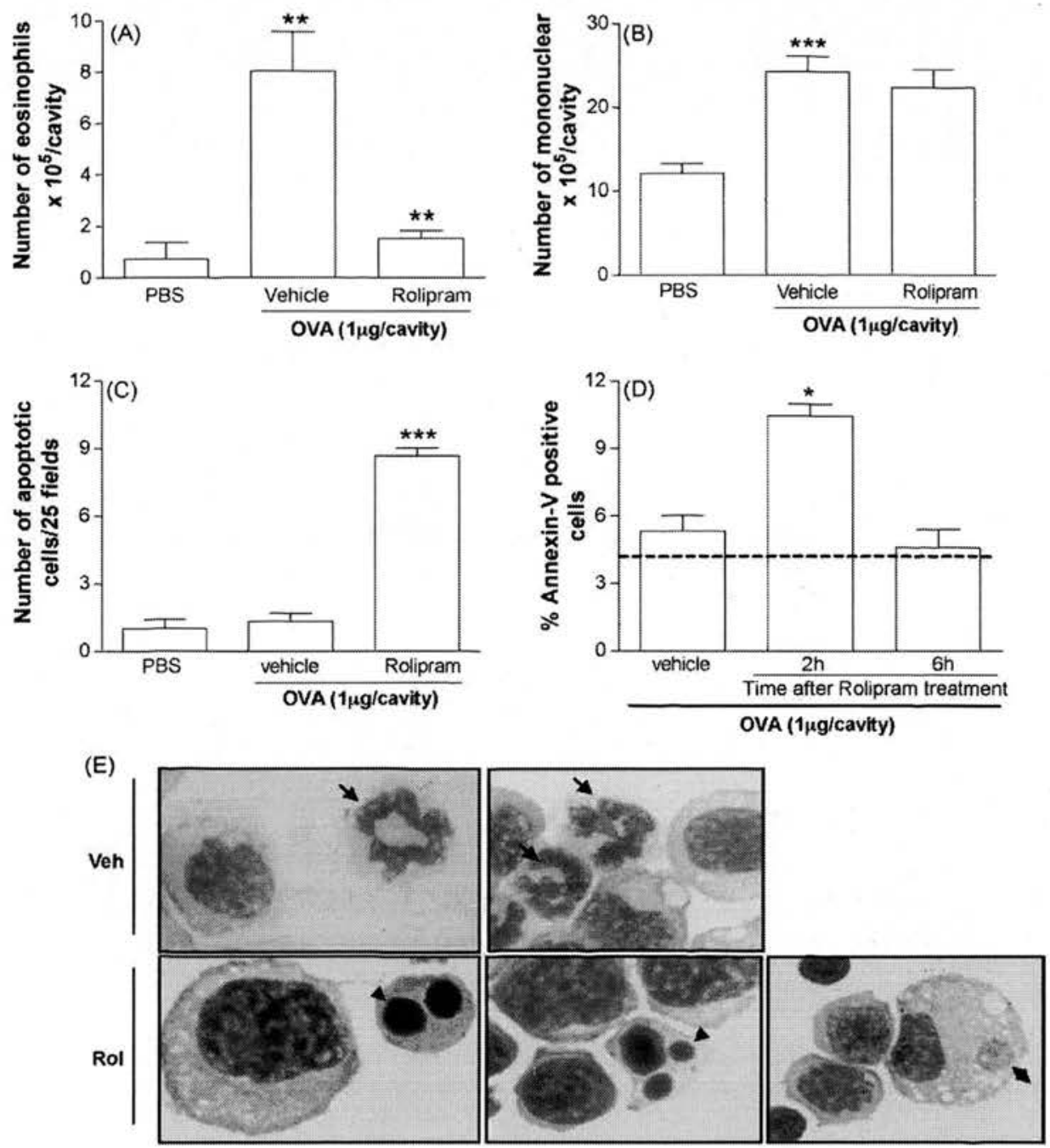

(F)

OVA (1 $\mu \mathrm{g} /$ cavity)

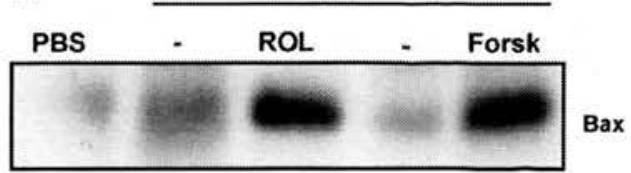

Fig. 2. Delayed treatment with rolipram resolves eosinophilic inflammation by promoting leukocyte apoptosis. Immunized mice were challenged with OVA or PBS and $24 \mathrm{~h} \mathrm{later.}$ received a systemic (s.c) injection of rolipram $(6 \mathrm{mg} / \mathrm{kg}$, i.p.) or drug vehicle. The number of eosinophils (A), mononuclear cells (B) and cells with distinctive apoptotic morphology (C) was assed $48 \mathrm{~h}$ after antigen-challenge as described in Section 2. Results are expressed as the number of cells per cavity, or number of cells with distinctive apoptotic morphology in 25 fields, and are shown as the mean + S.E.M of five mice in each group. $* P<0.01$ or $* * P<0.001$ when compared with PBS-injected mice or with vehicle treated OVAchallenged mice. In (D) $2.5 \times 10^{5}$ cells were collected $2 \mathrm{~h}$ and $6 \mathrm{~h}$ after drug or vehicle treatment and incubated with FITC-labeled annexin-V and propidium iodide. Results are expressed as the means \pm S.E.M of the percentage of cells staining with annexin-V-FITC alone. There were at least five mice in each group. " $P<0.05$, when compared with vehicle treated OVAchallenged mice. (E) Representative figures of apoptotic and non-apoptotic eosinophils. Vehicle (upper panels) and rolipram-treated (lower panels) animals are shown. Arrows indicate intact eosinophils, arrowheads indicate apoptotic eosinophils and double arrow indicates an apoptotic body inside a macrophage. In ( $F$ ), total extracts obtained of inflammatory cells harvested from the pleural cavity $2 \mathrm{~h}$ after drug treatments (i.e. $26 \mathrm{~h}$ after OVA-challenge) were subjected to Western blot to analysis Bax accumulation as described in Section 2. 
recruitment of neutrophil peaking at $4 \mathrm{~h} \mathrm{[29]} \mathrm{and} \mathrm{dropping} \mathrm{rapidly}$ to background levels by $24 \mathrm{~h}$ (Fig. 1D).

The next experiments were designed to investigate whether agents that promote increase of cAMP levels could interfere with eosinophil accumulation in the pleural cavity. We initially used rolipram, a selective PDE4 inhibitor [12]. Eosinophil influx was maximal at 24-48 h, with minor neutrophil contamination in the exudates at these times (compare Fig. 1C and D). Hence, we treated mice with rolipram $24 \mathrm{~h}$ after OVA-challenge, when inflammatory cell influx was already established, and performed the pleural lavage $24 \mathrm{~h}$ after rolipram treatment (i.e. $48 \mathrm{~h}$ after OVA-challenge) (See schematic representation in Fig. 1E).

Mice that were treated with rolipram showed a significant reduction in the accumulation of eosinophils in the pleural cavity at $48 \mathrm{~h}$ after challenge (Fig. 2A), without change in the number of mononuclear cells (Fig. 2B). The reduction of eosinophils was associated with an increase in the number of apoptotic cells at the pleural cavity, as demonstrated by morphologic criteria (Fig. 2C). The morphologic features of leukocytes at $24 \mathrm{~h}$ after treatment with rolipram are show in Fig. 2E. In agreement with the morphological assessment, there was a rapid increase in annexin- $\mathrm{V}^{+}$cells $2 \mathrm{~h}$ after treatment with rolipram, when compared with vehicle treated mice (Fig.2D). Treatment with rolipram also induced the expression of the pro-apoptotic protein Bax (Fig. 2F).

PDE4 inhibitors enhance intracellular levels of cAMP by inhibiting its degradation [12]. To investigate whether increases in cAMP by other means affected eosinophil apoptosis, we studied the effects of forskolin, an adenylate cyclase activator, and dbcAMP, a cell permeable cAMP analogue. The administration of forskolin or db-cAMP in the pleural cavity, when the inflammatory process was established, decreased eosinophil accumulation (Fig. 3A) and increased the number of apoptotic cells (Fig. 3B). Treatment with forskolin also enhanced Bax expression (Fig. 2F). A PKA inhibitor $\mathrm{H} 89$ prevented the resolution of eosinophilic inflammation caused by rolipram and db-AMP (Fig. 3C), implicating PKA as the cAMP effector in this resolving process.

\subsection{Resolution of OVA-induced pleurisy by rolipram is associated with inhibition of PI3K/Akt}

The PI3K/Akt pathway has been shown to mediate survival in many cell types [17]. Recently, we have demonstrated [8] that the PI3K/Akt pathway was important for the survival of eosinophils in vivo. With this in mind, we examined the levels of Akt phosphorylation after antigen-challenge and showed that there was a time-dependent increase of Akt phosphorylation in the inflammatory cells recovered from pleural cavity (Fig. 4A). The time-course of Akt phosphorylation mirrored the eosinophil influx into the pleural cavity (compare Fig. 4A and 1C). Treatment with rolipram $24 \mathrm{~h}$ after antigen-challenge $(24 \mathrm{~h}$ of $\mathrm{OVA}+2 \mathrm{~h}$ of rolipram) rapidly inhibited Akt phosphorylation to baseline levels (Fig. 4B). Similarly, treatment with db-cAMP or forskolin reduced Akt phosphorylation (data not shown). As a positive control, treatment with the PI3K inhibitor LY294002 also prevented Akt phosphorylation (Fig. 4B).

To explore the importance of the PI3K/Akt pathway for eosinophil recruitment/survival to the pleural cavity after antigen-challenge of immunized mice, we used the PI3K inhibitor LY294002 and the Akt inhibitor-IV. Treatment with the LY294002 or Akt inhibitor-IV reduced the number of eosinophils in the pleural cavity induced by antigen-challenge and increased the number of apoptotic cells (Fig. 4C and F). Altogether, these experiments show that inhibition of PDE4 or administration of cAMP mimetic induces clearance of eosinophils by preventing the phosphorylation of Akt, an important signal for eosinophil survival in the system.
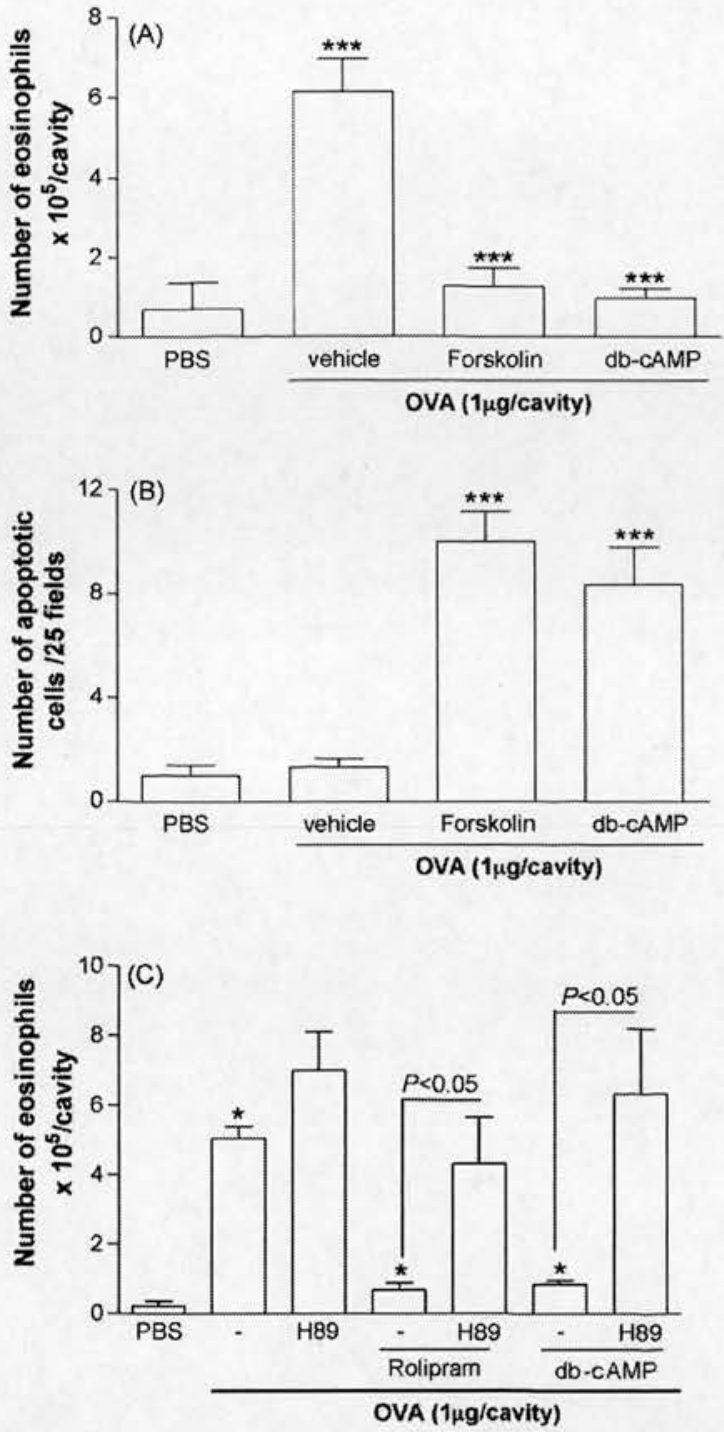

Fig. 3. Delayed treatment with cAMP elevating agents resolves eosinophilic inflammation in a PKA-dependent manner. Immunized mice were challenged with an intrapleural (i.pl.) injection of OVA $(1 \mu \mathrm{g} /$ cavity) or PBS and $24 \mathrm{~h}$ later were treated with a local (i.pl.) injection of forskolin $(10 \mu \mathrm{g} /$ mouse), db-cAMP $(100 \mu \mathrm{g} /$ mouse) or drug vehicle. In (C), mice were pre-treated by 30 min with $\mathrm{H} 89(60 \mu \mathrm{g}$ mouse, i.pl.) before receive rolipram or db-cAMP treatment. (A and C) Eosinophil recruitment and (B) number of apoptotic cells were evaluated $24 \mathrm{~h}$ after drug treatment, i.e, $48 \mathrm{~h}$ after antigen-challenge. Results are expressed as the number of eosinophils per cavity or as number of apoptotic cells in 25 fields and are shown as the mean \pm SEM of at least five mice in each group. ${ }^{*} P<0.05$ or ${ }^{* *} P<0.001$, when compared with PBS-injected mice or with vehicle treated OVA-challenged mice.

\subsection{Inhibition of $N F-\kappa B$ promotes resolution of established eosinophilic inflammation via induction of apoptosis}

The transcription factor nuclear factor kappa $B$ is a key regulator of several cellular functions, including leukocyte activation and survival [24-26]. The pro-survival/anti-apoptotic affects of Akt can be mediated by NF- $\mathrm{kB}$. For example, Akt may phosphorylate ІкB kinase leading to NF- $\kappa B$ activation $[27,28]$. To better characterize the involvement of NF- $\mathrm{KB}$ in allergic pleurisy, we determined the time-course and role of NF- $\mathrm{BB}$ activation in the model of OVAinduced pleurisy. As shown in Fig. 5, the kinetics of NF-kB activation in cells of pleural exudates, analyzed by NF-кB-DNAbinding activity (Fig. 5A), nuclear accumulation of the NF- $\kappa B$ p65 and 550 and IкB- $\alpha$ phosphorylation (Fig. 5B), paralleled the 
(A) OVA (1 $1 \mu \mathrm{g} /$ cavity)

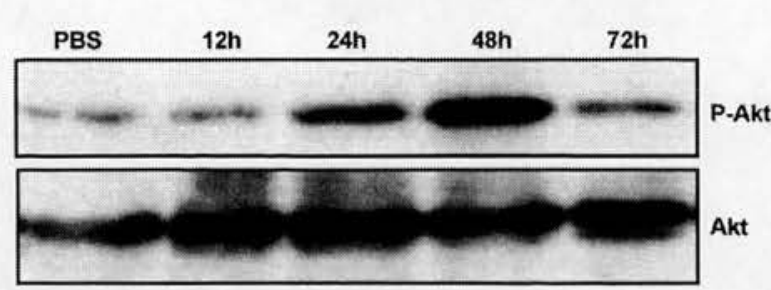

(B)

OVA (1 $1 \mu \mathrm{g} /$ cavity)
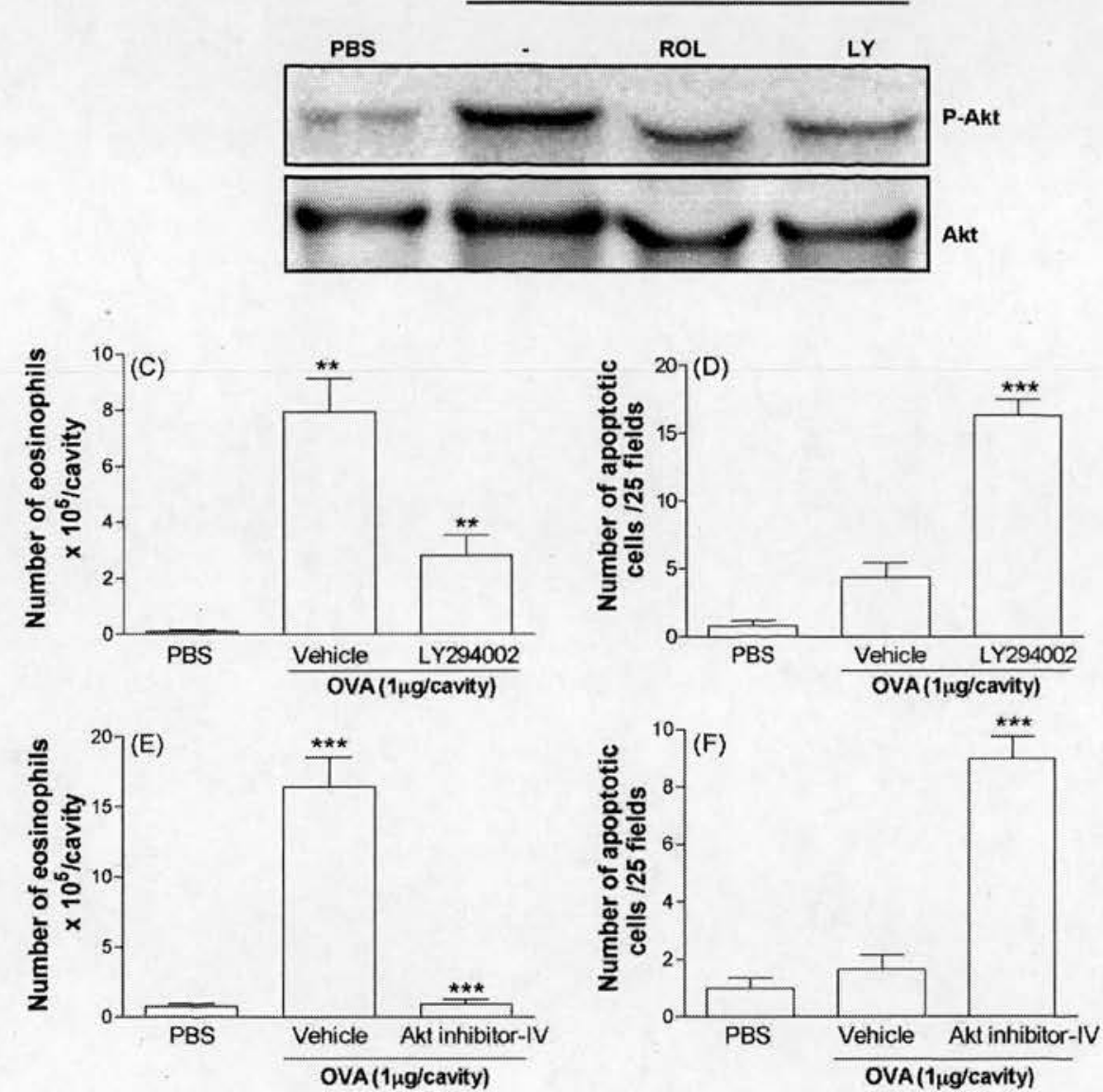

Fig. 4. Akt phosphorylation is decreased by rolipram and delayed treatment with inhibitors of the PI3K/Akt pathway resolve eosinophilic inflammation. Immunized mice were challenged with an intrapleural (i.pl.) injection of ovalbumin (OVA, $1 \mu \mathrm{g} /$ cavity) or PBS at indicated times (A) or were treatment with rolipram ( $6 \mathrm{mg} / \mathrm{kg}$, i.p.) or LY294002 (30 $\mu \mathrm{g} /$ mouse, i.pl.) $24 \mathrm{~h}$ after OVA-challenge (B). Akt phosphorylation was evaluated at indicated times (A) or after $26 \mathrm{~h}$ after antigen-challenge (24 h OVAchallenge $+2 \mathrm{~h}$ rolipram) (B) by Western blot analysis performed of total cell extracts obtained of pools of cells from at least five animals as shown in Section 2 . Data shown are representative of three independent experiments. In (C-F), the PI3K inhibitor LY294002 (30 $\mathrm{gg} / \mathrm{mouse}$, i.pl), Akt inhibitor-IV (10 $\mu \mathrm{g} / \mathrm{mouse}$, i.pl) or drug vehicle were administered in $24 \mathrm{~h}$-OVA-challenged mice and eosinophil recruitment ( $\mathrm{C}$ and $\mathrm{E}$ ) and number of apoptotic cells (D and F) were evaluated $24 \mathrm{~h}$ after drug treatment, i.e. $48 \mathrm{~h}$ after antigen-challenge. Results are expressed as the number of eosinophils per cavity or as number of apoptotic cells in 25 fields and are shown as the mean \pm SEM of at least five mice in each group. ${ }^{* P}>0.01$ or ${ }^{* * P} P<0.001$, when compared with PBS-injected mice or with vehicle treated OVA-challenged mice.

kinetics of total inflammatory cell influx into the pleural cavity, i.e. $\mathrm{NF}-\mathrm{\kappa B}$ activation was first detectable at $12 \mathrm{~h}$, peaked at $24-48 \mathrm{~h}$ of OVA-challenge and decreased thereafter $(72 \mathrm{~h})$.

We also evaluated whether the use of the NF-кB inhibitors given in the same way as cAMP elevating agents, i.e. at $24 \mathrm{~h}$ after antigen-challenge (Fig. 1E), could enhance resolution of eosinophilic inflammation. As seen in Fig. 6A, gliotoxin treatment given at $24 \mathrm{~h}$ after OVA-challenge drastically reduced the accumulation of eosinophils observed at $48 \mathrm{~h}$ but did not alter the number of mononuclear cells (PBS, $23.4 \pm 0.8 \times 10^{5}$ mononuclear per cavity; OVA + vehicle, $43 \pm 3.3 \times 10^{5}$ mononuclear per cavity; OVA + Glio,
$47.7 \pm 2.7 \times 10^{5}$ mononuclear per cavity; $n=5, P<0.001$ when compare PBS $\times$ OVA). The reduction of eosinophil number at $48 \mathrm{~h}$ was also seen when another structurally distinct NF- $\mathrm{BB}$ inhibitor, PDTC, was given at $24 \mathrm{~h}\left(\mathrm{OVA}+\right.$ vehicle, $10.9 \pm 1.5 \times 10^{5}$ eosinophils per cavity; OVA + PDTC, $2.5 \pm 0.8 \times 10^{5}$ eosinophils per cavity; $n=5$, $P<0.001$ ). For comparison, treatment with dexamethasone (Dexa), a potent anti-inflammatory drug with various cellular targets [31], at $24 \mathrm{~h}$ after challenge diminished the accumulation of eosinophils in the pleural cavity (Fig. 6A).

Next, we evaluated the efficacy of the compounds at blocking $\mathrm{NF}-\kappa \mathrm{B}$ activity at $2 \mathrm{~h}$ after compound administration (i.e. $26 \mathrm{~h}$ after 

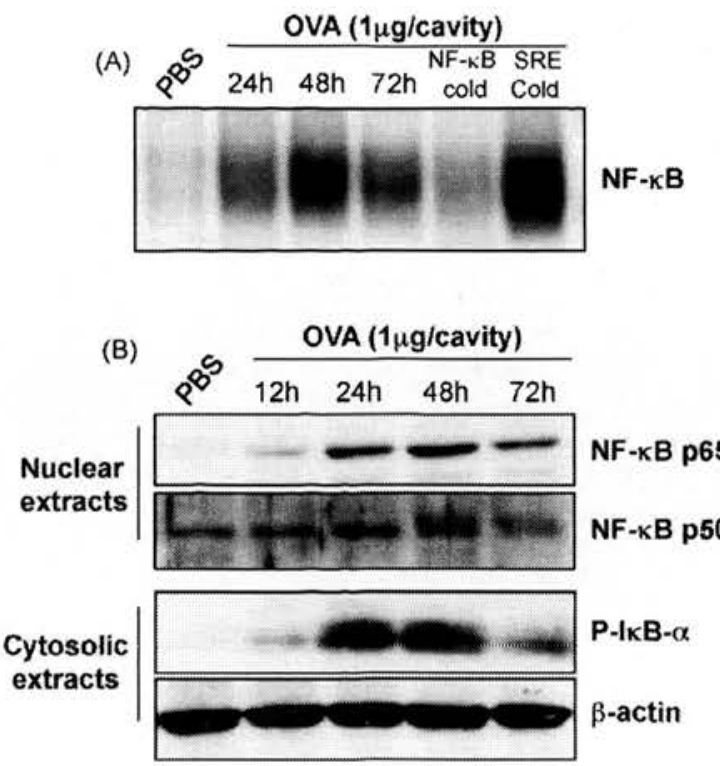

Fig. 5. Kinetics of NF- $\mathrm{KB}$ activation in allergic inflammation. Immunized mice were challenged with an i.pl. injection of OVA $(1 \mu \mathrm{g} /$ cavity) or PBS. The cells in the pleural cavity were colleted at indicated times and processed for protein extraction for EMSAs (A) and Western blot (B) analysis as described in Section 2. (A) EMSA was carried out of $10 \mu \mathrm{g}$ of nuclear protein incubated with an end-labeled probe containing the consensus NF- $\kappa$ B site. Specificity of the interactions was confirmed by competition of the probe with 100 -fold molar excess of the indicated cold oligodeoxynucleotide. (B) Nuclear extracts $(15 \mu \mathrm{g})$ or cytoplasmic extracts (40 $\mu \mathrm{g}$ ) were fractionated on $10 \%$ SDS-PAGE, transferred onto nitrocellulose membrane and then probed with anti p65, anti p50 or anti-phospho-IкB- $\alpha$ antibody. Re-probing of membrane with anti $\beta$-actin was used as control. Data are representative of three independent experiments in pools of cells from at least five animals.

antigen-challenge). As seen in Fig. 6B, treatment with gliotoxin inhibited OVA-induced NF-кB-DNA-binding activity and nuclear levels of $\mathrm{p} 65$.

The next experiments were performed in order to evaluate whether induction of apoptosis was involved in the ability of NF- $\mathrm{KB}$ inhibitors to resolve eosinophilic accumulation. To this end, apoptosis was assessed in several ways after the treatment with NF-кB inhibitors. The number of apoptotic cells, as accessed by morphologic criteria at $24 \mathrm{~h}$ after drug treatment, was markedly elevated in the pleural cavity of antigen-challenged mice treated with gliotoxin (Fig. 6C). Similarly, treatment with PDTC (OVA + vehicle, $0.5 \pm 0.8$ apoptotic cells/25 fields; OVA + PDTC, $6.0 \pm 0.5$ apoptotic cells $/ 25$ fields $n=5, P<0.01$ ) or dexamethasone (Fig. 6C) significantly increased the number of apoptotic events observed in the cavity of antigen-challenged mice. In agreement with the morphological assessment, there was a rapid increase in annexin- $V$ positive cells $2 \mathrm{~h}$ after treatment with gliotoxin or dexamethasone when compared with vehicle treated mice (Fig. 6D and E). Chromatin fragmentation assay showed a similar result (data not shown). Caspase activation may be involved in gliotoxin-induced apoptosis in granulocytes [24]. Consistent with the latter possibility, treatment with gliotoxin or dexamethasone increased caspase- 3 cleavage in cells of the pleural cavity of OVA-challenged mice, as analyzed $2 \mathrm{~h}$ after drug treatment (Fig. 6B). Altogether, the results suggest that inhibition of NF-KB induces inflammatory cell clearance from the pleural cavity of OVAchallenged mice by enhancing apoptosis of inflammatory cells.

\subsection{Resolution of OVA-induced pleurisy by rolipram is associated with inhibition of $N F-\kappa B$}

Next, we evaluated whether NF- $\mathrm{BB}$ inhibition was associated with rolipram-induced resolution. NF- $\mathrm{KB}$ activation was evaluated by EMSA and Western blot analysis for IкB- $\alpha$ in cells recovered from the pleural cavity. Treatment with rolipram or LY294002 $24 \mathrm{~h}$ after OVA-challenge greatly inhibited NF- $\mathrm{BB}$-DNA-binding activity and prevented IкB- $\alpha$ degradation (Fig. $7 \mathrm{~A}$ and B). Similarly, treatment with forskolin or db-cAMP also prevented the antigenassociated increased in IкB- $\alpha$ degradation (Fig. 7B).

\section{Discussion}

An understanding of the mechanisms involved in eosinophil recruitment, activation and survival in sites of allergic inflammation may be useful for the development of novel pharmacological therapies to control allergic diseases. In the present study, we demonstrate that increase of cAMP levels by means of PDE4 inhibition, adenylate cyclase activation or by mimicking cAMP action is effective at resolving eosinophilic inflammation after antigen-challenge of immunized mice. These agents induce the apoptosis of eosinophils resident in the pleural cavity in a PKAdependent manner and by preventing signaling via the PI3K/Akt pathway and, consequent, NF- $\kappa \mathrm{B}$ activation.

Treatment with the PDE4 inhibitor, forskolin or db-cAMP at peak of eosinophil accumulation greatly reduced the number of these cells. The reduction of eosinophil number was associated with an increase in the number of apoptotic events, as assessed by morphologic criteria, annexin- $\mathrm{V}$ binding and enhanced expression of Bax. Of note, eosinophil clearance was not associated with a decrease of mononuclear cells, suggesting that apoptotic cells were indeed eosinophils. Our findings that rolipram and forskolin treatment induced Bax accumulation is in agreement with previous findings showing that PDE4 inhibitors suppressed the expression of anti-apoptotic members of the Bcl-2 family and induced the pro-apoptotic protein Bax, thereby shifting the balance between pro- and anti-apoptotic members of the Bcl-2 family towards a pro-apoptotic direction in CLL cells $[34,35]$. In different cell lineages, cAMP-mediated signaling can be either antiapoptotic or pro-apoptotic. There have been conflicting reports on the effects of cAMP elevating agents on eosinophil survival/ apoptosis in vitro. In some experiments, cAMP has been shown to enhance survival and prevent apoptosis [36-38], whereas cAMP was shown to be involved in the induction of apoptosis in other experiments [39-41]. These discrepancies are probably due to differences in the source of eosinophils, dynamic abundance and distribution of intracellular cAMP effectors, previous priming of the cells and whether apoptosis-inducing agents were used or not. In our experiments, in vivo administration of compounds with different mechanism of action was clearly associated with resolution of eosinophilic inflammation. Hence, the net effect of cAMP elevation in the course of allergic inflammation is to resolve eosinophil, but not macrophage, accumulation.

Drugs that elevate cAMP may inhibit several eosinophil functions, including respiratory burst, degranulation, aggregation and lipid mediator production [12,42]. Because the agents were given to the whole animal and may have had access to several cell types in addition to the eosinophil, it is difficult to pin-point their major site of action. Known eosinophil survival factors such as GMCSF and IL-5 peak at $6 \mathrm{~h}$ after antigen-challenge $[8,29,30]$, hence much earlier than the schedule of administration (given at $24 \mathrm{~h}$ after challenge) of the compounds tested here. Moreover, treatment with anti-IL-5 or anti-GM-CSF at $24 \mathrm{~h}$ after challenge did not clear the eosinophils from the cavity (our unpublished observations). Of note, pre-treatment of mice with similar doses of these antibodies blocked OVA-induced eosinophil recruitment in the pleural cavity (our unpublished observations) suggesting that they act by mechanisms other than promoting survival in the system. Thus, administration of PDE4 inhibitors or other cAMP elevating agents may resolve eosinophilic inflammation by acting 


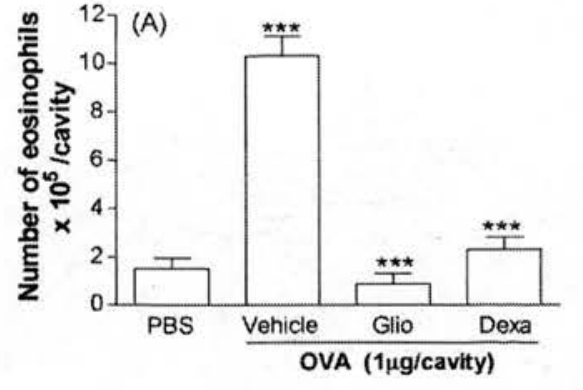

(B)
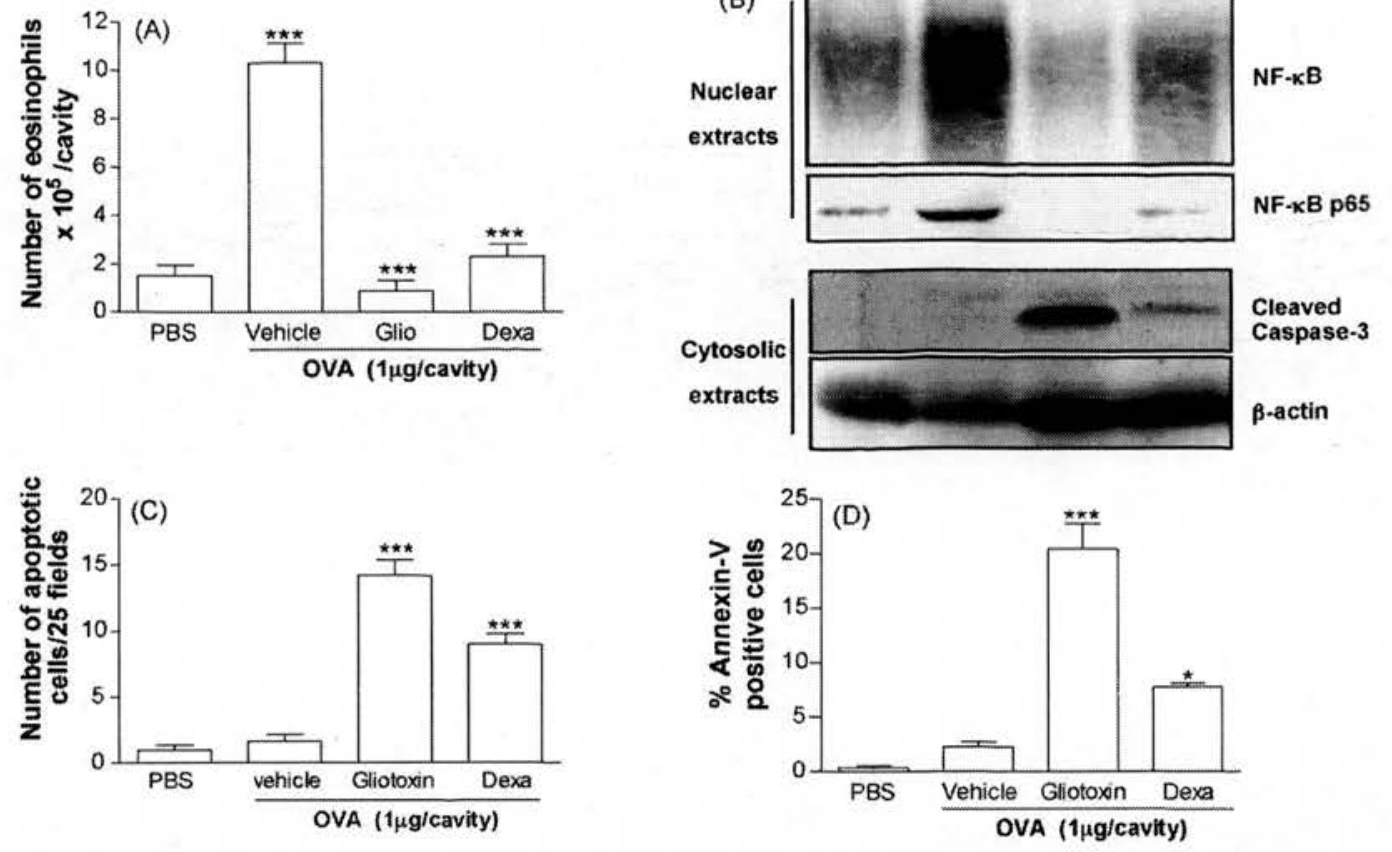

(E)
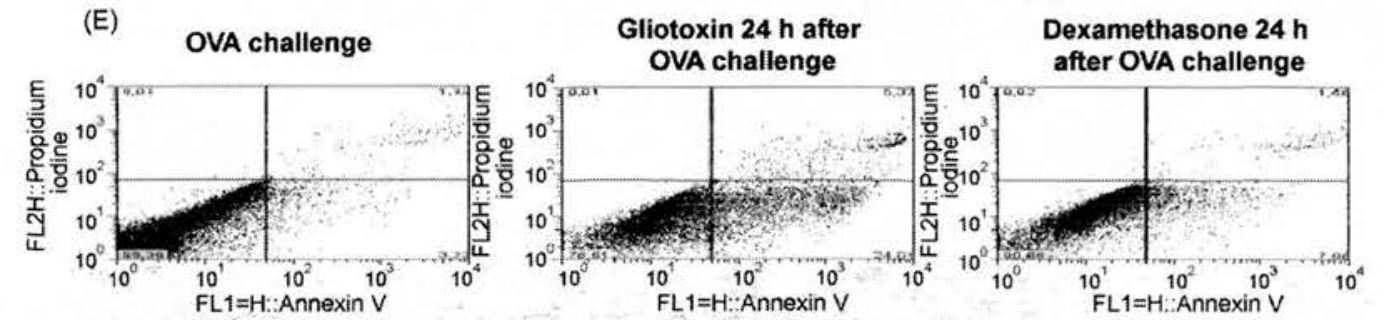

Fig. 6. Delayed treatment with NF- $\mathrm{KB}$ inhibitors reduces eosinophilic inflammation accumulation and induces apoptosis. Immunized mice were challenged with OVA or PBS and $24 \mathrm{~h}$ later, received a local (i.pl.) injection of gliotoxin (Glio) at dose of $20 \mu \mathrm{g} / \mathrm{mouse}(0.8 \mathrm{mg} / \mathrm{kg}$ ) or systemic (s.c) injection of dexamethasone (Dexa) at dose of $2.0 \mathrm{mg} / \mathrm{kg}$. or drug vehicle. In (A and C), cells were collected of the pleural cavity $48 \mathrm{~h}$ after OVA-challenge and were cyto-centrifuged, fixed, and stained with May-Grunwald-Giemsa. The proportion of cells with distinctive apoptotic morphology was evaluated in 25 fields. Results are expressed as the number of eosinophils per cavity or as number of apoptotic cells in 25 fields and are shown as the mean \pm SEM of at least five mice in each group. In (B), cytosolic and nuclear extracts were obtained of inflammatory cells recovered of pleural cavity $2 \mathrm{~h}$ after drugs treatments e subjected to EMSA and Western blot analysis as described in Section 2. In (D and E), apoptosis was accessed by flow cytometry. Cells $\left(2.5 \times 10^{5}\right)$ were collected $2 \mathrm{~h}$ after drugs or vehicle treatments and incubated with FrTC-labeled annexin-V and propidium iodide. Results are expressed as the means \pm SEM of the percentage of cells staining with annexin-V-FITC alone. There were at least five mice in each group. ${ }^{*} P<0.05$ or ${ }^{* *} P<0.001$, when compared with PBS-injected mice or with vehicle treated OVA-challenged mice.

on eosinophils themselves or by regulating the secretion of survival factors other than GM-CSF and IL-5.

The binding of cAMP to proteins such as PKA and Epac (exchange protein directly activated by cAMP) explain most of its functional activities but there are additional, less well-characterized effector proteins $[9,18]$. Although nonspecific effects of H89 may exist [43], this is a widely used tool to assess the role of PKA in in vitro and in vivo systems. In our model system, PKA inhibition by H89 limited cAMP-mediated eosinophil clearance, suggesting that PKA may be the cAMP effector.

In addition to their central role in cell proliferation and migration, class I PI3K has also been implicated in the prevention of apoptotic cell death. For example, studies have demonstrated that the PI3K/Akt pathway is constitutively activated in the majority of human pancreatic cancer cell lines [44] and use of selective inhibitors of PI3K could inhibit growth and survival of tumors [45]. The PI3K pathway has also been shown to be an important factor of survival in monocytes [46], neutrophils [47], and eosinophils $[8,48]$. We have previously demonstrated that treatment with
Wortmannin, a PI3K inhibitor, at the peak of eosinophilic inflammation decreased Akt phosphorylation and promoted eosinophil apoptosis [8]. Activation of Akt is a major mechanism by which PI3K provides survival signals [17]. Here, we find that antigen-challenge promoted Akt phosphorylation with a timecourse that was parallel to the influx of eosinophils into the pleural cavity. The importance of the Akt pathway for eosinophil survival was evidenced by experiments using PI3K and Akt inhibitors. Moreover, treatment with rolipram inhibited antigen-induced Akt phosphorylation, suggesting that Akt is relevant for eosinophil survival in vivo and is a site for the action of cAMP elevating agents. Our results are consistent with studies which demonstrate a crosstalk between cAMP-dependent and PI3K pathways [22,23]. Particularly, the studies of Smith and colleagues [23] showed that cAMP-mediated apoptosis in diffuse large B-cell lymphoma was associated with marked inhibition of PI3K/Akt pathway. Although it is not clear how cAMP modifies Akt activity, a recent report suggests that CAMP-dependent inhibition of Akt in thyroid cells is mediated by phosphatase $2 \mathrm{~A}$ involving both Epac and PKA CAMP 
(A)

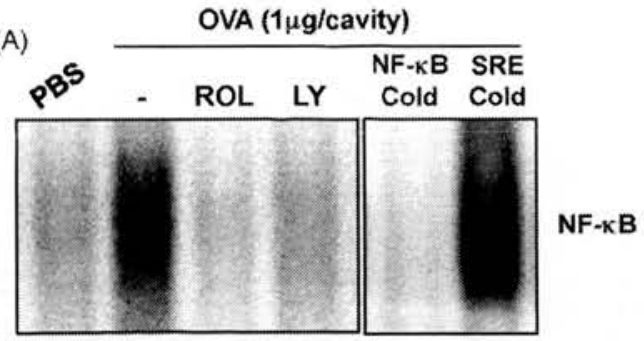

(B)

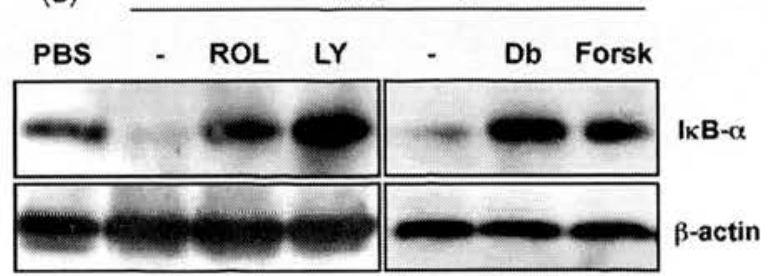

Fig. 7. Treatment with CAMP elevating agents and LY294002 prevents NF- $\kappa B$ activation in allergic inflammation. Immunized mice were challenged with an intrapleural (i.pl.) injection of OVA ( $1 \mu \mathrm{g} /$ cavity) or PBS and $24 \mathrm{~h}$ later were treated willı ıulipı dIII (6 my/kg. i.p.), LY294002 (30 $\mathrm{g} /$ mouse, i.pl.), forskolın ( $10 \mu \mathrm{g}$ / mouse), db-cAMP (100 $\mu \mathrm{g} /$ mouse) or drug vehicle. Nuclear and cytoplasmic extracts obtained of inflammatory cells harvested from the pleural cavity $2 \mathrm{~h}$ after drug treatments (i.e. $26 \mathrm{~h}$ after OVA-challenge) were subjected to EMSA (A) and Western blot (B) analysis, respectively. (A) EMSAs were carried out of $10 \mu \mathrm{g}$ of nuclear protein incubated with an end-labeled oligodeoxynucleotide probe containing the consensus NF- $\mathrm{KB}$ site. Specificity of the interactions was confirmed by competition of the probe with 100-fold molar excess of the indicated cold oligodeoxynucleotide. In (B) cytoplasmic extracts $(40 \mu \mathrm{g})$ were fractionated on $10 \%$ SDS-PAGE, transferred onto nitrocellulose membrane and then probed with anti-lкB- $\alpha$ antibody (upper panel) or with $\beta$-actin antibody (under panel). Data are representative of two independent experiments in pools of cells from at least five animals.

effectors [19]. Thus, cAMP may mediate its survival/pro-apoptotic effects by modifying PI3K/Akt. Observations of opposing effects of Epac and PKA on Akt activation can provide a potential mechanism for the apparent cell type-specific effects of cAMP [18].

Akt/PKB has direct effects on the apoptosis pathway, for example by phosphorylating pro-apoptotic proteins such as caspase-9 and BAD. Akt also have effects in transcription factors,

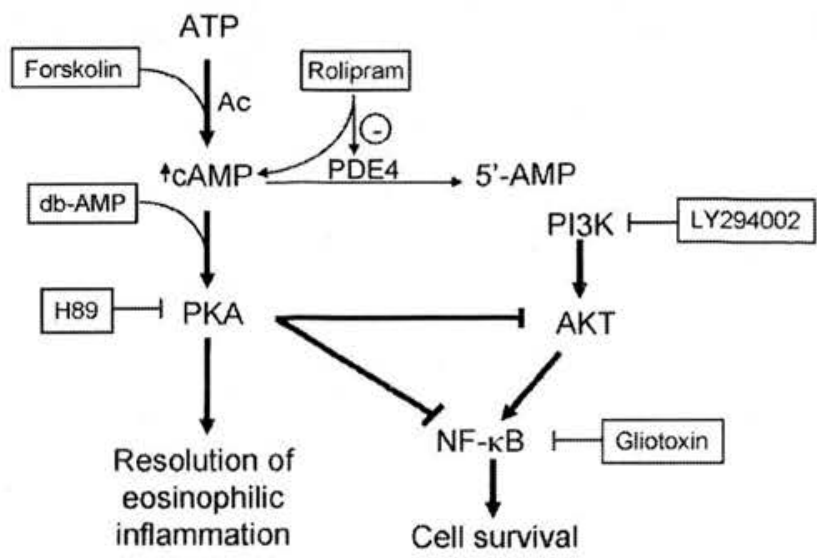

Fig. 8. Model for the resolution inducing effects of the delayed treatment with cAMP elevating agents in allergic eosinophilic inflammation. Increase in the intracellular levels of CAMP by means of the delayed treatment with an adenylate cyclase activator (forskolin), PDE4 inhibitor (rolipram) or db-cAMP promotes activation of a CAMP effector (PKA), which then inhibits signaling thought PI3K/Akt pathway. Down regulation of the Akt phosphorylation may itself lead to apoptosis or may prevent NF- $\kappa$ B activation, which is a relevant signal for eosinophil survival. Prevention of eosinophil survival leads to resolution of inflammation. including the Forkhead transcription factor and NF- $\kappa \mathrm{B}$ [17]. In this regard, Akt can induce cell survival by phosphorylating ІкB kinase and, consequently, activating NF- $\kappa B$ [27-28]. The activated NFmay then control cell survival via induction of the expression of anti-apoptotic genes [24-26]. In our experiments, NF- $\mathrm{KB}$ activation, as evaluated by DNA-binding activity, p65/p50 nuclear accumulation and IKB- $\alpha$ phosphorylation correlated temporally with the infiltration of leukocytes in the pleural cavity of antigenchallenge mice. Treatment with gliotoxin, PDTC or dexamethasone at doses that inhibited NF- $\mathrm{BB}$ activation, induced resolution of eosinophilic inflammation and increased leukocyte apoptosis without decreasing number of mononuclear cells. Importantly, cAMP elevation or PI3K inhibitors decreased antigen-induced NF$\kappa \mathrm{B}$ activation by preventing I $\kappa \mathrm{B}-\alpha$ degradation and NF- $\kappa \mathrm{B}-\mathrm{DNA}$ binding activity in vivo. Previous studies have shown that PDE4 inhibitors prevented NF- $\mathrm{B}$ B activation when given before or shortly after inflammatory stimulation [49-51], a finding consistent with the ability of PDE4 inhibitors to prevent leukocyte activation and recruitment [12]. However, our results are first to show the ability of delayed treatment with cAMP elevating agents to resolve eosinophilic inflammation and emphasize the importance of NF- $\mathrm{KB}$ for leukocyte survival in vivo. Moreover, our results are first to suggest that NF- $\mathrm{KB}$ activation is downstream of PI3K/ Akt activation and resolution inducing effects in vivo.

Taken together, our data demonstrate that cAMP elevating agents or mimetics promote resolution of established eosinophilic inflammation in a PKA-dependent manner and by inhibiting Akt phosphorylation and consequent NF-кB activation (see Fig. 8). To our knowledge, this is the first observation that cAMP promotes apoptosis in vivo via inhibition of a PI3K/Akt/NF-KB pathway. Hence, we suggest that elevation of cAMP in vivo may represent a powerful anti-inflammatory strategy for the treatment of diseases in which eosinophil accumulation is thought to play a relevant role.

\section{Acknowledgements}

We thank Valdinéria Borges and Ilma Marçal for technical assistance. This work was supported by grants from European Union FP6 (INNOCHEM, Grant number LSHB-CT-2005-518167), Fundação de Amparo a Pesquisa do Estado de Minas Gerais (FAPEMIG) and Conselho Nacional de Desenvolvimento Científico e Tecnológico (CNPq). Lirlândia P. Sousa was a recipient of a postdoctoral fellowship from CNPq.

\section{References}

[1] Cara DC, Negrao-Correa D, Teixeira MM. Mechanisms underlying eosinophil trafficking and their relevance in vivo. Histol Histopathol 2000:15:899-920.

[2] Lacy P, Moqbel R. Immune effector functions of eosinophils in allergic airway inflammation. Curr Opin Allergy Clin Immunol 2001;1:79-84.

[3] Kita H. Gleich GJ. Chemokines active on eosinophils: potential roles in allergic inflammation. J Exp Med 1996:183:2421-6.

[4] Rossi AG. Hallett JM, Sawatzky DA, Teixeira MM, Haslett C. Modulation of granulocyte apoptosis can influence the resolution of inflammation. Biochem Soc Trans 2007;35:288-91.

[5] Cohen JJ. Apoptosis. Immunol Today 1993:14:126-30.

[6] Sawatzky DA, Willoughby DA, Colville-Nash PR, Rossi AG. The involvement of the apoptosis-modulating proteins ERK $1 / 2 \mathrm{Bcl}-\mathrm{xL}$. and $\mathrm{Bax}$ in the resolution of acute inflammation in vivo. Am J Pathol 2006;168:33-41.

[7] Rossi AG, Sawatzky DA, Walker A, Ward C, Sheldrake TA, Riley NA, et al. Cyclindependent kinase inhibitors enhance the resolution of inflammation by promoting inflammatory cell apoptosis. Nat Med 2006;12:1056-64.

[8] Pinho V, Souza DG, Barsante MM, Hamer FP, De Freitas MS, Rossi AG, et al. Phosphoinositide-3 kinases critically regulate the recruitment and survival of eosinophils in vivo: importance for the resolution of allergic inflammation.J Leukoc Biol 2005; 77:800-10.

[9] Serezani CH, Ballinger MN, Aronoff DM, Peters-Golden M. Cyclic AMP: master regulator of innate immune cell function. Am J Respir Cell Mol Biol 2008;39:127-32.

[10] Soderling SH, Beavo JA. Regulation of CAMP and cGMP signaling: new phosphodiesterases and new functions. Curr Opin Cell Biol 2000;12:174-9. 
[11] Souness JE, Aldous D. Sargent C. Immunosuppressive and anti-inflammatory effects of cyclic AMP phosphodiesterase (PDE) type 4 inhibitors. Immunopharmacology 2000;47:127-62.

[12] Teixeira MM, Gristwood RW, Cooper N, Hellewell PG. Phosphodiesterase (PDE)4 inhibitors: anti-inflammatory drugs of the future? Trends Pharmacol Sci 1997:18:164-71.

[13] Alves AC, Pires AL, Cruz HN, Serra MF, Diaz BL, Cordeiro RS, et al. Selective inhibition of phosphodiesterase type IV suppresses the chemotactic responsiveness of rat eosinophils in vitro. Eur J Pharmacol 1996:312:89-96.

[14] Souza DG, Cassali GD, Poole S, Teixeira MM. Effects of inhibition of PDE4 and TNF-alpha on local and remote injuries following ischaemia and reperfusion injury, Br J Pharmacol 2001:134:985-94.

[15] Diaz BL, Serra MF, Alves AC, Cordeiro SB, Martins MA, e Silva PM. Local exposure to salbutamol or Bt2 cyclic AMP inhibits pleural exudation and leukocyte influx caused by antigen in rats. Eur J Pharmacol 1996:296:173-80.

[16] Hallsworth MP, Giembycz MA, Barnes PJ, Lee TH. Cyclic AMP-elevating agent prolong or inhibit eosinophil survival depending on prior exposure to GM-CSF. Br J Pharmacol 1996;117:79-86.

[17] Song G, Ouyang G, Bao S. The activation of Akt/PKB signaling pathway and cell survival. J Cell Mol Med 2005:9:59-71.

[18] Mei FC, Qiao J. Tsygankova OM, Meinkoth JL, Quilliam LA, Cheng X. Differential signaling of cyclic AMP: opposing effects of exchange protein directly activated by cyclic AMP and CAMP-dependent protein kinase on protein kinase B activation. I Biol Chem 2002;277:11497-504.

[19] Hong K, Lou L, Gupta S, Ribeiro-Neto F, Altschuler DL. A novel Epac-Rap-PP2A signaling module controls cAMP-dependent Akt regulation. J Biol Chem 2008:283:23129-38.

[20] Cass LA, Summers SA, Prendergast GV, Backer JM, Birnbaum M], Meinkoth JL. Protein kinase A-dependent and -independent signaling pathways contribute to cyclic AMP-stimulated proliferation. Mol Cell Biol 1999;19:5882-91.

[21] Cullen KA, McCool J. Anwer MS, Webster CR. Activation of cAMP-guanine exchange factor confers PKA-independent protection from hepatocyte apoptosis. Am J Physiol Gastrointest Liver Physiol 2004;287:G334-43.

[22] Kim S, Jee K, Kim D, Koh H, Chung J. Cyclic AMP inhibits Akt activity by blocking the membrane localization of PDK1. J Biol Chem 2001:276:12864-70.

[23] Smith PG, Wang F, Wilkinson KN, Savage KJ, Klein U, Neuberg DS, et al. The phosphodiesterase PDE4B limits CAMP-associated PI3K/AKT-dependent apoptosis in diffuse large B-cell lymphoma. Blood 2005;105:308-16.

[24] Ward C, Chilvers ER, Lawson MF, Pryde JG, Fujihara S, Farrow SN, et al. NFkappaB activation is a critical regulator of human granulocyte apoptosis in vitro. J Biol Chem 1999:274:4309-18.

[25] Ward C, Walker A, Dransfield I, Haslett C, Rossi AG. Regulation of granulocyte apoptosis by NF-kappaB. Biochem Soc Trans 2004:32:465-7.

[26] Dutta J, Fan Y, Gupta N, Fan G, Gelinas C. Current insights into the regulation of programmed cell death by NF-kappaB. Oncogene 2006:25:6800-16.

[27] Nair AS, Shishodia S, Ahn KS, Kunnumakkara AB, Sethi G, Aggarwal BB. Deguelin, an Akt inhibitor, suppresses IkappaBalpha kinase activation leading to suppression of NF-kappaB-regulated gene expression, potentiation of apoptosis, and inhibition of cellular invasion. J Immunol 2006:177:5612-22.

[28] Kane LP, Shapiro VS, Stokoe D, Weiss A. Induction of NF-kappaB by the Akt/PKB kinase. Curr Biol 1999;9:601-4.

[29] Klein A, Talvani A, Cara DC, Gomes KL, Lukacs NW, Teixeira MM. Stem cell factor plays a major role in the recruitment of eosinophils in allergic pleurisy in mice via the production of leukotriene B4. J Immunol 2000;164:4271-6.

[30] Klein A, Talvani A, Silva PM, Martins MA, Wells TN, Proudfoot A, et al. Stem cell factor-induced leukotriene B4 production cooperates with eotaxin to mediate the recruitment of eosinophils during allergic pleurisy in mice. J Immunol 2001:167:524-31.

[31] McColl A, Michlewska S, Dransfield I, Rossi AG. Effects of glucocorticoids on apoptosis and clearance of apoptotic cells. Sci World J 2007:7:1165-81.

[32] Sousa LP, Silva BM, Brasil BS, Nogueira SV, Ferreira PC, Kroon EG, et al. Plasminogen/plasmin regulates alpha-enolase expression through the MEK/ ERK pathway. Biochem Biophys Res Commun 2005;337:1065-71.
[33] Souza DG, Vieira AT, Pinho V, Sousa LP, Andrade AA, Bonjardim CA, et al. NFkappaB plays a major role during the systemic and local acute inflammatory response following intestinal reperfusion injury. $\mathrm{Br} \mathrm{J}$ Pharmacol 2005:145 246-54.

[34] Siegmund B, Welsch J, Loher F, Meinhardt G, Emmerich B, Endres S, et al. Phosphodiesterase type 4 inhibitor suppresses expression of anti-apoptotic members of the Bcl-2 family in B-CLL cells and induces caspase-dependent apoptosis. Leukemia 2001:15:1564-71.

[35] Moon EY, Lerner A. PDE4 inhibitors activate a mitochondrial apoptotic pathway in chronic lymphocytic leukemia cells that is regulated by protein phosphatase 2A. Blood 2003:101:4122-30.

[36] Machida K, Inoue H, Matsumoto K, Tsuda M, Fukuyama S, Koto H, et al. Activation of PI3K-Akt pathway mediates antiapoptotic effects of beta-adrenergic agonist in airway eosinophils. Am J Physiol Lung Cell Mol Physio 2005;288:L860-7,

[37] Parkkonen J, Hasala H, Moilanen E, Giembycz MA, Kankaanranta H. Phosphodiesterase 4 inhibitors delay human eosinophil and neutrophil apoptosis in the absence and presence of salbutamol. Pulm Pharmacol Ther 2008:21 499-506.

[38] Chang HS, Jeon KW, Kim YH, Chung IY, Park CS. Role of cAMP-dependent pathway in eosinophil apoptosis and survival. Cell Immunol 2000;203:29-38.

[39] Takeuchi M, Tatsumi Y, Kitaichi K, Baba K, Suzuki R, Shibata E, et al. Selective phosphodiesterase type 4 inhibitors reduce the prolonged survival of eosinophils stimulated by granulocyte-macrophage colony-stimulating factor. Bio Pharm Bull 2002:25:184-7.

[40] Wang W, Masu K, Tamura G, Suzuki K, Ohwada K, Okuyama K, et al. Inhibition of eosinophil survival by a selective inhibitor of phosphodiesterase 4 via the induction of apoptosis. Biol Pharm Bull 2005;28:515-9.

[41] Chung IY, Nam-Kung EK, Lee NM, Chang HS, Kim DJ. Kim YH, et al. The downregulation of $\mathrm{BCl}-2$ expression is necessary for theophylline-induced apoptosis of eosinophil. Cell Immunol 2000;203:95-102.

[42] Giembycz MA, Lindsay MA. Pharmacology of the eosinophil. Pharmacol Rev 1999;51:213-340.

[43] Murray AJ. Pharmacological PKA inhibition: all may not be what it seems. Sci Signal 2008; 1 :re4.

[44] Bondar VM, Sweeney-Gotsch B, Andreeff M, Mills GB, McConkey DJ. Inhibition of the phosphatidylinositol 3'-kinase-AKT pathway induces apoptosis in pancreatic carcinoma cells in vitro and in vivo. Mol Cancer Ther 2002:1 989-97.

[45] Krystal GW, Sulanke G, Litz J. Inhibition of phosphatidylinositol 3-kinase-Akt signaling blocks growth, promotes apoptosis, and enhances sensitivity of small cell lung cancer cells to chemotherapy. Mol Cancer Ther 2002;1 913-22.

[46] Kelley TW, Graham MM, Doseff AI, Pomerantz RW, Lau SM, Ostrowski MC et al. Macrophage colony-stimulating factor promotes cell survival through Akt/protein kinase B. J Biol Chem 1999;274:26393-8.

[47] Cowburn AS, Cadwallader KA, Reed BJ. Farahi N, Chilvers ER. Role of PI3kinase-dependent Bad phosphorylation and altered transcription in cytokinemediated neutrophil survival. Blood 2002;100:2607-16.

[48] Myou S, Leff AR, Myo S, Boetticher E, Tong J, Meliton AY, et al. Blockade of inflammation and airway hyperresponsiveness in immune-sensitized mice by dominant-negative phosphoinositide 3-kinase-TAT. J Exp Med 2003:198 1573-82.

[49] Sanchez AJ, Puerta C, Ballester S, Gonzalez P. Arriaga A, Garcia-Merino A. Rolipram impairs NF-kappaB activity and MMP-9 expression in experimental autoimmune encephalomyelitis. J Neuroimmunol 2005:168:13-20.

[50] Schmitz T, Souil E, Herve R, Nicco C, Batteux F, Germain G, et al. PDE4 inhibition prevents preterm delivery induced by an intrauterine inflammation. J Immunol 2007:178:1115-21.

[51] Kwak HJ, Song JS, Heo JY, Yang SD, Nam JY, Cheon HG. Roflumilast inhibits lipopolysaccharide-induced inflammatory mediators via suppression of nuclear factor-kappaB. p38 mitogen-activated protein kinase, and c-Jun NH2-terminal kinase activation. J Pharmacol Exp Ther 2005:315:1188-95. 


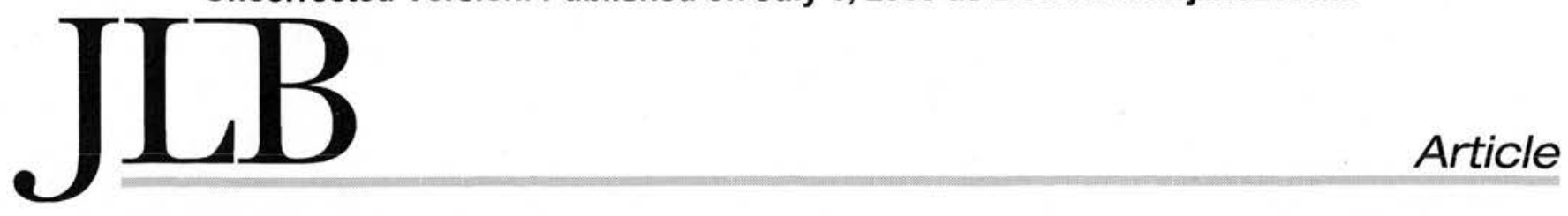

\title{
Secondary necrosis of apoptotic neutrophils induced by the human cathelicidin LL-37 is not proinflammatory to phagocytosing macrophages
}

\author{
Hsin-Ni Li, ${ }^{*}$ Peter G. Barlow, ${ }^{*}$ Johan Bylund, ${ }^{+}$Annie Mackellar, ${ }^{*}$ Åse Björstad, ${ }^{\dagger}$ \\ James Conlon, ${ }^{*}$ Pieter S. IIiemstra, ${ }^{\ddagger}$ Chris Haslett, ${ }^{*}$ Mohini Gray, ${ }^{*}$ A. John Simpson, ${ }^{*}$ \\ Adriano G. Rossi,* and Donald J. Davidson*,1 \\ *MRC/University of Edinburgh Centre for Inflammation Research, Queen's Medical Research Institute, Edinburgh, Scotland; \\ 'Rheumatology Department, University of Gothenburg, Sweden; and 'Department of Pulmonology, Leiden University Medical \\ Center, The Netherlands \\ KECEIVED FEBKUAKY 2, 2U09; KEVISED MAY 14, 2009; ACCEPTED MAY 16, 2009. DOI: 10.1189/j1b.0209050
}

\begin{abstract}
Cathelicidins are CHDP with essential roles in innate host defense but also more recently associated with the pathogenesis of certain chronic diseases. These peptides have microbicidal potential and the capacity to modulate innate immunity and inflammatory processes. PMN are key innate immune effector cells with pivotal roles in defense against infection. The appropriate regulation of PMN function, death, and clearance is critical to innate immunity, and dysregulation is implicated in disease pathogenesis. The efferocytosis of apoptotic PMN, in contrast to necrotic cells, is proposed to promote the resolution of inflammation. We demonstrate that the human cathelicidin LL-37 induced rapid secondary necrosis of apoptotic human PMN and identify an essential minimal region of $L L-37$ required for this activity. Using these LL-37-induced secondary necrotic PMN, we characterize the consequence for macrophage inflammatory responses. LL-37-induced secondary necrosis did not inhibit PMN ingestion by monocyte-derived macrophages and in contrast to expectation, was not proinflammatory. Furthermore, the anti-inflammatory effects of apoptotic PMN on activated macrophages were retained and even potentiated after LL-37-induced secondary necrosis. However, this process of secondary necrosis did induce the release of potentially harmful PMN granule contents. Thus, we suggest that LL-37 can be a potent inducer of PMN secondary necrosis during inflammation without promoting macrophage inflammation but may mediate host damage through PMN granule content release un-
\end{abstract}

Abbreviations: $A V=A n n e x i n V, B A L F=$ bronchoalveolar lavage fluid, $\mathrm{CD} 40 \mathrm{~L}=\mathrm{CD} 40$ ligand, $\mathrm{CHDP}=$ cationic host defense peptide(s). $\mathrm{DC}=$ dendritic cell, hCAP-18=human cationic antimicrobial protein, $\mathrm{LAL}=\mathrm{Limulus}$ amoebocyte $\mathrm{ysate}, \mathrm{mCRAMP}=$ mouse cathelicidin anti microbial peptide, $\mathrm{MDM}=$ monocyte-derived macrophage(s), $\mathrm{MPO}=$ myeloperoxidase, $\mathrm{Pl}=$ propidium iodide, $\mathrm{PMN}=$ polymorphonuclear granulocytes, TEM=transmission electron microscopy der chronic or dysregulated conditions. J. Leukoc. Biol. 86: 000-000; 2009.

\section{Introduction}

PMN are important first-line innate immune cells, which are mobilized rapidly in response to infection and injury. However, the arsenal of products used by PMN to destroy microbes is also potentially deleterious to host cells. Appropriate regulation of PMN influx, activation, death, and removal is therefore critical, and dysregulation of these processes has been implicated in the pathogenesis of chronic lung diseases [1, 2].

PMN have a short half-life and undergo spontaneous apoptosis. This programmed and regulated form of cell death enables recognition, ingestion, and removal by macrophages or DCs, a process involving multiple receptors and adaptors [3]. Efferocytosis (the uptake of apoptotic cells) can protect the host from the release of toxic PMN intracellular contents and induce anti-inflammatory and immunosuppressive effects.

These include dampening the LPS-induced, proinflammatory cytokine response and enhancing anti-inflammatory responses by macrophages [4-6]. These processes, in addition to an active switch in the lipid mediators generated to the production of lipoxins, resolvins, and protectins [7], can stimulate the resolution of inflammation. However, in the absence of efferocytosis, apoptotic PMN will undergo secondary necrosis with loss of membrane integrity. Although necrosis is typically regarded as proinflammatory [1], the effect of PMN secondary necrosis on inflammation and the mechanisms of clearance of these cells are relatively poorly understood. Further understanding of these issues is of great significance to evaluate the impact of delayed PMN clearance in the resolution of inflammation.

1. Correspondence: MRC/University of Edinburgh Centre for Inflammation
Research, Queen's Medical Research Institute, W2.05, 47 Little France CresResearch, Queen's Medical Research Institute, W2.05, 47 Little France Crcs.
cent, Edinburgh EH16 4TJ, Scotland. E-mail: donald.davidson@ed.ac.uk 
The survival and death of PMN can be modulated significantly by the inflammatory milieu, including cytokines such as TNF- $\alpha$ and GM-CSF, TLR agonists (e.g., LPS), and bacterial products (e.g., pyocyanin) [8], in addition to potential therapeutic modulators [9]. The human CHDP (also known as antimicrobial peptide) LL-37 can also modulate PMN death pathways in vitro $[10,11]$. We have demonstrated that LL-37 promotes PMN necrosis at the expense of apoptosis [10]. However, the mechanisms involved and the consequences for inflammation remain to be determined.

CHDP are key evolutionarily conserved components of innate host defense systems, which are being modified and developed as novel, antimicrobial therapeutic agents. The two major CHDP families in vertebrates are defensins and cathelicidins. LL-37 is the predominant, mature cationic peptide fragment of the sole human cathelicidin hCAP-18, which is found at high concentration in neutrophil specific granules and is cleaved to its active form by proteinase- $3[12,13]$. LL-37 is also produced by epithelial cells from a variety of tissues in response to infectious and inflammatory stimuli and is expressed by macrophages and other leukocytes [14]. In addition to direct microbicidal potential, LL-37 has a broad range of immunomodulatory functions including antiendotoxic activity and chemotactic function, modulation of chemokine and cytokine responses, promotion of cell wound-healing and angiogenesis, modulation of DC differentiation and function, and the capacity to modulate cell death pathways $[10,11,15-27]$. In vivo evidence in humans and mice supports a critical role in innate defense [28-31], hCAP-18/LL-37 concentration in BALF is increased significantly in human lung infections [32], and overexpression of LL-37 in the murine lung enhanced the clearance of infection [33]. However, increased levels of hCAP-18/LL-37 have also been associated with and implicated in chronic disease processes [34-36]. Thus, despite a fundamental role in innate defense, dysregulated control of LL-37 may be detrimental to the host. The key immunomodulatory properties that contribute to the physiological role of LL-37 in host defense and how these might contribute to disease pathogenesis when dysregulated remain unresolved.

We hypothesized that LL-37-induced necrosis of PMN could alter efferocytosis and the nature of the subsequent inflammatory response and could lead to the release of potentially damaging PMN intracellular contents. To examine the role of LL37-induced PMN necrosis on the inflammatory response, we characterized the dynamics of LL-37-induced PMN necrosis before evaluating the effect of LL-37-induced PMN necrosis on efferocytosis and macrophage inflammatory responses and the consequences for release of PMN intracellular contents.

\section{MATERIALS AND METHODS}

\section{Reagents}

rhGM-CSF was purchased from Research Diagnostics Inc. (Flanders, NJ, USA). R-Roscovitine was supplied by A. G. Scientific (San Diego, CA, USA). Ultra-pure LPS from the Escherichia coli 0111:B4 strain was purchased from InvivoGen (San Diego, CA, USA). Dexamethasone was purchased from Organon Laboratories Ltd. (Cambridge, UK). rhCD40L and IFN- $\gamma$ were purchased from PeproTech ED Ltd. (London, UK).

\section{LL-37 (LLGDFFRKSKEKIGKEFKRIVQRIKDFLRNLVPRTES) and} mCRAMP (GLLRKGGEKIGEKLKKIGQKIKNFFQKLVPQPEQ) were synthesized by $\mathrm{N}$-(9-fluorenyl) methoxycarbonyl chemistry at the Nucleic Acid/Protein Service Unit at the University of British Columbia (Canada), as described previously [10]. Peptides were purified by reverse-phase HPLC and were at least $98 \%$ pure. The concentration of the peptides in solution was de termined by amino acid analysis. Scrambled LL-37 (RSLEGTDRFPFVRLKNSRKLEFKDIKGIKREQFVKIL) was purchased from CSS-Albachem Ltd. (Glads muir, Scotland, UK), and the panel of 16 overlapping 22-mer partial LL-37 peptides (from N-terminal peptide LLGDFFRKSKEKIGKEFKRIVQ through to C-terminal peptide EFKRIVQRIKDFLRNLVPRTES) was synthesized by Jen W. Drijfhout at Leiden University Medical Center (The Netherlands) as described previously [37]. Peptides were dissolved in endotoxin-free water (Sigma-Aldrich, Poole, UK) and stored at $-20^{\circ} \mathrm{C}$ until further use. All reagents were tested using a LAL kinetic-quantitative chromogenic LAL assay (Cambrex, Walkersville, MD, USA) to ensure they were free of endotoxin and reconstituted in endotoxin-tree water.

\section{Isolation of human blood neutrophils}

Human venous blood was collected according to Lothian Research Ethics Committee approval \#08/S1103/38 or \#1702/95/4/72 using sodium citrate anticoagulant (Phoenix Pharma Ltd., Gloucester, UK), and cells were separated by Dextran sedimentation, followed by discontinuous, isotonic Percoll gradient centrifugation as described previously [10]. Granulocytes were washed in PBS without calcium or magnesium (PAA Laboratories, Somerset, UK) and resuspended in IMDM (PAA Laboratories) with $10 \%$ FCS (Biosera, East Sussex, UK). Purity was assessed by morphological criteria using cytocentrifuge preparations and FACS analyses and antibodies against human CD16 (Caltag-Medsystems Ltd., Towcester, UK) and CD66b (BD Biosciences, San Diego, CA, USA) to distinguish PMN from eosinophils and against CD14, CD4, CD8, and CD19 (Caltag-Medsystems Ltd.). Granulocyte purity of $>98 \%$ was yielded by this method, and granulocytes were typically 95-98\% PMN. However, high eosinophil donors were also studied, demonstrating no significant effect of eosinophil number on LL-37-induced secondary necrosis of apoptotic PMN. Total cell number was assessed by hemacytometer counts and by NucleoCounter YC-100 (ChemoMetec, Allerød, Denmark) automated cell number counting.

\section{Assessment of PMN death}

Freshly isolated $\mathrm{PMN}$ were incubated at $37^{\circ} \mathrm{C}, 5 \% \mathrm{CO}_{2}$, at $5 \times 10^{6} / \mathrm{ml}$ in IMDM with $10 \%(\mathrm{v} / \mathrm{v})$ FCS in the presence of LL-37, GM-CSF, R-Roscovitine, LPS, mCRAMP, scrambled LL-37, or partial LL-37 peptides at the stated concentrations or in control media in triplicate over the time periods detailed. To induce primary necrosis, freshly isolated cells were heated at $65^{\circ} \mathrm{C}$ for $30 \mathrm{~min}$. Cell death was assessed by light microscopic evaluation of apoptotic morphology as described [10] and also by examining cells stained at $4^{\circ} \mathrm{C}$ with FITC-labeled AV (Roche Applied Sciences, West Sussex, UK), diluted 1:2000 in HBSS with $5 \mathrm{mM} \mathrm{CaCl}_{2}$, and $3 \mu \mathrm{g} / \mathrm{ml}$ PI (Invitrogen Ltd., Paisley, UK) for flow cytometric evaluation using a FACSCalibur, counting $\geq 10,000$ cells, and analyzed using Flojo software (TreeStar Inc., Ashland, OR, USA). High concentrations of LL-37 can lead to a complete loss of cells from analysis $[10,38]$. To evaluate this accurately, the concentration-dependent induction of necrosis was determined by FACS, and the total detectable PMN numbers were determined using a NucleoCounter YC-100 (ChemoMetec) after $20 \mathrm{~h}$ culture over a range of LL-37 concentrations $(\leq 25 \mu \mathrm{g} / \mathrm{ml})$. An approximate $\mathrm{EC}_{50}=3.8 \mu \mathrm{g} / \mathrm{ml}$ was determined for LL-37-induced necrosis, and LL-37 $\geq 10 \mu \mathrm{g} / \mathrm{ml}(\sim 2 \mu \mathrm{M})$ was found to induce a significant and concentration-dependent loss in total detectable cell number (total cell number as a proportion of control sample $=81 \% \pm 5 \%$ at $20 \mathrm{~h}, \mathrm{LL}-3710 \mu \mathrm{g} / \mathrm{ml} ; P<0.01 ; n=20)$. In contrast, incubation with $\leq 5$ $\mu \mathrm{g} / \mathrm{ml} \mathrm{LL}-37$ did not result in a significant decrease, although a degree of cell loss was observed sometimes. LL-37 was therefore used at $\leq 5 \mu \mathrm{g} / \mathrm{ml}$ for further studies examining LL-37-mediated modulation of PMN cell death. 


\section{TEM}

Freshly isolated PMN were incubated with or without $5 \mu \mathrm{g} / \mathrm{ml} \mathrm{LL}-37$ for $20 \mathrm{~h}$, as described above, centrifuged at $250 \mathrm{~g}$ for $5 \mathrm{~min}$ at room temperature, and resuspended gently in $1 \mathrm{ml} \mathrm{2.5 \%}$ glutaraldehyde (Sigma-Aldrich) in $0.1 \mathrm{M}$ sodium cacodylate buffer, $\mathrm{pH} 7.3$ (Sigma-Aldrich), for $1 \mathrm{~h}$. Cells were washed in three $10-\mathrm{min}$ changes of $0.1 \mathrm{M}$ sodium cacodylate and postfixed in $1 \%$ osmium tetroxide in $0.1 \mathrm{M}$ sodium cacodylate for $45 \mathrm{~min}$ before three 10-min changes of $0.1 \mathrm{M}$ sodium cacodylate. Samples were then dehydrated in $50 \%, 70 \%, 90 \%$, and $100 \%$ normal grade acetones before two 10-min changes in analar acetone and embedded in Araldite resin. Toluidine blue-stained sections $(1 \mu \mathrm{m})$ were previewed before $60 \mathrm{~nm}$ ultrathin sections were cut from selected areas, stained in uranyl acetate and lead citrate, and then viewed in a Philips CM120 transmission electron microscope (FEI UK Ltd, Cambridge, UK). Images were taken on a Gatan Orius charged-coupled device camera (Gatan UK, Oxon, UK).

\section{Western immunoblotting}

Freshly isolated PMN were incubated as described above over a range of LL-37 concentrations. At Time $0 \mathrm{~h}$ or after $20 \mathrm{~h}$, cells were washed with PBS without $\mathrm{Ca}^{2+}$ and $\mathrm{Mg}^{2+}$, and proteins were extracted using Mammalian Protein Extraction Reagent (Pierce/Perbio Science UK, Cheshire, UK) containing $30 \mu \mathrm{l} / \mathrm{ml} \mathrm{Halt}{ }^{\mathrm{TM}}$ protease inhibitor cocktail, $30 \mu \mathrm{l} / \mathrm{ml} \mathrm{Halt}^{\mathrm{TM}}$ phosphatase inhibitor cocktail, $10 \mu \mathrm{l} / \mathrm{ml}$ EDTA solution (all Pierce/Perbio Science UK), $30 \mu \mathrm{M}$ pepstatin (Sigma-Aldrich), and $10 \mu \mathrm{M}$ lactacystin (Calbiochem/Merck Biosciences Ltd., Nottingham, UK). Total protein concentrations were determined by bicinchoninic acid assay (Pierce/Perbio Science UK). Equivalent total protein $(20-50 \mu \mathrm{g}$ ) was resolved in Pierce Precise gels (Pierce/Perbio Science UK) transferred to Immun-Blot polyvinylidene difluoride membranes (Bio-Rad Laboratories, Hercules, CA, USA) and immunoblotting performed as described [10] with anti-human cleaved caspase- 3 rabbit polyclonal antibody and reprobed with antipan-actin antibody (both Cell Signaling Technology, Beverly, MA, USA) for protein loading correction via densitometric analysis using ImageJ software (National Institutes of Health, Bethesda, MD, USA).

\section{Phagocytic studies}

Human PBMC were isolated by isotonic Percoll gradient centrifugation as described above and incubated at $4 \times 10^{6} / \mathrm{ml}$ in IMDM at $37^{\circ} \mathrm{C}, 5 \% \mathrm{CO}_{2}$, for $1 \mathrm{~h}$. Nonadherent cells were removed and adherent monocytes cultured for 5-7 days in IMDM and $10 \%$ autologous serum to generate MDM. For light microscopic analyses of phagocytosis, PMN were incubated previously for $20 \mathrm{~h}$, with or without LL-37 (or scrambled LL-37) at the stated concentrations (as described above), and the level of apoptosis was assessed as described. PMN $\left(2.5 \times 10^{6} ; \sim 2.5: 1\right.$ ratio of PMN:MDM) were overlaid onto HBSS-washed MDM monolayers for $1 \mathrm{~h}$ at $37^{\circ} \mathrm{C}$. MDM were then washed gently four times with IMDM to remove noningested PMN and fixed with $2.5 \%$ paraformaldehyde (Sigma-Aldrich) for $10 \mathrm{~min}$. PMN MPO was stained with $0.1 \mathrm{mg} / \mathrm{ml}$ dimethoxybenzidine (Sigma-Aldrich) and $0.03 \%(v / v) \mathrm{H}_{2} \mathrm{O}_{2}$ (Sigma-Aldrich) before analysis by light microscopy, counting at least $200 \mathrm{MDM}$ in five randomly selected fields of view to evaluate the proportion of MDM-containing peroxidase-positive cells, with $n=2$ replicates/experiment. Only MDM that had engulfed PMN clearly were scored as positive. For flow cytometric analyses of phagocytosis, freshly isolated PMN were stained with Cell Tracker Green (Molecular Probes, Eugene, OR, USA) at $37^{\circ} \mathrm{C}$ for $20 \mathrm{~min}$, according to the manufacturer's instructions, and then washed twice with PBS before incubation for $20 \mathrm{~h}$ in the presence or absence of LL-37, as described. The effects of LL-37 were not altered by Cell Tracker Green staining (data not shown). MDM were exposed to PMN as described above, but after $1 \mathrm{~h}$ incubation, cells were detached and collected using $0.05 \%$ trypsin/0.02\% EDTA solution (Cambrex). Samples were analyzed by FACSCalibur as described previously [39], with $n=2$ replicates/experiment. The number of Cell Tracker Green-positive events in the macrophage gate (based on forward-and side-scatter) represented the number of macrophages that had ingested PMN and was evaluated as a proportion of total number of MDM. As a positive control, wells of adher- ent monocytes were also cultured in the presence of $1 \mu \mathrm{M}$ dexamethasone for 5 days, a process demonstrated previously to up-regulate MDM phagocytosis of dead PMN [40].

\section{MDM cytokine production}

Freshly isolated PMN at $5 \times 10^{6} / \mathrm{ml}$ were incubated for $20 \mathrm{~h}$ in the presence or absence of LL-37, scrambled LL-37, or partial LL-37 peptides p1 (LLGDFFRKSKEKIGKEFKRIVQ), p2 (KIGKEFKRIVQRIKDFLRNLVP), or p3 (EFKRIVQRIKDFLRNLVPRTES) at the concentrations indicated in $10 \%$ FCS as described above. Samples $(1 \mathrm{ml})$ were collected and each split in two, and half of each sample was centrifuged at $230 \mathrm{~g}$ before collection of the supernatant and resuspension of the cells in $0.5 \mathrm{ml} \mathrm{X}$-vivo 10 media (Lonza Biologics, Slough, UK), with or without $10 \%$ FCS. Washed MDM, with or without concomitant exposure to $10 \mathrm{ng} / \mathrm{ml} \mathrm{E}$. coli 0111:B4 LPS or 3 $\mu \mathrm{g} / \mathrm{ml} \mathrm{rhCD} 40 \mathrm{~L} / 5 \mathrm{ng} / \mathrm{ml}$ rhIFN $-\gamma$, were then incubated at $37^{\circ} \mathrm{C}, 5 \% \mathrm{CO}_{2}$, for $18 \mathrm{~h}$ with $0.5 \mathrm{ml} \mathrm{PMN}$ in fresh X-vivo 10, PMN in the original IMDM with $10 \%$ FCS, IMDM with $10 \%$ FCS supernatant collected from overnightincubated PMN, or media alone. MDM alone and PMN alone in the presence of absence of LPS and/or LL-37 were also studied as controls. Supernatants were collected, centrifuged at $230 \mathrm{~g}$ for $6 \mathrm{~min}$ to remove cells and particulate debris, and then stored in aliquots at $-70^{\circ} \mathrm{C}$. The concentrations of TNF- $\alpha$, IL-6, IL-10, IL-12p70, and IL-1 $\beta$ in the supernatants were measured using BD Cytometric Bead Array human inflammation kits with a BD FACSArray (BD Biosciences) or Human TNF- $\alpha$ DuoSet ELISA (R\&D Systems, Minneapolis, MN, USA), according to the manufacturer's instructions.

\section{MPO release}

Freshly isolated PMN were incubated with different concentrations of L. -37 or scrambled peptide for $20 \mathrm{~h}$ as described above, centrifuged at 230 $\mathrm{g}$ for $6 \mathrm{~min}$, and placed immediately on ice, and the supernatants were collected. To detect MPO, $100 \mu \mathrm{l}$ supernatant samples (used neat or diluted in PBS) were incubated in the dark for $20 \mathrm{~min}$ at room temperature with $100 \mu \mathrm{l}$ substrate solution ( $1: 1$ mixture of $\mathrm{H}_{2} \mathrm{O}_{2}$ and tetramethylbenzidine; R\&D Systems), followed by addition of $50 \mu \mathrm{l} 2 \mathrm{~N} \mathrm{H}_{2} \mathrm{SO}_{4}$ stop solution and colorimetric analyses at $450 \mathrm{~nm}$ using a microplate reader. The concentration of MPO in the supernatants was evaluated as a percentage of MPO release after complete lysis of fresh control PMN using $0.1 \%$ Triton X-100 (Sigma-Aldrich).

\section{Statistical analysis}

GraphPad Prism 5 statistical software was used to determine statistical significance. One-way or two-way ANOVA with Bonferroni's multiple comparison post-tests or Student's t-tests was performed as appropriate. $P<0.05$ was considered significant. Values are expressed as mean \pm SEM.

\section{RESULTS}

\section{LL-37 induces the rapid secondary necrosis of apoptotic PMN}

We and others $[10,11]$ have demonstrated that the proportion of apoptotic $\left(\mathrm{AV}^{+} \mathrm{PI}^{-}\right)$cells detected in an in vitro model of spontaneous apoptosis of human PMN was decreased significantly when cells were cultured with LL-37. The most prominent consequence of this was an increased proportion of necrotic $\left(\mathrm{AV}^{+} \mathrm{PI}^{+}\right)$PMN [10]. To carefully characterize LL37-induced, necrotic PMN, with which to examine the consequences for macrophage inflammatory responses, a large donor pool ( $n=28$ different donors) was evaluated. This demonstrated the concentration-dependent capacity of LL-37 to promote PMN necrosis significantly at the expense of apoptosis, with no signifi- 
cant impact on the proportion of live $\left(\mathrm{AV}^{-} \mathrm{PI}^{-}\right)$cells at $\leq 5$ $\mu \mathrm{g} / \mathrm{ml} \mathrm{LL}-37$ using FACS (Fig. 1A) and morphological analyses. LL-37-induced necrotic PMN were observed to be similar to "late apoptotic" cells observed in control samples at $20 \mathrm{~h}$, with less-severe loss of membrane integrity than primary necrosis induced by heat treatment of cells (Fig. 1B). TEM was used to confirm the capacity of LL-37 to promote PMN necrosis after $20 \mathrm{~h}$ incubation when compared with control cells (Fig. $1, \mathrm{C}$ and $\mathrm{D})$.

We have demonstrated previously that LL-37 was not lytic to PMN [10]. Furthermore, no significant necrosis was observed at $0,1,4$, or $6 \mathrm{~h}$ (ref. [10], and data not shown). However, significant $(P \leq 0.001)$ induction of necrosis was observed at $12 \mathrm{~h}$ (data not shown) and thereafter, occurring at time-points at which substantial apoptosis was observed. In keeping with recent publications $[38,41]$, this suggested prior induction of apoptosis may be necessary and that LL-37 might induce secondary necrosis. To confirm this in our model system, PMN were exposed to early or late pulses of LL-37. PMN incubated with LL-37 ( $\leq 5 \mu \mathrm{g} / \mathrm{ml}$ ) for $4 \mathrm{~h}$, followed by washing and incubation in control media for $16 \mathrm{~h}$, had proportions of apoptotic, necrotic, and live cells identical to untreated controls (data not shown). In contrast, $20 \mathrm{~h}$ incubation of cells in control media, followed by exposure to LL-37 for just $10 \mathrm{~min}$ before FACS analyses, completely replicated the dose-dependent effect of $20 \mathrm{~h}$ incubation with LL-37 (Fig. 1E), demonstrating that LL-37 induced a rapid secondary necrosis of apoptotic PMN without affecting live cells.

\section{A}
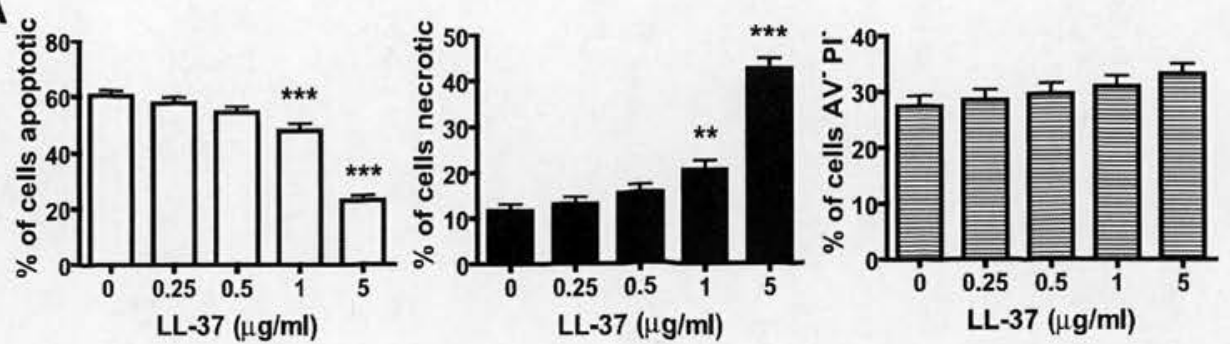

B
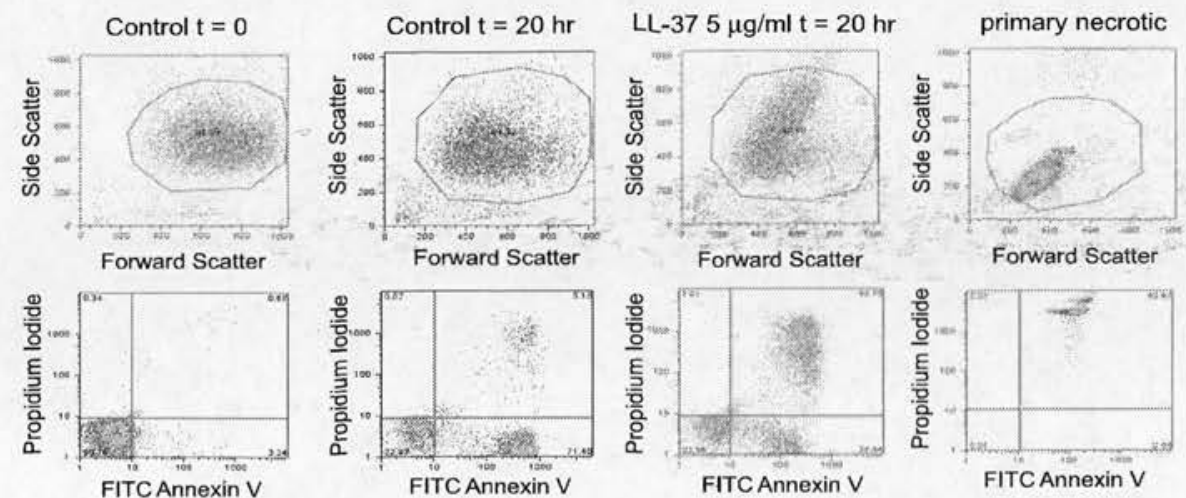

FITC Annexin V
C

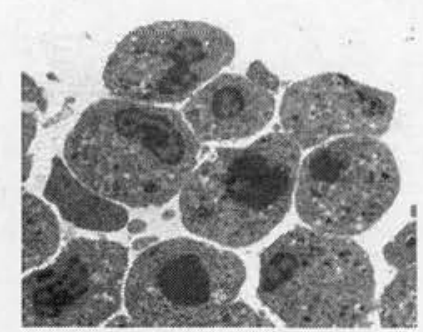

D

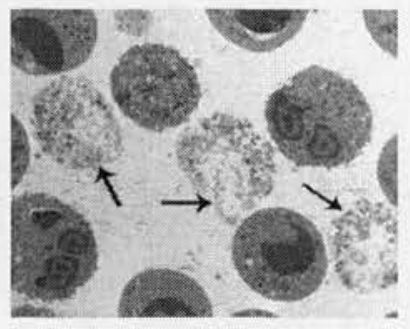

Figure 1. Induction of PMN necrosis by LL-37. (A-D) Freshly isolated human PMN were incubated for $20 \mathrm{~h}$ over a range of LL 37 concentrations before analyses. Primary necrosis was induced by heating at $65^{\circ} \mathrm{C}$ for $30 \mathrm{~min}$. Cell death was examined by FACS analyses and TEM morphology. (A) Apoptotic

(FITC-AV-positive, PI-negative), necrotic (FITC-AV-positive, PIpositive), and live (FITC-AVnegative, PI-negative) cells were enumerated. Figures represent mean values \pm SEM for $n=28$

different donors for each condition, performed in triplicate. Significance was assessed by one-way ANOVA with Bonferroni's multiple comparison test comparing each treatment with control; **, $P \leq 0.01$; ***, $P \leq 0.001$. (B) Representative FACS plots. (C and D) Representative TEM images of (C) untreated, apoptotic PMN and (D) PMN exposed to $5 \mu \mathrm{g} / \mathrm{ml} \mathrm{LL}-37$. Arrows indicate examples of necrotic PMN. (E) Freshly isolated human PMN were incubated for $20 \mathrm{~h}$ over a range of LL-37 concentrations ( $20 \mathrm{~h}$ samples) or incubated for $20 \mathrm{~h}$ in the absence of LL-37 followed by exposure to a range of LL-37 concentrations for $10 \mathrm{~min}$ (pulse samples) before FACS analyses of apoptotic, necrotic, and live cells. Panels represent mean values \pm SEM for $n=3$ donors performed in triplicate. Significance was assessed by two-way ANOVA with Bonferroni's post-test; **, $P \leq 0.01 ; * *, P \leq 0.001$, compared with untreated control. 


\section{Interaction of LL-37 with known modulators of PMN apoptosis}

To investigate whether LL-37 can interact with and modulate the effects of known modifiers of PMN apoptosis pathways, cells were incubated with LL-37 in the presence or absence of GM-CSF (a PMN survival factor [42]) or $R$-Roscovitine (a cyclin-dependent kinase inhibitor and inducer of PMN apoptosis [9]). GM-CSF reduced PMN apoptosis significantly at $20 \mathrm{~h}$, increasing the proportion of live cells with no effect on necrosis (Fig. 2A). Concomitant treatment with $5 \mu \mathrm{g} / \mathrm{ml} \mathrm{LL}-37$ had no effect on the capacity of GM-CSF to promote cell survival but significantly $(P<0.05)$ increased the proportion of necrotic cells with a reciprocal loss of apoptotic cells when compared with GM-CSF alone (Fig. 2A). R-Roscovitine induced PMN apoptosis significantly at $6 \mathrm{~h}$ with minimal necrosis (Fig. 2B). LL-37 alone $(\leq 25 \mu \mathrm{g} / \mathrm{ml})$ had no effect on cell death at this timepoint. Concomitant LL-37 treatment had no effect on the capacity of $R$-Roscovitine to induce cell death but significantly $(P<0.01)$ increased the proportion of necrotic cells in a dosedependent manner with a reciprocal loss of apoptotic cells when compared with $R$-Roscovitine alone (Fig. 2B). These data demonstrate no interaction with these direct modifiers of PMN apoptosis, but LL-37 induced secondary necrosis, relating in magnitude to the extent of induction of apoptosis.

In addition, studies were performed to assess the impact of LL-37 (a well-characterized antiendotoxic agent [15]) on the antiapoptotic effects of LPS in PMN [43]. LPS promoted PMN survival (Fig. 2C) significantly, inhibiting apoptosis without inducing necrosis (Fig. 2D). Concomitant incubation with LL-37 inhibited this effect significantly in a dose-dependent manner. LL-37 $(5 \mu \mathrm{g} / \mathrm{ml})$ blocked the pro-survival effects of LPS completely and significantly increased the proportion of dead cells that were necrotic $(P<0.01)$. However, the capacity of $5 \mu \mathrm{g} / \mathrm{ml} \mathrm{LL}-37$ to induce secondary necrosis was diminished significantly by LPS $(P<0.001)$. These data demonstrate LL-37mediated inhibition of the antiapoptotic effects of LPS and suggest a mutually inhibitory interaction with LPS diminishing LL-37-induced secondary necrosis.

\section{LL-37-mediated induction of secondary necrosis is conserved in mCRAMP and C-terminal partial peptides and is not primarily charge-dependent}

To examine the important characteristics of LL-37 for the capacity to induce secondary necrosis, FACS-based studies were performed using a scrambled LL-37 peptide (with conserved charge but altered amino acid sequence), the murine homologue mCRAMP, and a panel of sixteen 22-mer partial peptides spanning the sequence of LL-37 [37]. Incubation of PMN with scrambled LL-37 had no effect, whether exposed over $20 \mathrm{~h}$ or as a final 10 -min pulse (Fig. 3A). In contrast, mCRAMP exposure closely replicated the effects of LL-37 with significant, dose-dependent induction of secondary necrosis (Fig. 3B). Exposure to N-terminal 22-mer partial LL-37 peptides (from the peptide incorporating aa 1-22 of LL-37 through that spanning aa 10-31) had no significant effects (Fig. 3C). In contrast, C-terminal partial peptides (from the peptide incorporating aa 11-32 through that spanning aa 16$37)$ induced significant secondary necrosis $(P<0.01)$ in the absence of effects on live cells but to a lesser extent than fulllength LL-37. This identified a putative minimal core functional region for induction of secondary necrosis spanning aa 16-32. The effects of these peptides showed no correlation with charge, which ranged from +3 to +6 . These data demonstrate that the capacity to induce PMN secondary necrosis is evolutionarily conserved between mouse and human cathelicidin, is not primarily dependent on peptide charge, and is retained by C-terminal but not $\mathrm{N}$-terminal-truncated peptides of LL-37.
A

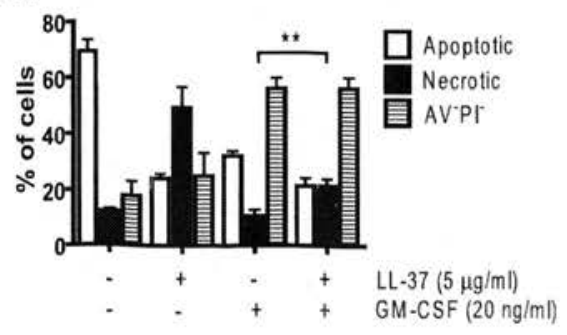

D

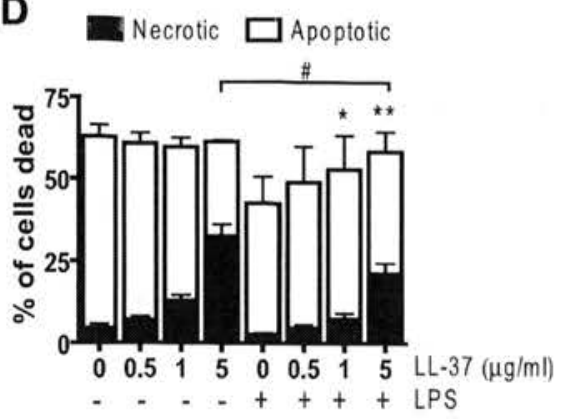

B

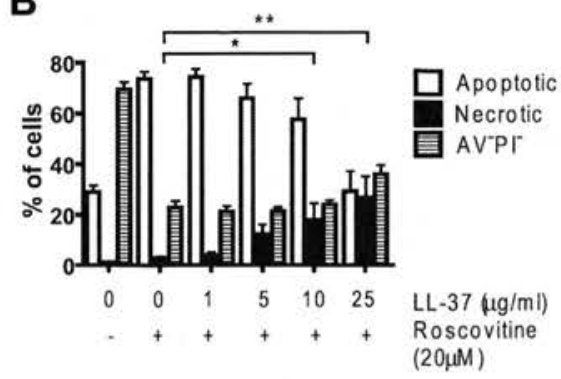

Figure 2. LL-37 exposure of PMN in the presence of modifiers of apoptotic pathways. Freshly isolated human PMN were incubated with LL-37 (at the concentrations indicated) for $20 \mathrm{~h}$ in the presence or absence of $20 \mathrm{ng} / \mathrm{ml}$ GM-CSF (A), for $6 \mathrm{~h}$ in the presence or absence of $20 \mu \mathrm{M}$ $R$-Roscovitine (B), or for $20 \mathrm{~h}$ in the presence or absence of $500 \mathrm{ng} / \mathrm{ml} \mathrm{E}$. coli LPS (C and D). Cell death was examined by FACS analyses. Figures represent mean values \pm SEM for $n \geq 3$ donors performed in triplicate. Significance was assessed by one-way ANOVA with Bonferroni's multiple comparison test comparing each treatment with control; *, $P \leq 0.05 ; * *, P \leq 0.01$, for necrotic (A, B, and D) or viable (C) cells in LL-37-treated samples compared with controls without LL-37 in the presence of (A) GM-CSF, (B) R-Roscovitine, or (C) LPS; ", $P<0.001$, for necrotic (D) or viable (C) cells in LPS-treated samples compared with controls without LPS in the comparable concentration of LL-37. 
Figure 3. Peptide specificity in the induction of PMN secondary necrosis. Freshly isolated human PMN were incubated with (A) a range of concentrations of scrambled LL-37 peptide for $20 \mathrm{~h}$, or for $10 \mathrm{~min}$ after $20 \mathrm{~h}$ incubation in the absence of peptide (pulse); $n=3$ donors performed in triplicate; (B) a range of concentrations of mCRAMP peptide; $n=$ 16 donors performed in triplicate; or (C) $5 \mu \mathrm{g} / \mathrm{ml}$ partial LL-37 peptides from a panel of 16 overlapping 22-mers using $\mathrm{N}$ terminal peptide (aa 1-22), then peptide spanning aa 2-23, and so on through $\mathrm{C}$-terminal peptide (aa 16-37) or full-length LL-37; $n=3$ donors performed in triplicale. Pe $\mu$ tides $\mu 1, \mu 2$, and $\mu^{3}$ are identified. Cell death was examined by FACS analyses as described. Figures represent mean values \pm SEM. Significance was assessed by one-way ANOVA with Bonferroni's multiple comparison test comparing each treatment with control; **, $P \leq 0.01$; ***, $P \leq 0.001$.
A
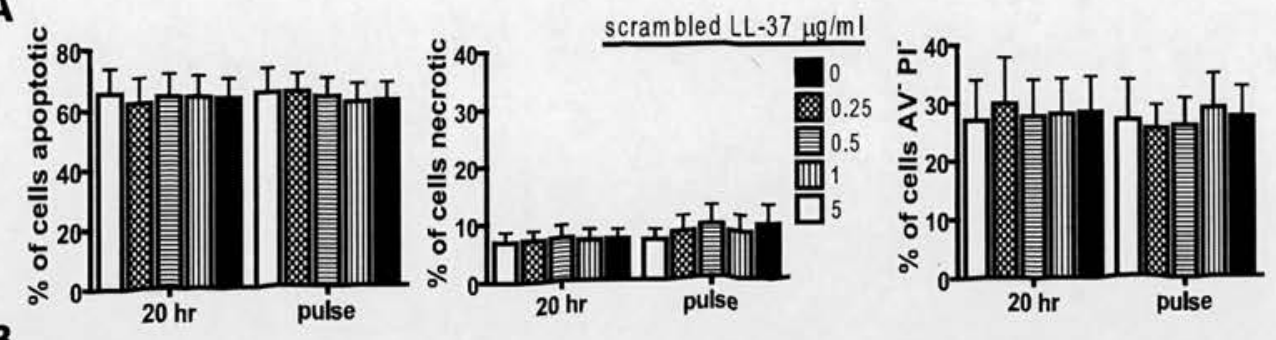

B
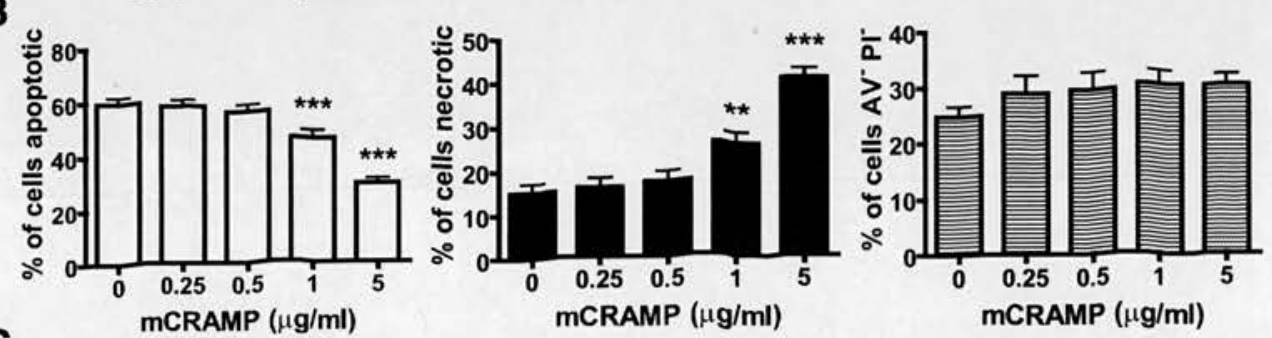

C
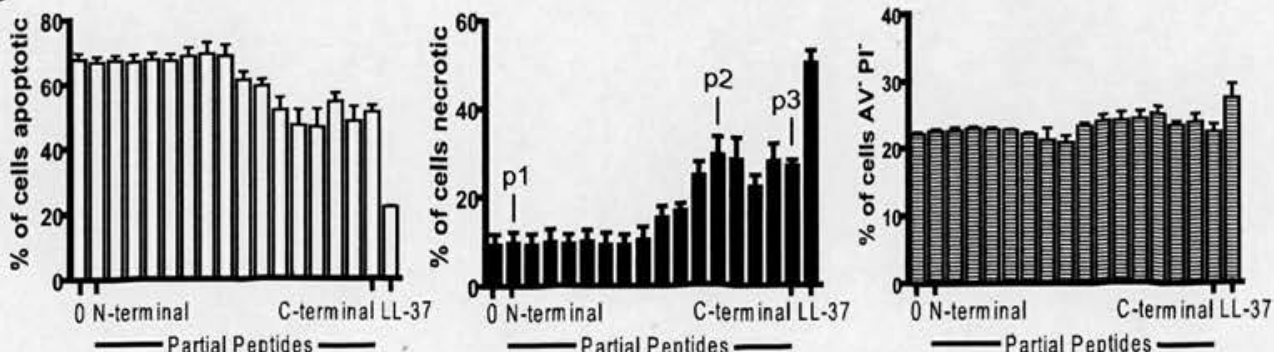

\section{LL-37-induced secondary necrosis of apoptotic PMN does not inhibit macrophage efferocytosis}

To determine whether macrophage ingestion of dead PMN was altered by LL-37-mediated secondary necrosis, ingestion of LL-37-treated PMN was studied using MDM by light microscopic enumeration (Fig. 4A). Confirmatory studies were performed using FACS analyses of MDM to determine ingestion of labeled PMN (Fig. 4B). In both studies, positive control dexamethasone-primed macrophages (shown previously to have increased capacity for apoptotic PMN ingestion [40]) were used. LL-37-mediated induction of secondary necrosis had no significant effects on the magnitude of PMN ingestion by MDM, even at $25 \mu \mathrm{g} / \mathrm{ml} \mathrm{LL}-37$ (a concentration at which $\sim 80 \%$ of dead cells are necrotic [10]).

\section{LL-37-induced secondarily necrotic PMN do not induce proinflammatory macrophage responses}

The interaction of apoptotic PMN and macrophages can induce anti-inflammatory macrophage responses and inhibit LPS-induced proinflammatory cytokine responses [5]. In contrast, necrotic cells are suggested to induce proinflammatory responses [44]. To examine the effects of LL-37-mediated PMN secondary necrosis, MDM cytokine responses to these cells were evaluated at $18 \mathrm{~h}$ after exposure. To separate the effects of any residual, functional LL-37 in the media from the effects of the dead PMN and of the cell bodies from the cellular contents released from secondarily necrotic cells, PMN were incubated for $20 \mathrm{~h}$ at a range of concentrations of LL-37, and MDM were then exposed to LL-37-treated PMN, resuspended in fresh serum-free media, unwashed LL-37-treated PMN in their original media, supernatant from LL-37-treated PMN alone, or media without PMN as a control.

MDM exposed to control apoptotic PMN, PMN incubated for $20 \mathrm{~h}$ with LL-37 ( $\leq 25 \mu \mathrm{g} / \mathrm{ml})$, or the supernatants from these cells did not produce detectable levels of TNF- $\alpha$, IL-6, IL-10, IL-12p70, or IL-1 $\beta$ (Fig. 5, and data not shown), and there were no significant differences between responses to LL37-treated and control apoptotic PMN. These data suggest that LL-37-induced secondarily necrotic PMN are not proinflammatory to macrophages nor release effective, proinflammatory contents into the supernatant.

\section{LL-37-induced secondarily necrotic PMN have anti- inflammatory effects on macrophages}

To determine the effect of these cells in the context of proinflammatory stimuli, MDM were exposed concomitantly to 10 $\mathrm{ng} / \mathrm{ml}$ E. coli LPS. Treatment of MDM with LPS alone stimulated significant production of TNF- $\alpha$, IL-6, and IL-10 (but not IL-12p70 or IL-1 $\beta$ ). In keeping with previous reports [5], the LPS-induced proinflammatory response was reduced by the addition of control apoptotic PMN in serum-free media with significant inhibition $(P<0.01)$ of TNF- $\alpha$ and IL-6 (Fig. 6A). However, these control apoptotic cells had no significant effect on LPS-induced IL-10 production (with considerable donor 

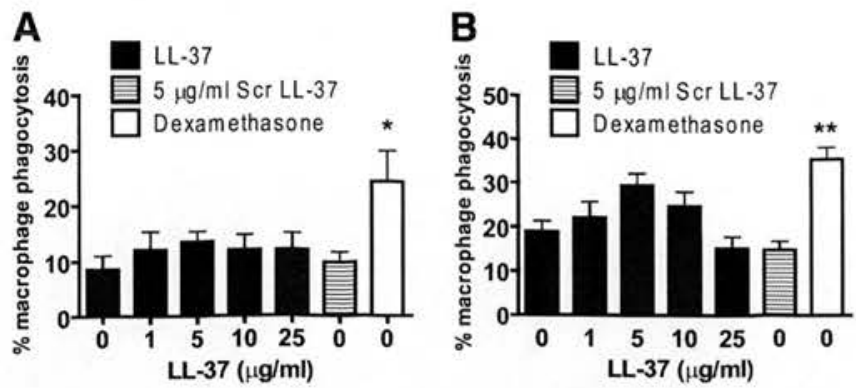

Figure 4. Macrophage phagocytosis of LL-37-induced secondarily necrotic PMN. PMN were incubated previously for $20 \mathrm{~h}$, with or without LL-37 (or scrambled LL-37) at the stated concentrations, and then incubated with $\mathrm{hMDM}$ for $1 \mathrm{~h}$ before removal of noningested PMN. Phagocytosis was assessed by (A) light microscopy, counting the proportion of MDM peroxidase-positive cells; $n=3$ donors for each condition and $n=2$ replicates/experiment; or (B) flow cytometric analyses, evaluating the proportion of MDM ingesting Cell Tracker Greenstained $\mathrm{PMN} ; n \geq 3$ donors for each condition, with $n=2$ replicates/ experiment. As a positive control, wells of adherent monocytes were also cultured in the presence of $1 \mu \mathrm{M}$ dexamethasone for 5 days to up-regulate MDM phagocytosis of dead PMN. Significance was assessed by one-way ANOVA with Bonferroni's multiple comparison test comparing each treatment with control; $*, P \leq 0.05 ; * *, P \leq 0.01$.

variation). Contrary to expectation, when PMN, incubated previously for $20 \mathrm{~h}$ with LL-37 to induce secondary necrosis, were resuspended in fresh, serum-free media and used, these cells also inhibited LPS-induced TNF- $\alpha$ and IL- 6 responses. The effect of cells incubated previously with $5 \mu \mathrm{g} / \mathrm{ml} \mathrm{LL}-37$ to induce secondary necrosis was not significantly different than control apoptotic cells (Fig. 6A). However, significantly greater inhibition of LPS-induced TNF- $\alpha(P<0.01)$ was observed in response to cells incubated previously with $25 \mu \mathrm{g} / \mathrm{ml} \mathrm{LL}-37$ (Fig. 6A), in which the largest induction of secondary necrosis is evident. In addition, in contrast to control apoptotic cells, a trend toward diminished, LPS-induced MDM IL-10 responses was observed in response to these secondarily necrotic cells (Fig. 6A). Exposure of MDM to control apoptotic PMN in their original supernatant (Fig. 6B) had no effect on the LPSinduced production of TNF- $\alpha$, IL- 6 , or IL-10 by MDM and was essentially the same as exposure to the supernatant alone (data not shown). In contrast, exposure to unwashed, LL-37treated PMN in their original supernatant did inhibit the LPSinduced production of TNF- $\alpha(P<0.001)$, IL-6 $(P<0.05)$, and IL-10 by MDM (Fig. 6B). This effect could be replicated using supernatants alone (data not shown) but was not observed using cells treated with scrambled LL-37 (Fig. 6B). In addition, fresh LL-37, added directly to LPS-stimulated MDM in the absence of PMN, was able to replicate this inhibitory effect (Fig. $6 \mathrm{~B})$, indicating that the antiendotoxic effects of remaining, active LL-37 in the original supernatant may be responsible for the results observed in these latter studies. To determine whether the loss of inhibitory activity of dead PMN in their original supernatant related to cellular products or serum within the original media, $20 \mathrm{~h}$ PMN, washed and resuspended in fresh serum-containing media, were used. These cells (control and LL-37-treated) lost the capacity to inhibit LPS-induced MDM TNF- $\alpha$ production (Fig. 7A), implicating an unidentified serum-factor and demonstrating that washed, LL-37-treated cells did not have sufficient residual LL 37 to be directly antiendotoxic.

To confirm that the anti-inflammatory effects of LL-37-induced secondarily necrotic PMN were independent of the antiendotoxic effects of the peptide, rCD40L and rIFN- $\gamma$ treatment was used to activate MDM, rather than LPS. Controlapoptotic and LL-37-induced secondarily necrotic PMN were capable of significantly $(P<0.001)$ inhibiting the resultant TNF- $\alpha$ production (Fig. 7B).

In addition, the impact of three 22-mer partial LL-37 peptides was evaluated. $\mathrm{N}$-terminal peptide $\mathrm{pl}$ induced no PMN secondary necrosis (Fig. 3C) and had no direct antiendotoxic effects when applied to LPS-treated MDM (Fig. 7C). C-terminal peptides p2 and p3 induced significant PMN secondary necrosis (Fig. 3C), but whereas p2 had direct, significant antiendotoxic effects $(P<0.05)$, p3 did not (Fig. 7C). When PMN, incubated previously for $20 \mathrm{~h}$ with these peptides, were resuspended in fresh serum-free media, these cells were all capable of mediating a significant $(P<0.01)$ inhibition of LPS-induced MDM TNF- $\alpha$ production, identical to control cells (Fig. 7D). However, when used in their original media, the effects of these cells replicated the direct, antiendotoxic capacity of the peptide with which they had been treated (Fig. 7C).
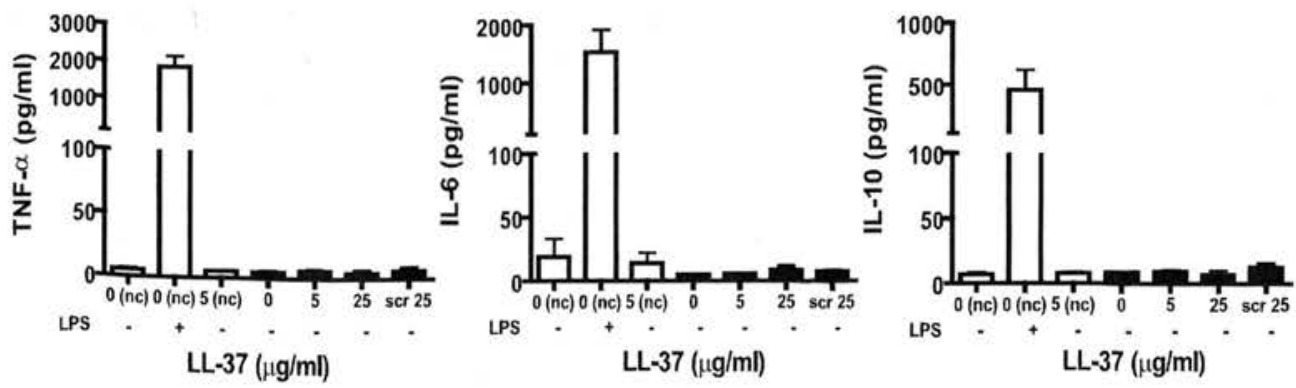

Figure 5. LL-37-induced secondarily necrotic $\mathrm{PMN}$ are not proinflammatory for macrophages. $\mathrm{Hu}$ man PMN were incubated for $20 \mathrm{~h}$ in the presence or absence of LL-37 or scrambled LL-37 at the concentrations indicated and used unwashed in the IMDM $+10 \%$ FCS media supernatant, in which they had been incubated. hMDM were incubated with these PMN or incubated without PMN (nc) in the presence or absence of LL.37 or 10 $\mathrm{ng} / \mathrm{ml}$ E. coli 0111:B4 LPS as a positive control. Supernatants were evaluated for cytokine responses. 
Figure 6. LL-37-induced secondarily necrotic PMN retain anti-inflammatory properties. Human PMN were incubated for $20 \mathrm{~h}$ in the presence or absence of LL-37 or scrambled LL-37 at the concentrations indicated. PMN were resuspended in serum-free X-vivo 10 media (A) or used unwashed in the IMDM $+10 \%$ FCS media supernatant, in which they were incubated overnight (B). MDM were incubated with these PMN, with concomitant exposure to $10 \mathrm{ng} / \mathrm{ml} \mathrm{E}$. coli 0111:B4 LPS for $18 \mathrm{~h}$. LPS treated MDM without PMN, in the presence or absence of LL_.37, were also studied as controls. Supernatants were evaluated for cytokine responses. Significance was assessed by one-way ANUVA with Bonferroni's multiple comparison test; $n=9$ donors (TNF- $\alpha$ ) $; n=5$ donors (IL-6/IL-10); *, $P \leq$ 0.05 ; **, $P \leq 0.01$; ***, $P \leq 0.001$, compared with MDM exposed to LPS in the absence of PMN under the same conditions; " ${ }^{\# \#,}, P \leq 0.01$; "\#\#", $P \leq 0.001$, compared with MDM exposed to control apoptotic PMN untreated with LL-37.
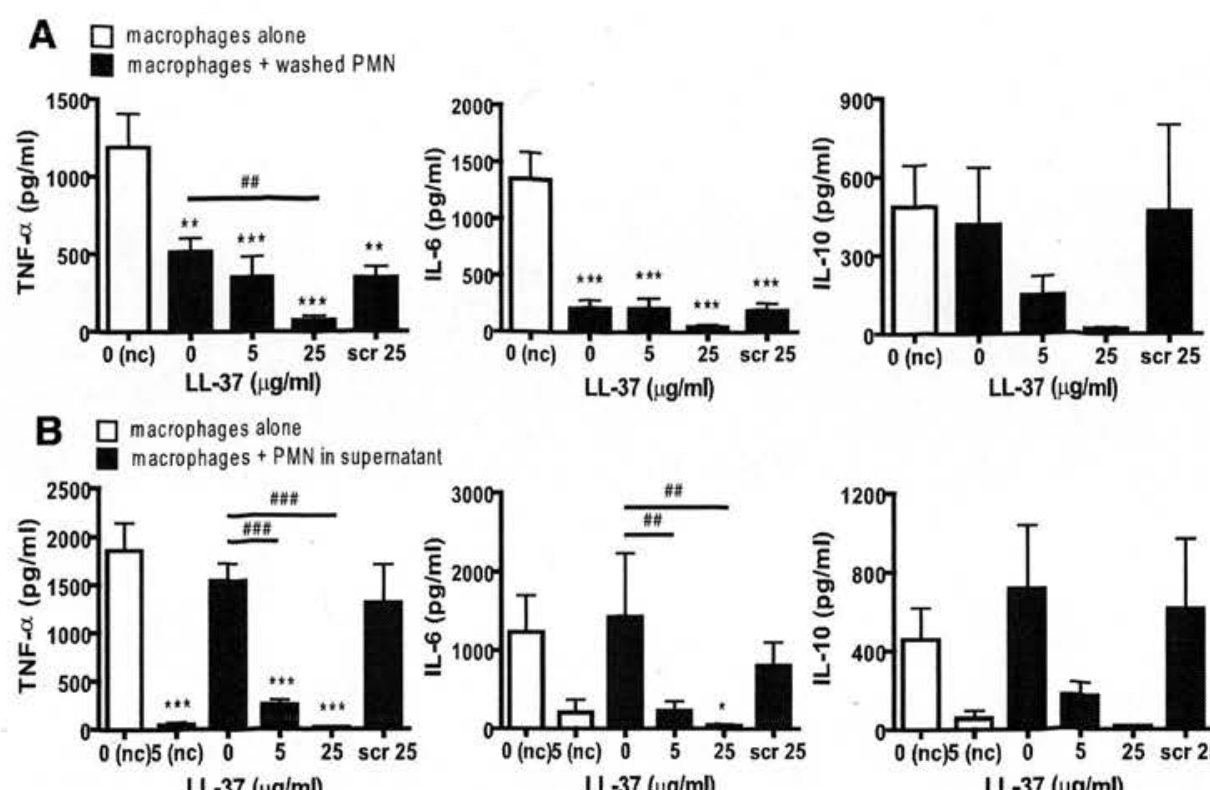

$\mathrm{LL}-37(\mu \mathrm{g} / \mathrm{ml})$

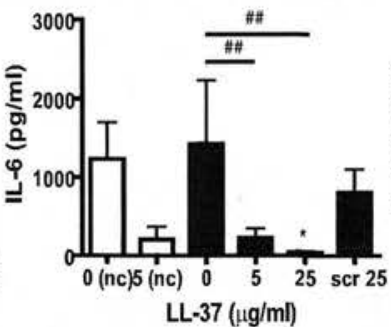

$\mathrm{LL}-37(\mu \mathrm{g} / \mathrm{ml})$

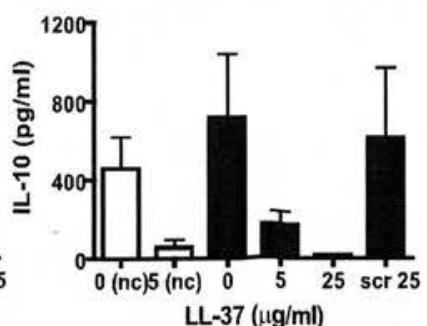

These data demonstrate the effect of residual peptide in the original media, show that functional peptide is not carried over in washed cells, and demonstrate that these dead PMN can inhibit LPS-induced MDM cytokine production, irrespective of the degree of peptide-induced secondary necrosis. These data demonstrate that the inhibition of activated MDM proinflammatory cytokine production mediated by apoptotic PMN is not inhibited and may even be enhanced by LL-37-mediated induction of secondary necrosis of these apoptotic PMN. Furthermore, the intracellular contents released by these cells are not actively proinflammatory or can be inhibited by the effects of LL-37.

\section{LL-37-mediated secondary necrosis can induce the release of PMN granule contents}

To determine the effect of LL-37-induced secondary necrosis of apoptotic PMN on the azurophilic (primary) granules, the release of MPO in response to LL-37 was quantified at $20 \mathrm{~h}$. MPO release was not detected above background for this timepoint following exposure to $\leq 10 \mu \mathrm{g} / \mathrm{ml} \mathrm{LL}-37$ (concentrations at which substantial secondary necrosis occurred) or in response to scrambled LL-37 (Fig. 8). However, significant levels of MPO were observed in response to $\geq 25 \mu \mathrm{g} / \mathrm{ml} \mathrm{LL}-37$ $(P<0.01)$ at levels approaching $40 \%$ of total MPO released by
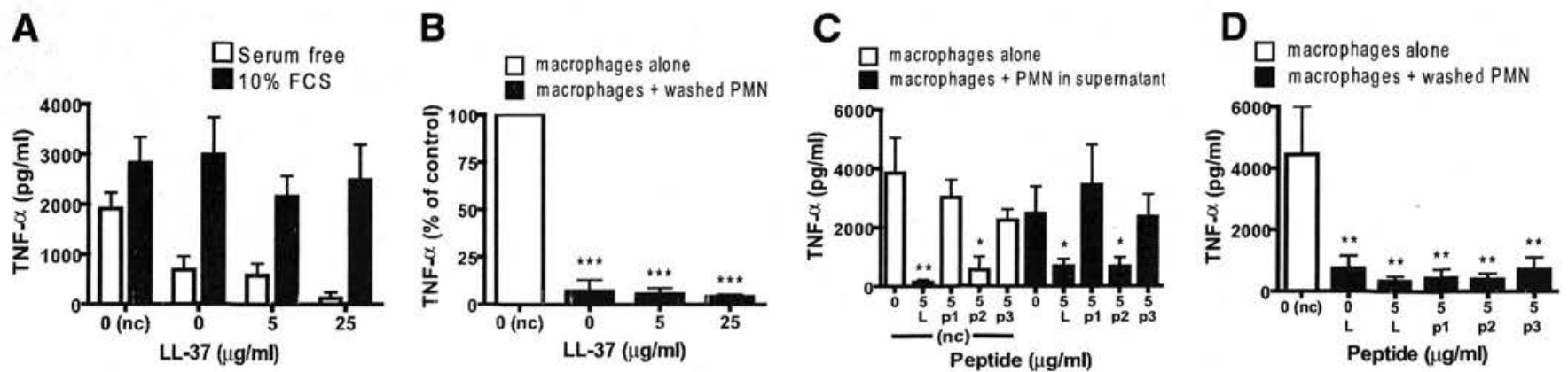

Figure 7. The anti-inflammatory properties LL-37-induced, secondarily necrotic PMN are independent of peptide antiendotoxic activity. Human PMN were incubated for $20 \mathrm{~h}$ in the presence or absence of LL-37 or partial peptides p1, p2, or p3 at the concentrations indicated. PMN were resuspended in X-vivo 10 media with $10 \%$ FCS (A), resuspended in serum-free X-vivo 10 media (B and D), or used unwashed in the IMDM + $10 \%$ FCS media supernatant, in which they were incubated overnight (C). MDM were incubated with these PMN, with concomitant exposure to $10 \mathrm{ng} / \mathrm{ml} \mathrm{E}$. coli 0111:B4 LPS (A, C, and D) or $3 \mu \mathrm{g} / \mathrm{ml}$ rhCD40L $+5 \mathrm{ng} / \mathrm{ml} \mathrm{rhIFN}-\gamma$ (B) for 18 h. Activated MDM without PMN, in the presence or absence of peptides, were also studied as controls and to evaluate direct antiendotoxic activity. Supernatants were evaluated for cytokine responses. Significance was assessed by one-way ANOVA with Bonferroni's multiple comparison test; $n \geq 3$ donors; *, $P \leq 0.05$; $* *, P \leq 0.01 ; * * *$, $P \leq 0.001$, compared with activated MDM in the absence of PMN under the same conditions. 


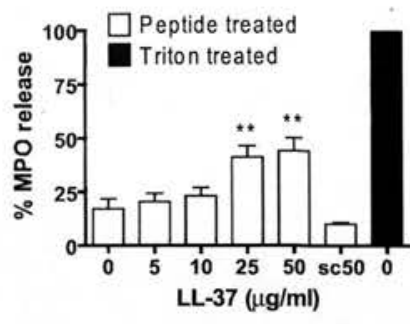

Figure 8. LL-37-induced secondary necrosis of PMN can release granule contents. Isolated human PMN were incubated for $20 \mathrm{~h}$ with a range of concentrations of LL-37 or $50 \mu \mathrm{g} / \mathrm{ml}$ scrambled LL-37 (sc50). The concentration of MPO in the supernatants was evaluated as a percentage of total MPO release after lysis of fresh control PMN using $0.1 \%$ Triton X-100. Significance was assessed by one-way ANOVA with Bonferroni's multiple comparison test; $n=7$ donors; **, $P \leq$ 0.01 , compared with untreated PMN.

lysis with Triton X-100. These data suggest that although LL37 -induced secondary necrosis of apoptotic PMN does not lead directly to the release of granule contents over this time scale, higher concentrations of LL-37 are able to destabilize granules and lead to the release of their potentially damaging contents.

\section{DISCUSSION}

PMN are key cells of the innate immune system. The appropriate regulation of PMN function, death, and clearance is critical to effective control of inflammation, and dysregulation of these processes has been implicated in disease pathogenesis. Cell death by apoptosis, followed by efferocytosis by professional phagocytic cells, is proposed to enable safe PMN removal without the release of harmful intracellular contents to inhibit proinflammatory responses and to promote anti-inflammatory responses to enhance resolution [3]. In contrast, cell death by necrosis (whether primary necrosis or secondary necrosis of apoptotic PMN that have not been cleared) is proposed to be proinflammatory. We demonstrate that the human cathelicidin LL-37 is a potent inducer of secondary necrosis in PMN, which have undergone prior apoptosis, and evaluate the impact of this process on macrophage inflammatory responses.

We demonstrate that the ingestion of LL-37-induced secondarily necrotic PMN was not a proinflammatory event. This process did not promote the release of proinflammatory cytokines by macrophages and was indistinguishable from the macrophage response to apoptotic cells. Furthermore, these secondarily necrotic PMN were actively anti-inflammatory, demonstrating that the capacity of apoptotic PMN to inhibit LPS-induced proinflammatory cytokine production by macrophages was undiminished by LL-37-induced secondary necrosis. Indeed, this anti-inflammatory effect was actually enhanced in response to PMN with the most extensive secondary necrosis. Although the anti-inflammatory activity of unwashed, LL-37treated apoptotic PMN was a result of the antiendotoxic properties of the remaining, functional LL-37 in the supernatant, washed cells retained the anti-inflammatory capacity of control apoptotic cells, even after treatment conditions $(25 \mu \mathrm{g} / \mathrm{ml}$
LL-37 for $20 \mathrm{~h}$ ) in which $\sim 80 \%$ of dead PMN have undergone secondary necrosis [10]. These data suggest functional equivalence, irrespective of membrane integrity, of apoptotic PMN and LL-37-induced secondarily necrotic cells in this system. A previous report has demonstrated potential functional dominance of apoptotic cells over primary necrotic cells [45]. We cannot exclude a dominant role for the remaining membraneintact apoptotic cells in our system. However, the significantly enhanced anti-inflammatory effects induced by PMN, with the greatest proportion of secondary necrosis, indicate that the anti-inflammatory activity of apoptotic PMN can in fact be potentiated by LL-37-induced secondary necrosis. Whether this applies to PMN necrosis induced in other ways and the mechanisms involved remains to be determined. However, exposure to a concentration of LL-37, sufficient to induce PMN granule content release, appeared to be necessary to generate secondary necrosis capable of potentiating the anti-inflammatory effects. This indicates that induction of necrosis per se is not sufficient and implicates a possible role for the release of endogenous LL-37 or other anti-inflammatory agents from PMN granules. Irrespective, it is clear that LL-37-induced secondary necrosis did not compromise the anti-inflammatory effects of the dead cells nor confer proinflammatory properties on them. Furthermore, any proinflammatory mediators released by secondarily necrotic PMN into the supernatant were unable to initiate or potentiate macrophage production of the cytokines studied in the presence of LL-37.

Whereas apoptosis has been viewed as anti-inflammatory and inducing immune-tolerance, necrosis has been considered a proinflammatory and immunogenic form of cell death. However, recent studies have suggested that this axiom is oversimplified [46]. Apoptotic Jurkat T cells are reported to be equally potent in modulating macrophage MAPK pathways, irrespective of membrane integrity [47], and the membranes of apoptotic and primary necrotic PMN have been shown to inhibit TNF- $\alpha$ production by activated macrophages [44]. In addition, apoptosis can neutralize potential intracellular danger signals, such as high-mobility group box-1 protein, via caspase-activated mitochondrial reactive oxygen species production [48]. Thus, the release of intracellular contents from apoptotic cells having undergone secondary necrosis may not be equivalent to the proinflammatory contents of primary necrotic cells, an hypothesis that would be compatible with our data.

We demonstrate that LL-37-induced PMN secondary necrosis had no impact on the magnitude of ingestion of dead PMN by macrophages, consistent with previous reports [44, 45]. Efferocytosis is a complex process with multiple receptors and adaptors proposed [3], independent from the anti-inflammatory effects initiated by apoptotic cells [49]. We cannot exclude the possibility that the mechanism of ingestion of these secondary necrotic PMN and apoptotic PMN may be different, but comparable levels of uptake suggest that LL-37-mediated induction of PMN secondary necrosis in vivo would not inhibit the clearance of dead PMN.

Irrespective of the impact of secondary necrosis on the clearance of dead PMN or macrophage cytokine responses, the loss of PMN membrane integrity presents the additional 
threat of the release of harmful intracellular contents, including granule contents such as neutrophil elastase, cathepsin G, MPO, and gelatinase [50]. In contrast to previous TEM-based observations [38], our data demonstrate that high concentrations of LL-37 induced the release of MPO from the azurophilic granules. Interestingly, this was not observed at lower LL-37 concentrations, at which significant induction of secondary necrosis was observed nevertheless. This suggests that LL37 -induced secondary necrosis did not disrupt granule integrity per se but that high concentrations of LL-37 ( $\geq 10 \mu \mathrm{g} / \mathrm{ml})$ had the capacity to damage granule membranes in addition to apoptotic outer membranes. The consequences of such an effect in a rapidly resolving acute infection may contrast with those in a chronic inflammatory process associated with high levels of LL-37. Indeed, increased hCAP-18/LL-37 concentrations in cystic fibrosis lung disease (detected at up to $\sim 15$ $\mu \mathrm{g} / \mathrm{ml}$ in BALF from patients without recent infectious exacerbation) were correlated with increased lung damage [34], and raised pulmonary LL-37 levels are associated with bronchiolitis obliterans syndrome in the recipients of lung transplants [36]. In addition, LL-37 has been proposed recently to contribute to the pathogenesis of psoriasis [35], and the concentration of hCAP-18/LL-37 was reported to be $\sim 1.5 \mathrm{mg} / \mathrm{ml}$ in psoriatic skin lesions [51]. These estimates of in vivo peptide concentrations do not take into account the recent observation that the ratio of active LL-37 to full-length precursor hCAP-18 varied considerably between donors in the inflamed lung [36]. Nevertheless, at these concentrations, extensive induction of secondary necrosis of apoptotic PMN, with the release of PMN granule contents, could be generated by exposure to LL-37 in vivo. Thus, the potentially detrimental effects of LL-37-mediated induction of PMN secondary necrosis could have significance in chronic disease processes.

The potential consequences of PMN granule release may be of particular significance for the ongoing development of CHDP as therapeutic agents for infectious and inflammatory diseases. A greater knowledge of the capacity of these peptides to modulate inflammation and the peptide attributes that confer them will have important implications. We have demonstrated recently that the capacity of LL- 37 to induce secondary necrosis of apoptotic cells is not specific to PMN [41] and now additionally define a core functional region and describe interaction with other modifiers of cell death.

We demonstrate that LL-37-induced secondary necrosis of PMN occurred rapidly, within minutes of exposure of an apoptotic PMN to the peptide. The specific membrane alterations that make these apoptotic cells acutely susceptible to damage by LL-37 and the nature of this interaction remain unclear, but the process has been proposed recently to be energy-independent [38] and independent of known LL-37 receptors [41]. Our data demonstrate that this property was conserved in the murine ortholog mCRAMP, and the effect was not dependent primarily on peptide charge (with no effects seen for a scrambled sequence peptide with the same charge) and was retained partially by C-terminal, but not $\mathrm{N}$-terminal partial LL-37 peptides. These latter studies, using truncated partial peptides, shown previously to be poorly antiendotoxic [37], identified a minimal core-functional region for induction of secondary necrosis by LL-37, spanning aa 16-32. This may indicate an important motif or be related to the more amphipathic nature of the peptides spanning this region. Interestingly, the region 17-32 has been identified previously as the core antimicrobial region [52]. This suggests a possible conservation of mechanism between the direct microbicidal effects of LL-37, for which amphipathicity is critical [52], and the capacity to induce PMN secondary necrosis. However, it is noteworthy that whereas core LL-37 peptide 17-32 had enhanced microbicidal function compared with full-length LL-37, our partial peptides had diminished capacity to induce secondary necrosis. This has important implications for the development of therapeutic peptides and in consideration of the possible in vivo activities of shorter-form cleavage products of hCAP-18, identified previously in the skin [53]. Further study of our partial peptides will enable dissection of the various properties of LL-37.

In conclusion, we demonstrate that the human cathelicidin LL-37 is a potent inducer of secondary necrosis of apoptotic PMN, with the potential to alter the profile of PMN cell death at sites of infection and inflammation. Our data give novel insights into the potential effects of PMN secondary necrosis, suggesting that LL-37-mediated secondary necrosis of PMN does not have proinflammatory effects on macrophages and can even potentiate the anti-inflammatory effects of efferocytosis. These data challenge the prevailing model that although apoptotic cells are anti-inflammatory to macrophages, necrotic cells (whether primary or secondary necrosis) are inherently proinflammatory. This has particular importance for the delayed clearance of the apoptotic cell hypothesis for chronic inflammation and implications for novel, anti-inflammatory strategies being developed based on our understanding the mechanisms controlling the resolution of inflammation. However, we also demonstrate that LL-37-induced secondary necrosis has the potential to induce the release of potentially harmful granule contents, which could have deleterious consequences for the host, particularly in chronic disease states. We demonstrate that this induction of secondary necrosis by LL-37 is not primarily charge-dependent, is partially retained by $\mathrm{C}$ terminal partial peptides, and is conserved in the murine ortholog. Further understanding of this mechanism and its physiological significance will assist the appropriate modification of synthetic analogs as novel therapeutics, illuminate possible roles in the pathogenesis of chronic disease, and advance our understanding of the impact of different cell death processes in the promotion and resolution of inflammation.

\section{ACKNOWLEDGMENTS}

This worked was funded by the Wellcome Trust and the Norman Salvesen Trust Emphysema Research Trust. D. J. D. is a Wellcome Trust Research Career Development Fellow (fellowship \#078265). The Swedish group was funded by grants from the Swedish Research Council, the King Gustav V 80-Year Foundation, and the Swedish State under the LUA/ALF agreement. The authors thank Stephen Mitchell, Fiona Rossi, Shonna Johnson, Katherine Miles, Tara Sheldrake, Jillian Ren- 
nie, Irini Bournazos, Stelios Bournazos, Dennis Ninaber, Sarah Fox, Andrew Leitch, Sylwia Michlewska, Sandra Franz, and Mark Marsden for technical assistance and Simon Brown, Ian Dransfield, Aisleen McColl, Robert Gray, Thomas Wilkinson, Kev Dhaliwal, Olga Lucia Moncayo Nieto, and Andy Conway Morris for valuable advice.

\section{REFERENCES}

1. Haslett, C. (1999) Granulocyte apoptosis and its role in the resolution and control of lung inflammation. Am. J. Respir. Crit. Care Med. 160 S5-11.

2. Leitch, A. E., Duffin, R., Haslett, C., Rossi, A. G. (2008) Relevance of granulocyte apoptosis to resolution of inflammation at the respiratory mucosa. Mucosal Tmmunol. 1, 350-363.

3. Savill, J., Dransfield, I., Gregory, C., Haslett, C. (2002) A blast from the past: clearance of apoptotic cells regulates immune responses. Nat. Rev. Immunol. 2, 965-975.

4. Voll, R. E., Herrmann, M., Roth, E. A., Stach, C., Kalden, J. R., Girkontaite, I. (1997) Immunosuppressive effects of apoptotic cells. Nature 390 $350-351$

5. Fadok, V. A., Bratton, D. L., Konowal, A., Freed, P. W., Westcott, J. Y., Henson, P. M. (1998) Macrophages that have ingested apoptotic cells in vitro inhibit proinflammatory cytokine production through autocrine/ paracrine mechanisms involving TGF- $\beta$, PGE2, and PAF. J. Clin. Invest. 101, 890-898.

6. Huynh, M. L., Fadok, V. A., Henson, P. M. (2002) Phosphatidylserinedependent ingestion of apoptotic cells promotes TGF- $\beta 1$ secretion and the resolution of inflammation. J. Clin. Invest. 109, 41-50.

7. Serhan, C. N., Savill, J. (2005) Resolution of inflammation: the beginning programs the end. Nat. Immunol. 6, 1191-1197.

8. Bianchi, S. M., Dockrell, D. H., Renshaw, S. A., Sabroe, I., Whyte, M. K. (2006) Granulocyte apoptosis in the pathogenesis and resolution of lung disease. Clin. Sci. (Lond.) 110, 293-304.

9. Rossi, A. G., Sawatzky, D. A., Walker, A., Ward, C., Sheldrake, T. A., Riley, N. A., Caldicott, A., Martinez-Losa, M., Walker, T. R., Duffin, R., Gray, M., Crescenzi, E., Martin, M. C., Brady, H. J., Savill, J. S., Dransfield, I., Haslett, C. (2006) Cyclin-dependent kinase inhibitors enhance the resolution of inflammation by promoting inflammatory cell apoptosis. Nat. Med. 12, 1056-1064.

10. Barlow, P. G., Li, Y., Wilkinson, T. S., Bowdish, D. M., Lau, Y. E., Cosseau, C., Haslett, C., Simpson, A. J., Hancock, R. E., Davidson, D. J. (2006) The human cationic host defense peptide LL-37 mediates contrasting effects on apoptotic pathways in different primary cells of the innate immune system. J. Leukoc. Biol. 80, 509-520.

11. Nagaoka, I., Tamura, H., Hirata, M. (2006) An antimicrobial cathelicidin peptide, human CAP18/LL-37, suppresses neutrophil apoptosis via the activation of formyl-peptide receptor-like 1 and P2X7. J. Immunol. 176, 3044-3052.

12. Sorensen, O., Arnljots, K., Cowland, J. B., Bainton, D. F., Borregaard, N. (1997) The human antibacterial cathelicidin, hCAP-18, is synthesized in myelocytes and metamyelocytes and localized to specific granules in neutrophils. Blood 90, 2796-2803.

13. Sorensen, O. E., Follin, P., Johnsen, A. H., Calafat, J., Tjabringa, G. S., Hiemstra, P. S., Borregaard, N. (2001) Human cathelicidin, hCAP-18, is processed to the antimicrobial peptide LL-37 by extracellular cleavage with proteinase 3. Blood 97, 3951-3959.

14. Bowdish, D. M., Davidson, D. J., Hancock, R. E. (2006) Immunomodulatory properties of defensins and cathelicidins. Curr. Top. Microbiol. Immunol. 306, 27-66.

15. Scott, M. G., Davidson, D. J., Gold, M. R., Bowdish, D., Hancock, R. E. (2002) The human antimicrobial peptide LL-37 is a multifunctional modulator of innate immune responses. J. Immunol 169, 3883-3891.

16. Fukumoto, K., Nagaoka, I., Yamataka, A., Kobayashi, H., Yanai, T., Kato, Y., Miyano, T. (2005) Effect of antibacterial cathelicidin peptide CAP18/ L. L-37 on sepsis in neonatal rats. Pediatr. Surg. Int. 21, 20-24.

17. De Yang, Chen, Q., Schmidt, A. P., Anderson, G. M., Wang, J. M., Wooters, J., Oppenheim, J. J., Chertov, O. (2000) LL-37, the neutrophil granule- and epithelial cell-derived cathelicidin, utilizes formyl peptide recep-
tor-like 1 (FPRL1) as a receptor to chemoattract human peripheral blood tor-like 1 (FPRL1) as a receptor to chemoattract human peripher
neutrophils, monocytes, and T cells. J. Exp. Med. 192, 1069-1074.

18. Kurosaka, K., Chen, Q., Yarovinsky, F., Oppenheim, J. J., Yang, D. (2005) Mouse cathelin-related antimicrobial peptide chemoattracts leukocytes using formyl peptide receptor-like 1 /mouse formyl peptide receptor-like 2 as the receptor and acts as an immune adjuvant. J. Immunol. 174, 62576265 .

19. Elssner, A., Duncan, M., Gavrilin, M., Wewers, M. D. (2004) A novel P2X7 receptor activator, the human cathelicidin-derived peptide LL37, induces II $-1 \beta$ processing and release. $J$. Immunol. 172, 49874994

20. Mookherjee, N., Brown, K. L., Bowdish, D. M., Doria, S., Falsafi, R., Hokamp, K., Roche, F. M., Mu, R., Doho, G. H., Pistolic, J., Powers, J. P.,
Bryan, J., Brinkman, F. S., Hancock, R. E. (2006) Modulation of the TLRmediated inflammatory response by the endogenous human host defense peptide LL-37. J. Immunol. 176, 2455-2464.

21. Niyonsaba, F., Someya, A., Hirata, M., Ogawa, H., Nagaoka, I. (2001) Evaluation of the effects of peptide antibiotics human $\beta$-defensins- $1 /-2$ and LL -37 on histamine release and prostaglandin D(2) production from mast cells. Eur. J. Immunol. 31, 1066-1075.

22. Heilborn, J. D., Nilsson, M. F., Kratz, G., Weber, G., Sorensen, O., Borre gaard, N., Stahle-Backdahl, M. (2003) The cathelicidin anti-microbial peptide LL-37 is involved in re-epithelialization of human skin wounds and is lacking in chronic ulcer epithelium. J. Invest. Dermatol. 120, 379389.

23. Koczulla, R., Von Degenfeld, G., Kupatt, C., Krotz, F., Zahler, S., Gloe, T., Issbrucker, K., Unterberger, P., Zaiou, M., Lebherz, C., Karl, A. Raake, P., Pfosser, A., Boekstegers, P., Welsch, U., Hiemstra, P. S., Vogelmeier, C., Gallo, R. L., Clauss, M., Bals, R. (2003) An angiogenic role for the human peptide antibiotic LL-37/hCAP-18. J. Clin. Invest. 111, 1665-1672.

24. Davidson, D. J., Currie, A. J., Reid, G. S., Bowdish, D. M., MacDonald, K. L., Ma, R. C., Hancock, R. E., Speert, D. P. (2004) The cationic an timicrobial peptide LL-37 modulates dendritic cell differentiation and dendritic cell-induced $\mathrm{T}$ cell polarization. I. Immunol. 172, 1146-1156.

25. An, L. L., Yang, Y. H., Ma, X. T., Lin, Y. M., Li, G., Song, Y. H., Wu, K. F. (2005) LL-37 enhances adaptive antitumor immune response in a murine model when genetically fused with M-CSFR(J6-1) DNA vaccine. Leuk. Res. 29, 535-543.

26. Lau, Y. E., Bowdish, D. M., Cosseau, C. C., Hancock, R. E., Davidson, D. J. (2006) Apoptosis of airway epithelial cells: human serum sensitive induction by the cathelicidin LL-37. Am. J. Respir. Cell Mol. Biol. 34, 399409.

27. Aarbiou, J., Tjabringa, G. S., Verhoosel, R. M., Ninaber, D. K., White, S. R., Peltenburg, L. T., Rabe, K. F., Hiemstra, P. S. (2006) Mechanisms of cell death induced by the neutrophil antimicrobial peptides $\alpha$-defensins and LL-37. Inflamm. Res. 55, 119-127.

28. Putsep, K., Carlsson, G., Boman, H. G., Andersson, M. (2002) Deficiency of antibacterial peptides in patients with morbus Kostmann: an observation study. Lancet 360, 1144-1149.

29. Nizet, V., Ohtake, T., Lauth, X., Trowbridge, J., Rudisill, J., Dorschner, R. A., Pestonjamasp, V., Piraino, J., Huttner, K., Gallo, R. L. (2001) Innate antimicrobial peptide protects the skin from invasive bacterial infection. Nature 414, 454-457.

30. Iimura, M., Gallo, R. L., Hase, K., Mivamoto, Y., Eckmann, L., Kagnoff, M. F. (2005) Cathelicidin mediates innate intestinal defense against colonization with epithelial adherent bacterial pathogens. J. Immunol. 174, 4901-4907.

31. Chromek, M., Slamova, Z., Bergman, P., Kovacs, L., Podracka, L., Ehren, I., Hokfelt, T., Gudmundsson, G. H., Gallo, R. L., Agerberth, B., Brauner, A. (2006) The antimicrobial peptide cathelicidin protects the urinary tract against invasive bacterial infection. Nat. Med. 12, $636-641$.

32. Schaller-Bals, S., Schulze, A., Bals, R. (2002) Increased levels of antimicrobial peptides in tracheal aspirates of newborn infants during infection. Am. J. Respir. Crit. Care Med. 165, 992-995.

33. Bals, R., Weiner, D. J., Moscioni, A. D., Meegalla, R. L., Wilson, J. M. (1999) Augmentation of innate host defense by expression of a cathelicidin antimicrobial peptide. Infect. Immun. 67, 6084-6089.

34. Chen, C. I., Schaller-Bals, S., Paul, K. P., Wahn, U., Bals, R. (2004) $\beta$-Defensins and LL-37 in bronchoalveolar lavage fluid of patients with cystic fibrosis. J. Cyst. Fibros. 3, 45-50.

35. Lande, R., Gregorio, J., Facchinetti, V., Chatterjee, B., Wang, Y. H., Homey, B., Cao, W., Wang, Y. H., Su, B., Nestle, F. O., Zal, T., Mellman, I., Schroder, J. M., Liu, Y. J., Gilliet, M. (2007) Plasmacytoid dendritic cells sense self-DNA coupled with antimicrobial peptide. Nature 449, $564-$ 569 .

36. Anderson, R. L., Hiemstra, P. S., Ward, C., Forrest, I. A., Murphy, D., Proud, D., Lordan, J., Corris, P. A., Fisher, A. J. (2008) Antimicrobial peptides in lung transplant recipients with bronchiolitis obliterans syndrome. Eur. Respir. J. 32, 670-677.

37. Nell, M. J., Tjabringa, G. S., Wafelman, A. R., Verrijk, R., Hiemstra, P. S. Drijfhout, J. W., Grote, J. J. (2006) Development of novel LL-37 derived antimicrobial peptides with LPS and LTA neutralizing and antimicrobial activities for therapeutic application. Peptides 27, 649-660.

38. Zhang, Z., Cherryholmes, G., Shively, J. E. (2008) Neutrophil secondary necrosis is induced by LL-37 derived from cathelicidin. J. Leukoc. Biol. 84, $780-788$.

39. Jersmann, H. P., Ross, K. A., Vivers, S., Brown, S. B., Haslett, C., Dransfield, I. (2003) Phagocytosis of apoptotic cells by human macrophages: analysis by multiparameter flow cytometry. Cytometry A 51, $7-15$

40. Liu, Y., Cousin, J. M., Hughes, J., Van Damme, J., Seckl, J. R., Haslett, C. Dransfield, I., Savill, J., Rossi, A. G. (1999) Glucocorticoids promote nonphlogistic phagocytosis of apoptotic leukocytes. J. Immunol. 162, 36393646 .

41. Bjorstad, A., Askarich, G., Brown, K. L., Christenson, K., Forsman, H., Onnheim, K., Li, H. N., Teneberg, S., Maier, O., Hoekstra, D., Dahlgren, 


\section{$\mathrm{JLB}$}

C., Davidson, D. J., Bylund, J. (2009) The host defence peptide LL-37 selectively permeabilizes apoptotic leukocytes. Antimicrob. Agents Che mother. 53, 1027-1038.

42. Klein, J. B., Rane, M. J., Scherzer, J. A., Coxon, P. Y., Kettritz, R. Mathiesen, J. M., Buridi, A., McLeish, K. R. (2000) Granulocyte-macrophage colony-stimulating factor delays neutrophil constitutive apoptosis through phosphoinositide 3-kinase and extracellular signal-regulated kinase pathways. J. Immunol. 164, 4286-4291.

43. Sabroe, I., Prince, L. R., Jones, E. C., Horsburgh, M. J., Foster, S. J., Vogel, S. N., Dower, S. K., Whyte, M. K. (2003) Selective roles for Toll-like receptor (TLR) 2 and TLR4 in the regulation of neutrophil activation and life span. J. Immunol. 170, 5268-5275.

44. Fadok, V. A., Bratton, D. L., Guthrie, L., Henson, P. M. (2001) Differential effects of apoptotic versus lysed cells on macrophage production of cytokines: role of proteases. J. Immunol. 166, 6847-6854.

45. Cocco, R. E., Ucker, D. S. (2001) Distinct modes of macrophage recognition for apoptotic and necrotic cells are not specified exclusively by phosphatidylserine exposure. Mol. Biol. Cell 12, 919-930.

46. Krysko, D. V., D'Herde, K., Vandenabeele, P. (2006) Clearance of apoptotic and necrotic cells and its immunological consequences. Apoplosis 11, 17091726.

47. Patel, V. A., Longacre, A., Hsiao, K., Fan, H., Meng, F., Mitchell, J. E., Rauch, J., Ucker, D. S., Levine, J. S. (2006) Apoptotic cells, at all stages of the death process, trigger characteristic signaling events that are divergent from and dominant over those triggered by necrotic cells: implications for the delayed clearance model of autoimmunity. J. Biol. Chem. 281, 4663-4670.
48. Kazama, H., Ricci, J. E., Herndon, J. M., Hoppe, G., Green, D. R., Ferguson, T. A. (2008) Induction of immunological tolerance by apoptotic cells requires caspase-dependent oxidation of high-mobility group box-1 protein. Immunity 29, 21-32.

49. Lucas, M., Stuart, L. M., Zhang, A., Hodivala-Dilke, K., Febbraio, M., Silverstein, R., Savill, J., Lacy-Hulbert, A. (2006) Requirements for apoptotic cell contact in regulation of macrophage responses. J. Immunol. 177, 4047-4054.

50. Borregaard, N., Cowland, J. B. (1997) Granules of the human neutrophilic polymorphonuclear leukocyte. Blood 89, 3503-3521.

51. Ong, P. Y., Ohtake, T., Brandt, C., Strickland, I., Boguniewicz, M., Ganz, T., Gallo, R. L., Leung, D. Y. (2002) Endogenous antimicrobial peptides and skin infections in atopic dermatitis. N. Engl. J. Med. 347, 1151-1160.

52. Li, X., Li, Y., Han, H., Miller, D. W., Wang, G. (2006) Solution structures of human LL-37 fragments and NMR-based identification of a minimal membrane-targeting antimicrobial and anticancer region. J. Am. Chem. Soc. 128, 5776-5785.

53. Murakami, M., Lopez-Garcia, B., Braff, M., Dorschner, R. A., Gallo, R. L (2004) Postsecretory processing generates multiple cathelicidins for enhanced topical antimicrobial defense. J. Immunol. 172, 3070-3077.

\section{KEY WORDS:}

cationic host defense peptide $\cdot$ antimicrobial peptide $\cdot$ innate immunity · inflammation · efferocytosis 


\title{
FEES
}

journal homepage: www.FEBSLetters.org

\section{The CDK inhibitor, R-roscovitine, promotes eosinophil apoptosis by down-regulation of Mcl-1}

\author{
Rodger Duffin ${ }^{\mathrm{a}, 1}$, Andrew E. Leitch ${ }^{\mathrm{a}, 1}$, Tara A. Sheldrake ${ }^{\mathrm{a}}$, John M. Hallett ${ }^{\mathrm{a}}$, Colette Meyer ${ }^{\mathrm{a}}$, \\ Sarah Fox ${ }^{a}$, Ana L. Alessandri ${ }^{a}$, Morag C. Martin ${ }^{a}$, Hugh J. Brady ${ }^{b}$, Mauro M. Teixeira ${ }^{c}$, \\ Ian Dransfield ${ }^{a}$, Christopher Haslett ${ }^{a}$, Adriano G. Rossi ${ }^{\mathrm{a}, *}$
}

${ }^{a}$ MRC Centre for Inflammation Research, The Queen's Medical Research Institute, University of Edinburgh Medical School, 47 Little France Crescent, Edinburgh, Scotland, UK
${ }^{\mathrm{b}}$ Section of Immunology and Infection, Division of Cell and Molecular Biology. Sir Alexander Fleming Building. Imperial College, South Kensington, London, UK

'Imunofarmacologia, Departamento de Bioquímica e Imunologia, Instituto de Ciências Biológicas, Universidade Federal de Minas Gerais, Belo Horizonte, Brazil

\section{A R T I C L E I N F O}

\section{Article history:}

Received 29 May 2009

Revised 10 July 2009

Accepted 13 July 2009

Available online 18 July 2009

Edited by Beat Imhof

\section{Keywords:}

Cyclin-dependent kinase

Eosinophil

Apoptosis

R-roscovitine

Inflammation

Mcl-1

Mitochondria

Caspase

\begin{abstract}
A B S T R A C T
Eosinophils are major players in inflammatory allergic diseases such as asthma, hay fever and eczema. Here we show that the cyclin-dependent kinase inhibitor (CDKi) R-roscovitine efficiently and rapidly induces human eosinophil apoptosis using flow cytometric analysis of annexin- $\mathrm{V} / \mathrm{propi}-$ dium iodide staining, morphological analysis by light microscopy, transmission electron microscopy and Western immunoblotting for caspase-3 cleavage. We further dissect these observations by demonstrating that eosinophils treated with R-roscovitine lose mitochondrial membrane potential and the key survival protein Mcl-1 is down-regulated. This novel finding of efficacious induction of eosinophil apoptosis by CDKi drugs has potential as a strategy for driving resolution of eosinophilic inflammation.
\end{abstract}

수 2009 Federation of European Biochemical Societies. Published by Elsevier B.V. All rights reserved.

\section{Introduction}

The eosinophil is a leukocyte of granulocyte lineage that confers resistance to parasitic infection [1]. In some societies levels of parasitic infection have significantly declined and in parallel, allergic, eosinophil-mediated, inflammatory diseases such as asthma, eczema and hay-fever have increased. In direct contrast, in those countries where parasitic infection has not declined, allergic inflammatory diseases are not prominent. It seems as though functional redundancy is not an option for eosinophils and allergic disease results from their inappropriate activation [2,3]. Eosinophils and their products have been demonstrated within airways, in lung parenchyma, at the site of eczematous skin lesions and in nasal mucosa. Evidence of their armamentarium has also been detected at sites of allergic inflammation and it has been shown that they contribute to airway epithelial damage and remodelling in asthma [1,4-7]. Therapeutic strategies that decrease eosinophil recruit-

\footnotetext{
- Corresponding author. Fax: +44 1312426578.

E-mail address: a.g.rossi@ed.ac.uk (A.G. Rossi).

R.D. and A.E.L. are joint first authors of this manuscript.
}

ment and activation or enhance resolution of inflammation by driving eosinophil apoptosis and clearance should ameliorate allergic inflammatory disease $[1,3,8]$.

An obvious target for the prevention of eosinophil growth, differentiation, survival [9-11] and, also to some extent recruitment and activation [12] is the cytokine IL- 5 but initial in vivo work has been disappointing suggesting a subordinate role or even redundancy for eosinophils in asthma [13]. Despite this, eosinophils have retained their place as key pathophysiological players in defined subsets of asthma following successful trials of anti-IL5 therapy [14-16]. If eosinophils can be driven towards apoptosis and efficiently cleared by phagocytes prior to membrane rupture, the damaging sequelae of degranulation and reactive oxygen species leakage should be avoided, thus protecting airway tissue and preventing detrimental remodelling $[5,17]$. Eosinophils undergo classical apoptosis in response to various treatments including: $\mathrm{NF}-\kappa \mathrm{B}$ inhibition, Fas-ligation, protein synthesis inhibition and exposure to corticosteroids. There is some controversy over the dominant mechanism by which eosinophils undergo apoptosis. Eosinophils are known to possess mitochondria which are important for apoptosis but probably not functional in terms of ATP 
generation. Caspases 3, 8 and 9 are constitutively expressed and have all been in implicated in dexamethasone driven apoptosis [7,18-20].

The cyclin-dependent kinase inhibitor (CDKi), R-roscovitine, was initially developed as an anti-cancer agent directed against cell-cycle proteins, promoting cancer cell death through effects on NF- $\mathrm{KB}, \mathrm{p} 53$ and key survival proteins $[21,22]$. It has been shown to promote apoptosis of myeloid cancer cells by down-regulation of the key survival protein Mcl-1 [23]. Eosinophils are terminally differentiated cells and as such should not require cell-cycling machinery such as CDKs. Nonetheless, like other terminally differentiated cells, including neutrophils $[24,25]$ and neurons [26], they have measurable expression of CDKs. Neutrophils are known to undergo apoptosis in response to CDK inhibitors and indeed work performed in our laboratory has shown that resolution of neutrophil-driven inflammation can be driven by this class of drugs [27]

In this paper, we are the first to show that CDK inhibition drives eosinophil apoptosis by the mitochondrial pathway, a novel finding with promising implications for the resolution of eosinophilic inflammation.

\section{Materials and methods}

\subsection{Eosinophil isolation}

Granulocytes were isolated from the peripheral venous blood of healthy adult donors (Lothian Research Ethics Committee approvals $\# 08 / S 1103 / 38$ or $\# 1702 / 95 / 4 / 72$ ) by dextran (Pharmacosmos) sedimentation followed by centrifugation through discontinuous PBS-Percoll (GE Healthcare) gradients [28,29]. Eosinophils were separated from contaminating neutrophils using an immunomagnetic separation step with sheep anti-mouse IgG-Dynabeads (Invitrogen) coated with the murine anti-neutrophil antibody 3G8 (anti-CD16; a gift fromDr.J.Unkeless, MountSinai MedicalSchool,NewYork)aspreviously described [30]. Eosinophil purity was routinely greater than $95 \%$.

\subsection{Apoptosis assessment}

Eosinophils were re-suspended in IMDM (PAA) with 10\% FBS (Biosera), penicillin $(100 \mathrm{U} / \mathrm{ml})$ and streptomycin $(100 \mathrm{U} / \mathrm{ml})$ (PAA). Cells were aliquoted $\left(5 \times 10^{6} \mathrm{cells} / \mathrm{ml}\right)$ into a 96 -well-flatbottomed-flexible-plate (BD Biosciences) in a final volume of $150 \mu \mathrm{l}$. They were incubated with R-roscovitine (A.G. Scientific, USA), Z-Val-Ala-DL-Asp(OMe)-fluoromethylketone (zVAD-fmk) (Bachem, Switzerland) or combinations at $37{ }^{\circ} \mathrm{C}$ with $5 \% \mathrm{CO}_{2}$. All stock reagents were initially dissolved in dimethylsulphoxide (DMSO) (Sigma) then diluted in buffer yielding a final DMSO concentration of $0.2 \%$; a corresponding DMSO control of $0.2 \%$ was assessed as an appropriate vehicle control. We assessed apoptosis by flow cytometry using annexin-V-FLUOS (Roche) in combination with propidium iodide (PI) (Sigma) on a BD FacsScan flow cytometer (Becton, Dickinson and Co.). Morphological apoptotic changes were assessed by light microscopy of DiffQuik ${ }^{\mathrm{MM}}$ (Dade Behring) stained cytocentrifuged cells.

\subsection{Mitochondrial transmembrane potential assessment}

MitoCapture $^{\mathrm{TM}}$ is a fluorescence-based tool for distinguishing between viable and apoptotic cells by detecting changes in the mitochondrial transmembrane potential. Eosinophils were isolated as above and incubated with appropriate reagent at a concentration of $5 \times 10^{4} \mathrm{ml}^{-1}$ in a flat-bottomed 96 -well plate at $37^{\circ} \mathrm{C}, 5 \%$ $\mathrm{CO}_{2}$. MitoCapture ${ }^{\mathrm{MM}}$ kit used as per manufacturer's instructions. Fluorescence microscopy was performed using a Zeiss Axiovert S100 microscope.

\subsection{Western blotting}

Cells were incubated in $2 \mathrm{ml}$ Eppendorf tubes at a concentration of $5 \times 10^{6}$ cells/ml with R-roscovitine, zVAD-fmk, MG-132 (Calbiochem) or combinations of these reagents for $4 \mathrm{~h}$ at $37^{\circ} \mathrm{C}$. Cells were lysed using 1\% IgepalCA-630 (Sigma) in TBS containing a protease inhibitor cocktail before centrifugation $\left(23100 \times \mathrm{g} ; 4^{\circ} \mathrm{C} ; 20 \mathrm{~min}\right.$ ) [24]. Protein samples (equivalent to $1.5 \times 10^{6}$ cells/lane) were resolved by SDS-PAGE ( $12 \%$ gel Thermo Scientific) then transferred to PVDF membranes (Millipore). Blots were blocked with $5 \%$ skimmed milk powder in TBS/0.1\% Tween-20 (Sigma) before probing with antibodies to Mcl-1 (Santa Cruz), caspase-3 (BD Biosciences), cleaved caspase-3 (Cell Signaling Technologies) and soluble $\beta$-actin (Sigma).

\subsection{Electron microscopy}

Cells were incubated with R-roscovitine $(20 \mu \mathrm{M})$ at $37{ }^{\circ} \mathrm{C}$ and $5 \%$ $\mathrm{CO}_{2}$ for $8 \mathrm{~h}$. Fixation was achieved by resuspension in 3\% gluteraldehyde/0.1 M sodium cacodylate buffer ( $\mathrm{pH} 7.4$ ) overnight. Photomicrographs were taken with a Phillips CM12 transmission electron microscope.

\subsection{Statistical methods}

Statistics shown are analysis of variance with post hoc testing by Student-Newman-Keuls using InStat software and data are expressed as means \pm S.E.M. unless otherwise stated.

\section{Results}

\section{1. $R$-roscovitine can drive primary human eosinophil apoptosis in a} concentration- and time-dependent manner

To explore whether R-roscovitine promotes similar pro-apoptotic effects on human eosinophils as those previously observed with neutrophils, cells were treated with increasing concentrations of 10,20 and $50 \mu \mathrm{M}$ R-roscovitine. Apoptosis was assessed at time points as early as $4 \mathrm{~h}$ by flow cytometric analysis and R-roscovitine was observed to induce apoptotic cell death in eosinophils. This contrasts with other pro-apoptotic agents such as dexamethasone where apoptosis is first detected at $12-24 \mathrm{~h}[30,31]$. In an early, preliminary experiment we included dexamethasone $(1 \mu \mathrm{M})$ as a positive control for induction of eosinophil apoptosis. We found that R-roscovitine $(30 \mu \mathrm{M})$ was more effective at inducing apoptosis as assessed by annexin- $V$ positivity. Interestingly, a combination of the two agonists did not yield higher levels of cell death than R-roscovitine alone. For example, after $20 \mathrm{~h}$ culture, control death was $12.5 \pm 0.8 \%$; dexamethasone was $22.1 \pm 2.2 \%$; $R$-roscovitine was $81.2 \pm 1.1 \%$ and a combination of dexamethasone and $R-$ roscovitine was $83.9 \pm 1.4 \%$ where values from duplicate samples obtained from a single donor are expressed as the means \pm S.D. Cells were further assessed for any morphological changes by light microscopy after cytocentrifugation and DiffQuik ${ }^{\mathrm{MM}}$ staining. The results showed a clear increase in annexin- $\mathrm{V} /$ propidium iodide staining, which occurred in a concentration- and time-dependent manner (Figs. 1A, B and 2A). Eosinophils progress through apoptosis to secondary necrosis (shown by joint staining with annexin- $\mathrm{V}$ and propidium iodide). This transition appears to occur more rapidly in eosinophils than in the other abundant granulocyte, neutrophils. At later time-points (up to $20 \mathrm{~h}$ ) we see increasing levels of apoptosis and a concomitant rise in necrosis again suggesting transition from apoptosis to secondary necrosis. Transmission electron microscopy of R-roscovitine treated eosinophils $(20 \mu \mathrm{M}, 8 \mathrm{~h})$ was utilised to ultimately characterise their cellular morphology. These 

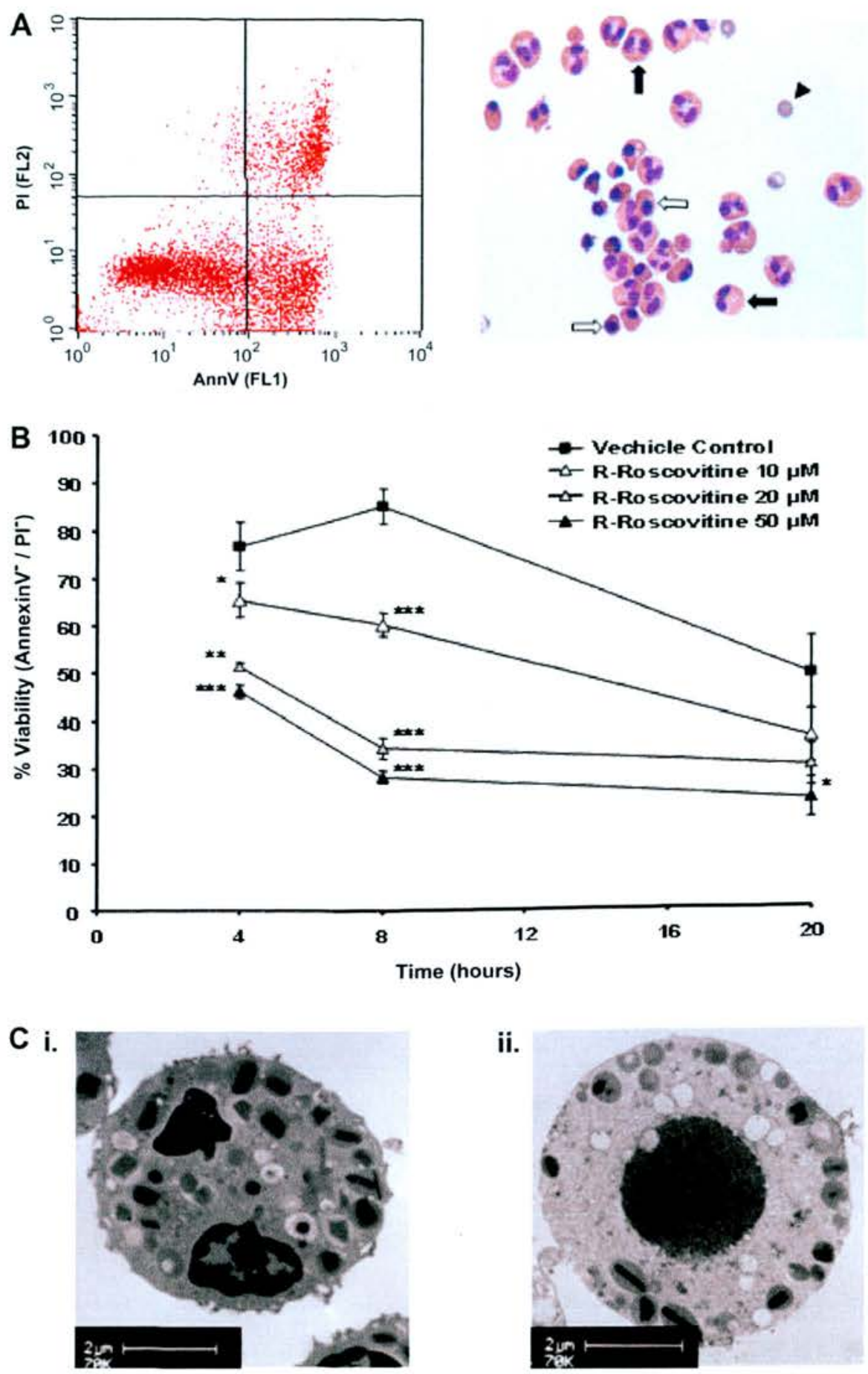

Fig. 1. Ability of increasing concentrations $(10,20$ and $50 \mu \mathrm{M})$ of R-roscovitine, to induce eosinophil cell death after $4 \mathrm{~h}$ treatment. (A) Flow cytometric profile of annexin-V/PI staining showing apoptotic cells in the lower right quadrant, necrotic cells in the top right quadrant and viable cells in the bottom left quadrant. Cytocentrifuge image (400x magnification) demonstrates cellular morphology. Black arrows indicate healthy, viable eosinophils and white arrows indicate apoptotic eosinophils. Black arrow head indicating an erythrocyte. (B) Cumulative flow cytometric analysis of the mean percentage annexin- $\mathrm{V}^{-} / \mathrm{PI}^{-}$cells. There were șignificant differences (using analysis of variance and post hoc Student-Newman-Keuls) between each treatment at the relevant time-point, $P$ values displayed are $P \leqslant 0.05$, $P \leqslant 0.01$, $P \leqslant 0.001$ and represent differences in levels of apoptosis against the DMSO control, $n=3$ independent experiments. (C) Transmission electron microscopy images of (i) a healthy non-apoptotic human eosinophil and (ii) an apoptotic eosinophil $8 \mathrm{~h}$ post-R-roscovitine $(20 \mu \mathrm{M})$ treatment. The latter cell is exhibiting characteristics typical of apoptosis, such as a condensed, round nucleus. Magnification $9500 \times$.

results clearly demonstrate the presence of classical apoptotic signs; namely the presence of chromatin condensation in the nucleus and granular rearrangement, when comparing R-roscovitine treated apoptotic eosinophils (Fig. 1C(ii)) with non-apoptotic cells (Fig. 1C(i)).
3.2. $R$-roscovitine driven eosinophil apoptosis can be delayed by caspase inhibition

Classical apoptosis is known to be a caspase-dependent phenomenon $[32,33]$. In order to confirm our finding that eosinophils 


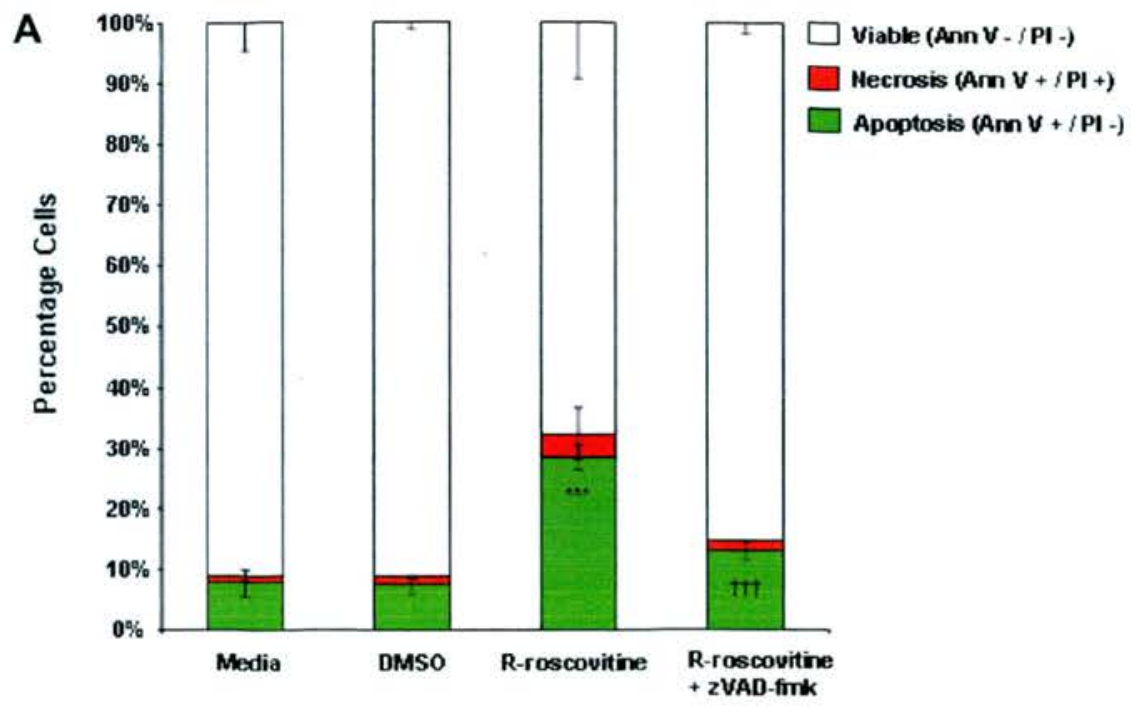

B

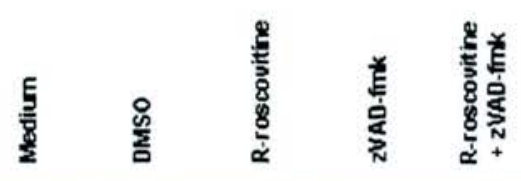

i $32 \mathrm{kDa}$

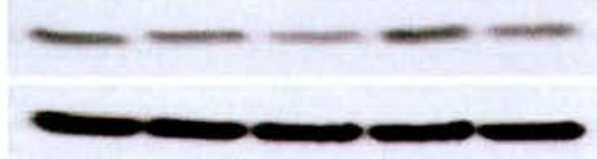

Caspase-3

$42 \mathrm{kDa}$

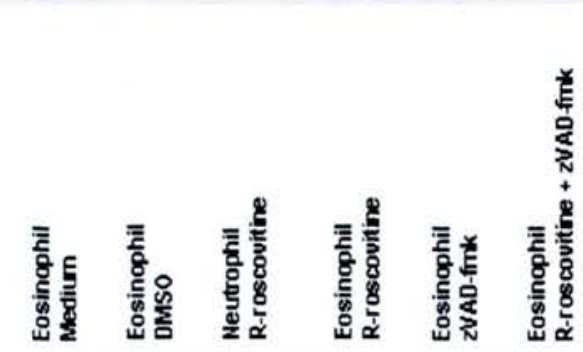

ii

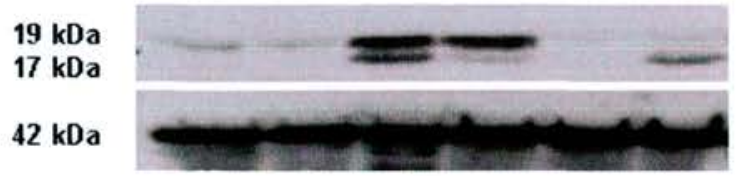

Cleaved caspase- 3

B-Actin

Fig. 2. Apoptosis induced $4 \mathrm{~h}$ post-treatment with R-roscovitine $(50 \mu \mathrm{M})$ was inhibited by $\mathrm{zVAD}$-fmk ( $100 \mu \mathrm{M})$, indicating that the mechanism of cell death is caspasedependent. (A) Cumulative flow cytometric profile analysis showing the mean percentagẹ of eosinophils in the viable (white bar), necrotic (red bar) or apoptotic (green bar) quadrants. $P$ values (using analysis of variance and post hoc Student-Newman-Keuls) of $P \leqslant 0.001$ represent difference in apoptosis compared to controls and $P$ values $" *$ $P \leqslant 0.001$ are compared to R-roscovitine treatment, $n=4$ independent experiments. (B) (i) Representative Western immunoblot image for caspase- 3 of the lysates from eosinophils treated with either R-roscovitine $(50 \mu \mathrm{M})$ alone or a co-treatment of R-roscovitine and zVAD-fmk (100 $\mu \mathrm{M})$ for $4 \mathrm{~h}$. (ii) Representative Western immunoblot image for cleaved caspase- 3 of the lysates from eosinophils treated with either R-roscovitine ( $50 \mu \mathrm{M})$ alone or a co-treatment of $\mathrm{R}$-roscovitine and $\mathrm{zVAD}$-fmk ( $100 \mu \mathrm{M})$ for $4 \mathrm{~h}$. Also included are the lysates of eosinophils treated with DMSO $(0.2 \%)$ as a vehicle control, culture medium alone, zVAD-fmk alone and lysates of R-roscovitine-treated human neutrophils (identical conditions) as a positive control. A $\beta$-actin blot is also included as a loading control.

undergo apoptosis following incubation with CDKis, we assessed caspase involvement using two different approaches. Initially, we co-incubated eosinophils with R-roscovitine $(50 \mu \mathrm{M})$ and $\mathrm{zVAD}$ fmk $(100 \mu \mathrm{M})$, the pan-caspase inhibitor and measured apoptosis, as before, using flow cytometry following annexin- $\mathrm{V}$ and propidium iodide staining. Co-incubation appeared to inhibit the early induction of apoptosis. At $4 \mathrm{~h}$ the level of apoptosis decreased significantly $(P \leqslant 0.001)$ by over $50 \%$ from $27.3 \pm 2.0 \%$ to $13.1 \pm 1.5 \%$ (Fig. 2A). To further demonstrate the role of caspases in R-roscovitine induced eosinophil apoptosis, we also performed Western blotting for caspase-3 (Fig. 2B(i)) and cleaved caspase-3
(Fig. 2B(ii)) on lysates from eosinophils, $4 \mathrm{~h}$ post-R-roscovitine with and without zVAD-fmk treatment. There were changes in the levels of caspase- 3 on R-roscovitine and zVAD-fmk treatment as detected by a specific caspase- 3 antibody and no evidence of cleaved caspase- 3 isoforms were detected with this antibody. These changes correlated with our subsequent finding using a specific cleaved caspase- 3 antibody. Cleaved casapase- 3 was easily detected in cells after R-roscovitine treatment with the use of a specific cleaved caspase- 3 antibody. However, co-treatment with R-roscovitine and zVAD-fmk reduced caspase-3 cleavage (Fig. 2B(ii)) thereby confirming the caspase-dependent nature of 
A

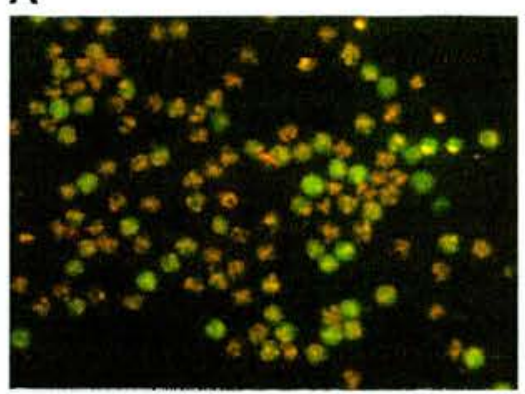

B

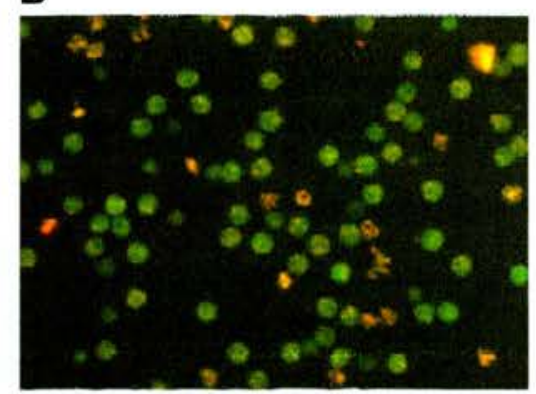

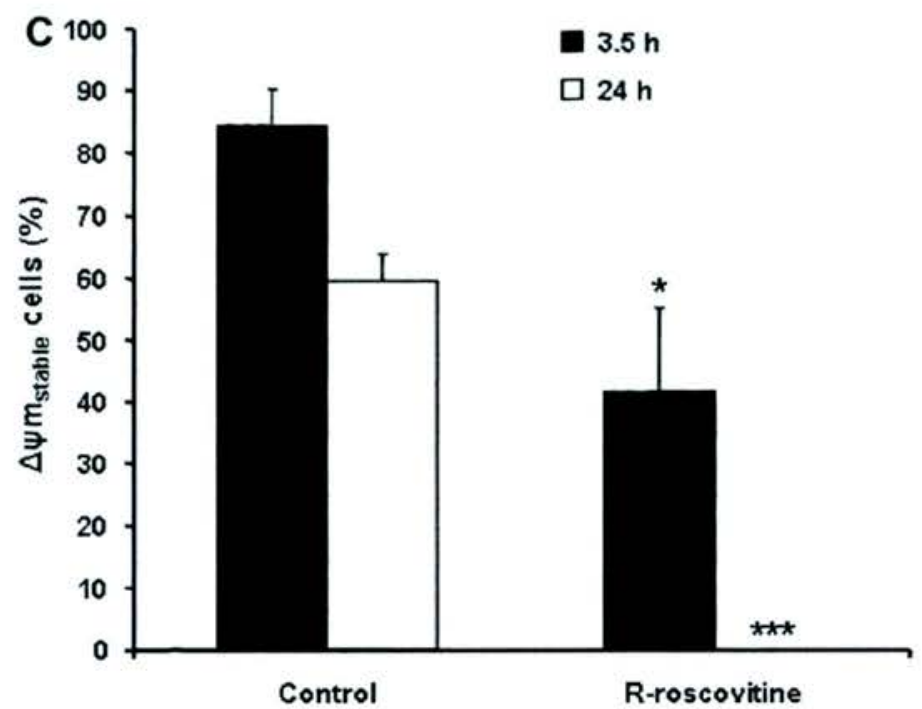

Fig. 3. Assessment of mitochondrial membrane potential using the MitoCapture ${ }^{\mathrm{m}}$ assay. (A) Eosinophils cultured in media supplemented with $\mathrm{FCS}$ at $37{ }^{\circ} \mathrm{C}, 5 \% \mathrm{CO}_{2}$ for $3.5 \mathrm{~h}$ were assayed. The vast majority of eosinophils retain mitochondrial membrane potential $\left(\Delta \psi_{\mathrm{m}}\right.$ stable) as identified by orange/red fluorescence. Representative images were taken $320 \times$ magnification. (B) Eosinophils cultured as above, however treated with R-roscovitine, $20 \mu \mathrm{M}$ for $3.5 \mathrm{~h}$. R-roscovitine treatment results in a significant increase in the proportion of eosinophils losing mitochondrial membrane potential as identified by green fluorescence within the cytoplasm. (C) Quantification of immunofluorescence șhowing the percentage cells in each mitochondrial membrane stage at both 3.5 and $24 \mathrm{~h}$. $P$ values (using analysis of variance and post hoc Student-Newman-Keuls) of $P \leqslant 0.05$ control vs $R$-roscovitine $3.5 \mathrm{~h}$ and $\quad P \leqslant 0.001$, control vs $R$-roscovitine $24 \mathrm{~h}$ are displayed. $n=3$ independent experiments with $>500$ cells counted per condition.

R-roscovitine induced cell death. Interestingly, despite an overall decrease in cleaved caspase-3 levels with combined R-roscovitine and $\mathrm{zVAD}-\mathrm{fmk}$ treatment there was a slight increase in the levels of the $17 \mathrm{kDa}$ cleaved caspase- 3 isoform. (The visible bands at MW 17 and $19 \mathrm{kDa}$ are cleaved caspase-3 isoforms (Fig. 2B(ii), inactive (non-cleaved) caspase- 3 is evident at $32 \mathrm{kDa}$ (Fig. 2B(i)).

\subsection{Apoptosis promoted by CDK inhibition is mediated by loss of mitochondrial membrane potential}

In order to further investigate our finding that eosinophils undergo apoptosis following treatment with R-roscovitine $(20 \mu \mathrm{M})$ we assessed mitochondrial membrane potential with the use of the mitochondria specific dye, MitoCapture ${ }^{\mathrm{TM}}$. This dye stains viable mitochondria orange/red as visualised by fluorescence microscopy, but dissipates into the cell cytoplasm and fluoresces green with loss of membrane potential. Loss of mitochondrial membrane potential $\left(\Delta \psi_{\mathrm{m}}\right)$ is a key event in the intrinsic pathway of apoptosis and wewere able to show that significantly more eosinophils lost mitochondrial membrane potential at 3.5 h post-R-roscovitine treatment compared to control (Fig. 3). At $24 \mathrm{~h}$ post-R-roscovitine treatment no eosinophils had intact mitochondria, whereas the majority of control eosinophils retained mitochondrial membrane potential (Fig. 3C).

\section{4. $R$-roscovitine down-regulates $M c l-1$, a key eosinophil survival} protein

Having found that eosinophil mitochondrial membrane potential was lost following treatment with R-roscovitine, we postulated that this might be due to the down-regulation of key survival proteins. The $\mathrm{Bcl}-2$ homologue, Mcl-1, has a short half-life because it can be ubiquitinated and degraded in the proteasome. It has previously been shown in neutrophil studies that treatment with MG-132, a proteasome inhibitor, preserves $\mathrm{Mcl}-1$ and prevents apoptosis at early time-points [30]. At late time-points other effects such as the preservation of $I \kappa B \alpha$ lead to acceleration of apoptosis [34]. Eosinophils co-incubated with both MG-132 (50 $\mu \mathrm{M})$ and R-roscovitine $(50 \mu \mathrm{M})$ for $4 \mathrm{~h}$ had enhanced viability compared to those treated with $\mathrm{R}$-roscovitine alone $(48.7 \pm 10.7 \%$ annexin$\mathrm{V}^{-} / \mathrm{PI}^{-}$vs $29.9 \pm 10.1 \%$ annexin- $\left.\mathrm{V}^{-} / \mathrm{PI}^{-} \quad P \leqslant 0.05, n=5\right)$. We demonstrated that this MG-132 survival effect correlated with preservation of $\mathrm{Mcl}-1$ protein in contrast to eosinophils incubated with R-roscovitine alone where Mcl-1 was significantly down-regulated (Fig. 4). This contrasted with cells co-incubated with R-roscovitine and zVAD-fmk $(100 \mu \mathrm{M})$ where no preservation of $\mathrm{Mcl}-1$ was apparent (to be expected as zVAD-fmk inhibits caspases which would normally function downstream of $\mathrm{Mcl}-1$ down-regulation). 


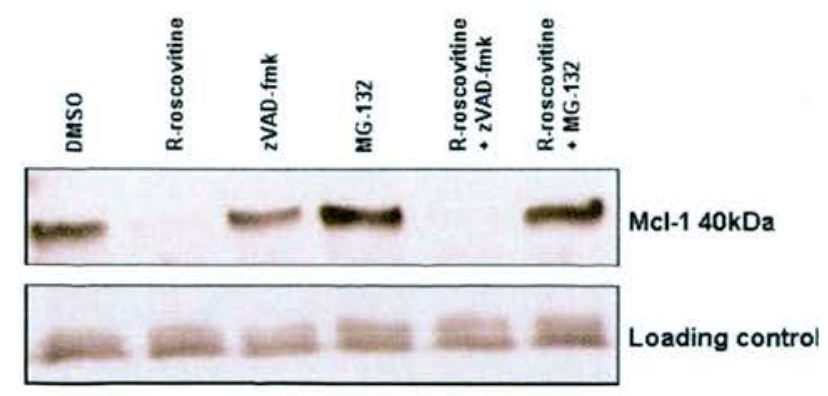

Fig. 4. Western immunoblotting of human eosinophil lysates for Mcl-1 (40 kDa) demonstrating significant down-regulation following $4 \mathrm{~h}$ R-roscovitine $(20 \mu \mathrm{M})$ treatment. The proteasome inhibitor, MG-132 $(50 \mu \mathrm{M})$ prevented R-roscovitine mediated down-regulation of $\mathrm{Mcl}-1$ whilst the caspase inhibitor, zVAD-fmk $(100 \mu \mathrm{M})$, did not. Loading control is a non-specific band at $20 \mathrm{kDa}$ on the same gel.

\section{Discussion}

We present the novel finding of rapid and efficacious induction of human eosinophil apoptosis, in vitro, with the CDKi drug. R-roscovitine. This is an important finding as eosinophils play a central role in the pathophysiology of common allergic diseases such as asthma and hay-fever as well as uncommon diseases such as eosinophilic oesophagitis, eosinophilic pneumonia and Churg-Strauss syndrome $[1,10,35]$. Eosinophils are also notoriously difficult to study for a number of reasons: their paucity in the peripheral blood of normal, healthy volunteer blood donors, the laborious isolation techniques required to ensure a pure population and their relative intractability to standard molecular biological techniques such as siRNA. Corticosteroids are known to induce significant eosinophil apoptosis, in vitro, but it is still debatable as to whether this effect is relevant in vivo [6,31,36-40]. Steroid drugs are extremely effective in the majority of asthmatic patients but some, at the more severe end of the spectrum, are resistant and others experience intolerable and unacceptable side-effects $[41-45]$. The initial disappointing results with therapy attempting to neutralise IL-5 had suggested that eosinophils were perhaps innocent bystanders in the disease process [13]. Two recent positive trials using the same approach with a carefully selected eosinophilic population of asthmatic patients suggest that eosinophils are central to a sub-population of asthmatic disease in what is increasingly recognised to be a heterogeneous patient group [14-16,45].

We have published results indicating that neutrophil apoptosis can be driven by CDKi drugs and that this can in turn promote resolution of inflammatory disease models [27]. We now show that this same drug-class can promote eosinophil apoptosis and we dissect the molecular mechanism by which this occurs. The mechanisms by which apoptosis occur in eosinophils remain controversial but it seems likely that the general paradigm of extrinsic (death-receptor mediated) and intrinsic (mitochondria mediated) pathways is applicable $[18,19,46,47]$. We have shown that eosinophils undergo apoptosis at early time-points $(4 \mathrm{~h})$ following treatment with CDKi and that this is preceded by loss of the key survival protein $\mathrm{Mcl}-1$. Mcl-1 is now generally accepted to be the pre-eminent $\mathrm{Bcl}-2$ homologue responsible for neutrophil survival and evidence is increasing for a central role in eosinophil survival. This evidence includes the granulopenic phenotype of the $\mathrm{MCl} 1^{-1-}$ mouse [48] and the finding that dexamethasone (a prototype corticosteroid) driven eosinophil apoptosis is associated with down-regulation of $\mathrm{Mcl}-1$ at the protein level [39]. These pro-survival $\mathrm{Bcl}-2$ homologues are short-lived compared to their pro-apoptotic counterparts, a feature which presumably biases granulocyte cells towards early, constitutive apoptosis. This is not the case in other cell types as, for example, the eponymous $\mathrm{Bcl}-2$ protein has a much longer half-life and therefore might be expected to protect cells from short-lived interruptions to survival protein production [49]. This may explain why we see little effect of $C D K$ inhibition on macrophage survival, a finding that protects our paradigm of therapeutic, selective apoptosis induction followed by efficient phagocytic clearance.

In keeping with our results suggesting down-regulation of survival proteins, we also show an early $(3.5 \mathrm{~h})$ loss of mitochondrial membrane potential. This immediately precedes significant caspase- 3 cleavage and supports our contention that we are inducing classical apoptosis mediated via the mitochondrial pathway as opposed to direct activation of the death-receptor pathway. We further support our data regarding caspase involvement, using the pan caspase inhibitor, zVAD-fmk at a concentration of $100 \mu \mathrm{M}$. This drug has been used over a concentration range of 10 $300 \mu \mathrm{M}$ in the literature and often at $100 \mu \mathrm{M}$ in granulocyte work $[47,48]$. At $300 \mu \mathrm{M}$ in combination with TNF accelerated apoptosis has been observed [49]. It is our experience working with primary human granulocytes that conventional low-dose zVAD-fmk is rapidly degraded by these cells. The capacity of granulocytes for zVAD-fmk degradation over short time-periods can lead to the erroneous conclusion that apoptosis, especially constitutive apoptosis, is caspase independent. Therefore, by necessity, we are forced to use higher concentrations than those routinely used by researchers working with other cell types. We are aware that non-specific effects on calpains have been described with caspase inhibitors but we feel that this pharmacological observation, together with the Western blotting analysis of caspase cleavage, strongly indicate that CDK inhibitor-induced apoptosis is indeed caspase-dependent. We have further demonstrated that the induction of apoptosis mediated by CDK inhibition is more powerful than that observed with dexamethasone. We are inclined to believe, given the data presented, that $\mathrm{CDK}$ inhibition mediates a more effective down-regulation of survival proteins. As CDK inhibition will promote neutrophil apoptosis in tandem with eosinophil apoptosis there is a risk of inducing neutropenia. There is evidence to suggest that activated neutrophils are preferentially targeted by CDKi (which may confer additional benefit in some asthma patients) but a strategy involving dual therapy with antibiotics might also be envisaged. The potential of this approach has been demonstrated in a recent paper by Koedel et al. [50] where combined CDKi and ceftriaxone therapy is used to resolve an experimental model of bacterial meningitis. Our novel finding, that CDKi drugs drive eosinophil apoptosis suggests that this approach may prove an effective resolution strategy for eosinophilic inflammation.

\section{Acknowledgements}

We thank the Medical Research Council, UK (G060481), Wellcome Trust (WT082181), Arthritis Research Council (16140), Asthma UK (01/042) and the Norman Salvesen Trust Emphysema Research Trust for financial support. The authors would like to thank Stephen Mitchell, Fiona Rossi, Shonna Johnson, and Mark E. Marsden for technical assistance, and Carol Ward, Annemieke Walker, Donald Davidson and John S. Savill for valuable advice over the years.

\section{References}

|1] Rothenberg. M.E. and Hogan. S.P. (2006) The eosinophil. Annu. Rev. Immunol 24, 147-174

[2] Giembycz, M.A. and Lindsay. M.A. (1999) Pharmacology of the eosinophil. Pharmacol. Rev. 51, 213-340.

[3] Leitch, A.E., Duffin, R., Haslett, C. and Rossi, A.G. (2008) Relevance of granulocyte apoptosis to resolution of inflammation at the respiratory mucosa. Mucosal. Immunol. 1, 350-363. 
[4] Duncan, C.J.. Lawrie, A., Blaylock, M.G., Douglas, J.G. and Walsh, G.M. (2003) Reduced eosinophil apoptosis in induced sputum correlates with asthma severity. Eur. Respir. J. 22, 484-490.

[5] Kankaanranta, H., Lindsay, M.A., Giembycz, M.A., Zhang, X., Moilanen, E. and Barnes, P.J. (2000) Delayed eosinophil apoptosis in asthma. J. Allergy Clin. Immunol. 106, 77-83.

[6] Walsh, G.M., Sexton, D.W. and Blaylock, M.G. (2003) Corticosteroids, eosinophils and bronchial epithelial cells: new insights into the resolution of inflammation in asthma. J. Endocrinol. 178, 37-43.

[7] Walsh, G.M. (2000) Eosinophil apoptosis: mechanisms and clinical relevance in asthmatic and allergic inflammation. Br. J. Haematol. 111, 61-67.

[8] Zhivotovsky, B. and Orrenius, S. (2009) Clinical perspectives of cell death: where we are and where to go. Apoptosis 14, 333-335.

[9] Dent, L.A., Strath, M., Mellor, A.L. and Sanderson, C.J. (1990) Eosinophilia in transgenic mice expressing interleukin 5. J. Exp. Med. 172, 1425-1431.

[10] Foster, P.S., Hogan, S.P., Ramsay, A.J., Matthaei, K.I. and Young. I.G. (1996) Interleukin 5 deficiency abolishes eosinophilia, airways hyperreactivity, and lung damage in a mouse asthma model. J. Exp. Med. 183, 195-201.

[11] Yamaguchi, Y., Hyashi, Y.I., Sugama, Y., Miura, Y., Kasahara, T., Kitamura, S., Torisuj. M., Mita, S., Tominaga, A., Takatsu, K. and Suda, T. (1988) Highly purified murine Interleukin 5 (IL-5) stimulates eosinophil function and prolongs in vitro survival. J. Exp. Med. 167. 1737-1742.

[12] Lopez, A.F., Sanderson, C.J., Gamble, J.R., Campbell, H.D., Young, I.G. and Vadas, M.A. (1988) Recombinant human interleukin 5 is a selective activator of human eosinophil function. J. Exp. Med. 167, 219-224.

[13] Flood-Page, P.T., Menzies-Gow, A.N., Kay, A.B. and Robinson, D.S. (2003) Eosinophil's role remains uncertain as anti-interleukin-5 only partially depletes numbers in asthmatic airway. Am. J. Respir. Crit. Care Med. 167. 199-204.

[14] Wenzel, S.E. (2009) Eosinophils in asthma-closing the loop or opening the door? N. Engl. J. Med. 360, 1026-1028.

[15] Nair, P., Pizzichini, M.M., Kjarsgaard, M., Inman, M.D., Efthimiadis, A., Pizzichini, E., Hargreave, F.E. and O'Byrne, P.M. (2009) Mepolizumab for prednisone-dependent asthma with sputum eosinophilia. N. Engl. J. Med. 360. 985-993.

[16] Haldar, P., Brightling, C.E., Hargadon, B., Gupta, S., Monteiro, W., Sousa, A., Marshall, R.P., Bradding. P., Green, R.H., Wardlaw, A.J. and Pavord, I.D. (2009) Mepolizumab and exacerbations of refractory eosinophilic asthma. N. Engl. J. Med. 360, 973-984.

[17] Kankaanranta, H., Moilanen, E. and Zhang, X. (2005) Pharmacological regulation of human eosinophil apoptosis. Curr. Drug Target Inflamm. Allergy 4, 433-445.

[18] Letuve, S., Druilhe, A., Grandsaigne, M., Aubier, M. and Pretolani, M. (2002) Critical role of mitochondria, but not caspases, during glucocorticosteroidinduced human eosinophil apoptosis. Am. J. Respir. Cell Mol. Biol. 26, 565571.

[19] Letuve, S., Druilhe, A., Grandsaigne, M., Aubier, M. and Pretolani, M. (2001) Involvement of caspases and of mitochondria in Fas ligation-induced eosinophil apoptosis: modulation by interleukin- 5 and interferon-gamma. J. Leukoc. Biol. 70, 767-775.

[20] Simon, H. and Alam, R. (1999) Regulation of eosinophil apoptosis: transduction of survival and death signals. Int. Arch. Allergy Immunol. 118. 7-14.

[21] McClue, S.J., Blake, D., Clarke, R., Cowan, A., Cummings, L., Fischer, P.M MacKenzie, M., Melville, J., Stewart, K., Wang, S., Zhelev, N. Zheleva, D. and Lane, D.P. (2002) In vitro and in vivo antitumor properties of the cyclin dependent kinase inhibitor CYC202 (R-roscovitine). Int. J. Cancer 102, 463468.

[22] Meijer, L. and Raymond, E. (2003) Roscovitine and other purines as kinase inhibitors. From starfish oocytes to clinical trials. Acc. Chem. Res. 36, 417-425.

[23] MacCallum, D.E., Melville, J., Frame, S., Watt, K., Anderson, S., GianellaBorradori, A., Lane, D.P. and Green, S.R. (2005) Seliciclib (CYC202, Rroscovitine) induces cell death in multiple myeloma cells by inhibition of RNA polymerase II-dependent transcription and down-regulation of Mcl-1. Cancer Res. 65, 5399-5407.

[24] Rosales, J.L. Ernst, J.D., Hallows, J. and Lee, K.Y. (2004) GTP-dependent secretion from neutrophils is regulated by Cdk5. J. Biol. Chem. 279, 5393253936.

[25] Leitch, A.E., Haslett, C., Rossi, A.G. (2009) Cyclin-dependent kinase inhibitor drugs as potential novel anti-inflammatory and pro-resolution agents. $\mathrm{Br}$. J. Pharmacol, in press.

[26] Blondel, M. and Meijer, L. (2007) Editorial: role of cyclin-dependent kinase-5 (Cdk5) in the central nervous system. Biotechnol. J. 2, 914-915.
[27] Rossi, A.G., Sawatzky, D.A., Walker, A., Ward, C., Sheldrake, T.A., Riley, N.A., Caldicott, A., Martinez-Losa, M., Walker, T.R., Duffin, R., Gray, M., Crescenzi, E., Martin, M.C., Brady, H.J., Savill, J.S., Dransfield, I. and Haslett, C. (2006) Cyclindependent kinase inhibitors enhance the resolution of inflammation by promoting inflammatory cell apoptosis. Nat. Med. 12, 1056-1064.

[28] Ward, C., Chilvers, E.R., Lawson, M.F., Pryde, J.G., Fujihara, S., Farrow, S.N., Haslett, C. and Rossi, A.G. (1999) NF-kappaB activation is a critical regulator of human granulocyte apoptosis in vitro. J. Biol. Chem. 274, 4309-4318.

[29] Haslett, C. Guthrie, L.A., Kopaniak, M.M., Johnston Jr., R.B. and Henson, P.M. (1985) Modulation of multiple neutrophil functions by preparative methods or trace concentrations of bacterial lipopolysaccharide. Am. J. Pathol. 119, 101-110.

[30] Meagher, L.C., Cousin, J.M., Seckl, J.R. and Haslett, C. (1996) Opposing effects of glucocorticoids on the rate of apoptosis in neutrophilic and eosinophilic granulocytes. J. Immunol. 156, 4422-4428.

[31] Fujihara, S., Ward, C., Dransfield, I., Hay, R.T., Uings, I.J., Hayes, B., Farrow, S.N., Haslett, C. and Rossi, A.G. (2002) Inhibition of nuclear factor-kappaB activation un-masks the ability of TNF-alpha to induce human eosinophil apoptosis. Eur. J. Immunol. 32, 457-466.

[32] Rossi, A.G., Haslett, C., Hirani, N., Greening. A.P., Rahman, I., Metz, C.N., Bucala, R. and Donnelly. S.C. (1998) Human circulating eosinophils secrete macrophage migration inhibitory factor (MIF). Potential role in asthma. J. Clin. Invest. 101, 2869-2874.

[33] Fesik, S.W. and Shi, Y. (2001) Structural biology. Controlling the caspases. Science 294, 1477-1478.

[34] Kohler, C., Orrenius, S. and Zhivotovsky, B. (2002) Evaluation of caspase activity in apoptotic cells. J. Immunol. Method 265, 97-110.

[35] Edwards, S.W., Derouet, M., Howse, M. and Moots, R.]. (2004) Regulation of neutrophil apoptosis by $\mathrm{Mcl}-1$. Biochem. Soc. Trans. 32, 489-492.

[36] Simon, H.U. (1998) Eosinophil apoptosis in allergic diseases-an emerging new issue. Clin. Exp. Allergy 28, 1321-1324.

[37] Adachi, T., Motojima, S., Hirata, A., Fukuda, T., Kihara, N., Kosaku, A., Ohtake, H. and Makino, S. (1996) Eosinophil apoptosis caused by theophylline, glucocorticoids, and macrolides after stimulation with IL-5. J. Allergy Clin. Immunol. 98, S207-S215.

[38] Gardai, S.J., Hoontrakoon, R., Goddard, C.D., Day, B.J., Chang, L.Y., Henson, P.M. and Bratton, D.L. (2003) Oxidant-mediated mitochondrial injury in eosinophil apoptosis: enhancement by glucocorticoids and inhibition by granulocytemacrophage colony-stimulating factor. J. Immunol. 170, 556-566.

[39] Sivertson, K.L., Seeds, M.C., Long, D.L., Peachman, K.K. and Bass, D.A. (2007) The differential effect of dexamethasone on granulocyte apoptosis involves stabilization of Mcl-1L in neutrophils but not in eosinophils. Cell Immunol. $246,34-45$

[40] Zhang, X., Moilanen, E. and Kankaanranta, H. (2000) Enhancement of human eosinophil apoptosis by fluticasone propionate, budesonide, and beclomethasone. Eur. J. Pharmacol. 406, 325-332.

[41] Holgate, S.T., Holloway. J., Wilson, S., Howarth, P.H., Haitchi, H.M., Babu, S. and Davies, D.E. (2006) Understanding the pathophysiology of severe asthma to generate new therapeutic opportunities. J. Allergy Clin. Immunol. 117, 496506.

[42] Hanania, N.A. (2008) Targeting airway inflammation in asthma: current and future therapies. Chest 133, 989-998.

[43] Busse, W.W., Banks-Schlegel, S. and Wenzel, S.E. (2000) Pathophysiology of severe asthma. J. Allergy Clin. Immunol. 106, 1033-1042.

[44] Barnes, P.J. (1996) Pathophysiology of asthma. Br. ]. Clin. Pharmacol. 42, 3-10.

[45] Wenzel, S.E. (2003) A different disease, many diseases or mild asthma gone bad? Challenges of severe asthma. Eur. Respir. J. 22, 397-398.

[46] Simon, H.U. (2001) Regulation of eosinophil and neutrophil apoptosissimilarities and differences. Immunol. Rev. 179. 156-162.

[47] Daigle, I. and Simon, H.U. (2001) Critical role for caspases 3 and 8 in neutrophil but not eosinophil apoptosis. Int. Arch. Allergy Immunol. 126, 147-156.

[48] Dzhagalov, I., St. J.A. and He, Y.W. (2007) The antiapoptotic protein Mcl-1 is essential for the survival of neutrophils but not macrophages. Blood 109 , $1620-1626$.

[49] Brustugun, O.T., Fladmark, K.E., Doskeland, S.O., Orrenius, S, and Zhivotovsky, B. (1998) Apoptosis induced by microinjection of cytochrome $c$ is caspasedependent and is inhibited by Bcl-2. Cell Death. Differ. 5, 660-668.

[50] Koedel, U., Frankenberg, T., Kirschnek, S., Obermaier, B. and Häcker, H. (2009) Apoptosis is essential for neutrophil functional shutdown and determines tissue damage in experimental pneumococcal meningitis. PLoS Pathog. 5 (5), e1000461. 
2

\section{Inflammation, Cell Injury, and Apoptosis}

\section{ADRIANO GIORGIO ROSSI and CHRISTOPHER HASLETT}

University of Edinburgh

Edinburgh, Scotland, United Kingdom

\section{Introduction}

Inflammation is normally a highly complex, interdependent biochemical and physiological protective response to tissue injury (e.g., due to bacterial infection or trauma). This process serves to destroy, dilute, partition off, or remove the injurious agent and the injured tissues, thereby promoting tissue repair. When this crucial and normally beneficial response occurs in an uncontrolled or exaggerated manner, the result is inappropriate or excessive tissue damage that often results in chronic inflammation. The products liberated by leukocytes recruited to sites of inflammation are primarily responsible for the tissue damage associated with these chronic inflammatory diseases. For example, leukocyte-derived reactive oxygen species (e.g., $\mathrm{O}_{2}-, \mathrm{H}_{2} \mathrm{O}_{2}$, $\mathrm{OH}^{-}, \mathrm{NO}$ ) and proteases (e.g., elastase and collagenase) can be deleterious to cells and tissues. Thus, the inflammatory response is also a fundamental process involved in the pathogenesis of many diseases, including asthma, chronic bronchitis, emphysema, rheumatoid arthritis, glomerulonephritis, and myocardial infarction and reperfusion injury, most of which are highly prominent, and on the increase, in developed societies. Understanding the mechanisms underlying the induction and resolution of the inflammatory response will have far-reaching implications for the prevention and treatment of inflammatory diseases. 


\section{The Inflammatory Response}

The gross clinical hallmarks of the acute inflammatory response were described at the beginning of the first millennium and include redness and swelling, with heat, pain, and often loss of function. The processes responsible for these changes have only really became apparent during the last century. The redness (erythema) and increased warmth occurring at sites of inflammation result mainly from vasodilatation leading to increased blood flow to the tissues. Vasodilatation of capillaries and arterioles produced by agents such as prostaglandins (e.g., $\mathrm{PGE}_{2}, \mathrm{PGI}_{2}, \mathrm{PGD}_{2}$ ), neuropeptides (e.g., calcitonin gene-related peptide, vasoactive intestinal peptide, substance $\mathrm{P}$ ), and nitric oxide cause an augmented intraluminal hydrostatic pressure downstream in postcapillary venules where plasma leakage occurs. These mediators act in concert with agents that cause an increase in postcapillary venule microvascular permeability, resulting in tissue fluid exudation (swelling or edema). Two main types of agents cause changes in microvascular permeability, those which act directly on endothelial cells (e.g., histamine, vasopressin, bradykinin, PAF) and those which cause increased microvascular permeability that depends on the recruitment of inflammatory cells. These latter mediators include complement-derived peptides (e.g., C5a and C3a), bacterial products (e.g., formylated peptides), lipids (e.g., LTB LT $_{4}$, and cytokines (e.g., IL-8) (1-3). The concerted action of these processes leads to the classical responses observed during inflammation.

\section{Mechanisms Underlying Inflammatory Cell Recruitment}

Over the last two decades a great deal of effort has been devoted to elucidating the mechanisms underlying recruitment of inflammatory cells to sites of inflammation $(2,3)$. Inflammatory cells, in order to reach sites of inflammation, have to (a) adhere loosely (tethering and rolling) to the postcapillary endothelial cells, a process mediated primarily by the selectins (E-, L- and P-selectin) and their respective ligands; (b) flatten and undergo firm adhesion to the endothelial cells, where the adhesive events are mediated mainly by the integrins (e.g., $\beta_{2}$ and $\beta_{1}$ integrins) and the immunoglobulin superfamily (e.g., ICAM-1, ICAM-2, VCAM-1); (c) traverse the endothelial cell layer of the blood vessel wall; (d) penetrate the basement membrane; and (e) migrate through the interstitial medium to reach the affected area (see Fig. 1). The site or the cause of the inflammatory response will often determine which type of inflammatory cell is recruited, possibly as a result of mediators generated (e.g., IL-8 for neutrophils and eotaxin for eosinophils) and adhesion molecules expressed (e.g. VLA-4 $\left[\alpha_{4} \beta_{1}\right]$ is highly expressed on eosinophils and lymphocytes but not on neutrophils). In addition, the structure and physiology of the vasculature may influence leukocyte recruitment to an inflammatory site. For example, in rheumatoid arthritis and in respiratory distress syndromes of the adult and neonate, the predominant leukocyte is the neutrophil; whereas in parasitic infection and in asthma, there is 


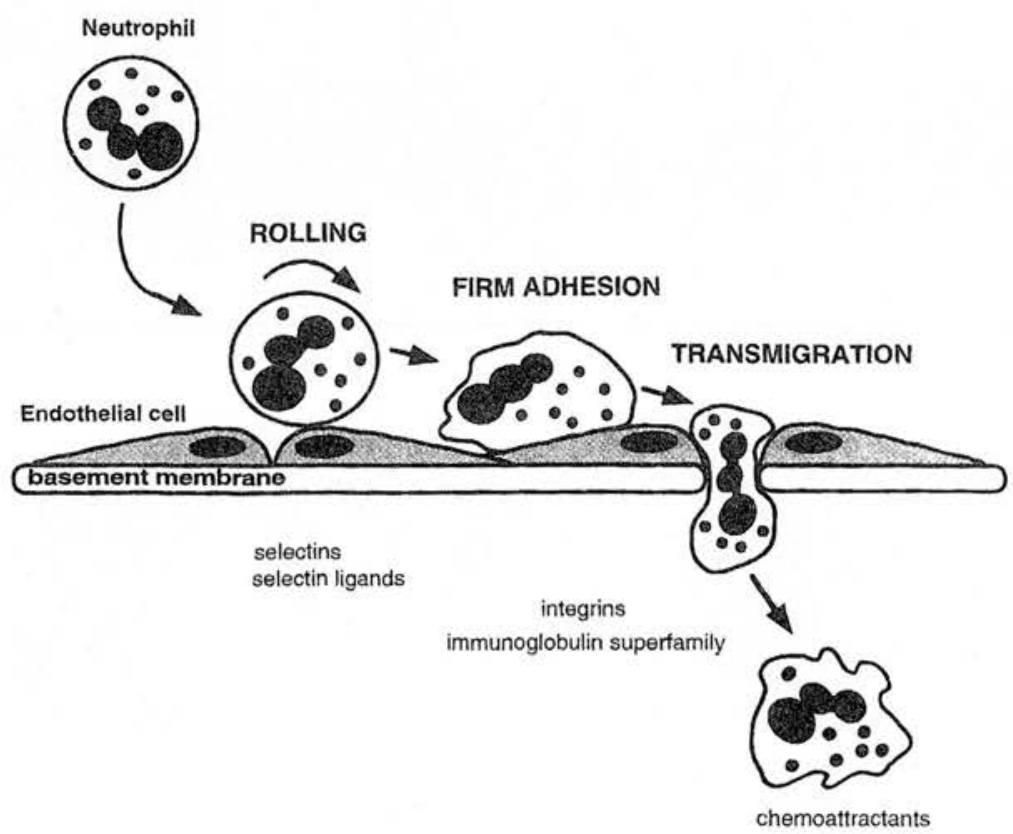

Figure 1 Schematic representation of the processes involved in neutrophil accumulation.

an abundance of recruited eosinophils. Evidence indicates that capillaries of the lung are the major site of leukocyte emigration, whereas postcapillary venules are the principal site in the systemic circulation (including the bronchial circulation). Intravascular pressure in the pulmonary circulation is approximately 10 -fold lower than in the systemic circulation, the blood flow is pulsatile, and the diameter of the pulmonary capillaries is somewhat smaller than the mean diameter of granulocytes. These cells therefore have to deform to pass through the pulmonary capillaries. Agents such as FMLP and C5a reduce neutrophil deformability, making them more rigid and favoring retention in the capillaries of the lung (see Refs. 3 and 4 for reviews).

Once inflammatory cells have been recruited to an inflammatory focus, if the concentration of secretagogue stimuli is sufficient, they become fully activated and, for example, phagocytose invading bacteria and liberate into the phagosome digestive enzymes and bactericidal oxygen metabolites. This scenario is perhaps akin to the phenomenon of "priming" observed in vitro, where cells such as the neutrophil and eosinophil in the presence of appropriate agents are rendered hyperresponsive to further activation by the same or other stimuli (5-7). The accumulation of leukocytes therefore depends on the complex interaction between inflammatory mediators, the expression of adhesion molecules and extracellular matrix proteins, and the physiology of the vasculature. 


\section{Resolution of Inflammation}

Although much attention has been directed toward understanding the mechanisms underlying the induction of inflammation, it is now apparent that the inflammatory process is regulated by a balance between its induction and its resolution (8). The remainder of this review will focus on this latter, rapidly developing area, with particular emphasis on the process of apoptosis and the removal of apoptotic cells.

\section{A. Inflammatory Mediator Dissipation}

At inflammatory foci, a plethora of inflammatory mediators are produced endogenously and/or introduced from exogenous sources. In order for inflammation to resolve, a number of mechanisms have evolved to control removal or inactivation of these pleiotropic mediators, including the following. (a) Vasodilatation and edema formation not only bring plasma proteins and leukocytes to inflammatory foci but also dilute, thereby reducing the effective concentrations, of inflammatory stimuli at the site. (b) The primary function of the accumulated leukocytes is to remove or destroy invading organisms; for example, eosinophils will neutralize parasites and neutrophils will phagocytose infecting bacteria. Removal of the source of exogenous mediators reduces the effective concentration of the inflammatory products. (c) Some inflammatory mediators will spontaneously become deactivated; for example, thromboxane $\mathrm{A}_{2}$ and nitric oxide can rapidly decompose to their relatively inactive metabolites, whereas others such as $\mathrm{C} 5 \mathrm{a}, \mathrm{PAF}$, and $\mathrm{LTB}_{4}$ are enzymatically rendered impotent. (d) A reduction in the synthesis and release, or an increase in the metabolism of a particular mediator, will also limit the concentration of the mediator. (e) Mediator-induced cellular or tissue responsiveness may also be reduced due to less well defined phenomena such as cellular or tissue desensitization. (f) Many mediators, generated at inflammatory sites, have opposing effects on effector cells and/or tissues; for example, C5a and IL-8 are potent neutrophil chemotactic agents, whereas prostaglandins can exert inhibitory effects on these cells by acting on specific cell surface receptors (9). Some inflammatory mediators (e.g., GM-CSF) prolong the survival of neutrophils by inhibiting apoptosis, whereas others (e.g., TNF- $\alpha$ ) can induce apoptosis (see below).

\section{B. Termination of Inflammatory Cell Recruitment}

Factors that control cessation of inflammatory cell accumulation remain ill defined. A number of in vivo studies have investigated the kinetics of accumulation of radiolabeled inflammatory cells in order to obtain information on the temporal emigration profiles of these cells from the blood to experimentally induced inflamed tissue. For example, in the acutely inflamed skin, lung, or joints, neutrophils accumulate within the first few hours (usually between $30 \mathrm{~min}$ and $4 \mathrm{hr}$ ), followed by a later, more protracted accumulation of monocytes. It was observed that the neutrophil influx in these acute inflammatory situations ceased remarkably quickly (10-13). In chronic inflammatory models or diseases where there is tissue damage and scarring, 
neutrophils and macrophages continue to accumulate for several days before resolution occurs (13). The mechanisms responsible for early cessation are obscure, but our study (14) suggests that dissipation of mediators may be a key element.

\section{Control of Inflammatory Cell Responses}

Undoubtedly, a number of mechanisms exist to limit the destructive capacity of inflammatory cells and allow resolution to occur, including: (a) a reduction in the effective concentration of proinflammatory stimuli; (b) an increase in antiinflammatory mediators; (c) an exhaustion of the responsiveness of inflammatory cells-i.e., cells may no longer have the capability to liberate destructive enzymes or toxic oxygen metabolites due to depletion of cellular resources or internal energy stores; (d) receptor or cellular desensitization; and (3) ultimately, the death and removal of the cell itself.

\section{Apoptosis of Inflammatory Cells}

Before the seminal work of Wyllie and colleagues $(15,16)$, who described a physiological mode of cell death termed apoptosis (programmed cell death), it was widely believed that cells simply died by necrosis in an uncontrolled manner (see Ref. 17). If this were the case, most cells, and especially inflammatory cells, would liberate their intracellular contents, many of which have major histotoxic and proinflammatory properties and would thus be expected to provoke an exaggerated inflammatory response. Virtually all cells under normal conditions undergo controlled physiological death by apoptosis, a process known to play a fundamental role in almost all aspects of life $(16)$, including the resolution of the inflammatory response $(8,18)$. Cells that have undergone apoptosis show remarkably similar structural, morphologi$\mathrm{cal}$, and biochemical changes. These similarities are perhaps indicative of a common underlying series of molecular mechanisms. Apoptotic cells are often smaller (due to cytoplasmic shrinkage), vacuolated, and exhibit major cell surface changes. Importantly, apoptotic cells remain intact, retaining their cytoplasmic granules and maintaining plasma membrane integrity such that they exclude vital dyes (e.g., trypan blue). It is the ultrastructural changes observed in the nucleus that are strikingly characteristic of an apoptotic cell; there is condensation of nuclear chromatin into dense, crescent-shaped aggregates, with the nucleolus becoming more conspicuous. In cells such as the neutrophil and eosinophil, where the nucleus is multilobed or bilobed, there is nuclear coalescence. Biochemically, apoptosis is typically characterized by endogenous endonuclease activation resulting in internucleosomal cleavage of chromatin. When DNA is extracted from apoptotic cells and separated electrophoretically on an agarose gel, there is a characteristic "ladder" pattern of DNA fragments, representing multimers of the 180-200 base pairs of DNA associated with nucleosomes. Most apoptotic cells express phosphatidylserine molecules on the external surface of their plasma membranes, a process which appears to be highly regulated (19-22), important in phagocyte recognition (see below), and useful for assessing apoptosis per se (23). Other cell surface changes may be unique to a 
particular cell system - for example, shedding of Fc $\gamma$ RIII (CD16) from neutrophils $(24,25)$. It has also been shown that apoptotic neutrophils are less responsive to external receptor-directed stimuli, in that neutrophil functions such as chemotaxis, phagocytosis, and degranulation are downregulated due to loss of cell surface receptors (e.g., FMLP receptors) and molecules important in phagocytosis (CD16) and adhesion (L-selectin) (24-27). These processes are likely to be important mechanisms in reducing the potential of cells to cause tissue damage, isolating apoptotic neutrophils from proinflammatory stimuli that would normally trigger effector function.

\section{E. Regulation of Inflammatory Cell Apoptosis}

It is now clear that apoptosis in granulocytes is regulated differently than in lymphocytes and thymocytes. Indeed, there are differences in the regulatory mechanisms for the closely related neutrophilic and eosinophilic granulocytes $(28,29)$. It is well established that certain inflammatory mediators prolong survival of inflammatory cells by inhibiting the rate of apoptosis (30-32). Furthermore, inflammatory stimuli such as GM-CSF, LPS, and C5a, by profoundly inhibiting neutrophil apoptosis, greatly prolong the functional longevity of these cells as assessed by chemotaxis and degranulation assays (26). Eosinophil apoptosis is also suppressed by GM-CSF and Il-5 (31), and by eotaxin (Rossi, Hannah, and Haslett, unpublished observations); IL-5 and eotaxin having no effect on neutrophil longevity. Suppression of apoptosis is not a universal phenomenon associated with all proinflammatory mediators; treatment of isolated human neutrophils in culture with TNF- $\alpha$ for $6 \mathrm{hr}$ will accelerate the rate of apoptosis; whereas at later time points ( $>18 \mathrm{hr}$ ), apoptosis is inhibited by this cytokine $(33,34)$. The mechanisms underlying TNF- $\alpha$ induction of apoptosis is unknown, but likely involves TNF receptor-associated proteins such as TRADD, FADD, and FLICE and may involve Fas (CD95/APO-1) and/or the sphingomyelinceramide pathways $(35,36)$. It has recently been demonstrated that anti-Fas-activating antibodies or Fas-L promote neutrophil and eosinophil apoptosis, an effect blocked by anti-Fas-blocking antibodies $(37,38)$. Whether Fas ligation plays an important role in inflammatory disease remains to be fully determined, although this pathway has been reported to induce apoptosis in experimental models in vivo (39). We have recently reported that nitric oxide donors induce granulocyte apoptosis via a cGMP-independent pathway, an observation that may explain part of the beneficial influence of nitric oxide in inflammation (40). Hypoxic conditions, which are found in chronically inflamed sites, inhibit neutrophil apoptosis, whereas apoptosis is induced in some cell lines (41). Other agents, such as the glucocorticoids (e.g., dexamethasone), inhibit neutrophil apoptosis concentration and steroid receptor dependently, but promote eosinophil apoptosis (28). Although the mechanisms underlying this divergent effect of glucocorticoids on granulocyte apoptosis are currently unknown, the effect may explain why steroid therapy is so efficacious in eosinophilic inflammation such as asthma. Interestingly, asthmatic patients who show an improvement in lung function after corticosteroid treatment also have an increased proportion of apoptotic eosinophils in their airway secretions (42). 
Using a pharmacological approach, the intracellular signaling pathways that control granulocyte apoptosis are slowly being unraveled. Neutrophil apoptosis is inhibited by elevation of intracellular free $\mathrm{Ca}^{2+}$ levels by calcium ionophores such as A23187 and ionomycin or by mobilization of intracellular stores using thapsigargin $(29,43)$, whereas eosinophil (29) and thymocyte (44) apoptosis is promoted. A key role for protein kinases is indicated by experiments in which apoptosis is promoted by inhibition of protein kinase $C$ by staurosporine and Ro-31,8220 (29) or delayed by activation of protein kinase A following treatment by agents that elevate cAMP $(25,45)$. Interestingly, elevation of cAMP promotes the rate of apoptosis in thymocytes (46) and in leukemic cell lines $(47,48)$. In addition, phosphatase inhibitors, okadaic acid and calyculin, inhibit or promote granulocytes apoptosis depending on the concentration used (49). Although intracellular kinase activity plays a key regulatory role in granulocyte apoptosis, the precise kinase(s) involved remain to be established. Why the intracellular signaling pathways that regulate neutrophil apoptosis are different from other cells, including the eosinophil, remains elusive but may be due to the differential expression of levels or types of protooncogenes shown to be involved in other cells systems $(50,51)$. Although we and others have demonstrated that neutrophils do not normally express $\mathrm{Bcl}-2$ protein, it is possible that other family members may be involved since it has been reported that overexpression of Bcl-2 in neutrophils prolongs neutrophil survival (52). It remains to be establișhed whether the recently characterized novel Bcl-2-like proteins are also expressed or play a role in neutrophil apoptosis (53). Protein synthesis inhibitors such as cycloheximide and actinomycin D, while inhibiting thymocyte apoptosis, promote neutrophil apoptosis, indicating endogenous synthesis of neutrophil survival proteins (54). Recent data also suggest that CD11b/CD18-dependent neutrophil phagocytosis can induce neutrophil apoptosis, a response that appears to be regulated by the formation of reactive oxygen intermediates (55). Despite the progress made during the last few years in identifying signaling mechanisms that control granulocyte apoptosis, it is obvious that much remains to be elucidated.

\section{F. Macrophage Phagocytosis of Senescent Apoptotic Cells}

Apoptotic cells at the inflammatory site would inevitably undergo disintegration (secondary necrosis) if macrophages were absent in situ, resulting in the release of their histotoxic contents and thereby causing further inflammation and the destruction of tissue cells. This highly undesirable scenario may occur in uncontrolled chronic inflammation, where the normally highly effective macrophage recognition and clearance mechanisms (see below) could be overwhelmed or defective. Indeed, it was once generally assumed that the majority of extravasated leukocytes met their ultimate fate at the inflamed site by necrosis, with removal of cell debris by tissue macrophages (17). Such a removal pathway would result in release of large quantities of leukocyte granular contents into the surrounding milieu, resulting in an exaggerated, potentially injurious inflammatory response. The majority of senescent cells at sites of inflammation are recognized by nearby phagocytes. Over 100 years ago, 
using recently developed intravital light microscopic techniques on a number of transparent invertebrates, the Russian biologist Elie Metchnikoff (56) first described phagocytosis of bacteria and senescent leukocytes by macrophages. Of particular relevance to the resolution of inflammation is the description of Reiter's cells in the inflamed joint fluid from people with Reiter's disease and other forms of acute arthritis. These Reiter's cells were shown to be macrophages containing apoptotic neutrophils $(57,58)$. Furthermore, in experimental inflammatory models where there is a large influx of neutrophils, the predominant mode of extravasated intact neutrophil removal is engulfment by the macrophages $(59,60)$, which subsequently leave the inflammatory site via the draining lymph nodes (61).

Although there have been numerous accounts of macrophage phagocytosis of neutrophils over the last century, it is only relatively recently that major developments regarding recognition of apoptotic cells have been described. Newman et al. (62) first showed that isolated neutrophils which had been aged in culture were recognized and ingested by inflammatory macrophages. The macrophages engorged themselves with senescent neutrophils but did not ingest freshly isolated neutrophils, indicating that aged neutrophils had undergone a fundamental process leading to recognition by macrophages. We subsequently showed that cells (e.g., neutrophils and eosinophils) had to undergo apoptosis before they were recognized and ingested by the macrophage - an extremely rapid and efficient process $(31,63,64)$. The macrophage can ingest several apoptotic cells at a time and, importantly, there is no detectable release of potentially injurious neutrophil contents (e.g., granule enzyme markers) into the surrounding medium $(65,66)$, effectively neutralizing any proinflammatory potential of apoptotic cells. Furthermore, when macrophages ingest particles such as zymosan (yeast cell walls) in vitro, the response is to liberate proinflammatory mediators (e.g., eicosanoids, granular enzymes, and cytokines). However, ingestion of apoptotic neutrophils does not result in such a proinflammatory mediator release from macrophages (67). We subsequently showed that this noninflammatory clearance mechanism was due to the molecular mechanisms by which macrophages recognize and ingest apoptotic cells (see 8,18 ).

\section{G. Mechanisms for Recognition and Clearance of Apoptotic Cells}

Phagocytes (usually macrophages) have developed highly efficient recognition mechanisms for the removal of apoptotic cells. Although it is the macrophage that is believed to remove the majority of apoptotic cells, other cells, such as fibroblasts (68), hepatocytes (69), endothelial cells (70), and glomelular mesangial cells (71), have a limited capacity for apoptotic cell recognition and removal. Perhaps the removal of apoptotic cells by these "semiprofessional" phagocytes plays a crucial role when local macrophages are overwhelmed by major waves of apoptotic cells. Surface changes on the apoptotic cell that may lead to phagocytic recognition remain poorly understood. The known molecular mechanisms of recognition of apoptotic cells are listed in Table 1, together with appropriate references [for a more detailed 
Table 1 Molecular Mechanisms of Recognition of Apoptotic Cells by Phagocytes

References

\section{Lectin-like receptors}

Specific carbohydrates on the surface of apoptotic cells are recognized by phagocytic cells containing lectin-like receptors such as the asialoglycoprotein, mannose or mannose/fucose receptor.

$$
\alpha_{v} \beta_{3} / \mathrm{CD} 36 / \text { thrombospondin (TSP) }
$$

It is hypothesized that a TSP-binding, moiety expressed on the surface of apoptotic cells binds to nearby TSP, which in turn acts as a bridging molecule between the apoptotic cell and the ingesting phagocyte. The phagocyte expresses two receptors on its surface, the $\alpha_{v} \beta_{3}$ "vitronectin receptor" integrin and an 88 -kDa monomer termed CD36, which cooperates to bind TSP.

Phosphatidylserine (PS) and PS receptors

Exposure of phosphatidylserine on the surface of apoptotic cells is believed to be recognized by putative phosphatidylserine receptors located on the surface of the ingesting phagocyte. Recently, it has been suggested that members of the scavenger receptor family may act as PS receptors.

$$
61 \mathrm{D} 3 \text { antigen }
$$

The mAb $61 \mathrm{D} 3$ can specifically attenuate the recognition of apoptotic cells by human monocyte-derived macrophages. The 61D3 antigen has not been fully characterized.

description of these mechanisms, we recommend the reviews by Savill et al. (18) and Hart et al. (72)]. Given that multiple genes are involved in the disposal of apoptotic bodies in the nematode Caenorhabditis elegans, it is likely that other unknown mechanisms exist in the recognition and phagocytosis of mammalian inflammatory cells.

\section{Relevance to Inflammatory Diseases: The Utilization of Apoptotic Mechanisms and Macrophage Clearance Mechanisms as Potential New Antiinflammatory Strategies}

Given that in chronic inflammatory diseases there is persistent accumulation of inflammatory cells which likely liberate their toxic intracellular products and thereby lead to tissue injury, scarring and architectural disruption with catastrophic loss of organ function, it seems likely that effective removal of these tissue-damaging inflammatory cells would be desirable. It is possible that in these diseases the inflammatory cell clearance mechanisms are either defective or overwhelmed, since in other examples of massive inflammatory reactions, such as lobar streptococcal 
pneumonia and acute gout, the lesions clear completely. The mechanisms by which inflammation normally resolves are not only essential for understanding of the persistent inflammatory states that characterize chronic inflammatory diseases, but should also provide novel approaches to antiinflammatory therapy. Significant differences in the intracellular regulation of inflammatory cells provide a special opportunity to induce apoptosis in inflammatory cell types selectively. Thus, by utilizing mechanisms of receptor-mediated induction of apoptosis of specific inflammatory cells (e.g., Fas ligation and glucocorticoid receptor activation) and by inhibition of the powerful survival signals found at inflammatory foci (e.g., GMCSF, LPS, prostaglandins, IL-5), it may be possible to accelerate apoptosis for therapeutic gain. In

NEUTROPHIL

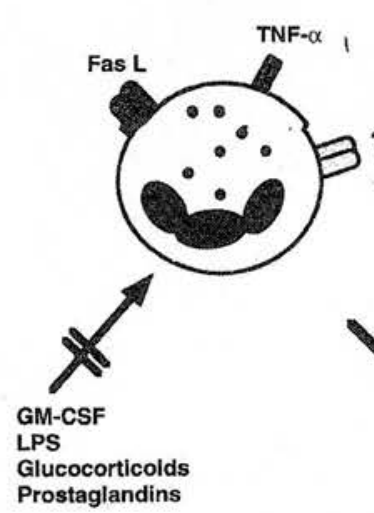

EOSINOPHIL

Glucocorticoids

Fas L
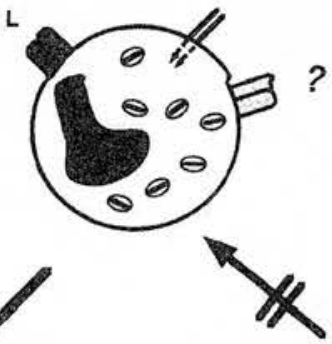

GM-CSF

IL-5

Prostaglandi

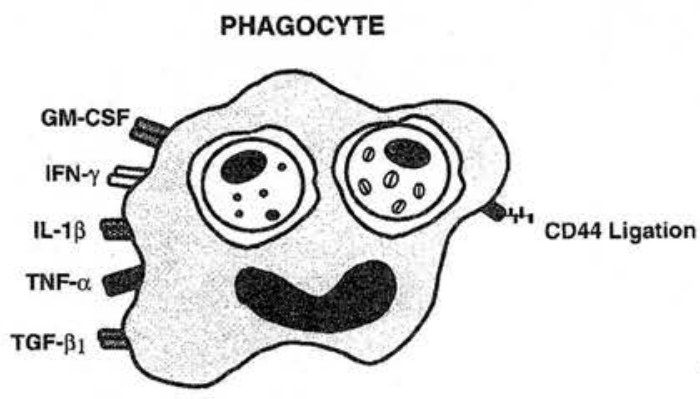

INCREASED RECOGNITION OR PHAGOCYTOSIS

Figure 2 Strategies for promotion of granulocyte apoptosis and removal of apoptotic cells by phagocytes (GM-CSF, granulocyte/macrophage-colony stimulating factor; LPS, lipopolysaccharide; TNF- $\alpha$, tumor necrosis factor- $\alpha$; IL- 5 , interleukin- 5 ; INF- $\gamma$, interferon- $\gamma$, IL-1 $\beta$, interleukin-1 $\beta$; TGF- $\beta_{1}$; transforming growth factor- $\beta_{1}$. 
combination with an effective therapeutic strategy for inducing apoptosis, development of mechanisms to specifically enhance apoptotic cell clearance by phagocytes such as the macrophage, or by "semiprofessional" phagocytes, may be equally desirable or indeed a prerequisite to preventing proinflammatory secondary necrosis (Fig. 2). It has recently been reported that certain cytokines (77), activation of macrophage protein kinase $\mathrm{C}$ (McCutcheon, Haslett, and Dransfield, unpublished observations), and ligation of macrophage CD44 (86) can enhance macrophage clearance of apoptotic cells.

\section{Concluding Remarks and Future Directions}

Although much progress has been made in understanding the mechanisms controlling the accumulation of inflammatory cells to sites of inflammation, we believe that elucidation of the mechanisms regulating the resolution of the inflammatory process can lead to novel approaches for therapeutic gain. Specifically, development of strategies to induce selective inflammatory cell apoptosis, together with augmentation of macrophage clearance mechanisms, will have far-reaching implications for the control of the many inflammatory diseases that are responsible for the heavy burden of morbidity and untimely deaths observed throughout the world.

\section{Acknowledgments}

We thank Dr. Ian Dransfield for helpful discussion and are grateful to the Medical Research Council (UK) for financial support.

\section{References}

1. Wedmore CV, Williams TJ. Control of vascular permeability by polymorphonuclear leukocytes in inflammation. Nature 1981; 289:646-650.

2. Showell HJ, Williams TJ. The neutrophil in inflammation. In: Roger TJ, Gilman SC, eds. Immunopharmacology. New York: Telford, 1989:23-63.

3. Rossi AG, Hellewell PG. Mechanisms of neutrophil accumulation in tissues. In: Hellewell PG, Williams TJ, eds. Immunopharmacology of Neutrophils. London: Academic Press, 1994:223-243.

4. MacNee W, Selby C. Neutrophil kinetics in the lung. Clin Sci 1990; 79:97-107.

5. O'Flaherty JT, Rossi AG. 5-Hydroxyicosatetraenoate stimulate neutrophils by a stereospecific, G-protein linked mechanism. J Biol Chem 1993; 268:14708-14714.

6. Rossi AG, MacIntyre DE, Jones CJP, McMillan RM. Stimulation of human neutrophil polmorphonuclear leukocytes by leukotriene $\mathrm{B}_{4}$ and platelet-activating factor: an ultrastructural and pharmacological study. J Leuk Biol 1993; 53:117-125.

7. Kitchen E, Rossi AG, Condliffe AM, Haslett C, Chilvers ER. Demonstration of reversible priming of human neutrophils using platelet-activating factor. Blood 1996; 88:43304337. 
8. Haslett C, Savill JS, Whyte MKB, Stern M, Dransfield I, Meagher LC. Granulocyte apoptosis and the control of inflammation. Phil Trans R Soc 1994; 345:327-333.

9. Rossi AG, O'Flaherty JT. Prostaglandin binding sites in human polymorphonuclear neutrophils. Prostaglandins 1989; 37:641-653.

10. Colditz IG, Movat HZ. Densensitisation of acute inflammatory lesions to chemotaxins and endotoxin. J Immunol 1984; 133:2163-2168.

11. Saverymuttu SH, Phillips G, Peters AM, Lavender JP. Indium-111 autologous leucocyte scanning in lobar pneumonia and lung abscesses. Thorax 1985; 40:925-930.

12. Clark RJ, Jones HA, Rhodes CG, Haslett C. Non-invasive assessment in self-limited pulmonary inflammation by external scintigraphy of 11 indium-labelled neutrophil influx and by measurement of the local metabolic response with positron emission tomography. Am Rev Respir Dis 1989; 139:A58.

13. Haslett C, Shen AS, Feldsien DC, Allen D, Henson PM, Cherniack RM. "1'Indiumlabelled neutrophil flux into the lungs of bleomycin-treated rabbits assessed noninvasively by external scintigraphy. Am Rev Respir Dis 1989; 140:756-763.

14. Haslett C, Jose PJ, Giclas PC, Williams TJ, Henson PM. Cessation of neutrophil influx in C5a-induced acute experimental arthritis is associated with loss of chemoattractant activity from joint spaces. J Immunol 1989; 142:3510-3517.

15. Kerr JFR, Wyllie AH, Currie AR. Apoptosis: a basic biological phenomenon with wide ranging implications in tissue kinetics. Br J. Cancer 1972; 26:239-257.

16. Wyllie AH, Kerr JFR, Currie AR. Cell death: the significance of apoptosis. Int Rev Cytol $1980 ; 68: 251-306$.

17. Hurley JV. Termination of acute inflammation. I. Resolution. In: Hurley JV, ed. Acute Inflammation. 2d ed. London: Churchill Livingstone, 1983:109-117.

18. Savill JS, Haslett C. Fate of neutrophils. In: Hellewell PG, Williams TJ, eds. Immunopharmacology of Neutrophils. London: Academic Press, 1994:295-314.

19. Fadok VA, Savill JS, Haslett C, Bratton DL, Doherty D, Campbell PA, Henson PM. Different populations of macrophages use either the vitronectin receptor or the phosphatidylserine receptor to recognize and remove apoptotic cells. J Immunol 1992; 149:40294035.

20. Fadok VA, Voelker DR, Campbell PA, Cohen JJ, Bratton DL, Henson PM. Exposure of phosphatidylserine on the surface of apoptotic lymphocytes triggers specific recognition and removal by macrophages. J Immunol 1992; 148:2207-2216.

21. Fadok VA, Laszlo DJ, Noble PW, Weinstein L, Riches DW, Henson PM. Particle digestibility is required for induction of the murine phosphatidylserine recognition mechanism used by murine macrophages to phagocytose apoptotic cells. J Immunol 1993; 151:4274-4285.

22. Fadok VA, Laszlo DJ, Noble PW, Weinstein L, Riches DWH, Henson PM. Particle digestibility is required for induction of the phosphatidylserine recognition mechanism used by murine macrophages to phagocytose apoptotic cells. J Immunol 1993; 151: 4274-4285.

23. Koopman G, Reutelingsperger CPM, Kuijten GAM, Keehnen RMJ, Pals ST, Van Oers MHJ. AnnexinV for flow cytometric detection of phosphatidylserine expression on B cells undergoing apoptosis. Blood 1994; 84:1415-1420.

24. Dransfield I, Buckle A-M, Savill JS, McDowall A, Haslett C, Hogg N. Neutrophil apoptosis is associated with a reduction in CD16 (FcyRIII) expression. J Immunol 1994; 153:1254-1263. 
25. Rossi AG, Cousin JM, Dransfield I, Lawson MF, Chilvers ER, Haslett C. Agents that elevate cAMP inhibit human neutrophil apoptosis. Biochem Biophys Res Commun $1995 ; 217: 892-899$.

26. Whyte MKB, Meagher LC, MacDermot J, Haslett C. Impairment of function in aging neutrophils is associated with apoptosis. J Immunol 1993; 150:5124-5134.

27. Dransfield I, Stocks SC, Haslett C. Regulation of cell adhesion molecule expression and function associated with neutrophil apoptosis. Blood 1995; 85:3264-3273.

28. Meagher L, Cousin JM, Seckl JR, Haslett C. Opposing effects of glucocorticoids on the rate of apoptosis in neutrophilic and eosinophilic granulocytes. J Immunol 1996; 156: $4422-4428$.

29. Cousin JM, Haslett C, Rossi AG. Regulation of granulocyte apoptosis by PKC inhibition and elevation of $\left[\mathrm{Ca}^{2+}\right]$ i. Biochem Soc Trans 1997; 25:243S.

30. Lee A, Whyte MBK, Haslett C. Prolonged in vitro lifespan and functional longevity of neutrophils induced by inflammatory mediators acting through inhibition of apoptosis. $\mathbf{J}$ Leuk Biol 1993; 54:283-288.

31. Stern M, Meagher L, Savill JS, Haslett C. Apoptosis in human eosinophils. Programmed cell death in the eosinophil leads to phagocytosis by macrophages and is modulated by IL-5. J Immunol 1992; 148:3543-3549.

32. Ward C, Hannah S, Chilvers ER, Farrow S, Haslett C, Rossi AG. Transforming growth factor- $\beta$ increases the inhibitory effect of GM-CSF and dexamethasone on neutrophil apoptosis. Biochem Soc Trans 1997; 25:244S.

33. Takeda Y, Watanabe H, Yonehara S, Yamashita T, Saito S, Sendo F. Rapid acceleration of neutrophil apoptosis by tumor necrosis factor. Int Immunol 1993; 5:691-694.

34. Murray J, Barbara JAJ, Dunkley SA, Lopez AF, Ostade XF, Condliffe AM, Dransfield I, Haslett C, Chilvers ER. Regulation of neutrophil apoptosis by tumour necrosis factor $\alpha$ : requirement for TNFR55 and TNFR-75 for induction of apoptosis in vitro. Blood 1997. In press.

35. Whyte MKB. A link between cell-surface receptors and ICE proteases. Trends Cell Biol 1996; 6:418.

36. McConkey DJ, Orrenius S. Signal transduction pathways to apoptosis. Trends Cell Biol 1996; 4:370-374.

37. Liles WC, Kiener PA, Ledbetter JA, Aruffo A, Klebenoff SJ. Differential expression of Fas (CD95) and Fas ligand on normal human phagocytes: implications for the regulation of apoptosis in neutrophils. J Exp Med 1996; 184:429-440.

38. Matsumoto K, Schleimet RP, Saito H, Iikura Y, Bochner BS. Induction of apoptosis in human eosinophils by anti-Fas antibody treatment in vitro. Blood 1995; 86:1437-1443.

39. Anderson GP. Resolution of chronic inflammation by therapeutic induction of apoptosis. TIPS 1996; 17:438-442.

40. Chilvers ER, Wong TH, Rahman I, Haslett C, Rossi AG. Nitric oxide stimulates neutrophil apoptosis via a cyclic-GMP-independent mechanisms. Keystone Symposia: Apoptosis and Programmed Cell Death, 1997; A109.

41. Hannah S, Mecklenburgh K, Rahman I, Bellingan GJ, Greening A, Haslett C, Chilvers ER. Hypoxia prolongs neutrophil survival in vitro. FEBS Lett 1995; 372:233-237.

42. Woolley KL, Gipson PG, Carty K, Wilson AJ, Twaddell SH, Woolley MJ. Eosinophil apoptosis and the resolution of airway inflammation in asthma. Am J Respir Crit Care 1996; 154:237-243.

43. Whyte MKB, Meagher LC, Hardwick SJ, Savill JS, Haslett C. Transient elevations of 
cytosolic free calcium retard subsequent apoptosis in neutrophils in vitro. $\mathrm{J}$ Clin Invest 1993; 92:446-455.

44. McConkey DJ, Nicotera P, Hartzell P, Bellomo G, Wyllie AH, Orrenius S. Glucocorticoids activate a suicide process in thymocytes through an elevation of cytosolic $\mathrm{Ca}^{2+}$ concentration. Arch Biochem Biophys 1989; 269:365-370.

45. Hallsworth MP, Geimbycz MA, Barnes PJ, Lee TH. Cyclic AMP-elevating agents prolong or inhibit eosinophil survival depending on prior exposure to GM-CSF. Br J Pharmacol 1996; 117:79-86.

46. McConkey DJ, Orrenius S, Okret S, Jondal M. Cyclic $\Lambda$ MP potentiates glucocorticoidinduced endogenous endonuclease activation in thymocytes. FASEB J 1993; 7:580-585.

47. Lanotte M, Riviere JB, Hermouet S, Houge G, Vintermyr OK, Gjertsen BT, Doskeland SO. Programmed cell death (apoptosis) is induced rapidly and with positive cooperativity by activation of cyclic adenosine monophosphate-kinase I in a myeloid leukemic cell line. J Cell Phys 1991; 146:73-80.

48. Gjertsen BT, Cressey LI, Ruchard S, Houge G, Lanotte M, Doskeland SO. Multiple apoptotic death types triggered through activation of separate pathways by CAMP and inhibitors of protein phosphatases in one (IPC leukemia) cell line. J Cell Sci 1994; 107: 3363-3377.

49. Cousin JM, Haslett C, Rossi AG. Effect of protein phosphatase inhibitors on dexamethasone-mediated inhibition of neutrophil apoptosis. Biochem Soc Trans 1997; 25:246S.

50. Yang E, Korsmeyer SJ. Molecular thanatopsis: a discourse on the BCL2 family and cell death. Blood 1996; 88:386-401.

51. Martins LM, Earnshaw WC. Apoptosis: alive and kicking in 1997. Trends Cell Biol 1997; 7:111-114.

52. Lagasse E, Weissman IL. Bcl-2 inhibits apoptosis of neutrophils but not their engulfment by macrophages. J Exp Med 1994; 179:1047-1051.

53. Gibson L, Holmgreen SP, Huang DCS, Bernard O, Copeland NG, Jenkins NA, Sutherland GR, Baker E, Adams JM, Cory S. bcl-w, a novel member of the bcl-2 family, promotes cell survival. Oncogene 1996; 13:665-675.

54. Whyte MKB, Meagher LC, Lee A, Haslett C. Coupling of neutrophil apoptosis to recognition by macrophages: co-ordinated acceleration by protein synthesis inhibitors. $\mathrm{J}$ Leuk Biol 1997. In press.

55. Coxon A, Rieu P, Barkalow FJ, Askari S, Sharpe AH, von Andrian UH, Arnaout MA, Mayadas TN. A novel role for the $\beta 2$ integrin $C D 11 \mathrm{~b} / \mathrm{CD} 18$ in neutrophil apoptosis: a homeostatic mechanism in inflammation. Immunity 1996; 5:653-666.

56. Metchnikoff $\mathrm{E}$. Lectures on the comparative pathology of inflammation. Lecture VII. Delivered at the Pasteur Institute in 1891 (transl Starling FA, Starling EH). London: Kegan, Paul, Trench, Trubner, 1968.

57. Pekin T, Malinin TI, Zwaifler R. Unusual synovial fluid findings in Reiter's syndrome. Ann Intern Med 1967; 66:677-684.

58. Spriggs RS, Boddington MM, Mowat AG. Joint fluid cytology in Reiter's syndrome. Ann Rheum Dis 1978; 37:557-560.

59. Sanui H, Yoshida S-I, Nomoto K, Ohhara R, Adachi Y. Peritoneal macrophages which phagocytose autologous polymorphonuclear leucocytes in guinea-pigs. $\mathrm{Br} \mathrm{J}$ Exp Pathol 1982; 63:278-285.

60. Chapes SK, Haskill S. Evidence for granulocyte-mediated macrophage activation after C. parvum immunization. Cell Immunol 1983; 75:367-377. 
61. Bellingan GJ, Caldwell H, Howie SEM, Dransfield I, Haslett C. In vivo fate of the inflammatory macrophage during the resolution of inflammation: inflammatory macrophages do not die locally but emigrate to the draining lymph nodes. J Immunol 1996; 157 : $2577-2585$.

62. Newman SL, Henson JE, Henson PM. Phagocytosis of senescent neutrophils by human monocyte-derived macrophages and rabbit inflammatory macrophages. J Exp Med 1982; $156: 430-442$.

63. Savill JS, Henson PM, Haslett C. Phagocytosis of aged human neutrophils by macrophages is mediated by a novel "charge-sensitive" recognition mechanism. J Clin Invest $1989 ; 84: 1518-1527$.

64. Stern M, Savill JS, Haslett C. Human monocyte-derived macrophage phagocytosis of senescent eosinophils undergoing apoptosis. Mediation by $\alpha_{\mathrm{v}} \beta_{3} / \mathrm{CD} 36 /$ thrombospondin recognition mechanism and lack of phlogistic response. Am J Pathol 1996; 149:911-921.

65. Savill JS, Wyllie AH, Henson JE, Walport MJ, Henson PM, Haslett C. Macrophage phagocytosis of aging neutrophils in inflammation: programmed cell death in the neutrophil leads to its recognition by macrophages. J Clin Invest 1989; 83:865-875.

66. Kar S, Ren Y, Savill JS, Haslett C. Inhibition of macrophage phagocytosis in vitro of aged neutrophils increases release of neutrophil contents (abstr). Clin Sci 1995; 85:27p.

67. Meagher LC, Savill JS, Baker A, Fuller RW, Haslett C. Phagocytosis of apoptotic neutrophils does not induce macrophage release of thromboxane $B_{2}$. J Leuk Biol 1992; 52:269-273.

68. Hall SE, Savill JS, Henson PM, Haslett C. Apoptotic neutrophils are phagocytosed by fibroblasts with participation of the fibroblast vitronectin receptor and involvement of a mannose/fucose-specific lectin. J Immunol 1994; 153:3218-3227.

69. Dini L, Autuori F, Lentini A, Oliverio S, Piacentini M. The clearance of apoptotic cells in the liver is mediated by the asialoglycoprotein receptor. FEBS Lett 1992; 296:174-178.

70. Dini L, Lentini A, Diez Diez G, Rocha M, Falasca L, Serafino L, Vidal-Vanaclocha F. Phagocytosis of apoptotic bodies by liver endothelial cells. J Cell Sci 1995; 108:967-973.

71. Savill JS, Smith J, Sarraf C, Ren Y, Abbott F, Rees A. Glomerular mesangial cells and inflammatory macrophages ingest neutrophils undergoing apoptosis. Kidney Int 1992; 42: 924-936.

72. Hart SP, Haslett C, Dransfield I. Recognition of apoptotic cells by phagocytes. Experientia 1996; 52:950-956.

73. Duvall E, Wyllie AH, Morris RG. Macrophage recognition of cells undergoing programmed cell death (apoptosis). Immunology 1985; 56:351-358.

74. Savill JS, Dransfield I, Hogg N, Haslett C. Vitronectin receptor-mediated phagocytosis of cells undergoing apoptosis. Nature 1990; 342:170-173.

75. Savill JS, Hogg N, Ren Y, Haslett C. Thrombospondin cooperates with CD 36 and the vitronectin receptor in macrophage recognition of neutrophils undergoing apoptosis. $\mathrm{J}$ Clin Invest 1992; 90:1513-1522.

76. Ren Y, Silverstein RL, Allen J, Savill JS. CD36 gene transfer confers capacity for phagocytosis of cells undergoing apoptosis. J Exp Med 1995; 181:1857-1862.

77. Ren Y, Savill JS. Proinflammatory cytokines potentiate thrombospondin-mediated phagocytosis of neutrophils undergoing apoptosis. J Immunol 1995; 154:2366-2374.

78. Martin SJ, Reutelingsperger CPM, McGahon AJ, Rader JA, van Schie RCAA, LaFace DM, Green DR. Early redistribution of plasma membrane phosphatidylserine is a general feature of apoptosis regardless of the initiating stimulus: inhibition by overexpression of bcl-2 and Abl. J Exp Med 1995; 182:1545-1556. 
79. Verhoven B, Schlegel RA, Williamson P. Mechanisms of phosphatidylserine exposure, a phagocyte recognition signal, on apoptotic T lymphocytes. J Exp Med 1995; 182:15971601.

80. Platt N, Suzuki H, Kurihara Y, Kodama T, Gordon S. Role of the class A macrophage scavenger receptor in the phagocytosis of apoptotic thymocytes in vitro. Proc Natl Acad Sci (USA) 1997; 93:12456-12460.

81. Ramprasad MP, Fischer W, Wiztum JL, Sambrano GR, Quehenberger O, Steinberg D. The 94-to 97-kDa mouse macrophage membrane protein that recognises oxidised low density lipoprotein and phosphatidylserine-rich liposomes is identical to macrosialin, the mouse homologue of human CD68. Proc Natl Acad Sci (USA) 1995; 92:9580-9584.

82. Rigotti A, Acton SL, Krieger M. The class B scavenger receptors SR-BI and CD36 are receptors for anionic phospholipids. J Biol Chem 1995; 270:16221-16224.

83. Sambrano GR, Parsatharathy S, Steinberg D. Recognition of oxidatively damaged erythrocytes by a macrophage receptor with specificity for oxidized low density lipoprotein. Proc Natl Acad Sci (USA) 1994; 91:3265-3269.

84. Sambrano GR, Steinberg D. Recognition of oxidatively damaged and apoptotic cells by an oxidized low density lipoprotein receptor on mouse peritoneal macrophages: role of membrane phosphatidylserine. Proc Natl Acad Sci (USA) 1995; 92:1396-1400.

85. Flora PK, Gregory GD. Recognition of apoptotic cells by human macrophages: inhibition by a monocyte/macrophage-specific monoclonal antibody. Eur J Immunol 1994; 24: $2625-2632$.

86. Hart SP, Dougherty GJ, Haslett C, Dransfield I. CD44 regulates phagocytosis of apoptotic neutrophil granulocytes, but not apoptotic lymphocytes, by human macrophages. J Immunol 1997; 159:919-925. 


\title{
Regulation of granulocyte apoptosis and implications for anti-inflammatory therapy
}

\author{
Edwin R Chilvers, Adriano G Rossi, Joanna Murray, Christopher Haslett
}

The elimination of unwanted cells is now regarded as an essential component of many normal biological programmes. These include embryonic remodelling, the removal of autoreactive thymic $\mathrm{T}$ cells, and the disposal of senescent enterocytes at the villus tip. Hence cell death, in particular apoptosis or "programmed cell death", appears to be a prerequisite for life. ${ }^{1}$ In the particular example of inflammation many of the unique features of apoptosis, such as the retention of plasma membrane integrity, the loss of the cytotoxic and secretory capacity of the cell, and the ability of apoptotic cells to be recognised and phagocytically removed, suggest that this remarkable process may play a key role in limiting tissue injury and facilitating the successful resolution of inflammation. ${ }^{2-5}$ Furthermore, the ingestion of apoptotic cells by macrophages, unlike the uptake of other biological and non-biological material, fails to induce a secretory or pro-inflammatory response. ${ }^{6}$ Although a number of different cells within the lung have the capacity to undergo apoptosis (including the bronchial epithelial cell and type II pneumocyte ${ }^{89}$ ), the central role of the granulocyte as the primary effector cell in most forms of lung and airway wall inflammation, coupled with the huge potential for these cells to induce tissue injury if not cleared, marks the apoptotic capacity of these cells as being of particular importance in lung inflammation.

Despite the considerable interest generated by the discovery that neutrophils and eosinophils undergo constitutive apoptosis when aged in vitro, ${ }^{20}$ a process that results in their ingestion by either professional ${ }^{211}$ or semi-professional phagocytes (including fibroblasts ${ }^{12}$ ), only a relatively small number of studies have addressed the importance of this process in vivo. This in part reflects the observation that apoptotic cells appear to be cleared extremely rapidly in tissues and hence are not readily apparent at any one time, and secondly that genetic manipulation of this process has been hampered by the global importance of apoptosis, especially in fetal development. Despite this, apoptotic granulocytes are observed at sites of inflammation ${ }^{45}$ and there is a substantial increase in the number of ingested and non-ingested apoptotic neutrophils in bronchoalveolar fluid in acute lung injury in neonates ${ }^{13}$ and in the recovery phase of experimental lipopolysaccharide induced alveolitis. ${ }^{14}$ More compelling evidence linking this event to the resolution of inflammation is the finding that the administration of corticosteroids in patients with acute severe asthma is associated with the appearance of apoptotic eosinophils in sputum, and this event parallels clinical improvement. ${ }^{15}$

An additional problem encountered in trying to track and quantify apoptosis in vivo is that many of the pro-inflammatory mediators and cytokines present at an inflamed site - for example, GM-CSF, C5a, IL-5-and the physicochemical characteristics of such an environmentfor example, hypoxia-cause profound inhibition of granulocyte apoptosis. ${ }^{10161718-21}$ Hence, eosinophil apoptosis is rarely encountered within nasal polyp tissue ${ }^{22}$ and neutrophil apoptosis is not readily apparent in fluid recovered from an empyema cavity. ${ }^{23}$ Moreover, it is now recognised that the inhibition of eosinophil apoptosis by agents such as IL-5, GM-CSF and other Th-2 derived cytokines represents a critical factor underlying the initial accumulation of these cells. ${ }^{5425}$ Such data also suggest that granulocyte induced tissue injury and chronic inflammation may reflect not only excessive granulocyte recruitment but also inhibition of normal apoptosis based clearance mechanisms. This concept is supported by data indicating that defective $\mathrm{T}$ cell killing, which involves autocrine or paracrine generation of Fas ligand (Fas-L) leading to activation of the death domain containing Fas receptor, may underlie chronic $\mathrm{T}$ cell activation and survival following antigen stimulation, ${ }^{26}$ and that bronchoalveolar lavage fluid from patients with ARDS prolongs the survival of normal human neutrophils in vitro. ${ }^{27}$

Such a hypothesis suggests that a therapeutic strategy based on stimulating granulocyte apoptosis may offer a novel approach to promoting the resolution of inflammation. To date, however, it has been difficult to identify agents that stimulate granulocyte apoptosis since, despite the relatively rapid rate of constitutive apoptosis observed when these cells are cultured in vitro, the majority of priming and activating agonists inhibit rather than accelerate this process. This list includes lipopolysaccharide and the pro-inflammatory mediators IL-5, C5a, GM-CSF, IL- $1 \beta$, IFN $\gamma$, and $\mathrm{LTB}_{4}{ }^{10}{ }^{16-20}$ Moreover, many of the well recognised routes to induce apoptosis in thymocytes and lymphocytes, such as the use of corticosteroids and the elevation of intracellular cAMP, have the opposite effect in neutrophils ${ }^{2829}$ - that is, they delay apoptosis - and it is possible that such observations explain in part the limited anti-inflammatory profile of $\beta_{2}$ adrenoceptor agonists and corticosteroids in certain "neutrophil dominant" forms of inflammation such as ARDS. ${ }^{30}$ Recent data, however, have indicated that it is possible to drive apoptosis in these cells-for example, with TNF $\alpha$, Fas- $\mathrm{L}$, the ingestion of $E$ coli or oil red particles ${ }^{19}{ }^{31-36}$-and, most intriguingly, theophylline ${ }^{37}$ which, unlike other agents that increase intracellular cAMP, causes a modest stimulation of neutrophil apoptosis. Hints that nature already utilises such a strategy to drive the removal of granulocytes from an inflamed site appear in studies showing that synovial fluid from patients with active rheumatoid arthritis, and bronchoalveolar fluid obtained from rabbits with experimental pneumococcal pneumonia, induce neutrophil apoptosis $^{38}$ (R Lawson and C Haslett, unpublished 
observations). Moreover, the potential to manipulate this process therapeutically is supported by the ability of anti-Fas monoclonal antibodies delivered to the lungs of mice with allergen induced airways eosinophilia to cause an increase in the numbers of peroxidase positive macrophages in lavage fluid and a substantial reduction in the number of eosinophils in the airways. ${ }^{33}$

The study of granulocyte apoptosis in vitro has also revealed some very important differences in the regulation of apoptosis in neutrophils and eosinophils. Most notably, it is now clear that glucocorticoids accelerate eosinophil apoptosis yet delay this process in the neutrophil. ${ }^{28}$ This observation may explain, at least in part, the ability of corticosteroids to resolve tissue eosinophilia in conditions such as asthma and pulmonary eosinophilia. ${ }^{14}$ Other differences also exist in the regulation of apoptosis between these two cell types. For example, the anti-apoptotic factor $\mathrm{Bcl}_{2}$ is readily detectable in eosinophils yet is absent from neutrophils, ${ }^{21}{ }^{30} 40$ and an increase in intracellular calcium enhances eosinophil apoptosis but delays neutrophil apoptosis. ${ }^{41}{ }^{12}$ Hence, despite the close proximity of these cells in phylogenetic terms, the prospect of being able to selectively target apoptosis in a particular inflammatory cell type appears to be a realistic goal.

Finally, while under in vitro conditions the uptake of apoptotic granulocytes by macrophages appears to be a rapid and highly efficient process, in certain pathological states this disposal system may become overwhelmed. Augmentation of the phagocytic capacity of inflammatory macrophages may therefore represent an additional option for intervention, and recent data demonstrating a major enhancement of macrophage ingestion of apoptotic neutrophils following CD44 ligation offers some support for this type of approach. ${ }^{13}$

Hence, the recognition that apoptotic cell death plays such a critical role in dictating the function and fate of inflammatory cells (and is amenable to exogenous regulation) has transformed our understanding of how "beneficial" and "deleterious" forms of inflammation may differ and offers a completely new avenue for therapeutic intervention in inflammation. Monitoring apoptosis in biological samples such as sputum should also provide a novel index of the success or otherwise of such strategies. The future goal of identifying the basis for the unique genetic control of granulocyte apoptosis may provide novel and selective therapeutic targets to drive this process and offer an explanation as to why the intensity, duration, and outcome of an inflammatory response differs so much between individuals.

Respiratory Medicine Unit, Department of Medicine,

E R CHILVERS

University of Cambridge School of Clinical Medicine,

Addenbrooke's Hospital, Cambridge CB2 2QQ, UK

A G ROSSI

J MURRAY

C HASLETT

Respiratory Medicine Unit, Department of Medicine (RIE),

University of Edinburgh Medical School, Teviot Place,

Edinburgh $\mathrm{EH} 89 \mathrm{AG}, \mathrm{UK}$

Correspondence to: Dr E R Chilvers.

1 Wyllie AH, Kerr JFR, Currie AR. Cell death: the significance of apoptosis. Int Rev Cytol 1980; 68:251-306. Savill JS, Wyllie AH, Henson JE, et al. Macrophage phagocytosis of aging
neutrophils in inflammation. FClin Invest 1989;83:865-75.

3 Whyte MKB, Meagher LC, MacDermot J, et al. Impairment of function in aging neutrophils is associated with apoptosis. Y Immunol 1993; 150:512434.

4 Haslett C, Savill JS, Whyte MKB, et al. Granulocyte apoptosis and control of inflammation. Phil Trans $R$ Soc Lond 1994; 345:327-33.

5 Haslett C. Granulocyte apoptosis and inflammatory disease. Br Med Bull 1997;53:669-83.
6 Meagher LC, Savill JS, Baker A, et al. Phagocytosis of apoptotic neutrophils does not induce macrophage release of thromboxane $\mathrm{B}_{2}$. F Leuk Biol 1992 52:269-73

7 Stern M, Savill JS, Haslett C. Human monocyte-derived macrophage phagocytosis of senescent eosinophils undergoing apoptosis. Am J Pathol $1996 ; 149: 911-2$

8 Aoshiba K, Rennard SI, Spurzem JR. Cell-matrix and cell-cell interactions modulate apoptosis of bronchial epithelial cells. Am f Physiol 1997 272:L.28-37.

Fine A, Anderson NL, Rothstein TL, at al. Fas expression in pulmonary alveolar type II cells. Am Y Physiol 1997; 273:L64-71

10 Stern M, Meagher L, Savill J, et al. Apoptosis in human eosinophils. Programmed cell death in the eosinophil leads to phagocytosis by

1 Cox G, Crossley J, Xing Z. Macrophage engulfment of apoptotic neutrophils contributes to the resolution of acute pulmonary inflammation in vivo. Am f Respir Cell Mol Biol 1995;12:232-7.

12 Hall SE, Savill JS, Henson PM, et al. Apoptotic neutrophils are phagocytosed by fibroblasts with participation of the fibroblast vitronectin receptor and involvement of a mannose/fucose-specific lectin. 7 Immuno 1994;153:3218-27.

13 Grigg JM, Savill JS, Sarraf C, et al. Neutrophil apoptosis and clearance from neonatal lungs. Lancet 1991;338:720-2

14 Cox G. IL-10 enhances resolution of pulmonary inflammation in vivo by promoting apoptosis of neutrophils. Am I Physiol 1996;271:L566-71.

15 Woolley KL, Gipson PG, Carty K, et al. Eosinophil apoptosis and the resolution of airway inflammation in asthma. Am I Respir Crit Care Med 1996; 154:237-43.

16 Her E, Frazer J, Austen F, et al. Eosinophil hematopoietins antagonise the programmed cell death of eosinophils. F Clin Imvest 1991;88:1982-7.

Colotta F, Re F, Polentarutti N, et al. Modulation of granulocyte survival and programmed cell death by cytokines and bacterial products. Blood
$1992 ; 80: 2012-20$.

18 Lee A, Whyte MKB, Haslett C. Inhibition of apoptosis and prolongation of neutrophil functional longevity by inflammatory mediators. I Lenk Biol 1993;54:283-8.

19 Murray J, Barbara JAJ, Dunkley SA, et al. Regulation of neutrophil apoptosis by tumor necrosis factor-a: requirement for TNFR 55 and TNFR75 for induction of apoptosis in vitro. Blood 1997;90:2772-83.

20 Hebert MJ, Takano T, Holthofer $\mathrm{H}$, et al. Sequential morphologic events during apoptosis of human neutrophils. Modulation by lipoxygenasederived eicosanoids. F Immunol 1996;157:3105-15.

21 Hannah S, Mecklenburgh K, Rahman I, et al. Hypoxia prolongs neutrophi survival in vitro. FEBS Lett 1995;372:233-

22 Simon HU, Yousefi S, Schranz C, et al. Direct demonstration of delayed eosinophil apoptosis as a mechanism causing tissue eosinophilia. J Immumo 1997; 158:3902-8.

23 Simon HU, Blaser K. Interdisciplinary aspects of pneumology. Report on the 23rd Ludwig-Heilmeyer Symposium of the Society for Advances in

24 Simon HU. Molecular mechanisms of defective eosinophil apoptosis in diseases associated with eosinophilia. Int Arch Allergy Imminol

5 Simon HU, Blaser K. Inhibition of programmed eosinophil death: a key pachogenic event for eosinophilia? Immuno Today 1995;16:53-5.

Vignaux F, Vivier $\mathrm{E}$, Malissen B, et al TCR CD3 coupling to Fas-based cytotoxicity. I Exp Med 1995;181:781-6.

Matute-Bello G, Liles WC, Radella F, et al. Neutrophil apoptosis in the acute respiratory distress syndrome. Am f Respir Crit Care Med 1997;156: 1969-77.

28 Meagher LC, Cousin JM, Seckl JR, et al. Opposing effects of glucocorticoids on the rate of apoptosis in neutrophilic and eosinophilic granulocytes. Imminol 1996;156:4422-8.

29 Rossi AG, Cousin JM, Dransfield I, et al. Agents that elevate cAMP inhibi human neutrophil apoptosis. Biochem Bioplys Res Commun 1995;271:892-

30 Donnelly SC, Haslett C. Cellular mechanisms of acute lung injury: implications for future treatment in the adult respiratory distress syndrome. Tho $\operatorname{rax} 1992 ; 47: 260-3$

31 Liles WC, Kiener PA, Ledbetter JA, et al. Differential expression of Fas (CD95) and Fas ligand on normal human phagocytes: implications for th 2 Matsumoto K, S chleimer RP, Saito H, al Induction . 29-40.

hum human eosin $1437-43$.

33 Tsuyuki S, Bertrand C, Erard F, et al. Activation of the Fas receptor on lun cosinophils leads to apoptosis and the resolution of tion of the airways. F Clin Imvest 1995:96:2924-31.

34 Druilhe A, Cai Z, Haile S, et al. Fas-mediated apoptosis in cultured human eosinophils. Blood 1996;87:2822-30.

35 Coxon A, Rieu P, Barkalow FJ, et al. A novel role for the $\beta_{2}$ integrin CD11b CD18 in neutrophil apoptosis: a homeostatic mechanism in inflammation Immumity 1996;5:653-66.

36 Watson RW, Redmond HP, Wang JH, et al. Neutrophils undergo apoptosi following ingestion of Escherichia coli. F Immenol 1996;156:3986-92.

37 Yasui K, Hu B, Nakazawa T, et al. Theophylline accelerates human granulocyte apoptosis not via phosphodiesterase inhibition. I Clin Invest 1997;100:

38 Nishioka K, Hasunuma T, Kato T, et al. Apoptosis in rheumatoid arthritis: a novel pathway in the regulation of synovial tissue. Arthritis Rherm 1998;41

9 Bell AL, Magill MK, McKane R, et al. Human blood and synovial neutrophils cultured in vitro undergo programmed cell death which is pro

10 moted by the addition of sy

Lagasse E, Weissman II. bcl-2 inhibits apoptosis of neutroph
engulfment by macrophages. 9 Exp Med 1994;179:1047-52

1 Whyte MKLB, Hardwick SJ, Meagher LC, \& al. Transient elevarions cytosolic free calcium retard subsequent apoptosis in neutrophils in vitro. Clin Invest 1993;92:446-55.

42 Cousin JM, Haslett C, Rossi AG. Regulation of granulocyte apoptosis by PKC inhibition and elevation of $[\mathrm{Ca} 2+]$. Biochem Soc Trans 1997;25:243S

43 Hart SP, Dougherty GJ, Haslett C, et al. CD44 regulates phagocytosis of apoptotic neutrophil granulocytes, but not apoptotic lymphocytes, by human macrophages. f Immunol 1997;159:919-25. 


\title{
You can lead a macrophage to an inflammatory site but can you make it eat?
}

\author{
S. P. Hart, A. G. Rossi and I. Dransfield'
}

The Rayne Laboratory, Respiratory Medicine Unit, University of Edinburgh Medical School, Teviot Place, Edinburgh EH8 9AG. U.K.

Neutrophil granulocytes and their products have been implicated in the pathogenesis of a variety of inflammatory diseases including the adult respiratory distress syndrome, idiopathic pulmonary fibrosis, ulcerative colitis, and rheumatoid arthritis [1]. Neutrophil apoptosis is associated with loss of expression of adhesion molecules [2] and greatly reduced responsiveness to external stimuli [3], so that these potentially destructive cells become functionally isolated from their environment. In contrast with necrosis, apoptosis is associated with preservation of plasma membrane integrity so that release of harmful neutrophil contents is limited, and as a consequence of poorly characterized surface changes apoptosis culminates in the recognition and phagocytosis of the dying cells by phagocytes $[4,5]$. In this way, apoptosis of neutrophils is a crucial process that promotes resolution of acute inflammation. If the rate of apoptotic cell death in the tissue is so high that phagocyte clearance capacity is exceeded, free apoptotic cells will undergo secondary necrosis, resulting in the release of harmful cellular contents and damage to the surrounding tissue. For example, treatment of mice with intraperitoneal Fas antibody triggered a massive wave of apoptosis in the liver, and the animals subsequently developed extensive hepatic necrosis and died [6]. Similarly, induction of apoptosis in the rat lung may culminate in pulmonary scarring [7]. The adverse outcomes in these experimental situations probably arose because of failure of the tissue phagocytes to clear the load of apoptotic cells with which they were confronted. In order that the load of apoptotic neutrophils at an inflammatory site is matched by appropriate clearance activity, macrophage capacity for apoptotic cell phagocytosis is likely to be closely regulated. Macrophage phagocyte capacity may be influenced by soluble mediators such as cytokines [8] and prostaglandins [9], and by adhesion interactions with the extracellular matrix components and neigh-

Abbreviations used: $\mathrm{PGE}_{2}$, prostaglandin $\mathrm{E}_{2}$; GM-CSF, granulocyte-macrophage colony-stimulating factor; IL, interleukin; TNF, tumour necrosis factor; TGF, transforming growth factor.

'To whom correspondence should be addressed. bouring cells. Following binding of a soluble mediator or matrix molecule to its surface receptor, intracellular signals are transduced which often involve activation of protein kinases and subsequent phosphorylation of intracellular proteins. These signalling events determine the capacity of the macrophage to phagocytose apoptotic cells by inducing reorganization of the cytoskeleton, a key process in cell adhesion, migration, and phagocytosis. In recent years, work by ourselves and others has begun to identify the potentially important ligand-receptor interactions and intracellular signalling pathways that are involved in the regulation of phagocytosis of apoptotic cells in mammals.

\section{Soluble mediators \\ Prostanoids}

Prostaglandins and rclated compounds play important roles in a variety of biological processes, including inflammation. We have demonstrated recently that transient elevation of cAMP within macrophages, for example following brief exposure to prostaglandin $\mathrm{E}_{2}\left(\mathrm{PGE}_{2}\right)$, resulted in reduced capacity for phagocytosis of apoptotic neutrophils [9]. This effect was specific for apoptotic cells: phagocytosis of IgG-opsonized particles was unaffected. Interestingly, inhibition of macrophage phagocytic capacity for apoptotic cells persisted for long after the intracellular cAMP concentration had declined to basal levels, implying that secondary signalling pathways had been initiated that ultimately determined the phagocytic response. There was no associated change in the level of expression of $\alpha_{v} \beta_{3}$ or the 61D3 epitope of CD14, or in the cellular distribution of $\beta_{3}$ integrins or CD36. In parallel with elevation of intracellular cAMP, we observed changes in macrophage morphology and redistribution of important cytoskeletal proteins. A variety of inflammatory mediators and pharmacological agents that stimulate adenylate cyclase and activate protein kinase $\mathrm{A}$ are likely to inhibit clearance of apoptotic neutrophils.

\section{Cytokines}

It has been shown previously that the cytokines granulocyte-macrophage colony-stimulat- 
ing factor (GM-CSF), interleukin (IL) $-1 \beta$, interferon $\gamma$, tumour necrosis factor (TNF) $\alpha$, or transforming growth factor (TGF) $\beta$ significantly augment macrophage phagocytosis of apoptotic neutrophils in vitro [10]. Although cytokineenhanced phagocytosis could be partly inhibited by antibodies against $\alpha_{v} \beta_{3}$ or CD36, cytokine exposure had no effect on macrophage surface expression of these molecules. [10]. In addition to modulating macrophage phagocytic capacity, GM-CSF plays a role in monocyte-macrophage maturation, and prolongs the lifespan of these cells by inhibiting the rate of apoptosis [11]; in turn this may regulate the number of neutrophils available for recognition by macrophages at a site of inflammation.

It is likely that a complex interplay of mediator release and autocrine/paracrine feedback regulates macrophage phagocytosis of apoptotic cells. Fadok and colleagues have demonstrated that in response to ingestion of apoptotic granulocytes, human monocyte-derived macrophages down-regulate secretion of a variety of proinflammatory cytokines including IL- $1 \beta$, IL-8, GM-CSF, and TNF- $\alpha[12]$. However, phagocytosis of apoptotic cells stimulated macrophages to release $\mathrm{PGE}_{2}, \mathrm{TGF}-\beta$, and platelet activating factor, which may have been partly responsible for the inhibition of pro-inflammatory cytokine production [12].

Furthermore, these mediators could exert feedback on local macrophages in differing ways, as the rapid down-regulation of phagocytosis of apoptotic cells by $\mathrm{PGE}_{2}$ would be opposed by the medium-term stimulatory effect of both TGF- $\beta$ and platelet activating factor.

\section{Exracellular matrix: role of phagocyte adhesion molecules}

Cell adhesion and phagocytosis are closely linked functions that are determined by organization of the cytoskeleton, and there are sevcral lines of evidence that suggest that macrophage capacity for phagocytosis of apoptotic cells may be modulated by the adhesive state of the macrophage. First, inflammatory responses to tissue injury are associated with remodelling of the extracellular matrix and sequential changes in the local concentrations of key molecules [13]. Second, macrophages adherent to plastic phagocytose apoptotic neutrophils much more readily than do those in suspension (I. Dransfield, unpublished work). Third, inhibition of recognition of apo- ptotic neutrophils is associated with changes in cell morphology and in the localization of the cytoskeletal proteins actin and talin within macrophages following elevation of intracellular cAMP [9].

\section{Integrin-mediated adhesion}

It has been recognized for some time that monocytes adherent to fibronectin are more efficient at phagocytosing Ig- and complement-opsonized particles than monocytes adherent to other substrata [14]. However, we have shown that the same is true for macrophage phagocytosis of apoptotic neutrophils [14a]. When allowed to adhere to fibronectin-coated wells for $30 \mathrm{~min}$ in vitro, the proportion of human monocyte-derived macrophages that were able to phagocytose apoptotic neutrophils was increased almost 2-fold compared with control conditions. The local concentration of fibronectin increases in the first few days following tissue injury [13], closely paralleling the influx of neutrophils from the circulation. By enhancing macrophage phagocytic capacity, adhesion of fibronectin may provide a mechanism for matching apoptotic neutrophil load to appropriate macrophage clearance capacity during the inflammatory response.

\section{CD44}

CD44 is a macrophage adhesion receptor that has been proposed to bind multiple ligands in the extracellular matrix, and has been implicated in cytoskeletal organization and intracellular signalling [15-17]. In support of a role for CD44 in the regulation of macrophage function in inflammation, we have demonstrated that phagocytosis of apoptotic neutrophils by human monocytederived macrophages was augmented following crosslinking of CD44 by bivalent antibodies in vitro [18]. This effect was rapid, occurred within minutes of adding the CD44 antibody, and was specific as there was no effect on the phagocytosis of zymosan- or immunoglobulin-opsonized erythrocytes. Interestingly, there was also no effect of CD44 antibody on phagocytosis of apoptotic lymphocytes, suggesting that cell typespecific surface changes may accompany apoptosis. These observations imply that CD44 may regulate the differential clearance of apoptotic leukocytes during evolution of inflammatory responses, representing a novel role for CD44 in inflammation and tissue repair. 


\section{Conclusion}

Macrophage phagocytosis of apoptotic cells is a vital process in a number of biological situations, but especially so in the inflammatory response where dying granulocytes have the potential to inflict serious tissue damage if ciearance is inadequate. We have described how macrophage capacity to phagocytose apoptotic cells may be regulated in vitro by a variety of soluble mediators and extracellular matrix components that bind to specific receptors on the macrophage surface. The relative importance of these different regulatory influences in vivo is not known at the present time, but exposure of phagocytes to different cytokines, prostanoids, hormones, matrix molecules, and neighbouring cells will vary over the course of the inflammatory response. It is tempting to speculate that in this way the tissue load of apoptotic cells is matched by appropriate phagocyte clearance capacity, so that release of histotoxic cell contents, especially from neutrophil granulocytes, is minimized. Furthermore, in the future it may be possible to induce neutrophil apoptosis to ameliorate neutrophil-mediated tissue damage in inflammatory diseases such as adult respiratory distress syndrome or rheumatoid arthritis. If this proves to be possible, then it will be important to augment phagocyte clearance capacity concurrently. Identification of the surface receptors and intracellular signalling pathways involved in regulation of phagocyte recognition of apoptotic cells may provide targets for therapeutic augmentation of clearance capacity in inflammatory diseases.

This work was supported by the Medical Research Council and Edinburgh University Faculty of Medicine (Fellowship to S.P.II.).

1 Weiss, S. J. (1989) N. Engl. J. Med. 320, 365-376

2 Dransfield, I., Stocks, S. C. and Haslett, C. (1995) Blood 85, 3264-3273
3 Whyte, M. K. B., Meagher, L. C., MacDermot, J. and Haslett, C. (1993) J. Immunol. 150, 5124-5134

4 Savill, J. S., Fadok, V. A., Henson, P. M. and Haslett, C. (1993) Immunol. Today 14, 131-136

5 Hart, S. P., Haslett, C. and Dransfield, I. (1996) Experientia 52, 950-956

6 Ogasawara, J., Watanabe-Fukunaga, R., Adachi, M., Matsuzawa, A., Kasugai, T., Kitamura, Y., Itoh, N., Suda, T. and Nagata, S. (1993) Nature (London) 364, 806-809

7 Hagimoto, N., Kuwano, K., Miyazaki, H., Kunitake, F., Fujuti, M., Kawasaki, M., Kaneko, Y. and Hara, N. (1997) Am. J. Respir. Cell. Mol. Biol. 17, 272-278

8 Ren, Y., Silverstein, R. L., Allen, J. and Savill, J. S. (1995) J. Exp. Med. 181, 1857-1862

9 Rossi, A. G., McCutcheon, J. C., Roy, N., Chilvers, E. R., Haslett, C. and Dransfield, I. (1998) J. Immunol. 160, 3562-3568

10 Ren, Y. and Savill, J. S. (1995) J. Immunol. 154, 2366-2374

11 Lee, A., Whyte, M. K. and Haslett, C. (1993) J. Leukoc. Biol. 54, 283-288

12 Fadok, V. A., Bratton, D. L., Konowal, A., Freed, P. W., Westcott, J. Y. and Henson, P. M. (1998) J. Clin. Invest. 101, 890-989

13 Gailit, J. and Clark, R. A. F. (1994) Curr. Opin. Cell Biol. 6, 717-725

14 Pommicr, C. G., Inada, S., Fries, L. F., Takahashi, T., Frank, M. M. and Brown, E. J. (1983) J. Exp. Med. 157, 1844-1854

14a McCutcheon, J. C., Hart, S. P., Canning, M., Ross, K., Humphries, M. J. and Dransfield, I. (1998) J. Leukoc. Biol., in the press

15 Lesley, J., Hyman, R. and Kincade, P. W. (1993) Adv. Immunol. 54, 271-335

16 Galandrini, R., Piccoli, M., Frati, L. and Santoni, A. (1996) Eur. J. Immunol. 26, 2807-2811

17 Taher, T. E. I., Smit, L., Griffioen, A. W., Schilder-Tol, E. J. M., Borst, J. and Pals, S. T. (1996) J. Biol. Chem. 271, 2863-2867

18 Hart, S. P., Dougherty, G. J., Haslett, C. and Dransfield, I. (1997) J. Immunol. 159, 919-925

Received 9 July 1998 


\title{
Role of neutrophil apoptosis in the resolution of pulmonary inflammation
}

\author{
K. Mecklenburgh*, J. Murray*, T. Brazil*, C. Ward*, \\ A.G. Rossi*, E.R. Chilvers**
}

ABSTRACT: Role of neutrophil apoptosis in the resolution of pulmonary inflammation. K. Mecklenburgh, J. Murray, $T$. Brazil, C. Ward, A.G. Rossi, E.R. Chilvers.

Neutrophil-mediated lung injury may result from one or more of the following possible causes: 1) loss of the normal mechanisms that regulate and switch off neutrophil influx, 2) inappropriate or uncontrolled neutrophil activation within the lung, 3 ) inhibition of neutrophil apoptosis, and 4) impairment or saturation of the normal macrophagedependent process for the removal of apoptotic neutrophils. Current in vitro data indicate that many factors operating at the inflamed site (e.g. cytokines, growth factors, chemotactic peptides, hypoxia, acidosis, etc.) serve a dual function in both priming and activating these cells, and delay apoptosis. The observation that the rate of eosinophil apoptosis can be accelerated by corticosteroid therapy in vivo suggests a novel mode of action for this drug and indicates that targeting this process in other granulocyte-dependent inflammatory conditions may offer a novel therapeutic approach in inflammatory lung disease.

Monaldi Arch Chest Dis 1999; 54: 4, 345-349.

Keywords: Acute respiratory distress syndrome, apoptosis, lung inflammation, neutrophils.

*Respiratory Medicine Unit, Dept of Medicine (RIE), University of Edinburgh Medical School, Edinburgh UK and **Respiratory Medicine Unit, Dept of Medicine, University of Cambridge School of Clinical Medicine, Addenbrooke's Hospital, Cambridge, UK.

Correspondence: E.R. Chilvers, Dept of Medicine, University of Cambridge School of Clinical Medicine, Box IS7, Level 5, Addenbrooke's Hospital, Hills Road, Cambridge CB2 2QQ, UK.

The authors' work is funded by the Wellcome Trust, the Medical Research Council and Papworth Hospital, National Health Service Trust.

Neutrophils are major effector cells of the immune system and play a central role in the pathogenesis of many inflammatory lung disorders. Hence, the accumulation and activation of these cells at an inflamed site, while important for host defence against invading micro-organisms, is also responsible for releasing into the lung highly toxic proteases and reactive oxygen radicals. This "unwanted" consequence of neutrophil activation has been implicated in causing the lung parenchymal abnormalities associated with acute respiratory distress syndrome (ARDS), the airway wall damage observed in acute severe asthma and the inflammatory damage seen in many chronic interstitial lung diseases [1-3]. It has long been recognized, however, that, in the setting of acute bacterial pneumonia, neutrophil influx can occur on such a scale as to completely fill and obliterate the normal alveolar airspace and yet this process can resolve rapidly with minimal or no residual tissue damage. This simple paradox implies that the presence of neutrophils per se at an inflamed site does not automatically equate with tissue injury, even within an organ as delicate as the lung, and that other factors dictate whether damage ensues or is avoided. To date, most research has focused on trying to explain such differences in terms of the duration of neutrophil influx into the lung, virulence issues relating to the infecting micro-organisms and the presence or absence of other costimulatory cells. However, the recognition that neutrophils, when aged in vitro, undergo rapid and spontaneous apoptosis or "programmed cell death" has suggested a novel and quite alternative explanation for such differences i.e. that the timely death and active removal of extravasated neutrophils may be a key issue determining whether granulocytic inflammation resolves ("beneficial inflammation") or persists ("detrimental inflammation").

\section{Neutrophil apoptosis and the resolution of inflammation}

The process of apoptosis (fig. 1) is important for two main reasons: first, it switches off the secretory capacity of the neutrophil [4] and, secondly, it results in the recognition and prompt removal of these cells by inflammatory macrophages [5]. Since the plasma membrane of the neutrophil remains intact following apoptosis and the phagocytic engulfment of such cells fails to incite macrophage activation, this event appears to represent an "inbuilt", efficient and safe mechanism for the removal of potentially hazardous cells and may explain how it is that neutrophil inflammation can so frequently resolve without causing tissue damage. Likewise, if the process of apoptosis is delayed or perverted, the potential for these cells to degranulate and/or release other more damaging histotoxic agents increases dramatically. The whole concept that cell death may be beneficial has revolutionized our understanding of the events that control 


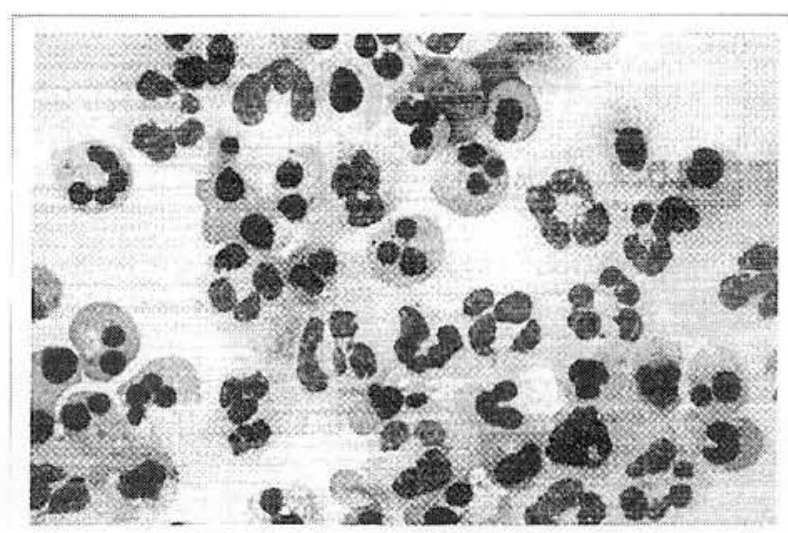

Fig. 1. - Morphological change associated with neutrophil apoptosis. Human neutrophils were purified from nuclear venous blood of healthy donors and cultured for $20 \mathrm{~h}$ in the presence of $10 \%$ autologous serum. Cytocentrifuge preparations were then performed revealing the clear morphological features of the apoptotic (denser nuclear staining) and non-apoptotic (normal) neutrophils. Identical preparations using freshly isolated neutrophils demonstrate no evidence of apoptosis.

neutrophil behaviour at the inflamed site and offers many exciting and novel opportunities for developing anti-inflammatory drugs which may target this process. Although there are a number of recent reviews detailing the biology of neutrophil apoptosis [6], the principal aim of this article is to address how this event relates to inflammation in the lung.

\section{Detection of neutrophil apoptosis in inflammatory lung disease}

Recent data suggest that bacterial infection in the lung triggers a rapid but surprisingly transient efflux of neutrophils from the blood into the alveolar wall and airspace $[2,7]$ and that, unlike in the systemic circulation, these cells exit at the the pulmonary capillary level rather than from postcapillary venules [8]. For example, in experimental pneumonia caused by Streptococcus pneumoniae, neutrophil efflux only occurs during the first $6 \mathrm{~h}$ following instillation of the organism, and "I'indium-labelled autologous neutrophils fail to accumulate in the lung in patients with bacterial pneumonia, even when studied within hours of presentation (see [2]). Although granulocyte influx undoubtedly occurs on a more sustained basis in conditions such as chronic bronchial sepsis [7], the above data predict that in the setting of acute inflammation, neutrophil influx is switched off early and that events influencing neutrophil survival within tissues are likely to dominate the subsequent clinical course.

Neutrophil apoptosis has now been observed in a number of human diseases and experimental models including neonatal lung injury [9], ARDS [10], and experimental pneumonia and lung injury in animals [11], and supports previous predictions made on the basis of in vitro work [2]. The animal studies have also demonstrated a close correlation between the onset of neutrophil apoptosis in the lung, the subsequent ingestion of apoptotic cells by alveolar and inflammatory macrophages, and the resolution of the alveolitis [11]. A similar correlation has been made for the eosinophil; apoptosis and the subsequent clearance of these cells from the airways has been demon- strated in patients with acute severe asthma treated with systemic corticosteroids [12]. In this latter study, clinical improvement was mirrored by the appearance of apoptotic eosinophils in the sputum. Hence, there is little doubt that granulocyte apoptosis occurs in vivo and is particularly prominent in situations in which spontaneous or drug-induced resolution of inflammation occurs. Likewise, mice that lack the major $\beta_{2}$ integrin cell surface adhesion molecule CDIIb/ CD18 (which is required for phagocytosis-induced apoptosis) are unable to effectively clear neutrophils from the inflamed peritoneal cavity [13].

\section{Functional consequences of neutrophil apoptosis}

Time-lapse video microscopy studies indicate that neutrophil apoptosis is an energetic and rapidly executed event. The major morphological changes that occur include cell shrinkage, nuclear condensation and cytoplasmic vacuolation. These events are associated with the shedding of a number of cell surface receptors, including CD 16 (Fc/RIII) and the tumour necrosis factor receptor (TNFR)-1, and the externalization of phosphatidylserine, which is normally located only on the inner leaflet of the plasma membrane. This dramatic change in the morphology of the neutrophil is associated with a "functional isolation" of the cell, and an abrupt reduction in the cell's capacity to respond to stimuli that normally induce chemotaxis, degranulation or superoxide anion generation [4].

The second major consequence of neutrophil apoptosis is the acquisition of a phenotype that allows these cells to be recognized and engulfed by macrophages [5]. Although alveolar macrophages and fibroblasts all display some ability to phagocytose apoptotic granulocytes [14], this capacity appears dwarfed by the ability of "professional" inflammatory macrophages to engulf and degrade these cells, and this "eating" capacity can be further enhanced by a range of inflammatory mediators including granulocyte-macrophage colony-stimulating factor (GM-CSF), interferon- $\gamma$, interleukin-(IL)- $1 \beta$ and transforming growth factor- $\beta 1$ [15]. Although the "ligand" on the apoptotic neutrophil cell surface that allows the senescent neutrophil to be recognized by macrophages is not known, the macrophage side of this interaction involves both the $\alpha_{1} \beta_{3}$ vitronectin receptor integrin and CD36, which bind thrombospondin, which engages the apoptotic neutrophil cell surface [5].

Macrophage uptake of apoptotic neutrophils has been observed at a number of inflammatory sites, including nonsuppurative arthritis, acute lung injury in neonates, ARDS, streptococcal lobar pneumonia, experimental peritonitis and resolving glomerulonephritis, and is thought to be the major disposal route of these cells in vivo. The crucial benefit of such a mechanism is that the phagocytic process removes cells that are potentially highly histotoxic in a "silent" and nonphlogistic manner [16]. Under in vitro conditions, blocking macrophage uptake using agents such as colchicine allows apoptotic cells to undergo secondary necrosis, causing the release of their granule contents, again underlining the need for neutrophil apoptosis to be co-ordinately linked to macrophage uptake [5]. 


\section{Regulation of neutrophil apoptosis}

The speed at which neutrophils undergo apoptosis when aged in vitro suggests the existence of a very strong and inbuilt apoptotic programme in these cells, which if triggered in vivo, could (and in streptococcal lobar pneumonia probably does) result in a very rapid and effective process for switching off and removing these cells from an inflamed site. Rather surprisingly therefore, it was revealed that the vast majority of input signals to the neutrophil (e.g. GM-CSF, lipopolysaccharide, complement factor 5 a and (C5a), IL-6 and IL-8; fig. 2) cause a profound inhibition of apoptosis [17-19] and, moreover, that many of the pharmacological manipulations shown to induce apoptosis in other cell types (including the thymocyte and the lymphocyte) have the opposite effect on the apoptotic programme in the neutrophil. The most striking example of this difference is the effect of raising intracellular calcium or cyclic adenasine monophosphate, since both stimulate thymocyte apoptosis but delay this process in neutrophils $[20,21]$. This early indication that neutrophils differ from most other inflammatory cells in their apoptotic programming was further supported by the observation that corticosteroids markedly delay apoptosis in the neutrophil yet clearly stimulate this process in the eosinophil [22]. This latter observation may explain, at least in part, the relative inability of corticosteroids to influence the clinical outcome of many neutrophil-dominated inflammatory diseases such as ARDS.

It has also been demonstrated that the rate of neutrophil apoptosis can be severely inhibited by hypoxia [23]. This influence is again contrary to the effect of hypoxia on apoptosis in other cells types, e.g. neurons and gut-derived epithelial tumour cells, and suggests that hypoxia may be a further factor operating to delay the resolution of neutrophil-mediated tissue inflammation.

In view of these data, recent studies have focused on how neutrophil apoptosis may be stimulated or, at the very least, how the above largely cytokinemediated survival effects may be blocked. This has proved a somewhat frustrating challenge since although agents such as TNF- $\alpha$ and Fas ligand (Fas-L) are both capable of inducing neutrophil apoptosis through binding to the cell membrane p55 and p75 TNF- $\alpha$ receptors and Fas respectively [24-27], the magnitude of the killing effect, at least in vitro, is small. Furthermore, neutrophils previously primed by agents such as platelet-activating factor, IL-8 or lipopolysaccharide, or cells isolated from an inflammatory focus, appear unresponsive to the same signals [26, 28, 29]. This implies that the early and often intense increase in TNF- $\alpha$ levels observed in many acute inflammatory lung states may be unable to promote effective neutrophil removal if the cells within the lung have been costimulated by other priming or activating agents. Early reports suggested that neutrophils actively produce and release Fas-L and thereby regulate their own survival via autocrine or paracrine Fas-L Fas interactions $[27,30]$. However, Fas-L/Fas-mediated apoptosis may not be as important in regulating neutrophil survival in viro since inflammatory neutrophils harvested from Fas-L $(g l d)$ and Fas (lpr)-deficient mice undergo apoptosis at an identical rate to cells from normal mice [31].

Other physiological stimuli known to enhance the rate of neutrophil apoptosis include human immunodeficiency virus infection [32], ingestion of Escherichia coli [33] and other particulate material [13], and, interestingly, migration of cells through an endothelial monolayer [34]. This latter observation may explain why apoptotic neutrophils are rarely if ever observed in circulating blood, and may relate to the previously noted ability of $\beta_{2}$ integrins to facilitate constitutive and TNF- $\alpha$-stimulated neutrophil apoptosis [13, 34]. There is also an early and fascinating suggestion that bronchoalveolar lavage fluid recovered from animals with experimental streptococcal (resolving) pneumonia contains a factor that drives neutrophil apoptosis in vitro and that this factor(s) is absent in E. coli (non-resolving) pneumonia [35].

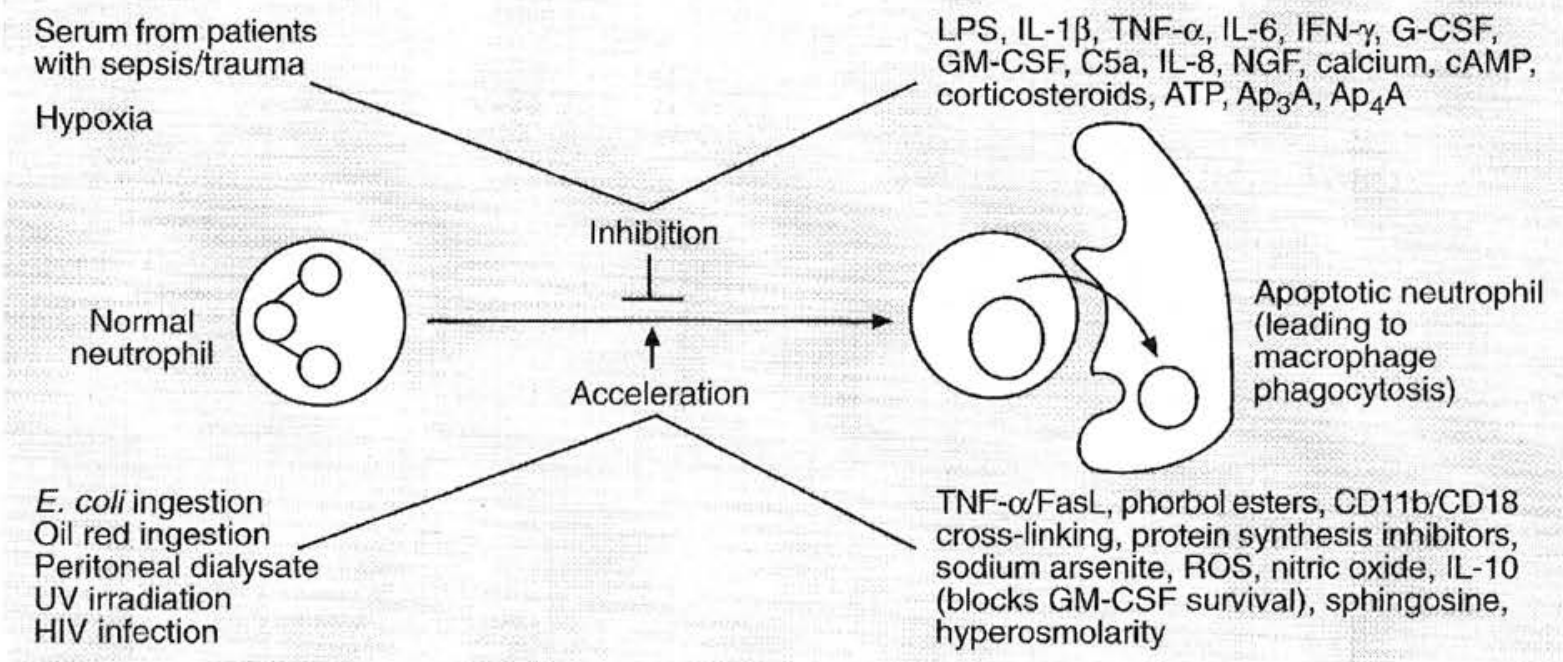

Fig. 2. - Regulation of neutrophil apoptosis. Summary of the physiological (left) and phatmacological (right) manipulations demonstrated to either inhibit or accelerate the rate of neutrophil apoptosis in vitro. E. coli: Escherichia coli; UV: ultraviolet; HIV: human immunodeficiency virus; LPS lipopolysaccharide; IL: interleukin; TNF- $\alpha$ : tumour necrosis factor- $\alpha$; IFN- $\gamma$, interferon- $\gamma$, G-CSF: granulocyte colony-stimulating factor; GM-CSF: granulocyte-macrophage colony-stimulating factor; C5a: complement factor 5a; NGF: nerve growth factor; cAMP: cyclic adenosine monophos phate; ATP: adenosine triphosphate; $A p_{3} A, A p_{A} A$ : diadenosine polyphosphates; FasL: Fas ligand; ROS: reactive oxygen species. 
Although the intracellular pathways mediating the above effects are uncertain, the proapoptotic effect of TNF- $\alpha$, unlike its priming/activation functions, are uniquely dependent on ligation of TNFR75 in addition to TNFR55, and both TNF- $\alpha$ and Fas-Linduced killing are blocked by compounds that inhibit the caspase family of cysteinyl proteases, which orchestrate cell death in other mammalian cells. The major nuclear and cytosolic targets of caspases in the neutrophil are, again, uncertain but appear to include gelsolin, which acts to sever actin filaments and induce cells to round up, detach and undergo nuclear fragmentation [36], and a $\mathrm{Ca}^{2+} / \mathrm{Mg}^{2+}$-independent acid endonuclease [37]. The ability of protein synthesis inhibitors to induce neutrophil apoptosis also implies that these normally short-lived cells are "preprogrammed" to die and that an actively produced antiapoptotic protein factor or factors exist to prevent premature cell death. Intriguingly, mature neutrophils do not possess $\mathrm{Bcl}_{2}$, a major antiapoptotic factor present in most other cells [29,38], and it has been proposed that the loss of this protein, which occurs during the final stages of granulocyte maturation, removes an important brake on the apoptotic capacity of the neutrophil prior to entry into the circulation. Current contenders for such survival proteins include other members of the $\mathrm{Bcl}_{2}$ family, namely Mcl-1 and Bax [39].

\section{Potential for therapeutic manipulation}

From the above line of argument, it follows that devising a therapeutic strategy based on driving programmed cell death or blocking the antiapoptotic effects of certain agents should, if executed at the appropriate stage of the inflammatory response, offer a novel and effective form of anti-inflammatory therapy. "Proof principle" for this is the well-recognized and major proapoptotic effect of glucocorticosteroids in eosinophils and the ability of Fas-L administration to induce resolution of eosinophilic inflammation in the airways [40]. Such selective targeting is not yet available for the neutrophil, but there are experimental data to support both of the above approaches. First, although the "solo" killing effect of TNF- $\alpha$ in neutrophils appear to be modest and are easily overcome by other costimuli, it is now possible to increase dramatically the efficacy of this response by coincubating neutrophils with the nuclear factor- $\mathrm{KB}$ inhibitor gliotoxin [41], which also unmasks a capacity for TNF- $\alpha$ to drive eosinophil apoptosis. Likewise, the protein synthesis inhibitor cycloheximide, used at a concentration that alone does not influence the rate of constitutive apoptosis, can also enhance TNF- $\alpha$ mediated neutrophil apoptosis [41]. Secondly, the antiinflammatory cytokine IL-10 has been shown to be able to block the survival (anti-apoptotic) effect of lipopolysaccharide in vitro [42], and a similar effect has been demonstrated with certain synthetic cellpermeable inhibitors that block certain intracellular second messenger phosphorylation pathways [43]. The very unusual and atypical apoptotic programme that exists in the neutrophil, and the ability to deliver drugs in a selective manner to the lungs, fuels current optimism that such an approach may be feasible.

\section{References}

1. Malech HL, Gallin JI. - Neutrophils in human diseases. N Engl J Med 1987; 317: 687-695.

2. Haslett C. - Granulocyte apoptosis and inflammatory disease. Br Med Bull 1997; 53: 669-683.

3. Sur S, Crotty TB, Kephart GM, et al. - Sudden-onset fatal asthma, a distinct entity with few eosinophils and relatively more neutrophils in the airway submucosa? Am Rev Respir Dis 1993; 148: 713-719.

4. Whyte MKB, Meagher LC, MacDermot J, Haslett C. Impairment of function in aging neutrophils is associated with apoptosis. I Immunol 1993; 150: 5124-5134.

5. Savill J. - Macrophage recognition of senescent neutrophils. Clin Sci 1992; 83: 649-655.

6. Homburg CHE, Roos D. - Apoptosis of neutrophils. Curr Opin Haematol 1996; 3: 94-99.

7. Jones HA, Sriskandan AM, Peters AM, et al. - Dissociation of neutrophil emigration and metabolic activity in lobar pneumonia and bronchiectasis. Eur Respir $J$ 1997; 10 : 795-803.

8. Downey GP, Worthen GS, Henson PM, Hyde DM. Neutrophil sequestration and migration in localized pulmonary inflammation. Capillary localization and migration across the interalveolar septum. Am Rev Respir Dis 1993; 147: 168-176.

9. Grigg JM, Savill JS, Sarraf C, Haslett C, Silverman M. - Neutrophil apoptosis and clearance from neonatal lungs. Lancet 1991; 338: 720-722.

10. Matute-Bello G, Liles WC, Radella F, et al. - Neutrophil apoptosis in the acute respiratory distress syndrome. Am J Respir Crit Care Med 1997; 156: 1969-1977.

11. Cox G, Crossley J, Xing Z. - Macrophage engulfment of apoptotic neutrophils contributes to the resolution of acute pulmonary inflammation in vivo. Am J Respir Cell Mol Biol 1995; 12: 232-237.

12. Woolley KL, Gipson PG, Carty K. - Eosinophil apoptosis and the resolution of airway inflammation in asthma. Am J Respir Crit Care Med 1996; 154: 237-243.

13. Coxon A, Rieu P, Barkalow FJ, et al. - A novel role for the $\beta_{2}$ integrin CD11b/CD18 in neutrophil apoptosis: a homeostatic mechanism in inflammation. Immunity 1996; 5: 653-666.

14. Hall SE, Savill JS, Henson P, Haslett C. - Apoptotic neutrophils are phagocytosed by fibroblasts with participation of the fibroblast vitronection receptor and involvement of a mannose/fucose-specific lectin. $J$ Immunol 1994; 153: 3218-3227.

15. Ren Y, Savill JS. - Proinflammatory cytokines potentiate thrombospondin-mediated phagocytosis of neutrophils undergoing apoptosis. J Immunol 1995; 154: 2366-2374

16. Meagher L, Savill JS, Baker A, Fuller RW, Haslett C. - Phagocytosis of apoptotic neutrophils does not induce macrophage release of thromboxane $\beta_{2}$.J Leukoc Biol 1992; 52: 269-273.

17. Colotta F, Polentarutti N, Sozzani S, Mantovani A. Modulation of granulocyte survival and programmed cell death by cytokines and bacterial products. Blood 1992; 80: 2012-2020.

18. Lee A, Whyte MKB, Haslett C. - Inhibition of apoptosis and prolongation of neutrophil functional longevity by inflammatory mediators. I Leukoc Biol 1993; 54: 283-288.

19. Biffl WL, Moore EE, Moore FA, Barnett CC. - Interleukin6 supression of neutrophil apopotis is neutrophil concentration dependent. J Leukoc Biol 1995; 58: 582-584.

20. Whyte MKB, Hardwick SJ, Meagher LC, Savill JS Haslett C. - Transient elevations of cytosolic free calcium retard subsequent apoptosis in neutrophils in vitro. $J$ Clin Invest 1993; 92: 446-455.

21. Rossi AG, Cousin JM, Dransfield I, Lawson MF, Chilvers ER, Haslett C. - Agents that elevate cAMP inhibit 
neutrophil apoptosis. Biochem Biophys Res Commun 1996; 217: 892-899.

22. Meagher LC, Cousin JM, Seckl JR, Haslett C. - Opposing effects of glucocorticosteroids on the rate of apoptosis in neutrophilic and eosinophilic granulocytes. J Immunol 1996; 156: 4422-4428.

23. Hannah S, Mecklenburgh K, Rahman I, et al. - Hypoxia prolongs neutrophil survival in vitro. FEBS Lett 1995; 372: 233-237.

24. Takeda Y, Watanabe H, Yonehara S, Yamashita T, Saito S, Sendo F. - Rapid acceleration of neutrophil apoptosis by tumor necrosis factor- $\alpha$. Int Immunol 1993; 5 : 691-694.

25. Gon S, Gatanaga T, Sendo F. - Involvement of two types of TNF receptor in TNF $\alpha$-induced neutrophil apoptosis. Microbiol Immunol 1996; 40: 463-465.

26. Murray J, Barbara J, Dunkley SA, et al. - Regulation of neutrophil apoptosis by tumour necrosis factor- $\alpha$ : requirement for CD120a (TNFR-55) and CD120b (TNFR75) for induction of apoptosis in vitro. Blood 1997; 90: 2772-2783.

27. Leuenroth S, Lee C, Grutkoski P, Keeping H, Simms HH. - Interleukin 8-induced suppression of polymophonuclear leukocyte apoptosis is mediated by suppressing CD95 (Fas/Apo-1) Fas-L interactions. Surgery 1998; 124: $409-417$.

28. Hachiya O, Takeda $\mathrm{Y}$, Miyata $\mathrm{H}$, Watanabe $\mathrm{H}$, Yamashita $\mathrm{T}$, Sendo F. - Inhibition by bacterial lipopolysaccharide of spontaneous and TNF- $\alpha$ induced human neutrophil apoptosis in vitro. Microbiol Immunol 1995; 39: 715-723.

29. Kettritz R, Gaido ML, Haller H, Luft FC, Jennette CJ, Falk RJ. - Interleukin- 8 delays spontaneous and tumor necrosis factor- $\alpha$-mediated apoptosis of human neucrophils. Kidney Int 1998; 53: 84-91.

30. Liles WC, Kiener PA, Ledbetter JA, Aruffo A, Klebanoff SL. - Differential expression of Fas (CD95) and Fasligand on normal human phagocytes: implications for the regulation of apoptosis in neutrophils. J Exp Med 1996; 184: 429-436.

31. Fecho K, Cohen PL. - Fas ligand ( $g l d)$ - and Fas (lpr)deficient mice do not show alterations in the extravasation or apoptosis of inflammatory neutrophils. $J$ Leukoc Biol 1998; 64: 373-383.
32. Pitrak DL, Tsai HC, Mullane KM, Sutton SH, Stevens P. - Accelerated neutrophil apoptosis in the acquired immunodeficiency syndrome. J Clin Invest 1996; 98: 2714-2719.

33. Watson RW, Redmond JP, Wang JH. - Neutrophils undergo apoptosis following ingestion of Escherichia coli. I Immunol 1996; 156: 3986-3992.

34. Walzog B, Jeblonski F, Zakrzewicz A, Gaehtgens P. $\beta_{2}$ integrins (CDII/CD18) promote apoptosis of human neutrophils. FASEB J 1997; 11: 1177-1186.

35. Lawson RA, Caldwell H, Usher L, Whyte MKB, Haslett C. - Modulation of neutrophil apoptosis during a model of bacterial pneumonia. Thorax 1998; 53: A36.

36. Kothakota S, Azuma T, Reinhard C, et al. - Caspase-3generated fragment of gelsonin: effector of morphological change in aoptosis. Science 1997; 278: 294-298.

37. Gottlieb RA, Giesing HA, Engler RL, Babior BM. The acid deoxyribonuclease of neutrophils: a possible participation in apoptosis-associated genome destruction. Blood 1995; 86: 2414-2418.

38. Delia D, Aiello A, Soligo D, et al. - Bcl-2 proto-oncogene expression in normal and neoplastic human myeloid cells. Blood 1992; 79: 1291-1298.

39. Moulding DA, Quayle JA, Hart CA, Edwards SW. Mcl-1 expression in human neutrophils: regulation by cytokines and correlation with cell survival. Blood 1998; 92: 2495-2502.

40. Tsuyuki S, Bertrand C, Erard F. - Activation of the Fas receptor on lung eosinophils leads to apoptosis and the resolution of eosinophilic inflammation of the airways. J Clin Invest 1995; 96: 2924-2931.

41. Ward C, Chilvers ER, Lawson MF, et al. - NF- $\kappa B$ activation is a critical regulator of human granulocyte apoptosis in vitro. J Biol Chem 1999; (in press).

42. Cox G, Gauldie J, Jordana M. - Bronchial epithelial cell-derived cytokines (G-CSF and GM-CSF) promote the survival of peripheral blood neutrophils in vitro. Am $J$ Respir Cell Mol Biol 1992; 7: 507-513.

43. Frasch SC, Nick JA, Fadok VA, Bratton DL, Worthen GS, Henson PM. - p38 mitogen-activated protein-kinase dependent and -independent intracellular signal transduction pathways leading to apoptosis in human neutrophils. J Biol Chem 1998; 273: 8389-8397.

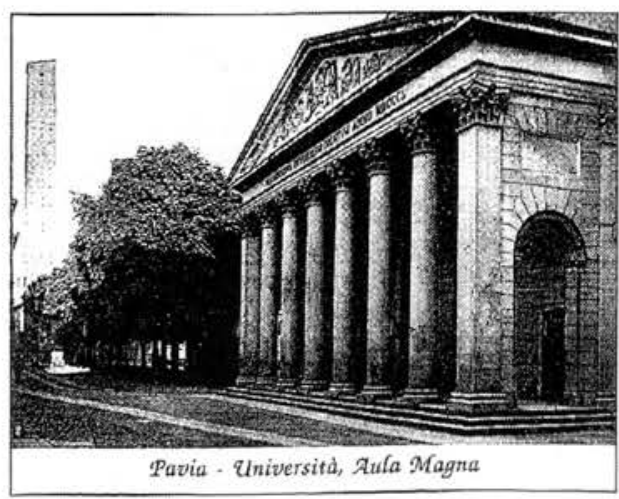




\section{Pharmacological manipulation of granulocyte apoptosis: potential therapeutic targets}

\author{
Carol Ward, Ian Dransfield, Edwin R. Chilvers, Chris \\ Haslett and Adriano G. Rossi
}

Resolution of inflammation involves the clearance of excess or effete inflammatory cells by a process of physiological programmed cell death (apoptosis) and the subsequent recognition and removal of apoptotic cells by phagocytes. The therapeutic induction of apoptosis for the resolution of chronic inflammation and the general pharmacology of apoptosis have become subjects of increasing interest. In this article, some of the unique and important differences in the control of apoptosis of various inflammatory cells (particularly neutrophil and eosinophil granulocytes) are highlighted. It is suggested that apoptosis can be specifically regulated pharmacologically and could be exploited to develop new drug therapies.

Granulocytes are recruited to inflammatory sites in response to infection or tissue injury. However, many defence mechanisms employed by these cells are potentially deleterious to host tissues; for example, excessive release of granule proteases or production of reactive oxygen species can damage tissue. Thus, it is important that granulocytes are effectively and rapidly destroyed concomitant with the removal of an inflammatory stimulus to avoid excessive tissue damage or chronic inflammation.

\section{Apoptosis and resolution of inflammation}

Necrosis of inflammatory cells involves a loss of membrane integrity, which leads to the release of potentially toxic intracellular contents, whereas apoptosis ${ }^{1-3}$ is an efficient, non-inflammatory method of removing aging leukocytes from inflammatory sites ${ }^{1}$. Apoptosis should ideally be matched by efficient recognition and removal of the apoptotic cells by phagocytes such as macrophages or, in certain situations, by cells with phagocytic potential such as fibroblasts or mesangial cells ${ }^{1}$. Apoptotic cells, if not recognized and removed, eventually undergo 'secondary necrosis' releasing damaging intracellular contents and amplifying the inflammatory response. This process might underlie the development of chronic inflammatory conditions in which apoptotic cell load and phagocytic clearance mechanisms are mismatched (Fig. 1). It is therefore prudent that interventions to increase the rate of apoptosis of inflammatory leukocytes as an anti-inflammatory strategy are developed in parallel with mechanisms that upregulate or preserve phagocytic clearance ${ }^{4-8}$. Macrophage recognition of apoptotic cells does not liberate proinflammatory agents ${ }^{6}$ but can release anti-inflammatory mediators such as interleukin 10 (IL-10) ${ }^{7}$ and transforming growth factor $\beta$ (TGF- $\beta)^{8}$, some of which might themselves affect the apoptotic programme of inflammatory cells (Figs 1,2). Thus, the removal process might also succumb to selective and specific modulation by pharmacological agents for therapeutic gain.

\section{Cell-specific signals and therapeutic strategies}

To develop useful therapeutic strategies, it is necessary to understand and exploit the underlying differences in the apoptotic control mechanisms that exist between specific cell types, as well as the common genes or proteins involved in the execution pathways. For example, although the activation of caspases is a crucial part of the effector limb of apoptosis, several common substrates for these proteases [e.g. poly (ADP-ribose) polymerase (PARP), nuclear/mitotic apparatus protein (NuMA), U1-70 kDa (small ribonucleoprotein), or the catalytic subunit of DNAdependent protein kinase (DNA-PK)], are not detected in neutrophils ${ }^{9}$. This suggests that some factors that regulate the execution phase of apoptosis are not universally applicable to all cells, and that different death factors and caspase subsets could be manipulated to interfere selectively with the apoptotic programme.

Selective pharmacological intervention to drive granulocyte apoptosis might be a realistic goal because manipulation of many of the physiological signalling pathways induces cell death in other cell types but fails to induce apoptosis in granulocytes. Granulocytes also show differential responses to apoptotic stimuli compared with other leukocytes. Importantly, there are also differences in the control of apoptosis between neutrophils and eosinophils ${ }^{10}$ (Table 1). During culture, $50-70 \%$ of neutrophils undergo constitutive apoptosis by $20 \mathrm{~h}$ (Refs 10,11); in eosinophils it can take up to two days to achieve equivalent rates of apoptosis ${ }^{10,11}$. This indicates that distinct regulatory mechanisms control apoptosis in these cells.

\section{Inhibition of survival pathways}

Many stimuli that prime or activate granulocytes and are present in abundance at inflammatory sites [e.g. granulocyte/macrophage colony-stimulating factor (GM-CSF), lipopolysaccharide (LPS) and complement fragment C5a] delay granulocyte apoptosis. Altered functional longevity influences the ability of granulocytes to migrate, degranulate or generate superoxide $\left(\mathrm{O}_{2}^{-}\right)$anions ${ }^{1}$. Specific inhibition is afforded by the presence of distinct receptors on the cell surface of granulocytes (e.g. binding of IL-5 to specific IL-5 receptors suppresses eosinophil apoptosis but does not affect neutrophil apoptosis $\left.{ }^{1}\right)$. Interestingly, the anti-inflammatory cytokine, IL-10 (Ref. 12) and the naturally occurring IL-1 receptor antagonist ${ }^{13}$ can suppress
C. Ward,

MRC Research Fellow, Email: c.ward(8) ed.ac.uk

I. Dransfield, Senior Lecturer, Email: i.dransfield@ ed.ac.uk

C. Haslett,

Professor and Head of Department of Respiratory Medicine, Email: chaslett@ ed.ac.uk

A. G. Rossi, Senior MRC Research Fellow, The Rayne Laboratory. Respiratory Medicine Unit, University of Edinburgh Medical School, Teviot Place, Edinburgh, UK EH89AG. Email: arossi@ ed.ac.uk and E. R. Chilvers, Professor, Respiratory Medicine Division, Department of Medicine, University of Cambridge School of Medicine, Box 157 Level 5, Addenbrooke's Hospital, Hills Road, Cambridge, UK CB2 200 Email: erc24@hermes. cam.ac.uk 


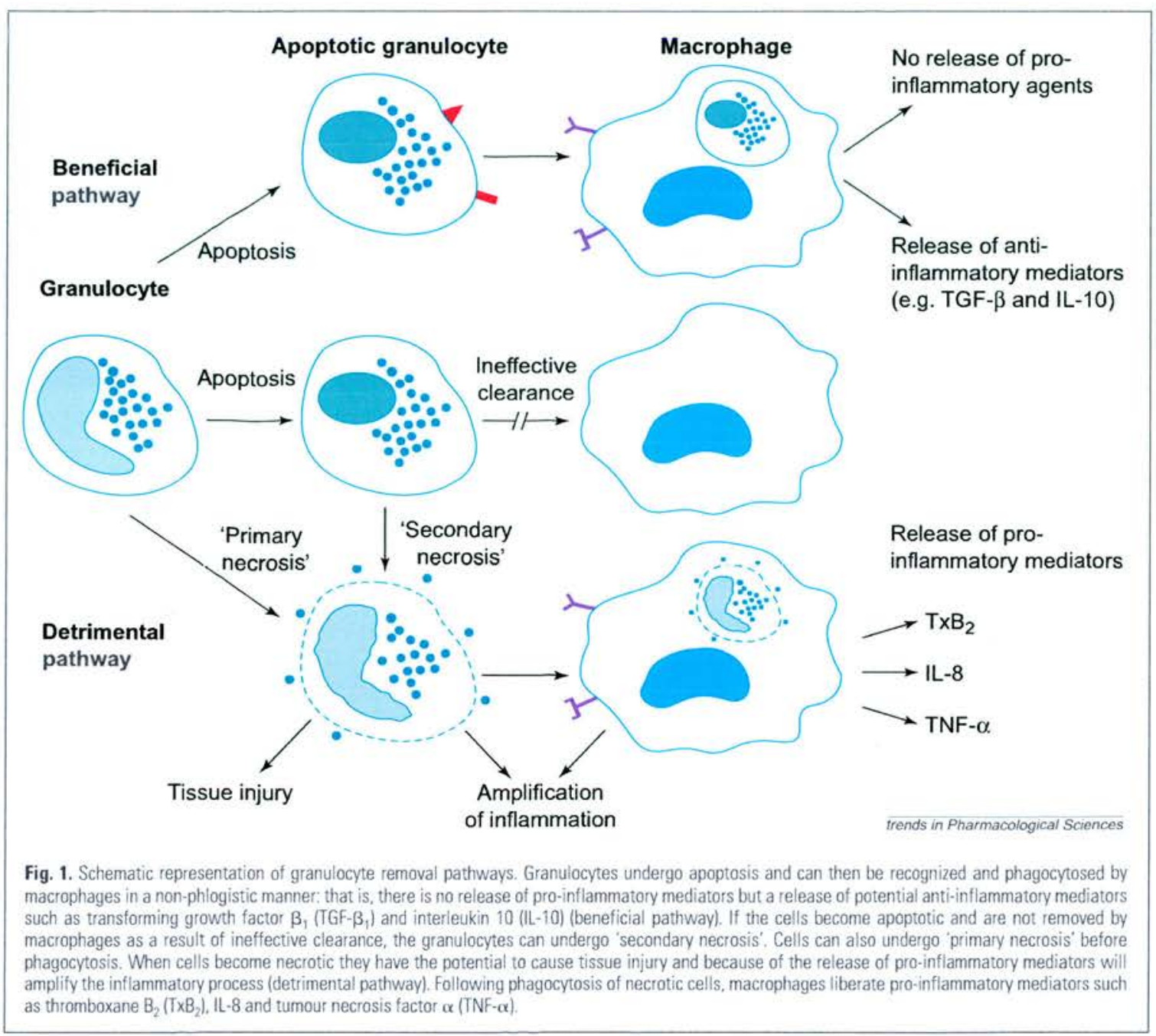

the anti-apoptotic effect of LPS and IL-1, respectively. Therefore, by using specific receptor antagonists or neutralizing antibodies, it should be possible to accelerate apoptosis. Alternatively, targeting receptor-mediated pathways that induce apoptosis in such cells might represent a more selective way of removing unwanted granulocytes at sites of inflammation (Fig. 2).

\section{Differential effects of glucocorticoids on granulocyte apoptosis}

Glucocorticoids are extensively used in treating patients with asthma ${ }^{14}$; they inhibit inflammatory cell recruitment, dampen cellular responsiveness and have diverse effects on apoptosis of inflammatory cells (e.g. glucocorticoids promote thymocyte and eosinophil apoptosis but inhibit apoptosis in neutrophils 1,10 ). Thus, glucocorticoids might exert part of their anti-inflammatory effect by affecting apoptosis. For example, patients with acute severe asthma who benefit from steroid therapy have increased numbers of apoptotic eosinophils in their sputum ${ }^{15}$. Although the glucocorticoid effect on apoptosis is steroid receptor ${ }^{14}$ and protein synthesis dependent ${ }^{16}$, and might involve certain transcription factors [e.g. activator protein-1 (AP-1) and nuclear factor $\kappa \mathrm{B}(\mathrm{NF}-\mathrm{\kappa B})]^{14}$, a clear picture of the underlying mechanisms has not yet emerged. Elevation of cytosolic free $\mathrm{Ca}^{2+}$ concentration $\left\{\left[\mathrm{Ca}^{2+}\right]_{i}\right\}$ in inflammatory cells mimics the apoptotic effects of glucocorticoids; that is, elevation of $\left[\mathrm{Ca}^{2+}\right]_{i}$ in thymocytes ${ }^{17}$ and eosinophils ${ }^{18}$ increases apoptosis whereas rises in $\left[\mathrm{Ca}^{2+}\right]_{\mathrm{i}}$ in the neutrophil delays apoptosis ${ }^{19}$, which suggests that glucocorticoids could regulate apoptosis by interfering with $\mathrm{Ca}^{2+}$ homeostasis ${ }^{17}$. Glucocorticoids also increase macrophage recognition of apoptotic leukocytes ${ }^{20}$. Together, these data provide compelling evidence that glucocorticoids not only affect the induction and propagation of inflammation but also influence resolution.

\section{Nitric oxide and oxidative changes}

Because nitric oxide (NO) has powerful effects on many cells, including smooth muscle and inflammatory cells, significant effort has focused on the role of NO in inflammation. Apoptosis can be modulated by NO in a concentration-dependent manner, with increased levels of NO having pro-apoptotic effects on neutrophils ${ }^{21-24}$, 


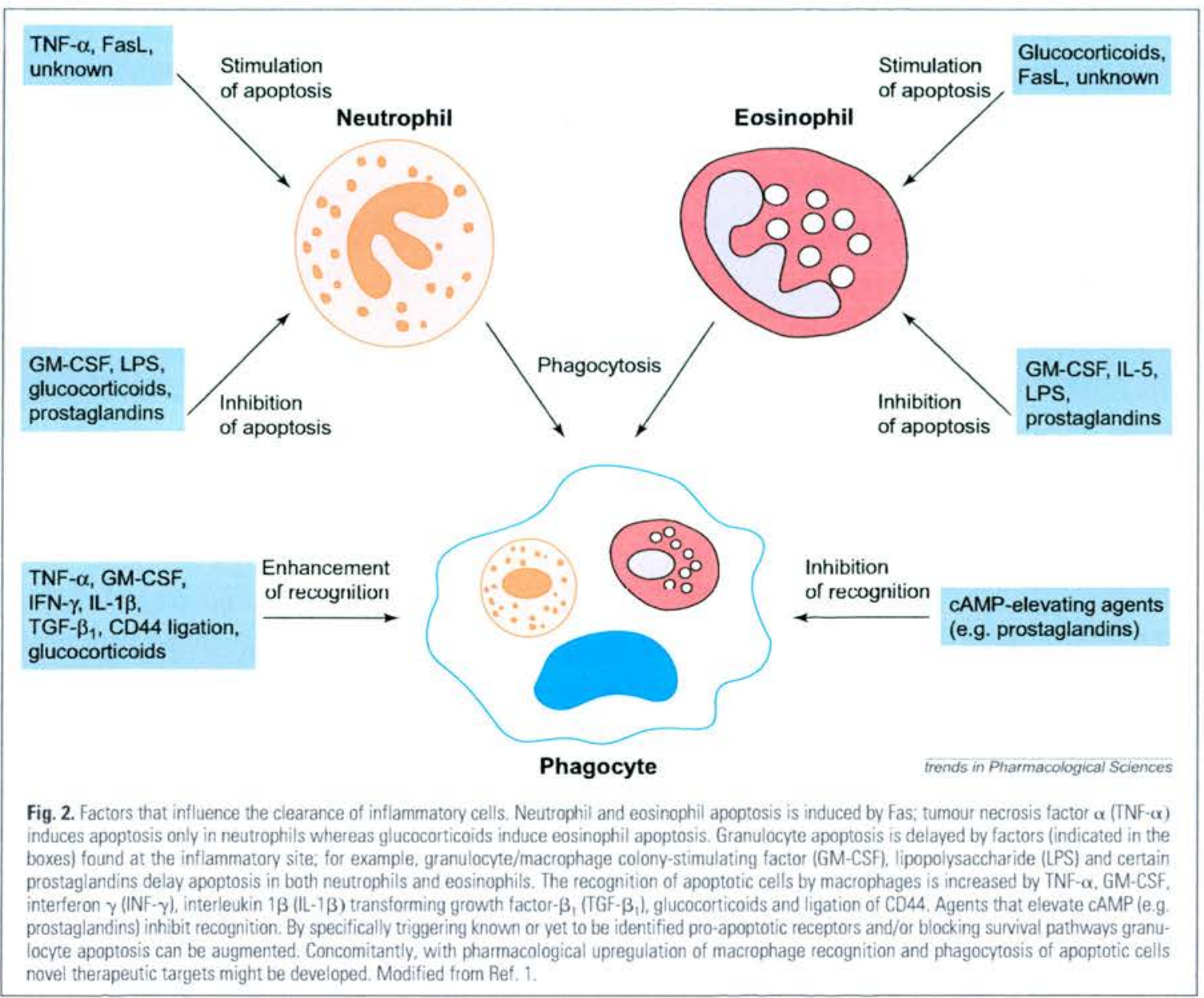

Table 1. Delay or acceleration of constitutive apoptosis in granulocytes by specific agents or treatments

\begin{tabular}{|c|c|c|}
\hline Granulocyte & Apoptosis delaying agents & Refs \\
\hline \multirow[t]{5}{*}{ Neutrophil } & Agents that increase cytosolic cAMP (e.g. db-cAMP, PGs) & $37-39,41$ \\
\hline & Agents that increase $\left[\mathrm{Ca}^{2+}\right]_{1}$ (e.g. A23187) & 18,19 \\
\hline & Glucocorticoids (e.g. dexamethasone) & 10,16 \\
\hline & Neutrophil agonists (e.g. LPS, FMLP, PAF, LTB, C5a, GM-CSF, IL-6, IL-8) & $1,11,28,58$ \\
\hline & Hypoxia & \\
\hline \multirow[t]{3}{*}{ Eosinophil } & Agents that increase cytosolic cAMP (e.g. db-cAMP, PGs) & 1,10 \\
\hline & Eosinophil agonists (e.g. IL-5, GM-CSF) & 40,41 \\
\hline & \multicolumn{2}{|l|}{ Apoptosis accelerating agents } \\
\hline \multirow[t]{5}{*}{ Neutrophil } & NO donors & $21-24$ \\
\hline & Activating Fas antibodies, FasL & $46-54$ \\
\hline & TNF- $\alpha$ & 11,58 \\
\hline & Protein synthesis inhibitors (e.g. actinomycin D and cycloheximide) & 10,67 \\
\hline & NF- $k B$ inhibitors (e.g. gliotoxin) & \\
\hline \multirow[t]{4}{*}{ Eosinophil } & Activating Fas antibodies, FasL & $41,47-50$ \\
\hline & Agents that increase $\left[\mathrm{Ca}^{2+}\right]$, (e.g. A23187) & 43 \\
\hline & Glucocorticoids (e.g. dexamethasone) & 10 \\
\hline & $N F-\kappa B$ inhibitors (e.g. gliotoxin) & 11 \\
\hline
\end{tabular}


whereas in eosinophils pharmacological augmentation of NO levels inhibits constitutive ${ }^{25}$ and Fas-induced apoptosis ${ }^{26}$. The mechanisms underlying these divergent effects are unknown but might involve the guanylate cyclase pathway, transcriptional regulation, direct DNA damage or oxidative pathways ${ }^{27}$. Although changes in oxidative metabolism can affect the rate of apoptosis in inflammatory cells, hypoxia, which induces cell death in many systems, inhibits neutrophil apoptosis ${ }^{28}$. Intriguingly, certain antioxidants such as pyrrolidine dithiocarbamate increase neutrophil apoptosis - an observation that might be related to other properties of these compounds ${ }^{11}$. Coxon et al. ${ }^{29}$ showed that phagocytosis of opsonized particles by human neutrophils induced apoptosis - a process that is dependent on the adhesion molecule Mac-1 (also known as CD11b/CD18) and can be blocked by anti-oxidants. A role for this adhesion molecule in the control of granulocyte apoptosis has been proposed $^{30,31}$, although specific cell culture conditions might influence these effects ${ }^{32}$.

\section{Manipulation of signalling pathways}

The adenylate cyclase-cAMP pathway can exert both pro-inflammatory and anti-inflammatory effects depending on concentration, site of action and route of administration of cAMP-elevating agents such as prostaglandins. This pathway is a target for the treatment of chronic inflammatory diseases [e.g. potential specific phosphodiesterase (PDE) inhibitors in asthma therapy $]^{33}$. Elevation of cAMP levels in inflammatory cells attenuates the cell's response to secretory agonists and has a cell-dependent effect on the rate of apoptosis, with apoptosis in thymocytes and leukaemic cell lines increasing in response to augmented intracellular concentrations of cAMP (Refs 34-36). By contrast, cAMP elevation delays apoptosis in granulocytes ${ }^{37-40}$. The cAMP-mediated inhibition of neutrophil apoptosis appears to be protein kinase A (PKA) dependent ${ }^{37-39}$ whereas it is PKA independent in eosinophils ${ }^{40}$. However, GM-CSF-mediated survival in eosinophils is paradoxically inhibited by cAMP via a mechanism involving PKA, which indicates that PKA is involved in regulating eosinophil apoptosis ${ }^{40}$. Recently, it has been reported that PDE4 inhibitors suppress eosinophil survival by elevating cAMP whereas theophylline, a relatively nonspecific PDE inhibitor, augments eosinophil apoptosis by a mechanism that is independent of its effects on the cAMP pathway ${ }^{41}$. This suggests that modulation of the adenylate cyclase-cAMP pathway might control the rate of apoptosis in specific inflammatory cells - an effect that must be considered when developing novel agents that interfere with cAMP signalling pathways.

A role for protein kinase $\mathrm{C}$ ( $\mathrm{PKC}$ ) in the regulation of granulocyte apoptosis is not fully established despite evidence that this enzyme is important in mediating granulocyte responsiveness ${ }^{42}$. The nonspecific PKC inhibitor staurosporine and the specific, but non-isoenzyme selective, inhibitor Ro318220 induce granulocyte apoptosis ${ }^{43}$ and recently, the PKC isoform PKC $\delta$ has been associated with neutrophil apoptosis ${ }^{44}$. However, in other inflammatory cells such as U937 myelomonocytic cells an increase in PKC $\beta$ and a decrease in PKC $\zeta$ occur during apoptosis ${ }^{45}$. Therefore, this area has potential for intervention strategies if different profiles of isoenzymes are involved in apoptosis or survival in specific cell types.

\section{Death-receptor-activated apoptosis}

Fas is a widely expressed membrane receptor, which, via interaction with its ligand FasL, is responsible for controlling immune responses. Engagement of the FasL-Fas pathway can initiate apoptosis both in vitro and in vivo but its role in granulocyte apoptosis remains controversial. Despite reports that Fas-mediated signals induce both neutrophil and eosinophil apoptosis ${ }^{46-49}$, the demonstration that production and release of FasL by granulocytes can effect constitutive apoptosis has not been convincing ${ }^{5051}$. Although Fas activating antibodies induce neutrophil cell death by apparent caspase- and mitochondrial-dependent mechanisms $s^{52}$, soluble FasL did not induce apoptosis but acted as a chemotaxin for neutrophils both in vitro ${ }^{53}$ and in vivo ${ }^{54}$. Furthermore, using FasL (gld)and Fas (lpr)-deficient mice it has been questioned whether FasL-Fas-mediated neutrophil apoptosis is important in controlling neutrophil longevity during acute inflammatory responses ${ }^{55}$. However, activation of the Fas receptor on lung eosinophils results in apoptosis and the resolution of eosinophilic inflammation of the airways ${ }^{47}$. It therefore remains possible that mechanisms involving the FasL-Fas pathway might be exploited to manipulate granulocyte death.

\section{Manipulation of survival proteins}

Granulocyte apoptosis in vitro is accelerated by inhibitors of protein synthesis, which suggests the existence of one or more survival proteins that might dictate the speed of onset of apoptosis ${ }^{56}$. In other cells, including thymocytes, inhibitors of protein synthesis prevent the onset of apoptosis induced by stimuli such as glucocorticoids, which suggests that in this case proteins must be actively synthesized before apoptosis can occur ${ }^{57}$. The identity, function and control of expression of these factors remain to be determined.

Tumour necrosis factor $\alpha$ (TNF- $\alpha$ ) might help to unravel the identity of this survival protein(s) in granulocytes. TNF- $\alpha$ induces apoptosis in many cells, but in neutrophils it exerts differential effects, accelerating apoptosis at early time points and inhibiting apoptosis after $18 \mathrm{~h}$ of culture $^{58}$. These divergent effects (i.e. rapid death of some neutrophils and enhanced survival of others) are lost when protein synthesis is blocked and instead the entire neutrophil population synchronously undergoes apoptosis within $2 \mathrm{~h}$ of exposure to TNF- $\alpha$ (Ref. 11). This suggests that the differential effect of TNF- $\alpha$ is mediated via the activation of an immediate 'pro-apoptotic pathway' together with the induction of a 'protective' protein(s) that protects the cells that 'evade' the initial apoptotic trigger. 
TNF- $\alpha$ signals through three main inter-related pathways: (1) activation of the caspase pathway via the interaction of the TNF-receptor-associated death domain protein (TRADD) with the death domain of TNF receptor I, which allows recruitment of Fas-associated death domain (FADD)/mediator of receptor induced toxicity (MORT1); (2) the p38-Jun N-terminal kinase (JNK) pathway (p38 and JNK represent two main families of mitogen-activated protein kinases); and (3) activation of the transcription factor NF-kB (Ref. 59). The caspase pathway is unlikely to be involved in TNF- $\alpha$-induced neutrophil survival, although it might be one target for an anti-apoptotic protein. There is still controversy over the role of activation of hoth p.38-JNK and NF-кB in cell death and survival. For example, p38-JNK activation has been linked to Fasinduced apoptosis ${ }^{60-62}$. By contrast, JNK and p38 might be involved in cell survival signals in response to TNF- $\alpha$ in a murine fibroblast cell line ${ }^{63}$. In neutrophils, there appears to be a p38-stress activated pro-apoptotic pathway and a spontaneous or Fas-inducible pathway that is p38 independent ${ }^{64}$. Clearly, the precise role of stress kinases in apoptosis or survival pathways should be fully assessed; they might play different roles in different cell types.

In neutrophils, survival factors such as LPS activate both the p38-JNK pathway and NF-кB (Ref. 11) ${ }^{65}$. By contrast, in HL60 cells and thymocytes NF-kB activation induces apoptosis and NF-kB inhibitors prevent cell death ${ }^{66,67}$. However, modulation of NF- $\kappa$ B activation can make certain cells more responsive to the cytotoxic effects of TNF- $\alpha$ (Ref. 59), which suggests that NF-kB is involved in determining how cells respond to TNF- $\alpha$. By blocking the activation of an inducible form of NF- $\mathrm{kB}$ in neutrophils, the entire cell population undergoes apoptosis within $2 \mathrm{~h}$ of exposure to TNF- $\alpha$ (Ref. 11). Although TNF- $\alpha$ alone has no significant effect on constitutive eosinophil apoptosis, in the presence of NF-kB inhibition almost $100 \%$ of the cell population was apoptotic within $4 \mathrm{~h}$ of exposure to TNF- $\alpha$ (Ref. 11). These data strongly suggest that NF- $\kappa B$ has an important role in regulating granulocyte survival (Fig. 3).

Many pro-inflammatory stimuli activate NF- $\mathrm{kB}$, which regulates the transcription of several genes encoding proinflammatory proteins. This regulatory mechanism acts as an amplification loop that might enhance the longevity of both neutrophils and eosinophils [e.g. via production of GM-CSF and G-CSF (Ref. 68)]. Although this makes physiological sense in an ongoing inflammatory situation, prolonged granulocyte activation together with inhibition of apoptosis could cause an excessive tissue load of granulocytes and the possibility of cell necrosis and chronic inflammation. Because most inflammatory responses resolve without complication, a physiological mechanism to limit the activation of this and other transcription factors involved in promoting cell survival must exist and can be overridden by pro-apoptotic factors. Thus, pharmacological control of NF-kB activation represents an attractive target for intervention in inflammatory conditions.

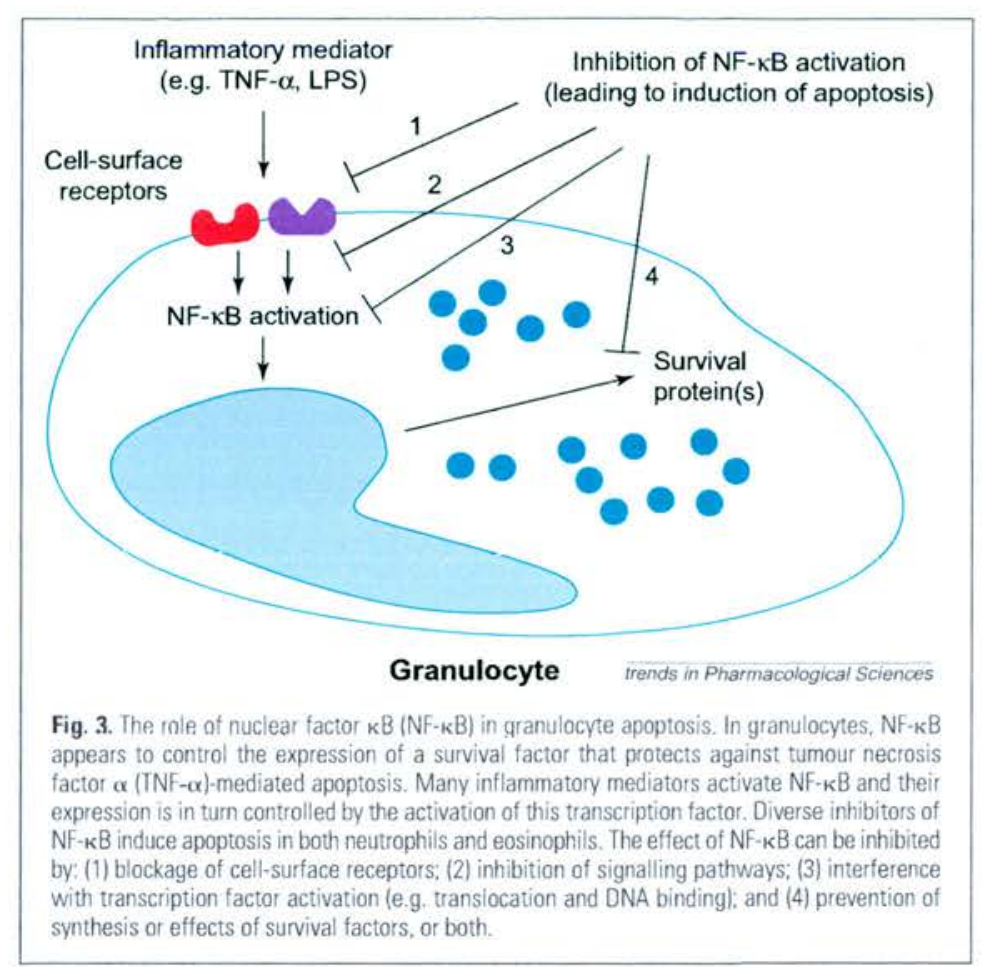

One strategy could be to develop compounds that interfere with the synthesis, metabolism or functions of putative proteins known to be regulated by NF-кB that are involved in controlling granulocyte survival. At present, the identity of such factors remains elusive but several possible candidates exist.

\section{BCL2 family members}

Control of apoptosis in various cells correlates with the expression of BCL2 family proteins, some of which are regulated, at least in part, by NF-кB (Refs 69-72). In cells in which activation of NF- $\mathrm{KB}$ leads to apoptosis, BCL2 suppresses NF- $\mathrm{B}$-dependent gene expression and inhibits apoptosis ${ }^{70}$; conversely, BCL2 can suppress apoptosis induced by disruption of NF-kB activation ${ }^{71}$. BCL2 is thought to control mitochondrial permeability transitions allowing the release of cytochrome c (Ref. 73) and apoptosis inducing factor $^{74}$, which are both involved in the execution phase of apoptosis. Mature human neutrophils do not express BCL2 but do express the pro-apoptotic protein BAX (Refs 75, 76), whereas eosinophils contain BCLXL, BAX and low levels of BCL2 (Ref. 77). Expression of MCL1, another BCL2 family member, decreases during neutrophil apoptosis but increases in response to LPS and GM-CSF, suggesting a link with survival in neutrophils 75 ; however, the association between MCL1 expression and eosinophil survival is more tenuous 77 . Neutrophils also express mRNA for A1, another BCL2 homologue with anti-apoptotic properties ${ }^{78}$. LPS and GM-CSF induce expression of A1 mRNA and inhibition of NF-kB activation blocks accumulation of A1 mRNA in some cells ${ }^{79}$. Neutrophil apoptosis is enhanced in mice that lack $A 1-a$, 


\begin{tabular}{|c|c|c|c|}
\hline Protein & $\begin{array}{l}\text { Present in } \\
\text { granulocytes }\end{array}$ & Details & Refs \\
\hline c-Myc & Yes & $\begin{array}{l}\text { Decreased in an NF-kB-dependent manner during apoptosis of HL-60 cells. In other cells, } \\
\text { increased c-Myc correlates with onset of apoptosis }\end{array}$ & 83,84 \\
\hline A20 & Unknown & $\begin{array}{l}\text { Specific protection against TNF- } \alpha \text {-induced apoptosis. Once synthesized, it can inhibit NF-kB. } \\
\text { Might represent a physiological feedback mechanism. Does not protect NF-kB-deficient } \\
\text { fibroblast from TNF- } \alpha\end{array}$ & $68,85-88$ \\
\hline CIAP & Unknown & $\begin{array}{l}\text { Induced by TNF- } \alpha \text {. Exogenous expression did not protect cells from this cytokine when } \\
\text { NF-kB was inhibited }\end{array}$ & 89 \\
\hline IEX-IL & Unknown & Confers resistance to TNF- $\alpha$-induced apoptosis & 90 \\
\hline
\end{tabular}

Abbreviations: NF-кB, nuclear factor $\kappa B$; TNF- $\alpha$, tumour necrosis factor $\alpha$.

a subtype of the $A 1$ gene $^{80}$, and LPS-induced inhibition of apoptosis is abolished. However, in these mice TNF- $\alpha$ induced apoptosis is unchanged, which suggests that A1 is involved in regulating some but not all neutrophil apoptotic pathways ${ }^{80}$. The presence of $\mathrm{A} 1$ in eosinophils has yet to be demonstrated convincingly ${ }^{81}$. BCLW, a BCL2 family member that promotes cell survival ${ }^{82}$, has not been shown in human granulocytes. Differences in the expression of BCL2 proteins in eosinophils and neutrophils might represent another specific checkpoint for therapeutic intervention. Other candidate survival proteins ${ }^{68,83-90}$ are shown in Table 2.

Activation of NF-кB depends on inhibitory factors IкBs, which are phosphorylated and ubiquitinated before degradation via the proteasome. Ubiquitination of one factor, I $\mathrm{B} \alpha$, can be prevented by the small ubiquitin-like protein SUMO-1 (also known as sentrin) ${ }^{91}$. This protein interacts with both TNF receptor subtype 1 and the Fas receptor, and protects against both TNF- $\alpha$ and Fas-mediated death signals $^{92}$. Whether SUMO- 1 is regulated via NF- $\mathrm{kB}$ is unclear, but it has the potential to both inhibit NF- $\mathrm{kB}$ activation (thus increasing TNF- $\alpha$-induced apoptosis) and to protect against the cytotoxic effects of this cytokine - a possibility that might explain the biphasic effects of TNF- $\alpha$ on neutrophil survival.

\section{Development of novel strategies}

Neutrophils are the main defence against bacteria and fungi, whereas eosinophils perform a mainly anti-parasitic role. Novel pro- and anti-apoptotic strategies could be developed by considering the mechanisms that pathogens have evolved to counter the immune attack posed by these cells. Some fungal metabolites (e.g. gliotoxin) have already proven to be powerful inducers of apoptosis in granulocytes ${ }^{11}$ by inhibition of NF-kB (Refs 11, 93). Bacteria can liberate agents (leukocidins) that can damage and kill granulocytes. For example, the bacteria Haemophilus sommus evades destruction by inducing neutrophil apoptosis $^{94}$ and Mycobacterium tuberculosis induces a rapid onset of neutrophil apoptosis in the presence of TNF- $\alpha$ (Ref. 95). The ingestion of Escherichia coli by human neutrophils induces apoptosis that is inhibited by antioxidants, which suggests that an oxygen-dependent mechanism is critically involved ${ }^{96}$. Bronchial lavage fluid obtained from animals with resolving experimental streptococcal pneumonia but not non-resolving $E$. coli pneumonia contains a powerful, yet unidentified, factor that accelerates neutrophil apoptosis ${ }^{97}$. Viruses can also induce granulocyte apoptosis; for example, respiratory syncytial virus ${ }^{98}$, EpsteinBarr virus ${ }^{99}$ and HIV (Ref. 100) all accelerate neutrophil apoptosis. This induction of apoptosis might offer advantages to the pathogen because, unlike damaged or necrotic cells, cleared apoptotic immune cells will not provoke an immune response. A thorough characterization of these naturally occurring promoters of granulocyte apoptosis and the elucidation of their mechanisms of action might help in the development of novel therapeutic agents.

\section{Concluding remarks}

The differences and similarities shown between eosinophils and neutrophils and other immune cells in response to pro-apoptotic and anti-apoptotic stimuli demonstrate that there is a potential for the development of agents with specificity to remove both cell types selectively. Achieving specificity could permit the resolution of inflammation using agents that promote apoptosis to become a therapeutic reality. Strategies for the development of such compounds require more fundamental research on the actual physiological mediators governing granulocyte apoptosis both in vitro and in vivo to decipher the precise underlying regulatory mechanisms. A similar strategy might also prove useful in the development of compounds for the selective deletion of cancer cells resulting in the dissolution of tumours without damaging healthy non-malignant tissues.

\section{Selected references}

1 Rossi, A. G. and Haslett, C. (1997) in Lung Biology in Health and Disease (Vol. 112) (Said, S. I., ed.), pp. 9-44, Marcel Dekker

2 Anderson, G. P. (1996) Trends Pharmacol. Sci. 17, 438-442

3 Kinloch, R. A., Treherne, J. M., Furness, L. M. and Hajimohamadreza, I. (1999) Trends Pharmacol. Sci. 20, 35-42

4 Hart, S. P., Haslett, C. and Dransfield, I. (1996) Experientia 52, 950-956

5 Savill, J. (1997) Br. Med. Bull, 53, 491-508

6 Hart, S. P., Dougherty, G. J., Haslett, C. and Dransfield, I. (1997) J. Immunol. 159,919-925

7 Voll, R. E. et al. (1997) Nature 390, 350-351

8 Fadok, V. A. et al. (1998) J. Clin. Invest. 101, 890-898 


\section{$\begin{array}{llllll}R & E & V & I & E & W\end{array}$}

9 Sanghavi, D. M., Thelen, M., Thornberry, N. A., Casciola-Rosen, L. and Rosen, A. (1998) FEBS Lett. 422, 179-184

10 Meagher, L. C., Cousin, J. M., Seckl, J. R. and Haslett, C. (1996) J. Immunol. 156, 4422-4428

11 Ward, C. et al. (1999) J. Biol. Chem. 274, 4309-4318

12 Cox, G. (1996) Am. J. Physiol. 271, L566-L571

13 Watson, R. W., Rotstein, O. D., Parodo, J., Bitar, R. and Marshall, J. C. (1998) J. Immunol. 161, 957-962

14 Barnes, P. J. and Adcock, I. (1993) Trends Pharmacol. Sci. 14, 436-441

15 Woolley, K. L. et al. (1996) Am. J. Respir. Crit. Care Med. 154, 237-243

16 Cox, G. and Austin, R. C. (1997) J. Leukocyte Biol. 61, 224-230

17 McConkey, D. J. et al. (1989) Arch. Biochem. Biophys. 269, 365-370

18 Cousin, J. M., Haslett, C. and Rossi, A. G. (1997) Biochem. Soc. Trans, $25,243 \mathrm{~S}$

19 Whyte, M. K. B., Meagher, L. C., Harkwick, S. J., Savill, J. S. and Haslett, C. (1993) J. Clin. Invest. 92, 446-455

20 Lui, Y. et al. (1999) J. Immunol. 162, 3639-3646

21 Wong, T., Rossi, A. G. and Chilvers, E. R. (1997) J. Clin. Sci, 92, 7P

22 Blaylock, M. G., Cuthbertson, B. H., Galley, H. F., Ferguson, N. R. and Webster, N. R. (1998) Free Radic. Biol. Med. 25, 748-752

23 Fortenberry, J. D., Owens, M. L., Brown, M. R., Atkinson, D. and Brown, L. A. (1998) Am. J. Respir. Cell. Mol. Biol. 18, 421-428

24 Ward, C. et al. Biochem. Pharmacol. (in press)

25 Reauvais, F, Michel, I. and Duhertret, I. (1995) FFRS Iftt 361, 229-232

26 Hebestreit, H. et al. (1998) J. Exp. Med. 187, 415-425

27 Clutton, S. (1997) Br. Med. Bull. 53, 662-668

28 Hannah, S. et al. (1995) FEBS Lett. 372, 233-237

29 Coxon, A. et al. (1996) Immunity 5, 653-666

30 Watson, R. W., Rotstein, O. D., Nathens, A. B., Parodo, J. and Marshall, J. C. (1997) J. Immunol. 158, 945-953

31 Walzog, B., Jeblonski, F., Zakrzewicz, A. and Gaehtgens, P. (1997) FASEB J.11, 1177-1186

32 Hannah, S. et al. (1998) FEBS Lett. 421, 141-146

33 Teixeira, M. M., Gristwood, R. W., Cooper, N. and Hellewell, P. G. (1997) Trends Pharmacol. Sci. 18, 164-171

34 McConkey, D. J., Orrenius, S. and Jondal, M. (1990) J. Immunol. 145, $1227-1230$

35 Lanotte, M. et al. (1991) J. Cell Physiol. 146, 73-80

36 Gjertsen, B. T. et al. (1994) J. Cell Sci. 107, 3363-3377

37 Rossi, A. G. et al. (1995) Biochem. Biophys. Res. Commun. 217, 892-899

38 Ottonello, L., Gonella, R., Dapino, P., Sacchetti, C. and Dallegri, F. (1998) Exp. Hematol. 26, 895-902

39 Parvathenani, L. K., Buescher, E. S., Chacon-Cruz, E. and Beebe, S. J. (1998) J. Biol. Chem. 273, 6736-6743

40 Hallsworth, M. P., Giembycz, M. A., Barnes, P. J. and Lee, T. H. (1996) Br. J. Pharmacol. 117, 79-86

41 Yasui, K., Hu, B., Nakazawa, T., Agematsu, K. and Komiyama, A. (1997) J. Clin. Invest. 100, 1677-1684

42 O'Flaherty, J. T., Jacobson, D. P., Redman, J. and Rossi, A. G. (1990) J. Biol. Chem. 265, 9146-9152

43 Cousin, J. M., Haslett, C. and Rossi, A. G. (1997) Biochem. Soc. Trans. $25,243 \mathrm{~S}$

44 Khwaja, A. and Tatton, L. (1999) Blood 94, 291-301

45 Pongracz, J. et al. (1995) Exp. Cell Res. 218, 430-438

46 Iwai, K. et al. (1994) Blood 84, 1201-1208

47 Tsuyuki, S. et al. (1995) J. Clin. Invest. 96, 2924-2931

48 Druilhe, A., Cai, Z., Haile, S., Chouaib, S. and Pretolani, M. (1996) Blood 87, 2822-2830

49 Matsumoto, K., Schleimer, R. P., Saito, H., likura, Y. and Bochner, B. S. (1995) Blood 86, 1437-1443

50 Liles, W. C., Kiener, P. A., Ledbetter, J. A., Aruffo, A. and Klebanoff, S. J. (1996) J. Exp. Med. 184, 429-440

51 Brown, S. B. and Savill, J. (1999) J. Immunol. 162, 480-485

52 Watson, R. W.et al. (1999) FEBS Lett. 453, 67-71

53 Ottenello, L., Tortolina, G., Amelotti, M. and Dallegri, F. (1999) J. Immunol. 162, 3601-3606

54 Miwa, K. et al. (1998) Nat. Med. 4, 1287-1292

55 Fecho, K. and Cohen, P. L. (1998) J. Leukocyte Biol. 64, 373-383

56 Whyte, M. K., Savill, J.S., Meagher, L. C., Lee, A. and Haslett, C. (1997) J. Leukocyte Biol. 62, 195-202

57 Wyllie, A. H. (1980) Nature 284, 555-556

58 Murray, J. et al. (1997) Blood 90, 2772-2783

59 Wallach, D. et al. (1999) Anmu. Rev. Immunol. 17, 331-367

60 Juo, P. et al. (1997) Mol. Cell. Biol. 1, 24-35

61 Ichijo, H. et al. (1997) Science 275, 90-94

62 Malinin, N. L., Boldin, M. P., Kovalenko, A. V. and Wallach, D. (1997) Nature $385,540-544$
63 Roulston, A., Reinhard, C., Amiri, P. and Williams, L. T. (1998) J. Biol. Chem. 273, 10232-10239

64 Frasch, S. C. et al. (1998) J. Biol. Chem. 273, 8389-8397

65 Nick, J. A., Avdi, N. J., Gerwins, P., Johnson, G. L. and Worthen, G. S. (1996) J. Immunol. 156, 4867-4875

66 Marinovich, M., Viviani, B., Corsini, E., Ghilardi, F. and Galli, C. L. (1996) Exp. Cell Res. 226, 98-104

67 Bessho, R. et al. (1994) Biochem. Pharmacol. 48, 1883-1889

68 Baeuerle, P. A. and Baltimore, D. (1996) Cell 87, 13-20

69 Anderson, G. P. (1997) Trends Pharmacol. Sci. 18, 51

70 Grimm, S., Bauer, M. K., Baeuerle, P. A. and Schulze-Osthoff, K. (1996) J. Cell Biol. 134, 13-23

71 Herrmann, J. L. et al. (1997) Exp. Cell Res. 237, 101-109

72 Dixon, E. P.,Stephenson, D. T., Clemens, J. A. and Little, S. P. (1997) Brain Res. 776, 222-229

73 Green, D. R. and Reed, J. C. (1998) Science 281, 1309-1312

74 Susin, S. A. et al. (1999) Nature 397, 441-446

75 Moulding, D. A., Quayle, J. A., Hart, C. A. and Edwards, S. W. (1998) Blood 92, 2495-2502

76 Delia, D. et al. (1992) Blood 79, 1291-1298

77 Druilhe, A., Arock, M., Le Goff, L. and Pretolani, M. (1998) Am. J. Respir. Cell. Mol. Biol. 18, 315-322

78 Chuang, P. I., Yee, E., Karsan, A., Winn, R. K. and Harlan, J. M. (1998) Biochem. Biophys. Res. Commun. 249, 361-365

$79 \mathrm{Hu}$, X., Yee, E., Harlan, J. M., Wong, F. and Karsan, A. (1998) Blood $92,2759-2765$

80 Hamasaki, A. et al. (1998) J. Exp. Med. 188, 1985-1992

81 Dibbert, B. et al. (1998) Blood 92, 778-783

82 Gibson, L. et al. (1996) Oncogene 13, 665-675

83 Kumakura, S. et al. (1996) Leuk. Lymiphoma 23, 383-394

84 Evan, G. I. et al. (1992) Cell 69, 119-128

85 Dixit, V. M., Green, M. S., Sarma, V. and Prochownik, E. V. (1990) J. Biol. Chem. 265, 2973-2978

86 Opipari, A. W. Jr, Hu, H. M., Yabkowitz, R. and Dixit, V. M. (1992) J. Biol. Chem. 267, 12424-12427

87 Kirikos, A., Laherty, C. D. and Dixit, V. M. (1992) J. Biol. Chem. 267, 17871-17976

88 Jaattela, M., Mourtizen, H., Elling, F. and Bastholm, L. (1996) J. Immunol. 156, 1166-1173

89 Chu, Z. et al. (1997) Proc. Natl. Acad. Sci. U. S. A. 94, 10057-10062

$90 \mathrm{Wu}$, M. X., Ao, Z., Prasad, K. V., Wu, R. and Schlossman, S. F. (1998) Science 281, 998-1001

91 Desterro, J. M. P., Rodriguez, M. S. and Hay, R. T. (1998) Mol. Cell 2, 1-20

92 Okura, T. et al. (1996) J. Immunol. 157, 4277-4281

93 Pahl, H. L. et al. (1996) J. Exp. Med. 183, 1829-1840

94 Yang, Y. F., Sylte, M. J. and Czuprynski, C. J. (1998) Microb. Pathol. 24, 351-359

95 Kasahara, K. et al. (1998) J. Infect. Dis. 178, 127-137

96 Watson, R. W. G., Redmond, H. P., Wang, J. H., Condron, C. and Bouchier-Hayes, D. (1996) J. Immunol. 156, 3986-3992

97 Lawson, R. A., Caldwell, H., Usher, L., Whyte, M. K. B. and Haslett, C. (1998) Thorax 53 (Suppl. 4), A36

98 Wang, S. Z. et al. (1998) Clin. Exp. Immunol. 114, 49-54

99 Larochelle, B., Flamand, L., Gourde, P., Beauchamp, D. and Gosselin, J. (1998) Blood 92, 291-299

100 Pitrak, D. L., Tsai, H. C., Mullane, K. M., Sutton, S. H. and Stevens, P. (1996) J. Clin. Invest. 98, 2714-2719

\section{Chemical name}

Ro318220: 1-[3-(amidinothio)propyl-1H-indol-3-yl]-3-(1methyl- $1 \mathrm{H}$-indol-3-yl)maleimide methane sulfonate

\section{Students}

Subscribe to TiPS at half price.

See the bound-in card for details.
Acknowledgements The work in the authors laboratory is funded by the MRC (Program Grant G9016491). E. R. C. is a Wellcome Trust Senior Research Fellow in Clinical Science. 


\section{Review}

\section{AN APPETITE FOR APOPTOTIC CELLS? CONTROVERSIES AND CHALLENGES}

The biochemical pathways involved in programmed cell death are broadly similar in phylogenetically diverse multicellular organisms, including the nematode worm Caenorhabditis elegans, Drosophila and mammals, suggesting that cell death and subsequent phagocytosis of apoptotic cells represents an important regulatory mechanism that has been conserved through evolution (Ellis et al, 1991; Hengartner, 1996).

During apoptosis, the cell activates intrinsic suicide mechanisms that lead rapidly (within hours) to the characteristic macroscopic features of cell shrinkage, chromatin condensation, membrane budding and, eventually, the formation of one or more apoptotic bodies (Kerr et al, 1972). Phagocytosis of senescent cells had been described in the late nineteenth century by the Russian biologist Elie Metchnikoff who observed that 'microphages' (neutrophil granulocytes) were 'englobed' by macrophages in injured tadpole fins, although the significance of these observations has only become apparent in the last few decades. It has now been established that apoptotic cells are swiftly recognized and ingested by neighbouring phagocytes without elicitation of pro-inflammatory responses from phagocytic cells (Meagher et al, 1992; Savill et al, 1993; Fadok et al, 1998). Apoptosis and the subsequent phagocytic clearance of senescent cells have central roles in many fundamental biological processes, including normal tissue turnover (Han et al, 1993), remodelling of embryological tissues (Hopkinson-Woolley et al, 1994), development of the immune system (Cohen, 1991) and the normal resolution processes of inflammation (Haslett et al, 1994). Macrophages are the principal phagocytes that are responsible for clearance of apoptotic cells in mammals, although cells with the potential for phagocytosis, e.g. endothelial cells (Dini et al, 1995: Hess et al, 1997), fibroblasts (Hall et al, 1994), glomerular mesangial cells (Hughes et al, 1997), hepatocytes (Dini et al, 1992), Sertoli cells (Shiratsuchi et al, 1997) and vascular smooth muscle cells (Bennett et al, 1995) may remove apoptotic cells in certain circumstances.

\section{GRANULOCYTE APOPTOSIS AND THE RESOLUTION OF INFLAMMATION}

There is increasing evidence that aberrant apoptosis and/or phagocytic clearance of apoptotic granulocytes may contribute to the pathogenesis of inflammatory diseases (Fig 1). Neutrophil granulocytes play a vital role in the body's

Correspondence: Dr I. Dransfield, The Rayne Laboratory, Respiratory Medicine Unit, University of Edinburgh Medical School, Teviot Place, Edinburgh EH8 9AG, UK. defence against infectious agents, but uncontrolled release of their formidable array of toxic substances may inflict 'friendly fire' damage on surrounding tissue and propagate the inflammatory response (Haslett et al, 1994). In contrast to the potentially harmful effects of necrotic cell death upon tissue integrity and organ function, deletion of cells by apoptosis represents a cellular removal pathway that does not provoke pro-inflammatory phagocyte responses. Indeed, dysregulated neutrophil and eosinophil granulocyte apoptosis has been implicated in the pathogenesis of the adult respiratory distress syndrome (ARDS), idiopathic pulmonary fibrosis, ulcerative colitis, rheumatoid arthritis, asthma and other allergic diseases (Weiss, 1989; Meagher et al, 1992; Haslett et al, 1994; Stern et al, 1996; Woolley et al, 1996). Uncoupling of neutrophil effector responses associated with apoptosis (Whyte et al, 1993), together with 'anti-inflammatory' clearance by phagocytes, provides a mechanism for the 'safe' disposal of potentially destructive inflammatory cells. Strategies for manipulation of cellular apoptosis programmes for therapeutic gain in inflammatory or allergic disease are likely to fail if the capacity for clearance of apoptotic cells is exceeded. Data from in vivo experimental models support this hypothesis. For example, induction of widespread apoptosis in the liver, after treatment of mice with intraperitoneal Fas antibody, led to extensive hepatic necrosis and death of the animals (Ogasawara et al, 1993). Similarly, induction of bronchial and alveolar epithelial cell apoptosis in the rat lung by repeated inhalation of aerosolized Fas antibody culminated in pulmonary inflammation and scarring (Hagimoto et al, 1997). Although previous strategies have identified several distinct families of molecules involved in apoptotic cell recognition, the precise mechanism of phagocytosis of apoptotic cells is still not known. Clearly, a detailed understanding of the mechanisms of phagocytic clearance of apoptotic cells and the underlying regulatory mechanisms are likely to have important implications for the design of therapeutic strategies for the treatment of inflammatory disease. In this review, we will illustrate some of the problems that need to be resolved and describe some of the challenges for future research on phagocyte recognition of apoptotic cells.

\section{PHAGOCYTE RECEPTORS FOR RECOGNITION OF APOPTOTIC CELLS}

At least seven distinct molecular families have been implicated in the recognition process. Table I summarizes the experimental data that support the involvement of the receptor pathways for the clearance of apoptotic cells shown schematically in Fig 2 (see also Savill et al, 1993; Hart et al, 


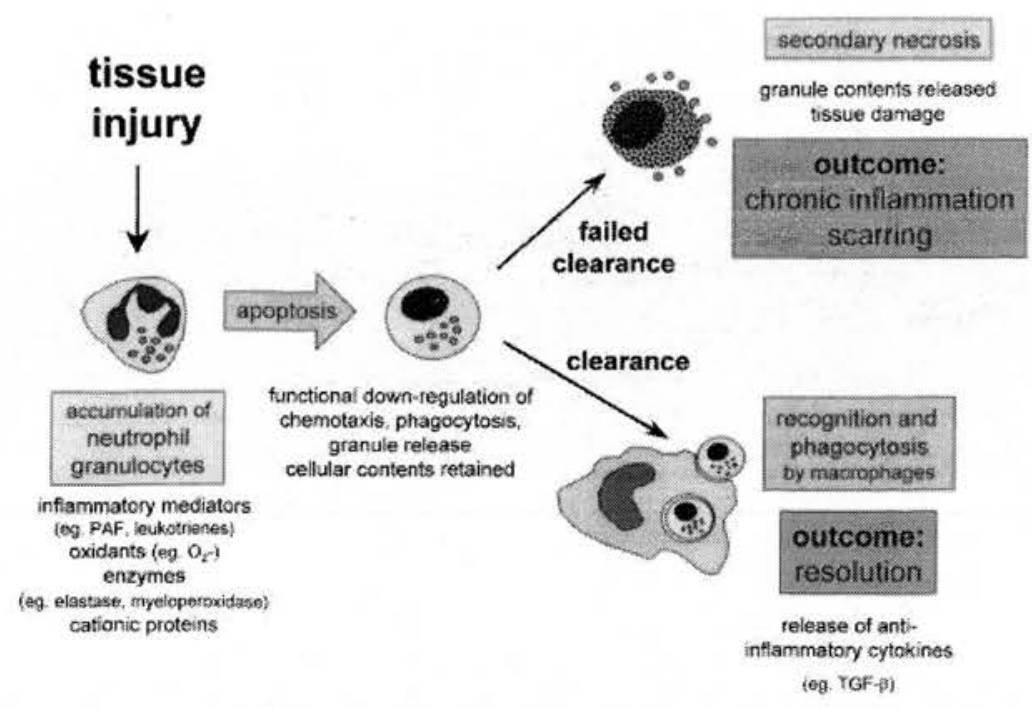

Fig 1. Schematic representation illustrating the proposed role of neutrophil apoptosis and phagocytic clearance by macrophages in the process of resolution of inflammation.
1996; Platt et al, 1998a). The first molecular pathway for the specific clearance of apoptotic neutrophils by human macrophages was identified on the basis of distinct patterns of inhibition by carbohydrates and sensitivity to altered $\mathrm{pH}$ (Savill et al, 1989). Subsequent analyses implicated the phagocyte integrin, $\alpha v \beta 3$ (Savill et al, 1990) and the class B scavenger receptor, $\mathrm{CD} 36$, as receptors of apoptotic cellassociated thrombospondin (Savill et al, 1992). Inhibition of phagocytosis of apoptotic cells after blockade of integrin function in murine bone marrow-derived macrophages indicated that similar pathways exist in other species (Fadok et al, 1992a). However, Fadok et al (1992b) also demonstrated that murine peritoneal macrophages did not use this pathway and characterized phosphatidylserine (PS) as a ligand for a putative PS receptor. In support of the suggestion that macrophages from different tissue sites use distinct pathways, Kupffer cells recognize altered carbohydrate present on apoptotic cells via the asialoglycoprotein receptor (Dini et al, 1995), whereas mouse thymic macrophages use class A scavenger receptors (Platt et al, 1998b). Furthermore, alteration of macrophage phenotype after stimulation (for example, with digestible particles) may drive switching of the dominant pathway used for clearance (Fadok et al, 1993; Pradhan et al, 1997). A number of alternative pathways for human macrophage clearance of apoptotic cells have been reported. Detailed characterization of macrophage recognition of apoptotic B cells (Flora \& Gregory, 1994) pointed to a role for phagocyte CD14 (Devitt et al, 1998) and apoptotic cell CD50 (Moffatt et al, 1999). Recent data have also suggested a role for elements of humoral immunity, specifically, complement components C1q (Botto et al, 1998) and C3bi (Mevorach et al, 1998). Specific opsonization of apoptotic cells may serve to accelerate apoptotic cell clearance. In addition, there are other receptors that have been identified to have a role in apoptotic cell clearance, e.g. ATP binding cassette transporter (Luciani \& Chimini, 1996). However, whether these molecules mediate phagocyte recognition of apoptotic cells directly is unclear. These data serve to illustrate the molecular complexity of phagocyte recognition of apoptotic cells and suggest considerable functional redundancy in molecular pathways that are used for phagocytic clearance, perhaps ensuring that clearance of apoptotic cells is not compromised by genetic mutations in any one pathway.

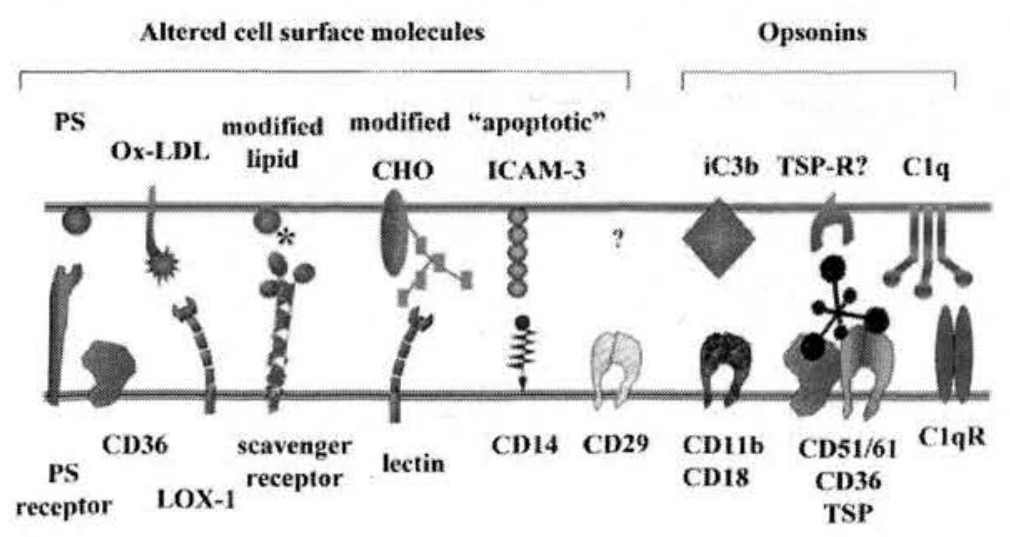

Fig 2. Cell surface molecules implicated in the uptake of apoptotic cells by phagocytes (see also Table $I$ and text). 
Review 3

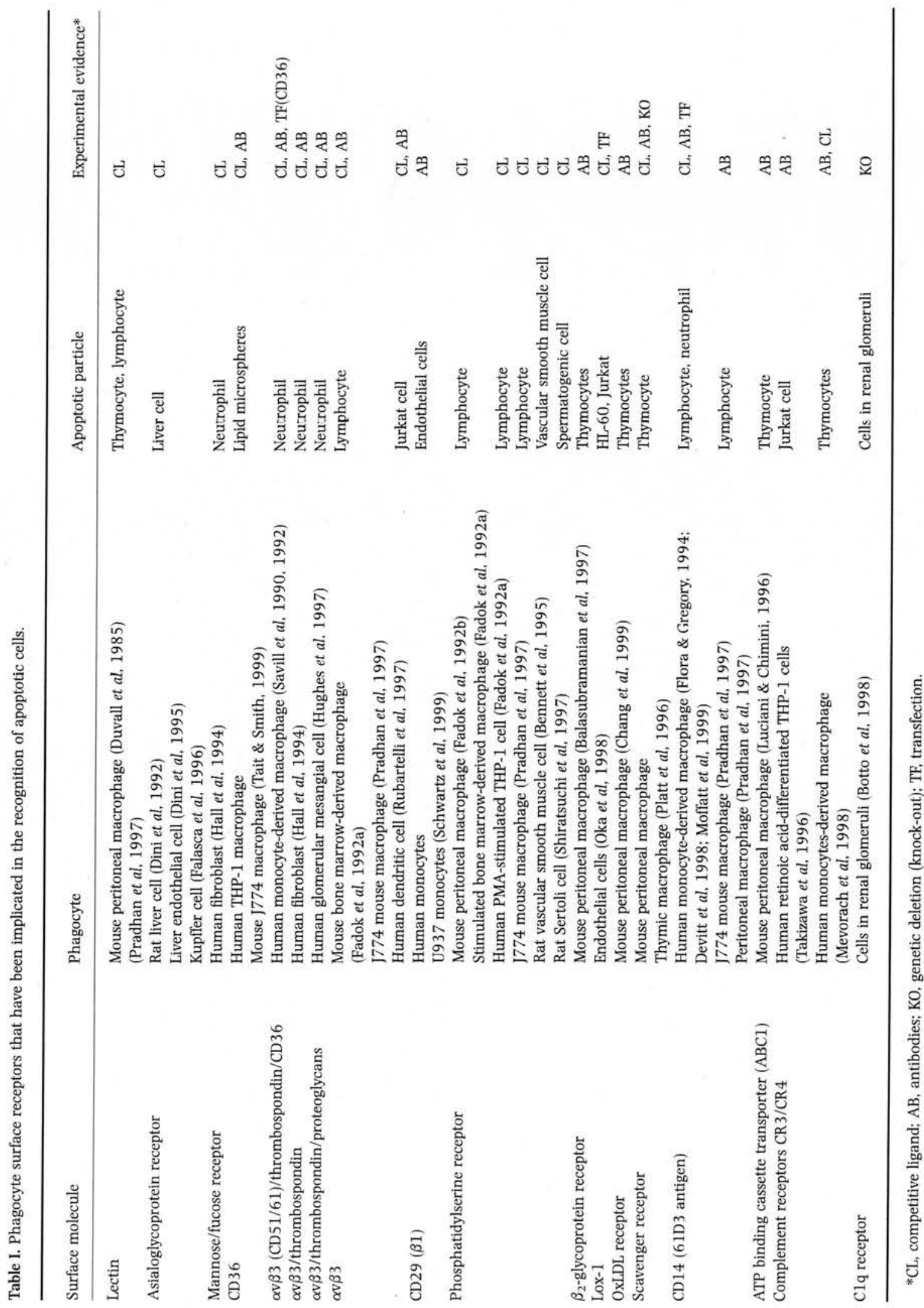

(C) 2000 Blackwell Science Ltd, British Journal of Haematology 109: 1-12 
Interpretation of results from studies of phagocyte clearance of apoptotic cells has been complicated by a number of factors in addition to the existence of tissuespecific clearance pathways. The use of 'apoptotic' cell populations that may contain cells at different stages of apoptosis (Hebert et al, 1996) and variable numbers of necrotic cells (Vermes et al, 1997) represents an additional, frequently overlooked variable in the study of phagocytic clearance. Phagocytosis of 'late' apoptotic and necrotic cells may be mediated by different mechanisms with distinct functional consequences. Furthermore, the experimental approaches that have been used to determine the involvement of specific molecules in recognition may give rise to further ambiguity. Identification of the molecular pathways involved in phagocytosis of apoptotic cells has relied to a large extent on the use of monoclonal antibodies (mAbs) and competitive ligands. One of the potential problems of competitive ligands is that, if the affinity of binding is low, high concentrations of ligand are necessary to compete with surface presentation of multivalent ligands on the surface of apoptotic cells, with the possibility of non-specific effects. Intact $\mathrm{mAb}$ or bivalent $\mathrm{F}\left(\mathrm{ab}^{\prime}\right)_{2}$ fragments have the potential for cross-linking macrophage surface receptors (including Fc receptors via intact $\mathrm{mAb}$ ) and the initiation of signalling cascades that may indirectly alter phagocyte function. For example, we have demonstrated previously that crosslinking CD44 with bivalent mAb augments phagocytosis of apoptotic cells (Hart et al, 1997). It is therefore possible that $\mathrm{mAb}$ may exert inhibitory effects without the recognized receptor necessarily being directly involved in the phagocytic process. It is notable that, with a few exceptions (Flora \& Gregory, 1994), confirmation of direct receptor involvement using $\mathrm{Fab}^{\prime}$ fragments has not been made. Specific genetic deletion of putative apoptotic cell receptors has provided somewhat equivocal data, perhaps reflecting the functional redundancy of receptor usage discussed above. For example, absence of thrombospondin-1 expression resulted in pneumonia, together with marked changes in lung homeostasis, which might have reflected altered potential for clearance of apoptotic cells. However, these effects were largely attributed to the ability of thrombospondin- 1 to activate the latent form of transforming growth factor (TGF)- $\beta 1$ (Crawford et al, 1998). Similarly, deletion of the class A scavenger receptor had profound effects upon phagocytosis of apoptotic thymocytes in vitro, but did not alter the architecture of the thymus significantly (Platt et al, 1998b). However, the absence of a structural abnormality in vivo may be accounted for by a large functional reserve for apoptotic cell clearance capacity in the healthy, 'pathogen-free' animal.

Although gene transfection into cell types lacking putative apoptotic cell receptors provides evidence suggestive of direct involvement via a 'gain of function' (Ren et al, 1995), these experimental approaches may be limited by the requirement for phagocytic machinery that may not present in the transfected cell type. Recent studies examining the binding of phospholipid vesicles have demonstrated a direct role for CD36 binding to anionic phospholipids (Tait \& Smith, 1999), and uptake of oxidized low-density lipoprotein (LDL) via scavenger receptor recognition of the lipid moiety rather than apoprotein B as described previously (Bird et al, 1999). Similar experimental approaches using binding of apoptotic cells to purified isolated cell surface receptors would provide definitive evidence for an apoptotic cell recognition receptor.

For each system described so far, it is also noteworthy that there is often considerable mismatch between observed levels of phagocytic responses in macrophage populations or transfected cells with the percentage of cells that express putative recognition molecules. For example, human monocytes express CD36 and CD14, yet they bind and ingest apoptotic cells very poorly, implying that these receptors may be necessary, but not sufficient, for efficient phagocytosis to occur. This suggestion is supported by the lack of complete inhibition of phagocytosis of apoptotic cells, even when multiple pathways are blocked, implying that further molecular pathways for removal of apoptotic cells remain to be identified.

One of the characteristics of apoptotic cell clearance mechanisms is the lack of induction of pro-inflammatory macrophage responses, which may be important for the silent clearance of apoptotic cells in normal physiological processes (Meagher et al, 1992; Savill et al, 1993; Fadok et al, 1998). In contrast, phagocyte clearance of 'post-apoptotic' or necrotic cells may trigger pro-inflammatory responses (Stern et al, 1996; Hughes et al, 1997). Fadok et al (1998) demonstrated that one potential mechanism for the inhibition of pro-inflammatory cytokine production after apoptotic cell phagocytosis might involve the production of TGF- $\beta$ and prostaglandins to actively suppress pro-inflammatory mediator production. Active modulation of inflammatory or immune responsiveness by macrophages after apoptotic cell phagocytosis is further supported by preliminary findings that interleukin (IL)-10 may be released from monocytes in a CD36-dependent manner after interaction with apoptotic cells (Voll et al, 1997). Recent data suggest that macrophage phagocytosis of apoptotic cells may also lead to the release of soluble Fas-ligand with the potential to influence inflammatory cell survival (Brown \& Savill, 1999). It is likely that progression of inflammatory responses and development of disease is profoundly influenced by the combination of these factors. Interestingly, analysis of interaction of type A scavenger receptors and $\mathrm{CD} 36$ with oxidized LDL (oxLDL) suggested that, in contrast to soluble oxLDL, surface-bound oxLDL induced macrophage secretion of $\mathrm{H}_{2} \mathrm{O}_{2}$. Inhibition studies indicated that $\mathrm{CD} 36 \mathrm{might}$ play a role in the promotion of secretion, suggesting that distinct $\mathrm{CD} 36$ ligands (apoptotic cells and oxLDL) might exert differential effects (Maxeiner et al, 1998). Similarly, CD14-mediated binding of lipopolysaccharide (LPS) has pro-inflammatory effects on macrophage behaviour, whereas apoptotic cell recognition via CD14 does not (Devitt et al, 1998). For many cellular molecular recognition processes (e.g. macrophage$\mathrm{T}$ cell interactions involved in antigen presentation), cooperative receptor interactions are required to ensure appropriate responses in the interacting cell types. One possibility is that phagocytes use a number of pathways in parallel to ensure the rapid identification of membrane changes of apoptotic cells and that the repertoire of receptor engagement during apoptotic cell recognition determines the phagocyte responses initiated. One of the challenges for 
future research on apoptotic cell recognition mechanisms is to define the pattern of utilization of distinct molecular pathways in vivo and the issue of functional redundancy or exclusivity using tissue-specific and temporal gene targeting strategies or double knock-out animals.

\section{PHAGOCYTOSIS: GENERAL MECHANISMS}

Understanding the basic cellular machinery required for apoptotic cell internalization and comparison with wellcharacterized [e.g. FcR- and complement receptor (CR)mediated] phagocytic mechanisms may generate useful clues for understanding the underlying control processes involved and provide the basis for novel strategies for manipulation of apoptotic cell clearance for therapeutic gain.

The dissociation of two distinct phases (initial binding to specific receptors on the phagocyte surface and subsequent internalization) during phagocytosis of particles that have been opsonized with immunoglobulin (Ig) or complement has allowed detailed dissection of the pathways at the molecular level. Binding of opsonized ligands via FcR or CR occurs in the absence of intact microfilaments or microtubules (Newman et al, 1991; Caron \& Hall, 1998). In contrast, attachment of apoptotic neutrophils appears to be both energy dependent and requires intact cytoskeletal elements. Little or no binding of apoptotic cells to human macrophages is observed at $4^{\circ} \mathrm{C}$ or when cytoskeletal elements are disrupted (our unpublished observations). These findings contrast with studies of scavenger receptor function, which show temperature-insensitive binding of lipid microspheres (Bird et al, 1999).

Particle internalization requires microfilament integrity in all three forms of phagocytosis. Fc phagocytosis is mediated by lamellipodia extension around the particle in a 'zipperlike' fashion (Griffin et al, 1975), and blockade of surface receptors after particle attachment inhibited internalization.

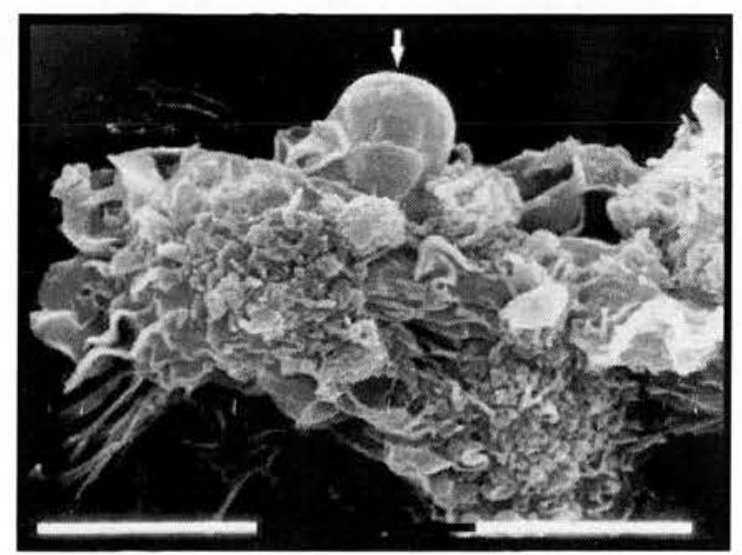

Fig 3. Scanning electron micrograph of the interaction between a human monocyte-derived macrophage and an apoptotic neutrophil in vitro. The apoptotic neutrophil has been bound to the ruffled membrane of the macrophage, and membrane projections can be seen extending around the apoptotic cell (arrowed). Bar $=10 \mu \mathrm{m}$.
Consistent with this model, inhibition of pseudopod extension by the PI-3 kinase inhibitor, wortmannin, prevented phagosome closure (Cox et al, 1999). CR1- and CR3mediated ingestion also requires intact microfilaments but, in contrast to FcR-mediated internalization, bound complement-opsonized particles appear to 'sink' into the phagocyte surface, suggesting that internalization may occur via a 'modified zippering' mechanism involving the formation of a phagocytic pit (Kaplan, 1977). Further differences were revealed after stabilization of microtubules by elevation of cGMP, which enhances CR-mediated phagocytosis (Newman et al, 1991). Examination of macrophages ingesting apoptotic cells by scanning electron microscopy suggests that apoptotic cell uptake may be similar to the zippering mechanism observed for FcR-mediated phagocytosis (Fig 3). In support of this assertion, stabilization of microtubules via elevation of cGMP did not affect phagocytosis of apoptotic neutrophils, whereas microfilament disruption after cAMP elevation agents prevented ingestion (Rossi et al, 1998).

Ultrastructural identification of differences in phagocytic uptake is supported by recent molecular analysis of the recruitment of cytoskeletal elements to nascent phagosomes and regulation of cytoskeletal integrity by the Rho family of GTPases. These findings suggest intriguing parallels with the recruitment of proteins to nascent phagosomes and the formation of actin-rich podosomes containing talin and tyrosine-phosphorylated proteins that have been described during macrophage adherence (Marchisio et al, 1987). During FcR-mediated phagocytosis, cytoskeletal proteins (actin, talin, paxillin and $\alpha$-actinin) and tyrosine-phosphorylated proteins are enriched near to the phagosome membrane in a diffuse manner, and phagocytosis is sensitive to tyrosine kinase inhibitors (Allen \& Aderem, 1996). In contrast, during CRmediated phagocytosis (which is insensitive to tyrosine kinase inhibition), discrete foci of these proteins are distributed over the phagosome surface. Consistent with the requirement for cytoskeletal elements described above, manipulation of Rho family GTPases does not alter FcR- or CR-mediated binding of particles. However, inhibition of formation of lamellipodial and filopodial membrane extensions by microinjection of dominant negative forms of Rho family GTPases, Rac and Cdc42, inhibits FcR-mediated phagocytosis (Caron \& Hall, 1998). In addition, Rac and Cdc42 control membrane ruffling in macrophages, causing cell rounding and loss of integrin-containing focal complexes with co-localized vinculin, paxillin and FAK (Allen et al, 1997). In contrast, injection of the Rho inhibitor C3 transferase into macrophages inhibited the assembly of mechanotransducing actomyosin filaments and prevented CR-mediated phagocytosis (Allen et al, 1997; Caron \& Hall, 1998). Distinct effects of Rho on cytoskeletal organization and adhesion were also observed with cell rounding after the injection of constitutively active Rho into macrophages, whereas inhibition of Rho led to loss of the presence of fine actin cables (Allen et al, 1997). These data demonstrate that there are distinct molecular mechanisms for phagocytosis via FcR and CR (termed type I and type II respectively), which may account for the observed differences in pro-inflammatory 
mediator production after particle internalization (Wright \& Silverstein, 1983; Yamamoto \& Johnston, 1984; Aderem et al, 1985; Scholl et al, 1992; Debets et al, 1998).

As yet, there has been no direct demonstration of a role for Rho or Rac in apoptotic cell uptake. However, recent studies of $C$. elegans mutants that display defective phagocytic removal of cellular 'corpses' have characterized a number of genes involved in this process (Liu \& Hengartner, 1998; Wu \& Horvitz, 1998a,b), which suggest an important role for cytoskeletal-associated proteins and regulators in apoptotic cell uptake. The ced- 6 gene product has sequences similar to phosphotyrosine-binding domains, and it may therefore act as an adaptor in the signalling pathways engaged after recognition and binding of apoptotic cells (Liu \& Hengartner, 1998). Our preliminary data indicate that there is an absolute requirement for tyrosine kinase activity in apoptotic cell uptake, in keeping with the involvement of tyrosine phosphorylation and ultrastructural evidence for a 'zippering'-like mechanism (Fig 3).

The protein encoded for by ced-5, a gene that is required for the orderly engulfment of cell corpses in C. elegans, shows homology to the human CRK-binding protein DOCK180, which has been implicated in cytoskeletal function and extension of cell surfaces (Hasegawa et al, 1996, Wu \& Horvitz, 1998a). We would suggest that phagocytosis of apoptotic cells shows characteristics that distinguish it from those involved in the clearance of opsonized particles, perhaps defining a novel 'type III' phagocytic mechanism. Further analysis of the basic phagocytic processes involved in the uptake of apoptotic cells will be required to identify the underlying control mechanisms.

\section{REGULATION OF PHAGOCYTOSIS OF APOPTOTIC CELLS}

Macrophage capacity for apoptotic cell phagocytosis is likely to be closely regulated in order that the tissue load of apoptotic cells is matched by appropriate clearance activity. Macrophage capacity for phagocytosis of apoptotic cells is influenced by soluble mediators, such as cytokines (Ren \& Savill, 1995), prostaglandins (Rossi et al, 1998) and glucocorticoids (Lui et al, 1999), and by adhesive interactions with the extracellular matrix (McCutcheon et al, 1998). After binding of ligand to cell surface receptors, intracellular signals are transduced that may influence the capacity of the macrophage to phagocytose apoptotic cells (Fig 4). The potentially important ligand-receptor interactions and intracellular signalling pathways that are involved in the regulation of phagocytosis of apoptotic cells are beginning to be identified. Furthermore, as mentioned previously, analysis of the proteins encoded by engulfment genes in $C$. elegans has provided additional clues relating to the role of intracellular signalling pathways and the cytoskeleton in the phagocytosis of apoptotic cells in mammals.

\section{SOLUBLE MEDIATORS}

Co-ordination of cellular behaviour during the inflammatory response involves a complex interplay of cytokines. It is perhaps not surprising that, in addition to the modulation of apoptotic pathways in leucocytes (Lee et al, 1993), incubation of human monocyte-derived macrophages with granulocyte-macrophage colony-stimulating factor (GMCSF), $\Pi-1 \beta$, interferon (IFN)- $\gamma$, tumour necrosis factor (TNF)- $\alpha$ or TGF- $\beta 1$ for $4 \mathrm{~h}$ was found to augment macrophage phagocytosis of apoptotic neutrophils significantly (Ren \& Savill, 1995). However, in rodent models, IFN- $\gamma$ appears to exert inhibitory effects upon the uptake of apoptotic neutrophils, suggesting possible species differences (Erwig et al, 1999). More recently, we have identified a novel role for glucocorticoids in 'reprogramming' the phagocyte for enhanced recognition and phagocytosis (Lui et al, 1999). One implication of these findings is that increased capacity for disposal of apoptotic cells may contribute to the powerful anti-inflammatory effects of glucocorticoids.

In contrast to the relatively slow effects of cytokines and glucocorticoids, transient elevation of cAMP and activation of protein kinase $\mathrm{A}$ after exposure to inflammatory mediators and pharmacological agents that stimulate adenylate cyclase (e.g. prostaglandins) rapidly and specifically inhibit macrophage phagocytosis of apoptotic neutrophils (Rossi

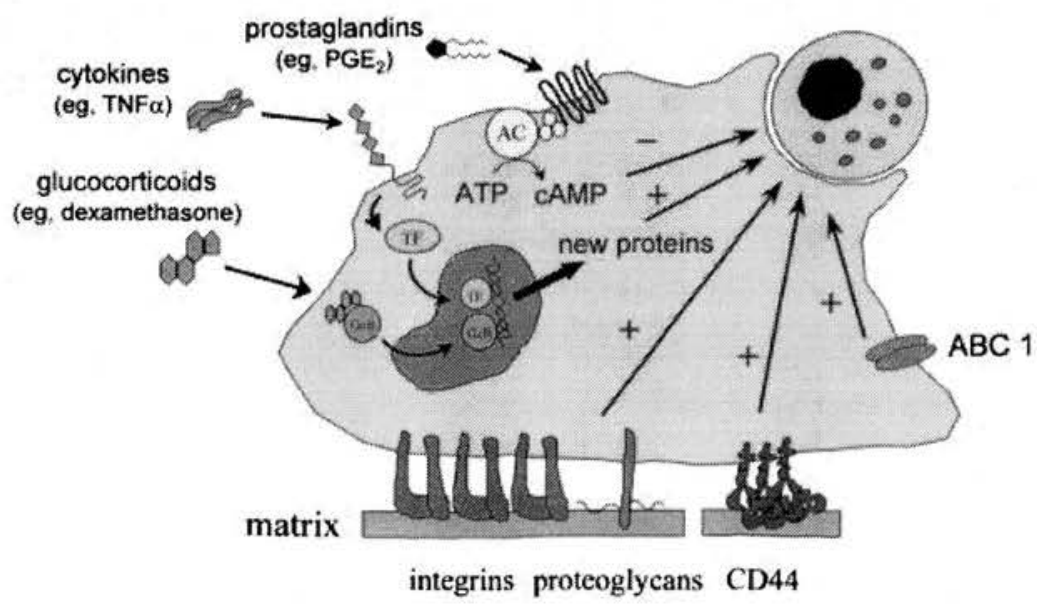

Fig 4. Regulation of macrophage phagocytic potential by soluble mediators, surface receptors and the concomitant intracellular signalling pathways (AC, adenylate cyclase; $\mathrm{ABC1}$, ATP binding cassette transporter 1; GcR, glucocorticoid receptor; TF, transcription factor). 
et al, 1998). Inhibition of macrophage phagocytic capacity for apoptotic cells persisted long after the intracellular cAMP concentration had fallen back to basal levels, implying that secondary signalling pathways had been initiated that ultimately determined phagocytic responses. Furthermore, activation of protein kinase $\mathrm{C}$ by phorbol esters enhanced macrophage phagocytosis of apoptotic neutrophils in vitro, while inhibition of tyrosine kinases with genestein had an inhibitory effect (unpublished observations). Together, these observations suggest that macrophage phagocytic capacity in the tissue may be dynamically 'fine-tuned' by the balance between the activity of different protein kinases.

\section{ADHESION MODULATION AND PHAGOCYTOSIS OF APOPTOTIC CELLS}

Phagocytosis shares mechanistic links with adhesion, spreading and migration, processes that require co-ordinated cytoskeletal reorganization, suggesting that regulatory factors may also have a role in the control of phagocytosis of apoptotic cells. Inhibition of apoptotic cell uptake after elevation of intracellular cAMP was associated with redistribution of cytoskeletal proteins, including actin, talin and paxillin, and profound morphological alterations (Rossi $e t$ al, 1998). In contrast, ligation of cell surface adhesion receptors by extracellular matrix (ECM) molecules leads to the assembly of cytoskeletal components and activation of intracellular signal transduction cascades (Giancotti, 1997), which, in concert with soluble chemical mediators, allows the behaviour of inflammatory cells to be co-ordinated (Nathan \& Sporn, 1991). Remodelling of the ECM and temporal alterations in the matrix composition after injury or infection (Alitalo et al, 1980; Gailit \& Clark, 1994) may therefore modulate the capacity for apoptotic cell phagocytosis during progression of the inflammatory response.

A precedent for the suggestion that adhesion receptor ligation may influence phagocyte capacity for clearance of apoptotic cells was provided by our demonstration that cross-linking of CD44 by bivalent antibodies rapidly (within minutes) augmented phagocytosis of apoptotic neutrophils by human monocyte-derived macrophages (Hart et al, 1997). CD44 is a cell surface glycoprotein that has been proposed to bind multiple ligands in the ECM (including hyaluronan and fibronectin) and has been implicated in cytoskeletal organization and intracellular signalling (Lesley et al, 1993; Tsukita et al, 1994; Galandrini et al, 1996; Taher et al, 1996). Previously defined inhibitors of apoptotic cell recognition did not affect CD44-augmented phagocytosis of apoptotic neutrophils, suggesting either that several pathways were engaged together or that unique molecular recognition pathways were involved. This latter possibility was supported by the finding that $\mathrm{CD} 44 \mathrm{mAb}$ failed to augment phagocytosis of apoptotic lymphocytes.

Although there is evidence that $\beta 1$ and $\beta 3$ integrins may directly mediate apoptotic cell recognition (Savill et al, 1990: Schwartz et al, 1999), integrins may have a broader role in the control of phagocytic processes. Generation of 'outside-in' signals via integrins allows 'sensing' of the local microenvironment and may influence phagocytic function.
In support of this suggestion, we have shown recently that adhesion of human monocyte-derived macrophages to fibronectin substantially augmented phagocytosis of apoptotic neutrophils (McCutcheon et al, 1998), an effect that was partially dependent on macrophage $\beta 1$ integrins. However, ligation of $\alpha_{v} \beta_{3}, \alpha_{1} \beta_{2}$ or $\alpha_{6} \beta_{1}$, after apoptotic cell uptake or with $\mathrm{mAb}$, was reported to inhibit subsequent phagocytosis when macrophages were rechallenged with apoptotic cells $48 \mathrm{~h}$ later (Erwig et al, 1999). Association of integrins with phosphatidylinositol 3-kinase (PI3K) (Lakkakorpi et al, 1997) or induction of tyrosine phosphorylation and activation of focal adhesion kinase-related molecule Pyk2 (Duong et al, 1998) after integrin-mediated binding of osteoclasts to matrix components illustrates how integrins might exert indirect effects.

It is interesting to note that other molecules implicated in apoptotic cell recognition have well-defined roles in adhesion processes. The macrophage scavenger receptor has a role in divalent cation-independent adhesion to serum-coated cell culture plastic (Fraser et al, 1993), and certain anti-CD36 mAbs may mimic many of the anti-adhesive effects of the ECM molecule thrombospondin (Dawson et al, 1997). Interestingly, CD36 may associate with tyrosine kinases Fyn, Yes and Lyn (Huang et al, 1991) and link directly with signal transduction cascades. Furthermore, ligation of cell surface molecules from diverse receptor families [e.g. urokinase-plasminogen activator receptor (CD87), integrinassociated protein (CD47), CD14, CD16, CD66 and CD31; Petty \& Todd, 1996; Stocks et al, 1996] act to regulate adhesion (Klemke et al, 1998; Schaffner-Reckinger et al, 1998). Further investigation into the role of indirect effects (e.g. via adhesion modulation) is required in order to understand the regulation of apoptotic cell phagocytosis. One of the challenges for future studies is to distinguish receptors that may act as accessory molecules (in a manner analogous to (CD11a/CD18) LFA-1 and ICAM-1 (CD54) in antigen presentation via the $\mathrm{T}$-cell receptor) from those that play a direct role in the recognition of apoptotic cells.

\section{APOPTOTIC CELL LIGANDS}

The final level of complexity concerning regulation of apoptotic cell uptake is the role of apoptotic cell ligands. Although it has been widely assumed that phagocytes are able to recognize alterations in the plasma membrane that distinguish apoptotic cells from their viable neighbours, it remains possible that phagocytes respond to a loss of signals that characterize interactions between viable cells. For example, in the absence of 'viable cell' signals, 'non-specific' phagocytosis mechanisms involved in the internalization of inert particles, such as latex microspheres or aldehyde-fixed cells, may be engaged. However, as discussed above, in contrast to apoptotic cell phagocytosis, uptake of inert particles elicits pro-inflammatory cytokine release in some phagocyte populations (Meagher et al, 1992). These data lend support to the concept that phagocytes have the capacity for recognition of determinants specific for apoptotic cells.

While a number of distinct phagocyte receptors involved in the recognition and subsequent internalization of dying 
cells have been identified, the determinants on the surface of the apoptotic cell that signal phagocyte recognition and removal remain poorly characterized. For the erythrocyte (which has an average lifespan of 120 days in the healthy adult human), post-translational modification of the anion transport protein band 3 may lead to binding of endogenous IgG autoantibodies and subsequent Fc receptor-mediated phagocytosis (Kay, 1981; Kay et al, 1996). In parallel, nonenzymatic glycosylation of surface proteins to form advanced glycosylation end-products (AGE) may allow the engagement of specific receptors (e.g. the immunoglobulin superfamily member RAGE; Schmidt et al, 1994) that trigger clearance. However, the kinetics of appearance of AGE on senescent cells is likely to be too slow to serve as a signal for apoptotic cell recognition by phagocytes. In addition, the patterns of inhibition of macrophage phagocytosis of apoptotic neutrophils using polyanionic ligands indicated that AGE and AGE receptors were unlikely to participate (Savill et al, 1989).

Recent data indicate that rapid redistribution of aminophospholipids, including PS (Martin et al, 1995) and phosphatidylethanolamine (Emoto et al, 1997), may represent a general feature of apoptosis in many cell types. In viable cells, PS is confined to the cytoplasmic face of the plasma membrane by the action of a phospholipid translocase and is specifically relocated (possibly via specific phospholipid scramblases) to the outer surface of the membrane during apoptosis (Verhoven et al, 1995). PS exposure has been implicated in the recognition of apoptotic leucocytes by mouse inflammatory macrophages, but not by human monocyte-derived macrophages, in vitro (Fadok et al, $1992 b$ ). Binding of the serum $\beta_{2}$-glycoprotein I to exposed PS residues on apoptotic thymocytes and lipid symmetric red blood cell ghosts may 'mark' them for uptake by both thioglycollate-elicited mouse peritoneal macrophages (Balasubramanian et al, 1997) and the human THP-1 macrophage cell line (Balasubramanian \& Schroit, 1998). Studies using the monocyte cell line THP-1 suggest that CD36 may act as a receptor for phosphatidylserine, with little or no specificity for other anionic phospholipids (phosphatidylinositol and phosphatidylglycerol). This activity appeared to involve sites in the receptor distinct from those involved in binding collagen or thrombospondin (Tait \& Smith, 1999). In addition, anti-oxLDL autoantibodies cloned from Apo-Edeficient mice specifically bound to apoptotic aortic endothelial cells and prevented phagocytosis by mouse peritoneal macrophages (Chang et al, 1999). It is therefore possible that scavenger receptor-mediated recognition of oxidized phospholipids and oxidized phospholipid protein complexes may provide a specific signal for phagocyte binding and recognition of damaged or apoptotic cells.

It has been reported recently that complement components $\mathrm{Clq}$ and $\mathrm{iC} 3 \mathrm{~b}$ bind to the surface of certain apoptotic cell types, facilitating their uptake by macrophage $\mathrm{Clq}$ receptors or CR3/CR4 (Takizawa et al, 1996; Korb \& Ahearn, 1997. Mevorach et al, 1998). These findings contrast with previous reports that CR3 was not involved in the recognition of apoptotic neutrophils by human monocyte-derived macrophages (Savill et al, 1989). Interestingly, cells in the late stages of apoptosis or blebs derived from apoptotic cells have been shown to contain self-antigens (Gilligan et al, 1996; Kalden, 1997), which are targets for autoantibodies found in autoimmune disease. One interpretation of these findings is that failure of tissue phagocytes to clear apoptotic cells may result in the development of autoimmune responses. Susceptibility of patients with $\mathrm{Clq}$ deficiency to the autoimmune disease systemic lupus erythematosus may be partly explained by the excess of free non-ingested apoptotic cells in the inflamed glomeruli seen in mice deficient in $\mathrm{Clq}$ (Botto et al, 1998).

Other molecular changes in the membrane of apoptotic cells have been studied using mAbs and lectins as probes. Early studies indicated that cellular microelectrophoretic mobility was reduced on apoptotic mouse thymocytes, suggestive of changes in the net negative charge of cell membranes (Morris et al, 1984). Reduced binding of lectins and $\mathrm{mAbs}$ observed in flow cytometric analysis corresponded closely with the degree of reduction in membrane surface area after apoptosis (Morris et al, 1984). It has been reported that high levels of mannose, $\mathrm{N}$-acetylgalactosamine and galactose can be detected on apoptotic human peripheral blood lymphocytes using fluoresceinated lectins (Dini $e t$ al, 1992). However, flow cytometric analysis of binding of a panel of conjugated lectins to apoptotic neutrophils suggests that exposure of these carbohydrate moieties may not be a general feature of apoptosis on all cell types (unpublished observations). Screening of a large panel of $m A b s$ revealed that blockade of certain epitopes of ICAM-3 (CD50) on apoptotic B cells was found to inhibit their phagocytosis by macrophages. These epitopes have been suggested as being associated with apoptotic cell-specific modifications of ICAM3 recognized by macrophage CD14 (Gregory et al, 1998; Moffatt et al, 1999). There is evidence that specific changes in surface protein expression accompany apoptosis (Dransfield et al, 1994, 1995), implying that mechanisms for the maintenance of receptor numbers are compromised during programmed cell death. Neutrophil apoptosis is associated with a marked downregulation of $\mathrm{Fc} \gamma \mathrm{RIII}$ (CD16) (Dransfield et al, 1994), leukosialin (CD43) and L-selectin (CD62 L) (Dransfield et al, 1995), which may provide a mechanism for functionally isolating apoptotic neutrophils from potentially pro-inflammatory signals. As these receptors are shed by proteolytic mechanisms during activation, one possibility is that there is activation of surface-associated proteases during apoptosis or, alternatively, release of functional constraints upon protease activity by as yet undefined mechanisms. Proteolytic shedding of surface receptors during apoptosis has been observed in myeloid cell lines (Brown et al, 1996), but whether this represents a fundamental feature of apoptosis in different cell types remains to be determined. One possibility is that the presence of proteolytic 'stubs' of shed receptors could contribute to a 'signal' for phagocytosis.

\section{CONCLUSION}

Phagocytosis of apoptotic cells is a vital process in cellular homeostasis in a wide range of tissues, but especially so in the inflammatory response in which apoptotic granulocytes 
have the potential to inflict tissue damage if they are not removed quickly and efficiently. Further work is required to identify cell surface molecules present on apoptotic cells that may trigger phagocyte recognition. Future research must resolve apparent differences in receptor usage in experimental models of apoptotic cell clearance in different species using different phagocyte and apoptotic cell populations. In addition, the role of macrophage receptors that may act as 'accessory' molecules that indirectly influence the recognition process needs to be defined. We would speculate that modulation of macrophage phagocytic capacity by a variety of cytokines, prostanoids, hormones, matrix molecules and possibly neighbouring cells may allow the tissue load of apoptotic cells to be matched by appropriate phagocyte clearance capacity, thereby minimizing release of histotoxic apoptotic cell contents. In contrast, phagocyte clearance capacity may be exceeded in pathological states. We suggest that therapeutic induction of cellular apoptosis to ameliorate tissue damage in inflammatory diseases, such as ARDS or rheumatoid arthritis, will require concurrent augmentation of phagocyte clearance capacity. Identification of the surface receptors involved in phagocyte recognition of apoptotic cells and definition of the contribution of regulatory influences in vivo will be important for the development of future therapeutic strategies for the treatment of inflammatory, allergic and autoimmune diseases.

\section{ACKNOWLEDGMENTS}

This work was supported by a University of Edinburgh Faculty of Medicine fellowship (to S.P.H.), the Wellcome Trust (K.M.G.) and the Medical Research Council.

The Rayne Laboratory, Respiratory Medicine Unit, University of Edinburgh Medical School,

Teviot Place,

Edinburgh EH8 9AG, UK.

\section{Katherine M. Giles SIMON P. HART Christopher Haslett Adriano G. Rossi IAN DRANSFIELD}

\section{REFERENCES}

Aderem, A.A., Wright, S.D., Silverstein, S.C. \& Cohn Z.A. (1985) Ligated complement receptors do not activate the arachidonic acid cascade in resident peritoneal macrophages. Journal of Experimental Medicine, 161, 617-622.

Alitalo, K., Hovi, T. \& Vaheri A. (1980) Fibronectin is produced by human macrophages. Journal of Experimental Medicine, 151, 602613.

Allen, L.A. \& Aderem A. (1996) Molecular definition of distinct cytoskeletal structures involved in complement- and Fc receptormediated phagocytosis in macrophages. Journal of Experimental Medicine, 184, 627-637.

Allen, W.E., Jones, G.E., Pollard, J.W. \& Ridley A.J. (1997) Rho, Rac and $\mathrm{Cdc} 42$ regulate actin organization and cell adhesion in macrophages. Journal of Cell Science, 110, 707-720.

Balasubramanian, K. \& Schroit A.J. (1998) Characterization of phosphatidylserine dependent $\beta_{2}$-glycoprotein I macrophage interactions. Journal of Biological Chemistry. 273, 29272-29277.

Balasubramanian, K., Chandra, J. \& Schroit A.J (1997) Immune clearance of phosphatidylserine-expressing cells by phagocytes. Journal of Biological Chemistry, 272, 31113-31117.

Bennett, M.R., Gibson, D.F., Schwartz, S.M. \& Tait J.F. (1995) Binding and phagocytosis of apoptotic vascular smooth muscle cells is mediated in part by exposure of phosphatidylserine. Circulation Research, 77, 1136-1142.

Bird, D.A., Gillotte, K.L., Horkko, S., Freidman, P., Dennis, E.A., Witztum, J.L. \& Steinberg D. (1999) Receptors for oxidised low-density lipoprotein on elicited mouse peritoneal macrophages can recognise both the modified lipid moieties and the modified protein moieties: implications with respect to macrophage recognition of apoptotic cells. Proceedings of the National Academy of Science USA, 96, 6347-6352.

Botto, M., Dell'Agnola, C., Bygrave, A.E., Thompson, E.M., Cook, H.T., Petry, F., Loos, M., Pandolfi, P.P. \& Walport M.J. (1998) Homozygous $\mathrm{Clq}$ deficiency causes glomerulonephritis associated with multiple apoptotic bodies. Nature Genetics, 19, 56-59.

Brown, S.B. \& Savill J. (1999) Phagocytosis triggers macrophage release of Fas ligand and induces apoptosis of bystander leukocytes. Journal of Immunology, 162, 480-485.

Brown, S.B., Kluck. R.M. \& Ellem K.A. (1996) Loss and shedding of surface markers from the leukemic myeloid monocytic line THP-1 induced to undergo apoptosis. Journal of Cellular Biochemistry. 60, 246-259.

Caron, B. \& Hall A. (1998) Identification of two distinct mechanisms of phagocytosis controlled by different Rho GTPases. Science, 282, 1717-1721.

Chang, M.K., Bergmark, C., Laurila, A., Horkko, S., Han, K.H., Friedman, P., Dennis, E.A. \& Witztum J.L. (1999) Monoclonal antibodies against oxidised low density lipoprotein bind to apoptotic cells and inhibit their phagocytosis by elicited macrophages: evidence that oxidation-specific epitopes mediate macrophage recognition. Proceedings of the National Academy of Science USA, 96, 6353-6358.

Cohen J.J. (1991) Programmed cell death in the immune system. Advances in Immunology, 50, 55-85.

Cox, D., Tseng, C.C., Bjekic, G. \& Greenberg S. (1999) A requirement for phosphatidylinositol 3-kinase in pseudopod extension. Journal of Biological Chemistry, , 274, 1240-1247.

Crawford, S.E., Stellmach, V., Murphy-Ullrich, J.E., Ribeiro, S.M., Lawler, J., Hynes, R.O., Boivin, G.P. \& Bouck N. (1998) Thrombospondin-1 is a major activator of TGF-betal in vivo. Cell, 93, 1159-1170.

Dawson, D.W., Pearce, S.F., Zhong, R., Silverstein, R.L., Frazier, W.A. \& Bouck N.P. (1997) CD36 mediates the in vitro inhibitory effects of thrombospondin-1 on endothelial cells. Journal of Cell Biology. 138, 707-717.

Debets, J.M., Van der Linden, C.J., Dieteren, I.E., Leeuwenberg, J.F. \& Buurman W.A. (1998) Fc-receptor cross-linking induces rapid secretion of tumor necrosis factor (cachectin) by human peripheral blood monocytes. Journal of Immunology, 141, 1197-1201.

Devitt, A., Moffatt, O.D., Raykundalia, C., Capra, J.D., Simmons, D.L. \& Gregory C.D. (1998) Human CD14 mediates recognition and phagocytosis of apoptotic cells. Nature, 392, 505-509.

Dini, L., Autuori, E., Lentini, A., Oliverio, S. \& Piacentini M. (1992) The clearance of apoptotic cells in the liver is mediated by the asialoglycoprotein receptor. FEBS Letters, 296, 174-178.

Dini, L., Lentini, A., Diez, G., Rocha, M., Falasca, L., Serafino, L. \& Vidal-Vanaclocha F. (1995) Phagocytosis of apoptotic bodies by liver endothelial cells. Journal of Cell Science, 108, 967-973.

Dransfield, I., Buckle, A.-M., Savill, J.S., McDowall, A., Haslett, C. \& Hogg N. (1994) Neutrophil apoptosis is associated with a reduction in CD16 (FcrRIII) expression. Journal of Immunology. 153, 1254-1263. 
Dransfield, I., Stocks, S.C. \& Haslett C. (1995) Regulation of cell adhesion molecule expression and function associated with neutrophil apoptosis. Blood, 85, 3264-3273.

Duong, L.T., Lakkakorpi, P.T., Nakamura, I., Machwate, M., Nagy, R.M. \& Rodan G.A. (1998) PYK2 in osteoclasts is an adhesion kinase, localized in the sealing zone, activated by ligation of alphavbeta3 integrin, and phosphorylated by src kinase. Journal of Clinical Investigation, 102, 881-892.

Duvall, E., Wyllie, A.H. \& Morris R.G. (1985) Macrophage recognition of cells undergoing programmed cell death (apoptosis). Immunology, 56, 351-358.

Ellis, R.E., Yuan, J. \& Horvitz H.R. (1991) Mechanisms and functions of cell death. Annual Review of Cell Biology, 7, 663-690.

Emoto, K., Toyama-Sorimachi, N., Karasuyama, H., Inoue, K. \& Umeda M. (1997) Exposure of phosphatidylethanolamine on the surface of apoptotic cells. Experimental Cell Research, 232, 430434

Erwig, L.P., Gordon, S., Walsh, G.M. \& Rees A.J. (1999) Previous uptake of apoptotic neutrophils or ligation of integrin receptors downmodulates the ability of macrophages to ingest apoptotic neutrophils. Blood, 93, 1406-1412.

Fadok, V.A., Savill, J.S., Haslett, C., Bratton, D.L., Doherty, D., Campbell, P.A. \& Henson P.M. (1992a) Different populations of macrophages use either the vitronectin receptor or the phosphatidylserine receptor to recognize and remove apoptotic cells. Journal of Immunology, 149, 4029-4035.

Fadok, V.A., Voelker, D.R., Campbell, P.A., Cohen, J.J., Bratton, D.L. \& Henson P.M. (1992b) Exposure of phosphatidylserine on the surface of apoptotic lymphocytes triggers specific recognition and removal by macrophages. Journal of Immunology, 148, 22072216.

Fadok, V.A., Laszlo, D.J., Noble, P.W., Weinstein, L., Riches, D.W.H. \& Henson P.M. (1993) Particle digestibility is required for induction of the phosphatidylserine recognition mechanism used by murine macrophages to phagocytose apoptotic cells. Journal of Immunology, 151, 4274-4285.

Fadok, V.A., Bratton, D.L., Konowal, A., Freed, P.W., Westcott, J.Y. \& Henson P.M. (1998) Macrophages that have ingested apoptotic cells in vitro inhibit proinflammatory cytokine production through autocrine/paracrine mechanisms involving TGF- $\beta$, $\mathrm{PGE}_{2}$, and PAF. Journal of Clinical Investigation, 101, 890-898.

Falasca, L., Bergamini, A., Serafino, L., Balabaud, C. \& Dini L. (1996) Human Kupffer cell recognition and phagocytosis of apoptotic peripheral blood lymphocytes. Experimental Cell Research, 224, 152-162.

Flora, P.K. \& Gregory G.D. (1994) Recognition of apoptotic cells by human macrophages: inhibition by a monocyte/macrophagespecific monoclonal antibody. European Journal of Immunology, 24, $2625-2632$

Fraser, I., Hughes, D. \& Gordon S. (1993) Divalent cationindependent macrophage adhesion inhibited by monoclonal antibody to murine scavenger receptor. Nature, 364, 343-346.

Gailit, J. \& Clark, R.A.F. (1994) Wound repair in the context of extracellular matrix. Current Opinion in Cell Biology. 6, 717-725.

Galandrini, R., Piccoli, M., Frati, L. \& Santoni A. (1996) Tyrosine kinase-dependent activation of human NK cell functions upon triggering through CD44 receptor. European Journal of Immunology. 26, 2807-2811.

Giancotti F.G. (1997) Integrin signaling: specificity and control of cell survival and cell cycle progression. Current Opinion in Cell Biology, 9, 691-700.

Gilligan, H.M., Bredy, B., Brady, H.R., Hebert, M.J., Slayter, H.S., Xu, Y., Rauch, J., Shia, M.A., Koh, J.S. \& Levine J.S. (1996) Antineutrophil cytoplasmic autoantibodies interact with primary granule constituents on the surface of apoptotic neutrophils in the absence of neutrophil priming. Journal of Experimental Medicine, 184, 2231-2240.

Gregory, C.D., Devitt, A. \& Moffatt O. (1998) Roles of ICAM-3 and CD14 in the recognition and phagocytosis of apoptotic cells by macrophages. Biochemical Society Transactions, 26, 644-649.

Griffin, E.M.J., Griffin, J.A., Leider, J.E. \& Silverstein S.C. (1975) Studies on the mechanism of phagocytosis. I. Requirements for circumferential attachment of particle-bound ligands to specific receptors on the macrophage plasma membrane. Journal of Experimental Medicine, 142, 1263-1282.

Hagimoto, N., Kuwano, K., Miyazaki, H., Kunitake, R., Fujuti, M. Kawasaki, M., Kaneko, Y. \& Hara N. (1997) Induction of apoptosis and pulmonary fibrosis in mice in response to ligation of Fas antigen. American Journal of Respiratory Cell and Molecular Biology $17,272-278$.

Hall, S.E., Savill, J.S., Henson, P.M. \& Haslett C. (1994) Apoptotic neutrophils are phagocytosed by fibroblasts with participation of the fibroblast vitronectin receptor and involvement of a mannose/ fucose-specific lectin. Journal of Immunology, 153, 3218-3227.

Han, H., Iwanaga, T., Uchiyama, Y. \& Fujuti T. (1993) Aggregation of macrophages in the tips of intestinal villi in guinea pigs: their possible role in the phagocytosis of effete epithelial cells. Cell and Tissue Research , 271, 407-416.

Hart, S.P., Haslett, C. \& Dransfield I. (1996) Recognition of apoptotic cells by phagocytes. Experientia, 52, 950-956.

Hart, S.P., Dougherty, G.J., Haslett, C. \& Dransfield I. (1997) CD44 regulates phagocytosis of apoptotic neutrophil granulocytes, but not apoptotic lymphocytes, by human macrophages. Journal of Immunology, 159, 919-925.

Hasegawa, H., Kiyokawa, E., Tanaka, S., Nagashima, K., Gotoh, N. Masabumi, S., Kurata, T. \& Matsuda M. (1996) DOCK180, a major CRK-binding protein, alters cell morphology upon translocation to the cell membrane. Molecular and Cellular Biology, 16, 17701776.

Haslett, C., Savill, J.S., Whyte, M.K., Stern, M., Dransfield, I. \& Meagher L.C. (1994) Granulocyte apoptosis and the control of inflammation. Philosophical Transactions of the Royal Society of London Series B Biological Science, 345, 327-333.

Hebert, M.J., Takano, T., Holthofer, H. \& Brady H.R. (1996) Sequential morphologic events during apoptosis of human neutrophils. Modulation by lipoxygenase-derived eicosanoids. Journal of Immunology, 157, 3105-3115.

Hengartner M.O. (1996) Programmed cell death in invertebrates. Current Opinion in Genetics and Development, 6, 34-38.

Hess, K.L., Tudor, K.S., Johnson, J.D., Osati-Ashtiani, K., Askew, D.S. \& Cook-Mills J.M. (1997) Human and murine high endothelial venule cells phagocytose apoptotic leukocytes. Experimental Cell Research, 236, 404-411.

Hopkinson-Woolley, J., Hughes, D., Gordon, S. \& Martin P. (1994) Macrophage recruitment during limb development and wound healing in the embryonic and foetal mouse. Journal of Cell Science. 107, 1159-1167.

Huang, M.M., Bolen, J.B., Barnwell, J.W., Shattil, S.J. \& Brugge J.S (1991) Membrane glycoprotein IV (CD36) is physically associated with the Fyn, Lyn, and Yes protein-tyrosine kinases in human platelets. Proceedings of the National Academy of Science USA, , 88 7844-7848.

Hughes, J., Liu, Y., Van Damme, J. \& Savill J. (1997) Human glomerular mesangial cell phagocytosis of apoptotic neutrophils: mediation by a novel CD36-independent vitronectin receptor/ thrombospondin recognition mechanism that is uncoupled from chemokine secretion. Journal of Immunology, 158, 4389-4397.

Kalden J.R. (1997) Defective phagocytosis of apoptotic cells: possible 
explanation for the induction of autoantibodies in SLE. Lupus, $\mathbf{6}$, 326-327.

Kaplan G. (1977) Differences in the mode of phagocytosis with Fc and C3 receptors in macrophages. Scandinavian Journal of Immunology, 6, 797-807.

Kay, M.M.B. (1981) Isolation of the phagocytosis-inducing IgGbinding antigen on senescent somatic cells. Nature, 289, 491494.

Kay, M.M., Rapcsak, S.Z., Bosman, G.J. \& Goodman J.R. (1996) Posttranslational modifications of brain and erythrocyte band 3 during aging and disease. Cellular and Molecular Biology 42, 919-944.

Kerr, J.F.R., Wyllie, A.H. \& Currie A.R. (1972) Apoptosis: a basic biological phenomenon with wide-ranging implications in tissue kinetics. British Journal of Cancer, 26, 239-257.

Klemke, R.L., Leng, J., Molander, R., Brooks, P.C., Vuori, K. \& Cheresh D.A. (1998) CAS/Crk coupling serves as a 'molecular switch' for induction of cell migration. Journal of Cell Biology. 140, 961-972.

Korb, L.C. \& Ahearn J.M. (1997) Clq binds directly and specifically to surface blebs of apoptotic human keratinocytes: complement deficiency and systemic lupus erythematosus revisited. Journal of Immunology, 158, 4525-4528.

Lakkakorpi, P.T., Wesolowski, G., Zimolo, Z., Rodan, G.A. \& Rodan S.B. (1997) Phosphatidylinositol 3-kinase association with the osteoclast cytoskeleton, and its involvement in osteoclast attachment and spreading. Experimental Cell Research, 237, 296-306.

Lee, A., Whyte, M.K. \& Haslett C. (1993) Inhibition of apoptosis and prolongation of neutrophil functional longevity by inflammatory mediators. Journal of Leukocyte Biology 54, 283-288.

Lesley, J., Hyman, R. \& Kincade P.W. (1993) CD44 and its interaction with the extracellular matrix. Advances in Immunologu 54, 271-335.

Liu, Q.A. \& Hengartner M.O. (1998) Candidate adaptor protein CED6 promotes the engulfment of apoptotic cells in C. elegans. Cell, 93, 961-972.

Luciani, M.F. \& Chimini G. (1996) The ATP binding cassette transporter $\mathrm{ABCl}$, is required for the engulfment of corpses generated by apoptotic cell death. European Molecular Biology Organization Journal, 15, 226-235.

Lui, Y., Cousin, J.M., Hughes, J., Van Damme, J., Seckl, J.R., Haslett, C., Dransfield, I., Savill, J. \& Rossi A.G. (1999) Glucocorticoids promote non-phlogistic phagocytosis of apoptotic leukocytes. Journal of Immunology, 162, 3639-3646.

McCutcheon, J.C., Hart, S.P., Canning, M., Ross, K., Humphries, M.J. \& Dransfield I. (1998) Regulation of macrophage phagocytosis of apoptotic cells by adhesion to fibronectin. Journal of Leukocyte Biology, 64, 600-607.

Marchisio, P.C., Cirillo, D., Teti, A., Zambonin-Zallone, A. \& Tarone G. (1987) Rous sarcoma virus-transformed fibroblasts and cells of monocytic origin display a peculiar dot-like organization of cytoskeletal proteins involved in microfilament-membrane interactions. Experimental Cell Research, 169, 202-214.

Martin, S.J., Reutelingsperger, C.P.M., McGahon, A.J., Rader, J.A., van Schie, R.C.A.A., LaFace, D.M. \& Green D.R. (1995) Early redistribution of plasma membrane phosphatidylserine is a general feature of apoptosis regardless of the initiating stimulus: inhibition by overexpression of bcl-2 and Abl. Journal of Experimental Medicine, 182, 1545-1556.

Maxeiner, H., Husemann, J., Thomas, C.A., Loike, J.D., El Khoury, J. \& Silverstein S.C. (1998) Complementary roles for scavenger receptor $\mathrm{A}$ and $\mathrm{CD} 36$ of human monocyte-derived macrophages in adhesion to surfaces coated with oxidized low-density lipoproteins and in secretion of $\mathrm{H}_{2} \mathrm{O}_{2}$. Journal of Experimental Medicine, 188, 2257-2265.

Meagher, L.C., Savill, J.S., Baker, A., Fuller, R.W. \& Haslett C. (1992)
Phagocytosis of apoptotic neutrophils does not induce macrophage release of thromboxane $\mathrm{B}_{2}$. Journal of Leukocyte Biology, 52, 269-273.

Mevorach, D., Mascarenhas, J.O., Gershov, D. \& Elkon K.B. (1998) Complement-dependent clearance of apoptotic cells by human macrophages. Journal of Experimental Medicine, 188 2313-2320

Moffatt, O.D., Devitt, A., Bell, E., Simmons, D.L. \& Gregory C.D. (1999) Macrophage recognition of ICAM-3 on apoptotic leukocytes. Journal of Immunology. 162, 6800-6810.

Morris, R.G., Hargreaves, A.D., Duvall, E. \& Wyllie A.H. (1984) Hormone-induced cell death. 2. Surface changes in thymocytes undergoing apoptosis. American Journal of Pathology. 115, 426436.

Nathan, C. \& Sporn M. (1991) Cytokines in context. Journal of Cell Biology, 113, 981-986.

Newman, S.L., Mikus, L.K. \& Tucci M.A. (1991) Differential requirements for cellular cytoskeleton in human macrophage complement receptor and $\mathrm{Fc}$ receptor-mediated phagocytosis. Journal of Immunology, 146, 967-974.

Ogasawara, J., Watanabe-Fukunaga, R., Adachi, M., Matsuzawa, A., Kasugai, T., Kitamura, Y., Itoh, N., Suda, T. \& Nagata S. (1993) Lethal effect of the anti-Fas antibody in mice. Nature, 364, 806809.

Oka, K., Sawamura, T., Kikuta, K.-I., Itokawa, S., Kume, N., Kita, T. \& Masaki T. (1998) Lectin-like oxidised low-density lipoprotein receptor 1 mediates phagocytosis of aged/apoptotic cells in endothelial cells. Proceedings of the National Academy of Science USA, 95, 9535-9540.

Petty, H.R. \& Todd R.F. (1996) Integrins as promiscuous signal transduction devices. Immunology Today. 17, 209-212.

Platt, N., da Silva, R.P. \& Gordon S. (1998a) Recognizing death: the phagocytosis of apoptotic cells. Trends in Cell Biology, 8, 365-372.

Platt, N., da Silva, R.P. \& Gordon S. (1998b) Class A scavenger receptors and the phagocytosis of apoptotic cells. Biochemical Society Transactions, 26, 639-644.

Platt, N., Suzuki, H., Kurihara, Y., Kodama, T. \& Gordon S. (1996) Role for the class A macrophage scavenger receptor in the phagocytosis of apoptotic thymocytes in vitro. Proceedings of the National Academy of Science USA, 93, 12456-12460.

Pradhan, D., Krahling, S., Williamson, P. \& Schlegel R.A. (1997) Multiple systems for recognition of apoptotic lymphocytes by macrophages. Molecular Biology of the Cell, 8, 767-778.

Ren, Y. \& Savill J.S. (1995) Proinflammatory cytokines potentiate thrombospondin-mediated phagocytosis of neutrophils undergoing apoptosis. Journal of Immunology, 154, 2366-2374.

Ren, Y., Silverstein, R.L., Allen, J. \& Savill J.S. (1995) CD36 gene transfer confers capacity for phagocytosis of cells undergoing apoptosis. Journal of Experimental Medicine, 181, 1857-1862.

Rossi, A.G., McCutcheon, J.C., Roy, N., Chilvers, E.R., Haslett, C. \& Dransfield I. (1998) Regulation of macrophage phagocytosis of apoptotic cells by cAMP. Journal of Immunology, 160, 3562-3568. Rubartelli, A., Poggi, A. \& Zoggi M.R. (1997) The selective engulfment of apoptotic bodies by dendritic cells is mediated by the $\alpha v \beta 3$ integrin and requires intracellular and extracellular calcium. European Journal of Immunology, 27, 1893-1900.

Savill, J.S., Henson, P.M. \& Haslett C. (1989) Phagocytosis of aged human neutrophils by macrophages is mediated by a novel 'charge-sensitive' recognition mechanism. Journal of Clinical Investigation, 84, 1518-1527.

Savill, J.S., Dransfield, I., Hogg, N. \& Haslett C. (1990) Vitronectin receptor-mediated phagocytosis of cells undergoing apoptosis. Nature, 342, 170-173.

Savill, J.S., Hogg, N., Ren, Y. \& Haslett C. (1992) Thrombospondin 


\section{Review}

cooperates with $\mathrm{CD} 36$ and the vitronectin receptor in macrophage recognition of neutrophils undergoing apoptosis. Journal of Clinical Investigation, 90, 1513-1522.

Savill, J.S., Fadok, V.A., Henson, P.M. \& Haslett C. (1993) Phagocyte recognition of cells undergoing apoptosis. Immunology Today. 14, 131-136.

Schaffner-Reckinger, E., Gouon, V., Melchior, C., Plancon, S. \& Kieffer N. (1998) Distinct involvement of beta3 integrin cytoplasmic domain tyrosine residues 747 and 759 in integrin-mediated cytoskeletal assembly and phosphotyrosine signaling. Journal of Biological Chemistry, 273, 12623-12632.

Schmidt, A.M., Mora, R., Cao, R., Yan, S.D., Brett, J., Ramakrishnan, R., Tsang, T.C., Simionescu, M. \& Stern D. (1994) The endothelial cell binding site for advanced glycation end products consists of a complex: an integral membrane protein and a lactoferrin-like polypeptide. Journal of Biological Chemistry, 269, 9882-9888.

Scholl, P.R., Ahern, D. \& Geha R.S. (1992) Protein tyrosine phosphorylation induced via the $\mathrm{IgG}$ receptors $\mathrm{Fc}$ gamma $\mathrm{Ri}$ and Fc gamma RII in the human monocytic cell line THP-1. Journal of Immunology, 149, 1751-1757.

Schwartz, B.R., Karsan, A., Bombeli, T. \& Harlan J.M. (1999) A novel $\beta_{1}$-integrin-dependent mechanism of leukocyte adherence to apoptotic cells. Journal of Immunology, 162, 4842-4842.

Shiratsuchi, A., Umeda, M., Ohba, Y. \& Nakanishi Y. (1997) Recognition of phosphatidylserine on the surface of apoptotic spermatogenic cells and subsequent phagocytosis by Sertoli cells of the rat. Journal of Biological Chemistry. 272, 2354-2358.

Stern, M., Savill, J.S. \& Haslett C. (1996) Human monocyte-derived macrophage phagocytosis of senescent eosinophils undergoing apoptosis: mediation by $\alpha_{v} \beta_{3} / \mathrm{CD} 36 /$ thrombospondin recognition mechanism and lack of phlogistic response. American Journal of Pathology, 149, 911-921.

Stocks, S.C., Ruchaud-Sparagano, M.H., Kerr, M.A., Grunert, F., Haslett, C. \& Dransfield I. (1996) CD66: role in the regulation of neutrophil effector function. European Journal of Immunology, 26, 2924-2932

Taher, T.E.I., Smit, L., Griffioen, A.W., Schilder-Tol, E.J.M., Borst, J. \& Pals S.T. (1996) Signaling through CD44 is mediated by tyrosine kinases. Journal of Biological Chemistry, 271, 2863-2867.

Tait, J. \& Smith C. (1999) Phosphatidylserine receptors: role of CD36 in binding of anionic phospholipid vesicles to monocytic cells. Journal of Biological Chemistry, 274, 3048-3054.

Takizawa, F., Tsuji, S. \& Nagasawa S. (1996) Enhancement of macrophage phagocytosis upon $\mathrm{iC} 3 \mathrm{~b}$ deposition on apoptotic cells FEBS Letters, 397, 269-272.

Tsukita, S., Oishi, K., Sato, N., Sagara, J., Kawai, A. \& Tsukita S. (1994) ERM family members as molecular linkers between the cell surface glycoprotein CD44 and actin-based cytoskeletons. Journal of Cell Biology. 126, 391-401.

Verhoven, B., Schlegel, R.A. \& Williamson P. (1995) Mechanisms of phosphatidylserine exposure, a phagocyte recognition signal, on apoptotic T lymphocytes. Journal of Experimental Medicine, 182, 1597-1601.

Vermes, I., Haanen, C., Richel, D.J., Schaafsma, M.R., KalsbeekBatenburg, E. \& Reutelingsperger C.P. (1997) Apoptosis and secondary necrosis of lymphocytes in culture. Acta Haematologica, 98, 8-13.

Voll, R.E., Herrmann, M., Roth, E.A., Stach, C., Kalden, J.R. \& Girkontaite I. (1997) Immunosuppressive effects of apoptotic cells (letter). Nature, 390, 350-351.

Weiss S.J. (1989) Mechanisms of disease: tissue destruction by neutrophils. New England Journal of Medicine, 320, 365-376.

Whyte, M.K.B., Meagher, L.C., MacDermot, J. \& Haslett C. (1993) Impairment of function in aging neutrophils is associated with apoptosis. Journal of Immunology, 150, 5124-5134.

Woolley, K.L., Gibson, P.G., Carty, K., Wilson, A.J., Twaddell, S.H. \& Woolley M.J. (1996) Eosinophil apoptosis and the resolution of airway inflammation in asthma. American Journal of Respiratory and Critical Care Medicine, 154, 237-243.

Wright, S.D. \& Silverstein S.C. (1983) Receptors for C3b and C3bi promote phagocytosis but not the release of toxic oxygen from human phagocytes. Journal of Experimental Medicine, 158, 20162023.

Wu, Y.C. \& Horvitz H.R. (1998a) The C. elegans cell corpse engulfment gene ced-7 encodes a protein similar to $\mathrm{ABC}$ transporters. Cell, 93, 951-960.

Wu, Y.C. \& Horvitz H.R. (1998b) C. elegans phagocytosis and cellmigration protein CED-5 is similar to human DOCK180. Nature, 392, 501-504.

Yamamoto, K. \& Johnston, R.B. Jr. (1984) Dissociation of phagocytosis from stimulation of the oxidative metabolic burst in macrophages. Journal of Experimental Medicine, 159, 405-416.

Keywords: apoptosis, phagocytosis, macrophage, inflammation, regulation. 


\section{Nitric oxide: a key regulator of myeloid inflammatory cell apoptosis}

\section{EL Taylor ${ }^{\star, 1}$, IL Megson ${ }^{2}$, C Haslett ${ }^{1}$ and AG Rossi ${ }^{1}$}

1 Centre for Inflammation Research, Rayne Laboratory, University of Edinburgh, Medical School, Teviot Place, Edinburgh EH8 9AG, UK

2 Centre for Cardiovascular Science, University of Edinburgh, Hugh Robson Building, George Square, Edinburgh EH8 9XD, UK

* Corresponding author: EL Taylor, Centre for Inflammation Research, Rayne Laboratory, University of Edinburgh, Medical School, Teviot Place, Edinburgh EH8 9AG, UK. Tel.: +44 131651 1323; Fax: +44 131650 4384;

E-mail: e.l.taylor-2@sms.ed.ac.uk

Received 30.4.02; revised 29.8.02; accepted 2.9.02 Edited by G. Melino

\begin{abstract}
Apoptosis of inflammatory cells is a critical event in the resolution of inflammation, as failure to undergo this form of cell death leads to increased tissue damage and exacerbation of the inflammatory response. Many factors are able to influence the rate of apoptosis in neutrophils, eosinophils, monocytes and macrophages. Among these is the signalling molecule nitric oxide (NO), which possesses both anti- and proapoptotic properties, depending on the concentration and flux of NO, and also the source from which NO is derived. This review summarises the differential effects of NO on inflammatory cell apoptosis and outlines potential mechanisms that have been proposed to explain such actions.

Cell Death and Differentiation (2003) 10, 418-430. doi:10.1038/ sj.cdd. 4401152
\end{abstract}

Keywords: nitric oxide; apoptosis; neutrophil; eosinophil; monocyte; macrophage

Abbreviations: NO, nitric oxide; NOS, nitric oxide synthase; SNP, sodium nitroprusside; SPER/NO, spermine diazeniumdiolate; DEA/NO, diethylamine diazeniumdiolate; DETA/NO, diethylenetriamine diazeniumdiolate; SIN-1, 3-morpholinosydnonimine; $\mathrm{O}_{2}^{-}$, superoxide anion; $\mathrm{ONOO}^{-}$, peroxynitrite; GEA 3162, 1,2,3,4-oxatriazolium, 5-amino-3-(3,4-dichorophenyl)-chloride; LPS, lipopolysaccharide; IFN, interferon; GSNO, $\mathrm{S}$-nitrosoglutathione; SNAP, S-nitroso- $\mathrm{N}$-acetylpenicillamine; SOD, superoxide dismutase; GSH, glutathione; PS, phosphatidylserine; SNOC, S-nitrosocysteine; IL, interleukin; cGMP, cyclic guanosine monophosphate; sGC, soluble guanylate cyclase; GC, glucocorticoid; cAMP, cyclic adenosine monophosphate; NF- $\mathrm{kB}$, nuclear factor kappa B; MPTP, mitochondrial permeability transition pore

\section{Introduction}

The free radical nitric oxide (NO) was first discovered as an endogenous vasodilator released from the endothelium to regulate vascular tone. ${ }^{1}$ However, it is now known that $\mathrm{NO}$ is a key mediator in a great number of physiological and pathophysiological processes (see Quinn). ${ }^{2}$ This ubiquitous signalling molecule can regulate the rate of apoptosis, or programmed cell death, in many cell types, including human inflammatory cells. Whether or not cells undergo apoptosis depends on the net balance of a large number of pro- versus antiapoptotic factors. Studies have revealed that NO has both pro and antiapoptotic properties, depending largely on the concentration and flux of NO, and the cell type under scrutiny (for reviews, see Nicotera et al. ${ }^{3}$ and Kim et al. $\left.{ }^{4}\right)$. It has been proposed that low concentrations of NO, derived from constitutively active endothelial and neuronal isoforms of NO synthase (eNOS and nNOS), usually have a protective effect on cells whereas higher concentrations derived from the inducible isoform (iNOS) are more likely to drive cell death. ${ }^{3}$

In stark contrast to cells undergoing necrosis, apoptotic inflammatory cells fail to release their proinflammatory and histotoxic contents. ${ }^{5}$ Furthermore, their clearance by professional phagocytes such as macrophages occurs via a nonphlogistic mechanism, which additionally aids the resolution of the inflammatory response (see Figure 1). ${ }^{6}$ Thus, apoptosis is generally regarded to be noninflammatory and is crucial for the successful resolution of inflammation. Chronic inflammatory conditions are frequently characterised by an apparent failure of myelocytic inflammatory cells to respond to apoptotic stimuli, or of phagocytes to remove apoptotic cells. Persistence of these cells leads to damage of the surrounding tissue and exacerbation of inflammation, as cells ultimately undergo either primary or secondary (following failed clearance of apoptotic cells) necrosis, an extremely proinflammatory form of cell death (see Figure 1). ${ }^{5,7}$ Manipulation of the rate of apoptosis in critical inflammatory effector cells, such as neutrophils, eosinophils, monocytes and macrophages, could therefore be of therapeutic benefit. ${ }^{7} \mathrm{NO}$ is capable of inducing inflammatory cell apoptosis and also possesses several other anti-inflammatory properties, including direct downregulation of leukocyte functions, such as neutrophil and monocyte adhesion, and neutrophil chemotaxis, degranulation and superoxide anion $\left(\mathrm{O}_{2}^{-}\right)$generation..$^{8-10}$ It also acts to maintain the impermeable nature of the vascular endothelium to leukocytes. ${ }^{9}$ Thus, manipulation of NO concentrations is a particularly promising candidate to alter leukocyte function and rates of apoptosis in inflammatory conditions.

A number of different $\mathrm{NO}$ donor classes have been utilised to investigate the role of $\mathrm{NO}$ and NO-related species in apoptotic processes. These fall into several broad categories defined by the species derived and the metabolic process 


\section{NON-INFLAMMATORY PATHWAY}

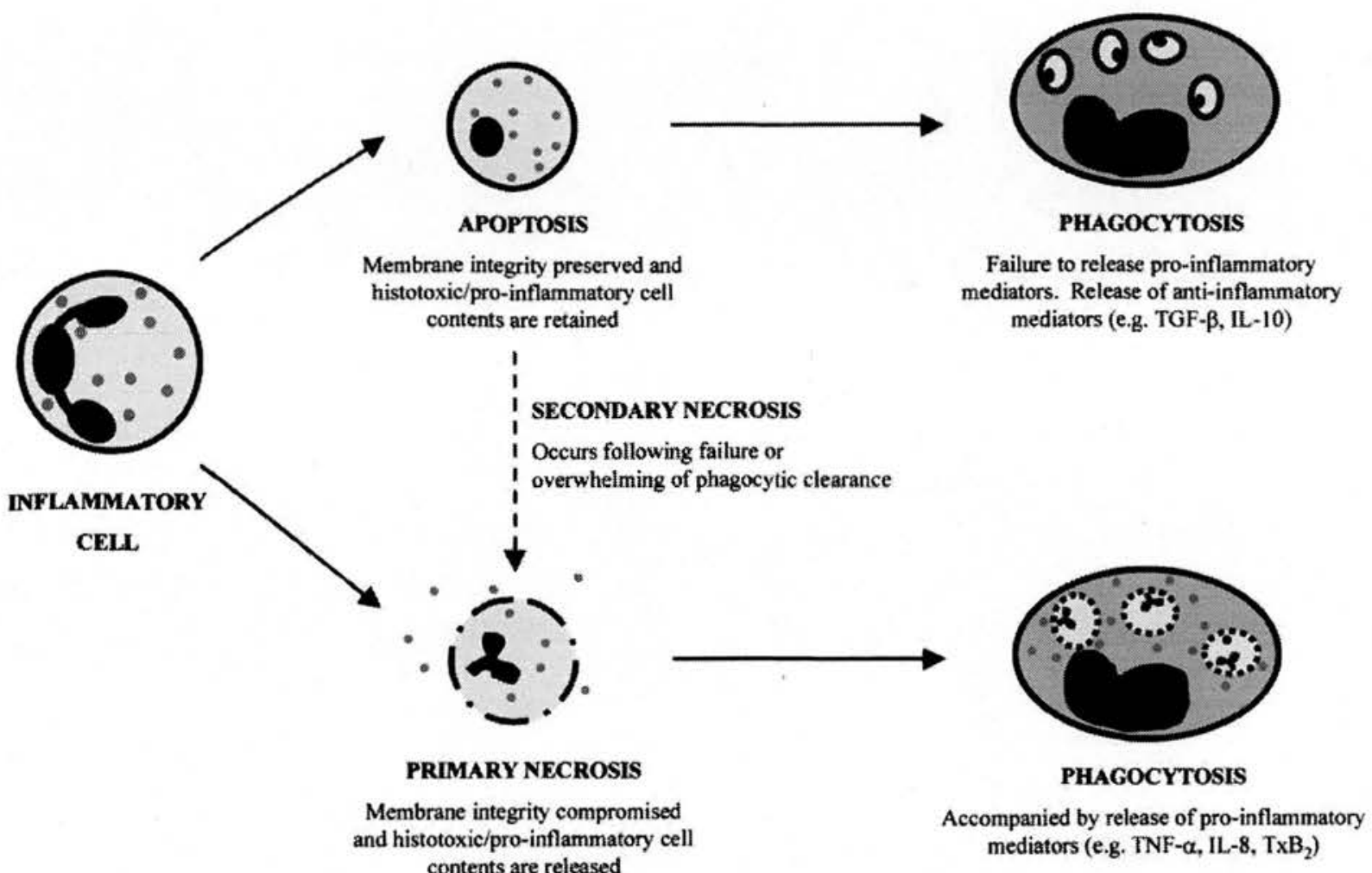

contents are released mediators (e.g. TNF- $\alpha$, IL-8, TxB $)$

\section{PRO-INFLAMMATORY PATHWAY}

Figure 1 Death pathways and phagocytic clearance of inflammatory cells. Cells may undergo apoptosis, the noninflammatory form of cell death, in which granule contents are retained within the cell, and cells are cleared by macrophages that release anti-inflammatory mediators. Alternatively, they may undergo primary necrosis, during which the cell membrane integrity is compromised and granule contents are lost, exacerbating inflammation and tissue injury. Phagocytosis of these cells leads to release of proinflammatory mediators from the macrophages. In the event of failure of phagocytosis, or if the capacity of the phagocytes to ingest cells is exceeded, then apoptotic cells may enter into secondary necrosis. Again, this leads to release of toxic granule contents and clearance by a proinflammatory mechanism. TGF, transforming growth factor; IL, interleukin; TNF, tumour necrosis factor; Tx, thromboxane

underlying NO release. ${ }^{11}$ Existing drugs such as sodium nitroprusside (SNP), azide and hydroxylamine require complex metabolism to generate intracellular NO. Release of NO from azide and hydroxylamine is catalase-dependent, while membrane-bound proteins are thought to have a role in NO generation from SNP. ${ }^{11,12}$ Another class of NO donors is the $S$-nitrosothiols, which can release NO spontaneously, probably both inside and outside the cell. However, they also have the ability to transfer $\mathrm{NO}^{+}$to free reduced thiols and cysteine residues in proteins, thus modulating enzyme activity, and this may constitute an important component of their cellular effects. ${ }^{12,13}$ The diazeniumdiolate, or 'NONOate', compounds are nucleophiles with two molecules of NO, which are spontaneously released in aqueous solution in a temperatureand $\mathrm{pH}$-dependent reaction. A wide variety of diazeniumdiolates are available, such as spermine (SPER/NO), diethylamine (DEA/NO) and diethylenetriamine (DETA/NO)-based compounds, each with a different rate of release of NO that depends on the nature of the nucleophile. ${ }^{14}$ Finally, although not strictly NO donors, the sydnonimines are frequently used in studies into the effects of NO. These compounds, such as $\mathrm{SIN}-1$, generate equal amounts of $\mathrm{NO}$ and $\mathrm{O}_{2}^{-}$, which combine rapidly to form peroxynitrite $\left(\mathrm{ONOO}^{-}\right)$; as a result, they are generally considered to be $\mathrm{ONOO}^{-}$donors. ${ }^{15}$ As the oxatriazole-5-imine derivative GEA 3162 is structurally similar to molsidomine, the precursor of SIN-1, it is possible that GEA 3162 generates $\mathrm{ONOO}^{-}$rather than pure $\mathrm{NO}$, although this issue remains to be resolved.

This review will examine our current understanding of the regulation of inflammatory cell apoptosis by NO and the mechanisms through which the process is mediated. Primary human inflammatory cells will constitute the main focus of the review since cell lines are usually transformed and, therefore, their apoptotic machinery is likely to be altered. The biology and chemistry of $\mathrm{NO},{ }^{2}$ the various classes of $\mathrm{NO}$ donor ${ }^{11}$ and the apoptotic process itself ${ }^{16}$ have all previously been extensively reviewed elsewhere and will not be covered in detail here. The regulation of apoptosis in distinct inflammatory cells of the myeloid lineage will be considered first, and summarised in Table 1, then potential mechanisms 
Table 1 Summary of the effects of NO on inflammatory cell apoptosis

\begin{tabular}{|c|c|c|c|c|c|}
\hline Cell type & Species/cell line & Source of NO & $\begin{array}{l}\text { Conc }^{N} \\
(\mu \mathrm{M} \text { unless otherwise stated) }\end{array}$ & $\begin{array}{l}\text { Effect on } \\
\text { apoptosis }\end{array}$ & Reference \\
\hline \multirow[t]{10}{*}{$\begin{array}{l}\text { Monocytes } \\
\text { and macrophages }\end{array}$} & Human $1^{\circ}$ monocytes & $\begin{array}{l}\text { SIN-1 } \\
\text { SNAP }\end{array}$ & $\begin{array}{l}250-2000 \\
500-2000\end{array}$ & $\begin{array}{l}\text { Induction } \\
\text { No effect }\end{array}$ & Adrie et $a l^{33}$ \\
\hline & Murine $1^{\circ}$ macrophages & iNOS & $?$ & Induction & Sarih et al. ${ }^{17}$ \\
\hline & $\begin{array}{l}\text { Murine } 1^{\circ} \text { peritoneal } \\
\text { macrophages }\end{array}$ & iNOS & $?$ & Induction & Albina et al. ${ }^{18}$ \\
\hline & $\begin{array}{l}\text { Murine } 1^{\circ} \text { peritoneal } \\
\text { macrophages/RAW } \\
264.7\end{array}$ & $\begin{array}{l}\text { iNOS } \\
\text { GSNO }\end{array}$ & $\begin{array}{l}? \\
1000\end{array}$ & Induction & Hortelano et al. ${ }^{23}$ \\
\hline & Human $1^{\circ} \mathrm{MDM} / \mathrm{RAW}$ & GSNO & 1000 & Induction & Von Knethen et al. ${ }^{25}$ \\
\hline & 264.7 & SPER/NO & 1000 & & \\
\hline & $\begin{array}{l}\text { Human canine LTMC } \\
\text { J774 }\end{array}$ & $\mathrm{SIN}-1$ & $0.006-0.6$ & Induction & Lee et $a l^{39}$ \\
\hline & $\begin{array}{l}\text { Murine macrophages in } \\
\text { vivo }\end{array}$ & L-arginine & 900 & Induction & Niebauer et al. ${ }^{75}$ \\
\hline & $\begin{array}{l}\text { Murine macrophages in } \\
\text { vivo }\end{array}$ & L-arginine & 1000 & Induction & Wang et al. ${ }^{74}$ \\
\hline & & SNP & 10 & & \\
\hline \multirow{20}{*}{$\begin{array}{l}\text { Myelomonocytic } \\
\text { cell lines }\end{array}$} & HL-60 & DETA/NO & $50-250$ & Induction & Yabuki et al. ${ }^{38}$ \\
\hline & $\mathrm{HL}-60$ & SNP & $1000-2000$ & Induction & Kwak et al. ${ }^{37}$ \\
\hline & $\mathrm{HL}-60$ & SNP & $500-5000$ & Induction & Kuo et al. ${ }^{36}$ \\
\hline & & SNAP & 100 & & \\
\hline & RAW 264.7 & iNOS & $?$ & Induction & Brune et $a l^{22}$ \\
\hline & & GSNO & $300-750$ & & \\
\hline & & SPER/NO & 500 & & \\
\hline & RAW 264.7 & SNP & $50-1000$ & Induction & Messmer and \\
\hline & & GSNO & $50-1000$ & & Brune $^{26}$ \\
\hline & & SPER/NO & $50-1000$ & & \\
\hline & & DEA/NO & $50-1000$ & & \\
\hline & & DETA/NO & $50-1000$ & & \\
\hline & RAW 264.7 & SNAP & 1000 & Induction & Gotoh et al. ${ }^{24}$ \\
\hline & RAW 264.7 & $\begin{array}{l}\text { L-arginine } \\
\text { iNOS }\end{array}$ & $\begin{array}{l}12000 \\
?\end{array}$ & Induction & Brockhaus and \\
\hline & & GSNO & 500 & & Brune $^{31}$ \\
\hline & & SPER/NO & 250 & & \\
\hline & & SIN-1 & 3000 & & \\
\hline & RAW 264.7 & GSNO & $200-1000$ & Induction & Scivitarro et al. ${ }^{27}$ \\
\hline & & $\begin{array}{l}\text { SPER/NO } \\
\text { ONOO- }^{-}\end{array}$ & $\begin{array}{l}200-2000 \\
30-50\end{array}$ & Inhibition & \\
\hline & & & & $\begin{array}{l}\text { (iNOS- } \\
\text { mediated } \\
\text { apoptosis) }\end{array}$ & is \\
\hline \multirow{7}{*}{$\begin{array}{l}\text { Myelomonocytic } \\
\text { cell lines }\end{array}$} & RAW 264.7 & GSNO & $250-1000$ & Induction & Callsen and Brune ${ }^{28}$ \\
\hline & RAW 264.7 & SPER/NO & $100-500$ & Induction & Boggs et al. ${ }^{29}$ \\
\hline & RAW 264.7 & $\mathrm{ONOO}^{-}$ & $10-300$ & Induction & Sandoval et al. ${ }^{30,116}$ \\
\hline & J774 & GSNO & 1000 & Induction & Borutaite et al. ${ }^{40}$ \\
\hline & & SNAP & 1000 & & \\
\hline & & DETA/NO & 1000 & & \\
\hline & U937/THP1 & DETA/NO & 1000 & Induction & Ferret et $a l^{35}$ \\
\hline \multirow[t]{8}{*}{ Neutrophils } & Human & $\begin{array}{l}\text { GEA } 3162 \\
\text { SIN-1 }\end{array}$ & $\begin{array}{l}10-100 \\
300-3000\end{array}$ & Induction & Wong et al. ${ }^{41}$ \\
\hline & Human & SNP & $8000-32000$ & Induction & Brennan et al. ${ }^{42}$ \\
\hline & Human & GEA 3162 & $10-100$ & Induction & Ward et al. ${ }^{10}$ \\
\hline & & SIN-1 & $300-3000$ & & \\
\hline & Human & GEA 3162 & 10 & Induction & Blaylock et al. ${ }^{46}$ \\
\hline & & SIN-1 & 1000 & No effect & \\
\hline & Human & NO gas & 20-50ppm & Induction & Fortenberry et al. ${ }^{43}$ \\
\hline & Human & GSNO & $100-5000$ & Induction & Fortenberry et al. ${ }^{45}$ \\
\hline
\end{tabular}


Table 1 (continued)

\begin{tabular}{|c|c|c|c|c|c|}
\hline Cell type & Species/cell line & Source of NO & $\begin{array}{l}\text { Conc }^{N} \\
\text { ( } \mu \mathrm{M} \text { unless otherwise stated) }\end{array}$ & $\begin{array}{l}\text { Effect on } \\
\text { apoptosis }\end{array}$ & Reference \\
\hline & Human & SNP & $125-500$ & Induction & Singhal et al. ${ }^{44}$ \\
\hline & Human & GEA 3162 & $30-100$ & Induction & Taylor et al. ${ }^{48}$ \\
\hline & & SPER/NO & $0.1-3$ & Inhibition & \\
\hline & & & $300-1000$ & Induction & \\
\hline & & DEA/NO & $0.1-30$ & Inhibition & \\
\hline & & & 1000 & Induction & \\
\hline \multirow[t]{10}{*}{ Eosinophils } & Human & Azide & 100 & Inhibition & Beauvais et al. ${ }^{29}$ \\
\hline & & Hydroxylamine & 300 & & \\
\hline & Human & SNAP & $1000-5000$ & Inhibition & Beauvais and Joly ${ }^{51}$ \\
\hline & & SIN-1 & $1000-5000$ & Inhibition & \\
\hline & & SNOC & $1000-5000$ & No effect & \\
\hline & & 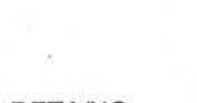 & $100-3000$ & $\begin{array}{l}\text { Induction } \\
\text { (IL-5 primed } \\
\text { cells) }\end{array}$ & \\
\hline & & DETA/NO & $300-3000$ & Induction (IL-5 & \\
\hline & & & & primed cells) & \\
\hline & Human & Azide & $20-1200$ & $\begin{array}{l}\text { Inhibition (Fas- } \\
\text { stimulated } \\
\text { Cells) }\end{array}$ & Hebestreit et al. ${ }^{50}$ \\
\hline & & $\begin{array}{l}\text { Hydroxylamine } \\
\text { SNAP }\end{array}$ & $\begin{array}{l}300-1200 \\
1-100\end{array}$ & & \\
\hline
\end{tabular}

SIN-1, 3-morpholino-sydnonimine; SNAP, S-nitroso-acetyl-penicillamine; iNOS, inducible nitric oxide synthase; GSNO, S-nitrosoglutathione; SPER/NO, spermine diazeniumdiolate (NONOate); SNP, sodium nitroprusside; DETA/NO, diethylenetriamine diazeniumdiolate (NONOate); ONOO- peroxynitrite; DEA/NO, diethylamine diazeniumdiolate (NONOate); SNOC, S-nitrosocysteine; IL, interleukin

through which NO may be acting will be discussed in general (Figure 2), as inevitably there seems to be a large degree of overlap between the mechanisms engaged in different cell types.

\section{Regulation of Inflammatory Cell Apoptosis by NO}

\section{Myelomonocytic cells}

It is well established that exogenous or endogenous iNOS-derived NO induces apoptosis in macrophages. Indeed, the macrophage was the first cell type in which NO-mediated apoptosis was demonstrated. ${ }^{17,18}$ Much of the work on this cell type has been carried out using cell lines, in particular, the murine macrophage cell line RAW 264.7. However, uncertainties have now been cast over the relevance of data obtained with animal cell lines to humans, as it has become apparent that species differences are more important than initially anticipated. Critical differences have been discovered in enzyme pathways in macrophages from different species, which may have a profound effect on NO production in these cells. It has been demonstrated that, whereas murine- and bovine-stimulated macrophages can produce copious amounts of NO, human, caprine, lapine and porcine cells may not be able to do so. ${ }^{19,20}$ Thus, although it is clear that stimulation of murine macrophages with LPS and IFN- $\gamma$ provokes NO production from iNOS, which is sufficient to induce apoptosis, ${ }^{21-24}$ it is by no means certain that this is also the case in human cells. Nevertheless, despite the relative inability of human macrophages to produce endogenous NO upon external stimulation, they appear to respond to exogenous sources of $\mathrm{NO}$ in much the same way as those from other species. NO from the $S$ nitrosothiol, S-nitrosoglutathione (GSNO) and the spontaneous NO generator, SPER/NO, increased apoptosis in both RAW 264.7 cells and primary human monocyte-derived macrophages. ${ }^{25}$ Therefore, observations using NO donors in murine cells could still be extrapolated to human cells, and may provide useful guidance on the potential therapeutic benefits of using NO in inflammatory conditions. Several NO donors have been demonstrated to elicit apoptosis in RAW 264.7 cells, including sodium nitroprusside (SNP), ${ }^{26} \mathrm{~S}$ nitroso- $N$-acetylpenicillamine (SNAP), ${ }^{24}$ GSNO ${ }^{21-23,26-28}$ and the NONOates SPER/NO, 21,22,26,27,29 DEA/NO and DETA/NO. ${ }^{26}$

In addition, Sandoval et al. ${ }^{30}$ demonstrated increased apoptosis in RAW 264.7 cells upon exposure to peroxynitrite (ONOO ${ }^{-} ; 100-300 \mu \mathrm{M}$ over $4 \mathrm{~h}$ and $10-100 \mu \mathrm{M}$ over $14 \mathrm{~h}$ ). Conversely, Scivittaro et al. ${ }^{27}$ observed that lower concentrations $(30-50 \mu \mathrm{M})$ of $\mathrm{ONOO}^{-}$had a protective effect against LPS and IFN- $\gamma$-induced apoptosis in these cells, although not against exogenous NO-mediated apoptosis. This discrepancy may well be the result of the different concentrations of $\mathrm{ONOO}^{-}$used in the above studies. In addition, Brune et $\mathrm{al}^{22}$ reported that RAW 264.7 cells that have higher levels of $\mathrm{O}_{2}^{-}$ are resistant to iNOS-mediated apoptosis, suggesting that $\mathrm{ONOO}^{-}$plays no role in mediating NO-induced apoptosis in murine macrophages, and indeed may have a protective role. Although Brockhaus and Brune ${ }^{31}$ showed that overexpression of superoxide dismutase (SOD) in RAW 264.7 macrophages inhibited NO-mediated apoptosis, which implies a role 


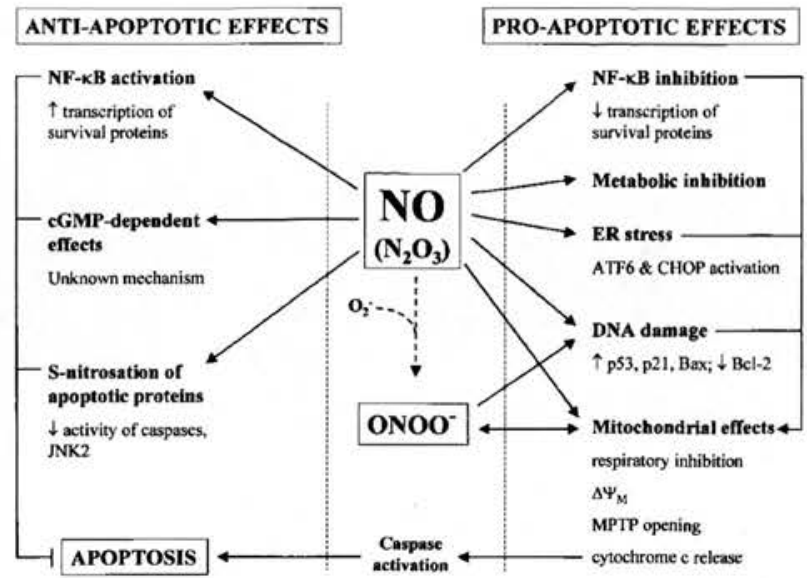

Figure 2 Proposed mechanisms for the effects of NO, its metabolites or byproducts on inflammatory cell apoptosis. Antiapoptotic effects are thought to be mediated through an increase in cGMP, S-nitrosation of apoptotic effector proteins or activation of the transcription factor NF- $\kappa \mathrm{B}$ by NO or reactive higher oxides of nitrogen, especially $\mathrm{N}_{2} \mathrm{O}_{3}$ the principal metabolite of $\mathrm{NO}$ which is likely to mediate many of its biological effects. A number of mechanisms have been suggested to explain the proapoptotic effects, which appear to converge on the release of cytochrome $c$ from mitochondria and subsequent activation of caspases, the central proteases of the apoptotic cascade. NF- $\kappa \mathrm{B}$, nuclear factor kappa B; cGMP, cyclic GMP; ER, endoplasmic reticulum; $\Delta \Psi_{m}$, change in mitochondrial membrane potential; MPTP, mitochondrial permeability transition pore

for $\mathrm{ONOO}^{-}$, on further investigation they found this not to be the case. The $\mathrm{ONOO}^{-}$scavenger, uric acid, failed to block apoptosis elicited by the NO donors GSNO and SPER/NO, while abolishing that induced by $\mathrm{SIN}-1$, thus ruling out $\mathrm{ONOO}^{-}$as a mediator of NO-induced apoptosis. Inhibition of murine monocyte/macrophage $\mathrm{J774}$ cell apoptosis by NO has also been reported when $\mathrm{O}_{2}^{-}$was scavenged using SOD. ${ }^{32}$ Boggs et al. ${ }^{29}$ found that a subset of RAW 264.7 cells that were resistant to NO-induced apoptosis had lower glutathione (GSH) levels and, therefore, higher oxidant status than nonresistant cells.

Little work has been carried out to examine the effects of NO on apoptosis in primary human monocytes that have not matured into macrophages. Adrie et al..$^{33}$ demonstrated that the peroxynitrite donor, $\mathrm{SIN}-1(0.25-2 \mathrm{mM})$, induced cell death (apoptosis and necrosis) in primary human monocytes from healthy volunteers, but that NO from $S$-nitroso- $N$ acetylpenicillamine (SNAP; $0.5-2 \mathrm{mM}$ ) was unable to do so. Muhl et al..$^{34}$ used U937 cells and peripheral blood mononuclear cells from human volunteers, and demonstrated an induction of apoptosis using DETA/NO, which has also been shown by Ferret et al. ${ }^{35}$ to induce apoptosis in U937 and THP1 cells. Some studies have also demonstrated NO-induced apoptosis in HL-60 cells, using DETA/NO, SNP and SNAP, ${ }^{36-}$ ${ }^{38}$ while $\mathrm{ONOO}^{-}$from SIN-1 can also induce apoptosis in human and canine long-term marrow cultures and $\mathrm{J} 774$ cells. ${ }^{39}$ Borutaite et al. ${ }^{40}$ demonstrated increased caspase activation in $\mathrm{J} 774$ cells treated with GSNO, SNAP and DETA $\mathrm{NO}(1 \mathrm{mM})$, which is generally accepted to be an indication of apoptosis. However, morphological apoptosis was not measured in these studies.

\section{Neutrophils}

In recent years, it has become clear that NO also has the ability to induce apoptosis in neutrophils. Wong et al. ${ }^{41}$ demonstrated that the oxatriazole-5-imine derivative, GEA 3162 , and SIN-1 increased the rate of apoptosis in human neutrophils. Simultaneously, Brennan et al..$^{42}$ found increased markers of DNA fragmentation treated with high $(8-32 \mathrm{mM})$ concentrations of SNP in these cells. However, this effect may have been the result of respiratory inhibition by cyanide derived from the liberation of NO from such high concentrations of this compound. Since then, a number of groups have validated these findings through the use of several different sources of NO. NO gas ( 20 and $50 \mathrm{ppm})$ reduces cell viability and augments DNA fragmentation over 2 or $24 \mathrm{~h}$ of culture, an effect that is particularly pronounced in the presence of $80 \%$ $\mathrm{O}_{2} \cdot{ }^{43}$ Whether this cell death comprises apoptosis or merely necrosis, however, remains unclear. An induction of neutrophil apoptosis has also been observed using both traditional NO donors such as SNP ${ }^{44}$ and different sources of NO such as GSNO $(0.5-5 \mathrm{mM}) .{ }^{45}$ Blaylock et $a l .{ }^{46}$ reported that GEA $3162(10 \mu \mathrm{M})$ increased neutrophil apoptosis as assessed by annexin $\mathrm{V}$ binding to exposed phosphatidylserine (PS) at 4 and $8 \mathrm{~h}$, but not at $16 \mathrm{~h}$. On the other hand, the $\mathrm{ONOO}^{-}$donor, SIN-1 (1 mM), showed no significant increase in PS exposure compared to control cells, although there was a small enhancement of annexin $V$ binding at $4 \mathrm{~h}$. The effects of these two compounds were also studied by Ward et al., ${ }^{10}$ who demonstrated that concentrations of $10-100 \mu \mathrm{M}$ GEA 3162 and $0.3-3 \mathrm{mM} \mathrm{SIN-1}$ enhanced caspase-dependent morphological neutrophil apoptosis at 6 and $20 \mathrm{~h}$, and 30 $100 \mathrm{mM}$ GEA 3162 increased annexin $\mathrm{V}$ binding at $6 \mathrm{~h}$. In contrast to Blaylock's study, however, the increase in morphological apoptosis by $10 \mu \mathrm{M}$ GEA 3162 was not significant at $6 \mathrm{~h}$, whereas Blaylock observed significant differences at just $4 \mathrm{~h}$. The principal methodological difference between the two studies is in the culture conditions. It has previously been demonstrated that the microenvironment, including cell density and concentration of plasma proteins, has a critical effect on the rate of neutrophil apoptosis in vitro. ${ }^{47}$ It must therefore be emphasised that the choice of culture conditions may subtly alter the effects of NO on neutrophils, and experimental design should receive careful consideration.

The early induction of PS exposure, but later absence of differences between control and GEA 3162-treated cells was confirmed and extended by Taylor et al. ${ }^{48}$ in 2001 . It was reported that 30-100 $\mu \mathrm{M}$ GEA $3162,300 \mu \mathrm{M}-1 \mathrm{mM}$ SPER/NO and $1 \mathrm{mM}$ DEA/NO all induced morphological evidence of neutrophil apoptosis over $20 \mathrm{~h}$. However, PS exposure was only increased from control levels at early time points in GEA 3162-treated neutrophils. The shedding of CD16 by apoptotic neutrophils equated well with morphological indications of apoptosis, but DNA fragmentation, as evidenced by propidium iodide intercalation and DNA ladders, was not necessarily observed alongside other markers of apoptosis. GEA 3162 $(30-100 \mu \mathrm{M})$ and SPER/NO $(1 \mathrm{mM})$, but not DEA/NO $(1 \mathrm{mM})$, produced a paradoxical inhibition of DNA fragmentation despite a clear induction of apoptosis as assessed by other techniques. 
Although there is a large body of evidence to demonstrate the proapoptotic effect of NO on neutrophils and the antiapoptotic effects in many cell types, it was not until recently that inhibition of neutrophil apoptosis by NO was reported. ${ }^{48} \mathrm{NO}$ derived spontaneously from low concentrations of the diazeniumdiolate compounds SPER/NO and DEA/NO reduced the rate of neutrophil apoptosis over a $20-\mathrm{h}$ time course. In contrast, equivalent concentrations of the oxatriazole derivative, GEA 3162, produced no such inhibition, suggesting that the antiapoptotic effects of NO may depend on the mechanism of NO liberation from donor drugs.

\section{Eosinophils}

Conflicting results have been reported for the effects of exogenous NO on eosinophil apoptosis. Beauvais et al. ${ }^{49}$ reported in 1995 that the catalase-dependent NO donors, azide and hydroxylamine, dramatically reduced the rate of apoptosis in this cell type, as evidenced by several apoptotic markers, with optimum concentrations for these effects being $100 \mu \mathrm{M}$ for azide and $300 \mu \mathrm{M}$ for hydroxylamine. In contrast, only a small inhibitory effect of azide $(0.02-1.2 \mathrm{mM})$, hydroxylamine $(300 \mu \mathrm{M}-1.2 \mathrm{mM})$ and the $S$-nitrosothiol, SNAP $(1 \mu \mathrm{M}-1 \mathrm{mM})$, on constitutive eosinophil apoptosis was observed by Hebestreit et al. ${ }^{50}$ However, Beauvais et al. ${ }^{51}$ later published that other sources of $\mathrm{NO}$, which are not dependent upon enzymatic activity to generate NO, fail to promote eosinophil survival. Although SNAP (1-5 mM) inhibits eosinophil apoptosis, it appears to drive the cells into a necrotic state; overall cell survival is virtually unchanged. Similar effects were also reported for SIN-1, but it appears that cell survival actually increased as expected on exposure to this compound, with a concomitant decrease in apoptosis. Treatment with S-nitroso-cysteine (SNOC), which has a very fast rate of NO release, produced little change in either cell survival or apoptosis. However, it is important to note that the concentrations used in this study were extremely high $(1-5 \mathrm{mM})$ and it appears that lower concentrations of SNOC $(1-3 \mathrm{mM})$ and SNAP $(1 \mathrm{mM})$ may in fact slightly increase apoptosis. It is therefore possible that submillimolar concentrations could induce eosinophil apoptosis, but that higher concentrations are sufficiently toxic to push the cells into necrosis. The increased eosinophil survival observed with azide and hydroxylamine was mimicked when 3-10 $\mu \mathrm{M}$ SNAP was added to the cells in the presence of $10 \mu \mathrm{M}$ haematin, the ferric form of haeme. On the other hand, when the cells were first primed using the cytokine IL-5 (which promotes eosinophil survival), a simultaneous inhibition of eosinophil survival and induction of apoptosis was observed with SNOC $(100 \mu \mathrm{M}-$ $3 \mathrm{mM})$ and DETA/NO $(300 \mu \mathrm{M}-3 \mathrm{mM})$. This difference from unstimulated cells was put down to the priming effect of IL-5. However, the concentrations used in these studies were considerably lower than in nonprimed cells, and as previously mentioned, submillimolar concentrations of these compounds could conceivably be able to induce apoptosis rather than necrosis, whether or not the cells are primed.

Alongside spontaneous eosinophil apoptosis, NO has also been reported to inhibit cell death stimulated by Fas receptor activation. Hebestreit et al. ${ }^{50}$ demonstrated that enhanced DNA fragmentation and PS exposure provoked by treatment with anti-Fas monoclonal antibody could be attenuated by NO derived from LPS and IFN- $\gamma$-stimulated human monocyte-like U937 cells. This inhibitory effect on Fas-mediated apoptosis could be blocked using inhibitors of NOS (L-NMMA; $1 \mathrm{mM}$ ) or the GC pathway (LY 83583; $10 \mu \mathrm{M})$, and was mimicked in a concentration-dependent manner by exogenous NO from $0.02-1.2 \mathrm{mM}$ azide, $0.3-1.2 \mathrm{mM}$ hydroxylamine and $1 \mu \mathrm{M}-$ $1 \mathrm{mM}$ SNAP.

\section{In vivo effects of $\mathrm{NO}$ and its therapeutic potential}

Delayed apoptosis of activated granulocytes has been reported to occur in a number of inflammatory conditions in humans or animal models, ${ }^{5,52}$ including rheumatoid arthritis, ${ }^{53}$ acute pancreatitis, ${ }^{54}$ bacterial pneumonia, ${ }^{55}$ inflammatory bowel disease, ${ }^{56}$ asthma, ${ }^{57-59}$ and following surgery. ${ }^{60,61}$ Failure of these cells to undergo programmed cell death and to be cleared by phagocytes allows persistent and inappropriate inflammation to occur, as activated granulocytes release a number of proinflammatory mediators, which may contribute significantly to the aetiology of the disease. Therefore, the induction of apoptosis in these cells is a potential target for therapeutic intervention, by removal of the inflammatory effector cells, thereby minimising tissue damage and oedema. As NO has a wide range of anti-inflammatory properties, including inhibition of platelet and neutrophil functions (see Granger and Kubes ${ }^{9}$ for review), this molecule could provide effective multitarget therapy against inflammation.

Although iNOS has been implicated in the pathogenesis of certain inflammatory diseases, such as arthritis, SLE and irritable bowel syndrome, ${ }^{62}$ a number of studies have demonstrated a protective effect of NO against several conditions characterised by inflammation, such as glomerulonephritis, ${ }^{63}$ acute hepatic necrosis, ${ }^{64}$ arthritis, ${ }^{65,66}$ endotoxaemia ${ }^{64}$ and acute lung injury ${ }^{67}$ in vivo (reviewed by Clancy and Abramson ${ }^{62}$ ). Most studies attribute these effects to the wide range of general anti-inflammatory properties of NO, as reviewed by Granger and Kubes, ${ }^{9}$ and are outwith the scope of this review. Of particular interest with regard to this review, however, is the increasing evidence that the protection afforded against inflammation and immunity by NO may be mediated in part through the induction of inflammatory cell apoptosis. As for apoptosis induction in vitro, the concentration of $\mathrm{NO}$ in the local environment, ${ }^{68,69}$ the timing of administration ${ }^{70,71}$ or the route of administration, and perhaps therefore the NOS isoform targeted ${ }^{72}$ appears to be critical. Studies have suggested that lower doses of NO may be detrimental but that higher doses may attenuate the inflammatory response, with some authors proposing a role for the cytotoxic effects of NO on myeloid inflammatory cells. ${ }^{69,73-75}$

NO appears to be particularly effective in autoimmune conditions, such as experimental allergic encephalomyelitis $(E A E)$, which serves as a model for human multiple sclerosis. ${ }^{76}$ In this model, several studies have reported that iNOS-deficient rats or mice that were immunised directly with myelin basic protein developed exacerbated disease, although results obtained following immunisation with myelin 
basic protein-specific T cells often contradict these findings. ${ }^{76}$ One proposed mechanism for the protective effect of iNOSderived NO is induction of apoptosis in macrophages or T cells. ${ }^{71,77}$

Other autoimmune disease models in which NO has been reported to be protective are the rat model of autoimmune interstitial nephritis and experimental autoimmune uveitis, as inhibition of NOS caused exacerbated injury in both models, although again other studies have produced conflicting results. ${ }^{76}$ Therefore, NO may be protective in a number of autoimmune conditions, although further research will be required to fully understand the apparent contradictory effects of NO.

A beneficial protective effect of NO has also been shown in the elicitation phase of contact hypersensitivity, as prolonged inflammatory reactions were observed in iNOS knockout mice. ${ }^{69}$ Again, it has been suggested that this effect may be partly because of the induction of apoptosis in infiltrating cells. In a human model similar to sunburn and psoriasis, Ormerod et al. ${ }^{68}$ described a cytotoxic effect of high concentrations of topically administered NO to immunocompetent cells, which was not seen with low concentrations. Aminoguanidine, an inhibitor of iNOS, prevented the impairment of renal vascular bed responses and reduced urine nitrate levels and apoptotic mononuclear cells in a rat model of experimental nephropathy. ${ }^{78}$ However, although inflammatory apoptosis might be expected to be beneficial, the effect of this form of cell death on prognosis was not studied within the late, sclerotic phase of the disease during this study. In the same model, Rangan et al. ${ }^{79}$ discovered that NOS inhibitors exacerbated progression of the disease, and tubulointerstitial injury was also found to be increased in the presence of NOS inhibitors in a model of thrombotic microangiopathy. ${ }^{80}$

Two in vivo studies were published in 1999, investigating the effects of $\mathrm{NO}$ on macrophage apoptosis in cardiovascular disease of cholesterol-fed rabbits. Niebauer et al. ${ }^{75}$ demonstrated that provision of L-arginine (the substrate for NOS) in drinking water could reduce the formation of inflammatory lesions following balloon angioplasty, while Wang et al. ${ }^{74}$ showed that it decreased existing atherosclerotic lesions, by inducing macrophage apoptosis.

Thus, there is a significant body of evidence to suggest that supplementation of NO may be beneficial in inflammatory diseases and that the induction of apoptosis in infiltrating cells may have a role in mediating this protection. However, these issues remain controversial, with much conflicting evidence. It is clear, however, that provision of NO may not be suitable for the treatment of all inflammatory conditions, and indeed may only be appropriate at certain stages of disease progression or disease provoked by a particular mechanism. Much further work is required to clarify these issues.

\section{Mechanisms of action of NO}

The mechanisms through which NO is able to both promote and delay inflammatory cell apoptosis still remain to be fully elucidated. However, it is generally believed that low concentrations tend to be antiapoptotic, acting through a rise in cyclic GMP or S-nitrosation of caspase enzymes. On the other hand, high concentrations of NO are generally more toxic, inducing either apoptosis or necrosis, with most reports suggesting that such actions occur independently of the SGC signalling pathway (see below).

Another possibility is that NO may indirectly interfere with alternative pathways that regulate inflammatory cell survival. For example, glucocorticoids (GCs) promote eosinophil apoptosis but inhibit neutrophil death. ${ }^{7,81}$ It has been shown that NO can inhibit GC receptor binding through S-nitrosation, ${ }^{82}$ and therefore could attenuate both the pro- and antiapoptotic effects of glucocorticoids on inflammatory cells.

\section{Antiapoptotic mechanisms}

The pathway by which inhibition of neutrophil apoptosis occurs has not yet been investigated. However, it is known that NO induces a rise in cGMP in neutrophils through activation of SGC. ${ }^{8,83}$ Given that the cell permeable analogues of CAMP (db-cAMP) and cGMP (db-cGMP) can delay constitutive neutrophil apoptosis, ${ }^{10}$ and that a rise in cGMP has been postulated to at least partially account for NOmediated inhibition of apoptosis in other cell types, ${ }^{84,85}$ it is possible that an increase in one or other of these cyclic nucleotides mediates the inhibition of apoptosis in neutrophils exposed to low concentrations of NO. A role for cyclic nucleotide (cGMP or CAMP) signalling has also been proposed in NO-mediated inhibition of both constitutive and Fas-triggered eosinophil apoptosis. Beauvais et al. ${ }^{49}$ observed that NO could only reduce eosinophil apoptosis when compounds that form nitrosyl-haeme in their liberation of NO were used, which was mimicked by the permeable cGMP analogue db-cGMP and reduced by the sGC inhibitor LY 83583. Pure NO donors alone, in contrast, failed to increase eosinophil survival, although inhibition of apoptosis could be seen if haematin was added alongside a pure NO donor to artificially create a nitrosyl-haeme complex. ${ }^{51}$ It has been suggested that such inhibition occurs through activation of sGC by the nitrosyl-haeme complex, ${ }^{51}$ which has been shown to occur in vitro using purified sGC, ${ }^{86}$ but as yet there is no evidence to suggest that this may occur in intact cells in vivo. However, the mechanism is not fully understood: the issue is complicated by the fact that haeme groups are known to scavenge $\mathrm{NO}$, and therefore might be expected to inhibit NOmediated effects. Inhibition of Fas-mediated apoptosis was reproduced by both $\mathrm{db}-\mathrm{cGMP}$ and db-cAMP, and potentiated by the phosphodiesterase inhibitor IBMX, ${ }^{50}$ again suggesting a role for cyclic nucleotides. This group localised the site of Fas receptor death pathway blockade to downstream of SMase activation and ceramide generation but upstream or around the level of JNK activation. So et al. ${ }^{87}$ showed inhibition of the stress protein JNK2 by NO in vitro via $S$ nitrosation.

Secondly, the transcription factor NF- $\kappa$ B is known to regulate neutrophil apoptosis as its inhibition leads to increased apoptosis. ${ }^{88}$ Agents such as LPS have been shown to delay apoptosis in this cell type through stimulation of $\mathrm{NF}-\kappa \mathrm{B}$ and subsequent caspase-1-dependent activation of $\mathrm{IL}-1 \beta .^{89}$ It has been demonstrated in several cell types that activation of NF- $\kappa \mathrm{B}$ leads to the transcriptional upregulation of survival factors such as the immediate early gene, IEX-1L, inhibitor of apoptosis proteins (IAPs), and members of the 
antiapoptotic $\mathrm{Bcl}-2$ gene family, including $\mathrm{Mcl}-1, \mathrm{Bfl} 1 / \mathrm{A} 1, \mathrm{Bcl}-$ $\mathrm{XL}$ and $\mathrm{Nr} 13$, potentially explaining the survival effects of $\mathrm{NO}$ which, at low concentrations, induces NF- $\mathrm{BB}$ activation in macrophage cell lines ${ }^{90,91}$ and in human peripheral blood mononuclear cells. ${ }^{92}$ Activation of NF- $\mathrm{KB}$ may occur through a cGMP-dependent mechanism, ${ }^{93}$ and NO has been demonstrated to modulate the expression of several proteins affecting the activity of Bcl-2 family members through sGC activation. A cGMP-dependent mechanism has been proposed to account for the NO-induced downregulation of BNIP3, a dominant proapoptotic Bcl-2 family member in hepatocytes. ${ }^{94}$ Levels of MAP kinase phosphatase-3 (MKP-3) mRNA, which causes degradation of Bcl-2 via ERK1/2 dephosphorylation, were seen to be decreased by NO, thus protecting Bcl-2 levels and promoting survival of endothelial cells. ${ }^{95}$ The proapoptotic adapter protein, p66shc, is also downregulated by NO via sGC activation. ${ }^{96}$ Furthermore, survival genes such as $\mathrm{Bcl}-2$ and $\mathrm{Bcl}-\mathrm{XL}$ have been shown to be upregulated in the presence of NO in endothelial cells ${ }^{97}$ and human neuroblastoma cells; ${ }^{96}$ therefore, it seems possible that there is a role for NF- $\mathrm{kB}$-mediated transcriptional regulation in the antiapoptotic effects of $\mathrm{NO}$ in inflammatory cells.

Contrasting studies in macrophage cell lines ${ }^{22,29,32}$ suggest that the redox status of the cell may partially determine the effects of NO. An inhibitory effect of endogenous NO on $\mathrm{J774}$ cell apoptosis can be unmasked when $\mathrm{O}_{2}^{-}$is scavenged, ${ }^{32}$ and over-expression of SOD also protects RAW 264.7 cells against apoptosis induced by endogenous or exogenously supplied NO. ${ }^{31}$ These studies suggest a role for $\mathrm{ONOO}^{-}$in mediating NO-induced apoptosis. Conflicting evidence suggests that RAW 264.7 cells that overproduce $\mathrm{O}_{2}^{-}$are resistant to NO-mediated apoptosis, ${ }^{22}$ and von Knethen et al. ${ }^{98}$ observed that $\mathrm{O}_{2}^{-}$activates $\mathrm{NF}-\kappa \mathrm{B}$, thus mediating survival in these cells. Furthermore, Brockhaus and Brune ${ }^{31}$ found that $\mathrm{ONOO}^{-}$had no role in NO-evoked apoptosis, despite the protective effect of SOD in RAW 264.7 cells. Others have found that such protection is observed when cellular thiols are depleted in RAW 264.7 cells. $^{29}$ It has been reported that endogenous antioxidant levels, ${ }^{99}$ or the balance between oxidative and nitrosative stress, ${ }^{100}$ can determine the cellular response to NO. It has been proposed that in low thiol concentrations, NO actually protects against cell death, whereas it induces death in cells with normal thiol levels. ${ }^{99}$ Exogenous glutathione has also been shown to enhance neutrophil apoptosis and increase $\mathrm{H}_{2} \mathrm{O}_{2}$ levels, possibly leading to hydroxyl radical- mediated damage. ${ }^{101}$

In the absence of large quantities of scavenger thiols such as glutathione, but in the presence of oxygen, it is possible that NO S-nitrosates critical effector molecules of apoptosis such as caspases, thus preventing their activation and having an inhibitory effect on the proteolytic cascade. It has been shown by several groups that NO can inhibit a number of apoptotic proteins, ${ }^{102}$ including caspase 3 (the protease responsible for the initiation of internucleosomal DNA fragmentation), ${ }^{103-108}$ caspase $8,{ }^{105,109,110}$ caspase $9,,^{111}$ caspase $1^{109,110}$ and caspases 2, 3, 4, 6 and $7^{105}$ activation via S-nitrosation. Inhibition of caspase 3 has been reported to involve two distinct mechanisms in hepatocytes - direct protein Snitrosation, and another mechanism, which has not yet been elucidated, but is dependent upon cGMP. ${ }^{108}$ Therefore, the cGMP-dependent antiapoptotic effects of $\mathrm{NO}$ in inflammatory cells may be mediated through an inhibitory effect on caspases.

\section{Proapoptotic mechanisms}

Studies have shown that apoptosis in neutrophils and macrophages proceeds via activation of caspase protease enzymes, ${ }^{10,112,113}$ part of the classical apoptotic effector cascade. However, the upstream mechanisms by which exposure to NO causes these enzymes to become activated has not been clarified, although several theories have been suggested.

$\mathrm{ONOO}^{-}$is considered to be one of the most likely candidates for the increased apoptosis of inflammatory cells observed with higher concentrations of NO, particularly in the case of neutrophils, ${ }^{10,43,46}$ which generate large quantities of $\mathrm{O}_{2}^{-}$that rapidly combines with $\mathrm{NO}$ to form $\mathrm{ONOO}^{-}$. Compounds that generate $\mathrm{ONOO}^{-}$, such as SIN-1, may promote neutrophil apoptosis similar to that evoked by NO, ${ }^{10}$ and primary human monocytes also undergo apoptosis in response to $\mathrm{ONOO}^{-}$by a mechanism that involves mitochondrial membrane depolarisation, release of cytochrome $c$ and caspase activation (see Figure 2). ${ }^{33} \mathrm{ONOO}^{-}$has been shown to reversibly or irreversibly inhibit a number of mitochondrial respiratory complexes as well as inducing mitochondrial swelling, depolarisation, calcium release and permeability transition. ${ }^{114,115}$

Macrophage apoptosis can also be induced by exposure to $\mathrm{ONOO}^{-}$, via oxidative stress, which can be reduced by antioxidants such as ascorbic acid or phytolens. ${ }^{30,116}$ Induction of apoptosis by NO in elicited murine macrophages or RAW 264.7 cells has also been attributed to the formation of $\mathrm{ONOO}^{-}$within mitochondria, as nitrotyrosine residues were detected in cytochrome $c^{23} \mathrm{ONOO}^{-}$and metabolites of NO (e.g. $\mathrm{N}_{2} \mathrm{O}_{3}$ ) can cause direct DNA damage or inhibit DNA repair enzymes, ${ }^{117}$ leading to an increase in the tumour suppressor protein $\mathrm{p} 53$, which has been shown to accumulate in NO-treated macrophages and may be the factor responsible for driving them towards apoptosis. ${ }^{22,25,118,119}$ The p53 protein promotes apoptosis through upregulation of the apoptotic proteins Bax and cyclin-dependent kinase p21, and downregulation of the antiapoptotic protein $\mathrm{Bcl}-2$ (Figure 2). ${ }^{85}$ However, Gotoh et al. ${ }^{24}$ measured no increase of $\mathrm{p} 53$ in NO-mediated apoptosis in RAW 264.7 cells stimulated with LPS/IFN- $\gamma$. Instead, this group proposed a role for the endoplasmic reticulum stress pathway involving the transcription factors ATF6 and CHOP leading to cytochrome $c$ release (Figure 2). Also, studies in murine macrophages ${ }^{31}$ suggest little or no involvement for $\mathrm{ONOO}^{-}$in NO-induced apoptosis in this cell type.

As previously described, the activation status of the survival factor, NF- $\kappa \mathrm{B}$, has been shown to play a role in regulation of the induction of inflammatory cell apoptosis. ${ }^{88}$ It has been demonstrated that high concentrations of $\mathrm{NO}$ can inhibit $\mathrm{NF}-\kappa \mathrm{B}$ activation in macrophage cell lines ${ }^{91}$ and human macrophages, ${ }^{120}$ monocytes ${ }^{121}$ and neutrophils. ${ }^{121,122}$ NO may inhibit NF- $\kappa$ B DNA binding through S-nitrosation of the p50 subunit of the transcription factor, as has been demon- 
strated in isolated NF- $\kappa \mathrm{B}$ protein ${ }^{123}$ and in human respiratory cells and murine macrophages. ${ }^{124}$ Alternatively, transcriptional induction and stabilisation of the inhibitory molecule I$\kappa \mathrm{B}$, that keeps $\mathrm{NF}-\kappa \mathrm{B}$ sequestered in the cytoplasm, may account for the inhibition of NF- $\kappa \mathrm{B}$ activity. ${ }^{125}$ Furthermore, it has been reported that NO inhibits NF- $\kappa$ B activation in rat vascular smooth muscle cells via a cGMP-independent inhibition of the phosphorylation and proteasomal degradation of $\mathrm{I}-\kappa \mathrm{B},{ }^{126}$ and inhibition of the proteasome by NO has additionally been demonstrated by Glockzin et $a l^{127}$ in macrophages. The result of such inhibition would be downregulation of survival factors under the control of this transcription factor, such as the antiapoptotic Bcl-2 family members. Indeed, this has been observed by a number of studies, as exogenous NO downregulates $\mathrm{Bcl}-2$ but upregulates the proapoptotic protein, Bax, in neurons, ${ }^{128,129}$ and upregulates $\mathrm{Bad}$ and $\mathrm{Bax}$, but downregulates $\mathrm{Bcl}-2$ in human colon adenocarcinoma cells. ${ }^{130}$

In nonsmall cell lung cancer cells, it has been shown that NF-KB inhibition leads to apoptosis by increasing mitochondrial permeability, thus allowing release of cytochrome $c$ and subsequent caspase activation (see Figure 2). ${ }^{131}$ This concurs with findings by Borutaite et al., ${ }^{40}$ who demonstrated increased mitochondrial permeability and cytochrome $c$ release from isolated rat mitochondria, and increased caspase activation in $\mathbf{J 7 7 4}$ cells treated with S-nitrosothiols (SNAP, GSNO) but not NONOates (DETA/NO), which activate caspases through an as yet unidentified alternative mechanism. As $S$-nitrosothiols readily transnitrosate endogenous cysteine residues, this supports the concept of $S$ nitrosation of the NF- $\kappa \mathrm{B}$ p50 subunit as the mechanism of inhibition.

In addition, the biphasic effects of NO on NF- $\mathrm{KB}$ activation reported by Connelly et al. are mirrored by its effects on the open probability of the mitochondrial permeability transition pore (MPTP). Low concentrations of NO donors (GEA 3162 , SNAP, SIN-1; $1-20 \mu \mathrm{M}$ ) delayed or had no effect on MPTP opening, while at higher concentrations $(20-100 \mu \mathrm{M})$, opening was enhanced. ${ }^{132}$ In this study, GEA 3162 was found to be particularly effective at inducing MPTP opening compared to the other two NO donors, and this drug induces neutrophil apoptosis at lower concentrations than NONOates ${ }^{48}$ or SIN1. ${ }^{10}$ Enhanced MPTP opening on exposure to NO was also reported by Hortelano et al. ${ }^{133}$ Therefore, there is growing evidence to suggest that the proapoptotic effects of NO in inflammatory cells may be mediated, at least in part, through inhibition of NF- $\kappa$ B.

Albina et al. ${ }^{18}$ proposed metabolic inhibition as a potential mechanism, as glucose starvation and inhibition of glycolysis or the TCA cycle all pushed macrophages into apoptosis. On the other hand, inhibition of the electron transport chain of respiration had no effect, ${ }^{18}$ and Messmer and Brune ${ }^{26}$ showed no reduction in $\mathrm{NAD}^{+}$or ATP levels in NO-induced apoptosis, suggesting that NO does not act through respiratory inhibition. In contrast, others have reported that NO inhibits mitochondrial respiration through two distinct pathways. ${ }^{114,115}$ Reversible inhibition of cytochrome oxidase was seen with low concentrations of $\mathrm{NO}$, whereas higher concentrations caused an inhibition of alternative respiratory chain complexes. ${ }^{115}$ Inhibition of complex IV was reversible, whereas inhibition of complex I was irreversible. ${ }^{114}$ Of course, the mechanisms described above may not be mutually exclusive. For example, respiratory inhibition by NO may enhance the production of reactive oxygen species by mitochondria, leading to the formation of $\mathrm{ONOO}^{-}$and providing an $\mathrm{ONOO}^{-}$-mediated pathway for NO-induced cytotoxicity (Figure 2). ${ }^{115}$

\section{Conclusion}

NO has a biphasic effect on apoptosis in many cell types, in which low concentrations delay but higher concentrations enhance this form of cell death, a pattern that has recently been confirmed in neutrophils. This correlates with the dichotomous action of NO on the activity of caspase enzymes responsible for the execution of apoptosis in vitro. Inhibition of caspases by S-nitrosation is a direct consequence of exposure to low concentrations of NO or, more likely, its oxidation products (e.g. $\mathrm{N}_{2} \mathrm{O}_{3}$ ). On the other hand, activation of these enzymes observed during the proapoptotic actions of higher concentrations represents a downstream event following initial effects on DNA or mitochondria, and can therefore be considered an indirect effect of NO.

Although the mechanism of inhibition has not yet been fully investigated, it is likely that cGMP production, NF-KB activation and subsequent expression of survival proteins or S-nitrosation of apoptotic proteins will play a major role. Inhibition of eosinophil apoptosis has been reported, but only with certain sources of NO that are capable of activating sGC with a consequent rise in cGMP. No such inhibitory effects have yet been demonstrated in monocytes or macrophages, and it remains to be seen whether these cell types are capable of producing such a response to low concentrations of NO.

It has been demonstrated that exogenous NO can induce apoptosis in all inflammatory cell types discussed in this review: monocytes, monocyte-derived macrophages, neutrophils and eosinophils. In addition, endogenous NO from iNOS also promotes apoptosis in macrophages. There still remains some controversy over the mechanism by which this molecule causes this form of cell death, although it involves activation of caspase proteases, and most agree that this occurs through a cGMP-independent pathway. Moreover, mitochondria appear to play a key role in the initiation of apoptosis by NO through release of cytochrome $c$, resulting in caspase activation.

It is clear that $\mathrm{ONOO}^{-}$derived from $\mathrm{SIN}-1$ or other agents has the ability to promote apoptosis in its own right, but its role in mediating NO-induced apoptosis remains controversial. Some groups have reported that NO-evoked cytotoxicity is likely to be effected through $\mathrm{ONOO}^{-}$formation, while studies by others have indicated little or no role for $\mathrm{ONOO}^{-}$. Modulation of the activation status of the transcription factor NF-KB has also been proposed to account for NO-induced apoptosis in neutrophils and macrophages, and there is an increasing body of evidence to support this theory. On the other hand, DNA damage (by $\mathrm{N}_{2} \mathrm{O}_{3}$ or $\mathrm{ONOO}^{-}$) has also been shown, leading to an accumulation of the proapoptotic molecule p53.

Differences may exist in the mechanisms by which NO causes apoptosis in different cell types that could potentially 
be exploited to target a particular inflammatory cell type in certain conditions. Despite the uncertainties and controversies surrounding the regulation of inflammatory cell apoptosis by NO, it is clear that the class and concentration of NOdonating compound used and the cell type are critical determinants of the response. Major differences between different classes of NO donors and opposing effects with low and high concentrations of certain NO donors are observed. Thus, the amount and rate of NO release and the redox status of the target cell appear to be key factors in the cellular response to NO exposure, and certain NO donors appear to be more effective than others in promoting inflammatory cell apoptosis.

It is also important to realise that the concentration of NO donor used may not necessarily reflect the concentration of $\mathrm{NO}$ to which the cells are exposed. Equivalent concentrations of different NO donors may liberate NO to different extents or at different rates, or may produce different reactive nitrogen species, such as $\mathrm{ONOO}^{-}$. Culture conditions may also affect NO levels; for example, plasma proteins such as albumin are able to scavenge NO through the formation of S-nitrosothiols. ${ }^{13,134,135}$ Therefore, the concentration of free NO in the vicinity of the cells at any given time may vary from compound to compound, and the NO concentration in the system needs to be measured in order to directly compare different NO donors.

The vast majority of work on this subject has been carried out using in vitro systems, often utilising animal cell lines. How the results obtained in these systems relate to the in vivo situation during inflammation in humans still largely remains to be determined, but two studies in rabbits show that NO is a promising candidate for treatment or prevention of inflammatory conditions such as atherosclerosis and restenosis, possibly by influencing apoptosis. ${ }^{74,75}$ Further studies are required to elucidate completely the mechanism of action of NO on inflammatory cell apoptosis, in order to identify potential targets for the treatment of human inflammatory conditions and to evaluate the sources of NO that provide greatest therapeutic potential.

\section{References}

1. Palmer RM, Ferrige AG and Moncada S (1987) Nitric oxide release accounts for the biological activity of endothelium-derived relaxing factor. Nature 327: 524-526

2. Quinn AC, Petros AJ and Vallance $P$ (1995) Nitric oxide: an endogenous gas. Br. J. Anaesth. 74: 443-451

3. Nicotera P, Brune B and Bagetta G (1997) Nitric oxide: inducer or suppressor of apoptosis?. Trends Pharmacol. Sci. 18: 189-190

4. Kim YM, Bombeck CA and Billiar TR (1999) Nitric oxide as a bifunctional regulator of apoptosis. Circ. Res. 84: 253-256

5. Haslett C (1997) Granulocyte apoptosis, and inflammatory disease. Br. Med. Bull. 53: 669-683

6. Meagher LC, Savill JS, Baker A, Fuller RW and Haslett C (1992) Phagocytosis of apoptotic neutrophils does not induce macrophage release of thromboxane B2. J. Leukoc. Biol. 52: 269-273

7. Ward C, Dransfield I, Chilvers ER, Haslett C and Rossi AG (1999) Pharmacological manipulation of granulocyte apoptosis: potential therapeutic targets. Trends Pharmacol. Sci. 20: 503-509
8. Moilanen $E$, Vuorinen $P$, Kankaanranta $H$, Metsa-Ketela $T$ and Vapaatalo $H$ (1993) Inhibition by nitric oxide donors of human polymorphonuclear leucocyte functions. Br. J. Pharmacol. 109: 852-858

9. Granger DN and Kubes P (1996) Nitric oxide as antiinflammatory agent. Methods Enzymol. 269: 434-442

10. Ward C, Wong TH, Murray J, Rahman I, Haslett C, Chilvers ER and Rossi AG (2000) Induction of human neutrophil apoptosis by nitric oxide donors: evidence for a caspase-dependent, cyclic-GMP-independent, mechanism. Biochem. Pharmacol. 59: 305-314

11. Megson IL (2000) Nitric oxide donor drugs. Drugs Fut. 25: 701-715

12. Feelisch $M$ (1991) The biochemical pathways of nitric oxide formation from nitrovasodilators: appropriate choice of exogenous NO donors, and aspects of preparation, and handling of aqueous NO solutions. J. Cardiovasc. Pharmacol. 17(Suppl. 3): S25-S33

13. Butler AR and Rhodes $P$ (1997) Chemistry, analysis, and biological roles of $S$ nitrosothiols. Anal. Biochem. 249: 1-9

14. Maragos CM, Morley D, Wink DA, Dunams TM, Saavedra JE, Hoffman A Bove AA, Isaac L, Hrabie JA and Keefer LK (1991) Complexes of NO with nucleophiles as agents for the controlled biological release of nitric oxide. Vasorelaxant effects. J. Med. Chem 34: 3242-3247

15. Feelisch M, Ostrowski J and NoackE. (1989) On the mechanism of NO release from sydnonimines. J. Cardiovasc. Pharmacol. 14(Suppl 11): S13-S22

16. Haunstetter A and Izumo S (1998) Apoptosis: basic mechanisms, and implications for cardiovascular disease. Circ. Res. 82: 1111-1129

17. Sarih M, Souvannavong V and Adam A (1993) Nitric oxide synthase induces macrophage death by apoptosis. Biochem. Biophys. Res. Commun. 191 503-508

18. Albina JE, Cui S, Mateo RB and Reichner JS (1993) Nitric oxide-mediated apoptosis in murine peritoneal macrophages. J. Immunol. 150: 5080-5085

19. Thomassen MJ and Kavuru MS (2001) Human alveolar macrophages, and monocytes as a source, and target for nitric oxide. Int. Immunopharmacol. 1 1479-1490

20. Schneemann M and Schoedon $G$ (2002) Species differences in macrophage NO production are important. Nat. Immunol. 3: 102

21. Messmer UK, Reed UK and Brune B (1996) Bcl-2 protects macrophages from nitric oxide-induced apoptosis. J. Biol. Chem. 271: 20192-20197

22. Brune B, Gotz C, Messmer UK, Sandau K, Hirvonen MR and Lapetina EG (1997) Superoxide formation, and macrophage resistance to nitric oxidemediated apoptosis. J. Biol. Chem. 272: 7253-7258

23. Hortelano S, Alvarez AM and Bosca L (1999) Nitric oxide induces tyrosine nitration, and release of cytochrome $c$ preceding an increase of mitochondrial transmembrane potential in macrophages. FASEB J. 13: 2311-2317

24. Gotoh T, Oyadomari S, Mori K and Mori M (2002) Nitric oxide-induced apoptosis in RAW 264.7 macrophages is mediated by endoplasmic reticulum stress pathway involving ATF6, and CHOP. J. Biol. Chem. 277: 12343-12350

25. von Knethen A, Brockhaus F, Kleiter I and Brune B (1999) NO-evoked macrophage apoptosis is attenuated by CAMP-induced gene expression. Mol. Med. 5: 672-684

26. Messmer UK and Brune B (1996) Nitric oxide (NO) in apoptotic Versus necrotic RAW 264.7 macrophage cell death: the role of NO-donor exposure, $\mathrm{NAD}+$ content, and p53 accumulation. Arch. Biochem. Biophys. 327: 1-10

27. Scivittaro V, Boggs S, Mohr S and Lapetina EG (1997) Peroxynitrite protects RAW 264.7 macrophage from lipopolysaccharide/interferon-gamma-induced cell death. Biochem. Biophys. Res. Commun. 241: 37-42

28. Callsen D and Brune B (1999) Role of mitogen-activated protein kinases in $S$ nitrosoglutathione-induced macrophage apoptosis. Biochemistry 38: 22792286

29. Boggs SE, McCormick TS and Lapetina EG (1998) Glutathione levels determine apoptosis in macrophages. Biochem. Biophys. Res. Commun. 247: 229-233

30. Sandoval M, Zhang XJ, Liu X, Mannick EE, Clark DA and Miller MJ (1997) Peroxynitrite-induced apoptosis in T84, and RAW 264.7 cells: attenuation by L-ascorbic acid. Free Radic. Biol. Med. 22: 489-495

31. Brockhaus F and Brune B (1999) Overexpression of CuZn superoxide dismutase protects RAW 264.7 macrophages against nitric oxide cytotoxicity. Biochem. J. 338(Part 2): 295-303

32. Vouldoukis I, Sivan V, Vozenin MC, Kamate C, Calenda A, Mazier D and Dugas B (2000) Fc-receptor-mediated intracellular delivery of Cu/Zn- 
superoxide dismutase (SOD1) protects against redox-induced apoptosis through a nitric oxide dependent mechanism. Mol. Med. 6: 1042-1053

33. Adrie C, Richter C, Bachelet M, Banzet N, Francois D, Dinh-Xuan AT, Dhainaut JF, Polla BS and Richard MJ (2000) Contrasting effects of NO and peroxynitrites on HSP70 expression, and apoptosis in human monocytes. Am. J. Physiol. Cell Physiol. 279: C452-C460

34. Muhl H, Nold M, Chang JH, Frank S, Eberhardt W and Pfeilschifter J (1999) Expression and release of chemokines associated with apoptotic cell death in human promonocytic U937 cells, and peripheral blood mononuclear cells. Eur. J. Immunol. 29: 3225-3235

35. Ferret PJ, Soum E, Negre O, Wollman EE and Fradelizi D (2000) Protective effect of thioredoxin upon NO-mediated cell injury in THP1 monocytic human cells. Biochem. J. 346(Part 3): 759-765

36. Kuo ML, Chau YP, Wang JH and Shiah SG (1996) Inhibitors of poly (ADPribose) polymerase block nitric oxide-induced apoptosis but not differentiation in human leukemia HL-60 cells. Biochem. Biophys. Res. Commun. 219: 502508

37. Kwak HJ, Jun CD, Pae HO, Yoo JC, Park YC, Choi BM, Na YG, Park RK, Chung HT, Chung HY, Park WY and Seo JS (1998) The role of inducible 70$\mathrm{kDa}$ heat shock protein in cell cycle control, differentiation, and apoptotic cell death of the human myeloid leukemic HL-60 cells. Cell Immunol. 187: 1-12

38. Yabuki M, Tsutsui K, Horton AA, Yoshioka T and Utsumi K (2000) Caspase activation, and cytochrome $c$ release during $\mathrm{HL}-60$ cell apoptosis induced by a nitric oxide donor. Free Radic. Res. 32: 507-514

39. Lee JW, Beckham C, Michel BR, Rosen H and Deeg HJ (1997) HLA-DRmediated signals for hematopoiesis, and induction of apoptosis involve but are not limited to a nitric oxide pathway. Blood 90: 217-225

40. Borutaite V, Morkuniene R and Brown GC (2000) Nitric oxide donors, nitrosothiols, and mitochondrial respiration inhibitors induce caspase activation by different mechanisms. FEBS Lett. 467: 155-159

41. Wong TH, Rossi AG and Chilvers ER (1997) Inhibition of neutrophil function, and survival by nitric oxide donors. Clin. Sci. 92: 7II

42. Brennan C, Stapleton PP, Redmond HP and Bouchier-Hayes D (1996) Nitric oxide-mediated neutrophil apoptosis. J. Leukoc. Biol. (Suppl): pp 39 , A157.

43. Fortenberry JD, Owens ML, Brown MR, Atkinson D and Brown LA (1998) Exogenous nitric oxide enhances neutrophil cell death, and DNA fragmentation. Am. J. Respir. Cell Mol. Biol. 18: 421-428

44. Singhal PC, Patel P, Nahar N, Franki N, Kapasi A, Reddy K, Shah N, Nwakoby IE and Mehrotra B (1999) Ethanol-induced neutrophil apoptosis is mediated through nitric oxide. J. Leukoc. Biol. 66: 930-936

45. Fortenberry JD, Owens ML and Brown LA (1999) S-nitrosoglutathione enhances neutrophil DNA fragmentation, and cell death. Am. J. Physiol. 276: L $435-\mathrm{L} 442$

46. Blaylock MG, Cuthbertson BH, Galley HF, Ferguson NR and Webster NR (1998) The effect of nitric oxide, and peroxynitrite on apoptosis in human polymorphonuclear leukocytes. Free Radic. Biol. Med. 25: 748-752

47. Hannah S, Nadra I, Dransfield I, Pryde JG, Rossi AG and Haslett C (1998) Constitutive neutrophil apoptosis in culture is modulated by cell density independently of beta2 integrin-mediated adhesion. FEBS Lett. 421 141-146

48. Taylor EL, Megson IL, Haslett C and Rossi AG (2001) Dissociation of DNA fragmentation from other hallmarks of apoptosis in nitric oxide-treated neutrophils: differences between individual nitric oxide donor drugs. Biochem. Biophys. Res. Commun. 289: 1229-1236

49. Beauvais F, Michel $L$ and Dubertret $L$ (1995) The nitric oxide donors, azide and hydroxylamine, inhibit the programmed cell death of cytokine-deprived human eosinophils. FEBS Lett. 361: 229-232

50. Hebestreit H, Dibbert B, Balatti I, Braun D, Schapowal A, Blaser K and Simon HU (1998) Disruption of fas receptor signaling by nitric oxide in eosinophils. J. Exp. Med. 187: 415-425

51. Beauvais $F$ and Joly $F$ (1999) Effects of nitric oxide on the eosinophil survival in vitro. A role for nitrosyl-heme. FEBS Lett. 443: $37-40$

52. Simon HU (1999) Apoptosis in inflammatory diseases. Int. Arch. Allergy Immunol. 118: 261-262

53. Ottonello L, Frumento G, Arduino N, Bertolotto M, Mancini M, Sottofattori E, Dallegri F and Cutolo M (2002) Delayed neutrophil apoptosis induced by synovial fluid in rheumatoid arthritis: role of cytokines, estrogens, and adenosine. Ann. N. Y. Acad. Sci. 966: 226-231
54. O'Neill S, O'Neill AJ, Conroy E, Brady HR, Fitzpatrick JM and Watson RW (2000) Altered caspase expression results in delayed neutrophil apoptosis in acute pancreatitis. J. Leukoc. Biol. 68: 15-20

55. Droemann D, Aries SP, Hansen F, Moellers M, Braun J, Katus HA and Dachoff K (2000) Decreased apoptosis, and increased activation of alveolar neutrophils in bacterial pneumonia. Chest 117: 1679-1684

56. Brannigan AE, O'Connell PR, Hurley $\mathrm{H}, \mathrm{O}^{\prime}$ Neill A, Brady HR, Fitzpatrick JM and Watson RW (2000) Neutrophil apoptosis is delayed in patients with inflammatory bowel disease. Shock 13: 361-366

57. Turlej RK, Fievez L, Sandersen CF, Dogne S, Kirschvink N, Lekeux $P$ and Bureau $F$ (2001) Enhanced survival of lung granulocytes in an animal model of asthma: evidence for a role of GM-CSF activated STAT5 signalling pathway. Thorax 56: 696-702

58. Woolley KL, Gibson PG, Carty K, Wilson AJ, Twaddell SH and Woolley MJ (1996) Eosinophil apoptosis, and the resolution of airway inflammation in asthma. Am. J. Respir. Crit. Care Med. 154: 237-243

59. Kankaanranta $H$, Lindsay MA, Giembycz MA, Zhang $X$, Moilanen $E$ and Barnes PJ (2000) Delayed eosinophil apoptosis in asthma. J. Allergy Clin. Immunol. 106: 77-83

60. Matsuda $T$, Saito $H$, Fukatsu $K$, Han I, Inoue T, Furukawa $S$, Ikeda $S$ and Hidemura A (2001) Cytokine-modulated inhibition of neutrophil apoptosis at local site augments exudative neutrophil functions, and reflects inflammatory response after surgery. Surgery 129: 76-85

61. Chello M, Mastroroberto P, Quirino A, Cuda G, Perticone F, Cirillo F and Covino $E$ (2002) Inhibition of neutrophil apoptosis after coronary bypass operation with cardiopulmonary bypass. Ann. Thorac. Surg. 73: 123-129

62. Clancy RM and Abramson SB (1995) Nitric oxide: a novel mediator of inflammation. Proc. Soc. Exp. Biol. Med. 210: 93-101

63. Heeringa $P$, van Goor $\mathrm{H}$, Itoh-Lindstrom $Y$, Maeda N, Falk RJ, Assmann KJ, Kallenberg CG and Jennette JC (2000) Lack of endothelial nitric oxide synthase aggravates murine accelerated anti-glomerular basement membrane glomerulonephritis. Am. J. Pathol. 156: 879-888

64. Billiar TR, Curran RD, Harbrecht BG, Stuehr DJ, Demetris AJ and Simmons RL (1990) Modulation of nitrogen oxide synthesis in vivo: NG-monomethyl-Larginine inhibits endotoxin-induced nitrate/nitrate biosynthesis while promoting hepatic damage. J. Leukoc. Biol. 48: 565-569

65. Veihelmann A, Landes J, Hofbauer A, Dorger M, Refior HJ, Messmer K and Krombach $F$ (2001) Exacerbation of antigen-induced arthritis in inducible nitric oxide synthase-deficient mice. Arthritis Rheum. 44: 1420-1427

66. McCartney-Francis NL, Song X, Mizel DE and Wahl SM (2001) Selective inhibition of inducible nitric oxide synthase exacerbates erosive joint disease. J. Immunol. 166: 2734-2740

67. Liu P, Xu B and Hock CE (2001) Inhibition of nitric oxide synthesis by L-name exacerbates acute lung injury induced by hepatic ischemia-reperfusion. Shock 16: 211-217

68. Ormerod AD, Copeland P, Hay I, Husain A and Ewen SW (1999) The inflammatory, and cytotoxic effects of a nitric oxide releasing cream on normal skin. J. Invest. Dermatol. 113: 392-397

69. Ross R and Reske-Kunz AB (2001) The role of NO in contact hypersensitivity. Int. Immunopharmacol. 1: 1469-1478

70. Okuda Y, Sakoda S, Fujimura H and Yanagihara T (1998) Aminoguanidine, a selective inhibitor of the inducible nitric oxide synthase, has different effects on experimental allergic encephalomyelitis in the induction, and progression phase. J. Neuroimmunol. 81: 201-210

71. Xu LY, Yang JS, Link H, and Xiao BG (2001) SIN-1, a nitric oxide donor, ameliorates experimental allergic encephalomyelitis in Lewis rats in the incipient phase: the importance of the time window. J. Immunol. 166: 5810 5816

72. Paul-dark MJ, Gilroy DW, Willis D, Willoughby DA and Tomlinson A (2001) Nitric oxide synthase inhibitors have opposite effects on acute inflammation depending on their route of administration. J. Immunol. 166: 1169-1177

73. Okuda Y, Sakoda S, Fujimura H and Yanagihara T (1997) Nitric oxide via an inducible isoform of nitric oxide synthase is a possible factor to eliminate inflammatory cells from the central nervous system of mice with experimental allergic encephalomyelitis. J. Neuroimmunol. 73: 107-116

74. Wang BY, Ho HK, Lin PS, Schwarzacher SP, Pollman MJ, Gibbons GH, Tsao PS and Cooke JP (1999) Regression of atherosclerosis: role of nitric oxide, and apoptosis. Circulation 99: 1236-1241 
75. Niebauer J, Schwarzacher SP, Hayase M, Wang B, Kernott RS, Cooke JP and Yeung AC (1999) Local L-arginine delivery after balloon angioplasty reduces monocyte binding, and induces apoptosis. Circulation 100: 18301835

76. Bogdan C (1998) The multiplex function of nitric oxide in (auto) immunity. J. Exp. Med. 187: 1361-1365

77. Puerta C, Martinez I, Baranda P, Blasco MR, Castejon R, Vargas JA and Garcia-Merino A (2000) Aminoguanidine reduces apoptosis of circulating $\checkmark$ Beta 8.2T lymphocytes in Lewis rats with actively induced experimental autoimmune encephalomyelitis. Association with persistent inflammation of the central nervous system, and lack of recovery. J. Neuroimmunol. 110: $140-150$

78. Ozen S, Usta Y, Sahin-Erdemli I, Orhan D, Gumusel B, Yang B, Gursoy Y, Tulunayo, Dalkara T, Bakkaloglu A and El-Nahas M (2001) Association of nitric oxide production, and apoptosis in a model of experimental nephropathy. Nephrol. Dial. Transplant. 16: 32-38

79. Rangan GK, Wang Y and Harris DC (2001) Pharmacologic modulators of nitric oxide exacerbate tubulointerstitial inflammation in proteinuric rats. J. Am. Soc. Nephrol. 12: 1696-1705

80. Shao J, Miyata T, Yamada K, Hanafusa N, Wada T, Gordon KL, Inagi R, Kurokawa K, Fujita T, Johnson RJ and Nangaku M (2001) Protective role of nitric oxide in a model of thrombotic microangiopathy in rats. J. Am. Soc. Nephrol. 12: 2088-2097

81. Meagher LC, Cousin JM, Seckl JR and Haslett C (1996) Opposing effects of glucocorticoids on the rate of apoptosis in neutrophilic, and eosinophilic granulocytes. J. Immunol. 156: 4422-4428

82. Galigniana MD, Piwien-Pilipuk G and Assreuy J (1999) Inhibition of glucocorticoid receptor binding by nitric oxide. Mol. Pharmacol. 55: 317-323

83. Kosonen O, Kankaanranta H, Malo-Ranta U and Moilanen E (1999) Nitric oxide-releasing compounds inhibit neutrophil adhesion to endothelial cells. Eur. J. Pharmacol. 382: 111-117

84. Li J and Billiar TR (1999) The anti-apoptotic actions of nitric oxide in hepatocytes. Cell Death Differ 6: 952-955

85. Kolb JP (2000) Mechanisms involved in the pro-, and anti-apoptotic role of NO in human leukemia. Leukemia 14: 1685-1694

86. Edwards JC, Barry BK, Gruetter DY, Ohlstein EH, Baricos WH and Ignarro LJ (1981) Activation of hepatic guanylate cyclase by nitrosyl-heme complexes. Comparison of unpurified, and partially purified enzyme. Biochem. Pharmacol. 30: 2531-2538

87. So HS, Park RK, Kim MS, Lee SR, Jung BH, Chung SY, Jun CD and Chung HT (1998) Nitric oxide inhibits c-Jun N-terminal kinase 2 (JNK2) via Snitrosylation. Biochem. Biophys. Res. Commun. 247: 809-813

88. Ward C, Chilvers ER, Lawson MF, Pryde JG, Fujihara S, Farrow SN, Haslett C and Rossi AG (1999) NF-kappaB activation is a critical regulator of human granulocyte apoptosis in vitro. J. Biol. Chem. 274: 4309-4318

89. Watson RW, Rotstein OD, Parodo J, Bitar R, Marshall JC, William R and Watson G (1998) The IL-1 beta-converting enzyme (caspase-1) inhibits apoptosis of inflammatory neutrophils through activation of IL-1 beta. J. Immunol. 161: 957-962

90. von Knethen A, Callsen D and Brune B (1999) NF-kappaB, and AP-1 activation by nitric oxide attenuated apoptotic cell death in RAW 264.7 macrophages. Mol. Biol. Cell 10: 361-372

91. Connelly L, Palacios-Callender M, Ameixa C, Moncada S and Hobbs AJ (2001) Biphasic regulation of NF-kappaB activity underlies the pro-, and antiinflammatory actions of nitric oxide. J. Immunol. 166: 3873-3881

92. Lander HM, Sehaipal P, Levine DM and Novogrodsky A (1993) Activation of human peripheral blood mononuclear cells by nitric oxide-generating compounds. J. Immunol. 50: 1509-1516

93. Kalra D, Baumgarten G, Dibbs Z, Seta Y, Sivasubramanian N and Mann DL (2000) Nitric oxide provokes tumor necrosis factor-alpha expression in adult feline myocardium through a cGMP-dependent pathway. Circulation 102: 1302-1307

94. Zamora R, Alarcon L, Vodovotz Y, Betten B, Kim PK, Gibson KF and Billiar TR (2001) Nitric oxide suppresses the expression of Bcl-2 binding protein BNIP3 in hepatocytes. J. Biol. Chem. 276: 46887-46895

95. Rossig L, Haendeler J, Hermann C, Malchow P, Urbich C, Zeiher AM and Dimmeler S (2000) Nitric oxide downregulates MKP-3 mRNA levels: involvement in endothelial cell protection from apoptosis. J. Biol. Chem. 275: 25502-25507

96. Andoh T, Lee SY and Chiueh CC (2000) Preconditioning regulation of bcl-2, and $\mathrm{p} 66 \mathrm{shc}$ by human NOSI enhances tolerance to oxidative stress. FASEB J. $14: 2144-2146$

97. Delikouras A, Hayes M, Malde P, Lechler RI and Dorling A (2001) Nitric oxidemediated expression of $\mathrm{Bcl}-2$, and $\mathrm{Bcl}-\mathrm{xl}$, and protection from tumor necrosis factor-alpha-mediated apoptosis in porcine endothelial cells after exposure to low concentrations of xenoreactive natural antibody. Transplantation 71:599605

98. von Knethen A, Callsen D and Brune B (1999) Superoxide attenuates macrophage apoptosis by NF-kappa B, and AP-1 activation that promotes cyclooxygenase-2 expression. J. Immunol. 163: 2858-2866

99. Rosenberg PA, LiY, Ali S, Altiok N, Back SA and Volpe JJ (1999) Intracellular redox state determines whether nitric oxide is toxic or protective to rat oligodendrocytes in culture. J. Neurochem. 73: 476-484

100. Espey MG, Miranda KM, Feelisch M, Fukuto J, Grisham MB, Vitek MP and Wink DA (2000) Mechanisms of cell death governed by the balance between nitrosative and oxidative stress. Ann. N. Y. Acad. Sci. 899: 209-221

101. Misso NL, Peacock CD, Watkins DN and Thompson PJ (2000) Nitrite generation, and antioxidant effects during neutrophil apoptosis. Free Radic Biol. Med. 28: 934-943

102. Melino G, Bernassola F, Knight RA, Corasaniti MT, Nistico G and Finazzi-Agro A (1997) S-nitrosylation regulates apoptosis. Nature 388: $432-433$

103. Mohr S, Zech B, Lapetina EG and Brune B (1997) Inhibition of caspase-3 by S-nitrosation and oxidation caused by nitric oxide. Biochem. Biophys. Res. Commun. 238: 387-391

104. Zech B, Wilm M, van Eldik R and Brune B (1999) Mass spectrometric analysis of nitric oxide-modified caspase-3. J. Biol. Chem. 274: 2093120936

105. Li J, Billiar TR, Talanian RV and Kim YM (1997) Nitric oxide reversibly inhibits seven members of the caspase family via S-nitrosylation. Biochem. Biophys. Res. Commun. 240: 419-424

106. Rossig L, Fichtlscherer B, Breitschopf K, Haendeler J, Zeiher AM, Mulsch A and Dimmeler S (1999) Nitric oxide inhibits caspase-3 by S-nitrosation in vivo. J. Biol. Chem. 274: 6823-6826

107. Kim YM, Kim TH, Seol DW, Talanian RV and Billiar TR (1998) Nitric oxide suppression of apoptosis occurs in association with an inhibition of Bcl-2 cleavage, and cytochrome $c$ release. J. Biol. Chem. 273: 31437 31441

108. Kim YM, Talanian RV and Billiar TR (1997) Nitric oxide inhibits apoptosis by preventing increases in caspase-3-like activity via two distinct mechanisms. J. Biol. Chem. 272: 31138-31148

109. Dimmeler S, Haendeler J, Nehls M and Zeiher AM (1997) Suppression of apoptosis by nitric oxide via inhibition of interleukin-1 beta-converting enzyme (ICE)-like, and cysteine protease protein (CPP)- 32-like proteases. J. Exp. Med. 185: 601-607

110. Dimmeler S, Haendeler J, Sause A and Zeiher AM (1998) Nitric oxide inhibits APO-1/Fas-mediated cell death. Cell Growth Differ. 9: 415-422

111. Torok NJ, Higuchi $H$, Bronk S and Gores GJ (2002) Nitric oxide inhibits apoptosis downstream of cytochrome $c$ release by nitrosylating caspase 9 . Cancer Res. 62: 1648-1653

112. Williams TE, Ayala A and Chaudry IH (1997) Inducible macrophage apoptosis following sepsis is mediated by cysteine protease activation, and nitric oxide release. J. Surg. Res. 70: 113-118

113. Knepper-Nicolai B, Savill J and Brown SB (1998) Constitutive apoptosis in human neutrophils requires synergy between calpains, and the proteasome downstream of caspases. J. Biol. Chem. 273: 30530-30536

114. Clementi E, Brown GC, Feelisch M and Moncada S (1998) Persistent inhibition of cell respiration by nitric oxide: crucial role of S-nitrosylation of mitochondrial complex I, and protective action of glutathione. Proc. Natl. Acad. Sci. USA 95: 7631-7636

115. Brown GC and Borutaite V (2001) Nitric oxide, mitochondria, and cell death IUBMB Life 52: 189-195

116. Sandoval M, Ronzio RA, Muanza DN, Clark DA and Miller MJ (1997) Peroxynitrite-induced apoptosis in epithelial (T84), and macrophage (RAW 264.7) cell lines: effect of legume-derived polyphenols (phytolens). Nitric Oxide 1: $476-483$ 
117. Wink DA, Grisham MB, Mitchell JB and Ford PC (1996) Direct and indirect effects of nitric oxide in chemical reactions relevant to biology. Methods Enzymol. 268: 12-31

118. Messmer UK, Ankarcrona M, Nicotera $P$ and Brune B (1994) p53 expression in nitric oxide-induced apoptosis. FEBS Lett. $355: 23-26$

119. Messmer UK and Brune B (1996) Nitric oxide-induced apoptosis: p53dependent, and p53-independent signalling pathways. Biochem. J. 319(Part 1): $299-305$

120. Raychaudhuri B, Dweik R, Connors MJ, Buhrow L, Malur A, Drazba J, Arroliga AC, Erzurum SC, Kavuru MS and Thomassen MJ (1999) Nitric oxide blocks nuclear factor-kappaB activation in alveolar macrophages. Am. J. Respir. Cell Mol. Biol. 21: $311-316$

121. Welters ID, Menzebach A, Goumon $Y$, Cadet $P$, Menges $T$, Hughes TK, Hempelmann G and Stefano GB (2000) Morphine inhibits NF-kappaB nuclear binding in human neutrophils and monocytes by a nitric oxide-dependent mechanism. Anesthesiology 92: 1677-1684

122. Fortenberry JD, Owens ML, Chen NX and Brown LA (2001) S nitrosoglutathione inhibits TNF-alpha-induced NFkappaB activation in neutrophils. Inflamm. Res. 50: 89-95

123. Matthews JR, Botting CH, Panico M, Morris HR and Hay RT (1996) Inhibition of NF-kappaB DNA binding by nitric oxide. Nucleic Acids Res. 24 2236-2242

124. Marshall HE and Stamler JS (2001) Inhibition of NF-kappa B by S nitrosylation. Biochemistry 40: 1688-1693

125. Peng HB, Libby P and Liao JK (1995) Induction, and stabilization of I kappa alpha by nitric oxide mediates inhibition of NF-kappa B. J. Biol. Chem. 270: 14214-14219

126. Katsuyama K, Shichiri M, Marumo F and Hirata $Y$ (1998) NO inhibits cytokine induced iNOS expression, and NF-kappaB activation by interfering with phosphorylation, and degradation of IkappaB-alpha. Arterioscler. Thromb. Vasc. Biol. 18: 1796-1802
127. Glockzin S, von Knethen A, Scheffner M and Brune B (1999) Activation of the cell death program by nitric oxide involves inhibition of the proteasome. J. Biol. Chem. 274: 19581-19586

128. Tamatani M, Ogawa S, Nunez G and Tohyama M (1998) Growth factors prevent changes in $\mathrm{Bcl}-2$, and Bax expression, and neuronal apoptosis induced by nitric oxide. Cell Death Differ. 5: 911-919

129. Matsuzaki $H$, Tamatani M, Mitsuda N, Namikawa K, Kiyama H, Miyake S and Tohyama M (1999) Activation of Akt kinase inhibits apoptosis, and changes in Bcl-2, and Bax expression induced by nitric oxide in primary hippocampal neurons. J. Neurochem. 73: 2037-2046

130. Ho YS, Liu HL, Duh JS, Chen RJ, Ho WL, Jeng JH, Wang YJ and Lin JK (1999) Induction of apoptosis by $\mathrm{S}$-nitrosoglutathione, and $\mathrm{Cu}^{2+}$ or $\mathrm{Ni}^{2+}$ ion through modulation of bax, bad, and bcl-2 proteins in human colon adenocarcinoma cells. Mol. Carcinogen. 26: 201-211

131. Jones DR, Broad RM, Comeau LD, Parsons SJ and Mayo MW (2002) Inhibition of nuclear factor kappaB chemosensitizes non-small cell lung cancer through cytochrome $c$ release, and caspase activation. J. Thorac. Cardiovasc. Surg. 123: $310-317$

132. Piantadosi CA, Tatro LG and Whorton AR (2002) Nitric oxide, and differential effects of ATP on mitochondrial permeability transition. Nitric Oxide 6: 45-60

133. Hortelano S, Dallaporta B, Zamzami N, Hirsch T, Susin SA, Marzo I, Bosca L and Kroemer G (1997) Nitric oxide induces apoptosis via triggering mitochondrial permeability transition. FEBS Lett. 410: 373-377

134. Stamler JS, Jaraki O, Osborne J, Simon DI, Keaney J, Vita J, Singel D, Valeri CR and Loscalzo J (1992) Nitric oxide circulates in mammalian plasma primarily as an S-nitroso adduct of serum albumin. Proc. Natl. Acad. Sci. USA 89: 7674-7677

135. Ramachandran N, Root $P$, Jiang XM, Hogg PJ and Mutus B (2001) Mechanism of transfer of NO from extracellular $S$-nitrosothiols into the cytosol by cell-surface protein disulfide isomerase. Proc. Natl. Acad. Sci. USA 98: 9539-9544 


\title{
Regulation of Granulocyte Apoptosis by Hemopoietic Growth Factors, Cytokines and Drugs: Potential Relevance to Allergic Inflammation
}

\author{
A. Walker, C. Ward, I. Dransfield, C. Haslett and A.G. Rossi*
}

\section{Respiratory Medicine Unit, Centre for Inflammation Research, University of Edinburgh Medical School, Teviot Place, Edinburgh, EH8 9AG, Scotland, UK}

\begin{abstract}
It has become apparent that the resolution of inflammation depends on the removal of unwanted inflammatory cells, a process governed by physiological apoptosis and non-inflammatory clearance of apoptotic cclls. Granulocytes are central to many of the pathophysiological consequences of uncontrolled inflammatory reactions. Hemopoietic factors and cytokines play a critical role in regulating the longevity of these cells in vitro and in vivo. Here we revicw the progress that has been made in the understanding of granulocyte apoptosis and the implications for immunotherapy and pharmacological strategies in the treatment of allergic inflammatory diseases for therapeutic gain.
\end{abstract}

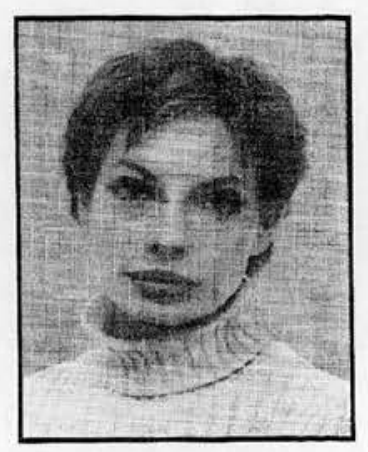

Keywords: eosinophil, neutrophil, inflammation, apoptosis, IL-5, GM-CSF, Glucocorticoids

\section{INTRODUCTION}

Common allergic diseases include asthma, allergic rhinitis, eczema, contact dermatitis and chronic sinusitis. Allergy is often considered to be an inappropriatc inflammatory response resulting in deleterious effects to the patient. Asthma affects 155 million people world wide and its incidence is on the increase [1]. Although, cosinophilic inflammation is strongly associated with asthma, increasingly in severe asthma neutrophilic inflammation is being implicated [2]. Initiation of an inflammatory response requires that large numbers of circulating leukocytes (e.g., neutrophils, eosinophils and mononuclear cells) are rapidly recruited to the affected site, a process requiring migration across the vascular endothelium (Fig. (1)) [3].

Migration of leukocytes from the blood vessels into the sitc of inflammation is dependent on the activation of adhesive molecules, on both the circulating cells and the endothelial cells [4]. Neutrophils bind to the vessel wall transiently, a form of adhesion known as 'rolling' and this is mediated by the reversible binding of selectins (e.g., Eselectin) to their ligands (e.g., PSGL-1) expressed on both neutrophils and vascular endothelial cells [5]. L-selectin is expressed on most circulating leukocytes and F- and Pselectin on endothclial cells lining the blood and lymph vessels [4]. Rolling adhesion on activated endothelial cell selectins allows adhesion of neutrophils via integrins (e.g., CD11/ CD18 complexes) and their counter ligands (e.g., ICAM-1, -2), particularly when chemokines are present to facilitate 'firm' adhesion to the endothelial cell and arrest from vascular flow [6]. Following adhesion, leukocytes undergo diapedesis and migrate by chemotaxis towards the site of inflammation [3]. At the inflammatory sitc, granulocytes can then phagocytose and destroy invading

*Address correspondence to this author at the Respiratory Medicine Unit, Centre for Inflammation Research, University of Edinburgh Medical School, Teviot Place, Edinburgh, EH8 9AG, Scotland, UK; Fax (+44) 131 650 4384; E-mail: a.g.rossi@ed.ac.uk microorganisms by releasing proteolytic enzymes, bactericidal proteins and toxic oxygen products into the phagolysosome (Fig. (1)).

Necrotic cell death or the inappropriate release of granulocyte granule contents is thought to be a major contributing factor to tissue damage occurring during inflammatory disease. Oxygen metabolites, produced by granulocytes to exert microbicidal function, can also injure surrounding host tissue. Although tissues contain protective anti-oxidant enzymes, e.g. superoxide dismutase and catalase, these enzyme systems may be overwhelmed during intense local production of toxic oxygen metabolites. Neutrophil granules also contain a large family of over 20 enzymes, of which the serine proteases, elastase and the two metalloproteinases-collagenase and gelatinase, seem to have the greatest potential to act as mediators of tissue destruction [7]. Similarly, eosinophil granule contents (e.g., major basic protein, eosinophil peroxidase, and eosinophil cationic protein), which are particularly effective against parasitic infection, are also liberated (Fig. (2)) [8,9].

Resolution of inflammation and the restoration of normal tissue architecture require the inflammatory stimulus to be removed, cessation of influx of inflammatory cells and elimination of inflammatory cells already present. The removal of granulocytes has been shown to be mediated by apoptosis [10-13] and their subscquent phagocytosis by macrophages or neighbouring cells, providing a mechanism for the safe removal of 'unwanted' cells without eliciting an inflammatory response [14]. However, if the inflammatory response becomes overwhelmed and either apoptotic granulocytes become too numerous for quick removal by macrophages or there is a defect in the phagocytic potential of macrophages then apoptotic granulocytes may undergo secondary necrosis and cause tissue damage. This is supported by the observation that apoptotic granulocytes incubated in vitro in the absence of macrophages spontaneously rclcasc their granule contents $[15,16]$. Thus it has become apparent that as well as the processes involved in the induction and propagation of the inflammatory 


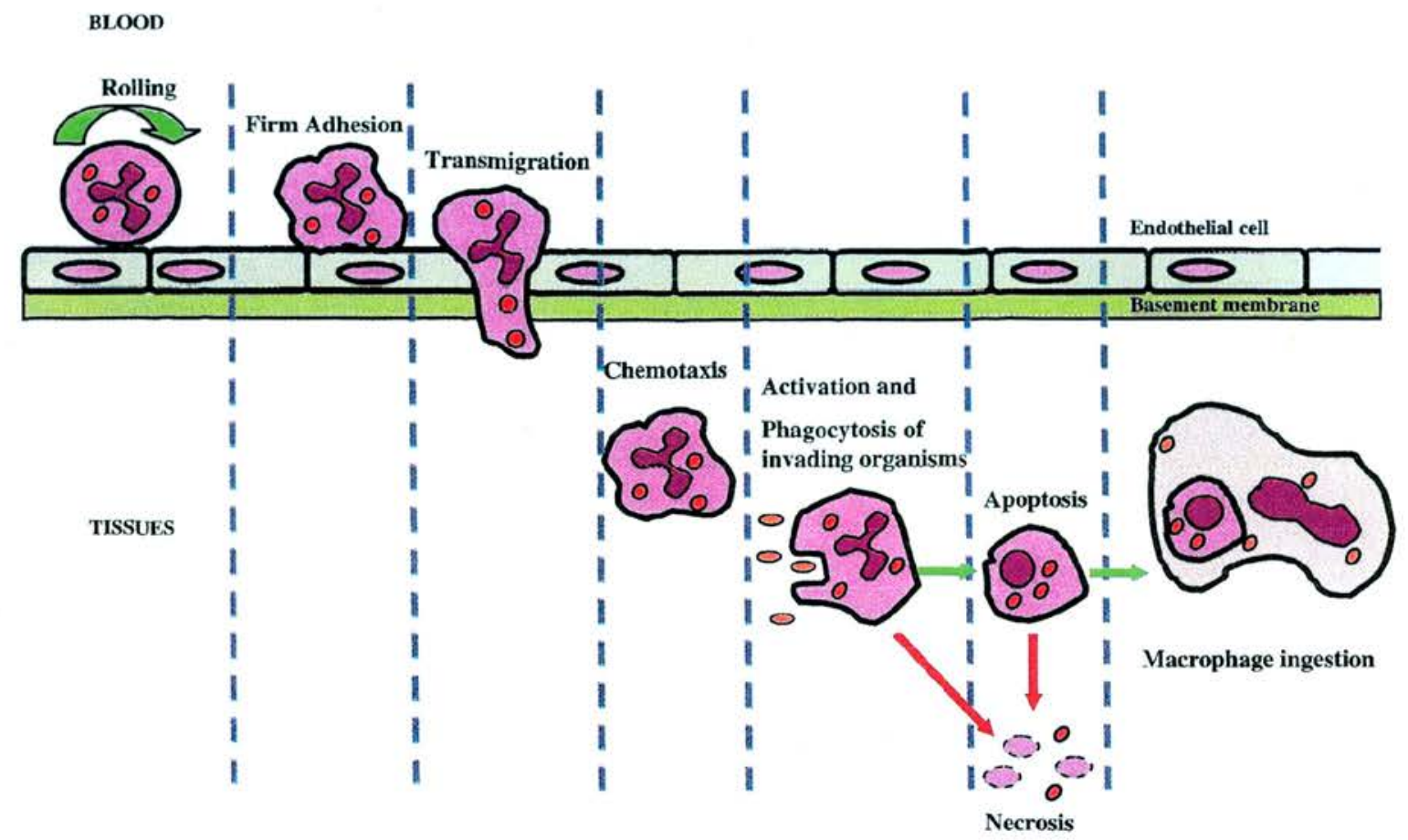

Fig. (1). Processes involved in leukocyte recruitment. Inflammatory cclls normally leave the blood by binding loosely to postcapillary venule endothelial cells before flattening and transmigrating to sites of infection through endothelial cell junctions and interstitial tissue. Invading organisms are then phagocytosed or destroyed by, for example, neutrophils, which then liberate their toxic contents into phagolysosomes. Once the useful function of recruited inflammatory cells has been fulfilled they normally die by the process of apoptosis. Apoptotic cells are then ingestcd and cleared by phagocytes such as macrophages using non-inflammatory mechanisms. Under non-physiological conditions inflammatory cells can undergo tissue damaging 'primary' necrosis or by 'secondary' necrosis in the case of failed phagocytic clearancc.

response, the mechanisms controlling its resolution (e.g. granulocyte apoptosis and phagocytosis of apoptotic granulocytes) are also critical in determining the ultimate outcome of the inflammatory response [17-19]. We therefore believe that by understanding the mechanisms underlying the resolution of the inflammatory response, novel highly effective therapies for the prevention and treatment of inflammatory diseases including allergic diseases such as asthma, allergic rhinitis, eczema, contact dermatitis and chronic sinusitis may be a reality. Our laboratory is focused on investigating the mechanisms controlling granulocyte apoptosis with a view to selectively induce apoptosis in neutrophilic and eosinophilic diseases for therapeutic benefit.

\section{Regulation of Granulocyte Apoptosis}

Seminal work in the early seventies described a physiological mode of cell death termed apoptosis [20]. Previously, it was believed that cell death occurred primarily by necrosis [21]; a process that would ultimately lead to the release of histotoxic intracellular contents provoking an exaggerated inflammatory response. Evolutionary conservation of death programs across a diverse range of species and tissue types means that in many cases the central pathways leading to cellular demise are common to different cell types. However, a thorough understanding of the subtle differences in the apoptotic program between different cells may be key for selective drug targeting strategies for the therapeutic manipulation of disease pathogenesis.

Fig. (3) provides an overview of the apoptotic pathways. The death receptor pathway may be triggered by binding of Fas ligand (FasL), inducing trimerisation of the Fas receptor (FasR). This results in the recruitment of procaspase-8 (procaspases are proteolytically cleaved to produce the active caspase form) via the adaptor molecule FADD, leading to activation of caspase-8. Caspase-8 (also known as FLICE) can be blocked by FLIP (FLICE-inhibitory protein). In socalled type II cells, caspase- 8 mediates cleavage of Bid to truncated Bid (tBID) which then acts on the mitochondria to induce apoptosis. In certain cells (type I cells) caspase- 8 can cleave procaspase- 3 directly to promote apoptosis. The milochondrial death pathway is induced by cellular stress which includes cytotoxic drugs, serum withdrawal or DNA damage. The death receptor and mitochondrial pathways converge at the point of caspase- 3 activation, which results in the cleavage of cellular substrates and apoptosis. Inhibitor of apoptosis proteins (IAPs) are thought to act as pseudosubstrates for caspases, binding to active caspases and neutralizing their activity. However, upon the induction of apoptosis, the inhibitory activity of IAPs is suppressed by a second mitochondria-derived activator of caspase (Smac/ DIABLO). Bcl-2 family members can be functionally divided into two classes, those that are anti-apoptotic (e.g. 


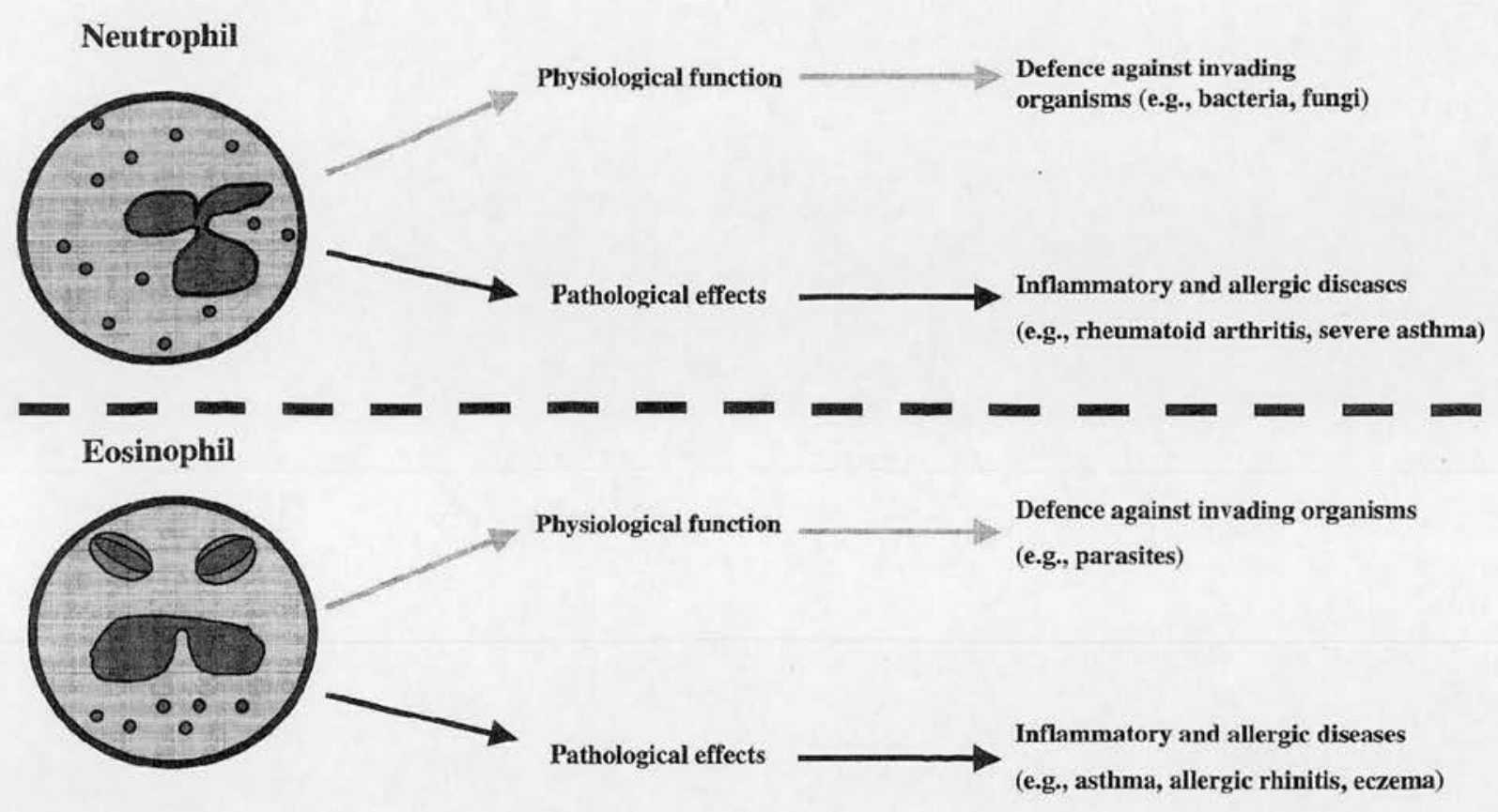

Fig. (2). Pathophysiological role of granulocytes. The physiological function of granulocytic neutrophils is to destroy invading organisms such as bacteria and fungi whereas eosinophils are particularly effective in the defense against parasitic infection. However inappropriate or exaggerated granulocytic recruitment or activation can lead to the release of tissue destructive granular contents, which contributes heavily toward the pathogenesis and propagation of chronic inflammatory conditions.

Bcl-2, Mcl-1) and those that promote apoptosis (e.g. Bax, $\mathrm{Bad})$. Many proteins have a C-terminal region that allows them to interact with the outer mitochondrial membranc. For example, Bax is normally cytosolic but upon the induction of apoptosis is inserted into the mitochondrial membrane. Activation of caspase- 9 requires the release of cytochrome $c$ and apoptosis protease-activating factor (Apaf-1) from the inner mitochondrial membrane. Binding of cytochrome $\mathrm{c}$ and ATP is thought to cause a conformational change in Apaf- 1 , which allows caspase- 9 to activate caspase- 3 (for review see [117]).

There are fundamental differences in apoptotic control mechanisms governing many cell types including thymocyte apoptosis compared to granulocyte apoptosis and indeed between eosinophil and neutrophil apoptosis [22, 23, 19].

Neutrophils undergo constitutive apoptosis during in vitro culture, but factors such as TNF- $\alpha$, IL-15, INF- $\gamma$, GCSF, GM-CSF, LPS and hypoxic conditions can inhibit this process $[22,19]$. On the other hand, while GM-CSF also prolongs eosinophil life-span, IL-5 has no effect on neutrophil survival/apoptosis. It is likely that granulocytes would encounter these stimuli at inflammatory sites in vivo, thereby prolonging their life-span and allowing them to fulfil their functional roles. Delayed apoptosis is however not a universal phenomenon associated with all proinflammatory mediators. For example TNF- $\alpha$ can induce neutrophil apoptosis following short term culture [24] and other agents with pro-inflammatory properties do not have a major effect on granulocyte apoptosis [25, 26]. Many death receptors belong to the TNF receptor family and contain homologous cytoplasmic regions termed "death domains" $[27,28]$. The signalling mcchanisms underlying TIJF- $\alpha$ mediated apoptosis/survival are unknown but likely involve receptor associated proteins such as TNF-receptor-associated death domain (TRADD), Fas-associated death domain (FADD) and caspase- 8 . Ligand binding results in the recruitment of the death-inducing signalling complex (DISC) when procaspase- 8 is activated [29]. Apoptosis proceeds by the direct activation of downstream caspases by caspase- 8 in type I cells; alternatively, it proceeds through caspase-8initiated modulation of $\mathrm{Bcl}-2$ family members, which are able to influence the mitochondrial pathway of caspase activation, in type II cells [30]. At present, it is still unclear which pathway is utilised by granulocytes.

The pro-apoptotic Bcl-2 family members, Bax, Bad, Bak and $\mathrm{Bid}$ are constitutively expressed in neutrophils and have a long half-life, perhaps explaining why on balance neutrophils are 'primed' for apoptosis [31]. Further, during constitutive neutrophil apoptosis, Bax translocates from the cytoplasm to the mitochondria resulting in caspase-3 activation [32]. In addition, translocation is not inhibited by $\mathrm{z}$-VAD-fmk, the broad range caspase inhibitor, suggesting that Bax redistribution is caspase-independent [33].

Eosinophils have similarly been shown to contain mitochondria which appear to have negligible respiratory chain function but instead appear to play a central role in the apoptotic process [34]. Differences in the activation of caspases -3 and -8 have also been noted in granulocytes where 


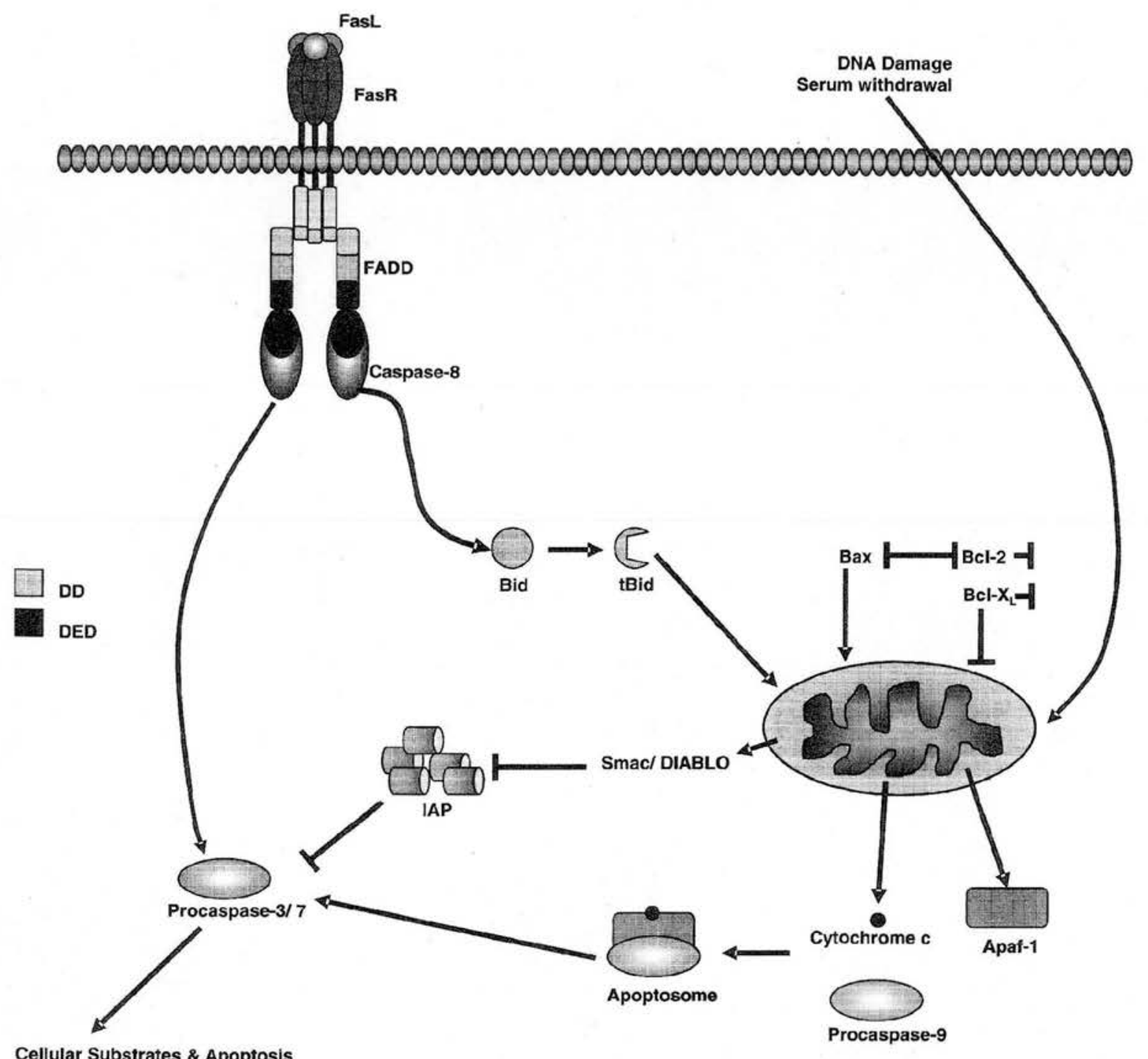

Fig. (3). General Overview of Apoptosis Pathway. The death reccptor pathway may be triggered by binding of Fas ligand (FasL), inducing trimerisation of the Fas receptor (FasR). This results in the recruitment of procaspase- 8 via the adaptor molecule FADD leading to activation of caspase- 8 . Caspase- 8 (or FLICE) can be blocked by FLIP (FLICE-inhibitory protein). In type II cells, caspase- 8 mediates cleavage of Bid to truncated Bid (tBID) which then acts on the mitochondria to induce apoptosis. In type I cells caspase- 8 can cleave procaspasc- 3 directly to promote apoptosis. The mitochondrial death pathway is induced by cellular stress. The death receptor and mitochondrial pathways converge at the point of caspase-3 activation, which results in the cleavage of cellular substrates and apoptosis. IAP proteins bind to active caspases and neutralize their activity. The inhibitory activity of IAPS is suppressed by Smac/ DIABLO. Bcl-2 family members can be functionally divided into two classes, those that are anti-apoptotic (e.g. $\mathrm{Bcl}-2, \mathrm{Mcl}-1$ ) and those that promote apoptosis (e.g. Bax, Bad). Bax is normally cytosolic but upon the induction of apoptosis is inserted into the mitochondrial membrane. Activation of caspasc-9 rcquires the release of cytochrome $c$ and Apaf-1 from the inner mitochondrial membrane. Binding of cytochrome $c$ and ATP is thought to cause a conformational change in Apaf-1, which allows caspase- 9 to activate caspase- 3 .

caspases -3 and -8 were reported to play a critical role in neutrophil but not eosinophil apoptosis [35] (see Fig. (3) for a schematic diagram showing the major pathway mediated by Fas-induced death). The signalling mechanisms governing granulocyte survival remain ill-defined but, depending on the survival factor involved, will involve complex interactions between various kinase cascades such as PI3K, MAPK and PKC $[19,22,23,36]$. Furthermore, transcriptional regulation has also been implicated in the control of granulocyte apoptosis, for examplc, inhibition of $\mathrm{NF}-\kappa \mathrm{B}$ dramatically enhances constitutive apoptosis and $N F-\kappa B$ activation critically regulates the retardation of apoptosis induced by LPS and TNF- $\alpha[37,38]$. 


\section{HEMOPOIETIC GROWTH FACTORS AND CYTOKINES AND THEIR EFFECTS ON APOPTOSIS}

Hemopoietic growth factors are a family of glycoproteins with multiple regulatory functions of hemopoietic progenitors. Some of them profoundly influence granulocyte production, differentiation, functional activation and longevity. The hemopoietic growth factors include erythropoietin, colony stimulating factors (CSF), various interleukins (IL-1 - IL-13) and stem-cell factor (SCF) [39].

Allergic diseases are commonly attributed to an 'inappropriate' immune response mediated by a polarised Th2 response [40]. Cytokines secreted by Th2 cclls include IL-3, IL-5 and GM-CSF [41] which are known to influence granulocyte survival [42]. This has led to the view that therapeutic intervention should be focused on modulating the levels and or activities of these cytokines.

\section{GM-CSF, IL-3 and IL-5}

GM-CSF, IL-3 and IL-5 stimulate granulocyte production from the bone marrow, cause activation and suppress apoptosis (except for IL-5 which does not affect neutrophils). Levels of these cytokines are often found to be elevated during allergy and are suggestive that they are key players in allergic disease [43]. Under normal circumstances GM-CSF is only found at very low levels in the circulation but becomes readily detectable in atopic individuals [44]. GM-CSF has long been known to enhance eosinophil and neutrophil survival [45] and one recent study has suggested that it more efficiently delays eosinophil apoptosis than either IL-3 or IL-5 [46] with which GM-CSF shares a receptor subunit $[24,37,47]$. The GM-CSF receptor belongs to the class 1 subgroup of the cytokine receptor superfamily and is expressed not only on cells of the granulocytemacrophage lineage, but also on endothelial cells where it is postulated to have a role in proliferation and migration [48, 49]. The GM-CSF receptor is composed of two subunits: the $\alpha$ chain, which binds GM-CSF with only low affinity by itself; and the $\beta$ chain, which does not bind ligand but is required for high-affinity binding in conjunction with the $\alpha$ chain [50-52]. While the $\alpha$ chain is specific for GM-CSF, the $\beta$ chain is common for the receptors of GM-CSF, IL-3 and IL-5. The role of the common $\beta$-chain in signalling has been relatively well characterised. It is known that the common $\beta$-chain is able to interact with various receptorassociated proteins that are important for the signalling downstream of the receptor [53-55]. In contrast, relatively little is known about the cytokine-specific signalling events mediated by the short cytoplasmic domains of the $\alpha$-chains.

One consequence of GM-CSF signalling in eosinophils is inhibition of Bax activation/translocation and maintenance of the X-Chromosome-linked inhibitors of apoptosis ( $\mathrm{x}$ IAPs) even in the face of glucocorticoid treatment (Fig. (3)). Prolonged JNK activation is necessary for Bax translocation in dexamethasone-treated eosinophils and while GM-CSF did not affect initial $\mathrm{JNK}$ activation, prolonged activation was abolished [56]. Similarly in neutrophils, GM-CSF was demonstrated to reducc intracellular Bax levels in vitro during culture, which correlated with enhanced neutrophil survival [57] and was also responsible for maintaining the level of the anti-apoptotic protein $\mathrm{Mcl}-1$ [31]. In addition, GM-CSF has been suggested to maintain the level of Bcl$\mathrm{X}_{\mathrm{L}}$ and to influence caspase- 3 activity [58].

GM-CSF acts on both eosinophils and neutrophils while IL-5 acts specifically on eosinophils. Increased levels of IL-5 have been detected in bronchoalveolar lavage (BAL) fluid and lung biopsies $[59,60]$ from asthmatic patients and the levels of IL-5 have also been shown to correlate to the number of eosinophils present in the lung [60]. Interestingly, IL-5 has long been known to increase eosinophil longevity $[61,62]$ although the intracellular survival mechanisms remain relatively ill defined. Ligand binding to the IL-5 receptor activates the Jak2/Stat pathway, MAPK cascades, Lyn and Syk tyrosine kinases [63] and Src homology 2 phosphatase 2 tyrosine phosphatase (SHPTP-2) [64]. However, how these signals integrate into the apoptotic pathway and prevent Bax translocation to the mitochondrion $[65]$ is unknown. IL-5 binding has becn shown to upregulate $\mathrm{Bcl}-\mathrm{X}_{\mathrm{L}}[66]$ and $\mathrm{Bcl}-2$ [67] demonstrating that eosinophil survival is protein synthesis dependent.

Considerable work has been carried out to investigate the therapeutic potential of removing IL-5 either by gene deletion or blocking antibodies from experimental models of allergic inflammation. In animal models of asthma there have been promising results. In a mouse model, administration of an antibody to IL-5 prevented airway hyper-reactivity (AHR) [68]; alternatively, in transgenic mice that constitutively express IL- 5 there was increased AHR [69]. Conversely, there are other reports of the use of monoclonal antibodies against IL- 5 in mouse models where IL- 5 significantly reduced eosinophilia but did not prevent AHR $[70,71]$. The expectation was that abrogation of IL-5 would reduce the number of eosinophils and thereby improve the clinical symptoms of asthma. However, in a human trial, paticnts with asthma were treated with a monoclonal antibody against IL-5 (mepolizumab) and although eosinophil counts were effectively reduced in blood and induced sputum, there was no improvement in AHR [72]. A recent study, however, showed that mepolizumab only reduced airway tissue and bone marrow eosinophils by $50 \%$. In addition, they demonstrated that eosinuphil degranulation was on going even after high doses of mepolizumab treatment in the residual airways [73]. Therefore, the authors concluded that the eosinophil couldn't yet be excluded as a therapeutic target in asthma. A further twist to the IL-5 story, however, is a recently published study using IL-5 transgenic mice, which when challenged with allergen induced a vigorous accumulation of eosinophils into their airways. Astonishingly, the eosinophilia protected against allergen-induced AHR, which the authors attributed to eosinophil TGF- $\beta$ production [74]. Interestingly, a recent study has shown that the IL-3, IL-5 and GM-CSF- $\alpha$ chain receptors are regulated differentially in eosinophils. All three cytokines mediate a reduction in IL-5$\alpha$ chain receptor expression but up-regulated IL-3- $\alpha$ chain receptor and only GM-CSF is able to regulate GM-CSF- $\alpha$ chain expression [75]. It may be, therefore, that a more effective therapeutic target would be the common $\beta$ chain receptor. Mice lacking the common $\beta$ chain receptor had reduced eosinophil responses to airway challenge [76] suggesting that while IL-3, IL-5 and GM-CSF are clearly 
critical to eosinophil recruitment and survival, they do not fully account for the role of granulocytes in allergic disease.

\section{G-CSF}

G-CSF is the major hematopoietc growth factor involved in the control of neutrophil development and is essential for neutrophil release from the bone marrow. G-CSF signals through a receptor of the hematopoietin superfamily, to activate a variety of signalling pathways including Jak/Stat, Ras-Raf-MAP Kinase and Src family MAP Kinase pathways [77]. G-CSF is the principal hemopoietic growth factor regulating neutrophil release from the bone marrow [78] and can also extend the life-span of mature neutrophils (Fig. (4)) $[79,31,57]$ and as such has a role in regulating the number of circulating neutrophils. Already G-CSF has been used clinically to stimulate neutrophil production as a therapy for neutropenia [80]. Whether G-CSF can be targeted therapeutically in allergy to influence the number of recruited or circulating neutrophils has not been fully investigated.

\section{IL-4 and IL-13}

While most research to date has been focused on IL-5, other hemopoietic factors are gaining attention. Key cytokines produced in many models of allergic inflammation, with effects on granulocytes, during a Th2type response include IL-4 and IL-13, which in addition to their role in immune regulation have also been shown to have a direct effect on granulocyte survival/apoptosis. IL-4 and $\mathrm{IL}-13$ both bind to the same receptor subunit IL-4R $\alpha$, explaining many of the overlapping biological activities of these cytokines [81]. Binding of ligand to IL- $4 \mathrm{R} \alpha$ leads to the recruitment of either $\gamma c$ (type 1 receptor) or IL-13R $\alpha 1$ (type 2 receptor). Type 1 receptor complexes can only be initiated by IL- 4 binding whereas type 2 receptor complexes can be mediated by both IL- 4 and IL-13. The heterodimeric receptor in the case of IL- 4 is formed by the initial binding of IL- 4 to IL $4 \mathrm{R} \alpha$ with the subsequent recruitment of IL$13 \alpha 1$, whereas IL-13 binds IL- $13 \alpha 1$ prior to recruiting IL$4 \mathrm{R} \alpha$ [82]. IL-4 was shown to induce apoptosis directly in both eosinophils [83] and neutrophils (Fig. (4)) [84] in vitro, although the signalling events have yet to be characterized. In the case of eosinophils, IL- 4 was not able to overcome the survival effects of IL-3, IL-5 or GM-CSF [83]. IL-13 on the other hand induced a variety of changes including morphological cell shape changes, protein synthesis and IL-8 production but there was no effect on neutrophil survival or apoptosis [25]. In eosinophils, IL-13 not only promoted activation but also enhanced eosinophil survival (Fig. (4)) [85]. In keeping with this, in transgenic mice overexpressing IL-13, eosinophilia, AIIR and airway

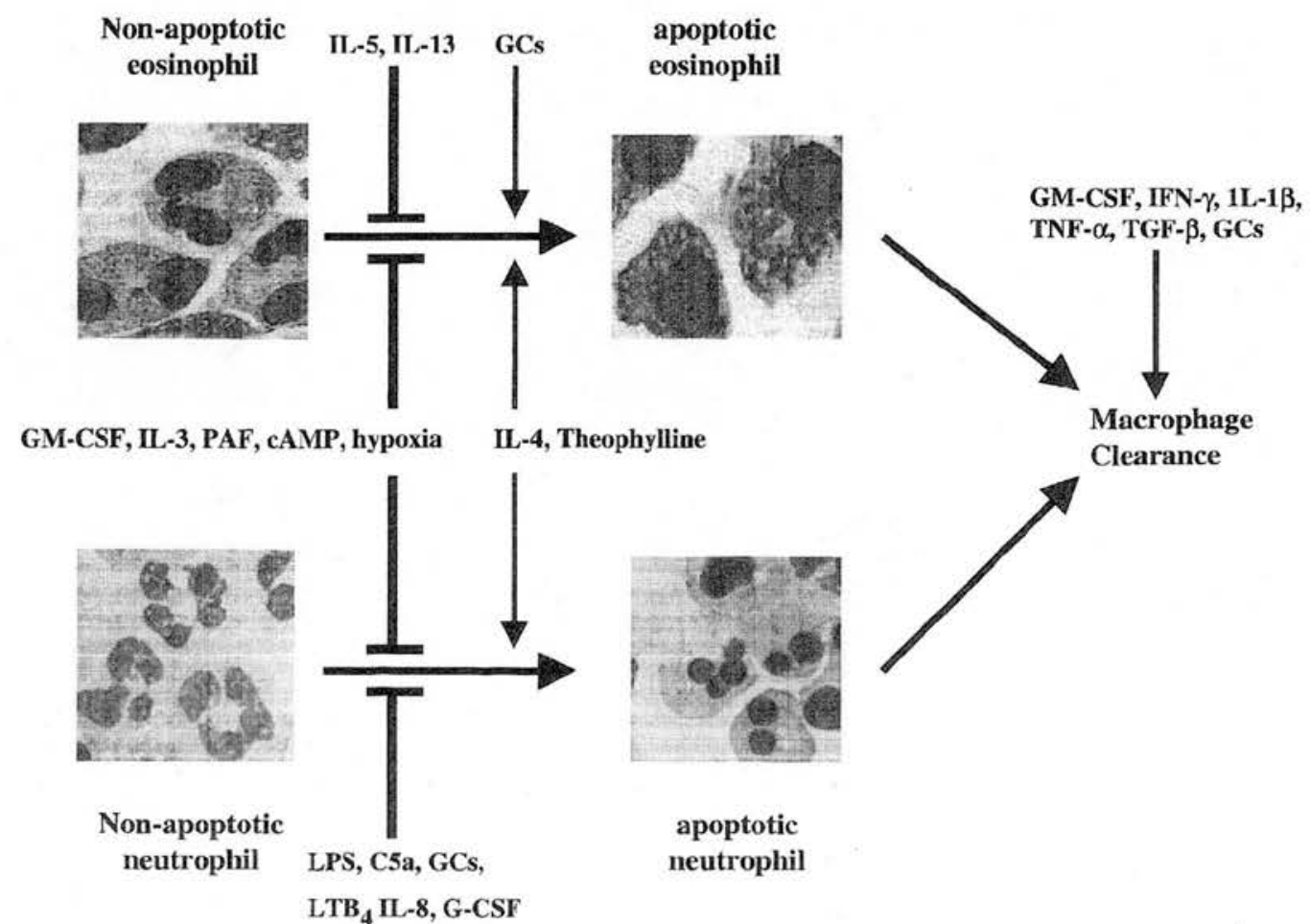

Fig. (4). Regulation of human granulocyte apoptosis by hemopoietic factors, cytokines and drugs. Functionally competent but potentially histotoxic granulocytes undergo constitutive apoptosis where the cells become hyporesponsive to stimulation and are recognizable by phagocytes such as macrophages. The rate of apoptosis can be altered dramatically by a number of factors. For example, certain hemopoetic factors and inflammatory mediators (e.g. GM-CSF, LPS, C5a, IL-1 $\beta$ INF- $\gamma$, LTB 4 , or PAF), environmental conditions (e.g. hypoxia, extracellular matrix proteins), increases in signalling molecule levels $\left(\mathrm{cAMP}^{\mathrm{AM}} \mathrm{Ca}^{2+}\right.$ ), as well as glucocorticoids can all alter the rate of apoptosis. Other stimuli such as TNF- $\alpha$, nitric oxide, Fas-L, and bacterial and fungal products can promote the onset of apoptosis. 
fibrosis were observed [86] and conversely in IL-13 knockout mice, AHR did not occur after challenge with allergen [87]. Studies targeting the blockade of IL-13 [88] have had promising results but whether the effects of IL-13 are due to its direct effects on granulocytes is debatable. Instead, IL-13 has been argued to primarily exert its effects through action on epithelial cells and smooth muscle cells [89].

\section{DRUGS USED IN THE TREATMENT OF ALLERGY AND THEIR EFFECTS ON APOPTOSIS}

Although there are several classes of drugs used in the treatment of allergy, very few have been investigated for their effects on granulocyte apoptosis. It is unknown, for example, whether the antihistamine class of drugs directly influence granulocyte survival or apoptosis, a possibility that should be further investigated. Therefore, we have focused on some of the major drugs used in allergic disease known to modulate granulocyte apoptosis.

\section{Glucocorticoids}

Glucocorticoids are one of the main anti-inflammatory classes of drugs widely used in allergic inflammation [90]. More recently of direct clinical importance is the discovery that glucocorticoids dramatically influence granulocyte apoptosis and also profoundly augment macrophage capacity for safe, non-inflammatory clearance of apoptotic granulocytes in vitro [91, 92] (Fig. (4)). However, while glucocorticoids accelerate eosinophil and lymphocyte apoptosis, they delay neutrophil apoptosis [93]. Although the mechanisms underlying this divergent effect of glucocorticoids on inflammatory cell apoptosis are currently unknown, the effect may explain why steroid therapy is so efficacious in eosinophilic inflammation such as asthma. Interestingly, asthmatic patients who show an improvement in lung function after corticosteroid treatment also have an increased proportion of apoptotic eosinophils in their airway secretions [94]. However, the enhancement of neutrophil survival by glucocorticoids (Fig. (4)) could be a concern in the treatment of chronic severe asthma where neutrophils play an important role $[2,95,96]$.

Glucocorticoids are cell permeablc and thus are able to bind directly to the cytosolic glucocorticoid receptor (GR). There are two GRs GR $\alpha$ and GR $\beta$, as a result of alternative splicing. GR $\alpha$ binds glucocorticoids with high affinity; in contrast, GR $\beta$ lacks a stcroid binding domain and is unable to bind glucocorticoids [97, 98]. Recently, it was shown that glucocorticoid sensitive mouse hybridoma cells stably transfected with GR $\beta$ became resistant to glucocorticoid treatment and it was suggested that increased expression of GR $\beta$ could contributc to chronic inflammatory diseases which are refractory to glucocorticoid treatment [99]. Interestingly, GR $\beta$ has becn shown to be the predominant isoform of GR in ncutrophils [100] and the inability of this receptor to inducc apoptosis may contribute to the pathogenesis of severe asthma. Therefore, further research delineating the molecular consequences of high GR $\beta$ levels is needed in order to target neutrophils for therapeutic advantage in severe asthma.

\section{Theophylline}

Theophylline has been used for over 60 years in the trcatment of asthma and yet its molecular mechanisms remain poorly defined [101]. Interestingly, theophylline is known as a phosphodiesterase (PDE) inhibitor and has becn shown to induce neutrophil and eosinophil apoptosis [102]. Elevation of intracellular cyclic-adenosine-monophosphate (cAMP) delays apoptosis in neutrophils (Fig. (4)) [103, 104] in contrast to inducing apoptosis in other cell types [105]. The effect of cAMP on eosinophil apoptosis is more complex since cAMP has been shown to delay constitutive apoptosis [106-108] but inhibits the survival induced by GM-CSF [106]. It is thereforc questionable whether the effect of theophylline on apoptosis is due to its effect on cAMP. Indeed, the induction of apoptosis in neutrophils appears to be via adenosine- $A_{2}$ receptors [109] whereas in lymphocytes effects occur via PDE inhibition [110, 105]. Clinical studies have shown that combination therapy of glucocorticoids and theophylline can be more effective in controlling mild to moderate asthma than doubling the dose of glucocorticoids [111, 112]. It is possible that one advantage of combination therapy would bc that theophylline is able to overcome glucocorticoid-mediated inhibition of neutrophil apoptosis and thereforc provide an effective method of removing both cosinophils and neutrophils from sites of inflammation.

\section{Leukotrienes}

The cysteinyl leukotrienes $\left(\mathrm{LTC}_{4}, \mathrm{LTD}_{4}, \mathrm{LTE}_{4}\right)$ are proinflammatory arachidonic acid derived mediators which have been shown to be involved in immediate hypersensitivity reactions, influence bronchoconstriction and vascular permeability and may partly mediatc cosinophilia associated with asthma [113]. Recently, LTD $_{4}$ receptor antagonists have becn successfully used in the treatment of asthma espccially in combination with glucocorticoid therapy $[114,115]$. Whether these agents will be useful in the management of other allergic diseases such as allergic rhinitis or eczema awaits investigation and the outcome of clinical trials. Although the cysteinyl LTs can influence eosinophil function, whether these inflammatory mediators and their antagonists can modulate apoptosis is less well defined. For example, despitc $\mathrm{LTD}_{4}$ bcing a potent stimulator for the clevation of eosinophil intracellular $\left[\mathrm{Ca}^{2+}\right]$, it does not scem to affect the longevity of these cells in vitro [26]. However, the activation status of the cells may bc important since the apoptotic program of eosinophils derived from asthmatic patients does appear to be inhibited by the cysteinyl LTs [116]. It may be that the cysteinyl LTs and/or the antagonists would influence granulocyte apoptosis when the apoptotic pathways have been altered by preexposure to other mediators such as GM-CSF, IL-3 or IL-5.

\section{CONCLUSION}

Although in recent years there has been some progress in unraveling the intracellular signalling mechanisms induced by hemopoietic factors and cytokines that influence the balance between life and death of granulocytes, further work is required. We believe that it is necessary to target neutrophil and eosinophil function and apoptosis pathways 
selectively for the treatment of diseases where these cells play a prominent role.

\section{ACKNOWLEDGEMENTS}

This work was supported by the Norman Salvesen Emphysema Research Trust and the National Asthma Campaign UK.

\section{ABBREVIATIONS}

\begin{tabular}{|c|c|c|}
\hline Apaf-1 & $=$ & Apoptotic Protease Activating Factor-1 \\
\hline $\mathrm{BAD}$ & $=$ & Bcl-XL /Bcl-2-associated Death Promoter \\
\hline BAL & $=$ & Bronchoalveolar Lavage \\
\hline $\mathrm{Bcl}$ & $=$ & B-cell Lymphoma \\
\hline DD & $=$ & Death Domain \\
\hline DED & $=$ & Death Effector Domain \\
\hline DISC & $=$ & Death Inducing Signalling Complex \\
\hline FADD & $=$ & Fas-Associated Death Domain \\
\hline fMLP & $=$ & $\mathrm{n}$-formylmethionylleucyl phenylalanine \\
\hline G-CSF & $=$ & Granulocyte Colony Stimulating Factor \\
\hline GM-CSF & $=$ & $\begin{array}{l}\text { Granulocyte Macrophage Colony } \\
\text { Stimulating Factor }\end{array}$ \\
\hline ICAM & $=$ & Intercellular Adhesion Molecule \\
\hline IFN & $=$ & Interferon \\
\hline IL & $=$ & Interleukin \\
\hline Jak & $=$ & Janus Kinase \\
\hline JNK & $=$ & Jun N-terminal Kinase \\
\hline$N F-\kappa B$ & $=$ & Nuclear Factor- $\kappa \mathrm{B}$ \\
\hline PDE & $=$ & Phosphodiesterase \\
\hline PI3-Kinase & $=$ & Phosphoinositide 3-Kinase \\
\hline PKC & $=$ & Protein Kinase C \\
\hline STAT & $=$ & $\begin{array}{l}\text { Signal Transducer and Activator of } \\
\text { Transcription }\end{array}$ \\
\hline TNF & $=$ & Tumour Necrosis Factor \\
\hline TNFR & $=$ & Tumour Necrosis Factor Receptor \\
\hline TRADD & $=$ & TNFR-associated Death Domain \\
\hline TRAF2 & $=$ & TNFR-Associated Death Domain \\
\hline X-IAP & $=$ & $\begin{array}{l}\text { X-Chromosome Linked Inhibitor of } \\
\text { Apoptosis }\end{array}$ \\
\hline z-VAD-fmk & $=$ & $\begin{array}{l}\text { N-benzyloxycarbonyl-Val-Ala-Asp- } \\
\text { fluoromethylketone; }\end{array}$ \\
\hline
\end{tabular}

\section{REFERENCES}

[1] Cookson, W. Nature 1999, 402, B5.

[2] Wenzel, S. E.; Szefler, S. J.; Leung, D. Y.; Sloan, S. I.; Rex, M. D.; Martin, R. J. Am. J. Respir. Crit. Care Med. 1997, 156, 737.

[3] Serghides, L.; Kain, K. C. J. Immunol. 2001, 166, 6742.

[4] Wagner, J. G.; Roth, R. A. Pharmacol. Rev. 2000, 52, 349.
[5] Crockett-Torabi, E.; Sulenbarger, B.; Smith, C. W.; Fantone, J. C. J. Immunol. 1995, I54, 2291.

[6] Rossi, A. G.; Hellewell, P. G. In Immunopharamacology of neutrophils. Eds. Hellewell P.G. \& Williams, T.J. London: Academic Press. 1994, Chapt. 10, 223-340.

[7] Borregaard, N.; Cowland, J. B. Blood 1997, 89, 3503.

[8] Giembycz, M. A.; Lindsay, M. A. Pharmacol. Rev. 1999, 51, 213.

[9] Crocker, P. R.; Varki, A. Trends Immunol. 2001, 22, 337.

[10] Haslett, C.; Shen, A. S.; Feldsien, D. C.; Allen, D.; Henson, P. M. Cherniack, R. M. Am. Rev. Respir. Dis. 1989, 140, 756.

[11] Haslett, C. Clin. Sci. (Lond) 1992, 83, 639.

[12] Savill, J. Kidney Int. 1992, 41, 607.

[13] Whyte, M. K.; Savill, J.; Meagher, L. C.; Lee, A.; Haslett, C. J. Leukoc. Biol. 1997, 62, 195.

[14] Savill, J.; Fadok, V.; Henson, P.; Haslett, C. Immunol. Today 1993, 14, 131.

[15] Meagher, L. C.; Savill, J. S.; Baker, A.; Fuller, R. W.; Haslett, C. J. Leukoc. Biol. 1992, 52, 269.

[16] Stern, M.; Savill, J.; Haslett, C. Am. J. Pathol. 1996, 149, 911.

[17] Haslett, C. Am. J. Respir. Crit. Care Med. 1999, 160, S5.

[18] Lawrence, T.; Willoughby, D. A.; Gilroy, D. W. Nat. Rev. Immunol. 2002, 2, 787.

[19] Ward, C.; Dransfield, I.; Chilvers, E. R.; Haslett, C.; Rossi, A. G. Trends Pharmacol. Sci. 1999, 20, 503.

[20] Kerr, J. F,; Wyllie, A. H.; Currie, A. R. Br. J. Cancer 1972, 26 , 239.

[21] Hurley, J. In Termination of Acute Inflammation I Resolution, $2^{\text {nd }}$ Ed. London, Churchill Livinstone, 1983, 109-117.

[22] Akgul, C.; Moulding, D. A.; Edwards, S. W. FEBS Lett. 2001, 487, 318.

[23] Walsh, G. M. Br. J. Haematol. 2000, 111, 61.

[24] Murray, J.; Barbara, J. A.; Dunkley, S. A.; Lopez, A. F.; Van, Ostade, X; Condliffe, A. M.; Dransfield, I.; Haslett, C.; Chilvers, E. R. Blood 1997, 90, 2772.

[25] Girard, D.; Paquin, R.; Naccache, P. H.; Beaulieu, A. D. J. Leukoc. Biol. 1996, 59, 412.

[26] Murray, J.; Ward, C.; O'Flaherty, J. T.; Dransfield, I.; Haslett, C.; Chilvers, E. R.; Rossi, A. G. Br. J. Pharmacol. 2003, 139, 388.

[27] Smith, C. A.; Farrah, T.; Goodwin, R. G. Cell 1994, 76, 959.

[28] Tartaglia, L. A.; Ayres, T. M.; Wong, G. H.; Goeddel, D. V. Cell 1993, 74, 845 .

[29] Ashkenazi, A.; Dixit, V. M. Science 1998, 281, 1305.

[30] Scaffidi, C.; Fulda, S.; Srinivasan, A.; Friesen, C.; Li, F.; Tomaselli, K. J.; Debatin, K. M.; Krammer, P. H.; Peter, M. E. EMBO J. $1998,17,1675$.

[31] Moulding, D. A.; Quayle, J. A.; Hart, C. A.; Edwards, S. W. Blood $1998,92,2495$

[32] Pryde, J. G.; Walker, A.; Rossi, A. G.; Hannah, S.; Haslett, C. J. Biol. Chem. 2000, 275, 33574.

[33] Maianski, N. A.; Mul, F. P.; van Buul, J. D.; Roos, D.; Kuijpers, T. W. Blood 2002, 99, 672 .

[34] Peachman, K. K.; Lyles, D. S.; Bass, D. A. Proc. Natl. Acad. Sci. USA 2001, 98, 1717.

[35] Daigle, I.; Simon, H. U. Int. Arch. Allergy Immunol. 2001, 126, 147.

[36] Simon, H. U. Immunol. Rev. 2001, 179, 156.

[37] Ward, C.; Chilvers, E. R.; Lawson, M. F.; Pryde, J. G.; Fujihara, S.; Farrow, S. N.; Haslett, C.; Rossi, A. G. J. Biol. Chem. 1999, 274, 4309.

[38] Fujihara, S.; Ward, C.; Dransfield, I.; Hay, R.; Uings, I.; Hayes, B.; Farrow, S.; Haslett, C.; Rossi, A. Eur. J. Immunol. 2002, 32, 457.

[39] Metcalf, D. Science 1985, 229, 16.

[40] Woodfolk, J. A.; Platts-Mills, T. A. Int. Arch. Allergy Immunol. $2002,129,277$

[41] Neurath, M. F.; Finotto, S.; Glimcher, L. H. Nat. Med. 2002, 8, 567.

[42] Umetsu, D. T.; McIntire, J. J.; Akbari, O.; Macaubas, C.; DeKruyff, R. H. Nat. Immunol. 2002, 3, 715.

[43] Ramshaw, H. S.; Woodcock, J. M.; Bagley, C. J.; McClure, B. J.; Hercus, T. R.; Iopez, A. F. Immunol. Cell Biol. 2001, 79, 154.

[44] Hamilton, J. A. Trends Immunol. 2002, 23, 403.

[45] Rossi, A. G.; Ilaslett, C., In Pro-infiammatory and antiinflammatory Peptides, Ed. Said S.I., 1998, in the Lung Biology in Health and Disease series (Ed. Lenfant, C.) Chapt. 2, 9-24. 
[46] Park, C. S.; Choi, Y. S.; Ki, S. Y.; Moon, S. H.; Jeong, S. W.; Uh, S. T.; Kim, Y. H. Eur. Respir. J. 1998, 12, 872.

[47] Ilannah, S.; Mecklenburgh, K.; Rahman, I.; Bellingan, G. J.; Greening, A.; Haslett, C.; Chilvers, E. R. FEBS Lett. 1995, 372, 233.

[48] Bussolino, F.; Ziche, M.; Wang, J. M.; Alessi, D.; Morbidelli, L.; Cremona, O.; Bosia, A.; Marchisio, P. C.; Mantovani, A. J. Clin. Invest 1991, 87, 986.

[49] Valdembri, D.; Serini, G.; Vacca, A.; Ribatti, D.; Bussolino, F. FASEB J. 2002, 16, 225.

[50] Gearing, D. P.; King, J. A.; Gough, N. M.; Nicola, N. A. EMBO J. $1989,8,3667$

[51] Haman, A.; Cadieux, C.; Wilkes, B.; Hercus, T.; Lopez, A.; Clark, S.; Hoang, T. J. Biol. Chem. 1999, 274, 34155.

[52] D'Andrea, R. J.; Gonda, T. J. Exp. Hematol. 2000, 28, 231.

[53] de Groot, R. P.; Coffer, P. J.; Koenderman, L. Cell Signal. 1998, 10,619.

[54] Aronica, S. M.; Broxmeyer, H. E. Curr. Opin. Hematol. 1996, 3, 185.

[55] Quelle, F. W.; Sato, N.; Witthuhn, B. A.; Inhorn, R. C.; Eder, M.; Miyajima, A.; Griffin, J. D.; Ihle, J. N. Mol. Cell. Biol. 1994, 14, 4335 .

[56] Gardai, S. J.; Hoontrakoon, R.; Goddard, C. D.; Day, B. J.; Chang, L. Y.; Henson, P. M.; Bratton, D. L. J. Immunol, 2003, 170, 556.

[57] Dibbert, B.; Weber, M.; Nikolaizik, W. H.; Vogt, P.; Schoni, M. H.; Blaser, K.; Simon, H. U. Proc. Natl. Acad. Sci. USA 1999, 96, 13330 .

[58] Weinmann, P.; Gaehtgens, P.; Walzog, B. Blood 1999, 93, 3106

[59] Oymar, K.; Elsayed, S.; Bjerknes, R. Pediatr. Allergy Immunol. $1996,7,180$.

[60] Bentley, A. M.; Meng, Q.; Rubinson, D. S.; Hamid, Q.; Kay, A. B.; Durham, S. R. Am. J. Respir. Cell Mol. Biol. 1993, 8, 35.

[61] Yamaguchi, Y.; Suda, T.; Ohta, S.; Tominaga, K.; Miura, Y.; Kasahara, T. Blood 1991, 78, 2542.

[62] Stern, M.; Meagher, L.; Savill, J.; Hasletı, C. J. Immunol. 1992, $148,3543$.

[63] Pazdrak, K.; Olszewska-Pazdrak, B.; Stafford, S.; Garofalo, R. P.; Alam, R. J. Exp. Med. 1998, 188, 421.

[64] Pazdrak, K.; Adachi, T.; Alam, R. J. Exp. Med. 1997, 186, 561

[65] Dewson, G.; Cohen, G. M.; Wardlaw, A. J. Blood 2001, 98, 2239.

[66] Dibbert, B.; Daigle, I.; Braun, D.; Schranz, C.; Weber, M.; Blaser, K.; Zangemeister-Wittke, U.; Akbar, A. N.; Simon, H. U. Blood $1998,92,778$.

[67] Ochiai, K.; Kagami, M.; Matsumura, R.; Tomioka, H. Clin. Exp. Immunol 1997, 107, 198.

[68] Hamelmann, E.; Oshiba, A.; Loader, J.; Larsen, G. L.; Gleich, G.; Lee, J.; Gelfand, E. W. Am. J. Respir. Crit. Care Med. 1997, 155, 819.

[69] Lee, J. J.; McGarry, M. P.; Farmer, S. C.; Denzler, K. L.; Larson, K. A.; Carrigan, P. E.; Brenneise, I. E.; Horton, M. A.; Haczku, A.; Gelfand, E. W.; Leikauf, G. D.; Lee, N. A. J. Exp. Med. 1997, $185,2143$.

[70] Hogan, S. P.; Matthaei, K. I.; Young, J. M.; Koskinen, A.; Young, I. G.; Foster, P. S. J. Immunol. 1998, 161, 1501

[71] Corry, D. B.; Grunig, G.; Hadeiba, H.; Kurup, V. P.; Warnock, M. L.; Sheppard, D.; Rennick, D. M.; Locksley, R. M. Mol. Med. $1998,4,344$

[72] Leckie, M. J.; ten Brinke, $\Lambda . ;$ Khan, J.; Diamant, Z.; O'Connor, B. J.; Walls, C. M.; Mathur, A. K.; Cowley, H. C.; Chung, K. F.; Djukanovic, R.; Hansel, T. T.; Holgate, S. T.; Sterk, P. J.; Barnes, P. J. Lancet 2000, 356, 2144

[73] Flood-Page, P. T.; Menzies-Gow, A. N.; Kay, A. B.; Robinson, D. S. Am. J. Respir. Crit. Care Med. 2003, 167, 199.

[74] Kobayashi, T.; Iijima, K.; Kita, H. J. Immunol. 2003, 170, 5756.

[75] Gregory, B.; Kirchem, A.; Phipps, S.; Gevaert, P.; Pridgeon, C.; Rankin, S. M.; Robinson, D. S. J. Immunol. 2003, 170, 5359.

[76] Nishinakamura, R.; Miyajima, A.; Mee, P. J.; Tybulewicz, V. L.; Murray, R. Blood 1996, 88, 2458.

[77] Avalos, B. R. Blond 1996, 88, 761 .

[78] Semerad, C. L.; Liu, F.; Gregory, A. D.; Stumpf, K.; Link, D. C. Immunity. 2002, 17, 413.

[79] Colotta, F.; Re, F.; Polentarutti, N.; Sozzani, S.; Mantovani, A. Blood 1992, 80, 2012.

[80] Dale, D. C.; Cottle, T. E.; Fier, C. J.; Bolyard, A. A.; Bonilla, M. A.; Boxer, L. A.; Cham, B.; Freedman, M. H.; Kannourakis, G.; Kinsey, S. E.; Davis, R.; Scarlata, D.; Schwinzer, B.; Zeidler, C.; Welte, K. Am. J. Hematol. 2003, 72, 82.
[81] Mueller, T. D.; Zhang, J. L.; Sebald, W.; Duschl, A. Biochim. Biophys. Acta 2002, 1592, 237.

[82] Nelms, K.; Keegan, A. D.; Zamorano, J.; Ryan, J. J.; Paul, W. E. Annu. Rev. Immunol. 1999, 17, 701.

[83] Wedi, B.; Raap, U.; Lewrick, H.; Kapp, A. J. Allergy Clin. Immunol. 1998, 102, 1013.

[84] Girard, D.; Paquin, R.; Beaulieu, A. D. Biochem. J. 1997, 325 ( Pt 1), 147 .

[85] Luttmann, W.; Knoechel, B.; Foerster, M.; Matthys, H.; Virchow, J. C., Jr.; Kroegel, C. J. Immunol. 1996, 157, 1678.

[86] Zhu, Z.; Homer, R. J.; Wang, Z.; Chen, Q.; Geba, G. P.; Wang, J.; Zhang, Y.; Elias, J. A. J. Clin. Invest. 1999, 103, 779.

[87] Walter, D. M.; McIntire, J. J.; Berry, G.; McKenzie, A. N.; Donaldson, D. D.; DeKruyff, R. H.; Umetsu, D. T. J. Immunol. 2001, 167, 4668

[88] Elias, J. A.; Lee, C. G.; Zheng, T.; Shim, Y.; Zhu, L. Am. J. Respir. Cell Mol. Biol. 2003, 28, 401.

[89] Wills-Karp, M.; Chiaramonte, M. Curr. Opin. Pulm. Med. 2003, 9, 21.

[90] Barnes, P. J. Allergy 2001, 56, 928.

[91] I.iu, Y.; Cousin, J. M.; Hughes, J.; Van Damme, J.; Seckl, J. R.; Haslett, C.; Dransfield, I.; Savill, J.; Rossi, A. G. J. Immunol. 1999, $162,3639$.

[92] Giles, K. M.; Ross, K.; Rossi, A. G.; Hotchin, N. A.; Haslett, C.; Dransfield, I. J. Immunol. 2001, 167,976.

[93] Meagher, L. C.; Cousin, J. M.; Seckl, J. R.; Haslett, C. J. Immunol. $1996,156,4422$.

[94] Woolley, K. L.; Gibson, P. G.; Carty, K.; Wilson, A. J.; Twaddell, S. H.; Woolley, M. J. Am. .J. Respir. Crit. Care Med. 1996, I54, 237.

[95] Jatakanon, A.; Uasuf, C.; Maziak, W.; Lim, S.; Chung, K. F.; Barnes, P. J. Am. J. Respir. Crit. Care Med. 1999, 160, 1532.

[96] Ennis, M. Curr. Allergy Asthma Rep. 2003, 3, 159.

[97] de Castro, M.; Elliot, S.; Kino, T.; Bamberger, C.; Karl, M.; Webster, E.; Chrousos, G. P. Mol. Med. 1996, 2, 597.

[981 Oakley, R. H.; Sar, M.; Cidlowski, J. A. J. Biol. Chem. 1996, 271, 9550.

[99] Hauk, P. J.; Goleva, E.; Strickland, I.; Vottero, A.; Chrousos, G. P.; Kisich, K. O.; Leung, D. Y. Am. J. Respir. Cell Mol. Biol. 2002, 27,361 .

[100] Strickland, I.; Kisich, K.; Hauk, P. J;; Vottero, A.; Chrousos, G. P.; Klemm, D. J.; Leung, D. Y. J. Exp. Med. 2001, 193, 585.

[101] Barnes, P. J. Am. J. Respir. Crit. Care Med. 2003, 167, 813.

[102] Yasui, K.; Hu, B.; Nakazawa, T.; Agematsu, K.; Komiyama, A. J. Clin. Invest. 1997, 100, 1677

[103] Rossi, A. G.; Cousin, J. M.; Dransfield, L.; Lawson, M. F.; Chilvers, E. R.; Haslell, C. Biochem. Biophys. Res. Commun. 1995, 217, 892.

[104] Martin, M. C.; Dransfield, I.; Haslett, C.; Rossi, A. G. J. Biol. Chem. 2001, 276, 45041.

105] Ohta, K.; Yamashita, N. J. Allergy Clin. Immunol. 1999, 104, 14.

[106] Hallsworth, M. P.; Giembycz, M. A.; Barnes, P. J.; Lee, T. H. Br. J. Pharmacol. 1996, /17, 79.

[107] Peacock, C. D.; Misso, N. L.; Watkins, D. N.; Thompson, P. J. J. Allergy Clin. Immunol. 1999, 104, 153.

[108] Ward, C.; Dransfield, I.; Murray, J.; Farrow, S. N.; Haslett, C.; Rossi, A. G. J. Immunol. 2002, 168, 6232.

[109] Yasui, K.; Agematsu, K.; Shinozaki, K.; Hokibara, S.; Nagumo, H.; Nakazawa, T.; Komiyama, A. J. Leukoc. Biol, 2000, 67, 529.

[110] Lee, R.; Wolda, S.; Moon, E.; Esselstyn, J.; Hertel, C.; Lerner, A. Cell Signal. 2002, 14, 277.

[111] Ukena, D.; Harnest, U.; Sakalauskas, R.; Magyar, P.; Vetter, N.; Steffen, H.; Leichtl, S.; Rathgeb, F.; Keller, A.; Steinijans, V. W. Eur. Respir. J. 1997, 10, 2754.

[112] Lim, S.; Jatakanon, A.; Gordon, D.; Macdonald, C.; Chung, K. F.; Barnes, P. J. Thorax 2000, 55, 837.

[113] Leff, A. R. Am. J. Respir. Crit. Care Med. 2000, 161, S125.

[114] Yurdakul, A. S.; Calisir, H. C.; Tunctan, B.; Ogretensoy, M. Respir. Med. 2002, 96, 322.

[115] Tohda, Y.; Fujimura, M.; Taniguchi, H.; Takagi, K.; Igarashi, T.; Yasuhara, H.; Takahashi, K.; Nakajima, S. Clin. Exp. Allergy 2002, 32, 1180.

[116] Lee, E.; Robertson, T.; Smith, J; Kilfeather, S. Am. J. Respir. Crit. Care Med. 2000, 161, 1881.

[117] Hengartner, M. O. Nature 2000, 407, 770. 


\title{
Apoptosis: future perspectives
}

\section{Carol Ward, Adriano G. Rossi, Christopher Haslett and Ian Dransfield ${ }^{1}$}

Centre for Inflammation Research, University of Edinburgh Medical School, Teviot Place, Edinburgh EH8 9AG, U.K.

\begin{abstract}
The field of apoptosis has expanded to include research in almost all subject areas of cell biology. Despite this growth, there are still many unanswered questions, some of which are fundamental to the development of therapeutic strategies. Most importantly, the underlying mechanisms involved in conferring specificity of apoptotic signals must be determined. This will allow the development of treatments to delete injured or transformed cells and minimize damage to other tissues. Such information is also necessary to combat degenerative diseases, to prevent critical loss of cells. In addition, it would be of therapeutic benefit to selectively induce apoptosis in specific cells where removal of unwanted cells is required (e.g. tumour cells in malignant tissue or inflammatory cells responsible for tissue damage in chronic inflammatory disease). Therefore we have to understand how the full complement of signalling pathways, and survival and apoptotic proteins determine the end effect of an apoptotic signal. Apoptotic cells are known to functionally influence phagocytes that ingest them, but little is known of the effect of apoptosis on neighbouring cells or of signals generated during the apoptotic process itself. Although the processes of mitosis and apoptosis would appear to

${ }^{1}$ To whom correspondence should be addressed (e-mail i.dransfield@ed.ac.uk).
\end{abstract}


be diametrically opposed, cell-cycle proteins are involved in apoptosis, and may be able to influence cell-death mechanisms; conversely, proliferation may be caspase-dependent in some cells. Further study is necessary to discover how these pathways are interlinked since such knowledge would be particularly useful in cancer treatment. The link between pathogen-survival strategies and apoptotic mechanisms will also reward further work. Viruses, in particular, exploit cellular survival and death pathways for replication purposes and may represent a targeting approach for specific treatments.

\section{Introduction}

The exponential growth in our knowledge of the biochemistry and cellular mechanisms of apoptosis has allowed development of new strategies for therapeutic treatment of discase. Since the realization in the early 1980 s that apoptosis or programmed cell death is an important physiological process in multicellular organisms, research into the underlying molecular mechanisms, cellular alterations and physiological consequences has yielded tremendous insights into the full significance of this process in homocostasis. Apoptosis plays a fundamental role in oncogenesis, development, inflammation and immune regulation; it is now clear that both the manner in which cells die and their subsequent recognition by neighbouring cells or phagocytes profoundly influence tissue integrity and generation of immune responses. It is therefore not surprising that defects in these processes underlie disease pathogenesis and developmental errors. Although we can now define pathways linking death-receptor signalling to caspase activation and changes in the mitochondrial membrane potential and we have a detailed understanding of the cell-surface membrane changes during apoptosis, there are still many facets of programmed cell death that remain ill-defined. The close association of celldeath regulation with signalling pathways and intracellular mechanisms for detecting cellular stress may give clues as to the origins of apoptotic mechanisms. In multicellular organisms, apoptosis represents a mechanism for deletion of cells that have accumulated genetic modification or DNA damage that threatens the existence of the whole organism. The overall frequency of tumours is below the levels that would be predicted given the rates of spontaneously arising mutations or those arising from genotoxic insults. It has been suggested that for a multicellular organism, cells are 'cheap' and that the default response to perturbation of cellular homoeostasis should be apoptosis. In vertebrates, excess cell production is critical for innate immune mechanisms, allowing 'disposable' cells (e.g. granulocytes) to be produced in large numbers in the fight against infectious organisms. Similarly, in the acquired immune response, cells with diverse receptor specificities can be generated and then those that are not required can be eliminated (e.g. cells that are autoreactive). Many intracellular pathogens or viruses interfere with homoeostatic mechanisms, thereby influencing apoptotic-effector pathways. 
Whereas some pathogens have exploited these mechanisms for survival advantage, deletion of infected cells and maintenance of tissue integrity was clearly a driving force in the development of molecular pathways involved in cell death. This chapter aims to discuss some of the unanswered questions and speculate on the future goals of apoptosis research.

\section{A matter of specificity}

One of the most pressing problems yet to be solved in programmed cell death research, which would allow full therapeutic bencfit to be realized, is the understanding of how and why defined apoptotic signals can kill one cell, but have no effect on another. At present, many therapeutic interventions will also inflict damage on bystander cells, resulting in complications and side effects when dealing with patients. Although genetic studies in Caenorhabditis elegans and other model organisms have allowed us to fit the key molecular regulators of apoptosis into a generalized model, there are additional levels of complexity associated with increased cellular diversity and tissue compartmentalization. Such levels of complexity are implicitly required in multicellular organisms. A simple apoptotic 'switch' would place unnecessary restrictions upon the development of complexity of multicellular organisms. Instead, multiple levels of regulation allow some cells to be deleted more readily, whereas cells with little capacity for proliferation must be protected from apoptosis. For example, some cell types lack caspases that are critical for apoptosis in C. elegans and there are distinct, tissuespecific profiles of pro-apoptotic and survival proteins. Furthermore, diverse patterns of receptor expression on different cell types may confer differential responses to apoptotic signals within a single organ or tissue. In addition, there may be interplay between pro-apoptotic and other intracellular signalling pathways that act to moderate the engagement of apoptotic effectors. This can be refined further by differential expression patterns and activation profiles of key signalling intermediates such that the repertoire of kinases, phosphatases, transcription factors and proteases that are produced in a particular cell confer additional flexibility of response. For example, TNF $\alpha$ (tumour necrosis factor $\alpha$ ) can drive apoptosis via the TNF-receptor-induced assembly of a death-inducing signalling complex. However, activation of the transcription factor nuclear factor $\kappa \mathrm{B}$ can override the apoptotic signal via the production of specific survival proteins. Therefore cellular responses to specific mediators of apoptosis can be interpreted in the context of micro-environmental cues.

Induction of apoptosis in specific cells would therefore be beneficial in a number of diseases. For example, in cancer therapy, one of the greatest breakthroughs would be complete induction of tumour-cell death leaving no resistant cells that would allow evasion of anti-tumour therapy. Additionally, it would be of paramount importance that therapy to induce tumour cell apoptosis did not detrimentally affect survival of healthy cells and tissues. In inflammatory diseases, where a particular cell is believed to contribute to tissue damage, it may be desir- 
able to specifically eliminate the offending cell by apoptosis. For example, in the asthmatic lung, where oeosinophils and/or lymphocytes are found in abundance and probably contribute to disease progression, a good therapeutic strategy would be to direct drugs to induce apoptosis in these cells without influencing the structural cells (c.g. epithelial cells) of the lung. In addition, such therapy would have to ensure that cells induced to undergo apoptosis are recognized and efficiently removed by macrophages without eliciting an inflammatory response. Failure to do this would cause detrimental inflammation due to liberation of histotoxic products from apoptotic cells that had undergone secondary necrosis due to failed clearance [1]. Indeed, it is possible that anti-asthma drugs already in clinical use may be using these mechanisms: for example, glucocorticoids induce apoptosis of cosinophils (and lymphocytes) and simultaneously enhance macrophage clearance of these apoptotic cells. Conversely, in degenerative discase (e.g. Alzheimer's disease, Parkinson's disease, Huntington's disease and amyotrophic lateral sclerosis), we need to define the factors that will prevent programmed cell death or convert necrotic to apoptotic death. 'Thus it seems likely that defining the fine-tuning of the death pathways in a specific cell or tissue will allow identification of targets that would modulate cell death for therapeutic gain.

\section{Signalling during apoptosis}

It is widely accepted that apoptotic cells become functionally senescent, but the question of whether apoptotic cells are really 'inert' remains open. In inflammatory loci, programmed cell death means that clearance occurs before cells become necrotic and cause tissue damage. Phagocytosis of apoptotic corpses influences macrophages causing them to release cytokines with antiinflammatory properties, such as transforming growth factor $\beta$ and interleukin 10. In addition, there is evidence that macrophages can direct apoptosis of surrounding cells. Studies of macrophage-clearance mechanisms indicate that recognition of apoptotic cells requires specific changes on the surface of the cell undergoing apoptosis. Thus apoptotic cells trigger signals generating an anti-inflammatory phenotype in the ingesting phagocytes, whereas a proinflammatory profile occurs when the phagocyte ingests non-apoptotic entities (e.g. necrotic cells or opsonized particles) [2]. It has been suggested that phagocyte recognition of apoptotic cells may be determined by a lack of signalling via the adhesion molecule CD31 [3]. It is possible that viable cells remain in constant dialogue with their neighbours through molecules such as CD31, representing another important homoeostatic mechanism. However, the possibility that apoptotic cells are not 'inert' remains to be fully explored.

Recent evidence suggests that cells undergoing apoptosis produce signals that can change the behaviour of other cells. Activation of caspase-3 initiated the release of lysophosphatidylcholine from apoptotic cell membranes which then acted as a chemotactic factor for macrophages [4]. Whether apoptotic cells can influence the behaviour of other neighbouring cells is not known and repre- 
sents a novel facet of cell-death research that is ripe for development. The intersection of signalling pathways from cell-adhesion molecules and those that influence cell survival is another example of how micro-environmental cues can determine cell survival. Recent studics have revealed that tumour-cell resistance to chemotherapeutic agents may be determined by the repertoire of adhesion receptors that are engaged. Another example of this phenomenon is the recent report that PECAM-1 (platelet-endothelial cell adhesion molecule)/CD31 acts as a powerful inhibitor of apoptosis, inhibiting Bax-induced cytochrome $c$ release from mitochondria [5]. Thus an alternative strategy for targeting tumour cell apoptosis may be to 'uncouple' adhesion signalling in combination with more traditional radiotherapy or chemotherapeutic drugs. Within tissues, cellular detachment from neighbouring cells or extracellular matrix may precede or accompany apoptosis, although the mechanisms involved are not known. One possibility is that disengagement of cellular adhesion associated with apoptosis may trigger proliferation or migration in those cells adjacent to the dying cell.

\section{Apoptosis versus mitosis}

Given the similarities between mitosis and apoptosis in terms of nuclear reorganization and organelle disassembly, the idea that apoptosis represented failed or aborted mitosis has been proposed. Whereas this view has lost favour over the years, new data would support the suggestion that proteins involved in cell-cycle control also have a role in the induction of apoptosis. Little is known of other potential functions of these cell-cycle proteins in addition to their role as 'checkpoints' that allow a cell to divide or act to inhibit proliferation. Cell division requires the activation of CDKs (cyclin-dependent kinases) by partner proteins called cyclins. Fas-induced death in Jurkat cells causes activation of CDK2 when caspase-3 cleaves the inhibitory kinase, Wee1 [6], although this does not involve general activation of mitotic mechanisms. In cultured cortical neurons, proteasome inhibitors induce apoptotic cell death in a manner that is dependent upon CDK2, CDK4 and CDK6 activation [7]. In mouse mesangial cells, differential localization of active CDK2 and cyclin A during proliferation (nuclear) and apoptosis (cytoplasmic) suggests that the subcellular location of CDK2 is an important determinant of whether a cell divides or dies [8]. Increased CDK activity occurred before mitochondrial involvement and did not involve caspases. Furthermore, some cell-cycle proteins such as $\mathrm{p} 21 \mathrm{Cip} / \mathrm{W}$ af1 and $\mathrm{p} 27 \mathrm{Kip} 1$ are known caspase targets and, as such, their cleavage could lead to CDK2 activation [9]. The CDK inhibitor $\mathrm{p} 21 \mathrm{Cip} / \mathrm{W}$ af1 protects some cells from $\mathrm{p} 53$-mediated apoptosis, but is pro-apoptotic in some systems and conditions [10]. Whether proteins involved in the cell cycle are able to interact with molecules identified in the classical pathway of programmed cell death during either the activation (mitochondrial) or execution (capsases) phases remains to be addressed. It is now emerging that cyclins have broader roles in regulation of cellular function and recent data suggest that integrin- 
dependent cell migration may require Cdc2/Cdk1 and cyclin D1 (see [11] for a commentary). Such diversity in the roles of cell-cycle proteins in a given cell type may provide additional novel targets for therapeutic intervention strategies. The links between the distinct processes of mitosis and apoptosis are further underlined by recent data demonstrating that caspases may also be involved in cell proliferation as well as cell death. Caspases 8 and 6 exhibit increased activity in B-cells treated with proliferative stimuli, and specific caspase inhibitors blocked B-cell division independently of apoptosis [12]. Similar results have also been reported for activated T-cells [13]. The mechanisms by which caspase activation can be uncoupled from the rest of the apoptotic execution pathways remains to be determined, and whether caspase activation is involved in other non-apoptotic cellular responses is not yet known. The extremely close relationship between the evolution of what would appear to be diametrically opposed processes of cell division and apoptosis may reflect the requirement for protection of the genome, with apoptosis representing the ultimate 'checkpoint' in cell division when DNA is damaged.

Our understanding of how complex systems develop is still poor perhaps the application of complex mathematical models will help us to understand the way in which organs in which there are repeating structural units formed from cells (e.g. lung, kidney and liver) or how development of vascular beds is achieved. This may be critical in understanding the response to tissue injury and for the therapeutic goal of restoration of tissue architecture.

\section{Pathogen-mediated interventions}

Researchers have long been aware that viral infection can modulate the apoptotic programme. Whether viruses have 'hijacked' control proteins as a way to manipulate host-cell survival to ensure completion of their replication cycle or whether the cellular repertoire of apoptotic proteins has evolved to combat viral infection is unknown. Many survival proteins have viral homologues which can act at almost any point in the cell death pathway (see [14] and references therein), which would ensure that replication occurs. Paradoxically, virus-induced cell death may also be advantageous, since apoptotic-body formation and phagocytic uptake by neighbouring cells facilitates the spread of viral progeny without causing activation of the immune response. A single virus may thercfore be able to produce mediators that can either promote or inhibit cell death according to the phase of the viral life cycle that is active. For example, the HIV-1 protein Nef can increase expression of cell-surface TNF $\alpha$, Tat can increase the expression of CD95, while Vpr induces cell-cycle arrest as a prelude to the induction of apoptosis. Conversely, both Tat and $\mathrm{Vpr}$ have been reported to inhibit cell death by increasing Bcl-2 levels. Interestingly, viruses can also manipulate apoptosis through interference with cell-cycle proteins such as $\mathrm{p} 53$ and pRb/p107/p130 [15]. Examination of virus-cell interactions will undoubtedly lead to a more comprehensive 
understanding of the mechanisms that underlie regulation of apoptosis. This may represent a starting point for the development of novel approaches to manipulation of cell death for therapeutic gain in either degenerative conditions or diseases in which cellular proliferative responses are dysregulared.

Other pathogens such as protozoa and bacteria have also been shown to affect apoptotic pathways (see [16] and references therein). For example, an important aspect of the pathogenic capacity of Mycobacterium tuberculosis is survival within host macrophages by active inhibition of phagosome-lysosome fusion. Stimulation of the $\mathrm{P}_{2} \mathrm{X}_{7}$ receptor on infected macrophages induces macrophage apoptosis, leading to phagosome-lysosome fusion and consequently killing of intracellular mycobacteria. Recent evidence suggests that ligation of Toll-like receptors may also lead to induction of macrophage apoptosis and killing of intracellular mycobacteria, although the cellular mechanism has yet to be elucidated. Definition of the regulatory mechanisms underlying macrophage apoptosis and macrophage ability to kill mycobacteria may also provide novel approaches for manipulation of disease. Whether we can use our knowledge of the control of apoptosis in eukaryotes to induce bacterial death as an alternative to antibiotics is an intriguing possibility that may be worthy of further exploration.

\section{Implications for the future}

Government statistics forecast that by 2020 at least $20 \%$ of the population will be over 65 . With the incidence of cancer and degenerative diseases increasing markedly with age, the manipulation of programmed cell death as a therapeutic strategy has obvious benefits. Although our understanding of this process has greatly increased in the last few years, there are still many questions that require answers. The importance of this field cannot and should not be underestimated; it is now poised to reap the rewards of years of research in terms of possible medical advances.

\section{Summary}

- Regulation of apoptosis is required for many homoeostatic mechanisms and failure to control cell death may underlie the development of tumours and chronic inflammatory disease.

- The signalling patbways involved in initiation of apoptosis are tightly controlled to ensure that cell death effector pathways are engaged following cellular damage and are closely linked to signalling pathways involved in cell activation.

- Similarities between the mechanisms underlying control of apoptosis and the cell cycle (particularly involvement of proteins of the cyclin family) suggest that cell death may have represented a form of checkpoint to ensure that damaged cells were deleted efficiently. 
- Determining the cellular specificities of agents that trigger death pathways will be crucial for targeted deletion of cells in inflammatory disease or within tumours without causing tissue damage.

- Defining how apoptotic cells influence neighbouring cells or phagocytes behaviour may offer new strategies for manipulating disease progression (e.g. autoimmunity, wound bealing).

\section{References}

I. Ward, C., Dransfield, I., Chilvers, E.R., Haslett, C. \& Rossi, A.G. (1999) Pharmacological manipulation of granulocyte apoptosis: potential therapeutic targets. Trends Phormacol. Sci. 20, 503-509

2. Giles, K.M., Hart, S.P., Haslett C., Rossi, A.G. \& Dransfield, I. (2000) An appetite for apoptotic cells: controversies and challenges. Br. f. Haematol. I09, I-12

3. Brown, S., Heinissch, I., Ross, E., Shaw, K., Buckley, C.D. \& Savill, J. (2002) Apoptosis disables CD31-mediated cell detachment from phagocytes promoting binding and engulfment. Nature (London) 418, 200-203

4. Lauber, K., Bohn, E., Krober, S.M., Xiao, Y., Blumenthal, S.G, Lindemann, R.K., Marini, P., Wiedig, C., Zobywalski, A., Baksh. S. et al. (2003) Apoptotic cells induce migration of phagocytes via caspase-3-mediated release of a lipid attraction signal. Cell I13, 717-730

5. Gao C., Sun, W.. Christofidou-Solomidou, M., Sawada, M., Newman, D.K., Bergom, C., Albelda, S.M.. Matsuyama, S. \& Newman, P.J. (2003) PECAM-I functions as a specific and potent inhibitor of mitochondrial-dependent apoptosis. Blood 102, 169-179

6. Zhou, B., Li, H., Yuan, J. \& Kirschner, M.W. (1998) Caspase-dependent activation of cyclindependent kinases during Fas-induced apoptosis in Jurkat cells. Proc. Natt. Acod. Sci. U.S.A. 95 , 6785-6790

7. Rideout, H.J., Wang, Q., Park, D.S. \& Stefanis, L. (2003) Cyclin-dependent kinase activity is required for apoptotic death but not inclusion formation in cortical neurons after proteasomal inhibition. J. Neurosci. 23, 1237-1245

8. Hiromura, K., Pippin, J.W., Blonski, M.J., Roberts, J.M. \& Shankland, S.J. (2002) The subceilular localization of cyclin dependent kinase 2 determines the fate of mesangial cells: role in apoptosis and proliferation. Oncogene 21, 1750-1758

9. Levkau, B., Koyama, H., Raines, E.W., Clurman, B.E., Herren B., Orthe K., Roberts, J.M. \& Ross, R. (1998) Cleavage of $21 \mathrm{Cip} / \mathrm{Wafl}$ and p27Kip I mediates apoptosis in endothelial cells through activation of Cdk2: role of a caspase cascade. Mol. Cell 1, 553-563

10. Gartel, A.L. \& Tyner, A.L. (2002) The role of the cyclin-dependent kinase inhibitor p2I in apoptosis. Mol. Cancer. Ther. I, 639-649

11. Juliano R (2003) Movin' on through with Cdc2. Nat. Cell Biol, 5, 589-590

12. Olson, N.E., Graves, J.D., Shu, G.L., Ryan, E.J. \& Clark, E.A. (2003) Caspase activity is required for stimulated B lymphocytes to enter the cell cycle. J. Immunol. 170, 6065-6072

13. Kennedy, N.J., Kataoka, T., Tschopp, J. \& Budd, R.C. (1999) Caspase activity is required for T cell proliferation. J. Exp. Med. 190, 1891-1896

14. Hay, S. \& Kannourakis, G. (2002) A time to kill: viral manipulation of the cell death program. J. Gen. Virol. 3, 1547-1564

15. Barry, M. \& McFadden, G. (1998) Apoptosis regulators from DNA viruses. Curr. Opin. Immunol. $10,422-430$

16. Häcker, G and Fischer, S.F. (2002) Bacterial anti-apoptotic activities. FEMS Microbiol. Lett. 2II, $1-6$ 


\title{
CH A P T E R 26 \\ Apoptosis of Inflammatory Cells
}

\author{
Adriano Giorgio Rossi, Carol Ward, Ian Dransfield, and Christopher Haslett
}

To fight infection and promote tissue repair, the inflammatory response normally serves to partition, dilute, destroy, or ultimately remove invading organisms (e.g., bacteria, fungi, parasites), injurious agents, or injured tissues. When this normally beneficial, highly complex, biochemical and physiologic response goes awry in an uncontrolled or exaggerated manner, inappropriate or excessive tissue damage can occur, culminating in chronic inflammation and loss of organ function. At sites of inflammation, recruited leukocytes (e.g., neutrophils, eosinophils, mononuclear cells) have the potential to liberate products such as reactive oxygen species (ROS; e.g., $\mathrm{O}_{2}^{-}, \mathrm{H}_{2} \mathrm{O}_{2}, \mathrm{OH}^{-}, \mathrm{NO}$ ) and proteases (e.g., elastase, collagenase) that are involved in the fight against infection but that also exhibit tissue-damaging properties. It is now generally accepted that to circumvent this potential tissue damage, leukocytes are removed in a noninflammatory manner.

This chapter discusses the cellular and molecular mechanisms involved in the resolution phase of the inflammatory response, focusing on the critical role of apoptosis in this process. Understanding the mechanisms underlying the resolution of the inflammatory response may lead to novel and highly effective therapies for the prevention and treatment of inflammatory diseases, including rheumatoid arthritis, glomerulonephritis, asthma, chronic bronchitis, emphysema, myocardial infarction, and reperfusion injury.

\section{THE ACUTE INFLAMMATORY RESPONSE}

Although the gross clinical hallmarks of the acute inflammatory response (redness and swelling with heat, pain, and frequently loss of function) were described at the beginning of the first millennium, the processes responsible for these changes have became apparent only relatively recently. Vasodilation resulting in augmented blood flow to the tissues is responsible for the redness (erythema) and increased warmth occurring at inflammatory foci. For example, vasodilation of capillaries and arterioles mediated by prostaglandins (PGs), neuropeptides, and nitric oxide (NO) cause increased intraluminal hydrostatic pressure downstream in postcapillary venules, where microvascular permeability occurs. These mediators act in synergy with agents that directly increase postcapillary venule microvascular permeability, resulting in tissue fluid exudation (swelling or edema). Agents act either directly on endothelial cells, such as histamine, vasopressin, bradykinin, and platelet-activating factor (PAF), or indirectly by requiring recruitment of inflammatory cells, including C5a, C3a, formylated bacterial peptides, leukotriene $\mathrm{B}_{4}$ $\left(\mathrm{LTB}_{4}\right)$, and interleukin-8 (IL-8), to increase microvacular permeability. Thus the concerted actions of these mediators and processes are responsible for the responses observed during inflammation. ${ }^{1}$

\section{INFLAMMATORY CELL RECRUITMENT}

To reach sites of inflammation, inflammatory cells must (1) adhere loosely (tethering and rolling) to endothelial cells, a process mediated by the selectins (E-, L-, and P-selectin) and their respective ligands; and (2) flatten and adhere firmly to the endothelial cells, primarily through the integrins (e.g., $\beta_{1}, \beta_{2}$ ) and immunoglobulin (Ig) superfamily members, including intercellular adhesion molecule-1 and -2 (ICAM-1, ICAM-2) and vascular cell adhesion molecule-1 (VCAM-1); (3) traverse the endothelium of the blood vessel wall; (4) penetrate the basement membrane; and (5) transmigrate through the interstitial tissue to reach the affected area ${ }^{2,3}$ (see Chapter 9). The inflammatory insult and inflammatory milieu determine which type of inflammatory cell is recruited; for example, specific chemokines produced in response to injury and infection promote selective recruitment of neutrophils (e.g., IL-8), eosinophils (e.g., eotaxin), or monocytes such as monocyte chemotactic protein-1 (MCP-1). In addition, cell adhesion molecule (CAM) expression is critical in determining the type of inflammatory cell recruited; for example, very late antigen-4 (VLA-4, or $\alpha_{4} \beta_{1}$ integrin) is highly expressed on eosinophils and monocytes but less well expressed on neutrophils.

The structure and physiology of the vasculature may influence leukocyte recruitment. In parasitic infection, allergy, and asthma, for example, there is an abundance of recruited eosinophils and lymphocytes, whereas in rheumatoid arthritis, adult respiratory distress syndrome (ARDS) and neonatal respiratory syndromes, the predominant leukocyte is the neutrophil. In inflammatory disease of the lung, evidence indicates that capillaries are the major sites of leukocyte emigration, whereas in other diseased organs (e.g., psoriasis), postcapillary venules are the principal site of leukocyte recruitment. ${ }^{1.4 .5}$ Leukocyte accumulation therefore depends on the complex 
interactions among inflammatory mediators, expression of CAMs and extracellular matrix (ECM) proteins, and physiology of the vasculature.

\section{RESOLUTION OF INFLAMMATION}

In addition to the processes involved in the induction and propagation of the inflammatory response, the mechanisms controlling its resolution are also critical in determining the ultimate outcome of the inflammatory response. ${ }^{6-8}$ The remainder of this chapter therefore focuses on this rapidly developing latter area, with particular emphasis on the process of apoptosis and the removal of apoptotic cells.

\section{Inflammatory Mediator Dissipation}

At inflammatory loci, many inflammatory mediators are produced endogenously and also are introduced from exogenous sources. A number of mechanisms have evolved that control the removal or inactivation of these pleiotropic mediators. First, vasodilation and edema formation not only help bring plasma proteins and leukocytes to inflammatory sites, but also reduce the effective concentrations of inflammatory stimuli. Second, extravasated leukocytes remove or destroy invading organisms, thereby directly removing the source of exogenous mediators and triggers of endogenously generated inflammatory products. Third, certain inflammatory mediators, such as thromboxane $\mathrm{A}_{2}\left(\mathrm{TxA}_{2}\right)$ and $\mathrm{NO}$, become deactivated spontaneously, whereas others, such as $\mathrm{C} 5 \mathrm{a}, \mathrm{PAF}$, and $\mathrm{LTB}_{4}$, are enzymatically rendered inactive. Fourth, reduced synthesis and release, or increased metabolism, of a particular mediator can limit its effective concentration. Fifth, mediatorinduced cellular or tissue desensitization can also limit the response. Sixth, many mediators, generated at inflammatory sites, have opposing effects on effector cells; for example, C5a and IL- 8 are potent neutrophil chemoattractants, whereas PGs can down-regulate cellular responsiveness by triggering inhibitory signals. In addition, some inflammatory mediators, such as granulocyte-macrophage colony-stimulating factor (GM-CSF), can prolong the survival of granulocytes by retarding apoptosis, whereas others such as tumor necrosis factor alpha (TNF- $\alpha$ ), can induce apoptosis (see later discussion).

\section{Termination of Cell Recruitment}

Mechanisms regulating the cessation of inflammatory cell accumulation remain poorly defined, although a number of in vivo studies investigating the kinetics of inflammatory cell accumulation from the blood to experimentally induced inflamed tissue have yielded useful information. In the acutely inflamed skin, lung, or joints, for example, neutrophils accumulate within the first few hours (usually 30 minutes to 4 hours), followed later by more protracted accumulation of mononuclear cells. In chronic inflammatory models or diseases associated with tissue damage and scarring, neutrophils and macrophages continue to accumulate for several days before resolution occurs. The mechanisms responsible for early cessation of neutrophil extravasation in acute inflammatory conditions are incompletely elucidated, but dissipation of inflammatory mediators and removal of effete cells by apoptosis likely are key events.

\section{Control of Inflammatory Responses}

The following mechanisms limit the destructive capacity of inflammatory cells and allow resolution to occur:

1. Reduction in the effective concentration of certain proinflammatory stimuli.

2. Increase in potential antiinflammatory mediators, such as interleukin-1 receptor antagonist (IL-1RA), interleukin-10 (IL-10), and transforming growth factor beta (TGF- $\beta$ ).

3 Exhaustion of the responsiveness of inflammatory cells; that is, cells may no longer have the capability to liberate further destructive enzymes or toxic oxygen metabolites due to depletion of cellular resources or internal energy stores.

4. Receptor or cellular desensitization.

5. Receptor shedding, leading to loss of cellular responsiveness and liberation of soluble receptors that can bind to ligands to prevent ligand binding to surface receptors.

6. Ultimately, apoptotic death of inflammatory cells and their subsequent removal by phagocytes.

\section{Apoptotic Cells}

In the early 1970 s, Kerr, Wyllie and Currie ${ }^{9}$ described a physiologic mode of cell death that they termed apoptosis (programmed cell death). ${ }^{10}$ It had been widely believed that cells disintegrated by uncontrolled necrosis. ${ }^{11}$ If this were the case, however, most cells and especially inflammatory cells would liberate their histotoxic intracellular contents, many of which would likely provoke an exaggerated inflammatory response. Virtually all cells can undergo controlled physiologic death by apoptosis, a process known to play a fundamental role in almost all aspects of life, including the resolution of the inflammatory response..$^{6,10,12}$

Intriguingly, apoptotic cells exhibit remarkably similar structural, morphologic, and biochemical changes, suggesting a common underlying series of molecular mechanisms. Apoptotic cells are usually smaller (due to cytoplasmic shrinkage), are vacuolated, and exhibit major cell surface changes. Importantly, apoptotic granulocytes remain intact, maintaining plasma membrane integrity (i.e., they exclude vital dyes such as trypan blue) and retaining their cytoplasmic granules. On the other hand, cells that have undergone necrosis will readily incorporate vital dyes and liberate cellular contents (e.g., lactate dehydrogenase), events that are easily measurable in the laboratory. Nuclear ultrastructural changes include condensation of chromatin into dense, crescent-shaped aggregates, with the nucleolus becoming more prominent. In multilobed or bilobed inflammatory cells, such as the eosinophil or neutrophil, there is nuclear coalescence (Figure 26-1).

Biochemically, endogenous endonuclease activation results in internucleosomal cleavage of chromatin typified by deoxyribonucleic acid (DNA) fragments of 180 to 200 base pairs (bp) in length; this type of DNA breakdown can be observed as a characteristic DNA ladder by gel electrophoresis. Most apoptotic cells express phosphatidylserine molecules on the external surface of the plasma membrane; this highly regulated process involves two enzymes, phospholipid scramblase and aminophospholipid translocase, and is important in phagocyte recognition. Phosphatidylserine exposure has been useful in assessing apoptosis because annexin $\mathrm{V}$ conjugated to fluorochromes (e.g., FITC, PE) binds 


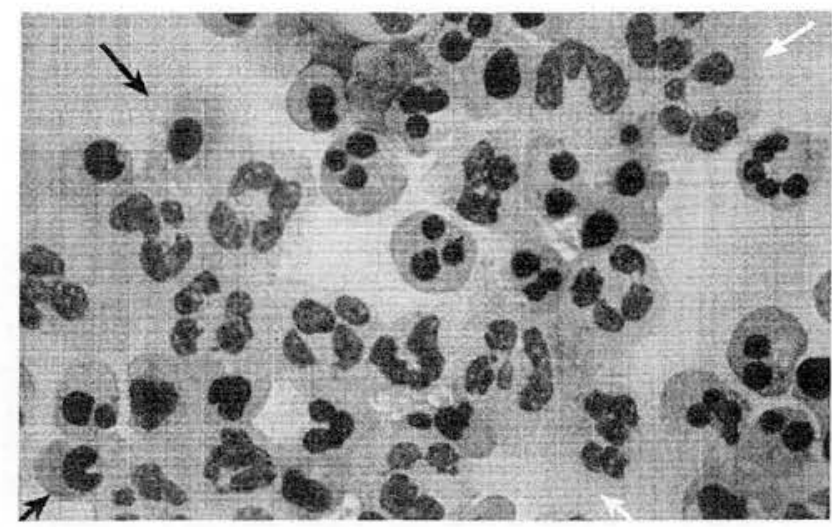

FIGLRE 26-1 Light micrograph of aged neutrophils. Isolated human neutrophils cultured in vitro for 20 hours undergo physiologic cell death, or apoptosis, in which approximaiely $60 \%$ of the cells usually become smaller, with visible, darkly stained nuclei (black arrows). Remaining nonapoptotic neutrophils (white arrows) eventually become apoptotic within next 20 hours of culture. Note that apoptotic cells have an intact plasma membrane that excludes vital dyes such as trypan blue.

phosphatidylserine and can be detected by flow cytometry. Other cell surface changes that may be particular to a specific cell type, such as loss of FcyRIII (CD16) from neutrophils, ${ }^{13}$ can also be used to assess apoptosis by flow cytometry. Apoptotic granulocytes are less responsive to external receptordirected stimuli, in that functions such as chemotaxis, phagocytosis, and degranulation are down-regulated due to loss of molecules important for activation (e.g., FMLP receptors), phagocytosis (CD16), and adhesion (L-selectin). ${ }^{13-15}$

When investigating cell death, it important to distinguish between apoptosis and necrosis, and a number of techniques have been adopted. One of the "gold standards" to demonstrate apoptosis convincingly is to view cells by light and especially electron microscopy and observe characteristic morphologic changes associated with apoptosis or to detect cell surface changes by flow cytometry. Use of dyes such as propidium iodide has been useful because uptake of such dyes by cells indicates necrosis has occurred. Alternatively, by deliberately making cells permeable, propidium iodide intercalates into the DNA, and when apoptosis has occurred, the DNA is stained in a characteristic manner, with a hypodiploid peak easily distinguishable by flow cytometry. Fluorescence microscopy is also particularly useful because dyes such as Hoechst 33342, acridine orange, and ethidium bromide can be used to determine the mode of cell death. Additional techniques to show evidence of apoptosis include measurement of the activity of key enzymes involved in executing apoptosis, such as the caspase family of proteases described later.

Detection of apoptosis in vivo has been more difficult; direct visualization by microscopy has been useful. The terminal deoxynucleotidyl transferase (TdT)-mediated $d$-uridine triphosphate (UTP) nick end labeling (TUNEL) technique involves staining and visualization of DNA of apoptotic cells in tissue sections and has been used with varying degrees of success, but care should be taken not to overestimate the amount of apoptosis occurring.

\section{Regulation of Apoptosis}

Although the characteristic features of apoptosis are remarkably similar in all cell types, regulation of the apoptotic process differs greatly from one cell to another. This phenomenon is especially apparent when control mechanisms are examined closely in inflammatory cells. For example, apoptosis in lymphocytes and thymocytes is regulated differently than in granulocytes. Indeed, there are even fundamental differences in the control of neutrophil and eosinophil apoptosis. ${ }^{7,16,17}$ Many inflammatory stimuli, such as GM-CSF, lipopolysaccharide, and C5a, by profoundly delaying neutrophil apoptosis, greatly prolong the functional longevity of these cells, as assessed by chemotaxis and degranulation assays ${ }^{15,17}$ (Figure 26-2). Eosinophil apoptosis is suppressed by GMCSF and interleukin-5 (IL-5), whereas IL-5 exerts no effect on neutrophil longevity. Delayed apoptosis, however, is not a universal phenomenon associated with all proinflammatory mediators; treatment of isolated human neutrophils in culture with TNF- $\alpha$ for 6 hours accelerates the rate of constitutive apoptosis, whereas at later time points ( $>16$ hours), apoptosis is delayed by this cytokine. ${ }^{18.19}$

Many death receptors belong to the TNF family and contain homologous cytoplasmic regions termed death domains $^{20,21}$ (Figure 26-3). The mechanisms underlying TNF- $\alpha$ induction of apoptosis is unknown but likely involves TNF receptor associated proteins such as TNF receptorassociated death domain (TRADD), Fas-associated death domain (FADD), and caspase- 8 and may involve pathways mediating Fas (CD95) and sphingomyelin ceramide signaling. Ligand binding causes association of cytoplasmic death domains that bind TRADD, allowing recruitment of FADD and procaspase- 8 , resulting in formation of a death-inducing signaling complex (DISC) when procaspase- 8 is activated. ${ }^{22,23}$ Apoptosis proceeds either by the direct activation of downstream caspases through caspase- 8 or by caspase- 8 causing modulation of $\mathrm{Bcl}-2$ family members, which influences the mitochondrial pathway of caspase activation. ${ }^{24}$ Caspase- 8 cleaves the Bcl-2 family member $\mathrm{Bid}$, producing a C-terminal fragment that interacts with the mitochondria, causing loss of mitochondrial transmembrane potential and the release of cytochrome $c$ and other proteins, such as apoptosis-inducing factor (AIF). ${ }^{25,26}$ Bid requires the presence of another proapoptotic Bcl-2 family protein, Bax, for this to occur. Bax has been shown to translocate to the mitochondria during neutrophil and eosinophil apoptosis. ${ }^{27}$ Cytochrome $c$, in the presence of adenosine triphosphate (ATP) or d-ATP, induces a conformational change in Apaf-1, a cytosolic adapter protein, forming a large complex known as the apoptosome, which binds and activates procaspase-9 to its mature form. ${ }^{28,29}$ Caspase- 9 then initiates processing of effector caspases 3 and $7,,^{30}$ which in turn activate caspases 6 and 2. Execution of this caspase cascade results in the morphologic and biochemical cellular changes of apoptosis. Both the caspase- 8 and the mitochondrial pathways have been identified in granulocytes and other inflammatory cells. ${ }^{7,27,31-35}$

Significant advances have been made in understanding the mechanisms controlling granulocyte apoptosis by a number of laboratories. For example, strong evidence indicates that spontaneous eosinophil apoptosis involves Bax translocation to the mitochondria, cytochrome $c$ release, caspase-independent perturbation of the mitochondrial membrane, and subsequent activation of caspases. ${ }^{32}$ Further, IL-5 inhibits spontaneous eosinophil apoptosis at a site upstream of Bax translocation and modulates the expression of $\mathrm{Bcl}-2{ }^{36}$ In their reports on control of granulocyte apoptosis, Simon et $\mathrm{al}^{37-41}$ provide 


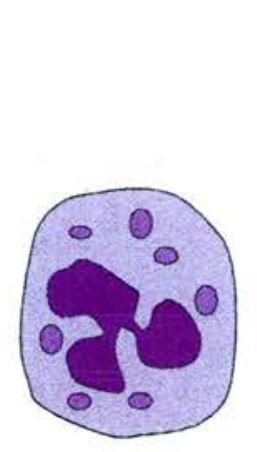

Functionally competent, potentially histotoxic

\section{GM-CSF, LPS, C5a, IL-1 13, IFN $\gamma, \mathrm{LTB}_{4}, \mathrm{PAF}$} hypoxia, $\uparrow\left[\mathrm{Ca}^{2+}\right]_{i}, \uparrow[\mathrm{CAMP}]_{\mathrm{l}}$, glucocorticoids

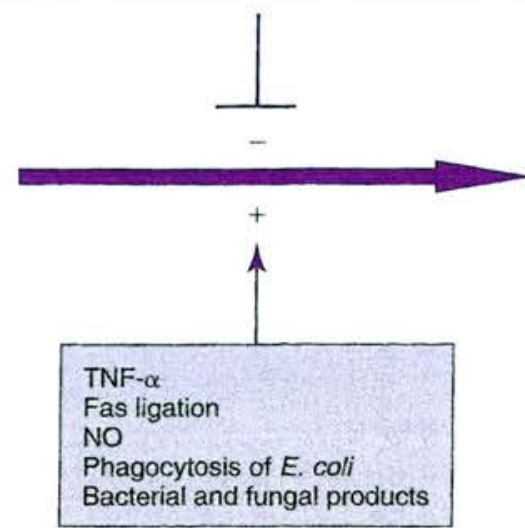

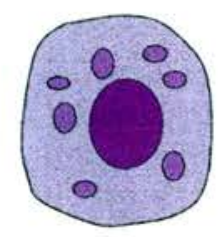

Functionally downregulated Hyporesponsive Recognized and cleared by macrophages

FIGURE 26-2 Regulation of human neutrophil apoptosis. Functionally competent but potentially histotoxic neutrophils undergo constitutive apoptosis, during which the cells become hyporesponsive to stimulation and are recognizable by phagocytes such as macrophages. Various factors can dramatically alter the rate of apoptosis. Inflammatory mediators (e.g., GM-CSF, LPS, C5a, IL-1 $\beta$, IFN- $\gamma, \mathrm{LTB}_{4}, \mathrm{PAF}$ ), environmental conditions (hypoxia, extracellular matrix proteins), increases in signaling molecule levels (e.g., cAMP, $\mathrm{Ca}^{2+}$ ), and glucocorticoids can all delay onset of apoptosis. Other stimuli (e.g., TNF- $\alpha$, NO, Fas ligand) and bacterial and fungal products can promote onset of apoptosis.

molecular and functional evidence for negative signaling by the TNF/nerve growth factor (NGF) family of death receptors. They showed that a conserved phosphotyrosine-containing motif within the death domain of these receptors can mediate inhibitory functions. The Src homology domain 2 (SH2)containing tyrosine phosphatase-1 (SHP-1), SHP-2, and SH2containing inositol phosphatase (SHIP) are bound to this motif in a caspase-independent but cell-dependent manner. Importantly, stimulation of death receptors disrupted anti-apoptosis pathways that were initiated (at least under certain conditions) by survival factors in neutrophils. In these cells, activation of the tyrosine kinase Lyn, an important anti-apoptotic event, was prevented as a consequence of death receptor stimulation, most likely through association of the receptor with activated SHP-1.42

It has been shown that anti-Fas-activating antibodies, or Fas ligand, promote neutrophil and eosinophil apoptosis. ${ }^{43,44}$ More recent studies have demonstrated that TNF apoptosisinducing ligand (TRAIL) can modulate granulocyte apoptosis. ${ }^{45}$ Whether these death receptors play important roles in inflammatory diseases remains to be fully determined, although the Fas-Fas ligand pathway has been reported to induce apoptosis in experimental models in vivo. ${ }^{46,47} \mathrm{NO}$ may also regulate inflammatory cell apoptosis. Indeed, we recently found that powerful caspase-dependent proapoptotic effects of NO donors are unaffected by guanylate cyclase inhibition, mimicked by exogenous peroxynitrite, and antagonized by superoxide dismutase..$^{48}$ Thus, NO-mediated apoptosis may be mediated by a cyclic guanosine monophosphate (cGMP)-independent mechanism that involves the concurrent generation of oxygen free radicals. NO therefore has a unique role in inflammatory responses; as well as having powerful properties on vasodilation, it can induce inflammatory cell apoptosis and downregulate cell activation. ${ }^{48}$ However, the ability of structurally diverse NO-donating compounds to delay or enhance neutrophil apoptosis and the apparent dissociation of DNA fragmentation from other changes occurring during apoptosis in NO-treated neutrophils renders the effects more complex than initially believed. ${ }^{49}$ Interestingly, the environmental milieu may also exert profound effects on the rate of inflammatory cell apoptosis; for example, a hypoxic environment, found in chronically inflamed sites, delays neutrophil ${ }^{50}$ and eosinophil ${ }^{51}$ apoptosis, whereas hypoxia induces apoptosis in most other cells types. ${ }^{52}$

Other agents used clinically for the treatment of inflammatory disease, such as glucocorticoids, delay neutrophil apoptosis but promote eosinophil and lymphocyte apoptosis. ${ }^{53}$ Although the mechanisms underlying this divergent effect of glucocorticoids on inflammatory cell apoptosis are currently unknown, the effect may explain why steroid therapy is so efficacious in eosinophilic inflammation such as asthma. Interestingly, asthmatic patients who show an improvement in lung function after corticosteroid treatment also have an increased proportion of apoptotic eosinophils in their airway secretions. ${ }^{54}$

Using a pharmacologic approach, the intracellular signaling pathways that control granulocyte apoptosis are slowly being unraveled. For example, neutrophil apoptosis is delayed by elevation of intracellular free cytosolic calcium $\left(\mathrm{Ca}^{2+}\right)$ levels using calcium ionophores such as A23187 and ionomycin or by mobilization of intracellular stores using thapsigargin, ${ }^{55.56}$ whereas eosinophil ${ }^{56}$ and thymocyte ${ }^{57}$ apoptosis is promoted. A key role for protein phosphorylation through protein kinase $\mathrm{C}$ (PKC), mitogen-activating protein (MAP) kinases, and tyrosine kinases are also thought to regulate apoptosis. Consequently, dephosphorylation through specific phosphatases is also likely to be important. Furthermore, apoptosis-delaying effects of inflammatory stimuli that increase cyclic adenosine monophosphate (cAMP) levels in granulocytes have been reported ${ }^{58,59}$ (Table 26-1). 


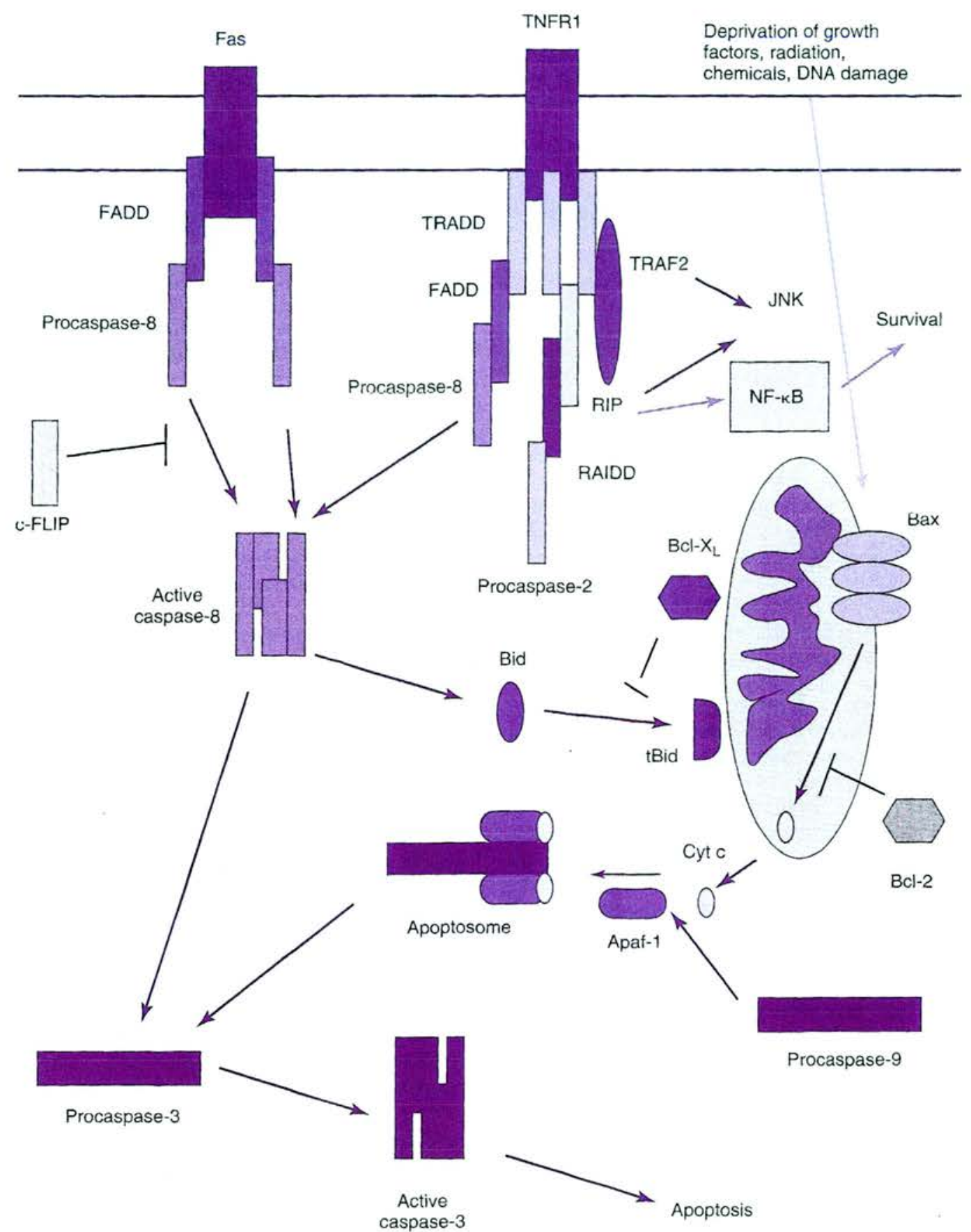

FIGURE 26-3 Simplified scheme depicting death signaling pathways. Fas or tumor necrosis factor receptor (TNFRI) ligation induces processing of caspase- 8 via death receptor-associated proteins and subsequent activation of effector caspases. Caspase- 8 cleavage of Bid as well as various stress and chemical induced proapoptotic stimuli (via Bax insertion into mitochondria) induces release of cytochrome $c$ from mitochondria, which induces a conformational change in the cytosolic adapter molecule (Apaf-1). Apaf-1 then is recruited and oligomerized to caspase- 9 causing processing of caspase- 9 within the apoptosome complex. Both caspase- 8 and caspase- 9 lead to processing and activation of caspase-3. The apoptotic pathway is tightiy regulated by $\mathrm{Bcl}-2$ and $\mathrm{Bcl}-$ 2-related proteins (e.g., Bcl-x, Bax) and c-FLIP, which prevents the recruitment of the caspase- 8 proenzyme into the receptor complex. Other signaling pathways can be triggered by death receptor ligation (e.g., JNK, NF-KB) and also regulate apoptotic pathways. FADD. Fas-associated death domain; TRADD. TNF receptor-associated death domain; RAIDD, receptor interacting protein (RIP)-associated ICH-1/CED-3-homologus protein with death domain; RIP, receptor interacting protein; TRAF2. TNF receptor associated factor- $2 ; J N K$, c-Jun $N$-terminal kinase; $N F-\kappa B$, nuclear factor kappa B; $c$-FLIP, cellular Fas-associated death domain (FADD)-like interleukin-1-converting enzyme (FLICE)-inhibitory protein. 
Table 26-1 Select Granulocyte Proapoptotic and Antiapoptotic Stimuli and Conditions

\begin{tabular}{lll}
\hline Granulocyte & Agents/Conditions & References \\
\hline Enhancement of Apoptosis & & \\
Neutrophil & Nitric oxide (NO) donors & $48,49,69,70$ \\
& Activating Fas antibodies, Fas ligand & $43,71,72$ \\
& Tumor necrosis factor alpha (TNF- $\alpha$ ) & $18,19,73$ \\
& TNF apoptosis-inducing ligand (TRAIL) & 45 \\
& Protein synthesis inhibitors (e.g., cycloheximide, & 65 \\
& actinomycin D) & \\
& NF- - B inhibitors (e.g., gliotoxin, MG-132) & 19,74 \\
& Antibiotics & 75 \\
Gosinophil & Actucocorticoids & 53,76 \\
& TNF- $\alpha$ & $44,46,77,78$ \\
& Agents that increase intracellular Ca ${ }^{2+}(\mathrm{e} . \mathrm{g} .$, A2.3187) & 67 \\
& Protein synthesis inhibitors & 56 \\
& NF- $k$ B inhibitors & 79,80 \\
& Transforming growth factor beta (TGF- $\beta$ ) & 19,67 \\
& CD45 & 81 \\
& Interleukin-4 (IL-4) & 82
\end{tabular}

\section{Delay in Apoptosis}

Neutrophil

Eosinophil

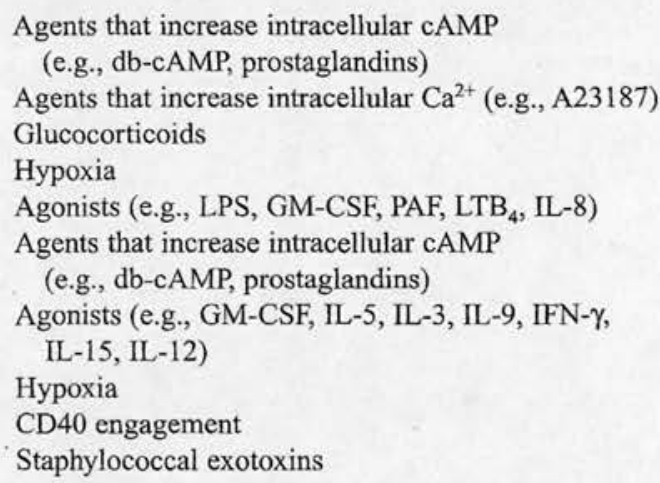

\author{
$31,58,86,87$ \\ 55 \\ 53,88 \\ 50,89 \\ 7,37 \\ $59,68,90$ \\ $16,37,91$ \\ $51,\left({ }^{*}\right)$ \\ 92 \\ 93
}

* Murray J, Ward C, Rossi AG: Unpublished observations, 2002.

$N F-\kappa B$, Nuclear factor kappa B; $C A M P$, cyclic adenosine monophosphate; $L P S$, lipopolysaccharide; GM-CSF, granulocyte-macrophage colony-stimulating factor; $P A F$, platelet-activating factor, $L T B_{4}$, leukotriene $\mathrm{B}_{4} ; I F N-\gamma$, interferon gamma.

Subsequently, the dramatic delay of the neutrophil apoptotic program by the cell-permeable analog of cAMP, $d b-c A M P$, may be mediated through a novel protein kinase A (PKA)-independent signaling pathway, involving maintenance of mitochondrial potential and inhibition of caspase activation. ${ }^{31}$ The survival effects of db-cAMP also appear to be independent of phosphatidylinositol-3 kinase (PI3K) and MAP kinase (MAPK) activation. These findings thus propose a potentially novel, reversible, transcriptionally independent mechanism of action of db-cAMP that may provide opportunities to shift the balance of proapoptotic and antiapoptotic proteins and thus accelerate clearance of granulocytes from inflamed sites. ${ }^{31}$ Interestingly, elevation of cAMP promotes the rate of apoptosis in many other cell systems, such as thymocytes and leukemic cell lines. ${ }^{60-62}$ Although intracellular kinase activity undoubtedly has a key regulatory role in granulocyte apoptosis, ${ }^{63}$ the precise kinase(s) involved remains to be established.

Why the intracellular signaling pathways that regulate neutrophil apoptosis are different from other cells, including the eosinophil, remains elusive but may be caused by the differential expression of levels or types of protooncogenes shown to be involved in other cell systems. ${ }^{17}$ Although we and others have demonstrated that neutrophils do not normally express $\mathrm{Bcl}-2$ protein, other $\mathrm{Bcl}-2$ family members may be involved because it has been reported that overexpression of $\mathrm{Bcl}-2$ in neutrophils prolongs their survival. ${ }^{64}$ It remains to be established whether the recently characterized novel Bcl-2-like proteins are also expressed or play a role in neutrophil apoptosis. ${ }^{17}$ Protein synthesis inhibitors such as 
cycloheximide and actinomycin D, while inhibiting thymocyte apoptosis, promote neutrophil apoptosis, indicting endogenous synthesis of neutrophil survival proteins. ${ }^{19,65}$

Together with the observation that bacterial lipopolysaccharide (LPS) profoundly delays apoptosis, ${ }^{19,66}$ researchers have started to dissect the complex signaling mechanisms upstream of transcriptional control that will also ultimately influence LPS-mediated survival of neutrophils. Most inflammatory cells sense LPS using a complex system that involves the interaction of LBP/CD14/MD-2 receptors and toll-like receptor-4 (TLR-4). When engaged, these receptors trigger interrelated signal transduction pathways, including the MAPK (ERK-1/-2, JNK, $\mathrm{p} 38), \mathrm{PI} 3 \mathrm{~K}$, and nuclear factor kappa $\mathrm{B}(\mathrm{NF}-\kappa \mathrm{B})$ pathways, to orchestrate innate immune responses, although systemic responses to LPS can also lead to septic shock or ARDS.

Furthermore, a role for specific transcriptional regulation of granulocyte apoptosis has been identified. As stated earlier, TNF- $\alpha$, a known trigger of NF- $\kappa$ B activation, was shown to delay apoptosis in those neutrophils (the majority) not induced into early death. ${ }^{18}$ Importantly, the possibility that NF- $\mathrm{KB}$ directed gene transcription and protein synthesis delayed apoptosis in "inflammatory" granulocytes was strongly supported by the proapoptotic effects of protein synthesis inhibitors. Indeed, recent studies have emphasized the capacity of inflammatory granulocytes to synthesize powerful cytokines (e.g., IL-8) that are regulated by NF- $\kappa$ B. Detailed investigation of the role of NF- $\kappa \mathrm{B}$ in granulocytes has revealed that both TNF- $\alpha$ and LPS indeed trigger NF- $\mathrm{KB}$ activation, as evidenced by the loss of cytoplasmic I $\mathrm{KB} \alpha$ (the inhibitory subunit of NF- $\kappa \mathrm{B}$ ) and by the rate of caspasedependent apoptosis in granulocytes being greatly accelerated by NF- $\mathrm{KB}$ inhibitors, such as the fungal metabolite gliotoxin. In neutrophils, TNF- $\alpha$ causes activation of NF- $\kappa B$, delaying apoptosis and limiting its early killing effect. NF- $\kappa B$ inhibitors and cycloheximide appear to unmask the ability of TNF- $\alpha$ to induce granulocyte apoptosis, suggesting that NF- $\kappa \mathrm{B}$ may regulate the production of proteins that protect these cells from the cytotoxic effects of TNF- $\alpha$.

Although regulation of eosinophil apoptosis is less well understood, NF- $\mathrm{\kappa B}$ activation also may play an important role. ${ }^{67}$ It has been shown that human eosinophils exposed to TNF- $\alpha$ rapidly lose their cytoplasmic $\operatorname{I\kappa B} \alpha$, resulting in $\mathrm{NF}-\kappa \mathrm{B}$ mobilization from the cytoplasm to the nucleus, as evidenced by tracking the NF- $\mathrm{KB}$ subunit $\mathrm{p} 65$ by immunofluorescence and Western blot analysis. Caspase-dependent proapoptotic properties of TNF- $\alpha$ in the eosinophil are unmasked by inhibition of TNF- $\alpha$-mediated I $\mathrm{I} \mathrm{B} \alpha$ degradation and inhibition of NF- $\kappa \mathrm{B}$ activation. Interestingly, TNF$\alpha$-triggered apoptosis may be more susceptible to NF- $\kappa B$ inhibition than constitutive apoptosis, leading to the possibility of the specific targeting of apoptosis in eosinophil subpopulations. ${ }^{67} \mathrm{NF}-\kappa \mathrm{B}$ activation is therefore likely to play a critical role in controlling granulocyte responsiveness and apoptosis, and select targeted inhibitors of NF- $\kappa$ B activation may ultimately provide alternative therapeutic agents for the treatment of eosinophilic disease (e.g., asthma, allergic rhinitis) and neutrophilic disease (e.g., chronic obstructive pulmonary disease). In addition, exciting new data also point to a key regulatory role for NF-kB-dependent genes in the pathways responsible for the metabolism of PGs in granulocytes.

Although many natural prostaglandins (e.g., $\mathrm{PGE}_{2}, \mathrm{PGA}_{2}$, $\mathrm{PGA}_{1}, \mathrm{PGF}_{2 \alpha}$ ) either delay granulocyte apoptosis or have no effect, $\mathrm{PGD}_{2}$ and its metabolite $\mathrm{PGJ}_{2}$ selectively induce eosinophil, but not neutrophil apoptosis. The sequential PGD metabolites $\Delta^{12} \mathrm{PGJ}_{2}$ and $15 \mathrm{dPGJ}_{2}$ were found to induce caspase-dependent apoptosis in both granulocytes. Despite $\Delta^{12} \mathrm{PGJ}_{2}$ and $15 \mathrm{dPGJ}_{2}$ being PPAR- $\gamma$ activators, apoptosis was not mimicked by synthetic PPAR- $\gamma$ and PPAR- $\gamma$ ligands and not blocked by an irreversible PPAR- $\gamma$ antagonist. It was found that $\Delta^{12} \mathrm{PGJ}_{2}$ and $15 \mathrm{dPGJ}_{2}$ inhibited LPS-induced and TNF- $\alpha$-induced $I \kappa B \alpha$ degradation in granulocytes and LPS-induced neutrophil survival. The powerful proapoptotic effects of $\Delta^{12} \mathrm{PGJ}_{2}$ and $15 \mathrm{dPGJ}_{2}$ in both granulocytes implies that differences in the ability of eosinophils and neutrophils to process and degrade PGs may be responsible for the differential effects of $\mathrm{PGD}_{2}$ on granulocyte survival. ${ }^{68}$ Despite this progress in identifying signaling mechanisms that control inflammatory cell apoptosis, much remains to be elucidated, providing difficult challenges for the future.

\section{Phagocytosis of Apoptotic Cells}

Apoptotic cells at an inflammatory site would inevitably undergo disintegration (secondary necrosis) if phagocytes (especially macrophages) were absent in situ, resulting in the release of their histotoxic contents and thereby causing further inflammation and the destruction of tissue cells (Figure 26-4). This undesirable scenario may occur in uncontrolled chronic inflammation. during which the normally highly effective macrophage recognition and clearance mechanisms (see below) are overwhelmed or defective. However, in resolving beneficial inflammation. the majority of senescent cells at sites of inflammation are recognized by nearby phagocytes. More than 100 years ago, using recently developed intravital light microscopic techniques on a number of transparent invertebrates, the Russian biologist Elie Metchnikoff described phagocytosis of bacteria and senescent leukocytes by macrophages. Of particular relevance to the resolution of inflammation is the description of Reiter's cells in the inflamed joint fluid from patients with acute arthritis. These Reiter's cells were shown to be macrophages containing apoptotic neutrophils. Furthermore, in experimental inflammatory models where there is a large influx of neutrophils, the predominant mode of extravasated intact neutrophil removal is engulfment by macrophages, which subsequently leave the inflammatory site through the draining lymph nodes. ${ }^{94}$

Although there have been numerous accounts of macrophage phagocytosis of neutrophils over the last century, only relatively recently have major developments regarding recognition of apoptotic cells been described. In 1982, Newman et $\mathrm{al}^{95}$ first showed that isolated neutrophils, which had been aged in culture, were recognized and ingested by inflammatory macrophages. The macrophages engorged themselves with senescent neutrophils but did not ingest freshly isolated neutrophils, indicating that aged neutrophils had undergone a fundamental process leading to recognition by macrophages. It was subsequently shown that cells including inflammatory cells had to undergo apoptosis before they were rapidly and efficiently recognized and ingested by macrophages. ${ }^{96}$ The macrophage can ingest several apoptotic cells at a time, and importantly, there is no detectable release of potentially injurious contents from the apoptotic target. If the target of the macrophage is a granulocyte, for example, there is no release of potentially histotoxic granule contents into the surrounding medium, thereby effectively neutralizing 


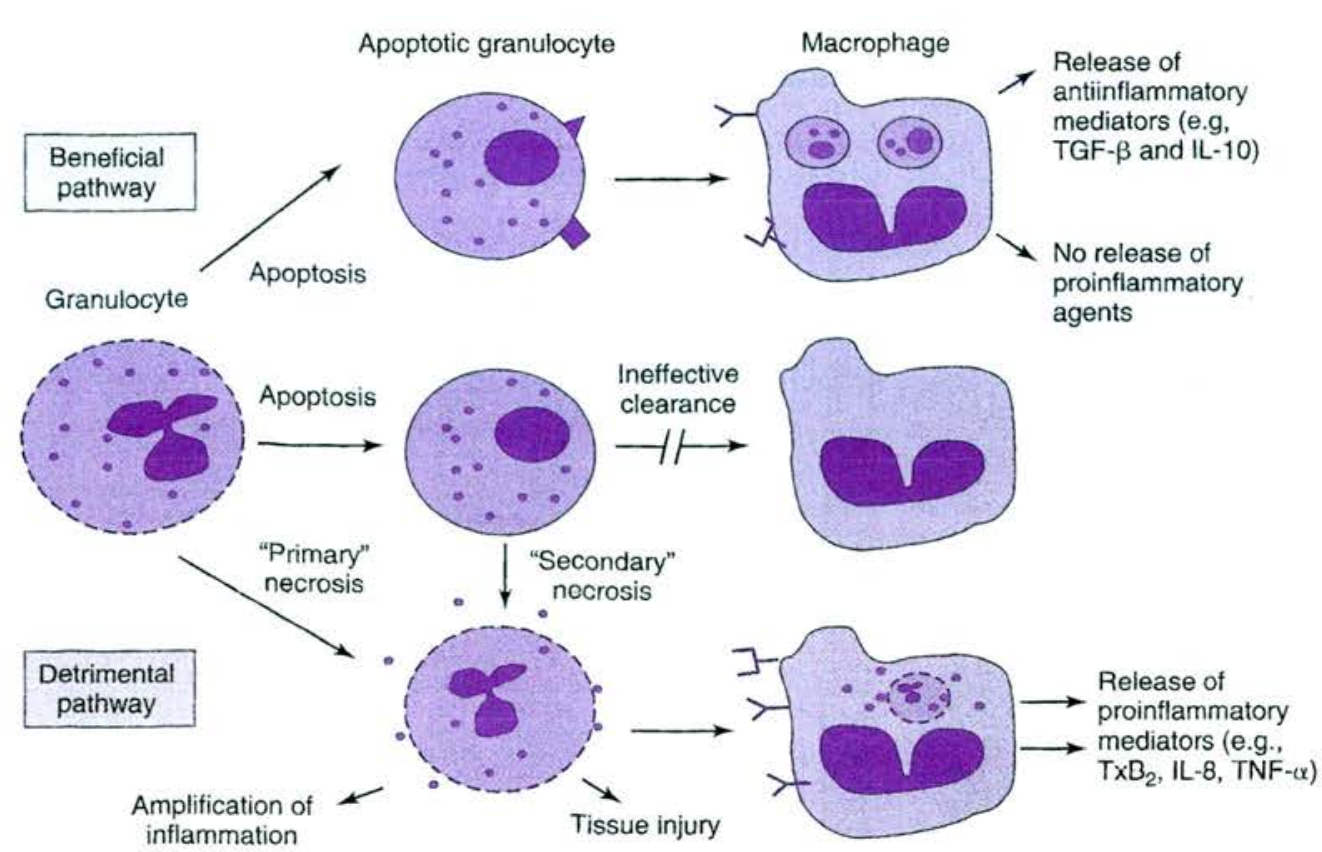

FIGURE 26-4 Granulocyte removal pathways. Under normal physiologic resolution of inflammation, a beneficial pathway occurs in which inflammatory cells (e.g., granulocytes) undergo apoptosis and are recognized and phagocytosed by phagocytes (e.g., macrophages) using specific mechanisms that do not incite an inflammatory response. There is no release of proinflammatory mediators, but rather a release of potential antiinflammatory mediators (e.g., TGF- $\beta$, IL-10). If recruited inflammatory cells undergo primary necrosis, or if apoptotic cells are not removed by macrophages as a result of ineffective clearance, the cells can undergo secondary necrosis. These necrotic cells can liberate their toxic contents and cause tissue injury and amplify the inflammatory process (detrimental pathway). Necrotic cells that are ingested result in the liberation of proinflammatory mediators (e.g., $\mathrm{TxB}_{2}, \mathrm{IL}-8$, TNF- $\alpha$ ) from the phagocyte.

any proinflammatory potential of the apoptotic cell. When phagocytes ingest particles such as zymosan (yeast cell walls) in vitro, they liberate proinflammatory mediators (e.g., eicosanoids, granular enzymes, cytokines). However, ingestion of apoptotic cells does not result in proinflammatory mediator release from macrophages. ${ }^{97}$ Indeed, increasing evidence suggests that macrophage phagocytosis of apoptotic cells triggers production and release of agents (TGF- $\beta$, IL-10) that have antiinflammatory capacity. ${ }^{98.99}$ This noninflammatory clearance mechanism is thought to be caused by the molecular mechanisms by which phagocytes recognize and ingest apoptotic cells.

\section{Molecular Mechanisms for Apoptotic Cell Recognition and Clearance}

Phagocytes (usually macrophages) use highly developed efficient recognition mechanisms for the removal of apoptotic cells. Although the macrophage is believed to remove the majority of apoptotic cells, other cells, such as fibroblasts, ${ }^{100}$ hepatocytes, ${ }^{101}$ endothelial cells, ${ }^{102}$ glomelular mesangial cells, ${ }^{103}$ vascular smooth muscle cells, ${ }^{104}$ Sertoli cells, ${ }^{105}$ and even lung epithelial cells, ${ }^{106}$ have a capacity for apoptotic cell recognition and clearance. Perhaps the removal of apoptotic cells by these "semiprofessional" phagocytes plays a crucial role when local macrophages are overwhelmed by major waves of apoptotic cells. Specific molecular mechanisms for recognition of apoptotic cells have been identified ${ }^{107,108}$ (Table 26-2). Given that multiple genes are involved in the disposal of apoptotic bodies in the nematode Caenorhabditis elegans, it is likely that other, as yet unidentified mechanisms exist in the recognition and phagocytosis of mammalian inflammatory cells.

\section{APOPTOTIC MECHANISMS AS ANTIINFLAMMATORY STRATEGIES}

In chronic inflammatory diseases, persistent accumulation of inflammatory cells likely liberates their toxic intracellular products, leading to tissue injury, scarring, and architectural disruption with catastrophic loss of organ function. Effective removal of these tissue-damaging inflammatory cells may be either defective or overwhelmed in these diseases; in other examples of massive inflammatory reactions, such as lobar streptococcal pneumonia and acute gout, the lesions clear completely. The mechanisms by which inflammation normally resolves not only are essential for understanding of the persistent inflammatory states that characterize chronic inflammatory diseases, but also should provide novel approaches to antiinflammatory therapy.

Significant differences in the intracellular regulation of inflammatory cells provide a special opportunity to induce apoptosis in inflammatory cell types selectively. Thus, by utilizing mechanisms of receptor-mediated induction of apoptosis of specific inflammatory cells (e.g., Fas ligation and glucocorticoid receptor activation) and by inhibition of the powerful survival signals found at inflammatory foci (e.g., GM-CSF, LPS, PGs, IL-5), it may be possible to accelerate apoptosis for therapeutic gain. In combination with an effective therapeutic strategy to induce apoptosis, development of 
Table 26-2 Major Surface Receptors and Molecular Mechanisms in Recognition of Apoptotic Cells by Phagocytes

\begin{tabular}{|c|c|c|}
\hline Receptors & Mechanisms & References \\
\hline $\begin{array}{l}\text { Lectinlike } \\
\text { receptors }\end{array}$ & $\begin{array}{l}\text { Receptors on phagocytes recognize carbohydrates on surface of } \\
\text { apoptotic cells } \\
\text { Include asialoglycoprotein, mannose, and mannose/fucose } \\
\text { receptors }\end{array}$ & $100,102,109-112$ \\
\hline $\begin{array}{l}\alpha_{v} \beta_{3} \text { integrin } \\
\text { CD36 } \\
\text { Thrombospondin } \\
\quad \text { (TSP) }\end{array}$ & $\begin{array}{l}\text { Moiety expressed on surface of apoptotic cells binds to TSP, } \\
\text { which acts as bridging molecule between apoptotic cell and } \\
\text { ingesting phagocyte } \\
\text { Phagocyte expresses } \alpha_{v} \beta_{3} \text { ("vitronectin receptor") and CD36 } \\
\text { (88-kD monomer), which cooperate to bind to TSP }\end{array}$ & $100,113-115$ \\
\hline CD29 ( $\beta 1$ integrins) & $\begin{array}{l}\text { CD29 on surface of phagocytes binds apoptotic cells by } \\
\text { poorly defined mechanism }\end{array}$ & 116 \\
\hline $\begin{array}{l}\text { Phosphatidylserine } \\
\text { (PS) and PS } \\
\text { receptors }\end{array}$ & $\begin{array}{l}\text { Exposure of PS un surface of apoptotic cells is recognized by } \\
\text { PS receptor on surface of ingesting phagocyte }\end{array}$ & $117-119$ \\
\hline $\begin{array}{l}\text { CD14 } \\
\text { ICAM-3 }\end{array}$ & $\begin{array}{l}\text { CD14, a GPI-anchored LPS-binding molecule on surface of } \\
\text { phagocytes, also binds } 5^{\prime} \text { domain immunoglobulin } \\
\text { superfamily member, intercellular adhesion molecule-3 } \\
\text { (ICAM-3), on surface of leukocytes }\end{array}$ & 120,121 \\
\hline $\begin{array}{l}\text { Adenosine } \\
\text { triphosphate } \\
\text { (ATP)-binding } \\
\text { cassette reporter } \\
\text { (ABC1) }\end{array}$ & $\begin{array}{l}\text { Rearrangement of plasma membrane lipids in both dying cell } \\
\text { and phagocyte by } \mathrm{ABC} 1 \text { contributes to recognition by poorly } \\
\text { defined mechanism }\end{array}$ & 122 \\
\hline $\begin{array}{l}\text { Complement } \\
\text { receptors } \mathrm{CR} 3 / \mathrm{CR} 4 \\
\text { and components } \\
\text { (Clq) }\end{array}$ & $\begin{array}{l}\text { May be involved in recognizing apoptotic cells } \\
\mathrm{Clq} \text { and related molecules mannose-binding lectin (MBL) } \\
\text { and pulmonary surfactant protein A (SPA) may opsonize } \\
\text { apoptotic cells; receptors for C1q may mediate uptake of } \\
\text { apoptotic cells in vitro and in vivo }\end{array}$ & $123-126$ \\
\hline $\begin{array}{l}\text { CD91 } \\
\text { Calreticulin }\end{array}$ & $\begin{array}{l}\mathrm{CD} 91\left(\alpha_{2} \text {-macroglobulin receptor) and calreticulin on }\right. \\
\text { phagocytes bind to entire collectin family of innate immune } \\
\text { proteins, including } \mathrm{C} 1 \mathrm{q} \text { and } \mathrm{MBL}\end{array}$ & 127 \\
\hline CD31 (PECAM-1) & $\begin{array}{l}\text { Novel mechanism whereby disabled apoptotic cell CD31 } \\
\text { binds tightly to CD31 (platelet-endothelial cell adhesion } \\
\text { molecule-1) present on phagocyte cells }\end{array}$ & 128 \\
\hline $\begin{array}{l}\text { Other receptor } \\
\text { molecules: } \\
\beta_{2} \text {-glycoprotein } \\
\text { receptor, Lox-1, } \\
\text { scavenger receptor, } \\
\text { oxidized low-density } \\
\text { lipoprotein receptor } 1 \\
\text { (OxLDLR1) }\end{array}$ & $\begin{array}{l}\text { Identified in a variety of systems involved in recognition of } \\
\text { apoptotic cells, but precise mechanisms and counterligands } \\
\text { involved remain to be fully elucidated }\end{array}$ & $129-134$ \\
\hline
\end{tabular}

mechanisms to specifically enhance apoptotic cell clearance by phagocytes such as the macrophage or by semiprofessional phagocytes may be equally desirable or even a prerequisite to prevent proinflammatory secondary necrosis.

In addition, the process of phagocytosis of apoptotic cells by macrophages can be specifically regulated by immunologic, pharmacologic, and molecular intervention. For example, certain cytokines, ${ }^{135}$ elevation of macrophage cAMP levels by prostaglandins ${ }^{136}$ (Figure 26-5, A), activation of macrophage PKC, ${ }^{137}$ and ligation of ECM receptors (e.g., integrins) ${ }^{138}$ have all been shown to modify macrophage capacity to ingest apoptotic cells. In addition, ligation of macrophage CD44, a transmembrane adhesion receptor and the major cell surface receptor for the nonsulfated glycosaminoglycan hyaluronan, can enhance macrophage clearance of apoptotic cells in vitro ${ }^{139}$ (Figure 26-5, B). Importantly, this receptor has now been shown to play a critical role in resolving lung inflammation. ${ }^{140}$ Of direct clinical importance is the recent discovery that glucocorticoids profoundly augment macrophage capacity for safe, noninflammatory clearance of apoptotic granulocytes in vitro ${ }^{141}$ and may be one of the targets for steroids in vivo. ${ }^{54}$ This important effect of glucocorticoids may result from their ability to "reprogram" monocyte protein synthesis and cytoskeletal function to yield macrophages with a highly phagocytic phenotype ${ }^{142}$ (Figure 26-5, C). In eosinophilic inflammation, this phenotype is perfectly coupled with glucocorticoids' ability to accelerate eosinophil apoptosis. 


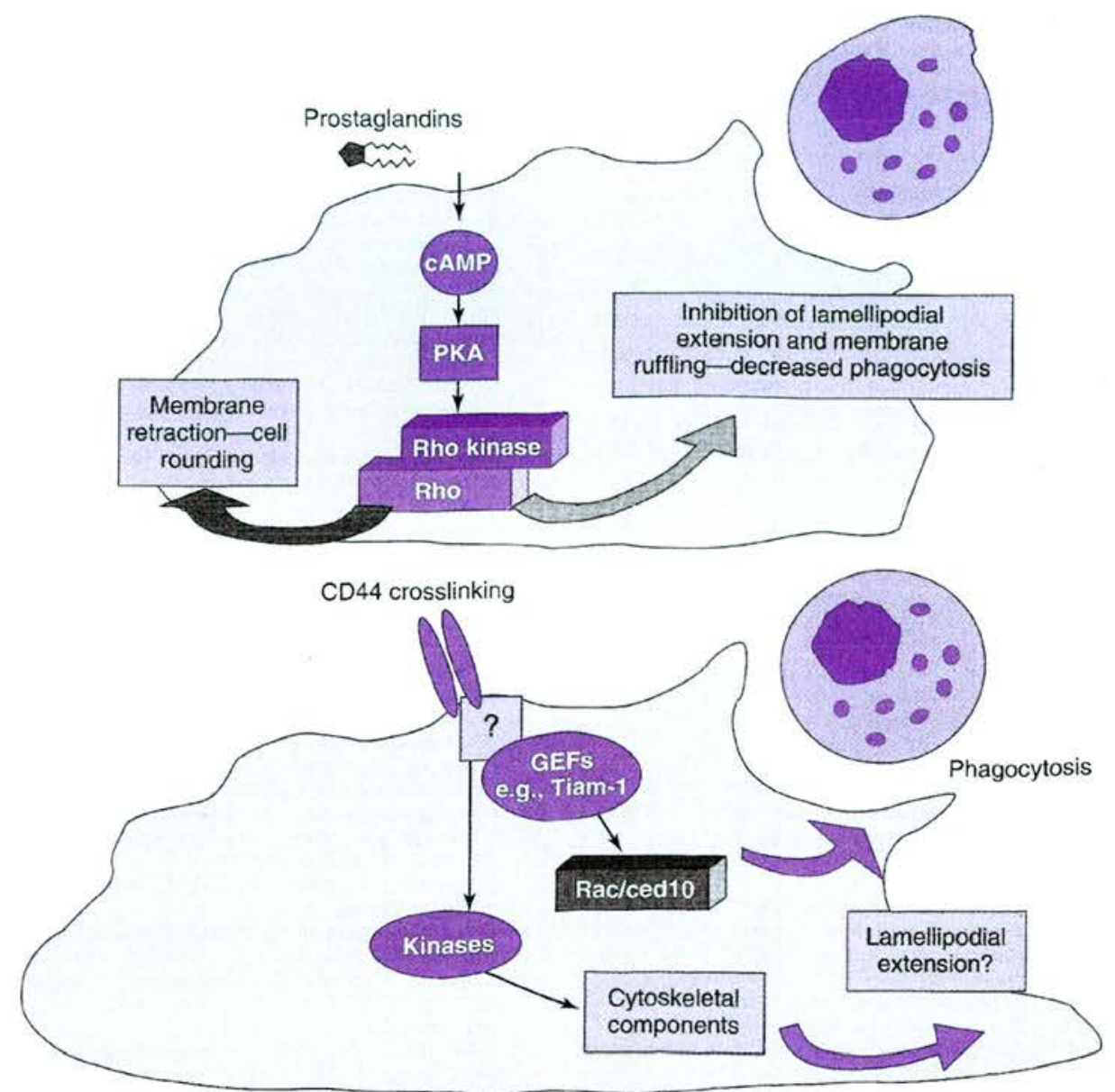

A

B

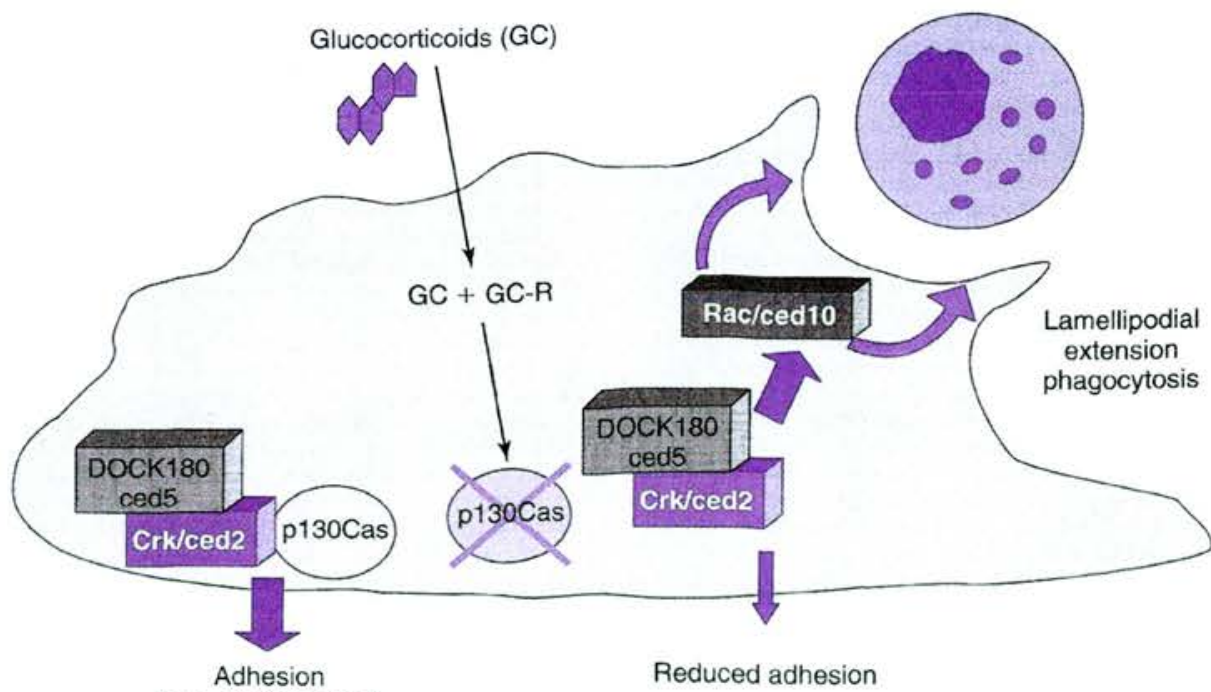

(e.g., via integrins)

FIGURE 26-5 Regulation of macrophage phagocytosis of apoptotic cells. A, Agents that elevate cyclic adenosine monophosphate ( $C A M P)$, such as prostaglandins, can inhibit macrophage phagocytosis of apoptotic cells by interfering with kinases, such as protein kinase A $(P K A)$ and Rho kinase, which in turn inhibit lamellipodial extention and membrane ruffling and cause cell rounding. B, Cross-linking of CD44 on the surface of macrophages also influences various kinases that control cytoskeletal components, resulting in augmentation of phagocytosis of apoptotic neutrophils. GEFs, Guanine nucleotide exchange factors. C, Interacting with specific GC receptors $(G C-R)$, glucocorticoids $(G C)$ cause a profound up-regulation of macrophage phagocytosis of apoptotic cells. GC augmentation of macrophage capacity for phagocytosis of apoptotic cells may be associated with reduced p130Cas expression, loss of paxillin/pyk2 phosphorylation, and high levels of active rac. 


\section{CONCLUSIONS}

Although much progress has been made in understanding the mechanisms controlling the extravasation of inflammatory cells to inflammatory foci, and given the recent increased interest in the resolution of inflammation, ${ }^{7,8,108,143-147}$ elucidation of the mechanisms regulating resolution of the inflammatory process should lead to the devclopment of novel therapeutic targets. In particular, development of strategies to induce apoptosis selectively in specific inflammatory cells, together with augmentation of macrophage clearance mechanisms, will have far-reaching implications for the control of inflammatory diseases, many of which are responsible for the heavy burden of morbidity and untimely deaths observed throughout the world.

\section{Aclinowledgment}

We thank the Medical Research Council (United Kingdom), the National Asthma Campaign, and the Wellcome Trust for financial support.

\section{REFERENCIES}

\section{The Acute Inflammatory Response}

1. Rossi AG, Hellewell PG: Mechanism of neutrophil accumulation in tissues. In Hellewell PG, Williams TJ, cditors: Immunopharmacology of neutmphils, London, 1994, Academic Press, p 223.

\section{Inflammatory Cell Recruitment}

2. Olson TS, Ley K: Chemokines and chemokine receptors in leukocyte trafficking, Am J Physiol Regul Integr Comp Physiol 283:R7, 2002.

3. Ley K: Integration of inflammatory signals by rolling neutrophils, Immunol Rev $186: 8,2002$.

4. Lee WL, Downey GP: Neutrcphil activation and acute lung injury, Curr Opin Crit Care 7:1, 2001.

5. Wagner JG, Roth RA: Ncutrophil migration mechanisms, with an emphasis on the pulmonary vasculature, Pharmacol Rev 52:349, 2000.

\section{Resolution of Inflammation}

6. Haslett $\mathrm{C}$ : Granulocyte apoptosis and its role in the resolution and control of lung inflammation, Am J Respir Crit Care Med 160:S5, 1999.

7. Ward I. Dransfield I, Chilvers ER, et al: Pharmacological manipulation of granulocyte apoptosis: potential therapeutic targets, Trends Pharmacol Sci 20:503, 1999.

8. Lawrence T, Willoughby DA, Gilroy DW: Anti-inflammatory lipid mediators and insights into the resolution of inflammation, Nat Rev immunoi 2:787, 2002.

9. Kerr JF, Wyllie AH, Currie AR: Apoptosis: a basic biological phenomenon with wide-ranging implications in tissue kinetic: $B r J$ Cancer 26:239, 1972

10. Wyllie AH, Kerr JF, Currie AR: Cell death: the significance of apoptosis, Int Rev Cytol 68:251, 1980.

11. Hurley J: Termination of acute inflammation. In Acute inflammation, London, 1983, Churchill Livingston, p 109.

12. Rossi AG, Haslett C: Inflammation, cell iujury and apoptosis. In Said S, editor: Proinflammatory and antiinfiammatory peptides. Vew York, 1998, Marcel Dekker, p 9.

13. Dransfield I, Buckle AM, Savill JS, ct al: Neutrophil apoptosis is associated with a reduction in CD16 (FcyRIII) expression, $J$ Immunol 153:1254, 1994.

14. Dransficld I, Stocks SC, Haslett C: Regulation of cell adhesion molecule expression and function associated with neutrophil apoptosis, Blood 85:3264, 1995.

15. Whyte MK, Meagher I.C, MacDermot J, Haslett C: Impairment of function in aging neutrophils is associated with apoptosis, J Immunol 150:5124, 1993.

16. Walsh GM: Eosinophil apoptosis: mechanisms and clinical relevance in asthmatic and allergic inflammation, Br J Hoematol 111:61. 2000.

17. Akgul C, Monlding DA, Edwards SW: Molecular control of neutrophil apoptosis, FEBS Lett $487: 318,2001$.

18. Murray J, Barbara JA, Dunkley SA, et al: Regulation of neutrophil apoptosis by tumor necrosis factor-alpha: requirement for TNFR55 and TNFR 75 for induction of apoptosis in vitro, Blood $90: 2772,1997$.

19. Ward C, Chilvers ER, Lawson MF, et al: NF- $\mathrm{kB}$ activation is a critical regulator of human granulocyte apoptosis in vitro, J Biol Chem 274:4309, 1999.

20. Smith CA, Farrah T, Goodwin RG: The TNF receptor superfamily of cellular and viral proteins: activation, costimulation, and death, Cell 76:959, 1994

21. Tartaglia LA, Ayres TM. Wong GH. Goeddel DV: A novel domain within the $55 \mathrm{kd}$ TNF receptor signals cell death, Cell 74:845, 1993.

22. Ashkenazi A, Dixit VM: Death receptors: signaling and modulation, Science 281:1305, 1998.

23. Kischkel FC, Lawrence DA, Tinel A, et al: Death receptor recruitment of endogenous caspase-10 and apoptosis initiation in the absence of caspase- $8, J$ Biol Chem $276: 46639,2001$.
24. Scaffidi C, Fulda S, Srinivasan A, et al: Two CD95 (APO-1/Fas) signaling pathways, EMBO J 17:1675, 1998

25. Kinloch RA, Treherne JM, Furness I.M, Hajimohamadreza 1: The pharmacology of apoptosis, Trends Pharmacol Sci 20:35, 1999.

26. Budihardjo I, Oliver H. Lutter M. et al: Biochemical pathways of caspase activation during apoptosis, Annu Rev Cell Dev Biol 15:269, 1999

27. Pryde JG, Walker A, Rossi AG, et al: Temperature-dependent arrest of neutrophil apoptosis: failure of $\mathrm{Bax}$ insertion into mitochondria at 15 degrees $\mathrm{C}$ prevents the release of cylochrome $c, J$ Biol Chem 275:33574, 2000.

28. Li P, Nijhawan D, Budihardjo I, et al: Cytochrome $c$ and dATP-dependent formation of Apaf-1/caspase-9 complex initiates an apoptotic protease cascade. Cell 91:479, 1997.

29. Hu Y, Benedict MA, Ding L, Nunez. G: Role of cytochrome $c$ and dATP/ATP hydrolysis in Apaf-1-mediated caspase-9 activation and apoptosis, EMBO J 18:3586, 1999.

30. Slee EA, Adrain C, Martin SJ: Executioner caspase-3, -6 , and -7 perform distinct. non-redundant roles during the demolition phase of apoptosis, $J$ Biol Chem $276: 7320,2001$

31. Martin MC, Dransfield I, Haslett C. Rossi AG: Cyclic AMP regulation of neutrophil apoptosis occurs via a novel protein kinase $\mathrm{A}$-independent signaling pathway, $J$ Biol Chein 276:45041, 2001.

32. Dewson G, Cohen GM, Wardlaw AJ: Interleukin-S inhibits translocation of Bax to the mitochondria, cytochrome $c$ release, and activation of caspases in human eosinophils, Blood 98:2239, 2001.

33. Peachman KK, Lyles DS, Bass DA: Mitochondria in eosinophils: functional rule in apoptosis but not respiration, Proc Natl Acad Sci USA 98:1717, 2001

34. Watson RW. O'Neill A, Brannigen AE, et al: Regulation of Fas antibody induced ncutrophil apoptosis is both caspase and mitochondrial dependent. FEBS Lett $453: 67,1999$.

35. Yamashita K. Takahashi A, Kobayashi S, et al: Caspases mediate tumor necrosis factor-alpha-induccd neutrophil apoptosis and downregulation of reactive oxygen. production. Blood 93:674, 1999.

36. Dewson G, Walsh GM, Wardlaw AJ: Expression of Bcl-2 and its homologues in human eosinophils: modulation by interleukin-5, Am J Respir Cell Mol Biol 20:720, 1999.

37. Simon HU: Regulation of eosinophil and neutrophil apoptosis: similarities and differences, Immunol Rev 179:156, 2001.

38. Simon HU: Eosinophil apoptosis: pathophysiologic and therapeutic implications. Allergy 55:910, 2000.

39. Simon HU: Molecular mechanisms of defcctive eosinophil apoptosis in diseases associated with eosinophilia. Int Arch Allergy Immuno! 113:206, 1997.

40. Simon HU, Youscfi S, Blaser K: Tyrosine phosphorylation regulates activation and inhibition of apoptosis in human eosinophils and neutrophils, Int Arch Allergy Immunol 107:338, 1995.

4I. Simon HU, Blaser K: Ithibition of programmed eosinophil death: a key pathogenic event for ensinophilia? Immunol Today 16:53, 1995.

42. Daigle I, Yousefi S, Colonna M, et al: Death receptors bind SHP-1 and block cytokine-induced anti-apoptotic signaling in neutrophils, Nat Med 8:61, 2002.

43. Liles WC, Klebanoff SJ: Regulation of apoptosis in neutrophils: Fas track to death? J Immunol 155:3289, 1995 .

44. Matsumoto $\mathrm{K}$, Schleimer RP, Saito $\mathrm{H}$, et al: Induction of apoptosis in human cosinophils by anti-Fas antibody treatment in vitro, Blood 86:1437, 1995.

45. Renshaw SA, Parmar JS, Singletun V, et al: Accelcration of human neutrophil apoptosis by TRAIL, J Immunol 170:1027, 2003

46. Tsuyuki S, Bertrand C, Erard F, et al: Activation of the Fas receptor on lung ensinophils leads to apoptosis and the resolution of eosinophilic inflammation of the airways, J Clin Invest 96:2924, 1995.

47. Ogasawara J, Watanabe-Fukunaga R, Adachi M, et al: Lcthal effect of the anti-Fa antibody in mice, Nature 364:806, 1993.

48. Ward C, Wong TH, Murray J, et al: Induction of human neutrophil apoptosis by nitric oxide donors: evicence for a caspase-dependent, cyclic-GMP-independent. mechanism, Biochem Pharmacol 59:305, 2000.

49. Taylor EL, Megson IL, Haslell C, Rossi AG: Dissociation of DNA fragmentation from other hallmarks of apoptosis in nitric oxide-treated neutrophils: differences between individual nitric oxide donor drugs, Biochem Biophys Res Commu 289:1229, 2001

50. Hannah S, Mecklenburgh K, Rahman I, et al: Hypoxia prolongs neutrophil survival in vitro, FEBS Lett 372:233, 1995.

51. Gardai SJ, Honntrakoon R. Goddard CD, et al: Oxidant-mediated mitochondrial injury in eosinophil apoptosis: enhancement by glucocorticoids and inhibition by granulocyte-macrophage colony-stimulating factor, J Immunol 170:556, 2003.

52. Riva C, Chauvin C, Pison C, Leverve X: Cellular physiology and molecular events in hypoxia-induced apoptosis, Anticencer Res 18:4729, 1998

53. Meagher IC, Cousin JM. Seckl JR, Hasiett C: Opposing effects of glucocorticoids on the rate of apoptosis in neutrophilic and eosinophilic granulocytes, $J$ Immuno 156:4422, 1996

54. Woolley KL, Gibson PG, Carty K. et al: Eosinophil apoptosis and the resolution of airway inflammation in asthma, Am J Respir Crit Care Med 154:237, 1996

55. Whyte MK. Hardwick SJ, Meagher LC, et al: Transient elevations of cytosolic frce calcium rctard subsequent apoptosis in neutrophils in vitro, $J$ Clin linvest 92 : 446, 1993.

56. Cousin JM, Haslett C, Rossi AG: Regulation of granulocyte apoptosis by PKC inhibition and elevation of $\left[\mathrm{Ca}^{2+}\right] \mathrm{i}$, Biochem Soc Trans 25:243S, 1997

57. McConkey DJ. Nicotera P, Hartzell P, et al: Glucocorticoids activate a suicide proccss in thymocytes through an elevation of cytosolic $\mathrm{Ca}^{2+}$ concentration, Anch Biochem Biophys 269:365, 1989. 
58. Rossi AG, Cousin JM, Dransfield I, et al: Agents that elevate cAMP inhibit human neutrophil apoptosis, Biochem Biophys Res Commun 217:892, 1995.

59. Hallsworth MP, Giembycz MA, Bames PJ, Lee TH: Cyclic AMP-elevating agents prolong or inhibit eosinophil survival depending on prior exposure to GM-CSF Br J Pharmacol 117:79, 1996.

60. McConkey DJ, Orrenius S, Jondal M: Agents that elevate cAMP stimulate DNA fragmentation in thymocytes, $J$ Immunol 145:1227, 1990.

61. Gjertsen BT, Cressey LI, Ruchaud S, et al: Multiple apoptotic death types triggered through activation of separate pathways by cAMP and inhibitors of protein phosphatases in one (IPC leukemia) cell line, J Cell Sci 107:3363, 1994.

62. Lanotte M, Riviere JB, Hermouet S, et al: Programmed cell death (apoptosis) is induced rapidly and with positive cooperativity by activation of cyclic adenosine monophosphate-kinase I in a myeloid leukemia cell line, J Cell Physiol 146:73, 1991

63. Webb PR, Wang KQ, Scheel-Toellner D, et al: Regulation of neutrophil apoptosis a role for protein kinase $\mathrm{C}$ and phosphatidylinositol-3-kinase, Apoptosis 5:451, 2000.

64. Lagasse E, Weissman IL: Bcl-2 inhibits apoptosis of neutrophils but not their enguifment by macrophages, J Exp Med 179:1047, 1994

65. Whyte MK, Savill J, Meagher LC, et al: Coupling of neutrophil apoptosis to recognition by macrophages: coordinated acceleration by protein synthesis inhibitors, J Leukoc Biol 62:195, 1997.

66. Lee A, Whyte MK, Haslett C: Inhibition of apoptosis and prolongation of neutrophi functional longevity by inflammatory mediators, J Leukoc Biol 54:283, 1993.

67. Fujihara S, Ward C, Dransfield I, et al: Inhibition of nuclear factor- $K B$ activation un-masks the ability of TNF- $\alpha$ to induce human eosinophil apoptosis, Eur $J$ Immunol 32:457, 2002.

68. Ward C, Dransfield I, Murray J, et al: Prostaglandin $\mathrm{D}_{2}$ and its metabolites induce caspase-dependent granulocyte apoptosis that is mediated via inhibition of $1 \times B O$ degradation using a peroxisome proliferator-activated receptor-gamma-independent mechanism, J Immunol 168:6232, 2002.

69. Fortenberry JD, Owens ML, Brown MR, et al: Exogenous nitric oxide enhances neutrophil cell death and DNA fragmentation, Am J Respir Cell Mol Biol 18:421, 1998.

70. Blaylock MG, Cuthbertson BH, Galley HF, et al: The effect of nitric oxide and peroxynitrite on apoptosis in human polymorphonuclear leukocytes, Free Radic Biol Med 25:748, 1998

71. Iwai K, Miyawaki T, Takizawa T, et al: Differential expression of bel-2 and susceptibility to anti-Fas-mediated cell death in peripheral blood lymphocytes, monocytes, and neutrophils, Blood 84:1201, 1994

72. Liles WC, Kiener PA, Ledbetter JA, et al: Differential expression of Fas (CD95) and Fas ligand on normal human phagocytes: implications for the regulation of apoptosis in neutrophils, J Exp Med 184:429, 1996.

73. Takeda Y, Watanabe H, Yonehara S, et al: Rapid acceleration of neutrophil apoptosis by tumor necrosis factor-alpha, Int Immunol 5:691, 1993.

74. Castro-Alcaraz S, Miskolci V, Kalasapudi B, et al: NF- $\mathrm{KB}$ regulation in human neutrophils by nuclear IאB $\alpha$ : correlation to apoptosis, J Immumol 169:3947, 2002

75. Chin AC, Lee WD, Murrin KA, et al: Tilmicosin induces apoptosis in bovine periph eral neutrophils in the presence or in the absence of Pasteurella haemolytica and promotes neutrophil phagocytosis by macrophages, Antimicrob Agents Chemother $44: 2465,2000$

76. Schleimer RP, Bochner BS: The effects of glucocorticoids on human eosinophils, J Allergy Clin Immunol 94:1202, 1994.

77. Druilhe A, Cai Z, Haile S, et al: Fas-mediated apoptosis in cultured human eosinophils, Blood 87:2822, 1996.

78. Hebestreit H, Yousefi S, Balatti I, et al: Expression and function of the Fas receptor on human blood and tissue eosinophils, Eur J Immunol 26:1775, 1996.

79. Sendo F, Kato T, Yazawa H: Modulation of neutrophil apoptosis by psychological stress and glucocorticoid, Int J Immunopharmacol 19:511, 1997.

80. Rossi AG, Ward C, Murray J, et al: Transcriptional regulation of eosinophil apoptosis, Respir Med 94:1259, 2000.

81. Alam R, Forsythe P, Stafford S, Fukuda Y: Transforming growth factor bet: abrogates the effects of hematopoietins on eosinophils and induces their apoptosis, J Exp Med 179:1041, 1994.

82. Blaylock MG, Sexton DW, Walsh GM: Ligation of CD45 and the isoforms CD45RA and CD45RB accelerates the rate of constitutive apoptosis in human eosinophils, J Allergy Clin Immunol 104:1244, 1999.

83. Wedi B, Raap U, Lewrick H, Kapp A: IL-4-induced apoptosis in peripheral blood eosinophils, J Allergy Clin Immunol 102:1013, 1998

84. Yasui K, Hu B, Nakazawa T, et al: Theophylline accelerates human granulocyte apoptosis not via phosphodiesterase inhibition, J Clin Invest 100:1677, 1997.

85. Walsh GM, Dewson G, Wardlaw AJ, et al: A comparative study of different methods for the assessment of apoptosis and necrosis in human eosinophils, $J$ Immunol Methods 217:153, 1998.

86. Parvathenani LK, Buescher ES, Chacon-Cruz E, Beebe SJ: Type I cAMP-dependent protein kinase delays apoptosis in human neutrophils at a site upstream of caspase-3, J Biol Chem 273:6736, 1998.

87. Ottonello L, Gonella R, Dapino P, et al: Prostaglandin $\mathrm{E}_{2}$ inhibits apoptosis in human neutrophilic polymorphonuclear leukocytes: role of intracellular cyclic AMP levels, Exp Hematol 26:895, 1998.

88. Cox G: Glucocorticoid treatment inhibits apoptosis in human neutrophils separation of survival and activation outcomes, J Immunol 154:4719, 1995.

89. Mecklenburgh KI, Walmsley SR, Cowburn AS, et al: Involvement of a ferroprotein sensor in hypoxia-mediated inhibition of neutrophil apoptosis, Blood 100:3008, 2002 .
90. Peacock CD, Misso NL, Watkins DN, Thompson PJ: PGE and dibutyryl cyclic adenosine monophosphate prolong eosinophil survival in vitro, $J$ Allergy Clin Immunol 104:153, 1999.

91. Simon HU: Eosinophil apoptosis: pathophysiologic and therapeutic implications, Allergy 55:910, 2000

92. Bureau F, Seumois G, Jaspar F, et al: CD40 engagement enhances eosinophil survival through induction of cellular inhibitor of apoptosis protein 2 expression: possible involvement in allergic inflammation, $J$ Allergy Clin Immunol 110: $443,2002$.

93. Wedi B, Wieczorek D, Stunkel T, et al: Staphylococcal exotoxins exert proinflammatory effects through inhibition of eosinophil apoptosis, increased surface antigen expression (CD11b, CD45, CD54, and CD69), and enhanced cytokine-activated oxidative burst, thereby triggering allergic inflammatory reactions, J Allergy Clin Immunol 109:477, 2002

94. Bellingan GJ, Caldwell H, Howie SE, et al: In vivo fate of the inflammatory macrophage during the resolution of inflammation: inflammatory macrophages do not die locally, but emigrate to the draining lymph nodes, J Immunol 157:2577. 1996.

95. Newman SL, Henson JE, Henson PM: Phagocytosis of senescent neutrophils by human monocyte-derived macrophages and rabbit inflammatory macrophages, $J$ Exp Med 156:430, 1982

96. Savill JS, Wyllie AH, Henson JE, et al: Macrophage phagocytosis of aging neutrophils in inflammation: programmed cell death in the neutrophil leads to its recognition by macrophages, J Clin Invest $83: 865,1989$.

97. Meagher LC, Savill JS, Baker A, et al: Phagocytosis of apoptotic neutrophils does not induce macrophage release of thromboxane B, J Leukoc Biol 52:269, 1992.

98. Fadok VA, Bratton DL, Konowal A, et al: Macrophages that have ingested apoptotic cells in vitro inhibit proinflammatory cytokine production through autocrine/ paracrine mechanisms involving TGF- $\beta, \mathrm{PGE}_{2}$, and PAF, $J$ Clin Invest 101:890, 1998.

99. Voll RE, Herrmann M, Roth EA, et al: Immunosuppressive effects of apoptotic cells, Nature 390:350, 1997

100. Hall SE, Savill JS, Henson PM, Haslett C: Apoptotic neutrophils are phagocytosed by fibroblasts with participation of the fibroblast vitronectin receptor and involvement of a mannose/fucose-specific lectin, J Immunol 153:3218, 1994.

101. Dini L, Pagliara P, Carla EC: Phagocytosis of apoptotic cells by liver: a morphological study, Micmsc Res Tech 57:530, 2002.

102. Dini L, Lentini A, Diez GD, et al: Phagocytosis of apoptotic bodies by liver endothelial cells, J Cell Sci 108:967, 1995.

103. Savill J, Smith J, Sarraf C, et al: Glomerular mesangial cells and inflammatory macrophages ingest neutrophils undergoing apoptosis, Kidney Int 42:924, 1992.

104. Bennett MR, Gibson DF, Schwartz SM, Tait JF: Binding and phagocytosis of apoptotic vascular smooth muscle cells is mediated in part by exposure of phosphatidylserine, Circ Res 77:1136, 1995.

105. Shiratsuchi A, Umeda M, Ohba Y, Nakanishi Y: Recognition of phosphatidylserine on the surface of apoptotic spermatogenic cells and subsequent phagocytosis by Sertoli cells of the rat, J Biol Chem 272:2354, 1997.

106. Walsh GM, Sexton DW, Blaylock MG, Convery CM: Resting and cytokinestimulated human small airway epithelial cells recognize and engulf apoptotic eosinophils, Blood 94:2827, 1999.

107. Giles KM, Hart SP, Haslett C, et al: An appetite for apoptotic cells? Controversies and challenges, Br J Haematol 109:1, 2000.

108. Savill J, Dransfield I, Gregory C, Haslett C: A blast from the past: clearance of apoptotic cells regulates immune responses, Nat Rev Immunol 2:965, 2002.

109. Duvall E, Wyllic AH, Morris RG: Macrophage recognition of cells undergoing programmed cell death (apoptosis), Immunology 56:351, 1985.

110. Pradhan D, Krahling S, Williamson P, Schlegel RA: Multiple systems for recognition of apoptotic lymphocytes by macrophages, Mol Biol Cell 8:767, 1997.

111. Dini L, Autuori F, Lentini A, et al: The clearance of apoptotic cells in the liver is mediated by the asialoglycoprotein receptor, FEBS Lett 296:174, 1992

112. Dini L: Asialoglycoprotein (ASGP) receptors induced by apoptosis: their immunohistochemical localization in neonatal rat hepatocytes, Rev Biol 90:423, 1997

113. Savill J, Dransfield I, Hogg N, Haslett C: Vitronectin receptor-mediated phagocytosis of cells undergoing apoptosis, Nature 343:170, 1990.

114. Savill J, Hogg N, Ren Y, Haslett C: Thrombospondin cooperates with CD36 and the vitronectin receptor in macrophage recognition of neutrophils undergoing apoptosis, J Clin Invest 90:1513, 1992.

115. Stern M, Savill J, Haslett C: Human monocyte-derived macrophage phagocytosis of senescent eosinophils undergoing apoptosis: mediation by $\alpha_{v} \beta_{3} / C D 36 /$ thrombospondin recognition mechanism and lack of phlogistic response, Am J Pathol 149:911, 1996.

116. Schwartz BR, Karsan A, Bombeli T, Harlan JM: A novel $\beta_{1}$ integrin-dependent mechanism of leukocyte adherence to apoptotic cells, J Immunol 162:4842, 1999.

117. Hoffmann PR, deCathelineau AM, Ogden CA, et al: Phosphatidylserine (PS) induces PS receptor-mediated macropinocytosis and promotes clearance of apoptotic cells, J Cell Biol 155:649, 2001.

118. Fadok VA, Voelker DR, Campbell PA, et al: Exposure of phosphatidylserine on the surface of apoptotic lymphocytes triggers specific recognition and removal by macrophages, J Immunol 148:2207, 1992.

119. Fadok VA, Bratton DL, Rose DM, et al: A receptor for phosphatidylserine-specific clearance of apoptotic cells, Nature 405:85, 2000.

120. Devitt A, Moffatt OD, Raykundalia C, et al: Human CD14 mediates recognition and phagocytosis of apoptotic cells, Nature 392:505, 1998.

121. Gregory CD, Devitt A, Moffatt O: Roles of ICAM-3 and CD14 in the recognition and phagocytosis of apoptotic cells by macrophages, Biochem Soc Trans 26:644, 1998. 
122. Luciani MF, Chimini $\mathrm{G}$ : The ATP binding cassette transporter $\mathrm{ABCl}$, is required for the engulfment of corpses generated by apoptotic cell death, EMBO J 15:226, 1996.

123. Takizawa F, Tsuji S, Nagasawa S: Enhancement of macrophage phagocytosis upon iC3b deposition on apoptotic cells, FEBS Lett 397:269, 1996.

124. Mevorach D, Mascarenhas JO, Gershov D, Elkon KB: Complement-dependent clearance of apoptotic cells by human macrophages, J Exp Med 188:2313, 1998.

125. Fishelson Z, Attali G, Mevorach D: Complement and apoptosis, Mol Immunol 38:207, 2001.

126. Taylor PR, Carugati A, Fadok VA, et al: A hierarchical role for classical pathway complement proteins in the clearance of apoptotic cells in vivo, $J$ Exp Med $192: 359,2000$

127. Vandivier RW, Ogden CA, Fadok VA, et al: Role of surfactant proteins A, D, and $\mathrm{Clq}$ in the clearance of apoptotic cells in vivo and in vitro: calreticulin and CD91 as a common collectin receptor complex, J Immunol 169:3978, 2002.

128. Brown S, Heinisch I, Ross E, et al: Apoptosis disables CD31-mediated cel detachment from phagocytes promoting binding and engulfment, Nature 418:200, 2002.

129. Balasubramanian K, Schroit AJ: Characterization of phosphatidylserine-dependen $\beta_{2}$-glycoprotein I macrophage interactions: implications for apoptotic cell clearance by phagocytes, J Biol Chem 273:29272, 1998.

130. Balasubramanian K, Chandra J, Schroit AJ: Immune clearance of phosphatidylserine-expressing cells by phagocytes: the role of $\beta_{2}$-glycoprotein $I$ in macrophage recognition, $J$ Biol Chem 272:31113, 1997.

131. Oka K, Sawamura T, Kikuta K, et al: Lectin-like oxidized low-density lipoprotein receptor 1 mediates phagocytosis of aged/apoptotic cells in endothelial cells, Proc Natl Acad Sci USA 95:9535, 1998.

132. Chang MK, Bergmark C, Laurila A, et al: Monoclonal antibodies against oxidized low-density lipoprotein bind to apoptotic cells and inhibit their phagocytosis by elicited macrophages: evidence that oxidation-specific epitopes mediate macrophage recognition, Proc Natl Acad Sci USA 96:6353, 1999.

133. Platt N, Suzuki H, Kurihara $\mathrm{Y}$, et al: Role for the class A macrophage scavenger receptor in the phagocytosis of apoptotic thymocytes in vitro, Proc Natl Acad Sci U S A 93:12456, 1996

134. Platt N, Gordon S: Is the class A macrophage scavenger receptor (SR-A) multifunctional? The mouse's tale, J Clin Invest 108:649, 200

\section{Apoptotic Mechanisms as Antiinflammatory Strategies}

135. Ren Y, Savill J: Proinflammatory cytokines potentiate thrombospondinmediated phagocytosis of neutrophils undergoing apoptosis, $J$ Immunol 154:2366. 1995.

136. Rossi AG, McCutcheon JC, Roy N, et al: Regulation of macrophage phagocytosis of apoptotic cells by cAMP, $J$ Immumol 160:3562, 1998.

137. McCutcheon JC: Studies on mechanisms involved in macrophage recognition of apoptotic neutrophils (PhD thesis), University of Edinburgh, UK, 1994.

138. McCutcheon JC, Hart SP, Canning M, et al: Regulation of macrophage phagocytosis of apoptotic neutrophils by adhesion to fibronectin. J Leukoc Biol 64:600. 1998.

139. Hart SP, Dougherty GJ, Haslett C. Dransfield I: CD44 regulates phagocytosis of apoptotic neutrophil granulocytes, but not apoptotic lymphocytes, by human macrophages, J Immunol 159:919, 1997.

140. Teder P, Vandivier RW, Jiang D, et al: Resolution of lung inflammation by CD44. Science 296:155, 2002.

141. Liu Y, Cousin JM, Hughes J, et al: Glucocorticoids promote nonphlogistic phagocytosis of apoptotic leukocytes, J Immunol 162:3639, 1999.

142. Giles KM, Ross K, Rossi AG, et al: Glucocorticoid augmentation of macrophage capacity for phagocytosis of apoptotic cells is associated with reduced p130Cas expression, loss of paxillin/pyk2 phosphorylation, and high levels of active Rac. J Immunol 167:976, 2001.

\section{Conclusions}

143. Willoughby DA, Moore AR, Colville-Nash PR. Gilroy D: Resolution of inflammation, Int J Immunopharmacol 22:1131, 2000.

144. Serhan CN: Lipoxins and aspirin-triggered 15-epi-lipoxin biosynthesis: an update and role in anti-inflammation and pro-resolution, Prostaglandins Other Lipid Mediat 68/69:433, 2002.

145. Perretti M, Chiang N, La M, et al: Endogenous lipid- and peptide-derived anti-inflammatory pathways generated with glucocorticoid and aspirin treatment activate the lipoxin A4 receptor, Nat Med 8:1296, 2002.

146. Levy $\mathrm{BD}, \mathrm{Clish} \mathrm{CB}, \mathrm{Schmidt} \mathrm{B}$, et al: Lipid mediator class switching during acute inflammation: signals in resolution, Nat Immunol 2:612, 2001.

147. Fierro IM, Serhan CN: Mechanisms in anti-inflammation and resolution: the role of lipoxins and aspirin-triggered lipoxins, Braz J Med Biol Res 34:555, 2001. 


\title{
MECHANISMS OF STEROID ACTION AND RESISTANCE IN INFLAMMATION
}

\section{Glucocorticoid-mediated regulation of granulocyte apoptosis and macrophage phagocytosis of apoptotic cells: implications for the resolution of inflammation}

\author{
S J Heasman, K M Giles ${ }^{1}$, C Ward, A G Rossi, C Haslett and \\ I Dransfield
}

MRC Centre for Inflammation Research, University of Edinburgh Medical School, Teviot Place, Edinburgh EH8 9AG, UK

'Leukocyte Adhesion Laboratory, Cancer Research UK, Lincoln's Inn Fields, London WC2A 3PX, UK

(Requests for offprints should be addressed to I Dransfield; Email: I.dransfield@ed.ac.uk)

\begin{abstract}
Glucocorticoids represent one of the most effective clinical treatments for a range of inflammatory conditions, including severe acute inflammation. Although glucocorticoids are known to affect processes involved in the initiation of inflammation, the influence of glucocorticoids on the mechanisms by which acute inflammation normally resolves have received less attention. Apoptosis of granulocytes present at inflamed sites leads to their rapid recognition and internalisation by macrophages, a process which may be important for resolution of inflammation. However, if clearance of either eosinophils or neutrophils is impaired, these cells rapidly undergo secondary necrosis leading to release of pro-inflammatory mediators from the phagocyte, potentially prolonging inflammatory responses.
\end{abstract}

Physiologically relevant concentrations of glucocorticoids accelerate eosinophil apoptosis whilst delaying neutrophil apoptosis during in vitro culture. Here we discuss key pathways regulating the granulocyte apoptotic programme and summarise the effects of glucocorticoids on monocyte differentiation and the consequent changes to apoptotic cell clearance capacity. Definition of the mechanisms underlying resolution of inflammatory responses following glucocorticoid treatment may unveil new targets for modulation of inflammatory disease, allowing co-ordinated augmentation of granulocyte apoptosis together with increased macrophage capacity for clearance of apoptotic cells.

Journal of Endocrinology (2003) 178, 29-36

\section{Introduction}

Whilst the effects of glucocorticoids on the events associated with the initiation of inflammation have been studied extensively (Pitzalis et al. 2002, Webster et al. 2002), the influence of glucocorticoids on the mechanisms by which acute inflammation normally resolves have received less attention. Over the past few years, we have been studying the process of resolution of inflammation, hypothesising that definition of the underlying mechanisms may allow development of new therapeutic approaches aimed at promoting the safe resolution of inflammatory responses which underlie a heavy burden of disease in the lung and other organs (Haslett et al. 1994) (see Fig. 1). It is now clear that neutrophil granulocytes undergo constitutive apoptosis (Savill et al. 1989a) at inflamed sites, a process associated with the 'disabling' of their potentially injurious secretion responses and other effector functions including adhesion and phagocytosis (Whyte et al. 1993a). Surface molecular alterations, including marked down-regulation of expression of Fc $\gamma$ R III (Dransfield et al. 1994), L-selectin and uncoupling of integrins of the $\beta 2$ family (Dransfield et al. 1995), contribute to attenuation of functional responsiveness. In addition, apoptosis-associated cell surface alterations, including phosphatidylserine exposure (Homburg et al. 1995), are thought to provide cues that lead to rapid recognition and internalisation of apoptotic cells by macrophages (Savill et al. 1989b). Importantly, phagocytic clearance of apoptotic granulocytes, in contrast to other phagocytic pathways, fails to promote the release of pro-inflammatory mediators from macrophages (Meagher et al. 1992), which may be important for the 'normal' resolution process. In addition, our studies have shown that macrophages (Savill et al. 1990, 1992) 


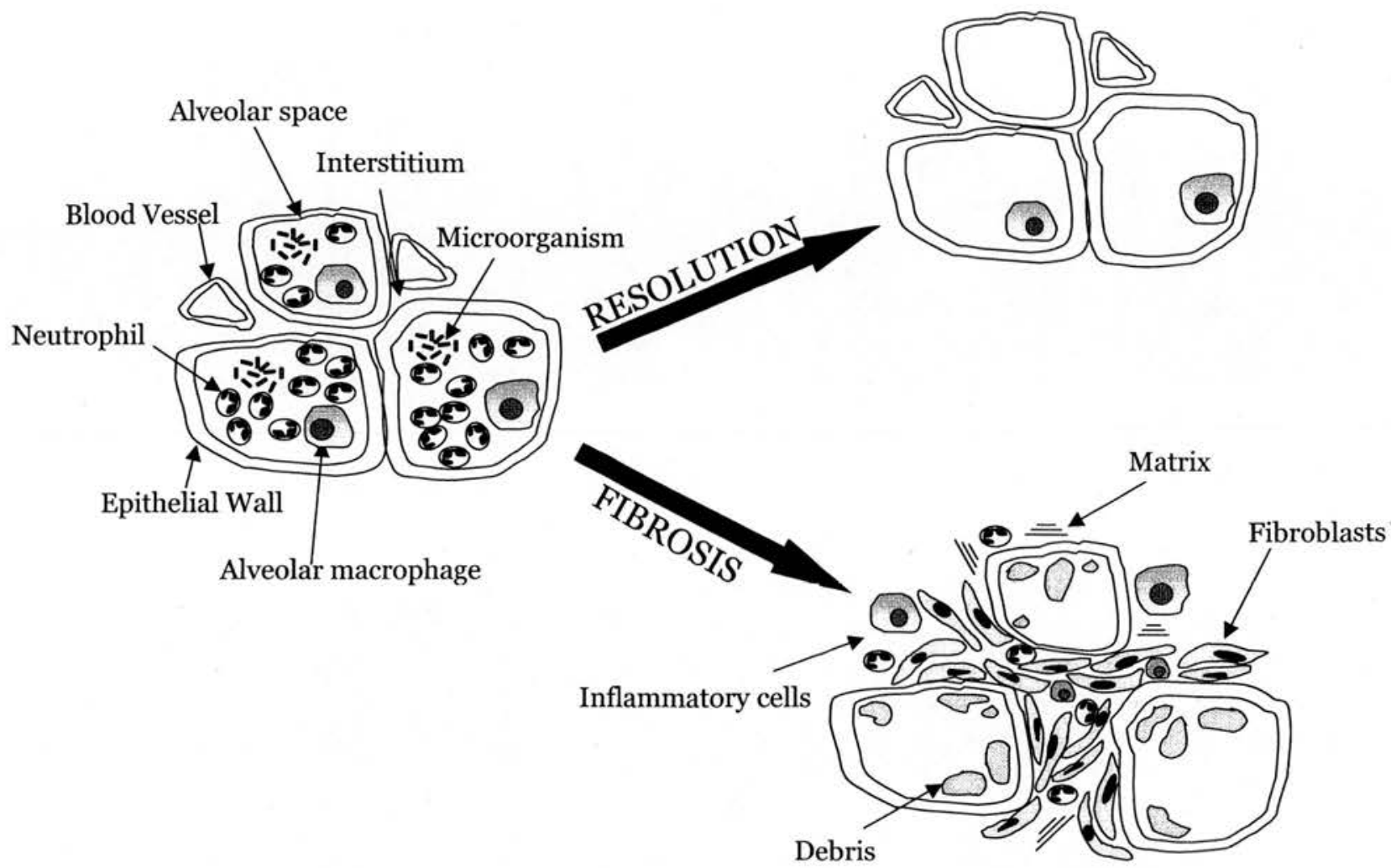

Figure 1 Schematic representation of cellular changes associated with either resolution of inflammation or development of fibrosis. Microbial infection is accompanied by infiltration of alveoli with inflammatory cells, predominantly neutrophils. Resolution of inflammation is associated with removal of microbial infection and return of tissue architecture to normal. In contrast, failure to resolve inflammation results in chronic recruitment, matrix deposition and fibroblast migration and proliferation, leading to loss of gas exchange capacity.

subsequently emigrate from the inflamed site to draining lymph nodes (Bellingan et al. 1996), where they may have the potential to influence activation of cells of the acquired immune system. Similarly, eosinophil granulocytes, which have been strongly implicated in tissue injury in allergic acute inflammation, are also programmed to die by constitutive apoptosis. Whilst the rate of constitutive eosinophil apoptosis is slower than that of the neutrophil granulocyte, apoptosis also leads to rapid, noninflammatory phagocytic clearance by macrophages (Stern et al. 1992). However, if clearance of either eosinophils or neutrophils is impaired, these cells rapidly undergo secondary necrosis. In addition to the release of potentially toxic intracellular contents associated with necrosis, one important consequence is that macrophage phagocytosis of post-apoptotic cells leads to the release of pro-inflammatory mediators, potentially prolonging inflammatory responses (Stern et al. 1996).

Granulocyte deletion by apoptosis has been shown to be amenable to external regulation by lineage-specific inflammatory signals (Haslett et al. 1991, Stern et al. 1992, Lee et al. 1993, Whyte et al. 1993b, Hannah et al. 1995, Murray et al. 1997, Ward et al. 1997, Coxon et al. 1999,
Hofman et al. 2000), providing an opportunity for targeted therapeutic intervention. However, if triggering of apoptosis is to be considered as a therapeutic target, failure to match the apoptotic cell load to the tissue clearance capacity at an inflamed site may have deleterious consequences in terms of resolution of inflammation. Macrophage capacity for phagocytosis of apoptotic granulocytes can be rapidly regulated by exogenous factors e.g. following ligation of extracellular matrix receptors such as CD44 (Hart et al. 1997, McCutcheon et al. 1998) or prostaglandin receptors (Rossi et al. 1998). Thus, co-ordinated acceleration of granulocyte apoptosis at inflamed sites together with augmentation of macrophage capacity to clear apoptotic cells may be a realistic therapeutic goal (Ward et al. 1999a).

Glucocorticoids (GCs) represent one of the most effective clinical treatments for a range of inflammatory conditions, including severe acute inflammation. GCs have profound effects, both on granulocyte apoptotic programmes (Cox 1995) and macrophage phagocytic function (Liu et al. 1999). In particular, physiologically relevant concentrations of GCs, acting via the GC receptor, were found to accelerate eosinophil apoptosis whilst 


\section{Circulating neutrophil}

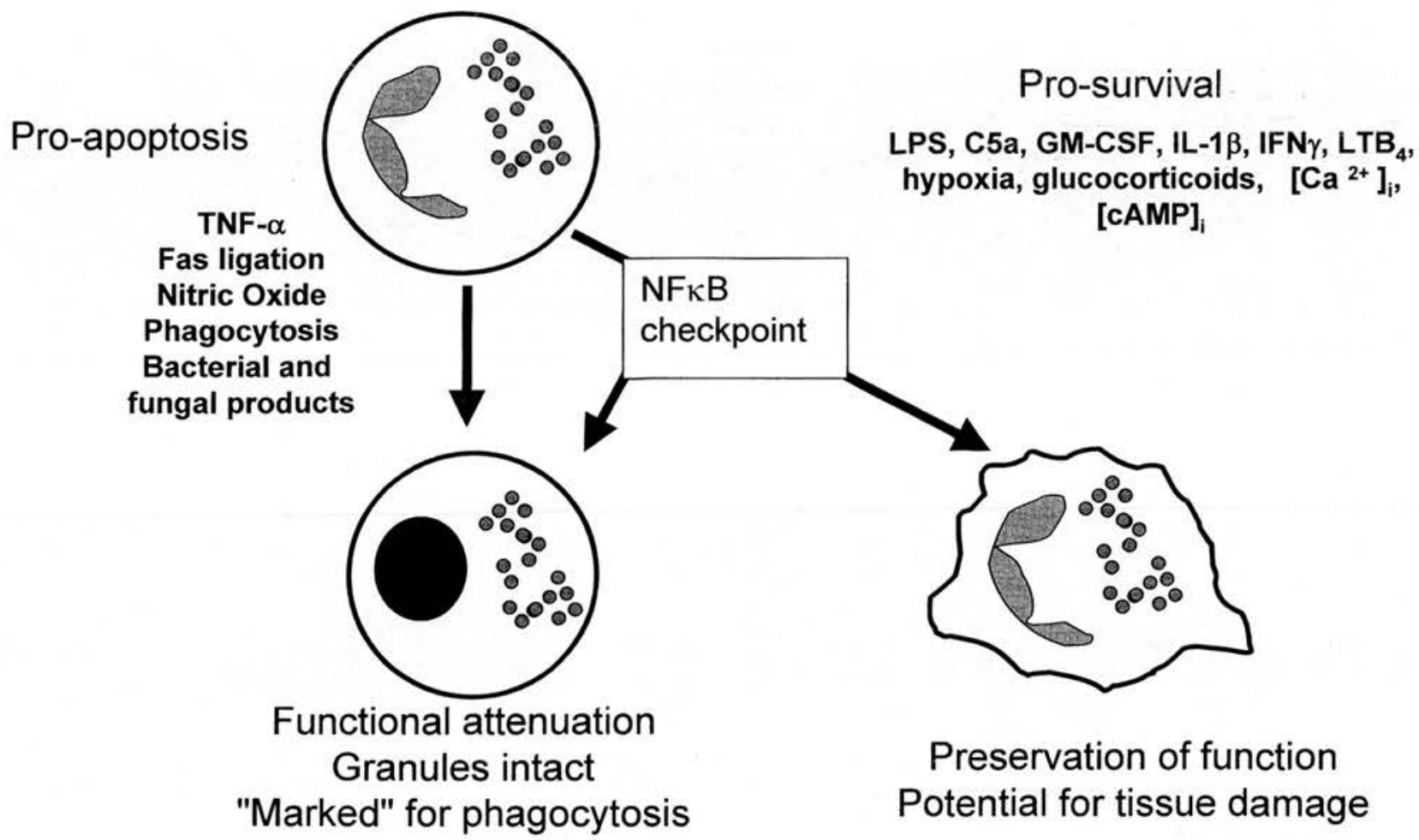

Figure 2 Neutrophils are poised on a 'knife edge' decision of life or death. A range of extracellular stimuli can promote apoptosis or survival. For those stimuli that drive survival, NFKB activation acts as a checkpoint that can result in engagement of cell death pathways, leading to functional downregulation and preservation of membrane integrity. Apoptotic cells can then be swiftly cleared by phagocytes. IFN $\gamma$, interferon $\gamma$. LTB-4, leukotriene B4.

delaying neutrophil apoptosis during in vitro culture (Meagher et al. 1996). In this article, we will discuss key pathways regulating the granulocyte apoptotic programme following treatment with GCs, and describe recent data relating to the effects of $\mathrm{GC}$ on monocyte differentiation and the consequent changes to apoptotic cell clearance capacity.

\section{Granulocytes are poised on a knife edge of nuclear} factor kappaB (NFкB)-mediated survival

It is now well established that at an inflammatory site there are many cytokines and growth factors present that provide survival signals for granulocytes with the potential to over-ride granulocyte apoptotic pathways (see Fig. 2). We have demonstrated important apoptosis-inhibiting effects of inflammatory stimuli that increase cyclic AMP levels in neutrophils (Rossi et al. 1995). The dramatic delay of the caspase-dependent apoptosis in neutrophils by dibutyryl cAMP (dbcAMP) was found to occur via a novel protein kinase A (PKA)-independent signalling pathway involving maintenance of mitochondrial potential (Martin et al. 2001). The survival effects of dbcAMP were independent of phosphatidylinositol-3 kinase (PI3K) and MAP kinase (MAPK) activation and our data point to a novel, reversible, transcriptionally independent mechanism of action of dbcAMP that may provide opportunities to shift the balance of pro-apoptotic and anti-apoptotic proteins and hence accelerate clearance of granulocytes from inflamed sites.

One of the most potent agents known to modulate granulocyte apoptosis is bacterial lipopolysaccharide (LPS) (Lee et al. 1993). Most inflammatory cells sense LPS using a complex system that involves the interaction of LBP/ CD14/MD-2 and Toll-like receptor 4 (TLR4) (O'Neill \& Dinarello 2000, Triantafilou \& Triantafilou 2002) receptors which, when engaged, trigger inter-related signal transduction pathways, including the MAPK (ERK1/2, JNK and p38), PI3K and NFKB pathways to orchestrate innate immune responses. Detailed investigation of the role of NFKB in control of granulocyte survival has revealed that inflammatory mediators such as LPS and granulocyte macrophage colony stimulating factor 
(GM-CSF) downregulate susceptibility of neutrophils to Fas-directed death (L Murray, S O'Dea, D Harrison, C Haslett \& A G Rossi, unpublished data) implying that specific pro-apoptotic regulatory pathways are overridden by NFKB signalling pathways (Fig. 2). Although both tumour necrosis factor- $\alpha$ (TNF- $\alpha$ ) and LPS act to trigger NFKB activation in neutrophils, TNF $\alpha$-mediated activation of NFKB delays apoptosis in the majority of neutrophils not induced into early apoptosis (Murray et al. 1997, Ward et al. 1999b). The pronounced effects of protein synthesis inhibitors upon granulocyte survival supports the suggestion that NFkB-directed gene transcription and protein synthesis of anti-apoptotic factors and powerful cytokines delays apoptosis in 'inflammatory' granulocytes.

We have demonstrated that the rate of constitutive apoptosis in both neutrophils and eosinophils was greatly accelerated by the NFkB inhibitor and fungal metabolite gliotoxin. This effect was reproduced using other NFkB inhibitors and suggests TNF- $\alpha$-induced activation of $\mathrm{NF} \kappa \mathrm{B}$ and production of survival proteins limits proapoptotic effects and may delay apoptosis at later time points. Similarly, for human eosinophils exposed to TNF- $\alpha$, cytoplasmic levels of I $\kappa B \alpha$, the inhibitory subunit of NFKB are rapidly reduced and NFKB is mobilised from the cytoplasm to the nucleus (Fujihara et al. 2002). Inhibition of TNF- $\alpha$-mediated $\mathrm{I} \kappa \mathrm{B} \alpha$ degradation and NFkB activation by gliotoxin treatment of eosinophils reveals caspase-dependent pro-apoptotic properties of TNF- $\alpha$. Selective inhibition of eosinophil NFKB activation may therefore represent an alternative target for inducing specific deletion of eosinophils in diseases including asthma and allergic rhinitis.

NFKB-dependent genes may also have a key regulatory role in the pathways responsible for the metabolism of prostaglandins (PGs) in granulocytes. Although many natural prostaglandins (e.g. $\mathrm{PGE}_{2}, \mathrm{PGA}_{2}, \mathrm{PGA}_{1}, \mathrm{PGF}_{2 \alpha}$ ) act either to delay apoptosis or have no effect, $\mathrm{PGD}_{2}$ and its metabolite $\mathrm{PGJ}_{2}$ selectively induced eosinophil apoptosis (Ward et al. 2002). In contrast, the sequential $\mathrm{PGD}_{2}$ metabolites $\Delta^{12} \mathrm{PGJ}_{2}$ and $15 \mathrm{dPGJ}_{2}$ were found to induce caspase-dependent apoptosis in both eosinophils and neutrophils. Despite $\Delta^{12} \mathrm{PGJ}_{2}$ and $15 \mathrm{dPGJ}_{2}$ being known activators of peroxisome proliferator-activated receptor (PPAR) $-\gamma$, apoptosis was not mimicked by synthetic PPAR $-\gamma$ and PPAR $-\alpha$ ligands nor blocked by an irreversible PPAR $-\gamma$ antagonist, suggesting a PPAR $-\gamma$ independent mechanism (Ward et al. 2002). We found that $\Delta^{12} \mathrm{PGJ}_{2}$ and $15 \mathrm{dPGJ}_{2}$ inhibited LPS-induced I $\kappa \mathrm{B} \alpha$ degradation and NFKB activation, thereby triggering apoptosis. The powerful pro-apoptotic effects of $\Delta^{12} \mathrm{PGJ}_{2}$ and $15 \mathrm{dPGJ}_{2}$ in both eosinophils and neutrophils implies that differences in the ability of eosinophils and neutrophils to process and degrade prostaglandins may be responsible for the differential effects of $\mathrm{PGD}_{2}$ upon granulocyte survival.
One potential limitation to the effectiveness of GCs in treatment of inflammatory diseases is that they undesirably prolong neutrophil survival (Cox 1995), increasing the potential for secretion of pro-inflammatory granule contents during inflammatory episodes. We believe that definition of mechanisms by which glucocorticoiddirected survival of neutrophils may be 'disengaged' may improve the efficacy of GCs in neutrophilic inflammatory diseases. Our preliminary data indicate that glucocorticoid-mediated delay of neutrophil apoptosis is reversed by inhibition of protein synthesis and inhibited by blockade of NFKB (C Ward \& A G Rossi, unpublished data). We suggest that GCs engage NFאB-directed synthesis of 'survival proteins' that may be targeted to make neutrophils respond to GCs in the same way that eosinophils do.

\section{Macrophages can be enabled for phagocytosis of apoptotic granulocytes}

Apoptotic cells have potentially toxic cellular contents and autoantigens may be revealed or generated within apoptotic cells. Thus, defects in clearance of apoptotic cells would be predicted to be associated with spontaneous and/or persistent inflammatory responses and evidence of autoimmunity to intracellular antigens (Lorenz et al. 2000, Beutler 2001, Botto 2001, Greidinger 2001, Stuart \& Hughes 2002). In support of this suggestion, spontaneous/persistent tissue inflammation and autoimmunity is observed in mutant mice with proven and probable defects in clearance of dying cells (Botto et al. 1998). Indeed, some patients with systemic lupus erythematosus exhibit (as yet uncharacterised) defects in macrophage phagocytosis of apoptotic cells (Baumann et al. 2002). As discussed above, we would predict that upregulation of macrophage capacity for 'safe' phagocytosis of apoptotic granulocytes will represent an important aspect of therapeutic strategies aimed at promoting the resolution of inflammation.

We and others have shown that macrophage phagocytosis may be rapidly modulated in response to extracellular environmental signals (Fig. 3). For example, elevation of intracellular cAMP in human monocyte-derived macrophages using the cell permeable cAMP analogue, dbcAMP, specifically reduced the phagocytosis of apoptotic neutrophils without affecting FcR-mediated phagocytosis (Rossi et al. 1998). Treatment of macrophages with $\mathrm{PGE}_{2}$ resulted in rapid, transient increases in levels of intracellular CAMP and induced PKA-dependent morphological alterations indicative of changes in the adhesive status of the macrophage, including cell rounding and disassembly of 'podosome' adhesion structures containing actin, vinculin and talin that represent points of contact with extracellular matrix (Rossi et al. 1998). Consistent with the suggestion that adhesive interactions may influence 


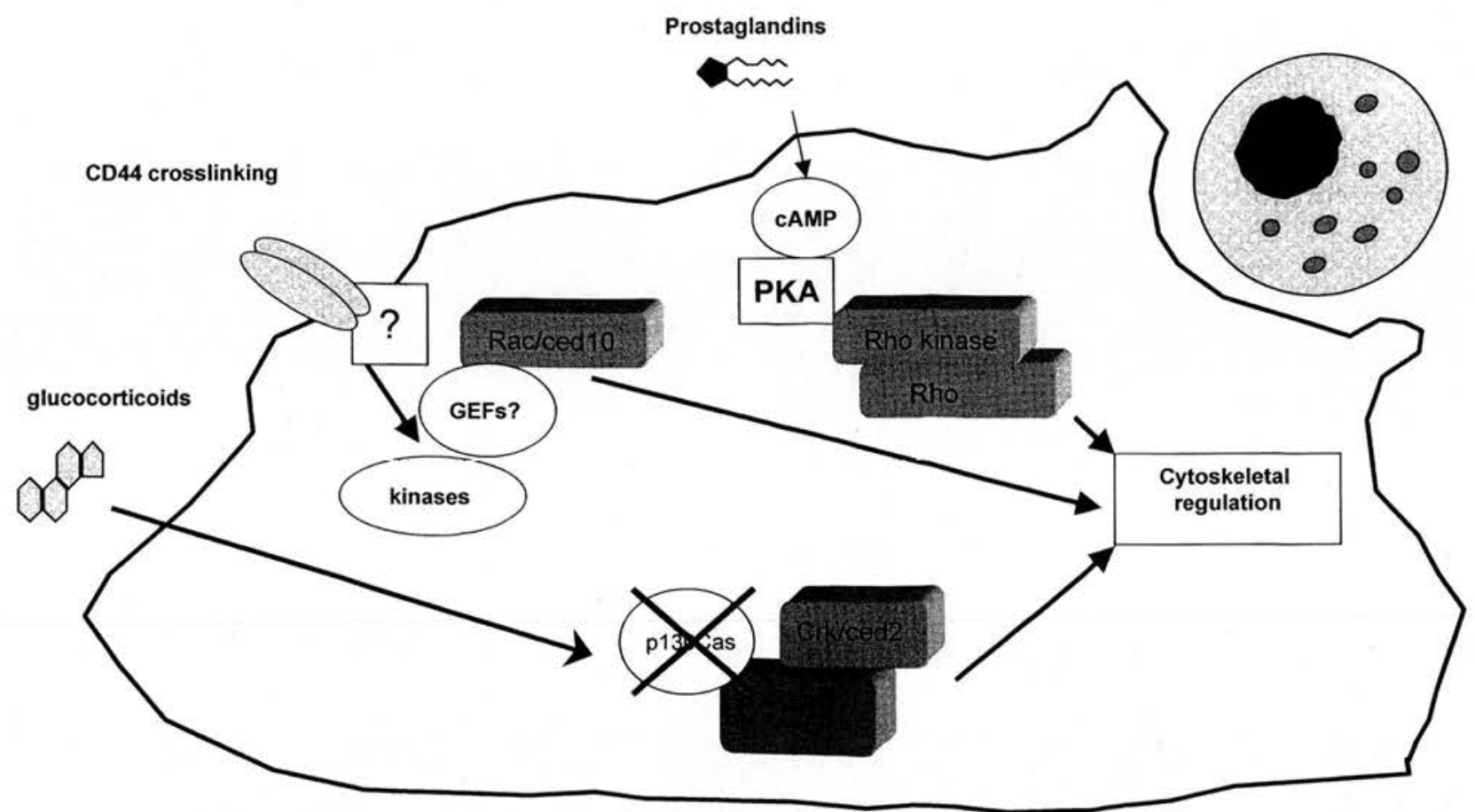

Figure 3 Schematic representation of mechanisms that regulate macrophage phagocytosis. Cross linking of CD44/matrix receptors or engagement of lipoxin receptors leads to rapid augmentation of phagocytic activity. In contrast, binding of prostaglandins to specific receptors causes cytoskeletal changes that inhibit phagocytic function. Alternatively, glucocorticoids act via glucocorticoid receptors to alter the pattern of gene expression (via either specific transactivation or transrepression) to alter cytoskeletal regulation within macrophages.

macrophage phagocytic capacity, we demonstrated that ligation of the matrix receptor CD44 rapidly and specifically increases apoptotic neutrophil internalisation (Hart et al. 1997). We now have very clear evidence that CD44 cross-linking is associated with the generation of intracellular signals that specifically augment clearance of apoptotic neutrophils. First, binding of Fab' fragments of CD44 antibodies does not promote phagocytosis, indicating that these reagents do not mask sites that are normally involved in negatively regulating cellular interactions in a manner analogous to the sialomucin, CD43. Furthermore, there does not appear to be 'capping' of CD44 within the membrane e.g. within uropod-like structures following cross-linking. Detailed temporal analysis of the CD44 cross-linking effects provides evidence that CD44 acts as an 'enabler' of macrophage phagocytosis, recruiting otherwise non-responsive cells. Recent data from studies of lung injury in CD44-deficient mice adds further weight to a role for CD44 in the regulation of macrophage clearance of apoptotic neutrophils in the resolution of inflammation (Teder et al. 2002). Our preliminary evidence indicates that engagement of specific signal transduction events following CD44 cross-linking leads to rapid changes in cytoskeletal regulation. We are currently investigating whether CD44 initiates signals that influence cytoskeletal regulatory molecules e.g. membrane recruitment of Rac GTPase via guanine nucleotide exchange factors (GEFs) such as Tiam1 which have been shown to associate with CD44 (Bourguignon et al. 2000).

\section{Glucocorticoids facilitate clearance of apoptotic cells, favouring resolution of inflammation}

In contrast to the rapid effects of CD44 ligation, [cAMP] elevation, or lipoxins (Godson et al. 2000), we found that exposure of macrophages to GCs for 24 hours specifically enhanced the uptake of apoptotic leukocytes by both human and murine macrophage populations (Liu et al. 1999). These observations establish the capacity of GCs to promote phagocytosis of cells undergoing apoptosis, raising the possibility that anti-inflammatory effects of GCs may involve pro-phagocytic effects. Importantly, glucocorticoidmediated enhancement of macrophage phagocytosis of apoptotic cells was not achieved by costly loss of the teleogically appropriate lack of pro-inflammatory response, failing to stimulate monocyte chemoattractant protein-1 (MCP-1) production and down-regulating interleukin (IL)-8 release by the phagocyte (Liu et al. 1999). 


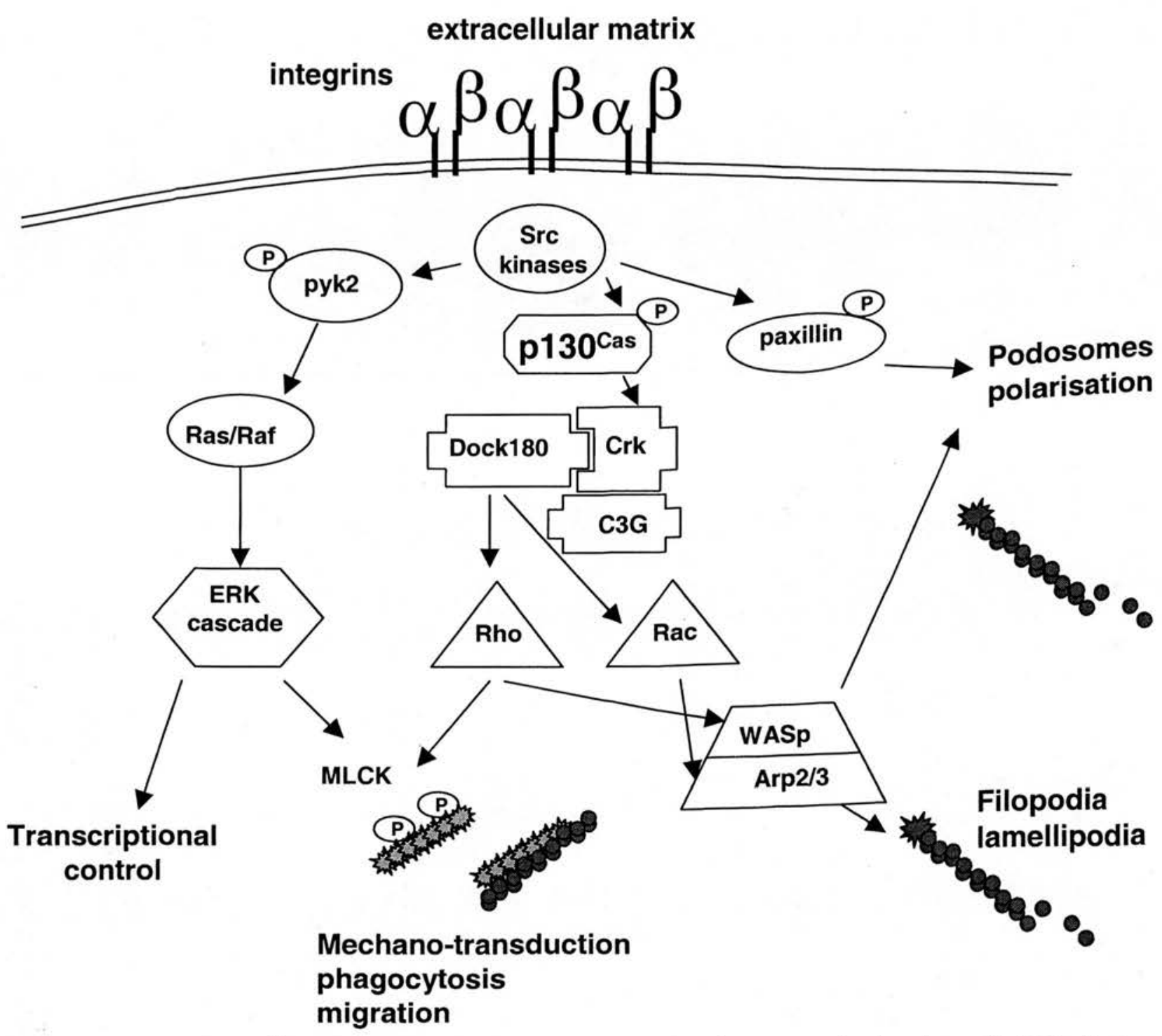

Figure $4 \mathrm{~A}$ central role for $\mathrm{p} 130^{\mathrm{Cas}}$ in adhesion signalling. Schematic representation of events occurring downstream of integrin adhesion

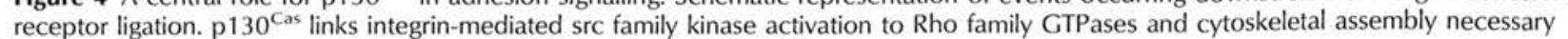
for adhesion, migration and phagocytosis. MLCK, myosin light chain kinase.

More recently, we have found that exposure of peripheral blood monocytes to GCs during the first 24 hours of the 5-day culture period induced a highly phagocytic monocyte-derived macrophage (MDM $\varphi$ ) phenotype. This GC-MDM $\varphi$ phenotype was characterised by a marked morphological appearance, consisting of smaller, more 'rounded' cells with more homogeneous laser scatter properties in flow cytometric analysis (Giles et al. 2001). Functional and morphological homogeneity was matched by cell surface phenotype, including specific induction of expression of the haemoglobin scavenger receptor, CD163 following GC treatment. Our data indicate that GCs acting via GC receptors have the potential to re-programme monocyte differentiation towards an 'anti-inflammatory' phenotype. In light of recent studies of apoptotic cell clearance in Caenorhabditis elegans (Ellis et al. 1991, Liu \& Hengartner 1998, Wu \& Horvitz 1998a,b, Chung et al. 2000, Reddien \& Horvitz 2000, Gumienny et al. 2001, May 2001), we next examined key intracellular components that regulate cytoskeletal coupling following adhesion. Alterations in the morphology of GC-MDM $\varphi$ were mirrored by changes in 
cytoskeletal organisation, with a loss of paxillin and actin containing podosome structures. Tyrosine phosphorylation of paxillin and pyk2, proteins that are recruited to adhesion contacts, were not phosphorylated in GC$\operatorname{MDM} \varphi$. A particularly striking change was that GC$\mathrm{MDM} \varphi$ showed decreased expression of $\mathrm{p} 130^{\text {Cas }}$ (Giles et al. 2001), an adaptor protein that links integrin receptors to Rho family GTPases and the MAPK pathway (see Fig. 4). Reduced expression of $\mathrm{p} 130^{\mathrm{Cas}}$ would be predicted to disrupt Crk/DOCK180/ELMO complexes, which together with reduced phosphorylation of paxillin and pyk2 may have implications for control of the turnover of adhesion structures in macrophages.

Specific recruitment of $\mathrm{p} 130^{\mathrm{Cas}}$ to focal contacts following adhesion to matrix may mimic loss of $\mathrm{p} 130^{\text {Cas }}$ observed in dexamethasone-treated cells and thus influence the availability of other components to drive cytoskeletal re-organisation necessary for phagocytosis. We propose that the repertoire of adhesion receptors that are engaged on the macrophage surface might therefore control phagocytic potential indirectly by releasing or sequestering key regulatory molecules like $\mathrm{p} 130^{\text {Cas }}$ from focal adhesion complexes. Time-lapse video microscopy analysis revealed that despite the small rounded appearance of GC-MDM $\varphi$ these cells are able to rapidly extend and retract cellular processes. Thus, although recruitment of proteins such as paxillin to podosome adhesion signalling complexes does not occur in the absence of $\mathrm{p} 130^{\text {Cas }}$, Rac may still drive the extension and retraction of processes required for phagocytosis. One possibility is that other p $130^{\text {Cas }}$-like adapters such as HEF1 and Efts/Sin, present in macrophages may allow the recruitment of Rac/Crk/ DOCK180 specifically to membranes in a manner that facilitates phagocytosis of apoptotic cells and possibly other particles. Importantly, these data raise the possibility that expression or phosphorylation of $\mathrm{p} 130^{\mathrm{Ca}}$ may have a negative regulatory role upon macrophage phagocytic potential. Our recent studies examining the effect of the cytokine environment on GC-mediated monocyte differentiation have shown that the Th1 cytokine interferon- $\gamma$ reverses the augmentation of phagocytosis seen in the GC-MDM $\varphi$ (K M Giles, SJ Heasman \& I Dransfield, unpublished data). The reduction in phagocytic ability is not accompanied by morphological changes, indicating that adhesion status and the capacity for phagocytosis can be dissociated. Further understanding of the interplay between cytokine environment and GCs in inflammation may allow the tailoring of therapies that facilitate the resolution of inflammatory disease.

\section{Acknowledgements}

This work was supported by the MRC Programme Grant G9016491 and the National Asthma Campaign $(01 / 042)$. S JH and KMG were funded by the Wellcome Trust.

\section{References}

Baumann I, Kolowos W, Voll RE, Manger B, Gaipl U, Neuhuber WL, Kirchner T, Kalden JR and Herrmann M 2002 Impaired uptake of apoptotic cells into tingible body macrophages in germinal centers of patients with systemic lupus erythematosus. Arthritis and Rheumatism 46 191-201.

Bellingan GJ, Caldwell H, Howie SE, Dransfield I and Haslett C 1996 In vivo fate of the inflammatory macrophage during the resolution of inflammation: inflammatory macrophages do not die locally, but emigrate to the draining lymph nodes. Joumal of Immunology 157 2577-2585.

Beutler B 2001 Autoimmunity and apoptosis: the Crohn's connection. Immunity 15 5-14.

Botto M 2001 Links between complement deficiency and apoptosis. Arthritis Research 3 207-210.

Botto M, Dell'Agnola C, Bygrave AE, Thompson EM, Cook HT, Petry F, Loos M, Pandolfi PP \& Walport MJ 1998 Homozygous $\mathrm{C} 1 \mathrm{q}$ deficiency causes glomerulonephritis associated with multiple apoptotic bodies. Nature Genetics 19 56-59.

Bourguignon LY, Zhu H, Shao L \& Chen YW 2000 CD44 interaction with tiam1 promotes Rac1 signaling and hyaluronic acid-mediated breast tumor cell migration. Joumal of Biological Chemistry 275 1829-1838.

Chung S, Gumienny TL, Hengartner MO \& Driscoll M 2000 A common set of engulfment genes mediates removal of both apoptotic and necrotic cell corpses in C. elegans. Nature Cell Biology 2 931-937.

Cox G 1995 Glucocorticoid treatment inhibits apoptosis in human neutrophils. Separation of survival and activation outcomes. Joumal of Immunology 154 4719-4725.

Coxon A, Tang T \& Mayadas TN 1999 Cytokine-activated endothelial cells delay neutrophil apoptosis in vitro and in vivo. A role for granulocyte/macrophage colony-stimulating factor. Joumal of Experimental Medicine 190 923-934.

Dransfield I, Buckle AM, Savill JS, McDowall A, Haslett C \& Hogg N 1994 Neutrophil apoptosis is associated with a reduction in CD16 Fc gamma RIII expression. Joumal of Immunology 153 1254-1263.

Dransfield I, Stocks SC \& Haslett C 1995 Regulation of cell adhesion molecule expression and function associated with neutrophil apoptosis. Blood 85 3264-3273.

Ellis RE, Jacobson DM \& Horvitz HR 1991 Genes required for the engulfment of cell corpses during programmed cell death in Caenorhabditis elegans. Genetics 129 79-94.

Fujihara S, Ward C, Dransfield I, Hay R, Uings I, Hayes B, Farrow S, Haslett C \& Rossi A 2002 Inhibition of nuclear factor-kappaB activation un-masks the ability of TNF-alpha to induce human eosinophil apoptosis. European Journal of Immunology 32 457-466.

Giles KM, Ross K, Rossi AG, Hotchin NA, Haslett C \& Dransfield I 2001 Glucocorticoid augmentation of macrophage capacity for phagocytosis of apoptotic cells is associated with reduced p $130^{\text {Cas }}$ expression, loss of paxillin/pyk2 phosphorylation, and high levels of active Rac. Joumal of Immunology 167 976-986.

Godson C, Mitchell S, Harvey K, Petasis NA, Hogg N \& Brady HR 2000 Cutting edge: lipoxins rapidly stimulate nonphlogistic phagocytosis of apoptotic neutrophils by monocyte-derived macrophages. Joumal of Immunology 164 1663-1667.

Greidinger EL 2001 Apoptosis in lupus pathogenesis. Frontiers in Bioscience 6 D1392-D1402.

Gumienny TL, Brugnera E, Tosello-Trampont AC, Kinchen JM, Haney LB, Nishiwaki K, Walk SF, Nemergut ME, Macara IG, Francis R, Schedl T, Qin Y, Van Aelst L, Hengartner MO \& Ravichandran KS 2001 CED-12/ELMO, a novel member of the CrkII/Dock180/Rac pathway, is required for phagocytosis and cell migration. Cell $10727-41$.

Hannah S, Mecklenburgh K, Rahman I, Bellingan GJ, Greening A, Haslett C \& Chilvers ER 1995 Hypoxia prolongs neutrophil survival in vitro. FEBS Letters 372 233-237. 
Hart SP, Dougherty GJ, Haslett C \& Dransfield I 1997 CD44 regulates phagocytosis of apoptotic neutrophil granulocytes, but not apoptotic lymphocytes, by human macrophages. Joumal of Immunology 159 919-925.

Haslett C, Lee A, Savill JS, Meagher L \& Whyte MK 1991 Apoptosis programmed cell death and functional changes in aging neutrophils. Modulation by inflammatory mediators. Chest $996 \mathrm{~S}$.

Haslett C, Savill JS, Whyte MK, Stern M, Dransfield I \& Meagher LC 1994 Granulocyte apoptosis and the control of inflammation. Philosophical Transactions of the Royal Society of London 345 327-333.

Hofman P, Piche M, Far DF, Le Negrate G, Selva E, Landraud L, Alliana-Schmid A, Boquet P \& Rossi B 2000 Increased Escherichia coli phagocytosis in neutrophils that have transmigrated across a cultured intestinal epithelium. Infection and Immunity 68 449-455.

Homburg CH, de Haas M, dem Borne AE, Verhoeven AJ,

Reutelingsperger CP \& Roos D 1995 Human neutrophils lose their surface Fc ganma RIII and accuire Annexin V binding sites during apoptosis in vitro. Blood 85 532-540.

Lee A, Whyte MK \& Haslett C 1993 Inhibition of apoptosis and prolongation of neutrophil functional longevity by inflammatory mediators. Joumal of Leukocyte Biology 54 283-288.

Liu QA \& Hengartner MO 1998 Candidate adaptor protein CED-6 promotes the engulfment of apoptotic cells in $\mathrm{C}$. elegans. Cell 93 961-972.

Liu Y, Cousin JM, Hughes J, Van Damme J, Seckl JR, Haslett C, Dransfield I, Savill J \& Rossi AG 1999 Glucocorticoids promote nonphlogistic phagocytosis of apoptotic leukocytes. Joumal of Immunology 162 3639-3646.

Lorenz HM, Herrmann M, Winkler T, Gaipl U \& Kalden JR 2000 Role of apoptosis in autoimmunity. Apoptosis 5 443-449.

McCutcheon JC, Hart SP, Canning M, Ross K, Humphries MJ \& Dransfield I 1998 Regulation of macrophage phagocytosis of apoptotic neutrophils by adhesion to fibronectin. Joumal of Leukocyte Biology 64 600-607.

Martin MC, Dransfield I, Haslett C \& Rossi AG 2001 Cyclic AMP regulation of neutrophil apoptosis occurs via a novel protein kinase A-independent signaling pathway. Joumal of Biological Chemistry 276 45041-45050.

May RC 2001 Phagocytosis in C. elegans: CED-1 reveals its secrets. Trends in Cell Biology 11150.

Meagher LC, Savill JS, Baker A, Fuller RW \& Haslett C 1992 Phagocytosis of apoptotic neutrophils does not induce macrophage release of thromboxane B2. Journal of Leukocyte Biology 52 269-273.

Meagher LC, Cousin JM, Seckl JR \& Haslett C 1996 Opposing effects of glucocorticoids on the rate of apoptosis in neutrophilic and eosinophilic granulocytes. Joumal of Immunology 156 4422-4428.

Murray J, Barbara JA, Dunkley SA, Lopez AF, Van OX, Condliffe AM, Dransfield I, Haslett C \& Chilvers ER 1997 Regulation of neutrophil apoptosis by tumor necrosis factor-alpha: requirement for TNFR 55 and TNFR75 for induction of apoptosis in vitro. Blood 90 2772-2783.

O'Neill LA \& Dinarello CA 2000 The IL-1 receptor/toll-like receptor superfamily: crucial receptors for inflammation and host defense. Immunology Today 21 206-209.

Pitzalis C, Pipitone N \& Perretti M 2002 Regulation of leukocyte-endothelial interactions by glucocorticoids. Annals of the New York Academy of Sciences 966 108-118.

Reddien PW \& Horvitz HR 2000 CED-2/CrkII and CED-10/Rac control phagocytosis and cell migration in Caenorhabditis elegans. Nature Cell Biology 2 131-136.

Rossi AG, Cousin JM, Dransfield I, Lawson MF, Chilvers ER \& Haslett C 1995 Agents that elevate cAMP inhibit human neutrophil apoptosis. Biochemical and Biophysical Research Communications 217 892-899.

Rossi AG, McCutcheon JC, Roy N, Chilvers ER, Haslett C \& Dransfield I 1998 Regulation of macrophage phagocytosis of apoptotic cells by cAMP Joumal of Immunology 160 3562-3568.
Savill JS, Wyllie AH, Henson JE, Walport MJ, Henson PM \& Haslett C 1989a Macrophage phagocytosis of aging neutrophils in inflammation. Programmed cell death in the neutrophil leads to its recognition by macrophages. Joumal of Clinical Investigation 83 865-875.

Savill JS, Henson PM \& Haslett C 1989b Phagocytosis of aged human neutrophils by macrophages is mediated by a novel 'charge-sensitive' recognition mechanism. Joumal of Clinical Investigation 84 1518-1527.

Savill J, Dransfield I, Hogg N \& Haslett C 1990 Vitronectin receptormediated phagocytosis of cells undergoing apoptosis. Nature $\mathbf{3 4 2}$ 170-173.

Savill J, Hogg N, Ren Y \& Haslett C 1992 Thrombospondin cooperates with CD36 and the vitronectin receptor in macrophage recognition of neutrophils undergoing apoptosis. Joumal of Clinical Investigation 90 1513-1522.

Stern M, Meagher L, Savill J \& Haslett C 1992 Apoptosis in human eosinophils. Programmed cell death in the eosinophil leads to phagocytosis by macrophages and is modulated by IL-5. Joumal of Immunology. 148 3543-3549.

Stern M, Savill J \& Haslett C 1996 Human monocyte-derived macrophage phagocytosis of senescent eosinophils undergoing apoptosis. Mediation by alpha $\mathrm{v}$ beta $3 / \mathrm{CD} 36 /$ thrombospondin recognition mechanism and lack of phlogistic response. American Joumal of Pathology 149 911-921.

Stuart L \& Hughes J 2002 Apoptosis and autoimmunity. Nephrology, Dialysis, Transplantation 17 697-700.

Teder P, Vandivier RW, Jiang D, Liang J, Cohn L, Pure E, Henson PM \& Noble PW 2002 Resolution of lung inflammation by CD44. Science 296 155-158.

Triantafilou M \& Triantafilou K 2002 Lipopolysaccharide recognition: CD14, TLRs and the LPS-activation cluster. Trends in Immunology 23 301-304.

Ward C, Hannah S, Chilvers ER, Farrow S, Haslett C \& Rossi AG 1997 Transforming growth factor-beta increases the inhibitory effects of GM-CSF and dexamethasone on neutrophil apoptosis. Biochemical Society Transactions 25 244S.

Ward C, Dransfield I, Chilvers ER, Haslett C \& Rossi AG 1999a Pharmacological manipulation of granulocyte apoptosis: potential therapeutic targets. Trends in Pharmacological Sciences 20 503-509.

Ward C, Chilvers ER, Lawson MF, Pryde JG, Fujihara S, Farrow SN, Haslett C \& Rossi AG 1999b NF-kappaB activation is a critical regulator of human granulocyte apoptosis in vitro. Joumal of Biological Chemistry 274 4309-4318.

Ward C, Dransfield I, Murray J, Farrow SN, Haslett C \& Rossi AG 2002 Prostaglandin $D_{2}$ and its metabolites induce caspase-dependent granulocyte apoptosis that is mediated via inhibition of I kappa B alpha degradation using a peroxisome proliferator-activated receptor- gamma-independent mechanism. Joumal of Immunology 168 6232-6243.

Webster JI, Tonelli L \& Sternberg EM 2002 Neuroendocrine regulation of immunity. Annual Review of Immunology 20 125-163.

Whyte MK, Meagher LC, MacDermot J \& Haslett C 1993a Impairment of function in aging neutrophils is associated with apoptosis. Journal of Immunology 150 5124-5134.

Whyte MK, Hardwick SJ, Meagher LC, Savill JS \& Haslett C 1993 b Transient elevations of cytosolic free calcium retard subsequent apoptosis in neutrophils in vitro. Joumal of Clinical Investigation 92 446-455.

Wu YC \& Horvitz HR 1998a C. elegans phagocytosis and cell-migration protein CED-5 is similar to human DOCK180. Nature 392 501-504.

Wu YC \& Horvitz HR $1998 b$ The C. elegans cell corpse engulfment gene ced-7 encodes a protein similar to ABC transporters. Cell 93 951-960.

Received 16 December 2002

Accepted 24 March 2003 


\title{
INFLAMMATORY RESOLUTION: NEW OPPORTUNITIES FOR DRUG DISCOVERY
}

\author{
Derek W. Gilroy ${ }^{*}$ Toby Lawrence ${ }^{\ddagger}$, Mauro Perretti* and Adriano G. Rossi ${ }^{\lessgtr}$ \\ Treatment of inflammatory diseases today is largely based on interrupting the synthesis or action \\ of mediators that drive the host's response to injury. Non-steroidal anti-inflammatories, steroids \\ and antihistamines, for instance, were developed on this basis. Although such small-molecule \\ inhibitors have provided the main treatment for inflammatory arthropathies and asthma, they are \\ not without their shortcomings. This review offers an alternative approach to the development of \\ novel therapeutics based on the endogenous mediators and mechanisms that switch off acute \\ inflammation and bring about its resolution. It is thought that this strategy will open up new \\ avenues for the future management of inflammation-based diseases.
}

*William Harvey Research Institute, St. Bartholomew's and the Royal London School of Medicine and Dentistry, Charterhouse Square, London ECIM 6BQ, UK. 'Laboratory of Gene Regulation and Signal Transduction, Department of Pharmacology, School of Medicine, University of California at San Diego, 9500 Gilman Drive, La Jolla, California 92093-0636, USA ${ }^{6}$ Respiratory Medicine Unit, Centre for Inflammation Research, University of Edinburgh Medical School, Teviot Place, Edinburgh EH8 9AG, UK.

Correspondence to D.W.G. e-mail:

d.w.gilroy@qmul.ac.uk doi: $10.1038 /$ nrd 1383
We have come a long way in our understanding of inflammation since the days of Celsus (about $30 \mathrm{BC}$ to $38 \mathrm{AD}$ ) and his original description of the inflammatory response in terms of cardinal signs - heat, redness, swelling and pain. Dotted throughout its colourful history, inflammation research has had innumerable individuals who have described many of the mechanisms associated with tissue injury. Among these are Lewis (1881-1945), who described the Triple Response; Cohnheim and Samuel (late 1800s), who reported on leukocyte emigration and vascular permeability; and Metchnikoff (1845-1916), who described phagocytosis, to name but a few ${ }^{1}$. However, even until the midtwentieth century our understanding of the chemical mediators that bring about these events - in particular, changes that lead to Vascular LEAKage, pain and cell accumulation - was lacking. Indeed, Wilhelm, writing in the mid-1960s, commented that "Half a century of work on the identification of substances increasing vascular permeability and the other vascular events in the inflammatory process has resulted in little reliable information concerning relevant chemical mediators"'. The subsequent 40 years of research have made up for this shortfall by revealing many of the factors that we now know propagate the initial response to tissue injury (BOX 1). However, once the inflammatory leukocyte and the exudate have entered the inflammatory site and neutralized the inciting agent, they must be eliminated and cleared in a NON-PHLOGISIIC manner. This is acute inflammatory resolution, a burgeoning area of inflammation research that might help to understand the aetiology of chronic inflammation. Like Wilhelm and colleagues in the 1960 s, who lamented the lack of understanding of the mediators that drive acute inflammation, we stand now in the new millennium knowing little of what switches it off. In much the same way as pharmacologists developed drugs based on blocking what initiates and drives inflammation, perhaps now we can develop new anti-inflammatory drugs based on mimicking those molecules that resolve the problem.

\section{What is inflammatory resolution?}

It is a popular misconception that once the inflammatory response has neutralized an injurious stimulus, inflammation somehow fizzles out, possibly from proinflammatory mediator catabolism. On the contrary, the resolution of acute inflammation is a highly coordinated and active process that is controlled by endogenous 'pro-resolving' mediators. These factors switch off leukocyte trafficking to the inflamed site, reverse vasodilation and vascular permeability, and bring 


\section{Box 1 | Mediators of the acute inflammatory response}

Mediators known to drive the acute inflammatory response

- The clotting system products (plasmin, fibrinopeptides)

- Fibrinolytic system products (fibrin)

- Kinins (bradykinin)

-Vasoactive amines (histamine and 5-hydroxytryptamine)

- Substance P

- Complement system by-products

- Eicosanoids (prostaglandins, leukotrienes and platelet activation factor)

- Cell-adhesion molecules

- Cytokines

- Chemokines

- Oxygen-derived free radicals

- Nitric oxide

Mediators recently found to be involved in pro-resolution

- Cyclopentenone prostaglandins

- Lipoxins/resolvins

- NF-kB (p50/p50)

- Mediators of apoptosis (caspases, CD44, etc.)

-Annexin-1

VASCUIAR IEAKAGE The process of the escape of plasma and plasma proteins, along with white blood cells, from the vessel is known as exudation. This inflammatory exudate accounts for an increase in the volume of interstitial fluid (oedema) and tissue swelling at the local site of injury

\section{NON-PHLOGISTIC}

Non-inflammatory. This term is used to describe the clearance of leukocytes in a manner that does not elicit an inflammatory response.

NF-KB

(Nuclear factor of $\kappa \mathrm{B}$ ). A group of sequence-specific

transcription factors that are best known as key regulator of the innate and adaptive inflammatory responses, cell survival and ancogenesis. In mammals, NF- $\mathrm{kB}$ consists of five structurally related and functionally conserved proteins: RELA (p65), RELB, $c-R E L, N F-\kappa B 1$ (p105 and p50) and $\mathrm{NF}-\mathrm{KB} 2(\mathrm{p} 100$ and $\mathrm{p} 52)$ about the safe disposal of inflammatory leukocytes, exudate and fibrin, thereby leading to the restoration of the inflamed tissue to its prior physiological function. Importantly, successful resolution will limit excessive tissue injury and give little opportunity for the development of chronic, immune-mediated inflammation ${ }^{2}$. However, if the host is unable to neutralize the injurious agent and/or there is a failure of endogenous pro-resolving mediators to invoke resolution, then acute inflammation might perpetuate, resulting in varying degrees of tissue injury. If tissue injury is mild, necrotic parenchymal cells will be replaced by new cells of the same type in a process known as regeneration. If, however, tissue damage is extensive, or when fibrin is not rapidly cleared after acute inflammation, the process of healing is by repair. This involves the in-growth from the surrounding connective tissue of an initially vascular tissue containing capillary loops, fibroblasts and leukocytes, and is known as granulation tissue. With time, the fibroblasts lay down collagen and the capillaries disappear, leaving an avascular area of fibrosis or scar. Repair by granulation and fibrosis occurs in many parts of the body where a deposit of clot, exudate or dead tissue occurs and is given the general name of organization ${ }^{3}$ (FIG. 1). Undoubtedly, this is also a form of inflammatory resolution associated with tissue damage. As in rheumatoid arthritis and asthma, for example, there will be continuous or repeated bouts of acute inflammation, resulting in ongoing tissue damage. Attempts at wound healing would result in granulomatous tissue formation, angiogenesis, fibrosis and scar formation, all occurring concurrently ${ }^{2}$. This is chronic inflammation and might be defined as a continuous inflammatory disease state that could be driven by the development of an immune response to an endogenous antigen (autoimmunity) ${ }^{4-7}$. This review will not consider aspects of wound healing or chronic inflammation but will focus on the mediators and mechanisms that regulate the fate of inflammatory leukocytes during the resolution of acute inflammation.

\section{Why develop drugs based on resolution?}

A range of therapies exist for the treatment of inflammation-driven diseases such as asthma, rheumatoid arthritis, psoriatic arthritis, systemic lupus erythematosus, Crohn's disease, multiple sclerosis and systemic vasculitis. However, such therapeutics have undesirable side effects. For instance, steroids can cause osteoporosis ${ }^{8}$ and impair wound healing ${ }^{9}$, whereas novel selective inducible cyclooxygenase (COX) 2 inhibitors might reduce protective vascular prostacyclin synthesis, leading to an increased risk of thrombosis ${ }^{10}$. Indeed, experience with tumour necrosis factor- $\alpha$ (TNF- $\alpha$ )-neutralizing therapy has also revealed several complications ${ }^{11}$. Clinical studies indicate that TNF- $\alpha$-neutralizing therapy should not be given to patients with cardiac failure or a history of demyelinating disease. In addition, increased infection rates can occur, especially the activation of latent tuberculosis, although other opportunistic infections such as listeriosis, pneumonia and aspergillosis have also been reported. These unwanted side effects stem from the inhibition of essential endogenous factors that have a role in normal physiology or, in the case of anti-TNF- $\alpha$, by dampening down the host's ability to adequately deal with infection, given that the innate inflammatory response is a beneficial defensive event. Our view is that detailed characterization of the biochemical pathways leading to resolution might lead to the identification of novel targets that can be exploited for innovative antiinflammatory drug discovery. We foresee that novel compounds based on the mechanism of action of a given pro-resolving mediator will be modulatory in their action and likely to produce a lower burden of side effects.

\section{Mediators and mechanisms of resolution}

Studies on the resolution of acute inflammation have already revealed novel mediators with potent antiinflammatory properties. Determining their basic structure and function might help in the development of unique anti-inflammatory therapeutics. So far, these pro-resolving mediators have been shown to exert powerful anti-inflammatory effects in various experimental models of inflammatory diseases. Of these, lipid-mediator derivatives of the $\operatorname{COX} 2$ and lipoxygenase (LOX)/LOX interaction pathways of arachidonic acid, eicosapentaenoic acid (EPA) and docosahexaenoic acid (DHA) metabolism, as well as inducible haem-oxygenase ( $\mathrm{HO}-1$ ), will be described in this review. We will also discuss the fate of inflammatory leukocytes after their job is done (apoptosis), reveal a virtuous side to nuclear factor $(\mathrm{NF})-\mathrm{KB}$ and discuss how old workhorses of inflammation (glucocorticoids and annexins) cross paths with the new (lipoxins). 

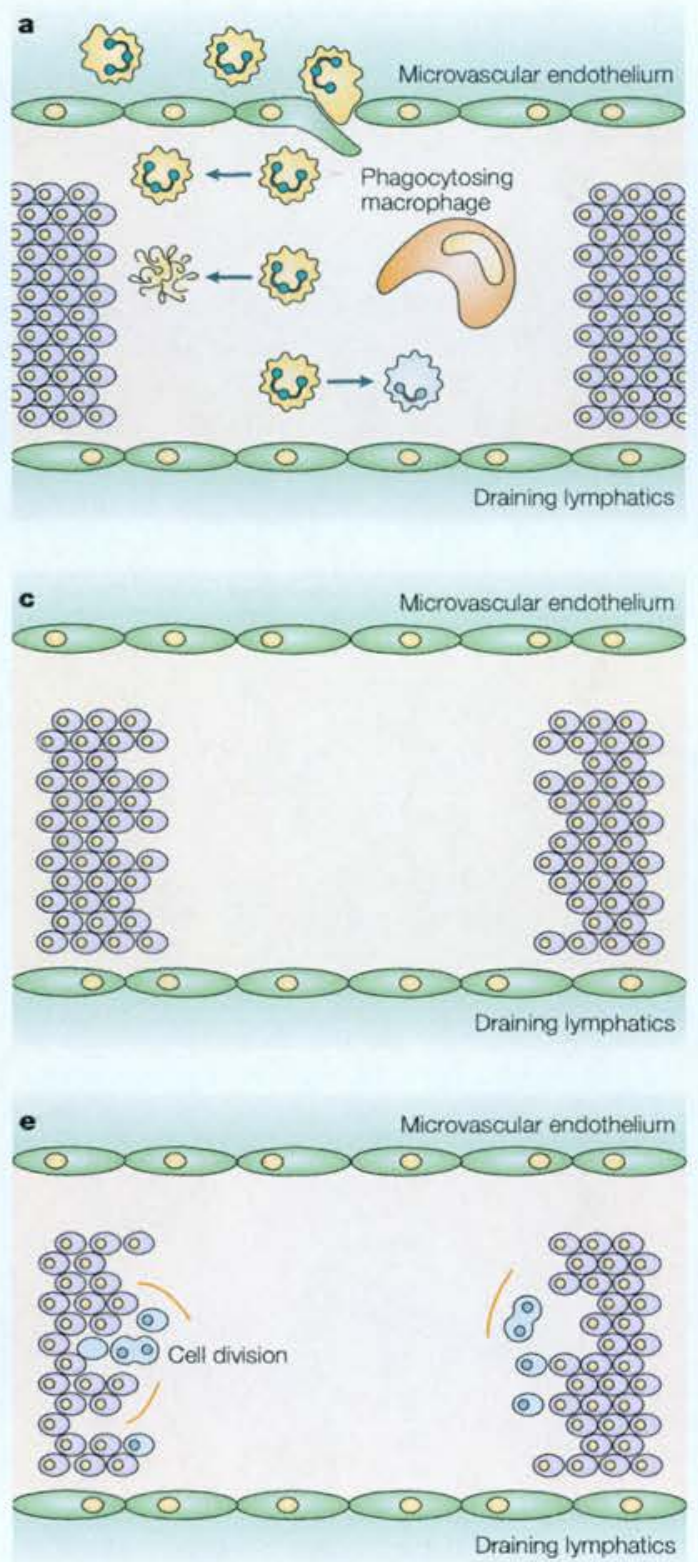

Figure 1 | Scheme of events depicting the various consequences to acute inflammation. In the first instance, inflammatory leukocytes migrate to the inflamed site or area of tissue damage, with PMNs or eosinophils being the first cell types at the scene (a). Although in some circumstances PMNs are cleared from the inflamed site by returning to the circulation, the most common fate of PMNs is apoptosis, after which they are phagocytosed by newly migrated macrophages (macrophages containing effete leukocytes are called Reiter cells). Macrophages, in turn, might apoptose locally or be cleared by lymphatic drainage (b), thereby allowing this acute inflammatory response to resolve and the inflamed tissue to return to its normal physiological function (c). Invariably, however, there is some evidence of parenchymal tissue damage (d), with some cells dying by necrosis but, in turn, being replaced by local tissue regeneration and some evidence of residual tissue scarring (e). If, however, the surrounding tissue damage is extensive, the process of healing is by repair. This involves the in-growth from the surrounding connective tissue of an initially vascular tissue containing endothelial cells, fibroblasts, myofibroblasts and macrophages, and is known as granulation tissue (f). After some time, the number of macrophages and myofibroblasts decline. Eventually, fibroblasts lay down collagen and the capillaries disappear, leaving a dense avascular, hypocellular extracellular matrix (g). b

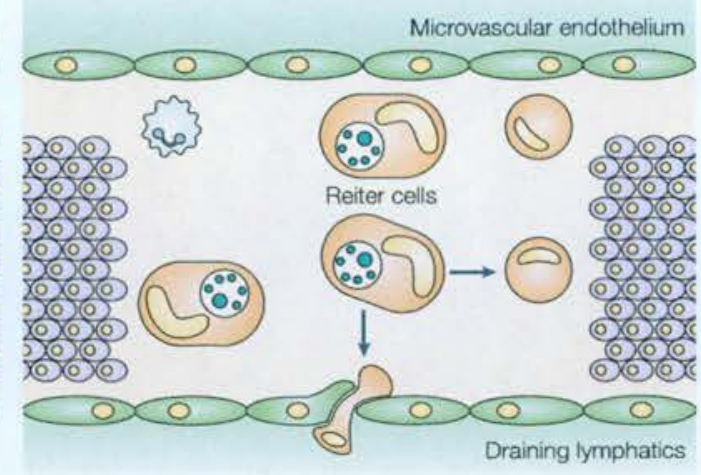

d

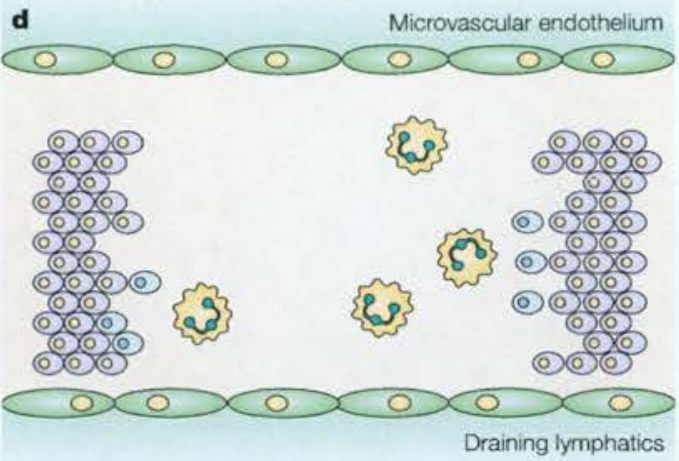

f $0 \times 0 \times 0 \times 0 \times 0 \times 0$

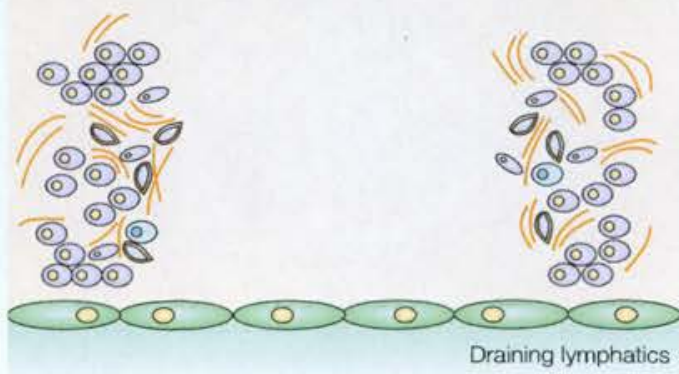

g Microvascular endothelium $0 \times 0 \times 0 \times 0 \times 0 \times 0$

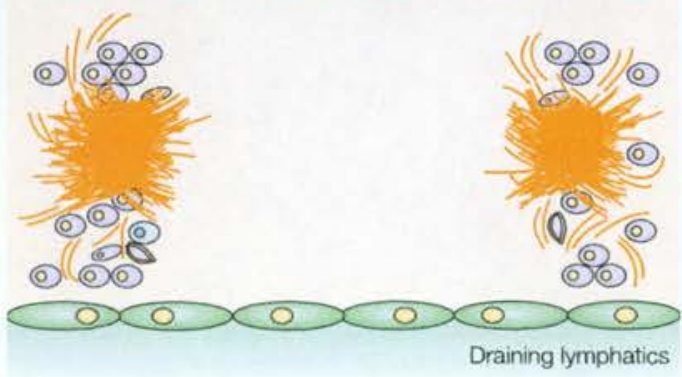

(0) Tissue parenchyma $\left\{\begin{array}{c}\text { A } \\ \text { Apoptotic PMNs }\end{array}\right.$ द्रूद्ड Necrotic PMNs Apoptotic macrophage 
PEROXISOME PROLIFERATORACTIVATED RECEPTORS (PPARs). Members of the nuclear receptor family that regulate the transcription of genes involved in lipid and lipoprotein metabolism, glucose and energy homeostasis, as well as cellular differentiation. They consist of three isotypes: alpha (NRICl), gamma (NRIC3) and beta/dedta ( $\mathrm{NRC1C2}$, with a differential tissue distribution.

IGNAL TRANSDUCERS AND ACTIVATORS OF TRANSCRIPTION Signal transducers and activators of transcription (STAT) proteins are intracellular effector molecules of cytokinemodulated signalling.

POLYMORPHONUCAEAR IFUKOCYTES (PMNs). Discovered by Paul Ehrlich who used contemporary fixing and staining techniques to identify the lobulated nucleus and the granules that typify cells that we now dassify as cosinophils, basophils and neutrophils. In the context of this review, PMNs refer to neutrophils.

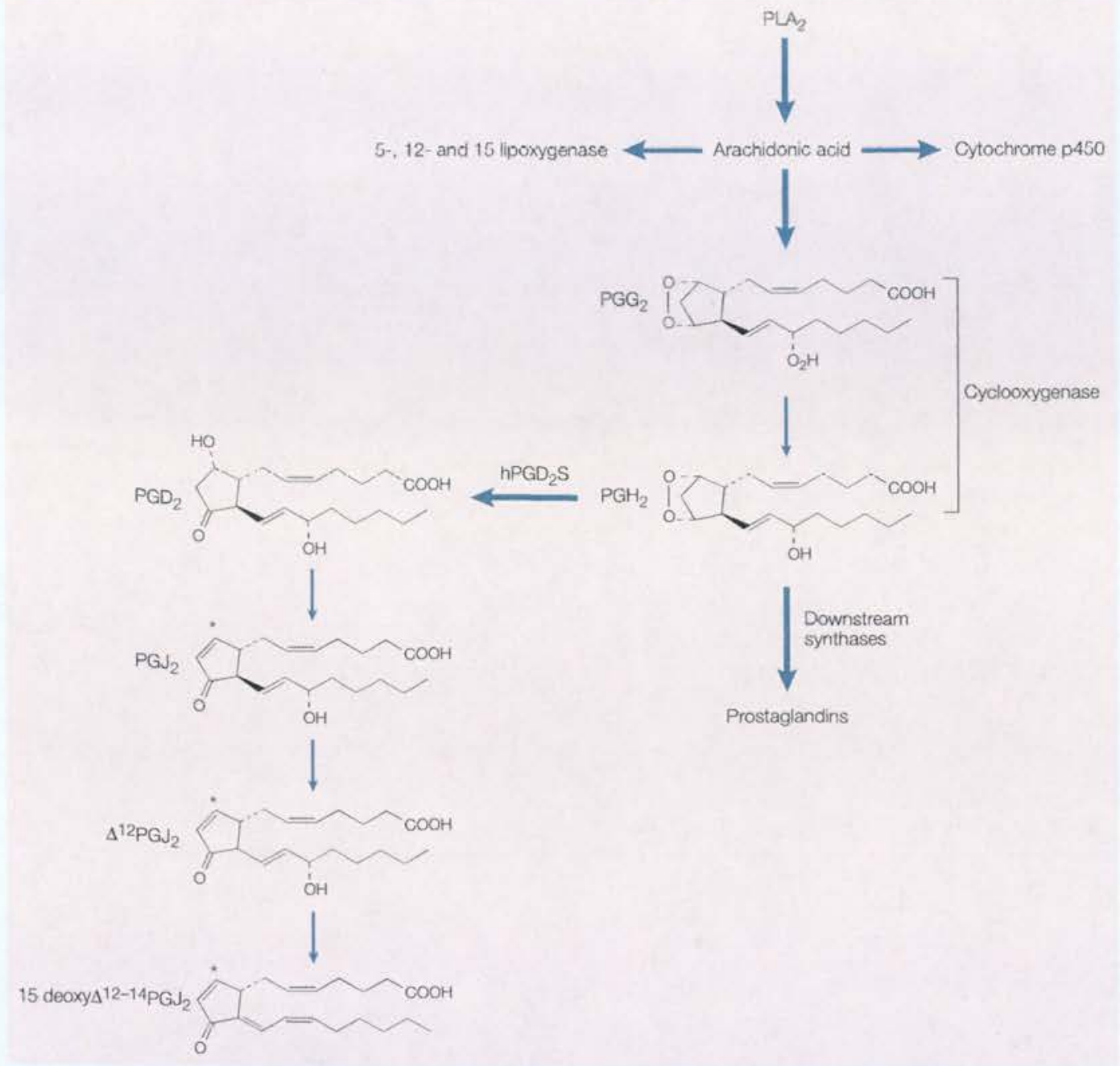

Figure 2 Arachidonic metabolism by COX, as well as the LOX and cytochrome p450 family of enzymes. Arachidonic acid is released by either cytosolic, secretory or calcium-independent phospholipase $A_{2}$. The phospholipase $A_{2}$ involved is cell-type-and stimulus-specific. Liberated arachidonic acid is metabolized by cyclooxygenase $(C O X) 1$ or COX2 to PGG and then $\mathrm{PGH}_{2}$. $\mathrm{PGH}_{2}$

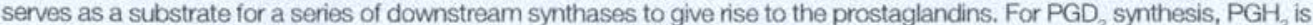
metabolized by haematopoietic prostaglandin $\mathrm{D}_{2}$ synthase. $\mathrm{PGD}_{2}$, in turn, is non-enzymically broken down to the cyPGs. $\mathrm{PGD}_{2}$, prostaglandin $\mathrm{D}_{2} ; \mathrm{PGG}_{2}$, prostaglandin $\mathrm{G}_{2} ; \mathrm{PGH}_{2}$, prostaglandin $\mathrm{H}_{2}$.

\section{COX2-derived cyclopentenone prostaglandins}

Prostaglandin (PG) $\mathrm{D}_{2}$ is a major product from a COXcatalysed reaction in a variety of tissues and cells, including those of the immune system, such as mast cells, platelets, T cells, dendritic cells and macrophages. $\mathrm{PGD}_{2}$ has significant effects on a number of biological processes, including inhibition of platelet aggregation, vascular and nonvascular smooth-muscle relaxation, as well as sleep-wake regulation and inflammation ${ }^{12}$. PGD undergoes dehydration in vivo and in vitro to yield biologically active PGs of the $\mathrm{J}_{2}$ series, including PGJ, $\Delta^{12,14}-\mathrm{PGJ}_{2}$ and 15-deoxy- $\Delta^{2} 12,14-\mathrm{PGJ}_{2}\left(15 \mathrm{~d}-\mathrm{PGJ}_{2}\right)$, which are characterized by the presence of a reactive $\alpha, \beta$-unsaturated ketone in the cyclopentenone ring ${ }^{13}$ (FIG. 2). Although 15d-PGJ was initially identified as a high-affinity natural ligand for PEROXISOME PROLIFERATORACTIVATED RECTPTOR gamma (PPAR $\gamma)^{14,15}$, it is now thought to exert its effects through PPAR $\gamma$-dependent as well as -independent mechanisms ${ }^{16}$, resulting in the suppression of various pro-inflammatory signalling pathways. These include pathways that operate through NF- $\mathrm{KB}$, APl and SIGNAL TRANSDUCFRS AND ACTIVATORS OF TRANSCRIPTION (STATs) $^{14,15,17-19}$, as well as the suppression of inducible nitric oxide synthase, interleukin (IL)- $1 \beta$, TNF- $\alpha$ and IL-12 in macrophages ${ }^{14,20}$, microglial cells ${ }^{21,22}$ and dendritic cells ${ }^{23} .15 \mathrm{~d}-\mathrm{PG} \mathrm{J}_{2}$ also preferentially inhibits monocyte rather than POLYMORPHONUCIEAR LEUKOCYTE (PMN) trafficking through the differential regulation of celladhesion molecule and chemokine expression ${ }^{24-27}$, indicating that cyclopentenone PGs (cyPGs) do not control the onset phase of acute inflammation, in which PMNs predominate, but tightly regulate the chronicity of the inflammatory response. In support of this, we have shown that COX2 has a dual role in acute pleuritis ${ }^{28,29}$. 


\section{Box 2 Cytokines and the resolution of acute inflammation}

In unpublished experiments, we found a peak in interleukin (IL)-10 and transforming growth factor (TGF)- $\beta 1$ expression during the resolving phase of acute inflammation, indicating an important role for these cytokines in pro-resolution. We suspect that they are synthesized by macrophages following the recognition of apoptotic polymorphonuclear leukocytes (PMNs; see the in vitro work in REFS 81,138,140). In addition, is was recently reported that under the control of interferon (IFN)- $\gamma, \mathrm{IL}-6$ and its soluble receptor controls $\mathrm{PMN}$ trafficking in peritoneal inflammation by suppressing CXC and CC chemokine expression and hence further PMN recruitment ${ }^{146,147}$. Furthermore, IL-6 and its soluble receptor promotes $\mathrm{PMN}$ apoptosis and clearance. This highlights some of the early signals that might influence the longevity of PMNs in self-limiting inflammation.

\section{DELAYED-TYPE}

HYPERSENSITIVTTY

(DTH). A delayed-type hypersensitivity or type IV

hypersensitivity is mediated by T lymphocytes and not by antibody-antigen complexes (arthus or type III hypersensitivity). Typically, this response occurs $24-72$ hours after the sensitized host is exposed to the offending antigen. For example, a DTH reaction might be set up experimentally by sensitizing to methylated bovine serum albumin in Freund's complete adjuvant and challenging 12 days later with the same antigen.

$\mathrm{T}_{\mathrm{H}^{1}}$

The $\mathrm{T}_{\mathrm{H}} 1 / \mathrm{T}_{\mathrm{H}} 2$ hypothesis came to prominence in the late $1980 \mathrm{~s}$, indicating that mouse $\mathrm{T}$-helper $\left(\mathrm{T}_{H}\right.$ ) cells broadly express differing cytokine profiles. Whereas T-helper $1\left(\mathrm{~T}_{\mathrm{H}} 1\right)$ cells secrete IFN- $\gamma$ and TNF- $\alpha$ : $\mathrm{T}_{H}{ }^{2}$ cells secrete IL-4, IL-5 and II- 13 . In addition, $\mathrm{T}_{\mathrm{H}} 3$ and regulatory $\mathrm{CD} 25^{+} \mathrm{CD} 4^{+} \mathrm{T}$ cells exist that produce TGF- $\beta$ and II- 10 , respectively. scavenging of free radicals.
This enzyme not only contributes to the onset of the reaction through the synthesis of pro-inflammatory $\mathrm{PGE}_{2}$, but also to its resolution through the preferential synthesis of $\mathrm{PGD}_{2}$ and $15 \mathrm{~d}-\mathrm{PGJ}$. Moreover, we recently identified the phospholipases (PLAs) that release arachidonic acid for these dual phases of eicosanoid metabolism with calcium-independent PLA, and to a lesser extent secretory PLA, which is necessary for the onset phase switching to a requirement for cytosolic PLA coupling to $\mathrm{COX} 2$ during resolution for the generation of pro-resolving $\mathrm{PGD}_{2}$ (REF. 30). It seems that $\mathrm{PGD}_{2}$ and $15 \mathrm{~d}-\mathrm{PGJ}_{2}$, bring about resolution, in part, by inducing PMN and, surprisingly, macrophage apoptosis ${ }^{31}$, albeit a contentious issue. Indeed, of all the leukocytes, macrophages tend to be very resistant to undergoing programmed cell death. Yet, $15 \mathrm{~d}-\mathrm{PGJ} \mathrm{J}_{2}$ has previously been reported to induce granulocyte $\mathrm{e}^{32}$, macrophage $\mathrm{e}^{33}$ and, more recently, myofibroblast apoptosis ${ }^{34}$. These studies indicate that the cyPGs might not only be important for controlling the fate of inflammatory leukocytes during the resolution of acute inflammation, but also in preventing complications arising from excessive tissue fibrosis and wound healing.

That 15d-PGJ ${ }_{2}$ has both PPAR $\gamma$-dependent and -independent effects and that it is formed by nonenzymic dehydration from the parent eicosanoid, PGD , makes tying down the precise contribution of $15 \mathrm{~d}-\mathrm{PGJ} \mathrm{J}_{2}$ to the inflammatory response problematic. However, we can begin to get some idea about this by examining the inflammatory response in mice that are deficient in haematopoietic PGD $\mathrm{P}_{2}$ synthase, the enzyme that synthesises $\mathrm{PGD}_{2}$ from its $\mathrm{COX}$-derived $\mathrm{PGH}_{2}$ precursor. Therefore, in an immune-mediated DELAYFD-TYPE HYPERSENSITIVITY reaction, mice that are deficient in haematopoietic $\mathrm{PGD}_{2}$ synthase showed an exaggerated inflammatory response that failed to resolve readily. Lymphocytes from these animals proliferated uncontrollably, producing a $\mathrm{T}_{\mathrm{H}} 1$-type cytokine profile and showed enhanced binding or pro-inflammatory NF- $\kappa B$ activity (D.W.G., J. Newson, S. Gor and P.R. ColvilleNash, manuscript in preparation; see BOX 2). Despite the questions surrounding the PPAR $\gamma$-dependent or independent effects of $15 \mathrm{~d}-\mathrm{PGJ}$, these results are in general agreement with those obtained from PPAR $\gamma^{+/-}$ mice with a type IV hypersensitivity reaction ${ }^{36}$. Similarly, when fibroblasts expressing retrovirally transfected haematopoietic PGD, synthase complementary DNA
(cDNA) are introduced to mice bearing bleomycininduced lung injury, significantly reduced lung oedema, leukocyte infiltration in bronchoalveolar lavage fluid and pulmonary collagen content is observed, in addition to prolonging the lifespan of the transfected mice ${ }^{37}$. These results, as well as those showing a protective role for $15 \mathrm{~d}-\mathrm{PGJ}_{2}$ in models of ischaemia reperfusion injury ${ }^{38,39}$, inflammatory bowel disease ${ }^{38}$, adjuvantinduced arthritis ${ }^{40}$ and experimental autoimmune encephalomyelitis ${ }^{41}$, provide support for $15 \mathrm{~d}-\mathrm{PGJ}_{2}$ as a novel therapeutic agent against inflammatory diseases. $\mathrm{PGD}_{2}$ and the cyPGs might contribute to the resolution of inflammation through various mechanisms that include the inhibition of the expression of proinflammatory genes, the induction of apoptosis in activated macrophages and myofibroblasts, and the activation of PPAR $\gamma$. However, stable therapeutics based on $\mathrm{PGD}_{2}$ and its metabolites might not be a panacea for all inflammatory processes. For instance, in the lung there is mounting evidence that $\mathrm{PGD}_{2}$ might contribute significantly to the symptoms and pathogenesis associated with asthma ${ }^{42,43}$,indicating that although such putative endogenous PGs might be protective at one site of tissue injury, it might be detrimental at another, reflecting differing organ-specific aetiologies of disease processes in terms of onset and resolution.

\section{Effect of haem oxygenase induction}

There is still much debate over the differential effects of $15 \mathrm{~d}_{-} \mathrm{PGJ}_{2}$ on pro-inflammatory cytokine synthesis. However, a closer look at the literature reveals that this disparity arises from the concentrations of 15d-PGJ (ng or $\mu \mathrm{g}$ ) used on different cell types (peripheral blood monocytes-macrophages, J774 macrophages or A549 cells) that are stimulated differently (such as LPS, PMA and TNF- $\alpha)^{14,26,44}$. However, one interesting observation from these studies was the delayed but sustained inhibition of LPS-stimulated TNF- $\alpha$ release achieved by low concentration $15 \mathrm{~d}-\mathrm{PGJ}_{2}(0.5 \mu \mathrm{M})$ on LPS-treated J774 macrophages, which was attributed to the induction of HO-1 by $15 \mathrm{~d}-\mathrm{PGJ}{ }_{2}$ (REF. 44). HO-1 is a stress-inducible enzyme that catalyses the degradation of haem to liberate free iron, carbon monoxide, BIIVERDIN and BLIRUBrN in mammalian cells ${ }^{45}$ and was one of the original endogenous factors identified as playing a crucial role in the resolution of acute pleuritis ${ }^{46}$, with current studies indicating that $\mathrm{HO}-1$ might also have a role during the onset phase of acute inflammation by regulating PMN trafficking ${ }^{47}$. 15d-PGJ induction of $\mathrm{HO}-1$ and its associated suppression of pro-inflammatory gene expression is certainly consistent with its role in acute inflammatory resolution, but the interaction between HO-1 and $15 \mathrm{~d}-\mathrm{PGJ}_{2}$ during this phase has yet to be clarified. Numerous studies have since reported an important role for $\mathrm{HO}-1$ as a defence mechanism against oxidative insults through the antioxidant properties of biliverdin and bilirubin, as well as the anti-inflammatory action of carbon monoxide ${ }^{48}$. Moreover, in mouse-to-rat transplantation, induction of $\mathrm{HO}-1$ by cobra venom factor and cyclosporine A in endothelial and smoothmuscle cells of cardiac xenografts prior to transplantation 
a

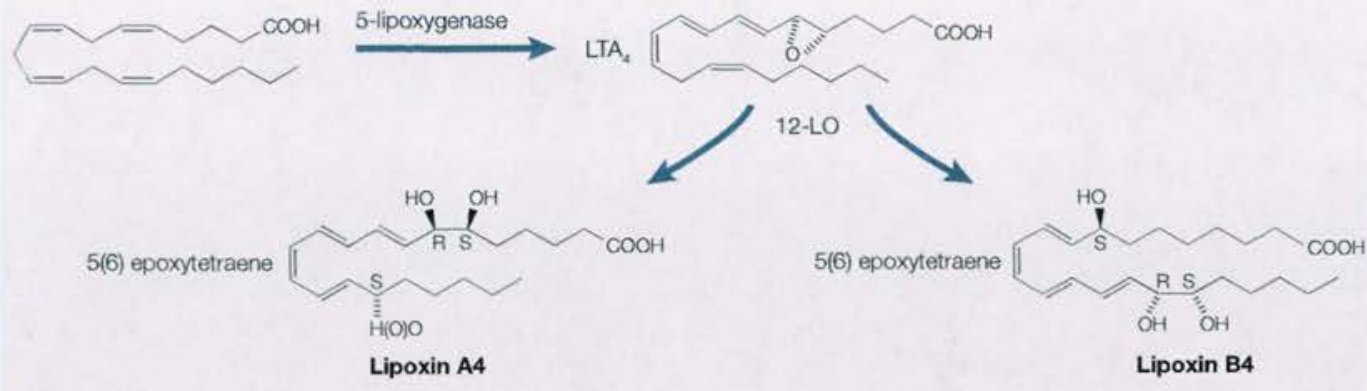

b

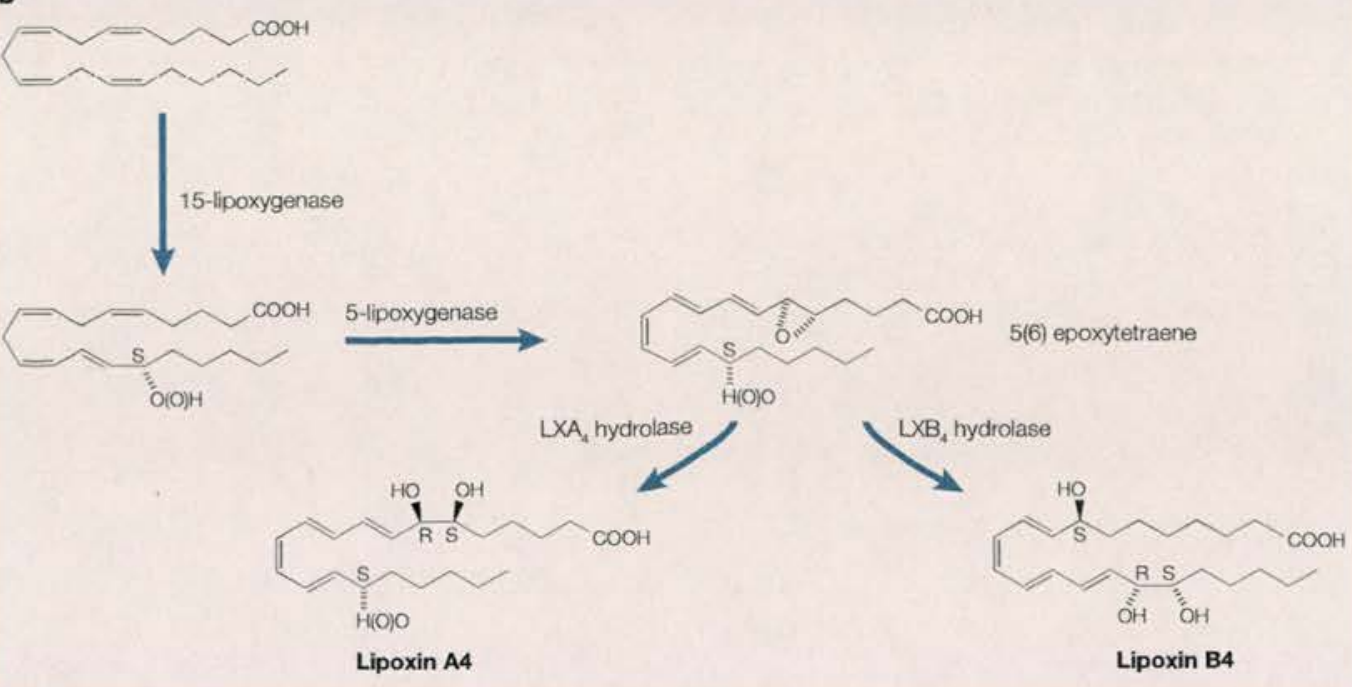

c Arachidonic acid

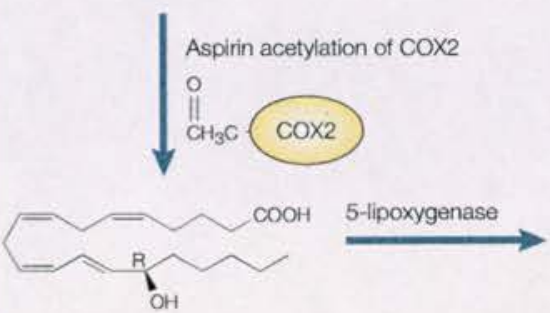

15R-HETE<smiles>CCCCCC[B]P(O)O</smiles>

15-epi-lipoxin A4
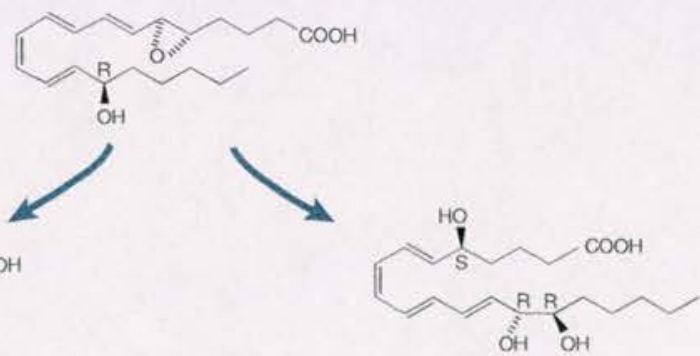

15-epi-lipoxin B4

Figure 3 | Biosynthesis of LXs occurs through three distinct biosynthetic pathways. a | The first is via platelet-PMN (polymorphonuclear leukocyte) interactions, whereby 5-LOX (lipoxygenase) in PMNs metabolizes arachidonic acid to leukotriene $A_{4}\left(L X A_{4}\right)$, which is taken up by platelets in a transcellular manner and converted by platelet 12-LOX to LXA $($ REFS 142,143). b | The second route of lipoxin (LX) formation is via the action of monocytes or epithelial-cell 15-LOX. Molecular oxygen is inserted into carbon 15 of arachidonic acid, yielding 15(S)hydroperoxyeicosatetraenoic acid, which can then serve as a substrate for PMN 5-LOX to generate 5,6-epoxytetraene. This unstable epoxide intermediate is converted to LXA, and LXB, by epoxide hydrolases in leukocytes ${ }^{144}$. A final and unorthodox route for LX metabolism involves aspirin ${ }^{145} . \mathbf{c} \mid$ Aspirin acetylates the active site of cyclooxygenase (COX)2, resulting not in the inhibition of COX2 enzyme activity but in the conversion of arachidonic acid to $15(R)$-hydroxyeicosatetraenoic, which when released from endothelial and epithelial cells might be transformed by leukocyte 5-LOX to generate 15-epi-LXA, or 15-epi-LXB, 
prolongs the survival rate of these organs ${ }^{49}$. Upregulation of $\mathrm{HO}-1$ with anti-CD40L monoclonal antibody also protects mouse allografts against transplant rejection ${ }^{50}$, whereas induction of $\mathrm{HO}-1$ by cobalt-protoporphyrin IX protects against murine acute graft-versus-host disease $^{51}$. So, compounds that regulate HO-1 might represent yet another strategy for the treatment of inflammatory diseases.

\section{The lipoxins and resolvins}

Lipoxins. Transcellular metabolism of arachidonic acid by LOX/LOX interaction pathways gives rise to the lipoxin (LX) family of eicosanoid metabolites (FIG.3). LXs display selective actions on leukocytes that include inhibition of PMN chemotaxis ${ }^{52}$, PMN adhesion to and transmigration through endothelial cells $\mathrm{s}^{53}$, as well as PMN-mediated increases in vascular permeability ${ }^{54}$. In contrast to their effects on PMN and eosinophils, LXs are potent stimuli for peripheral blood monocytes, stimulating monocyte chemotaxis and adherence ${ }^{55,56}$ without causing degranulation or release of reactive oxygen species $^{57}$. In fact, LXs and their stable analogues accelerate the resolution of allergic pleural oedem $\mathrm{a}^{58}$ and enhance phagocytosis of apoptotic PMNs by monocyte-derived macrophages in a non-phlogistic fashion ${ }^{59,60}$, paving the way for a return to tissue normality. $\mathrm{LXA}_{4}$ and aspirintriggered 15-epi-LXA, as well as their stable analogues, act with high affinity at a G-protein-coupled receptor, $\mathrm{LXA}_{4}$ receptor (ALXR; also referred to as formyl peptide receptor-like 1 or FPRL1). FPRL1 is a member of the family of seven transmembrane G-coupled receptors, which has at least two other members - FPRL2 and the formyl-Met-Leu-Phe receptor (FPR). By contrast, $\mathrm{LXB}_{4}$ does not bind the ALXR and, although functional studies have indicated the existence of a receptor that is activated by $\mathrm{LXB}_{4}$, this receptor has not been cloned ${ }^{61}$. As with the cyPGs, the LXs have also been identified as being expressed during and being crucially important for the resolution of acute inflammation. In a model of rat allergic oedema, for instance, LXA was identified along with $\mathrm{PGE}_{2}$ as being present during the clearance of oedema in this model ${ }^{62}$. Inhibition of their synthesis prolonged oedema clearance, which was rescued using stable analogues of these eicosanoids. A recent analysis of eicosanoid synthesis in a murine dorsal AIR-POUCH MODEI of acute inflammation elicited by TNF- $\alpha$ has revealed a switch in lipid-class metabolism reminiscent of that found in the rat carrageenin-induced pleurisy. In response to TNF- $\alpha$, levels of leukotriene $B_{4}$ increased rapidly, followed by PMN infiltration, which coincided with a rise in inflammatory exudate PGE, (REF. 52). Concomitant with the eventual reduction in PMN numbers and $\mathrm{PGE}_{2}$ was an increase in $\mathrm{LXA}_{4}$. It was concluded that $\mathrm{PGE}$ induced a switch in lipid mediator synthesis from predominantly 5 -LOX-generated leukotriene $\mathrm{B}_{4}$ to

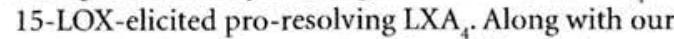
findings in the rat carrageenin-induced pleurisy in terms of PG metabolism ${ }^{29,30}$, this work indicates that, in acute inflammation, lipid-mediator biosynthesis is biphasic, with a role for eicosanoids in the initiation as well as termination of the inflammatory response.
Resolvins. Arachidonic acid is not the only fatty-acid substrate that can be transformed by COXs and LOXs to bioactive mediators with roles in anti-inflammation and resolution. DHA and EPA - omega- 3 fatty-acid constituents of fish oils - were shown recently to be metabolized during the resolving phase of an aspirintreated TNF- $\alpha$-induced inflammation to potent antiinflammatory products, aptly named resolvins ${ }^{63,64}$ (FIG.4). For instance, endothelial cells expressing COX2 and treated with aspirin convert EPA to $18 R$-hydroxyeicosapentaenoic acid (HEPE) and 15R-HEPE. Both are subsequently used by PMNs to generate separate classes of novel trihydroxy-containing mediators that potently inhibit human PMN transendothelial migration ${ }^{63}$. Similarly, aspirin-acetylated COX2 converts DHA to 17R-HDHA, which is subsequently transformed by PMNs into two sets of novel di- and trihydroxy products that can inhibit microglial-cell cytokine expression and ameliorate experimental models of dermal inflammation and leukocyte accumulation in peritonitis at nanogram doses ${ }^{64}$. Even in the absence of aspirin, human whole blood converts DHA to $17 \mathrm{~S}$ series resolvins as well as novel dihydroxy-containing docosanoids. DHA-loaded glial cells stimulated with zymosan also release docosanoids, with these novel resolvins possessing such potent anti-inflammatory effects as inhibiting leukocyte trafficking in vivo and pro-inflammatory cytokine release by stimulated human glial cells ${ }^{65}$.

Collectively, the LXs and resolvins represent novel classes of anti-inflammatory agents that are tightly associated with the resolution of acute inflammation and shown to be implicated in the pathogenesis of disease processes, including atherosclerosis ${ }^{66}$, periodontitis ${ }^{67}$, chronic liver disease ${ }^{68}$ and asthma ${ }^{69}$. Moreover, LXs and their analogues are proving to be highly effective therapeutics in a range of experimental disease models, including immune-mediated glomerulonephritis ${ }^{70}$ and renal ischaemia-reperfusion injury ${ }^{71}$, a range of skin inflammatory-like diseases ${ }^{72}$ and gastritis ${ }^{73}$. Lipid mediators of this sort are not only natural and essential components of acute inflammatory resolution, but show that when applied to inflammatory disease processes are highly effective, thereby providing the rationale for the development of compliant and stable mimetics that target key aspects of chronic inflammation, either ongoing or recurrent, forcing them down a revolving pathway and into remission.

\section{Apoptosis and phagocytosis}

What is the fate of inflammatory leukocytes after they have carried out their role? There are a number of routes available for the efficient clearance of these cells, including systemic re-circulation ${ }^{74,75}$, lymphatic drainage ${ }^{76,77}$, or death by either apoptosis or necrosis followed by phagocytosis, with apoptosis followed by phagocytosis being considered the most desirable for successful resolution ${ }^{78}$. It is clear that apoptosis has a fundamental role in almost all biological processes and there is increasing evidence to indicate that dysregulated apoptosis is likely to be an important factor in the pathogenesis and progression of a number of diseases ${ }^{79,80}$. 


\section{a 17R: D-series resolvins}

10, 17R-diH DHA

16, 17R-diH DHA

Epoxidation
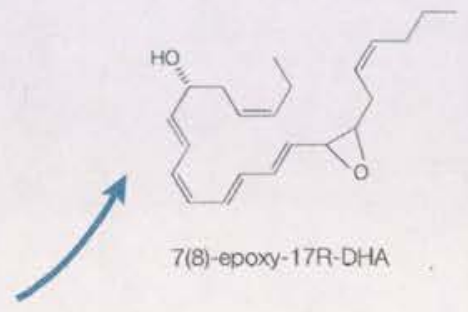

7(8)-epoxy-17R-DHA

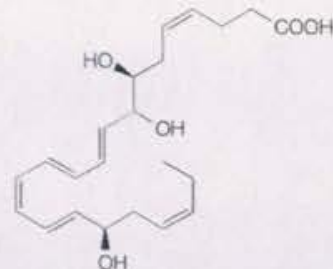

DHA

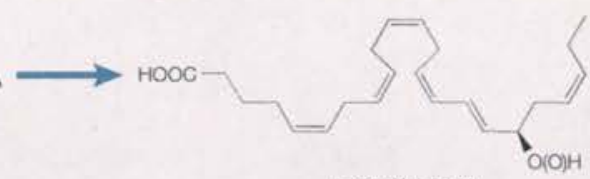

17R-H(p) DHA

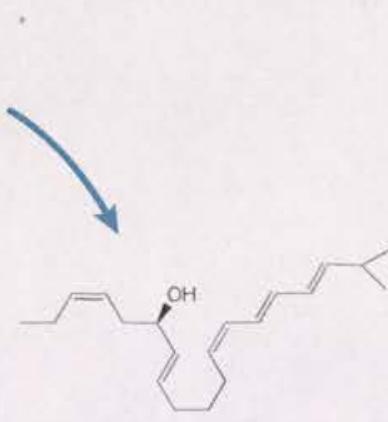

4(5)-epoxy-17R-HDHA
7S, 8, 17R-triHDHA

Resolvin D1
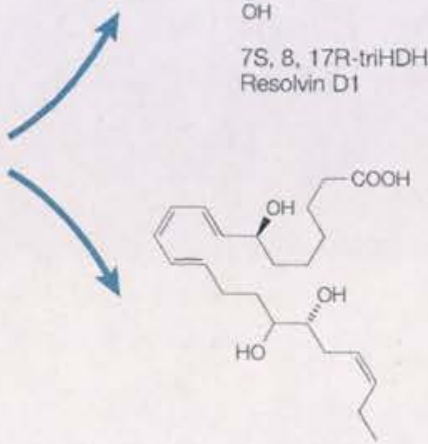

7S, 16, 17R-triHDHA Rocolvin D2

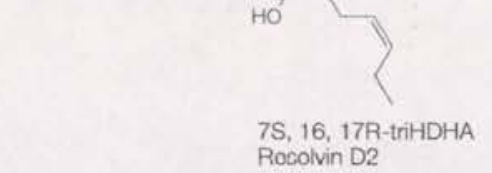


Enhanced undesirable apoptosis occurs in many neurological diseases, such as Alzheimer's disease, Parkinson's disease, Huntington's disease and multiple sclerosis. Furthermore, inappropriate inflammatory responses or dysfunctional vascular effects leading to tissue damage with increased apoptosis have been observed. So, there is good evidence of cell or tissue apoptosis during myocardial infarction, stroke or sepsis. Consequently, a therapeutic strategy to delay or inhibit apoptosis would seem a viable option assuming that cell specificity can be achieved.

On the other hand, there is much evidence indicating that reduced apoptosis occurs in most cancers. Essentially, as well as uncontrolled cell division or proliferation, there is an apparent failure of cancerous cells to undergo apoptosis. It has also been proposed that in many inflammatory diseases (for example, rheumatoid arthritis, atopic dermatitis, Crohn's disease, asthma and chronic obstructive pulmonary disease) there might be delayed apoptosis of key inflammatory cells, thereby prolonging the functional responsiveness of these potential histotoxic cells. A strategy to specifically promote death of cancer cells or tissue-damaging inflammatory cells is therefore likely to be therapeutically beneficial. However, any attempts to induce cell, especially inflammatory-cell, apoptosis must be matched by effective non-inflammatory clearance by phagocytic cells (for example, macrophages) ${ }^{60,78,81}$. Failure to remove these apoptotic cells would undoubtedly lead to the cells becoming necrotic, thereby increasing the potential for tissue damage occurring. Great progress has been achieved in recent years in the elucidation of the complex mechanisms that are involved in recognition of apoptotic cells (or apoptotic bodies) by phagocytes (at least 10 recognition mechanisms have been identified so far $)^{82}$. Furthermore, phagocytosis of apoptotic cells has been shown to be a highly regulatable process and therefore likely to be amenable to pharmacological manipulation ${ }^{83,84}$. It has been shown, for example, that elevation of cAMP by $\mathrm{PGs}^{85}$ can downregulate macrophage capacity to ingest apoptotic cells, whereas treatment of phagocytes with glucocorticoids ${ }^{86,87}, \mathrm{LXs}^{59}$ or even certain cytokines ${ }^{88}$ can markedly increase macrophage clearance of apoptotic cells. Interestingly, the environment in which phagocytes are likely to reside can also upregulate apoptotic-cell clearance. For example, interactions with extracellular-matrix components such as fibronectin ${ }^{89}$, and ligation of macrophage $\mathrm{CD} 44$ with crosslinking antibodies ${ }^{90}$, can augment the capacity of phagocytes to engulf apoptotic cells. So, clearance of apoptotic cells in a noninflammatory manner by phagocytes is a therapeutic possibility. Indeed, there is already evidence in animal models that CD44 has an important role in resolving lung inflammation"1 and that glucocorticoids might exert some of their therapeutic beneficial anti-inflammatory effects in patients with asthma by influencing apoptosis and apoptotic-cell clearance ${ }^{92}$. Some of these novel developments have led to the design of drugs that have even gone into clinical trials ${ }^{79,93}$.
One extremely active area of drug development that targets apoptosis is the identification of small-molecule caspase inhibitors. Caspases are a family of cysteinyl aspartate-specific proteases that are of fundamental importance in the initiation and execution of apoptosis, ultimately being responsible for the dismantling of the cell during apoptosis ${ }^{94,95}$. A number of small-molecule inhibitors of caspases (for example, ZVAD-fmk) have already been tested in animal models of human disease with remarkable success. For example, specific caspase inhibitors have been shown to be effective in preventing or reducing the effects of ischaemia (for example, organ failure and death) in various animal models ${ }^{79,96,97}$. These inhibitors of apoptosis are now being tested pre-clinically or have reached clinical trials for hepatic disease, acute myocardial infarction and sepsis ${ }^{93}$. A good example of a novel specific broad-spectrum caspase inhibitor that has been successfully used pre-clinically is IDN-6556 (REE 98). This compound, administered by a number of routes, was shown to be potent and efficient in reducing signs of liver damage in in vivo rodent models of liver disease. It was shown that the compound seemed to exert its activity by effectively inhibiting caspase activity ${ }^{99}$. Furthermore, this drug has successfully undergone Phase II clinical trials for liver disease in which it was well tolerated and reduced plasma alanine aminotransferease activity, a marker for hepatic injury, in patients with liver impairment ${ }^{100}$. There are a number of other drugs that are in development for inhibiting apoptosis, but so far the most promising therapeutic development is in the induction of apoptosis, especially for the treatment of a number of cancers. The strategy for inducing apoptosis has been to block powerful survival pathways by, for example, inhibiting BCL2-mediated survival using antisense oligonucleotides, and interference of survival pathways that are mediated by NF- $\kappa B$, phosphoinositol-3-kinase and tyrosine kinase activation. Another strategy is to directly induce apoptosis by engaging death-receptor pathways (such as FASR, TNFR and TRAILR) or by other less welldefined mechanisms ${ }^{93}$.

\section{NF-KB}

The NF- $\kappa B$ pathway is pivotal in the inflammatory response by regulating the expression of pro-inflammatory cytokines, chemokines and inducible enzymes ${ }^{101}$. Furthermore, NF- $\mathrm{\kappa B}$ controls the expression of antiapoptotic genes that might protect inflammatory leukocytes from programmed cell death ${ }^{102}$, an important determinant of inflammatory resolution. Given the central role of this pathway in the initiation of the inflammatory response and preservation of leukocyte longevity, it seems that factors that threaten the pro-inflammatory or anti-apoptotic status of NF- $\kappa B$ would hasten the resolution of inflammation. Some insight into this was provided by the PPAR $y$-independent inhibitory effects of $15 \mathrm{~d}-\mathrm{PGJ} \mathrm{J}_{2}$ on NF- $\kappa \mathrm{B}$ activation (see BOX 3 ). For example, the NF- $\kappa B$ pathway regulates the expression of the anti-apoptotic protein BCL- $\mathrm{X}_{\mathrm{L}}$, the promoter activation of which is inhibited by $15 \mathrm{~d}_{-} \mathrm{PGJ} \mathrm{2}_{2}$. This leads to programmed cell death of CD28 co-stimulated 
a

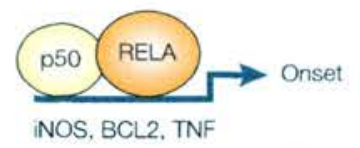

b
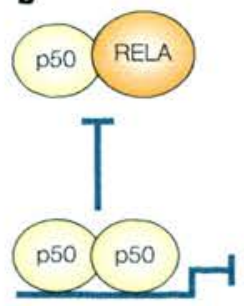

iNOS, BCL2, TNF

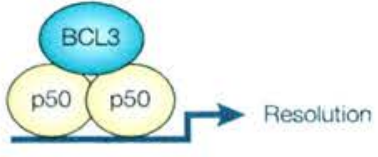

COX2, BAX, p53

Figure 5 | The profile of NF- $\kappa$ B subunit binding from onset to resolution in acute inflammation. a | Inhibition of nuclear factor of $\mathrm{kB}(\mathrm{NF}-\mathrm{\kappa B})$ at the onset of the inflammatory response resulted in the inhibition of acute inflammation due to the binding of the proinflammatory NF-kB subunits p50/RELA. b | At resolution, however, there was predominance of p50-p50 homodimers, which is responsible for pro-inflammatory mediator suppression and the induction of pro-apoptotic gene expression leading to the onset of resolution (c). The inhibition of $\mathrm{NF}-\mathrm{kB}$ during this phase prevented resolution.

primary human $\mathrm{CD} 4+\mathrm{T}$ cells in vitro ${ }^{103}$. We recently described the induction of caspase-dependent apoptosis in both PMN and eosinophil granulocytes by $15 \mathrm{~d}-\mathrm{PGJ}$ through the inhibition of NF- $\kappa \mathrm{B}$ independent of $\operatorname{PPAR} \gamma^{32}$. Previous studies from our laboratory have also shown that the specific inhibition of NF- $\kappa \mathrm{B}$ can induce granulocyte apoptosis directly and prevent the delay of programmed cell death in cytokine-stimulated granulocytes $^{104}$. Although a direct causal link between $15 \mathrm{~d}-\mathrm{PGJ}_{2}-$ mediated inhibition of NF- $\mathrm{\kappa B}$ and the resolution of inflammation has yet to be proven, these studies show that inhibiting NF- $\mathrm{KB}$ speeds up leukocyte apoptosis, which is good news for resolution. This can be taken one step forward by suggesting that selective inhibition of leukocyte NF-кB in inflammatory disease processes might also signal the onset of resolution.

In contrast to pharmacologically manipulating the well-documented pro-inflammatory pathway of NF- $\mathrm{\kappa B}$ to control inflammation, we recently found that NF- $\mathrm{KB}$ also has inherent anti-inflammatory properties that mediate the resolution of acute experimental pleuritis ${ }^{105}$. This involves the recruitment of alternative DNA-binding

complexes such as p50-p50 homodimers, which might antagonize NF- $\kappa \mathrm{B}$-dependent pro-inflammatory gene expression while simultaneously promoting the expression of genes that are required for the resolution of inflammation. Broad-spectrum inhibitors of the NF- $\kappa B$ pathway, such as antioxidants and proteasome inhibitors, had the expected anti-inflammatory actions during the initiation of inflammation. However, when administered after the onset of inflammation, these inhibitors prevented resolution, which was associated with the inhibition of leukocyte apoptosis. We hypothesize that this alternative NF- $\kappa \mathrm{B}$ pathway promotes leukocyte apoptosis through the recruitment of NF-kB DNA-binding complexes that lack transactivation domains and act as dominant-negative inhibitors of anti-apoptotic gene expression (see FIG. 5). Indeed, the persistence of p50-p50 homodimers during the resolution of inflammation is perhaps not surprising considering the reported role of p50-p50 in the repression of pro-inflammatory gene transcription ${ }^{106,107}$. Transgenic mice that express $\mathrm{p} 50$ but not the inhibitory carboxyl terminus of the p105 molecule showed increased p50-p50 DNA-binding activity ${ }^{106}$. These mice show reduced expression of cytokine genes in resident macrophages and impaired leukocyte recruitment, indicating p50-mediated suppression of inflammation in vivo. These studies also revealed that in certain cell types p 50-p50 might enhance gene transcription, particularly when complexed with the IкB-like protein $B C L 3$. This might help explain the unlikely expression of COX 2 during resolution as RELA-p50 and p50-p50 complexes bind to distinct $\mathrm{\kappa B}$ response elements in the COX 2 promoter, whereas the $\mathrm{p} 50$ - $\mathrm{p} 50$ complex alone can also upregulate COX2 transcription through association with transcriptional co-activators of the C/EBP family.

These studies might also help explain, at the molecular level, how NF- $\kappa$ B controls leukocyte clearance during resolution. We found that inhibition of p105 processing to $\mathrm{p} 50$ and of NF- $\mathrm{kB}$ DNA-binding activity by the proteosome inhibitor MG132 reduced the

\section{Box $3 \mid$ PPAR $\gamma$-independent inhibitory effects of $15 d-\mathbf{P G}_{\mathbf{2}}$ on NF- $\mathrm{K}$ B activation}

Although $15 \mathrm{~d}-\mathrm{PGI}_{2}$ exerts some of its anti-inflammatory effects through peroxisome proliferator-activated receptor gamma (PPAR $\gamma$ ) activation, resulting in, for example, the inhibition of nuclear factor of $\kappa \mathrm{B}$ (NF- $\kappa \mathrm{B}) \mathrm{DNA}$ binding ${ }^{15}$, it seems that $15 \mathrm{~d}-\mathrm{PGJ}{ }_{2}$ has more of an effect on the inflammatory response by altering the activation of NF-KB directly. Through Michael addition reactions, the highly reactive electrophilic carbon atom in the unsaturated carbonyl group of the cyclopentane ring can react with nucleophiles, such as the free sulphydryl groups of glutathione (GSH) and cysteine residues that form disulphide bonds in proteins ${ }^{17,148}$. In this respect, cyclopentenone PGs (cyPGs) can modify conserved cysteine residues located in both the trans-acting DNA-binding proteins of NF- $\kappa \mathrm{B}$ and elements of the upstream signalling complex that activates NF- $\mathrm{KB}$ in response to pro-inflammatory cytokines (IKB kinase-IKK) ${ }^{17}$. Therefore, $15 \mathrm{~d}_{-\mathrm{PGJ}}{ }_{2}$ can alkylate Cys 179 of IKK $\beta$, which is located in the kinase activation loop ${ }^{149}$. The DNA-binding subunits of NF- $\kappa B$, p50 and RELA might also be alkylated at Cys62 and Cys38, respectively, by $15 \mathrm{dPGJ}_{2}$ (REF. 150). These residues are located in the DNA-binding domains of the proteins and their alkylation prevents the activation of gene expression representing multiple targets for the inactivation of NF-KB by $15 \mathrm{~d}-\mathrm{PGJ}_{2}$. It was shown recently that $15 \mathrm{~d}-\mathrm{PGJ}{ }_{2}$ was not detectable during adipocyte differentiation, throughout the time course of LPS-administration to humans or in the synovial fluid of arthritic patients, questioning the status of $15 \mathrm{~d}-\mathrm{PGJ} \mathrm{J}_{2}$ as an endogenous PPAR $\gamma$ receptor ligand ${ }^{15 !}$. However, given its short half-life and propensity to bind avidly to sulphydryl groups, one possible reason why $15 \mathrm{~d}-\mathrm{PGJ}{ }_{2}$ is not readily detectable in biological fluids is because it is bound to pro-inflammatory signalling proteins. Perhaps a more appropriate strategy is to look for intracellular $15 \mathrm{~d}-\mathrm{PGJ} / 2$ protein complexes. 
expression of the pro-apoptotic proteins BAX and p 53 in leukocytes during the resolution of acute inflammation ${ }^{105}$. Finding BAX and p53 expressed during resolution in this model is highly significant in itself as it identifies the signalling pathways that mediate leukocyte apoptosis during acute resolution in vivo. Although the BAX promoter contains both $\kappa \mathrm{B}$ and $\mathrm{p} 53$ response elements, $\mathrm{BAX}$ is thought to be a target gene for $\mathrm{p} 53$ rather than NF- $\kappa B$. As p50-p50 homodimers are known to positively regulate the $\mathrm{p} 53$ promoter, it is interesting to speculate that $\mathrm{p} 53$ and NF- $\mathrm{\kappa B}$ (p50-p50) cooperate to regulate $\mathrm{BAX}$ expression and leucocyte apoptosis during resolution, which would certainly explain why $\mathrm{p} 53$ and $\mathrm{BAX}$ expression is diminished after NF- $\kappa B$ inhibition during this period. Signal-induced processing of p105 to $\mathrm{p} 50$ is dependent on inducible phosphorylation and proteosome-mediated limited proteolysis, which is dependent in turn on the IKK complex. However, although the IKK complex is essential for inducible p105 processing, IKK $\beta$ is redundant in this pathway. It could be hypothesized that endogenous anti-inflammatory mediators such as cyPGs repress the activation of IKK $\beta$, leading to a predominance of anti-inflammatory signalling pathways that are regulated independently of IKK or by an alternative IKK complex that mediates p105 processing. It should be noted that recent data with tissue-specific knockout experiments of IKK $\beta$ have illustrated the possible adverse side effects of global IKK $\beta$ inhibition ${ }^{108}$. It was shown that inhibition of IKK $\beta$ expression in colonic epithelial cells increases apoptosis in response to ischaemia reperfusion injury, leading to a loss of barrier function and considerable intestinal damage. This indicates a tissue-specific role for IKK $\beta$ in inflammation: IKK $\beta$ inhibition might reduce pro-inflammatory cytokine production and leukocyte recruitment, and inhibition of anti-apoptotic pathways might result in increased tissue injury. This might be particularly important in epithelial cells at mucosal surfaces that have a barrier function, such as the lung and the intestine. Understanding of these molecular mechanisms that terminate pro-inflammatory signalling pathways and promote the resolution of inflammation will enable the design and development of more specific and appropriate pharmacological agents with therapeutic potential for the treatment of chronic inflammatory diseases.

\section{Glucocorticoids and annexin}

Glucocorticoids are endogenous potent anti-inflammatory mediators that are released within minutes in response to stress and tissue injury to control the severity of the inflammatory response, functioning as internal brakes against potentially exaggerated inflammatory reactions mounted by the host ${ }^{109}$. Accordingly, removal of this potent and primary braking mechanism is associated with augmented and prolonged inflammation, including, in some cases, lethality ${ }^{110,111}$. Although probably not released into the circulation during the resolution of acute inflammation, glucocorticoids might act, in part, by speeding up resolution in certain disease processes. For instance, glucocorticoids selectively induce eosinophil apoptosis and, like the LXs, also enhance macrophage phagocytosis of apoptotic bodies. This indicates that the role of steroids in the treatment of chronic inflammation, such as asthma, might be to reprogramme this ongoing inflammatory lesion down a pro-resolution pathway.

It is now clear that the unbound glucocorticoid receptor (GR) is associated within the cytoplasm in a non-active oligomeric complex with regulatory proteins such as heat-shock proteins 90 and 70, p59 immuno-philin and p23 phosphoprotein ${ }^{112}$. Receptor engagement leads to GR activation, which occurs in at least two distinct ways. Following ligand binding, the GR undergoes a conformational change leading to dissociation from specific proteins and the formation of glucocorticoid/GR complex monomers or dimers. In the latter case, the complex translocates into the nucleus to control gene transcription. Monomer glucocorticoid/GR, however, might also regulate several specific biochemical pathways, including the RELA subunit of NF- $\kappa B$ and the JUN and FOS components of AP1 (REFS 101,113-115) - so-called 'non-genomic' effects of glucocorticoids. This is an area of active research for the identification of glucocorticoids with distinct genomic versus non-genomic properties. No studies have yet addressed the question of whether these non-genomic actions of glucocorticoids can 'modulate' specific signal-transduction pathways that favour resolution.

As well as binding to cytoplasmic steroid hormone receptors, glucocorticoids also modulate the expression and secretion of regulatory proteins that have antiinflammatory actions, of which the peptide annexin-1 (lipocortin-1) has been well described in vitro and in vivo ${ }^{116-119}$. Of a protein more than 300 amino-acids long, small peptides drawn for the amino-terminal region of annexin-1 retain biological activity. As the annexin-1 amino terminus is not homologous to other members of the family, it is likely that these peptides reflect solely the biological effects of annexin-1 (REFS $41,120,121$ ). The availability of a biologically active peptide sequence could certainly be the starting point for the development of new anti-inflammatory entities. However, as small molecules are certainly more appealing for drug discovery, identification of the precise target for anti-inflammatory actions of annexin- 1 has long been required. Recent data showed an unexpected twist in this area, showing that annexin-1 and LXA share the same ALX receptor ${ }^{122}$. Human recombinant annexin-1 and annexin-1-derived peptides competed with $\mathrm{LXA}_{4}$ for receptor binding not only on engineered cells but also on human PMNs. These results, and the fact that the ALXR binds other endogenous proteins ${ }^{123}$ (including serum amyloid protein A), broadens our understanding of the endogenous control of acute inflammation by suggesting that this anti-inflammatory receptor has been evolutionarily kept for multi-use, that the ALXR is pivotal for controlling the inflammatory response from start to finish and that the host economizes by using the same receptors with different agonists. 
In support of this, a distinct profile of endogenous LXA $_{4}$ and annexin-1 generation was found in the cellfree exudates of a mouse air-pouch model of resolving inflammation, with $\mathrm{LXA}_{4}$ peaking at 4 hours but higher annexin-1 levels detectable at 20 hours post-insult, occurring at the same time as reduced PMN influx ${ }^{124}$. Consistently, passive immunization of mice with neutralizing anti-annexin-1 sera did not exacerbate PMN recruitment but prolonged it ${ }^{125}$. In this study, several parameters of the acute inflammatory response were altered by blockade of endogenous annexin-1, including the profile of cytokine and proteolytic enzyme release. The recent generation of annexin-1null mice confirm these findings. Several markers of acute inflammation are exacerbated in these animals, including the oedema (vascular) response and PMN recruitment ${ }^{126}$. Annexin-1-null mice also have an altered susceptibility of some of these inflammatory responses to glucocorticoid treatment ${ }^{126}$. It is important that similar observations have also been produced in a model of chronic joint inflammation ${ }^{127}$, thereby highlighting potential therapeutic application of annexin-1 mimetics. So, the discovery that annexin-1 and its antiinflammatory peptido-mimetics bind to ALX can open the way to the development of novel small-molecule entities based on annexin-1 (REF. 128), thereby, at least in part, retaining some of the anti-inflammatory properties of glucocorticoids that signal the resolution of ongoing inflammatory lesions.

\section{Current drugs that may trigger resolution}

To the author's knowledge, there are no drugs in the clinic that are purposefully based on the elicitation of proresolving pathways, with the exception of those drugs that target apoptosis ${ }^{93}$. There are, however, drugs in current usage that mediate their effects, at least in part, by triggering the synthesis of endogenous anti-inflammatory

\section{Box 4 | Examples of non-resolving inflammatory disease processes}

This box includes known drugs that mediate some anti-inflammatory effects through the induction of endogenous anti-inflammatory mediators. In doing so, they might control ongoing inflammation by triggering resolution, a possibility that requires further clarification. The shortness of this list reflects the scarcity of drugs developed on the basis of pro-resolution properties.

Non-resolving inflammatory disorders

- Arthritis

- Nephritis

- Asthma

- Bronchitis

- Chronic granulomatous disease

- Psoriasis

Drugs that might mediate resolution

- Methotrexate

- Sulphasalazine

-FK506

- Drugs designed for the regulation of apoptosis (see REF. 93 for a detailed description of the disease targets and mechanisms of action mediators in a manner that might be related to proresolution. These include methotrexate, sulphasalazine and FK506, which are thought to exert their effects by increasing adenosine release $\mathrm{e}^{129}$. Adenosine works through four G-protein-coupled receptors that are designated $A_{A}, A_{2 A}, A_{2 B}$ and $A_{3}$, and generally signals through the cAMP pathway to dampen inflammation ${ }^{130}$. Methotrexate, for instance, is a widely used and highly effective drug for the treatment of rheumatoid arthritis and other inflammatory diseases. Data from in vitro studies have shown that methotrexate increases adenosine release from co-cultures of stimulated PMNs and umbilical-vein endothelial cells or stimulated PMNs and fibroblasts by inhi-bitingAICAR( 5 aminoimidazole-4-carboxamidoribonucleotide) transformylase, resulting in the accumulation of AICAR, which is the nucleoside precursor that has previously been shown to cause adenosine release from ischaemic cardiac tissue ${ }^{131}$. Moreover, in vivo studies showed that adenosine, working through the $\mathrm{A}$, receptors, mediates the anti-inflammatory effects of methotrexate in mice ${ }^{132}$, an observation that has been confirmed in adenosine$\mathrm{A}_{2 \mathrm{~A}}$ - and adenosine- $\mathrm{A}_{3}$-receptor-deficient mice ${ }^{133}$. Adenosine fits well with our theme of resolution as it not only prevents leukocyte trafficking but it might also switch off acute inflammation by downregulating macrophage activation ${ }^{134}$ and by favouring $\mathrm{T}_{\mathrm{H}} 2$ - versus $\mathrm{T}_{\mathrm{H}} 1$-cell development ${ }^{135}$. Therefore, adenosine or adenosine receptor occupancy on cells of the immune system presents a potential target for pro-resolving therapy, with the exception of asthma and chronic obstructive pulmonary disease in which there are continuously high endogenous levels of adenosine, which has been suggested to contribute to the chronic nature of the disease ${ }^{136}$. Similar considerations, although not yet supported by drugs in clinical use, could be put forward for another family of five G-protein-coupled receptors that signal through the cAMP - the melanocortin receptors ${ }^{137}$. There are many chronic inflammatory diseases (BOX 4) that could benefit from a treatment regimen that, somehow, leads to resolution of an ongoing inflammatory response. Drugs such as methotrexate, sulphasalazine and FK506, which trigger the synthesis of endogenous factors with established anti-inflammatory and possibly pro-resolving properties, provide support for the concept of activating pro-resolution pathways to treat chronic inflammation.

\section{Future directions}

Developing drugs based on endogenous mediators that are inherent to resolution might represent a new strategy in anti-inflammatory therapy. So far, a number of factors have been identified, including the LXs and the resolvins, which although are unstable with short half-lives in vivo, exert powerful alleviating effects when used in their native form or, particularly, as stable analogues to treat experimental inflammatory diseases. Therefore, the development of compliant mimetics based on their structure shows great promise for clinical usage. Indeed, efforts in LX research have identified that the LX receptor, in addition to binding some of 
the LX family of LOX/LOX interaction products, also binds other endogenous factors, including the glucocorticoid-inducible annexin-1 (REF. 122). This has consequences for understanding the nature of the endogenous control of resolving inflammation, showing how anti-inflammatory mediators of differing structure and metabolic origin can exert their antiinflammatory effects through the same receptor. On this theme, our ongoing challenge is to identify novel receptors, the activation of which is integral to resolving inflammation, thereby presenting alternative targets for drug development ${ }^{128}$. Other potential pro-resolving lipid mediators for drug targeting are $\mathrm{PGD}_{2}$ metabolites. We found a crucially important role for these eicosanoids in controlling the severity and progression of an experimental T-lymphocyte-mediated delayed-type hypersensitivity, indicating that stable analogues of these eicosanoids might prove beneficial in the management of certain immune-mediated diseases, such as rheumatoid arthritis or encephalomyelitis. However, as already mentioned, PGD, metabolites seem to exacerbate allergic lung diseases, almost certainly indicating organ-specific pathways of inflammatory resolution. This is important for drug development for resolving inflammation as not all organs have the same pro-resolving machinery. A good example of this is in the kidney: a model of glomerulonephritis showed that only a small percentage of influxing PMNs are phagocytosed after undergoing apoptosis, with most being cleared from the inflamed glomeruli by returning to the circulation ${ }^{74}$. This indicates that although the end result is resolution, the cellular mechanisms that ultimately switch off acute inflammation might be organ specific.

Seminal work on the fate of inflammatory leukocytes has revealed that not only is apoptosis of PMNs and eosinophils crucial as a basic mechanism of cell disposal, but that the apoptotic cells themselves express on their surface a repertoire of signals, of which phosphatidylserine is well characterized ${ }^{138}$. These signals facilitate their phagocytosis, and hence clearance, by macrophages, while at the same time programming the phagocytosing macrophage into an anti-inflammatory or pro-resolution phenotype. Interestingly, rapid membrane translocation of annexin-1 in T cells entering into apoptosis also favours phagocytosis by macrophages and other cleaning cells ${ }^{139}$. It is therefore not surprising that injection of apoptotic PMNs or phosphatidylserine

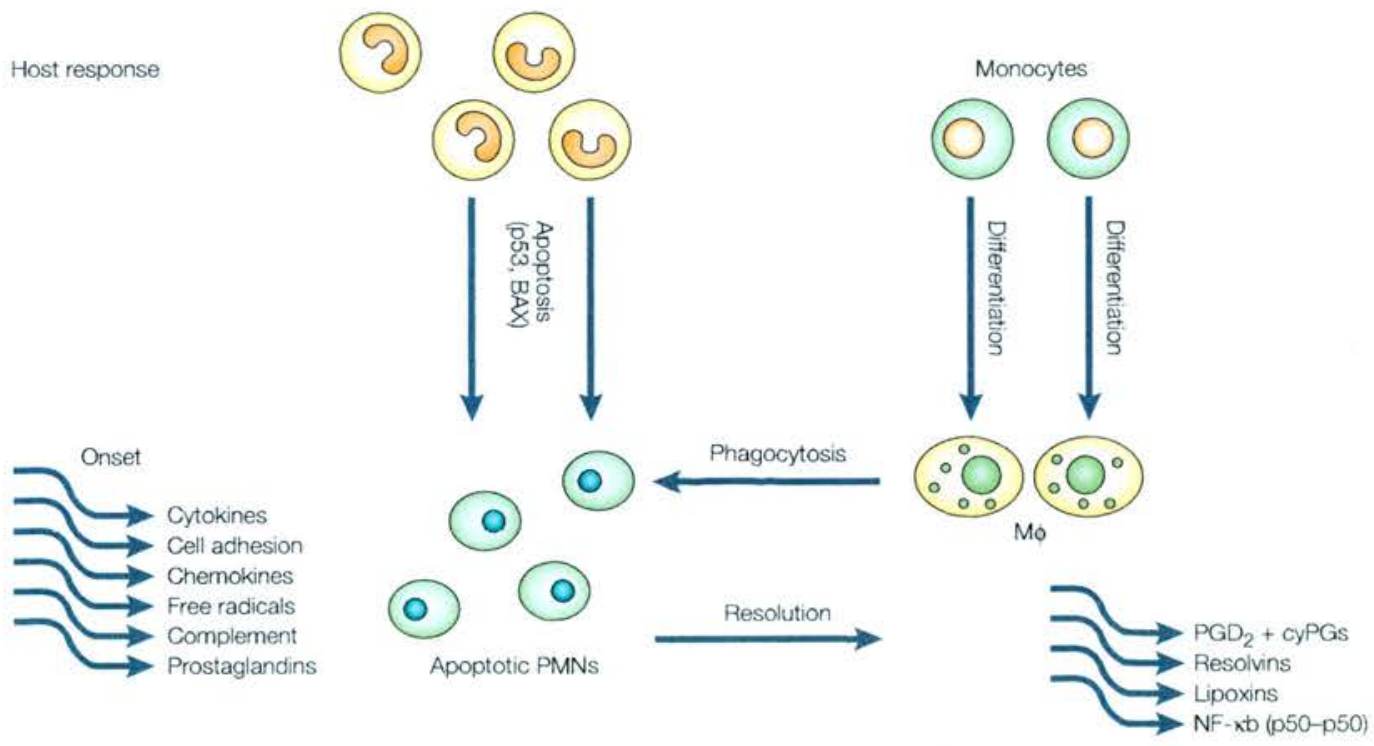

Time up to 72 hours

Figure 6 $\mid$ A general schematic summarizing the mediators and mechanisms that drive and switch off acute innate inflammation. During the early phases of acute inflammation and under the control of endogenous cytokines, chemokines, cell-adhesion molecules and other pro-inflammatory stimuli, polymorphonuclear leukocytes (PMNs) or eosinophils (depending on the nature of the stimulus) migrate into the inflamed site and, along with oedema, begin the task of neutralizing the injurious agent. These leukocytes undergo apoptosis or programmed cell death, which, in vivo, can take from 24-48 hours for PMNs or much longer for eosinophils. From our studies, it seems that BAX and $\mathrm{p} 53$ have a role in PMN apoptosis during resolution. Within time (anything from 6-24 hours, depending again on the nature and 'quantity' of the stimulus), monocytes are recruited, which differentiate to larger, more granular macrophages. These macrophages, which are generally fewer in number than the PMN infiltrate, set about phagocytosing apoptotic PMNs or eosinophils. During this phase of active resolution. Reiter cells (macrophages containing, usually, 2-4 effete leukocytes) can be observed histologically. When the apoptotic leukocytes are cleared, macrophages must also be disposed of in order to prevent, for instance, granuloma tissue formation. The fate of macrophages is either local-cell apoptosis or lymphatic drainage. The events controlling leukocyte apoptosis, phagocytosis and macrophage clearance are not well understood, but it is known so far that the lipoxins, resolvins, annexin-1, PGD (prostaglandin $\mathrm{D}_{2}$ ) metabolites and nuclear factor of $\mathrm{\kappa B}(\mathrm{NF}-\mathrm{\kappa B})$ subunits $(\mathrm{p} 50-\mathrm{p} 50)$ are crucial in resolution. 
liposomes in a model of peritonitis accelerates resolution ${ }^{140}$ and a similar intervention inhibits onset of experimental arthritis ${ }^{141}$. This could provide the impetus to develop drugs that mimic the signals displayed by apoptosing cells and enhancing the capacity of infiltrating or resident macrophages to phagocytose, with the LXs and the glucocorticoids showing particular effect in this area. As well as soluble mediators, the NF- $\mathrm{\kappa B}$ pathway has sprung some unexpected surprises namely, its role in pro-resolution through the preferential binding of the p $50-$ p50 anti-inflammatory subunits. Understanding the signals that preferentially favour this subunit complex formation will help in the development of drugs that speed up resolution through the induction of apoptosis. A temporal profile of the events leading to resolution and mediators that control them, as we understand them today, is shown in FIG. 6 .

\section{Summary}

In conclusion, we believe that failure of acute inflammation to adequately resolve might contribute to the development of chronic inflammation and even autoimmunity. We therefore suggest developing drugs that mimic the actions of mediators that are essential for resolution to treat chronic inflammatory diseases, such as rheumatoid arthritis, asthma and encephalomyelitis, for example. Such therapeutics might be based on the mechanisms of action of cyPGs, lipoxins or lipoxin-receptor agonists, or on factors that trigger leukocyte apoptosis and stimulate macrophage phagocytosis of apoptotic cells. In doing so, it might be possible to 'trick' ongoing inflammation into resolution. Unfortunately, such therapeutics, with the exception of those that regulate leukocyte apoptosis ${ }^{93}$, are far from the clinic. This reflects the relative immaturity of this field of research. However, further investigation into the mediators and mechanisms that are central to resolution will bring us into a new era of inflammation research, which, if approached with creativity and persistence, might provide numerous benefits for those suffering from inflammationmediated diseases.
. Forey, H. W. General Pathology (Lloyd-Luke Ltd, London, 1970).

Provides a fantastic historical perspective of inflammation research as well as detailed accounts of some of the classic experiments that have contibuted to our current understanding of derstanding of the inflammatory response.

2. Cotran, R. S., Kumar, V. \& Collins, T. (eds) in Robbins Pathologic Basis of Disease Vol. $650-88$ (W. B. Saunders Company, Philadelphia, 1999).

3. Cotran, R. S., Kumar, V. \& Collins, T. (eds) in Robbins Pathologic Basis of Disease Vol. 689-112 (W. B Saunders and Company, Philadelphia, 1999).

4. Dumonde, D. C. \& Glynn, L.E. The production of arthritis in rabbits by an immunological reaction to fibrin. Br. J. Exp. Pathol, 43, 373 (1962)

5. Dumonde, D. C. \& Glymn, L.E. The reaction of guinea-pigs to autologous and heterologous fibrin implants. J. Pathol. Bacteniol. 90, 649-657 (1965).

6. Weissmann, G., Becher, G., Wiedermann, G. \& Bernheimer, A. W. Studies on lysozymes. VII. Acute and chronic arthritis produced by intra-articular injections of streptolysin 'S' in rabbits. Am. J. Pathol. 46, 129 (1965).

7. Whoughby, D. A. \& Ryan, G. B. Evidence for a possible endogenous antigen in chronic inflammation. J. Pathol. 101, 233-239 (1970)

8. Recommendations for the prevention and treatment of glucocorticoid-induced osteoporosis: 2001 update. American College of Rheumatology Ad Hoc Committee on Glucocoritcoid-Induced Osteoporosis. Arthritis Rheum. 44. 1496-1503 (2001)

9. Beer, H. D., Fassler, R. \& Werner, S. Glucocorticoidregulated gene expression during cutaneous wound repair. Vitam, Horm, 59, 217-239 (2000).

10. Mukheriee, D. Selective cyclooxygenase-2 (COX-2) inhibitors and potential nisk of cardiovascular events. Biochem. Pharmacol. 63, 817-821 (2002).

11. Antoni, C. \& Braun, J. Side effects of ant-TNF therapy: curren knowedge, Cin. Exp. Pheumatol. 20, S152-S157 (2002).

12. Urade, $Y, \&$ Hayaishi, O. Prostaglandin D synthase: structure and function. Vitam. Horm. 58, 89-120 (2000).

13. Straus, D. S. \& Glass, C. K. Cyclopentenone prostaglandins: new insights on biological activities and cellular targets. Med. Res. Rev. 21, 185-210 (2001)

This is a comprehensive review of cyclopentenone prostaglandins that covers their synthesis, structure and role in pathophysiology.

14. Jiang, C., Ting, A T. \& Seed, B. PPAR-gamma agonists inhibit production of monocyle inflammatory cytokines. Nature 391, 82-86 (1998).

15. Picote, M., L. A. C., Willson, T. M., Kelly, C. J. \& Glass, C. K. The peroxisome proliferator-activated receptor-gamma is a negative regulator of macrophage activation. Nature 391 79-82 (1998).
References 14 and 15 report on the receptor-mediated actions of the cyclopentenone prostaglandins that lead to a suppression of inflammation.

16. Nosjean, O. \& Boutin, J. A. Natural ligands of PPARgamma: are prostaglandin $\mathrm{J}(2)$ derivatives really playing the part? Cell Signal. 14, 573-583 (2002)

17. Straus, D. S, et al. 15-deoxy-delta 12,14-prostaglandin J2 inhibits multiple steps in the NF-kappa B signaling pathway. Proc. Natl Acad. Sci. USA 97, 4844-4849 (2000).

18. Perez-Sala, D., Cemuda-Morollon, E. \& Canada, F. J. Molecular basis for the direct inhibition of AP-1 DNA binding by 15-deoxy-Detta 12,14-prostaglandin J2. J. Biol. Chem. 278, 51251-51260 (2003)

19. Chen, C. W., Chang, Y. H., Tsi, C. J. \& Lin, W. W. Inhibition of IFN-gamma-mediated inducible nitric oxide synthase induction by the peroxisome proliferator-activated receptor garmma agonist, 15-deoxy-delta 12, 14-prostaglandin $\mathrm{J} 2$, involves inhibition of the upstream Janus kinase/STAT1 signaing pathway. J. Immunol. 171, 979-988 (2003).

20. Maggi, L. B. Jr et al, Anti-inflammatory actions of 15-deoxydelta 12,14-prostaglandin $\mathrm{J} 2$ and trogitazone: evidence for heat shock-dependent and-independent inhibition of cytokine-induced inducible nitric oxide synthase expression. Diabetes 49, 346-355 (2000).

21. Petrova, T. V., Akama, K. T. \& Van Elik , L. J. Cyclopentenone prostaglandins suppress activation of microglia: down-regulation of inducible nitric-oxide synthase by 15-deoxy-Delta12,14-prostaglandin J2. Proc. Natt Acad. Sci. USA 96, 4668-4673 (1999)

22. Bemardo, A., Levi, G. \& Minghetti, L. Role of the peroxisome proliferator-activated receptor-gamma (PPAR-gamma) and its natural ligand 15-deoxy-Delta12, 14-prostaglandin $\mathrm{J} 2$ in the regulation of microglial functions. Eur. J. Neurosci. 12 2215-2223 (2000)

23. Gosset, P. et al. Prostaglandin D2 affects the maturation of human monocyte-derived dendritic cells: consequence on the polarization of naive Th cells. J. Immunol. 170 494349-494352 (2003).

24. Jackson, S. M. et al. Peroxisome proliferator-activated receptor activators target human endothelial cells to inhibit leukocyte-endothelial cell interaction. Artenioscler. Thromb. Vasc. Biol. 19, 2094-2104 (1999)

25. Pasceri, V., Wu, H. D., Willerson, J. T. \& Yeh, E. T. Modulation of vascular inflammation in vitro and in vivo by peroxisome proliferator-activated receptor-gamma activators. Circulation 101, 235-238 (2000).

26. Thang, X., Wang, J. M., Gong, W. H., Mukaida, N. \& Young. H. A. Differential regulation of chemokine gene expression by 15-deoxy-delta 12,14 prostaglandin J2. J. Immunol, 166, 7104-7111 (2001).

27. Zemecke, A., Er, W., Fraemohs, L., Lietz, M. \& Weber, C. Suppression of endothelial adhesion molecule up-requlation with cydopentenone prostaglandins is dissociated from
IkappaB-alpha kinase inhibition and cell death induction. FASEB J. 17. 1099-1101 (2003)

28. Gilroy, D. W., Tomlinson, A. \& Willoughby, D. A. Differentia effects of inhibitors of cydooxygenase (cyclooxygenase -1 and cydooxygenase 2) in acute inflammation. Eur. $J$ Pharmacol. 355, 211-217 (1998)

29. Gitroy, D. W. et al. Inducible cyclooxygenase may have antiinflammatory properties. Nature Med. 5, 698-701 (1999). This paper originally described the synthesis of antiinflammatory eicosanoids during acute inflammatory resolution, thereby highlighting that the host actively manufactures soluble mediators to switch off acute inflammation and limit tissue damage.

30. Giroy, D. W.. Newson, J., Sawmynaden, P., Willoughby, D. A. $\&$ Croxtall, J. D. A novel role for phospholipase A2 isoforms in the checkpoint control of acute inflammation. FASEB J. $18,489-498$ (2004)

31. Gilroy, D. W. et al, Inducible cyclooxygenase-derived 15-deoxy(Delta) 12-14PG 2 brings about acute inflammatory resolution in rat pleurisy by inducing neutrophil and macrophage apoptosis. FASEB J. 17, 2269-2271 (2003)

32. Ward, C. et al. Prostaglandin D2 and its metabolites induce caspase-dependent granulocyle apoptosis that is mediater via inhibition of I kappa $B$ alpha degradation using a peroxiscme proliferator-activated receptor-gamma-independent mechanism. J. Immunol. 168, 6232-6243 (2002).

33. Castrillo, $\mathrm{A}$, et al, Potentiation of protein kinase $\mathrm{C}$ zeta activity by 15 -deoxy-delta(12,14)-prostaglandin $J(2)$ induces an imbalance between mitogen-activated protein kinases and NF-kappaB that promotes apoptosis in macrophages. Mol. Cell Biol. 23, 1196-1208 (2003).

34. Li, L. et al. 15-deoxy-delta 12,14-prostaglandin J2 induces apoptosis of human hepatic myofibroblasts. A pathway mvalving oxidative stress independently of peroxisomeproliferator-activated receptors. J. Biol. Chem. 276 38152-38158 (2001)

35. Rieference deleted in proof

36. Setoguchi, K. et al. Peroxiscme proliferator-activated receptor-gamma haploinsufficiency enhances B cell proliferative responses and exacerbates experimentally induced arthritis. J. Clin. Invest. 108, 1667-1675 (2001).

37. Ando, M. et af. Retrovirally introduced prostaglandin D2 synthase suppresses lung injury induced by bleomycin. Am. J. Respir. Cell. Mol. Biol. 28, 582-591 (2003).

38. Cuzzocrea, S. et al. The cyclopentenone prostaglandir 15-deoxy-delta(12,14)-PGJ atterisates the development of colon injury caused by dinitrobenzene sulphonic acid in the rat. Br. J. Pharmacol. 138, 678-688 (2003).

39. Chatteriee, P. K. et al. The cyclopentenone prostaglandin 15-deoxy-Delta(12,14)-prostaglandin J(2) amelicrates ischenic acute renal falure. Cardiovasc. Res, 61, 630-643 (2004).

40. Kawahito, Y. et al. 15-deoxy-delta(12,14)-PGJ(2) induces synoviocyte apoptosis and suppresses adjuvantinduced arthritis in rats. J. Clin. Ifwest. 106, 189-197 (2000). 
41. Diab. A. et al. Peroxisome proliferator-activated receptorjamma agonist 15-deoxy-delta(12,14)-prostaglandin J(2) unencrates expenmental autoimmune encephalomyeifis. J. Immunol. 168, 2508-2515 (2002).

42. Matsucka, T. et al. Prostaglandin D2 as a mediator of allergic asthma. Science 287, 2013-2017 (2000).

43. Honda, $K$. et al. Prostaglandin D2 reinforces $T_{3} 2$ type inflammatory responses of airways to low-dose antigen through bronchial expression of macrophage-derived chemokine. J. Exp. Med. 198, 533-543 (2003).

44. Lee, T. S., Tsai, H. L. \& Chau, L. Y. Induction of heme oxygenase-1 expression in murine macrophages is essentia for the anti-inflammatory effect of low dose 15-deoxy-delta 12,14-prostaglandin J2. J. Biol. Chem. 278, 19325-19330 (2003).

45. Ryler, S. W., Otterbein, L. E., Morse, D. \& Choi, A. M. Heme oxygenase/carbon monoxide signaling pathways: regulation and functional significance. Mol. Cell. Biochem. 234-235, 249-263 (2002).

46. Willis, D. M. A.. Frederick, R. \& Willoughby, D. A. Heme oxygenase: a novel target for the modulation of the inflammatory response. Nature Med. 2, 87-90 (1996).

47. Graca-Souza, A. V., Amuda, M. A., de Freitas, M. S., Barja-Fidalgo, C. \& Olveira, P. L. Neutrcphil activation by herne: implications for inflammatory processes. Blood 99 $4160-4165$ (2002).

48. Acaraz, M. J., Fernandez, P. \& Guillen, M. L. Antiinflammatory actions of the heme oxygenase-1 pathway Curr. Pharm. Des. 9, 2541-2551 (2003)

49. Sato, $K$. et at. Carbon monoxide generated by heme oxygenase-1 suppresses the rejection of mouse-lu-tal cardiac transplants. J. Immunol. 166, 4185-4194 (2001).

50. Hancock, W. W., Buelow, R., Sayegh, M. H. \& Turka, L. A. Antibody-induced transplant arteriosclerosis is prevented by graft expression of anti-oxidant and anti-apoptotic genes. Nature Med. 4. 1392-1396 (1998),

51. Woo, J., lyer, S., Mori, N. \& Buelow, R. Alleviation of graft-versus-host disease after conditioning with cobalt-protoporphyrin, an inducer of heme oxygenase-1. Transplantation 69, 623-633 (2000).

52. Levy, B. D., Cish, C. B., Schmidt, B., Gronert, K. \& Serhan, C. N. Lipid mediator class switching during acute inflammation: signals in resolution. Nature Immunol. 2 , 612-619 (2001).

In addition to reference 25 , this paper reinforces the concept of lipid mediator class switching from proinflammatory at the onset of acute inflammation to pro-resolving at resolution.

53. Papayianni, A., Serhan, C. N. \& Brady, H. R. Lipoxin A4 and $B 4$ inhibit leukotriene-stimulated interactions of humar neutrophils and endothelial cells. J. Immunol. 156, 2264-2272 (1996).

54. Serhan, C. N., Takano, T., Clish, C. B., Gronert, K. \& Petasis, N. Aspirin-triggered 15-epi-lipoxin A4 and novel Fpoxin B4 stable analogs inhibit neutrophil-mediated changes in vascular pemeablity. Adv. Exp. Med. Biol. 469 287-293 (1999)

55. Maddox, J. F. \& Serhan, C. N. Lipoxin A4 and B4 are poten stimuli for human monocyte migration and adhesion: selective inactivation by dehydrogenation and reduction. J. Exp. Med, 183, 137-146 (1996).

56. Maddox, J. F. et al. Lipoxin A4 stable analogs are potent mimetics that stimulate human monocyles and THP-1 cells via a G-protein-linked lipoxin A4 receptox. J. Biol. Chem. 272, 6972-6978 (1997)

57. Jozsef, L., Zouki, C., Petasis, N. A., Serhan, C. N. \& Flep, J. G. Lipoxin A4 and aspirin-triggered 15-epi-lipoxin A4 inhibit peroxynitrite formation, NF-kappa B and AP-1 activation, and IL-8 gene expression in human leukocytes. Proc. Natt Acad. Sci. USA 99, 13266-13271 (2002).

58. Bandeira-Melo, C. et al. Cydooxygenase-2-derived prostaglandin E2 and lipoxin A4 accelerate reschution of allergic edema in Angiostrongylus costaricensis-infected rats: relationship with concurrent eosincphilia. J. Immunol. 164. 1029-1036 (2000).

59. Godson, C. et al. Cutting edge: lipoxins rapidly stimulate nonphlogistic phagocytosis of apoptotic neutrophils by monocyte-derived macrophages. J. Immunol. 164. 1663-1667 (2000)

60. Mitchell, S. et al. Lipoxins, aspirin-triggered epi-lipoxins, poxin stable analogues, and the resolution of inflammation: stimulation of macrophage phagocytosis of apoptotic neutrophils in vivo. J. Am. Soc. Nephrol. 13, 2497-2507 (2002)

61. Brink, C. et al. International Union of Phamacology $X \times X \mathrm{VI}$. Nomenclature for leukotriene and lipoxin receptors. Pharmacol. Rev. 55, 195-227 (2003)
62. Bandeira-Melo, C. et al. Cutting edge: lipoxin (LX) A4 and aspinin-triggered 15-epi-LXA4 block allergen-induced eosinophil trafficking. J. Immunol. 164, 2267-2271 (2000).

63. Serhan, C. N. et al. Novel functional sets of lipid-derived mediators with antinilammatory actions generated from omega-3 fatty acids via cyclooxygenase 2-nonsteroidal antinflammatory drugs and transcellular processing. J. Exp. Med. 192, 1197-1204 (2000).

64. Serhan, C. N. et al. Resolvins: a family of bicactive products of omega-3 fatty acid transformation circuits initiated by aspirin treatment that counter proinflammation signals. J. Exp. Med. 196, 1025-1037 (2002).

\section{References 63 and 64 report on the role of aspirin-} triggered lipid mediator metabolites of polyunsaturated fatty acids and their spontaneous synthesis during the resolving phase of acute inflammation

65. Hong, S., Gronert, K., Devchand, P. R., Moussignac, R. L \& Serhan, C. N. Novel docosatrienes and 17S-resolvins generated from docosahexaenoic acid in murine brain, human blood and glial cells. Autacoids in anti-inflammation. J. Biol, Chem. 278, 14677-14687 (2003).

66. Brezinski, D. A., Nesto, R. W. \& Serhan, C. N. Angicplasty triggers intracoronary leukotrienes and lipoxin A4. Impact of aspinn therapy. Circulation 86, 56-63 (1992)

67. Van Dyke, T.E. \& Serhan, C. N. Resolution of inflammation: a new paradigm for the pathogenesis of periodontal diseases. J. Dent. Res. 82, 82-90 (2003).

68. Claria, J. et al. Attered biosynthesis of leukotrienes and lipoxins and host defense discrders in patients with cinhosis and ascites. Gastroenterology 115, 147-156 (1998).

69. Levy, B. D. el al. Mulli-prunged int ibilion of airway hyperresponsiveness and inflammation by lipoxin $\mathrm{A}(4)$. Nature Med. 8, 1018-1023 (2002).

70. Munger, K. A. et al. Transfection of rat kidney with human 15-lipoxygenase suppresses inflammation and preserves function in experimental glomerulonephritis. Proc. Natt Acad. Sci. USA 96, 13375-13380 (1999)

71. Leonard, M. O. et al. 15-Epi-16-(para-fuorophenoxy)-lipoxin A(4)-methyl ester, a synthetic analogue of 15-epi-lipoxin A(4), is protective in expenmental ischemic acute renal falure. J. Am. Soc. Nephrol, 13, 1657-1662 (2002)

72. Schottefius, A. J. et al. An aspirin-triggered lipoxin A4 stable analog displays a unique topical anti-inflammatory profile, J. Immunol. 169, 7063-7070 (2002)

73. Souza, M. H., de Lima, O. M., Zamuner, S. R., Fionucci, S \& Wallace, J. L. Gastritis increases resistance to aspirininduced mucosal injury via COX-2-mediated lipoxin synthesis. Am. J. Physiol. Gastrointest. Liver Physiol. 285, G54-G61 (2003).

74. Hughes, J. et al. Neutrophil fate in experimental glomenular capillary iniury in the rat. Emigration exceeds in situ dearance by apoptosis. Am. J. Pathol. 150, 223-234 (1997).

75. Martin, C. et al. Chemokines acting via CXCR2 and CXCR4 control the release of neutrophils from the bone marrow and their retum following senescence. Immunity 19, 583-593 (2003).

76. Bellingan, G. J., Caldwell, H., Howie, S. E., Dransfield, I. \& Haslett, C. In vivo fate of the inflammatory macrophage during the resolution of inflammation: inflammatory macrophages do not die locally, but emigrate to the draining lymph nodes. J. Immunol. 157, 2577-2585 (1996).

77. Bellingan, G. J. et al. Adhesion molecule-dependent mechanisms regulate the rate of macrophage clearance during the resolution of peritoneal inflammation. J. Exp. Med. 196, 1515-1521 (2002). References 76 and 77 show comprehensively that in resolving peritonitis, the fate of macrophage clearance is through lymphatic drainage.

78. Savill, J. \& Fadok, V. Corpse dearance defines the meaning of cell death. Nature 407, 784-788 (2000). A concise review highlighting the concept of apoptosis and phagocytosis and their relevance to inflammatory disease processes.

79. Nicholson, D. W. From bench to dinic with apoptosis-based therapeutic agents. Nature 407, 810-816 (2000).

80. Brunner, T. \& Mueller, C, Apoptosis in disease: about shorlage and excess. Essays Biochem. 39, 119-130 (2003).

81. Fadok, V. A., Bratton, D. L. \& Henson, P. M. Phagocyte receptors for apoptotic cells: recogrition, uptake, and consequences. J. Clin. Invest. 108, 957-962 (2001).

82. Savill, J., Dransfield, I., Gregory, C. \& Haslett, C. A blast from the past: clearance of apoptotic cells regulates immune responses. Nature Rev. Immunol. 2, 965-975 (2002).

83. Ward, C., Dransfield, L., Chilvers, E. R., Haslett, C. \& Rossi, A. Pharmacological manipulation of granulocyte apoptosis: potential therapeutic targets. Trends Phamacol. Sci. 20, 503-509 (1999).
84. Giles, K. M. Hart, S. P. Haclett, C., Rossi, A. G. \& Dransfield, I. An appetite for apoptotic cells? Controversies and challenges. Br. J. Haematol. 109, 1-12 (2000).

85. Rossi, A. G. et al. Regulation of macrophage phagocytosis of apoptotic cells by cAMP. J. Immunol. 160, 3562-3568 (1988)

86. Liu, Y. et al. Glucocorticoids promote nonphlogistic phagocytosis of apoptotic leukocyles. J. Immund. 162, $3639-3646$ (1999)

87. Giles, K. M. ef al. Glucocorticoid augmentation of macrophage capacity for phagocylosis of apoptotic cells is associated with reduced p130Cas expression, loss of paxilin/pyk2 phosphonjation, and high levels of active Rac. 1. Immunol. 167, 976-986 (2001).

88. Ren, Y. \& Savill, J. Proinflammatory cytokines potentiate thrombospondin-mediated phagocytosis of neutrophils undergoing apoptosis. J. Immunol. 154, 2366-2374 (1995).

89. McCutcheon, J. C. et al. Regulation of macrophage phagocylosis of apoptotic neutrophils by adhesion to fibronectin. J. Leukoc. Biol. 64, 600-607 (1998).

90. Hart, S. P., Dougherty, G. J., Haslett, C. \& Dransfield, I. $\mathrm{CD} 44$ regulates phagocytosis of apoptotic neutrophil granulocytes, but not apoptotic lymphocytes, by human macrophages. J. Immunol. 159, 919-925 (1997).

91. Teder, P. et al. Resolution of lung inflammation by $\mathrm{CD} 44$ Science 296, 155-158 (2002)

92. Woolley, K. L. et al. Eosinophil apoptosis and the resolution of airway inflammation in asthma. Am. J. Respir. Crit. Care Med. 154, 237-243 (1996)

83. Murphy, F. J., Seery, L. T. \& Hayes, I. Therapeutic approaches to the modulation of apoptosis. tssays Biochem. 39, 131-153 (2003).

94. Degterev, A., Boyce, M. \& Yuan, J. A decade of caspases. Oncogene 22, 8543-8567 (2003)

95. Eamshaw, W. C. Martins, L. M. \& Kaufmarnn, S. H. Mammalian caspases: structure, activation, substrates, and functions during apoptosis. Annu. Rev. Biochem. 68, 383-424 (1999).

96. Graczyk, P. P. Caspase inhibitors as anti-inflammatory and antiapoptotic agents. Prog. Med. Chem. 39, 1-72 (2002).

97. I wata, A. Harlan, J. M., Vedder, N. B. \& Winn, R. K. The caspase inhibitor $z-V A D$ is more effective than $C D 18$ achesion blockade in reducing muscle ischemia-repertusion injury: implication for clinical trials. Blood 100, 2077-2080 (2002)

98. Natori. S., Higuchi, H., Contreras, P. \& Gores, G. J. The caspase inhibitor IDN-6556 prevents caspase activation and apoptosis in sinusoidal endothelial cells during liver preservation injury. Liver Transpl. 9, 278-284 (2003).

99. Hoglen, N. C. et al. Characterization of IDN-6556: a livertargeted caspase inhibitor. J. Pharmacol. Exp. Ther. 23 Jan 2004 [epub ahead of print]

100. Valentino, K. L., Gutierrez, M., Sanchez, R., Winship, M. J. \& Shapiro, D. A. First clinical trial of a novel caspase inhibitor: anti-apoptotic caspase inhibitor, IDN-6556, improves liver enzymes. Int. J. Clin. Pharmacol. Ther. 41, 441-449 (2003).

101. Karin, M. New twists in gene regulation by glucocorticoid receptor: is DNA binding dispensable? Cell $93,487-490$ (1998).

102. Ghosh, S., May, M. J. \& Kopp, E. B. NF-kappa B and Re proteins: evolutionanly conserved mediators of immune responses. Annu. Rev. Immunol. 16, 225-260 (1998).

103. Khoshnan, A. et al. The NF-kappa B cascade is important in $B C-x L$ expression and for the anti-apoptotic effects of the CD28 receptor in primary human $\mathrm{CD} 4+$ lymphocyles. J. Immunol, 165, 1743-1754 (2000).

104. Ward, C. et al. NF-kappaB activation is a critical regulator of human granulocyle apoptosis in vitro. J. Biol. Chem. 274. 4309-4318 (1999).

105. Lawrence, T., Gilroy. D. W., Colwille-Nash, P. R. \& Willoughby, D. A. Possible new role for NF-kappaB in the resolution of inflammation. Nature Med. 7, 1291-1297 (2001). Shows an important role for $\mathrm{NF}-\mathrm{kB}$ in the resolution of acute inflammation that underpins a novel protective role for this otherwise pro-inflammatory factor.

106. Ishikawa, H. et al. Chronic inflammation and susceptibility to bacterial infections in mice lacking the polypeptide (p) 105 precursor (NF-kappaB1) but expressing p50. J. Exp. Med. $187,985-996$ (1998)

107. Bohustav, J. et al. Regulation of an essential innate immune response by the p 50 subunit of NF-kappaB. J. Clin. Invest 102, 1645-1652 (1998)

108. Chen, L. W. et al. The two faces of IKK and NF-kappaB inhibition: prevention of systemic inflammation but increased local injury following intestinal ischemia-reperfusion. Nature Med. 9, 575-581 (2003)

109. Munck, A., Guyre, P. M. \& Holbrook, N. J. Physiological functions of glucocorticoids in stress and their relation to pharmacological actions. Endocr. Rev. 5, 25-44 (1984). 
10. Fower, R. J., Parente, L., Persico, P. \& Salmon, J. A. A companson of the acute inflammatory response in idrenaiectomised and sham-operated rats. Br.J. Pharmacol. 87, 57-62 (1986).

111. Perretti, M., Bechenucci, C., Scapigliat, G. \& Parente, L The effect of adrenalectomy on interleukin-1 release in vitro and in vivo. Br. J. Phamacol. 98, 1137-1142 (1989).

112. Adcock, I. M. Molecular mechanisms of glucocorticosteroid actions. Pulm. Pharmacol. Ther. 13, 115-126 (2000).

113. Auphan, N., DiDonato, J. A., Rosette, C., Heimberg, A. \& Karin, M. Immunosuppression by glucocorticoids: inhibition of NF-kappa B activity through induction of I kappa B synthesis. Science 270, 286-290 (1995).

114. Buttgereit, F., Burmester, G. R. \& Brand, M. D. Bioenergetics of immune functions: fundamental and therapeutic aspects. Immunol. Today 21, 192-199 (2000).

115. Nissen, R. M. \& Yamamoto, K. R. The glucocorticoid receptor inhibits NFkappaB by interfering with serine-2 phosphorylation of the RiNA polymerase Il carboxy-terninal domain. Genes Dev. 14, 2314-2329 (2000).

116. Fower, R. J. Eleventh Gaddum memorial lecture. Lipocortin and the mechanism of action of the glucocorticoids. Br. J. Pharmacol. 94, 987-1015 (1988).

117. Croxtall, J. D. \& Flower, R. J. Lipocortin 1 mediates dexamethasone-induced growth arest of the A549 lung adenocarcinoma cell line. Proc. Natl Acad. Sci. USA 89, 3571-3575 (1992)

118. De Caterina, R. et al, Macrophage-specific eicosanoid synthesis inhibition and lipocortin-1 induction by glucocorticoids. J. Appl. Physiol. 75, 2368-2375 (1993).

119. Mancuso, F., Fower, R. J. \& Ferrett, M. Leukocyte transmigration, but not rolling or adhesion, is selectively inhibited by dexamethasone in the hamster post-capillary venule. Involverment of endogenous lipocortin 1. J. Immunol 155, $377-386$ (1995)

120. Perretti, M., Ahluwalia, A., Harris, J. G., Goulding, N. J. \& Flower, R. J. Lipocortin-1 fragments inhibit neutrophil accumulation and neutrophil-dependent edema in the mouse. A qualitative comparison with an anti-CD11b monodonal antibody 1 Immund $151,4306-4314$ (1993).

121. Gerke, V. \& Moss, S. E. Annexins: from structure to function Physiol. Rev. 82, 331-371 (2002)

122. Perrett, M. et al. Endogenous lipid- and peptide-derived anti-inflammatory pathways generaled with glucocorticoid and aspinin treatment activate the lipoxin A4 receptor. Nature Med. 8, 1296-1302 (2002).

Demonstrates, for the first time, the biochemical and functional link between endogenous and exogenous annexin-1 and ALX. Experiments of binding, immunoprecipitation and in vivo analyses of PMN recruitment support this novel idea.

123. Le, Y., Murphy, P. M. \& Wang, J. M. Formyl-peptide receptors revisited. Trends immunol. 23, 541-548 (2002)

124. Perretti, M. \& Gavins, F. N. Annexin 1: an endogenous antiinflammatory protein. News Physiol. Sci, 18, $60-64$ (2003).

125. Perretti, M. et al. Acute inflammatory response in the mouse: exacerbation by immunoneutralization of lipocortin 1. Br. J. Pharmacol. 117, 1145-1154 (1996).

126. Hannon, $R$, et al. Aberrant inflammation and resistance to glucocorticoids in annexin 1-/-mouse. FASEB J. 17. $253-255$ (2003).
127. Yang, Y. H. et al, Modulation of inflammation and response lo dexamethasone by Annexin 1 in antigen-induced arthritis. Arthritis fineum. 50, 976-984 (2004).

References 126 and 127 were the first studies with the newly generated annexin-1-null mice to confirm the crucial, not redundant, role that this anti-inflammatory protein plays in prolonging the acute inflammatory response as well as in exacerbating joint inflammation in a model of arthritis.

128. Perretti, M. The annexin 1 receptoris): is the plot unraveling? Trends Phamacol. Sci. 24, 574-579 (2003).

A concise review highlighting the opportunities that derive from the identification of the molecular targets that are responsible for annexin-1 actions.

129. Hasko, G. \& Cronstein, B. N. Adenosine: an endogenous regulator of innate immunity. Trends immunol. 25, 33-39 (2004).

130. Sitkovsky, M. V. Use of the A(2A) adenosine receptor as a physiological immunosuppressor and to engineer inflarnmation in vivo. Biochem. Phamacol. 65, 493-501 (2003).

131. Cronstein, B. N., Eberle, M. A., Gruber, H. E. \& Levin, R. I. Methotrexate inhibits neutrophil function by stimulating adenosine release from connective tissue cells. Proc. Nat Acad. Sci. USA 88, 2441-2445 (1991)

132. Cronstein, B. N., Naime, D. \& Ostad, E. The antinflammatory mechanism of methotrexate, Increased adenosine release at inflamed sites diminishes leukocyte accumulation in an in vivo model of inflammation. J. Clin. Invest. 92, 2675-2682 (1993).

133. Montesinos, M. C. et al. Adenosine A2A or A3 receptors are required for inhibition of inflammation by methotrexat and its analog MX-68. Arthritis Rheum. 48, 240-247 (2003).

134. Xaus, J. et al. IFN-gamma up-regulates the A2B adenosine receptor expression in macrophages: a mechanism of macrophage deactivation. J. Immunol. 162, 3607-3614 (1999).

135. Hasko, G., Deitch, E. A., Szabo, C., Nemeth, Z. H. \& Vizi, E.S. Adenosine: a potential mediator of immunosuppression in multiple organ failure. Curr. Opin. Pharmacol, 2, 440-444 (2002)

136. Blackbum, M. R. Too much of a good thing: adenosine overload in adenosine-deaminase-deficient mice. Trends Pharmacol. Sci. 24, 66-70 (2003)

137. Catania, A., Gatti, S., Colombo, G. \& Lipton, J. M. Targeting melanocortin receptors as a novel strategy to control inflammation. Pharmacol. Riev. 56, 1-29 (2004).

138. Fadok, V. A., Bratton, D. L., Frasch, S. C., Wamer, M. L. \& Henson, P. M. The role of phosphatidy/serine in recognition of apoptotic cells by phagocytes. Cell Death Differ. 5 551-562 (1998).

139. Avr, S. et al. Avnexin I is an endogenous ligand that mediates apcoptotic cell engulfiment. Dev. Cell 4, 587-598 (2003).

140. Huynh, M. L., Fadok, V. A. \& Henson, P. M. Phosphatidylserine-dependent ingestion of apoptotic cells promotes TGF-beta1 secretion and the resolution of inflammation. J. Clin. Invest, 109, 11-50 (2002)

141. Biom, A. B., van Lent, P. L., Holthuysen, A. E. \& van den Berg, W. B. Skewed balances in regulation of stimulating and inhibitory $\mathrm{FC}$ gamma receptors on macrophages of $\mathrm{CIA}$ sensitive mice. Inflamm. Res. 50 (Suppl. 3), S155 (2001).
142. Edenius, C... Haeggstrom, J. \& Lindgren, J. A. Transcellular conversion of endogenous arachidonic acid to lipoxins in mixed human platelet-granulocyte suspensions. Biochem. Biophys. Res. Commun. 157, 801-807 (1988).

143. Fiore, S. \& Serhan, C. N. Formation of lipoxins and leukotrienes during receptor-mediated interactions of human platelets and recombinant human granulocyte/macrophage colony-stimulating factor-primed neutrophils. J. Exp. Med. 172, 1451-1457 (1990).

144. Serhan, C. N., Hamberg, M. \& Samuelsson, B. Lipoxins: novel series of bidogically active compounds formed from arachidonic acid in human leukocyles. Proc. Natl Acad. Sci USA 81, 5335-5339 (1984).

145. Claria, J. \& Serhan, C. N. Aspirin triggers previousty undescribed bioactive eicosancids by human endothelial cell-leukocyle interactions. Proc. Nat Acad. Sci. USA 92 9475-9479 (1995)

146. McLoughlin, R. M. et al. Interplay between IFN-gamm and IL-6 signaling govems neutrophil trafficking and apoptosis during acute inflammation. J. Clin. Invest. 112 598-607 (2003)

147. Hurst, S. M. et al. II-6 and its soluble receptor orchestrate a temporal switch in the pattem of leukocyle recruitment seen during acute inflammation. Immunity 14, 705-714 (2001).

148. Rossi, A. et al. Anti-inflammatory cyclopentenone prostaglandins are direct inhibitors of lkappaB kinase. Nature 403, 103-108 (2000)

149. Castrillo, A., Diaz-Guerra, M. J., Hortelano, S., Martin-Sanz, P. \& Bosca, L. Inhibition of lkappaB kinase and lkappaB phosphorytation by 15-deoxy-delta(12,14)-prostaglandin $\mathrm{J}(2)$ in activated murine macrophages. Mol, Cell, Biol, 20 1692-1698 (2000)

150. Cernuda-Morclion, E., Pineda-Molina, E., Canada, F. J. \& Perez-Sala, D. 15-Deoxy-delta 12,14-prostaglandin J2 inhibition of NF-kappaB-DNA binding through covalent modification of the $\mathrm{p} 50$ subunit. J. Biol. Chem. 276 , $35530-35536$ (2001).

References 148-150 show that the cyclopentenone prostaglandins have potent inhibitory signalling effects by directly binding to and altering NF- $\mathrm{kB}$ activation in addition to their modes of action discussed in references 14 and 15.

151. Bell-Parkh, L. C. ef al. Biosynthesis of 15-deoxy detta12,14-PGJ2 and the ligation of PPARgamma. J. Clin invest. 112, 945-955 (2003).

Competing interests statement

The authors decdare that they have no competing financial interests.

\section{(5) Online links}

\section{DATABases}

The following terms in this article are linked online to:

LocusLink: http://www.ncbi.nlm.nih.gov/Locus Link ALXR / annexin-1 | BAX | BCL2 2 BCL3 | CD4OL $\mid$ CD $44|\mathrm{COX} 2|$ FASR |FOS |FPRL 2 |LL-1 $\beta$ |LL-12 | JUN | NF-kB |p50|p53 | PPARYIRELAITNF-A

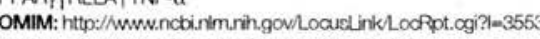
Alzheimer's disease | asthma | atopic dermatitis | Huntingdon's disease | multiple sclerosis | Parkinson's disease | meumatoid arthritis

Access to this links box is available online. 


\title{
Apoptosis in Myeloid Cells: Molecular Insights into Disease Processes
}

\section{Granulocyte apoptosis: who would work with a 'real' inflammatory cell?}

\author{
I. Dransfield' and A.G. Rossi \\ MRC Centre for Inflammation Research, University of Edinburgh Medical School, Teviot Place, Edinburgh EH8 9AG, U.K.
}

\begin{abstract}
The neutrophil granulocyte is a key factor in cellular innate defence mechanisms against infection or tissue damage. Granulocyte apoptosis is now acknowledged to have a critical role in progression of inflammatory responses. Granulocytes are preprogrammed to die with important physiological mechanisms for noninflammatory clearance. Shutdown of secretory capacity represents an important aspect of the programme of biochemical events that accompany neutrophil apoptosis together with surface molecular changes that serve to identify apoptotic cells as targets for phagocytic removal. Defining the underlying regulatory mechanisms together with the changes in patterns of gene/protein expression associated with granulocyte death remains a challenge. Use of novel strategies for inducing cell death will allow biochemical approaches to dissect the underlying pathways. Although study of granulocyte cell death has especial difficulties when compared with other cell types, there are clearly potential benefits for new therapeutic approaches to treat inflammatory diseases.
\end{abstract}

\section{Historical considerations}

Polymorphonuclear leucocytes, particularly the neutrophil granulocyte, are a key factor in cellular innate defence mechanisms against invading micro-organisms or tissue damage [1]. Rapid recruitment of granulocytes to sites of injury or infection is a hallmark of the inflammatory response and is required for effective host defence against pathogenic organisms. In leucocyte adhesion deficiency, genetic mutations in the $\beta 2$ integrin adhesion receptor lead to decreased capacity for emigration of neutrophils from peripheral blood to inflamed sites. In cases of severe deficiency, patients rarely survive childhood, succumbing to overwhelming bacterial infection [2]. Granulocytes are equipped with an impressive arsenal of degradative enzymes contained within intracellular granules and have the potential to produce toxic metabolites that act to limit microbial growth and hasten destruction of pathogens [3]. However, it is only in the last decade or so that

Key words: apoptosis, granulocyte, inflammation, neutrophil.

Abbreviations used: FOXO, forkhead box, class 0 ; NF- $\kappa$ B, nuclear factor $\wedge$; ; PI3K, phosphoinositide 3 -kinase.

'To whom correspondence should be addressed (email i.dransfield@ed.ac.uk). we have begun to understand the important contribution of granulocyte apoptosis in determining the progression and ultimate resolution of inflammatory responses [4]. In this short paper, we will consider recent progress in our understanding of granulocyte apoptosis and the implications for development of new approaches to treat inflammatory disease.

A large number of granulocytes $\left(4 \times 10^{9}\right)$ are released from the bone marrow every hour; studies with infused radiolabelled granulocytes suggest that they circulate in peripheral blood with a half-life of approx. $6 \mathrm{~h}$ before localizing to the spleen, bone marrow or liver [5]. In the absence of defined exogenous signals, granulocytes undergo constitutive apoptosis when cultured in vitro, suggesting that they are preprogrammed to die [6]. Approx. $50 \%$ of the neutrophils remain viable after culture for $15 \mathrm{~h}$. In view of the discrepancy between the circulating half-lives and the survival times in vitro and lack of evidence for significant levels of neutrophil apoptosis occurring in the peripheral circulation, it seems probable that granulocytes may be selectively sequestered in those tissues in which they ultimately meet their fate. Recent studies have demonstrated that the expression of 
CXCR4 (CXC chemokine receptor 4) is induced on neutrophils during in vitro culture, raising the possibility that stromal cell-derived factor can guide 'older' neutrophils from the circulation before these cells undergo apoptosis [7]. However, for extravasated granulocytes present at inflamed sites, it is now clear that the physiological mechanisms for removal must be efficient and non-inflammatory to ensure that effete granulocytes do not cause host tissue damage through the inappropriate release of intracellular contents [4]. Whether stromal cells can specifically trigger the apoptosis of granulocytes to facilitate their clearance remains to be demonstrated.

Granulocytes are competent phagocytes and are capable of recognizing material that has become opsonized by immunoglobulin or complement components, swiftly internalizing and degrading the particulate material in phagolysosomes [8]. Although it is often considered that the granulocyte has little capacity for re-programming its destructive potential, many studies on granulocytes have demonstrated that this is not the case. Indeed, engagement of granulocyte effector functions is exquisitely controlled, and inappropriate neutrophil activation is considered to be an underlying pathogenic feature of many inflammatory diseases [9]. In chronic granulomatous disease, in which assembly of the NADPH oxidase system for production of reactive oxygen species is lost, patients are not able to deal effectively with infectious microorganisms [10]. Uncontrolled release of this same destructive potential may also lead to host tissue damage. It has now been established that an important aspect of the programme of biochemical events that accompany neutrophil apoptosis is that they act to limit the potential for tissue damage via shutdown of the secretory capacity [11], together with surface molecular changes that serve to identify apoptotic cells as targets for phagocytic removal [12-14].

\section{Investigation of granulocyte function in vitro}

Most functional and biochemical studies on granulocytes, including studies on apoptosis, require the isolation of granulocytes from peripheral blood samples, raising issues relating to cell fractionation, including contaminating cell types and the conditions under which cells are cultured. In addition, while blood represents a convenient source of granulocytes, the relevance of in vitro studies on the mechanisms involved in apoptosis of circulating cells to the situation at inflammatory sites is worth considering. Although much effort has been made to ensure 'minimal' activation of isolated cells [15], a number of studies indicate that expression profiles of surface receptors are different on Percoll ${ }^{\mathrm{TM}}$ gradient isolated cells when compared with whole blood, with significantly decreased expression of L-selectin, a sensitive marker of activation, and increased expression of CD11b, a $\beta 2$ integrin family member present in granules within the neutrophil [16]. Inclusion of additional steps to lyse contaminating erythrocytes also acted to compound these effects.
Most of the granulocytes present within tissues would have to migrate across an activated endothelial-cell monolayer. Recent evidence suggests that after transendothelial neutrophil migration, the functional status of the endothelial cell is altered [17]. Probably, neutrophils within tissues also show specific functional alterations after transmigration, particularly in terms of neutrophil survival $[18,19]$. Selectin receptor engagement has been suggested to trigger neutrophil effector function $[20,21]$ and subsequent arrest from vascular flow and transmigration requires the activation of integrin function via G-protein-coupled receptor activity [22]. Thus granulocytes present within tissues are unlikely to be functionally equivalent to the circulating non-activated cells that most researchers strive to isolate. Although the quoted purities of granulocyte preparations appear to be satisfactory (a minimum of $95 \%$ neutrophils), recent studies have highlighted that the presence of low percentages (approx. 1\%) of monocytes can have a marked effect on the responses of granulocytes to inflammatory stimuli [23]. In our experience, mononuclear cells are not often present at these levels in iso-osmotic Percoll ${ }^{\mathrm{TM}}$ gradient preparations. However, similar considerations regarding cell purity must apply to contaminating eosinophils, which can represent up to $5 \%$ of the cells present in a 'neutrophil' preparation. Immunomagnetic cell deletion techniques, as used by Sabroe et al. [23], should perhaps be a prerequisite for functional studies as well as PCR or gene array techniques.

Although cell isolation can be a divisive issue among researchers investigating granulocyte function, the conditions for cell culture also show some variation between research groups. Early studies by the Haslett and Henson group [6], investigating the effects of culturing on granulocyte function, used a glucose-rich medium with autologous platelet-rich plasma-derived 'serum' and Teflon-based culture system that was optimized for cell recovery and survival. While these conditions have been extensively used for the study of the effects of inflammatory mediators, it is clear that cell-culture conditions can influence the 'rate' of apoptosis.

The microenvironment of inflammatory sites is probably quite extreme with low $\mathrm{pH}$ values and decreased oxygen tension and glucose levels when compared with 'normal' physiological settings. Thus established findings regarding factors that have been proposed to regulate apoptosis under normal culture conditions may require re-examination. For example, hypoxic conditions markedly delay neutrophil apoptosis [24] and recent studies have provided strong evidence that neutrophils may be particularly adapted to functioning under low oxygen conditions [25]. Hypoxia induces the transcription factor HIF1 $\alpha$ (hypoxia-inducible factor $1 \alpha$ ) to promote NF- $\kappa \mathrm{B}$ (nuclear factor $\kappa \mathrm{B}$ )-dependent gene expression, which has previously been shown to have a central role in the regulation of granulocyte survival [26]. Although the precise molecular mechanisms by which hypoxia exerts these regulatory effects remain to be defined, exogenous factors may have distinct effects under hypoxic conditions. 


\section{Control of granulocyte apoptosis}

Therapeutically, we and others believed previously that selective induction of granulocyte apoptosis (when combined with accelerated clearance pathways) may have beneficial effects with respect to progression of inflammation, favouring the resolution of chronic inflammatory conditions [27]. Molecular characterization of the proteins involved in granulocyte apoptosis has been problematic in view of the difficulties associated with transfection of these cells. One exciting possibility is that protein-transduction approaches will allow the manipulation of protein levels within neutrophils and a definitive demonstration of a regulatory role [28]. It has long been known that treatment with translation inhibitors (e.g. cycloheximide) or transcription inhibitors (e.g. actinomycin D) accelerate the constitutive rate of apoptosis [29]. Indeed, these treatments can drive apoptosis triggered by ligation of death domain-containing receptors such as Fas or TNFR1 and TNFR2 [26]. Together, these results strongly suggest that granulocyte survival requires the production of a labile survival protein. The precise identity of the survival protein(s) generated in neutrophils has not been identified. One strong candidate protein with a key role in neutrophil apoptosis has been identified by biochemical examination of the role of bcl-2 family members in neutrophils. Edwards and co-workers [30] have identified Mcl-1; unlike Bax, $\mathrm{Bak}$ or Bik, $\mathrm{Mcl}-1$ is a rapidly turned over anti-apoptotic protein and shows regulation by cytokines such as GM-CSF (granulocyte/macrophage colony-stimulating factor), which act to delay the constitutive rate of apoptosis in granulocytes [30]. It is possible that the levels of key regulatory proteins are controlled entirely at the post-transcriptional level, e.g. via a proteolytic mechanism as described for Mcl-1.

Although non-transcriptional mechanisms certainly play a role in the control of granulocyte survival, it is clear that the neutrophil is not silent in terms of the capacity for gene transcription. Indeed, the central role identified for NF- $\kappa$ B [26] would be consistent with an important contribution for altered patterns of gene expression. Recent studies have identified a role for the FOXO (forkhead box, class $\mathrm{O}$ ) subfamily of transcription factors in the control of myeloid cell differentiation and apoptosis. FOXO transcription factors are targets for PI3K/Akt (where PI3K stands for phosphoinositide 3-kinase) and their activity is regulated by phosphorylation status. Constitutively active FOXO can induce the expression of Bim and drive apoptosis in neuronal cells [31]. More recently, studies on fMLP ( $N$-formylmethionylleucylphenylalanine)-induced activation in neutrophils suggest that FOXO may bind Mcl-1 and support survival [32]. One of the problems with microarray approaches to identify the genes involved in control of apoptosis is the sheer number of altered gene products that can be defined. For example, the studies by DeLeo and co-workers $[33,34]$ have shown more than 500 gene products that exhibit significant changes after phagocytosis-induced apoptosis in human neutrophils. Although defining the meaningful changes in the patterns of gene expression associated with granulocyte death remains a challenge, potential insights into the underlying control mechanisms will undoubtedly be worth the effort.

\section{Signalling for cell death}

Time-lapse video microscopy analysis of granulocyte cell death reveals the very active nature of the membrane and cytoskeletal alterations that either accompany or precede nuclear morphological changes or surface membrane alterations. In view of the well-defined signalling pathways associated with agents that act to either induce or delay apoptosis, studies with pharmacological inhibitors suggest a key role for intracellular kinases, including PI3K and protein kinase $\mathrm{C} \delta[35]$. PI3K inhibition reversed the GMCSF-mediated survival responses that were associated with phosphorylation and cytosolic localization of Bad [36]. Surprisingly, when we dissected the effects of increase in intracellular cAMP with dibutyryl cAMP on delay of neutrophil apoptosis, we found that activation of protein kinase A could not account for the observed effect. Instead, our results suggested a non-transcriptional survival mechanism, with a critical role for proteasome activity [37]. One possible mechanism could be that targeted degradation of a death protein is part of the normal granulocyte survival pathway, with reversal of the cAMP effect after inhibition of proteasome activity. The interesting recent results of Lord and co-workers [38] suggest that DISCs (death-inducing signalling complexes) may be associated with lipid rafts, possibly even in the absence of ligands. Careful biochemical analysis suggests that aggregation of receptors within lipid microdomains may allow spontaneous DISC formation and activation of downstream caspase execution pathways.

\section{Synchronous apoptosis}

One of the problems with biochemical analysis of neutrophil apoptosis is the relatively heterogeneous manner in which neutrophils enter apoptosis during constitutive apoptosis. Although it may be possible to investigate biochemical changes associated with 'end-stage' apoptosis, the molecular events occurring during the active phase of cell death are more difficult. Thus, although certain treatments can accelerate the rate of granulocyte apoptosis, the percentage of cells actually undergoing apoptosis at any point in time is relatively low. We have begun to define new culturing methods that allow rapid and synchronous induction of apoptosis. Ligation of death receptors while blocking either protein synthesis or NF- $\kappa \mathrm{B}$ activation results in rapid and synchronous apoptosis [26]. Another novel experimental approach is the use of temperature shift to induce 'synchronous' apoptosis. Neutrophils are incubated at low temperature $\left(15^{\circ} \mathrm{C}\right)$ for several hours before rewarming to $37 \mathrm{C}$, which is accompanied by a rapid burst of apoptosis [39]. These methods may allow the identification of novel regulatory factors and provide further insights into the mechanisms that underlie the clearance of apoptotic cells from tissues. However, one major problem for biochemical analysis of 
neutrophil functional responses is that the proteolytic activity of granule contents can degrade proteins after cellular lysis. We and others have found that the most potent anti-protease strategies (e.g. di-isopropyl phosphorofluoridate + 'extra strong' protease inhibitor cocktails) are required to ensure that the most labile proteins, e.g. NF- $\kappa$ B and STAT (signal transduction and activators of transcription) proteins, can be detected. Whether the low levels of cytochrome $c$ detected in neutrophils were a consequence of proteolysis or merely reflected the decreased number of mitochondria containing cytochrome $c$ is not clear.

The role of mitochondria in neutrophils is still somewhat controversial. Mitochondria can be readily detected with Mitotracker dyes and the presence of mitochondria can be validated by quantification of mitochondrial DNA and proteins such as porin $[40,41]$. However, these mitochondria may not be functional in terms of generation of ATP, using neutrophil production of ATP by the process of glycolysis. In addition, biochemical analysis of the components present in neutrophil mitochondria suggests very low levels of cytochrome $c$ and other well-characterized mitochondrial proteins, e.g. heat-shock protein 60 [42]. One interesting approach to examine the mechanisms of neutrophil cell death is the use of cytoplasts that are free of nuclear mitochondria. Using this approach, Kuijpers and co-workers [40] have shown that other proapoptotic factors that can be released from mitochondria, such as SMAC/Diablo, may have a critical role in neutrophil apoptosis [40].

\section{Surface membrane changes associated with granulocyte death}

One well-established characteristic of apoptosis is exposure of phosphatidylserine on the outer leaflet of the plasma membrane, a consequence of loss of phospholipid translocase activity [12]. Some years ago, we reported that expression of CD16 was markedly down-regulated on the surface of neutrophils that had undergone apoptosis, suggesting that there may also be cell-type-specific membrane alterations [14]. Subsequent studies suggested a co-ordinated programme of surface changes that accompany neutrophil death, including shedding of CD62L and down-regulation of a number of other molecules on the neutrophil plasma membrane that may uncouple pro-inflammatory responses of neutrophils to exogenous stimuli [43]. Activity of receptors such as integrins may be down-regulated independent of changes in receptor expression, again consistent with attenuation of neutrophil function. Whether the repertoire of surface changes mark the apoptotic neutrophil for recognition by phagocytes is not clear. Early studies indicated that molecular changes in glycoproteins constitutively expressed on the surface of leucocytes may confer phagocyte recognition. For example, ICAM-3 (intercellular cell-adhesion molecule 3), which acts as a ligand for LFA-1 (lymphocyte function associated antigen 1)-mediated adhesion, ceases to bind LFA-1 after induction of apoptosis [44]. We have recently demonstrated that Fc $\gamma$ RII is 'enabled' after apoptosis, allowing the binding of immunocomplexes, which may promote proinflammatory macrophage responses after phagocytosis [45].

In conclusion, the investigation of the mechanisms of granulocyte cell death is difficult when compared with other cell types. However, the benefits of working with this important inflammatory cell type in terms of its therapeutic potential for inflammatory disease outweigh the disadvantages.

\section{References}

1 Haslett, C., Savill, J.S. and Meagher, L. (1989) Curr. Opin. Immunol. 2 10-18

2 Anderson, D.C. and Springer, T.A. (1987) Annu. Rev. Med. 38, 175-194

3 Borregaard, N. and Cowland, J.B. (1997) Blood 89, 3503-3521

4 Haslett, C., Savill, I.S. Whyte, M.K. Stern, M., Dransfield, I. and Meagher, L.C. (1994) Philos. Trans. R. Soc. Lond. B 345, 327-333

5 Haslett, C., Shen, A.S., Feldsien, D.C., Allen, D., Henson, P.M. and Cherniack, R.M. (1989) Am. Rev. Respir. Dis, 140, 756-763

6 Savill, J.S., Wyllie, A.H., Henson, J.E., Walport, M.J., Henson, P.M. and Haslett, C. (1989) J. Clin. Invest. 83, 865-875

7 Martin, C., Burdon, P.C., Bridger, G., Gutierrez-Ramos, J.C., Williams, T.J and Rankin, S.M. (2003) Immunity 19, 583-593

8 Stossel, T.P. (1978) Ann. Intern. Med. 89, 398-402

9 Weiss, S.J. (1989) N. Engl. J. Med. 320, 365-376

10 Segal, A.W. (1996) Mol. Med. Today 2, 129-135

11 Whyte, M.K., Meagher, L.C., MacDermot, J. and Haslett, C. (1993) J. Immunol. 150, 5124-5134

12 Homburg, C.H., de Haas, M., dem Borne, A.E., Verhoeven, A.J., Reutelingsperger, C.P. and Roos, D. (1995) Blood 85, 532-540

13 Hart, S.P., Ross, J.A., Ross, K., Haslett, C. and Dransfield, I. (2000) Cell Death Differ. 7, 493-503

14 Dransfield, I., Buckle, A.M., Savill, J.S., McDowall, A., Haslett, C. and Hogg, N. (1994) J. Immunol. 153, 1254-1263

15 Haslett, C., Guthrie, L.A., Kopaniak, M.M., Johnston, Jr, R.B. and Henson, P.M. (1985) Am. J. Pathol. 119, 101-110

16 Macey, M.G., McCarthy, D.A., Vordermeier, S., Newland, A.C. and Brown, K.A. (1995) J. Immunol. Methods 181, 211-219

17 Cepinskas, G., Savickiene, J., lonescu, C.V. and Kvietys, P.R. (2003) J. Cell Biol. 161, 641-651

18 Coxon, A., Tang, T. and Mayadas, T.N. (1999) J. Exp. Med. 190, 923-934

19 Watson, R.W., Rotstein, 0.D., Nathens, A.B., Parodo, J. and Marshall, J.C. (1997) J. Immunol. 158, 945-953

20 Ruchaud-Sparagano, M.H., Drost, E.M., Donnelly, S.C., Bird, M.I., Haslett, C. and Dransfield, I. (1998) Eur. J. Immunol. 28, 80-89

21 Simon, S.I., Hu, Y., Vestweber, D. and Smith, C.W. (2000) J. Immunol 164, $4348-4358$

22 Tonnesen, M.G., Anderson, D.C., Springer, T.A., Knedler, A., Avdi, N. and Henson, P.M. (1989) J. Clin. Invest. 83, 637-646

23 Sabroe, I., Jones, E.C., Usher, L.R., Whyte, M.K. and Dower, S.K. (2002) J. Immunol. 168, 4701-4710

24 Hannah, S., Mecklenburgh, K., Rahman, I., Bellingan, G.J., Greening, A. Haslett, C. and Chilvers, E.R. (1995) FEBS Lett. 372, 233-237

25 Cramer, T, and johnson, R.S. (2003) Cell Cycle 2, 192-193

26 Ward, C., Chilvers, E.R., Lawson, M.F., Pryde, J.G., Fujihara, S., Farrow, S.N., Haslett, C. and Rossi, A.G. (1999) J. Biol. Chem. 274, 4309-4318

27 Ward, C., Dransfield, I., Chilvers, E.R., Haslett, C. and Rossi, A.G. (1999) Trends Pharmacol. Sci. 20, 503-509

28 Han, H., Fuortes, M. and Nathan, C. (2003) J. Exp. Med. 197, 63-75

29 Whyte, M.K., Savill, J., Meagher, L.C., Lee, A. and Haslett, C. (1997) J. Leukoc. Biol. 62, 195-202

30 Moulding, D.A., Quayle, J.A., Hart, C.A. and Edwards, S.W. (1998) Blood 92, 2495-2502

31 Gilley, J., Coffer, P.J. and Ham, J. (2003) J. Cell Biol. 162, 613-622

32 Crossley, L.J. (2003) J. Leukoc. Biol. 74, 583-592

33 Kobayashi, S.D., Voyich, J.M., Somerville, G.A., Braughton, K.R., Malech, H.L. Musser, J.M. and Deleo, F.R. (2003) J. Leukoc. Biol. 73, 315-322

34 Kobayashi, S.D., Braughton, K.R., Whitney, A.R., Voyich, J.M., Schwan, T.G. Musser, J.M. and Deleo, F.R. (2003) Proc. Natl. Acad. Sci. U.S.A. 100, 10948-10953

35 Pongracz, J., Webb, P., Wang, K., Deacon, E., Lunn, 0.J. and Lord, J.M. (1999) J. Biol. Chem. 274, 37329-37334

36 Cowburn, A.S., Cadwallader, K.A., Reed, B.J., Farahi, N. and Chilvers, E.R (2002) Blood 100, 2607-2616 
37 Martin, M.C., Dransfield, I., Haslett, C. and Rossi, A.G. (2001) J. Biol. Chem. 276, 45041-45050

38 Scheel-Toellner, D., Wang, K., Singh, R., Majeed, S., Raza, K., Curnow, S.J., Salmon, M. and Lord, J.M. (2002) Biochem. Biophys. Res. Commun. 297, 876-879

39 Pryde, J.G., Walker, A., Rossi, A.G., Hannah, S. and Haslett, C. (2000) J. Biol. Chem. 275, 33574-33584

40 Maianski, N.A., Geissler, J., Srinivasula, S.M., Alnemri, E.S., Roos, D. and Kuijpers, T.W. (2004) Cell Death Differ. 11, 143-153

41 Fossati, G., Moulding, D.A., Spiller, D.G., Moots, R.J., White, M.R. and Edwards, S.W. (2003) J. Immunol. 170, 1964-1972
42 Murphy, B.M., O'Neill, A.J., Adrain, C., Watson, R.W. and Martin, S.J. (2003) J. Exp. Med. 197, 625-632

43 Dransfield, I., Stocks, S.C. and Haslett, C. (1995) Blood 85, 3264-3273

44 Moffatt, O.D., Devitt, A., Bell, E.D., Simmons, D.L. and Gregory, C.D. (1999) J. Immunol. 162, 6800-6810

45 Hart, S.P., Jackson, C., Kremmel, L.M., McNeill, M.S., Jersmann, H., Alexander, K.M., Ross, J.A. and Dransfield, I. (2003) Am. J. Pathol. 162, $1011-1018$

Received 9 December 2003 


\title{
Getting to grips with the granulocyte: manipulation of granulocyte behaviour and apoptosis by protein transduction methods
}

\author{
A.G. Rossi ${ }^{1}$, C. Ward and I. Dransfield \\ MRC Centre for Inflammation Research, University of Edinburgh Medical School, Teviot Place, Edinburgh EH8 9AG, U.K.
}

\begin{abstract}
Human granulocytes clearly play an important role in host defence against invading organisms, however they have also been implicated in the pathogenesis and progression of many chronic inflammatory diseases. In addition, these cells have been paramount in gaining a better understanding of many key-signalling pathways regulating fundamental biological processes. Since granulocytes are terminally differentiated and undergo relatively rapid constitutive apoptosis it has been difficult to manipulate intracellular events by transfection or transduction procedures. It has been shown in recent years that the HIV-TAT protein transduction system can be successfully used in granulocytes to manipulate key signalling mechanisms regulating functional responsiveness and survival. In this paper, we review recent literature highlighting important developments using this system in isolated human granulocytes and in inflammatory process in vivo where these cells play a prominent role.
\end{abstract}

The human polymorphonuclear neutrophil has been an invaluable and excellent cell for studies elucidating signal transduction mechanisms, especially signalling pathways involving phospholipid turnover (e.g. phosphatidylinositol metabolism, phospholipase $\mathrm{C}$ and $\mathrm{D}$ activity) and kinase systems [e.g. protein kinase C and PI3K (phosphoinositide 3kinase)]. The primary reasons for their use are that they are relatively easy to purify and high numbers can be readily obtained from blood of human volunteers. Neutrophils have obvious direct relevance to inflammatory processes, and findings obtained using these cells have clinical implications for the development of therapy for chronic inflammatory diseases, such as chronic obstructive pulmonary disease, rheumatoid arthritis, glomerulonephritis, myocardial reperfusion injury etc. However, there are some variables and factors that have to be considered. For example, variations in cellular responses are often observed using cells isolated from different donors and even from cells isolated at different times from the same donor. The culture conditions used can also determine the outcome of cellular responses. In particular, the density of neutrophils in culture can influence the rate of constitutive apoptosis [1]. The culture medium used is also an important factor that needs to be considered, e.g. cation (e.g. $\mathrm{Ca}^{2+}$ and $\mathrm{Mg}^{2+}$ ) concentrations, the presence of proteins (e.g. bovine albumin or serum versus human albumin or serum) and other constituents (e.g. Hepes, phenol red, nutrients etc.) may significantly affect cellular activity. The mode of cell isolation is also critically

Key words: apoptosis, eosinophil, granulocyte, HIV-TAI, neutrophil, protein transduction. Abbreviations used: ERK, extracellular-signal-regulated kinase; LPL, t-plastin; NF- $\kappa$ B, nudear factor $\kappa$ B; PI3K, phosphoinositide 3-kinase; PID, protein transduction domain; Pyk2, proline-rich tyrosine kinase.

'To whom correspondence should be addressed (email a.g.rossi@ed.ac.uk). important since methods often require red cell lysis, changes of temperature during centrifugation steps, different reagents used during sedimentation (e.g. using gelatin, dextran etc.) and centrifugation (through gradients made with Percoll, Hypaque-Ficoll etc.) that can influence the biological activity of these cells. Recently, it has been shown that low-level contamination of other cells especially monocytes or eosinophils in neutrophil preparations may influence cellular function [2]. To remove contaminating cells, an extra procedure has to be introduced that uses antibody-dependent positive selection of the contaminating cells, a process that requires careful quality control assessment ensuring that neutrophil function remains unaffected. Another important issue that has to be addressed is the problem associated with protein degradation due to the high amounts of proteases inherently found in neutrophils and eosinophils. This has largely been remedied using techniques that use cocktails containing high concentrations of very powerful proteases $[3,4]$. Another consideration when working with granulocytes is the difficulty in transfecting these cells, since they are terminally differentiated and do not undergo cell division. However, it is now emerging that it is possible to manipulate key processes in granulocytes using efficient and rapid protein transduction techniques. It is crucial that any technique used to introduce proteins into granulocytes is fast since these cell die rapidly by apoptosis, a process that is underway within hours if not minutes once these cells have left the bone marrow. Furthermore, it is critical that techniques used to introduce proteins into these cells do not interfere nonspecifically with biological processes especially since cellular responsiveness of granulocytes can be easily influenced. In this paper, we have highlighted the attempts made by researchers to introduce proteins into granulocytes to 
Figure 1| Protein transduction in granulocytes using PTDs For the transduction of proteins into granulocytes, the protein of interest is linked to positively charged PIDs (usually the 11-aminoacid HIV-TAT peptide). The protein enters the cell and/or nucleus via a predominately passive interaction with the negatively charged outer or nuclear membrane to exert biological effects.

\section{Granulocyte}

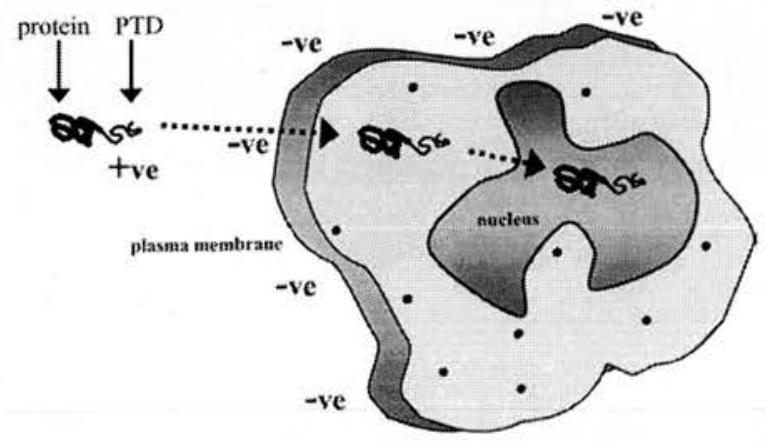

manipulate key signalling events and processes involved in regulating functional responsiveness and survival. However, there are several recent excellent reviews describing protein transduction technology in general that provide up to date information [5-9].

Unless a specific transporter system is active, all viable cells that have an intact cell membrane will exclude nonspecific entry of macromolecules ( $>500 \mathrm{Da}$ ). There have been a number of techniques that have attempted to address this issue to introduce molecules into granulocytes to target specific intracellular targets. These techniques (including hypo-osmotic shock, electroporation, digitonin or saponin permeabilization and use of lipofectin) have had some limited success but in general it is likely that these procedures may non-specifically affect granulocyte function and therefore their usefulness is somewhat limited. More recently, it has been recognized that there are a relatively small number of proteins that are capable of entering directly into cells using as yet ill-defined mechanisms [5-9]. There are three well- established techniques and systems that have been successfully used to transduce proteins or peptides, compounds, nucleic acids and particles into both transformed and primary cells. All these techniques involve the use of small peptides or proteins that possess the ability to traverse the plasmalemma, or indeed nuclear membranes, using mechanisms that are generally believed to involve a passive mechanism that does not involve active transport, although there is evidence that the mode of entry may depend on the protein involved. Essentially, the three main systems involve the HIV-TAT (transactivator of transcription) protein transduction system, the Drosophila Antennapedia homoeotic transcription factor and the herpes simplex virus-1 DNAbinding protein, VP22. The protein segment responsible for actual cell entry has been termed the PTD (protein transduction domain). HIV-TAT technology has been the most successful mode of protein transduction in human granulocytes. The technique involves the synthesis of a fusion protein whereby the protein of interest is linked to the HIVTAT transduction domain (a small peptide comprising the 11 amino acids YGRKKRRQRRR) using bacterial expression vectors. Procedures involve sonication of the bacterial pellet and denaturing the recombinant protein. The protein is then purified, usually involving $\mathrm{Ni}^{2+}$-nitrilotriacetateagarose affinity column procedures, often followed by further purification and quality control steps. Cells in culture can then be treated directly with the purified protein. Protein transduction is concentration-dependent and highly efficient with reports of almost $100 \%$ of cells being transduced within $10 \mathrm{~min}$. In addition, TAT-fusion proteins have been successfully used in experimental in vivo scenarios (see Figure 1).

There is now good recent evidence showing that HIV-TAT protein transduction in neutrophils and eosinophils is viable and highly successful (Table 1). One of the first reports of this technology being successfully used in human neutrophils was described by Jones et al. [10]. These authors investigated whether LPL (L-plastin), a leucocyte-specific actin-bundling protein, was involved in leucocyte integrin function. For this, a synthetic LPL-TAT peptide was developed, which was derived from the region of the LPL that contains a serine residue required for phosphorylation and linked to the

Table 1 | The use of HIV-TAT-linked peptides in human granulocytes

\begin{tabular}{|c|c|c|c|}
\hline Granulocyte type & HIV-TAT linked peptide target & Primary functions investigated & Reference \\
\hline Neutrophil & LPL (an actin bundling protein) & Adhesion & {$[10]$} \\
\hline Neutrophil & $\beta 3$-integrin cytoplasmic tail & Chemotaxis and transmigration & {$[11]$} \\
\hline Neutrophil & Pyk2 & Spreading, respiratory burst, degranulation and killing of bacteria & {$[12]$} \\
\hline Neutrophil & NEMO (IKK $\gamma$ ) binding domain & Apoptosis and survival & {$[15]$} \\
\hline Eosinophil & $\begin{array}{l}\text { RhoA (a Ras superfamily member of } \\
\text { GIP-binding proteins) }\end{array}$ & Cell adhesion and detachment & {$[16]$} \\
\hline Eosinophil & H-Ras & Survival & {$[17]$} \\
\hline Eosinophil & Y-box-binding factor 1 & Apoptosis and survival & [18] \\
\hline Eosinophil & Ras & Adhesion, airway inflammation and hyperresponsiveness & {$[19,21]$} \\
\hline Eosinophil & $\mathrm{PI} 3 \mathrm{~K}$ & PLA2 activation, airway inflammation and hyperresponsiveness & {$[20,22]$} \\
\hline
\end{tabular}


TAT domain. These authors showed that these LPL-derived proteins rapidly (within $15 \mathrm{~min}$ ) enter human leucocytes (neutrophils and monocytes) and induce leucocyte integrinmediated adhesion. Interestingly, substitution of alanine for the serine abolished the ability of the peptide to induce adhesion. Thus by using such TAT-linked peptides, as well as other supportive evidence, these authors concluded that there is a novel role for LPL phosphorylation in the regulation of leucocyte integrin-mediated adhesion. The role of integrins in neutrophil responses was further developed by Bruyninckx et al. [11], who used HIV-TAT peptides corresponding to the $\beta 3$-integrin cytoplasmic tail with modified tyrosine residues and identified the functional tyrosine residue responsible for its activation.

An important recent study by $\mathrm{Han}$ et al. [12], used a dominant-negative protein where the C-terminus of Pyk2 (proline-rich tyrosine kinase) fused to a TAT peptide but not other regions of Pyk2, inhibited specifically the respiratory burst of neutrophil responding to tumour necrosis factor, Salmonella or Listeria but not the response induced by the phorbol ester PMA. This study describes in detail the problems that can be encountered using this technology. They assessed uptake of fluorescently labelled TAT-tagged proteins using flow cytometric techniques and demonstrated that uptake depended on the method used to purify the peptides. For example, purification by ion exchange followed by gel permeation chromatography led to efficient and maximal uptake by $30 \mathrm{~min}$. Importantly, other factors eluting from the column could induce toxic responses in the cells. However, if the peptides were desalted by membrane filtration they were not toxic. Uptake also increased with time over a period of $120 \mathrm{~min}$ at $37^{\circ} \mathrm{C}$. Surprisingly perhaps, and unlike some other TAT peptides, there was no detectable uptake at $4 \mathrm{C}$. In addition, there was no saturation of uptake with concentrations of TAT peptides up to $1 \mu \mathrm{M}$ and fluorescence was almost entirely intracellular. Interestingly, evidence was presented showing that lysosomal degradation can limit the accumulation and intracellular retention of the fluorescently tagged TAT protein. This study showed that optimal uptake required the presence of cations $\mathrm{Ca}^{2+}$ and $\mathrm{Mg}^{2+}$ and was insensitive to $\mathrm{pH}$ over the range 6.8-7.4. The authors chose peptides desalted by membrane filtration and a loading time of $30 \mathrm{~min}$ as this efficiently labelled the cells by approx. $90 \%$. It was also noted that the endosomal compartment degraded the large amount of peptide that entered the neutrophils with only minute amounts reaching the cytosol. These authors attempted to introduce proteins using the Antennapedia system or a carrier peptide modelled on the sequence of the $\beta 3$ integrin shown to enter myeloid cells directly. The authors were successful with only the HIVTAT system and concluded that TAT-based transduction was the only approach that worked consistently in human neutrophils. Another important issue raised by Han et al. [12] is that, as mentioned above, the way the peptides are purified will also determine their relative toxicity and the fact that the peptides are prepared by bacterial expression systems means there is a realistic risk of introducing LPS (lipopolysaccharide) contamination. This latter point is particularly relevant in the investigation of neutrophil biology since LPS can stimulate human neutrophil responsiveness as well as influence rate of apoptosis and survival.

Recently, the HIV-TAT system has been successfully used in neutrophils and eosinophils to investigate signalling mechanisms regulating survival. We originally showed that $\mathrm{NF}-\kappa \mathrm{B}$ (nuclear factor $\kappa \mathrm{B}$ ) plays a critical role in human granulocyte apoptosis $[13,14]$, since blockade of NF- $\kappa \mathrm{B}$ activation by pharmacological inhibitors induced apoptosis in granulocytes and sensitized these cells to apoptosis induced by tumour necrosis factor $\alpha$. These observations have recently been confirmed using the HIV-TAT protein transduction approach. Choi et al. [15] developed a specific inhibitor of NF- $\kappa$ B linked to a TAT-PTD to shuttle the inhibitor into human neutrophils. The peptide generated contained a NEMO $\left(\mathrm{NF}_{\kappa} \mathrm{B}\right.$ essential modulator)-binding domain that selectively inhibited IKK $\gamma(\mathrm{NEMO}) / \mathrm{IKK} \beta$ interaction to prevent agonistinduced NF- $\kappa \mathrm{B}$ activation. Using this TAT peptide, constitutive apoptosis was accelerated and LPS-induced delay of apoptosis was attenuated. These authors carefully assessed the uptake of the TAT peptides using a combination of flow cytometry and confocal microscopy quality control checks. It was observed that almost $100 \%$ of the neutrophils were rapidly (within 10-20 min) stained with FITC-TAT, FITC-NEMO-binding domain and a FITC-control mutant peptide, with no non-specific effects of the TAT peptides. Interestingly, these authors reported (results not shown) that FITC-TAT peptides are effectively transduced into the entire neutrophil population in whole blood. This latter observation opens up the possibility of manipulation of intracellular pathways without the issues raised above concerning isolation procedures. To confirm our observation describing a role of $\mathrm{NF}-\kappa \mathrm{B}$ in eosinophil survival, we have recently generated a TAT peptide fused to a mutant of $\mathrm{I} \kappa \mathrm{B} \alpha$ (S32A and S36A) that is resistant to phosphorylation and proteasomal degradation. This TAT peptide efficiently blocked nuclear translocation of $\mathrm{NF}-\kappa \mathrm{B}$ in eosinophils and accelerated eosinophil apoptosis (S. Fujihara, R.T. Hay and A.G. Rossi, unpublished work).

There are now a number of publications demonstrating successful use of the HIV-TAT technology in eosinophils. The first publication to our knowledge to use a TAT peptide in eosinophils was by Alblas et al. [16]. These authors introduced, into human eosinophils, a TAT-fusion construct containing active RhoA, a member of the Ras superfamily of small GTP-binding proteins that controls actin stress fibre formation and focal adhesions. It was reported that in the presence of the chemoattractant platelet-activating factor the construct could stimulate detachment. Although the effect of the construct increased with time as concentrations of the peptide accumulated in the cells, the effect was lost after $45 \mathrm{~min}$. The precise reason for this rapid loss of activity is unknown; however, it is possible that TAT peptides may be leaching out of the cell or that they are prone to proteolytic degradation. Interestingly, it was noted that in a small number of experiments the TAT construct had no effect. Although no explanation was presented, it is possible that the activation 
status of the eosinophils or donor variability may have been responsible.

It was subsequently shown that a TAT-linked dominantnegative $\mathrm{H}$-Ras introduced into eosinophils inhibited ERK (extracellular-signal-regulated kinase) activation and interleukin-5-induced cell viability [17]. The TAT peptide entered eosinophils in a concentration- and time-dependent manner with $>95 \%$ efficiency. Indeed, it was demonstrated that this TAT construct entered eosinophils within $20 \mathrm{~min}$ of treatment and effectively blocked the activation of ERK1 and ERK2 for at least $1 \mathrm{~h}$. Interestingly, eosinophil viability prolonged by interleukin- 5 was blocked by the construct even when measured after $60 \mathrm{~h}$ of eosinophil culture. In addition, another study investigated the mechanisms controlling GM-CSF (granulocyte/monocyte colony-stimulating factor) induced by effective use of the HIV-TAT technology [18]. A human Y-box-binding factor 1 linked to TAT, but not a control peptide (TAT- $\beta$-galactosidase), promoted eosinophil survival by stabilizing GM-CSF mRNA. Myuo and coworkers [19-22] have taken advantage of the HIV-TAT system to investigate a number of biological processes in eosinophils in vitro and inflammatory processes involving eosinophils in vivo. For example, they have used an HIVTAT dominant-negative Ras to block focal clustering and active conformation in $\beta 2$-integrin-mediated adhesion of eosinophils to intercellular cell-adhesion molecule 1 [19]. In addition, these authors have used an HIV-TAT-linked dominant-negative form of class $1 \mathrm{~A}$ PI3K adapter subunit to block fMLP ( $N$-formylmethionyl-leucylphenylalanine)stimulated phosphorylation of the downstream target of PI3K, protein kinase B, in human eosinophils [20]. Such effects helped to demonstrate that group IV cytosolic phospholipase $\mathrm{A}_{2}$ activation occurs by PI3K through a mitogenactivated protein kinase independent pathway [20]. Importantly, this group $[21,22]$ has now shown that these HIV-TAT constructs can be used successfully in vivo as has been described previously using other TAT constructs $[23,24]$. These proteins were administered $(3-10 \mathrm{mg} / \mathrm{kg}$ ) intraperitoneally into mice to show that Ras and PI3K are key signalling molecules involved in airway inflammation and bronchial hyper-responsiveness. Importantly, relevant control peptides did not have significant effects on airway inflammation [21,22].

In conclusion, the HIV-TAT system has been successfully used to transduce proteins into isolated granulocytes to provide incisive evidence for the involvement of specific signalling pathways. Furthermore, HIV-TAT technology can be used in vivo to investigate inflammatory processes where these cells play an important role. Thus use of this technology will probably increase exponentially and undoubtedly will provide exciting advances in understanding granulocyte cell biology in vitro and inflammatory processes in vivo.

\section{References}

1 Hannah, S., Nadra, I., Dransfield, I., Pryde, J.G., Rossi, A.G. and Haslett, C. (1998) FEBS Lett. 421, 141-146

2 Sabroe, I., Jones, E.C., Usher, L.R., Whyte, M.K. and Dower, S.K. (2002) J. Immunol. 168, 4701-4710

3 McDonald, P.P., Bovolenta, C. and Cassatella, M.A. (1998) Biochemistry 37, 13165-13173

4 Ward, C., Dransfield, I., Murray, J., Farrow, S.N., Haslett, C. and Rossi, A.G. (2002) J. Immunol. 168, 6232-6243

5 Green, I., Christison, R., Voyce, C.J., Bundell, K.R. and Lindsay, M.A. (2003) Trends Pharmacol. Sci. 24, 213-215

6 Vives, E., Richard, J.P., Rispal, C. and Lebleu, B. (2003) Curr. Protein Pept. Sci. 4, 125-132

7 Lindsay, M.A. (2002) Curr. Opin. Pharmacol. 2, 587594

8 Ford, K.G., Souberbielle, B.E., Darling, D. and Farzaneh, F. (2001) Gene Ther. 8, 1-4

9 Snyder, E.L. and Dowdy, S.F. (2001) Curr. Opin. Mol. Ther, 3, 147-152

10 Jones, S.L., Wang, J., Turck, C.W. and Brown, E.J. (1998) Proc. Natl. Acad. Sci. U.S.A. 95, 9331-9336

11 Bruyninckx, W.J., Comerford, K.M., Lawrence, D.W. and Colgan, S.P. (2001) Blood 97, 3251-3258

12 Han, H., Fuortes, M. and Nathan, C. (2003) J. Exp. Med. 197, 63-75

13 Ward, C., Chilvers, E.R., Lawson, M.F., Pryde, J.G., Fujihara, S., Farrow, S.N. Haslett, C. and Rossi, A.G. (1999) J. Biol. Chem. 274, 4309-4318

14 Fujihara, S., Ward, C., Dransfield, I., Hay, R.T., Uings, I.J., Hayes, B., Farrow, S.N., Haslett, C. and Rossi, A.G. (2002) Eur. J. Immunol. 32, 457-466

15 Choi, M., Rolle, S., Wellner, M., Cardoso, M.C., Scheidereit, C., Luft, F.C. and Kettritz, R. (2003) Blood 102, 2259-2267

16 Alblas, J., Ulfman, L., Hordijk, P. and Koenderman, L. (2001) Mol. Biol. Cell 12, 2137-2145

17 Hall, D.J., Cui, J., Bates, M.E., Stout, B.A., Koenderman, L., Coffer, P.J. and Bertics, P.J. (2001) Blood 98, 2014-2021

18 Capowski, E.E., Esnault, S., Bhattacharya, S. and Malter, J.S. (2001) J. Immunol. 167, 5970-5976

19 Myou, S., Zhu, X., Boetticher, E., Myo, S., Meliton, A., Lambertino, A., Munoz, N.M. and Leff, A.R. (2002) J. Immunol. 169, 2670-2676

20 Myou, S., Leff, A.R., Myo, S., Boetticher, E., Meliton, A.Y., Lambertino, A.T., Liu, J., Xu, C., Munoz, N.M. and Zhu, X. (2003) J. Immunol. 171, 4399-4405

21 Myou, S., Zhu, X., Myo, S., Boetticher, E., Meliton, A.Y., Liu, J., Munoz, N.M. and Leff, A.R. (2003) J. Immunol. 171, 4379-4384

22 Myou, S., Leff, A.R., Myo, S., Boetticher, E., Tong, J., Meliton, A.Y., Liu, J., Munoz, N.M. and Zhu, X. (2003) J. Exp. Med. 198, 1573-1582

23 Schwarze, S.R. and Dowdy, S.F. (2000) Trends Pharmacol. Sci. 21, 45-48

24 Schwarze, S.R., Ho, A., Vocero-Akbani, A. and Dowdy, S.F. (1999) Science 285, 1569-1572

Received 11 December 2003 


\title{
Regulation of granulocyte apoptosis by NF- $\kappa$ B
}

\author{
C. Ward', A. Walker, I. Dransfield, C. Haslett and A.G. Rossi \\ Respiratory Medicine Unit, MRC Centre for Inflammation Research, University of Edinburgh Medical School, Teviot Place, Edinburgh EH8 9AG, U.K.
}

\begin{abstract}
Granulocyte apoptosis is a crucial part of the successful resolution of inflammation. In vitro results show that activation of $\mathrm{NF}-\kappa \mathrm{B}$ (nuclear factor $\kappa \mathrm{B}$ ) in granulocytes is a survival mechanism. NF- $\kappa \mathrm{B}$ inhibitors increase the rate of constitutive apoptosis in neutrophils and eosinophils and cause these cells to respond to the pro-apoptotic effects of TNF- $\alpha$ (tumour necrosis factor- $\alpha$ ). Results from both in vivo and in vitro experiments suggest that there are at least two important waves of NF- $\kappa$ B activation in inflammatory loci, which increase the expression of COX-2 (cyclooxygenase-2), itself an NF- $\kappa$ B controlled gene. The first wave causes the production of inflammatory mediators such as $\mathrm{PGE}_{2}$ (prostaglandin $\mathrm{E}_{2}$ ), allowing the establishment of inflammation. The second wave causes the synthesis of $\mathrm{PGD}_{2}$ and its metabolites that induce granulocyte apoptosis by inhibiting NF- $\kappa$ B activation. These metabolites may therefore be important physiological mediators controlling the resolution of inflammation. Although $\mathrm{NF}-\kappa \mathrm{B}$ is an important target for anti-inflammatory therapy, the timing of inhibition in vivo may be crucial, to ensure that production of $\mathrm{PGD}_{2}$ and its sequential metabolites can occur.
\end{abstract}

\section{Introduction}

Apoptosis of neutrophils and eosinophils probably plays a prominent role in the resolution of inflammation. The process of programmed cell death closes down the functional activity of granulocytes and allows their removal by phagocytes without the release of histotoxic contents that have the potential to cause ongoing tissue damage and chronic inflammation. Macrophage ingestion of apoptotic cells clears effete granulocytes from the inflammatory site and potentiates the secretion of anti-inflammatory cytokines such as interleukin10 and transforming growth factor- $\beta$, promoting resolution and healing [1,2]. Although both neutrophils and eosinophils readily undergo constitutive cell death within hours of isolation from whole blood, most mediators that influence granulocyte function including cytokines and chemokines, which are found in an inflammatory foci, actually delay the onset of apoptosis in in vitro models $[3,4]$. Therefore it is difficult to understand which mechanisms are involved in allowing apoptosis and resolution to occur in vivo.

There was some indication of a possible mechanism when TNF- $\alpha$ (tumour necrosis factor- $\alpha$ ), a cytokine previously supposed to induce neutrophil survival, was found to induce apoptosis at early time points of $6 \mathrm{~h}$ or less [5]. Why TNF- $\alpha$ was a pro-death stimulus for some cells in the same culture, but was pro-survival in others, was not understood. Most of the signalling pathways activated by TNF- $\alpha$, such as extracellular-signal-regulated kinase, p38 and phosphatidylinositol 3 kinase, had been associated with survival in most of the cell types. The role of $\mathrm{c}$-Jun kinase in TNF- $\alpha$ signalling in neutrophils is controversial, and little is known about

Key words: apoptosis, eosinophil, inflammation, neutrophil, prostaglandin, resolution. Abbreviations used: $A A$, arachidonic acid; COX, cyclooxygenase; $N F-\kappa B$, nuclear factor $\kappa B$; $I k B$, inhibitor of $N F-k B ; P G$, prostaglandin; TNF, tumour necrosis factor.

'To whom correspondence should be addressed (email c.ward@ed.ac.uk). the effect of NF- $\kappa \mathrm{B}$ (nuclear factor $\kappa \mathrm{B}$ ), although activation of this transcription factor had been associated with the induction of apoptosis in some systems (see [6] and references therein).

\section{The transcription factor NF- $\kappa$ B}

$\mathrm{NF}-\kappa \mathrm{B}$ is an important mediator of innate immune responses. Many pro-inflammatory genes such as cytokines, chemokines, adhesion molecules as well as stress-response proteins are regulated by NF- $\kappa \mathrm{B}$ activation. This transcription factor is normally found in the cytoplasm held by inhibitory proteins called $\mathrm{I} \kappa \mathrm{B}$ (inhibitor of $\mathrm{NF}-\kappa \mathrm{B}$ ). NF- $\kappa \mathrm{B}$ is activated by various stimuli, which converge at the IKK ( $\mathrm{I} \kappa \mathrm{B}$ kinase) complex. IKK phosphorylates $\mathrm{I} \kappa \mathrm{B}$ leading to its ubiquitination, followed by proteosomal degradation. The release of $\mathrm{I} \kappa \mathrm{B}$ from the NF- $\kappa \mathrm{B}$ complex uncovers the nuclear localization sequence and allows active $\mathrm{NF}-\kappa \mathrm{B}$ to translocate into the nucleus and bind to consensus sites in the DNA of responsive genes [7] (see Figure 1).

$\mathrm{NF}-\kappa \mathrm{B}$ controls the expression of many survival genes such as Bcl-2 family members (e.g. Bcl-xL and A1), the inhibitor of apoptosis proteins and X-ray-inducible immediate early response factor-1 long. Such anti-apoptotic genes will enhance the functional lifespan of cells in inflammatory conditions, although mouse knockout models illustrate that the precise identities of these key survival proteins remain to be determined. However, NF- $\kappa$ B activation should cause enhanced survival in most cells through increased expression of these survival proteins.

\section{The involvement of NF- $\kappa$ B in granulocyte survival}

In granulocytes, inhibition of $\mathrm{NF}-\kappa \mathrm{B}$ is a powerful inducer of apoptosis, and activators of $\mathrm{NF}-\kappa \mathrm{B}$ provide a strong 
Figure 1| Pathway of $\mathrm{NF}-\kappa \mathrm{B}$ activation

$\mathrm{NF}-\kappa \mathrm{B}$ is held in the cytoplasm as an inactive complex by the inhibitory subunit $\boldsymbol{}_{\kappa} \mathrm{B}$. On activation, $I_{\kappa} \mathrm{B}$ is phosphorylated and ubiquitinated resulting in proteasomal degradation. This allows the active complex of p65 and p50 to translocate to the nucleus to regulate gene expression. The $\mathrm{PGD}_{2}$ metabolites, $\Delta^{12} P \mathrm{PJ}_{2}$ and 15 -deoxy- $\left.\Delta^{12,14}-\mathrm{PG}\right)_{2}$, block the degradation of $I_{\kappa} B$ in granulocytes.

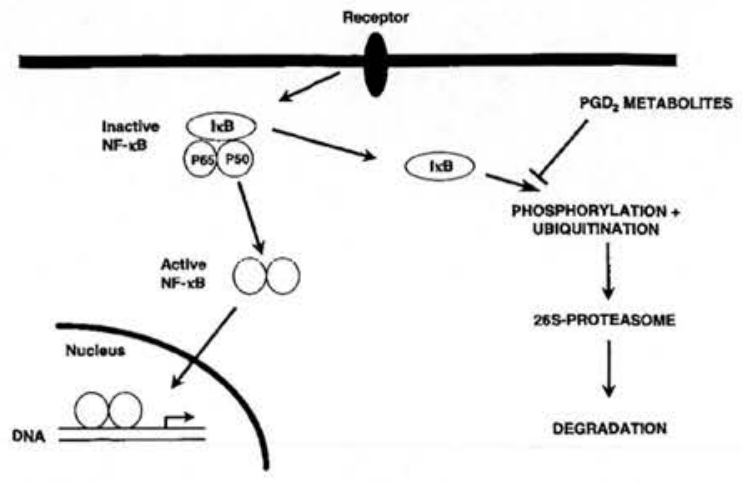

survival signal. When NF- $\kappa \mathrm{B}$ is inhibited, both neutrophils and eosinophils respond to TNF- $\alpha$ treatment with a massive increase in apoptosis. Within $2 \mathrm{~h}$, almost $100 \%$ of neutrophils undergo cell death in response to TNF- $\alpha$, compared with a control rate of almost zero. The results are similar in eosinophils, but the induction of apoptosis occurs between 3 and $4 \mathrm{~h}$ [8]. These results can be mimicked by protein synthesis inhibition, suggesting that the response of granulocytes to TNF- $\alpha$ is dependent on the presence or absence of NF- $\kappa$ Bcontrolled survival proteins with a short half-life [9]. This in vitro result suggests that, in an inflammatory lesion, if $\mathrm{NF}-\kappa \mathrm{B}$ activation is prevented, TNF- $\alpha$ can rapidly induce granulocyte cell death allowing clearance and resolution to begin. This initial work using pharmacological inhibitors as $\mathrm{NF}-\kappa \mathrm{B}$ suppressors is further supported by the use of HIV-Tat to transfect neutrophils and eosinophils with proteins that prevent NF- $\kappa$ B activation ([10]; S. Fujihara, R.T. Hay and A.G. Rossi, unpublished work). Similar increases in constitutive apoptosis and enhanced pro-apoptotic effects of TNF- $\alpha$ also occur when NF- $\kappa$ B is blocked in eosinophils.

\section{Is there a physiological NF- $\kappa$ B inhibitor?}

No physiological inflammatory mediators have been recognized that could inhibit NF- $\kappa$ B. Since most cytokines and chemokines had already been characterized as either inhibitors of granulocyte apoptosis or having no effect, they were therefore unlikely to inhibit NF- $\kappa \mathrm{B}$ activation. We examined the role of PGs (prostaglandins). Cells can metabolize AA (arachidonic acid) into five main PG subtypes depending on the enzymes present in the cell (Figure 2) [11]. When examined, all these PGs had either no effect or delayed granulocyte apoptosis with the exception of $\mathrm{PGD}_{2}$ that specifically induced apoptosis in eosinophils. $\mathrm{PGD}_{2}$ can be further metabolized to $\mathrm{PGJ}_{2}, \Delta^{12} \mathrm{PGJ}_{2}$ and 15-deoxy-

\section{Figure 2 | PG synthesis from AA}

$P G$ are formed from AA. COX converts AA to $P G_{2}$, which is in turn converted to $\mathrm{PGH}_{2}$ by peroxidase. The five main isoforms of $P G$ are synthesized from $\mathrm{PGH}_{2}$ and this depends on the synthases present in the cell. $P G D_{2}$ is further metabolized to $P G J_{2}$ and ultimately to $\Delta^{12} P G_{2}$ and 15-deoxy- $\Delta^{12,14}-P G_{2}$. TXA $A_{2}$, thromboxane $A_{2}$.

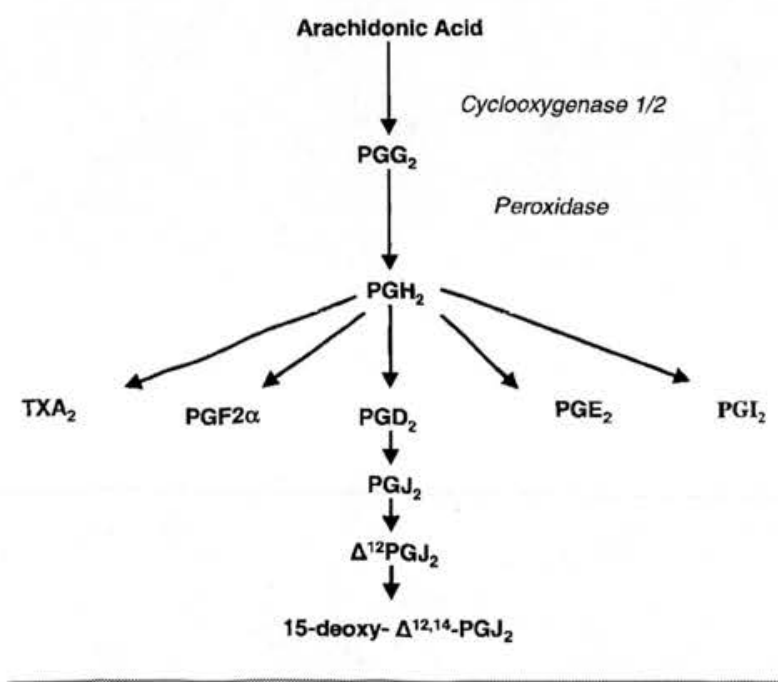

$\Delta^{12,14}-\mathrm{PGJ}_{2} . \mathrm{PGJ}_{2}$ also selectively induced eosinophil apoptosis, but the rates achieved were much reduced when compared with $\mathrm{PGD}_{2} . \mathrm{PGJ}_{2}$ is a fairly selective agonist on the DP receptor, which transduces signals from $\mathrm{PGD}_{2}$ binding, and is thus additional supporting evidence that this is not the signalling pathway responsible for the induction of cell death. The sequential $\mathrm{PGD}_{2}$ metabolites $\Delta^{12} \mathrm{PGJ}_{2}$ and 15 -deoxy- $\Delta^{12,14}-\mathrm{PGJ}_{2}$ are powerful inducers of caspasedependent apoptosis in both neutrophils and eosinophils. These metabolites are well-known ligands for the PPAR- $\gamma$ (peroxisome proliferator-activated receptor- $\gamma$ ), a transducer protein that belongs to the steroid/thyroid/retinoid receptor superfamily. However, neither PPAR $-\gamma$ agonists nor antagonists affected the induction of apoptosis by these metabolites suggesting that cell death does not depend on this nuclear receptor, in agreement with a study in other cell types [12]. Further study showed that both $\Delta^{12} \mathrm{PGJ}_{2}$ and 15-deoxy- $\Delta^{12,14}-\mathrm{PGJ}_{2}$ could inhibit the activation of NF- $\kappa$ B by lipopolysaccharide and TNF- $\alpha$ in granulocytes [13]. Could $\mathrm{PGD}_{2}$ and its metabolites be the physiological factors involved in inflammatory resolution?

PGs are formed via the action of COX (cyclooxygenase) enzymes [11]. COX-2 is an inducible form which is increased in inflammation and whose expression is controlled by $\mathrm{NF}-\kappa \mathrm{B}$. Studies have demonstrated that COX -2 expression is involved in the onset of the inflammatory process by producing the pro-inflammatory $\mathrm{PGE}_{2}$. However, during the resolution phase, there is another wave of COX-2 synthesis which causes a preferential synthesis of $\mathrm{PGD}_{2}$ allowing production of its metabolites $\Delta^{12} \mathrm{PGJ}_{2}$ and 15 -deoxy- $\Delta^{12,14}$ $\mathrm{PGJ}_{2}$ to occur. This has been demonstrated in vivo (see [14] and references therein). Further work has shown that 
during the resolution phase, if COX-2 expression is inhibited, resolution is impaired probably because synthesis of $\mathrm{PGD}_{2}$ does not occur [15].

Although the results clearly demonstrate a therapeutic potential for NF- $\kappa$ B blockade, they also raise a note of caution. Since NF- $\kappa$ B inhibition does have the ability to enhance inflammation, if synthesis of COX-2 is prevented during the resolution phase, the timing of any therapeutic intervention involving NF- $\kappa$ B inhibition will be crucial for successful treatment of inflammatory conditions.

This work was funded by the Medical Research Council Programme Grant (G9016491) and the National Asthma Campaign (01/042).

\section{References}

1 Huynh, M.L., Fadok, V.A. and Henson, P.M. (2002) J. Clin. Invest. 109 41-50

2 Voll, R.E., Herrmann, M., Roth, E.A., Stach, C., Kalden, J.R. and Grikontaite, I. (1998) Nature (London) 390, 350-351

3 Ward, C., Dransfield, I., Chilvers, E.R., Haslett, C. and Rossi, A.G. (1999) Trends Pharmacol. Sci. 20, 503-509
4 Walker, A., Ward, C., Dransfield, I., Haslett, C. and Rossi, A.G. (2003) Curr. Drug Targets - Inflam. Allergy 2, 339-347

5 Murray, J., Barbara, J.A.J., Dunkley, S.A., Lopez, A.F., Van Ostade, X. Condliffe, A.M., Dransfield, I., Haslett, C. and Chilvers, E.R. (1997) Blood 90, 2772-2783

6 Wallach, D., Varfolomeev, E.E., Malinin, N.L., Goltsev, Y.V., Kovalenko, A.V. and Boldin, M.P. (1999) Annu. Rev. Immunol. 17, 331-367 7 Gilmore, T.D. (1999) Oncogene 18, 6842-6844

8 Fujihara, S., Ward, C., Dransfield, I., Hay, R.T., Uings, I. ., Hayes, B. Farrow, S.N., Haslett, C. and Rossi, A.G. (2002) Eur. J. Immunol. 32 457-466

9 Ward, C., Chilvers, E.R., Lawson M.F., Pryde, J.G., Fujihara, S., Farrow, S.N., Haslett, C. and Rossi, A.G. (1999) J. Biol. Chem. 274, 4309-4318

10 Choi, M., Rolle, S., Wellner, M., Cardoso, C., Scheidereit, C., Luft, F.C. and Kettritz, R. (2003) Blood 102, 2259-2267

11 Vane, J.R., Bakhle, Y.S. and Botting, R.M. (1998) Annu. Rev Pharmacol. Toxicol. 38, 97-120

12 Rossi, A., Kapahi, P., Natoli, G., Takahashi, T., Chen, Y., Karin, M. and Santoro, M.G. (2000) Nature (London) 403, 103108

13 Ward, C., Dransfield, I., Murray, J., Farrow, S.N., Haslett, C. and Rossi, A.G. (2002) J. Immunol. 168, 6232-6243

14 Lawrence, T., Willoughby, D.A. and Gilroy, D.W. (2002) Nat. Rev. Immunol. 2, 787-795

15 Lawrence, T., Gilroy, D.W., Colville-Nash, P.R. and Willoughby, D.A (2001) Nat. Med. 7, 1291-1297

Received 11 December 2003 


\title{
EDITORIAL
}

\section{Eating their way out of trouble: selective uptake of apoptotic eosinophils by bronchial epithelial cells}

\author{
N. Farahi, A. S. Cowburn, A. G. Rossi and E. R. Chilvers
}

\section{Role of eosinophils in allergic inflammation and asthma}

Central to the inflammatory hypothesis in asthma is the role of the eosinophil and its capacity to elaborate and release a series of highly cytotoxic and pro-inflammatory mediators [1]. The regulation of eosinophil production and function has been extensively studied and much is now known about its selective recruitment, priming, activation and prolonged survival by cytokines predominantly encoded on chromosome $5 \mathrm{q}_{31-34}$ (the IL-4 gene cluster) $[2,3]$. Three major eosinophilopoietic cytokines (IL-3, IL-5, GM-CSF (granulocyte macrophage-colony stimulating factor)) lead to the maturation and terminal differentiation of eosinophils within the bone marrow [4] and influence their recruitment to sites of inflammation. This latter process involves adhesion to endothelial cells, which is mediated via an interaction between vascular cell adhesion molecule-1 (VCAM-1) and the eosinophil-restricted very late activation antigen-4 [5] and intracellular adhesion molecule-1 (ICAM-1) with lymphocyte function associated antigen [6]. Eosinophils migrate in response to a range of chemoattractants, including chemokines, complement components and lipid mediators $[2,5,7]$, and express the cognate receptors for a variety of factors including IL-8, regulated on activation, normal $\mathrm{T}$ cell expressed and secreted (RANTES), eotaxin, IL-1, IL-2, IL3 , IL-4, IL-5, IL-9, IL-16, GM-CSF, IFN- $\gamma$, Ig and TNF- $\alpha$ [8]. These cells are thus equipped to respond to a wide range of inflammatory stimuli. Eosinophil activation and/or cytolysis leads to the release of a unique repertoire of highly histotoxic substances including cationic proteins (eosinophilderived neurotoxin, eosinophil peroxidase, eosinophilic cationic protein, major basic protein) [9] and metalloproteases and eosinophils are a major source of cysteinyl-leukotrienes that contribute to bronchoconstriction, microvascular leak and bronchial hyper-responsiveness [10].

\section{The resolution of eosinophil inflammation}

The elimination of airway eosinophils is thought to rely on two major processes. Firstly, it is clear that antigen challenge can induce a rapid and highly co-ordinated exit of large numbers of eosinophils into the airway lumen (estimated at up to 35000 cells $/ \mathrm{min} / \mathrm{cm}^{2}$ ) [11]. This event is likely to be driven by the polarized release of chemoattractants from the apical surface of epithelial cells [12] and appears to cause little if any immediate damage to the epithelium $[11,13,14]$.

In addition, attention has focussed on the potential role of cytolysis and in particular apoptosis in the resolution of eosinophilic inflammation [15-17]. In this respect it is of interest that many cytokines that are associated with tissue eosinophilia in vivo can inhibit the rate of constitutive cell death in vitro [18]. The hypothesis that the inhibition of apoptotic cell death may underlie certain forms of chronic eosinophilic inflammation is supported by the observation that corticosteroids are potent inducers of eosinophil apoptosis both in vitro and in vivo $[19,20]$. In contrast, neutrophils are resistant to the pro-apoptotic effects of corticosteroids, an effect that may relate to the differential expression of glucocorticoid- $\alpha$ and $-\beta$ (GR- $\alpha$ and GR- $\beta$ ) mRNA between these two cell types [21] and the capacity of GR- $\beta$ to act as a dominant negative inhibitor of GR- $\alpha$ activity [22].

Under in vitro conditions apoptotic eosinophils are rapidly engulfed by inflammatory macrophages, a process that would be predicted to prevent secondary necrosis and result in the resolution of airway inflammation $[15,23]$. This view has been challenged, however, by in vivo studies where eosinophil apoptosis could be detected only in cells present in the airway lumen [14]. Moreover, corticosteroids were shown to have no effect on the extent of eosinophil apoptosis within the airway wall and failed to affect the rate of eosinophil entry into the lumen. While these data may not take full account of the very rapid and efficient coupling of granulocyte apoptosis to engulfment in vivo [24], they suggest the possibility that transendothelial migration of eosinophils or residency within the airway wall may confer an 'apoptosis-resistant' phenotype on these cells. This process would be predicted to be relatively selective for the eosinophil since apoptotic neutrophils are readily apparent in a number of inflammatory conditions [25].

\section{Modulation of eosinophil survival by endothelial cells}

Endothelial cells were initially thought to play a passive 'barrier' role in vascular physiology, with their active functions limited to aiding coagulation. However, numerous studies have now demonstrated a pivotal role for these cells in regulating metabolism (e.g. angiotensin converting enzyme expression, serotonin degradation), inflammation (e.g. TNF$\alpha$, VCAM and ICAM expression) and vascular tone (e.g. NO (nitric oxide), prostacylcin and endothelin production) [26]. Their strategic positioning also enables endothelial cells to influence the priming and activation status of peripheral blood leucocytes as they transmigrate into an inflammatory focus $[6,27]$. This effect is mediated in part through the ability of endothelial cells to express a wide range of cytokines including IL-1, IL-5, IL-6, IL-8, monocyte chemotactic 
protein and the colony-stimulating factors GM-CSF, G-CSF and M-CSF [28]. In conjunction with other endothelial cellderived stimuli (e.g. platelet activating factor, eicosanoids and nitric oxide), these factors are thought to play a central role in modulating the inflammatory process.

With reference to the transmigrating eosinophil, recent preliminary data indicate that human pulmonary artery endothelial cells produce a factor(s) that delays eosinophil apoptosis in vitro [29]. This factor, which is discrete from GM-CSF, IL-5 and IL-3, has a similar efficacy in survival terms to GM-CSF but is entirely eosinophil-selective in that it has no effect on neutrophil longevity. The precise identity of this survival factor and whether it is elaborated in vivo remains to be determined. These studies do, however, emphasize the intimate cross-talk that exists between structural 'barrier' cells in the lung and the influxing inflammatory cells. Indeed, such interactions may be crucial to the regulation of chronic inflammatory [30]. They also illustrate the fundamental differences that exist between the apoptotic programming of neutrophils and eosinophils.

\section{Modulation of eosinophil function and fate by bronchial epithelial cells}

The capacity of eosinophils to damage bronchial epithelial cells is well recognized and is believed to be one of the major factors contributing to airway damage in asthma [1]. A number of studies suggest that this interaction is not all one sided and that bronchial epithelial cells can also influence eosinophil function. Hence bronchial epithelial cells elaborate many pro-inflammatory molecules including GM-CSF, eotaxin, RANTES and macrophage inflammatory protein- $1 \alpha$, all of which are powerful chemoattractants for eosinophils [31]. Likewise, eosinophils bind directly to bronchial epithelial cells and in certain circumstances this interaction leads to marked degranulation [32]. Bronchial epithelial cells have also been shown to prolong eosinophil longevity again through the production of GM-CSF [33].

So, how does the bronchial epithelial cell defend itself against such attack and can it fight back? The capacity of these cells to propel inflammatory cells towards the larynx by mucociliary transport may well help [34], as will the production of several anti-inflammatory molecules, including prostaglandin $\mathrm{E}_{2}$ and $\alpha 1$-antitrypsin [35]. Bronchial epithelial cells also express Fas- $\mathrm{L}$ and other agents such as TNF- $\alpha$ that are capable of inducing eosinophil apoptosis [36]. This suggests that bronchial epithelial cells have evolved a fairly extensive raft of barrier, humeral and cell:cell interaction mechanisms to defend themselves against granulocyte attack.

In the current issue of Clinical and Experimental Allergy, Sexton et al. [37] provide a further insight into the relationship between airway epithelial cells and eosinophils. Firstly, using confocal microscopy, scanning and transmission electron microscopy (EM) they confirm their earlier observation that bronchial epithelial cells cultured from either large or small airways display a capacity to recognize and phagocytose apoptotic eosinophils [38]. While the phagocytic capacity of epithelial cells has been described in a number of other settings (e.g. in the renal tubule and involuting mouse mammary gland $[39,40])$ the apparent specificity of this event is intriguing. Hence, the current paper reveals that when tested under identical conditions, quiescent- and cytokinestimulated bronchial epithelial cells are unable to recognize or ingest apoptotic neutrophils. This effect is ascribed to the lack of expression of the mannose receptor on epithelial cells. As might be predicted, the phagocytic uptake of apoptotic eosinophils involves integrin $(\alpha v \beta 3, \alpha 5 \beta 5, \mathrm{CD} 36)$, phosphatidylserine receptor- and lectin-dependent interactions [37, 38, 41]. Of note, the avarice of epithelial cells for apoptotic eosinophils can be further enhanced by prior treatment with the corticosteroid dexamethasone [41], IL-1, TNF- $\alpha$ or ligation of CD44 [37], effects all similar to those reported by Dransfield and colleagues examining macrophage uptake of apoptotic neutrophils [42, 43]. The study by Sexton and colleagues also confirms that monocyte-derived macrophages ingest apoptotic eosinophils more avidly than apoptotic neutrophils [42].

Taken together these studies have a number of important implications for our understanding of eosinophilic inflammation. First, they suggest that the transmigration of eosinophils across the vascular endothelium and then across the epithelial surface into the airway lumen may have a major influence on the apoptotic programming of eosinophils. Indeed, the 'prosurvival' environment within the micro-compartment of the airway wall may be so intense as to prevent apoptotic cell death entirely. A different balance appears to exist, however, in the airway lumen where apoptotic eosinophils are seen in far greater numbers especially during the resolution phase of an asthma exacerbation [44]. A number of factors may contribute to this, not least a relative defect in the capacity of macrophages within the lumen to ingest apoptotic cells. It is possible therefore that bronchial epithelial cells have developed the capacity to phagocytose apoptotic eosinophils to provide an additional tier of defence against these highly toxic cells; if left, secondary necrosis may ensue creating further havoc.

The limitations of Sexton's study relate firstly to its in vitro setting and the use of a monolayer cell culture system. The stringency of the wash protocol employed following the initial eosinophil and bronchial epithelial cell co-culture and the inability of trypsin to affect the phagocytic index suggest that eosinophil adhesion, as opposed to phagocytosis, has been fully excluded. Studies examining the capacity of other nonprofessional phagocytes (e.g. fibroblasts, smooth muscle cells, dendritic cells and hepatocytes) to engulf apoptotic neutrophils [45] have proven difficult to replicate and hence it is important that independent validation of this work is provided. Moreover, this study does not address the relative contribution of bronchial epithelial cells and other nonprofessional phagocytes to the removal of apoptotic cells in vivo [15]. If phagocytic uptake of apoptotic eosinophils really does represent a major clearance pathway for these cells, this should be apparent in bronchial biopsy specimens.

As with all good studies, the work by Sexton and coworkers opens up more questions than it answers; not least the relative contribution of bronchial epithelial cell phagocytosis of apoptotic eosinophils to the clearance of airway lumen eosinophils in vivo, whether bronchial epithelial cells can ingest apoptotic eosinophils if presented at their basal or baso-lateral (non-luminal) surface and the (presumably profound) effects of epithelial damage on this process. 
Whether the increased phagocytic uptake induced by corticosteroid treatment underlies any of the therapeutic efficacy of these agents is also unclear. However, the fact that the uptake of apoptotic eosinophils by bronchial epithelial cells is seemingly so selective and can be regulated gives encouragement that this pathway may offer a useful therapeutic target.

\section{Acknowledgements}

The work in the authors' laboratory is supported by the Wellcome Trust, MRC, Asthma UK, British Lung Foundation, Papworth Hospital R\&D Committee and the Isaac Newton Trust.

\section{References}

1 Gleich GJ. Mechanisms of eosinophil-associated inflammation. J Allergy Clin Immunol 2000; 105:651-63.

2 Collins PD, Marleau S, Griffiths-Johnson DA, Jose PJ, Williams TJ. Cooperation between interleukin-5 and the chemokine eotaxin to induce cosinophil accumulation in vivo. J Exp Med 1995; 182:1169-74.

3 Lee TH. Cytokine networks in the pathogenesis of bronchial asthma: implications for therapy. J R Coll Physicians Lond 1998; 32:56-64.

4 Clutterbuck EJ, Hirst EM, Sanderson CJ. Human interleukin-5 (IL-5) regulates the production of eosinophils in human bone marrow cultures: comparison and interaction with IL-1, IL-3, IL-6, and GM-CSF. Blood 1989; 73:1504-12.

5 Broide D, Sriramarao P. Eosinophil trafficking to sites of allergic inflammation. Immunol Rev 2001; 179:163-72.

6 Bochner BS, Luscinskas FW, Gimbrone M-AJ et al. Adhesion of human basophils, eosinophils, and neutrophils to interleukin 1activated human vascular endothelial cells: contributions of endothelial cell adhesion molecules. J Exp Med 1991; 173:1553-7.

7 Palframan RT, Collins PD, Williams TJ, Rankin SM. Eotaxin induces a rapid releases of eosinophils and their progenitors from the bone marrow. Blood 1998; 91:2240-8.

8 Weller PF. Cytokine regulation of eosinophil function. Clin Immunol Immunopathol 1992; 62:S55-9.

9 Egesten A, Calafat J, Weller PF et al. Localization of granule proteins in human eosinophil bone marrow progenitors. Int Arch Allergy Immunol 2001; 124:267-71.

10 Busse WW. Leukotrienes and inflammation. Am J Respir Crit Care Med 1998; 157:S210-3.

11 Erjefält JS, Uller L, Malm-Erjefält M, Persson CG. Rapid and efficient clearance of airway tissue granulocytes through transepithelial migration. Thorax 2004; 59:136-43.

12 McCormick BA, Parkos CA, Colgan SP et al. Apical secretion of a pathogenelicited epithelial chemoattractant activity in response to surface colonization of intestinal epithelia by Salmonella typhimurium. J Immunol 1998; 160:455-66.

13 Erjefält JS, Persson CG. New aspects of degranulation and fates of airway mucosal eosinophils. Am J Respir Crit Care Med 2000; 161:2074-85.

14 Uller L, Persson CG, Kallstrom L et al. Lung tissue eosinophils may be cleared through luminal entry rather than apoptosis: effects of steroid treatment. Am J Respir Crit Care Med 2001; 164: 1948-56.

15 Stern M, Meagher L, Savill J, Haslett C. Apoptosis in human eosinophils. Programmed cell death in the eosinophil leads to phagocytosis by macrophages and is modulated by IL-5. J Immunol 1992; 148:3543-9.
16 Simon HU, Blaser K. Inhibition of programmed eosinophil death: a key pathogenic event for eosinophilia? Immunol Today 1995; 16:53-5.

17 Walsh GM. Eosinophil apoptosis: mechanisms and clinical relevance in asthmatic and allergic inflammation. $\mathrm{Br} \mathrm{J}$ Haematol 2000; 111:61-7.

18 Yousefi S, Blaser K, Simon HU. Activation of signaling pathways and prevention of apoptosis by cytokines in eosinophils. Int Arch Allergy Immunol 1997; 112:9-12.

19 Meagher LC, Cousin JM, Seckl JR, Haslett C. Opposing effects of glucocorticoids on the rate of apoptosis in neutrophilic and eosinophilic granulocytes. J Immunol 1996; 156:4422-8.

20 Nittoh T, Fujimori H, Kozumi Y, Ishihara K, Mue S, Ohuchi K. Effects of glucocorticoids on apoptosis of infiltrated eosinophils and neutrophils in rats. Eur J Pharmacol 1998; 354:73-81.

21 Pujols L, Mullol J, Perez M et al. Expression of the human glucocorticoid receptor alpha and beta isoforms in human respiratory epithelial cells and their regulation by dexamethasone. Am J Respir Cell Mol Biol 2001; 24:49-57.

22 Gagliardo R, Chanez P, Vignola AM et al. Glucocorticoid receptor alpha and beta in glucocorticoid dependent asthma. Am J Respir Crit Care Med 2000; 162:7-13.

23 Haslett C. Granulocyte apoptosis and its role in the resolution and control of lung inflammation. Am J Respir Crit Care Med 1999; 160:S5-11.

24 Hughes J, Johnson RJ, Mooney A, Hugo C, Gordon K, Savill J. Neutrophil fate in experimental glomerular capillary injury in the rat. Emigration exceeds in situ clearance by apoptosis. Am J Pathol 1997; 150:223-34.

25 Kotecha S, Mildner RJ, Prince LR et al. The role of neutrophil apoptosis in the resolution of acute lung injury in newborn infants. Thorax 2003; 58:961-7.

26 Luscher TF. Vascular protection: current possibilities and future perspectives. Int J Clin Pract Suppl 2001; (117):3-6.

27 Yamamoto H, Sedgwick JB, Vrtis RF, Busse WW. The effect of transendothelial migration on eosinophil function. Am J Respir Cell Mol Biol 2000; 23:379-88.

28 Krishnaswamy G, Kelley J, Yerra L, Smith JK, Chi DS. Human endothelium as a source of multifunctional cytokines: molecular regulation and possible role in human disease. J Interferon Cytokine Res 1999; 19:91-104.

29 Farahi N, Upton P, Deighton J, Morrell NW, Chilvers ER, Cowburn AS. Effect of conditioned media from human pulmonary artery endothelial cells on eosinophil apoptosis in vitro. Am J Respir Crit Care Med 2002; 165:275.

30 Pearlman DS. Pathophysiology of the inflammatory response. J Allergy Clin Immunol 1999; 104:S132-7.

31 Walsh GM. Eosinophil-epithelial cell interactions: a special relationship? Clin Exp Allergy 2001; 31:351-4.

32 Takafuji S, Ohtoshi T, Takizawa $\mathrm{H}$ et al. Eosinophil degranulation in the presence of epithelial cells. Effect of cytokines and role of adhesion. J Immunol 1996; 156:3980-5.

33 Soloperto M, Mattoso VL, Fasoli A, Mattoli S. A bronchial epithelial cell-derived factor in asthma that promotes eosinophil activation and survival as GM-CSF. Am J Physiol 1991; 260:L530-8.

34 Zhang P, Summer WR, Bagby GJ et al. Innate immunity and pulmonary host defense. Immunol Rev 2000; 173:39-51.

35 Johansson B, Malm J, Persson T et al. Alpha-1-antitrypsin is present in the specific granules of human eosinophilic granulocytes. Clin Exp Allergy 2001; 31:379-86.

36 Tsuyuki S, Bertand C, Erard F et al. Activation of the Fas receptor on lung eosinophils leads to apoptosis and the resolution of cosinophilic inflammation of the airways. J Clin Invest 1995; 96:2924-31.

37 Sexton DW, Al-Rabia M, Blaylock MG, Walsh GM. Phagocytosis of apoptotic eosinophils but not apoptotic neutrophils by bronchial epithelial cells. Clin Exp Allergy 2004; 34:1514-24. 
38 Walsh GM, Sexton DW, Blaylock MG et al. Resting and cytokinestimulated human small airway epithelial cells recognise and ingest apoptotic eosinophils. Blood 1999; 94:2827-35.

39 Gobé CG, Axelsen RA. Genesis of renal tubular atrophy in experimental hydronephrosis in the rat. Role of apoptosis. Lab Invest $1987 ; 56: 273-82$.

40 Monks J, Geske FJ, Lehman L, Fadok VA. Do inflammatory cells participate in mammary gland involution. $\mathbf{J}$ Mammary Gland Biol Neoplasia 2002; 7:163-76.

41 Sexton DW, Blaylock MG, Walsh GM. Human alveolar epithelial cells engulf apoptotic eosinophils via integrin- and phosphatidylserine-dependent mechanisms: a process unregulated by dexamethasone. J Allergy Clin Immunol 2001; 108:962-9.

42 Liu YQ, Cousin JM, Hughes J et al. Glucocorticoids promote nonphlogistic phagocytosis of apoptotic leukocytes. J Immunol 1999; 162:3639-46.

43 Hart SP, Dougherty GJ, Haslett C, Dransfield I. CD44 regulates phagocytosis of apoptotic neutrophil granulocytes, but not apoptotic lymphocytes, by human macrophages. J Immunol 1997; 159:919-25.

44 Woolley KL, Gibson PG, Carty K, Wilson AJ, Twaddell SH, Woolley MJ. Eosinophil apoptosis and the resolution of airway inflammation in asthma. Am J Respir Crit Care Med 1996; 154:237-43.

45 Hall SE, Savill JS, Henson PM, Haslett C. Apoptotic neutrophils are phagocytosed by fibroblasts with participation of the fibroblast vitronectin receptor and involvement of a mannose/fucose-specific lectin. J Immunol 1994; 153:3218-27.

N. Farahi*, A. S. Cowburn*, A. G. Rossi† and E. R. Chilvers* *Respiratory Medicine Division Department of Medicine University of Cambridge School of Clinical Medicine Box 157, Level 5 Addenbrooke's and Papworth Hospitals Hills Road, Cambridge CB2 $2 Q Q$

E-mail: erc24@cam.ac.uk $\dagger$ Respiratory Medicine Unit $M R C$ Centre for Inflammation University of Edinburgh Medical School Teviot Place Edinburgh UK 


\title{
Neutrophils and Apoptosis
}

\author{
Annemieke Walker, Carol Ward, Magdalena \\ Martinez-Losa, Adriano G. Rossi*
}

\begin{abstract}
Neutrophils are key effector cells involved in host defence against invading organisms such as bacteria and fungi. Their over-recruitment, uncontrolled activation and defective removal contribute to the initiation and propagation of many chronic inflammatory conditions. Neutrophil apoptosis is a physiological process that terminates the cells' functional responsiveness and induces phenotypic changes that render them recognizable by phagocytes (e.g. macrophages). Evidence indicates that neutrophil apoptosis and the subsequent removal of these cells by macrophages occur via mechanisms that do not elicit an inflammatory response and that these processes are fundamental for the successful resolution of inflammation. The molecular mechanisms regulating apoptosis in neutrophils are being elucidated and consequently it is now believed that selective induction of neutrophil is a potential target for therapeutic intervention.
\end{abstract}

Keywords: neutrophil; apoptosis; resolution; macrophage; clearance; caspase

\footnotetext{
*Correspondence to: Adriano G. Rossi. Centre for Inflammation Research, University of Edinburgh Medical School, Teviot Place, Edinburgh, EH8 9AG, UK; e-mail: a.g.rossi@ed.ac.uk.
} 


\section{The Neutrophils}

\section{INTRODUCTION}

As highlighted in other chapters, the neutrophil is highly developed for its principal role of fighting invading organisms (e.g. bacteria or fungi). Thus, the neutrophil has evolved a great capacity to generate toxic oxygen metabolites (e.g. $\mathrm{O}_{2}^{-}, \mathrm{H}_{2} \mathrm{O}_{2}, \mathrm{OH}^{-}, \mathrm{NO}$ ) and an ability to liberate products (e.g. elastase and collagenase) located within different cytoplasmic granules that can destroy or render invading organisms impotent. ${ }^{1}$ However, when this normally beneficial response becomes dysregulated, neutrophil-derived toxic products can cause severe tissue damage resulting in the development of chronic inflammatory scenarios. There is now good evidence showing that once invading organisms have been eliminated, neutrophils have to be removed by mechanisms that limit their capacity to cause tissue injury and allow resolution of the inflammatory process to occur. For example, mechanisms exist to reduce or stop neutrophil recruitment and activation, decrease the concentrations of proinflammatory stimuli and augment the generation of mediators with anti-inflammatory potential (e.g. IL-1 receptor antagonist, IL-10, TGF- $\beta$ ). However, it has become apparent that the key mechanisms involved in the successful resolution of inflammation are neutrophil apoptosis and the subsequent removal of these cells by phagocytes. ${ }^{2,3}$ In this chapter, we will focus on these processes and describe the mechanisms controlling this previously under-investigated aspect of neutrophil biology.

\section{NEUTROPHIL APOPTOSIS}

Although researchers have been aware of cell death, especially by necrosis, for many decades, it was only in the early seventies that apoptosis, or programmed cell death, was first formally described as a physiological and highly regulated form of cell death. ${ }^{45}$ Apoptosis has now been shown to be responsible for the physiological death of virtually all cells in every organ and is therefore believed to be important in all aspects of biology. Apoptosis also plays a fundamental role in regulating neutrophilmediated inflammation and inflammatory diseases, as well as in fundamental processes such as embryological morphogenesis and tissue remodeling. 
When cells, including neutrophils, become apoptotic, a chain of highly regulated molecular events occur that result in distinct structural, morphological and biochemical phenotypes. Additionally, apoptotic cells maintain plasma membrane integrity and retain their cytoplasmic granules. ${ }^{4,5}$ This is especially important in the neutrophil, since death by necrosis would result in the release of histotoxic intracellular contents that can augment the inflammatory response. Thus, the intact membrane of apoptotic cells renders them still capable of excluding vital dyes such as trypan blue, an important consideration when attempting to evaluate apoptosis experimentally. Apoptotic neutrophils tend to be smaller but more vacuolated as a result of cyloplasmic changes and the typical multilobed nucleus observed in nonapoptotic neutrophils coalesces into one or sometimes several distinct visually disconnected nuclear lobes. The nuclear chromatin condenses into dense, crescent-shaped structures with the nucleolus becoming more prominent. Endogenous endonuclease activation is responsible for internucleosomal cleavage into characteristic DNA fragments of $180-200$ base pairs of the chromatin. Like all cells, there are major cell surface changes that occur in neutrophils undergoing apoptosis. For example, the distribution of plasma membrane phospholipid changes dramatically. Phosphatidylserine normally located on the inner leaflet of the plasma membrane of nonapoptotic cells flips onto the external surface of apoptotic cells by the combined efforts of two enzymes (phospholipid scramblase and aminophospholipid translocase). ${ }^{6}$

Although membrane phosphatidylserine redistribution is a general phenomenon and has been utilized for assessing apoptosis, there are other cell surface changes restricted to particular cell types. The neutrophil, for example, sheds its surface FcyRIII (CD16) when undergoing apoptosis. ${ }^{7,8}$ Importantly, as neutrophils become apoptotic their ability to respond to agonists (e.g. fMLP) is dramatically reduced so that they are no longer capable of undergoing chemotaxis and degranulation and their phagocytic abilities are impaired. ${ }^{9}$ Although the biochemical mechanisms responsible for this functional down-regulation remain ill defined, it is thought that loss of molecules important for activation (e.g. specific receptors), phagocytosis (CD16) and adhesion (L-selectin) $)^{7,10}$ as well as changes in secretory pathways are important. ${ }^{9,10} \mathrm{It}$ is changes such as these that are responsible for limiting the potential for neutrophils to cause tissue damage and for the 
ability of phagocytes such as macrophages to recognize and phagocytose apoptotic cells from inflammatory sites using mechanisms that do not incite an inflammatory response (see below). Thus human neutrophils in vivo, or when isolated from blood, have a limited lifespan and are destined to die within hours of leaving the bone marrow. In vivo, it has been estimated that neutrophils have a half-life of approximately 6 hours and that neutrophils that leave the vasculature die by apoptosis within 48 hours once their useful inflammatory purposes have been fulfilled. ${ }^{11}$ Recently, a novel mechanism for the removal of senescent neutrophils from the circulation has been postulated. It has been demonstrated that aging neutrophils that remain in the circulation upregulate their CXCR4 expression and acquire an ability to migrate towards SDF- $1 \alpha$, a process involved in preferential neutrophil homing to the bone marrow in vivo. ${ }^{12}$ In tissue culture conditions, isolated human neutrophils undergo constitutive apoptosis with approximately $40-70 \%$ of the cells being apoptotic within 20 hours. By 40 hours, virtually the entire population exhibits characteristics of apoptosis. The rate at which these cells die can either be decreased or enhanced by specific treatments and therefore appears to be highly regulated. ${ }^{13,14}$

\section{REGULATION OF NEUTROPHIL APOPTOSIS}

Since many of the characteristic features of apoptosis are similar in all cell types, there are many evolutionally conserved mechanisms responsible for many of these changes. However, it is apparent that control of the apoptotic process differs from one cell to another and can depend on the types of receptors expressed on the cell. Thus, the control of apoptosis can be highly specific in that neutrophil apoptosis can be regulated without influencing apoptosis of closely related inflammatory cells (e.g. eosinophils) ${ }^{8,13-16}$ (Fig. 1).

\subsection{Internal Control Mechanisms}

\subsubsection{Caspases}

Arguably the most important enzymes responsible for the cellular events occurring during apoptosis are the caspase (for cysteinyl aspartate-specific protease) family of enzymes. Since the initial discovery of caspase 1 (also 


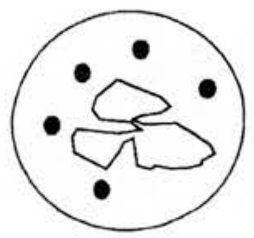

Non-apoptotic neutrophil

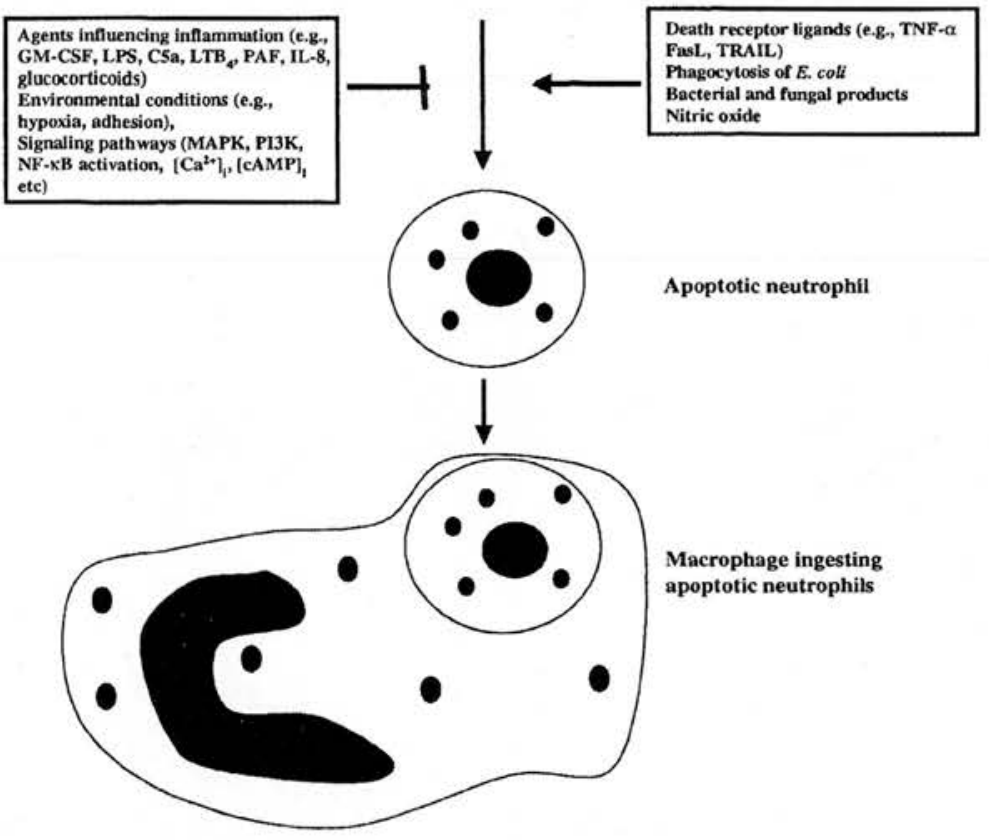

Fig 1 Regulation of neutrophil apoptosis and their subsequent removal by macrophages. Once functionally competent non-apoptotic neutrophils have fulfilled their defense against invading organisms, they undergo constitutive apoptosis, a process that can be delayed or enhanced by a number of factors. The apoptotic neuirophil is then rapidly and efficiently ingested by neighboring tissue resident macrophages using specific recognition mechanisms that do not elicit an inflammatory response. Failure of this beneficial physiological process can lead to undesirable enhanced inflammation as apoptotic neutrophils can eventually undergo necrosis with subsequent liberation of their histotoxic contents.

called interleukin-1 $\beta$-converting enzyme) there have been a total of at least 14 mammalian caspases (caspases 1-14) identified so far.

Essentially, one group of caspases (collectively referred to as the caspase- 1 subfamily of caspases and consisting of caspases-1, $-4,-5$, 
$-11,-12,13$, and -14) are primarily involved in inflammatory processes while the other group referred to as the CED-3 subfamily (consisting of caspases-2, $-3,-6,-7,-8,-9,-10)$ are primarily involved in cell death processes. ${ }^{17-19}$

More recently, caspases of both divisions have been identified in neutrophils, including caspases- $1,-3,-4,-6,-7,-8,-9$ and $-14 .{ }^{20}$ Of the caspases involved in apoptosis, there are two main types: initiator caspases (e.g. caspase- $8,-9,-10$ ) and effector caspases (e.g. caspases $-3,-6$ and -7 ).

The initiator caspases possess long prodomains containing unique motifs, including death effector domains (DED) and CARDS (caspase recruitment domains), allowing association with adapter molecules involved in apoptosis (e.g. DED associates with adapter molecules such as Fas-associated death domain (FADD)). These prodomains also permit oligomerization, culminating in their activation by autocatalysis. ${ }^{17}$ It is these initiator caspases that leads to downstream activation of the effector caspases. The primary function of the effector caspases is the cleavage of key proteins that leads to the characteristic features of apoptotic cells. ${ }^{19}$ The effector caspases may also be activated by the formation of the "apoptosome," which consists of a complex of cytochrome c, ATP, apoptosis protease-activating factor (Apaf-1) and caspase-9. Apaf-1, like cytochrome $c$, is released from the inner mitochondrial membrane when an apoptotic signal is received (through the intrinsic or extrinsic pathway). Apoptosome formation allows the activation of caspase-9, which in turn cleaves and activates caspase- $3 .{ }^{21}$ Although many caspase substrates have been identified, the number identified in neutrophils is relatively few. The most notable ones identified in neutrophils include PKC- $\delta$, 22 lamin B, fodrin ${ }^{23}$ and gelsolin. ${ }^{24}$

\subsubsection{Initiation of apoptosis}

Although there are many triggers that can initiate the apoptotic caspasecascade, two principal interrelated pathways exist for the initiation of the apoptotic cascade in all cells. In the neutrophil, apoptotic cell death occurs constitutively, as neutrophils will undergo spontaneous cell death when cultured in vitro, with rates of apoptosis at the time of isolation from the blood being extremely low (usually below $5 \%$ of the neutrophil 
population being apoptotic), and by approximately 20 hours of culture, the rates of apoptosis can be in excess of $70 \% .^{25}$ The precise role of caspases in constitutive apoptosis remains ill-defined, with some groups reporting that constitutive apoptosis proceeds by mechanisms independent of caspase activation. ${ }^{26,27}$ Apoptosis of neutrophils can be accelerated or triggered by specific ligation of the so called "death receptors" (e.g. Fas, TNFR1, Trail-R1), or through the intrinsic death pathway in response to cellular injury or stress.

Although ligation of Fas in neutrophils clearly induces apoptosis, the precise biochemical pathway regulating this death has not been fully defined in neutrophils. ${ }^{28}$ From studies in other cell types, it is believed that Fas ligation results in receptor trimerization and death initiating signaling complex (DISC) formation through interaction of the receptor with its adapter protein FADD. ${ }^{29}$ Procaspase- 8 is then recruited and is activated by autocatalysis. There is good evidence that this process is regulated by a competitive inhibitor of procaspase- 8 , named c-FLIP that acts by binding to the DISC. ${ }^{29}$ Depending on the cell type, active caspase8 may cleave procaspase- 3 directly (type I cells), or cleave the Bcl-2 family member Bid (type II cells), to form truncated Bid (tBid) which subsequently acts on the mitochondria to promote the release of cytochrome c. ${ }^{29}$ The category in which neutrophils fall is unknown but work by Watson and colleagues ${ }^{30}$ suggested that these cells may be type II cells. This group demonstrated that caspase- 8 failed to activate procaspase- 3 in the presence of the mitochondrial stabilizing agent bonkrekic acid.

The classical pro-inflammatory cytokine TNF- $\alpha$, as well as promoting cytoskeletal rearrangement and enhancing neutrophil degranulation responses, has been shown to influence the rate of neutrophil apoptosis. Careful examination of neutrophil apoptosis at early time points (4- 8 hours) during culture, in the presence of TNF- $\alpha$, revealed an enhanced rate of apoptosis, whereas at later time points (18-24 hours), apoptosis was delayed. ${ }^{31}$ It appears that ligation of TNFR1 by TNF results in interactions between TNF receptor-associated proteins, such as TRADD (TNF-receptor-associated death domain), FADD (Fas-associated death domain), and caspase 8 and may involve Fas (CD95) and sphingomyelin-ceramide signaling pathways. It has also been demonstrated in 
neutrophils that TNF inducing ligand (TRAIL) can also induce apoptosis by interacting with TRAIL receptors. ${ }^{32,33}$ Although the pathways triggered by ligation of the TRAIL receptors have been partially defined in other cells, they still have to be elucidated in neutrophils.

\subsubsection{Mitochondria and $\mathrm{BCl}-2$ family proteins}

The central role of cytochrome $\mathrm{c}$ in apoptosis is now well established, but due to the scarcity and morphological irregularity of neutrophil mitochondria and the inability of groups to detect this molecule in neutrophils, ${ }^{20,34-36}$ there has been some debate concerning the role of cytochrome $\mathrm{c}$ in these cells. ${ }^{20,36}$ However, $\mathrm{we}^{36}$ and more recently Murphy and colleague ${ }^{20}$ have shown that while cytochrome $c$ still remained illusive at the protein level, cytochrome $c$ is, however, necessary to trigger apoptosis. Further evidence for the role of mitochondria in neutrophils came from work by Altznauer, et al. (2003), ${ }^{37}$ who showed that Smac/ DIABLO release from mitochondria could be inhibited by blocking calpain activity during Fas-induced and spontaneous apoptosis. Smac/ DIABLO blocks the activity of inhibitor of apoptosis proteins (IAPs) present in the cytoplasm, which in some cases bind directly to caspases, inhibiting their activity. ${ }^{38}$

The Bcl-2 family proteins have a major role in regulating neutrophil apoptosis. Interestingly, however, there appears to be no Bcl-2 in neutrophils 28,39 despite the presence of other main Bcl-2 family members, such as Bcl- $\mathrm{X}_{\mathrm{L}}, \mathrm{Mcl}-1, \mathrm{~A} 1, \mathrm{Bax}, \mathrm{Bad}$ and Bid, being identified in these cells. $^{8} \mathrm{Bax}$ is arguably the most studied pro-apoptotic Bcl-2 member in mature neutrophils. During apoptosis, Bax translocates from the cytoplasm to the mitochondria and this occurs independently of caspases, since incubation of cells with $\mathrm{z}$-VAD-fmk in the presence of an apoptosisinducing agent does not prevent translocation. ${ }^{36}$ In the absence of prosurvival signals, neutrophils appear to be primed for apoptosis and this correlates with the Bcl-2 family member profiles. The pro-apoptotic Bcl-2 family members Bax, Bad and Bak are constitutively expressed in neutrophils, ${ }^{40,41}$ whereas the anti-apoptotic members A1 and Mcl-1 are only maintained or increased by inflammatory mediators that delay neutrophil apoptosis. ${ }^{42-46}$ 


\subsection{Regulation of Neutrophil Apoptosis by External Mediators}

Many of the central apoptotic pathways are common to all cells, but discrete differences in their control and implementation allow a window for therapeutically targeting specific cell types. Neutrophils undergo constitutive apoptosis in vitro, but this may be delayed by inflammatory mediators. For example, GM-CSF, G-CSF, LPS, $\mathrm{LTB}_{4}$, PAF, C5a, IL-1 $\beta$, IL-2, IL-8, TNF- $\alpha$, IL-15, and IFN- $\gamma^{13,14,16,47,48}$ can promote neutrophil survival, suggesting that while neutrophils are on balance primed to die, activation of certain critical survival pathways can overcome this "default" state. Thus at inflammatory sites, neutrophils exposed to such mediators would survive longer to aid their defense against invading organisms. Much progress has been made in understanding the signaling mechanisms likely to regulate this process and it appears that these control mechanisms differ somewhat from many other cell types. For example, elevation of intracellular free $\mathrm{Ca}^{2+}$ levels using calcium ionophores (e.g. A23187 and ionomycin) or by mobilization of intracellular $\mathrm{Ca}^{2+}$ stores using thapsigargin ${ }^{9,49}$ promotes neutrophil longevity, whereas similar treatment in other cells (e.g. eosinophils) induces apoptosis. ${ }^{49}$ Similarly, unlike most cell types, increasing intracellular cyclic AMP (cAMP) also delays neutrophil apoptosis ${ }^{50}$ and seems to be mediated through a mechanism involving altering the balance of pro-survival versus pro-apoptotic proteins and not via protein kinase A (PKA), PI-3 kinase (PI3K), MAPK or mechanisms dependent on transcription. ${ }^{51}$ The control of neutrophil apoptosis is likely to be a reflection of the neutrophil's specialized immune purpose and the environmental milieu in which it must function. For example, a hypoxic environment, found in chronically inflamed sites, delays neutrophil apoptosis, ${ }^{26,52,53}$ whereas hypoxia induces apoptosis in most other cells types. ${ }^{54}$ Not surprisingly, bacterial lipopolysaccharide (LPS) delays neutrophil apoptosis, ${ }^{14,55}$ an event that can be enhanced by low levels $(<1 \%)$ of contaminating monocytes in neutrophil preparations ${ }^{56}$ Activation of several signal transduction pathways has been identified in LPS-treated neutrophils through the LBP/CD14/MD-2 and Toll-like receptors (TLR4) ${ }^{57}$ triggering MAPK (ERK $1 / 2$ and p38), ${ }^{58}$ PI3K ${ }^{59}$ and NF- $\mathrm{KB}$ signaling. ${ }^{60}$ Thus the precise involvement of the 
signaling pathways appears to depend on the specific survival factor under investigation. Consequently, many signaling pathways have been implicated in the regulation of neutrophil apoptosis, including protein kinase C, MAP kinases, PI3K and tyrosine kinases as well as transcriptional pathways. ${ }^{13,16,60}$ Furthermore, the likely cross talk between these pathways in neutrophils still remains to be elucidated.

Agents used clinically for the treatment of inflammatory disease can also influence neutrophil apoptosis. For example, glucocorticoids, as well as influencing inflammatory cell recruitment and activation, and inflammatory mediator generation can delay neutrophil apoptosis but promote eosinophil and lymphocyte apoptosis. ${ }^{61}$ This effect may explain why steroid therapy is so efficacious in eosinophilic inflammation, such as asthma, but not in chronic obstructive pulmonary disease (COPD), where inflammation appears to be neutrophil driven. Although the mechanisms underlying the divergent effect of glucocorticoids on inflammatory cell apoptosis are currently unknown, it has been suggested that glucocorticoids may differentially influence cytosolic $\mathrm{Ca}^{2+}$ concentrations in these cells, ${ }^{62}$ or that the ratios of glucocorticoid receptor isoforms may be different in neutrophils as compared to other cells. ${ }^{63}$

Other agents which have been shown to induce neutrophil apoptosis, include anti-Fas antibodies/Fas-L, TNF and nitric oxide. It is clear that activation of the Fas pathway promotes neutrophil apoptosis, but whether Fas-L/Fas pathway plays a role in neutrophil apoptosis in vivo remains controversial. ${ }^{64}$ Nitric oxide (NO), which has powerful vasodilatory effects, also influences neutrophil apoptosis. Recently, we and others have shown that NO donating compounds promote neutrophil apoptosis. ${ }^{65-67}$ Although the mechanisms involved have yet to be fully elucidated, it appears that NO exerts its effects via a cGMP-independent mechanism that involves the simultaneous release of oxygen free radicals. ${ }^{65}$ Oxidative dependent mechanisms have also been implicated in neutrophil apoptosis following adhesion molecule-dependent phagocytosis of bacteria or by certain bacterial products, ${ }^{68-70}$ and in apoptosis induced by factors liberated by bacteria ${ }^{71}$ or fungi. ${ }^{14}$

NF- $\mathrm{kB}$ is activated in response to a wide variety of agents in the neutrophil and appears to be a central "switch" in determining life or death. ${ }^{60}$ Perhaps the best example of the role of NF-kB in neutrophil survival is 
during TNF signaling. As mentioned above, TNF enhances neutrophil apoptosis at early time points $(\sim 6 \mathrm{~h})$, but at later times $(20 \mathrm{~h})$ promotes neutrophil survival. ${ }^{31}$ This early apoptotic effect was dramatically increased if neutrophils were co-incubated with the fungal metabolite gliotoxin, a reported powerful inhibitor of NF- $\mathrm{kB}$ activation. ${ }^{60}$ Subsequently, it was shown that TNF activates NF- $\mathrm{kB}$, limiting early apoptosis and promoting survival of neutrophils at later time points. NF- $\mathrm{kB}$ activation results in the transcription of anti-apoptotic genes necessary if neutrophil longevity is to be prolonged. This is confirmed by incubation of neutrophils with protein synthesis inhibitors in the presence of NF- $\kappa B$ activating agents, since ncutrophil apoptosis still occurs readily. NF-кB targeting may therefore provide a powerful therapeutic target for the treatment of neutrophil-mediated disease. In line with this, recent studies indicate that NF- $\mathrm{KB}$ may play a role in the apoptotic effects of certain arachidonic acid metabolites. The prostaglandin $\mathrm{D}_{2}\left(\mathrm{PGD}_{2}\right)$ metabolites $\Delta^{12} \mathrm{PGJ}_{2}$ and $15 \mathrm{dPGJ}_{2}$ are powerful inducers of apoptosis in neutrophils. $\Delta^{12} \mathrm{PGJ}_{2}$ and $15 \mathrm{dPGJ}_{2}$ are PPAR- $\gamma$ agonists, yet in the neutrophil, synthetic PPAR- $\gamma$ and PPAR- $\alpha$ ligands could not replicate the effects nor could they be blocked PPAR- $\gamma$ antagonists. LPS and TNF- $\alpha$ mediated longevity is readily overcome by $\triangle^{12} \mathrm{PGJ}_{2}$ and $15 \mathrm{dPGJ}_{2}$, and this is mediated by inhibit-

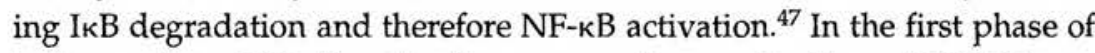
inflammation, NF- $\mathrm{KB}$ activation causes the production of COX-2, an enzyme which controls the formation of PGs, including $\mathrm{PGD}_{2}$ and its metabolites. During the resolution phase, these metabolites can inhibit the activation of NF- $\mathrm{\kappa B}$, production of pro-inflammatory cytokines and ultimately inducing neutrophil apoptosis. Therefore, emerging data suggest that both NF- $\mathrm{KB}$ activation and the formation of $\mathrm{PGD}_{2}$ metabolites may promote the resolution of inflammation by influencing inflammatory cell (including neutrophil) apoptosis. ${ }^{47,72-76}$

\section{CLEARANCE OF APOPTOTIC NEUTROPHILS}

Once the beneficial function of recruited neutrophils into inflammatory sites has been fulfilled, it is evident that failure of neutrophil removal could lead to tissue damage as these cells would inevitably undergo disintegration and liberate their histotoxic contents. Thus, mechanisms have 
evolved to efficiently remove these cells in order to limit potential tissue damage and development of chronic inflammation. A key mechanism for limiting potential neutrophil mediated damage is rapid, efficient and noninflammatory recognition and phagocytosis by macrophages of neutrophils that have undergone apoptosis (Fig. 1). Indeed, it is believed that failure of phagocytes such as the macrophage to scavenge apoptotic cells, may be directly responsible, in part, for the pathogenesis and progression of inflammatory conditions. There is now good evidence in in vitro, experimental inflammatory models and human disease that clearance of apoptotic neutrophils occurs by this process. It was Newman, et al. $(1982)^{77}$ who first reported that aged senescent, but not fresh, isolated human neutrophils were recognized and ingested by inflammatory macrophages. It took several years before it was realized that the process of apoptosis had to have occurred before the neutrophils could be engulfed by macrophages. ${ }^{78}$ Importantly, it was subsequently shown that macrophage ingestion of apoptotic neutrophils does not result in the liberation of histotoxic neutrophil granule contents and generation of macrophage-derived pro-inflammatory mediators. ${ }^{79}$ Indeed, this highly efficient and specific process results in the generation and release of macrophage-derived mediators with potential anti-inflammatory properties (e.g. TGF- $\alpha$ and IL-10). ${ }^{80-82}$ It has become apparent that there exist a number of molecular mechanisms by which phagocytes recognize and ingest apoptotic neutrophils. ${ }^{83-85}$

\section{CONCLUDING REMARKS}

Due to the potential for the development of novel therapeutic targets afforded by specifically inducing neutrophil apoptosis and augmenting macrophage clearance of apoptotic neutrophils, a great deal of effort is being invested in deciphering the precise molecular mechanisms underlying these processes. We believe that the fruits of these efforts will lead to a greater appreciation of the physiological mechanisms involved in the resolution of inflammation and help identify novel therapies aimed at influencing neutrophil apoptosis with the ultimate goal of treating chronic inflammatory diseases where the neutrophil plays a prominent role. 


\section{REFERENCES}

1. Borregaard N, Cowland JB. Blood 1997; 89:3503-3521.

2. Haslett C. Br Med Bull 1997; 53:669-683.

3. Rossi AG, Haslett C. Inflammation, Cell Injury, and Apoptosis. In: ProInflammatory and Anti-Inflammatory Peptides (ed. Said SI) in the Lung Biology in Health and Disease Series (ed. Haslett C); 112:9-24.

4. Kerr JF, Wyllie AH, Currie AR. Br J Cancer 1972; 26:239-257.

5. Wyllie AH. Br Med Bull 1997; 53:451-465.

6. Fadok VA, Bratton DL, Frasch SC, et al. Cell Death Differ 1998; 5:551-562.

7. Dransfield I, Buckle AM, Savill JS, et al. J Immunol 1994; 153: 1254-1263.

8. Akgul C, Moulding DA, Edwards SW. FEBS Lett 2001; 487:318-322.

9. Whyte MK, Meagher LC, MacDermot J, Haslett C. J Immunol 1993; 150: 5124-5134.

10. Dransfield I, Stocks SC, Haslett C. Blood 1995; 85:3264-3273.

11. Cannistra SA, Griffin JD. Semin Hematol 1988; 25:173-188.

12. Martin C, Burdon PC, Bridger G, et al. Immunity 2003; 19:583-593.

13. Walker A, Ward C, Dransfield I, et al. Curr Drug Targets Inflamm Allergy 2003; 2:339-347.

14. Ward C, Dransfield I, Chilvers ER, et al. Trends Pharmacol Sci 1999; 20:503-509.

15. Walsh GM. Br J Haematol 2000; 111:61-67.

16. Simon HU. Immunol Rev 2003; 193:101-110.

17. Kumar S. Cell Death Differ 1999; 6:1060-1066.

18. Leist M, Jaattela M. Nat Rev Mol Cell Biol 2001; 2:589-598.

19. Nicholson DW. Cell Death Differ 1999; 6:1028-1042.

20. Murphy BM, O'Neill AJ, Adrain C, et al. J Exp Med 2003; 197:625-632.

21. Hengartner MO. Nature 2000; 407:770-776.

22. Pongracz J, Webb P, Wang K, et al. J Biol Chem 1999; 274:37329-37334.

23. Sanghavi DM, Thelen M, Thornberry NA, et al. FEBS Lett 1998; 422:179-184.

24. Kothakota S, Azuma T, Reinhard C, et al. Science 1997; 278:294-298. 
25. Savill JS, Wyllie AH, Henson JE, et al. J Clin Invest 1989; 83:865-875.

26. Mecklenburgh KI, Walmsley SR, Cowburn AS, et al. Blood 2002; 100: 3008-3016.

27. Harter L, Keel M, Hentze H, et al. J Trauma 2001; 50:982-988.

28. Iwai K, Miyawaki T, Takizawa T, et al. Blood 1994; 84:1201-1208.

29. Tibbetts MD, Zheng L, Lenardo MJ. Nat Immunol 2003; 4:404-409.

30. Watson RW, O'Neill A, Brannigen AE, et al. FEBS Lett 1999; 453:67-71.

31. Murray J, Barbara JA, Dunkley SA, et al. Blood 1997; 90:2772-2783.

32. Daigle I, Simon HU. Swiss Med Wkly 2001; 131:231-237.

33. Renshaw SA, Parmar JS, Singleton V, et al. J Immunol 2003; 170:1027-1033.

34. Fossati G, Moulding DA, Spiller DG, et al. J Immunol 2003; 170:1964-1972.

35. Maianski NA, Mul FP, van Buul JD, et al. Blood 2002; 99:672-679.

36. Pryde JG, Walker A, Rossi AG, et al. J Biol Chem 2000; 275:33574-33584.

37. Altznauer F, Conus S, Cavalli A, et al. J Biol Chem 2004; 279:5947-5957.

38. Salvesen GS, Duckett CS. Nat Rev Mol Cell Biol 2002; 3:401-410.

39. Lagasse E, Weissman IL. J Exp Med 1994; 179:1047-1052.

40. Moulding DA, Akgul C, Derouet M, et al. J Leukoc Biol 2001; 70:783-792.

41. Cowburn AS, Cadwallader KA, Reed BJ, et al. Blood 2002; 100:2607-2616.

42. Chuang PI, Yee E, Karsan A, et al. Biochem Biophys Res Commun 1998; 249: 361-365.

43. Epling-Burnette PK, Zhong B, Bai F, et al. J Immunol 2001; 166:7486-7495.

44. Fulop T Jr, Larbi A, Linteau A, et al. Ann NY Acad Sci 2002; 973:305-308.

45. Moulding DA, Quayle JA, Hart CA, Edwards SW. Blood 1998; 92:2495-2502.

46. Wang JM, Chao JR, Chen W, et al. Mol Cell Biol 1999; 19:6195-6206.

47. Ward C, Dransfield I, Murray J, et al. J Immunol 2002; 168:6232-6243.

48. Edwards SW, Moulding DA, Derouet M, Moots RJ. Chem Immunol Allergy 2003; 83:204-224.

49. Cousin JM, Haslett C, Rossi AG. Biochem Soc Trans 1997; 25:243S.

50. Rossi AG, Cousin JM, Dransfield I, et al. Biochem Biophys Res Commun 1995; 217:892-899. 
51. Martin MC, Dransfield I, Haslett C, Rossi AG. J Biol Chem 2001; 276:45041-45050.

52. Hannah S, Mecklenburgh K, Rahman I, et al. FEBS Lett 1995; 372:233-237.

53. Murray J, Walmsley SR, Mecklenburgh KI, et al. Ann NY Acad Sci 2003; 1010:417-425.

54. Riva C, Chauvin C, Pison C, Leverve X. Anticancer Res 1998; 18:4729-4736.

55. Lee A, Whyte MK, Haslett C. J Leukoc Biol 1993; 54:283-288.

56. Sabroe I, Jones EC, Usher LR, et al. J Immunol 2002; 168:4701-4710.

57. Sabroe I, Prince LR, Jones EC, et al. J Immunol 2003; 170:5268-5275.

58. Nolan B, Duffy A, Paquin L, et al. Surgery 1999; 126:406-412.

59. Klein JB, Buridi A, Coxon PY, et al. Cell Signal 2001; 13:335-343.

60. Ward C, Chilvers ER, Lawson MF, et al. J Biol Chem 1999; 274:4309-4318.

61. Meagher LC, Cousin JM, Seckl JR, Haslett C. J Immunol 1996; 156:4422-4428.

62. Distelhorst CW. Cell Death Differ 2002; 9:6-19.

63. Strickland I, Kisich K, Hauk PJ, et al. J Exp Med 2001; 193:585-593.

64. Ottonello L, Tortolina G, Amelotti M, Dallegri F. J Immunol 1999; 162: 3601-3606.

65. Ward C, Wong TH, Murray J, et al. Biochem Pharmacol 2000; 59:305-314.

66. Taylor EL, Megson IL, Haslett C, Rossi AG. Biochem Biophys Res Commun $2001 ; 289: 1229-1236$.

67. Taylor EL, Megson IL, Haslett C, Rossi AG. Cell Death Differ 2003; 10:418-430.

68. Coxon A, Rieu P, Barkalow FJ, et al. Immunity 1996; 5:653-666.

69. Kagan VE, Borisenko GG, Serinkan BF, et al. Am J Physiol Lung Cell Mol Physiol 2003; 285:L1-17.

70. Watson RW. Antioxid Redox Signal 2002; 4:97-104.

71. Usher LR, Lawson RA, Geary I, et al. J Immunol 2002; 168:1861-1868.

72. Lawrence T, Willoughby DA, Gilroy DW. Nat Rev Immunol 2002; 2:787-795.

73. Lawrence T, Gilroy DW, Colville-Nash PR, Willoughby DA. Nat Med 2001; 7:1291-1297.

74. Gilroy DW, Colville-Nash PR. J Mol Med 2000; 78:121-129.

75. Gilroy DW, Colville-Nash PR, McMaster S, et al. FASEB J 2003; 17:2269-2271. 
168 The Neutrophils

76. Colville-Nash PR, Gilroy DW. Drug News Perspect 2000; 13:587-597.

77. Newman SL, Henson JE, Henson PM. J Exp Med 1982; 156:430-442.

78. Savill JS, Wyllie AH, Henson JE, et al. J Clin Invest 1989; 83:865-875.

79. Meagher LC, Savill JS, Baker A, et al. J Leukoc Biol 1992; 52:269-273.

80. Fadok VA, Bratton DL, Konowal A, et al. J Clin Invest 1998; 101:890-898.

81. Voll RE, Herrmann M, Roth EA, et al. Nature 1997; 390:350-351.

82. Liu Y, Cousin JM, Hughes J, et al. J Immunol 1999; 162:3639-3646.

83. Giles KM, Ross K, Rossi AG, et al. J Immunol 2001; 167:976-986.

84. Savill J, Fadok V. Nature 2000; 407:784-788.

85. Savill J, Dransfield I, Gregory C, Haslett C. Nat Rev Immunol 2002; 2:965-975. 


\title{
Nitric oxide and the resolution of inflammation: implications for atherosclerosis
}

\author{
Catherine A Shaw, Emma L Taylor*, Ian L Megson, Adriano G Rossi*/+ \\ Centre for Cardiovascular Science, Hugh Robson Building, George Square *MRC Centre for Inflammation Research, \\ University of Edinburgh, Teviot Place, Edinburgh, Scotland, UK
}

The ubiquitous free radical, nitric oxide (NO), plays an important role in many biological processes including the regulation of the inflammatory response. Alterations in NO synthesis by endogenous systems likely influence inflammatory processes occurring in a wide range of diseases including many in the cardiovascular system (e.g. atherosclerosis). Progression of inflammatory conditions depends not only upon the recruitment and activation of inflammatory cells but also upon their subsequent removal from the inflammatory milieu. Apoptosis, or programmed cell death, is a fundamental process regulating inflammatory cell survival and is critically involved in ensuring the successful resolution of an inflammatory response. Apoptosis results in shutdown of secretory pathways and renders effete, but potentially highly histotoxic, cells instantly recognisable for non-inflammatory clearance by phagocytes (e.g., macrophages). However; dysregulation of apoptosis and phagocytic clearance mechanisms can have drastic consequences for development and resolution of inflammatory processes. In this review we highlight the complexities of NO-mediated regulation of inflammatory cell apoptosis and clearance by phagocytes and discuss the molecular mechanisms controlling these $\mathrm{NO}$ mediated effects. We believe that manipulation of pathways involving NO may have previously unrecognised therapeutic potential for limiting or resolving inflammatory and cardiovascular disease.

Key words: nitric oxide - apoptosis - inflammation - resolution

The inorganic free radical, nitric oxide (NO), was first identified as an endothelium-derived endogenous messenger responsible for the regulation of vascular tone (Furchgott \& Zawadzki 1980, Palmer et al. 1987). However, since then it has become clear that NO is the signalling molecule responsible for several diverse physiologi$\mathrm{cal}$ and pathophysiological processes. Synthesised from $\mathrm{L}$-arginine by three isoforms of the enzyme nitric oxide synthase (NOS), NO is now known to control vascular smooth muscle tone, inhibit platelet and inflammatory cell adhesion and activation, and to be a transmitter at nonadrenergic non-cholinergic (NANC) synapses (Moncada et al. 1991, Quinn et al. 1995). Recent studies have revealed that NO can also modulate apoptosis, or programmed cell death, in a variety of cell types, including human inflammatory cells (Taylor et al. 2003). Apoptosis of inflammatory cells is a highly regulated process whereby cellular death occurs without the disruption of the cell membrane and subsequent release of the pro-inflammatory and histotoxic contents of the dying cell (Haslett 1997, Rossi et al. 2003). Apoptotic cells are instantly recognised and ingested by phagocytes, such as macrophages, using mechanisms that down-regulate pro-inflammatory mediator release and increase the release of agents with anti-inflammatory potential from the ingesting cell (Meagher et al. 1992, Fadok et al. 1998, Liu et al. 1999). Hence, apoptosis represents a non-inflammatory

\footnotetext{
${ }^{+}$Corresponding author. E-mail: a.g.rossi@ed.ac.uk Received 8 November 2004 Accepted 30 December 2004
}

mechanism to remove potentially damaging pro-inflammatory cells from the site of inflammation and is therefore critical to the successful resolution of the inflammatory response. Pharmacological manipulation of the rate of apoptosis in inflammatory cells, such as granulocytes and macrophages, may represent a potential therapeutic strategy for the treatment of chronic inflammatory disorders (Ward et al. 1999, Gilroy et al. 2004).

NO can be both pro- and anti-apoptotic, depending on local concentrations and the specific cell type in question (Quinn et al. 1995, Kim et al. 1999, Taylor et al. 2003). Current evidence suggests that lower concentrations of NO produced by the constitutive endothelial and neuronal isoforms of NOS (eNOS and nNOS) are cytoprotective, whilst supraphysiological concentrations produced by the inducible NOS isoform (iNOS) trigger cell death (Nicotera et al. 1997). This paradox may be explained, at least in part, by the free radical nature of NO and hence the ease with which it will react with other radicals, particularly reactive oxygen species, present in the milieu to form various NO-related species in vivo. For example, NO combines rapidly with inflammatory cell derived superoxide anions $\left(\mathrm{O}_{2}^{-}\right)$to form highly cytotoxic peroxynitrite (ONOO-) (Maxwell \& Lip 1997).

\section{NO as a mediator of inflammatory cell apoptosis}

The pro- and anti-apoptotic actions of NO have been well documented in many cell systems. For example, high concentrations of either exogenous or endogenous iNOSderived NO have been shown to induce apoptosis in murine macrophage cell lines (Albina et al. 1993, Sarih et al. 1993). However, pre-treatment with low concentrations of exogenous NO protects RAW 264 cells against cell death upon subsequent exposure to higher concentra- 
tions of NO which would normally be cytotoxic (Yoshioka et al. 2003). However, despite the apparent reduced capacity of human macrophages in comparison to murine macrophages (Albina 1995, Schneemann \& Schoedon 2002), to generate iNOS derived NO (Thomassen \& Kavuru 2001), human macrophages do undergo apoptosis in response to exogenous NO. For example, the NO donors, S-nitrosoglutathione (GSNO), and spermine diazenium diolate (SPER/NO) induce apoptosis in primary human monocyte-derived macrophages (von Knethen et al. 1999). Exogenously delivered NO from NO donors (e.g., sodium nitroprusside; SNP and GSNO) induce apoptosis in human neutrophils (Fortenberry et al. 1999, Singhal et al. 1999). However, it has also been established that NO may have anti-apoptotic potential in neutrophils; low concentrations of NO generated from the spontaneous NO donors, SPER/NO and DEA/NO, reduce the rate of neutrophil apoptosis (Taylor et al. 2001). In contrast, the same study showed that the oxatriazole derivative, GEA3162 , at equivalent concentrations produced no such inhibition. However, it was demonstrated that GEA-3162 decomposes to co-generate both $\mathrm{NO}$ and $\mathrm{O}_{2}^{-}$, which then react to form $\mathrm{ONOO}^{-}$(Taylor et al. 2004). This suggests that the pro- or anti-apoptotic effects of NO may be critically governed by the specific NO-related species generated.

Interestingly, the production of $\mathrm{ONOO}^{-}$may be of particular importance at sites of inflammation where the concentration of reactive oxygen species is likely to be elevated (Crow \& Beckman 1995). However, the precise role of $\mathrm{ONOO}^{-}$in inflammatory cell apoptosis remains to be fully elucidated. There is some evidence to suggest that $\mathrm{ONOO}^{-}$at high concentrations increases apoptosis in murine RAW 264.7 cells (Sandoval et al. 1997), whilst at lower concentrations it may have a protective effect against lipopolysaccharide (LPS) and interferon (IFN)- $\gamma$ induced apoptosis in these cells (Scivittaro et al. 1997). A scavenger of $\mathrm{ONOO}^{-}$, uric acid, had no effect on apoptosis induced by the NO donors GSNO or SPER/NO in RAW 264.7 macrophages, but abolished apoptosis induced by the $\mathrm{ONOO}^{-}$generator $\mathrm{SIN}-1$, suggesting that $\mathrm{ONOO}^{-}$is a mediator of apoptosis, at least not in this cell type (Brockhaus \& Brune 1999).

As is the case with macrophages, there are conflicting reports about the ability of $\mathrm{ONOO}^{-}$to induce or suppress apoptosis in neutrophils. Several investigators have demonstrated that SIN-1 and GEA-3162 increases the rate of apoptosis in human neutrophils (Blaylock et al. 1998, Ward et al. 2000, Taylor et al. 2004). Conversely, Blaylock et al. (1998) reported SIN-1 produced no significant increase in neutrophil apoptosis. However, this may be due to experimental differences and the exact amounts of $\mathrm{ONOO}^{-}$ present rather than a true difference in the effect of $\mathrm{ONOO}^{-}$ (Taylor et al. 2003).

\section{NO and apoptosis in the resolution of inflammation}

The ability of NO to induce apoptosis is particularly relevant during the resolution phase of inflammation. In a mouse model of kidney inflammation, activated macrophages have been shown to induce apoptosis in neighbouring mesangial cells prior to their ingestion by phago- cytes (Duffield et al. 2000). The ability of these activated macrophages to induce apoptosis is greatly reduced in the presence of the NOS inhibitor N-É-monomethyl-L-arginine (L-NMMA), suggesting that macrophage-directed apoptosis of mesangial cell apoptosis occurs via a NOdependent mechanism (Duffield et al. 2001). Similarly, several studies have demonstrated that activated macrophages infiltrating murine tumours induce apoptosis via a NO-dependent pathway in both activated anti-tumour T cells and in the tumour cells themselves (Saio et al. 2001, Chattopadhyay et al. 2002). Thus, it appears that macrophages have the capacity to induce apoptosis of nearby cells by the liberation of NO to enhance the clearance of apoptotic cells and thereby promote the resolution phase of inflammation (Figure).

\section{Mechanism of action of NO}

The classical pathway by which NO exerts many of its actions is via activation of the enzyme soluble guanylate cyclase (sGC) (Moncada et al. 1991) and resultant conversion of guanosine 5'-triphosphate (GTP) to the second messenger 3', 5'-cyclic guanosine monophosphate (cGMP) (Ignarro et al. 1999). However, recent studies have established that NO can also act via cGMP-independent pathways in various systems, particularly during the inhibition of platelet aggregation and regulation of inflammatory cell apoptosis (Gordge et al. 1998, Sogo et al. 2000, Ward et al. 2000, Crane et al. 2002).

It is generally thought that lower concentrations of NO inhibit apoptosis via cGMP-dependent mechanisms, whilst higher concentrations are cytotoxic on account of cGMP-independent signalling. For example, Yoshoka et al. (2003) demonstrated that pre-treatment of RAW 264 cells with a low concentration of the NO donor SNP, inhibited cell death upon subsequent exposure to higher concentrations of NO. This protection was negated in the presence of sGC inhibitors and could be mimicked by cGMP analogues, suggesting that the cellular protection was conferred by cGMP.

Conversely, at higher concentrations, NO has been shown to induce apoptosis in rabbit macrophages - an effect which was unaffected by antagonism of cGMP-dependent kinases and not mimicked by cGMP analogues, suggesting that the pro-apoptotic action of NO is cGMPindependent (Wang et al. 1999). The peroxynitrite generators, SIN-1 and GEA-3162, have also been shown to produce a marked concentration-dependent induction of apoptosis in isolated human neutrophils (Ward et al. 2000). Again, this induction was unaffected by inhibitors of sGC, and cGMP analogues failed to elicit a pro-apoptotic response suggesting that a mechanism independent of cGMP signalling also featured in neutrophils. Interestingly, superoxide dismutase (SOD), the enzyme responsible for converting $\mathrm{O}_{2}^{-}$to hydrogen peroxide $\left(\mathrm{H}_{2} \mathrm{O}_{2}\right)$, antagonised the actions of SIN-1 and GEA-3162, whilst "authentic" peroxynitrite mimicked their effects. This result may, therefore, highlight the critical importance of NO-related species in determining an anti- or pro-apoptotic response, with the final outcome depending on the balance between reactive oxygen and nitrogen species. 


\section{Atherosclerosis}

Atherosclerosis is a multi-factorial condition with a complicated aetiology, and, in combination with the associated cardiovascular syndromes, such as myocardial infarction and stroke, is a major cause of morbidity and mortality. However, it is now widely recognised that there is an inflammatory component to the disease pathogenesis and progression (Ross 1999a, b, Ludewig et al. 2002).

Atherosclerosis is characterised by the development of lipid-rich atherosclerotic plaques in the subendothelial space of conduit vessels, such as the coronary artery and aorta (Badimon et al. 1993). These plaques are usually eccentric, with the lipid rich core encapsulated by a fibrous, collagen-rich cap of smooth muscle cells and extracellular matrix (Davies 1997). The underlying causes of atherogenesis remain largely unknown, although a critical early stage is thought to be an insult to the endothelium, either physical or through oxidative stress. The consequences of this insult are multiple; firstly, in contrast to the situation in healthy endothelium, the injured endothelium becomes dysfunctional and production of $\mathrm{NO}$ by eNOS decreases, promoting vasoconstriction and platelet and inflammatory cell adhesion. Secondly, a protective inflammatory response is triggered. However, depending on the nature and duration of the insult, this protective response becomes excessive and over a period of years, comes to constitute the disease process itself(Ross 1999a, b). The inflammatory process begins with the expression of chemotactic and adhesion molecules for monocytes and lymphocytes, such as vascular cell adhesion molecule 1 (VCAM-1), on dysfunctional endothelial cells. Circulating monocytes adhere to the site of endothelial damage and translocate to the sub-endothelial space (Vogel 1997). Colony stimulating factors secreted from areas of endothelial damage induce monocytes to differentiate into macrophages, which then express scavenger receptors on their membranes, facilitating the internalisation of oxidised low density lipoprotein (ox-LDL). The accumulation of ox-LDL continues unchecked as, unlike LDL receptors, scavenger receptors are not downregulated by cells in the cholesterol-replete state (Maxwell \& Lip 1997). In this lipid-laden state, macrophages are known as foam cells and it is an aggregation of these foam cells in the vessel intima which form the earliest recognisable lesion of atherosclerosis - the fatty streak (Ross 1993). The plaque continues to grow via the accumulation of further macrophage foam cells and eventually becomes overlaid with a layer of smooth muscle cells forming a fibrous, collagen-rich cap. The cap serves to keep the highly thrombogenic contents of the plaque separate from the circulation. However, if the plaque cap is compromised and the contents exposed to the circulation, platelets are rapidly recruited and activated resulting in thrombus formation, leading to the more serious acute cardiovascular syndromes (Badimon et al. 1993).

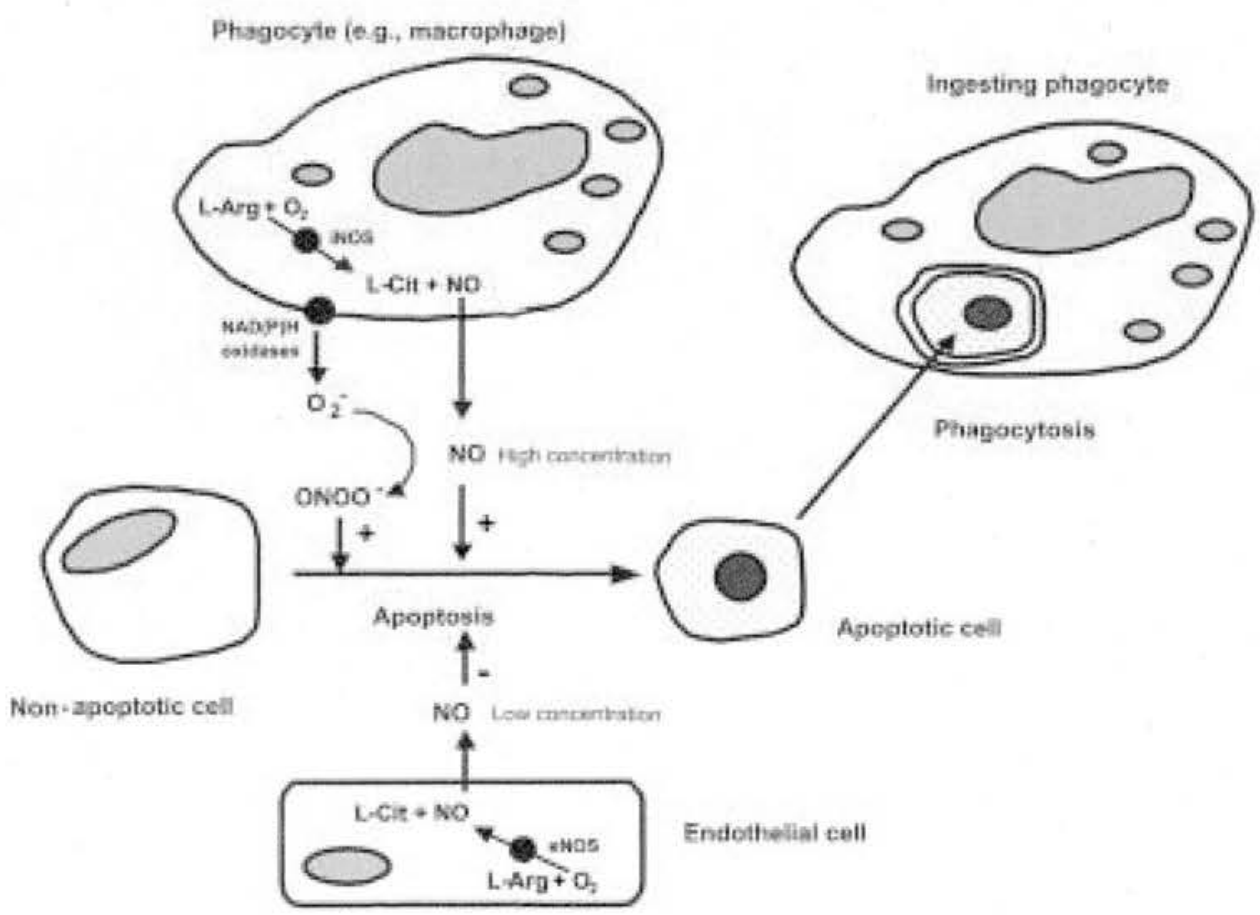

High concentrations of nitric oxide (NO) synthesised by iNOS in phagocytes, such as macrophages, induce apoptosis in neighbouring cells. In addition, apoptosis can also be induced by $\mathrm{ONOO}^{-}$generated as $\mathrm{O}_{2}^{-}$produced by phagocytes reacts with $\mathrm{NO}$. Apoptotic cells are subsequently recognised and ingested by phagocytes, thus aiding the resolution of inflammation. Conversely, low concentrations of NO produced constitutively by eNOS in endothelial cells can inhibit apoptosis. 


\section{Inflammatory cell apoptosis in atherosclerosis}

Recruitment of inflammatory cells, particularly monocytes and macrophages, is the major driving force behind plaque growth and development. However, the plaque is dynamic and inflammatory cells are constantly turning over within the core. It is well established that apoptotic cells, particularly macrophages, are present in atherosclerotic plaques in both human and animal models of the disease. Apoptotic macrophages and smooth muscle cells have been identified by TUNEL staining in sections from human plaques by various authors (Bjorkerud \& Bjorkerud 1996, Haunstetter \& Izumo 1998). Because apoptotic cells are ingested by phagocytes without initiating any further proinflammatory response, it has been suggested that apoptosis may represent a mechanism to regress the plaque. NO is a particularly promising candidate for this strategy because, as well as the pro-apoptotic actions discussed above, it has several other powerful anti-atherogenic characteristics including a powerful inhibitory effect on platelet and inflammatory cell activation (Moncada et al. 1991, Armstrong 2001). Evidence is emerging in support of this hypothesis. For example, administration of Larginine (the substrate for NOS) to hypercholesterolemic rabbits increases the number of apoptotic macrophages in intimal lesions by three fold. This increase in apoptosis was associated with a regression of the plaque, suggesting that manipulation of the NO synthase pathway may well represent a therapeutic approach to resolving the inflammatory response in the vessel wall (Wang et al. 1999). However, care must by exercised when considering this approach because NO is also known to induce apoptosis in smooth muscle cells (Labelle et al. 2004). Loss of cells from the fibrous cap during the latter stages of atherosclerosis may well be detrimental, destabilising the plaque and promoting rupture (Kockx \& Knaapen 2000)

\section{Conclusion}

Apoptosis of inflammatory cells is a tightly regulated process whereby cells are removed from the site of inflammation without triggering a subsequent pro-inflammatory response that would instigate further tissue injury. Pharmacological manipulation of apoptosis during chronic inflammatory conditions, such as atherosclerosis, may aid the resolution of inflammation and hence halt, or delay, disease progression. The ubiquitous signalling molecule and inducer of apoptosis, $\mathrm{NO}$, is a likely candidate for such manipulation and may represent a novel therapeutic target for the treatment of such conditions.

\section{REFERENCES}

Albina JE 1995. On the expression of nitric oxide synthase by human macrophages. Why no NO? J Leukoc Biol 58: 643649 .

Albina JE, Cui S et al. 1993. Nitric oxide-mediated apoptosis in murine peritoneal macrophages. J Immunol 150: 5080-5085.

Armstrong R 2001. The physiological role and pharmacological potential of nitric oxide in neutrophil activation. Int Immunopharmacol 1: 1501-1512.

Badimon JJ, Fuster V et al. 1993. Coronary atherosclerosis. A multifactorial disease. Circulation 87: 113-16.
Bjorkerud S, Bjorkerud B 1996. Apoptosis is abundant in human atherosclerotic lesions, especially in inflammatory cells (macrophages and T cells), and may contribute to the accumulation of gruel and plaque instability. Am J Pathol 149: 367-380.

Blaylock MG, Cuthbertson BH et al. 1998. The effect of nitric oxide and peroxynitrite on apoptosis in human polymorphonuclear leukocytes. Free Radic Biol Med 25: 748-752.

Brockhaus F, Brune B 1999. Overexpression of CuZn superoxide dismutase protects RAW 264.7 macrophages against nitric oxide cytotoxicity. Biochem J 338 ( Pt 2): 295-303.

Chattopadhyay S, Das T et al. 2002. Protein A-activated macrophages induce apoptosis in Ehrlich's ascites carcinoma through a nitric oxide-dependent pathway. Apoptosis 7: 4957.

Crane MS, Ollosson R et al. 2002. Novel role for low molecular weight plasma thiols in nitric oxide-mediated control of platelet function. J Biol Chem 277: 46858-46863.

Crow JP, Beckman JS 1995. Reactions between nitric oxide, superoxide, and peroxynitrite: footprints of peroxynitrite in vivo. Adv Pharmacol 34: 17-43.

Davies MJ 1997. The composition of coronary-artery plaques. $N$ Engl J Med 336: 1312-1314.

Duffield JS, Erwig LP et al. 2000. Activated macrophages direct apoptosis and suppress mitosis of mesangial cells. $J$ Immunol 164: 2110-2119.

Duffield JS, Ware CF et al. 2001. Suppression by apoptotic cells defines tumor necrosis factor-mediated induction of glomerular mesangial cell apoptosis by activated macrophages. Am J Pathol 159: 1397-1404.

Fadok VA, Bratton DL et al. 1998. Macrophages that have ingested apoptotic cells in vitro inhibit proinflammatory cytokine production through autocrine/paracrine mechanisms involving TGF-beta, PGE2, and PAF. J Clin Invest 101: 890-898.

Fortenberry JD, Owens ML et al. 1999. S-nitrosoglutathione enhances neutrophil DNA fragmentation and cell death. $\mathrm{Am}$ J Physiol 276: L435-442.

Furchgott RF, Zawadzki JV 1980. The obligatory role of endothelial cells in the relaxation of arterial smooth muscle by acetylcholine. Nature 288: 373-376.

Gilroy DW, Lawrence T et al. 2004. Inflammatory resolution: new opportunities for drug discovery. Nat Rev Drug Discov 3: 401-416.

Gordge MP, Hothersall JS et al. 1998. Evidence for a cyclic GMP-independent mechanism in the anti-platelet action of S-nitrosoglutathione. Br J Pharmacol 124: 141-148.

Haslett C 1997. Granulocyte apoptosis and inflammatory disease. $\mathrm{Br}$ Med Bull 53: 669-683.

Haunstetter A, Izumo S 1998. Apoptosis: basic mechanisms and implications for cardiovascular disease. Circ Res 82 : 1111-1129

Ignarro LJ, Cirino G et al. 1999. Nitric oxide as a signaling molecule in the vascular system: an overview. J Cardiovasc Pharmacol 34: 879-886.

Kim YM, Bombeck CA et al. 1999. Nitric oxide as a bifunctional regulator of apoptosis. Circ Res 84: 253-256. 
Kockx MM, Knaapen MW 2000. The role of apoptosis in vascular disease. J Pathol 190: 267-280.

Labelle M, Beaulieu M et al. 2004. Integrating clinical practice guidelines into daily practice: impact of an interactive workshop on drafting of a written action plan for asthma patients. J Contin Educ Health Prof 24: 39-49.

Liu Y, Cousin JM et al. 1999. Glucocorticoids promote nonphlogistic phagocytosis of apoptotic leukocytes. $J$ Immunol 162: 3639-3646.

Ludewig B, Zinkernagel RM et al. 2002. Arterial inflammation and atherosclerosis. Trends Cardiovasc Med 12: 154-159.

Maxwell SR, Lip GY 1997. Free radicals and antioxidants in cardiovascular disease. Br J Clin Pharmacol 44: 307-317.

Meagher LC, Savill JS et al. 1992. Phagocytosis of apoptotic neutrophils does not induce macrophage release of thromboxane B2. J Leukoc Biol 52: 269-273.

Moncada S, Palmer RM et al. 1991. Nitric oxide: physiology, pathophysiology, and pharmacology. Pharmacol Rev 43: 109-142.

Nicotera P, Brune B et al. 1997. Nitric oxide: inducer or suppressor of apoptosis? Trends Pharmacol Sci 18: 189-190.

Palmer RM, Ferrige AG et al. 1987. Nitric oxide release accounts for the biological activity of endothelium-derived relaxing factor. Nature 327 : 524-526.

Quinn AC, Petros AJ et al. 1995. Nitric oxide: an endogenous gas. BrJ Anaesth 74: 443-451.

Ross R 1993. The pathogenesis of atherosclerosis: a perspective for the 1990s. Nature 362: 801-809.

Ross R 1999a. Atherosclerosis is an inflammatory disease. Am Heart J 138: S419-420.

Ross R 1999b. Atherosclerosis is an inflammatory disease. $N$ Engl J Med 340: 115-126.

Rossi AG, Ward C, Dransfield I, Haslett C 2003. Apoptosis of inflammatory cells. In NF Adkinson et al. (eds), Middleton's Allergy: Principles and Practice, Chapt 26, 6th ed., MosbyHarcourt, p. 412-424.

Saio M, Radoja S et al. 2001. Tumor-infiltrating macrophages induce apoptosis in activated CD8 $(+) \mathrm{T}$ cells by a mechanism requiring cell contact and mediated by both the cellassociated form of TNF and nitric oxide. J Immunol 167: 5583-5593.

Sandoval M, Ronzio RA et al. 1997. Peroxynitrite-induced apoptosis in epithelial (T84) and macrophage (RAW 264.7) cell lines: effect of legume-derived polyphenols (phytolens). Nitric Oxide 1: 476-483.

Sarih M, Souvannavong V et al. 1993. Nitric oxide synthase induces macrophage death by apoptosis. Biochem Biophys
Res Commun 191: 503-508.

Schneemann M, Schoedon G 2002. Species differences in macrophage NO production are important. Nat Immunol 3: 102.

Scivittaro V, Boggs S et al. 1997. Peroxynitrite protects RAW 264.7 macrophage from Lipopolysaccharide/interferongamma-induced cell death. Biochem Biophys Res Commun 241: 37-42.

Singhal PC, Patel P et al. 1999. Ethanol-induced neutrophil apoptosis is mediated through nitric oxide. J Leukoc Biol 66: 930-936.

Sogo N, Magid KS et al. 2000. Inhibition of human platelet aggregation by nitric oxide donor drugs: relative contribution of cGMP-independent mechanisms. Biochem Biophys Res Commun 279: 412-419.

Taylor EL, Megson IL et al. 2001. Dissociation of DNA fragmentation from other hallmarks of apoptosis in nitric oxide-treated neutrophils: differences between individual nitric oxide donor drugs. Biochem Biophys Res Commun 289: 1229-1236.

Taylor EL, Megson IL et al. 2003. Nitric oxide: a key regulator of myeloid inflammatory cell apoptosis. Cell Death Differ 10: 418-430.

Taylor EL, Rossi AG et al. 2004. GEA 3162 decomposes to cogenerate nitric oxide and superoxide and induces apoptosis in human neutrophils via a peroxynitrite-dependent mechanism. Br J Pharmacol 143: 179-185.

Thomassen MJ, Kavuru MS 2001. Human alveolar macrophages and monocytes as a source and target for nitric oxide. Int Immunopharmacol 1: 1479-1490.

Vogel RA 1997. Coronary risk factors, endothelial function, and atherosclerosis: a review. Clin Cardiol 20: 426-432.

von Knethen A, Brockhaus F et al. 1999. NO-Evoked macrophage apoptosis is attenuated by cAMP-induced gene expression. Mol Med 5: 672-684.

Wang BY, Ho HK et al. 1999. Regression of atherosclerosis: role of nitric oxide and apoptosis. Circulation 99: 12361241.

Ward C, Dransfield I et al. 1999. Pharmacological manipulation of granulocyte apoptosis: potential therapeutic targets. Trends Pharmacol Sci 20: 503-509.

Ward C, Wong TH et al. 2000. Induction of human neutrophil apoptosis by nitric oxide donors: evidence for a caspasedependent, cyclic-GMP-independent, mechanism. Biochem Pharmacol 59: 305-314.

Yoshioka Y, Yamamuro A et al. 2003. Nitric oxide at a low concentration protects murine macrophage RAW264 cells against nitric oxide-induced death via cGMP signaling pathway. Br J Pharmacol 139: 28-34. 


\title{
Regulation of Neutrophil Apoptosis and Removal of Apoptotic Cells
}

\author{
Annemieke Walker, Carol Ward, Emma L. Taylor, Ian Dransfield, Simon P. Hart, \\ Christopher Haslett and Adriano G. Rossi*
}

\section{MRC Centre for Inflammation Research, The Queen's Medical Research Institute, University of Edinburgh, 47 Little France Crescent, Edinburgh, EHI6 4TJ, UK}

\begin{abstract}
The accumulation of neutrophils during inflammation is essential for the destruction and removal of invading microorganisms. However, for resolution of inflanumation to occur, neutrophils must also be removed from the inflammatery site since these cells are capable of releasing tissue toxic molecules. Neutrophil removal has becn shown to occur via apoptosis and phagocytc clcarance of apoptotic cells. Thereforc, manipulation of these processes is likely to be a key therapeutic strategy in the management of inflammatory disease. In this review, we examine mediators of neutrophil survival and apoptosis and the signalling pathways that regulate the halance between life and death in these cells.
\end{abstract}

\section{INTRODUCTION}

Neutrophils have been implicated in many of the pathologies associated with inflammatory discasc. During inflammation, high numbers of neutrophils are often recruited as part of the innatc immunc responsc. They are often held accountable for tissue damage which occurs when the inflammatory response goes awry $[1,2]$. The cytoplasm of the neutrophi contains high numbers of granules full of toxic agents, normally reserved for killing and digestion of microorganisms, which may be released inappropriately resulting in damage to the surrounding tissucs [3] However, ncutrophils are vital to the immune response as they provide a first-linc-of-defence against invading organisms. To illustrate this, patients infections [4]. According to the inflammatory insult or disease, inflammation may be predominantly eosinophil or neutrophil-dependent. For example, while asthma is usually associated with eosinophil recruitment, severe or chronic asthma is characterised by neutrophilic inflammation [5-7]. Other diseases where neutrophils play a prominent role include emphysema, chronic bronchitis, rheumatoid arthritis, myocardial reperfusion injury and inflammatory bowel disease [8].

During self-resolving inflammation, neutrophils are removed from the inflammatory site by undergoing apoptosis and subsequently being phagocytosed by nearby macrophages [1]. Apoptosis is a noninflammatory cell death process since one of its hallmarks is the
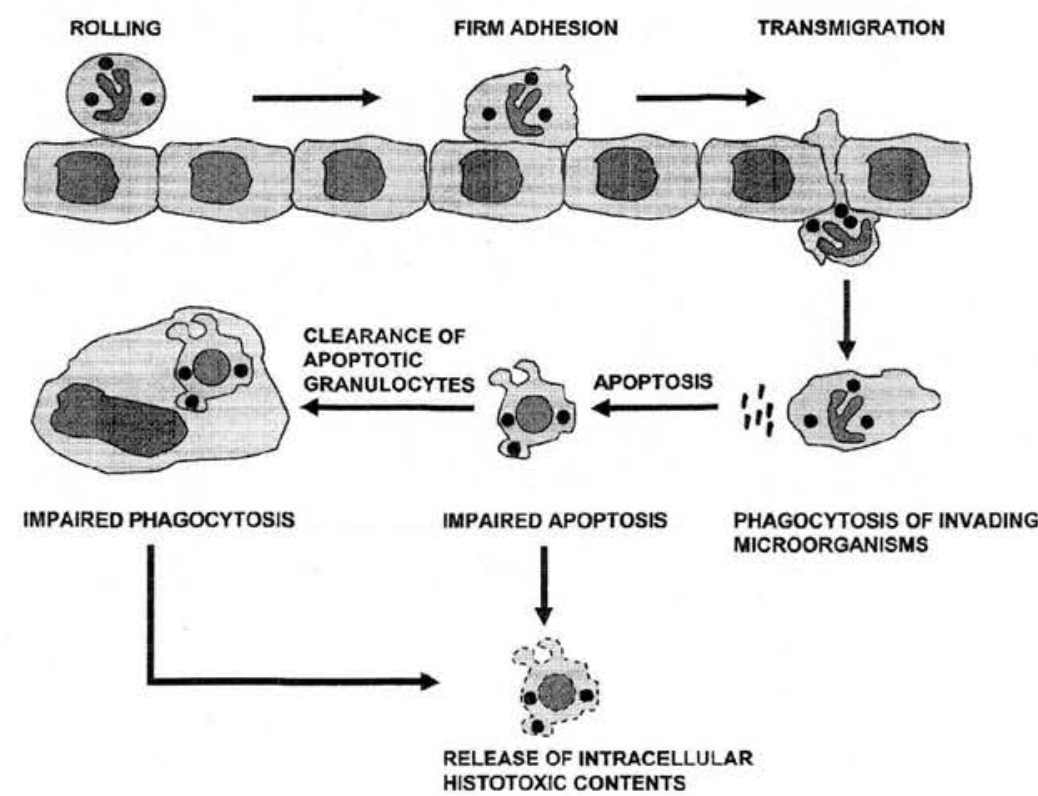

Fig. (1). The Main Stages of the Inflammatory Response.

Neutrophils bind loosely (rolling) to endothelial cells of the blood vessel wall before adhering and transmigrating to the site of injuty or infection. Neutrophils then phagocytose and destroy invading bacteria before undergoing apoptosis. Tissue-resident macrophages then ingest apoptotic neutrophils. Either impaired neutrophil apoptosis or inefficient clearance of apoptotic neutrophils can lead to the inappropriate release of toxic neutrophil granule contents, resulting in tissue injury often seen in chronic inflammatory disease.

who have a defect in neutrophil function, such as in chronic granulomatous disease or leukocyte adhesion deficiency, suffer repeated

-Address correspondence to this author at the MRC Centre for Inflammation Research. The Queen's Medical Research Institute, University of Edinburgh, 47 Little France Crescent, Edinburgh, EH16 4TJ, UK; Tel: +44-131-650-6948; Fax 144-131-650-4384; E-mail: a.g.rossi@ed ac.uk maintenance of cell membrane integrity [9], preventing leakage of the toxic contents of neutrophil intracellular granules. However, apoptotic granulocytes must be rapidly cleared from the site of inflammation since eventual cellular breakdown or secondary necrosis will allow escape of neutrophil toxic compounds. Macrophages and other cells with phagocytic capacity (e.g. fibroblasts, dendritic cells, epithelial cells, etc.) are known to remove apoptotic granulocytes by phagocytosis in a non-inflammatory manner [10]. Therefore, there are at least two areas where the process of self-resolving inflammation may become dysregulated. Firstly, neutrophils 
may fail to undergo apoptosis appropriately, and secondly, tissue resident macrophages may not clear the apoptotic neutrophils efficiently to prevent leakage of tissue toxic agents from granulocytes which have undergone apoptosis [11]. See Fig. 1 for an overview of neutrophil recruitment and removal during inflammation. In addition, we refer the reader to other relevant reviews of the subject matter $[12-23]$ and due to space constraints, apologisc for omitting other important manuscripts describing mechanisms regulating neutrophil apoptosis and their subsequent clearance by phagocytes.

\section{Morphological \& Molecular Changes during Apoptosis}

The characteristic morphological features of apoptosis of virtually all cells including neutrophils are chromatin condensation, DNA fragmentation, cytoplasmic shrinkage and membrane blebbing, where the intracellular contents are contained $[9,24]$. These features are due to an evolutionary conserved apoptotic death pathway $[24,25]$ on which several apoptotic stimuli convergc. A detailcd understanding of regulatory mechanisms and cell type specific engagement of conserved apoptotic pathways is crucial, if this phenomenon is to be exploited to maximum potential for therapeutic gain.

\section{Caspases}

The central effectors of the apoptotic pathway are the cysteinedependent aspartate-specific protease (caspase) family (see below for their activation and regulation). The caspases contain cystcinc residucs within their active sites and cleave target proteins at aspartic acid residues [26]. At least 14 mammalian caspases have been identified and can be broadly divided into 2 groups: those involved in apoptosis and those involved in cytokine processing (see Table 1). Neutrophils have been shown to possess caspases $-1,-3,-4,-6,-7,-8,-9-10$ and $-14[17,21,27$. 30]. Those caspases centrally involved in apoptosis may further be divided in terms of function into two categories: initiator caspases and effector caspases. Initiator caspases (e.g. caspase-8, 9, 10) have long prodomains containing death effector domains (DED), allowing association with adapter molecules such as Fas-associated death domain (FADD). The long prodomains of initiator caspascs also allow oligomerisation, resulting in their activation by autocatalysis [31]. The initiator caspases are thought to be primarily upstream activators of the effector caspases. Once activated initiator caspases process the effector caspases $-3,-6$ and -7 , whose primary function is thought to be cleavage of cellular proteins to disassemble the cell [32]. Many caspase substrates have been identified with cleavage resulting in their inactivation or activation [see[33]] Comparatively few caspase targets have been identified in neutrophils although fodrin [34], protein kinase $C-\delta(\mathrm{PKC}-\delta)[35]$ and more recently the pro-apoptotic Bic2 family member BID [36] have been identified as caspase substrates in neutrophils.

Table 1. Functional Division of Caspases

\begin{tabular}{|c|c|}
\hline Apoptosis & Cytokine Processing \\
\hline \hline Caspase-2 & Caspase-1 \\
\hline Caspase-3 & Caspase-4 \\
\hline Caspase-6 & Caspasc-5 \\
\hline Caspase-7 & Caspase-11 \\
\hline Caspase-8 & Caspase-13 \\
\hline Caspase-9 & \\
\hline Caspase-10 & \\
\hline Caspase-12 & \\
\hline Caspase-14 & \\
\hline
\end{tabular}

The induction of the apoptotic caspase-cascade can occur via ligation of death receptors (e.g. Fas, TNFR, TRAIL) or cellular stress (e.g. cytotoxic drugs) and is reliant upon the activation of caspasc- 9 and appears to be mitochondria dependent. However, there is considerable overlap between death receptor and stress induced pathways depending on the cell type and apoptotic stimulus, and in many cases, receptormediated apoptosis is also mitochondria-dependent [31]. Activation of caspase-9 requires the release of cytochrome $c$ and apoptosis proteaseactivating factor (Apaf-1) from the inner mitochondrial membrane and the presence of ATP or dATP. This complex of cytochrome c, ATP and procaspase- 9 , the apoptosome, is believed to allow the proximity-induced processing of procaspase-9, which can then activate procaspase- 3 [37,38]. Cytochrome c release was shown to be involved in the induction of apoptosis and caspase- 3 activation in neutrophils, and this was later independently confirmed $[29,39]$. See Fig. 2 for the role of caspases in ncutrophil apoptosis.

\section{Initiation of Apoptosis}

Two principal pathways exist for the initiation of the apoptotic cascade; firstly, engagement of 'death receptors' such as TNF and Fas and secondly, through the intrinsic death pathway in response to cellular injury or stress. TNF $-\alpha$ is unusual in that it mediates both pro- and antiapoptotic signals in neutrophils [40]. Fas ligation also has dual capabilities: regulating chemotaxis and the induction of apoptosis in neutrophils $[41,42]$.

Stimulation of the Fas death pathway results in rapid apoptosis in neutrophils [41]. Studies in other cell types have shown that activation of Fas induces receptor trimerisation and formation of the death initiating signalling complex (DISC) through interaction of the receptor with its adapter protein FADD [43]. This complex can then recruit procaspase-8, which in turn is proteolytically cleaved to form the active caspase-8, although c-FLIP acts as a competitive inhibitor of procaspase- 8 binding to the DISC and therefore has anti-apoptotic effects [43]. Once active, caspase -8 can cleave procaspases $-3,-6$ and -7 to their respective active effector caspases, which are responsible for the vast majority of proteolytic cleavage in the apoptotic process [43]. In some cell types, caspase- 8 can cleave procaspase- 3 directly (type I cells) and in type II cells caspase- 8 initiates the mitochondrial pathway by cleaving the $\mathrm{Bcl}-2$ family member Bid. Truncated Bid (tBid) then translocates to the mitochondria and promotes the release of cytochrome $c$ and the formation of the apoptosome. Whether caspase- 8 cleaves procaspase- 3 directly or cxerts its effects via the mitochondrial pathway in neutrophils is unknown. However, data in a study by Watson el al., [44] suggested that in neutrophils, caspase- 8 exerts its effects via the mitochondria. The mitochondrial stabilising agent bongkrekic acid inhibited caspase- 3 activation and apoptosis, but did not affect caspase- 8 activity Interestingly, they also showed that bongkrekic acid, and inhibition of caspase- 3 and caspase- 8 did not inhibit spontaneous apoptosis

\section{Mitochondria \& Bcl-2 Family Proteins}

Cytochrome $\mathrm{c}$ is important for the formation of the apoptosome, yet this molecule has remained elusive in neutrophils. We and others have demonstrated that neutrophils possess mitochondria but fluorescence staining revealed the relative scarcity of mitochondria within these cells $[29,39,45,46]$. It is clear that pro- and anti-apoptotic members of the bcl- 2 family of proteins play a role in regulating the rate of spontaneous neutrophil apoptosis. Translocation of pro-apoptotic protcin Bax to the mitochondria during neutrophil apoptosis has been observed, thus activating the mitochondrial apoptotic pathway and causing caspase-3 activation (Fig. 2) [39,47-49]. In addition, Bax translocation was not inhibited by $z$-VAD-fmk, the broad range caspase inhibitor, suggesting that $\mathrm{Bax}$ redistribution is caspase-independent. In addition, BID, another bcl-2 pro-apoptotic family member, has been shown to be important in the regulation of human neutrophil apoptosis. It appears that BID also translocates to the mitochondria and becomes truncated via a caspase dependent mechanism [36,50]. $\mathrm{Bcl}-2$ is absent from mature neutrophils, but its homologue $\mathrm{Al}$ and also $\mathrm{Mcl}-1$ have been found, which represent the two major anti-apoptotic bcl-2 family proteins present in neutrophils and their levels have been shown to be maintained or increased by inflammatory mediators that delay neutrophil apoptosis (Fig. 2) [51,52]. In contrast, the pro-apoptotic Bcl-2 family members, Bax, Bad and Bak are constitutively expressed in neutrophils and have a long half-life $[52,53]$. perhaps explaining why on balance, neutrophils are primed for apoptosis.

\section{Inhibitors of Apoptosis \& Calpains}

Inhibitor of apoptosis (IAP) proteins are evolutionarily conserved and can interfere with caspase activity by, in some cases, binding directly both effector and initiator caspases. However, upon perturbation of the mitochondrial membrane, IAP activity can be neutralised by the release of Second Mitochondria-derived Activator of Caspase/Direct Inhibitor of Apoptosis Binding Protein with Low pI (Smac/DIABLO) from the mitochondrial membrane, which in turn binds IAPs, preventing their caspase-inhihitory activity [54]. Only recently have studies investigated a possible role for IAPs in neutrophil apoptosis (Fig. 2). Kobayashi et al., [55] first demonstrated the presence of $x$-linked inhibitor of apoptosis 




Fig. (2). General Overview of Neutrophil Life vs. Death Signalling. See text for details.

protein (XIAP), the most potent inhibitor of caspases [54]. Furthermorc, XIAP was shown to be degraded by calpains, previously shown to be involved in the dissociation of actin-binding proteins, including $\alpha$-actin and ezrin [56], at the onset of apoptosis, and inhibition of calpains impaired the induction of apoptosis in neutrophils [55]. Recently, cIAP1 and cIAP2 have also been detected in neutrophils. cIAP2 was selectively upregulated in response to granulocyte-colony stimulating factor (G-CSF) but not granulocyte macrophage-colony stimulating factor (GM-CSF) and interestingly, neutrophils from a patient with chronic neutrophilic leukaemia had markedly upregulated levels of clAP2 mRNA along with prolonged survival in vitro [57]. Calpains are cysteine proteases and are comparatively little understood but are ubiquitously expressed and have roles in apoptosis, proliferation and cell migration [58]. Very recently, a role for calpain-1 in neutrophil apoptosis was demonstrated by Altznauer et al., [59]. These authors showed that inhibition of calpain-1 activity blocked both Fas-induced apoptosis and spontaneous apoptosis Furthermore, calpain-1 activity was necessary to generate an $18 \mathrm{kDa}$ Bax fragment with enhanced apoptotic activity, perhaps because it could not associate with $\mathrm{Bcl}-\mathrm{x}_{\mathrm{L}}$ in this form, in both constitutive and Fas-mediated neutrophil apoptosis. Inhibition of calpain also blocked Smac/ DIABLO release from the mitochondria and interestingly, prevented the full processing of caspase -3 trom the $20 \mathrm{kDa}$ form, which was inactive to the $17 \mathrm{kDa}$ form.

\section{Life or Death: Intracellular Signalling}

Neutrophils undergo constitutive apoptosis during in vitro culture, but IL-1 $\beta$, IL- 2 , TNF- $\alpha$, IL-15, IFN- $\gamma$, G-CSF, GM-CSF, LPS and hypoxic conditions can inhibit this process $[12-23]$. It is likely that neutrophils would encounter these stimuli at inflammatory sites in vivo, thereby prolonging their life-span and allowing them to fulfil their role in clearance of microbes. For example, hypoxic conditions have been observed in wound tissues [60-63], which may contribute to enhanced neutrophil survival $[64,65]$ mediated via hypoxic inducible factor-1 (HIF1) [66]. In contrast, stimulation of neutrophils with an antibody against the Fas receptor in vitro increases their rate of apoptosis [41]. In addition, agents that inhibit protein synthesis or transcription such as cycloheximide and actinomycin D have been shown to promote neutrophil apoptosis $[14,67]$. The observed induction of apoptosis implies that de novo synthesis of survival proteins is required for neutrophil survival. Here we focus on the 'internal battle' within the neutrophil that must occur in the face of pro-inflammatory mediators and/or pro-apoptotic stimuli. See (Fig.2) for a signalling overview.

\section{PI3 Kinase}

Phosphoinositide 3-Kinase (PI3K) activity is critical to many of the inflammatory mediators which enhance neutrophil survival, such as GMCSF [53], TNF- $\alpha[68]$, IFN- $\beta$ [69] and LPS [70]. The PI3K family of enzymes exist as heterodimers and can be divided into three classes: class I, class II and class III. Class 1 PI3Ks are further subdivided into classes
IA and IB, depending upon the composition of regulatory and catalytic subunits and mode of activation. Class IA PI3Ks consist of a p85 $\alpha, \mathrm{p} 85 \beta$, or p55 $\gamma$ regulatory subunit and a p1 $10 \alpha$, p1 $10 \alpha$ or p1 108 catalytic subunit and are activated by interaction with tyrosine-phosphorylated molecules [71]. The class IA isoform p85/p110 PI3K $\alpha$ has been identified in neutrophils [72]. In class IB PI3Ks there is only one catalytic subunit p110\% and one regulatory subunit pIOI, which are mainly activated via $\mathrm{G}$ protein-coupled receptors [71]; pl 10 $\gamma$ has been demonstrated to play an important role in neutrophil survival, chemotaxis and activation $[70,73]$.

PI3K survival effects in neutrophils are mediated via activation of Akt, NF- $\mathrm{kB}$ and $\mathrm{c} \Lambda \mathrm{MP}$ response element binding protcin (CREB). In murine $\mathrm{PI} 3 \mathrm{~K} \gamma-/-$ neutrophils, NF- $\mathrm{KB}$ nuclear translocation was reduced, as was phosphorylation of CREB [73]. This downregulation coincided with decreased expression of the survival proteins $\mathrm{Mcl}-1$ and $\mathrm{Bcl}-\mathrm{X}_{\mathrm{L}}$. This study also demonstrated that Akt phosphorylated and inactivated the proapoptotic Bad, Forkhead (FKR) and GSK- $\beta \beta$ proteins. Akt plays a central role in neutrophil apoptosis since CREB (Mcl-1 expression) and nuclear factor $-\kappa \mathrm{B}(\mathrm{NF}-\kappa \mathrm{B})\left(\mathrm{Bcl}-\mathrm{X}_{\mathrm{L}}\right.$ expression) anti-apoptotic transcription factors are directly under its control [73].

TNF- $\alpha$ has a PI3K-dependent pro-survival effect on rates of neutrophil apoptosis at late time points $(20 \mathrm{~h})$; at early time points, TNF- $\alpha$ (0-6h) actually induces apoptosis [40,53]. Interestingly, Bad mRNA leveis were shown to be reduced at early time points and increased at later time points in TNF- $\alpha$ treated neutrophils [53]. GM-CSF-mediated survival also appears to be critically dependent on PI3K, since the PI3K inhibitor LY2942002 is able to block the enhanced survival of neutrophils treated with this cytokine [74]. In addition, phosphorylation of Bad in GM-CSF treated neutrophils requires $\mathrm{Akt} /$ protein kinasc $\mathrm{B}(\mathrm{PKB})$ activity [74]; work was later confirmed by Cowburn et al., [53]. Cowburn et al., [53] further showed that Bad mRNA levels were reduced in GM-CSF treated neutrophils via a PI3K-dependent mechanism. In addition to Bad, another $\mathrm{Bcl}-2$ family member, $\mathrm{Mcl}-1$ is upregulated at the protein level in GM-CSF treated neutrophils $[51,52]$; the expression of which is under the control of the CREB transcription factor [75].

In IFN- $\beta$ treated neutrophils, Wang et al.. [76] showed that PKC- $\delta$ translocated to the cell membrane, where when activated by lipid second messengers, its role is anti-apoptotic. Furthermore, both PKC- $\delta$ and $\mathrm{Pl} 3 \mathrm{~K}$ signalling were essential for NF- $\mathrm{KB}$ activation and survival, since inhibition of either enzyme resulted in apoptosis. NF- $\mathrm{KB}$ activation is also crucial to LPS-mediated ncutrophil survival $[77,78]$ and its activity is dependent on upstream PI3K [70]

\section{$N F-x B$}

NF- $\kappa B$ plays a pivotal role in deciding the fate of neutrophils. Many of the agents which prolong neutrophil survival, do so via the NF- $\kappa B$ pathway (e.g. TNF- $\alpha$, LPS). Under normal conditions, NF- $K B$ is kept sequestered in the cytoplasm by inhibitory I $\mathrm{\kappa} B$ proteins such as $I \kappa B \alpha[79]$. An activating stimulus causes phosphorylation of $I \kappa B$ by $І \kappa B$ kinase (IKK), making I $\kappa$ B 
susceptible to proteolytic degradation, leaving NF- $\mathrm{kB}$ free to translocate to the nucleus and bind to DNA. There, it induces transcription of target genes that promote cell survival in most cell types [79]. The cell survival effects of $\mathrm{NF} \kappa \mathrm{B}$ are largely due to upregulation of anti-apoptotic genes [80].

Work by Ward et al., [77] first demonstrated the importance of NF- $\kappa$ B in neutrophil survival and apoptosis. The NF- $\mathrm{KB}$ inhibitor gliotoxin (a fungal metabolite) was able to inhibit the survival effect of LPS and moreover, increase the apoptotic effect of TNF- $\alpha$ at early time points [77]. The critical role of NF- $K B$ in deciding life or death in the neutrophil was later confirmed hy other studies [81-83]. Furthermore, the prostaglandin $D_{2}$ metabolites, $\Delta^{12}$ prostaglandin $\mathrm{J}_{2}\left(\Delta^{12} \mathrm{PGJ}_{2}\right)$ and 15 deoxy- $\Delta^{12,14} \mathrm{PGJ}_{2}$, were shown to inhibit I $\mathrm{kB}-\alpha$ degradation and therefore increase constitutive neutrophil apoptosis as well as overcome LPSmediated survival of these cells [84]. Interestingly, it has been subsequently and elegantly shown that $\mathrm{PGD}_{2}$ metabolites bring about acute inflammatory resolution in rat pleurisy by inducing neutrophil and macrophage apoptosis [85].

\section{MAPK}

There are three subfamilies of mitogen-activated protein kinases (MAPKs) which are regulated by phosphorylation by upstream kinases. These subfamilies, extracellular-signal-related kinases (ERKs), p38 and stress-activated protein kinase/c-Jun amino-terminal kinase (SAPK/JNK) are ubiquitously expressed and are involved in survival decisions in most cells. MAPKs are involved in the signalling pathways of the cytokincs and inflammatory mediators which increase neutrophil lifespan. Many such mediators activate more than one MAPK, and the resulting biological effect will be dependent on crosstalk between pathways.

The pro-survival effect of GM-CSF, and IL-8 are both, in part, mediated via ERK activation [74], bcing partially reversed by the inhibitor PD98059. This inhibitor has no effect on constitutive levels of apoptosis in aged neutrophils ([74], and our unpublished observations), suggesting that inactivation of ERK is not a prerequisite for neutrophils to undergo spontaneous apoptosis. Other survival agents such as G-CSF, LPS, IL-15 and C5a also activate ERK [86-89]

The role of p38 in constitutive neutrophil apoptosis and in enhancement of survival is controversial. For example, there is evidence that the p38 MAPK may be involved in stress induced (UV, hyperosmolarity, sphingosine) but not in constitutive or FAS induced neutrophil apoptosis [90]. Others have shown that p38 is not phosphorylated in response to G-CSF in ncutrophils [87], IL-15, an inhibitor of neutrophil apoptosis does activate p38 [88], and while inhibition of p38 by SB203580 has no effect on GM-CSF-induced neutrophil survival [74], sphingosine-1-phosphate inhibition of neutrophil apoptosis can be reversed by SB203580 [91]. Although LPS has been reported to activate p38 in neutrophils [86], the p38 inhibitor does not reverse LPS-inhibition of neutrophil apoptosis ([92] and our unpublished data). Furthermore, one study found that the p38 inhibitor could increase the inhibition of apoptosis induced by LPS via a mechanism that involves activation of ERK [93]. Recent data have demonstrated that p38 can phosphorylate and inhibit caspases-8 and 3 in human neutrophils [94], suggesting a role in survival

Controversy also surrounds the role of SAPK/JNK in neutrophils. This has in part been caused by the lack of specific pharmacological inhibitors of JNK, and the difficulty in transfecting end terminally differentiated cells with a short life span such as neutrophils. Also few stimuli have been shown to directly active JNK in these cells. For example, it has been reported that JNK is not activated by LPS, fMLP, PMA, TNF- $\alpha$, or GMCSF in neutrophils $[86,95]$. Recent data suggest that JNK in neutrophils is not activated under most circumstances, except in response to TNF- $\alpha$, and then only when the cells are treated under very specific circumstances and are adherent [95-97]. These data have been supported by use of SP 600125 [98], a JNK inhibitor which has recently become available: however, this inhibitor did not affect pro-inflammatory cytokine release in treated activated neutrophils suggesting no role for JNK in this process. New data do however suggest that LPS can activate JNK in nonsuspended neutrophils [99]. Therefore, the importance of JNK activation in neutrophil survival may change as new studies are reported.

Interestingly, both ERK and p38 may be cleaved and degraded indirectly by caspase activation during neutrophil apoptosis [100], and this may explain the non-responsiveness of apoptotic cells to activation stimuli. G-CSF inhibits this cleavage, therefore such a mechanism may also act to inhibit cell death in neutrophils [100]. On the other hand, MEKK-1, an MAPK kinase, is cleaved during apoptosis forming a constitutively active fragment which activates JNK [101]. Some controversy therefore arises as to whether JNK activation is a cause of neutrophil apoptosis or a consequence of it.

\section{Other Key Regulators}

Listed below is a non-exhaustive list of other regulators of neutrophil apoptosis many of which may use the above molecular and signalling mechanisms.

In recent years, several studies have demonstrated that nitric oxide (NO) has the ability to influence apoptosis in many cells (see [102-104] including ncutrophils [105-109]). The mcchanisms through which NO is able to both promote, and in some cases, delay inflammatory cell apoptosis still remain to be fully elucidated. However, it is generally believed that low concentrations tend to be anti-apoptotic, possibly acting through a rise in cyclic Guanosine Mono Phosphate (cGMP) or S-nitrosation of caspaseenzymes [105,107]. High concentrations of NO have been shown to induce cell death in neutrophils either by apoptosis or necrosis [106-108] by mechanisms that may involve generation of peroxynitrite $[107,110]$. Another possibility is that NO may indirectly interfere with pathways that regulate inflammatory cell survival including the NF-kB pathway [82].

GM-CSF causes a profound delay of both neutrophil and eosinophil apoptosis. There is strong evidence that GM-CSF signalling also results in the tyrosine phosphorylation of janus kinase 2 (JAK2), which in turn phosphorylates signal transducer and activator of transcription 1 (STAT1), STAT3 and STAT5, promoting DNA binding complexes, independent of lyn activation [111-113]. The precise role of lyn is still controversial, since another report showed that inhibition of lyn kinase overrides GM-CSF mediated survival in neutrophils [114].

Recently, Src Homology Domain 2 (SH2)-Containing Tyrosine Phosphatase-1 (SHP-1) has been demonstrated to play a role in downregulating survival signals upon stimulation of Fas in neutrophils [115]. The authors showed that in SHP-1 deficient mice, Fas-mediated inhibition of GM-CSF survival did not occur and interestingly, these mice presented with a persistent neutrophil type inflammation. Furthermore, they were able to show that SHP-1 interacted with Lyn, suggesting that SHP-I might dephosphorylate and inactivate Lyn. Other targets of SHP-1 however remain to be identified in neutrophils.

Leukotriene $\mathrm{B}_{4}\left(\mathrm{LTB}_{4}\right)$ has long been known to be a powerful activator of ncutrophils $[116,117]$, and many stimuli of ncutrophil responses also delay apoptosis in these cells [118-121]. LTB $_{4}$ is thought to bind to BLT1/ 2 receptors and may also signal via the peroxisome proliferators-activated receptor- $\alpha$ (PPAR- $\alpha$ ) group of receptors which regulated target genes by binding to PPAR response elements [122-124]. Recently, $\mathrm{LTB}_{4}$ was shown to enhance neutrophil longevity not via the PPAR $\alpha$ receptor but via BLT1 [121].

Glucocorticoids (GC) are widely used in the treatment of inflammatory disease. Yet in the case of neutrophilic-mediated inflammation, the use of GCs may be counter-productive. GCs dramatically prolong neutrophil survival in vitro and therefore may act similarly in vivo [125-128]. The signalling pathways involved in GCmediated survival have not been elucidated in neutrophils, although the $\beta$ isoform of the $\mathrm{GC}$ receptor (GCR) is the predominant form in neutrophils [129]. There are two isoforms of the GCR as a result of alternative splicing: GR $\alpha$ and GR $\beta$. GR $\beta$ lacks a steroid binding domain and therefore cannot bind to glucocorticoids $[130,131]$. It has been suggested that the ratio of GR $\alpha$ : GR $\beta$ receptors may determine a cells susceptibility to GC induced apoptosis [129]. Interestingly, the sex steroids progesterone and estradiol also delay neutrophil apoptosis, although this effect is reversed by induction of the Fas death pathway [132].

The proinflammatory cytokine, migration inhibitory factor (MIF) $[133,134]$ has been implicated in a variety of diseases including sepsis $[133,135,136]$ and asthma $[137,138]$. It is of interest that recently this cytokine has been shown to cause neutrophil survival by inhibiting the mitochondrial dependent death pathway [36].

\section{REMOVAL OF APOPTOTIC CELLS}

For tissues to return to normal and the inflammatory reaction to cease, inflammatory granulocytes must be removed from the site of injury. Removal of granulocytes from the inflammatory site requires that 1 . granulocytes undergo apoptosis (see above for neutrophil apoptosis) and 2. apoptotic granulocytes are efficiently phagocytosed by tissue-resident macrophages (for excellent recent reviews on this subject matter see $[18,21,22,139-142]$. 
Table 2. Major Receptors Involved in the Recognition of Apoptotic Cells

\begin{tabular}{|c|c|c|}
\hline Receptors & Proposed Mechanisms & References \\
\hline $\begin{array}{l}\text { Adenosine triphosphate (ATP)-binding } \\
\text { cassette reporter }(\mathrm{ABC1})\end{array}$ & $\begin{array}{l}\text { Disruption of asymmetric phospholipid distribution on plasma membrane in both } \\
\text { apoptotic and phagocytic cells. }\end{array}$ & [167] \\
\hline$\# \alpha \mathrm{V} \beta 3 / \mathrm{CD} 36$ & Receptors for apoptotic cell associated thrombospondin. & [168-171] \\
\hline \# CD14 & $\begin{array}{l}\text { Binds apoptotic cells without the inflammatory effects associated with LPS/LBP binding } \\
\text { which also acts via this receptor. }\end{array}$ & {$[157]$} \\
\hline CD29 (integrin $\beta 1$ subunit) & Present on phagocytes and allows binding to apoptotic cells. & {$[172]$} \\
\hline$\# \mathrm{CD} 31$ & Becomes 'disabled' during apoptosis allowing binding also via $\mathrm{CD} 31$ on the phagocyte. & [173] \\
\hline CD91/Calreticulin & $\begin{array}{l}\text { Surfactant Protcin A (SP-A) and SP-D involved in apoptotic cell uptakc dependent on } \\
\text { CD91/calreticulin. }\end{array}$ & {$[174]$} \\
\hline \# Complement: CR3/CR4 & $\mathrm{Cl} 1$ /C3 may act as opsonin for apoptotic cell uptake via $\mathrm{CR} 3 / \mathrm{CR} 4$ or calreticulin/CD91. & [175-178] \\
\hline \# Lectin-like Receptors & Recognise carbohydrates on apoptotic cells. & {$[154,155,168,179,180]$} \\
\hline \# PS Receptor & $\begin{array}{l}\text { PS is rapidly mobilised to cell surface during apoptosis. PS then allows apoptotic cell } \\
\text { recognition via the PS receptor on the phagocyte. }\end{array}$ & {$[181-183]$} \\
\hline \# Scavenger Receptor & $\begin{array}{l}\text { Present on myeloid cells and some endothelial cells. Recognises scavenger receptor } \\
\text { ligands on apoptotic cells. }\end{array}$ & {$[184]$} \\
\hline
\end{tabular}

Please note that these studies have used different systems, phagncytes and apoptotic targets so the precise recngnition receptors and mechanism that apply to phagncytosis of apoptotic neutrophils is Please note that these studies have used different systems, phagncytes and apoptotic targets so the precise recngnition
currently unknown however $\#$ indicates receptors identified as being involved in clearance of apoptotic neutrophils.

A major focus of our laboratory has been to investigate exactly how apoptotic neutrophils are recognised by macrophages [143-149]. The apoptotic neutrophil may be capable of releasing phagocyte recruitment signals [150] as well as undergoing several cell surface changes, which may lead to the 'eat me' signals required for ingestion by macrophages (see Table 2). The phosphatidylserine receptor (PSR) system has been proposed as a core mechanism for clearing apoptotic cells in the lung and during development. Studies by $\mathrm{Li}$ and colleagues [151] showed that in PSR-deficient mice, there was an accumulation of apoptotic cells and these mice died shortly after birth due to respiratory failure. However, many different receptors have been identified as being important in phagocytosis (Table 2), and perhaps the reason for this is that mechanisms of cell clearance may be tissue-specific [152-158]. In addition to these direct cell-to-cell interactions, macrophage phagocytic capacity may be modulated by pharmacological and immunological intervention. For example, $\mathrm{PGE}_{2}$-treated macrophages show a marked transient increase in intracellular cAMP levels, activating PKA which resulted in altered adhesion patterns [144]. These macrophages as a consequence were less efficient at ingesting apoptotic neutrophils, suggesting that adhesion is intimately linked with the phagocytic capacity of the macrophage [144]. In support of this, ligation of the matrix receptor CD44 results in increased apoptotic cell uptake [143] and later, Teder et al., [159] showed that in CD44-deficient mice, there was reduced uptake of apoptotic neutrophils by macrophages contributing to lung injury.

The recent discovery that anti-inflammatory glucocorticoids influence the phagocytic potential of macrophages provided key insights into their mode of action. The phagocytic capacity of macrophages treated with glucocorticoids for $24 \mathrm{~h}$ was markedly upregulated and importantly, this was accompanied by a lack of inflammatory mediator release [146]. Later it was also shown that monocyte-derived macrophages cultured in the presence of GCs over 5 days were highly phagocytic and had altered morphology associated with molecular changes in cytoskeletal organisation [147]. In addition, it appears that GCs have the capability to 'reprogram' monocyte differentiation towards a non-inflammatory state [147]. Interestingly, the Thl cytokine IFN- $\gamma$ appears to reverse the enhanced phagocytic capacity of GC monocyte-derived-macrophages without eliciting any morphological changes [148]. Presumably therefore, the efficacy of GC in the treatment of various inflammatory diseases will also be influenced by the cytokine environment. The ability of GCs to accelerate eosinophil and lymphocyte apoptosis, as well as enhance macrophage phagocytosis, may contribute to the success of GC in the treatment of many inflammatory diseases. However, one possibility is that GCs are less successful in the treatment of neutrophilic inflammation, since they actually prolong survival in these cells (see above). Therefore, a combination drug therapy may be more desirable in neutrophilic inflammation, where GCs would enhance phagocytosis but another therapeutic agent would uncouple GC-mediated neutrophil survival. Other modulators of human apoptotic neutrophil uptake by phagocytes include lipoxins $[160,161]$, modified matrix proteins by cigarette smoke and lipid peroxidation [149], fetuin/alpha2-HS glycoprotein [162], immune complexes $[163,164]$ and complement proteins $[165,166]$.

\section{CONCLUDING REMARKS}

While much progress has been made in unravelling the control mechanisms that exist in neutrophil survival and apoptosis, research has perhaps been slower than in other cell types due to the inherent difficulties of working with these cells. Nevertheless, understanding neutrophil survival/apoptosis is crucial to revealing potential drug targets in the treatment of inflammatory disease and more research on primary neutrophil biology is core to this. Linked to this is the efficient removal of neutrophils from the inflammatory site for resolution to occur where huge efforts are currently being undertaken to understand the mechanism behind this. Together, research into these two fundamental processes of inflammation should yield therapeutic strategies for the treatment of inflammatory disease.

\section{ACKNOWLEDGEMENTS}

We thank the Arthritis Research Campaign UK, Asthma UK, the Medical Research Council UK and the Wellcome Trust for financial support.

\section{ABBREVIATIONS}

$\begin{array}{ll}\text { AIF } & =\text { Apoptosis Inducing Factor } \\ \text { Akt } & =\text { Apaf-1 = Apoptotic Protease Activating Factor } \\ \text { BAD } & =\text { Bcl-XL/ Bcl-2-associated Death Promoter } \\ \text { Bax } & =\text { Bcl-2-associatcd x Protcin } \\ \text { Bak } & =\text { Bcl-2 Homologous Antagonist Killer } \\ \text { Bcl-2 } & =\text { B-cell Lymphoma-2 } \\ \text { Bcl-XL } & =\text { B Cell Leukaemia-x long } \\ \text { BID } & =\text { BH3 Interacting Domain Death Agonist } \\ \text { Caspase } & =\text { Cysteine-dependent Aspartate-specific Protease } \\ \text { c-FLIP } & =\text { Cellular-flice-inhibitory-protein } \\ \text { cGMP } & =\text { cyclic Guanosine Mono Phosphate } \\ \text { CREB } & =\text { AMP Response Element Binding Protein } \\ \text { CR3 } & =\text { Complement Receptor Type 3 (CD11b/CD18) } \\ \text { CR4 } & =\text { Complement Receptor Type 4 (CD11 } / C D 18)\end{array}$




\begin{tabular}{|c|c|c|}
\hline DD & $=$ & Death Domain \\
\hline DED & $=$ & Death Effector Domain \\
\hline DIABLO & $=$ & Direct Inhibitor of Apoptosis Binding Protein with Low pI \\
\hline DISC & $=$ & Death Inducing Signalling Complex \\
\hline ERK & $=$ & Extracellular-signal-regulated Kinase \\
\hline FADD & $=$ & Fas-Associated Death Domain \\
\hline fMLP & $=$ & $\mathrm{N}$-formylmethionylleucyl phenylalanine \\
\hline GC & $=$ & Glucocorticoid \\
\hline $\mathrm{G}-\mathrm{CSF}$ & $=$ & Granulocyte Colony Stimulating Factor \\
\hline GM-CSF & $=$ & Granulocyte Macrophage Colony Stimulating Factor \\
\hline HIF-1 $\alpha$ & $=$ & Hypoxia Inducible Factor- $1 \alpha$ \\
\hline IFN & $=$ & Interferon \\
\hline I $\mathrm{kB}$ & - & Inhibitor of $\mathrm{kB}$ \\
\hline IKK & $=$ & IкB Kinase \\
\hline IL & $=$ & Interleukin \\
\hline JAK & - & Janus Kinase \\
\hline JNK & $=$ & c-Jun N-terminal Kinase \\
\hline LPS & $=$ & Lipopolysaccharide \\
\hline $\mathrm{LTB}_{4}$ & $=$ & Leukotriene $\mathrm{B}_{4}$ \\
\hline MAPK & $=$ & Mitogen-activated Protein Kinase \\
\hline Mcl-1 & $=$ & Myeloid Cell Leukemia-1 \\
\hline MEK & $=$ & MAPK/ERK Activating Kinase \\
\hline $\mathrm{NF}-\mathrm{\kappa B}$ & $=$ & Nuclear Factor-kB \\
\hline NO & $=$ & Nitric Oxide \\
\hline PI3K & $=$ & Phosphoinositide 3-Kinase \\
\hline PKB & $=$ & Protein Kinase B \\
\hline PKC & $=$ & Protein Kinase C \\
\hline PPAR- $\gamma$ & $=$ & Peroxisome Proliferator-activated Receptor- $\gamma$ \\
\hline PGJ2 & $=$ & Prostaglandin J2 \\
\hline PS & $=$ & Phosphatidylserine \\
\hline TNF & $=$ & Tumour Necrosis Factor \\
\hline TRAIL & $=$ & TNF Related Apoptosis-inducing Ligand \\
\hline $\mathrm{SH} 2$ & $=$ & Src Homology Domain 2 \\
\hline SHP-1 & $=$ & SH2-Containing Tyrosine Phosphatase-1 \\
\hline Smac & $=$ & Second Mitochondria-derived Activator of Caspase \\
\hline STAT & $=$ & Signal Transducer and Activator of Transcription \\
\hline
\end{tabular}

\section{REFERENCES}

1] Haslett, C. Clin. SCi. (lond), 1992, 83,639

[2] Chilvers, E. R.; Rossi, A. G.; Murray, J.; Haslett, C. Thorax, 1998, 53, 533 .

Borregaard. N.; Cowland. J. B. Blood. 1997, 89. 3503.

Borregaard. N.; Cowland. J, B. Blood. 1997, 89, 3503

Wenzel, S. E.; Szefler, S. J; Leung, D. Y.; Sloan, S. I.; Rex, M. D.; Martin, R. J. Am. J. Respir. Crit Care Med., 1997, 156, 737.

Jatakanon, A.; Uasuf, C.; Maziak, W.; Lim, S.; Chung, K. F.; Barnes, P. J. Am. J. Respir. Cril Care Med., 1999, 160, 1532

Ennis, M. Curr. Allergy Asthma Rep., 2003, 3, 159

Rossi, A. G. Lung Biol. Hlth. Dis., 1998, 112, 9

Kerr, J. F.; Wyllie, A. H.; Currie, A. R. Br. J. Cancer, 1972, 26, 239

Haslett, C. Br. Med. Bull., $1997,53,669$.

Savill, J.: Haslett, C. Semin. Cell Biol., 1995, 6, 385.

Hornburg, C. H; Roos, D. Curr. Opin. Hematol. 1996, 3, 94

Haslett, C. Am. J. Respir. Crit Care Med., 1999, 160, S5.

Ward, C.; Dransfield, I.; Chilvers, E. R.; Haslett, C.; Rossi, A. G. Trends Pharmacol. Sci. 1999, 20, 503.

[15] Akgul, C.; Moulding, D. A.; Edwards, S. W. FEBS Lett., 2001, 487, 318.

[16] Simon, H. U. Immunol. Rev., 2003, 193, 101.

[17] Edwards, S. W; Moulding, D. A.; Derouet, M; Moots, R. J. Chem. Immunol. Allergy, 2003, 83, 204.

[18] Fadeel, B.; Kagan, V. E. Redox. Rep., 2003, 8, 143

[19] Maianski, N. A.; Maianski, A. N.; Kuijpers, T. W: Roos, D. Acta Haematol, 2004, III, 56 .

[20] Walker, A.; Ward, C.; Dransfield, I.; Haslctt, C.; Rossi, A. G. Curr. Drug Targets. Infiamm. Allergy, 2003, 2, 339

[21] Hofman, P. Curr. Drug Targets. Inflamm. Ailergy, 2004, 3, 1.
[22] Gilroy, D. W.; Lawrence, T.; Perretti, M.; Rossi, A. G. Nat. Rev. Drug Discov.. 2004, 3, 401.

[23] DeLeo, F. R. Apoptosis, 2004, 9, 399

[24] Wyllie, A. H.; Kerr, J. F.; Currie, A. R. Int. Rev. Cytol., 1980, 68, 251

[25] Leist, M.; Jaattela, M. Nat. Rev. Mol. Cell Biol., 2001, 2, 589.

[26] Martin, S. J.; Green, D. R, Cell, 1995, 82, 349.

[27] Daigle, 1,; Simon, H. U. Int. Arch. Allergy Immunol, 2001, 126, 147

[28] Zhang, B.; Hirahashi, J.; Cullere, X.; Mayadas, T. N. J. Biol. Chem. $2003,278,28443$

[29] Murphy, B. M.; O'Ncill, A. J.; Adrain, C.; Watson, R. W.; Martin, S. J. J. Exp. Med., 2003, 197, 625 .

[30] Goepel, F.; Weinmann, P.; Schymeinsky, J.; Walzog, B. J. Leukoc. Biol., 2004, 75, 836.

[31] Kumar, S. Cell Death. Differ., 1999, 6, 1060

[32] Nicholson, D. W. Cell Dealh. Differ., 1999, 6, 1028.

[33] Fischer, U.; Janicke, R. U.; Schulze-Osthoff, K. Cell Death. Differ. $2003,10,76$.

[34] Sanghavi, D. M.; Thelen, M.; Thornberry, N. A.; Casciola-Rosen, L.; Rosen, A. FEBS Lett., 1998, 422, 179.

[35] Pongracz, J.; Webb, P.; Wang, K.; Deacon, E.; Lunn, O. J.; Lord, J. M

[36] Baumann, R. Casaulta, C.; Simon, D.; Conus, S.; Yousefi, S.; Simon, H. U. FASEB J., 2003, 17, 2221.

[37] Srinivasula, S. M.; Ahmad, M.; Fernandes-Alnemri, T.; Alnemri, E. S. Mol. Cell, 1998, I, 949.

[38] Hu, Y.; Benedict, M. A.; Ding, L.; Nunez, G. FMBO I, 1999, 18, 3586

[39] Pryde, J. G.; Walker, A.; Rossi, A. G.; Hannah, S.; Haslett, C. J. Biol. Chem., 2000, 275, 33574

[40] Murray, J.; Barbara, J. A.; Dunkley, S. A.; Lopez, A. F; Van, O., X; Condliffe, A. M.; Dransfield, I.; Haslett, C.; Chilvers, E. R. Blood, 1997, $90,2772$.

[41] Iwai, K.; Miyawaki, T.; Takizawa, T.; Konno, A.; Ohta, K.; Yachie, A.; Seki, H.; Taniguchi, N. Blood, 1994, 8-, 1201.

[42] Ottonello, L.: Tortolina, G.; Amelotti, M.; Dallegri, F. J. Immunol., 1999. 162,3601 .

[43] Tibbetts, M. D.; Zheng, L.; Lenardo, M. J. Nal. Immunol., 2003, \&, 404

[14] Watson, R. W. O'Neill, A.; Brannigen, A. E. Coffey, R.; Marshall, J. C.; Brady, H. R.; Fitzpatrick, J. M. FEBS Lett., 1999, +53, 67

[45] Maianski, N. A.; Mul, F. P.; van Buul, J. D.; Ruos, D.; Kuijpers, T. W. Blood, 2002, 99, 672

[46] Fossati, G.; Moulding, D. A.; Spiller, D. G.; Moots, R. J.; White, M. R.; Edwards, S. W. J. Immunol, 2003, i70, 1964

[47] Dibbert, B. Weber. M. Nikolaizik, W. H. Vogt P.; Schoni, M. H. Blaser, K.; Simon, H. U. Proc. Natl. Acad. Sci. USA, 1999, 96, 13330.

[48] Fulop, T., Jr; Larbi, A.; Linteau, A; Desgeorges, S.; Douziech, N. Ann. N. Y. Acad. Sci., 2002, 973,305

[49] Altznaucr, F.; Conus, S.; Cavalli, A.; Folkcrs, G.; Simon, H. U. J. Biol Chem., 2003, 297, 5947.

[50] Maianski, N. A.; Roos, D.; Kuijpers, T. W. J. Immunol., 2004, 172, 7024.

[51] Moulding, D. A.; Quayle, J. A.; Hart, C. A.; Edwards, S. W. Blood, $1998,92,2495$.

[52] Moulding, D. A.; Akgul, C.; Derouet, M.; White, M. R.; Edwards, S. W. J. Leuknc. Biol, 2001, 70, 783 .

[53] Cowburn, A. S.; Cadwallader, K A.; Reed, B. J.; Farahi, N.; Chilvers, E. R. Blood, 2002, 100,2607

[54] Salvesen, G. S.; Duckett, C. S. Nat. Rev. Mol. Cell Biol., 2002, 3, 401.

[55] Kobayashi, S.; Yamashita, K.; Takeoka, T.; Ohtsuki, T.; Suzuki, Y. Takahashi, R.; Yamamoto, K.; Kaufmann, S. H.; Uchiyama, T.; Sasada, M.; Takahashı, A. J. Biol. Chem., 2002, 277, 33968

[56] Knepper-Nicolai, B.; Savill, J.; Brown, S. B. J. Biol. Chem., 1998, 273. 30530 .

[57] Hasegawa, T.: Suzuki, K.; Sakamoto, C.: Ohta, K.: Nishiki. S.: Hino, M.: Tatsumi, N.; Kitagawa, S Blood, 2003,101, 1164

[58] Perrin, B. J.; Huttenlocher, A. Int. J. Biochem. Cell Biol., 2002, 3t, 722.

[59] Altznaucr, F. Conus, S.; Cavalli, A.; Folkers, G.; Simon, H. U. J. Biol. Chem., 2004, 279, 5947

[60] Hunt, T. K.; Twomey, P.; Zederfeldt, B.; Dunphy, J. E. Am. J. Surg., $1967,11+, 302$.

[61] Niinikoski, J.; Hunt, T. K.; Dunphy, J. E. Am. J. Surg., 1972, 123, 247

[62] Cramer, T.; Johnson, R. S. Cell Cycle, 2003, 2, 192

[63] Cramer, T; Yamanishi, Y; Clausen, B F; Forster, I; Pawlinski, R.; Mackman, N.; Haase, V. H.; Jaenisch, R; Corr, M.; Nizet, V.; Firestein, G. S.; Gerber, H. P.; Ferrara, N.; Johnson, R. S. Cell, 2003, 112, 645.

[64] Ilannah, S.; Mecklenburgh, K.; Rahman, I.; Bellingan, G. J.; Greening, A.; Haslett, C.; Chilvers, E. R. FEBS Lett., 1995, 372, 233.

[65] Murray, J.; Walmsley, S. R.; Mecklenburgh, K. I.; Cowburn, A. S.; White, J. F.; Rossi, A. G.; Chilvers, E. R. Ann. N. Y. Acad. Sci., 2003, 1010,417

[66] Mecklenburgh, K. I; Walmsley, S. R.; Cowburn, A. S.; Wiesener, M.; Reed, B. J.; Upton, P. D.; Deighton, J.; Greening, A. P.; Chilvers, E. R. Blood, 2002, 100, 3008 .

[67] Whyte, M. K.; Savill, J.; Meagher, L. C.; Lee, A.; Haslett, C. J. Leukoc, Biol., 1997, 62, 195. 
[68] Kilpatrick, L. E.; Lee, J. Y.; Haines, K. M.; Campbell, D. E.; Sullivan, K. E.; Korchak, H. M. Am. J. Physiol. Cell Physiol., 2002, 283, C48

[69] Scheel-Toellner, D; Wang, K.; Henricuez, N. V.; Webb, P. R. Craddock, R.; Pilling, D.; Akbar, A. N.; Salmon, M.; Lord, J M. Fur. J. Immunol., 2002, 32, 486 .

[70] Yum, H. K.; Arcaroli, J.; Kupfner, J.; Shenkar, R.; Penninger, I M Sasaki, T.; Yang, K. Y., Park, J. S.; Abraham, E. J. Immunol., 2001 167,6601 .

[71] Vanhaesebroeck, B.; Leevers, S. J.; Ahmadi, K.; Timms, J.; Katso, R. Drisco.I, P. C.; Woscholski, R.; Parker, P. J.; Waterfield, M. D. Annu. Rev. Biochem., 2001, 70, 535.

72] Thelen, M. Didichenko, S. A. Ann N Y Acad Sci., 1997, 832, 368

[73] Yang, K. Y.; Arcaroli, J.; Kupfner, J.; Pitts, T. M.; Park, J. S.; Strasshiem. D.; Perng, R. P.; Abraham, E. Ceil Signal., 2003, 15, 225

[74] Klein, J. B.; Rane, M. J.; Scherzer, J. A ; Coxon, P. Y.; Kettritz, R.; Mathiesen, J. M.: Buridi, A.; McLeish, K. R. J. Immunol., 2000, 164, 4286 .

[75] Wang, J. M. Chao, J. R.; Chen, W.; Kuo, M. L.; Yen, J. J.; Yang-Yen, H. F. Mol. Cell Biol. 1999, 19, 6195 .

[76] Wang, K.; Scheel-Toellner, D.; Wong, S. H.; Craddock, R.; Caamano, J.; Akhar, A. N; Salmon, M; Lord, J. M. J. Immunol., 2003, /7I, 1035.

[77] Ward, C.: Chilvers, E. R.; Lawson, M. F.; Pryde, J. G.; Fujihara, S. Farrow, S. N.; Haslett, C.; Rossi, A. G. J. Biol, Chem., 1999, 274, 4309

[78] Ward, C.; Walker, A.; Dransficld, I.; Haslett, C.; Rossi, A. G. Biochem. Soc. Trans., 2004, 32, 465

[79] Barkett, M.; Gilmore, T. D. Oncogene, 1999, 18, 6910.

[80] Pahl, H. L. Oncogene, 1999, 18, 6853.

[81] Niwa, M.; Hara, A.: K.anamori, Y.; Hatakeyama, D.; Saio, M.: Takami, T. Matsuno, H.; Kozawa, O.; Uematsu, T. Eur. J. Pharmacol., 2000, 407 , 211

[82] Fortenberry, J. D.; Owens, M. L.; Chen, N. X.; Brown, L. A. Inflamm. Res., 2001, 50, 89.

[83] Choi, M.; Rolle, S.; Wellner, M.; Cardoso, M. C.: Scheidereit, C.; Luft. F. C.: Kettritz, R. Blood, 2003, 102, 2259

[84] Ward, C.; Dransfield, I.; Murray, J.; Farrow, S. N.; Haslett, C.; Rossi, A G. $J$ Immunol., 2002, 168,6232

[85] Gilroy, D. W.; Colville-Nash, P. R.; McMaster, S.; Sawatzky, D. A.; Willoughby, D. A.; Lawrence, T. FASEB J., 2003, /7, 2269

[86] Nolan, B; Duffy, A.; Paquin, L.; De, M.; Collette, H.; Graziano, C. M.. Bankey, P. Surgery, 1999, 126, 406

[87] Suzuki, K.; Hlino, M.; Hato, F.; Tatsumi, N.; Kitagawa, S. Blood, 1999, 93, 341 .

[88] Pelletier, M.; Ratthe, C.; Girard, D. FlEBS Lett., 2002, 532, 164

[89] Perianayagam, M. C.; Balakrishnan, V. S.; Pereira, B. J.; Jaber, B. L. Eur J. Clin. Invest., 2004, 34, 50

[90] Frasch, S. C.; Nick, J. A.; Fadok, V. A.; Bratton, D. L.; Worthen, G. S.; Henson, P M J. Biol, Chem, 1998, 273,8389.

[91] Chihab, R., Porn-Ares, M. 1.; Alvarado-Kristensson, M.; Andersson, T. Cell Mol. Life Scl., 2003, 60, 776.

[92] Harter, L.; Keel, M.; Steckholzer, U.; Ungethuem, U.; Trentz, O.; Ertel, W Shock, 2002, 18, 401

[93] Sheth, K.; Fricl, J.; Nolan, B.; Bankey, P. Surgery, 2001, 130, 242.

[94] Alvarado-Kristensson, M.; Melander, F.; Leandersson, K; Ronnstrand, L.; Wernstedt, C.; Andersson, T. J. Exp. Med., 2004, 199, 449.

[95] Cloutier, A.; Ear, T.; Borissevitch, O; Larivec, P.; McDonald, P. P. J Immunol, $2003,17 i, 3751$

[96] Avdi, N. J.; Nick, J. A.; Whitlock, B. B.; Billstrom, M. A.; Henson, P. M.; Johnson, G. L.; Worthen, G. S. J. Biol. Chem., 2001, 276, 2189

[97] Avdi, N. J.; Malcolm, K. C.; Nick, J. A.; Worthen, G. S.J. Biol Chem Avdi, N. J., Macolm

[98] Bennett, B. L.; Sasaki, D. T., Murray, B. W; O'Leary, E. C.; Sakata, S T.; Xu, W.; Leisten, J. C; Motiwala, A.; Pierce, S.; Satoh, Y.; Bhagwat, S. S.; Manning, A. M.; Anderson, D. W. Proc. Nati. Acad. Sci. USA 2001, 98, 13681.

[99] Arndt, P. G.; Suzuki, N.; Avdi, N. J.; Malcolm, K. C.; Worthen, G. S. Biol. Chem., 2004, 279, 10883.

[100] Suzuki, K.: Hasegawa, T.: Sakamoto, C.: Zhou, Y. M.: Hato, F.: Hino, M.; Tatsumi, N.; Kitagawa, S. J. Immunol., 2001, 166, 1185

[101] Cardone, M. H.; Salvesen, G. S.; Widmann, C.; Johnson, G.; Frisch, S. M. Cell, 1997, 90, 315.

[102] Taylor, E. L.; Megson, I. L.; Haslett, C.; Rossi, A. G. Cell Death. Differ. $2003,10,418$.

[103] Brune, B.; Zhou, J; von Knethen, A. Kidney Int. Suppl, 2003, S22.

[101] Kim, P. K.; Zamora, R.; Petrosko, P.; Billiar, T. R. In Immunopharmacol., 2001, I, 1421

[105] Fortenberry, J. D.; Owens, M. L.; Brown, L. A. Am. J. Physiol., 1999. 276, L 435

[106] Blaylock, M. G.: Cuthbertson, B. H.; Galley, H. F.; Ferguson, N. R.; Webster, N. R. Free Radic. Bial. Med., 1998, 25, 748

[107] Ward, C.; Wong, T. H.; Murray, J.; Rahman, I; Haslett, C.; Chilvers, E. R. Rossi, A. G. Biochem. Pharmacol., 2000,59, 305.

[108] Taylor, E. L.; Megson, I. L.; Haslett, C.; Rossi, A. G. Biochem. Biophys. Res. Commun., 2001, 289, 1229

[109] Secco, D. D.; Paron, J. A.; de Oliveira, S. H.; Ferreira, S. H.; Silva, J. S.; Cunha, F. Q. Nitric Oxide, $2003,9,153$.
[110] Fortenberry, J. D; Owens, M. I.; Brown, M. R.; Atkinson, D.; Brown, L. A. Am. J. Respir. Cell Mol. Biol., 1998, 18, 421 .

[111] Briczi, M. F.; Aronica, M. G; Rosso, A.; Bagnara, G. P; Yarden, Y; Pegoraro, L. J. Biol. Chem., 1996, 271, 3562.

[112] McDonald, P. P., Bovolenta, C.; Cassatella, M. A. Biochemistry, 1998, 37. 13165 .

[113] Al Shami, A.; Mahanna, W.; Naccache, P. H. J. Biol Chem. 1998, 273, 1058 .

[114] Wei, S.; Liu, J. H.; Epling-Burnette, P. K., Gamero, A. M.; Ussery, D.; Pearson, E. W.; Elkabani, M. E.; Diaz, J. I,; Djeu, J. Y. J. Immunol., $1996,157,5155$

[115] Daigle, L.; Yousefi, S: Colonna, M.: Green, D. R.; Simon, H. U. Nat. Med., 2002, 8, 61

[116] Ford-Hutchinson, A. W.; Bray, M. A.; Doig, M. V.; Shipley, M. E.; Smith, M. J. Nature, $1980,286,264$.

[117] Rossi, A. G.; MacIntyre, D. E; Jones, C. J.; McMillan, R. M. J. Leukoc. Biol., 1993, 53, 117 .

[118] Hebert, M. J.; Takano, T:; Holthofer, H: Brady, H. R f. Immunol. $1996,157,3105$.

[119] Stankova, J.; Turcotte, S.; Harris, J.; Rola-Pleszczynski, M, J. Immunol., $2002,168,3570$

[120] Lee, E.; Lindo, T.; Jackson, N.; Meng-Choong, L.; Reynolds, P.; Hill, A.; Haswell, M.; Jackson, S.; Kilfeather, S. Am. J. Respir. Crit. Care Med., 1999, 160, 2079

[121] Murray, J.; Ward, C.; O'Flaherty, J. T.; Dransficld, I.; Haslett, C.; Chilvers, E. R.; Rossi, A. G. Br. J. Pharmacol., 2003, 139, 388.

[122] Devchand, P. R.; Keller, H.; Peters, J. M.; Vazquez, M.; Gonzalez, F. J.; Wahli, W. Naiure, 1996, 384, 39.

[123] Yokomizo, T.; Izumi, T; Chang, K; Takuwa, Y; Shimizu, T. Nature, $1997,387,620$.

[124] Tager, A. M.; Luster, A. D. Prostaglandins Leukot. Essent. Fatty Acids, $2003,69,123$

[125] Cox, G. J. Immunol., 1995, 154, 4719

[126] Kato, T.; Takeda, Y; Nakada, T.; Sendo, F. Nat. Immun., 1995, If, 198.

[127] Liles, W. C.; Dalc, D. C.; Klebanoff, S. J. Blood, 1995, 86, 3181.

[128] Meagher, L. C.; Cousin, J. M.; Seckl, J. R.; Haslett, C. J. Immunol., $1996,156,4422$

[129] Strickland, I.; Kisich, K; Hauk, P. J; Vottero, A; Chrousos, G. P.; Klemm, D. J.; Leung, D. Y. J. Exp. Med., 2001, 193, 585

[130] de Castro, M.; Elliot, S.; Kino, T.; Bamberger, C.; Karl, M.; Webster, E. Chrousos, G. P. Mol. Med., 1996, 2, 597.

[131] Oakley, R. H.; Sar, M; Cidlowski, J. A. J. Biol. Chem., 1996, 271, 9550 .

[132] Molloy, E. J.; O'Neill, A. J.: Grantham, J. J.; Sheridan-Pereira, M.; Fitzpatrick, J. M.; Webb, D. W; Watson, R. W. Blond, 2003, 102 . 2653.

[133] Calandra, T.; Roger, T. Nat. Rev. Immunol, 2003, 3, 791

[134] Baugh, J. A; Donnelly, S. C. J. Endocrmol., 2003, 179, 15

[135] Bozza, M.; Satoskar, A. R.; Lin, G; Lu, B.; Humbles, A. A; Gerard, C.; David, J. R. J. Exp. Med., 1999, 189, 341.

[136] Martin, T. R. Nat. Med, 2000, 6, 140

[137] Rossi, A. G.; Haslett, C.; Hirani, N.; Greening, A. P. Rahman, I.; Metz, C. N.; Bucala, R.; Donnelly, S. C. J. Clin. Invest., 1998, 101, 2869.

[138] Yamaguchi, E; Nishihira, J.; Shimizu, T; Takahashi, T.; Kitashiro, N.; Hizawa, N.; Kamishima, K.; Kawakami, Y. Clin. Exp. Aliergy, 2000, 30, 1244 .

[139] Hart, S. P.; Rossi, A. G.; Dransfield, I. Biochem. Soc. Trans., 1998, 26, 650 .

[140] Savill, J.; Fadok, V. Nature, 2000, 407, 784.

[141] Giles, K. M.; Hart, S. P.: Haslett, C.; Kossi, A. G.: Dransfield, I. Br. J. Haemaiol., 2000, 109, 1 .

[142] Savill, J.; Dransfield, I.; Gregory, C.: Haslett, C. Nat. Rev. Immunol,, $2002,2,965$

[143] Hart, S. P.; Dougherty, G. J., Haslett, C.; Dransfield, I. J. Immunol., $1997,159,919$.

[144] Rossi, A. G.; McCutcheon, J. C.; Roy, N.; Chilvers, E. R.; Haslett, C.; Dransfield, I. J. Immunol., 1998, 160, 3562.

[145] McCutcheon, J. C.; Hart, S P.; Canning, M.; Ross, K.; Humphries, M. J.; Dransfield, I. J. Leukoc. Biol., 1998, 64, 600 .

[146] Liu, Y.; Cousin, J. M.; Hughes, J.; Van Damme, J., Seckl, J. R.; Haslett, C.; Dransfield, 1.; Savill, J.; Rossi, A. G. J. Immunol., 1999, I62, 3639.

[147] Giles, K. M.; Ross, K.; Rossi, A. G.; Hotchin, N. A.; Haslett, C.; 1)ransfield, I. I. Immunol., 2001, 167. 976.

[148] Heasman, S. J.; Giles, K. M.; Rossi, A. G.; Allen, J. E.; Haslett, C. Dransfield, 1. Eur. J. Immunoi, 2004, 3t, 1752 .

[149] Kirkham, P. A.; Spooner, G.; Rahman, I.; Rossi, A. G. Biochem. Biophys. Res. Commun., 2004, 318, 32.

[150] Ravichandran, K. S. Cell, 2003, /13,817

[151] Li, M. O,: Sarkisian, M. R.; Mehal, W Z.; Rakic, P; Flavell, R. A. Science, 2003, 302, 1560 ,

[152] Fadok, V, A.; Laszlo, D. J.; Noble, P. W.; Weinstein, L.; Riches, D. W ; Henson, P. M. J. Imminol., 1993, 151, 4274.

[153] Flora, P. K. Gregory, C. D. Eur. J. Immunol., 1994, 24, 2625.

[154] Dini, L; Lentini, A.; Diez, G. D.: Rocha, M.; Falasca, L.; Serafino, L.; Vidal-Vanaclocha, F, J Cell Sci. 1995, 108 (Pt. 3), 967 
[155] Pradhan, D.; Krahling, S.; Williamson, P.; Schlegel, R. A. Mol. Biol Cell, 1997, 8, 767

[156] Platt, N.; da Silva, R. P.; Gordon, S. Trends Cell Biol., 1998, 8, 365.

[157] Devitt, A.; Moffatt, O. D.; Raykundalia, C.; Capra, J. D.; Simmons, D. L.: Gregory, C. D. Nature, 1998, 392, 505.

[158] Moffatt, O. D.; Devitt, A.; Bell, E. D.; Simmons, D. L.; Gregory, C. D. $J$ Immunol., 1999, 162, 6800 .

[159] Teder, P.; Vandivier, R. W; Jiang, D.; Liang, J.; Cohn, L.; Pure, E. Henson, P. M.; Noble, P. W. Science, 2002, 296, 155

[160] Godson, C.; Mitchell, S.; Harvey, K.; Petasis, N. A.; Hogg, N.; Brady, H. R. J. Immunol., 2000, 164, 1663 .

[161] Mitchell, S.; Thomas, G.; Harvey, K.; Cottell, D.; Reville, K.; Berlasconi, G.; Petasis, N. A.; Erwig, L.; Rees, A. J.; Savill, J.; Brady, H. R.; Godson, C. J. Am. Soc. Nephrol., 2002, 13, 2497.

[162] Jersmann, H. P.; Dransfield, I.; Hart, S. P. Clin. Sci. (Lond), 2003, 105, 273.

[163] Hart, S. P.; Jackson, C.; Kremmel, L. M.; McNeill, M. S.; Jersmann, H. Alexander, K. M.; Ross, J. A.; Dransfield, I. Am. J. Pathol., 2003, 162, Alexander, K. M., Ross, J. A.; Dransfield, I. Am. J. Paihol., 2003, 162,
1011.

[164] Hart, S. P.; Alexander, K. M.; Dransfield, I. J. Immunol., 2004, 172, 1882 .

[165] Korb, L. C.; Ahearn, J. M. J. Immunol., 1997, 158, 4525,

[166] Mevorach, D.; Mascarenhas, J. O.; Gershov, D.; Elkon, K. B. J. Exp. Med., 1998, 188, 2313.

[167] Luciani, M. F.; Chimini, G. EMBO J., 1996, 15, 226

[168] Hall, S. E.; Savill, J. S.; Henson, P. M.; Haslett, C. J. Immunol., 1994, 153, 3218 .

[169] Savill, J.; Dransfield, I.; Hogg, N.; Haslett, C. Nature, 1990, 3+3, 170
[170] Savill, J.; Hogg, N.; Ren, Y.; Haslett, C. J. Clin. Invest., 1992, 90 , 1513.

[171] Stern, M.; Savill, J.; Haslett, C. Am. J. Pathol., 1996, I49, 911

[172] Schwartz, B. R.; Karsan, A.; Bombeli, T.; Harlan, J. M. J. Immunol, $1999,162,4842$

[173] Brown, S.; Heinisch, I.; Ross, E.; Shaw, K.; Buckley, C. D.; Savill, J. Nature, 2002, 418, 200

[174] Vandivier, R. W.; Ogden, C. A.; Fadok, V. A.; Hoffmann, P. R.; Brown, K. K.; Botto, M.; Walport, M. J.; Fisher, J. H.; Henson, P. M.; Greene, K. E. J. Immunol., 2002, 169, 3978 .

[175] Fishelson, Z; Attali, G.; Mevorach, D. Mol. Immunol, 2001, 38, 207.

[176] Mevorach, D.; Mascarenhas, J. O.; Gershov, D.; Elkon, K. B. J. Exp. Med., 1998, 188, 2313.

[177] Takizawa, F.; Tsuji, S.; Nagasawa, S. FEBS Lett., 1996, 397, 269.

[178] Taylor, P. R.; Carugati, A.; Fadok, V. A.; Cook, H. T.; Andrews, M. Carroll, M. C.; Savill, J. S.; Henson, P. M.; Botto, M.; Walport, M. J. J Exp. Med., 2000,192, 359

[179] Dini, L.; Autuori, F.; Lentini, A.; Oliverio, S.; Piacentini, M. FEBS Lett. $1992,296,174$

[180] Duvall, E.; Wyllie, A. H.; Morris, R. G. Immunology, 1985, 56, 351.

[181] Fadok, V. A.; Voelker, D. R.; Campbell, P. A.; Cohen, J. J.; Bratton, D. L.; Henson, P. M. J. Immunol., 1992, I48, 2207.

[182] Fadok, V. A.; Bratton, D. L.; Rose, D. M.; Pearson, A.; Ezekewitz, R. A Henson, P. M. Nature, 2000, 405,85

[183] Hoffmann, P. R.; deCathelineau, A. M.; Ogden, C. A.; Leverrier, Y. Bratton, D. L.; Daleke, D. L.; Ridley, A. J.; Fadok, V. A.; Henson, P. M J. Cell Biol., 2001, I55, 649.

[184] Plall, N.; Suzuki, H.; Kurihara, Y.; Kodama, T.; Gordon, S. Proc. Natl. Acad. Sci. USA, 1996, 93, 12456. 


\section{Neutrophils: dead or effete? Cell surface phenotype and implications for phagocytic clearance}

\author{
I Dransfield ${ }^{*, 1}$, AG Rossi ${ }^{1}$, SB Brown ${ }^{1}$ and SP Hart ${ }^{1}$ \\ ${ }^{1}$ MRC Centre for Inflammation Research, University of Edinburgh Medical \\ School, Teviot Place, Edinburgh EH8 9AG, UK \\ - Corresponding author. I Dransfield, MRC Centre for Inflammation Research, \\ University of Edinburgh Medical School, Teviot Place, Edinburgh EH8 9AG, \\ UK. Tel: + 44131 6506948; Fax: + 441316504384 ; \\ E-mail: i.dransfield@ed.ac.uk
}

Cell Death and Differentiation (2005) 12, 1363-1367. doi:10.1038/s..cdd.4401695; published online 17 June 2005

\section{Neutrophil apoptosis - not all cells are equal}

The neutrophil granulocyte provides a versatile cellular defence against invading pathogens. This cell type is produced in large numbers in the bone marrow ( $10^{11}$ cells/ day) and represents the principal leukocyte subset that is present in the circulation. Neutrophils can be rapidly recruited to sites of tissue injury or infection, transmigrating endothelial barriers to enter tissues. In terms of effector function, the neutrophil is principally a phagocytic cell, armed with a diverse repertoire of receptors for potential opsonins of pathogens, a large array of anti-microbial compounds, degradative enzymes and the ability to produce reactive oxygen species that ultimately allow destruction of infectious organisms. ${ }^{1}$ Although the neutrophil may be thought of as a cellular 'thug' with little capacity for modulated destructive function, in vitro studies have revealed the exquisite control of every aspect of neutrophil behaviour, including adhesion, migration, phagocytosis and granule release. In addition, it is now apparent that neutrophils are also capable of limited production of important inflammatory mediators that may influence development or progression of an inflammatory response. Finally, the ultimate demise of neutrophils through the process of programmed cell death or apoptosis is also closely regulated. ${ }^{2}$

The interaction of the neutrophil with the local microenvironment occurs through the cell surface receptors that are expressed at the plasma membrane. Many of the key molecular mediators of adhesion, migration and phagocytosis are maintained within intracellular granule compartments, the azurophilic and specific granules. In response to exposure to a variety of stimuli derived either from pathogens (e.g. formyl methionyl-leucyl-phenylalanine) or from the host (a range of cytokines and growth factors), the neutrophil is able to rapidly mobilise these granule contents to the plasma membrane. Coupled with this, the neutrophil can control the levels of surface receptor expression through the rapid and specific proteolytic 'shedding' of the ecto-domain of specific receptors through the action of metalloproteases. Together these processes combine to generate a very different receptor profile that is optimal for destructive capacity. Indeed, the functional repertoire and cell surface receptor profile of neutrophils that have been exposed to these different stimuli is distinct and has been used to define neutrophil functional status. $^{3}$

\section{Regulation of apoptosis}

We have been particularly interested in the molecular mechanisms that underlie the engagement of apoptotic programmes in neutrophils. As a terminally differentiated cell type, the neutrophil constitutively undergoes apoptosis when cultured in vitro. It is also clear that apoptosis is influenced by neutrophil functional activity, including adhesion and phagocytic activity. For example, apoptosis is delayed in the CD11b deficient animals due to reduced phagocytic capability and activation of the NADPH oxidase. ${ }^{4}$ In view of the importance of neutrophil apoptosis in determining the numbers of neutrophils that will be present within inflammatory sites, many studies have investigated the signalling pathways that exert regulatory effects upon the rate at which neutrophils undergo apoptosis. These studies indicate that a wide variety of stimuli can control neutrophil survival during in vitro culture, including growth factors (e.g. GM-CSF), cytokines (e.g. TNF$\alpha)$ and inflammatory mediators (e.g. prostaglandin $\left.E_{2}\right)^{5,6}$

The rates of neutrophil apoptosis that are derived from in vitro studies are likely to be too low to account for the rapid removal of large numbers of neutrophils from inflammatory sites in vivo. For example, in murine peritonitis models, 5-10 million recruited neutrophils are cleared within $24 \mathrm{~h}$, whereas in vitro data relating to constitutive apoptosis would predict that a small proportion of that number would be removed. Under physiological conditions, neutrophils may be removed by a process of assisted suicide. In addition, there are other internal control mechanisms that can augment apoptosis of neutrophils including ligation of death receptors (e.g. Fas, TNFR and TRAILR). Thus, cellular interactions between neutrophils and phagocytes or stromal cells may engage the proapoptotic signalling receptors, allowing initiation of neutrophil apoptosis in close proximity to cells involved in their phagocytic removal. ${ }^{7}$ In addition, apoptosis can be specifically accelerated following interactions of neutrophils with a number of pathogens including Escherichia coli, Streptococcus pneumoniae, Mycobacterium tuberculosis and Neisseia gonorrhoeae. Interestingly, mapping the neutrophil transcriptome following pathogen-induced apoptosis suggests that there may be pathogen-specific alterations in gene expression profiles associated with apoptosis. ${ }^{8}$ The induction of neutrophil apoptosis by pathogens may represent a mechanism for ensuring that highly phagocytic neutrophils containing 
pathogenic organisms are 'shut down' and subsequently cleared.

One possible explanation for the experimental evidence in vitro for involvement of many different molecular pathways in the recognition and internalisation of apoptotic cells ${ }^{9}$ is that the assumption that all apoptotic cells are recognised in a similar manner by phagocytes is flawed. In the study of apoptotic cell clearance there has been a reluctance to address the heterogeneity of apoptotic cell populations that are used in studies of phagocytosis. In particular, distinct subpopulations of apoptotic neutrophils can be readily identified with monoclonal antibodies or by use of specific soluble extracellular proteins. ${ }^{10}$ This may go some way towards explaining apparently contradictory findings, for example, the question of serum dependency. As an example, Table 1 shows data relating to the effects of serum on phagocytosis of apoptotic neutrophils. These data clearly show that the proportion of necrotic cells present in the target cell population may determine the extent of observed dependency upon the presence of serum for efficient phagocytosis. Furthermore, while we may be able lo define in vitro culture conditions that yield reproducible apoptotic neutrophil phenotypes, it is likely that under conditions present at inflamed sites, neutrophils may have been exposed to multiple stimuli with either synergistic or antagonistic effects upon the apoptotic process and also upon cell surface phenotype.

\section{Apoptosis-induced surface receptor alterations}

Investigation of the surface receptor alterations that are associated with long-term exposure of neutrophils to cytokines (including GM-CSF and interferon- $\gamma$ ) revealed that for the glycophosphatidylinositol-anchored IgG receptor, Fc $\gamma$ RIII (CD16), the overall levels of expression were much higher in GM-CSF-treated neutrophils. ${ }^{11}$ Careful analysis indicated that two distinct subpopulations of neutrophils were present in cultured cells in terms of levels of CD16 expression. Subsequent studies demonstrated that the Fc $\gamma$ RIII high expressing neutrophils had normal nuclear morphology, whereas those with lower levels of membrane Fc $y$ RIII displayed morphological alterations that were characteristic of apoptosis. ${ }^{12}$ This observation represented one of the first demonstrations that specific alterations in membrane receptor expression accompanied the programme of morphological changes associated with apoptosis. Our data suggested that the nature of the membrane anchor was not responsible for the profound loss ( $>90 \%$ of surface receptors) of Fc $\gamma$ RIII.
A number of other specific membrane receptors also exhibited marked down-regulation as neutrophils underwent apoptosis, notably CD62L, CD44, CD43 and TNFRI (CD120a). ${ }^{13,10}$ In these studies it was difficult to detect neutrophils with an intermediate level of expression of these receptors when either single or dual immunofluorescence (with labelled annexin V to mark apoptotic neutrophils) profiles were examined. This observation would be consistent with the suggestion that the levels of receptor expression reflect a 'catastrophic' event rather than a gradual loss of receptors over time. Since of these receptors can be shed via the action of metalloproteases during neutrophil activation, it is possible that the intense membrane re-organisation (blebbing) gives rise to metalloprotease activity resulting in receptor loss. However, the importance of the cytoskeleton in regulation of these processes should not be underestimated.

It was notable that many surface receptors exhibit a smaller reduction in surface expression (by about $30 \%$ ) on apoptotic neutrophils when compared with non-apoptotic neutrophils. ${ }^{14}$ This may reflect the loss of plasma membrane that is associated with membrane blebbing in the earliest stages of apoptosis. Alternatively, studies from a number of laboratories have suggested that the disruption of the Golgi represents an early event in the apoptotic process. ${ }^{15}$ Loss of the ER-Golgiplasma membrane secretory transport pathway during apoptosis might be predicted to contribute to the net loss of receptors from the plasma membrane. A corollary of this suggestion is that equivalent or increased levels of receptor expression upon apoptotic neutrophils may require some mobilisation of intracellular stores of receptors during the blebbing phase. Interestingly, recent data suggest that bacterially loaded apoptotic neutrophils, in contrast with UVinduced apoptotic cells upregulate expression of heat shock proteins (HSP), notably HSP-60 and HSP-70. ${ }^{16}$ The expression of HSPs by pathogen-induced apoptotic neutrophils has the potential to provoke pro-inflammatory cytokine production by macrophages and clearance of pathogen-loaded apoptotic neutrophils may influence the development of acquired immunity through processing and presentation of antigen in the context of costimulatory molecule expression. In addition, proteins that exhibit altered patterns of glycosylation, perhaps due to incomplete processing in the Golgi, become expressed on the plasma membrane during membrane blebbing. It is also possible that there is altered activity of carbohydrate modifying enzymes that accompany apoptosis, for example, sialidases. Analysis of the changes in protein glycoforms that are displayed by apoptotic neutrophils using a panel of labelled lectins did not reveal a global loss of binding of lectins derived from Maackia amurensis and Sambrucus

Table 1 Effect of serum as an opsonin on macrophage phagocytosis of apoptotic neutrophils

\begin{tabular}{|c|c|c|c|c|}
\hline Stimulus & $\%$ Apoptosis & $\%$ Necrosis & \%Phagocytosis-Serum & \%Phagocytosis+Serum \\
\hline $\begin{array}{l}\text { None } \\
\text { Fas } \\
\text { UV }\end{array}$ & $\begin{array}{l}42 \pm 8 \\
68 \pm 12 \\
70 \pm 13\end{array}$ & $\begin{array}{r}4 \pm 2 \\
7 \pm 2 \\
21 \pm 7\end{array}$ & $\begin{array}{l}20 \pm 4 \\
24 \pm 6 \\
24 \pm 5\end{array}$ & $\begin{array}{l}25 \pm 4 \\
23 \pm 6 \\
45 \pm 9\end{array}$ \\
\hline
\end{tabular}

Neutrophils were cultured in the absence of serum to undergo spontaneous apoptosis (None), or induced to undergo apoptosis with the addition of $\mathrm{CH} 11$ (anti-Fas) antibody (Fas) or irradiation with ultraviolet (UV). Apoptosis and necrosis were determined by flow cytometry using annexin V and propidium iodide and phagocytosis determined by flow cytometric analysis. Results shown are mean \pm standard deviation for at least 3 experiments 
nigra that bind specific sialic acid linkages. Nor did increased binding of lectins such as peanut agglutinin that recognises galactose occur, implying that carbohydrate modifications that accompany apoptosis do not involve mass desialylation. $^{10}$

One of the important aspects of membrane changes associated with neutrophil apoptosis is that there is functional uncoupling, effectively isolating the apoptotic cell from stimuli that might trigger destructive responses. It is extremely likely that the activity of caspases and calpains may simply disrupt the assembly and localisation of signalling complexes that are required to translate receptor occupancy into a functional response. Furthermore, disruption of cytoskeletal integrity through cleavage of actin or other binding proteins such as ezrin, fodrin or band 4.1 would be predicted to inhibit neutrophil capacity for adhesion, migration and degranulation. ${ }^{17}$ In addition to this somewhat nonselective loss of receptor function, we reported specific loss of $\beta 2$ integrin ligand binding function despite maintained levels of surface expression. ${ }^{13}$ Since the $\beta 2$ integrins are known to show regulated ligand binding function through a process known as 'inside-out' signalling, it is possible that this observation also reflects dysregulation of key signalling pathways following apoptosis. However, an active conformation of either cell surface expressed or purified $\beta 2$ integrins can be forced using the divalent cation $\mathrm{Mn}^{2+}$. Surprisingly, our experiments revealed that $\beta 2$ integrin function could not be promoted by $\mathrm{Mn}^{2+}$ on apoptotic cells, implying that the membrane lipid ${ }^{18}$ and cytoskeletal alterations associated with apoptosis may pose additional restrictions upon receptor function that ensure a lack of neutrophil response to environmental signals.

\section{Apoptotic cell subsets}

In the course of screening monoclonal antibodies derived from several independent fusions for antibodies that bound specifically to apoptotic cells, it was notable that there were many clones producing antibodies that bound to subsets of neutrophils that had been cultured in vitro. Typical dual fluorescence profiles from a recent screen are shown in Figure 1. At least two distinct staining patterns can be identified. First, some antibodies bind a subset of apoptotic (annexin $\mathrm{V}$ positive) neutrophils that exhibit reduced forward/side scatter properties. Further analysis reveals that these cells are distinct from apoptotic cells with loss of membrane integrity and exposure of nuclear material (propidium iodide positive). The proportion of cells with this phenotype increases when neutrophils are cultured for prolonged periods or following conditions of stress, although evidence that they are derived from early apoptotic cells is lacking. Since these cells do not bind isotype-matched antibody non-specifically, one implication is that certain antigens become accessible to antibody when neutrophils lose membrane integrity.

A second identifiable staining profile observed is binding of antibodies to a subset of neutrophils that are annexin $\mathrm{V}$ positive, yet distinct from membrane permeable cells (Figure 1). We have previously described that this population represents 'late' apoptotic cells, with distinctive morphological appearance. Our data indicate that progression of neutrophils from early apoptosis to this late apoptotic phenotype is a relatively slow process. In contrast, lymphocytes rapidly acquire the late apoptotic phenotype following induction of apoptosis. Electron micrographs of affinity isolated late apoptotic neutrophils, show extensive nuclear degradation (see representative transmission electron micrograph in Figure 1). Whilst it was possible to select neutrophils from this population that exhibited intact plasma membranes, many of the cells showed one or more points of loss of integrity. Whether this represents the fragility of these cells during processing for electron microscopy is not clear. However, what is notable is that the extensive retention of intracellular granules within these neutrophils. Mechanisms for clearing late apoptotic cells may represent a backup pathway for ensuring that failure to clear early apoptotic cells does not lead to cells entering a necrotic phase. Surprisingly, given the potential importance of apoptotic cell removal in so many diverse processes, there have been few studies that have compared molecular mechanisms and functional consequences of phagocyte clearance of early and late apoptotic cells.

Interestingly, late apoptotic neutrophils have been shown to bind a number of plasma proteins that are importance in homeostatic regulation. Binding of these molecules does not appear to represent nonspecific protein binding capacity since other proteins (e.g. labelled bovine serum albumin) do not bind to these cells. These cells bind the matricellular protein thrombospondin and also bind C-reactive protein and C1q. ${ }^{10,19}$ At present, it is not clear whether these bound proteins are accessible to phagocytes or not. However, the specific binding of proteins that have been implicated in the recognition and subsequent phagocytic clearance of apoptotic cells may have profound importance in determining how these cells may be removed if they are present at inflammatory sites. From the information we have relating to the potential for opsonisation of late apoptotic cells, it seems likely that their clearance may differ both in terms of phagocyte recognition pathways engaged and in efficiency of internalisation.

\section{Apoptosis enabled receptors?}

Recently, we have described a novel mechanism whereby apoptotic neutrophils become opsonised by immune complexes. This finding arose from the characterisation of a monoclonal antibody that exhibited a unique binding profile for neutrophils. After extensive characterisation, we found that this murine IgG1 antibody rapidly formed immune complexes in the presence of the antigen (a serum protein) and bound to apoptotic neutrophils via an interaction of the Fc portion to Fc $\gamma$ RII. ${ }^{20}$ Surprisingly, antibody-antigen complexes did not bind, or bound weakly to freshly isolated or cytokine/ chemokine activated neutrophils, despite abundant expression of Fc $\gamma$ RII on these cells. We believe that this alteration in ligand binding activity is the first example of a molecule that shows reduced expression on apoptotic cells, yet exhibits enhanced function. The molecular mechanism(s) 


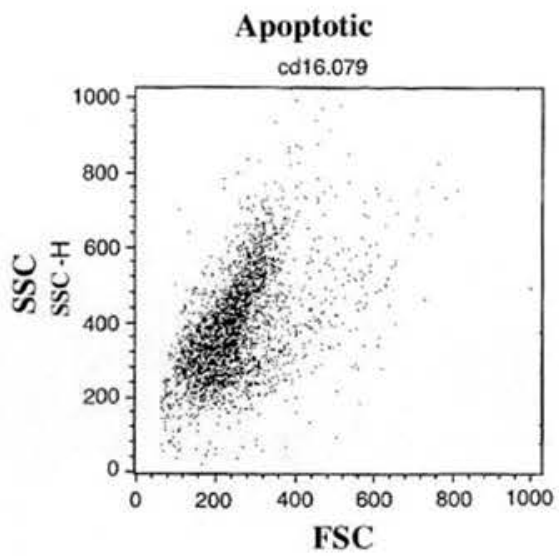

Late Apoptotic

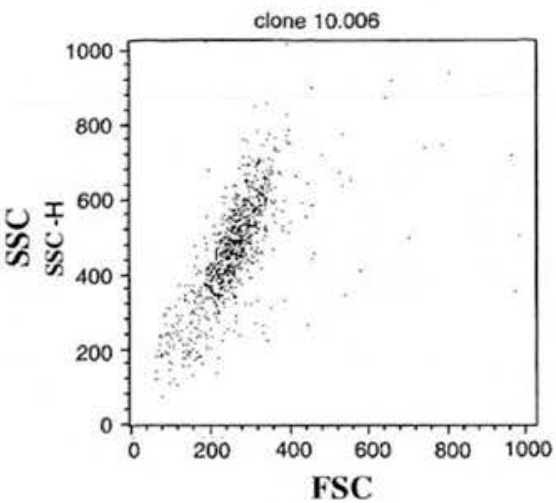

Necrotic

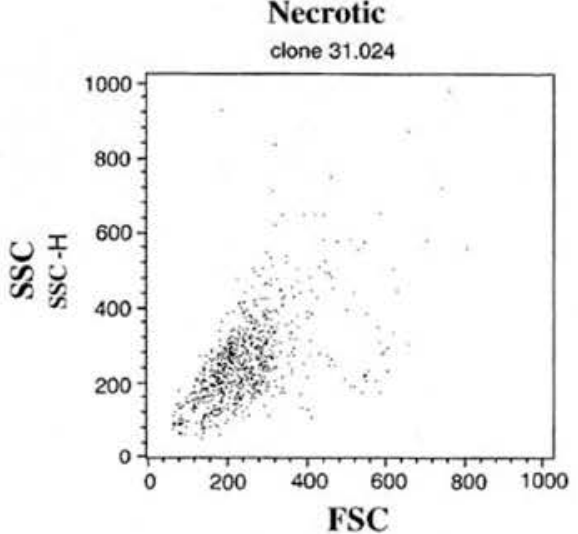

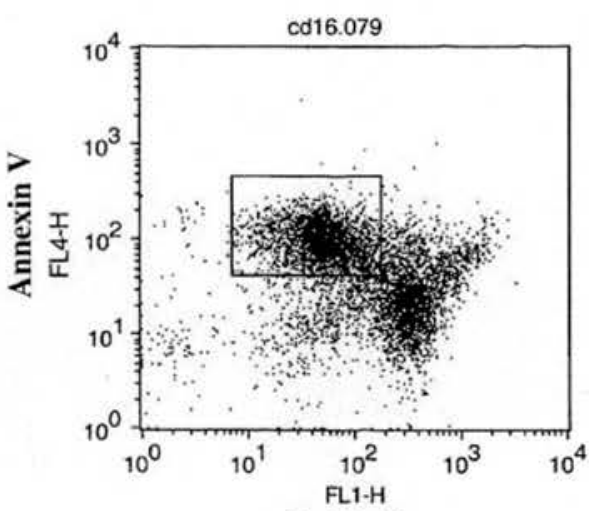

mAb binding
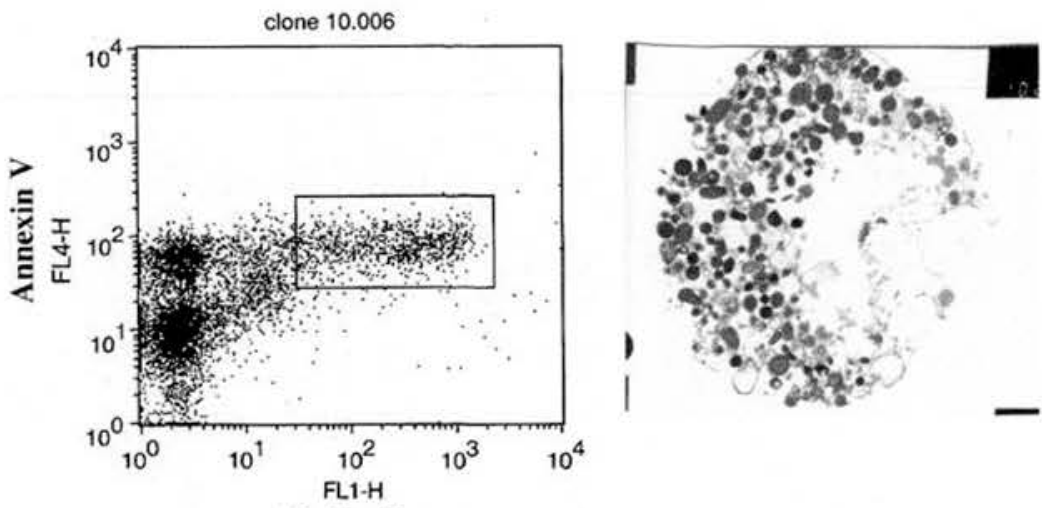

mAb binding

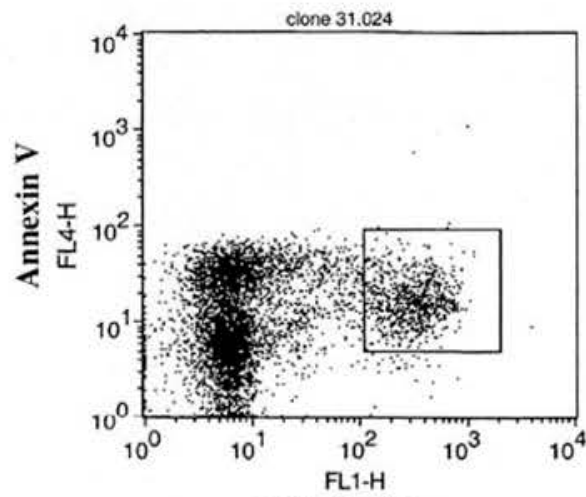

mAb binding

Figure 1 Subpopulations of in vitro cultured neutrophils defined by monoclonal antibodies. Human neutrophils were cultured in vitro for $20 \mathrm{~h}$ in Iscove's DMEM containing $10 \%$ autologous serum. Cells were labelled with murine mAb and FITC-conjugated $F\left(a^{\prime}\right)_{2}$ anti-mouse lgG (FL1) together with annexin V APC (FL4); CD16 $\mathrm{mAb} 3 G 8$ was used to define apoptotic neutrophils Dransfield et $\mathrm{al}^{12}$, uncharacterised mAb (clone 10) as an example of binding to 'late' apoptotic cells, and clone 31 as an example of binding to necrotic cells. Representative histograms show laser scatter properties; forward scatter (FSC) and $90^{\circ}$ scatter (SSC) for the populations defined by the mAb. Typical two colour immunofluorescence profiles are shown to illustrate gates used for laser scatter plots. A representative electron micrograph of a "late' apoptotic cell affinity isolated using $\mathrm{mAb} B O B 78^{10}$ showing extensive nuclear degradation, lack of intact plasma membrane and retention of granule contents

responsible for this effect remains to be defined. It is possible that other, as yet unidentified, surface receptors may behave in a similar manner. Although the significance of opsonisation of apoptotic neutrophils by immune complexes during inflammation is not known, it is likely that subsequent phagocytic clearance will be affected. ${ }^{21}$ 


\section{Summary}

In conclusion, since the first observation that neutrophils undergo programmed cell death in $1989,{ }^{22}$ there has been tremendous progress in defining the underlying regulatory mechanisms and the consequences in terms of gene expression patterns, functional activity and membrane receptor alterations. However, the issues relating to heterogeneity of apoptotic cell phenotype discussed here have profound implications for future studies of phagocyte recognition, uptake and, crucially, phagocyte responses following phagocytosis of apoptotic neutrophils.

\section{Acknowledgements}

This work was supported by the Medical Research Council (Clinician Scientist award to SPH), the Arthritis Research Campaign (grant R0622) and the Wellcome Trust. We thank Professor Christopher Haslett and Professor John Savill for incisive, constructive comments and Professor David Gray for providing hybridoma culture supernatants for screening purposes.
1. Haslett C et al. (1989) Curr. Opin. Immunol. 2: 10-18

2. Haslett C et al. (1994) Philos. Trans. R. Soc. Lond. B. Biol. Sci. 345: 327-333

3. Condliffe AM et al. (1996) Immunology 89: 105-111

4. Coxon A et al. (1996) Immunity 5: 653-666

5. Derouet $M$ et al. (2004) J. Biol. Chem. 279: 26915-26921

6. Ward C et al. (1999) Trends Pharmacol. Sci. 20: 503-509

7. Brown SB et al. (1999) J. Immunol. 162: 480-485

8. Kobayashi SD et al. (2003) Proc. Natt. Acad. Sci. USA 100: 10948-10953

9. Savill J et al. (2002) Nat. Rev. Immunol. 2: 965-975

10. Hart SP et al. (2000) Cell Death Differ. 7: 493-503

11. Buckle AM et al. (1989) J. Immunol. 143: 2295-2301

12. Dransfield I et al. (1994) J. Immunol. 153: 1254-1263

13. Dransfield I et al. (1995) Blood 85: 3264-3273

14. Jones J et al. (1995) Immunology 86: 651-660

15. Walker A et al. (2004) Biochem. Biophys. Res. Commun. 316: 6-11

16. Zheng $L$ et al. (2004) J. Immunol. 173: 6319-6326

17. Brown SB et al. (1997) Biochem. J. 323 (Part 1): 233-237

18. Sheriff A et al. (2004) Cytometry A 62: 75-80

19. Gaipl US et al. (2001) Cell Death Differ. 8: $327-334$

20. Hart SP et al. (2003) Am. J. Pathol. 162: 1011-1018

21. Hart SP et al. (2004) J. Immunol. 172: 1882-1887

22. Savill JS et al. (1989) J. Clin. Invest. 83: 865-875 


\title{
Granulocyte Apoptosis and Macrophage Clearance of Apoptotic Cells as Targets for Pharmacological Intervention in Inflammatory Diseases
}

\author{
Nicola A. Riley, Carol Ward, Deborah A. Sawatzky, Tara A. Sheldrake, Ian Dransfield, \\ Christopher Haslett and Adriano G. Rossi*
}

\begin{abstract}
MRC Centre for Inflammation Research, The Queen's Medical Research Institute, University of Edinburgh, 47 Little France Crescent, Edinburgh, EHI6 4TJ, UK
\end{abstract}

\begin{abstract}
A subset of leukocytes, known as the granulocytes, are the body's first line of innate immune defense. The granulocytes are comprised of neutrophils, eosinophils and basophils of which the former two will be the focus of this review. Neutrophils defend the body against bacterial and fungal infection whilst eosinophils are thought to defend against parasitic invasions. Granulocytes are recruited to the site of infection or tissue damage where their relatively short half-life can be extended by regulatory external factors including hypoxic environments or agents that activate signaling pathways, such as NF- $\mathrm{KB}$ which is implicated in the up-regulation of anti-apoptotic genes. Granulocytes release various proteins, proteolytic enzymes and toxic oxygen products into the phagolysosome or surrounding environment destroying the invading organism. However, in order for inflammation to be resolved it is essential that granulocytes die by apoptosis and are phagocytosed by macrophages in a non-inflammatory fashion. This prevents the release of the cell's histotoxic contents into the extracellular milieu thereby reducing the potential for tissue damage. In instances when granulocytes fail to appropriately enter apoptosis or a defect in phagocytic clearance occurs the inflammatory response can be perpetuated, potentially resulting in the development and promotion of inflammatory disorders such as asthma or rheumatoid arthritis. Thus, selective enhancement of apoptosis and augmentation of macrophage clearance could allow targeting of inflammatory resolution to provide potential novel therapeutic agents for the treatment of inflammatory disorders.
\end{abstract}

\section{INTRODUCTION}

Neutrophil, eosinophil and basophil leukocytes are collectively known as granulocytes and are the main cells involved in innate immune defense. Neutrophils make up the highest proportion of the leukocytes in the circulation (approximately $50-70 \%$ ) defending the body against bacterial and fungal infections. Eosinophils, on the other hand, usually comprise only $1-4 \%$ of the circulating leukocytes with their main function being to defend against parasitic infection. Basophil granulocytes are numerically very scarce $(<1 \%$ of circulating leukocytes), are thought to be involved in parasitic infection and allergic disease, and can undergo regulated cell death by apoptosis, however these cells will not be the focus of this review (see [1,2] for excellent reviews). Granulocytes derive their name from their abundant cytoplasmic granules that contain toxic agents (e.g., proteases) which play a pivotal role in the innate immune response. In response to the presence of foreign organisms or tissue damage, granulocytes and mononuclear cells are recruited to the site of damage. Here the granulocytes destroy and eliminate invading organisms by releasing bactericidal proteins, proteolytic enzymes and toxic oxygen products into the phagolysosome or surrounding environment, where they bring about the destruction of the invading microorganism. In order for the inflammation to be resolved, the granulocytes must undergo apoptosis; a highly regulated physiolo-

*Address correspondence to this author at the MRC Centre for Inflammation Research, The Queen's Medical Research Institute, University of Edinburgh, 47 Little France Crescent, Edinburgh, EHI6 4TJ, UK; Tel: +44-131242-6659; Fax: +44-131-242-6578; E-mail: a.g.rossi@ed.ac.uk gical form of cell death that results in loss of responsiveness to external stimuli and ultimate functional shut-down [3-5]. Apoptosis, rather than necrosis, is essential as the cellular membrane of the granulocytes remain intact throughout therefore preventing the uncontrolled release of their histotoxic contents into the extracellular milieu. Secondly apoptotic granulocytes are rapidly recognized and removed from the site of inflammation via phagocytosis by neighboring cells or macrophages using mechanisms that dampen inflammation $[3,6-8]$. Failure of this process causes granulocytes to undergo necrosis where the cell membrane ruptures allowing the cytotoxic contents to be released into the extracellular milieu resulting in damage to surrounding tissue and an exacerbation of the inflammatory process. Thus, failure of granulocytes to appropriately enter apoptosis at a suitable rate or, alternatively, a defect in phagocytic clearance of the apoptotic granulocytes can lead to a disrupted inflammatory response. In such instances this could prevent effective resolution of inflammation resulting in development or promotion of various inflammatory disorders. The granulocytes have therefore been implicated in diseases where inflammatory resolution is disrupted. For example, eosinophils are involved in allergic type diseases (e.g., asthma and allergic rhinitis) and neutrophils are involved in diseases such as rheumatoid arthritis and inflammatory bowel disease. Therefore, strategic use of therapeutic agents that increase selective granulocyte apoptosis in conjunction with those that upregulate macrophage phagocytosis, could pose a realistic approach to developing novel anti-inflammatory treatments [9-11]. 


\section{STRUCTURAL AND CELLULAR CHANGES OCCU- RRING DURING APOPTOSIS}

Apoptosis is an evolutionary conserved pathway and like virtually all cells granulocytes undergo this form of programmed cell death [12]. Granulocytes having left the bone marrow enter the circulation (it is estimated that the half life of neutrophils in the circulation is approximately 6 hours) and in response to appropriate stimuli enter the tissues. Once their physiological role (e.g., killing and digestion of invading organisms) has been accomplished the cells are destined to undergo spontaneous or constitutive apoptosis in the tissues. Isolated human neutrophils under optimal in vitro culture conditions can survive up to 20-40 hours whereas eosinophils in vitro tend to survive longer ( $40-80$ hours) before apoptosis is engaged. Apoptosis can be delayed significantly by a number of environmental factors and mediators. However, once the process of apoptosis has been initiated (usually by the activation of caspases - see below) there is a reduction in granulocyte volume, their chromatin condenses and their DNA is cleaved internucleosomally. Most cell types fragment, resulting in the release of many membrane encapsulated apoptotic bodies; however granulocytes tend to stay intact resulting in the formation of relatively few apoptotic bodies per cell. The apoptotic bodies are membrane enclosed thereby preventing release of the intracellular contents into the extracellular milieu, and preventing damage to neighboring cells and tissues. Apoptotic cells are then rapidly recognized and engulfed by phagocytes (e.g., macrophages) in response to the exposure of phagocytosis signals (e.g., phosphatidylserine (PS)) on the surface of the apoptotic cell $[8,13]$. Manipulation of this process therefore poses huge potential for the treatment of inflammatory disorders. However in order for this to occur it is essential that every aspect of apoptosis from cell type specific variations to general regulatory mechanisms are fully understood.

\section{APOPTOTIC PATHWAYS}

There are a number of signaling pathways that can regulate the rate of apoptosis in granulocytes, like most other cells, apoptosis can be initiated via two conserved pathways, commonly known as the extrinsic or the intrinsic pathways.

\section{EXTRINSIC PATHWAY}

The extrinsic pathway triggers apoptosis through the stimulation of cell surface 'death receptors' for example TNFR, Fas, and TRAILR (all of which are present on human neutrophils [14] and eosinophils [15]) by specific counter ligands. Interestingly the ligands for these receptors not only initiate apoptotic pathways but can also impact upon other signaling pathways that influence cellular responsiveness. The role of TNF $\alpha$ receptors in neutrophils is particularly notable as they have the capacity to 'prime' the cells for subsequent enhanced stimulation by other agonists (e.g., formylated peptides) and infer both pro-apoptotic and antiapoptotic signaling depending upon the length of the incubation period with TNF $\alpha$. Neutrophils cultured in the presence of TNF $\alpha$ for 12 hours or longer has been reported to have a delayed rate of apoptosis whilst conversely periods of culture of 8 hours or less appear to trigger apoptosis in subpopulations of neutrophils [16]. The TNF $\alpha$ pro-apoptotic and anti-apoptotic pathways are activated upon binding of TNF $\alpha$ to TNFR1 (TNF receptor 1 ) and TNFR2 $[16,17]$. The receptors trimerise inducing proximity of their death domains, consequently allowing the death domains of TNFRassociated death domain-containing proteins (TRADDs) to bind. TRADD can also induce apoptosis through binding to FADD subsequently activating pro-caspase- 8 and has the ability to activate the transcription factors NF- $\mathrm{KB}$ and AP-1 via binding to secondary adaptor molecules such as TNFRassociated factor-2 and Receptor-Interacting Protein (RIP), which may implicate TNF $\alpha$ as also having a role in protecting the cell from apoptosis as NF- $\mathrm{KB}$ is known to induce anti-apoptotic factors.

Activation of Fas is also thought to stimulate dual properties within neutrophils, with membrane bound FasL being postulated to be a more effective inducer of apoptosis than soluble FasL. Soluble FasL (sFasL) may play a more pivotal role in cell migration as it has been exhibited to possess chemotactic properties, which are thought to be due to activation of signaling pathways separate to those involved in the induction of apoptosis [14]. Once activated Fas receptors trimerise allowing interaction with the FADD adapter protein and pro-caspase- 8 creating the Death Initiating Signaling Complex (DISC) to yield active caspase 8 through induced proximity, which then initiates the cascade of caspase activation, activating either pro-caspases 3,6 or 7 . The binding of c-FLIP a competitive inhibitor of pro-caspase-8, can inhibit the activation of the DISC complex. Similarly ligation of Fas on eosinophils leads to enhanced rates of apoptosis $[10,18]$.

TRAIL and multiple TRAIL-receptors (TRAIL-R) 1-4 have been identified as cell surface death receptors. So far it is known that human neutrophils express TRAIL, TRAIL-R2 and TRAIL-R4. Of these TRAIL receptors, TRAIL-2 has been identified as having apoptosis inducing ability in some cell types as it contains a death domain, however TRAIL-4 lacks a death domain rendering it incapable of inducing apoptosis. As a result of this it is hypothesized that TRAIL-4 acts as a decoy receptor with anti-apoptotic capacity. However, TRAIL is thought not to be involved in the regulation of constitutive apoptosis but instead in the removal of neutrophils from inflammatory sites $[14,15,19,20]$. Interestingly TRAIL-R1, 3 and 4 have been found on purified human eosinophils and surprisingly stimulation of eosinophils by TRAIL caused survival in some cells using undefined mechanisms [15].

\section{INTRINSIC PATHWAY}

Cellular stress or injury triggered by, for example, UV irradiation or withdrawal of growth or survival factors activates the intrinsic apoptotic pathway. In this instance the key players in the initiation of apoptosis are a family of proteins called the Bcl-2 family (see below), which control the release of cytochrome $\mathrm{C}$ from the mitochondria by increasing outer membrane permeability. This allows the release of proapoptotic factors from the intermembrane space into the cytoplasm; for example, cytochrome $\mathrm{C}$ release is responsible for the formation of the apoptosome (a complex comprising apoptosis protease activating factor-1 (Apaf-1), caspase-9, caspase-3), Smac/Diablo and $\mathrm{Htra2} / \mathrm{Omi}$, which obstruct the 
Inhibitor of Apoptosis (IAP) family of caspase inhibitors, Alt/endonuclease $\mathrm{G}$ which are important in caspaseindependent nuclear changes in apoptosis and Heat Shock protein 60 [21].

\section{THE BCL-2 FAMILY - REGULATORS OF APOP- TOSIS AND THE ROLE OF THE MITOCHONDRIA}

Members of the Bcl-2 family are a series of cell death regulatory proteins, of which there have been over $20 \mathrm{mem}$ bers identified so far [22]. They are divided into three subgroups depending upon their function and the number of $\mathrm{Bcl}$ 2 Homology $(\mathrm{BH})$ domains they contain, of which there are four in total. These subgroups are the anti-apoptotic Bcl-2 family and the pro-apoptotic family, which is further sub classified into multi-domain pro-apoptotic $\mathrm{Bcl}-2$ members and $\mathrm{BH} 3$ domain only pro-apoptotic Bcl-2 members. Neutrophils and eosinophils are known to contain various members of the $\mathrm{Bcl}-2$ family which play an essential role in regulating their apoptotic processes. For example, neutrophils constitutively express multi-domain pro-apoptotic members $\mathrm{Bax}$ and $\mathrm{Bak}$ and the $\mathrm{BH} 3$ only domain proapoptotic members Bid and Bad [23]. Bax, which has a relatively long half life, undergoes translocation to the mitochondria during constitutive apoptosis resulting in the activation of the intrinsic apoptotic pathway culminating in the activation of caspase 3 [24-28]. The translocation of Bax is thought to be caspase independent due to its non-inhibition by the broad range caspase inhibitor z-VAD-fmk. Furthermore, it is proposed that Bid, which also has a long half life, also undergoes translocation to the mitochondria where it is truncated via the caspases $[29,30]$. As previously mentioned cytochrome $\mathrm{C}$ is an essential molecule in the apoptotic process due to its necessity for the formation of the apoptosome (cytochrome $\mathrm{C}$ however remains hard to detect in neutrophils). Yet neutrophils are known to contain some (albeit relatively few) mitochondria which are a major source of cytochrome $\mathrm{C}$. The key role of mitochondria in both neutrophils and eosinophils appears to be in regulation of apoptosis as opposed to respiration [29,31-37].

Anti-apoptotic members of the Bcl-2 family are also present in granulocytes. Neutrophils, whilst lacking $\mathrm{Bcl}-2$, are known to express the $\mathrm{Bcl}-2$ homologue $\mathrm{Al}$ and the antiapoptotic proteins $\mathrm{Mcl}-1, \mathrm{Bcl}-\mathrm{X}_{\mathrm{L}}$ and X-IAP. The best studied of these proteins in neutrophils is $\mathrm{Mcl}-1$. $\mathrm{Mcl}-1$ protein levels in blood circulating neutrophils decrease preceding the cell entering apoptosis. Inflammatory mediators that postpone neutrophil apoptosis do this (at least in part) through maintaining or increasing levels of $\mathrm{Mcl}-1$ and/or A1 enabling neutrophils to carry out their vital defensive functions. The half lives of both Al and Mcl-1 mRNA along with the Mcl-1 protein are known to be short in contrast to the long half lives of the pro-apoptotic $\mathrm{Bcl}-2$ members. This offers an explanation as to why neutrophils are 'primed' for apoptosis when in the circulation as de novo synthesis of $\mathrm{Mcl}-1$ and $\mathrm{Al}$ is absent, therefore allowing the effect of long lived proapoptotic proteins to pre-dominate $[23,38,39]$. Survival signals in the form of cytokines and other mediators found at sites of inflammation induce de novo synthesis of the antiapoptotic proteins, demonstrated through the use of agents that inhibit protein synthesis (e.g., cycloheximide and actinomycin D) preventing increased granulocyte survival and directly inducing apoptosis [40,41]. One such cytokine known to increase neutrophil survival that is found at elevated levels at sites of inflammation is granulocytemacrophage colony-stimulating factor (GM-CSF). GM-CSF is known to increase levels of Mcl-1 protein through stimulating transcription of the $\mathrm{Mcl}-1$ gene [38]. However this may not be the sole way through which GM-CSF influences Mcl-1 levels. Interestingly, it has also been shown that GM$\mathrm{CSF}$ increases the cellular concentration of $\mathrm{Mcl}-1$ in neutrophils to a more significant level than those achieved through enhanced de novo synthesis of the $\mathrm{Mcl}-1$ protein by increasing the stability of the $\mathrm{Mcl}-1$ protein and reducing the rate with which it is turned over via the proteasome [38].

X-IAP, a member of the inhibitor of apoptosis (IAP) family, inhibits caspases directly [42-44]. X-IAP is known to inhibit caspase 3, 7 and 9, and like Mcl-1 and A1, levels of $\mathrm{X}$-IAP decrease in the presence of stress inducing and proapoptotic stimuli. It has been proposed by Henson et al. that in neutrophils activation of the ERK/MAPK pathway can protect against stress induced apoptosis by preventing a decrease in levels of X-IAP (either through inhibiting degradation or inducing X-IAP synthesis). In addition, oxidants found at sites of inflammation are hypothesized to block ERK activity via activation of $\mathrm{P} 38$, which promotes a decrease in X-IAP thereby enhancing apoptosis [45].

\section{CYSTEINE-DEPENDENT ASPARTATE-SPECIFIC PROTEASE FAMILY (CASPASES)}

As has been mentioned above much of the initiation and execution of apoptosis is mediated via the caspase family of enzymes. The caspases are a family of death promoting proteolytic enzymes that become activated through the cleavage of their precursor molecule to generate an active caspase during apoptosis. They derive their name from ubiquitously contained cysteine residues within their active sites, which upon activation of the enzyme, cleave aspartic acid residues in their target proteins eliciting a cascade of destruction that ultimately brings about the demise of the cell. Caspases involved in apoptosis can be divided into two classes: initiator caspases and effector caspases. Initiator caspases (caspases 8,9 and 10) as their name suggests bring about the initiation of the extrinsic (e.g., death receptor) apoptotic pathway. Procaspases 8 and 10 do this via binding to adaptor molecules for example Fas-associated death domain (FADD) using their long death effector domain (DED) containing prodomains. In addition initiator caspases can be activated by autocatalysis, which is also dependent on their prodomains, as in this instance they act to bring about the oligomerization of the initiator pro-caspases aiding autoactivation. Consequently, initiator caspases can proceed to activate the downstream effector caspases (caspases 3,6 and 7) via cleavage of the effector procaspases resulting in morphological changes. Neutrophils and eosinophils have been shown to possess both initiator and effector caspases $[46,47]$.

\section{CROSS TALK BETWEEN INTRINSIC AND EXTRI- NSIC PATHWAYS}

Although in most circumstances the two pathways described above occur independently, they can be integrated by the pro-apoptotic Bcl-2 family member Bid. The crosstalk 
phenomenon is initiated via caspase 8 cleaving Bid to form truncated Bid (tBid) which then translocates to the mitochondria increasing the permeability of its outer membrane resulting in the release of cytochrome $\mathrm{C}$. Cytochrome $\mathrm{C}$ together with Apaf-1 forms the apoptosome resulting in induced proximity of procaspase- 9 molecules. This results in activation of caspase- 9 to activate procaspase- 3 resulting in the cleavage of substrate proteins, inducing the morphological changes associated with apoptosis. Cells in which cross talk occurs are referred to as type Il cells (in type I cells caspase- 8 directly cleaves pro-caspase 3 ) and it has been proposed that neutrophils fall within the type II classification.

\section{INTRACELLULAR SIGNALING AFFECTING GRAN- ULOCYTE SURVIVAL}

Whilst the basic structural and cellular characteristics occurring during apoptosis vary only slightly between cell types, the mechanisms controlling apoptosis differ greatly. There are key differences in the mechanisms regulating lymphocytes and granulocytes and even between types of granulocytes $[9,10]$. It has been revealed through in vitro observations that inflammatory stimuli such as IL-1 $\beta$, IL-2, IL-8 IL-15, TNF $\alpha$ (>12hr incubation), IFN- $\gamma$, GM-CSF, GCSF, LPS, C5a and hypoxic conditions all have the ability to delay neutrophil apoptosis. These pro-survival factors allow neutrophils the opportunity to persist at the site of infection ensuring efficient removal of invading pathogens. GM-CSF also acts to delay eosinophil apoptosis, while IL-5 another suppressor of eosinophil apoptosis has no effect on neutrophil life span [10]. The fate of the granulocyte at the inflammatory site can therefore be viewed in terms of a fine balance between pro-apoptotic and the anti-apoptotic signals from local pro-inflammatory mediators. Some of the pathways known to influence apoptosis will be considered.

\section{NUCLEAR FACTOR-KB (NF-KB)}

The transcription factor NF- $\mathrm{KB}$ is a key component in regulating the fate of the granulocyte as it is implicated in up-regulating anti-apoptotic genes. It is through NF- $\mathrm{KB}$ that pro-inflammatory mediators such as LPS and TNF $\alpha$ are thought to extend neutrophil survival. The role of NF- $\mathrm{KB}$ on neutrophil and eosinophil survival has been investigated using pharmacological tools such as the NF- $\kappa B$ inhibitor gliotoxin, which when used in conjunction with exposure to LPS, was found to inhibit the anti-apoptotic effects of LPS. Additionally, at early time points gliotoxin dramatically enhances the apoptotic action of TNF $\alpha$ [48]. The importance of $\mathrm{NF}-\kappa \mathrm{B}$ in neutrophil and eosinophil survival has also been demonstrated using physiological NF- $\kappa \mathrm{B}$ inhibitors, namely the prostaglandin $D_{2}$ metabolites $\Delta^{12}$ prostaglandin $J_{2}$ $\left(\Delta^{12} \mathrm{PGJ}_{2}\right)$ and 15-deoxy- $\Delta^{12,14}$ prostaglandin $\mathrm{J}_{2} \quad\left(15 \mathrm{dPGJ}_{2}\right)$ [49]. These also increased granulocyte apoptosis and prevented mediator induced survival. In addition $\mathrm{PGD}_{2}$ metabolites have been shown to possess therapeutic benefits for the treatment of rat pleurisy where they were found to induce neutrophil and macrophage apoptosis to bring about resolution of acutely inflamed tissue [50]. Although less is known about the pathways mediating eosinophil apoptosis, NF- $\mathrm{KB}$ is thought to be involved $[48,51,52]$. When incubated with $\mathrm{TNF} \alpha$, eosinophils appear to lose cytoplasmic $\mathrm{I} \kappa \mathrm{B} \alpha$ allow- ing the released NF- $\mathrm{KB}$ to travel to the nucleus. In addition, there is evidence suggesting that TNF $\alpha$ triggered eosinophil apoptosis may be more sensitive to NF- $\kappa \mathrm{B}$ inhibition than constitutive apoptosis [52]. Recently, using a cell permeable HIV-tat linked super-repressor of the NF-KB inhibitor molecule $I \kappa B \alpha$ we have confirmed a role for NF- $\kappa B$ in the regulation of eosinophil apoptosis as this inhibitor induced direct eosinophil apoptosis [51]. Other groups have now verified the importance of NF- $\mathrm{KB}$ activation in the regulation of human granulocyte apoptosis [53-58].

However, there is a dichotomy in terms of a role for NF$\kappa \mathrm{B}$ in inflammation where NF- $\mathrm{KB}$ also can have an antiinflammatory/pro-resolving role during the resolution of acute inflammation [59]. The development of therapeutics that specifically manipulate the anti-inflammatory effects of $\mathrm{NF}-\kappa \mathrm{B}$ are currently being investigated. Although $\mathrm{I} \kappa \mathrm{B} \alpha$ is an endogenous negative regulator of NF- $\mathrm{KB}$, therapeutic administration of this molecule itself would not be suitable due to its rapid biodegradation in vivo. However, recent studies have shown that a stable chimeric form of this molecule, comprising the super-repressor I $\mathrm{B} B \alpha$ fused to a membranetransducing domain of HIV-tat protein, can promote the resolution of inflammation in an acute rat carrageenaninduced pleurisy model [60]. Furthermore, IKK $\alpha$, an upstream regulator of $\mathrm{I} \kappa \mathrm{B} \alpha$ and $\mathrm{NF}-\kappa \mathrm{B}$, has recently been shown to negatively regulate macrophage activation, local inflammation and innate immunity to bacterial infections [61]. IKK $\alpha$ inhibits NF- $\kappa B$ activity by inducing the NF- $\kappa B$ subunits RelA and c-Rel and suppressing their interaction with pro-inflammatory gene promoters. In addition, genetically modified mice that have inactivated IKK $\alpha$ have an exaggerated inflammatory response in a model of LPS-induced septic shock [61].

\section{PHOSPHOINOSITIDE 3-KINASE (PI3K)}

PI3K is another vital signaling molecule that is likely to regulate many of the pro-inflammatory and anti-apoptotic pathways triggered by the inflammatory mediators (e.g., LPS, GM-CSF and TNF $\alpha$ ). There are three classes of the PI3K family, class I, class II and class III, of which class I PI3Ks are subdivided again according to the organization of their catalytic and regulatory subunits along with their mode of action into classes $1 \mathrm{~A}$ and $1 \mathrm{~B}$. The class $1 \mathrm{~A}$ p $85 / \mathrm{p} 110$ isoform of PI3K appears to be involved in the delay of neutrophil apoptosis induced by L.PS, GM-CSF and TNF $\alpha$. However, inhibition of PI3K signaling using specific PI3K inhibitors do not influence constitutive rates of neutrophil apoptosis [62]. PI3K affects apoptosis via controlling the activation of Akt, NF- $\mathrm{KB}$ and the cAMP response element binding protein (CREB). For -example, in PI3K $\gamma$ knockout mice translocation of NF- $\mathrm{KB}$ to the nucleus and phosphorylation of CREB were decreased. This correlated with reduced expression of the anti-apoptotic proteins $\mathrm{Bcl}-\mathrm{X}_{\mathrm{L}}$ and $\mathrm{Mcl}-1$. It was also found that Akt inactivated Bad, Forkhead and GSK-3 $\beta$ through phosphorylation, with Akt now known to be an integral enzyme in neutrophil apoptosis as it controls Mcl-1 expression through CREB as well as NF- $\mathrm{KB}$ and $\mathrm{Bcl}-$ $\mathrm{X}_{\mathrm{L}}$ [63]. PI3K is also implicated in the regulation of eosinophil recruitment and survival in vivo. Recent studies, using PI3K $\gamma$-defient mice and specific PI3K inhibitors, have demonstrated that PI3Ks critically regulate the recruitment and 
survival of eosinophils in a model of allergic pleurisy. PI3K inhibitors administered during post antigen challenge resulted in a significant increase in apoptotic events and clearance of eosinophils. It was concluded that $\mathrm{PI} 3 \mathrm{~K} \gamma$ is necessary for maintenance of eosinophilic inflammation in vivo and that other isoforms of PI3K may be relevant for eosinophil recruitment [64].

\section{MITOGEN ACTIVATED PROTEIN KINASES (MAPK)}

Upstream kinases regulate the three subfamilies of MAPKs by phosphorylation. These three types of ubiquitously expressed enzymes are p38, extracellular-signalregulated kinases (ERKs) and stress-activated protein kinase/c-jun amino-terminal kinase (SAPK/JNK); in most cell types they play a pivotal role in the regulation of survival. Inflammatory mediators activate several MAPKs therefore it is proposed that crosstalk between MAPKs may be responsible for increasing granulocyte survival. ERK pathways are known to be activated by agents that affect granulocyte responsiveness and apoptosis (e.g., GM-CSF, LPS, IL-8, IL-15 and C5a). Using MEK inhibitors (especially PD098959) it has been suggested that ERK activation is not essential for controlling constitutive apoptosis but that it is likely to be important for maintaining survival induced by certain inflammatory mediators (e.g., GM-CSF) (see [65]).

Our recent studies have shown that the pro-survival molecules pERK $1 / 2$ and $\mathrm{Bcl}-\mathrm{x}_{\mathrm{L}}$ are present in inflammatory cells that are predominant at the onset of inflammation and become down-regulated during the resolution phase of the inflammatory response (Sawatzky et al. 2005; Am.J.Pathol. (in press)). Furthermore, we have qualitatively shown that inducing granulocyte apoptosis with the ERK $1 / 2$ inhibitor PD98059 promotes the resolution of inflammation when given at the peak of the inflammatory response in a rat carrageenan-induced pleurisy in vivo model. This study shows that the induction of neutrophil apoptosis halts the progression of the inflammatory response and pro-actively initiates the onset of resolution.

The importance of p38 and SAPK/JNK in neutrophil survival however remains more controversial. p38 may be an important factor in stress induced apoptosis, yet is thought to have no involvement in Fas or constitutive apoptosis. In addition in GM-CSF treated neutrophils it has been reported that $\mathrm{p} 38$ does not play a role in increasing neutrophil survival as it fails to become phosphorylated. This is supported by the observation that inhibition of p38 by SB203580 fails to affect the anti-apoptotic capacities of GM-CSF. However a possible role for $\mathrm{p} 38$ in neutrophil survival could be provided by its apparent ability to phosphorylate and thereby inhibit caspases- 3 and $8[45,66-68]$. It has been proposed that once the caspases become activated upon the neutrophil entering apoptosis they can result in the cleavage and subsequent degradation of ERK and $\mathrm{p} 38$. This cleavage of MAPKs is thought to be prevented by G-CSF therefore this could pose a possible pathway through which apoptosis is inhibited in neutrophils $[69,70]$.

There is a lack of conclusive evidence regarding the role of JNK in neutrophil apoptosis. However, the limited data suggest that inflammatory mediators such LPS and TNF $\alpha$ have effects on the JNK pathway to influence function. There is some debate however as to whether neutrophil apoptosis is caused by activation of JNK or if it is in fact merely a consequence of it. This is because it has been reported that MAPK kinase kinase and MEKK-1 are cleaved when the cell undergoes apoptosis and that consequently they can activate JNK $[71,72]$.

\section{CYCLIC ADENOSINE MONO PHOSPHATE (CAMP)}

Granulocyte apoptosis is also delayed through an increase in cAMP brought about via some inflammatory mediators (e.g., prostaglandins) and pharmacological agents that elevate cAMP (e.g., the cell permeable analogue db-cAMP) $[36,73-76]$. The precise mechanism of action of cAMP mediated delay of neutrophil apoptosis remains to be elucidated. It has been suggested that this effect may be mediated by both a PKA-dependent [77,78] and PKA-independent mechanism [36]. Similarly cAMP elevation delays eosinophil apoptosis however the mechanisms by which this occurs still remains to be fully elucidated.

\section{CALCIUM}

Use of agents (e.g. A23187 and thapsigargin) that raise cytosolic free calcium in granulocytes have shown that this important second messenger molecule not only regulates granulocyte function but also apoptosis. In neutrophils, raised cytosolic calcium levels have been found to delay apoptosis [79-81] whereas increased calcium levels in eosinophils appear to enhance rates of apoptosis [80]. The reasons for such differential regulation is currently not known and it is also apparent that there is a dissociation between raised elevation of cytosolic calcium and rates of apoptosis [81]

\section{GLUCOCORTICOIDS}

Glucocorticoids are one of the major forms of treatment for inflammatory diseases and, whilst they prove invaluable especially in lymphocytic and eosinophilic dominant diseases such as asthma they appear to be less beneficial in neutrophilic-mediated inflammation. This may be because whilst glucocorticoids act to up-regulate the phagocytic ability of macrophages $[7,82,83]$ and increase eosinophil [84] and lymphocyte [85-87] apoptosis they extend neutrophil life span $[84,88,89]$ therefore slowing their removal from the site of inflammation. It is plausible that the same effect may occur in vivo. There are two known isoforms of the glucocorticoid receptor (GR), GR $\alpha$ and GR $\beta$. The latter of which lacks a steroid binding domain rendering it unable to bind with glucocorticoids. Whilst it is known that the predominant isoform of the glucocorticoid receptor in neutrophils is the $\alpha$-isoform, little is known about the glucocorticoid survival pathway in neutrophils. One theory is that the ability of glucocorticoids to induce apoptosis in a cell, revolves around the ratio of $\mathrm{GR} \alpha$ to $\mathrm{GR} \beta$ [90]. Interestingly it has also been shown that other steroids can also affect neutrophil apoptosis. For example, oestradiol and progesterone have an antiapoptotic effect on neutrophils, a response that is reversed by activating the Fas pathway [91].

\section{NITRIC OXIDE}

Nitric oxide is not only important in the processes of vasodilatation and neurotransmission as it also plays a regu- 
latory role in granulocyte apoptosis $[92,93]$. Its role however is not straightforward, as it appears to have both the ability to promote, as wcll as dclay granulocyte apoptosis. The general trend in neutrophils however appears to be that high concentrations of nitric oxide have a pro-apoptotic/necrotic effect possibly via peroxynitrite generation [94]. In addition, it is speculated that pathways that influence granulocyte survival such as NF- $\kappa \mathrm{B}$ may be disrupted indirectly by the generation of nitric oxide [95]. On the contrary, low concentrations of nitric oxide favour an anti-apoptotic effect that is proposed to involve S-nitrosation of caspase enzymes or an increase in cGMP [94].

\section{MISCELLANEOUS}

There are many other mediators, drugs and agents that influence granulocyte apoptosis that have not been specifically covered in detail in this review. Others include, oxidative stress, bacteria and their products, and cytokines such as macrophage migration inhibitory factor (MIF), many of which have been described in other reviews [10,65,96-98]. For example, MIF an inflammatory cytokine that is released by monocytes/macrophages T-cells and eosinophils, delays neutrophil apoptosis via inhibiting the apoptotic pathway upstream of the mitochondria ultimately preventing activation of caspase-3 [30]. MIF has also been proposed to be involved in eosinophil-dependent inflammatory disorders, such as asthma. Eosinophils have been shown to be an important source of MIF and bronchoalveolar lavage fluid samples from asthma sufferers demonstrating increased levels of MIF when compared to samples obtained from healthy non-asthmatics [99]. It is now recognized that the inflammatory environment is hypoxic (often with a $\mathrm{p}_{2}<3 \%$ ). Granulocytes function efficiently under these conditions and it is believed that adaptive responses to hypoxia are controlled mainly by the transcription factor HIF- $1 \alpha$. Indeed it has recently been shown that HIF-I $\alpha$ is essential for myeloid cell-mediated inflammation in vitro and in vivo [100]. Hypoxic conditions profoundly delay neutrophil [101-104] and cosinophil (Ward and Rossi, unpublished) apoptosis. The precise mechanism underlying the hypoxia-mediated neutrophil survival is unknown but good evidence suggests that $\mathrm{HIF}-1 \alpha$-dependent $\mathrm{NF}-\kappa \mathrm{B}$ activity is involved [104].

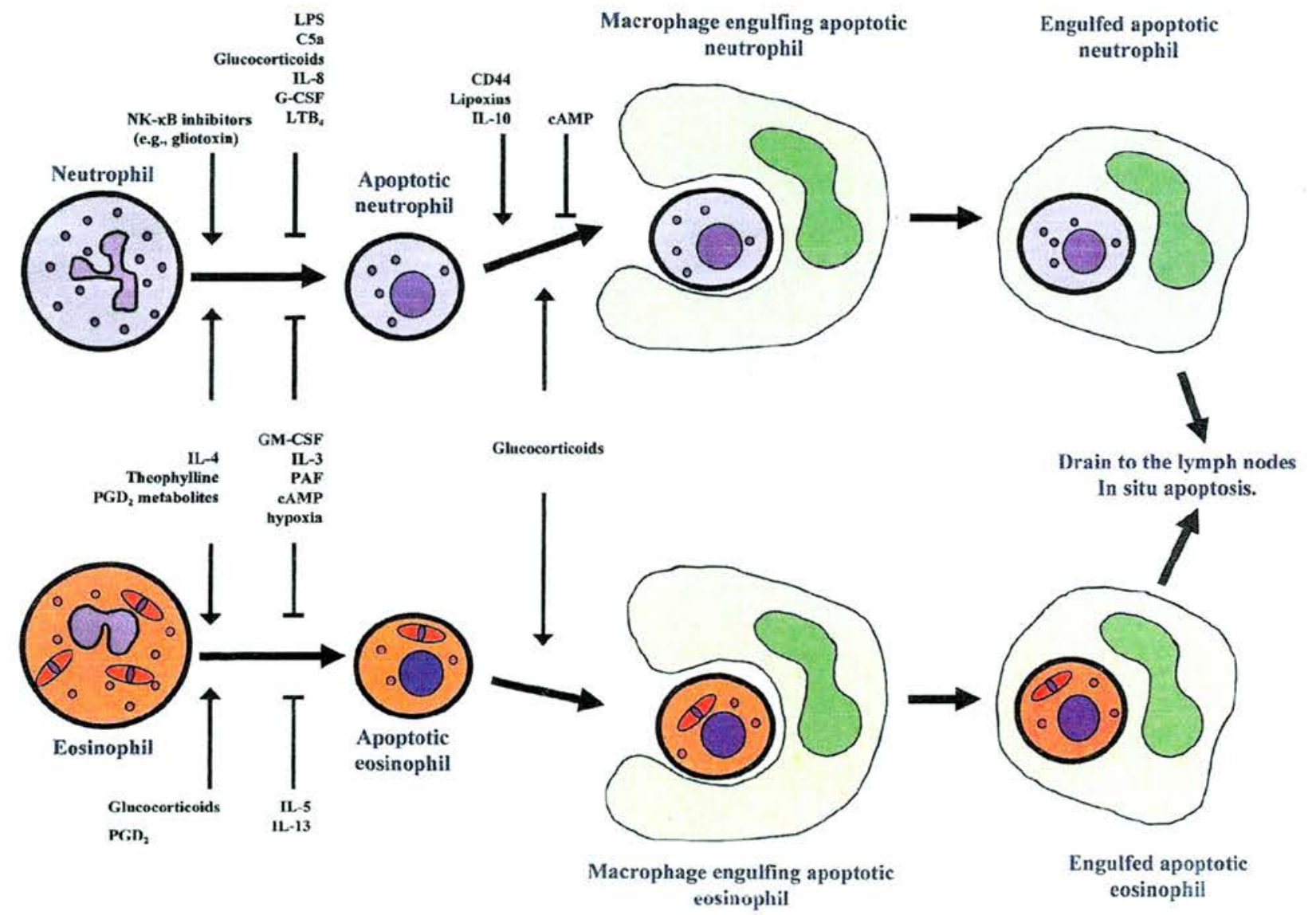

Fig. (1). Factors influencing human granulocyte apoptosis and macrophage clearance of apoptotic granulocytes. The life span of granulocytes is regulated at sites of inflammation by numerous factors that can increase or decrease rates of apoptosis. Some of these include agents such as GM-CSF, IL-3, PAF, cAMP and conditions such as a hypoxic environment which can delay both neutrophil and eosinophil apoptosis, whereas agents such as glucocorticoids can enhance eosinophil apoptosis but conversely delay neutrophil apoptosis. Specific agents can also act to modulate the rate at which apoptotic cells are recognized and phagocytosed by professional phagocytes. Glucocorticoids, for example, augment the ability of phagocytic removal of apoptotic cells. 


\section{MACROPHAGE REMOVAL OF APOPTOTIC GRAN- ULOCYTES- TARGETS FOR PHARMACOLOGICAL INTERVENTION}

Resolution of inflammation requires that granulocytes are removed from the site of cellular damage by a process involving granulocyte apoptosis and subsequent disposal via phagocytosis by tissue resident macrophages or other cells with a capacity for phagocytosis (e.g., fibroblasts, endothelial cells). Importantly, there is much evidence suggesting that phagocytes when ingesting apoptotic cells increase their expression and release of anti-inflammatory pro-resolution mediators and down-regulate release of pro-inflammatory mediators [6,7]. Apoptotic cells experience changes to their cell membrane allowing recognition and ingestion of the apoptotic cells by phagocytes. One such signal suggested to be a key mechanism behind the removal of apoptotic cells is the recognition of PS on the apoptotic cell surface by specific PS receptors. However, PS is not thought to be the sole 'eat me' signal responsible for the removal of apoptotic neutrophils (see $[8,13,105])$. Therefore it has been proposed that different mechanisms may be responsible for signaling removal of apoptotic cells in different tissue types. Interestingly, it has been suggested that apoptotic cells also have the capacity to liberate specific agents that promote the recruitment of cells capable of their removal [106].

If this process is defective, it is believed that tissue damage can ensue due to the accumulation of apoptotic cells and subsequent liberation of histotoxic contents. Manipulation of the resolution phase of the inflammatory process is a promising area to provide novel anti-inflammatory therapies, selectively accelerating apoptosis in specific inflammatory cells and enhancing their subsequent clearance by exploiting their differences in intracellular regulation. Inhibition of the pro-survival signals found at the inflammatory site (for example GM-CSF and IL-5) and selective induction of specific receptors such as Fas to bring about apoptosis in chosen inflammatory cells would increase the rate of apoptosis at inflammatory sites thereby aiding the resolution process. Another optimistic area for pharmacological intervention is the regulation of the uptake of the apoptotic cells by phagocytes. It has been demonstrated that the ability of macrophages to internalize apoptotic cells can be adjusted, for example by using prostaglandins such as $\mathrm{PGE}_{2}$ to elevate macrophage levels of cAMP, thereby activating PKA and transforming adhesion patterns. Macrophages treated as such have a reduced capacity to uptake apoptotic neutrophils therefore implying a possible link between adhesion and macrophage phagocytic abilities [107]. Furthermore the internalization of apoptotic neutrophils by macrophages can be upregulated via ligating the transmembrane adhesion receptor CD44 [108]. This molecule has been found to be of particular importance in the resolution of lung inflammation [109]. Other means which have also been found to alter macrophage ability to internalize apoptotic cells are; by activating macrophage signaling pathways (e.g., PKC) [110-112], ligating extracellular matrix receptors $[108,113]$, use of certain cytokines (e.g., IL-10, GM-CSF, TGF- $\beta$ ) $[114,115]$ or eicosanoids (e.g., lipoxins)[116,117]. Recent breakthroughs regarding the ability of glucocorticoids to increase the phagocytic potential of macrophages to remove apoptotic cells by innocuous noninflammatory means have proved invaluable in aiding the understanding of their action $[7,83,118]$. This could be linked to studies revealing that glucocorticoids have the ability to transform the cytoskeletal organization and the protein generating ability of monocytes driving them to become highly phagocytic macrophages [82]. Interestingly, it has been proposed that the cytokine environment present at the site of inflammation may influence the effectiveness of glucocorticoids having implications on their therapeutic use. For example, IFN- $\gamma$ has the ability to counteract the increased phagocytic ability of macrophages obtained from glucocorticoid treated monocytes without altering their morphology [83]. This increase in the phagocytic ability of macrophages through exposure to glucocorticoids complements glucocorticoids capacity to up-regulate eosinophil and lymphocyte apoptosis, possibly explaining why the use of glucocorticoids proves to be a successful treatment for a range of inflammatory diseases.

\section{THERAPEUTIC APPLICATIONS AND DRUG DIS- COVERY}

The safe engulfment and removal of apoptotic cells is an emerging target for the treatment of inflammatory diseases. Indeed, this mechanism has now been attributed to many of the drugs that are already in clinical use, such as the glucocorticoids and NSAIDs. Glucocorticoids have been shown to induce apoptosis of inflammatory cells (except neutrophils), and importantly, to promote phagocytosis of these effete cells ensuring their safe removal thus limiting tissue damage and injury. In addition, there are many endogenous 'brake' mechanisms that halt inflammation and actively drive the resolution of inflammation. These include the cyclopentenone prostaglandins, NF- $\mathrm{\kappa B}$ p50/50, lipoxins/resolvins, annexin 1 (formerly known as lipocortin 1) and caspases, as previously discussed in this review. These have been shown to induce apoptosis and/or phagocytosis in addition to their other known anti-inflammatory properties $[11,50]$. Stable analogues and structurally related analogues of cyclopentanone prostaglandins and lipoxins show some promise in various in vivo models of inflammation and may prove useful in the treatment of non-resolving inflammatory disorders, such as arthritis, psoriasis and asthma [11]. There are now a number of novel anti-inflammatory strategies under development that target the regulation of apoptosis. In particular there are promising drugs that interfere with caspase activation to influence apoptosis already in phase I and phase II clinical trials for the treatment of rheumatoid arthritis, sepsis, hepatitis and stroke [119-121].

The forced selective induction of apoptosis is thought to be beneficial for the treatment of inflammatory diseases due to a variety of reasons. Primarily, the removal of potentially cytotoxic cells in a safe manner that maintains membrane integrity prevents potential damage to surrounding tissues. Also, phagocytosis of apoptotic cells leads to the generation of anti-inflammatory cytokines, such as IL-10 and TGF- $\beta_{1}$ and also may have other unknown endogenous pro-resolving mechanisms that aid resolution of inflammation. Indeed, previous research has shown that the exogenous administration of apoptotic cells induces resolution in an experimental model of inflammation [122]. Interestingly, autologous administration of apoptotic cells has also been clinically proven to prevent heart, kidney and lung transplant rejection [123] 
and limit the need for immunosuppressive drugs, which have many associated side effects and weakens the patient's immune system to kill invading pathogens and bacteria. Recent research shows that the engulfment of the apoptotic cells induces tolerogenic changes of immature dendritic cells, which then stimulates inhibitory $T$ regulatory cells to suppress immune rejection [124]. This clearly shows that the administration of apoptotic cells and their safe engulfment in humans has a role in the adaptive and the innate immune system by improving tolerance and promoting the resolution of inflammation.

Autoimmunity may occur due to the failure to clear apoptotic cells resulting in necrosis with the loss of cell membranc integrity, which causes the release of cytotoxic contents that initiates damage to surrounding tissues. This has been shown in approximately half of the patients with systemic lupus erythematosis where failed clearance leads to the prevalence of apoptotic cells, which has been correlated with discase severity [125]. It wonld be beneficial to assess if the clearance defects of the phagocytic cells could be overcome in order to promote the safe removal of these unwanted cells and what outcome this strategy could have on limiting disease progression or give an improved prognosis for these patients. It is thus becoming clearer that the induction of apoptosis of inflammatory cells could be a beneficial target for the treatment of inflammatory diseases, not only to ensure their safe removal preventing tissue damage but also to elicit an anti-inflammatory cascade of events that promote the resolution of inflammation

\section{CONCLUSION}

Granulocyte apoptosis is a highly regulated process in that there are many complex interrelated signaling pathways and external stimuli controlling this cell death program. There are many similarities and differences between the control of neutrophil and eosinophil apoptosis. We are beginning to understand the biochemical and molecular checkpoints that control granulocyte apoptosis and it is our belief that they may be exploited for therapeutic gain for the treatment of chronic inflammatory diseases. It would therefore be advantageous to enhance the resolution phase of established inflammation by modifying appropriately apoptosis of specific inflammatory cells and enhancing the clearance of apoptotic cells by phagocytes.

\section{ACKNOWLEDGEMENTS}

We thank the Arthritis Research Campaign UK, $\Lambda$ sthma UK, The Medical Research Council UK and the Wellcome Trust for financial support.

\section{REFERENCES}

[1] Falcone, F. H.; Haas, H.; Gibbs, B. F. Blood, 2000, 96, 4028

[2] Kawakami, T.; Galli, S. J. Nat. Rev. Immunol, 2002, 2, 773.

[3] Savill, J. S.; Wyllie, A. H.; Henson, J. E.; Walport, M. J.; Henson, P. M. Haslett, C. J. Clin. Invest., 1989, 83, 865

[4] Whyte, M. K.; Meagher, L. C.; MacDermot, J.; Haslett, C. J. Immunol., 1993, 150, 5124 .

[5] Stern, M.; Savill, J.; Haslett, C. Am. J. Pathol., 1996, 149, 911

[6] Fadok, V. A.; Bratton, D. L.; Konowal, A; Freed, P. W.; Westcott J. Y.; Henson, P. M. J. Clin. Invest., 1998, 101,890.
Liu, Y.; Cousin, J. M.; Hughes, J.; Van Damme, J.; Seckl, J. R Haslett, C.; Dransfield, 1.; Savill, J.; Rossi, A. G. J. Immunol. $1999,162,3639$

[8] Savill, J.; Dransfield, I.; Gregory, C.; Haslett, C. Nat. Rev. Immunol., 2002, 2, 965

[9] Haslett, C. Br. Med. Bull., 1997, 53, 669

[10] Ward, C.; Dransfield, I.; Chilvers, E. R.; Haslett, C.; Rossi, A. G Trends Pharmacol. Sci., 1999, 20, 503

[11] Gilroy, D. W.; Lawrence, T.; Perretti, M,; Rossi, A. G. Nat. Rev Drug Discov., 2004, 3, 401

[12] Wyllie, A. H.; Kerr, J. F.; Currie, A. R. Int. Rev. Cytol., 1980, 68, 251.

[13] Gregory, C. D.; Devitt, A. Immunology, 2004, 113, 1

[14] Akgul, C.; Edwards, S. W. Cell Mol. Life Sci., 2003, 60, 2402

[15] Daigle, 1.; Simon, H. U. Swiss. Med. Wkly., 2001, 131, 231

[16] Murray, J.; Barbara, J. A.; Dunkley, S. A.; Lopez, A. F.; Van, O. X; Condliffe, A. M.; Dransfield, I.; Haslett, C.; Chilvers, E. R Blood, 1997, 90, 2772.

[17] Walmsley, S. R.; Cowburn, A. S.; Sobolewski, A.; Murray, J.; Farahi, N.; Sabroe, I.; Chilvers, E. R. Biochem. Soc. Trans., 2004, 32,456 .

[18] Simon, H. U. Immunol. Rev., 2001, 179, 156

[19] Renshaw, S. A.; Parmar, J. S; Singleton, V.; Rowe, S. J; Dockrell D. H.; Dower, S. K.; Bingle, C. D.; Chilvers, E. R.; Whyte, M. K. J. Immunol., 2003, 170, 102\%

[20] Kamohara, H.; Matsuyama, W; Shimozato, O; Abe, K.; Galligan, C.; Hashimoto, S.; Matsushima, K.; Yoshimura, T. Immunology, 2004, $I I I, 186$

[2I] Maianski, N. A.; Maianski, A. N.; Kuijpers, T. W.; Roos, D. Acta Haematol., 2004, $111,56$.

[22] Baliga, B. C.; Kumar, S. Hematol. Oncol,, 2002, $20,63$.

[23] Moulding, D. A.; Quayle, J. A.; Hart, C. A.; Edwards, S. W. Blood, $1998,92,2495$

[24] Altznauer, F.; Conus, S; Cavalli, A.; Folkers, G.; Simon, H. U. J. Biol. Chem., 2004, 279, 5947 .

[25] Dewson, G.; Cohen, G. M; Wardlaw, A. J. Blood, 2001, 98, 2239

[26] Dibbert, B.; Weber, M.; Nikolaizik, W. H.; Vogt, P.; Schoni, M. H.; Blaser, K.; Simon, H. U. Proc. Natl. Acad. Sci. USA, 1999, 96, 13330.

[27] lulop, T., Jr.; Larbi, A.; Linteau, A.; Desgeorges, S.; Douziech, N. Ann. N. Y. Acad. Sci., 2002, 973,305

[28] Pryde, J. G.; Walker, A.; Rossi, A. G.; Hannah, S.; Haslett, C. J. Biol. Chem., 2000, 275, 33574 .

[29] Maianski, N. A.; Roos, D.; Kuijpers, T. W. J. Immunol., 2004, 172, 7024.

[30] Baumann, R.; Casaulta, C.: Simon, D.; Conus, S.; Yousefi, S. Simon, H. U. FASEB J., 2003, 17, 2221

[31] Druilhe, A.; Letuye, S.; Pretolani, M. Apoptosis, 2003, 8, 481.

[32] Letuve, S.; Druilhe, A.; Grandsaigne, M.; Aubier, M.; Pretolani, M. Am. J. Respir. Cell Mol. Biol., 2002, 26, 565.

[33] Letuve, S.; Druilhe, A.; Grandsaigne, M.; Aubier, M.; Pretolani, M. J. Leukoc. Biol., 2001, 70, 767

[34] Maianski, N. A.; Mul, F. P.; van Buul, J. D.; Roos, D.; Kuijpers, T W. Blood, 2002, 99,672 .

[35] Maianski, N. A.; Maianski, A. N.; Kuijpers, T. W.; Roos, D. Acta Haematol., 2004, $111,56$.

[36] Martin, M. C.; Dransfield, I.; Haslett, C.; Rossi, A. G. J. Biol. Chem., 2001, 276, 45041 .

[37] Peachman, K. K.; Lyles, D. S.; Bass, D. A. Proc. Natl. Acad. Sci. USA, 2001, 98, 1717.

[38] Derouet, M.; Thomas, L.; Cross, A.; Moots, R. J.; Edwards, S. W J. Biol. Chem., 2004, 279, 26915

[39] Edwards, S. W.; Derouet, M.; Howse, M.; Moots, R. J. Biochem. Soc. Trans., 2004, 32, 489

[40] Whyte, M. K.; Savill, J.; Meagher, L. C.; Lee, A.; Haslett, C. J Leukoc. Biol., 1997, 62, 195

[41] Rossi, A. G.; Ward, C.; Murray, J.; Martin, M. C.; Fujihara, S. Dransfield, I; Haslett, C. Resp. Med. 2000, 94, 1259.

[42] Holcik, M.; Gibson, H; Korneluk, R. G. Apoptosis, 2001, 6, 253.

[43] Liston, P.; Young, S. S.; MacKenzie, A. E.; Korneluk, R. G. Apoptosis, 1997, 2, 423 .

[44] Yang, Y. L.; Li, X. M. Cell Res., 2000, I0, 169

[45] Gardai, S. J.; Whitlock, B. B.; Xiao, Y. Q.; Bratton, D. B ; Henson, P. M. J. Biol. Chem., 2004, 279, 44695

[46] Riedl, S. J.; Shi, Y. Nat. Rev. Mol. Cell Biol., 2004, 5, 897 
[47] Creagh, E. M.; Conroy, H.; Martin, S. J. Immunol. Rev., 2003, 193, 10.

[48] Ward, C.; Chilvers, E. R.; Lawson, M. F.; Pryde, J. G.; Fujihara, S.; Farrow, S. N.; Haslett, C.; Rossi, A. G. J. Biol. Chem., 1999, 274, 4309.

[49] Ward, C.; Dransfield, I.; Murray, J.; Farrow, S. N.; Haslett, C.; Rossi, A. G. J. Immunol., 2002, 168, 6232.

[50] Gilroy, D. W.; Colville-Nash, P. R.; McMaster, S.; Sawatzky, D. A.; Willoughby, D. A.; Lawrence, T. FASEB J., 2003, 17, 2269.

[51] Fujihara, S.; Jaffray, E.; Farrow, S. N.; Rossi, A. G.; Haslett, C.; Hay, R. T. Biochem. Biophys. Res. Commun., 2005, 326, 632.

[52] Fujihara, S.; Ward, C.; Dransfield, I.; Hay, R. T.; Uings, I. J.; Hayes, B.; Farrow, S. N.; Haslett, C.; Rossi, A. G. Eur. J. Immunol., 2002, 32, 457 .

[53] Arruda, M. A.; Rossi, A. G.; de Freitas, M. S.; Barja-Fidalgo, C. Graca-Souza, A. V. J. Immunol., 2004, 173, 2023.

[54] Choi, M.; Rolle, S.; Wellner, M.; Cardoso, M. C.; Scheidereit, C.; Luft, F. C.; Kettritz, R. Blood, 2003, 102, 2259.

[55] Francois, S.; El Benna, J.; Dang, P. M.; Pedruzzi, E.; GougerotPocidalo, M. A.; Elbim, C. J. Immunol., 2005, 174, 3633.

[56] Wang, K.; Scheel-Toellner, D.; Wong, S. H.; Craddock, R. Caamano, J.; Akbar, A. N.; Salmon, M.; Lord, J. M. J. Immunol., 2003, 171,1035 .

[57] Castro-Alcaraz, S.; Miskolci, V.; Kalasapudi, B.; Davidson, D. Vancurova, I. J. Immunol., 2002, 169, 3947.

[58] Vancurova, I.; Miskolci, V.; Davidson, D. J. Biol. Chem., 2001, 276,19746

[59] Lawrence, T.; Gilroy, D. W.; Colville-Nash, P. R.; Willoughby, D. A. Nat. Med., 2001, 7, 1291

[60] Blackwell, N. M.; Sembi, P.; Newson, J. S.; Lawrence, T.; Gilroy, D. W.; Kabouridis, P. S. Arthritis Rheum., 2004, 50, 2675.

[61] Lawrence, T.; Bebien, M.; Liu, G. Y.; Nizet, V.; Karin, M. Nature, $2005,434,1138$

[62] Lindemans, C. A.; Coffer, P. J. Biochem. Soc. Trans., 2004, 32, 480.

[63] Yang, K. Y.; Arcaroli, J.; Kupfner, J.; Pitts, T. M.; Park, J. S.; Strasshiem, D.; Perng, R. P.; Abraham, E. Cell Signal., 2003, 15, 225.

[64] Pinho, V.; Souza, D. G.; Barsante, M. M.; Hamer, F. P.; de Freitas, M. S.; Rossi, A. G.; Teixeira, M. M. J. Leukoc. Biol., 2005, 77, 800 .

[65] Walker, A.; Ward, C.; Dransfield, 1.; Haslett, C.; Rossi, A. G. Curr Drug Targets. Inflamm. Allergy, 2003, 2, 339.

[66] Alvarado-Kristensson, M.; Porm-Ares, M. I.; Grethe, S.; Smith, D.; Zheng, L.; Andersson, T. FASEB J., 2002, 16, 129.

[67] Frasch, S. C.; Nick, J. A.; Fadok, V. A.; Bratton, D. L.; Worthen, G. S.; Henson, P. M. J. Biol. Chem., 1998, 273, 8389

[68] Sheth, K.; Friel, J.; Nolan, B.; Bankey, P. Surgery, 2001, 130, 242.

[69] Alvarado-Kristensson, M.; Melander, F.; Leandersson, K.; Ronnstrand, L.; Wernstedt, C.; Andersson, T. J. Exp. Med., 2004, 199,449

[70] Suzuki, K.; Hasegawa, T.; Sakamoto, C.; Zhou, Y. M.; Hato, F.; Hino, M.; Tatsumi, N.; Kitagawa, S. J. Immunol., 2001, 166, 1185.

[71] Nolan, B.; Duffy, A.; Paquin, L.; De, M.; Collette, H.; Graziano, C M.; Bankey, P. Surgery, 1999, 126, 406

[72] Avdi, N. J.; Nick, J. A.; Whitlock, B. B.; Billstrom, M. A.; Henson, P. M.; Johnson, G. L.; Worthen, G. S. J. Biol. Chem., 2001, 276, 2189.

[73] Hallsworth, M. P.; Giembycz, M. A.; Barnes, P. J.; Lee, T. H. Br. J. Pharmacol., 1996, 117, 79 .

[74] Peacock, C. D.; Misso, N. L.; Watkins, D. N.; Thompson, P. J. J. Allergy Clin. Immunol., 1999, 104, 153.

[75] Rossi, A. G.; Cousin, J. M.; Dransfield, I.; Lawson, M. F.; Chilvers, E. R.; Haslett, C. Biochem. Biophys. Res. Commun., 1995, 217,892

[76] Tortorella, C.; Piazzolla, G.; Spaccavento, F.; Antonaci, S. Mediators. Inflamm., 1998, 7, 391.

[77] Krakstad, C.: Christensen, A. E.; Doskeland, S. O. J. Leukoc. Biol., 2004, 76, 641 .

[78] Parvathenani, L. K.; Buescher, E. S.; Chacon-Cruz, E.; Beebe, S. J. J. Biol. Chem., 1998, 273, 6736.

[79] Whyte, M. K.; Hardwick, S. J.; Meagher, L. C.; Savill, J. S.; Haslett, C. J. Clin. Invest., 1993, 92, 446.

[80] Cousin, J. M.; Haslett, C.; Rossi, A. G. Biochem. Soc. Trans., 1997, $25,243 \mathrm{~S}$.
[81] Murray, J.; Ward, C.; O'Flaherty, J. T.; Dransfield, I.; Haslett, C.; Chilvers, E. R.; Rossi, A. G. Br. J. Pharmacol., 2003, 139, 388.

[82] Giles, K. M.; Ross, K.; Rossi, A. G.; Hotchin, N. A.; Haslett, C. Dransfield, 1. J. Immunol., 2001, 167, 976.

[83] Heasman, S. J.; Giles, K. M.; Rossi, A. G.; Allen, J. E.; Haslett, C. Dransfield, I. Eur. J. Immunol, 2004, 34, 1752.

[84] Meagher, L. C.; Cousin, J. M.; Seckl, J. R.; Haslett, C. J. Immunol., $1996,156,4422$.

[85] McConkey, D. J.; Nicotera, P.; Hartzell, P.; Bellomo, G.; Wyllie, A. H.; Orrenius, S. Arch. Biochem. Biophys., 1989, 269, 365.

[86] Wyllie, A. H.; Morris, R. G.; Smith, A. L.; Dunlop, D. J. Pathol., $1984,142,67$.

[87] Wyllie, A. H.; Morris, R. G. Am. J. Pathol., 1982, 109, 78

[88] Cox, G.; Austin, R. C. J. Leukoc. Biol., 1997, 61, 224.

[89] Cox, G. J. Immunol., 1995, 154, 4719.

[90] Strickland, I.; Kisich, K.; Hauk, P. J.; Vottero, A.; Chrousos, G. P.; Klemm, D. J.; Leung, D. Y. J. Exp. Med., 2001, 193, 585 .

[91] Molloy, E. J.; O'Neill, A. J.; Grantham, J. J.; Sheridan-Pereira, M. Fitzpatrick, J. M.; Webb, D. W.; Watson, R. W. Blood, 2003, 102, 2653.

[92] Ward, C.; Wong, T. H.; Murray, J.; Rahman, I.; Haslett, C.; Chilvers, E. R.; Rossi, A. G. Biochem. Pharmacol., 2000, 59, 305.

[93] Taylor, E. L.; Megson, I. L.; Haslett, C.; Rossi, A. G. Cell Death. Differ., 2003, 10,418.

[94] Taylor, E. L.; Megson, I. L.; Haslett, C.; Rossi, A. G. Biochem Biophys. Res. Commun., 2001, 289, 1229.

[95] Fortenberry, J. D.; Owens, M. L.; Chen, N. X.; Brown, L. A. Inflamm. Res., 2001, 50, 89.

[96] Akgul, C.; Moulding, D. A.; Edwards, S. W. FEBS Lett., 2001, 487,318 .

[97] Simon, H. U. Immunol. Rev., 2003, 193, 101

[98] Hofman, P. Curr. Drug Targets. Inflamm. Allergy, 2004, 3, 1

[99] Rossi, A. G.; Haslett, C.; Hirani, N.; Greening, A. P.; Rahman, L. Metz, C. N.; Bucala, R.; Donnelly, S. C. J. Clin. Invest., 1998, 101, 2869.

[100] Cramer, T.; Yamanishi, Y.; Clausen, B. E.; Forster, I.; Pawlinski, R.; Mackman, N.; Haase, V. H.; Jaenisch, R.; Corr, M.; Nizet, V.; Firestein, G. S.; Gerber, H. P.; Ferrara, N.; Johnson, R. S. Cell, 2003, 112,645 .

[101] Hannah, S.; Mecklenburgh, K.; Rahman, I.; Bellingan, G. J.; Greening, A.; Haslett, C.; Chilvers, E. R. FEBS Lett., 1995, 372, 233.

[102] Mecklenburgh, K. I.; Walmsley, S. R.; Cowburn, A. S.; Wiesener, M.; Reed, B. J.; Upton, P. D.; Deighton, J.; Greening, A. P.; Chilvers, E. R. Blood, 2002, 100, 3008.

[103] Murray, J.; Walmsley, S. R.; Mecklenburgh, K. I.; Cowburn, A. S.; White, J. F.; Rossi, A. G.; Chilvers, E. R. Ann. N. Y. Acad. Sci., 2003, 1010, 417

[104] Walmsley, S. R.; Print, C.; Farahi, N.; Peyssonnaux, C.; Johnson, R. S.; Cramer, T.; Sobolewski, A.; Condliffe, A. M.; Cowburn, A. S.; Johnson, N.; Chilvers, E. R. J. Exp. Med., 2005, 20I, 105.

[105] Giles, K. M.; Hart, S. P.; Haslett, C.; Rossi, A. G.; Dransfield, I. Br. J. Haematol., 2000, 109, 1.

[106] Lauber, K.; Bohn, E.; Krober, S. M.; Xiao, Y. J.; Blumenthal, S. G.; Lindemann, R. K.; Marini, P.; Wiedig, C.; Zobywalski, A.; Baksh, S.; Xu, Y.; Autenrieth, I. B.; Schulze-Osthoff, K.; Belka, C.; Stuhler, G.; Wesselborg, S. Cell, 2003, 113, 717.

[107] Rossi, A. G.; McCutcheon, J. C.; Roy, N.; Chilvers, E. R.; Haslett, C.; Dransfield, I. J. Immunol., 1998, 160, 3562.

[108] Hart, S. P.; Dougherty, G. J.; Haslett, C.; Dransfield, I. J. Immunol., $1997,159,919$.

[109] Teder, P.; Vandivier, R. W.; Jiang, D.; Liang, J.; Cohn, L.; Pure, E.; Henson, P. M.; Noble, P. W. Science, 2002, 296, 155.

[110] Hu, B.; Punturieri, A.; Todt, J.; Sonstein, J.; Polak, T.; Curtis, J. L. J. Leukoc. Biol., 2002, 71, 881

[111] Todt, J. C.; Hu, B.; Curtis, J. L. J. Leukoc. Biol., 2004, 75, 705.

[112] Todt, J. C.; Hu, B.; Punturieri, A.; Sonstein, J.; Polak, T.; Curtis, J. L. J. Biol. Chem., 2002, 277, 35906

[113] Hart, S. P.; Rossi, A. G.; Dransfield, I. Biochem. Soc. Trans., 1998, 26,650 .

[114] Ren, Y.; Savill, J. J. Immunol., 1995, 154, 2366

[115] Ogden, C. A.; Pound, J. D.; Batth, B. K.; Owens, S.; Johannessen, I.; Wood, K.; Gregory, C. D. J. Immunol., 2005, 174, 3015

[116] Godson, C.; Mitchell, S.; Harvey, K.; Petasis, N. A.; Hogg, N. Brady, H. R. J. Immunol., 2000, 164, 1663. 
[117] Mitchell, S.; Thomas, G.; Harvey, K.; Cottell, D.; Reville, K.; Berlasconi, G.; Petasis, N. A.; Erwig, L.; Rees, A. J.; Savill, J.; Brady, H. R.; Godson, C. J. Am. Soc. Nephrol., 2002, 13, 2497.

[118] Giles, K. M.; Ross, K.; Rossi, A. G.; Hotchin, N. A.; Haslett, C.; Dransfield, I. J. Immunol., 2001, 167, 976.

[119] Murphy, F. J.; Seery, L. T.; Hayes, I. Essays Biochem., 2003, 39, 131 .

[120] Murphy, F. J.; Hayes, I.; Cotter, T. G. Curr. Opin. Pharmacol., $2003,3,412$.
[121] Reed, J. C. Nat. Rev. Drug Discov., 2002, 1, 111

[122] Huynh, M. L.; Fadok, V. A.; Henson, P. M. J. Clin. Invest., 2002, 109,41 .

[123] Morelli, A. E.; Thomson, A. W. Immunol. Rev., 2003, 196, 125

[124] Lamioni, A.; Parisi, F.; Isacchi, G.; Giorda, E.; Di Cesare, S.: Landolfo, A.; Cenci, F.; Bottazzo, G. F.; Carsetti, R Transplantation, 2005, 79, 846.

[125] Mevorach, D. Clin. Rev. Allergy Immunol., 2003, 25, 49. 


\title{
Apoptosis and Atherosclerosis: The Role of Nitric Oxide
}

\author{
Catherine A. Shaw ${ }^{1}$, Ian L. Megson ${ }^{1}$, and Adriano G. Rossi, ${ }^{2, *}$ \\ 'Centre for Cardiovascular Science and ${ }^{2}$ MRC Centre for Inflammation Research, The Queens Medical Research Insti- \\ tute, University of Edinburgh, 47 Little France Crescent, Edinburgh, EHI6 4TJ, UK
}

\begin{abstract}
Atherosclerosis, and its associated complications, are a major cause of morbidity and mortality, and it is now recognised as a chronic inflammatory disorder. Progression of inflammation depends on the balance between recruitment of inflammatory cells and their subsequent removal from a site of inflammation. Apoptosis, or programmed cell death, is a fundamental process governing cell survival and is a major determinant of the resolution of the inflammatory response. Apoptotic cells are instantly recognised for non-inflammatory clearance by phagocytes (e.g. macrophages) and removed from the vicinity of inflammation without the release of their pro-inflammatory cell contents. Nitric oxide (NO) plays an important role in many biological processes and has several anti-atherogenic properties including vasodilatation, inhibition of platelet activation and aggregation, and the regulation of apoptosis in a variety of cell types involved in atherogenesis. A critical early event during atherogenesis is injury to the endothelium. The ensuing damage results in endothelial dysfunction, including a reduction in the capacity of the endothelium to generate NO. Decreased NO bioavailability is likely to influence many cellular processes occurring within atherosclerotic lesions, including apoptosis. Modulation of apoptosis is a novel target for therapeutic intervention in the treatment of chronic inflammatory disorders, such as atherosclerosis. This modulation may help limit or resolve inflammation without the concomitant recruitment of subsequent inflammatory cells, thereby reducing the potential for further tissue damage. NO is a possible candidate for manipulation of atherosclerotic processes due to both its powerful anti-atherogenic characteristics and ability to affect apoptosis. This review highlights the role of apoptosis in atherosclerosis and discusses the therapeutic potential of NO to limit and/or resolve vascular inflammation.
\end{abstract}

Keywords: Atherosclerosis, nitric oxide, inflammation

\section{INTRODUCTION}

Atherosclerosis and its associated secondary complications, such as myocardial infarction and stroke, remain a major cause of morbidity and mortality in industrialised nations. Atherosclerosis is a multifactorial disease process with a complicated aetiology. However, it is now widely recognised that there is a chronic inflammatory component to the disease process, which is characterised by the formation of lipid-rich plaques in the wall of major conduit vessels such as the coronary arteries and aorta [1-4]. These lesions are usually eccentric and made up of a necrotic corc of lipidladen inflammatory cells encapsulated by a fibrous, collagen-rich cap consisting of vascular smooth muscle cells (VSMC) and extracellular matrix [5]. In this state, the plaque is considered 'stable'; its physical presence results in partial occlusion of the vessel, but because the vessel is dynamic rather than static, it can often compensate for this occlusion and accommodate for the presence of the plaque without a decrease in lumen diameter [6]. However, if the cap is subject to mechanical breakdown or erosion, the plaque can become 'unstable' and rupture. When the plaque cap is compromised, the highly thrombogenic contents of the core are released into the circulation. Platelets are rapidly recruited and activated resulting in thrombus formation. Plaque rupture can occur several times and remain sub-clinical with the

*Address correspondence to this author at the MRC Centre for Inflammation Research, The Queen's Medical Research Institute, University of Edinburgh; 47 Little France Crescent, Edinburgh EH16 4TJ, UK; Tel: +44-131242-6659; Fax: +44-131-242-6578; E-mail: a.g.rossi@cd.ac.uk
VSMC cap reforming, or healing, over the top of the thrombus which becomes incorporated into the lesion, resulting in a layering effect within the plaque [7]. This process can further occlude the vessel in situ, or the thrombus can detach from the plaque surface, and the resulting embolus occlude smaller vessels downstream, leading to the more serious acute cardiovascular syndromes, such as myocardial infarction and stroke [8]. The determinants of plaque vulnerability to destabilisation and rupture have yet to be fully identified, but a growing body of evidence is emerging that points toward a critical role for both the thickness of the VSMC layer overlaying the core [9] and to inflammatory processes occurring within the plaque [10-12].

\section{APOPTOSIS}

Inflammatory cell apoptosis, or programmed cell death, is a highly regulated process whereby cellular death and subsequent phagocytosis occur without disruption of the cell membrane and ensuing release of the histotoxic and proinflammatory mediators from the cytoplasm [13-15]. Apoptosis therefore represents a non-inflammatory mechanism for the removal of cells from a site of tissue damage, and hence, is critical to the successful resolution of the inflammatory response. Pharmacological manipulation of apoptosis in a variety of cell types, particularly inflammatory cells, may represent a novel therapeutic strategy for the treatment of chronic inflammatory disorders, including atherosclerosis $[16,17]$. 
Apoptosis can be modulated by the endogenous messenger nitric oxide (NO), which can be both pro- and antiapoptotic depending on local concentration, the specific cell type in question and the NO-related species generated in vivo $[18,19]$. The aim of this review is to examine the role of apoptosis in atherosclerosis and to discuss the potential of this process as a target for therapeutic intervention by NO donor drugs, whereby disease progression may be stabilised in an effort to prevent the acute cardiovascular syndromes.

\section{ATHEROSCLEROSIS}

Despite arduous research in both humans and animal models of the process, the underlying causes of atherogenesis remain largely unknown. It is widely accepted that a critical early event of atheroma development is endothelial cell injury and damage. This can occur by several mechanisms: chemical injury can occur through oxidative stress, including oxidation of circulating low density lipids (LDL) resulting in the formation of damaging oxidised LDL (ox-LDL); and physical damage to the endothelium can result from shear stress within the vessel, with plaques tending to form at sites usually subjected to elevated shear stress, such as bifurcation points in the arterial tree or where low shear stress results in reduced stimulation of NO synthase and NO generation [20]. The consequences of an insult to the endothelium are numrous: firstly, the damaged endothelium becomes dysfunctional and the net production of NO by eNOS decreases, promoting vasoconstriction. This is reflected in patients with risk factors for atherosclerosis, such as hypercholesterolaemia. In hypercholesterolaemic individuals, vasodilatation in response to the endothelium-dependent vasodilator, acetylcholine (which acts by triggering NO release from the endothelium), is impaired in forearm resistance vessels compared to normocholesterolaemic controls. However, vasodilatation in response to the endothelium-independent NO donor, sodium nitroprusside (SNP), is unaffected. This suggests that endothelial injury resulting from the hypercholesterolaemic state decreases the normal NO-producing capacity of the endothelium [21]. Although resistance vessels are not usually susceptible to plaque formation, they are widely accepted to reflect changes occurring globally throughout the arterial tree.

In addition, in response to the insult to the endothelium, an inflammatory response is triggered. Depending on the nature and duration of the insult, this response can become excessive and, over a period of years, constitutes the disease process itself $[2,3]$. The inflammatory process begins with the expression of chemotactic and adhesion molecules for monocytes and lymphocytes, including vascular cell adhesion molecule I (VCAM-1), on the surface of dysfunctional endothelial cells. Circulating monocytes adhere to the site of endothelial damage and translocate to the sub-endothelial space where they accumulate [22]. Colony stimulating factors secreted from arcas of endothelial damage induce monocytes to differentiate into macrophages, which then begin to express scavenger receptors, facilitating the internalisation of oxidised low density lipoprotein (ox-LDL) [23]. Cultured macrophages do not accumulate native LDL on account of downregulation of LDL receptors in the cholesterol-replete state. However, once LDL has been modificd by oxidation, accumulation of the resultant ox-LDL by macrophages con- tinues unchecked because scavenger receptors are not downregulated by cholesterol accumulation. The mechanism of lipid peroxidation is still not fully understood, but free radicals such as superoxide $\left(\mathrm{O}_{2}{ }^{-}\right)$and hydroxyl radicals $(\mathrm{OH})$, undoubtedly have a role to play $[24,25]$. Peroxynitrite (ONOO), formed by the rapid reaction of $\mathrm{NO}$ with $\mathrm{O}_{2}^{-}$, can also initiate lipid peroxidation, both in vitro, and in membrane lipids and lipoprotcins [26, 27]. In the lipid-laden condition, macrophages are known as foam cells and it is an accumulation of these cells in the vessel intima which forms the earliest recognisable lesion of atherosclerosis - the fatty streak (Fig. 1A) [20,28]. Fatty streaks have been observed in the vessels of young adults and children (including neonates), suggesting that athcrosclerosis may be initiated early in life but remains sub-clinical unless vessel occlusion and/or plaque rupture lead to diagnosis [29].

Once the fatty streak is established, the plaque grows in size as the ox-LDL accumulated in macrophage-derived foam cells causes both further endothelial damage and is chemoattractant for circulating monocytes, which freely migrate through the endothelium, accumulate in the subendothelial space, and differentiate into macrophages which then go on to become foam cells [30]. This establishes a perpetual cycle of endothelial damage, leading to monocyte recruitment and accumulation of ox-LDL, which encourages further endothelial damage (Fig. 1). Activated macrophages in fatty streaks secrete numerous cytokines and growth factors including platelet derived growth factor (PDGF), basic fibroblast growth factor (bFGF), interleukin-1 (IL-1), tumour necrosis factor- $\alpha$ (TNF- $\alpha$ ), and transforming growth factor- $\beta$ $(\mathrm{TGF}-\beta)[20]$. All of these factors and cytokines induce vascular smooth muscle cell (VSMC) hypertrophy andior hyperplasia. This represents a change in phenotype for VSMCs, from the usual adult vascular contractile phenotype to the synthetic phenotype usually seen only in developing vessels. However, characterisation of VSMCs into only two distinct phenotypes is a vast over-simplification. A wide spectrum of diverse intermediary phenotypes exist under different physiological and pathophysiological conditions, without a clear distinction between phenotypes. This is especially true when VSMCs are undergoing phenotypic transition and there may, in fact, be several sub-populations of VSMCs present within the plaque at any given time [31]. In a synthetic phenotype, VSMCs have the ability to proliferate and synthesise large amounts of collagen. Proliferation begins in the media of the vesscl, but after a time the cells begin to migrate, invade the intima and form a layer over the top of the fatty streak. This layer of VSMCs secretes large amounts of extracellular matrix and connective tissue consisting of glycosaminoglycans, dermatan sulfate, and collagen. These molecules form a mesh over the fatty streak, which becomes calcified and forms the fibrous cap of the plaque, encapsulating the highly thrombogenic lipid core and maintaining a barrier between the plaque contents and the circulation (Fig. 1A) $[4,5]$.

\section{APOPTOSIS IN ATHEROSCLEROSIS}

\section{Inflammatory Cells}

Recruitment of inflammatory cells, particularly monocytes and macrophages, is the major driving force behind 
plaque growth and development. Apoptosis of inflammatory cells within an atherosclerotic lesion may result in their subsequent phagocytosis and removal from the plaque core without the concomitant recruitment of additional inflammatory cells (Fig. 1B). The identity of the cells responsible for clearing the apoptotic macrophages from the plaque remains to be confirmed. It is currently unclear whether activated macrophages can themselves phagocytose populations of apoptotic inflammatory cells, or if this is done by a separate subset of specialised phagocytes.

Apoptotic macrophages have been located by TUNEL staining in plaques from animal models of atherosclerosis and in plaques excised from human vessels [32-34]. The apoptotic macrophages tend to be clustered in areas of the plaque most vulnerable to rupture, most notably the base, or shoulder, regions where the VSMC prevalence is also decreased [35]. It is currently unclear why these areas of the plaque are particularly susceptible to rupture, however, reduced VSMC localisation in these areas of vulnerable plaques may indicate that apoptosis of a variety of cell types, including VSMCs, may play a role in plaque susceptibility to rupture (Fig. 1C) [36]. Recently, it has been suggested that the macrophage enzyme, myeloperoxidase (MPO), could also have an important role in determining plaque vulnerability. MPO, a member of the heme peroxidase superfamily, generates reactive oxidants including hypochlorous acid $(\mathrm{HOCl})$ as part of its normal function in innate host defences [37, 38]. Sugiyama et al. have described a strong colocalisation between macrophage MPO expression and $\mathrm{HOCl}$-modified proteins at sites of lesion rupture in patients who suffer acute cardiac events [39]. MPO-generated $\mathrm{HOCl}$ at relatively high physiological concentrations, but still within the range expected to be produced at areas of vascular inflammation $(30-50 \mu \mathrm{M})$, has been shown to promote endothelial cell death by stimulating apoptotic pathways including rapid caspase-3 activation and DNA fragmentation [40]. This observation suggests that, prior to undergoing apoptosis themselves, activated macrophages may induce apoptosis of neighbouring endothelial cells through MPO expression, resulting ultimately in plaque rupture.

\section{Vascular Smooth Muscle Cells}

As previously mentioned, loss of VSMCs from the protective plaque cap is a major determinant of plaque rupture. Because healthy endothelial cells secrete factors that promote VSMC survival, a consequence of activated macro-

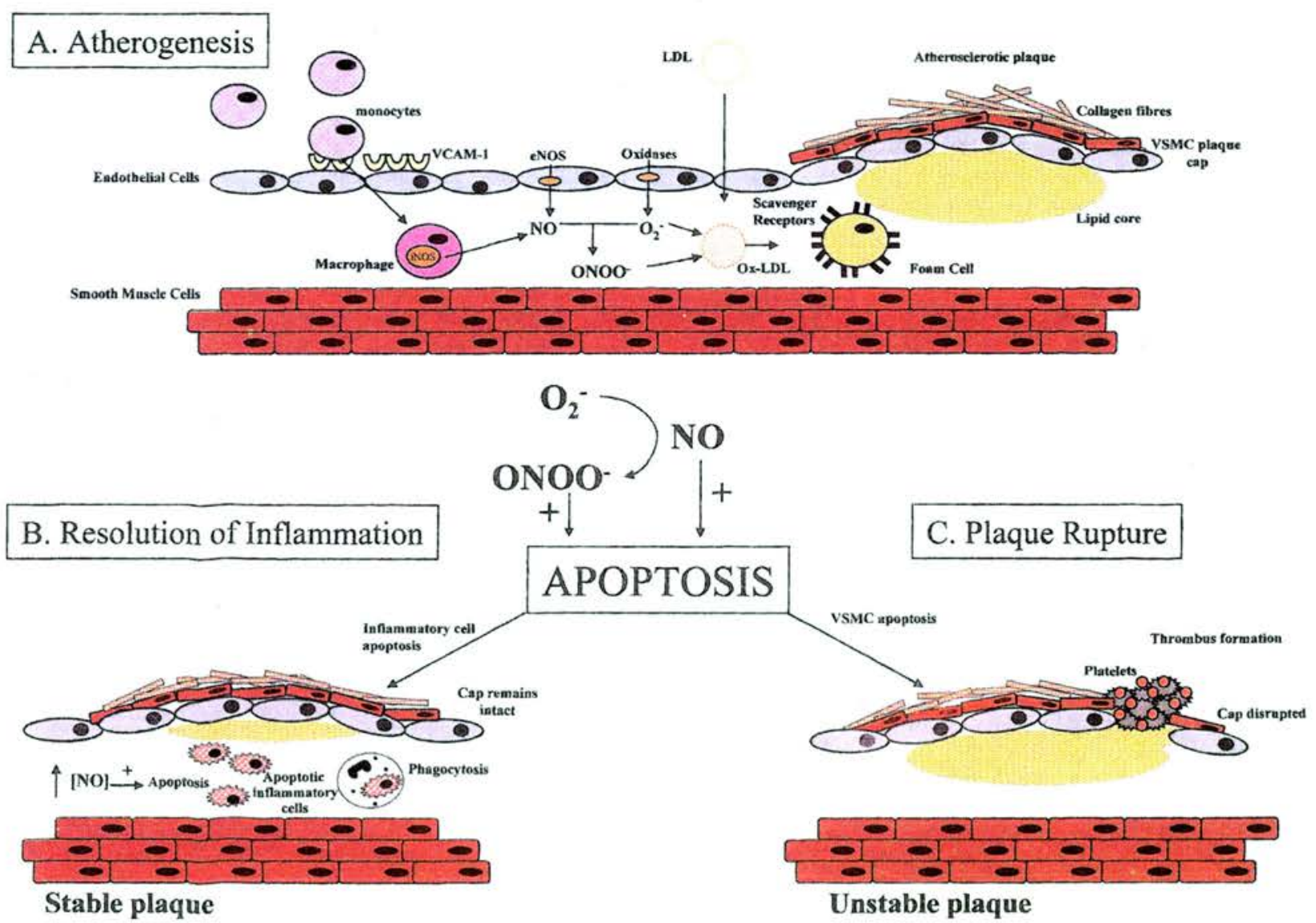

Fig. (1). During atherogenesis circulating monocyes translocate to the subendothelial space where they accumulate modified lipids and form an atherosclerotic plaque, overlain with a fibrous cap of VSMCs (A). Apoptosis can be induced in a variety of cell types by NO. Apoptotic inflammatory cells are cleared by phagocytes, aiding resolution of the inflammatory response, and ultimately facilitating plaque regression (B). However, apoptosis of VSMC may be detrimental, resulting in degradation of the plaque cap and leading to plaque rupture, particularly in the shoulder regions, and ultimately to thrombus formation $(C)$. 
phage-induced endothelial cell death is an increase in VSMC death [41]. Direct induction of VSMC apoptosis in the apolipoprotein $\mathrm{E}$ null murine model of atherosclerosis induces both rupture and thrombosis of the plaque [42]. In addition to removing the protective presence of the endothelium, macrophages can also influence VSMC apoptosis directly. Activated human monocytes/macrophages have been found to be responsible for the death of human VSMCs in vitro $[43,44]$. Seshiah et al. have hypothesised that macrophage colony-stimulating factor (M-CSF), a haematopoietic growth factor supporting survival and differentiation of monocytes, is secreted from VSMCs resulting in macrophage activation, ultimately triggering subsequent VSMC apoptosis [44]. The exact mechanism of this process remains to be fully understood but is thought that macrophages prime VSMCs to respond to apoptotic stimuli, for example by triggering the expression of death receptor ligands such as TNF- $\alpha$ on the cell surface [45].

\section{NITRIC OXIDE}

NO was first identified as the biological messenger responsible for endothelium dependent vasodilatation [46, 47]. Since then, it has become increasingly clear that NO has many diverse actions beyond that of control of vascular tone. Synthesised by three isoforms of the enzyme NO synthase (NOS), NO is now known to inhibit platelet and inflammatory cell adhesion and activation, to act as a transmitter at non-adrenergic non-cholinergic (NANC) neurones, and to exhibit both pro- and anti-apoptotic effects depending on the NO-related species, concentration and cell type in question [48].

The classical pathway by which NO exerts its effects is via activation of the enzyme soluble guanylate cyclase (sGC) and resultant conversion of guanosine 5'-triphosphate (GTP) to 3', 5'-cyclic guanosine monophosphate (cGMP) $[48,49]$. However, recent studies have established that NO can act $v i a$, as yet unidentified, cGMP-independent pathways in various systems, particularly during the inhibition of platelet aggregation [50-53] and regulation of inflammatory cell apoptosis [51-54].

There is currently much debate as to whether all of the effects of $\mathrm{NO}$ are mediated by the NO radical per se, or by other NO-related species formed in situ. Due to its free radical status, NO is highly reactive, and combines readily with reactive oxygen species (ROS) to form a variety of NOrelated species. For example, superoxide anions $\left(\mathrm{O}_{2}{ }^{-}\right)$, often generated at sites of inflammation by the inflammatory cell enzyme NADPH oxidase [55], react rapidly with NO to produce toxic peroxynitrite (ONOO-). This reaction is usually prevented in vivo by the battery of antioxidants that inactivate ROS. For instance, the enzyme superoxide dismutase (SOD) removes $\mathrm{O}_{2}^{-}$by catalysing its conversion to hydrogen peroxide $\left(\mathrm{H}_{2} \mathrm{O}_{2}\right)$, which is subsequently further inactivated by catalase [56]. However, if the production of ROS is such that the antioxidant capacity is overcome, then any free NO present will react rapidly with the $\mathrm{O}_{2}^{-}$to form $\mathrm{ONOO}^{-}$. NO can also form S-nitrosothiols via the transfer of $\mathrm{NO}^{+}$from higher oxides of nitrogen (e.g. $\mathrm{N}_{2} \mathrm{O}_{3}$ ) to reduced thiol groups on proteins such as albumin, resulting in the formation of Snitrosoalbumin $[57,58]$. It has recently been hypothesised that the formation of S-nitrosothiols may act as a slowrelease NO store in vivo [50]. S-nitrosation of a range of cellular proteins is now considered to be one of a number of post-translational modifications that can alter protein function, and these modifactions are likely to be responsible for many of the cGMP-independent effects of NO, including inhibition of caspase- 3 activation $[59,60]$.

\section{NO AS A MEDIATOR OF APOPTOSIS}

The pro- and anti-apoptotic actions of NO have been well documented in many cell systems. Current evidence suggests that lower concentrations of NO produced constitutively by endothelial and neuronal NOS (eNOS and nNOS) are cytoprotective via primarily cGMP-dependent mechanisms, whilst higher, supraphysiological concentrations generated in some pathologies by inducible NOS (iNOS) mediate apoptosis via mechanisms independent of cGMP signalling [61]. For example, high concentrations of either exogenous or endogenous iNOS-derived NO have been shown to induce apoptosis in murine and rabbit macrophage cell lines [62, 63]. Apoptosis in rabbit macrophages was unaffected by inhibition of cGMP-dependent kinases and not mimicked by cGMP analogs, suggesting that the pro-apoptotic actions of $\mathrm{NO}$ are cGMP-independent [64]. Conversely, pre-treatment with relatively low concentrations of exogenous NO, delivered by the synthetic NO donor sodium nitroprusside (SNP) protects RAW 264.7 cells (a mouse macrophage cell line) against cell death upon subsequent exposure to higher concentrations of NO which would normally be cytotoxic [65]. Furthermore, this protection was mimicked by cGMP analogs but negated in the presence of sGC inhibitors, suggesting that the cellular protection was conferred by cGMP. Similarly, high levels of NO produced by activated macrophages as a consequence of iNOS upregulation may also induce VSMC apoptosis through DNA damage and subsequent p53 activation [66].

This dual role, and apparent paradox of NO, may be explained, at least in part, by the free radical nature of NO and the ease with which it will form various NO-related species in vivo. As already mentioned, $\mathrm{NO}$ will react rapidly with $\mathrm{O}_{2}{ }^{-}$ to form $\mathrm{ONOO}^{-}$. The production of $\mathrm{ONOO}^{-}$may be of particular importance at sites of vascular inflammation such as atherosclerotic lesions, where the concentration of reactive oxygen is likely to be elevated [67]. The precise role of ONOO $^{-}$in inflammatory cell apoptosis remains to be elucidated. There is some evidence to suggest that at high concentrations $(100 \mu \mathrm{M}-300 \mu \mathrm{M})$, ONOO $^{-}$induces apoptosis in RAW 264.7 cells [68], whilst at lower concentrations (30 $\mu \mathrm{M}-50 \mu \mathrm{M})$, it may have a protective effect against lipopolysaccharide (LPS) and interferon (IFN)- $\gamma$-induced apoptosis in these cells [69]. Brockhaus et al. [70] have demonstrated that overexpression of copper/zinc SOD (CuZnSOD) can protect RAW 264.7 cells against apoptosis initiated by NO, either exogenous or iNOS-derived. This implies a role for $\mathrm{ONOO}^{-}$as the mediator of NO-initiated apoptosis. However, whilst a specific scavenger of $\mathrm{ONOO}^{\circ}$, uric acid, effectively abolishes apoptosis induced by the $\mathrm{ONOO}^{\circ}$ generator SIN-1, it left apoptosis induced by the NO donors S-nitrosoglutathione (GSNO) and spermine diazeniumdiolate SPER/ $\mathrm{NO}$ unaltered, suggesting that $\mathrm{NO}$ and NO-related species 
may be able to activate several pathways when initiating apoptosis [70].

Whilst endogenous macrophage iNOS-derived NO has been shown to induce apoptosis in animal models, this is not necessarily the case in human macrophages, which might not produce any NO at all. [71-73]. However, despite this reduced capability to produce endogenous NO, human macrophages still undergo apoptosis in response to exogenous NO and NO-related species. For example, the NO donors GSNO and SPER/NO induce apoptosis in primary human monocyte-derived macrophages [74].

\section{NO AND APOPTOSIS IN THE RESOLUTION OF INFLAMMATION}

Apoptosis is now thought to be key to the resolution of the inflammatory response. Pharmacological manipulation of the rate of apoptosis during chronic inflammatory disorders such as atherosclerosis, may aid the resolution of inflammation and delay disease progression. NO is a promising candidate for such manipulation, because its ability to induce apoptosis and aid inflammatory resolution has already been demonstrated in several animal models. In a mouse model of kidney inflammation, activated macrophages have been shown to induce apoptosis in neighbouring mesangial cells prior to ingestion by phagocytes [75]. The ability of the activated macrophages to induce apoptosis is greatly reduced in the presence of the NOS inhibitor, $\mathrm{N}^{\mathrm{G}}$-monomethyl-Larginine (L-NMMA), indicating that macrophage-directed apoptosis of mesangial cell apoptosis occurs via NOdependent mechanism [76]. Similarly, several studies have demonstrated that activated macrophages infiltrating murine tumours induce apoptosis via a NO-dependent pathway in both activated anti-tumour $\mathrm{T}$ cells and in the tumour cells themselves [77, 78]. Thus, it appears that macrophages have the capacity to induce apoptosis of nearby cells by the liberation of $\mathrm{NO}$, or a related species, to enhance the clearance of apoptotic cells.

Inducing apoptosis in inflammatory cells within an atherosclerotic lesion is an attractive prospect as it may represent a mechanism to stabilise the plaque or to resolve the vascular inflammation and lead to regression of the plaque, thereby halting disease progression. Reducing the number of activated macrophages present in the plaque would have multiple consequences. Firstly, the physical presence of the plaque in the vessel wall would be decreased, reducing stenosis. Secondly, because activated macrophages induce apoptosis in neighbouring endothelial cells and VSMCs, reducing the number of activated macrophages may help to preserve endothelial function and maintain the integrity of the plaque cap.

$\mathrm{NO}$ as a mechanism to regress atherosclerosis is an appealing possibility because in addition to the pro-apoptotic properties described above, NO also has a number of other powerful anti-atherogenic characteristics including inhibition of platelet and inflammatory cell activation [48, 79]. Animal studies are emerging to support the hypothesis that manipulation of apoptosis could be used to reverse atherosclerosis. For example, L-arginine (the substrate for NOS) or the NO donor SNP, administered to hypercholesterolaemic rabbits increases the number of apoptotic macrophages in intimal lesions by three fold [64]. This increase in apoptosis was accompanied by a significant reduction in lesion surface area, suggesting that manipulation of the NO synthase pathway, or delivery of exogenous $\mathrm{NO}$, may be a way to boost NO availabilty in order to stabilise, or even regress, the plaque via an apoptotic mechanism. However, the treatments used in this study are by no means selective for macrophages, and as already discussed, NO will also induce apoptosis in endothelial cells and VSMCs. This could have several serious detrimental consequences for the plaque; firstly, additional loss of endothelial function would occur, leading to further exacerbation of the disease process. Secondly, because VSMCs are essential for maintaining the integrity of the plaque cap, loss of this population of cells in vulnerable areas of the lesion could de-stabilise the plaque resulting in plaque rupture which could result in myocardial infarction or stroke. The potential benefit of regressing the plaque in this way must also be offset against the cost of reducing the size of the macrophage population available for scavenging existing apoptotic macrophages, endothelial cells and VSMC. If left in situ, these apoptotic cells will undergo secondary necrosis, thereby increasing the thrombogenicity of the plaque as a whole. In the study by Wang et al. [64] discussed above, apoptosis is quantified by the number of apoptotic nuclei per area of plaque, rather than as a percentage of the total macrophage population. Therefore, it is not possible to draw any conclusions as to whether the phagocytosis capacity of the plaque has been effected in this case.

Although human macrophages appear unable to generate the supraphysiological concentrations that murine cells produce, human macrophages will respond to exogenous NO delivered by synthetic NO donor compounds, suggesting that NO could potentially be used to manipulate rates of apoptosis in human atherosclerosis. However, it is essential to target any therapeutic intervention to specific cell types within the plaque, and in particular, to appropriate cell types within plaques vulnerable to rupture. Indiscriminate pro-apoptotic events may have serious adverse consequences for the plaque dynamic.

At present, existing NO donor drugs are not selective for particular cell types. Common clinically used organic nitrates, such as nitroglycerin (NTG), tend to have an unfavourable selectivity profile when considered in the context of atherosclerosis, they are more selective for veins (which are not subject to plaque formation) than arteries, and more selective for arteries than platelets (which play a major role in thrombus formation following plaque ruture). In order to use NO donor drugs as effectors of apoptosis in atherosclerosis, compounds which are able to selectively act on, for example, macrophages but not endothelial cells or VSMC, will have to be developed in the future. This however, may be difficult given that macrophages are relatively resistant to nxidative stress induced apoptosis.

In addition to selectivity, a major consideration when contemplating the use of NO donor drugs for the treatment of atherosclerosis is that of delivery. Global, non-selective release of NO throughout the circulation may have various anti-atherosclerotic actions, but dosing will be limited by concurrent vasodilatation resulting in systemic hypotension. The challenge therefore, is to generate high local concentra- 
tions in the vicinity of a plaque. One possible means of achieving this is to employ NO donor drugs which are selective for areas of endothelial damage. S-nitrosothiols are generally accepted to be platelet-selective NO donor drugs [80] and have been shown in vitro to have vasodilator actions which are selective for areas of experimentally denuded endothelium [81]. Furthermore, in a rabbit balloon angioplasty model of vascular injury, the S-nitrosothiol, S-nitroso- $N$ valerylpenicillamine (SNVP), in contrast to the traditional organic nitrate NTG, reduced the adhesion of radio-labelled platelets in areas of endothelial damage without significantly affecting systemic blood pressure [82]. In this study, the NO donors were delivered directly into the carotid artery via a cannula; obviously this is not practical in humans as a long term therapy, but a possible alternative is to deliver NO via drug-eluting stents. There has been some success in humans with the use of sirolimus (rapamycin)-eluting stents in preventing neointimal proliferation (which can lead to restenosis) following angioplasty, although long-term effectiveness ( $>1$ year) remains to be established [83]. A further potential method of delivering high local concentrations of NO directly to the interior of the plaque may be to exploit the lipid nature of the plaque core by developing novel lipophilic NO donor drugs designed to induce apoptosis in the lipid-laden macrophages within the core.

\section{CONCLUSION}

Atherosclerosis is now considered a chronic vascular inflammatory disorder involving complicated interactions between many cell types. Apoptosis plays a key role in determining both turnover of cells within the plaque, and plaque vulnerability to rupture. Because apoptotic cells are removed from the site of inflammation without triggering a subsequent pro-inflammatory response, inflammatory cell apoptosis is a promising target for therapeutic intervention in the treatment of this disease. The ability of NO to induce apoptosis, combined with the additional anti-atherogenic properties of NO, points to this molecule being a powerful tool in the treatment of atherosclerosis. The challenge will be to deliver NO in the appropriate chemical form and to balance any benefit in reducing inflammatory cell number within the plaque against the potential deleterious effects of destabilising the plaque cap and promoting plaque rupture.

\section{REFERENCES}

[1] Ludewig, B.; Zinkernagel, R.M.; Hengartner, H. Trends Cardiovasc. Med., 2002, 12, 154

[2] Ross, R. Am. Heart J., 1999, 138, S419.

[3] Ross, R. N. Engl. J. Med., 1999, 340, 115

[4] Badimon, J.J.; Fuster, V.; Chesebro, J.H.; Badimon, L. Circulation, 1993,87, II3.

[5] Davies, M.J. N. Engl. J. Med., 1997, 336, 1312

[6] Glagov, S.; Weisenberg, E.; Zarins, C.K.; Stankunavicius, R.; Kolettis, G.J. N. Engl. J. Med., 1987, 316, 1371

[7] Mann, J.; Davies, M.J. Heart, 1999, 82(3), 265.

[8] Mitra, A.K.; Dhume, A.S; Agrawal, D.K. Can. J. Physiol. Pharmacol, 2004, 82, 860

[9] Leskinen, M.J.; Kovanen, P.T.; Lindstedt, K.A. Biochem. Pharmacol. 2003, 66, 1493

[10] Lombardo, A.; Biasucci, L.M.; Lanza, G.A.; Coli, S.; Silvestri, P.; Cianflone, D.; Liuzzo, G.; Burzotta, F.; Crea, F.; Maseri, A. Circulation, 2004, 109, 3158 .

[11] Robbins, M.; Topo!, E.J. Cleve Clin. J. Med., 2002, 6, SII130.

[12] Libby, P. Nature, 2002, 420(6917), 868-74.
[13] Taylor, E.L.; Megson, I.L.; Haslett, C.; Rossi, A.G. Cell Death Differ., 2003, 10, 418

[14] Haslett, C. Br. Med. Bull., 1997, 53, 669

[15] Rossi, A.G.; Ward, C.; Dransfield, I Biochem. Soc. Trans., 2004, 32,452 .

[16] Gilroy, D.W.; Lawrence, T; Perretti, M.; Rossi, A.G. Nat. Rev. Drug Discov., 2004, 3, 401

[17] Ward, C.; Dransfield, I.; Chilvers, E.R.; Haslett, C.; Rossi, A.G. Trends Pharmacol. Sci., 1999, 20, 503.

[18] Kim, Y.M.; Bombeck, C.A.; Billiar, T.R. Circ. Res., 1999, 84, 253

[19] Quinn, A.C.; Petros, A.J.; Vallance, P. Br. J. Anaesth., 1995, 74, 443.

[20] Ross, R. Nature, 1993, 362, 801

[21] Chowienczyk, P.J.; Watts, G.F.; Cockcroft, J.R.; Ritter, J.M. Lancet, 1992, 340, 1430.

[22] Vogel, R.A. Clin. Cardiol., 1997, 20, 426

[23] Henriksen, T;; Mahoney, E.M; Steinberg, D. Proc. Natl. Acad. Sci. USA, 1981, 78, 6499 .

[24] Maxwell, S.R.; Lip, G.Y. Br. J. Clin. Pharmacol., 1997, 44, 307

[25] Heinecke, J.W. Curr. Opin. Lipidol., 1997, 8, 268.

[26] Darley-Usmar, V.M.; Hogg, N.; O'Leary, V.J.; Wilson, M.T.; Moncada, S. Free Radic. Res. Commun., 1992, 17, 9.

[27] Radi, R.; Beckman, J.S.; Bush, K.M.; Freeman, B.A. Arch. Biochem. Biophys., 1991, 288, 481.

[28] Schwartz, C.J.; Valente, A.J.; Sprague, E.A.; Kellcy, J.L.; Nerem, R.M. Clin. Cardiol., 1991, 14, II.

[29] McGill, H.C. Jr.; McMahan, C.A.; Herderick, E.E.; Malcom, G.T.; Tracy, R.E.; Strong, J.P. Am. J. Clin. Nutr, 2000, 72, 1307S.

[30] Simon, B.C.; Cunningham, L.D.; Cohen, R.A. J. Clin. Invest., $1990,86,75$

[31] Owens, G.K.; Kumar, M.S.; Wamhoff, B.R. Physiol. Rev., 2004. 84,767 .

[32] Bjorkerud, S.; Bjorkerud, B. Am. J. Pathol., 1996, 149, 367.

[33] Haunstetter, A.; lzumo, S. Circ. Res., 1998, 82, 1111.

[34] Han, D.K.; Haudenschild, C.C.; Hong, M.K.; Tinkle, B.T.; Leon, M.B.; Liau, G. Am. J. Pathol., 1995, 147, 267.

[35] Kolodgie, F.D.; Narula, J.; Burke, A.P.; Haider, N.; Farb, A.; HuiLiang, Y.; Smialek, J.; Virmani, R. Am. J. Pathol., 2000, 157, 1259.

[36] Kockx, M.M.; Herman, A.G. Eur. Heart J., 1998, 19, G23.

[37] Hazen, S.L.; Heinecke, J.W. J. Clin. Invest., 1997, 99, 2075

[38] Klebanoff, S.J. Ann. Intern. Med., 1980, 93, 480.

[39] Sugiyama, S.; Okada, Y.; Sukhova, G.K.; Virmani, R.; Heinecke, J.W.; Libby, P. Am. J. Pathol., 2001, 158, 879.

[40] Sugiyama, S.; Kugiyama, K.; Aikawa, M.; Nakamura, S.; Ogawa, H.; Libby, P. Arterioscler. Thromb. Vasc. Biol., 2004, 24, 1309.

[41] Gordon, D.; Reidy, M.A.; Benditt, E.P.; Schwartz, S.M. Proc. Natl. Acad. Sci. USA, 1990, 87, 4600.

[42] von der Thusen, J.H.; van Vlijmen, B.J.; Hoeben, R.C.; Kockx, M.M.; Havekes, L.M.; van Berkel, T.J.; Biessen, E.A. Circulation, 2002, 105, 2064

[43] Vasudevan, S.S.; Lopes, N.H.; Seshiah, P.N.; Wang, T.; Marsh, C.B.; Kereiakes, D.J.; Dong, C.; Goldschmidt-Clermont, P.J. Cardiovasc. Res., 2003, 59, 723 .

[44] Seshiah, P.N.; Kereiakes, D.J; Vasudevan, S.S.; Lopes, N.; Su, B.Y.; Flavahan, N.A.; Goldschmidt-Clermont, P.J. Circulation, 2002, 105, 174 .

[45] Mayr, M.; Xu, Q. Exp. Gerontol., 2001, 36, 969

[46] Furchgott, R.F.; Zawadzki, J.V. Nature, 1980, 288, 373.

[47] Palmer, R.M.; Ferrige, A.G.; Moncada, S. Nature, 1987, 327, 524.

[48] Moncada, S.; Palmer, R.M.; Higgs, E.A. Pharmacol. Rev., 1991, 43, 109 .

[49] Ignarro, L.J.; Cirino, G.; Casini, A.; Napoli, C. J. Cardiovasc. Pharmacol., 1999, 34, 879 .

[50] Crane, M.S.; Rossi, A.G.; Megson, I.L. Br. J. Pharmacol., 2005 144,849 .

[51] Crane, M.S.; Ollosson, R.; Moore, K.P.; Rossi, A.G.; Megson, L.L. J. Biol. Chem., 2002, 277, 46858

[52] Gordge, M.P.; Hothersall, J.S.; Noronha-Dutra, A.A. Br. J. Pharmacol., 1998, 124, 141.

[53] Sogo, N.; Magid, K.S.; Shaw, C.A.; Webb, D.J.; Megson, I.L. Biochem. Biophys, Res. Commun, 2000, 279, 412.

[54] Ward, C.; Wong, T.H.; Murray, J.; Rahman, I.; Haslett, C.; Chilvers, E.R.; Rossi, A.G. Biochem. Pharmacol., 2000, 59, 305.

[55] Forman, H.J.; Torres, M. Mol. Aspects Med., 2001, 22, 189.

[56] Beckman, J.S.; Koppenol, W.H. Am. J. Physiol., 1996, 271, C1424. 
[57] Minamiyama, Y.; Takemura, S.; Inoue, M. Biochem. Biophys. Res. Commun., 1996, 225, 112 .

[58] Stamler, J.S.; Jaraki, O.; Osborne, J.; Simon, D.I.; Keaney, J.; Vita, J.; Singel, D.; Valeri, C.R.; Loscalzo, J. Proc. Natl. Acad. Sci. USA, 1992, 89, 7674-7.

[59] Mohr, S.; Zech, B.; Lapetina, E.G.; Brune, B. Biochem. Biophys. Res. Commun., 1997, 238, 387.

[60] Melino, G.; Bernassola, F.; Knight, R.A.; Corasaniti, M.T.; Nistico, G.; Finazzi-Agro, A. Nature, 1997, 388, 432

[61] Nicotera, P.; Brune, B.; Bagetta, G. Trends Pharmacol. Sci., 1997, 18, 189.

[62] Albina, J.E.; Cui, S.; Mateo, R.B.; Reichner, J.S. J. Immunol., $1993,150,5080$

[63] Sarih, M.; Souvannavong, V.; Adam, A. Biochem. Biophys. Res. Commun., 1993, 19I, 503

[64] Wang, B.Y.; Ho, H.K.; Lin, P.S.; Schwarzacher, S.P.; Pollman, M.J.; Gibbons, G.H.; Tsao, P.S.; Cooke, J.P. Circulation, 1999, 99 1236.

[65] Yoshioka, Y.; Yamamuro, A.; Maeda, S. Br. J. Pharmacol., 2003, 139, 28.

[66] Bennett, M.R; Boyle, J.J. Atherosclerosis, 1998, 138, 3.

[67] Crow, J.P.; Beckman, J.S. Adv. Pharmacol., 1995, 34, 17.

[68] Sandoval, M.; Ronzio, R.A.; Muanza, D.N.; Clark, D.A.; Miller, M.J. Nitric Oxide, 1997, $1,476$.

[69] Scivittaro, V.; Boggs, S.; Mohr, S.; Lapetina, E.G. Biochem. Biophys. Res. Commun., 1997, 241, 37.

[70] Brockhaus, F.; Brune, B. Biochem. J., 1999, 338, 295.
[71] Albina, J.E. J. Leukoc. Biol., 1995, 58, 643.

[72] Schneemann, M.; Schoedon, G. Nat. Immunol., 2002, 3, 102.

[73] Thomassen, M.J.; Kavuru, M.S. Int. Immunopharmacol., 2001, I 1479.

[74] von Knethen, A.; Brockhaus, F.; Kleiter, I.; Brune, B. Mol. Med., $1999,5,672$.

[75] Duffield, J.S.; Erwig, L.P.; Wei, X.; Liew, F.Y.; Rees, A.J.; Savill, J.S. J. Immunol., 2000, 164, 2110

[76] Duffield, J.S.; Ware, C.F.; Ryffel, B.; Savill, J. Am. J. Pathol. 2001, 159, 1397.

[77] Chattopadhyay, S.; Das, T; Sa, G.; Ray, P.K. Apoptosis, 2002, 7 , 49.

[78] Saio, M.; Radoja, S.; Marino, M.; Frey, A.B. J. Immunol., 2001, 167,5583 .

[79] Armstrong, R. Int. Immumopharmacol, 2001, I, 1501

[80] de Belder, A.J.; MacAllister, R.; Radomski, M.W.; Moncada, S.; Vallance, P.J. Cardiovasc. Res., 1994, 28, 691

[81] Megson, I.L.; Morton, S.; Greig, 1.R.; Mazzei, F.A.; Field, R.A. Butler, A.R.; Caron, G.; Gasco, A.; Fruttero, R.; Webb, D.J. Br. J. Pharmacol., 1999, 126, 639.

[82] Miller, M.R.; Hanspal, I.S.; Hadoke, P.W.; Newby, D.E.; Rossi, A.G.; Webb, D.J.; Megson, I.L. Cardiovasc. Res., 2003, 57, 853.

[83] Sousa, J.E.; Costa, M.A.; Abizaid, A.C.; Rensing, B.J.; Abizaid, A.S.; Tanajura, L.F.; Kozuma, K.; Van Langenhove, G.; Sousa, A.G.; Falotico, R ; Jaeger, J.; Popma, J.J.; Serruys, P.W. Circulation, 2001, 104, 2007. 


\title{
Resolution of inflammation: state of the art, definitions and terms
}

\author{
Charles N. Serhan, ${ }^{*}, 1$ Sue D. Brain, ${ }^{\dagger}$ Christopher D. Buckley, ${ }^{\ddagger}$ Derek W. Gilroy, ${ }^{\S}$ \\ Christopher Haslett, ${ }^{\|}$Luke A. J. O'Neill, ${ }^{\mathbb{T}}$ Mauro Perretti, ${ }^{* *}$ Adriano G. Rossi, \\ and John L. Wallace ${ }^{\dagger+}$ \\ *Center for Experimental Therapeutics and Reperfusion Injury, Brigham and Women's Hospital and \\ Harvard Medical School, Department of Oral Medicine, Infection and Immunity, Harvard School of \\ Dental Medicine, Boston, Massachusetts, USA; ${ }^{\dagger}$ Cardiovascular Division, King's College, New Hunt's \\ House, Guy's Campus, London, UK; ${ }^{\ddagger}$ Division of Immunity and Infection, MRC Center for Immune \\ Regulation, University of Birmingham Medical School, Edgbaston, UK; ${ }^{8}$ Centre for Clinical \\ Pharmacology and Therapeutics, Division of Medicine, University College London, London, UK; \\ "University of Edinburgh, MRC/Centre for Inflammation Research, The Queen's Medical Research \\ Institute, Edinburgh, UK; ${ }^{\mathbf{T}}$ School of Biochemistry and Immunology, Trinity College Dublin, Ireland; \\ **William Harvey Research Institute, Queen Mary University of London, Charterhouse Square, \\ London, UK; ${ }^{+1}$ Inflammation Research Network, University of Calgary, Calgary, Alberta, Canada
}

\begin{abstract}
A recent focus meeting on Controlling Acute Inflammation was held in London, April 27-28, 2006, organized by D.W. Gilroy and S.D. Brain for the British Pharmacology Society. We concluded at the meeting that a consensus report was needed that addresses the rapid progress in this emerging field and details how the specific study of resolution of acute inflammation provides leads for novel anti-inflammatory therapeutics, as well as defines the terms and key components of interest in the resolution process within tissues as appreciated today. The inflammatory response protects the body against infection and injury but can itself become dysregulated with deleterious consequences to the host. It is now evident that endogenous biochemical pathways activated during defense reactions can counter-regulate inflammation and promote resolution. Hence, resolution is an active rather than a passive process, as once believed, which now promises novel approaches for the treatment of inflammation-associated diseases based on endogenous agonists of resolution.-Serhan, C. N., Brain, S. D., Buckley, C. D., Gilroy, D. W., Haslett, C., O'Neill, L. A. J., Perretti, M., Rossi, A. G., Wallace, J. L. Resolution of inflammation: state of the art, definitions and terms. FASEB J. 21, 325-332 (2007)
\end{abstract}

Key Words: anti-inflammatories - leukocytes · lipid mediators - chemokines cylokines

\section{KEY CELL TYPES: THE CELLULAR PLAYERS IN THE STAGE OF RESOLUTION}

In response to injury or infection, specialized, "frontline" leukocytes (polymorphonuclear neutrophils (PMNs) and eosinophils) migrate to infected/damaged sites to neutralize and eliminate potentially injurious stimuli. This requirement is perhaps the most obvious but undeniably critical one for acute inflammation to resolve. Dispensing with the inciting stimulus will halt further pro-inflammatory mediator synthesis (eicosanoids, chemokines, cytokines, cell adhesion molecules, etc.) and lead to their catabolism and the curtailment of pro-inflammatory signaling pathways (Fig. 1). Toll-like receptors are now held to play essential roles in the recognition of many of these invading organisms (vide infra). This, coupled with the release of factors that prevent ongoing $\mathrm{PMN} /$ eosinophil trafficking and edema formation, hails the beginning of the end-namely resolution of the acute inflammatory response and return to normal homeostasis.

One traditional view argued that pro-inflammatory mediator catabolism was sufficient for inflammation to switch off and the response subsequently just "fizzled out" (1). This is only part of the process at the tissue level, as PMN or eosinophils if left unchecked could do untold harm to an already inflamed site and must be disposed of in a controlled and effective manner. Thus, next in the sequence of events is cell clearance. The exit routes available to inflammatory leukocytes include systemic recirculation (less well described) or local death followed by their phagocytosis by recruited monocyte-derived macrophages. Once phagocytosis is complete, macrophages exit the inflamed site by lymphatic drainage with evidence that a small population may die locally by apoptosis. If all of these pathways are strictly followed, then acute inflammation will resolve

\footnotetext{
${ }^{1}$ Correspondence: Center for Experimental Therapeutics and Reperfusion Injury, Brigham and Women's Hospital and Harvard Medical School, Department of Oral Medicine, Infection and Immunity, Harvard School of Dental Medicine, 75 Francis St., Thorn 724, Boston, MA, USA 02115. E-mail: cnserhan @zeus.bwh.harvard.edu

doi: $10.1096 /$ fj.06-7227rev
} 
Figure 1. Schematic depicting the cellular and molecular components of the inflammatory response and the requirements for resolution. A) (adapted from Cotran; see ref. 1) depicts the cellular and histological changes that occur in tissues following an inflammatory insult. Acute inflammation is characterized by the extravascular accumulation of neutrophils (PMN) and edema formation early in the response. Later during the response, mononuclear cells and macrophages accumulate and help prepare the tissue for resolution. $B, C$ ) Represent the role that specific molecular mediators play in these events. In $(B)$, we highlight that early on in the inflammatory response, immediate and early sequential predominately pro-inflammatory mediators are released, which initiate and augment the acute-phase of the response (green lights). However, this is counterbalanced by endogenous anti-inflammatory signals such as corticosterone, which serve to temper the severity and limit the duration of the early onset phase. As inflammation progresses, certain "stop signals" at appropriate "checkpoints" ptevent further leukocyte traffic into tissuc These stop signals include the lipoxins, Resolvins, and prostaglandins (PGs) of the D series and pave the way for monocyte migration and their differentiation to phagocytosing macrophages, which remove dead cells and then exit the site of inflammation. Stromal cells such as fibroblasts also contribute to the resolution of inflammation by the withdrawal of survival signals and the normalization of chemokine gradients, thereby allowing infiltrating leukocytes to undergo apoptosis or leave the tissue through the draining lymphatics. This sequential set of responses leads to complete resolution and, importantly, the restoration of the inflamed tissue to its prior physiological functioning. This is the ideal sequence of events in physiological inflammation, which contrast to the situation in patho-

logical inflammation $(B)$ where some of the factors that initiate the resolution program lead to the inappropriate accumulation of leukocytes in the wrong place at the wrong time.

without causing excessive tissue damage and give little opportunity for the development of acute, ongoing inflammation and its associated complications.

The last but equally essential aspect in the quest for tissue resolution and homeostasis is that the parenchy$\mathrm{mal} /$ stromal cells that hosted the inflammatory event revert back to a non-inflammatory phenotype (2). Most current therapies target immune cells in an attempt to inhibit the production of pro-inflammatory chemical mediators. However, an equally important target is the active induction of proresolution programs by stromal cells such as fibroblasts within the inflamed tissues (2).

As with the onset phase of acute inflammation, each of the above steps in the quest for resolution is also highly coordinated and under the tight control of what may be called "proresolution" factors (3). These factors and their importance in controlling inflammation have become apparent only in the past few years (Fig. 1A, $B$ ). Here, we discuss the state of the research in this field as
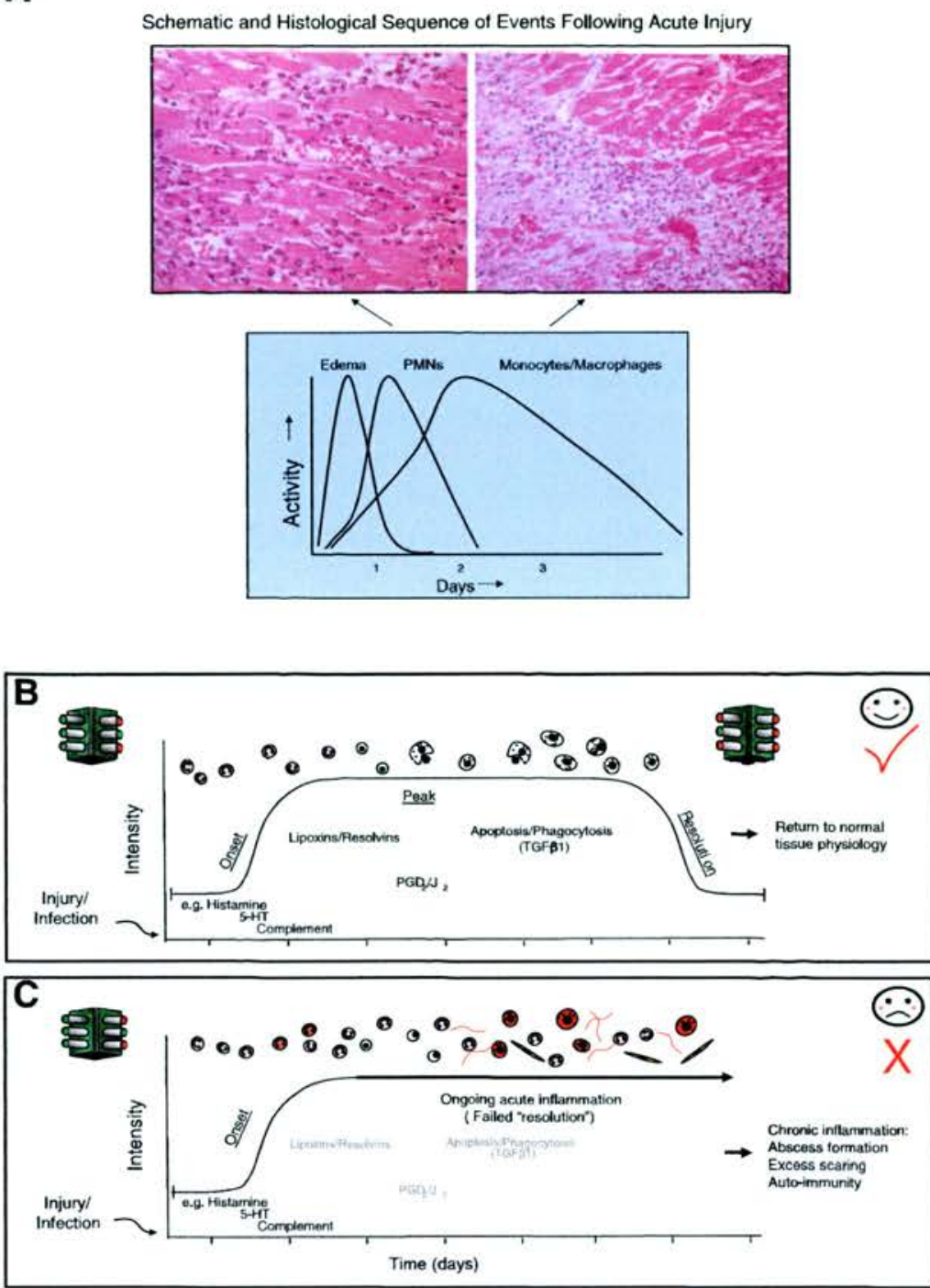

its stands today and highlight the virtues of "resolution" and why we trust that understanding it in molecular terms may help us in the quest for new drugs to treat inflammatory diseases.

\section{CHEMICAL MEDIATORS IN RESOLUTION}

Indeed, a number of recent reports have heightened the awareness that resolution is an active process, one that requires activation of endogenous programs that enable the host tissue to maintain homeostasis (3-5). In this paper, we shall underscore current topics and definitions of components in resolution as well as outline where gaps in information lie within this new field. It is apparent that inflammation plays an important role in the pathophysiology of many common diseases that affect Western civilizations, including some degenerative diseases not previously thought to 
have inflammatory components, such as Alzheimer's and atherosclerosis. Thus, the cellular mechanisms by which resolution occurs and the key biochemical pathways associated (8) with the return to homeostasis/ catabasis (return from disease) clearly open many new avenues for potential therapeutic interventions in a wide range of diseases associated with unresolved inflammation. These will surely aid in our understanding of innate immunity and clearance of microbes.

\section{"ANTI-INFLAMMATORY" VS. "PRO-RESOLUTION"}

The notion that the inflammatory response generates its own regulators in tandem with the better-known pro-inflammatory mediators and pathways makes sense teleologically: From the cybernetic viewpoint it is easier to control a process with both positive and negative regulatory inputs (Fig. $1 B$ ). Or perhaps a finer level of control can be achieved; for example, consider the car metaphor of hitting the brakes to stop or the accelerator to go. Indeed, several endogenous regulators of the inflammatory response have already been elucidated (for reviews, see refs. 3-5), adding support to the idea that this is a widely employed mechanism. Clearly, disturbances in such counter-regulatory circuits could lead to exacerbated inflammatory responses just as effectively (although perhaps less obviously) than excessive activation of the pro-inflammatory cascades (6).

Noteworthy, one of the most widely used classes of anti-inflammatory and immunosuppressive drugs, the glucocorticoids, have been developed from the pioneering work of Philip Hench and represents the first successful exploitation of an endogenous anti-inflammatory mediator, cortisol.

\section{RESOLUTION OF ACUTE INFLAMMATION: WHAT WE KNOW TODAY}

Although resolution in cellular and molecular terms has been known to pathologists at the tissue level for more than 100 years, only recently have we begun to take note. Resolution of acute inflammation, or its ideal outcome, would be complete resolution. The return to homeostasis by the tissue was thought to occur by passive mechanisms. Expressly, on surgical trauma, tissue, or chemical injury, the liberated chemical mediators (exogenous and/or endogenous) would evoke leukocyte chemotaxis into tissue. The decrease in chemotactic gradients or "the burning out" of the initial signals was thought to eventually dissipate depending on the magnitude of the invading microbes and/or injury. Resolution of acute inflammation via the exodus of neutrophils from tissues after their infiltration and involvement in host defense, namely, after the job is done, was thought to be a passive series of events (Fig. 1). The uncovering of several distinct biochemical pathways that are actively turned on during inflammation in the resolution phase, i.e., when the numbers of neutrophils infiltrating from the tissues are dropping and are actively pushed back by the mediators produced (3), provides clear evidence for the role of active biochemical pathways in resolution.

The resolution phase can be defined at the histological level as the interval from maximum neutrophilic infiltration to the point when they are lost from the tissue. Concomitantly, mononuclear cells are then introduced in a nonphlogistic fashion and play a key role in tissue repair. They, too, are eventually lost from the tissue and are not found in tissue sections following neutralization of the insult. These cellular terms and temporal relationships $(1,7)$ have now called for the need to introduce quantitative indices, which enable us to define the precise changes in leukocytic traffic and biochemical pathways activated in exudates, as well as determine the impact of various endogenous mediators, exogenous compounds, and potential drugs within the resolution phase $(8,9)$. Along with these temporal changes in the quantity and quality of the leukocyte infiltrate, additional new approaches are needed to define how infiltrating cells change the stromal microenvironment and thereby affect the timing of tissue repair and remodeling (10).

\section{TOLL-LIKE RECEPTORS, NOD-LIKE RECEPTORS, AND RESOLUTION}

The initiation of the inflammatory response during infection or in response to sterile tissue injury involves two families of receptors, the toll-like receptors (TLRs) and nod-like receptors (NLRs) (11). TLRs recognize a range of microbial products, the best characterized is TLR4, which senses lipopolysaccharide (LPS), and TLR2, which senses bacterial lipoproteins. NLRs also sense bacterial products, the best characterized are NOD1 and NOD2, which sense peptidoglycan breakdown products, and Nalp3 (also termed cryopyrin), which is required for caspase-1 activation by several types of bacteria. Nalp3 occurs in an inflammasome complex with caspase-1, ASC, cardinal, and Nalp2 (12). The activation of the inflammasome requires TLR priming. TLRs and NLRs also sense products of inflamed tissue, and notable examples are the sensing of low MW hyaluronic acid fragments by TLR2 and TLR 4 (13) and the sensing of uric acid by Nalp3 (14). Uric acid is the causative agent of gout but is also released by damaged cells, possibly as a general signal for inflammation (15). All of these represent substantial progress toward understanding innate immunity and inflammation.

Regarding resolution of inflammation, in the past two years more than 25 inhibitors of TLR and NLR action have been described, which are all induced by TLRs and therefore act as negative-feedback inhibitors (16). In effect, TLRs sow the seeds of their own destruction, and the role of negative regulators clearly indicates how robust the TLR system is. Examples include splice variants of signaling pro- 
teins such as MyD88s, cell surface receptors acting as decoys such as ST2 and SIGIRR, protein phosphatases such as MKP-1, and proteins such as Triad3a that promote degradation of TLRs (16). Deletion of genes encoding these proteins leads to a hyperinflamed state, as revealed for example by increased infiltration of neutrophils following LPS challenge in MKP-1 deficient mice (17). Similarly, inhibitors of NLRs have been found, such as caspase recruitment domain (CARD)-only protein (COP) or inhibitor of pro-caspase-1 activation (ICEBERG).

Once TLRs or NLRs are triggered, their effects will ultimately be limited, allowing resolution to proceed. It is also possible that inflammation will become chronic, however, if the system is somehow dysregulated. An example here is the work of Noble and colleagues, who have shown that polymeric hyaluronic acid binds TLR2 and TLR4 and generates a protective signal in the epithelium in lung (13). Fragments of hyaluronic acid, however, generated in response to tissue injury, become inflammatory, again via TLR2 and TLR4 via an unknown mechanism. This in turn will lead to the further breakdown of hyaluronic acid via induction of hyaluronidases, promoting further inflammation. This might turn into a vicious circle, leading to chronicity, particularly if there is a defect in negative regulation due to polymorphisms in the negative regulator.

Another example concerns regulatory $\mathrm{T}$ cells ( $\mathrm{T}$ regs) $(18,19)$. These cells inhibit immunity and also suppress inflammation. During bacterial infection, TLR2 ligands are sensed by TLR2 on both T regs and macrophages. The $\mathrm{T}$ regs expand but are kept inhibited. TLR2 in macrophages promotes host defense, which leads to bacterial clearance. Once the TLR2 ligands have been removed, the "brake" is removed from the $\mathrm{T}$ regs. They produce IL10, limit inflammation, presumably promote resolution, and importantly prevent autoimmunity. These recent results provide insight into the resolution process and how it might become dysregulated, leading to chronic inflammation.

\section{HOW TARGETING RESOLUTION RATHER THAN INHIBITING INFLAMMATION WOULD AFFECT DRUG DISCOVERY PROGRAMS}

Current anti-inflammatory therapy arises from our need to control the cardinal signs of inflammation. Most of the anti-inflammatories studied to date block/ antagonize key pro-inflammatory mediator pathways that are elicited on the initiation of an acute inflammatory response. Thus, most of the anti-inflammatories in use block key biochemical pathways and/or block the signaling of key pro-inflammatory mechanisms.

With the past few years has been a growing recognition that, although targeting infiltrating immune cells can control the inflammatory response, it does not lead to remission or permanent resolution. Inflammatory reactions occur within distinct micro-environments composed of tissue-specific cells, such as fibroblasts, endothelial cells, and macrophages, along with their highly specialized extracellular matrix (ECM) components. Evidence now exists that such stromal cells can determine the nature and duration of leukocyte infiltrates (20). At the conclusion of the inflammatory response, stromal cells also contribute to resolution of inflammation by $i$ ) the withdrawal of survival signals, ii) the normalization of chemokine gradients, and iii) the induction of resolution programs that allow infiltrating cells to undergo apoptosis or exit the inflamed tissue through draining lymphatics. The subversion of these pathways would lead to persistent inflammation, which can be remarkably stable. The relative lack of reagents that target the stromal cells may account for the failure of current treatments to affect a permanent cure.

Embracing resolution would require a cultural shift in emphasis from depletion therapy to replacement therapy (i.e., as in endocrinology where a missing agent, e.g., insulin, is replaced). Currently, anti-inflammatory approaches target chemical mediators generated during the resolution phase (as defined above, temporally from surrounding tissues or by the traffic of specific leukocytes, neutrophils, monocytes, and macrophages in and out of the tissue). Resolution therapies act quite differently in that they serve as activators, e.g., receptor agonists, which turn on protective mechanisms that lead to the return of homeostasis.

Consistently, agonists at receptor targets utilized by endogenous anti-inflammatory proresolving mediators exert an anti-inflammatory effect when added back into experimental models/specific settings in the laboratory $(4,5)$. We envision that new drugs specifically designed to promote resolving mediators and biochemical pathways will have a much better compliance and be "homeostatic and modulatory" in their actions, mimicking the way inflammation naturally subsides in the body. Hence, it is highly likely that these new proresolving drugs would be better tolerated.

\section{"RESOLUTION TOXIC" AND "RESOLUTION FRIENDLY” DRUGS ALREADY IN OUR MIDST}

It is now very apparent that certain widely used drugs, as well as those in experimental settings, are "resolution-toxic" in that they derange or impair timely and/or complete resolution. Since no widely established in vivo models monitored the key components in resolution, a set of resolution indices were recently introduced to help set benchmarks for assessing the impact of proresolving agents and their impact within resolution (8). Many currently used drugs and potential therapeutic compounds without undesirable effects have slipped by underappreciated.

This is most notable in the case of the inhibition of COX-2 with selective COX-2 inhibitors, where these inhibitors block the production of $\mathrm{PGE}_{2}$ and $\mathrm{PGD}_{2}$, which play important roles in bringing about resolution (21). Their findings also underscore the role of COX-2 and its products in both the initiation of acute inflam- 
matory response as well as its resolution. Thus, temporal and spatial positioning of these biochemical pathways can dictate the actions of their local mediators. Hence, it is important to develop drugs that spare the resolution process. Besides COX-2 inhibitors, another example may be anti-TNF therapy. It has been proposed that the poor clinical outcome observed in SIRS patients is related to the derailment of a proper temporal anti-inflammatory proresolving response.

An ideal drug to treat inflammatory disease would be able to both blunt the inflammatory response as well as activate the resolution program. Glucocorticoids, in addition to their anti-inflammatory properties, also promote resolution by stimulating macrophages in a nonphlogistic proresolving fashion to stimulate the uptake of apoptotic neutrophils (22), possibly by altering the phenotype of macrophages during their differentiation via a mechanism involving reorganization of key cytoskeletal elements (23) and/or acting via another endogenous homeostatic anti-inflammatory factor, annexin 1 (24).

Additionally, the widely used aspirin jump-starts resolution by making up for its loss in prostaglandin production, which plays key roles in resolution, by instead generating the epimeric version of lipid mediators (25), such as the aspirin-triggered lipoxins (recently reviewed in ref. 26), as well as specific epimeric versions of the omega- 3 fatty acid-derived resolvins and protectins. Perhaps these drugs are so widely used today because of a perceived reduction in the cardinal signs of inflammation and ergo their anti-inflammatory actions. However, in addition they spare and/or enhance resolution. Thus, it is important for us to clearly define the earlier signs of resolution in molecular terms, which at present do not appear to be effectively captured in clinical endpoints. This will be key in appreciating and selecting the most appropriate anti-inflammatory therapies to use in a given clinical scenario.

Recent evidence has shown aspirin-triggered lipoxins and glucocorticoid-induced annexin 1 converging onto an identical receptor target, a specific 7-transmembrane spanning G-protein coupled receptor (27). Thus, as in the case of pro-inflammatory mediators and the long list of cascades interlinking them (think of cytokines and chemokines), very recent work highlights the existence of an anti-inflammatory network, whereby distinct proresolving mediators and pathways are interconnected to ensure the correct resolution of inflammation and return of homeostasis. Besides lipoxins and annexin 1, another example is that of melanocortins or nuclear receptor agonists and heme oxygenase-1. These endogenous mediators qualify as agonists of key steps in resolution. Hence, they are proresolving mediators with anti-inflammatory properties in vivo. It is likely that other drugs in the general practitioner's armamentarium would act, at least in part, by promoting or mimicking proresolution pathways, but this point might have been totally overlooked because it has not been investigated at all!

Currently no drugs in the clinic are purposefully based on the elicitation of proresolving pathways, although compounds that target apoptotic pathway may have potential. Recently, it was shown in a rat carrageenan-induced pleurisy model of resolving inflammation that manipulation of key signaling pathways influenced resolution (28). In that study, an inhibitor (PD98059) of MAPK (ERK1/2), administered at the peak of inflammation, promoted resolution by enhancing inflammatory cell apoptosis. Conversely, administration of a specific inhibitor (V5) of the proapoptotic Bcl-2 family member, Bax, attenuated resolution by inhibiting inflammatory cell apoptosis. In another study (29), it was demonstrated that inhibitors of phosphoinositide-3 kinase, namely wortmannin and LY294002, administered at the peak of a resolving model of allergic pleurisy in mice markedly enhanced the clearance of eosinophils by promoting their apoptosis and their subsequent removal. However, we would like to stress that if apoptosis is induced specifically in inflammatory cells, it must be accompanied with efficient macrophage clearance of the apoptotic cells. If this did not occur, it is likely that failed clearance of the apoptotic cells would result in secondary necrosis and result in unwanted tissue damage. It may be that macrophages, or other cells with phagocytic potential (e.g., fibroblasts, epithelial cells, etc.), have the capacity to deal with induced apoptosis. Fortunately, macrophage phagocytosis is regarded as a highly efficient and regulatable process and can therefore be modulated by pharmacological means as occurs with glucocorticoids and lipoxins $(3,24)$.

There are, however, drugs that mediate their effects, at least in part, by triggering the synthesis of endogenous anti-inflammatory mediators, counteracting the activity of early pro-inflammatory "go signals" to prepare for or facilitate resolution. These include methotrexate, sulfasalazine, and FK506, which are thought to exert their effects by increasing adenosine synthesis, another endogenous mediator released very early in the inflammatory response and acting through the $\mathrm{A} 2 \mathrm{~A}$ receptor to control leukocyte trafficking by elevating intracellular levels of cAMP (30). However, glucocorticoids, while acting on many facets of the inflammatory response, traditionally by curtailing the severity of onset, may also exert hitherto unknown bona fide proresolution effects through their ability to hasten apoptosis in certain leukocytes (e.g., eosinophils and lymphocytes) while concomitantly enhancing the ability of monocyte-derived macrophages to phagocytose apoptotic cells. One may argue that, given that steroids are used mainly after the inflammatory event has started, their therapeutic benefits arise largely from eliciting proresolution pathways.

\section{CLINICAL IMPLICATIONS OF RESOLUTION}

What are the clinical implications of nonresolving inflammation? Traditionally, clinicians have considered certain forms of inflammation nonresolving, as in the 
case of chronic Pseudomonas infections experienced in cystic fibrosis, a classic nonresolving inflammation/ infection (1). However, in many clinical conditions infection does not seem to play a role in the persistence of the disease (i.e., chronic persistent infection is not the same as chronic persistent inflammation) (7). These include most if not all of the immune-mediated inflammatory diseases: rheumatoid arthritis, asthma, inflammatory bowel disease, and psoriasis. In these conditions, the persistence of the inflammatory response appears to have become divorced from the inciting agent. Classic anti-inflammatory agents only provide symptomatic relief and do not alter the course of the disease.

It is highly likely that this is because the naturally occurring resolution programs have been subverted in such diseases. The problem is that current clinical measurements do not adequately capture or even measure how inflammation differs from repair and resolution (8). Most clinical parameters take account of disease activity and accumulated damage, but very few can measure resolution and repair. This finding suggests that we have been using the wrong measurements and that new clinical and pathological indices will need to be developed to measure these parameters effectively. It is possible that a number of useful drugs have been discarded because they did not show efficacy using classic anti-inflammatory readouts; yet they may have been very effective in promoting resolution. Thus, the temporal phase of the disease will determine whether a drug is likely to work. For example, consider that TGF- $\beta$ is anti-inflammatory during the initial phase of an inflammatory response yet at the later phases it prolongs inflammation, so that replacement might be more important than blockade depending on the phase of the disease.

\section{KINETICS AND THE INVESTIGATOR}

An experimental setting at time zero with addition of a pro-inflammatory agonist and/or a challenge permits us as experimentalists to document precise kinetic events and their temporal relationships in animal disease models in vivo. It is difficult to capture these models with isolated cell systems in order to get a handle on the components of resolution or, for example, determine whether compounds are proresolving $(8,20-24)$. Moreover, it is difficult to spot these in clinical situations because patients with active disease do not arrive in the clinic at time zero. Therefore, it is important to recognize early molecular components to study them in these individuals for cause-and-effect relationships.

It is becoming increasingly clear that inflammation has a number of tactically placed checkpoints that limit the magnitude and duration of the response. Defects in these endogenous anti-inflammatory pathways will arguably predispose the host to chronic inflammatory diseases (Fig. 1C). Of these endogenous controllers, the cyclooxygenase and lipoxygenase (LOX) pathways have both protective and pro-inflammatory roles in inflammation, with the precise function each enzyme plays depending on the inflammatory stimulus, the phase of the acute inflammatory response when these enzymes are expressed (31), and whether it is resolving or nonresolving inflammation. This raises a number of important matters pertaining to anti-inflammatory drug development. We need to be mindful that during inflammation endogenous pathways may attempt to limit or switch off the ongoing response. These pathways must not be inadvertently altered by anti-inflammatory drugs (Fig. $1 B, C$ ). The flip-side of this biological scenario is the realization that factors expressed during resolution could be mimicked and used to push ongoing inflammation down a proresolution pathway $(8,31)$. Such an approach would mean developing molecules that exert multiple effects at various phases of the inflammatory response-limiting leukocyte trafficking, hastening cell clearance, and helping to restore inflamed stromal tissue to its prior state. Though a chemist's nightmare, it could arguably exert striking effects on ongoing inflammatory diseases. Although we don't develop drugs along these lines, it is provocative to think that existing anti-inflammatory agents such as steroids, whose development was based on an entirely different strategy, may exert potent proresolution effects arising from their proapoptotic (eosinophils) and prophagocytic properties.

The potential side effect of developing "proresolvers" (and it seems that not all treatments are without side effects) is that inflammation could be prematurely resolved before it has a chance to deal with the insulting agent, the very essence of the inflammatory response (26) in the first instance. This brings us to underlining the fact that all inflammatory responses are not the same and that developing "pan-inflammatory inhibitors" is not necessarily the most effective approach to treating inflammation-driven diseases. Thus, we need to foster a greater understanding of the nature of the response that drives each individual inflammatory event and its resolution and tailor treatments accordingly. To achieve this goal we must improve our understanding of the mechanisms in resolution of inflammation and identify possible approaches to promote this process, perhaps in combination with antiinflammatory therapy. Of interest, pharmacological induction of caspase-dependent inflammatory cell apoptosis by selective cyclin-dependent kinase (CDK) inhibitors can augment the resolution of established inflammation (32). It was shown that human neutrophils contain functionally active CDKs (CDK1, 2, and 5) and that different CDK inhibitor drugs induce neutrophil apoptosis per se and override the retardation of apoptosis induced by survival factor such as GM-CSF. In addition, the specific CDK inhibitor, R-roscovitine (the anti-cancer agent), enhanced inflammatory resolution in three in vivo mouse models of established neutrophil-dependent inflammation (carrageenan-elicited acute pleurisy, bleomycin-induced lung injury, and 
passively-induced arthritis). It was also demonstrated that the CDK inhibitor drug-induced enhanced resolution of established pleurisy was mediated by caspasedependent apoptosis of inflammatory cells. Thus, it is possible that CDK inhibitor drugs may provide a novel strategy for enhancing the resolution of inflammation and may have potential for the treatment of inflammatory disorders.

At present, it appears that possible deficiencies in resolution pathways and/or precursors of proresolving mediators, such as dietary essential omega- 3 fatty acids, which are precursors to resolvins and protectins (Fig. $1 C$ ), may lead to prolonged inflammation and the inability for the tissue to return to homeostasis (26) and complete the full life-cycle per se of the neutrophils and mononuclear cells recruited to the tissue.

Therefore, it is very likely that the diffused application of unbiased wide-spectrum approaches (i.e., microarrays, lipid mediator informatics, proteomics) in experimental (8) and clinical trials will unveil important and "unexpected" links between novel therapeutics still under development-and likely old drugs, too-and induction of endogenous anti-inflammatory mediators. We are at the dawn of a new era of drug discovery for inflammatory diseases, which finally exploit natural endogenous mechanisms operating in our body to terminate the inflammatory response. The present consensus will hopefully provide a framework to facilitate our appreciation of these resolution components and their structure-function that are key to this process for navigating resolution to improve health. FJ

The authors acknowledge grant support for their respective research efforts from the National Institutes of Health (U.S.A.) and Medical Research Councils (U.K., Ireland, Canada). We also thank Mary Haim Small and Stacey Lindberg (CET\&RI, Brigham and Women's Hospital) for their expert assistance with the preparation of this manuscript.

\section{REFERENCES}

1. Cotran, R. S., Kumar, V., and Collins, T., eds. (1999) Robbins Pathologic Basis of Disease, W.B. Saunders Co., Philadelphia, Pennsylvania, USA

2. Filer, A., Pitzalis, C., and Buckley, C. D. (2006) Targeting the stromal microenvironment in chronic inflammation. Cur. Opin. Pharmacol. 6, 393-400

3. Serhan, C. N. (2004) A search for endogenous mechanisms of anti-inflammation uncovers novel chemical mediators: missing links to resolution. Histochem. Cell Biol. 122, 305-321

4. Gilroy, D. W., Lawrence, T., Perretti, M., and Rossi, A. G. (2004) Inflammatory resolution: new opportunities for drug discovery. Nat. Rev. Drug. Discov. 3, 401-416

5. Perretti, M. (1997) Endogenous mediators that inhibit the leukocyte-endothelium interaction. Trends Pharmacol. Sci. 18, $418-425$

6. Weissmann, G., Smolen, J. E., and Korchak, H. M. (1980) Release of inflammatory mediators from stimulated neutrophils. N. Engl. J. Med. 303, 27-34

7. Majno, G., and Joris, I. (2004) Cells, Tissues, and Disease: Principles of General Pathology, Oxford Univ. Press, New York, New York, USA

8. Bannenberg, G. L., Chiang, N., Ariel, A., Arita, M., Tjonahen, E., Gotlinger, K. H., Hong, S., and Serhan, C. N. (2005) Molecular circuits of resolution: Formation and actions of resolvins and protectins. J. Immunol. 174, 4345-4355

9. Damazo, A. S., Yona, S., Flower, R. J., Perretti, M., and Oliani, S. M. (2006) Spatial and temporal profiles for anti-inflammatory gene expression in leukocytes during a resolving model of peritonitis. J. Immunol. 176, 4410-4418

10. Parsonage, G., Filer, A. D., Haworth, O., Nash, G. B., Rainger, G. E., Salmon, M., and Buckley, C. D. (2005) A stromal address code defined by fibroblasts. Trends. Immunol. 26, 150-156

11. Creagh, E., and O'Neill, L. A. (2006) TLRs, NLRs and RLRs: a trinity of pathogen sensors that co-operate in innate immunity. Trends Immunol. 352-357

12. Agostini, L., Martinon, F., Burns, K., McDermott, M. F., Hawkins, P. N., and Tschopp, J. (2004) NALP3 forms an IL-1beta-processing inflammasome with increased activity in Muckle-Wells autoinflammatory disorder. Immunity 20, 319-325

13. Jiang, D., Liang, J., Fan, J., Yu, S., Chen, S., Luo, Y., Prestwich, G. D., Mascarenhas, M. M., Garg, H. G., Quinn, D. A., et al. (2005) Regulation of lung injury and repair by toll-like receptors and hyaluronan. Nature Med. 11, 1173-1179

14. Martinon, F., Petrilli, V., Mayor, A., Tardivel, A., and Tschopp, J. (2006) Gout-associated uric acid crystals activate the NALP3 inflammasome. Nature 440, 237-241

15. Shi, Y., Evans, J. E., and Rock, K. L. (2003) Molecular identification of a danger signal that alerts the immune system to dying cells. Nature $425,516-52\rceil$

16. Liew, F. Y., Xu, D., Brint, E. K., and O'Neill, L. A. (2005) Negative regulation of toll-like receptor-mediated immune responses. Nat. Rev. Immunol. 5, 446-458

17. Sonya, M. A., et al. (2006) Anti-inflammatory effects of dexamethasone are partly dependent on induction of dual specificity phosphatise-1 (DUSP1). J. Exp. Med., in press

18. Liu, H., Komai-Koma, M., Xu, D., and Liew, F. Y. (2006) Toll-like receptor 2 signalling modulates the functions of CD4+ CD25+ regulatory T cells. Proc. Natl. Acad. Sci. U. S. A. 103, 7048-7053

19. Sutmuller, R. P., den Brok, M. H., Kramer, M., Bennink, E. J., Toonen, L. W., Kullberg, B. J., Joosten, L. A., Akira, S., Netea, M. G., and Adema, G. J. (2006) Toll-like receptor-2 controls expansion and function of regulatory T cells. J. Clin. Invest. 116, $485-494$

20. Buckley, C. D., Pilling, D., Lord, J. M., Akbar, A. N., ScheelToellner, D., and Salmon, M. (2001) Fibroblasts regulate the switch from acute resolving to chronic persistent inflammation. Trends. Immunol. 22, 199-204

21. Gilroy, D. W., Colville-Nash, P. R., Willis, D., Chivers, J., Paul-Clark, M. J., and Willoughby, D. A. (1999) Inducible cycloxygenase may have anti-inflammatory properties. Nature Med. 5, 698-701

22. Liu, Y., Cousin, J. M., Hughes, J., Van Damme, J., Seckl, J. R., Haslett, C., Dransfield, I., Savill, J., and Rossi, A. G. (1999) Glucocorticoids promote nonphlogistic phagocytosis of apoptotic leukocytes. J. Immunol. 162, 3639-3646

23. Giles, K. M., Ross, K., Rossi, A. G., Hotchin, N. A., Haslett, C., and Dransfield, I. (2001) Glucocorticoid augmentation of macrophage capacity for phagocytosis of apoptotic cells is associated with reduced p130Cas expression, loss of paxillin/pyk2 phosphorylation, and high levels of active Rac. J. Immunol. 167, 976-986

24. Maderna, P., Yona, S., Perretti, M., and Godson, C. (2005) Modulation of phagocytosis of apoptotic neutrophils by supernatant from dexamethasone-treated macrophages and annexinderived peptide Ac(2-26). J. Immunol. 174, 3727-3733

25. Serhan, C. N., Hong, S., Gronert, K., Colgan, S. P., Devchand, P. R., Mirick, G., and Moussignac, R.-L. (2002) Resolvins: a family of bioactive products of omega-3 fatty acid transformation circuits initiated by aspirin treatment that counter proinflammation signals. J. Exp. Med. 196, 1025-1037

26. Serhan, C. N., and Savill, J. (2005) Resolution of inflammation: the beginning programs the end. Nature Immunol. 6, 1191-1197

27. Perretti, M., Chiang, N., La, M., Fierro, I. M., Marullo, S. Getting, S. J., Solito, E., and Serhan, C. N. (2002) Endogenous lipid- and peptide-derived anti-inflammatory pathways generated with glucocorticoid and aspirin treatment activate the lipoxin A(4) receptor. Nat. Med. 8, 1296-1302

28. Sawatzky, D. A., Willoughby, D. A., Colville-Nash, P. R., and Rossi, A. G. (2006) The involvement of the apoptosis-modulating proteins ERK 1/2, Bcl-xL and Bax in the resolution of acute inflammation in vivo. Am. J. Pathol. 168, 33-41 
29. Pinho, V., Souza, D. G., Barsante, M. M., Hamer, F. P., De Freitas, M. S., Rossi, A. G., and Teixeira, M. M. (2005) Phosphoinositide- 3 kinases critically regulate the recruitment and survival of eosinophils in vivo: importance for the resolution of allergic inflammation. J. Leukoc. Biol. 77, 800-810

30. Cronstein, B. N. (2006) Going with the flow: methotrexate, adenosine, and blood flow. Ann. Rheum. Dis. 65, 421-422

31. Levy, B. D., Clish, C. B., Schmidt, B., Gronert, K., and Serhan, C. N. (2001) Lipid mediator class switching during acute inflammation: signals in resolution. Nature. Immunol. 2, 612-619
32. Rossi, A. G., Sawatzky, D. A., Walker, A., Ward, C., Sheldrake, T. A., Riley, N. A., Caldicott, A., Martinez-Losa, M., Walker, T. R., Duffin, R., et al. (2006) Cyclin-dependent kinase inhibitors enhance the resolution of inflammation by promoting inflammatory cell apoptosis. Nat. Med. 12, 1056-1064

Received for publication August 28, 2006. Accepted for publication September 22, 2006. 


\title{
The role of the purinergic $\mathbf{P} 2 \mathbf{X}_{\mathbf{7}}$ receptor in inflammation Martin F Lister ${ }^{1}$, John Sharkey ${ }^{2}$, Deborah A Sawatzky ${ }^{1}$, Joseph P Hodgkiss ${ }^{2}$, Donald J Davidson ${ }^{1}$, Adriano G Rossi*1 and Keith Finlayson ${ }^{2}$
}

\author{
Address: 'MRC Centre for Inflammation Research, The Queen's Medical Research Institute, The University of Edinburgh, 47 Little France Crescent, \\ Edinburgh, EH16 4TJ, UK and ${ }^{2}$ Astellas CNS Research in Edinburgh, The Chancellor's Building, The University of Edinburgh, 49 Little France \\ Crescent, EH16 4SB, UK \\ Email: Martin F Lister - M.F.Lister@sms.ed.ac.uk; John Sharkey - j.sharkey@ed.ac.uk; Deborah A Sawatzky - D.A.Sawatzky@ed.ac.uk; \\ Joseph P Hodgkiss - joseph.hodgkiss@ed.ac.uk; Donald J Davidson - Donald.Davidson@ed.ac.uk; Adriano G Rossi* - a.g.rossi@ed.ac.uk; \\ Keith Finlayson-Keith.Finlayson@ed.ac.uk \\ - Corresponding author
}

This is an Open Access article distributed under the terms of the Creative Commons Attribution License (http://creativecommons.org/licenses/by/2.0), which permits unrestricted use, distribution, and reproduction in any medium, provided the original work is properly cited.

\begin{abstract}
The inflammatory process, orchestrated against a variety of injurious stimuli, is composed of three inter-related phases; initiation, propagation and resolution. Understanding the interplay between these three phases and harnessing the beneficial properties of inflammation whilst preventing its damaging effects, will undoubtedly lead to the advent of much needed therapies, particularly in chronic disease states. The $P 2 X_{7}$ receptor $\left(P 2 X_{7} R\right)$ is increasingly recognised as an important cell surface regulator of several key inflammatory molecules including IL-I $\beta, I L-18$, TNF- $\alpha$ and IL-6. Moreover, as $\mathrm{P} 2 \mathrm{X}_{7} \mathrm{R}$-dependent cytokine production is driven by activating the inflammasome, antagonists of this receptor are likely to have therapeutic potential as novel anti-inflammatory therapies. The function of the $P 2 X_{7} R$ in inflammation, immunity and its potential role in disease will be reviewed and discussed.
\end{abstract}

\section{Background}

Inflammation is an important physiological reaction which occurs in response to a wide variety of injurious agents (e.g. bacterial infection or physical trauma) ultimately aiming to perform the dual function of limiting damage and promoting tissue repair [1]. The inflammatory process is often viewed as being comprised of three closely linked phases: - initiation, propagation and resolution, with current anti-inflammatory therapies designed to limit or prevent the initiation and propagation phases. However, it is increasingly recognised that therapies aimed at enhancing the resolution phase will be important in limiting the damage associated with persistent inflammatory disease states such as rheumatoid arthritis, chronic obstructive pulmonary diseases and artherosclerosis [2].

In recent years, the role of ATP and its cognate receptors in the inflammatory process has been recognised. In particular, the $\mathrm{P} 2 \mathrm{X}_{7}$ receptor $\left(\mathrm{P} 2 \mathrm{X}_{7} \mathrm{R}\right)$ which is expressed primarily (though not exclusively) on cells of haemopoietic origin [3] is thought to play an important role in macrophage/microglial and granulocyte function by regulating cytokine production and apoptosis. Moreover, as the $\mathrm{P} 2 \mathrm{X}_{7} \mathrm{R}$ is known to be up-regulated during inflammation, antagonists of this receptor may serve as novel antiinflammatory agents. In this review we summarise recent advances in the understanding of the role of the $\mathrm{P} 2 \mathrm{X}_{7} \mathrm{R}$ in inflammatory processes and highlight the potential of 
$\mathrm{P} 2 \mathrm{X}_{7} \mathrm{R}$ ligands for the treatment of chronic inflammatory diseases, focusing particularly on tuberculosis and cancer.

\section{2. $\mathbf{P} 2 X_{7}$ Receptor Pharmacology}

Extracellular ATP is known to activate two classes of membrane-bound receptors; the metabotropic P2Y (P2Y $\mathrm{P}_{2} \mathrm{Y}_{2}, \mathrm{P} 2 \mathrm{Y}_{4}, \mathrm{P} 2 \mathrm{Y}_{6}$ and $\left.\mathrm{P} 2 \mathrm{Y}_{11-14}\right)$, and ionotropic P2X $\left(\mathrm{P} 2 \mathrm{X}_{1-7}\right)$ receptors with the pharmacology, distribution and putative functions of these receptors extensively reviewed [4-6]. Of the $\mathrm{P} 2$ receptors, the $\mathrm{P} 2 \mathrm{X}_{7} \mathrm{R}$ has attracted considerable interest as a consequence of its unique biological properties. Brief activation of the $\mathrm{P} 2 \mathrm{X}_{7} \mathrm{R}$ by ATP or its stable analogue 2',3'-O-(benzoyl-4-benzoyl)-ATP (BzATP) results in the opening of a non-selective cationic channel. However, upon prolonged stimulation, the $\mathrm{P} 2 \mathrm{X}_{7} \mathrm{R}$ forms an aqueous pore that allows the passage of hydrophilic molecules of up to $900 \mathrm{Da}$, which can ultimately lead to cell death [7], probably by colloido-osmotic lysis [8]. In contrast, transient receptor activation can induce pseudoapoptosis, a process which is readily reversible [9]. The activation of this receptor has now been associated with the stimulation of a plethora of downstream signalling cascades resulting in the release of a number of inflammatory mediators. Principle amongst these is interleukin-1 $\beta$ (IL-1 $\beta$ ), the processing and release of which is critically dependent upon $\mathrm{P} 2 \mathrm{X}_{7} \mathrm{R}$ activation and is discussed extensively below. As with all $\mathrm{P} 2 \mathrm{X}$ receptors, elucidating the role of the $\mathrm{P} 2 \mathrm{X}_{7} \mathrm{R}$ has been hampered by a paucity of receptor selective agonists and antagonists. BzATP, widely viewed as a selective agonist of the $\mathrm{P} 2 \mathrm{X}_{7} \mathrm{R}$, exhibits greater potency for other $\mathrm{P} 2 \mathrm{X}$ and $\mathrm{P} 2 \mathrm{Y}$ receptors [10-12]. Similarly, it is important to appreciate that oxidised ATP (oATP), although often presented as a P2 $\mathrm{X}_{7} \mathrm{R}$ specific antagonist, can attenuate pro-inflammatory signalling by mechanisms distinct from $\mathrm{P} 2 \mathrm{X}_{7} \mathrm{R}$ activation $[13,14]$. Although a number of putatively selective $P 2 X_{7} R$ antagonists have recently been described [15-17], the effects of these agents in animal models of disease has yet to be published.

\section{The role of the $P 2 X_{7} R$ in inflammatory cell function}

Since nucleotides (such as ATP) are normally retained within the cytoplasm of a cell, their presence in the external milieu (e.g. during the process of cytolysis [7]) are thought to provide 'danger' signals, inducing antigen presenting cells to initiate the innate immune response [18]. Importantly, innate immunity can be initiated by a variety of cytokines such as IL- $1 \beta$, IL-18, IL- 6 and tumour necrosis factor- $\alpha$ (TNF- $\alpha$ ), all of which can be produced by $\mathrm{P} 2 \mathrm{X}_{7} \mathrm{R}$ activation (vide infra). In contrast, chronic exposure to low-dose ATP activates dendritic cells and macrophages to secrete anti-inflammatory cytokines (IL-10 and IL-1 receptor antagonist (IL-1RA)) suppressing inflammation and favouring the development of a Th2 response [18]. These observations suggest that the immune and/or inflammatory response can be redirected when deemed to be detrimental to the host. The putative role of the $\mathrm{P} 2 \mathrm{X}_{7} \mathrm{R}$ in such processes is discussed below.

\section{I. $P 2 X_{7} R$ regulation of cytokine production in haemopoietic cells}

It has been clear since the cloning of the $\mathrm{P} 2 \mathrm{X}_{7} \mathrm{R} 10$ years ago [19], that this channel is predominantly expressed on cells of haemopoietic origin such as monocytes, macrophages and microglia. More importantly, as activation of these cell types is associated with increased expression of the $\mathrm{P} 2 \mathrm{X}_{7} \mathrm{R}$, this ultimately leads to an amplification of the downstream production of the pro-inflammatory cytokines IL- $1 \beta$ and IL-18, and in turn IL-6, IL- 8 and TNF$\alpha$. As over-production of these cytokines is detrimental, particularly in chronic disease states, and underlies the pathophysiology of a range of peripheral and central disorders, controlling their release is paramount.

\section{I.I. The role of $P 2 X_{7} R$ in IL-I $\beta$ production}

In recent years, a great deal of attention has been devoted to elucidating the mechanisms of release of the proinflammatory leaderless cytokine IL-1 from monocytes and macrophages. Originally produced as $31-\mathrm{kDa}$ precursors, the two IL- 1 isoforms, pro-IL- $1 \alpha$ and pro-IL- $1 \beta$, are subsequently cleaved by interleukin-converting enzyme (ICE; also known as caspase-1 [20]) to produce the mature $17-\mathrm{kDa}$ forms [21]. IL- $1 \alpha$ and IL- $1 \beta$ are thought to have identical biological actions, although IL-1 $\beta$, unlike $\mathrm{IL}-1 \alpha$, is inactive in its immature form [21]. The mechanism of IL- $1 \beta$ release has been extensively studied in vitro, although there are only a limited number of molecules capable of inducing controlled release, and whether these processes reflect the in vivo situation remains unclear. Upon release, IL- $1 \beta$ is known to elicit diverse responses, including the activation of macrophages, T-cells and signalling cascades, as well as the induction of cyclooxygenase type 2 (COX-2) and fever [22]. IL-1 has been shown to be important in many diseases including rheumatoid arthritis [23], multiple sclerosis [24], asthma [25] and chronic obstructive pulmonary disease [26]. It is therefore clear that IL- $1 \beta$ is of particular importance in the initiation and propagation of an inflammatory response, with its functions and therapeutic potential extensively reviewed $[22,27]$.

Originally, cell death by apoptosis was reported to stimulate the production and release of mature IL-1 $\beta$, although the mechanism was not identified [28]. The release of mature IL-1 $\beta$ appeared to require two consecutive stimuli [29], with LPS stimulation in monocytes only producing pro-ICE and pro-IL-1 $\beta$ [30]. The latter authors reported that ATP-stimulated $\mathrm{K}^{+}$efflux was important for the release of mature IL-1 $\beta[30]$, with Ferrari and colleagues 
subsequently suggesting that it was $\mathrm{P} 2 \mathrm{X}_{7} \mathrm{R}$-mediated, and independent of apoptosis [31]. This was latterly confirmed in pharmacological [32] studies and those using $\mathrm{P} 2 \mathrm{X}_{7} \mathrm{R}$ knockout mice $[33,34]$, with the activation of the $\mathrm{P} 2 \mathrm{X}_{7} \mathrm{R}$ by ATP producing a fall in cytoplasmic $\mathrm{K}^{+}$concentration which in turn stimulates processing of pro-ICE to ICE, and thereby inducing release of mature IL- $1 \beta$ (Figure $1 ;[35])$. Indeed, in an elegant series of studies Surprenant and colleagues have subsequently demonstrated that ATPinduced activation of the $\mathrm{P} 2 \mathrm{X}_{7} \mathrm{R}$ results in the shedding of microvesicles which contain mature IL-1 $\beta$ [36] and more recently IL-1RA [37]. With high concentrations (0.5-5 $\mathrm{mM}$ ) of ATP required for optimal activation of $\mathrm{P} 2 \mathrm{X}_{7} \mathrm{R}$ mediated IL- $1 \beta$ release in vitro [38], alternative endogenous agonists that could produce significant $\mathrm{P} 2 \mathrm{X}_{7} \mathrm{R}$ stimulation have been sought. Interestingly, several cationic host defence peptides (CHDP; also known as antimicrobial peptides) have recently been shown to mediate posttranslational processing of IL- $1 \beta$ in LPS-primed monocytes. Although the mechanisms of action of the porcine CHDP protegrin-1 and -3 have been shown to be $P 2 X_{7} R$ independent [39], three studies have now proposed that $\mathrm{P} 2 \mathrm{X}_{7} \mathrm{R}$ activation underlies some of the immunomodulatory effects of the human CHDP, LL-37 [38,40,41]. LL-37 is the major active cleavage product of the only human cathelicidin hCAP18, is upregulated in infection and inflammation $[42,43]$, and in addition to broad-spectrum antimicrobial activity and direct anti-endotoxic effects, LL-37 has a number of immunomodulatory roles [44]. LL37 has now been shown to induce caspase-1 activation and secretion of mature IL- $1 \beta$ in LPS-primed monocytes, in the absence of cytotoxicity, through $\mathrm{P} 2 \mathrm{X}_{7} \mathrm{R}$ activation [38]. Furthermore, recent studies have demonstrated that concentrations of LL-37 as low as $250 \mathrm{ng} / \mathrm{ml}$, and well within the physiological range, can inhibit apoptosis in human neutrophils, in a $\mathrm{P} 2 \mathrm{X}_{7} \mathrm{R}$-dependent manner involving the PI3-kinase pathway $[40,41]$. Such studies indicate that in addition to extracellular ATP, the endogenous, inducible CHDP, LL-37 may activate the $\mathrm{P} 2 \mathrm{X}_{7} \mathrm{R}$ on key innate immune effector cells to modulate cytokine release. Finally, as compounds such as Tenidap, which is being evaluated for its anti-inflammatory and antiarthritic properties also appear to inhibit the release of IL$1 \beta[45]$, whilst sensitising the $P 2 X_{7} R$ on macrophages to the cytotoxic effects of ATP [46], future studies may show that the $\mathrm{P} 2 \mathrm{X}_{7} \mathrm{R}$ could be regulated by a range of ligands.

The importance of, and the mechanisms through which the $\mathrm{P} 2 \mathrm{X}_{7} \mathrm{R}$ regulates the production of the pro-inflammatory cytokines IL- $1 \beta$ and IL-18, and potentially the innate immune response, was recently and beautifully described by Mariathasan and colleagues [47]. These authors showed that the P2X $\mathrm{X}_{7} \mathrm{R}$ is up-stream of the inflammasome, an important complex of cytosolic proteins that are known to regulate caspase- 1 activation and ultimately the processing of IL-1 $\beta$ and IL-18. With inflammasome dysregulation known to produce inflammatory disorders such as Muckle-Wells syndrome and neonatal onset multisystem inflammatory disease, it is clear that inhibiting inflammasome activation with $\mathrm{P} 2 \mathrm{X}_{7} \mathrm{R}$ antagonists could affect the outcome of a range of inflammatory disorders [47]. However, one must remember that the $\mathrm{P} 2 \mathrm{X}_{7} \mathrm{R}$ may not be the only purinergic receptor involved in $\mathrm{IL}-1 \beta$ release. A recent study has shown ATP-dependent $\mathrm{Ca}^{2+}$ release from intracellular stores (endoplasmic reticulum) is also involved in the secretion of pro-IL-1 $\beta$, although it was not independently capable of releasing mature IL- $1 \beta$ [48]. As discussed above, $\mathrm{K}^{+}$efflux was also reported to be necessary for the release of mature IL- $1 \beta$, with Brough and colleagues (2003) proposing that ATP may stimulate both $\mathrm{P} 2 \mathrm{X}$ and P2Y receptors [48]. The importance of P2Y receptor stimulation and $\mathrm{Ca}^{2+}$ release from intracellular stores remains to be determined.

$\mathrm{P} 2 \mathrm{X}_{7} \mathrm{R}$-mediated regulation of $\mathrm{IL}-1 \beta$ has also been demonstrated within the central nervous system where microglia are the resident monocytic cells. In a seminal study in 1997, Ferrari et al [49] reported that ATP induced IL-1 $\beta$ production in cultured microglial cells through the activation of the $\mathrm{P} 2 \mathrm{X}_{7} \mathrm{R}$. Subsequent studies showing that cultured microglia from $\mathrm{P} 2 \mathrm{X}_{7} \mathrm{R}$ knockout mice do not release $\mathrm{IL}-1 \beta$ following exposure to LPS and ATP [50] support the role for $\mathrm{P} 2 \mathrm{X}_{7} \mathrm{R}$ in IL- $1 \beta$ production, albeit in vitro. $\mathrm{P} 2 \mathrm{X}_{7} \mathrm{R}$ up-regulation has been observed in response to a variety of inflammatory brain insults, underpinning the view that $\mathrm{P} 2 \mathrm{X}_{7} \mathrm{R}$ antagonists may be of therapeutic use for the treatment of several disorders including stroke, traumatic brain injury (TBI), multiple sclerosis and Alzheimer's disease $[3,51-53]$. Since IL-1 $\beta$ has been reported to induce COX-2 in various tissues including glia, it has been proposed that a vicious cycle occurs whereby ATP release (from cell death for example) leads to $\mathrm{P} 2 \mathrm{X}_{7} \mathrm{R}$ activation, IL- $1 \beta$ release, COX- 2 induction and further cell death with consequent ATP release; this type of self-perpetuating cycle may underlie lesion expansion particularly in stroke and TBI. Once selective P2X $\mathrm{X}_{7} \mathrm{R}$ antagonists become commercially available it will be possible to test the importance of this receptor in these processes. However, it is interesting to note that non-specific antagonism of P2X receptors by PPADS, and the inhibition of $\mathrm{IL}-1 \beta$, and COX-2, have all been reported to be effective in animal models of stroke and other neurodegenerative disorders $[51,54]$. Intriguingly, another function attributed to the $\mathrm{P} 2 \mathrm{X}_{7} \mathrm{R}$ that is important in neuropathology is microglial production of superoxide anion [55]. The significance of $\mathrm{P} 2 \mathrm{X}_{7} \mathrm{R}$ regulation of superoxides was underlined by the observation that $\mathrm{P} 2 \mathrm{X}_{7} \mathrm{R}$ expression was up-regulated around $\beta$-amyloid plaques in a mouse model of Alzheimer's disease [55]. It was also subsequently shown that in human microglia, $\beta$-amyloid-induced cytokine release 
1.

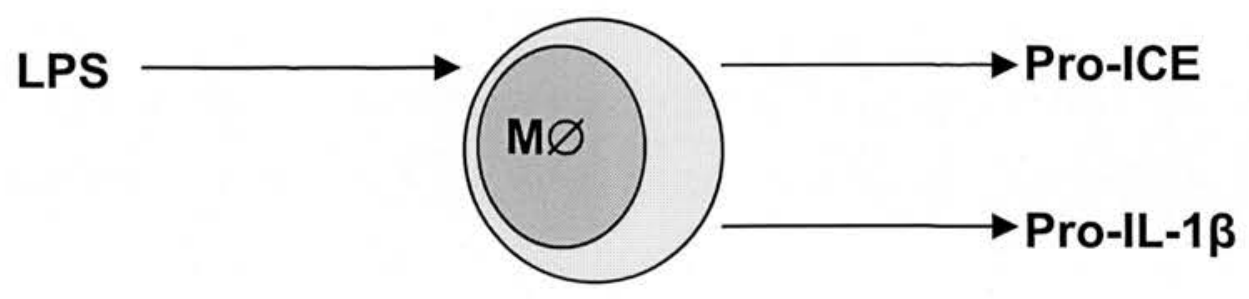

2.

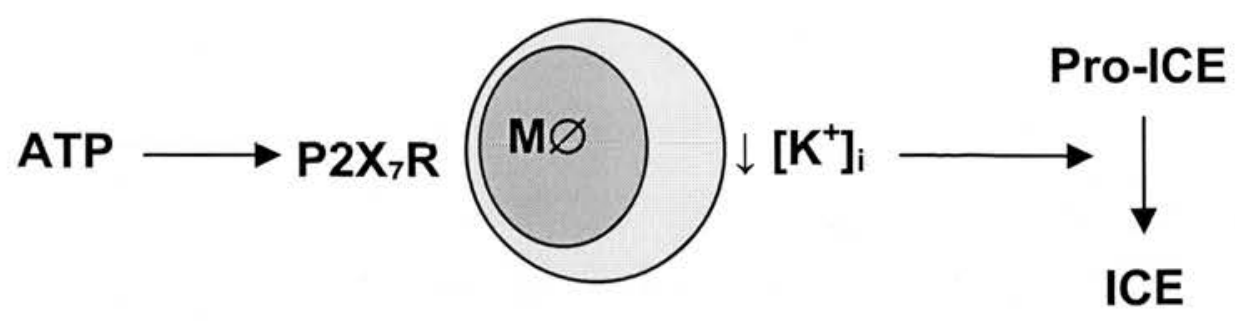

3.

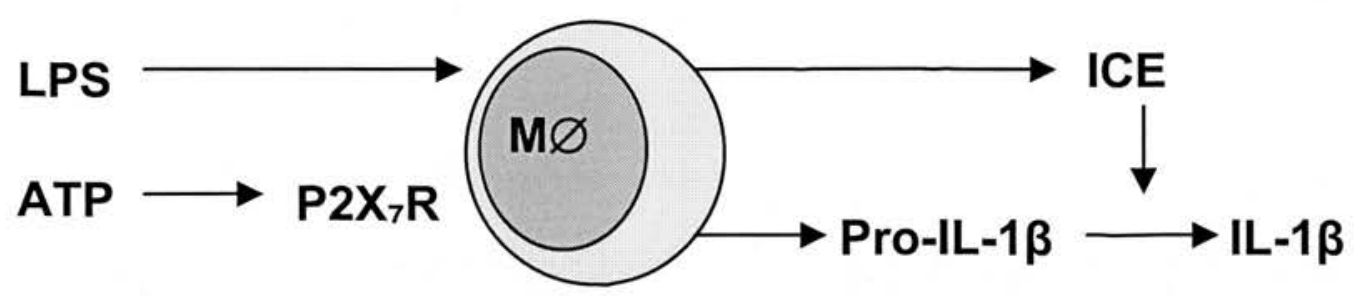

Figure I

Summary of the production of active IL-I $\beta$. This process can be divided into 3 stages. Stage I: LPS stimulates monocytes/ macrophages $(M \varnothing)$ to produce pro-ICE and pro-IL-I $\beta$. Stage 2: ATP stimulates the P2X $X_{7} R$ expressed on $M \varnothing$ to cause a fall in intracellular $\mathrm{K}^{+}$concentration $\left(\left[\mathrm{K}^{+}\right]_{\mathrm{i}}\right)$ which in turn converts pro-ICE to ICE. Stage 3 : LPS-primed M $\varnothing$ following ATP stimulation results in activated ICE which converts inactive pro-IL- $\beta$ to active IL-I $\beta$. It should be noted that this process is intracellular and the figure is for illustrative purposes only (see text for references).

(e.g. IL-1 $\beta$ ) was found to be modulated by ATP, probably via the $\mathrm{P} 2 \mathrm{X}_{7} \mathrm{R}[56]$.

Understandably, polymorphisms in the genes encoding IL-1, its receptor, and IL-1RA have been found to be associated with a range of diseases including rheumatoid arthritis, systemic lupus erythematosus, atherosclerosis and tuberculosis [57]. As a result of the importance of the $\mathrm{P} 2 \mathrm{X}_{7} \mathrm{R}$ in $\mathrm{IL}-1 \beta$ processing and release, polymorphisms in this unique ion channel have been investigated and to date, in excess of 260 polymorphisms have been identified for the P2X 7 R $[58,59]$. One such polymorphism is the single nucleotide substitution at position 1513 of the $\mathrm{P} 2 \mathrm{X}_{7} \mathrm{R}$ gene which changes a glutamic acid to an alanine at amino acid position 496 (Glu ${ }^{496} \mathrm{Ala}$ ), and leads to loss of function of the receptor [60]. It is interesting to note that this polymorphism decreased the ATP-induced $\mathrm{K}^{+}$ efflux subsequently delaying the ATP-induced release of $\mathrm{IL}-1 \beta$. The fact that $\mathrm{IL}-1 \beta$ release was delayed rather than abrogated indicates that there are compensatory or redundant mechanisms present [61]. However there is now evidence from $\mathrm{P} 2 \mathrm{X}_{7} \mathrm{R}$ polymorphism studies, that those associated with a loss of function mutation have a reduced sensitivity to inflammation [62].

In the absence of commercially available potent and selective $\mathrm{P} 2 \mathrm{X}_{7} \mathrm{R}$ antagonists, $\mathrm{P} 2 \mathrm{X}_{7} \mathrm{R}$ knockout mice have provided new insights into the in vivo role of this receptor. 
Labasi and colleagues [34] reported that peritoneal macrophages from $\mathrm{P} 2 \mathrm{X}_{7} \mathrm{R}$ deficient mice were unable to produce mature IL- $1 \beta$ in response to LPS, or ATP application, or with a combination of both stimuli. This study also compared the induction of monoclonal anti-collageninduced arthritis in $\mathrm{P} 2 \mathrm{X}_{7} \mathrm{R}$-deficient mice and wild-type littermates, with the former group demonstrating reduced susceptibility to, and severity of disease [34]. It was therefore suggested that, in normal mice, endogenous ATP is present in sufficient concentrations at sites of inflammation to activate the $\mathrm{P} 2 \mathrm{X}_{7} \mathrm{R}$ [34], (an area that has attracted some scepticism based on in vitro work with the addition of exogenous ATP [38]). However, as described earlier, care must now be taken in interpreting results observed in vivo, as although ATP was originally thought to be the only endogenous agonist of the $\mathrm{P} 2 \mathrm{X}_{7} \mathrm{R}$, recently other physiological agents such as LL37 (see above) and NAD [63] have been reported to activate the $\mathrm{P} 2 \mathrm{X}_{7} \mathrm{R}$ at lower concentrations. New studies in $\mathrm{P} 2 \mathrm{X}_{7} \mathrm{R}$ knockout mice continue to indicate that this receptor plays a role in a number of conditions in addition to arthritis and include multiple sclerosis, hepatitis and pain [34,64-66].

\section{I.2. The role of $P 2 X_{7} R$ in IL- 18 production}

In addition to IL- $1 \beta$ secretion, the $P 2 X_{7} R$ has been implicated in the synthesis and release of the related leaderless cytokine IL-18 (interferon- $\gamma$-inducing factor), which is also produced through cleavage of pro-IL- 18 by ICE $[47,59,67]$, although it has not yet been extensively studied. In contrast to IL-1 $\beta$, secretion of IL- 18 was found to be less dependent on LPS-priming [68], although conflicting data was presented by Mehta et al who found IL-18 production to be LPS-dependent [69]. Indeed it has been shown that individuals expressing the Glu ${ }^{496} \mathrm{Ala}$ P2X $\mathrm{X}_{7} \mathrm{R}$ polymorphism produce significantly less IL-18 when their monocytes are stimulated by ATP [61]. We have also shown that in LPS primed, BzATP stimulated, human monocytic THP- 1 cells, both IL- $1 \beta$ and IL- 18 release is inhibited by $\mathrm{P} 2 \mathrm{X}_{7} \mathrm{R}$ antagonists (Finlayson et al., unpublished observations). The importance of IL-18 in general inflammatory processes, and its suitability as a therapeutic target have been extensively discussed [70], however the simultaneous inhibition of both IL- $1 \beta$ and IL-18 by $\mathrm{P} 2 \mathrm{X}_{7} \mathrm{R}$ antagonism has its obvious attractions.

\subsubsection{The role of $P 2 X_{7} R$ in TNF- $\alpha$ production}

In general, TNF- $\alpha$ is regarded as a pro-inflammatory cytokine that is produced in response to injury, exerting a number of important roles in the immune system and during inflammatory responses. It is of particular interest in neuropathology where this dual role is most clear, with TNF- $\alpha$ having both neurotoxic and neuroprotective effects [71-74]. It appears that microglia, the principal immune cells of the central nervous system, have enhanced $\mathrm{P} 2 \mathrm{X}_{7} \mathrm{R}$ expression following inflammatory insults (see above)
$[3,75]$. However, as mentioned previously, ATP may act as a 'danger' signal, which recruits microglia to damaged areas of the brain through $\mathrm{P} 2 \mathrm{Y}$ rather than $\mathrm{P} 2 \mathrm{X}$ receptors [76]. In a rat model of neuronal injury, stimulation of the P $2 \mathrm{X}_{7} \mathrm{R}$ by ATP has been shown to protect neurones by releasing TNF- $\alpha$ [77]. In contrast to TNF- $\alpha$ release in rat microglia, Kucher and Neary reported that the $\mathrm{P} 2 \mathrm{X}_{7} \mathrm{R}$ was probably responsible for the inhibition of TNF- $\alpha$ release in rat LPS-stimulated astrocytes [78]. Indeed, these authors proposed that this could be a mechanism to sense the severity of damage and alter the inflammatory response appropriately. There are also some reports by Perregaux et al [68] that show ATP alters TNF- $\alpha$ production in human monocytes. As the effects of TNF- $\alpha$ in the CNS will be dependent upon the circumstances of its release, and may differ during the acute response to injury versus the long-term recovery from injury [79], it is vital to understand these effects to facilitate the development of novel therapeutic agents.

In addition to the effects that $\mathrm{P} 2 \mathrm{X}_{7} \mathrm{R}$ polymorphisms have on IL- $1 \beta$ production, it has also been noted that individuals harbouring such polymorphisms have reduced plasma TNF- $\alpha$ levels (but higher levels of the anti-inflammatory cytokine IL-10) relative to normal subjects [62]. Results from this study suggested that during infectious perturbations, $15 \%$ of healthy individuals exhibited anti-inflammatory mediator responses, which was correlated with the level of $\mathrm{P} 2 \mathrm{X}_{7} \mathrm{R}$ pore activity. While normal pore activity appeared to increase microbial clearance, reduced pore activity may provide some protection from autoimmune disorders as those with an anti-inflammatory cytokine profile are less likely to mount an adaptive immune response to self tissues [62]). Since the $\mathrm{P} 2 \mathrm{X}_{7} \mathrm{R}$ is important in the production of both TNF- $\alpha$ and IL- $1 \beta$ and as inhibitors of both are in clinical use for the treatment of rheumatoid arthritis [80] and other inflammatory conditions, such observations possibly underlie why AstraZeneca, Pfizer and Abbot amongst others are currently developing $\mathrm{P} 2 \mathrm{X}_{7} \mathrm{R}$ antagonists.

\section{I.4. The role of $P 2 X_{7} R$ in IL-6 Production}

In rheumatoid arthritis ATP is found in the synovial fluid where a number of $\mathrm{P} 2 \mathrm{X}_{7} \mathrm{R}$-expressing cells including macrophages are present $[81,82]$. In joint diseases such as rheumatoid arthritis and in other conditions such as atherosclerosis the $\mathrm{P} 2 \mathrm{X}_{7} \mathrm{R}$ has also been implicated in the secretion of the pro-inflammatory cytokine IL- 6 from fibroblasts [83]. In atherosclerosis fibroblasts are likely to be exposed to increased concentrations of ATP because of its secretion from platelets and at sites of chronic inflammation [84]. In a more recent study the same authors have shown that fibroblasts from type-2 diabetic patients have increased sensitivity to ATP, which is likely to contribute to diabetic vascular disease [85]. Furthermore, although 
mast cells have received little attention with regard to the $\mathrm{P} 2 \mathrm{X}_{7} \mathrm{R}$, it has been known for some time that these cells express this unique receptor (originally described as the $\mathrm{P} 2 \mathrm{Z}$ receptor) along with several other $\mathrm{P} 2 \mathrm{X}$ and $\mathrm{P} 2 \mathrm{Y}$ receptors [86]. In addition to inducing cell death, ATP-stimulation of the $\mathrm{P} 2 \mathrm{X}_{7} \mathrm{R}$ on murine mast cells has been shown to increase the expression of several pro-inflammatory cytokines, including IL- 6 and TNF- $\alpha$ [87]. Considering the role of mast cells, especially in allergic inflammation, it would appear pertinent to re-examine the role of the $\mathrm{P} 2 \mathrm{X}_{7} \mathrm{R}$ given its therapeutic potential in this area. Finally, new in vivo evidence has been presented supporting the use of $\mathrm{P} 2 \mathrm{X}_{7} \mathrm{R}$ antagonists as anti-inflammatory and antipyretic agents (where excessive pro-inflammatory cytokine production or high fever is harmful to the host [88]). These authors provided important new insights into LPS-induced febrile response in rats, and showed that the ATP released from activated immune cells stimulated cytokine release which then initiated the febrile response [88]. These authors suggested that the $\mathrm{P} 2 \mathrm{X}_{7} \mathrm{R}$ plays a central role [88], which is perhaps unsurprising given that the cytokines IL- 6, IL- $1 \beta$ and TNF- $\alpha$ all act as endogenous pyrogens [89].

\section{2. $P 2 X_{7} R$ regulation of granulocyte function and cell death}

It is well known that granulocytes play a critical role in acute inflammation, with polymorphonuclear neutrophils (PMNs; 95\% of circulating granulocytes) and eosinophils of particular interest. PMNs are phagocytic cells that play a critical role in the host defence against bacterial and fungal infections, whereas eosinophils are primarily involved in the host defence against parasites, and function in the pathogenesis of allergic and immunological disease. In general, granulocytes are recruited to sites of inflammation where they release inflammatory mediators such as leukotriene $\mathrm{B}_{4}$, platelet activating factor and IL-8. However in the event of the failed clearance of apoptotic PMNs these inflammatory mediators can lead to tissue destruction and are thought to underlie the pathophysiology of diseases such as asthma, rheumatoid arthritis and atopic dermatitis [90-92].

\subsection{1. $P 2 X_{7} R$ mediated modulation of apoptosis in PMNs}

The process of cell death is fundamental to many aspects of physiology and pathophysiology, and of great importance to the regulation of inflammation. Apoptosis is a process of controlled cell death in which cells undergo well characterised morphological changes, including the classical features of chromatin condensation, cell shrinkage, and the formation of apoptotic bodies [93]. In contrast to necrotic cell death, apoptotic cell death is a predominantly non-inflammatory process in which the membranes of cells remain intact. This allows the cytotoxic granule contents of cells such as PMN to remain enclosed within the cytoplasmic membrane while the cell is phagocytosed, thereby minimising tissue damage. Furthermore, phagocytosis of apoptotic cells, unlike other particles, has been shown to inhibit the release of proinflammatory mediators including IL- $1 \beta$, IL- 8 and TNF- $\alpha$ [94]. However, failure of rapid phagocytosis can result in secondary necrosis of the apoptotic cell leading to tissue damage and inflammatory infiltrate (Figure 2). Thus, regulation of innate immune effector cell apoptosis, in particular that of short-lived granulocytes, is critical to the induction, maintenance and resolution of inflammatory processes [95]. Apoptosis is regulated at a cellular level by the expression and activation of the Bcl-2 family of proteins and the components of the caspase pathways, which dictate the lifespan and mode of cell death in such cells [96]. Importantly, recent studies indicate that $P 2 X_{7} R$ activation may modulate a number of cell death processes through effects upon these key regulators of apoptosis.

As described above, the human cathelicidin LL-37 inhibited PMN apoptosis in a $\mathrm{P} 2 \mathrm{X}_{7} \mathrm{R}$-dependent manner $[40,41]$. Stimulation of PMN with LL-37 was shown to upregulate expression of the Bcl-2 family protein $\mathrm{Mcl}-1$, a key rapid response component which promotes PMN survival [97], and to inhibit the cleavage and activation of the critical apoptotic regulator pro-caspase-3 $[98,99]$. Interestingly, whereas lower levels of LL-37 acted primarily as a neutrophil survival factor, higher levels appeared to promote necrotic cell death while inhibiting apoptosis [41]. Thus stimulation of the $\mathrm{P} 2 \mathrm{X}_{7} \mathrm{R}$ has the capacity to exert a potent effect upon neutrophil survival. These data indicate that PMN express functional $\mathrm{P} 2 \mathrm{X}_{7} \mathrm{R}$, but the cellular localisation of these receptors in this cell type remains unclear. $\mathrm{P} 2 \mathrm{X}_{7} \mathrm{R}$ expression on human cells has been demonstrated on PMN, HL- 60 promyelocytes and granulocytic differentiated cells, and is reported to increase with granulocytic differentiation [100]. However, one report has suggested that human PMN have an intracellular pool of $\mathrm{P} 2 \mathrm{X}_{7} \mathrm{R}$, with little or no surface expression [101]. Irrespective, these studies suggest that $\mathrm{P} 2 \mathrm{X}_{7} \mathrm{R}$ activation might extend the lifespan of PMN at sites of infection and inflammation, and modulate the mechanism of cell death in these cells.

In contrast to the effects observed in neutrophils, prolonged $\mathrm{P} 2 \mathrm{X}_{7} \mathrm{R}$ activation with extracellular ATP has been shown to induce apoptosis in other cell types, including mast cells and epithelial cells $[9,102,103]$. In addition, murine whole blood exposed to ATP demonstrated a near complete loss of monocytes, and a decrease in lymphocytes, but no change in PMN numbers [34]. This effect was not seen in $\mathrm{P} 2 \mathrm{X}_{7} \mathrm{R}$-deficient mice, indicating a $\mathrm{P} 2 \mathrm{X}_{7} \mathrm{R}$ mediated induction of cell death in these cells [34]. This induction of apoptosis has been proposed to involve the opening of cation-selective membrane pores, and to be a 


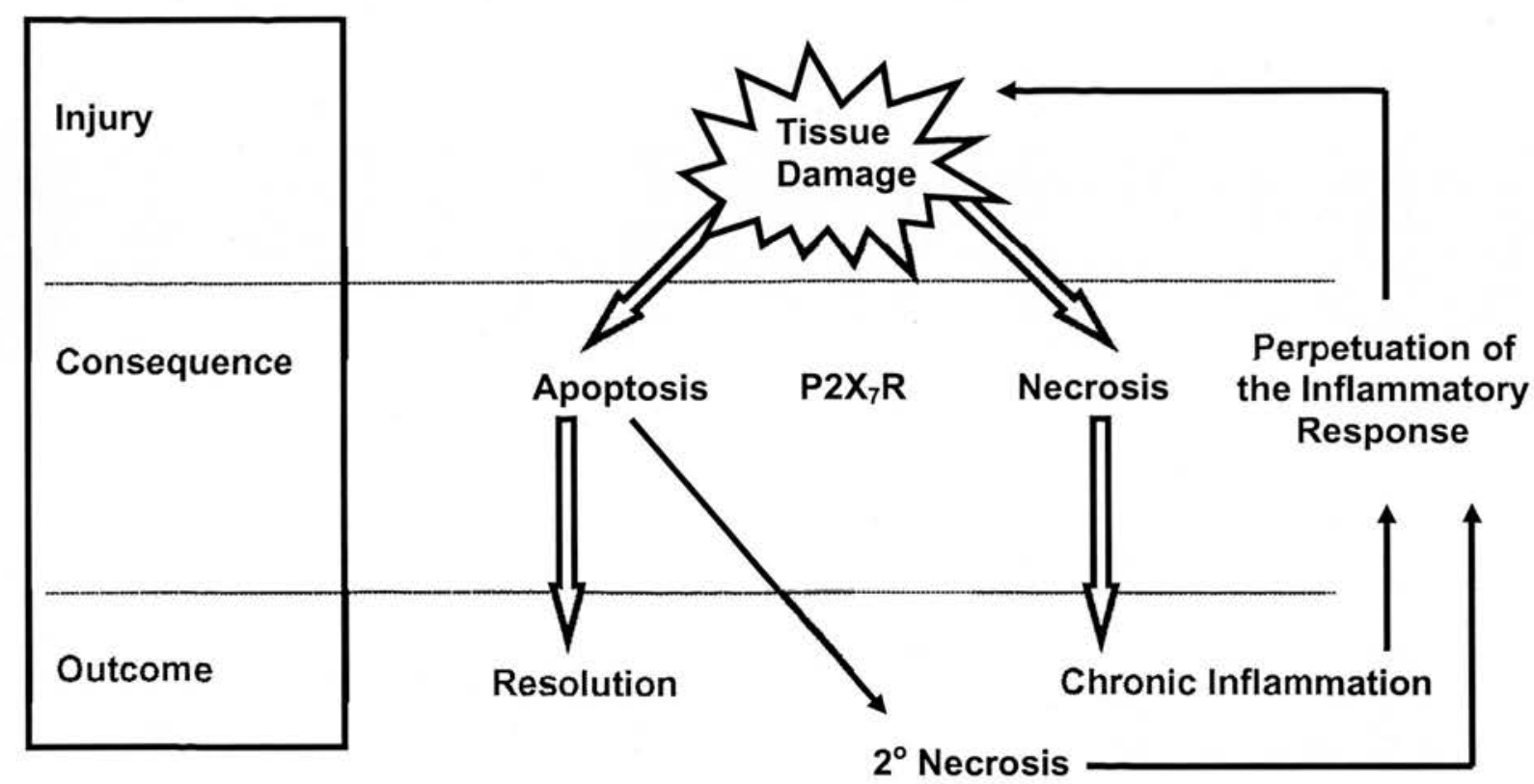

Figure 2

Possible outcomes of an inflammatory response. Tissue damage (inflammation initiation) can lead to cell death by apoptosis or necrosis. The balance between these two types of cell death can determine the outcome of the inflammatory response e.g. propagation (leading to chronic inflammation) or resolution. Resolution is more common when cell death is predominantly apoptotic, however, the phagocytosis of apoptotic or necrotic cells is also an important determinant of the outcome of inflammation. As can be seen, the $\mathrm{P} 2 \mathrm{X}_{7} \mathrm{R}$ may be critical to determining the outcome of an inflammatory response.

calcium-independent, ROCK-1-dependent pathway [9]. Whereas prolonged or excessive $\mathrm{P} 2 \mathrm{X}_{7} \mathrm{R}$ activation with ATP induces apoptosis, transient activation induces a state of pseudoapoptosis in epithelial cells [9]. Under these conditions, $\mathrm{P} 2 \mathrm{X}_{7} \mathrm{R}$ activation results in a series of very rapid and reversible effects, including calcium-dependent translocation of plasma membrane phosphatidylserine, loss of mitochondrial membrane potential (without cytochrome c release), disruption of the actin filament/microtubule network and membrane blebbing. These data suggest that the $\mathrm{P} 2 \mathrm{X}_{7} \mathrm{R}$ can be associated with two different pathways, inducing pseudoapoptosis or apoptosis in epithelial cells. These effects on cell death, assuming the physiological ligand is ATP, are most likely to occur at sites of tissue damage where ATP is released in considerable quantities [104]. Interestingly, LL-37 has also been shown to induce eukaryotic membrane permeability [38] and been implicated in the induction of apoptosis in epithelial cells [105]. Thus, although the possible role for $\mathrm{P} 2 \mathrm{X}_{7} \mathrm{R}$ in mediating these latter effects remains to be determined, it is tempting to speculate that alternative agonists such as LL-37 could induce $\mathrm{P} 2 \mathrm{X}_{7} \mathrm{R}$-dependent apoptosis, and the safe removal of infected cells in an inflammatory environment, even in the absence of high concentrations of ATP.

Thus, an intriguing contrast exists between the effects of $\mathrm{P} 2 \mathrm{X}_{7} \mathrm{R}$ stimulation on cell death pathways in different host innate immune effector cells. Nevertheless, the consequences in each case may enhance the inflammatory response and the clearance of infection in acute infection, but have potentially deleterious effects in chronic inflammatory conditions. Indeed, Chen and Brosnan have shown $\mathrm{P} 2 \mathrm{X}_{7} \mathrm{R}$ knockout mice to be more susceptible to autoimmune encephalomyelitis (a model for multiple sclerosis), attributing this susceptibility to reduced apoptotic activity in lymphocytes [64]. A further understanding of these processes is anticipated to facilitate the development of novel therapeutic agents capable of modulating inflammation via $\mathrm{P} 2 \mathrm{X}_{7} \mathrm{R}$-mediated effects on cell death pathways.

\subsection{2. $P 2 X_{7} R$ and cytokine production in eosinophils}

Ferrari et al [106] were the first to show that the $\mathrm{P} 2 \mathrm{X}_{7} \mathrm{R}$ was present on eosinophils, with Mohanty et al [107] showing one year later that this expression was dependent upon 
stimulation by interferon- $\gamma$ (IFN- $\gamma$ ). This stimulationdependent expression contrasts with a more recent study which showed functional $\mathrm{P} 2 \mathrm{X}_{7} \mathrm{R}$ were expressed endogenously on eosinophils and that inhibition of the $\mathrm{P} 2 \mathrm{X}_{7} \mathrm{R}$, abrogated agonist (BzATP) induced IL-8 release from eosinophils [108]. This is interesting in light of the observation that asthmatics secrete more IL-8 from their peripheral blood eosinophils than normal individuals [109]. Furthermore, as IL-8 is chemotatic for neutrophils [110] and CD16 + natural killer cells [111] this suggests a role for IL- 8 in the initiation and propagation of the inflammatory response [108]. As ATP can be released upon tissue damage [104] and in response to inflammatory stimuli [49] (both of which may be present in asthma) it is possible that the $\mathrm{P} 2 \mathrm{X}_{7} \mathrm{R}$ would be activated, resulting in IL-8 production and propagation of the immune response (Figure 3 ). This simplified description of part of the interplay between inflammatory cells and the mediators released, again suggests that the $\mathrm{P} 2 \mathrm{X}_{7} \mathrm{R}$ may be a potential target for therapeutic intervention: however, these complex interactions are not yet fully understood. A better understanding of the basic pathophysiology of the initiation of inflammation will allow us to determine whether more specific therapies such as $\mathrm{P} 2 \mathrm{X}_{7} \mathrm{R}$ regulation would prevent excessive inflammatory reactions, suppress acute inflammatory reactions and possibly augment the healing process following tissue damage [112].

\section{Therapies directed at influencing the $P \mathbf{2} X_{7} R$}

To date the majority of studies have focused on inhibiting the $\mathrm{P} 2 \mathrm{X}_{7} \mathrm{R}$ to abrogate its downstream production of proinflammatory cytokines, with a number of reports now highlighting the potential benefit of $\mathrm{P} 2 \mathrm{X}_{7} \mathrm{R}$ antagonists. Inhibiting the production of the undesirable excess of pro-inflammatory mediators such as IL- $1 \beta$ and TNF- $\alpha$ which cause the inflammatory state in many immune disorders is likely to be advantageous. In other circumstances, such as $M$. tuberculosis infection, activating the $\mathrm{P} 2 \mathrm{X}_{7} \mathrm{R}$ may prove beneficial in bacilli eradication by encouraging infected macrophages to die by apoptosis rather than necrosis. This could introduce a number of problems, most notably being a systemic increase in inflammatory mediators and increased apoptosis in all cells expressing the $\mathrm{P} 2 \mathrm{X}_{7} \mathrm{R}$. A contrasting problem could exist for $\mathrm{P} 2 \mathrm{X}_{7} \mathrm{R}$ antagonists, as the suppression of any natural $\mathrm{P} 2 \mathrm{X}_{7} \mathrm{R}$-dependent apoptosis could result in an increased susceptibility to autoimmune disease and carcinogenesis (vide infra). However, $\mathrm{P} 2 \mathrm{X}_{7} \mathrm{R}$-deficient mice have been described as having generally suppressed immune responses, without being immunocompromised [34]. Only when selective agonists and antagonists are widely available can any such assertions be addressed, although it is important to consider them as part of the broader recognition of the $\mathrm{P} 2 \mathrm{X}_{7} \mathrm{R}$ as a potential therapeutic target.

\section{I. The $P 2 X_{7} R$, multinucleated giant cells and tuberculosis} In granulomatous disorders, monocytes or macrophages often fuse to form multinucleated giant cells (MGCs) [113], which results in increased cytokine production, non-phagocytic antigen internalisation, and disposal of infected or damaged monocytes. The antimicrobial activity of monocytes actually decreases with maturation to macrophages [114], whereas it is enhanced upon MGC formation [115]. An early study showed that the $\mathrm{P} 2 \mathrm{X}_{7} \mathrm{R}$ may be important in the formation of MGCs [116], with Falzoni et al [117] speculating later that the $\mathrm{P} 2 \mathrm{X}_{7} \mathrm{R}$ is involved in the final step of MGC formation (membrane fusion), as the receptor was found to cluster at sites of cellto-cell interactions. They also showed that the $\mathrm{P} 2 \mathrm{X}_{7} \mathrm{R}$ does not affect chemotaxis, cell aggregation or the expression of adhesion molecules and indicated that other factors may play an important role in the earlier stages of MGC formation [117]. However, there is new evidence to suggest that ICAM-1, in association with the $\mathrm{P} 2 \mathrm{X}_{7} \mathrm{R}$, may be important in this process [118-120].

Tuberculosis is a granulomatous disease caused by infection with Mycobacterium tuberculosis (M. tuberculosis), with the pathogen residing and replicating within macrophages. It still represents a major health burden, as a consequence of the emergence of antibiotic-resistant strains and co-infection with the human immunodeficiency virus (HIV) [121]. Following infection, part of the host immune response involves the initiation of a T-helper cell response against $M$. tuberculosis, with the subsequent activation of macrophages enabling them to become mycobactericidal $[122,123]$. This T-helper response also stimulates the formation of granulomas, which, as noted above, are characterised by $\mathrm{P} 2 \mathrm{X}_{7} \mathrm{R}$-expressing MGCs. In 1994 Molloy et al observed that apoptosis of an infected macrophage, but not necrosis, resulted in decreased mycobacterial viability [114] and that $M$. Tuberculosisinfected macrophages undergo apoptosis by a TNF- $\alpha$ dependent mechanism $[124,125]$. However, pathogenic strains have been shown to reduce this TNF- $\alpha$ effect by increasing IL-10 production [126]. This anti-inflammatory cytokine then induces the release of soluble TNF- $\alpha$ receptor 2 (sTNFR2) from alveolar macrophages which inactivates TNF- $\alpha$, thus inhibiting TNF- $\alpha$-dependent apoptosis and ultimately favouring mycobacterial growth [126]. Interfering with this mechanism could therefore lead to the development of a new therapeutic strategy aimed at treating tuberculosis.

With $\mathrm{P} 2 \mathrm{X}_{7} \mathrm{R}$ activation known to be associated with cell death, Lammas et al [127] suggested that the $\mathrm{P} 2 \mathrm{X}_{7} \mathrm{R}$ may play a role in the apoptosis of infected macrophages and 


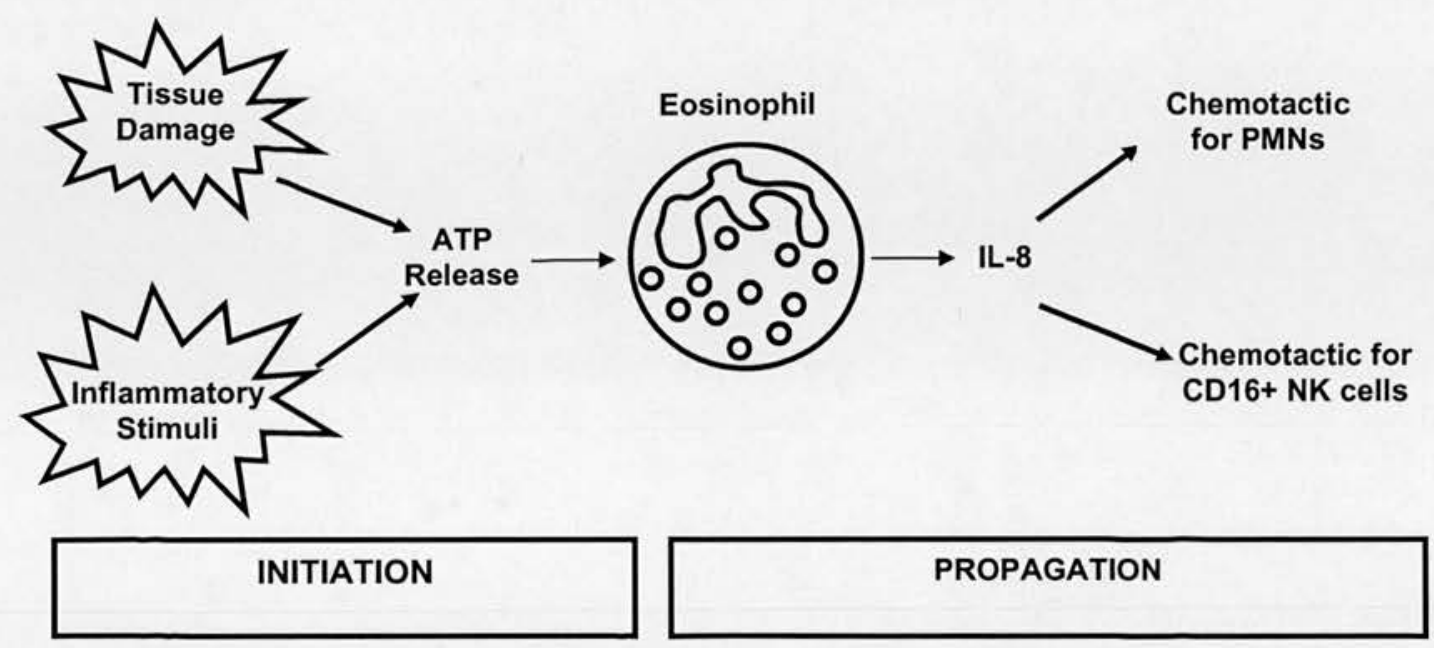

Figure 3

Diagrammatic representation of the interplay between inflammatory mediators and cells. Tissue damage or inflammatory stimuli results in ATP release which activates the P2 $X_{7} R$ causing eosinophils to release IL- 8 which amplifies the initial inflammatory response.

the accompanying mycobacterial death. The authors clearly showed that ATP-induced mycobacterial death was not a consequence of reactive oxygen or nitrogen species production, membrane disruption, or via any direct toxic effect [127]. The finding that apoptosis of infected macrophages is TNF- $\alpha$ dependent may provide an explanation as to why $\mathrm{P} 2 \mathrm{X}_{7} \mathrm{R}$ are involved in mycobacterial death, however, to date, $\mathrm{P} 2 \mathrm{X}_{7} \mathrm{R}$-dependent TNF- $\alpha$ production has not been investigated in alveolar macrophages. Further evidence for involvement of the $\mathrm{P} 2 \mathrm{X}_{7} \mathrm{R}$ in apoptosis of infected macrophages was provided in a study utilising $\mathrm{P} 2 \mathrm{X}_{7} \mathrm{R}$ knockout mice [128]. However, again it was noted in this study that there are likely to be additional purinergic receptors that contribute to loss of mycobacterial viability, confirming an earlier observation by Sikora et al [129]. In 2000, it was found that extracellular ATP promoted the killing of virulent $M$. Tuberculosis in a phospholipase D (PLD) dependent manner [130], with further research suggesting that the mycobactericidal activity was due to $M$. tuberculosis-containing phagosomes fusing with lysosomes. ATP appeared to act through both $\mathrm{P} 2 \mathrm{X}_{7} \mathrm{R}$ dependent and independent mechanisms, with this process dependent upon increased cytosolic calcium and PLD [131]. More recently it has been shown that infection with the attenuated strain $M$. tuberculosis H37Ra inhibited $\mathrm{P} 2 \mathrm{X}_{7} \mathrm{R}$ signalling [132] and in the same study cyclosporin A (an inhibitor of mitochondrial permeability transition (MPT), which is associated with increased mitochondrial cytochrome c release, necrotic macrophage death with resultant uncontrolled mycobacterial replication) was shown to re-establish $\mathrm{P} 2 \mathrm{X}_{7} \mathrm{R}$ function in infected macrophages, and restore the antimycobacterial mechanisms associated with apoptosis [132].

Further evidence highlighting the potential importance of the $\mathrm{P} 2 \mathrm{X}_{7} \mathrm{R}$ in tuberculosis has been provided by looking at receptor polymorphisms. Loss-of-function $\mathrm{P}_{2} \mathrm{X}_{7} \mathrm{R}$ polymorphisms have been shown to contribute to the variability in susceptibility to mycobacterial infections [133], perhaps through abolition of ATP-mediated killing of mycobacteria [134]. It appears that infected macrophages from individuals with polymorphisms in the $\mathrm{P} 2 \mathrm{X}_{7} \mathrm{R}$ gene were resistant to apoptosis, which, as noted above, is important in the killing of intracellular mycobacteria $[135,136]$. It is therefore clear that the $\mathrm{P} 2 \mathrm{X}_{7} \mathrm{R}$ should be investigated as a potential new therapy for treating tuberculosis.

\subsection{The role of the $P 2 X_{7} R$ in cancer}

The connection between inflammation and cancer was first described by Rudolf Virchow in 1863 (see reference [137] and references therein), with the interplay having been studied extensively since. For example, it has now been shown that there is an increased likelihood of a cancer developing at a site of chronic inflammation [138]. A polymorphism in the TNF- $\alpha$ promoter resulting in 
enhanced plasma TNF- $\alpha$ has been associated with an increased incidence of prostate cancer [139], while a polymorphism increasing IL-1 $\beta$ production conferred a greater susceptibility to gastric cancer $[140,141]$. Given the importance of the $\mathrm{P} 2 \mathrm{X}_{7} \mathrm{R}$ in regulating cell death and cytokine production it is perhaps unsurprising it may play a role in cancer. Therefore, the development of either $\mathrm{P} 2 \mathrm{X}_{7} \mathrm{R}$ agonists or antagonists may be useful anti-cancer agents, as agonists could kill cells, whereas antagonists would perhaps stop proliferation.

In 1996, T lymphocytes were found to express a purinergic receptor (suggested to be the $\mathrm{P} 2 \mathrm{X}_{7} \mathrm{R}$ ) which when inhibited, severely decreased cell proliferation [142]. Three years later these authors extended their findings by reporting that $\mathrm{P} 2 \mathrm{X}_{7} \mathrm{R}$ transfection into lymphoid cells (lacking endogenous receptor expression), sustained their growth in serum-free medium [143]. They suggested that an ATPbased autocrine/paracrine loop existed which supported lymphoid cell proliferation in the absence of growth factors normally present in serum [143]. In isolation this was an important finding because one of the six alterations (the 'Hallmarks of cancer') thought to be essential in the transformation of a normal cell into a cancerous cell is 'self-sufficiency' in growth signals [144]. Recently it was shown that $\mathrm{P} 2 \mathrm{X}_{7} \mathrm{R}$ transfection increased cellular energy stores (i.e. ATP) and the resting mitochondrial potential of transfected cells both of which gave the cells a growth advantage [145]. As mitochondrial dysfunction is important in apoptosis [146], any increase in resting mitochondrial potential would be expected to make cells resistant to apoptosis, thus providing them with a growth advantage [145] a further alteration thought to be essential in carcinogenesis - 'evasion of apoptosis' [144]. These observations are of clear importance given the earlier observation that the $\mathrm{P} 2 \mathrm{X}_{7} \mathrm{R}$ is over expressed in several cancers [147].

In addition, the Glu ${ }^{496} \mathrm{Ala} P 2 \mathrm{X}_{7} \mathrm{R}$ polymorphism discussed earlier produced a lack of agonist-mediated apoptosis in some patients with chronic lymphoblastic leukaemia [60]. In contrast, another report found that this polymorphism did not cause an increased risk of chronic lymphoblastic leukaemia [148], however the situation is clearly complex with different $\mathrm{P} 2 \mathrm{X}_{7} \mathrm{R}$ polymorphisms found to contribute to the clinical outcome of chronic lymphoblastic leukaemia [149]. It is important that $\mathrm{P} 2 \mathrm{X}_{7} \mathrm{R}$ polymorphisms and their associations with cancer be clarified, so that their potential as a prognostic tool can be determined. A new paper by Carta et al [150] has suggested that histone deacetylase (HDAC) inhibitors (novel agents currently being developed as pleiotropic anti-cancer agents) may have potential for development as antiinflammatory agents as they reduced ATP-stimulated IL$1 \beta$ production via the $\mathrm{P} 2 \mathrm{X}_{7} \mathrm{R}$. The potential role of $\mathrm{P} 2 \mathrm{X}_{7} \mathrm{R}$ ligands in the treatment of cancer appears exciting and will undoubtedly be the subject of many future investigations.

\section{Conclusion}

In the 10 years since the purinergic $\mathrm{P} 2 \mathrm{X}_{7} \mathrm{R}$ was cloned it is now clear that this receptor plays a number of important functions in the immune system. The importance of the $\mathrm{P} 2 \mathrm{X}_{7} \mathrm{R}$ on macrophages is best understood, with the $\mathrm{P} 2 \mathrm{X}_{7} \mathrm{R}$ playing an important role in the formation of MGCs and in macrophage intracellular killing of mycobacteria, such as M. tuberculosis. Moreover, the $\mathrm{P} 2 \mathrm{X}_{7} \mathrm{R}$ is clearly involved in secretion of cytokines by macrophages (and other cells such as monocytes and microglia), particularly IL- $1 \beta$, IL- 18 , TNF- $\alpha$ and IL- 6 , all of which play an important role in mediating inflammatory responses. The $\mathrm{P} 2 \mathrm{X}_{7} \mathrm{R}$ has been shown to regulate the release of IL- 8 from eosinophils and may be expressed on PMNs, potentially influencing their function. Although there is currently less evidence that the $\mathrm{P} 2 \mathrm{X}_{7} \mathrm{R}$ regulates cytokine production in granulocytes, it appears to play a pivotal role in regulating apoptosis and cell death. Therefore, the P2X $\mathrm{X}_{7} \mathrm{R}$ represents an exciting target for regulating peripheral and central inflammation and given the appropriate disease state, $\mathrm{P} 2 \mathrm{X}_{7} \mathrm{R}$ antagonists may serve as a new class of anti-inflammatory compounds, capable of not only inhibiting the initiation of inflammation, but also potentially enhancing resolution.

\section{Abbreviations}

ATP Adenosine 5'-triphosphate

BzATP 2', 3'-O-(benzoyl-4-benzoyl)-ATP

COX-2 Cyclooxygenase type 2

ICE Interleukin-converting enzyme

IL Interleukin

IL-1RA Interleukin 1 receptor antagonist

INF- $\gamma$ Interferon- $\gamma$

LPS Lipopolysaccharide

MGC Multinucleated giant cell

oATP Oxidised adenosine 5'-triphosphate

P2 $\mathrm{X}_{7} \mathrm{R}$ P2 $\mathrm{X}_{7}$ receptor

PMN Polymorphonuclear neutrophil

TBI Traumatic brain injury 


\section{TNF- $\alpha$ Tumour necrosis factor- $\alpha$}

\section{Authors' contributions}

MFL performed the literature review, wrote the first draft of the review and provided ideas and discussion related to the topic. DAS, DJD and JPH provided intellectual input and contributed to the writing of the review. AGR conceived the idea of writing a review, and along with JS and KF contributed to the structure and writing and provided significant editorial contributions to the content of the review.

\section{References}

1. Nathan C: Points of control in inflammation. Nature 2002, 420:846-852.

2. Gilroy DW, Lawrence T, Perretti M, Rossi AG: Inflammatory resolution: new opportunities for drug discovery. Nat Rev Drug Discov 2004, 3:401-416.

3. Collo G, Neidhart S, Kawashima E, Kosco-Vilbois M, North RA, Buell G: Tissue distribution of the $\mathbf{P} 2 \times \mathbf{7}$ receptor. Neuropharmacology 1997, 36:1277-1283.

4. Abbracchio MP, Boeynaems JM, Barnard EA, Boyer JL, Kennedy C, Miras-Portugal MT, King BF, Gachet C, Jacobson KA, Weisman GA, Burnstock G: Characterization of the UDP-glucose receptor (re-named here the P2Y 14 receptor) adds diversity to the P2Y receptor family. Trends in Pharmacological Sciences 2003, 24:52-55.

5. North RA: Molecular Physiology of P2X Receptors. Physiol Rev 2002, 82:1013-1067.

6. Burnstock G: Purinergic signalling--an overview. Novartis Found Symp 2006, 276:26-48.

7. Di Virgilio F: The $\mathbf{P 2 Z}$ purinoceptor: an intriguing role in immunity, inflammation and cell death. Immunology Today 1995, 16:524-528.

8. Falzoni S, Munerati M, Ferrari D, Spisani S, Moretti S, Di Virgilio F: The purinergic $\mathbf{P} 2 Z$ receptor of human macrophage cells. Characterization and possible physiological role. J Clin Invest 1995, 95:1207-1216.

9. Mackenzie AB, Young MT, Adinolfi E, Surprenant A: Pseudoapoptosis induced by brief activation of ATP-gated $P 2 X 7$ receptors. I Biol Chem 2005, 280:33968-33976.

10. Hodgkiss JP McLuckie.J..Sharkey.J.\& Finlayson,K.: Characterisation of intracellular calcium responses in HEK293 cells stably expressing human $\mathbf{P} 2 \mathbf{X}$ receptors. 4th International Symposium of nucleosides and nucleotides 2004, 38T.

11. Bianchi BR, Lynch KJ, Touma E, Niforatos W, Burgard EC, Alexander KM, Park HS, Yu H, Metzger R, Kowaluk E: Pharmacological characterization of recombinant human and rat $\mathrm{P} 2 \mathrm{X}$ receptor subtypes. European Journal of Phormocology 1999, 376:127-138.

12. Communi D, Robaye B, Boeynaems JM: Pharmacological characterization of the human P2YII receptor. 1999 128:1199-1206.

13. Beigi RD, Kertesy SB, Aquilina G, Dubyak GR: Oxidized ATP (oATP) attenuates proinflammatory signaling via $\mathbf{P 2}$ receptor-independent mechanisms. 2003, 140:507-519.

14. Di Virgilio F: Novel data point to a broader mechanism of action of oxidized ATP: the P2X7 receptor is not the only target. 2003, 140:441-443.

15. Alcaraz L, Baxter A, Bent J., Bowers K, Braddock M, Cladingboel D, Donald D, Fagura M, Furber M, Laurent C, Lawson M, Mortimore M, McCormick M, Roberts N, Robertson M: Novel P2X7 receptor antagonists. Bioorg Med Chem Lett 2003, I 3:4043-4046.

16. Baxter A, Bent J, Bowers K, Braddock M, Brough S, Fagura M, Lawson M, Mclnally T, Mortimore M, Robertson M, Weaver R, Webborn P. Hit-to-Lead studies: the discovery of potent adamantane amide P2X7 receptor antagonists. Bioorg Med Chem Lett 2003, 13:4047-4050.

17. Merriman GH, Ma L, Shum P, McGarry D, Volz F, Sabol IS, Gross A Zhao Z, Rampe D, Wang L, Wirtz-Brugger F, Harris BA, Macdonald $D$ : Synthesis and SAR of novel 4,5-diarylimidazolines as potent P2X7 receptor antagonists. Bioorg Med Chem Lett 2005 . 15:435-438.

18. Ia Sala A, Ferrari D, Di Virgilio F, Idzko M, Norgauer J, Girolomoni G: Alerting and tuning the immune response by extracellular nucleotides. J Leukoc Biol 2003, 73:339-343.

19. Surprenant A, Rassendren F, Kawashima E, North RA, Buell G: The Cytolytic P2Z Receptor for Extracellular ATP Identified as a P2X Receptor (P2X7). Science 1996, 272:735-738.

20. Thornberry NA, Bull HG, Calaycay JR, Chapman KT, Howard AD, Kostura MJ, Miller DK, Molineaux SM, Weidner JR, Aunins J, Elliston KO, Ayala JM, Casano FJ, Chin J, Ding GJF, Egger LA, Gaffney EP, Limjuco G, Palyha OC, Raju SM, Rolando AM, Salley JP, Yamin TT, Lee TD, Shively JE, MacCross M, Mumford RA, Schmidt JA, Tocci MJ: A novel heterodimeric cysteine protease is required for inter leukin-I[beta]processing in monocytes. Nature 1992. 356:768-774.

21. Mosley B, Urdal DL, Prickett KS, Larsen A, Cosman D, Conlon PJ, Gillis S, Dower SK: The interleukin-I receptor binds the human interleukin-I alpha precursor but not the interleukin-I beta precursor. J Biol Chem 1987, 262:294|-2944.

22. Dinarello CA: The IL-I family and inflammatory diseases. Clin Exp Rheumatol 2002, 20:S1-13.

23. Goldblatt F, Isenberg DA: New therapies for rheumatoid arthritis. Clin Exp Immunol 2005, 140:195-204

24. Moynagh PN: The interleukin-I signalling pathway in astrocytes: a key contributor to inflammation in the brain. I Anot 2005, 207:265-269.

25. Shore SA, Moore PE: Effects of cytokines on contractile and dilator responses of airway smooth muscle. Clin Exp Pharmacol Physiol 2002, 29:859-866.

26. Chung KF: Cytokines in chronic obstructive pulmonary disease. Eur Respir J Suppl 2001, 34:50s-55s.

27. Braddock M, Quinn A: Targeting IL-I in inflammatory disease: new opportunities for therapeutic intervention. Not Rev Drug Discov 2004, 3:330-340.

28. Hogquist KA, Nett MA, Unanue ER, Chaplin DD: Interleukin I is Processed and Released During Apoptosis. PNAS 1991, 88:8485-8489.

29. Chin J, Kostura MJ: Dissociation of IL-I beta synthesis and secretion in human blood monocytes stimulated with bacterial cell wall products. J Immunol 1993, I5 I:5574-5585.

30. Perregaux D, Gabel CA: Interleukin-I beta maturation and release in response to $A T P$ and nigericin. Evidence that potassium depletion mediated by these agents is a necessary and common feature of their activity. I Biol Chem 1994, 269:15195-15203

31. Ferrari D, Chiozzi P, Falzoni S, Dal Susino M, Melchiorri L, Baricord OR, Di Virgilio F: Extracellular ATP triggers IL-I beta release by activating the purinergic $P 2 Z$ receptor of human macrophages. J Immunol 1997, 159:1451-1458.

32. Sanz JM, Virgilio FD: Kinetics and Mechanism of ATP-Dependent IL-I \{beta\} Release from Microglial Cells. J Immunol 2000 164:4893-4898.

33. Solle M, Labasi J, Perregaux DG, Stam E, Petrushova N, Koller BH Griffiths RJ, Gabel CA: Altered Cytokine Production in Mice Lacking P2X7 Receptors. J Biol Chem 2001, 276:125-1 32.

34. Labasi JM, Petrushova N, Donovan C, McCurdy S, Lira P, Payette MM Brissette W, Wicks JR, Audoly L, Gabel CA: Absence of the P2X7 Receptor Alters Leukocyte Function and Attenuates an Inflammatory Response. J Immunol 2002, 168:6436-6445.

35. Kahlenberg JM, Dubyak GR: Mechanisms of caspase-I activation by $\mathbf{P} \mathbf{2 X 7}$ receptor-mediated $\mathrm{K}+$ release. Am J Physiol Cell Physio 2004, 286: CII 00-CII08.

36. MacKenzie A, Wilson HL, Kiss-Toth E, Dower SK, North RA, Surprenant A: Rapid secretion of interleukin-I beta by microvesicle shedding. Immunity 2001, 15:825-835.

37. Wilson HL, Francis SE, Dower SK, Crossman DC: Secretion of Intracellular IL-I Receptor Antagonist (Type I) Is Dependent on P2X7 Receptor Activation. J Immunol 2004, 173:1202-1208

38. Elssner A, Duncan M, Gavrilin M, Wewers MD: A Novel P2X Receptor Activator, the Human Cathelicidin-Derived Peptide LL37, Induces IL-I \{beta\} Processing and Release. J Immunol 2004, 172:4987-4994

39. Perregaux DG, Bhavsar K, Contillo L, Shi J. Gabel CA: Antimicrobial Peptides Initiate IL-I beta\} Posttranslational Process 
ing: A Novel Role Beyond Innate Immunity. J Immunol 2002, 168:3024-3032.

40. Nagaoka I, Tamura H, Hirata M: An Antimicrobial Cathelicidin Peptide, Human CAPI8/LL-37, Suppresses Neutrophil Apoptosis via the Activation of Formyl-Peptide ReceptorLike I and P2X7. IImmunol 2006, 176:3044-3052

41. Barlow PG, LiY, Wilkinson TS, Bowdish DME, Lau YE, Cosseau C Haslett C, Simpson AJ, Hancock REW, Davidson DJ: The human cationic host defense peptide LL-37 mediates contrasting effects on apoptotic pathways in different primary cells of the innate immune system. J Leukoc Biol 2006, 80:509-520.

42. Zanetti M: Cathelicidins, multifunctional peptides of the innate immunity. J Leukoc Biol 2004, 75:39-48.

43. SCHALLER-BALS SUSA, SCHULZE ANDR, BALS ROBE: Increased Levels of Antimicrobial Peptides in Tracheal Aspirates of Newborn Infants during Infection. Am J Respir Crit Care Med 2002, 165:992-995.

44. Bowdish DME, Davidson DJ, Lau YE, Lee K, Scott MG, Hancock REW: Impact of LL-37 on anti-infective immunity. J Leukoc Biol 2005, 77:451-459

45. Laliberte R, Perregaux D, Svensson L, Pazoles Cl, Gabel CA: Tenidap modulates cytoplasmic $\mathrm{pH}$ and inhibits anion transport in vitro. II. Inhibition of IL-I beta production from ATP. treated monocytes and macrophages. I Immunol 1994. 153:2168-2179.

46. Sanz JM. Chiozzi P, Di Virgilio F: Tenidap enhances P2Z/P2X7 receptor signalling in macrophages. European Journal of Pharmacology 1998, 355:235-244.

47. Mariathasan S, Weiss DS, Newton K, McBride J, O'Rourke K, RooseGirma M, Lee WP, Weinrauch Y, Monack DM, Dixit VM: Cryopyrin activates the inflammasome in response to toxins and ATP. Nature 2006, 440:228-232.

48. Brough D, Le Feuvre RA, Wheeler RD, Solovyova N, Hilfiker S, Rothwell NJ, Verkhratsky A: Ca2 + Stores and Ca2+ Entry Differentially Contribute to the Release of IL-I \{beta\} and IL-I \{alpha\} from Murine Macrophages. J Immunol 2003, 170:3029-3036.

49. Ferrari D, Chiozzi P, Falzoni S, Hanau S, Di áVirgilio F: Purinergic Modulation of Interleukin-I beta Release from Microglial Cells Stimulated with Bacterial Endotoxin. J Exp Med 1997. 185:579-582.

50. Brough D, Le Feuvre RA, Iwakura Y, Rothwell NJ: Purinergic ( $P 2 \times 7)$ receptor activation of microglia induces cell death via an interleukin-I-independent mechanism. Mol Cell Neurosci 2002, 19:272-280.

51. Franke H, Gunther A, Grosche J, Schmidt R, Rossner S, Reinhardt R, Faber-Zuschratter H, Schneider D, Illes P: P2X7 receptor expression after ischemia in the cerebral cortex of rats. J Neuropatho Exp Neurol 2004, 63:686-699.

52. Franke $H$, Krugel U, Illes $P$ : $\mathbf{P 2}$ receptors and neuronal injury. Pflugers Arch 2006, 452:622-644.

53. Yiangou Y, Facer P, Durrenberger P, Chessell IP, Naylor A, Bountra $C$, Banati RR, Anand P: COX-2, CB2 and P2X7-immunoreactivities are increased in activated microglial cells/macrophages of multiple sclerosis and amyotrophic lateral sclerosis spinal cord. BMC Neurol 2006, 6:12

54. Le Feuvre RA, Brough D, Touzani O, Rothwell NJ: Role of P2X7 receptors in ischemic and excitotoxic brain injury in vivo. Cereb Blood Flow Metob 2003, 23:381-384.

55. Parvathenani LK, Tertyshnikova S, Greco CR, Roberts SB, Robertson B, Posmantur R: P2X7 Mediates Superoxide Production in Primary Microglia and Is Up-regulated in a Transgenic Mouse Model of Alzheimer's Disease. J Biol Chem 2003. 278: 13309-13317.

56. Rampe $D$, Wang $L$, Ringheim GE: $\mathbf{P} \mathbf{2 X 7}$ receptor modulation of [beta]-amyloid- and LPS-induced cytokine secretion from human macrophages and microglia. Journol of Neuroimmunology 2004, 147:56-61.

57. Vamvakopoulos J, Green C. Metcalfe S: Genetic control of IL I beta bioactivity through differential regulation of the IL-I receptor antagonist. Eur J Immunol 2002, 32:2988-2996.

58. Cabrini G, Falzoni S, Forchap SL, Pellegatti P, Balboni A, Agostini P, Cuneo A, Castoldi G, Baricordi OR, Di Virgilio F: A His-155 to Tyr Polymorphism Confers Gain-of-Function to the Human P2X7 Receptor of Human Leukemic Lymphocytes. J Immunol 2005, 175:82-89.
59. Ferrari D, Pizzirani C, Adinolfi E, Lemoli RM, Curti A, Idzko M, Panther E, Di Virgilio F: The P2X7 Receptor: A Key Player in IL-1 Processing and Release. J Immunol 2006, 176:3877-3883.

60. Gu BJ, Zhang W, Worthington RA, Sluyter R, Dao-Ung P, Petrou S, Barden JA, Wiley JS: A Glu-496 to Ala Polymorphism Leads to Loss of Function of the Human P2X7 Receptor. J Biol Chem 2001, 276:111135-11142

61. Sluyter R, Shemon AN, Wiley JS: Glu496 to Ala Polymorphism in the P2X7 Receptor Impairs ATP-Induced IL-I \{beta\} Release from Human Monocytes. J Immunol 2004, 1 72:3399-3405.

62. Denlinger LC, Angelini G, Schell K, Green DN, Guadarrama AG, Prabhu U, Coursin DB, Bertics PJ, Hogan K: Detection of Human P2X7 Nucleotide Receptor Polymorphisms by a Novel Monocyte Pore Assay Predictive of Alterations in Lipopolysaccharide-Induced Cytokine Production. J Immunol 2005, 174:4424-4431.

63. Haag F, Freese D, Scheublein F, Ohlrogge W, Adriouch S, Seman M, Koch-Nolte $F$ : $T$ cells of different developmental stages differ in sensitivity to apoptosis induced by extracellular NAD. De Immunol 2002, 9:197-202

64. Chen L Brosnan CF: Exacerbation of Experimental Autoim mune Encephalomyelitis in P2X7R-/- Mice: Evidence for Loss of Apoptotic Activity in Lymphocytes. I Immunol 2006, 176:3115-3126.

65. Kawamura H, Aswad F, Minagawa M, Govindarajan S, Dennert G: P2X7 Receptors Regulate NKT Cells in Autoimmune Hepatitis. J Immunol 2006, I 76:2152-2160.

66. Chessell IP, Hatcher JP, Bountra C, Michel AD, Hughes JP, Green P, Egerton J. Murfin M, Richardson J. Peck WL, Grahames CB, Casula MA, Yiangou Y, Birch R, Anand P, Buell GN: Disruption of the P2X7 purinoceptor gene abolishes chronic inflammatory and neuropathic pain. Pain 2005, II 4:386-396.

67. Gu Y, Kuida K, Tsutsui H, Ku G, Hsiao K, Fleming MA, Hayashi N, Higashino K, Okamura H, Nakanishi K, Kurimoto M, Tanimoto T, Flavell RA, Sato V, Harding MW, Livingston DJ, Su MSS: Activation of Interferon-gamma Inducing Factor Mediated by InterleukinI beta Converting Enzyme. Science 1997, 275:206-209.

68. Perregaux DG, McNiff P, Laliberte R, Conklyn M, Gabel CA: ATP Acts as an Agonist to Promote Stimulus-Induced Secretion of IL-I fbeta\} and IL-18 in Human Blood. I Immunol 2000, 165:4615-4623

69. Mehta VB, Hart J. Wewers MD: ATP-stimulated Release of Interleukin (IL)-Ibeta and IL-18 Requires Priming by Lipopolysaccharide and Is Independent of Caspase-I Cleavage. I Biol Chem 2001, 276:3820-3826.

70. Muhl H, Pfeilschifter J: Interleukin-18 bioactivity: a novel target for immunopharmacological anti-inflammatory intervention. Eur / Pharmacol 2004, 500:63-71.

71. Arnett HA, Mason J. Marino M, Suzuki K, Matsushima GK, Ting JPY: TNF[alpha] promotes proliferation of oligodendrocyte progenitors and remyelination. Nat Neurosci 2001, 4:1116-1122.

72. Fontaine V, Mohand-Said S, Hanoteau N, Fuchs C, Pfizenmaier K, Eise $\mathrm{U}$ : Neurodegenerative and neuroprotective effects of tumor Necrosis factor (TNF) in retinal ischemia: opposite roles of TNF receptor I and TNF receptor 2. I Neurosci 2002. 22(7):RC216.

73. Combs CK, Karlo JC, Kao SC, Landreth GE: \{beta\}-Amyloid Stim ulation of Microglia and Monocytes Results in TNF\{alpha\} Dependent Expression of Inducible Nitric Oxide Synthase and Neuronal Apoptosis. J Neurosci 2001, 21:1179-1188.

74. Suzuki T, Hide I, Ido K, Kohsaka S, Inoue K, Nakata Y: Production and Release of Neuroprotective Tumor Necrosis Factor by P2X7 Receptor-Activated Microglia. J Neurosci 2004, 24:1-7.

75. James G, Butt AM: $\mathbf{P 2 Y}$ and $\mathbf{P 2 X}$ purinoceptor mediated $\mathrm{Ca2+}$ signalling in glial cell pathology in the central nervous system. European Journal of Pharmacology 2002, 447:247-260.

76. Honda S, Sasaki Y, Ohsawa K, Imai Y, Nakamura Y, Inoue K, Kohsaka S: Extracellular ATP or ADP Induce Chemotaxis of Cultured Microglia through Gi/o-Coupled P2Y Receptors. J Neurosci 2001, 21:1975-1982.

77. Hide I, Tanaka M, Inoue A, Nakajima K, Kohsaka S, Inoue K, Nakata Y: Extracellular ATP Triggers Tumor Necrosis FactorRelease from Rat Microglia. Journal of Neurochemistry 2000, 75:965-972.

78. Kucher BM, Neary JT: Bi-functional effects of ATP/P2 receptor activation on tumor necrosis factor-alpha release in lipopol- 
ysaccharide-stimulated astrocytes. Journal of Neurochemistry 2005, 92:525-535.

79. Scherbel U, Raghupathi R, Nakamura M, Saatman KE, Trojanowski JQ, Neugebauer E, Marino MW, Mclntosh TK: Differential acute and chronic responses of tumor necrosis factor-deficient mice to experimental brain injury. PNAS 1999, 96:8721-8726.

80. Botsios C: Safety of tumour necrosis factor and interleukin-I blocking agents in rheumatic diseases. Autoimmunity Reviews 2005, 4: $162-170$.

81. Tran CN, Lundy SK, Fox DA: Synovial biology and $T$ cells in rheumatoid arthritis. Pathophysiology 2005, 12:183-189.

82. Ryan LM, Rachow JW, McCarty DJ: Synovial fluid ATP: a potential substrate for the production of inorganic pyrophosphate. J Rheumatol 1991, 18:716-720.

83. Solini A, Chiozzi P, Morelli A, Fellin R, Di Virgilio F: Human primary fibroblasts in vitro express a purinergic $\mathbf{P} 2 \times 7$ receptor coupled to ion fluxes, microvesicle formation and IL-6 release. I Cell Sci 1999, I I 2:297-305.

84. Beigi R, Kobatake E, Aizawa M, Dubyak GR: Detection of local ATP release from activated platelets using cell surfaceattached firefly luciferase. Am J Physiol 1999, 276(I Pt 1):C267-C278.

85. Solini A, Chiozzi P, Morelli A, Adinolfi E, Rizzo R, Baricordi OR, Di Virgilio F: Enhanced P2X7 Activity in Human Fibroblasts From Diabetic Patients: A Possible Pathogenetic Mechanism for Vascular Damage in Diabetes. Arterioscler Thromb Vasc Biol 2004, 24:1240-1245.

86. Gordon JL: Extracellular ATP: effects, sources and fate. Biochem J 1986, 233:309-319.

87. Bulanova E, Budagian V, Orinska Z, Hein M, Petersen F, Thon L, Adam D, Bulfone-Paus S: Extracellular ATP Induces Cytokine Expression and Apoptosis through P2X7 Receptor in Murine Mast Cells. J Immunol 2005, 174:3880-3890.

88. Gourine AV, Poputnikov DM, Zhernosek N, Melenchuk EV, Gerstberger R, Spyer KM, Gourine VN: P2 receptor blockade attenuates fever and cytokine responses induced by lipopolysaccharide in rats. 2005, 146:139-145.

89. Kluger MJ: Fever: role of pyrogens and cryogens. Physiol Rev 1991, 71:93-127.

90. Kasama $T$, Miwa $Y$, Isozaki $T$, Odai $T$, Adachi $M$, Kunkel SL: Neutrophil-derived cytokines: potential therapeutic targets in inflammation. Curr Drug Targets Inflamm Allergy 2005, 4:273-279.

91. Walker A, Ward C, Taylor EL, Dransfield I, Hart SP, Haslett C, Rossi AG: Regulation of neutrophil apoptosis and removal of apoptotic cells. Curr Drug Targets Inflamm Allergy 2005, 4:447-454.

92. Gompertz S, Stockley RA: Inflammation--role of the neutrophil and the eosinophil. Semin Respir Infect 2000, 15:14-23.

93. Kerr JF, Wyllie AH, Currie AR: Apoptosis: a basic biological phenomenon with wide-ranging implications in tissue kinetics. Br J Cancer 1972, 26:239-257.

94. Fadok VA, Bratton DL, Konowal A, Freed PW, Westcott JY, Henson PM: Macrophages That Have Ingested Apoptotic Cells In Vitro Inhibit Proinflammatory Cytokine Production Through Autocrine/Paracrine Mechanisms Involving TGFbeta , PGE2, and PAF. J Clin Invest 1998, 101:890-898.

95. Savill J. Dransfield I, Gregory C, Haslett C: A blast from the past: clearance of apoptic cells regulates immune responses. Nat Rev Immunol 2002, 2:965-975.

96. Sprick MR, Walczak $\mathrm{H}$ : The interplay between the $\mathbf{B c l - 2}$ family and death receptor-mediated apoptosis. Biochim Biophys Acto 2004, 1 644:125-132.

97. Michels J, Johnson PW, Packham G: Mcl-I. Int J Biochem Cell Biol 2005, 37:267-271.

98. Kerr LE, McGregor AL, Amet LE, Asada T, Spratt C, Allsopp TE, Harmar AJ, Shen S, Carlson G, Logan N, Kelly JS, Sharkey J: Mice overexpressing human caspase 3 appear phenotypically normal but exhibit increased apoptosis and larger lesion volumes in response to transient focal cerebral ischaemia. Cell Death Differ 2004, II:1 102-1111.

99. Young JW, Kerr LE, Kelly JS, Marston HM, Spratt C, Finlayson K, Sharkey J: The odour span task: A novel paradigm for assessing working memory in mice. Neuropharmacology 2007, 52(2):634-645.

100. Suh BC, Kim JS, Namgung U, Ha H, Kim KT: P2X7 Nucleotide Receptor Mediation of Membrane Pore Formation and
Superoxide Generation in Human Promyelocytes and Neutrophils. J Immunol 200I, 1 66:6754-6763.

101. Gu BJ. Zhang WY, Bendall LI, Chessell IP, Buell GN, Wiley IS: Expression of $\mathbf{P} 2 \times 7$ purinoceptors on human lymphocytes and monocytes: evidence for nonfunctional $P 2 X 7$ receptors. Am J Physiol Cell Physiol 2000, 279:CI I89-CII 97.

102. Bulanova E, Budagian V, Orinska Z, Hein M, Petersen F, Thon L, Adam $D$, Bulfone-Paus S: Extracellular ATP induces cytokine expression and apoptosis through $\mathbf{P} 2 \times 7$ receptor in murine mast cells. J Immunol 2005, 174:3880-3890.

103. Wang Q, Wang L, Feng YH, Li X, Zeng R, Gorodeski GI: P2X7 receptor-mediated apoptosis of human cervical epithelial cells. Am J Physiol Cell Physiol 2004, 287:CI349-CI358.

104. Cook SP, McCleskey EW: Cell damage excites nociceptors through release of cytosolic ATP. Pain 2002, 95:41-47.

105. Lau YE, Bowdish DM, Cosseau C, Hancock RE, Davidson DJ: Apoptosis of airway epithelial cells: human serum sensitive induction by the cathelicidin LL-37. Am J Respir Cell Mol Biol 2006. 34:399-409.

106. Ferrari D, Idzko M, Dichmann S, Purlis D, Virchowjr. C, Norgauer J, Chiozzi P, Di Virgilio F, Luttmann W: P2 purinergic receptors of human eosinophils: characterization and coupling to oxygen radical production. FEBS Letters 2000, 486:217-224.

107. Mohanty JG, Raible DG, McDermott LJ. Pelleg A, Schulman ES Effects of purine and pyrimidine nucleotides on intracellular $\mathrm{Ca2}+$ in human eosinophils: Activation of purinergic P2Y receptors. J Allergy Clin Immunol 200I, 107(5):849-855.

108. Idzko M, Panther E, Bremer HC, Sorichter S, Luttmann W, Virchow CJ). Di Virgilio F, Herouy Y, Norgauer J, Ferrari D: Stimulation of P2 purinergic receptors induces the release of eosinophil cationic protein and interleukin-8 from human eosinophils. 2003, 138: 1244-1250.

109. Yousefi S, Hemmann S, Weber M, Holzer C, Hartung K, Blaser K, Simon HU: IL-8 is expressed by human peripheral blood eosinophils. Evidence for increased secretion in asthma. J Immuno 1995, 154:5481-5490.

110. Teran LM, Carroll MP, Frew AJ, Redington AE, Davies DE, Lindley I, Howarth PH, Church MK, Holgate ST: Leukocyte recruitment after local endobronchial allergen challenge in asthma. Relationship to procedure and to airway interleukin-8 release. Am J Respir Crit Care Med 1996, 154:469-476.

1II. Campbell Jj, Qin S, Unutmaz D, Soler D, Murphy KE, Hodge MR, Wu L, Butcher EC: Unique Subpopulations of CD56+ NK and NKT Peripheral Blood Lymphocytes Identified by Chemokine Receptor Expression Repertoire. I Immunol 200I, 166:6477-6482.

1 12. Ferrari D, la Sala A, Panther E, Norgauer J, Di Virgilio F, Idzko M: Activation of human eosinophils via $P 2$ receptors: novel findings and future perspectives. I Leukoc Biol 2006, 79:7-15.

1 13. Okamoto H, Mizuno K, Horio T: Monocyte-derived multinucleated giant cells and sarcoidosis. Journal of Dermatological Science 2003, 31:119-128.

114. Molloy A, Laochumroonvorapong P, Kaplan G: Apoptosis, but not necrosis, of infected monocytes is coupled with killing of intracellular bacillus Calmette-Guerin. I Exp Med 1994, 180:1499-1509.

115. Most J, Spotl L, Mayr G, Gasser A, Sarti A, Dierich MP: Formation of Multinucleated Giant Cells In Vitro Is Dependent on the Stage of Monocyte to Macrophage Maturation. Blood 1997. 89:662-671.

116. Chiozzi P, Sanz JM, Ferrari D, Falzoni S, Aleotti A, Buell GN, Collo G, Virgilio FD: Spontaneous Cell Fusion in Macrophage Cultures Expressing High Levels of the P2Z/P2X7 Receptor. J Cell Bio 1997, 138:697-706.

117. Falzoni S, Chiozzi P, Ferrari D, Buell G, Di Virgilio F: P2X7 Receptor and Polykarion Formation. Mol Biol Cell 2000, II:3169-3176.

118. Namazi MR: Cetirizine and allopurinol as novel weapons against cellular autoimmune disorders. Int Immunopharmacol 2004, 4:349-353.

119. Mizuno K, Okamoto H, Horio T: Inhibitory influences of xanthine oxidase inhibitor and angiotensin l-converting enzyme inhibitor on multinucleated giant cell formation from monocytes by downregulation of adhesion molecules and purinergic receptors. British Journal of Dermatology 2004, 150:205-210.

120. Lemaire I, Falzoni S, Leduc N, Zhang B, Pellegatti P, Adinolfi E, Chiozzi P. Di Virgilio F: Involvement of the Purinergic P2X7 Receptor 
in the Formation of Multinucleated Giant Cells. I Immunol 2006, 177:7257-7265.

121. DeAngelis CD, Flanagin A: Tuberculosis--a global problem requiring a global solution. JAMA 2005, 293:2793-2794.

122. Flynn JAL, Chan J: Immunology of tuberculosis. Annual Review of Immunology 2001, 19:93-129.

123. Flynn JAL: Immunology of tuberculosis and implications in vaccine development. Tuberculosis 2004, 84:93-101.

124. Keane J, Balcewicz-Sablinska MK, Remold HG, Chupp GL, Meek BB, Fenton MJ. Kornfeld H: Infection by Mycobacterium tuberculosis promotes human alveolar macrophage apoptosis. Infect Immun 1997, 65:298-304.

125. Keane J, Remold HG, Kornfeld H: Virulent Mycobacterium tuberculosis Strains Evade Apoptosis of Infected Alveolar Macrophages. J Immunol 2000, 164:2016-2020.

126. Balcewicz-Sablinska MK, Keane J, Kornfeld H, Remold HG: Pathogenic Mycobacterium tuberculosis Evades Apoptosis of Host Macrophages by Release of TNF-R2, Resulting in Inactivation of TNF-\{alpha\}. J Immunol 1998, 161:2636-2641.

127. Lammas DA, Stober C, Harvey CJ, Kendrick N, Panchalingam S, Kumararatne DS: ATP-Induced Killing of Mycobacteria by Human Macrophages Is Mediated by Purinergic P2Z(P2X7) Receptors. Immunity 1997, 7:433-444.

128. Fairbairn IP, Stober CB, Kumararatne DS, Lammas DA: ATP-Mediated Killing of Intracellular Mycobacteria by Macrophages Is a P2X7-Dependent Process Inducing Bacterial Death by Phagosome-Lysosome Fusion. J Immunol 200I, 167:3300-3307.

129. Sikora A, Liu J, Brosnan C, Buell G, Chessel I, Bloom BR: Cutting Edge: Purinergic Signaling Regulates Radical-Mediated Bacterial Killing Mechanisms in Macrophages Through a P2X7. Independent Mechanism. J Immunol 1999, 163:558-561.

130. Kusner DJ, Adams J: ATP-Induced Killing of Virulent Mycobacterium tuberculosis Within Human Macrophages Requires Phospholipase D. J Immunol 2000, 164:379-388.

131. Kusner DJ, Barton JA: ATP Stimulates Human Macrophages to Kill Intracellular Virulent Mycobacterium tuberculosis Via Calcium-Dependent Phagosome-Lysosome Fusion. J Immunol 2001, 167:3308-3315.

132. Gan H, He X, Duan L, Mirabile-Levens E, Kornfeld H, Remold HG: Enhancement of antimycobacterial activity of macrophages by stabilization of inner mitochondrial membrane potential. J Infect Dis 2005, 191:1292-1300.

133. Li CM, Campbell SJ, Kumararatne DS, Bellamy R, Ruwende C, McAdam KP. Hill AV, Lammas DA: Association of a polymorphism in the $P 2 X 7$ gene with tuberculosis in a Gambian population. J Infect Dis 2002, 186:1458-1462.

134. Saunders BM, Fernando SL, Sluyter R, Britton WJ, Wiley JS: A Lossof-Function Polymorphism in the Human P2X7 Receptor Abolishes ATP-Mediated Killing of Mycobacteria. J Immunol 2003, 171:5442-5446.

135. Fernando SL, Saunders BM, Sluyter R, Skarratt KK, Wiley JS, Britton $W]$ : Gene dosage determines the negative effects of polymorphic alleles of the $P 2 X 7$ receptor on adenosine triphosphate-mediated killing of mycobacteria by human macrophages. J Infect Dis 2005, 192:149-155.

136. Shemon AN, Sluyter R, Fernando SL, Clarke AL, Dao-Ung LP, Skarratt KK, Saunders BM, Tan KS, Gu BJ. Fuller SJ, Britton W]. Petrou S, Wiley JS: A Thr357 to Ser Polymorphism in Homozygous and Compound Heterozygous Subjects Causes Absent or Reduced P2X7 Function and Impairs ATP-induced Mycobacterial Killing by Macrophages. J Biol Chem 2006, 281:2079-2086.

137. Pearce JM: Rudolf Ludwig Karl Virchow (I821-1902). J Neurol 2002, 249:492-493.

138. Marx ]: CANCER RESEARCH: Inflammation and Cancer: The Link Grows Stronger. Science 2004, 306:966-968.

139. Oh BR, Sasaki M, Perinchery G, Ryu SB, Park YI, Carroll P, Dahiya R: Frequent genotype changes at -308 , and 488 regions of the tumor necrosis factor-alpha (TNF-alpha) gene in patients with prostate cancer. J Urol 2000, 163:1584-1587.

140. El Omar EM, Carrington M. Chow WH, McColl KEL, Bream JH, Young HA, Herrera J, Lissowska J, Yuan CC, Rothman N, Lanyon G, Martin M, Fraumeni JF, Rabkin CS: Interleukin-I polymorphisms associated with increased risk of gastric cancer. Nature 2000. 404:398-402

141. El OmarEmad M, CarringtonMary, ChowWong H, McCollKenneth EL, BreamJay $\mathrm{H}$, YoungHoward A, Herrerajesus, Lissowskajolanta,
YuanChiu C, RothmanNathaniel, LanyonGeorge, MartinMaureen, Fraumeniloseph F, RabkinCharles S: correction: The role of interleukin-I polymorphisms in the pathogenesis of gastric cancer. Nature 2001, 41 2:99-99.

142. Baricordi OR, Ferrari D, Melchiorri L, Chiozzi P, Hanau S, Chiari E, Rubini M, Di Virgilio F: An ATP-activated channel is involved in mitogenic stimulation of human T lymphocytes. Blood 1996, 87:682-690.

143. Baricordi OR, Melchiorri L, Adinolfi E, Falzoni S, Chiozzi P, Buell G, Di Virgilio F: Increased Proliferation Rate of Lymphoid Cells Transfected with the P2X7 ATP Receptor. J Biol Chem 1999, 274:33206-33208.

144. Hanahan D, Weinberg RA: The Hallmarks of Cancer. Cell 2000, 100:57-70.

145. Adinolfi E, Callegari MG, Ferrari D, Bolognesi C, Minelli M, Wieckowski MR, Pinton P, Rizzuto R, Di Virgilio F: Basal Activation of the P2X7 ATP Receptor Elevates Mitochondrial Calcium and Potential, Increases Cellular ATP Levels, and Promotes Serum-independent Growth. Mol Biol Cell 2005, 16:3260-3272.

146. Bernardi P, Petronilli V, Di Lisa F, Forte M: A mitochondrial perspective on cell death. Trends in Biochemical Sciences 2001, 26:112-117.

147. Wiley JS, Dao-Ung LP, Gu BJ. Sluyter R, Shemon AN, Li C, Taper J, Gallo J, Manoharan A: A loss-of-function polymorphic mutation in the cytolytic $\mathbf{P 2 X 7}$ receptor gene and chronic lymphocytic leukaemia: a molecular study. Lancet 2002 359(93/2): $1114-1119$.

148. Sellick GS, Rudd M, Eve P, Allinson R, Matutes E, Catovsky D, Houlston RS: The P2X7 Receptor Gene A1513C Polymorphism Does Not Contribute to Risk of Familial or Sporadic Chronic Lymphocytic Leukemia. Cancer Epidemiol Biomarkers Prev 2004 13:1065-1067.

149. Thunberg U, Tobin G, Johnson A, Soderberg O, Padyukov L, Hultdin M, Klareskog L, Enblad G, Sundstrom C, Roos G, Rosenquist R: Polymorphism in the $P 2 \times 7$ receptor gene and survival in chronic lymphocytic leukaemia. Lancet 2002. 360(9349): 1935-1939.

150. Carta S, Tassi S, Semino C, Fossati G, Mascagni P, Dinarello CA Rubartelli A: Histone deacetylase inhibitors prevent exocytosis of interleukin-I \{beta\}-containing secretory lysosomes: role of microtubules. Blood 2006.
Publish with Bio Med Central and every scientist can read your work free of charge

"BioMed Central will be the most significant development for disseminating the results of biomedical research in our lifetime. "

Sir Paul Nurse, Cancer Research UK

Your research papers will be:

- available free of charge to the entire biomedical community

- peer reviewed and published immediately upon acceptance

- cited in PubMed and archived on PubMed Central

- yours - you keep the copyright
BioMedcentral 


\title{
Modulation of granulocyte apoptosis can influence the resolution of inflammation
}

\author{
A.G. Rossi*1, J.M. Hallett ${ }^{*}$, D.A. Sawatzky*, M.M. Teixeiraf and C. Haslett* \\ "MRC Centre for Inflammation Research, Queen's Medical Research Institute, University of Edinburgh Medical School, 47 Little France Crescent, Edinburgh \\ EH16 4TJ, U.K., and †Immunopharmacology, Departamento de Bioquímica e Imunologia, ICB, Universidade Federal de Minas Gerais, Belo Horizonte, Minas \\ Gerais, Brazil
}

\begin{abstract}
Apoptosis of granulocytes and the subsequent clearance of apoptotic cells are important processes for the successful resolution of inflammation. Signalling pathways, including those involving NF- $\kappa$ B (nuclear factor $\kappa B$ ), MAPK (mitogen-activated protein kinase) and PI3K (phosphoinositide 3-kinase) have been shown to be key regulators of inflammatory cell survival and apoptosis in vitro. In addition, manipulation of such pathways in vivo has indicated that they also play a role in the resolution of inflammation. Furthermore, manipulation of proteins directly involved in the control of apoptosis, such as Bcl-2 family members and caspases, can be targeted in vivo to influence inflammatory resolution. Recently, it has been shown that CDK (cyclin-dependent kinase) inhibitor drugs induce caspase-dependent human neutrophil apoptosis possibly by altering levels of the anti-apoptotic $\mathrm{BCl}-2$ family member, $\mathrm{MCl}-1$. Importantly, $\mathrm{CDK}$ inhibitor drugs augment the resolution of established 'neutrophil-dominant' inflammation by promoting apoptosis of neutrophils. Thus manipulation of apoptotic pathways, together with ensuring macrophage clearance of apoptotic cells, appears to be a viable pharmacological target for reducing established inflammation.
\end{abstract}

\section{Introduction}

Granulocytic neutrophils and eosinophils play a prominent role in defence against invading organisms and are likely to contribute to tissue destruction in many inflammatory diseases. Apoptosis of granulocytes and their subsequent clearance by phagocytes [1,2] have been suggested to be key processes that are involved in the resolution of beneficial inflammation and their dysregulation probably contributes to inflammatory disease pathogenesis and progression [3-5]. For example, delayed apoptosis resulting in enhanced functional activity of granulocytes and/or failed clearance of apoptotic cells will result in further pro-inflammatory responses and augmented tissue damage associated with diseases such as rheumatoid arthritis and chronic obstructive pulmonary disease $[3,4,6]$. Like macrophage clearance [6-13], apoptosis of granulocytes is highly regulated, with many diverse stimuli controlling rates of cell longevity [3,14]. There is increasing evidence that apoptosis-modulating pharmacological agents administered in vivo, which have the capacity to promote apoptosis of inflammatory cells, enhance the resolution of established inflammation $[15,16]$. The converse has also been demonstrated, whereby preventing apoptosis using pharmacological inhibitors in vivo results in delayed resolution of inflammation $[15,16]$. Thus modulation of pathways asso-

Key words: apoptosis, eosinophil, inflammation, neutrophil, resolution of inflarnmatory response. Abbreviations used: COK, cyclin-dependent kinase; ERK, extracellular-signal-regulated kinase; GM-CSF, granulocyle/macrophage colony-slimulating factor; $1 \kappa B$, inhibitory $\kappa B$; IKK, $\mid<B$ kinase, IL, interleukin; JNK, C-Jun N-terminal kinase; LPS, lipopolysaccharide; MAPK, mitogen-activated protein kinase; NEMO, nuclear factor $\kappa B$ essential modifier; NF- $\kappa$ B, nuclear factor $\kappa B$; PIIK, phosphoinositide 3-kinase; $\operatorname{TNF} \alpha$, tumour necrosis lactor $\alpha$.

'To whom correspondence should be addressed (email a.g.rossi@ed.ac.uk). ciated with apoptosis (discussed below) provides possible novel therapeutic targets for the treatment of inflammatory diseases.

\section{NF- $\kappa$ B (nuclear factor $\kappa$ B) as a critical regulator of granulocyte apoptosis}

The ubiquitous transcription factor $\mathrm{NF}-\kappa \mathrm{B}$ regulates the synthesis of many pro-inflammatory mediators [e.g. TNF $\alpha$ (tumour necrosis factor $\alpha$ ), IL-8 (interleukin 8)] that are involved in the immune response and its selective inhibition is therefore a major target for pharmacological intervention. However, NF- $\kappa \mathrm{B}$ also controls the expression of many prosurvival products such as $\mathrm{Bcl}-2$ family members [e.g. Bcl- $\mathrm{X}_{\mathrm{L}}$, XIAP (X-linked inhibitor of apoptosis), A1] and activation or inhibition of $\mathrm{NF}-\kappa \mathrm{B}$ has been shown generally to enhance or inhibit survival of inflammatory cells respectively. There is now considerable evidence that $\mathrm{NF}-\kappa \mathrm{B}$ plays an important role in the regulation of granulocyte apoptosis [17]. Much of the evidence is derived from experimental inhibition of $\mathrm{NF}-\kappa \mathrm{B}$ using pharmacological inhibitors. The first demonstration by Ward et al. in 1999 [18] showed that a panel of $\mathrm{NF}-\kappa \mathrm{B}$ inhibitors including the fungal metabolite gliotoxin increased the rate of apoptosis in human neutrophils and eosinophils in a caspase-dependent manner. Importantly, inhibition of NF- $\kappa$ B also augmented dramatically the early pro-apoptotic effects of TNF $\alpha$ in neutrophils and rendered TNF $\alpha$ a powerful inducer of eosinophil apoptosis $[18,19]$. In addition, inhibition of protein synthesis by cycloheximide also augmented TNF $\alpha$-induced neutrophil apoptosis, indicating that NF- $\kappa \mathrm{B}$ controls the synthesis of protein(s) which 
protect granulocytes from the pro-apoptotic effects of TNF $\alpha$ [18]. It was also demonstrated that the pro-resolution cyclopentenone prostaglandins [e.g. 15-dPGJ ${ }_{2}$ (15-deoxy- $\Delta^{12,14}$ prostaglandin $\left.\left.\mathrm{J}_{2}\right)\right][20]$ induced caspase-dependent granulocyte apoptosis via inhibition of NF- $\kappa \mathrm{B}[21]$ by a mechanism that does not involve the peroxisome-proliferator-activated receptor- $\gamma$ [21], but is likely to involve direct inhibition of IKK [ $\mathrm{I} \kappa \mathrm{B}$ (inhibitory $\kappa \mathrm{B}$ ) kinase] [22]. The importance of the NF- $\kappa$ B pathway in the regulation of neutrophil apoptosis was confirmed by delivery of biologically active peptides into granulocytes using HIV-tat tagged protein transduction techniques [23]. Using a tat-NEMO (NF- $\kappa$ B essential modifier)binding domain that selectively inhibits the IKK $\gamma$ (NEMO)IKK $\beta$ interaction in neutrophils [24], and an HIV-tat-I $\kappa \mathrm{B} \alpha$ (S32A, S36A) superrepressor in eosinophils [25] to inhibit $\mathrm{NF}-\kappa \mathrm{B}$ activation augmented apoptosis and suppressed NF$\kappa \mathrm{B}$-mediated survival. Many other studies have reinforced the importance of $\mathrm{NF}-\kappa \mathrm{B}$ in the regulation of granulocyte apoptosis. Although the precise role of NF- $\kappa \mathrm{B}$ in inflammation in vivo is undoubtedly complex and critically dependent on the stage of the inflammatory response $[26,27]$, it is possible that targeting of the NF- $\kappa \mathrm{B}$ pathway in specific inflammatory cells would influence apoptosis and consequent resolution of inflammation. However, owing to the ubiquitous nature of this signalling pathway and the possibility of overwhelming the apoptotic cell clearance capacity, caution must be taken into consideration when adopting this strategy.

\section{The role of MAPK (mitogen-activated protein kinase) pathways in granulocyte apoptosis}

The MAPK family of signalling molecules are involved in cell differentiation, apoptosis, stress responses and inflammation. There are three main MAPK homologues that are released via MAPK kinases \{MEK [MAPK/ERK (extracellular-signalregulated kinase) kinase] 1-7\}: ERK (p42, p44, MAPK), JNKs (c-Jun N-terminal kinases) and p38 MAPK [28]. Given the considerable evidence for a role for the MAPK pathways in many aspects of the inflammatory response (e.g. inflammatory cell recruitment, activation and apoptosis) and, in inflammatory diseases, this pathway is a major target that may be exploited for therapeutic benefit. There is evidence that ERK [29,30], p38 [31-33] and JNK [34] are all involved in the regulation of granulocyte apoptosis. The ERK pathway has been best examined, and it has been shown that neutrophil survival factors such as LPS (lipopolysaccharide) and GM-CSF (granulocyte/macrophage colony-stimulating factor) trigger ERK activation and pharmacological inhibitors can prevent survival induced by these factors $[29,30,35]$. Recently, a role for the ERK pathway in the resolution of a rat carrageenan-induced pleurisy model has been reported whereby the specific ERK1/2 inhibitor PD98059 enhanced the resolution of inflammation. Specifically, PD98059, administered at the peak of inflammation, significantly decreased the total number of macrophages and neutrophils in the pleural cavity, mainly by increasing the rate of neutrophil apoptosis, as measured by annexin V labelling and morphological analysis. Consequently, the inflammatory response in the pleural cavity induced by carrageenan resolved much quicker when ERK was inhibited.

\section{Role of the PI3K (phosphoinositide 3-kinase) pathway in granulocyte apoptosis}

$\mathrm{PI} 3 \mathrm{~K}$ is a family of lipid kinases that is subdivided into three subfamilies according to structure and substrate specificity and has been recognized as an important signalling system that regulates many biological processes, especially leucocyte trafficking and activation [36,37]. PI3K generates the important signalling lipid $\operatorname{PtdIns}(3,4,5) P_{3}$, which in turns leads to the activation of Akt. Use of specific PI3K inhibitors has also implicated PI3K in the regulation of neutrophil and eosinophil longevity and apoptosis [33,38-40], although the precise subfamilies involved requires establishing further investigation. In vivo, PI $3 \mathrm{~K}$ clearly regulates leucocyte accumulation and it is now emerging that PI $3 \mathrm{~K}$ may also play a role in the resolution phase of inflammation by influencing granulocyte apoptosis. For example, Pinho et al. [41] have evaluated the involvement of PI $3 \mathrm{~K}$ in the recruitment and survival of eosinophils in a model of allergic pleurisy in mice. Following induction of allergic pleurisy, it was found that eosinophil accumulation in PI3K $\gamma$-deficient mice was not different at earlier time points ( 6 and $24 \mathrm{~h}$ ) as compared with wild-type mice; however, at a later time point $(48 \mathrm{~h})$, there was a marked reduction in eosinophil numbers. It was shown in experiments of adoptive transfer of bone marrow that it was $\mathrm{PI} 3 \mathrm{~K} \gamma$ present in eosinophils and not in non-bonemarrow-derived cells that was responsible for the eosinophil accumulation. Specific, but non-PI3K subtype-selective, PI3K inhibitors (wortmannin and LY294002) administered systematically before antigen challenge decreased Akt phosphorylation, IL-5 production and eosinophil release from the bone marrow and, consequently, prevented the recruitment of eosinophils. Importantly, local (intrapleural) treatment with the PI3K inhibitors $24 \mathrm{~h}$ after antigen challenge (at the peak of inflammation) enhanced the clearance of accumulated eosinophils, an effect associated with inhibition of Akt phosphorylation and an increased number of apoptotic events (assessed microscopically and by annexin $\mathrm{V}$ binding). This study suggested an important role for PI $3 \mathrm{~K}$ in the accumulation and survival of eosinophils in a model of allergic pleurisy. It appears that $\mathrm{PI} 3 \mathrm{~K} \gamma$ may not play a prominent part in eosinophil recruitment, but is important for maintenance (possibly by influencing eosinophil apoptosis) of eosinophilic inflammation in vivo, whereas other isoforms of PI3K may be responsible for the recruitment process.

\section{Targeting Bcl-2 family members in inflammation resolution}

Bcl-2 family proteins are major regulators of apoptosis signalling pathways and are potential therapeutic targets for 
the many diseases where apoptosis is likely to be dysregulated, including inflammatory disorders $[42,43]$. It has been shown that the expression of Bcl-2 family members (assessed by Western blotting of pleural inflammatory cell lysates) changes dramatically during the resolution phase of a rat carrageenan-induced pleurisy model [16]. Specifically, expression of the anti-apoptotic protein $\mathrm{Bcl}-\mathrm{X}_{\mathrm{L}}$ decreases to almost negligible levels as resolution occurs, whereas expression of pro-apoptotic Bax increases. When Bax was targeted deliberately by locally (intrapleural) administering a specific inhibitor of Bax (V5) at the peak of inflammation (36 h after carrageenan), inflammatory cell numbers were increased ( $48 \mathrm{~h}$ after carrageenan), indicating that, by preventing apoptosis in vivo, resolution of inflammation can be delayed [16]. Thus using manipulation of apoptosis, by interfering with antiapoptotic and pro-apoptotic Bcl-2 family members, it may be possible to influence inflammatory resolution.

\section{A novel mechanism for enhancing the resolution of inflammation}

CDKs (cyclin-dependent kinases) have traditionally been thought of as key regulators of the cell cycle allowing proliferation to progress once activated and complexed with their associated cyclin partners [44]. For this reason, it is thought that inhibition of CDKs by specific CDK inhibitors in cancer cells may limit or prevent tumour progression. Indeed, CDK inhibitor drugs are currently undergoing clinical trials for the treatment for a number of different cancers $[45,46]$. It has become increasingly apparent, with much experimental evidence, that $\mathrm{CDKs}$ also regulate apoptosis $[46,47]$. These findings were usually limited to proliferating cells and it was generally thought that CDKs would have no or little effect on non-proliferating or terminally differentiated cells. However, evidence indicating that CDKs also modulate the rate of apoptosis of terminally differentiated neurons has emerged [48]. In a recent study, we have demonstrated that different CDK inhibitor drugs (i.e. R-roscovitine, NG75 and hymenialdisine) induce time-, concentration- and caspase-dependent apoptosis of isolated (terminally differentiated) human neutrophils [15]. Importantly, the CDK inhibitor drugs overcame the delayed apoptosis induced by powerful survival factors (e.g. GM-CSF, dibutyryl-cAMP and LPS) [15]. Furthermore, possible targets for the inhibitor drugs (e.g. CDK1, CDK2 and CDK5) have been found to be present and functionally active in human neutrophils $[15,49]$. The precise molecular mechanism of action of the CDK inhibitor drug-mediated induction of apoptosis has not been fully elucidated, but may involve a reduction in the expression of the Bcl-2 family member and anti-apoptotic protein, $\mathrm{Mcl}-1$. Importantly, $\mathrm{R}$-roscovitine markedly promoted the resolution of established neutrophil-dependent inflammation in a carrageenan-elicited acute pleurisy; an effect that was blocked by the caspase inhibitor zVAD-fmk (benzyloxycarbonyl-Val-Ala-DL-Asp-fluoromethylketone). These data indicate that $\mathrm{R}$-roscovitine augments resolution by inducing inflammatory cell apoptosis in vivo. Furthermore,
Figure 1| Modulation of inflammatory resolution by influencing granulocyte apoptosis

During a resolving inflammatory response (solid arrow and box), administration of pharmacological agents at the peak of established inflammation that enhance granulocyte apoptosis (e.g. inhibitors of CDK, ERK1/2, PI3K) (dotted arrows and box) or delay apoptosis [e.g. zVAD-fmk, Bax inhibitory peptide (V5)] (dashed arrows and box), resolution can be promoted or inhibited respectively.

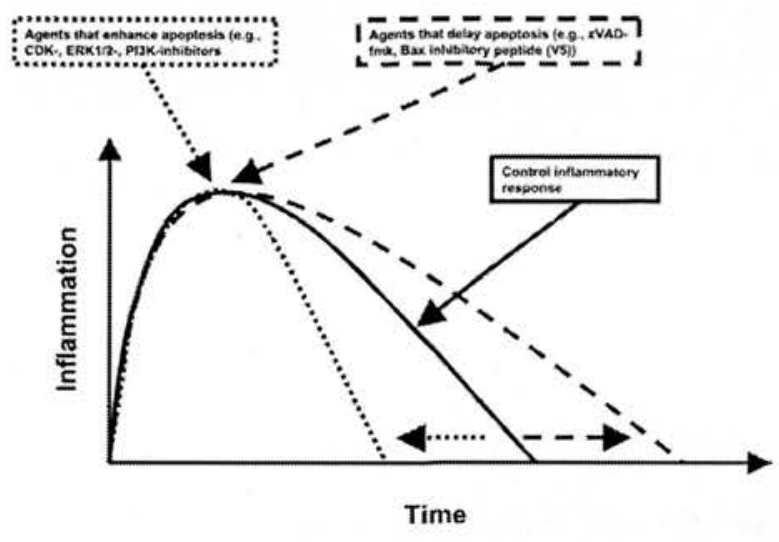

it was demonstrated that R-roscovitine similarly enhanced resolution in two other different experimental models in mice (bleomycin-induced lung injury and passively induced arthritis) [15]. It was therefore concluded that CDK inhibitor drugs enhance the resolution of established neutrophildominant inflammation by promoting inflammatory cell apoptosis, and that these drugs may exhibit a hitherto unrecognized potential for the treatment of inflammatory diseases

\section{Conclusions}

Clearly, the rate of neutrophil or eosinophil apoptosis can be increased or reduced in vitro by a number of agents. There is now good evidence emerging that inhibition or promotion of granulocyte apoptosis by pharmacological agents in vivo can delay or enhance the resolution of acute inflammation respectively (Figure 1). Consequently, it is possible that apoptosis, provided of course that clearance of the apoptotic cells is matched, may be targeted as a strategy for the development of novel anti-inflammatory agents.

\section{References}

1 Savill, J.S., Wyllie, A.H., Henson, J.E., Walport, M.J., Henson, P.M. and Haslett, C. (1989) J. Clin. Invest. 83, 865-875

2 Stern, M., Meagher, L., Savill, J. and Haslett, C. (1992) J. Immunol. 148 , 3543-3549

3 Ward, C., Dransfield, I., Chilvers, E.R., Haslett, C. and Rossi, A.G. (1999) Trends Pharmacol. Sci. 20, 503-509

4 Gilroy, D.W., Lawrence, T., Perretti, M. and Rossi, A.G. (2004) Nat. Rev. Drug Discovery 3, 401-416

5 Serhan, C.N., Brain, S.D., Buckley, C.D., Gilroy, D.W., Haslett, C., O'Neill, L.A., Perretti, M., Rossi, A.G. and Wallace, J.L. (2007) FASEB I. 21, 325-332 6 Serhan, C.N. and Savill, J. (2005) Nat, Immunol. 6, 1191-1197 
7 Hart, S.P., Dougherty, G.J., Haslett, C. and Dransfield, I. (1997) J. Immunol. 159, 919-925

8 Rossi, A.G., McCutcheon, J.C., Roy, N., Chilvers, E.R., Haslett, C. and Dransfield, I. (1998) J. Immunol. 160, 3562-3568

9 Liu, Y., Cousin, J.M., Hughes, J., Van Damme, J., Seckl, J.R., Haslett, C., Dransfield, I., Savill, J. and Rossi, A.G. (1999) J. Immunol. 162, 3639-3646

10 Godson, C., Mitchell, S., Harvey, K., Petasis, N.A., Hogg, N. and Brady, H.R. (2000) J. Immunol. 164, 1663-1667

11 Kirkham, P.A., Spooner, G., Rahman, I. and Rossi, A.G. (2004) Biochem. Biophys. Res. Commun. 318, 32-37

12 Hart, S.P., Alexander, K.M. and Dransfield, I. (2004) J. Immunol. 172 1882-1887

13 Maderna, P., Yona, S., Perretti, M. and Godson, C. (2005) J. Immunol. 174, 3727-3733

14 Riley, N.A., Ward, C., Sawatzky, D.A., Sheldrake, T.A., Dransfield, I., Haslett, C. and Rossi, A.G. (2006) Anti-Inflammatory Anti-Allergy Agents Med. Chem. 5, 3-12

15 Rossi, A.G., Sawatzky, D.A., Walker, A., Ward, C., Sheldrake, T.A., Riley, N.A., Caldicott, A., Martinez-Losa, M., Walker, T.R., Duffin, R. et al. (2006) Nat. Med. 12, 1056-1064

16 Sawatzky, D.A., Willoughby, D.A., Colville-Nash, P.R. and Rossi, A.G. (2006) Am. J. Pathol, 168, 33-41

17 Ward, C., Walker, A., Dransfield, I., Haslett, C. and Rossi, A.G. (2004) Biochem. Soc. Trans. 32, 465-467

18 Ward, C., Chilvers, E.R., Lawson, M.F., Pryde, J.G., Fujihara, S., Farrow, S.N., Haslett, C. and Rossi, A.G. (1999) J. Biol. Chem. 274, 4309-4318

19 Fujihara, S., Ward, C., Dransfield, I., Hay, R.T., Uings, I.j., Hayes, B., Farrow, S.N., Haslett, C. and Rossi, A.G. (2002) Eur. J. Immunol. 32, 457-466

20 Gilroy, D.W., Colville-Nash, P.R., McMaster, S., Sawatzky, D.A Willoughby, D.A. and Lawrence, I. (2003) FASEB J. 17, 2269-2271

21 Ward, C., Dransfield, I., Murray, J., Farrow, S.N., Haslett, C. and Rossi, A.G. (2002) J. Immunol. 168, 6232-6243

22 Rossi, A., Kapahi, P., Natoli, G., Takahashi, I., Chen, Y., Karin, M. and Santoro, M.G. (2000) Nature 403, 103-108

23 Rossi, A.G., Ward, C. and Dransfield, I. (2004) Biochem. Soc. Irans. 32 452-455

24 Choi, M., Rolle, S., Wellner, M., Cardoso, M.C., Scheidereit, C., Luft, F.C. and Kettritz, R. (2003) Blood 102, 2259-2267

25 Fujihara, S., Jaffray, E., Farrow, S.N., Rossi, A.G., Haslett, C. and Hay, R.T. (2005) Biochem. Biophys. Res. Commun. 326, 632-637

26 Gilroy, D.W. and Colville-Nash, P.R. (2000) J. Mol. Med. 78, 121-129

27 Lawrence, T., Gilroy, D.W., Colville-Nash, P.R. and Willoughby, D.A. (2001) Nat. Med. 7, 1291-1297
28 Kaminska, B. (2005) Biochim. Biophys. Acta 1754, 253-262

29 Downey, G.P., Butler, J.R., Tapper, H., Fialkow, L., Saltiel, A.R., Rubin, B.B. and Grinstein, S. (1998) J. Immunol. 160, 434-443

30 Nolan, B., Duffy, A., Paquin, L., De, M., Collette, H., Graziano, C.M. and Bankey, P. (1999) Surgery 126, 406-412

31 Frasch, S.C. Nick, J.A., Fadok, V.A., Bratton, D.L., Worthen, G.S. and Henson, P.M. (1998) J. Biol. Chem. 273, 8389-8397

32 Sheth, K., Friel, J., Nolan, B. and Bankey, P. (2001) Surgery 130 , 242-248

33 Arruda, M.A., Rossi, A.G., de Freitas, M.S., Barja-Fidalgo, C. and Graca-Souza, A.V. (2004) J. Immunol. 173, 2023-2030

34 Avdi, N.J., Nick, J.A., Whitlock, B.B., Billstrom, M.A., Henson, P.M., Johnson, G.L. and Worthen, G.S. (2001) J. Biol. Chem. 276, 2189-2199

35 Ward, C., Murray, J., Clugston, A., Dransfield, I., Haslett, C. and Rossi, A.G. (2005) Eur. J. Immunol. 35, 2728-2737

36 Sotsios, Y. and Ward, S.G. (2000) Immunol, Rev. 177, 217-235

37 Ward, S.G. (2006) Trends Immunol. 27, 80-87

38 Martin, M.C., Dransfield, I., Haslett, C. and Rossi, A.G. (2001) J. Biol. Chem. 276, 45041-45050

39 Cowburn, A.S., Cadwallader, K.A., Reed, B.J., Farahi, N. and Chilvers, E.R. (2002) Blood 100, 2607-2616

40 Derouet, M., Thomas, L., Cross, A., Moots, R.J. and Edwards, S.W. (2004) J. Biol. Chem. 279, 26915-26921

41 Pinho, V., Souza, D.G., Barsante, M.M., Hamer, F.P., de Freitas, M.S., Rossi, A.G. and Teixeira, M.M. (2005) J. Leukocyte Biul. 77, 800-810

42 Droin, N.M. and Green, D.R. (2004) Biochim. Biophys. Acta 1644, 179-188

43 Sorenson, C.M. (2004) Biochim. Biophys. Acta 1644, 169-177

44 Vermeulen, K., Van Bockstaele, D.R. and Berneman, Z.N. (2003) Cell Prolif. 36, 131-149

45 Knockaert, M., Greengard, P. and Meijer, L. (2002) Trends Pharmacol. Sci. 23, 417-425

46 Fischer, P.M., Endicott, J. and Meijer, L. (2003) Prog. Cell Cycle Res. 5 , 235-248

47 Vermeulen, K., Berneman, Z.N. and Van Bockstaele, O.R. (2003) Cell Prolif. 36, 165-175

48 Monaco, III, E.A. and Vallano, M.L. (2003) Curr. Med. Chem. 10, 367-379

49 Rosales, J.L., Ernst, J.D., Hallows, J. and Lee, K.Y. (2004) J. Biol. Chem. 279, 53932-53936

Received 9 January 2007 


\title{
Clearance of dying cells and autoimmunity
}

\author{
SYLWIA MICHLEWSKA ${ }^{1}$, AISLEEN MCCOLL ${ }^{1}$, ADRIANO G ROSSI $^{1}$, IAN L MEGSON ${ }^{2}$, \& \\ IAN DRANSFIELD ${ }^{1}$
}

${ }^{1}$ The Queen's Medical Research Institute, MRC Centre for Inflammation Research, University of Edinburgh Medical School, 47 Little France Crescent, Edinburgh EH16 4TF, Scotland, UK, and ${ }^{2}$ UHI Millennium Institute, The Green House, Beechwood Business Park North, Inverness IV2 3BL, Scotland, UK

\begin{abstract}
Phagocytic clearance of apoptotic cells is an important physiologic homeostatic mechanism that is associated with noninflammatory or anti-inflammatory sequalae. Disruption of the process of apoptotic cell clearance may contribute to development of a number of inflammatory and autoimmune diseases. In this review, we summarize the molecular pathways that have been suggested to account for phagocytic clearance of apoptotic cells. We discuss potential mechanisms for regulation of phagocytosis and the implications for development of autoimmunity.
\end{abstract}

Keywords: Phagocytosis, apoptosis, macrophage, inflammation, autoimmunity

\section{Introduction}

Apoptosis, in contrast to necrosis (cell disintegration), does not provoke pro-inflammatory responses and plays a fundamental role in almost all physiological processes [1,2]. Apoptotic cells are characterised by rapid shrinkage of the cytoplasm, nuclear coalescence, membrane budding and formation of one or more apoptotic bodies. Importantly, membrane integrity during apoptosis is maintained preventing liberation of intracellular histotoxic contents thereby limiting the potential for propagation and exaggeration of inflammatory processes. To avoid cell disintegration (secondary necrosis), apoptotic cells must be efficiently and rapidly removed by macrophages [3], dendritic cells (DC) [4] or in certain circumstances other cell types such as endothelial cells [5], vascular smooth muscle cells [6], and fibroblasts [7]. Moreover, clearance of apoptotic cells results in diminished pro-inflammatory mediator release and an augmented secretion of anti-inflammatory cytokines TGF- $\beta$, IL$10,[8]$, whereas uptake of necrotic cells including secondarily necrotic cells derived from nonengulfed apoptotic cells, stimulates release of pro-inflammatory mediators (TNF $\alpha, \mathrm{IL}-1 \beta$ and $\mathrm{NO}$ ) [9]. It is now widely accepted that failed clearance of apoptotic cells and consequent secondary necrosis may lead to the development of diseases that have an autoimmune component such as systemic lupus erythematosus (SLE) [10], type II diabetes [11], cystic fibrosis [12] and chronic obstructive pulmonary disease (COPD) [13].

\section{Recognition of apoptotic cells by phagocytes}

Removal of apoptotic cells usually involves three central elements: (1) attraction of phagocytes via soluble "find me" signals, (2) recognition and phagocytosis via displayed "eat me" and absence of "don't eat me" signals, and (3) altered production of pro- and anti-inflammatory cytokines. In higher organisms secretion of specialised chemotactic signals by apoptotic prey appears to be an important factor in recruiting phagocytic populations and preventing secondary necrosis. Although "find me" signals are so far poorly characterised, recent studies demonstrated that apoptotic bodies secrete the phospholipid lysophosphatidylcholine (LPC) attracting phagocytic cells to apoptotic cells in a caspase-3-dependent 
fashion [14]. Other examples of recruitment signals include S19 (ribosomal protein dimer) [15], split human tyrosyl-tRNA synthetase [16] and thrombospondin-1 (TSP-1) [17]. Recognition and engulfment of apoptotic particles involves multiple ligandreceptor interactions including "eat me" signals (molecules enabling recognition of apoptotic cells by phagocytes), bridging molecules and phagocytic receptors. One of the most extensively studied "eat me" signal is loss of phospholipid asymmetry and translocation of phosphatidylserine (PS) to the outer leaflet of the plasma membrane during apoptosis [18]. Inhibition of the flippase that normally confines PS to the inner leaflet together with activation of a bidirectional non-specific phospholipid scramblase may lead to loss of lipid asymmetry [19]. In addition the ATP binding cassette transporter ABC1 has also been reported to have a role in phospholipid redistribution [20]. Masking of external PS on the apoptotic cell surface with annexin $\mathrm{V}$ usually blocks phagocytosis [21]. However, some viable cells, such as CD45RB low T lymphocytes [22], neutrophils in Barth syndrome [23] or activated B cells [24] also expose PS on the outer leaflet of the cell membrane and these cells fail to be ingested by either amateur or professional phagocytes.

Attempts to identify a phagocyte receptor that recognises PS using phage display and monoclonal antibody 217 (mAb 217) that inhibited binding of PS liposomes and engulfment of apoptotic cells by macrophages lead to cloning of this putative phosphatidylserine receptor (PSR) [25]. Although interaction of PS and this putative PSR promoted engulfment of apoptotic targets by phagocytes [26] Bose et al. showed that the ablation of putative PSR function resulted in developmental abnormalities during embryogenesis but failed to affect macrophage capacity to remove apoptotic cells both in vitro and in vivo. Furthermore, no differences in expression of the antigen recognised by $\mathrm{mAb} 217$ was found between wild-type and knockout mice whilst blotting with commercially generated antibody against PSR showed that this protein did disappear from knockout mice [27]. In addition, Cui et al. demonstrated nuclear localisation of GFT-tagged putative PSR which was consistent with the presence of five nuclear localisation signals [28]. One conclusion is that a phage display approach has the potential to identify weak cross-reacting epitopes present on the nuclear protein belonging to iron-oxidase family [29] and that the real PS receptor remains to be identified [30].

- Recently it has been shown that PS interacts with a number of bridging molecules including annexin I (Anx I or lipocortin) [31], milk-fat-globule-EGFfactor 8 (MFG-E8), growth-arrest-specific 6 (Gas 6) and $\beta 2$-glycoprotein-I ( $\beta 2-$ GPI) [32]. These interactions may facilitate PS interaction with other phagocyte receptors, e.g. $\alpha_{\mathrm{v}} \beta_{3}$-integrin, the receptor-tyrosine kinase Mer, and the $\beta 2$-GPI receptor by acting as a bridge between the apoptotic cell and the phagocyte [33]. Interestingly, a recent manuscript has demonstrated that the macrophage class B scavenger receptor $\mathrm{CD} 36$ interacts with membrane associated oxidised PS (oxPS) on the surface of apoptotic cells [34].

Apart from translocation of PS (or oxPS), apoptotic cells are characterised by surface exposure of oxidised low-density lipoproteins (oxLDL) [35] recognised by diverse scavenger receptors: SR-A (class A macrophage scavenger receptor) [36], lectin-like oxLDLreceptor-1 (LOX-1) [37], CD-68 [38] and CD36 [39]. Another class of rather poorly characterised "eat me" signals are sites binding TSP-1 [40], complement proteins $\mathrm{C} 1 \mathrm{q}$ or $\mathrm{C} 3 \mathrm{~b} / \mathrm{bi}$ [41] or collectins like mannose binding lectin (MBL) or lung surfactant proteins-A and -D (SP-A and SP-D) [42]. CD36 and integrin $\alpha_{v} \beta_{3}$ bind apoptotic cells to the phagocyte via TSP-1 $[43,44]$, integrins $\alpha_{\mathrm{m}} \beta_{2}$ and $\alpha_{\mathrm{x}} \beta_{2}$ (complement receptors: CR3, CR4) recognise complement protein $\mathrm{C} 3 \mathrm{~b} / \mathrm{bi}$ [45], whereas scavenger complex calreticulin (CRT)-CD91 interacts with lung surfactant proteins-A and -D (SP-A and SP-D), MBL and collectin-like complement protein C1q [46]. Exposure of CRT on the cell surface increases during apoptosis and this is consistent with a potential role in phagocytic clearance. Removal of apoptotic CRT-deficient cells by macrophages is impaired and this effect is reversed by addition of soluble CRT. Moreover CRT interacts with CD91 also known as low-density lipoprotein receptor-related protein (LRP1) or $\alpha 2$ macroglobulin receptor which is a highly effective internalisation receptor on phagocytes [47]. Since CRT may be exposed on the apoptotic cell or the phagocyte surface, CRT activation of LRP may occur either in trans or cis action [48]. The differential anti-inflammatory or pro-inflammatory outcomes of clearance of apoptotic and late apoptotic/secondarily necrotic cells may be a consequence of engagement of specific receptors and adaptor molecules. For example, CD36 triggers strong anti-inflammatory responses from both monocytes and macrophages including inhibition of TNF $\alpha$, IL-1 1 , IL-12 production and up-regulation of IL-10 or TGF- $\beta$ $[49,50]$. In contrast, complement components $\mathrm{C} 1 \mathrm{q}$, C3 and C4 seem to bind only to primary or secondary necrotic cells, opsonising those cells not cleared by other mechanisms [51]. C1q cooperates with serum DNase 1 in the degradation of chromatin from necrotic cells, enabling uptake of chromatin fragments by monocyte-derived phagocytes [52] and C-reactive protein (CRP) fails to bind to early apoptotic neutrophils but binds to late apoptotic cells, membrane-permeable cells [53]. Some receptors including 
TSP-CD36- $\alpha_{v} \beta 3$ complex, CD14 and putative PSR may also be involved in uptake of both apoptotic and necrotic cells $[54,55]$.

The presence of "eat me" signals on viable cells raises the possibility that inhibitory signals, termed "don't eat me" signals, prevent phagocytosis of those cells [56]. One paradigm for this might be the expression of inhibitory receptors by NK cells, e.g. killer cell Ig-like receptor (KIR) which binds self major histocompatibility complex (MHC) class 1 molecules and subsequently suppress elimination of target cells by NK cells. This effect may be due to recruitment of srchomology 2 -containing protein tyrosine phosphatase-1 (SHP-1) and SHP-2 to two immunoreceptor tyrosine-based inhibitory motifs (ITIMs) [57]. A similar mechanism may also be operative in macrophage activation and viable cells may express surface molecules that bind to macrophage inhibitory receptors such as signal regulatory protein $\alpha$ (SIRP $\alpha)$ display markers of "self" in form of ubiquitously expressed surface molecules that bind to macrophage inhibitory receptors such as signal regulatory protein $\alpha$ $(\operatorname{SIRP} \alpha)[58]$.

-Brown et al. demonstrated that homophilic interaction of CD31 promotes detachment of viable cells from phagocytes, whereas apoptotic cells are unable to detach and become internalised by macrophages. In addition CD31 expressed by apoptotic cells failed to associate with the cytoplasmic signalling molecules SHP-1 and SHP-2 indicating disruption in CD31mediated signalling in apoptotic cells [59]. CD47 may also suppress removal of "self" viable cells when binding to extracellular domain of $\operatorname{SIRP} \alpha$ on macrophages [60]. CD47 acts as a "don't eat me" signal on normal red blood cells (RBC) [61] and is also highly express on the surface of some cancer cells (i.e. ovarian cancer cells) and virally-infected cells leading to impaired immune defence [62].

\section{Engulfment pathways}

Studies in the nematode Caenorhabditis elegans, fruit fly Drosophila melanogaster and mammalian cells revealed conservation of the engulfment machinery $[63,64]$ involving two signalling pathways influencing the balance of membrane Rho family GTPases activity [65]. RhoA and Rac-1 regulate phagocytosis of apoptotic cells in opposite manner-RhoA inhibits this process while Rac-1 is obligatorily required for engulfment [66]. Assembly of a trimolecular complex trimolecular complex of ELMO, CrkII and DOCK180 in mammals (known respectively as CED-12, CED-2 and CED-5 in the worm) [67] activates the guanine nucleotide guanine nucleotide exchange activity of DOCK180 for Rac (CED-10), which in turn promotes cytoskeletal rearrangements crucial for the uptake of target particles [68]. The upstream components required for Rac activation are different. One pathway identified in both mammalian cells and worms involves RhoG/MIG-2 and the guanine nucleotide exchange factor TRIO/UNC-73 [69], although the surface receptor remains unknown. The second pathway involves the cell surface receptor proteins $A B C 1 / C E D-7$ and $C D-91 / C E D 1$ and the adaptor protein GULP/CED-6. ABC1/CED-7 downstream ligands have not been identified yet [70].

\section{Pharmacological modulation of phagocytosis}

Our group and others have shown that macrophage phagocytic capacity is modulated by pharmacological or immunological intervention. For example, antiinflammatory glucocorticoids augment the ability of macrophages to phagocytose apoptotic cells in a concentration-and time-dependent manner via the glucocorticoid receptor [71]. Glucocorticoid treatment was suggested to reprogram macrophage differentiation towards a highly phagocytic phenotype with high levels of active Rac, increased formation of lamellipodia and cellular extensions as assessed by time lapse video microscopy and decreased cellular adhesion [72,73]. Furthermore, one important mediator of glucocorticoid action, the anti-inflammatory protein Anx-1, has been suggested to be essential for phagocytosis. Macrophages from Anx-1 null mice have reduced phagocytic capacities in vitro and in vivo associated with decreased expression of CD11b receptor protein [74].

Extracellular matrix (ECM) proteins such as fibronectin and collagen may also influence macrophage phagocytic abilities. Their interaction with macrophages through $\beta 1$ integrin receptor was shown to upregulate phagocytosis [75]. However, exposure to cigarette smoke causes post-translational carbonyl modification of ECM proteins as well as lipid peroxidation resulting in increased macrophage adherence but decreased macrophage uptake of apoptotic neutrophils [76].

CD44 has been demonstrated to act as an adhesion receptor for ECM molecules and mediates a number of cellular functions such as adhesion and proliferation of lymphocytes, cytocidal activity of NK cells and tumor metastasis [77]. Cross linking of human monocytederived macrophage CD44 with bivalent antibodies rapidly and specifically augmented uptake of apoptotic neutrophils in vitro [78] suggesting the possibility that ligation of CD44 in vivo may selectively promote clearance of apoptotic neutrophils and subsequently resolution of inflammation. Studies on CD44 deficient mice administrated with bleomycin to induce inflammation in alveolar interstitium, confirmed this suggestion. A 13-fold increase in the number of apoptotic cells was detected in lung tissue of CD44 deficient animals in comparison to control, indicating impaired clearance of apoptotic cells resulting in development of lung injury in these mice [79]. 
The close relationship between cell adhesion and phagocytosis is further revealed in studies with agents that elevate intracellular cAMP levels such as prostaglandins (PG). Pretreatment of macrophages with $\mathrm{PGE}_{2}$ or $\mathrm{PGD}_{2}$ or cAMP stable analogues such as dibutyryl-cAMP and 8-bromo-cAMP significantly reduced the proportion of macrophages that phagocytosed apoptotic cells and caused alterations in macrophage adhesion, loss in membrane activity and cell locomotion [80]. It has been reported that variety of inflammatory mediators, including PG, that stimulate activity of adenylate cyclase and PKA are likely to inhibit clearance of apoptotic cells [81]. Furthermore, PKA is able to phosphorylate Rho, a key negative regulator of cytoskeletal organisation, indicating that CAMP may play a pivotal role in regulation of the phagocytic process [82]. All Rho family GTPases require prenylation (covalent attachment of lipid adducts) and subsequent membrane insertion for activity. Since the cholesterol lowering drugs (statins) inhibit activity of HMG-CoA reductase they also reduce production of prenylation substrates and it has been recently reported that lovastatin may enhance phagocytosis of apoptotic cells via suppression of prenylation and membrane localization of RhoA, altering the RhoA-Rac-1 membrane balance towards Rac-1 - a positive regulator of phagocytosis [83].

The lipoxins represent further arachidonic acid metabolites that play an important role in resolution of inflammation. Their strong inhibitory effects on neutrophil chemotaxis and adhesion is well documented, but more recent data show that $\mathrm{LXA}_{4}$ and its stable synthetic analogues augmented clearance of apoptotic neutrophils in a concentration-dependent manner [84]. It has been also demonstrated that Anx-1 and $\mathrm{LXA}_{4}$ converge onto the same specific membrane lipoxin $\mathrm{A}_{4}$ receptor [85]. In addition the stable cAMP analogue 8-bromo-cAMP attenuated and the PKA inhibitor, Rp-cAMP, mimicked $\mathrm{LXA}_{4}$ effects indicating that lipoxin may inhibit PKA activity. The lack of additive effects of Rp-cAMP and $\mathrm{LXA}_{4}$ suggested they act through the same pathway. Interestingly, $\mathrm{LXA}_{4}$ was shown to inhibit PKA activity induced by $\mathrm{PGE}_{2}$, consistent with an important role for PKA in regulation of phagocytosis. One potential substrate for PKA is scavenger receptor CD36 [86] and loss of CD36 phosphorylation in platelets results in increased cytoadhesion [87]. Monoclonal antibodies against CD36 blocked macrophage phagocytosis of apoptotic neutrophils induced by $\mathrm{LXA}_{4}$ raising the possibility that $\mathrm{LXA}_{4}$-mediated dephosphorylation of CD36 may promote phagocyteapoptotic cell recognition [88].

\section{Clearance deficiency and autoimmunity}

Impairment of apoptotic cell clearance may result in exacerbation of inflammation and tissue injury, together with loss of production of cytokines (i.e. IL10) that maintain an anti-inflammatory environment [89]. On the contrary, enhancement of phagocyte clearance capacity by IL-10 [90] may further promote an environment of tissue repair and resolution of inflammation. There is now a wealth of evidence that support the hypothesis that defects in clearance of apoptotic cells have a role in pathogenesis of human autoimmune diseases. For an instance in SLE patients phagocytic activity is impaired [91], including clearance of autologous apoptotic material $[92,93]$.

Apoptotic cells undergoing secondary necrosis within tissue represent a source of pro-inflammatory stimuli and potentially immunogenic autoantigens. As shown at Figure 1 presentation of autoantigens by DC and macrophages may drive production of autoantibodies [94]. Nucleosomal proteins released from DNA as a result of internucleosomal cleavage during apoptosis represent one of the most abundant class of autoantigens found in patients with autoimmune disorders [95]. Antibodies against nucleosomes are a serological hallmark of SLE but also other autoimmune diseases. Additionally, delayed uptake of apoptotic cells may generate caspase-derived neoantigenic peptides that are capable of triggering autoimmune responses, inducing loss of tolerance by B cells and formation of immune complexes (IC) [96]. Autoantibodies opsonising apoptotic corpses may further alter phagocytic clearance pathways. It has been demonstrated that anti-phospholipid antibodies may bind to externalised PS on apoptotic cell surfaces and promote Fc receptordependent phagocytosis which may have pro-inflammatory sequelae [97]. It could be postulated that whilst low number of apoptotic cells are disposed of in a noninflammatory manner, problems may arise when tissue phagocytes are faced with an overwhelming load of apoptotic cells following infection or serious tissue injury. We have recently demonstrated that IC bind to "enabled" FcyRIIA on apoptotic human neutrophils [98]. Surprisingly, opsonisation of apoptotic neutrophils with IgG-containing IC allowed their rapid clearance by macrophages without production of proinflammatory cytokines, thus providing a mechanism for preventing release of intracellular antigens (particularly nuclear constituents) even in the face of a heavy apoptotic cell load. Paradoxically, therefore, IC may have beneficial effects, acting to limit development of autoimmunity in some circumstances.

Impairment of apoptotic cell removal by macrophages in peripheral or lymphoid tissues may result in late apoptotic cells become accessible to tissue or follicular DC. The capacity of DC to induce tolerance or immune activation is strongly related to the levels of "maturation" and expression of co-stimulatory molecules. Thus, cross-presentation of self or foreign antigens to $\mathrm{CD}^{+}$cytotoxic $\mathrm{T}$ lymphocytes (CTL) and stimulation of production of antibodies by B cells may depend on local micro-environmental conditions that 


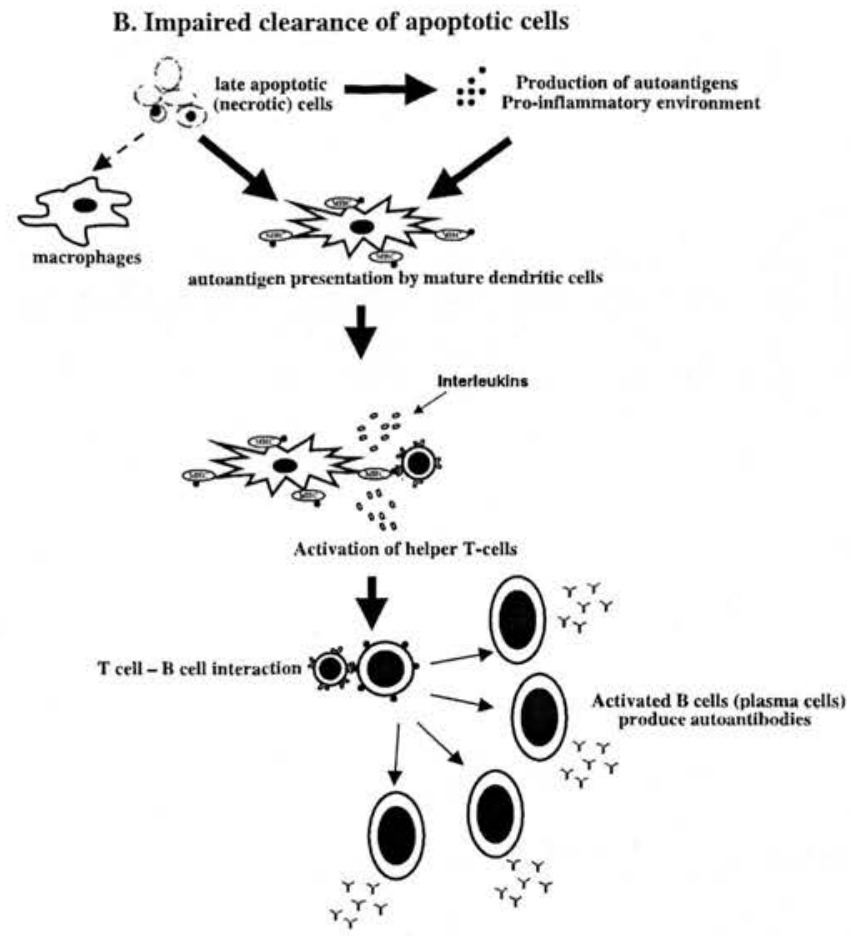

Figure 1. Diagram illustrating cascade of events resulting from efficient (A) or impaired (B) clearance of apoptotic cells. Efficient clearance of apoptotic cells prevents cell necrosis and stimulates production of anti-inflammatory mediators by phagocytes. In anti-inflammatory environment maturation of antigen presenting cells (i.e. DC) and immune response is inhibited. On the contrary, when clearance of apoptotic cells is affected, accumulated apoptotic cells undergo secondary necrosis resulting in production of autoantigens and pro-inflammatory mediators. In the presence of pro-inflammatory mediators maturation of DC is induced. Mature DC, after gaining access to autoantigens, present them to $\mathrm{T}$ helper cells followed by subsequent $\mathrm{B}$ cell production of autoantibodies and development of autoimmunity.

regulate levels of DC maturation [99]. Pro-inflammatory signals such as LPS, TNF $\alpha$, IL- $1 \beta$ or IFN- $\alpha$ as well as viral or bacterial proteins, high antigen load or uptake of necrotic cells appear to be very powerful inductors of DC maturation and activators of immune response [100]. On the other hand, anti-inflammatory cytokines such as TGF- $\beta$ or IL- 10 or more importantly uptake of apoptotic cells strongly suppress maturation of DC and promotes peripheral tolerance [100]. It has been shown that engulfment of apoptotic material by DC may inhibit even such strong pro-maturation signal as LPS, indirectly reducing DC capacity to stimulate $\mathrm{T}$ cells [101]. Furthermore, uptake of apoptotic cells by DC fails to stimulate effective antigen presentation to lymphocytes. In contrast, uptake of necrotic cells induces maturation of DC and a break in peripheral tolerance $[102,103]$. Therefore, the current model for the development of autoimmunity involves two important factors: (i) production of autoantigens, and (ii) a strongly pro-inflammatory environment, both of which are closely linked to deficiency in clearance of apoptotic cells.

However, although this association is supported by some studies using mice lacking apoptotic cell recognition molecules, e.g. $\mathrm{C} 1 \mathrm{q}$, in some cases defective clearance of apoptotic cells may have no consequences in terms of development of autoantibodies and autoimmunity, for example, CD-14 deficient animals show a defect in the clearance of apoptotic cells in the absence of an overt autoimmune phenotype [104]. There is convincing evidence linking complement protein $\mathrm{C} 1 \mathrm{q}$ deficiency with development of SLE. It has been reported that lack of complement pathway proteins results in accumulation of apoptotic material and elevated levels of self-antigens and IC, and consequently in direct induction of tissue inflammation, maturation of DC and production of autoantibodies. Complement fragments also deliver regulatory signals to $\mathrm{T}$ and $\mathrm{B}$ lymphocytes maintaining peripheral tolerance to self-antigens and absence of stimulation results in decrease thresholds of activation for T and B cells [100,105].

Another example of condition with an autoimmune component and clearance deficiency is cystic fibrosis characterised by massive recruitment of inflammatory cells and release of intracellular sources into the lung. Patients are also characterised by increased number of apoptotic cells in airways and it is highly probable that impaired clearance may result from elastase-mediated inhibition of phagocytosis causing chronic inflammation and progressive lung tissue damage [106]. 


\section{S. Michlewska et al.}

Type- 1 diabetes is another disease condition in which autoimmunity and clearance of dying cells play a crucial role. This autoimmune disease usually emerges in genetically predisposed children, most likely in response to an environmental insult. Humoral and cellmediated immunities trigger chronic apoptotic cell death amongst the insulin-secreting $\beta$-cells of the pancreatic islets of Langerhans [107]. It has since been postulated that the apoptotic $\beta$-cells themselves constitute a trigger for autoimmunity and that impaired clearance of apoptotic cells is central to development of the disease, perhaps on account of harmful autoantigens released during the apoptotic process and resultant infiltration of T-cells [108]. Results from animal studies suggest that the underlying cause of impaired clearance is likely to be due to a deficiency in the macrophages themselves [109] but the mechanism has yet to be fully elucidated.

\section{Closing remarks}

Apoptosis and subsequent clearance by professional or non-professional phagocytes is a complex and dynamic process crucial for normal tissue homeostasis and modulation of immune responses and development of autoimmunity. Uptake of late apoptotic or necrotic cells engages different receptors and signalling pathways than early apoptotic cells with profound implications for regulation of immunity. As our knowledge of the phagocytic clearance mechanisms and the potential for modulation of macrophage phagocytosis increases, we may uncover new ways to treat patients with autoimmune conditions arising from defective removal of apoptotic cells.

\section{Acknowledgements}

The Funding support of the Wellcome Trust and the Medical Research Council is gratefully acknowledged.

\section{References}

[1] Haslett C. Am J Respir Crit Care Med 1999;160:S5.

[2] Wyllie AH, Kerr JF, Currie AR. Int Rev Cytol 1980;68:251.

[3] Savill JS, et al. J Clin Invest 1989;83:865.

[4] Albert ML, et al. J Exp Med 1998;188:1359.

[5] Dini L, Rossi L, Lentini A, De MA, Rotilio G. Cell Mol Biol (Noisy-le-grand) 1995;41:1051.

[6] Bennett MR, Gibson DF, Schwartz SM, Tait JF. Circ Res 1995;77:1136.

[7] Hall SE, Savill JS, Henson PM, Haslett C. J Immunol 1994; 153:3218,

[8] Fadok VA, et al. J Clin Invest 1998;101:890.

[9] Voll RE, et al. Nature 1997;390:350.

[10] Ren Y, et al. Arthritis Rheum 2003;48:2888.

[11] O'Brien BA, Fieldus WE, Field CJ, Finegood DT. Cell Death Differ 2002;9:457.

[12] Vandivier RW, et al. J Clin Invest 2002;109:661.

[13] Hodge S, Hodge G, Scicchitano R, Reynolds PN, Holmes M. Immunol Cell Biol 2003;81:289.
[14] Lauber K, et al. Cell 2003;113:717.

[15] Horino K, et al. Lab Invest 1998;78:603.

[16] Wakasugi K, Schimmel P. J Biol Chem 1999;274:23155.

[17] Moodley Y, et al. Am J Pathol 2003;162:771.

[18] Fadok VA, et al. J Immunol 1992;148:2207.

[19] Williamson P, Schlegel RA. Biochim Biophys Acta 2002;1585:53.

[20] Luciani MF, Chimini G. EMBO J 1996;15:226.

[21] Krahling S, Callahan MK, Williamson P, Schlegel RA. Cell Death Differ 1999;6:183.

[22] Elliott JI, et al. Nat Cell Biol 2005;7:808.

[23] Kuijpers TW, et al. Blood 2004;103:3915.

[24] Dillon SR, Constantinescu A, Schlissel MS. J Immunol 2001; 166:58.

[25] Fadok VA, et al. Nature 2000;405:85.

[26] Hoffmann PR, et al. J Cell Biol 2001;155:649.

[27] Bose J, et al. J Biol 2004;3:15.

[28] Cui P, Qin B, Liu N, Pan G, Pei D. Exp Cell Res 2004; 293:154.

[29] Cikala M, et al. Cell Biol 2004;5:26.

[30] Williamson P, Schlegel RA. J Biol 2004;3:14.

[31] Arur S, et al. Dev Cell 2003;4:587.

[32] Lauber K, Blumenthal SG, Waibel M, Wesselborg S. Mol Cell 2004;14:277.

[33] Lauber K, Blumenthal SG, Waibel M, Wesselborg S. Mol Cell 2004;14:277.

[34] Greenberg ME, et al. J Exp Med 2006;203:2613.

[35] Chang MK, et al. Proc Natl Acad Sci USA 1999;96:6353.

[36] Platt N, Suzuki H, Kurihara Y, Kodama T, Gordon S. Proc Natl Acad Sci USA 1996;93:12456.

[37] Oka K, et al. Proc Natl Acad Sci USA 1998;95:9535.

[38] Erdosova B, Hlavkova L, Prochazkova J, Lichnovsky V. Biomed Pap Med Fac Univ Palacky Olomouc Czech Repub 2002;146:41.

[39] Ren Y, Silverstein RL, Allen J, Savill J. J Exp Med 1995; 181:1857.

[40] Stern M, Savill J, Haslett C. Am J Pathol 1996;149:911.

[41] Takizawa F, Tsuji S, Nagasawa S. FEBS Lett 1996; 397:269.

[42] Vandivier RW, et al. J Immunol 2002;169:3978.

[43] Savill J, Dransfield I, Hogg N, Haslett C. Nature 1990; 343:170.

[44] Savill J, Hogg N, Ren Y, Haslett C. J Clin Invest 1992;90:1513.

[45] Takizawa F, Tsuji S, Nagasawa S. FEBS Lett 1996;397:269.

[46] Vandivier RW, et al. J Immunol 2002;169:3978.

[47] Gardai SJ, et al. Cell 2005;123:321.

[48] Gardai SJ, Bratton DL, Ogden CA, Henson PM. J Leukoc Biol 2006;79:896,

[49] Voll RE, et al. Nature 1997;390:350.

[50] Fadok VA, et al. J Clin Invest 1998;101:890.

[51] Gaipl US, et al. Cell Death Differ 2001;8:327.

[52] Gaipl US, et al. Arthritis Rheum 2004;50:640.

[53] Hart SP, Alexander KM, MacCall SM, Dransfield I. J Inflamm (Lond) 2005;2:5

[54] Devitt A, et al. Nature 1998;392:505.

[55] Bottcher A, et al. Arthritis Rheum 2006;54:927.

[56] Lauber K, Blumenthal SG, Waibel M, Wesselborg S. Mol Cell 2004;14:277.

[57] Yusa S, Catina TL, Campbell KS. J Immunol 2004;172:7385.

[58] Kharitonenkov A, et al. Nature 1997;386:181.

[59] Brown S, et al. Nature 2002;418:200.

[60] Oldenborg PA, Gresham HD, Lindberg FP. J Exp Med 2001; 193:855.

[61] Oldenborg PA, et al. Science 2000;288:2051.

[62] Campbell IG, Freemont PS, Foulkes W, Trowsdale J. Cancer Res 1992;52:5416.

[63] Reddien PW, Horvitz HR. Nat Cell Biol 2000;2:131.

[64] Gumienny TL, Hengartner MO. Cell Death Differ 2001; 8:564. 
[65] Gardai SJ, Bratton DL, Ogden CA, Henson PM. J Leukoc Biol 2006;79:896.

[66] Morimoto K, et al. J Immunol 2006;176:7657.

[67] Henson PM. Curr Biol 2005;15:R29.

[68] Krysko DV, D’Herde K, Vandenabeele P. Apoptosis 2006; 11:1709.

[69] deBakker CD, et al. Curr Biol 2004;14:2208.

[70] Zhou Z, Hartwieg E, Horvitz HR. Cell 2001;104:43.

[71] Liu Y, et al. J Immunol 1999;162:3639.

[72] Giles KM, et al. J Immunol 2001;167:976.

[73] Heasman SJ, et al. J Endocrinol 2003;178:29.

[74] Yona S, et al. J Pharmacol 2006;148:469.

[75] McCutcheon JC, et al. J Leukoc Biol 1998;64:600.

[76] Kirkham PA, Spooner G, Rahman I, Rossi AG. Biochem Biophys Res Commun 2004;318:32.

[77] Henke CA, Roongta U, Mickelson DJ, Knutson JR, McCarthy JB. J Clin Invest 1996;97:2541.

[78] Hart SP, Dougherty GJ, Haslett C, Dransfield I. J Immunol 1997;159:919.

[79] Teder P, et al. Science 2002;296:155.

[80] Rossi AG, et al. J Immunol 1998;160:3562.

[81] Oropeza-Rendon RL, Speth V, Hiller G, Weber K, Fischer H. Exp Cell Res 1979;119:365.

[82] Lang P, et al. EMBO J 1996;15:510.

[83] Morimoto K, et al. J Immunol 2006;176:7657.

[84] Godson C, et al. J Immunol 2000;164:1663.

[85] Gavins FN, Sawmynaden P, Chatterjee BE, Perretti M. Prostaglandins Leukot Essent Fatty Acids 2005;73:211.

[86] Hatmi M, Gavaret JM, Elalamy I, Vargaftig BB, Jacquemin C. J Biol Chem 1996;271:24776.

[87] Asch AS, et al. Science 1993;262:1436.
[88] Godson C, et al. J Immunol 2000;164:1663.

[89] Voll RE, et al. Nature 1997;390:350.

[90] Xu W, et al. Blood 2006;107:4930.

[91] Hurst NP, Nuki G, Wallington T. Clin Exp Immunol 1984; 55:303.

[92] Herrmann M, et al. Arthritis Rheum 1998;41:1241.

[93] Baumann I, et al. Arthritis Rheum 2002;46:191.

[94] Mahoney JA, Rosen A. Curr Opin Immunol 2005;17:583.

[95] Baumann I, et al. Arthritis Rheum 2002;46:191.

[96] Casiano CA, Martin SJ, Green DR, Tan EM. J Exp Med 1996;184:765.

[97] Price BE, et al. J Immunol 1996;157:2201.

[98] Hart SP, Alexander KM, Dransfield I. J Immunol 2004;172: 1882.

[99] Savill J, Dransfield I, Gregory C, Haslett C. Nat Rey Immunol 2002;2:965.

[100] Manderson AP, Botto M, Walport MJ. Annu Rev Immunol 2004;22:431.

[101] Chen W, Frank ME, Jin W, Wahl SM. Immunity 2001;14:715.

[102] Sato K, Yamashita N, Matsuyama T. Cell Immunol 2002; 215:186.

[103] Sauter B, et al. J Exp Med 2000;191:423.

[104] Devitt A, et al. J Cell Biol 2004;167:1161.

[105] Munoz LE, et al. Rheumatology (Oxford) 2005;44:1101.

[106] Vandivier RW, et al. J Clin Invest 2002;109:661.

[107] Eisenbarth GS. N Engl J Med 1986;314:1360.

[108] O'Brien BA, Fieldus WE, Field CJ, Finegood DT. Cell Death Differ 2002;9:457.

[109] O'Brien BA, Fieldus WE, Field CJ, Finegood DT. Cell Death Differ 2002;9:457. 


\section{TheScientificWorldJOURNAL}

\author{
whw.thescientificworld.com
}

\title{
Effects of Glucocorticoids on Apoptosis and Clearance of Apoptotic Cells
}

\author{
Aisleen McColl, Sylwia Michlewska, Ian Dransfield, and Adriano G. Rossi* \\ MRC Centre for Inflammation Research, The Queen's Medical Research Institute, \\ University of Edinburgh Medical School, 47 Little France Crescent, Edinburgh, EH16 \\ 4TJ, Scotland, U.K. \\ E-mail: A.G.Rossi@ed.ac.uk
}

Received May 28, 2007; Revised July 30, 2007; Accepted August 1, 2007; Published August 17, 2007

The glucocorticoid (GC) drugs are one of the most commonly prescribed and effective anti-inflammatory agents used for the treatment of many inflammatory disorders through their ability to attenuate phlogistic responses. The glucocorticoid receptor (GCR) primarily mediates GC actions via activation or repression of gene expression. GCs directly induce the expression of proteins displaying anti-inflammatory activities. However, the likely predominant effect of GCs is the repression of multiple inflammatory genes that invariably are overexpressed during nonresolving chronic inflammation. Although most GC actions are mediated through regulation of transcription, rapid nongenomic actions have also been reported. In addition, GCs modulate inflammatory cell survival, inducing apoptosis in immature thymocytes and eosinophils, while delaying constitutive neutrophil apoptosis. Importantly, GCs promote noninflammatory phagocytosis of apoptotic cell targets, a process important for the successful resolution of inflammation. Here, the effects and mechanisms of action of GC on inflammatory cell apoptosis and phagocytosis will be discussed.

KEYWORDS: glucocorticoids, apoptosis, inflammation, macrophage phagocytosis

\section{INTRODUCTION}

Inflammation is an important physiological host defence mechanism against infection and injury. Granulocytes, such as neutrophils and eosinophils, are crucial in innate immune defence against bacterial and parasitic infections, respectively[1]. However, the persistent recruitment and/or enhanced survival of granulocytes at inflamed sites may result from dysregulated expression of proinflammatory genes, such as cytokines (IL-1, TNF $\alpha$, GM-CSF, etc.), chemokines (IL-8, IL-5, MIP-1 $\alpha$ ), and adhesion molecules (ICAM-1 and E-selectin)[2]. Uncontrolled leukocyte responsiveness will lead to release of inflammatory mediators, such as eicosanoids (prostaglandins, leukotrienes, and thromboxanes), cytokines (IL-8, etc.), reactive oxygen/nitrogen species $\left(\mathrm{O}_{2}^{-}, \mathrm{NO}\right)$, and granular enzymes (e.g., elastase)[3], resulting in damage to the surrounding tissue and propagation of the inflammatory response. This likely contributes to persistent dysregulated inflammation, resulting in the pathogenesis of disorders such as chronic obstructive pulmonary disease (COPD) and bronchial asthma[4]. 
Apoptosis is a programmed form of cell death[5] that regulates the number and fate of leukocytes at inflamed sites and, in contrast to necrosis, is associated with maintenance of cell membrane integrity and damage limitation[6]. Granulocytes are terminally differentiated cells and undergo constitutive apoptosis during in vitro culture[6,7], which is amenable to lineage-specific modulation by intrinsic and extrinsic factors including cAMP[8], IL-5[7], GM-CSF[9], prostaglandins[10], and TNF $\alpha[11]$. Synthetic glucocorticoids (GCs), such as dexamethasone, initially demonstrated to induce apoptosis of immature thymocytes[12], also accelerate apoptosis in eosinophils while, surprisingly, prolong neutrophil survival[13,14]. This observation has led to speculation that GCs may be exerting part of their beneficial effect in eosinophil-dominant diseases (e.g., asthma) by inducing apoptosis of lymphocytes and eosinophils while maintaining beneficial neutrophil-dependent antimicrobial responses. Consequently, it is possible that direct pharmacological triggering of cell-specific apoptosis may be a novel therapeutic strategy in the treatment of inflammatory disorders[15,16]. However, for this to be considered as a successful tactic, efficient removal of the apoptotic leukocytes is also required. Failure to do so may lead to apoptotic cells undergoing secondary necrosis with deleterious consequences in terms of tissue damage and the outcome of the inflammatory response. An additional anti-inflammatory effect of GCs is the profound potentiation of phagocytic clearance of intact apoptotic leukocytes described in vitro $[17,18]$ and possibly in vivo[19]. These recently described effects of GCs, together with limiting inflammatory cell recruitment and activation, may prevent further injury through the release of noxious intracellular contents and may be important for promoting the resolution of inflammation. However, there are potential limitations to the application of GCs in disease, particularly as a consequence of the undesirable side effects associated with long-term treatment. Delineating the precise mechanisms of GC action would provide a significant insight into the anti-inflammatory role of GCs and allow the development of novel strategies that are more selective in their action.

\section{THE GLUCOCORTICOID RECEPTOR}

GCs mediate most of their effects by binding to glucocorticoid receptors (GCRs). The GCR is a member of the nuclear receptor superfamily, which includes receptors for mineralocorticoids and sex hormones[20]. Alternative splicing of the gcr gene generates two or more GCR isoforms with distinct functions. Although GCR $\alpha$ is the predominant isoform and is responsible for GC binding, GCR $\beta$ is a cterminally truncated variant that cannot bind $\mathrm{GC}$ or regulate transcription, but can form heterodimers with GCR $\alpha$ to modulate GCR $\alpha$ function[21,22]. Inactive GCR resides in the cytoplasm as part of a multiprotein complex, being bound to chaperone molecules including heat shock proteins (HSP90) and immunophilin[23]. HSP90 forms interactions with GCR in the c-terminal domain essential for maintaining the correct configuration of GCR and also masks a nuclear localisation signal to prevent translocation of the unoccupied GCR to the nucleus[24]. Upon binding of GC to GCR, these chaperone proteins dissociate from the GCR, unmasking the nuclear localisation signal required for the activated GC-GCR complex to translocate to the nucleus[24]. Here, GCR can directly or indirectly modulate the transcription of multiple target genes.

\section{MECHANISMS OF GC ANTI-INFLAMMATORY ACTION}

\section{Transactivation: Induction of Anti-Inflammatory Gene Expression}

One mechanism whereby GCs can mediate their action is via direct binding of GCRs to DNA to increase the transcription of anti-inflammatory genes, a process known as transactivation (Table 1). Ligandactivated GCRs translocate to the nucleus and bind specifically to palindromic glucocorticoid-responsive elements (GREs) found in the promoter region of GC-responsive genes[25,26]. The central domain of the GCR contains two zinc fingers essential for GCR dimerisation and binding to GRE sequences[26,27], 
with a point mutation in the D-loop of the central domain abolishing transactivation[28]. Thus, transcription of GC-responsive genes requires GCRs to be in the homodimeric form. GCR "switching on" of gene transcription also requires the recruitment of specific transcriptional coactivator proteins, which are important for localised chromatin remodelling and stabilisation of the basal transcriptional machinery[29].

This mechanism directly modulates the expression of secretory leukocyte proteinase inhibitor (SLPI)[30], IL-1 receptor antagonist[29], and C1q [31]. Annexin I (or Lipocortin 1) is an additional GCinducible protein thought to mediate many of the anti-inflammatory actions of GCs. This is confirmed by studies in deficient mice, which have altered expression of annexins, COX-2, and cPLA2; exaggerated responses to carrageenin- or zymosan-induced inflammation; and partial resistance to the antiinflammatory effects of GCs[32]. Administration of exogenous annexin I confers anti-inflammatory activity in some models of inflammatory disease[33]. Conversely, infusion of annexin I antibodies neutralises the effect of annexin I and abrogates the anti-inflammatory activities of GCs[33]. It has been suggested that autoantibodies to annexin I may contribute to GC resistance and the pathogenesis found in inflammatory diseases where GCs may be used as a treatment, such as rheumatoid arthritis and systemic lupus erythematosus (SLE)[34]. Additionally, annexin I-derived peptides have been shown to mimic some of the anti-inflammatory effects of endogenous annexin I, including a role in phagocytic clearance of apoptotic cells[35]. GCs also enhance the expression of other anti-inflammatory proteins that may be important in switching off signalling pathways engaged during persistant inflammation. Mitogenactivated protein kinase (MAPK) phosphatase 1 (MKP-1) dephosphorylates and inactivates MAPKs, such as p38 MAPK and c-Jun terminal kinase (JNK), which contribute to enhanced expression of proinflammatory mediators[36]. The suppressive effect of GCs on MAPK signalling is impaired in MKP1 knockout mice, which show enhanced expression of proinflammatory genes, including COX2, TNF $\alpha$ and IL-1[36]. Thus, the anti-inflammatory action of GCs via transactivation may be important in aiding the resolution of inflammation and in boosting innate immune defence through the induction of protective proteins. Nevertheless, it is doubtful that all the effects mediated by pleiotropic GCs could be explained by enhanced production of a small number of proteins with anti-inflammatory properties.

\section{Transrepression: Inhibition of Proinflammatory Gene Expression}

Although a small number of genes can be regulated directly, many more genes are regulated indirectly by the GCR via suppression of gene expression, a process known as transrepression (Table 1). Originally, this repression was thought to result from homodimeric GCRs binding to putative negative GRE sites in the promoter regions of proinflammatory genes to switch off their transcription[37]. However, the majority of genes repressed by GCs do not contain negative GRE sequences; therefore, alternative mechanisms to DNA binding must be engaged.

Ligand-activated GCRs can "switch off" inflammatory gene expression by a DNA-independent mechanism through direct protein-protein interactions with activated NFkB and AP-1 transcription factors $[38,39]$. Since $\mathrm{NF} \kappa \mathrm{B}$ and $\mathrm{AP}-1$ induce the transcription of multiple inflammatory and immune genes characteristic of various inflammatory disorders[40,41], this mechanism of GC action is extremely important and probably accounts for most of the inhibitory effects of GCs on inflammation. Although cytoplasmic interaction of ligand-activated GCR with transcriptional regulators has been reported, more recent data indicate that GCR may interfere with $\mathrm{NF \kappa B}$ and AP-1 at a later stage after they have bound to DNA to influence transcription[39,42]. Reichardt and colleagues introduced a point mutation A458T into the GCR gene by a gene-targeting technique involving Cre/loxP recombination[28,42,43]. Specifically, the mutation was "knocked in" to the D-loop of the central domain of the GCR, preventing DNA binding, GCR dimerisation $\left(\mathrm{GCR}^{\mathrm{dim}}\right)$, and transactivation. However, transrepression of NF $\kappa \mathrm{B}-$ and AP-1-mediated gene expression remained intact, indicating that this process is mediated by monomeric GCR. Application of the $\mathrm{GCR}^{\mathrm{dim}}$ mouse model would allow investigation into the process of transrepression in the absence 
of transactivation and allow elucidation of GC mechanism of action in various processes during inflammation in vivo. Interestingly, higher concentrations of GCs are required for induction of antiinflammatory gene expression, while gene repression by the GCR can occur at much lower, more clinically relevant concentrations of GC[24]. Indeed, it has been questioned whether DNA binding is required at all for physiological GCR function[44]. Thus, although GCs can induce anti-inflammatory gene expression, the repression of activated proinflammatory transcription factors appears to be the dominant mechanism of GC action. Moreover, these modes of GC effect may even occur concurrently, where the induction of $\mathrm{I} \kappa \mathrm{B} \alpha$ and glucocorticoid-inducible leucine zipper (GILZ) may contribute to later onset inhibition of $\mathrm{NF} \kappa \mathrm{B}$ and $\mathrm{AP}-1$, respectively[20].

Table 1.

Mechanisms of Glucocorticoid Anti-Inflammatory Action

\begin{tabular}{ll} 
Mechanism of GC Action & Genes Affected \\
\hline $\begin{array}{l}\text { Transactivation: Induction of anti-inflammatory } \\
\text { gene expression }\end{array}$ & SLPI, IL-1 receptor antagonist, C1q, annexin I, MKP-1, \\
Transrepression: Suppression of proinflammatory & Cytokines (IL-1, TNF $\alpha$, GM-CSF), chemokines (IL-8, \\
gene expression & MIP-1 $\alpha$ ), adhesion molecules (ICAM-1, E-selectin)
\end{tabular}

\section{Chromatin Remodelling, HATs, and HDACs}

It is now apparent that an additional DNA binding-independent mechanism of GC action may involve reversible alterations in chromatin structure and histone acetylation. Gene expression and repression are associated with alterations in chromatin structure through modification of core histones[45] (Fig. 1). In a resting cell, the DNA is tightly coiled around core histone proteins and is inaccessible to transcriptional regulators, a configuration referred to as "closed" and is associated with gene silencing[46]. Initiation of transcription by $\mathrm{NF} \kappa \mathrm{B}$ and $\mathrm{AP}-1$ requires slackening of chromatin and unwinding of DNA for transcriptional cofactors and RNA polymerase II to gain access to genes. Upon binding to DNA, NFKB and AP-1 recruit and activate coactivator proteins, such as cAMP response element binding (CREB) binding protein (CBP) and p300/CBP-associated factor, which have intrinsic histone acetyltransferase (HAT) activity[46]. Acetylation of key lysine residues in histones by coactivator proteins initiates chromatin remodelling required for gene transcription[45]. Reversal of histone acetylation results in tightening of chromatin around DNA and hinders binding of NFKB and AP-1. Deacetylation is controlled by corepressor proteins, such as histone deacetylases (HDACs) and nuclear receptor corepressor (NcoR), and is associated with gene repression[29]. This process may be important for "switching off" genes once the stimulus is removed and inflammation is no longer required, and failure to do so could contribute to the development of persistent or chronic inflammation.

\section{GCR Interacts with HATs and HDACs to Regulate Their Function}

GCR can directly interact with NFKB and AP-1 to suppress the expression of proinflammatory genes, a process that may involve reversal of histone acetylation[39] (Fig. 1). The exact mechanism involved here is uncertain, although a competitive role between GCR, NFKB, and AP-1 for binding to coactivators remains controversial[47]. GCRs may carry out their repressive function by directly suppressing the HAT 
activity of coactivators[2]. Alternatively, activated GCRs may recruit corepressors, such as HDAC2, to the transcriptional complex to reverse histone acetylation[48].

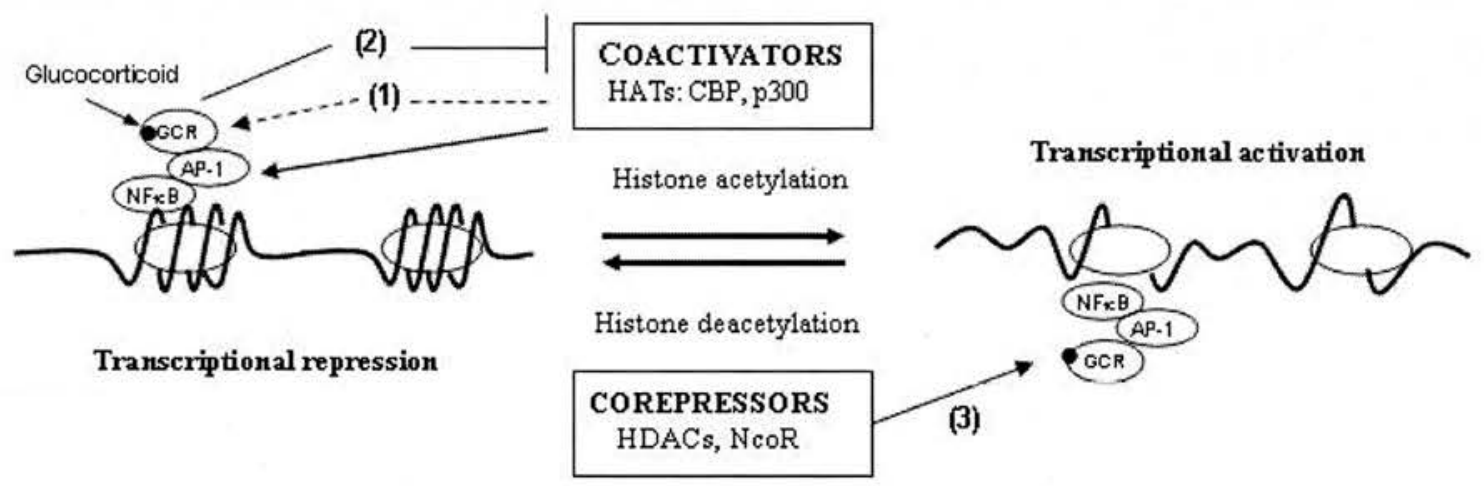

FIGURE 1. Effect of GCR on chromatin remodelling, HATs, and HDACs. Gene expression and repression are regulated by modification of core histones. In the resting state, DNA is tightly coiled around histones and is inaccessible to transcriptional cofactors and RNA polymerase II. Upon binding to DNA, NFKB and AP-1 recruit and activate coactivator proteins, such as CBP and p300/CBP-associated factor, which have intrinsic HAT activity. Histone acetylation results in slackening of chromatin and unwinding of DNA, allowing the transcriptional machinery to gain access to genes and initiate transcription. Deacetylation is controlled by corepressor proteins, such as HDACs and NcoR, and is associated with gene repression. Activated GCRs may interact with HATs and HDACs to regulate their function. Suggested mechanisms of GR transrepression include (1) GCR competes with NFKB and AP-1 for binding to coactivators, (2) direct suppression of HAT activity of coactivators by GCR, (3) recruitment of corepressors by GCR to reverse histone acetylation.

\section{Nongenomic Effects}

The genomic effects of GCs have a lag period of around $30 \mathrm{~min}$ to a few hours/days[20], result from GC binding to cytoplasmic GCR, and are blocked by inhibitors of transcription and translation, such as cycloheximide[49]. Some data indicate that GCs may exert more rapid actions (seconds to minutes) that are most likely transcriptional independent[50]. Furthermore, it has been suggested that some immediate GC effects may be GCR independent, for instance, inhibition of superoxide production by macrophages[51] or alteration of plasma membrane properties[50]. Other investigations have implicated GCR-dependent nongenomic effects of GCs via post-transcriptional regulation, both at the level of mRNA and protein[52]. This may provide an immediate inhibitory effect of GCs on the expression, synthesis, and release of proinflammatory mediators. For this, a putative membrane-associated form of the GCR (mGCR) may bind GC and transduce signals via MAPKs, PI-3K, and PKC, which mediate the rapid effects[49,53]. Investigations by Croxtall and colleagues have implicated GCR-dependent effects of dexamethasone via altered phosphorylation status of annexin I, leading to inhibition of cPLA2 activation and arachidonic acid release[54]. Whether any of these nongenomic effects described occur at physiological concentrations of GC and have a contributory role to the anti-inflammatory effect of these steroids remains controversial. 


\section{GC RESISTANCE}

GCs are pleiotropic steroids with the ability to attenuate many phlogistic responses, making them the most effective clinical treatment for various inflammatory disorders, e.g., asthma. However, a small proportion of asthmatic patients do not respond well to GC treatment and may require high doses of GC[55]. Several mechanisms have been postulated to contribute to GC resistance. Increased expression of GCR $\beta$ has been suggested to interfere with GCR $\alpha$ function[21,22]. Interestingly, the level of GCR $\beta$ is increased by proinflammatory cytokines[56] and in peripheral blood mononuclear cells from patients with GC-resistant rheumatoid arthritis[57]. The level of intracellular GC is much lower than the extracellular concentration due to expulsion of GC from the cell by members of the $\mathrm{ABC}$ family of transporter molecules[52]. Overexpression of these proteins may contribute to the development of GC resistance. COPD is an inflammatory disorder where GCs are not usually a beneficial treatment and patients are relatively resistant to these steroids even when given high doses[24]. This ineffectiveness has been linked to factors such as smoking and oxidative stress[58].

\section{GC SIDE EFFECTS}

Despite being the mainstay therapy for treatment of inflammatory disease, long-term GC use is associated with many undesirable side effects, the molecular mechanisms of which are extremely complex and are not entirely understood. Endocrine and metabolic side effects leading to diabetes and osteoporosis have been linked to transactivation of genes by the ligand-activated GCR[59]. For example, GCs may increase glucose synthesis through transactivation of enzymes involved in gluconeogenesis[59]. GCR ${ }^{\text {dim }}$ mutants, which are transactivation deficient, retain many of their beneficial anti-inflammatory effects through transrepression of NFKB- and AP-1-mediated gene expression[28,42,43]. Hence, identification of "dissociated steroids" that can mediate transrepression of proinflammatory genes without induction of transactivation would represent an attractive therapeutic strategy. Such compounds may provide a safer approach to treatment of inflammatory diseases through this ability to convey the beneficial actions of GCs with reduced adverse effects[60]. However, some side effects, such as skin atrophy and suppression of the hypothalamic-pituitary-adrenal axis, may be mediated by the transrepressive function of the GCR[59] or may even involve a combination of both GCR mechanisms that would complicate the use of dissociated steroids in therapy. It is imperative, therefore, that the specific molecular mechanisms involved in GC-induced side effects are delineated to allow the development of more selective therapies.

\section{GCS AS A THERAPY FOR INFLAMMATORY DISEASE}

\section{Inflammation is Normally Self-Resolving}

Under normal circumstances, inflammation is beneficial to the host and is self-resolving, requiring controlled clearance of granulocytes from inflamed sites[6]. Apoptosis of granulocytes, in contrast to necrosis, is associated with maintenance of membrane integrity[6] and down-regulation of potentially injurious secretory responses[61]. For granulocytes, functional attenuation is also achieved by surface changes during the apoptotic process, including down-regulation of FcyRIII (CD16)[62] and L-selectin expression[63], and uncoupling $\beta 2$ integrins[63]. Cells undergoing apoptosis display specific surface alterations important for signalling rapid recognition and internalisation by phagocytes $[64,65,66]$. Many receptor mechanisms have been acknowledged in the direct recognition and engulfment of apoptotic cells by phagocytes[67,68,69], including the vitronectin receptor[70], scavenger receptors[71], CD31[72], putative phosphatidylserine receptor (PSR)[73], C1q receptor calreticulin[74], and the tyrosine kinase receptor, MER[75]. Blocking individual receptors on phagocytes only partially inhibits phagocytosis of apoptotic cells, suggesting redundancy in the engulfment pathways and underlining the importance of 
phagocytic clearance of apoptotic cells. During resolving inflammation, macrophages (or other cells with phagocytic capacity) are required to remove the potentially histotoxic apoptotic cells rapidly and efficiently[76]. Phagocytic removal of apoptotic cells normally occurs early on in the death process when the dying cell is still intact[6] and, in contrast to phagocytosis of necrotic cells, fails to induce the release of proinflammatory mediators, such as eicosanoids, GM-CSF, or IL-8, and MCP-1 chemoattractants from the phagocytic cell[ $77,78,79]$. Engulfment of apoptotic cells also actively induces anti-inflammatory and immunosuppressive effects in the phagocyte by, for example, inducing the production of IL-10, TGF $\beta$, PGE2, and platelet-activating factor[ 80,81$]$. Thus, rapid recognition, ingestion, and degradation of dying cells by phagocytes are nonphlogistic and equally as vital as the process of apoptosis itself. Both apoptosis and subsequent phagocytic clearance are prerequisites for efficient clearance of granulocytes and other inflammatory cells from tissues and, ultimately, for the resolution of inflammation.

\section{Disease: Failure of Natural Resolution Process?}

Neutrophils and eosinophils have been implicated in the pathogenesis of many inflammatory and allergic disorders, such as COPD and bronchial asthma. Accumulation of leukocytes reflects a mismatch between their infiltration into the inflamed site in response to cytokines, chemokines, and adhesion molecules, which are overexpressed during chronic inflammation and the mechanisms required for their clearance. Defective apoptosis or failure to remove intact apoptotic cells efficiently before lysis may play a contributory role in the disease pathogenesis through release of histotoxic granulocyte contents that have the capacity to damage the surrounding tissue and by stimulating proinflammatory macrophage secretory responses. Indeed, impaired phagocytosis of apoptotic cell material may contribute to the severity of disease in human SLE[82,83]. Mouse models of C1q deficiency develop spontaneous/persistant inflammation that resembles an SLE-like disease and may be due to defective clearance of apoptotic cells $[84,85]$. In both cases, a systemic autoimmunity is developed against autoantigens that may be displayed on or released by the noningested apoptotic cells. This underlines the importance for "safe" and efficient removal of these potentially harmful cells and the therapeutic value of agents that promote this. Our laboratory and others have identified GCs as important regulators of both apoptosis and phagocytic removal of apoptotic cells in diseases where these processes may be inefficient or dysregulated. Indeed, it has recently been demonstrated that manipulation of apoptosis in vivo may result in enhanced resolution of inflammation[86,87], thereby highlighting the potential to manipulate processes involved in the resolution of inflammation for therapeutic gain $[15,16]$. Consequently, the effects of glucorticoids on the resolution process, in our opinion, are highly clinically relevant.

\section{GLUCOCORTICOID REGULATION OF APOPTOSIS IN INFLAMMATORY CELLS}

\section{Internal Controls of Apoptosis}

Apoptosis plays a crucial role in regulation of the inflammatory response. Neutrophils have a short life span with a circulating half-life of $6-10 \mathrm{~h}$, which is delayed by redundant mediators present at inflamed sites (24-48 h)[88]. Eosinophil apoptosis occurs at a much slower rate than in neutrophils[7]. Despite their derivation from a common precursor cell, apoptosis in these granulocytes is controlled quite differently. Many of the inflammatory mediators present at an inflamed site can exert either anti- or proapoptotic influences. For example, G-CSF, GM-CSF, C5a, and LPS profoundly inhibit neutrophil apoptosis[14,89,90], as does increased cAMP[8] or a hypoxic environment[91]. Eosinophil survival is promoted in response to GM-CSF, IL-3, and IL-5[92], and $\mathrm{PGD}_{2}[10]$.

$\mathrm{T}$ cells are an essential component of the adaptive immune system, and apoptosis is important for their development and maintenance. Apoptosis is fundamental in regulating T-cell development in the thymus during selection of developing thymocytes bearing a functional TCR[93]. Regulation of apoptosis 
in T cells differs from that in granulocytes. Thymocytes undergo apoptosis upon elevation of intracellular calcium concentrations in response to calcium ionophores[94], whereas increased levels of intracellular calcium delays eosinophil apoptosis[10]. Additionally, cycloheximide accelerates the constitutive rate of apoptosis in both neutrophils[95] and eosinophils[13], but not T cells[4]. Apoptosis is also essential for maintenance of mature $\mathrm{T}$ cells in the periphery. Upon encounter with cognate antigen, mature $\mathrm{T}$ cells undergo significant clonal expansion and removal of these expanded cells once the stimulus has been cleared is by activation-induced cell death[93], which involves activation of the extrinsic pathway of apoptosis by ligation of death receptors such as FasL[96]. Hence, apoptosis is an indispensable process to avoid autoimmunity.

From the knowledge gained on the internal controls of apoptosis, it may be possible to induce apoptotic death of specific inflammatory cells involved in the pathogenesis of diverse inflammatory diseases. In support of this suggestion, in vitro studies suggest that apoptosis in the closely related neutrophil and eosinophil granulocytes appears to be controlled by different mechanisms.

\section{GC Modulation of Apoptosis}

Physiological concentrations of GCs can regulate the intrinsic rate of apoptosis in many leukocytes and their efficacy depends upon a number of modulatory factors, including the GC type and concentration. GC regulation of granulocyte survival is dose dependent and occurs through the GCR (blocked by mifepristone) $[13,14]$. The GCs dexamethasone, methylprednisolone, and hydrocortisone all prolonged neutrophil viability from 12 to $48 \mathrm{~h}$, however non-GC progesterone failed to inhibit development of apoptosis[14,97], indicating this is not due to nonspecific effects of high dose GCs. Endogenous cortisone also mediates these effects on granulocyte survival, albeit to a lesser extent[13]. Differential effects of GCs on granulocyte apoptosis have also been shown in a rat peritoneal model[98], where various GCs induce eosinophil apoptosis, but delay neutrophil apoptosis with most significant effects observed at GC concentrations of $10^{-6}$ and $10^{-8} \mathrm{M}$, respectively. Thus, GCs used at optimal concentrations for eosinophil apoptosis prolong neutrophil survival, indicating that the beneficial effects of GC treatment for asthma may involve suppressing eosinophilic airway inflammation while maintaining antimicrobial responses. This is a more specific therapeutic approach to treatment of inflammatory diseases compared to general nonspecific immunosuppressive agents, such as cyclosporine. However, the anti-inflammatory action of GCs is not reproducible for COPD, a neutrophil-dependent disease. Here, GC-mediated neutrophil survival may contribute to tissue damage with increased potential for release of intracellular contents. $N_{F} \mathrm{~B}$ plays a vital role in the regulation of granulocyte apoptosis by inducing the expression of prosurvival genes[99] and targeted inhibition may be an approach to induce neutrophil apoptosis in COPD. Indeed, the $\mathrm{NF} \kappa \mathrm{B}$ inhibitor gliotoxin induces apoptosis in granulocytes alone or synergistically with TNF $\alpha[99]$.

GCs can also induce apoptosis in thymocytes[12] and, hence, are used in the treatment of lymphoid diseases. Interestingly, thymocyte sensitivity to GCs seems to be dependent upon their developmental stage where GCs induce apoptosis in $\mathrm{CD}^{+} \mathrm{CD} 8^{+}$double positive thymocytes, but not upon maturation to single positive thymocytes[93]. In contrast, mature $\mathrm{T}$ cells are relatively resistant to $\mathrm{GC}$-induced apoptosis, and some investigations have even reported GCs to prevent activation-induced cell death in these cells[100]. The effect of GCs on monocyte viability is more controversial. Although GCs have been suggested to induce apoptosis in monocytes[101], a recent study by Ehrchen et al. showed that monocytes were protected from staurosporine-induced apoptosis, mediated by reactive oxygen species (ROS), by increasing the expression of antioxidant molecules upon GC treatment[102].

GC influence on cell survival is initiated by GC binding to the cytoplasmic GCR and involves alterations in gene expression. However, there has been much controversy over the mechanism used by the GCR, whether by transactivation of death genes, transrepression of survival genes or both. 


\section{MECHANISMS OF GC EFFECTS ON APOPTOSIS}

\section{Evidence for Transrepression}

Helmberg and colleagues[103] have suggested that interference with proinflammatory signalling through the transrepressional activity of GCR is an important mechanism of GC-induced apoptosis. This was derived from studies using T-cell leukaemia cell lines expressing mutant GCRs that were DNA-binding and transactivation defective, but retained the ability to repress $\mathrm{NF \kappa B}$ and AP-1 transcriptional capability. These mutants were sufficient to induce apoptosis in these cells in response to GCs, implicating a role for GCs in apoptosis via the transrepression of prosurvival genes. Transrepression by GCR is suggested to be the main mechanism of suppressing the expression of cytokines and other inflammatory mediators that modulate granulocyte survival, and this may contribute to GC-induced apoptosis. In a recent study, Novac and colleagues provide data that support the role of GCR repression of gene expression in GC-induced apoptosis[104]. Here, GCs transiently suppress the level of the death receptor FasL and subsequently inhibit activation-induced T-cell apoptosis. Regulation of FasL expression was suggested to be a result of GCR binding directly to DNA, specifically to negative GREs that overlap an NFkB site. Hence, repression of FasL by GCR binding in cis may be due to competition for a common binding site and their rationalisation of this mechanism is that it would slow down T-cell apoptosis so that macrophages are not overwhelmed with a massive apoptotic cell load.

\section{Evidence for Transactivation}

Ramdas and Harmon[105] used a transactivation-defective mutant GCR that retained transrepressional activity, but did not mediate GC-induced apoptosis in human leukaemic T cells, suggesting that DNA binding by the GCR is important. Similarly, Reichardt and coworkers used a GCR ${ }^{\mathrm{dim}}$ mutant to show the requirement for transactivation in GC-induced thymocyte apoptosis[28]. New protein synthesis has been implicated in the regulation of apoptosis and, indeed, GC-induced thymocyte apoptosis and neutrophil survival can be abolished with cycloheximide[106]. This indicates that neutrophil survival requires ongoing gene expression and continuous synthesis of a prosurvival factor(s), a process that requires continued presence of GC[106], whereas GC-induced thymocyte apoptosis may be due to transactivation of death genes. Alternatively, GC induction of IкB expression may block proinflammatory signalling and contribute to induction of apoptosis in responsive cells[105].

\section{Effects of GCs on the Mitochondrial Pathway-Regulated Apoptosis}

Regulation of apoptosis may occur at the level of the mitochondria, an intracellular organelle with a central role in the apoptotic process. During the early stages of apoptosis, loss of mitochondrial membrane potential and increase in outer mitochondrial membrane permeability allow the release of factors such as Cytochrome C[8], which initiates downstream effects culminating in caspase activation and ultimately apoptosis[107]. Human eosinophils stimulated with dexamethasone show morphological changes characteristic of apoptosis, including DNA fragmentation, caspase-3 activation, and loss of mitochondrial membrane permeability[107]. Inhibition of mitochondrial permeabilisation, but not caspase-3 activity, prevented both mitochondrial disruption and apoptosis in response to dexamethasone. This suggests that GC-induced eosinophil apoptosis occurs through activation of the mitochondrial pathway, possibly resulting from the release of caspase-independent apoptosis-inducing factor (AIF)[107]. Loss of mitochondrial membrane potential and induction of eosinophil apoptosis may result from oxidant-induced mitochondrial injury, a process enhanced by GCs through production of ROS and sustained activation of proapoptotic JNK[3]. Ruiz and coworkers used the glucose-glucose oxidase system to achieve a constant production of hydrogen peroxide as a source of ROS to stimulate oxidative 
stress in neutrophils and, hence, apoptosis[108]. Dexamethasone delayed ROS-induced apoptosis in a concentration-dependent manner, with most significant inhibition at $10^{-6} \mathrm{M}$ dexamethasone at 12 and $24 \mathrm{~h}$ time points[108].

Although some studies described above do not implicate a role for caspases, GC-induced apoptosis in thymocytes and lymphoma cells can be blocked by pharmacological inhibition of caspases (z-VADfmk)[109], showing an additional level of apoptosis regulation, at least in these cell types, by GCs.

\section{Involvement of Bcl-2 Family Members in GC-Induced Apoptosis}

Bcl-2 proteins play a crucial role in apoptosis by regulating mitochondrial membrane stability[88]. Neutrophils express proapoptotic (Bak and Bax) and antiapoptotic (A1 and Mcl-1) members of the bcl-2 family[88]. GCs may delay neutrophil apoptosis resulting from mitochondrial dysfunction by increasing the abundance of antiapoptotic A1 mRNA, while decreasing the level of proapoptotic Bak mRNA in a GCR-dependent manner[88]. GC-induced thymocyte apoptosis may also result from mitochondrial dysfunction[110] as a consequence of alterations in the levels of pro- and antiapoptotic bcl-2 factors. Indecd, thymocyte apoptosis in response to dexamethasone is accelerated by bcl-2 deficiency[111], whereas bcl-2 overexpression inhibits GC-induced lymphoma cell apoptosis[112]. Furthermore, alterations in the phosphorylation status of bcl-2 family members in response to GCs may lead to mitochondrial injury and subsequent eosinophil apoptosis[3]. Thus, the intrinsic mitochondrial pathway may contribute significantly to determining the fate of cells by integrating signals from pro- and antiapoptotic members of the bcl-2 family of proteins and this may be regulated by GCs. This apoptotic pathway is also inhibited in human neutrophils by a cell permeable analogue of cAMP, dibutyryl cAMP (db-cAMP), resulting in neutrophil survival[8], whereas proinflammatory mediators G-CSF and GM-CSF increase the expression of proapoptotic bcl-2 proteins and, hence, may promote apoptosis[88].

\section{MODULATION OF PHAGOCYTIC REMOVAL OF APOPTOTIC CELLS BY MACROPHAGES}

In situations where cells are stimulated to undergo apoptosis at a high rate (for example, when there are overwhelming proapoptotic signals), the tissue load of apoptotic cells may be in danger of exceeding the removal capacity by phagocytes and noningested apoptotic cells may then undergo secondary necrosis with detrimental consequences[113]. Thus, the phagocytic clearance of cells dying by apoptosis plays a pivotal role in determining the inflammatory outcome and may be a prerequisite for effective resolution of inflammation. Hence, in order for deliberate induction of apoptotic cell death to be considered as a therapeutic strategy, parallel strategies for their efficient removal will also be required.

\section{Pharmacological Modulation}

Our laboratory is interested in the way inflammation resolves and we have made attempts to modulate macrophage phagocytic potential via pharmacological means. Increased levels of cAMP in human monocyte-derived macrophages (MDM $\phi)$, using db-cAMP, specifically inhibits phagocytosis of apoptotic neutrophils[114], whereas ligation of macrophage surface CD44 rapidly and specifically augments phagocytic uptake of apoptotic neutrophils, but not apoptotic lymphocytes[115]. Adhesion to the matrix protein fibronectin also rapidly increases macrophage capacity for internalisation of apoptotic neutrophils[116]. Particular focus is being made now on interpreting the influence of GCs on the mechanisms by which acute inflammation normally resolves. We have demonstrated that GCs augment phagocytic potential for nonphlogistic clearance of apoptotic leukocytes, a process that is essential for the resolution of inflammation. Pretreatment of 5-day human MDM $\phi$ for the first $24 \mathrm{~h}$ of culture with GCs 
methylprednisolone, dexamethasone, and hydrocortisone induced a prophagocytic phenotype displaying augmented phagocytosis of neutrophils undergoing apoptosis[17]. GCs also enhance macrophage capacity for uptake of other apoptotic targets, including Jurkat $\mathrm{T}$ cells and eosinophils, and also promote uptake of apoptotic neutrophils by alternative phagocytes, including human glomerular mesangial cells. GC action on phagocytosis is specific, as non-GCs aldosterone, estradiol, and progesterone did not exert this effect and GCs did not promote ingestion of Ig-opsonised erythrocytes. These observed effects are GCR dependent, being inhibited by the GCR antagonist mifepristone, and are reversed by cycloheximide, indicating the requirement for new protein synthesis.

\section{Reprogramming of Monocyte Differentiation by GCs}

Freshly isolated monocytes lack the capacity to ingest apoptotic targets, an ability that is gradually acquired during in vitro culture as adherent monocytes differentiate into macrophages[117]. GCs potentiate the phagocytic capacity of $\mathrm{MDM} \phi$ cultured for 5 days in vitro, with a greater potentiation observed the earlier monocytes were exposed to GCs during maturation[17]. Furthermore, we have demonstrated that long-term exposure (5 days) of monocytes to GCs induced differentiation of monocytes into $\mathrm{MDM} \phi$ displaying enhanced phagocytosis of apoptotic cells (up to threefold) and, hence, represents a proresolution phenotype[18]. Macrophages that had been treated with GC for this period were a more homogenous population of smaller, more rounded, and less well-spread cells with a phenotype characterised by reduced phosphorylation and, hence, recruitment of paxillin and pyk2 to sites of adhesion, with consequential loss of actin- and paxillin-containing podosomes, and loss of p130cas expression, an important adaptor molecule in integrin adhesion signalling through the DOCK 180/Crk/p130cas pathway. Despite their altered adhesion, time-lapse video microscopy revealed that GC-treated MDM $\phi$ remained membrane active with extension and retraction of cellular process, probably as a result of increased levels of active Rac. GC-treated MDM $\phi$ also displayed homogeneity in surface receptor expression, as shown by their laser scatter properties from flow cytometry, which identified a more uniform expression of HLA-DR, CD14, and CD44, consistent with reprogramming during monocyte differentiation. Although GC-treated MDM $\phi$ exhibit elevated surface expression of the haemoglobin scavenger receptor, CD163, no single surface receptor was identified that would define a prophagocytic macrophage phenotype. Combinatorial treatment with GC and the "classical activator" IFN $\gamma$ abolished GC potentiation of macrophage phagocytic capacity for apoptotic cells[118]. The first 24 $\mathrm{h}$ of culture with GC was critical for acquisition of a prophagocytic phenotype as this was overridden by subsequent treatment with IFN $\gamma$, with less pronounced inhibition the later IFN $\gamma$ was added. Interestingly, IFN $\gamma$ did not have an observed effect on GC-mediated morphology or surface receptor expression indicating that GC-treated MDM $\phi$ adhesion status can be dissociated from phagocytic capacity. This has implications for the application of GC therapy in Th1-mediated diseases characterised by high levels of IFN $\gamma$.

\section{Induction of an Anti-Inflammatory Macrophage Phenotype by GCs}

GCs are associated with induction of an anti-inflammatory macrophage phenotype. Importantly, GC potentiated phagocytosis of apoptotic cells did not stimulate the release of proinflammatory mediators, including MCP-1 and IL-8 chemokines[17]. Therefore, GCs promote "safe" clearance of cells dying by apoptosis and may directly contribute to the resolution of inflammation. The GC-inducible protein annexin I undergoes caspase-dependent recruitment from the cytosol to the outer plasma membrane during apoptosis to colocalise with phosphatidylserine and may be required for efficient clearance of dying cells[33]. Alternatively, GC-induced expression of annexin I in macrophages and subsequent release may enhance apoptotic cell uptake[35]. Indeed, macrophages from annexin I knockout mice show 
defective phagocytosis of apoptotic cells acting through the FPRL1[35]. This has been furthered by a recent study by Scannell et al., who report that annexin I is a prophagocytic factor released by apoptotic cells and actively promotes the FPRL1-dependent clearance of apoptotic cells by macrophages[119]. Thus, transactivation of annexin I expression by the GCR may contribute to enhanced recognition and internalisation of apoptotic cells in response to GC treatment.

$\mathrm{C} 1 \mathrm{q}$ is an important subcomponent of complement $\mathrm{C}$, which activates the classical pathway upon binding to immune complexes or CRP and its expression in monocytes/macrophages can be induced by GCs or inhibited by IFN $\gamma$ and LPS[31,120]. Clq binds to apoptotic cells[121] and may aid in their removal by phagocytes. Interestingly, Botto and colleagues demonstrated $\mathrm{Clq}$ deficiency in mice to cause an SLE-like disease with high titres of autoantibodies and accumulation of apoptotic cells in glomeruli[85] and defective clearance of apoptotic cells is also observed in human SLE patients[82,83]. Thus, C1q may protect against development of SLE by targeting apoptotic cells for clearance. This has been supported by in vitro studies where Clq-deficient human macrophages show impaired phagocytosis of apoptotic cells, and this is restored with purified human $\mathrm{C1q}$ [84]. Hence, induction of innate proteins by GCs may contribute to their anti-inflammatory effect by enhancing removal of dying cells.

A recent study by Ehrchen and coworkers used a microarray system to analyse the expression profile in human monocytes treated with GCs[102]. Their data signify the multitude of GC effects, where the expression of over 100 genes were GC regulated with a more pronounced induction of genes that are important in monocyte/macrophage functions such as phagocytosis, apoptosis, and adhesion. Important to the process of phagocytosis, there was induced expression of CD163, FPR1, and MER tyrosine kinase receptors, and MFG-E8 and C1q serum proteins, however, the relevance of this in GC-potentiated phagocytic capacity is unclear. These results indicate that GC effects on monocytes are not simply immunosuppressive, but also immunomodulatory. GCs promote induction of an anti-inflammatory phenotype important for the resolution of inflammation, challenging the concept that transrepression is the dominant mechanism of GC action on monocytes.

\section{CONCLUSION}

Apoptosis is a fundamental process in cell and tissue homeostasis that, in contrast to necrosis, is associated with maintenance of cell membrane integrity and noninflammatory clearance by phagocytes. Failure or inefficient apoptosis and/or phagocytosis may result in necrosis with detrimental proinflammatory consequences. GCs modulate inflammatory cell survival and promote nonphlogistic phagocytosis of apoptotic cell targets in vitro, a situation that could be deemed proresolution with consequent implications in the clinical setting where unresolving chronic inflammatory diseases cause considerable morbidity and untimely death. Thus, considerable effort to elucidate the precise molecular and cellular mechanisms of action of GCs, as well as improve existing GCs, is being made.

\section{ACKNOWLEDGEMENTS}

We thank the Medical Research Council UK (MRC), The Wellcome Trust, and the Arthritis Research Campaign (16140) for financial support throughout recent years. AM is an MRC-funded PhD student and SM is a Wellcome Trust-funded PhD student.

\section{REFERENCES}

1. Haslett, C., Savill, J.S., Whyte, M.K., Stern, M., Dransfield, I., and Meagher, L.C. (1994) Granulocyte apoptosis and the control of inflammation. Philos. Trans. R. Soc. Lond. B Biol. Sci. 345, 327-333.

2. Cosio, B.G., Torrego, A., and Adcock, I.M. (2005) [Molecular mechanisms of glucocorticoids]. Arch. Bronconeumol. 41, 34-41.

3. Gardai, S.J., Hoontrakoon, R., Goddard, C.D., Day, B.J., Chang, L.Y., Henson, P.M., and Bratton, D.L. (2003) 
Oxidant-mediated mitochondrial injury in eosinophil apoptosis: enhancement by glucocorticoids and inhibition by granulocyte-macrophage colony-stimulating factor. J. Immunol. 170, 556-566.

4. Haslett, C. (1999) Granulocyte apoptosis and its role in the resolution and control of lung inflammation. Am. J. Respir. Crit. Care Med. 160, S5-11.

5. Kerr, J.F., Wyllie, A.H., and Currie, A.R. (1972) Apoptosis: a basic biological phenomenon with wide-ranging implications in tissue kinetics. Br. J. Cancer 26, 239-257.

6. Savill, J.S., Wyllie, A.H., Henson, J.E., Walport, M.J., Henson, P.M., and Haslett, C. (1989) Macrophage phagocytosis of aging neutrophils in inflammation. Programmed cell death in the neutrophil leads to its recognition by macrophages. J. Clin. Invest. 83, 865-875.

7. Stern, M., Meagher, L., Savill, J., and Haslett, C. (1992) Apoptosis in human eosinophils. Programmed cell death in the eosinophil leads to phagocytosis by macrophages and is modulated by IL-5. J. Immunol. 148, 3543-3549.

8. Martin, M.C., Dransfield, I., Haslett, C., and Rossi, A.G. (2001) Cyclic AMP regulation of neutrophil apoptosis occurs via a novel protein kinase A-independent signaling pathway. J. Biol. Chem. 276, 45041-45050.

9. Ward, C., Murray, J., Clugston, A., Dransfield, I., Haslett, C., and Rossi, A.G. (2005) Interleukin-10 inhibits lipopolysaccharide-induced survival and extracellular signal-regulated kinase activation in human neutrophils. Eur. J. Immunol. 35, 2728-2737.

10. Ward, C., Dransfield, I., Murray, J., Farrow, S.N., Haslett, C., and Rossi, A.G. (2002) Prostaglandin D2 and its metabolites induce caspase-dependent granulocyte apoptosis that is mediated via inhibition of I kappa B alpha degradation using a peroxisome proliferator-activated receptor-gamma-independent mechanism. J. Immunol. 168, $6232-6243$

11. Murray, J., Barbara, J.A., Dunkley, S.A., Lopez, A.F., Van Ostade, X., Condliffe, A.M., Dransfield, I., Haslett, C., and Chilvers, E.R. (1997) Regulation of neutrophil apoptosis by tumor necrosis factor-alpha: requirement for TNFR55 and TNFR75 for induction of apoptosis in vitro. Blood 90, 2772-2783.

12. Wyllie, A.H. (1980) Glucocorticoid-induced thymocyte apoptosis is associated with endogenous endonuclease activation. Nature 284, 555-556.

13. Meagher, L.C., Cousin, J.M., Seckl, J.R., and Haslett, C. (1996) Opposing effects of glucocorticoids on the rate of apoptosis in neutrophilic and eosinophilic granulocytes. J. Immunol. 156, 4422-4428.

14. Cox, G. (1995) Glucocorticoid treatment inhibits apoptosis in human neutrophils. Separation of survival and activation outcomes. J. Immunol. 154, 4719-4725.

15. Serhan, C.N., Brain, S.D., Buckley, C.D., Gilroy, D.W., Haslett, C., O'Neill, L.A., Perretti, M., Rossi, A.G., and Wallace, J.L. (2007) Resolution of inflammation: state of the art, definitions and terms. FASEB J. 21, 325-332.

16. Rossi, A.G., Hallett, J.M., Sawatzky, D.A., Teixeira, M.M., and Haslett, C. (2007) Modulation of granulocyte apoptosis can influence the resolution of inflammation. Biochem. Soc. Trans. 35, 288-291.

17. Liu, Y., Cousin, J.M., Hughes, J., Van Damme, J., Seckl, J.R., Haslett, C., Dransfield, I., Savill, J., and Rossi, A.G. (1999) Glucocorticoids promote nonphlogistic phagocytosis of apoptotic leukocytes. J. Immunol. 162, 3639-3646.

18. Giles, K.M., Ross, K., Rossi, A.G., Hotchin, N.A., Haslett, C., and Dransfield, I. (2001) Glucocorticoid augmentation of macrophage capacity for phagocytosis of apoptotic cells is associated with reduced p130Cas expression, loss of paxillin/pyk2 phosphorylation, and high levels of active Rac. J. Immunol. 167, 976-986.

19. Woolley, K.L., Gibson, P.G., Carty, K., Wilson, A.J., Twaddell, S.H., and Woolley, M.J. (1996) Eosinophil apoptosis and the resolution of airway inflammation in asthma. Am. J. Respir. Crit. Care Med. 154, 237-243.

20. Goulding, N.J. (2004) The molecular complexity of glucocorticoid actions in inflammation - a four-ring circus. Curr. Opin. Pharmacol. 4, 629-636.

21. Bamberger, C.M., Bamberger, A.M., de Castro, M., and Chrousos, G.P. (1995) Glucocorticoid receptor beta, a potential endogenous inhibitor of glucocorticoid action in humans. J. Clin. Invest. 95, 2435-2441.

22. Oakley, R.H., Jewell, C.M., Yudt, M.R., Bofetiado, D.M., and Cidlowski, J.A. (1999) The dominant negative activity of the human glucocorticoid receptor beta isoform. Specificity and mechanisms of action. J. Biol. Chem. 274, 2785727866.

23. Pratt, W.B. and Toft, D.O. (1997) Steroid receptor interactions with heat shock protein and immunophilin chaperones. Endocr. Rev. 18, 306-360.

24. Barnes, P.J. (2006) Corticosteroids: the drugs to beat. Eur. J. Pharmacol. 533, 2-14.

25. Chandler, V.L., Maler, B.A., and Yamamoto, K.R. (1983) DNA sequences bound specifically by glucocorticoid receptor in vitro render a heterologous promoter hormone responsive in vivo. Cell 33, 489-499.

26. Drouin, J., Sun, Y.L., Tremblay, S., Lavender, P., Schmidt, T.J., de Lean, A., and Nemer, M. (1992) Homodimer formation is rate-limiting for high affinity DNA binding by glucocorticoid receptor. Mol. Endocrinol. 6, 1299-1309.

27. Dahlman-Wright, K., Wright, A., Gustafsson, J.A., and Carlstedt-Duke, J. (1991) Interaction of the glucocorticoid receptor DNA-binding domain with DNA as a dimer is mediated by a short segment of five amino acids. J. Biol. Chem. 266, 3107-3112.

28. Reichardt, H.M., Kaestner, K.H., Tuckermann, J., Kretz, O., Wessely, O., Bock, R., Gass, P., Schmid, W., Herrlich, P., Angel, P., and Schutz, G. (1998) DNA binding of the glucocorticoid receptor is not essential for survival. Cell 93, 531-541.

29. Barnes, P.J. (1998) Anti-inflammatory actions of glucocorticoids: molecular mechanisms. Clin. Sci. (Lond.) 94, 557572. 
30. Abbinante-Nissen, J.M., Simpson, L.G., and Leikauf, G.D. (1995) Corticosteroids increase secretory leukocyte protease inhibitor transcript levels in airway epithelial cells. Am. J. Physiol. 268, L601-606.

31. Faust, D., Akoglu, B., Zgouras, D., Scheuermann, E.H., Milovic, V., and Stein, J. (2002) Anti-inflammatory drugs modulate $\mathrm{Clq}$ secretion in human peritoneal macrophages in vitro. Biochem. Pharmacol. 64, 457-462.

32. Hannon, R., Croxtall, J.D., Getting, S.J., Roviezzo, F., Yona, S., Paul-Clark, M.J., Gavins, F.N., Perretti, M., Morris, J.F., Buckingham, J.C., and Flower, R.J. (2003) Aberrant inflammation and resistance to glucocorticoids in annexin 1-/-mouse. FASEB J. 17, 253-255.

33. Arur, S., Uche, U.E., Rezaul, K., Fong, M., Scranton, V., Cowan, A.E., Mohler, W., and Han, D.K. (2003) Annexin I is an endogenous ligand that mediates apoptotic cell engulfment. Dev. Cell 4, 587-598.

34. Roviezzo, F., Getting, S.J., Paul-Clark, M.J., Yona, S., Gavins, F.N., Perretti, M., Hannon, R., Croxtall, J.D., Buckingham, J.C., and Flower, R.J. (2002) The annexin-1 knockout mouse: what it tells us about the inflammatory response. J. Physiol. Pharmacol. 53, 541-553.

35. Maderna, P., Yona, S., Perretti, M., and Godson, C. (2005) Modulation of phagocytosis of apoptotic neutrophils by supernatant from dexamethasone-treated macrophages and annexin-derived peptide Ac(2-26). J. Immunol. 174, 37273733.

36. Abraham, S.M., Lawrence, T., Kleiman, A., Warden, P., Medghalchi, M., Tuckermann, J., Saklatvala, J., and Clark, A.R. (2006) Antiinflammatory effects of dexamethasone are partly dependent on induction of dual specificity phosphatase 1. J. Exp. Med. 203, 1883-1889.

37. Sakai, D.D., Helms, S., Carlstedt-Duke, J., Gustafsson, J.A., Rottman, F.M., and Yamamoto, K.R. (1988) Hormonemediated repression: a negative glucocorticoid response element from the bovine prolactin gene. Genes Dev. 2, 1144 1154.

38. De Bosscher, K., Vanden Berghe, W., Vermeulen, L., Plaisance, S., Boone, E., and Haegeman, G. (2000) Glucocorticoids repress NF-kappaB-driven genes by disturbing the interaction of p65 with the basal transcription machinery, irrespective of coactivator levels in the cell. Proc. Natl. Acad. Sci. U. S. A. 97, 3919-3924.

39. Karin, M. and Chang, L. (2001) AP-1--glucocorticoid receptor crosstalk taken to a higher level. J. Endocrinol. 169, $447-451$

40. Tak, P.P. and Firestein, G.S. (2001) NF-kappaB: a key role in inflammatory diseases. J. Clin. Invest. 107, 7-11.

41. Rahman, I. and MacNee, W. (1998) Role of transcription factors in inflammatory lung diseases. Thorax 53, 601-612.

42. Reichardt, H.M., Tuckermann, J.P., Gottlicher, M., Vujic, M., Weih, F., Angel, P., Herrlich, P., and Schutz, G. (2001) Repression of inflammatory responses in the absence of DNA binding by the glucocorticoid receptor. EMBO J. 20, $7168-7173$.

43. Tuckermann, J.P., Reichardt, H.M., Arribas, R., Richter, K.H., Schutz, G., and Angel, P. (1999) The DNA bindingindependent function of the glucocorticoid receptor mediates repression of AP-1-dependent genes in skin. J. Cell Biol. $147,1365-1370$

44. Karin, M. (1998) New twists in gene regulation by glucocorticoid receptor: is DNA binding dispensable? Cell 93, 487-490.

45. Urnov, F.D. and Wolffe, A.P. (2001) Chromatin remodeling and transcriptional activation: the cast (in order of appearance). Oncogene 20, 2991-3006.

46. Adcock, I.M., Ito, K., and Barnes, P.J. (2004) Glucocorticoids: effects on gene transcription. Proc. Am. Thorac. Soc. $1,247-254$.

47. De Bosscher, K., Vanden Berghe, W., and Haegeman, G. (2001) Glucocorticoid repression of AP-1 is not mediated by competition for nuclear coactivators. Mol. Endocrinol. 15, $219-227$.

48. Ito, K., Barnes, P.J., and Adcock, I.M. (2000) Glucocorticoid receptor recruitment of histone deacetylase 2 inhibits interleukin-lbeta-induced histone $\mathrm{H} 4$ acetylation on lysines 8 and 12. Mol. Cell. Biol. 20, 6891-6903.

49. Norman, A.W., Mizwicki, M.T., and Norman, D.P. (2004) Steroid-hormone rapid actions, membrane receptors and a conformational ensemble model. Nat. Rev. Drug Discov. 3, 27-41.

50. Buttgereit, F. and Scheffold, A. (2002) Rapid glucocorticoid effects on immune cells. Steroids 67, 529-534.

51. Long, F., Wang, Y.X., Liu, L., Zhou, J., Cui, R.Y., and Jiang, C.L. (2005) Rapid nongenomic inhibitory effects of glucocorticoids on phagocytosis and superoxide anion production by macrophages. Steroids 70, 55-61.

52. Buckingham, J.C. (2006) Glucocorticoids: exemplars of multi-tasking. Br. J. Pharmacol. 147(Suppl 1), S258-268.

53. Chen, Y.Z. and Qiu, J. (1999) Pleiotropic signaling pathways in rapid, nongenomic action of glucocorticoid. Mol. Cell Biol. Res. Commun. 2, 145-149.

54. Croxtall, J.D., Choudhury, Q., and Flower, R.J. (2000) Glucocorticoids act within minutes to inhibit recruitment of signalling factors to activated EGF receptors through a receptor-dependent, transcription-independent mechanism. $\mathrm{Br}$. J. Pharmacol. 130, 289-298.

55. Barnes, P.J. and Adcock, I.M. (2003) How do corticosteroids work in asthma? Ann. Intern. Med. 139, 359-370.

56. Webster, J.C., Oakley, R.H., Jewell, C.M., and Cidlowski, J.A. (2001) Proinflammatory cytokines regulate human glucocorticoid receptor gene expression and lead to the accumulation of the dominant negative beta isoform: a mechanism for the generation of glucocorticoid resistance. Proc. Natl. Acad. Sci. U. S. A. 98, 6865-6870.

57. Kozaci, D.L., Chernajovsky, Y., and Chikanza, I.C. (2007) The differential expression of corticosteroid receptor isoforms in corticosteroid-resistant and -sensitive patients with rheumatoid arthritis. Rheumatology (Oxford) 46, 579-585. 
58. Ito, K., Lim, S., Caramori, G., Chung, K.F., Barnes, P.J., and Adcock, I.M. (2001) Cigarette smoking reduces histone deacetylase 2 expression, enhances cytokine expression, and inhibits glucocorticoid actions in alveolar macrophages. FASEB J. 15, 1110-1112.

59. Schacke, H., Docke, W.D., and Asadullah, K. (2002) Mechanisms involved in the side effects of glucocorticoids. Pharmacol. Ther. 96, 23-43.

60. Catley, M. (2007) Dissociated steroids. TheScientificWorldJOURNAL 7, 421-430.

61. Whyte, M.K., Meagher, L.C., MacDermot, J., and Haslett, C. (1993) Impairment of function in aging neutrophils is associated with apoptosis. J. Immunol. 150, 5124-5134.

62. Dransfield, I., Buckle, A.M., Savill, J.S., McDowall, A., Haslett, C., and Hogg, N. (1994) Neutrophil apoptosis is associated with a reduction in CD16 (Fc gamma RIII) expression. J. Immunol. 153, 1254-1263.

63. Dransfield, I., Stocks, S.C., and Haslett, C. (1995) Regulation of cell adhesion molecule expression and function associated with neutrophil apoptosis. Blood 85, 3264-3273.

64. Savill, J.S., Henson, P.M., and Haslett, C. (1989) Phagocytosis of aged human neutrophils by macrophages is mediated by a novel "charge-sensitive" recognition mechanism. J. Clin. Invest. 84, 1518-1527.

65. Hart, S.P., Ross, J.A., Ross, K., Haslett, C., and Dransfield, I. (2000) Molecular characterization of the surface of apoptotic neutrophils: implications for functional downregulation and recognition by phagocytes. Cell Death Differ. 7, 493-503.

66. Fadok, V.A., Voelker, D.R., Campbell, P.A., Cohen, J.J., Bratton, D.L., and Henson, P.M. (1992) Exposure of phosphatidylserine on the surface of apoptotic lymphocytes triggers specific recognition and removal by macrophages. J. Immunol. 148, 2207-2216.

67. Gregory, C.D. and Devitt, A. (2004) The macrophage and the apoptotic cell: an innate immune interaction viewed simplistically? Immunology 113, 1-14.

68. Liu, G., Wu, C., Wu, Y., and Zhao, Y. (2006) Phagocytosis of apoptotic cells and immune regulation. Scand. J. Immunol. 64, 1-9.

69. Michlewska, S., McColl, A., Rossi, A.G., Megson, I.L., and Dransfield, I. (2007) Clearance of dying cells and autoimmunity. Autoimmunity 40, 267-273.

70. Savill, J., Hogg, N., Ren, Y., and Haslett, C. (1992) Thrombospondin cooperates with CD36 and the vitronectin receptor in macrophage recognition of neutrophils undergoing apoptosis. J. Clin. Invest. 90, 1513-1522.

71. Platt, N., Suzuki, H., Kurihara, Y., Kodama, T., and Gordon, S. (1996) Role for the class A macrophage scavenger receptor in the phagocytosis of apoptotic thymocytes in vitro. Proc. Natl. Acad. Sci. U. S. A. 93, 12456-12460.

72. Brown, S., Heinisch, I., Ross, E., Shaw, K., Buckley, C.D., and Savill, J. (2002) Apoptosis disables CD31-mediated cell detachment from phagocytes promoting binding and engulfment. Nature 418, 200-203.

73. Fadok, V.A., Bratton, D.L., Rose, D.M., Pearson, A., Ezekewitz, R.A., and Henson, P.M. (2000) A receptor for phosphatidylserine-specific clearance of apoptotic cells. Nature 405, 85-90.

74. Ogden, C.A., deCathelineau, A., Hoffmann, P.R., Bratton, D., Ghebrehiwet, B., Fadok, V.A., and Henson, P.M. (2001) Clq and mannose binding lectin engagement of cell surface calreticulin and CD91 initiates macropinocytosis and uptake of apoptotic cells. J. Exp. Med. 194, 781-795.

75. Scott, R.S., McMahon, E.J., Pop, S.M., Reap, E.A., Caricchio, R., Cohen, P.L., Earp, H.S., and Matsushima, G.K. (2001) Phagocytosis and clearance of apoptotic cells is mediated by MER. Nature 411, 207-211.

76. Savill, J. (1997) Recognition and phagocytosis of cells undergoing apoptosis. Br. Med. Bull. 53, 491-508.

77. Meagher, L.C., Savill, J.S., Baker, A., Fuller, R.W., and Haslett, C. (1992) Phagocytosis of apoptotic neutrophils does not induce macrophage release of thromboxane B2. J. Leukoc. Biol. 52, 269-273.

78. Hughes, J., Liu, Y., Van Damme, J., and Savill, J. (1997) Human glomerular mesangial cell phagocytosis of apoptotic neutrophils: mediation by a novel CD36-independent vitronectin receptor/thrombospondin recognition mechanism that is uncoupled from chemokine secretion. J. Immunol. 158, 4389-4397.

79. Stern, M., Savill, J., and Haslett, C. (1996) Human monocyte-derived macrophage phagocytosis of senescent eosinophils undergoing apoptosis. Mediation by alpha $\mathrm{v}$ beta 3/CD36/thrombospondin recognition mechanism and lack of phlogistic response. Am. J. Pathol. 149, 911-921.

80. Voll, R.E., Herrmann, M., Roth, E.A., Stach, C., Kalden, J.R., and Girkontaite, I. (1997) Immunosuppressive effects of apoptotic cells. Nature 390, 350-351.

81. Fadok, V.A., Bratton, D.L., Konowal, A., Freed, P.W., Westcott, J.Y., and Henson, P.M. (1998) Macrophages that have ingested apoptotic cells in vitro inhibit proinflammatory cytokine production through autocrine/paracrine mechanisms involving TGF-beta, PGE2, and PAF. J. Clin. Invest. 101, 890-898.

82. Gaipl, U.S., Kuhn, A., Sheriff, A., Munoz, L.E., Franz, S., Voll, R.E., Kalden, J.R., and Herrmann, M. (2006) Clearance of apoptotic cells in human SLE. Curr. Dir. Autoimmun. 9, 173-187.

83. Tas, S.W., Quartier, P., Botto, M., and Fossati-Jimack, L. (2006) Macrophages from patients with SLE and rheumatoid arthritis have defective adhesion in vitro, while only SLE macrophages have impaired uptake of apoptotic cells. Ann. Rheum. Dis. 65, 216-221.

84. Taylor, P.R., Carugati, A., Fadok, V.A., Cook, H.T., Andrews, M., Carroll, M.C., Savill, J.S., Henson, P.M., Botto, M., and Walport, M.J. (2000) A hierarchical role for classical pathway complement proteins in the clearance of apoptotic cells in vivo. J. Exp. Med. 192, 359-366.

85. Botto, M., Dell'Agnola, C., Bygrave, A.E., Thompson, E.M., Cook, H.T., Petry, F., Loos, M., Pandolfi, P.P., and 
Walport, M.J. (1998) Homozygous Clq deficiency causes glomerulonephritis associated with multiple apoptotic bodies. Nat. Genet. 19, 56-59.

86. Rossi, A.G., Sawatzky, D.A., Walker, A., Ward, C., Sheldrake, T.A., Riley, N.A., Caldicott, A., Martinez-Losa, M., Walker, T.R., Duffin, R., Gray, M., Crescenzi, E., Martin, M.C., Brady, H.J., Savill, J.S., Dransfield, I., and Haslett, C. (2006) Cyclin-dependent kinase inhibitors enhance the resolution of inflammation by promoting inflammatory cell apoptosis. Nat. Med. 12, 1056-1064.

87. Sawatzky, D.A., Willoughby, D.A., Colville-Nash, P.R., and Rossi, A.G. (2006) The involvement of the apoptosismodulating proteins ERK 1/2, Bcl-xL and Bax in the resolution of acute inflammation in vivo. Am. J. Pathol. 168, $33-41$.

88. Madsen-Bouterse, S.A., Rosa, G.J., and Burton, J.L. (2006) Glucocorticoid modulation of Bcl-2 family members A1 and Bak during delayed spontaneous apoptosis of bovine blood neutrophils. Endocrinology 147, 3826-3834.

89. Colotta, F., Re, F., Polentarutti, N., Sozzani, S., and Mantovani, A. (1992) Modulation of granulocyte survival and programmed cell death by cytokines and bacterial products. Blood 80, 2012-2020.

90. Lee, A., Whyte, M.K., and Haslett, C. (1993) Inhibition of apoptosis and prolongation of neutrophil functional longevity by inflammatory mediators. J. Leukoc. Biol. 54, 283-288.

91. Hannah, S., Mecklenburgh, K., Rahman, I., Bellingan, G.J., Greening, A., Haslett, C., and Chilvers, E.R. (1995) Hypoxia prolongs neutrophil survival in vitro. FEBS Lett. 372, 233-237.

92. Walsh, G.M. (1997) Mechanisms of human eosinophil survival and apoptosis. Clin. Exp. Allergy 27, $482-487$

93. Herold, M.J., McPherson, K.G., and Reichardt, H.M. (2006) Glucocorticoids in T cell apoptosis and function. Cell. Mol. Life Sci. 63, 60-72.

94. McConkey, D.J., Hartzell, P., Jondal, M., and Orrenius, S. (1989) Inhibition of DNA fragmentation in thymocytes and isolated thymocyte nuclei by agents that stimulate protein kinase C. J. Biol. Chem. 264, 13399-13402.

95. Whyte, M.K., Savill, J., Meagher, L.C., Lee, A., and Haslett, C. (1997) Coupling of neutrophil apoptosis to recognition by macrophages: coordinated acceleration by protein synthesis inhibitors. J. Leukoc. Biol. 62, 195-202.

96. Chilvers, E.R., Rossi, A.G., Murray, J., and Haslett, C. (1998) Regulation of granulocyte apoptosis and implications for anti-inflammatory therapy. Thorax 53, 533-534.

97. Liles, W.C., Dale, D.C., and Klebanoff, S.J. (1995) Glucocorticoids inhibit apoptosis of human neutrophils. Blood 86, $3181-3188$

98. Nittoh, T., Fujimori, H., Kozumi, Y., Ishihara, K., Mue, S., and Ohuchi, K. (1998) Effects of glucocorticoids on apoptosis of infiltrated eosinophils and neutrophils in rats. Eur. J. Pharmacol. 354, 73-81.

99. Ward, C., Chilvers, E.R., Lawson, M.F., Pryde, J.G., Fujihara, S., Farrow, S.N., Haslett, C., and Rossi, A.G. (1999) NF-kappaB activation is a critical regulator of human granulocyte apoptosis in vitro. J. Biol. Chem. 274, 4309-4318.

100. Ashwell, J.D., Lu, F.W., and Vacchio, M.S. (2000) Glucocorticoids in T cell development and function. Annu. Rev. Immunol. 18, 309-345.

101. Schmidt, M., Pauels, H.G., Lugering, N., Lugering, A., Domschke, W., and Kucharzik, T. (1999) Glucocorticoids induce apoptosis in human monocytes: potential role of IL-1 beta. J. Immunol. 163, 3484-3490.

102. Ehrchen, J., Steinmuller, L., Barczyk, K., Tenbrock, K., Nacken, W., Eisenacher, M., Nordhues, U., Sorg, C., Sunderkotter, C., and Roth, J. (2007) Glucocorticoids induce differentiation of a specifically activated, antiinflammatory subtype of human monocytes. Blood 109, 1265-1274.

103. Helmberg, A., Auphan, N., Caelles, C., and Karin, M. (1995) Glucocorticoid-induced apoptosis of human leukemic cells is caused by the repressive function of the glucocorticoid receptor. EMBO J. 14, 452-460.

104. Novac, N., Baus, D., Dostert, A., and Heinzel, T. (2006) Competition between glucocorticoid receptor and NFkappaB for control of the human FasL promoter. FASEB J. 20, 1074-1081

105. Ramdas, J. and Harmon, J.M. (1998) Glucocorticoid-induced apoptosis and regulation of NF-kappaB activity in human leukemic T cells. Endocrinology 139, 3813-3821.

106. Cox, G. and Austin, R.C. (1997) Dexamethasone-induced suppression of apoptosis in human neutrophils requires continuous stimulation of new protein synthesis. J. Leukoc. Biol. 61, 224-230.

107. Letuve, S., Druilhe, A., Grandsaigne, M., Aubier, M., and Pretolani, M. (2002) Critical role of mitochondria, but not caspases, during glucocorticosteroid-induced human eosinophil apoptosis. Am. J. Respir. Cell Mol. Biol. 26, 565-571.

108. Ruiz, L.M., Bedoya, G., Salazar, J., Garcia de, O.D., and Patino, P.J. (2002) Dexamethasone inhibits apoptosis of human neutrophils induced by reactive oxygen species. Inflammation 26, 215-222.

109. Distelhorst, C.W. (2002) Recent insights into the mechanism of glucocorticosteroid-induced apoptosis. Cell Death Differ. 9, 6-19.

110. Petit, P.X., Lecoeur, H., Zorn, E., Dauguet, C., Mignotte, B., and Gougeon, M.L. (1995) Alterations in mitochondrial structure and function are early events of dexamethasone-induced thymocyte apoptosis. J. Cell Biol. 130, 157-167.

111. Veis, D.J., Sorenson, C.M., Shutter, J.R., and Korsmeyer, S.J. (1993) Bcl-2-deficient mice demonstrate fulminant lymphoid apoptosis, polycystic kidneys, and hypopigmented hair. Cell 75, 229-240.

112. McColl, K.S., He, H., Zhong, H., Whitacre, C.M., Berger, N.A., and Distelhorst, C.W. (1998) Apoptosis induction by the glucocorticoid hormone dexamethasone and the calcium-ATPase inhibitor thapsigargin involves Bcl-2 regulated caspase activation. Mol. Cell. Endocrinol. 139, 229-238.

113. Ogasawara, J., Watanabe-Fukunaga, R., Adachi, M., Matsuzawa, A., Kasugai, T., Kitamura, Y., Itoh, N., Suda, T., and Nagata, S. (1993) Lethal effect of the anti-Fas antibody in mice. Nature 364, 806-809. 
114. Rossi, A.G., McCutcheon, J.C., Roy, N., Chilvers, E.R., Haslett, C., and Dransfield, I. (1998) Regulation of macrophage phagocytosis of apoptotic cells by cAMP. J. Immunol. 160, 3562-3568.

115. Hart, S.P., Dougherty, G.J., Haslett, C., and Dransfield, I. (1997) CD44 regulates phagocytosis of apoptotic neutrophil granulocytes, but not apoptotic lymphocytes, by human macrophages. J. Immunol. 159, 919-925.

116. McCutcheon, J.C., Hart, S.P., Canning, M., Ross, K., Humphries, M.J., and Dransfield, I. (1998) Regulation of macrophage phagocytosis of apoptotic neutrophils by adhesion to fibronectin. J. Leukoc. Biol. 64, 600-607.

117. Newman, S.L., Henson, J.E., and Henson, P.M. (1982) Phagocytosis of senescent neutrophils by human monocytederived macrophages and rabbit inflammatory macrophages. J. Exp. Med. 156, 430-442.

118. Heasman, S.J., Giles, K.M., Rossi, A.G., Allen, J.E., Haslett, C., and Dransfield, I. (2004) Interferon gamma suppresses glucocorticoid augmentation of macrophage clearance of apoptotic cells. Eur. J. Immunol. 34, 1752-1761.

119. Scannell, M., Flanagan, M.B., deStefani, A., Wynne, K.J., Cagney, G., Godson, C., and Maderna, P. (2007) Annexin1 and peptide derivatives are released by apoptotic cells and stimulate phagocytosis of apoptotic neutrophils by macrophages. J. Immunol. 178, 4595-4605.

120. Armbrust, T., Nordmann, B., Kreissig, M., and Ramadori, G. (1997) C1Q synthesis by tissue mononuclear phagocytes from normal and from damaged rat liver: up-regulation by dexamethasone, down-regulation by interferon gamma, and lipopolysaccharide. Hepatology 26, 98-106.

121. Korb, L.C. and Ahearn, J.M. (1997) Clq binds directly and specifically to surface blebs of apoptotic human keratinocytes: complement deficiency and systemic lupus erythematosus revisited. J. Immunol. 158, 4525-4528.

\section{This article should be cited as follows:}

McColl, A., Michlewska, S., Dransfield, I., and Rossi, A.G. (2007) Effects of glucocorticoids on apoptosis and clearance of apoptotic cells. TheScientificWorldJOURNAL 7, 1165-1181. DOI 10.1100/tsw.2007.224. 


\title{
Analysis of Neutrophil Apoptosis
}

\author{
Emma L. Taylor, Adriano G. Rossi, Ian Dransfield, and Simon P. Hart
}

\begin{abstract}
Summary
Neutrophil-derived granule enzymes, oxidants, and mediators have been implicated in the pathogenesis of a variety of inflammatory diseases. Neutrophil apoptosis is associated with the loss of expression of adhesion molecules and greatly reduced responsiveness to external stimuli, so that these cells become functionally isolated from their environment. In contrast with necrosis, apoptosis is associated with preservation of plasma membrane integrity, so that release of harmful neutrophil contents is limited, and the inert neutrophils are phagocytosed by local macrophages. Furthermore, phagocytosis of apoptotic neutrophils by human macrophages in vitro suppresses release of macrophage-derived pro-inflammatory mediators. In this way, by downregulating neutrophil functions and triggering "silent" clearance by phagocytes, apoptosis provides a mechanism for the safe disposal of potentially destructive inflammatory cells. Many of the molecular events involved in the apoptosis pathway have been identified and several complementary methods may be employed to identify and quantitate neutrophil apoptosis. This chapter will discuss analysis of neutrophil morphology, DNA fragmentation, membrane changes, mitochondrial alterations, caspase activation, and phagocytosis of apoptotic neutrophils by macrophages.
\end{abstract}

Key Words: Apoptosis; caspase; necrosis; DNA fragmentation; phagocytosis.

\section{Introduction}

Once recruited in large numbers to a site of tissue damage, neutrophils die locally by undergoing apoptosis, which is the process of programmed cell death in vertebrates. The removal of apoptotic neutrophils is thought to be an important step in preventing the release of granule contents and chemotactic factors into the extracellular fluid, thereby halting further injury during resolution of inflammation. The characteristic morphological features of apoptosis in human neutrophils were first reported by Savill et al. in 1989 (1). Until that time, it 
was widely believed that neutrophils died by undergoing necrosis, the classical mode of cell death that follows irreversible cell injury (2), but which is associated with disruption of the plasma membrane and release of intracellular contents, which in the case of the neutrophil may promote further tissue injury. Savill et al. demonstrated that when human neutrophils were aged in culture for $10 \mathrm{~h}$ at $37^{\circ} \mathrm{C}$ a proportion of cells displayed densely condensed nuclei and vacuolated cytoplasm. Electron microscopy of the same cell population revealed chromatin condensation, nucleolar prominence, vacuolated cytoplasm, and intact cytoplasmic granules. In contrast to apoptosis in other cell lineages, substantial cell shrinkage, membrane blebbing, and formation of apoptotic bodies are not prominent features of apoptotic neutrophils when observed by light microscopy. Neutrophils are terminally differentiated cells and undergo constitutive apoptosis, so that after culture in vitro for $20 \mathrm{~h}$, typically $>50 \%$ of the cells will have undergone apoptosis. In vitro aging of neutrophils has been successfully used to demonstrate the pro-apoptotic or anti-apoptotic effects of numerous mediators, bacterial products, and pharmaceutical agents (3-5). Potential apoptosis-regulating agents are incubated with a suspension of freshly isolated human neutrophils and apoptosis is assessed over time. Early time points (e.g., $4 \mathrm{~h}$ ) are used to look for induction of apoptosis, and later time points (e.g., $>20 \mathrm{~h}$ ) for assessing inhibition of apoptosis. It should be appreciated that the methods of neutrophil isolation and culture can profoundly influence rates of constitutive apoptosis and the response to external apoptosis-modulating reagents. Human neutrophil isolation may involve multiple steps including leukocyte sedimentation (using Percoll, Hypaque-Ficoll, gelatine, etc.), many centrifugation steps, temperature changes, and red cell lysis. Each step has the potential to influence neutrophil responsiveness and apoptosis. Furthermore, contamination of neutrophil preparations, even with low numbers of monocytes or eosinophils, can influence rates of neutrophil apoptosis. In order to overcome this variable, some researchers have recommended neutrophil isolation protocols based on antibody-dependent depletion of contaminating cells (6), which in turn requires careful quality-control steps. Other variables that must be taken into account when investigating human neutrophil apoptosis include the $\mathrm{pH}$ of media, glucose concentration, oxygen tension, and cell density during culture $(7,8)$.

Substantial research activity has identified many of the molecular events involved in the apoptosis pathway, which in turn have led to a variety of different techniques that may be used to identify and quantify apoptotic neutrophils in the laboratory. The inevitable consequence of initiation of the apoptosis pathway is disassembly of the intracellular machinery which is mediated principally by effector caspases, a family of mammalian cysteine proteases which cleave specific aspartate-containing sites in a variety of target proteins. Caspases 
exist constitutively in the cell as zymogens, and upon an initiating pro-apoptotic stimulus they are sequentially activated in a tightly regulated, irreversible cascade. Caspases have been classified into initiators (caspases 2, 8, 9, 10,12) and effectors (caspases $3,6,7,14)(9)$. Initiation of the biochemical pathways of apoptotic cell death can broadly be defined as occurring via either intrinsic or extrinsic mechanisms, although it is now recognized that a certain amount of cross-talk occurs between the two. The intrinsic pathway is recruited in response to cell damage or stress, and involves changes at the level of the mitochondria including processing and activation of pro-apoptotic Bcl-2 family member proteins (e.g., Bax), release of cytochrome $\mathrm{c}$ from mitochondria, formation of the apoptosome (composed of cytochrome c, APAF-1, and procaspase 9), and activation of caspase 9. The extrinsic pathway of apoptosis results from engage ment of cell surface death receptors (e.g., Fas [CD95], tumor necrosis factor [TNF] receptor) leading to formation of death-inducing signaling complex (DISC), and involves cleavage and activation of procaspase 8 as the initiator caspase. Both mechanisms result in downstream processing and activation of caspase 3, which is the predominant effector caspase responsible for the cleavage of cell components (10). Normal cell membranes exhibit marked phospholipid asymmetry, with phosphatidylcholine and sphingomyelin predominantly on the external layer and most of the phosphatidylethanolamine and phosphatidylserine on the inner layer. Apoptosis is associated with loss of normal asymmetrical distribution of aminophospholipids, and in particular phosphatidylserine becomes exposed on the exterior of the cell, where it may mediate binding of opsonins and permit recognition of apoptotic cells by phagocytes (11). Activation of an endonuclease during apoptosis leads to internucleosomal digestion of DNA into monomers or oligomers or nucleosomal DNA of 200 base pairs and multiples thereof, and a regular "ladder-type" pattern of oligonucleosomal DNA fragmentation on agarose gel electrophoresis is commonly considered to define apoptosis. Apoptotic neutrophils are recognized and ingested by macrophages (11), and it has been shown that macrophage phagocytic capacity for apoptotic neutrophils may be influenced by cytokines (12), prostaglandins (13), glucocorticoid hormones (14), and interaction of surface adhesion molecules with neighboring cells and extracellular matrix components (15). Furthermore, apoptotic cells may become opsonised, whereby naturally occurring soluble opsonins bind to the cell surface and initiate phagocytosis (16).

Neutrophil apoptosis may be assessed in the laboratory by analysis of cell morphology by microscopy, assessing plasma membrane alterations, measuring loss of mitochondrial membrane potential, looking for evidence of caspase activation, and gel electrophoresis of genomic DNA. Methods for quantifying macrophage phagocytosis of apoptotic neutrophils are also described in this chapter. 


\section{Materials}

\subsection{Cytocentrifuge Preparations for Light Microscopy}

1. Cytocentrifuge chambers, filter cards, glass slides, and coverslips.

2. Methanol, Diff-Quik ${ }^{\mathrm{TM}}$ stains (Baxter Healthcare), and distrene-80, plasticizer, xylene.

3. Iscove's modified Dulbecco's medium (IMDM) (Invitrogen).

\subsection{Plasma Membrane Alterations}

1. 96-well U-bottom flexible polyvinyl chloride plate (Becton Dickinson Labware).

2. Annexin binding buffer: $140 \mathrm{mmol} \mathrm{NaCl}, \mathrm{pH} 7.4,20 \mathrm{mM}$ HEPES, $4 \mathrm{mM} \mathrm{CaCl}$.

3. Stock solution of $1 \mathrm{mg} / \mathrm{mL}$ propidium iodide in phosphate-buffered saline (PBS) (Sigma-Aldrich) (see Note 1).

4. Cytometry buffer: cation-free PBS containing $0.1 \%$ bovine serum albumin. Store at $4^{\circ} \mathrm{C}$.

5. For CD16 staining: $3 \mathrm{G} 8$ (anti-Fc $\gamma \mathrm{RIIIB}) \mathrm{F}\left(\mathrm{ab}^{\prime}\right)_{2}$ fragments (Ancell, Bayport, $\mathrm{MN}$ ); fluorescein isothiocyanate (FITC)-conjugated $\mathrm{F}\left(\mathrm{ab}^{\prime}\right)_{2}$ goat anti-mouse immunoglobulin ( $\mathrm{Ig}$ ) (Dako); nonbinding murine $\mathrm{IgG}_{1}$ control (Dako).

\subsection{Mitochondrial Permeability}

Mit-E- $\Psi$ mitochondria permeability detection kit (Biomol International): contains lyophilized Mit-E- $\psi$ reagent, to be reconstituted in $125 \mu \mathrm{L}$ of dimethylsulfoxide (DMSO) per vial then stored at $-20^{\circ} \mathrm{C}$ (avoid repeated freezethawing), and $10 \mathrm{X}$ assay buffer, to be diluted before use. Once diluted, assay buffer can be stored at $4^{\circ} \mathrm{C}$ for up to $7 \mathrm{~d}$.

\subsection{Western Blotting for Caspases}

1. Tris-buffered saline (TBS): dissolve $8 \mathrm{~g}$ of $\mathrm{NaCl}, 0.2 \mathrm{~g}$ of $\mathrm{KCl}$, and $3 \mathrm{~g}$ Trizma base in $800 \mathrm{~mL}$ of distilled water. Adjust the $\mathrm{pH}$ to 7.4 with $\mathrm{HCl}$ and add distilled water to $1 \mathrm{~L}$. Autoclave aliquots and store at room temperature.

2. Protease inhibitor buffer (see Note 2): to $0.3 \mathrm{~mL}$ of TBS add $0.5 \mathrm{~mL}$ of protease inhibitor cocktail for use with mammalian cell and tissue extracts (1:50 dilution in TBS) (Sigma-Aldrich), supplemented with 4-(2-aminoethyl)-benzenesulfonylfluoride (AEBSF; $20 \mu \mathrm{L}$ of $400 \mathrm{mM}$ stock in $\left.\mathrm{H}_{2} \mathrm{O}\right)$, aprotinin $(20 \mu \mathrm{L}$ of $0.15 \mu M$ stock in $\mathrm{H}_{2} \mathrm{O}$ ), leupeptin $\left(20 \mu \mathrm{L}\right.$ of $20 \mathrm{mM}$ stock in $\left.\mathrm{H}_{2} \mathrm{O}\right)$, pepstatin $\mathrm{A}(40 \mu \mathrm{L}$ of $0.75 \mu M$ stock in methanol), sodium orthovanadate ( $20 \mu \mathrm{L}$ of $1 M$ stock, $\mathrm{pH} 10$, boiled), benzamidine ( $20 \mu \mathrm{L}$ of $0.5 \mathrm{M}$ stock), levamisole ( $20 \mu \mathrm{L}$ of $2 \mathrm{M}$ stock) and $\beta$-glycerophosphate ( $60 \mu \mathrm{L}$ of $3.33 \mathrm{M}$ stock in $\mathrm{H}_{2} \mathrm{O}$ ) (all from Sigma-Aldrich).

3. $10 \%$ NP-40 in TBS.

4. Sample Buffer (for $10 \mathrm{~mL}$ of $4 X$ Sample Buffer): $5.5 \mathrm{~mL}$ of $50 \%$ glycerol, $2 \mathrm{~mL}$ of $20 \%$ sodium dodecyl sulfate (SDS), $2.5 \mathrm{~mL}$ of $0.5 \mathrm{M}$ Tris- $\mathrm{HCl}$ (pH 6.8), $20 \mu \mathrm{L}$ of $1 \%$ (w/v in ethanol) bromophenol blue. For reducing buffer, add $100 \mu \mathrm{L}$ of $\beta$ mercaptoethanol in a fume hood. 
5. $12 \%$ acrylamide separating gel (see Note 3): $2.5 \mathrm{~mL}$ of $1.5 \mathrm{M}$ Tris- $\mathrm{HCl}(\mathrm{pH} 8.8), 4$ $\mathrm{mL}$ of $30 \%$ acrylamide/bis-acrylamide solution (37.5:1 ratio), $3.3 \mathrm{~mL}$ of $\mathrm{dH}_{2} \mathrm{O}, 100$ $\mu \mathrm{L}$ of $10 \%$ (w/v) SDS. Add $30 \mu \mathrm{L}$ of $N, N, N^{\prime}, N^{\prime}$-tetramethylethylenediamine (TEMED) and $60 \mu \mathrm{L}$ of $10 \%(\mathrm{w} / \mathrm{v})$ ammonium persulphate to initiate polymerization.

6. $\mathrm{dH}_{2} \mathrm{O}$-saturated butanol: mix approx $75 \mathrm{~mL}$ of butan-2-ol with approx $25 \mathrm{~mL}$ of distilled water and shake vigorously. Allow to separate, and store at room temperature. Use the top layer.

7. $4.5 \%$ acrylamide stacking gel: $2.5 \mathrm{~mL}$ of $0.5 M$ Tris- $\mathrm{HCl}(\mathrm{pH} 6.8), 1.5 \mathrm{~mL}$ of $30 \%$ acrylamide/bis-acrylamide solution (37.5:1 ratio), $5.8 \mathrm{~mL}$ of $\mathrm{dH}_{2} \mathrm{O}, 100 \mu \mathrm{L}$ of $10 \%$ (w/v) SDS. Add $30 \mu \mathrm{L}$ of TEMED and $60 \mu \mathrm{L}$ of $10 \%(\mathrm{w} / \mathrm{v})$ ammonium persulphate to initiate polymerization.

8. Running buffer: $25 \mathrm{mM}$ Tris, $192 \mathrm{mM}$ glycine $\mathrm{pH} 8.3,0.1 \%$ SDS.

9. Transfer buffer: $25 \mathrm{mM}$ Tris, $192 \mathrm{mM}$ glycine pII $8.3,20 \%$ methanol.

10. Hybond-P polyvinylidene difluoride (PVDF) membrane (Amersham Biosciences).

11. Benchmark ${ }^{\mathrm{TM}}$ prestained molecular weight standards (Invitrogen).

12. Blocking buffer: TBS containing $0.1 \%$ Tween 20 (polysorbat 20$)^{\oplus}$ and $5 \%$ Marvel dried milk powder.

13. Primary antibody: anti-caspase-3 (active) polyclonal rabbit antibody (Biovision Research Products).

14. Secondary antibody: horseradish peroxidase (HRP)-conjugated polyclonal goat antirabbit Ig (Dako).

15. Enhanced chemiluminescence (ECL) reagents (Amersham Biosciences).

\subsection{Fluorimetric Caspase Kit}

Homogeneous Caspases Assay Kit (Roche Diagnostics) (see Note 4): contains $1 \mathrm{X}$ incubation buffer, stock caspase substrate solution (500 $\mu M$ DEVDR110 in DMSO), positive control (lysate from apoptotic camptothecin-treated U937 cells), and R110 standard for calibration curve construction (1 $\mathrm{mM}$ in DMSO).

\subsection{Caspase Profiling Plate}

1. ApoAlert ${ }^{\mathrm{TM}}$ Caspase Profiling Plate (Clontech): contains 96-well microplate with immobilized substrates for caspase 2 (VDVAD-AMC), caspase 3 (DEVD-AMC), caspase 8 (IETD-AMC), and caspase 9 (LEHD-AMC) in 24 wells each, lysis Buffer, 2X Reaction Buffer, 100X dithiothreitol (DTT) solution, and inhibitors of caspases $2,3,8$, and 9 .

2. $10 \%$ NP-40 in TBS.

\subsection{Gel Electrophoresis for DNA Fragmentation}

1. Wizard ${ }^{\circledR}$ genomic DNA purification kit (Promega).

2. $0.5 \mathrm{X}$ TBE running buffer: Make a $5 \mathrm{X}$ stock $(\mathrm{pH} 8.3$ ) with $54 \mathrm{~g}$ Trizma base, 27.5 $\mathrm{g}$ boric acid, and $20 \mathrm{~mL} 0.5 \mathrm{M}$ ethylenediamine tetraacetic acid (EDTA) (pH 8.0 ) in $1 \mathrm{~L}$ of $\mathrm{dH}_{2} \mathrm{O}$. 
3. SeaKem LE agarose (Cambrex) for DNA electrophoresis (see Note 5).

4. $10 \mathrm{mg} / \mathrm{mL}$ ethidium bromide solution (Sigma-Aldrich).

5. $6 \mathrm{X}$ blue/orange loading dye (Promega).

\subsection{Propidium lodide Intercalation into DNA}

1. 96-well flat-bottom flexible polyvinyl chloride plate (Becton Dickinson Labware).

2. $70 \%$ ethanol in distilled $\mathrm{H}_{2} \mathrm{O}$.

3. $0.5 \mathrm{mg} / \mathrm{mL}$ solution of RNase A (Sigma-Aldrich).

4. Solution of $0.1 \mathrm{mg} / \mathrm{mL}$ propidium iodide in PBS (Sigma-Aldrich).

\subsection{Terminal Deoxynucleotidyltransferase-Mediated dUTP Nick End Labeling Staining}

1. 96-well flat-bottom flexible polyvinyl chloride plate (Becton Dickinson Labware).

2. In Situ Cell Death Detection Kit, Fluorescein (Roche Diagnostics): contains 10X enzyme solution (terminal deoxynucleotidyltransferase [TdT]) in storage buffer and $1 \mathrm{X}$ labeled nucleotide mixture in reaction buffer. This protocol also requires PBS (wash buffer), $3 \% \mathrm{H}_{2} \mathrm{O}_{2}$ in methanol (blocking solution), $4 \%$ paraformaldehyde in PBS at $\mathrm{pH} 7.4$ (fixation buffer; freshly prepared), and $0.1 \%$ Triton X-100 in $0.1 \%$ sodium citrate (permeabilization buffer; freshly prepared).

\subsection{Plate-Based Phagocytosis Assay}

1. $2.5 \%$ glutaraldehyde (Sigma-Aldrich) in PBS.

2. $0.1 \mathrm{mg} / \mathrm{mL}$ dimethoxybenzidine (o-diansidine) (Sigma-Aldrich) in PBS, made up fresh from $1 \mathrm{mg} / \mathrm{mL}$ frozen stock.

3. $30 \%$ hydrogen peroxide solution (Sigma-Aldrich).

4. Costar 48-well TC-treated microplates (Fisher Scientific).

\subsection{Flow Cytometry-Based Phagocytosis Assay}

1. 5-chloromethylfluorescein diacetate (CMFDA; CellTracker ${ }^{\mathrm{TM}}$ Green; Molecular Probes).

2. $0.25 \%$ trypsin/1 $\mathrm{m} M$ EDTA solution (Invitrogen).

\section{Methods}

\subsection{Analysis of Neutrophil Morphology by Light Microscopy}

1. Suspend freshly-isolated peripheral blood neutrophils (at least $97 \%$ purity) at $4 \times$ $10^{6}$ cells $/ \mathrm{mL}$ in IMDM supplemented with $10 \%$ autologous serum (see Note 6).

2. Add $135 \mu \mathrm{L}$ of neutrophil suspension to wells of a 96-well, flat-bottom flexible plate. To each well, add $15 \mu \mathrm{L}$ of apoptosis-modifying agents (10X concentration) or buffer control, cover with a lid, and incubate at $37^{\circ} \mathrm{C}$ in a $5 \% \mathrm{CO}_{2}$ incubator for the desired length of time.

3. Load a cytospin chamber with $100 \mu \mathrm{L}$ of aged neutrophil suspension.

4. Cytocentrifuge at $300 \mathrm{rpm}(30 \mathrm{~g})$ for $3 \mathrm{~min}$.

5. Air-dry for $5 \mathrm{~min}$. 


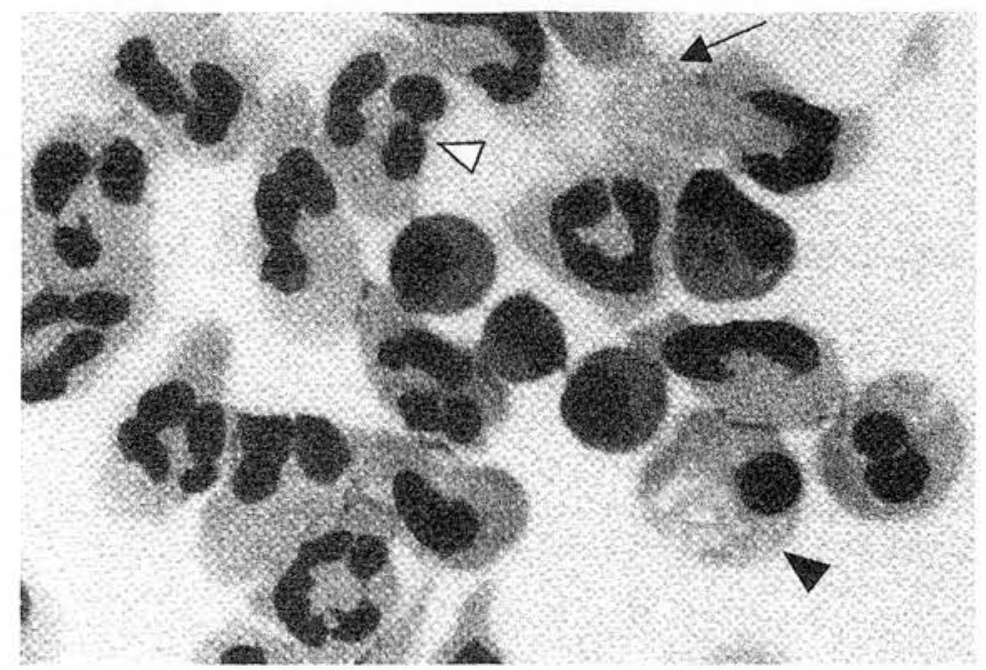

Fig. 1. A cytocentrifuge preparation of human neutrophils aged for $22 \mathrm{~h}$ in vitro. Nonapoptotic neutrophils exhibit characteristic multilobed nuclei and an open pattern of chromatin (open arrowhead). Apoptotic neutrophils show condensation of nuclear material into one to three dark-staining nuclear bodies with prominent nucleoli, and often demonstrate cytoplasmic vacuolation (closed arrowhead). Late apoptotic neutrophils appear as "ghosts" with little or no nuclear material (closed arrow).

6. Fix in methanol for $1 \mathrm{~min}$. Drain.

7. Stain in Diff Quik ${ }^{\mathrm{TM}}$ solution 1 or equivalent acid dye for $1 \mathrm{~min}$. Drain.

8. Stain in Diff Quik solution 2 or equivalent basic dye for $1 \mathrm{~min}$. Drain and rinse with distilled water.

9. Allow to dry, mount with a drop of DPX, and apply a coverslip. View using a light microscope with a $\times 40$ or $\times 100$ objective and count $>200$ cells per slide. Apoptotic neutrophils can be identified by their characteristic condensation of nuclear material, prominent nucleoli, and frequently vacuolation of the cytoplasm (Fig. 1) (see Note 7).

\subsection{Annexin V Staining}

Apoptosis is associated with the loss of phospholipid asymmetry and the exposure of phosphatidylserine on the outer surface of the plasma membrane. Annexin $\mathrm{V}$ is a protein that binds specifically to phophatidylserine in the presence of $\mathrm{Ca}^{2+}$, and its fluorescent derivatives can be used to identify apoptotic cells using a simple and rapid flow cytometric method.

1. Suspend freshly isolated peripheral blood neutrophils (at least $97 \%$ purity) at $4 \times$ $10^{6} \mathrm{cells} / \mathrm{mL}$ in IMDM supplemented with $10 \%$ autologous serum (see Note 6). 
2. Add $135 \mu \mathrm{L}$ of neutrophil suspension to wells of a 96-well, flat-bottom flexible plate.

3. Add $15 \mu \mathrm{L}$ of apoptosis-modifying agents (10X concentration) or buffer control to each well.

4. Cover plate, and incubate at $37^{\circ} \mathrm{C}$ in a $5 \% \mathrm{CO}_{2}$ incubator for the desired length of time.

5. Resuspend cells by gentle pipetting and transfer $100 \mu \mathrm{L}$ of the cell suspension to a 96-well, U-bottom flexible plate ( room temperature. Discard the supernatants.

6. Vortex the plate for $5 \mathrm{~s}$ to disrupt the cell pellet.

7. Resuspend the cells in $50 \mu \mathrm{L}$ of recombinant human Annexin V-FITC (Caltag) freshly diluted 1:50 in annexin binding buffer (see Note 9).

8. Incubate for $5 \mathrm{~min}$ at room temperature. No wash is required.

9. Co-label membrane permeable necrotic or late apoptotic cells by staining with the membrane impermeant nuclear dye propidium iodide (PI; $5 \mu \mathrm{g} / \mathrm{mL}$ ) 30 s prior to running the sample.

10. Analyze by flow cytometry using FL-1/FL-2 channel analysis following appropriate compensation (see Note 10).

11. Live cells are annexin V-negative and PI-negative; apoptotic cells are annexin Vpositive and PI-negative; necrotic cells are both annexin V-positive and PI-positive (Fig. 2).

\subsection{Loss of CD16 Expression}

Neutrophil apoptosis is associated with a marked downregulation of Fc $\gamma$ RIII (CD16) (21). A simple flow cytometric method using an anti-CD16 monoclonal antibody will reliably discriminate apoptotic from non-apoptotic neutrophils in a mixed cell population.

1. Suspend freshly isolated peripheral blood neutrophils (at least $97 \%$ purity) at $4 \times$ $10^{6} \mathrm{cells} / \mathrm{mL}$ in IMDM supplemented with $10 \%$ autologous serum (see Note 6).

2. Add $90 \mu \mathrm{L}$ of neutrophil suspension to wells of a 96-well, flat-bottom flexible plate.

3. Add $10 \mu \mathrm{L}$ of apoptosis-modifying agents (10X concentration) or buffer control to each well.

4. Cover plate, and incubate at $37^{\circ} \mathrm{C}$ in a $5 \% \mathrm{CO}_{2}$ incubator for the desired length of time.

5. Resuspend cells by gentle pipetting and transfer $100 \mu \mathrm{L}$ of the cell suspension to a 96-well U-bottom flexible plate ( $4^{\circ} \mathrm{C}$. Discard the supernatants.

6. Vortex the plate for $5 \mathrm{~s}$ to disrupt the cell pellet.

7. Incubate neutrophils with $20 \mu \mathrm{g} / \mathrm{mL}$ monoclonal murine $\operatorname{IgG}_{1}$ anti-CD16 (clone 3G8) or nonbinding control $\mathrm{IgG}_{1}$ in $50 \mu \mathrm{L}$ cytometry buffer on ice for $30 \mathrm{~min}$.

8. Add $75 \mu \mathrm{L}$ of cytometry buffer. Centrifuge at $200 \mathrm{~g}$ for $2 \mathrm{~min}$ at $4^{\circ} \mathrm{C}$. Discard the supernatants and vortex the plate for $5 \mathrm{~s}$ to disrupt the cell pellet. 


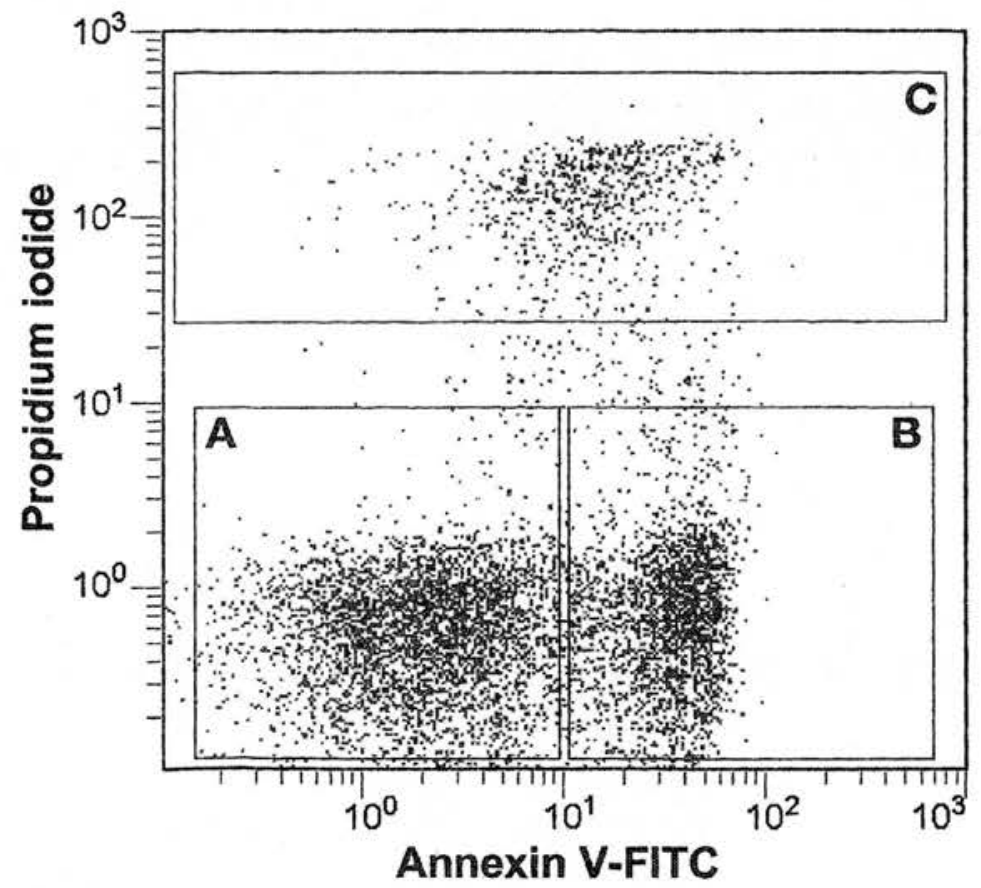

Fig. 2. Annexin V staining. An example two-colour flow cytometry dot plot of aged human neutrophils stained with annexin V-fluorescein isothiocyanate (FL-1, $x$-axis) and propidium iodide (FL-2, y-axis) is shown. The regions indicated represent (A) nonapoptotic neutrophils, (B) apoptotic neutrophils, and (C) late apoptotic or necrotic neutrophils.

9. Add $125 \mu \mathrm{L}$ of cytometry buffer. Centrifuge at $200 \mathrm{~g}$ for $2 \mathrm{~min}$ at $4{ }^{\circ} \mathrm{C}$. Discard the supernatants and vortex the plate for $5 \mathrm{~s}$ to disrupt the cell pellet.

10. Add $50 \mu \mathrm{L}$ of $1: 40 \mathrm{~F}\left(\mathrm{ab}^{\prime}\right)_{2}$ goat anti-mouse Ig-FITC in cytometry buffer on ice for $30 \mathrm{~min}$.

11. Repeat steps 8 and 9 . Resuspend the cells in $50 \mu \mathrm{L}$ of cytometry buffer.

12. Analyse cells using a flow cytometer. Apoptotic neutrophils have low FL-1 channel fluorescence; non-apoptotic neutrophils have high FL-1 fluorescence (see Note 11).

\subsection{Measurement of Mitochondrial Membrane Potential Using JC-1.}

Upon activation of apoptosis-promoting proteins such as Bid, Bax, and Bak, the potential across the mitochondrial membrane $\left(\Delta \Psi_{M}\right)$ is lost and pores are formed which allow the passage of proteins from the mitochondria into the cytosol, leading to activation of caspase 9 and subsequently caspase 3 (22). Changes 
in neutrophil mitochondrial membrane potential may be measured using JC-1 (5, 5',6,6'-tetrachloro-1, 1', 3, 3'-tetra-ethyl-benzimidazocarbo-cyaniniodide; Molecular Probes), a cationic dye which exhibits membrane potential-dependent accumulation in mitochondria indicated by a fluorescence emission shift from green $(525 \mathrm{~nm})$ to red $(590 \mathrm{~nm})$. In viable cells with intact mitochondrial membrane potential, JC-1 is able to enter mitchondria and polymerise, where it fluoresces in the red (FL-2) channel. However, when $\Delta \Psi_{\mathrm{M}}$ is lost during apoptosis, JC-1 is unable to penetrate the mitochondrial membrane and it remains in monomeric form in the cytosol and fluoresces in the green (FL-1) channel. Apoptosisassociated mitochondrial depolarisation is indicated by a decrease in the red/ green fluorescence intensity ratio (23) (see Note 12). Although not described here, the change in fluorescence of $\mathrm{JC}-1$ associated with apoptosis can also be quantified flow cytometrically.

1. This protocol assumes the use of a Mit-E- $\Psi$ mitochondria permeability detection kit (Biomol International UK, Exeter, UK)

2. Suspend freshly isolated peripheral blood neutrophils (at least $97 \%$ purity) at $2 \times$ $10^{6}$ cells $/ \mathrm{mL}$ in IMDM supplemented with $10 \%$ autologous serum (see Note 6).

3. Dispense $1 \mathrm{~mL}$ of neutrophil suspension $\left(2 \times 10^{6}\right.$ cells) into $2-\mathrm{mL}$ round-bottomed polypropylene tubes and incubate \pm apoptosis-modifying agents at $37^{\circ} \mathrm{C}$ for the desired length of time (see Note 13).

4. Centrifuge at $220 \mathrm{~g}$ for $5 \mathrm{~min}$ and discard the supernatants.

5. Gently resuspend each cell pellet in $1 \mathrm{~mL}$ of freshly prepared Mit-E- $\Psi$ (JC-1) solution and incubate at $37^{\circ} \mathrm{C}$ for $10 \mathrm{~min}$.

6. Add $2 \mathrm{~mL}$ of assay buffer added to each tube and centrifuge at $220 \mathrm{~g}$ for $5 \mathrm{~min}$. Discard the supernatants.

7. Resuspend the cell pellets in $1 \mathrm{~mL}$ of assay buffer and centrifuge at $220 \mathrm{~g}$ for $5 \mathrm{~min}$. Discard the supernatants.

8. Resuspend the cell pellets in $1 \mathrm{~mL}$ of assay buffer.

9. Transfer duplicate aliquots of $100 \mu \mathrm{L}$ from each sample to a black 96-well plate.

10. Measure fluorescence using a fluorescence microtiter plate reader (excitation of 485 $\mathrm{nm}$; emission of $590 \mathrm{~nm}$ for red fluorescence and $530 \mathrm{~nm}$ for green fluorescence).

11. Calculate the ratio of red:green fluorescence.

\subsection{Caspase Activation}

Caspase 3 is the archetypal effector caspase which has received the most attention in studies of apoptosis. Caspase 3 is synthesized as an inactive zymogen $(32 \mathrm{kDa})$ that is cleaved in cells undergoing apoptosis into large (17 kDa) and small ( $12 \mathrm{kDa}$ ) subunits that associate to form the active enzyme. Western blotting can be used to observe the increase in active caspase or disappearance of inactive procaspase on treatment with an apoptotic stimulus. For studying caspase 3, several antibodies are appropriate for Western blot analysis; a rabbit polyclonal antibody that detects only the large (p17) cleaved fragment of active 


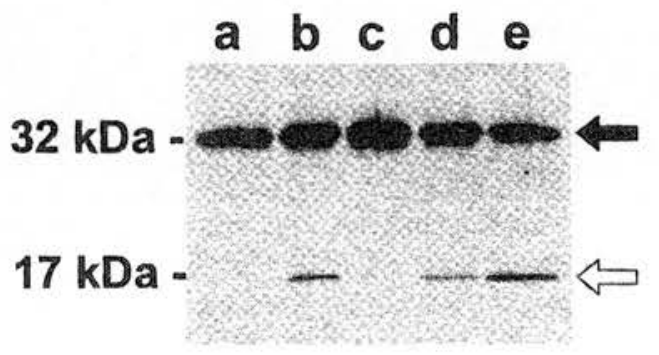

Fig. 3. Western blotting for caspase 3. Human neutrophils were incubated in Iscove's modified Dulbecco's medium $+10 \%$ autologous serum at $37^{\circ} \mathrm{C}$ under the following conditions: (A) medium only ( $2 \mathrm{~h}$ ); (B) $10 \mathrm{ng} / \mathrm{mL}$ tumor necrosis factor (TNF)- $\gamma(2 \mathrm{~h})$; (C) $0.1 \mu \mathrm{g} / \mathrm{mL}$ gliotoxin $(2 \mathrm{~h})$; (D) $0.1 \mu \mathrm{g} / \mathrm{mL}$ gliotoxin $+10 \mathrm{ng} / \mathrm{mL}$ TNF- $\gamma(2 \mathrm{~h})$; (E) medium only $(20 \mathrm{~h})$. Cells were lysed and subjected to sodium dodecyl sulfate-polyacrylamide gel electrophoresis and Western blotting and probed for whole caspase 3 (closed arrow) and cleaved active caspase 3 (open arrow).

caspase 3, a mouse monoclonal antibody that detects only the procaspase (p32) form, and a mouse monoclonal antibody and a rabbit polyclonal antibody to detect both the p32 zymogen and the p17 active subunit (Biovision Research Products). An example of Western blotting for active caspase 3 is shown in Fig. 3 .

\subsubsection{Western Blotting for Caspases}

1. These instructions assume the use of a minigel apparatus such as the Atto Corporation Dual Mini Slab Chamber (GRI Ltd, Braintree, UK) or the mini-PROTEAN electrophoresis system (BioRad Laboratories).

2. Suspend freshly-isolated peripheral blood neutrophils (at least $97 \%$ purity) at $5 \times$ $10^{6}$ cells/mL in IMDM supplemented with $10 \%$ autologous serum (see Note 6).

3. Dispense $1 \mathrm{~mL}$ of neutrophil suspension $\left(5 \times 10^{6}\right.$ cells $)$ into $2-\mathrm{mL}$ round-bottomed polypropylene tubes and incubate \pm apoptosis-modifying agents at $37^{\circ} \mathrm{C}$ for the desired length of time (see Note 13).

4. Prepare and pour a $12 \%$ acrylamide gel. Overlay with approx $0.5 \mathrm{~mL}$ of dH $\mathrm{H}_{2} \mathrm{O}$-saturated butanol whilst polymerization occurs (allow $30 \mathrm{~min}$ ). Rinse off butanol with $\mathrm{dH}_{2} \mathrm{O}$ and pour a $4.5 \%$ acrylamide stacking gel on top and insert comb. Allow the stacking gel to polymerise, then assemble the electrophoresis apparatus and remove the comb.

5. Centrifuge the neutrophil suspensions at $220 \mathrm{~g}$ for $5 \mathrm{~min}$ at $4^{\circ} \mathrm{C}$. Discard the supernatants.

6. Resuspend the cell pellets in protease inhibitor buffer $\left(90 \mu \mathrm{L}\right.$ per $5 \times 10^{6}$ cells $)$ and incubate on ice for $10 \mathrm{~min}$ (see Note 2 ).

7. Add $10 \% \mathrm{NP}-40$ in TBS $\left(10 \mu \mathrm{L}\right.$ per $5 \times 10^{6}$ cells $)$, immediately vortex for $5 \mathrm{~s}$, and incubate for a further $10 \mathrm{~min}$ on ice. 
8. Centrifuge at $20,000 \mathrm{~g}$ for $20 \mathrm{~min}$ at $4^{\circ} \mathrm{C}$, and retain the detergent-soluble supernatant containing the cytosolic and membrane fractions.

9. Transfer the supernatants to fresh tubes on ice and add $33 \mu \mathrm{L}$ (per $100 \mu \mathrm{L}$ ) $4 \mathrm{X}$ sample buffer. Heat at $95^{\circ} \mathrm{C}$ for $5 \mathrm{~min}$. Samples may be stored at $-20^{\circ} \mathrm{C}$ prior to SDSpolyacrylamide gel electrophoresis (PAGE).

10. Load samples ( $20 \mu \mathrm{L}$ per lane for a minigel) onto a $12 \%$ polyacrylamide gel alongside molecular weight standards and run at $80 \mathrm{~V}$ for approx $15 \mathrm{~min}$ to allow samples to migrate through the stacking gel. Increase the voltage to $160 \mathrm{~V}$ through the separating gel until the dye front reaches the bottom of the gel.

11. Transfer proteins from the gel to the PVDF membrane at $70 \mathrm{~V}$ for $1 \mathrm{~h}$ at room temperature.

12. Wash the membrane in TBS $/ 0.1 \%$ Tween 20 for $5 \mathrm{~min}$.

13. Block the membrane in $10 \mathrm{~mL}$ of $5 \%$ Marvel dried milk powder in TBS/ $0.1 \%$ Tween 20 at room temperature on a rocking platform for $30 \mathrm{~min}$.

14. Wash the membrane in TBS $/ 0.1 \%$ Tween 20 for $5 \mathrm{~min}$.

15. Incubate with the primary antibody (anti-caspase-3 [active] polyclonal rabbit antibody) at $1 \mu \mathrm{g} / \mathrm{mL}$ in TBS $/ 0.1 \%$ Tween 20 containing $5 \%$ Marvel dried milk powder. Incubate in a covered hydrophobic tray (just large enough to accommodate the blot) for $2 \mathrm{~h}$ at room temperature on a rocking platform. Use sufficient volume to just cover the blot.

16. Wash membrane three times in TBS $/ 0.1 \%$ Tween 20 each for $5 \mathrm{~min}$ at room temperature on a rocking platform.

17. Incubate with the secondary antibody (HRP-conjugated polyclonal goat anti-rabbit Ig) diluted 1:2500 in TBS/0.1\% Tween 20 containing 5\% Marvel dried milk powder. Incubate in a covered hydrophobic tray (just large enough to accommodate the blot) for $2 \mathrm{~h}$ at room temperature on a rocking platform. Use sufficient volume to just cover the blot.

18. Wash membrane three times in TBS/ $/ 0.1 \%$ Tween 20 each for $5 \mathrm{~min}$ at room temperature on a rocking platform.

19. Develop using ECL according to the manufacturer's instructions.

\subsubsection{Fluorometric Homogeneous Caspase Assay}

Apoptosis usually occurs via caspase-dependent processes, and an increase in caspase activity gives a general indication of the occurrence of apoptosis. Commercial kits are available in which total caspase activity can be measured (homogeneous caspase assay). However, these kits do not dissect out precisely which caspases are active. They utilize cleavage of a fluorescence-conjugated, modified nonspecific caspase substrate (e.g., VAD-fmk, DEVD) to generate a fluorescent product (e.g., FITC, rhodamine 110), and fluorescence correlates with the extent of total caspase activity.

1. These instructions assume the use of a Homogeneous Caspases Assay Kit (Roche Diagnostics). 
2. Suspend freshly isolated peripheral blood neutrophils (at least $97 \%$ purity) at $10^{6}$ cells $/ \mathrm{mL}$ in IMDM supplemented with $10 \%$ autologous serum (see Note 6).

3. Plate out neutrophils $\left(10^{5}\right.$ per well; see Note 14) and incubate at $37^{\circ} \mathrm{C} \pm$ apoptosismodifying agents for the required time in a black 96 -well microplate (total volume $100 \mu \mathrm{L})$.

4. Dilute stock caspase substrate 1:10 in incubation buffer. Add $100 \mu \mathrm{L}$ of freshly prepared caspase substrate to each well, plus duplicate wells containing medium alone (negative control) and duplicate wells containing positive control lysate.

5. Cover the plate and incubate at $37^{\circ} \mathrm{C}$ for at least $1 \mathrm{~h}$.

6. Measure fluorescence with a plate reader (excitation, $470-500 \mathrm{~nm}$; emission, 500 $560 \mathrm{~nm})$

\subsubsection{Caspase Profiling Assay}

Fluorometric assays for specific caspases work in a similar way to the homogeneous caspase assays, but exploit a certain degree of specificity between the substrates of individual caspases or groups of caspases. Fluorescence-conjugated substrates specific for certain caspases (e.g., 2, 3, 8, and 9) are immobilized in a 96-well plate. When cell lysates are added to the wells and incubated with the substrates, the amount of fluorescence generated correlates with the activation of that particular caspase. They can therefore be used to study particular pathways of apoptosis by looking for activity of a caspase protease that is specific for a particular pathway or cell type, e.g., caspase 8 in death receptor-mediated death.

1. These instructions assume the use of the ApoAlert ${ }^{\mathrm{TM}}$ Caspase Profiling Plate (see Notes 15 and 16).

2. Suspend freshly-isolated peripheral blood neutrophils (at least $97 \%$ purity) at $2 \times$ $10^{6}$ cells $/ \mathrm{mL}$ in IMDM supplemented with $10 \%$ autologous serum (see Note 6).

3. Dispense $1 \mathrm{~mL}$ of neutrophil suspension $\left(2 \times 10^{6}\right.$ cells $)$ into 2 - $\mathrm{mL}$ round-bottomed polypropylene tubes and incubate \pm apoptosis-modifying agents at $37^{\circ} \mathrm{C}$ for the desired length of time (see Note 13).

4. Centrifuge at $220 \mathrm{~g}$ for $5 \mathrm{~min}$ at $4^{\circ} \mathrm{C}$. Discard the supernatants.

5. Resuspend the cells in $400 \mu \mathrm{L}$ ice-cold $1 \mathrm{X}$ lysis buffer and incubate for $10 \mathrm{~min}$ on ice.

6. Meanwhile, add $10 \mu \mathrm{L}$ of DTT per $1 \mathrm{~mL}$ of $2 \mathrm{X}$ reaction buffer, then add $50 \mu \mathrm{L}$ to each well of the 96-well caspase profiling plate.

7. Cover the plate with film and incubated for $5 \mathrm{~min}$ at $37^{\circ} \mathrm{C}$.

8. Vortex the neutrophil lysates, then add $50 \mu \mathrm{L}$ from each lysate to duplicate wells of each caspase substrate.

9. Cover the plate with film and incubate for $2 \mathrm{~h}$ at $37^{\circ} \mathrm{C}$.

10. Measure fluorescence using a plate reader (excitation, $380 \mathrm{~nm}$; emission, $460 \mathrm{~nm}$ ).

\subsection{Gel Electrophoresis of DNA}

A characteristic event of apoptosis is the endonuclease-mediated cleavage of DNA at regular intervals along its length, thus generating single-nucleosome 


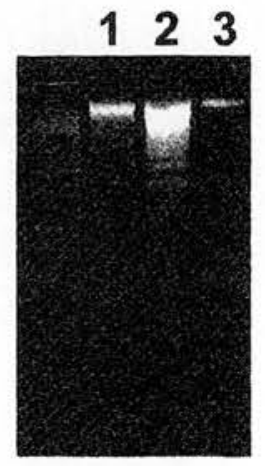

Fig. 4. Internucleosomal DNA fragmentation in neutrophils. Neutrophils were incubated for $8 \mathrm{~h}$ in the presence of phosphate-buffered saline (control, lane 1), $0.1 \mu \mathrm{g} / \mathrm{m}$ gliotoxin (inducer of neutrophil apoptosis, lane 2), or $0.2 \mathrm{mM}$ dibutyryl-cAMP (inhibitor of neutrophil apoptosis, lane 3). DNA was extracted and run on a $2 \%$ agarose gel containing ethidium bromide and the characteristic laddering pattern of apoptosis visualised under ultraviolet light.

fragments of around $180 \mathrm{bp}$, or oligonucleosomal fragments at multiples thereof, following earlier large-scale (50-200 kbp) degradation (26). Such ordered fragmentation produces discretely sized lengths of DNA, which produce a distinct "laddering" pattern on electrophoresis, in contrast to necrotic cell death in which DNA is cleaved randomly, thus producing a smear on a DNA gel.

1. Suspend freshly-isolated peripheral blood neutrophils (at least $97 \%$ purity) at $5 \times$ $10^{6}$ cells/mL in IMDM supplemented with $10 \%$ autologous serum (see Note 6).

2. Dispense $1 \mathrm{~mL}$ of neutrophil suspension $\left(5 \times 10^{6}\right.$ cells $)$ into 2 -mL round-bottomed polypropylene tubes and incubate \pm apoptosis-modifying agents at $37^{\circ} \mathrm{C}$ for the desired length of time (see Note 13).

3. Extract the genomic DNA using a Wizard Genomic DNA Purification Kit.

4. Run the DNA ( $23 \mu \mathrm{L}$ of DNA plus $7 \mu \mathrm{L}$ of loading dye) on a $2 \%$ agarose gel containing ethidium bromide $(2.5 \mu \mathrm{g} / \mathrm{mL})$ in TBE buffer at $10 \mathrm{~V} / \mathrm{cm}$.

5. Visualize gel under ultraviolet illumination. DNA from apoptotic cells exhibits a characteristic ladder pattern (Fig. 4).

\subsection{Hypodiploid DNA Content}

DNA fragmentation also leads to an apparent reduction in nuclear DNA content of ethanol-permeabilised cells, so that staining with a DNA-intercalating dye such as propidium iodide allows detection of a "hypodiploid" cell population. This technique works particularly well with neutrophils, because they are terminally differentiated cells which do not undergo proliferation, and consequently generate only two peaks when DNA content is measured: diploid (viable) cells and hypodiploid (apoptotic) cells. 


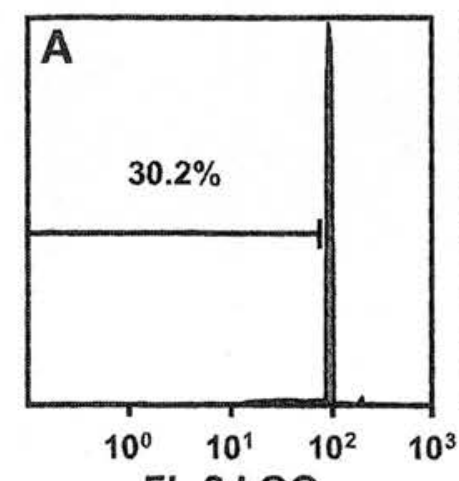

FL-2 LOG

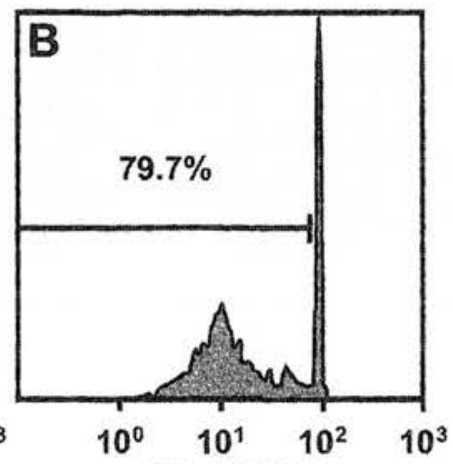

FL-2 LOG

Fig. 5. Propidium iodide intercalation into DNA of neutrophils. Neutrophils were incubated at $37^{\circ} \mathrm{C}$ for (A) $8 \mathrm{~h}$ or (B) $20 \mathrm{~h}$, then assessed by flow cytometry to determine the percentage of permeabilised cells with hypodiploid DNA content. After $8 \mathrm{~h}$ in culture $30.2 \%$ of the cells had undergone apoptosis associated with internucleosomal DNA fragmentation. After $20 \mathrm{~h}$ in culture, $79.7 \%$ of the cells had undergone apoptosis.

1. Suspend freshly isolated peripheral blood neutrophils (at least $97 \%$ purity) at $5 \times$ $10^{6}$ cells $/ \mathrm{mL}$ in IMDM supplemented with $10 \%$ autologous serum (see Note 6).

2. Add $90 \mu \mathrm{L}$ of neutrophil suspension to wells of a 96 -well, flat-bottom flexible plate. To each well add $10 \mu \mathrm{L}$ of apoptosis-modifying agents (10X concentration) or buffer control, cover with a lid, and incubate at $37^{\circ} \mathrm{C}$ in a $5 \% \mathrm{CO}_{2}$ incubator for the desired length of time.

3. Harvest cells by pipetting and transfer to 96-well, U-bottomed flexiwell plate (see Note 8).

4. Centrifuge the plate at $200 \mathrm{~g}$ for $2 \mathrm{~min}$ at $4^{\circ} \mathrm{C}$ and discard the supernatants. Vortex the plate for $5 \mathrm{~s}$ to disrupt the cell pellets.

5. Resuspend the cells in $70 \%$ ethanol and incubate on ice for $10 \mathrm{~min}$ to permeabilize them.

6. Centrifuge the plate at $200 \mathrm{~g}$ for $3 \mathrm{~min}$ at $4^{\circ} \mathrm{C}$ and discard the supernatants.

7. Wash the cells three times by adding $100 \mu \mathrm{L}$ of PBS per well, centrifuging the plate at $200 \mathrm{~g}$ for $3 \mathrm{~min}$ at $4^{\circ} \mathrm{C}$, and discarding the supernatants.

8. Resuspend the cells in $60 \mu \mathrm{L}$ of RNase $\mathrm{A}(0.5 \mathrm{mg} / \mathrm{mL})$ followed by $60 \mu \mathrm{L}$ of propidium iodide $(0.1 \mathrm{mg} / \mathrm{mL})$

9. Incubate at room temperature for $25 \mathrm{~min}$ in the dark.

10. Transfer the cells to flow cytometry tubes, add $100 \mu \mathrm{L}$ of PBS, and analyze by flow cytometry (FL2 channel) to determine the percentage of cells with hypodiploid DNA content (Fig. 5).

\subsection{TdT-Mediated dUTP Nick End Labeling Staining for DNA Breaks}

The presence of DNA strand breaks can be assessed by enzymatic methods, because DNA breaks create acceptor sites for enzymes such as TdT. Addition 
of TdT together with fluorescein-12-2'deoxyuridine-5'-triphosphate is used to reveal DNA fragmentation in the TdT-mediated dUTP nick end labeling (TUNEL) technique.

1. These instructions assume the use of the In Situ Cell Death Detection Kit.

2. Prepare freshly-isolated peripheral blood neutrophils (at least $97 \%$ purity) resuspended at $2 \times 10^{7}$ cells $/ \mathrm{mL}$ in IMDM supplemented with $10 \%$ autologous serum (see Note 6).

3. Add $90 \mu \mathrm{L}$ of neutrophil suspension to wells of a 96 -well, flat-bottom flexible plate. To each well, add $10 \mu \mathrm{L}$ of apoptosis-modifying agents (10X concentration) or buffer control.

4. Cover plate, and incubate at $37^{\circ} \mathrm{C}$ in a $5 \% \mathrm{CO}_{2}$ incubator for the desired length of time.

5. Transfer $100 \mu \mathrm{L}$ of neutrophil suspension to a 96-well, U-bottom flexible plate (see Note 8) and centrifuge at $200 \mathrm{~g}$ for $2 \mathrm{~min}$ at $4^{\circ} \mathrm{C}$. Discard the supernatants.

6. Wash the cells three times by adding $100 \mu \mathrm{L}$ of PBS per well, centrifuging the plate at $200 \mathrm{~g}$ for $3 \mathrm{~min}$ at $4^{\circ} \mathrm{C}$, discarding the supernatants, and vortexing the plate for $5 \mathrm{~s}$.

7. Add $100 \mu \mathrm{L}$ of fixation solution to each well.

8. Incubate on a shaker for $60 \mathrm{~min}$ at room temperature.

9. Add $200 \mu \mathrm{L}$ of PBS to each well then centrifuge the plate at $200 \mathrm{~g}$ for $10 \mathrm{~min}$ at $4^{\circ} \mathrm{C}$ and discard the supernatants.

10. Resuspend the cells in permeabilization solution for $2 \mathrm{~min}$ on ice.

11. Add $50 \mu \mathrm{L}$ of nucleotide mixture to the two negative control wells. The TUNEL reaction mixture is then made up by mixing the enzyme solution $(50 \mu \mathrm{L})$ with the remaining $450 \mu \mathrm{L}$ of nucleotide mixture.

12. The two positive control wells are treated for $10 \mathrm{~min}$ at room temperature with DNase I to introduce DNA strand breaks.

13. Wash twice in PBS ( $200 \mu \mathrm{L}$ per well) then resuspend in TUNEL reaction mixture ( $50 \mu \mathrm{L}$ per well).

14. Cover the plate and incubate at $37^{\circ} \mathrm{C}$ for $60 \mathrm{~min}$ in the dark.

15. Wash twice in PBS ( $200 \mu \mathrm{L}$ per well) then transfer to flow cytometry tubes for analysis of fluorescence levels (FL-1).

\subsection{Plate-Based Assay for Phagocytosis of Apoptotic Neutrophils}

Macrophage phagocytosis of apoptotic neutrophils may be assessed using minor modifications of a serum-free phagocytosis assay first described by Newman et al. in $1982(\mathbf{1 , 2 7 , 2 8 )}$. This method uses adherent human monocytederived macrophages, which are most efficient at ingesting apoptotic cells, but it has also been used successfully with murine peritoneal and bone marrowderived monocytes and human apoptotic neutrophils.

1. This method assumes the use of adherent macrophages in Costar 48-well TC-treated microplates.

2. Suspend $10^{8}$ freshly-isolated peripheral blood neutrophils (at least $97 \%$ purity) in $20 \mathrm{~mL}$ of IMDM supplemented with $10 \%$ autologous serum (see Note 6). 
3. Dispense the neutrophil suspension into a $75-\mathrm{cm}^{2}$ cell culture flask and stand the flask on its end in an incubator for $20 \mathrm{~h}$ at $37^{\circ} \mathrm{C}$ in a $5 \% \mathrm{CO}_{2}$ atmosphere.

4. Harvest the neutrophil suspension into a $50-\mathrm{mL}$ conical polypropylene tube and wash twice in warm IMDM ( $50 \mathrm{~mL}$ volume per wash) by centrifuging at $220 \mathrm{~g}$ for $5 \mathrm{~min}$ and discarding the supernatant. After each wash, gently resuspend the neutrophil pellet in $1 \mathrm{~mL}$ of warm IMDM using a plastic pipet to avoid clumping of the cells.

5. Count the cells using a haemocytometer and finally resuspend the aged neutrophils at $4 \times 10^{6} \mathrm{cells} / \mathrm{mL}$ in warm $\left(37^{\circ} \mathrm{C}\right) \mathrm{IMDM}$ (no serum).

6. Rinse the macrophage monolayer with warm $\left(37^{\circ} \mathrm{C}\right) \mathrm{IMDM}$ to remove nonadherent cells.

7. Overlay the macrophage monolayer with $0.5 \mathrm{~mL}\left(2 \times 10^{6}\right.$ cells $)$ of the suspension of aged neutrophils in IMDM (serum-free), and incubate for $60 \mathrm{~min}$ at $37^{\circ} \mathrm{C}$ in a $5 \% \mathrm{CO}_{2}$ atmosphere.

8. Wash each well with ice-cold PBS (without cations). Use an inverted microscope to check that noningested neutrophils have been largely removed.

9. Repeat the wash as necessary, continually checking by microscopy to ensure that the macrophage monolayer is not disrupted.

10. Fix in $2.5 \%$ glutaraldehyde for $30 \mathrm{~min}$ and rinse off with PBS.

11. Stain for myeloperoxidase (MPO) with $0.1 \mathrm{mg} / \mathrm{mL}$ dimethoxybenzidine and $0.03 \%$ $(\mathrm{v} / \mathrm{v})$ hydrogen peroxide in PBS for about $60 \mathrm{~min}$ at room temperature.

12. Count the percentage of macrophages (MPO-negative) that have phagocytosed one or more apoptotic neutrophils (MPO-positive) by examination with an inverted microscope of at least five fields (minimum 400 cells), and record as the mean percent phagocytosis of duplicate or triplicate wells (Fig. 6).

\subsection{Flow Cytometry-Based Phagocytosis Assay}

This modification of the plate-based phagocytosis assay utilizes a fluorescent chloromethyl dye that diffuses freely through plasma membranes to label the cytoplasm of live neutrophils. This method avoids the need for vigorous washing of the macrophage monolayer following interaction with aged neutrophils and eliminates potential observer bias when counting macrophages. It is less laborious than the plate-based counting method and so is particularly suitable for investigating a large number of conditions. However, we recommend that new investigators validate this method against the "gold standard" plate-based counting assay in their own laboratories (29).

1. This method assumes the use of adherent macrophages in 48-well, TC-treated microplates.

2. Suspend $10^{8}$ freshly isolated peripheral blood neutrophils (at least $97 \%$ purity) in $5 \mathrm{~mL}$ of warm IMDM in a 50-mL Falcon ${ }^{\circledR}$ conical polypropylene tube.

3. Add $10 \mu \mathrm{L}$ of $10 \mathrm{~m} M$ CMFDA ( $20 \mu M$ final concentration), mix gently, and incubate at $37^{\circ} \mathrm{C}$ for $15 \mathrm{~min}$. 


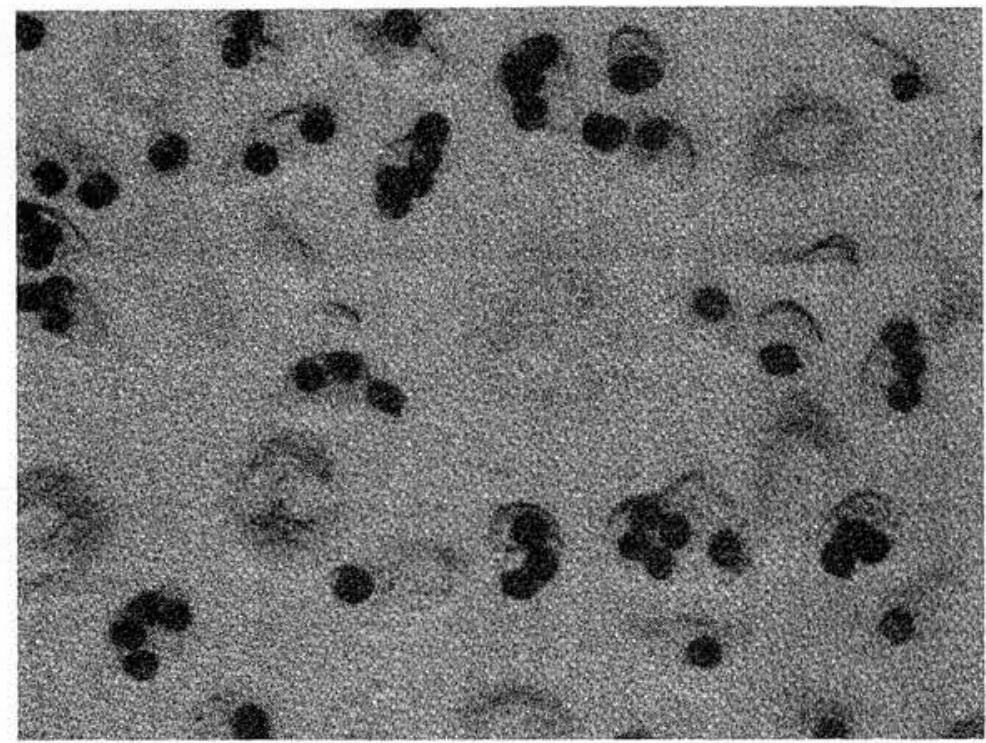

Fig. 6. Phagocytosis assay with human monocyte-derived macrophages and aged neutrophils. Following $60 \mathrm{~min}$ incubation at $37^{\circ} \mathrm{C}$, the macrophage monolayer was washed with cold phosphate-buffered saline, fixed with glutaraldehyde, and stained for myeloperoxidase (MPO). Ingested neutrophils are MPO-positive (dark reaction product), whereas macrophages are MPO-negative.

4. Dilute the labeled neutrophils by adding $15 \mathrm{~mL}$ of warm IMDM supplemented with $10 \%$ autologous serum (see Note 6).

5. Dispense the neutrophil suspension into a $75-\mathrm{cm}^{2}$ cell culture flask and stand the flask on its end in an incubator for $20 \mathrm{~h}$ at $37^{\circ} \mathrm{C}$ in a $5 \% \mathrm{CO}_{2}$ atmosphere.

6. Harvest the neutrophil suspension into a $50-\mathrm{mL}$ Falcon conical polypropylene tube and wash twice in warm IMDM ( $50 \mathrm{~mL}$ per wash) by centrifuging at $220 \mathrm{~g}$ for $5 \mathrm{~min}$ and discarding the supernatant. After each wash, gently resuspend the neutrophil pellet in $1 \mathrm{~mL}$ of warm IMDM using a plastic pipet to avoid clumping of the cells.

7. Count the cells using a hemocytometer and finally resuspend the aged neutrophils at $4 \times 10^{6}$ cells $/ \mathrm{mL}$ in warm $\left(37^{\circ} \mathrm{C}\right)$ IMDM (no serum).

8. Rinse the macrophage monolayer with warm $\left(37^{\circ} \mathrm{C}\right) \mathrm{IMDM}$ to remove nonadherent cells.

9. Overlay the macrophage monolayer with $0.5 \mathrm{~mL}\left(2 \times 10^{6}\right.$ cells $)$ of the suspension of labelled aged neutrophils in IMDM (serum-free), and incubate for $60 \mathrm{~min}$ at $37^{\circ} \mathrm{C}$ in a $5 \% \mathrm{CO}_{2}$ atmosphere.

10. After a $60 \mathrm{~min}$ incubation at $37^{\circ} \mathrm{C}$, aspirate the neutrophil suspension from the wells.

11. Detach the macrophages by incubation with $300 \mu \mathrm{L}$ of $0.25 \%$ trypsin/ $1 \mathrm{~m} M$ EDTA solution (Invitrogen) for $20 \mathrm{~min}$ at $37^{\circ} \mathrm{C}$ followed by $20 \mathrm{~min}$ at $4^{\circ} \mathrm{C}$ (see Note 17). 


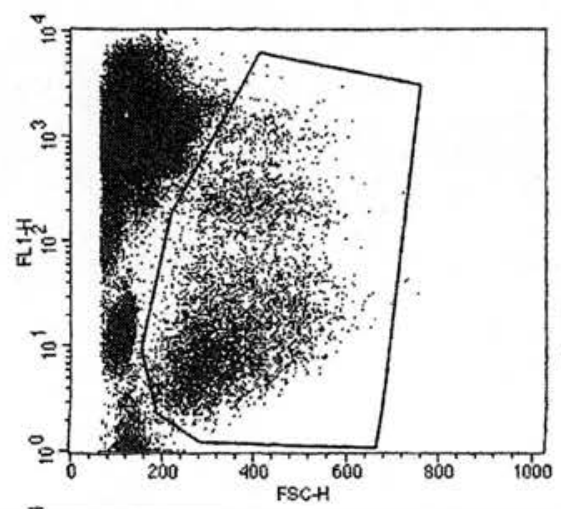

\section{Untreated}
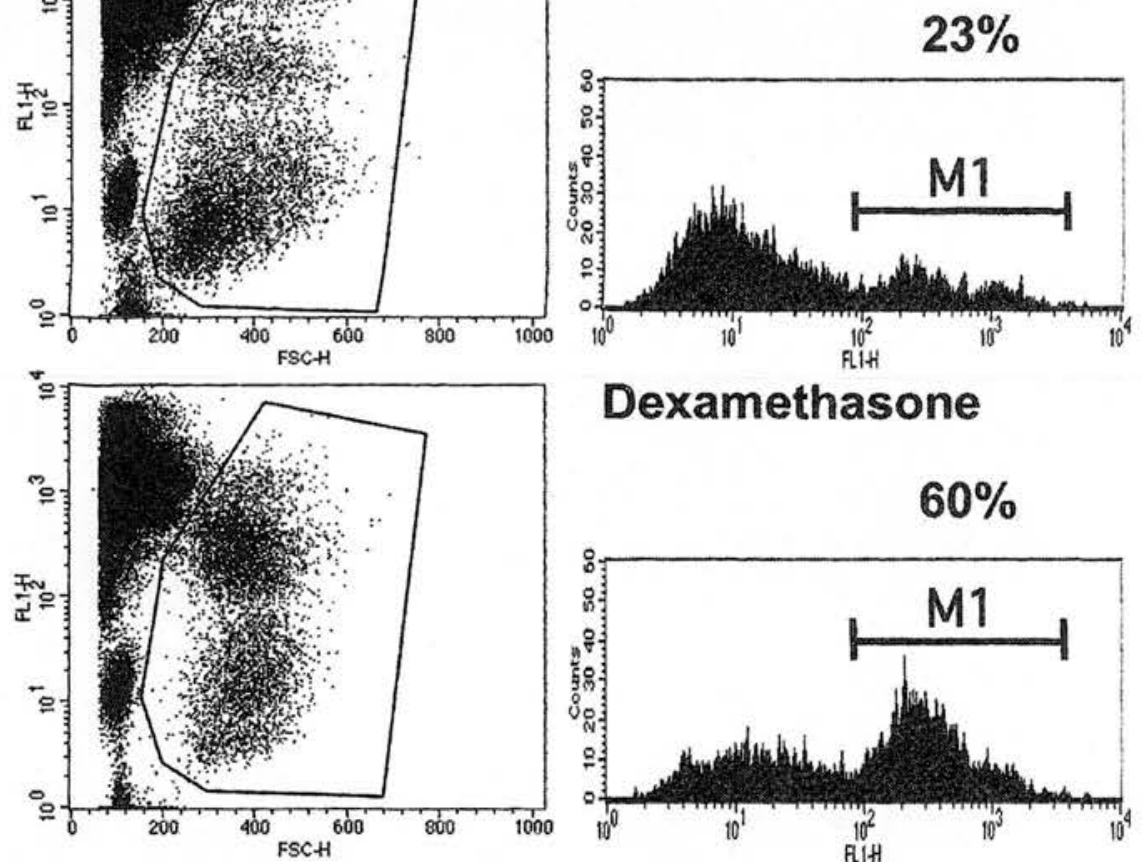

\section{Dexamethasone}

\section{$60 \%$}

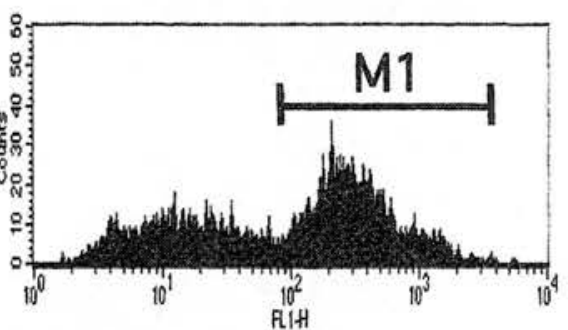

Fig. 7. Flow cytometry-based phagocytosis assay. Aged human neutrophils were labeled with 5-chloromethylfluorescein diacetate (CMFDA) and incubated with monocyte-derived macrophages cultured in medium alone (Untreated) or in the presence of $1 \mu M$ dexamethasone as indicated. Macrophages were detached and analysed by flow cytometry. Macrophages were distinguished by their typical high forward-scatter (FSC) characteristics, and macrophages which had ingested CMFDA-labeled apoptotic neutrophils were FL1-positive. Dexamethasone substantially increased the percentage of macrophages that phagocytosed labeled apoptotic neutrophils.

12. Harvest the detached macrophages by forceful pipetting. Analyze the entire 300 $\mu \mathrm{L}$ of sample (unfixed) immediately using a FACSCalibur flow cytometer (Becton Dickinson), aiming to collect at least 5000 events in the macrophage gate.

13. Apoptotic cells and macrophage populations are identified by their distinct laserscatter properties. The number of the FL-1-positive events in the macrophage gate is divided by the total number of macrophages to obtain the proportion of macrophages that have internalised apoptotic cells. (Fig. 7).

14. Treatment of monocytes for 5-7 days with $1 \mu M$ dexamethasone can be used to increase the percentage of macrophages that phagocytose apoptotic neutrophils (Fig. 7). 


\section{Notes}

1. $1 \mu M$ TO-PRO3 (Molecular Probes) may also be used to identify necrotic cells, and has the advantage that fluorescence is measured in the FL-4 channel, which requires minimal compensation when used alongside FL-1.

2. Neutrophil granules contain high concentrations of proteases. Extreme care must be taken to keep all samples on ice during the preparation of neutrophil lysates in order to prevent the protein of interest being degraded before blotting. Higher protease inhibitor concentrations are required for neutrophils than for other cell types. The protocol describes high protease inhibitor concentrations that we have used successfully in our laboratory; however, some experimentation with regard to manipulation of concentrations may be required. Recent evidence demonstrates that pre-incubation of neutrophils with $1 \mathrm{~m} M$ phenylmethylsulfonylfluoride (PMSF; Sigma-Aldrich) for up to $1 \mathrm{~h}$ prior to lysis may be required to prevent neutrophil protease-mediated cleavage of intracellular proteins following addition of lysis buffer (17).

3. The $12 \%$ gel recommended in this protocol is based on blotting for cleaved caspase $3(14-21 \mathrm{kDa})$. However, gel percentages may be altered according to the size of the protein of interest. In general, low-percentage gels are used to blot for highmolecular-weight proteins, and high-percentage gels for low-molecular-weight proteins. Therefore, the composition of the gel may have to be altered depending on the size of the protein of interest. Some proteins that are important in apoptosis are of similarly low size to cleaved caspase 3 , e.g., Bax $(21 \mathrm{kDa})$ and truncated Bid ( $15 \mathrm{kDa})$, and therefore a similar percentage gel will probably be appropriate, whereas other proteins such as procaspase $8(55 \mathrm{kDa})$ and procaspase $3(32 \mathrm{kDa})$ are larger and may require a lower percentage gel for optimal resolution.

4. The protocol described is based on the homogeneous caspase kit available from Roche Diagnostics, and represents the simplest method for fluorimetric detection of total caspase activity. However, other commercial kits are available, which may be based on either the same or slightly different protocol.

5. We have found that SeaKem LE agarose is effective for separation of DNA fragments. It forms strong gels with high electrophoretic mobility, and shows very low background levels when stained with ethidium bromide.

6. We culture neutrophils in IMDM containing $10 \%$ serum. We routinely use autologous platelet-rich plasma-derived serum, prepared from platelet-rich plasma (harvested after centrifugation of citrate-anticoagulated blood) by recalcification with $20 \mathrm{mM} \mathrm{CaCl}{ }_{2}$ for $1 \mathrm{~h}$ at $37^{\circ} \mathrm{C}$ in glass tubes. However, bovine serum may also be used. Alternatively, neutrophils may be cultured in the absence of serum with a small amount of added protein (e.g., $0.5 \%[\mathrm{w} / \mathrm{v}]$ serum albumin), but apoptosis will proceed more rapidly. Cell-saver pipet tips should be used when pipetting neutrophils to minimize cell damage.

7. Some investigators add extra serum to the cells in the cytospin chamber to avoid artifacts caused by cell breakage during centrifugation. We have observed that adding serum reduces the effectiveness of deposition of neutrophils that have progressed 
to a late stage of apoptosis (late apoptotic or postapoptotic cells), so potentially underestimating the true rate of neutrophil apoptosis. Late apoptotic neutrophils appear as cell ghosts with little or no evidence of nuclear staining, having undergone "nuclear evanescence" (18-20). Furthermore, late apoptotic cell ghosts in a cell population may be overlooked because the lack of nuclear material means that they stain very faintly with Romanowsky stains such as Diff Quik, and in the past late apoptotic cells may have been gated out as "debris" when analyzed by flow cytometry.

8. The flexible plate is best trimmed and mounted in a standard 96-well tissue culture plate for support. Alternatively, for small numbers of samples, 2-mL roundbottomed polypropylene vials may be used.

9. Dilutions prior to flow cytometry should be performed using Annexin binding buffer, because in the absence of $\mathrm{Ca}^{2+}$ Annexin $\mathrm{V}$ will rapidly dissociate from PS on the apoptotic cell surface.

10. Flow cytometers interpret the laser forward-scatter (FS) signal in different ways. For example, the Coulter Epics XL measures integral (area) FS signals, whereas the Becton-Dickinson FACSCalibur records height signals from the FS pulse. This difference significantly affects how late apoptotic neutrophils appear in a standard FS vs side-scatter dot plot. On a Coulter Epics XL, late apoptotic neutrophils appear as a distinct low-FS population that can be gated separately from the population of nonapoptotic/early apoptotic neutrophils. This has the advantage that a nuclear stain such as propidium iodide is not routinely required to identify the membrane permeable late apoptotic cells. On a FACSCalibur cytometer, the late apoptotic neutrophils appear within a single population along with the nonapoptotic and early apoptotic cells. Digital cytometers such as the FACSVantage analyze the area of the FS pulse similarly to the Coulter machine.

11. Eosinophils present in the granulocyte population also have low levels of CD16 expression, so eosinophil counts (by analysis of cell morphology in cytocentrifuge preparations or CD16 expression) should be performed at baseline.

12. Another way of analyzing mitochondrial changes associated with apoptosis is Western blotting of the Bcl-2 family proteins that are important in the control of the intrinsic (mitochondrial) apoptotic pathway. The expression profile of Bcl-2 family proteins in peripheral blood neutrophils has been analyzed and shows expression of Bak, Bad, Bcl-w, and Bfl-1 in these cells, but relatively little Bcl-2, Bcl-xL, Bik, and Bax (24). Therefore, the more abundant proteins should be considered first for analysis for roles in neutrophil apoptosis, although some proteins, such as Bax, may be transcriptionally upregulated in response to certain apoptotic stimuli under the control of p53, and may therefore still be important in neutrophil apoptosis. The Western blotting protocol is identical to that described for caspases.

13. For short-term culture, tubes may be incubated in a shaking heated block set to $37^{\circ} \mathrm{C}$. For longer incubation periods, a hole is made in the top of each polypropylene round-bottomed tube, and the tubes are placed in an incubator in a $5 \% \mathrm{CO}_{2}$ atmosphere at $37^{\circ} \mathrm{C}$. 
14. The protocol for this kit recommends a cell number of $4 \times 10^{4}$ cells per well in a volume of $100 \mu \mathrm{L}$. However, in assays involving neutrophils cell numbers may be increased in order to amplify the signals obtained from the assay particularly as neutrophils are so abundant. On the other hand, cell density may affect the rate of spontaneous apoptosis of these cells during culture, with a high cell density promoting survival, especially at densities of $8 \times 10^{6} \mathrm{cells} / \mathrm{mL}$ and above (25). Therefore, some experimentation may be required to manipulate the cell density, volume, and time of culture in order to gain the best results with this assay.

15. Colorimetric assays for single-caspase activity are also available. These are similar to the fluorometric assays, and follow a similar protocol, but instead utilize cleavage of a chromophore (e.g., p-nitroanalide) from caspase substrates as a measure of caspase activation. Development of colour can be monitored using a spectrophotometer or microplate reader ( $405 \mathrm{~nm}$ wavelength light), and activity quantified by comparison with a calibration curve constructed using known standards.

16. This protocol applies to any cell type, as caspase activation is a general event of apoptosis and is not unique to neutrophils. However, differences may exist between the expression profiles of the various caspases in different cell types, and this must be borne in mind when selecting an assay to use in neutrophils. Caspases 1, 3, 4, and 7-10 are expressed in neutrophils (24). In contrast, it has been reported that caspase 2 is absent from peripheral blood neutrophils, although it is expressed in HL-60 cells.

17. Treatment with trypsin-EDTA may lead to clumping of cells leading to blockage of the flow cytometer's sample intake nozzle. Clumping may be minimised by adding $50 \mu \mathrm{L}$ of bovine serum to each well following incubation with trypsin-EDTA.

\section{Acknowledgments}

This work was funded by a Wellcome Trust Research Fellowship (E. T.) and a Medical Research Council Clinician Scientist Fellowship (S. P. H.). The Western blot demonstrating activation of caspase 3 (Fig. 3) was kindly provided by Tara Sheldrake. We are grateful to Shonna MacCall for her helpful comments.

\section{References}

1. Savill, J. S., Wyllie, A. H., Henson, J. E., Walport, M. J., Henson, P. M., and Haslett, C. (1989) Macrophage phagocytosis of aging neutrophils in inflammation: programmed cell death in the neutrophil leads to its recognition by macrophages. J. Clin. Invest. 83, 865-875.

2. Hurley, J. V. (1983) Terminations of acute inflammation I. Resolution, in Acute Inflammation (Hurley, J. V., ed.), Churchill-Livingstone, London, pp. 109-117.

3. Ward, C., Chilvers, E. R., Lawson, M. F., et al. (1999) NF-kappaB activation is a critical regulator of human granulocyte apoptosis in vitro. J. Biol. Chem. 274, 43094318. 
4. Taylor, E. L., Megson, I. L., Haslett, C., and Rossi, A. G. (2001) Dissociation of DNA fragmentation from other hallmarks of apoptosis in nitric oxide-treated neutrophils: differences between individual nitric oxide donor drugs. Biochem. Biophys. Res. Commun. 289, 1229-1236.

5. Walker, A., Ward, C., Taylor, E. L., et al. (2005) Regulation of neutrophil apoptosis and removal of apoptotic cells. Curr. Drug Targets Inflamm. Allergy 4, 447-454.

6. Sabroe, I., Jones, E. C., Usher, L. R., Whyte, M. K. B., and Dower, S. K. (2002) Toll-like receptor (TLR)2 and TLR4 in human peripheral blood granulocytes: a critical role for monocytes in leukocyte lipopolysaccharide responses. J. Immunol. 168, 4701-4710.

7. Dransfield, I. and Rossi, A. G. (2004) Granulocyte apoptosis: who would work with a 'real' inflammatory cell? Biochem. Soc. Trans. 32, 447-451.

8. Rossi, A. G., Ward, C., and Dransfield, I. (2004) Getting to grips with the granulocyte: manipulation of granulocyte behaviour and apoptosis by protein transduction methods. Biochem. Soc. Trans. 32, 452-455.

9. Wyllie, A. H. (1997) Apoptosis: an overview. Br. Med. Bull. 53, 451-465.

10. Zimmermann, K. C., Bonzon, C., and Green, D. R. (2001) The machinery of programmed cell death. Pharmacol. Ther. 92, 57-70.

11. Savill, J., Dransfield, I., Gregory, C., and Haslett, C. (2002) A blast from the past: clearance of apoptotic cells regulates immune responses. Nat. Rev. Immunol. 2, 965-975.

12. Ren, Y. and Savill, J. S. (1995) Proinflammatory cytokines potentiate thrombospondin-mediated phagocytosis of neutrophils undergoing apoptosis. J. Immunol. 154, 2366-2374.

13. Rossi, A. G., McCutcheon, J. C., Roy, N., Chilvers, E. R., Haslett, C., and Dransfield, I. (1998) Regulation of macrophage phagocytosis of apoptotic cells by cAMP. J. Immunol. 160, 3562-3568.

14. Liu, Y., Cousin, J. M., Hughes, J., et al. (1999) Glucocorticoids promote nonphlogistic phagocytosis of apoptotic leukocytes. J. Immunol. 162, 3639-3646.

15. McCutcheon, J. C., Hart, S. P., Canning, M., Ross, K., Humphries, M. J., and Dransfield, I. (1998) Regulation of macrophage phagocytosis of apoptotic cells by adhesion to fibronectin. J. Leukoc. Biol. 64, 1-8.

16. Hart, S. P., Smith, J. R., and Dransfield, I. (2004) Phagocytosis of opsonized apoptotic cells: roles for 'old-fashioned' receptors for antibody and complement. Clin. Exp. Immunol. 135, 181-185.

17. Kato, T., Sakamoto, E., Kutsuna, H., Kimura-Eto, A., Hato, F., and Kitagawa, S. (2004) Proteolytic conversion of STAT3alpha to STAT3gamma in human neutrophils: role of granule-derived serine proteases. J. Biol. Chem. 279, 31,076-31,080.

18. Hebert, M. J., Takano, T., Holthofer, H., and Brady, H. R. (1996) Sequential morphologic events during apoptosis of human neutrophils. Modulation by lipoxygenase-derived eicosanoids. J. Immunol. 157, 3105-3115.

19. Ren, Y., Stuart, L., Lindberg, F. P., et al. (2001) Nonphlogistic clearance of late apoptotic neutrophils by macrophages: efficient phagocytosis independent of beta 2 integrins. J. Immunol. 166, 4743-4750. 
20. Hart, S. P., Alexander, K. M., MacCall, S. M., and Dransfield, I. (2005) C-reactive protein does not opsonize early apoptotic human neutrophils, but binds only membrane-permeable late apoptotic cells and has no effect on their phagocytosis by macrophages. J. Inflamm. (Lond) 2,5 .

21. Dransfield, I., Buckle, A.-M., Savill, J. S., McDowall, A., Haslett, C., and Hogg, N. (1994) Neutrophil apoptosis is associated with a reduction in CD16 (FcgRIII) expression. J. Immunol. 153, 1254-1263.

22. Harada, H. and Grant, S. (2003) Apoptosis regulators. Rev. Clin. Exp. Hematol. 7, 117-138.

23. Martin, M. C., Dransfield, I., Haslett, C., and Rossi, A. G. (2001) Cyclic AMP regulation of neutrophil apoptosis occurs via a novel protein kinase A-independent signaling pathway. J. Biol. Chem. 276, 45041-45050.

24. Santos-Beneit, A. M. and Mollinedo, F. (2000) Expression of genes involved in initiation, regulation, and execution of apoptosis in human neutrophils and during neutrophil differentiation of HL-60 cells. J. Leukoc. Biol. 67, 712-724.

25. Hannah, S., Nadra, I., Dransfield, I., Pryde, J. G., Rossi, A. G., and Haslett, C. (1998) Constitutive neutrophil apoptosis in culture is modulated by cell density independently of beta2 integrin-mediated adhesion. FEBS Lett. 421, 141-146.

26. Nagata, S. (2005) DNA degradation in development and programmed cell death. Annu. Rev. Immunol. 23, 853-875.

27. Newman, S. L., Henson, J. E., and Henson, P. M. (1982) Phagocytosis of senescent neutrophils by human monocyte-derived macrophages and rabbit inflammatory macrophages. J. Exp. Med. 156, 430-442.

28. Hart, S. P., Dougherty, G. J., Haslett, C., and Dransfield, I. (1997) CD44 regulates phagocytosis of apoptotic neutrophil granulocytes, but not apoptotic lymphocytes, by human macrophages. J. Immunol. 159, 919-925.

29. Jersmann, H. P., Ross, K. A., Vivers, S., Brown, S. B., Haslett, C., and Dransfield, I. (2003) Phagocytosis of apoptotic cells by human macrophages: Analysis by multiparameter flow cytometry. Cytometry $\mathbf{5 1 A}, 7-15$. 


\title{
Phagocytosis of apoptotic cells
}

\author{
Simon P. Hart, Ian Dransfield, Adriano G. Rossi * \\ MRC Centre for Inflammation Research, University of Edinburgh, Queen's Medical Research Institute, \\ 47 Little France Crescent, Edinburgh EHI6 4TJ, UK
}

Accepted 10 November 2007

\begin{abstract}
Removal of apoptotic cells by phagocytes plays an important role in many biological processes, including embryological development and tissue remodelling. In addition, it has become apparent that one of the key mechanisms for the successful resolution of inflammation is the orchestrated clearance of apoptotic inflammatory cells by phagocytes (e.g., macrophages and dendritic cells) and other cells known to have phagocytic capacity (e.g., hepatocytes, endothelial cells, epithelial cells, etc.). Furthermore, phagocytosis of apoptotic cells is an active and highly regulated process that not only serves to remove potentially histotoxic cells from the inflammatory milieu, but also directs the phenotype of the phagocytic cell to be anti-inflammatory. Convincing evidence has been presented that reduced or dysregulated phagocytosis of apoptotic cells contributes to the development and propagation of inflammatory disorders. Conversely, enhanced phagocytosis of apoptotic cells may be exploited for therapeutic gain. Indeed, powerful anti-inflammatory drugs such as the glucocorticoids have been shown to augment clearance of apoptotic cells which may contribute to their therapeutic effectiveness. In this chapter, we describe methods for studying phagocytosis of apoptotic cells.
\end{abstract}

(c) 2007 Elsevier Inc. All rights reserved.

Keywords: Apoptosis; Inflammation; Macrophage; Neutrophil; Phagocytosis

\section{Introduction}

Phagocytic clearance of apoptotic cells plays a vital role in many fundamental biological processes, including normal tissue turnover [1], remodelling of embryological tissues [2], development of the immune system [3], and resolution of inflammation [4]. Phagocytosis of senescent cells was first described in the late 19 th century by the Russian biologist Elie Metchnikoff who, using a simple light microscope, observed that "microphages" (neutrophil granulocytes) were "englobed" by macrophages in injured tadpole fins. Macrophages are tissue cells that are highly specialised for endocytosis and intracellular digestion, and their traditional role as scavenger cells has been known for over a century. Apoptotic cells are swiftly recognised and ingested by macrophages and other mononuclear phagocytes (dendritic cells). There is also evidence that in

\footnotetext{
- Corresponding author.

E-mail address: a.g.rossi@ed.ac.uk (A.G. Rossi).
}

certain situations apoptotic cells may be removed by nonprofessional phagocytes such as hepatocytes [5], endothelial cells [6], epithelial cells [7], glomerular mesangial cells [8], and fibroblasts [9].

We have studied phagocytosis of apoptotic neutrophils as a paradigm for apoptotic cell clearance. During the acute inflammatory response huge numbers of neutrophils are mobilised and recruited to the tissues, where they survive for only a short time before undergoing apoptosis. Apoptotic neutrophils exhibit greatly reduced responsiveness to external stimuli [10], but retain plasma membrane integrity so that release of harmful cellular contents is limited. Apoptotic neutrophils are recognised and ingested by macrophages [11], which are thought to be important steps in preventing the release of toxic granules and chemotactic factors into the extracellular fluid, thereby halting further injury and allowing resolution to occur. Apoptotic cells when recognised and phagocytosed by macrophages, provoke an anti-inflammatory cytokine response. For example, stimulated production and release of pro-inflammatory 
mediators such as TNF- $\alpha$ is suppressed $[12,13]$ and production of the anti-inflammatory cytokine TGF- $\beta$ is stimulated [4]. In this way, by down-regulating neutrophil functions and triggering their non-inflammatory clearance by phagocytes, apoptosis provides a mechanism for the safe disposal of potentially destructive inflammatory cells. Evidence for a role for leukocyte apoptosis and their subsequent clearance by phagocytes in the resolution of inflammation is supported by the observation that apoptotic granulocytes can be identified within phagocytic vacuoles in macrophages from inflamed joints [11], airways, [14], and lungs [15].

It has been assumed that macrophages and other phagocytes must be able to recognise changes on the surface of apoptotic cells that distinguish them from healthy live cells, but intensive research has failed to identify a single dominant phagocyte receptor responsible for apoptotic cell clearance. Inhibition studies using ligands and monoclonal antibodies have implicated many different and often unrelated phagocyte receptors for apoptotic cells, including the integrins $\alpha v \beta 3$ [16] and $\alpha v \beta 5$ [17], CD36 [18], a phosphatidylserine (PS) receptor [see 19], various lectins $[5,20]$, a scavenger receptor [21], CD14 [22], an ATP binding cassette transporter [23], LOX-1 [24], and CD68 [25]. It is notable that complete inhibition of phagocytosis of apoptotic cells has never been achieved in experimental systems, even when inhibitory antibodies or ligands have been used in combination. Furthermore, the molecular alterations on the surface of apoptotic cells that are responsible for phagocyte recognition remain to be fully characterised. We and others have shown that a number of specific alterations in the protein and carbohydrate composition of the plasma membrane are associated with apoptosis $[10,26,27]$. It has also become apparent that apoptosis is associated with membrane alterations that confer specific binding of naturally occurring soluble opsonins that bind to the cell surface and initiate phagocytosis. Classical serum opsonins such as antibodies and complement proteins may bind to apoptotic cells and mediate phagocytosis, providing a mechanism for clearance that does not require a novel apoptotic cell receptor $[28,29]$.

In order that the load of apoptotic neutrophils at an inflammatory site is matched by appropriate clearance activity, macrophage capacity for apoptotic cell phagocytosis is likely to be closely regulated. Macrophage phagocytic capacity may be influenced by soluble mediators such as cytokines [30], prostaglandins [31], or glucocorticoid hormones [32]. In addition, interaction of surface adhesion molecules with neighbouring cells and extracellular matrix components may profoundly influence many aspects of cellular behaviour, including phagocytosis $[33,34]$. If the rate of cell death by apoptosis is such that macrophage clearance capacity is exceeded, apoptotic cells may become necrotic, resulting in release of harmful cellular contents and damage to the surrounding tissue. In support of this suggestion, treatment of mice with Fas antibody triggered a massive wave of apoptosis in the liver, and the animals developed extensive hepatic necrosis and died [35]. Similarly, induction of apoptosis in the rat lung led to pulmonary fibrosis [36]. In these situations, we believe the local macrophages were unable to clear the load of apoptotic cells with which they were faced. However, careful, specific and timed induction of granulocyte apoptosis at the peak of inflammatory cell influx has demonstrated that, providing the clearance capacity of phagocytes is not overwhelmed, enhanced resolution of inflammation can be achieved [37-39]. Potential future therapeutic approaches may need to specifically augment of the clearance efficiency of local macrophages in parallel with apoptosis induction. Here, we describe some of the main techniques that may be used to observe and quantify phagocytic ingestion of apoptotic cells, using macrophage phagocytosis of apoptotic neutrophils as a model.

\section{Description of method}

\subsection{Plate-based assay for phagocytosis of apoptotic neutrophils}

Macrophage phagocytosis of apoptotic cells may be assessed in vitro using minor modifications of a serum-free phagocytosis assay first described by Newman et al. [40]. This method uses adherent human monocyte-derived macrophages which are most efficient at ingesting apoptotic cells, but it has also been used successfully with murine peritoneal and bone marrow-derived monocytes and human apoptotic neutrophils. In practice, any cells adherent to tissue culture plastic may be used in the phagocytosis assay, including non-professional phagocytes such as fibroblasts or epithelial cells.

In serum-free phagocytosis assays such as this, macrophages specifically recognise and ingest neutrophils that have undergone spontaneous apoptosis during culture in $10 \%$ serum $[11,40]$. In vitro serum-free assays have been the cornerstone of most subsequent studies of the phagocytosis of apoptotic neutrophils and other apoptotic cells, and it has been naturally assumed that serum is not required for apoptotic cell clearance. On the other hand, several studies have reported a requirement for serum for phagocytosis of apoptotic cells in vitro [41-43]. Although we have found that serum present during an in vitro phagocytosis assay has no significant effect on macrophage phagocytosis of apoptotic neutrophils (our unpublished observations), it is possible that apoptotic cells might become opsonised during culture in the presence of serum, prior to their use in phagocytosis assays. Even when apoptotic cells are cultured in the absence of serum, macrophage-derived opsonins such as complement protein iC $3 \mathrm{~b}$ [44], Clq, and MFG-E8 [45] may be important [46]. When investigating ways of modulating phagocytosis of apoptotic cells, specificity may be demonstrated by comparison of the capacity for phagocytosis of another particle, such as immunoglobulin G-opsonized erythrocytes (EIgG) or serum-opsonized zymosan. 
Specifically, mononuclear cells are isolated from human peripheral blood by dextran sedimentation and discontinuous Percoll gradient centrifugation [47,48]. Monocytes are then enriched by selective adherence to $\operatorname{Costar}^{\otimes} 48$ well TC-treated microplates (Fisher Scientific, Loughborough, UK) during incubation at $37^{\circ} \mathrm{C}$ for $1 \mathrm{~h}$. Adherent cells (>90\% CD14-positive monocytes) are washed twice in PBS, and monocyte-derived macrophages differentiated by culture (at $37^{\circ} \mathrm{C} ; 5 \% \mathrm{CO}_{2}$ ) for 5-7 days in Iscove's medium (Invitrogen, Paisley, UK) containing 10\% (v/v) autologous serum in the presence or absence of reagents of interest. Similarly, adherent monolayers of murine macrophages or other phagocytes may be used.

To prepare apoptotic neutrophils as an apoptotic cell target, human peripheral blood neutrophils are isolated by dextran sedimentation and discontinuous Percoll gradient centrifugation using Dransfield's modification of the method described by Haslett et al. [26,49]. Purity should be assessed by examining stained cytocentrifuge preparations, or by flow cytometric analysis of laser scatter properties and CD16 staining to exclude significant contamination by mononuclear cells, erythrocytes, or eosinophils. Neutrophils should ideally be cultured in vitro at a final cell concentration of $4 \times 10^{6} / \mathrm{ml}$ in Iscove's medium containing $10 \%(\mathrm{v} / \mathrm{v})$ autologous serum or bovine serum for $20 \mathrm{~h}$ at $37^{\circ} \mathrm{C}, 5 \% \mathrm{CO}_{2}$, during which time 40 $60 \%$ of the cells will undergo spontaneous apoptosis. Other apoptotic cell targets commonly used included dexamethasone-treated murine thymocytes, or $\gamma$-irradiated lymphocytes. The percentage of apoptotic cells within the cell population is best assessed by flow cytometry following staining with FITC-conjugated annexin $\mathrm{V}$ and propidium iodide to identify apoptotic and necrotic cells respectively; especially important as the mechanisms for phagocytosis of apoptotic and necrotics cells are likely to be different.

Prior to the phagocytosis assay, harvest the neutrophil suspension into a $50 \mathrm{ml}$ Falcon ${ }^{\otimes}$ conical polypropylene tube (Becton-Dickinson Labware, Franklin Lakes, NJ, USA) and wash twice in warm $\left(37^{\circ} \mathrm{C}\right)$ Iscove's medium ( $50 \mathrm{ml}$ volume per wash) by centrifuging at $220 \mathrm{~g}$ for $5 \mathrm{~min}$ and discarding the supernatant. After each wash, gently resuspend the neutrophil pellet in $1 \mathrm{ml}$ of warm $\left(37^{\circ} \mathrm{C}\right)$ Iscove's medium using a plastic pipette to avoid clumping of the cells. Count the cells using a haemocytometer and finally resuspend the aged neutrophils at $4 \times 10^{6}$ cells $/ \mathrm{ml}$ in warm $\left(37^{\circ} \mathrm{C}\right)$ Iscove's medium (no serum). Rinse the macrophage monolayer with warm $\left(37^{\circ} \mathrm{C}\right)$ medium to remove non-adherent cells, and then overlay the macrophages with $0.5 \mathrm{ml}\left(2 \times 10^{6}\right.$ cells $)$ of the suspension of aged neutrophils, and incubate for $60 \mathrm{~min}$ at $37^{\circ} \mathrm{C}$ in a $5 \% \mathrm{CO}_{2}$ atmosphere. Wash each well in an identical fashion with cold PBS (without cations) and use an inverted microscope to check that non-ingested neutrophils have been largely removed, and repeat the wash as necessary, continually checking by microscopy to ensure that the macrophage monolayer is not disrupted. Fix in $2.5 \%$ glutaraldehyde for $30 \mathrm{~min}$ and rinse off with PBS, and stain for myeloperoxidase (MPO) by covering the base of each well with PBS containing $0.1 \mathrm{mg} / \mathrm{ml}$ dimethoxybenzidine $(o-$ diansidine) (Sigma-Aldrich Company Ltd., Gillingham, UK) and $0.03 \%(v / v)$ hydrogen peroxide. Leave for about $60 \mathrm{~min}$ at room temperature and monitor for development of a brown colour. Rinse with PBS. Count the percentage of macrophages (MPO-negative) that have phagocytosed one or more apoptotic neutrophils (MPO-positive) by examination with an inverted microscope of at least five fields (minimum 400 cells), and record as the mean percent phagocytosis of duplicate or triplicate wells (Fig. 1). The phagocytic index may also be recorded, which is the number of apoptotic neutrophils ingested per 100 macrophages. Plates may be stored dry at $4^{\circ} \mathrm{C}$ for a number of weeks to several months.

The phagocytic nature of the interaction may be confirmed by examining cytocentrifuge preparations of unfixed macrophages detached with $0.05 \%$ trypsin $/ 0.02 \%$ ethylenediaminetetraacetic acid (EDTA) solution, or by transmission electron microscopy. Macrophages are detached by incubation with $0.05 \%$ trypsin $/ 0.02 \%$ EDTA for $10 \mathrm{~min}$ at $37^{\circ} \mathrm{C}$, and $100 \mu \mathrm{l}$ the cell suspension from each well cytocentrifuged at $30 \mathrm{~g}$ onto a glass slide. Examination by light microscopy of slides fixed in methanol and stained with Diff-Quik $^{\mathrm{TM}}$ permits identification of apoptotic cells within macrophage phagocytic vacuoles (Fig. 2). This method is also appropriate for assessing phagocytosis of apoptotic lymphocytes of other target cells that cannot be stained specifically for MPO.

To prepare erythrocytes coated with IgG (EIgG) as a control particle, wash erythrocytes from human blood and incubate them with a polyclonal rabbit anti-human erythrocyte antibody for $1 \mathrm{~h}$ using a concentration of antibody that does not result in erythrocyte aggregation. In order to maximise the chance of observing any augmentation or inhibition of phagocytosis, a concentration of opsonizing antibody should be chosen that, in preliminary experiments, results in phagocytosis of EIgG by $40-50 \%$

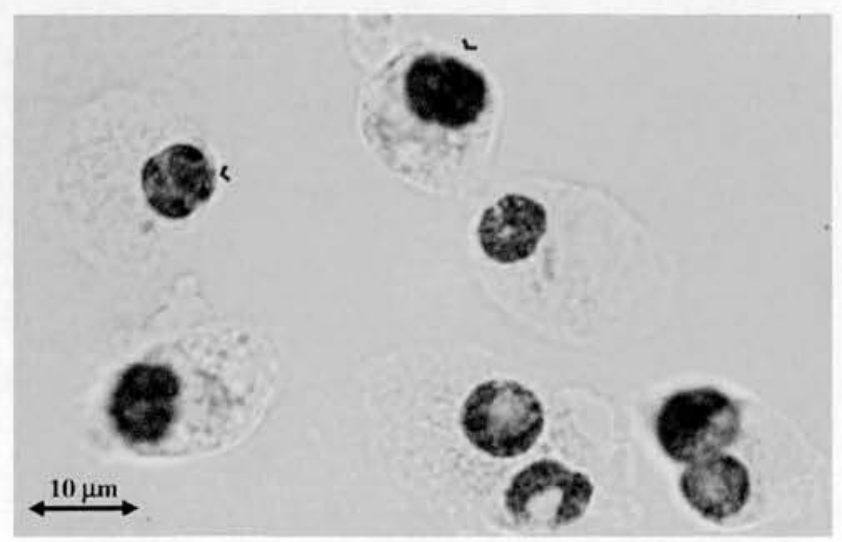

Fig. 1. Plate-based phagocytosis assay. Phagocytosis of apoptotic human neutrophils (arrowheads; stained brown for MPO) by adherent human monocyte-derived macrophages (unstained). 


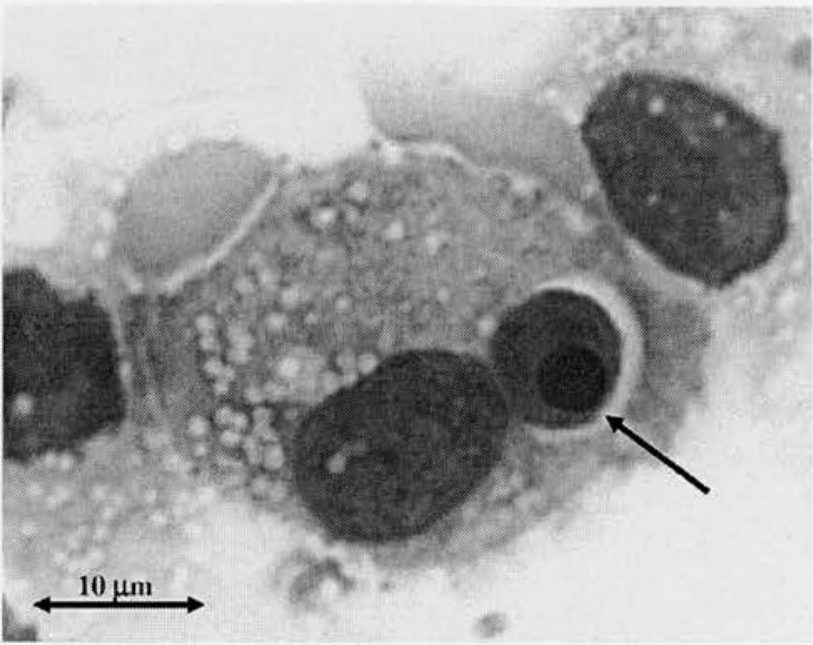

Fig. 2. Cytocentrifuge preparation of an apoptotic human neutrophil within the phagocytic vacuole (arrow) of a monocyte-derived macrophage.

of macrophages. The EIgG are washed twice in PBS and suspended in Iscove's medium at $4 \times 10^{6} / \mathrm{ml}$. Assessment of phagocytosis of EIgG is performed as described for aged neutrophils. After incubation and washing, non-phagocytosed EIgG may be lysed with a $10 \mathrm{~s}$ application of distilled water before immediate fixation in $2.5 \%$ glutaraldehyde. Ingested EIgG may be stained for MPO in the same way as neutrophils.

\subsection{Flow cytometry-based phagocytosis assay}

This modification of the plate-based phagocytosis assay utilises a fluorescent chloromethyl dye that diffuses freely through plasma membranes to label the cytoplasm of live cells. This method avoids the need for vigorous washing of the macrophage monolayer following interaction with aged neutrophils and eliminates potential observer bias when counting macrophages. It is less laborious than the plate-based counting method and so is particularly suitable for high throughput experiments. It is recommended that investigators validate this method against the plate-based counting assay in their own laboratories [50].

This method assumes the use of adherent macrophages in Costar ${ }^{\circledR} 48$ well TC-treated microplates (Fisher Scientific, Loughborough, UK). To label neutrophils, suspend $10^{8}$ freshly-isolated peripheral blood neutrophils (at least $97 \%$ purity) in $5 \mathrm{ml}$ of warm $\left(37^{\circ} \mathrm{C}\right)$ Iscove's medium in a $50 \mathrm{ml} \mathrm{Falcon}{ }^{\circledR}$ conical polypropylene tube (Becton-Dickinson Labware, Franklin Lakes, NJ, USA). Add $10 \mu \mathrm{l}$ of $10 \mathrm{mM}$ 5-chloromethylfluorescein diacetate (CMFDA; CellTracker $^{\text {TM }}$ Green, Molecular Probes, Oregon, USA; $20 \mu \mathrm{M}$ final concentration), mix gently, and incubate at $37^{\circ} \mathrm{C}$ for $15 \mathrm{~min}$. Dilute the labelled neutrophils by adding $15 \mathrm{ml}$ of warm $\left(37^{\circ} \mathrm{C}\right)$ Iscove's medium and supplemented with $10 \%(\mathrm{v} / \mathrm{v})$ autologous serum. Dispense the neutrophil suspension into a Costar ${ }^{\otimes} 75 \mathrm{~cm}^{2}$ cell culture flask (Fisher
Scientific, Loughborough, UK) and stand the flask on its end in an incubator for $20 \mathrm{~h}$ at $37^{\circ} \mathrm{C}$ in a $5 \% \mathrm{CO}_{2}$ atmosphere. Prior to the phagocytosis assay assess the degree of apoptosis by examining Diff-Quik ${ }^{\mathrm{TM}}$-stained methanolfixed cytocentrifuge preparations. Harvest the neutrophil suspension and wash twice in warm $\left(37^{\circ} \mathrm{C}\right)$ medium ( $50 \mathrm{ml}$ volume per wash) by centrifuging at $220 \mathrm{~g}$ for $5 \mathrm{~min}$ and discarding the supernatant. After each wash, gently resuspend the neutrophil pellet in $1 \mathrm{ml}$ of warm $\left(37^{\circ} \mathrm{C}\right)$ IMDM using a plastic pipette to avoid clumping of the cells. Count the cells using a haemocytometer and finally resuspend the aged neutrophils at $4 \times 10^{6}$ cells $/ \mathrm{ml}$ in warm $\left(37^{\circ} \mathrm{C}\right)$ Iscove's medium without serum. Rinse the macrophage monolayer with warm $\left(37^{\circ} \mathrm{C}\right)$ medium to remove non-adherent cells.

Overlay the macrophage monolayer with $0.5 \mathrm{ml}\left(2 \times 10^{6}\right.$ cells) of the suspension of CMFDA-labelled aged neutrophils and incubate for $60 \mathrm{~min}$ at $37^{\circ} \mathrm{C}$ in a $5 \% \mathrm{CO}_{2}$ atmosphere. After $60 \mathrm{~min}$ of incubation at $37^{\circ} \mathrm{C}$, aspirate the neutrophil suspension from each well. Detach the macrophages by incubation with $300 \mu \mathrm{l} 0.05 \%$ trypsin/0.02\% EDTA solution (Invitrogen, Paisley, UK) for $10 \mathrm{~min}$ at $37^{\circ} \mathrm{C}$ followed by $10 \mathrm{~min}$ at $4{ }^{\circ} \mathrm{C}$. Harvest the detached macrophages by forceful pipetting. Analyse the entire $300 \mu \mathrm{l}$ sample (unfixed) immediately using a FACSCalibur $^{\mathrm{TM}}$ flow cytometer (Becton-Dickinson, Oxford, UK), aiming to collect at least 5000 events in the macrophage gate. Apoptotic cells and macrophage populations are identified by their distinct laser scatter properties. The number of the FL-1-positive events in the macrophage gate is divided by the total number of macrophages to obtain the proportion of macrophages that have internalised apoptotic cells (Fig. 3).

\subsection{Phagocytosis of apoptotic cells in vivo}

Similar methods have been used successfully to assess apoptotic cell phagocytosis in vivo. Potter and colleagues used a "peritoneal test tube" model to investigate clearance of apoptotic thymocytes by resident peritoneal macrophages, whereby mice received an intraperitoneal injection of $10^{7}$ murine thymocytes which had undergone apoptosis in response to $1 \mu \mathrm{M}$ dexamethasone for $3 \mathrm{~h}$. After $30 \mathrm{~min}$ peritoneal cells were recovered by lavage with ice-cold buffer, and phagocytosis was assessed on stained cytocentrifuge preparations [51]. Apoptotic cells may also be labelled with CMFDA prior to injection, and lavage cells analysed by flow cytometry in after staining with a macrophage marker e.g., F4/80 mAb.

Clearance of native apoptotic cells can be assessed by careful microscopy of stained tissue sections or lavage samples. For example, following experimental sterile peritonitis induced by thioglycollate, peritoneal exudate cells are harvested by peritoneal lavage with ice-cold buffer and analysed by microscopy of stained cytocentrifuged preparations [51]. In the thymus, Devitt and colleagues identified apoptotic thymocytes in tissue sections by in situ end labelling of 

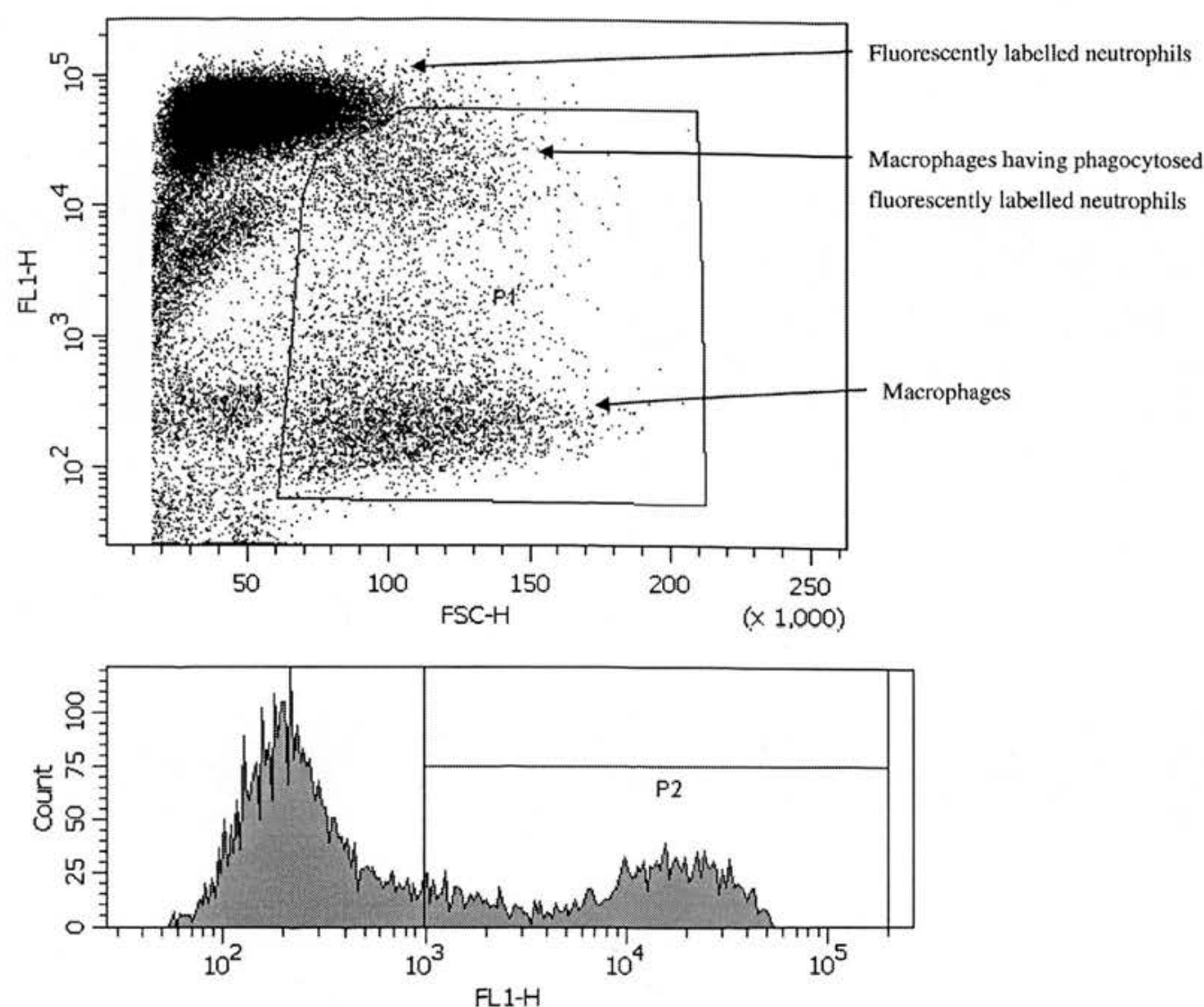

Fig. 3. Flow cytometry-based phagocytosis assay. The dot-plot (upper panel) and histogram (lower panel) demonstrate phagocytosis of CMFDA-labelled apoptotic human neutrophils by human macrophages. Phagocytic macrophages have high FL-1 fluorescence. In this experiment, $36 \%$ of macrophages have phagocytosed apoptotic neutrophils (figure courtesy S. Bournazos).

DNA breaks. Detailed morphological analyses of toluidine blue-stained, resin-embedded sections along with F4/80 co-staining allowed apoptotic cells to be defined as either "free" or "macrophage-associated" [52].

\section{Concluding remarks}

Phagocytosis of apoptotic cells is clearly an important physiological mechanism to remove effete cells before they disgorge their contents of potential histotoxic products. We have highlighted a number of easy, rapid and reliable techniques for measuring this phenomenon. The challenges ahead include optimising assessment of phagocytic clearance in vivo perhaps non-invasively using state-of-the-art, real time in vivo imaging techniques, understanding the molecular mechanisms involved and developing pharmacological strategies to manipulate this process for therapeutic gain.

\section{Acknowledgments}

Figure 3 was kindly provided by S. Bournazos. We acknowledge the MRC for funding this work.

\section{References}

[1] H. Han, T. Iwanaga, Y. Uchiyama, T. Fujuti, Cell Tissue Res. 271 (1993) 407-416.

[2] J. Hopkinson-Woolley, D. Hughes, S. Gordon, P. Martin, J.Cell Sci. 107 (1994) 1159-1167.

[3] J.J. Cohen, Adv. Immunol. 50 (1991) 55-85.

[4] J. Savill, I. Dransfield, C. Gregory, C. Haslett, Nat. Rev. Immunol. 2 (2002) 965-975.

[5] L. Dini, F. Autuori, A. Lentini, S. Oliverio, M. Piacentini, FEBS Lett. 296 (1992) 174-178.

[6] L. Dini, A. Lentini, G. Diez Diez, M. Rocha, L. Falasca, L. Serafino, F. Vidal-Vanaclocha, J. Cell Sci. 108 (1995) 967-973.

[7] G.M. Walsh, D.W. Sexton, M.G. Blaylock, C.M. Convery, Blood 94 (1999) 2827-2835.

[8] J.S. Savill, J. Smith, C. Sarraf, Y. Ren, F. Abbot, A. Rees, Kidney Int. 42 (1992) 924-936.

[9] S.E. Hall, J.S. Savill, P.M. Henson, C. Haslett, J. Immunol. 153 (1994) 3218-3227.

[10] I. Dransfield, S.C. Stocks, C. Haslett, Blood 85 (1995) 3264-3273.

[11] J.S. Savill, A.H. Wyllie, J.E. Henson, M.J. Walport, P.M. Henson, C. Haslett, J. Clin. Invest. 83 (1989) 865-875.

[12] M. Stern, J.S. Savill, C. Haslett, Am. J. Pathol 149 (1996) 911-921.

[13] V.A. Fadok, D.L. Bratton, A. Konowal, P.W. Freed, J.Y. Westcott, P.M. Henson, J. Clin. Invest. 101 (1998) 890-898.

[14] K.L. Woolley, P.G. Gibson, K. Carty, A.J. Wilson, S.H. Twaddell, M.J. Woolley, Am. J. Respir. Crit. Care Med. 154 (1996) 237-243. 
[15] S. Kotecha, R.J. Mildner, L.R. Prince, J.R. Vyas, A.E. Currie, R.A. Lawson, M.K. Whyte, Thorax 58 (2003) 961-967.

[16] J.S. Savill, I. Dransfield, N. Hogg, C. Haslett, Nature 342 (1990) 170 173.

[17] M.L. Albert, J.I. Kim, R.B. Birge, Nat. Cell Biol. 2 (2000) 899-905.

[18] J.S. Savill, N. Hogg, Y. Ren, C. Haslett, J. Clin. Invest. 90 (1992) $1513-1522$.

[19] K.S. Ravichandran, U. Lorenz, Nat. Rev. Immunol. 7 (2007) 964-978.

[20] E. Duvall, A.H. Wyllie, R.G. Morris, Immunology 56 (1985) 351358.

[21] N. Platt, H. Suzuki, Y. Kurihara, T. Kodama, S. Gordon, Proc. Natl. Acad. Sci. USA 93 (1996) 12456-12460.

[22] A. Devitt, O.D. Moffatt, C. Raykundalia, J.D. Capra, D.L. Simmons, C.D. Gregory, Nature 392 (1998) 505-509.

[23] M.-F. Luciani, G. Chimini, EMBO J. 15 (1996) 226-235.

[24] K. Oka, T. Sawamura, K. Kikuta, S. Itokawa, N. Kume, T. Kita, T. Masaki, Proc. Natl. Acad. Sci. USA 95 (1998) 9535-9540.

[25] M.P. Ramprasad, W. Fischer, J.L. Witztum, G.R. Sambrano, O. Quehenberger, D. Steinberg, Proc. Natl. Acad. Sci. USA (1995) 9580 9584.

[26] 1. Dransfield, A.-M. Buckle, J.S. Savill, A. McDowall, C. Haslett, N. Hogg, J. Immunol. 153 (1994) 1254-1263.

[27] S.P. Hart, J.A. Ross, K. Ross, C. Haslett, I. Dransfield, Cell Death Differ. 7 (2000) 493-503.

[28] S.P. Hart, J.R. Smith, I. Dransfield, Clin. Exp. Immunol. 135 (2004) 181-185.

[29] S.P. Hart, K.M. Alexander, I. Dransfield, J. Immunol. 172 (2004) 1882-1887.

[30] Y. Ren, R.L. Silverstein, J. Allen, J.S. Savill, J. Exp. Med. 181 (1995) 1857-1862.

[31] A.G. Rossi, J.C. McCutcheon, N. Roy, E.R. Chilvers, C. Haslett, I. Dransfield, J. Immunol. 160 (1998) 3562-3568.

[32] Y. Liu, J.M. Cousin, J. Hughes, J.V. Damme, J.R. Seckl, C. Haslett, I. Dransfield, J. Savill, A.G. Rossi, J. Immunol. 162 (1999) 3639 3646.

[33] S.P. Hart, G.J. Dougherty, C. Haslett, I. Dransfield, J. Immunol. 159 (1997) 919-925.

[34] J.C. McCutcheon, S.P. Hart, M. Canning, K. Ross, M.J. Humphries, I. Dransfield, J. Leukoc. Biol. 64 (1998) 1-8.
[35] J. Ogasawara, R. Watanabe-Fukunaga, M. Adachi, A. Matsuzawa, T. Kasugai, Y. Kitamura, N. Itoh, T. Suda, S. Nagata, Nature 364 (1993) 806-809.

[36] N. Hagimoto, K. Kuwano, H. Miyazaki, R. Kunitake, M. Fujuti, M. Kawasaki, Y. Kaneko, N. Hara, Am. J. Respir. Cell Mol. Biol. 17 (1997) 272-278.

[37] D.A. Sawatzky, D.A. Willoughby, P.R. Colville-Nash, A.G. Rossi, Am. J. Pathol. 168 (2006) 33-41.

[38] A.G. Rossi, D.A. Sawatzky, A. Walker, C. Ward, T.A. Sheldrake, N.A. Riley, A. Caldicott, M. Martinez-Losa, T.R. Walker, R. Duffin, M. Gray, E. Crescenzi, M.C. Martin, H.J. Brady, J.S. Savill, I. Dransfield, C. Haslett, Nat. Med. 12 (2006) 1056-1064.

[39] A.G. Rossi, J.M. Hallett, D.A. Sawatzky, M.M. Teixeira, C. Haslett, Biochem. Soc. Trans. 35 (2007) 288-291.

[40] S.L. Newman, J.E. Henson, P.M. Henson, J. Exp. Med. 156 (1982) $430-442$.

[41] J. Hughes, Y. Liu, J. Van Damme, J. Savill, J. Immunol. 158 (1997) 4389-4397.

[42] D. Mevorach, J.O. Mascarenhas, D. Gershov, K.B. Elkon, J. Exp. Med. 188 (1998) 2313-2320.

[43] D. Mevorach, Ann. N.Y. Acad. Sci. 926 (2000) 226-235.

[44] R.A.B. Ezekowitz, R.B. Sim, G.G. MacPherson, S. Gordon, J. Clin. Invest. 76 (1985) 2368-2376.

[45] R. Hanayama, M. Tanaka, K. Miwa, A. Shinohara, A. Iwamatsu, S. Nagata, Nature 417 (2002) 182-187.

[46] R.A. Ezekowitz, Nat. Immunol. 3 (2002) 510-512.

[47] S.K. Ackerman, S.D. Douglas, J. Immunol. 120 (1978) 1372-1374.

[48] L.J. Partridge, I. Dransfield, in: G. Gallagher, R.C. Rees, C.W. Reynolds (Eds.), Tumour Immunobiology: A Practical Approach, Oxford University Press, Oxford, UK, 1993, pp. 91-118.

[49] C. Haslett, L.A. Guthrie, M.M. Kopaniak, R.B. Johnston Jr., P.M. Henson, Am. J. Pathol. 119 (1985) 101-110.

[50] H.P. Jersmann, K.A. Ross, S. Vivers, S.B. Brown, C. Haslett, I. Dransfield, Cytometry 51A (2003) 7-15.

[51] P.K. Potter, J. Cortes-Hernandez, P. Quartier, M. Botto, M.J. Walport, J. Immunol. 170 (2003) 3223-3232.

[52] A. Devitt, K.G. Parker, C.A. Ogden, C. Oldreive, M.F. Clay, L.A. Melville, C.O. Bellamy, A. Lacy-Hulbert, S.C. Gangloff, S.M. Goyert, C.D. Gregory, J. Cell Biol. 167 (2004) 1161-1170. 


\title{
Novel pharmacological strategies for driving inflammatory cell apoptosis and enhancing the resolution of inflammation
}

\author{
John M. Hallett*, Andrew E. Leitch", Nicola A. Riley, Rodger Duffin, \\ Christopher Haslett and Adriano G. Rossi
}

MRC Centre for Inflammation Research, Queen's Medical Research Institute, University of Edinburgh Medical School, 47 Little France Crescent, Edinburgh EH16 4TJ, UK

\begin{abstract}
Resolution of inflammation requires the effective downregulation of key inflammatory cells such as neutrophils and eosinophils, which normally undergo programmed cell death (apoptosis) to enable their detection and removal by phagocytes such as macrophages. Dysregulation of this process is thought to contribute to the pathogenesis and progression of chronic inflammatory disorders such as chronic obstructive pulmonary disease, asthma, rheumatoid arthritis, allergic rhinitis and inflammatory bowel disease. Importantly, knowledge of the signalling pathways responsible for the induction and execution of granulocyte apoptosis and the phagocytic removal of apoptotic cells continues to increase and, with it, the potential for incisive pharmacological intervention. In this article, we highlight pharmacological strategies that could be used to drive the resolution of inflammation by augmenting apoptosis of inflammatory cells.
\end{abstract}

\section{Introduction}

The inflammatory process is a complex series of interrelated, ideally tightly controlled, cellular and biochemical events that has evolved to eliminate or contain infectious agents and to repair damaged tissue. An ineffective or uncontrolled inflammatory response contributes to the cellular dysfunction, tissue damage and inadequate repair that occurs in many chronic inflammatory diseases. Currently, knowledge of the triggers and progression of the inflammatory process exceeds that of the events responsible for the termination and ultimate resolution of inflammation $[1,2]$.

A central paradigm of inflammation research has been that the resolution of inflammation depends on apoptosis of inflammatory cells (e.g. neutrophils and eosinophils) and their subsequent clearance by phagocytes (especially macrophages) [3]. This process is synchronized by an interconnecting web of signalling pathways that not only downregulates the inflammatory response but also generates agents capable of driving its resolution. In this review, we

Corresponding author: Rossi, A.G. (a.g.rossi@ed.ac.uk). Authors contributed equally. focus on the granulocyte, which we consider to be the archetypal inflammatory cell, and discuss the pharmacological interventions that have stemmed from a greater understanding of its role [4].

\section{The granulocyte life cycle}

Granulocytes - the collective name given to neutrophil, eosinophil and basophil leukocytes - have a prominent role in immune defence [5]. As the most abundant circulating granulocyte in human blood, neutrophils have a key role in the defence against bacterial, fungal and viral infections. Eosinophils account for $<5 \%$ of the circulating granulocytes and confer resistance to parasitic invasion, in addition to their involvement, with basophils (the third, and numerically scarce, type of granulocyte), in the allergic response [6].

Granulocytes are derived from pluripotent haematopoietic stem cells in the bone marrow. If a cell is destined to become a granulocyte, it must differentiate from a common myeloid progenitor cell to become a common granulocyte progenitor cell, which, following appropriate stimulation, can produce any granulocyte lineage. Granulocytes are terminally differentiated and, having effectively completed the cell cycle, they remain in the G0 phase of growth for the remainder of their relatively short lives [7]. It is believed that, once they have discharged their function, extravasated granulocytes in the tissues die by apoptosis (see next section), whereas non-migratory, circulating granulocytes leave the blood and return to the liver, spleen or bone marrow, where they meet an illdefined fate (possibly apoptosis) [8]. The life cycle of the granulocyte and its differentiation in particular are under increasing scrutiny. A new pharmacological intervention cyclin-dependent kinase inhibition - targets the cell-cycle machinery and drives granulocyte apoptosis [9].

\section{Granulocyte apoptosis}

Apoptosis is a complex physiological mechanism in which a cell undergoes programmed death as a result of survivalfactor withdrawal or exposure to pro-apoptotic signals (Box 1). The alternative to granulocyte apoptosis (Figure 1) is necrosis, which can be a primary event or which can 


\section{Box 1. Apoptotic pathways and the role of caspases}

\section{The extrinsic pathway}

The extrinsic apoptotic pathway is initiated by stimulation of the TNF receptor (TNFR) superfamily of cell-surface 'death receptors', which includes TNF-related apoptosis-inducing ligand receptor, TNFR and Fas. On binding to TNFR1, TNF- $\alpha$ induces receptor trimerization, which co-localizes death domains and enables further binding to TNFR-associated death domain-containing proteins (TRADDs). TRADD mediates clustering of procaspase-8 molecules and their proximity to one another initiates an autocatalytic activation cascade leading to apoptosis. (Similarly, Fas-associated death domain protein, FADD, mediates the same reaction following Fas-receptor ligation.) Interestingly, via binding through TNFRassociated factor- 2 and receptor-interacting protein, TRADD can activate the transcription factors $A P-1$ and $N F-\kappa B$, with the latter having a pivotal role in the immune response. Caspase- 8 can either directly cleave procaspase- 3 to caspase- 3 (the terminal step in the cascade preceding cleavage of DNA and chromatin) or-in an example of cross-talk between extrinsic and intrinsic pathwaystruncate the Bcl-2 family member Bid: a step that contributes to MOMP $[73,74]$. CD137 is another member of the TNFR family (despite lacking a death domain), which seems to have varying effects on eosinophil and neutrophil survival signalling [65].

\section{The intrinsic pathway}

MOMP, which is mediated by a predominance of pro-apoptotic over anti-apoptotic Bcl-2 family members, is the central event in the intrinsic pathway, which is stimulated by genotoxic stress (e.g. cytotoxic agents, UV radiation or oxidative stress). Cytochrome $c$, apoptotic-protease-activating factor-1, apoptosis-inducing factor, endonuclease $\mathrm{G}$ and Smac/DIABLO are released following MOMP. $\mathrm{Smac} / \mathrm{DIABLO}$ is required for the release of procaspase-9 from IAPs, enabling formation of the apoptosome and activation of caspase-3, which initiates apoptosis. It has been suggested that, whereas caspase- 9 is essential for neutrophil and eosinophil apoptosis, caspase- 3 and caspase- 8 could have less significance in eosinophil apoptosis - a difference that was hoped might have potential for exploitation. It should be noted, however, that the importance of caspases to constitutive apoptosis has not yet been satisfactorily confirmed $[21,66-68]$.

supersede apoptosis when phagocytosis is delayed. Apoptosis, in contrast to necrosis, ensures that the cell membrane retains its integrity, preventing spillage of the inherently pro-inflammatory and, especially in the case of neutrophils, histotoxic contents of the cell into the surrounding tissues. The release of histotoxic products [e.g. proteases or reactive oxygen species (ROS)] from overrecruited, inappropriately activated and/or necrotic granulocytes probably contributes to the tissue damage observed in chronic inflammatory and autoimmune diseases $[2-4,10]$. However, there is growing evidence that potentially harmful neutrophil granule contents such as ROS or cathepsin G have important pro-apoptotic roles in the successful resolution of inflammation $[10,11]$. Another important component of granulocyte apoptosis is the preparation of the cell for recognition and removal by phagocytes. During apoptosis, receptors for pro-inflammatory signals are removed from the cell membrane, whereas 'eat me' signals [including phosphatidylserine (PS) residues], which are recognized by phagocytes, are displayed. A standard tool for the identification of apoptotic cells involves annexin-V binding to PS residues. Interestingly, a putative phagocyte receptor for PS has proved difficult to define, and three recently identified receptors (Tim4, stabilin2 and BAI1) indicate that the complexity of this
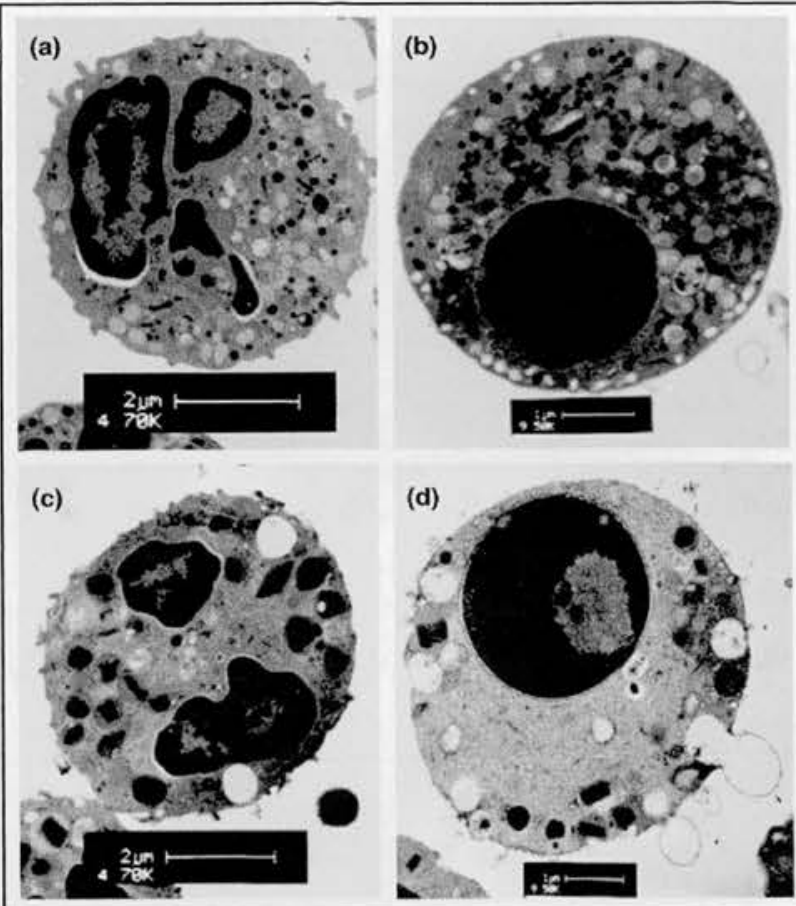

Figure 1. Electron micrographs of granulocytes. (a) A healthy neutrophil, identifiable by its three-lobed nuclei and heavily granular structure (with a $2-\mu \mathrm{m}$ scale bar taken at $4700 \times$ magnification). (b) An apoptotic neutrophil with dense chromatin condensation and smooth cell membrane (with a $1-\mu \mathrm{m}$ scale bar taken at $9500 \times$ magnification). (c) A healthy eosinophil with two-lobed nuclei, large granules and Charcot-Leyden crystals (crystals comprising eosinophil lysophospholipase: a hallmark of eosinophilic infiltration) (with a $2-\mu \mathrm{m}$ scale bar taken at $4700 \times$ magnification). (d) An apoptotic eosinophil showing chromatin condensation, peripheral granular distribution, smooth cell membrane and vacuolated appearance (with a $1-\mu \mathrm{m}$ scale bar taken at $9500 \times$ magnification).

process might have been underestimated [12-16]. Phagocytosis should be non-phlogistic, implying that a professional phagocyte such as the macrophage - which, in other circumstances, can propagate inflammation - on recognizing an apoptotic cell, is programmed to upregulate the production of anti-inflammatory mediators such as transforming growth factor (TGF)- $\beta$ and interleukin (IL)10 [17]. Developing an understanding of granulocyte behaviour has been a research priority, and has indicated that a therapeutically tractable approach to the modulation of granulocyte functional responsiveness might be to influence the longevity of these cells by inducing apoptosis [18]. Additionally, research is generating an increasingly detailed picture of the apoptotic machinery at a molecular level. This information is crucial for the accurate identification of apoptotic cells and could provide novel opportunities for intervention.

\section{Key signalling pathways as targets for pharmacological intervention}

Granulocyte apoptosis is a sensitive, responsive and highly regulated process. These features are conferred by various signalling pathways. Each pathway is responsive to specific endogenous stimuli and mediates pro- or antiapoptotic effects. As such, a key area for pharmacological intervention is the inhibition or augmentation of key granulocyte signalling pathways (Table 1 ). 
Table 1. Current pharmacological agents proven to drive granulocyte apoptosis or resolution of inflammation

\begin{tabular}{|c|c|c|c|c|c|}
\hline Drug & Action & Outcome & $\begin{array}{l}\text { Influence on granulocyte } \\
\text { apoptosis }\end{array}$ & $\begin{array}{l}\text { Resolution of } \\
\text { inflammation }\end{array}$ & Refs \\
\hline Gliotoxin & NF-kB inhibition & Decreased NF-kB activation & Yes & $\begin{array}{l}\text { Possible, but probably } \\
\text { nonspecific toxicity }\end{array}$ & [20] \\
\hline MG132 & Proteasome inhibition & $\begin{array}{l}\text { Increased Mcl-1, decreased } \\
\text { NF-KB activation }\end{array}$ & No & No & {$[36,69]$} \\
\hline $\mathrm{PGD}_{2}$ metabolites & Block 1 kB degradation & Decreased NF-kB activation & Yes & Yes & {$[22,70-72]$} \\
\hline PD098059 & $\begin{array}{l}\text { Decreased ERK } \\
\text { phosphorylation }\end{array}$ & ERK inhibition & Yes & Yes & {$[31,32]$} \\
\hline SB203580 & p38 MAPK inhibition & p38 MAPK inhibition & No & No & {$[33-36]$} \\
\hline Sodium salicylate & p38 MAPK activation & Decreased Mcl-1 & Yes & Yes & [36] \\
\hline SP600125 & JNK inhibition & TNF- $\alpha$-mediated apoptosis & Yes & Yes & {$[37-39]$} \\
\hline Wortmannin & PI3K inhibition & $\begin{array}{l}\text { Decreased Akt } \\
\text { phosphorylation and IL-5 }\end{array}$ & Possible & $\begin{array}{l}\text { Possible, but probably } \\
\text { nonspecific toxicity }\end{array}$ & [44] \\
\hline Ly294002 & PI3K inhibition & $\begin{array}{l}\text { Decreased Akt } \\
\text { phosphorylation and IL-5 }\end{array}$ & Possible & $\begin{array}{l}\text { Possible, but probably } \\
\text { nonspecific toxicity }\end{array}$ & [44] \\
\hline IC87114 & PI3K- $\delta$ inhibition & Decreased chemotaxis & No & Possible & [45] \\
\hline AS605240 & PI3K- $\gamma$ inhibition & Decreased chemotaxis & No & Possible & [45] \\
\hline CDKi & $?$ & Decreased Mcl-1-X-IAP & Yes & Yes & {$[9,58-62]$} \\
\hline Smac-DIABLO mimetics & Decreased X-IAP & Increased ease of apoptosis & Unknown & Unknown & {$[51,52]$} \\
\hline Glucocorticoids & $\begin{array}{l}\text { Decreased Mcl-1 in } \\
\text { eosinophils }\end{array}$ & Eosinophil apoptosis & Eosinophil apoptosis only & Eosinophilic inflammation & {$[1,3,49]$} \\
\hline
\end{tabular}

\section{NF- $\kappa$ B: a crucial regulator of granulocyte apoptosis}

The transcription factor nuclear factor-кB $(\mathrm{NF}-\kappa \mathrm{B})$ has a pivotal role in the inflammatory response, and a plethora of molecules - including cytokines, chemokines, adhesion molecules and stress response proteins - is dependent on it for synthesis. The synthesis of several proteins under the direct transcriptional control of NF-кB are anti-apoptotic in nature, including B-cell lymphoma-2 [Bcl-2 (which is less relevant in neutrophils, in which it is not expressed)], inhibitor of apoptosis proteins (IAPs) and Xray-inducible immediate-early-response factor-1-long [19]. Specific inhibition of NF- $\mathrm{kB}$ is an attractive pharmacological target that is made viable by the ever-increasing understanding of its activity and regulation.

Inactive NF-kB is held in the cytoplasm bound to its inhibitor protein, inhibitor of $\kappa \mathrm{B}\left(\mathrm{I}_{\kappa} \mathrm{B}\right)$. However, once activated by pro-inflammatory stimuli such as tumour necrosis factor (TNF)- $\alpha$ or lipopolysaccharide (LPS), the I $\mathrm{B}$ kinase (IKK) complex phosphorylates I $\mathrm{B}$. I $\mathrm{kB}$ then dissociates from NF- $\mathrm{kB}$ to be ubiquitylated and targeted for proteasomal degradation. As a result of $I_{\kappa} B$ degradation, nuclear-localization sequences (NLSs) on NF-кB are revealed and $\mathrm{NF}-\mathrm{\kappa B}$ subunits are free to translocate to the nucleus and begin the process of protein transcription. From a pharmacological perspective, an obvious interven-

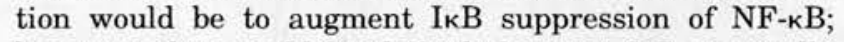
however, this would be difficult because I $\mathrm{B}$ is rapidly biodegraded [18,19].

Several pharmacological inhibitors of NF-кB are available and have been shown to enhance apoptosis when applied to neutrophils in vitro. These inhibitors include cell-permeable inhibitory peptide of NF- $\mathrm{kB}, \mathrm{SN}-50$, curcumin, pyrrolidine-dithiocarbamate and MG132 (a proteasome inhibitor the impact of which could be considered nonspecific). A widely studied inhibitor is the fungal metabolite gliotoxin, which induces neutrophil apoptosis in vitro and can overcome the neutrophil survival effect conferred by LPS. The effects of TNF- $\alpha$ on granulocyte apoptosis are perplexing, although it has been shown that, in combination with gliotoxin, it induces marked neutrophil and eosinophil apoptosis [20]. In addition, cycloheximide-mediated inhibition of protein synthesis augments the effect of TNF- $\alpha$, implying the presence of a TNF- $\alpha$-induced survival protein that is under the transcriptional control of NF- $\mathrm{kB}$. In the search for physiological inhibitors of NF-kB, it was noted that arachidonic acid metabolites and the cyclopentenone prostaglandin $(\mathrm{PG}) \mathrm{D}_{2}$ could inhibit $\mathrm{NF}-\mathrm{kB}$ activation by LPS or TNF- $\alpha$ and induce caspase-dependent granulocyte apoptosis. This effect was independent of the peroxisomeproliferator-activated-receptor- $\gamma$ and probably occurred by direct IKK inhibition $[19,21]$. There is now in vivo evidence, from a rat pleurisy model, that $\mathrm{PGD}_{2}$ can contribute to the resolution of inflammation and could be a natural retardant of inflammation progression [22].

An innovative and encouraging use of the HIV tat protein transduction method has facilitated the transduction of neutrophils with HIV-tat-linked NF-кB essential modulator domain (NEMO), which is a specific inhibitor of the IKK $\gamma$-IKK $\beta$ interaction [23]. A similar approach was used to transduce eosinophils with an HIV-tat-IкB $\alpha$ super-repressor that is resistant to phosphorylation and proteasomal degradation [24]. This highly specific inhibition resulted in the augmentation of apoptosis and the suppression of NF-кB-mediated survival, lending credence to the pursuit of NF-kB as a pharmacological target.

However, an apparent contradiction is emerging in the literature because NF- $\mathrm{KB}$ has been shown to have an antiinflammatory and pro-resolution role in acute inflammation. The contradiction revolves around detailed analysis of IKK $\alpha$ inhibition of NF- $\mathrm{kB}$ activity, in which induction of the NF- $\mathrm{kB}$ subunits RelA and c-Rel using a super-repressor $I_{\kappa} B \alpha$ fused to a membrane-transducing domain of HIV tat protein suppressed NF- $\mathrm{B}$ interaction with pro-inflammatory gene promoters in vivo. It is now recognized that there are two phases of NF- $\mathrm{kB}$ activation. The first phase, which occurs within $6 \mathrm{~h}$ of the onset of inflammation, is coincident with the formation of complexed inducible nitric oxide synthase (iNOS) and cRelp50 heterodimer; these bind to and regulate the mouse iNOS promoter, and macrophages from knockout mice 
show increased iNOS expression in response to NF- $\mathrm{B}$ during the induction of inflammation in vivo. During the resolution phase, however, there is no expression of iNOS or cRel-p50 heterodimers despite substantial NF-кB activation, which indicates a switch to a second, pro-resolution phase [25-27].

It seems that the dominant impact of NF-кB inhibition is to drive granulocyte apoptosis and the resolution of inflammation, but a note of caution is warranted because it is conceivable that inhibition of the later, pro-resolution phase of $\mathrm{NF}-\kappa \mathrm{B}$ activation might prolong and/or prevent the successful resolution of inflammation.

\section{The role of MAPK pathways in granulocyte apoptosis}

There is considerable evidence of the involvement of the mitogen-activated protein kinase (MAPK) signalling cascade in diverse aspects of the inflammatory response, including cell recruitment, activation and apoptosis. The three main MAPK subtypes activated by MAPK kinases (MEKs) 1-7 are extracellular-signal-related kinase [ERK (which includes p42/p44 MAPK)], c-Jun N-terminal kinases (JNKs) and p38 MAPK. All have been implicated in the regulation of granulocyte apoptosis [28-30].

The ERK pathway is involved in negotiating survival conferred by inflammatory mediators such as granulocyte macrophage-colony stimulating factor (GM-CSF), IL-8, IL15, C5a and LPS but it is non-essential for the regulation of constitutive apoptosis. PD098059 (2'-amino- $3^{\prime}$-methoxyflavone) is a specific pharmacological ERK inhibitor that mediates its effect by binding to the ERK-specific MAPkinase, thereby preventing the phosphorylation of ERK1/2 (p44/p42 MAPK) by MEK1. PD098059 has been used in vitro to reverse GM-CSF-mediated and LPS-mediated neutrophil survival, and its in vivo efficacy has been demonstrated in a rat carrageenan-induced pleurisy model, in which it enhanced the resolution of inflammation by promoting neutrophil apoptosis within the pleural cavity. It is likely that the pro-apoptotic effects of this compound are conferred by inhibition of survival-factor-induced antiapoptotic effects because it seems to have no direct effect on constitutive neutrophil apoptosis in non-inflammatory conditions $[31,32]$.

The p38 MAPK and JNK cascades are involved in the cellular response to stresses, including heat shock, hyperosmolarity, UV radiation, protein-synthesis inhibitor drugs and inflammatory cytokines. p38 MAPK might also have a role in superoxide anion production and chemotaxis [33]. There is indirect evidence of a pro-survival role for p38 MAPK because it has been shown to inhibit, by phosphorylation, caspase- 3 and -8 and it is specifically cleaved in mature neutrophils undergoing apoptosis [34]. However, it is unclear whether it is sufficiently influential to be considered a pharmacological target in inflammatory states because its inhibition (by the pyridinyl imidazole inhibitor SB203580) fails to overcome cell-survival signals from GM-CSF. It has been suggested that this controversial pathway could generate a neutrophil death signal through the reduction of Mcl-1, as observed when apoptosis is triggered by sodium salicylate. Perhaps there are further opportunities for the pharmacological augmentation of p38 MAPK to promote neutrophil apoptosis. There is certainly an opportunity for further study of this pathway, which seems to have been under-investigated compared with other signalling systems $[35,36]$.

The JNK pathway is also controversial and insufficiently investigated, but researchers have demonstrated that the activation of JNK signalling is a component of LPS- and TNF- $\alpha$-mediated neutrophil apoptosis. Additionally, an in vivo rat model of smoke inhalation injury responded favourably to JNK inhibition by SP600125 \{anthra [1,9-cd]pyrazol-6(2H)-one\}, with decreased airway inflammation and increased rates of survival [37-39].

PI3K pathway as a regulator of granulocyte apoptosis The phosphoinositide-3-kinase (PI3K) pathway is an important cell-surface-receptor-controlled signal-transduction pathway that catalyses phosphorylation of the $3^{\prime}-\mathrm{OH}$ position of the inositol ring of phosphoinositides, resulting in the formation of the $3^{\prime}$-phosphatidylinositol (PtdIns) lipids. PI3K is involved in the regulation of all major leukocyte events, including growth, proliferation, recruitment, activation and survival [40]. From the perspective of granulocyte survival, PI3K has a role in mediating the anti-apoptotic-pro-inflammatory signals triggered by LPS, GM-CSF and TNF- $\alpha$. It generates the important signalling lipid PtdIns $(3,4,5) P_{3}$, which activates Akt and influences NF-kB and cAMP-response-elementbinding protein (CREB); these produce anti-apoptotic signals in granulocytes [41-43].

Pharmacological inhibition was initially achieved with the use of wortmannin and LY294002, which are general PI3K family inhibitors. Work is in progress to determine which isoforms of PI3K are responsible for its diversity of function and to delineate their mechanisms of action. To this end, PI3K-isoform-specific knockout mice have been developed alongside new pharmacological inhibitors. For example, the involvement of PI3K in the recruitment and survival of eosinophils in a model of allergic pleurisy in PI3K $\gamma$-deficient mice with the use of wortmannin and LY294002 has been evaluated [44]. They found that, following the induction of allergic pleurisy, eosinophil accumulation was not different from that in wild-type mice at earlier time-points ( 6 and $24 \mathrm{~h}$ ). However, there was a marked reduction in eosinophil numbers at a later timepoint $(48 \mathrm{~h})$. Wortmannin and LY294002 administered systematically before antigen challenge led to a decrease in Akt phosphorylation, IL-5 production and eosinophil release from the bone marrow and, consequently, prevented the recruitment of eosinophils. Importantly, local (intrapleural) treatment with the PI3K inhibitors $24 \mathrm{~h}$ after antigen challenge (at the peak of inflammation) enhanced the clearance of accumulated eosinophils - an effect associated with the inhibition of Akt phosphorylation and an increased number of apoptotic events (assessed microscopically and using annexin-V binding). This study highlights an important role for the PI3K family in the accumulation and survival of eosinophils and implies a specific role in the maintenance (possibly by influencing eosinophil apoptosis) of eosinophilic inflammation in vivo. The same group has now used the specific PI3K- $\delta$ inhibitor IC87114 and the PI3K- $\gamma$ inhibitor AS605240 to demonstrate that, whereas chemokine receptor (CXCR)2 requires 
both isoforms to recruit neutrophils to the alveolar space, $\mathrm{C} 5 \mathrm{a}$ and formyl-methionyl-leucyl-phenylalanine (fMLP) require only $\mathrm{PI} 3 \mathrm{~K} \gamma[45]$.

Pharmacological inhibitors of specific PI3K isoforms and the development of knockout mice have demonstrated the importance of this signalling pathway to the granulocyte and the wider inflammatory response.

\section{The Bcl-2 family}

The Bcl-2 family, which contains $>20$ members, comprises a series of cell-death-receptor proteins. These are further classified into three subgroups depending on their function and the number of Bcl-2 homology domains that they contain: the multidomain pro-apoptotic members, the Bcl-2 homology 3 (BH3)-only domain pro-apoptotic members and the anti-apoptotic family. Neutrophils and eosinophils express the multidomain pro-apoptotic family members Bcl-2-associated X protein (Bax) and Bcl-2 homologous antagonist/killer (Bak), and the BH3-only domain pro-apoptotic members BH3-interacting domain death agonist (Bid) and Bcl-2-associated death promoter (Bad), which are essential components of the apoptotic machinery. Neutrophils also express the pro-apoptotic BH3-only domain proteins Bim and p53 upregulated modulator of apoptosis (PUMA). These pro-apoptotic Bcl-2 family proteins are thought to function either by translocation to the mitochondria, where they can influence mitochondrial outer-membrane permeabilization (MOMP), or by binding to and downregulating pro-survival proteins. In fact, the role of mitochondria in neutrophils and eosinophils seems to be geared predominantly towards the induction of apoptosis rather than cellular respiration [46].

Anti-apoptotic members of the Bcl-2 family are also present in granulocytes. Neutrophils express the Bcl-2 homologue A1 and the anti-apoptotic proteins myeloid cell leukaemia-1 (Mcl-1), Bcl- $\mathrm{X}_{\mathrm{L}}$ and X-linked mammalian IAP (X-IAP). Survival signals, in the form of cytokines and other mediators found at sites of inflammation, induce de novo synthesis of these anti-apoptotic proteins $[47,48]$.

Mcl-1 was initially isolated from a human myeloblastic leukaemia cell line and it is increasingly recognized as a key regulator of immune-cell proliferation and longevity. In human neutrophils, Mcl-1 is thought to be important for cytokine-regulated survival but does not seem to be transcribed by NF-кB, which (as discussed) is one of the most important inflammatory transcription factors. Levels of Mcl-1 in human neutrophils correlate closely with neutrophil survival kinetics. Mcl-1 levels are elevated in viable cells and decrease before the induction of apoptosis. This protein also has the fastest turnover rate and, therefore, the shortest half-life of the anti-apoptotic members of the Bcl-2 family: a feature that is compatible with the necessarily short half-life of the neutrophil. Mcl-1 is targeted for degradation in the proteasome following ubiquitylation and undergoes significant post-translational modification, providing further opportunities for pharmacological intervention. Glucocorticoids are well-established anti-inflammatory agents that promote eosinophil apoptosis but extend neutrophil longevity. They are important in the management of eosinophil-dominant diseases such as asthma but are less effective in neutrophil-dominant diseases such as chronic obstructive pulmonary disease. Partial efficacy in neutrophil-dominant disease is probably achieved by effects on the downregulation of inflammatory cytokines and the enhancement of macrophage phagocytosis of apoptotic neutrophils [75]. The glucocorticoid dexamethasone has recently been shown to stabilize Mcl-1 levels in neutrophils but to reduce levels in eosinophils, which might account for this dichotomous effect on granulocytes [50]. An important development in Mcl-1 research has been the advent of a myeloid-specific Mcl-1-knockout mouse, which has a normal, functional macrophage population but which lacks neutrophils demonstrating the pivotal survival role of Mcl-1 in neutrophils [51]. A putative inhibitor should induce neutrophil apoptosis but enable the effective clearance of apoptotic cells by macrophages. As such, Mcl-1 could be a key pharmacological target. Our experience with the cyclindependent kinase (CDK) inhibitor R-roscovitine indicates that it adequately fulfils the criteria [9].

Members of the IAP family of proteins, of which X-IAP seems to be the most potent, inhibit both caspase- 9 (an initiator caspase) and the effector caspases-3 and -7, thereby promoting cell survival. In addition, overexpression of X-IAP protects cells from apoptosis (see Refs [5154]). X-IAP is negatively regulated by X-IAP-associated factor 1 , which is found predominantly in the nucleus, and by Smac/DIABLO (second-mitochondria-derived-activator of caspase/direct inhibitor of apoptosis-binding protein with low pI), which is located in the mitochondria $[52,53]$. Using NMR and X-ray crystallography, recent characterization of the Smac-IAP interaction has enabled the design and testing of Smac/DIABLO mimetics that have in vitro and in vivo activity $[52,53]$. The implications for granulocyte apoptosis have not yet been established. Interestingly, stabilization of X-IAP by the ERK pathway has been described, and this effect could be reversed by oxidant-induced p38 MAPK [54]. This provides an important insight into the signalling pathway cross-talk that is present at inflammatory loci [54].

$\mathrm{Bcl}-\mathrm{X}_{\mathrm{L}}$ is a Bcl-2 homologue that conserves all four $\mathrm{BH}$ domains. Experiments using in vivo rat carrageenaninduced pleurisy models have shown that, during the onset of inflammation, increased expression of $\mathrm{Bcl}-\mathrm{X}_{\mathrm{L}}$ is accompanied by significantly decreased levels of Bax expression. Conversely, during resolution of inflammation, Bax expression was increased and negligible levels of Bcl- $\mathrm{X}_{\mathrm{L}}$ were found. In the same experiment, the inhibition of Bax by V5 (a Bax inhibitory peptide) resulted in prolonged inflammation, supporting the hypothesis that Bax has a crucial role in the induction of neutrophil apoptosis, and thereby facilitates the resolution of inflammation [31]. There is clearly a delicate balance between the influence of prosurvival and anti-apoptotic proteins within the granulocyte, whereby processes that mediate the effective clearance of inflammatory stimuli compete with those that result in a counter-productive prolongation of inflammation.

\section{Lipoxins, resolvins and protectins: pro-resolution lipid} mediators

Polyunsaturated fatty acids can be metabolized to arachidonic acid, eicosapentaenoic acid and docosahexaenoic 
acid, which can be processed to lipoxins, resolvins and protectins, respectively. These lipid mediators are increasingly recognized for their potent anti-inflammatory and proresolution roles and might have a modulatory role in several inflammatory conditions such as atherosclerosis, chronic liver disease, inflammatory bowel disease, glomerulonephritis and asthma [1]. Lipoxins have diverse actions, including the inhibition of neutrophil chemotaxis, adhesion and transmigration. Additionally, they mediate normalization of vascular permeability, non-phlogistic recruitment of mononuclear cells and stimulation of macrophage phagocytosis of apoptotic neutrophils. Aspirin-triggered lipoxins seem to target the same seven-transmembrane G-proteincoupled receptor as does glucocorticoid-induced annexin-1. Annexin-1 induces granulocyte apoptosis but is also released by both neutrophils and macrophages to enhance the phagocytosis of apoptotic cells. This is interesting because it implies that lipoxins might participate in an interconnected anti-inflammatory network and pro-resolution cascade $[55,56]$. Resolvins and protectins share some of the actions of lipoxins and have protective roles in many organ systems. Importantly, the resolution of inflammation has been reported in vitro and in a mouse peritonitis model with resolvin E1 and protectin D1 [57]. The anti-inflammatory and pro-resolution effects of this family of mediators have been demonstrated in vitro and, latterly, in vivo, indicating that it will be an important target for novel anti-inflammatory pharmacotherapeutics [49].

\section{CDK inhibition as a novel anti-inflammatory approach} CDK inhibitors have been used for the selective induction of apoptosis in actively proliferating cells for several years. Several CDK inhibitors, including R-roscovitine, flavopiridol and SU9516, can induce apoptosis in cancer cell lines. R-roscovitine alone reduces the proliferation index of 19 distinct cancer cell lines in vitro [58]. The induction of apoptosis by CDK inhibitors seems to be mediated by the modulation of Bcl-2 family members and to be executed in a caspase-dependent manner. A wealth of research has meant that confidence in CDK inhibitor safety and efficacy has increased to the extent that clinical trials are underway for conditions such as Bcell malignancy, non-small-cell lung cancer and breast cancer. So far, the side-effect profile of CDK inhibitors has been promising but it includes tolerable gastrointestinal disturbance, minor hepatic dysfunction and hypokalaemia [59-61].

Logically, in terminally differentiated cells such as granulocytes, CDK inhibitors should have no effect because the cell-cycle machinery ought to be redundant. In fact, in post-proliferation neurons, CDK inhibitors have been shown to have a protective effect against apoptosis [62]. Nonetheless, we recently demonstrated in vitro that a panel of CDK inhibitors induces neutrophil apoptosis in a time- and concentration-dependent manner and that this effect is sufficient to overcome the anti-apoptotic effects of survival factors such as GM-CSF, dibutyryl-cAMP and LPS [9]. When neutrophils were treated with R-roscovitine and the caspase inhibitor zVAD-fmk ( $N$-benzyloxycarbonyl-Val-Ala-Asp-fluoromethylketone) cells failed to enter apoptosis, indicating that R-roscovitine functions in a caspase-dependent manner. Furthermore, in vitro studies have demonstrated the presence of CDK1 and CDK2 in human neutrophils and have shown that levels of both remain constant throughout ageing, treatment with GMCSF or gliotoxin, which indicates that these kinases are not targeted for degradation during apoptosis [9]. In addition, a reduction in $\mathrm{Mcl}-1$ levels after treatment of neutrophils with R-roscovitine was noted, offering a tantalizing insight into its mechanism of action [9]. The same study [9] assessed the impact of R-roscovitine on models of neutrophil-dominant inflammation, including carrageenan-induced pleurisy, bleomycin lung injury and arthritis in mice. The results indicated a resolution of inflammation - as assessed by several parameters, including cytokine levels, inflammatory cell numbers, histology and arthritis scores. Caspase inhibition in vivo with $\mathrm{zVAD}-\mathrm{fmk}$, which reversed the pro-resolution effect, confirmed the integral role of granulocyte apoptosis in resolution. In addition, recent studies have identified a role for CDK5 in neutrophils, and CDK inhibition has been shown to influence inflammatory cell recruitment $[63,64]$. Further elucidation of the mechanism of action of CDK inhibitors could enable this therapy to be refined and might highlight further targets for pharmacological intervention.

\section{Concluding remarks}

Research into granulocyte apoptosis and its role in the resolution of inflammation has revealed a complex web of signal-transduction pathways. Work has begun on the pharmacological manipulation of these pathways to provide novel treatments for inflammatory disorders. Clearly, the rate of neutrophil or eosinophil apoptosis can be increased or reduced in vitro by several agents. Importantly, this rate can be matched by appropriate clearance of apoptotic cells. There is now conclusive evidence that inhibition or promotion of granulocyte apoptosis by pharmacological agents in vivo can delay or enhance the resolution of acute inflammation, respectively. Ways to limit the toxicity of some of these agents must be found but we can be confident of the transition of others, in the near future, to clinical trials.

\section{Acknowledgment}

We thank the Wellcome Trust (WT082181] and the Medical Research Council (G0601481) for their support of the work that has enabled us to contribute to this review, and our friends and colleagues within the field whose work constitutes the bulk of the information provided.

\section{References}

1 Gilroy, D.W. et al. (2004) Inflammatory resolution: new opportunities for drug discovery. Nat. Rev. Drug Discov. 3, 401-416

2 Lawrence, T. and Gilroy, D.W. (2007) Chronic inflammation: a failure of resolution? Int. J. Exp. Pathol. 88, 85-94

3 Ward, C. et al. (1999) Pharmacological manipulation of granulocyte apoptosis: potential therapeutic targets. Trends Pharmacol. Sci. 20, 503-509

4 Serhan, C.N. et al. (2007) Resolution of inflammation: state of the art, definitions and terms. FASEB J. 21, 325-332

5 Dransfield, I. and Rossi, A.G. (2004) Granulocyte apoptosis: who would work with a 'real' inflammatory cell? Biochem. Soc. Trans. 32, 447-451

6 Rossi, A.G. et al. (2007) Modulation of granulocyte apoptosis can influence the resolution of inflammation. Biochem. Soc. Trans. 35 , 288-291 
7 Opferman, J.T. (2007) Life and death during hematopoietic differentiation. Curr. Opin. Immunol. 19, 497-502

8 Martin, C, et al. (2003) Chemokines acting via CXCR2 and CXCR4 control the release of neutrophils from the bone marrow and their return following senescence. Immunity 19, 583-593

9 Rossi, A.G. et al. (2006) Cyclin-dependent kinase inhibitors enhance the resolution of inflammation by promoting inflammatory cell apoptosis. Nat. Med. 12, 1056-1064

10 Conus, S. et al. (2008) Caspase- 8 is activated by cathepsin D initiating neutrophil apoptosis during the resolution of inflammation. J. Exp. Med. 205, 685-698

11 Fialkow, L. et al. (2007) Reactive oxygen and nitrogen species as signaling molecules regulating neutrophil function. Free Radic. Biol. Med. 42, 153-164

12 Savill, J. et al. (1992) Thrombospondin co-operates with CD36 and the vitronectin receptor in macrophage recognition of aged neutrophils. J. Clin. Invest. 90, 1513-1529

13 Miyanishi, M. et al. (2007) Identification of Tim4 as a phosphatidylserine receptor. Nature 450, 435-439

14 Park, S.Y. et al. (2007) Rapid cell corpse clearance by stabilin-2, a membrane phosphatidylserine receptor. Cell Death Differ. 15, 192201

15 Park, D. et al. (2007) BAI1 is an engulfment receptor for apoptotic cells upstream of the ELMO/Dock180/Rac module. Nature 450, 430-434

16 Fadok, V.A. et al. (2000) A receptor for phosphatidylserine-specific clearance of apoptotic cells. Nature 405, 85-90

17 Fadok, V.A. et al. (1998) Macrophages that have ingested apoptotic cells in vitro inhibit proinflammatory cytokine production through autocrine/paracrine mechanisms involving TGF $\beta, \mathrm{PGE}_{2}$ and $\mathrm{PAF}$. J. Clin. Invest. 101, 890-898

18 Riley, N.A. et al. (2006) Granulocyte apoptosis and macrophage clearance of apoptotic cells as targets for pharmacological intervention in inflammatory diseases. Anti-inflammatory \& Anti Allergy Agents in Medicinal Chemistry 5, 3-12

19 Ward, C. et al. (2004) Regulation of granulocyte apoptosis by NF-кB. Biochem. Soc. Trans, 32, 465-467

20 Ward, C. et al. (1999) NF-kB activation is a critical regulator of human granulocyte apoptosis in vitro. J. Biol. Chem. 274, 4309-4318

21 Simon, H.U. (2001) Regulation of eosinophil and neutrophil apoptosis similarities and differences. Immunol. Rev. 179, 156-162

22 Lawrence, T. et al. (2002) Anti-inflammatory lipid mediators and insights into the resolution of inflammation. Nat. Rev. Immunol. 2 , 787-795

23 Choi, M. et al. (2003) Inhibition of NF-kB by a TAT-NEMO-binding domain peptide accelerates constitutive apoptosis and abrogates LPS delayed neutrophil apoptosis. Blood 102, 2259-2267

24 Fujihara, S. et al. (2005) Inhibition of $\mathrm{NF}-\mathrm{\kappa B}$ by a cell permeable form of IкB $\alpha$ induces apoptosis in eosinophils. Biochem. Biophys. Res. Commun. 326, 632-637

25 Lawrence, T. et al. (2001) Possible new role for NF-кB in the resolution of inflammation. Nat. Med. 7, 1291-1297

26 Lawrence, T. et al. (2005) IKK $\alpha$ limits macrophage NF-kB activation and contributes to the resolution of inflammation. Nature 434, 1138 1143

27 Gilmore, T.D. (1999) The Rel/NF-кB signal transduction pathway: introduction. Oncogene 18, 6842-6844

28 Kumar, S. et al. (2003) p38 MAP kinases: key signalling molecules as therapeutic targets for inflammatory diseases. Nat. Rev. Drug Discov. 2, 717-726

29 Pearson, G. et al. (2001) Mitogen-activated protein (MAP) kinase pathways: regulation and physiological functions. Endocr. Rev. 22, 153-183

30 Suzuki, K. et al. (1999) Cytokine-specific activation of distinct mitogen activated protein kinase subtype cascades in human neutrophils stimulated by granulocyte colony-stimulating factor, granulocytemacrophage colony-stimulating factor, and tumor necrosis factor- $\alpha$. Blood 93, 341-349

31 Sawatzky, D.A. et al. (2006) The involvement of the apoptosismodulating proteins ERK 1/2, Bcl-xL and Bax in the resolution of acute inflammation in vivo. Am. J. Pathol. 168, 33-41

32 Ward, C. et al. (2005) Interleukin-10 inhibits lipopolysaccharideinduced survival and extracellular signal-regulated kinase activation in human neutrophils. Eur. J. Immunol. 35, 2728-2737
33 Frasch, S.C. et al. (1998) p38 mitogen-activated protein kinasedependent and -independent intracellular signal transduction pathways leading to apoptosis in human neutrophils. J. Biol. Chem. $273,8389-8397$

34 Alvarado-Kristensson, M. et al. (2004) p38-MAPK signals survival by phosphorylation of caspase- 8 and caspase- 3 in human neutrophils. J. Exp. Med. 199, 449-458

35 Suzuki, K. et al. (2001) Cleavage of mitogen-activated protein kinases in human neutrophils undergoing apoptosis: role in decreased responsiveness to inflammatory cytokines. J. Immunol. 166, 1185-1192

36 Derouet, M. et al. (2006) Sodium salicylate promotes neutrophi apoptosis by stimulating caspase-dependent turnover of Mcl-1. J. Immunol. 176, 957-965

37 Syrkina, O.L. et al. (2007) Inhibition of JNK activation prolongs survival after smoke inhalation from fires. Am. J. Physiol. Lung Cell Mol. Physiol. 292, L984-L991

38 Nolan, B. et al. (1999) Mitogen-activated protein kinases signal inhibition of apoptosis in lipopolysaccharide-stimulated neutrophils. Surgery 126, 406-412

39 Avdi, N.J. et al. (2001) Tumor necrosis factor- $\alpha$ activation of the c-Jun $\mathrm{N}$-terminal kinase pathway in human neutrophils. Integrin involvement in a pathway leading from cytoplasmic tyrosine kinases apoptosis. J. Biol. Chem. 276, 2189-2199

40 Hawkins, P.T. (2006) Signalling through class I PI3Ks in mammalian cells. Biochem. Soc. Trans, 34, 647-662

41 Lindemans, C.A. and Coffer, P.J. (2004) Regulation of granulocyte apoptosis by phosphatidylinositol 3-kinase. Biochem. Soc. Trans. 32 , 480-484

42 Yang, K.Y. et al. (2003) Involvement of phosphatidylinositol 3-kinase $\gamma$ in neutrophil apoptosis. Cell. Signal. 15, 225-233

43 Cowburn, A.S. et al. (2004) The survival effect of TNF- $\alpha$ in human neutrophils is mediated via NF-kB-dependent $\mathrm{IL}-8$ release. Eur. J. Immunol. 34, 1733-1743

44 Pinho, V. et al. (2005) Phosphoinositide-3 kinases critically regulate the recruitment and survival of eosinophils in vivo: importance for the resolution of allergic inflammation. J. Leukoc. Biol. 77, 800810

45 Pinho, V. et al. (2007) Tissue- and stimulus-dependent role of phosphatidylinositol 3-kinase isoforms for neutrophil recruitment induced by chemoattractants in vivo. J. Immunol. 179, 7891-7898

46 Moulding, D.A. et al. (2001) BCL-2 family expression in human neutrophils during delayed and accelerated apoptosis. J. Leukoc. Biol. 70, 783-792

47 Edwards, S.W. et al. (2004) Regulation of neutrophil apoptosis by Mcl1. Biochem. Soc. Trans. 32, 489-492

48 Bianchi, S.M. et al. (2006) Granulocyte apoptosis in the pathogenesis and resolution of lung disease. Clin. Sci. (Lond.) 110, 293-304

49 Serhan, C.N. (2007) Resolution phase of inflammation: novel endogenous anti-inflammatory and proresolving lipid mediators and pathways. Annu. Rev. Immunol. 25, 101-137

50 Sivertson, K.L. et al. (2007) The differential effect of dexamethasone on granulocyte apoptosis involves stabilization of Mcl-1L in neutrophils but not in eosinophils. Cell Immunol. 246, 34-45

51 Dzhagalov, I. et al. (2007) The antiapoptotic protein Mcl-1 is essential for the survival of neutrophils but not macrophages. Blood 109, 16201626

$52 \mathrm{Liu}$, Z. et al. (2000) Structural basis for binding of Smac/DIABLO to the XIAP BIR3 domain. Nature 408, 1004-1008

53 Oost, T.K. et al. (2004) Discovery of potent antagonists of the antiapoptotic protein XIAP for the treatment of cancer. J. Med. Chem. 47, 4417-4426

54 Gardai, S.J. et al. (2004) Oxidants inhibit ERK/MAPK and prevent its ability to delay neutrophil apoptosis downstream of mitochondrial changes and at the level of XIAP. $J$. Biol. Chem 279, 44695-44703

55 Perretti, M. and Solito, E. (2004) Annexin 1 and neutrophil apoptosis. Biochem. Soc. Trans. 32, 507-510

56 Scannell, M. et al. (2007) Annexin 1 and peptide derivatives are released by apoptotic cells and stimulate phagocytosis of apoptotic neutrophils by macrophages. J. Immunol. 178, 4595-4605

57 Schwab, J.M. et al. (2007) Resolvin E1 and protectin D1 activate inflammation-resolution programmes. Nature 447, 869-874

58 MacCallum, D.E. et al. (2005) Seliciclib (CYC202, R-roscovitine) induces cell death in multiple myeloma cells by inhibition of RNA 
polymerase II-dependent transcription and down-regulation of Mcl-1. Cancer Res. 65, 5399-5407

$59 \mathrm{McClue}$, S.J. et al. (2002) In vitro and in vivo antitumor properties of the cyclin dependent kinase inhibitor CYC202 (R-roscovitine). Int. J. Cancer 102, 463-468

60 Meijer, L. and Raymond, E. (2003) Roscovitine and other purines as kinase inhibitors. From starfish oocytes to clinical trials. Acc. Chem. Res. $36,417-425$

61 Knockaert, M. et al. (2002) Pharmacological inhibitors of cyclindependent kinases. Trends Pharmacol. Sci. 23, 417-425

62 Liu, L. et al. (2008) Cyclin-dependent kinase inhibitors block leukocyte adhesion and migration. J. Immunol. 180, 1808-1817

63 Rosales, J.L. and Lee, K.Y. (2006) Extraneuronal roles of cyclindependent kinase 5. Bioessays 28, 1023-1034

64 Park, D.S. et al. (1996) Inhibitors of cyclin-dependent kinases promote survival of post-mitotic neuronally differentiated PC12 cells and sympathetic neurons. J. Biol. Chem. 271, 8161-8169

65 Simon, H.U. (2003) Neutrophil apoptosis pathways and their modifications in inflammation. Immunol. Rev. 193, 101-110

66 Green, D.R. and Kroemer, G. (2004) The pathophysiology of mitochondrial cell death. Science 305, 626-629

67 Daigle, I. and Simon, H.U. (2001) Critical role for caspases 3 and 8 in neutrophil but not eosinophil apoptosis. Int. Arch. Allergy Immunol. $126,147-156$
68 Yamashita, K. et al. (1999) Caspases mediate tumor necrosis factor- $\alpha$ induced neutrophil apoptosis and downregulation of reactive oxygen production. Blood 93, 674-685

69 Goldberg, A.L. (2007) Functions of the proteasome: from protein degradation and immune surveillance to cancer therapy. Biochem. Soc. Trans. 35, 12-17

70 Rossi, A. et al. (2000) Anti-inflammatory cyclopentenone prostaglandins are direct inhibitors of IкB kinase. Nature 403, 103-108

71 Ward, C. et al. (2002) Prostaglandin $\mathrm{D}_{2}$ and its metabolites induce caspase-dependent granulocyte apoptosis that is mediated via inhibition of $\mathrm{I}_{\kappa} \mathrm{B} \alpha$ degradation using a peroxisome proliferatoractivated receptor- $\gamma$-independent mechanism. J. Immunol. 168, $6232-6243$

72 Gilroy, D.W. et al. (2003) Inducible cyclooxygenase-derived 15 deoxy $(\delta) 12-14 \mathrm{PGJ}_{2}$ brings about acute inflammatory resolution in rat pleurisy by inducing neutrophil and macrophage apoptosis. FASEB J. 17, 2269-2271

73 Susin, S.A. et al. (2000) Two distinct pathways leading to nuclear apoptosis. J. Exp. Med. 192, 571-580

74 Roy, S. and Nicholson, D.W. (2000) Cross-talk in cell death signaling. J. Exp. Med. 192, F21-F25

75 Savill, J.S. et al. (1989) Macrophage phagocytosis of aging neutrophils in inflammation. Programmed cell death in the neutrophil leads to its recognition by macrophages. J. Clin. Invest. $83,865-875$

\section{Five things you might not know about Elsevier}

\section{1.}

Elsevier is a founder member of the WHO's HINARI and AGORA initiatives, which enable the world's poorest countries to gain free access to scientific literature. More than 1000 journals, including the Trends and Current Opinion collections and Drug Discovery Today, are now available free of charge or at significantly reduced prices.

2.

The online archive of Elsevier's premier Cell Press journal collection became freely available in January 2005. Free access to the recent archive, including Cell, Neuron, Immunity and Current Biology, is available on ScienceDirect and the Cell Press journal sites 12 months after articles are first published.

3.

Have you contributed to an Elsevier journal, book or series? Did you know that all our authors are entitled to a $30 \%$ discount on books and stand-alone CDs when ordered directly from us? For more information, call our sales offices:

+18007824927 (USA) or +18004603110 (Canada, South and Central America) or +44 (0) 1865474010 (all other countries)

\section{4.}

Elsevier has a long tradition of liberal copyright policies and for many years has permitted both the posting of preprints on public servers and the posting of final articles on internal servers. Now, Elsevier has extended its author posting policy to allow authors to post the final text version of their articles free of charge on their personal websites and institutional repositories or websites.

5.

The Elsevier Foundation is a knowledge-centered foundation that makes grants and contributions throughout the world. A reflection of our culturally rich global organization, the Foundation has,

for example, funded the setting up of a video library to educate for children in Philadelphia, provided storybooks to children in Cape Town, sponsored the creation of the Stanley L. Robbins

Visiting Professorship at Brigham and Women's Hospital, and given funding to the 3rd International Conference on Children's Health and the Environment. 


\title{
"Relevance of granulocyte apoptosis to resolution of inflammation at the respiratory mucosa"
}

\begin{abstract}
AE Leitch ${ }^{1}$, R Duffin $^{1}$, C Haslett $^{1}$ and AG Rossi ${ }^{1}$
The respiratory mucosa is responsible for gas exchange and is therefore, of necessity, exposed to airborne pathogens, allergens, and foreign particles. It has evolved a multi-faceted, physical and immune defense system to ensure that in the majority of instances, potentially injurious invaders are repelled. Inflammation, predominantly mediated by effector cells of the granulocyte lineage including neutrophils and eosinophils, is a form of immune defense. Where inflammation proves unable to remove an inciting stimulus, chronic inflammatory disease may supervene because of the potential for tissue damage conferred by the presence of large numbers of frustrated, activated granulocytes. Successful recovery from inflammatory disease and resolution of inflammation rely on the clearance of these cells. Ideally, they should undergo apoptosis prior to phagocytosis by macrophage, dendritic, or epithelial cells. The outcome of inflammation can have serious sequelae for the integrity of the respiratory mucosa leading to disease. Therapeutic strategies to drive resolution of inflammation may be directed at the induction of granulocyte apoptosis and the enhancement of granulocyte clearance.
\end{abstract}

\section{RESPIRATORY SYSTEM AND GRANULOCYTE-MEDIATED MUCOSAL INFLAMMATION}

The respiratory mucosa is the largest body surface area to be exposed to, and require defense from, the external environment. It is not surprising, therefore, that a robust, vigorous, and rapidly responsive immune defense system has evolved over millennia of exposure to diverse and increasingly sophisticated microbes. In addition, air-borne irritant particulate matter such as allergens, biopersistent fibers such as asbestos and combustionderived nanoparticles (e.g., diesel exhaust particles) must be overcome to maintain a high level of function. The architecture of the respiratory system provides a first layer of defense against air-borne microorganisms and particulate matter, effectively blocking the lower airways from anything greater than $5 \mu \mathrm{m}$ in diameter. Inhaled air is warmed and filtered through the nasal hair and nasopharyngeal passages before negotiating the larynx (which ordinarily prevents any intrusion from matter destined for the gastrointestinal system and allows phonation) down the large tubular trachea and into the upper bronchi. The initially large bronchial airways repeatedly bifurcate until they reach an extremely narrow gauge and form alveoli at which point gas exchange with the capillary network that lines the alveolar wall becomes possible. Pseudostratified columnar-ciliated epithelium predominates in the trachea and bronchi forming the muco- ciliary escalator. Any intruding microorganism or misplaced particulate matter slipping out of the air stream in the upper airways is trapped in the muco-ciliary escalator and forced up and out by the regular beating motion of the respiratory cilia.

Should these initial defenses be breached then an intruder must encounter an uninviting but nonspecific chemical milieu including lysozyme, endogenous antimicrobial agents, adverse $\mathrm{pH}, \mathrm{IgA}$, and surfactants. ${ }^{1}$ Should this fail then a more goaldirected system is brought on line. The respiratory mucosa has developed a sensitive and specific recognition strategy that allows it to identify microbe-specific protein patterns such as lipopolysaccharide, lipotechoic acid, formylated peptides, flagellin, and non-methylated DNA. Once detected, these galvanize resident alveolar macrophages and initiate a variety of proinflammatory pathways that instigate the classical elements of inflammation (calor (heat), rubor (redness), dolor (pain), tumor (swelling), and loss of function as described by Celsus and (debatably) Virchow $)^{2}$ driven by increased vascular permeability leading to a proteinaceous infiltrate and leucocyte recruitment. The Tolllike receptors (TLRs) mediate most of this form of recognition and though they each have a specific role, TLRs can function in unison to expand their powers of recognition. ${ }^{3}$ Resident alveolar macrophages deal with the majority of insults that trigger this 
alert system but if they are overwhelmed recruited leucocytes including granulocytes lend assistance.

The invading microbes are rendered highly visual to recruited granulocytes by comprehensive opsonization mediated by both complement-dependent and -independent means. They are then ingested (phagocytosed) by neutrophils or, if they should prove resistant to this because of size or learned subversion, are subjected to a chemical onslaught (exocytosis) as neutrophils forcibly externalize toxic granule substances such as lactoferrin and myeloperoxidase as well as reactive oxygen species (ROS). ${ }^{4,5}$ A subset of neutrophils (and other leucocytes potentially ${ }^{6}$ ) employs web-like neutrophil extracellular traps to ensnare and kill resistant organisms but must themselves die in the process. ${ }^{7}$ In beneficial neutrophil-dominant inflammation, the organisms or foreign particles are detected and phagocytosed by neutrophils, which then undergo an organized, nonprovocative programmed cell death (apoptosis) that promotes their own recognition and removal by macrophages or dendritic cells. At this point, the interface between the innate and adaptive immune systems occurs as macrophages, which migrate to the lympho-reticular system following ingestion of apoptotic neutrophils, act as antigen-presenting cells allowing the lymphocyte population to complete the resolution and remembrance process. The next time this particular organism is encountered a preprepared, specific response should be available to ensure it has less opportunity to make an impact. ${ }^{8}$

The lungs are the arena for another type of granulocyte-driven inflammatory response. Eosinophils are present in larger numbers within the lungs of asthma sufferers and are recruited in greater numbers in response to sensitizing allergens such as pollen, house dust mite, and animal dander. This response has no obvious beneficial effects and it is still unclear why it should occur. The eosinophil is a useful and active defender against parasitic infection and has an armament specific to that end. It has been noted that as parasitic infections have been largely eradicated in Western society, the incidence of this abnormal eosinophil response (termed allergy or atopy) has increased and it seems that eosinophilic inflammation occurs almost as an outlet for redundancy. ${ }^{1}$

Inflammation does not always resolve neatly and, unfortunately, for diverse reasons, pulmonary inflammatory disease is one of the biggest drains on health resources in this and many other countries (respiratory disease cost in the United Kingdom was $£ 6.6$ billion in 2004). 9,10 Non-resolving or chronic inflammation is established when an acute inflammatory response fails to counter an instigating stimulus. In pulmonary infection, this may occur because a microorganism can subvert host defense by surviving either within inflammatory cells (Mycobacterium tuberculosis) or inside a protective micro-environment (Pseudomonas aeruginosa). ${ }^{11,12}$ Persistent inflammation may occur because of a recurrent stimulus like tobacco smoke (chronic obstructive pulmonary disease (COPD)), allergens (asthma), or long $(>20 \mu \mathrm{m})$ biopersistent fibers (asbestosis). ${ }^{13}$ Inflammation is also chronic where the host immune system malfunctions and becomes under- or overactive or misdirected as occurs in autoimmune disease (rheumatoid arthritis, systemic sclerosis), immune deficiency syndromes (chronic granulomatous disease, severe combined immunodeficiency syndrome), cystic fibrosis (CF), or adult respiratory distress syndrome/acute lung injury (ARDS/ALI). In some cases, the etiology is unknown and subject to debate (idiopathic pulmonary fibrosis (IPF)). Regardless of the cause, it is apparent that the over-recruitment, ineffective clearance, and hence accumulation and misdirected or frustrated activation of neutrophils and eosinophils lead to tissue damage and an inciting feedback loop of inflammation that perpetuates chronicity ${ }^{13}$ (Figure 1).

It is evident that granulocytes play an important role in the pathogenesis of pulmonary inflammation but other cell types such as macrophages, dendritic cells, mast cells, and lymphocytes are equally and potentially more influential in subsets of inflammatory respiratory disease. In this review, our focus will be on granulocyte research as expert reviewers have addressed the role of other cell types across the spectrum of respiratory disease and the interplay of different cell types within single diseases. Our aim will be to address current understanding of granulocyte biology in relation to respiratory inflammatory diseases and prospects for driving resolution of inflammation at the respiratory mucosa to develop effective treatments for lung-based disease.

\section{GRANULOCYTES}

Pluripotent hematopoietic stem cells in the bone marrow generate the common myeloid progenitor cell. This cell differentiates into a common granulocyte progenitor cell, which when given appropriate stimulation can produce any granulocyte lineage. Potential granulocyte lineages include neutrophils, eosinophils, and basophils. Basophils are the least well studied and least numerous of the granulocytes, and their physiological function seems to overlap somewhat with that of eosinophils. They are probably meant to counter parasites but their relevance to Western society is in the production of numerous mediators necessary for the incitement of the asthmatic response. Neutrophils and eosinophils are discussed in greater detail below. Granulocytes are terminally differentiated and having therefore exited the cell cycle, they remain in the $G_{0}$ phase of growth for the duration of their lives. However, a recent report suggests that postmitotic neutrophils are capable of radical phenotypic switching to take on major characteristics of macrophages when stimulated with growth factors, whereas another study shows that mouse neutrophils can be switched to a dendritic cell phenotype following in vitro incubation with colony-stimulating factor-1. ${ }^{14,15}$ Extravasated granulocytes recruited to the tissues ought to die by apoptosis whilst non-migratory, circulating granulocytes are removed by the combined efforts of the bone marrow, liver, and spleen. There is renewed interest in granulocyte life cycle and differentiation as pharmacological inhibitors of the cell-cycle machinery have been shown to promote granulocyte apoptosis. ${ }^{16,17}$

\section{NEUTROPHILS}

Neutrophils are $12-15 \mu \mathrm{m}$ in diameter and account for $70 \%$ of the circulating leucocyte population, which corresponds 


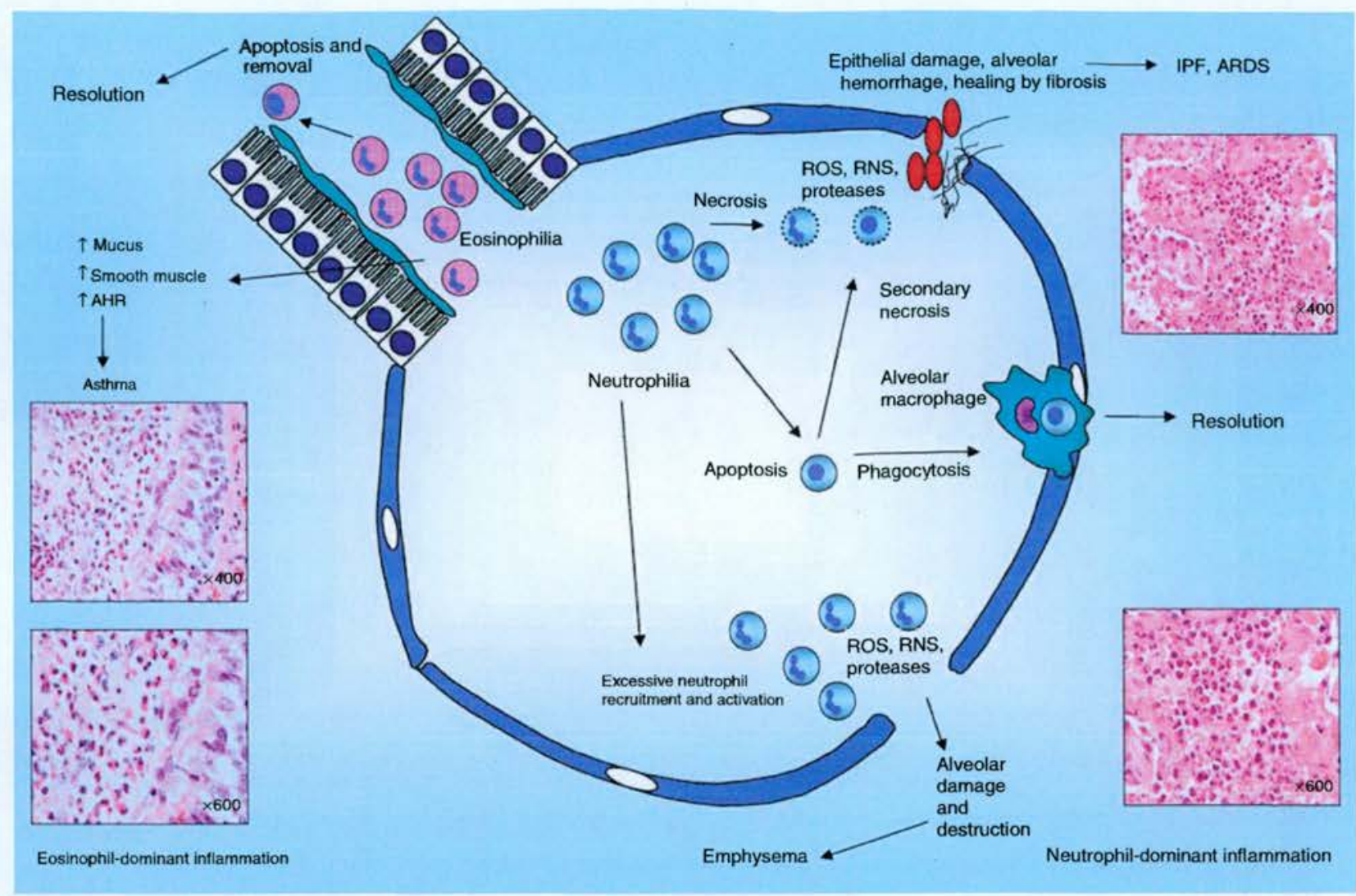

Figure 1 Diagram showing both resolution and failure of resolution of eosinophil- and neutrophil-dominant inflammatory processes. Neutrophilia at the respiratory mucosa is resolved by apoptosis of infiltrating neutrophils and phagocytic clearance by macrophages. It fails to resolve where neutrophils are in great excess or are not efficiently cleared and undergo secondary necrosis following apoptosis. This leads to alveolar damage and destruction followed by fibrotic healing. Histology on the right-hand side of the diagram shows neutrophil-dominant inflammation. Eosinophil-dominant inflammation is resolved by the same mechanisms and fails to resolve for the same reasons. The effects of eosinophil-dominant inflammation in the asthmatic airway are depicted and an example of the histology demonstrated on the left-hand side of the diagram. Histology was kindly provided by William Wallace (Pathology Department, Edinburgh Royal Infirmary).

to approximately $2.5-7.5 \times 10^{9} / \mathrm{h}$ neutrophils. They survive in the circulation for approximately $7-10 \mathrm{~h}$ but if compelled by inflammatory circumstance or chemical persuasion can extend their life span up to $48 \mathrm{~h}$ and beyond. This population turnover requires efficient production by the bone marrow and prompt clearance by that same organ with help from the spleen and liver. Neutrophils possess at least four different types of granules termed: primary (azurophilic), secondary (specific), gelatinase, and secretory; each carries a specific arsenal of toxic chemicals that the neutrophil uses against non-host elements. The neutrophil is a key effector cell at the front line of immune defense and is efficacious (and lifesaving) in the majority of instances because of its versatility. Neutrophils prove to be effective phagocytes because on accomplishment of this task they are programmed to die a prompt, quiet and importantly, contained, apoptotic death. This limits the time available to internalized microorganisms and prevents hijacking of cellular controls to enable subversion (as is thought to occur when $M$. tuberculosis is internalized by macrophages ${ }^{18}$ ). This timely death also signals larger scale phagocytes such as macrophages, dendritic cells, and epithelial cells to initiate a phagocytic response. If a neutrophil is unable to phagocytose an invader then it will disgorge granule contents into the surrounding environment causing tissue damage and amplifying the inflammatory response. This should not be construed as a purely detrimental response as it serves to block potential routes of entry for invading organisms as tissue damage causes capillary shutdown and collapses lymphatics, sends a strong signal of imperiled defense, and allows time for an alternative immune strategy to be adopted. ${ }^{8}$

Neutrophils may also employ extracellular traps to ensnare and kill resistant organisms. Neutrophil extracellular trap formation involves the extrusion of fine chromatin and granulecoated tendrils that are microbicidal and fungicidal. This effort proves fatal to the neutrophil. ${ }^{19}$ Finally, neutrophils are not just blunt effectors but also smooth operators responsible for negotiating the recruitment and education of other arms of the immune system. It is becoming appreciated that there is a false division between the innate and adaptive immune response systems and that a more realistic model involves a continual interplay between constituents of these systems. Neutrophils not only alert antigen-presenting cells and lymphocytes to danger but regulate their response to it and in return receive counter regulation. Appropriate resolution of an inflammatory immune response is finely balanced..$^{5,8}$ 
It is not surprising, given the constant exposure of the vast respiratory mucosa to threat, the fine balance of the immune response and the pivotal inflammatory role played by neutrophils, that neutrophil-dominant inflammation has been implicated in the pathophysiology of numerous inflammatory respiratory diseases including pneumonia, COPD, IPF, CF, and ARDS.

\section{EOSINOPHILS}

Eosinophils are approximately $12-17 \mu \mathrm{m}$ in size and, under normal circumstances, account for less than $5 \%$ of the circulating leucocyte population. They may survive for up to $12 \mathrm{~h}$ in the circulation but have the ability to extend their longevity to over a week if required. Not only are they bigger than neutrophils but they also wear more flamboyant colors when stained by the Romanowsky method (methylene blue and eosin), which accounts for their name. Eosinophils may also be distinguished by their production of Charcot-Leyden crystals (manufactured from lysophospholipase, an eosinophil-derived enzyme), which are often visible in their cytoplasm. Eosinophils, like neutrophils, are supplied with numerous granules though their constituents differ including major basic protein, eosinophil cationic protein, eosinophil peroxidase, and eosinophil-derived neurotoxin. This array is certainly capable of tissue damage and probably evolved to combat helminthic infection. Increasingly, new roles for eosinophils are being identified and it seems likely that they have a role in combating viral infection as eosinophil cationic protein and eosinophil-derived neurotoxin have been shown to degrade single-stranded RNA viruses. They are said to play an early role in innate immunity by production of important cytokines such as interleukin (IL)- 4 though this is probably only physiologically important in the gastrointestinal tract. They can also modulate adaptive immunity by specific activation of T cells. ${ }^{20}$

Eosinophils are implicated in a different spectrum of disease from neutrophils, of which the most common variety is allergic/ atopic disease, which includes asthma, allergic rhinitis, and eczema. In other countries, parasitic infection is still common including schistosomiasis, dranunculoriasis, ascariasis, filariasis, and hookworm. Eosinophil numbers may be increased in various cancers as well as in rare conditions such as ChurgStrauss syndrome, aspergillosis, and eosinophilic pneumonia. In eosinophil-dominant disease, it has been consistently demonstrated that eosinophil excess due to excessive recruitment, apoptosis avoidance, and failed clearance has a correlation with disease pathology.

\section{GRANULOCYTE APOPTOSIS}

Neutrophils and to a lesser extent eosinophils are short-lived cells, a feature that may help to limit their potential for causing damage by ensuring that they cannot be subverted by pathogens. They are explosively reactive cells and it is therefore surprising that their death is a model of contained self-restraint. Apoptosis is a physiological marvel that allows cells with an incendiary cargo (granules) to package it efficiently and safely (in plasma membrane) so that the cellular environment is protected. In addition, the cell nucleus condenses and chromatin is chopped up and re-organized into packages termed nucleosomes. Throughout this process, the cell membrane is retained intact though glycoprotein and phospholipid signals are displayed to attract macrophages and facilitate interaction and uptake (efferocytosis). Fluorescently-tagged Annexin-V is often used to label phosphatidylserine residues, which are characteristically flipped to the outer membrane of apoptotic cells thereby enabling flow cytometric identification of apoptosis. Another member of this family, Annexin-1, has been shown to induce granulocyte apoptosis but is also released by both neutrophils and macrophages to enhance phagocytosis of apoptotic cells. ${ }^{21,22}$ The whole process is amplified by many orders of magnitude during inflammation whether it be neutrophil or eosinophil dominant. Unfortunately, apoptotic cells cannot stay that way forever, and if they are not cleared by phagocytes then they undergo secondary necrosis and all the good work is undone. Large-scale granulocyte recruitment must necessarily be followed by large-scale granulocyte apoptosis and clearance by macrophages. It is extremely important that this is taken into account when plotting to drive granulocyte apoptosis to promote resolution of inflammation. ${ }^{4,23}$

Neutrophil and eosinophil apoptosis are similar but not identical processes. It is generally believed, though not universally, ${ }^{24}$ that there are two pathways by which apoptosis proceeds, both of which are ultimately dependent on the caspase family (Figure 2). The intrinsic pathway occurs when the cell faces withdrawal of growth/survival factors, genotoxic stress, or ultraviolet irradiation. This pathway relies on proapoptotic members of the Bcl-2 family, which escape regulation by their antiapoptotic counterparts and translocate to the mitochondria facilitating liberation of cytochrome $c$. The various components of the apoptosome (Apaf-1, cytochrome $c$ etc.) then assemble to cleave the inactive zymogen, procaspase- 9 , to active caspase9 , which inexorably commits the cell to caspase-3-mediated apoptosis. The extrinsic pathway proceeds through external cell-membrane death receptors such as the tumor necrosis factor receptor (TNFR), the Fas receptor (FasR), and TNF-related apoptosis-inducing ligand receptor (TRAILR). Ligand activation of these receptors promotes clustering of receptors and association with their internal adaptor proteins (TNFR-associated death domain protein and Fas-associated death domain protein) in the lipid-raft. Multiple procaspase- 8 molecules assemble at the adaptor proteins (formation of the death-inducing signaling complex), and their physical approximation generates an autocatalytic reaction initiating the caspase cascade. The death receptor CD137 has also been implicated in neutrophil and eosinophil apoptosis but it is currently unclear what its physiological role is. ${ }^{25}$ In neutrophils the importance of caspase action in death receptor-mediated apoptosis has previously been a matter of debate but evidence of caspase-independent apoptosis has been effectively countered by work demonstrating that culture conditions and concentrations of caspase inhibitor had not been optimally utilized in these studies. ${ }^{26}$

To further complicate the story, it is apparent that there is a degree of cross talk between the intrinsic and extrinsic pathways. Caspase- 8 may alternatively cleave Bid, which can translocate 


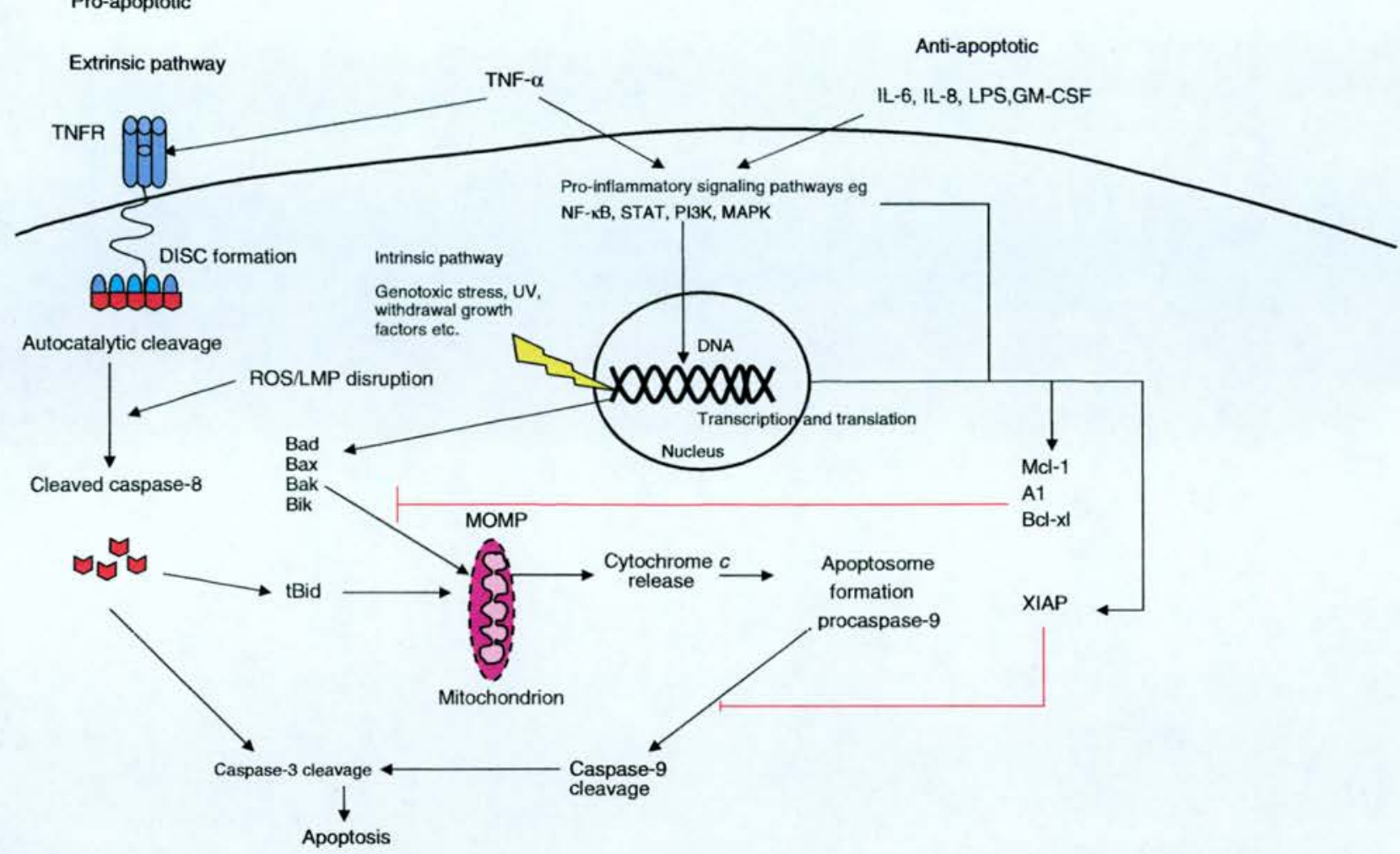

Figure 2 Neutrophil apoptosis. The intrinsic and extrinsic pathways are shown along with some examples of pro and antiapoptotic signaling. Proinflammatory signaling pathways may directly influence transcription of survival proteins e.g., NF-kB promotes transcription of XIAP or increase protein stability e.g., ERK (of the MAPK family) maintains XIAP levels. TNF- $\alpha$ may have both early proapoptotic action and late antiapoptotic effects. TNFR is the TNF receptor. DISC refers to the death-inducing signaling complex, which is composed of TNFR-associated death domain protein (TRADD), Fas-associated death domain protein (FADD), and procaspase-8. MOMP is mitochondrial outer membrane permeabilization. ERK, extracellular signal-regulated kinase; LMP, Lysosomal membrane permeabilisation; NF- $\mathrm{B}$, nuclear factor- $\mathrm{kB}$; TNF- $\alpha$, tumor necrosis factor- $\alpha$; TNFR, tumor necrosis factor receptor.

to the mitochondrial membrane and permeabilize it allowing apoptosis to proceed through intrinsic pathway apoptotic machinery. ${ }^{23,25}$

Eosinophil apoptosis most likely occurs along approximately the same lines; however, controversy still reigns with regard to the caspase family. It has previously been stated that caspase- 3 , -8 , and -9 have no demonstrable role in eosinophil apoptosis. Subsequently, it has been suggested that perhaps caspase- 9 plays a role and that therefore the mitochondrial pathway is of importance. This work was confirmed by observations with regard to the effects of known eosinophil apoptosis-inducing agents such as glucocorticoids, which appear to mediate their effects through mitochondrial permeabilization, with caspase activation merely a downstream effect. In contrast, Fas ligation has been shown to promote eosinophil apoptosis in a caspase-3-and -8-dependent manner, and although mitochondrial integrity was disrupted it proved non-essential for apoptotic progression. ${ }^{21,27-30}$

Support for the predominance of mitochondria-driven granulocyte apoptosis is provided by the increasing evidence that survival proteins are key determinants of neutrophil longevity. The antiapoptotic Bcl-2 family member, Mcl-1, is present in both neutrophils and eosinophils, but other family members show contrasting expression. Neutrophils express A1, eosinophils express Bcl-xL but Bcl-2 has not been shown to be constitutively present in either cell at demonstrable levels. Interestingly, however, in eosinophils, Bcl-2 expression can be stimulated by IL-5. ${ }^{31}$ These survival proteins mediate their effects by marshaling proapoptotic Bcl-2 family counterparts away from the mitochondrial membrane. Proapoptotic Bcl-2 family members are capable of causing permeabilization of the outer mitochondrial membrane (MOMP) when numbers predominate over those of their chaperones. Bax is perhaps the best-characterized member of this family and appears to play a pivotal role in neutrophil apoptosis. In eosinophils, however, Bax fails to be downregulated by antiapoptotic survival factors weakening its position as a key player in apoptosis. ${ }^{32}$ The role of $\mathrm{Mcl}-1$ in neutrophil apoptosis has received a great deal of attention and it appears that this protein is an essential component of neutrophil viability; a contention that is supported by the neutropenic phenotype of the $\mathrm{Mcl}-1$ knockout mouse in comparison with the increased apoptotic phenotype of the $A 1$ knockout mouse. ${ }^{33,34}$

\section{Modulation of granulocyte apoptosis}

Granulocyte longevity is necessarily highly regulated and consequently highly variable from a matter of hours up to several days for neutrophils and extending to 2 weeks for eosinophils. 
Persistent markers of bacterial infection such as lipopolysaccharide, pathogen-derived molecules that act as ligands for TLR-2, -4 and -9 , and inflammatory cytokines such as IL-6, IL-8, granulocyte macrophage-colony stimulating factor, and TNF can delay neutrophil apoptosis whereas resolving infection indicated by successful neutrophil phagocytosis of bacteria, removal of bacterial products, and downregulation of inflammatory cytokines will promote apoptosis. ${ }^{3,35}$

Granulocyte longevity is also extremely dependent on intracellular signaling pathways. Perhaps the most important of which is that controlled by the pivotal transcription factor of inflammatory cytokines, nuclear factor (NF) $-\kappa B$. NF- $\kappa B$ can be activated by lipopolysaccharide, and is known to control the survival proteins XIAP (an inhibitor of caspase- $-3,-8$, and -9) and $\mathrm{Bcl}-\mathrm{xl}$ thus enhancing neutrophil and eosinophil longevity by tangible means. Pharmacological inhibitors of NF- $\kappa B$ such as gliotoxin promote neutrophil apoptosis and, in combination with TNF- $\alpha$, cause dramatically enhanced apoptosis. This feature may partially explain why TNF- $\alpha$ has opposing effects on neutrophils at early and late time points. ${ }^{36,37}$ Other important signaling pathways include the phosphoinositide 3-kinase pathway, which can be stimulated by granulocyte macrophagecolony stimulating factor to promote longevity by upregulation of Mcl-1 in neutrophils and which has an emerging role in the maintenance of eosinophilic inflammation. ${ }^{38,39}$ Similarly, the extracellular signal-regulated kinase pathway is likely to be important for survival factor-mediated antiapoptotic effects at sites of inflammation. However, direct inhibition of either of these pathways will not promote granulocyte apoptosis perse. 4,40

Perhaps the most interesting and clinically relevant modulation of granulocyte apoptosis is that achieved with the use of glucocorticosteroids. These drugs extend neutrophil life span but promote eosinophil apoptosis. This effect seems to correlate with their ability to upregulate Mcl-1 in neutrophils but downregulate the same protein in eosinophils. ${ }^{41}$ Dexamethasone has also been shown to promote macrophage phagocytosis of apoptotic neutrophils. ${ }^{42}$ The impact of putative inflammation-resolving agents on clearance of apoptotic neutrophils is of paramount importance as prompt removal is essential to avoid secondary necrosis and loss of toxic contents. This is perhaps why the discovery of a new class of endogenous lipid-derived proresolution agents has been so exciting. The lipoxin family produced by neutrophils and macrophages limits the recruitment of neutrophils to inflammatory sites and enhances macrophage phagocytosis of apoptotic neutrophils. This occurs as part of a natural brake on progression of inflammation and there is hope that enhancement of this pathway may provide a novel therapeutic strategy to counter inflammatory disease, a hypothesis that has already shown promise in several animal models. ${ }^{43}$ Another exciting prospect for therapeutic modulation of granulocyte apoptosis has emerged with the discovery of active cell-cycle machinery in neutrophils, which can be inhibited to promote apoptosis. Cyclin-dependent kinase inhibitor drugs promote neutrophil apoptosis and drive resolution of inflammation in animal models. Given that these drugs are already in use for the treatment of cancer, it is possible that they could make the transition from bench to bedside for the management of inflammatory disease in the near future. ${ }^{17,44}$

\section{ALVEOLAR MACROPHAGES AND PHAGOCYTOSIS OF APOPTOTIC GRANULOCYTES}

In normal healthy lungs, the predominant alveolar leucocyte is the macrophage, a cell that becomes resident following differentiation from a bone marrow-produced circulating precursor monocyte. Alveolar macrophages occupy a unique position at the interface between inhaled air (and hence the external environment) and the circulation (in the form of the alveolar capillary network, which is closely juxtaposed to the alveolus to promote efficient gaseous exchange) and because key macrophage functions include chemotaxis, phagocytosis, and cytotoxicity they are lynch pins of immune defense. Importantly, during pulmonary infective or inflammatory disease, a key role in resolution of inflammation falls to both alveolar and recruited circulating macrophages. These cells are responsible for the removal of apoptotic neutrophils and to some extent eosinophils (though epithelial cells may be equally important for eosinophil removal) by efferocytosis. In order for an apoptotic cell to be recognized by a macrophage it must display specific signals. The most obvious change in the apoptotic granulocyte's plasma membrane is the externalization of phosphatidylserine residues but this in itself is not sufficient to expedite phagocytosis. The search for a macrophage-docking receptor on granulocytes has been exhaustive but inconclusive and currently a combination of various glycoproteins and phospholipids is of postulated importance. These putative receptors include the recently identified Tim, ${ }^{5,45}$ stabilin-2, ${ }^{46}$ and BAIl $1{ }^{47}$ The process of efferocytosis, literally meaning "burying the dead", is a proresolution strategy in itself. Macrophages that consume apoptotic neutrophils switch to a resolution phenotype that allows them to secrete TGF- $\beta$ and IL- $10^{48}$ as opposed to proinflammatory cytokines such as IL-6, IL- 8 , and TNF- $\alpha$. This is in contrast to macrophages that have consumed necrotic neutrophils where the opposite is true and inflammation is actively propagated. In addition, the proresolution phenotype includes the production and enhanced responsiveness to lipoxins, protectins, and resolvins. These mediators enhance macrophage phagocytosis and promote proresolution cytokine production. ${ }^{43}$

\section{DYSREGULATION OF GRANULOCYTE APOPTOSIS/ PHAGOCYTOSIS AND INFLAMMATORY DISEASES INTHE RESPIRATORYTRACT}

There has been a tendency, certainly in clinical circles, to question the validity of inflammation-based hypotheses for various diseases based on the efficacy or lack of efficacy of glucocorticosteroid medications. Given the complexity of the inflammatory response and the relative bluntness of this therapeutic tool, it is overly simplistic to make such assumptions. Steroid medications have certainly been a paradigmatic therapy in the treatment of inflammatory disease but they do not and will not drive resolution of inflammation in all settings. With increased understanding of the mechanisms of the inflammatory 
response and a new focus on its resolution, it is hoped that novel incisive or pleiotropic therapy combinations may be developed to address the inflammatory lung diseases discussed below.

\section{COPD}

In most cases of COPD ( $\alpha$-1-antitrypsin-deficient patients are a notable exception), the respiratory mucosa is damaged by repeated exposure to inhaled toxic chemicals leading to chronic inflammation, reduced immunity, and susceptibility to respiratory infections. COPD is a prevalent, largely smoking-related (though there is an increasingly recognized occupational contribution) disease in this country (in others it is related to the burning of bio-fuels), which presents with increasing breathlessness and a productive cough. It is an obstructive airways disease but unlike asthma this obstruction is irreversible with inhaled therapies. The mainstay of current treatment is with inhaled or oral corticosteroids and antibiotics when exacerbations are judged infective. (For interestcd readers, the refs. ${ }^{1,23,49-51}$ give an overview of COPD pathophysiology.)

Neutrophils are likely to play an important role in this condition and they are found in increased numbers throughout the respiratory tract. The highest concentrations of neutrophils are found in sputum and bronchoalveolar lavage fluid (BAL) (which is perhaps representative of rapid airway-directed migration) but numbers of neutrophils are also increased in lung parenchyma and airway smooth muscle. Neutrophilic inflammation is characteristic of COPD exacerbations and there is a correlation between the resting burden of lung-based neutrophils and severity of disease phenotype. Neutrophilic inflammation appears to be driven by the irritant force as smoking drives alveolar macrophages and epithelial cells to express increased levels of IL-8, a potent neutrophil chemoattractant. IL-8 also stimulates neutrophils to release myeloperoxidase whereas TNF- $\alpha$ and leukotriene $\mathrm{B}_{4}$ (also produced by epithelial cells, mast cells, and $\mathrm{T}$ lymphocytes) cause neutrophil activation (degranulation, reactive oxygen species production). Meanwhile, macrophage phagocytic function is impaired by cigarette smoking and cannot keep pace with the increased neutrophil burden. ${ }^{52}$ Enhanced neutrophil recruitment and activity combined with decreased macrophage phagocytosis weigh the scales heavily toward neutrophil-mediated tissue damage. COPD lungs are subjected to high levels of toxic neutrophil products including reactive oxygen species, elastase, and proteinases. These toxic substances overload the capacity of native antiproteinases and antioxidants to neutralize them, which leads to damaged epithelial cilia and decreased mucociliary clearance. Damage to the respiratory mucosa means that alveolar cells are replaced by goblet cells that increase mucous production. Furthermore, airways are progressively remodeled by the reparative process so that they become thicker, less-efficient conductors of air. There is some controversy with regard to the longevity of neutrophils isolated from the airways of COPD patients. Studies have shown both enhanced longevity and no enhancement of longevity whereas peripheral blood neutrophils appear to have an increased life span in keeping with systemic inflammation.
Chronic obstructive pulmonary disease is notoriously resistant to glucocorticoid therapy perhaps because these drugs promote neutrophil longevity by upregulating the survival protein $\mathrm{Mcl}-1{ }^{41} \mathrm{It}$ is also known that smoking promotes dysfunction of histone deacetylase- 2 , an enzyme that is usually recruited by glucocorticoid receptors to switch off the transcription of proinflammatory genes. The small benefits attributable to steroid therapy in COPD may stem from enhancement of macrophage phagocytosis of apoptotic cells. Of other therapies currently in use, theophylline (a nonspecific adenosine antagonist and phosphodiesterase inhibitor) is known to restore histone deacetylase- 2 function, reversing corticosteroid resistance and reducing IL- 8 concentrations and sputum neutrophilia. Longacting $\beta_{2}$ agonists (originally employed to relax smooth muscle in peripheral airways) have now been shown to inhibit neutrophilic inflammation as measured by sputum or BAL analysis, and they are reported to drive neutrophil apoptosis though the circumstances of this are difficult to ascertain. It is clear that potential therapies must consider targeting neutrophil recruitment as well as striving to drive neutrophil apoptosis and removal. ${ }^{49,50,53,54}$

\section{Asthma}

In asthma, the respiratory mucosa is hypersensitive to exposures that are comfortably processed under normal conditions and a characteristic inflammatory response ensues. It is part of a spectrum of atopic or allergic disorders, where an enhanced sensitivity to particular antigens or environmental conditions results in a disease flare. Exacerbations of asthma are characterized by narrowing of the airways (bronchoconstriction), which leads to shortness of breath, wheezing, and cough. (For interested readers, these refs. ${ }^{1,55-60}$ give an overview of asthma pathophysiology.)

Recent work has suggested that there may be many subtle mucosal abnormalities that contribute to the pathogenesis of asthma. For example, it appears that exacerbations of asthma caused by rhinovirus may be facilitated by a defect in interferon $\lambda$ induction, which increases susceptibility to invasive disease whereas viral exacerbations or damage caused by chronic inflammatory disease facilitate epidermal growth factor receptor signaling (a wound repair response), which enhances neutrophil function and chemotaxis. ${ }^{61,62}$

The allergy arm of this disease is mediated by IgE, which is produced by $\mathrm{B}$ cells in response to an initial exposure to a given antigen. IgE attaches to mast cells and basophils priming them to release histamine, leukotrienes, and ILs at subsequent exposures. Traditionally, asthma is an eosinophil-dominant disease and eosinophils are heavily recruited by the same TH2 cell type signaling (IL-4, IL-5, IL-6) responsible for the production of IgE. This process was thought to be under the direct control of IL-5 but as anti-IL-5 therapy has proved ineffective, it is suspected that there is a greater redundancy in the system. Anti-IL-5 therapy failed to significantly reduce eosinophil numbers in the asthmatic airway but did decrease deposition of extracellular matrix proteins suggesting a role for Il-5 in airway remodeling. ${ }^{63,64}$ Interestingly, an IL-4 analog (pitrakinra), which 
prevents binding of IL- 4 and IL- 13 to IL- $4 \alpha$ receptor complex, attenuated the late-phase response (bronchoconstriction measured by reduction in Forced Expiratory Volume in $1 \mathrm{~s}$ ) to allergen challenge. It is as yet unclear whether this success was due to inhibition of IL-4 alone, IL-13 alone, or due to combined inhibition. ${ }^{65}$ In addition, a role for TH17 cells has been postulated particularly in forms of asthma where neutrophil recruitment contributes to pathogenesis. ${ }^{66}$

Regardless of the super-intending mechanism, it is clear that a heavy burden of apoptosis-resistant eosinophils concomitantly resistant to macrophage phagocytosis, yet capable of disgorging their formidable armament onto the respiratory mucosa, have significant potential to cause tissue damage and airway remodeling. Thankfully, asthma is usually responsive to glucocorticoid therapy partly because steroids are effective at promoting eosinophil apoptosis. ${ }^{67}$ This apoptosis may, in direct contrast to experience in neutrophils, be mediated by downregulation of Mcl-1. Rarely, asthma may be steroid resistant and there are different theories as to how resistance might arise.

First, it is clear that smokers with asthma respond poorly to steroids because of the defective action of histone deacetylase2 (discussed earlier with regard to COPD patients). Second, it is apparent that a subset of asthmatics as well as end-stage or severe asthmatics manifests a neutrophil-dominant inflammation for reasons that are unknown. It has been postulated that these patients may represent a different disease or disease phenotype. ${ }^{68}$ Third, it is known that airway epithelial damage results in smooth muscle hypertrophy causing significant luminal narrowing without a requirement for a significant bronchoconstrictor stimulus and finally, it is postulated that resistant asthmatics might express gene polymorphisms encoding defective caspase machinery. ${ }^{60}$

Regardless of efficacy, the side effect profile (adrenal suppression, osteoporosis, peptic ulcer disease) of higher dose steroid therapy in the subset of resistant patients makes it less than ideal as they potentially require prolonged or maintenance treatment. It should be emphasized, however, that maintenance therapy with routine doses of inhaled steroids has established symptom control and reduced mortality in the majority of patients without these side effects becoming a problem. Anti-IgE therapy is showing promise in a subset of patients, and increased eosinophil apoptosis and downregulation of inflammatory cytokines have been demonstrated in peripheral blood samples taken from asthmatics treated with omalizumab (a recombinant humanized monoclonal antibody that selectively binds IgE). Future developments will probably include anti-IL-4, anti-IL-13, and potentially anti-Th17-based therapies, now that there is a rationale for their development. However, it is not always best practice to attempt a one-hit, magic-bullet cure especially in inflammatory disease where there is considerable redundancy in the cytokine system. If an agent was developed that could promote eosinophil apoptosis without detriment to macrophage phagocytosis and with a negligible side effect profile, it would be an extremely useful addition to the pharmacopoeia.

\section{Idiopathic pulmonary fibrosis}

Idiopathic pulmonary fibrosis is a condition characterized by the devastation of the respiratory mucosa though its pathophysiology is complex. There is a great deal of debate about the relative importance of inflammation vs. aberrant wound repair in the pathogenesis of IPF. There is not sufficient space to address that debate in this article, suffice to say that there is enough evidence to justify an argument in support of a significant neutrophilderived component to the disease model. (For interested readers, these references give an overview of the pathophysiology of IPF and a flavor of the debate. ${ }^{1,69-73}$ )

The argument is that the initial and subsequent "exacerbatory" insults are acute and inflammatory even if the characteristic phenotype is conveyed by a disordered "healing" process. Healing by fibrosis results in significant loss of lung architecture and vital respiratory mucosa to scarring. Neutrophils are known to be present in BAL from IPF patients and are also significantly increased within lung tissue. In addition, there is evidence of the toxic chemicals produced by neutrophils including myeloperoxidase, elastase, collagenases, and proteases. The importance of this chemical insult is supported by the elastase knockout mouse, which is significantly protected against lung injury and also fails to upregulate TGF- $\beta .^{74} \mathrm{~A}$ recent clinical paper has shown that significant neutrophilia within the BAL fluid of IPF patients correlates with increased mortality. ${ }^{75}$ It has been argued that the terminal scarring process of IPF occurs because of a significant loss of lung architecture and that where lung architecture is preserved there is potential for reversal of the remodeling process and resolution. ${ }^{72}$ It has also been suggested that the fibrotic response may in a sense be driven by a persistent but ineffective proresolution of inflammation phenotype. This hypothesis is drawn from the observation that TGF- $\beta$ is a key proresolution molecule but is also intimately involved in fibrogenesis. In a disease model where non-resolving low-level inflammation results in sustained TGF- $\beta$ production, it is possible to conceive that a fibrotic healing phenotype might evolve. Therapeutic strategies that drive neutrophils toward apoptosis will certainly have something to add to treatment of this disease if they can prevent or ameliorate the inflammatory insult (whether it be acute, chronic, or relapsing) that must be responsible for such extreme distortion of lung architecture. ${ }^{69,70}$

\section{ARDS/ALI}

Adult respiratory distress syndrome/acute lung injury is the pulmonary component of the multi-organ dysfunction syndrome (MODS) and represents a global failing of lung function in response to a specific, nonspecific, or unidentifiable stimulus. Effectively, these conditions are the result of an aggressive mucosal inflammatory immune response. (The refs. ${ }^{1,76-80}$ cover the wider pathophysiology of ARDS/ALI.).

Characterized by the pathological reaction termed diffuse alveolar damage, ARDS/ALI seems to be a neutrophil dominant disease (interestingly, diffuse alveolar damage is also seen in the terminal stages of IPF). Analysis of BAL fluid from patients with early-stage ARDS demonstrates increased numbers of activated neutrophils and their numbers correlate with severity of 
lung injury. Indeed, persistence of BAL neutrophilia at day 7 is associated with increased mortality. The pulmonary circulation contains a large sequestered neutrophil population termed the "marginated" pool that does not normally circulate but is loosely adhered to the vessel walls. This population can be mobilized into the circulation by steroid therapy or by exercise. Neutrophils are subject to slow transit through the pulmonary microvasculature where blood vessel diameters are smaller than their own. The ability of neutrophils to progress depends on their considerable properties of distensibility. In ARDS/ALI, neutrophils are recruited early in large numbers, and an alteration in their rheological properties (they become less deformable) means that they struggle to maneuver through vessels whereas cytokine excess encourages their adhesion to vessel walls and subsequent translocation into the lung parenchyma and airways. The influx of huge numbers of neutrophils and proteinaceous inflammatory edema fluid from permeabilized vessels impairs gas exchange and makes adequate ventilation extremely difficult.

Neutrophil influx is driven by alveolar macrophage production of IL-8, and excessive levels of this cytokine have been found to be predictive of progression to ARDS in susceptible patients. ${ }^{81}$ There is no effective, specific therapy for this condition and yet some patients survive with supportive therapy alone, implying that successful resolution is possible and can be mediated by physiological mechanisms. Neutrophils isolated from the BAL of ARDS patients have enhanced longevity, are activated, and may cause insurmountable damage to the respiratory mucosa leading to healing by fibrosis and permanent scarring with loss of lung architecture and function. In experimental models where neutrophils are depleted, ALI caused by endotoxin is attenuated. In other studies, enhancement of neutrophil apoptosis also reduced inflammatory parameters and tissue damage. The picture is complicated by apparent ARDS/ALI in neutropenic human patients. It is also clear that given the extravagant neutrophil influx associated with this condition, it would likely require a dual strategy of enhancing both neutrophil apoptosis and neutrophil clearance to ensure that secondary necrosis (and tissue damage) is prevented by prompt removal. ${ }^{76,82}$

\section{Pneumonia}

Pneumonia is the term given to an infection within the lower respiratory tract that is sufficiently significant to result in visible changes on a chest radiograph. ${ }^{83}$ There are many potential causative organisms including viruses and bacteria. Invading pathogens that overcome immediate host defenses are nonetheless recognized as foreign by their non-host constituents such as lipopolysaccharide or formylated peptides in their outer membranes. TLRs, CD14, and G-protein coupled receptors (e.g., FPR) found on macrophages and other respiratory mucosal cells are responsible for this detection and subsequently stimulate an acute inflammatory reaction by the production of cytokines such as TNF- $\alpha$, IL-1, IL-6, and IL-8. This proinflammatory milieu prolongs neutrophil life span and promotes neutrophil activation to allow effective microbial phagocytosis and killing. ${ }^{3,35,84}$ If the inflammatory reaction is successful in containing the pathogens and they are cleared by phagocytosis or killed, then the inflammatory reaction remains localized to a single lobe or segment of the lung. The upregulation of anti-inflammatory, proresolution molecules should ensure containment of inflammation. Neutrophil phagocytosis of bacteria and the induction of proresolution cytokines such as IL-10 and TGF- $\beta$ promote neutrophil apoptosis and removal by macrophages. ${ }^{85}$

The alternative is non-resolution with enhanced neutrophil longevity and local tissue damage (resulting in abscess, empyema, or bronchiectasis) and/or loss of containment resulting in global lung inflammation characterized by ARDS or spread through the circulation to other organs (sepsis) with the potential for MODS. Streptococcal pneumonia (the pathogen responsible is Streptococcus pneumoniae) is often cited as a paradigm of resolving inflammation as despite an acute inflammatory response characterized by a massive neutrophil influx (giving the pathological appearance referred to as "red hepatization") it is possible for the lung to achieve complete recovery. ${ }^{13}$ There is, however, a sub-population of severe pneumonia that behaves aggressively or is not contained by the patient's immune system and antibiotic therapy resulting in admission to intensive care and potentially MODS, sepsis, terminal decline, and death.

Antibiotics are central to the treatment of pneumonia but they are not always sufficient and it is possible that addressing acute inflammation associated with pneumonia may prove a successful adjuvant therapy. In this setting, it is unclear whether the ideal strategy is to dampen down the inflammatory response or to augment it in the hope that a supra-physiological immune reaction will prove successful in eradicating the organism responsible. Glucocorticoid therapy has been used as an adjuvant to antibiotics in this kind of patient with varying success rates in different trials. ${ }^{86}$ The theory is that steroids should dampen inflammation through downregulation of NF- $\kappa \mathrm{B}$ activation and consequently the inflammatory cytokines under its direct transcriptional control. A recent study that utilized a physiological steroid dose (supra-physiological doses had been employed in previous studies) has shown a significant reduction in length of hospital stay and mortality in Intensive Care Unit patients with severe community-acquired pneumonia. ${ }^{87}$ It is possible therefore that in a subgroup of patients with pneumonia, strategies to aid resolution of inflammation (in combination with antibiotic therapy) may be warranted.

\section{Cystic fibrosis}

Cystic fibrosis is the commonest inherited disease in Caucasian populations affecting one in 2,500 births. It seems surprising that a defect in salt transport (the genetic abnormality affects the cystic fibrosis transmembrane regulator gene (CFTR), which encodes a chloride channel) should be responsible for such widespread organ pathology including pancreatic insufficiency, bronchiectasis, liver dysfunction, infertility, and gut defects. Mortality (on average at age 34 in Western society) is most frequently from respiratory failure caused by aggressive bronchiectasis.

There are competing hypotheses as to the root cause of the repeated infective exacerbations that lead to bronchiectasis in $\mathrm{CF}$. It is postulated that a relative dehydration of the respiratory 
Table 1 Selected rodent models of inflammatory lung disease

\begin{tabular}{|c|c|c|c|c|c|c|c|}
\hline $\begin{array}{l}\text { Disease } \\
\text { modelled }\end{array}$ & Technique & $\begin{array}{l}\text { Strategy/basis } \\
\text { for technique }\end{array}$ & $\begin{array}{l}\text { Phenotype/ } \\
\text { cytokine } \\
\text { expression }\end{array}$ & Advantage & Disadvantage & Outcome & References \\
\hline \multirow[t]{6}{*}{ IPF } & $\begin{array}{l}\text { Bleomycin } \\
\text { lung injury }\end{array}$ & $\begin{array}{l}\text { Known pulmo- } \\
\text { nary toxi- } \\
\text { city causing } \\
\text { fibrosis in } \\
\text { human cancer } \\
\text { patients }\end{array}$ & $\begin{array}{l}\text { T-cell-independ- } \\
\text { ent, CCL2 and } \\
12 \text { required, } \\
\text { inflammatory } \\
\text { cell recruitment, } \\
\text { TGF- } \beta\end{array}$ & $\begin{array}{l}\text { Well-known, } \\
\text { characterized, } \\
\text { quick (14-28 } \\
\text { days), multi- } \\
\text { ple routes of } \\
\text { administration }\end{array}$ & $\begin{array}{l}\text { Disease resolves } \\
\text { in mice but not in } \\
\text { humans. Variable } \\
\text { response between } \\
\text { mouse strains. }\end{array}$ & $\begin{array}{l}\text { Dependent } \\
\text { on time point } \\
\text { (inflammation } \\
\text { vs. fibrosis) }\end{array}$ & $94,96,97$ \\
\hline & FITC & $\begin{array}{l}\text { Direct chemi- } \\
\text { cal injury }\end{array}$ & $\begin{array}{l}\text { T-cell-independ- } \\
\text { ent, inflammatory } \\
\text { cell and fibrocyte } \\
\text { recruitment, } \\
\text { IL-13 }\end{array}$ & $\begin{array}{l}\text { Visualization as } \\
\text { FITC deposi- } \\
\text { tion denoted } \\
\text { by green } \\
\text { immuno- } \\
\text { fluorescence, } \\
\text { persistent }\end{array}$ & $\begin{array}{l}\text { Variable efficacy } \\
\text { of dose }\end{array}$ & $\begin{array}{l}\text { Lymphocyte- } \\
\text { independent } \\
\text { pulmonary } \\
\text { fibrosis by } \\
\text { day } 21\end{array}$ & 93,94 \\
\hline & Irradiation & $\begin{array}{l}\text { Radiation } \\
\text { injury }\end{array}$ & $\begin{array}{l}\text { Monocyte/lym- } \\
\text { phocyte-derived } \\
\text { lymphotactin, } \\
\text { RANTES, CCL-2 } \\
\text { and } 7, \text { CXCL-10, } \\
\text { TGF- } \beta\end{array}$ & $\begin{array}{l}\text { Different } \\
\text { susceptibil- } \\
\text { ity of mouse } \\
\text { strains allows } \\
\text { genetic study }\end{array}$ & Slow (24 weeks) & $\begin{array}{l}\text { Model of radi- } \\
\text { ation fibrosis }\end{array}$ & 93,94 \\
\hline & Silica & $\begin{array}{l}\text { Resistant, } \\
\text { fibrogenic } \\
\text { particles } \\
\text { administered } \\
\text { intratracheally }\end{array}$ & $\begin{array}{l}\text { Inflammatory } \\
\text { cell recruitment, } \\
\text { IL-1, TNF- } \alpha \text {, } \\
\text { IL-10, TH2 }\end{array}$ & Persistent & $\begin{array}{l}\text { Specialized } \\
\text { aerosoliza- } \\
\text { tion equipment } \\
\text { required } \\
\text { (non-essential), } \\
\text { lengthy ( } 60 \text { days) }\end{array}$ & $\begin{array}{l}\text { Inflammatory } \\
\text { injury followed } \\
\text { by fibrosis } \\
\text { after min } \\
30 \text { days }\end{array}$ & 94 \\
\hline & $\begin{array}{l}\text { Transgenic } \\
\text { TGF- } \alpha\end{array}$ & $\begin{array}{l}\text { TGF- } \alpha \\
\text { increased in } \\
\text { IPF patients' } \\
\text { BAL }\end{array}$ & $\begin{array}{l}\text { TGF- } \alpha \text { over- } \\
\text { expression. } \\
\text { Fibrosis without } \\
\text { inflammation }\end{array}$ & $\begin{array}{l}\text { Incisive sin- } \\
\text { gle-cytokine } \\
\text { system }\end{array}$ & $\begin{array}{l}\text { Not representa- } \\
\text { tive of complexity } \\
\text { of actual disease } \\
\text { state }\end{array}$ & $\begin{array}{l}\text { Pulmonary } \\
\text { fibrosis at } 4 \\
\text { days }\end{array}$ & 98 \\
\hline & $\begin{array}{l}\text { Adenovirus } \\
\text { delivery of } \\
\text { GMCSF, TNF, } \\
\text { TGF- } \beta \text {, IL- } 1 \mathrm{~b}\end{array}$ & $\begin{array}{l}\text { Overexpression } \\
\text { of important } \\
\text { cytokines }\end{array}$ & Various & $\begin{array}{l}\text { Incisive sin- } \\
\text { gle-cytokine } \\
\text { system }\end{array}$ & $\begin{array}{l}\text { As before, } \\
\text { vigorous immune } \\
\text { response to virus, } \\
\text { epithelium trophic }\end{array}$ & $\begin{array}{l}\text { Cytokine } \\
\text { dependent }\end{array}$ & 99-102 \\
\hline $\begin{array}{l}\text { IPF and } \\
\text { asthma }\end{array}$ & $\begin{array}{l}\text { Transgenic } \\
\text { IL-13 }\end{array}$ & $\begin{array}{l}\text { IL-13 elevated } \\
\text { in IPF patients } \\
\text { and asthmatics }\end{array}$ & $\begin{array}{l}\text { IL-13, CCR1,-2,- } \\
\text { 5,-10, TGF- } \beta \text {,IL- } \\
11, \text { MMP-1,VEGF }\end{array}$ & $\begin{array}{l}\text { More } \\
\text { complex- } \\
\text { cytokine } \\
\text { pattern }\end{array}$ & $\begin{array}{l}\text { As above plus TH2 } \\
\text { phenotype }\end{array}$ & $\begin{array}{l}\text { Eosinophil- } \\
\text { rich inflamma- } \\
\text { tion followed } \\
\text { by fibrotic foci } \\
\text { long term }\end{array}$ & 103 \\
\hline Asthma & $\begin{array}{l}\text { Ovalbumin/ } \\
\text { HDM/cock- } \\
\text { roach/ } \\
\text { ragweed } \\
\text { sensitization }\end{array}$ & $\begin{array}{l}\text { Allergen } \\
\text { sensitization }\end{array}$ & $\begin{array}{l}\text { TH2, IgE, } \\
\text { eosinophilia, } \\
\text { airway hyper- } \\
\text { responsiveness }\end{array}$ & $\begin{array}{l}\text { Models TH2 } \\
\text { inflammatory } \\
\text { response, } \\
\text { quick }\end{array}$ & $\begin{array}{l}\text { High-dose, infre- } \\
\text { quent exposure } \\
\text { as opposed to } \\
\text { low-dose frequent } \\
\text { allergen exposure } \\
\text { in human disease, } \\
\text { eosinophils less } \\
\text { likely to degranu- } \\
\text { late. Effective } \\
\text { mouse therapies } \\
\text { do not necessar- } \\
\text { ily translate (e.g., } \\
\text { anti-ll-5) }\end{array}$ & $\begin{array}{l}\text { Eosinophilic } \\
\text { inflammation }\end{array}$ & 92,104 \\
\hline \multirow[t]{2}{*}{ COPD } & $\begin{array}{l}\text { Inhalation } \\
\text { of smoke. } \\
\text { Chronic } \\
\text { smoke } \\
\text { exposure }\end{array}$ & $\begin{array}{l}\text { Smoking caus- } \\
\text { ative of COPD }\end{array}$ & $\begin{array}{l}\text { Mild COPD } \\
\text { (Gold 1,2) }\end{array}$ & $\begin{array}{l}\text { Simple design. } \\
\text { Relevant to } \\
\text { etiology of } \\
\text { disease in } \\
\text { humans }\end{array}$ & $\begin{array}{l}\text { Time-consum- } \\
\text { ing, humans tend } \\
\text { to have more } \\
\text { advanced disease } \\
\text { at presentation }\end{array}$ & $\begin{array}{l}\text { Mild COPD } \\
\text { model }\end{array}$ & 105 \\
\hline & $\begin{array}{l}\text { Neutrophil } \\
\text { elastase KO } \\
\text { mouse }\end{array}$ & $\begin{array}{l}\text { Elastase a } \\
\text { key neutrophil } \\
\text { product }\end{array}$ & $\begin{array}{l}\text { Smoke damage } \\
\text { resistant }\end{array}$ & Incisive & Simplistic & $\begin{array}{l}59 \% \text { protec- } \\
\text { tion from } \\
\text { emphysema }\end{array}$ & 74 \\
\hline
\end{tabular}


Table 1 continued

\begin{tabular}{|c|c|c|c|c|c|c|c|}
\hline $\begin{array}{l}\text { Disease } \\
\text { modelled }\end{array}$ & Technique & $\begin{array}{l}\text { Strategy/ } \\
\text { basis for } \\
\text { technique }\end{array}$ & $\begin{array}{l}\text { Phenotype/ } \\
\text { cytokine } \\
\text { expression }\end{array}$ & Advantage & Disadvantage & Outcome & References \\
\hline & $\begin{array}{l}\text { Variety of } \\
\text { transgenic } \\
\text { KO mice } \\
\text { plus smoke } \\
\text { exposure } \\
\text { (MMP1,9,12, } \\
\text { TNFR } 1+2 \text { ) }\end{array}$ & $\begin{array}{l}\text { Relevance } \\
\text { of MMPs in } \\
\text { COPD devel- } \\
\text { opment }\end{array}$ & Various & $\begin{array}{l}\text { Examine } \\
\text { importance } \\
\text { of a single } \\
\text { chemokine } \\
\text { to COPD and } \\
\text { smoke-related } \\
\text { inflammation }\end{array}$ & $\begin{array}{l}\text { Difficult } \\
\text { technique } \\
\text { requiring } \\
\text { expertise. }\end{array}$ & $\begin{array}{l}\text { KO depend- } \\
\text { ent }\end{array}$ & 106,107 \\
\hline & $\begin{array}{l}\alpha 1-A T \text { "Pallid } \\
\text { mouse" }\end{array}$ & $\begin{array}{l}\alpha 1-A T \text { defi- } \\
\text { ciency } \\
\text { predisposes } \\
\text { to emphysema } \\
\text { in humans }\end{array}$ & $\begin{array}{l}\text { CD4+ cells } \\
\text { significantly } \\
\text { increased in tis- } \\
\text { sue }\end{array}$ & $\begin{array}{l}\text { Has human } \\
\text { corollary in } \alpha 1 \text { - } \\
\text { AT deficiency } \\
\text { phenotype }\end{array}$ & $\begin{array}{l}\text { Small minority } \\
\text { human COPD } \\
\text { due to } \alpha 1-A T \\
\text { deficiency }\end{array}$ & $\begin{array}{l}\text { Panlobular } \\
\text { emphysema }\end{array}$ & 106,107 \\
\hline & $\begin{array}{l}\text { Itgb6 null } \\
\text { mice }\end{array}$ & $\begin{array}{l}\text { Alteration } \\
\text { in TGF- } \beta \\
\text { responsive- } \\
\text { ness }\end{array}$ & $\begin{array}{l}\text { TGF- } \beta \text { deplete, } \\
\text { MMP12 over- } \\
\text { activity, age- } \\
\text { dependent } \\
\text { emphysema }\end{array}$ & $\begin{array}{l}\text { Chronic pro- } \\
\text { gressive model }\end{array}$ & Complex & $\begin{array}{l}\text { Age-depend- } \\
\text { ent emphy- } \\
\text { sema }\end{array}$ & 106,107 \\
\hline $\mathrm{CF}$ & $\begin{array}{l}\text { CFTR gene } \\
\text { knockouts } \\
\text { (various, } \\
\text { approx. } 11 \\
\text { models) }\end{array}$ & $\begin{array}{l}\text { CF single- } \\
\text { gene disease }\end{array}$ & $\begin{array}{l}\text { Failed muco- } \\
\text { ciliary clearance, } \\
\text { inflammatory } \\
\text { cell recruitment, } \\
\text { parenchymal } \\
\text { interstitial thicken- } \\
\text { ing, pseudomo- } \\
\text { nal susceptibility }\end{array}$ & $\begin{array}{l}\text { Multiple } \\
\text { phenotypes } \\
\text { generated by } \\
\text { different CFTR } \\
\text { mutations }\end{array}$ & $\begin{array}{l}\text { Phenotypes } \\
\text { not directly } \\
\text { applicable to } \\
\text { human } \\
\text { genotypes }\end{array}$ & Various & 91 \\
\hline $\begin{array}{l}\text { ALI/ } \\
\text { ARDS }\end{array}$ & Hyperoxia & $\begin{array}{l}\text { Exposure } \\
\text { to } 95 \% \mathrm{O}_{2}\end{array}$ & $\begin{array}{l}\text { TNF, IFN- } \gamma \text {, } \\
\text { ROS,IL-12, IL-18 }\end{array}$ & Quick & $\begin{array}{l}\mathrm{O}_{2} \text { chamber } \\
\text { required. }\end{array}$ & $\begin{array}{l}\text { Hyperoxic } \\
\text { lung injury }\end{array}$ & 108 \\
\hline \multirow[t]{3}{*}{. } & LPS IT & $\begin{array}{l}\text { Sepsis } \\
\text { associated } \\
\text { with ARDS }\end{array}$ & $\begin{array}{l}\text { ROS, NF-kB, } \\
\text { IL-6, IL-8 }\end{array}$ & $\begin{array}{l}\text { Widely } \\
\text { used, well- } \\
\text { characterized }\end{array}$ & Overly simplistic & $\begin{array}{l}\text { Model of } \\
\text { sepsis-related } \\
\text { ARDS/ALI }\end{array}$ & 109 \\
\hline & $\begin{array}{l}\text { Hemorrhage/ } \\
\text { resus lung } \\
\text { injury }\end{array}$ & $\begin{array}{l}\text { Venesection } \\
\text { to shock } \\
+ \text { - -resus }\end{array}$ & $\begin{array}{l}\text { CREB, ROS, } \\
\text { NF-kB, IL-6, IL-8 }\end{array}$ & $\begin{array}{l}\text { Models } \\
\text { clinical } \\
\text { events }\end{array}$ & $\begin{array}{l}\text { Technically } \\
\text { difficult }\end{array}$ & $\begin{array}{l}\text { Model of } \\
\text { traumatic } \\
\text { ARDS/ALI }\end{array}$ & 110,111 \\
\hline & Infective & $\begin{array}{l}\text { Respiratory } \\
\text { reovirus } 1 / \mathrm{L} \\
\text { induction of } \\
\text { diffuse alveolar } \\
\text { damage }\end{array}$ & $\begin{array}{l}\text { Overlapping } \\
\text { phases of exu- } \\
\text { dation includ- } \\
\text { ing hyaline } \\
\text { membranes, } \\
\text { regeneration, } \\
\text { and healing via } \\
\text { resolution and } \\
\text { or repair with } \\
\text { fibrosis. }\end{array}$ & $\begin{array}{l}\text { Fibro-repara- } \\
\text { tive phase } \\
\text { modeled as } \\
\text { well as initial } \\
\text { insult }\end{array}$ & $\begin{array}{l}\text { Technically } \\
\text { difficult }\end{array}$ & $\begin{array}{l}\text { Neutrophilic } \\
\text { inflammation }\end{array}$ & 112 \\
\hline
\end{tabular}

Abbreviations: ARDS/ALI, adult respiratory distress syndrome/acute lung injury; CF, cystic fibrosis; COPD, chronic obstructive pulmonary disease; IPF, idiopathic pulmonary fibrosis.

mucosa means that the mucociliary escalator is compromised by viscid mucus and that altered mucosal $\mathrm{pH}$ results in malfunction of host antimicrobial peptides. There may also be a failure of normal bacterial internalization processes and it has been suggested that an intrinsic proinflammatory phenotype is conferred by CFTR malfunction. The proinflammatory phenotype theory postulates that an overburdened endosomal system encumbered by the processing of faulty CFTR signals distress, which leads to activation of NF- $\mathrm{kB}$. Production of IL-8 is stimulated resulting in enhanced neutrophil recruitment. Regardless, the net result is a failure of mucosal immunity, and a succession of bacterial infections occur (initially, Staphylococcus aureus and/or Haemophilus influenzae but subsequently and devastatingly $P$. aeruginosa, Burkholderia cepacia, and Stenotrophomonas maltophilia). With bacterial colonization, innate defense is roused and a significant neutrophil influx occurs. This is usually thwarted, initially by microbial resistance and subsequently by distorted lung architecture. This results in an insurmountable accumulation of inflammatory cells and toxic damage from chemicals eluted by their necrotic carcasses. Elastase, reactive oxygen species, and myeloperoxidase cause further lung damage, and the clearance of inflammatory cells is so poor that 
the lungs literally become clogged with inflammatory cell DNA. Eventually, there is a terminal paucity of functional gas-exchange equipment, and respiratory failure and death supervene if lung transplant is not possible. ${ }^{88}$

Recent work on $P$. aeruginosa has provided an interesting insight into the mechanisms by which this organism thwarts immune defense. It was known that $P$. aeruginosa could effectively hide from the immune system in a protective and impenetrable bio-film. A more subtle effect mediated by pseudomonas produced pyocyanin has now been recognized. This chemical promotes neutrophil death but inhibits macrophage clearance resulting in neutrophil death by necrosis. This leads to the spillage of cathepsin $\mathrm{G}$ and elastase, which can cleave the important chemokine receptor CXCR1 on other neutrophils. This receptor would usually allow IL-8-mediated enhancement of neutrophil killing prowess, which enabling neutrophils to kill pseudomonas. In CF patients, neutrophils have little CXCR1 for the above reason and are consequently effectively crippled as well as out-maneuvered. Even more detrimental, fragments of this receptor stimulate the TLR system resulting in enhanced neutrophil recruitment and prolongation of the inflammatory response. ${ }^{89}$

Anti-inflammatory therapy in the form of non-steroidal anti-inflammatory drugs has been used to mild benefit in CF but it appears that the early introduction of a potent anti-inflammatory proresolution therapeutic strategy might be of enhanced benefit. Neutrophils in CF patients are dysfunctional and ineffective at restraining or removing typical CF pathogens, so driving their apoptosis and removal might be of more benefit than allowing them to remain in the hope that they are contributing to defense against these microbes. ${ }^{88}$

\section{ANIMAL MODELS OF GRANULOCYTE-MEDIATED PULMONARY INFLAMMATORY DISEASE}

There are many methods available for the production of relevant animal models of inflammatory disease. These include direct chemical/other injury, constitutive and inducible transgenics, viral vector delivery of relevant genes, adoptive cell transfer, and direct infection strategies. The advantage of the animal model is that it reproduces the complexity of the wider immune/inflammatory response on an organ or system-wide basis as opposed to cell-line strategies that can only examine individual cellular responses. A selection of key rodent models and their major advantages/disadvantages are shown in Table 1. A more comprehensive discussion of this field is not possible due to space limitations but there are excellent reviews available in this area. ${ }^{90-95}$ Clearly, reliable animal models are central to the development of efficacious and safe pharmaceutical agents that drive granulocyte apoptosis and clearance to enhance resolution of inflammation.

\section{CONCLUSION}

The respiratory mucosa is frequently threatened with microbial invasion because it is responsible for gas exchange. Consequently, it has developed a sophisticated, sensitive surveillance system and a rapid-response immune policing arm to ensure that it usually prevails in a given interaction. In inflammatory disease, this system is thwarted or dysfunctional with devastating results. The respiratory mucosal immune and inflammatory response can be volatile and damaging for lung function and architecture. To understand respiratory inflammatory disease, it is essential to fully appreciate the biology of archetypal inflammatory cells such as granulocytes. To drive granulocyte apoptosis and enhance granulocyte clearance is a research priority that will hopefully enable amelioration or cure of prevalent (and obscure) respiratory inflammatory conditions.

\section{ACKNOWLEDGMENTS}

We thank the Wellcome Trust (WT082181) and the Medical Research Council (G0601481) for their support of the work that has enabled us to contribute to this review, and our friends and colleagues within the field whose work constitutes the bulk of the information provided.

\section{REFERENCES}

1. Seaton, A., Seaton, D. \& Leitch, A.G. Crofton and Douglas's respiratory diseases. 5th edn, (Blackwell Science, 2000).

2. Rather, L.J. Disturbance of function (functio laesa): the legendary fifth cardinal sign of inflammation, added by Galen to the four cardinal signs of celsus. Bull NY Acad. Med. 47, 303-322 (1971).

3. Sabroe, I. \& Whyte, M.K. Toll-like receptor (TLR)-based networks regulate neutrophilic inflammation in respiratory disease. Biochem. Soc. Trans. 35, 1492-1495 (2007).

4. Rossi, A.G., Hallett, J.M., Sawatzky, D.A., Teixeira, M.M. \& Haslett, C. Modulation of granulocyte apoptosis can influence the resolution of inflammation. Biochem. Soc. Trans. 35, 288-291 (2007).

5. Serhan, C.N. et al. Resolution of inflammation: state of the art, definitions and terms. FASEB. J. 21, 325-332 (2007).

6. von Kockritz-Blickwede, M. et al. Phagocytosis-independent antimicrobial activity of mast cells by means of extracellular trap formation. Blood 111, 3070-3080 (2008).

7. Brinkmann, V. et al. Neutrophil extracellular traps kill bacteria. Science 303, 1532-1535 (2004).

8. Nathan, C. Neutrophils and immunity: challenges and opportunities. Nat. Rev. Immunol. 6, 173-182 (2006).

9. Holgate, S.T. The future of lung research in the UK. Thorax 62 , 1028-1032 (2007).

10. British Thoracic Society. Health Select Committee inquiry into Health InequalitiesSubmission from the British Thoracic Society-08.01.08 (2008).

11. Lachmann, P.J. Microbial subversion of the immune response. Proc. Natl. Acad. Sci. USA. 99, 8461-8462 (2002).

12. Prince, L.R. et al. Subversion of a lysosomal pathway regulating neutrophil apoptosis by a major bacterial toxin, pyocyanin. J. Immunol. 180, 3502-3511 (2008)

13. Haslett, C. Granulocyte apoptosis and its role in the resolution and control of lung inflammation. Am. J. Respir. Crit. Care. Med. 160, S5-11 (1999).

14. Sasmono, R.T. et al. Mouse neutrophilic granulocytes express mRNA encoding the macrophage colony-stimulating factor receptor (CSF-1R) as well as many other macrophage-specific transcripts and can transdifferentiate into macrophages in vitro in response to CSF-1. J. Leukoc. Biol. 82, 111-123 (2007).

15. Araki, H. et al. Reprogramming of human postmitotic neutrophils into macrophages by growth factors. Blood 103, 2973-2980 (2004).

16. Opferman, J.T. Life and death during hematopoietic differentiation. Curr. Opin. Immunol. 19, 497-502 (2007).

17. Rossi, A.G. et al, Cyclin-dependent kinase inhibitors enhance the resolution of inflammation by promoting inflammatory cell apoptosis. Nat. Med. 12, 1056-1064 (2006).

18. Sundaramurthy, V. \& Pieters, J. Interactions of pathogenic mycobacteria with host macrophages. Microbes Infect. 9, 1671-1679 (2007).

19. Brinkmann, V. \& Zychlinsky, A. Beneficial suicide: why neutrophils die to make NETs. Nat. Rev. Microbiol. 5, 577-582 (2007).

20. Rothenberg, M.E. \& Hogan, S.P. The eosinophil. Annu. Rev. Immunol. $24,147-174(2006)$ 
21. Perretti, M. \& Solito, E. Annexin 1 and neutrophil apoptosis. Biochem. Soc. Trans. 32, 507-510 (2004).

22. Scannell, M. et al. Annexin-1 and peptide derivatives are released by apoptotic cells and stimulate phagocytosis of apoptotic neutrophils by macrophages. J. Immunol. 178, 4595-4605 (2007).

23. Bianchi, S.M., Dockrell, D.H., Renshaw, S.A., Sabroe, I. \& Whyte, M.K. Granulocyte apoptosis in the pathogenesis and resolution of lung disease. Clin. Sci. 110, 293-304 (2006).

24. Maianski, N.A., Roos, D. \& Kuijpers, T.W. Tumor necrosis factor alpha induces a caspase-independent death pathway in human neutrophils. Blood 101, 1987-1995 (2003).

25. Simon, H.U. Neutrophil apoptosis pathways and their modifications in inflammation. Immunol. Rev. 193, 101-110 (2003).

26. Cowburn, A.S., White, J.F., Deighton, J., Walmsley, S.R. \& Chilvers, E.R. z-VAD-fmk augmentation of TNF alpha-stimulated neutrophil apoptosis is compound specific and does not involve the generation of reactive oxygen species. Blood 105, 2970-2972 (2005).

27. Simon, H.U. Regulation of eosinophil and neutrophil apoptosissimilarities and differences. Immunol. Rev. 179, 156-162 (2001).

28. Simon, H.U. Molecules involved in the regulation of eosinophil apoptosis. Chem. Immunol. Allergy 91, 49-58 (2006).

29. Simon, H. \& Alam, R. Regulation of eosinophil apoptosis: transduction of survival and death signals. Int. Arch. Allergy Immunol. 118, 7-14 (1999).

30. Daigle, I. \& Simon, H.U. Critical role for caspases 3 and 8 in neutrophil but not eosinophil apoptosis. Int. Arch. Allergy Immunol. 126, 147-156 (2001).

31. Ochiai, K., Kagami, M., Matsumura, R. \& Tomioka, H. IL-5 but not interferon-gamma (IFN-gamma) inhibits eosinophil apoptosis by up-regulation of bcl-2 expression. Clin. Exp. Immunol. 107, 198-204 (1997).

32. Weinmann, P., Gaehtgens, P. \& Walzog, B. Bcl-Xl-and Bax-alphamediated regulation of apoptosis of human neutrophils via caspase- 3 . Blood 93, 3106-3115 (1999).

33. Dzhagalov, I., St John, A. \& He, Y.W. The antiapoptotic protein Mcl-1 is essential for the survival of neutrophils but not macrophages. Blood 109, 1620-1626 (2007).

34. Hamasaki, A. et al. Accelerated neutrophil apoptosis in mice lacking A1-a, a subtype of the bcl-2-related A1 gene. J. Exp. Med. 188, 1985-1992 (1998)

35. Sabroe, I., Dower, S.K. \& Whyte, M.K. The role of Toll-like receptors in the regulation of neutrophil migration, activation, apoptosis. Clin. Infect. Dis. 41 (Suppl 7), S421-S426 (2005).

36. Ward, C. et al. NF-kappaB activation is a critical regulator of human granulocyte apoptosis in vitro. J. Biol. Chem. 274, 4309-4318 (1999).

37. Lawrence, T., Gilroy, D.W., Colville-Nash, P.R. \& Willoughby, D.A. Possible new role for NF-kappaB in the resolution of inflammation. Nat. Med. 7, 1291-1297 (2001).

38. Pinho, V. et al. Phosphoinositide-3 kinases critically regulate the recruitment and survival of eosinophils in vivo: importance for the resolution of allergic inflammation. J. Leukoc. Biol. 77, 800-810 (2005).

39. Pinho, V. et al. Tissue- and stimulus-dependent role of phosphatidylinositol 3-kinase isoforms for neutrophil recruitment induced by chemoattractants in vivo. J. Immunol. 179, 7891-7898 (2007).

40. Savill, J. \& Haslett, C. Granulocyte clearance by apoptosis in the resolution of inflammation. Semin. Cell. Biol. 6, 385-393 (1995).

41. Sivertson, K.L., Seeds, M.C., Long, D.L., Peachman, K.K. \& Bass, D.A. The differential effect of dexamethasone on granulocyte apoptosis involves stabilization of $\mathrm{Mcl}-1 \mathrm{~L}$ in neutrophils but not in eosinophils. Cell. Immunol. 246, 34-45 (2007).

42. Heasman, S.J., Giles, K.M., Ward, C., Rossi, A.G., Haslett, C. \& Dransfield, I. Glucocorticoid-mediated regulation of granulocyte apoptosis and macrophage phagocytosis of apoptotic cells: implications for the resolution of inflammation. J. Endocrinol. 178, 29-36 (2003).

43. Serhan, C.N. Resolution phase of inflammation: novel endogenous anti-inflammatory and proresolving lipid mediators and pathways. Annu. Rev. Immunol. 25, 101-137 (2007).

44. Hallett, J.M., Leitch, A.E., Riley, N.A., Duffin, R., Haslett, C. \& Rossi, A.G. Novel pharmacological strategies for driving inflammatory cell apoptosis and enhancing the resolution of inflammation. Trends Pharmacol. Sci. $29,250-257$ (2008).
45. Kobayashi, N. et al. TIM-1 and TIM-4 glycoproteins bind phosphatidylserine and mediate uptake of apoptotic cells. Immunity 27, 927-940 (2007).

46. Park, S.Y. et al. Rapid cell corpse clearance by stabilin-2, a membrane phosphatidylserine receptor. Cell Death Differ. 15, 192-201 (2008).

47. Park, D. et al. BAl1 is an engulfment receptor for apoptotic cells upstream of the ELMO/Dock180/Rac module. Nature 450, 430-434 (2007).

48. Fadok, V.A., Bratton, D.L., Konowal, A., Freed, P.W., Westcott, J.Y. \& Henson, P.M. Macrophages that have ingested apoptotic cells in vitro inhibit proinflammatory cytokine production through autocrine/paracrine mechanisms involving TGF-beta, PGE2, PAF. J. Clin. Invest. 101, 890-898 (1998).

49. Barnes, P.J. Alveolar macrophages in chronic obstructive pulmonary disease (COPD). Cell. Mol. Biol. 50, Online Pub:OL627-OL637 (2004).

50. Barnes, P.J. New molecular targets for the treatment of neutrophilic diseases. J. Allergy Clin. Immunol. 119, 1055-1062 (2007).

51. MacNee, W. Pathogenesis of chronic obstructive pulmonary disease. Clin. Chest Med. 28, 479-513 (2007).

52. Kirkham, P.A., Spooner, G., Rahman, I. \& Rossi, A.G. Macrophage phagocytosis of apoptotic neutrophils is compromised by matrix proteins modified by cigarette smoke and lipid peroxidation products. Biochem. Biophys. Res. Commun. 318, 32-37 (2004)

53. Barnes, P.J., Ito, K. \& Adcock, I.M. Corticosteroid resistance in chronic obstructive pulmonary disease: inactivation of histone deacetylase. Lancet 363, 731-733 (2004).

54. Quint, J.K. \& Wedzicha, J.A. The neutrophil in chronic obstructive pulmonary disease. J. Allergy Clin. Immunol. 119, 1065-1071 (2007)

55. Barnes, P.J. Pathophysiology of asthma. Br. J. Clin. Pharmacol. 42, 3-10 (1996).

56. Busse, W.W., Banks-Schlegel, S. \& Wenzel, S.E. Pathophysiology of severe asthma. J. Allergy Clini. Immunol. 106, 1033-1042 (2000).

57. Hanania, N.A. Targeting airway inflammation in asthma: current and future therapies. Chest 133, 989-998 (2008).

58. Holgate, S.T. et al. Understanding the pathophysiology of severe asthma to generate new therapeutic opportunities. J. Allergy Clin. Immunol. 117, 496-506 (2006).

59. Maddox, L. \& Schwartz, D.A. The pathophysiology of asthma. Annu. Rev. Med. 53, 477-498 (2002).

60. Walsh, G.M., Sexton, D.W. \& Blaylock, M.G. Corticosteroids, eosinophils and bronchial epithelial cells: new insights into the resolution of inflammation in asthma. J. Endocrinol. 178, 37-43 (2003).

61. Contoli, M. et al. Role of deficient type Ill interferon-[lambda] production in asthma exacerbations. Nat. Med. 12, 1023-1026 (2006).

62. Uddin, M., Seumois, G., Lau, L.C., Rytila, P., Davies, D.E. \& Djukanovic, R. Enhancement of neutrophil function by the bronchial epithelium stimulated by epidermal growth factor. Eur. Respir. J. 31, 714-724 (2008).

63. Flood-Page, P. et al. Anti-IL-5 treatment reduces deposition of ECM proteins in the bronchial subepithelial basement membrane of mild atopic asthmatics. J. Clin. Invest. 112, 1029-1036 (2003).

64. Flood-Page, P.T., Menzies-Gow, A.N., Kay, A.B. \& Robinson, D.S Eosinophil's role remains uncertain as anti-interleukin- 5 only partially depletes numbers in asthmatic airway. Am. J. Respir. Crit. Care. Med. 167, 199-204 (2003)

65. Wenzel, S., Wilbraham, D., Fuller, R., Getz, E.B. \& Longphre, M. Effect of an interleukin- 4 variant on late phase asthmatic response to allergen challenge in asthmatic patients: results of two phase 2 a studies. Lancet 370, 1422-1431 (2007).

66. Bullens, D.M. et al. IL-17 mRNA in sputum of asthmatic patients: linking T cell driven inflammation and granulocytic influx? Respir. Res. 7, 135-13 (2006).

67. Meagher, L.C., Cousin, J.M., Seckl, J.R. \& Haslett, C. Opposing effects of glucocorticoids on the rate of apoptosis in neutrophilic and eosinophilic granulocytes. J. Immunol. 156, 4422-4428 (1996).

68. Wenzel, S.E. A different disease, many diseases or mild asthma gone bad? Challenges of severe asthma. Eur. Respir. J. 22, 397-398 (2003).

69. Collard, H.R. et al. Acute exacerbations of idiopathic pulmonary fibrosis. Am. J. Respir. Crit. Care. Med. 176, 636-643 (2007).

70. Du Bois, R. \& King, T.E. Jr Challenges in pulmonary fibrosis $\times 5$ : the NSIP/NIP debate. Thorax 62, 1008-1012 (2007). 
71. Hunninghake, G.W. \& Schwarz, M.I. State of the Art. Does current knowledge explain the pathogenesis of idiopathic pulmonary fibrosis? a perspective. Proc. Am. Thorac. Soc. 4, 449-452 (2007).

72. Wallace, W.A., Fitch, P.M., Simpson, A.J. \& Howie, S.E. Inflammationassociated remodelling and fibrosis in the lung - a process and an end point. Int. J. Exp. Pathol. 88, 103-110 (2007).

73. Strieter, R.M. What differentiates normal lung repair and fibrosis? Inflammation, resolution of repair, fibrosis. Proc. Am. Thorac. Soc. 5 , 305-310 (2008).

74. Chua, F. et al. Mice lacking neutrophil elastase are resistant to bleomycin-induced pulmonary fibrosis. Am. J. Pathol. 170, 65-74 (2007).

75. Kinder, B.W., Brown, K.K., Schwarz, M.I., Ix, J.H., Kenvitsky, A. \& King, T.E. Jr Baseline BAL neutrophilia predicts early mortality in idiopathic pulmonary fibrosis. Chest 133, 226-232 (2008).

76. Abraham, E. Neutrophils and acute lung injury. Crit. Care. Med. 31 , S195-S199 (2003).

77. Bellingan, G.J. The pulmonary physician in critical care * 6 : The pathogenesis of ALI/ARDS. Thorax 57, 540-546 (2002).

78. Leaver, S.K. \& Evans, T.W. Acute respiratory distress syndrome. BMJ 335, 389-394 (2007)

79. Martin, T.R. Interactions between mechanical and biological processes in acute lung injury. Proc. Am. Thorac. Soc. 5, 291-296 (2008).

80. Matthay, M.A. \& Zimmerman, G.A. Acute lung injury and the acute respiratory distress syndrome: four decades of inquiry into pathogenesis and rational management. Am. J. Respir. Cell. Mol. Biol. 33, 319-327 (2005).

81. Donnelly, S.C. et al. Interleukin-8 and development of adult respiratory distress syndrome in at-risk patient groups. Lancet 341, 643-647 (1993).

82. Oeckler, R.A. \& Hubmayr, R.D. Ventilator-associated lung injury: a search for better therapeutic targets. Eur. Respir. J. 30, 1216-1226 (2007).

83. British Thoracic Society. BTS Guidelines for the Management of Community Acquired Pneumonia in Adults. Thorax 56 (Suppl 4), IV1-I64 (2001).

84. Droemann, D. et al. Decreased apoptosis and increased activation of alveolar neutrophils in bacterial pneumonia. Chest 117, 1679-1684 (2000).

85. Watson, R.W., Redmond, H.P., Wang, J.H., Condron, C. \& BouchierHayes, D. Neutrophils undergo apoptosis following ingestion of Escherichia coli. J. Immunol. 156, 3986-3992 (1996).

86. Cazzola, M., Matera, M.G. \& Pezzuto, G. Inflammation--a new therapeutic target in pneumonia. Respiration 72, 117-126 (2005).

87. Confalonieri, M. et al. Hydrocortisone infusion for severe communityacquired pneumonia: a preliminary randomized study. Am. J. Respir. Crit. Care. Med. 171, 242-248 (2005).

88. Elizur, A., Cannon, C.L. \& Ferkol, T.W. Airway inflammation in cystic fibrosis. Chest 133, 489-495 (2008).

89. Sabroe, I. \& Whyte, M.K. Incapacitating the immune system in cystic fibrosis. Nat. Med. 13, 1417-1418 (2007)

90. Brodmerkel, C.M. \& Vaddi, K. Transgenic animals in inflammatory disease models. Curr. Opin. Biotechnol. 14, 652-658 (2003).

91. Guilbault, C., Saeed, Z., Downey, G.P. \& Radzioch, D. Cystic fibrosis mouse models. Am. J. Respir. Cell. Mol. Biol. 36, 1-7 (2007).

92. Kips, J.C. et al. Murine models of asthma. Eur. Respir. J. 22, 374-382 (2003).
93. Moeller, A. et al. Models of pulmonary fibrosis. Drug Discov. Today Dis. Models 3, 243-249 (2006).

94. Moore, B.B. \& Hogaboam, C.M. Murine models of pulmonary fibrosis. Am. J. Physiol. Lung Cell. Mol. Physiol. 294, L152-L160 (2008).

95. Renshaw, S.A., Loynes, C.A., Elworthy, S., Ingham, P.W. \& Whyte, M.K. Modeling inflammation in the zebrafish: how a fish can help us understand lung disease. Exp. Lung Res. 33, 549-554 (2007).

96. Harrison, J.H. Jr \& Lazo, J.S. High dose continuous infusion of bleomycin in mice: a new model for drug-induced pulmonary fibrosis. J. Pharmacol. Exp. Ther. 243, 1185-1194 (1987).

97. Moeller, A., Ask, K., Warburton, D., Gauldie, J. \& Kolb, M. The bleomycin animal model: a useful tool to investigate treatment options for idiopathic pulmonary fibrosis? Int. J. Biochem. Cell. Biol, 40, 362-382 (2008).

98. Hardie, W.D., Le Cras, T.D., Jiang, K., Tichelaar, J.W., Azhar, M. \& Korfhagen, T.R. Conditional expression of transforming growth factoralpha in adult mouse lung causes pulmonary fibrosis. Am. J. Physiol. Lung. Cell. Mol. Physiol. 286, L741-L749 (2004).

99. Sime, P.J., Xing, Z., Graham, F.L., Csaky, K.G. \& Gauldie, J. Adenovector-mediated gene transfer of active transforming growth factor-beta 1 induces prolonged severe fibrosis in rat lung. J. Clin. Investig. 100, 768-776 (1997).

100. Xing, Z. et al. Gene transfer for cytokine functional studies in the lung: the multifunctional role of GM-CSF in pulmonary inflammation. J. Leukoc. Biol. 59, 481-488 (1996).

101. Kolb, M., Margetts, P.J., Anthony, D.C., Pitossi, F. \& Gauldie, J. Transient expression of IL-1 beta induces acute lung injury and chronic repair leading to pulmonary fibrosis. J. Clin. Investig. 107, 1529-1536 (2001).

102. Miyazaki, Y. et al. Expression of a tumor-necrosis-factor-alpha transgene in murine lung causes lymphocytic and fibrosing alveolitis - a mouse model of progressive pulmonary fibrosis. J. Clin. Investig. 96, 250-259 (1995).

103. Elias, J.A. et al. Transgenic modeling of interleukin-13 in the lung. Chest 123, 339S-345S (2003).

104. Zosky, G.R. \& Sly, P.D. Animal models of asthma. Clin. Exp. Allergy 37, 973-988 (2007).

105. Churg, A., Cosio, M. \& Wright, J.L. Mechanisms of cigarette smokeinduced COPD: insights from animal models. Am. J. Physiol. Lung. Cell. Mol. Physiol. 294, L612-L631 (2008).

106. Fujita, M. \& Nakanishi, Y. The pathogenesis of COPD: lessons learned from in vivo animal models. Med. Sci. Monit. 13, RA19-RA24 (2007).

107. Shapiro, S.D. Transgenic and gene-targeted mice as models for chronic obstructive pulmonary disease. Eur. Respir. J. 29, 375-378 (2007)

108. Dos Santos, C.C. Hyperoxic acute lung injury and ventilator-induced/ associated lung injury: new insights into intracellular signaling pathways. Crit. Care. 11, 126-12 (2007).

109. Kabir, K. et al. Characterization of a murine model of endotoxin-induced acute lung injury. Shock 17, 300-303 (2002).

110. Moine, P., Shenkar, R., Kaneko, D., Le Tulzo, Y. \& Abraham, E. Systemic blood loss affects NF-kappa B regulatory mechanisms in the lungs. Am. J. Physiol. Lung. Cell. Mol. Physiol. 273, L185-L192 (1997).

111. Bahrami, S. et al. Small-volume fluid resuscitation with hypertonic saline prevents inflammation but not mortality in a rat model of hemorrhagic shock. Shock. 25, 283-289 (2006).

112. London, L., Majeski, E.I., Paintlia, M.K., Harley, R.A. \& London, S.D. Respiratory reovirus $1 / \mathrm{L}$ induction of diffuse alveolar damage: a model of acute respiratory distress syndrome. Exp. Mol. Pathol. 72, 24-36 (2002). 


\title{
THEMED SECTION: MEDIATORS AND RECEPTORS IN THE RESOLUTION OF INFLAMMATION
}

\section{REVIEW}

\section{Cyclin-dependent kinase inhibitor drugs as potential novel anti-inflammatory and pro-resolution agents}

\author{
AE Leitch, C Haslett and AG Rossi \\ MRC Centre for Inflammation Research, The Queen's Medical Research Institute, University of Edinburgh Medical School, 47 \\ Little France Crescent, Edinburgh, UK
}

\begin{abstract}
The cyclin-dependent kinase inhibitor (CDKi) drugs such as R-roscovitine have emerged as potential anti-inflammatory, pharmacological agents that can influence the resolution of inflammation. Usually, once an inciting inflammatory stimulus has been eliminated, resolution proceeds by prompt, safe removal of dominant inflammatory cells. This is accomplished by programmed cell death (apoptosis) of prominent effector, inflammatory cells typified by the neutrophil. Apoptosis of neutrophils ensures that toxic neutrophil granule contents are securely packaged in apoptotic bodies and expedites phagocytosis by professional phagocytes such as macrophages. A panel of CDKi drugs have been shown to promote neutrophil apoptosis in a concentration- and time-dependent manner and the archetypal CDKi drug, R-roscovitine, overrides the anti-apoptotic effects of powerful survival factors [including lipopolysaccharide (LPS) and granulocyte macrophage-colony stimulating factor (GMCSF)]. Inflammatory cell longevity and survival signalling is integral to the inflammatory process and any putative antiinflammatory agent must unravel a complex web of redundancy in order to be effective. CDKi drugs have also been demonstrated to have significant effects on other cell types including lymphocytes and fibroblasts indicating that they may have pleiotropic anti-inflammatory, pro-resolution activity. In keeping with this, CDKi drugs like R-roscovitine have been reported to be efficacious in resolving established animal models of neutrophil-dominant and lymphocyte-driven inflammation. However, the mechanism of action behind these powerful effects has not yet been fully elucidated. CDKs play an integral role in the regulation of the cell cycle but are also recognized as participants in processes such as apoptosis and transcriptional regulation. Neutrophils have functional CDKs, are transcriptionally active and demonstrate augmented apoptosis in response to CDKi drugs, while lymphocyte proliferation and secretory function are inhibited. This review will discuss current understanding of the processes of inflammation and resolution but will focus on CDKis and their potential mechanisms of action.
\end{abstract}

British Journal of Pharmacology (2009) 158, 1004-1016; doi:10.1111/j.1476-5381.2009.00402.x; published online 23 September 2009

This article is part of a themed issue on Mediators and Receptors in the Resolution of Inflammation. To view this issue visit http://www3.interscience. wiley.com/journal/121548564/issueyear?year=2009

Keywords: cyclin-dependent kinase; resolution of Inflammation; apoptosis; neutrophil; anti-inflammatory drugs; R-roscovitine; transcriptional regulation

Abbreviations: AA, arachidonic acid; CDKi, cyclin-dependent kinase inhibitor; COX, cyclo-oxygenase; CTD, C-terminal domain; ERK, extracellular regulated kinase; GM-CSF, granulocyte macrophage-colony stimulating factor; IL, interleukin; JAK, Jun N-terminal Kinase; LPS, lipopolysaccharide; MAPK, mitogen activated protein kinase; MCl-1, myeloid cell leukaemia-1; MCP-1, monocyte chemoattractant protein-1; MMP-3, matrix metalloproteinase-3; NF-kB, nuclear factor-kappaB; NSAIDs, non-steroidal anti-inflammatory drugs; PCNA, proliferating cell nuclear antigen; PG, prostaglandin; PI3K, phosphoinositide-3-kinase; RNA Pol II, RNA polymerase II; siRNA, small interfering RNA; STAT, signal transducers and activators of transcription; TGF- $\beta$, transforming growth factor beta; TNF- $\alpha$, tumour necrosis factor alpha; TXA $A_{2}$, thromboxane $A_{2}$; VEGF, vascular endothelial growth factor; zVAD-fmk, Z-Val-Ala-Asp(OMe)-CH2F 


\section{Introduction}

Cyclin-dependent kinase inhibitor (CDKi) drugs

CDKi drugs such as R-roscovitine have emerged as potential, anti-inflammatory agents that augment neutrophil apoptosis (Rossi et al., 2006) and suppress lymphocyte proliferation and secretory function (Obligado et al., 2008). This former finding seems counter-intuitive because the neutrophil is a terminally differentiated cell and its cell cycle machinery ought to be effectively vestigial. Further research is required before we develop a functional understanding of the mechanism of action of CDKi drugs in inflammation, but already, some tantalizing clues are emerging. This review will provide background material on inflammation research in general but will focus on the CDKs, their inhibitors, the role of CDK inhibition in inflammation and areas for further research. We anticipate that CDK inhibition will provide the basis for the development of novel therapeutic agents that drive resolution of inflammation and counter inflammatory disease.

\section{Inflammation}

Inflammation is part of the beneficial anti-microbial, immune defence system that has been honed and conserved by evolution over millions of years (Marchalonis et al., 2002). The system has become increasingly sophisticated because of the breadth of micro-organisms the human body has encountered and because of the mechanisms these organisms have evolved to enable evasion of its front-line defences. Ideally, following prompt detection of a micro-organism by immune mechanisms, an inflammatory reaction should contain and destroy the organism before it multiplies, spreads, becomes established or causes harm. Self-regulation and limitation are the key, final components of the response as the system must actively drive resolution of inflammation to restore tissue homeostasis (Lawrence and Gilroy, 2007).

Granulocytes are the foot soldiers of the inflammatory response and are dispatched in large numbers to overcome many challenges to the host organism. Neutrophils and eosinophils are leucocytes of the granulocyte lineage that are key players in the immune response to bacteria, fungi and parasites. They are attracted by, and are believed to follow a concentration gradient of, chemotactic stimuli released by invading pathogens or tissues under challenge. They migrate from the circulation across post-capillary venule endothelial cells (or capillary endothelial cells in pulmonary inflammation) (Downey et al., 1993) and employ a formidable armamentarium to overcome their adversaries. Granulocytes are named for the numerous granules within their cytoplasm (e.g. neutrophilic granulocytes contain at least four different types of granules). Each of these granules contains a range of toxic products such as proteases, lysozyme and lactoferrin. Eosinophils have an armament (including major basic protein, eosi-

Correspondence: Professor Adriano G. Rossi, MRC Centre for Inflammation Research, The Queen's Medical Research Institute, University of Edinburgh Medical School, 47 Little France Crescent, Edinburgh. E-mail: a.g.rossi@ ed.ac.uk

Received 6 April 2009; accepted 29 April 2009 nophil cationic protein and eosinophil peroxidase) more specific to the killing and digestion of parasites but, in asthma, respond to allergens or airway irritants in a concerted reaction which involves IgE, mast cells, lymphocytes, basophils (the third member of the granulocyte lineage) and smooth muscle. Neutrophils can either phagocytose an offending organism and then drown it in toxic chemicals (e.g. granule products and reactive oxygen species (ROS) such as superoxide anion) within the phagolysosome or, particularly when frustrated by an indigestible opponent, disgorge the same array of chemicals in the general direction of the target (exocytosis). A fascinating recent development has been the identification of neutrophil extracellular traps (Brinkmann and Zychlinsky, 2007; Fuchs et al., 2007). It appears that a proportion of activated neutrophils can extrude web-like fibres of granule constituents and chromatin in order to ensnare, disarm and kill invading pathogens. This appears to be a terminal event for the neutrophil occurring coincidentally with cell membrane rupture and death. Similarly, eosinophils can catapult (by an unidentified but ROS-independent mechanism) mitochondrial DNA which is also loxic to various pathogens but does not result in eosinophil death (Yousefi et al., 2008). In this review, we will focus on the effects of CDKi drugs on neutrophil granulocytes and, to a lesser degree, lymphocytes.

Assuming the influx of neutrophils is successful in overcoming a threat, the next stage is to remove the invading organisms, the neutrophils themselves (alive, dying and dead) and other cellular debris from the inflammatory site. Neutrophils are short-lived cells that leave the circulation within hours but can be influenced by survival factors present at inflammatory sites to extend their longevity to days. During neutrophil apoptosis (Figure 1), cell membrane integrity is maintained (ensuring toxic granule contents are secured) and specific phospholipid or glycoprotein signals are displayed on the outer membrane that are recognized by professional phagocytes. Macrophages, the most common professional phagocyte, are triggered by these signals to engulf the apoptotic neutrophil (Savill et al., 1989; Haslett et al., 1990). Following engulfment, macrophages express a pro-resolution phenotype which allows them to switch from secretion of pro-inflammatory stimuli such as TNF- $\alpha$ to tissue repair mediators such as TGF- $\beta$ and IL-10 (Fadok et al., 1998; Girkontaite et al., 2007). Additionally, lymphocytes of the adaptive immune response which had previously served to focus (via B-cell-mediated antibody opsonization of non-self antigens) and amplify (via T-cell-mediated interferon- $\gamma$ secretion leading to macrophage activation) the inflammatory reaction now aid in resolution as interferon- $\gamma$ dampens down the production of pro-inflammatory chemokines by macrophages (Schultz, 1987; Chung, 2001; Ozato et al., 2002). Streptococcal lobar pneumonia can be paradigmatic of a beneficial inflammatory response as despite a massive, neutrophildominant inflammatory reaction, resolution of inflammation occurs (and did so in the majority of cases in the preantibiotic era) with no pathological damage (Haslett, 1999).

The common alternative to granulocyte apoptosis is necrosis where membrane integrity is lost and cell contents are free to spill out into the tissues, initiating all the damaging sequelae associated with chronic inflammatory and autoimmune disease. This may occur where neutrophils are 

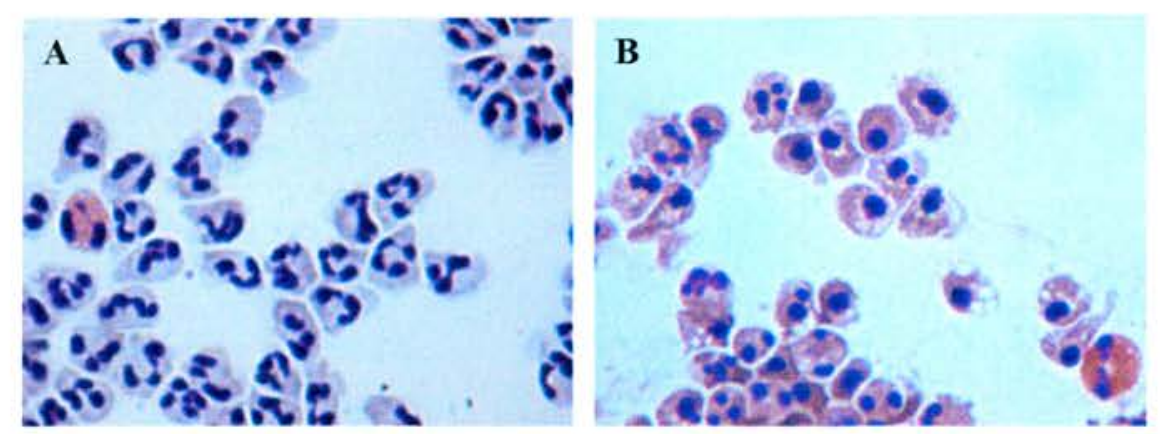

Figure 1 In (A), untreated neutrophils cultured for $8 \mathrm{~h}$ (and one eosinophil) are shown at $\times 200$ magnification displaying typical viable neutrophil morphology including polymorphic, multilobed nuclei and pale cytoplasm. In (B), neutrophils (and one eosinophil) treated with the CDK inhibitor R-roscovitine for $8 \mathrm{~h}$ are shown at $200 \times$ magnification displaying typical apoptotic morphology including condensed, rounded nuclei, darker cytoplasm and blebbing.
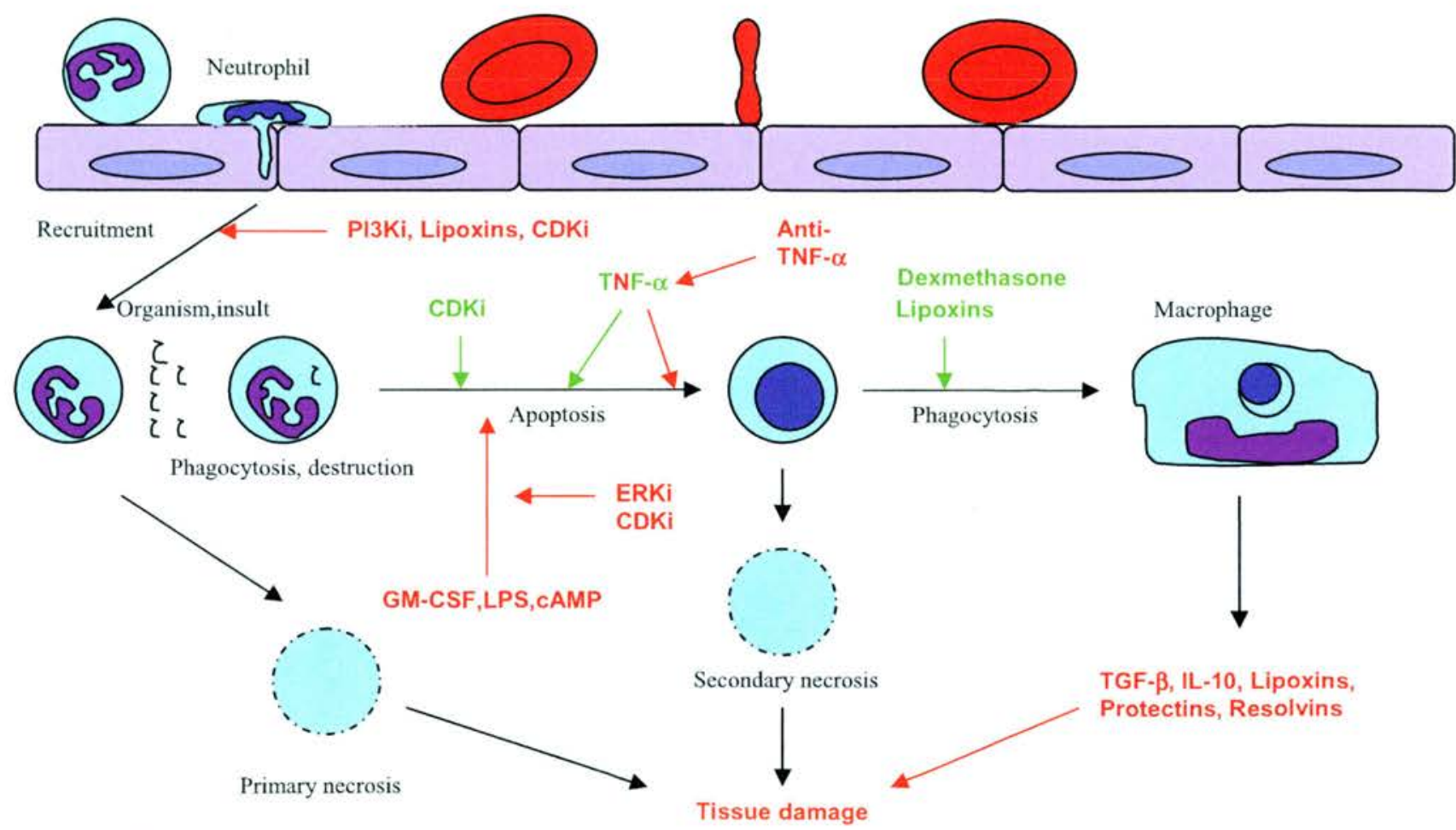

Figure 2 The effects of pro-resolution of inflammation agents on chronic inflammation.

overproduced, over-recruited, have inappropriate longevity or are inadequately cleared. It has been a central tenet of our research that an ideal anti-inflammatory, pro-resolution of inflammation pharmacological agent should drive neutrophil apoptosis without overburdening, or causing detriment to, the ability of professional phagocytes to phagocytose and remove apoptotic neutrophils (Figure 2). There is now abundant evidence that inflammatory cell- (especially neutrophil) programmed cell death followed by non-phlogistic clearance of apoptotic cells by phagocytes such as macrophages plays a key role in ensuring efficient (and in the majority complete) resolution of inflammation (Hallett et al., 2008; Leitch et al., 2008). It is clear that resolution of inflammation may also be driven by strategies that prevent excessive amplification and prolongation of inflammation by the adaptive immune response and allow stop signals and pro-resolution signals to predominate.

\section{Inflammatory disease}

Inflammatory diseases arise for a number of reasons; some of which are not fully understood. If the inflammatory system cannot destroy organisms or foreign particles (such as asbestos fibre and silica), it malfunctions, initiating excessive or prolonged inflammatory reactions which are likely to lead to irreversible fibrosis and scarring. Alternatively, reactions may be sited where none are apparently needed or self-antigens 
become recognized as foreign. In these settings, chronic inflammatory and autoimmune disease may supervene. The outcome of excessive or persistent (chronic) inflammation is tissue damage caused by the secretion or loss of histotoxic products from overactivated, under-regulated or necrotic inflammatory cells (Rossi et al., 2007). Autoimmune disease is thought to occur by a combination of inappropriate, excessive proliferation and activation of B and T-lymphocytes and the generation of autoantibodies to endogenous antigens. It is possible that endogenous antigens externalized on apoptotic cells that fail to be efficiently cleared contribute to this mechanism (Munoz et al., 2005). The spectrum of inflammatory disease includes all the major organ systems and, increasingly, we are recognizing a role for inflammation in other disease processes such as cancer (Coussens and Werb, 2002), neurological disorders (Gilgun-Sherki et al., 2006), atheroma (Hansson et al., 2006) and menstrual dysfunction (Critchley et al., 2001). There are many similarities between inflammatory diseases but also important differences which may occur even within the same organ system. For example, in lung-based disease, inflammation in asthma is usually eosinophil dominant (although neutrophil-dominant sub-types exist), while in chronic obstructive pulmonary disease (COPD) and idiopathic pulmonary fibrosis, arguably, neutrophils play a major role, although a plethora of other inflammatory cells have been implicated (Bianchi et al., 2006).

There are always competing hypotheses with regard to putative key cellular determinants of given diseases and it is, perhaps, therefore important (therapeutically, if less helpful scientifically) that novel pro-resolution of inflammation therapies should have pleiotropic actions across the different inflammatory cell populations. Work with CDKi drugs, so far, indicates that they influence not only neutrophils but also lymphocytes, suggesting that they may have a role to play in the management of a number of inflammatory diseases.

\section{Current anti-inflammatory therapies}

Many inflammatory diseases are treated with glucocorticosteroid drugs either independently or in combination regimens. Glucocorticoids are very successful at treating eosinophil-dominant inflammatory disease such as asthma, while they have had more modest success with neutrophildominant diseases such as COPD. It is now known that glucocorticoids promote eosinophil apoptosis but actually prolong neutrophil survival (Meagher et al., 1996). The latter effect is, presumably, partially compensated by glucocorticoid-mediated, enhanced macrophage phagocytosis of apoptotic neutrophils (Liu et al., 1999; Heasman et al., 2003; Michlewska et al., 2009). The development of nonsteroidal anti-inflammatory drugs (NSAIDs) and subsequently cyclo-oxygenase (COX)-2- specific NSAIDs demonstrates further the difficulties associated with combating a wellestablished, integral host response such as inflammation. NSAIDs work mainly by inhibiting the COX enzymes 1 and 2 . COX-1 is constitutively expressed in all tissues, while COX-2 is largely an inducible enzyme subject to various regulatory factors. These enzymes convert arachidonic acid to prostaglandin (PG) $-\mathrm{H}_{2}$ which can be converted by isomerase enzymes to: $\mathrm{PGE}_{2}, \mathrm{PGF}_{20}, \mathrm{PGD}_{2}$, prostacyclin $\left(\mathrm{PGI}_{2}\right)$, and thromboxane (TX) $A_{2}$. PGE 2 is responsible for some of the classic features of inflammation. Traditional NSAIDs inhibited COX-1 and -2 resulting in side effects related to sites where COX-1 has constitutive housekeeping duties (such as in the gut leading to peptic ulcer disease); COX-2-specific inhibitors were an attempt to avoid these side effects. Unfortunately, this specific inhibition appeared to confer a greater risk of vascular thrombotic events (such as heart attack and stroke) thought to be related to enhanced suppression of $\mathrm{PGI}_{2}$, an atheroprotective agent, in comparison to COX-1-derived $\mathrm{TXA}_{2}$, a proaggregatory and vasoconstrictor mediator. However, recent work has suggested that it may be possible to exploit the anti-inflammatory and pro-resolution effects of $\mathrm{PGD}_{2}$ metabolites (usually responsible for negative feedback inhibition of the inflammatory response) which inhibit NF- $\mathrm{kB}$ and have been shown to resolve inflammation in animal models (Ward et al., 1999; Rossi et al., 2000; Lawrence et al., 2001). The lipidderived mediators of the lipoxin, resolvin and protectin family may provide a novel therapeutic approach. These compounds have demonstrable pro-resolution properties in vivo including inhibition of neutrophil chemotaxis, recruitment of mononuclear cells and enhanced phagocytosis. In addition, aspirin-triggered lipoxins utilize the same receptor as glucocorticoid-induced annexin 1 suggesting an intriguing interconnectivity between pro-resolution strategies (Lawrence et al., 2002; Perretti and Solito, 2004; Serhan, 2007). A significant barrier to the successful design of novel antiinflammatory agents has been the complex, multilayered redundancy that characterizes the inflammatory system. Where one cytokine pathway is knocked down, another may compensate and maintain the inflammatory response. Only the removal of certain essential cytokines or pathways will allow resolution. Recent anti-inflammatory approaches have utilized 'biological therapies' in the targeting of specific cytokines. Anti-TNF therapy is the standout success of this genre and there is now considerable evidence that TNF- $\alpha$ is the sought-after essential cytokine in inflammatory disease, although other agents are showing promise (Feldmann and Maini, 2002; Williams et al., 2007). It is clear that with such redundancy in the system, there will always be room for new pharmacological agents that have novel targets within the inflammatory system (Hallett et al., 2008).

\section{CDK}

CDKs are serine/threonine kinases and part of the diverse protein kinase family. They are essential facilitators of life at the molecular level via their ubiquitous phosphorylation reactions. The majority of CDKs identified rely on binding partners called cyclins for their activation. In all, there are 13 CDKs and 25 identified cyclins so far and although there is a high level of sequence and structural homology between them, knowledge of their function varies. A typical protein kinase consists of a small N-terminal domain formed of $\beta$-sheets and a large C-terminal domain (CTD) formed of $\alpha$-helices; between the terminals, there is an ATP-binding pocket and this pattern is also observed in CDKs. CDKs switch to an active conformation on binding to cyclin proteins 
which reveals enzymatic elements crucial to the catalytic function of their ATP-binding clefts. CDKs were named for this interaction with cyclin proteins which facilitates passage of cells through the G1, S, G2 and M phases of the cell division cycle (Meijer and Raymond, 2003). Progression of the cell cycle is, of necessity, heavily regulated as cancers arise from its dysfunction. This regulation is more dependent on the concentrations of endogenous CDKi (e.g. the Ink 4 and Cip/Kip families) and cyclins than on the relatively stable CDK population. Extra layers of regulation are provided by phosphorylation and dephosphorylation of CDKs. We commented earlier that CDKi drug action on neutrophils appears counter-intuitive but given the diversity of function that CDKs have been demonstrated to possess, it is perhaps not surprising that another role for them has been found. Although initially identified as key components of the cell cycle machinery, they have subsequently been shown to play roles in cell differentiation, cell death (especially apoptosis), transcription and neuronal function. These alternative roles are mediated by different CDKs, an important factor in the drive to develop increasingly specific pharmacological CDKi drugs (Knockaert et al., 2002).

\section{Endogenous CDKis}

There are two major families of endogenous CDKis, the Ink4 family (which includes p15, p16, p18 and p19) and the Cip/ Kip family (including p21, p27 and p57). Ink4 family proteins are responsible for the inhibition of cyclin D-associated CDK activity and can therefore inhibit CDK4 and CDK6 to cause G1 arrest. They achieve inhibition by competing with D-type cyclins at CDK-binding sites. Of the Ink4 family, the tumour suppressor, p16, has perhaps been studied in most detail and the p16 knockout mouse develops normally but has an increased susceptibility to development of tumours. This is in keeping with the known importance of p16 to the induction of senescence and anoikis (a form of programmed cell death caused by detachment of anchorage-dependent cells from surrounding extracellular matrix) and negative impact on various tumour activities including angiogenesis, cell spreading, vascular endothelial growth factor expression and cell growth. The Cip/Kip family inhibit the kinase activity of cyclin-CDK complexes, in particular, those formed by CDK2 leading once again to G1 arrest. p21 has been implicated in p53-dependent DNA damage-induced cell cycle cessation and also binds proliferating cell nuclear antigen leading to inhibition of DNA synthesis without affecting DNA repair. Interestingly, it appears that the $\mathrm{p} 21$ may be induced by both the signal transducers and activators of transcription (STAT) signal transduction pathway and TGF- $\beta$ and negatively regulates TGF- $\beta$-induced apoptosis. Additionally, p21 has been shown to bind pro-caspase- 3 in some cell types keeping a check on apoptosis (Vidal and Koff, 2000; Lee and Yang, 2001). This has led researchers to suggest the induction of p21 as a therapeutic strategy to prevent epithelial cell loss due to inflammatory injury. Paradoxically, however, another group has cited $\mathrm{p} 21$ as a pro-inflammatory mediator and have noted that the $p 21$ knockout mouse is less susceptible to inflammatory injury caused by cigarette smoke. They attributed these findings to decreased NF- $\mathrm{BB}$ activation and decreased ROS release due to down-modulation of p21-activated kinase (Yao et al., 2008). Possible therapeutic applications of endogenous CDKis will be discussed later in the text, but it is clear that the Byzantine complexity of regulation of cyclins, CDKs and CDKi and their differing expression between cells and tissues will mean that conflicting results are almost to be expected.

\section{CDKs 1 and 2}

CDKs 1 and 2 have integral roles in the cell cycle, putative roles in transcriptional regulation and a controversial role in apoptosis (Golsteyn, 2005). Traditionally, it has been believed that CDK2 interaction with cyclin E facilitates G1/S transition, although this role has recently been called into question by knockout mouse studies suggesting that the complex is dispensable for transition, although it does have a role in centrosome duplication (Roberts and Sherr, 2003). A second CDK2 complex, CDK2-cyclin A, phosphorylates multiple substrates to inactivate G1 transcription factors and allow DNA replication. CDK1 binds cyclin $\mathrm{A}$ at $\mathrm{S} / \mathrm{G} 2$ transition but binds cyclin B to trigger $\mathrm{G} 2 / \mathrm{M}$ transition and, subsequently, has a role in the completion of mitosis by facilitating transition to anaphase (Golsteyn, 2005). CDKs 1 and 2 have been implicated in induction and facilitation of the apoptotic process in some experimental scenarios, while in others, it is clear that their inhibition promotes apoptosis. CDK2 knockout in HeLa cells and CDK1 knockout in an FT210 cell line resulted in a reduction in apoptosis, while levels of CDK1-cyclin complexes were noted to be elevated during apoptosis in HL-60 cells, YAC lymphoma cells and Jurkat cells. This observation had led some investigators to speculate that apoptosis might represent a form of 'failed mitosis' (Golsteyn, 2005). Additionally, CDK inhibition with R-roscovitine appeared to prevent apoptosis in human hepatoma cell lines and rat cerebellar neurons. In the latter model, it was suggested that CDK1 phosphorylates BAD (a pro-apoptotic bcl-2 homologue) which facilitates its liberation from sequestration by 14-3-3 proteins. This allows BAD to translocate to the mitochondrial membrane where it mediates apoptosis (Konishi et al., 2002). This work is in complete contrast to experimental experience with CDKi in various cancer cell lines where induction of apoptosis has been demonstrated (MacCallum et al., 2005).

Several CDKs have roles in transcriptional regulation which is perhaps not surprising given the extensive degree of regulation that the transcription process receives from protein kinases. CDK2 has been implicated in cisplatin-related acute kidney injury through involvement in the release of E2F transcriptional machinery by phosphorylation of Retinoblastoma (Rb) protein which promotes transcription of pro-apoptotic genes (Obligado et al., 2008). Additionally, CDK2 inhibition has been implicated in the induction of apoptosis in diffuse large B-cell lymphoma where CDK2 was linked to transcription of Mcl-1 (an important survival protein). Apoptosis induced by CDK2 inhibition was associated with downregulation of Mcl-1 and reduced phosphorylation of the enzyme responsible for $\mathrm{Mcl}-1$ gene transcription, RNA polymerase II (RNA Pol II) (Faber and Chiles, 2007). CDK1mediated phosphorylation of the CTD of RNA Pol II has also 
been reported in vitro (Bregman et al., 2000). Despite a degree of controversy, the involvement of CDKs 1 and 2 in apoptosis and transcription (particularly of an important neutrophil survival protein) remains interesting when trying to define a role for CDK inhibition in the resolution of inflammation.

\section{CDK5}

CDK5 is perhaps most renowned as an important player in regulation of neuronal cytoarchitecture and migration, although evidence for its extra-neuronal effects is increasing. So far, there is literature describing involvement in apoptosis, transcription, differentiation and endocytosis. Interestingly, there is no evidence that it has any role to play in the cell cycle and as it can function independently of cyclin proteins, it is essentially misnamed. CDK5 has been implicated in the progression of apoptosis in mouse models of ovarian follicle degeneration, embryonic limb interdigital web apoptosis and digoxin-mediated prostate apoptosis (Rosales and Lee, 2006). CDK5 has also been shown to phosphorylate the important transcription factor STAT3 whose gene products include $c$-fos and $j u n-b$ as well as monocyte non-specific esterase. Of particular relevance to inflammation and apoptosis CDK5 complexed to p35 was expressed in HL60 cells induced to differentiate towards a monocytic phenotype. Specifically, this complex was shown to confer monocyte morphologydefining features such as CD14 and non-specific esterase suggesting a role for CDK5 in myeloid differentiation. Additionally, a key neutrophil function, GTP-dependent secretion, was shown to depend on CDK5-p35 (Rosales et al., 2004). Numerous CDK5-p35 substrates were found within neutrophil granules, while neutrophils treated with a CDKi drug lost GTP-dependent secretory function as well as CD63 and CD66b expression. Evidence of CDK5 involvement in transcriptional regulation, apoptosis and specific effects on inflammatory cells appears to be convincing (Dhavan and Tsai, 2001).

\section{CDKs 4 and 6}

CDKs 4 and 6 are required for progression through G1 phase and are activated by cyclin D in the presence of appropriate growth factors. As we have previously noted, neutrophils are terminally differentiated granulocytes. This irreversible cell cycle arrest is mediated by withdrawal of CDK4 and 6 and up-regulation of p27kip1 (an endogenous CDKi). CDK4 and 6, like CDK2, phosphorylate Rb to relieve E2F transcription factors that initiate the switch to S-phase transcription (Klausen et al., 2004). CDK4 and 6 have been implicated in inflammatory events within the rheumatoid joint where they are said to modulate rheumatoid synovial fibroblast production of MMP-3 and MCP-1 via Rb-dependent and -independent mechanisms (Nonomura et al., 2006). Importantly, cyclin D/CDK4 complexes have been shown to activate the STAT transcription pathway independently of JAK in Drosophila and CDK4 has been shown to be potentially important in leukocyte adhesion and migration (discussed later) (Silver and Montell, 2003; Liu et al., 2008). CDK4 and 6 appear to have roles in inflammatory cell differentiation, adhesion and recruitment as well as inflammatory cytokine production and possibly inflammatory signalling (Silver and Montell, 2003; Klausen et al., 2004; Nonomura et al., 2006; Liu et al., 2008). Targeting these CDKs would be facilitated by the current availability of specific pharmacological inhibitors of CDK4 and 6 .

\section{CDKs 7 and 9}

CDK7 is a CDK-activating kinase responsible for enhancing the kinase activity of CDK1 and 2 by phosphorylation of the activation segment or T-loop. CDK7 associated with cyclin $\mathrm{H}$ and MAT1 is responsible for initiation of transcription by the holoenzyme RNA Pol II and mediates this effect by phosphorylation of the CTD. This is a function it shares with CDK9 which, when associated with cyclin T, is responsible for transcriptional elongation by RNA Pol II (Oelgeschlager, 2002). CDK7 and 9 have been implicated in transcription during early infection by CMV and are known to be important in activation of HIVI transcription (Fisher, 2005; Kapasi and Spector, 2008). Perhaps, most interestingly, CDK7 and 9 with their respective binding partners have been shown to play an integral role in the aberrant survival of multiple myeloma cells. This effect is thought to be mediated by RNAPol II transcription of the survival protein Mcl-1. R-roscovitine, a CDKi which has specificity for CDKs $1,2,5,7$ and 9, was able to promote apoptosis in these cells which was associated with down-regulation of Mcl-1 (MacCallum et al., 2005). Additionally CDK9 has also been shown to bind TRAF2, a protein that is of known importance in the activation of NF- $\mathrm{KB}$ mediated by TNF- $\alpha$ (MacLachlan et al., 1998; Wang and Fischer, 2008). Regulation of transcription of Mcl-1 (a key neutrophil survival protein) and involvement in pivotal inflammatory signalling via NF- $\kappa B$ are functions of CDKs 7 and 9 that might be applicable to the regulation of neutrophil apoptosis in inflammatory resolution.

\section{CDKs $3,8,10$ and 11}

These CDKs are considered last because they are perhaps the least well studied to date. CDK3 has been implicated in G0 exit and G1/S phase transition in the cell cycle and binds cyclins C and E2 (van den and Harlow, 1993; Ren and Rollins, 2004). CDK8 has been shown to phosphorylate the CTD of RNA Pol II negatively affecting the activity of CDK7 but also sterically inhibits the interaction between mediator complex and RNA Pol II to repress transcription (Tassan et al., 1995; Elmlund et al., 2006). CDK10 which has a role in transition from $G 2$ to $\mathrm{M}$ phase has been shown to bind the Ets 2 transcription factor and has been identified as a gene with potential significance in the aetiology of non-small cell lung cancer (Kasten and Giordano, 2001; Sun et al., 2005). CDK11 associates with cyclin L and cyclin D3 and has potentially dual roles in transcription that do not involve direct phosphorylation of RNA Pol II CTD. CDK11 has been linked to creatine kinase-2 (CK2)-mediated phosphorylation of RNAPol II CTD and also may increase the histone-acetyltransferase activity of HBO1 (Trembley et al., 2003; Zong et al., 2005). Interestingly, CDK11 has been implicated in melanoma cell apoptosis, a contribution that may be 
mediated by phosphorylation of death substrates and/or direct effects on mitochondrial permeability (Trembley et al., 2003). As more information becomes available, inhibition or activation of these CDKs may well prove important scientific and therapeutic targets.

\section{CDKs in granulocytes}

As neutrophils do not undergo cell division and can progress no further in the cell cycle (they are thought to remain in G0 phase), CDKs had not been thought relevant to their biology. There is, therefore, a paucity of information about CDKs, their interactions and potential roles in these cells. CDK1 and CDK2 proteins had been identified in neutrophils, but there was little indication of their function. No change in CDK1 or CDK2 protein expression was found in a variety of neutrophil populations including fresh human neutrophils, aged human neutrophils, survival factor-treated neutrophils at various time points and CDKi drug-treated neutrophils (Rossi et al., 2006). This is perhaps nol a surprising finding as levels of CDKs 1 and 2 are relatively constant throughout the cell cycle, but changes in expression of their binding partner cyclins and endogenous CDKis modulate effects on cell cycle progression (Knockaert et al., 2002). It cannot be assumed, therefore, that these CDKs are not important in the induction of apoptosis even though they are not targets for degradation. As previously discussed, CDK1 and CDK2 can bind to various cyclin sub-types, whereas CDK5 binds non-cyclin partners including $\mathrm{p} 35$ and $\mathrm{p} 39$ regulatory proteins. In addition, CDK5 is expressed in human neutrophils as evidenced by detection of CDK5 mRNA and protein (Rosales et al., 2004). Evidence of CDK1, CDK2 and CDK5 activity in neutrophils is scarce but available. Our group has demonstrated a prompt reduction in CDK1 activity during induction of apoptosis by the activating Fas antibody CH11. The CDK-binding partners and regulatory proteins in neutrophils remain to be identified (Rossi et al., 2006). It is known that despite the terminally differentiated status of neutrophils, they are capable of phenotypic alteration. Indeed, it has been postulated following work with the 'MacGreen' mouse that mouse neutrophils sufficiently stimulated with CSF-1 in vitro can trans-differentiate into dendritictype cells (Sasmono et al., 2007). This has implications for the role of neutrophils in the resolution of inflammation as it may significantly extend their capability to positively contribute. Another phenotypic switch from generation of pro-inflammatory mediators to anti-inflammatory and/or pro-resolving mediators such as lipoxins and resolvins has also been identified (Serhan, 2007). This raises the possibility that a transcriptional effect might be mediated by up- or down-regulation of protein kinases such as the CDKs. More information is required about CDKs, cyclins, endogenous CDKis and their function in inflammatory cells.

\section{CDKs in lymphocytes}

Lymphocytes would be expected to possess a full complement of functional cell cycle machinery given their retained capacity to proliferate and differentiate in response to the presence or withdrawal of mitogenic factors. CDK2 is central to T-lymphocyte proliferation and it has previously been shown that in combination with antigenic stimulation, $\mathrm{IL}-2$ can down-regulate the endogenous $\mathrm{CDKi}, \mathrm{p} 27$ to promote peripheral T-lymphocyte proliferation. By contrast, the immunosuppressant drug rapamycin mediates some of its effects through stabilization of p27, thus preventing IL-2-mediated T-lymphocyte proliferation. It is increasingly clear that CDKs have a pivotal role in the regulation of T-cell immune responsiveness versus anergy (Wells, 2007). p21 knockout mice have been shown to have an autoimmune phenotype including excessive T-cell activation and proliferation with reactance to self-nuclear antigens. This is said to resemble the autoimmune disease lupus, and reconstitution of p21 functionality was shown to halt disease progression (Zoja et al., 2007). It is expected that targeting CDK2 may have dual therapeutic applicability first in promoting immune tolerance in transplant recipients and secondarily by switching off auto-reactive T-cell responses in autoimmune disease. B-cell development, proliferation and differentiation are also dependent on cell cycle control. The archetypal adaptive immune response, antibody generation, requires activation and expansion of antigen-specific B-cells and their differentiation into plasma cells. Plasma cells arrest at G1 phase of the cell cycle exiting as the major arm of the humoral immune response. p18, an endogenous $\mathrm{CDKi}$, has been implicated in both cell cycle exit and differentiation of antibody-secreting plasma cells from plasmacytoid cells (Morse et al., 1997; Chen-Kiang, 2003). Cell cycle competent lymphocytes may be beneficially affected by exposure to CDKi drugs. Experience with animal models and applicability of CDKi drugs will be discussed later in the review.

\section{Pharmacological CDK inhibition}

Pioneering work on the cell cycle in starfish oocytes in the 1990 s, driven by the realization that anti-mitotic agents would make effective anti-cancer drugs, led to the discovery of CDKi drugs (for a fascinating account of this discovery, see Meijer and Raymond, 2003). The widely available, non-specific kinase inhibitors staurosporine, $6 \mathrm{D}-\mathrm{MAP}$ and isopentanyladine were found to potently inhibit CDK1/cyclin B but were too nonspecific in action to yield useful information about the functional impact. A laborious screening process subsequently uncovered olomoucine, a purine analogue that showed enhanced specificity for CDKs but some action against MAPKs. This compound was shown to mediate its inhibitory effect by competing with ATP for a binding site on CDK1. Further structural analysis of this ATP-binding site interaction identified the 2,6,9 trisubstituted purine family as likely candidates for effective CDK inhibition. This was the development that brought R-roscovitine and Purvalanol B into the frame and led to the identification of a family of approximately $50 \mathrm{CDKi}$ drugs (Meijer and Raymond, 2003) (Table 1).

CDKi drugs are universally (to date) flat, hydrophobic heterocycles of low molecular weight that mediate binding to the ATP pocket via hydrophobic interactions and hydrogen bonds. Surprisingly, despite this uniformity, it is possible to categorize the CDKi drugs on the basis of their relative specificity for CDKs: (i) non-specific (Flavopiridol); (ii) CDKs 1,2,5,7-specific 
Table 1 IC50 values for selected CDK inhibitor drugs. (Knockaert et al., 2002; Obligado et al., 2008), (Krystof et al., 2002; Bettayeb et al., 2007)

\begin{tabular}{|c|c|c|c|c|c|c|c|c|c|}
\hline \multirow[t]{2}{*}{ CDKi } & \multicolumn{9}{|c|}{ ICSO values for kinase inhibited by CDKi $(\mu M)$} \\
\hline & CDK1/cyclinb & CDK2/cyclinE & CDK4 & CDKS & $C D K 7$ & CDK9 & GSK & ERK1 & ERK2 \\
\hline R-roscovitine & $0.45,2.7$ & $0.13,0.7$ & $14.2,14.7,>100$ & 0.16 & 0.49 & $0.74,0.78$ & 130 & 34 & 14 \\
\hline Olomoucine & 7 & 7 & $>1000$ & 3 & & & 100 & 30 & 50 \\
\hline Olomoucine II & 7.6 & 0.1 & 19.8 & & 0.45 & 0.06 & $>100$ & & 32 \\
\hline Purvalanol B & 0.006 & 0.009 & $>10$ & 0.006 & & & $>10$ & 3.33 & \\
\hline Flavopiridol & $0.06,0.4$ & 0.15 & 0.4 & 0.17 & 0.3 & 0.006 & 0.45 & & \\
\hline Aminopurvalanol & 0.033 & 0.028 & & 0.02 & & & & 12 & 2.4 \\
\hline Hymenialdisine & 0.022 & 0.04 & 0.6 & 0.028 & & ${ }^{\prime}$ & 0.01 & 0.47 & 2 \\
\hline Fascaplysin & $>100$ & $>50$ & 0.35 & 20 & & & & & \\
\hline OL567 & 0.23 & & & & & & & & \\
\hline $\mathrm{H} 717$ & 0.23 & 0.05 & & & & & & & \\
\hline NU2058 & 5 & 12 & & & & & & & \\
\hline NU6027 & 2.5 & 1.3 & & & & & & & \\
\hline Staurosporine & 0.006 & 0.007 & $<10$ & & & & & 0.02 & 0.02 \\
\hline PD183812 & $>40$ & 0.17 & 0.008 & & & & & & \\
\hline Meriolin 1 & 0.78 & 0.09 & & 0.51 & & 0.026 & 0.63 & & \\
\hline SU 9516 & 0.04 & & 0.2 & & & & & & \\
\hline Alsterpaullone & 0.035 & 0.2 & $>10$ & 0.04 & & & 0.004 & 22 & 4.5 \\
\hline
\end{tabular}

(Olomoucine, R-roscovitine and Purvalanol B); (iii) CDK 4,6specific (fascaplysin, PD0183812). It is clear that any discussion of CDKi drug specificity for individual CDKs can only be couched in relative terms (Table 1). Additionally, there are some non-CDK targets of these inhibitors identified on random screening which include ERK, GSK3 and pyridoxal kinase (Knockaert et al., 2002). Given the range of activities performed by CDKs on a variety of vital cell processes, a lack of incisive specificity attributable to an inhibitor makes it extremely difficult to assign outcomes to specific actions on defined targets. The basis for this review is that CDKi drugs have proven efficacy in models of inflammation, but the reality is that these effects may not relate to any action on CDKs themselves. An obvious point of interest then is the inhibitory effect of certain CDKi drugs on the ERK signalling pathway. Recent work has shown that specific ERK inhibitors can enhance the resolution of carrageenan-induced pleurisy in rats, an accepted model of acute inflammation (Sawatzky et al., 2006). However, it seems likely that the inhibitory effect of CDKi drugs on the ERK pathway would not account for the anti-inflammatory actions of R-roscovitine. In addition, the biological effect of CDKi and ERK inhibitors on neutrophil apoptosis differs markedly. For example, the ERK inhibitor PD98059 does not induce neutrophil apoptosis per se, whereas CDKi drugs do; R-roscovitine reverses lipopolysaccharide (LPS), granulocyte macrophage-colony stimulating factor (GM-CSF) and db-cAMP-mediated prolongation of neutrophil survival, whereas PD98059 only reverses LPS and GM-CSF survival; finally, PD98059 completely inhibits LPS-induced phospho-ERK expression in neutrophils, whereas R-roscovitine does not (N. Riley, A.E. Leitch, A.G. Rossi, unpubl. data). Regardless of the difficulties associated with non-specificity, the ability of CDKi drugs to selectively induce apoptosis in actively proliferating cells has meant that research into their use as anti-cancer agents has progressed rapidly.

CDKi drugs including R-roscovitine, flavopiridol and SU9516 have anti-cancer actions and R-roscovitine alone is known to reduce the proliferation index of 19 distinct cancer cell lines in vitro (McClue et al., 2002). The mechanism of action of CDKi drugs in cancer cell lines, while not directly applicable to inflammatory cells, is of great interest to those interested in targeting CDKs to resolve inflammation. CDKi drugs were an exciting therapeutic prospect because CDKs interact with the $\mathrm{Rb}$ family widely regarded as the master switch of the cell cycle. CDKs are over-abundant in some cancers resulting in excessive phosphorylation and functional inactivation of $\mathrm{Rb}$, allowing unregulated proliferation Johnson et al., 1994). However, it was noted that R-roscovitine was capable not only of inducing cell cycle arrest, but of active promotion of apoptosis. The induction of apoptosis by R-roscovitine in myeloma cell lines has been reported to be promoted by CDK modulation of RNA Pol II-mediated transcription of Mcl-1. Additionally, downregulation of XIAP, another $\mathrm{Bcl}-2$ homologous survival protein, was observed in chronic lymphatic leukaemia cells, and apoptosis in both cell lines was found to be caspase dependent (Hahntow et al., 2004; MacCallum et al., 2005). A recent paper has shown inhibition of the well-known inflammatory transcription factor, NF-кB by R-roscovitine in A549, 293, H1299 and ARN8 cancer cell lines (Dey et al., 2008). It is tempting to directly extrapolate from these findings to inflammation studies but given the sometimes directly contrasting effects of pharmacological agents in different cell types (e.g. the anti-apoptotic effects of CDK inhibition in cerebellar neuronal cells), we must await further definitive studies. The availability of this mechanistic information and encouraging safety profiles in animal studies have allowed progression to clinical trials of CDKi drugs in conditions such as B-cell malignancy, non-small cell lung cancer and breast cancer. The side-effect profile has been promising but includes tolerable and limited gastrointestinal disturbance, skin rash, reversible transaminitis and hypokalaemia (Senderowicz, 2003). This transition to human studies makes an elucidation of the mechanism of action of CDKi drugs in inflammation imperative, as trials of these drugs in inflammatory disease are likely to be on the near horizon. 


\section{CDK inhibitors in inflammation}

The research discussed above led our group to investigate a role for CDKi drugs in inflammation. Our research paradigm has been that inflammation should be driven down resolution pathways by the induction of apoptosis in granulocytes followed by their effective clearance by professional phagocytes such as macrophages (Leitch et al., 2008). We initially examined the in vitro effects of R-roscovitine, NG-75 and hymenialdisine on human neutrophils at different time points and drug concentrations. These CDKi drugs are structurally diverse but their uniform effect was to promote neutrophil apoptosis in a time- and concentration-dependent manner as evidenced by annexin- $\mathrm{V}$ binding and morphological assessment. Neutrophils treated with R-roscovitine and the caspase inhibitor zVAD-fmk failed to enter apoptosis, suggesting that $\mathrm{R}$-roscovitine was acting in a caspase-dependent manner. This was evident at $4 \mathrm{~h}$ post-incubation when caspase 3 cleavage was already detectable. The most intriguing in vitro result was the ability of the CDKi drugs to overcome diverse survival factors including db $\mathrm{C} \Lambda \mathrm{MP}, \mathrm{GM}-\mathrm{CSF}$ and LPS. These survival factors utilize the major inflammatory signalling pathways: PI3K, NF- $\kappa B$, JAK/STAT and MAPK to augment neutrophil survival. Given that the major hurdle to development of anti-inflammatory agents is the redundancy conferred by these same signalling pathways, this result underlined the potential of CDKi therapy for treatment of inflammatory disease. Inflammation research demands in vivo experimentation as it is impossible to recreate the inflammatory milieu in vitro and hence to predict the efficacious translation of an agent that has been successful in vitro. R-roscovitine was investigated in three mouse models of neutrophil-dominant inflammation including: carrageenan-induced pleurisy, bleomycin lung injury and passively induced arthritis. In the carrageenan pleurisy model, $100 \mathrm{mg} \cdot \mathrm{kg}^{-1}$ of R-roscovitine administered intra-peritoneally reduced an established inflammatory infiltrate to near-basal levels consistent with those found in an untreated mouse pleural cavity. There was a reduction in populations of inflammatory cells (including neutrophils, monocytes and macrophages), oedema formation and pro-inflammatory cytokines. This effect was reversed in vivo by administration of the caspase inhibitor $\mathrm{ZVAD}-\mathrm{fmk}$. In mice with established bleomycin lung injury, there was a reduction in BAL neutrophil numbers assessed after 3 days, a reduction in histopathological lung inflammation after 7 days and an effect on bleomycin-induced lethality. Finally, in mice with established passively induced arthritis, there was an improvement in clinical scores of arthritis following R-roscovitine administration (Rossi et al., 2006). These in vivo findings suggest encouraging pleiotropic effects of CDKi drug on granulocyte recruitment, survival and removal. Pleiotropic effects of CDKi drug are supported by work from Liu et al. who have shown that CDK4 is important in leukocyte recruitment and adhesion. They studied CDK4 $-1-$ knockout mice with bleomycin lung injury and utilized siRNA to CDK4 and CDKi to show that CDK4 inhibition inhibited leukocyte recruitment in the mouse model and leukocyte adhesion in EC matrix models (Liu et al., 2008). In addition, Sekine et al. have now demonstrated positive effects of flavopiridol and a specific CDK4,6 inhibitor on animal models of rheumatoid arthritis
(Sekine et al., 2008). Their findings suggest lymphocyteindependent effects of CDKi drugs (including downregulation of fibroblast proliferation and growth) responsible for improvement in joint histology and clinical arthritis scores in various mouse models. Findings in inflammatory joint and lung disease models are mirrored in kidney disease models where the CDKi drug R-roscovitine has entered phase $1 \mathrm{~b}$ clinical trials for inflammatory kidney disease. Glomerulonephritides are characterized by inflammation and progressive, scarring destruction of key functional kidney units leading to renal dysfunction and failure. Preclinically, CDKi drugs have been shown to protect renal tubular epithelium from enhanced apoptosis while inhibiting the abnormal proliferation of tubular epithelial and mesangial cells. $I n$ vitro and in vivo work has demonstrated that $\mathrm{R}$-roscovitine can restore normal kidney function in animal models of IgA-mediated glomerulonephritis, crescentic glomerulonephritis, lupus nephritis and collapsing glomerulonephropathy (Gherardi et al., 2004; Milovanceva-Popovska et al., 2005; Zoja et al., 2007; Obligado et al., 2008). The work with NZBxNZW mice affected by early or established proliferative lupus nephritis is particularly interesting, as while leucocyte-driven inflammation was shown to be reduced, there was also evidence of a direct effect of CDKi against autoimmune T- and B-lymphocyte responses. It is perhaps less surprising that CDKi should work in this setting, given the proliferative potential/state of differentiation of lymphocytes, but nonetheless, the possibility of pleiotropic action against autoimmune inflammation is an exciting one.

Further evidence for the utility of CDK inhibition has been provided by research into the properties of endogenous CDKis. The physiological CDKi p21 (WAF1, SD1, Cip1), a specific inhibitor of CDK2, 4 and 6 , has been shown to negatively regulate macrophage activation by reducing TNF- $\alpha$ and IL- $1 \beta$ production in response to LPS. Additionally, p21-/- mice have an increased susceptibility to LPSinduced shock which is associated with elevated levels of IL-1 $\beta$ (Lloberas and Celada, 2009; Scatizzi et al., 2009). In an inflammatory lung disease model, p21 was over-expressed in the lungs of mice subjected to bleomycin injury by an intratracheal adenoviral transfer method (Inoshima et al., 2004). p21 expression in lung epithelial cells led to a reduction in lung inflammation, preservation of epithelial cells and reduced fibrosis. In rheumatoid arthritis patients, p21 gene transfer was shown to down-regulate expression of inflammatory mediators and tissue-degrading proteinases such as: IL-6, -8, type I IL-1 receptor, monocyte chemoattractant protein-1 (MCP-1), macrophage inflammatory protein3alpha, cathepsins B and $\mathrm{K}$, and matrix metalloproteinases-1 and -3 (Nonomura et al., 2003).

In summary, CDK inhibition promotes neutrophil apoptosis in vitro even in the presence of powerful survival factors and promotes resolution of inflammation, in vivo, in various animal models of neutrophil-dominant inflammation. Neutrophil apoptosis has been shown to be central to the resolution of inflammation by caspase inhibition which reversed the beneficial impact of CDKi drugs. In addition, the antiproliferative and anti-apoptotic effects of CDKi drugs protect epithelia against inflammatory insult. CDKi drugs also prevent lymphocyte proliferation and pro-inflammatory 
signalling indicating potential effects against chronic and autoimmune, inflammatory disease. An understanding of the mechanism of action by which these powerful antiinflammatory effects are achieved may allow optimization of CDK inhibition and suggest further targets for pharmacological intervention.

\section{Challenges associated with $\mathrm{CDK}$ inhibition as a therapeutic strategy}

Apart from the side-effect profile associated with known CDKi drugs and already discussed, there are some potential drawbacks to CDKi therapy. The most obvious of these drawbacks is the degree of redundancy apparent in the cell cycle machinery. The best example of this is the CDK2 knockout mouse which survives (it is subsequently sterile) despite the central cell cycle role attributed to CDK2 (Berthet et al., 2003). The complicated redundancy of the inflammatory system means that single-hit therapies may prove ineffectual, which may be the case with CDK targeting. However, it is proving difficult to achieve more than pan-selectivity for individual CDKs, which may be a blessing in disguise. An expected side effect of the aggressive induction of neutrophil apoptosis would be neutropenia and although this was not reported in early trials, it appears that an early (though recovering) neutropenia is a feature. While this may not be beneficial in cancer patients, in patients with neutrophil-dominant inflammation, it would be hoped that this effect could be dose limited and controlled, especially given some data that suggest that stimulated inflammatory neutrophils are more susceptible to CDKi than unstimulated. It would of course be important to ensure that infective aetiologies for disease had been excluded and antibiotic prophylaxis might be appropriate in some circumstances. Finally, neutrophil apoptosis must not overload macrophage capacity for phagocytosis or the sequelae of secondary necrosis could occur. It may be that combination therapy with phagocytosis-enhancing drugs (e.g., steroids) might prevent this occurrence.

\section{Concluding remarks: Potential mechanisms for anti-inflammatory action of the CDK inhibitors and the future of $\mathrm{CDK}$ targeting}

The indications from the available CDK/CDKi literature are that CDKs may be important targets of CDKi antiinflammatory action. They are known to have key roles in inflammatory cell differentiation, in neutrophil function and in influencing established inflammatory signalling pathways. Additionally, they are intimately involved in the regulation of apoptosis and gene transcription in a number of cell lines. There are also strong links between cancer and inflammation as highlighted by a number of recent reviews and CDK inhibition is an efficacious therapeutic approach in a number of cancers (Coussens and Werb, 2002; Hussain and Harris, 2007). Currently, the role of CDKs in granulocyte apoptosis and the resolution of inflammation are unclear, but a number of plausible hypotheses are apparent.

\section{Gene transcription and survival proteins}

It is known that R-roscovitine promotes apoptosis in multiple myeloma cell lines via inhibition of CDK- mediated phosphorylation of RNA Pol II which down-regulates Mcl-1 expression. CDKs $1,2,7,8$ and 9 are all capable of RNA Pol II CTD phosphorylation. The applicability of this to neutrophil biology is unclear; however, it is established that Mcl-1 is a key survival protein in neutrophils (Edwards et al., 2004; Dzhagalov et al., 2007). Our own experiments have shown a rapid reduction in $\mathrm{Mcl}-1$ protein following R-roscovitine treatment of neutrophils that occurs even in the presence of powerful survival factors (in fact, its reduction is enhanced in the presence of survival factors, unpubl. data). We have not as yet established the mechanism by which this occurs and Mcl-1 is a highly regulated protein, so it is possible or even probable that there is an independent effect at work. It is also possible that R-roscovitine has a more direct effect on Mcl-1 RNA processing or protein expression. A splice variant of Mcl-1 has potentially pro-apoptotic properties and interference with the balance of competing variants of Mcl-1 could result in rapid apoptosis. Mcl-1 protein is subject to ubiquitination with subsequent turnover by the proteasome. Varying levels of phosphorylation have been demonstrated to either postpone or facilitate this process. Different pharmacological agents have been demonstrated to stabilize or destabilize Mcl-1 with down-regulation occurring by both proteasome and caspase-dependent mechanisms (Derouet et al., 2004; Mandelin and Pope, 2007).

\section{CDK4 and leucocyte recruitment}

At least part of the pro-resolution of inflammation effect of CDKi may be explained by the impact of CDK4 knockout on leukocyte recruitment and adhesion, although this would not explain the ability of CDKi drugs to promote granulocyte apoptosis, and given that CDK4 is not within the specificity of R-roscovitine, it would not explain the anti-inflammatory effects of this compound (Liu et al., 2008).

CDK balance and apoptosis. An alternative hypothesis is that the complement of CDKs, cyclins and endogenous CDKi is important in the maintenance of neutrophil survival. As the specificity of R-roscovitine extends across CDKs 1,2,5,7 and 9, it is not currently possible to attribute the pro-apoptotic effect to one CDK, but it may be that effects on multiple CDKs are important.

\section{Inflammatory signalling mechanisms}

Signalling pathways such as MAPK, NF- $\mathrm{\kappa B}$ and PI3K are extremely important in the regulation of the inflammatory response and in neutrophil survival (Suzuki et al., 1999; Ward et al., 1999; Lindemans and Coffer, 2004). At high concentrations, R-roscovitine is known to have a direct inhibitory effect on ERK 1 and 2 (Meijer and Raymond, 2003) and we know from work in our laboratory that specific ERK inhibitors are antiinflammatory in vitro and in vivo. It is possible that this effect makes an important contribution to the actions of R-roscovitine. NF- $\kappa \mathrm{B}$ inhibition was recently identified as playing a role in the anti-cancer actions of R-roscovitine and 


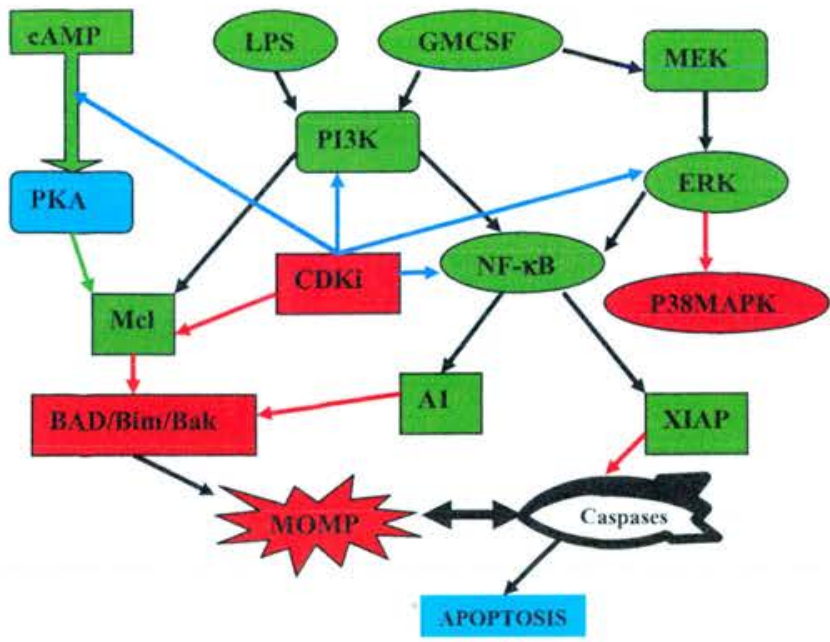

Figure 3 Known and potential CDKi effects on neutrophil survival signalling in inflammation.

$\mathrm{NF}-\mathrm{kB}$ as well as being central to the inflammatory signalling system is a powerful regulator of neutrophil survival (Ward et al., 1999). However, our own experimental results suggest that this effect is not reproduced in neutrophils (unpubl. data). PI3K has importance in neutrophil survival and recruitment and is implicated in the effects of CDK inhibition on xenopus oocyte maturation and the lethal effects of CDK inhibition on the human leukaemia cell lines: U937, HL-60, Jurkat, Raji, and NB4 (Hehl et al., 2001; Yu et al., 2003; Pinho et al., 2005).

An effect on one or multiple inflammatory signalling systems is a potential explanation for observations with regard to CDKi drugs and inflammation. In many respects, it would be ideal if CDKi drugs acted in a pleiotropic manner to counter the inherent redundancy of the inflammatory system (Figure 3).

It is clear that CDKs hold promise as novel pharmacological targets in inflammation, although further research is required to confirm this hypothesis. The development of new and more specific CDKi drugs continues apace and is increasingly supported by structural elucidation and mechanistic studies. While incisive specificity may not prove beneficial in the treatment of disease, it would certainly prove an important addition to our scientific tool kit. The future of CDK targeting must lie in a human trial of efficacy against neutrophildominant or autoimmune inflammatory disease and we expect this to occur in the near future.

\section{Note added in proof}

A very recent, important and elegant study by Koedel et al. (2009) has shown that roscovitine induction of neutrophil apoptosis in vivo promoted the resolution of inflammation of mice treated with antibiotics following experimental pneumococcal meningitis.

\section{Conflict of interest}

None.

\section{References}

Berthet C, Aleem E, Coppola V, Tessarollo L., Kaldis P (2003). Cdk2 knockout mice are viable. Curr Biol 13: 1775-1785.

Bettayeb K, Tirado OM, Marionneau-Lambot S, Ferandin Y, Lozach O, Morris JC et al. (2007). Meriolins, a new class of cell death inducing kinase inhibitors with enhanced selectivity for cyclin-dependent kinases. Cancer Res 67: 8325-8334.

Bianchi SM, Dockrell DH, Renshaw SA, Sabroe I, Whyte MK (2006). Granulocyte apoptosis in the pathogenesis and resolution of lung disease. Clin Sci 110: 293-304.

Bregman DB, Pestell RG, Kidd VJ (2000). Cell cycle regulation and RNA polymerase II. Front Biosci 5: D244-D257.

Brinkmann V, Zychlinsky A (2007). Beneficial suicide: why neutrophils die to make NETs. Nat Rev Microbiol 5: 577-582.

Chen-Kiang S (2003). Cell-cycle control of plasma cell differentiation and tumorigenesis. Immumol Rev 194: 39-47.

Chung F (2001). Anti-inflammatory cytokines in asthma and allergy: interleukin-10, interleukin-12, interferon-gamma. Mediators Inflamm 10: 51-59.

Coussens I.M, Werb Z (2002). Inflammation and cancer. Nature 420: $860-867$

Critchley HO, Kelly KW, Brenner KM, Baird DI (2001). The endocrinology of menstruation - a role for the immune system. Clin Endocrinol 55: 701-710.

Derouet M, Thomas L, Cross A, Moots RJ, Edwards SW (2004). Granulocyte macrophage colony-stimulating factor signaling and proteasome inhibition delay neutrophil apoptosis by increasing the stability of Mcl-1. J Biol Chem 279: 26915-26921.

Dey A, Wong ET, Cheok CF, Tergaonkar V, Lane DP (2008). R-Roscovitine simultaneously targets both the p53 and NF-kappaB pathways and causes potentiation of apoptosis: implications in cancer therapy. Cell Death Differ 15: 263-273.

Dhavan R, Tsai LH (2001). A decade of CDK5. Nat Rev Mol Cell Biol 2: 749-759.

Downey GP, Worthen GS, Henson PM, Hyde DM (1993). Neutrophil sequestration and migration in localized pulmonary inflammation. Capillary localization and migration across the interalveolar septum. Am Rev Respir Dis 147: 168-176.

Dzhagalov I, St. John A, He YW (2007). The antiapoptotic protein Mcl-1 is essential for the survival of neutrophils but not macrophages. Blood 109: 1620-1626.

Edwards SW, Derouet M, Howse M, Moots RJ (2004). Regulation of neutrophil apoptosis by Mcl-1. Biochem Soc Trans 32: 489492.

Elmlund H, Baraznenok V, Lindahl M, Samuelsen CO, Koeck PJ, Holmberg 5 et al. (2006). The cyclin-dependent kinase 8 module sterically blocks Mediator interactions with RNA polymerase II. Proc Natl Acad Sci USA 103: 15788-15793.

Faber AC, Chiles TC (2007). Inhibition of cyclin-dependent kinase-2 induces apoptosis in human diffuse large B-cell lymphomas. Cell Cycle 6: 2982-2989.

Fadok VA, Bratton DL, Konowal A, Freed PW, Westcott JY, Henson PM (1998). Macrophages that have ingested apoptotic cells in vitro inhibit proinflammatory cytokine production through autocrine/ paracrine mechanisms involving TGF-beta, PGE2, and PAF. I Clin Invest 101: 890-898.

Feldmann M, Maini RN (2002). Discovery of TNF-alpha as a therapeutic target in rheumatoid arthritis: preclinical and clinical studies. Joint Bone Spine 69: 12-18.

Fisher RP (2005). Secrets of a double agent: CDK7 in cell-cycle control and transcription. I Cell Sci 118: $5171-5180$.

Fuchs TA, Abed U, Goosmann C, Hurwitz R, Schulze I, Wahn V et al. (2007). Novel cell death program leads to neutrophil extracellular traps. I Cell Biol 176: 231-241.

Gherardi D, D'Agati V, Chu TH, Barnett A, Gianella-Borradori A, Gelman IH et al. (2004). Reversal of collapsing glomerulopathy in 
mice with the cyclin-dependent kinase inhibitor CYC202. I Am Soc Nephrol 15: 1212-1222.

Gilgun-Sherki Y, Melamed E, Offen D (2006). Anti-inflammatory drugs in the treatment of neurodegenerative diseases: current state Curr Pharm Des 12: 3509-3519.

Girkontaite I, Urbonaviciute V, Maseda D, Neubert K, Herrmann M, Voll RE (2007). Apoptotic cells selectively suppress the Th1 cytokine interferon gamma in stimulated human peripheral blood mononuclear cells and shift the Th1/Th2 balance towards Th2. Autoimmunity 40: 327-330.

Golsteyn RM (2005). Cdk1 and Cdk2 complexes (cyclin dependent kinases) in apoptosis: a role beyond the cell cycle. Cancer Lett 217 129-138.

Hahntow IN, Schneller F, Oelsner M, Weick K, Ringshausen I, Fend F et al. (2004). Cyclin-dependent kinase inhibitor Roscovitine induces apoptosis in chronic lymphocytic leukemia cells. Leukemia 18: 747-755.

Hallett JM, Leitch AE, Riley NA, Duffin R, Haslett C, Rossi AG (2008) Novel pharmacological strategies for driving inflammatory cell apoptosis and enhancing the resolution of inflammation. Trend: Pharmacol Sci 29: 250-257.

Hansson GK, Robertson AK, Soderberg-Naucler C (2006). Inflammation and atherosclerosis. Ammu Rev Pathol 1: 297-329.

Haslett C (1999). Granulocyte apoptosis and its role in the resolution and control of lung inflammation. Am J Respir Crit Care Med 160 : S5-11.

Haslett C, Savill J, Meagher L (1990). Macrophage recognition of senescent granulocytes. Biochem Soc Trans 18: 225-227.

Heasman SJ, Giles KM, Ward C, Rossi AG, Haslett C, Dransfield I (2003). Glucocorticoid-mediated regulation of granulocyte apoptosis and macrophage phagocytosis of apoptotic cells: implications for the resolution of inflammation. J Endocrinol 178: 29-36.

Hehl S, Stoyanov B, Oehrl W, Schonherr R, Wetzker R, Heinemann SH (2001). Phosphoinositide 3-kinase-gamma induces Xenopus oocyte maturation via lipid kinase activity. Biochem J 360: 691-698.

Hussain SP, Harris CC (2007). Inflammation and cancer: an ancient link with novel potentials. Int I Cancer 121: 2373-2380.

Inoshima I, Kuwano K, Hamada N, Yoshimi M, Maeyama T, Hagimoto $\mathrm{N}$ et al. (2004). Induction of CDK inhibitor $\mathrm{p} 21$ gene as a new therapeutic strategy against pulmonary fibrosis. Am J Physiol Lung Cell Mol Physiol 286: L727-L733.

Johnson DG, Ohtani K, Nevins JR (1994). Autoregulatory control of E2F1 expression in response to positive and negative regulators of cell cycle progression. Genes Dev 8: 1514-1525.

Kapasi AJ, Spector DH (2008). Inhibition of the cyclin-dependent kinases at the beginning of human cytomegalovirus infection specifically alters the levels and localization of the RNA polymerase II carboxyl-terminal domain kinases cdk9 and cdk7 at the viral transcriptosome. J Virol 82: 394-407.

Kasten M, Giordano A (2001). Cdk10, a Cdc2-related kinase, associates with the Ets 2 transcription factor and modulates its transactivation activity. Oncogene 20: 1832-1838.

Klausen P, Bjerregaard MD, Borregaard N, Cowland JB (2004). Endstage differentiation of neutrophil granulocytes in vivo is accompanied by up-regulation of p27kip1 and down-regulation of CDK2 CDK4, and CDK6. J Leukoc Biol 75: 569-578.

Knockaert M, Greengard P, Meijer L (2002). Pharmacological inhibitors of cyclin-dependent kinases. Trends Pharmacol Sci 23: 417425 .

Koedel U, Frankenberg T, Kirschnck S, Obermaier B, Häcker H, Paul R, et al. (2009). Apoptosis is essential for neutrophil functional shutdown and determines tissue damage in experimental pneumococcal meningitis. PLoS Pathog 5: e1000461.

Konishi Y, Lehtinen M, Donovan N, Bonni A (2002). Cde2 phosphorylation of BAD links the cell cycle to the cell death machinery. Mol Cell 9: 1005-1016.

Krystof V, Lenobel R, Havlicek L, Kuzma M, Strnad M (2002). Synthe- sis and biological activity of olomoucine II. Bioorg Med Chem Lett 12: 3283-3286.

Lawrence T, Gilroy DW (2007). Chronic inflammation: a failure of resolution? Int J Exp Pathol 88: 85-94.

Lawrence T, Gilroy DW, Colville-Nash PR, Willoughby DA (2001). Possible new role for NF-kappaB in the resolution of inflammation. Nat Med 7: 1291-1297.

Lawrence T, Willoughby DA, Gilroy DW (2002). Anti-inflammatory lipid mediators and insights into the resolution of inflammation. Nat Rev Immunol 2: 787-795.

Lee MH, Yang HY (2001). Negative regulators of cyclin-dependent kinases and their roles in cancers. Cell Mol Life Sci 58: 1907-1922.

Leitch AE, Duffin R, Haslett C, Rossi AG (2008). Relevance of granulocyte apoptosis to resolution of inflammation at the respiratory mucosa. Mucosal Immunol 1: 350-363.

Lindemans CA, Coffer PJ (2004). Regulation of granulocyte apoptosis by phosphatidylinositol 3-kinase. Biochem Soc Trans 32: 480484 .

Liu L, Schwartz B, Tsubota Y, Raines E, Kiyokawa H, Yonekawa K et al. (2008). Cyclin-dependent kinase inhibitors block leukocyte adhesion and migration. I Immunol 180: 1808-1817.

Liu Y, Cousin JM, Hughes J, Van DJ, Seckl JR, Haslett C et al. (1999). Glucocorticoids promote nonphlogistic phagocytosis of apoptotic leukocytes. I Immunol 162: 3639-3646.

Lloberas J, Celada A (2009). p21(waf1/CIP1), a CDK inhibitor and a negative feedback system that controls macrophage activation. Eur I Immunol 39: 691-694.

MacCallum DE, Melville J, Frame S, Watt K, Anderson S, GianellaBorradori A et al. (2005). Seliciclib (CYC202, R-Roscovitine) induces cell death in multiple myeloma cells by inhibition of RNA polymerase II-dependent transcription and down-regulation of Mcl-1. Cancer Res 65: 5399-5407.

MacLachlan TK, Sang N, De LA, Puri PL, Levrero M, Giordano A (1998). Binding of CDK9 to TRAF2. J Cell Biochem 71: 467-478.

Mandelin AM, Pope RM (2007). Myeloid cell leukemia-1 as a therapeutic target. Expert Opin Ther Targets 11: 363-373.

Marchalonis JJ, Kaveri S, Lacroix-Desmazes S, Kazatchkine MD (2002). Natural recognition repertoire and the evolutionary emergence of the combinatorial immune system. FASEB J 16: 842-848.

McClue SJ, Blake D, Clarke R, Cowan A, Cummings L, Fischer PM et al. (2002). In vitro and in vivo antitumor properties of the cyclin dependent kinase inhibitor CYC202 (R-roscovitine). Int I Cancer 102: 463-468.

Meagher LC, Cousin JM, Seckl JR, Haslett C (1996). Opposing effects of glucocorticoids on the rate of apoptosis in neutrophilic and eosinophilic granulocytes. J Immumol 156: 4422-4428.

Meijer L, Raymond E (2003). Roscovitine and other purines as kinase inhibitors. From starfish oocytes to clinical trials. Acc Chem Res 36: 417-425.

Michlewska S, Dransfield 1, Megson IL, Rossi AG (2009). Macrophage phagocytosis of apoptotic neutrophils is critically regulated by the opposing actions of pro-inflammatory and anti-inflammatory agents: key role for TNF-alpha. FASEB J 23: 844-854.

Milovanceva-Popovska M, Kunter U, Ostendorf T, Petermann A, Rong S, Eitner F et al. (2005). R-roscovitine (CYC202) alleviates renal cell proliferation in nephritis without aggravating podocyte injury. Kidney Int 67: 1362-1370.

Morse L, Chen D, Franklin D, Xiong Y, Chen-Kiang S (1997). Induction of cell cycle arrest and B cell terminal differentiation by CDK inhibitor p18(INK4c) and IL-6. Immunity 6: 47-56.

Munoz LE, Gaipl US, Franz S, Sheriff A, Voll RE, Kalden JR et al. (2005). SLE - a disease of clearance deficiency? Rheumatology 44: 11011107.

Nonomura Y, Kohsaka H, Nagasaka K, Miyasaka N (2003). Gene transfer of a cell cycle modulator exerts anti-inflammatory effects in the treatment of arthritis. I Immumol 171: 4913-4919.

Nonomura Y, Nagasaka K, Hagiyama H, Sekine C, Nanki T, Tamamori- 
Adachi M et al. (2006). Direct modulation of rheumatoid inflammatory mediator expression in retinoblastoma protein-dependent and -independent pathways by cyclin-dependent kinase $4 / 6$. Arthritis Rheum 54: 2074-2083.

Obligado SH, Ibraghimov-Beskrovnaya O, Zuk A, Meijer L, Nelson PJ (2008). CDK/GSK-3 inhibitors as therapeutic agents for parenchymal renal diseases. Kidney Int 3 (6): 684-690.

Oelgeschlager T (2002). Regulation of RNA polymerase II activity by CTD phosphorylation and cell cycle control. I Cell Physiol 190: 160-169.

Ozato K, Tsujimura H, Tamura T (2002). Toll-like receptor signaling and regulation of cytokine gene expression in the immune system. Biotechmiques 33: S66-S75.

Perretti M, Solito E (2004). Annexin 1 and neutrophil apoptosis. Biochem Soc Trans 32: 507-510.

Pinho V, Souza DG, Barsante MM, Hamer FP, De Freitas MS, Rossi AG et al. (2005). Phosphoinositide-3 kinases critically regulate the recruitment and survival of eosinophils in vivo: importance for the resolution of allergic inflammation. I Leukoc Biol 77: 800810.

Ren S, Rollins BJ (2004). Cyclin C/cdk3 promotes Rb-dependent GO exit. Cell 117: 239-251.

Roberts JM, Sherr CJ (2003). Bared essentials of CDK2 and cyclin E. Nat Genet 35: 9-10.

Rosales JL, Lee KY (2006). Extraneuronal roles of cyclin-dependent kinase 5. Bioessays 28: 1023-1034.

Rosales JL, Ernst JD, Hallows J, Lee KY (2004). GTP-dependent secretion from neutrophils is regulated by Cdk5. J Biol Chem 279: 53932 53936.

Rossi A, Kapahi P, Natoli G, Takahashi T, Chen Y, Karin M et al. (2000). Anti-inflammatory cyclopentenone prostaglandins are direct inhibitors of IkappaB kinase. Nature 403: 103-108.

Rossi AG, Hallett JM, Sawatzky DA, Teixeira MM, Haslett C (2007). Modulation of granulocyte apoptosis can influence the resolution of inflammation. Biochem Soc Trans 35: 288-291.

Rossi AG, Sawatzky DA, Walker A, Ward C, Sheldrake TA, Riley NA et al. (2006). Cyclin-dependent kinase inhibitors enhance the resolution of inflammation by promoting inflammatory cell apoptosis. Nat Med 12: 1056-1064.

Sasmono RT, Ehrnsperger A, Cronau SL, Ravasi T, Kandane R, Hickey MJ et al. (2007). Mouse neutrophilic granulocytes express mRNA encoding the macrophage colony-stimulating factor receptor (CSF$1 \mathrm{R})$ as well as many other macrophage-specific transcripts and can transdifferentiate into macrophages in vitro in response to CSF-1. I Leukoc Biol 82: 111-123.

Savill JS, Wyllie AH, Henson JE, Walport MJ, Henson PM, Haslett C (1989). Macrophage phagocytosis of aging neutrophils in inflammation. Programmed cell death in the neutrophil leads to its recognition by macrophages. J Clin Invest 83: 865-875.

Sawatzky DA, Willoughby DA, Colville-Nash PR, Rossi AG (2006). The involvement of the apoptosis-modulating proteins ERK $1 / 2, \mathrm{Bcl}$-xL and Bax in the resolution of acute inflammation in vivo. Am J Pathol 168: $33-41$.

Scatizzi JC, Mavers M, Hutcheson J, Young B, Shi B, Pope RM et al. (2009). The CDK domain of p21 is a suppressor of IL-1betamediated inflammation in activated macrophages. Eur I Immunol 39: $820-825$.

Schultz RM (1987). Interleukin 1 and interferon-gamma: cytokines that provide reciprocal regulation of macrophage and T cell function. Toxicol Pathol 15: 333-337.

Sekine C, Sugihara T, Miyake S, Hirai H, Yoshida M, Miyasaka N et al. (2008). Successful treatment of animal models of rheumatoid arthritis with small-molecule cyclin-dependent kinase inhibitors. I Immumol 180: 1954-1961.

Senderowicz AM (2003). Novel small molecule cyclin-dependent kinases modulators in human clinical trials. Cancer Biol Ther 2: S84-S95.

Serhan CN (2007). Resolution phase of inflammation: novel endogenous anti-inflammatory and proresolving lipid mediators and pathways. Anmu Rev Immunol 25: 101-137.

Silver DL, Montell DJ (2003). A new trick for Cyclin-Cdk: activation of STAT. Dev Cell 4: 148-149.

Sun M, Li Z, Zhang Y, Zheng Q Sun CC (2005). Homology modeling and docking study of cyclin-dependent kinase (CDK) 10. Bioorg Med Chem Lett 15: 2851-2856.

Suzuki K, Hino M, Hato F, Tatsumi N, Kitagawa S (1999). Cytokinespecific activation of distinct mitogen-activated protein kinase subtype cascades in human neutrophils stimulated by granulocyte colony-stimulating factor, granulocyte-macrophage colonystimulating factor, and tumor necrosis factor-alpha. Blood 93: 341349.

Tassan JP, Jaquenoud M, Leopold P, Schultz SJ, Nigg EA (1995). Identification of human cyclin-dependent kinase 8 , a putative protein kinase partner for cyclin C. Proc Natl Acad Sci USA 92: 8871-8875.

Trembley JH, Hu D, Slaughter CA, Lahti JM, Kudd VJ (2003). Casein kinase 2 interacts with cyclin-dependent kinase 11 (CDK11) in vivo and phosphorylates both the RNA polymerase II carboxyl-terminal domain and CDK11 in vitro. J Biol Chem 278: 2265-2270.

van den HS, Harlow E (1993). Distinct roles for cyclin-dependent kinases in cell cycle control. Science 262: 2050-2054.

Vidal A, Koff A (2000). Cell-cycle inhibitors: three families united by a common cause. Gene 247: 1-15.

Wang S, Fischer PM (2008). Cyclin-dependent kinase 9: a key transcriptional regulator and potential drug target in oncology, virology and cardiology. Trends Pharmacol Sci 29: 302-313.

Ward C, Chilvers ER, Lawson MF, Pryde JG, Fujihara S, Farrow SN et al. (1999). NF-kappaB activation is a critical regulator of human granulocyte apoptosis in vitro. I Biol Chem 274: 4309-4318.

Wells AD (2007). Cyclin-dependent kinases: molecular switches controlling anergy and potential therapeutic targets for tolerance. Semin Immunol 19: 173-179.

Williams RO, Paleolog E, Feldmann M (2007). Cytokine inhibitors in rheumatoid arthritis and other autoimmune diseases. Curr Opin Pharmacol 7: 412-417.

Yao H, Yang SR, Edirisinghe I, Rajendrasozhan S, Caito S, Adenuga D et al. (2008). Disruption of p21 attenuates lung inflammation induced by cigarette smoke, LPS, and fMLP in mice. Am J Respir Cell Mol Biol 39: 7-18.

Yousefi S, Gold JA, Andina N, Lee J, Kelly AM, Kozlowski E et al. (2008). Catapult-like release of mitochondrial DNA by eosinophils contributes to antibacterial defense. Nat Med 14: 949-953.

Yu C, Rahmani M, Dai Y, Conrad D, Krystal G, Dent P et al. (2003). The lethal effects of pharmacological cyclin-dependent kinase inhibitors in human leukemia cells proceed through a phosphatidylinositol 3-kinase/Akt-dependent process. Cancer Res 63: 18221833.

Zoja C, Casiraghi F, Conti S, Corna D, Rottoli D, Cavinato RA et al. (2007). Cyclin-dependent kinase inhibition limits glomerulonephritis and extends lifespan of mice with systemic lupus. Arthritis Rheum 56: 1629-1637.

Zong $\mathrm{H}$, Li Z, Liu L, Hong Y, Yun X, Jiang J et al. (2005). Cyclindependent kinase 11(p58) interacts with $\mathrm{HBO} 1$ and enhances its histone acetyltransferase activity. FEBS Lett 579: 3579-3588. 